\title{
Quality Control Summary Report for the RFI/RI Assessment of the Submerged Sediment Core Samples taken at Par Pond, Pond C, and L Lake
}

(U)

Revision date: December 1996

\section{MASTER}

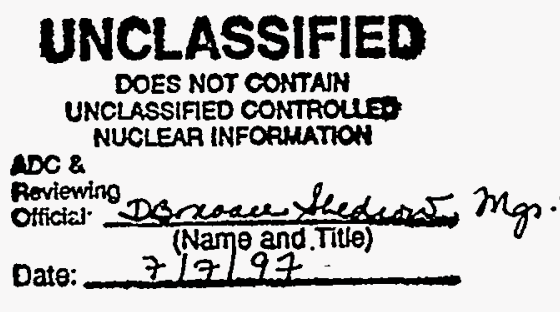

ETIRIBUTION OF THIS DOCUMENT IS UNLMTTED 


\section{DISCLAIMER}

This report was prepared as an account of work sponsored by an agency of the United States Government. Neither the United States Government nor any agency thereof, nor any of their employees, makes any warranty, express or implied, or assumes any legal liability or responsibility for the accuracy, completeness, or usefulness of any information, apparatus, product, or process disclosed, or represents that its use would not infringe privately owned rights. Reference herein to any specific commercial product, process, or service by trade name, trademark, manufacturer, or otherwise does not necessarily constitute or imply its endorsement, recommendation, or favoring by the United States Government or any agency thereof. The views and opinions of authors expressed herein do not necessarily state or reflect those of the United States Government or any agency thereof.

This report has been reproduced directly from the best available copy.

Available to DOE and DOE contractors from the Office of Scientific and Technical Information, P.O. Box 62, Oak Ridge, TN 37831; prices available from (615) 576-8401.

Available to the public from the National Technical Information Service, U.S. Department of Commerce; 5285 Port Royal Road, Springfield, VA 22161.
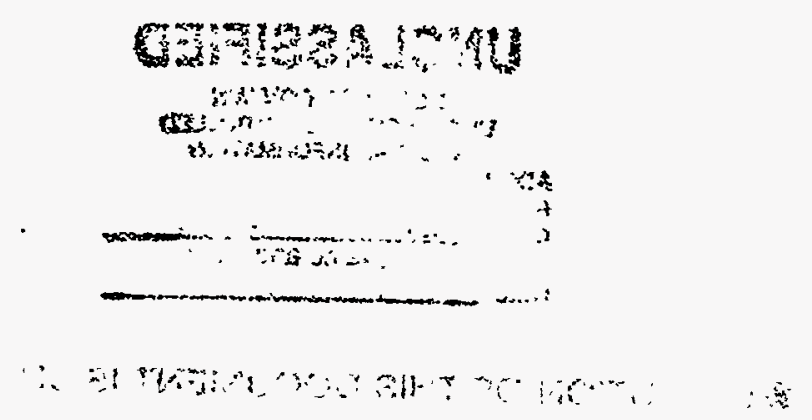


\section{DISCLAIMER}

Portions of this document may be illegible electronic image products. Images are produced from the best available original document. 


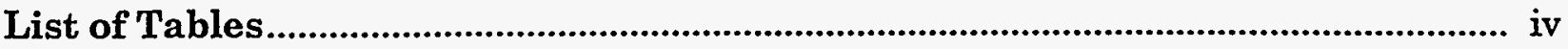

Figure ........................................................................................................................ vi

Figure 1. Submerged Sediment Core Samples taken at Par Pond, Pond C and

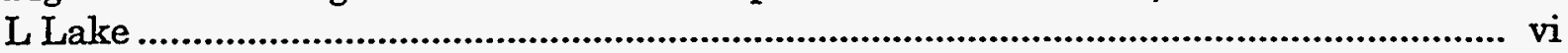

Executive Summary ....................................................................................................... 1

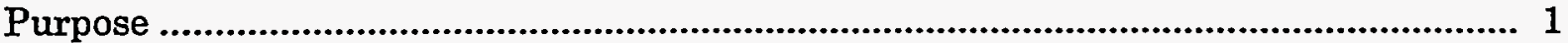

Scope of the Project............................................................................................................. 1

Process and Documentation ....................................................................................... 1

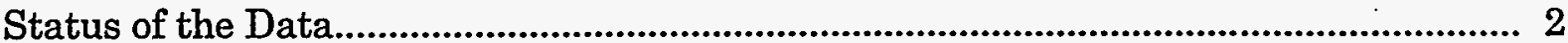

Other Data Considerations................................................................................... 3

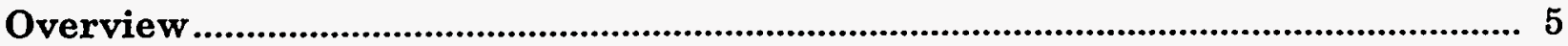

Site History ................................................................................................................... 5

Sample Identification Numbers for the Submerged Sediment Core Samples taken at

Par Pond, Pond C and L Lake ............................................................................................ 6

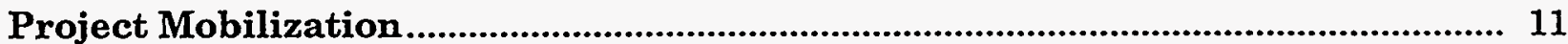

Summary of the Work Plan Assessment ......................................................................... 11

Changes Made to the Sample List and Constituents...................................................... 11

Sampling Methodology ...................................................................................................... 11

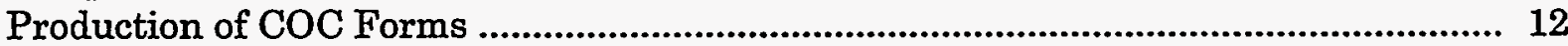

Decisions to be Made in the Field ............................................................................... 13

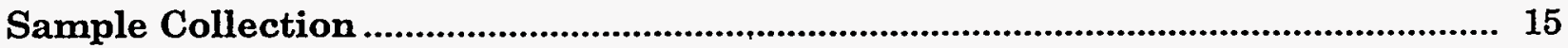

Sampling Objectives ...................................................................................................... 15

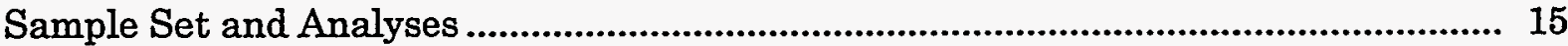

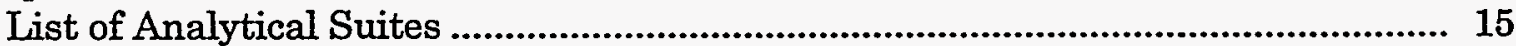

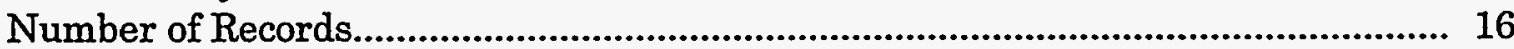

Changes to the Work Plan................................................................................................ 16

Particle Size Distribution...................................................................................................... 16

Description of Sample Collection ..................................................................................... 16

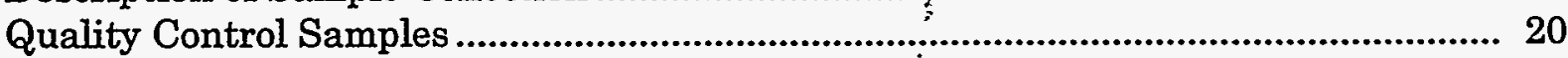

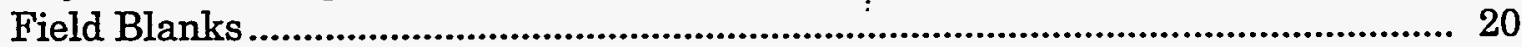

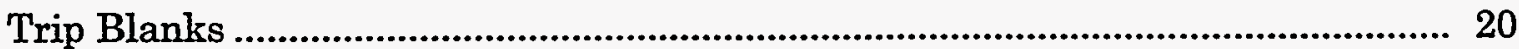

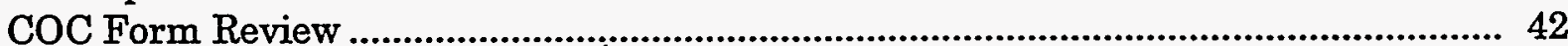

Sampling Discrepancies ..................................................................................................... 46

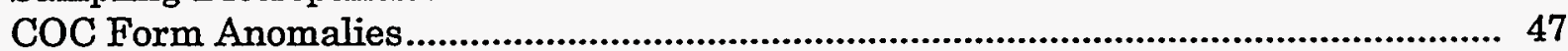

SRTC Project Manager ............................................................................................... 47

General Engineering Analytical Laboratories, Inc.................................................. 47

Sample Preservation ........................................................................................... 48

Preservation Requirements .............................................................................. 48

Adherence to Requirements ...................................................................................... 48 
Contract Laboratory Evaluation

The Laboratories.

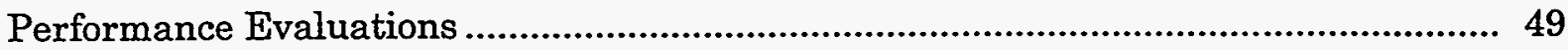

Quality Control Standards .................................................................................... 51

Laboratory Quality Assurance/Quality Control .................................................... 52

Performance Reviews .................................................................................... 52

General Engineering Laboratories, Inc., Performance Review ............................. 52

Roy F. Weston, Inc., Performance Review ......................................................... 53

Validation and Verification: Objective and Qualification Policy ......................... 57

Objective ...................................................................................................... 57

Qualification Policy ......................................................................................... 57

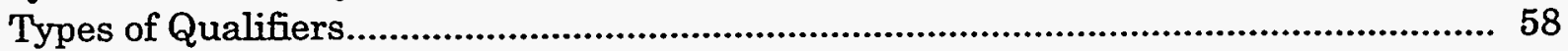

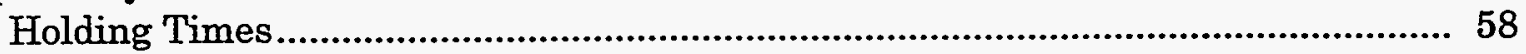

Sample Preservation................................................................................... 59

Instrument Calibration, Analyte Identification, and Analyte Quantitation ........... 59

Trip Blanks ................................................................................................... 59

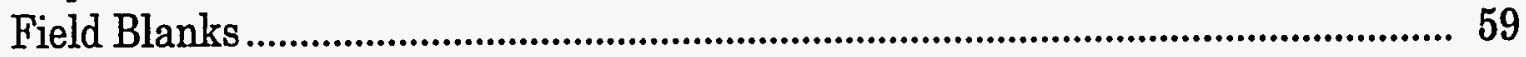

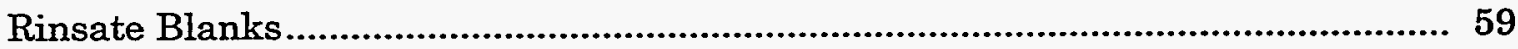

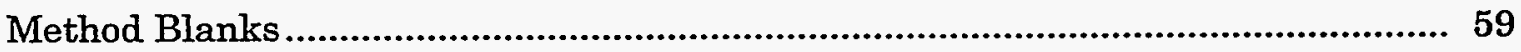

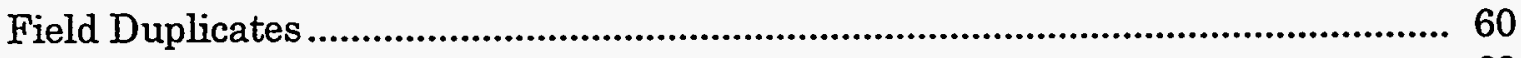

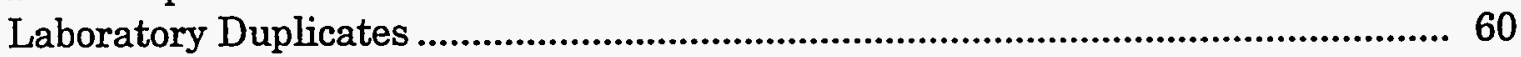

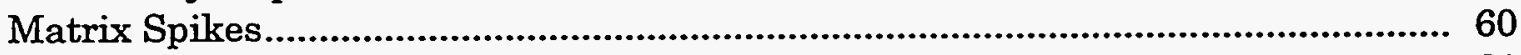

Laboratory Control Samples and Blank Spikes................................................. 61

Surrogate Recovery ........................................................................................ 61

Validation and Verification: Process ................................................................ 63

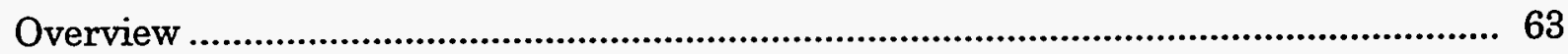

Data Quality Objectives ...................................................................................... 63

Analytical Methods and Quantitation Limits..................................................... 64

Review of the Electronic Data Files.................................................................... 64

Sample Collection, Shipping, and Analytical Data............................................ 64

Between-Records Validity ............................................................................. 65

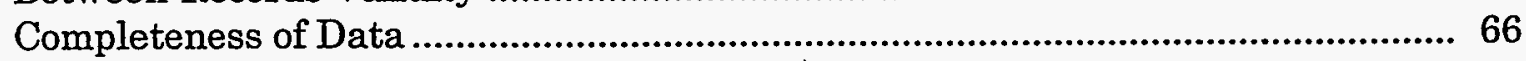

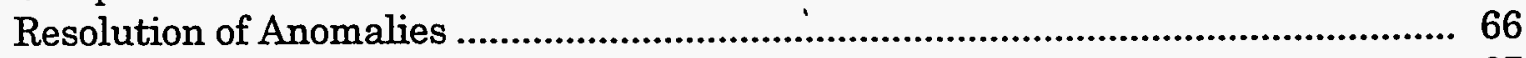

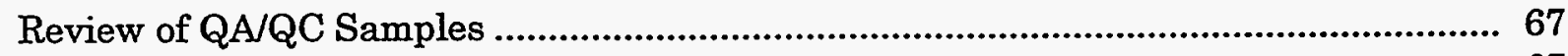

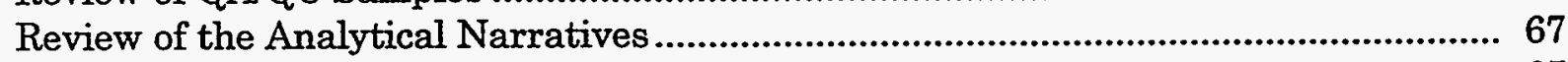

Laboratory Data Records Reviews ...................................................................... 67

Validation and Verification: Findings ....................................................... 69

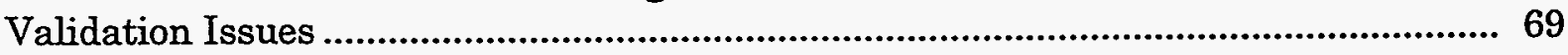

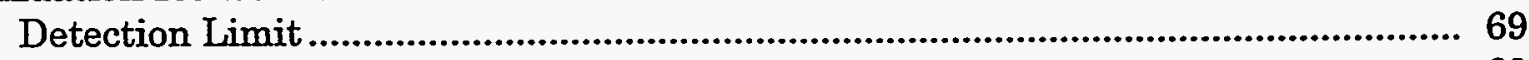

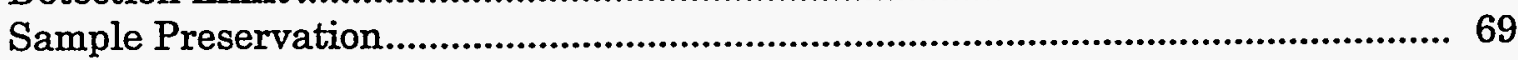

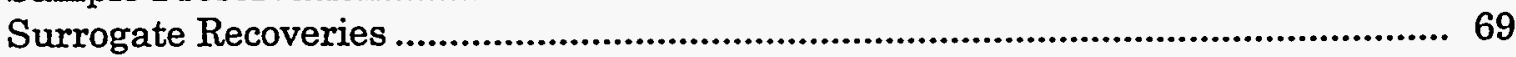

Types and Numbers of QA/QC Samples ....................................................... 70

Qualifiers Applied Following Review of the Electronic Data Deliverables and

Narratives .......................................................................................................... 70

Review of the Analytical Narratives ................................................................ 80 
Roy F. Weston Analytical Laboratories, Inc. .................................................... 80

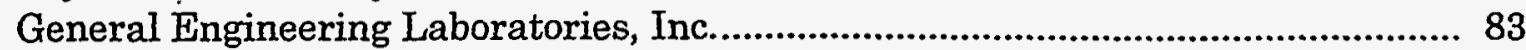

Laboratory Data Records Reviews ...................................................................... 90

Technical Issues................................................................................... 93

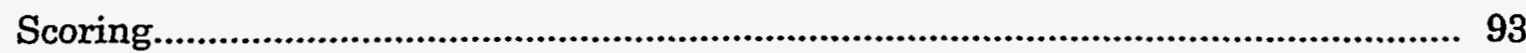

Qualification of Analyses ........................................................................... 95

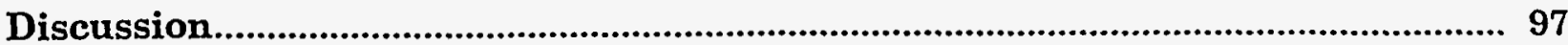

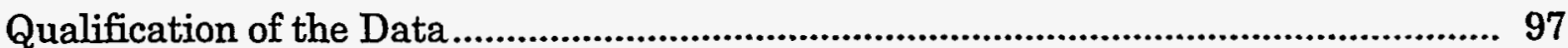

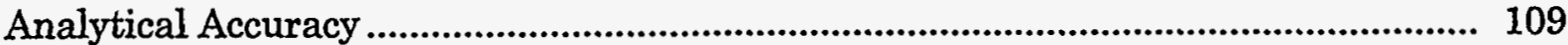

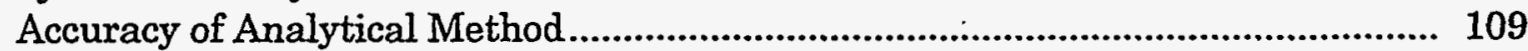

Laboratory Control Samples ....................................................................... 109

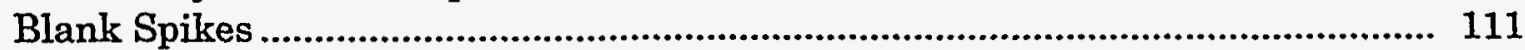

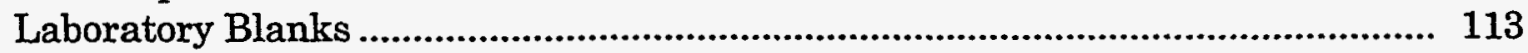

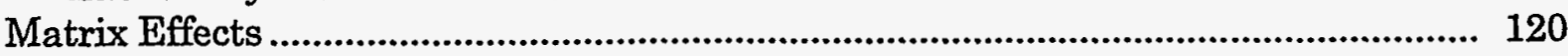

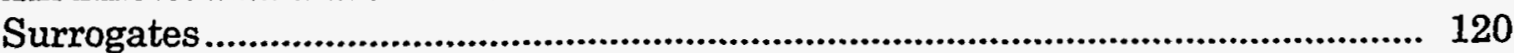

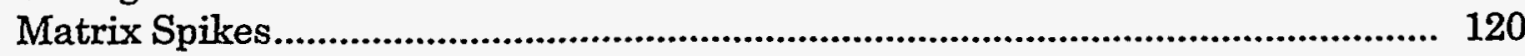

Effects of Sampling and Transport .................................................................. 125

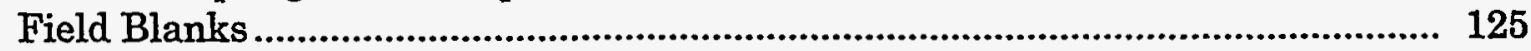

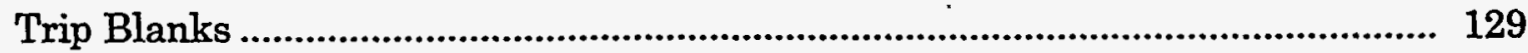

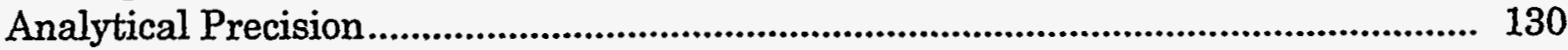

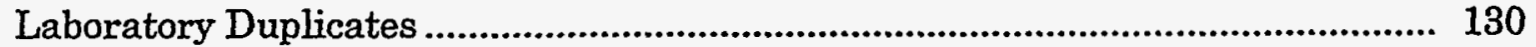

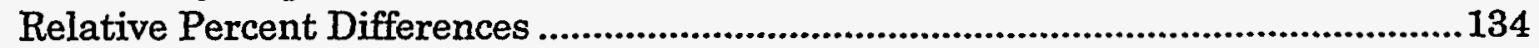

Sampling Effects and Inter-Sample Heterogeneity ........................................... 144

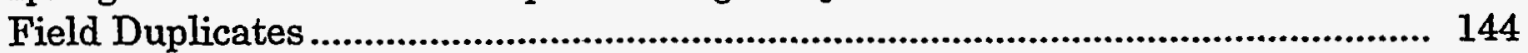

Quality Control Samples .............................................................................................. 157

Replicate and Duplicate Analyses of Samples................................................. 157

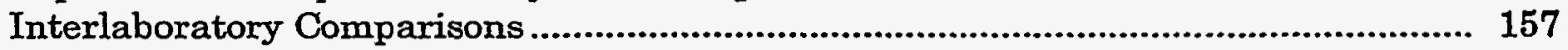

Normalizing Data to the Reference Detection Limit................................................ 158

Comments on the Replicate and Duplicate Analyses................................................. 158

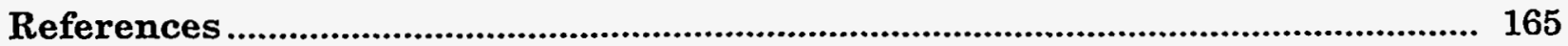

\section{Appendices}

Appendix A: Constituents Lists ..................................................................... A-1

Appendix B: Laboratory Performance Evaluation Results ..................................... B-1

Appendix C: Formats of the Data Files ........................................................... C-1

Appendix D: Data Tables ............................................................................... D 1

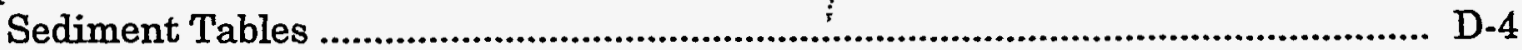

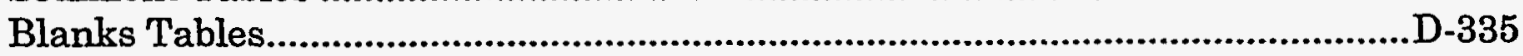

Appendix E: Data Management/Administrative Files .......................................... E-1

Appendix F: Analytical Methods and Quantitation Limits................................... F-1

Appendix G: Particle Size Distribution Charts ...................................................... G-1 
Table 1A. Par Pond Sample Identification Numbers .....................................................6

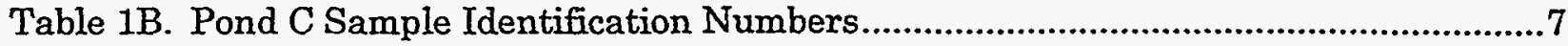

Table 1C. L Lake Sample Identification Numbers .........................................................7

Table 1D. Background Sample Identification Numbers ................................................8

Table 1E. Field Blank and Trip Blank Sample Identification Numbers ............................9

Table 2. Sediment Sample Set (Excluding Blanks Samples) ........................................17

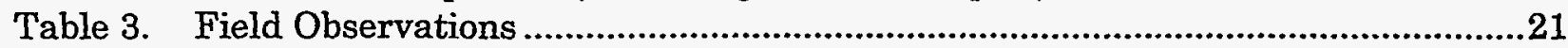

Table 4. Cooler Numbers, Temperatures, and Trip Blanks......................................... 43

Table 5. Comments/Observations from the COC Forms...............................................46

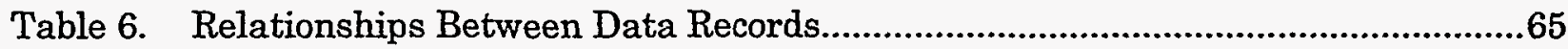

Table 7. Samples Assigned Qualifiers Due to RPDs Outside Range for GE.....................70

Table 8. Contaminated Laboratory Blanks Listed by Analyte and Preparation Batch for GE and Roy F. Weston Analytical Laboratories, Inc. (WA) .........................73

Table 9. Matrix Spikes Qualified with R4 for GE and WA.........................................76

Table 10. Matrix Spikes Qualified with R5 for GE and WA............................................76

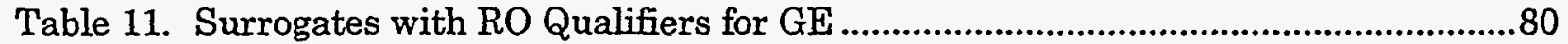

Table 12. Laboratory Data Records Review Methods/Analyses .......................................91

Table 13. Summary of Samples Reviewed ..................................................................91

Table 14. Number of Qualified Samples.......................................................................98

Table 15. Laboratory Control Samples Analyzed with Sediment Samples........................ 109

Table 16. Laboratory Control Samples Analyzed with Water Samples ...........................110

Table 17. Blank Spikes for Sediment Samples .........................................................111

Table 18 Blank Spikes for Water Samples.............................................................. 112

Table 19. Analytes Not Detected in Laboratory Blanks Analyzed with Sediment Samples

Table 20. Analytes Not Detected in Laboratory Blanks Analyzed with Water Samples

Table 21. Analytes Detected in Laboratory Blanks Analyzed with Sediment Samples .... 118

Table 22. Analytes Detected in Laboratory Blanks Analyzed with Water Samples..........119

Table 23. Surrogate Recoveries for Sediment Samples .................................................121

Table 24. Surrogate Recoveries for Water Samples.....................................................121

Table 25. Matrix Spike Recoveries for Sediment Samples ...............................................122

Table 26. Matrix Spike Recoveries for Water Samples...................................................124

Table 27. Analytes Not Detected in Field Blanks for Water Samples ............................125

Table 28. Analytes Detected in Field Blanks for Water Samples ..................................127

Table 29. Analytes Not Detected in Trip Blanks .........................................................129

Table 30. Analytes Detected in Trip Blanks ............................................................129

Table 31. Analytes Not Detected in Laboratory Duplicates for Sediment Samples .......... 130

Table 32. Analytes Not Detected in Laboratory Duplicates for Water Samples................ 133

Table 33. RPDs for Analytes Detected in Laboratory Duplicates for Sediment Samples

Table 34. RPDs for Analytes Detected in Laboratory Duplicates for Water Samples.......143

Table 35. Analytes Not Detected in Field Duplicates for Sediment Samples....................144

Table 36. RPDs for Analytes Detected in Field Duplicates for Sediment Samples ...........146 
Table 37. Samples Used for Interlaboratory Comparisons............................................ 158

Table 38. The Number of Determinations of Analytes Not Showing Measurable Concentrations above Detection Limits in Any Replicated Samples between GE and Weston

Table 39. Interlaboratory MRD and t-test Results for Analytes with at Least One Pair of Results above the RDL for GE and Weston

Table 40. Analytes with One Laboratory's Result Greater than Twice the Result from the Other Laboratory between the Primary and QC Laboratories

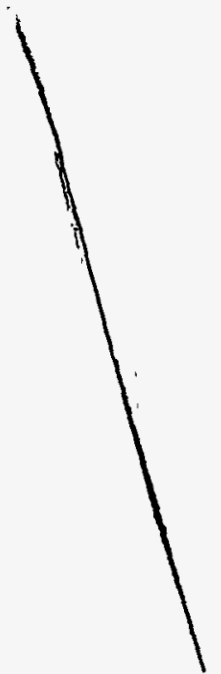




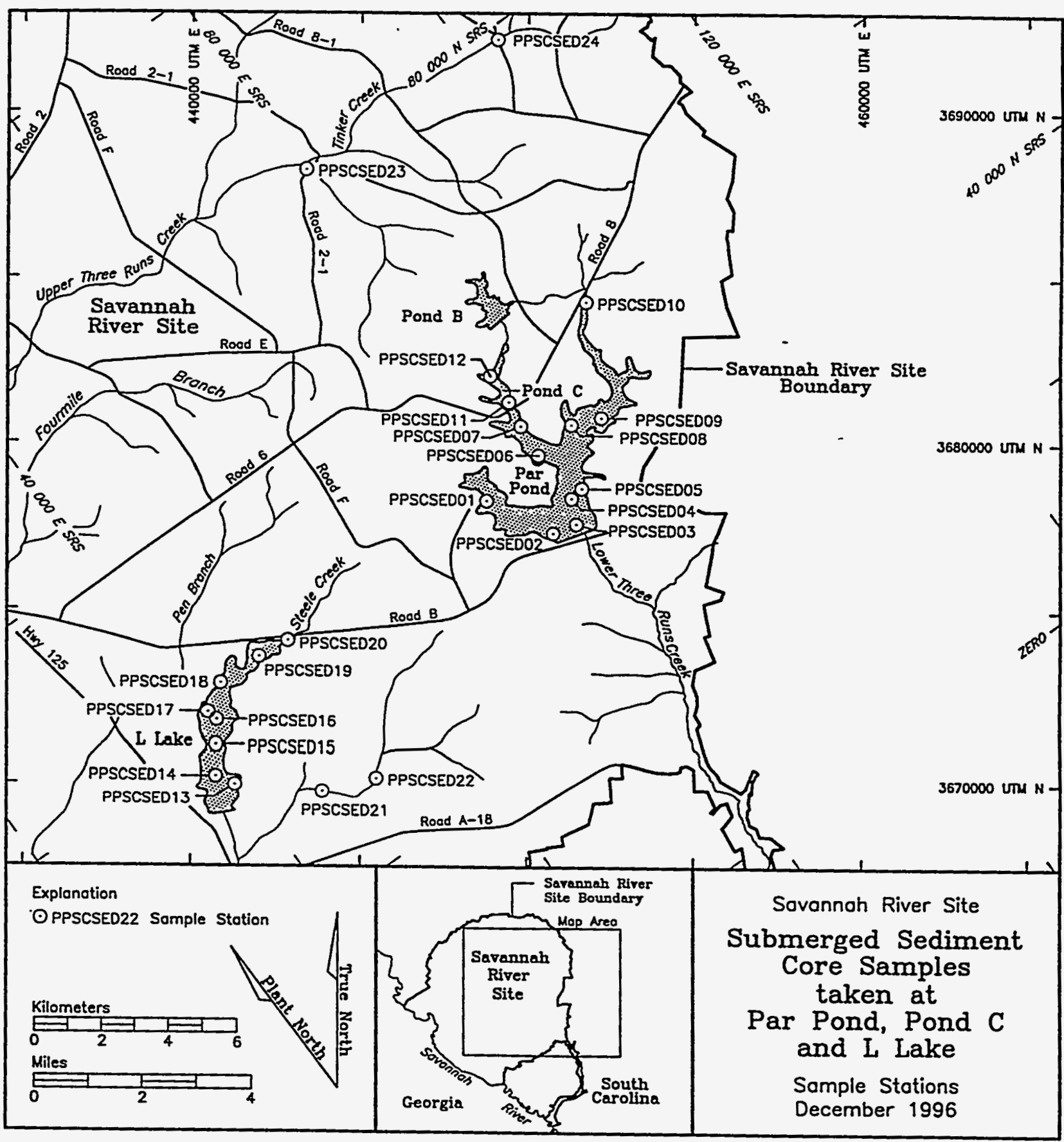

Figure 1. Submerged Sediment Core Samples taken at Par Pond, Pond C, and L Lake 


\section{Executive Summary}

\section{Purpose}

This report presents a summary of the sediment characterization performed under the direction of the Westinghouse Savannah River Company's (WSRC) Environmental Protection Department/Environmental Monitoring Section (EPD/EMS) in support of Par Pond, Pond C, and L Lake. This characterization will be a screening study and will enable the Environmental Sciences Section (ESS) to develop a defensible contaminants of concern list for more extensive characterization of the Par Pond, Pond C, and L Lake.

\section{Scope of the Project}

Exploration Resources, Inc., of Athens, GA, validated and verified the sample collection, shipping, and laboratory information compiled for this project. The information compiled, reviewed, and maintained includes the following:

- sample documentation

- chains of custody

- sample holding times

- initial and continuing instrument calibration

- analyte identification

- analyte quantitation

- analytical error

- analysis of blanks

- laboratory performance evaluations

- detection limits

The validation process began with project mobilization and continued through the delivery of electronic data deliverables (EDDs) and this report.

\section{Process and Documentation}

Sample documentation and maintenance of chain of custody (COC) were reviewed by examination of the field logs and COC forms. See the Sample Collection and Contract Laboratory Evaluation sections of this report for more information. 
Sample holding times were checked by comparing the time between sample collection and analysis with a look-up table of required holding times. Analytical instrument calibration was reviewed as part of the Laboratory Data Records Review (LDRR). See the Laboratory Data Records Reviews subsection in the Validation and Verification: Findings section of this report.

Analyte identification and analyte quantitation were verified as part of the computerized checking of the EDDs, during review of the analytical narratives, and as part of the LDRR. Anomalies have been clarified with the laboratories wherever possible, and records not meeting criteria have been qualified. See the appropriate subsection of the Validation and Verification: Findings section of this report for further information.

Field-generated and laboratory-generated blanks were examined. See the Sample Collection and Contract Laboratory Evaluation sections for an overview of the sampling plan regarding field-generated blanks, as well as the Discussion section for a list of all constituents detected in field-generated blanks. Laboratory-generated (method) blanks were performed in ratios of more than one for every twenty samples.

In evaluating analytical error, percent recoveries for Quality Assurance/Quality Control (QA/QC) samples were reviewed, and the QA/QC sample and associated project sample data were qualified when necessary. The criteria for qualification are described in the Validation and Verification: Objective and Qualification Policy section of this report. Relative percent differences between duplicate sample analyses were calculated.

Laboratory performance evaluations are detailed in the Contract Laboratory Evaluation section and in Appendix $B$ of this report.

\section{Status of the Data}

The data are final and complete except as noted in this subsection. Data validation is also complete.

All analytes requested in the work plan were reported except for the following:

- General Engineering Laboratories cannot separate and, therefore, cannot quantitate certain analytes. However, they report two indistinguishable compounds as combined analytes as shown in this table.

\begin{tabular}{|l|l|}
\hline Requested Analyte & Reported Analyte \\
\hline Plutonium-239 & Plutonium-239/240 \\
\hline Plutonium-240 & Plutonium-239/240 \\
\hline 4-Methylphenol & 3- and 4-Methylphenol \\
\hline
\end{tabular}

The analytical data file contains records for WSRC samples not covered by the workplan for this project. These records were submitted by the laboratory for QA/QC purposes only.

Some of the data did not meet all criteria delineated in the Data Quality Objectives Process for Superfund (USEPA, 1993). The data are as follows: 
- Because the field measurements are not analyte specific, they are designed to meet the criteria for screening data, and definitive data criteria are not applicable.

- Analytical error determination (a measurement of method precision) has not been calculated; however, precision can be evaluated by referring to tables in the Discussion section of this report.

- Total measurement error determination (measurement of overall precision from sample acquisition through analysis) was not performed because it requires independently collected samples from the same location, and this practice does not fit into the scope of the project work plan. Each sampling location is used for one sampling event.

- No adjustments to sample values were made for bias or precision.

- One hundred thirty-two analytical records were rejected by the laboratory. All laboratory-rejected data records are laboratory QA samples. The reasons for record rejection by the laboratory were that matrix spikes could not be quantitated due to matrix interference, the matrix spike sample concentration was four times greater than the amount of spike added, or surrogate spikes were diluted out.

- Through the use of criteria defined in Data Quality Objectives Process for Superfund (USEPA,1993), matrix spike recoveries for an entire project are averaged and all associated sample data are qualified on the basis of this average. Because soil matrices are heterogeneous, matrix spike-based qualification for this project was done on a sample-by-sample basis, using criteria in the Qualification Policy subsection of the Validation and Verification: Objective and Qualification Policy section of this report.

- In order to meet contract requirements, the primary laboratory generated QA/QC samples from WSRC samples which were not within the scope of the project plan. These samples served as matrix spikes, matrix spike duplicates, and/or laboratory duplicates and were included only for purposes of estimating laboratory precision. Only sample records having sample identification numbers 104600 through 104699 and 105100 through 105120 will be represented in the data tables included with this report. However, all QA/QC samples, regardless of sample identification number, will be included on the data disk which accompanies this report.

\section{Other Data Considerations}

It is Environmental Geochemistry Group (EGG) policy to ship all volatile organic compound sample bottles in one cooler, accompanied by a trip blank. Bottles containing samples for other analyses are shipped in a separate cooler. One COC form accompanies each sample, and each cooler has a COC seal. As long as the COC and all the bottles for any sample travel in the same shipment, COC procedure has been maintained according to EGG policy. 
This page was left blank intentionally. 


\section{Site History}

Par Pond is a 2,640-acre reservoir that was constructed in 1958 by impounding the headwaters of Lower Three Runs Creek with an earthen emankment. Through 1963, Par Pond served as a recirculating cooling reservoir for $P$ and $R$ Reactors (see Figure 1 for location). After $R$ Reactor was shut down in 1964, Par Pond was used to cool effluents from $P$ Reactor only. Par Pond received thermal effluent from R-Reactor through a pre-cooler pond, Pond C, from August 1958 to October 1961. All thermal discharge to Par Pond ceased when reactor operations were stopped in 1988 (WSRC, 1993).

A 1991 inspection of the Par Pond dam revealed a depression on the downstream slope. Subsequent investigations revealed erosion around the outside of the buried conduits and of the dam's fill material. Although there was no imminent danger of dam failure, precautionary measures were taken in 1991 to minimize the potential for failure and decrease the magnitude of associated flooding in the case of failure. These precautions involved draining Par Pond from its normal level of $200 \mathrm{ft}$ above mean sea level to a level of $181 \mathrm{ft}$ above msl (mean sea level), a nearly two-thirds reduction in the original volume of the pond. The 19-ft water level decrease exposed sediments known to have been contaminated with low levels of cesium-137 and other radionuclides (WSRC, 1993). In addition to the drawdown, a detailed investigation of the dam's safety was performed and Congressional funding to repair the dam was authorized (WSRC, 1993).

During the drawdown of Par Pond, the sediments exposed along the water line were sampled to determine contamination levels. After Par Pond was lowered to $195 \mathrm{ft}$, core samples were collected from 17 locations in the pond on each of four separate sampling events after every five ft lowering of the water level. An additional five locations at Pond $\mathrm{C}$ (see the figure for location) were also sampled as part of this initial risk assessment. A total of 348 core segments from 73 cores were analyzed for gamma emitters, total strontium, plutonium-238, and plutonium-239 (WSRC, 1993).

Most of the samples, 330 of 348, exhibited elevated levels of cesium-137 with the majority of activity present in the top two inches of the cores and evenly distributed throughout Par Pond. Strontium was detected in 159 of the 348 core segments with the majority of the activity occurring in the four to eight-inch depth of the cores. It appears the south arm of Par Pond contains more detectable strontium activity, as well as generally higher strontium concentrations, than any other area of the pond. Three samples from this area of Par Pond showed plutonium-238 and two samples showed plutonium-239 concentrations above the laboratory's nominal detection limits (WSRC, 1993).

Remote sensing data (i.e., aerial helicopter gamma surveys) were collected during and after the Par Pond drawdown. The gamma data outlined the distribution of man-made radionuclides, primarily cesium-137, along the exposed shoreline of Par Pond. A two percent increase in activity was noted between October 1991 and August 1992 and was attributed to sediment drying (WSRC, 1993). 
An underwater high purity germanium detector was used to provide a refined analysis of cesium-137 in Par Pond sediments during 1991. About 200 measurements were taken around the pond and a cesium-137 "hot" spot was detected at the mouth of the pond's middle arm. Improved information for dose estimates resulted from the above analysis and indicated that the maximum cesium-137 mass activity is at the sediment surface (WSRC, 1993).

Mercury also has been identified as a contaminant in the pond and is believed to have been introduced into the Par Pond system by water pumped from the Savannah River, which is known to be contaminated (WSRC, 1992f). The fish population in Par Pond have been shown to contain high levels of mercury, in the form of methylmercury, while the sediments, normally considered to be a mercury sink in aquatic environments, exhibit natural levels (Schindler, et. al., n.d.).

\section{Sample Identification Numbers for the Submerged Sediment Core Samples taken at Par Pond, Pond $\mathrm{C}$ and L-Lake}

The following samples were taken in July and August 1995 to further characterize the contamination in the Par Pond, Pond $\mathrm{C}$ and L Lake. These samples provide the basis of this report. Table 1 presents the samples analyzed for this project.

Table 1A: Par Pond Sample Identification Numbers

\begin{tabular}{|l|l|l|l|}
\hline Survey Station ID & Sample ID & Laboratory ID & Sample Type \\
\hline PPSC0101 & 104600 & $9507128-21$ & Sediment \\
\hline PPSC0102 & 104601 & $9507128-22$ & Sediment \\
\hline PPSC0103 & 104602 & $9507128-23$ & Sediment \\
\hline PPSC0201 & 104604 & $9507128-01$ & Sediment \\
\hline PPSC0202 & 104605 & $9507128-02$ & Sediment \\
\hline PPSC0203 & 104606 & $9507128-03$ & Sediment \\
\hline PPSC0204 & 104607 & $9507128-04$ & Sediment \\
\hline PPSC0205 & 104608 & $9507128-05$ & Sediment \\
\hline PPSC0301 & 104609 & $9507128-06$ & Sediment \\
\hline PPSC0302 & 104610 & $9507128-07$ & Sediment \\
\hline PPSC0303 & 104611 & $9507128-08$ & Sediment \\
\hline PPSC0304 & 104612 & $9507128-09$ & Sediment \\
\hline PPSC0501 & 104613 & $9507128-10$ & Sediment \\
\hline PPSC0502 & 104614 & $9507128-11$ & Sediment \\
\hline PPSC0503 & 104615 & $9507128-12$ & Sediment \\
\hline PPSC0601 & 104616 & $9507128-13$ & Sediment \\
\hline PPSC0602 & 104617 & $9507128-14$ & Sediment \\
\hline PPSC0603 & 104618 & $9507128-15$ & Sediment \\
\hline PPSC0604 & 104619 & $9507128-16$ & Sediment \\
\hline PPSC0701 & 104620 & $9507128-17$ & Sediment \\
\hline
\end{tabular}


Table 1A: Par Pond Sample Identification Numbers, Continued

\begin{tabular}{|l|l|l|l|}
\hline Survey Station ID & Sample ID & Laboratory ID & Sample Type \\
\hline PPSC0702 & 104621 & $9507128-18$ & Sediment \\
\hline PPSC0703 & 104622 & $9507128-19$ & Sediment \\
\hline PPSC0704 & 104623 & $9507128-20$ & Sediment \\
\hline PPSC1001 & 104625 & $9507199-01$ & Sediment \\
\hline PPSC1002 & 104626 & $9507199-02$ & Sediment \\
\hline PPSC1003 & 104627 & $9507199-03$ & Sediment \\
\hline PPSC1004 & 104628 & $9507199-04$ & Sediment \\
\hline PPSC1005 & 104629 & $9507160-03$ & Sediment \\
\hline PPSC0801 & 104630 & $9507160-04$ & Sediment \\
\hline PPSC0802 & 104632 & $9507160-05$ & Sediment \\
\hline PPSC0803 & 104633 & $9507160-06$ & Sediment \\
\hline PPSC0901 & 104635 & $9507199-05$ & Sediment \\
\hline PPSC0902 & 104636 & $9507199-06$ & Sediment \\
\hline PPSC0903 & 104637 & $9507199-07$ & Sediment \\
\hline PPSC0401 & 104643 & $9507226-01$ & Sediment \\
\hline PPSC0402 & 104644 & $9507226-02$ & Sediment \\
\hline PPSC0403 & 104645 & $9507226-03$ & Sediment \\
\hline PPSC0404 & 104646 & $9507226-04$ & Sediment \\
\hline
\end{tabular}

Table 1B: Pond C Sample Identification Numbers

\begin{tabular}{|l|l|l|l|}
\hline Survey Station ID & Sample ID & Laboratory ID & Sample Type \\
\hline PPSC1101 & 104652 & $9507281-01$ & Sediment \\
\hline PPSC1102 & 104653 & $9507281-02$ & Sediment \\
\hline PPSC1103 & 104655 & $9507281-03$ & Sediment \\
\hline PPSC1201 & 104656 & $9507281-04$ & Sediment \\
\hline PPSC1202 & 104658 & $9507281-06$ & Sediment \\
\hline PPSC1203 & 104660 & $9507281-07$ & Sediment \\
\hline
\end{tabular}

Table 1C: L Lake Sample Identification Numbers

\begin{tabular}{|l|l|l|l|}
\hline Survey Station ID & Sample ID & Laboratory ID & Sample Type \\
\hline PPSC1301 & 104664 & $9507343-01$ & Sediment \\
\hline PPSC1302 & 104665 & $9507343-04$ & Sediment \\
\hline PPSC1303 & 104666 & $9507343-03$ & Sediment \\
\hline PPSC1304 & 104667 & $9507343-02$ & Sediment \\
\hline PPSC1801 & 104668 & $9507343-07$ & Sediment \\
\hline PPSC1802 & 104669 & $9507343-06$ & Sediment \\
\hline
\end{tabular}


Table 1C: L Lake Sample Identification Numbers, Continued

\begin{tabular}{|l|l|l|l|}
\hline Survey Station ID & Sample ID & Laboratory ID & Sample Type \\
\hline PPSC1803 & 104670 & $9507343-05$ & Sediment \\
\hline PPSC1901 & 104671 & $9507381-01$ & Sediment \\
\hline PPSC1902 & 104672 & $9507381-02$ & Sediment \\
\hline PPSC1903 & 104673 & $9507381-03$ & Sediment \\
\hline PPSC1904 & 104674 & $9507381-04$ & Sediment \\
\hline PPSC1905 & 104675 & $9507381-05$ & Sediment \\
\hline PPSC2001 & 104676 & $9507381-06$ & Sediment \\
\hline PPSC2002 & 104677 & $9507381-07$ & Sediment \\
\hline PPSC2003 & 104678 & $9507381-08$ & Sediment \\
\hline PPSC1701 & 104693 & $9507509-01$ & Sediment \\
\hline PPSC1702 & 104694 & $9507509-02$ & Sediment \\
\hline PPSC1703 & 104695 & $9507509-03$ & Sediment \\
\hline PPSC1704 & 104696 & $9507509-04$ & Sediment \\
\hline PPSC1601 & 104697 & $9507509-08$ & Sediment \\
\hline PPSC1602 & 104698 & $9507509-07$ & Sediment \\
\hline PPSC1603 & 105100 & $9507509-05$ & Sediment \\
\hline PPSC1604 & 105101 & $9507509-06$ & Sediment \\
\hline PPSC1605 & 105102 & $9507509-09$ & Sediment \\
\hline PPSC1501 & 105104 & $9507546-11$ & Sediment \\
\hline PPSC1502 & 105105 & $9507546-13$ & Sediment \\
\hline PPSC1503 & 105106 & $9507546-12$ & Sediment \\
\hline PPSC1504 & 105107 & $9507546-14$ & Sediment \\
\hline PPSC1505 & 105108 & $9507546-15$ & Sediment \\
\hline PPSC1401 & 105109 & $9507546-09$ & Sediment \\
\hline PPSC1402 & 105110 & $9507546-10$ & Sediment \\
\hline PPSC1403 & 105111 & $9507546-16$ & Sediment \\
\hline & & & \\
\hline
\end{tabular}

Table 1D: Background Sample Identification Numbers

\begin{tabular}{|l|l|l|l|}
\hline Survey Station ID & Sample ID & Laboratory ID & Sample Type \\
\hline PPSC2101 & 104680 & $9507448-01$ & Sediment \\
\hline PPSC2102 & 104681 & $9507448-02$ & Sediment \\
\hline PPSC2103 & 104682 & $9507448-03$ & Sediment \\
\hline PPSC2201 & 104683 & $9507448-04$ & Sediment \\
\hline PPSC2202 & 104684 & $9507448-05$ & Sediment \\
\hline PPSC2203 & 104685 & $9507448-06$ & Sediment \\
\hline PPSC2204 & 104686 & $9507448-07$ & Sediment \\
\hline PPSC2301 & 104687 & $9507448-08$ & Sediment \\
\hline
\end{tabular}


Table 1D: Background Sample Identification Numbers, Continued

\begin{tabular}{|l|l|l|l|}
\hline Survey Station ID & Sample ID & Laboratory ID & Sample Type \\
\hline PPSC2302 & 104688 & $9507448-09$ & Sediment \\
\hline PPSC2303 & 104689 & $9507448-10$ & Sediment \\
\hline PPSC2304 & 104690 & $9507448-11$ & Sediment \\
\hline PPSC2305 & 104691 & $9507448-12$ & Sediment \\
\hline PPSC2401 & 105117 & $9507546-05$ & Sediment \\
\hline PPSC2402 & 105118 & $9507546-06$ & Sediment \\
\hline PPSC2403 & 105119 & $9507546-07$ & Sediment \\
\hline PPSC2404 & 105120 & $9507546-08$ & Sediment \\
\hline PPSC2501 & 105113 & $9507546-01$ & Sediment \\
\hline PPSC2502 & 105114 & $9507546-02$ & Sediment \\
\hline PPSC2503 & 105115 & $9507546-03$ & Sediment \\
\hline PPSC2504 & 105116 & $9507546-04$ & Sediment \\
\hline PPSC2601A & 104638 & $9507199-08$ & Duplicate \\
\hline PPSC2602D & 104639 & $9507 L 559-001$ & Split \\
\hline PPSC2603A & 104640 & $9507199-09$ & Duplicate \\
\hline PPSC2701D & 104647 & $9507 L 559-002$ & Split \\
\hline PPSC2702A & 104648 & $9507226-06$ & Duplicate \\
\hline PPSC2703D & 104649 & $9507 L 559-003$ & Split \\
\hline PPSC2704A & 104650 & $9507226-05$ & Duplicate \\
\hline PPSC2801A & 104657 & $9507281-05$ & Duplicate \\
\hline PPSC2802D & 104659 & $9507 L 581-001$ & Split \\
\hline PPSC2803A & 104661 & $9507281-08$ & Duplicate \\
\hline
\end{tabular}

Table 1E: Field Blank and Trip Blank Sample Identification Numbers

\begin{tabular}{|l|l|l|l|}
\hline Survey Station ID & Sample ID & Laboratory ID & Sample Type \\
\hline PPSCFB01C & 104603 & $9507128-30$ & Field Blank \\
\hline PPSCTB01B & 104624 & $9507128-31$ & Trip Blank \\
\hline PPSCFB03C & 104631 & $9507160-02$ & Field Blank \\
\hline PPSCTB02B & 104634 & $9507160-01$ & Trip Blank \\
\hline PPSCTB03B & 104641 & $9507199-10$ & Trip Blank \\
\hline PPSCTB15B & 104642 & $9507 L 559-004$ & Trip Blank \\
\hline PPSCTB04B & 104651 & $9507226-07$ & Trip Blank \\
\hline PPSCFB02C & 104654 & $9507281-09$ & Field Blank \\
\hline PPSCTB05B & 104662 & $9507281-10$ & Trip Blank \\
\hline PPSCTB16B & 104663 & $9507 L 581-002$ & Trip Blank \\
\hline PPSCTB06B & 104679 & $9507381-09$ & Trip Blank \\
\hline PPSCTB07B & 104692 & $9507448-13$ & Trip Blank \\
\hline
\end{tabular}


Table 1E: Field Blank and Trip Blank Sample Identification Numbers, Continued

\begin{tabular}{|l|l|l|l|}
\hline Survey Station ID & Sample ID & Laboratory ID & Sample Type \\
\hline PPSCFB05C & 104699 & $9507509-10$ & Field Blank \\
\hline PPSCTB08B & 105103 & $9507509-11$ & Trip Blank \\
\hline PPSCTB09B & 105112 & $9507546-17$ & Trip Blank \\
\hline
\end{tabular}




\section{Project Mobilization}

\section{Summary of the Work Plan Assessment}

The work plan assessment for the submerged sediment core samples taken at Par Pond, Pond C and L Lake (PPSC) was presented in a Westinghouse Savannah River Company (WSRC) memo (Nichols, C.E., 1995) from the Project Technical Manager, Chester E. Nichols, to the Savannah River Technology Center (SRTC) Project Manager and Subcontract Technical Representative, James Koch. This work plan assessment was based on information from the Sampling Plan (WSRC, 1995a).

\section{Changes Made to the Sample List and Constituents}

During mobilization, Quality Assurance/Quality Control (QA/QC) samples were added to the sediment sample list. Splits and duplicates were taken at a rate of one per 10 samples collected and were taken from the Hot Dam, Cold Dam, and North Arm sample locations within Par Pond (Nichols, C.E., 1995). Thirteen duplicates/splits were required and seven duplicates and six splits were planned. The following table provides a list of the samples taken.

\begin{tabular}{|l|l|l|}
\hline Sample Type & Survey Station ID & Location \\
\hline Duplicate & PPSC0901A & Adjacent to PPSC09 \\
\hline Split & PPSC0902D & Adjacent to PPSC09 \\
\hline Duplicate & PPSC0903A & Adjacent to PPSC09 \\
\hline Split & PPSC0401D & Adjacent to PPSC04 \\
\hline Duplicate & PPSC0402A & Adjacent to PPSC04 \\
\hline Split & PPSC0403D & Adjacent to PPSC04 \\
\hline Duplicate & PPSC0404A & Adjacent to PPSC04 \\
\hline Duplicate & PPSC1201A & Adjacent to PPSC12 \\
\hline Split & PPSC1202D & Adjacent to PPSC12 \\
\hline Duplicate & PPSC1203A & Adjacent to PPSC12 \\
\hline
\end{tabular}

Field blanks were planned at a rate of one per 20 samples. Trip blanks were planned at a rate of one per day of VOA sample collection at the rate of one trip blank per cooler for nine primary lab coolers and two secondary lab coolers.

See Appendix $A$ for a list of the constituents analyzed; see Appendix $F$ for a list of the analytical methods used and quantitation limits.

\section{Sampling Methodology}

Sampling methodology used for this project was defined according to Guidance for Data Useability in Risk Assessment (USEPA, 1990c). The work plan assessment (Nichols, C.E., 
1995) further describes the sampling procedure for the QC samples described in the following paragraphs.

Splits were sent to a different laboratory as a cross-check of data quality. Trip blanks consisted of deionized water provided by the laboratory and were placed in a cooler with other bottles for analysis of volatile organic compounds. Field blanks, which consist of distilled-deionized water placed in a stainless steel bowl and exposed to the air while the sample is being collected from the opened core, were analyzed for target compound list (TCL) and target analyte list (TAL) inorganics only to monitor major pathways of contamination. Sampling equipment was dedicated, therefore, no rinsate blanks were required.

The sampling plan does not require chain of custody (COC) forms or sample containers for radiological screening samples. As has been the Environmental Monitoring Section's practice to date, it was the responsibility of the SRTC Project Manager to provide field and onset radiological screening of samples. One purpose of these screenings was to determine handling and shipping requirements for shipment to the offsite analytical laboratory. In order to inform laboratory personnel of the radiological characteristics of the samples, the SRTC Project Manager filled out the Radiological Screening Compilation Form and included it with the sample shipments.

Two cores were collected at each location in an attempt to provide sufficient sample volume for analysis. Each core was subdivided into five equal sections. TCL VOA samples were collected from the center of each individual core section as soon as the core was split open. The individual core sections were then homogenized and the non-VOA samples were collected. At locations designated for collection of duplicates and splits, four cores were taken to provide enough sediment for analysis.

\section{Production of COC Forms}

COC forms containing the list of requested analyses for each planned sample were printed during mobilization. A tear-off stub attached to the COC form indicated each sample location. Information filled in on the COC form includes the following:

- sample ID number from the sample logbook page for that sample type

- laboratory sample ID (to be completed by the analytical laboratory)

- sample collection date

- shipping information for dates and signatures of each person handling the sample

- cooler number and temperature upon arrival at the analytical laboratory

In addition, a peel-off section of each bottle label, printed with the bottle number, was affixed to each line of requested analyses. COC forms were generated based on the maximum number of projected samples. Unused COC forms were marked as "Not Sampled" and returned to the subcontract technical representative. The oversight geologist entered "Bottom of Hole" in the comments section of the sample logbook for the last sample interval in each boring. 


\section{Decisions to be Made in the Field}

The submerged sediment core sampling program depends upon correct decision making in the field. The decisions to be made in the field for this project included the following:

- which COC forms were appropriate to each sampling point

- when to generate a trip blank for each cooler

- when to collect field blanks to ensure a rate of one per 20 samples

- whether a duplicate or split sample would require additional sampling to recover adequate material

See the next section of this report, Sample Collection, for details on the adherence of field activities to the work plan assessment. 
This page left blank intentionally. 


\section{Sample Collection}

Field activities for this project were conducted by the Ecology Group, the Environmental Sciences Section of the Savannah River Technology Center (SRTC), at the Westinghouse Savannah River Company (WSRC). The project was overseen by the SRTC project manager, Jim Koch. The objective of this project was to provide a plan to collect soil core samples needed to determine contamination levels in sediment core samples of Par Pond, Pond $\mathrm{C}$, and L Lake. Activities performed involved the collection and analysis of deep and shallow water sediment cores using vibracoring equipment.

\section{Sampling Objectives}

The assessment of Par Pond, Pond C, and L Lake was designed to provide additional data for conducting human and ecological risk assessment, corrective measures assessment, and remedial action, if necessary. The planned objectives for the assessment at the Par Pond, Pond $\mathrm{C}$ and L Lake are as follows:

- identify all of the contaminants of concern and determine their extent and levels of contamination in the submerged Par Pond, Pond C, and L Lake sediments

- support a screening study to enable WSRC to develop a defensible contaminants-ofconcern list for more extensive characterization of the Par Pond, Pond C, and L Lake sediments, when such a list is required

\section{Sample Set and Analyses}

\section{List of Analytical Suites}

Chain-of-custody $(\mathrm{COC})$ forms were sent to the laboratories requesting the following analyses for sediment samples:

- Particle size distribution and cation exchange capacity

- Radionuclides (gross alpha, nonvolatile beta, gamma PHA, strontium-90, plutonium series)

- Specified analytes

- Target analyte list (TAL) inorganics

- Target compound list (TCL) with tentatively identified compounds (TICs)

- TCL volatile organic analysis (VOA)

Trip blanks were analyzed for TCL VOA and field blanks were analyzed for TCL organics and TAL inorganics. The sediment and quality assurance/quality control (QA/QC) samples were analyzed by General Engineering Analytical Laboratories, Inc. (GE), and the splits were analyzed by Roy F. Weston Analytical Laboratories, Inc. (WA).

Refer to Appendix $A$ for constituents analyzed in each suite. 


\section{Number of Records}

Included with the data for this project are 121 sample logbook records, $121 \mathrm{COC}$ form records, and 25 sample survey station records. The 23,531 analytical records consist of 15,417 sample records; 8,114 laboratory blanks, blank spikes, and other laboratorygenerated QA/QC records.

The result qualifier $R$ was assigned to 129 analytical records, all of which were QA/QC records. The $J$ result qualifier was assigned to 1,584 analytical data records, which consisted of 1,147 sample records and $437 \mathrm{QA} / \mathrm{QC}$ records. The $U J$ result qualifier was assigned to 2,198 analytical data records, which consisted of 1,917 sample records and 281 $\mathrm{QA} / \mathrm{QC}$ records.

Refer to Appendix $C$ for the data file formats for the analytical data, COC data, sample data, and survey station data.

\section{Changes to the Work Plan}

- The sampling plan did not require COC forms or sample containers for radiological screening samples. As has been the practice of the Environmental Protection Department/Environmental Monitoring Section (EPD/EMS) to date, the project manager is responsible for providing field and on-site radiological screening of samples.

- The work plan assessment (Nichols, C.E., 1995) required two cores to be collected at each location to provide sufficient soil volume for analysis.

- The sampling plan required two rinsate blanks for the project. According to the work plan assessment, sampling equipment was dedicated and rinsate blanks were not required.

\section{Particle Size Distribution}

Particle size distribution charts plotting the grain size against cumulative weight are included in Appendix $G$ of this report. These charts reflect the content of the soil, i.e., the amount of sand, silt, and clay. Contaminants sorb to the clay materials mixed in the soil. During review of the charts, it was observed that most of the Par Pond, Pond C, and L Lake sampling sites contain mostly sand. The precipitous curves on the particle size distribution charts reflect variance between the medium and fine sand zones. A high sand content in the samples indicates a textural classification of sand to loamy sand for most of the samples analyzed (Soil Survey Staff, 1990).

\section{Description of Sample Collection}

The sampling plan and work plan assessment specified that soil cores would be collected from the surface of the basin at various depths on Par Pond, Pond C, and L Lake. Sample cores were collected from a total of 25 locations using the vibracoring sampling method. Cores were also collected from five uncontaminated reference sites that served as background. 
Samples were sent to GE under contract AA07208N. Splits were sent to WA under contract AA07210N.

The following table lists the sediment samples taken (with the exception of blanks samples) for this project.

Táble 2. Sediment Sample Set (Excluding Blanks Samples)

\begin{tabular}{|c|c|c|c|c|c|}
\hline $\begin{array}{l}\text { Survey } \\
\text { Station ID }\end{array}$ & Sample ID & $\begin{array}{l}\text { Sample } \\
\text { Type }\end{array}$ & $\begin{array}{l}\text { Sample Date } \\
\text { and Time }\end{array}$ & $\begin{array}{l}\text { Sampling } \\
\text { Depth (ft) }\end{array}$ & $\begin{array}{l}\text { Analyses }^{1} \\
\text { Requested }\end{array}$ \\
\hline PPSC0101 & 104600 & Sediment & $07 / 7 / 95,11: 35$ & $0.0-1.0$ & $0,1,2,3$ \\
\hline PPSC0102 & 104601 & Sediment & $07 / 7 / 95,12: 05$ & $1.0-2.0$ & $0,1,2,3$ \\
\hline PPSC0103 & 104602 & Sediment & $07 / 7 / 95,12: 25$ & $2.0-3.1$ & $0,1,2,3$ \\
\hline PPSC0201 & 104604 & Sediment & $07 / 7 / 95,16: 20$ & $0.0-1.0$ & $0,1,2$ \\
\hline PPSC0202 & 104605 & Sediment & $07 / 7 / 95,16: 40$ & $1.0-2.0$ & $0,1,2$ \\
\hline PPSC0203 & 104606 & Sediment & $07 / 7 / 95,17: 00$ & $2.0-4.0$ & $0,1,2$ \\
\hline PPSC0204 & 104607 & Sediment & $07 / 7 / 95,17: 30$ & $4.0-6.0$ & $0,1,2$ \\
\hline PPSC0205 & 104608 & Sediment & $07 / 7 / 95,17: 40$ & $6.0-7.1$ & $0,1,2$ \\
\hline PPSC0301 & 104609 & Sediment & $07 / 8 / 95,12: 30$ & $0.0-1.0$ & $0,1,2$ \\
\hline PPSC0302 & 104610 & Sediment & $07 / 8 / 95,12: 45$ & $1.0-2.0$ & $0,1,2$ \\
\hline PPSC0303 & 104611 & Sediment & 07/8/95, 13:00 & $2.0-4.0$ & $0,1,2$ \\
\hline PPSC0304 & 104612 & Sediment & 07/8/95, 13:15 & $4.0-6.0$ & $0,1,2$ \\
\hline PPSC0501 & 104613 & Sediment & $07 / 8 / 95,16: 20$ & $0.0-1.0$ & $0,1,2$ \\
\hline PPSC0502 & 104614 & Sediment & $07 / 8 / 95,16: 30$ & $1.0-2.0$ & $0,1,2$ \\
\hline PPSC0503 & 104615 & Sediment & $07 / 8 / 95,16: 45$ & $2.0-4.0$ & $0,1,2$ \\
\hline PPSC0601 & 104616 & Sediment & $07 / 9 / 95,12: 40$ & $0.0-1.0$ & $0,1,2$ \\
\hline PPSC0602 & 104617 & Sediment & $07 / 9 / 95,13: 00$ & $1.0-2.0$ & $0,1,2$ \\
\hline PPSC0603 & 104618 & Sediment & $07 / 9 / 95,13: 10$ & $2.0-4.0$ & $0,1,2$ \\
\hline PPSC0604 & 104619 & Sediment & $07 / 9 / 95,13: 30$ & $4.0-6.0$ & $0,1,2$ \\
\hline PPSC0701 & 104620 & Sediment & $07 / 9 / 95,15: 30$ & $0.0-1.0$ & $0,1,2$ \\
\hline PPSC0702 & 104621 & Sediment & 07/9/95, 15:45 & $1.0-2.0$ & $0,1,2$ \\
\hline PPSC0703 & 104622 & Sediment & $07 / 9 / 95,16: 00$ & $2.0-4.0$ & $0,1,2$ \\
\hline PPSC0704 & 104623 & Sediment & $07 / 9 / 95,16: 15$ & $4.0-5.8$ & $0,1,2$ \\
\hline PPSC1001 & 104625 & Sediment & $07 / 10 / 95,13: 20$ & $0.0-1.0$ & $0,1,2$ \\
\hline PPSC1002 & 104626 & Sediment & $07 / 10 / 95,13: 40$ & $1.0-2.0$ & $0,1,2$ \\
\hline PPSC1003 & 104627 & Sediment & $07 / 10 / 95,14: 00$ & $2.0-4.0$ & $0,1,2$ \\
\hline PPSC1004 & 104628 & Sediment & $07 / 10 / 95,14: 25$ & $4.0-6.0$ & $0,1,2$ \\
\hline
\end{tabular}

$10=$ TAL for inorganics

$1=$ Specified analytes

$2=$ Radionuclides (gross alpha, nonvolatile beta, gamma PHA, strontium-90, and plutonium series)

$3=$ TCL with TICs 
Table 2. Sediment Sample Set (Excluding Blank Samples), Continued

\begin{tabular}{|c|c|c|c|c|c|}
\hline $\begin{array}{l}\text { Survey } \\
\text { Station ID }\end{array}$ & Sample ID & $\begin{array}{l}\text { Sample } \\
\text { Type }\end{array}$ & $\begin{array}{l}\text { Sample Date } \\
\text { and Time }\end{array}$ & $\begin{array}{l}\text { Sampling } \\
\text { Depth (ft) }\end{array}$ & $\begin{array}{l}\text { Analyses } 1 \\
\text { Requested }\end{array}$ \\
\hline PPSC1005 & 104629 & Sediment & $07 / 10 / 95,14: 45$ & $6.0-8.0$ & $0,1,2$ \\
\hline PPSC0801 & 104630 & Sediment & $07 / 10 / 95,16: 55$ & $0.0-1.0$ & $0,1,2$ \\
\hline PPSC0802 & 104632 & Sediment & $07 / 10 / 95,17: 30$ & $1.0-2.0$ & $0,1,2$ \\
\hline PPSC0803 & 104633 & Sediment & $07 / 10 / 95,17: 50$ & $2.0-3.0$ & $0,1,2$ \\
\hline PPSC0901 & 104635 & Sediment & $07 / 11 / 95,14: 00$ & $0.0-1.0$ & $0,1,2,3$ \\
\hline PPSC0902 & 104636 & Sediment & $07 / 11 / 95,14: 15$ & $1.0-2.0$ & $0,1,2,3$ \\
\hline PPSC0903 & 104637 & Sediment & $07 / 11 / 95,14: 30$ & $2.0-4.0$ & $0,1,2,3$ \\
\hline PPSC2601A & 104638 & Duplicate & 07/11/95, 16:15 & $0.0-1.0$ & $0,1,2,3$ \\
\hline PPSC2602D & 104639 & Split & $07 / 11 / 95,16: 15$ & $1.0-2.0$ & $0,1,2,3$ \\
\hline PPSC2603A & 104640 & Duplicate & $07 / 11 / 95,16: 15$ & $2.0-4.0$ & $0,1,2,3$ \\
\hline PPSC0401 & 104643 & Sediment & $07 / 12 / 95,17: 15$ & $0.0-1.0$ & $0,1,2,3$ \\
\hline PPSC0402 & 104644 & Sediment & $07 / 12 / 95,17: 30$ & $1.0-2.0$ & $0,1,2,3$ \\
\hline PPSC0403 & 104645 & Sediment & $07 / 12 / 95,17: 45$ & $2.0-4.0$ & $0,1,2,3$ \\
\hline PPSC0404 & 104646 & Sediment & $07 / 12 / 95,18: 00$ & 4.0-5.8 & $0,1,2,3$ \\
\hline PPSC2701D & 104647 & Split & $07 / 12 / 95,17: 15$ & $0.0-1.0$ & $0,1,2,3$ \\
\hline PPSC2702A & 104648 & Duplicate & $07 / 12 / 95,17: 30$ & $1.0-2.0$ & $0,1,2,3$ \\
\hline PPSC2703D & 104649 & Split & $07 / 12 / 95,17: 45$ & $2.0-4.0$ & $0,1,2,3$ \\
\hline PPSC2704A & 104650 & Duplicate & $07 / 12 / 95,18: 00$ & $4.0-5.8$ & $0,1,2,3$ \\
\hline PPSC1101 & 104652 & Sediment & 07/14/95, 10:45 & $0.0-1.0$ & $0,1,2,3$ \\
\hline PPSC1102 & 104653 & Sediment & $07 / 14 / 95,11: 00$ & $1.0-2.0$ & $0,1,2,3$ \\
\hline PPSC1103 & 104655 & Sediment & $07 / 14 / 95,11: 30$ & $2.0-4.0$ & $0,1,2,3$ \\
\hline PPSC1201 & 104656 & Sediment & $07 / 14 / 95,14: 00$ & $0.0-1.0$ & $0,1,2,3$ \\
\hline PPSC2801A & 104657 & Duplicate & $07 / 14 / 95,14: 10$ & $0.0-1.0$ & $0,1,2,3$ \\
\hline PPSC1202 & 104658 & Sediment & $07 / 14 / 95,14: 20$ & $1.0-2.0$ & $0,1,2,3$ \\
\hline PPSC2802 & 104659 & Split & 07/14/95, 14:30 & $1.0-2.0$ & $0,1,2,3$ \\
\hline PPSC1203 & 104660 & Sediment & $07 / 14 / 95,14: 40$ & $2.0-4.0$ & $0,1,2,3$ \\
\hline PPSC2803A & 104661 & Duplicate & $07 / 14 / 95,14: 50$ & $2.0-4.0$ & $0,1,2,3$ \\
\hline PPSC1301 & 104664 & Sediment & $07 / 18 / 95,13: 40$ & $0.0-1.0$ & $0,1,2$ \\
\hline PPSC1302 & 104665 & Sediment & 07/18/95, 14:00 & $1.0-2.0$ & $0,1,2$ \\
\hline PPSC1303 & 104666 & Sediment & $07 / 18 / 95,14: 20$ & $2.0-4.0$ & $0,1,2$ \\
\hline PPSC1304 & 104667 & Sediment & $07 / 18 / 95,14: 45$ & $4.0-6.0$ & $0,1,2$ \\
\hline PPSC1801 & 104668 & Sediment & $07 / 18 / 95,17: 45$ & $0.0-1.0$ & $0,1,2$ \\
\hline PPSC1802 & 104669 & Sediment & $07 / 18 / 95,18: 05$ & $1.0-2.0$ & $0,1,2$ \\
\hline PPSC1803 & 104670 & Sediment & $07 / 18 / 95,18: 30$ & $2.0-3.8$ & $0,1,2$ \\
\hline PPSC1901 & 104671 & Sediment & $07 / 19 / 95,13: 00$ & $0.0-1.0$ & $0,1,2,3$ \\
\hline PPSC1902 & 104672 & Sediment & $07 / 19 / 95,13: 00$ & $1.0-2.0$ & $0,1,2,3$ \\
\hline PPSC1903 & 104673 & Sediment & $07 / 19 / 95,13: 00$ & $2.0-4.0$ & $0,1,2,3$ \\
\hline
\end{tabular}


Table 2. Sediment Sample Set (Excluding Blank Samples), Continued

\begin{tabular}{|c|c|c|c|c|c|}
\hline $\begin{array}{l}\text { Survey } \\
\text { Station ID }\end{array}$ & Sample ID & $\begin{array}{l}\text { Sample } \\
\text { Type }\end{array}$ & $\begin{array}{l}\text { Sample Date } \\
\text { and Time }\end{array}$ & $\begin{array}{l}\text { Sampling } \\
\text { Depth (ft) }\end{array}$ & $\begin{array}{l}\text { Analyses }^{1} \\
\text { Requested }\end{array}$ \\
\hline PPSC1904 & 104674 & Sediment & $07 / 19 / 95,13: 00$ & $4.0-6.0$ & $0,1,2,3$ \\
\hline PPSC1905 & 104675 & Sediment & $07 / 19 / 95,13: 00$ & $6.0-8.0$ & $0,1,2,3$ \\
\hline PPSC2001 & 104676 & Sediment & $07 / 19 / 95,17: 00$ & $0.0-1.0$ & $0,1,2$ \\
\hline PPSC2002 & 104677 & Sediment & $07 / 19 / 95,17: 00$ & $1.0-2.0$ & $0,1,2$ \\
\hline PPSC2003 & 104678 & Sediment & $07 / 19 / 95,17: 00$ & $2.0-4.0$ & $0,1,2$ \\
\hline PPSC2101 & 104680 & Sediment & $07 / 21 / 95,08: 30$ & $0.0-1.0$ & $0,1,2,3$ \\
\hline PPSC2102 & 104681 & Sediment & $07 / 21 / 95,08: 30$ & $1.0-2.0$ & $0,1,2,3$ \\
\hline PPSC2103 & 104682 & Sediment & $07 / 21 / 95,08: 30$ & $2.0-4.0$ & $0,1,2,3$ \\
\hline PPSC2201 & 104683 & Sediment & $07 / 21 / 95,11: 00$ & $0.0-1.0$ & $0,1,2,3$ \\
\hline PPSC2202 & 104684 & Sediment & $07 / 21 / 95,11: 00$ & $1.0-2.0$ & $0,1,2,3$ \\
\hline PPSC2203 & 104685 & Sediment & $07 / 21 / 95,11: 00$ & $2.0-4.0$ & $0,1,2,3$ \\
\hline PPSC2204 & 104686 & Sediment & $07 / 21 / 95,11: 00$ & $4.0-6.0$ & $0,1,2,3$ \\
\hline PPSC2301 & 104687 & Sediment & $07 / 21 / 95,14: 00$ & $0.0-1.0$ & $0,1,2,3$ \\
\hline PPSC2302 & 104688 & Sediment & $07 / 21 / 95,14: 00$ & $1.0-2.0$ & $0,1,2,3$ \\
\hline PPSC2303 & 104689 & Sediment & $07 / 21 / 95,14: 00$ & $2.0-4.0$ & $0,1,2,3$ \\
\hline PPSC2304 & 104690 & Sediment & $07 / 21 / 95,14: 00$ & $4.0-6.0$ & $0,1,2,3$ \\
\hline PPSC2305 & 104691 & Sediment & $07 / 21 / 95,14: 00$ & $6.0-7.1$ & $0,1,2,3$ \\
\hline PPSC1701 & 104693 & Sediment & $07 / 25 / 95,10: 30$ & $0.0-1.0$ & $0,1,2,3$ \\
\hline PPSC1702 & 104694 & Sediment & $07 / 25 / 95,10: 30$ & $1.0-2.0$ & $0,1,2,3$ \\
\hline PPSC1703 & 104695 & Sediment & $07 / 25 / 95,10: 30$ & $2.0-4.0$ & $0,1,2,3$ \\
\hline PPSC1704 & 104696 & Sediment & $07 / 25 / 95,10: 30$ & $4.0-6.0$ & $0,1,2,3$ \\
\hline PPSC1601 & 104697 & Sediment & $07 / 25 / 95,16: 30$ & $0.0-1.0$ & $0,1,2$ \\
\hline PPSC1602 & 104698 & Sediment & $07 / 25 / 95,16: 30$ & $1.0-2.0$ & $0,1,2$ \\
\hline PPSC1603 & 105100 & Sediment & $07 / 25 / 95,16: 30$ & $2.0-4.0$ & $0,1,2$ \\
\hline PPSC1604 & 105101 & Sediment & $07 / 25 / 95,16: 30$ & $4.0-6.0$ & 0,$1 ; 2$ \\
\hline PPSC1605 & 105102 & Sediment & $07 / 25 / 95,16: 30$ & $6.0-8.0$ & $0,1,2$ \\
\hline PPSC1501 & 105104 & Sediment & $07 / 26 / 95,10: 30$ & $0.0-1.0$ & $0,1,2$ \\
\hline PPSC1502 & 105105 & Sediment & $07 / 26 / 95,10: 30$ & $1.0-2.0$ & $0,1,2$ \\
\hline PPSC1503 & 105106 & Sediment & $07 / 26 / 95,10: 30$ & $2.0-4.0$ & $0,1,2$ \\
\hline PPSC1504 & 105107 & Sediment & $07 / 26 / 95,10: 30$ & $4.0-6.0$ & $0,1,2$ \\
\hline PPSC1505 & 105108 & Sediment & $07 / 26 / 95,10: 30$ & $6.0-8.0$ & $0,1,2$ \\
\hline PPSC1401 & 105109 & Sediment & 07/26/95, 15:00 & $0.0-1.0$ & $0,1,2,3$ \\
\hline PPSC1402 & 105110 & Sediment & $07 / 26 / 95,15: 00$ & $1.0-2.0$ & $0,1,2,3$ \\
\hline PPSC1403 & 105111 & Sediment & $07 / 26 / 95,15: 00$ & $2.0-2.7$ & $0,1,2,3$ \\
\hline PPSC2501 & 105113 & Sediment & $07 / 26 / 95,17: 30$ & $0.0-1,0$ & $0,1,2,3$ \\
\hline PPSC2502 & 105114 & Sediment & $07 / 26 / 95,17: 30$ & $1.0-2.0$ & $0,1,2,3$ \\
\hline PPSC2503 & 105115 & Sediment & $07 / 26 / 95,17: 30$ & $2.0-4.0$ & $0,1,2,3$ \\
\hline
\end{tabular}

Par Pond, Pond C, and L Lake, Revision 2 
Table 2. Sediment Sample Set (Excluding Blank Samples), Continued

\begin{tabular}{|l|l|l|l|l|l|}
\hline $\begin{array}{l}\text { Survey } \\
\text { Station ID }\end{array}$ & Sample ID & $\begin{array}{l}\text { Sample } \\
\text { Type }\end{array}$ & $\begin{array}{l}\text { Sample Date } \\
\text { and Time }\end{array}$ & $\begin{array}{l}\text { Sampling } \\
\text { Depth (ft) }\end{array}$ & $\begin{array}{l}\text { Analyses } \\
\text { Requested }\end{array}$ \\
\hline PPSC2504 & 105116 & Sediment & $07 / 26 / 95,17: 30$ & $4.0-5.7$ & $0,1,2,3$ \\
\hline PPSC2401 & 105117 & Sediment & $07 / 26 / 95,20: 00$ & $0.0-1.0$ & $0,1,2,3$ \\
\hline PPSC2402 & 105118 & Sediment & $07 / 26 / 95,20: 00$ & $1.0-2.0$ & $0,1,2,3$ \\
\hline PPSC2403 & 105119 & Sediment & $07 / 26 / 95,20: 00$ & $2.0-4.0$ & $0,1,2,3$ \\
\hline PPSC2404 & 105120 & Sediment & $07 / 26 / 95,20: 00$ & $4.0-5.1$ & $0,1,2,3$ \\
\hline
\end{tabular}

\section{Quality Control Samples}

The work plan assessment (Nichols, C.E., 1995) stated that QC samples would be placed in the sample list with the following frequencies. Sample duplicates and splits were planned at a combined rate of at least one per 10 samples. Field blanks were planned at a rate of one per 20 samples, and trip blanks were planned at a rate of one per day of VOA sample collection. Sampling equipment was dedicated; therefore, no rinsate blanks were required.

As part of the characterization, field blanks and trip blanks were collected. The field blanks were used to measure the contamination in the environment surrounding the sample. One field blank was collected every four days of the sampling event. The trip blanks accompanied each cooler containing a bottle for volatiles analysis.

\section{Field Blanks}

Field blanks were used to monitor contamination introduced during field activities from either local or regional external environmental sources. They were planned to be collected at a rate of one per 20 samples. Field blanks were analyzed for TCL and TAL inorganics to monitor major pathways for contamination. The field blanks consisted of distilleddeionized water placed in a stainless steel bowl and exposed to the air while the sample was collected from the opened core. The blank was located in the vicinity of the sample but not close enough to be accidentally contaminated by it. The time that the blank was opened and closed was recorded in the sample logbook.

Field blanks were assigned to assure proper statistical and sample equipment type coverage. Field personnel were encouraged to optimize use of these samples by adjusting their collection during the day to better test field conditions, such as during conditions of increased smoke, pollen, or dust. The SRTC Project Manager was responsible for ensuring field personnel record any sampling modifications on the COC forms.

\section{Trip Blanks}

Trip blanks were used to monitor contamination of samples by volatile constituents during shipping and handling. They were planned to be collected at a rate of one per day of VOA sample collection. Trip blanks were analyzed for TCL VOA only. A blank consisted of distilled-deionized water provided by the laboratory and was placed in the cooler with VOA samples at a rate of one trip blank per cooler. They were provided only for those samples that required VOA analyses. In the field, VOA samples were separated from the rest of that sample's bottles and combined in one cooler with a trip blank for shipment to the lab. 
To minimize the number of trip blanks used, every effort was made to place all volatile samples collected during each day in one cooler. Approximately 10 trip blanks were used for this project.

Table 3 contains comments from the samplers that relate to field decisions and includes descriptions of sample composition. The information in the table has been taken verbatim from the samplers' logbooks; no changes or corrections have been made except.obvious spelling errors, which have been corrected.

\section{Table 3. Field Observations}

\begin{tabular}{|c|c|}
\hline Sample ID & Comments/Observations \\
\hline 104600 & $\begin{array}{l}\text { Composition: Upper } 0.3 \mathrm{ft} \text { organic rich silty fine sand; remainder fine to } \\
\text { medium sand with varying silty/clay. } \\
\text { Description: (ML) Sandy silt, dusky yellowish brown, wet, v. fine to fine } \\
\text { sand, organic rich (0-0.3 ft.); } 0.3-1.0 \mathrm{ft} \text {; sand (SP), medium, grayish orange, } \\
\text { damp. } \\
\text { List any bottles not filled: see below. } \\
\text { Comments: VOAs for } 104600,104601 \text {, and } 104602 \text { all taken at the same } \\
\text { time because all three intervals were in one sample tube. TAL inorganics } \\
\text { container half-filled; } 1 \text { gamma PHA not filled; } 1 \text { CEC and } 1 \text { particle size } \\
\text { container filled. }\end{array}$ \\
\hline 104601 & $\begin{array}{l}\text { Composition: Sand with silt/clay } \\
\text { Description: Poorly graded sand with silt/clay SP-SM or SP-SC, light } \\
\text { brown, fine to medium sand, dense, moist, moderate plasticity. } \\
\text { List any bottles not filled: None } \\
\text { Comments: VOA containers filled at 11:10. Sample remained in bowl on } \\
\text { table from 11:10-12:05. All bottles filled. }\end{array}$ \\
\hline 104602 & $\begin{array}{l}\text { Composition: Sand with silt/clay } \\
\text { Description: Poorly graded sand with silt (SP-SM), mottled, moderate } \\
\text { reddish brown, dark yellowish orange and v. pale orange, firm, dry to moist, } \\
\text { some granules, mostly sand; typical mottled "B" Sediment horizon } \\
\text { List any bottles not filled: } 1 \text { gamma PHA; } 1 \text { CEC; } 2 \text { particle size } \\
\text { distribution. } \\
\text { Comments: VOA containers for this interval filled at } 11: 10 \text {. Remainder of } \\
\text { sample stayed in bowl until } 12: 25 \text { when compositing and remaining } \\
\text { containerization took place. Sampler refused at } 3.0 \text { feet on tube \#1 and } 2.25 \\
\mathrm{ft} \text { on tube \#2 prevented this interval from being } 2.0-4.0 \mathrm{ft} \text {. There are two } \\
\text { tubes per interval to try to get sufficient volume. }\end{array}$ \\
\hline 104603 & $\begin{array}{l}\text { Blank and standard descriptions: Field blank consisting of deionized water. } \\
\text { Composition: Deionized water plus miscellaneous preservatives. } \\
\text { List any bottles not filled: None } \\
\text { Comments: This field blank applies to } 104604-104608 \text { because it was } \\
\text { exposed while the tubes for all five intervals were being cut. }\end{array}$ \\
\hline 104604 & Blank and standard descriptions: 104603 is a field blank associated with \\
\hline
\end{tabular}


Table 3. Field Observations, Continued

\begin{tabular}{|c|c|}
\hline Sample ID & Comments/Observations \\
\hline & $\begin{array}{l}\text { this interval } \\
\text { Composition: Sand, mostly quartz } \\
\text { Description: Poorly graded sand (SP), dark yellowish orange 10YR } 6 / 6 \text { to } \\
\text { pale yellowish brown 10YR 6/2, medium to coarse, subangular to } \\
\text { subrounded, trace heavies, wet, loose. } \\
\text { List any bottles not filled: } 1 \text { CEC container; } 2 \text { particle size distributions. } \\
\text { Comments: Insufficient volume in 0-0.1 ft interval for all containers. } \\
\text { Followed work plan assessment and dropped one CEC and two particle size } \\
\text { distribution. Sample tubes for } 104604-104608 \text { were opened at } 15: 30 \text {. }\end{array}$ \\
\hline 104605 & $\begin{array}{l}\text { Blank and standard descriptions: } 104603 \text { is a field blank associated with } \\
\text { this interval } \\
\text { Composition: Quartz sand } \\
\text { Description: Poorly graded sand (SP), dark yellowish orange, medium to } \\
\text { coarse, subangular to subrounded, trace heavies, loose, moist. } \\
\text { List any bottles not filled: None. } \\
\text { Comments: Sample tubes for } 104604-104608 \text { were opened at } 15: 30 \text {. }\end{array}$ \\
\hline 104606 & $\begin{array}{l}\text { Blank and standard descriptions: } 104603 \text { is a field blank associated with } \\
\text { this sample. } \\
\text { Composition: Quartz sand } \\
\text { Description: Poorly graded sand (SP), dark yellowish orange, medium to } \\
\text { coarse, subangular to subrounded, damp, loose; color grades to v. pale } \\
\text { orange at } 3.5 \mathrm{ft} \text {. } \\
\text { List any bottles not filled: None; } 2 \text { particle size distributions collected; COC } \\
\text { calls for } 3 \text {. } \\
\text { Comments: Sample tubes for } 104604-104608 \text { were opened at } 15: 30 \text {. }\end{array}$ \\
\hline 104607 & $\begin{array}{l}\text { Blank and standard descriptions: Field Blank } 104603 \text { is associated with } \\
\text { this interval } \\
\text { Composition: Quartz sand w/ trace fines. } \\
\text { Description: Well graded sand (SW), dark yellowish orange, v. fine to v. } \\
\text { coarse, subrounded, damp, trace fines, slightly cohesive, firm (4.8-6.0 ft); } \\
\text { 4.0-4.8 ft is sand (SP), v. pale orange. } \\
\text { List any bottles not filled: None; one particle size distribution not filled. } \\
\text { Comments: Sample tubes for intervals } 104604-104608 \text { were opened at } \\
\text { 15:30. }\end{array}$ \\
\hline
\end{tabular}


Table 3. Field Observations, Continued

\begin{tabular}{|c|c|}
\hline Sample ID & Comments/Observations \\
\hline 104608 & $\begin{array}{l}\text { Blank and standard descriptions: Field blank } 104603 \text { is associated with } \\
\text { this interval } \\
\text { Composition: Quartz sand with trace clay } \\
\text { Description: Well graded sand (SW), faintly mottled, dark yellowish orange } \\
\text { to moderate reddish brown, v. fine to v. coarse, subrounded, trace fines, } \\
\text { firm, slightly cohesive, damp. } \\
\text { List any bottles not filled: One particle size distribution not filled. } \\
\text { Comments: Last interval for } 07 / 07 / 95 \text {. This interval was planned for } 6.0 \text { - } \\
8.0 \mathrm{ft} \text { but due to refusal of sampling device (hard sediment)-bottom of hole } \\
\text { was } 7.1 \mathrm{ft} \text {. }\end{array}$ \\
\hline 104609 & $\begin{array}{l}\text { Composition: Quartz sand. } \\
\text { Description: Poorly graded sand (SP), coarse to v. coarse, light gray, } \\
\text { subangular to subrounded grains, trace heavy minerals, loose, moist to wet; } \\
\text { top } 0.1 \mathrm{ft} \text { is organic rich, dark gray mud. } \\
\text { List any bottles not filled: One gamma PHA half-filled, one CEC deleted, } \\
\text { two partial size distribution deleted. } \\
\text { Comments: Sample tube \#1 at PPSC-SED03 was collected at 09:30. } \\
\text { Sample tube \#2 was collected at } 11: 30 \text {. Both tubes were iced on the boat } \\
\text { and returned to shore at } 12: 00 \text {. Tubes were opened at 12:10. }\end{array}$ \\
\hline 104610 & $\begin{array}{l}\text { Composition: Quartz sand } \\
\text { Description: Poorly graded sand (SP), coarse to v. coarse, light gray, } \\
\text { subangular to subrounded grains, trace heavy minerals, loose, moist, } \\
\text { coarsening w/ depth. } \\
\text { List any bottles not filled: One particle size distribution deleted. } \\
\text { Comments: See comments from } 104609 .\end{array}$ \\
\hline 104611 & $\begin{array}{l}\text { Composition: Quartz sand } \\
\text { Description: } 2.0 \text { to } 2.7 \mathrm{ft} \text {, same as } 104610 ; 2.7 \text { to } 4.0 \mathrm{ft} \text {. Poorly graded sand } \\
\text { (SP), medium to coarse, grayish orange } 10 \mathrm{YR} 7 / 4 \text {, trace fines, moist, loose. } \\
\text { List any bottles not filled: Deleted one particle size distribution. } \\
\text { Comments: See comments from } 104609 \text {. }\end{array}$ \\
\hline 104612 & $\begin{array}{l}\text { Composition: Quartz sand, mica, silt, clay } \\
\text { Description: Clayey sand (SC), v. fine to medium, fining w/ depth, light } \\
\text { gray w/ some dark yellowish orange mottling, firm, 5-20\% clay, low to } \\
\text { moderate plasticity, moist, some mica evident, trace heavy minerals. } \\
\text { List any bottles not filled: Deleted one particle size distribution. } \\
\text { Comments: See comments for } 104609 \text {. }\end{array}$ \\
\hline 104613 & $\begin{array}{l}\text { Composition: Quartz sand } \\
\text { Description: Poorly graded sand (SP), coarse, pale yellowish brown, } \\
\text { subangular to angular grains, loose, moist; top } 0.2 \text { feet is organic rich, dark, } \\
\text { rooted. } \\
\text { List any bottles not filled: Deleted two particle size distribution, one CEC; } \\
\text { half-filled one gamma PHA }\end{array}$ \\
\hline
\end{tabular}


Table 3. Field Observations, Continued

\begin{tabular}{|c|c|}
\hline Sample ID & Comments/Observations \\
\hline & $\begin{array}{l}\text { Comments: PPSC-SED05 Tube No. } 1 \text { was retrieved at } 14: 45 \text { and placed in } \\
\text { the on-board cooler. Tube No. } 2 \text { was collected between } 15: 30 \text { and } 15: 45 \text { and } \\
\text { placed in the cooler. Both tubes returned to staging area at 16:00. }\end{array}$ \\
\hline 104614 & $\begin{array}{l}\text { Composition: Quartz sand } \\
\text { Description: Poorly graded sand (SP), coarse, pale yellowish brown, } \\
\text { subangular to angular grains, loose, moist. } \\
\text { List any bottles not filled: Deleted one particle size distribution. } \\
\text { Comments: See comments for } 104613 \text {. }\end{array}$ \\
\hline 104615 & $\begin{array}{l}\text { Composition: Quartz sand, silt } \\
\text { Description: } 2.0 \text { to } 2.8 \text {, same as } 104614 ; 2.8 \text { to } 3.4 \text { is sandy silt (ML), dark } \\
\text { yellowish brown, moist, soft, low strength, organic rich, low to moderate } \\
\text { plasticity, } 40 \% \text { fine to medium sand. } \\
\text { List any bottles not filled: Deleted one particle size distribution. } \\
\text { Comments: See comments for } 104613 \text {. Bottom of hole for PPSC-SED05 is } \\
4.3 \text { feet. From } 4.0 \text { to } 4.3 \mathrm{ft} \text {. was discarded for both tubes. }\end{array}$ \\
\hline 104616 & $\begin{array}{l}\text { Composition: Quartz sand } \\
\text { Description: Poorly graded sand (SP), medium to coarse, pale yellowish } \\
\text { brown, angular grains, loose, wet, trace heavy minerals; top } 0.2 \text { feet is } \\
\text { organic rich and rooted but still sandy } \\
\text { List any bottles not filled: One gamma PHA half filled, one CEC deleted, } \\
\text { two particle size deleted. } \\
\text { Comments: Tube No. } 1 \text { collected at } 09: 45 \text { and placed in cooler. Tube No. } 2 \\
\text { collected at 10:50 and placed in cooler. Tubes delivered to staging area at } \\
\text { 11:20. Tubes opened at 12:15. SED06-01 containerized at 12:40. }\end{array}$ \\
\hline 104617 & $\begin{array}{l}\text { Composition: Quartz sand } \\
\text { Description: Poorly graded sand (SP), medium to coarse, pale yellowish } \\
\text { brown, angular grains, loose, damp, trace heavy minerals. } \\
\text { List any bottles not filled: One (1) particle size distribution deleted. } \\
\text { Comments: See comment from } 104616 .\end{array}$ \\
\hline 104618 & $\begin{array}{l}\text { Composition: Quartz sand, silt } \\
\text { Description: } 2.0-2.5 \text {, same as } 104617 ; 2.5-3.2 \text { is silty sand (SM), coarse, } \\
\text { moderate yellowish brown, } 20-30 \% \text { silt, soft, moderate plasticity, moist to } \\
\text { wet, trace roots (old lake bottom); } 3.2-4.0 \text { is silty sand (SM), mottled, firm. } \\
\text { List any bottles not filled: One (1) particle size distribution deleted. } \\
\text { Comments: See comments from } 104616 \text {. }\end{array}$ \\
\hline 104619 & $\begin{array}{l}\text { Composition: Quartz sand, silt } \\
\text { Description: Silty sand, (SM), medium to v. coarse, mottled, moderate } \\
\text { reddish brown and dark yellowish orange, } 20-30 \% \text { silt (and/or clay), firm to } \\
\text { hard, damp to dry; appears to be "B" Sediment horizon (non lacustrine) } \\
\text { List any bottles not filled: One (1) particle size distribution deleted. } \\
\text { Comments: See comments for } 104616 \text {. PPSC-SED06-04 is a composite of }\end{array}$ \\
\hline
\end{tabular}


Table 3. Field Observations, Continued

\begin{tabular}{|c|c|}
\hline Sample ID & Comments/Observations \\
\hline & $\begin{array}{l}\text { 4.0-6.0 from Tube No. } 1 \text { and } 4.0 \text { to } 5.0 \text { from Tube No. } 2 \text {. Tube No. } 1 \text { was } 6.5 \\
\mathrm{ft} \text { overall and 6.0-6.5 was discarded. }\end{array}$ \\
\hline 104620 & $\begin{array}{l}\text { Composition: Quartz sand, organic material (decayed), roots } \\
\text { Description: } 0.0 \text { to } 0.5 \mathrm{ft} \text {. silty sand (SM), v. fine to fine, greenish black, } \\
\text { wet, soft, organic rich, heavily rooted; } 0.5 \text { to } 1.0 \mathrm{ft} \text {. is sand (SP), medium to } \\
\text { coarse, dark yellowish brown, wet, loose, rooted. } \\
\text { list any bottles not filled: One (1) gamma PHA deleted, one (1) CEC } \\
\text { deleted, three (3) particle size deleted. } \\
\text { Comments: PPSC-SED07 Tube No. } 1 \text { was collected between } 13: 00 \text { and } \\
\text { 13:30. Tube No. } 2 \text { was collected at } 14: 30 \text {. Both tubes were placed in the on- } \\
\text { board cooler. The boat returned with the tubes at } 14: 50 \text {. The core tubes } \\
\text { were opened at 15:00. }\end{array}$ \\
\hline 104621 & $\begin{array}{l}\text { Composition: Quartz sand, silt/clay } \\
\text { Description: } 1.0 \text { to } 1.5 \mathrm{ft} \text {, sand (SP), medium to coarse, dark yellowish } \\
\text { brown, subangular to subrounded grains, wet, soft, organic material } \\
\text { present; } 1.5 \text { to } 2.0 \mathrm{ft} \text {. becomes silty or clayey (SM or SC), moderate } \\
\text { yellowish brown, } 10-20 \% \text { fines. } \\
\text { List any bottles not filled: One (1) CEC deleted, two (2) particle size } \\
\text { distribution deleted. } \\
\text { Comments: See comments for } 104620 \text {. }\end{array}$ \\
\hline 104622 & $\begin{array}{l}\text { Composition: Quartz sand, silt or clay, roots. } \\
\text { Description: Silty or clayey sand (SM or SC), medium to coarse, moderate } \\
\text { yellowish brown, soft, wet, } 10-20 \% \text { fines, root material to } 3.0 \mathrm{ft}: 4.0 \mathrm{ft} \text { is } \\
\text { possibly the base of a former lake bottom. } \\
\text { List any bottles not filled: One (1) particle size distribution deleted. } \\
\text { Comments: See comments for } 104620 \text {. }\end{array}$ \\
\hline 104623 & $\begin{array}{l}\text { Blank and standard descriptions: } 104624 \text { is a trip blank associated with } \\
\text { this sample } \\
\text { Composition: Quartz sand, clay } \\
\text { Description: Clayey sand (SC), fine grained, mottled moderate yellowish } \\
\text { brown, moderate reddish brown, and light gray, } 30-40 \% \text { clay (confirmed } \\
\text { clay w/ floc test), firm, moist, moderate to high plasticity; apparent "B" } \\
\text { Sediment horizon. } \\
\text { List any bottles not filled: One (1) particle size distribution deleted } \\
\text { Comments: See comments for 104620. Bottom of SED07 was } 5.8 \text { feet. } \\
\text { Both tubes contained } 5.8 \text { feet of sample. }\end{array}$ \\
\hline 104624 & $\begin{array}{l}\text { Blank and standard descriptions: Trip blank } \\
\text { Composition: Deionized water with HCl preservative } \\
\text { Comments: Trip blanks dated by GE 06/27/95, 15:00. }\end{array}$ \\
\hline 104625 & $\begin{array}{l}\text { Composition: Quartz sand, silt, water, roots, decaying organic material } \\
\text { Description: Silty sand (SM), v. fine grained, greenish black, v. soft, high } \\
\text { silt content ( } 50 \% \text { ?), organic rich, rooted, saturated. }\end{array}$ \\
\hline
\end{tabular}


Table 3. Field Observations, Continued

\begin{tabular}{|c|c|}
\hline Sample ID & Comments/Observations \\
\hline & $\begin{array}{l}\text { List any bottles not filled: One (1) gamma PHA container half-filled, one (1) } \\
\text { CEC deleted; two (2) particle size deleted. } \\
\text { Comments: PPSC-SED10, Tube No. } 1 \text { was retrieved at 10:00 a.m. and } \\
\text { placed in the on-board cooler. Tube No. } 2 \text { was collected at 10:50 a.m. and } \\
\text { placed in the on-board cooler. Tubes were cut and opened at staging area at } \\
\text { 12:00. First interval processed at 13:20. }\end{array}$ \\
\hline 104626 & $\begin{array}{l}\text { Composition: Sand, silt, roots } \\
\text { Description: } 1.0 \text { to } 1.5 \mathrm{ft} \text {. silty sand (SM), v. fine grained, greenish black, } \\
\text { soft, saturated, rooted; } 1.5 \text { to } 2.0 \mathrm{ft} ., \text { silty sand (SM), medium, greenish } \\
\text { black, moist, loose, rooted, silt content }<10 \% \text {. } \\
\text { List any bottles not filled: Two (2) particle size distribution deleted, one (1) } \\
\text { CEC deleted. } \\
\text { Comments: See comments for } 104625 \text {. }\end{array}$ \\
\hline 104627 & $\begin{array}{l}\text { Composition: Quartz sand, silt, roots } \\
\text { Description: Silty sand (SM), fine to medium, greenish black, soft, moist, } \\
\text { heavily rooted, silt content <10\%. Removed root fragment } \sim 1.0 \mathrm{ft} \text {. long by } 2 \text { " } \\
\text { diameter. } \\
\text { List any bottles not filled: One (1) particle size distribution not filled. } \\
\text { Comments: See comments from } 104625 \text {. }\end{array}$ \\
\hline 104628 & $\begin{array}{l}\text { Composition: Quartz sand, silt, roots } \\
\text { Description: Silty sand (SM), fine to medium, greenish black, soft, moist, } \\
\text { rooted, silt content <10\%; discarded large root fragment. } \\
\text { List any bottles not filled: One (1) particle size distribution deleted } \\
\text { Comments: See comments from } 104625 \text {. }\end{array}$ \\
\hline 104629 & $\begin{array}{l}\text { Composition: Quartz sand, silt, roots. } \\
\text { Description: } 6.0 \text { to } 6.9 \mathrm{ft} \text {, same as } 104628 ; 6.9 \text { to } 8.0 \mathrm{ft} ., \text { sand (SP) medium } \\
\text { to coarse; dark yellowish brown, subrounded grains, soft, moist, rooted. } \\
\text { List any bottles not filled: One (1) particle size distribution deleted } \\
\text { Comments: See comments from } 104625 \text {. PPSC-SED10-05 is a composite of } \\
6.0 \text { to } 8.0 \mathrm{ft} \text {. from tube No. } 1 \text { and } 6.0 \text { to } 7.3 \mathrm{ft} \text { from Tube No. } 2 \text {. From } 8.0 \text { to } \\
10.3 \mathrm{ft} \text {. of Tube No. } 1 \text { was discarded in accordance with the project scope of } \\
\text { work. }\end{array}$ \\
\hline 104630 & $\begin{array}{l}\text { Blank and standard descriptions: } 104631 \text { is a field blank associated with } \\
\text { this sample. } \\
\text { Composition: Quartz sand, silt, clay. bivalve shells } \\
\text { Description: Silty sand (SM), v. fine to fine, greenish black, <20\% fines, v. } \\
\text { soft, subrounded to rounded grains, saturated, small ( } 5-10 \mathrm{~mm} \text { ) bi-valve } \\
\text { shells present. } \\
\text { List any bottles not filled: One (1) gamma PHA deleted; one (1) CEC } \\
\text { deleted; two (2) particle size distribution deleted. } \\
\text { Comments: PPSC-SED08 samples collected between } 14: 30 \text { and 16:00 hrs. } \\
\text { Boat returned at 16:35. Core tubes cut and opened at 16:40. }\end{array}$ \\
\hline
\end{tabular}


Table 3. Field Observations, Continued

\begin{tabular}{|c|c|}
\hline Sample ID & Comments/Observations \\
\hline 104631 & $\begin{array}{l}\text { Blank and standard descriptions: Field blank } \\
\text { Composition: Deionized water with various preservatives. The deionized } \\
\text { water was obtained from the Par Pond SRTC ESS Lab. This is a routinely } \\
\text { maintained system. } \\
\text { Comments: This field blank was prepared by filling two stainless steel } \\
\text { bowls and leaving them on the sample table while PPSC-SED08-01 was } \\
\text { processed. }\end{array}$ \\
\hline 104632 & $\begin{array}{l}\text { Composition: Qtz. sand, silt, clay } \\
\text { Description: Silty or clayey sand (SC or SM), fine to coarse (well graded), } \\
\text { moderate yellowish brown, soft, cohesive, moist to wet, } 20-30 \% \text { fines } \\
\text { List any bottles not filled: One (1) particle size distribution deleted } \\
\text { Comments: See comments from } 104630 .\end{array}$ \\
\hline 104633 & $\begin{array}{l}\text { Composition: Clay, quartz sand } \\
\text { Description: Clayey sand (SC), well graded, v. fine to coarse, mottled, } \\
\text { moderate reddish brown and dark yellowish orange, } 20-30 \% \text { clay, firm to } \\
\text { hard, "B" Sediment horizon. } \\
\text { List any bottles not filled: One (1) particle size distribution deleted. } \\
\text { Comments: See comments from } 104630 \text {. PPSC-SED08-03 is a composite of } \\
1.0 \text { to } 2.6 \mathrm{ft} \text { from Tube No. } 1 \text { and } 1.0 \text { to } 3.0 \mathrm{ft} \text { from Tube No. } 2 \text {. }\end{array}$ \\
\hline 104634 & $\begin{array}{l}\text { Blank and standard descriptions: Trip blank } \\
\text { Composition: Deionized water plus HCL } \\
\text { Comments: This trip blank associated with 104625-104653. Trip blank } \\
\text { dated by GE 06/27/95 @ 15:00. }\end{array}$ \\
\hline 104635 & $\begin{array}{l}\text { Composition: Quartz sand } \\
\text { Description: Poorly graded sand (SP), coarse, olive black } 5 Y 2 / 1 \text {, } \\
\text { subangular to angular grains, loose, wet; top } 0.1 \text { feet is organic rich, rooted, } \\
\text { v. soft. } \\
\text { List any bottles not filled: One (1) gamma PHA deleted, one (1) CEC } \\
\text { deleted, two (2) particle size deleted. . } \\
\text { Comments: PPSC-SED09-01 through } 03 \text { collected from } 11: 25 \text { (Tube No. } 1 \text { ) } \\
\text { to } 12: 00 \text { (Tube No. 2). Both tubes placed on ice in the on-board cooler. Tube } \\
\text { No. } 1 \text { was cut and opened at } 13: 45 \text {. Containers for VOAs from all three } \\
\text { intervals were immediately filled. At } 14: 00 \text {, tube No. } 2 \text { was opened on the } \\
\text { table and } 0 \text { to } 1.0 \mathrm{ft} \text { from both tubes was composited. }\end{array}$ \\
\hline 104636 & $\begin{array}{l}\text { Composition: Quartz sand } \\
\text { Description: Poorly graded sand (SP), coarse, moderate yellowish brown, } \\
\text { subangular to angular grains, loose, moist. } \\
\text { List any bottles not filled: Two (2) particle size distribution deleted; one (1) } \\
\text { CEC deleted } \\
\text { Comments: See comments for } 104635 \text {. }\end{array}$ \\
\hline 104637 & $\begin{array}{l}\text { Composition: Quartz sand, clay } \\
\text { Description: Clayey sand (SC), coarse, dark yellowish orange, soft, }\end{array}$ \\
\hline
\end{tabular}


Table 3. Field Observations, Continued

\begin{tabular}{|c|c|}
\hline Sample ID & Comments/Observations \\
\hline & $\begin{array}{l}\text { cohesive, moist, clay content } 10-20 \% \text { ( } 2.0 \text { to } 3.0 \mathrm{ft}) ; 3.0 \text { to } 4.0 \mathrm{ft} \text {, abrupt } \\
\text { change to light gray, increased clay content } 30-40 \% \text {, firm, coarse to v. } \\
\text { coarse. } \\
\text { List any bottles not filled: One (1) particle size distribution deleted. } \\
\text { Comments: See comments for } 104635 \text {. Bottom of hole } 4.6 \mathrm{ft} \text {. }\end{array}$ \\
\hline 104638 & $\begin{array}{l}\text { Composition: Same as } 104635 \\
\text { Description: Same as } 104635 \\
\text { List any bottles not filled: One (1) gamma PHA deleted, one (1) CEC } \\
\text { deleted, two (2) particle size deleted } \\
\text { Comments: This sample is the duplicate for } 104635 \text {. See comments for } \\
104635 \text {. }\end{array}$ \\
\hline 104639 & $\begin{array}{l}\text { Composition: Same as } 104636 \\
\text { Description: Same as } 104636 \\
\text { List any bottles not filled: Particle size only partially filled. } \\
\text { Comments: This sample is the split to } 104636 \text {. See comments for } 104635 \text {. }\end{array}$ \\
\hline 104640 & $\begin{array}{l}\text { Composition: Same as } 104637 \\
\text { Description: Same as } 104637 \\
\text { List any bottles not filled: One (1) particle size deleted } \\
\text { Comments: This sample is the duplicate for } 104637 \text {. See comments for } \\
104635 \text {. }\end{array}$ \\
\hline 104641 & $\begin{array}{l}\text { Blank and standard descriptions: Trip blank } \\
\text { Composition: Deionized water from GE. } \\
\text { Comments: Trip blanks dated by GE 06/27/95, 15:00. }\end{array}$ \\
\hline 104642 & $\begin{array}{l}\text { Blank and standard descriptions: Trip blank } \\
\text { Composition: Deionized water from WA. } \\
\text { Comments: This is a WA trip blank that goes with the WA splits. }\end{array}$ \\
\hline 104643 & $\begin{array}{l}\text { Composition: Quartz sand } \\
\text { Description: Sand (SP), mostly fine to medium (90\%) w/ few coarse (10\%), } \\
\text { pale to dark yellowish brown, subangular fine to medium grains, } \\
\text { subrounded coarse grains, loose, saturated. } \\
\text { List any bottles not filled: One (1) gamma PHA not filled; one (1) CEC } \\
\text { deleted; three ( } 3 \text { ) particle size deleted. } \\
\text { Comments: Four cores collected at the SED04 location including samples } \\
\text { for duplicates and splits (SED27). Cores collected between 11:30 and 16:20. } \\
\text { All cores placed on ice in on-board cooler, as retrieved. Core tubes opened } \\
\text { at 16:40. VOA samples for all intervals including duplicates and splits } \\
\text { collected first and immediately. Remaining material composited and } \\
\text { containerized beginning with interval SED04-01 and SED27-01D. }\end{array}$ \\
\hline 104644 & $\begin{array}{l}\text { Composition: Quartz sand, clay } \\
\text { Description: Sand with clay (SP-SC), fine to medium, yellowish gray, } \\
\text { subangular grains, } 5-10 \% \text { clay, moderately cohesive, soft. }\end{array}$ \\
\hline
\end{tabular}


Table 3. Field Observations, Continued

\begin{tabular}{|c|c|}
\hline Sample ID & Comments/Observations \\
\hline & $\begin{array}{l}\text { List any bottles not filled: One (1) CEC deleted, One (1) particle size } \\
\text { deleted } \\
\text { Comments: See comments for } 104643 \text {. }\end{array}$ \\
\hline 104645 & $\begin{array}{l}\text { Composition: Quartz sand, clay } \\
\text { Description: } 2.0 \text { to } 3.0 \text {, same as } 104644,3.0 \text { to } 4.0 \text {, sand (SP), medium to } \\
\text { coarse w/ trace v. coarse, v. pale orange, subangular to subrounded grains, } \\
\text { trace heavy minerals, loose, moist. } \\
\text { List any bottles not filled: One (1) particle size deleted } \\
\text { Comments: See comments for } 104643 \text {. }\end{array}$ \\
\hline 104646 & $\begin{array}{l}\text { Composition: Quartz sand. } \\
\text { Description: Sand (SP), medium to coarse w/trace v. coarse, v. pale orange, } \\
\text { subangular to subrounded grains, trace heavy minerals, loose, moist. } \\
\text { List any bottles not filled: One (1) gamma PHA deleted; one (1) CEC } \\
\text { deleted; two (2) particle size deleted. } \\
\text { Comments: See comments for } 104643 \text {. The longest of four cores collected } \\
\text { was } 5.8 \mathrm{ft} \text {. }\end{array}$ \\
\hline 104647 & $\begin{array}{l}\text { Composition: Same as } 104643 \\
\text { Description: Same as } 104643 \\
\text { List any bottles not filled: One (1) particle size distribution deleted } \\
\text { Comments: This sample is a split of SED04-01. See comments for } 104643 \\
\text { for additional information. }\end{array}$ \\
\hline 104648 & $\begin{array}{l}\text { Composition: See } 104644 \\
\text { Description: See } 104644 \\
\text { List any bottles not filled: One (1) CEC deleted; two (2) particle size } \\
\text { deleted. } \\
\text { Comments: This sample is a duplicate of SED04-02. See comments for } \\
104643 \text { for additional information. }\end{array}$ \\
\hline 104649 & $\begin{array}{l}\text { Composition: Same as } 104645 \\
\text { Description: Same as } 104645 \\
\text { Comments: This sample is a split of PPSC-SED04-03. See comments for } \\
104643 \text { for additional information. }\end{array}$ \\
\hline 104650 & $\begin{array}{l}\text { Composition: Quartz sand } \\
\text { Description: Same as } 104646 \\
\text { List any bottles not filled: One (1) gamma PHA deleted; one (1) CEC } \\
\text { deleted, two (2) particle size deleted. } \\
\text { Comments: See comments for } 104643 \text { and } 104646 . \text { This sample is a } \\
\text { duplicate of SED04-04. }\end{array}$ \\
\hline 104651 & $\begin{array}{l}\text { Blank and standard descriptions: Deionized water with HCL preservatives } \\
\text { from GE. Trip blank. } \\
\text { Composition: See above } \\
\text { Comments: This trip blank is dated } 06 / 27 / 95,15: 00 \text { by GE. }\end{array}$ \\
\hline
\end{tabular}


Table 3. Field Observations, Continued

\begin{tabular}{|c|c|}
\hline Sample ID & Comments/Observations \\
\hline 104652 & $\begin{array}{l}\text { Composition: Organic ooze, clay, silt } \\
\text { Description: Organic Sediment (OL/OH), greenish black, very loose, soupy } \\
\text { consistency, <20\% fine sand, saturated. } \\
\text { List any bottles not filled: One (1) TCL Semivol, one (1) gamma PHA, two } \\
\text { (2) CEC, three (3) particle size. } \\
\text { Comments: The one gamma PHA submitted is only } 1 / 5 \text { full. Sample Tube } \\
\text { No. } 1 \text { collected at } 16: 00 \text { on } 07 / 13 / 95 \text { and placed in a cooler overnight (PAR } \\
\text { Pond Walk-In ). Tube No. } 2 \text { collected at } 08: 45 \text { on 07/14/95. Both tubes were } \\
\text { cut and opened at 09:40 on 07/14/95. VOAs were immediately collected from } \\
\text { tube No. } 2 \text { (the more recent tube) from all three intervals, SED11-01, -02 } \\
\text { and -03. Following VOA sample collection each interval was composited } \\
\text { and containerized according to the times shown on the following pages. }\end{array}$ \\
\hline 104653 & $\begin{array}{l}\text { Composition: Quartz sand } \\
\text { Description: Poorly graded sand (SP), mostly medium, little coarse (<20\%), } \\
\text { pale yellowish brown, subangular grains, loose, moist. } \\
\text { List any bottles not filled: One (1) TCL Semivol, one (1) gamma PHA, one } \\
\text { (1) CEC, three (3) particle size deleted. } \\
\text { Comments: One CEC container submitted is less than half full. TAL } \\
\text { inorganics container is only half full. See comments also for } 104652 \text {. }\end{array}$ \\
\hline 104654 & $\begin{array}{l}\text { Blank and standard descriptions: Field blank } \\
\text { Composition: Deionized water collected from SRC -ESD par pond lab. } \\
\text { System is regularly maintained. } \\
\text { Comments: Placed two stainless steel bowls filled with deionized water on } \\
\text { the sampling table while PPSC-SED11-02 was being composited and } \\
\text { containerized. Following containerization of SED11-02, the field blank } \\
\text { containers were filled. }\end{array}$ \\
\hline 104655 & $\begin{array}{l}\text { Composition: Qtz. sand and clay } \\
\text { Description: } 2.0 \text { to } 2.5 \text { is same as } 104653 ; 2.5 \text { to } 4.0 \text { is clayey sand (SC), } \\
\text { mostly medium grained, moderate reddish brown and dark yellowish } \\
\text { orange, } 10-20 \% \text { clay, v. firm, grains are subrounded, sample is dry to moist. } \\
\text { List any bottles not filled: One (1) particle size deleted. } \\
\text { Comments: See comments for } 104652 \text {. }\end{array}$ \\
\hline
\end{tabular}


Table 3. Field Observations, Continued

\begin{tabular}{|c|c|}
\hline Sample ID & Comments/Observations \\
\hline 104656 & $\begin{array}{l}\text { Composition: Organic material, clay, silt } \\
\text { Description: Organic Sediment (OL/OH), brownish black to greenish black, } \\
\text { clay/silt present in unknown quantity, soupy to sludge-like consistency, } \\
\text { strong organic odor. } \\
\text { List any bottles not filled: One (1) TCL Semivol, One (1) TAL Inorganics } \\
\text { partially filled, One (1) Gamma PHA deleted, Two (2) Cation Exchange \& } \\
\text { Three (3) Particle Size Deleted. } \\
\text { Comments: Four cores collected at SED-12 location between 10:15 and } \\
\text { 12:40 on 07/14/95. VOA samples for all intervals including duplicates and } \\
\text { splits, collected from Core No. 4. Remaining material composited and } \\
\text { containerized beginning with SED12-01 at 14:00. }\end{array}$ \\
\hline 104657 & $\begin{array}{l}\text { Composition: Same as } 104656 \\
\text { Description: Same as } 104656 \\
\text { List any bottles not filled: One (1) TCL Semivol deleted, One (1) TAL } \\
\text { Inorganics partially filled, One (1) Gamma PHA partially filled, Two (2) } \\
\text { Cation Exchange \& Three (3) Particle Size Distribution deleted } \\
\text { Comments: This interval is a duplicate of PPSC-SED12-01. See comments } \\
\text { for } 104656 .\end{array}$ \\
\hline 104658 & $\begin{array}{l}\text { Composition: Organic Sediment, quartz sand } \\
\text { Description: } 1.0 \text { to } 1.4 \text { same as } 104656 ; 1.4 \text { to } 2.0 \text { sand (SP), medium, pale } \\
\text { yellowish brown, subangular grains, soft, moist. } \\
\text { List any bottles not filled: One (1) TCL Semivol deleted, One (1) TAL } \\
\text { Inorganics partially filled, Only One (1) Gamma PHA partially filled, Two } \\
\text { (2) Cation Exchange \& Three (3) Particle Size deleted } \\
\text { Comments: See comments for } 104656 \text {. }\end{array}$ \\
\hline 104659 & $\begin{array}{l}\text { Composition: Same as } 104658 \\
\text { Description: Same as } 104658 \\
\text { List any bottles not filled: One (1) TCL Semivol deleted, One (1) TAL } \\
\text { Inorganics partially filled, One (1) Pu series partially filled, One (1) Gamma } \\
\text { PHA \& One (1) Particle Size deleted } \\
\text { Comments: This sample is a split of SED12-02. See comments for } 104656 .\end{array}$ \\
\hline 104660 & $\begin{array}{l}\text { Composition: Quartz sand, clay } \\
\text { Description: } 2.0 \text { to } 2.8 \text {, sand (SP), medium, pale yellowish brown, } \\
\text { subangular, moist, } 2.8 \text { to } 3.2 \text {, clayey sand (SC), mostly fine to medium } \\
\text { w/few coarse, dark yellowish orange, }<20 \% \text { clay, soft; } 3.2 \text { to } 4.0 \text {, sand (SP), } \\
\text { medium, trace to few clay, grades into clay, light gray. } \\
\text { List any bottles not filled: One (1) Particle Size deleted } \\
\text { Comments: Core lengths ranged from } 4.4 \text { to } 5.0 \mathrm{ft} \text { of recovery. All material } \\
\text { in excess of } 4.0 \mathrm{ft} \text { was discarded. All four cores bottomed out in light gray } \\
\text { clay. See comments for } 104656 \text { for additional information. }\end{array}$ \\
\hline 104661 & $\begin{array}{l}\text { Composition: Same as } 104660 \\
\text { Description: Same as } 104660\end{array}$ \\
\hline
\end{tabular}


Table 3. Field Observations, Continued

\begin{tabular}{|c|c|}
\hline Sample ID & Comments/Observations \\
\hline & $\begin{array}{l}\text { List any bottles not filled: One (1) Particle Size deleted } \\
\text { Comments: This sample is a split from SED12-03. See comments for } \\
104656 \text {. }\end{array}$ \\
\hline 104662 & $\begin{array}{l}\text { Blank and standard descriptions: Trip blank } \\
\text { Composition: Deionized water from GE } \\
\text { Comments: Trip blank dated } 06 / 27 / 95,15: 00 \text { hrs by GE. }\end{array}$ \\
\hline 104663 & $\begin{array}{l}\text { Blank and standard descriptions: Trip blank } \\
\text { Composition: Deionized water from WA. } \\
\text { Comments: This trip blank associated with split sample PPSC-SED28-02D }\end{array}$ \\
\hline 104664 & $\begin{array}{l}\text { Composition: Qtz sand, roots, plant matter. } \\
\text { Description: Poorly graded sand (SP), fine to coarse, brownish gray, } \\
\text { subrounded grains, moist, loose, few roots. } \\
\text { List any bottles not filled: One (1) particle size distribution deleted } \\
\text { Comments: At location PPSC-SED13-01, Tube No. } 1 \text { was retrieved at } 09: 45 \\
\text { and tube No. } 2 \text { was retrieved at 10:15. Both tubes were immediately placed } \\
\text { on ice in the onboard cooler. At the boat ramp, tubes were placed in an air } \\
\text { conditioned van and transported to } 704-B \text {. From } 12: 00 \text { until } 13: 15 \text { tubes } \\
\text { were unrefrigerated but in the shade of } 704-B \text {. At } 13: 15 \text { the tubes, } \\
\text { representing PPSC-SED13-01, }-02,-03 \text {, and }-04 \text {, were opened and sample } \\
\text { processing begun. }\end{array}$ \\
\hline 104665 & $\begin{array}{l}\text { Composition: Quartz sand } \\
\text { Description: Poorly graded sand (SP), fine to coarse, brownish gray, } \\
\text { subrounded grains, moist, loose, few roots } \\
\text { List any bottles not filled: One (1) particle size distribution deleted. } \\
\text { Comments: See comments for } 104664 \text {. }\end{array}$ \\
\hline 104666 & $\begin{array}{l}\text { Composition: Quartz sand, roots } \\
\text { Description: } 2.0-3.3 \text { same as } 104665 ; 3.3-4.0 \text {, poorly graded sand (SP), } \\
\text { medium to coarse, grayish orange } 10 \mathrm{YR} 7 / 4 \text { to dark yellowish orange } 10 \mathrm{YR} \\
6 / 6 \text {, subrounded grains, moist, loose, soft, few roots. } \\
\text { List any bottles not filled: One (1) particle size distribution deleted. } \\
\text { Comments: See comments for } 104664 \text {. }\end{array}$ \\
\hline 104667 & $\begin{array}{l}\text { Composition: Quartz sand } \\
\text { Description: Poorly graded sand (SP), medium to coarse, grayish orange to } \\
\text { dark yellowish orange, subrounded grains, moist, loose, soft. } \\
\text { List any bottles not filled: One (1) Particle Size Deleted } \\
\text { Comments: See comments for } 104664 \text {. Bottom of hole at PPSC-SED13 is } \\
7.1 \mathrm{ft} \text {. Tube No. } 1 \text { was } 7.1 \mathrm{ft} \text {. and Tube No. } 2 \text { was } 6.0 \mathrm{ft} \text {. From } 6.0 \text { to } 7.1 \mathrm{ft} \text {. } \\
\text { of Tube No. } 1 \text { was discarded. }\end{array}$ \\
\hline 104668 & $\begin{array}{l}\text { Composition: Quartz sand, organics } \\
\text { Description: Sand (SP), mostly fine, few medium, grayish brown, wet, v. } \\
\text { soft, loose, quartzitic with abundant black (organic?) grains. }\end{array}$ \\
\hline
\end{tabular}


Table 3. Field Observations, Continued

\begin{tabular}{|c|c|}
\hline Sample ID & Comments/Observations \\
\hline & $\begin{array}{l}\text { List any bottles not filled: Two (2) particle size deleted; one (1) CEC } \\
\text { deleted. } \\
\text { Comments: Cores from PPSC-SED18 were collected between 15:20 and } \\
\text { 17:00. Core tubes were placed on ice in the on board cooler. From the boat } \\
\text { ramp, cores were transported via truck to 704-B. At 17:30 the tubes were } \\
\text { cut and opened and sample processing began. }\end{array}$ \\
\hline 104669 & $\begin{array}{l}\text { Composition: Quartz sand } \\
\text { Description: Sand (SP), medium, moderate yellowish brown, moist, soft, } \\
\text { loose, quartzitic, subangular grains. } \\
\text { List any bottles not filled: One (1) particle size distribution deleted. } \\
\text { Comments: See comments for } 104668 \text {. }\end{array}$ \\
\hline 104670 & $\begin{array}{l}\text { Composition: Quartz sand } \\
\text { Description: Sand (SP), mostly medium to coarse, few to little v. coarse to } \\
\text { pebble size, faintly mottled, dark yellowish orange, light brown, and } \\
\text { moderate reddish brown, subangular to subrounded grains, soft to firm, } \\
\text { moist. } \\
\text { List any bottles not filled: One (1) particle size deleted. } \\
\text { Comments: See comments for } 104668 \text {. Bottom of boring } 3.75 \mathrm{ft} \text {. No trip } \\
\text { blanks this date because no VOA analyses. }\end{array}$ \\
\hline 104671 & $\begin{array}{l}\text { Composition: Quartz sand, clay, organic matter } \\
\text { Description: Poorly graded sand with clay (SP-SC), v. fine to fine, }<10 \% \\
\text { clay, brownish gray, organic rich, soft, cohesive, saturated, rooted. } \\
\text { List any bottles not filled: One (1) gamma PHA, two (2) CEC, three (3) } \\
\text { particle size deleted. } \\
\text { Comments: PPSC SED } 19 \text { cores collected between } 09: 30 \text { and } 10: 30 \text { on } \\
\text { 07/19/95. Cores were placed on ice in on-board cooler. Transported by air } \\
\text { conditioned van to 704-B, arriving at 11:30. Cores cut open at 12:30. } \\
\text { Processing began at 13:00 with VOAs from all intervals collected first prior } \\
\text { to compositing and containerizing remaining samples. All SED } 19 \text { intervals } \\
\text { are logged in this book as 13:00. }\end{array}$ \\
\hline 104672 & $\begin{array}{l}\text { Composition: Quartz sand } \\
\text { Description: Sand (SP), mostly coarse, pale yellowish brown, subangular to } \\
\text { subrounded grains, moist, loose. } \\
\text { List any bottles not filled: Two (2) particle size deleted; one (1) CEC } \\
\text { deleted. } \\
\text { Comments: See comments for } 104671 \text {. }\end{array}$ \\
\hline 104673 & $\begin{array}{l}\text { Composition: Quartz sand } \\
\text { Description: } 2.0 \text { to } 2.5 \text {, sand (SP), coarse, pale yellowish brown, subangular } \\
\text { to subrounded, moist, loose; } 2.5 \text { to } 4.0 \text {, sand (SP), coarse, light brown, } \\
\text { subrounded to rounded grains, loose, moist. } \\
\text { List any bottles not filled: One (1) particle size deleted } \\
\text { Comments: See comments for } 104671 \text {. }\end{array}$ \\
\hline
\end{tabular}

Par Pond, Pond C, and L Lake, Revision 2 
Table 3. Field Observations, Continued

\begin{tabular}{|c|c|}
\hline Sample ID & Comments/Observations \\
\hline 104674 & $\begin{array}{l}\text { Composition: Quartz sand } \\
\text { Description: Sand (SP), coarse, light brown, subrounded to rounded grains, } \\
\text { loose, moist. } \\
\text { List any bottles not filled: One (1) particle size distribution not filled. } \\
\text { Comments: See comments for } 104671 \text {. }\end{array}$ \\
\hline 104675 & $\begin{array}{l}\text { Composition: Quartz sand, } \mathrm{MnO}_{2} \text { or organic splotches. } \\
\text { Description: } 6.0 \text { to } 7.7 \text {, sand (SP), coarse, light brown, subrounded to } \\
\text { rounded grains, loose, moist; } 7.7 \text { to } 8.0 \text {, sand (SP), coarse, dark yellowish } \\
\text { orange with organic or } \mathrm{MnO}_{2} \text { splotches. } \\
\text { List any bottles not filled: One (1) particle size deleted. } \\
\text { Comments: See comments for } 104671 \text {. Core No. } 1 \text { for SED19 was } 10.0 \mathrm{ft} \text {. } \\
\text { long and core No. } 2 \text { was } 9.1 \mathrm{ft} \text {. long. }\end{array}$ \\
\hline 104676 & $\begin{array}{l}\text { Composition: Sand, organic material, mud } \\
\text { Description: Sand (SP), v. fine to fine, with clay, brownish gray, v. soft } \\
\text { (soupy), saturated, organic rich. } \\
\text { List any bottles not filled: One (1) gamma PHA, one (1) CEC, and three (3) } \\
\text { particle size deleted. } \\
\text { Comments: PPSC-SED20 cores collected between 13:30 and 16:00. Cores } \\
\text { placed on ice in on-board cooler. Transported from L-lake to 704-B } \\
\text { (processing facility) on ice, in cooler. Arrived at 704-b at 16:30. } \\
\text { Immediately opened and composited/containerized. }\end{array}$ \\
\hline 104677 & $\begin{array}{l}\text { Composition: Quartz sand } \\
\text { Description: Sand (SP), mostly coarse, few rounded pebbles, light brownish } \\
\text { gray, subangular to subrounded grains, trace to few heavy minerals or } \\
\text { black organic grains, loose, moist. } \\
\text { List any bottles not filled: One (1) CEC and two (2) particle size deleted. } \\
\text { Comments: See comments for } 104676 \text {. }\end{array}$ \\
\hline 104678 & $\begin{array}{l}\text { Composition: Quartz sand, terrigenous clastic pebbles } \\
\text { Description: Sand (SP), mostly coarse, few rounded pebbles, some pebbles } \\
\text { to } 40 \mathrm{~mm} \text {, light brownish gray, subangular to subrounded sand grains, trace } \\
\text { to few heavy minerals or black organic grains, loose, moist. } \\
\text { List any bottles not filled: One (1) particle size deleted. } \\
\text { Comments: See comments for } 104676 \text {. This interval (SED20-03) is a } \\
\text { composite of } 2.0 \text { to } 2.9 \mathrm{ft} \text {. from Tube No. } 1 \text { and } 2.0 \text { to } 4.0 \text { from Tube No. } 2 \text {. } \\
\text { Bottom of corehole was } 4.3 \mathrm{ft} \text {. }\end{array}$ \\
\hline 104679 & $\begin{array}{l}\text { Blank and standard descriptions: Trip blank } \\
\text { Composition: Deionized water in } 40 \mathrm{ml} \text { vials provided by GE. } \\
\text { Comments: Trip blanks dated by GE 06/27/95, 15:00. }\end{array}$ \\
\hline 104680 & $\begin{array}{l}\text { Composition: Quartz sand. } \\
\text { Description: Sand (SP), coarse, pale brown to dark yellowish brown, quartz, } \\
\text { subangular to subrounded grains, loose, saturated. }\end{array}$ \\
\hline
\end{tabular}


Table 3. Field Observations, Continued

\begin{tabular}{|c|c|}
\hline Sample ID & Comments/Observations \\
\hline & $\begin{array}{l}\text { List any bottles not filled: One (1) gamma PHA, one (1) CEC, and three (3) } \\
\text { particle size deleted. } \\
\text { Comments: The PPSC-SED21 location was cored on 07/20/95. Tube No. } 1 \\
\text { was extracted at } 13: 30 \text { and Tube No. } 2 \text { was extracted at 14:15. Both tubes } \\
\text { were placed on ice in a large cooler while PPSC-SED22 was cored. At 17:00, } \\
\text { the tubes were placed in the PAR Pond cooler for overnight storage. They } \\
\text { were retrieved at 08:00 on 07/21/95 and opened for sample processing at } \\
08: 30 \text { on 07/21/95. Therefore, all intervals from SED21 are dated 07/21/95. }\end{array}$ \\
\hline 104681 & $\begin{array}{l}\text { Composition: Qtz. sand and pebbles } \\
\text { Description: Well graded sand (SW) with gravel, coarse to pebbles, grayish } \\
\text { orange pink, loose, moist, pebbles to } 30 \mathrm{~mm} \text {. } \\
\text { List any bottles not filled: One (1) gamma PHA, one (1) CEC, and two (2) } \\
\text { particle size deleted. } \\
\text { Comments: See comments for } 104680 \text {. }\end{array}$ \\
\hline 104682 & $\begin{array}{l}\text { Composition: Clay, quartz sand, organic matter } \\
\text { Description: Clay (CL), organic rich, dark gray to brownish black, high } \\
\text { plasticity, soft, moist, organic fibers, roots throughout, grades to quartz } \\
\text { sand from } 3.0 \text { to } 4.0 \mathrm{ft} \text {, same color, rooted. } \\
\text { List any bottles not filled: One (1) particle size deleted. } \\
\text { Comments: See comments for } 104680 \text {. SED21-03 is a composite of } 2.0 \text { to } \\
3.2 \text { from Tube No. } 1 \text { and } 2.0 \text { to } 4.0 \text { from Tube No. } 2 \text {. } 4.0 \text { to } 4.4 \mathrm{ft} \text {. of Tube } \\
\text { No. } 2 \text { was discarded. }\end{array}$ \\
\hline 104683 & $\begin{array}{l}\text { Composition: Quartz sand, clay } \\
\text { Description: } 0.0-0.5 \text { sand (SP), coarse, light brown, trace heavy minerals, } \\
\text { loose, saturated, 0.5-1.0 sand with clay (SP-SC), medium, light greenish } \\
\text { gray, moderately cohesive, } 10 \% \text { clay. } \\
\text { List any bottles not filled: One (1) CEC and two (2) particle size deleted. } \\
\text { Comments: PPSC-SED22, cores collected on } 07 / 20 / 95 \text { at } 16: 00 \text {. Tubes place } \\
\text { in PAR Pond cooler for overnight storage. Tubes retrieved at 08:00 on } \\
\text { 07/21/95 and carried to bldg. 704-B where they remained on ice in a large } \\
\text { cooler until 10:30 on 07/21/95. }\end{array}$ \\
\hline 104684 & $\begin{array}{l}\text { Composition: Quartz sand } \\
\text { Description: Sand (SP), medium, yellowish gray to pale yellowish orange, } \\
\text { some dark organic layers, trace heavy minerals, loose, soft. } \\
\text { List any bottles not filled: One (1) particle size deleted. } \\
\text { Comments: See comments for } 104683 \text {. }\end{array}$ \\
\hline 104685 & $\begin{array}{l}\text { Composition: Quartz sand } \\
\text { Description: Sand (SP), medium, yellowish gray to dark yellowish orange, } \\
\text { some dark organic layers, trace heavy minerals, loose, soft. } \\
\text { List any bottles not filled: One (1) particle size deleted. } \\
\text { Comments: See comments for } 104683 \text {. }\end{array}$ \\
\hline
\end{tabular}


Table 3. Field Observations, Continued

\begin{tabular}{|c|c|}
\hline Sample ID & Comments/Observations \\
\hline 104686 & $\begin{array}{l}\text { Composition: Clay, quartz sand } \\
\text { Description: Clayey sand (SC), fine to coarse, dark yellowish orange, to } \\
\text { mod. yellowish brown, cohesive, } 10-15 \% \text { clay, convoluted appearance, micro } \\
\text { parting (root traces?), moist. } \\
\text { List any bottles not filled: One (1) CEC and two (2) particle size deleted. } \\
\text { Comments: See comments from } 104683 \text {. SED22-04 is from } 4.0-6.0 \mathrm{ft} \text { of } \\
\text { Tube No. } 2 \text {. Tube No. } 1 \text { was only } 3.9 \mathrm{ft} \text {. long. TD of boring was } 6.5 \mathrm{ft} \text { and } \\
\text { from } 6.0 \text { to } 6.5 \mathrm{ft} \text {. was discarded }\end{array}$ \\
\hline 104687 & $\begin{array}{l}\text { Composition: Quartz sand, organic material. } \\
\text { Description: } 0.0 \text { to } 0.7 \text { sand (SP) medium to coarse, dark yellowish brown, } \\
\text { subrounded quartz grains, loose, saturated; } 0.7 \text { to } 1.0 \text {, organic Sediment } \\
\text { (OL/OH), mostly medium sand, brownish black, some fines, some organic } \\
\text { material (decaying leaves, roots, twigs). } \\
\text { List any bottles not filled: One (1) gamma PHA, one (1) CEC, and two (2) } \\
\text { particle size deleted. } \\
\text { Comments: PPSC-SED23 cores were collected between } 10: 00 \text { and } 11: 00 \text { on } \\
\text { 07/21/95. They arrived on ice at 704-B at } 11: 30 \text {. Cores were cut open at } \\
\text { 13:30. Sample processing began immediately with VOA containerization } \\
\text { for all intervals preceding compositing and remaining sample processing. } \\
\text { All intervals in this log for SED23 correspond to } 14: 00 \text {. }\end{array}$ \\
\hline 104688 & $\begin{array}{l}\text { Composition: Quartz sand, clay, decaying organic matter. } \\
\text { Description: } 1.0 \text { to } 1.6 \text {, sand (SP), medium to coarse, dark yellowish brown, } \\
\text { subrounded, loose, saturated; } 1.6 \text { to } 2.0 \text {, clayey sand (SC), fine to medium, } \\
\text { clayey (<20\%), brownish black, organic rich, soft, cohesive. } \\
\text { List any bottles not filled: One (1) CEC and two (2) particle size deleted. } \\
\text { Comments: See comments for } 104687 \text {. }\end{array}$ \\
\hline 104689 & $\begin{array}{l}\text { Composition: Quartz sand, gravel, decaying organic matter } \\
\text { Description: } 2.0 \text { to } 3.5 \text {, clayey sand (SC), fine to medium, }<20 \% \text { clay, } \\
\text { brownish black, organic rich, soft, cohesive, saturated, } 3.5 \text { to } 4.0 \text {, sand (SP), } \\
\text { coarse to v. coarse, light gray to v. light gray, gravelly at base with rounded } \\
\text { granules and pebbles } \\
\text { List any bottles not filled: One (1) particle size deleted } \\
\text { Comments: See comments for } 104687 \text {. }\end{array}$ \\
\hline 104690 & $\begin{array}{l}\text { Composition: Quartz sand, pebbles. } \\
\text { Description: } 4.0 \text { to } 5.6 \text {, sand (SP), coarse to v. coarse, light gray to v. light } \\
\text { gray, subrounded grains with rounded gravel at base, moist, loose, } 5.6 \text { to } \\
6.0 \text {, sand (SP), medium, olive gray, dry to moist, soft, loose. } \\
\text { List any bottles not filled: One (1) particle size deleted. } \\
\text { Comments: See comments for } 104687 \text {. }\end{array}$ \\
\hline 104691 & $\begin{array}{l}\text { Composition: Quartz sand, mica. } \\
\text { Description: } 6.0 \text { to } 6.8 \text {, sand (SP), coarse to v. coarse, light to v. light gray, } \\
\text { subrounded, rounded gravel at base, moist, loose; } 6.8 \text { to } 7.1 \text {, sand (SP), }\end{array}$ \\
\hline
\end{tabular}


Table 3. Field Observations, Continued

\begin{tabular}{|c|c|}
\hline Sample ID & Comments/Observations \\
\hline & $\begin{array}{l}\text { v. fine, to medium dark greenish gray, firm, dry, with mica. } \\
\text { List any bottles not filled: One (1) gamma PHA, one (1) CEC, and two (2) } \\
\text { particle size deleted. } \\
\text { Comments: See comments for } 104687 \text {. PPSC-SED23 cores were } 6.5 \text { and } 7.1 \\
\text { ft. respectively. Interval -05 is a composite of } 6.0 \text { to } 6.5 \text { and } 6.0 \text { to } 7.1 \mathrm{ft} \text {. }\end{array}$ \\
\hline 104692 & $\begin{array}{l}\text { Blank and standard descriptions: Trip blank } \\
\text { Composition: Deionized water in two } 40 \mathrm{ml} \text { vials provided by GE. } \\
\text { Comments: Trip blanks dated by GE } 06 / 27 / 95,15: 00 \text {. }\end{array}$ \\
\hline 104693 & $\begin{array}{l}\text { Composition: Quartz sand } \\
\text { Description: Sand (SP), medium to coarse, light brownish gray, subangular } \\
\text { grains, saturated, loose. } \\
\text { List any bottles not filled: One (1) gamma PHA, one (1) CEC, and two (2) } \\
\text { particle size deleted. } \\
\text { Comments: PPSC-SED17 cores were collected between 10:45 and 12:00 on } \\
\text { 07/24/95. Cores were kept on ice until 17:20, then transported to the PAR } \\
\text { Pond walk-in cooler for overnight storage. Cores were retrieved at 09:00 on } \\
07 / 25 / 95 \text { and cut open for sampling at 10:10. Sample processing began at } \\
\text { 10:30 and therefore all SED17 intervals bear this time. }\end{array}$ \\
\hline 104694 & $\begin{array}{l}\text { Composition: Quartz sand } \\
\text { Description: Sand (SP),-medium to coarse, light brownish gray, subangular } \\
\text { grains, saturated, loose. } \\
\text { List any bottles not filled: One (1) CEC and two (2) particle size deleted. } \\
\text { Comments: See comments for 104693. }\end{array}$ \\
\hline 104695 & $\begin{array}{l}\text { Composition: Quartz sand } \\
\text { Description: } 2.0 \text { to } 2.4 \text { same as } 104694 ; 2.4-3.6 \text { is sand (SP), fine to } \\
\text { medium, brownish gray, subrounded grains, moist; } 3.6-4.0 \text { is clayey sand } \\
\text { (SC), medium to coarse, light gray w/ dark yellowish orange streaks, } 20 \text { - } \\
30 \% \text { clay, soft, cohesive, moist. } \\
\text { List any bottles not filled: One (1) particle size deleted. } \\
\text { Comments: See comments for } 104693 \text {. }\end{array}$ \\
\hline 104696 & $\begin{array}{l}\text { Composition: Quartz sand, clay } \\
\text { Description: Clayey sand (SC), medium to coarse, light gray with dark } \\
\text { yellowish orange iron staining streaks, } 20-30 \% \text { clay, soft, cohesive, moist. } \\
\text { List any bottles not filled: One (1) particle size deleted. } \\
\text { Comments: See comments for } 104693 \text {. For SED } 17 \text {, core lengths were } 5.7 \\
\text { and } 6.8 \mathrm{ft} \text {. For interval }-04 \text {, from } 4.0 \text { to } 5.7 \text { and } 4.0 \text { to } 6.0 \text { were composited. } \\
\text { For the longer core (core No. } 1 \text { ) from } 6.0 \text { to } 6.8 \mathrm{ft} \text { were discarded. }\end{array}$ \\
\hline 104697 & $\begin{array}{l}\text { Composition: Sand, roots, decaying organic matter. } \\
\text { Description: Sandy organic Sediment }(\mathrm{OL} / \mathrm{OH}) \text {, mostly v. fine to fine sand } \\
\text { w/ few fines, pale yellowish brown, trace mica, abundant roots, fibers, etc., } \\
\text { saturated, v. soft, no density. }\end{array}$ \\
\hline
\end{tabular}


Table 3. Field Observations, Continued

\begin{tabular}{|c|c|}
\hline Sample ID & Comments/Observations \\
\hline & $\begin{array}{l}\text { List any bottles not filled: One (1) CEC and three (3) particle size deleted } \\
\text { Comments: PPSC-SD16 was sampled on } 07 / 24 / 95 \text { at } 14: 00 \text {. Cores were } \\
\text { taken to PAR Pond walk-in cooler for overnight storage. Cores were } \\
\text { retrieved at } 15: 00 \text { on } 07 / 25 / 95 \text { and cut open at } 16: 10 \text {. Sample processing } \\
\text { began at } 16: 30 \text { and all of the SED16 intervals bear this time. }\end{array}$ \\
\hline 104698 & $\begin{array}{l}\text { Composition: Sand, roots, decaying organic matter } \\
\text { Description: } 1.0-1.6 \text { sand organic Sediment (OL/OH), mostly v. fine to fine } \\
\text { sand, few fines, pale yellowish brown, trace mica, abundant roots, fibers, } \\
\text { etc., saturated, v. soft, no density, } 1.6-2.0 \mathrm{ft} \text { sand, mostly medium, light } \\
\text { brownish gray } \\
\text { List any bottles not filled: One (1) CEC and three (3) particle size deleted. } \\
\text { Comments: Collected a field blank (104699) while processing this sample. } \\
\text { See comments for } 104697 \text {. }\end{array}$ \\
\hline 104699 & $\begin{array}{l}\text { Blank and standard descriptions: Field blank } \\
\text { Composition: Deionized water from Well Bldg source. } \\
\text { Comments: Filled two stainless steel bowls (while compositing and } \\
\text { containerizing PPSC-SED16-02) with deionized water, on the sample } \\
\text { processing table. Poured the water into the appropriate containers from the } \\
\text { SS bowls. }\end{array}$ \\
\hline 105100 & $\begin{array}{l}\text { Composition: Sand, decaying organic matter } \\
\text { Description: Sand, (SP), fine to coarse, mostly medium, light brownish } \\
\text { gray, rooted. } \\
\text { List any bottles not filled: One (1) particle size deleted. } \\
\text { Comments: SED16 cores were collected on } 07 / 24 / 95 \text { between } 13: 45 \text { and } \\
\text { 15:15. Cores were immediately placed on ice in the on-board cooler. At } \\
\text { 17:20, the cores were taken to PAR pond for overnight storage in the walk- } \\
\text { in cooler. Cores were retrieved at 15:00 on 07/25/95 and brought to sample } \\
\text { processing area at } 704-B \text {. Cores were opened at } 16: 10 \text { and all five intervals } \\
\text { were composited and containerized starting with SED16-01. All SED16 } \\
\text { intervals bear the time } 16: 30 \text {. }\end{array}$ \\
\hline 105101 & $\begin{array}{l}\text { Composition: Quartz sand } \\
\text { Description: Sand (SP), medium to coarse, few fine, light greenish gray, } \\
\text { trace silt/clay, trace heavy minerals, firm, moist, subangular to subrounded } \\
\text { grains. } \\
\text { List any bottles not filled: One (1) particle size deleted } \\
\text { Comments: See comments for } 105100 \text {. }\end{array}$ \\
\hline
\end{tabular}


Table 3. Field Observations, Continued

\begin{tabular}{|c|c|}
\hline Sample ID & Comments/Observations \\
\hline 105102 & $\begin{array}{l}\text { Composition: Clay (CL), } \mathrm{d} \\
\text { Description: Clay (CL), dark yellowish orange, firm, dense, laminated, } \\
\text { breaks along laminae, high plasticity, moist, } \mathrm{MnO}_{2} \text { splotches; "Tan Clay" of } \\
\text { Dry Branch Fm. } \\
\text { List any bottles not filled: One (1) particle size deleted } \\
\text { Comments: See comments for } 105100 \text {. Core from } 8.0 \text { to } 8.4 \text { and } 8.0 \text { to } 9.2 \\
\text { was discarded. }\end{array}$ \\
\hline 105103 & $\begin{array}{l}\text { Blank and standard descriptions: Trip Blank } \\
\text { Composition: Deionized water, cold and preserved, filled at lab (GE). } \\
\text { Comments: Trip blanks dated by GE 06/27/95 15:00. }\end{array}$ \\
\hline 105104 & $\begin{array}{l}\text { Composition: Quartz sand, clay, roots, mica } \\
\text { Description: Clayey sand (SC), v. fine to fine, pale yellowish brown, mostly } \\
\text { quartz, few mica, root fibers and roots throughout, v. soft, cohesive, } \\
\text { "gummy" texture, saturated } \\
\text { List any bottles not filled: One (1) gamma PHA, one (1) CEC, and three (3) } \\
\text { particle size deleted. } \\
\text { Comments: PPSC-SED15 cores were collected on 07/25/95 between 09:40 } \\
\text { and 10:30. Both cores placed on ice in on-board cooler. Taken to Par Pond } \\
\text { at day's end and placed in walk-in cooler for overnight storage. Cores } \\
\text { retrieved from Par Pond on 07/26/95 at 09:45. Cut and opened at 10:00. } \\
\text { Composited and containerized each SED15 interval starting with -01. All } \\
\text { SED15 intervals bear the time 10:30. }\end{array}$ \\
\hline 105105 & $\begin{array}{l}\text { Composition: Quartz sand, mica, clay, roots } \\
\text { Description: Clayey sand (SC), v. fine to fine, mostly quartz, few mica, pale } \\
\text { yellowish brown, v. soft, "gummy" texture, cohesive, saturated. } \\
\text { List any bottles not filled: One (1) CEC and two (2) particle size deleted. } \\
\text { Comments: See comments for } 105104 .\end{array}$ \\
\hline 105106 & $\begin{array}{l}\text { Composition: Quartz sand, clay, roots, mica } \\
\text { Description: } 2.0 \text { to } 3.6 \text {, same as } 105105 ; 3.6 \text { to } 4.0 \text {, sand (SP), medium to } \\
\text { coarse, brownish black, subrounded grains, extremely rooted and organic } \\
\text { rich, soft, cohesive, moist. } \\
\text { List any bottles not filled: One (1) particle size deleted } \\
\text { Comments: See comments for } 105104 \text {. }\end{array}$ \\
\hline 105107 & $\begin{array}{l}\text { Composition: Quartz sand, roots } \\
\text { Description: Sand, (SP), medium to coarse, brownish black, subrounded } \\
\text { grains, extremely rooted and organic rich, soft, cohesive, moist. } \\
\text { List any bottles not filled: One (1) particle size deleted. } \\
\text { Comments: See comments for } 105104 \text {. }\end{array}$ \\
\hline 105108 & $\begin{array}{l}\text { Composition: Quartz sand, clay } \\
\text { Description: } 6.0 \text { to } 7.0 \text {, same as } 105107 ; 7.0 \text { to } 8.0 \text {, sand (SP), medium to } \\
\text { coarse, yellowish gray, interbedded with clay, brownish gray, rooted, soft, }\end{array}$ \\
\hline
\end{tabular}


Table 3. Field Observations, Continued

\begin{tabular}{|c|c|}
\hline Sample ID & Comments/Observations \\
\hline & $\begin{array}{l}\text { cohesive, moist. } \\
\text { List any bottles not filled: One (1) particle size deleted. } \\
\text { Comments: See comments for 105104. SED15 Core No. } 1 \text { was } 8.0 \mathrm{ft} \text {. long } \\
\text { and Core No. } 2 \text { was } 10.9 \mathrm{ft} \text { long. From } 8.0 \text { to } 10.9 \mathrm{ft} \text { of Core No. } 2 \text { was } \\
\text { discarded. }\end{array}$ \\
\hline 105109 & $\begin{array}{l}\text { Composition: Quartz sand } \\
\text { Description: Sand (SP), medium to coarse, pale yellowish brown, } \\
\text { subangular to subrounded grains, loose moist. } \\
\text { List any bottles not filled: One (1) CEC and two (2) particle size not filled } \\
\text { (deleted) } \\
\text { Comments: PPSC-SED14 cores were collected on 07/25/95 between } 14: 20 \\
\text { and 16:10. Cores were immediately placed on ice in the on-board cooler. At } \\
\text { day's end ( 17:00), cores were taken to Par Pond for overnight storage in } \\
\text { the walk-in cooler. Cores were retrieved from Par Pond and transported to } \\
\text { the sample processing station ( } 704-B) \text { at } 1430 \text {. Sample tubes (cores) were } \\
\text { cut and opened and VOA samples for all SED14 intervals were immediately } \\
\text { collected. Remaining material was composited and containerized starting } \\
\text { with interval-01. All intervals bear time } 1500 \text { hrs. }\end{array}$ \\
\hline 105110 & $\begin{array}{l}\text { Composition: Quartz sand } \\
\text { Description: Sand (SP), medium to coarse, pale yellowish brown, } \\
\text { subangular to subrounded grains, loose, moist. } \\
\text { List any bottles not filled: One (1) gamma PHA, one (1) CEC, and two (2) } \\
\text { particle size deleted } \\
\text { Comments: See comments for } 105109 .\end{array}$ \\
\hline 105111 & $\begin{array}{l}\text { Composition: Quartz sand, organic material } \\
\text { Description: } 2.0 \text { to } 2.5 \text {, sand (SP), medium to coarse, pale yellowish brown, } \\
\text { subangular to subrounded grains, loose, moist; } 2.5 \text { to } 2.7 \text {, brownish black, } \\
\text { organic rich, sand (same as above). } \\
\text { List any bottles not filled: Two (2) gamma PHA, two (2) CEC, and three (3) } \\
\text { particle size deleted. } \\
\text { Comments: See comments for } 105109 \text {. SED14-03 is a composite of } 2.0 \text { to } \\
2.6 \text { and } 2.0 \text { to } 2.7 \text { from the two cores. }\end{array}$ \\
\hline 105112 & $\begin{array}{l}\text { Blank and standard descriptions: Trip blank } \\
\text { Composition: Deionized water; cold and preserved in } 40 \mathrm{ml} \text { vials by GE. } \\
\text { Comments: Trip blanks dated by GE, } 06 / 27 / 95,1500 \mathrm{hrs} \text {. }\end{array}$ \\
\hline 105113 & $\begin{array}{l}\text { Composition: Quartz sand, organic fibrous material } \\
\text { Description: } 0.0 \text { to } 0.5 \text {, sand (SP), coarse to v. coarse, v. pale orange, } \\
\text { subangular to subrounded grains, loose, moist; } 0.5 \text { to } 1.0 \text {, sand (SP), } \\
\text { medium to coarse, black, organic rich, abundant fibrous material, moist. } \\
\text { List any bottles not filled: One (1) gamma PHA, one (1) CEC, and two (2) } \\
\text { particle size deleted. } \\
\text { Comments: PPSC-SED25 was a background location at Lake Marion. Two }\end{array}$ \\
\hline
\end{tabular}


Table 3. Field Observations, Continued

\begin{tabular}{|c|c|}
\hline Sample ID & Comments/Observations \\
\hline & $\begin{array}{l}\text { cores were collected between } 13: 30 \text { and } 14: 30 \text { on } 07 / 26 / 95 \text { and immediately } \\
\text { placed on ice in a large cooler. Cores arrived at the sample processing area } \\
\text { (704-B) at 17:00. Cores were cut and opened at 17:20. VOA samples were } \\
\text { immediately collected for all SED25 intervals. Remaining material was } \\
\text { composited and containerized beginning with interval-01. All SED25 } \\
\text { intervals bear the time of } 17: 30 \text {. }\end{array}$ \\
\hline 105114 & $\begin{array}{l}\text { Composition: Quartz sand, pebbles, fibrous organic material. } \\
\text { Description: } 1.0 \text { to } 1.2 \text {, sand (SP), medium to coarse, black, organic rich, } \\
\text { abundant fibrous material, moist; } 1.2 \text { to } 2.0 \text {, sand (SP), mostly coarse, few } \\
\text { v. coarse, few to little granules and rounded pebbles, moderate yellowish } \\
\text { brown, loose, moist. } \\
\text { List any bottles not filled: One (1) CEC and two (2) grain size deleted; one } \\
\text { (1) gamma PHA partial. } \\
\text { Comments: See comments for } 105113 \text {. }\end{array}$ \\
\hline 105115 & $\begin{array}{l}\text { Composition: Quartz sand, granules, pebbles } \\
\text { Description: Sand (SP), mostly coarse, few v. coarse, few to little granules } \\
\text { and rounded pebbles, pale to moderate yellowish brown, 10YR 6/2 to } 10 \mathrm{YR} \\
\text { 5/4, loose, moist. } \\
\text { List any bottles not filled: One (1) particle size deleted. } \\
\text { Comments: See comments for } 105113 \text {. }\end{array}$ \\
\hline 105116 & $\begin{array}{l}\text { Composition: Quartz sand, silt, clay } \\
\text { Description: } 4.0 \text { to } 4.6 \text {, sand (SP), mostly coarse, few v. coarse, few to little } \\
\text { granules and rounded pebbles; } 4.6 \text { to } 5.7 \text {, clayey or silty sand (SC) or (SM), } \\
\text { v. fine to coarse, pale yellowish brown, cohesive, <20\% silt/clay, soft, moist. } \\
\text { List any bottles not filled: One (1) particle size deleted } \\
\text { Comments: See comments for } 105113 \text {. PPSC-SED25-04 is a composite of } \\
4.0 \text { to } 5.4 \mathrm{ft} \text { from Core No. } 2 \text { and } 4.0 \text { to } 5.7 \mathrm{ft} \text {. from Core No. } 1 \text {. }\end{array}$ \\
\hline 105117 & $\begin{array}{l}\text { Composition: Quartz sand } \\
\text { Description: Sand (SP), coarse, light brownish gray w/ occasional black, } \\
\text { dipping laminae, subangular grains, loose, saturated } \\
\text { List any bottles not filled: One (1) gamma PHA, one (1) CEC, and three (3) } \\
\text { particle size deleted. } \\
\text { Comments: PPSC SED } 24 \text { cores were collected between 18:00 and 19:00 on } \\
\text { 07/26/95. The cores were cut and opened at 19:30. VOAs were immediately } \\
\text { collected for all four sampled intervals. Remaining material was } \\
\text { composited and containerized beginning with SED24-01. All SE24 intervals } \\
\text { bear the time of 20:00. SED24 is the last location sampled on the PPSC } \\
\text { project. }\end{array}$ \\
\hline
\end{tabular}


Table 3. Field Observations, Continued

\begin{tabular}{|l|l|}
\hline Sample ID & Comments/Observations \\
\hline 105118 & $\begin{array}{l}\text { Composition: Quartz sand } \\
\text { Description: Sand (SP), coarse, light brownish gray with occasional black } \\
\text { dipping laminae, also black splotches, subangular grains, loose, saturated. } \\
\text { List any bottles not filled: One (1) CEC and two (2) particle size deleted. } \\
\text { Comments: See comments for 105117 }\end{array}$ \\
\hline 105119 & $\begin{array}{l}\text { Composition: Quartz sand, granules, pebbles } \\
\text { Description: 2.0 to 2.6 sand (SP) with gravel, v. coarse sand, pebbles to } \\
\text { 10mm, light brown, loose, saturated; } 2.6 \text { to 3.4, same w/ thin gravel beds } \\
\text { and thin beds of fine to medium sand, grayish orange; 3.4 to 4.0, sand (SP), } \\
\text { fine to medium, light gray. } \\
\text { List any bottles not filled: One (1) particle size deleted } \\
\text { Comments: See comments for 105117 }\end{array}$ \\
\hline $\begin{array}{l}\text { Composition: Quartz sand, gravel, mica } \\
\text { Description: 4.0 to 4.1, thin bed of flat rounded qtz. pebbles; } 4.1 \text { to 5.1, sand } \\
\text { (SP), v. fine to fine, mica and quartz, light greenish gray, and dark } \\
\text { yellowish orange, laminated, moist, slightly cohesive; base of Santee Fm. } \\
\text { List any bottles not filled: One (1) CEC and two (2) particle size deleted } \\
\text { Comments: Cores for SED24 were } 4.9 \mathrm{ft} \text { and } 5.1 \mathrm{ft} \text { respectively. Therefore, } \\
\text { interval -04 is a composite of 4.0 to } 4.9 \text { and 4.0 to 5.1 ft. See comments for } \\
\text { 105117 for additional information. }\end{array}$ \\
\hline
\end{tabular}

\section{COC Form Review}

The COC records were maintained to document the possession of samples from the time they were collected until they were received at the analytical laboratory and analyzed. A COC form accompanied each sample shipment sent to the laboratory and accompanied the sample during analysis and any other time the sample was moved.

The COC seal ensures that the transfer of custody is maintained. For this project, there was insufficient documentation to guarantee the integrity of the COC seal.

During data validation, the COC forms were audited for completeness and maintenance of the chain of custody and were reviewed for comments written on them by the samplers and laboratory personnel. It was observed that some of the bottles requested had been marked through; therefore, fewer bottles were collected. Many samples had more than one cooler number and/or more than one temperature on the chain-of-custody. GE said that they had no way to verify which cooler actually contained the sample since their cooler receipt log had the multiple coolers listed. By process of elimination and using the preparation batch and associating the trip blanks with their respective VOA coolers, the samples were then matched with their coolers.

Table 4 gives the cooler numbers, temperatures, and trip blank numbers for all samples received by the laboratories. 
Table 4. Cooler Numbers, Temperatures, and Trip Blanks

\begin{tabular}{|c|c|c|c|c|c|}
\hline $\begin{array}{l}\text { Survey } \\
\text { Station ID }\end{array}$ & $\begin{array}{l}\text { Sample } \\
\text { ID }\end{array}$ & $\begin{array}{l}\text { Sample Date } \\
\text { and Time }\end{array}$ & $\begin{array}{l}\text { Cooler } \\
\text { Number }\end{array}$ & Trip Blank & $\begin{array}{l}\text { Temperature } \\
\text { at Lab }\left({ }^{\circ} \mathrm{C}\right)\end{array}$ \\
\hline PPSC0101 & 104600 & 07/07/95, 11:35 & 119168 & 104624 & 4 \\
\hline PPSC0102 & 104601 & $07 / 07 / 95,12: 05$ & 119168 & 104624 & 4 \\
\hline PPSC0103 & 104602 & $07 / 07 / 95,12: 25$ & 119168 & 104624 & 4 \\
\hline PPSCFB01C & 104603 & 07/07/95, 15:30 & 119168 & 104624 & 4 \\
\hline PPSC0201 & 104604 & $07 / 07 / 95,16: 20$ & 176 & N/A & 6 \\
\hline PPSC0202 & 104605 & $07 / 07 / 95,16: 40$ & 119324 & N/A & 3 \\
\hline PPSC0203 & 104606 & 07/07/95, 17:00 & 119328 & N/A & 3 \\
\hline PPSC0204 & 104607 & $07 / 07 / 95,17: 30$ & 119324 & N/A & 3 \\
\hline PPSC0205 & 104608 & $07 / 07 / 95,17: 40$ & 119324 & N/A & 3 \\
\hline PPSC0301 & 104609 & $07 / 08 / 95,12: 30$ & 119168 & 104624 & 4 \\
\hline PPSC0302 & 104610 & $07 / 08 / 95,12: 45$ & 119324 & $\mathrm{~N} / \mathrm{A}$ & 3 \\
\hline PPSC0303 & 104611 & 07/08/95, $13: 00$ & 119324 & N/A & 3 \\
\hline PPSC0304 & 104612 & $07 / 08 / 95,13: 15$ & 119324 & N/A & 3 \\
\hline PPSC0501 & 104613 & $07 / 08 / 95,16: 20$ & 119328 & N/A & 3 \\
\hline PPSC0502 & 104614 & 07/08/95, 16:30 & 119319 & N/A & 4 \\
\hline PPSC0503 & 104615 & $07 / 08 / 95,16: 45$ & 119319 & N/A & 4 \\
\hline PPSC0601 & 104616 & $07 / 09 / 95,12: 40$ & 119319 & N/A & 4 \\
\hline PPSC0602 & 104617 & 07/09/95, 13:00 & 119319 & N/A & 4 \\
\hline PPSC0603 & 104618 & $07 / 09 / 95,13: 10$ & 119319 & $\mathrm{~N} / \mathrm{A}$ & 4 \\
\hline PPSC0604 & 104619 & 07/09/95, 13:30 & 119319 & $\mathrm{~N} / \mathrm{A}$ & 4 \\
\hline PPSC0701 & 104620 & 07/09/95, 15:30 & 119328 & $\mathrm{~N} / \mathrm{A}$ & 3 \\
\hline PPSC0702 & 104621 & $07 / 09 / 95,15: 45$ & 119328 & N/A & 3 \\
\hline PPSC0703 & 104622 & 07/09/95, 16:00 & 119328 & N/A & 3 \\
\hline PPSC0704 & 104623 & 07/09/95, 16:15 & 119328 & N/A & 3 \\
\hline PPSCTB01B & 104624 & $07 / 09 / 95,17: 00$ & 119168 & -2 & 4 \\
\hline PPSC1001 & 104625 & 07/10/95, 13:20 & 119324 & N/A & 5 \\
\hline PPSC1002 & 104626 & 07/10/95, 13:40 & 119324 & N/A & 5 \\
\hline PPSC1003 & 104627 & 07/10/95, 14:00 & $119324^{*}$ & N/A & 5 \\
\hline PPSC1004 & 104628 & $07 / 10 / 95,14: 25$ & 119321 & $\mathrm{~N} / \mathrm{A}$ & 2 \\
\hline
\end{tabular}

1 N/A = not applicable. No trip blank was required because the sample was not analyzed for volatile organic compounds

$2_{-}=$trip blank 
Table 4. Cooler Numbers, Temperatures, and Trip Blanks, Continued

\begin{tabular}{|c|c|c|c|c|c|}
\hline $\begin{array}{l}\text { Survey } \\
\text { Station ID }\end{array}$ & $\begin{array}{l}\text { Sample } \\
\text { ID }\end{array}$ & $\begin{array}{l}\text { Sample Date } \\
\text { and Time }\end{array}$ & $\begin{array}{l}\text { Cooler } \\
\text { Number }\end{array}$ & Trip Blank & $\begin{array}{l}\text { Temperature } \\
\text { at Lab }\left({ }^{\circ} \mathrm{C}\right)\end{array}$ \\
\hline PPSC1005 & 104629 & $07 / 10 / 95,14: 45$ & 119315 & 104634 & 2 \\
\hline PPSC0801 & 104630 & $07 / 10 / 95,16: 55$ & 119315 & 104634 & 2 \\
\hline PPSCFB03C & 104631 & $07 / 10 / 95,17: 10$ & 119315 & 104634 & 2 \\
\hline PPSC0802 & 104632 & 07/10/95, 17:30 & 53 & N/A & 7 \\
\hline PPSC0803 & 104633 & $07 / 10 / 95,17: 50$ & 53 & N/A & 7 \\
\hline PPSCTB02B & 104634 & $07 / 10 / 95,18: 30$ & 119315 & - & 2 \\
\hline PPSC0901 & 104635 & 07/11/95, 14:00 & 119325 & 104641 & 3 \\
\hline PPSC0902 & 104636 & $07 / 11 / 95,14: 15$ & 119325 & 104641 & 3 \\
\hline PPSC0903 & 104637 & $07 / 11 / 95,14: 30$ & 119325 & 104641 & 3 \\
\hline PPSC2601A & 104638 & $07 / 11 / 95,16: 15$ & 119325 & 104641 & 3 \\
\hline PPSC2602D & 104639 & $07 / 11 / 95,16: 15$ & 836 & 104642 & 8.1 \\
\hline PPSC2603A & 104640 & $07 / 11 / 95,16: 15$ & 119325 & 104641 & 3 \\
\hline PPSCTB03B & 104641 & $07 / 11 / 95,17: 00$ & 119325 & - & 3 \\
\hline PPSCTB15B & 104642 & $07 / 11 / 95,17: 00$ & 836 & - & 8.1 \\
\hline PPSC0401 & 104643 & 07/12/95, 17:15 & 328 & 104651 & 13 \\
\hline PPSC0402 & 104644 & $07 / 12 / 95,17: 30$ & 328 & 104651 & 13 \\
\hline PPSC0403 & 104645 & $07 / 12 / 95,17: 45$ & 328 & 104651 & 13 \\
\hline PPSC0404 & 104646 & $07 / 12 / 95,18: 00$ & 328 & 104651 & 13 \\
\hline PPSC2701D & 104647 & $07 / 12 / 95,17: 15$ & 836 & 104642 & 8.1 \\
\hline PPSC2702A & 104648 & 07/12/95, 17:30 & 328 & 104651 & 13 \\
\hline PPSC2703D & 104649 & $07 / 12 / 95,17: 45$ & 836 & 104642 & 8.1 \\
\hline PPSC2704A & 104650 & $07 / 12 / 95,18: 00$ & 328 & 104651 & 13 \\
\hline PPSCTB04B & 104651 & $06 / 27 / 95,15: 00$ & 328 & - & 13 \\
\hline PPSC1101 & 104652 & $07 / 14 / 95,10: 45$ & 119309 & 104662 & 13 \\
\hline PPSC1102 & 104653 & 07/14/95, 11:00 & 119309 & 104662 & 13 \\
\hline PPSCFB02C & 104654 & $07 / 14 / 95,11: 15$ & 119309 & 104662 & 13 \\
\hline PPSC1103 & 104655 & 07/14/95, 11:30 & 119309 & 104662 & 13 \\
\hline PPSC1201 & 104656 & $07 / 14 / 95,14: 00$ & 119309 & 104662 & 13 \\
\hline PPSC2801A & 104657 & 07/14/95, 14:10 & 119309 & $\cdot 104662$ & 13 \\
\hline PPSC1202 & 104658 & 07/14/95, 14:20 & 119309 & 104662 & 13 \\
\hline PPSC2802D & 104659 & 07/14/95, 14:30 & 840 & 104663 & 5.7 \\
\hline PPSC1203 & 104660 & $07 / 14 / 95,14: 40$ & 119309 & 104662 & 13 \\
\hline PPSC2803A & 104661 & $07 / 14 / 95,14: 50$ & 119309 & 104662 & 13 \\
\hline PPSCTB05B & 104662 & $07 / 14 / 95,16: 45$ & 119309 & - & 13 \\
\hline PPSCTB16B & 104663 & $07 / 14 / 95,16: 45$ & 840 & - & 5.7 \\
\hline PPSC1301 & 104664 & $07 / 18 / 95,13: 40$ & 119314 & N/A & 5 \\
\hline PPSC1302 & 104665 & 07/18/95, 14:00 & 19 & N/A & 6 \\
\hline
\end{tabular}


Table 4. Cooler Numbers, Temperatures, and Trip Blanks, Continued

\begin{tabular}{|c|c|c|c|c|c|}
\hline $\begin{array}{l}\text { Survey } \\
\text { Station ID }\end{array}$ & $\begin{array}{l}\text { Sample } \\
\text { ID }\end{array}$ & $\begin{array}{l}\text { Sample Date } \\
\text { and Time }\end{array}$ & $\begin{array}{l}\text { Cooler } \\
\text { Number }\end{array}$ & Trip Blank & $\begin{array}{l}\text { Temperature } \\
\text { at Lab }\left({ }^{\circ} \mathrm{C}\right)\end{array}$ \\
\hline PPSC1303 & 104666 & $07 / 18 / 95,14: 20$ & 19 & N/A & 6 \\
\hline PPSC1304 & 104667 & $07 / 18 / 95,14: 45$ & 19 & N/A & 6 \\
\hline PPSC1801 & 104668 & $07 / 18 / 95,17: 45$ & 119314 & N/A & 5 \\
\hline PPSC1802 & 104669 & 07/18/95, 18:05 & 119314 & N/A & 5 \\
\hline PPSC1803 & 104670 & 07/18/95, 18:30 & 119314 & N/A & 5 \\
\hline PPSC1901 & 104671 & 07/19/95, 13:00 & 168 & 104679 & 6 \\
\hline PPSC1902 & 104672 & 07/19/95, 13:00 & 168 & 104679 & 6 \\
\hline PPSC1903 & 104673 & 07/19/95, 13:00 & 168 & 104679 & 6 \\
\hline PPSC1904 & 104674 & 07/19/95, 13:00 & 168 & 104679 & 6 \\
\hline PPSC1905 & 104675 & $07 / 19 / 95,13: 00$ & 168 & 104679 & 6 \\
\hline PPSC2001 & 104676 & $07 / 19 / 95,17: 00$ & 168 & 104679 & 6 \\
\hline PPSC2002 & 104677 & $07 / 19 / 95,17: 00$ & 168 & 104679 & 6 \\
\hline PPSC2003 & 104678 & $07 / 19 / 95,17: 00$ & 119318 & N/A & 6 \\
\hline PPSCTB06B & 104679 & 07/19/95, 18:00 & 168 & - & 6 \\
\hline PPSC2101 & 104680 & $07 / 21 / 95,08: 30$ & 195 & 104692 & 5 \\
\hline PPSC2102 & 104681 & $07 / 21 / 95,08: 30$ & 195 & 104692 & 5 \\
\hline PPSC2103 & 104682 & $07 / 21 / 95,08: 30$ & 195 & 104692 & 5 \\
\hline PPSC2201 & 104683 & $07 / 21 / 95,11: 00$ & 195 & 104692 & 5 \\
\hline PPSC2202 & 104684 & $07 / 21 / 95,11: 00$ & 195 & 104692 & 5 \\
\hline PPSC2203 & 104685 & $07 / 21 / 95,11: 00$ & 195 & 104692 & 5 \\
\hline PPSC2204 & 104686 & $07 / 21 / 95,11: 00$ & 195 & 104692 & 5 \\
\hline PPSC2301 & 104687 & $07 / 21 / 95,14: 00$ & 195 & 104692 & 5 \\
\hline PPSC2302 & 104688 & $07 / 21 / 95,14: 00$ & 195 & 104692 & 5 \\
\hline PPSC2303 & 104689 & $07 / 21 / 95,14: 00$ & 195 & 104692 & 5 \\
\hline PPSC2304 & 104690 & $07 / 21 / 95,14: 00$ & 195 & 104692 & 5 \\
\hline PPSC2305 & 104691 & $07 / 21 / 95,14: 00$ & 195 & 104692 & 5 \\
\hline PPSCTB07B & 104692 & $07 / 21 / 95,15: 30$ & 195 & - & 5 \\
\hline PPSC1701 & 104693 & $07 / 25 / 95,10: 30$ & 119311 & 105103 & 6 \\
\hline PPSC1702 & 104694 & $07 / 25 / 95,10: 30$ & 119311 & 105103 & 6 \\
\hline PPSC1703 & 104695 & $07 / 25 / 95,10: 30$ & 119311 & 105103 & 6 \\
\hline PPSC1704 & 104696 & $07 / 25 / 95,10: 30$ & 119311 & 105103 & 6 \\
\hline PPSC1601 & 104697 & 07/25/95, 16:30 & 119311 & 105103 & 6 \\
\hline PPSC1602 & 104698 & $07 / 25 / 95,16: 30$ & 119311 & 105103 & 6 \\
\hline PPSCFB05C & 104699 & $07 / 25 / 95,16: 30$ & 119311 & 105103 & 6 \\
\hline PPSC1603 & 105100 & $07 / 25 / 95,16: 30$ & 119311 & 105103. & 6 \\
\hline PPSC1604 & 105101 & $07 / 25 / 95,16: 30$ & 119311 & 105103 & 6 \\
\hline PPSC1605 & 105102 & $07 / 25 / 95,16: 30$ & 119311 & 105103 & 6 \\
\hline
\end{tabular}


Table 4. Cooler Numbers, Temperatures, and Trip Blanks, Continued

\begin{tabular}{|l|l|l|l|l|l|}
\hline $\begin{array}{l}\text { Survey } \\
\text { Station ID }\end{array}$ & $\begin{array}{l}\text { Sample } \\
\text { ID }\end{array}$ & $\begin{array}{l}\text { Sample Date } \\
\text { and Time }\end{array}$ & $\begin{array}{l}\text { Cooler } \\
\text { Number }\end{array}$ & Trip Blank & $\begin{array}{l}\text { Temperature } \\
\text { at Lab ( }{ }^{\circ} \text { ) }\end{array}$ \\
\hline PPSCTB08B & 105103 & $07 / 25 / 95,18: 00$ & 119311 & - & 6 \\
\hline PPSC1501 & 105104 & $07 / 26 / 95,10: 30$ & 119313 & N/A & 4 \\
\hline PPSC1502 & 105105 & $07 / 26 / 95,10: 30$ & 119313 & N/A & 4 \\
\hline PPSC1503 & 105106 & $07 / 26 / 95,10: 30$ & 168 & N/A & 2 \\
\hline PPSC1504 & 105107 & $07 / 26 / 95,10: 30$ & 119313 & N/A & 4 \\
\hline PPSC1505 & 105108 & $07 / 26 / 95,10: 30$ & 168 & N/A & 2 \\
\hline PPSC1401 & 105109 & $07 / 26 / 95,15: 00$ & 119317 & 105112 & 4 \\
\hline PPSC1402 & 105110 & $07 / 26 / 95,15: 00$ & 119317 & 105112 & 4 \\
\hline PPSC1403 & 105111 & $07 / 26 / 95,15: 00$ & 119317 & 105112 & 4 \\
\hline PPSCTB09B & 105112 & $07 / 26 / 95,16: 00$ & 119317 & - & 4 \\
\hline PPSC2501 & 105113 & $07 / 26 / 95,17: 30$ & 119317 & 105112 & 4 \\
\hline PPSC2502 & 105114 & $07 / 26 / 95,17: 30$ & 119317 & 105112 & 4 \\
\hline PPSC2503 & 105115 & $07 / 26 / 95,17: 30$ & 119317 & 105112 & 4 \\
\hline PPSC2504 & 105116 & $07 / 26 / 95,17: 30$ & 119317 & 105112 & 4 \\
\hline PPSC2401 & 105117 & $07 / 26 / 95,20: 00$ & 119317 & 105112 & 4 \\
\hline PPSC2402 & 105118 & $07 / 26 / 95,20: 00$ & 119317 & 105112 & 4 \\
\hline PPSC2403 & 105119 & $07 / 26 / 95,20: 00$ & 119317 & 105112 & 4 \\
\hline PPSC2404 & 105120 & $07 / 26 / 95,20: 00$ & 119317 & 105112 & 4 \\
\hline
\end{tabular}

Many samples had cooler temperatures in excess of the required $4^{\circ} \mathrm{C} \pm 2^{\circ} \mathrm{C}$ when received by the laboratories. The data from these samples were assigned a $Y$ qualifier because the temperatures exceeded preservation requirements established by EPD/EMS.

Table 5. Comments/Observations from the COC Forms

\begin{tabular}{|l|l|}
\hline Sample ID & Comments/Observations \\
\hline $\begin{array}{l}105104, \\
105106-105108\end{array}$ & No ice is recorded in the cooler temperature box. \\
\hline $\begin{array}{l}105110, \\
105115-105119\end{array}$ & $\begin{array}{l}\text { No ice is recorded in the cooler temperature box. Radiological is } \\
\text { recorded in the cooler number box. }\end{array}$ \\
\hline
\end{tabular}

\section{Sampling Discrepancies}

The work plan assessment (Nichols, C. E., 1995) established priority for planned analyses and specific steps to be taken if complete samples were not collected. According to the work plan assessment, one filled bottle for gamma PHA may be submitted. One bottle of gamma PHA submitted for sample 104652 was only $1 / 5$ full. 


\section{COC Form Anomalies}

The SRTC project manager and the laboratory project manager were queried by Exploration Resources, Inc., (ExR) regarding all anomalies reflected in the COC forms.

\section{SRTC Project Manager}

On September 29, 1995, ExR sent a query letter to the SRTC project manager.

The first question concerned the station name abbreviation, SED, which is included in the sampling point. ExR stated that their data entry format only allows for two characters in the sampling point. Therefore, the SED abbreviation was removed on 10/7/95 as requested by the SRTC project manager.

The second question concerned duplicate and split samples. The sampling portions of the station names were changed to be consistent with those of associated samples. Samples 104638-40, 104647-50, 104657, 104659, and 104661 were changed to aid future users of the data who wish to associate duplicates and splits with parent samples.

The third question concerned the improper sample date for sample PPSC TB04B, sample ID 104651. The COC and analytical data showed the sample date as 06/27/95, while the sample date given in the sample logbook was 07/12/95. Normally, the sample date for a trip blank is the date the laboratory prepared the blank, not the date the blank was placed in the VOA cooler for shipment. The sampler's comments state the date of preparation as $06 / 27 / 95$; therefore the sample logbook has been changed to reflect this date.

\section{General Engineering Analytical Laboratories, Inc.}

On October 4, 1995, ExR sent a query letter to GE.

The first question concerned inconsistent laboratory ID numbers for sample 104607. The COC shows the laboratory ID to be 9506128-04, but the analytical data show the laboratory ID to be 9507128-04. GE was asked to review the sample and provide an explanation for the inconsistency.

The second question also concerned inconsistency in laboratory ID for sample 104608. The COC shows the laboratory ID to be 9506128-05, but the analytical data show the laboratory ID to be 9507128-05. GE was asked to review the sample and provide an explanation for the inconsistency.

The third question concerned multiple cooler numbers and/or cooler temperatures for the following samples: 104625-27, 104635-37, 104644-46, 104648, 104650, 104652-58, 104660-61, 104671-104675, 104678, 104680-91, 104693-105106, 105108-11, and 105113105119. GE was asked to review the samples and provide an explanation for the inconsistency.

The fourth question dealt with the lack of signatures or dates on the COC for sample 104637, laboratory ID 9507199-07. GE was asked to review the sample and provide an explanation for the inconsistency. 
The fifth question concerned sample 104643. This sample's COC was missing laboratory ID, cooler temperature, cooler number, signatures, and dates. GE was asked to address this problem.

The sixth question concerned a discrepancy in laboratory ID for sample 104622. The COC shows the laboratory ID to be 9507281-100, but the analytical data show the laboratory ID to be 9507281-10. ExR believes the laboratory ID was written incorrectly on the COC and should be changed to 9507281-10. GE was asked to review and verify this change.

GE verified a "received by" date of 7/27/95 which was incorrectly recorded as 7/26/95 on the COC form for sample 105106 (9507546-12).

\section{Sample Preservation}

\section{Preservation Requirements}

Sample preservation requirements are determined by regulatory guidance, method specifications, and laboratory protocols. General requirements follow.

\section{Sediments}

Inorganics: Inorganic analyses for metals require that soil samples be kept at $4^{\circ} \mathrm{C} \pm 2^{\circ} \mathrm{C}$.

Organics: Organic analyses for chlorinated hydrocarbons, haloethers, PCBs, pesticides, phenols, phthalate esters, purgeable aromatic hydrocarbons, purgeable halocarbons, tetrachlorodibenzo-p-dioxin isomers, and total organic carbon also require that soil samples be kept at $4^{\circ} \mathrm{C} \pm 2^{\circ} \mathrm{C}$. Sediment samples to be analyzed for nitroaromatics and isophorone, nitrosamines, or polynuclear aromatic hydrocarbons must be stored in the dark at temperatures of $4^{\circ} \mathrm{C} \pm 2^{\circ} \mathrm{C}$.

\section{Adherence to Requirements}

The laboratory recorded the temperatures of the samples as they were received. All cooler temperatures are noted in the COC Form Review subsection of this section.

Both soil and water samples for base/neutral/acid extractables, including polynuclear aromatic hydrocarbons, are shipped in amber bottles.

The pH of samples received by the laboratory, any adjustment of $\mathrm{pH}$ done by the laboratory, and any other preservatives added by the laboratory after sample receipt are not reported. 


\section{Contract Laboratory Evaluation}

\section{The Laboratories}

Analyses were performed by General Engineering Laboratories, Inc. (GE), of Charleston, SC, and Roy F. Weston, Inc. (WA), of Lionville, PA. Both laboratories have been certified by the South Carolina Department of Health and Environmental Control (SCDHEC).

\section{Performance Evaluations}

GE and WA participated in the Environmental Protection Agency (EPA) Water Pollution Laboratory Performance Evaluation study, WP033, for which results were reported in February 1995. EPA conducts the water pollution study biannually to certify laboratories for specific analyses. EPA's Environmental Monitoring Systems Laboratory (EMSL) of Cincinnati, $\mathrm{OH}$, prepares water samples spiked with known concentrations of constituents found in polluted waters and submits them to all laboratories seeking certification to analyze wastewater. EMSL evaluates the results, using limits statistically based on the performance of approximately 100 top-rated laboratories that analyze each constituent by the same procedure as the laboratory under evaluation.

Table B-1 in Appendix B contains GE's results of the February 1995 WP033 study. The only result outside the acceptable range was for one sample of nitrate as nitrogen. Although acceptable, results for one sample each of lead, manganese, and orthophosphate and two samples of fluoride were near the acceptance limits. GE was instructed to check for error.

Table B-2 in Appendix B contains WA's results of the February 1995 WP033 study. Results were outside the acceptable range for one sample each of alkalinity, chloride, $\mathrm{pH}$, selenium, sodium, specific conductance, and total residual chlorine; and two samples each of calcium, dibromochloromethane, magnesium, and oil and grease. Although acceptable, results for one sample each of bromoform, chloride, 1,2-dichlorobenzene, lead, potassium, sodium, specific conductance, sulfate, total phenolics, and total residual chlorine were near the acceptance limits. WA was instructed to check for error.

GE and WA also participated in EPA Water Supply Laboratory Performance Evaluation studies, WS035 and WS034. Table B-3 in Appendix B contains GE's results for WS035, for which results were reported in April 1995. Results for one sample each of chlordane and methoxychlor were outside the acceptable range. Table B-4 in Appendix B contains WA's results of the October 1994 WS034 study. Results for one sample each of alkalinity (as $\mathrm{CaCO}_{3}$ ), hexachlorobenzene, nitrate as nitrogen, $\mathrm{pH}$, sodium, sulfate, total chlordane, 1,2,3trichloropropane, and trifluralin were outside the acceptable range. The laboratory was instructed to check for error. 
WA responded to the findings of Performance Evaluation (PE) WS034 with the following corrective actions:

- The high 1,2,3-trichloropropane results occurred because the compound coeluted with the surrogate (1-chloroheptane) that was used during those analyses. WA discarded the surrogate and will not use it in the future. This was the only time that 1-chloroheptane was used. Alternate surrogate compounds are now in use when 1,2,3-trichloropropane is a target compound. If the reported result for 1,2,3-trichloropropane is adjusted for the coelution, the result becomes $17.3 \mu \mathrm{g} / \mathrm{L}$, which meets the acceptance limits.

- The reported value of chlordane $(13.9 \mu \mathrm{g} / \mathrm{L})$ that was outside the acceptance limits of $3.99-10.5 \mu \mathrm{g} / \mathrm{L}$ was calculated incorrectly by the gas chromatograph (GC) analyst. The analyst assumed a $10 \mathrm{~mL}$ final volume instead of the correct $5 \mathrm{~mL}$ final volume. The properly calculated result of $6.97 \mu \mathrm{g} / \mathrm{L}$ falls within the acceptance limits. To prevent this from happening in the future, WA informed the analysts to be aware of final volume differences and not to assume a $10 \mathrm{~mL}$ final volume. A more extensive final review by the unit leader, or someone appointed by the unit leader, will also insure that this problem will no longer occur.

- Hexachlorobenzene and trifluralin results were reported outside the acceptance limits. After further review of the data, no notable problems were identified. All method criteria, including surrogate and spike recoveries, were met. Calculations for standard concentrations were also verified. WA verified their standards with an independent second source (two different vendors).

- One nitrate value was reported below the acceptance limits at $0.403 \mathrm{mg} / \mathrm{L}$. Based on review of the nitrate data, the calculations, and the associated quality control (QC) checks, the analysis appears acceptable. A two-fold dilution was performed to bring the sample result within the working calibration range. A replicate was analyzed with a value of $0.446 \mathrm{mg} / \mathrm{L}$, which is within acceptance limits. WA suspects an error in the dilution. In the future, the wet-chemistry unit leader and/or section manager will review the analyst's technique and monitor for sources of error.

- The $\mathrm{pH}$ was reported low and was outside the acceptance limits at 8.84 versus a true value of 9.12. Prior to sample analysis, the $\mathrm{pH}$ buffer checks at 4, 7, and 10 were performed and were within the control windows of $+1-0.05$. WA suspects that the samples were exposed longer than usual and not analyzed promptly. WA will have the wet-chemistry unit leader insure that analysts analyze the $\mathrm{pH}$ of samples immediately.

- Alkalinity was outside the acceptance limits at $70.0 \mathrm{mg} / \mathrm{L}$ versus a true value of 33.0 $\mathrm{mg} / \mathrm{L}$. Based on a review of the alkalinity data, the calculations, and the associated QC checks, the analysis appears acceptable. The bench log indicates a $50 \mathrm{~mL}$ aliquot was taken for titration against $0.2 \mathrm{~N}$ sulfuric acid. However, for low-level titrations, a 100 to $200 \mathrm{~mL}$ sample is typically used on alkalinity according to standard operating procedure. The analyst, who is no longer with the laboratory and not available for questioning, may have analyzed a $100 \mathrm{~mL}$ aliquot but mistakenly recorded a $50 \mathrm{~mL}$ volume. The wet-chemistry unit leader will review accurate documentation procedures with the current analyst. 
- Sodium was outside the acceptance limits at $10.1 \mathrm{mg} / \mathrm{L}$ versus a true value of 15.2 $\mathrm{mg} / \mathrm{L}$. Based on review of the reported inductively coupled plasma (ICP) data, the calculations, and the associated QC checks, the analysis appears acceptable. A reference standard was analyzed prior to the PE sample and was biased low, perhaps due to an irregular aspiration. The reference standard was analyzed again following the PE sample and was recovered within acceptance limits. The PE sample was not reanalyzed and should have been according to proper procedures. In the future, the section manager and metals unit leader will ensure such instances are evaluated properly and re-analyzed.

- Sulfate was outside the acceptance limits at $462 \mathrm{mg} / \mathrm{L}$ versus the true value of 400 $\mathrm{mg} / \mathrm{L}$. Based on review of the sulfate data, calculations, and all associated $\mathrm{QC}$ checks, the analysis appears acceptable. A reference standard was analyzed and recovered within limits. The source of error was not apparent. It is possible that suspended matter was introduced into the sample and interfered with the analysis. WA will have the wet-chemistry unit leader re-evaluate the analyst's technique and monitor for sources of positive bias.

The PEs discussed later in this section are another $\mathrm{QC}$ check. The validation and verification sections in this report provide information on evaluation of the laboratories with respect to the specific analyses performed for this project.

\section{Quality Control Standards}

During the February 1995 study, EPD/EMS conducted quality assessments of GE and WA laboratories. Each laboratory received a set of certified environmental quality control standards from Environmental Resource Associates (ERA) of Arvada, CO (lot numbers 426, 563, 3212, 3412, 8917, and 9964). Each laboratory's results were compared with the ERAcertified values and performance acceptance limits (PALs). The PALs are listed as guidelines for acceptable analytical results given the limitations of the EPA methodologies used to determine these parameters. The PALs closely approximate the $95 \%$ confidence interval. The laboratories' results and the certified values and ranges are listed in tables B-5 (GE) and B-6 (WA) in Appendix B.

GE reported $\mathrm{m} / \mathrm{p}$-Cresol as $\mathrm{p}$-Cresol due to the inability to separate $\mathrm{m}$-Cresol and p-Cresol completely. In addition, the volume of the oil and grease vial allowed for only one analysis. Due to the lack of sample in the herbicides/pesticides vial, all the requested compounds could not be analyzed; the base/neutrals/acid target compound list was performed instead.

WA analyzed oil and grease by the gravimetric method and total petroleum hydrocarbons by the infrared method. In addition, WA did not analyze alachlor, ammonia nitrogen, atrazine, dalapon, dinoseb, hexachlorocyclopentadiene, picloram, simazine, or strontium because these analytes are not in the current contract.

Of 102 analyses reported by GE, 87 analyses or $85 \%$, were within the advisory range. Of 98 analyses reported by WA, 73 analyses, or $75 \%$, were within the advisory range. 


\section{Laboratory Quality Assurance/Quality Control}

The laboratories follow their own quality assurance/quality control (QA/QC) plans, which are designed to measure and document the accuracy and precision for the various analytical methods they perform. The laboratories also are guided by published methodspecific QA/QC requirements as well as other regulatory and contract-required QA procedures.

\section{Performance Reviews}

The laboratories providing analytical services to EPD/EMS are evaluated periodically for adherence to their QA plans and to ensure that the analytical work is being performed according to contract-prescribed laboratory procedure, method execution, documentation, record keeping, and data reporting. Performance reviews, or annual evaluations, are executed to identify potential problems at the laboratories or with the contract and are part of continuing efforts to maintain high-quality defensible data for environmental monitoring and assessment at the Savannah River Site.

\section{General Engineering Laboratories, Inc., Performance Review}

Westinghouse Savannah River Company (WSRC) personnel visited GE on June 15-17, 1994, to review GE's implementation of its QA plan and to verify that GE, as a supplier, had met the requirements of applicable WSRC specifications. The audit team reviewed documentation and observed processes associated with sample receipt; organic, inorganic, and radiological sample preparation and analysis; sample residue and laboratory waste handling; and administration of the QA program.

Although seven findings were issued, the audit team concluded that GE has both the technical and quality capability to meet the requirements of the current contract. The findings were primarily in administration and documentation.

\section{Findings}

1. Documentation of minimum qualifications for job positions was incomplete.

2. GE had not developed a program for defining technical and QA requirements applicable to their subcontracting laboratories and waste disposal services, and had not reviewed subcontracting laboratory documentation for its conformance to all specified WSRC requirements. Although audits were performed on subcontract laboratories, the audit documentation did not address the requirements of EPA's methods document Test Methods for Evaluating Solid Waste (SW-846) (USEPA, 1986).

3. Chain of custody was not tracked completely for toxicity characteristic leaching procedure samples, which are outside GE's Laboratory Information Management System.

4. WSRC had requested during past reviews that GE bracket all WSRC samples with a blank to prevent sample-to-sample contamination during preparation, extraction, and analysis when WSRC samples are run in sequence with other customers' samples. GE had not complied with these requests. 
5. Instrument maintenance logs were not filled out completely.

6. GE had not implemented an internal performance audit program, and corrective action for audit findings issued by external reviewers has not been adequately documented.

7. There was insufficient documentation of the handling of WSRC sample residue.

\section{Observations}

Cleaning procedures for bottles that contained semivolatile samples and for equipment used in soils preparation were not developed or were inadequate.

\section{Roy F. Weston, Inc., Performance Review}

WSRC visited WA's Analytics Division of Lionville, PA, on September 7-9, 1994, to review WA's implementation of its QA plan and to verify that WA, as a supplier, had met applicable WSRC requirements. The audit team reviewed the documentation for analyses performed for WSRC and for the QA program and examined the processes associated with organic and inorganic chemistry.

The audit team also completed a statistical evaluation of WA's performance in EPD/EMS blind replicates versus duplicates and split samples during 1993 and its performance during EPD/EMS' implementation of the adjusted mean relative difference statistics for data near quantitation limits. The evaluation revealed high variations in reporting aluminum, iron, total dissolved solids, total organic carbon, and zinc. EPD/EMS technical personnel discussed the problems with WA, and a corrective action will follow to identify the source of these biases.

This audit indicated that over the last year WA's technical performance has been acceptable, but their QA program has been problematic. As a result, the following six findings and 10 observations were issued.

\section{Findings}

1. The following problems were identified during the review of analyst training files:

a) For the files reviewed, evidence was not available that the analyst had completed the required subjects identified as "Core Training."

b) The program did not require or provide for a review of "Core Training" to assure it was completed prior to certification of the analyst.

c) The training folders did not contain a clear statement of qualification.

d) The results of analyses performed for qualification were not included in many of the training files.

2. Procedure manuals in the laboratories contained out-of-date and superseded revisions of controlled procedures. Areas reviewed included sample receiving, metals, digestion, ICP, and GC. 
3. Many of the corrective action documents had no response in the two-week time required by the WA procedure. Two documents were over six months old without a response, and no action had been taken to contact higher management for a response.

4. Several files for 1993 and 1994 system audits were incomplete (missing checklists and/or reports). One 1993 audit was identified on the schedule as having been conducted and notes were in the file, but the report was never issued (deficiencies were identified in the notes).

5. Expired chemicals were used in the wet-chemistry laboratory to calibrate the cyanide standard. Additionally, many chemicals that had no expiration dates were between five and 10 years old.

6. a) SRS samples were not bracketed by laboratory blanks when run in batches with other customers' samples; therefore, WA could not report the results of the laboratory blanks as required.

b) GC analysis did not follow the specification requirements to run one replicate with every 20 samples. WA currently is running a matrix spike and matrix spike duplicate in its place.

\section{Observations}

1. Section 15 of revision seven of the QA manual requires regular and periodic reports to management defining the status of the program. This requirement has been in the manual for several years. The first report was to be issued by the end of October 1994 .

2. The contract documents for subcontract laboratories did not specify the correct revision of the supplier's QA program.

3. Audit reports for subcontract laboratories did not state the revision number of the QA manual in effect at the time the audit was conducted.

4. QC charts were being prepared as required. However, these charts apparently were not used because computer programming errors rendered the information on the reports unusable.

5. The method currently used to track between the run logs and the preparation logs in the wet-chemistry area was very difficult to follow. Sometimes, if the analyst responsible for a specific log was not present in the lab, the information was not available.

6. Occasionally, chemicals and standards were not labeled with the concentrations or expiration dates.

7. The drying-oven log showed temperatures between 90 and $110^{\circ} \mathrm{C}$. The procedure requires the temperature to be $104 \pm 2^{\circ} \mathrm{C}$.

8. When opened, coolers did not fit completely under the fume hood in the radioactive materials area.

9. The top portions of the bound refrigerator logs were not completed prior to readings being taken for the month. The portions from past months were completed. 
10. The organics laboratory had difficulty locating manufacturers' certifications of chemicals and standards used in the calibration of analytical equipment and in QC.

Noteworthy Practices

The audit team identified the following two areas as noteworthy:

1. The corrective action system developed and implemented for gas chromatography/mass spectrometry and defined in WA Procedure 21-06-105,6 is an excellent example of "Good Laboratory Practices."

2. WA has developed an excellent program for the qualification of subcontractors providing waste treatment, storage, transportation, and disposal services to the laboratory. 
This page left blank intentionally. 


\section{Validation and Verification: Objective and Qualification Policy}

\section{Objective}

Definitive data, as defined in the Environmental Protection Agency's (EPA) Data Quality Objectives Process for Superfund (USEPA, 1993), has been determined to be the most appropriate type of data to meet the data quality objectives of this investigation.

Validation and verification activities were undertaken to assess the data against these standards, particularly with regard to the following issues:

- Sample documentation

- Maintenance of chain of custody

- Adherence to sample holding-time requirements

- Achievement of contract-required detection limits

- Analytical instrument calibration

- Analyte identification

- Analyte quantitation

Evaluation of the laboratories' performance in analyte identification and quantitation included examination of the following:

- Analytical error

- Contamination of blanks

- Laboratory performance in analyzing independent standards

\section{Qualification Policy}

This section describes the Environmental Monitoring Section's (EMS) data validation policy for assigning qualifiers to Resource Conservation and Recovery Act (RCRA) Facility Investigation/Remedial Investigation (RFI/RI) analytical data. The qualification policy is based on EPA's Contract Laboratory Program, Statement of Work for Organics Analysis (CLP SOW) (USEPA, 1991b), Functional Guidelines for Evaluating Organics Analyses (USEPA, 1988b), Functional Guidelines for Evaluating Inorganics Analyses (USEPA, 1988a), Test Methods for Evaluating Solid Waste (SW-846) (USEPA, 1986), Data Validation Procedures for Radiochemical Analyses (WHC, not dated), and Westinghouse Savannah River Company's (WSRC) contract-specific requirements.

Qualification criteria for instrument calibration, analyte identification, and analyte quantitation are given in the EMS informal Procedures for Qualifying Data Based on Laboratory Data Records Reviews (LDRRs). Refer to the front matter of Appendix D for complete qualifier definitions. 


\section{Types of Qualifiers}

In the analytical records, qualifiers are used by laboratory personnel or by validation personnel to provide non-numerical information about results. The three types of qualifiersresult, analysis, and bias-are described as follows:

- Result qualifiers indicate the usability of a reported result and address additional considerations necessary to interpret the result correctly. Result qualifiers separate all the data into the following categories:

(no qualifier)-indicates the result can be used for the most rigorous evaluations.

$J$ qualifier-indicates the results are estimated for data that are less than reliable.

$N$ qualifier-denotes results for data whose identity is only tentative.

$R$ qualifier-denotes rejected data.

$U$ and $U I$ qualifiers-indicate that non-detected analytes could be present at less than the quantitation limit.

Result qualifiers are an integral part of the result. The numerical value field is worthless to the data user if the result qualifier field is missing from the record.

- Analysis qualifiers provide the rationale for assigning a result qualifier by describing laboratory conditions, particularly quality assurance/quality control (QA/QC) measures associated with the analysis, that cause data to be estimated or rejected. Occasionally; analysis qualifiers are used without associated result qualifiers. In these cases, the qualifiers indicate minor deviations from perfect analytical conditions that are not believed to affect the usability of a given result.

- Bias qualifiers indicate that a result is believed to be higher or lower than the actual concentration of an analyte or that the analytical record is associated with QA/QC results that create uncertainty about the precision of the result. This qualifier usually is assigned when the laboratory personnel or the validator believes the reported result has been prejudiced and the data could be misinterpreted if the bias is not indicated.

\section{Holding Times}

\section{Criteria}

Refer to EPA holding times for extraction and analysis in 40 CFR Part 136 (USEPA, 1994a).

\section{Action}

Assign Result Qualifier $(\mathrm{RQ})=J$ and Analysis Qualifier $(\mathrm{AQ})=Q$ to all analyses for which holding time has been exceeded. 


\section{Sample Preservation}

\section{Criteria}

Temperature must be maintained between $2^{\circ}$ and $6^{\circ} \mathrm{C}$ for organics and non-metal inorganics; applicable to both soil and water samples.

$\mathrm{pH}$ of water samples:

- $\quad 2$ for volatiles, metals, radionuclides, nitrate, phosphate, phenols by 420.2 , ammonia, dissolved organic carbon, tentatively identified compounds, total carbon, total organic carbon, total organic halogens, total organic nitrogen, total phenols, and oil and grease.

- $>12$ for cyanide and sulfide.

Action

Assign $\mathrm{AQ}=Y$ for all samples that do not meet criteria.

Instrument Calibration, Analyte Identification, and Analyte Quantitation

Criteria

See the LDRR checklists for each analytical method.

Action

See the EMS informal Procedures for Qualifying Data Based on LDRRs for each analytical method.

\section{Trip Blanks}

No qualification is based on trip blank results.

\section{Field Blanks}

No qualification is based on field blank results.

\section{Rinsate Blanks}

No qualification is based on rinsate blank results.

\section{Method Blanks}

\section{Criteria}

Refer to the Contract Laboratory Program (USEPA, 1991b) 5x/10x rule.

\section{Action}

If an analyte is detected in a method blank, assign $A Q=V$ to that analyte in the blank and to every sample of the preparation batch having positive results, including QC duplicate samples. If the analyte concentration in a sample is less than five times that in the blank (less than 10 times for common laboratory contaminants), assign $R Q=U$ and $A Q=V$ to 
the analyte in the sample. A bias code $=H$ should be used only if the laboratory believes the sample is contaminated.

\section{Field Duplicates}

No qualification is based on field duplicate results.

\section{Laboratory Duplicates}

\section{Criteria}

Inorganics: Functional Guidelines for Evaluating Inorganics Analyses (USEPA, 1988a), page 10.

Organics: No qualification is performed on this basis.

Radiologicals: Data Validation Procedures for Radiochemical Analyses (WHC, not dated).

\section{Action}

For soil samples, if duplicate analytical results for a particular analyte fall outside the control limits for the Relative Percent Difference (RPD), assign $A Q=X$ to that analyte in the parent and duplicate samples.

For water samples, if the RPD for a particular analyte is outside the appropriate control limits, assign $R Q=J$ and $A Q=X$ to that analyte in the parent and duplicate samples.

\section{Matrix Spikes}

\section{Criteria}

Dioxins: Acceptable recovery $=50-110 \%$.

Inorganics: Functional Guidelines for Evaluating Inorganics Analyses (USEPA, 1988a), page 11.

Pesticides: CLP SOW (USEPA, 1991b), page D-61 PEST.

Semivolatiles: CLP SOW (USEPA, 1991b), Table 7, page D-59 SV.

Volatiles: CLP SOW (USEPA, 1991b), Table 7, page D-55 VOA.

\section{Action}

If the spike nominal concentration is less than or equal to $25 \%$ of the sample result, assign $\mathrm{RQ}=R$ and $\mathrm{AQ}=5$ to the spike record. If the laboratory believes poor recoveries are due to matrix interferences, they should assign $\mathrm{RQ}=R$ and $\mathrm{AQ}=4$ to the spike record. The record should not be used for qualification or any further determinations. If a matrix spike fails criteria, $\mathrm{AQ}=I$ qualifiers are added to the failed analyte in the matrix spike, and the failed analyte in associated samples for the analyte. For organics, assign $R Q=J, A Q=I, B$ $=L$ or $H$. For inorganics, assign $\mathrm{RQ}$ according to criteria, $\mathrm{AQ}=I, \mathrm{~B}=L$ or $H$.

If the laboratory fails to supply matrix spike data for non-radiological analyses, flag all analytes in all samples in the appropriate preparation batch. Assign $\mathrm{RQ}=P$ and $\mathrm{AQ}=I$. 
The matrix spike, the parent sample, and all the samples in the same preparation batch are qualified for water samples for the same analyte and method as the matrix spike. For soil samples, only the matrix spike and parent sample are qualified.

\section{Laboratory Control Samples and Blank Spikes}

\section{Criteria}

Inorganics: $80-120 \%$ recovery.

Organics: Same as matrix spike criteria.

Radiologicals: $80-120 \%$ recovery; following Data Validation Procedures for Radiochemical Analyses (WHC, not dated).

\section{Action}

Assign $\mathrm{AQ}=C$ to the failed analyte(s) in the Laboratory Control Samples/Blank Spikes (LCS/BS). Assign $\mathrm{RQ}=J$ and $\mathrm{AQ}=C$ to the failed analyte(s) in every sample in the same preparation batch and method as the LCS/BS.

LCS/BS were not reported by the primary analytical laboratory for this project. LCS/BS recoveries are a review criterion during LDRRs.

\section{Surrogate Recovery}

\section{Criteria}

Dioxins: Not applicable.

Pesticides by Method 8080: 60-150\% from CLP SOW (USEPA, 1988a), page D-56 PEST.

Semivolatiles by Method 8270: SW-846 (USEPA, 1986), Table 8, page 8270-31.

Volatiles by Method 8240: SW-846 (USEPA, 1986), Table 8, page 8240-38.

Volatiles by Method 8260: $S W-846$ (USEPA, 1986), Table 9, page 8260-44.

Action

Volatiles and Pesticides: If one or more surrogates fail acceptance criteria, assign $R Q=J$ or $U J$ and $\mathrm{AQ}=O$ to all analytes in the sample. If all the failed surrogates have low recoveries, assign bias $=L$. If all the failed surrogates have high recoveries, assign bias = $H$. If one surrogate is high and another is low, do not assign a bias code. Also, assign $A Q=$ $O$ to the failed surrogate(s).

Semivolatiles: If only one surrogate fails and its recovery is less than $10 \%$, take no action. If more than one surrogate fails, assign $\mathrm{RQ}=J$ or $U J$ and $\mathrm{AQ}=O$ to all analytes in the sample. If a recovery is negative, assign $R$. If all the failed surrogates have low or high recoveries, assign $L$ for low bias or $H$ for high bias. If one surrogate is high and another is low, do not assign a bias. Also, assign $\mathrm{AQ}=O$ to the failed surrogate(s). 
Surrogates were not reported by the primary analytical laboratory for this project. Surrogate recoveries are a review criterion during LDRRs, and recovery failures are reported in the analytical case narratives. 


\section{Overview}

The validation and verification process conducted by the Environmental Geochemistry Group includes examining the electronic data files and paper records and conducting onsite reviews of the laboratory records. The validation and verification operations are summarized in this section.

The laboratories include result and analysis qualifiers in the electronic data deliverables (EDDs). Laboratory data records reviews (LDRRs), analytical narratives, and EDDs verify the qualification conducted by the laboratories and identify additional qualification required by information not available to the laboratory. Due to contractual restraints, some of the qualification that could be conducted by the laboratories is done during data review. The changes and data qualification identified by data review are incorporated into the data validation logbook and the electronic data files. The project technical manager is responsible for overseeing and controlling this process.

\section{Data Quality Objectives}

The U.S. Environmental Protection Agency (USEPA) defines two analytical data quality categories, Screening Data with Definitive Confirmation and Definitive Data, in Data Quality Objectives Process for Superfund (USEPA, 1993).

- Screening Data with Definitive Confirmation may include health and safety measurements, field screening for organic vapors and radiation, and measurements of $\mathrm{pH}$, temperature, and specific conductance. The definitive confirmation process confirms at least $10 \%$ of the screening data using analytical methods and quality assurance/quality control (QA/QC) procedures and criteria associated with definitive data. For the Par Pond Sedimentation Characterization project, screening data included measurements of $\mathrm{pH}$, temperature, and specific conductance. These analyses are listed in the data tables in Appendix $D$ but are not discussed elsewhere in this report. Definitive confirmation for the screening data was not performed.

- Definitive Data applies to all analyte-specific laboratory data. The objective of using definitive data is to provide a level of confidence formaking decisions involving pollutant source identification, delineation of contaminants, site remediation and/or removal of pollutants, health risk or environmental impact, and similar issues. Exploration Resources, Inc. (ExR), of Athens, GA, verified and validated the data from this project against the definitive data requirements. 


\section{Analytical Methods and Quantitation Limits}

See Appendix $F$ for tables of analytical methods and quantitation limits used for this project. Generally, the analytical methods used for this project were those specified in the work plan assessment (Nichols, C.E., 1995).

The estimated quantitation limit (EQL) is the minimum concentration of a substance that can be reliably measured and reported within specified limits of precision and accuracy during routine laboratory operating conditions.

The quantitation limits reported in the data tables of this report are the sample-specific estimated quantitation limits (ssEQLs). The EQL multiplied by factors of concentration, dilution, aliquot size, and percent solids defines the ssEQL. Radiological analyses are reported with the sample-specific minimum detectable concentration.

\section{Review of the Electronic Data Files}

\section{Sample Collection, Shipping, and Analytical Data}

Data reviewers used computer programs and look-up tables to verify the EDDs of sample collection, shipping, and analytical data as described below.

- The following fields were checked to ensure they were not blank: Westinghouse Savannah River Company (WSRC) sample identification number, sample collection date, laboratory receipt date, analysis date, analysis time, analytical method, laboratory code, laboratory sample identification number, analyte code (test name), result units, nominal concentration, number of dilutions, dilution factor, instrument, instrument batch, and analyst's initials.

The following fields were checked to ensure the data were within a range of values:

- WSRC sample identification number (between 100,000 and 999,999)

- Sample collection date (after January 1, 1994, and before the date the computer program was run)

- Laboratory receipt date (between the sample collection date and the program run date except for laboratory blanks, which have no laboratory receipt dates, and toxicity characteristic leaching procedure [TCLP] samples; for TCLP samples, identified by a $T$ in the sample fraction field, the laboratory receipt date must be no later than the sample collection date)

- Analysis date (same as or later than the laboratory receipt date and before the date the program was run)

- Analysis time (an integer between zero and 2,359 or blank)

- Quantitation limit (greater than zero)

- Analytical result (a real number if the result qualifier is not $M$ or $T$; blank if the result qualifier field contains $M$ or $T$ )

- Accuracy (a real number or blank) 
- Residual weight (a positive number for radionuclide analyses; blank for others)

- Number of dilutions (zero or a positive integer)

- Dilution factor (if the dilution factor is one, then the number of dilutions must be zero; if the number of dilutions is greater than zero, the dilution factor cannot equal one)

- Nominal concentration (a positive number or blank; cannot be zero if the result unit is percent recovery), percent solids (less than or equal to 100 or blank)

- Bottle number (an integer equal to or between 10,000,000 and 99,999,999)

- The following fields were checked against a look-up table or a list of valid codes to ensure acceptability: laboratory code, laboratory duplicate code, analyte code, analytical method, result qualifier, analysis qualifier, analysis bias, result units, and sample fraction.

- Sample holding times were checked from the laboratory EDDs. The sample collection date was subtracted from the date the analysis was performed, and the result was checked against a look-up table of maximum holding times. Any analysis performed after the holding time was exceeded was qualified with a $Q$ in the analysis qualifier field and $\mathrm{a} J$ in the result qualifier field.

- Reported quantitation limits were checked against a look-up table of quantitation limits to determine if they were correctly adjusted to reflect dilution factors.

\section{Between-Records Validity}

Additionally, a computer program identified duplicate records and verified relationships between records as shown in the following table.

Table 6. Relationships Between Data Records

\begin{tabular}{|c|c|c|c|c|c|}
\hline $\begin{array}{l}\text { Sample } \\
\text { Bottle Data }\end{array}$ & $\begin{array}{l}\text { Chain-of- } \\
\text { Custody Data }\end{array}$ & $\begin{array}{l}\text { Planned } \\
\text { Analyses Data }\end{array}$ & $\begin{array}{l}\text { Sample } \\
\text { Data }\end{array}$ & $\begin{array}{l}\text { Analytical } \\
\text { Data }\end{array}$ & $\begin{array}{l}\text { Station } \\
\text { Data }\end{array}$ \\
\hline \multirow[t]{4}{*}{$\begin{array}{l}\text { WSRC } \\
\text { sample } \\
\text { identification } \\
\text { number }\end{array}$} & $\begin{array}{l}\text { WSRC sample } \\
\text { identification } \\
\text { number }\end{array}$ & . & $\begin{array}{l}\text { WSRC } \\
\text { sample } \\
\text { identification } \\
\text { number }\end{array}$ & $\begin{array}{l}\text { WSRC } \\
\text { sample } \\
\text { identification } \\
\text { number }\end{array}$ & \\
\hline & Sample date & & Sample date & Sample date & \\
\hline & $\begin{array}{l}\text { Laboratory } \\
\text { sample } \\
\text { identification } \\
\text { number }\end{array}$ & & & $\begin{array}{l}\text { Laboratory } \\
\text { sample } \\
\text { identification } \\
\text { number }\end{array}$ & \\
\hline & & Analyte code & & Analyte code & \\
\hline \multirow[t]{3}{*}{$\begin{array}{l}\text { Number of } \\
\text { bottles }\end{array}$} & & & $\begin{array}{l}\text { Number of } \\
\text { bottles }\end{array}$ & & \\
\hline & & & Actual station & & Station \\
\hline & & Planned station & $\begin{array}{l}\text { Planned } \\
\text { station }\end{array}$ & & \\
\hline
\end{tabular}


The data record relationships must meet the following criteria:

- The WSRC sample identification number in each sample bottle record must match the WSRC sample identification number in the corresponding sample record.

- The number of bottles record for each WSRC sample identification number must match the number of bottles field on the matching sample record.

- Except for laboratory blanks and laboratory-generated QA/QC samples, each analytical record must correspond to the WSRC sample identification number, sample date, and laboratory sample identification number fields of a chain-of-custody (COC) record.

- Each sample record must have at least one planned analyses record. The planned analyses record(s) and the sample record should have the same entries in the planned station fields.

- There must be a sample record for each COC record with the same WSRC sample identification number and sample date.

- Except for laboratory standards and blanks, a sample record must correspond to each analytical record in the WSRC sample identification number field and the sample date field (except for TCLP records).

- The station field in the station records should be identical to the planned station field in the planned analyses records. (The station field is a concatenation of facility identifier and sampling point. Although, as can be seen in Table 6 above, there is no direct correspondence between planned analyses data and station data, the planned station rarely differs from the actual station.) Except for field-generated blanks, the actual station field in each sample record must correspond to the station field in a station record.

\section{Completeness of Data}

The data must meet the following criteria for completeness:

- Except for laboratory standards and blanks, each analytical record must link with the WSRC sample identification number and sample date fields of corresponding COC records and sample records.

- There must be a planned analyses record for each analytical record except for laboratory standards and blanks. Because there is no common sample-specific field between planned analyses records and analytical records, this examination requires connection through sample data. Planned analyses data connects to sample data at planned station, while sample data connects to analytical data at WSRC sample identification number and sample date. Once associated, the analytical record and planned analyses record should have the same entries in the analyte field.

\section{Resolution of Anomalies}

The data reviewers clarified anomalies with the laboratory wherever possible and qualified records that did not meet the criteria. Some anomalies were recorded as unresolvable. 


\section{Review of QA/QC Samples}

QA/QC samples include field-generated blanks and duplicates and laboratory-generated blanks, duplicates, matrix spikes, surrogates, internal standards, and others. Both fieldand laboratory-generated blanks were checked for contamination that may affect sample results, and samples were qualified when contamination in blanks was detected above established levels.

Laboratory duplicates were compared with the original sample for relative percent difference (RPD). The recoveries obtained by the laboratory for matrix spikes and surrogates were examined, and the number of matrix spikes was checked for adherence to the data quality objectives. See the Discussion section of this report for precision and accuracy determinations from QA/QC sample results.

Calibration standards for a portion of the samples were examined during LDRRs. Samples were qualified when calibration standards exceeded criteria.

\section{Review of the Analytical Narratives}

Generally, the analytical narrative, with COC forms attached, and EDD arrive from the laboratory and are logged in together. If the analytical narrative is missing, it is requested from the laboratory. The analytical narrative contains a summary of problems encountered by the laboratory (e.g., QA samples that do not meet the criteria specified by the analytical method, problems with matrix interference, sample-specific adjustments to the method caused by high concentrations of some analytes, problems with sample preservation and holding time, instrument calibration problems, and contaminated blanks) and additional information about the analytical data and COC forms. The analytical narrative is used in data review and is referred to throughout the data validation process. Discrepancies between the narrative and the analytical data or the COC forms must be resolved by the laboratory.

Analytical narratives from General Engineering Laboratories, Inc. (GE), and Roy F. Weston Analytical Laboratories, Inc. (WA), were reviewed for this project.

\section{Laboratory Data Records Reviews}

On October 5-6, 1995, laboratory data records for GE and Environmental Physics, Inc., were reviewed for organics, inorganics, and radiological analyses associated with the Par Pond Sediment Characterization soil samples. The review incorporated 55 worksheets and was conducted by Raymond M. Keller, ExR. Since WA was chosen as a secondary laboratory to analyze the split samples, an LDRR was not conducted at WA because the number of records did not warrant a data records review.

The purpose of the reviews was to investigate validation issues not adequately addressed by computer checking of the EDDs and reviewing the analytical narratives. These issues include instrument calibration, analyte identification, and analyte quantitation. Criteria used for sample selection included samples identified as critical by the project manager, samples with analytical results near an EPA action level, and representatives from all large sample preparation batches. 
Validation checklists were used to assess the instrument printouts associated with particular analyses. These checklists consisted of 12 to 19 questions and were prepared from these sources: Quality Assurance/Quality Control Guidance for Removal Activities, Sampling QA/QC Plan and Data Validation Procedures (USEPA, 1990b), Test Methods for Evaluating Solid Waste (SW-846) (USEPA, 1986), Laboratory Data Validation Functional Guidelines for Evaluating Inorganics Analyses (USEPA, 1988a), Laboratory Data

Validation Functional Guidelines for Evaluating Organics Analyses (USEPA, 1988b), Data Validation Procedures for Radiochemical Analyses (WHC, not dated), and discussions with laboratory personnel.

For the findings from the reviews, see the Laboratory Data Records Review subsection in the Validation and Verification: Findings section of this report. 


\section{Validation and Verification: Findings}

\section{Validation Issues}

The data provided with this report are final and complete.

Most of the problems detected during validation and verification were resolved through communication with the samplers and laboratories. Changes made to the data are recorded in the project's data review logbooks. Quality assurance/quality control (QA/QC) anomalies remaining in the data were qualified according to the guidelines described in the Qualification Policy subsection of the Validation and Verification: Objective and Qualification Policy section of this report.

\section{Detection Limit}

The estimated quantitation limit (EQL) is the minimum concentration of a substance that can be reliably measured and reported within specified limits of precision and accuracy during routine laboratory operating conditions.

The quantitation limits reported in the data tables of this report are the sample-specific estimated quantitation limits (ssEQLs). The EQL multiplied by factors of concentration, dilution, aliquot size, and percent solids defines the ssEQL. Radiological analyses are reported with the sample-specific minimum detectable concentration.

\section{Sample Preservation}

Under the current contract, the laboratory does not report the $\mathrm{pH}$ value upon receipt of samples requiring preservation. Thus, no assessment of the preservation criterion could be made on this basis. However, samples were qualified based on temperature upon receipt by the laboratory.

\section{Surrogate Recoveries}

In addition to the analysis qualifier $O$, a bias qualifier of $L$ or $H$ has been added to surrogate results with recoveries outside the control limits as listed in the Qualification Policy subsection of the Validation and Verification: Objective and Qualification Policy section of this report. Associated samples have been qualified as described in this same subsection of this report.

A number of pesticide samples required dilution. Due to these dilutions, surrogate spikes were either diluted out, or recoveries were adversely affected. It was determined that poor recoveries were due to dilution and not indicative of poor analytical precision. Therefore, no qualification to diluted samples was performed on the basis of surrogate spike recovery. Fourteen surrogate spikes were rejected and assigned the result qualifier $R$ and analysis qualifier $O$. Thirty-three surrogate spikes received the analysis qualifier $O$. Bias qualifiers were assigned based on high or low recovery rates. 
Types and Numbers of QA/OC Samples

U.S. Environmental Protection Agency (EPA) methods, used for all of the analyses for this project, require specific types and numbers of QA/QC analyses. Tables in the Discussion section of this report provide details on the laboratories' performances for this project.

It was not possible to obtain from General Engineering Laboratories, Inc. (GE), all QA/QC analyses required for proper qualification. For preparation batch 68857 , no duplicate analysis for target analyte list (TAL) inorganics was performed. For preparation batch 69377, no duplicate analysis for target compound list (TCL) pesticides/PCBs was performed. For preparation batch 69539 , the parent sample was not provided for the duplicate analysis performed for mercury. For preparation batch 69460 , the parent sample was not provided for the duplicate analysis performed for cyanide. Without the analytical information, relative percent differences (RPDs) could not be calculated in order to check laboratory precision. Therefore, no qualifications were performed on the preparation batches.

Other missing QA/QC materials did not affect the qualification of the results because soil matrices are nonhomogeneous. Qualification extends only to the QA/QC sample and its associated sample. For TCL pesticides/PCBs and TCL semivolatiles, several parent samples results for matrix spikes or laboratory replicates were missing. Perhaps the parent sample was included in a preparation batch different from the QA/QC analyses. In most cases, the missing parent sample is a Westinghouse Savannah River Company sample that has been associated with a separate project.

\section{Qualifiers Applied Following Review of the Electronic Data Deliverables and Narratives}

The following tables give details of qualification based on the review of the Electronic Data Deliverables (EDDs) and narratives.

The maximum RPD criteria for soil and water samples are $35 \%$ and $20 \%$, respectively, and samples that failed the appropriate criteria were assigned the analysis qualifier $(A Q)=X$. Other AQs used were $Y$ (unpreserved or improperly preserved sample), $I$ (spike recovery not within control limits), $B$ (result is below the sample quantitation limit and above or equal to the instrument quantitation limit), $C$ (laboratory control sample criteria were not met), and $V$ (the analyte was detected in both the sample and associated method blank). Result qualifiers (RQs) used were $J$ (the analytical result is an estimated quantity) and $U$ (the material analyzed for was not detected). Table 7 lists soil and water samples that were assigned qualifiers due to RPDs outside range.

Table 7. Samples Assigned Qualifiers Due to RPDs Outside Range for GE

\begin{tabular}{|l|l|l|l|l|l|l|}
\hline Sample ID & Laboratory ID & Analyte & Result & Units & $\begin{array}{l}\text { Result } \\
\text { Qualifier }\end{array}$ & $\begin{array}{l}\text { Analysis } \\
\text { Qualifier }\end{array}$ \\
\hline 104600 & $9507128-21$ & Aluminum & 2860 & $\mathrm{mg} / \mathrm{kg}$ & & $\mathrm{X}$ \\
\hline 104600 & QC290283 & Aluminum & 4220 & $\mathrm{mg} / \mathrm{kg}$ & & $\mathrm{X}$ \\
\hline 104600 & $9507128-21$ & Copper & 1.54 & $\mathrm{mg} / \mathrm{kg}$ & & $\mathrm{X}$ \\
\hline
\end{tabular}


Table 7. Samples Assigned Qualifiers Due to RPDs Outside Range for GE, Continued

\begin{tabular}{|c|c|c|c|c|c|c|}
\hline Sample ID & Laboratory ID & Analyte & Result & Units & \begin{tabular}{|l} 
Result \\
Qualifier
\end{tabular} & $\begin{array}{l}\text { Analysis } \\
\text { Qualifier }\end{array}$ \\
\hline 104600 & QC290283 & Copper & 4.63 & $\mathrm{mg} / \mathrm{kg}$ & & $\mathrm{X}$ \\
\hline 104600 & $9507128-21$ & Manganese & 6.4 & $\mathrm{mg} / \mathrm{kg}$ & & $\mathrm{X}$ \\
\hline 104600 & QC290283 & Manganese & 23.3 & $\mathrm{mg} / \mathrm{kg}$ & & $\mathrm{X}$ \\
\hline 104600 & $9507128-21$ & Zinc & 2.42 & $\mathrm{mg} / \mathrm{kg}$ & & $\mathrm{X}$ \\
\hline 104600 & QC290283 & Zinc & 6.1 & $\mathrm{mg} / \mathrm{kg}$ & & $\mathrm{X}$ \\
\hline 104614 & $9507128-11$ & Magnesium & 39.1 & $\mathrm{mg} / \mathrm{kg}$ & $\mathrm{J}$ & IX \\
\hline 104614 & $\mathrm{QC290280}$ & Magnesium & 65.3 & $\mathrm{mg} / \mathrm{kg}$ & $\mathrm{J}$ & IX \\
\hline 104625 & 9507199-01 & Magnesium & 256 & $\mathrm{mg} / \mathrm{kg}$ & $\mathrm{J}$ & IX \\
\hline 104625 & QC290292 & Magnesium & 171 & $\mathrm{mg} / \mathrm{kg}$ & $\mathrm{J}$ & IX \\
\hline 104650 & 9507226-05 & Barium & 6.56 & $\mathrm{mg} / \mathrm{kg}$ & & $\mathrm{X}$ \\
\hline 104650 & QC290985 & Barium & 9.48 & $\mathrm{mg} / \mathrm{kg}$ & & $\mathrm{X}$ \\
\hline 104654 & 9507281-09 & Aluminum & 0.025 & $\mathrm{mg} / \mathrm{L}$ & $\mathrm{U}$ & $\mathrm{x}$ \\
\hline 104667 & $9507343-02$ & Aluminum & 3810 & $\mathrm{mg} / \mathrm{kg}$ & $\mathrm{J}$ & $\mathrm{CX}$ \\
\hline 104667 & QC293001 & Aluminum & 2510 & $\mathrm{mg} / \mathrm{kg}$ & $\mathrm{J}$ & $\mathrm{CX}$ \\
\hline 104667 & $9507343-02$ & Iron & 4680 & $\mathrm{mg} / \mathrm{kg}$ & $\mathrm{J}$ & VCX \\
\hline 104667 & QC293001 & Iron & 2650 & $\mathrm{mg} / \mathrm{kg}$ & $\mathrm{J}$ & $\mathrm{VCX}$ \\
\hline 104667 & 9507343-02 & Magnesium & 86 & $\mathrm{mg} / \mathrm{kg}$ & $\mathrm{J}$ & IX \\
\hline 104667 & QC293001 & Magnesium & 55.4 & $\mathrm{mg} / \mathrm{kg}$ & $\mathrm{J}$ & IX \\
\hline 104667 & $9507343-02$ & Vanadium & 11 & $\mathrm{mg} / \mathrm{kg}$ & & $\mathrm{x}$ \\
\hline 104667 & QC293001 & Vanadium & 5.94 & $\mathrm{mg} / \mathrm{kg}$ & & $\mathrm{x}$ \\
\hline 104674 & $9507381-04$ & Gross alpha & 11.7 & $\mathrm{pCi} / \mathrm{g}$ & & $\mathrm{X}$ \\
\hline 104674 & QC292827 & Gross alpha & 25.6 & $\mathrm{pCi} / \mathrm{g}$ & & $\mathrm{x}$ \\
\hline 104680 & 9507448-01 & Chromium & 7.02 & $\mathrm{mg} / \mathrm{kg}$ & & $\mathrm{x}$ \\
\hline 104680 & QC295806 & Chromium & 1.86 & $\mathrm{mg} / \mathrm{kg}$ & & $\mathrm{x}$ \\
\hline 104681 & $9507448-02$ & Radium-228 & 0.522 & $\mathrm{pCi} / \mathrm{g}$ & & $\mathrm{x}$ \\
\hline 104681 & QC293374 & Radium-228 & 4910 & $\mathrm{pCi} / \mathrm{g}$ & & $\mathrm{X}$ \\
\hline 104683 & 9507448-04 & Aluminum & 5270 & $\mathrm{mg} / \mathrm{kg}$ & & $\mathrm{X}$ \\
\hline 104683 & QC296585 & Aluminum & 3590 & $\mathrm{mg} / \mathrm{kg}$ & & $\mathrm{x}$ \\
\hline 104683 & 9507448-04 & Vanadium & 4.89 & $\mathrm{mg} / \mathrm{kg}$ & & $\mathrm{x}$ \\
\hline 104683 & QC296585 & Vanadium & 3.34 & $\mathrm{mg} / \mathrm{kg}$ & & $\mathrm{X}$ \\
\hline 104693 & $9507509-01$ & Manganese & 21 & $\mathrm{mg} / \mathrm{kg}$ & & $\mathrm{x}$ \\
\hline 104693 & QC296588 & Manganese & 12.9 & $\mathrm{mg} / \mathrm{kg}$ & & $\mathrm{X}$ \\
\hline 104699 & $9507509-10$ & Zinc & 0.005 & $\mathrm{mg} / \mathrm{L}$ & $\mathrm{U}$ & $\mathrm{x}$ \\
\hline 105110 & $9507546-10$ & Magnesium & 24.4 & $\mathrm{mg} / \mathrm{kg}$ & & $x$ \\
\hline 105110 & QC297413 & Magnesium & 36 & $\mathrm{mg} / \mathrm{kg}$ & & $\mathrm{X}$ \\
\hline 105113 & $9507546-01$ & Aluminum & 1180 & $\mathrm{mg} / \mathrm{kg}$ & & VX \\
\hline
\end{tabular}


Table 7. Samples Assigned Qualifiers Due to RPDs Outside Range for GE, Continued

\begin{tabular}{|l|l|l|l|l|l|l|}
\hline Sample ID & Laboratory ID & Analyte & Result & Units & $\begin{array}{l}\text { Result } \\
\text { Qualifier }\end{array}$ & $\begin{array}{l}\text { Analysis } \\
\text { Qualifier }\end{array}$ \\
\hline 105113 & QC297379 & Aluminum & 2740 & $\mathrm{mg} / \mathrm{kg}$ & & $\mathrm{VX}$ \\
\hline 105113 & $9507546-01$ & Barium & 8.45 & $\mathrm{mg} / \mathrm{kg}$ & $\mathrm{J}$ & $\mathrm{CX}$ \\
\hline 105113 & $\mathrm{QC297379}$ & Barium & 13.6 & $\mathrm{mg} / \mathrm{kg}$ & $\mathrm{J}$ & $\mathrm{CX}$ \\
\hline 105113 & $9507546-01$ & Calcium & 276 & $\mathrm{mg} / \mathrm{kg}$ & $\mathrm{J}$ & $\mathrm{VIX}$ \\
\hline 105113 & $\mathrm{QC297379}$ & Calcium & 437 & $\mathrm{mg} / \mathrm{kg}$ & $\mathrm{J}$ & $\mathrm{VIX}$ \\
\hline 105113 & $9507546-01$ & Iron & 967 & $\mathrm{mg} / \mathrm{kg}$ & $\mathrm{J}$ & $\mathrm{CX}$ \\
\hline 105113 & $\mathrm{QC297379}$ & Iron & 1490 & $\mathrm{mg} / \mathrm{kg}$ & $\mathrm{J}$ & $\mathrm{CX}$ \\
\hline 105113 & $9507546-01$ & Magnesium & 69.9 & $\mathrm{mg} / \mathrm{kg}$ & $\mathrm{J}$ & $\mathrm{IX}$ \\
\hline 105113 & $\mathrm{QC297379}$ & Magnesium & 137 & $\mathrm{mg} / \mathrm{kg}$ & $\mathrm{J}$ & $\mathrm{IX}$ \\
\hline 105113 & $9507546-01$ & Manganese & 11.1 & $\mathrm{mg} / \mathrm{kg}$ & & $\mathrm{X}$ \\
\hline 105113 & $\mathrm{QC297379}$ & Manganese & 22.2 & $\mathrm{mg} / \mathrm{kg}$ & & $\mathrm{X}$ \\
\hline 105113 & $9507546-01$ & Vanadium & 2.85 & $\mathrm{mg} / \mathrm{kg}$ & $\mathrm{J}$ & $\mathrm{CX}$ \\
\hline 105113 & QC297379 & Vanadium & 4.59 & $\mathrm{mg} / \mathrm{kg}$ & $\mathrm{J}$ & $\mathrm{CX}$ \\
\hline 105113 & $9507546-01$ & Zinc & 2.51 & $\mathrm{mg} / \mathrm{kg}$ & & $\mathrm{VX}$ \\
\hline 105113 & QC297379 & Zinc & 5.65 & $\mathrm{mg} / \mathrm{kg}$ & & $\mathrm{VX}$ \\
\hline
\end{tabular}

Laboratory blanks with analytical results showing concentrations greater than zero are considered to be contaminated. The laboratory blanks are assigned $\mathrm{AQ}=V$, and all samples in the same preparation batch are qualified based on the policy outlined in the Validation and Verification: Objective and Qualification Policy section of this report. Table 8 lists contaminated laboratory blanks for soil and water samples by analyte and preparation batch. 
Table 8. Contaminated Laboratory Blanks Listed by Analyte and Preparation Batch for GE and Roy F. Weston Analytical Laboratories, Inc. (WA)

\begin{tabular}{|c|c|c|c|c|}
\hline Analyte & Preparation Batch & $\begin{array}{l}\text { Detection } \\
\text { Limit }\end{array}$ & $\begin{array}{l}\text { Analytical } \\
\text { Result }\end{array}$ & Units \\
\hline Acetone & 70069 & 5 & 6.74 & $\mu \mathrm{g} / \mathrm{kg}$ \\
\hline Acetone & 95LVC143 & 10 & 1.33 & $\mu \mathrm{g} / \mathrm{kg}$ \\
\hline Acetone & 95LVC144 & 10 & 0.549 & $\mu \mathrm{g} / \mathrm{L}$ \\
\hline Silver & 69121 & 1.25 & 0.51 & $\mathrm{mg} / \mathrm{kg}$ \\
\hline Aluminum & 69121 & 12.5 & 1.595 & $\mathrm{mg} / \mathrm{kg}$ \\
\hline Aluminum & 69360 & 12.5 & 1.615 & $\mathrm{mg} / \mathrm{kg}$ \\
\hline Aluminum & 69557 & 0.025 & 0.0177 & $\mathrm{mg} / \mathrm{L}$ \\
\hline Aluminum & 70665 & 12.5 & 0.1 .16 & $\mathrm{mg} / \mathrm{kg}$ \\
\hline Aluminum & 71499 & 0.02 & 0.0219 & $\mathrm{mg} / \mathrm{L}$ \\
\hline $\begin{array}{l}\text { Bis(2-ethylhexyl) } \\
\text { phthalate }\end{array}$ & 69116 & 1 & 12.4 & $\mu \mathrm{g} / \mathrm{L}$ \\
\hline $\begin{array}{l}\text { Bis(2-ethylhexyl) } \\
\text { phthalate }\end{array}$ & 95LE1489 & 33 & 13.3 & $\mu \mathrm{g} / \mathrm{kg}$ \\
\hline Barium & 71499 & 0.003 & 0.000111 & $\mathrm{mg} / \mathrm{L}$ \\
\hline Benzoic acid & 95LE1489 & 165 & 55 & $\mu \mathrm{g} / \mathrm{kg}$ \\
\hline Calcium & 68857 & 12.5 & 2.3 & $\mathrm{mg} / \mathrm{kg}$ \\
\hline Calcium & 69360 & 12.5 & 1.21 & $\mathrm{mg} / \mathrm{kg}$ \\
\hline Calcium & 69557 & 0.25 & 0.0233 & $\mathrm{mg} / \mathrm{L}$ \\
\hline Calcium & 69699 & 12.5 & 2.21 & $\mathrm{mg} / \mathrm{kg}$ \\
\hline Calcium & 70665 & 12.5 & 2.73 & $\mathrm{mg} / \mathrm{kg}$ \\
\hline Calcium & 70670 & 12.5 & 0.84 & $\mathrm{mg} / \mathrm{kg}$ \\
\hline Calcium & 95L5711 & 22 & 3.4 & $\mathrm{mg} / \mathrm{kg}$ \\
\hline Cadmium & 71499 & 0.002 & 0.000172 & $\mathrm{mg} / \mathrm{L}$ \\
\hline $\begin{array}{l}\text { Dichloromethane } \\
\text { (Methylene chloride) } \\
\end{array}$ & 69617 & 0.5 & 1.37 & $\mu \mathrm{g} / \mathrm{L}$ \\
\hline $\begin{array}{l}\text { Dichloromethane } \\
\text { (Methylene chloride) } \\
\end{array}$ & 69770 & 0.5 & 5.66 & $\mu \mathrm{g} / \mathrm{kg}$ \\
\hline $\begin{array}{l}\text { Dichloromethane } \\
\text { (Methylene chloride) }\end{array}$ & 70069 & 0.5 & 1.12 & $\mu \mathrm{g} / \mathrm{kg}$ \\
\hline $\begin{array}{l}\text { Dichloromethane } \\
\text { (Methylene chloride) }\end{array}$ & 70272 & 0.5 & 0.56 & $\mu \mathrm{g} / \mathrm{kg}$ \\
\hline $\begin{array}{l}\text { Dichloromethane } \\
\text { (Methylene chloride) }\end{array}$ & 70327 & 0.5 & 0.95 & $\mu \mathrm{g} / \mathrm{L}$ \\
\hline $\begin{array}{l}\text { Dichloromethane } \\
\text { (Methylene chloride) }\end{array}$ & 70735 & 0.5 & 0.81 & $\mu \mathrm{g} / \mathrm{kg}$ \\
\hline $\begin{array}{l}\text { Dichloromethane } \\
\text { (Methylene chloride) }\end{array}$ & 70736 & 0.5 & 0.81 & $\mu \mathrm{g} / \mathrm{L}$ \\
\hline
\end{tabular}


Validation and Verification: Findings

Table 8. Contaminated Laboratory Blanks Listed by Analyte and Preparation Batch for GE and Roy F. Weston Analytical Laboratories, Inc. (WA), Continued

\begin{tabular}{|l|l|l|l|l|}
\hline Analyte & Preparation Batch & $\begin{array}{l}\text { Detection } \\
\text { Limit }\end{array}$ & $\begin{array}{l}\text { Analytical } \\
\text { Result }\end{array}$ & Units \\
\hline Cobalt & 71499 & 0.004 & 0.00045 & $\mathrm{mg} / \mathrm{L}$ \\
\hline Chromium & 68857 & 1.25 & 0.865 & $\mathrm{mg} / \mathrm{kg}$ \\
\hline Carbon disulfide & $95 \mathrm{LVC143}$ & 2 & 0.17 & $\mu \mathrm{g} / \mathrm{kg}$ \\
\hline Carbon disulfide & $95 \mathrm{LVC144}$ & 2 & 0.274 & $\mu \mathrm{gg} / \mathrm{L}$ \\
\hline Copper & 68857 & 1.25 & 0.6 & $\mathrm{mg} / \mathrm{kg}$ \\
\hline Copper & 69699 & 1.25 & 0.865 & $\mathrm{mg} / \mathrm{kg}$ \\
\hline Cyanide & $95 \mathrm{LC104A}$ & 0.92 & 0.158 & $\mathrm{mg} / \mathrm{kg}$ \\
\hline Di-n-butyl phthalate & 70236 & 32.4 & 44.7 & $\mu \mathrm{g} / \mathrm{kg}$ \\
\hline Di-n-butyl phthalate & $95 \mathrm{LE} 1489$ & 33 & 55 & $\mu \mathrm{g} / \mathrm{kg}$ \\
\hline Iron & 68857 & 5 & 2.435 & $\mathrm{mg} / \mathrm{kg}$ \\
\hline Iron & 69360 & 5 & 1.32 & $\mathrm{mg} / \mathrm{kg}$ \\
\hline Iron & 69699 & 5 & 0.775 & $\mathrm{mg} / \mathrm{kg}$ \\
\hline Iron & 70482 & 5 & 0.5 & $\mathrm{mg} / \mathrm{kg}$ \\
\hline Iron & 70670 & 5 & 0.915 & $\mathrm{mg} / \mathrm{kg}$ \\
\hline Mercury & 68862 & 0.0002 & 0.000023 & $\mathrm{mg} / \mathrm{L}$ \\
\hline Mercury & 69539 & 0.0002 & 0.000232 & $\mathrm{mg} / \mathrm{L}$ \\
\hline Mercury & 69542 & 0.0002 & 0.000083 & $\mathrm{mg} / \mathrm{L}$ \\
\hline Mercury & 69746 & 0.0334 & 0.00551 & $\mathrm{mg} / \mathrm{kg}$ \\
\hline Mercury & 69747 & 0.0334 & 0.00534 & $\mathrm{mg} / \mathrm{kg}$ \\
\hline Mercury & 70759 & 0.0334 & 0.0579 & $\mathrm{mg} / \mathrm{kg}$ \\
\hline Potassium-40 & 68912 & 0.0811 & 0.0917 & $\mathrm{pCi} / \mathrm{g}$ \\
\hline Potassium-40 & 69781 & 0.046 & 0.0762 & $\mathrm{pCi} / \mathrm{g}$ \\
\hline Potassium-40 & 70113 & 0.0457 & 0.103 & $\mathrm{pCi} / \mathrm{g}$ \\
\hline Toluene & 69770 & 0.5 & 0.18 & $\mu \mathrm{gg} / \mathrm{kg}$ \\
\hline Methyl ethyl ketone & 69617 & 1 & 4.78 & $\mu \mathrm{L} / \mathrm{L}$ \\
\hline Methyl ethyl ketone & 69770 & 1 & 0.95 & $\mu \mathrm{g} / \mathrm{kg}$ \\
\hline Methyl ethyl ketone & 70069 & 1 & 1.24 & $\mu \mathrm{g} / \mathrm{kg}$ \\
\hline Methyl ethyl ketone & $95 \mathrm{LVC143}$ & 10 & 0.174 & $\mu \mathrm{gg} / \mathrm{kg}$ \\
\hline Magnesium & 68857 & 5 & 1.58 & $\mathrm{mg} / \mathrm{kg}$ \\
\hline Magnesium & 69360 & 5 & 1.54 & $\mathrm{mg} / \mathrm{kg}$ \\
\hline Manganese & 68857 & 0.5 & 0.219 & $\mathrm{mg} / \mathrm{kg}$ \\
\hline Sodium & $95 \mathrm{~L} 5711$ & 60 & 60 & $\mathrm{mg} / \mathrm{kg}$ \\
\hline Nitrate-nitrite as & $95 \mathrm{LNS} 102$ & 0.6 & $\mathrm{mg} / \mathrm{kg}$ \\
\hline neadtrogen & & 0.00925 & 0.0231 & $\mathrm{pCi} / \mathrm{g}$ \\
\hline Lead-212 & 68912 & 0.0178 & $\mathrm{pCi} / \mathrm{g}$ \\
\hline
\end{tabular}


Table 8. Contaminated Laboratory Blanks Listed by Analyte and Preparation Batch for GE and Roy F. Weston Analytical Laboratories, Inc. (WA), Continued

\begin{tabular}{|c|c|c|c|c|}
\hline Analyte & Preparation Batch & $\begin{array}{l}\text { Detection } \\
\text { Limit }\end{array}$ & $\begin{array}{l}\text { Analytical } \\
\text { Result }\end{array}$ & Units \\
\hline Lead & 69115 & 0.005 & 0.00308 & $\mathrm{mg} / \mathrm{L}$ \\
\hline Plutonium-238 & 9507075 & 0.06 & 0.14 & $\mathrm{pCi} / \mathrm{g}$ \\
\hline Plutonium-239/240 & 68823 & 0.00817 & 0.00848 & $\mathrm{pCi} / \mathrm{g}$ \\
\hline Radium-226 & 69935 & 0.00962 & 0.0136 & $\mathrm{pCi} / \mathrm{g}$ \\
\hline Selenium & 70145 & 0.005 & 0.00176 & $\mathrm{mg} / \mathrm{L}$ \\
\hline Strontium-90 & 69334 & 0.0123 & 0.163 & $\mathrm{pCi} / \mathrm{g}$ \\
\hline Strontium-90 & 69940 & 0.0368 & 0.37 & $\mathrm{pCi} / \mathrm{g}$ \\
\hline Strontium-90 & 71514 & 0.0632 & 0.15 & $\mathrm{pCi} / \mathrm{g}$ \\
\hline Strontium-90 & 9507075 & 0.76 & 0.7 & $\mathrm{pCi} / \mathrm{g}$ \\
\hline Thorium-234 & 68912 & 0.202 & 0.315 & $\mathrm{pCi} / \mathrm{g}$ \\
\hline Thorium-234 & 69935 & 0.206 & 0.476 & $\mathrm{pCi} / \mathrm{g}$ \\
\hline Thorium-234 & 70113 & 0.163 & 0.183 & $\mathrm{pCi} / \mathrm{g}$ \\
\hline Thorium-234 & 71004 & 0.186 & 0.295 & $\mathrm{pCi} / \mathrm{g}$ \\
\hline Thallium & 69121 & 15 & 2.43 & $\mathrm{mg} / \mathrm{kg}$ \\
\hline Thallium & 95L5710 & 1.6 & 0.19 & $\mathrm{mg} / \mathrm{kg}$ \\
\hline Total organic carbon & 70130 & 100 & 56.7 & $\mathrm{mg} / \mathrm{kg}$ \\
\hline Total organic carbon & 71397 & 100 & 44 & $\mathrm{mg} / \mathrm{kg}$ \\
\hline Total organic carbon & 71400 & 100 & 30.3 & $\mathrm{mg} / \mathrm{kg}$ \\
\hline Total organic carbon & 71402 & 100 & 51.4 & $\mathrm{mg} / \mathrm{kg}$ \\
\hline Total organic carbon & 95LTZ15A & 8.4 & 0.2 & $\mathrm{mg} / \mathrm{kg}$ \\
\hline Total organic nitrogen & 95LAM062 & 125 & 22.2 & $\mathrm{mg} / \mathrm{kg}$ \\
\hline 2-Hexanone & 69617 & 1 & 5.44 & $\mu \mathrm{g} / \mathrm{L}$ \\
\hline 2-Hexanone & 69770 & 1 & 1.95 & $\mu \mathrm{g} / \mathrm{kg}$ \\
\hline 2-Hexanone & 70216 & 1 & 3.18 & $\mu \mathrm{g} / \mathrm{kg}$ \\
\hline 2-Hexanone & 70217 & 1 & 3.18 & $\mu \mathrm{g} / \mathrm{L}$ \\
\hline 2-Hexanone & 70272 & 1 & 2.79 & $\mu \mathrm{g} / \mathrm{kg}$ \\
\hline 2-Hexanone & 70526 & 1 & 2 & $\mu \mathrm{g} / \mathrm{kg}$ \\
\hline 2-Hexanone & 70735 & 1 & 1.72 & $\mu \mathrm{g} / \mathrm{kg}$ \\
\hline 2-Hexanone & 70736 & 1 & 1.72 & $\mu \mathrm{g} / \mathrm{L}$ \\
\hline Zinc & 68857 & 1 & 0.219 & $\mathrm{mg} / \mathrm{kg}$ \\
\hline Zinc & 69121 & 1 & 0.2695 & $\mathrm{mg} / \mathrm{kg}$ \\
\hline Zinc & 69699 & 1 & 0.4875 & $\mathrm{mg} / \mathrm{kg}$ \\
\hline Zinc & 70665 & 1 & 0.171 & $\mathrm{mg} / \mathrm{kg}$ \\
\hline
\end{tabular}


Tables 9 and 10 list matrix spikes for soil and water samples with their assigned qualifiers. These rejected records were not to be used for any further qualification of calculations. RQ $=R$ and $\mathrm{AQ}=4$ when there were matrix interferences. $\mathrm{AQ}=Y$ if the results were obtained from unpreserved or improperly preserved samples.

Table 9. Matrix Spikes Qualified with $R 4$ for GE and WA

\begin{tabular}{|l|l|l|l|l|l|l|}
\hline Sample ID & Analyte & $\begin{array}{l}\text { Sample } \\
\text { Conc. }\end{array}$ & $\begin{array}{l}\text { Nominal Conc. } \\
\text { (Spiked Amount) }\end{array}$ & Units & RQ & AQ \\
\hline 104602 & Sulfide & 270 & 43.2 & $\mathrm{mg} / \mathrm{kg}$ & $\mathrm{R}$ & 4 \\
\hline 104623 & Orthophosphate & 30.3 & 15.1 & $\mathrm{mg} / \mathrm{kg}$ & $\mathrm{R}$ & 4 \\
\hline 104630 & Sulfide & 342 & 54.8 & $\mathrm{mg} / \mathrm{kg}$ & $\mathrm{R}$ & 4 \\
\hline 104637 & Sulfide & 294 & 47.1 & $\mathrm{mg} / \mathrm{kg}$ & $\mathrm{R}:$ & 4 \\
\hline 104639 & Arsenic & & 4.6 & $\mathrm{mg} / \mathrm{kg}$ & $\mathrm{R}$ & $\mathrm{Y}$ \\
\hline 104639 & Selenium & & 1.2 & $\mathrm{mg} / \mathrm{kg}$ & $\mathrm{R}$ & $\mathrm{Y}$ \\
\hline 104672 & Aldrin & 49.6 & 3.97 & $\mu \mathrm{g} / \mathrm{kg}$ & $\mathrm{R}$ & 4 \\
\hline 104672 & Dieldrin & 99.2 & 9.92 & $\mu \mathrm{g} / \mathrm{kg}$ & $\mathrm{R}$ & 4 \\
\hline 104672 & Endrin & 64.6 & 9.92 & $\mu \mathrm{g} / \mathrm{kg}$ & $\mathrm{R}$ & 4 \\
\hline 104672 & Heptachlor & 99.2 & 3.97 & $\mu \mathrm{g} / \mathrm{kg}$ & $\mathrm{R}$ & 4 \\
\hline 104672 & Lindane & 49.6 & 3.97 & $\mu \mathrm{g} / \mathrm{kg}$ & $\mathrm{R}$ & 4 \\
\hline 104672 & p,p'-DDT & 198 & 9.92 & $\mu \mathrm{g} / \mathrm{kg}$ & $\mathrm{R}$ & 4 \\
\hline 105101 & Phenols & 369 & 922 & $\mu \mathrm{g} / \mathrm{kg}$ & $\mathrm{R}$ & 4 \\
\hline 105102 & Orthophosphate & 13 & 6.49 & $\mathrm{mg} / \mathrm{kg}$ & $\mathrm{R}$ & 4 \\
\hline 105110 & Total phosphates & 7.22 & 2.15 & $\mathrm{mg} / \mathrm{kg}$ & $\mathrm{R}$ & 4 \\
\hline & (as P) & & & & & \\
\hline
\end{tabular}

If the nominal concentration (spiked amount) of a sample was less than or equal to $25 \%$ of the sample result, $\mathrm{RQ}=R$ and $\mathrm{AQ}=5$ for the matrix spike. $\mathrm{AQ}=Y$ if the results were obtained from unpreserved or improperly preserved samples.

Table 10. Matrix Spikes Qualified with $R 5$ for GE and WA

\begin{tabular}{|c|c|c|c|c|c|c|}
\hline Sample ID & Analyte & $\begin{array}{l}\text { Standard } \\
\text { Conc. }\end{array}$ & $\begin{array}{l}\text { Nominal Conc. } \\
\text { (Spiked Amount) }\end{array}$ & Units & $\mathbf{R Q}$ & AQ \\
\hline 103622 & Iron & 2.67 & 0.2 & $\mathrm{mg} / \mathrm{L}$ & $\mathrm{R}$ & 5 \\
\hline 103622 & Iron & 2.67 & 0.2 & $\mathrm{mg} / \mathrm{L}$ & $R$ & 5 \\
\hline 103624 & Nonvolatile beta & 189 & 42.4 & $\mathrm{pCi} / \mathrm{g}$ & $\mathrm{R}$ & 5 \\
\hline 103632 & Aluminum & 1330 & 49 & $\mathrm{mg} / \mathrm{kg}$ & $\mathrm{R}$ & 5 \\
\hline 103632 & Aluminum & 1330 & 49 & $\mathrm{mg} / \mathrm{kg}$ & $\mathrm{R}$ & 5 \\
\hline 103632 & Iron & 1450 & 49 & $\mathrm{mg} / \mathrm{kg}$ & $\mathrm{R}$ & 5 \\
\hline 103632 & Iron & 1450 & 49 & $\mathrm{mg} / \mathrm{kg}$ & $\mathrm{R}$ & 5 \\
\hline 104600 & Aluminum & 2860 & 51.1 & $\mathrm{mg} / \mathrm{kg}$ & $\mathrm{R}$ & 5 \\
\hline 104600 & Aluminum & 2860 & 48.9 & $\mathrm{mg} / \mathrm{kg}$ & $\mathrm{R}$ & 5 \\
\hline
\end{tabular}


Table 10. Matrix Spikes Qualified with $R 5$ for GE and WA, Continued

\begin{tabular}{|c|c|c|c|c|c|c|}
\hline Sample ID & Analyte & $\begin{array}{l}\text { Standard } \\
\text { Conc. }\end{array}$ & $\begin{array}{l}\text { Nominal Conc. } \\
\text { (Spiked Amount) }\end{array}$ & Units & RQ & $\mathbf{A Q}$ \\
\hline 104600 & Iron & 3400 & 51.1 & $\mathrm{mg} / \mathrm{kg}$ & $\mathrm{R}$ & 5 \\
\hline 104600 & Iron & 3400 & 48.9 & $\mathrm{mg} / \mathrm{kg}$ & $\mathrm{R}$ & 5 \\
\hline 104602 & $\begin{array}{l}\text { Total phosphates } \\
\text { (as P) }\end{array}$ & 136 & 5.92 & $\mathrm{mg} / \mathrm{kg}$ & $\mathrm{R}$ & 5 \\
\hline 104605 & Aluminum & 3410 & 53.7 & $\mathrm{mg} / \mathrm{kg}$ & $R$ & 5 \\
\hline 104605 & Aluminum & 3410 & 57.5 & $\mathrm{mg} / \mathrm{kg}$ & $\mathrm{R}$ & 5 \\
\hline 104605 & Iron & 2470 & 53.7 & $\mathrm{mg} / \mathrm{kg}$ & $R$ & 5 \\
\hline 104605 & Iron & 2470 & 57.5 & $\mathrm{mg} / \mathrm{kg}$ & $\mathrm{R}$ & 5 \\
\hline 104613 & $\begin{array}{l}\text { Total phosphates } \\
\text { (as P) }\end{array}$ & 76.5 & 10.8 & $\mathrm{mg} / \mathrm{kg}$ & $\mathrm{R}$ & 5 \\
\hline 104614 & Aluminum & 1550 & 45.3 & $\mathrm{mg} / \mathrm{kg}$ & $\mathrm{R}$ & 5 \\
\hline 104614 & Aluminum & 1550 & 49.8 & $\mathrm{mg} / \mathrm{kg}$ & $R$ & 5 \\
\hline 104614 & Iron & 975 & 45.3 & $\mathrm{mg} / \mathrm{kg}$ & $\mathrm{R}$ & 5 \\
\hline 104614 & Iron & 975 & 49.8 & $\mathrm{mg} / \mathrm{kg}$ & $\mathrm{R}$ & 5 \\
\hline 104623 & $\begin{array}{l}\text { Total phosphates } \\
\text { (as P) }\end{array}$ & 115 & 5.81 & $\mathrm{mg} / \mathrm{kg}$ & $\mathrm{R}$ & 5 \\
\hline 104625 & Aluminum & 15900 & 121 & $\mathrm{mg} / \mathrm{kg}$ & $\mathrm{R}$ & 5 \\
\hline 104625 & Aluminum & 15900 & 121 & $\mathrm{mg} / \mathrm{kg}$ & $R$ & 5 \\
\hline 104625 & Iron & 7570 & 121 & $\mathrm{mg} / \mathrm{kg}$ & $\mathrm{R}$ & 5 \\
\hline 104625 & Iron & 7570 & 121 & $\mathrm{mg} / \mathrm{kg}$ & $R$ & 5 \\
\hline 104639 & Aluminum & 2230 & 230 & $\mathrm{mg} / \mathrm{kg}$ & $R$ & Y5 \\
\hline 104639 & Iron & 6610 & 115 & $\mathrm{mg} / \mathrm{kg}$ & $\mathrm{R}$ & Y5 \\
\hline 104648 & $\begin{array}{l}\text { Total phosphates } \\
\text { (as P) }\end{array}$ & 54.9 & 10.9 & $\mathrm{mg} / \mathrm{kg}$ & $\mathrm{R}$ & 5 \\
\hline 104650 & Aluminum & 2860 & 47.6 & $\mathrm{mg} / \mathrm{kg}$ & $\mathrm{R}$ & 5 \\
\hline 104650 & Aluminum & 2860 & 48.1 & $\mathrm{mg} / \mathrm{kg}$ & $\mathrm{R}$ & 5 \\
\hline 104650 & Iron & 450 & 47.6 & $\mathrm{mg} / \mathrm{kg}$ & $\mathrm{R}$ & 5 \\
\hline 104650 & Iron & 450 & $48.1:$ & $\mathrm{mg} / \mathrm{kg}$ & $\mathrm{R}$ & 5 \\
\hline 104657 & $\begin{array}{l}\text { Total phosphates } \\
\text { (as P) }\end{array}$ & 195 & 35 & $\mathrm{mg} / \mathrm{kg}$ & $R$ & 5 \\
\hline 104664 & Aluminum & 2380 & 56 & $\mathrm{mg} / \mathrm{kg}$ & $\mathrm{R}$ & 5 \\
\hline 104664 & Aluminum & 2380 & 57.1 & $\mathrm{mg} / \mathrm{kg}$ & $\mathrm{R}$ & 5 \\
\hline 104664 & Iron & 918 & 56 & $\mathrm{mg} / \mathrm{kg}$ & $\mathrm{R}$ & 5 \\
\hline 104664 & Iron & 918 & 57.1 & $\mathrm{mg} / \mathrm{kg}$ & $\mathrm{R}$ & 5 \\
\hline 104667 & Aluminum & 3810 & 57.6 & $\mathrm{mg} / \mathrm{kg}$ & $\mathrm{R}$ & 5 \\
\hline 104667 & Aluminum & 3810 & 58.1 & $\mathrm{mg} / \mathrm{kg}$ & $\mathrm{R}$ & 5 \\
\hline 104667 & Iron & 4680 & 57.6 & $\mathrm{mg} / \mathrm{kg}$ & $\mathrm{R}$ & 5 \\
\hline
\end{tabular}


Table 10. Matrix Spikes Qualified with $R 5$ for GE and WA, Continued

\begin{tabular}{|c|c|c|c|c|c|c|}
\hline Sample ID & Analyte & $\begin{array}{l}\text { Standard } \\
\text { Conc. }\end{array}$ & $\begin{array}{l}\text { Nominal Conc. } \\
\text { (Spiked Amount) }\end{array}$ & Units & $\mathbf{R Q}$ & $\mathbf{A Q}$ \\
\hline 104667 & Iron & 4680 & 58.1 & $\mathrm{mg} / \mathrm{kg}$ & $R$ & 5 \\
\hline 104668 & \begin{tabular}{|l} 
Total phosphates \\
(as P)
\end{tabular} & 151 & 23.4 & $\mathrm{mg} / \mathrm{kg}$ & $\mathrm{R}$ & 5 \\
\hline 104671 & Aluminum & 11100 & 81.9 & $\mathrm{mg} / \mathrm{kg}$ & $\mathrm{R}$ & 5 \\
\hline 104671 & Aluminum & 11100 & 88.6 & $\mathrm{mg} / \mathrm{kg}$ & $\mathrm{R}$ & 5 \\
\hline 104671 & Iron & 23500 & 81.9 & $\mathrm{mg} / \mathrm{kg}$ & $\mathrm{R}$ & 5 \\
\hline 104671 & Iron & 23500 & 88.6 & $\mathrm{mg} / \mathrm{kg}$ & $\mathrm{R}$ & 5 \\
\hline 104671 & Magnesium & 1410 & 81.9 & $\mathrm{mg} / \mathrm{kg}$ & $R$ & 5 \\
\hline 104671 & Magnesium & 1410 & 88.6 & $\mathrm{mg} / \mathrm{kg}$ & $\mathrm{R}$ & 5 \\
\hline 104676 & $\begin{array}{l}\text { Nitrogen by } \\
\text { Kjeldahl method }\end{array}$ & 831 & 35.2 & $\mathrm{mg} / \mathrm{kg}$ & $\mathrm{R}$ & 5 \\
\hline 104680 & Aluminum & 1060 & 56.8 & $\mathrm{mg} / \mathrm{kg}$ & $\mathrm{R}$ & 5 \\
\hline 104680 & Aluminum & 1060 & 58.5 & $\mathrm{mg} / \mathrm{kg}$ & $\mathrm{R}$ & 5 \\
\hline 104680 & Iron & 887 & 56.8 & $\mathrm{mg} / \mathrm{kg}$ & $\mathrm{R}$ & 5 \\
\hline 104680 & Iron & 887 & 58.5 & $\mathrm{mg} / \mathrm{kg}$ & $\mathrm{R}$ & 5 \\
\hline 104683 & Aluminum & 5270 & 58.1 & $\mathrm{mg} / \mathrm{kg}$ & $\mathrm{R}$ & 5 \\
\hline 104683 & Aluminum & 5270 & 59.2 & $\mathrm{mg} / \mathrm{kg}$ & $\mathrm{R}$ & 5 \\
\hline 104683 & Iron & 2000 & 58.1 & $\mathrm{mg} / \mathrm{kg}$ & $\mathrm{R}$ & 5 \\
\hline 104683 & Iron & 2000 & 59.2 & $\mathrm{mg} / \mathrm{kg}$ & $\mathrm{R}$ & 5 \\
\hline 104683 & Magnesium & 627 & 58.1 & $\mathrm{mg} / \mathrm{kg}$ & $\mathrm{R}$ & 5 \\
\hline 104683 & Magnesium & 627 & 59.2 & $\mathrm{mg} / \mathrm{kg}$ & R & 5 \\
\hline 104687 & $\begin{array}{l}\text { Nitrogen by } \\
\text { Kjeldahl method }\end{array}$ & 466 & 42.4 & $\mathrm{mg} / \mathrm{kg}$ & $\mathrm{R}$ & 5 \\
\hline 104691 & $\begin{array}{l}\text { Total phosphates } \\
\text { (as P) }\end{array}$ & 222 & 18.8 & $\mathrm{mg} / \mathrm{kg}$ & $\mathrm{R}$ & 5 \\
\hline 104693 & Aluminum & 5100 & 54.6 & $\mathrm{mg} / \mathrm{kg}$ & $\mathrm{R}$ & 5 \\
\hline 104693 & Aluminum & 5100 & 53.6 & $\mathrm{mg} / \mathrm{kg}$ & $\mathrm{R}$ & 5 \\
\hline 104693 & Iron & 1550 & 54.6 & $\mathrm{mg} / \mathrm{kg}$ & $\mathrm{R}$ & 5 \\
\hline 104693 & Iron & 1550 & 53.6 & $\mathrm{mg} / \mathrm{kg}$ & $\mathrm{R}$ & 5 \\
\hline 104736 & Aluminum & 19100 & 49.5 & $\mathrm{mg} / \mathrm{kg}$ & $\mathrm{R}$ & 5 \\
\hline 104736 & Aluminum & 19100 & 47.6 & $\mathrm{mg} / \mathrm{kg}$ & $\mathrm{R}$ & 5 \\
\hline 104736 & Iron & 20000 & 49.5 & $\mathrm{mg} / \mathrm{kg}$ & $\mathrm{R}$ & 5 \\
\hline 104736 & Iron & 20000 & 47.6 & $\mathrm{mg} / \mathrm{kg}$ & $\mathrm{R}$ & 5 \\
\hline 104736 & Magnesium & 255 & 49.5 & $\mathrm{mg} / \mathrm{kg}$ & $R$ & 5 \\
\hline 104736 & Magnesium & 255 & 47.6 & $\mathrm{mg} / \mathrm{kg}$ & $\mathrm{R}$ & 5 \\
\hline 105101 & $\begin{array}{l}\text { Nitrogen by } \\
\text { Kjeldahl method }\end{array}$ & 141 & 24.1 & $\mathrm{mg} / \mathrm{kg}$ & $\mathrm{R}$ & 5 \\
\hline 105102 & Total phosphates & 408 & 24.7 & $\mathrm{mg} / \mathrm{kg}$ & $\mathrm{R}$ & 5 \\
\hline
\end{tabular}

Par Pond, Pond C, and L Lake, Revision 2 
Table 10. Matrix Spikes Qualified with $R 5$ for GE and WA, Continued

\begin{tabular}{|c|c|c|c|c|c|c|}
\hline Sample ID & Analyte & $\begin{array}{l}\text { Standard } \\
\text { Conc. }\end{array}$ & $\begin{array}{l}\text { Nominal Conc. } \\
\text { (Spiked Amount) }\end{array}$ & Units & $\mathbf{R Q}$ & $\mathbf{A Q}$ \\
\hline & (as P) & & & & & \\
\hline 105110 & Aluminum & 927 & 55.7 & $\mathrm{mg} / \mathrm{kg}$ & $\mathrm{R}$ & 5 \\
\hline 105110 & Aluminum & 927 & 56.3 & $\mathrm{mg} / \mathrm{kg}$ & $\mathrm{R}$ & 5 \\
\hline 105110 & Iron & 1210 & 55.7 & $\mathrm{mg} / \mathrm{kg}$ & $\mathrm{R}$ & 5 \\
\hline 105110 & Iron & 1210 & 56.3 & $\mathrm{mg} / \mathrm{kg}$ & $\mathrm{R}$ & 5 \\
\hline 105111 & $\begin{array}{l}\text { Nitrogen by } \\
\text { Kjeldahl method }\end{array}$ & 209 & 23.2 & $\mathrm{mg} / \mathrm{kg}$ & $R$ & 5 \\
\hline 105111 & $\begin{array}{l}\text { Total phosphates } \\
\text { (as P) }\end{array}$ & 6.47 & 2.07 & $\mathrm{mg} / \mathrm{kg}$ & $\mathrm{R}$ & 5 \\
\hline 105113 & Aluminum & 1180 & 58.4 & $\mathrm{mg} / \mathrm{kg}$ & $\mathrm{R}$ & 5 \\
\hline 105113 & Aluminum & 1180 & 58.7 & $\mathrm{mg} / \mathrm{kg}$ & $\mathrm{R}$ & 5 \\
\hline 105113 & Iron & 967 & 58.4 & $\mathrm{mg} / \mathrm{kg}$ & $\mathrm{R}$ & 5 \\
\hline 105113 & Iron & 967 & 58.7 & $\mathrm{mg} / \mathrm{kg}$ & $R$ & 5 \\
\hline 105237 & Benzene & 0.595 & 2 & $\mu \mathrm{g} / \mathrm{kg}$ & $R$ & 5 \\
\hline 105237 & Benzene & 0.595 & 2 & $\mu \mathrm{g} / \mathrm{kg}$ & $\mathrm{R}$ & 5 \\
\hline 105237 & Chlorobenzene & 0.0595 & 2 & $\mu \mathrm{g} / \mathrm{kg}$ & $\mathrm{R}$ & 5 \\
\hline 105237 & Chlorobenzene & 0.0595 & 2 & $\mu \mathrm{g} / \mathrm{kg}$ & $R$ & 5 \\
\hline 105237 & Toluene & 0.452 & 2 & $\mu \mathrm{g} / \mathrm{kg}$ & $\mathrm{R}$ & 5 \\
\hline 105237 & Toluene & 0.452 & 2 & $\mu \mathrm{g} / \mathrm{kg}$ & $R$ & 5 \\
\hline 105237 & Trichloroethylene & 0.0595 & 2 & $\mu \mathrm{g} / \mathrm{kg}$ & $R$ & 5 \\
\hline 105237 & Trichloroethylene & 0.0595 & 2 & $\mu \mathrm{g} / \mathrm{kg}$ & $\mathrm{R}$ & 5 \\
\hline 105237 & $\begin{array}{l}1,1- \\
\text { Dichloroethylene }\end{array}$ & 0.0595 & 2 & $\mu \mathrm{g} / \mathrm{kg}$ & $\mathrm{R}$ & 5 \\
\hline 105237 & $\begin{array}{l}\text { 1,1- } \\
\text { Dichloroethylene }\end{array}$ & 0.0595 & 2 & $\mu \mathrm{g} / \mathrm{kg}$ & $\mathrm{R}$ & 5 \\
\hline FSB 93 & Calcium & 27.9 & 1 & $\mathrm{mg} / \mathrm{L}$ & $\mathrm{R}$ & 5 \\
\hline FSB 93 & Calcium & 27.9 & 1 & $\mathrm{mg} / \mathrm{L}$ & $\mathrm{R}$ & 5 \\
\hline FSB 93 & Magnesium & 8.35 & 1 & $\mathrm{mg} / \mathrm{L}$ & $\mathrm{R}$ & 5 \\
\hline FSB 93 & Magnesium & 8.35 & 1 & $\mathrm{mg} / \mathrm{L}$ & $\mathrm{R}$ & 5 \\
\hline FSB105 & Aluminum & 6.96 & 0.5 & $\mathrm{mg} / \mathrm{L}$ & $\mathrm{R}$ & 5 \\
\hline FSB105 & Aluminum & 6.96 & 0.5 & $\mathrm{mg} / \mathrm{L}$ & $\mathrm{R}$ & 5 \\
\hline
\end{tabular}


Table 11 lists surrogates for soil samples that were outside acceptance criteria and assigned $R O$ qualifiers. The surrogates were rejected because sample quantitation required dilutions that resulted in poor surrogate recovery. The surrogates were assigned $\mathrm{RQ}=R$ and $\mathrm{AQ}=O$. No qualification of the samples was required.

Table 11. Surrogates with RO Qualifiers for GE

\begin{tabular}{|l|l|l|l|l|l|}
\hline Sample ID & Analyte & Units & $\begin{array}{l}\text { Nominal Conc. } \\
\text { (Spiked Amount) }\end{array}$ & RQ & AQ \\
\hline 104648 & Dibutylchlorendate & $\mu \mathrm{g} / \mathrm{kg}$ & 20 & $\mathrm{R}$ & $\mathrm{O}$ \\
\hline 104648 & Tetrachloro-m-xylene & $\mu \mathrm{g} / \mathrm{kg}$ & 20 & $\mathrm{R}$ & $\mathrm{O}$ \\
\hline 104652 & Dibutylchlorendate & $\mu \mathrm{g} / \mathrm{kg}$ & 100 & $\mathrm{R}$ & $\mathrm{O}$ \\
\hline 104652 & Tetrachloro-m-xylene & $\mu \mathrm{g} / \mathrm{kg}$ & 100 & $\mathrm{R}$ & $\mathrm{O}$ \\
\hline 104655 & Tetrachloro-m-xylene & $\mu \mathrm{g} / \mathrm{kg}$ & 10 & $\mathrm{R}$ & $\mathrm{O}$ \\
\hline 104656 & Dibutylchlorendate & $\mu \mathrm{g} / \mathrm{kg}$ & 20 & $\mathrm{R}$ & $\mathrm{O}$ \\
\hline 104656 & Tetrachloro-m-xylene & $\mu \mathrm{g} / \mathrm{kg}$ & 20 & $\mathrm{R}$ & $\mathrm{O}$ \\
\hline 104657 & Dibutylchlorendate & $\mu \mathrm{g} / \mathrm{kg}$ & 20 & $\mathrm{R}$ & $\mathrm{O}$ \\
\hline 104657 & Tetrachloro-m-xylene & $\mu \mathrm{g} / \mathrm{kg}$ & 20 & $\mathrm{R}$ & $\mathrm{O}$ \\
\hline 104658 & Dibutylchlorendate & $\mu \mathrm{g} / \mathrm{kg}$ & 20 & $\mathrm{R}$ & $\mathrm{O}$ \\
\hline 104658 & Tetrachloro-m-xylene & $\mu \mathrm{g} / \mathrm{kg}$ & 20 & $\mathrm{R}$ & $\mathrm{O}$ \\
\hline 104673 & Dibutylchlorendate & $\mu \mathrm{g} / \mathrm{kg}$ & 50 & $\mathrm{R}$ & $\mathrm{O}$ \\
\hline 105117 & Dibutylchlorendate & $\mu \mathrm{g} / \mathrm{kg}$ & 50 & $\mathrm{R}$ & $\mathrm{O}$ \\
\hline 105117 & Tetrachloro-m-xylene & $\mu \mathrm{g} / \mathrm{kg}$ & 50 & $\mathrm{R}$ & $\mathrm{O}$ \\
\hline
\end{tabular}

\section{Review of the Analytical Narratives}

The analytical laboratories provided the following narratives concerning samples they analyzed for the Par Pond sediment characterization project. The excerpts provided here are as stated by the laboratories and contain only information that may affect the sample analysis or results.

\section{Roy F. Weston Analytical Laboratories, Inc.}

The following entries reflect the analytical narratives compiled by WA. The italicized identifiers following the analytical suite refer to the laboratory batch number.

\section{Metals}

\section{$9507 L 559$}

- All preparation blank results were below method detection limits (MDL) with the exception of calcium, sodium, and thallium.

- All matrix spike recoveries were within the control limits with the exception of aluminum, arsenic, iron, and selenium. 
- Relative percent differences (RPDs) for replicate results were within control limits with the exception of cobalt, lead, and thallium.

- The post digestion spike recoveries for arsenic in sample ID 104649 and selenium in sample ID 104639 were outside the control limits of $85-115 \%$, while the samples' absorbance/concentration was less than $50 \%$ of the spike absorbance/concentration.

- The inductively coupled plasma (ICP) serial dilution for aluminum, iron, and manganese for sample ID 104639 was outside the 10\% difference criteria.

\section{L581}

- All preparation blank results were below the MDL with the exception of calcium, sodium, and thallium.

- The post-digestion spike recovery for selenium for sample ID 104659 was outside the control limits of $85-115 \%$, while the sample absorbance/concentration was less than $50 \%$ of the spike absorbance/concentration.

\section{Inorganics}

$9507 L 559$

- Method blanks were within method criteria with the exception of cyanide for lab ID 95LC104A-MB1 and total organic carbon for lab ID 95LTZ017-MB1.

- Matrix spike recoveries were within the 75-125\% control limits with the exception of orthophosphate for sample ID 104647 . Recovery results of $4.2 \%$ and $4.3 \%$ (measured $7 / 26 / 95$ and 08/01/95, respectively) were obtained for orthophosphate for this sample. The probable cause of poor recoveries is matrix interference. Matrix spike duplicates were within the $20 \%$ RPD control limits.

- Replicate analyses were within the $20 \%$ RPD control limits with the exception of phenol for sample ID 104647.

- The results reported for phenols are based on five gram aliquots of sample used for the distillation preparation.

- All samples were received at a temperature higher than $6^{\circ} \mathrm{C}$.

9507L581

- All method blanks were within method criteria with the exception of cyanide for lab ID 95LC104A-MB1, nitrate-nitrite for lab ID 95LNS102-MB1, total organic nitrogen for lab ID 95LAM062-MB1, and total organic carboin for lab ID 95LTZ15A-MB1.

- Replicate analyses were within the $20 \%$ RPD control limits with the exception of phenol.

- The results reported for phenols are based on five gram aliquots of sample used for the distillation preparation.

\section{Pesticides/PCBs}

9507L559

- Two of 14 surrogate recoveries were outside acceptance criteria.

- All samples were received at a temperature higher than $6^{\circ} \mathrm{C}$. 


\section{GC/MS Volatiles}

$9507 L 559$

- Tentatively identified compounds (TICs) were detected in the samples.

- Seven of 27 surrogate recoveries were outside EPA quality control (EPA QC) limits. Sample IDs 104642, 104639, and 104649 were reanalyzed on 07/25/95 and reported. The out-of-limit recovery in method blank laboratory ID 94LVCB43-MB1 was only slightly high, and the data were not adversely affected.

- The method blanks contained the common laboratory contaminant acetone and the target compounds 2-butanone and carbon disulfide at levels less than the contractrequired quantitation level (CRQL).

- The samples were received at a temperature higher than $6^{\circ} \mathrm{C}$.

- The water sample in this batch was analyzed under the same analytical conditions and calibrations as the soil samples.

$9507 L 581$

- TICs were detected in the samples.

- The method blanks contained the common laboratory contaminant acetone and the target compound carbon disulfide at levels less than CRQL. The method blank laboratory ID 95LVC143-MB1 also contained the target compound 2-butanone at a level less than the CRQL.

- The water sample in this batch was analyzed under the same analytical conditions and calibrations as the soil sample.

\section{GC Volatiles}

$9507 L 559$

- The samples were not packaged and stored as specified in the method protocol.

- All samples were received at a temperature higher than $6^{\circ} \mathrm{C}$.

\section{Semivolatiles}

\section{$9507 L 559$}

- Method 8270 was modified to achieve lower reporting limits by concentrating the extracts to $1 / 10$ the specified final volume (with appropriate adjustment to spiking levels).

- TICs were detected in the samples.

- One of 11 matrix spike recoveries was outside EPA QC limits.

- The method blank contained the common laboratory contaminants di-n-butylphthalate and bis(2-ethylhexyl)phthalate at levels less than two times the CRQL and the target compound benzoic acid at a level less than the CRQL.

- All samples were received at a temperature higher than $6^{\circ} \mathrm{C}$. 


\section{$9507 L 581$}

- Method 8270 was modified to achieve lower reporting limits by concentrating the extracts to $1 / 10$ the specified final volume (with appropriate adjustment to spiking levels).

- TICs were detected in the samples.

- The method blank contained the common laboratory contaminants di-n-butylphthalate and bis(2-ethylhexyl)phthalate at levels less than two times the CRQL and the target compound benzoic acid at a level less than the CRQL.

\section{General Engineering Laboratories, Inc.}

The following entries reflect the analytical narratives compiled by General Engineering Laboratories, Inc. (GE). The italicized identifiers following the analytical suite refer to the electronic data file delivered to the Environmental Geochemistry Group (EGG) at WSRC.

\section{Inorganics}

\section{SC082895.DT6}

- Matrix spike recoveries for antimony (sample IDs 104600 MS, 104600 MS DUP, 104605 MS, 104605 MS DUP, 104614 MS, and 104614 MS DUP), magnesium (sample IDs 104605 MS, 104614 MS, 104614 MS DUP, 104600 MS, and 104600 MS DUP), sulfide (sample ID 104609 MS), and cyanide (sample ID 104613 MS) were not within the $75-125 \%$ guidance limits; however, the LCS associated with the batch was within control limits.

- Due to matrix interferences, matrix spike recoveries were outside the $\mathbf{7 5 - 1 2 5 \%}$ guidance limits for orthophosphate (sample IDs 104613 MS, 104623 MS, and 104602 MS), sulfide (sample ID $104602 \mathrm{MS}$ ), and cyanide (sample ID 104602).

- An R 5 qualifier was assigned to the following samples because performance requirements in the sample or the associated QC analyses were not met: aluminum (sample IDs 104600 MS, 104600 MS DUP, 104605 MS, 104605 MS DUP, $104614 \mathrm{MS}$, and 104614 MS DUP), iron (sample IDs 104600 MS, 104600 MS DUP, 104605 MS, 104605 MS DUP, $104614 \mathrm{MS}$, and $104614 \mathrm{MS}$ DUP), magnesium (sample ID $104605 \mathrm{MS}$ DUP), and total phosphate (sample IDs $104602 \mathrm{MS}, 104613 \mathrm{MS}$, and $104623 \mathrm{MS}$ ).

\section{SC082995.DT3}

- Matrix spike recoveries for antimony (sample IDs 103632 MS and 103632 MS DUP) and orthophosphate (sample ID $104636 \mathrm{MS}$ ) were not within the 75-125\% guidance limits; however, the laboratory control sample (LCS) associated with the batch was within control limits.

- Due to matrix interferences, matrix spike recoveries were outside the $75-125 \%$ guidance limits for total phosphate (sample ID $104644 \mathrm{MS}$ ) and sulfide (sample IDs $104630 \mathrm{MS}, 104637 \mathrm{MS}, 104664 \mathrm{MS}$, and $104672 \mathrm{MS}$ ).

- An R 5 qualifier was assigned to the following samples because performance requirements in the sample or the associated QC analyses were not met: iron (sample IDs 
$103622 \mathrm{MS}, 103622 \mathrm{MS}$ DUP, $103632 \mathrm{MS}$, and $103632 \mathrm{MS}$ DUP), aluminum (sample IDs $103632 \mathrm{MS}$ and $103632 \mathrm{MS}$ DUP), and total phosphate (sample ID $104648 \mathrm{MS}$ ).

SC083095.DAT, SC083095.DT4

- Matrix spike recoveries for antimony (sample IDs 104625 MS, 104625 MS DUP, 104630 MS, 104630 MS DUP, 103632 MS, 103632 MS DUP, 104650 MS, 104650 MS DUP, 104736 MS, 104736 MS DUP, 103631 MS, and 103631 MS DUP), magnesium (sample IDs 104625 MS, 104650 MS, 103631 MS, and 103631 MS DUP), cyanide (sample ID 104735 MS), vanadium (sample ID 104630 MS DUP), and orthophosphate (sample ID $104636 \mathrm{MS}$ ) were not within the 75-125\% guidance limits; however, the LCS associated with the batch was within control limits.

- Due to matrix interferences, matrix spike recoveries were outside the $75-125 \%$ guidance limits for total phosphate (sample ID 104644 MS) and sulfide (sample IDs 104637 MS, $104672 \mathrm{MS}, 104664 \mathrm{MS}$, and $104630 \mathrm{MS})$.

- An R 5 qualifier was assigned to the following samples because performance requirements in the sample or the associated QC analyses were not met: aluminum (sample IDs 103632 MS, 103632 MS DUP, 104625 MS, 104625 MS DUP, 103631 MS, 103631 MS DUP, 104650 MS, 104650 MS DUP, 104736 MS, 104736 MS DUP, 104630 MS, and $104630 \mathrm{MS}$ DUP), iron (sample IDs $103632 \mathrm{MS}, 103632 \mathrm{MS}$ DUP, $104625 \mathrm{MS}$, 104625 MS DUP, 103631 MS, 103631 MS DUP, 104650 MS, 104650 MS DUP, 104736 MS, 104736 MS DUP, $104630 \mathrm{MS}$, and $104630 \mathrm{MS}$ DUP), magnesium (sample IDs 104630 MS, 104630 MS DUP, 104736 MS, and 104736 MS DUP), manganese (sample ID $103631 \mathrm{MS}$ ), and total phosphate (sample IDs $104648 \mathrm{MS}, 104657 \mathrm{MS}$, and 104668 MS).

SC083195.DT5

- Matrix spike recoveries for antimony (sample IDs $103631 \mathrm{MS}, 103631 \mathrm{MS}$ DUP, 104650 MS, 104650 MS DUP, HSB 104C MS, and HSB 104C MS DUP), magnesium (sample IDs $104650 \mathrm{MS}, 103631 \mathrm{MS}$, and $103631 \mathrm{MS}$ DUP), sulfide (sample ID $104655 \mathrm{MS}$ ), selenium (sample ID FSB 99A MS), and orthophosphate (sample ID $104661 \mathrm{MS}$ ) were not within the 75-125\% guidance limits; however, the LCS associated with the batch was within control limits.

- An R 5 qualifier was assigned to the following samples because performance requirements in the sample or the associated QC analyses were not met: aluminum (sample IDs $103631 \mathrm{MS}, 103631$ MS DUP, $104650 \mathrm{MS}$, and 104650 MS DUP), iron (sample IDs $103631 \mathrm{MS}, 103631 \mathrm{MS}$ DUP, $104650 \mathrm{MS}, 104650$ MS DUP, TBG 5B MS, and TBG 5B MS DUP), magnesium (sample IDs FSB 93C MS and FSB 93C MS DUP), manganese (sample ID $103631 \mathrm{MS}$ ), calcium (sample IDs FSB 93C MS and FSB 93C MS DUP), sodium (sample IDs FSB 93C MS and FSB 93C MS DUP), and cyanide (non-WSRC sample).

SC090595.D12

- Matrix spike recoveries for antimony (sample IDs 104664 MS, 104664 MS DUP, $104667 \mathrm{MS}$, and $104667 \mathrm{MS}$ DUP), magnesium (sample IDs $104664 \mathrm{MS}, 104667 \mathrm{MS}$, and 104667 MS DUP), sulfide (sample ID $104664 \mathrm{MS}$ ), and manganese (sample ID 
$104680 \mathrm{MS})$ were not within the 75-125\% guidance limits; however, the LCS associated with the batch was within control limits.

- Due to matrix interferences, matrix spike recoveries were outside the $75-125 \%$ guidance limits for sulfide (sample IDs $104672 \mathrm{MS}, 104637 \mathrm{MS}$, and $104630 \mathrm{MS}$ ).

- An R 5 qualifier was assigned to the following samples because performance requirements in the sample or the associated QC analyses were not met: aluminum (sample IDs 104667 MS, 104667 MS DUP, 104664 MS, 104664 MS DUP, 104680 MS, and 104680 MS DUP), iron (sample IDs 104667 MS, 104667 MS DUP, 104664 MS, 104664 MS DUP, $104680 \mathrm{MS}$, and $104680 \mathrm{MS}$ DUP), nitrogen by Kjeldahl method (sample ID $104676 \mathrm{MS}$ ), and total phosphate (sample IDs $104657 \mathrm{MS}$ and $104668 \mathrm{MS}$ ).

- Chloride for sample IDs $104665,104666,104668,104669$, and 104670 was not analyzed within the regulatory holding times required in the $40 \mathrm{CFR}$, Part 136 (USEPA, 1994a).

\section{SC090795.DT5}

- Matrix spike recoveries for antimony (sample IDs 104671 MS, 104671 MS DUP, $104664 \mathrm{MS}$, and 104664 MS DUP), sulfide (sample ID $104664 \mathrm{MS}$ ), and manganese (sample IDs $104664 \mathrm{MS}$ and $104680 \mathrm{MS}$ ) were not within the 75-125\% guidance limits; however, the LCS associated with the batch was within control limits.

- Due to matrix interferences, matrix spike recoveries were outside the $75-125 \%$ guidance limits for sulfide (sample IDs 104672 MS and 104637 MS) and total phosphate (sample ID 104681 MS).

- An R 5 qualifier was assigned to the following samples because performance requirements in the sample or the associated $\mathrm{QC}$ analyses were not met: aluminum (sample IDs 104664 MS, 104664 MS DUP, 104680 MS, 104680 MS DUP, 104671 MS, $104671 \mathrm{MS}$ DUP, $105113 \mathrm{MS}$, and $105113 \mathrm{MS}$ DUP), iron (sample IDs $104664 \mathrm{MS}$, 104664 MS DUP, 104680 MS, 104680 MS DUP, 104671 MS, 104671 MS DUP, 105113 MS, and 105113 MS DUP), magnesium (sample IDs $104671 \mathrm{MS}$ and $104671 \mathrm{MS}$ DUP), manganese (sample IDs 104671 MS and 104671 MS DUP), nitrogen by Kjeldahl method (sample IDs $104676 \mathrm{MS}$ and $104687 \mathrm{MS}$ ), and total phosphate (sample IDs $104691 \mathrm{REP}$ and $104691 \mathrm{MS}$ ).

- Chloride for sample IDs 104671 through 104678 was not analyzed within the regulatory holding times required in the 40 CFR, Part 136 (USEPA, 1994a).

SC091395.DAT, SC091395.DT2

- Matrix spike recoveries for antimony (sample IDs 104683 MS, 104683 MS DUP, 104693 MS, 104693 MS DUP, 104664 MS, and 104664 MS DUP), sulfide (sample IDs $104680 \mathrm{MS}, 105120 \mathrm{MS}, 105110 \mathrm{MS}$, and $104697 \mathrm{MS}$ ), mercury (sample IDs 104680 MS and 104680 MS DUP), magnesium (sample IDs 104693 MS and 104693 MS DUP), calcium (sample ID 104683 MS DUP), potassium (sample IDs 104683 MS DUP and 104693 MS DUP), orthophosphate (sample ID $104690 \mathrm{MS}$ ), ammonia nitrogen (sample ID $104689 \mathrm{MS}$ ), and manganese (sample ID $104680 \mathrm{MS}$ ) were not within the 75-125\% guidance limits; however, the LCS associated with the batch was within control limits. 
- Due to matrix interferences, matrix spike recoveries were outside the $75-125 \%$ guidance limits for total phosphate (sample ID 104681 MS).

- An R 5 qualifier was assigned to the following samples because performance requirements in the sample or the associated QC analyses were not met: aluminum (sample IDs 104664 MS, 104664 MS DUP, 104680 MS, 104680 MS DUP, 104683 MS, 104683 MS DUP, $104693 \mathrm{MS}$, and 104693 MS DUP), iron (sample IDs $104664 \mathrm{MS}, 104664 \mathrm{MS}$ DUP, 104680 MS, 104680 MS DUP, 104683 MS, 104683 MS DUP, 104693 MS, and 104693 MS DUP), magnesium (sample IDs 104683 MS and 104683 MS DUP), nitrogen by Kjeldahl method (sample ID $104687 \mathrm{MS}$ ), and total phosphate (sample IDs $104691 \mathrm{MS}$ and $105102 \mathrm{MS}$ ).

- Chloride for sample IDs 104690 and 104691 was not analyzed within the regulatory holding times required in the 40 CFR, Part 136 (USEPA, 1994a).

- For sample ID $104691 \mathrm{MS}$, the percent recovery of total phosphate is reported as zero because of dilutions performed on the sample.

SC091895.DT2, SC091895.DT3

- Matrix spike recovery for mercury (sample ID $103651 \mathrm{MS}$ ) was not within the 75$125 \%$ guidance limits; however, the LCS associated with the batch was within control limits.

- An R 5 qualifier was assigned to the following samples because performance requirements in the sample or the associated $\mathrm{QC}$ analyses were not met: aluminum (sample IDs 104683 MS, 104683 MS DUP, 104693 MS, 104693 MS DUP, 104671 MS, 104671 MS DUP, 105110 MS, 105110 MS DUP, 105113 MS, 105113 MS DUP, 105200 MS, 105200 MS DUP, FSB 91C MS, FSB 91C MS DUP, FSB105DR MS, and FSB105DR MS DUP), iron (sample IDs 104683 MS, 104683 MS DUP, 104693 MS, 104693 MS DUP, 104671 MS, 104671 MS DUP, 105110 MS, 105110 MS DUP, 105113 MS, 105113 MS DUP, $105200 \mathrm{MS}$, and 105200 MS DUP), magnesium (sample IDs $104671 \mathrm{MS}, 104671$ MS DUP, 104683 MS, 104683 MS DUP, FSB 91C MS, and FSB 91C MS DUP), calcium (sample IDs FSB 91C MS and FSB 91C MS DUP), sodium (sample IDs FSB 91C MS and FSB 91C MS DUP), manganese (sample IDs 104671 MS and 104671 MS DUP), nitrogen by Kjeldahl method (sample IDs $105101 \mathrm{MS}$ and $105111 \mathrm{MS}$ ), and total phosphate (sample IDs $105101 \mathrm{MS}$ and $105111 \mathrm{MS}$ ).

- Chloride (sample IDs 104697 and 105102) and total organic carbon (sample ID 105111) were not analyzed within the regulatory holding times required in the 40 CFR, Part 136 (USEPA, 1994a).

\section{Pesticides/PCBs}

\section{SC082895.DT6}

- Results for sample ID 104602 were reported as being analyzed out of holding time due to the need for re-extraction or re-analysis. The original analysis was performed within holding time. 


\section{SC083095.DAT, SC083095.DT4}

- Matrix spike recoveries for sample ID 104635 MS for aldrin, heptachlor, lindane, endrin, and dieldrin were not within the 75-125\% guidance limits but were within the laboratory's control limits.

- Sample IDs 104638 and 104640 were diluted for analysis due to matrix interferences. SC083195.DT5

- Sample IDs 104652, 104656, 104657, 104658, 104653, 104655, and 104660 were diluted for analysis due to matrix interferences.

SC090795.DT5

- Sample ID 104672 MS received an R 5 qualifier for aldrin and endrin because performance requirements in the sample or the associated $\mathrm{QC}$ analyses were not met.

- An R 5 qualifier was assigned to the surrogate spikes tetrachloro-m-xylene and dibutylchorendate for sample ID 104749 because performance requirements in the sample or the associated QC analyses were not met.

- Sample IDs 104671 through 104678 were diluted for analysis due to matrix interferences.

- Due to matrix interference in sample ID $104672 \mathrm{MS}$, matrix spike recoveries were outside the 75-125\% guidance limits for aldrin, dieldrin, heptachlor, lindane, and p,p'DDT.

SC091395.DAT, SC091395.DT2

- Sample IDs 104691, 104680, 104681, 104682, 104683, 104687, 104688, 104689, and 104690 were diluted for analysis due to matrix interferences.

- Matrix spike recoveries for sample ID 104689 MS for aldrin and p,p'-DDT were not within the 75-125\% guidance limits, but were within the laboratory's control limits.

SC091895.DT2, SC091895.DT3

- Sample IDs 104693, 104694, 104695, 104696, 105113, 105117, 105118, 105109, and 105110 were diluted for analysis due to matrix interferences.

- Matrix spike recoveries for sample ID $105110 \mathrm{MS}^{5}$ for aldrin, dieldrin and p,p'-DDT were not within the $75-125 \%$ guidance limits, but were within the laboratory's control limits.

\section{GC/MS Volatiles}

SC082895.DT6

- Matrix spike recovery for sample ID 104600 MS for 1,1-dichloroethylene did not fall within the $75-125 \%$ guidance limit but was within the laboratory's control limit.

SC083095.DAT, SC083095.DT4

- Sample ID 104651 was not analyzed within the regulatory holding times required in 40 CFR, Part 136 (USEPA, 1994a), but the sample was analyzed within the contractrequired holding times. 
- Matrix spike recoveries for sample ID 104603 MS and 104603 MSD for trichloroethylene, benzene, and 1,1-dichloroethylene were not within the 75-125\% guidance limits but were within the laboratory's control limits.

SC083195.DT5

- Sample ID 104655 was not analyzed within the regulatory holding times required in 40 CFR, Part 136 (USEPA, 1994a), but the sample was analyzed within the contractrequired holding times.

- Matrix spike recovery for sample ID 104742 MS for benzene was not within the 75$125 \%$ guidance limits but was within the laboratory's control limits.

SC091395.DAT, SC091395.DT2

- Matrix spike recovery for sample ID 104748 MS for toluene was not within the 75$125 \%$ guidance limits but was within the laboratory's control limits.

- Sample ID 104687 had low results for the internal standards; re-analysis confirmed the original results.

SC091895.DT2, SC091895.DT3

- Matrix spike recoveries on sample IDs 105111 MS for toluene, 105120 for benzene and 1,1-dichloroethylene, and 105120 MS DUP for 1,1-dichloroethylene were not within the 75-125\% guidance limits but were within the laboratory's control limits.

- Results for acetone for sample IDs 105109, 105110, 105111, and 105119 and vinyl acetate for sample ID 105109 were reported as being analyzed out of holding times due to the need for re-extraction or re-analysis. The original analyses were performed within holding times.

\section{Semivolatiles}

SC083095.DAT, SC083095.DT4

- Matrix spike recoveries for sample ID 104636 MS for N-nitrosodipropylamine, acenaphthene, phenol, and 2-chlorophenol were not within the 75-125\% guidance limits but were within the laboratory's control limits.

- Matrix spike recovery for sample ID 104644 MS for phenol was not within the 75$125 \%$ guidance limits but was within the laboratory's control limits

SC091395.DAT, SC091395.DT2

- Results for sample IDs 104681 through 104684, 104686, and 104688 through 104691 were reported as being analyzed out of holding times due to the need for re-extraction or re-analysis. The original analyses were performed within holding times.

- Matrix spike recovery on sample ID 104691 MS for phenol was not within the 75$125 \%$ guidance limits but was within the laboratory's control limits.

SC091895.DT2, SC091895.DT3

- Results for sample IDs 104693 through 104696, and 104699 were reported as being analyzed out of holding times due to the need for re-extraction or re-analysis. The original analyses were performed within holding times. 


\section{Radionuclides}

SC082895.DT6

- The following analytes were not quantified due to low abundance or interference: europium-155 (sample IDs 104600, 104601, 104602, 104604,104605, 104606, 104607, 104608, and 104610 through 104623), zirconium-95 (sample IDs 104601, 104604, $104608,104610,104611,104613,104619,104621$, and 104623), promethium-146 (sample IDs 104615 and 104622), zinc-65 (sample ID 104622), cobalt-60 (sample ID 104600), and neptunium-239 (sample IDs 104600, 104601, and 104602).

\section{SC083095.DAT, SC083095.DT4}

- The following analytes were not quantified due to low abundance or interference: europium-155 (sample IDs 104625, 104626, 104627, 104628,104635, 104636, 104637, and 104638), zirconium-95 (sample IDs 104626, 104627, 104628, 104635, 104636, 104637, and 104638), and promethium-146 (sample IDs 104627, 104628, 104635, 104636, 104637, and 104638).

\section{SC083195.DT5}

- The following analytes were not quantified due to low abundance or interference: europium-155 (sample IDs 104653, 104655, 104658, and 104660) and promethium-146 (sample ID 104655).

\section{SC090595.D12}

- The following analytes were not quantified due to low abundance or interference: europium-155 (sample IDs 104667, 104666, 104665, 104670, 104669, and 104668), zirconium-95 (sample IDs 104665, 104670, 104669, and 104668), promethium-146 (sample IDs 104666, 104665, 104670, 104669, and 104668), cobalt-60 (sample IDs 104664, 104667, 104666, 104665, 104670, 104669, and 104668), and neptunium-239 (sample IDs 104669 and 104668).

- Neptunium-239 failed the abundance test in the gamma-PHA analysis of sample ID 104668.

\section{SC090795.DT5}

- The following analytes were not quantified due to low abundance or interference: europium-155 (sample IDs 104671, 104672, 104673, 104674, 104675, 104676, 104677, and 104678), zirconium-95 (sample IDs 104679, 104676, 104677, and 104678), promethium-146 (sample ID 104678), and antimony-125 (sample IDs 104674, 104675, 104676, 104677, and 104678).

\section{SC091395.DAT, SC091395.DT2}

- The following analytes were not quantified due to low abundance or interference: europium-155 (sample IDs 104691, 104681, 104682, 104683, 104685, 104686, 104688, 104689, and 104690), zirconium-95 (sample IDs 104691, 104680, 104688, 104689, and 104690), promethium-146 (sample IDs 104691, 104681, 104682, and 104690), zinc-65 (sample ID 104690), manganese-54 (sample ID 104683), potassium-40 (sample ID 104690), yttrium-88 (sample ID 104689), and neptunium-239 (sample IDs 104688 and 104690). 
SC091895.DT2, SC091895.DT3

- The following analytes were not quantified due to low abundance or interference: europium-155 (sample IDs 104693, 104694, 104695, 104696, 104698, 105100, 105101, $105102,105113,105114, \cdot 105115,105116,105117$, and 105119), zirconium-95 (sample IDs 104693, 104696, 104697, 105100, 105113, and 105119), promethium-146 (sample IDs 105100, 105102, 105113, 105116, and 105119), zinc-65 (sample ID 104693), barium-133 (sample ID 104696), sodium-22 (sample ID 104698), plutonium-238 (sample ID 105116), and neptunium-239 (sample IDs 104694, 105100, and 105116).

- Sample ID 105119 was noted as being non-homogeneous during the gross alpha/nonvolatile beta analysis.

\section{Laboratory Data Records Reviews}

On October 5, 1995, laboratory data records were reviewed for first quarter 1995 inorganics, organics, and radiological analyses associated with the Par Pond Sediment Characterization Resource Conservation and Recovery Act Facility Investigation/Remedial Investigation (RFI/RI) Project at GE and Environmental Physics, Inc., Charleston, South Carolina. The review was conducted by Raymond M. Keller of Exploration Resources, Athens, Georgia.

The purpose of this review was to investigate technical validation issues discussed in the Quality Assurance/Quality Control Guidance for Removal Activities, Sampling QA/QC Plan and Data Validation Procedures (USEPA, 1990b), which are not adequately addressed by computer checking of the EDD files, review of the analytical narratives, or review of the chain-of-custody (COC) forms. These technical issues include instrument calibration, analyte identification, and analyte quantitation. The issues are addressed by comparing the instrument print outs associated with particular analyses against validation checklists. These checklists consist of 12 to 20 questions each and were prepared from the following sources: Data Validation Procedures for Radiochemical Analysis (WHC, not dated), discussions with laboratory personnel, Environmental Physics, Inc. standard operating procedures, GE standard operating procedures, Laboratory Data Validation Functional Guidelines for Evaluating Inorganics Analyses (USEPA, 1988a), Laboratory Data Validation Functional Guidelines for Evaluating Organics Analyses (USEPA, 1988b), Quality Assurance/Quality Control Guidance for Removal Activities, Sampling QA/QC Plan and Data Validation Procedures (USEPA, 1990b), and Test Methods for Evaluating Solid Waste (SW-846) (USEPA, 1986).

For each analytical method, approximately $10 \%$ of the samples analyzed were chosen for review.

In all, 55 worksheets were completed during this review. Each worksheet represents the review of one sample for one analytical method; i.e., one sample/method combination (SMC). An inventory of the number of worksheets and the methods reviewed are given in Table 12. 
Table 12. Laboratory Data Records Review Methods/Analyses

\begin{tabular}{|c|l|l|l|}
\hline Number of SMCs & Method & Analyte/Analyte Suite & Checklist Used \\
\hline 6 & EPA 335:3 & Cyanide & 335 \\
\hline 2 & EPA 353.1 & Nitrate & 353 \\
\hline 6 & EPA 6010 & ICP Metals & 6010 \\
\hline 3 & EPA 7041 & $\begin{array}{l}\text { Antimony (Atomic Absorption) } \\
\text { (AA) }\end{array}$ & 7000 \\
\hline 1 & EPA 7060 & Arsenic (AA) & 7000 \\
\hline 1 & EPA 7421 & Lead (AA) & 7000 \\
\hline 1 & EPA 7470 & Mercury (Cold vapor) & 7000 \\
\hline 6 & EPA 7471 & Mercury (Cold vapor) & 7000 \\
\hline 1 & EPA 7740 & Selenium (AA) & 7000 \\
\hline 1 & EPA 7841 & Thallium (AA) & 7000 \\
\hline 3 & EPA 8080 & Pesticides & 8080 \\
\hline 4 & EPA 8260 & GC/MS Volatiles & $8240 / 8260$ \\
\hline 3 & EPA 8270 & GC/MS Semivolatiles & 8270 \\
\hline 4 & EPIA-001B & $\begin{array}{l}\text { Gross Alpha / Nonvolatile } \\
\text { Beta }\end{array}$ & Gas flow proportional \\
\hline 4 & EPIA-004 & Strontium-89/90 & Gas flow proportional \\
\hline 5 & EPIA-012B & Plutonium-239/240 & Alpha Spectroscopy \\
\hline 4 & EPIA-013 & Cesium-137, Lead-212 & Gamma Spectroscopy \\
\hline Total $=55$ & & & \\
\hline & & & \\
\hline
\end{tabular}

Table 13 provides a summary of the samples reviewed. Run date and run time refer to the date and time the analysis was performed in the laboratory.

Table 13. Summary of Samples Reviewed

\begin{tabular}{|l|l|l|l|l|l|}
\hline Method & $\begin{array}{l}\text { Laboratory } \\
\text { ID }\end{array}$ & Analyte & $\begin{array}{l}\text { Preparation } \\
\text { Batch }\end{array}$ & $\begin{array}{l}\text { Run } \\
\text { Date }\end{array}$ & $\begin{array}{l}\text { Run } \\
\text { Time }\end{array}$ \\
\hline EPA335.3 & $\mathbf{9 5 0 7 1 2 8 - 1 8}$ & Cyanide & 68870 & $7 / 14 / 95$ & $19: 44$ \\
\hline EPA335.3 & $9507128-23$ & Cyanide & 68869 & $7 / 14 / 95$ & $19: 22$ \\
\hline EPA335.3 & $9507160-02$ & Cyanide & 69460 & $7 / 21 / 95$ & $11: 56$ \\
\hline EPA335.3 & $9507281-03$ & Cyanide & 69211 & $7 / 20 / 95$ & $12: 03$ \\
\hline EPA335.3 & $9507343-04$ & Cyanide & 69481 & $7 / 31 / 95$ & $10: 40$ \\
\hline EPA335.3 & $9507381-06$ & Cyanide & 69619 & $7 / 31 / 95$ & $11: 04$ \\
\hline EPA353.1 & $9507226-03$ & Nitrate-nitrite as nitrogen & 69693 & $7 / 25 / 95$ & $11: 30$ \\
\hline EPA353.1 & $9507226-05$ & Nitrate-nitrite as nitrogen & 69693 & $7 / 25 / 95$ & $11: 30$ \\
\hline EPA6010 & $9507128-16$ & Selenium, total recoverable & 69120 & $7 / 18 / 95$ & $15: 33$ \\
\hline EPA6010 & $9507160-03$ & Cadmium, total recoverable & 69360 & $7 / 21 / 95$ & $10: 37$ \\
\hline EPA6010 & $9507281-01$ & Copper, total recoverable & 69278 & $7 / 24 / 95$ & $10: 51$ \\
\hline EPA6010 & $9507381-01$ & Selenium, total recoverable & 70665 & $8 / 11 / 95$ & $10: 22$ \\
\hline
\end{tabular}


Table 13. Summary of Samples Reviewed, Continued

\begin{tabular}{|c|c|c|c|c|c|}
\hline Method & $\begin{array}{l}\text { Laboratory } \\
\text { ID }\end{array}$ & Analyte & $\begin{array}{l}\text { Preparation } \\
\text { Batch }\end{array}$ & $\begin{array}{l}\text { Run } \\
\text { Date }\end{array}$ & $\begin{array}{l}\text { Run } \\
\text { Time }\end{array}$ \\
\hline EPA6010 & 9507381-05 & Sodium, total recoverable & 70665 & $8 / 11 / 95$ & $10: 58$ \\
\hline EPA6010 & $9507381-06$ & Zinc, total recoverable & 70316 & $8 / 09 / 95$ & $11: 21$ \\
\hline EPA7041 & $9507128-30$ & Antimony, total recoverable & 69023 & $7 / 28 / 95$ & $02: 54$ \\
\hline EPA7041 & $9507160-02$ & Antimony, total recoverable & 69115 & $8 / 02 / 95$ & $16: 13$ \\
\hline EPA7041 & $9507281-09$ & Antimony, total recoverable & 70145 & $8 / 15 / 95$ & $13: 46$ \\
\hline EPA7060 & $9507160-02$ & Arsenic, total recoverable & 69115 & $8 / 02 / 95$ & $21: 54$ \\
\hline EPA7421 & 9507281-09 & Lead, total recoverable & 70145 & $8 / 16 / 95$ & $00: 05$ \\
\hline EPA7470 & $9507160-02$ & Mercury, total recoverable & 69539 & $7 / 29 / 95$ & 19:39 \\
\hline EPA7471 & 9507128.08 & Mercury, total recoverable & 69744 & $7 / 28 / 95$ & $02: 21$ \\
\hline EPA7471 & $9507128-20$ & Mercury, total recoverable & 69744 & $7 / 28 / 95$ & $02: 59$ \\
\hline EPA7471 & $9507160-03$ & Mercury, total recoverable & 69746 & $7 / 28 / 95$ & 03:50 \\
\hline EPA7471 & $9507199-01$ & Mercury, total recoverable & 69747 & $7 / 28 / 95$ & $04: 51$ \\
\hline EPA7471 & 9507281-04 & Mercury, total recoverable & 69750 & $7 / 28 / 95$ & 09:00 \\
\hline EPA7471 & 9507381-01 & Mercury, total recoverable & 70269 & $8 / 10 / 95$ & $00: 09$ \\
\hline EPA7740 & $9507160-02$ & Selenium, total recoverable & 69115 & $8 / 02 / 95$ & 19:00 \\
\hline EPA7841 & $9507128-30$ & Thallium, total recoverable & 69023 & $7 / 26 / 95$ & $18: 37$ \\
\hline EPA8080 & 9507199-06 & Heptachlor epoxide & 69701 & $8 / 08 / 95$ & $04: 50$ \\
\hline EPA8080 & 9507281-04 & Dieldrin & 69701 & $8 / 14 / 95$ & $.23: 28$ \\
\hline EPA8080 & 9507381-01 & delta-Benzene hexachloride & 70186 & $8 / 17 / 95$ & $06: 33$ \\
\hline EPA8260 & 9507128-21 & Toluene & 69090 & $7 / 14 / 95$ & $14: 25$ \\
\hline EPA8260 & $9507226-04$ & Dichloromethane & 69770 & $7 / 25 / 95$ & $19: 32$ \\
\hline EPA8260 & 9507281-10 & Methyl ethyl ketone & 69617 & $7 / 24 / 95$ & $02: 14$ \\
\hline EPA8260 & 9507381-09 & Acetone & 70217 & $8 / 01 / 95$ & $19: 48$ \\
\hline EPA8270 & $9507160-02$ & Diethyl phthalate & 69116 & $7 / 21 / 95$ & 22:47 \\
\hline EPA8270 & 9507281-04 & Di-n-butyl phthalate & 69790 & $7 / 27 / 95$ & $18: 14$ \\
\hline EPA8270 & 9507281-09 & Bis(2-ethylhexyl) phthalate & 69292 & $7 / 21 / 95$ & 18:01 \\
\hline EPIA-001B & 9507199-09 & Gross alpha & 68993 & $7 / 28 / 95$ & $10: 46$ \\
\hline EPIA-001B & $9507226-04$ & Gross alpha & 69297 & $8 / 01 / 95$ & 13:57 \\
\hline EPIA-001B & $9507381-01$ & Gross alpha & 69660 & $8 / 03 / 95$ & $12: 22$ \\
\hline EPIA-001B & 9507381-06 & Gross alpha & 69669 & $8 / 15 / 95$ & $20: 09$ \\
\hline EPIA-004 & $9507226-04$ & Strontium-90 & 69334 & $8 / 08 / 95$ & 05:57 \\
\hline EPIA-004 & $9507281-03$ & Strontium-90 & 69334 & $8 / 08 / 95$ & 05:59 \\
\hline EPIA-004 & $9507343-04$ & Strontium-90 & 69940 & $8 / 10 / 95$ & $10: 07$ \\
\hline EPIA-004 & 9507381-02 & Strontium-90 & 69940 & $8 / 10 / 95$ & 10:07 \\
\hline EPIA-012B & 9507128.09 & Plutonium-238 & 68823 & $8 / 01 / 95$ & 09:15 \\
\hline EPIA-012B & $9507128-17$ & Plutonium-238 & 68823 & $8 / 01 / 95$ & $10: 33$ \\
\hline EPIA-012B & 9507226.03 & Plutonium-239/240 & 69301 & $8 / 02 / 95$ & $12: 46$ \\
\hline EPAI-012B & $9507343-01$ & Plutonium-239/240 & 69751 & $8 / 08 / 95$ & $13: 49$ \\
\hline EPIA-012B & 9507381-01 & Plutonium-239/240 & 69751 & $8 / 08 / 95$ & 13:53 \\
\hline EPIA-013 & $9507160-04$ & Cesium-137 & 68912 & $8 / 15 / 95$ & 11:14 \\
\hline
\end{tabular}

Par Pond, Pond C, and L Lake, Revision 2 
Table 13. Summary of Samples Reviewed, Continued

\begin{tabular}{|l|l|l|l|l|l|}
\hline Method & $\begin{array}{l}\text { Laboratory } \\
\text { ID }\end{array}$ & Analyte & $\begin{array}{l}\text { Preparation } \\
\text { Batch }\end{array}$ & $\begin{array}{l}\text { Run } \\
\text { Date }\end{array}$ & $\begin{array}{l}\text { Run } \\
\text { Time }\end{array}$ \\
\hline EPIA-013 & $9507199-09$ & Cobalt-57 & 68912 & $8 / 16 / 95$ & $11: 15$ \\
\hline EPIA-013 & $9507281-08$ & Cerium-144 & 71004 & $8 / 18 / 95$ & $12: 46$ \\
\hline EPIA-013 & $9507381-05$ & Europium-152 & 69781 & $8 / 20 / 95$ & $12: 21$ \\
\hline
\end{tabular}

\section{Technical Issues}

No issues of concern were discovered during this review.

\section{Scoring}

\section{EPA353.3 Cyanide}

One of the six samples reviewed, laboratory ID 9507128-23, did not meet the validation checklist criterion for question 11 because the matrix spike recovery was not within $\pm 25 \%$ of the true value. Matrix interference was evident with this sample, which had matrix spike and matrix spike duplicate recovery results of zero percent.

\section{EPA353.1 Nitrate-Nitrite as Nitrogen}

All validation checklist criteria were met in the two reviewed samples.

\section{EPA6010 ICP Metals}

All validation checklist criteria were met in the six reviewed samples.

EPA7041 Antimony by AA

All validation checklist criteria were met in the three reviewed samples.

EPA7060 Arsenic by AA

All validation checklist criteria were met in the reviewed sample.

EPA7421 Lead by AA

All validation checklist criteria were met in the reviewed sample.

EPA7470 Mercury by Cold Vapor

All validation checklist criteria were met in the reviewed sample.

EPA7471 Mercury by AA

All validation checklist criteria were met in the six reviewed samples.

EPA7740 Selenium by $A A$

All validation checklist criteria were met in the reviewed sample. 
EPA7841 Thallium by AA

All validation checklist criteria were met in the reviewed sample.

\section{EPA8080 Pesticides}

Two of the three samples reviewed did not meet the validation checklist criterion for question 12 because the surrogate recovery was not within normal QC limits for decachlorobiphenyl (DCB) and tetrachloro-m-xylene (TCMX).

\begin{tabular}{|l|c|l|l|}
\hline Laboratory ID & $\begin{array}{l}\text { TCMX } \\
\text { Recovery (\%) }\end{array}$ & $\begin{array}{l}\text { DCB } \\
\text { Recovery (\%) }\end{array}$ & Instrument Batch \\
\hline $9507381-01$ & $0^{1}$ & $0^{1}$ & 70186 \\
\hline $9507281-04$ & $0^{2}$ & $0^{2}$ & 69701 \\
\hline
\end{tabular}

1 This sample was analyzed at a 1:50 dilution. The surrogate was diluted out.

2 This sample was analyzed at a 1:20 dilution. The surrogate was diluted out.

One of the three samples reviewed, laboratory ID 9507199-06 (instrument batch 69701), had large matrix interference on the non-polar column (the confirmation column) which was not properly integrated. The value reported for heptachlor epoxide was exaggerated by more than $100 \%$. If the chromatogram was properly integrated, heptachlor epoxide would not be confirmed in the sample. Therefore, the reported value of heptachlor epoxide at $1.36 \mu \mathrm{g} / \mathrm{kg}$ was rejected and assigned a $U$ result qualifier instead of its present $J$ result qualifier.

\section{EPA8260 GC/MS Volatiles}

All validation checklist criteria were met in the four reviewed samples.

\section{EPA8270 Semivolatiles}

All validation checklist criteria were met in the three reviewed samples.

\section{EPIA-001 Gross Alpha/Nonvolatile Beta}

Two of the four samples reviewed did not meet the validation checklist criterion for question 15 because the duplicate result was greater than five times the contract required detection limit (CRDL) and the RPD was greater than $20 \%$.

\begin{tabular}{|l|l|l|l|}
\hline Laboratory ID & Date and Time of Analysis & RPD (\%) & $\begin{array}{l}\text { Instrument } \\
\text { Batch }\end{array}$ \\
\hline $9507226-04$ & $08 / 01 / 9513: 57$ & 28 & 69297 \\
\hline $9507381-01$ & $08 / 03 / 9512: 22$ & 75 & 69660 \\
\hline
\end{tabular}


EPIA-004 Strontium-90

Two of the four samples reviewed did not meet the validation checklist criterion for question 10 because the method blank result was not below the CRDL.

\begin{tabular}{|l|l|l|l|}
\hline Laboratory ID & $\begin{array}{l}\text { Sample Quantitation } \\
\text { Limit }\end{array}$ & Blank Result & Instrument Batch \\
\hline $9507343-04$ & $0.111 \mathrm{pC} / \mathrm{g}$ & $0.37 \pm 0.06 \mathrm{pC} / \mathrm{g}$ & 69940 \\
\hline $9507381-02$ & $0.111 \mathrm{pC} / \mathrm{g}$ & $0.37 \pm 0.06 \mathrm{pC} / \mathrm{g}$ & 69940 \\
\hline
\end{tabular}

EPIA-012 Plutonium-239/240 and Thorium-228

All validation checklist criteria were met in the five reviewed samples.

EPIA-013 Lead-212

All validation checklist criteria were met in the four reviewed samples.

\section{Qualification of Analyses}

\section{EPA335.3 Cyanide}

One sample, laboratory ID 9507128-23, for cyanide failed question 11 and each sample in the preparation batch was assigned an $I$ analysis qualifier and a $J$ result qualifier if it was detected and an $R$ result qualifier if it was not detected.

EPA8080 Pesticides

\begin{tabular}{|l|l|l|l|l|}
\hline Laboratory ID & Analyte(s) & $\begin{array}{l}\text { Result } \\
\text { Qualifier }\end{array}$ & $\begin{array}{l}\text { Analysis } \\
\text { Qualifier }\end{array}$ & Failed Question(s) \\
\hline $9507381-01$ & All & J & O & 12 \\
\hline $9507281-04$ & All & J & O & 12 \\
\hline $9507199-06$ & Heptachlor epoxide & UJ & I & Improper integration \\
\hline
\end{tabular}

EPIA-001 Gross Alpha/Nonvolatile Beta

\begin{tabular}{|l|l|l|l|l|}
\hline Laboratory ID & Analyte(s) & $\begin{array}{l}\text { Result } \\
\text { Qualifier }\end{array}$ & $\begin{array}{l}\text { Analysis } \\
\text { Qualifier }\end{array}$ & Failed Question(s) \\
\hline $9507226-04$ & Gross alpha & $\mathrm{J}^{1}$ & $\mathrm{X}^{2}$ & 15 \\
\hline $9507381-01$ & Gross alpha & $\mathrm{J}^{1}$ & $\mathrm{X}^{2}$ & 15 \\
\hline
\end{tabular}

1 Applies only to duplicate and parent samples.

2 Applies to all samples in the preparation batch. 
EPIA-004 Strontium-90

One sample, laboratory ID 9507343-04, for strontium-90 failed question 12 and a $U$ result qualifier was assigned to all samples in the preparation batch with a result of $>1.85 \mathrm{pC} / \mathrm{g}$. A $V$ analysis qualifier was applied to all samples in the preparation batch. 


\section{Discussion}

\section{Qualification of the Data}

Table 14 presents the samples requested, received, and qualified for each analyte. Analytical results for both the primary laboratory and the laboratory receiving quality assurance (QA) samples are included in the table. Surrogates, matrix spikes, and other laboratory quality control $(\mathrm{QC})$ results are not included in the table.

- The Samples Requested column contains the number of times the laboratory received a chain-of-custody and its associated sample (environmental samples, field-generated duplicates, field blanks, and trip blanks) requesting a particular analysis be performed.

- The Samples Received column contains the total number of samples for which at least one analytical record was reported. Missing analyses are discussed in the Sample Collection and Validation and Verification: Findings sections of this report. Percent solids was analyzed routinely for all soil samples but was not requested on the chain-ofcustody (COC) forms.

- The Analyses Reported column contains the total number of reported results for the analyte. This number includes laboratory-initiated duplicate analyses and re-analysis results; it does not include analyses of associated laboratory blanks.

- The remaining columns in the table list the number of analyses that were qualified for meeting specified conditions. The column labeled Rejected contains the number of records for which the result qualifier (RQ) was $R$. Samples having a rejected result also may have an acceptable re-analysis result.

- The column labeled Out of Holding contains the number of records for which the time between sample collection and analysis exceeded the maximum allowed by the analytical method. These records have an analysis qualifier (AQ) of $Q$. All laboratory $\mathrm{pH}$ measurements exceed holding time, regardless of the laboratory's efficiency.

- The column labeled $Q A$ Problems contains the number of records containing an $\mathrm{AQ}$ of $C, G, H, I, O, R, V$, or $X$ because at least one associated laboratory $\mathrm{QC}$ sample did not meet the required standards.

- The column labeled Preservation contains the number of records with preservation problems. These records have an $\mathrm{AQ}=Y$. In most of these cases, the samples were not maintained at a cold enough temperature.

- The number of records with identified calibration problems is listed in the column labeled Calibration. These records have an $\mathrm{AQ}=L$. 
Table 14. Number of Qualified Samples

\begin{tabular}{|c|c|c|c|c|c|c|c|c|}
\hline Analyte & $\begin{array}{l}\text { Samples } \\
\text { Requested }\end{array}$ & $\begin{array}{l}\text { Samples } \\
\text { Received }\end{array}$ & $\begin{array}{l}\text { Analyses } \\
\text { Reported }\end{array}$ & $\begin{array}{l}\text { Records } \\
\text { Rejected }\end{array}$ & $\begin{array}{l}\text { Out of } \\
\text { Holding }\end{array}$ & $\begin{array}{l}\text { QA } \\
\text { Problems }\end{array}$ & $\begin{array}{l}\text { Preser- } \\
\text { vation }\end{array}$ & $\begin{array}{l}\text { Cali- } \\
\text { bration }\end{array}$ \\
\hline \multicolumn{9}{|c|}{ Physical Parameters and Specified Analyses } \\
\hline Ammonia nitrogen & & 50 & 56 & 0 & 0 & 4 & 0 & 0 \\
\hline Cation exchange capacity & 106 & 102 & 116 & 0 & 0 & 0 & 4 & 0 \\
\hline Chloride & 106 & 106 & 117 & 0 & 19 & 0 & 4 & 0 \\
\hline Cyanide & 110 & 110 & 121 & 0 & 0 & 29 & 3 & 0 \\
\hline Nitrate-nitrite as nitrogen & 106 & 106 & 117 & 0 & 0 & 13 & 3 & 0 \\
\hline Nitrogen by Kjeldahl method & & 50 & 55 & 0 & 0 & 0 & 0 & 0 \\
\hline Orthophosphate & 106 & 106 & 120 & 0 & 0 & 14 & 4 & 0 \\
\hline Phenols & 106 & 106 & 118 & 0 & 0 & 51 & 4 & 0 \\
\hline Sulfide & 106 & 106 & 121 & 0 & 0 & 118 & 4 & 0 \\
\hline Total organic carbon & 106 & 106 & 122 & 0 & 2 & 44 & 4 & 0 \\
\hline Total organic nitrogen & 106 & 106 & 108 & 0 & 0 & 2 & 4 & 0 \\
\hline Total phosphates (as P) & 106 & 106 & 119 & 0 & 0 & 6 & 4 & 0 \\
\hline \multicolumn{9}{|c|}{ Total Metals (metals are total recoverable) } \\
\hline Aluminum & 110 & 110 & 124 & 0 & 0 & 48 & 4 & 0 \\
\hline Antimony & 110 & 110 & 123 & 0 & 0 & 26 & 4 & 0 \\
\hline Arsenic & 110 & 110 & 123 & 0 & 0 & 2 & 4 & 0 \\
\hline Barium & 110 & 110 & 124 & 0 & 0 & 17 & 4 & 0 \\
\hline Beryllium & 110 & 110 & 124 & 0 & 0 & 14 & 4 & 0 \\
\hline Cadmium & 110 & 110 & 124 & 0 & 0 & 1 & 4 & 0 \\
\hline Calcium & 110 & 110 & 124 & 0 & 0 & 54 & 4 & 0 \\
\hline Chromium & 110 & 110 & 124 & 0 & 0 & 12 & 4 & 0 \\
\hline Cobalt & 110 & 110 & 124 & 0 & 0 & 0 & 4 & 0 \\
\hline Copper & 110 & 110 & 124 & 0 & 0 & 18 & 4 & 0 \\
\hline
\end{tabular}


Table 14. Number of Qualified Samples, Continued

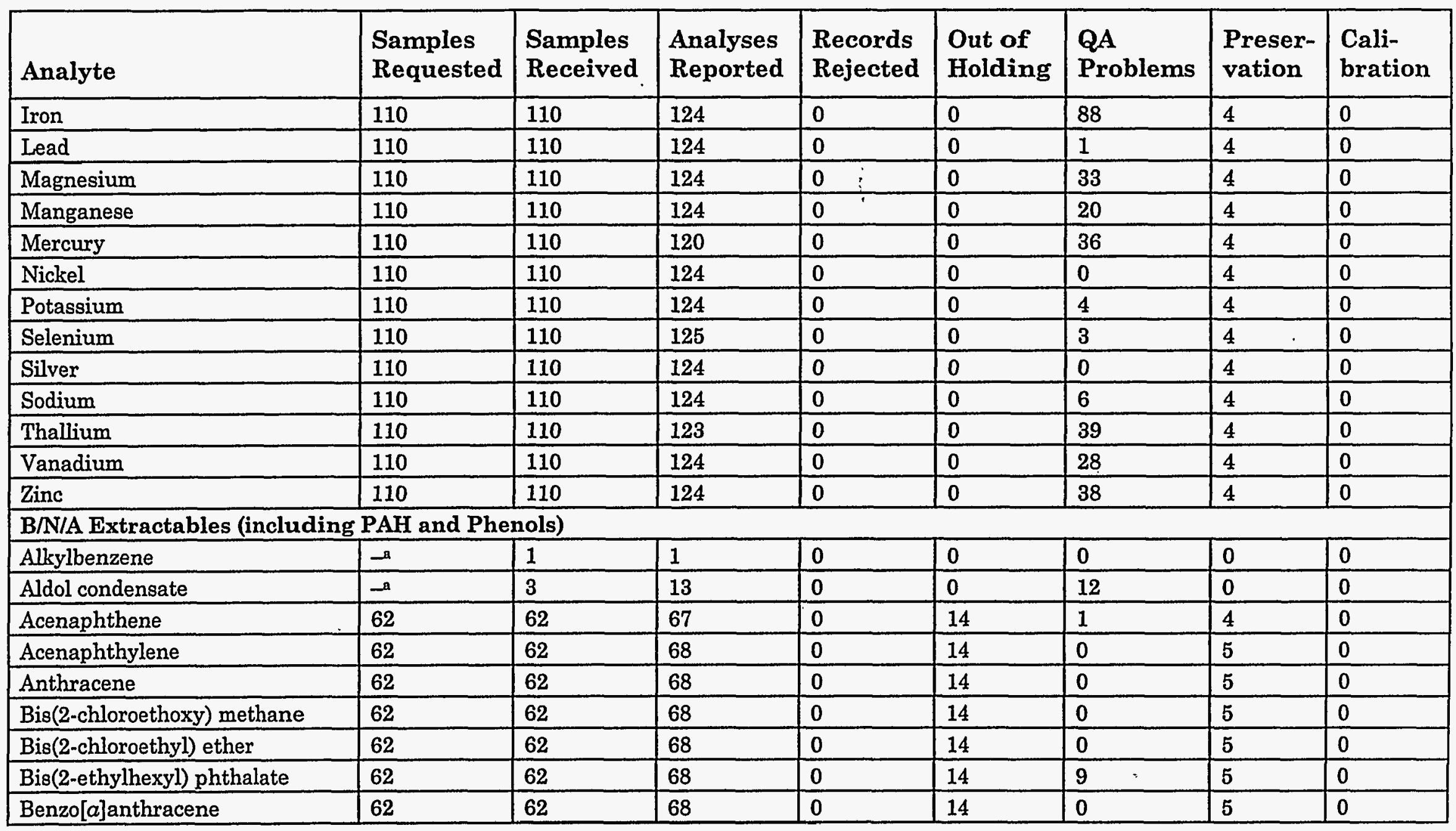

a Tentatively identified compounds (TICs) are requested but not reported in the requested column. 
Table 14. Number of Qualified Samples, Continued

\begin{tabular}{|c|c|c|c|c|c|c|c|c|}
\hline Analyte & $\begin{array}{l}\text { Samples } \\
\text { Requested }\end{array}$ & $\begin{array}{l}\text { Samples } \\
\text { Received }\end{array}$ & $\begin{array}{l}\text { Analyses } \\
\text { Reported }\end{array}$ & $\begin{array}{l}\text { Records } \\
\text { Rejected }\end{array}$ & $\begin{array}{l}\text { Out of } \\
\text { Holding }\end{array}$ & $\begin{array}{l}\text { QA } \\
\text { Problems }\end{array}$ & $\begin{array}{l}\text { Preser- } \\
\text { vation }\end{array}$ & $\begin{array}{l}\text { Cali- } \\
\text { bration }\end{array}$ \\
\hline Benzo[a]pyrene & 62 & 62 & 68 & 0 & 14 & 0 & 5 & 0 \\
\hline Benzo[b]fluoranthene & 62 & 62 & 68 & 0 & 14 & 0 & 5 & 0 \\
\hline Benzo[g,h,i]perylene & 62 & 62 & 68 & 0 & 14 & 0 & 5 & 0 \\
\hline Benzo[k]fluoranthene & 62 & 62 & 68 & 0 & 14 & 0 & 5 & 0 \\
\hline Benzoic acid & 62 & 62 & 68 & 0 & 14 & 5 & 5 & 0 \\
\hline Benzyl alcohol & 62 & 62 & 68 & 0 & 14 & 0 & 5 & 0 \\
\hline Beta-Amyrin & $-a$ & 1 & 1 & 0 & 0 & 0 & 0 & 0 \\
\hline Bis(2-chloroisopropyl) ether & 62 & 62 & 68 & 0 & 14 & 0 & 5 & 0 \\
\hline Butylbenzyl phthalate & 62 & 62 & 68 & 0 & 14 & 0 & 5 & 0 \\
\hline Caryophyllene oxide & & 1 & 1 & 0 & 0 & 0 & 0 & 0 \\
\hline Chrysene & 62 & 62 & 68 & 0 & 14 & 0 & 5 & 0 \\
\hline Cinnamyl cinnamate & & 1 & 1 & 0 & 0 & 0 & 0 & 0 \\
\hline Copaene & & 1 & 1 & 0 & 0 & 0 & 0 & 0 \\
\hline Cyclotetracosane & & 1 & 1 & 0 & 0 & 0 & 0 & 0 \\
\hline Di-n-butyl phthalate & 62 & 62 & 68 & 0 & 14 & 33 & 5 & 0 \\
\hline Di-n-octyl phthalate & 62 & 62 & 68 & 0 & 14 & 0 & 5 & 0 \\
\hline Dibenz $[a, h]$ anthracene & 62 & 62 & 68 & 0 & 14 & 0 & 5 & 0 \\
\hline Dibenzofuran & 62 & 62 & 68 & 0 & 14 & 0 & 5 & 0 \\
\hline Diethyl phthalate & 62 & 62 & 68 & 0 & 14 & 0 & 5 & 0 \\
\hline Dimethyl phthalate & 62 & 62 & 68 & 0 & 14 & 0 & 5 & 0 \\
\hline Docosane & & 5 & 6 & 0 & 0 & 0 & 0 & 0 \\
\hline Ergostanol & $-\mathbf{a}$ & 3 & 3 & 0 & 0 & 0 & 0 & 0 \\
\hline Fluoranthene & 62 & 62 & 68 & 0 & 14 & 0 & 5 & 0 \\
\hline Fluorene & 62 & 62 & 68 & 0 & 14 & 0 & 5 & 0 \\
\hline Friedoolean & & 1 & 1 & 0 & 0 & 0 & 0 & 0 \\
\hline Heneicosane & & 1 & 1 & 0 & 0 & 0 & 0 & 0 \\
\hline
\end{tabular}


Table 14. Number of Qualified Samples, Continued

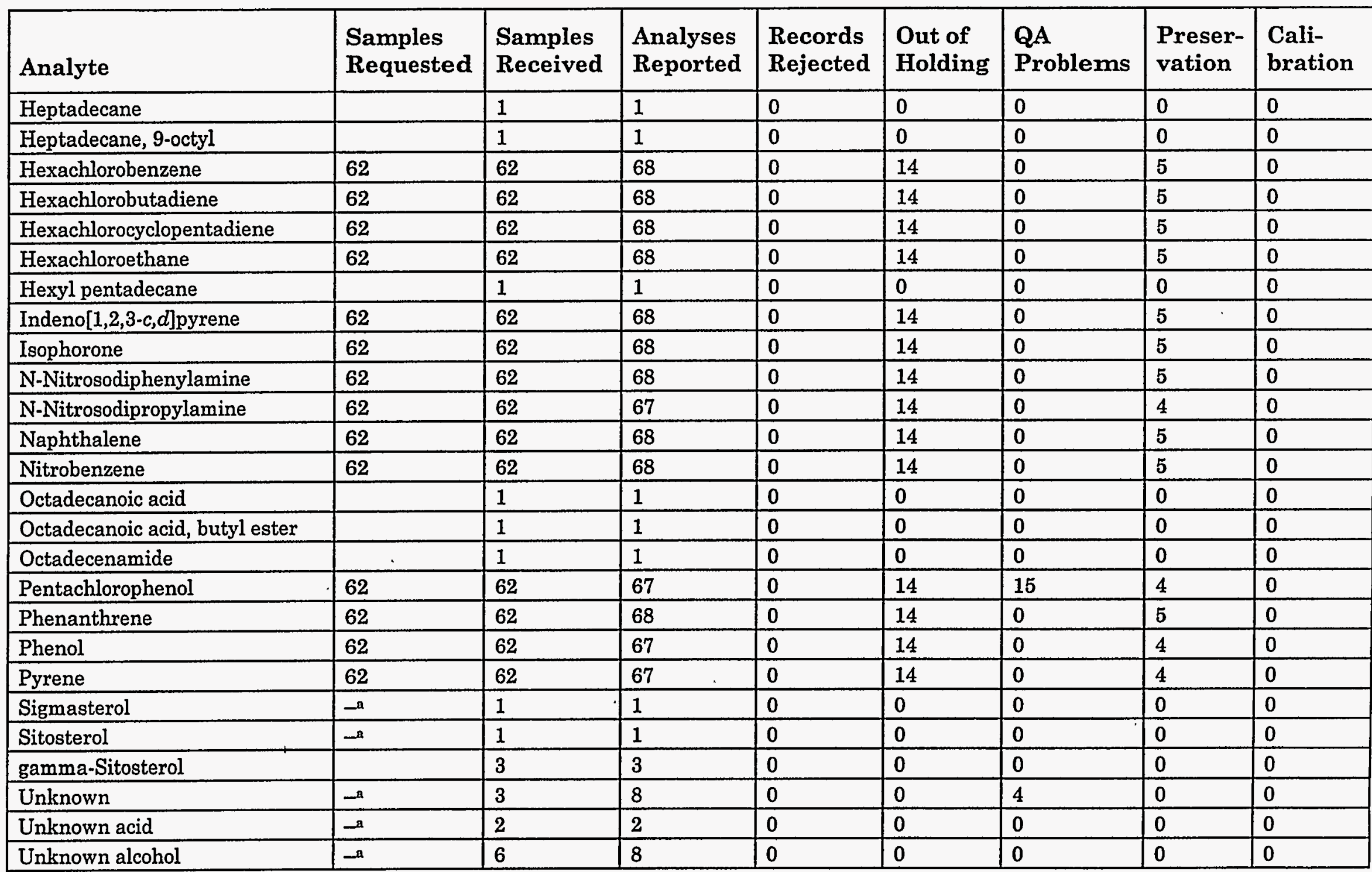


Table 14. Number of Qualified Samples, Continued

\begin{tabular}{|c|c|c|c|c|c|c|c|c|}
\hline Analyte & $\begin{array}{l}\text { Samples } \\
\text { Requested }\end{array}$ & $\begin{array}{l}\text { Samples } \\
\text { Received }\end{array}$ & $\begin{array}{l}\text { Analyses } \\
\text { Reported }\end{array}$ & $\begin{array}{l}\text { Records } \\
\text { Rejected }\end{array}$ & $\begin{array}{l}\text { Out of } \\
\text { Holding }\end{array}$ & $\begin{array}{l}\text { QA } \\
\text { Problems }\end{array}$ & $\begin{array}{l}\text { Preser- } \\
\text { vation }\end{array}$ & $\begin{array}{l}\text { Cali- } \\
\text { bration }\end{array}$ \\
\hline Unknown aldehyde & $-a$ & 1 & 1 & 0 & 0 & 0 & 0 & 0 \\
\hline Unknown alkane & $-\mathrm{a}$ & 4 & 5 & 0 & 0 & 0 & 0 & 0 \\
\hline Unknown alkene & $-\mathrm{a}$ & 2 & 2 & 0 & 0 & 0 & 0 & 0 \\
\hline Unknown amine & $-a$ & 1 & 1 & 0 & 0 & 0 & 0 & 0 \\
\hline Unknown azulene & $-a$ & 1 & 1 & 0 & 0 & 0 & 0 & 0 \\
\hline Unknown hydrocarbon & $-a$ & 10 & 13 & 0 & 0 & 0 & 0 & 0 \\
\hline Unknown naphthalene & $-a$ & 2 & 2 & 0 & 0 & 0 & 0 & 0 \\
\hline Unknown silane compound & $-\mathrm{a}$ & 1 & 1 & 0 & 0 & 0 & 0 & 0 \\
\hline Unknown vitamin & $-a$ & 1 & 1 & 0 & 0 & 0 & 0 & 0 \\
\hline 1,2,4-Trichlorobenzene & 62 & 62 & 67 & 0 & 14 & 0 & 4 & 0 \\
\hline 1,2-Dichlorobenzene & 62 & 62 & 68 & 0 & 14 & 0 & 5 & 0 \\
\hline 1,3-Dichlorobenzene & 62 & 62 & 68 & 0 & 14 & 0 & 5 & 0 \\
\hline 1,4-Dichlorobenzene & 62 & 62 & 67 & 0 & 14 & 0 & 4 & 0 \\
\hline 2,4,5-Trichlorophenol & 62 & 62 & 68 & 0 & 14 & 0 & 5 & 0 \\
\hline 2,4,6-Trichlorophenol & 62 & 62 & 68 & 0 & 14 & 0 & 5 & 0 \\
\hline 2,4-Dichlorophenol & 62 & 62 & 68 & 0 & 14 & 0 & 5 & 0 \\
\hline 2,4-Dimethyl phenol & 62 & 62 & 68 & 0 & 14 & 0 & 5 & 0 \\
\hline 2,4-Dinitrophenol & 62 & 62 & 68 & 0 & 14 & 1 & 5 & 0 \\
\hline 2,4-Dinitrotoluene & 62 & 62 & 67 & 0 & 14 & 51 & 4 & 0 \\
\hline 2,6-Dinitrotoluene & 62 & 62 & 68 & 0 & 14 & 0 & 5 & 0 \\
\hline 2-Chlorophenol & 62 & 62 & 67 & 0 & 14 & 1 & 4 & 0 \\
\hline 2-Chloronaphthalene & 62 & 62 & 68 & 0 & 14 & 0 & 5 & 0 \\
\hline 2-Methylnaphthalene & 62 & 62 & 68 & 0 & 14 & 0 & 5 & 0 \\
\hline o-Cresol (2-Methylphenol) & 62 & 62 & 68 & 0 & 14 & 0 & 5 & 0 \\
\hline o-Nitroaniline & 62 & 62 & 68 & 0 & 14 & 0 & 5 & 0 \\
\hline 2-Nitrophenol & 62 & 62 & 68 & 0 & 14 & 0 & 5 & 0 \\
\hline
\end{tabular}

Par Pond, Pond , C, and L Lake, Revision 2 
Table 14. Number of Qualified Samples, Continued

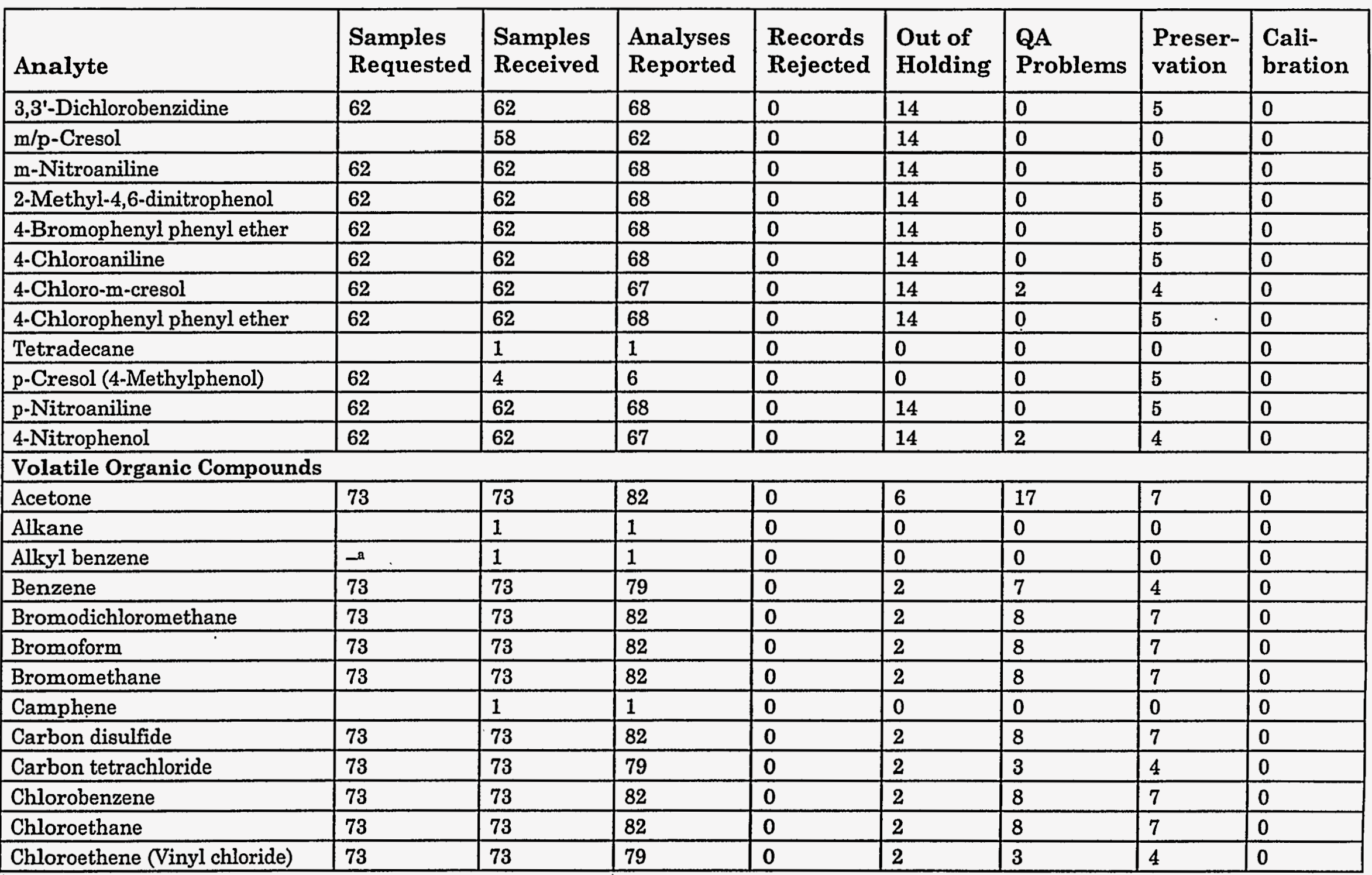


Table 14. Number of Qualified Samples, Continued

\begin{tabular}{|c|c|c|c|c|c|c|c|c|}
\hline Analyte & $\begin{array}{l}\text { Samples } \\
\text { Requested }\end{array}$ & $\begin{array}{l}\text { Samples } \\
\text { Received }\end{array}$ & $\begin{array}{l}\text { Analyses } \\
\text { Reported }\end{array}$ & $\begin{array}{l}\text { Records } \\
\text { Rejected }\end{array}$ & $\begin{array}{l}\text { Out of } \\
\text { Holding }\end{array}$ & $\begin{array}{l}\text { QA } \\
\text { Problems }\end{array}$ & $\begin{array}{l}\text { Preser- } \\
\text { vation }\end{array}$ & $\begin{array}{l}\text { Cali- } \\
\text { bration }\end{array}$ \\
\hline Chloroform & 73 & 73 & 82 & 0 & 2 & 8 & 7 & 0 \\
\hline Chloromethane & 73 & 73 & 82 & 0 & 2 & 8 & 7 & 0 \\
\hline Dibromochloromethane & 73 & 73 & 82 & 0 & 2 & 8 & 7 & 0 \\
\hline Dichloromethane & 73 & 73 & 79 & 0 & 2 & 59 & 4 & 0 \\
\hline Ethylbenzene & 73 & 73 & 79 & 0 & 2 & 3 & 4 & 0 \\
\hline Hexanol & & 1 & 1 & 0 & 0 & 0 & 0 & 0 \\
\hline Isocamphene & & 6 & 6 & 0 & 0 & 0 & 0 & 0 \\
\hline Isopropyl alcohol & & 1 & 1 & 0 & 0 & 0 & 0 & 0 \\
\hline Methyl cyclopentane & & 8 & 8 & 0 & 0 & 0 & 0 & 0 \\
\hline Methyl ethyl ketone & 73 & 73 & 82 & 0 & 2 & 78 & 7 & 0 \\
\hline Methyl isobutyl ketone & 73 & 73 & 82 & 0 & 2 & 8 & 7 & 0 \\
\hline Methyl pentane & & 2 & 2 & 0 & 0 & 0 & 0 & 0 \\
\hline Propyl benzene & & 3 & 3 & 0 & 0 & 0 & 0 & 0 \\
\hline Styrene & 73 & 73 & 79 & 0 & 2 & 3 & 4 & 0 \\
\hline Tetrachloroethylene & 73 & 73 & 82 & 0 & 2 & 8 & 7 & 0 \\
\hline Toluene & 73 & 73 & 79 & 0 & 2 & 34 & 4 & 0 \\
\hline Trichloroethylene & 73 & 73 & 79 & 0 & 2 & 3 & 4 & 0 \\
\hline Unknown & & 3 & 7 & 0 & 0 & 0 & 0 & 0 \\
\hline Unknown acid & & 1 & 1 & 0 & 0 & 0 & 0 & 0 \\
\hline Unknown alcohol & & 10 & 10 & 0 & 0 & 0 & 0 & 0 \\
\hline Unknown alkane & & 2 & 2 & 0 & 0 & 0 & 0 & 0 \\
\hline Unknown alkylbenzene & & 1 & 1 & 0 & 0 & 0 & 0 & 0 \\
\hline Unknown hydrocarbon & & 3 & 3 & 0 & 0 & 0 & 0 & 0 \\
\hline Vinyl acetate & 73 & 73 & 82 & 0 & 3 & 8 & 7 & 0 \\
\hline 1,1,2,2-Tetrachloroethane & 73 & 73 & 79 & 0 & 2 & 3 & 4 & 0 \\
\hline 1,1,1-Trichloroethane & 73 & 73 & 82 & 0 & 2 & 8 & 7 & 0 \\
\hline
\end{tabular}


Table 14. Number of Qualified Samples, Continued

\begin{tabular}{|c|c|c|c|c|c|c|c|c|}
\hline Analyte & $\begin{array}{l}\text { Samples } \\
\text { Requested }\end{array}$ & $\begin{array}{l}\text { Samples } \\
\text { Received }\end{array}$ & $\begin{array}{l}\text { Analyses } \\
\text { Reported }\end{array}$ & $\begin{array}{l}\text { Records } \\
\text { Rejected }\end{array}$ & $\begin{array}{l}\text { Out of } \\
\text { Holding }\end{array}$ & $\begin{array}{l}\text { QA } \\
\text { Problems }\end{array}$ & $\begin{array}{l}\text { Preser- } \\
\text { vation }\end{array}$ & $\begin{array}{l}\text { Cali- } \\
\text { bration }\end{array}$ \\
\hline 1,1,2-Trichloroethane & 73 & 73 & 82 & 0 & 2 & 8 & 7 & 0 \\
\hline 1,1-Dichloroethane & 73 & 73 & 82 & 0 & 2 & 8 & 7 & 0 \\
\hline 1,2-Dichloroethylene & 73 & 73 & 82 & 0 & 2 & 8 & 7 & 0 \\
\hline 1,2-Dichloroethane & 73 & 73 & 82 & 0 & 2 & 8 & 7 & 0 \\
\hline trans-1,3-Dichloropropene & 73 & 73 & 82 & 0 & 2 & 8 & 7 & 0 \\
\hline Methyl(methylethyl)cyclohexane & & 2 & 2 & 0 & 0 & $\mathbf{0}$ & 0 & 0 \\
\hline 2-Hexanone & 73 & 73 & 82 & 0 & 2 & 51 & 7 & 0 \\
\hline Xylenes & 73 & 73 & 82 & 0 & 2 & 8 & 7 & 0 \\
\hline \multicolumn{9}{|c|}{ Pesticides/PCBs and Dioxins/Furans } \\
\hline alpha-Chlordane & 62 & 62 & 66 & 0 & 1 & 17 & 5 & 0 \\
\hline gamma-Chlordane & 62 & 62 & 66 & 0 & 1 & 17 & 5 & 0 \\
\hline$p, p^{\prime}-\mathrm{DDD}$ & 62 & 62 & 66 & 0 & 1 & 17 & 5 & 0 \\
\hline p,p'-DDE & 62 & 62 & 66 & 0 & 1 & 17 & 5 & 0 \\
\hline $\mathrm{p}, \mathrm{p}^{\prime}-\mathrm{DDT}$ & 62 & 62 & 65 & 0 & 1 & 18 & 4 & 0 \\
\hline Dieldrin & 62 & 62 & 65 & 0 & 1 & 17 & 4 & 0 \\
\hline Endrin ketone & 62 & 62 & 66 & 0 & 1 & 17 & 5 & 0 \\
\hline Endrin & 62 & 62 & 65 & 0 & 1 & 17 & 4 & 0 \\
\hline Endosulfan I & 62 & 62 & 66 & 0 & 1 & 17 & 5 & 0 \\
\hline Endosulfan II & 62 & 62 & 66 & 0 & 1 & 17 & 5 & 0 \\
\hline
\end{tabular}


Table 14. Number of Qualified Samples, Continued

\begin{tabular}{|c|c|c|c|c|c|c|c|c|}
\hline Analyte & $\begin{array}{l}\text { Samples } \\
\text { Requested }\end{array}$ & $\begin{array}{l}\text { Samples } \\
\text { Received }\end{array}$ & $\begin{array}{l}\text { Analyses } \\
\text { Reported }\end{array}$ & $\begin{array}{l}\text { Records } \\
\text { Rejected }\end{array}$ & $\begin{array}{l}\text { Out of } \\
\text { Holding }\end{array}$ & $\begin{array}{l}\text { QA } \\
\text { Problems }\end{array}$ & $\begin{array}{l}\text { Preser- } \\
\text { vation }\end{array}$ & $\begin{array}{l}\text { Cali- } \\
\text { bration }\end{array}$ \\
\hline Endosulfan sulfate & 62 & 62 & 66 & 0 & 1 & 17 & 5 & 0 \\
\hline Heptachlor & 62 & 62 & 65 & 0 & 1 & 17 & 4 & 0 \\
\hline Heptachlor epoxide & 62 & 62 & 66 & 0 & 1 & 17 & 5 & 0 \\
\hline Lindane & 62 & 62 & 65 & 0 & 1 & 17 & 4 & 0 \\
\hline Methoxychlor & 62 & 62 & 66 & 0 & 1 & 17 & 5 & 0 \\
\hline PCB 1016 & 62 & 62 & 66 & 0 & 1 & 17 & 5 & 0 \\
\hline PCB 1221 & 62 & 62 & 66 & 0 & 1 & 17 & 5 & 0 \\
\hline PCB 1232 & 62 & 62 & 66 & 0 & 1 & 17 & 5 & 0 \\
\hline PCB 1242 & 62 & 62 & 66 & 0 & 1 & 17 & 5 & 0 \\
\hline PCB 1248 & 62 & 62 & 66 & 0 & 1 & 17 & 5 & 0 \\
\hline PCB 1254 & 62 & 62 & 66 & 0 & 1 & 17 & 5 & 0 \\
\hline PCB 1260 & 62 & 62 & 66 & 0 & 1 & 17 & 5 & 0 \\
\hline Toxaphene & 62 & 62 & 66 & 0 & 1 & 17 & 5 & 0 \\
\hline \multicolumn{9}{|c|}{ Radionuclide Indicators and Radionuclides } \\
\hline Actinium-228 & 106 & 105 & 111 & 0 & 0 & 0 & 0 & 0 \\
\hline Antimony-124 & 106 & 105 & 111 & 0 & 0 & 0 & 0 & 0 \\
\hline Antimony-125 & 106 & 105 & 111 & 0 & 0 & 0 & 0 & 0 \\
\hline Barium-133 & 106 & 105 & 111 & 0 & 0 & 0 & 0 & 0 \\
\hline Cerium-144 & 106 & 105 & 111 & 0 & 0 & 0 & 0 & 0 \\
\hline Cesium-134 & 106 & 105 & 111 & 0 & 0 & 0 & 0 & 0 \\
\hline Cesium-137 & 106 & 105 & 111 & 0 & 0 & 0 & 0 & 0 \\
\hline Cobalt-57 & 106 & 105 & 111 & 0 & 0 & 0 & 0 & 0 \\
\hline Cobalt-58 & 106 & 105 & 111 & 0 & 0 & 0 & 0 & 0 \\
\hline Cobalt-60 & 106 & 105 & 111 & 0 & 0 & 0 & 0 & 0 \\
\hline Europium-152 & 106 & 105 & 111 & 0 & 0 & 0 & 0 & 0 \\
\hline Europium-154 & 106 & 105 & 111 & 0 & 0 & 0 & 0 & 0 \\
\hline
\end{tabular}


Table 14. Number of Qualified Samples, Continued

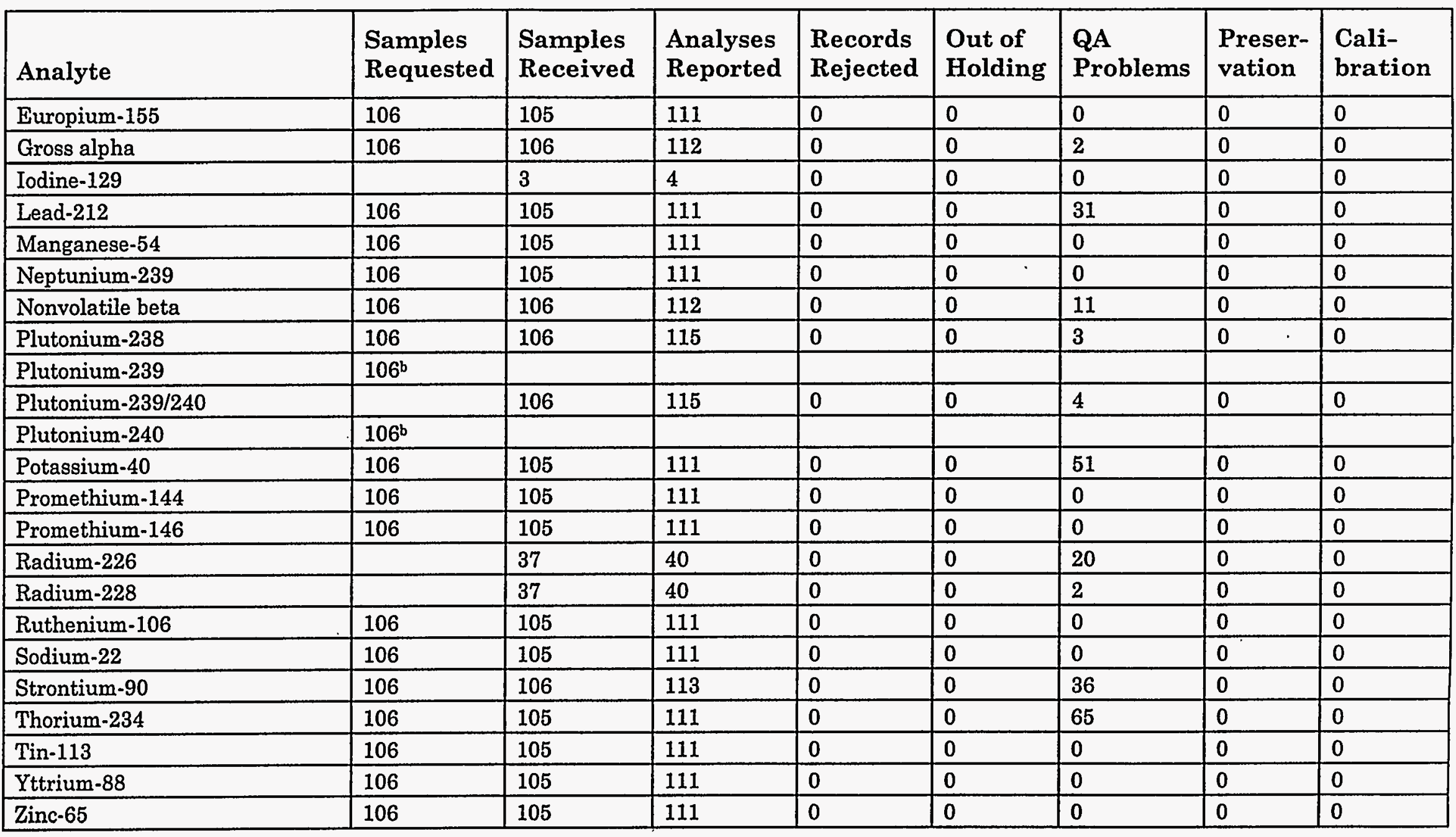

b Plutonium-239 and plutonium-240 were requested separately but were reported as plutonium-239/240. 
Table 14. Number of Qualified Samples, Continued

\begin{tabular}{|l|l|l|l|l|l|l|l|l|}
\hline Analyte & $\begin{array}{l}\text { Samples } \\
\text { Requested }\end{array}$ & $\begin{array}{l}\text { Samples } \\
\text { Received }\end{array}$ & $\begin{array}{l}\text { Analyses } \\
\text { Reported }\end{array}$ & $\begin{array}{l}\text { Records } \\
\text { Rejected }\end{array}$ & $\begin{array}{l}\text { Out of } \\
\text { Holding }\end{array}$ & $\begin{array}{l}\text { QA } \\
\text { Problems }\end{array}$ & $\begin{array}{l}\text { Preser- } \\
\text { vation }\end{array}$ & $\begin{array}{l}\text { Cali- } \\
\text { bration }\end{array}$ \\
\hline Zirconium-95 & 106 & 105 & 111 & 0 & 0 & 0 & 0 & 0 \\
\hline
\end{tabular}




\section{Analytical Accuracy}

This report examines the analytical results from laboratory control samples, blank spikes, and laboratory blanks to determine how accurate the results are and whether the results are biased. Only results from the primary laboratory are considered in this evaluation. Recoveries in the tables in this section are given as percentages.

\section{Accuracy of Analytical Method}

The accuracy of the laboratory's performance with each analytical method is evaluated by reviewing the results of the analyses of laboratory blanks and spiked blank samples (laboratory control samples and blank spikes).

\section{Laboratory Control Samples}

Laboratory control samples are deionized water samples that are spiked with the target analyte, digested, and analyzed along with the regular samples for inorganic analyses. Laboratory control samples monitor the overall performance of all steps in the analysis, including sample preparation. See Tables 15 and 16 for the laboratory control samples analyzed with sediment and water samples, respectively.

Table 15. Laboratory Control Samples Analyzed with Sediment Samples

\begin{tabular}{|l|l|l|l|l|l|}
\hline & $\begin{array}{l}\text { Number } \\
\text { Analyzed }\end{array}$ & $\begin{array}{l}\text { Mean } \\
\text { Recovery }\end{array}$ & $\begin{array}{l}\text { Std. } \\
\text { Dev. }\end{array}$ & $\begin{array}{l}\text { Min. } \\
\text { Recovery }\end{array}$ & $\begin{array}{l}\text { Max. } \\
\text { Recovery }\end{array}$ \\
\hline Method EPA6010A (inorganics are total recoverable) & \multicolumn{3}{l|}{} \\
\hline Aluminum & 22 & 92 & 14 & 68.5 & 117 \\
\hline Antimony & 22 & 93.8 & 10 & 68 & 111 \\
\hline Arsenic & 22 & 108.1 & 6.3 & 94.4 & 119 \\
\hline Barium & 22 & 104.7 & 8.1 & 83.2 & 121 \\
\hline Beryllium & 21 & 105.7 & 5.9 & 95.6 & 121 \\
\hline Cadmium & 22 & 103.8 & 6.2 & 90.6 & 115 \\
\hline Calcium & 22 & 102.2 & 5.3 & 87.3 & 112 \\
\hline Chromium & 22 & 106.4 & 5.4 & 97.6 & 120 \\
\hline Cobalt & 22 & 102.8 & 4.4 & 94.3 & 113 \\
\hline Copper & 22 & 103.5 & 5.3 & 92.6 & 118 \\
\hline Iron & 22 & 81.8 & 13.4 & 58.1 & 111 \\
\hline Lead & 22 & 97.5 & 4.4 & 89.2 & 106 \\
\hline Magnesium & 22 & 102.8 & 4.7 & 94.7 & 114 \\
\hline Manganese & 22 & 96.9 & 3.6 & 91.1 & 107 \\
\hline Nickel & 22 & 105.1 & 5.1 & 97.3 & 118 \\
\hline Potassium & 22 & 97.8 & 4.6 & 89.9 & 109 \\
\hline Selenium & 22 & 94.2 & 4.4 & 85.5 & 103 \\
\hline Silver & 22 & 109.1 & 4.8 & 98.8 & 120 \\
\hline Sodium & 22 & 90.5 & 3.4 & 85.2 & 101 \\
\hline Thallium & 22 & 112.6 & 9 & 92.8 & 125 \\
\hline
\end{tabular}


Table 15. Laboratory Control Samples Analyzed with Sediment Samples, Continued

\begin{tabular}{|l|l|l|l|l|l|}
\hline & $\begin{array}{l}\text { Number } \\
\text { Analyzed }\end{array}$ & $\begin{array}{l}\text { Mean } \\
\text { Recovery }\end{array}$ & $\begin{array}{l}\text { Std. } \\
\text { Dev. }\end{array}$ & $\begin{array}{l}\text { Min. } \\
\text { Recovery }\end{array}$ & $\begin{array}{l}\text { Max. } \\
\text { Recovery }\end{array}$ \\
\hline Vanadium & 22 & 110.4 & 7.1 & 93.5 & 125 \\
\hline Zinc & 22 & 94.8 & 5.7 & 80.4 & 106 \\
\hline Method EPA7471 (total recoverable) & 99.9 & 4.5 & 93.9 & 112 \\
\hline Mercury & 16 & & \\
\hline
\end{tabular}

Table 16. Laboratory Control Samples Analyzed with Water Samples

\begin{tabular}{|c|c|c|c|c|c|}
\hline Analyte & $\begin{array}{l}\text { Number } \\
\text { Analyzed }\end{array}$ & $\begin{array}{l}\text { Mean } \\
\text { Recovery }\end{array}$ & $\begin{array}{l}\text { Std. } \\
\text { Dev. }\end{array}$ & $\begin{array}{l}\text { Min. } \\
\text { Recovery }\end{array}$ & $\begin{array}{l}\text { Max. } \\
\text { Recovery }\end{array}$ \\
\hline \multicolumn{6}{|c|}{ Method EPA6010A (inorganics are total recoverable) } \\
\hline Aluminum & 6 & 101.3 & 5 & 96.9 & 111 \\
\hline Antimony & 2 & 97.4 & 2.3 & 95.7 & 99 \\
\hline Barium & 6 & 98.6 & 3.5 & 94.8 & 105 \\
\hline Beryllium & 6 & 99.6 & 3.1 & 95.1 & 104 \\
\hline Cadmium & 6 & 95.2 & 2.7 & 92.7 & 100 \\
\hline Calcium & 6 & 100.8 & 5.5 & 92.1 & 106 \\
\hline Chromium & 6 & 98.8 & 5.1 & 92 & 106 \\
\hline Cobalt & 6 & 99.3 & 5 & 92.4 & 106 \\
\hline Copper & 6 & 99.6 & 3.7 & 95.2 & 106 \\
\hline Iron & 6 & 100.8 & 4.4 & 94.6 & 107 \\
\hline Lead & 6 & 99.5 & 7.3 & 90.3 & 108 \\
\hline Magnesium & 6 & 97.1 & 5.7 & 89.4 & 105 \\
\hline Manganese & 6 & 99.5 & 4 & 93.7 & 105 \\
\hline Nickel & 6 & 101.8 & 6.5 & 92.6 & 109 \\
\hline Potassium & 6 & 92.4 & 1.9 & 91.1 & 96.1 \\
\hline Silver & 6 & 98.2 & 4.1 & 93 & 104 \\
\hline Sodium & 6 & 97.1 & 2.6 & 94.1 & 101 \\
\hline Vanadium & 6 & 100 & 4 & 94.3 & 106 \\
\hline Zinc & 6 & 100 & 6.7 & 88.6 & 107 \\
\hline \multicolumn{6}{|c|}{ Method EPA7041 (total recoverable) } \\
\hline Antimony & 6 & 94.5 & 12.3 & 80 & 110 \\
\hline \multicolumn{6}{|c|}{ Method EPA7060 (total recoverable) } \\
\hline Arsenic & 6 & 92.6 & 3.5 & 88.5 & 97.5 \\
\hline \multicolumn{6}{|c|}{ Method EPA7421 (total recoverable) } \\
\hline Lead & 6 & 95.6 & 15.1 & 85 & 124 \\
\hline \multicolumn{6}{|c|}{ Method EPA7470 (total recoverable) } \\
\hline Mercury, total recoverable & 8 & 98.2 & 2.7 & 93 & 101 \\
\hline \multicolumn{6}{|c|}{ Method EPA7740 (total recoverable) } \\
\hline Selenium & 6 & 94.7 & 8.2 & 86.5 & 106 \\
\hline
\end{tabular}

Par Pond, Pond C, and L Lake, Revision 2 

Table 17. Blank Spikes for Sediment Samples, Continued

\begin{tabular}{|c|c|c|c|c|c|}
\hline Analyte & $\begin{array}{l}\text { Number } \\
\text { Analyzed }\end{array}$ & $\begin{array}{l}\text { Mean } \\
\text { Recovery }\end{array}$ & $\begin{array}{l}\text { Std. } \\
\text { Dev. }\end{array}$ & $\begin{array}{l}\text { Min. } \\
\text { Recovery }\end{array}$ & $\begin{array}{l}\text { Max. } \\
\text { Recovery }\end{array}$ \\
\hline Lindane & 12 & 96.2 & 11.3 & 75.4 & 112 \\
\hline \multicolumn{6}{|l|}{ Method EPA8270 } \\
\hline Acenaphthene & 14 & 80.4 & 6.1 & 70.3 & 91.1 \\
\hline N-Nitrosodipropylamine & 14 & 80.9 & 8.2 & 69.7 & 95.5 \\
\hline Pentachlorophenol & 14 & 97.8 & 13 & 70.8 & 119 \\
\hline Phenol & 14 & 64.7 & 5.3 & 57 & 72.1 \\
\hline Pyrene & 14 & 103.1 & 12.3 & 81.6 & 115 \\
\hline 2,4-Dinitrotoluene & 14 & 88.4 & 9.6 & 68.7 & 98.1 \\
\hline 2-Chlorophenol & 14 & 75.5 & 6.2 & 65.3 & 87.5 \\
\hline 4-Chloro-m-cresol & 14 & 85.7 & 7.4 & 69.8 & 93.7 \\
\hline 4-Nitrophenol & 14 & 90.7 & 9.6 & 76.2 & 107 \\
\hline \multicolumn{6}{|l|}{ Method EPA9081 } \\
\hline Cation exchange capacity & 20 & 94.5 & 4.7 & 86.3 & 104 \\
\hline \multicolumn{6}{|l|}{ Method EPIA-001B } \\
\hline Gross alpha & 8 & 107.3 & 12.6 & 83.3 & 118 \\
\hline Nonvolatile beta & 8 & 116 & 7.2 & 100 & 124 \\
\hline \multicolumn{6}{|l|}{ Method EPIA-004 } \\
\hline Strontium-90 & 7 & 99.4 & 9.4 & 80.3 & 110 \\
\hline \multicolumn{6}{|l|}{ Method EPIA-012B } \\
\hline Plutonium-238 & 7 & 96.5 & 3.8 & 93.7 & 104 \\
\hline \multicolumn{6}{|l|}{ Method EPIA-013B } \\
\hline Cesium-137 & 7 & 116.3 & 1.7 & 114 & 118 \\
\hline
\end{tabular}

Table 18. Blank Spikes for Water Samples

\begin{tabular}{|l|l|l|l|l|l|}
\hline \multicolumn{2}{|l|}{$\begin{array}{l}\text { Number } \\
\text { Analyzed }\end{array}$} & $\begin{array}{l}\text { Mean } \\
\text { Recovery }\end{array}$ & $\begin{array}{l}\text { Std. } \\
\text { Dev. }\end{array}$ & $\begin{array}{l}\text { Min. } \\
\text { Recovery }\end{array}$ & $\begin{array}{l}\text { Max. } \\
\text { Recovery }\end{array}$ \\
\hline Method EPA335.3 & 8 & 95.8 & 1.4 & 94.2 & 98 \\
\hline Cyanide & 8 & \multicolumn{5}{l|}{} \\
\hline Method EPA8080 & 6 & 99.5 & 9 & 88.6 & 111 \\
\hline Aldrin & 6 & 110.5 & 34.8 & 78.8 & 164 \\
\hline p,p'-DDT & 6 & 105.6 & 17.5 & 89.2 & 132 \\
\hline Dieldrin & 6 & 94.8 & 18.5 & 81.6 & 120 \\
\hline Endrin & 6 & 94.1 & 8 & 81.1 & 104 \\
\hline Heptachlor & 6 & 97.5 & 18.8 & 79.5 & 123 \\
\hline Lindane & 6 & & & 78.8 \\
\hline Method EPA8270 & 6 & 70.5 & 8.4 & 59.7 & 84.5 \\
\hline Acenaphthene & 6 & 76.3 & 6.1 & 66.5 & \\
\hline N-Nitrosodipropylamine &
\end{tabular}


Table 18. Blank Spikes for Water Samples, Continued

\begin{tabular}{|l|l|l|l|l|l|}
\hline Analyte & $\begin{array}{l}\text { Number } \\
\text { Analyzed }\end{array}$ & $\begin{array}{l}\text { Mean } \\
\text { Recovery }\end{array}$ & $\begin{array}{l}\text { Std. } \\
\text { Dev. }\end{array}$ & $\begin{array}{l}\text { Min. } \\
\text { Recovery }\end{array}$ & $\begin{array}{l}\text { Max. } \\
\text { Recovery }\end{array}$ \\
\hline Pentachlorophenol & 6 & 90.6 & 5.2 & 84.1 & 96.9 \\
\hline Phenol & 6 & 39.1 & 8 & 26.5 & 46.3 \\
\hline Pyrene & 6 & 100.2 & 10.1 & 92.2 & 116 \\
\hline 2,4-Dinitrotoluene & 6 & 90.1 & 4.2 & 82.2 & 94 \\
\hline 2-Chlorophenol & 6 & 71 & 7 & 58.5 & 78.3 \\
\hline 4-Chloro-m-cresol & 6 & 79.4 & 4.1 & 73.1 & 83.5 \\
\hline 4-Nitrophenol & 6 & 49.2 & 9.6 & 39.2 & 61.7 \\
\hline
\end{tabular}

\section{Laboratory Blanks}

Laboratory blanks are portions of the water used for dilutions that are analyzed along with the samples. Laboratory blanks are used to determine the existence and magnitude of contamination problems within the laboratory. See Tables 19 and 21 for analytes not detected and detected in laboratory blanks of sediment samples, respectively. See Tables 20 and 22 for the analytes not detected and detected in laboratory blanks of water samples, respectively.

Table 19. Analytes Not Detected in Laboratory Blanks Analyzed with Sediment Samples

\begin{tabular}{|l|l|}
\hline Analyte & Number \\
\hline Method EPA300.0 & 8 \\
\hline Chloride & 22 \\
\hline Method EPA335.3 & 8 \\
\hline Cyanide & 8 \\
\hline Method EPA350.1 & 5 \\
\hline Ammonia nitrogen & 13 \\
\hline Method EPA351.2 \\
\hline Nitrogen by Kjeldahl method & 5 \\
\hline Method EPA365.2 & 12 \\
\hline Orthophosphate & 13 \\
\hline Method EPA365.4 & 12 \\
\hline Total phosphates (as P) & 11 \\
\hline Method EPA376.2 & 10 \\
\hline Sulfide & 12 \\
\hline Method EPA420.2 & \\
\hline Phenols & \\
\hline Method EPA6010A (total recoverable) \\
\hline Antimony \\
\hline Arsenic \\
\hline Barium & 11 \\
\hline
\end{tabular}

\begin{tabular}{|l|l|}
\hline Analyte & Number \\
\hline Beryllium & 11 \\
\hline Cadmium & 11 \\
\hline Cobalt & 11 \\
\hline Lead & 11 \\
\hline Nickel & 11 \\
\hline Potassium & 11 \\
\hline Selenium & 11 \\
\hline Sodium & 11 \\
\hline Vanadium & 11 \\
\hline Method EPA8080 & \multicolumn{1}{|l|}{} \\
\hline Aldrin & 6 \\
\hline alpha-Benzene hexachloride & 6 \\
\hline beta-Benzene hexachloride & 6 \\
\hline delta-Benzene hexachloride & 6 \\
\hline alpha-Chlordane & 6 \\
\hline gamma-Chlordane & 6 \\
\hline p,p'-DDD & 6 \\
\hline p,p'-DDE & 6 \\
\hline p,p'-DDT & 6 \\
\hline Dieldrin & 6 \\
\hline
\end{tabular}


Table 19. Analytes Not Detected in Laboratory Blanks Analyzed with Sediment Samples, Continued

\begin{tabular}{|c|c|}
\hline Analyte & Number \\
\hline Endosulfan I & 6 \\
\hline Endosulfan II & 6 \\
\hline Endosulfan sulfate & 6 \\
\hline Endrin & 6 \\
\hline Endrin ketone & 6 \\
\hline Heptachlor & 6 \\
\hline Heptachlor epoxide & 6 \\
\hline Lindane & 6 \\
\hline Methoxychlor & 6 \\
\hline PCB 1016 & 6 \\
\hline PCB 1221 & 6 \\
\hline PCB 1232 & 6 \\
\hline PCB 1242 & 6 \\
\hline PCB 1248 & 6 \\
\hline PCB 1254 & 6 \\
\hline PCB 1260 & 6 \\
\hline Toxaphene & 6 \\
\hline \multicolumn{2}{|l|}{ Method EPA8260 } \\
\hline Benzene & 8 \\
\hline Bromodichloromethane & 8 \\
\hline Bromoform & 8 \\
\hline $\begin{array}{l}\text { Bromomethane } \\
\text { (Methyl bromide) }\end{array}$ & 8 \\
\hline Carbon disulfide & 8 \\
\hline Carbon tetrachloride & 8 \\
\hline Chlorobenzene & 8 \\
\hline Chloroethane & 8 \\
\hline Chloroethene (Vinyl chloride) & 8 \\
\hline Chloroform & 8 \\
\hline $\begin{array}{l}\text { Chloromethane } \\
\text { (Methyl chloride) }\end{array}$ & 8 \\
\hline Dibromochloromethane & 8 \\
\hline Ethylbenzene & 8 \\
\hline Styrene & 8 \\
\hline Tetrachloroethylene & 8 \\
\hline Trichloroethylene & 8 \\
\hline Vinyl acetate & 8 \\
\hline Xylenes & 8 \\
\hline 1,1,2,2-Tetrachloroethane & 8 \\
\hline 1,1,1-Trichloroethane & 8 \\
\hline
\end{tabular}

\begin{tabular}{|c|c|}
\hline Analyte & Number \\
\hline 1,1,2-Trichloroethane & 8 \\
\hline 1,1-Dichloroethylene & 8 \\
\hline 1,1-Dichloroethane & 8 \\
\hline 1,2-Dichloroethylene & 8 \\
\hline 1,2-Dichloroethane & 8 \\
\hline 1,2-Dichloropropane & 8 \\
\hline cis-1,3-Dichloropropene & 8 \\
\hline trans-1,3-Dichloropropene & 8 \\
\hline \multicolumn{2}{|l|}{ Method EPA8270 } \\
\hline Acenaphthene & 7 \\
\hline Acenaphthylene & 7 \\
\hline Anthracene & 7 \\
\hline Benzo[a]anthracene & 7 \\
\hline Benzo[a]pyrene & 7 \\
\hline Benzo[b]fluoranthene & 7 \\
\hline Benzo[g, $h, i]$ perylene & 7 \\
\hline Benzo[k]fluoranthene & 7 \\
\hline Benzoic acid & 7 \\
\hline Benzyl alcohol & 7 \\
\hline Bis(2-chloroethoxy) methane & 7 \\
\hline Bis(2-chloroethyl) ether & 7 \\
\hline Bis(2-chloroisopropyl) ether & 7 \\
\hline Bis(2-ethylhexyl) phthalate & 7 \\
\hline Butylbenzyl phthalate & 7 \\
\hline Chrysene & 7 \\
\hline Di-n-octyl phthalate & 6 \\
\hline Dibenz $[a, h]$ anthracene & 7 \\
\hline Dibenzofuran & 7 \\
\hline Dimethyl phthalate & 7 \\
\hline Fluoranthene & 7 \\
\hline Fluorene & 7 \\
\hline Hexachlorobenzene & 7 \\
\hline Hexachlorobutadiene & 7 \\
\hline Hexachlorocyclopentadiene & 7 \\
\hline Hexachloroethane & 7 \\
\hline Indeno $[1,2,3-c, d]$ pyrene & 7 \\
\hline Isophorone & 7 \\
\hline N-Nitrosodiphenylamine & 7 \\
\hline N-Nitrosodipropylamine & 7 \\
\hline Naphthalene & 7 \\
\hline
\end{tabular}


Table 19. Analytes Not Detected in Laboratory Blanks Analyzed with Sediment Samples, Continued

\begin{tabular}{|l|l|}
\hline Analyte & Number \\
\hline Nitrobenzene & 7 \\
\hline Pentachlorophenol & 7 \\
\hline Phenanthrene & 7 \\
\hline Phenol & 7 \\
\hline Pyrene & 7 \\
\hline 1,2,4-Trichlorobenzene & 7 \\
\hline 1,2-Dichlorobenzene & 7 \\
\hline 1,3-Dichlorobenzene & 7 \\
\hline 1,4-Dichlorobenzene & 7 \\
\hline 2,4,5-Trichlorophenol & 7 \\
\hline 2,4,6-Trichlorophenol & 7 \\
\hline 2,4-Dichlorophenol & 7 \\
\hline 2,4-Dimethyl phenol & 7 \\
\hline 2,4-Dinitrophenol & 7 \\
\hline 2,4-Dinitrotoluene & 7 \\
\hline 2,6-Dinitrotoluene & 7 \\
\hline 2-Chlorophenol & 7 \\
\hline 2-Chloronaphthalene & 7 \\
\hline 2-Methylnaphthalene & 7 \\
\hline o-Cresol (2-Methylphenol) & 7 \\
\hline o-Nitroaniline & 7 \\
\hline 2-Nitrophenol & 7 \\
\hline 3,3'-Dichlorobenzidine & 7 \\
\hline m/p-Cresol & 7 \\
\hline m-Nitroaniline & 7 \\
\hline 2-Methyl-4,6-dinitrophenol & 7 \\
\hline 4-Bromophenyl phenyl ether & 7 \\
\hline 4-Chloroaniline & 7 \\
\hline 4-Chloro-m-cresol & 7 \\
\hline 4-Chlorophenyl phenyl ether & 7 \\
\hline p-Nitroaniline & 7 \\
\hline
\end{tabular}

\begin{tabular}{|c|c|}
\hline Analyte & Number \\
\hline 4-Nitrophenol & 7 \\
\hline \multicolumn{2}{|l|}{ Method EPA9081 } \\
\hline Cation exchange capacity & 16 \\
\hline \multicolumn{2}{|l|}{ Method EPIA-D01B } \\
\hline Actinium-228 & 7 \\
\hline Antimony-124 & 7 \\
\hline Antimony-125 & 7 \\
\hline Barium-133 & 7 \\
\hline Cerium-144 & 7 \\
\hline Cesium-134 & 7 \\
\hline Cesium-137 & 7 \\
\hline Cobalt-57 & 7 \\
\hline Cobalt-58 & 7 \\
\hline Cobalt-60 & 7 \\
\hline Europium-152 & 7 \\
\hline Europium-154 & 7 \\
\hline Europium-155 & 7 \\
\hline Gross alpha & 8 \\
\hline Manganese-54 & 7 \\
\hline Neptunium-239 & 7 \\
\hline Nonvolatile beta & 8 \\
\hline Plutonium-238 & 7 \\
\hline Promethium-144 & 7 \\
\hline Promethium-146 & 7 \\
\hline Radium-228 & 4 \\
\hline Ruthenium-106 & 7 \\
\hline Sodium-22 & 7 \\
\hline Tin-113 & 7 \\
\hline Yttrium-88 & 7 \\
\hline Zinc-65 & 7 \\
\hline Zirconium-95 & 7 \\
\hline
\end{tabular}


Table 20. Analytes Not Detected in Laboratory Blanks Analyzed with Water Samples

\begin{tabular}{|c|c|}
\hline Analyte & Number \\
\hline \multicolumn{2}{|l|}{ Method EPA335.3 } \\
\hline Cyanide & 8 \\
\hline \multicolumn{2}{|c|}{ Method EPA6010A (total recoverable) } \\
\hline Antimony & 1 \\
\hline Arsenic & 1 \\
\hline Beryllium & 3 \\
\hline Chromium & 3 \\
\hline Copper & 3 \\
\hline Iron & 3 \\
\hline Lead & 3 \\
\hline Magnesium & 3 \\
\hline Manganese & 3 \\
\hline Nickel & 3 \\
\hline Potassium & 3 \\
\hline Selenium & 1 \\
\hline Silver & 3 \\
\hline Sodium & 3 \\
\hline Thallium & 1 \\
\hline Vanadium & 3 \\
\hline Zinc & 3 \\
\hline \multicolumn{2}{|l|}{ Method EPA7041 } \\
\hline Antimony, total recoverable & 3 \\
\hline \multicolumn{2}{|l|}{ Method EPA7060 } \\
\hline Arsenic, total recoverable & 3 \\
\hline \multicolumn{2}{|l|}{ Method EPA7841 } \\
\hline Thallium, total recoverable & 3 \\
\hline \multicolumn{2}{|l|}{ Method EPA8080 } \\
\hline Aldrin & 3 \\
\hline alpha-Benzene hexachloride & 3 \\
\hline beta-Benzene hexachloride & 3 \\
\hline delta-Benzene hexachloride & 3 \\
\hline alpha-Chlordane & 3 \\
\hline gamma-Chlordane & 3 \\
\hline p,p'-DDD & 3 \\
\hline $\mathrm{p}, \mathrm{p}^{\prime}-\mathrm{DDE}$ & 3 \\
\hline p,p'-DDT & 3 \\
\hline Dieldrin & 3 \\
\hline Endrin ketone & 3 \\
\hline Endrin & 3 \\
\hline Endosulfan I & 3 \\
\hline
\end{tabular}

\begin{tabular}{|c|c|}
\hline Analyte & Number \\
\hline Endosulfan II & 3 \\
\hline Endosulfan sulfate & 3 \\
\hline Heptachlor & 3 \\
\hline Heptachlor epoxide & 3 \\
\hline Lindane & 3 \\
\hline Methoxychlor & 3 \\
\hline PCB 1016 & 3 \\
\hline PCB 1221 & 3 \\
\hline PCB 1232 & 3 \\
\hline PCB 1242 & 3 \\
\hline PCB 1248 & 3 \\
\hline PCB 1254 & 3 \\
\hline PCB 1260 & 3 \\
\hline Toxaphene & 3 \\
\hline \multicolumn{2}{|l|}{ Method EPA8260 } \\
\hline Acetone & 5 \\
\hline Benzene & 5 \\
\hline Bromodichloromethane & 5 \\
\hline Bromoform & 5 \\
\hline $\begin{array}{l}\text { Bromomethane (Methyl } \\
\text { bromide) }\end{array}$ & 5 \\
\hline Carbon disulfide & 5 \\
\hline Carbon tetrachloride & 5 \\
\hline Chlorobenzene & 5 \\
\hline Chloroethane & 5 \\
\hline Chloroethene (Vinyl chloride) & 5 \\
\hline Chloroform & 5 \\
\hline $\begin{array}{l}\text { Chloromethane (Methyl } \\
\text { chloride) }\end{array}$ & 5 \\
\hline Dibromochloromethane - & 5 \\
\hline Ethylbenzene & 5 \\
\hline Styrene & 5 \\
\hline Tetrachloroethylene & 5 \\
\hline Trichloroethylene & 5 \\
\hline Vinyl acetate & 5 \\
\hline Xylenes & 5 \\
\hline 1,1,2,2-Tetrachloroethane & 5 \\
\hline 1,1,1-Trichloroethane & 5 \\
\hline 1,1,2-Trichloroethane & 5 \\
\hline 1,1-Dichloroethylene & 5 \\
\hline
\end{tabular}


Table 20. Analytes Not Detected in Laboratory Blanks Analyzed with Water Samples, Continued

\begin{tabular}{|l|l|}
\hline Analyte & Number \\
\hline 1,1-Dichloroethane & 5 \\
\hline 1,2-Dichloroethylene & 5 \\
\hline 1,2-Dichloroethane & 5 \\
\hline 1,2-Dichloropropane & 5 \\
\hline cis-1,3-Dichloropropene & 5 \\
\hline trans-1,3-Dichloropropene & 5 \\
\hline Method EPA8270 & \\
\hline Acenaphthene & 3 \\
\hline Acenaphthylene & 3 \\
\hline Anthracene & 3 \\
\hline Benzo[a]anthracene & 3 \\
\hline Benzo[a]pyrene & 3 \\
\hline Benzo[b]fluoranthene & 3 \\
\hline Benzo[g,h,i]perylene & 3 \\
\hline Benzo[ $k]$ fluoranthene & 3 \\
\hline Benzoic acid & 3 \\
\hline Benzyl alcohol & 3 \\
\hline Bis(2-chloroethoxy) methane & 3 \\
\hline Bis(2-chloroethyl) ether & 3 \\
\hline Bis(2-chloroisopropyl) ether & 3 \\
\hline Butylbenzyl phthalate & 3 \\
\hline Chrysene & 3 \\
\hline Di-n-octyl phthalate & 3 \\
\hline Dibenz[a,h]anthracene & 3 \\
\hline Dibenzofuran & 3 \\
\hline Diethyl phthalate & 3 \\
\hline Dimethyl phthalate & 3 \\
\hline Fluoranthene & 3 \\
\hline Fluorene & 3 \\
\hline Hexachlorobenzene & 3 \\
\hline Hexachlorobutadiene & 3 \\
\hline Hexachlorocyclopentadiene & 3 \\
\hline Hexachloroethane & 3 \\
\hline Indeno[1,2,3-c,d]pyrene & 3 \\
\hline N-Nitrosodiphenylamine & 3 \\
\hline N-Nitrosodipropylamine & 3 \\
\hline
\end{tabular}

\begin{tabular}{|l|l|}
\hline Analyte & Number \\
\hline Naphthalene & 3 \\
\hline Nitrobenzene & 3 \\
\hline Pentachlorophenol & 3 \\
\hline Phenanthrene & 3 \\
\hline Phenol & 3 \\
\hline Pyrene & 3 \\
\hline 1,2,4-Trichlorobenzene & 3 \\
\hline 1,2-Dichlorobenzene & 3 \\
\hline 1,3-Dichlorobenzene & 3 \\
\hline 1,4-Dichlorobenzene & 3 \\
\hline 2,4,5-Trichlorophenol & 3 \\
\hline 2,4,6-Trichlorophenol & 3 \\
\hline 2,4-Dichlorophenol & 3 \\
\hline 2,4-Dimethyl phenol & 3 \\
\hline 2,4-Dinitrophenol & 3 \\
\hline 2,4-Dinitrotoluene & 3 \\
\hline 2,6-Dinitrotoluene & 3 \\
\hline 2-Chlorophenol & 3 \\
\hline 2-Chloronaphthalene & 3 \\
\hline 2-Methylnaphthalene & 3 \\
\hline o-Cresol (2-Methylphenol) & 3 \\
\hline o-Nitroaniline & 3 \\
\hline 2-Nitrophenol & 3 \\
\hline 3,3'-Dichlorobenzidine & 3 \\
\hline m/p-Cresol & 3 \\
\hline m-Nitroaniline & 3 \\
\hline 2-Methyl-4,6-dinitrophenol & 3 \\
\hline 4-Bromophenyl phenyl ether & 3 \\
\hline 4-Chloroaniline & 3 \\
\hline 4-Chloro-m-cresol & 3 \\
\hline 4-Chlorophenyl phenyl ether & 3 \\
\hline p-Nitroaniline & 3 \\
\hline 4-Nitrophenol & 3 \\
\hline
\end{tabular}


Table 21. Analytes Detected in Laboratory Blanks Analyzed with Sediment Samples

\begin{tabular}{|c|c|c|c|c|c|c|c|}
\hline Analyte & $\begin{array}{l}\text { Number } \\
\text { Detected }\end{array}$ & $\begin{array}{l}\text { Number } \\
\text { Not } \\
\text { Detected }\end{array}$ & $\begin{array}{l}\text { Mean } \\
\text { Detected } \\
\text { Result }\end{array}$ & $\begin{array}{l}\text { Std. } \\
\text { Dev. }\end{array}$ & $\begin{array}{l}\text { Min. } \\
\text { Result }\end{array}$ & $\begin{array}{l}\text { Max. } \\
\text { Result }\end{array}$ & Units \\
\hline \multicolumn{8}{|c|}{ Method EPA415.1 } \\
\hline $\begin{array}{l}\text { Nitrate-nitrite as } \\
\text { nitrogen }\end{array}$ & 1 & 12 & 0.03 & & 0.03 & 0.03 & $\mathrm{mg} / \mathrm{kg}$ \\
\hline $\begin{array}{l}\text { Total organic } \\
\text { carbon }\end{array}$ & 5 & 12 & 42.54 & 12.05 & 30.3 & 56.7 & $\mathrm{mg} / \mathrm{kg}$ \\
\hline \multicolumn{8}{|c|}{ Method EPA6010A (inorganics are total recoverable) } \\
\hline Aluminum & 3 & 8 & 1456.7 & 257.12 & 1160 & 1615 & $\mu \mathrm{g} / \mathrm{kg}$ \\
\hline Calcium & 5 & 6 & 1858 & 796.22 & 840 & 2730 & $\mu \mathrm{g} / \mathrm{kg}$ \\
\hline Chromium & 1 & 10 & 865 & & 865 & 865 & $\mu \mathrm{g} / \mathrm{kg}$ \\
\hline Copper & 2 & 9 & 732.5 & 187.38 & 600 & 865 & $\mu \mathrm{g} / \mathrm{kg}$ \\
\hline Iron & 5 & 6 & 1189 & 756.78 & 500 & 2435 & $\mu \mathrm{g} / \mathrm{kg}$ \\
\hline Magnesium & 2 & 9 & 1560 & 28.284 & 1540 & 1580 & $\mu \mathrm{g} / \mathrm{kg}$ \\
\hline Manganese & 1 & 10 & 218.5 & & 218.5 & 218.5 & $\mu \mathrm{g} / \mathrm{kg}$ \\
\hline Silver & 1 & 10 & 510 & & 510 & 510 & $\mu \mathrm{g} / \mathrm{kg}$ \\
\hline Thallium & 2 & 9 & 2490 & 84.853 & 2430 & 2550 & $\mu \mathrm{g} / \mathrm{kg}$ \\
\hline Zinc & 4 & 7 & 286.63 & 139.88 & 170.5 & 487.5 & $\mu g / \mathrm{kg}$ \\
\hline \multicolumn{8}{|c|}{ Method EPA7471 (total recoverable) } \\
\hline Mercury & $3 \cdot$ & 5 & 22.935 & 30.323 & 5.344 & 57.949 & $\mu \mathrm{g} / \mathrm{kg}$ \\
\hline \multicolumn{8}{|c|}{ Method EPA8260 } \\
\hline Acetone & 1 & 7 & 6.74 & & 6.74 & 6.74 & $\mu \mathrm{g} / \mathrm{kg}$ \\
\hline $\begin{array}{l}\text { Dichloromethane } \\
\text { (Methylene } \\
\text { chloride) }\end{array}$ & 6 & 2 & 1.4767 & 2.0703 & 0.33 & 5.66 & $\mu \mathrm{g} / \mathrm{kg}$ \\
\hline $\begin{array}{l}\text { Methyl ethyl } \\
\text { ketone }\end{array}$ & 7 & 1 & 0.7914 & 0.2958 & 0.33 & 1.24 & $\mu \mathrm{g} / \mathrm{kg}$ \\
\hline $\begin{array}{l}\text { Methyl isobutyl } \\
\text { ketone }\end{array}$ & 2 & 6 & 0.4 & 0.2121 & 0.25 & 0.55 & $\mu \mathrm{g} / \mathrm{kg}$ \\
\hline Toluene & 4 & 4 & 0.12 & 0.0535 & 0.05 & 0.18 & $\mu \mathrm{g} / \mathrm{kg}$ \\
\hline 2-Hexanone & 5 & 3 & 2.328 & 0.6244 & 1.72 & 3.18 & $\mu \mathrm{g} / \mathrm{kg}$ \\
\hline \multicolumn{8}{|c|}{ Method EPA8270 } \\
\hline Diethyl phthalate & 1 & 6 & 0.0186 & & 0.0186 & 0.0186 & $\mathrm{mg} / \mathrm{kg}$ \\
\hline $\begin{array}{l}\text { Di-n-butyl } \\
\text { phthalate }\end{array}$ & 4 & 3 & 0.0299 & 0.01 & 0.0236 & 0.0447 & $\mathrm{mg} / \mathrm{kg}$ \\
\hline \multicolumn{8}{|l|}{ Method EPIA-004 } \\
\hline Strontium-90 & 3 & 4 & 0.2277 & 0.1234 & 0.15 & 0.37 & $\mathrm{pCi} / \mathrm{g}$ \\
\hline
\end{tabular}


Table 21. Analytes Detected in Laboratory Blanks Analyzed with Sediment Samples, Continued

\begin{tabular}{|c|c|c|c|c|c|c|c|}
\hline Analyte & $\begin{array}{l}\text { Number } \\
\text { Detected }\end{array}$ & $\begin{array}{l}\text { Number } \\
\text { Not } \\
\text { Detected }\end{array}$ & $\begin{array}{l}\text { Mean } \\
\text { Detected } \\
\text { Result }\end{array}$ & $\begin{array}{l}\text { Std. } \\
\text { Dev. }\end{array}$ & $\begin{array}{l}\text { Min. } \\
\text { Result }\end{array}$ & $\begin{array}{l}\text { Max. } \\
\text { Result }\end{array}$ & Units \\
\hline \multicolumn{8}{|c|}{ Method EPIA-012B } \\
\hline $\begin{array}{l}\text { Plutonium- } \\
239 / 240\end{array}$ & $\overline{1}$ & 5 & 0.0085 & & 0.0085 & 0.0085 & $\mathrm{pCi} / \mathrm{g}$ \\
\hline \multicolumn{8}{|c|}{ Method EPLA-013B } \\
\hline Lead-212 & 2 & 5 & 0.0205 & 0.0037 & 0.0178 & 0.0231 & $\mathrm{pCi} / \mathrm{g}$ \\
\hline Potassium-40 & 3 & 4 & 0.0903 & 0.0135 & 0.0762 & 0.103 & $\mathrm{pCi} / \mathrm{g}$ \\
\hline Radium-226 & 1 & 3 & 0.0136 & & 0.0136 & 0.0136 & $\mathrm{pCi} / \mathrm{g}$ \\
\hline Thorium-234 & 4 & 3 & 0.3173 & 0.1207 & 0.183 & 0.476 & $\mathrm{pCj} / \mathrm{g}$ \\
\hline
\end{tabular}

Table 22. Analytes Detected in Laboratory Blanks Analyzed with Water Samples

\begin{tabular}{|c|c|c|c|c|c|c|c|}
\hline Analyte & $\begin{array}{l}\text { Number } \\
\text { Detected }\end{array}$ & $\begin{array}{l}\text { Number } \\
\text { Not } \\
\text { Detected }\end{array}$ & $\begin{array}{l}\text { Mean } \\
\text { Detected } \\
\text { Result }\end{array}$ & $\begin{array}{l}\text { Std. } \\
\text { Dev. }\end{array}$ & $\begin{array}{l}\text { Min. } \\
\text { Result }\end{array}$ & $\begin{array}{l}\text { Max. } \\
\text { Result }\end{array}$ & Units \\
\hline \multicolumn{8}{|c|}{ Method EPA6010A (inorganics are total recoverable) } \\
\hline Aluminum & 2 & 1 & 19.8 & 2.9698 & 17.7 & 21.9 & $\mu \mathrm{g} / \mathrm{L}$ \\
\hline Barium & 1 & 2 & 0.111 & & 0.111 & 0.111 & $\mu \mathrm{g} / \mathrm{L}$ \\
\hline Cadmium & 1 & 2 & 0.172 & & 0.172 & 0.172 & $\mu \mathrm{g} / \mathrm{L}$ \\
\hline Calcium & 1 & 2 & 23.3 & & 23.3 & 23.3 & $\mu \mathrm{g} / \mathrm{L}$ \\
\hline Cobalt & 1 & 2 & 0.45 & & 0.45 & 0.45 & $\mu \mathrm{g} / \mathrm{L}$ \\
\hline \multicolumn{8}{|c|}{ Method EPA7421 (total recoverable) } \\
\hline Lead & 1 & 2 & 3.08 & & 3.08 & 3.08 & $\mu \mathrm{g} / \mathrm{L}$ \\
\hline \multicolumn{8}{|c|}{ Method EPA7470 (total recoverable) } \\
\hline Mercury & 3 & 1 & 0.1127 & 0.1076 & 0.023 & 0.232 & $\mu \mathrm{g} / \mathrm{L}$ \\
\hline \multicolumn{8}{|c|}{ Method EPA7740 (total recoverable) } \\
\hline Selenium & 1 & 2 & 1.76 & & 1.76 & 1.76 & $\mu \mathrm{g} / \mathrm{L}$ \\
\hline \multicolumn{8}{|c|}{ Method EPA8260 } \\
\hline 2-Hexanone & 4 & 1 & 2.695 & 2.1452 & 0.44 & 5.44 & $\mu \mathrm{g} / \mathrm{L}$ \\
\hline $\begin{array}{l}\text { Dichloromethane } \\
\text { (Methylene } \\
\text { chloride) }\end{array}$ & 3 & 2 & 1.0433 & 0.2914 & 0.81 & 1.37 & $\mu \mathrm{g} / \mathrm{L}$ \\
\hline $\begin{array}{l}\text { Methyl ethyl } \\
\text { ketone }\end{array}$ & 5 & 0 & 1.448 & 1.8818 & 0.33 & 4.78 & $\mu \mathrm{g} / \mathrm{L}$ \\
\hline $\begin{array}{l}\text { Methyl isobutyl } \\
\text { ketone }\end{array}$ & 1 & 4 & 0.25 & & 0.25 & 0.25 & $\mu \mathrm{g} / \mathrm{L}$ \\
\hline Toluene & 2 & 3 & 0.135 & 0.0212 & $0: 12$ & 0.15 & $\mu g / L$ \\
\hline
\end{tabular}


Table 22. Analytes Detected in Laboratory Blanks Analyzed with Water Samples, Continued

\begin{tabular}{|l|l|l|l|l|l|l|l|l|}
\hline & \multicolumn{2}{|l|}{$\begin{array}{l}\text { Number. } \\
\text { Detected }\end{array}$} & $\begin{array}{l}\text { Number } \\
\text { Not } \\
\text { Detected }\end{array}$ & $\begin{array}{l}\text { Mean } \\
\text { Detected } \\
\text { Result }\end{array}$ & $\begin{array}{l}\text { Std. } \\
\text { Dev. }\end{array}$ & $\begin{array}{l}\text { Min. } \\
\text { Result }\end{array}$ & $\begin{array}{l}\text { Max. } \\
\text { Result }\end{array}$ & Units \\
\hline Method EPA8270 & $\begin{array}{l}\text { Rets(2-ethylhexyl) } \\
\text { phthalate }\end{array}$ & 2 & 1 & 0.0065 & 0.0083 & 0.0006 & 0.0124 & $\mathrm{mg} / \mathrm{L}$ \\
\hline $\begin{array}{l}\text { Di-n-butyl } \\
\text { phthalate }\end{array}$ & 2 & 1 & 0.0007 & 0.0002 & 0.0005 & 0.0009 & $\mathrm{mg} / \mathrm{L}$ \\
\hline Isophorone & 1 & 2 & 0.001 & & 0.001 & 0.001 & $\mathrm{mg} / \mathrm{L}$ \\
\hline
\end{tabular}

\section{Matrix Effects}

The matrix of a sample can interfere with accurate determinations during the analytical process. This report examines the results of surrogates and matrix spike recoveries to evaluate the interference caused by the matrix.

\section{Surrogates}

All samples for organic analysis are spiked with surrogates prior to sample preparation to examine the laboratory's performance. Sample results may be affected due to factors such as interferences and high concentrations of analytes. See Tables 23 and 24 for the surrogates analyzed with sediment and water samples, respectively. 
Table 23. Surrogate Recoveries for Sediment Samples

\begin{tabular}{|l|l|l|l|l|l|}
\hline Analyte & Number & $\begin{array}{l}\text { Mean } \\
\text { Recovery }\end{array}$ & $\begin{array}{l}\text { Std. } \\
\text { Dev. }\end{array}$ & $\begin{array}{l}\text { Min. } \\
\text { Recovery }\end{array}$ & $\begin{array}{l}\text { Max. } \\
\text { Recovery }\end{array}$ \\
\hline Method EPA8080 & 55 & 95.7 & 61.8 & 21.8 & 453.0 \\
\hline Dibutyl chlorendate & 55 & 82.0 & 48.4 & 28.2 & 216.0 \\
\hline Tetrachloro-m-xylene & \multicolumn{5}{|l|}{} \\
\hline Method EPA8260 & 73 & 70.7 & 20.4 & 13.0 & 115.0 \\
\hline 1,4-Dichlorobenzene-D4 & 73 & 103.5 & 4.8 & 92.0 & 115.0 \\
\hline Dibromofluoromethane & 73 & 104.6 & 8.5 & 84.4 & 132.0 \\
\hline Toluene-d8 & \multicolumn{5}{|l|}{} \\
\hline Method EPA8270 & 65 & 75.8 & 10.1 & 42.0 & 90.9 \\
\hline Nitrobenzene-d5 & 65 & 84.8 & 9.0 & 60.4 & 107.0 \\
\hline Phenol-d6 & 65 & 97.9 & 12.1 & 68.6 & 129.0 \\
\hline p-Terphenyl-d14 & 66 & 96.1 & 13.7 & 62.8 & 137.0 \\
\hline 2,4,6-Tribromophenol (surr) & 65 & 81.3 & 11.7 & 44.5 & 113.0 \\
\hline 2-Fluorobiphenyl & 65 & 80.4 & 9.4 & 51.7 & 98.8 \\
\hline 2-Fluorophenol &
\end{tabular}

Table 24. Surrogate Recoveries for Water Samples

\begin{tabular}{|c|c|c|c|c|c|}
\hline Analyte & Number & $\begin{array}{l}\text { Mean } \\
\text { Recovery }\end{array}$ & $\begin{array}{l}\text { Std. } \\
\text { Dev. }\end{array}$ & $\begin{array}{l}\text { Min. } \\
\text { Recovery }\end{array}$ & $\begin{array}{l}\text { Max. } \\
\text { Recovery }\end{array}$ \\
\hline \multicolumn{6}{|l|}{ Method EPA8080 } \\
\hline Dibutylchlorendate & 8 & 74.3 & 20.6 & 46.0 & 99.6 \\
\hline Tetrachloro-m-xylene & 8 & 89.2 & 25.5 & 67.8 & 147.0 \\
\hline \multicolumn{6}{|l|}{ Method EPA8260 } \\
\hline 1,4-Dichlorobenzene-D4 & 28 & 89.5 & 16.1 & 60.8 & 112.0 \\
\hline Dibromofluoromethane & 28 & 101.8 & 6.6 & 92.2 & 109.0 \\
\hline Toluene-d8 & 28 & 98.5 & 6.2 & 80.2 & 105.0 \\
\hline \multicolumn{6}{|l|}{ Method EPA8270 } \\
\hline Nitrobenzene-d5 & 8 & 67.2 & 11.4 & 52.3 & 86.8 \\
\hline Phenol-d6 & 8 & 44.2 & $\hat{11.2}$ & 29.2 & 60.5 \\
\hline p-Terphenyl-d14 & 8 & 86.7 & 6.3 & 78.3 & 94.8 \\
\hline 2,4,6-Tribromophenol (surr) & 8 & 81.0 & 7.0 & 72.4 & 89.3 \\
\hline 2-Fluorobiphenyl & 8 & 70.7 & 7.8 & 60.2 & 82.8 \\
\hline 2-Fluorophenol & 8 & 55.8 & 12.8 & 37.7 & 73.7 \\
\hline
\end{tabular}

\section{Matrix Spikes}

Selected samples are spiked to provide information about the effect of each sample matrix on the digestion and measurement methodology. Matrix spikes are rejected if the concentration of the analyte in the sample is more than four times the amount of the spike. 
Percent bias in the table below is the difference between $100 \%$ and the mean recovery; a negative value indicates that the mean recovery was below 100\%. See Tables 25 and 26 for the matrix spikes analyzed with sediment and water samples, respectively.

Table 25. Matrix Spike Recoveries for Sediment Samples

\begin{tabular}{|c|c|c|c|c|c|c|}
\hline Analyte & Number & Bias & $\begin{array}{l}\text { Mean } \\
\text { Recovery }\end{array}$ & $\begin{array}{l}\text { Std. } \\
\text { Dev. }\end{array}$ & $\begin{array}{l}\text { Min. } \\
\text { Recovery }\end{array}$ & $\begin{array}{l}\text { Max. } \\
\text { Recovery }\end{array}$ \\
\hline \multicolumn{7}{|l|}{ Method EPA300.0 } \\
\hline Chloride & 24 & -14.8 & 85.2 & 2.7 & 81.6 & 91.7 \\
\hline \multicolumn{7}{|l|}{ Method EPA335.3 } \\
\hline Cyanide & 15 & -14.6 & 85.4 & 21.4 & 17.7 & 105.0 \\
\hline \multicolumn{7}{|l|}{ Method EPA350.1 } \\
\hline Ammonia nitrogen & 6 & 6.2 & 106.2 & 24.2 & 71.5 & 140 \\
\hline \multicolumn{7}{|l|}{ Method EPA351.2 } \\
\hline $\begin{array}{l}\text { Nitrogen by Kjeldahl } \\
\text { method }\end{array}$ & 1 & -0.4 & 99.6 & & 99.6 & 99.6 \\
\hline \multicolumn{7}{|l|}{ Method EPA353.1 } \\
\hline $\begin{array}{l}\text { Nitrate-nitrite as } \\
\text { nitrogen }\end{array}$ & 11 & -1.3 & 98.7 & 6.9 & 84.3 & 108 \\
\hline \multicolumn{7}{|l|}{ Method EPA365.2 } \\
\hline Orthophosphate & 11 & -24 & 76 & 35.7 & 10.7 & 135 \\
\hline \multicolumn{7}{|l|}{ Method EPA365.4 } \\
\hline Total phosphates (as P) & 2 & 54.5 & 154.5 & 145 & 52 & 257 \\
\hline \multicolumn{7}{|l|}{ Method EPA376.2 } \\
\hline Sulfide & 11 & -50.7 & 49.3 & 21.2 & 15.8 & 80.8 \\
\hline \multicolumn{7}{|l|}{ Method EPA415.1 } \\
\hline Total organic carbon & 15 & -1 & 99 & 7.3 & 86.8 & 112 \\
\hline \multicolumn{7}{|l|}{ Method EPA420.2 } \\
\hline Phenols & 11 & 2.4 & 102.4 & 14.2 & 78.5 & 126 \\
\hline \multicolumn{7}{|c|}{ Method EPA6010A (inorganics are total recoverable) } \\
\hline Antimony & 30 & -44.2 & 55.8 & 15.5 & 20 & 80.3 \\
\hline Arsenic & 30 & -8.7 & 91.3 & 6 & 82.7 & 105 \\
\hline Barium & 30 & -1 & 99 & 7.5 & 85.8 & 117 \\
\hline Beryllium & 30 & -2.7 & 97.3 & 4.9 & 90.6 & 115 \\
\hline Cadmium & 30 & -5.2 & 94.8 & 3.9 & 90.2 & 109 \\
\hline Calcium & 30 & 2 & 102.1 & 19.2 & 70.4 & 191 \\
\hline Chromium & 30 & -3 & 97 & 5.6 & 86.8 & 114 \\
\hline Cobalt & 32 & -4.3 & 95.7 & 4 & 90.8 & 111 \\
\hline Copper & 30 & -3.1 & 96.9 & 4.9 & 91.5 & 113 \\
\hline Lead & 30 & -3.8 & 96.2 & 5.1 & 85.5 & 110 \\
\hline Magnesium & 24 & 10.3 & 110.3 & 56.9 & -14.4 & 241 \\
\hline Manganese & 30 & -2.1 & 97.9 & 13.5 & 70.3 & 145 \\
\hline Nickel & 30 & -2.1 & 97.9 & 4.2 & 93.1 & 113 \\
\hline
\end{tabular}


Table 25. Matrix Spike Recoveries for Sediment Samples, Continued

\begin{tabular}{|c|c|c|c|c|c|c|}
\hline Analyte & Number & Bias & $\begin{array}{l}\text { Mean } \\
\text { Recovery }\end{array}$ & $\begin{array}{l}\text { Std. } \\
\text { Dev. }\end{array}$ & $\begin{array}{l}\text { Min. } \\
\text { Recovery }\end{array}$ & $\begin{array}{l}\text { Max. } \\
\text { Recovery }\end{array}$ \\
\hline Potassium & 30 & -10.9 & 89.1 & 15.8 & 19.8 & 109 \\
\hline Selenium & 32 & -5.6 & 94.4 & 5.7 & 87.4 & 110 \\
\hline Silver & 30 & -1 & 99 & 6 & 88 & 121 \\
\hline Sodium & 30 & -1.7 & 98.3 & 4.7 & 92 & 115 \\
\hline Thallium & 32 & -1.6 & 98.4 & 6.3 & 89.6 & 119 \\
\hline Vanadium & 34 & 0.3 & 100.3 & 7.3 & 90.2 & 124 \\
\hline Zinc & 32 & -8.9 & 91.1 & 4.2 & 84.8 & 103 \\
\hline \multicolumn{7}{|c|}{ Method EPA7471 (total recoverable) } \\
\hline Mercury & 18 & 5.7 & 105.7 & 14 & 95.8 & 133 \\
\hline \multicolumn{7}{|l|}{ Method EPA8080 } \\
\hline Aldrin & 5 & 18.4 & 118.4 & 45.4 & 55.6 & 179 \\
\hline p,p'-DDT & 5 & 29.2 & 129.2 & 31.7 & 89.2 & 168 \\
\hline Dieldrin & 5 & 18.9 & 118.9 & 21.3 & 86.4 & 141 \\
\hline Endrin & 5 & 14.7 & 114.7 & 18.8 & 87.6 & 140 \\
\hline Heptachlor & 5 & 18.9 & 118.9 & 18.1 & 99.6 & 144 \\
\hline Lindane & 5 & 11.7 & 111.7 & 21.7 & 89.1 & 142 \\
\hline \multicolumn{7}{|l|}{ Method EPA8260 } \\
\hline Benzene & 14 & 7.0 & 107.0 & 14.9 & 75.5 & 127.0 \\
\hline Chlorobenzene & 14 & 3.8 & 103.8 & 9.6 & 92.5 & 128.0 \\
\hline Toluene & 14 & -11.5 & 88.5 & 34.7 & 5.5 & 123.0 \\
\hline Trichloroethylene & 14 & -5.2 & 94.8 & 10.5 & 75.5 & 112.0 \\
\hline 1,1-Dichloroethylene & 14 & 10.1 & 110.1 & 19.1 & 68.5 & 135.0 \\
\hline \multicolumn{7}{|l|}{ Method EPA8270 } \\
\hline Acenaphthene & 7 & -12.4 & 87.6 & 12.5 & 70.4 & 111.0 \\
\hline N-Nitrosodipropylamine & 7 & -14.7 & 85.3 & 5.8 & 74.8 & 92.6 \\
\hline Pentachlorophenol & 7 & -0.2 & 99.8 & 14.4 & 82.9 & 119.0 \\
\hline Phenol & 7 & -32.5 & 67.5 & 3.5 & 62.3 & 73.0 \\
\hline Pyrene & 7 & 7.7 & 107.7 & 12.9 & 90.1 & 126.0 \\
\hline 2,4-Dinitrotoluene & 7 & -3.1 & 96.9 & 10.6 & 76.8 & 109.0 \\
\hline 2-Chlorophenol & 7 & -20.1 & 79.9 & 6.4 & 66.8 & 86.6 \\
\hline 4-Chloro-m-cresol & 7 & -4.0 & 96.0 & 9.0 & 77.7 & 104.0 \\
\hline 4-Nitrophenol & 7 & 2.7 & 102.7 & 14.3 & 86.9 & 132.0 \\
\hline \multicolumn{7}{|l|}{ Method EPIA-001B } \\
\hline Gross alpha & 8 & 7.3 & 107.3 & 13.5 & 82.6 & 124 \\
\hline Nonvolatile beta & 7 & 12.7 & 112.7 & 13.5 & 84.2 & 124 \\
\hline \multicolumn{7}{|l|}{ Method EPIA-004 } \\
\hline Strontium-90 & 7 & 5.3 & 105.3 & 19.3 & 81.1 & 125 \\
\hline \multicolumn{7}{|l|}{ Method EPIA-012B } \\
\hline Plutonium-239/240 & 7 & -11.6 & 88.4 & 7.1 & 76.4 & 96 \\
\hline
\end{tabular}

Par Pond, Pond C, and L Lake, Revision 2 
Table 26. Matrix Spike Recoveries for Water Samples

\begin{tabular}{|c|c|c|c|c|c|c|}
\hline Analyte & Number & Bias & $\begin{array}{l}\text { Mean } \\
\text { Recovery }\end{array}$ & $\begin{array}{l}\text { Std. } \\
\text { Dev. }\end{array}$ & $\begin{array}{l}\text { Min. } \\
\text { Recovery }\end{array}$ & $\begin{array}{l}\text { Max. } \\
\text { Recovery }\end{array}$ \\
\hline \multicolumn{7}{|c|}{ Method EPA335.3 } \\
\hline Cyanide & 4 & 8.5 & 108.5 & 4.4 & 105.0 & 114.0 \\
\hline \multicolumn{7}{|c|}{ Method EPA6010A (inorganics are total recoverable) } \\
\hline Aluminum & 6 & 2.6 & 102.6 & 9.2 & 92.5 & 114 \\
\hline Antimony & 2 & 0.3 & 100.3 & 1.1 & 99.5 & 101 \\
\hline Barium & 8 & 2.5 & 102.5 & 8.8 & 96 & 117 \\
\hline Beryllium & 4 & 1 & 101 & 0.9 & 99.8 & 102 \\
\hline Cadmium & 8 & -4.9 & 95.1 & 2.5 & 92.4 & 100 \\
\hline Calcium & 6 & 3.6 & 103.6 & 7.7 & 96.5 & 116 \\
\hline Chromium & 8 & -0.3 & 99.8 & 1.6 & 97.5 & 102 \\
\hline Cobalt & 8 & -0.2 & 99.8 & 2.3 & 95.3 & 102 \\
\hline Copper & 8 & -0.5 & 99.5 & 3 & 95 & 105 \\
\hline Iron & 6 & -0.1 & 99.9 & 5.3 & 91.9 & 104 \\
\hline Lead & 8 & -0.1 & 99.9 & 1.3 & 97.7 & 102 \\
\hline Magnesium & 6 & -3.4 & 96.6 & 1.1 & 95.1 & 98 \\
\hline Manganese & 8 & 0.9 & 100.9 & 5.2 & 93.9 & 109 \\
\hline Nickel & 8 & 0.5 & 100.5 & 3.6 & 96.3 & 106 \\
\hline Potassium & 8 & -5.6 & 94.4 & 3.5 & 91 & 101 \\
\hline Silver & 8 & -1 & 99 & 2.2 & 96.1 & 103 \\
\hline Sodium & 8 & 95.3 & 195.3 & 176.6 & 91.4 & 488 \\
\hline Vanadium & 8 & 0.8 & 100.8 & 1.2 & 99.1 & 102 \\
\hline Zinc & 7 & -0.4 & 99.6 & 3.5 & 94.1 & 106 \\
\hline \multicolumn{7}{|c|}{ Method EPA7041 (total recoverable) } \\
\hline Antimony & 6 & -5.9 & 94.2 & 12.9 & 77.2 & 108 \\
\hline \multicolumn{7}{|c|}{ Method EPA7060 (total recoverable) } \\
\hline Arsenic & 8 & -15.2 & 84.8 & 6.5 & 76.2 & 94.5 \\
\hline \multicolumn{7}{|c|}{ Method EPA7421 (total recoverable) } \\
\hline Lead & 8 & -13.2 & 86.9 & 5 & 79.4 & 95.8 \\
\hline \multicolumn{7}{|c|}{ Method EPA7470 (total recoverable) } \\
\hline Mercury & 8 & 0.8 & 100.8 & 9 & 90.1 & 120 \\
\hline \multicolumn{7}{|c|}{ Method EPA7471 (total recoverable) } \\
\hline Mercury & 2 & -4.9 & 95.1 & 0.9 & 94.4 & 95.7 \\
\hline \multicolumn{7}{|c|}{ Method EPA7740 (total recoverable) } \\
\hline Selenium & 6 & -12.8 & 87.2 & 11.7 & 73.5 & 101 \\
\hline \multicolumn{7}{|c|}{ Method EPA7841 (total recoverable) } \\
\hline Thallium & 6 & -14.9 & 85.1 & 3.3 & 80.9 & 89 \\
\hline \multicolumn{7}{|c|}{ Method EPA8080 } \\
\hline Aldrin & 3 & -2.9 & 97.1 & 24.3 & 74.8 & 123 \\
\hline p,p'-DDT & 3 & -6.6 & 93.4 & 55.8 & 46.4 & 155 \\
\hline
\end{tabular}


Table 26. Matrix.Spike Recoveries for Water Samples, Continued

\begin{tabular}{|c|c|c|c|c|c|c|}
\hline Analyte & Number & Bias & $\begin{array}{l}\text { Mean } \\
\text { Recovery }\end{array}$ & $\begin{array}{l}\text { Std. } \\
\text { Dev. }\end{array}$ & $\begin{array}{l}\text { Min. } \\
\text { Recovery }\end{array}$ & $\begin{array}{l}\text { Max. } \\
\text { Recovery }\end{array}$ \\
\hline Dieldrin & 3 & -2.2 & 97.8 & 33.3 & 64.4 & 131 \\
\hline Endrin & 2 & -5.6 & 94.4 & 33.4 & 70.8 & 118 \\
\hline Heptachlor & 3 & -4.5 & 95.5 & 22.9 & 76.8 & 121 \\
\hline Lindane & 3 & -16.5 & 83.5 & 32.5 & 63.4 & 121 \\
\hline \multicolumn{7}{|l|}{ Method EPA8260 } \\
\hline Benzene & 10 & 3.8 & 103.8 & 16.3 & 74.0 & 121.0 \\
\hline Chlorobenzene & 10 & -0.7 & 99.3 & 8.5 & 89.0 & 114.0 \\
\hline Toluene & 10 & -15.0 & 85.1 & 6.3 & 74.5 & 93.5 \\
\hline Trichloroethylene & 10 & -8.7 & 91.3 & 10.1 & 74.0 & 106.0 \\
\hline 1,1-Dichloroethylene & 10 & 1.5 & 101.5 & 20.6 & 63.0 & 121.0 \\
\hline \multicolumn{7}{|l|}{ Method EPA8270 } \\
\hline Acenaphthene & 3 & -32.8 & 67.2 & 12.5 & 53.1 & 77.2 \\
\hline N-Nitrosodipropylamine & 3 & -34.1 & 65.9 & 9.2 & 57.4 & 75.6 \\
\hline Pentachlorophenol & 3 & -10.4 & 89.6 & 13.5 & 77.1 & 104.0 \\
\hline Phenol & 3 & -64.4 & 35.6 & 7.3 & 28.0 & 42.5 \\
\hline Pyrene & 3 & -5.6 & 94.4 & 10.2 & 86.8 & 106.0 \\
\hline 2,4-Dinitrotoluene & 3 & -19.1 & 80.9 & 3.0 & 78.4 & 84.2 \\
\hline 2-Chlorophenol & 3 & -35.7 & 64.3 & 5.2 & 58.8 & 69.2 \\
\hline 4-Chloro-m-cresol & 3 & -22.5 & 77.5 & 14.2 & 62.4 & 90.5 \\
\hline 4-Nitrophenol & 3 & -60.7 & 39.3 & 17.4 & 20.4 & 54.7 \\
\hline
\end{tabular}

\section{Effects of Sampling and Transport}

Sampling and sample transport can add variability to the sample results. Field blanks and trip blanks are used to detect contamination or false positive results.

\section{Field Blanks}

Field blanks are deionized water sealed in sample bottles prior to sampling, opened in the field during sampling, resealed, and shipped to the laboratory with the samples. Positive results from field blanks can result from analytical bias, contaminated sample bottles, contaminated deionized water, vapors in the air during sampling, or contamination during shipping or analysis. See Tables 27 and 28 for the analytes not detected and detected for the field blanks.

Table 27. Analytes Not Detected in Field Blanks for Water Samples

\begin{tabular}{|l|l|}
\hline Analyte & Number \\
\hline Method EPA6010A (total recoverable) \\
\hline Antimony & 1 \\
\hline Arsenic & 1 \\
\hline Cadmium & 4 \\
\hline Chromium & 4 \\
\hline
\end{tabular}

\begin{tabular}{|l|l|}
\hline Analyte & Number \\
\hline Cobalt & 4 \\
\hline Lead & 1 \\
\hline Nickel & 4 \\
\hline Potassium & 4 \\
\hline Selenium & 1 \\
\hline
\end{tabular}


Table 27. Analytes Not Detected in Field Blanks for Water Samples, Continued

\begin{tabular}{|l|l|}
\hline Analyte & Number \\
\hline Silver & 4 \\
\hline Thallium & 1 \\
\hline Vanadium & 4 \\
\hline Method EPA7041 (total recoverable) \\
\hline Antimony & 3 \\
\hline Method EPA7421 (total recoverable) \\
\hline Lead & 3 \\
\hline Method EPA7470 (total recoverable) \\
\hline Mercury & 4 \\
\hline Method EPA7740 (total recoverable) \\
\hline Selenium & 3 \\
\hline Method EPA7841 (total recoverable) \\
\hline Thallium & 3 \\
\hline Method EPA8080 & \\
\hline Aldrin & 4 \\
\hline alpha-Benzene hexachloride & 4 \\
\hline beta-Benzene hexachloride & 4 \\
\hline delta-Benzene hexachloride & 4 \\
\hline alpha-Chlordane & 4 \\
\hline gamma-Chlordane & 4 \\
\hline p,p'-DDD & 4 \\
\hline p,p'-DDE & 4 \\
\hline p,p'-DDT & 4 \\
\hline Dieldrin & 4 \\
\hline Endrin ketone & 4 \\
\hline Endrin & 4 \\
\hline Endosulfan I & 4 \\
\hline Endosulfan II & 4 \\
\hline Endosulfan sulfate & 4 \\
\hline Heptachlor & 4 \\
\hline Heptachlor epoxide & 4 \\
\hline Lindane & 4 \\
\hline Methoxychlor & 4 \\
\hline PCB 1016 & 4 \\
\hline PCB 1221 & 4 \\
\hline PCB 1232 & 4 \\
\hline PCB 1242 & 4 \\
\hline PCB 1248 & 4 \\
\hline PCB 1254 & 4 \\
\hline PCB 1260 & 4 \\
\hline Toxaphene & 4 \\
\hline
\end{tabular}

\begin{tabular}{|c|c|}
\hline Analyte & Number \\
\hline \multicolumn{2}{|l|}{ Method EPA8260 } \\
\hline Acetone & 4 \\
\hline Benzene & 4 \\
\hline Bromodichloromethane & 4 \\
\hline Bromoform & 4 \\
\hline $\begin{array}{l}\text { Bromomethane (Methyl } \\
\text { bromide) }\end{array}$ & 4 \\
\hline Carbon disulfide & 4 \\
\hline Carbon tetrachloride & 4 \\
\hline Chloroethane & 4 \\
\hline Chloroethene (Vinyl chloride) & 4 \\
\hline Chloroform & 4 \\
\hline $\begin{array}{l}\text { Chloromethane (Methyl } \\
\text { chloride) }\end{array}$ & 4 \\
\hline Dibromochloromethane & 4 \\
\hline $\begin{array}{l}\text { Dichloromethane (Methylene } \\
\text { chloride) }\end{array}$ & 4 \\
\hline Ethylbenzene & 4 \\
\hline Methyl ethyl ketone & 4 \\
\hline Styrene & 4 \\
\hline Tetrachloroethylene & 4 \\
\hline Toluene & 4 \\
\hline Trichloroethylene & 4 \\
\hline Vinyl acetate & 4 \\
\hline Xylenes & 4 \\
\hline 1,1,2,2-Tetrachloroethane & 4 \\
\hline 1,1,1-Trichloroethane & 4 \\
\hline 1,1,2-Trichloroethane & 4 \\
\hline 1,1-Dichloroethylene & 4 \\
\hline 1,1-Dichloroethane & 4 \\
\hline 1,2-Dichloroethylene & 4 \\
\hline 1,2-Dichloroethane & 4 \\
\hline 1,2-Dichloropropane & 4 \\
\hline cis-1,3-Dichloropropene & 4 \\
\hline trans-1,3-Dichloropropene & 4 \\
\hline 2-Hexanone & 4 \\
\hline \multicolumn{2}{|l|}{ Method EPA8270 } \\
\hline Acenaphthene & 4 \\
\hline Acenaphthylene & 4 \\
\hline Anthracene & 4 \\
\hline Benzo $[a]$ anthracene & 4 \\
\hline Benzo[a]pyrene & 4 \\
\hline
\end{tabular}


Table 27. Analytes Not Detected in Field Blanks for Water Samples, Continued

\begin{tabular}{|l|l|}
\hline Analyte & Number \\
\hline Benzo[b]fluoranthene & 4 \\
\hline Benzo[g,h,i]perylene & 4 \\
\hline Benzo[k]fluoranthene & 4 \\
\hline Benzoic acid & 4 \\
\hline Benzyl alcohol & 4 \\
\hline Bis(2-chloroethoxy) methane & 4 \\
\hline Bis(2-chloroethyl) ether & 4 \\
\hline Bis(2-chloroisopropyl) ether & 4 \\
\hline Butylbenzyl phthalate & 4 \\
\hline Chrysene & 4 \\
\hline Di-n-octyl phthalate & 4 \\
\hline Dibenz[a,h]anthracene & 4 \\
\hline Dibenzofuran & 4 \\
\hline Dimethyl phthalate & 4 \\
\hline Fluoranthene & 4 \\
\hline Fluorene & 4 \\
\hline Hexachlorobenzene & 4 \\
\hline Hexachlorobutadiene & 4 \\
\hline Hexachlorocyclopentadiene & 4 \\
\hline Hexachloroethane & 4 \\
\hline Indeno[1,2,3-c,d]pyrene & 4 \\
\hline Isophorone & 4 \\
\hline N-Nitrosodiphenylamine & 4 \\
\hline N-Nitrosodipropylamine & 4 \\
\hline Naphthalene & 4 \\
\hline Nitrobenzene & 4 \\
\hline Pentachlorophenol & 4 \\
\hline Phenanthrene & 4 \\
\hline Phenol & 4 \\
\hline
\end{tabular}

\begin{tabular}{|l|l|}
\hline Analyte & Number \\
\hline Pyrene & 4 \\
\hline 1,2,4-Trichlorobenzene & 4 \\
\hline 1,2-Dichlorobenzene & 4 \\
\hline 1,3-Dichlorobenzene & 4 \\
\hline 1,4-Dichlorobenzene & 4 \\
\hline 2,4,5-Trichlorophenol & 4 \\
\hline 2,4,6-Trichlorophenol & 4 \\
\hline 2,4-Dichlorophenol & 4 \\
\hline 2,4-Dimethyl phenol & 4 \\
\hline 2,4-Dinitrophenol & 4 \\
\hline 2,4-Dinitrotoluene & 4 \\
\hline 2,6-Dinitrotoluene & 4 \\
\hline 2-Chlorophenol & 4 \\
\hline 2-Chloronaphthalene & 4 \\
\hline 2-Methylnaphthalene & 4 \\
\hline o-Cresol (2-Methylphenol) & 4 \\
\hline o-Nitroaniline & 4 \\
\hline 2-Nitrophenol & 4 \\
\hline 3,3'-Dichlorobenzidine & 4 \\
\hline m/p-Cresol & 4 \\
\hline m-Nitroaniline & 4 \\
\hline 2-Methyl-4,6-dinitrophenol & 4 \\
\hline 4-Bromophenyl phenyl ether & 4 \\
\hline 4-Chloroaniline & 4 \\
\hline 4-Chloro-m-cresol & 4 \\
\hline 4-Chlorophenyl phenyl ether & 4 \\
\hline p-Nitroaniline & 4 \\
\hline 4-Nitrophenol & 4 \\
\hline & \\
\hline
\end{tabular}

Table 28. Analytes Detected in Field Blanks for Water Samples

\begin{tabular}{|l|l|l|l|l|l|l|l|}
\hline & $\begin{array}{l}\text { Number } \\
\text { Detected }\end{array}$ & $\begin{array}{l}\text { Number } \\
\text { Not } \\
\text { Detected }\end{array}$ & $\begin{array}{l}\text { Mean } \\
\text { Detected } \\
\text { Result }\end{array}$ & $\begin{array}{l}\text { Std. } \\
\text { Dev. }\end{array}$ & $\begin{array}{l}\text { Min. } \\
\text { Result }\end{array}$ & $\begin{array}{l}\text { Max. } \\
\text { Result }\end{array}$ & Units \\
\hline Method EPA335.3 & \multicolumn{5}{|l|}{} \\
\hline Cyanide & 1 & 3 & 3.93 & 3.93 & 3.93 & $\mu \mathrm{g} / \mathrm{L}$ \\
\hline Method EPA6010A (all inorganics are total recoverable) & 23.2 &. & 23.2 & 23.2 & $\mu \mathrm{g} / \mathrm{L}$ \\
\hline Aluminum & 1 & 3 & 1.14 & & 1.14 & 1.14 & $\mu \mathrm{g} / \mathrm{L}$ \\
\hline Barium & 1 & 3 & & &
\end{tabular}


Table 28. Analytes Detected in Field Blanks, Continued

\begin{tabular}{|c|c|c|c|c|c|c|c|}
\hline Analyte & $\begin{array}{l}\text { Number } \\
\text { Detected }\end{array}$ & $\begin{array}{l}\text { Number } \\
\text { Not; } \\
\text { Detected }\end{array}$ & $\begin{array}{l}\text { Mean } \\
\text { Detected } \\
\text { Result }\end{array}$ & $\begin{array}{l}\text { Std. } \\
\text { Dev. }\end{array}$ & $\begin{array}{l}\text { Min. } \\
\text { Result }\end{array}$ & $\begin{array}{l}\text { Max. } \\
\text { Result }\end{array}$ & Units \\
\hline Beryllium & 1 & 3 & 0.0669 & & 0.0669 & 0.0669 & $\mu \mathrm{g} / \mathrm{L}$ \\
\hline Calcium & 3 & 1 & 47.133 & 14.324 & 35.7 & 63.2 & $\mu \mathrm{g} / \mathrm{L}$ \\
\hline Copper & 1 & 3 & 2.13 & & 2.13 & 2.13 & $\mu \mathrm{g} / \mathrm{L}$ \\
\hline Iron & 1 & 3 & 13 & & 13 & 13 & $\mu \mathrm{g} / \mathrm{L}$ \\
\hline Magnesium & 1 & 3 & 4.64 & & 4.64 & 4.64 & $\mu \mathrm{g} / \mathrm{L}$ \\
\hline Manganese & 3 & 1 & 4.5653 & 4.3101 & 0.596 & 9.15 & $\mu \mathrm{g} / \mathrm{L}$ \\
\hline Sodium & 1 & 3 & 54 & & 54 & 54 & $\mu \mathrm{g} / \mathrm{L}$ \\
\hline Zinc & 3 & 1 & 11.497 & 6.403 & 4.69 & 17.4 & $\mu \mathrm{g} / \mathrm{L}$ \\
\hline \multicolumn{8}{|c|}{ Method EPA7060 } \\
\hline Arsenic & 1 & 2 & 1.16 & & 1.16 & 1.16 & $\mu \mathrm{g} / \mathrm{L}$ \\
\hline \multicolumn{8}{|c|}{ Method EPA8260 } \\
\hline Chlorobenzene & 3 & 1 & 0.1833 & 0.0231 & 0.17 & 0.21 & $\mu \mathrm{g} / \mathrm{L}$ \\
\hline $\begin{array}{l}\text { Methyl isobutyl } \\
\text { ketone }\end{array}$ & 1 & 3 & 0.27 & & 0.27 & 0.27 & $\mu \mathrm{g} / \mathrm{L}$ \\
\hline \multicolumn{8}{|c|}{ Method EPA8270 } \\
\hline $\begin{array}{l}\text { Bis(2-ethylhexyl) } \\
\text { phthalate }\end{array}$ & 1 & 3 & 0.0005 & & 0.0005 & 0.0005 & $\mathrm{mg} / \mathrm{L}$ \\
\hline $\begin{array}{l}\text { Diethyl } \\
\text { phthalate }\end{array}$ & 2 & 2 & 0.0006 & $141 \mathrm{E}-7$ & 0.0006 & 0.0006 & $\mathrm{mg} / \mathrm{L}$ \\
\hline $\begin{array}{l}\text { Di-n-butyl } \\
\text { phthalate }\end{array}$ & 1 & 3 & 0.0009 & & 0.0009 & 0.0009 & $\mathrm{mg} / \mathrm{L}$ \\
\hline
\end{tabular}




\section{Trip Blanks}

Trip blanks are used to detect contamination during shipping, primarily due to depressurization during air transport. Trip blanks are analyzed only for volatile organic compounds (VOCs). See Tables 29 and 30 for the analytes not detected and detected for the trip blanks. In Table 30, all nine trip blanks analyzed at General Engineering Laboratories, Inc., had a measurable concentration for trichloroethylene but no samples associated with these trip blanks had results above the quantitation limit.

Table 29. Analytes Not Detected in Trip Blanks

\begin{tabular}{|c|c|}
\hline Analyte & Number \\
\hline \multicolumn{2}{|l|}{ Method EPA8260 } \\
\hline Benzene & 9 \\
\hline Bromodichloromethane & 9 \\
\hline Bromoform & 9 \\
\hline Carbon disulfide & 9 \\
\hline Carbon tetrachloride & 9 \\
\hline Chlorobenzene & 9 \\
\hline Chloroethane & 9 \\
\hline $\begin{array}{l}\text { Chloroethene (Vinyl } \\
\text { chloride) }\end{array}$ & 9 \\
\hline Chloroform & 9 \\
\hline Dibromochloromethane & 9 \\
\hline Ethylbenzene & 9 \\
\hline Methyl isobutyl ketone & 9 \\
\hline
\end{tabular}

\begin{tabular}{|l|l|}
\hline Analyte & Number \\
\hline Styrene & 9 \\
\hline Tetrachloroethylene & 9 \\
\hline Vinyl acetate & 9 \\
\hline $1,1,2,2-$ Tetrachloroethane & 9 \\
\hline 1,1,1-Trichloroethane & 9 \\
\hline 1,1,2-Trichloroethane & 9 \\
\hline 1,1-Dichloroethylene & 9 \\
\hline 1,1-Dichloroethane & 9 \\
\hline 1,2-Dichloroethylene & 9 \\
\hline 1,2-Dichloroethane & 9 \\
\hline 1,2-Dichloropropane & 9 \\
\hline cis-1,3-Dichloropropene & 9 \\
\hline trans-1,3-Dichloropropene & 9 \\
\hline
\end{tabular}

Table 30. Analytes Detected in Trip Blanks

\begin{tabular}{|c|c|c|c|c|c|c|c|}
\hline Analyte & $\begin{array}{l}\text { Number } \\
\text { Detected }\end{array}$ & $\begin{array}{l}\text { Number } \\
\text { Not } \\
\text { Detected }\end{array}$ & $\begin{array}{l}\text { Mean } \\
\text { Detected } \\
\text { Result }\end{array}$ & $\begin{array}{l}\text { Std. } \\
\text { Dev. }\end{array}$ & $\begin{array}{l}\text { Min. } \\
\text { Result }\end{array}$ & $\begin{array}{l}\text { Max. } \\
\text { Result }\end{array}$ & Units \\
\hline \multicolumn{8}{|l|}{ Method EPA8260 } \\
\hline Acetone & 9 & 0 & 5.6733 & 2.1834 & 3.25 & 10.6 & $\mu \mathrm{g} / \mathrm{L}$ \\
\hline $\begin{array}{l}\text { Bromomethane } \\
\text { (Methyl bromide) }\end{array}$ & 1 & 8 & 0.16 & & 0.16 & 0.16 & $\mu \mathrm{g} / \mathrm{L}$ \\
\hline $\begin{array}{l}\text { Chloromethane } \\
\text { (Methyl chloride) }\end{array}$ & 1 & 8 & 0.11 & & 0.11 & 0.11 & $\mu \mathrm{g} / \mathrm{L}$ \\
\hline $\begin{array}{l}\text { Dichloromethane } \\
\text { (Methylene } \\
\text { chloride) }\end{array}$ & 3 & 6 & 0.9933 & 0.2491 & 0.83 & 1.28 & $\mu \mathrm{g} / \mathrm{L}$ \\
\hline $\begin{array}{l}\text { Methyl ethyl } \\
\text { ketone }\end{array}$ & 1 & 8 & 8.34 & & 8.34 & 8.34 & $\mu \mathrm{g} / \mathrm{L}$ \\
\hline Toluene & 4 & 5 & 0.1475 & 0.0427 & 0.12 & 0.21 & $\mu \mathrm{g} / \mathrm{L}$ \\
\hline Trichloroethylene & 9 & 0 & 0.1678 & 0.0644 & 0.1 & 0.27 & $\mu \mathrm{g} / \mathrm{L}$ \\
\hline
\end{tabular}

Par Pond, Pond C, and L Lake, Revision 2 
Table 30. Analytes Detected in Trip Blanks, Continued

\begin{tabular}{|l|l|l|l|l|l|l|l|}
\hline & $\begin{array}{l}\text { Number } \\
\text { Detected }\end{array}$ & $\begin{array}{l}\text { Number } \\
\text { Not } \\
\text { Detected }\end{array}$ & $\begin{array}{l}\text { Mean } \\
\text { Detected } \\
\text { Result }\end{array}$ & $\begin{array}{l}\text { Std. } \\
\text { Dev. }\end{array}$ & $\begin{array}{l}\text { Min. } \\
\text { Result }\end{array}$ & $\begin{array}{l}\text { Max. } \\
\text { Result }\end{array}$ & Units \\
\hline Xylenes & 2 & 7 & 0.175 & 0.0778 & 0.12 & 0.23 & $\mu \mathrm{g} / \mathrm{L}$ \\
\hline 2-Hexanone & 2 & 7 & 0.285 & 0.0495 & 0.25 & 0.32 & $\mu \mathrm{g} / \mathrm{L}$ \\
\hline
\end{tabular}

\section{Analytical Precision}

The precision of each analytical method is evaluated by reviewing the variability of the results of the analysis of laboratory duplicates.

\section{Laboratory Duplicates}

The laboratory routinely analyzes $10 \%$ of the samples twice and reports both results. The relative percent difference (RPD) of the results reflect the precision of the analyses. RPDs were not calculated for pairs of samples with both results below detection. If only one result was below the quantitation limit, the quantitation limit was used to calculate the RPD. Tables 31 and 32 list the results of laboratory duplicates for sediment and water samples, respectively.

Table 31. Analytes Not Detected in Laboratory Duplicates for Sediment Samples

\begin{tabular}{|c|c|}
\hline Analyte & $\begin{array}{l}\text { Number } \\
\text { of Pairs }\end{array}$ \\
\hline \multicolumn{2}{|l|}{ Method EPA376.2 } \\
\hline Sulfide & 14 \\
\hline \multicolumn{2}{|l|}{ Method EPA6010A } \\
\hline Cadmium & 12 \\
\hline Silver & 12 \\
\hline Thallium & 12 \\
\hline \multicolumn{2}{|l|}{ Method EPA8080 } \\
\hline Aldrin & 2 \\
\hline alpha-Chlordane & 2 \\
\hline gamma-Chlordane & 2 \\
\hline alpha-Benzene hexachloride & 2 \\
\hline beta-Benzene hexachloride & 2 \\
\hline delta-Benzene hexachloride & 2 \\
\hline p,p'-DDD & 2 \\
\hline p,p'-DDE & 2 \\
\hline $\mathrm{p}, \mathrm{p}^{\prime}$-DDT & 2 \\
\hline Dieldrin & 2 \\
\hline Endrin ketone & 2 \\
\hline Endrin & 2 \\
\hline Endosulfan I & 2 \\
\hline
\end{tabular}

\begin{tabular}{|l|l|}
\hline Analyte & $\begin{array}{l}\text { Number } \\
\text { of Pairs }\end{array}$ \\
\hline Endosulfan II & 2 \\
\hline Endosulfan sulfate & 2 \\
\hline Heptachlor & 2 \\
\hline Heptachlor epoxide & 2 \\
\hline Lindane & 2 \\
\hline Methoxychlor & 2 \\
\hline PCB 1016 & 2 \\
\hline PCB 1221 & 2 \\
\hline PCB 1232 & 2 \\
\hline PCB 1242 & 2 \\
\hline PCB 1248 & 2 \\
\hline PCB 1254 & 2 \\
\hline PCB 1260 & 2 \\
\hline Toxaphene & 2 \\
\hline Method EPA8260 & 3 \\
\hline Benzene & 3 \\
\hline Bromodichloromethane & 3 \\
\hline Bromoform & 3 \\
\hline $\begin{array}{l}\text { Bromomethane (Methyl } \\
\text { bromide) }\end{array}$ & \\
\hline
\end{tabular}

Par Pond, Pond C, and L Lake, Revision 2 
Table 31. Analytes Not Detected in Laboratory Duplicates for Sediment Samples, Continued

\begin{tabular}{|c|c|}
\hline Analyte & $\begin{array}{l}\text { Number } \\
\text { of Pairs }\end{array}$ \\
\hline Carbon tetrachloride & 3 \\
\hline Chlorobenzene & 3 \\
\hline Chloroethane & 3 \\
\hline Chloroethene (Vinyl chloride) & 3 \\
\hline Chloroform & 3 \\
\hline $\begin{array}{l}\text { Chloromethane (Methyl } \\
\text { chloride) }\end{array}$ & 3 \\
\hline Dibromochloromethane & 3 \\
\hline $\begin{array}{l}\text { Dichloromethane (Methylene } \\
\text { chloride) }\end{array}$ & 3 \\
\hline Ethylbenzene & 3 \\
\hline Methyl ethyl ketone & 3 \\
\hline Methyl isobutyl ketone & 3 \\
\hline Tetrachloroethylene & 3 \\
\hline Trichloroethylene & 3 \\
\hline Vinyl acetate & 3 \\
\hline Xylenes & 3 \\
\hline 1,1,2,2-Tetrachloroethane & 3 \\
\hline 1,1,1-Trichloroethane & 3 \\
\hline 1,1,2-Trichloroethane & 3 \\
\hline 1,1-Dichloroethylene & 3 \\
\hline 1,1-Dichloroethane & 3 \\
\hline 1,2-Dichloroethylene & 3 \\
\hline 1,2-Dichloroethane & 3 \\
\hline 1,2-Dichloropropane & 3 \\
\hline cis-1,3-Dichloropropene & 3 \\
\hline trans-1,3-Dichloropropene & 3 \\
\hline 2-Hexanone & 3 \\
\hline \multicolumn{2}{|l|}{ Method EPA8270 } \\
\hline Acenaphthene & 3 \\
\hline Acenaphthylene & 3 \\
\hline Anthracene & 3 \\
\hline Benzo[a]anthracene & 3 \\
\hline Benzo[a]pyrene & 3 \\
\hline Benzo[b]fluoranthene & 3 \\
\hline Benzo $[g, h, i]$ perylene & 3 \\
\hline Benzo[k]fluoranthene & 3 \\
\hline Benzyl alcohol & 3 \\
\hline Bis(2-chloroethoxy) methane & 3 \\
\hline Bis(2-chloroethyl) ether & 3 \\
\hline
\end{tabular}

\begin{tabular}{|c|c|}
\hline Analyte & $\begin{array}{l}\text { Number } \\
\text { of Pairs }\end{array}$ \\
\hline Bis(2-chloroisopropyl) ether & 3 \\
\hline Butylbenzyl phthalate & 3 \\
\hline Chrysene & 3 \\
\hline Di-n-octyl phthalate & 3 \\
\hline Dibenz $[a, h]$ anthracene & 3 \\
\hline Dibenzofuran & 3 \\
\hline Dimethyl phthalate & 3 \\
\hline Fluoranthene & 3 \\
\hline Fluorene & 3 \\
\hline Hexachlorobenzene & 3 \\
\hline Hexachlorobutadiene & 3 \\
\hline Hexachlorocyclopentadiene & 3 \\
\hline Hexachloroethane & 3 \\
\hline Indeno[1,2,3-c,d]pyrene & 3 \\
\hline Isophorone & 3 \\
\hline N-Nitrosodiphenylamine & 3 \\
\hline N-Nitrosodipropylamine & 3 \\
\hline Naphthalene & 3 \\
\hline Nitrobenzene & 3 \\
\hline Pentachlorophenol & 3 \\
\hline Phenanthrene & 3 \\
\hline Phenol & 3 \\
\hline 1,2,4-Trichlorobenzene & 3 \\
\hline 1,2-Dichlorobenzene & 3 \\
\hline 1,3-Dichlorobenzene & 3 \\
\hline 1,4-Dichlorobenzene & 3 \\
\hline 2,4,5-Trichlorophenol & 3 \\
\hline 2,4,6-Trichlorophenol & 3 \\
\hline 2,4-Dichlorophenol & 3 \\
\hline 2,4-Dimethyl phenol & 3 \\
\hline 2,4-Dinitrophenol & 3 \\
\hline 2,4-Dinitrotoluene & 3 \\
\hline 2,6-Dinitrotoluene & 3 \\
\hline 2-Chlorophenol & 3 \\
\hline 2-Chloronaphthalene & 3 \\
\hline 2-Methylnaphthalene & 3 \\
\hline o-Cresol (2-Methylphenol) & 3 \\
\hline o-Nitroaniline & 3 \\
\hline 2-Nitrophenol & 3 \\
\hline
\end{tabular}


Table 31. Analytes Not Detected in Laboratory Duplicates for Sediment Samples, Continued

\begin{tabular}{|l|l|}
\hline Analyte & $\begin{array}{l}\text { Number } \\
\text { of Pairs }\end{array}$ \\
\hline 3,3'-Dichlorobenzidine & 3 \\
\hline m/p-Cresol & 3 \\
\hline m-Nitroaniline & 3 \\
\hline 2-Methyl-4,6-dinitrophenol & 3 \\
\hline 4-Bromophenyl phenyl ether & 3 \\
\hline 4-Chloroaniline & 3 \\
\hline 4-Chloro-m-cresol & 3 \\
\hline 4-Chlorophenyl phenyl ether & 3 \\
\hline p-Nitroaniline & 3 \\
\hline 4-Nitrophenol & 3 \\
\hline Method EPIA-012B & \\
\hline Plutonium-238 & 7 \\
\hline Plutonium-239/240 & 7 \\
\hline Method EPIA-013B & \\
\hline Antimony-124 & 5 \\
\hline Antimony-125 & 5 \\
\hline Barium-133 & 5 \\
\hline
\end{tabular}

\begin{tabular}{|l|l|}
\hline Analyte & $\begin{array}{l}\text { Number } \\
\text { of Pairs }\end{array}$ \\
\hline Cerium-144 & 5 \\
\hline Cesium-134 & 5 \\
\hline Cobalt-57 & 5 \\
\hline Cobalt-58 & 5 \\
\hline Cobalt-60 & 5 \\
\hline Europium-152 & 5 \\
\hline Europium-154 & 5 \\
\hline Europium-155 & 5 \\
\hline Promethium-144 & 5 \\
\hline Promethium-146 & 5 \\
\hline Ruthenium-106 & 5 \\
\hline Sodium-22 & 5 \\
\hline Tin-113 & 5 \\
\hline Yttrium-88 & 5 \\
\hline Zinc-65 & 5 \\
\hline
\end{tabular}


Table 32. Analytes Not Detected in Laboratory Duplicates for Water Samples

\begin{tabular}{|c|c|}
\hline Analyte & $\begin{array}{l}\text { Number } \\
\text { of Pairs }\end{array}$ \\
\hline \multicolumn{2}{|c|}{ Method EPA6010A (total recoverable) } \\
\hline Barium & 1 \\
\hline Beryllium & 1 \\
\hline Cadmium & 1 \\
\hline Chromium & 1 \\
\hline Cobalt & 1 \\
\hline Copper & 1 \\
\hline Iron & 1 \\
\hline Magnesium & 1 \\
\hline Nickel & 1 \\
\hline Potassium & 1 \\
\hline Silver & 1 \\
\hline Sodium & 1 \\
\hline Vanadium & 1 \\
\hline \multicolumn{2}{|c|}{ Method EPA7421 (total recoverable) } \\
\hline Lead & 1 \\
\hline \multicolumn{2}{|l|}{ Method EPA8260 } \\
\hline Bromodichloromethane & 3 \\
\hline Bromoform & 3 \\
\hline $\begin{array}{l}\text { Bromomethane (Methyl } \\
\text { bromide) }\end{array}$ & 3 \\
\hline Carbon disulfide & 3 \\
\hline Carbon tetrachloride & 3 \\
\hline Chloroethane & 3 \\
\hline Chloroethene (Vinyl chloride) & 3 \\
\hline Chloroform & 3 \\
\hline $\begin{array}{l}\text { Chloromethane (Methyl } \\
\text { chloride) }\end{array}$ & 3 \\
\hline Dibromochloromethane & 3 \\
\hline $\begin{array}{l}\text { Dichloromethane (Methylene } \\
\text { chloride) }\end{array}$ & 3 \\
\hline Ethylbenzene & 3 \\
\hline Styrene & 3 \\
\hline Tetrachloroethylene & 3 \\
\hline Vinyl acetate & 3 \\
\hline 1,1,2,2-Tetrachloroethane & 3 \\
\hline 1,1,1-Trichloroethane & 3 \\
\hline 1,1,2-Trichloroethane & 3 \\
\hline 1,1-Dichloroethylene & 3 \\
\hline 1,1-Dichloroethane & 3 \\
\hline
\end{tabular}

\begin{tabular}{|l|l|}
\hline Analyte & $\begin{array}{l}\text { Number } \\
\text { of Pairs }\end{array}$ \\
\hline 1,2-Dichloroethylene & 3 \\
\hline 1,2-Dichloroethane & 3 \\
\hline 1,2-Dichloropropane & 3 \\
\hline cis-1,3-Dichloropropene & 3 \\
\hline trans-1,3-Dichloropropene & 3 \\
\hline 2-Hexanone & 3 \\
\hline Method EPA8270 & \\
\hline Acenaphthene & 1 \\
\hline Acenaphthylene & 1 \\
\hline Anthracene & 1 \\
\hline Benzo[a]anthracene & 1 \\
\hline Benzo[a]pyrene & 1 \\
\hline Benzo[b]fluoranthene & 1 \\
\hline Benzo[g,h,i]perylene & 1 \\
\hline Benzo[k]fluoranthene & 1 \\
\hline Benzoic acid & 1 \\
\hline Benzyl alcohol & 1 \\
\hline Bis(2-chloroethoxy) methane & 1 \\
\hline Bis(2-chloroethyl) ether & 1 \\
\hline Bis(2-chloroisopropyl) ether & 1 \\
\hline Bis(2-ethylhexyl) phthalate & 1 \\
\hline Chrysene & 1 \\
\hline Di-n-butyl phthalate & 1 \\
\hline Di-n-octyl phthalate & 1 \\
\hline Dibenz[a,h]anthracene & 1 \\
\hline Dibenzofuran & 1 \\
\hline Dimethyl phthalate & 1 \\
\hline Fluoranthene & 1 \\
\hline Fluorene & 1 \\
\hline Hexachlorobenzene & 1 \\
\hline Hexachlorobutadiene & 1 \\
\hline Hexachlorocyclopentadiene & 1 \\
\hline Hexachloroethane & 1 \\
\hline Indeno[1,2,3-c,d]pyrene & 1 \\
\hline Isophorone & 1 \\
\hline N-Nitrosodiphenylamine & 1 \\
\hline N-Nitrosodipropylamine & 1 \\
\hline Naphthalene & 1 \\
\hline Nitrobenzene & 1 \\
\hline
\end{tabular}


Table 32. Analytes Not Detected in Laboratory Duplicates for Water Samples, Continued

\begin{tabular}{|l|l|}
\hline Analyte & $\begin{array}{l}\text { Number } \\
\text { of Pairs }\end{array}$ \\
\hline Pentachlorophenol & 1 \\
\hline Phenanthrene & 1 \\
\hline Phenol & 1 \\
\hline Pyrene & 1 \\
\hline 1,2,4-Trichlorobenzene & 1 \\
\hline 1,2-Dichlorobenzene & 1 \\
\hline 1,3-Dichlorobenzene & 1 \\
\hline 1,4-Dichlorobenzene & 1 \\
\hline 2,4,5-Trichlorophenol & 1 \\
\hline 2,4,6-Trichlorophenol & 1 \\
\hline 2,4-Dichlorophenol & 1 \\
\hline 2,4-Dimethyl phenol & 1 \\
\hline 2,4-Dinitrophenol & 1 \\
\hline 2,4-Dinitrotoluene & 1 \\
\hline 2,6-Dinitrotoluene & 1 \\
\hline 2-Chlorophenol & 1 \\
\hline
\end{tabular}

\begin{tabular}{|l|l|}
\hline Analyte & $\begin{array}{l}\text { Number } \\
\text { of Pairs }\end{array}$ \\
\hline 2-Chloronaphthalene & 1 \\
\hline 2-Methylnaphthalene & 1 \\
\hline o-Cresol (2-Methylphenol) & 1 \\
\hline o-Nitroaniline & 1 \\
\hline 2-Nitrophenol & 1 \\
\hline 3,3'-Dichlorobenzidine & 1 \\
\hline m/p-Cresol & 1 \\
\hline m-Nitroaniline & 1 \\
\hline 2-Methyl-4,6-dinitrophenol & 1 \\
\hline 4-Bromophenyl phenyl ether & 1 \\
\hline 4-Chloroaniline & 1 \\
\hline 4-Chloro-m-cresol & 1 \\
\hline 4-Chlorophenyl phenyl ether & 1 \\
\hline p-Nitroaniline & 1 \\
\hline 4-Nitrophenol & 1 \\
\hline
\end{tabular}

\section{Relative Percent Difference}

In the tables of RPDs that follow, the $J$ and $U$ qualifiers in the results columns mean "estimated" and "undetected," respectively. The $I$ qualifier indicates that the value in the result field is the instrument reading, not the sample quantitation limit. See Tables 33 and 34 for the RPDs for analytes detected in laboratory duplicates for sediment and water samples, respectively.

Table 33. RPDs for Analytes Detected in Laboratory Duplicates for Sediment Samples

\begin{tabular}{|c|c|c|c|c|c|c|c|}
\hline Analyte & Sample & RPD & \multicolumn{2}{|c|}{ First Result } & \multicolumn{2}{|c|}{$\begin{array}{l}\text { Second } \\
\text { Resült }\end{array}$} & Units \\
\hline \multicolumn{8}{|c|}{ Method EPA300.0 } \\
\hline Chloride & 104625 & 21.1 & & 7.8 & & 9.64 & $\mathrm{mg} / \mathrm{kg}$ \\
\hline Chloride & 104643 & 11.1 & $\mathrm{~J}$ & 1.61 & $J$ & 1.44 & $\mathrm{mg} / \mathrm{kg}$ \\
\hline Chloride & 104665 & 53.5 & $\mathrm{~J}$ & 4.24 & $\mathrm{~J}$ & 2.45 & $\mathrm{mg} / \mathrm{kg}$ \\
\hline Chloride & 104681 & 51.4 & $J$ & 1.65 & $\mathrm{U}$ & 2.79 & $\mathrm{mg} / \mathrm{kg}$ \\
\hline Chloride & 104697 & 20.6 & $\mathrm{~J}$ & 5.93 & $\mathrm{~J}$ & 7.29 & $\mathrm{mg} / \mathrm{kg}$ \\
\hline Chloride & 105104 & 6.5 & & 12.8 & & 12 & $\mathrm{mg} / \mathrm{kg}$ \\
\hline \multicolumn{8}{|c|}{ Method EPA335.3 } \\
\hline Cyanide & 104602 & 3.9 & $\mathrm{~J}$ & 3400 & $\mathrm{~J}$ & 3270 & $\mu \mathrm{g} / \mathrm{kg}$ \\
\hline Cyanide & 104625 & 120.2 & $\mathrm{UJ}$ & 2410 & $J$ & 601 & $\mu \mathrm{g} / \mathrm{kg}$ \\
\hline
\end{tabular}

Par Pond, Pond C, and L Lake, Revision 2 
Table 33. RPDs for Analytes Detected in Laboratory Duplicates for Sediment Samples, Continued

\begin{tabular}{|c|c|c|c|c|c|c|c|}
\hline Analyte & Sample & $\mathbf{R P D}$ & \multicolumn{2}{|c|}{ First Result } & \multicolumn{2}{|c|}{$\begin{array}{l}\text { Second } \\
\text { Result }\end{array}$} & Units \\
\hline \multicolumn{8}{|l|}{ Method EPA350.1 } \\
\hline Ammonia nitrogen & 104676 & 14.6 & $\mathrm{~J}$ & 24.2 & $J$ & 28 & $\mathrm{mg} / \mathrm{kg}$ \\
\hline Ammonia nitrogen & 104689 & 1.6 & $\mathrm{~J}$ & 31.8 & $\mathrm{~J}$ & 32.3 & $\mathrm{mg} / \mathrm{kg}$ \\
\hline Ammonia nitrogen & 105104 & 5.9 & & 87.2 & & 92.5 & $\mathrm{mg} / \mathrm{kg}$ \\
\hline \multicolumn{8}{|l|}{ Method EPA351.2 } \\
\hline $\begin{array}{l}\text { Nitrogen by Kjeldahl } \\
\text { method }\end{array}$ & 104676 & 8.6 & & 831 & & 906 & $\mathrm{mg} / \mathrm{kg}$ \\
\hline $\begin{array}{l}\text { Nitrogen by Kjeldahl } \\
\text { method }\end{array}$ & 104687 & 6 & & 466 & & 439 & $\mathrm{mg} / \mathrm{kg}$ \\
\hline $\begin{array}{l}\text { Nitrogen by Kjjeldahl } \\
\text { method }\end{array}$ & 105101 & 8.9 & & 141 & & 129 & $\mathrm{mg} / \mathrm{kg}$ \\
\hline $\begin{array}{l}\text { Nitrogen by Kjeldahl } \\
\text { method }\end{array}$ & 105111 & 4.7 & & 209 & & 219 & $\mathrm{mg} / \mathrm{kg}$ \\
\hline $\begin{array}{l}\text { Nitrogen by Kjeldahl } \\
\text { method }\end{array}$ & 105118 & 3.7 & . & 36.1 & & 34.8 & $\mathrm{mg} / \mathrm{kg}$ \\
\hline \multicolumn{8}{|l|}{ Method EPA353.1 } \\
\hline $\begin{array}{l}\text { Nitrate-nitrite } \\
\text { as nitrogen } \\
\end{array}$ & 104664 & 3.6 & $J$ & 0.602 & $J$ & 0.624 & $\mathrm{mg} / \mathrm{kg}$ \\
\hline $\begin{array}{c}\text { Nitrate-nitrite } \\
\text { as nitrogen }\end{array}$ & 104674 & 20.2 & $\bar{J}$ & 0.45 & $\mathrm{~J}$ & 0.551 & $\mathrm{mg} / \mathrm{kg}$ \\
\hline $\begin{array}{c}\text { Nitrate-nitrite } \\
\text { as nitrogen } \\
\end{array}$ & 104683 & 17.5 & $\mathrm{~J}$ & 0.354 & $J$ & 0.422 & $\mathrm{mg} / \mathrm{kg}$ \\
\hline $\begin{array}{l}\text { Nitrate-nitrite } \\
\text { as nitrogen } \\
\end{array}$ & 105117 & 106 & $\bar{U}$ & 1.25 & $J$ & 0.384 & $\mathrm{mg} / \mathrm{kg}$ \\
\hline \multicolumn{8}{|l|}{ Method EPA365.2 } \\
\hline Orthophosphate & 104613 & 51.5 & $\mathrm{~J}$ & 9.57 & $\mathrm{~J}$ & 5.65 & $\mathrm{mg} / \mathrm{kg}$ \\
\hline Orthophosphate & 104629 & 0.6 & $\mathrm{~J}$ & 5 & $\mathrm{~J}$ & 5.03 & $\mathrm{mg} / \mathrm{kg}$ \\
\hline Orthophosphate & 104636 & 5.7 & $\mathrm{~J}$ & 4.1 & $\mathrm{~J}$ & 4.34 & $\mathrm{mg} / \mathrm{kg}$ \\
\hline Orthophosphate & 105102 & 17 & $\mathrm{~J}$ & 3.19 & $J$ & 2.69 & $\mathrm{mg} / \mathrm{kg}$ \\
\hline \multicolumn{8}{|l|}{ Method EPA365.4 } \\
\hline Total phosphates (as P) & 104602 & 15 & $J$ & 136 & $\mathrm{~J}$ & 117 & $\mathrm{mg} / \mathrm{kg}$ \\
\hline Total phosphates (as P) & 104613 & 7 & $J$ & 76.5 & $J$ & 71.3 & $\mathrm{mg} / \mathrm{kg}$ \\
\hline Total phosphates (as P) & 104623 & 13.8 & $\mathrm{~J}$ & 115 & $\mathrm{~J}$ & 132 & $\mathrm{mg} / \mathrm{kg}$ \\
\hline Total phosphates (as P) & 104644 & 14.2 & $J$ & 15 & $\mathrm{~J}$ & 17.3 & $\mathrm{mg} / \mathrm{kg}$ \\
\hline Total phosphates (as P) & 104648 & 27.2 & $\mathrm{~J}$ & 54.9 & $\mathrm{~J}$ & 72.2 & $\mathrm{mg} / \mathrm{kg}$ \\
\hline Total phosphates (as P) & 104657 & 1 & $\mathrm{~J}$ & 195 & $\mathrm{~J}$ & 193 & $\mathrm{mg} / \mathrm{kg}$ \\
\hline Total phosphates (as P) & 104668 & 9 & $\mathrm{~J}$ & 151 & $J$ & 138 & $\mathrm{mg} / \mathrm{kg}$ \\
\hline Total phosphates (as P) & 104681 & 7.1 & $J$ & 31.4 & $\cdot \mathrm{J}$ & 33.7 & $\mathrm{mg} / \mathrm{kg}$ \\
\hline Total phosphates (as P) & 104691 & 33.1 & $J$ & 222 & $\mathrm{~J}$ & 159 & $\mathrm{mg} / \mathrm{kg}$ \\
\hline Total phosphates (as P) & 105102 & 3.7 & $J$ & 408 & $\mathrm{~J}$ & 393 & $\mathrm{mg} / \mathrm{kg}$ \\
\hline
\end{tabular}


Table 33. RPDs for Analytes Detected in Laboratory Duplicates for Sediment Samples, Continued

\begin{tabular}{|c|c|c|c|c|c|c|c|}
\hline \multirow{2}{*}{$\begin{array}{l}\text { Analyte } \\
\text { Total phosphates (as P) }\end{array}$} & \multirow{2}{*}{$\begin{array}{l}\text { Sample } \\
105110 \\
\end{array}$} & \multirow{2}{*}{$\begin{array}{l}\text { RPD } \\
24.6 \\
\end{array}$} & \multicolumn{2}{|c|}{ First Result } & \multicolumn{2}{|c|}{$\begin{array}{l}\text { Second } \\
\text { Result }\end{array}$} & \multirow{2}{*}{$\begin{array}{l}\text { Units } \\
\mathrm{mg} / \mathrm{kg} \\
\end{array}$} \\
\hline & & & $\mathrm{J}$ & 4.17 & $\mathrm{~J}$ & 5.34 & \\
\hline Total phosphates (as P) & 105111 & 27.7 & $\mathrm{~J}$ & 6.47 & $\mathrm{~J}$ & 8.55 & $\mathrm{mg} / \mathrm{kg}$ \\
\hline \multicolumn{8}{|l|}{ Method EPA415.1 } \\
\hline Total organic carbon & 104604 & 14 & & 1260 & & 1450 & $\mathrm{mg} / \mathrm{kg}$ \\
\hline Total organic carbon & 104612 & 13.2 & & 832 & & 729 & $\mathrm{mg} / \mathrm{kg}$ \\
\hline Total organic carbon & 104621 & 11.7 & & 2820 & & 3170 & $\mathrm{mg} / \mathrm{kg}$ \\
\hline Total organic carbon & 104629 & 10.8 & & 29000 & & 32300 & $\mathrm{mg} / \mathrm{kg}$ \\
\hline Total organic carbon & 104635 & 1.9 & & 4200 & & 4120 & $\mathrm{mg} / \mathrm{kg}$ \\
\hline Total organic carbon & 104644 & 3.4 & & 842 & & 871 & $\mathrm{mg} / \mathrm{kg}$ \\
\hline Total organic carbon & 104658 & 13.1 & & 11100 & & 9740 & $\mathrm{mg} / \mathrm{kg}$ \\
\hline Total organic carbon & 104669 & 13.7 & & 3000 & & 3440 & $\mathrm{mg} / \mathrm{kg}$ \\
\hline Total organic carbon & 104681 & 0.9 & & 889 & & 897 & $\mathrm{mg} / \mathrm{kg}$ \\
\hline Total organic carbon & 104684 & 6.6 & & 469 & & 439 & $\mathrm{mg} / \mathrm{kg}$ \\
\hline Total organic carbon & 104693 & 3 & & 1310 & & 1350 & $\mathrm{mg} / \mathrm{kg}$ \\
\hline Total organic carbon & 104694 & 7.6 & & 1400 & & 1510 & $\mathrm{mg} / \mathrm{kg}$ \\
\hline Total organic carbon & 105110 & 14 & $\mathrm{~J}$ & 2730 & $\mathrm{~J}$ & 3140 & $\mathrm{mg} / \mathrm{kg}$ \\
\hline Total organic carbon & 105111 & 5 & $J$ & 7850 & $J$ & 8250 & $\mathrm{mg} / \mathrm{kg}$ \\
\hline Total organic carbon & 105113 & 18.8 & & 3150 & & 2610 & $\mathrm{mg} / \mathrm{kg}$ \\
\hline \multicolumn{8}{|l|}{ Method EPA420.2 } \\
\hline Phenols & 104636 & 117.2 & $\mathrm{~J}$ & 89.5 & UJ & 343 & $\mu \mathrm{g} / \mathrm{kg}$ \\
\hline Phenols & 104674 & 7.6 & $J$ & 114 & $\mathrm{~J}$ & 123 & $\mu \mathrm{g} / \mathrm{kg}$ \\
\hline Phenols & 104689 & 112.9 & $\mathrm{U}$ & 417 & $\mathrm{~J}$ & 116 & $\mu \mathrm{g} / \mathrm{kg}$ \\
\hline \multicolumn{8}{|c|}{ Method EPA6010A (inorganics are total recoverable) } \\
\hline Aluminum & 104600 & 38.4 & & 2860 & & 4220 & $\mathrm{mg} / \mathrm{kg}$ \\
\hline Aluminum & 104614 & 31.1 & & 1550 & & 2120 & $\mathrm{mg} / \mathrm{kg}$ \\
\hline Aluminum & 104625 & 34.7 & & 15900 & & 11200 & $\mathrm{mg} / \mathrm{kg}$ \\
\hline Aluminum & 104650 & 20.1 & & 2860 & & 3500 & $\mathrm{mg} / \mathrm{kg}$ \\
\hline Aluminum & 104664 & 8.3 & & 2380 & & 2190 & $\mathrm{mg} / \mathrm{kg}$ \\
\hline Aluminum & 104667 & 41.1 & $\mathrm{~J}$ & 3810 & $\mathrm{~J}$ & 2510 & $\mathrm{mg} / \mathrm{kg}$ \\
\hline Aluminum & 104671 & 19.4 & & 11100 & & 9140 & $\mathrm{mg} / \mathrm{kg}$ \\
\hline Aluminum & 104680 & 13.7 & & 1060 & & 924 & $\mathrm{mg} / \mathrm{kg}$ \\
\hline Aluminum & 104683 & 37.9 & & 5270 & & 3590 & $\mathrm{mg} / \mathrm{kg}$ \\
\hline Aluminum & 104693 & 17.5 & & 5100 & & 4280 & $\mathrm{mg} / \mathrm{kg}$ \\
\hline Aluminum & 105110 & 24.8 & $\mathrm{~J}$ & 927 & $\mathrm{~J}$ & 1190 & $\mathrm{mg} / \mathrm{kg}$ \\
\hline Aluminum & 105113 & 79.6 & & 1180 & & 2740 & $\mathrm{mg} / \mathrm{kg}$ \\
\hline Antimony & 104671 & 9.7 & $J$ & 7.62 & $\mathrm{UJ}$ & 8.4 & $\mathrm{mg} / \mathrm{kg}$ \\
\hline Arsenic & 104671 & 22.3 & $\mathrm{~J}$ & 5.15 & $J$ & 6.44 & $\mathrm{mg} / \mathrm{kg}$ \\
\hline Barium & 104600 & 2.8 & & 8.93 & & 8.68 & $\mathrm{mg} / \mathrm{kg}$ \\
\hline Barium & 104614 & 22.5 & & 8.62 & & 10.8 & $\mathrm{mg} / \mathrm{kg}$ \\
\hline
\end{tabular}


Table 33. RPDs for Analytes Detected in Laboratory Duplicates for Sediment Samples, Continued

\begin{tabular}{|c|c|c|c|c|c|c|c|}
\hline \multirow{2}{*}{$\frac{\text { Analyte }}{\text { Barium }}$} & \multirow{2}{*}{$\begin{array}{l}\text { Sample } \\
104625 \\
\end{array}$} & \multirow{2}{*}{$\begin{array}{l}\text { RPD } \\
26.1 \\
\end{array}$} & \multicolumn{2}{|c|}{ First Result } & \multicolumn{2}{|c|}{$\begin{array}{l}\text { Second } \\
\text { Result }\end{array}$} & \multirow{2}{*}{$\frac{\text { Units }}{\mathrm{mg} / \mathrm{kg}}$} \\
\hline & & & & 69.2 & & 53.2 & \\
\hline Barium & 104650 & 36.4 & & 6.56 & & 9.48 & $\mathrm{mg} / \mathrm{kg}$ \\
\hline Barium & 104664 & 5.3 & & 14.8 & & 15.6 & $\mathrm{mg} / \mathrm{kg}$ \\
\hline Barium & 104667 & 10.3 & & 25.6 & & 23.1 & $\mathrm{mg} / \mathrm{kg}$ \\
\hline Barium & 104671 & 9 & $\mathrm{~J}$ & 106 & $\mathrm{~J}$ & 96.9 & $\mathrm{mg} / \mathrm{kg}$ \\
\hline Barium & 104680 & 9.6 & & 5.63 & & 6.2 & $\mathrm{mg} / \mathrm{kg}$ \\
\hline Barium & 104683 & 22.7 & & 12.2 & & 9.71 & $\mathrm{mg} / \mathrm{kg}$ \\
\hline Barium & 104693 & 12.5 & & 13.6 & & 12 & $\mathrm{mg} / \mathrm{kg}$ \\
\hline Barium & 105110 & 23.6 & & 5.6 & & 7.1 & $\mathrm{mg} / \mathrm{kg}$ \\
\hline Barium & 105113 & 46.7 & $\mathrm{~J}$ & 8.45 & $J$ & 13.6 & $\mathrm{mg} / \mathrm{kg}$ \\
\hline Beryllium & 104600 & 3.2 & $\mathrm{~J}$ & 0.0576 & $J$ & 0.0558 & $\mathrm{mg} / \mathrm{kg}$ \\
\hline Beryllium & 104614 & 26 & $\mathrm{~J}$ & 0.0446 & $\mathrm{~J}$ & 0.0579 & $\mathrm{mg} / \mathrm{kg}$ \\
\hline Beryllium & 104625 & 16.3 & & 1.33 & $\mathrm{~J}$ & 1.13 & $\mathrm{mg} / \mathrm{kg}$ \\
\hline Beryllium & 104650 & 25.2 & $\mathrm{~J}$ & 0.0451 & $J$ & 0.0581 & $\mathrm{mg} / \mathrm{kg}$ \\
\hline Beryllium & 104664 & 10 & $J$ & 0.0811 & $\mathrm{~J}$ & 0.0896 & $\mathrm{mg} / \mathrm{kg}$ \\
\hline Beryllium & 104667 & 15.8 & $\mathrm{~J}$ & 0.123 & $J$ & 0.105 & $\mathrm{mg} / \mathrm{kg}$ \\
\hline Beryllium & 104671 & 1 & $\mathrm{~J}$ & 1.05 & $\mathrm{~J}$ & 1.04 & $\mathrm{mg} / \mathrm{kg}$ \\
\hline Beryllium & 104680 & 20.7 & $\mathrm{~J}$ & 0.0494 & $J$ & 0.0608 & $\mathrm{mg} / \mathrm{kg}$ \\
\hline Beryllium & 104683 & 24 & $\mathrm{~J}$ & 0.154 & $J$ & 0.121 & $\mathrm{mg} / \mathrm{kg}$ \\
\hline Beryllium & 104693 & 11.3 & $\mathrm{~J}$ & 0.0459 & $\mathrm{~J}$ & 0.041 & $\mathrm{mg} / \mathrm{kg}$ \\
\hline Beryllium & 105110 & 7.6 & $\mathrm{~J}$ & 0.0433 & $J$ & 0.0467 & $\mathrm{mg} / \mathrm{kg}$ \\
\hline Beryllium & 105113 & 48.6 & $J$ & 0.0615 & $J$ & 0.101 & $\mathrm{mg} / \mathrm{kg}$ \\
\hline Calcium & 104600 & 13.8 & & 47.3 & & 54.3 & $\mathrm{mg} / \mathrm{kg}$ \\
\hline Calcium & 104614 & 15.1 & & 41 & & 47.7 & $\mathrm{mg} / \mathrm{kg}$ \\
\hline Calcium & 104625 & 9.5 & & 835 & & 759 & $\mathrm{mg} / \mathrm{kg}$ \\
\hline Calcium & 104650 & 23.2 & & 26 & & 20.6 & $\mathrm{mg} / \mathrm{kg}$ \\
\hline Calcium & 104664 & 6.9 & & 62.6 & & 58.4 & $\mathrm{mg} / \mathrm{kg}$ \\
\hline Calcium & 104667 & 28.6 & & 101 & & 75.7 & $\mathrm{mg} / \mathrm{kg}$ \\
\hline Calcium & 104671 & 0 & & 349 & & 349 & $\mathrm{mg} / \mathrm{kg}$ \\
\hline Calcium & 104680 & 23.6 & & 112 & & 142 & $\mathrm{mg} / \mathrm{kg}$ \\
\hline Calcium & 104683 & 3.9 & $\mathrm{~J}$ & 552 & $J$ & 531 & $\mathrm{mg} / \mathrm{kg}$ \\
\hline Calcium & 104693 & 0.7 & & 43.1 & & 42.8 & $\mathrm{mg} / \mathrm{kg}$ \\
\hline Calcium & 105110 & 1.4 & & 22 & & 21.7 & $\mathrm{mg} / \mathrm{kg}$ \\
\hline Calcium & 105113 & 45.2 & $\mathrm{~J}$ & 276 & $\mathrm{~J}$ & 437 & $\mathrm{mg} / \mathrm{kg}$ \\
\hline Chromium & 104600 & 2.2 & & 4.97 & & 4.86 & $\mathrm{mg} / \mathrm{kg}$ \\
\hline Chromium & 104614 & 26.2 & & 1.59 & & 2.07 & $\mathrm{mg} / \mathrm{kg}$ \\
\hline Chromium & 104625 & 21.4 & & 25.4 & & 20.5 & $\mathrm{mg} / \mathrm{kg}$ \\
\hline Chromium & 104650 & 7.4 & & 4.03 & & 4.34 & $\mathrm{mg} / \mathrm{kg}$ \\
\hline Chromium & 104664 & 1.7 & & 1.83 & & 1.8 & $\mathrm{mg} / \mathrm{kg}$ \\
\hline
\end{tabular}


Discussion

Table 33. RPDs for Analytes Detected in Laboratory Duplicates for Sediment Samples, Continued

\begin{tabular}{|c|c|c|c|c|c|c|c|}
\hline \multirow{2}{*}{$\begin{array}{l}\text { Analyte } \\
\text { Chromium }\end{array}$} & \multirow{2}{*}{\begin{tabular}{|l|} 
Sample \\
104667 \\
\end{tabular}} & \multirow{2}{*}{$\begin{array}{l}\text { RPD } \\
56.3\end{array}$} & \multicolumn{2}{|c|}{ First Result } & \multicolumn{2}{|c|}{$\begin{array}{l}\text { Second } \\
\text { Result }\end{array}$} & \multirow{2}{*}{\begin{tabular}{|l} 
Units \\
$\mathrm{mg} / \mathrm{kg}$
\end{tabular}} \\
\hline & & & & 4.14 & & 2.32 & \\
\hline Chromium & 104671 & 8.6 & & 34.1 & & 31.3 & $\mathrm{mg} / \mathrm{kg}$ \\
\hline Chromium & 104680 & 116.2 & & 7.02 & & 1.86 & $\mathrm{mg} / \mathrm{kg}$ \\
\hline Chromium & 104683 & 32.7 & & 8.86 & & 6.37 & $\mathrm{mg} / \mathrm{kg}$ \\
\hline Chromium & 104693 & 17.1 & & 3.69 & & 3.11 & $\mathrm{mg} / \mathrm{kg}$ \\
\hline Chromium & 105110 & 23.6 & & 1.83 & & 2.32 & $\mathrm{mg} / \mathrm{kg}$ \\
\hline Chromium & 105113 & 54.7 & & 1.62 & & 2.84 & $\mathrm{mg} / \mathrm{kg}$ \\
\hline Cobalt & 104600 & 22.7 & $\mathrm{~J}$ & 0.274 & $\mathrm{~J}$ & 0.344 & $\mathrm{mg} / \mathrm{kg}$ \\
\hline Cobalt & 104614 & 44.7 & $\mathrm{~J}$ & 0.243 & $\mathrm{~J}$ & 0.383 & $\mathrm{mg} / \mathrm{kg}$ \\
\hline Cobalt & 104625 & 13.4 & & 7.75 & & 6.78 & $\mathrm{mg} / \mathrm{kg}$ \\
\hline Cobalt & 104650 & 81.7 & $\mathrm{U}$ & 0.543 & $\mathrm{~J}$ & 0.228 & $\mathrm{mg} / \mathrm{kg}$ \\
\hline Cobalt & 104664 & 38.1 & $\mathrm{~J}$ & 0.378 & & 0.556 & $\mathrm{mg} / \mathrm{kg}$ \\
\hline Cobalt & 104667 & 3.4 & & 0.891 & & 0.861 & $\mathrm{mg} / \mathrm{kg}$ \\
\hline Cobalt & 104671 & 11.5 & . & 7.82 & & 6.97 & $\mathrm{mg} / \mathrm{kg}$ \\
\hline Cobalt & 104680 & 3.1 & & 0.723 & & 0.701 & $\mathrm{mg} / \mathrm{kg}$ \\
\hline Cobalt & 104683 & 89.7 & $\mathrm{~J}$ & 0.217 & $\mathrm{U}$ & 0.57 & $\mathrm{mg} / \mathrm{kg}$ \\
\hline Cobalt & 104693 & 10.4 & $\mathrm{~J}$ & 0.232 & $\mathrm{~J}$ & 0.209 & $\mathrm{mg} / \mathrm{kg}$ \\
\hline Cobalt & 105110 & 4.2 & $\mathrm{~J}$ & 0.212 & $\mathrm{~J}$ & 0.221 & $\mathrm{mg} / \mathrm{kg}$ \\
\hline Cobalt & 105113 & 30.9 & $\mathrm{~J}$ & 0.468 & & 0.639 & $\mathrm{mg} / \mathrm{kg}$ \\
\hline Copper & 104600 & 100.2 & & 1.54 & & 4.63 & $\mathrm{mg} / \mathrm{kg}$ \\
\hline Copper & 104614 & 3.6 & $\mathrm{~J}$ & 0.933 & $\mathrm{~J}$ & 0.967 & $\mathrm{mg} / \mathrm{kg}$ \\
\hline Copper & 104625 & 31.3 & & 7.03 & & 5.13 & $\mathrm{mg} / \mathrm{kg}$ \\
\hline Copper & 104650 & 31.1 & $\mathrm{~J}$ & 0.532 & $\mathrm{~J}$ & 0.728 & $\mathrm{mg} / \mathrm{kg}$ \\
\hline Copper & 104664 & 68.1 & & 2.54 & $\mathrm{~J}$ & 1.25 & $\mathrm{mg} / \mathrm{kg}$ \\
\hline Copper & 104667 & 14.8 & & 2.1 & & 1.81 & $\mathrm{mg} / \mathrm{kg}$ \\
\hline Copper & 104671 & 12.5 & & 14.5 & & 12.8 & $\mathrm{mg} / \mathrm{kg}$ \\
\hline Copper & 104680 & 31.2 & $\mathrm{~J}$ & 1.17 & $\mathrm{~J}$ & 0.854 & $\mathrm{mg} / \mathrm{kg}$ \\
\hline Copper & 104683 & 96 & & 1.42 & $\mathrm{~J}$ & 0.499 & $\mathrm{mg} / \mathrm{kg}$ \\
\hline Copper & 104693 & 70 & & 2.72 & $\mathrm{~J}$ & 1.31 & $\mathrm{mg} / \mathrm{kg}$ \\
\hline Copper & 105110 & 3.3 & $\mathrm{~J}$ & 0.804 & $\mathrm{~J}$ & 0.778 & $\mathrm{mg} / \mathrm{kg}$ \\
\hline Copper & 105113 & 83.3 & $\mathrm{~J}$ & 1.03 & & 2.5 & $\mathrm{mg} / \mathrm{kg}$ \\
\hline Iron & 104600 & 19.4 & & 3400 & & 2800 & $\mathrm{mg} / \mathrm{kg}$ \\
\hline Iron & 104614 & 19 & & 975 & & 1180 & $\mathrm{mg} / \mathrm{kg}$ \\
\hline Iron & 104625 & 27.3 & $\mathrm{~J}$ & 7570 & $\mathrm{~J}$ & 5750 & $\mathrm{mg} / \mathrm{kg}$ \\
\hline Iron & 104650 & 32.2 & & 450 & & 623 & $\mathrm{mg} / \mathrm{kg}$ \\
\hline Iron & 104664 & 11.5 & $\mathrm{~J}$ & 918 & $\mathrm{~J}$ & 1030 & $\mathrm{mg} / \mathrm{kg}$ \\
\hline Iron & 104667 & 55.4 & $\mathrm{~J}$ & 4680 & $\mathrm{~J}$ & 2650 & $\mathrm{mg} / \mathrm{kg}$ \\
\hline Iron & 104671 & 7 & $\mathrm{~J}$ & 23500 & $\mathrm{~J}$ & 21900 & $\mathrm{mg} / \mathrm{kg}$ \\
\hline Iron & 104680 & 6.4 & $\mathrm{~J}$ & 887 & $\mathrm{~J}$ & 946 & $\mathrm{mg} / \mathrm{kg}$ \\
\hline
\end{tabular}


Table 33. RPDs for Analytes Detected in Laboratory Duplicates for Sediment Samples, Continued

\begin{tabular}{|c|c|c|c|c|c|c|c|}
\hline \multirow{2}{*}{$\begin{array}{l}\text { Analyte } \\
\text { Iron }\end{array}$} & \multirow{2}{*}{$\begin{array}{l}\text { Sample } \\
104683 \\
\end{array}$} & \multirow{2}{*}{$\begin{array}{l}\text { RPD } \\
20.4 \\
\end{array}$} & \multicolumn{2}{|c|}{ First Result } & \multicolumn{2}{|c|}{$\begin{array}{l}\text { Second } \\
\text { Result }\end{array}$} & \multirow{2}{*}{$\frac{\text { Units }}{\mathrm{mg} / \mathrm{kg}}$} \\
\hline & & & & 2000 & & 1630 & \\
\hline Iron & 104693 & 11.6 & & 1550 & & 1380 & $\mathrm{mg} / \mathrm{kg}$ \\
\hline Iron & 105110 & 20.7 & $\mathrm{~J}$ & 1210 & $\mathrm{~J}$ & 1490 & $\mathrm{mg} / \mathrm{kg}$ \\
\hline Iron & 105113 & 42.6 & $\mathrm{~J}$ & 967 & $\mathrm{~J}$ & 1490 & $\mathrm{mg} / \mathrm{kg}$ \\
\hline Lead & 104600 & 16.6 & $\mathrm{~J}$ & 2.7 & $\mathrm{~J}$ & 3.19 & $\mathrm{mg} / \mathrm{kg}$ \\
\hline Lead & 104625 & 47.8 & & 17.9 & $\bar{J}$ & 11 & $\mathrm{mg} / \mathrm{kg}$ \\
\hline Lead & 104650 & 0.2 & $\mathbf{J}$ & 4.27 & $\mathrm{~J}$ & 4.26 & $\mathrm{mg} / \mathrm{kg}$ \\
\hline Lead & 104664 & 47.5 & $\bar{J}$ & 3.44 & $\bar{J}$ & 2.12 & $\mathrm{mg} / \mathrm{kg}$ \\
\hline Lead & 104671 & 14.4 & & 18.6 & & 16.1 & $\mathrm{mg} / \mathrm{kg}$ \\
\hline Lead & 104680 & 3.4 & $\mathrm{~J}$ & 2.66 & $\mathrm{~J}$ & 2.57 & $\mathrm{mg} / \mathrm{kg}$ \\
\hline Lead & 104693 & 71.5 & $J$ & 4.31 & $\mathrm{~J}$ & 2.04 & $\mathrm{mg} / \mathrm{kg}$ \\
\hline Lead & 104736 & 28.8 & & 9.53 & & 7.13 & $\mathrm{mg} / \mathrm{kg}$ \\
\hline Lead & 105110 & 79.1 & $\mathrm{~J}$ & 2.44 & $U$ & 5.63 & $\mathrm{mg} / \mathrm{kg}$ \\
\hline Lead & 105113 & 53.5 & $\mathrm{U}$ & 6.21 & $\mathrm{~J}$ & 3.59 & $\mathrm{mg} / \mathrm{kg}$ \\
\hline Magnesium & 104600 & 5 & $\mathrm{~J}$ & 50.3 & $\mathrm{~J}$ & 52.9 & $\mathrm{mg} / \mathrm{kg}$ \\
\hline Magnesium & 104614 & 50.2 & $\mathrm{~J}$ & 39.1 & $\mathrm{~J}$ & 65.3 & $\mathrm{mg} / \mathrm{kg}$ \\
\hline Magnesium & 104625 & 39.8 & $\mathrm{~J}$ & 256 & $\mathrm{~J}$ & 171 & $\mathrm{mg} / \mathrm{kg}$ \\
\hline Magnesium & 104650 & 15.9 & & 45.8 & & 53.7 & $\mathrm{mg} / \mathrm{kg}$ \\
\hline Magnesium & 104664 & 29.5 & & 44.2 & & 59.5 & $\mathrm{mg} / \mathrm{kg}$ \\
\hline Magnesium & 104667 & 43.3 & $\mathrm{~J}$ & 86 & $\mathrm{~J}$ & 55.4 & $\mathrm{mg} / \mathrm{kg}$ \\
\hline Magnesium & 104671 & 12 & & 1410 & & 1250 & $\mathrm{mg} / \mathrm{kg}$ \\
\hline Magnesium & 104680 & 7.6 & & 16.3 & & 15.1 & $\mathrm{mg} / \mathrm{kg}$ \\
\hline Magnesium & 104683 & 32.9 & & 627 & & 450 & $\mathrm{mg} / \mathrm{kg}$ \\
\hline Magnesium & 104693 & 10.8 & $J$ & 147 & $J$ & 132 & $\mathrm{mg} / \mathrm{kg}$ \\
\hline Magnesium & 105110 & 38.4 & & 24.4 & & 36 & $\mathrm{mg} / \mathrm{kg}$ \\
\hline Magnesium & 105113 & 64.9 & $\mathrm{~J}$ & 69.9 & $\mathrm{~J}$ & 137 & $\mathrm{mg} / \mathrm{kg}$ \\
\hline Manganese & 104600 & 113.8 & & 6.4 & & 23.3 & $\mathrm{mg} / \mathrm{kg}$ \\
\hline Manganese & 104614 & 40.2 & & 7.72 & & 11.6 & $\mathrm{mg} / \mathrm{kg}$ \\
\hline Manganese & 104625 & 14.7 & & 117 & & 101 & $\mathrm{mg} / \mathrm{kg}$ \\
\hline Manganese & 104650 & 9 & & 3.41 & & 3.73 & $\mathrm{mg} / \mathrm{kg}$ \\
\hline Manganese & 104664 & 20.2 & $\mathrm{~J}$ & 55.7 & $\mathrm{~J}$ & 45.5 & $\mathrm{mg} / \mathrm{kg}$ \\
\hline Manganese & 104667 & 7.6 & & 63.2 & & 58.6 & $\mathrm{mg} / \mathrm{kg}$ \\
\hline Manganese & 104671 & 3.7 & & 360 & & 347 & $\mathrm{mg} / \mathrm{kg}$ \\
\hline Manganese & 104680 & 0.3 & $\mathrm{~J}$ & 65.1 & $\mathrm{~J}$ & 65.3 & $\mathrm{mg} / \mathrm{kg}$ \\
\hline Manganese & 104683 & 26.1 & & 18.6 & & 14.3 & $\mathrm{mg} / \mathrm{kg}$ \\
\hline Manganese & 104693 & 47.8 & & 21 & & 12.9 & $\mathrm{mg} / \mathrm{kg}$ \\
\hline Manganese & 105110 & 1 & & 10.3 & & 10.2 & $\mathrm{mg} / \mathrm{kg}$ \\
\hline Manganese & 105113 & 66.7 & & 11.1 & & 22.2 & $\mathrm{mg} / \mathrm{kg}$ \\
\hline Nickel & 104600 & 14.6 & $J$ & 0.818 & $\mathrm{~J}$ & 0.947 & $\mathrm{mg} / \mathrm{kg}$ \\
\hline
\end{tabular}


Discussion

Table 33. RPDs for Analytes Detected in Laboratory Duplicates for Sediment

Samples, Continued

\begin{tabular}{|c|c|c|c|c|c|c|c|}
\hline \multirow{2}{*}{$\begin{array}{l}\text { Analyte } \\
\text { Nickel }\end{array}$} & \multirow{2}{*}{$\frac{\text { Sample }}{104614}$} & \multirow{2}{*}{$\frac{\text { RPD }}{22.4}$} & \multicolumn{2}{|c|}{ First Result } & \multicolumn{2}{|c|}{$\begin{array}{l}\text { Second } \\
\text { Result }\end{array}$} & \multirow{2}{*}{$\frac{\text { Units }}{\mathrm{mg} / \mathrm{kg}}$} \\
\hline & & & $J$ & 0.617 & $J$ & 0.773 & \\
\hline Nickel & 104625 & 23 & & 7.82 & & 6.21 & $\mathrm{mg} / \mathrm{kg}$ \\
\hline Nickel & 104650 & 36.2 & $\mathrm{~J}$ & 0.691 & $\mathrm{~J}$ & 0.996 & $\mathrm{mg} / \mathrm{kg}$ \\
\hline Nickel & 104664 & 35.4 & $\mathrm{~J}$ & 0.825 & $\mathrm{~J}$ & 1.18 & $\mathrm{mg} / \mathrm{kg}$ \\
\hline Nickel & 104667 & 35.5 & & 1.69 & $\mathrm{~J}$ & 1.18 & $\mathrm{mg} / \mathrm{kg}$ \\
\hline Nickel & 104671 & 19.9 & & 7.74 & & 6.34 & $\mathrm{mg} / \mathrm{kg}$ \\
\hline Nickel & 104680 & 132.8 & & 2.49 & $\mathrm{~J}$ & 0.503 & $\mathrm{mg} / \mathrm{kg}$ \\
\hline Nickel & 104683 & 31.7 & & 1.79 & $J$ & 1.3 & $\mathrm{mg} / \mathrm{kg}$ \\
\hline Nickel & 104693 & 5.8 & $J$ & 1.33 & & 1.41 & $\mathrm{mg} / \mathrm{kg}$ \\
\hline Nickel & 105110 & 2.9 & $\mathrm{~J}$ & 0.639 & $\mathrm{~J}$ & 0.658 & $\mathrm{mg} / \mathrm{kg}$ \\
\hline Nickel & 105113 & 46.3 & $\mathrm{~J}$ & 0.867 & $\mathrm{~J}$ & 1.39 & $\mathrm{mg} / \mathrm{kg}$ \\
\hline Potassium & 104600 & 18.1 & $J$ & 47.5 & $\mathrm{~J}$ & 39.6 & $\mathrm{mg} / \mathrm{kg}$ \\
\hline Potassium & 104614 & 30.4 & $\mathrm{~J}$ & 32.6 & $\mathrm{~J}$ & 44.3 & $\mathrm{mg} / \mathrm{kg}$ \\
\hline Potassium & 104625 & 44.2 & $J$ & 185 & $\mathrm{~J}$ & 118 & $\mathrm{mg} / \mathrm{kg}$ \\
\hline Potassium & 104650 & 4.3 & $\mathrm{~J}$ & 27.5 & $\mathrm{~J}$ & 28.7 & $\mathrm{mg} / \mathrm{kg}$ \\
\hline Potassium & 104664 & 31.8 & $\mathrm{~J}$ & 39.4 & $\mathrm{~J}$ & 54.3 & $\mathrm{mg} / \mathrm{kg}$ \\
\hline Potassium & 104667 & 38.9 & $\mathrm{~J}$ & 81 & $J$ & 54.6 & $\mathrm{mg} / \mathrm{kg}$ \\
\hline Potassium & 104671 & 14.1 & & 796 & & 691 & $\mathrm{mg} / \mathrm{kg}$ \\
\hline Potassium & 104680 & 21.2 & $\mathrm{~J}$ & 21.4 & $\mathrm{~J}$ & 17.3 & $\mathrm{mg} / \mathrm{kg}$ \\
\hline Potassium & 104683 & 25.9 & $\mathrm{~J}$ & 379 & $J$ & 292 & $\mathrm{mg} / \mathrm{kg}$ \\
\hline Potassium & 104693 & 3.6 & $\mathrm{~J}$ & 94.4 & $\mathrm{~J}$ & 97.9 & $\mathrm{mg} / \mathrm{kg}$ \\
\hline Potassium & 105110 & 2.8 & $\mathrm{~J}$ & 18.2 & $\mathrm{~J}$ & 17.7 & $\mathrm{mg} / \mathrm{kg}$ \\
\hline Potassium & 105113 & 40.6 & $\mathrm{~J}$ & 49.1 & $\mathrm{~J}$ & 74.1 & $\mathrm{mg} / \mathrm{kg}$ \\
\hline Selenium & 104671 & 107.9 & $\mathrm{~J}$ & 7.54 & $\mathrm{U}$ & 25.2 & $\mathrm{mg} / \mathrm{kg}$ \\
\hline Sodium & 104625 & 10.3 & $\mathrm{~J}$ & 30.7 & $J$ & 27.7 & $\mathrm{mg} / \mathrm{kg}$ \\
\hline Sodium & 104667 & 144.5 & $J$ & 8.83 & $\mathrm{U}$ & 54.8 & $\mathrm{mg} / \mathrm{kg}$ \\
\hline Sodium & 104671 & 0.4 & $\mathrm{~J}$ & 23.1 & $\mathrm{~J}$ & 23 & $\mathrm{mg} / \mathrm{kg}$ \\
\hline Sodium & 105113 & 148.4 & $\mathrm{U}$ & 62.1 & $\mathrm{~J}$ & 9.2 & $\mathrm{mg} / \mathrm{kg}$ \\
\hline Vanadium & 104600 & 17 & & 9.25 & & 7.8 & $\mathrm{mg} / \mathrm{kg}$ \\
\hline Vanadium & 104614 & 21.5 & & 2.7 & & 3.35 & $\mathrm{mg} / \mathrm{kg}$ \\
\hline Vanadium & 104625 & 22.9 & & 30.2 & & 24 & $\mathrm{mg} / \mathrm{kg}$ \\
\hline Vanadium & 104650 & 15.1 & & 3.68 & & 4.28 & $\mathrm{mg} / \mathrm{kg}$ \\
\hline Vanadium & 104664 & 10.9 & & 2.42 & & 2.7 & $\mathrm{mg} / \mathrm{kg}$ \\
\hline Vanadium & 104667 & 59.7 & & 11 & & 5.94 & $\mathrm{mg} / \mathrm{kg}$ \\
\hline Vanadium & 104671 & 5.4 & $\mathrm{~J}$ & 43.5 & $\mathrm{~J}$ & 41.2 & $\mathrm{mg} / \mathrm{kg}$ \\
\hline Vanadium & 104680 & 10.1 & & 1.67 & & 1.51 & $\mathrm{mg} / \mathrm{kg}$ \\
\hline Vanadium & 104683 & 37.7 & & 4.89 & & 3.34 & $\mathrm{mg} / \mathrm{kg}$ \\
\hline Vanadium & 104693 & 9.5 & & 5.6 & & 5.09 & $\mathrm{mg} / \mathrm{kg}$ \\
\hline Vanadium & 105110 & 26.2 & & 3.39 & & 4.41 & $\mathrm{mg} / \mathrm{kg}$ \\
\hline
\end{tabular}


Table 33. RPDs for Analytes Detected in Laboratory Duplicates for Sediment Samples, Continued

\begin{tabular}{|c|c|c|c|c|c|c|c|}
\hline \multirow{2}{*}{$\begin{array}{l}\text { Analyte } \\
\text { Vanadium }\end{array}$} & \multirow{2}{*}{$\begin{array}{l}\text { Sample } \\
105113\end{array}$} & \multirow{2}{*}{$\begin{array}{l}\text { RPD } \\
46.8 \\
\end{array}$} & \multicolumn{2}{|c|}{ First Result } & \multicolumn{2}{|c|}{$\begin{array}{l}\text { Second } \\
\text { Result }\end{array}$} & \multirow{2}{*}{$\frac{\text { Units }}{\mathrm{mg} / \mathrm{kg}}$} \\
\hline & & & $\mathrm{J}$ & 2.85 & $J$ & 4.59 & \\
\hline Zinc & 104600 & 86.4 & & 2.42 & & 6.1 & $\mathrm{mg} / \mathrm{kg}$ \\
\hline Zinc & 104614 & 29.1 & & 1.73 & & 2.32 & $\mathrm{mg} / \mathrm{kg}$ \\
\hline Zinc & 104625 & 17.8 & & 31.8 & & 26.6 & $\mathrm{mg} / \mathrm{kg}$ \\
\hline Zinc & 104650 & 11.5 & & 1.81 & & 2.03 & $\mathrm{mg} / \mathrm{kg}$ \\
\hline Zinc & 104664 & 35.1 & & 3.18 & & 2.23 & $\mathrm{mg} / \mathrm{kg}$ \\
\hline Zinc & 104667 & 17.9 & & 2.74 & & 2.29 & $\mathrm{mg} / \mathrm{kg}$ \\
\hline Zinc & 104671 & 8.3 & & 38.8 & & 35.7 & $\mathrm{mg} / \mathrm{kg}$ \\
\hline Zinc & 104680 & 4 & & 2.57 & & 2.47 & $\mathrm{mg} / \mathrm{kg}$ \\
\hline Zinc & 104683 & 26.1 & & 5.38 & & 4.14 & $\mathrm{mg} / \mathrm{kg}$ \\
\hline Zinc & 104693 & 37.4 & & 5.52 & & 3.78 & $\mathrm{mg} / \mathrm{kg}$ \\
\hline Zinc & 105110 & 5.6 & & 1.47 & & 1.39 & $\mathrm{mg} / \mathrm{kg}$ \\
\hline Zinc & 105113 & 77 & & 2.51 & & 5.65 & $\mathrm{mg} / \mathrm{kg}$ \\
\hline \multicolumn{8}{|c|}{ Method EPA7471 (total recoverable) } \\
\hline Mercury & 104600 & 32.7 & & 0.0366 & & 0.0509 & $\mathrm{mg} / \mathrm{kg}$ \\
\hline Mercury & 104604 & 8.4 & $\mathrm{~J}$ & 0.0186 & $\mathrm{~J}$ & 0.0171 & $\mathrm{mg} / \mathrm{kg}$ \\
\hline Mercury & 104613 & 55.2 & $\mathrm{~J}$ & 0.0161 & $\mathrm{~J}$ & 0.0091 & $\mathrm{mg} / \mathrm{kg}$ \\
\hline Mercury & 104625 & 1 & & 0.0846 & & 0.0838 & $\mathrm{mg} / \mathrm{kg}$ \\
\hline Mercury & 104652 & 27.5 & & 0.0766 & & 0.101 & $\mathrm{mg} / \mathrm{kg}$ \\
\hline Mercury & 104664 & 158.6 & $J$ & 0.0044 & $\mathrm{U}$ & 0.0378 & $\mathrm{mg} / \mathrm{kg}$ \\
\hline Mercury & 105100 & 58 & $J$ & 0.0104 & $\mathrm{~J}$ & 0.0189 & $\mathrm{mg} / \mathrm{kg}$ \\
\hline \multicolumn{8}{|l|}{ Method EPA8260 } \\
\hline Acetone & 104635 & 33.5 & $\mathrm{U}$ & 44.2 & & 62 & $\mu \mathrm{g} / \mathrm{kg}$ \\
\hline Acetone & 105120 & 64.1 & $\mathrm{U}$ & 39.2 & & 76.2 & $\mu \mathrm{g} / \mathrm{kg}$ \\
\hline Carbon disulfide & 105120 & 125.3 & $\mathrm{U}$ & 1.41 & $J$ & 0.324 & $\mu g / \mathrm{kg}$ \\
\hline Styrene & 104635 & 66.7 & & 0.138 & & 0.276 & $\mu \mathrm{g} / \mathrm{kg}$ \\
\hline Toluene & 104600 & 10 & & 3.05 & & 2.76 & $\mu \mathrm{g} / \mathrm{kg}$ \\
\hline \multicolumn{8}{|l|}{ Method EPA8270 } \\
\hline Benzoic acid & 104691 & 14.5 & $\mathrm{~J}$ & 28.7 & $\mathrm{U}$ & 33.2 & $\mu \mathrm{g} / \mathrm{kg}$ \\
\hline $\begin{array}{l}\text { Bis(2-ethylhexyl) } \\
\text { phthalate }\end{array}$ & 104640 & 55.8 & $\mathrm{U}$ & 39.2 & $J$ & 22.1 & $\mu \mathrm{g} / \mathrm{kg}$ \\
\hline $\begin{array}{l}\text { Bis(2-ethylhexyl) } \\
\text { phthalate }\end{array}$ & 104645 & 25.3 & $\mathrm{~J}$ & 29.4 & $\mathrm{U}$ & 37.9 & $\mu \mathrm{g} / \mathrm{kg}$ \\
\hline Diethyl phthalate & 104640 & 60.6 & $J$ & 20.7 & $\mathrm{U}$ & 38.7 & $\mu \mathrm{g} / \mathrm{kg}$ \\
\hline Di-n-butyl phthalate & 104640 & 7.9 & $J$ & 25.1 & $\mathrm{~J}$ & 23.2 & $\mu \mathrm{g} / \mathrm{kg}$ \\
\hline Di-n-butyl phthalate & 104691 & 54.9 & $\mathrm{~J}$ & 23.8 & $\mathrm{U}$ & 41.8 & $\mu \mathrm{g} / \mathrm{kg}$ \\
\hline \multicolumn{8}{|l|}{ Method EPA9081 } \\
\hline Cation exchange capacity & 104618 & 37.8 & & 133 & & 195 & $\mathrm{meq} / 100 \mathrm{~g}$ \\
\hline Cation exchange capacity & 104623 & 31.7 & & 91.5 & & 126 & meq $/ 100 \mathrm{~g}$ \\
\hline
\end{tabular}


Discussion

Table 33. RPDs for Analytes Detected in Laboratory Duplicates for Sediment Samples, Continued

\begin{tabular}{|c|c|c|c|c|c|c|c|}
\hline \multirow{2}{*}{$\begin{array}{l}\text { Analyte } \\
\text { Cation exchange capacity }\end{array}$} & \multirow{2}{*}{$\begin{array}{l}\text { Sample } \\
104637\end{array}$} & \multirow{2}{*}{$\frac{\text { RPD }}{13.9}$} & \multicolumn{2}{|c|}{ First Result } & \multicolumn{2}{|c|}{$\begin{array}{l}\text { Second } \\
\text { Result }\end{array}$} & \multirow{2}{*}{$\begin{array}{l}\text { Units } \\
\text { meq/100g } \\
\end{array}$} \\
\hline & & & & 100 & & 87 & \\
\hline Cation exchange capacity & 104648 & 1.2 & & 48.5 & & 49.1 & $\mathrm{meq} / 100 \mathrm{~g}$ \\
\hline Cation exchange capacity & 104650 & 7.1 & & 68.8 & & 73.9 & $\mathrm{meq} / 100 \mathrm{~g}$ \\
\hline Cation exchange capacity & 104669 & 3.7 & & 58.7 & & 60.9 & $\mathrm{meq} / 100 \mathrm{~g}$ \\
\hline Cation exchange capacity & 104677 & 72.3 & & 18.3 & & 39 & meq $/ 100 \mathrm{~g}$ \\
\hline Cation exchange capacity & 104678 & 54.7 & $U$ & 5 & & 8.76 & meq $/ 100 \mathrm{~g}$ \\
\hline Cation exchange capacity & 104688 & 6.4 & & 77 & & 82.1 & $\mathrm{meq} / 100 \mathrm{~g}$ \\
\hline Cation exchange capacity & 105100 & 1.8 & & 67.8 & & 69 & meq $/ 100 \mathrm{~g}$ \\
\hline Cation exchange capacity & 105107 & 37.5 & & 130 & & 190 & meq $/ 100 \mathrm{~g}$ \\
\hline Cation exchange capacity & 105118 & 21.9 & & 8.67 & & 10.8 & meq $/ 100 \mathrm{~g}$ \\
\hline \multicolumn{8}{|l|}{ Method EPIA-001B } \\
\hline Gross alpha & 104620 & 0.9 & & 21.3 & & 21.5 & $\mathrm{pCi} / \mathrm{g}$ \\
\hline Gross alpha & 104633 & 20.1 & & 22.4 & & 18.3 & $\mathrm{pCi} / \mathrm{g}$ \\
\hline Gross alpha & 104674 & 74.5 & & 11.7 & & 25.6 & $\mathrm{pCi} / \mathrm{g}$ \\
\hline Gross alpha & 105111 & 47.2 & & 12.8 & & 20.7 & $\mathrm{pCi} / \mathrm{g}$ \\
\hline Nonvolatile beta & 104620 & 19.6 & & 14.7 & & 17.9 & $\mathrm{pCi} / \mathrm{g}$ \\
\hline Nonvolatile beta & 104633 & 8.5 & & 10.1 & & 11 & $\mathrm{pCi} / \mathrm{g}$ \\
\hline Nonvolatile beta & 104674 & 39.2 & $\mathrm{~J}$ & 6.69 & $\mathrm{~J}$ & 9.95 & $\mathrm{pCi} / \mathrm{g}$ \\
\hline Nonvolatile beta & 105111 & 36.6 & & 6.81 & & 9.86 & $\mathrm{pCi} / \mathrm{g}$ \\
\hline \multicolumn{8}{|l|}{ Method EPIA-004 } \\
\hline Strontium-90 & 104623 & 83 & & 0.0711 & & 0.172 & $\mathrm{pCi} / \mathrm{g}$ \\
\hline \multicolumn{8}{|l|}{ Method EPIA-013B } \\
\hline Actinium-228 & 104623 & 0.9 & & 2.19 & & 2.21 & $\mathrm{pCi} / \mathrm{g}$ \\
\hline Actinium-228 & 104633 & 4.1 & & 1.51 & & 1.45 & $\mathrm{pCi} / \mathrm{g}$ \\
\hline Actinium-228 & 104681 & 6.1 & & 0.522 & & 0.491 & $\mathrm{pCi} / \mathrm{g}$ \\
\hline Actinium-228 & 105102 & 6.8 & & 1.22 & & 1.14 & $\mathrm{pCi} / \mathrm{g}$ \\
\hline Actinium-228 & 105108 & 1.4 & 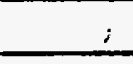 & 1.45 & & 1.43 & $\mathrm{pCi} / \mathrm{g}$ \\
\hline Cesium-137 & 104681 & 7 & & 0.0152 & & 0.0163 & $\mathrm{pCi} / \mathrm{g}$ \\
\hline Cesium-137 & 105102 & 35.1 & & 0.0101 & & 0.0144 & $\mathrm{pCi} / \mathrm{g}$ \\
\hline Cesium-137 & 105108 & 200 & & 0.015 & UI & 0 & $\mathrm{pCi} / \mathrm{g}$ \\
\hline Lead-212 & 104623 & 2.2 & & 2.29 & & 2.34 & $\mathrm{pCi} / \mathrm{g}$ \\
\hline Lead-212 & 104633 & 2.6 & & 1.49 & & 1.53 & $\mathrm{pCi} / \mathrm{g}$ \\
\hline Lead-212 & 104681 & 2.2 & & 0.545 & & 0.533 & $\mathrm{pCi} / \mathrm{g}$ \\
\hline Lead-212 & 105102 & 0.8 & & 1.22 & & 1.23 & $\mathrm{pCi} / \mathrm{g}$ \\
\hline Lead-212 & 105108 & 2.7 & & 1.51 & & 1.47 & $\mathrm{pCi} / \mathrm{g}$ \\
\hline Manganese-54 & 104623 & 28 & & 0.0289 & & 0.0383 & $\mathrm{pCi} / \mathrm{g}$ \\
\hline Manganese-54 & 104633 & 5.7 & & 0.0251 & & 0.0237 & $\mathrm{pCi} / \mathrm{g}$ \\
\hline Manganese-54 & 104681 & 54.2 & & 0.0102 & & 0.0059 & $\mathrm{pCi} / \mathrm{g}$ \\
\hline Manganese-54 & 105102 & 47.6 & & 0.0157 & & 0.0255 & $\mathrm{pCi} / \mathrm{g}$ \\
\hline
\end{tabular}


Table 33. RPDs for Analytes Detected in Laboratory Duplicates for Sediment Samples, Continued

\begin{tabular}{|c|c|c|c|c|c|c|c|}
\hline \multirow{2}{*}{$\begin{array}{l}\text { Analyte } \\
\text { Manganese-54 }\end{array}$} & \multirow{2}{*}{$\begin{array}{l}\text { Sample } \\
105108 \\
\end{array}$} & \multirow{2}{*}{$\begin{array}{l}\text { RPD } \\
11.5 \\
\end{array}$} & \multicolumn{2}{|c|}{ First Result } & \multicolumn{2}{|c|}{$\begin{array}{l}\text { Second } \\
\text { Result }\end{array}$} & \multirow{2}{*}{$\frac{\text { Units }}{\mathrm{pCi} / \mathrm{g}}$} \\
\hline & & & & 0.0221 & & 0.0248 & \\
\hline Neptunium-239 & 105108 & 200 & UI & 0 & & 0.0765 & $\mathrm{pCi} / \mathrm{g}$ \\
\hline Potassium-40 & 104623 & 2.6 & & 1.54 & & 1.58 & $\mathrm{pCi} / \mathrm{g}$ \\
\hline Potassium-40 & 104633 & 7.8 & & 0.87 & & 0.805 & $\mathrm{pCi} / \mathrm{g}$ \\
\hline Potassium-40 & 105102 & 7.7 & & 2.01 & & 2.17 & $\mathrm{pCi} / \mathrm{g}$ \\
\hline Potassium-40 & 105108 & 3.7 & & 1.39 & & 1.34 & $\mathrm{pCi} / \mathrm{g}$ \\
\hline Potassium-40 & 105203 & 20 & & 0.49 & & 0.599 & $\mathrm{pCi} / \mathrm{g}$ \\
\hline Radium-226 & 104681 & 1.3 & & 0.309 & & 0.305 & $\mathrm{pCi} / \mathrm{g}$ \\
\hline Radium-226 & 105102 & 0.3 & & 0.768 & & 0.77 & $\mathrm{pCi} / \mathrm{g}$ \\
\hline Radium-226 & 105108 & 2.4 & & 0.908 & & 0.93 & $\mathrm{pCi} / \mathrm{g}$ \\
\hline Radium-228 & 104681 & 200 & & 0.522 & & 4910 & $\mathrm{pCi} / \mathrm{g}$ \\
\hline Radium-228 & 105102 & 6.8 & & 1.22 & & 1.14 & $\mathrm{pCi} / \mathrm{g}$ \\
\hline Radium-228 & 105108 & 1.4 & & 1.45 & & 1.43 & $\mathrm{pCi} / \mathrm{g}$ \\
\hline Thorium-234 & 104623 & 50.4 & & 2.16 & & 1.29 & $\mathrm{pCi} / \mathrm{g}$ \\
\hline Thorium-234 & 104633 & 49.1 & & 1.75 & $\mathrm{U}$ & 1.06 & $\mathrm{pCi} / \mathrm{g}$ \\
\hline Thorium-234 & 104681 & 13.4 & & 0.639 & & 0.559 & $\mathrm{pCi} / \mathrm{g}$ \\
\hline Thorium-234 & 105108 & 7.5 & & 1.8 & & 1.67 & $\mathrm{pCi} / \mathrm{g}$ \\
\hline Zirconium-95 & 104633 & 75.4 & UI & 0.0243 & & 0.0537 & $\mathrm{pCi} / \mathrm{g}$ \\
\hline
\end{tabular}

Table 34. RPDs for Analytes Detected in Laboratory Duplicates for Water Samples

\begin{tabular}{|c|c|c|c|c|c|c|c|}
\hline Analyte & Sample & RPD & \multicolumn{2}{|c|}{ First Result } & \multicolumn{2}{|c|}{$\begin{array}{l}\text { Second } \\
\text { Result }\end{array}$} & Units \\
\hline \multicolumn{8}{|c|}{ Method EPA6010A (all inorganics are total recoverable) } \\
\hline Aluminum & 104631 & 7.5 & $\mathrm{~J}$ & 0.0232 & $\mathrm{U}$ & 0.025 & $\mathrm{mg} / \mathrm{L}$ \\
\hline Calcium & 104631 & 125.4 & $\mathbf{J}$ & 0.0425 & $\bar{J}$ & 0.0097 & $\mathrm{mg} / \mathrm{L}$ \\
\hline Manganese & 104631 & 0.8 & $\mathbf{J}$ & 0.004 & $\bar{J}$ & 0.0039 & $\mathrm{mg} / \mathrm{L}$ \\
\hline Zinc & 104631 & 11.1 & $\mathrm{~J}$ & 0.0124 & $\bar{J}$ & 0.0111 & $\mathrm{mg} / \mathrm{L}$ \\
\hline \multicolumn{8}{|l|}{ Method EPA8260 } \\
\hline Acetone & 104692 & 45.5 & & 7.36 & $\mathrm{U}$ & 4.63 & $\mu g / L$ \\
\hline Acetone & 105112 & 48 & & 5.65 & & 9.22 & $\mu \mathrm{g} / \mathrm{L}$ \\
\hline Benzene & 105112 & 112.5 & $\mathrm{U}$ & 0.5 & $\mathrm{~J}$ & 0.14 & $\mu \mathrm{g} / \mathrm{L}$ \\
\hline Chlorobenzene & 104603 & 11.1 & $\mathbf{J}$ & 0.17 & & 0.19 & $\mu \mathrm{g} / \mathrm{L}$ \\
\hline Toluene & 104692 & 62.5 & $J$ & 0.21 & $\mathrm{U}$ & 0.11 & $\mu \mathrm{g} / \mathrm{L}$ \\
\hline Toluene & 105112 & 8 & $\mathrm{~J}$ & 0.12 & $\mathrm{~J}$ & 0.13 & $\mu \mathrm{g} / \mathrm{L}$ \\
\hline Methyl ethyl ketone & 104692 & 156.8 & & 8.34 & $\mathrm{U}$ & 1.01 & $\mu \mathrm{g} / \mathrm{L}$ \\
\hline Methyl ethyl ketone & 105112 & 77.4 & $\bar{U}$ & 1.53 & & 3.46 & $\mu \mathrm{g} / \mathrm{L}$ \\
\hline
\end{tabular}


Table 34. RPDs for Analytes Detected in Laboratory Duplicates for Water Samples, Continued

\begin{tabular}{|c|c|c|c|c|c|c|c|}
\hline \multirow{2}{*}{$\begin{array}{l}\text { Analyte } \\
\text { Methyl isobutyl ketone }\end{array}$} & \multirow{2}{*}{$\begin{array}{l}\text { Sample } \\
104603 \\
\end{array}$} & \multirow{2}{*}{$\begin{array}{l}\text { RPD } \\
115 \\
\end{array}$} & \multicolumn{2}{|c|}{ First Result } & \multicolumn{2}{|c|}{$\begin{array}{l}\text { Second } \\
\text { Result }\end{array}$} & \multirow{2}{*}{$\frac{\text { Units }}{\mu \mathrm{g} / \mathrm{L}}$} \\
\hline & & & $\overline{\mathrm{J}}$ & 0.27 & $\mathrm{U}$ & 1 & \\
\hline Trichloroethylene & 104692 & 4.4 & & 0.23 & $\mathrm{U}$ & 0.22 & $\mu \mathrm{g} / \mathrm{L}$ \\
\hline Trichloroethylene & 105112 & 4.1 & & 0.24 & & 0.25 & $\mu \mathrm{g} / \mathrm{L}$ \\
\hline Xylenes & 104692 & 42.1 & & 0.23 & $\mathrm{U}$ & 0.15 & $\mu \mathrm{g} / \mathrm{L}$ \\
\hline \multicolumn{8}{|l|}{ Method EPA8270 } \\
\hline Diethyl phthalate & 104631 & 7.5 & $\mathrm{~J}$ & 0.55 & $\mathrm{~J}$ & 0.51 & $\mu \mathrm{g} / \mathrm{L}$ \\
\hline
\end{tabular}

\section{Sampling Effects and Inter-Sample Heterogeneity}

The effects of sampling and inter-sample heterogeneity on precison are evaluated by examining the variability of the results of field duplicate samples.

\section{Field Duplicates}

Field duplicates are generated by splitting samples during collection and then sending the two samples to the same laboratory. The laboratory is not informed which samples are field duplicates. The RPDs of the results reflect the precision of the analyses. RPDs are not calculated for pairs of samples with both results below detection. If one result is below the quantition limit, the quantitation limit is used to calculate the RPD. See Tables 35 and 36 for the analytes not detected and RPDs for analytes detected in field duplicates for sediment samples, respectively.

Table 35. Analytes Not Detected in Field Duplicates for Sediment Samples

\begin{tabular}{|c|c|}
\hline Analyte & $\begin{array}{l}\text { Number } \\
\text { of Pairs }\end{array}$ \\
\hline \multicolumn{2}{|l|}{ Method EPA335.3 } \\
\hline Cyanide & 6 \\
\hline \multicolumn{2}{|l|}{ Method EPA376.2 } \\
\hline Sulfide & 6 \\
\hline \multicolumn{2}{|c|}{ Method EPA6010A (total recoverable) } \\
\hline Antimony & 6 \\
\hline Arsenic & 6 \\
\hline Cadmium & 6 \\
\hline Selenium & 6 \\
\hline Silver & 6 \\
\hline \multicolumn{2}{|l|}{ Method EPA8080 } \\
\hline Aldrin & 6 \\
\hline alpha-Benzene hexachloride & 6 \\
\hline beta-Benzene hexachloride & 6 \\
\hline
\end{tabular}

\begin{tabular}{|l|l|}
\hline Analyte & $\begin{array}{l}\text { Number } \\
\text { of Pairs }\end{array}$ \\
\hline delta-Benzene hexachloride & 6 \\
\hline alpha-Chlordane & 6 \\
\hline gamma-Chlordane & 6 \\
\hline p,p'-DDD & 6 \\
\hline p,p'-DDE & 6 \\
\hline p,p'-DDT & 6 \\
\hline Dieldrin & 6 \\
\hline Endrin ketone & 6 \\
\hline Endrin & 6 \\
\hline Endosulfan I & 6 \\
\hline Endosulfan II & 6 \\
\hline Endosulfan sulfate- & 6 \\
\hline Heptachlor & 6 \\
\hline Heptachlor epoxide & 6 \\
\hline
\end{tabular}


Table 35. Analytes Not Detected in Field Duplicates for Sediment Samples, Continued

\begin{tabular}{|c|c|}
\hline Analyte & $\begin{array}{l}\text { Number } \\
\text { of Pairs }\end{array}$ \\
\hline Lindane & 6 \\
\hline Methoxychlor & 6 \\
\hline PCB 1016 & 6 \\
\hline PCB 1221 & 6 \\
\hline PCB 1232 & 6 \\
\hline PCB 1242 & 6 \\
\hline PCB 1248 & 6 \\
\hline PCB 1254 & 6 \\
\hline PCB 1260 & 6 \\
\hline Toxaphene & 6 \\
\hline \multicolumn{2}{|l|}{ Method EPA8260 } \\
\hline Bromodichloromethane & 6 \\
\hline Bromoform & 6 \\
\hline $\begin{array}{l}\text { Bromomethane (Methyl } \\
\text { bromide) }\end{array}$ & 6 \\
\hline Carbon tetrachloride & 6 \\
\hline Chlorobenzene & 6 \\
\hline Chloroethane & 6 \\
\hline Chloroethene (Vinyl chloride) & 6 \\
\hline $\begin{array}{l}\text { Chloromethane (Methyl } \\
\text { chloride) }\end{array}$ & 6 \\
\hline Dibromochloromethane & 6 \\
\hline $\begin{array}{l}\text { Dichloromethane (Methylene } \\
\text { chloride) }\end{array}$ & 6 \\
\hline Ethylbenzene & 6 \\
\hline Tetrachloroethylene & 6 \\
\hline Toluene & 6 \\
\hline Trichloroethylene & 6 \\
\hline Vinyl acetate & 6 \\
\hline 1,1,2,2-Tetrachloroethane & 6 \\
\hline 1,1,1-Trichloroethane & 6 \\
\hline 1,1,2-Trichloroethane & 6 \\
\hline 1,1-Dichloroethylene & 6 \\
\hline 1,1-Dichloroethane & 6 \\
\hline 1,2-Dichloroethylene & 6 \\
\hline 1,2-Dichloroethane & 6 \\
\hline 1,2-Dichloropropane & 6 \\
\hline cis-1,3-Dichloropropene & 6 \\
\hline trans-1,3-Dichloropropene & 6 \\
\hline 2-Hexanone & 6 \\
\hline
\end{tabular}

\begin{tabular}{|c|c|}
\hline Analyte & $\begin{array}{l}\text { Number } \\
\text { of Pairs }\end{array}$ \\
\hline \multicolumn{2}{|l|}{ Method EPA8270 } \\
\hline Acenaphthene & 6 \\
\hline Acenaphthylene & 6 \\
\hline Anthracene & 6 \\
\hline Benzo[a]anthracene & 6 \\
\hline Benzo[a]pyrene & 6 \\
\hline Benzo[b]fluoranthene & 6 \\
\hline Benzo[g,h,i]perylene & 6 \\
\hline Benzo $[k]$ fluoranthene & 6 \\
\hline Benzyl alcohol & 6 \\
\hline Bis(2-chloroethoxy) methane & 6 \\
\hline Bis(2-chloroethyl) ether & 6 \\
\hline Bis(2-chloroisopropyl) ether & 6 \\
\hline Butylbenzyl phthalate & 6 \\
\hline Chrysene & 6 \\
\hline Di-n-octyl phthalate & 6 \\
\hline Dibenz $[a, h]$ anthracene & 6 \\
\hline Dibenzofuran & 6 \\
\hline Dimethyl phthalate & 6 \\
\hline Fluoranthene & 6 \\
\hline Fluorene & 6 \\
\hline Hexachlorobenzene & 6 \\
\hline Hexachlorobutadiene & 6 \\
\hline Hexachlorocyclopentadiene & 6 \\
\hline Hexachloroethane & 6 \\
\hline Indeno $[1,2,3-c, d]$ pyrene & 6 \\
\hline Isophorone & 6 \\
\hline N-Nitrosodiphenylamine & 6 \\
\hline N-Nitrosodipropylamine & 6 \\
\hline Naphthalene & 6 \\
\hline Nitrobenzene & 6 \\
\hline Pentachlorophenol & 6 \\
\hline Phenanthrene & 6 \\
\hline Phenol & 6 \\
\hline Pyrene & 6 \\
\hline 1,2,4-Trichlorobenzene & 6 \\
\hline 1,2-Dichlorobenzene & 6 \\
\hline 1,3-Dichlorobenzene & 6 \\
\hline 1,4-Dichlorobenzene & 6 \\
\hline
\end{tabular}


Table 35. Analytes Not Detected in Field Duplicates for Sediment Samples, Continued

\begin{tabular}{|l|l|}
\hline Analyte & $\begin{array}{l}\text { Number } \\
\text { of Pairs }\end{array}$ \\
\hline 2,4,5-Trichlorophenol & 6 \\
\hline 2,4,6-Trichlorophenol & 6 \\
\hline 2,4-Dichlorophenol & 6 \\
\hline 2,4-Dimethyl phenol & 6 \\
\hline 2,4-Dinitrophenol & 6 \\
\hline 2,4-Dinitrotoluene & 6 \\
\hline 2,6-Dinitrotoluene & 6 \\
\hline 2-Chlorophenol & 6 \\
\hline 2-Chloronaphthalene & 6 \\
\hline 2-Methylnaphthalene & 6 \\
\hline o-Cresol (2-Methylphenol) & 6 \\
\hline o-Nitroaniline & 6 \\
\hline 2-Nitrophenol & 6 \\
\hline 3,3'-Dichlorobenzidine & 6 \\
\hline m/p-Cresol & 6 \\
\hline m-Nitroaniline & 6 \\
\hline 2-Methyl-4,6-dinitrophenol & 6 \\
\hline 4-Bromophenyl phenyl ether & 6 \\
\hline 4-Chloroaniline & 6 \\
\hline 4-Chloro-m-cresol & 6 \\
\hline 4-Chlorophenyl phenyl ether & 6 \\
\hline
\end{tabular}

\begin{tabular}{|l|l|}
\hline Analyte & $\begin{array}{l}\text { Number } \\
\text { of Pairs }\end{array}$ \\
\hline p-Nitroaniline & 6 \\
\hline 4-Nitrophenol & 6 \\
\hline Method EPIA-013B & \\
\hline Antimony-124 & 6 \\
\hline Antimony-125 & 6 \\
\hline Barium-133 & 6 \\
\hline Cerium-144 & 6 \\
\hline Cesium-134 & 6 \\
\hline Cobalt-57 & 6 \\
\hline Cobalt-58 & 6 \\
\hline Europium-152 & 6 \\
\hline Europium-154 & 6 \\
\hline Neptunium-239 & 6 \\
\hline Promethium-144 & 6 \\
\hline Promethium-146 & 6 \\
\hline Ruthenium-106 & 6 \\
\hline Sodium-22 & 6 \\
\hline Tin-113 & 6 \\
\hline Yttrium-88 & 6 \\
\hline Zinc-65 & 6 \\
\hline
\end{tabular}

Table 36. RPDs for Analytes Detected in Field Duplicates for Sediment Samples

\begin{tabular}{|l|l|l|l|l|l|l|l|}
\hline Analyte & Sample & RPD & First Result & Second Result & Units \\
\hline Method EPA300.0 & 104635 & 78.1 & $\mathrm{~J}$ & 1.24 & $\mathrm{U}$ & 2.83 & $\mathrm{mg} / \mathrm{kg}$ \\
\hline Chloride & 104637 & 63.4 & $\mathrm{U}$ & 2.72 & $\mathrm{~J}$ & 1.41 & $\mathrm{mg} / \mathrm{kg}$ \\
\hline Chloride & 104644 & 33.2 & $\mathrm{~J}$ & 1.65 & $\mathrm{~J}$ & 1.18 & $\mathrm{mg} / \mathrm{kg}$ \\
\hline Chloride & 104646 & 12.3 & $\mathrm{~J}$ & 1.64 & $\mathrm{~J}$ & 1.45 & $\mathrm{mg} / \mathrm{kg}$ \\
\hline Chloride & 104656 & 37.9 & $\mathrm{~J}$ & 4.55 & $\mathrm{~J}$ & 3.1 & $\mathrm{mg} / \mathrm{kg}$ \\
\hline Chloride & 104660 & 71.7 & $\mathrm{~J}$ & 1.28 & $\mathrm{U}$ & 2.71 & $\mathrm{mg} / \mathrm{kg}$ \\
\hline Chloride & \multicolumn{7}{|l|}{} \\
\hline Method EPA351.2 & 104635 & 46.4 & $\mathrm{C}$ & 78.9 & $\mathrm{C}$ & 49.2 & $\mathrm{mg} / \mathrm{kg}$ \\
\hline Total organic nitrogen & 104637 & 17.1 & $\mathrm{C}$ & 73.1 & $\mathrm{C}$ & 86.8 & $\mathrm{mg} / \mathrm{kg}$ \\
\hline Total organic nitrogen & 104644 & 4.7 & $\mathrm{C}$ & 103 & $\mathrm{C}$ & 98.3 & $\mathrm{mg} / \mathrm{kg}$ \\
\hline Total organic nitrogen & 104646 & 40.9 & $\mathrm{C}$ & 41.8 & $\mathrm{C}$ & 63.3 & $\mathrm{mg} / \mathrm{kg}$ \\
\hline Total organic nitrogen & 104656 & 12 & $\mathrm{C}$ & 385 & $\mathrm{C}$ & 434 & $\mathrm{mg} / \mathrm{kg}$ \\
\hline Total organic nitrogen & \multicolumn{7}{|l|}{}
\end{tabular}




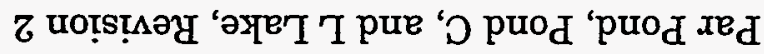

\begin{tabular}{|c|c|c|c|c|c|c|c|}
\hline 8ిम/8ूu & $08 L 9$ & & $08 \mp L$ & & $8 \cdot 6$ & $\$ \varpi 970 \mathrm{I}$ & unuṭunโF \\
\hline 8Вम/8ిm & 0888 & & 0699 & & $8^{\circ} L E$ & LE970I & unnutunty \\
\hline 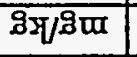 & 0808 & & $0 \angle Z 6$ & & $\varepsilon^{\circ} 0 \varepsilon$ & GE970I & unuțumiV \\
\hline \multicolumn{8}{|c|}{ 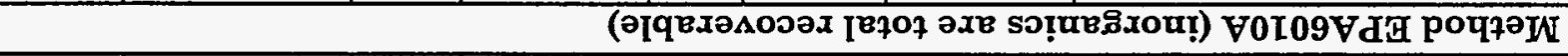 } \\
\hline 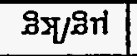 & $\varepsilon \mp \varepsilon$ & $\mathrm{P} \cap$ & $8 \varepsilon 8$ & $\mathfrak{R} \Omega$ & & 09970I & slouəud \\
\hline 8ิ//3ి & 989 & $\stackrel{\rho}{\rho}$ & LLG & $\mathrm{Pn}$ & & $99970 \mathrm{I}$ & sтourəपd \\
\hline 8ิস/बిగ & $97 \varepsilon$ & $\rho \Omega$ & $8 \pi \varepsilon$ & $\rho n$ & & $97970 \mathrm{I}$ & sT०uәपू \\
\hline 8ิ耳/द्र & 678 & $\rho \cap$ & $L \overline{L E}$ & $\Re \cap$ & & $77970 I$ & sโоนəЧૃ \\
\hline 8ม//ి & 098 & $\rho \cap$ & 978 & $\rho \cap$ & & $\angle 8970 \mathrm{I}$ & sTouəपd \\
\hline 8ิ:/8ి & $8 \varepsilon \varepsilon$ & $\rho \Omega$ & OII & $\mathcal{L}$ & $8^{\bullet} \mathrm{IOI}$ & 989ð0I & sTouəपd \\
\hline \multicolumn{8}{|r|}{ 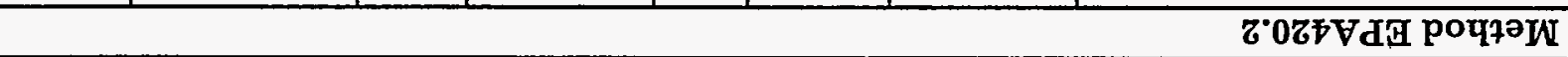 } \\
\hline 8श/9m & $\angle 06$ & & 696 & & $9^{\circ} 9$ & $09970 \mathrm{I}$ & 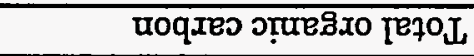 \\
\hline 8Y/9m & 007\&I & & 00TGL & & $6 . I I$ & $99970 \mathrm{I}$ & 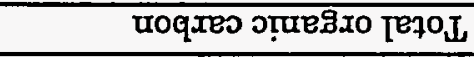 \\
\hline 8श/8m & ISG & & IZ8 & & $\overline{708}$ & $97970 \mathrm{I}$ & 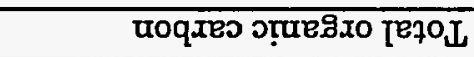 \\
\hline 8ีy/8ిm & 608 & & 678 & & $\overline{7}$ & Ђ7970I & 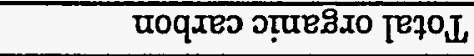 \\
\hline 8Y/814 & 968 & & 090I & & $8 \cdot 9 I$ & LE970I & uoquxes oṇuegixo [e70 \\
\hline 8ిप/8ిజ & 0ZZI & & 0077 & & OII & 98970I & 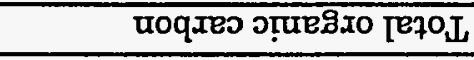 \\
\hline \multicolumn{8}{|r|}{ 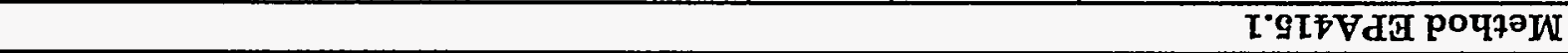 } \\
\hline 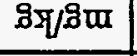 & $8^{\circ} 89$ & $\mathcal{f}$ & 909 & $\rho$ & $\varepsilon^{\prime} q$ & 09970I & (d se) so7eq dsoqd [e70 $\mathrm{J}$ \\
\hline 8.7/8ूu & G6I & $\overline{\mathcal{L}}$ & $\angle Z Z$ & $\mathcal{f}$ & $\overline{Z^{*} G I}$ & 99970I & (d se) sә7eqdsoqd Ieq0ل \\
\hline 3ีम/3ిm & $\tau: L 8$ & $\mathcal{P}$ & E.I6 & $\bar{p}$ & $L i$ & $97970 \mathrm{I}$ & $(\mathrm{d}$ se) seqeqdsoqd Ieq0 $\mathrm{L}$ \\
\hline 8स/3m & 6.79 & 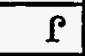 & SI & $\bar{\rho}$ & $Z^{\prime}$ DII & $79970 \mathrm{I}$ & (d SE) seqeq dsoqd Teq0L \\
\hline 8y/81m & $\bar{g} \mathbf{g}$ & $\mathcal{f}$ & ZZL & $\rho$ & 6.96 & L8970I & (d se) seqeqdisoqd jeq0 $\mathrm{L}$ \\
\hline $8 \mathrm{~g} / 8 \mathrm{gm}$ & $9^{\circ} 76$ & $\rho$ & $8 L I$ & $\rho$ & $\varepsilon^{-19}$ & 98970I & (d se) so7eqdsoqd jeq0J \\
\hline \multicolumn{8}{|r|}{ 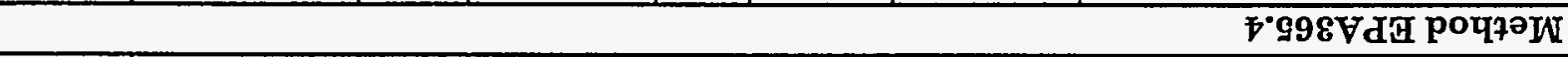 } \\
\hline 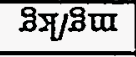 & $\varphi^{\circ} I T$ & $\Omega$ & DIII & $\Omega$ & & 09970I & 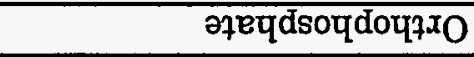 \\
\hline 9श/810 & $6 . \mathrm{LI}$ & $\Omega$ & $\mathrm{Z}^{*} 6 \mathrm{I}$ & $\Omega$ & & $99970 \mathrm{I}$ & әqeqdsoqdouqxo \\
\hline 8प/8m & $\varepsilon^{\circ} I I$ & $\Omega$ & $\varepsilon \tilde{D}^{\circ} \varepsilon$ & $\mathcal{L}$ & $6.90 \mathrm{I}$ & 97970I & 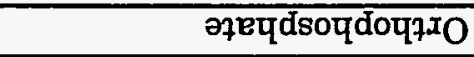 \\
\hline 8Y/81m & $9^{\circ} \mathrm{II}$ & $\Omega$ & $\varepsilon^{*} I I$ & $\Omega$ & & $78970 I$ & 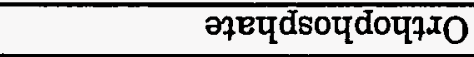 \\
\hline 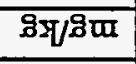 & $G^{*} I I$ & $\bar{\Omega}$ & $99 \%$ & $\rho$ & ZLZI & LE970I & 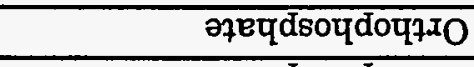 \\
\hline 8ีभ/8ిய & II & $\Omega$ & $69^{\circ} \mathrm{L}$ & $\bar{f}$ & $\overline{7} 98$ & SE970I & 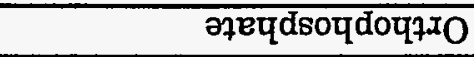 \\
\hline \multicolumn{8}{|r|}{$\mathrm{Z}^{\circ}$ 998VdH poq7әW } \\
\hline 8स/8జس & $907^{\circ} 0$ & $\mathcal{L}$ & $67^{\circ} 0$ & $\mathcal{f}$ & $L 8 I$ & 09970I & 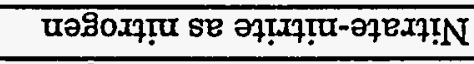 \\
\hline 8म/8m & $709^{\circ} 0$ & $\rho$ & $969^{\circ} 0$ & $\rho$ & $9^{\circ} 9 \mathrm{I}$ & 99970I & 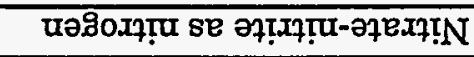 \\
\hline 8ีम/81 & GI'I & & $889^{\circ} 0$ & $\mathfrak{L}$ & IG & $97970 I$ & 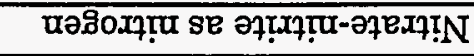 \\
\hline 9श/3ిm & $879^{\circ} 0$ & $\rho$ & $\mathbf{8 T 8 ^ { \circ } 0}$ & $\rho$ & $Z^{*} \varepsilon Z$ & Ð7970I & 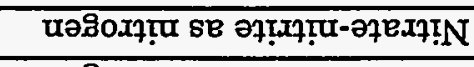 \\
\hline 9भ/8ిm & $868^{\circ} 0$ & $\Omega$ & $898^{\circ} 0$ & $\Omega$ & & L8970I & 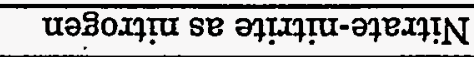 \\
\hline 98/81m & $60^{\circ} \mathrm{I}$ & 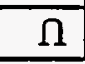 & ZI'I & $\Omega$ & & 98970I & 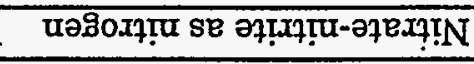 \\
\hline \multicolumn{8}{|r|}{ 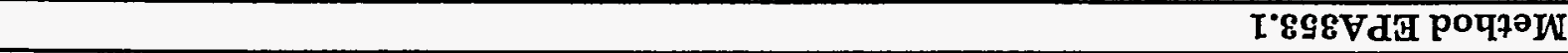 } \\
\hline 9y/8m & 899 & $\mathrm{D}$ & $9.9 L$ & 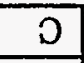 & 7.TS & $09970 \mathrm{I}$ & 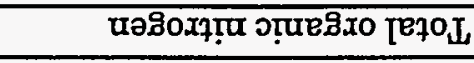 \\
\hline sq!u $\Omega$ & \multicolumn{2}{|c|}{ 7[nsəy puooəs } & \multicolumn{2}{|c|}{ 7[nsəม 7Sג!. } & वдม & ə[đưes & 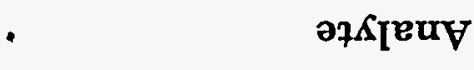 \\
\hline
\end{tabular}

pənựนuoD

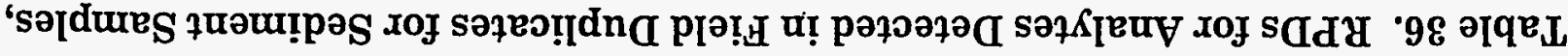


Table 36. RPDs for Analytes Detected in Field Duplicates for Sediment Samples, Continued

\begin{tabular}{|c|c|c|c|c|c|c|c|}
\hline \multirow{2}{*}{$\frac{\text { Analyte }}{\text { Aluminum }}$} & \multirow{2}{*}{$\begin{array}{l}\text { Sample } \\
104646\end{array}$} & \multirow{2}{*}{$\frac{\text { RPD }}{57.4}$} & \multicolumn{2}{|c|}{ First Result } & \multicolumn{2}{|c|}{ Second Result } & \multirow{2}{*}{$\frac{\text { Units }}{\mathrm{mg} / \mathrm{kg}}$} \\
\hline & & & & 5160 & & 2860 & \\
\hline Aluminum & 104656 & 40 & & 18000 & & 12000 & $\mathrm{mg} / \mathrm{kg}$ \\
\hline Aluminum & 104660 & 4.9 & & 4800 & & 4570 & $\mathrm{mg} / \mathrm{kg}$ \\
\hline Barium & 104635 & 27.1 & & 17.2 & & 13.1 & $\mathrm{mg} / \mathrm{kg}$ \\
\hline Barium & 104637 & 135.6 & & 147 & & 28.2 & $\mathrm{mg} / \mathrm{kg}$ \\
\hline Barium & 104644 & 10 & & 15.7 & & 14.2 & $\mathrm{mg} / \mathrm{kg}$ \\
\hline Barium & 104646 & 74.2 & & 14.3 & & 6.56 & $\mathrm{mg} / \mathrm{kg}$ \\
\hline Barium & 104656 & 31.6 & & 86.1 & & 62.6 & $\mathrm{mg} / \mathrm{kg}$ \\
\hline Barium & 104660 & 24 & & 7.11 & & 9.05 & $\mathrm{mg} / \mathrm{kg}$ \\
\hline Beryllium & 104635 & 47 & $\mathrm{~J}$ & 0.133 & $\mathrm{~J}$ & 0.0824 & $\mathrm{mg} / \mathrm{kg}$ \\
\hline Beryllium & 104637 & 98.6 & $\mathrm{~J}$ & 0.486 & $\mathrm{~J}$ & 0.165 & $\mathrm{mg} / \mathrm{kg}$ \\
\hline Beryllium & 104644 & 4.4 & $\mathrm{~J}$ & 0.0642 & $\mathrm{~J}$ & 0.0671 & $\mathrm{mg} / \mathrm{kg}$ \\
\hline Beryllium & 104646 & 63.3 & $\mathrm{~J}$ & 0.0869 & $J$ & 0.0451 & $\mathrm{mg} / \mathrm{kg}$ \\
\hline Beryllium & 104656 & 26.2 & $\mathrm{~J}$ & 0.772 & $J$ & 0.593 & $\mathrm{mg} / \mathrm{kg}$ \\
\hline Beryllium & 104660 & 22.7 & $\mathrm{~J}$ & 0.0897 & $\mathrm{~J}$ & 0.0714 & $\mathrm{mg} / \mathrm{kg}$ \\
\hline Calcium & 104635 & 64.2 & & 92.6 & & 47.6 & $\mathrm{mg} / \mathrm{kg}$ \\
\hline Calcium & 104637 & 94.3 & & 112 & & 40.2 & $\mathrm{mg} / \mathrm{kg}$ \\
\hline Calcium & 104644 & 53.7 & & 125 & & 72.1 & $\mathrm{mg} / \mathrm{kg}$ \\
\hline Calcium & 104646 & 8 & & 24 & & 26 & $\mathrm{mg} / \mathrm{kg}$ \\
\hline Calcium & 104656 & 9.5 & & 817 & & 743 & $\mathrm{mg} / \mathrm{kg}$ \\
\hline Calcium & 104660 & 0.7 & & 42.8 & & 43.1 & $\mathrm{mg} / \mathrm{kg}$ \\
\hline Chromium & 104635 & 64.4 & & 1.83 & & 3.57 & $\mathrm{mg} / \mathrm{kg}$ \\
\hline Chromium & 104637 & 14.3 & & 13.5 & & 11.7 & $\mathrm{mg} / \mathrm{kg}$ \\
\hline Chromium & 104644 & 29.5 & & 8.79 & & 6.53 & $\mathrm{mg} / \mathrm{kg}$ \\
\hline Chromium & 104646 & 58.2 & & 7.34 & & 4.03 & $\mathrm{mg} / \mathrm{kg}$ \\
\hline Chromium & 104656 & 14 & & 23.7 & & 20.6 & $\mathrm{mg} / \mathrm{kg}$ \\
\hline Chromium & 104660 & 41.7 & & 13.4 & & 8.78 & $\mathrm{mg} / \mathrm{kg}$ \\
\hline Cobalt & 104635 & 67.1 & & 0.643 & $J$ & 0.32 & $\mathrm{mg} / \mathrm{kg}$ \\
\hline Cobalt & 104637 & 94.8 & $J$ & 0.206 & $\mathrm{U}$ & 0.577 & $\mathrm{mg} / \mathrm{kg}$ \\
\hline Cobalt & 104644 & 29.5 & $\mathrm{~J}$ & 0.364 & $\mathrm{~J}$ & 0.49 & $\mathrm{mg} / \mathrm{kg}$ \\
\hline Cobalt & 104646 & 76.3 & $\mathrm{~J}$ & 0.243 & $\mathrm{U}$ & 0.543 & $\mathrm{mg} / \mathrm{kg}$ \\
\hline Cobalt & 104656 & 34.3 & & 3.45 & & 2.44 & $\mathrm{mg} / \mathrm{kg}$ \\
\hline Cobalt & 104660 & 24.5 & $J$ & 0.348 & $\mathrm{~J}$ & 0.272 & $\mathrm{mg} / \mathrm{kg}$ \\
\hline Copper & 104635 & 69.3 & & 1.8 & & 3.71 & $\mathrm{mg} / \mathrm{kg}$ \\
\hline Copper & 104637 & 3 & & 2.27 & & 2.34 & $\mathrm{mg} / \mathrm{kg}$ \\
\hline Copper & 104644 & 26 & & 2.13 & & 1.64 & $\mathrm{mg} / \mathrm{kg}$ \\
\hline Copper & 104646 & 86.9 & $\mathrm{~J}$ & 1.35 & $\mathrm{~J}$ & 0.532 & $\mathrm{mg} / \mathrm{kg}$ \\
\hline Copper & 104656 & 3.1 & & 7.92 & & 7.68 & $\mathrm{mg} / \mathrm{kg}$ \\
\hline Copper & 104660 & 33 & & 2.68 & & 1.92 & $\mathrm{mg} / \mathrm{kg}$ \\
\hline Iron & 104635 & 0.7 & $J$ & 1460 & $\mathrm{~J}$ & 1450 & $\mathrm{mg} / \mathrm{kg}$ \\
\hline
\end{tabular}

Par Pond, Pond C, and L Lake, Revision 2 
Table 36. RPDs for Analytes Detected in Field Duplicates for Sediment Samples, Continued

\begin{tabular}{|c|c|c|c|c|c|c|c|}
\hline \multirow{2}{*}{$\begin{array}{l}\text { Analyte } \\
\text { Iron }\end{array}$} & \multirow{2}{*}{$\begin{array}{l}\text { Sample } \\
104637\end{array}$} & \multirow{2}{*}{$\frac{\text { RPD }}{36.6}$} & \multicolumn{2}{|c|}{ First Result } & \multicolumn{2}{|c|}{ Second Result } & \multirow{2}{*}{$\frac{\text { Units }}{\mathrm{mg} / \mathrm{kg}}$} \\
\hline & & & $\mathrm{J}$ & 4690 & $\mathrm{~J}$ & 3240 & \\
\hline Iron & 104644 & 11.8 & $\mathrm{~J}$ & 1120 & & 1260 & $\mathrm{mg} / \mathrm{kg}$ \\
\hline Iron & 104646 & 103.7 & $\mathrm{~J}$ & 1420 & & 450 & $\mathrm{mg} / \mathrm{kg}$ \\
\hline Iron & 104656 & 11.3 & & 14000 & & 12500 & $\mathrm{mg} / \mathrm{kg}$ \\
\hline Iron & 104660 & 65.8 & & 9450 & & 4770 & $\mathrm{mg} / \mathrm{kg}$ \\
\hline Lead & 104635 & 82.6 & $\mathrm{U}$ & 5.63 & $\mathrm{~J}$ & 2.34 & $\mathrm{mg} / \mathrm{kg}$ \\
\hline Lead & 104637 & 47.7 & $\mathrm{~J}$ & 3.84 & $\mathrm{~J}$ & 2.36 & $\mathrm{mg} / \mathrm{kg}$ \\
\hline Lead & 104644 & 2.9 & & 6.03 & & 6.21 & $\mathrm{mg} / \mathrm{kg}$ \\
\hline Lead & 104646 & 6 & $\mathrm{~J}$ & 4.02 & $\mathrm{~J}$ & 4.27 & $\mathrm{mg} / \mathrm{kg}$ \\
\hline Lead & 104656 & 21.4 & & 18.1 & & 14.6 & $\mathrm{mg} / \mathrm{kg}$ \\
\hline Lead & 104660 & 5.7 & $\mathrm{~J}$ & 4.48 & $\mathrm{~J}$ & 4.23 & $\mathrm{mg} / \mathrm{kg}$ \\
\hline Magnesium & 104635 & 2.3 & & 75.4 & & 73.7 & $\mathrm{mg} / \mathrm{kg}$ \\
\hline Magnesium & 104637 & 51 & & 157. & & 93.2 & $\mathrm{mg} / \mathrm{kg}$ \\
\hline Magnesium & 104644 & 11.9 & & 142 & & 160 & $\mathrm{mg} / \mathrm{kg}$ \\
\hline Magnesium & 104646 & 52.9 & & 78.7 & & 45.8 & $\mathrm{mg} / \mathrm{kg}$ \\
\hline Magnesium & 104656 & 30 & & 376 & & 278 & $\mathrm{mg} / \mathrm{kg}$ \\
\hline Magnesium & 104660 & 22 & & 46.9 & & 58.5 & $\mathrm{mg} / \mathrm{kg}$ \\
\hline Manganese & 104635 & 25.6 & & 60.8 & & 47 & $\mathrm{mg} / \mathrm{kg}$ \\
\hline Manganese & 104637 & 54.9 & & 5.2 & & 2.96 & $\mathrm{mg} / \mathrm{kg}$ \\
\hline Manganese & 104644 & 32.9 & & 17 & & 12.2 & $\mathrm{mg} / \mathrm{kg}$ \\
\hline Manganese & 104646 & 54.3 & & 5.95 & & 3.41 & $\mathrm{mg} / \mathrm{kg}$ \\
\hline Manganese & 104656 & 1.1 & & 181 & & 179 & $\mathrm{mg} / \mathrm{kg}$ \\
\hline Manganese & 104660 & 34.5 & & 6.8 & & 4.8 & $\mathrm{mg} / \mathrm{kg}$ \\
\hline Nickel & 104635 & 41 & & 1.48 & $J$ & $0: 976$ & $\mathrm{mg} / \mathrm{kg}$ \\
\hline Nickel & 104637 & 20.5 & & 1.56 & $J$ & 1.27 & $\mathrm{mg} / \mathrm{kg}$ \\
\hline Nickel & 104644 & 0.5 & & 2.1 & & 2.11 & $\mathrm{mg} / \mathrm{kg}$ \\
\hline Nickel & 104646 & 69.7 & & 1.43 & $\mathrm{~J}$ & 0.691 & $\mathrm{mg} / \mathrm{kg}$ \\
\hline Nickel & 104656 & 19.9 & & 5.25 & & 4.3 & $\mathrm{mg} / \mathrm{kg}$ \\
\hline Nickel & 104660 & 20.8 & $\mathrm{~J}$ & 0.852 & $\mathrm{~J}$ & 1.05 & $\mathrm{mg} / \mathrm{kg}$ \\
\hline Potassium & 104635 & 19.5 & $\mathrm{~J}$ & 48.5 & $J$ & 39.9 & $\mathrm{mg} / \mathrm{kg}$ \\
\hline Potassium & 104637 & 46.9 & & 155 & $\mathrm{~J}$ & 96.1 & $\mathrm{mg} / \mathrm{kg}$ \\
\hline Potassium & 104644 & 6.6 & $\mathrm{~J}$ & 102 & $J$ & 109 & $\mathrm{mg} / \mathrm{kg}$ \\
\hline Potassium & 104646 & 79.3 & $\mathrm{~J}$ & 63.6 & $\mathrm{~J}$ & 27.5 & $\mathrm{mg} / \mathrm{kg}$ \\
\hline Potassium & 104656 & 27.8 & & 320 & & 242 & $\mathrm{mg} / \mathrm{kg}$ \\
\hline Potassium & 104660 & 32.8 & $\mathrm{~J}$ & 34.9 & $\mathrm{~J}$ & 48.6 & $\mathrm{mg} / \mathrm{kg}$ \\
\hline Sodium & 104635 & & $\mathrm{U}$ & 56.3 & $\mathrm{U}$ & 55.7 & $\mathrm{mg} / \mathrm{kg}$ \\
\hline Sodium & 104637 & 146.1 & $J$ & 8.99 & $\mathrm{U}$ & 57.7 & $\mathrm{mg} / \mathrm{kg}$ \\
\hline Sodium & 104644 & 42.2 & $\mathrm{~J}$ & 17.5 & $\mathrm{~J}$ & 11.4 & $\mathrm{mg} / \mathrm{kg}$ \\
\hline Sodium & 104646 & 135.7 & $\mathrm{~J}$ & 10.4 & $\mathrm{U}$ & 54.3 & $\mathrm{mg} / \mathrm{kg}$ \\
\hline Sodium & 104656 & 26.1 & $\mathrm{~J}$ & 20.8 & $\mathrm{~J}$ & 16 & $\mathrm{mg} / \mathrm{kg}$ \\
\hline
\end{tabular}

Par Pond, Pond C, and L Lake, Revision 2 
Discussion

Table 36. RPDs for Analytes Detected in Field Duplicates for Sediment Samples, Continued

\begin{tabular}{|c|c|c|c|c|c|c|c|}
\hline \multirow{2}{*}{$\begin{array}{l}\text { Analyte } \\
\text { Sodium }\end{array}$} & \multirow{2}{*}{$\begin{array}{l}\text { Sample } \\
104660 \\
\end{array}$} & \multirow[t]{2}{*}{ RPD } & \multicolumn{2}{|c|}{ First Result } & \multicolumn{2}{|c|}{ Second Result } & \multirow{2}{*}{$\frac{\text { Units }}{\mathrm{mg} / \mathrm{kg}}$} \\
\hline & & & $\mathrm{U}$ & 55.7 & $\mathrm{U}$ & 53.7 & \\
\hline Thallium & 104635 & 137.5 & $\mathrm{~J}$ & 3.09 & UJ & 16.7 & $\mathrm{mg} / \mathrm{kg}$ \\
\hline Thallium & 104637 & & $\mathrm{UJ}$ & 17.3 & $\mathrm{UJ}$ & 17.3 & $\mathrm{mg} / \mathrm{kg}$ \\
\hline Thallium & 104644 & & UJ & 16.5 & $\mathrm{U}$ & 16.8 & $\mathrm{mg} / \mathrm{kg}$ \\
\hline Thallium & 104646 & & $\mathrm{UJ}$ & 16.5 & $\mathrm{U}$ & 16.3 & $\mathrm{mg} / \mathrm{kg}$ \\
\hline Thallium & 104656 & 140.6 & $\mathrm{~J}$ & 4.59 & $\mathrm{U}$ & 26.3 & $\mathrm{mg} / \mathrm{kg}$ \\
\hline Thallium & 104660 & 122.2 & $\mathrm{~J}$ & 3.89 & $\mathrm{U}$ & 16.1 & $\mathrm{mg} / \mathrm{kg}$ \\
\hline Vanadium & 104635 & 32.3 & & 2.93 & & 4.06 & $\mathrm{mg} / \mathrm{kg}$ \\
\hline Vanadium & 104637 & 46.4 & & 16.2 & & 26 & $\mathrm{mg} / \mathrm{kg}$ \\
\hline Vanadium & 104644 & 17.3 & & 10.9 & & 9.16. & $\mathrm{mg} / \mathrm{kg}$ \\
\hline Vanadium & 104646 & 66.4 & & 7.34 & & 3.68 & $\mathrm{mg} / \mathrm{kg}$ \\
\hline Vanadium & 104656 & 11.8 & & 40.4 & & 35.9 & $\mathrm{mg} / \mathrm{kg}$ \\
\hline Vanadium & 104660 & 64.8 & & 42.3 & & 21.6 & $\mathrm{mg} / \mathrm{kg}$ \\
\hline Zinc & 104635 & 36 & & 3.65 & & 5.25 & $\mathrm{mg} / \mathrm{kg}$ \\
\hline Zinc & 104637 & 41.1 & & 3.96 & & 2.61 & $\mathrm{mg} / \mathrm{kg}$ \\
\hline Zinc & 104644 & 28.8 & & 3.81 & & 5.09 & $\mathrm{mg} / \mathrm{kg}$ \\
\hline Zinc & 104646 & 64.7 & & 3.54 & & 1.81 & $\mathrm{mg} / \mathrm{kg}$ \\
\hline Zinc & 104656 & 14.9 & & 19.5 & & 16.8 & $\mathrm{mg} / \mathrm{kg}$ \\
\hline Zinc & 104660 & 17.8 & & 2.57 & & 2.15 & $\mathrm{mg} / \mathrm{kg}$ \\
\hline \multicolumn{8}{|c|}{ Method EPA7471 (total recoverable) } \\
\hline Mercury & -104635 & & $\mathrm{U}$ & 0.0206 & $\mathrm{U}$ & 0.012 & $\mathrm{mg} / \mathrm{kg}$ \\
\hline Mercury & 104637 & 11.6 & $\mathrm{~J}$ & 0.0357 & $\mathrm{~J}$ & 0.0318 & $\mathrm{mg} / \mathrm{kg}$ \\
\hline Mercury & 104644 & 22.8 & $\mathrm{~J}$ & 0.0381 & $\mathrm{~J}$ & 0.0303 & $\mathrm{mg} / \mathrm{kg}$ \\
\hline Mercury & 104646 & & $\mathrm{U}$ & 0.017 & $\mathrm{U}$ & 0.0214 & $\mathrm{mg} / \mathrm{kg}$ \\
\hline Mercury & 104656 & 25.3 & $\mathrm{~J}$ & 0.0397 & & 0.0512 & $\mathrm{mg} / \mathrm{kg}$ \\
\hline Mercury & 104660 & 45.1 & $\mathrm{~J}$ & 0.0139 & $\mathrm{~J}$ & 0.022 & $\mathrm{mg} / \mathrm{kg}$ \\
\hline \multicolumn{8}{|c|}{ Method EPA8260 } \\
\hline Acetone & 104635 & 9.7 & $\mathrm{U}$ & 44.2 & & 40.1 & $\mu \mathrm{g} / \mathrm{kg}$ \\
\hline Acetone & 104637 & 50.8 & & 93.3 & & 55.5 & $\mu \mathrm{g} / \mathrm{kg}$ \\
\hline Acetone & 104644 & & $\mathrm{U}$ & 42.6 & $\mathrm{U}$ & 14.8 & $\mu \mathrm{g} / \mathrm{kg}$ \\
\hline Acetone & 104646 & & $\mathrm{U}$ & 24.1 & $\mathrm{U}$ & 19.9 & $\mu \mathrm{g} / \mathrm{kg}$ \\
\hline Acetone & 104656 & 16.9 & & 73.1 & $\mathrm{UJ}$ & 61.7 & $\mu \mathrm{g} / \mathrm{kg}$ \\
\hline Acetone & 104660 & 60.3 & & 111 & $\mathrm{U}$ & 59.6 & $\mu \mathrm{g} / \mathrm{kg}$ \\
\hline Benzene & 104635 & & $\mathrm{U}$ & 0.575 & $U$ & 0.568 & $\mu \mathrm{g} / \mathrm{kg}$ \\
\hline Benzene & 104637 & & $U$ & 0.588 & $\mathrm{U}$ & 0.588 & $\mu \mathrm{g} / \mathrm{kg}$ \\
\hline Benzene & 104644 & & $U$ & 0.581 & $\mathrm{U}$ & 0.581 & $\mu \mathrm{g} / \mathrm{kg}$ \\
\hline Benzene & 104646 & & $\mathrm{U}$ & 0.588 & $\mathrm{U}$ & 0.581 & $\mu \mathrm{g} / \mathrm{kg}$ \\
\hline Benzene & 104656 & 0.6 & $\mathrm{~J}$ & 0.923 & $\mathrm{~J}$ & 0.929 & $\mu \mathrm{g} / \mathrm{kg}$ \\
\hline Benzene & 104660 & 2.5 & & 0.648 & & 0.632 & $\mu g / \mathrm{kg}$ \\
\hline
\end{tabular}


Table 36. RPDs for Analytes Detected in Field Duplicates for Sediment Samples, Continued

\begin{tabular}{|c|c|c|c|c|c|c|c|}
\hline \multirow{2}{*}{$\begin{array}{l}\text { Analyte } \\
\text { Carbon disulfide }\end{array}$} & \multirow{2}{*}{$\begin{array}{l}\text { Sample } \\
104635\end{array}$} & \multirow[t]{2}{*}{ RPD } & \multicolumn{2}{|c|}{ First Result } & \multicolumn{2}{|c|}{ Second Result } & \multirow{2}{*}{$\frac{\text { Units }}{\mu \mathrm{g} / \mathrm{kg}}$} \\
\hline & & & $\mathrm{U}$ & 1.15 & $\mathrm{U}$ & 1.14 & \\
\hline Carbon disulfide & 104637 & 35.5 & $J$ & 0.824 & $\mathrm{U}$ & 1.18 & $\mu \mathrm{g} / \mathrm{kg}$ \\
\hline Carbon disulfide & 104644 & 66.5 & $J$ & 0.465 & $\mathbf{J}$ & 0.233 & $\mu \mathrm{g} / \mathrm{kg}$ \\
\hline Carbon disulfide & 104646 & & $\mathrm{U}$ & 1.18 & $\mathrm{U}$ & 1.16 & $\mu \mathrm{g} / \mathrm{kg}$ \\
\hline Carbon disulfide & 104656 & 35.4 & $J$ & 0.462 & $\mathrm{~J}$ & 0.661 & $\mu \mathrm{g} / \mathrm{kg}$ \\
\hline Carbon disulfide & 104660 & 70.9 & & 2.05 & & 0.977 & $\mu \mathrm{g} / \mathrm{kg}$ \\
\hline Chloroform & 104635 & & $\mathrm{U}$ & 0.0575 & $\mathrm{U}$ & 0.0568 & $\mu \mathrm{g} / \mathrm{kg}$ \\
\hline Chloroform & 104637 & & U & 0.0588 & $U$ & 0.0588 & $\mu \mathrm{g} / \mathrm{kg}$ \\
\hline Chloroform & 104644 & & $\mathrm{U}$ & 0.0581 & $\mathrm{U}$ & 0.0581 & $\mu \mathrm{g} / \mathrm{kg}$ \\
\hline Chloroform & 104646 & & $\mathrm{U}$ & 0.0588 & $\mathrm{U}$ & 0.0581 & $\mu \mathrm{g} / \mathrm{kg}$ \\
\hline Chloroform & 104656 & 2.4 & & 0.75 & $\mathrm{~J}$ & 0.768 & $\mu \mathrm{g} / \mathrm{kg}$ \\
\hline Chloroform & 104660 & 7.3 & & 0.545 & & 0.586 & $\mu \mathrm{g} / \mathrm{kg}$ \\
\hline Methyl ethyl ketone & 104635 & & $\mathrm{U}$ & 0.782 & $\mathrm{U}$ & 1.28 & $\mu \mathrm{g} / \mathrm{kg}$ \\
\hline Methyl ethyl ketone & 104637 & & $\mathrm{U}$ & 3.12 & $\mathrm{U}$ & 3.25 & $\mu \mathrm{g} / \mathrm{kg}$ \\
\hline Methyl ethyl ketone & 104644 & & $\mathrm{U}$ & 0.837 & $U$ & 0.779 & $\mu \mathrm{g} / \mathrm{kg}$ \\
\hline Methyl ethyl ketone & 104646 & & $\mathrm{U}$ & 1.16 & $U$ & 1.08 & $\mu \mathrm{g} / \mathrm{kg}$ \\
\hline Methyl ethyl ketone & 104656 & & $\mathrm{U}$ & 2.52 & $\mathrm{UJ}$ & 2.61 & $\mu \mathrm{g} / \mathrm{kg}$ \\
\hline Methyl ethyl ketone & 104660 & 77.9 & & 25.5 & $\mathrm{U}$ & 11.2 & $\mu \mathrm{g} / \mathrm{kg}$ \\
\hline Methyl isobutyl ketone & 104635 & & $\mathrm{U}$ & 1.15 & $\mathrm{U}$ & 1.14 & $\mu \mathrm{g} / \mathrm{kg}$ \\
\hline Methyl isobutyl ketone & 104637 & & $\mathrm{U}$ & 1.18 & $\mathrm{U}$ & 1.18 & $\mu \mathrm{g} / \mathrm{kg}$ \\
\hline Methyl isobutyl ketone & 104644 & & $\mathrm{U}$ & 1.16 & $\mathrm{U}$ & 1.16 & $\mu \mathrm{g} / \mathrm{kg}$ \\
\hline Methyl isobutyl ketone & 104646 & & $\mathrm{U}$ & 1.18 & $U$ & 1.16 & $\mu \mathrm{g} / \mathrm{kg}$ \\
\hline Methyl isobutyl ketone & 104656 & & $\mathrm{U}$ & 1.92 & UJ & 1.79 & $\mu \mathrm{g} / \mathrm{kg}$ \\
\hline Methyl isobutyl ketone & 104660 & 0.9 & $\mathrm{~J}$ & 0.557 & $\mathbf{J}$ & 0.552 & $\mu \mathrm{g} / \mathrm{kg}$ \\
\hline Styrene & 104635 & 83.4 & & 0.138 & $\mathrm{U}$ & 0.0568 & $\mu \mathrm{g} / \mathrm{kg}$ \\
\hline Styrene & 104637 & & $\mathrm{U}$ & 0.0588 & $\mathrm{U}$ & 0.0588 & $\mu \mathrm{g} / \mathrm{kg}$ \\
\hline Styrene & 104644 & & $\mathrm{U}$ & 0.0581 & $\mathrm{U}$ & 0.0581 & $\mu \mathrm{g} / \mathrm{kg}$ \\
\hline Styrene & 104646 & & $\mathrm{U}$ & 0.0588 & $\mathrm{U}$ & 0.0581 & $\mu \mathrm{g} / \mathrm{kg}$ \\
\hline Styrene & 104656 & & $\mathrm{U}$ & 0.0962 & UJ & 0.0893 & $\mu \mathrm{g} / \mathrm{kg}$ \\
\hline Styrene & 104660 & 89.6 & $\mathrm{U}$ & 0.0568 & & 0.149 & $\mu \mathrm{g} / \mathrm{kg}$ \\
\hline Xylenes & 104635 & & $\mathrm{U}$ & 0.172 & $\mathrm{U}$ & 0.17 & $\mu \mathrm{g} / \mathrm{kg}$ \\
\hline Xylenes & 104637 & & $\mathrm{U}$ & 0.176 & $\mathrm{U}$ & 0.176 & $\mu \mathrm{g} / \mathrm{kg}$ \\
\hline Xylenes & 104644 & & $\mathrm{U}$ & 0.174 & $\mathrm{U}$ & 0.174 & $\mu \mathrm{g} / \mathrm{kg}$ \\
\hline Xylenes & 104646 & & $\mathrm{U}$ & 0.176 & $\mathrm{U}$ & 0.174 & $\mu \mathrm{g} / \mathrm{kg}$ \\
\hline Xylenes & 104656 & 16.4 & $\mathrm{~J}$ & 0.231 & $\mathrm{~J}$ & 0.196 & $\mu \mathrm{g} / \mathrm{kg}$ \\
\hline Xylenes & 104660 & 1.2 & $\mathrm{U}$ & 0.17 & $\mathrm{~J}$ & 0.172 & $\mu \mathrm{g} / \mathrm{kg}$ \\
\hline
\end{tabular}


Table 36. RPDs for Analytes Detected in Field Duplicates for Sediment Samples, Continued

\begin{tabular}{|c|c|c|c|c|c|c|c|}
\hline Analyte & Sample & RPD & \multicolumn{2}{|c|}{ First Result } & \multicolumn{2}{|c|}{ Second Result } & Units \\
\hline \multicolumn{8}{|l|}{ Method EPA8270 } \\
\hline Benzoic acid & 104635 & & $\mathrm{U}$ & 37.4 & $\mathrm{U}$ & 37.6 & $\mu \mathrm{g} / \mathrm{kg}$ \\
\hline Benzoic acid & 104637 & & $\mathrm{U}$ & 38.3 & $\mathrm{U}$ & 39.2 & $\mu \mathrm{g} / \mathrm{kg}$ \\
\hline Benzoic acid & 104644 & & $\mathrm{U}$ & 38.7 & $\mathrm{U}$ & 38.5 & $\mu \mathrm{g} / \mathrm{kg}$ \\
\hline Benzoic acid & 104646 & & $\mathrm{U}$ & 38.8 & $\mathrm{U}$ & 38.3 & $\mu \mathrm{g} / \mathrm{kg}$ \\
\hline Benzoic acid & 104656 & 131.8 & $\mathrm{U}$ & 63.3 & & 308 & $\mu \mathrm{g} / \mathrm{kg}$ \\
\hline Benzoic acid & 104660 & & $\mathrm{U}$ - & 36.9 & $\mathrm{U}$ & 37.6 & $\mu \mathrm{g} / \mathrm{kg}$ \\
\hline Bis(2-ethylhexyl) phthalate & 104635 & 0.9 & $\mathrm{~J}$ & 22.8 & $\mathrm{~J}$ & 22.6 & $\mu \mathrm{g} / \mathrm{kg}$ \\
\hline Bis(2-ethylhexyl) phthalate & 104637 & & $\mathrm{U}$ & 38.3 & $\mathrm{U}$ & 39.2 & $\mu \mathrm{g} / \mathrm{kg}$ \\
\hline Bis(2-ethylhexyl) phthalate & 104644 & & $\mathrm{U}$ & 38.7 & $U$ & 38.5 & $\mu \mathrm{g} / \mathrm{kg}$ \\
\hline Bis(2-ethylhexyl) phthalate & 104646 & 60.8 & $\mathrm{U}$ & 38.8 & $\mathbf{J}$ & 20.7 & $\mu \mathrm{g} / \mathrm{kg}$ \\
\hline Bis(2-ethylhexyl) phthalate & 104656 & & $\mathrm{U}$ & 63.3 & $\mathrm{U}$ & 58.4 & $\mu \mathrm{g} / \mathrm{kg}$ \\
\hline Bis(2-ethylhexyl) phthalate & 104660 & & $\mathrm{U}$ & 36.9 & $\mathrm{U}$ & 37.6 & $\mu \mathrm{g} / \mathrm{kg}$ \\
\hline Di-n-butyl phthalate & 104635 & 13.5 & $J$ & 28.4 & $J$ & 24.8 & $\mu \mathrm{g} / \mathrm{kg}$ \\
\hline Di-n-butyl phthalate & 104637 & 17.8 & $J$ & 21 & $\mathrm{~J}$ & 25.1 & $\mu \mathrm{g} / \mathrm{kg}$ \\
\hline Di-n-butyl phthalate & 104644 & & $\mathrm{U}$ & 42.9 & $\mathrm{U}$ & 45 & $\mu \mathrm{g} / \mathrm{kg}$ \\
\hline Di-n-butyl phthalate & 104646 & & $\mathrm{U}$ & 44.3 & $\mathrm{U}$ & 50.2 & $\mu \mathrm{g} / \mathrm{kg}$ \\
\hline Di-n-butyl phthalate & 104656 & & $\mathrm{U}$ & 55.7 & $\mathrm{U}$ & 52.6 & $\mu \mathrm{g} / \mathrm{kg}$ \\
\hline Di-n-butyl phthalate & 104660 & 30.3 & $\mathrm{~J}$ & 27.7 & $\mathrm{U}$ & 37.6 & $\mu \mathrm{g} / \mathrm{kg}$ \\
\hline Diethyl phthalate & 104635 & & $\mathrm{U}$ & 37.4 & $\mathrm{U}$ & 37.6 & $\mu \mathrm{g} / \mathrm{kg}$ \\
\hline Diethyl phthalate & 104637 & 59.7 & $\mathrm{U}$ & 38.3 & $\mathrm{~J}$ & 20.7 & $\mu \mathrm{g} / \mathrm{kg}$ \\
\hline Diethyl phthalate & 104644 & & $\mathrm{U}$ & 38.7 & $\mathrm{U}$ & 38.5 & $\mu \mathrm{g} / \mathrm{kg}$ \\
\hline Diethyl phthalate & 104646 & & $\mathrm{U}$ & 38.8 & $U$ & 38.3 & $\mu g / \mathrm{kg}$ \\
\hline Diethyl phthalate & 104656 & & $\mathrm{U}$ & 63.3 & $\mathrm{U}$ & 58.4 & $\mu \mathrm{g} / \mathrm{kg}$ \\
\hline Diethyl phthalate & 104660 & & $\mathrm{U}$ & 36.9 & $\mathrm{U}$ & 37.6 & $\mu g / \mathrm{kg}$ \\
\hline \multicolumn{8}{|l|}{ Method EPA9081 } \\
\hline Cation exchange capacity & 104635 & 83.4 & & 29.4 & & 12.1 & $\mathrm{meq} / 100 \mathrm{~g}$ \\
\hline Cation exchange capacity & 104637 & 37.8 & & 100 & & 68.2 & $\mathrm{meq} / 100 \mathrm{~g}$ \\
\hline Cation exchange capacity & 104644 & 0.4 & & 48.3 & & 48.5 & meq $/ 100 \mathrm{~g}$ \\
\hline Cation exchange capacity & 104646 & 29.5 & & 51.1 & & 68.8 & $\mathrm{meq} / 100 \mathrm{~g}$ \\
\hline Cation exchange capacity & 104660 & 0.3 & & 58.1 & & 58.3 & $\mathrm{meq} / 100 \mathrm{~g}$ \\
\hline \multicolumn{8}{|l|}{ Method EPIA-001B } \\
\hline Gross alpha & 104635 & 81.2 & & 13.9 & & 32.9 & $\mathrm{pCi} / \mathrm{g}$ \\
\hline Gross alpha & 104637 & 7.2 & & 30.8 & & 33.1 & $\mathrm{pCi} / \mathrm{g}$ \\
\hline Gross alpha & 104644 & 115.3 & & 16.7 & & 62.2 & $\mathrm{pCi} / \mathrm{g}$ \\
\hline Gross alpha & 104646 & 52.6 & & 52.1 & & 30.4 & $\mathrm{pCi} / \mathrm{g}$ \\
\hline Gross alpha & 104656 & 28.8 & & 17.8 & & 23.8 & $\mathrm{pCi} / \mathrm{g}$ \\
\hline Gross alpha & 104660 & 13.5 & & 22.9 & & 20 & $\mathrm{pCi} / \mathrm{g}$ \\
\hline
\end{tabular}


Table 36. RPDs for Analytes Detected in Field Duplicates for Sediment Samples, Continued

\begin{tabular}{|c|c|c|c|c|c|c|c|}
\hline \multirow{2}{*}{$\frac{\text { Analyte }}{\text { Nonvolatile beta }}$} & \multirow{2}{*}{$\frac{\text { Sample }}{104635}$} & \multirow{2}{*}{$\frac{\text { RPD }}{46.5}$} & \multicolumn{2}{|c|}{ First Result } & \multicolumn{2}{|c|}{ Second Result } & \multirow{2}{*}{$\frac{\text { Units }}{\mathrm{pCi} / \mathrm{g}}$} \\
\hline & & & $\mathrm{J}$ & 7.97 & $\mathrm{~J}$ & 12.8 & \\
\hline Nonvolatile beta & 104637 & 32.9 & $J$ & 11.7 & $\mathrm{~J}$ & 16.3 & $\mathrm{pCi} / \mathrm{g}$ \\
\hline Nonvolatile beta & 104644 & 41.3 & & 9.73 & & 14.8 & $\mathrm{pCi} / \mathrm{g}$ \\
\hline Nonvolatile beta & 104646 & 74.5 & & 23.4 & & 10.7 & $\mathrm{pCi} / \mathrm{g}$ \\
\hline Nonvolatile beta & 104656 & 33.9 & & 21.8 & & 30.7 & $\mathrm{pCi} / \mathrm{g}$ \\
\hline Nonvolatile beta & 104660 & 22.8 & & 9.2 & & 7.32 & $\mathrm{pCi} / \mathrm{g}$ \\
\hline \multicolumn{8}{|l|}{ Method EPIA-004 } \\
\hline Strontium-90 & 104635 & & UI & -0.076 & UI & -0.08 & $\mathrm{pCi} / \mathrm{g}$ \\
\hline Strontium-90 & 104637 & 72.9 & UI & 0.061 & & 0.131 & $\mathrm{pCi} / \mathrm{g}$ \\
\hline Strontium-90 & 104644 & 92.2 & & 0.995 & $\mathrm{U}$ & 0.367 & $\mathrm{pCi} / \mathrm{g}$ \\
\hline Strontium-90 & 104646 & 52.3 & $\mathrm{U}$ & 0.679 & & 1.16 & $\mathrm{pCi} / \mathrm{g}$ \\
\hline Strontium-90 & 104656 & 21.6 & & 1.49 & & 1.2 & $\mathrm{pCi} / \mathrm{g}$ \\
\hline Strontium-90 & 104660 & 3.8 & & 1.61 & & 1.55 & $\mathrm{pCi} / \mathrm{g}$ \\
\hline \multicolumn{8}{|l|}{ Method EPIA-012B } \\
\hline Plutonium-238 & 104635 & 262.9 & & 0.0159 & UI & -0.002 & $\mathrm{pCi} / \mathrm{g}$ \\
\hline Plutonium-238 & 104637 & & UI & 0.0016 & UI & 0.0043 & $\mathrm{pCi} / \mathrm{g}$ \\
\hline Plutonium-238 & 104644 & & UI & 0.0013 & UI & $-1.20 \mathrm{E}-04$ & $\mathrm{pCi} / \mathrm{g}$ \\
\hline Plutonium-238 & 104646 & & UI & 0.0149 & UI & 0.0236 & $\mathrm{pCi} / \mathrm{g}$ \\
\hline Plutonium-238 & 104656 & & UI & 0.0121 & UI & 0.0172 & $\mathrm{pCi} / \mathrm{g}$ \\
\hline Plutonium-238 & 104660 & 164 & UI & 0.0174 & & 0.176 & $\mathrm{pCi} / \mathrm{g}$ \\
\hline Plutonium-239/240 & 104635 & & UI & 0.0029 & UI & 0.0016 & $\mathrm{pCi} / \mathrm{g}$ \\
\hline Plutonium-239/240 & 104637 & & UI & 5.51E-05 & UI & 0.0013 & $\mathrm{pCi} / \mathrm{g}$ \\
\hline Plutonium-239/240 & 104644 & & UI & -0.007 & UI & 0 & $\mathrm{pCi} / \mathrm{g}$ \\
\hline Plutonium-239/240 & 104646 & & UI & 0.012 & UI & 0.0063 & $\mathrm{pCi} / \mathrm{g}$ \\
\hline Plutonium-239/240 & 104656 & 59.1 & & 0.171 & & 0.093 & $\mathrm{pCi} / \mathrm{g}$ \\
\hline Plutonium-239/240 & 104660 & 195.5 & UI & 0.0013 & & 0.119 & $\mathrm{pCi} / \mathrm{g}$ \\
\hline \multicolumn{8}{|l|}{ Method EPIA-013B } \\
\hline Actinium-228 & 104635 & 11.7 & & 0.544 & & 0.484 & $\mathrm{pCi} / \mathrm{g}$ \\
\hline Actinium-228 & 104637 & 3.7 & & 1.05 & & 1.09 & $\mathrm{pCi} / \mathrm{g}$ \\
\hline Actinium-228 & 104644 & 11.4 & & 1.08 & & 0.964 & $\mathrm{pCi} / \mathrm{g}$ \\
\hline Actinium-228 & 104646 & 16.5 & & 1.71 & & 1.45 & $\mathrm{pCi} / \mathrm{g}$ \\
\hline Actinium-228 & 104656 & 19.2 & & 1.71 & & 1.41 & $\mathrm{pCi} / \mathrm{g}$ \\
\hline Actinium-228 & 104660 & 4.7 & & 0.834 & & 0.874 & $\mathrm{pCi} / \mathrm{g}$ \\
\hline Cesium-137 & 104635 & 8.8 & & 0.543 & & 0.497 & $\mathrm{pCi} / \mathrm{g}$ \\
\hline Cesium-137 & 104637 & & UI & 0.003 & UI & 0.0026 & $\mathrm{pCi} / \mathrm{g}$ \\
\hline Cesium-137 & 104644 & 13.3 & & 0.0775 & & 0.0885 & $\mathrm{pCi} / \mathrm{g}$ \\
\hline Cesium-137 & 104646 & & UI & -0.004 & UI & 0.0002 & $\mathrm{pCi} / \mathrm{g}$ \\
\hline Cesium-137 & 104656 & 2.5 & & 24 & & 23.4 & $\mathrm{pCi} / \mathrm{g}$ \\
\hline Cesium-137 & 104660 & 16.3 & & 0.0569 & & 0.067 & $\mathrm{pCi} / \mathrm{g}$ \\
\hline Cobalt- 60 & 104635 & & UI & 0.0048 & UI & 0.0004 & $\mathrm{pCi} / \mathrm{g}$ \\
\hline
\end{tabular}


Table 36. RPDs for Analytes Detected in Field Duplicates for Sediment Samples, Continued

\begin{tabular}{|c|c|c|c|c|c|c|c|}
\hline \multirow{2}{*}{$\begin{array}{l}\text { Analyte } \\
\text { Cobalt-60 }\end{array}$} & \multirow{2}{*}{$\begin{array}{l}\text { Sample } \\
104637\end{array}$} & \multirow[t]{2}{*}{ RPD } & \multicolumn{2}{|c|}{ First Result } & \multicolumn{2}{|c|}{ Second Result } & \multirow{2}{*}{$\begin{array}{l}\text { Units } \\
\mathrm{pCi} / \mathrm{g} \\
\end{array}$} \\
\hline & & & UI & -0.002 & UI & -0.002 & \\
\hline Cobalt- 60 & 104644 & 354.4 & UI & -0.004 & & 0.0135 & $\mathrm{pCi} / \mathrm{g}$ \\
\hline Cobalt-60 & 104646 & 147.2 & UI & 0.0029 & & 0.0188 & $\mathrm{pCi} / \mathrm{g}$ \\
\hline Cobalt- 60 & 104656 & 7.6 & & 0.415 & & 0.448 & $\mathrm{pCi} / \mathrm{g}$ \\
\hline Cobalt-60 & 104660 & & UI & 0.0018 & $\mathrm{UI}$ & 0.0003 & $\mathrm{pCi} / \mathrm{g}$ \\
\hline Europium-155 & 104635 & & UI & 0.0254 & $\mathrm{UI}$ & 0.0226 & $\mathrm{pCi} / \mathrm{g}$ \\
\hline Europium-155 & 104637 & 1.8 & & 0.0797 & & 0.0783 & $\mathrm{pCi} / \mathrm{g}$ \\
\hline Europium-155 & 104644 & & UI & 0 & UI & 0 & $\mathrm{pCi} / \mathrm{g}$ \\
\hline Europium-155 & 104646 & & UI & 0 & UI & 0 & $\mathrm{pCi} / \mathrm{g}$ \\
\hline Europium-155 & 104656 & & UI & 0.0703 & $\mathrm{UI}$ & 0.0525 & $\mathrm{pCi} / \mathrm{g}$ \\
\hline Europium-155 & 104660 & & UI & 0 & UI & 0 & $\mathrm{pCi} / \mathrm{g}$ \\
\hline Lead-212 & 104635 & 6.6 & & 0.545 & & 0.51 & $\mathrm{pCi} / \mathrm{g}$ \\
\hline Lead-212 & 104637 & 0.9 & & 1.12 & & 1.13 & $\mathrm{pCi} / \mathrm{g}$ \\
\hline Lead-212 & 104644 & 7.5 & & 1.11 & & 1.03 & $\mathrm{pCi} / \mathrm{g}$ \\
\hline Lead-212 & 104646 & 12.8 & & 1.75 & & 1.54 & $\mathrm{pCi} / \mathrm{g}$ \\
\hline Lead-212 & 104656 & 9.4 & & 1.78 & & 1.62 & $\mathrm{pCi} / \mathrm{g}$ \\
\hline Lead-212 & 104660 & 4.2 & & 0.909 & & 0.872 & $\mathrm{pCi} / \mathrm{g}$ \\
\hline Manganese-54 & 104635 & 67.8 & & 0.0095 & UI & 0.0047 & $\mathrm{pCi} / \mathrm{g}$ \\
\hline Manganese-54 & 104637 & 5.4 & & 0.0233 & & 0.0246 & $\mathrm{pCi} / \mathrm{g}$ \\
\hline Manganese-54 & 104644 & 7.1 & & 0.0136 & & 0.0146 & $\mathrm{pCi} / \mathrm{g}$ \\
\hline Manganese-54 & 104646 & 40.2 & & 0.043 & & 0.0286 & $\mathrm{pCi} / \mathrm{g}$ \\
\hline Manganese-54 & 104656 & & UI & 0.0144 & UI & -0.006 & $\mathrm{pCi} / \mathrm{g}$ \\
\hline Manganese-54 & 104660 & 6.6 & & 0.0132 & & 0.0141 & $\mathrm{pCi} / \mathrm{g}$ \\
\hline Potassium-40 & 104635 & 3.1 & & 0.486 & & 0.471 & $\mathrm{pCi} / \mathrm{g}$ \\
\hline Potassium-40 & 104637 & 5.4 & & 1.32 & & 1.25 & $\mathrm{pCi} / \mathrm{g}$ \\
\hline Potassium-40 & 104644 & 33 & & 0.912 & & 0.654 & $\mathrm{pCi} / \mathrm{g}$ \\
\hline Potassium-40 & 104646 & 2.6 & & 0.76 & & 0.78 & $\mathrm{pCi} / \mathrm{g}$ \\
\hline Potassium-40 & 104656 & 8.4 & & 3.22 & & 2.96 & $\mathrm{pCi} / \mathrm{g}$ \\
\hline Potassium-40 & 104660 & 25.5 & & 0.601 & & 0.777 & $\mathrm{pCi} / \mathrm{g}$ \\
\hline Thorium-234 & 104635 & & $\mathrm{U}$ & 0.628 & UI & 0.362 & $\mathrm{pCi} / \mathrm{g}$ \\
\hline Thorium-234 & 104637 & & $\mathrm{U}$ & 1.25 & $\mathrm{U}$ & 1.47 & $\mathrm{pCi} / \mathrm{g}$ \\
\hline Thorium-234 & 104644 & & $\mathrm{U}$ & 0.823 & $\mathrm{U}$ & 1.26 & $\mathrm{pCi} / \mathrm{g}$ \\
\hline Thorium-234 & 104646 & 10 & & 1.57 & $\mathrm{U}$ & 1.42 & $\mathrm{pCi} / \mathrm{g}$ \\
\hline Thorium-234 & 104656 & 25 & & 2.77 & & 3.56 & $\mathrm{pCi} / \mathrm{g}$ \\
\hline Thorium-234 & 104660 & & $\mathrm{U}$ & 1.21 & $\mathrm{U}$ & 0.706 & $\mathrm{pCi} / \mathrm{g}$ \\
\hline Zirconium-95 & 104635 & & UI & 0.014 & UI & 0 & $\mathrm{pCi} / \mathrm{g}$ \\
\hline Zirconium-95 & 104637 & 200 & & 0.0654 & UI & 0 & $\mathrm{pCi} / \mathrm{g}$ \\
\hline Zirconium-95 & 104644 & & UI & 0.016 & UI & 0 & $\mathrm{pCi} / \mathrm{g}$ \\
\hline Zirconium-95 & 104646 & & UI & 0 & UI & 0 & $\mathrm{pCi} / \mathrm{g}$ \\
\hline Zirconium-95 & 104656 & & UI & -0.046 & UI & -0.032 & $\mathrm{pCi} / \mathrm{g}$ \\
\hline
\end{tabular}


Table 36. RPDs for Analytes Detected in Field Duplicates for Sediment Samples, Continued

\begin{tabular}{|l|l|l|l|l|l|l|l|}
\hline Analyte & Sample & RPD & \multicolumn{2}{|l|}{ First Result } & Second Result & Units \\
\hline Zirconium-95 & 104660 & & UI & 0.0085 & UI & 0 & pCi/g \\
\hline
\end{tabular}


This page was left blank intentionally. 


\section{Quality Control Samples}

\section{Replicate and Duplicate Analyses of Samples}

Blind replicate and duplicate samples are analyzed in order to establish the precision of the analyses. The replicate and duplicate analytical results are used to generate mean relative difference (MRD) indices, which are used to evaluate the laboratories' performance.

\section{Interlaboratory Comparisons}

The MRD was developed by R. C. Tuckfield of the Applied Statistics Group at the Savannah River Technology Center, in conjunction with M. M. Khalil of the Environmental Protection Department/Environmental Monitoring Section (EPD/EMS), to assess the reproducibility of identical chemical analyses. For interlaboratory comparisons, the MRD is calculated as the average absolute difference between the laboratories for the $i^{t_{h}}$ sample expressed as a percentage of the mean of both laboratories. For these comparisons, $x_{i}$ and $y_{i}$ represent the mean analyte concentrations for the $i^{i h}$ sample; $x_{i}$ represents the mean from one laboratory and $y_{i}$ represents the mean from the other. The means are calculated from the known sample results and the EPD blind replicate results.

$$
M R D=\left\{\frac{\sum_{i=1}^{n}\left(\left|x_{i}-y_{i}\right| /\left[\left(x_{i}+y_{i}\right) / 2\right]\right)}{n}\right\} x 100
$$

where

$x_{i}=$ the mean analyte concentration in a sample for the $i^{\text {th }}$ sample location

$y_{i}=$ the mean analyte concentration in the replicate or duplicate

$n=$ the number of pairs of observations

Generally, the closer the original results and their replicate or duplicate results are to each other, the lower the MRD. 


\section{Normalizing Data to the Reference Detection Limit}

Because some detection limits may be anomalously high (because of dilution or other effects, for example), it is necessary to use a reference detection limit (RDL) in the MRD calculations. This is set as the 90th percentile value of the detection limits of the non-detected samples. All the results less than the RDL are adjusted up to that value. Results that are detection limit values above the RDL are eliminated from the MRD index calculations and from the $t$ tests. By definition, fewer than $10 \%$ of the detection limit values are above the RDL.

In addition to the interlaboratory MRD calculations, paired $t$-tests are performed to see if the difference between the mean concentrations of an analyte from the same sample reported by each laboratory is significant. The $t$-test tests the null hypothesis that there is no significant difference in the concentrations reported by the two laboratories. The MRD and the $t$-test for analytes with at least one pair of results above the interlaboratory RDL are listed in Table 39. The MRD and $t$-test were not conducted due to the insufficient pairs of samples.

\section{Comments on the Replicate and Duplicate Analyses}

Table 37 lists the sample identification numbers and associated sample identification numbers for samples used as replicates.

Table 37. Samples Used for Interlaboratory Comparisons

\begin{tabular}{|l|l|}
\hline Sample & Associated Sample \\
\hline 104636 & 104639 \\
\hline 104643 & 104647 \\
\hline 104645 & 104649 \\
\hline 104658 & 104659 \\
\hline
\end{tabular}

Certain analytes were not present in concentrations above detection limits in any samples having replicates. These analytes are not considered in further evaluation of replicate analyses and are listed in Table 38.

Table 38. The Number of Determinations of Analytes Not Showing Measurable Concentrations above Detection Limits in Any Replicated Samples between GE and Weston

\begin{tabular}{|l|l|l|}
\hline Analyte & GE & WA \\
\hline $\begin{array}{l}\text { Physical Parameters and Specified } \\
\text { Analyses }\end{array}$ \\
\hline Chloride & 5 & 6 \\
\hline Cyanide & 4 & 4 \\
\hline Orthophosphate & 5 & 5 \\
\hline Phenols & 5 & 6 \\
\hline Sulfide & 1 & 3 \\
\hline Inorganics (total recoverable) \\
\hline
\end{tabular}

\begin{tabular}{|l|l|l|}
\hline Analyte & GE & WA \\
\hline Antimony & 4 & 5 \\
\hline Arsenic & 4 & $\mathbf{5}$ \\
\hline Beryllium & 4 & 5 \\
\hline Cadmium & 4 & 5 \\
\hline Mercury & 4 & 5 \\
\hline Potassium & 4 & 5 \\
\hline Selenium & 4 & 5 \\
\hline Silver & 4 & 5 \\
\hline
\end{tabular}

Par Pond, Pond C, and L Lake, Revision 2 
Table 38. The Number of Determinations of Analytes Not Showing Measurable Concentrations above Detection Limits in Any Replicated Samples between GE and Weston, Continued

\begin{tabular}{|l|l|l|}
\hline Analyte & GE & WA \\
\hline Sodium & 4 & 5 \\
\hline Thallium & 4 & 5 \\
\hline B/N/A Extractables & 5 & 5 \\
\hline Acenaphthene & 5 & 6 \\
\hline Acenaphthylene & 5 & 6 \\
\hline Anthracene & 5 & 6 \\
\hline Benzo[a]anthracene & 5 & 6 \\
\hline Benzo[b]fluoranthene & 5 & 6 \\
\hline Benzo[k]fluoranthene & 5 & 6 \\
\hline Benzoic acid & 5 & 6 \\
\hline Benzo[g,h,i]perylene & 5 & 6 \\
\hline Benzo[a]pyrene & 5 & 6 \\
\hline Benzyl alcohol & 5 & 6 \\
\hline $\begin{array}{l}\text { Bis(2-chloroethoxy) } \\
\text { methane }\end{array}$ & & \\
\hline Bis(2-chloroethyl) ether & 5 & 6 \\
\hline Bis(2-chloroisopropyl) ether & 5 & 6 \\
\hline Bis(2-ethylhexyl) phthalate & 5 & 6 \\
\hline $\begin{array}{l}\text { 4-Bromophenyl phenyl } \\
\text { ether }\end{array}$ & 5 & 6 \\
\hline Butylbenzyl phthalate & 5 & 6 \\
\hline 4-Chloroaniline & 5 & 6 \\
\hline 4-Chloro-m-cresol & 5 & 5 \\
\hline 2-Chloronaphthalene & 5 & 6 \\
\hline 2-Chlorophenol & 5 & 5 \\
\hline $\begin{array}{l}\text { 4-Chlorophenyl phenyl } \\
\text { ether }\end{array}$ & 5 & 6 \\
\hline Chrysene & 5 & 6 \\
\hline o-Cresol (2-Methylphenol) & 5 & 6 \\
\hline p-Cresol (4-Methylphenol) & - & 3 \\
\hline Dibenz[a,h]anthracene & 5 & 6 \\
\hline Dibenzofuran & 5 & 6 \\
\hline Di-n-butyl phthalate & 5 & 6 \\
\hline 1,2-Dichlorobenzene & 5 & 6 \\
\hline 1,3-Dichlorobenzene & 5 & 6 \\
\hline 1,4-Dichlorobenzene & 5 & 5 \\
\hline 3,3'-Dichlorobenzidine & 5 & 6 \\
\hline 2,4-Dichlorophenol & 5 & 6 \\
\hline Diethyl phthalate & 5 & 6 \\
\hline
\end{tabular}

\begin{tabular}{|l|l|l|}
\hline Analyte & GE & WA \\
\hline 2,4-Dimethyl phenol & 5 & 6 \\
\hline Dimethyl phthalate & 5 & 6 \\
\hline 2,4-Dinitrophenol & 5 & 6 \\
\hline 2,4-Dinitrotoluene & 5 & 5 \\
\hline 2,6-Dinitrotoluene & 5 & 6 \\
\hline Di-n-octyl phthalate & 5 & 6 \\
\hline Fluoranthene & 5 & 6 \\
\hline Fluorene & 5 & 6 \\
\hline Hexachlorobenzene & 5 & 6 \\
\hline Hexachlorobutadiene & 5 & 6 \\
\hline Hexachlorocyclopentadiene & 5 & 6 \\
\hline Hexachloroethane & 5 & 6 \\
\hline Indeno[1,2,3-c,d]pyrene & 5 & 6 \\
\hline Isophorone & 5 & 6 \\
\hline 2-Methyl-4,6-dinitrophenol & 5 & 6 \\
\hline 2-Methylnaphthalene & 5 & 6 \\
\hline Naphthalene & 5 & 6 \\
\hline m-Nitroaniline & 5 & 6 \\
\hline o-Nitroaniline & 5 & 6 \\
\hline p-Nitroaniline & 5 & 6 \\
\hline Nitrobenzene & 5 & 6 \\
\hline 2-Nitrophenol & 5 & 6 \\
\hline 4-Nitrophenol & 5 & 5 \\
\hline N-Nitrosodiphenylamine & 5 & 6 \\
\hline N-Nitrosodipropylamine & 5 & 6 \\
\hline Pentachlorophenol & 5 & 5 \\
\hline Phenanthrene & 5 & 6 \\
\hline Phenol & 5 & 5 \\
\hline Pyrene & 5 & 5 \\
\hline 1,2,4-Trichlorobenzene & 5 & 5 \\
\hline 2,4,5-Trichlorophenol & 5 & 6 \\
\hline 2,4,6-Trichlorophenol & 5 & 6 \\
\hline Volatile Organic Compounds & 4 \\
\hline Bromodichloromethane & 4 & 6 \\
\hline Bromoform & 6 \\
\hline $\begin{array}{l}\text { Bromomethane (Methyl } \\
\text { bromide) }\end{array}$ & 6 \\
\hline Carbon tetrachloride & 4 & 4 \\
\hline & & \\
\hline & & \\
\hline & 5 & 5 \\
\hline
\end{tabular}


Table 38. The Number of Determinations of Analytes Not Showing Measurable Concentrations above Detection Limits in Any Replicated Samples between GE and Weston, Continued

\begin{tabular}{|l|l|l|}
\hline Analyte & GE & WA \\
\hline Chlorobenzene & 4 & 6 \\
\hline Chloroethane & 4 & 6 \\
\hline $\begin{array}{l}\text { Chloroethene (Vinyl } \\
\text { chloride) }\end{array}$ & 4 & 4 \\
\hline Dibromochloromethane & 4 & 6 \\
\hline 1,1-Dichloroethane & 4 & 6 \\
\hline 1,2-Dichloroethane & 4 & 6 \\
\hline 1,1-Dichloroethylene & 4 & 6 \\
\hline 1,2-Dichloroethylene & 4 & 6 \\
\hline $\begin{array}{l}\text { Dichloromethane } \\
\text { (Methylene chloride) }\end{array}$ & 4 & 4 \\
\hline 1,2-Dichloropropane & 4 & 4 \\
\hline cis-1,3-Dichloropropene & 4 & 6 \\
\hline trans-1,3-Dichloropropene & 4 & 6 \\
\hline Ethylbenzene & 4 & 4 \\
\hline 2-Hexanone & 4 & 6 \\
\hline Methyl ethyl ketone & 4 & 6 \\
\hline Methyl isobutyl ketone & 4 & 6 \\
\hline Styrene & 4 & 4 \\
\hline 1,1,2,2-Tetrachloroethane & 4 & 4 \\
\hline Tetrachloroethylene & 4 & 6 \\
\hline 1,1,1-Trichloroethane & 4 & 6 \\
\hline 1,1,2-Trichloroethane & 4 & 6 \\
\hline Trichloroethylene & 4 & 4 \\
\hline Vinyl acetate & 4 & 6 \\
\hline Xylenes & 4 & 6 \\
\hline Pesticides/PCBs and Dioxins/Furans \\
\hline Aldrin & 5 & 6 \\
\hline $\begin{array}{l}\text { alpha-Benzene } \\
\text { hexachloride }\end{array}$ & 5 & 6 \\
\hline beta-Benzene hexachloride & 5 & 6 \\
\hline delta-Benzene hexachloride & 5 & 6 \\
\hline alpha-Chlordane & 5 & 6 \\
\hline gamma-Chlordane & 5 & 6 \\
\hline p,p'-DDD & 5 & 6 \\
\hline p,p'-DDE & 5 & 6 \\
\hline p,p'-DDT & 5 \\
\hline Dieldrin & 5 \\
\hline Endosulfan sulfate & 6 \\
\hline
\end{tabular}

\begin{tabular}{|l|l|l|}
\hline Analyte & GE & WA \\
\hline Endosulfan I & 5 & 6 \\
\hline Endosulfan II & 5 & 6 \\
\hline Endrin & 5 & 5 \\
\hline Endrin ketone & 5 & 6 \\
\hline Heptachlor & 5 & 5 \\
\hline Heptachlor epoxide & 5 & 6 \\
\hline Lindane & 5 & 5 \\
\hline Methoxychlor & 5 & 6 \\
\hline PCB 1016 & 5 & 6 \\
\hline PCB 1221 & 5 & 6 \\
\hline PCB 1232 & 5 & 6 \\
\hline PCB 1242 & 5 & 6 \\
\hline PCB 1248 & 5 & 6 \\
\hline PCB 1254 & 5 & 6 \\
\hline PCB 1260 & 5 & 6 \\
\hline Toxaphene & 5 & 6 \\
\hline $\begin{array}{l}\text { Radionuclide Indicators and } \\
\text { Radionuclides }\end{array}$ & & \\
\hline Antimony-124 & 3 & 4 \\
\hline Antimony-125 & 3 & 4 \\
\hline Cobalt-57 & 3 & 4 \\
\hline Cobalt-58 & 3 & 4 \\
\hline Europium-152 & 3 & 4 \\
\hline Europium-154 & 3 & 4 \\
\hline Iodine-129 & - & 2 \\
\hline Neptunium-239 & 3 & 4 \\
\hline Promethium-144 & 3 & 4 \\
\hline Promethium-146 & 3 & 4 \\
\hline Ruthenium-106 & 3 & 4 \\
\hline Sodium-22 & 3 & 4 \\
\hline Yttrium-88 & 4 \\
\hline Zinc-65 & \multicolumn{2}{|l}{} \\
\hline Zirconium-95 & 3 \\
\hline & & 4 \\
\hline
\end{tabular}


Analytes with significance-of-probability values less than .050 in Table 39 have a $95 \%$ chance that one laboratory's results are significantly higher than those from the other laboratory.

Table 39. Interlaboratory MRD and $t$-test Results for Analytes with at Least One Pair of Results above the RDL for GE and Weston

\begin{tabular}{|c|c|c|c|c|}
\hline Analyte & RDL & Unit & MRD & $\begin{array}{l}t \text {-test } \\
\text { Probabilitya }\end{array}$ \\
\hline \multicolumn{5}{|c|}{ Physical Parameters and Specified Analyses } \\
\hline Cation exchange capacity & 25.8 & $\mathrm{mg} / \mathrm{kg}$ & 0 & \\
\hline Nitrate-nitrite as nitrogen & 1.24 & $\mathrm{mg} / \mathrm{kg}$ & 11.4 & 0.416 \\
\hline Total organic carbon & $t$ & & 96.8 & 0.215 \\
\hline Total organic nitrogen & 125 & $\mathrm{mg} / \mathrm{kg}$ & 8.85 & 0.391 \\
\hline Total phosphates (as P) & 414 & $\mathrm{mg} / \mathrm{kg}$ & 0 & \\
\hline \multicolumn{5}{|l|}{ Total Metals } \\
\hline Aluminum & $\dagger$ & & 51.8 & 0.142 \\
\hline Barium & 4.46 & $\mathrm{mg} / \mathrm{kg}$ & 47 & 0.01 \\
\hline Calcium & $t$ & & 18.1 & 0.573 \\
\hline Chromium & 7.91 & $\mathrm{mg} / \mathrm{kg}$ & 0 & \\
\hline Cobalt & 5.9 & $\mathrm{mg} / \mathrm{kg}$ & 0 & \\
\hline Copper & 3.99 & $\mathrm{mg} / \mathrm{kg}$ & 0 & \\
\hline Iron & $\dagger$ & & 57.5 & 0.54 \\
\hline Lead & 5.63 & $\mathrm{mg} / \mathrm{kg}$ & 2.13 & 0.391 \\
\hline Magnesium & $\dagger$ & & 33.3 & 0.063 \\
\hline Manganese & $t$ & & 33 & 0.081 \\
\hline Nickel & 9.29 & $\mathrm{mg} / \mathrm{kg}$ & 0 & \\
\hline Vanadium & 4.26 & $\mathrm{mg} / \mathrm{kg}$ & 37.9 & 0.517 \\
\hline Zinc & 5.4 & $\mathrm{mg} / \mathrm{kg}$ & 0 & \\
\hline \multicolumn{5}{|l|}{ Volatile Organic Compounds } \\
\hline Acetone & 20.7 & $\mu \mathrm{g} / \mathrm{kg}$ & 55.4 & 0.194 \\
\hline Benzene & 0.588 & $\mu \mathrm{g} / \mathrm{kg}$ & 2.23 & 0.391 \\
\hline Carbon disulfide & 2.4 & $\mu \mathrm{g} / \mathrm{kg}$ & 31.8 & 0.391 \\
\hline Chloroform & 0.24 & $\mu \mathrm{g} / \mathrm{kg}$ & 18.6 & 0.391 \\
\hline Chloromethane (Methyl chloride) & 0.36 & $\mu \mathrm{g} / \mathrm{kg}$ & 0 & \\
\hline
\end{tabular}

a Values less than .050 indicate a probability of less than 1 in 20 that the results for that analyte are the same from both laboratories. Note: The MRD and t-test were not performed due to insufficient pairs of samples.

$\dagger$ Replicate or duplicate results below quantitation or no quantitation limit. 
Table 39. Interlaboratory MRD and $t$-test Results for Analytes with at Least One Pair of Results above the RDL for GE and Weston, Continued

\begin{tabular}{|c|c|c|c|c|}
\hline Analyte & RDL & Unit & MRD & $\begin{array}{l}t \text {-test } \\
\text { Probability }\end{array}$ \\
\hline Toluene & 0.595 & $\mu \mathrm{g} / \mathrm{kg}$ & 0 & \\
\hline \multicolumn{5}{|c|}{ Radionuclide Indicators and Radionuclides } \\
\hline Actinium-228 & $t$ & & 35.1 & 0.084 \\
\hline Barium-133 & 0.12 & $\mathrm{pCi} / \mathrm{g}$ & 24.6 & 0.423 \\
\hline Cesium-134 & 0.1 & $\mathrm{pCi} / \mathrm{g}$ & 15.4 & 0.423 \\
\hline Cesium-137 & 0.06 & $\mathrm{pCi} / \mathrm{g}$ & 18.6 & 0.409 \\
\hline Cobalt- 60 & 0.1 & $\mathrm{pCi} / \mathrm{g}$ & 0 & \\
\hline Europium-155 & 0.27 & $\mathrm{pCi} / \mathrm{g}$ & 6.67 & 0.423 \\
\hline Gross alpha & $t$ & & 37.2 & 0.716 \\
\hline Lead-212 & $\dagger$ & & 37.4 & 0.092 \\
\hline Manganese-54 & 0.1 & $\mathrm{pCi} / \mathrm{g}$ & 0 & \\
\hline Nonvolatile beta & $t$ & & 52.2 & 0.4 \\
\hline Plutonium-238 & 0.22 & $\mathrm{pCi} / \mathrm{g}$ & 17.6 & 0.518 \\
\hline Plutonium-239/240 & 0.23 & $\mathrm{pCi} / \mathrm{g}$ & 0 & \\
\hline Potassium-40 & 0.5 & $\mathrm{pCi} / \mathrm{g}$ & 26.7 & 0.195 \\
\hline Strontium-90 & 1.1 & $\mathrm{pCi} / \mathrm{g}$ & 16.7 & 0.178 \\
\hline Thorium-234 & 1.9 & $\mathrm{pCi} / \mathrm{g}$ & 29.3 & 0.321 \\
\hline Tin-113 & 0.12 & $\mathrm{pCi} / \mathrm{g}$ & 0 & \\
\hline
\end{tabular}

Table 40 lists analytes and samples where a result from one laboratory was more than twice the corresponding result from the other laboratory.

Table 40. Analytes with One Laboratory's Result Greater than Twice the Result from the Other Laboratory between the Primary and QC Laboratories

\begin{tabular}{|l|l|}
\hline Analyte & Samples \\
\hline Physical Parameters and Other Analyses & \multicolumn{2}{|l|}{} \\
\hline Total organic carbon & $104636,104643,104658$ \\
\hline Total Metals & 104645 \\
\hline Aluminum & 104645 \\
\hline Barium & 104636,104645 \\
\hline Iron & 104645 \\
\hline Manganese & 104636 \\
\hline Vanadium & \\
\hline
\end{tabular}


Table 40. Analytes with One Laboratory's Result Greater than Twice the Result from the Other Laboratory between Primary and QC Laboratories, Continued

\begin{tabular}{|l|l|}
\hline Analyte & \multicolumn{2}{l|}{ Samples } \\
\hline Volatile Organic Compounds & 104636,104658 \\
\hline Acetone & 104636 \\
\hline Carbon disulfide & 104658 \\
\hline Chloroform & \\
\hline Radionuclide Indicators and Radionuclides & 104636 \\
\hline Barium-133 & 104636 \\
\hline Lead-212 & 104643,104645 \\
\hline Nonvolatile beta & 104643 \\
\hline Thorium-234 &
\end{tabular}


This page was left blank intentionally. 
ASTM (American Society for Testing and Materials), 1988. ASTM Standards on Precision and Bias for Various Applications, 3rd edition. Philadelphia, PA.

DOE (U.S. Department of Energy), 1992. Environmental Measurements Laboratory Procedures Manual, HASL-300, 27th edition. Environmental Measurements Laboratory, New York, NY.

EPD/EMS (Environmental Protection Department/Environmental Monitoring Section), 1995. AN95 Format, January 1, 1995. Westinghouse Savannah River Company, Savannah River Site, Aiken, SC.

Keith, L. H., ed., 1991. Compilation of EPA's Sampling and Analysis Methods. Boca Raton, FL.

Keller, R. M., 1995. P \& R-Area Reactor Cooling Pond Sediment Characterization RFI/RI Project Technical Issues Report of General Engineering Labòratories, Inc., and Environmental Physics, Inc. (U), ESH-EMS-950788, October 11, 1995. Prepared by Exploration Resources, Inc., for Westinghouse Savannah River Company, Savannah River Site, Aiken, SC.

Koch, J. W., 1995. Scoping Activity Plan: Par Pond and L Lake Sediment Core Sampling, memorandum SRT-ESS-95-0150, Rev.0., May 1, 1995. Environmental Restoration, Westinghouse Savannah River Company, Savannah River Site, Aiken, SC.

Krieger, H. L., and E. L. Whitaker, 1980. Prescribed Procedures for Measurement of Radioactivity in Drinking Water, EPA-600/4-80-032. Environmental Monitoring and Support Laboratory, U.S. Environmental Protection Agency, Cincinnati, OH.

Nichols, C. E., 1995. Work Plan Assessment, Par Pond Sediment Characterization, memorandum ESH-EMS-950364, May 15, 1995. Prepared by Science Applications International Corporation for Westinghouse Savannah River Company, Savannah River Site, Aiken, SC.

Pfaff, J. D., C. A. Brockhoff, and J. W. O'Dell, 1991. Test Method: The Determination of Inorganic Anions in Water by Ion Chromatography-Method 300.0. Environmental Monitoring and Systems Laboratory, U:S. Environmental Protection Agency, Cincinnati, $\mathrm{OH}$.

Schindler, J. E., D. J. Williams, and J. J. Alberts, not dated. A Report on the Methylmercury Situation in Par Pond. Department of Zoology, University of Georgia, Athens, GA.

Soil Survey Staff, 1990. Keys to Soil Taxonomy. Soil Management Support Services Technical Monograph No. 6, Fourth Edition. Department of Crop and Soil Environmental Sciences, Virginia Polytechnic Institute and State University, Blacksburg, VA. 
USEPA (U.S. Environmental Protection Agency), 1983. Methods for Chemical Analysis of Water and Wastes, EPA-600/4-79-020. Environmental Monitoring and Support Laboratory, Cincinnati, $\mathrm{OH}$.

USEPA (U.S. Environmental Protection Agency), 1986. Test Methods for Evaluating Solid Waste (SW-846), Volume I, November 1986. Office of Solid Waste Management and Emergency Response, Washington, DC.

USEPA (U.S. Environmental Protection Agency), 1987. Data Quality Objectives for Remedial Response Activities: Development Process, EPA 540/G-87/003.

Washington, DC.

USEPA (U.S. Environmental Protection Agency), 1988a. Laboratory Data Validation Functional Guidelines for Evaluating Inorganics Analyses. Hazardous Site Evaluation Division, Washington, DC.

USEPA (U.S. Environmental Protection Agency), 1988b. Laboratory Data Validation Functional Guidelines for Evaluating Organics Analyses. Hazardous Site Evaluation Division, Washington, DC.

USEPA (U.S. Environmental Protection Agency), 1989a. Method 1620: Metals by Inductively Coupled Plasma Atomic Emission Spectroscopy and Atomic Absorption Spectroscopy, September 1989, draft. Office of Water Regulations and Standards, Industrial Technology Division, Washington, DC.

USEPA (U.S. Environmental Protection Agency), 1989b. Risk Assessment Guidance for Superfund Human Health Evaluation Manual, Vol. 1, EPA-540/1-89/002, December 1989. Washington, DC.

USEPA (U.S. Environmental Protection Agency), 1990a. Guidance for Data Useability in Risk Assessment, EPA-540/G-90/008, October 1990. Washington, DC.

USEPA (U.S. Environmental Protection Agency), 1990b. Quality Assurance/Quality Control Guidance for Removal Activities, Sampling QAVQC Plan and Data Validation Procedures, EPA 540/G-90/004. Washington, DC.

USEPA (U.S. Environmental Protection Agency), 1990c. Guidance for Data Usability in Risk Assessment, EPA/540/G-90/008. Office of Emergency and Remedial Response, Washington, DC.

USEPA (U.S. Environmental Protection Agency), 1991à. National Functional Guidelines for Organic Data Review, EPA/540/R-94/012, June 1991, draft. USEPA Contract Laboratory Program. Washington, DC.

USEPA (U.S. Environmental Protection Agency), 1991b. USEPA Contract Laboratory Program, Statement of Work (CLP SOW) for Organics Analysis, Multi-Media, Multi-Concentration, OLMO1.0. Washington, DC.

USEPA (U.S. Environmental Protection Agency), 1993. Data Quality Objectives Process for Superfund, EPA 540/R-93/071. Washington, DC. 
USEPA (U.S. Environmental Protection Agency), 1994a. Guidelines Establishing Test Procedures for the Analysis of Pollutants, Code of Federal Regulations, Title 40, Part 136. Washington, DC.

USEPA (U.S. Environmental Protection Agency), 1994b. Identification and Listing of Hazardous Waste, Code of Federal Regulations, Title 40, Part 261. Washington, DC.

USEPA (U.S. Environmental Protection Agency), 1994c. Requirements for Authorization of State Hazardous Waste Programs, Code of Federal Regulations, Title 40, Part 271. Washington, DC.

WHC (Westinghouse Hanford Company), not dated. Data Validation Procedures for Radiochemical Analyses, WHC-SD-EN-SPP-001, Rev. 1. Richland, WA.

WSRC (Westinghouse Savannah River Company), 1992a. 1992 Renewal Application for a RCRA Part B Permit, Volume I, Appendix 6, WSRC-IM-91-53. Savannah River Site, Aiken, SC.

WSRC (Westinghouse Savannah River Company), 1992b. Soil Boring Investigations, Hydrogeologic Data Collection Procedures and Speciications, Manual 3Q5, Rev. 1, Chapter 6. Savannah River Site, Aiken, SC.

WSRC (Westinghouse Savannah River Company), 1992c. Sampling Groundwater Monitoring Wells, Hydrogeologic Data Collection Procedures and Specifications, Manual 3Q5, Rev. 1, Chapter 15. Savannah River Site, Aiken, SC.

WSRC (Westinghouse Savannah River Company), 1992d. Sampling Soils for Analytical Purposes, Hydrogeologic Data Collection Procedures and Specifications, Manual 3Q5, Rev. 1, Chapter 16. Savannah River Site, Aiken, SC.

WSRC (Westinghouse Savannah River Company), 1992e. Approved Analytical Procedures, Hydrogeologic Data Collection Procedures and Specifications, Manual 3Q5, Rev. 1, Chapter 17. Savannah River Site, Aiken, SC.

WSRC (Westinghouse Savannah River Company), 1992f. Baseline Risk Assessment Using Existing Data for Par Pond (U), WSRC-MS-92-448X, April 1992. Savannah River Site, Aiken, SC.

WSRC (Westinghouse Savannah River Company), 1993. Savannah River Site Environmental Report for 1992, WSRC-TR-93-075. Savannah River Site, Aiken, SC.

WSRC (Westinghouse Savannah River Company), 1995a. Par Pond Sediment Characterization Sampling Plan, draft. Savannah River Site, Aiken, SC.

WSRC (Westinghouse Savannah River Company), 1995b. Procurement Specifications for Coring Services in Support of Par Pond and L Lake Characterization Studies, G-SPP-G-00061, Rev. 0, January 1995. Savannah River Site, Aiken, SC. 
This page was left blank intentionally. 


\section{Appendix A: Constituents List}

Table A-1. Soil and Water Constituents List

\begin{tabular}{|l|l|}
\hline $\begin{array}{l}\text { Target Compound List (TCL): Semivolatile organic compounds (B/N/A } \\
\text { extractables), including polycyclic aromatic hydrocarbons (PAHs) and phenols } \\
\text { with Tentatively Identified Compounds (TIC) }\end{array}$ \\
\hline Acenaphthene & Acenaphthylene \\
\hline Anthracene & Benzo[a]anthracene \\
\hline Benzo[b]fluoranthene & Benzo[k]fluoranthene \\
\hline Benzoic acid & Benzo[g,h,i]perylene \\
\hline Benzo[a]pyrene & Benzyl alcohol \\
\hline Bis(2-chloroethoxy) methane & Bis(2-chloroisopropyl) ether \\
\hline Bis(2-chloroethyl) ether & 4-Bromophenyl phenyl ether \\
\hline Bis(2-ethylhexyl) phthalate & Butylbenzyl phthalate \\
\hline 4-Chloroaniline & 4-Chloro-m-cresol \\
\hline 2-Chloronaphthalene & 2-Chlorophenol \\
\hline 4-Chlorophenyl phenyl ether & Chrysene \\
\hline m-cresol (3-Methylphenol) & o-Cresol (2-Methylphenol) \\
\hline p-Cresol (4-Methylphenol) & Dibenz[a,h]anthracene \\
\hline Dibenzofuran & Di-n-butyl phthalate \\
\hline 1,2-Dichlorobenzene & $1,3-$ Dichlorobenzene \\
\hline 1,4-Dichlorobenzene & $3,3^{\prime}$-Dichlorobenzidine \\
\hline 2,4-Dichlorophenol & Diethyl phthalate \\
\hline 2,4-Dimethyl phenol & Dimethyl phthalate \\
\hline 2,4-Dinitrophenol & 2,4-Dinitrotoluene \\
\hline 2,6-Dinitrotoluene & Di-n-octyl phthalate \\
\hline Fluoranthene & Fluorene \\
\hline Hexachlorobenzene & Hexachlorobutadiene \\
\hline Hexachlorocyclopentadiene & Hexachloroethane \\
\hline Indeno[1,2,3-c,d]pyrene & Isophorone \\
\hline 2-Methyl-4,6-dinitrophenol & 2-Methylnaphthalene \\
\hline Naphthalene & m-Nitroaniline \\
\hline o-Nitroaniline & p-Nitroaniline \\
\hline Nitrobenzene & 2-Nitrophenol \\
\hline 4-Nitrophenol & N-Nitrosodiphenylamine \\
\hline N-Nitrosodipropylamine & Pentachlorophenol \\
\hline Phenanthrene & Phenol \\
\hline Pyrene & $1,2,4-$ Trichlorobenzene \\
\hline & \\
\hline
\end{tabular}


Table A-1. Soil and Water Constituents List, Continued

\begin{tabular}{|c|c|}
\hline 2,4,5-Trichlorophenol & 2,4,6-Trichlorophenol \\
\hline \multicolumn{2}{|c|}{ TCL: Volatile organic compounds with TIC } \\
\hline Acetone & Benzene \\
\hline Bromodichloromethane & Bromoform \\
\hline Bromomethane (Methyl bromide) & Carbon disulfide \\
\hline Carbon tetrachloride & Chlorobenzene \\
\hline Chlorodibromomethane & Chloroethane \\
\hline Chloroethene (Vinyl chloride) & Chloroform \\
\hline Chloromethane (Methyl chloride) & 1,1-Dichloroethane \\
\hline 1,2-Dichloroethane & 1,1-Dichloroethylene \\
\hline 1,2-Dichloroethylene & Dichloromethane (Methylene chloride) \\
\hline 1,2-Dichloropropane & cis-1,3-Dichloropropene \\
\hline trans-1,3-Dichloropropene & Ethylbenzene \\
\hline 2-Hexanone & Methyl ethyl ketone \\
\hline Methyl isobutyl ketone & Styrene \\
\hline 1,1,2,2-Tetrachloroethane & Tetrachloroethylene \\
\hline Toluene & 1,1,1-Trichloroethane \\
\hline 1,1,2-Trichloroethane & Trichloroethylene \\
\hline Vinyl acetate & Xylenes (Total) \\
\hline \multicolumn{2}{|l|}{ TCL: Pesticides/PCBs } \\
\hline Aldrin & alpha-Benzene hexachloride \\
\hline beta-Benzene hexachloride & delta-Benzene hexachloride \\
\hline alpha-Chlordane & gamma-Chlordane \\
\hline $\mathrm{p}, \mathrm{p}^{\prime}-\mathrm{DDD}$ & $\mathrm{p}, \mathrm{p}^{\prime}-\mathrm{DDE}$ \\
\hline $\mathrm{p}, \mathrm{p}^{\prime}$-DDT & Dieldrin \\
\hline Endosulfan I & Endosulfan II \\
\hline Endosulfan sulfate & Endrin \\
\hline Endrin ketone & Heptachlor \\
\hline Heptachlor epoxide & Lindane, \\
\hline Methoxychlor & PCB 1016 \\
\hline PCB 1221 & PCB 1232 \\
\hline PCB 1242 & PCB 1248 \\
\hline PCB 1254 & PCB 1260 \\
\hline \multicolumn{2}{|l|}{ Toxaphene } \\
\hline \multicolumn{2}{|l|}{ TAL: Inorganics } \\
\hline Aluminum & Antimony \\
\hline Arsenic & Barium \\
\hline
\end{tabular}


Table A-1. Soil and Water Constituents List, Continued

\begin{tabular}{|l|l|}
\hline Beryllium & Cadmium \\
\hline Calcium & Chromium \\
\hline Cobalt & Copper \\
\hline Cyanide & Iron \\
\hline Lead & Magnesium \\
\hline Manganese & Mercury \\
\hline Nickel & Potassium \\
\hline Selenium & Silver \\
\hline Sodium & Thallium \\
\hline Vanadium & Zinc \\
\hline Radionuclide Indicators & \multicolumn{2}{|l|}{} \\
\hline Gamma PHA & Gross alpha \\
\hline Nonvolatile beta & \\
\hline Radionuclide Species & \multicolumn{2}{|l|}{} \\
\hline Plutonium-238 & Plutonium-239 \\
\hline Plutonium-240 & Strontium-90 \\
\hline Specified Analyses & \multicolumn{2}{|l|}{} \\
\hline Cation exchange capacity & Chloride \\
\hline Nitrate/Nitrite & Orthophosphate \\
\hline Particle size distribution & Phenols \\
\hline Sulfide & Total organic carbon \\
\hline Total organic nitrogen & Total phosphates (as P) \\
\hline
\end{tabular}


This page was left blank intentionally. 
Table B-1. General Engineering Laboratory Performance Evaluation, Water Pollution Study WP033

\begin{tabular}{|c|c|c|c|}
\hline Analyte & $\begin{array}{l}\text { Reported } \\
\text { Value }\end{array}$ & $\begin{array}{l}\text { True } \\
\text { Value }\end{array}$ & $\begin{array}{l}\text { Acceptance } \\
\text { Limits }\end{array}$ \\
\hline \multicolumn{4}{|c|}{ Trace Metals (mg/L) } \\
\hline \multirow[t]{2}{*}{ Aluminum } & 133 & 130 & $93.8-175$ \\
\hline & 590 & 610 & $498-719$ \\
\hline \multirow[t]{2}{*}{ Antimony } & 225 & 233 & $154-281$ \\
\hline & 648 & 660 & $467-801$ \\
\hline \multirow[t]{2}{*}{ Arsenic } & 310 & 311 & $250-374$ \\
\hline & 734 & 743 & $598-891$ \\
\hline \multirow[t]{2}{*}{ Beryllium } & 350 & 350 & 294-395 \\
\hline & 876 & 850 & $716-957$ \\
\hline \multirow[t]{2}{*}{ Cadmium } & 89.2 & 86.8 & $73.4-101$ \\
\hline & 742 & 750 & $638-870$ \\
\hline \multirow[t]{2}{*}{ Chromium } & 44.0 & 43.5 & $35.2-51.1$ \\
\hline & 530 & 529 & $454-608$ \\
\hline \multirow[t]{2}{*}{ Cobalt } & 536 & 530 & $468-577$ \\
\hline & 925 & 931 & $827-1,030$ \\
\hline \multirow[t]{2}{*}{ Copper } & 33.3 & 33.6 & $28.1-38.3$ \\
\hline & 175 & 187 & $164-208$ \\
\hline \multirow[t]{2}{*}{ Iron } & 64.6 & 64.5 & $48.6-81.6$ \\
\hline & 1,290 & 1,300 & $1,150-1,450$ \\
\hline \multirow[t]{2}{*}{ Lead } & 581 & 645 & $546-721$ \\
\hline & $2,570^{b}$ & 2,900 & $2,540-3,240$ \\
\hline \multirow[t]{2}{*}{ Manganese } & 179 & 177 & $158-195$ \\
\hline & $4,230^{b}$ & 3,900 & $3,520-4,280$ \\
\hline \multirow[t]{2}{*}{ Mercury } & 5.62 & 5.81 & $4.04-7.33$ \\
\hline & 27.6 & 27.1 & $20.6-32.5$ \\
\hline \multirow[t]{2}{*}{ Molybdenum } & 107 & 106 & $84.9-128$ \\
\hline & 385 & 380 & $308-455$ \\
\hline \multirow[t]{2}{*}{ Nickel } & 270 & 265 & $234-295$ \\
\hline & 1,070 & 1,080 & $959-1,200$ \\
\hline \multirow[t]{2}{*}{ Selenium } & 602 & 625 & $422-767$ \\
\hline & 1,320 & 1,380 & $933-1,690$ \\
\hline
\end{tabular}


Table B-1. General Engineering Laboratory Performance Evaluation, Water Pollution Study WP033, Continued

\begin{tabular}{|c|c|c|c|}
\hline Analyte & $\begin{array}{l}\text { Reported } \\
\text { Value }\end{array}$ & $\begin{array}{l}\text { True } \\
\text { Value }\end{array}$ & $\begin{array}{l}\text { Acceptance } \\
\text { Limits }\end{array}$ \\
\hline \multirow[t]{2}{*}{ Silver } & 86.1 & 84.0 & $70.0-98.1$ \\
\hline & 423 & 410 & $342-479$ \\
\hline \multirow[t]{2}{*}{ Strontium } & 16.2 & 15.9 & $13.0-18.5$ \\
\hline & 195 & 193 & $162-220$ \\
\hline \multirow[t]{2}{*}{ Thallium } & 183 & 170 & $131-197$ \\
\hline & 744 & 680 & $535-776$ \\
\hline \multirow[t]{2}{*}{ Titanium } & 69.3 & 72.1 & $59.3-83.6$ \\
\hline & 190 & 192 & $160-220$ \\
\hline \multirow[t]{2}{*}{ Vanadium } & 416 & 415 & $364-461$ \\
\hline & 1,230 & 1,220 & $1,070-1,350$ \\
\hline \multirow[t]{2}{*}{ Zinc } & 34.1 & 31.9 & $23.9-40.3$ \\
\hline & 706 & 726 & $640-813$ \\
\hline \multicolumn{4}{|l|}{ Minerals (mg/L, except as noted) } \\
\hline \multirow[t]{2}{*}{ Alkalinity (as $\mathrm{CaCO}_{3}$ ), total } & 28.2 & 28.5 & $24.2-33.1$ \\
\hline & 81.0 & 86.4 & $76.2-95.0$ \\
\hline \multirow[t]{2}{*}{ Calcium } & 12.6 & 12.0 & $10.7-13.6$ \\
\hline & 92.3 & 89.0 & 77.7--98.1 \\
\hline \multirow[t]{2}{*}{ Chloride } & 58.3 & 59.2 & $53.5-64.1$ \\
\hline & 164 & 170 & $155-182$ \\
\hline \multirow[t]{2}{*}{ Fluoride } & $0.293^{b}$ & 0.240 & $0.168-0.310$ \\
\hline & $4.38^{b}$ & 4.00 & $3.50-4.46$ \\
\hline \multirow[t]{2}{*}{ Hardness (as $\left.\mathrm{CaCO}_{3}\right)$, total } & 88.4 & 83.5 & $74.2-91.0$ \\
\hline & 248 & 239 & $216-259$ \\
\hline \multirow[t]{2}{*}{ Magnesium } & 13.8 & 13.0 & $11.4-14.9$ \\
\hline & 4.30 & 4.10 & $3.57-4.74$ \\
\hline \multirow[t]{2}{*}{ pH (pH units) } & $5: 28$ & 5.26 & $5.21-5.36$ \\
\hline & 4.08 & 4.11 & $4.02-4.16$ \\
\hline \multirow[t]{2}{*}{ Potassium } & 14.7 & 14.0 & $12.1-16.0$ \\
\hline & 5.56 & 5.20 & $4.24-6.23$ \\
\hline \multirow[t]{2}{*}{ Sodium } & 16.3 & 15.7 & $13.7-18.2$ \\
\hline & 94.5 & 94.2 & $85.7-101$ \\
\hline \multirow[t]{2}{*}{ Specific conductance $\left(\mu \mathrm{S} / \mathrm{cm}\right.$ at $\left.25^{\circ} \mathrm{C}\right)$} & 316 & 309 & $284-337$ \\
\hline & 964 & 937 & $873-1,020$ \\
\hline
\end{tabular}

Par Pond, Pond C, and L Lake, Revision 2 
Table B-1. General Engineering Laboratory Performance Evaluation, Water Pollution Study WP033, Continued

\begin{tabular}{|c|c|c|c|}
\hline Analyte & $\begin{array}{l}\text { Reported } \\
\text { Value }\end{array}$ & $\begin{array}{l}\text { True } \\
\text { Value }^{\mathrm{a}}\end{array}$ & $\begin{array}{l}\text { Acceptance } \\
\text { Limits }\end{array}$ \\
\hline \multirow[t]{2}{*}{ Sulfate } & 21.0 & 22.0 & $17.6-25.4$ \\
\hline & 103 & 110 & $88.6-127$ \\
\hline \multirow[t]{2}{*}{ Total dissolved solids at $180^{\circ} \mathrm{C}$} & 165 & 184 & $124-200$ \\
\hline & 545 & 561 & $441-701$ \\
\hline \multicolumn{4}{|l|}{ Nutrients (mg/L) } \\
\hline \multirow[t]{2}{*}{ Ammonia-nitrogen } & 5.56 & 6.30 & $4.98-7.50$ \\
\hline & 0.46 & 0.55 & $0.346-0.772$ \\
\hline \multirow[t]{2}{*}{ Kjeldahl-nitrogen } & 1.82 & 1.60 & $0.863-2.38$ \\
\hline & 12.3 & 12.0 & $8.84-14.7$ \\
\hline \multirow[t]{2}{*}{ Nitrate as nitrogen } & 0.870 & 0.860 & $0.654-1.05$ \\
\hline & $0.407^{c}$ & 0.270 & $0.177-0.362$ \\
\hline \multirow[t]{2}{*}{ Orthophosphate } & $4.32^{b}$ & 5.00 & $4.28-5.67$ \\
\hline & 0.109 & 0.120 & $0.0883-0.152$ \\
\hline \multirow[t]{2}{*}{ Total phosphorus } & 0.190 & 0.250 & $0.137-0.328$ \\
\hline & 4.44 & 5.40 & $3.98-6.40$ \\
\hline \multicolumn{4}{|l|}{ Demands (mg/L) } \\
\hline \multirow[t]{2}{*}{ 5-day Biochemical oxygen demand } & 9.90 & 12.1 & $5.36-18.8$ \\
\hline & 46.5 & 54.7 & $26.0-83.5$ \\
\hline \multirow[t]{2}{*}{ Chemical oxygen demand } & 18.4 & 19.5 & $9.75-28.9$ \\
\hline & 85.0 & 88.6 & $66.8-103$ \\
\hline \multirow[t]{2}{*}{ Total organic carbon } & 8.05 & 7.70 & $6.08-9.23$ \\
\hline & 36.6 & 35.0 & $27.3-41.5$ \\
\hline \multicolumn{4}{|l|}{ PCBs (mg/L) } \\
\hline PCB (Aroclor) 1232 & 3.11 & 3.67 & $0.717-6.07$ \\
\hline PCB (Aroclor) 1254 & 4.58 & 5.40 & $2.57-7.06$ \\
\hline \multicolumn{4}{|l|}{ PCBs in Oil (mg/kg) } \\
\hline PCB 1016/1242 & 19.6 & 24.6 & $4.00-35.9$ \\
\hline PCB 1254 & 30.4 & 38.4 & $9.07-51.1$ \\
\hline
\end{tabular}


Table B-1. General Engineering Laboratory Performance Evaluation, Water Pollution Study WP033, Continued

\begin{tabular}{llll}
\hline Analyte & $\begin{array}{l}\text { Reported } \\
\text { Value }\end{array}$ & $\begin{array}{l}\text { True } \\
\text { Value }^{\mathrm{a}}\end{array}$ & $\begin{array}{l}\text { Acceptance } \\
\text { Limits }\end{array}$ \\
\hline Pesticides (mg/L) & & & 1 \\
Aldrin & 0.252 & 0.286 & $0.0663-0.378$ \\
Chlordane & 1.86 & 2.16 & $0.653-2.89$ \\
p,p'-DDD & 5.82 & 7.41 & $3.31-10.5$ \\
& 2.37 & 2.54 & $1.50-3.18$ \\
p,p'-DDE & 1.37 & 1.52 & $0.611-2.29$ \\
p,p'-DDT & 2.63 & 2.68 & $1.31-3.72$ \\
& 1.10 & 1.37 & $0.612-1.89$ \\
Dieldrin & 2.67 & 3.14 & $1.63-4.16$ \\
& 1.46 & 1.66 & $0.738-2.31$ \\
Heptachlor & 3.33 & 3.55 & $1.87-4.77$ \\
& 1.03 & 1.24 & $0.643-1.70$ \\
Heptachlor epoxide & 2.55 & 2.87 & $1.49-3.94$ \\
& 0.287 & 0.330 & $0.067-0.466$ \\
& 1.76 & 1.87 & $0.543-2.54$ \\
& 0.271 & 0.316 & $0.169-0.401$ \\
& 1.61 & 1.72 & $0.945-2.28$
\end{tabular}

Volatile Halocarbons (mg/L)

Bromodichloromethane

16.7

58.8

Bromoform

19.2

52.9

Carbon tetrachloride

Chlorobenzene

Chloroform

Dibromochloromethane

1,2-Dichloroethane
12.2

54.1

18.6

48.9

13.4

47.9

18.0

63.5

19.0

45.9
16.2

58.3

18.7

48.5

11.7

53.6

18.3

48.3

12.6

47.3

17.3

62.0

17.0

42.8
11.3-21.6

41.1-78.0

11.1-26.0

30.0-67.9

8.52-15.3

34.2-77.2

13.6-22.7

35.4-60.0

$8.92-16.2$

31.1-62.4

11.7-22.6

41.3-82.7

12.5-21.8

29.8-55.7 
Table B-1. General Engineering Laboratory Performance Evaluation, Water Pollution Study WP033, Continued

\begin{tabular}{llll}
\hline Analyte & $\begin{array}{l}\text { Reported } \\
\text { Value }\end{array}$ & $\begin{array}{l}\text { True } \\
\text { Value }\end{array}$ & $\begin{array}{l}\text { Acceptan } \\
\text { Limits }\end{array}$ \\
\hline Dichloromethane & 18.3 & 18.1 & $11.1-25.9$ \\
Tetrachloroethylene & 65.0 & 63.2 & $38.7-89.2$ \\
1,1,1-Trichloroethane & 12.5 & 12.4 & $8.72-16.0$ \\
& 59.9 & 63.5 & $42.1-80.7$ \\
Trichloroethylene & 15.6 & 15.7 & $10.3-20.3$ \\
& 54.7 & 56.3 & $35.1-74.7$ \\
& 17.6 & 17.6 & $12.2-22.5$ \\
& 46.5 & 48.0 & $30.8-63.6$
\end{tabular}

Volatile Aromatics (mg/L)

Benzene

67.7

69.6

48.4-92.4

1,2-Dichlorobenzene

18.1

18.3

$12.6-24.9$

52.6

55.7

37.0-73.0

16.7

16.3

11.1-21.0

1,3-Dichlorobenzene

51.6

53.8

$36.5-65.3$

18.3

17.3

12.2-22.2

1,4-Dichlorobenzene

60.7

64.0

$41.6-84.6$

15.5

14.7

9.74-19.7

Ethylbenzene

70.5

72.5

47.2-94.5

13.2

12.5

8.14-16.9

Toluene

54.4

56.5

$40.5-71.2$

16.3

15.6

$11.1-19.8$

Miscellaneous Parameters (mg/L)

Cyanide, total

0.509

0.490

$0.348-0.622$

0.214

0.210

$0.139-0.271$

Nonfilterable residue

61.9

72.0

54.6-79.6

Oil and grease

95.5

100

76.2-107

38.5

43.0

$25.1-50.6$

12.7

14.0

7.79-17.9

Phenolics, total

1.10

1.48

$0.868-2.10$

0.715

0.913

$0.540-1.29$ 
Table B-1. General Engineering Laboratory Performance Evaluation, Water Pollution Study WP033, Continued

\begin{tabular}{llll}
\hline Analyte & $\begin{array}{l}\text { Reported } \\
\text { Value }\end{array}$ & $\begin{array}{l}\text { True } \\
\text { Value }\end{array}$ & $\begin{array}{l}\text { Acceptance } \\
\text { Limits }\end{array}$ \\
\hline Residual chlorine, total & & & 0.839 \\
& 0.868 & 4.30 & $0.632-0.996$ \\
& 4.66 & $3.50-5.00$ \\
\hline
\end{tabular}

a Based on gravimetric calculations or a reference value when necessary.

b Acceptable, but near limits. The laboratory was warned to check for error.

c Not acceptable. 
Table B-2. Roy F. Weston Analytical Laboratory Performance Evaluation, Water Pollution Study WP033

\begin{tabular}{|c|c|c|c|}
\hline Analyte & $\begin{array}{l}\text { Reported } \\
\text { Value } \\
\end{array}$ & $\begin{array}{l}\text { True } \\
\text { Value }^{\mathrm{a}}\end{array}$ & $\begin{array}{l}\text { Acceptance } \\
\text { Limits }\end{array}$ \\
\hline \multicolumn{4}{|c|}{ Trace Metals (mg/L) } \\
\hline \multirow[t]{2}{*}{ Aluminum } & 123 & 130 & 93.8-175 \\
\hline & 639 & 610 & $498-719$ \\
\hline \multirow[t]{2}{*}{ Antimony } & 242 & 233 & $154-281$ \\
\hline & 711 & 660 & $467-801$ \\
\hline \multirow[t]{2}{*}{ Arsenic } & 280 & 311 & $250-374$ \\
\hline & 821 & 743 & $598-891$ \\
\hline \multirow[t]{2}{*}{ Beryllium } & 336 & 350 & 294-395 \\
\hline & 823 & 850 & $716-957$ \\
\hline \multirow[t]{2}{*}{ Cadmium } & 95.1 & 86.8 & $73.4-101$ \\
\hline & 805 & 750 & $638-870$ \\
\hline \multirow[t]{2}{*}{ Chromium } & 45.2 & 43.5 & $35.2-51.1$ \\
\hline & 546 & 529 & $454-608$ \\
\hline \multirow[t]{2}{*}{ Cobalt } & 554 & 530 & $468-577$ \\
\hline & 970 & 931 & $827-1,030$ \\
\hline \multirow[t]{2}{*}{ Copper } & 36.1 & 33.6 & $28.1-38.3$ \\
\hline & 195 & 187 & $164-208$ \\
\hline \multirow[t]{2}{*}{ Iron } & 65.0 & 64.5 & $48.6-81.6$ \\
\hline & 1,309 & 1,300 & $1,150-1,450$ \\
\hline \multirow[t]{2}{*}{ Lead } & $720^{b}$ & 645 & $546-721$ \\
\hline & 3,130 & 2,900 & $2,540-3,240$ \\
\hline \multirow[t]{2}{*}{ Manganese } & 184 & 177 & $158-195$ \\
\hline & 4,091 & 3,900 & $3,520-4,280$ \\
\hline \multirow[t]{2}{*}{ Mercury } & 5.58 & 5.81 & $4.04-7.33$ \\
\hline & 26.1 & 27.1 & $20.6-32.5$ \\
\hline \multirow[t]{2}{*}{ Molybdenum } & 115 & 106 & $84.9-128$ \\
\hline & 414 & 380 & $308-455$ \\
\hline \multirow[t]{2}{*}{ Nickel } & 283 & 265 & $234-295$ \\
\hline & 1,148 & 1,080 & $959-1,200$ \\
\hline \multirow[t]{2}{*}{ Selenium } & $776^{c}$ & 625 & $422-767$ \\
\hline & 1,455 & 1,380 & $933-1,690$ \\
\hline \multirow[t]{2}{*}{ Silver } & 90.3 & 84.0 & $70.0-98.1$ \\
\hline & 443 & 410 & $342-479$ \\
\hline
\end{tabular}


Table B-2. Roy F. Weston Analytical Laboratory Performance Evaluation, Water Pollution Study WP033, Continued

\begin{tabular}{|c|c|c|c|}
\hline Analyte & $\begin{array}{l}\text { Reported } \\
\text { Value } \\
\end{array}$ & $\begin{array}{l}\text { True } \\
\text { Value }^{\mathbf{a}}\end{array}$ & $\begin{array}{l}\text { Acceptance } \\
\text { Limits }\end{array}$ \\
\hline \multirow[t]{2}{*}{ Strontium } & 16.6 & 15.9 & $13.0-18.5$ \\
\hline & 205 & 193 & $162-220$ \\
\hline \multirow[t]{2}{*}{ Thallium } & 157 & 170 & $131-197$ \\
\hline & 669 & 680 & $535-776$ \\
\hline \multirow[t]{2}{*}{ Titanium } & 74.7 & 72.1 & $59.3-83.6$ \\
\hline & 200 & 192 & $160-220$ \\
\hline \multirow[t]{2}{*}{ Vanadium } & 438 & 415 & $364-461$ \\
\hline & 1,276 & 1,220 & $1,070-1,350$ \\
\hline \multirow[t]{2}{*}{ Zinc } & 36.0 & 31.9 & $23.9-40.3$ \\
\hline & 773 & 726 & $640-813$ \\
\hline \multicolumn{4}{|l|}{ Minerals (mg/L, except as noted) } \\
\hline \multirow[t]{2}{*}{ Alkalinity (as $\left.\mathrm{CaCO}_{3}\right)$, total } & 28.0 & 28.5 & $24.2-33.1$ \\
\hline & $76.0^{c}$ & 86.4 & $76.9-95.0$ \\
\hline \multirow[t]{2}{*}{ Calcium } & $14.2^{\mathrm{c}}$ & 12.0 & $10.7-13.6$ \\
\hline & $112.9^{c}$ & 89.0 & $77.7-98.1$ \\
\hline \multirow[t]{2}{*}{ Chloride } & $64.1^{\mathrm{b}}$ & 59.2 & $53.5-64.1$ \\
\hline & $190^{c}$ & 170 & $155-182$ \\
\hline \multirow[t]{2}{*}{ Fluoride } & 0.227 & 0.240 & $0.168-0.310$ \\
\hline & 4.08 & 4.00 & $3.50-4.46$ \\
\hline \multirow[t]{2}{*}{ Hardness (as $\mathrm{CaCO}_{3}$ ), total } & 83.3 & 83.5 & $74.2-91.0$ \\
\hline & 229 & 239 & $216-259$ \\
\hline \multirow[t]{2}{*}{ Magnesium } & $15.2^{\mathrm{c}}$ & 13.0 & $11.4-14.9$ \\
\hline & $5.32^{\mathrm{C}}$ & 4.10 & $3.57-4.74$ \\
\hline \multirow[t]{2}{*}{$\mathrm{pH}$ (pH units) } & $5.43^{c}$ & 5.26 & $5.21-5.36$ \\
\hline & 4.11 & 4.11 & $4.02-4.16$ \\
\hline \multirow[t]{2}{*}{ Potassium } & $15.9^{b}$ & 14.0 & $12.1-16.0$ \\
\hline & 5.65 & 5.20 & $4.24-6.23$ \\
\hline \multirow[t]{2}{*}{ Sodium } & $18.1^{b}$ & 15.7 & $13.7-18.2$ \\
\hline & $107.7^{\mathrm{c}}$ & 94.2 & $85.7-101$ \\
\hline \multirow[t]{2}{*}{ Specific conductance $\left(\mu \mathrm{S} / \mathrm{cm}\right.$ at $\left.25^{\circ} \mathrm{C}\right)$} & $285^{\mathrm{b}}$ & 309 & $284-337$ \\
\hline & $870^{c}$ & 937 & $873-1,020$ \\
\hline \multirow[t]{2}{*}{ Sulfate } & 21.0 & 22.0 & $17.6-25.4$ \\
\hline & $91.1^{\mathrm{b}}$ & 110 & $88.6-127$ \\
\hline
\end{tabular}


Table B-2. Roy F. Weston Analytical Laboratory Performance Evaluation, Water Pollution Study WP033, Continued

\begin{tabular}{|c|c|c|c|}
\hline Analyte & $\begin{array}{l}\text { Reported } \\
\text { Value } \\
\end{array}$ & $\begin{array}{l}\text { True } \\
\text { Value }^{a}\end{array}$ & $\begin{array}{l}\text { Acceptance } \\
\text { Limits } \\
\end{array}$ \\
\hline \multirow[t]{2}{*}{ Total dissolved solids at $180^{\circ} \mathrm{C}$} & 161 & 184 & $124-200$ \\
\hline & 568 & 561 & $441-701$ \\
\hline \multicolumn{4}{|l|}{ Nutrients (mg/L) } \\
\hline \multirow[t]{2}{*}{ Ammonia-nitrogen } & 6.44 & 6.30 & $4.98-7.50$ \\
\hline & 0.564 & 0.550 & $0.346-0.772$ \\
\hline \multirow[t]{2}{*}{ Kjeldahl-nitrogen } & 1.63 & 1.60 & $0.863-2.38$ \\
\hline & 11.4 & 12.0 & $8.84-14.7$ \\
\hline \multirow[t]{2}{*}{ Nitrate as nitrogen } & 0.851 & 0.860 & $0.654-1.05$ \\
\hline & 0.265 & 0.270 & $0.177-0.362$ \\
\hline \multirow[t]{2}{*}{ Orthophosphate } & 5.45 & 5.00 & $4.28-5.67$ \\
\hline & 0.117 & 0.120 & $0.0883-0.152$ \\
\hline \multirow[t]{2}{*}{ Total phosphorus } & 0.219 & 0.250 & $0.137-0.328$ \\
\hline & 4.94 & 5.40 & $3.98-6.40$ \\
\hline \multicolumn{4}{|l|}{ Demands (mg/L) } \\
\hline \multirow[t]{2}{*}{ 5-day Biochemical oxygen demand } & 14.0 & 12.1 & $5.36-18.8$ \\
\hline & 45.0 & 54.7 & $26.0-83.5$ \\
\hline \multirow[t]{2}{*}{ Chemical oxygen demand } & 22.0 & 19.5 & $9.75-28.9$ \\
\hline & 94.8 & 88.6 & $66.8-103$ \\
\hline \multirow[t]{2}{*}{ Total organic carbon } & 7.80 & 7.70 & $6.08-9.23$ \\
\hline & 35.8 & 35.0 & $27.3-41.5$ \\
\hline \multicolumn{4}{|l|}{ PCBs (mg/L) } \\
\hline PCB (Aroclor) 1232 & 4.00 & 3.67 & $0.717-6.07$ \\
\hline PCB (Aroclor) 1254 & 4.70 & 5.40 & $2.57-7.06$ \\
\hline \multicolumn{4}{|l|}{ PCBs in Oil (mg/kg) } \\
\hline PCB 1016/1242 & 18.0 & 24.6 & $4.00-35.9$ \\
\hline PCB 1254 & 29.0 & 38.4 & $9.07-51.1$ \\
\hline \multicolumn{4}{|l|}{ Pesticides (mg/L) } \\
\hline \multirow[t]{2}{*}{ Aldrin } & 0.220 & 0.286 & $0.0663-0.378$ \\
\hline & 1.46 & 2.16 & $0.653-2.89$ \\
\hline
\end{tabular}


Table B-2. Roy F. Weston Analytical Laboratory Performance Evaluation, Water Pollution Study WP033, Continued

\begin{tabular}{|c|c|c|c|}
\hline Analyte & $\begin{array}{l}\text { Reported } \\
\text { Value } \\
\end{array}$ & $\begin{array}{l}\text { True } \\
\text { Value } \\
\end{array}$ & $\begin{array}{l}\text { Acceptance } \\
\text { Limits } \\
\end{array}$ \\
\hline \multirow[t]{2}{*}{ Chlordane } & 6.61 & 7.41 & $3.31-10.5$ \\
\hline & 2.72 & 2.54 & $1.50-3.18$ \\
\hline \multirow[t]{2}{*}{$p, p^{\prime}-D D D$} & 1.26 & 1.52 & $0.611-2.29$ \\
\hline & 2.21 & 2.68 & $1.31-3.72$ \\
\hline \multirow[t]{2}{*}{$\mathrm{p}, \mathrm{p}^{\prime}-\mathrm{DDE}$} & 1.16 & 1.37 & $0.612-1.89$ \\
\hline & 2.36 & 3.14 & $1.63-4.16$ \\
\hline \multirow{2}{*}{$\mathrm{p}, \mathrm{p}^{\prime}-\mathrm{DDT}$} & 1.32 & 1.66 & $0.738-2.31$ \\
\hline & 2.82 & 3.55 & $1.87-4.77$ \\
\hline \multirow[t]{2}{*}{ Dieldrin } & 1.04 & 1.24 & $0.643-1.70$ \\
\hline & 2.15 & 2.87 & $1.49-3.94$ \\
\hline \multirow[t]{2}{*}{ Heptachlor } & 0.280 & 0.330 & $0.067-0.466$ \\
\hline & 1.42 & 1.87 & $0.543-2.54$ \\
\hline \multirow[t]{2}{*}{ Heptachlor epoxide } & 0.270 & 0.316 & $0.169-0.401$ \\
\hline & 1.38 & 1.72 & $0.945-2.28$ \\
\hline \multicolumn{4}{|c|}{ Volatile Halocarbons (mg/L) } \\
\hline \multirow[t]{2}{*}{ Bromodichloromethane } & 19.9 & 16.2 & $11.3-21.6$ \\
\hline & 71.2 & 58.3 & $41.1-78.0$ \\
\hline \multirow[t]{2}{*}{ Bromoform } & 23.7 & 18.7 & $11.1-26.0$ \\
\hline & $65.2^{b}$ & 48.5 & $30.0-67.9$ \\
\hline \multirow[t]{2}{*}{ Carbon tetrachloride } & 11.9 & 11.7 & $8.52-15.3$ \\
\hline & 55.8 & 53.6 & $34.2-77.2$ \\
\hline \multirow[t]{2}{*}{ Chlorobenzene } & 19.3 & 18.3 & $13.6-22.7$ \\
\hline & 51.9 & 48.3 & $35.4-60.0$ \\
\hline \multirow[t]{2}{*}{ Chloroform } & 12.2 & 12.6 & $8.92-16.2$ \\
\hline & 47.6 & 47.3 & $31.1-62.4$ \\
\hline \multirow[t]{2}{*}{ Dibromochloromethane } & $23.1^{\mathrm{c}}$ & 17.3 & $11.7-22.6$ \\
\hline & $84.0^{c}$ & 62.0 & $41.3-82.7$ \\
\hline \multirow[t]{2}{*}{ 1,2-Dichloroethane } & 17.5 & 17.0 & $12.5-21.8$ \\
\hline & 44.6 & 42.8 & $29.8-55.7$ \\
\hline \multirow[t]{2}{*}{ Dichloromethane } & 19.2 & 18.1 & $11.1-25.9$ \\
\hline & 67.8 & 63.2 & $38.7-89.2$ \\
\hline \multirow[t]{2}{*}{ Tetrachloroethylene } & 12.9 & 12.4 & $8.72-16.0$ \\
\hline & 67.1 & 63.5 & $42.1-80.7$ \\
\hline 1,1,1-Trichloroethane & 15.4 & 15.7 & $10.3-20.3$ \\
\hline
\end{tabular}


Table B-2. Roy F. Weston Analytical Laboratory Performance Evaluation, Water Pollution Study WP033, Continued

\begin{tabular}{|c|c|c|c|}
\hline Analyte & $\begin{array}{l}\text { Reported } \\
\text { Value } \\
\end{array}$ & $\begin{array}{l}\text { True } \\
\text { Value } \\
\end{array}$ & $\begin{array}{l}\text { Acceptance } \\
\text { Limits }\end{array}$ \\
\hline \multirow{3}{*}{ Trichloroethylene } & 55.8 & 56.3 & $35.1-74.7$ \\
\hline & 17.5 & 17.6 & $12.2-22.5$ \\
\hline & 48.8 & 48.0 & $30.8-63.6$ \\
\hline \multicolumn{4}{|c|}{ Volatile Aromatics (mg/L) } \\
\hline \multirow[t]{2}{*}{ Benzene } & 71.5 & 69.6 & $48.4-92.4$ \\
\hline & 17.2 & 18.3 & $12.6-24.9$ \\
\hline \multirow[t]{2}{*}{ 1,2-Dichlorobenzene } & $71.2^{\mathrm{b}}$ & 55.7 & $37.0-73.0$ \\
\hline & 19.1 & 16.3 & $11.1-21.0$ \\
\hline \multirow[t]{2}{*}{ 1,3-Dichlorobenzene } & 56.7 & 53.8 & $36.5-65.3$ \\
\hline & 16.9 & 17.3 & $12.2-22.2$ \\
\hline \multirow[t]{2}{*}{ 1,4-Dichlorobenzene } & 71.1 & 64.0 & $41.6-84.6$ \\
\hline & 15.2 & 14.7 & $9.74-19.7$ \\
\hline \multirow[t]{2}{*}{ Ethylbenzene } & 77.5 & 72.5 & $47.2-94.5$ \\
\hline & 12.6 & 12.5 & $8.14-16.9$ \\
\hline \multirow[t]{2}{*}{ Toluene } & 62.1 & 56.5 & $40.5-71.2$ \\
\hline & 15.3 & 15.6 & $11.1-19.8$ \\
\hline \multicolumn{4}{|c|}{ Miscellaneous Parameters (mg/L) } \\
\hline \multirow[t]{2}{*}{ Cyanide, total } & 0.483 & 0.490 & $0.348-0.622$ \\
\hline & 0.209 & 0.210 & $0.139-0.271$ \\
\hline \multirow[t]{2}{*}{ Nonfilterable residue } & 64.0 & 72.0 & $54.6-79.6$ \\
\hline & 88.0 & 100 & $76.2-107$ \\
\hline \multirow[t]{2}{*}{ Oil and grease } & $187 \mathrm{c}$ & 43.0 & $25.1-50.6$ \\
\hline & $199 c$ & 14.0 & $7.79-17.9$ \\
\hline \multirow[t]{2}{*}{ Phenolics, total } & 1.89 & 1.48 & $0.868-2.10$ \\
\hline & $1.21^{b}$ & 0.913 & $0.540-1.29$ \\
\hline \multirow[t]{2}{*}{ Residual chlorine, total } & $0.980^{\mathrm{b}}$ & 0.839 & $0.632-0.996$ \\
\hline & $2.90^{c}$ & 4.30 & $3.50-5.00$ \\
\hline
\end{tabular}

a Based on gravimetric calculations or a reference value when necessary.

b Acceptable, but near limits. The laboratory was warned to check for error.

c Not acceptable. 
Table B-3. General Engineering Laboratory Performance Evaluation, Water Supply Study WS035

\begin{tabular}{|c|c|c|c|}
\hline Analyte & $\begin{array}{l}\text { Reported } \\
\text { Value } \\
\end{array}$ & $\begin{array}{l}\text { True } \\
\text { Value } \\
\end{array}$ & $\begin{array}{l}\text { Acceptance } \\
\text { Limits } \\
\end{array}$ \\
\hline \multicolumn{4}{|l|}{ Insecticides (mg/L) } \\
\hline Aldrin & 0.930 & 1.23 & $0.479-1.61$ \\
\hline Chlordane & $23.4^{\mathrm{b}}$ & 13.6 & $7.48-19.7$ \\
\hline Dieldrin & 0.804 & 0.857 & $0.605-1.07$ \\
\hline Endrin & 0.545 & 0.649 & $0.454-0.844$ \\
\hline Heptachlor & 2.22 & 2.54 & $1.40-3.68$ \\
\hline Heptachlor epoxide & 1.27 & 1.49 & $0.820-2.16$ \\
\hline Lindane & 0.486 & 0.543 & $0.299-0.787$ \\
\hline Methoxychlor & $25.7^{\mathrm{b}}$ & 62.6 & $34.4-90.8$ \\
\hline Toxaphene & 14.1 & 18.3 & $10.1-26.5$ \\
\hline \multicolumn{4}{|l|}{ Herbicides (mg/L) } \\
\hline $2,4-\mathrm{D}$ & 56.3 & 52.4 & $26.2-78.6$ \\
\hline 2,4,5-TP (Silvex) & 18.6 & 17.6 & $8.80-26.4$ \\
\hline \multicolumn{4}{|l|}{ Trihalomethanes (mg/L) } \\
\hline Bromodichloromethane & 12.6 & 13.8 & $11.0-16.6$ \\
\hline Bromoform & 16.8 & 16.4 & $13.1-19.7$ \\
\hline Chlorodibromomethane & 18.9 & 18.6 & $14.9-22.3$ \\
\hline Chloroform & 11.8 & 12.0 & $9.60-14.4$ \\
\hline Trihalomethane, total & 60.1 & 60.8 & $48.6-73.0$ \\
\hline \multicolumn{4}{|c|}{ Volatile Organic Compounds (mg/L) } \\
\hline Benzene & 14.1 & 14.0 & $11.2-16.8$ \\
\hline Carbon tetrachloride & 10.9 & 10.8 & $8.64-13.0$ \\
\hline Chlorobenzene & 12.1 & 12.4 & $9.92-14.9$ \\
\hline 2-Chlorotoluene & 15.0 & 13.3 & $10.2-16.0$ \\
\hline 1,2-Dichlorobenzene & 13.4 & 12.5 & $10.0-15.0$ \\
\hline 1,4-Dichlorobenzene & 18.4 & 16.7 & $13.4-20.0$ \\
\hline 1,2-Dichloroethane & 15.0 & 14.1 & $11.3-16.9$ \\
\hline 1,1-Dichloroethylene & 15.1 & 13.9 & $11.1-16.7$ \\
\hline cis-1,2-Dichloroethylene & 5.83 & 6.69 & $4.01-9.37$ \\
\hline trans-1,2-Dichloroethylene & 8.91 & 8.41 & $5.05-11.8$ \\
\hline Dichloromethane & 6.39 & 5.83 & $3.50-8.16$ \\
\hline
\end{tabular}


Table B-3. General Engineering Laboratory Performance Evaluation, Water Supply Study WS035, Continued

\begin{tabular}{|c|c|c|c|}
\hline Analyte & $\begin{array}{l}\text { Reported } \\
\text { Value }\end{array}$ & $\begin{array}{l}\text { True } \\
\text { Value }^{\mathrm{a}} \\
\end{array}$ & $\begin{array}{l}\text { Acceptance } \\
\text { Limits }\end{array}$ \\
\hline 1,2-Dichloropropane & 7.66 & 9.00 & $5.40-12.6$ \\
\hline 1,3-Dichloropropane & 12.1 & 11.8 & $9.43-14.1$ \\
\hline cis-1,3-Dichloropropene & 18.5 & 23.4 & $18.8-25.0$ \\
\hline trans-1,3-Dichloropropene & 7.30 & 10.5 & $6.80-10.9$ \\
\hline Ethylbenzene & 13.9 & 13.6 & $10.9-16.3$ \\
\hline Hexachlorobutadiene & 13.9 & 12.6 & $7.14-17.1$ \\
\hline Styrene & 16.8 & 16.7 & $13.4-20.0$ \\
\hline 1,1,1,2-Tetrachloroethane & 16.6 & 17.0 & $13.0-20.6$ \\
\hline Tetrachloroethylene & 11.8 & 11.6 & $9.28-13.9$ \\
\hline Toluene & 9.69 & 9.92 & $5.95-13.9$ \\
\hline 1,2,4-Trichlorobenzene & 19.0 & 18.4 & $14.7-22.1$ \\
\hline 1,1,1-Trichloroethane & 8.37 & 8.78 & $5.27-12.3$ \\
\hline 1,1,2-Trichloroethane & 13.0 & 12.8 & $10.2-15.4$ \\
\hline Trichloroethylene & 6.41 & 6.13 & $3.68-8.58$ \\
\hline 1,2,3-Trichloropropane & 14.8 & 15.5 & $7.62-21.6$ \\
\hline Vinyl chiloride & 5.42 & 4.91 & $2.95-6.87$ \\
\hline Xylenes, total & 18.2 & 17.4 & $13.9-20.9$ \\
\hline \multicolumn{4}{|c|}{ Miscellaneous Analytes (mg/L, except as noted) } \\
\hline Alkalinity (as $\mathrm{CaCO}_{3}$ ) & 47.8 & 48.3 & $44.9-53.7$ \\
\hline Calcium (as $\mathrm{CaCO}_{3}$ ) & 121 & 118 & $110-128$ \\
\hline Corrosivity (Langelier Ind. at $20^{\circ} \mathrm{C}$ ) & 0.635 & 0.940 & $0.616-1.23$ \\
\hline Cyanide, total & 0.515 & 0.480 & $0.360-0.600$ \\
\hline Filterable residue, total & 249 & 306 & $210-454$ \\
\hline $\mathrm{pH}$ (pH units) & 9.08 & 9.13 & $8.89-9.35$ \\
\hline Residual-free chlorine & 3.09 & 3.00 & $2.54-3.33$ \\
\hline Sodium & 23.6 & 22.2 & $20.2-24.3$ \\
\hline Sulfate & 7.55 & 6.40 & $4.39-8.16$ \\
\hline Turbidity (NTU) & 3.56 & 3.50 & $3.05-4.07$ \\
\hline
\end{tabular}

a Based on gravimetric calculations or a reference value when necessary.

b Not acceptable. 
Table B-4. Roy F. Weston Analytical Laboratory Performance Evaluation, Water Supply Study WS034

\begin{tabular}{|c|c|c|c|}
\hline Analyte & $\begin{array}{l}\text { Reported } \\
\text { Value } \\
\end{array}$ & $\begin{array}{l}\text { True } \\
\text { Value }^{\mathrm{a}}\end{array}$ & $\begin{array}{l}\text { Acceptance } \\
\text { Limits }\end{array}$ \\
\hline \multicolumn{4}{|l|}{ Trace Metals (mg/L) } \\
\hline Aluminum & 167 & 151 & $128-172$ \\
\hline Antimony & 23.4 & 23.7 & $16.6-30.8$ \\
\hline Arsenic & 117 & 140 & $117-158$ \\
\hline Barium & 865 & 859 & $730-988$ \\
\hline Beryllium & 5.68 & 5.33 & $4.53-6.13$ \\
\hline Boron & 134 & 115 & $43.8-239$ \\
\hline Cadmium & 24.5 & 23.0 & $18.4-27.6$ \\
\hline Chromium & 11.0 & 11.6 & $9.86-13.3$ \\
\hline Copper & 431 & 420 & $378-462$ \\
\hline Lead & 26.0 & 27.0 & $18.9-35.1$ \\
\hline Manganese & 602 & 590 & $546-625$ \\
\hline Mercury & 4.88 & 5.09 & $3.56-6.62$ \\
\hline Molybdenum & 22.2 & 24.0 & $17.9-30.2$ \\
\hline Nickel & 273 & 270 & $230-311$ \\
\hline Selenium & 47.9 & 51.8 & $41.4-62.2$ \\
\hline Silver & 40.9 & 38.1 & $33.0-42.4$ \\
\hline Thallium & 6.0 & 6.19 & $4.33-8.05$ \\
\hline Zinc & 440 & 419 & $385-452$ \\
\hline \multicolumn{4}{|c|}{ Fluoride, Nitrate, and Nitrite (mg/L) } \\
\hline Fluoride & 1.06 & 1.10 & $0.99-1.21$ \\
\hline Nitrate as nitrogen & $0.403^{b}$ & 0.460 & $0.414-0.506$ \\
\hline Nitrite as nitrogen & 0.715 & 0.680 & $0.578-0.782$ \\
\hline \multicolumn{4}{|l|}{ Insecticides (mg/L) } \\
\hline Alachlor & 3.12 & 3.43 & $1.89-4.97$ \\
\hline Atrazine & 5.08 & 5.38 & $2.96-7.80$ \\
\hline Bromacil & 27.8 & 32.7 & D.L. -53.9 \\
\hline Chlordane, total & $13.9^{b}$ & 7.26 & $3.99-10.5$ \\
\hline Endrin & 0.154 & 0.143 & $0.10-0.186$ \\
\hline Heptachlor & 0.840 & 0.914 & $0.503-1.33$ \\
\hline Heptachlor epoxide & 0.570 & 0.550 & $0.303-0.798$ \\
\hline Hexachlorobenzene & $0.315^{b}$ & 3.57 & $1.15-4.72$ \\
\hline
\end{tabular}


Table B-4. Roy F. Weston Analytical Laboratory Performance Evaluation, Water Supply Study WS034, Continued

\begin{tabular}{|c|c|c|c|}
\hline Analyte & $\begin{array}{l}\text { Reported } \\
\text { Value } \\
\end{array}$ & $\begin{array}{l}\text { True } \\
\text { Value }^{\text {a }} \\
\end{array}$ & $\begin{array}{l}\text { Acceptance } \\
\text { Limits } \\
\end{array}$ \\
\hline Hexachlorocyclopentadiene & 2.78 & .2 .14 & $0.181-2.86$ \\
\hline Lindane & 0.277 & 0.264 & $0.145-0.383$ \\
\hline Methoxychlor & 12.7 & 14.2 & $7.81-20.6$ \\
\hline Metolachlor & 13.5 & 14.4 & $4.82-20.9$ \\
\hline Metribuzin & 8.04 & 11.2 & $2.19-14.8$ \\
\hline Prometon & 14.4 & 16.2 & $1.49-26.2$ \\
\hline Simazine & 5.12 & 6.27 & $1.03-10.8$ \\
\hline Toxaphene & 5.11 & 5.37 & $2.95-7.79$ \\
\hline Trifluralin & $0.770^{b}$ & 2.36 & $0.856-3.13$ \\
\hline \multicolumn{4}{|l|}{ Herbicides (mg/L) } \\
\hline $2,4-\mathrm{D}$ & 27.5 & 32.8 & $16.4-49.2$ \\
\hline Dalapon & 10.2 & 24.3 & D.L. -37.1 \\
\hline Dicamba & 10.2 & 14.7 & D.L. -25.9 \\
\hline Dinoseb & 4.75 & 9.33 & D.L. -14.9 \\
\hline Pentachlorophenol & 15.4 & 22.6 & $11.3-33.9$ \\
\hline Picloram & 6.91 & 13.2 & $0.0792-22.4$ \\
\hline 2,4,5-TP (Silvex) & 7.43 & 9.28 & $4.64-13.9$ \\
\hline \multicolumn{4}{|l|}{ Adipate/Phthalates (mg/L) } \\
\hline Bis(2-ethylhexyl) adipate & 19.7 & 24.9 & $1.73-39.2$ \\
\hline Bis(2-ethylhexyl) phthalate & 18.1 & 21.3 & $2.0-34.8$ \\
\hline Butylbenzyl phthalate & 28.8 & 19.6 & D.L. -38.4 \\
\hline Diethyl phthalate & 21.1 & 15.7 & $4.72-23.1$ \\
\hline Dimethyl phthalate & 10.8 & 17.3 & $1.27-23.1$ \\
\hline \multicolumn{4}{|c|}{$\begin{array}{l}\text { Polycyclic Aromatic Hydrocarbons } \\
\text { (mg/L) }\end{array}$} \\
\hline Benzo[a]pyrene & 0.62 & 0.751 & $0.0539-1.04$ \\
\hline Fluorene & 7.37 & 6.23 & $2.56-8.57$ \\
\hline Indeno $[1,2,3-c, d]$ pyrene & 0.34 & 0.606 & $0.07-0.812$ \\
\hline Naphthalene & 18.2 & 17.1 & $2.42-26.6$ \\
\hline Pyrene & 1.60 & 1.46 & $0.849-1.75$ \\
\hline
\end{tabular}


Table B-4. Roy F. Weston Analytical Laboratory Performance Evaluation, Water Supply Study WS034, Continued

\begin{tabular}{|c|c|c|c|}
\hline Analyte & $\begin{array}{l}\text { Reported } \\
\text { Value } \\
\end{array}$ & $\begin{array}{l}\text { True } \\
\text { Value }^{a}\end{array}$ & $\begin{array}{l}\text { Acceptance } \\
\text { Limits }\end{array}$ \\
\hline \multicolumn{4}{|l|}{ PCBs (mg/L) } \\
\hline Decachlorobiphenyl & 0.980 & 1.08 & D.L. -2.16 \\
\hline \multicolumn{4}{|l|}{ Trihalomethanes (mg/L) } \\
\hline Bromodichloromethane & 31.5 & 31.1 & $24.9-37.3$ \\
\hline Bromoform & 12.5 & 14.4 & $11.5-17.3$ \\
\hline Chlorodibromomethane & 20.3 & 22.9 & $18.3-27.5$ \\
\hline Chloroform & 39.8 & 42.9 & $34.3-51.5$ \\
\hline \multicolumn{4}{|c|}{ Volatile Organic Compounds (mg/L) } \\
\hline Benzene & 4.94 & 4.94 & $2.96-6.92$ \\
\hline Carbon tetrachloride & 5.54 & 6.27 & $3.76-8.78$ \\
\hline Chlorobenzene & 5.91 & 6.41 & $3.85-8.97$ \\
\hline 1,2-Dibromo-3-chloropropane & 0.306 & 0.363 & $0.218-0.508$ \\
\hline 1,2-Dichlorobenzene & 6.37 & 6.98 & $4.19-9.77$ \\
\hline 1,4-Dichlorobenzene & 4.66 & 5.78 & $3.47-8.09$ \\
\hline 1,2-Dichloroethane & 12.6 & 12.1 & $9.68-14.5$ \\
\hline 1,1-Dichloroethylene & 6.87 & 7.64 & $4.58-10.7$ \\
\hline cis-1,2-Dichloroethylene & 8.24 & 9.41 & $5.65-13.2$ \\
\hline trans-1,2-Dichloroethylene & 5.07 & 6.77 & $4.06-9.48$ \\
\hline Dichloromethane & 15.8 & 18.4 & $14.7-22.1$ \\
\hline 1,2-Dichloropropane & 11.7 & 12.3 & $9.84-14.8$ \\
\hline cis-1,3-Dichloropropene & 19.5 & 19.7 & $15.8-23.6$ \\
\hline trans-1,3-Dichloropropene & 7.60 & 7.89 & $4.73-11.0$ \\
\hline Ethylbenzene & 6.89 & 7.41 & $4.45-10.4$ \\
\hline Ethylene dibromide & 0.385 & 0.406 & $0.244-0.568$ \\
\hline Hexachlorobutadiene & 16.8 & 17.2 & $13.8-20.6$ \\
\hline Styrene & 13.6 & 14.7 & $11.8-17.6$ \\
\hline 1,1,1,2-Tetrachloroethane & 14.3 & 14.4 & $11.5-17.3$ \\
\hline Tetrachloroethylene & 14.9 & 16.5 & $13.2-19.8$ \\
\hline Toluene & 14.4 & 15.6 & $12.5-18.7$ \\
\hline 1,2,4-Trichlorobenzene & 21.8 & 22.9 & $18.3-27.5$ \\
\hline 1,1,1-Trichloroethane & 4.90 & 5.73 & $3.44-8.02$ \\
\hline 1,1,2-Trichloroethane & 8.36 & 8.50 & $5.10-11.9$ \\
\hline Trichloroethylene & 8.87 & 8.89 & $5.33-12.4$ \\
\hline
\end{tabular}


Table B-4. Roy F. Weston Analytical Laboratory Performance Evaluation, Water Supply Study WS034, Continued

\begin{tabular}{llll}
\hline Analyte & $\begin{array}{l}\text { Reported } \\
\text { Value }\end{array}$ & $\begin{array}{l}\text { True } \\
\text { Value }\end{array}$ & $\begin{array}{l}\text { Acceptance } \\
\text { Limits }\end{array}$ \\
\hline 1,2,3-Trichloropropane & $24.9^{\mathrm{b}}$ & 20.5 & $16.4-24.6$ \\
Vinyl chloride & 12.4 & 14.1 & $8.46-19.7$ \\
Xylenes, total & 11.5 & 13.1 & $10.5-15.7$ \\
& & & \\
Miscellaneous Analytes (mg/L, except as noted) & & \\
Alkalinity (as $\mathrm{CaCO}_{3}$ ) & $70.0^{\mathrm{b}}$ & 33.0 & $30.7-37.2$ \\
Calcium (as CaCO3) & 187 & 180 & $169-193$ \\
Corrosivity (Langelier Ind. at 20C) & 1.0 & 0.943 & $0.611-1.23$ \\
Cyanide, total & 0.12 & 0.14 & $0.105-0.175$ \\
Filterable residue, total & 452 & 319 & $216-536$ \\
pH (pH units) & $8.84^{\mathrm{b}}$ & 9.12 & $8.86-9.27$ \\
Residual-free chlorine & 1.30 & 1.60 & $1.23-1.73$ \\
Sodium & $10.1^{\mathrm{b}}$ & 15.2 & $13.7-16.6$ \\
Sulfate & $462^{\mathrm{b}}$ & 400 & $364-435$ \\
Turbidity (NTU) & 0.823 & 0.72 & $0.446-1.12$ \\
\hline
\end{tabular}

a Based on theoretical calculations or a reference value when necessary.

b Not acceptable. 
Table B-5. Quality Control Standards for Selected Analyses for General Engineering (GE) Laboratory

\begin{tabular}{|c|c|c|c|c|}
\hline Analyte & $\begin{array}{l}\text { Certified } \\
\text { Value } \\
\end{array}$ & $\begin{array}{l}\text { Advisory } \\
\text { Range } \\
\end{array}$ & $\begin{array}{l}\text { GE } \\
\text { Result }\end{array}$ & Qualifier \\
\hline \multicolumn{5}{|l|}{ Acids (Lot 563) } \\
\hline 4-Chloro-m-cresol (mg/L) & 89.7 & $46.1-102$ & 80.5 & $\mathrm{RQ}$ \\
\hline $\mathrm{m} / \mathrm{p}$-Cresol $(\mathrm{mg} / \mathrm{L})$ & 24.6 & $10.6-27.6$ & 25.2 & RQ \\
\hline o-Cresol (mg/L) & 75.2 & $23.0-89.5$ & 55.8 & RQ \\
\hline Pentachlorophenol (mg/L) & 118 & $36.8-149$ & 111 & $\mathrm{RQ}$ \\
\hline 2,4,6-Trichlorophenol (mg/L) & 50.6 & $22.2-56.7$ & 49.8 & RQ \\
\hline \multicolumn{5}{|l|}{ Base/Neutrals (Lot 563) } \\
\hline Anthracene $(\mathrm{mg} / \mathrm{L})$ & 111 & $51.9-129$ & 88.5 & $\mathrm{RQ}$ \\
\hline Benzo[a]anthracene (mg/L) & 36.3 & $15.8-42.5$ & 31.2 & $R Q$ \\
\hline Benzo[k]fluoranthene $(\mathrm{mg} / \mathrm{L})$ & 27.2 & $9.93-34.8$ & 30.2 & $\mathrm{RQ}$ \\
\hline Bis(2-ethylhexyl) phthalate (mg/L) & 55.6 & $21.4-71.2$ & 63.6 & $\mathrm{RQ}$ \\
\hline Butylbenzyl phthalate (mg/L) & 54.7 & $20.9-64.0$ & $69.2^{\mathrm{b}}$ & $R Q$ \\
\hline Chrysene (mg/L) & 75.2 & $34.8-91.7$ & 64.3 & $\mathrm{RQ}$ \\
\hline Dibenzofuran (mg/L) & 40.9 & $19.6-47.0$ & 39.5 & RQ \\
\hline 1,2-Dichlorobenzene $(\mathrm{mg} / \mathrm{L})$ & 119 & $31.3-134$ & 92.0 & $\mathrm{RQ}$ \\
\hline 1,3-Dichlorobenzene (mg/L) & 97.3 & $23.3-113$ & 24.5 & JQ \\
\hline 2,4-Dinitrotoluene (mg/L) & 30.2 & $11.0-35.9$ & 31.5 & RQ \\
\hline 2,6-Dinitrotoluene (mg/L) & 52.8 & $26.1-60.2$ & $60.4^{\mathrm{b}}$ & $\mathrm{RQ}$ \\
\hline Fluorene (mg/L) & 72.1 & $35.5-88.7$ & 62.9 & RQ \\
\hline Hexachlorobenzene (mg/L) & 31.9 & $17.4-37.0$ & 24.3 & $R Q$ \\
\hline Naphthalene (mg/L) & 148 & $57.6-172$ & 101 & $\mathrm{RQ}$ \\
\hline Pyrene (mg/L) & 104 & $45.9-132$ & 109 & $\mathrm{RQ}$ \\
\hline 1,2,4-Trichlorobenzene (mg/L) & 26.1 & $8.43-30.8$ & 25.3 & $\mathrm{RQ}$ \\
\hline \multicolumn{5}{|l|}{ Cations (Lot 426) } \\
\hline Calcium (mg/L) & 84,800 & $72,900-96,700$ & 82,200 & \\
\hline Magnesium (mg/L) & 108,000 & $92,900-123,000$ & 107,000 & \\
\hline Potassium (mg/L) & 28,800 & $24,500-33,100$ & $12,600^{\mathrm{b}}$ & \\
\hline Sodium (mg/L) & 208,000 & $177,000-239,000$ & 220,000 & \\
\hline \multicolumn{5}{|l|}{ Cyanide and Phenol (Lot 9964) } \\
\hline Cyanide (mg/L) & 105 & $76.9-134$ & 104 & JQC \\
\hline Phenols (mg/L) & 218 & $166-270$ & 205 & JC \\
\hline
\end{tabular}


Table B-5. Quality Control Standards for Selected Analyses for General Engineering (GE) Laboratory, Continued

\begin{tabular}{|c|c|c|c|c|}
\hline Analyte & $\begin{array}{l}\text { Certified } \\
\text { Value }\end{array}$ & $\begin{array}{l}\text { Advisory } \\
\text { Range } \\
\end{array}$ & $\begin{array}{l}\text { GE } \\
\text { Result }\end{array}$ & Qualifier \\
\hline \multicolumn{5}{|l|}{ Inorganics (Lot 3412) } \\
\hline Alkalinity (as $\left.\mathrm{CaCO}_{3}\right)(\mathrm{mg} / \mathrm{L})$ & 131,000 & $111,000-151,000$ & 140,000 & JQ \\
\hline Chloride (mg/L) & 129,000 & $115,000-144,000$ & 123,000 & JQ \\
\hline Fluoride $(\mathrm{mg} / \mathrm{L})$ & 6,470 & $5,820-7,120$ & 6,100 & JQ \\
\hline Nitrate as nitrogen (mg/L) & 8,770 & $7,890-9,650$ & 8,950 & RQ \\
\hline $\mathrm{pH}$ (pH units) & 9.14 & $8.94-9.34$ & 9.13 & RQ \\
\hline Potassium (mg/L) & 37,800 & $32,100-43,800$ & 32,200 & \\
\hline Sodium $(\mathrm{mg} / \mathrm{L})$ & 197,000 & $167,000-227,000$ & 209,000 & \\
\hline Specific conductance (mS/cm) & 1,030 & $876-1,180$ & 1,040 & JQ \\
\hline Sulfate (mg/L) & 110,000 & $94,600-127,000$ & 102,000 & JQ \\
\hline Total dissolved solids (mg/L) & 771,000 & $632,000-864,000$ & 746,000 & $\mathrm{JQ}$ \\
\hline \multicolumn{5}{|l|}{ Nutrients (Lot 9964) } \\
\hline Ammonia nitrogen (mg/L) & 8,780 & $7,370-10,200$ & 7,700 & \\
\hline Nitrate-nitrite as nitrogen (mg/L) & 5,490 & $4,880-6,090$ & 5,480 & JIC \\
\hline Total phosphates (as P) (mg/L) & 6,640 & $5,650-7,640$ & 6,280 & JI \\
\hline \multicolumn{5}{|l|}{ Oil and Grease (Lot 9964) } \\
\hline $\begin{array}{l}\text { Oil and grease, gravimetric } \\
\text { (mg/bottle) }\end{array}$ & 74.1 & $44.5-92.6$ & 70.3 & JQ \\
\hline \multicolumn{5}{|l|}{ PCBs (Lot 563) } \\
\hline PCB 1254 (mg/L) & 2.72 & $1.84-3.16$ & 1.98 & JQ \\
\hline \multicolumn{5}{|l|}{ Pesticides (Lot 563) } \\
\hline Aldrin (mg/L) & 4.22 & $2.16-5.02$ & 3.75 & JQCO \\
\hline alpha-Benzene hexachloride (mg/L) & 4.85 & $2.57-6.26$ & 4.45 & JQO \\
\hline beta-Berizene hexachloride (mg/L) & 8.65 & $4.50-10.8$ & 8.09 & JQO \\
\hline alpha-Chlordane $(\mathrm{mg} / \mathrm{L})$ & 4.99 & $2.51-6.34$ & 4.76 & JQO \\
\hline p,p'-DDD (mg/L) & 3.73 & $2.42-4.89$ & 3.8 & JQO \\
\hline $\mathrm{p}, \mathrm{p}^{\prime}-\mathrm{DDE}(\mathrm{mg} / \mathrm{L})$ & 6.27 & $3.47-8.03$ & 5.8 & JQO \\
\hline $\mathrm{p}, \mathrm{p}^{\prime}-\mathrm{DDT}(\mathrm{mg} / \mathrm{L})$ & 2.30 & $1.34-2.90$ & 2.26 & JQCO \\
\hline Dieldrin (mg/L) & 2.08 & $1.24-2.75$ & 2.1 & JQCO \\
\hline Endrin (mg/L) & 2.02 & $1.06-2.61$ & 1.9 & JQCO \\
\hline Heptachlor (mg/L) & 8.72 & $3.99-11.0$ & 8.17 & JQCO \\
\hline
\end{tabular}


Table B-5. Quality Control Standards for Selected Analyses for General Engineering (GE) Laboratory, Continued

\begin{tabular}{lllll}
\hline Analyte & $\begin{array}{l}\text { Certified } \\
\text { Value }\end{array}$ & $\begin{array}{l}\text { Advisory } \\
\text { Range }\end{array}$ & $\begin{array}{l}\text { GE } \\
\text { Result }\end{array}$ & Qualifi \\
\hline Heptachlor epoxide (mg/L) & 1.51 & $0.939-1.84$ & 1.5 & JQO \\
Pesticides/Herbicides (Lot 3212) & & & & \\
Alachlor (mg/L) & 1.99 & $1.09-2.89$ & $-\mathrm{a}$ & \\
Atrazine (mg/L) & 3.14 & $1.73-4.55$ & $-\mathrm{a}$ & \\
2,4-Dichlorophenoxyacetic acid & 6.47 & $3.24-9.71$ & $-\mathrm{a}$ & \\
(mg/L) & & & & \\
Dalapon (mg/L) & 9.54 & $3.91-13.0$ & $-\mathrm{a}$ & \\
Dinoseb (mg/L) & 7.03 & $1.76-8.86$ & $-\mathrm{a}$ & \\
Endrin (mg/L) & 2.02 & $1.41-2.63$ & 1.9 & JQCO \\
Heptachlor (mg/L) & 0.400 & $0.220-0.580$ & $8.17^{\mathrm{b}}$ & JQCO \\
Heptachlor epoxide (mg/L) & 0.200 & $0.110-0.290$ & $1.5^{\mathrm{b}}$ & JQO \\
Hexachlorobenzene (mg/L) & 1.00 & $0.550-1.45$ & 0.83 & RQ \\
Hexachlorocyclopentadiene (mg/L) & 8.74 & $4.81-12.7$ & 5.11 & RQ \\
Lindane (mg/L) & 0.211 & $0.116-0.306$ & $<0.025^{\mathrm{b}}$ & JQCO \\
Methoxychlor (mg/L) & 7.15 & $3.93-10.4$ & $<0.5^{\mathrm{b}}$ & JQO \\
Pentachlorophenol (mg/L) & 1.02 & $0.510-1.53$ & $<1.0$ & RQ \\
Picloram (mg/L) & 8.24 & $4.70-11.9$ & $-\mathrm{a}$ & \\
Simazine (mg/L) & 4.08 & $3.02-5.06$ & $-\mathrm{a}$ & \\
2,4,5-TP (Silvex) (mg/L) & 6.60 & $3.30-9.90$ & $-\mathrm{a}$ &
\end{tabular}

Total Petroleum Hydrocarbons (Lot 8917)

Standard 1 fatty acids not present $\quad 96.0$ gravimetric (mg/bottle)

$63.2-124$

99.0

JQ

Toxaphene (Lot 3212)

Toxaphene (mg/L)

Trace Metals (Lot 3412)

Aluminum (mg/L)

313

266-360

316

Antimony (mg/L)

68.8

$48.2-89.4$

69.1

Arsenic (mg/L)

75.0

$60.0-90.0$

75.7

Barium (mg/L)

650

553-748

613

Beryllium (mg/L)

37.5

$31.9-43.1$

36.4

Boron (mg/L)

93.8

76.9-111

102

JE 
Table B-5. Quality Control Standards for Selected Analyses for General Engineering (GE) Laboratory, Continued

\begin{tabular}{|c|c|c|c|c|}
\hline Analyte & $\begin{array}{l}\text { Certified } \\
\text { Value } \\
\end{array}$ & $\begin{array}{l}\text { Advisory } \\
\text { Range }\end{array}$ & $\begin{array}{l}\text { GE } \\
\text { Result }\end{array}$ & Qualifier \\
\hline Cadmium (mg/L) & 31.3 & $25.0-37.6$ & 31.6 & \\
\hline Calcium (mg/L) & 50,100 & $45,100-55,100$ & 49,700 & \\
\hline Chromium (mg/L) & 213 & $181-245$ & 212 & \\
\hline Copper (mg/L) & 875 & $788-963$ & 867 & \\
\hline Iron (mg/L) & 150 & $113-179$ & 147 & \\
\hline Lead (mg/L) & 81.3 & $56.9-106$ & 77.5 & \\
\hline Manganese (mg/L) & 87.5 & $74.4-101$ & 85.4 & \\
\hline $\operatorname{Mercury}(\mathrm{mg} / \mathrm{L})$ & 2.50 & $1.75-3.25$ & 1.99 & JQ \\
\hline Molybdenum (mg/L) & 106 & $86.9-125$ & 109 & \\
\hline Nickel (mg/L) & 150 & $128-173$ & 145 & \\
\hline Selenium (mg/L) & 37.5 & $30.0-45.0$ & 36.4 & \\
\hline Silver $(\mathrm{mg} / \mathrm{L})$ & 68.8 & $58.5-79.1$ & 67.1 & \\
\hline Thallium (mg/L) & 31.3 & $21.9-40.7$ & 28.5 & \\
\hline $\operatorname{Zinc}(\mathrm{mg} / \mathrm{L})$ & 775 & $659-891$ & 742 & \\
\hline Hardness (as $\left.\mathrm{CaCO}_{3}\right)(\mathrm{mg} / \mathrm{L})$ & 125,000 & $106,000-144,000$ & 124,000 & \\
\hline \multicolumn{5}{|l|}{ Turbidity (Lot 3412) } \\
\hline Turbidity (NTU) & 0.650 & $0.585-0.748$ & 0.68 & $\mathrm{RQ}$ \\
\hline \multicolumn{5}{|l|}{ Volatiles (Lot 563) } \\
\hline Benzene $(\mathrm{mg} / \mathrm{L})$ & 61.4 & $47.5-76.1$ & 48.2 & $\mathrm{JQ}$ \\
\hline Bromodichloromethane (mg/L) & 61.8 & $47.6-77.3$ & 53.1 & $\mathrm{JQ}$ \\
\hline Bromoform (mg/L) & 72.1 & $52.6-93.0$ & 54.7 & $\mathrm{JQ}$ \\
\hline Carbon tetrachloride $(\mathrm{mg} / \mathrm{L})$ & 68.3 & $49.1-86.7$ & $45.8^{\mathrm{b}}$ & $\mathrm{JQ}$ \\
\hline Chlorobenzene $(\mathrm{mg} / \mathrm{L})$ & 34.7 & $26.5-42.0$ & 27.6 & JQ \\
\hline Chlorodibromomethane (mg/L) & 44.3 & $33.5-54.9$ & $-a$ & \\
\hline Chloroform (mg/L) & 124 & $93.5-153$ & 107 & JQ \\
\hline 1,2-Dichlorobenzene (mg/L) & 44.3 & $33.6-53.6$ & $32.1^{\mathrm{b}}$ & $\mathrm{JQ}$ \\
\hline 1,3-Dichlorobenzene (mg/L) & 34.8 & $26.0-42.5$ & $24.5^{b}$ & $\mathrm{JQ}$ \\
\hline 1,4-Dichlorobenzene (mg/L) & 73.2 & $55.6-90.8$ & $47.1^{\mathrm{b}}$ & $\mathrm{JQ}$ \\
\hline 1,2-Dichloroethane (mg/L) & 76.5 & $59.0-95.6$ & 69.6 & JQ \\
\hline Dichloromethane (mg/L) & 81.9 & $55.6-109$ & 69.7 & JQ \\
\hline Ethylbenzene (mg/L) & 71.2 & $52.5-88.3$ & 52.6 & JQ \\
\hline Methyl isobutyl ketone (mg/L) & 34.2 & $19.7-47.5$ & 26.5 & $\mathrm{JQ}$ \\
\hline Tetrachloroethylene (mg/L) & 60.2 & $43.9-73.4$ & $37.7^{\mathrm{b}}$ & JQ \\
\hline
\end{tabular}


Table B-5. Quality Control Standards for Selected Analyses for General Engineering (GE) Laboratory, Continued

\begin{tabular}{lllll}
\hline Analyte & $\begin{array}{l}\text { Certified } \\
\text { Value }\end{array}$ & $\begin{array}{l}\text { Advisory } \\
\text { Range }\end{array}$ & $\begin{array}{l}\text { GE } \\
\text { Result }\end{array}$ & Qualifier \\
\hline & & & & \\
Toluene (mg/L) & 89.1 & $68.7-108$ & $66.8^{\mathrm{b}}$ & $\mathrm{JQ}$ \\
1,1,1-Trichloroethane (mg/L) & 29.1 & $20.4-34.9$ & $19.2^{\mathrm{b}}$ & $\mathrm{JQ}$ \\
Trichloroethylene (mg/L) & 74.4 & $55.3-90.0$ & $50.8^{\mathrm{b}}$ & $\mathrm{JQ}$ \\
\hline
\end{tabular}

a Result out of range.

b Result not received.

\section{Result Qualifiers}

C Criteria were not met.

E The result is estimated because of interference, indicated by high recoveries.

$J$ Result is an estimated quantity.

O Surrogate spike recoveries are out of specification.

Q Sample held beyond normal holding time.

$\mathrm{V}$ Laboratory blanks contaminated. The analyte was detected in both the sample and the associated method blank.

Note: GE reported the results for metals as total recoverable metals. 
Table B-6. Quality Control Standards for Selected Analyses for Roy F. Weston Analytical Laboratory

\begin{tabular}{|c|c|c|c|c|}
\hline Analyte & $\begin{array}{l}\text { Certified } \\
\text { Value } \\
\end{array}$ & $\begin{array}{l}\text { Advisory } \\
\text { Range } \\
\end{array}$ & $\begin{array}{l}\text { Weston } \\
\text { Result } \\
\end{array}$ & Qualifier \\
\hline \multicolumn{5}{|l|}{ Acids (Lot 563) } \\
\hline 4-Chloro-m-cresol $(\mu \mathrm{g} / \mathrm{L})$ & 89.7 & $46.1-102$ & 68.4 & JYQ \\
\hline $\mathrm{m} / \mathrm{p}-\mathrm{Cresol}(\mu \mathrm{g} / \mathrm{L})$ & 24.6 & $10.6-27.6$ & $-\mathrm{a}$ & \\
\hline o-Cresol $(\mu \mathrm{g} / \mathrm{L})$ & 75.2 & $23.0-89.5$ & 49.9 & JYQ \\
\hline p-Cresol $(\mu \mathrm{g} / \mathrm{L})$ & 86.3 & 29.1-99.2 & 56.9 & JYQ \\
\hline Pentachlorophenol $(\mu \mathrm{g} / \mathrm{L})$ & 118 & $36.8-149$ & 108 & JYQ \\
\hline 2,4,6-Trichlorophenol $(\mu \mathrm{g} / \mathrm{L})$ & 50.6 & $22.2-56.7$ & 38.0 & JYQ \\
\hline \multicolumn{5}{|l|}{ Base/Neutrals (Lot 563) } \\
\hline Anthracene $(\mu \mathrm{g} / \mathrm{L})$ & 111 & $51.9-129$ & 81.9 & JYQ \\
\hline Benzo $[a]$ anthracene $(\mu \mathrm{g} / \mathrm{L})$ & 36.3 & $15.8-42.5$ & 24.1 & JYQ \\
\hline Benzo[ $k]$ fluoranthene $(\mu \mathrm{g} / \mathrm{L})$ & 27.2 & $9.93-34.8$ & 19.3 & JYQ \\
\hline Bis(2-ethylhexyl) phthalate $(\mu g / L)$ & 55.6 & $21.4-71.2$ & 42.3 & JYQV \\
\hline Butylbenzyl phthalate $(\mu \mathrm{g} / \mathrm{L})$ & 54.7 & $20.9-64.0$ & 36.8 & JYQ \\
\hline Chrysene $(\mu g / L)$ & 75.2 & $34.8-91.7$ & 57.8 & JYQ \\
\hline Dibenzofuran $(\mu \mathrm{g} / \mathrm{L})$ & 40.9 & $19.6-47.0$ & 23.4 & JYQ \\
\hline 1,2-Dichlorobenzene $(\mu \mathrm{g} / \mathrm{L})$ & 119 & $31.3-134$ & 44.5 & $\mathrm{Y}$ \\
\hline 1,3-Dichlorobenzene $(\mu \mathrm{g} / \mathrm{L})$ & $\mathbf{9 7 . 3}$ & $23.3-113$ & 36.7 & $\mathrm{Y}$ \\
\hline 2,4-Dinitrotoluene ( $\mu \mathrm{g} / \mathrm{L})$ & 30.2 & $11.0-35.9$ & 19.0 & JYQ \\
\hline 2,6-Dinitrotoluene ( $\mu \mathrm{g} / \mathrm{L})$ & 52.8 & $26.1-60.2$ & 31.2 & JYQ \\
\hline Fluorene $(\mu \mathrm{g} / \mathrm{L})$ & 72.1 & $35.5-88.7$ & 41.2 & JYQ \\
\hline Hexachlorobenzene $(\mu \mathrm{g} / \mathrm{L})$ & 31.9 & $17.4-37.0$ & 22.4 & JYQ \\
\hline Naphthalene $(\mu \mathrm{g} / \mathrm{L})$ & 148 & $57.6-172$ & $48.9^{b}$ & JYQ \\
\hline Pyrene $(\mu \mathrm{g} / \mathrm{L})$ & 104 & $45.9-132$ & 81.8 & JYQ \\
\hline 1,2,4-Trichlorobenzene $(\mu \mathrm{g} / \mathrm{L})$ & 26.1 & $8.43-30.8$ & $5.94^{b}$ & JYQE \\
\hline \multicolumn{5}{|l|}{ Cations (Lot 426) } \\
\hline Calcium $(\mu \mathrm{g} / \mathrm{L})$ & 84,800 & $72,900-96,700$ & 83,800 & $\mathrm{Y}$ \\
\hline Magnesium $(\mu \mathrm{g} / \mathrm{L})$ & 108,000 & $92,900-123,000$ & 111,000 & $\mathrm{Y}$ \\
\hline Potassium $(\mu \mathrm{g} / \mathrm{L})$ & 28,800 & $24,500-33,100$ & 32,800 & $\mathrm{Y}$ \\
\hline Sodium $(\mu g / L)$ & 208,000 & $177,000-239,000$ & 216,000 & $\mathrm{Y}$ \\
\hline
\end{tabular}


Table B-6. Quality Control Standards for Selected Analyses for Roy F. Weston Analytical Laboratory, Continued

\begin{tabular}{|c|c|c|c|c|c|}
\hline Analyte & $\begin{array}{l}\text { Certified } \\
\text { Value } \\
\end{array}$ & $\begin{array}{l}\text { Advisory } \\
\text { Range } \\
\end{array}$ & $\begin{array}{l}\text { Weston } \\
\text { Result }\end{array}$ & Qualifier & \\
\hline \multicolumn{5}{|l|}{ Cyanide and Phenol (Lot 9964) } & \\
\hline Cyanide $(\mu \mathrm{g} / \mathrm{L})$ & 105 & $76.9-134$ & 106 & $\mathrm{Y}$ & \\
\hline Phenols $(\mu \mathrm{g} / \mathrm{L})$ & 218 & $166-270$ & 220 & $\mathrm{Y}$ & \\
\hline \multicolumn{6}{|l|}{ Inorganics (Lot 3412) } \\
\hline Alkalinity (as $\left.\mathrm{CaCO}_{3}\right)(\mu \mathrm{g} / \mathrm{L})$ & 131,000 & $111,000-151,000$ & 137,000 & $\mathrm{Y}$ & \\
\hline Chloride $(\mu \mathrm{g} / \mathrm{L})$ & 129,000 & $115,000-144,000$ & 131,000 & $\mathrm{Y}$ & \\
\hline Fluoride $(\mu \mathrm{g} / \mathrm{L})$ & 6,470 & $5,820-7,120$ & 6,250 & YV & \\
\hline Nitrate as nitrogen $(\mu \mathrm{g} / \mathrm{L})$ & 8,770 & $7,890-9,650$ & 8,680 & $\mathrm{Y}$ & \\
\hline $\mathrm{pH}$ (pH units) & 9.14 & $8.94-9.34$ & 9.13 & JYQ & \\
\hline Potassium $(\mu \mathrm{g} / \mathrm{L})$ & 37,800 & $32,100-43,800$ & 40,600 & $\mathrm{Y}$ & \\
\hline Sodium $(\mu \mathrm{g} / \mathrm{L})$ & 197,000 & $167,000-227,000$ & 204,000 & $\mathrm{Y}$ & \\
\hline Specific conductance $(\mu \mathrm{S} / \mathrm{cm})$ & 1,030 & $876-1,180$ & 1,080 & YV & \\
\hline Sulfate $(\mu g / L)$ & 110,000 & $94,600-127,000$ & 109,000 & $\mathrm{Y}$ & \\
\hline Total dissolved solids ( $\mu \mathrm{g} / \mathrm{L})$ & 771,000 & $632,000-864,000$ & 744,000 & $J Y Q V$ & \\
\hline \multicolumn{6}{|l|}{ Nutrients (Lot 9964) } \\
\hline Ammonia nitrogen $(\mu \mathrm{g} / \mathrm{L})$ & 8,780 & $7,370-10,200$ & $-\mathbf{a}$ & & \\
\hline Nitrate-nitrite as nitrogen $(\mu \mathrm{g} / \mathrm{L})$ & 5,490 & $4,880-6,090$ & 5,410 & & \\
\hline \multirow[t]{2}{*}{ Total phosphates (as P) $(\mu \mathrm{g} / \mathrm{L}$ ) } & 6,640 & $5,650-7,640$ & $-a$ & & \\
\hline & - & & & & \\
\hline \multicolumn{6}{|l|}{ Oil and Grease (Lot 9964) } \\
\hline $\begin{array}{l}\text { Oil and grease, gravimetric } \\
\text { (mg/bottle) }\end{array}$ & 74.1 & $44.5-92.6$ & 68.0 & & \\
\hline \multicolumn{6}{|l|}{ PCBs (Lot 563) } \\
\hline PCB $1254(\mu \mathrm{g} / \mathrm{L})$ & 2.72 & $1.84-3.16$ & 3.01 & $\mathrm{Y}$ & \\
\hline \multicolumn{6}{|l|}{ Pesticides (Lot 563) } \\
\hline Aldrin $(\mu \mathrm{g} / \mathrm{L})$ & 4.22 & $2.16-5.02$ & 3.96 & $\mathrm{Y}$ & \\
\hline alpha-Benzene hexachloride $(\mu \mathrm{g} / \mathrm{L})$ & 4.85 & $2.57-6.26$ & 5.45 & $\mathrm{Y}$ & \\
\hline beta-Benzene hexachloride $(\mu \mathrm{g} / \mathrm{L})$ & 8.65 & $4.50-10.8$ & 9.72 & $\mathrm{Y}$ & \\
\hline alpha-Chlordane $(\mu \mathrm{g} / \mathrm{L})$ & 4.99 & $2.51-6.34$ & 5.50 & $\mathrm{Y}$ & \\
\hline $\mathrm{p}, \mathrm{p}^{\prime}-\mathrm{DDD}(\mu \mathrm{g} / \mathrm{L})$ & 3.73 & $2.42-4.89$ & 3.62 & $\mathrm{Y}$ & \\
\hline
\end{tabular}


Table B-6. Quality Control Standards for Selected Analyses for Roy F. Weston Analytical Laboratory, Continued

\begin{tabular}{|c|c|c|c|c|}
\hline Analyte & $\begin{array}{l}\text { Certified } \\
\text { Value } \\
\end{array}$ & $\begin{array}{l}\text { Advisory } \\
\text { Range }\end{array}$ & $\begin{array}{l}\text { Weston } \\
\text { Result }\end{array}$ & Qualifier \\
\hline $\mathrm{p}, \mathrm{p}^{\prime}-\mathrm{DDE}(\mu \mathrm{g} / \mathrm{L})$ & 6.27 & $3.47-8.03$ & 7.09 & $\mathrm{Y}$ \\
\hline $\mathrm{p}, \mathrm{p}^{\prime}-\mathrm{DDT}(\mu \mathrm{g} / \mathrm{L})$ & 2.30 & $1.34-2.90$ & 2.51 & $\mathrm{Y}$ \\
\hline Dieldrin $(\mu \mathrm{g} / \mathrm{L})$ & 2.08 & $1.24-2.75$ & 2.32 & $\mathrm{Y}$ \\
\hline Endrin $(\mu \mathrm{g} / \mathrm{L})$ & 2.02 & $1.06-2.61$ & 2.31 & $\mathrm{Y}$ \\
\hline Heptachlor $(\mu \mathrm{g} / \mathrm{L})$ & 8.72 & $3.99-11.0$ & 8.51 & $\mathrm{Y}$ \\
\hline Heptachlor epoxide $(\mu \mathrm{g} / \mathrm{L})$ & 1.51 & $0.939-1.84$ & 1.61 & $\mathrm{Y}$ \\
\hline \multicolumn{5}{|l|}{ Pesticides/Herbicides (Lot 3212) } \\
\hline Alachlor $(\mu \mathrm{g} / \mathrm{L})$ & 1.99 & $1.09-2.89$ & $-a$ & \\
\hline Atrazine $(\mu \mathrm{g} / \mathrm{L})$ & 3.14 & $1.73-4.55$ & $\rightarrow$ & \\
\hline $\begin{array}{l}\text { 2,4-Dichlorophenoxyacetic acid } \\
(\mu \mathrm{g} / \mathrm{L})\end{array}$ & 6.47 & $3.24-9.71$ & $\rightarrow$ & \\
\hline Dalapon $(\mu \mathrm{g} / L)$ & 9.54 & $3.91-13.0$ & $\rightarrow$ & \\
\hline Dinoseb $(\mu \mathrm{g} / \mathrm{L})$ & 7.03 & $1.76-8.86$ & $-\mathrm{a}$ & \\
\hline Endrin $(\mu \mathrm{g} / \mathrm{L})$ & 2.02 & $1.41-2.63$ & 2.31 & $\mathrm{Y}$ \\
\hline Heptachlor $(\mu \mathrm{g} / \mathrm{L})$ & 0.400 & $0.220-0.580$ & 0.400 & $\mathrm{Y}$ \\
\hline Heptachlor epoxide $(\mu \mathrm{g} / \mathrm{L})$ & 0.200 & $0.110-0.290$ & 0.270 & $\mathrm{Y}$ \\
\hline Hexachlorobenzene $(\mu \mathrm{g} / \mathrm{L})$ & 1.00 & $0.550-1.45$ & $22.4^{\mathrm{b}}$ & JYQ \\
\hline Hexachlorocyclopentadiene $(\mu \mathrm{g} / \mathrm{L})$ & 8.74 & $4.81-12.7$ & $-\mathrm{a}$ & \\
\hline Lindane $(\mu \mathrm{g} / \mathrm{L})$ & 0.211 & $0.116-0.306$ & 0.220 & $\mathrm{Y}$ \\
\hline Methoxychlor $(\mu \mathrm{g} / \mathrm{L})$ & 7.15 & $3.93-10.4$ & 8.99 & $\mathrm{Y}$ \\
\hline Pentachlorophenol $(\mu \mathrm{g} / \mathrm{L})$ & 1.02 & $0.510-1.53$ & $108^{b}$ & JYQ \\
\hline Picloram $(\mu \mathrm{g} / \mathrm{L})$ & 8.24 & $4.70-11.9$ & $-\mathrm{a}$ & \\
\hline Simazine $(\mu \mathrm{g} / \mathrm{L})$ & 4.08 & $3.02-5.06$ & $-\mathrm{a}$ & \\
\hline 2,4,5-TP (Silvex) ( $\mu \mathrm{g} / \mathrm{L})$ & 6.60 & $3.30-9.90$ & $-\mathrm{a}$ & \\
\hline \multicolumn{5}{|c|}{ Total Petroleum Hydrocarbons (Lot 8917) } \\
\hline $\begin{array}{l}\text { Standard } 1 \text {, fatty acids not present, } \\
\text { gravimetric (mg/bottle) }\end{array}$ & 96.0 & $63.2-124$ & 90.5 & $\mathrm{Y}$ \\
\hline \multicolumn{5}{|l|}{ Toxaphene (Lot 3212) } \\
\hline Toxaphene $(\mu \mathrm{g} / \mathrm{L})$ & 8.20 & $4.51-11.9$ & 4.65 & $\mathrm{Y}$ \\
\hline
\end{tabular}


Table B-6. Quality Control Standards for Selected Analyses for Roy F. Weston Analytical Laboratory, Continued

\begin{tabular}{|c|c|c|c|c|}
\hline Analyte & $\begin{array}{l}\text { Certified } \\
\text { Value } \\
\end{array}$ & $\begin{array}{l}\text { Advisory } \\
\text { Range }\end{array}$ & $\begin{array}{l}\text { Weston } \\
\text { Result } \\
\end{array}$ & Qualifier \\
\hline \multicolumn{5}{|l|}{ Trace Metals (Lot 3412) } \\
\hline Aluminum $(\mu \mathrm{g} / \mathrm{L})$ & 313 & $266-360$ & $649^{b}$ & $Y$ \\
\hline Antimony $(\mu \mathrm{g} / \mathrm{L})$ & 68.8 & $48.2-89.4$ & $141^{\mathrm{b}}$ & $\mathrm{Y}$ \\
\hline Arsenic $(\mu g / L)$ & 75.0 & $60.0-90.0$ & $151^{b}$ & $\mathrm{Y}$ \\
\hline Barium ( $\mu g / L)$ & 650 & $553-748$ & $1,300^{\mathrm{b}}$ & $\mathrm{Y}$ \\
\hline Beryllium ( $\mu \mathrm{g} / \mathrm{L})$ & 37.5 & $31.9-43.1$ & $73.6^{\mathrm{b}}$ & $\mathrm{Y}$ \\
\hline Boron $(\mu \mathrm{g} / \mathrm{L})$ & 93.8 & $76.9-111$ & $212^{\mathrm{b}}$ & $\mathrm{Y}$ \\
\hline Cadmium $(\mu \mathrm{g} / \mathrm{L})$ & 31.3 & $25.0-37.6$ & $64.4^{\mathrm{b}}$ & $\mathrm{Y}$ \\
\hline Calcium $(\mu \mathrm{g} / \mathrm{L})$ & 50,100 & $45,100-55,100$ & $83,800^{b}$ & $\mathrm{Y}$ \\
\hline Chromium $(\mu \mathrm{g} / \mathrm{L})$ & 213 & $181-245$ & $425^{b}$ & $\mathrm{Y}$ \\
\hline Copper $(\mu \mathrm{g} / \mathrm{L})$ & 875 & $788-963$ & $1,720^{\mathrm{b}}$ & $\mathrm{Y}$ \\
\hline Iron $(\mu \mathrm{g} / \mathrm{L})$ & 150 & $113-179$ & $295^{b}$ & $\mathrm{Y}$ \\
\hline Lead $(\mu \mathrm{g} / \mathrm{L})$ & 81.3 & $56.9-106$ & $164^{\mathrm{b}}$ & $\mathrm{Y}$ \\
\hline Manganese $(\mu \mathrm{g} / \mathrm{L})$ & 87.5 & $74.4-101$ & $176^{b}$ & $\mathrm{Y}$ \\
\hline Mercury $(\mu \mathrm{g} / \mathrm{L})$ & 2.50 & $1.75-3.25$ & $4.8^{b}$ & $\mathrm{Y}$ \\
\hline Molybdenum $(\mu \mathrm{g} / \mathrm{L})$ & 106 & $86.9-125$ & $214^{b}$ & $\mathrm{Y}$ \\
\hline Nickel $(\mu \mathrm{g} / \mathrm{L})$ & 150 & $128-173$ & $307^{b}$ & $\mathrm{Y}$ \\
\hline Selenium $(\mu \mathrm{g} / \mathrm{L})$ & 37.5 & $30.0-45.0$ & $75.4^{\mathrm{b}}$ & $\mathrm{Y}$ \\
\hline Silver $(\mu \mathrm{g} / \mathrm{L})$ & 68.8 & $58.5-79.1$ & $135^{b}$ & $\mathrm{Y}$ \\
\hline Thallium ( $\mu \mathrm{g} / \mathrm{L})$ & 31.3 & $21.9-40.7$ & $59.7^{b}$ & $\mathrm{Y}$ \\
\hline $\operatorname{Zinc}(\mu \mathrm{g} / \mathrm{L})$ & 775 & $659-891$ & $1,550^{\mathrm{b}}$ & $\mathrm{Y}$ \\
\hline Hardness (as $\left.\mathrm{CaCO}_{3}\right)(\mu \mathrm{g} / \mathrm{L})$ & 125,000 & $106,000-144,000$ & $240,000^{b}$ & $\mathrm{Y}$ \\
\hline \multicolumn{5}{|l|}{ Turbidity (Lot 3412) } \\
\hline Turbidity (NTU) & 0.650 & $0.585-0.748$ & 0.701 & JYQ \\
\hline \multicolumn{5}{|l|}{ Volatiles (Lot 563) } \\
\hline Benzene $(\mu \mathrm{g} / \mathrm{L})$ & 61.4 & $47.5-76.1$ & 61.4 & $\mathrm{Y}$ \\
\hline Bromodichloromethane $(\mu \mathrm{g} / \mathrm{L})$ & 61.8 & $47.6-77.3$ & 61.4 & $\mathrm{Y}$ \\
\hline Bromoform $(\mu \mathrm{g} / \mathrm{L})$ & 72.1 & $52.6-93.0$ & 71.5 & $\mathrm{Y}$ \\
\hline Carbon tetrachloride $(\mu \mathrm{g} / \mathrm{L})$ & 68.3 & $49.1-86.7$ & 69.4 & $\mathrm{Y}$ \\
\hline Chlorobenzene $(\mu \mathrm{g} / \mathrm{L})$ & 34.7 & $26.5-42.0$ & 37.7 & $\mathrm{Y}$ \\
\hline Chlorodibromomethane $(\mu \mathrm{g} / \mathrm{L})$ & 44.3 & $33.5-54.9$ & $-a$ & \\
\hline
\end{tabular}


Table B-6. Quality Control Standards for Selected Analyses for Roy F. Weston Analytical Laboratory, Continued

\begin{tabular}{lllll}
\hline Analyte & $\begin{array}{l}\text { Certified } \\
\text { Value }\end{array}$ & $\begin{array}{l}\text { Advisory } \\
\text { Range }\end{array}$ & $\begin{array}{l}\text { Weston } \\
\text { Result }\end{array}$ & Qualifier \\
\hline Chloroform $(\mu \mathrm{g} / \mathrm{L})$ & 124 & $93.5-153$ & 125 & $\mathrm{Y}$ \\
1,2-Dichlorobenzene $(\mu \mathrm{g} / \mathrm{L})$ & 44.3 & $33.6-53.6$ & 44.5 & $\mathrm{Y}$ \\
1,3-Dichlorobenzene $(\mu \mathrm{g} / \mathrm{L})$ & 34.8 & $26.0-42.5$ & 36.7 & $\mathrm{Y}$ \\
1,4-Dichlorobenzene $(\mu \mathrm{g} / \mathrm{L})$ & 73.2 & $55.6-90.8$ & 64.9 & $\mathrm{Y}$ \\
1,2-Dichloroethane $(\mu \mathrm{g} / \mathrm{L})$ & 76.5 & $59.0-95.6$ & 78.0 & $\mathrm{Y}$ \\
Dichloromethane $(\mu \mathrm{g} / \mathrm{L})$ & 81.9 & $55.6-109$ & 92.9 & $\mathrm{YV}$ \\
Ethylbenzene $(\mu \mathrm{g} / \mathrm{L})$ & 71.2 & $52.5-88.3$ & 68.3 & $\mathrm{Y}$ \\
Methyl isobutyl ketone $(\mu \mathrm{g} / \mathrm{L})$ & 34.2 & $19.7-47.5$ & $-\mathrm{a}$ & \\
Tetrachloroethylene $(\mu \mathrm{g} / \mathrm{L})$ & 60.2 & $43.9-73.4$ & 59.5 & $\mathrm{Y}$ \\
Toluene $(\mu \mathrm{g} / \mathrm{L})$ & 89.1 & $68.7-108$ & 85.0 & $\mathrm{Y}$ \\
1,1,1-Trichloroethane $(\mu \mathrm{g} / \mathrm{L})$ & 29.1 & $20.4-34.9$ & 27.2 & $\mathrm{Y}$ \\
Trichloroethylene $(\mu \mathrm{g} / \mathrm{L})$ & 74.4 & $55.3-90.0$ & 73.4 & $\mathrm{Y}$ \\
\hline
\end{tabular}

a Result not received.

b Result out of range.

\section{Result Qualifiers}

E The result is estimated because of interference, indicated by high recoveries.

$J$ The result is an estimated quantity.

Q Sample held beyond normal holding time.

$\mathrm{V}$ The analyte was detected in both the sample and associated method blank.

Note: Weston reported the results for metals as total recoverable metals. 
This page was left blank intentionally. 


\section{Appendix C: Formats of the Data Files}

The following table describes the SAM93 format for coding and transmitting sampling data records. This format has been established by the Environmental Monitoring Section of the Environmental Protection Department (EPD/EMS) of the Westinghouse Savannah River Company (WSRC).

Sample Data Format SAM93

\begin{tabular}{|c|c|c|c|}
\hline Name & Columns & Type & Description \\
\hline Record format & $1-8$ & Alphanumeric & SAM93 (the format of the data record) \\
\hline $\begin{array}{l}\text { Sample } \\
\text { identification } \\
\text { number }\end{array}$ & $9-14$ & Integer & $\begin{array}{l}\text { The number of the sample printed on the } \\
\text { page of the sample logbook }\end{array}$ \\
\hline $\begin{array}{l}\text { Sample collection } \\
\text { date }\end{array}$ & $15-20$ & Numeric & $\begin{array}{l}\text { Sample collection date recorded using the } \\
\text { MMDDYY format }\end{array}$ \\
\hline $\begin{array}{l}\text { Sample collection } \\
\text { time }\end{array}$ & $21-24$ & Integer & $\begin{array}{l}\text { Sample collection time recorded as military } \\
\text { time }\end{array}$ \\
\hline Sample type & 25 & Numeric & $\begin{array}{l}\text { Sample type coded as } \\
1=\text { normal } \\
2=\text { trip blank } \\
3=\text { field blank } \\
4=\text { split } \\
5=\text { rinsate } \\
6=\text { duplicate } \\
7=\text { other }\end{array}$ \\
\hline Sample matrix & 26 & Numeric & $\begin{array}{l}\text { Sample matrix coded as } \\
1=\text { soil } \\
2=\text { rock } \\
3=\text { sludge } \\
4=\text { water } \\
5=\text { sediment } \\
6=\text { other }\end{array}$ \\
\hline Delay check & 27 & Alpha & $\begin{array}{l}\text { Enter a } Y \text { if there was an unusual delay in } \\
\text { bottling samples }\end{array}$ \\
\hline Sampler's initials & $28-30$ & Alpha & Sampler's initials \\
\hline Associated sample & $31-36$ & Integer & $\begin{array}{l}\text { Sample identification of associated sample if } \\
\text { this sample is a duplicate, split, rinsate, etc. }\end{array}$ \\
\hline $\begin{array}{l}\text { Planned sample } \\
\text { name }\end{array}$ & $37-46$ & Alphanumeric & $\begin{array}{l}\text { Identification of the sample as it appears in } \\
\text { the site work plan }\end{array}$ \\
\hline
\end{tabular}

- Alpha and alphanumeric entries must be justified left in the records; numeric entries must be justified right in the records. There will be no leading zeros except in dates and times. 


\section{Sample Data Format SAM93, Continued}

\begin{tabular}{|c|c|c|c|}
\hline Name & Columns & Type & Description \\
\hline Sample name & $47-56$ & Alphanumeric & $\begin{array}{l}\text { Identification of the actual sample (includes } \\
\text { any modification made in the field) }\end{array}$ \\
\hline $\begin{array}{l}\text { Planned top of } \\
\text { interval }\end{array}$ & $57-62$ & Numeric & $\begin{array}{l}\text { Depth from the ground to the top of the } \\
\text { planned sampling interval to the nearest } \\
0.01 \mathrm{ft}\end{array}$ \\
\hline Top of interval & $63-68$ & Numeric & $\begin{array}{l}\text { Depth from the ground to the top of the } \\
\text { actual sampling interval to the nearest } 0.01 \\
\mathrm{ft}\end{array}$ \\
\hline $\begin{array}{l}\text { Planned bottom of } \\
\text { interval }\end{array}$ & $69-74$ & Numeric & $\begin{array}{l}\text { Depth from the ground to the bottom of the } \\
\text { planned sampling interval to the nearest } \\
0.01 \mathrm{ft}\end{array}$ \\
\hline Bottom of interval & $75-80$ & Numeric & $\begin{array}{l}\text { Depth from the ground to the bottom of the } \\
\text { actual sampling interval to the nearest } 0.01 \\
\text { ft }\end{array}$ \\
\hline Soil moisture & 81 & Numeric & $\begin{array}{l}\text { Soil moisture coded as: } \\
1=\text { dry } \\
2=\text { damp } \\
3=\text { wet } \\
4=\text { sludge } \\
5=\text { liquid }\end{array}$ \\
\hline Munsell color & $82-91$ & Alphanumeric & Munsell color \\
\hline Soil classification & $92-93$ & Alphanumeric & Unified Soil Classification \\
\hline Number of bottles & $94-96$ & Integer & $\begin{array}{l}\text { The number of bottle label numbers used } \\
\text { during collection }\end{array}$ \\
\hline $\begin{array}{l}\text { Insufficient } \\
\text { recovery check }\end{array}$ & 97 & Alpha & $\begin{array}{l}\text { Enter an } R \text { if insufficient material was } \\
\text { recovered to perform all analyses }\end{array}$ \\
\hline $\begin{array}{l}\text { Improper procedure } \\
\text { check }\end{array}$ & 98 & Alpha & $\begin{array}{l}\text { Enter a } P \text { if an improper sampling } \\
\text { procedure was followed }\end{array}$ \\
\hline
\end{tabular}


This is the description of the SAM93A format for coding and transmitting sample bottle data records.

\section{Sample Bottle Data Format SAM93A}

\begin{tabular}{|l|l|l|l|}
\hline Name & Columns & Type & Description \\
\hline Record format* & $1-8$ & Alphanumeric & SAM93A (the format of the data record) \\
\hline $\begin{array}{l}\text { Sample identification } \\
\text { number }\end{array}$ & $9-14$ & Integer & $\begin{array}{l}\text { The number of the sample printed on } \\
\text { the page of the sample logbook }\end{array}$ \\
\hline Bottle label number & $15-23$ & Integer & $\begin{array}{l}\text { The number of the bottle label attached } \\
\text { to the bottle }\end{array}$ \\
\hline
\end{tabular}

- Alpha and alphanumeric entries must be justified left in the records; numeric entries must be justified right in the records. There will be no leading zeros except in dates and times. 
This is the description of the STA93 format for coding and transmitting station data records.

\section{Station Data Format STA93}

\begin{tabular}{|l|l|l|l|}
\hline Name & Columns & Type & Description \\
\hline Record format* & $1-8$ & Alphanumeric & STA93 (the format of the data record) \\
\hline Station & $9-18$ & Alphanumeric & $\begin{array}{l}\text { The identification of the station (the } \\
\text { facility identification and sampling } \\
\text { point) }\end{array}$ \\
\hline $\begin{array}{l}\text { Savannah River Site } \\
\text { (SRS) north coordinate }\end{array}$ & $20-27$ & Numeric & $\begin{array}{l}\text { The SRS grid north coordinate of the } \\
\text { station reported in feet }\end{array}$ \\
\hline SRS east coordinate & $29-36$ & Numeric & $\begin{array}{l}\text { The SRS grid east coordinate of the } \\
\text { station reported in feet }\end{array}$ \\
\hline Coordinate error & $38-41$ & Numeric & $\begin{array}{l}\text { Estimated maximum error reported in } \\
\text { feet in the reported site coordinates. }\end{array}$ \\
\hline Ground elevation & $43-48$ & Numeric & $\begin{array}{l}\text { Ground elevation reported in feet } \\
\text { above median sea level }\end{array}$ \\
\hline $\begin{array}{l}\text { Universal Transverse } \\
\text { Mercator Zone 17 (UTM) } \\
\text { north coordinate }\end{array}$ & $51-58$ & Numeric & $\begin{array}{l}\text { The UTM grid north coordinate of the } \\
\text { station reported in meters }\end{array}$ \\
\hline UTM east coordinate & $60-67$ & Numeric & $\begin{array}{l}\text { The UTM grid east coordinate of the } \\
\text { station reported in meters }\end{array}$ \\
\hline
\end{tabular}

- Alpha and alphanumeric entries must be justified left in the records; numeric entries must be justified right in the records. There will be no leading zeros except in dates and times. 
This is the description of the format (COC93) for coding and transmitting chain-of-custody data records. This format has been established by EPD/EMS.

\section{Chain-Of-Custody Data Format COC93}

\begin{tabular}{|l|l|l|l|}
\hline Name & Columns & Type & Description \\
\hline Record format* & $1-8$ & Alphanumeric & COC93 (the format of the data record) \\
\hline $\begin{array}{l}\text { Sample identification } \\
\text { number }\end{array}$ & $9-18$ & Integer & $\begin{array}{l}\text { The number of the sample printed on the } \\
\text { page of the sample logbook }\end{array}$ \\
\hline $\begin{array}{l}\text { Laboratory sample } \\
\text { identification }\end{array}$ & $19-33$ & Alphanumeric & $\begin{array}{l}\text { Identifier used for the sample by the } \\
\text { analytical laboratory }\end{array}$ \\
\hline Sample collection date & $34-39$ & Numeric & $\begin{array}{l}\text { Sample collection date recorded using the } \\
\text { MMDDYY format }\end{array}$ \\
\hline Shipping date & $40-45$ & Numeric & $\begin{array}{l}\text { Sample shipping date recorded using the } \\
\text { MMDDYY format }\end{array}$ \\
\hline Cooler identification & $46-53$ & Alphanumeric & $\begin{array}{l}\text { The number assigned to the cooler in which } \\
\text { the samples are shipped to the analytical } \\
\text { laboratory }\end{array}$ \\
\hline $\begin{array}{l}\text { Sample temperature } \\
\text { upon receipt }\end{array}$ & $\mathbf{5 4 - 5 7}$ & Numeric & $\begin{array}{l}\text { The temperature of the samples upon } \\
\text { receipt at the analytical laboratory in } \\
\text { degrees centigrade }\end{array}$ \\
\hline
\end{tabular}

- Alpha and alphanumeric entries must be justified left in the records; numeric entries must be justified right in the records. There will be no leading zeros except in dates and times. 
This is the description of the format (AN95) for coding and transmitting analytical data records. This format has been established by the EPD/EMS of WSRC.

\section{Analytical Data Format AN95}

\begin{tabular}{|c|c|c|c|}
\hline Name & Columns & Type & Description \\
\hline WSRC identifier ${ }^{*} \dagger$ & $1-12$ & Alphanumeric & WSRC identifier for the sample \\
\hline COC number & $13-17$ & Alphanumeric & $\begin{array}{l}\text { Identifier of the sample's chain-of-custody } \\
\text { form (Required field for groundwater } \\
\text { samples; not used for soil samples) }\end{array}$ \\
\hline Sample collection date ${ }^{\dagger}$ & 18-23 & Numeric & $\begin{array}{l}\text { Date that the sample was collected. For } \\
\text { laboratory blanks and QA samples, enter } \\
\text { the preparation date. For TCLP samples, } \\
\text { enter the date of completion of TCLP } \\
\text { sample generation. }\end{array}$ \\
\hline Sample collection time & 24-27 & Numeric & Time of sample collection or generation \\
\hline Lab receipt date ${ }^{\dagger}$ & $28-33$ & Numeric & $\begin{array}{l}\text { Date that the sample was received by the } \\
\text { laboratory; leave blank for laboratory } \\
\text { blanks and QA samples. }\end{array}$ \\
\hline Extraction date & $34-39$ & Numeric & $\begin{array}{l}\text { Date that the sample was extracted; leave } \\
\text { blank unless the holding time is dependent } \\
\text { upon the extraction time. }\end{array}$ \\
\hline Extraction time & $40-43$ & Numeric & $\begin{array}{l}\text { Time that the sample extraction was } \\
\text { started; leave blank unless the holding } \\
\text { time is dependent upon the extraction time. }\end{array}$ \\
\hline $\begin{array}{l}\text { Extraction/digestion } \\
\text { method }\end{array}$ & $44-56$ & Alphanumeric & $\begin{array}{l}\text { The coded identifier for the method used to } \\
\text { perform the extraction or digestion (e.g., } \\
\text { EPA \#.\#) }\end{array}$ \\
\hline Analysis date ${ }^{\dagger}$ & $57-62$ & Numeric & $\begin{array}{l}\text { Date that the analyte concentration was } \\
\text { determined }\end{array}$ \\
\hline Analysis timet & $63-66$ & Numeric & $\begin{array}{l}\text { Time that the analyte concentration was } \\
\text { determined }\end{array}$ \\
\hline Analytical method ${ }^{\dagger}$ & $67-79$ & Alphanumeric & $\begin{array}{l}\text { The coded identifier for the method used to } \\
\text { perform the analysis (e.g., EPA\#.\#) }\end{array}$ \\
\hline Preparation batch & $80-87$ & Alphanumeric & $\begin{array}{l}\text { Identification of the sample preparation } \\
\text { batch }\end{array}$ \\
\hline Laboratory ${ }^{\dagger}$ & $88-89$ & Alphanumeric & $\begin{array}{l}\text { Code assigned by EPD/EMS to identify the } \\
\text { laboratory performing the analysis }\end{array}$ \\
\hline $\begin{array}{l}\text { Laboratory sample } \\
\text { identification }{ }^{\dagger}\end{array}$ & $90-104$ & Alphanumeric & $\begin{array}{l}\text { Identifier used for the sample by the } \\
\text { analytical laboratory }\end{array}$ \\
\hline
\end{tabular}

*Alpha and alphanumeric entries must be justified left in the records; numeric entries must be justified right in the records. There will be no leading zeros except in dates and times. Dates should be in MMDDYY format; times in HHMM format.

tThis field must have an entry for every record. 
Analytical Data Format AN95, Continued

\begin{tabular}{|c|c|c|c|}
\hline Name & Columns & Type & Description \\
\hline Laboratory replicate & $105-107$ & Alphanumeric & $\begin{array}{l}\text { Indicates that the sample is a non-blind } \\
\text { replicate analysis (codes on pages } \mathrm{C}-9 \text { and } \\
\mathrm{C}-10 \text { ) }\end{array}$ \\
\hline Analytet & $108-117$ & Alphanumeric & $\begin{array}{l}\text { The coded identifier (provided by } \\
\text { EPD/EMS) for the analyte }\end{array}$ \\
\hline Quantitation limit & $118-125$ & Numeric & $\begin{array}{l}\text { Both GE and Weston reported the sample- } \\
\text { specific estimated quantitation limit } \\
\text { (ssEQL) for non-radiochemistry analyses } \\
\text { All labs reported the minimum detectable } \\
\text { activity for radiochemistry analyses }\end{array}$ \\
\hline Result qualifier & $126-128$ & Alphanumeric & $\begin{array}{l}\text { The coded field used to qualify the } \\
\text { analytical result (codes in Appendix } D \text { ) }\end{array}$ \\
\hline Analysis qualifier & $129-131$ & Alphanumeric & $\begin{array}{l}\text { The coded field used to qualify the analysis } \\
\text { (codes in Appendix } D \text { ) }\end{array}$ \\
\hline Bias of analysis & 132 & Alphanumeric & $\begin{array}{l}\text { The coded field for data having result } \\
\text { qualifiers of } J \text { or } R \text { specifying the expected } \\
\text { bias (codes in Appendix } D \text { ) }\end{array}$ \\
\hline Analytical result & $133-142$ & Numeric & $\begin{array}{l}\text { The concentration of the analyte } \\
\text { For non-detects, the ssEQL is reported } \\
\text { Results were reported on a dry weight basis } \\
\text { for all analyses reported in per mass units }\end{array}$ \\
\hline Result units ${ }^{\dagger}$ & $143-146$ & Alpha & $\begin{array}{l}\text { The coded identifier (provided by } \\
\text { EPD/EMS) of the units in which the } \\
\text { analytical result is expressed }\end{array}$ \\
\hline Accuracy & $147-154$ & Numeric & $\begin{array}{l}\text { The statistically determined value, } 95 \% \\
\text { confidence level, representing a plus or } \\
\text { minus value for the result and reported in } \\
\text { the same units as the analytical result; } \\
\text { required for all radiochemical analyses } \\
\text { above the detection limit but not generally } \\
\text { reported for non-radiochemical analyses. } \\
\text { Do not report more figures than are } \\
\text { significant. }\end{array}$ \\
\hline Residual weight & $155-160$ & Numeric & $\begin{array}{l}\text { The weight of material in milligrams on the } \\
\text { planchet after evaporation; used for } \\
\text { radionuclide determinations. }\end{array}$ \\
\hline $\begin{array}{l}\text { Nominal } \\
\text { concentrationt }\end{array}$ & $161-168$ & Numeric & $\begin{array}{l}\text { The concentration of analyte added to the } \\
\text { sample prior to analysis; reported in the } \\
\text { same units as the analytical result; the } \\
\text { value of zero must be entered if the sample } \\
\text { was not spiked. }\end{array}$ \\
\hline Percent recovery & $169-174$ & Numeric & $\begin{array}{l}\text { Recovery of the spiked constituent reported } \\
\text { as a percentage }\end{array}$ \\
\hline
\end{tabular}


Analytical Data Format AN95, Continued

\begin{tabular}{|c|c|c|c|}
\hline Name & Columns & Type & Description \\
\hline Number of dilutionst & 175 & Numeric & $\begin{array}{l}\text { The number of steps required to reach the } \\
\text { final dilution factor; zero if the sample was } \\
\text { not diluted }\end{array}$ \\
\hline Dilution factor ${ }^{\dagger}$ & $176-181$ & Numeric & $\begin{array}{l}\text { The dilution used in the analysis; a value of } \\
\text { one indicates the sample was not diluted. }\end{array}$ \\
\hline Instrument ${ }^{\dagger}$ & $182-189$ & Alphanumeric & $\begin{array}{l}\text { The coded identifier for the instrument } \\
\text { used to perform the analysis; each } \\
\text { laboratory develops its own codes and } \\
\text { provides them to EPD/EMS. }\end{array}$ \\
\hline Instrument batch ${ }^{\dagger}$ & $190-197$ & Alphanumeric & $\begin{array}{l}\text { The number assigned by the analytical } \\
\text { laboratory to the analysis batch (group of } \\
\text { samples analyzed together in a single } \\
\text { instrument run along with the associated } \\
\text { QC samples) }\end{array}$ \\
\hline Analyst's initials ${ }^{\dagger}$ & $198-200$ & Alpha & The initials of the analyst \\
\hline Percent solids & $201-205$ & Numeric & $\begin{array}{l}\text { The percentage of the sample that is dry } \\
\text { solids; required field if result is reported in } \\
\text { per mass units. Not applicable for water } \\
\text { samples. }\end{array}$ \\
\hline Bottle label number & $206-215$ & Integer & $\begin{array}{l}\text { The number on the bottle label; required } \\
\text { field if bottle label is numbered. }\end{array}$ \\
\hline Sample fraction & 216-219 & Alphanumeric & $\begin{array}{l}\text { The coded identifier (provided by } \\
\text { EPD/EMS) for the fraction of the sample } \\
\text { analyzed. }\end{array}$ \\
\hline Validation status & $220-221$ & Alphanumeric & The lab will report a zero ("0"). \\
\hline EPD/EMS receipt date & $222-227$ & Numeric & $\begin{array}{l}\text { Date that the analytical data is received by } \\
\text { EPD/EMS from the laboratory. The } \\
\text { laboratory will leave this blank. }\end{array}$ \\
\hline
\end{tabular}


When making entries in the laboratory replicate field (columns 105-107), use only the codes listed below.

\section{Codes for Laboratory Replicates}

\begin{tabular}{|l|l|}
\hline Remark & Definition \\
\hline (blank) & Data not remarked; the result is a routine analysis of a sample. \\
\hline 1 & Laboratory replicate \\
\hline $2 \mathrm{~A}$ & Matrix spike \\
\hline $2 \mathrm{~B}$ & Matrix spike duplicate \\
\hline $2 \mathrm{C}$ & Surrogate spike \\
\hline $2 \mathrm{D}$ & Blank spike \\
\hline $2 \mathrm{E}$ & Blank spike duplicate \\
\hline 3 & Laboratory blank \\
\hline 4 & Re-analysis of a sample at the request of EPD/EMS \\
\hline CCB & Continuing calibration blank \\
\hline $\mathrm{CCV}$ & Continuing calibration verification sample \\
\hline ICB & Initial calibration blank \\
\hline ICS & Interference check sample \\
\hline ICV & Initial calibration verification sample \\
\hline
\end{tabular}

\section{WSRC Identifier for Groundwater Samples}

\begin{tabular}{|c|l|l|l|}
\hline Name & Columns & Type & Description \\
\hline Well name & & & Alpha \\
\hline Series name & $1-3$ & $\begin{array}{l}\text { Name of the well series (except 241-H) } \\
\text { from which the sample was collected; } \\
\text { QA/QC laboratory deionized water samples } \\
\text { will have LB (the first character is blank) } \\
\text { as well series designation. }\end{array}$ \\
\hline Cluster number & $4-6$ & Numeric & Number of the cluster (except 241-H) \\
\hline Well within cluster & $7-8$ & Alphanumeric & Can be blank \\
\hline & $9-10$ & Blank & Unused \\
\hline $\begin{array}{l}\text { Duplicate } \\
\text { identification }\end{array}$ & 11 & Alphanumeric & $\begin{array}{l}\text { Assigned by field personnel to identify } \\
\text { different sampling events occurring in one } \\
\text { day }\end{array}$ \\
\hline Secondary identifier & 12 & Alphanumeric & $\begin{array}{l}\text { Used as an alternate identifier by the } \\
\text { Groundwater Monitoring group }\end{array}$ \\
\hline
\end{tabular}


WSRC Identifier for Sediment, Core, and Surface Water Samples

\begin{tabular}{|l|l|l|l|}
\hline Name & Columns & Type & Description \\
\hline $\begin{array}{l}\text { Sample identification } \\
\text { number }\end{array}$ & $1-6$ & Numeric & The sample logbook page number \\
\hline
\end{tabular}




\section{Data Table Generation}

The data tables in this appendix are provided as an adjunct to the electronic data files. Selected sampling, station, and analytical data are included for all samples. The tables are ordered by sample designation specified in the test plan. Results for laboratory blanks, surrogates, matrix spikes, and other laboratory QA/QC samples are not presented in the printed data tables.

Table generation does not in any way alter the data in the electronic data files.

The tables are generated from the validated data files by a SAS program that combines and processes the data into a form suitable for word-processing software. The SAS program's output is a combination of sampling, station, and analytical data, which are otherwise maintained as separate data files. For presentation in the tables, analytical data were rounded to two significant digits.

For each sample, the header provides the sample name and COC number. If the sample is an environmental sample, the header further provides the sampling location in SRS coordinates. If the sample is a duplicate, blank, etc., the header specifies the sample type and identifies the primary sample with which the sample is associated.

The table includes columns for constituent names, result qualifiers, analysis qualifiers, bias codes, results, units of measurement, detection limits, and analytical methods. The constituents are sorted first by analytical suite and then within each suite according to accepted chemical nomenclature as specified by the American Chemical Society. Analytical suites are set off by identifying headers.

\section{Abbreviations Used in the Tables}

\begin{tabular}{|l|l|}
\hline MSL & Mean sea level \\
\hline $\mathrm{RQ}$ & Result qualifier \\
\hline $\mathrm{AQ}$ & Analysis qualifier \\
\hline $\mathrm{B}$ & Bias qualifier \\
\hline D. Limit & Detection limit (sample quantitation limit) \\
\hline
\end{tabular}




\section{Qualifier Codes}

Result Qualifiers

\begin{tabular}{|l|l|}
\hline (blank) & Data not remarked. Number should be interpreted exactly as reported. \\
\hline $\mathrm{C}^{*}$ & $\begin{array}{l}\text { Calculated. Analytical result reported was not measured directly but was } \\
\text { calculated from other data available. This does not apply to conversions, nor } \\
\text { does it apply to calculations made as part of an analytical procedure. }\end{array}$ \\
\hline $\mathrm{I}$ & $\begin{array}{l}\text { The value in the result field is the instrument reading, not the sample. } \\
\text { quantitation limit. Always use with the result qualifier } U .\end{array}$ \\
\hline $\mathrm{J}^{* *}$ & The analytical result is an estimated quantity. \\
\hline $\mathrm{R}^{*}$ & $\begin{array}{l}\text { Result was rejected because performance requirements in the sample or } \\
\text { associated QC analysis were not met. The analyte may or may not be present. }\end{array}$ \\
\hline $\mathrm{U}^{* *}$ & $\begin{array}{l}\text { Material analyzed for but not detected. The analyte concentration is less than } \\
\text { the sample-specific estimated quantitation limit (ssEQL). NOTE: when both } \\
\text { the result qualifier } U \text { and the result qualifier } I \text { are reported, the numerical } \\
\text { value in the result field is the instrument reading rather than the ssEQL. }\end{array}$ \\
\hline
\end{tabular}

\section{Analysis Qualifiers}

\begin{tabular}{|c|c|}
\hline $\mathrm{C}$ & $\begin{array}{l}\text { Laboratory Control Sample } \\
\text { Criteria were not met. }\end{array}$ \\
\hline $\mathrm{D}$ & $\begin{array}{l}\text { Inductively Coupled Plasma (ICP) Serial Dilution } \\
\text { Criteria were not met. }\end{array}$ \\
\hline $\mathrm{E}$ & $\begin{array}{l}\text { The detected result is between the ssEQL and the method detection limit. } \\
\text { Report the actual result detected. Use with } J \text {. }\end{array}$ \\
\hline $\mathrm{G}$ & $\begin{array}{l}\text { Pesticides Instrument Performance } \\
\text { Use for all conditions outside criteria other than those flagged } N \text { (see code } N \\
\text { below). }\end{array}$ \\
\hline $\mathrm{I}$ & $\begin{array}{l}\text { Matrix Spike/Matrix Spike Duplicate } \\
\text { Matrix Spike Sample Analysis } \\
\text { Spike recovery was not within control limits. }\end{array}$ \\
\hline $\mathrm{K}$ & $\begin{array}{l}\text { Tentatively Identified Compounds (TICs) } \\
\text { A tentatively identified compound is a suspected aldol-condensation product. } \\
\text { Use with both } J \text { result and } N \text { analysis qualifiers. }\end{array}$ \\
\hline $\mathrm{N}$ & $\begin{array}{l}\text { Tentatively Identified Compounds } \\
\text { All TIC results. Use with } J \text {. }\end{array}$ \\
\hline 0 & $\begin{array}{l}\text { Surrogate Recovery } \\
\text { Surrogate spike recovery results are not within control limits. }\end{array}$ \\
\hline $\mathrm{Q}$ & $\begin{array}{l}\text { Holding Time } \\
\text { Sample held beyond normal holding time. }\end{array}$ \\
\hline
\end{tabular}




\section{Analysis Qualifiers, continued}

\begin{tabular}{|c|c|}
\hline $\mathrm{V}$ & $\begin{array}{l}\text { Method Blank Contamination } \\
\text { Indicates the analyte was detected in both the sample and associated method } \\
\text { blank. Use alone or with } U \text { in cases where the sample falls within the CLP } 5 \times \\
\text { and } 10 \times \text { rule. Use with } U J \text { if the laboratory adjusts a sample result to equal } \\
5 \times \text { or } 10 \times \text { the result in the method blank. }\end{array}$ \\
\hline $\mathrm{W}$ & $\begin{array}{l}\text { Furnace Atomic Absorption QC } \\
\text { Post-digestion spike recovery for furnace atomic absorption (AA) analysis is not } \\
\text { within control limits while sample absorbance is less than } 50 \% \text { of spike } \\
\text { absorbance. }\end{array}$ \\
\hline $\bar{X}$ & $\begin{array}{l}\text { Matrix Spike/Matrix Spike Duplicates } \\
\text { Laboratory Duplicate Sample Analysis } \\
\text { Duplicate analysis relative percent difference (RPD) is not within control } \\
\text { limits. }\end{array}$ \\
\hline $\bar{Y}$ & $\begin{array}{l}\text { Result obtained from unpreserved or improperly preserved sample. Data may } \\
\text { not be accurate. }\end{array}$ \\
\hline 4 & Matrix interference. Value cannot be determined. Use with $R$. \\
\hline 5 & $\begin{array}{l}\text { The analytical value was four times higher than the standard concentration, } \\
\text { and percent recovery cannot be determined. Use with } R \text {. }\end{array}$ \\
\hline 8 & $\begin{array}{l}\text { Indicates the analyte was detected in both the sample and associated trip } \\
\text { blank. Use alone or with } U \text { in cases where the sample falls within the CLP } 5 \times \\
\text { and } 10 \times \text { rule. }\end{array}$ \\
\hline
\end{tabular}

\section{Bias Codes}

\begin{tabular}{|l|l|}
\hline $\mathrm{H}$ & $\begin{array}{l}\text { Analytical factor causing bias. The associated result may overestimate the true } \\
\text { value. }\end{array}$ \\
\hline $\mathrm{L}$ & $\begin{array}{l}\text { Analytical factor causing bias. The associated result may underestimate the } \\
\text { true value. }\end{array}$ \\
\hline $\mathrm{P}$ & $\begin{array}{l}\text { Analytical factor causing poor precision. The associated result may be of high } \\
\text { variability. }\end{array}$ \\
\hline
\end{tabular}

\footnotetext{
- This code is based on the STORET codes from the U.S. Environmental Protection Agency (EPA).

" This code is based on the CLP Laboratory Data Qualifiers from the U.S. EPA.
} 
Survey location: 44879E 3678269N (SRS coordinates)

Depth of core interval: 0.00 to $1.00 \mathrm{ft}$

Sample type: Normal

Sample matrix: Soil
Percent solids: 84.00

Unit

D. Limit Method

Physical Parameters and Specified Analyses

Cation exchange capacity
Chloride
Cyanide
Nitrate-nitrite as nitrogen
Orthophosphate
Phenols
Sulfide
Total organic carbon
Total organic nitrogen
Total phosphates (as P)

Metals (total recoverable)

Aluminum

Aluminum

Antimony

Antimony

Arsenic

Arsenic

Barium

Barium

Beryllium

Beryllium

Cadmium

Cadmium

Calcium

Calcium

Chromium

Chromium

Cobalt

Cobalt

Copper

Copper

Iron

Iron

Lead

Lead

Magnesium

Magnesium

Manganese

Manganese

Mercury

Mercury

Nickel

Nickel

Potassium

Potassium

Selenium

Selenium

$\begin{array}{lll} & & 80.1 \\ \mathrm{~J} & \mathrm{E} & 1.61 \\ \mathrm{U} & & \mathbf{0 . 9 0 9} \\ \mathrm{J} & & 1.04 \\ \mathrm{U} & \mathrm{E} & 4.28 \\ \mathrm{UJ} & \mathrm{C} & 381 \\ & & 264 \\ \mathrm{C} & & 3700 \\ \mathrm{~J} & \mathrm{E} & \mathbf{1 7 5}\end{array}$

$\begin{array}{ll}\mathrm{meq} / 100 \mathrm{~g} & 5 \\ \mathrm{mg} / \mathrm{kg} & 2.95 \\ \mathrm{mg} / \mathrm{kg} & 0.909 \\ \mathrm{mg} / \mathrm{kg} & 1.04 \\ \mathrm{mg} / \mathrm{kg} & 20.6 \\ \mu \mathrm{g} / \mathrm{kg} & 381 \\ \mathrm{mg} / \mathrm{kg} & 264 \\ \mathrm{mg} / \mathrm{kg} & 119 \\ \mathrm{mg} / \mathrm{kg} & 0.238 \\ \mathrm{mg} / \mathrm{kg} & 425\end{array}$

EPA9081

EPA300.0

EPA335.3

EPA353:1

EPA365.2

EPA420.2

EPA376.2

EPA415.1

EPA351.2

EPA365.4

\begin{tabular}{|c|c|c|c|}
\hline & $X$ & & 2860 \\
\hline & $X$ & & 4220 \\
\hline UJ & I & L & 4.46 \\
\hline UJ & I & $\mathbf{L}$ & 5.31 \\
\hline $\mathbf{U}$ & & & 13.4 \\
\hline$U$ & & & 15.9 \\
\hline & & & 8.93 \\
\hline & & & 8.68 \\
\hline$J$ & $\mathrm{E}$ & & 0.0576 \\
\hline$J$ & $\mathrm{E}$ & & 0.0558 \\
\hline $\mathrm{U}$ & & & 0.223 \\
\hline $\mathrm{U}$ & & & 0.265 \\
\hline & & & 47.3 \\
\hline & & & 54.3 \\
\hline & & & 4.97 \\
\hline & & & 4.86 \\
\hline$J$ & $\mathrm{E}$ & & 0.274 \\
\hline$J$ & $\mathrm{E}$ & & 0.344 \\
\hline & $X$ & & 1.54 \\
\hline & $X$ & & 4.63 \\
\hline & & & 3400 \\
\hline & & & 2800 \\
\hline $\mathrm{J}$ & $\mathrm{E}$ & & 2.7 \\
\hline $\mathrm{J}$ & $\mathbf{E}$ & & 3.19 \\
\hline$J$ & $I$ & $\mathrm{H}$ & 50.3 \\
\hline$J$ & I & $\mathrm{H}$ & 52.9 \\
\hline & $X$ & & 6.4 \\
\hline & $\mathrm{X}$ & & 23.3 \\
\hline & $\mathrm{V}$ & & 0.0366 \\
\hline & $\mathrm{V}$ & & 0.0509 \\
\hline $\mathrm{J}$ & $\mathbf{E}$ & & 0.818 \\
\hline$J$ & $\mathrm{E}$ & & 0.947 \\
\hline $\boldsymbol{J}$ & $\mathrm{E}$ & & 47.5 \\
\hline $\mathrm{J}$ & $E$ & & 39.6 \\
\hline $\mathrm{U}$ & & & 13.4 \\
\hline $\mathrm{U}$ & & & 15.9 \\
\hline
\end{tabular}

\begin{tabular}{lll}
$\mathrm{mg} / \mathrm{kg}$ & 11.2 & EPA6010A \\
$\mathrm{mg} / \mathrm{kg}$ & 13.3 & EPA6010A \\
$\mathrm{mg} / \mathrm{kg}$ & 4.46 & EPA6010A \\
$\mathrm{mg} / \mathrm{kg}$ & 5.31 & EPA6010A \\
$\mathrm{mg} / \mathrm{kg}$ & 13.4 & EPA6010A \\
$\mathrm{mg} / \mathrm{kg}$ & 15.9 & EPA6010A \\
$\mathrm{mg} / \mathrm{kg}$ & 1.12 & EPA6010A \\
$\mathrm{mg} / \mathrm{kg}$ & 1.33 & EPA6010A \\
$\mathrm{mg} / \mathrm{kg}$ & 0.446 & EPA6010A \\
$\mathrm{mg} / \mathrm{kg}$ & 0.531 & EPA6010A \\
$\mathrm{mg} / \mathrm{kg}$ & 0.223 & EPA6010A \\
$\mathrm{mg} / \mathrm{kg}$ & 0.265 & EPA6010A \\
$\mathrm{mg} / \mathrm{kg}$ & 11.2 & EPA6010A \\
$\mathrm{mg} / \mathrm{kg}$ & 13.3 & EPA6010A \\
$\mathrm{mg} / \mathrm{kg}$ & 1.12 & EPA6010A \\
$\mathrm{mg} / \mathrm{kg}$ & 1.33 & EPA6010A \\
$\mathrm{mg} / \mathrm{kg}$ & 0.446 & EPA6010A \\
$\mathrm{mg} / \mathrm{kg}$ & 0.531 & EPA6010A \\
$\mathrm{mg} / \mathrm{kg}$ & 1.12 & EPA6010A \\
$\mathrm{mg} / \mathrm{kg}$ & 1.33 & EPA6010A \\
$\mathrm{mg} / \mathrm{kg}$ & 4.46 & EPA6010A \\
$\mathrm{mg} / \mathrm{kg}$ & 5.31 & EPA6010A \\
$\mathrm{mg} / \mathrm{kg}$ & 4.46 & EPA6010A \\
$\mathrm{mg} / \mathrm{kg}$ & 5.31 & EPA6010A \\
$\mathrm{mg} / \mathrm{kg}$ & 4.46 & EPA6010A \\
$\mathrm{mg} / \mathrm{kg}$ & 5.31 & EPA6010A \\
$\mathrm{mg} / \mathrm{kg}$ & 0.446 & EPA6010A \\
$\mathrm{mg} / \mathrm{kg}$ & 0.531 & EPA6010A \\
$\mathrm{mg} / \mathrm{kg}$ & 0.028 & EPA7471 \\
$\mathrm{mg} / \mathrm{kg}$ & 0.0377 & EPA7471 \\
$\mathrm{mg} / \mathrm{kg}$ & 1.12 & EPA6010A \\
$\mathrm{mg} / \mathrm{kg}$ & 1.33 & EPA6010A \\
$\mathrm{mg} / \mathrm{kg}$ & 89.2 & EPA6010A \\
$\mathrm{mg} / \mathrm{kg}$ & 106 & EPA6010A \\
$\mathrm{mg} / \mathrm{kg}$ & 13.4 & EPA6010A \\
\hline
\end{tabular}


SURVEY ID: PPSC 0101 (continued)

Constituent

Metals (total recoverable)
Sample ID: 104600

RQ AQ B Result Unit D. Limit Method

\begin{tabular}{|c|c|c|c|c|c|}
\hline Silver & U & 1.12 & $\mathrm{mg} / \mathrm{kg}$ & 1.12 & EPA6010A \\
\hline Silver & $\mathrm{U}$ & 1.33 & $\mathrm{mg} / \mathrm{kg}$ & 1.33 & EPA6010A \\
\hline Sodium & $\mathrm{U}$ & 44.6 & $\mathrm{mg} / \mathrm{kg}$ & 44.6 & EPA6010A \\
\hline Sodium & $\mathrm{U}$ & 53.1 & $\mathrm{mg} / \mathrm{kg}$ & 53.1 & EPA6010A \\
\hline Thallium & $\mathrm{U}$ & 13.4 & $\mathrm{mg} / \mathrm{kg}$ & 13.4 & EPA6010A \\
\hline Thallium & U & 15.9 & $\mathrm{mg} / \mathrm{kg}$ & 15.9 & EPA6010A \\
\hline Vanadium & & 9.25 & $\mathrm{mg} / \mathrm{kg}$ & 0.446 & EPA6010A \\
\hline Vanadium & & 7.8 & $\mathrm{mg} / \mathrm{kg}$ & 0.531 & EPA6010A \\
\hline Zinc & $\mathrm{X}$ & 2.42 & $\mathrm{mg} / \mathrm{kg}$ & 0.892 & EPA6010A \\
\hline Zinc & $\mathrm{X}$ & 6.1 & $\mathrm{mg} / \mathrm{kg}$ & 1.06 & EPA6010A \\
\hline
\end{tabular}

$B / N / A$ Extractables (including $P A H$ and phenols)

\begin{tabular}{|c|c|c|c|}
\hline Acenaphthene & U & & 39.3 \\
\hline Acenaphthylene & $U$ & & 39.3 \\
\hline Anthracene & $\mathrm{U}$ & & 39.3 \\
\hline Benzo[a] anthracene & $U$ & & 3.93 \\
\hline Benzo[b]fluoranthene & $\mathrm{U}$ & & 3.93 \\
\hline Benzo $[k]$ fluoranthene & $U$ & & 3.93 \\
\hline Benzoic acid & $\mathrm{U}$ & & 39.3 \\
\hline Benzo[g,h,i]perylene & U & & 39.3 \\
\hline Benzo[a]pyrene & $\mathrm{U}$ & & 3.93 \\
\hline Benzyl alcohol & $\mathrm{U}$ & & 39.3 \\
\hline Bis(2-chloroethoxy) methane & $\mathrm{U}$ & & 39.3 \\
\hline Bis(2-chloroethyl) ether & U & & 39.3 \\
\hline Bis(2-chloroisopropyl) ether & $\mathrm{U}$ & & 39.3 \\
\hline Bis(2-ethylhexyl) phthalate & $\vec{J}$ & $\mathbf{E}$ & 29.1 \\
\hline 4-Bromophenyl phenyl ether & $\mathrm{U}$ & & 39.3 \\
\hline Butylbenzyl phthalate & $\mathrm{U}$ & & 39.3 \\
\hline 4-Chloroaniline & $\mathrm{U}$ & & 39.3 \\
\hline 4-Chloro-m-cresol & $\mathrm{U}$ & & 39.3 \\
\hline 2-Chloronaphthalene & $\mathrm{U}$ & & 39.3 \\
\hline 2-Chlorophenol & $\mathrm{U}$ & & 39.3 \\
\hline 4-Chlorophenyl phenyl ether & $\mathrm{U}$ & & 39.3 \\
\hline Chrysene & $\mathrm{U}$ & & 3.93 \\
\hline $\mathrm{m} / \mathrm{p}$-Cresol & U & & 393 \\
\hline o-Cresol (2-Methylphenol) & $\mathrm{U}$ & & 39.3 \\
\hline Dibenz $[\alpha, h]$ anthracene & $\mathbf{U}$ & & 3.93 \\
\hline Dibenzofuran & $\mathrm{U}$ & & 39.3 \\
\hline Di-n-butyl phthalate & $\mathrm{U}$ & $\mathrm{V}$ & 63.6 \\
\hline 1,2-Dichlorobenzene & $\mathrm{U}$ & & 39.3 \\
\hline 1,3-Dichlorobenzene & $\mathrm{U}$ & & 39.3 \\
\hline 1,4-Dichlorobenzene & $\mathrm{U}$ & & 39.3 \\
\hline 3,3'-Dichlorobenzidine & $\mathbf{U}$ & & 39.3 \\
\hline 2,4-Dichlorophenol & U & & 39.3 \\
\hline Diethyl phthalate & $\mathrm{U}$ & & 39.3 \\
\hline 2,4-Dimethyl phenol & $\mathrm{U}$ & & 39.3 \\
\hline Dimethyl phthalate & $\mathrm{U}$ & & 39.3 \\
\hline 2,4-Dinitrophenol & $\mathrm{U}$ & & 393 \\
\hline 2,4-Dinitrotoluene & UJ & C & 3.93 \\
\hline 2,6-Dinitrotoluene & $U$ & & 3.93 \\
\hline Di-n-octyl phthalate & $\mathrm{U}$ & & 39.3 \\
\hline Ergostanol & $J$ & $N$ & 220 \\
\hline Fluoranthene & $\mathrm{U}$ & & 39.3 \\
\hline Fluorene & $\mathrm{U}$ & & 39.3 \\
\hline
\end{tabular}

\begin{tabular}{|c|c|c|}
\hline$\mu g / k g$ & 39.3 & EPA8270 \\
\hline$\mu \mathrm{g} / \mathrm{kg}$ & 39.3 & EPA8270 \\
\hline$\mu \mathrm{g} / \mathrm{kg}$ & 39.3 & EPA8270 \\
\hline$\mu \mathrm{g} / \mathrm{kg}$ & 3.93 & EPA8270 \\
\hline$\mu \mathrm{g} / \mathrm{kg}$ & 3.93 & EPA8270 \\
\hline$\mu g / \mathrm{kg}$ & 3.93 & EPA8270 \\
\hline$\mu \mathrm{g} / \mathrm{kg}$ & 39.3 & EPA8270 \\
\hline$\mu \mathrm{g} / \mathrm{kg}$ & 39.3 & EPA8270 \\
\hline$\mu \mathrm{g} / \mathrm{kg}$ & 3.93 & EPA8270 \\
\hline$\mu \mathrm{g} / \mathrm{kg}$ & 39.3 & EPA8270 \\
\hline$\mu \mathrm{g} / \mathrm{kg}$ & 39.3 & EPA8270 \\
\hline$\mu \mathrm{g} / \mathrm{kg}$ & 39.3 & EPA8270 \\
\hline$\mu \mathrm{g} / \mathrm{kg}$ & 39.3 & EPA8270 \\
\hline$\mu \mathrm{g} / \mathrm{kg}$ & 39.3 & EPA8270 \\
\hline$\mu \mathrm{g} / \mathrm{kg}$ & 39.3 & EPA8270 \\
\hline$\mu \mathrm{g} / \mathrm{kg}$ & 39.3 & EPA8270 \\
\hline$\mu \mathrm{g} / \mathrm{kg}$ & 39.3 & EPA8270 \\
\hline$\mu \mathrm{g} / \mathrm{kg}$ & 39.3 & EPA8270 \\
\hline$\mu \mathrm{g} / \mathrm{kg}$ & 39.3 & EPA8270 \\
\hline$\mu \mathrm{g} / \mathrm{kg}$ & 39.3 & EPA8270 \\
\hline$\mu \mathrm{g} / \mathrm{kg}$ & 39.3 & EPA8270 \\
\hline$\mu \mathrm{g} / \mathrm{kg}$ & 3.93 & EPA8270 \\
\hline$\mu \mathrm{g} / \mathrm{kg}$ & 393 & EPA8270 \\
\hline$\mu \mathrm{g} / \mathrm{kg}$ & 39.3 & EPA 8270 \\
\hline$\mu \mathrm{g} / \mathrm{kg}$ & 3.93 & EPA8270 \\
\hline$\mu \mathrm{g} / \mathrm{kg}$ & 39.3 & EPA8270 \\
\hline$\mu \mathrm{g} / \mathrm{kg}$ & 39.3 & EPA8270 \\
\hline$\mu \mathrm{g} / \mathrm{kg}$ & 39.3 & EPA8270 \\
\hline$\mu \mathrm{g} / \mathrm{kg}$ & 39.3 & EPA8270 \\
\hline$\mu \mathrm{g} / \mathrm{kg}$ & 39.3 & EPA8270 \\
\hline$\mu \mathrm{g} / \mathrm{kg}$ & 39.3 & EPA8270 \\
\hline$\mu \mathrm{g} / \mathrm{kg}$ & 39.3 & EPA8270 \\
\hline$\mu \mathrm{g} / \mathrm{kg}$ & 39.3 & EPA8270 \\
\hline$\mu \mathrm{g} / \mathrm{kg}$ & 39.3 & EPA8270 \\
\hline$\mu \mathrm{g} / \mathrm{kg}$ & 39.3 & EPA8270 \\
\hline$\mu \mathrm{g} / \mathrm{kg}$ & 393 & EPA8270 \\
\hline$\mu \mathrm{g} / \mathrm{kg}$ & 3.93 & EPA8270 \\
\hline$\mu \mathrm{g} / \mathrm{kg}$ & 3.93 & EPA8270 \\
\hline$\mu \mathrm{g} / \mathrm{kg}$ & 39.3 & EPA8270 \\
\hline$\mu \mathrm{g} / \mathrm{kg}$ & & EPA8270 \\
\hline$\mu \mathrm{g} / \mathrm{kg}$ & 39.3 & EPA8270 \\
\hline$\mu \mathrm{g} / \mathrm{kg}$ & 39.3 & EPA8270 \\
\hline
\end{tabular}


Appendix D: Sediment Tables

D-6

SURVEY ID: PPSC 0101 (continued)

Sample ID: 104600

Constituent

RQ AQ B Result

Unit

D. Limit Method

$B / N / A$ Extractables (including PAH and phenols)

Hexachlorobenzene
Hexachlorobutadiene
Hexachlorocyclopentadiene
Hexachloroethane
Indeno[1,2,3-c,d]pyrene
Isophorone
2-Methyl-4,6-dinitrophenol
2-Methylnaphthalene
Naphthalene
m-Nitroaniline
o-Nitroaniline
p-Nitroaniline
Nitrobenzene
2-Nitrophenol
4-Nitrophenol
N-Nitrosodiphenylamine
N-Nitrosodi-n-propylamine
Octadecenamide
Pentachlorophenol
Phenanthrene
Phenol
Pyrene
Stigmasterol
1,2,4-Trichlorobenzene
2,4,5-Trichlorophenol
2,4,6-Trichlorophenol
Unknown acid
Unknown alkane
Unknown hydrocarbon
Unknown hydrocarbon

$\begin{array}{lll}\text { U } & & 3.93 \\ \text { U } & & 39.3 \\ U & & 39.3 \\ U & & 3.93 \\ U & & 3.93 \\ U & & 39.3 \\ U & & 393 \\ U & & 39.3 \\ U & & 39.3 \\ U & & 39.3 \\ \text { U } & & 39.3 \\ \text { U } & & 39.3 \\ \text { U } & & 39.3 \\ \text { U } & & 39.3 \\ \text { U } & & 39.3 \\ \text { U } & & 39.3 \\ \text { U } & & 39.3 \\ \text { J } & \text { N } & 200 \\ \text { UJ } & \text { C } & 39.3 \\ \text { U } & & 39.3 \\ \text { U } & & 39.3 \\ \text { U } & & 39.3 \\ \text { J } & \text { N } & 200 \\ \text { U } & & 3.93 \\ \text { U } & & 39.3 \\ \text { U } & & 39.3 \\ \text { J } & \text { N } & 200 \\ \text { J } & \text { N } & 540 \\ \text { J } & \text { N } & 600 \\ \text { J } & \text { N } & 180 \\ & & \end{array}$

$\begin{array}{lll}\mu \mathrm{g} / \mathrm{kg} & 3.93 & \text { EPA8270 } \\ \mu \mathrm{g} / \mathrm{kg} & 39.3 & \text { EPA8270 } \\ \mu \mathrm{g} / \mathrm{kg} & 39.3 & \text { EPA8270 } \\ \mu \mathrm{g} / \mathrm{kg} & 3.93 & \text { EPA8270 } \\ \mu \mathrm{g} / \mathrm{kg} & 3.93 & \text { EPA8270 } \\ \mu \mathrm{g} / \mathrm{kg} & 39.3 & \text { EPA8270 } \\ \mu \mathrm{g} / \mathrm{kg} & 393 & \text { EPA8270 } \\ \mu \mathrm{g} / \mathrm{kg} & 39.3 & \text { EPA8270 } \\ \mu \mathrm{g} / \mathrm{kg} & 39.3 & \text { EPA8270 } \\ \mu \mathrm{g} / \mathrm{kg} & 39.3 & \text { EPA8270 } \\ \mu \mathrm{g} / \mathrm{kg} & 39.3 & \text { EPA8270 } \\ \mu \mathrm{g} / \mathrm{kg} & 39.3 & \text { EPA8270 } \\ \mu \mathrm{g} / \mathrm{kg} & 39.3 & \text { EPA8270 } \\ \mu \mathrm{g} / \mathrm{kg} & 39.3 & \text { EPA8270 } \\ \mu \mathrm{g} / \mathrm{kg} & 39.3 & \text { EPA8270 } \\ \mu \mathrm{g} / \mathrm{kg} & 39.3 & \text { EPA8270 } \\ \mu \mathrm{g} / \mathrm{kg} & 39.3 & \text { EPA8270 } \\ \mu \mathrm{g} / \mathrm{kg} & & \text { EPA8270 } \\ \mu \mathrm{g} / \mathrm{kg} & 39.3 & \text { EPA8270 } \\ \mu \mathrm{g} / \mathrm{kg} & 39.3 & \text { EPA8270 } \\ \mu \mathrm{g} / \mathrm{kg} & 39.3 & \text { EPA8270 } \\ \mu \mathrm{g} / \mathrm{kg} & 39.3 & \text { EPA8270 } \\ \mu \mathrm{g} / \mathrm{kg} & & \text { EPA8270 } \\ \mu \mathrm{g} / \mathrm{kg} & 3.93 & \text { EPA8270 } \\ \mu \mathrm{g} / \mathrm{kg} & 39.3 & \text { EPA8270 } \\ \mu \mathrm{g} / \mathrm{kg} & 39.3 & \text { EPA8270 } \\ \mu \mathrm{g} / \mathrm{kg} & & \text { EPA8270 } \\ \mu \mathrm{g} / \mathrm{kg} & & \text { EPA8270 } \\ \mu \mathrm{g} / \mathrm{kg} & & \text { EPA8270 } \\ \mu \mathrm{g} / \mathrm{kg} & & \text { EPA8270 }\end{array}$

Volatile Organic Compounds

\begin{tabular}{|c|c|c|c|}
\hline Acetone & $\mathbf{U}$ & 8 & 25 \\
\hline Acetone & $\mathrm{U}$ & 8 & 24.3 \\
\hline Benzene & $U$ & & 0.595 \\
\hline Benzene & $\mathrm{U}$ & & 0.595 \\
\hline Bromodichloromethane & $\mathrm{U}$ & & 0.0595 \\
\hline Bromodichloromethane & $\mathrm{U}$ & & 0.0595 \\
\hline Bromoform & $\mathrm{U}$ & & 0.0595 \\
\hline Bromoform & $\mathrm{U}$ & & 0.0595 \\
\hline Bromomethane (Methyl bromide) & $\mathrm{U}$ & & 0.119 \\
\hline Bromomethane (Methyl bromide) & $\mathrm{U}$ & & 0.119 \\
\hline Carbon disulfide & $\mathrm{U}$ & & 1.19 \\
\hline Carbon disulfide & $\mathrm{U}$ & & 1.19 \\
\hline Carbon tetrachloride & $\mathrm{U}$ & & 0.0595 \\
\hline Carbon tetrachloride & $\mathrm{U}$ & & 0.0595 \\
\hline Chlorobenzene & $\mathrm{U}$ & & 0.0595 \\
\hline Chlorobenzene & $\mathrm{U}$ & & 0.0595 \\
\hline Chloroethane & $\mathrm{U}$ & & 0.119 \\
\hline Chloroethane & $\mathrm{U}$ & & 0.119 \\
\hline Chloroethene (Vinyl chloride) & $\mathrm{U}$ & & 0.119 \\
\hline Chloroethene (Vinyl chloride) & $\mathrm{U}$ & & 0.119 \\
\hline Chloroform & $U$ & & 0.0595 \\
\hline & U & & 0.0595 \\
\hline
\end{tabular}

$\begin{array}{lll}\mu g / k g & 5.95 & \text { EPA8260 } \\ \mu \mathrm{g} / \mathrm{kg} & 5.95 & \text { EPA8260 } \\ \mu \mathrm{g} / \mathrm{kg} & 0.595 & \text { EPA8260 } \\ \mu \mathrm{g} / \mathrm{kg} & 0.595 & \text { EPA8260 } \\ \mu \mathrm{g} / \mathrm{kg} & 0.0595 & \text { EPA8260 } \\ \mu \mathrm{g} / \mathrm{kg} & 0.0595 & \text { EPA8260 } \\ \mu \mathrm{g} / \mathrm{kg} & 0.0595 & \text { EPA8260 } \\ \mu \mathrm{g} / \mathrm{kg} & 0.0595 & \text { EPA8260 } \\ \mu \mathrm{g} / \mathrm{kg} & 0.119 & \text { EPA8260 } \\ \mu \mathrm{g} / \mathrm{kg} & 0.119 & \text { EPA8260 } \\ \mu \mathrm{g} / \mathrm{kg} & 1.19 & \text { EPA8260 } \\ \mu \mathrm{g} / \mathrm{kg} & 1.19 & \text { EPA8260 } \\ \mu \mathrm{g} / \mathrm{kg} & 0.0595 & \text { EPA8260 } \\ \mu \mathrm{g} / \mathrm{kg} & 0.0595 & \text { EPA8260 } \\ \mu \mathrm{g} / \mathrm{kg} & 0.0595 & \text { EPA8260 } \\ \mu \mathrm{g} / \mathrm{kg} & 0.0595 & \text { EPA8260 } \\ \mu \mathrm{g} / \mathrm{kg} & 0.119 & \text { EPA8260 } \\ \mu \mathrm{g} / \mathrm{kg} & 0.119 & \text { EPA8260 } \\ \mu \mathrm{g} / \mathrm{kg} & 0.119 & \text { EPA8260 } \\ \mu \mathrm{g} / \mathrm{kg} & 0.119 & \text { EPA8260 } \\ \mu \mathrm{g} / \mathrm{kg} & 0.0595 & \text { EPA8260 } \\ \mu \mathrm{g} / \mathrm{kg} & 0.0595 & \text { EPA8260 }\end{array}$


SURVEY ID: PPSC 0101 (continued)

Constituent

Volatile Organic Compounds

Chloromethane (Methyl chloride)

Chloromethane (Methyl chloride)

Dibromochloromethane

Dibromochloromethane

1,1-Dichloroethane

1,1-Dichloroethane

1,2-Dichloroethane

1,2-Dichloroethane

1,1-Dichloroethylene

1,1-Dichloroethylene

1,2-Dichloroethylene

1,2-Dichloroethylene

Dichloromethane (Methylene chloride)

Dichloromethane (Methylene chloride)

1,2-Dichloropropane

1,2-Dichloropropane

cis-1,3-Dichloropropene

cis-1,3-Dichloropropene

trans-1,3-Dichloropropene

trans-1,3-Dichloropropene

Ethylbenzene

Ethylbenzene

2-Hexanone

2-Hexanone

Methyl cyclopentane

Methyl ethyl ketone

Methyl ethyl ketone

Methyl isobutyl ketone

Methyl isobutyl ketone

Styrene

Styrene

1,1,2,2-Tetrachloroethane

1,1,2,2-Tetrachloroethane

Tetrachloroethylene

Tetrachloroethylene

Toluene

Toluene

1,1,1-Trichloroethane

1,1,1-Trichloroethane

1,1,2-Trichloroethane

1,1,2-Trichloroethane

Trichloroethylene

Trichloroethylene

Vinyl acetate

Vinyl acetate

Xylenes

Xylenes
Sample ID: 104600

RQ AQ B Result

Unit

D. Limit Method

$\begin{array}{lll}\text { U } & & 0.119 \\ U & & 0.119 \\ U & & 0.0595 \\ U & & 0.0595 \\ U & & 0.0595 \\ U & & 0.0595 \\ U & & 0.0595 \\ U & & 0.0595 \\ U & & 0.0595 \\ U & & 0.0595 \\ U & & 0.119 \\ U & & 0.119 \\ U & 8 & 1.13 \\ U & 8 & 0.976 \\ U & & 0.0595 \\ U & & 0.0595 \\ U & & 0.0595 \\ U & & 0.0595 \\ U & & 0.0595 \\ U & & 0.0595 \\ U & & 0.0595 \\ U & & 0.0595 \\ U & 8 & 1.02 \\ U J & I 8 & 0.69 \\ J & N & 3 \\ U & V & 2.52 \\ U & V & 2.29 \\ U & & 1.19 \\ U & & 1.19 \\ U & & 0.0595 \\ U & & 0.0595 \\ U & & 0.0595 \\ U & & 0.0595 \\ U & & 0.0595 \\ U & & 0.0595 \\ & V & 3.05 \\ U & V & 2.76 \\ U & & 0.0595 \\ U & & 0.0595 \\ U & & 0.0595 \\ U & & 0.0595 \\ U & & 0.0595 \\ U & & 1.19 \\ U & & \mathbf{1 . 1 9} \\ U & & 0.179 \\ U & & 0.179\end{array}$

\begin{tabular}{|c|c|c|}
\hline$\mu \mathrm{g} / \mathrm{kg}$ & 0.119 & EPA8260 \\
\hline$\mu \mathrm{g} / \mathrm{kg}$ & 0.119 & EPA8260 \\
\hline$\mu \mathrm{g} / \mathrm{kg}$ & 0.0595 & EPA8260 \\
\hline$\mu \mathrm{g} / \mathrm{kg}$ & 0.0595 & EPA8260 \\
\hline$\mu \mathrm{g} / \mathrm{kg}$ & 0.0595 & EPA8260 \\
\hline$\mu \mathrm{g} / \mathrm{kg}$ & 0.0595 & EPA8260 \\
\hline$\mu \mathrm{g} / \mathrm{kg}$ & 0.0595 & EPA8260 \\
\hline$\mu \mathrm{g} / \mathrm{kg}$ & 0.0595 & EPA8260 \\
\hline$\mu \mathrm{g} / \mathrm{kg}$ & 0.0595 & EPA8260 \\
\hline$\mu \mathrm{g} / \mathrm{kg}$ & 0.0595 & EPA8260 \\
\hline$\mu \mathrm{g} / \mathrm{kg}$ & 0.119 & EPA8260 \\
\hline$\mu \mathrm{g} / \mathrm{kg}$ & 0.119 & EPA8260 \\
\hline$\mu \mathrm{g} / \mathrm{kg}$ & 0.595 & EPA8260 \\
\hline$\mu \mathrm{g} / \mathrm{kg}$ & 0.595 & EPA8260 \\
\hline$\mu g / k g$ & 0.0595 & EPA8260 \\
\hline$\mu \mathrm{g} / \mathrm{kg}$ & 0.0595 & EPA8260 \\
\hline$\mu \mathrm{g} / \mathrm{kg}$ & 0.0595 & EPA8260 \\
\hline$\mu \mathrm{g} / \mathrm{kg}$ & 0.0595 & EPA8260 \\
\hline$\mu \mathrm{g} / \mathrm{kg}$ & 0.0595 & EPA8260 \\
\hline$\mu \mathrm{g} / \mathrm{kg}$ & 0.0595 & EPA8260 \\
\hline$\mu \mathrm{g} / \mathrm{kg}$ & 0.0595 & EPA8260 \\
\hline$\mu \mathrm{g} / \mathrm{kg}$ & 0.0595 & EPA8260 \\
\hline$\mu \mathrm{g} / \mathrm{kg}$ & 1.19 & EPA8260 \\
\hline$\mu \mathrm{g} / \mathrm{kg}$ & 1.19 & EPA8260 \\
\hline$\mu \mathrm{g} / \mathrm{kg}$ & & EPA8260 \\
\hline$\mu \mathrm{g} / \mathrm{kg}$ & 1.19 & EPA8260 \\
\hline$\mu \mathrm{g} / \mathrm{kg}$ & 1.19 & EPA8260 \\
\hline$\mu \mathrm{g} / \mathrm{kg}$ & 1.19 & EPA8260 \\
\hline$\mu \mathrm{g} / \mathrm{kg}$ & 1.19 & EPA8260 \\
\hline$\mu \mathrm{g} / \mathrm{kg}$ & 0.0595 & EPA8260 \\
\hline$\mu \mathrm{g} / \mathrm{kg}$ & 0.0595 & EPA8260 \\
\hline$\mu \mathrm{g} / \mathrm{kg}$ & 0.0595 & EPA8260 \\
\hline$\mu \mathrm{g} / \mathrm{kg}$ & 0.0595 & EPA8260 \\
\hline$\mu \mathrm{g} / \mathrm{kg}$ & 0.0595 & EPA8260 \\
\hline$\mu \mathrm{g} / \mathrm{kg}$ & 0.0595 & EPA8260 \\
\hline$\mu \mathrm{g} / \mathrm{kg}$ & 0.595 & EPA8260 \\
\hline$\mu \mathrm{g} / \mathrm{kg}$ & 0.595 & EPA8260 \\
\hline$\mu \mathrm{g} / \mathrm{kg}$ & 0.0595 & EPA8260 \\
\hline$\mu \mathrm{g} / \mathrm{kg}$ & 0.0595 & EPA8260 \\
\hline$\mu \mathrm{g} / \mathrm{kg}$ & 0.0595 & EPA8260 \\
\hline$\mu \mathrm{g} / \mathrm{kg}$ & 0.0595 & EPA8260 \\
\hline$\mu \mathrm{g} / \mathrm{kg}$ & 0.0595 & EPA8260 \\
\hline$\mu \mathrm{g} / \mathrm{kg}$ & 0.0595 & EPA8260 \\
\hline$\mu \mathrm{g} / \mathrm{kg}$ & 1.19 & EPA8260 \\
\hline$\mu \mathrm{g} / \mathrm{k}$ & 1.19 & EPA8260 \\
\hline$\mu \mathrm{g} / \mathrm{kg}$ & 0.179 & EPA8260 \\
\hline$\mu \mathrm{g} / \mathrm{kg}$ & 0.179 & EPA8260 \\
\hline
\end{tabular}


SURVEY ID: PPSC 0101 (continued)

Constituent

Pesticides/PCBs and Dioxins/Furans

Aldrin

alpha-Benzene hexachloride

beta-Benzene hexachloride

delta-Benzene hexachloride

alpha-Chlordane

gamma-Chlordane

$p, p^{\prime}-D D D$

$p, p^{\circ}-D D E$

$\mathrm{p}, \mathrm{p}^{\prime}-\mathrm{DDT}$

Dieldrin

Endosulfan sulfate

Endosulfan I

Endosulfan II

Endrin

Endrin ketone

Heptachlor

Heptachlor epoxide

Lindane

Methoxychlor

PCB 1016

PCB 1221

PCB 1232

PCB 1242

PCB 1248

PCB 1254

PCB 1260

Toxaphene

\section{Radionuclides}

Actinium-228

Antimony-124

Antimony-125

Barium-133

Cerium-144

Cesium-134

Cesium-137

Cobalt-57

Cobalt-58

Cobalt-60

Europium-152

Europium-154

Europium-155

Gross alpha

Lead-212

Manganese-54

Neptunium-239

Nonvolatile beta

Plutonium-238

Plutonium-239/240

Potassium-40

Promethium-144

Promethium-146

Ruthenium-106

Sodium-22

Sample ID: 104600

RQ AQ B Result Unit D. Limit Method

$\begin{array}{llll}0.983 & \mu \mathrm{g} / \mathrm{kg} & 0.983 & \text { EPA8080 } \\ 0.983 & \mu \mathrm{g} / \mathrm{kg} & 0.983 & \text { EPA8080 } \\ 1.97 & \mu \mathrm{g} / \mathrm{kg} & 1.97 & \text { EPA8080 } \\ 1.97 & \mu \mathrm{g} / \mathrm{kg} & 1.97 & \text { EPA8080 } \\ 1.97 & \mu \mathrm{g} / \mathrm{kg} & 1.97 & \text { EPA8080 } \\ 1.97 & \mu \mathrm{g} / \mathrm{kg} & 1.97 & \text { EPA8080 } \\ 3.93 & \mu \mathrm{g} / \mathrm{kg} & 3.93 & \text { EPA8080 } \\ 1.97 & \mu \mathrm{g} / \mathrm{kg} & 1.97 & \text { EPA8080 } \\ 3.93 & \mu \mathrm{g} / \mathrm{kg} & 3.93 & \text { EPA8080 } \\ 1.97 & \mu \mathrm{g} / \mathrm{kg} & 1.97 & \text { EPA8080 } \\ 3.93 & \mu \mathrm{g} / \mathrm{kg} & 3.93 & \text { EPA8080 } \\ 1.97 & \mu \mathrm{g} / \mathrm{kg} & 1.97 & \text { EPA8080 } \\ 3.93 & \mu \mathrm{g} / \mathrm{kg} & 3.93 & \text { EPA8080 } \\ 1.97 & \mu \mathrm{g} / \mathrm{kg} & 1.97 & \text { EPA8080 } \\ 3.93 & \mu \mathrm{g} / \mathrm{kg} & 3.93 & \text { EPA8080 } \\ 1.97 & \mu \mathrm{g} / \mathrm{kg} & 1.97 & \text { EPA8080 } \\ 1.97 & \mu \mathrm{g} / \mathrm{kg} & 1.97 & \text { EPA8080 } \\ 0.983 & \mu \mathrm{g} / \mathrm{kg} & 0.983 & \text { EPA8080 } \\ 19.7 & \mu \mathrm{g} / \mathrm{kg} & 19.7 & \text { EPA8080 } \\ 4.91 & \mu \mathrm{g} / \mathrm{kg} & 4.91 & \text { EPA8080 } \\ 4.91 & \mu \mathrm{g} / \mathrm{kg} & 4.91 & \text { EPA8080 } \\ 4.91 & \mu \mathrm{g} / \mathrm{kg} & 4.91 & \text { EPA8080 } \\ 4.91 & \mu \mathrm{g} / \mathrm{kg} & 4.91 & \text { EPA8080 } \\ 4.91 & \mu \mathrm{g} / \mathrm{kg} & 4.91 & \text { EPA8080 } \\ 4.91 & \mu \mathrm{g} / \mathrm{kg} & 4.91 & \text { EPA8080 } \\ 4.91 & \mu \mathrm{g} / \mathrm{kg} & 4.91 & \text { EPA8080 } \\ 39.3 & \mu \mathrm{g} / \mathrm{kg} & 39.3 & \text { EPA8080 }\end{array}$

$1.24 \mathrm{E}+00 \pm 6.15 \mathrm{E}-02 \mathrm{pCi} / \mathrm{g}$

$-1.84 \mathrm{E}-03 \pm 8.90 \mathrm{E}-03 \mathrm{pCi} / \mathrm{g}$

$5.85 \mathrm{E}-03 \pm 2.12 \mathrm{E}-02 \mathrm{pCi} / \mathrm{g}$

$-4.35 \mathrm{E}-03 \pm 9.94 \mathrm{E}-03 \mathrm{pCj} / \mathrm{g}$

$-2.92 \mathrm{E}-02 \pm 4.97 \mathrm{E}-02 \mathrm{pCi} / \mathrm{g}$

$-1.93 \mathrm{E}-03 \pm 6.80 \mathrm{E}-03 \mathrm{pCi} / \mathrm{g}$

$2.17 \mathrm{E}+00 \pm 3.64 \mathrm{E}-02 \mathrm{pCi} / \mathrm{g}$

$1.22 \mathrm{E}-03 \pm 6.17 \mathrm{E}-03 \mathrm{pCi} / \mathrm{g}$

$-8.54 \mathrm{E}-03 \pm 7.03 \mathrm{E}-03 \mathrm{pCi} / \mathrm{g}$

$0.00 \mathrm{E}+00$ $\mathrm{pCi} / \mathrm{g}$

$-8.99 \mathrm{E}-04 \pm 2.06 \mathrm{E}-02 \mathrm{pCi} / \mathrm{g}$

$-3.90 \mathrm{E}-03 \pm 4.51 \mathrm{E}-02 \mathrm{pCi} / \mathrm{g}$

$0.00 \mathrm{E}+00 \quad \mathrm{pCj} / \mathrm{g}$

$1.96 \mathrm{E}+01 \pm 2.81 \mathrm{E}+00 \mathrm{pCi} / \mathrm{g}$

$1.30 \mathrm{E}+00 \pm 3.02 \mathrm{E} .02 \mathrm{pCi} / \mathrm{g}$

$2.32 \mathrm{E}-02 \pm 7.00 \mathrm{E}-03 \mathrm{pCi} / \mathrm{g}$

$0.00 \mathrm{E}+00 \quad \mathrm{pCi} / \mathrm{g}$

$9.14 \mathrm{E}+00 \pm 1.61 \mathrm{E}+00 \mathrm{pCi} / \mathrm{g}$

$1.72 \mathrm{E}-02 \pm 1.67 \mathrm{E}-02 \mathrm{pCi} / \mathrm{g}$

$3.01 \mathrm{E}-03 \pm 9.30 \mathrm{E}-03 \mathrm{pCi} / \mathrm{g}$

$6.64 \mathrm{E}-01 \pm 1.22 \mathrm{E}-01 \mathrm{pCi} / \mathrm{g}$

$-3.20 \mathrm{E}-03 \pm 5.87 \mathrm{E}-03 \mathrm{pCi} / \mathrm{g}$

$6.93 \mathrm{E}-03 \pm 1.37 \mathrm{E}-02 \mathrm{pCi} / \mathrm{g}$

$-7.72 \mathrm{E}-03 \pm 5.73 \mathrm{E}-02 \mathrm{pCj} / \mathrm{g}$

$1.15 \mathrm{E}-03 \pm 4.91 \mathrm{E}-03 \mathrm{pCi} / \mathrm{g}$
3.08E-02 EPLA-013B

1.54E-02 EPIA-013B

3.50E-02 EPIA-013B

1.44E-02 EPIA-013B

8.46E-02 EPIA-013B

1.03E-02 EPIA-013B

1.13E-02 EPIA-013B

1.07E-02 EPIA-013B

1.14E-02 EPIA-013B

9.37E-03 EPIA-013B

3.40E-02 EPIA-013B

7.72E-02 EPIA-013B

4.21E-02 EPIA-013B

9.73E-01 EPIA-001B

2.08E-02 EPIA-013B

8.76E-03 EPIA-013B

7.41E-02 EPIA-013B

$2.22 \mathrm{E}+00$ EPIA-001B

2.66E-02 EPIA-012B

1.81E-02 EPIA-012B

8.54E-02 EPIA-013B

9.94E-03 EPIA-013B

1.77E-02 EPIA-013B

9.91E-02 EPLA-013B

8.54E-03 EPIA-013B 
SURVEY ID: PPSC 0101 (continued)

Constituent

RQ AQ B Result

Radionuclides

Strontium-90

Thorium-234

Tin-113

Yttrium-88

Zinc-65

Zirconium-95
Unit

D. Limit Method

Sample ID: 104600

5.50E-02 EPIA-004

5.34E-01 EPIA-013B

1.90E-02 EPIA-013B

1.37E-02 EPIA-013B

1.84E-02 EPIA-013B

2.55E-02 EPIA-013B

SURVEY ID: PPSC 0102

Sample ID: 104601

Survey location: 44879E 3678269N (SRS coordinates)

Depth of core interval: 1.00 to $2.00 \mathrm{ft}$

Sample type: Normal

Sample matrix: Soil

Percent solids: 83.00

Constituent

RQ AQ B Result

Unit

D. Limit Method

Physical Parameters and Specified Analyses

Cation exchange capacity
Chloride
Cyanide
Nitrate-nitrite as nitrogen
Orthophosphate
Phenols
Sulfide
Total organic carbon
Total organic nitrogen
Total phosphates (as P)

Metals (total recoverable)

$\begin{array}{llll}\text { Aluminum } & & & 18200 \\ \text { Antimony } & \text { U } & & 5.09 \\ \text { Arsenic } & \text { J } & \text { E } & 7.67 \\ \text { Barium } & & & 16 \\ \text { Beryllium } & \text { J } & \text { E } & 0.11 \\ \text { Cadmium } & \text { U } & & 0.254 \\ \text { Calcium } & & & 119 \\ \text { Chromium } & & & 23 \\ \text { Cobalt } & \mathrm{U} & & \mathbf{0 . 5 0 9} \\ \text { Copper } & & & 4.94 \\ \text { Iron } & & & 24900 \\ \text { Lead } & & & 9.37 \\ \text { Magnesium } & & & 282 \\ \text { Manganese } & & & 9.15 \\ \text { Mercury } & & \mathrm{V} & \mathbf{0 . 0 6 6 8} \\ \text { Nickel } & & & 3.29 \\ \text { Potassium } & & & 177 \\ \text { Selenium } & \mathrm{J} & \mathrm{E} & 4.67 \\ \text { Silver } & \mathrm{J} & \mathrm{E} & 0.841 \\ \text { Sodium } & \mathrm{J} & \mathrm{E} & 23.6 \\ \text { Thallium } & \mathrm{U} & & 15.3 \\ \text { Vanadium } & & & 48.9 \\ \text { Zinc } & & & 6\end{array}$

$\begin{array}{lll}\mathrm{meq} / 100 \mathrm{~g} & \mathbf{5} & \text { EPA9081 } \\ \mathrm{mg} / \mathrm{kg} & 2.92 & \text { EPA300.0 } \\ \mathrm{mg} / \mathrm{kg} & 1.15 & \text { EPA335.3 } \\ \mathrm{mg} / \mathrm{kg} & 0.937 & \text { EPA353.1 } \\ \mathrm{mg} / \mathrm{kg} & 29.3 & \text { EPA365.2 } \\ \mu \mathrm{g} / \mathrm{kg} & 443 & \text { EPA420.2 } \\ \mathrm{mg} / \mathrm{kg} & 272 & \text { EPA376.2 } \\ \mathrm{mg} / \mathrm{kg} & 120 & \text { EPA415.1 } \\ \mathrm{mg} / \mathrm{kg} & 0.241 & \text { EPA351.2 } \\ \mathrm{mg} / \mathrm{kg} & 379 & \text { EPA365.4 }\end{array}$

$\begin{array}{lll}\mathrm{mg} / \mathrm{kg} & 12.7 & \text { EPA6010A } \\ \mathrm{mg} / \mathrm{kg} & 5.09 & \text { EPA6010A } \\ \mathrm{mg} / \mathrm{kg} & 15.3 & \text { EPA6010A } \\ \mathrm{mg} / \mathrm{kg} & 1.27 & \text { EPA6010A } \\ \mathrm{mg} / \mathrm{kg} & \mathbf{0 . 5 0 9} & \text { EPA6010A } \\ \mathrm{mg} / \mathrm{kg} & 0.254 & \text { EPA6010A } \\ \mathrm{mg} / \mathrm{kg} & 12.7 & \text { EPA6010A } \\ \mathrm{mg} / \mathrm{kg} & 1.27 & \text { EPA6010A } \\ \mathrm{mg} / \mathrm{kg} & 0.509 & \text { EPA6010A } \\ \mathrm{mg} / \mathrm{kg} & 1.27 & \text { EPA6010A } \\ \mathrm{mg} / \mathrm{kg} & 5.09 & \text { EPA6010A } \\ \mathrm{mg} / \mathrm{kg} & 5.09 & \text { EPA6010A } \\ \mathrm{mg} / \mathrm{kg} & 5.09 & \text { EPA6010A } \\ \mathrm{mg} / \mathrm{kg} & 0.509 & \text { EPA6010A } \\ \mathrm{mg} / \mathrm{kg} & 0.035 & \text { EPA7471 } \\ \mathrm{mg} / \mathrm{kg} & 1.27 & \text { EPA6010A } \\ \mathrm{mg} / \mathrm{kg} & 102 & \text { EPA6010A } \\ \mathrm{mg} / \mathrm{kg} & 15.3 & \text { EPA6010A } \\ \mathrm{mg} / \mathrm{kg} & 1.27 & \text { EPA6010A } \\ \mathrm{mg} / \mathrm{kg} & 50.9 & \text { EPA6010A } \\ \mathrm{mg} / \mathrm{kg} & 15.3 & \text { EPA6010A } \\ \mathrm{mg} / \mathrm{kg} & 0.509 & \text { EPA6010A } \\ \mathrm{mg} / \mathrm{kg} & 1.02 & \text { EPA6010A }\end{array}$


$B / N / A$ Extractables (including PAH and phenols)

\begin{tabular}{|c|c|c|c|}
\hline Acenaphthene & $U$ & & 40 \\
\hline Acenaphthylene & $\mathrm{U}$ & & 40 \\
\hline Anthracene & $\mathrm{U}$ & & 40 \\
\hline Benzo[a]anthracene & $\mathrm{U}$ & & 4 \\
\hline Benzo[b]fluoranthene & $U$ & & 4 \\
\hline Benzo[k]fluoranthene & $\mathrm{U}$ & & 4 \\
\hline Benzoic acid & $\mathrm{U}$ & & 40 \\
\hline Benzo $[g, h, i]$ perylene & $\mathrm{U}$ & & 40 \\
\hline Benzo[a]pyrene & $U$ & & 4 \\
\hline Benzyl alcohol & $U$ & & 40 \\
\hline $\operatorname{Bis}(2$-chloroethoxy) methane & $\mathrm{U}$ & & 40 \\
\hline Bis(2-chloroethyl) ether & $\mathrm{U}$ & & 40 \\
\hline Bis(2-chloroisopropyl) ether & $\mathrm{U}$ & & 40 \\
\hline Bis(2-ethylhexyl) phthalate & $\mathbf{J}$ & $\mathbf{E}$ & 21.2 \\
\hline 4-Bromophenyl phenyl ether & $\mathrm{U}$ & & 40 \\
\hline Butylbenzyl phthalate & $\mathbf{J}$ & $\mathbf{E}$ & 36.4 \\
\hline 4-Chloroaniline & $U$ & & 40 \\
\hline 4-Chloro-m-cresol & $\mathrm{U}$ & & 40 \\
\hline 2-Chloronaphthalene & $\mathrm{U}$ & & 40 \\
\hline 2-Chlorophenol & $U$ & & 40 \\
\hline 4-Chlorophenyl phenyl ether & $U$ & & 40 \\
\hline Chrysene & $\mathrm{U}$ & & 4 \\
\hline $\mathrm{m} / \mathrm{p}$-Cresol & $\mathrm{U}$ & & 400 \\
\hline o-Cresol (2-Methylphenol) & $\mathrm{U}$ & & 40 \\
\hline Dibenz $[a, h]$ anthracene & $\mathrm{U}$ & & 4 \\
\hline Dibenzofuran & $U$ & & 40 \\
\hline Di-n-butyl phthalate & $\mathrm{U}$ & & 40 \\
\hline 1,2-Dichlorobenzene & $\mathrm{U}$ & & 40 \\
\hline 1,3-Dichlorobenzene & $\mathrm{U}$ & & 40 \\
\hline 1,4-Dichlorobenzene & $U$ & & 40 \\
\hline 3,3'-Dichlorobenzidine & $U$ & & 40 \\
\hline 2,4-Dichlorophenol & U & & 40 \\
\hline Diethyl phthalate & $\mathrm{U}$ & & 40 \\
\hline 2,4-Dimethyl phenol & $U$ & & 40 \\
\hline Dimethyl phthalate & $\mathrm{U}$ & & 40 \\
\hline 2,4-Dinitrophenol & U & & 400 \\
\hline 2,4-Dinitrotoluene & UJ & $\mathrm{C}$ & 4 \\
\hline 2,6-Dinitrotoluene & $\mathrm{U}$ & & 4 \\
\hline Di-n-octyl phthalate & $U$ & & 40 \\
\hline Fluoranthene & $\mathrm{U}$ & & 40 \\
\hline Fluorene & $\mathrm{U}$ & & 40 \\
\hline Hexachlorobenzene & $\mathrm{U}$ & & 4 \\
\hline Hexachlorobutadiene & $\mathrm{U}$ & & 40 \\
\hline Hexachlorocyclopentadiene & $\mathrm{U}$ & & 40 \\
\hline Hexachloroethane & $\mathrm{U}$ & & 4 \\
\hline Indeno $[1,2,3-c, d]$ pyrene & U & & 4 \\
\hline Isophorone & $U$ & & 40 \\
\hline 2-Methyl-4,6-dinitrophenol & $U$ & & 400 \\
\hline 2-Methylnaphthalene & $\mathrm{U}$ & & 40 \\
\hline Naphthalene & $\mathrm{U}$ & & 40 \\
\hline m-Nitroaniline & $\mathrm{U}$ & & 40 \\
\hline
\end{tabular}

\begin{tabular}{|c|c|c|}
\hline$\mu \mathrm{g} / \mathrm{kg}$ & 40 & EPA8270 \\
\hline$\mu \mathrm{g} / \mathrm{kg}$ & 40 & EPA8270 \\
\hline$\mu \mathrm{g} / \mathrm{kg}$ & 40 & EPA8270 \\
\hline$\mu \mathrm{g} / \mathrm{kg}$ & 4 & EPA8270 \\
\hline$\mu \mathrm{g} / \mathrm{kg}$ & 4 & EPA8270 \\
\hline$\mu \mathrm{g} / \mathrm{kg}$ & 4 & EPA8270 \\
\hline$\mu \mathrm{g} / \mathrm{kg}$ & 40 & EPA8270 \\
\hline$\mu \mathrm{g} / \mathrm{kg}$ & 40 & EPA8270 \\
\hline$\mu \mathrm{g} / \mathrm{kg}$ & 4 & EPA8270 \\
\hline$\mu \mathrm{g} / \mathrm{kg}$ & 40 & EPA8270 \\
\hline$\mu \mathrm{g} / \mathrm{kg}$ & 40 & EPA8270 \\
\hline$\mu \mathrm{g} / \mathrm{kg}$ & 40 & EPA8270 \\
\hline$\mu \mathrm{g} / \mathrm{kg}$ & 40 & EPA8270 \\
\hline$\mu \mathrm{g} / \mathrm{kg}$ & 40 & EPA8270 \\
\hline$\mu \mathrm{g} / \mathrm{kg}$ & 40 & EPA8270 \\
\hline$\mu \mathrm{g} / \mathrm{kg}$ & 40 & EPA8270 \\
\hline$\mu \mathrm{g} / \mathrm{kg}$ & 40 & EPA8270 \\
\hline$\mu \mathrm{g} / \mathrm{kg}$ & 40 & EPA8270 \\
\hline$\mu \mathrm{g} / \mathrm{kg}$ & 40 & EPA8270 \\
\hline$\mu \mathrm{g} / \mathrm{kg}$ & 40 & EPA8270 \\
\hline$\mu \mathrm{g} / \mathrm{kg}$ & 40 & EPA8270 \\
\hline$\mu \mathrm{g} / \mathrm{kg}$ & 4 & EPA8270 \\
\hline$\mu \mathrm{g} / \mathrm{kg}$ & 400 & EPA8270 \\
\hline$\mu \mathrm{g} / \mathrm{kg}$ & 40 & EPA8270 \\
\hline$\mu \mathrm{g} / \mathrm{kg}$ & 4 & EPA8270 \\
\hline$\mu \mathrm{g} / \mathrm{kg}$ & 40 & EPA8270 \\
\hline$\mu \mathrm{g} / \mathrm{kg}$ & 40 & EPA8270 \\
\hline$\mu \mathrm{g} / \mathrm{kg}$ & 40 & EPA8270 \\
\hline$\mu \mathrm{g} / \mathrm{kg}$ & 40 & EPA8270 \\
\hline$\mu \mathrm{g} / \mathrm{kg}$ & 40 & EPA8270 \\
\hline$\mu \mathrm{g} / \mathrm{kg}$ & 40 & EPA8270 \\
\hline$\mu \mathrm{g} / \mathrm{kg}$ & 40 & EPA8270 \\
\hline$\mu \mathrm{g} / \mathrm{kg}$ & 40 & EPA8270 \\
\hline$\mu \mathrm{g} / \mathrm{kg}$ & 40 & EPA8270 \\
\hline$\mu \mathrm{g} / \mathrm{kg}$ & 40 & EPA8270 \\
\hline$\mu \mathrm{g} / \mathrm{kg}$ & 400 & EPA8270 \\
\hline$\mu \mathrm{g} / \mathrm{kg}$ & 4 & EPA8270 \\
\hline$\mu \mathrm{g} / \mathrm{kg}$ & 4 & EPA8270 \\
\hline$\mu \mathrm{g} / \mathrm{kg}$ & 40 & EPA8270 \\
\hline$\mu \mathrm{g} / \mathrm{kg}$ & 40 & EPA8270 \\
\hline$\mu \mathrm{g} / \mathrm{kg}$ & 40 & EPA8270 \\
\hline$\mu \mathrm{g} / \mathrm{kg}$ & 4 & EPA8270 \\
\hline$\mu \mathrm{g} / \mathrm{kg}$ & 40 & EPA8270 \\
\hline$\mu \mathrm{g} / \mathrm{kg}$ & 40 & EPA8270 \\
\hline$\mu \mathrm{g} / \mathrm{kg}$ & 4 & EPA8270 \\
\hline$\mu \mathrm{g} / \mathrm{kg}$ & 4 & EPA8270 \\
\hline$\mu \mathrm{g} / \mathrm{kg}$ & 40 & EPA8270 \\
\hline$\mu \mathrm{g} / \mathrm{kg}$ & 400 & EPA8270 \\
\hline$\mu \mathrm{g} / \mathrm{kg}$ & 40 & EPA8270 \\
\hline$\mu \mathrm{g} / \mathrm{kg}$ & 40 & EPA8270 \\
\hline$\mu \mathrm{g} / \mathrm{kg}$ & 40 & EPA8270 \\
\hline
\end{tabular}


SURVEY ID: PPSC 0102 (continued)

Constituent

RQ AQ B Result

$B / N / A$ Extractables (including $P A H$ and phenols)
Unit

D. Limit Method

\begin{tabular}{|c|c|c|c|}
\hline 0-Nitroaniline & $\mathrm{U}$ & & 40 \\
\hline p-Nitroaniline & $\mathrm{U}$ & & 40 \\
\hline Nitrobenzene & U & & 40 \\
\hline 2-Nitrophenol & $\mathrm{U}$ & & 40 \\
\hline 4-Nitrophenol & $\mathrm{U}$ & & 40 \\
\hline N-Nitrosodiphenylamine & $\mathrm{U}$ & & 40 \\
\hline N-Nitrosodi-n-propylamine & U & & 40 \\
\hline Pentachlorophenol & UJ & C & 40 \\
\hline Phenanthrene & $\mathrm{U}$ & & 40 \\
\hline Phenol & $\mathrm{U}$ & & 40 \\
\hline Pyrene & $\mathrm{U}$ & & 40 \\
\hline 1,2,4-Trichlorobenzene & $\mathrm{U}$ & & 4 \\
\hline 2,4,5-Trichlorophenol & $\mathbf{U}$ & & 40 \\
\hline 2,4,6-Trichlorophenol & $U$ & & 40 \\
\hline Unknown hydrocarbon & $\mathbf{J}$ & $\mathrm{N}$ & 280 \\
\hline
\end{tabular}

Volatile Organic Compounds

Acetone
Benzene
Bromodichloromethane
Bromoform
Bromomethane (Methyl bromide)
Carbon disulfide
Carbon tetrachloride
Chlorobenzene
Chloroethane
Chloroethene (Vinyl chloride)
Chloroform
Chloromethane (Methyl chloride)
Dibromochloromethane
1,1-Dichloroethane
1,2-Dichloroethane
1,1-Dichloroethylene
1,2-Dichloroethylene
Dichloromethane (Methylene chloride)
1,2-Dichloropropane
cis-1,3-Dichloropropene
trans-1,3-Dichloropropene
Ethylbenzene
2-Hexanone
Methyl cyclopentane
Methyl ethyl ketone
Methyl isobutyl ketone
Methyl pentane
Styrene
1,1,2,2-Tetrachloroethane
Tetrachloroethylene
Toluene
1,1,1-Trichloroethane
1,1,-Trichloroethane
Trichloroethylene
Vinyl acetate
Xylenes

$\begin{array}{lll}\text { U } & 8 & 26.6 \\ U & & 0.602 \\ U & & 0.0602 \\ U & & 0.0602 \\ U & & 0.12 \\ U & & 1.2 \\ U & & 0.0602 \\ U & & 0.0602 \\ U & & 0.12 \\ U & & 0.12 \\ U & & 0.0602 \\ U & & 0.12 \\ U & & 0.0602 \\ U & & 0.0602 \\ U & & 0.0602 \\ U & & 0.0602 \\ U & & 0.12 \\ U & & 0.602 \\ U & & 0.0602 \\ U & & 0.0602 \\ U & & 0.0602 \\ U & & 0.0602 \\ U & 8 & 0.265 \\ \text { J } & N & 3 \\ U & V & 1.01 \\ \text { J } & \text { E } & 0.398 \\ \text { J } & \text { N } & 2 \\ U & & 0.0602 \\ U & & 0.0602 \\ U & & 0.0602 \\ U & V & 0.145 \\ U & & 0.0602 \\ U & & 0.0602 \\ U & & 0.0602 \\ U & & 1.2 \\ U & & 0.181 \\ \text { U } & & \end{array}$

$\begin{array}{lll}\mu \mathrm{g} / \mathrm{kg} & 40 & \text { EPA8270 } \\ \mu \mathrm{g} / \mathrm{kg} & 40 & \text { EPA8270 } \\ \mu \mathrm{g} / \mathrm{kg} & 40 & \text { EPA8270 } \\ \mu \mathrm{g} / \mathrm{kg} & 40 & \text { EPA8270 } \\ \mu \mathrm{g} / \mathrm{kg} & 40 & \text { EPA8270 } \\ \mu \mathrm{g} / \mathrm{kg} & 40 & \text { EPA8270 } \\ \mu \mathrm{g} / \mathrm{kg} & 40 & \text { EPA8270 } \\ \mu \mathrm{g} / \mathrm{kg} & 40 & \text { EPA8270 } \\ \mu \mathrm{g} / \mathrm{kg} & 40 & \text { EPA8270 } \\ \mu \mathrm{g} / \mathrm{kg} & 40 & \text { EPA8270 } \\ \mu \mathrm{g} / \mathrm{kg} & 40 & \text { EPA8270 } \\ \mu \mathrm{g} / \mathrm{kg} & 4 & \text { EPA8270 } \\ \mu \mathrm{g} / \mathrm{kg} & 40 & \text { EPA8270 } \\ \mu \mathrm{g} / \mathrm{kg} & 40 & \text { EPA8270 } \\ \mu \mathrm{g} / \mathrm{kg} & & \text { EPA8270 }\end{array}$

$\begin{array}{lll}\mu \mathrm{g} / \mathrm{kg} & 6.02 & \text { EPA8260 } \\ \mu \mathrm{g} / \mathrm{kg} & 0.602 & \text { EPA8260 } \\ \mu \mathrm{g} / \mathrm{kg} & 0.0602 & \text { EPA8260 } \\ \mu \mathrm{g} / \mathrm{kg} & 0.0602 & \text { EPA8260 } \\ \mu \mathrm{g} / \mathrm{kg} & 0.12 & \text { EPA8260 } \\ \mu \mathrm{g} / \mathrm{kg} & 1.2 & \text { EPA8260 } \\ \mu \mathrm{g} / \mathrm{kg} & 0.0602 & \text { EPA8260 } \\ \mu \mathrm{g} / \mathrm{kg} & 0.0602 & \text { EPA8260 } \\ \mu \mathrm{g} / \mathrm{kg} & 0.12 & \text { EPA8260 } \\ \mu \mathrm{g} / \mathrm{kg} & 0.12 & \text { EPA8260 } \\ \mu \mathrm{g} / \mathrm{kg} & 0.0602 & \text { EPA8260 } \\ \mu \mathrm{g} / \mathrm{kg} & 0.12 & \text { EPA8260 } \\ \mu \mathrm{g} / \mathrm{kg} & 0.0602 & \text { EPA8260 } \\ \mu \mathrm{g} / \mathrm{kg} & 0.0602 & \text { EPA8260 } \\ \mu \mathrm{g} / \mathrm{kg} & 0.0602 & \text { EPA8260 } \\ \mu \mathrm{g} / \mathrm{kg} & 0.0602 & \text { EPA8260 } \\ \mu \mathrm{g} / \mathrm{kg} & 0.12 & \text { EPA8260 } \\ \mu \mathrm{g} / \mathrm{kg} & 0.602 & \text { EPA8260 } \\ \mu \mathrm{g} / \mathrm{kg} & 0.0602 & \text { EPA8260 } \\ \mu \mathrm{g} / \mathrm{kg} & 0.0602 & \text { EPA8260 } \\ \mu \mathrm{g} / \mathrm{kg} & 0.0602 & \text { EPA8260 } \\ \mu \mathrm{g} / \mathrm{kg} & 0.0602 & \text { EPA8260 } \\ \mu \mathrm{g} / \mathrm{kg} & 1.2 & \text { EPA8260 } \\ \mu \mathrm{g} / \mathrm{kg} & & \text { EPA8260 } \\ \mu \mathrm{g} / \mathrm{kg} & 1.2 & \text { EPA8260 } \\ \mu \mathrm{g} / \mathrm{kg} & 1.2 & \text { EPA8260 } \\ \mu \mathrm{g} / \mathrm{kg} & & \text { EPA8260 } \\ \mu \mathrm{g} / \mathrm{kg} & 0.0602 & \text { EPA8260 } \\ \mu \mathrm{g} / \mathrm{kg} & 0.0602 & \text { EPA8260 } \\ \mu \mathrm{g} / \mathrm{kg} & 0.0602 & \text { EPA8260 } \\ \mu \mathrm{g} / \mathrm{kg} & 0.602 & \text { EPA8260 } \\ \mu \mathrm{g} / \mathrm{kg} & 0.0602 & \text { EPA8260 } \\ \mu \mathrm{g} / \mathrm{kg} & 0.0602 & \text { EPA8260 } \\ \mu \mathrm{g} / \mathrm{kg} & 0.0602 & \text { EPA8260 } \\ \mu \mathrm{g} / \mathrm{kg} & 1.2 & \text { EPA8260 } \\ \mu \mathrm{g} / \mathrm{kg} & 0.181 & \text { EPA8260 }\end{array}$


SURVEY ID: PPSC 0102 (continued)

Constituent

Pesticides/PCBs and Dioxins/Furans

Aldrin

alpha-Benzene hexachloride

beta-Benzene hexachloride

delta-Benzene hexachloride

alpha-Chlordane

gamma-Chlordane

$\mathrm{p}, \mathrm{p}^{\circ}-\mathrm{DDD}$

$\mathrm{p}, \mathrm{p}^{\prime}-\mathrm{DDE}$

p,p'-DDT

Dieldrin

Endosulfan sulfate

Endosulfan I

Endosulfan II

Endrin

Endrin ketone

Heptachlor

Heptachlor epoxide

Lindane

Methoxychlor

PCB 1016

PCB 1221

PCB 1232

PCB 1242

PCB 1248

PCB 1254

PCB 1260

Toxaphene

Radionuclides

Actinium-228

Antimony-124

Antimony-125

Barium-133

Cerium-144

Cesium-134

Cesium-137

Cobalt-57

Cobalt-58

Cobalt-60

Europium-152

Europium-154

Europium-155

Gross alpha

Lead-212

Manganese-54

Neptunium-239

Nonvolatile beta

Plutonium-238

Plutonium-239/240

Potassium-40

Promethium-144

Promethium-146

Ruthenium-106

Sodium-22
Sample ID: 104601

RQ AQ B Result Unit D. Limit Method

$\begin{array}{lllll}\mathrm{U} & 0.993 & \mu \mathrm{g} / \mathrm{kg} & 0.993 & \text { EPA8080 } \\ \mathrm{U} & 0.993 & \mu \mathrm{g} / \mathrm{kg} & 0.993 & \text { EPA8080 } \\ \mathrm{U} & 1.99 & \mu \mathrm{g} / \mathrm{kg} & 1.99 & \text { EPA8080 } \\ \mathrm{U} & 1.99 & \mu \mathrm{g} / \mathrm{kg} & 1.99 & \text { EPA8080 } \\ \mathrm{U} & 1.99 & \mu \mathrm{g} / \mathrm{kg} & 1.99 & \text { EPA8080 } \\ \mathrm{U} & 1.99 & \mu \mathrm{g} / \mathrm{kg} & 1.99 & \text { EPA8080 } \\ \mathrm{U} & 3.97 & \mu \mathrm{g} / \mathrm{kg} & 3.97 & \text { EPA8080 } \\ \mathrm{U} & 1.99 & \mu \mathrm{g} / \mathrm{kg} & 1.99 & \text { EPA8080 } \\ \mathrm{U} & 3.97 & \mu \mathrm{g} / \mathrm{kg} & 3.97 & \text { EPA8080 } \\ \mathrm{U} & 1.99 & \mu \mathrm{g} / \mathrm{kg} & 1.99 & \text { EPA8080 } \\ \mathrm{U} & 3.97 & \mu \mathrm{g} / \mathrm{kg} & 3.97 & \text { EPA8080 } \\ \mathrm{U} & 1.99 & \mu \mathrm{g} / \mathrm{kg} & 1.99 & \text { EPA8080 } \\ \mathrm{U} & 3.97 & \mu \mathrm{g} / \mathrm{kg} & 3.97 & \text { EPA8080 } \\ \mathrm{U} & 1.99 & \mu \mathrm{g} / \mathrm{kg} & 1.99 & \text { EPA8080 } \\ \mathrm{U} & 3.97 & \mu \mathrm{g} / \mathrm{kg} & 3.97 & \text { EPA8080 } \\ \mathrm{U} & 1.99 & \mu \mathrm{g} / \mathrm{kg} & 1.99 & \text { EPA8080 } \\ \mathrm{U} & 1.99 & \mu \mathrm{g} / \mathrm{kg} & 1.99 & \text { EPA8080 } \\ \mathrm{U} & 0.993 & \mu \mathrm{g} / \mathrm{kg} & 0.993 & \text { EPA8080 } \\ \mathrm{U} & 19.9 & \mu \mathrm{g} / \mathrm{kg} & 19.9 & \text { EPA8080 } \\ \mathrm{U} & 4.97 & \mu \mathrm{g} / \mathrm{kg} & 4.97 & \text { EPA8080 } \\ \mathrm{U} & 4.97 & \mu \mathrm{g} / \mathrm{kg} & 4.97 & \text { EPA8080 } \\ \mathrm{U} & 4.97 & \mu \mathrm{g} / \mathrm{kg} & 4.97 & \text { EPA8080 } \\ \mathrm{U} & 4.97 & \mu \mathrm{g} / \mathrm{kg} & 4.97 & \text { EPA8080 } \\ \mathrm{U} & 4.97 & \mu \mathrm{g} / \mathrm{kg} & 4.97 & \text { EPA8080 } \\ \mathrm{U} & 4.97 & \mu \mathrm{gg} / \mathrm{kg} & 4.97 & \text { EPA8080 } \\ \mathrm{U} & 4.97 & \mu \mathrm{g} / \mathrm{kg} & 4.97 & \text { EPA8080 } \\ \mathrm{U} & 39.7 & \mu \mathrm{g} / \mathrm{kg} & 39.7 & \text { EPA8080 }\end{array}$

$1.70 \mathrm{E}+00 \pm 7.97 \mathrm{E}-02 \mathrm{pCi} / \mathrm{g}$ $-6.39 \mathrm{E}-03 \pm 1.05 \mathrm{E}-02 \mathrm{pCj} / \mathrm{g}$ $1.48 \mathrm{E}-02 \pm 1.97 \mathrm{E}-02 \mathrm{pCi} / \mathrm{g}$ $4.19 \mathrm{E}-03 \pm 1.01 \mathrm{E}-02 \mathrm{pCi} / \mathrm{g}$ $-3.20 \mathrm{E}-02 \pm 5.73 \mathrm{E}-02 \mathrm{pCi} / \mathrm{g}$ $-4.65 \mathrm{E}-04 \pm 8.10 \mathrm{E}-03 \mathrm{pCi} / \mathrm{g}$ $4.66 \mathrm{E}-03 \pm 9.04 \mathrm{E}-03 \mathrm{pCi} / \mathrm{g}$ $3.22 \mathrm{E}-03 \pm 7.21 \mathrm{E}-03 \mathrm{pCi} / \mathrm{g}$ $-8.63 \mathrm{E}-03 \pm 9.76 \mathrm{E}-03 \mathrm{pCi} / \mathrm{g}$ 7.13E-04 $6.44 \mathrm{E}-03 \mathrm{pCi} / \mathrm{g}$ $-1.26 \mathrm{E}-03 \pm 2.19 \mathrm{E}-02 \mathrm{pCi} / \mathrm{g}$ $-4.12 \mathrm{E}-02 \pm 6.51 \mathrm{E}-02 \mathrm{pCi} / \mathrm{g}$ $0.00 \mathrm{E}+00 \quad \mathrm{pCi} / \mathrm{g}$ $2.39 \mathrm{E}+01 \pm 3.44 \mathrm{E}+00 \mathrm{pCi} / \mathrm{g}$

$\mathrm{V} \quad 1.73 \mathrm{E}+00 \pm 3.59 \mathrm{E}-02 \mathrm{pCi} / \mathrm{g}$ $1.89 \mathrm{E}-02 \pm 1.05 \mathrm{E}-02 \mathrm{pCi} / \mathrm{g}$ $0.00 \mathrm{E}+00 \quad \mathrm{pCi} / \mathrm{g}$ $1.39 \mathrm{E}+01 \pm 1.78 \mathrm{E}+00 \mathrm{pCi} / \mathrm{g}$ $-3.94 \mathrm{E}-03 \pm 1.31 \mathrm{E}-02 \mathrm{pCi} / \mathrm{g}$ $3.08 \mathrm{E}-03 \pm 4.40 \mathrm{E}-03 \mathrm{pCi} / \mathrm{g}$ $1.37 \mathrm{E}+00 \pm 1.72 \mathrm{E}-01 \mathrm{pCi} / \mathrm{g}$ $1.05 \mathrm{E}-03 \pm 7.84 \mathrm{E}-03 \mathrm{pCi} / \mathrm{g}$ $9.18 \mathrm{E}-03 \pm 1.43 \mathrm{E}-02 \mathrm{pCi} / \mathrm{g}$ 2.81E-02 $+6.62 \mathrm{E}-02 \mathrm{pCi} / \mathrm{g}$ $5.79 \mathrm{E}-04 \pm 7.14 \mathrm{E}-03 \mathrm{pCi} / \mathrm{g}$
4.06E-02 EPIA-013B 1.75E-02 EPIA-013B 3.49E-02 EPIA-013B 1.49E-02 EPIA-013B 9.68E-02 EPIA-013B 1.20E-02 EPIA-013B 1.38E-02 EPIA-013B

1.24E-02 EPIA-013B 1.57E-02 EPIA-013B 1.13E-02 EPIA-013B

3.56E-02 EPIA-013B

9.51E-02 EPIA-013B 5.08E-02 EPIA-013B

1.70E $\div 00$ EPLA-001B

2.13E-02 EPIA-013B

1.37E-02 EPIA-013B

8.68E-02 EPIA-013B

2.06E+00 EPIA-001B

2.79E-02 EPIA-012B

4.62E-03 EPIA-012B

1.03E-01 EPIA-013B

1.33E-02 EPIA-013B

$1.68 \mathrm{E}-02$ EPIA-013B

1.15E-01 EPIA-013B

1.25E-02 EPIA-013B 
SURVEY ID: PPSC 0102 (continued)

Constituent

RQ AQ

Sample ID: 104601

Radionuclides

Strontium-90

Thorium-234

Tin-113

Yttrium-88

Zinc-65

Zirconium-95

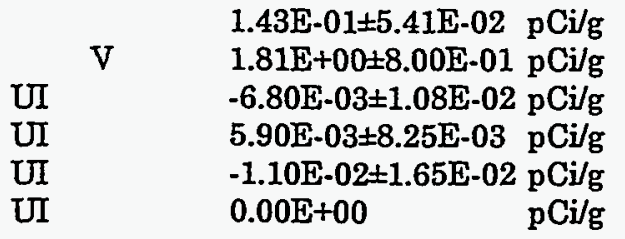

Sample ID: 104602
SURVEY ID: PPSC 0103
B Result

Unit

6.87E-02 EPIA-004

6.65E-01 EPIA-013B

1.85E-02 EPIA-013B

1.57E-02 EPIA-013B

2.38E-02 EPIA-013B

3.49E-02 EPIA-013B

Survey location: $44879 \mathrm{E}$ 3678269N (SRS coordinates)

Depth of core interval: 2.00 to $3.10 \mathrm{ft}$

Sample type: Normal

Sample matrix: Soil

Percent solids: 82.00

$\begin{array}{lllll}\text { Constituent } & \text { RQ AQ B Result Unit Dimit Method }\end{array}$

Physical Parameters and Specified Analyses

Cation exchange capacity

Chloride

Cyanide

Cyanide

Nitrate-nitrite as nitrogen

Orthophosphate

Phenols

Sulfide

Sulfide

Total organic carbon

Total organic nitrogen

Total phosphates (as P)
Orthophosphate

Total phosphates (as P)

$\begin{array}{llll} & & & 183 \\ \text { J } & \text { I } & \text { L } & 4.78 \\ \text { J } & \text { I } & \text { L } & 3.27 \\ \text { U } & & & 1.29 \\ \text { UJ } & \text { I } & \text { L } & 30.4 \\ \text { UJ } & \text { I } & \text { L } & 28.6 \\ \text { U } & & & 415 \\ \text { UJ } & \text { C } & & 286 \\ \text { UJ } & \text { C } & & 288 \\ & & & 3320 \\ \text { C } & & & 84.5 \\ \text { J } & \text { E } & & 136 \\ \text { J } & \text { E } & & 117\end{array}$

Metals (total recoverable)

$\begin{array}{lll}\mathrm{meq} / 100 \mathrm{~g} & \mathbf{5} & \text { EPA9081 } \\ \mathrm{mg} / \mathrm{kg} & 3.04 & \text { EPA300.0 } \\ \mathrm{mg} / \mathrm{kg} & \mathbf{0 . 9 3 8} & \text { EPA335.3 } \\ \mathrm{mg} / \mathrm{kg} & \mathbf{0 . 9 0 3} & \text { EPA335.3 } \\ \mathrm{mg} / \mathrm{kg} & 1.29 & \text { EPA353.1 } \\ \mathrm{mg} / \mathrm{kg} & \mathbf{3 0 . 4} & \text { EPA365.2 } \\ \mathrm{mg} / \mathrm{kg} & 28.6 & \text { EPA365.2 } \\ \mu \mathrm{gg} / \mathrm{kg} & 415 & \text { EPA420.2 } \\ \mathrm{mg} / \mathrm{kg} & 286 & \text { EPA376.2 } \\ \mathrm{mg} / \mathrm{kg} & 288 & \text { EPA376.2 } \\ \mathrm{mg} / \mathrm{kg} & 122 & \text { EPA415.1 } \\ \mathrm{mg} / \mathrm{kg} & 0.244 & \text { EPA351.2 } \\ \mathrm{mg} / \mathrm{kg} & 413 & \text { EPA365.4 } \\ \mathrm{mg} / \mathrm{kg} & 474 & \text { EPA365.4 }\end{array}$

$\begin{array}{lll}\mathrm{mg} / \mathrm{kg} & 12.9 & \text { EPA6010A } \\ \mathrm{mg} / \mathrm{kg} & 5.14 & \text { EPA6010A } \\ \mathrm{mg} / \mathrm{kg} & 15.4 & \text { EPA6010A } \\ \mathrm{mg} / \mathrm{kg} & 1.29 & \text { EPA6010A } \\ \mathrm{mg} / \mathrm{kg} & 0.514 & \text { EPA6010A } \\ \mathrm{mg} / \mathrm{kg} & 0.257 & \text { EPA6010A } \\ \mathrm{mg} / \mathrm{kg} & 12.9 & \text { EPA6010A } \\ \mathrm{mg} / \mathrm{kg} & 1.29 & \text { EPA6010A } \\ \mathrm{mg} / \mathrm{kg} & \mathbf{0 . 5 1 4} & \text { EPA6010A } \\ \mathrm{mg} / \mathrm{kg} & 1.29 & \text { EPA6010A } \\ \mathrm{mg} / \mathrm{kg} & 5.14 & \text { EPA6010A } \\ \mathrm{mg} / \mathrm{kg} & 5.14 & \text { EPA6010A } \\ \mathrm{mg} / \mathrm{kg} & 5.14 & \text { EPA6010A } \\ \mathrm{mg} / \mathrm{kg} & 0.514 & \text { EPA6010A } \\ \mathrm{mg} / \mathrm{kg} & 0.0394 & \text { EPA7471 } \\ \mathrm{mg} / \mathrm{kg} & 1.29 & \text { EPA6010A } \\ \mathrm{mg} / \mathrm{kg} & 103 & \text { EPA6010A } \\ \mathrm{mg} / \mathrm{kg} & 15.4 & \text { EPA6010A }\end{array}$


SURVEY ID: PPSC 0103 (continued)

Constituent

RQ AQ B Result

Unit

D. Limit Method

Metals (total recoverable)

Silver

Sodium

Thallium

Vanadium

Zinc

$\begin{array}{lll}\mathrm{J} & \mathrm{E} & 0.535 \\ \mathrm{~J} & \mathrm{E} & 17.4 \\ \mathrm{U} & & 15.4 \\ & & 50.4 \\ & & 4.14\end{array}$

$B / N / A$ Extractables (including $P A H$ and phenols)

Acenaphthene

Acenaphthylene

Anthracene

Benzo[a]anthracene

Benzo[b]fluoranthene

Benzo[k]fluoranthene

Benzoic acid

Benzo[g,h,i]perylene

Benzo[a]pyrene

Benzyl alcohol

Bis(2-chloroethoxy) methane

Bis(2-chloroethyl) ether

Bis(2-chloroisopropyl) ether

Bis(2-ethylhexyl) phthalate

4-Bromophenyl phenyl ether

Butylbenzyl phthalate

4-Chloroaniline

4-Chloro-m-cresol

2-Chloronaphthalene

2-Chlorophenol

4-Chlorophenyl phenyl ether

Chrysene

$\mathrm{m} / \mathrm{p}$-Cresol

o-Cresol (2-Methylphenol)

Dibenz $[a, h]$ anthracene

Dibenzofuran

Di-n-butyl phthalate

1,2-Dichlorobenzene

1,3-Dichlorobenzene

1,4-Dichlorobenzene

3,3'-Dichlorobenzidine

2,4-Dichlorophenol

Diethyl phthalate

2,4-Dimethyl phenol

Dimethyl phthalate

2,4-Dinitrophenol

2,4-Dinitrotoluene

2,6-Dinitrotoluene

Di-n-octyl phthalate

Eluoranthene

Fluorene

Hexachlorobenzene

Hexachlorobutadiene

Hexachlorocyclopentadiene

Hexachloroethane

Indeno[1,2,3-c,d]pyrene

Isophorone

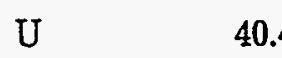

U 40.4

$\mathrm{U} \quad 40.4$

U $\quad 4.04$

U 4.04

U $\quad 4.04$

$\mathrm{U} \quad 40.4$

$\mathrm{U} \quad 40.4$

U $\quad 4.04$

U $\quad 40.4$

U $\quad 40.4$

$\begin{array}{ll}\mathrm{U} & 40.4 \\ \mathrm{U} & 40.4\end{array}$

J $\quad \mathrm{E} \quad 21$

$\begin{array}{lll}\mathrm{U} & \mathrm{E} & 21 \\ \mathrm{U} & & 40.4\end{array}$

U $\quad 40.4$

$\mathrm{U} \quad 40.4$

U $\quad 40.4$

U $\quad 40.4$

U $\quad 40.4$

U $\quad 40.4$

U $\quad 4.04$

$\mathrm{U} \quad 404$

U $\quad 40.4$

U $\quad 4.04$

U $\quad 40.4$

U $\quad \mathrm{V} \quad 38.4$

$U \quad 40.4$

U $\quad 40.4$

U $\quad 40.4$

U $\quad 40.4$

U $\quad 40.4$

U $\quad 40.4$

U $\quad 40.4$

$\mathrm{U} \quad 40.4$

U 404

UJ C 4.04

U 4.04

U $\quad 40.4$

U $\quad 40.4$

U $\quad 40.4$

U $\quad 4.04$

U $\quad 40.4$

U $\quad 40.4$

U $\quad 4.04$

$\mathrm{U} \quad 4.04$

$\mathrm{U} \quad 40.4$

$\begin{array}{lll}\mathrm{mg} / \mathrm{kg} & 1.29 & \text { EPA6010A } \\ \mathrm{mg} / \mathrm{kg} & 51.4 & \text { EPA6010A } \\ \mathrm{mg} / \mathrm{kg} & 15.4 & \text { EPA6010A } \\ \mathrm{mg} / \mathrm{kg} & 0.514 & \text { EPA6010A } \\ \mathrm{mg} / \mathrm{kg} & 1.03 & \text { EPA6010A }\end{array}$

$\mu \mathrm{g} / \mathrm{kg} \quad 40.4 \quad$ EPA8270

$\mu \mathrm{g} / \mathrm{kg} \quad 40.4 \quad$ EPA8270

$\mu \mathrm{g} / \mathrm{kg} \quad 40.4 \quad$ EPA8270

$\mu \mathrm{g} / \mathrm{kg} \quad 4.04 \quad \mathrm{EPA8270}$

$\mu g / \mathrm{kg} \quad 4.04 \quad$ EPA8270

$\mu \mathrm{g} / \mathrm{kg} \quad 4.04 \quad$ EPA8270

$\mu \mathrm{g} / \mathrm{kg} \quad 40.4 \quad$ EPA8270

$\mu \mathrm{g} / \mathrm{kg} \quad 40.4 \quad$ EPA8270

$\mu \mathrm{g} / \mathrm{kg} \quad 4.04 \quad$ EPA8270

$\mu \mathrm{g} / \mathrm{kg} \quad 40.4 \quad$ EPA8270

$\mu \mathrm{g} / \mathrm{kg} \quad 40.4 \quad$ EPA8270

$\mu \mathrm{g} / \mathrm{kg} \quad 40.4 \quad$ EPA8270

$\mu \mathrm{g} / \mathrm{kg} \quad 40.4 \quad$ EPA8270

$\mu \mathrm{g} / \mathrm{kg} \quad 40.4 \quad$ EPA8270

$\mu \mathrm{g} / \mathrm{kg} \quad 40.4 \quad$ EPA8270

$\mu \mathrm{g} / \mathrm{kg} \quad 40.4 \quad$ EPA8270

$\mu \mathrm{g} / \mathrm{kg} \quad 40.4 \quad$ EPA8270

$\mu \mathrm{g} / \mathrm{kg} \quad 40.4 \quad$ EPA8270

$\mu \mathrm{g} / \mathrm{kg} \quad 40.4 \quad$ EPA8270

$\mu \mathrm{g} / \mathrm{kg} \quad 40.4 \quad$ EPA8270

$\mu \mathrm{g} / \mathrm{kg} \quad 40.4 \quad$ EPA8270

$\mu \mathrm{g} / \mathrm{kg} \quad 4.04 \quad$ EPA8270

$\mu \mathrm{g} / \mathrm{kg} \quad 404 \quad$ EPA8270

$\mu \mathrm{g} / \mathrm{kg} \quad 40.4 \quad$ EPA8270

$\mu g / \mathrm{kg} \quad 4.04 \quad$ EPA8270

$\mu \mathrm{g} / \mathrm{kg} \quad 40.4 \quad$ EPA8270

$\mu \mathrm{g} / \mathrm{kg} \quad 40.4 \quad$ EPA8270

$\mu \mathrm{g} / \mathrm{kg} \quad 40.4 \quad$ EPA8270

$\mu g / \mathrm{kg} \quad 40.4 \quad$ EPA8270

$\mu g / k g \quad 40.4 \quad$ EPA8270

$\mu \mathrm{g} / \mathrm{kg} \quad 40.4 \quad$ EPA8270

$\mu g / \mathrm{kg} \quad 40.4 \quad$ EPA8270

$\mu \mathrm{g} / \mathrm{kg} \quad 40.4 \quad$ EPA8270

$\mu \mathrm{g} / \mathrm{kg} \quad 40.4 \quad$ EPA8270

$\mu \mathrm{g} / \mathrm{kg} \quad 40.4 \quad$ EPA8270

$\mu g / k g \quad 404 \quad$ EPA8270

$\mu \mathrm{g} / \mathrm{kg} \quad 4.04 \quad$ EPA8270

$\mu \mathrm{g} / \mathrm{kg} \quad 4.04 \quad$ EPA8270

$\mu \mathrm{g} / \mathrm{kg} \quad 40.4 \quad$ EPA8270

$\mu \mathrm{g} / \mathrm{kg} \quad 40.4 \quad$ EPA8270

$\mu \mathrm{g} / \mathrm{kg} \quad 40.4 \quad$ EPA8270

$\mu \mathrm{g} / \mathrm{kg} \quad 4.04 \quad$ EPA8270

$\mu \mathrm{g} / \mathrm{kg} \quad 40.4 \quad$ EPA8270

$\mu \mathrm{g} / \mathrm{kg} \quad \cdot 40.4 \quad$ EPA8270

$\mu \mathrm{g} / \mathrm{kg} \quad 4.04 \quad$ EPA8270

$\mu \mathrm{g} / \mathrm{kg} \quad 4.04 \quad$ EPA8270

$\mu \mathrm{g} / \mathrm{kg} \quad 40.4 \quad$ EPA8270 
SURVEY ID: PPSC 0103 (continued)

Constituent

$\mathbf{R Q}$

AQ B

$B / N / A$ Extractables (including PAH and phenols)
B Result

Sample ID: 104602

Unit

D. Limit Method

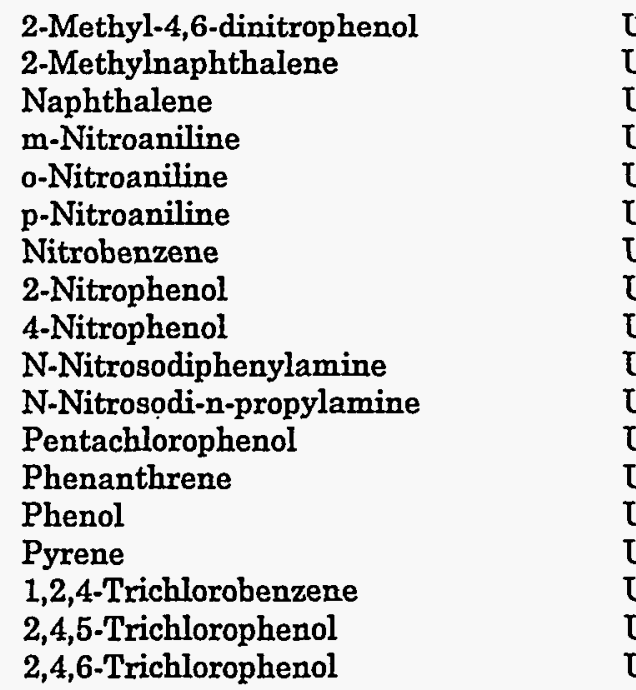

Volatile Organic Compounds

Acetone
Benzene
Bromodichloromethane
Bromoform
Bromomethane (Methyl bromide)
Carbon disulfide
Carbon tetrachloride
Chlorobenzene
Chloroethane
Chloroethene (Vinyl chloride)
Chloroform
Chloromethane (Methyl chloride)
Dibromochloromethane
1,1-Dichloroethane
1,2-Dichloroethane
1,1-Dichloroethylene
1,2-Dichloroethylene
Dichloromethane (Methylene chloride)
1,2-Dichloropropane
cis-1,3-Dichloropropene
trans-1,3-Dichloropropene
Ethylbenzene
2-Hexanone
Methyl cyclopentane
Methyl ethyl ketone
Methyl isobutyl ketone
Methyl pentane
Styrene
1,1,2,2-Tetrachloroethane
Tetrachloroethylene
Toluene
1,1,1-Trichloroethane
1,1,2-Trichloroethane
Trichloroethylene

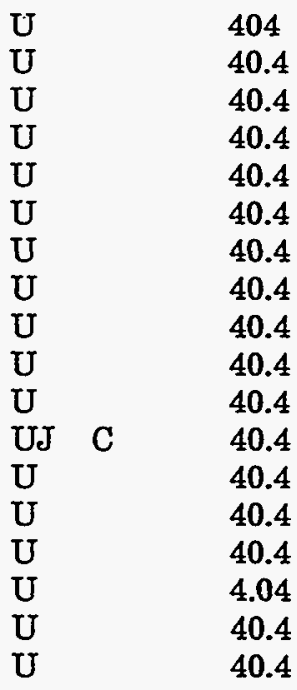

$\begin{array}{lll}\mu \mathrm{g} / \mathrm{kg} & 404 & \text { EPA8270 } \\ \mu \mathrm{g} / \mathrm{kg} & 40.4 & \text { EPA8270 } \\ \mu \mathrm{g} / \mathrm{kg} & 40.4 & \text { EPA8270 } \\ \mu \mathrm{g} / \mathrm{kg} & 40.4 & \text { EPA8270 } \\ \mu \mathrm{g} / \mathrm{kg} & 40.4 & \text { EPA8270 } \\ \mu \mathrm{g} / \mathrm{kg} & 40.4 & \text { EPA8270 } \\ \mu \mathrm{g} / \mathrm{kg} & 40.4 & \text { EPA8270 } \\ \mu \mathrm{g} / \mathrm{kg} & 40.4 & \text { EPA8270 } \\ \mu \mathrm{g} / \mathrm{kg} & 40.4 & \text { EPA8270 } \\ \mu \mathrm{g} / \mathrm{kg} & 40.4 & \text { EPA8270 } \\ \mu \mathrm{g} / \mathrm{kg} & 40.4 & \text { EPA8270 } \\ \mu \mathrm{g} / \mathrm{kg} & 40.4 & \text { EPA8270 } \\ \mu \mathrm{g} / \mathrm{kg} & 40.4 & \text { EPA8270 } \\ \mu \mathrm{g} / \mathrm{kg} & 40.4 & \text { EPA8270 } \\ \mu \mathrm{g} / \mathrm{kg} & 40.4 & \text { EPA8270 } \\ \mu \mathrm{g} / \mathrm{kg} & 4.04 & \text { EPA8270 } \\ \mu \mathrm{g} / \mathrm{kg} & 40.4 & \text { EPA8270 } \\ \mu \mathrm{g} / \mathrm{kg} & 40.4 & \text { EPA8270 }\end{array}$

U $8 \quad 16$

$\mathrm{U} \quad 0.61$

U $\quad 0.061$

U $\quad 0.061$

U $\quad 0.122$

$\mathrm{U} \quad 1.22$

U $\quad 0.061$

U $\quad 0.061$

$\mathrm{U} \quad 0.122$

U $\quad 0.122$

U $\quad 0.061$

U $\quad 0.122$

$\mathrm{U} \quad 0.061$

$\mathrm{U} \quad 0.061$

U $\quad 0.061$

U $\quad 0.061$

$\mathrm{U} \quad 0.122$

$\begin{array}{lll}U & 8 & 0.646\end{array}$

U $\quad 0.061$

U $\quad 0.061$

U $\quad 0.061$

U $\quad 0.061$

$\begin{array}{lll}U & 8 & 1.38\end{array}$

J $\quad \mathrm{N} \quad 3$

$\begin{array}{lll}\mathrm{U} & \mathrm{V} & 1.01\end{array}$

$\begin{array}{lll}J & \mathrm{E} & 0.841\end{array}$

J $\quad \mathrm{N} \quad 1.5$

$\mathrm{U} \quad 0.061$

U $\quad 0.061$

U $\quad 0.061$

$\begin{array}{lll}\mathrm{U} & \mathrm{V} & 0.134\end{array}$

U $\quad 0.061$

$\mathrm{U} \quad 0.061$

0.061

$\begin{array}{lll}\mu \mathrm{g} / \mathrm{kg} & 6.1 & \text { EPA8260 } \\ \mu \mathrm{g} / \mathrm{kg} & 0.61 & \text { EPA8260 } \\ \mu \mathrm{g} / \mathrm{kg} & 0.061 & \text { EPA8260 } \\ \mu \mathrm{g} / \mathrm{kg} & 0.061 & \text { EPA8260 } \\ \mu \mathrm{g} / \mathrm{kg} & 0.122 & \text { EPA8260 } \\ \mu \mathrm{g} / \mathrm{kg} & 1.22 & \text { EPA8260 } \\ \mu \mathrm{g} / \mathrm{kg} & 0.061 & \text { EPA8260 } \\ \mu \mathrm{g} / \mathrm{kg} & 0.061 & \text { EPA8260 } \\ \mu \mathrm{g} / \mathrm{kg} & 0.122 & \text { EPA8260 } \\ \mu \mathrm{g} / \mathrm{kg} & 0.122 & \text { EPA8260 } \\ \mu \mathrm{g} / \mathrm{kg} & 0.061 & \text { EPA8260 } \\ \mu \mathrm{g} / \mathrm{kg} & 0.122 & \text { EPA8260 } \\ \mu \mathrm{g} / \mathrm{kg} & 0.061 & \text { EPA8260 } \\ \mu \mathrm{g} / \mathrm{kg} & 0.061 & \text { EPA8260 } \\ \mu \mathrm{g} / \mathrm{kg} & 0.061 & \text { EPA8260 } \\ \mu \mathrm{g} / \mathrm{kg} & 0.061 & \text { EPA8260 } \\ \mu \mathrm{g} / \mathrm{kg} & 0.122 & \text { EPA8260 } \\ \mu \mathrm{g} / \mathrm{kg} & 0.61 & \text { EPA8260 } \\ \mu \mathrm{g} / \mathrm{kg} & 0.061 & \text { EPA8260 } \\ \mu \mathrm{g} / \mathrm{kg} & 0.061 & \text { EPA8260 } \\ \mu \mathrm{g} / \mathrm{kg} & 0.061 & \text { EPA8260 } \\ \mu \mathrm{g} / \mathrm{kg} & 0.061 & \text { EPA8260 } \\ \mu \mathrm{g} / \mathrm{kg} & 1.22 & \text { EPA8260 } \\ \mu \mathrm{g} / \mathrm{kg} & & \text { EPA8260 } \\ \mu \mathrm{g} / \mathrm{kg} & 1.22 & \text { EPA8260 } \\ \mu \mathrm{g} / \mathrm{kg} & 1.22 & \text { EPA8260 } \\ \mu \mathrm{g} / \mathrm{kg} & & \text { EPA8260 } \\ \mu \mathrm{g} / \mathrm{kg} & 0.061 & \text { EPA8260 } \\ \mu \mathrm{g} / \mathrm{kg} & 0.061 & \text { EPA8260 } \\ \mu \mathrm{g} / \mathrm{kg} & 0.061 & \text { EPA8260 } \\ \mu \mathrm{g} / \mathrm{kg} & 0.61 & \text { EPA8260 } \\ \mu \mathrm{g} / \mathrm{kg} & 0.061 & \text { EPA8260 } \\ \mu \mathrm{g} / \mathrm{kg} & 0.061 & \text { EPA8260 } \\ \mu \mathrm{g} / \mathrm{kg} & 0.061 & \text { EPA8260 }\end{array}$


SURVEY ID: PPSC 0103 (continued)

Constituent

Volatile Organic Compounds

Vinyl acetate

Xylenes

U

U

1.22

0.183

$\mu \mathrm{g} / \mathrm{kg}$

$\mu \mathrm{g} / \mathrm{kg}$

1.22

0.183

EPA8260

EPA8260

Pesticides/PCBs and Dioxins/Furans

Aldrin

alpha-Benzene hexachloride

beta-Benzene hexachloride

delta-Benzene hexachloride

alpha-Chlordane

gamma-Chlordane

p,p'-DDD

p,p'-DDE

p,p'-DDT

Dieldrin

Endosulfan sulfate

Endosulfan I

Endosulfan II

Endrin

Endrin ketone

Heptachlor

Heptachlor epoxide

Lindane

Methoxychlor

PCB 1016

PCB 1221

PCB 1232

PCB 1242

PCB 1248

PCB 1254

PCB 1260

Toxaphene

Radionuclides

Actinium-228

Antimony-124

Antimony-125

Barium-133

Cerium-144

Cesium-134

Cesium-137

Cobalt-57

Cobalt-58

Cobalt-60

Europium-152

Europium-154

Europium-155

Gross alpha

Lead-212

Manganese-54

Neptunium-239

Nonvolatile beta

Plutonium-238

Plutonium-238

$\begin{array}{lll}\text { UJ } & \mathbf{Q} & \mathbf{1 . 0 1} \\ \text { UJ } & \mathbf{Q} & \mathbf{1 . 0 1} \\ \text { UJ } & \mathbf{Q} & 2.02 \\ \text { UJ } & \mathbf{Q} & 2.02 \\ \text { UJ } & \mathbf{Q} & \mathbf{2 . 0 2} \\ \text { UJ } & \mathbf{Q} & \mathbf{2 . 0 2} \\ \text { UJ } & \mathbf{Q} & 4.04 \\ \text { UJ } & \mathbf{Q} & \mathbf{2 . 0 2} \\ \text { UJ } & \mathbf{Q} & 4.04 \\ \text { UJ } & \mathbf{Q} & 2.02 \\ \text { UJ } & \mathbf{Q} & 4.04 \\ \text { UJ } & \mathbf{Q} & \mathbf{2 . 0 2} \\ \text { UJ } & \mathbf{Q} & 4.04 \\ \text { UJ } & \mathbf{Q} & \mathbf{2 . 0 2} \\ \text { UJ } & \mathbf{Q} & 4.04 \\ \text { UJ } & \mathbf{Q} & \mathbf{2 . 0 2} \\ \text { UJ } & \mathbf{Q} & \mathbf{2 . 0 2} \\ \text { UJ } & \mathbf{Q} & \mathbf{1 . 0 1} \\ \text { UJ } & \mathbf{Q} & \mathbf{2 0 . 2} \\ \text { UJ } & \mathbf{Q} & \mathbf{5 . 0 6} \\ \text { UJ } & \mathbf{Q} & \mathbf{5 . 0 6} \\ \text { UJ } & \mathbf{Q} & \mathbf{5 . 0 6} \\ \text { UJ } & \mathbf{Q} & \mathbf{5 . 0 6} \\ \text { UJ } & \mathbf{Q} & \mathbf{5 . 0 6} \\ \text { UJ } & \mathbf{Q} & \mathbf{5 . 0 6} \\ \text { UJ } & \mathbf{Q} & \mathbf{5 . 0 6} \\ \text { UJ } & \mathbf{Q} & \mathbf{4 0 . 4}\end{array}$

$\begin{array}{lll}\mu \mathrm{g} / \mathrm{kg} & 1.01 & \text { EPA8080 } \\ \mu \mathrm{g} / \mathrm{kg} & 1.01 & \text { EPA8080 } \\ \mu \mathrm{g} / \mathrm{kg} & 2.02 & \text { EPA8080 } \\ \mu \mathrm{g} / \mathrm{kg} & 2.02 & \text { EPA8080 } \\ \mu \mathrm{g} / \mathrm{kg} & 2.02 & \text { EPA8080 } \\ \mu \mathrm{g} / \mathrm{kg} & 2.02 & \text { EPA8080 } \\ \mu \mathrm{g} / \mathrm{kg} & 4.04 & \text { EPA8080 } \\ \mu \mathrm{g} / \mathrm{kg} & 2.02 & \text { EPA8080 } \\ \mu \mathrm{g} / \mathrm{kg} & 4.04 & \text { EPA8080 } \\ \mu \mathrm{g} / \mathrm{kg} & 2.02 & \text { EPA8080 } \\ \mu \mathrm{g} / \mathrm{kg} & 4.04 & \text { EPA8080 } \\ \mu \mathrm{g} / \mathrm{kg} & 2.02 & \text { EPA8080 } \\ \mu \mathrm{g} / \mathrm{kg} & 4.04 & \text { EPA8080 } \\ \mu \mathrm{g} / \mathrm{kg} & 2.02 & \text { EPA8080 } \\ \mu \mathrm{g} / \mathrm{kg} & 4.04 & \text { EPA8080 } \\ \mu \mathrm{g} / \mathrm{kg} & 2.02 & \text { EPA8080 } \\ \mu \mathrm{g} / \mathrm{kg} & 2.02 & \text { EPA8080 } \\ \mu \mathrm{g} / \mathrm{kg} & 1.01 & \text { EPA8080 } \\ \mu \mathrm{g} / \mathrm{kg} & 20.2 & \text { EPA8080 } \\ \mu \mathrm{g} / \mathrm{kg} & 5.06 & \text { EPA8080 } \\ \mu \mathrm{g} / \mathrm{kg} & 5.06 & \text { EPA8080 } \\ \mu \mathrm{g} / \mathrm{kg} & 5.06 & \text { EPA8080 } \\ \mu \mathrm{g} / \mathrm{kg} & 5.06 & \text { EPA8080 } \\ \mu \mathrm{g} / \mathrm{kg} & 5.06 & \text { EPA8080 } \\ \mu \mathrm{g} / \mathrm{kg} & 5.06 & \text { EPA8080 } \\ \mu \mathrm{g} / \mathrm{kg} & 5.06 & \text { EPA8080 } \\ \mu \mathrm{g} / \mathrm{kg} & 40.4 & \text { EPA8080 }\end{array}$

$1.85 \mathrm{E}+00 \pm 8.93 \mathrm{E}-02 \mathrm{pCi} / \mathrm{g}$

$-8.78 \mathrm{E}-03 \pm 1.14 \mathrm{E}-02 \mathrm{pCi} / \mathrm{g}$ $-7.73 \mathrm{E}-03 \pm 2.33 \mathrm{E}-02 \mathrm{pCi} / \mathrm{g}$ $2.91 \mathrm{E}-03 \pm 1.10 \mathrm{E}-02 \mathrm{pCi} / \mathrm{g}$ $8.26 \mathrm{E}-02 \pm 8.13 \mathrm{E}-02 \mathrm{pCi} / \mathrm{g}$ $-1.01 \mathrm{E}-01 \pm 1.07 \mathrm{E}-02 \mathrm{pCi} / \mathrm{g}$ $-2.07 \mathrm{E}-02 \pm 1.08 \mathrm{E}-02 \mathrm{pCi} / \mathrm{g}$ $2.48 \mathrm{E}-03 \pm 7.70 \mathrm{E}-03 \mathrm{pCi} / \mathrm{g}$ $1.09 \mathrm{E}-04 \pm 1.07 \mathrm{E}-02 \mathrm{pCi} / \mathrm{g}$ $-3.94 \mathrm{E}-03 \pm 7.23 \mathrm{E}-03 \mathrm{pCi} / \mathrm{g}$ $-1.56 \mathrm{E}-02 \pm 2.33 \mathrm{E}-02 \mathrm{pCi} / \mathrm{g}$ $3.40 \mathrm{E}-03 \pm 7.48 \mathrm{E}-02 \mathrm{pCi} / \mathrm{g}$ $0.00 \mathrm{E}+00 \quad \mathrm{pCj} / \mathrm{g}$ $2.79 \mathrm{E}+01 \pm 3.87 \mathrm{E}+00 \mathrm{pCi} / \mathrm{g}$ $1.85 \mathrm{E}+00 \pm 3.91 \mathrm{E}-02 \mathrm{pCi} / \mathrm{g}$ $3.67 \mathrm{E}-02 \pm 1.23 \mathrm{E}-02 \mathrm{pCi} / \mathrm{g}$ $0.00 \mathrm{E}+00 \quad \mathrm{pCi} / \mathrm{g}$ $1.18 \mathrm{E}+01 \pm 1.68 \mathrm{E}+00 \mathrm{pCi} / \mathrm{g}$ $-2.30 \mathrm{E}-04 \pm 2.31 \mathrm{E}-02 \mathrm{pCi} / \mathrm{g}$ 2.72E-02 $\pm 3.50 \mathrm{E}-02 \mathrm{pCi} / \mathrm{g}$
4.42E-02 EPIA-013B

1.92E-02 EPIA-013B

3.78E-02 EPIA-013B

1.64E-02 EPIA-013B

1.05E-01 EPIA-013B

1.36E-02 EPIA-013B

1.49E-02 EPIA-013B .

1.33E-02 EPIA-013B

1.62E-02 EPIA-013B

1.24E-02 EPIA-013B

3.78E-02 EPIA-013B

1.12E-01 EPIA-013B

5.53E-02 EPIA-013B

$1.85 \mathrm{E}+00$ EPIA-001B

2.32E-02 EPIA-013B

1.49E-02 EPIA.013B

9.12E-02 EPIA-013B

$2.01 \mathrm{E}+00$ EPIA.001B

$5.54 \mathrm{E}-02$ EPIA.012B

6.43E-02 EPIA-012B 
SURVEY ID: PPSC 0103 (continued)

Constituent

Radionuclides

Plutonium-239/240

Plutonium-239/240

Potassium -40

Promethium-144

Promethium-146

Ruthenium-106

Sodium-22

Strontium-90

Thorium-234

Tin-113

Yttrium-88

Zinc-65

Zirconium-95
Sample ID: 104602

RQ AQ B Result Unit D. Limit Method
SURVEY ID: PPSC 0201

Survey location: 45079E 3677305N (SRS coordinates)

Depth of core interval: 0.00 to $1.00 \mathrm{ft}$

Sample type: Normal

\begin{tabular}{|c|c|}
\hline UI & $-3.83 \mathrm{E}-05 \pm 9.40 \mathrm{E}-03 \mathrm{pCi} / \mathrm{g}$ \\
\hline UI & $-7.54 \mathrm{E}-03 \pm 8.70 \mathrm{E}-03 \mathrm{pCi} / \mathrm{g}$ \\
\hline \multirow[t]{2}{*}{$\mathrm{V}$} & $1.54 \mathrm{E}+00 \pm 1.86 \mathrm{E}-01 \mathrm{pCi} / \mathrm{g}$ \\
\hline & $1.05 \mathrm{E}-04 \pm 8.05 \mathrm{E}-03 \mathrm{pCi} / \mathrm{g}$ \\
\hline UI & $1.07 \mathrm{E}-02 \pm 1.29 \mathrm{E}-02 \mathrm{pCi} / \mathrm{g}$ \\
\hline UI & $2.56 \mathrm{E}-02 \pm 7.18 \mathrm{E}-02 \mathrm{pCi} / \mathrm{g}$ \\
\hline UI & $.5 .40 \mathrm{E}-03 \pm 7.14 \mathrm{E}-03 \mathrm{pCi} / \mathrm{g}$ \\
\hline & $1.22 \mathrm{E}-01 \pm 4.56 \mathrm{E}-02 \mathrm{pCi}$ \\
\hline V & $1.92 \mathrm{E}+00 \pm 7.66 \mathrm{E}-01 \mathrm{pCi} / \mathrm{g}$ \\
\hline UI & $-9.21 \mathrm{E}-03 \pm 1.28 \mathrm{E}-02 \mathrm{pCi} / \mathrm{g}$ \\
\hline UI & $-3.59 \mathrm{E}-04 \pm 8.29 \mathrm{E}-03 \mathrm{pCi} / \mathrm{g}$ \\
\hline UI & $1.18 \mathrm{E}-02 \pm 1.70 \mathrm{E}-02 \mathrm{pCi} /$ \\
\hline $\mathrm{IIT}$ & $3.37 \mathrm{E}-02 \pm 3.46 \mathrm{E}-02 \mathrm{pCi} /$ \\
\hline
\end{tabular}

2.94E-02 EPIA-012B

4.34E-02 EPIA-012B

1.23E-01 EPIA-013B

1.39E-02 EPIA-013B

1.90E-02 EPIA-013B

1.26E-01 EPIA-013B

1.21E-02 EPIA-013B

5.77E-02 EPIA-004

7.12E-01 EPIA-013B

2.06E-02 EPIA-013B

1.47E-02 EPIA-013B

2.68E-02 EPIA-013B

3.80E-02 EPIA-013B
Constituent
RQ AQ B Result
Unit
D. Limit Method

Physical Parameters and Specified Analyses

Cation exchange capacity

Chloride

Chloride

Cyanide

Nitrate-nitrite as nitrogen

Orthophosphate

Phenols

Sulfide

Total organic carbon

Total organic carbon

Total organic nitrogen

Total phosphates (as P)

Metals (total recoverable)

$\begin{array}{lll} & & 15.8 \\ U & & 2.76 \\ U & & 2.73 \\ U & & 1.17 \\ \text { J } & E & 0.581 \\ U & & 27.5 \\ U & & 414 \\ \text { UJ } & \text { C } & 249 \\ & & 1260 \\ & & 1450 \\ \text { C } & & 66.5 \\ \text { J } & \text { E } & 107\end{array}$

3250

5.53

16.6

12.6

0.068

V $\quad 47.1$

$\begin{array}{lll}\mathrm{U} & \mathrm{V} & 2.69\end{array}$

0.672

$\begin{array}{lll}\mathrm{U} & \mathrm{V} & 2.04\end{array}$

V $\quad 1790$

J E $\quad 3.77$
Percent solids: $\mathbf{8 7 . 0 0}$

$\begin{array}{lll}\mathrm{meq} / 100 \mathrm{~g} & 5 & \text { EPA9081 } \\ \mathrm{mg} / \mathrm{kg} & 2.76 & \text { EPA300.0 } \\ \mathrm{mg} / \mathrm{kg} & 2.73 & \text { EPA300.0 } \\ \mathrm{mg} / \mathrm{kg} & 1.17 & \text { EPA335.3 } \\ \mathrm{mg} / \mathrm{kg} & 1.16 & \text { EPA353.1 } \\ \mathrm{mg} / \mathrm{kg} & 27.5 & \text { EPA365.2 } \\ \mu \mathrm{g} / \mathrm{kg} & 414 & \text { EPA420.2 } \\ \mathrm{mg} / \mathrm{kg} & 249 & \text { EPA376.2 } \\ \mathrm{mg} / \mathrm{kg} & 115 & \text { EPA415.1 } \\ \mathrm{mg} / \mathrm{kg} & 115 & \text { EPA415.1 } \\ \mathrm{mg} / \mathrm{kg} & 0.23 & \text { EPA351.2 } \\ \mathrm{mg} / \mathrm{kg} & 400 & \text { EPA365.4 }\end{array}$

$\begin{array}{lll}\mathrm{mg} / \mathrm{kg} & 13.8 & \text { EPA6010A } \\ \mathrm{mg} / \mathrm{kg} & 5.53 & \text { EPA6010A } \\ \mathrm{mg} / \mathrm{kg} & 16.6 & \text { EPA6010A } \\ \mathrm{mg} / \mathrm{kg} & 1.38 & \text { EPA6010A } \\ \mathrm{mg} / \mathrm{kg} & 0.553 & \text { EPA6010A } \\ \mathrm{mg} / \mathrm{kg} & 0.276 & \text { EPA6010A } \\ \mathrm{mg} / \mathrm{kg} & 13.8 & \text { EPA6010A } \\ \mathrm{mg} / \mathrm{kg} & 1.38 & \text { EPA6010A } \\ \mathrm{mg} / \mathrm{kg} & 0.553 & \text { EPA6010A } \\ \mathrm{mg} / \mathrm{kg} & 1.38 & \text { EPA6010A } \\ \mathrm{mg} / \mathrm{kg} & 5.53 & \text { EPA6010A } \\ \mathrm{mg} / \mathrm{kg} & 5.53 & \text { EPA6010A } \\ \mathrm{mg} / \mathrm{kg} & 5.53 & \text { EPA6010A }\end{array}$


SURVEY ID: PPSC 0201 (continued)

Constituent

Metals (total recoverable)
Sample ID: 104604

RQ AQ B Result Unit D. Limit Method

$\begin{array}{llllll} & \mathrm{V} & 23.1 & \mathrm{mg} / \mathrm{kg} & 0.553 & \text { EPA6010A } \\ \mathrm{J} & \mathrm{E} & 0.0186 & \mathrm{mg} / \mathrm{kg} & 0.0341 & \text { EPA7471 } \\ \mathrm{J} & \mathrm{E} & 0.0171 & \mathrm{mg} / \mathrm{kg} & 0.0342 & \text { EPA7471 } \\ & & 1.45 & \mathrm{mg} / \mathrm{kg} & 1.38 & \text { EPA6010A } \\ \mathrm{J} & \mathrm{E} & 71.7 & \mathrm{mg} / \mathrm{kg} & 111 & \text { EPA6010A } \\ \mathrm{U} & & 16.6 & \mathrm{mg} / \mathrm{kg} & 16.6 & \text { EPA6010A } \\ \mathrm{U} & & 1.38 & \mathrm{mg} / \mathrm{kg} & 1.38 & \text { EPA6010A } \\ \mathrm{U} & & 55.3 & \mathrm{mg} / \mathrm{kg} & 55.3 & \text { EPA6010A } \\ \mathrm{UJ} & \mathrm{C} & 16.6 & \mathrm{mg} / \mathrm{kg} & 16.6 & \text { EPA6010A } \\ \mathrm{J} & \mathrm{C} & 4.2 & \mathrm{mg} / \mathrm{kg} & 0.553 & \text { EPA6010A } \\ & \mathrm{V} & 6.48 & \mathrm{mg} / \mathrm{kg} & 1.11 & \text { EPA6010A }\end{array}$

\section{Radionuclides}

Actinium-228

Antimony-124

Antimony-125

Barium-133

Cerium-144

Cesium-134

Cesium-137

Cobalt-57

Cobalt-58

Cobalt-60

Europium-152

Europium-154

Europium-155

Gross alpha

Lead-212

Manganese-54

Neptunium-239

Nonvolatile beta

Plutonium-238

Plutonium-239/240

Potassium -40

Promethium-144

Promethium-146

Ruthenium-106

Sodium-22

Strontium-90

Thorium-234

Tin-113

Yttrium-88

Zinc-65

Zirconium-95
UI

UI

UI

UI

UI

UI

UI

UI

UI

UT

UI

UI

UI

UI

UI

UI

UI

UI

UI

UI

UI

UI

UI
5.49E-01 $\pm 4.52 \mathrm{E}-02 \mathrm{pCi} / \mathrm{g}$ 9.76E-04 $7.43 \mathrm{E}-03 \mathrm{pCi} / \mathrm{g}$ 8.09E-03 $\pm 2.17 \mathrm{E}-02 \mathrm{pCi} / \mathrm{g}$ $-2.17 \mathrm{E}-03 \pm 9.64 \mathrm{E}-03 \mathrm{pCi} / \mathrm{g}$ $3.42 \mathrm{E}-03 \pm 4.54 \mathrm{E}-02 \mathrm{pCi} / \mathrm{g}$ $5.69 \mathrm{E}-04 \pm 5.50 \mathrm{E}-03 \mathrm{pCi} / \mathrm{g}$ $3.44 \mathrm{E}+00 \pm 4.64 \mathrm{E}-02 \mathrm{pCi} / \mathrm{g}$ 2.91E-04 $5.27 \mathrm{E}-03 \mathrm{pCi} / \mathrm{g}$ $-1.60 \mathrm{E}-03 \pm 5.20 \mathrm{E}-03 \mathrm{pCi} / \mathrm{g}$ $3.52 \mathrm{E}-03 \pm 4.03 \mathrm{E}-03 \mathrm{pCi} / \mathrm{g}$ $6.85 \mathrm{E}-04 \pm 1.92 \mathrm{E}-02 \mathrm{pCi} / \mathrm{g}$ $3.10 \mathrm{E}-02 \pm 3.50 \mathrm{E}-02 \mathrm{pCi} / \mathrm{g}$ $0.00 \mathrm{E}+00 \quad \mathrm{pCi} / \mathrm{g}$ $6.15 \mathrm{E}+00 \pm 1.55 \mathrm{E}+00 \mathrm{pCi} / \mathrm{g}$ $5.81 \mathrm{E}-01 \pm 2.35 \mathrm{E}-02 \mathrm{pCi} / \mathrm{g}$ $1.13 \mathrm{E}-02 \pm 5.88 \mathrm{E}-03 \mathrm{pCj} / \mathrm{g}$ $-2.82 \mathrm{E}-02 \pm 3.74 \mathrm{E}-02 \mathrm{pCi} / \mathrm{g}$ $8.64 \mathrm{E}+00 \pm 1.53 \mathrm{E}+00 \mathrm{pCi} / \mathrm{g}$ $1.38 \mathrm{E}-02 \pm 1.98 \mathrm{E}-02 \mathrm{pCi} / \mathrm{g}$ $6.97 \mathrm{E}-03 \pm 1.01 \mathrm{E}-02 \mathrm{pCi} / \mathrm{g}$ $5.11 \mathrm{E}-01 \neq 9.86 \mathrm{E}-02 \mathrm{pCi} / \mathrm{g}$ $1.74 \mathrm{E}-03 \pm 4.23 \mathrm{E}-03 \mathrm{pCi} / \mathrm{g}$ $-8.30 \mathrm{E}-04 \pm 1.24 \mathrm{E}-02 \mathrm{pCi} / \mathrm{g}$ $7.34 \mathrm{E}-02 \pm 5.19 \mathrm{E}-02 \mathrm{pCi} / \mathrm{g}$ $1.15 \mathrm{E}-03 \pm 3.86 \mathrm{E}-03 \mathrm{pCi} / \mathrm{g}$ $-1.07 \mathrm{E}-01 \pm 1.36 \mathrm{E}-02 \mathrm{pCi} / \mathrm{g}$ 7.87E-01 $4.71 \mathrm{E}-01 \mathrm{pCi} / \mathrm{g}$ 4.70E-04 $\pm 1.09 \mathrm{E}-02 \mathrm{pCi} / \mathrm{g}$ $1.66 \mathrm{E}-03 \pm 4.96 \mathrm{E}-03 \mathrm{pCi} / \mathrm{g}$ $-1.43 \mathrm{E}-03 \pm 8.97 \mathrm{E}-03 \mathrm{pCi} / \mathrm{g}$ $0.00 \mathrm{E}+00 \quad \mathrm{pCi} / \mathrm{g}$

$\begin{array}{ll}2.32 \mathrm{E}-02 & \text { EPIA-013B } \\ 1.30 \mathrm{E}-02 & \text { EPIA-013B } \\ 3.57 \mathrm{E}-02 & \text { EPIA-013B } \\ 1.40 \mathrm{E}-02 & \text { EPIA-013B } \\ 7.02 \mathrm{E}-02 & \text { EPIA-013B } \\ 8.55 \mathrm{E}-03 & \text { EPIA-013B } \\ 9.97 \mathrm{E}-03 & \text { EPIA-013B } \\ 9.09 \mathrm{E}-03 & \text { EPIA-013B } \\ 8.91 \mathrm{E}-03 & \text { EPIA-013B } \\ 7.40 \mathrm{E}-03 & \text { EPIA-013B } \\ 3.18 \mathrm{E}-02 & \text { EPIA-013B } \\ 6.01 \mathrm{E}-02 & \text { EPIA-013B } \\ 3.63 \mathrm{E}-02 & \text { EPIA-013B } \\ 1.21 \mathrm{E}+00 & \text { EPIA-001B } \\ 1.74 \mathrm{E}-02 & \text { EPIA-013B } \\ 7.89 \mathrm{E}-03 & \text { EPIA-013B } \\ 6.37 \mathrm{E}-02 & \text { EPIA-013B } \\ 2.10 \mathrm{E}+00 & \text { EPIA-001B } \\ 3.76 \mathrm{E}-02 & \text { EPIA-012B } \\ 1.76 \mathrm{E}-02 & \text { EPIA-012B } \\ 6.05 \mathrm{E}-02 & \text { EPIA-013B } \\ 7.55 \mathrm{E}-03 & \text { EPIA-013B } \\ 1.79 \mathrm{E}-02 & \text { EPIA-013B } \\ 9.52 \mathrm{E}-02 & \text { EPIA-013B } \\ 7.22 \mathrm{E}-03 & \text { EPIA-013B } \\ 5.13 \mathrm{E}-02 & \text { EPIA-004 } \\ 4.32 \mathrm{E}-01 & \text { EPIA-013B } \\ 1.80 \mathrm{E}-02 & \text { EPIA-013B } \\ 9.45 \mathrm{E}-03 & \text { EPIA-013B } \\ 1.34 \mathrm{E}-02 & \text { EPIA-013B } \\ 2.07 \mathrm{E}-02 & \text { EPIA-013B }\end{array}$

2.32E-02 EPIA-013B

3.57E-02 EPIA-013B

1.40E-02 EPIA-013B

7.02E-02 EPIA-013B

8.55E-03 EPIA.013B

9.97E-03 EPIA-013B

9.09E-03 EPIA-013B

8.91E-03 EPIA-013B

7.40E-03 EPIA-013B

3.18E-02 EPIA-013B

6.01E-02 EPIA-013B

3.63E-02 EPIA-013B

1.21E+00 EPIA-001B

1.74E-02 EPIA-013B

7.89E-03 EPIA-013B

$2.10 \mathrm{E}+00$ EPIA-001B

3.76E-02 EPIA-012B

1.76E-02 EPIA-012B

6.05E-02 EPIA-013B

7.55E-03 EPIA-013B

1.79202 EPIA $013 \mathrm{~B}$

7.22E-03 EPIA-013B

5.13E-02 EPIA-004

4.32E-01 EPIA-013B

9.45E-03 EPIA-013B

1.34E-02 EPIA-013B

Sample ID: 104605

\section{SURVEY ID: PPSC 0202}

\section{es)}

Survey location: $45079 \mathrm{E} 3677305 \mathrm{~N}$ (SR
Depth of core interval: 1.00 to $2.00 \mathrm{ft}$

Sample type: Normal

Sample matrix: Soil

Percent solids: 87.00 
SURVEY ID: PPSC 0202 (continued)

Constituent
Sample ID: 104605

RQ AQ B Result

Unit

D. Limit Method

Physical Parameters and Specified Analyses

Cation exchange capacity

Chloride

Cyanide

Nitrate-nitrite as nitrogen

Orthophosphate

Phenols

Sulfide

Total organic carbon

Total organic nitrogen

Total phosphates (as P)

Metals (total recoverable)

$\begin{array}{lll} & & 16.8 \\ \text { U } & & 2.82 \\ \text { U } & & 0.891 \\ \text { J } & \text { E } & 0.347 \\ \text { U } & & 25.3 \\ \text { U } & & 345 \\ \text { UJ } & \text { C } & 246 \\ & & 814 \\ \text { C } & & 41.6 \\ \text { J } & \text { E } & 144\end{array}$

$\begin{array}{llll} & & & 3410 \\ \text { UJ } & \text { I } & \text { L } & \mathbf{5 . 7 5} \\ \text { U } & & & 17.2 \\ & & & 16.4 \\ \text { J } & \text { E } & & \mathbf{0 . 0 8 9 7} \\ \text { U } & & & 0.287 \\ & \text { V } & & \mathbf{5 1 . 3} \\ \text { U } & \text { V } & & 2.53 \\ & & & \mathbf{0 . 6 2 8} \\ \text { U } & \text { V } & & 1.52 \\ & \text { V } & & 2470 \\ \text { U } & & & 5.75 \\ \text { J } & \text { IV } & \text { H } & 118 \\ & \text { V } & & 20.8 \\ \text { J } & \text { E } & & \mathbf{0 . 0 1 3 4} \\ & & & 1.57 \\ \text { J } & \text { E } & & 79.6 \\ \text { U } & & & 17.2 \\ \text { U } & & & 1.44 \\ \text { U } & & & \mathbf{5 7 . 5} \\ \text { UJ } & \text { C } & & 17.2 \\ \text { J } & \text { C } & 5.07 \\ & \text { V } & 4.13\end{array}$

$\begin{array}{lll}\mathrm{meq} / 100 \mathrm{~g} & 5 & \text { EPA9081 } \\ \mathrm{mg} / \mathrm{kg} & 2.82 & \text { EPA300.0 } \\ \mathrm{mg} / \mathrm{kg} & 0.891 & \text { EPA335.3 } \\ \mathrm{mg} / \mathrm{kg} & 1.16 & \text { EPA353.1 } \\ \mathrm{mg} / \mathrm{kg} & 25.3 & \text { EPA365.2 } \\ \mu \mathrm{g} / \mathrm{kg} & 345 & \text { EPA420.2 } \\ \mathrm{mg} / \mathrm{kg} & 246 & \text { EPA376.2 } \\ \mathrm{mg} / \mathrm{kg} & 115 & \text { EPA415.1 } \\ \mathrm{mg} / \mathrm{kg} & 0.23 & \text { EPA351.2 } \\ \mathrm{mg} / \mathrm{kg} & 393 & \text { EPA365.4 }\end{array}$

$\begin{array}{lll}\mathrm{mg} / \mathrm{kg} & 14.4 & \text { EPA6010A } \\ \mathrm{mg} / \mathrm{kg} & 5.75 & \text { EPA6010A } \\ \mathrm{mg} / \mathrm{kg} & 17.2 & \text { EPA6010A } \\ \mathrm{mg} / \mathrm{kg} & 1.44 & \text { EPA6010A } \\ \mathrm{mg} / \mathrm{kg} & 0.575 & \text { EPA6010A } \\ \mathrm{mg} / \mathrm{kg} & 0.287 & \text { EPA6010A } \\ \mathrm{mg} / \mathrm{kg} & 14.4 & \text { EPA6010A } \\ \mathrm{mg} / \mathrm{kg} & 1.44 & \text { EPA6010A } \\ \mathrm{mg} / \mathrm{kg} & 0.575 & \text { EPA6010A } \\ \mathrm{mg} / \mathrm{kg} & 1.44 & \text { EPA6010A } \\ \mathrm{mg} / \mathrm{kg} & 5.75 & \text { EPA6010A } \\ \mathrm{mg} / \mathrm{kg} & 5.75 & \text { EPA6010A } \\ \mathrm{mg} / \mathrm{kg} & 5.75 & \text { EPA6010A } \\ \mathrm{mg} / \mathrm{kg} & 0.575 & \text { EPA6010A } \\ \mathrm{mg} / \mathrm{kg} & 0.0383 & \text { EPA7471 } \\ \mathrm{mg} / \mathrm{kg} & 1.44 & \text { EPA6010A } \\ \mathrm{mg} / \mathrm{kg} & 115 & \text { EPA6010A } \\ \mathrm{mg} / \mathrm{kg} & 17.2 & \text { EPA6010A } \\ \mathrm{mg} / \mathrm{kg} & 1.44 & \text { EPA6010A } \\ \mathrm{mg} / \mathrm{kg} & 57.5 & \text { EPA6010A } \\ \mathrm{mg} / \mathrm{kg} & 17.2 & \text { EPA6010A } \\ \mathrm{mg} / \mathrm{kg} & 0.575 & \text { EPA6010A } \\ \mathrm{mg} / \mathrm{kg} & 1.15 & \text { EPA6010A }\end{array}$

Radionuclides

Actinium-228

Antimony-124

Antimony-125

Barium-133

Cerium-144

Cesium-134

Cesium-137

Cobalt-57

Cobalt-58

Cobalt-60

Europium-152

Europium-154
6.76E-01士4.61E-02 pCi/g

$-7.51 \mathrm{E}-03 \pm 6.12 \mathrm{E}-03 \mathrm{pCi} / \mathrm{g}$

$-7.98 \mathrm{E}-03 \pm 1.23 \mathrm{E}-02 \mathrm{pCi} / \mathrm{g}$

$1.57 \mathrm{E}-03 \pm 5.68 \mathrm{E}-03 \mathrm{pCi} / \mathrm{g}$

$-4.51 \mathrm{E}-02 \pm 3.37 \mathrm{E}-02 \mathrm{pCi} / \mathrm{g}$

$-4.67 \mathrm{E}-02 \pm 5.80 \mathrm{E}-03 \mathrm{pCi} / \mathrm{g}$

$3.25 \mathrm{E}-02 \pm 7.24 \mathrm{E}-03 \mathrm{pCi} / \mathrm{g}$

1.21E-03+4.30E-03 pCi/g

$-8.43 \mathrm{E}-04 \pm 5.26 \mathrm{E}-03 \mathrm{pCi} / \mathrm{g}$

$-1.77 \mathrm{E}-03 \pm 4.00 \mathrm{E}-03 \mathrm{pCi} / \mathrm{g}$

$5.32 \mathrm{E}-03 \pm 1.24 \mathrm{E}-02 \mathrm{pCi} / \mathrm{g}$

$-9.52 \mathrm{E}-03 \pm 3.69 \mathrm{E}-02 \mathrm{pCi} / \mathrm{g}$
2.44E-02 EPIA-013B

1.01E-02 EPIA-013B

1.98E-02 EPIA-013B

8.63E-03 EPIA-013B

5.65E-02 EPIA-013B

6.85E-03 EPIA-013B

7.73E-03 EPIA-013B

7.49E-03 EPLA-013B

9.13E-03 EPIA-013B

7.01E-03 EPIA-013B

2.11E-02 EPIA-013B

5.49E-02 EPIA-013B 
SURVEY ID: PPSC 0202 (continued)

Constituent

Radionuclides

Europium-155

Gross alpha

Lead-212

Manganese-54

Neptunium-239

Nonvolatile beta

Plutonium-238

Plutonium-239/240

Potassium-40

Promethium-144

Promethium-146

Ruthenium-106

Sodium-22

Strontium-90

Thorium-234

Tin-113

Yttrium-88

Zinc-65

Zirconium-95
Sample ID: 104605

$\begin{array}{lllll}\text { RQ AQ B Result Unit } & \text { D. Limit Method }\end{array}$

UI

$\begin{array}{ll}0.00 \mathrm{E}+00 & \mathrm{pCi} / \mathrm{g} \\ 1.69 \mathrm{E}+01 \pm 3.16 \mathrm{E}+00 \mathrm{pCi} / \mathrm{g} \\ 6.70 \mathrm{E}-01 \pm 2.11 \mathrm{E}-02 \mathrm{pCi} / \mathrm{g} \\ 1.46 \mathrm{E}-02 \pm 7.44 \mathrm{E}-03 \mathrm{pCi} / \mathrm{g} \\ 3.63 \mathrm{E}-03 \pm 3.24 \mathrm{E}-02 \mathrm{pCi} / \mathrm{g} \\ 5.74 \mathrm{E}+00 \pm 1.28 \mathrm{E}+00 \mathrm{pCi} / \mathrm{g} \\ -1.38 \mathrm{E}-03 \pm 1.39 \mathrm{E}-02 \mathrm{pCi} / \mathrm{g} \\ 0.00 \mathrm{E}+00 & \mathrm{pCi} / \mathrm{g} \\ 7.94 \mathrm{E}-01 \pm 1.10 \mathrm{E}-01 & \mathrm{pCi} / \mathrm{g} \\ 2.99 \mathrm{E}-03 \pm 4.35 \mathrm{E}-03 & \mathrm{pCi} / \mathrm{g} \\ 6.09 \mathrm{E}-03 \pm 5.76 \mathrm{E}-03 & \mathrm{pCi} / \mathrm{g} \\ -1.25 \mathrm{E}-03 \pm 3.93 \mathrm{E}-02 & \mathrm{pCi} / \mathrm{g} \\ -1.03 \mathrm{E}-03 \pm 4.22 \mathrm{E}-03 \mathrm{pC} / \mathrm{g} \\ -4.66 \mathrm{E}-02 \pm 2.61 \mathrm{E}-02 \mathrm{pCi} / \mathrm{g} \\ 7.23 \mathrm{E}-01 \pm 4.18 \mathrm{E}-01 \mathrm{pCi} / \mathrm{g} \\ 3.80 \mathrm{E}-04 \pm 6.93 \mathrm{E}-03 \mathrm{pCi} / \mathrm{g} \\ 4.77 \mathrm{E}-03 \pm 5.14 \mathrm{E}-03 \mathrm{pCi} / \mathrm{g} \\ 3.27 \mathrm{E}-03 \pm 1.04 \mathrm{E}-02 & \mathrm{pCi} / \mathrm{g} \\ 4.55 \mathrm{E}-03 \pm 1.21 \mathrm{E}-02 & \mathrm{pCi} / \mathrm{g}\end{array}$

3.03E-02 EPIA-013B

$1.84 \mathrm{E}+00$ EPIA-001B

1.29E-02 EPIA-013B

6.04E-03 EPIA-013B

$5.08 \mathrm{E}-02$ EPIA-013B

$1.94 \mathrm{E}+00$ EPIA-001B

3.47E-02 EPIA-012B

7.45E-03 EPIA-012B

5.78E-02 EPIA-013B

7.87E-03 EPIA-013B

1.00E-02 EPIA-013B

6.91E-02 EPIA-013B

7.10E-03 EPIA-013B

6.58E-02 EPIA-004

3.99E-01 EPIA-013B

1.16E-02 EPIA-013B

9.52E-03 EPIA-013B

1.62E-02 EPIA-013B

2.15E-02 EPIA-013B

\section{SURVEY ID: PPSC 0203}

Sample ID: 104606

Survey location: $45079 \mathrm{E} 3677305 \mathrm{~N}$ (SRS coordinates)

Depth of core interval: 2.00 to $4.00 \mathrm{ft}$

Sample type: Normal

Sample matrix: Soil

Percent solids: $\mathbf{8 8 . 0 0}$

Constituent

RQ AQ B Result

Unit

D. Limit Method

Physical Parameters and Specified Analyses

Cation exchange capacity

Chloride

Cyanide

Nitrate-nitrite as nitrogen

Orthophosphate

Phenols

Sulfide

Total organic carbon

Total organic nitrogen

Total phosphates (as P)

Metals (total recoverable)

Aluminum

Antimony

Arsenic

Barium

Beryllium

Cadmium

Calcium

Chromium

Cobalt

$\begin{array}{lll} & & 14.3 \\ \text { U } & & 2.73 \\ \text { U } & & 0.916 \\ \text { J } & \text { E } & 0.422 \\ \text { U } & & 24.6 \\ \text { U } & & 341 \\ \text { UJ } & \text { C } & 252 \\ & & 563 \\ \text { C } & & 40 \\ \text { J } & \text { E } & 109\end{array}$

3120

5.21

15.6

12.2

$\begin{array}{lll}\mathrm{J} & \mathrm{E} & \mathbf{0 . 0 7 0 9} \\ \mathrm{U} & & 0.261\end{array}$

$\begin{array}{ll}\mathrm{V} & 52.8 \\ \mathrm{~V} & 2.96\end{array}$

$\begin{array}{lll}\mathrm{U} & \mathrm{V} & 2.96 \\ \mathrm{~J} & \mathrm{E} & 0.48\end{array}$

$\begin{array}{lll}\mathrm{meq} / 100 \mathrm{~g} & 5 & \text { EPA9081 } \\ \mathrm{mg} / \mathrm{kg} & 2.73 & \text { EPA300.0 } \\ \mathrm{mg} / \mathrm{kg} & \mathbf{0 . 9 1 6} & \text { EPA335.3 } \\ \mathrm{mg} / \mathrm{kg} & 1.05 & \text { EPA353.1 } \\ \mathrm{mg} / \mathrm{kg} & 24.6 & \text { EPA365.2 } \\ \mu \mathrm{g} / \mathrm{kg} & 341 & \text { EPA420.2 } \\ \mathrm{mg} / \mathrm{kg} & 252 & \text { EPA376.2 } \\ \mathrm{mg} / \mathrm{kg} & 114 & \text { EPA415.1 } \\ \mathrm{mg} / \mathrm{kg} & 0.227 & \text { EPA351.2 } \\ \mathrm{mg} / \mathrm{kg} & 385 & \text { EPA365.4 }\end{array}$

$\mathrm{mg} / \mathrm{kg}$

$\mathrm{mg} / \mathrm{kg}$

$\mathrm{mg} / \mathrm{kg}$

$\mathrm{mg} / \mathrm{kg}$

$\mathrm{mg} / \mathrm{kg}$

$\mathrm{mg} / \mathrm{kg}$

$\mathrm{mg} / \mathrm{kg}$

$\mathrm{mg} / \mathrm{kg}$

mg/kg

$\begin{array}{ll}13 & \text { EPA6010A } \\ 5.21 & \text { EPA6010A } \\ 15.6 & \text { EPA6010A } \\ 1.3 & \text { EPA6010A } \\ 0.521 & \text { EPA6010A } \\ 0.261 & \text { EPA6010A } \\ 13 & \text { EPA6010A } \\ 1.3 & \text { EPA6010A } \\ 0.521 & \text { EPA6010A }\end{array}$


SURVEY ID: PPSC 0203 (continued)

Constituent

RQ AQ B Result

$\begin{array}{lll}\text { U } & \text { V } & 1.82 \\ & \text { V } & 2290 \\ J & \text { E } & 2.11 \\ & \text { V } & 106 \\ & \text { V } & 21.6 \\ \text { J } & \text { E } & 0.0187 \\ & & 1.49 \\ \text { J } & \text { E } & 87.8 \\ \text { U } & & 15.6 \\ \text { U } & & 1.3 \\ \text { U } & & 52.1 \\ \text { UJ } & \text { C } & 15.6 \\ \text { J } & \text { C } & 4.95 \\ & \text { V } & 5.49\end{array}$

Copper

Iron

Lead

Magnesium

Manganese

Mercury

Nickel

Potassium

Selenium

Silver

Sodium

Thallium

Vanadium

Zinc

\section{Radionuclides}

Actinium-228

Antimony-124

Antimony-125

Barium-133

Cerium-144

Cesium-134

Cesium-137

Cobalt-57

Cobalt-58

Cobalt-60

Europium-152

Europium-154

Europium-155

Gross alpha

Lead-212

Manganese-54

Neptunium-239

Nonvolatile beta

Plutonium-238

Plutonium-239/240

Potassium-40

Promethium-144

Promethium-146

Ruthenium-106

Sodium-22

Strontium-90

Thorium-234

Tin-113

Yttrium-88

Zinc-65

Zirconium-95

UI
Sample ID: 104606

Unit D. Limit Method

$\begin{array}{lll}\mathrm{mg} / \mathrm{kg} & 1.3 & \text { EPA6010A } \\ \mathrm{mg} / \mathrm{kg} & 5.21 & \text { EPA6010A } \\ \mathrm{mg} / \mathrm{kg} & 5.21 & \text { EPA6010A } \\ \mathrm{mg} / \mathrm{kg} & 5.21 & \text { EPA6010A } \\ \mathrm{mg} / \mathrm{kg} & 0.521 & \text { EPA6010A } \\ \mathrm{mg} / \mathrm{kg} & 0.0377 & \text { EPA7471 } \\ \mathrm{mg} / \mathrm{kg} & 1.3 & \text { EPA6010A } \\ \mathrm{mg} / \mathrm{kg} & 104 & \text { EPA6010A } \\ \mathrm{mg} / \mathrm{kg} & 15.6 & \text { EPA6010A } \\ \mathrm{mg} / \mathrm{kg} & 1.3 & \text { EPA6010A } \\ \mathrm{mg} / \mathrm{kg} & 52.1 & \text { EPA6010A } \\ \mathrm{mg} / \mathrm{kg} & 15.6 & \text { EPA6010A } \\ \mathrm{mg} / \mathrm{kg} & 0.521 & \text { EPA6010A } \\ \mathrm{mg} / \mathrm{kg} & 1.04 & \text { EPA6010A }\end{array}$

6.02E-01 $14.34 \mathrm{E}-02 \mathrm{pCi} / \mathrm{g}$

UI $\quad-2.87 \mathrm{E}-03 \pm 5.71 \mathrm{E}-03 \mathrm{pCi} / \mathrm{g}$

UI $\quad-2.03 \mathrm{E}-03 \pm 1.05 \mathrm{E}-02 \mathrm{pCi} / \mathrm{g}$

UI $\quad-1.11 \mathrm{E}-03 \pm 5.46 \mathrm{E}-03 \mathrm{pCi} / \mathrm{g}$

UI $\quad-1.32 \mathrm{E}-02 \pm 3.09 \mathrm{E}-02 \mathrm{pCi} / \mathrm{g}$

UI $6.39 \mathrm{E}-04 \pm 4.20 \mathrm{E}-03 \mathrm{pCi} / \mathrm{g}$

UI $\quad-5.07 \mathrm{E}-04 \pm 4.29 \mathrm{E}-03 \mathrm{pCi} / \mathrm{g}$

UI $3.31 \mathrm{E}-04 \pm 5.33 \mathrm{E}-03 \mathrm{pCi} / \mathrm{g}$

UI 2.00E-04 $2.83 \mathrm{E}-03 \mathrm{pCi} / \mathrm{g}$

UI $\quad-4.09 \mathrm{E}-03 \pm 4.08 \mathrm{E}-03 \mathrm{pCi} / \mathrm{g}$

UI $\quad-3.68 \mathrm{E}-03 \pm 1.13 \mathrm{E}-02 \mathrm{pCi} / \mathrm{g}$

UI $\quad 1.42 \mathrm{E}-02 \pm 3.44 \mathrm{E}-02 \mathrm{pCi} / \mathrm{g}$

$0.00 \mathrm{E}+00$ $\mathrm{pCi} / \mathrm{g}$

$1.69 \mathrm{E}+01 \pm 2.46 \mathrm{E}+00 \mathrm{pCi} / \mathrm{g}$

$6.47 \mathrm{E}-01 \pm 2.00 \mathrm{E}-02 \mathrm{pCi} / \mathrm{g}$

$1.14 \mathrm{E}-02 \pm 4.78 \mathrm{E}-03 \mathrm{pCi} / \mathrm{g}$

UI $\quad-7.56 \mathrm{E}-03 \pm 2.77 \mathrm{E}-02 \mathrm{pCi} / \mathrm{g}$

$7.80 \mathrm{E}+00 \pm 1.42 \mathrm{E}+00 \mathrm{pCi} / \mathrm{g}$ $0.00 \mathrm{E}+00 \quad \mathrm{pCi} / \mathrm{g}$

UI $\quad 1.31 \mathrm{E}-03 \pm 5.91 \mathrm{E}-03 \mathrm{pCi} / \mathrm{g}$ $5.24 \mathrm{E}-01 \pm 8.23 \mathrm{E}-02 \mathrm{pCi} / \mathrm{g}$

UI $\quad-5.03 \mathrm{E}-04 \pm 3.99 \mathrm{E}-03 \mathrm{pCi} / \mathrm{g}$

UI $\quad 3.00 \mathrm{E}-03 \pm 4.81 \mathrm{E}-03 \mathrm{pCi} / \mathrm{g}$

UI $\quad 4.56 \mathrm{E}-03 \pm 3.53 \mathrm{E}-02 \mathrm{pCi} / \mathrm{g}$

UI $\quad-1.40 \mathrm{E}-04 \pm 3.55 \mathrm{E}-03 \mathrm{pCi} / \mathrm{g}$ $8.06 \mathrm{E}-02 \pm 4.05 \mathrm{E}-02 \mathrm{pCj} / \mathrm{g}$

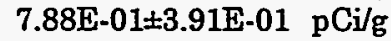
1.91E-03 $\pm 5.70 \mathrm{E}-03 \mathrm{pCi} / \mathrm{g}$ 1.01E-04 $3.90 \mathrm{E}-03 \mathrm{pCi} / \mathrm{g}$ $-6.62 \mathrm{E}-03 \pm 8.78 \mathrm{E}-03 \mathrm{pCi} / \mathrm{g}$ $6.25 \mathrm{E}-03 \pm 1.24 \mathrm{E}-02 \mathrm{pCi} / \mathrm{g}$
2.01E-02 EPIA-013B 9.60E-03 EPIA-013B

1.82E-02 EPIA-013B

7.88E-03 EPLA-013B

5.24E-02 EPIA-013B

6.36E-03 EPIA-013B

7.30E-03 EPIA-013B

6.57E-03 EPIA-013B

8.28E-03 EPIA-013B

5.42E-03 EPIA-013B

1.84E-02 EPIA-013B

5.62E-02 EPIA-013B

2.74E-02 EPIA-013B

1.33E+00 EPIA-001B

1.15E-02 EPIA-013B

7.75E-03 EPIA-013B

4.74E-02 EPIA-013B

$1.87 \mathrm{E}+00$ EPIA-001B

$0.00 \mathrm{E}+00$ EPIA-012B

1.66E-02 EPIA-012B

6.04E-02 EPIA-013B

6.78E-03 EPIA-013B

8.61E-03 EPIA-013B

6.13E-02 EPIA-013B

6.28E-03 EPIA-013B

5.65E-02 EPIA-004

3.50E-01 EPIA-013B

1.01E-02 EPIA-013B

7.31E-03 EPIA-013B

1.25E-02 EPIA-013B

1.91E-02 EPIA-013B

SURVEY ID: PPSC 0204

Sample ID: 104607

Survey location: $45079 \mathrm{E} 3677305 \mathrm{~N}$ (SRS coordinates)

Depth of core interval: 4.00 to $6.00 \mathrm{ft}$

Sample type: Normal

Sample matrix: Soil

Percent solids: $\mathbf{8 9 . 0 0}$ 
Appendix D: Sediment Tables

D-22

SURVEY ID: PPSC 0204 (continued)

Constituent

RQ AQ B Result

Sample ID: 104607

Physical Parameters and Specified Analyses

Cation exchange capacity

Chloride

Cyanide

Nitrate-nitrite as nitrogen

Orthophosphate

Phenols

Sulfide

Total organic carbon

Total organic nitrogen

Total phosphates (as P)

Metals (total recoverable)

Aluminum

Antimony

Arsenic

Barium

Beryllium

Cadmium

Calcium

Chromium

Cobalt

Copper

Iron

Lead

Magnesium

Manganese

Mercury

Nickel

Potassium

Selenium

Silver

Sodium

Thallium

Vanadium

Zinc

Radionuclides

Actinium-228

Antimony-124

Antimony-125

Barium-133

Cerium-144

Cesium-134

Cesium-137

Cobalt-57

Cobalt-58

Cobalt-60

Europium-152

Europium-154

Europium-155

Gross alpha

Lead-212

$\begin{array}{lll} & & 90.8 \\ \text { U } & & 2.73 \\ \text { U } & & 1.06 \\ \text { U } & & 1.06 \\ \text { U } & & 22.6 \\ \text { U } & & 337 \\ \text { UJ } & \text { C } & 243 \\ & & 479 \\ \text { C } & & 39.1 \\ \text { J } & \text { E } & 105\end{array}$

$\begin{array}{ll}\mathrm{meq} / 100 \mathrm{~g} & 5 \\ \mathrm{mg} / \mathrm{kg} & 2.73 \\ \mathrm{mg} / \mathrm{kg} & 1.06 \\ \mathrm{mg} / \mathrm{kg} & 1.06 \\ \mathrm{mg} / \mathrm{kg} & 22.6 \\ \mu \mathrm{g} / \mathrm{kg} & 337 \\ \mathrm{mg} / \mathrm{kg} & 243 \\ \mathrm{mg} / \mathrm{kg} & 112 \\ \mathrm{mg} / \mathrm{kg} & 0.225 \\ \mathrm{mg} / \mathrm{kg} & 428\end{array}$

EPA9081

EPA300.0

EPA335.3

EPA353.1

EPA365.2

EPA420.2

EPA376.2

EPA415.1

EPA351.2

EPA365.4

$\begin{array}{lll}\mathrm{mg} / \mathrm{kg} & 12.9 & \text { EPA6010A } \\ \mathrm{mg} / \mathrm{kg} & 5.15 & \text { EPA6010A } \\ \mathrm{mg} / \mathrm{kg} & 15.5 & \text { EPA6010A } \\ \mathrm{mg} / \mathrm{kg} & 1.29 & \text { EPA6010A } \\ \mathrm{mg} / \mathrm{kg} & 0.515 & \text { EPA6010A } \\ \mathrm{mg} / \mathrm{kg} & 0.258 & \text { EPA6010A } \\ \mathrm{mg} / \mathrm{kg} & 12.9 & \text { EPA6010A } \\ \mathrm{mg} / \mathrm{kg} & 1.29 & \text { EPA6010A } \\ \mathrm{mg} / \mathrm{kg} & 0.515 & \text { EPA6010A } \\ \mathrm{mg} / \mathrm{kg} & 1.29 & \text { EPA6010A } \\ \mathrm{mg} / \mathrm{kg} & 5.15 & \text { EPA6010A } \\ \mathrm{mg} / \mathrm{kg} & 5.15 & \text { EPA6010A } \\ \mathrm{mg} / \mathrm{kg} & 5.15 & \text { EPA6010A } \\ \mathrm{mg} / \mathrm{kg} & 0.515 & \text { EPA6010A } \\ \mathrm{mg} / \mathrm{kg} & 0.0367 & \text { EPA7471 } \\ \mathrm{mg} / \mathrm{kg} & 1.29 & \text { EPA6010A } \\ \mathrm{mg} / \mathrm{kg} & 103 & \text { EPA6010A } \\ \mathrm{mg} / \mathrm{kg} & 15.5 & \text { EPA6010A } \\ \mathrm{mg} / \mathrm{kg} & 1.29 & \text { EPA6010A } \\ \mathrm{mg} / \mathrm{kg} & 51.5 & \text { EPA6010A } \\ \mathrm{mg} / \mathrm{kg} & 15.5 & \text { EPA6010A } \\ \mathrm{mg} / \mathrm{kg} & 0.515 & \text { EPA6010A } \\ \mathrm{mg} / \mathrm{kg} & 1.03 & \text { EPA6010A }\end{array}$

$8.70 \mathrm{E}-01 \pm 5.00 \mathrm{E}-02 \mathrm{pCi} / \mathrm{g}$

$-7.86 \mathrm{E}-04 \pm 6.42 \mathrm{E}-03 \mathrm{pCi} / \mathrm{g}$

$-4.31 \mathrm{E}-03 \pm 1.25 \mathrm{E}-02 \mathrm{pCj} / \mathrm{g}$

3.28E-04 $\pm 6.13 \mathrm{E}-03 \mathrm{pCi} / \mathrm{g}$

$4.85 \mathrm{E}-02 \pm 4.34 \mathrm{E}-02 \mathrm{pCi} / \mathrm{g}$

$-7.66 \mathrm{E}-04 \pm 4.80 \mathrm{E}-03 \mathrm{pCi} / \mathrm{g}$

$5.33 \mathrm{E}-03 \pm 6.20 \mathrm{E}-03 \mathrm{pCi} / \mathrm{g}$

$1.78 \mathrm{E}-04 \pm 4.88 \mathrm{E}-03 \mathrm{pCi} / \mathrm{g}$

$-1.34 \mathrm{E}-03 \pm 5.98 \mathrm{E}-03 \mathrm{pCi} / \mathrm{g}$

$2.65 \mathrm{E}-04 \pm 3.71 \mathrm{E}-03 \mathrm{pCi} / \mathrm{g}$

$2.67 \mathrm{E}-03 \pm 1.32 \mathrm{E}-02 \mathrm{pCi} / \mathrm{g}$

$1.04 \mathrm{E}-02 \pm 3.80 \mathrm{E}-02 \mathrm{pCi} / \mathrm{g}$

$0.00 \mathrm{E}+00 \quad \mathrm{pCi} / \mathrm{g}$

$1.57 \mathrm{E}+01 \pm 2.47 \mathrm{E}+00 \mathrm{pCi} / \mathrm{g}$

9.23E-01 $\pm 2.46 \mathrm{E}-02 \mathrm{pCi} / \mathrm{g}$

$\begin{array}{ll}2.36 \mathrm{E}-02 & \text { EPIA-013B } \\ 1.08 \mathrm{E}-02 & \text { EPIA-013B } \\ 2.13 \mathrm{E}-02 & \text { EPIA-013B } \\ 9.40 \mathrm{E}-03 & \text { EPIA-013B } \\ 6.51 \mathrm{E}-02 & \text { EPIA-013B } \\ 7.02 \mathrm{E}-03 & \text { EPIA-013B } \\ 7.34 \mathrm{E}-03 & \text { EPIA-013B } \\ 8.17 \mathrm{E}-03 & \text { EPIA-013B } \\ 9.91 \mathrm{E}-03 & \text { EPIA-013B } \\ 6.56 \mathrm{E}-03 & \text { EPIA-013B } \\ 2.30 \mathrm{E}-02 & \text { EPIA-013B } \\ 6.02 \mathrm{E}-02 & \text { EPIA-013B } \\ 3.25 \mathrm{E}-02 & \text { EPIA-013B } \\ 1.04 \mathrm{E}+00 & \text { EPIA-001B } \\ 1.43 \mathrm{E}-02 & \text { EPIA-013B }\end{array}$

UI

UI 
SURVEY ID: PPSC 0204 (continued)

Constituent

Radionuclides

Manganese-54

Neptunium-239

Nonvolatile beta

Plutonium-238

Plutonium-239/240

Potassium-40

Promethium-144

Promethium-146

Ruthenium-106

Sodium-22

Strontium-90

Thorium-234

Tin-113

Yttrium-88

Zinc-65

Zirconium-95
Sample ID: 104607
RQ
AQ B Result
Unit
D. Limit Method

8.64E-03 EPIA-013B

5.68E-02 EPIA-013B

$2.22 \mathrm{E}+00$ EPIA-001B

4.38E-02 EPIA-012B

8.31E-03 EPIA-012B

6.77E-02 EPIA-013B

7.90E-03 EPIA-013B

1.05E-02 EPIA-013B

7.11E-02 EPIA-013B

6.27E-03 EPIA-013B

5.20E-02 EPIA-004

4.17E-01 EPIA-013B

1.17E-02 EPIA-013B

9.67E-03 EPIA-013B

1.63E-02 EPIA-013B

2.15E-02 EPIA-013B

SURVEY ID: PPSC 0205

Sample ID: 104608

Survey location: $45079 \mathrm{E} 3677305 \mathrm{~N}$ (SRS coordinates)

Depth of core interval: 6.00 to $7.10 \mathrm{ft}$

Sample type: Normal

Sample matrix: Soil

Percent solids: $\mathbf{8 9 . 0 0}$

Constituent

RQ AQ B Result

Unit

D. Limit Method

Physical Parameters and Specified Analyses

Cation exchange capacity

Chloride

Cyanide

Nitrate-nitrite as nitrogen

Orthophosphate

Phenols

Sulfide

Total organic carbon

Total organic nitrogen

Total phosphates (as P)

Metals (total recoverable)

Aluminum

Antimony

Arsenic

Barium

Beryllium

Cadmium

Calcium

Chromium

Cobalt

Copper

Iron

Lead

$\begin{array}{lll} & & 68.9 \\ \text { U } & & 2.75 \\ \text { U } & & 1.02 \\ \text { J } & \text { E } & \\ \text { U } & & \mathbf{0 . 3 4 2} \\ \text { U } & & 25.4 \\ \text { UJ } & \text { C } & 337 \\ & & 245 \\ \text { C } & & 642 \\ \text { J } & \text { E } & 40.2 \\ & & 148\end{array}$

$\begin{array}{lll} & & 9160 \\ \text { U } & & 5.3 \\ \text { U } & & 15.9 \\ & & 9.57 \\ \text { J } & \text { E } & 0.0599 \\ \text { U } & & 0.265 \\ & \text { V } & 50.3 \\ & \text { V } & 9.05 \\ \text { J } & \text { E } & 0.281 \\ & \text { V } & 4.51 \\ & \text { V } & 9280 \\ \text { J } & \text { E } & 4.18\end{array}$

$\begin{array}{lll}\mathrm{meq} / 100 \mathrm{~g} & 5 & \text { EPA9081 } \\ \mathrm{mg} / \mathrm{kg} & 2.75 & \text { EPA300.0 } \\ \mathrm{mg} / \mathrm{kg} & 1.02 & \text { EPA335.3 } \\ \mathrm{mg} / \mathrm{kg} & 1.14 & \text { EPA353.1 } \\ \mathrm{mg} / \mathrm{kg} & 25.4 & \text { EPA365.2 } \\ \mu \mathrm{g} / \mathrm{kg} & 337 & \text { EPA420.2 } \\ \mathrm{mg} / \mathrm{kg} & 245 & \text { EPA376.2 } \\ \mathrm{mg} / \mathrm{kg} & 112 & \text { EPA415.1 } \\ \mathrm{mg} / \mathrm{kg} & 0.225 & \text { EPA351.2 } \\ \mathrm{mg} / \mathrm{kg} & 441 & \text { EPA365.4 }\end{array}$

$\mathrm{mg} / \mathrm{kg}$

$\mathrm{mg} / \mathrm{kg}$

$\mathrm{mg} / \mathrm{kg}$

$\mathrm{mg} / \mathrm{kg}$

$\mathrm{mg} / \mathrm{kg}$

$\mathrm{mg} / \mathrm{kg}$

$\mathrm{mg} / \mathrm{kg}$

$\mathrm{mg} / \mathrm{kg}$

$\mathrm{mg} / \mathrm{kg}$

$\mathrm{mg} / \mathrm{kg}$

$\mathrm{mg} / \mathrm{kg}$

$\mathrm{mg} / \mathrm{kg}$
13.2

5.3

15.9

1.32

0.53

0.265

13.2

1.32

0.53

- 1.32

5.3

5.3
EPA6010A

EPA6010A

EPA6010A

EPA6010A

EPA6010A

EPA6010A

EPA6010A

EPA6010A

EPA6010A

EPA6010A

EPA6010A

EPA6010A 
SURVEY ID: PPSC 0205 (continued)

Constituent

Metals (total recoverable)

Magnesium

Manganese

Mercury

Nickel

Potassium

Selenium

Silver

Sodium

Thallium

Vanadium

Zinc

Radionuclides

Actinium-228

Antimony-124

Antimony-125

Barium-133

Cerium-144

Cesium-134

Cesium-137

Cobalt-57

Cobalt-58

Cobalt-60

Europium-152

Europium-154

Europium-155

Gross alpha

Lead-212

Manganese-54

Neptunium-239

Nonvolatile beta

Plutonium-238

Plutonium-239/240

Potassium-40

Promethium-144

Promethium-146

Ruthenium-106

Sodium-22

Strontium-90

Thorium-234

Tin-113

Yttrium-88

Zinc-65

Zirconium-95
RQ AQ B Result

Sample ID: 104608

$\begin{array}{lll} & \text { V } & 146 \\ & \text { V } & 9.34 \\ J & \text { E } & 0.0253 \\ & & 1.51 \\ & & 114 \\ \text { U } & & 15.9 \\ \text { U } & & 1.32 \\ \text { U } & & 53 \\ \text { UJ } & \text { C } & 15.9 \\ J & \text { C } & 27.7 \\ & \text { V } & 5.6\end{array}$

$\begin{array}{lll}\mathrm{mg} / \mathrm{kg} & 5.3 & \text { EPA6010A } \\ \mathrm{mg} / \mathrm{kg} & 0.53 & \text { EPA6010A } \\ \mathrm{mg} / \mathrm{kg} & 0.0369 & \text { EPA7471 } \\ \mathrm{mg} / \mathrm{kg} & 1.32 & \text { EPA6010A } \\ \mathrm{mg} / \mathrm{kg} & 106 & \text { EPA6010A } \\ \mathrm{mg} / \mathrm{kg} & 15.9 & \text { EPA6010A } \\ \mathrm{mg} / \mathrm{kg} & 1.32 & \text { EPA6010A } \\ \mathrm{mg} / \mathrm{kg} & 53 & \text { EPA6010A } \\ \mathrm{mg} / \mathrm{kg} & 15.9 & \text { EPA6010A } \\ \mathrm{mg} / \mathrm{kg} & 0.53 & \text { EPA6010A } \\ \mathrm{mg} / \mathrm{kg} & 1.06 & \text { EPA6010A }\end{array}$

$1.06 \mathrm{E}+00 \pm 6.21 \mathrm{E}-02 \mathrm{pCi} / \mathrm{g}$ $-4.80 \mathrm{E}-03 \pm 7.90 \mathrm{E}-03 \mathrm{pCi} / \mathrm{g}$ $4.64 \mathrm{E}-03 \pm 1.56 \mathrm{E}-02 \mathrm{pCi} / \mathrm{g}$ $-5.42 \mathrm{E}-04 \pm 7.72 \mathrm{E}-03 \mathrm{pCi} / \mathrm{g}$ 1.41E-02 $44.49 \mathrm{E}-02 \mathrm{pCi} / \mathrm{g}$ $5.78 \mathrm{E}-03 \pm 5.70 \mathrm{E}-03 \mathrm{pCi} / \mathrm{g}$ 4.00E-03 $\pm 7.18 \mathrm{E}-03 \mathrm{pCi} / \mathrm{g}$ $-1.75 \mathrm{E}-03 \pm 5.54 \mathrm{E}-03 \mathrm{pCi} / \mathrm{g}$ 4.99E-03 $\pm 7.83 \mathrm{E}-03 \mathrm{pCi} / \mathrm{g}$ $5.56 \mathrm{E}-04 \pm 4.83 \mathrm{E}-03 \mathrm{pCi} / \mathrm{g}$ $-3.82 \mathrm{E}-03 \pm 1.66 \mathrm{E}-02 \mathrm{pCi} / \mathrm{g}$ $-2.08 \mathrm{E}-02 \pm 5.19 \mathrm{E}-02 \mathrm{pCi} / \mathrm{g}$ $0.00 \mathrm{E}+00 \quad \mathrm{pCi} / \mathrm{g}$ $1.37 \mathrm{E}+01 \pm 2.40 \mathrm{E}+00 \mathrm{pCi} / \mathrm{g}$ $1.15 \mathrm{E}+00 \pm 2.92 \mathrm{E}-02 \mathrm{pCi} / \mathrm{g}$ $1.69 \mathrm{E}-02 \pm 9.51 \mathrm{E}-03 \mathrm{pCi} / \mathrm{g}$ 1.68E-02+4.31E-02 pCi/g $6.75 \mathrm{E}+00 \pm 1.40 \mathrm{E}+00 \mathrm{pCi} / \mathrm{g}$ $1.27 \mathrm{E}-02 \pm 1.47 \mathrm{E} .02 \mathrm{pCi} / \mathrm{g}$ $-1.45 \mathrm{E}-03 \pm 7.50 \mathrm{E}-03 \mathrm{pCi} / \mathrm{g}$ $7.28 \mathrm{E}-01 \pm 1.15 \mathrm{E}-01 \mathrm{pCi} / \mathrm{g}$ $-2.30 \mathrm{E}-03 \pm 5.72 \mathrm{E}-03 \mathrm{pCi} / \mathrm{g}$ $6.06 \mathrm{E}-03 \pm 8.29 \mathrm{E}-03 \mathrm{pCi} / \mathrm{g}$ $3.07 \mathrm{E}-02 \pm 5.29 \mathrm{E}-02 \mathrm{pCi} / \mathrm{g}$ 2.32E-03 $44.84 \mathrm{E}-03 \mathrm{pCi} / \mathrm{g}$ $1.65 \mathrm{E}-02 \pm 2.81 \mathrm{E}-02 \mathrm{pCj} / \mathrm{g}$ 9.57E-01 $\pm 5.82 \mathrm{E}-01 \mathrm{pCi} / \mathrm{g}$ $-8.27 \mathrm{E}-03 \pm 8.89 \mathrm{E}-03 \mathrm{pCi} / \mathrm{g}$ 1.13E-03 $46.35 \mathrm{E}-03 \mathrm{pCi} / \mathrm{g}$ $3.33 \mathrm{E}-03 \pm 1.24 \mathrm{E}-02 \mathrm{pCi} / \mathrm{g}$ $0.00 \mathrm{E}+00 \quad \mathrm{pCi} / \mathrm{g}$
D. Limit Method

2.96E-02 EPIA-013B

1.34E-02 EPIA-013B

2.60E-02 EPIA-013B

1.14E-02 EPIA-013B

7.75E-02 EPIA-013B

9.18E-03 EPIA-013B

9.50E-03 EPIA-013B

1.26E-02 EPIA-013B

8.80E-03 EPIA-013B

2.74E-02 EPIA-013B

7.51E-02 EPLA-013B

3.90E-02 EPIA-013B

1.33E+00 EPIA-001B

1.66E-02 EPIA-013B

9.55E-03 EPIA-013B

6.77E-02 EPIA-013B

1.97E+00 EPIA-001B

2.46E-02 EPIA-012B

2.46E-02 EPIA-012B

8.17E-02 EPIA-013B

9.73E-03 EPIA-013B

1.26E-02 EPIA-013B

9.46E-02 EPIA-013B

9.07E-03 EPIA-013B

4.88E-02 EPLA-004

4.94E-01 EPIA-013B

1.42E-02 EPIA-013B

1.17E-02 EPIA-013B

1.92E-02 EPIA-013B

2.93E-02 EPIA-013B
9.30E-03 EPIA-013B

SURVEY ID: PPSC 0301

Sample ID: 104609

Survey location: $45149 \mathrm{E} 3677579 \mathrm{~N}$ (SRS coordinates)

Depth of core interval: 0.00 to $1.00 \mathrm{ft}$

Sample type: Normal

Sample matrix: Soil

Percent solids: $\mathbf{8 5 . 0 0}$ 
SURVEY ID: PPSC 0301 (continued)

Constituent

Sample ID: 104609
RQ AQ B Result
Unit
D. Limit Method

Physical Parameters and Specified Analyses

Cation exchange capacity

Chloride

Cyanide

Nitrate-nitrite as nitrogen

Orthophosphate

Phenols

Phenols

Sulfide

Sulfide

Total organic carbon

Total organic nitrogen

Total phosphates (as P)

Metals (total recoverable)

Aluminum

Antimony

Arsenic

Barium

Beryllium

Cadmium

Calcium

Chromium

Cobalt

Copper

Iron

Lead

Magnesium

Manganese

Mercury

Nickel

Potassium

Selenium

Silver

Sodium

Thallium

Vanadium

Zinc

Radionuclides

Actinium-228

Antimony-124

Antimony-125

Barium-133

Cerium-144

Cesium-134

Cesium-137

Cobalt-57

Cobalt-58

Cobalt-60

$\begin{array}{lll}\cdot & & 9.3 \\ \text { U } & & 2.66 \\ \text { U } & & 1.11 \\ \text { U } & & 1.18 \\ \text { U } & & 27.8 \\ \text { U } & & 353 \\ \text { U } & & 367 \\ \text { UJ } & \text { CI } & 253 \\ \text { UJ } & \text { CI } & 275 \\ & & 965 \\ \text { C } & & 13.1 \\ \text { J } & \text { E } & 59.8\end{array}$

$\begin{array}{ll}\mathrm{meq} / 100 \mathrm{~g} & 5 \\ \mathrm{mg} / \mathrm{kg} & 2.66 \\ \mathrm{mg} / \mathrm{kg} & 1.11 \\ \mathrm{mg} / \mathrm{kg} & 1.18 \\ \mathrm{mg} / \mathrm{kg} & 27.8 \\ \mu \mathrm{g} / \mathrm{kg} & 353 \\ \mu \mathrm{g} / \mathrm{kg} & 367 \\ \mathrm{mg} / \mathrm{kg} & 253 \\ \mathrm{mg} / \mathrm{kg} & 275 \\ \mathrm{mg} / \mathrm{kg} & 118 \\ \mathrm{mg} / \mathrm{kg} & 0.235 \\ \mathrm{mg} / \mathrm{kg} & 118\end{array}$

EPA9081

EPA300.0

EPA335.3

EPA353.1

EPA365.2

EPA420.2

EPA420.2

EPA376.2

EPA376.2

EPA415.1

EPA351.2

EPA365.4

$\begin{array}{lll}\mathrm{mg} / \mathrm{kg} & 14.6 & \text { EPA6010A } \\ \mathrm{mg} / \mathrm{kg} & 5.82 & \text { EPA6010A } \\ \mathrm{mg} / \mathrm{kg} & 17.5 & \text { EPA6010A } \\ \mathrm{mg} / \mathrm{kg} & 1.46 & \text { EPA6010A } \\ \mathrm{mg} / \mathrm{kg} & 0.582 & \text { EPA6010A } \\ \mathrm{mg} / \mathrm{kg} & 0.291 & \text { EPA6010A } \\ \mathrm{mg} / \mathrm{kg} & 14.6 & \text { EPA6010A } \\ \mathrm{mg} / \mathrm{kg} & 1.46 & \text { EPA6010A } \\ \mathrm{mg} / \mathrm{kg} & 0.582 & \text { EPA6010A } \\ \mathrm{mg} / \mathrm{kg} & 1.46 & \text { EPA6010A } \\ \mathrm{mg} / \mathrm{kg} & 5.82 & \text { EPA6010A } \\ \mathrm{mg} / \mathrm{kg} & 5.82 & \text { EPA6010A } \\ \mathrm{mg} / \mathrm{kg} & 5.82 & \text { EPA6010A } \\ \mathrm{mg} / \mathrm{kg} & 0.582 & \text { EPA6010A } \\ \mathrm{mg} / \mathrm{kg} & 0.0292 & \text { EPA7471 } \\ \mathrm{mg} / \mathrm{kg} & 1.46 & \text { EPA6010A } \\ \mathrm{mg} / \mathrm{kg} & 116 & \text { EPA6010A } \\ \mathrm{mg} / \mathrm{kg} & 17.5 & \text { EPA6010A } \\ \mathrm{mg} / \mathrm{kg} & 1.46 & \text { EPA6010A } \\ \mathrm{mg} / \mathrm{kg} & 58.2 & \text { EPA6010A } \\ \mathrm{mg} / \mathrm{kg} & 17.5 & \text { EPA6010A } \\ \mathrm{mg} / \mathrm{kg} & 0.582 & \text { EPA6010A } \\ \mathrm{mg} / \mathrm{kg} & 1.16 & \text { EPA6010A }\end{array}$

$1.03 \mathrm{E}+00 \pm 6.49 \mathrm{E}-02 \mathrm{pCi} / \mathrm{g}$ 9.24E-04 $\pm 8.90 \mathrm{E}-03 \mathrm{pCi} / \mathrm{g}$ $6.71 \mathrm{E}-03 \pm 2.16 \mathrm{E}-02 \mathrm{pCi} / \mathrm{g}$ $-1.43 \mathrm{E}-02 \pm 9.61 \mathrm{E}-03 \mathrm{pCi} / \mathrm{g}$ $1.98 \mathrm{E}-03 \pm 4.93 \mathrm{E}-02 \mathrm{pCi} / \mathrm{g}$ $-4.81 \mathrm{E}-03 \pm 6.90 \mathrm{E}-03 \mathrm{pCi} g$ $1.39 \mathrm{E}+00 \pm 3.40 \mathrm{E}-02 \mathrm{pCi} / \mathrm{g}$ $4.81 \mathrm{E}-03 \pm 6.22 \mathrm{E}-03 \mathrm{pCi} / \mathrm{g}$ $-1.86 \mathrm{E}-03 \pm 7.82 \mathrm{E}-03 \mathrm{pCi} / \mathrm{g}$ 1.02E-02 7.06E-03 pCig $\begin{array}{ll}3.17 \mathrm{E}-02 & \text { EPIA-013B } \\ 1.56 \mathrm{E}-02 & \text { EPIA-013B } \\ 3.59 \mathrm{E}-02 & \text { EPIA-013B } \\ 1.52 \mathrm{E}-02 & \text { EPIA-013B } \\ 8.49 \mathrm{E}-02 & \text { EPIA-013B } \\ 1.01 \mathrm{E}-02 & \text { EPIA-013B } \\ 1.34 \mathrm{E}-02 & \text { EPIA-013B } \\ 1.09 \mathrm{E}-02 & \text { EPIA-013B } \\ 1.18 \mathrm{E}-02 & \text { EPIA-013B } \\ 9.49 \mathrm{E}-03 & \text { EPIA-013B }\end{array}$ 
SURVEY ID: PPSC 0301 (continued)

Constituent

RQ AQ B Result

UI

Europium-152

Europium-154

Europium-155

Gross alpha

Lead-212

Manganese-54

Neptunium-239

Nonvolatile beta

Plutonium-238

Plutonium-239/240

Potassium-40

Promethium-144

Promethium-146

Ruthenium-106

Sodium-22

Strontium-90

Thorium-234

Tin-113

Yttrium-88

Zinc-65

Zirconium-95
Sample ID: 104609

Unit D. Limit Method
3.36E-02 EPIA-013B
8.17E-02 EPIA-013B
4.23E-02 EPIA-013B
1.27E+00 EPIA-001B
1.99E-02 EPIA-013B
9.30E-03 EPIA-013B
7.39E-02 EPIA-013B
1.97E+00 EPIA-001B
4.28E-02 EPIA-012B
3.44E-02 EPIA-012B
8.64E-02 EPIA-013B
1.02E-02 EPIA-013B
1.77E-02 EPIA-013B
1.08E-01 EPIA-013B
9.71E-03 EPIA-013B
7.17E-02 EPIA-004
5.41E-01 EPIA-013B
1.89E-02 EPIA-013B
1.19E-02 EPIA-013B
2.01E-02 EPIA-013B
2.80E-02 EPIA-013B

SURVEY ID: PPSC 0302

Sample ID: 104610

Survey location: $45149 \mathrm{E}$ 3677579N (SRS coordinates)

Depth of core interval: 1.00 to $2.00 \mathrm{ft}$

Sample type: Normal

Sample matrix: Soil

Percent solids: $\mathbf{8 9 . 0 0}$

$\begin{array}{lllll}\text { Constituent } & \text { RQ AQ B Result Dit Limit Method }\end{array}$

Physical Parameters and Specified Analyses

Cation exchange capacity

Cation exchange capacity

Chloride

Cyanide

Nitrate-nitrite as nitrogen

Orthophosphate

Phenols

Sulfide

Total organic carbon

Total organic nitrogen

Total phosphates (as P)

Metals (total recoverable)

Aluminum

Antimony

Arsenic

Barium

Beryllium

Cadmium

$\begin{array}{lll}\text { U } & & 5 \\ \text { U } & & 5.62 \\ \text { U } & & 2.54 \\ \text { U } & & 1.05 \\ \text { U } & & 0.99 \\ \text { U } & & 26.9 \\ \text { J } & \text { E } & 138 \\ \text { UJ } & \text { C } & 250 \\ & & 351 \\ \text { C } & & 12.6 \\ \text { J } & \text { E } & 81.3\end{array}$

$\begin{array}{ll}\mathrm{meq} / 100 \mathrm{~g} & 5 \\ \mathrm{meq} / 100 \mathrm{~g} & 5.62 \\ \mathrm{mg} / \mathrm{kg} & 2.54 \\ \mathrm{mg} / \mathrm{kg} & 1.05 \\ \mathrm{mg} / \mathrm{kg} & 0.99 \\ \mathrm{mg} / \mathrm{kg} & 26.9 \\ \mu \mathrm{g} / \mathrm{kg} & 337 \\ \mathrm{mg} / \mathrm{kg} & 250 \\ \mathrm{mg} / \mathrm{kg} & 112 \\ \mathrm{mg} / \mathrm{kg} & 0.225 \\ \mathrm{mg} / \mathrm{kg} & 225\end{array}$

EPA9081

EPA9081

EPA300.0

EPA335.3

EPA353.1

EPA365.2

EPA420.2

EPA376.2

EPA415.1

EPA351.2

EPA365.4
EPA6010A
EPA6010A
EPA6010A
EPA6010A
EPA6010A
EPA6010A 
SURVEY ID: PPSC 0302 (continued)

Constituent

Metals (total recoverable)

Calcium

Chromium

Cobalt

Copper

Iron

Lead

Magnesium

Manganese

Mercury

Nickel

Potassium

Selenium

Silver

Sodium

Thallium

Vanadium

Zinc

\section{Radionuclides}

Actinium-228

Antimony-124

Antimony-125

Barium-133

Cerium-144

Cesium-134

Cesium-137

Cobalt-57

Cobalt-58

Cobalt-60

Europium-152

Europium-154

Europium-155

Gross alpha

Lead-212

Manganese-54

Neptunium-239

Nonvolatile beta

Plutonium-238

Plutonium-239/240

Potassium-40

Promethium-144

Promethium-146

Ruthenium-106

Sodium-22

Strontium-90

Thorium-234

Tin-113

Yttrium-88

Zinc-65

Zirconium-95
Sample ID: 104610

RQ AQ B Result

Unit

D. Limit Method

$\begin{array}{lll}\mathrm{mg} / \mathrm{kg} & 13.4 & \text { EPA6010A } \\ \mathrm{mg} / \mathrm{kg} & 1.34 & \text { EPA6010A } \\ \mathrm{mg} / \mathrm{kg} & 0.535 & \text { EPA6010A } \\ \mathrm{mg} / \mathrm{kg} & 1.34 & \text { EPA6010A } \\ \mathrm{mg} / \mathrm{kg} & 5.35 & \text { EPA6010A } \\ \mathrm{mg} / \mathrm{kg} & 5.35 & \text { EPA6010A } \\ \mathrm{mg} / \mathrm{kg} & 5.35 & \text { EPA6010A } \\ \mathrm{mg} / \mathrm{kg} & 0.535 & \text { EPA6010A } \\ \mathrm{mg} / \mathrm{kg} & \mathbf{0 . 0 3 3} & \text { EPA7471 } \\ \mathrm{mg} / \mathrm{kg} & 1.34 & \text { EPA6010A } \\ \mathrm{mg} / \mathrm{kg} & 107 & \text { EPA6010A } \\ \mathrm{mg} / \mathrm{kg} & 16.1 & \text { EPA6010A } \\ \mathrm{mg} / \mathrm{kg} & 1.34 & \text { EPA6010A } \\ \mathrm{mg} / \mathrm{kg} & 53.5 & \text { EPA6010A } \\ \mathrm{mg} / \mathrm{kg} & 16.1 & \text { EPA6010A } \\ \mathrm{mg} / \mathrm{kg} & 0.535 & \text { EPA6010A } \\ \mathrm{mg} / \mathrm{kg} & 1.07 & \text { EPA6010A }\end{array}$

$1.05 \mathrm{E}+00 \pm 5.59 \mathrm{E}-02 \mathrm{pCi} / \mathrm{g}$ $-1.37 \mathrm{E}-03 \pm 7.01 \mathrm{E}-03 \mathrm{pCi} / \mathrm{g}$ $9.62 \mathrm{E}-04 \pm 1.42 \mathrm{E}-02 \mathrm{pCi} / \mathrm{g}$ $5.75 \mathrm{E}-03 \pm 7.33 \mathrm{E}-03 \mathrm{pCi} / \mathrm{g}$ $7.38 \mathrm{E}-03 \pm 4.04 \mathrm{E}-02 \mathrm{pCi} / \mathrm{g}$ $3.54 \mathrm{E}-03 \pm 5.20 \mathrm{E}-03 \mathrm{pCi} / \mathrm{g}$ $4.79 \mathrm{E}-01 \pm 1.91 \mathrm{E}-02 \mathrm{pCi} / \mathrm{g}$ $2.41 \mathrm{E}-03 \pm 5.18 \mathrm{E}-03 \mathrm{pCi} / \mathrm{g}$ $1.81 \mathrm{E}-03 \pm 6.92 \mathrm{E}-03 \mathrm{pCi} / \mathrm{g}$ $-1.11 \mathrm{E}-03 \pm 5.05 \mathrm{E}-03 \mathrm{pCi} / \mathrm{g}$ $-5.68 \mathrm{E}-03 \pm 1.58 \mathrm{E}-02 \mathrm{pCi} / \mathrm{g}$ $-3.77 \mathrm{E}-02 \pm 3.82 \mathrm{E}-02 \mathrm{pCi} / \mathrm{g}$ $0.00 \mathrm{E}+00 \quad \mathrm{pCi} / \mathrm{g}$ $2.10 \mathrm{E}+01 \pm 2.70 \mathrm{E}+00 \mathrm{pCi} / \mathrm{g}$ $1.07 \mathrm{E}+00 \pm 2.65 \mathrm{E}-02 \mathrm{pCi} / \mathrm{g}$ $1.73 \mathrm{E}-02 \pm 8.98 \mathrm{E}-03 \mathrm{pCi} / \mathrm{g}$ UI $\quad-1.68 \mathrm{E} \cdot 02 \pm 3.59 \mathrm{E} \cdot 02 \mathrm{pCi} / \mathrm{g}$ $9.21 \mathrm{E}+00 \pm 1.53 \mathrm{E}+00 \mathrm{pCi} / \mathrm{g}$ $0.00 \mathrm{E}+00 \quad \mathrm{pCi} / \mathrm{g}$ $3.37 \mathrm{E}-02 \pm 2.09 \mathrm{E}-02 \mathrm{pCi} / \mathrm{g}$ $6.44 \mathrm{E} \cdot 01 \pm 1.05 \mathrm{E}-01 \mathrm{pCi} / \mathrm{g}$ $-3.45 \mathrm{E}-03 \pm 5.16 \mathrm{E}-03 \mathrm{pCi} / \mathrm{g}$ $3.26 \mathrm{E}-03 \pm 6.98 \mathrm{E}-03 \mathrm{pCi} / \mathrm{g}$ $3.95 \mathrm{E}-02 \pm 4.96 \mathrm{E}-02 \mathrm{pCi} / \mathrm{g}$ $1.23 \mathrm{E}-03 \pm 4.39 \mathrm{E}-03 \mathrm{pCi} / \mathrm{g}$ $1.99 \mathrm{E}-02 \pm 2.76 \mathrm{E}-02 \mathrm{pCi} / \mathrm{g}$ $8.00 \mathrm{E}-01 \pm 4.44 \mathrm{E}-01 \mathrm{pCi} / \mathrm{g}$ $-5.28 \mathrm{E}-03 \pm 7.73 \mathrm{E}-03 \mathrm{pCi} / \mathrm{g}$ $3.97 \mathrm{E}-03 \pm 5.62 \mathrm{E}-03 \mathrm{pCi} / \mathrm{g}$ $-1.47 \mathrm{E}-02 \pm 1.20 \mathrm{E}-02 \mathrm{pCi} / \mathrm{g}$ $0.00 \mathrm{E}+00 \quad \mathrm{pCi} / \mathrm{g}$
2.55E-02 EPIA-013B

1.19E-02 EPIA-013B

2.48E-02 EPIA-013B

1.10E-02 EPIA-013B

6.90E-02 EPIA-013B

8.11E-03 EPIA-013B

9.44E-03 EPIA-013B

8.93E-03 EPIA-013B

1.05E-02 EPIA-013B

7.48E-03 EPIA-013B

2.55E-02 EPIA-013B

6.37E-02 EPIA-013B

3.64E-02 EPIA-013B

$1.50 \mathrm{E}+00$ EPIA-001B

1.57E-02 EPIA-013B

8.97E-03 EPIA-013B

6.11E-02 EPLA.013B

2.00E+00 EPIA-001B

0.00E+00 EPIA-012B

2.16E-02 EPIA-012B

7.15E-02 EPIA-013B

8.50E-03 EPIA-013B

1.22E-02 EPIA-013B

8.09E-02 EPIA-013B

7.86E-03 EPIA-013B

4.69E-02 EPIA-004

4.58E-01 EPIA-013B

1.32E-02 EPIA-013B

1.08E-02 EPIA-013B

1.64E-02 EPIA-013B

2.47E-02 EPIA-013B 
Survey location: $45149 \mathrm{E} 3677579 \mathrm{~N}$ (SRS coordinates)

Depth of core interval: 2.00 to $4.00 \mathrm{ft}$

Sample type: Normal

Constituent

RQ AQ B Result

Unit

D. Limit Method

Physical Parameters and Specified Analyses

Cation exchange capacity

Chloride

Cyanide

Nitrate-nitrite as nitrogen

Orthophosphate

Phenols

Sulfide

Total organic carbon

Total organic nitrogen

Total phosphates (as $\mathrm{P}$ )

$\begin{array}{lll} & & 10.4 \\ \text { U } & & 2.76 \\ \text { U } & & 1.14 \\ \text { U } & & 1.03 \\ \text { J } & \text { E } & 11.9 \\ \text { U } & & 341 \\ \text { UJ } & \text { C } & 242 \\ & & 388 \\ \text { C } & & 18.9 \\ \text { J } & \text { E } & 65.1\end{array}$

Metals (total recoverable)

$\begin{array}{lll} & & 1260 \\ \text { U } & & 5.31 \\ \text { U } & & 15.9 \\ & & 4.86 \\ \text { J } & \text { E } & 0.0361 \\ \text { U } & & 0.266 \\ & \text { V } & 30.9 \\ \text { U } & \text { V } & 2.47 \\ \text { J } & \text { E } & 0.222 \\ \text { U } & \text { V } & 0.54 \\ & \text { V } & 1360 \\ \text { J } & \text { E } & 2.5 \\ & \text { V } & 25.9 \\ & \text { V } & 9.81 \\ \text { J } & \text { E } & 0.0107 \\ \text { J } & \text { E } & 0.473 \\ \text { U } & & 106 \\ \text { U } & & 15.9 \\ \text { U } & & 1.33 \\ \text { U } & & 53.1 \\ \text { UJ } & \text { C } & 15.9 \\ \text { J } & \text { C } & 7.15 \\ & \text { V } & 1.16\end{array}$

$\begin{array}{lll}\mathrm{meq} / 100 \mathrm{~g} & 5 & \text { EPA9081 } \\ \mathrm{mg} / \mathrm{kg} & 2.76 & \text { EPA300.0 } \\ \mathrm{mg} / \mathrm{kg} & 1.14 & \text { EPA335.3 } \\ \mathrm{mg} / \mathrm{kg} & 1.03 & \text { EPA353.1 } \\ \mathrm{mg} / \mathrm{kg} & 26.2 & \text { EPA365.2 } \\ \mu \mathrm{g} / \mathrm{kg} & 341 & \text { EPA420.2 } \\ \mathrm{mg} / \mathrm{kg} & 242 & \text { EPA376.2 } \\ \mathrm{mg} / \mathrm{kg} & 114 & \text { EPA415.1 } \\ \mathrm{mg} / \mathrm{kg} & 0.227 & \text { EPA351.2 } \\ \mathrm{mg} / \mathrm{kg} & 223 & \text { EPA365.4 }\end{array}$

$\begin{array}{lll}\mathrm{mg} / \mathrm{kg} & 13.3 & \text { EPA6010A } \\ \mathrm{mg} / \mathrm{kg} & 5.31 & \text { EPA6010A } \\ \mathrm{mg} / \mathrm{kg} & 15.9 & \text { EPA6010A } \\ \mathrm{mg} / \mathrm{kg} & 1.33 & \text { EPA6010A } \\ \mathrm{mg} / \mathrm{kg} & 0.531 & \text { EPA6010A } \\ \mathrm{mg} / \mathrm{kg} & 0.266 & \text { EPA6010A } \\ \mathrm{mg} / \mathrm{kg} & 13.3 & \text { EPA6010A } \\ \mathrm{mg} / \mathrm{kg} & 1.33 & \text { EPA6010A } \\ \mathrm{mg} / \mathrm{kg} & 0.531 & \text { EPA6010A } \\ \mathrm{mg} / \mathrm{kg} & 1.33 & \text { EPA6010A } \\ \mathrm{mg} / \mathrm{kg} & 5.31 & \text { EPA6010A } \\ \mathrm{mg} / \mathrm{kg} & 5.31 & \text { EPA6010A } \\ \mathrm{mg} / \mathrm{kg} & 5.31 & \text { EPA6010A } \\ \mathrm{mg} / \mathrm{kg} & 0.531 & \text { EPA6010A } \\ \mathrm{mg} / \mathrm{kg} & 0.0374 & \text { EPA7471 } \\ \mathrm{mg} / \mathrm{kg} & 1.33 & \text { EPA6010A } \\ \mathrm{mg} / \mathrm{kg} & 106 & \text { EPA6010A } \\ \mathrm{mg} / \mathrm{kg} & 15.9 & \text { EPA6010A } \\ \mathrm{mg} / \mathrm{kg} & 1.33 & \text { EPA6010A } \\ \mathrm{mg} / \mathrm{kg} & 53.1 & \text { EPA6010A } \\ \mathrm{mg} / \mathrm{kg} & 15.9 & \text { EPA6010A } \\ \mathrm{mg} / \mathrm{kg} & 0.531 & \text { EPA6010A } \\ \mathrm{mg} / \mathrm{kg} & 1.06 & \text { EPA6010A }\end{array}$

Radionuclides

Actinium-228

Antimony-124

Antimony-125

Barium-133

Cerium-144

Cesium-134

Cesium-137

Cobalt-57

Cobalt-58

Cobalt-60
$8.60 \mathrm{E}-01 \pm 4.90 \mathrm{E}-02 \mathrm{pCi} / \mathrm{g}$ $-1.66 \mathrm{E}-03 \pm 6.05 \mathrm{E}-03 \mathrm{pCi} / \mathrm{g}$ $1.00 \mathrm{E}-02 \pm 1.24 \mathrm{E}-02 \mathrm{pCi} / \mathrm{g}$ $2.53 \mathrm{E}-03 \pm 6.02 \mathrm{E}-03 \mathrm{pCi} / \mathrm{g}$ $-1.55 \mathrm{E}-02 \pm 3.60 \mathrm{E}-02 \mathrm{pCi} / \mathrm{g}$ $-1.49 \mathrm{E}-04 \pm 4.50 \mathrm{E}-03 \mathrm{pCi} / \mathrm{g}$ $1.07 \mathrm{E}-01 \pm 1.22 \mathrm{E}-02 \mathrm{pCi} / \mathrm{g}$ -2.05E-04 $44.38 \mathrm{E}-03 \mathrm{pCi} / \mathrm{g}$ $-2.81 \mathrm{E}-03 \pm 5.48 \mathrm{E}-03 \mathrm{pCi} / \mathrm{g}$ $-2.13 \mathrm{E} \cdot 03 \pm 4.30 \mathrm{E}-03 \mathrm{pCi} / \mathrm{g}$
2.42E-02 EPIA.013B

1.05E.02 EPIA.013B

2.11E-02 EPIA-013B

9.10E-03 EPIA-013B

6.13E-02 EPIA-013B

6.93E-03 EPIA.013B

8.92E-03 EPIA-013B

7.55E-03 EPLA.013B

9.22E-03 EPIA-013B

6.38E-03 EPIA-013B 
SURVEY ID: PPSC 0303 (continued)

Constituent

Radionuclides

Europium-152

Europium-154

Europium-155

Gross alpha

Lead-212

Manganese-54

Neptunium-239

Nonvolatile beta

Plutonium-238

Plutonium-239/240

Potassium-40

Promethium-144

Promethium-146

Ruthenium-106

Sodium-22

Strontium-90

Thorium-234

Tin-113

Yttrium-88

Zinc-65

Zirconium-95
Sample ID: 104611

RQ AQ B Result Unit D. Limit Method
2.10E-02 EPIA-013B

6.03E-02 EPIA-013B

3.05E-02 EPIA-013B

1.50E+00 EPIA-001B

1.33E-02 EPIA-013B

7.27E-03 EPIA-013B

5.32E-02 EPIA.013B

2.02E+00 EPIA-001B

3.73E-02 EPIA-012B

2.13E-02 EPIA-012B

6.18E-02 EPIA-013B

8.10E-03 EPIA-013B

1.03E-02 EPIA-013B

6.73E-02 EPIA-013B

6.51E-03 EPIA-013B

4.92E-02 EPIA-004

3.90E-01 EPIA-013B

1.10E-02 EPIA-013B

8.67E-03 EPIA-013B

1.43E-02 EPIA-013B

2.19E-02 EPIA-013B

\section{SURVEY ID: PPSC 0304}

Sample ID: 104612

Survey location: $45149 \mathrm{E}$ 3677579N (SRS coordinates)

Depth of core interval: 4.00 to $6.00 \mathrm{ft}$

Sample type: Normal

Percent solids: $\mathbf{8 9 . 0 0}$

Constituent

RQ AQ B Result

Unit

D. Limit Method

Physical Parameters and Specified Analyses

Cation exchange capacity

Chloride

Cyanide

Nitrate-nitrite as nitrogen

Orthophosphate

Phenols

Sulfide

Total organic carbon

Total organic carbon

Total organic nitrogen

Total phosphates (as P)

Metals (total recoverable)

$\begin{array}{lll}\mathrm{meq} / 100 \mathrm{~g} & 5 & \text { EPA9081 } \\ \mathrm{mg} / \mathrm{kg} & 2.69 & \text { EPA300.0 } \\ \mathrm{mg} / \mathrm{kg} & 1.07 & \text { EPA335.3 } \\ \mathrm{mg} / \mathrm{kg} & 1.06 & \text { EPA353.1 } \\ \mathrm{mg} / \mathrm{kg} & 23.1 & \text { EPA365.2 } \\ \mu \mathrm{g} / \mathrm{kg} & 337 & \text { EPA420.2 } \\ \mathrm{mg} / \mathrm{kg} & 250 & \text { EPA376.2 } \\ \mathrm{mg} / \mathrm{kg} & 112 & \text { EPA415.1 } \\ \mathrm{mg} / \mathrm{kg} & 112 & \text { EPA415.1 } \\ \mathrm{mg} / \mathrm{kg} & 0.225 & \text { EPA351.2 } \\ \mathrm{mg} / \mathrm{kg} & 220 & \text { EPA365.4 }\end{array}$

$\begin{array}{ll}\mathrm{mg} / \mathrm{kg} & 14 \\ \mathrm{mg} / \mathrm{kg} & 5.62 \\ \mathrm{mg} / \mathrm{kg} & 16.9 \\ \mathrm{mg} / \mathrm{kg} & 1.4 \\ \mathrm{mg} / \mathrm{kg} & 0.562 \\ \mathrm{mg} / \mathrm{kg} & 0.281\end{array}$

EPA6010A EPA6010A EPA6010A EPA6010A EPA6010A EPA6010A 
SURVEY ID: PPSC 0304 (continued)

Constituent

RQ AQ B Result

Unit

D. Limit Method

Metals (total recoverable)

Calcium

Chromium

Cobalt

Copper

Iron

Lead

Magnesium

Manganese

Mercury

Nickel

Potassium

Selenium

Silver

Sodium

Thallium

Vanadium

Zinc

Radionuclides

Actinium-228

Antimony-124

Antimony-125

Barium-133

Cerium-144

Cesium-134

Cesium-137

Cobalt-57

Cobalt-58

Cobalt-60

Europium-152

Europium-154

Europium-155

Gross alpha

Lead-212

Manganese-54

Neptunium-239

Nonvolatile beta

Plutonium-238

Plutonium-239/240

Potassium- 40

Promethium-144

Promethium-146

Ruthenium-106

Sodium-22

Strontium-90

Thorium-234

Tin-113

Yttrium-88

Zinc-65

Zirconium-95

$\begin{array}{lll} & \text { V } & 84.6 \\ & \text { V } & 4.72 \\ \text { J } & \text { E } & 0.191 \\ \text { U } & \text { V } & 1.16 \\ & \text { V } & 2330 \\ J & \text { E } & 4.27 \\ & \text { V } & 58.6 \\ & \text { V } & 20.6 \\ \text { J } & \text { E } & \mathbf{0 . 0 1 7 9} \\ \text { J } & \text { E } & 0.642 \\ \text { J } & \text { E } & 59.5 \\ \text { U } & & 16.9 \\ \text { U } & & 1.4 \\ \text { U } & . & 56.2 \\ \text { UJ } & \text { C } & 16.9 \\ \text { J } & \text { C } & 12.2 \\ & \text { V } & 1.61\end{array}$

$\begin{array}{lll}\mathrm{mg} / \mathrm{kg} & 14 & \text { EPA6010A } \\ \mathrm{mg} / \mathrm{kg} & 1.4 & \text { EPA6010A } \\ \mathrm{mg} / \mathrm{kg} & 0.562 & \text { EPA6010A } \\ \mathrm{mg} / \mathrm{kg} & 1.4 & \text { EPA6010A } \\ \mathrm{mg} / \mathrm{kg} & 5.62 & \text { EPA6010A } \\ \mathrm{mg} / \mathrm{kg} & 5.62 & \text { EPA6010A } \\ \mathrm{mg} / \mathrm{kg} & 5.62 & \text { EPA6010A } \\ \mathrm{mg} / \mathrm{kg} & 0.562 & \text { EPA6010A } \\ \mathrm{mg} / \mathrm{kg} & 0.0342 & \text { EPA7471 } \\ \mathrm{mg} / \mathrm{kg} & 1.4 & \text { EPA6010A } \\ \mathrm{mg} / \mathrm{kg} & 112 & \text { EPA6010A } \\ \mathrm{mg} / \mathrm{kg} & 16.9 & \text { EPA6010A } \\ \mathrm{mg} / \mathrm{kg} & 1.4 & \text { EPA6010A } \\ \mathrm{mg} / \mathrm{kg} & 56.2 & \text { EPA6010A } \\ \mathrm{mg} / \mathrm{kg} & 16.9 & \text { EPA6010A } \\ \mathrm{mg} / \mathrm{kg} & 0.562 & \text { EPA6010A } \\ \mathrm{mg} / \mathrm{kg} & 1.12 & \text { EPA6010A }\end{array}$

UI

UI

UI

UI

UI

UI

UI

UI

UI

UI

UI

UI

UI

UI

UI

UI

UI

UI

UI

UI

UI

UI

UI
$1.31 \mathrm{E}+00 \pm 6.63 \mathrm{E}-02 \mathrm{pCi} / \mathrm{g}$ $-3.81 \mathrm{E}-03 \pm 7.58 \mathrm{E}-03 \mathrm{pCi} / \mathrm{g}$ $1.30 \mathrm{E}-02 \pm 1.65 \mathrm{E}-02 \mathrm{pCi} / \mathrm{g}$ $3.42 \mathrm{E}-03 \pm 7.56 \mathrm{E}-03 \mathrm{pCi} / \mathrm{g}$ $-2.53 \mathrm{E}-02 \pm 4.48 \mathrm{E}-02 \mathrm{pCi} / \mathrm{g}$ $-2.46 \mathrm{E}-03 \pm 5.70 \mathrm{E}-03 \mathrm{pCi} / \mathrm{g}$ $1.44 \mathrm{E}-03 \pm 6.70 \mathrm{E}-03 \mathrm{pCi} / \mathrm{g}$ $-2.82 \mathrm{E}-03 \pm 5.55 \mathrm{E}-03 \mathrm{pCi} / \mathrm{g}$ $1.04 \mathrm{E}-03 \pm 6.96 \mathrm{E}-03 \mathrm{pCi} / \mathrm{g}$ $-3.47 \mathrm{E}-03 \pm 5.87 \mathrm{E}-03 \mathrm{pCi} / \mathrm{g}$ $-1.69 \mathrm{E}-03 \pm 1.65 \mathrm{E}-02 \mathrm{pCi} / \mathrm{g}$ $-9.42 \mathrm{E}-03 \pm 5.05 \mathrm{E}-02 \mathrm{pCi} / \mathrm{g}$ $0.00 \mathrm{E}+00 \quad \mathrm{pCi} / \mathrm{g}$ $2.16 \mathrm{E}+01 \pm 2.92 \mathrm{E}+00 \mathrm{pCi} / \mathrm{g}$ 1.43E+00 $13.00 \mathrm{E}-02 \mathrm{pCi} / \mathrm{g}$ 2.91E-02 $\pm 8.65 \mathrm{E}-03 \mathrm{pCi} / \mathrm{g}$ $-3.41 \mathrm{E}-02 \pm 3.97 \mathrm{E}-02 \mathrm{pCi} / \mathrm{g}$ $1.10 \mathrm{E}+01 \pm 1.61 \mathrm{E}+00 \mathrm{pCi} / \mathrm{g}$ 9.13E-02 $\pm 3.56 \mathrm{E}-02 \mathrm{pCi} / \mathrm{g}$ 2.62E-03 $18.39 \mathrm{E}-03 \mathrm{pCi} / \mathrm{g}$ $1.66 \mathrm{E}+00 \pm 1.65 \mathrm{E}-01 \mathrm{pCi} / \mathrm{g}$ $-1.25 \mathrm{E}-04 \pm 5.94 \mathrm{E}-03 \mathrm{pCi} / \mathrm{g}$ $5.45 \mathrm{E}-03 \pm 7.48 \mathrm{E}-03 \mathrm{pCi} / \mathrm{g}$ $-3.99 \mathrm{E}-02 \pm 5.21 \mathrm{E}-02 \mathrm{pCi} / \mathrm{g}$ 1.10E-03 $\pm 5.61 \mathrm{E}-03 \mathrm{pCi} / \mathrm{g}$ 5.69E-02+3.88E-02 pCi/g $1.60 \mathrm{E}+00 \pm 6.30 \mathrm{E}-01 \mathrm{pCi} / \mathrm{g}$ $-3.50 \mathrm{E}-03 \pm 8.82 \mathrm{E}-03 \mathrm{pCi} / \mathrm{g}$ $6.20 \mathrm{E}-03 \pm 6.05 \mathrm{E}-03 \mathrm{pCi} / \mathrm{g}$ 1.63E-02 $1.12 \mathrm{E}-02 \mathrm{pCi} / \mathrm{g}$ 1.74E-02 $1.75 \mathrm{E}-02 \mathrm{pCi} / \mathrm{g}$
3.03E-02 EPIA-013B 1.30E-02 EPIA-013B

2.78E-02 EPIA-013B

1.14E-02 EPIA-013B

7.62E-02 EPIA-013B

8.59E-03 EPIA-013B

1.03E-02 EPIA-013B

9.50E-03 EPIA-013B

1.21E-02 EPIA-013B

8.64E-03 EPIA-013B

2.73E-02 EPIA-013B

7.47E-02 EPIA-013B

3.88E-02 EPIA-013B

$1.59 \mathrm{E}+00$ EPIA-001B

1.87E-02 EPIA-013B

9.40E-03 EPIA-013B

6.76E-02 EPIA-013B

1.94E+00 EPIA-001B

3.70E-02 EPIA-012B

2.03E-02 EPIA-012B

8.26E-02 EPIA-013B

1.02E-02 EPIA-013B

1.26E-02 EPIA-013B

8.80E-02 EPIA-013B

8.49E-03 EPIA-013B

5.87E-02 EPIA-004

4.98E-01 EPIA-013B

1.44E-02 EPIA-013B

1.18E-02 EPIA-013B

1.87E-02 EPIA-013B

2.79E-02 EPIA-013B 
Survey location: $45135 \mathrm{E} 3678345 \mathrm{~N}$ (SRS coordinates)

Depth of core interval: 0.00 to $1.00 \mathrm{ft}$

Sample type: Normal

Constituent

Unit

D. Limit Method

Physical Parameters and Specified Analyses

Cation exchange capacity

Chloride

Chloride

Cyanide

Nitrate-nitrite as nitrogen

Orthophosphate

Phenols

Sulfide

Total organic carbon

Total organic nitrogen

Total phosphates (as P)

Metals (total recoverable)

$\begin{array}{lll} & & 17.5 \\ J & \mathrm{E} & 1.61 \\ J & \mathrm{E} & 1.44 \\ \mathrm{U} & & 1.16 \\ J & \mathrm{E} & 0.711 \\ \mathrm{~J} & \mathrm{E} & 5.78 \\ \mathrm{UJ} & \mathrm{C} & 345 \\ \text { UJ } & \mathrm{C} & 294 \\ & & 1100 \\ \mathrm{C} & & 88.2 \\ \mathrm{~J} & \mathrm{E} & 60.2\end{array}$

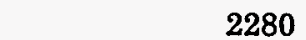

Aluminum

Antimony

Barium

Beryllium

Cadmium

Calcium

Chromium

Cobalt

Copper

Iron

Lead

Magnesium

Manganese

Mercury

Nickel

Potassium

Selenium

Silver

Sodium

Thallium

Vanadium

Zinc

\section{$B / N / A$ Extractables (including $P A H$ and phenols)}

Acenaphthene

Acenaphthylene

Anthracene

Benzo[a]anthracene

Benzo[b]fluoranthene

Benzo $[k]$ fluoranthene

Benzoic acid

Benzo[g,h,i]perylene

Benzo[a]pyrene

$\begin{array}{ll}\mathrm{U} & 38.6 \\ \mathrm{U} & 38.6 \\ \mathrm{U} & 38.6 \\ \mathrm{U} & 3.86 \\ \mathrm{U} & 3.86 \\ \mathrm{U} & 3.86 \\ \mathrm{U} & 38.6 \\ \mathrm{U} & 38.6 \\ \mathrm{U} & 3.86\end{array}$

2280
5.45
16.3
9.15
0.031
0.272
60.4
3.05
0.244
0.867
874
2.38
62.1
15.7
0.018
0.877
46.9
16.3
1.36
54.5
16.3
3.85
2.04

$\mathrm{mg} / \mathbf{k g}$

$\mathrm{mg} / \mathrm{kg}$

$\mathrm{mg} / \mathrm{kg}$

$\mathrm{mg} / \mathrm{kg}$

$\mathrm{mg} / \mathrm{kg}$

$\mathrm{mg} / \mathrm{kg}$

$\mathrm{mg} / \mathrm{kg}$

$\mathrm{mg} / \mathrm{kg}$

$\mathrm{mg} / \mathrm{kg}$

$\mathrm{mg} / \mathrm{kg}$

$\mathrm{mg} / \mathrm{kg}$

$\mathrm{mg} / \mathrm{kg}$

$\mathrm{mg} / \mathrm{kg}$

$\mathrm{mg} / \mathrm{kg}$

$\mathrm{mg} / \mathrm{kg}$

$\mathrm{mg} / \mathrm{kg}$

$\mathrm{mg} / \mathrm{kg}$

$\mathrm{mg} / \mathrm{kg}$

$\mathrm{mg} / \mathrm{kg}$

$\mathrm{mg} / \mathrm{kg}$

$\mathrm{mg} / \mathrm{kg}$

$\mathrm{mg} / \mathrm{kg}$

$\mathrm{mg} / \mathrm{kg}$
EPA9081

EPA300.0

EPA300.0

EPA335.3

EPA353.1

EPA365.2

EPA420.2

EPA376.2

EPA415.1

EPA351.2

EPA365.4

$\begin{array}{ll}\mu \mathrm{g} / \mathrm{kg} & 38.6 \\ \mu \mathrm{g} / \mathrm{kg} & 38.6 \\ \mu \mathrm{g} / \mathrm{kg} & 38.6 \\ \mu \mathrm{g} / \mathrm{kg} & 3.86 \\ \mu \mathrm{g} / \mathrm{kg} & 3.86 \\ \mu \mathrm{g} / \mathrm{kg} & 3.86 \\ \mu \mathrm{g} / \mathrm{kg} & 38.6 \\ \mu \mathrm{g} / \mathrm{kg} & 38.6 \\ \mu \mathrm{g} / \mathrm{kg} & 3.86\end{array}$

EPA6010A

EPA6010A

EPA6010A

EPA6010A

EPA6010A

EPA6010A

EPA6010A

EPA6010A

EPA6010A

EPA6010A

EPA6010A

EPA6010A

EPA6010A

EPA6010A

EPA7471

EPA6010A

EPA6010A

EPA6010A

EPA6010A

EPA6010A

EPA6010A

EPA6010A

EPA6010A

0.545

1.09

EPA8270

EPA8270

EPA8270

EPA8270

EPA8270

EPA8270

EPA8270

EPA8270

EPA8270 
SURVEY ID: PPSC 0401 (continued)

Constituent
Sample ID: 104643

RQ AQ B Result

Unit

D. Limit Method

$B / N / A$ Extractables (including PAH and phenols)

Benzyl alcohol

Bis(2-chloroethoxy) methane

$\mathrm{Bis}$ (2-chloroethyl) ether

Bis(2-chloroisopropyl) ether

Bis(2-ethylhexyl) phthalate

4-Bromophenyl phenyl ether

Butylbenzyl phthalate

4-Chloroaniline

4-Chloro-m-cresol

2-Chloronaphthalene

2-Chlorophenol

4-Chlorophenyl phenyl ether

Chrysene

$\mathrm{m} / \mathrm{p}$-Cresol

o-Cresol (2-Methylphenol)

Dibenz $[a, h]$ anthracene

Dibenzofuran

Di-n-butyl phthalate

1,2-Dichlorobenzene

1,3-Dichlorobenzene

1,4-Dichlorobenzene

3,3'-Dichlorobenzidine

2,4-Dichlorophenol

Diethyl phthalate

2,4-Dimethyl phenol

Dimethyl phthalate

2,4-Dinitrophenol

2,4-Dinitrotoluene

2,6-Dinitrotoluene

Di-n-octyl phthalate

Fluoranthene

Fluorene

Hexachlorobenzene

Hexachlorobutadiene

Hexachlorocyclopentadiene

Hexachloroethane

Indeno[1,2,3-c, $d]$ pyrene

Isophorone

2-Methyl-4,6-dinitrophenol

2-Methylnaphthalene

Naphthalene

m-Nitroaniline

o-Nitroaniline

p-Nitroaniline

Nitrobenzene

2-Nitrophenol

4-Nitrophenol

N-Nitrosodiphenylamine

N-Nitrosodi-n-propylamine

Pentachlorophenol

Phenanthrene

Phenol

Pyrene

1,2,4-Trichlorobenzene

2,4,5-Trichlorophenol

2,4,6-Trichlorophenol
38.6

U $\quad 38.6$

U $\quad 38.6$

$\begin{array}{lll}J & E & 30.5\end{array}$

U $\quad 38.6$

U $\quad 38.6$

$\mathrm{U} \quad 38.6$

U $\quad 38.6$

U $\quad 38.6$

U $\quad 38.6$

U $\quad 38.6$

U $\quad 3.86$

U 386

$\mathrm{U} \quad 38.6$

$\mathrm{U} \quad 3.86$

U 38.6

J E 24.3

$\mathrm{U} \quad 38.6$

U $\quad 38.6$

38.6

38.6

38.6

38.6

38.6

38.6

386

3.86

3.86

38.6

38.6

38.6

3.86

38.6

38.6

3.86

3.86

38.6

386

38.6

38.6

38.6

38.6

38.6

38.6

38.6

38.6

38.6

38.6

38.6

38.6

38.6

38.6

3.86

38.6

38.6

\begin{tabular}{|c|c|c|}
\hline$\mu \mathrm{g} / \mathrm{kg}$ & 38.6 & EPA8270 \\
\hline$\mu \mathrm{g} / \mathrm{kg}$ & 38.6 & EPA8270 \\
\hline$\mu \mathrm{g} / \mathrm{kg}$ & 38.6 & EPA8270 \\
\hline$\mu \mathrm{g} / \mathrm{kg}$ & 38.6 & EPA8270 \\
\hline$\mu \mathrm{g} / \mathrm{kg}$ & 38.6 & EPA8270 \\
\hline$\mu \mathrm{g} / \mathrm{kg}$ & 38.6 & EPA8270 \\
\hline$\mu \mathrm{g} / \mathrm{kg}$ & 38.6 & EPA8270 \\
\hline$\mu \mathrm{g} / \mathrm{kg}$ & 38.6 & EPA8270 \\
\hline$\mu \mathrm{g} / \mathrm{kg}$ & 38.6 & EPA8270 \\
\hline$\mu \mathrm{g} / \mathrm{kg}$ & 38.6 & EPA8270 \\
\hline$\mu \mathrm{g} / \mathrm{kg}$ & 38.6 & EPA8270 \\
\hline$\mu \mathrm{g} / \mathrm{kg}$ & 38.6 & EPA8270 \\
\hline$\mu \mathrm{g} / \mathrm{kg}$ & 3.86 & EPA8270 \\
\hline$\mu \mathrm{g} / \mathrm{kg}$ & 386 & EPA8270 \\
\hline$\mu \mathrm{g} / \mathrm{kg}$ & 38.6 & EPA8270 \\
\hline$\mu \mathrm{g} / \mathrm{kg}$ & 3.86 & EPA8270 \\
\hline$\mu \mathrm{g} / \mathrm{kg}$ & 38.6 & EPA8270 \\
\hline$\mu \mathrm{g} / \mathrm{kg}$ & 38.6 & EPA8270 \\
\hline$\mu \mathrm{g} / \mathrm{kg}$ & 38.6 & EPA8270 \\
\hline$\mu \mathrm{g} / \mathrm{kg}$ & 38.6 & EPA8270 \\
\hline$\mu \mathrm{g} / \mathrm{kg}$ & 38.6 & EPA8270 \\
\hline$\mu \mathrm{g} / \mathrm{kg}$ & 38.6 & EPA8270 \\
\hline$\mu \mathrm{g} / \mathrm{kg}$ & 38.6 & EPA8270 \\
\hline$\mu \mathrm{g} / \mathrm{kg}$ & 38.6 & EPA8270 \\
\hline$\mu \mathrm{g} / \mathrm{kg}$ & 38.6 & EPA8270 \\
\hline$\mu \mathrm{g} / \mathrm{kg}$ & 38.6 & EPA8270 \\
\hline$\mu \mathrm{g} / \mathrm{kg}$ & 386 & EPA8270 \\
\hline$\mu \mathrm{g} / \mathrm{kg}$ & 3.86 & EPA8270 \\
\hline$\mu \mathrm{g} / \mathrm{kg}$ & 3.86 & EPA8270 \\
\hline$\mu \mathrm{g} / \mathrm{kg}$ & 38.6 & EPA8270 \\
\hline$\mu \mathrm{g} / \mathrm{kg}$ & 38.6 & EPA8270 \\
\hline$\mu \mathrm{g} / \mathrm{kg}$ & 38.6 & EPA8270 \\
\hline$\mu \mathrm{g} / \mathrm{kg}$ & 3.86 & EPA8270 \\
\hline$\mu \mathrm{g} / \mathrm{kg}$ & 38.6 & EPA8270 \\
\hline$\mu \mathrm{g} / \mathrm{kg}$ & 38.6 & EPA8270 \\
\hline$\mu \mathrm{g} / \mathrm{kg}$ & 3.86 & EPA8270 \\
\hline$\mu \mathrm{g} / \mathrm{kg}$ & 3.86 & EPA8270 \\
\hline$\mu \mathrm{g} / \mathrm{kg}$ & 38.6 & EPA8270 \\
\hline$\mu \mathrm{g} / \mathrm{kg}$ & 386 & EPA8270 \\
\hline$\mu \mathrm{g} / \mathrm{kg}$ & 38.6 & EPA8270 \\
\hline$\mu \mathrm{g} / \mathrm{kg}$ & 38.6 & EPA8270 \\
\hline$\mu \mathrm{g} / \mathrm{kg}$ & 38.6 & EPA8270 \\
\hline$\mu \mathrm{g} / \mathrm{kg}$ & 38.6 & EPA8270 \\
\hline$\mu \mathrm{g} / \mathrm{kg}$ & 38.6 & EPA8270 \\
\hline$\mu \mathrm{g} / \mathrm{kg}$ & 38.6 & EPA8270 \\
\hline$\mu \mathrm{g} / \mathrm{kg}$ & 38.6 & EPA8270 \\
\hline$\mu \mathrm{g} / \mathrm{kg}$ & 38.6 & EPA8270 \\
\hline$\mu \mathrm{g} / \mathrm{kg}$ & 38.6 & EPA8270 \\
\hline$\mu \mathrm{g} / \mathrm{kg}$ & 38.6 & EPA8270 \\
\hline$\mu \mathrm{g} / \mathrm{kg}$ & 38.6 & EPA8270 \\
\hline$\mu \mathrm{g} / \mathrm{kg}$ & 38.6 & EPA8270 \\
\hline$\mu \mathrm{g} / \mathrm{kg}$ & 38.6 & EPA8270 \\
\hline$\mu \mathrm{g} / \mathrm{kg}$ & 38.6 & EPA8270 \\
\hline$\mu \mathrm{g} / \mathrm{kg}$ & 3.86 & EPA8270 \\
\hline$\mu \mathrm{g} / \mathrm{kg}$ & 38.6 & EPA8270 \\
\hline$\mu \mathrm{g} / \mathrm{kg}$ & 38.6 & EPA8270 \\
\hline
\end{tabular}


Sample ID: 104643
SURVEY ID: PPSC 0401 (continued)

Volatile Organic Compounds

Acetone
Benzene
Bromodichloromethane
Bromoform
Bromomethane (Methyl bromide)
Carbon disulfide
Carbon tetrachloride
Chlorobenzene
Chloroethane
Chloroethene (Vinyl chloride)
Chloroform
Chloromethane (Methyl chloride)
Dibromochloromethane
1,1-Dichloroethane
1,2-Dichloroethane
1,1-Dichloroethylene
1,2-Dichloroethylene
Dichloromethane (Methylene chloride)
1,2-Dichloropropane
cis-1,3-Dichloropropene
trans-1,3-Dichloropropene
Ethylbenzene
2-Hexanone
Methyl ethyl ketone
Methyl isobutyl ketone
Styrene
1,1,2,2-Tetrachloroethane
Tetrachloroethylene
Toluene
1,1,1-Trichloroethane
1,1,2-Trichloroethane
Trichloroethylene
Vinyl acetate
Xylenes
(n)

RQ AQ B Result Unit D. Limit Method

\begin{tabular}{|c|c|c|c|c|c|}
\hline$U$ & 8 & 20.7 & $\mu \mathrm{g} / \mathrm{kg}$ & 5.88 & EPA8260 \\
\hline $\mathrm{U}$ & & 0.588 & $\mu \mathrm{g} / \mathrm{kg}$ & 0.588 & EPA8260 \\
\hline $\mathrm{U}$ & & 0.0588 & $\mu g / k g$ & 0.0588 & EPA8260 \\
\hline $\mathrm{U}$ & & 0.0588 & $\mu \mathrm{g} / \mathrm{kg}$ & 0.0588 & EPA8260 \\
\hline $\mathrm{U}$ & & 0.118 & $\mu \mathrm{g} / \mathrm{kg}$ & 0.118 & EPA8260 \\
\hline$J$ & $\mathrm{E}$ & 0.153 & $\mu \mathrm{g} / \mathrm{kg}$ & 1.18 & EPA8260 \\
\hline $\mathrm{U}$ & & 0.0588 & $\mu \mathrm{g} / \mathrm{kg}$ & 0.0588 & EPA8260 \\
\hline $\mathrm{U}$ & & 0.0588 & $\mu \mathrm{g} / \mathrm{kg}$ & 0.0588 & EPA8260 \\
\hline $\mathrm{U}$ & & 0.118 & $\mu \mathrm{g} / \mathrm{kg}$ & 0.118 & EPA8260 \\
\hline $\mathrm{U}$ & & 0.118 & $\mu g / k g$ & 0.118 & EPA8260 \\
\hline $\mathrm{U}$ & & 0.0588 & $\mu \mathrm{g} / \mathrm{kg}$ & 0.0588 & EPA8260 \\
\hline $\mathrm{U}$ & & 0.118 & $\mu \mathrm{g} / \mathrm{kg}$ & 0.118 & EPA8260 \\
\hline $\mathrm{U}$ & & 0.0588 & $\mu \mathrm{g} / \mathrm{kg}$ & 0.0588 & EPA8260 \\
\hline $\mathrm{U}$ & & 0.0588 & $\mu g / k g$ & 0.0588 & EPA8260 \\
\hline $\mathrm{U}$ & & 0.0588 & $\mu \mathrm{g} / \mathrm{kg}$ & 0.0588 & EPA8260 \\
\hline $\mathrm{U}$ & & 0.0588 & $\mu \mathrm{g} / \mathrm{kg}$ & 0.0588 & EPA8260 \\
\hline $\mathrm{U}$ & & 0.118 & $\mu \mathrm{g} / \mathrm{kg}$ & 0.118 & EPA8260 \\
\hline $\mathrm{U}$ & V & 9.29 & $\mu \mathrm{g} / \mathrm{kg}$ & 0.588 & EPA8260 \\
\hline $\mathrm{U}$ & & 0.0588 & $\mu g / k g$ & 0.0588 & EPA8260 \\
\hline $\mathrm{U}$ & & 0.0588 & $\mu g / \mathrm{kg}$ & 0.0588 & EPA8260 \\
\hline $\mathrm{U}$ & & 0.0588 & $\mu g / k g$ & 0.0588 & EPA8260 \\
\hline $\mathrm{U}$ & & 0.0588 & $\mu g / k g$ & 0.0588 & EPA8260 \\
\hline $\mathrm{U}$ & V & 0.353 & $\mu \mathrm{g} / \mathrm{kg}$ & 1.18 & EPA8260 \\
\hline $\mathrm{U}$ & V & 0.6 & $\mu g / \mathrm{kg}$ & 1.18 & EPA8260 \\
\hline $\mathrm{U}$ & & 1.18 & $\mu \mathrm{g} / \mathrm{kg}$ & 1.18 & EPA8260 \\
\hline U & & 0.0588 & $\mu g / \mathrm{kg}$ & 0.0588 & EPA8260 \\
\hline $\mathrm{U}$ & & 0.0588 & $\mu \mathrm{g} / \mathrm{kg}$ & 0.0588 & EPA8260 \\
\hline $\mathrm{U}$ & & 0.0588 & $\mu g / \mathbf{k g}$ & 0.0588 & EPA8260 \\
\hline $\mathrm{U}$ & V & 0.129 & $\mu \mathrm{g} / \mathrm{kg}$ & 0.588 & EPA8260 \\
\hline U & & 0.0588 & $\mu \mathrm{g} / \mathrm{kg}$ & 0.0588 & EPA8260 \\
\hline $\mathrm{U}$ & & 0.0588 & $\mu \mathrm{g} / \mathrm{kg}$ & 0.0588 & EPA8260 \\
\hline $\mathrm{U}$ & & 0.0588 & $\mu g / \mathrm{kg}$ & 0.0588 & EPA8260 \\
\hline $\mathrm{U}$ & & 1.18 & $\mu \mathrm{g} / \mathrm{kg}$ & 1.18 & EPA8260 \\
\hline $\mathrm{U}$ & & 0.176 & $\mu \mathrm{g} / \mathrm{kg}$ & 0.176 & EPA8260 \\
\hline
\end{tabular}

Pesticides/PCBs and. Dioxins/Furans

Aldrin
alpha-Benzene hexachloride
beta-Benzene hexachloride
delta-Benzene hexachloride
alpha-Chlordane
gamma-Chlordane
p,p'-DDD
p,p'-DDE
p,p'-DDT
Dieldrin
Endosulfan sulfate
Endosulfan I
Endosulfan II
Endrin

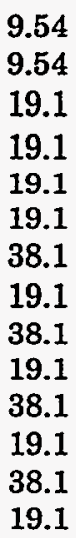

\section{Constituent}

RQ AQ B Result

$\begin{array}{ll}\mathrm{U} & 9.54 \\ \mathrm{U} & 9.54 \\ \mathrm{U} & 19.1 \\ \mathrm{U} & 19.1 \\ \mathrm{U} & 19.1 \\ \mathrm{U} & 19.1 \\ \mathrm{U} & 38.1 \\ \mathrm{U} & 19.1 \\ \mathrm{U} & 38.1 \\ \mathrm{U} & 19.1 \\ \mathrm{U} & 38.1 \\ \mathrm{U} & 19.1 \\ \mathrm{U} & 38.1 \\ \mathrm{U} & 19.1\end{array}$


SURVEY ID: PPSC 0401 (continued)

Constituent

Pesticides/PCBs and Dioxins/Furans

Endrin ketone

Heptachlor

Heptachlor epoxide

Lindane

Methoxychlor

PCB 1016

PCB 1221

PCB 1232

PCB 1242

PCB 1248

PCB 1254

PCB 1260

Toxaphene

Radionuclides

Actinium-228

Antimony-124

Antimony-125

Barium-133

Cerium-144

Cesium-134

Cesium-137

Cobalt-57

Cobalt-58

Cobalt-60

Europium-152

Europium-154

Europium-155

Gross alpha

Lead-212

Manganese-54

Neptunium-239

Nonvolatile beta

Plutonium-238

Plutonium-239/240

Potassium-40

Promethium-144

Promethium-146

Ruthenium-106

Sodium-22

Strontium-90

Thorium-234

Tin-113

Yttrium-88

Zinc-65

Zirconium-95
Sample ID: 104643
RQ AQ B Result
Unit
D. Limit Method

38.1

$\mu \mathrm{g} / \mathrm{kg} \quad 38.1 \quad$ EPA8080

19.1

19.1

9.54

191

47.7

47.7

47.7

47.7

47.7

47.7

47.7

$\mu \mathrm{g} / \mathrm{kg}$

$\mu \mathrm{g} / \mathrm{kg}$

19.1

EPA8080

$\quad 9.54 \quad$ EPA8080

$\mu g / \mathrm{kg} \quad 191 \quad$ EPA8080

$\mu \mathrm{g} / \mathrm{kg} \quad 47.7 \quad$ EPA8080

$\mu \mathrm{g} / \mathrm{kg} \quad 47.7 \quad$ EPA8080

$\mu \mathrm{g} / \mathrm{kg} \quad 47.7 \quad$ EPA8080

$\mu \mathrm{g} / \mathrm{kg} \quad 47.7 \quad$ EPA8080

$\mu \mathrm{g} / \mathrm{kg} \quad 47.7 \quad$ EPA8080

$\mu \mathrm{g} / \mathrm{kg}$

$\mu \mathrm{g} / \mathrm{kg}$

$\mu \mathrm{g} / \mathrm{kg}$

47.7

47.7

EPA8080

EPA8080

EPA8080

$1.05 \mathrm{E}+00 \pm 5.82 \mathrm{E}-02 \mathrm{pCi} / \mathrm{g}$ $-1.27 \mathrm{E}-04 \pm 8.66 \mathrm{E}-03 \mathrm{pCi} / \mathrm{g}$ $1.91 \mathrm{E}-03 \pm 2.12 \mathrm{E}-02 \mathrm{pCi} / \mathrm{g}$ 4.58E-03 $\pm 9.83 \mathrm{E}-03 \mathrm{pCi} / \mathrm{g}$ 6.93E-03 $\pm 4.77 \mathrm{E}-02 \mathrm{pCi} / \mathrm{g}$ $-3.60 \mathrm{E}-03 \pm 6.87 \mathrm{E}-03 \mathrm{pCi} / \mathrm{g}$ $1.43 \mathrm{E}+00 \pm 3.41 \mathrm{E}-02 \mathrm{pCi} / \mathrm{g}$ $-2.42 \mathrm{E}-03 \pm 6.02 \mathrm{E}-03 \mathrm{pCi} / \mathrm{g}$ $-1.37 \mathrm{E}-03 \pm 6.59 \mathrm{E}-03 \mathrm{pCi} / \mathrm{g}$ $1.39 \mathrm{E}-02 \pm 8.26 \mathrm{E}-03 \mathrm{pCi} / \mathrm{g}$ $-2.31 \mathrm{E}-03 \pm 1.96 \mathrm{E}-02 \mathrm{pCi} / \mathrm{g}$ $3.57 \mathrm{E}-03 \pm 5.53 \mathrm{E}-02 \mathrm{pCi} / \mathrm{g}$ $0.00 \mathrm{E}+00 \quad \mathrm{pCi} / \mathrm{g}$ $1.58 \mathrm{E}+01 \pm 2.93 \mathrm{E}+00 \mathrm{pCi} / \mathrm{g}$

$\mathrm{V} \quad 1.11 \mathrm{E}+00 \pm 3.02 \mathrm{E}-02 \mathrm{pCi} / \mathrm{g}$ 1.48E-02+7.97E-03 pCi/g $-5.67 \mathrm{E}-02 \pm 4.32 \mathrm{E}-02 \mathrm{pCi} / \mathrm{g}$ $8.28 \mathrm{E}+00 \pm 1.80 \mathrm{E}+00 \mathrm{pCj} / \mathrm{g}$ $-4.06 \mathrm{E}-03 \pm 4.70 \mathrm{E}-03 \mathrm{pCi} / \mathrm{g}$ $-1.38 \mathrm{E}-03 \pm 7.12 \mathrm{E}-03 \mathrm{pCi} / \mathrm{g}$ $7.18 \mathrm{E}-01 \pm 1.11 \mathrm{E}-01 \mathrm{pCi} / \mathrm{g}$ $-1.35 \mathrm{E}-03 \pm 5.92 \mathrm{E}-03 \mathrm{pCi} / \mathrm{g}$ $3.73 \mathrm{E}-03 \pm 1.21 \mathrm{E}-02 \mathrm{pCi} / \mathrm{g}$ $-6.25 \mathrm{E}-02 \pm 5.70 \mathrm{E}-02 \mathrm{pCi} / \mathrm{g}$ $-2.53 \mathrm{E}-03 \pm 5.45 \mathrm{E}-03 \mathrm{pCi} / \mathrm{g}$

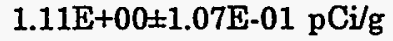
7.92E-01 $\pm 5.40 \mathrm{E}-01 \mathrm{pCi} / \mathrm{g}$ $-8.44 \mathrm{E}-03 \pm 1.09 \mathrm{E}-02 \mathrm{pCi} / \mathrm{g}$ $1.61 \mathrm{E}-03 \pm 6.61 \mathrm{E}-03 \mathrm{pCi} / \mathrm{g}$ $6.83 \mathrm{E}-03 \pm 1.27 \mathrm{E}-02 \mathrm{pCj} / \mathrm{g}$ $6.62 \mathrm{E}-03 \pm 1.69 \mathrm{E}-02 \mathrm{pCi} / \mathrm{g}$
3.07E-02 EPIA-013B

1.51E-02 EPIA-013B

3.50E-02 EPIA-013B

1.48E-02 EPIA-013B

8.24E-02 EPIA-013B

1.03E-02 EPIA-013B

1.26E-02 EPIA-013B

1.03E-02 EPIA-013B

1.14E-02 EPIA-013B

8.90E-03 EPIA-013B

3.25E-02 EPIA-013B

8.44E-02 EPIA-013B

4.30E-02 EPIA-013B

2.14E+00 EPIA-001B

1.93E-02 EPIA-013B

1.09E-02 EPIA-013B

7.31E-02 EPIA-013B

2.47E+00 EPIA-001B

2.34E-02 EPIA-012B

2.34E-02 EPIA-012B

8.08E-02 EPIA-013B

1.02E-02 EPIA-013B

1.79E-02 EPIA-013B

9.50E-02 EPIA-013B

8.96E-03 EPIA-013B

5.75E-02 EPIA-004

5.43E-01 EPIA-013B

1.76E-02 EPIA-013B

1.23E-02 EPIA-013B

2.03E-02 EPIA-013B

2.64E-02 EPIA-013B 
SURVEY ID: PPSC 0401D

Sample type: Split

Constituent
Sample ID: 104647

Associated sample: 104643

Physical Parameters and Specified Analyses

Cation exchange capacity
Chloride
Chloride
Cyanide
Nitrate-nitrite as nitrogen
Orthophosphate
Orthophosphate
Phenols
Phenols
Sulfide
Sulfide
Total organic carbon
Total organic nitrogen
Total organic nitrogen
Total phosphates (as P)
Total phosphates (as P)

\begin{tabular}{|c|c|c|}
\hline$U$ & $\mathrm{Y}$ & 25.5 \\
\hline $\mathbf{J}$ & YE & 14.8 \\
\hline $\mathrm{J}$ & YE & 12.5 \\
\hline \multirow[t]{2}{*}{$\mathrm{U}$} & YV & 0.146 \\
\hline & $\mathrm{Y}$ & 1.18 \\
\hline$J$ & YEI L & 0.138 \\
\hline $\mathbf{J}$ & YEI L & 0.149 \\
\hline $\mathrm{J}$ & YE & 145 \\
\hline $\mathrm{J}$ & $\mathrm{YE}$ & 229 \\
\hline UJ & YI L & 28900 \\
\hline \multirow[t]{2}{*}{ UJ } & YI L & 29000 \\
\hline & YV & 464 \\
\hline $\mathbf{J}$ & YE & 71.5 \\
\hline \multirow[t]{3}{*}{$\mathbf{J}$} & YE & 78.5 \\
\hline & $\mathrm{Y}$ & 32.8 \\
\hline & $\mathrm{Y}$ & 33.9 \\
\hline
\end{tabular}

$\begin{array}{lll}\mathrm{mg} / \mathrm{kg} & 25.5 & \text { EPA9081 } \\ \mathrm{mg} / \mathrm{kg} & 20.1 & \text { EPA300.0 } \\ \mathrm{mg} / \mathrm{kg} & 20.1 & \text { EPA300.0 } \\ \mathrm{mg} / \mathrm{kg} & 1.07 & \text { EPA9010 } \\ \mathrm{mg} / \mathrm{kg} & 0.7 & \text { EPA353.2 } \\ \mathrm{mg} / \mathrm{kg} & 1.16 & \text { EPA365.2 } \\ \mathrm{mg} / \mathrm{kg} & 1.16 & \text { EPA365.2 } \\ \mu \mathrm{g} / \mathrm{kg} & 1190 & \text { EPA420.2 } \\ \mu \mathrm{g} / \mathrm{kg} & 1190 & \text { EPA420.2 } \\ \mu \mathrm{g} / \mathrm{kg} & 28900 & \text { SW9030 } \\ \mu \mathrm{g} / \mathrm{kg} & 29000 & \text { SW9030 } \\ \mathrm{mg} / \mathrm{kg} & 8.4 & \text { LLOYDKAHN } \\ \mathrm{mg} / \mathrm{kg} & 125 & \text { EPA351.4 } \\ \mathrm{mg} / \mathrm{kg} & 125 & \text { EPA351.4 } \\ \mathrm{mg} / \mathrm{kg} & 3.36 & \text { EPA365.2 } \\ \mathrm{mg} / \mathrm{kg} & 3.21 & \text { EPA365.2 } \\ & & \end{array}$

Metals (total recoverable)

\begin{tabular}{|c|c|c|c|}
\hline Aluminum & & $\mathrm{Y}$ & 1300 \\
\hline Antimony & $\mathrm{U}$ & $\mathrm{Y}$ & 47 \\
\hline Arsenic & $U$ & $\mathrm{Y}$ & 0.696 \\
\hline Barium & & $\mathrm{Y}$ & 6.3 \\
\hline Beryllium & $U$ & $\mathrm{Y}$ & 0.348 \\
\hline Cadmium & $\mathrm{U}$ & $\mathrm{Y}$ & 2.69 \\
\hline Calcium & & YV & 75.6 \\
\hline Chromium & $J$ & $\mathrm{YE}$ & 1.6 \\
\hline Cobalt & $\mathrm{U}$ & $\mathrm{Y}$ & 5.45 \\
\hline Copper & $\mathbf{J}$ & $\mathrm{YE}$ & 0.568 \\
\hline Iron & & $\mathrm{Y}$ & 536 \\
\hline Lead & $J$ & $\mathrm{YE}$ & 1.2 \\
\hline Magnesium & & $\mathrm{Y}$ & 39.9 \\
\hline Manganese & & $\mathrm{Y}$ & 14.8 \\
\hline Mercury & $U$ & $\mathrm{Y}$ & 0.696 \\
\hline Nickel & $\mathbf{U}$ & $\mathrm{Y}$ & 8.58 \\
\hline Potassium & U & $\mathrm{Y}$ & 1020 \\
\hline Selenium & $\mathrm{U}$ & $\mathrm{Y}$ & 1.74 \\
\hline Silver & $\mathrm{U}$ & $\mathrm{Y}$ & 5.66 \\
\hline Sodium & $\mathrm{U}$ & YV & 19.1 \\
\hline Thallium & $\mathbf{U}$ & $\mathrm{Y}$ & 1.86 \\
\hline Vanadium & $J$ & $\mathrm{YE}$ & 2.3 \\
\hline Zinc & $J$ & YE & 1.3 \\
\hline
\end{tabular}

$\begin{array}{lll}\mathrm{mg} / \mathrm{kg} & 47 & \text { CLP-MOD } \\ \mathrm{mg} / \mathrm{kg} & 47 & \text { CLP-MOD } \\ \mathrm{mg} / \mathrm{kg} & 0.696 & \text { CLP-MOD } \\ \mathrm{mg} / \mathrm{kg} & 4.42 & \text { CLP-MOD } \\ \mathrm{mg} / \mathrm{kg} & 0.348 & \text { CLP-MOD } \\ \mathrm{mg} / \mathrm{kg} & 2.69 & \text { CLP-MOD } \\ \mathrm{mg} / \mathrm{kg} & 26 & \text { CLP-MOD } \\ \mathrm{mg} / \mathrm{kg} & 7.31 & \text { CLP-MOD } \\ \mathrm{mg} / \mathrm{kg} & \mathbf{5 . 4 5} & \text { CLP-MOD } \\ \mathrm{mg} / \mathrm{kg} & 3.69 & \text { CLP-MOD } \\ \mathrm{mg} / \mathrm{kg} & 23 & \text { CLP-MOD } \\ \mathrm{mg} / \mathrm{kg} & 3.83 & \text { CLP-MOD } \\ \mathrm{mg} / \mathrm{kg} & 27 & \text { CLP-MOD } \\ \mathrm{mg} / \mathrm{kg} & 1.12 & \text { CLP-MOD } \\ \mathrm{mg} / \mathrm{kg} & \mathbf{0 . 6 9 6} & \text { CLP-MOD } \\ \mathrm{mg} / \mathrm{kg} & 8.58 & \text { CLP-MOD } \\ \mathrm{mg} / \mathrm{kg} & 1020 & \text { CLP-MOD } \\ \mathrm{mg} / \mathrm{kg} & 1.74 & \text { CLP-MOD } \\ \mathrm{mg} / \mathrm{kg} & 5.66 & \text { CLP-MOD } \\ \mathrm{mg} / \mathrm{kg} & 70 & \text { CLP-MOD } \\ \mathrm{mg} / \mathrm{kg} & 1.86 & \text { CLP-MOD } \\ \mathrm{mg} / \mathrm{kg} & 4.22 & \text { CLP-MOD } \\ \mathrm{mg} / \mathrm{kg} & 4.99 & \text { CLP-MOD }\end{array}$

$B / N / A$ Extractables (including $P A H$ and phenols)

$\begin{array}{llll}\text { Acenaphthene } & \mathrm{U} & \mathrm{Y} & 38 \\ \text { Acenaphthene } & \mathrm{U} & \mathrm{Y} & 38 \\ \text { Acenaphthylene } & \mathrm{U} & \mathrm{Y} & 38 \\ \text { Acenaphthylene } & \mathrm{U} & \mathrm{Y} & 38 \\ \text { Acenaphthylene } & \mathrm{U} & \mathrm{Y} & 38 \\ \text { Aldol condensate } & \text { UJ } & \mathrm{NKV} & 900 \\ \text { Aldol condensate } & \mathrm{UJ} & \mathrm{NKV} & \mathbf{2 0 0}\end{array}$

$\begin{array}{rrr}\mu g / k g & 38 & \text { EPA8270 } \\ \mu g / k g & 38 & \text { EPA8270 } \\ \mu g / k g & 38 & \text { EPA8270 } \\ \mu g / k g & 38 & \text { EPA8270 } \\ \mu g / k g & 38 & \text { EPA8270 } \\ \mu g / k g & & \text { EPA8270 } \\ \mu g / k g & & \text { EPA8270 }\end{array}$


SURVEY ID: PPSC 0401D (continued)

Sample ID: 104647

Constituent

RQ AQ B Result

Unit

D. Limit Method

$B / N / A$ Extractables (including $P A H$ and phenols)

\begin{tabular}{|c|c|c|c|c|c|c|}
\hline Aldol condensate & UJ & NKV & 200 & $\mu g / k g$ & & EPA8270 \\
\hline Aldol condensate & UJ & NKV & 600 & $\mu \mathrm{g} / \mathrm{kg}$ & & EPA8270 \\
\hline Aldol condensate & UJ & NKV & 100 & $\mu \mathrm{g} / \mathrm{kg}$ & & EPA8270 \\
\hline Aldol condensate & UJ & NKV & 100 & $\mu \mathrm{g} / \mathrm{kg}$ & & EPA8270 \\
\hline Anthracene & $\mathrm{U}$ & $\mathrm{Y}$ & 38 & $\mu \mathrm{g} / \mathrm{kg}$ & 38 & EPA8270 \\
\hline Anthracene & $\mathrm{U}$ & $\mathrm{Y}$ & 38 & $\mu \mathrm{g} / \mathrm{kg}$ & 38 & EPA8270 \\
\hline Anthracene & $\mathrm{U}$ & $\mathrm{Y}$ & 38 & $\mu \mathrm{g} / \mathrm{kg}$ & 38 & EPA8270 \\
\hline Benzo[a]anthracene & $\mathrm{U}$ & $\mathrm{Y}$ & 38 & $\mu \mathrm{g} / \mathrm{kg}$ & 38 & EPA8270 \\
\hline Benzo[a]anthracene & $\mathrm{U}$ & $\mathrm{Y}$ & 38 & $\mu \mathrm{g} / \mathrm{kg}$ & 38 & EPA8270 \\
\hline Benzo[a]anthracene & $\mathrm{U}$ & $\mathrm{Y}$ & 38 & $\mu \mathrm{g} / \mathrm{kg}$ & 38 & EPA8270 \\
\hline Benzo[b]fluoranthene & $U$ & $\mathrm{Y}$ & 38 & $\mu \mathrm{g} / \mathrm{kg}$ & 38 & EPA8270 \\
\hline Benzo[b]fluoranthene & $\mathrm{U}$ & $\mathrm{Y}$ & 38 & $\mu \mathrm{g} / \mathrm{kg}$ & 38 & EPA8270 \\
\hline Benzo $[b]$ fluoranthene & $\mathrm{U}$ & $\mathrm{Y}$ & 38 & $\mu \mathrm{g} / \mathrm{kg}$ & 38 & EPA8270 \\
\hline Benzo[k]fluoranthene & $\mathrm{U}$ & $\mathrm{Y}$ & 38 & $\mu \mathrm{g} / \mathrm{kg}$ & 38 & EPA8270 \\
\hline Benzo[k]fluoranthene & $\mathrm{U}$ & $\mathrm{Y}$ & 38 & $\mu \mathrm{g} / \mathrm{kg}$ & 38 & EPA8270 \\
\hline Benzo[k]fluoranthene & $U$ & $\mathrm{Y}$ & 38 & $\mu \mathrm{g} / \mathrm{kg}$ & 38 & EPA8270 \\
\hline Benzoic acid & $\mathrm{U}$ & YV & 52.6 & $\mu \mathrm{g} / \mathrm{kg}$ & 190 & EPA8270 \\
\hline Benzoic acid & $\mathrm{U}$ & YV & 13 & $\mu \mathrm{g} / \mathrm{kg}$ & 190 & EPA8270 \\
\hline Benzoic acid & $\mathrm{U}$ & YV & 17.9 & $\mu \mathrm{g} / \mathrm{kg}$ & 190 & EPA8270 \\
\hline Benzo $[g, h, i]$ perylene & $\mathrm{U}$ & $\mathrm{Y}$ & 38 & $\mu \mathrm{g} / \mathrm{kg}$ & 38 & EPA8270 \\
\hline Benzo $[g, h, i]$ perylene & $\mathrm{U}$ & $\mathrm{Y}$ & 38 & $\mu \mathrm{g} / \mathrm{kg}$ & 38 & EPA8270 \\
\hline Benzo[g,h,i]perylene & U & $\mathrm{Y}$ & 38 & $\mu \mathrm{g} / \mathrm{kg}$ & 38 & EPA8270 \\
\hline Benzo[a]pyrene & $U$ & $\mathrm{Y}$ & 38 & $\mu \mathrm{g} / \mathrm{kg}$ & 38 & EPA8270 \\
\hline Benzo[a]pyrene & $\mathrm{U}$ & $\mathrm{Y}$ & 38 & $\mu \mathrm{g} / \mathrm{kg}$ & 38 & EPA8270 \\
\hline Benzo[a]pyrene & $\mathrm{U}$ & $\mathrm{Y}$ & 38 & $\mu \mathrm{g} / \mathrm{kg}$ & 38 & EPA8270 \\
\hline Benzyl alcohol & $\mathrm{U}$ & $\bar{Y}$ & 38 & $\mu \mathrm{g} / \mathrm{kg}$ & 38 & EPA8270 \\
\hline Benzyl alcohol & $U$ & $\bar{Y}$ & 38 & $\mu \mathrm{g} / \mathrm{kg}$ & 38 & EPA8270 \\
\hline Benzyl alcohol & $\mathrm{U}$ & $\mathrm{Y}$ & 38 & $\mu \mathrm{g} / \mathrm{kg}$ & 38 & EPA8270 \\
\hline Bis(2-chloroethoxy) methane & $U$ & $\mathrm{Y}$ & 38 & $\mu \mathrm{g} / \mathrm{kg}$ & 38 & EPA8270 \\
\hline Bis(2-chloroethoxy) methane & $\mathrm{U}$ & $\mathrm{Y}$ & 38 & $\mu \mathrm{g} / \mathrm{kg}$ & 38 & EPA8270 \\
\hline Bis(2-chloroethoxy) methane & $\mathrm{U}$ & $\mathrm{Y}$ & 38 & $\mu \mathrm{g} / \mathrm{kg}$ & 38 & EPA8270 \\
\hline Bis(2-chloroethyl) ether & $\mathrm{U}$ & $\mathrm{Y}$ & 38 & $\mu g / \mathrm{kg}$ & 38 & EPA8270 \\
\hline Bis(2-chloroethyl) ether & $\mathrm{U}$ & $\mathrm{Y}$ & 38 & $\mu \mathrm{g} / \mathrm{kg}$ & 38 & EPA8270 \\
\hline Bis(2-chloroethyl) ether & $U$ & $\mathrm{Y}$ & 38 & $\mu g / k g$ & 38 & EPA8270 \\
\hline Bis(2-chloroisopropyl) ether & $\mathrm{U}$ & $\mathrm{Y}$ & 38 & $\mu g / \mathrm{kg}$ & 38 & EPA8270 \\
\hline Bis(2-chloroisopropyl) ether & $\mathrm{U}$ & $\ddot{Y}$ & 38 & $\mu \mathrm{g} / \mathrm{kg}$ & 38 & EPA8270 \\
\hline Bis(2-chloroisopropyl) ether & $\mathrm{U}$ & $\mathrm{Y}$ & 38 & $\mu g / \mathrm{kg}$ & 38 & EPA8270 \\
\hline Bis(2-ethylhexyl) phthalate & $\mathrm{U}$ & YV & 37.9 & $\mu \mathrm{g} / \mathrm{kg}$ & 38 & EPA8270 \\
\hline Bis(2-ethylhexyl) phthalate & $U$ & YV & 18.3 & $\mu g / \mathrm{kg}$ & 38 & EPA8270 \\
\hline Bis(2-ethylhexyl) phthalate & $\mathrm{U}$ & YV & 30.7 & $\mu g / \mathrm{kg}$ & 38 & EPA8270 \\
\hline 4-Bromophenyl phenyl ether & $\mathrm{U}$ & $\mathrm{Y}$ & 38 & $\mu \mathrm{g} / \mathrm{kg}$ & 38 & EPA8270 \\
\hline 4-Bromophenyl phenyl ether & $\mathrm{U}$ & $\mathrm{Y}$ & 38 & $\mu \mathrm{g} / \mathrm{kg}$ & 38 & EPA8270 \\
\hline 4-Bromophenyl phenyl ether & $\mathrm{U}$ & $\mathrm{Y}$ & 38 & $\mu \mathrm{g} / \mathrm{kg}$ & 38 & EPA8270 \\
\hline Butylbenzyl phthalate & $\mathrm{U}$ & $\mathrm{Y}$ & 38 & $\mu \mathrm{g} / \mathrm{kg}$ & 38 & EPA8270M \\
\hline Butylbenzyl phthalate & $\mathrm{U}$ & $\mathrm{Y}$ & 38 & $\mu \mathrm{g} / \mathrm{kg}$ & 38 & EPA8270M \\
\hline Butylbenzyl phthalate & $\mathrm{U}$ & $\mathrm{Y}$ & 38 & $\mu \mathrm{g} / \mathrm{kg}$ & 38 & EPA8270M \\
\hline 4-Chloroaniline & $\mathrm{U}$ & $\mathrm{Y}$ & 38 & $\mu \mathrm{g} / \mathrm{kg}$ & 38 & EPA8270 \\
\hline 4-Chloroaniline & $\mathrm{U}$ & $\mathrm{Y}$ & 38 & $\mu \mathrm{g} / \mathrm{kg}$ & 38 & EPA8270 \\
\hline 4-Chloroaniline & $U$ & $\bar{Y}$ & 38 & $\mu \mathrm{g} / \mathrm{kg}$ & 38 & EPA8270 \\
\hline 4-Chloro-m-cresol & $\mathrm{U}$ & $\mathrm{Y}$ & 38 & $\mu \mathrm{g} / \mathrm{kg}$ & 38 & EPA8270M \\
\hline 4-Chloro-m-cresol & $\mathrm{U}$ & $\mathrm{Y}$ & 38 & $\mu \mathrm{g} / \mathrm{kg}$ & 38 & EPA8270M \\
\hline 2-Chloronaphthalene & $\mathrm{U}$ & $\mathrm{Y}$ & 38 & $\mu \mathrm{g} / \mathrm{kg}$ & 38 & EPA8270 \\
\hline 2-Chloronaphthalene & $\mathrm{U}$ & $\mathrm{Y}$ & 38 & $\mu \mathrm{g} / \mathrm{kg}$ & 38 & EPA8270 \\
\hline 2-Chloronaphthalene & $U$ & $\mathrm{Y}$ & 38 & $\mu \mathrm{g} / \mathrm{kg}$ & 38 & EPA8270 \\
\hline 2-Chlorophenol & $\mathrm{U}$ & $\mathrm{Y}$ & 38 & $\mu g / \mathrm{kg}$ & 38 & EPA8270M \\
\hline
\end{tabular}


SURVEY ID: PPSC 0401D (continued)

Sample ID: 104647

Constituent

RQ AQ B Result

Unit

D. Limit Method

$B / N / A$ Extractables (including PAH and phenols)

2-Chlorophenol

4-Chlorophenyl phenyl ether

4-Chlorophenyl phenyl ether

4-Chlorophenyl phenyl ether

Chrysene

Chrysene

Chrysene

o-Cresol (2-Methylphenol)

o-Cresol (2-Methylphenol)

o-Cresol (2-Methylphenol)

p-Cresol (4-Methylphenol)

p-Cresol (4-Methylphenol)

p-Cresol (4-Methylphenol)

Dibenz $[a, h]$ anthracene

Dibenz $[a, h]$ anthracene

Dibenz $[a, h]$ anthracene

Dibenzofuran

Dibenzofuran

Dibenzofuran.

Di-n-butyl phthalate

Di-n-butyl phthalate

Di-n-butyl phthalate

1,2-Dichlorobenzene

1,2-Dichlorobenzene

1,2-Dichlorobenzene

1,3-Dichlorobenzene

1,3-Dichlorobenzene

1,3-Dichlorobenzene

1,4-Dichlorobenzene

1,4-Dichlorobenzene

3,3'-Dichlorobenzidine

3,3'.Dichlorobenzidine

3,3'-Dichlorobenzidine

2,4-Dichlorophenol

2,4-Dichlorophenol

2,4-Dichlorophenol

Diethyl phthalate

Diethyl phthalate

Diethyl phthalate

2,4-Dimethyl phenol

2,4-Dimethyl phenol

2,4-Dimethyl phenol

Dimethyl phthalate

Dimethyl phthalate

Dimethyl phthalate

2,4-Dinitrophenol

2,4-Dinitrophenol

2,4-Dinitrophenol

2,4-Dinitrotoluene

2,4-Dinitrotoluene

2,6-Dinitrotoluene

2,6-Dinitrotoluene

2,6-Dinitrotoluene

Di-n-octyl phthalate

Di-n-octyl phthalate

\begin{tabular}{|c|c|c|}
\hline $\mathrm{U}$ & $\mathrm{Y}$ & 38 \\
\hline U & $\mathrm{Y}$ & 38 \\
\hline $\mathrm{U}$ & $Y$ & 38 \\
\hline $\mathbf{U}$ & $\mathrm{Y}$ & 38 \\
\hline $\mathbf{U}$ & $\overline{\mathrm{Y}}$ & 38 \\
\hline U & $\mathrm{Y}$ & 38 \\
\hline $\mathrm{U}$ & $\mathrm{Y}$ & 38 \\
\hline $\mathbf{U}$ & $\mathrm{Y}$ & 38 \\
\hline $\mathrm{U}$ & $\mathrm{Y}$ & 38 \\
\hline $\mathrm{U}$ & $Y$ & 38 \\
\hline $\mathrm{U}$ & $\overline{\mathrm{Y}}$ & 38 \\
\hline $\mathrm{U}$ & $\bar{Y}$ & 38 \\
\hline$U$ & $\mathrm{Y}$ & 38 \\
\hline $\mathbf{U}$ & $\mathrm{Y}$ & 38 \\
\hline$U$ & $\mathrm{Y}$ & 38 \\
\hline $\mathrm{U}$ & $\mathrm{Y}$ & 38 \\
\hline$U$ & $\mathrm{Y}$ & 38 \\
\hline U & $Y$ & 38 \\
\hline $\mathrm{U}$ & $\mathrm{Y}$ & 38 \\
\hline $\mathrm{U}$ & YV & 144 \\
\hline $\mathrm{U}$ & YV & 71.2 \\
\hline $\mathrm{U}$ & $Y V$ & 104 \\
\hline$U$ & $Y$ & 38 \\
\hline $\mathrm{U}$ & $Y$ & 38 \\
\hline $\mathrm{U}$ & $\mathrm{Y}$ & 38 \\
\hline $\mathrm{U}$ & $\mathrm{Y}$ & 38 \\
\hline $\mathrm{U}$ & $\mathrm{Y}$ & 38 \\
\hline $\mathrm{U}$ & $\mathrm{Y}$ & 38 \\
\hline$U$ & $\mathrm{Y}$ & 38 \\
\hline $\mathrm{U}$ & $\mathrm{Y}$ & 38 \\
\hline $\mathrm{U}$ & $\mathrm{Y}$ & 76 \\
\hline $\mathrm{U}$ & $\mathrm{Y}$ & 76 \\
\hline U & $\mathrm{Y}$ & 76 \\
\hline $\mathrm{U}$ & $\mathrm{Y}$ & 38 \\
\hline U & $\mathrm{Y}$ & 38 \\
\hline $\mathrm{U}$ & $\mathrm{Y}$ & 38 \\
\hline $\mathrm{J}$ & $\mathrm{YE}$ & 7.36 \\
\hline $\mathrm{U}$ & $\mathrm{Y}$ & 38 \\
\hline$J$ & $\mathrm{YE}$ & 4.44 \\
\hline $\mathrm{U}$ & $\mathrm{Y}$ & 38 \\
\hline U & $\mathrm{Y}$ & 38 \\
\hline$U$ & $\mathrm{Y}$ & 38 \\
\hline $\mathrm{U}$ & $\mathrm{Y}$ & 38 \\
\hline$U$ & $Y$ & 38 \\
\hline$U$ & $\mathrm{Y}$ & 38 \\
\hline U & $\mathrm{Y}$ & 190 \\
\hline $\mathrm{U}$ & $\mathrm{Y}$ & 190 \\
\hline UJ & YI H & 190 \\
\hline $\mathrm{U}$ & $\mathrm{Y}$ & 38 \\
\hline $\mathrm{U}$ & $\mathrm{Y}$ & 38 \\
\hline U & $\mathrm{Y}$ & 38 \\
\hline $\mathrm{U}$ & $\mathrm{Y}$ & 38 \\
\hline U & $\mathrm{Y}$ & 38 \\
\hline U & $\mathrm{Y}$ & 38 \\
\hline U & $\mathrm{Y}$ & 38 \\
\hline
\end{tabular}

\begin{tabular}{|c|c|c|}
\hline$\mu \mathrm{g} / \mathrm{kg}$ & 38 & EPA8270M \\
\hline$\mu \mathrm{g} / \mathrm{kg}$ & 38 & EPA8270 \\
\hline$\mu \mathrm{g} / \mathrm{kg}$ & 38 & EPA8270 \\
\hline$\mu \mathrm{g} / \mathrm{kg}$ & 38 & EPA8270 \\
\hline$\mu \mathrm{g} / \mathrm{kg}$ & 38 & EPA8270 \\
\hline$\mu \mathrm{g} / \mathrm{kg}$ & 38 & EPA8270 \\
\hline$\mu \mathrm{g} / \mathrm{kg}$ & 38 & EPA8270 \\
\hline$\mu \mathrm{g} / \mathrm{kg}$ & 38 & EPA8270 \\
\hline$\mu \mathrm{g} / \mathrm{kg}$ & 38 & EPA8270 \\
\hline$\mu \mathrm{g} / \mathrm{kg}$ & 38 & EPA8270 \\
\hline$\mu \mathrm{g} / \mathrm{kg}$ & 38 & EPA8270 \\
\hline$\mu \mathrm{g} / \mathrm{kg}$ & 38 & EPA8270 \\
\hline$\mu \mathrm{g} / \mathrm{kg}$ & 38 & EPA8270 \\
\hline$\mu \mathrm{g} / \mathrm{kg}$ & 38 & EPA8270 \\
\hline$\mu \mathrm{g} / \mathrm{kg}$ & 38 & EPA8270 \\
\hline$\mu \mathrm{g} / \mathrm{kg}$ & 38 & EPA8270 \\
\hline$\mu \mathrm{g} / \mathrm{kg}$ & 38 & EPA8270 \\
\hline$\mu \mathrm{g} / \mathrm{kg}$ & 38 & EPA8270 \\
\hline$\mu \mathrm{g} / \mathrm{kg}$ & 38 & EPA8270 \\
\hline$\mu \mathrm{g} / \mathrm{kg}$ & 38 & EPA8270M \\
\hline$\mu \mathrm{g} / \mathrm{kg}$ & 38 & EPA8270M \\
\hline$\mu \mathrm{g} / \mathrm{kg}$ & 38 & EPA8270M \\
\hline$\mu \mathrm{g} / \mathrm{kg}$ & 38 & EPA8270 \\
\hline$\mu \mathrm{g} / \mathrm{kg}$ & 38 & EPA8270 \\
\hline$\mu \mathrm{g} / \mathrm{kg}$ & 38 & EPA8270 \\
\hline$\mu \mathrm{g} / \mathrm{kg}$ & 38 & EPA8270 \\
\hline$\mu \mathrm{g} / \mathrm{kg}$ & 38 & EPA8270 \\
\hline$\mu \mathrm{g} / \mathrm{kg}$ & 38 & EPA8270 \\
\hline$\mu \mathrm{g} / \mathrm{kg}$ & 38 & EPA8270 \\
\hline$\mu \mathrm{g} / \mathrm{kg}$ & 38 & EPA8270 \\
\hline$\mu \mathrm{g} / \mathrm{kg}$ & 76 & EPA8270 \\
\hline$\mu \mathrm{g} / \mathrm{kg}$ & 76 & EPA8270 \\
\hline$\mu \mathrm{g} / \mathrm{kg}$ & 76 & EPA8270 \\
\hline$\mu \mathrm{g} / \mathrm{kg}$ & 38 & EPA8270M \\
\hline$\mu \mathrm{g} / \mathrm{kg}$ & 38 & EPA8270M \\
\hline$\mu \mathrm{g} / \mathrm{kg}$ & 38 & EPA8270M \\
\hline$\mu \mathrm{g} / \mathrm{kg}$ & 38 & EPA8270 \\
\hline$\mu \mathrm{g} / \mathrm{gg}$ & 38 & EPA8270 \\
\hline$\mu \mathrm{g} / \mathrm{kg}$ & 38 & EPA8270 \\
\hline$\mu \mathrm{g} / \mathrm{kg}$ & 38 & EPA8270M \\
\hline$\mu \mathrm{g} / \mathrm{kg}$ & 38 & EPA8270M \\
\hline$\mu \mathrm{g} / \mathrm{kg}$ & 38 & EPA8270M \\
\hline$\mu \mathrm{g} / \mathrm{kg}$ & 38 & EPA8270M \\
\hline$\mu \mathrm{g} / \mathrm{kg}$ & 38 & EPA8270M \\
\hline$\mu \mathrm{g} / \mathrm{kg}$ & 38 & EPA8270M \\
\hline$\mu \mathrm{g} / \mathrm{kg}$ & 190 & EPA8270M \\
\hline$\mu \mathrm{g} / \mathrm{kg}$ & 190 & EPA8270M \\
\hline$\mu \mathrm{g} / \mathrm{kg}$ & 190 & EPA8270M \\
\hline$\mu \mathrm{g} / \mathrm{kg}$ & 38 & EPA8270 \\
\hline$\mu \mathrm{g} / \mathrm{kg}$ & 38 & EPA8270 \\
\hline$\mu \mathrm{g} / \mathrm{kg}$ & 38 & EPA8270 \\
\hline$\mu \mathrm{g} / \mathrm{kg}$ & 38 & EPA8270 \\
\hline$\mu \mathrm{g} / \mathrm{kg}$ & 38 & EPA8270 \\
\hline$\mu g / \mathrm{kg}$ & 38 & EPA8270 \\
\hline & 38 & EPA8270 \\
\hline
\end{tabular}


Appendix D: Sediment Tables

SURVEY ID: PPSC 0401D (continued)

Sample ID: 104647

Constituent

RQ AQ B Result

Unit

D. Limit Method

$B / N / A$ Extractables (including $P A H$ and phenols)

$\begin{array}{llll}\text { Di-n-octyl phthalate } & \mathrm{U} & \mathrm{Y} & 38 \\ \text { Fluoranthene } & \mathrm{U} & \mathrm{Y} & 38 \\ \text { Fluoranthene } & \mathrm{U} & \mathrm{Y} & 38 \\ \text { Fluoranthene } & \mathrm{U} & \mathrm{Y} & 38 \\ \text { Fluorene } & \mathrm{U} & \mathrm{Y} & 38 \\ \text { Fluorene } & \mathrm{U} & \mathrm{Y} & 38 \\ \text { Fluorene } & \mathrm{U} & \mathrm{Y} & 38 \\ \text { Hexachlorobenzene } & \mathrm{U} & \mathrm{Y} & 38 \\ \text { Hexachlorobenzene } & \mathrm{U} & \mathrm{Y} & 38 \\ \text { Hexachlorobenzene } & \mathrm{U} & \mathrm{Y} & 38 \\ \text { Hexachlorobutadiene } & \mathrm{U} & \mathrm{Y} & 38 \\ \text { Hexachlorobutadiene } & \mathrm{U} & \mathrm{Y} & 38 \\ \text { Hexachlorobutadiene } & \mathrm{U} & \mathrm{Y} & 38 \\ \text { Hexachlorocyclopentadiene } & \mathrm{U} & \mathrm{Y} & 38 \\ \text { Hexachlorocyclopentadiene } & \mathrm{U} & \mathrm{Y} & 38 \\ \text { Hexachlorocyclopentadiene } & \mathrm{U} & \mathrm{Y} & 38 \\ \text { Hexachloroethane } & \mathrm{U} & \mathrm{Y} & 38 \\ \text { Hexachloroethane } & \mathrm{U} & \mathrm{Y} & 38 \\ \text { Hexachloroethane } & \mathrm{U} & \mathrm{Y} & 38 \\ \text { Indeno[1,2,3-c,d]pyrene } & \mathrm{U} & \mathrm{Y} & 38 \\ \text { Indeno[1,2,3-c,d]pyrene } & \mathrm{U} & \mathrm{Y} & 38 \\ \text { Indeno[1,2,3-c,d]pyrene } & \mathrm{U} & \mathrm{Y} & 38 \\ \text { Isophorone } & \mathrm{U} & \mathrm{Y} & 38 \\ \text { Isophorone } & \mathrm{U} & \mathrm{Y} & 38 \\ \text { Isophorone } & \mathrm{U} & \mathrm{Y} & 38 \\ \text { 2-Methyl-4,6-dinitrophenol } & \mathrm{U} & \mathrm{Y} & 190 \\ \text { 2-Methyl-4,6-dinitrophenol } & \mathrm{U} & \mathrm{Y} & 190 \\ \text { 2-Methyl-4,6-dinitrophenol } & \mathrm{U} & \mathrm{Y} & 190 \\ \text { 2-Methylnaphthalene } & \mathrm{U} & \mathrm{Y} & 38 \\ \text { 2-Methylnaphthalene } & \mathrm{U} & \mathrm{Y} & 38 \\ \text { 2-Methylnaphthalene } & \mathrm{U} & \mathrm{Y} & 38 \\ \text { Naphthalene } & \mathrm{U} & \mathrm{Y} & 38 \\ \text { Naphthalene } & \mathrm{U} & \mathrm{Y} & 38 \\ \text { Naphthalene } & \mathrm{U} & \mathrm{Y} & 38 \\ \text { m-Nitroaniline } & \mathrm{U} & \mathrm{Y} & 190 \\ \text { m-Nitroaniline } & \mathrm{U} & \mathrm{Y} & 190 \\ \text { m-Nitroaniline } & \mathrm{U} & \mathrm{Y} & 190 \\ \text { o-Nitroaniline } & \mathrm{U} & \mathrm{Y} & 190 \\ \text { o-Nitroaniline } & \mathrm{U} & \mathrm{Y} & 190 \\ \text { 0-Nitroaniline } & \mathrm{U} & \mathrm{Y} & 190 \\ \text { p-Nitroaniline } & \mathrm{U} & \mathrm{Y} & 190 \\ \text { p-Nitroaniline } & \mathrm{U} & \mathrm{Y} & 190 \\ \text { p-Nitroaniline } & \mathrm{U} & \mathrm{Y} & 190 \\ \text { Nitrobenzene } & \mathrm{U} & \mathrm{Y} & 38 \\ \text { Nitrobenzene } & \mathrm{U} & \mathrm{Y} & 38 \\ \text { Nitrobenzene } & \mathrm{U} & \mathrm{Y} & 38 \\ \text { 2-Nitrophenol } & \mathrm{U} & \mathrm{Y} & 38 \\ \text { 2-Nitrophenol } & \mathrm{U} & \mathrm{Y} & 38 \\ \text { 2-Nitrophenol } & \mathrm{U} & \mathrm{Y} & 38 \\ \text { 4-Nitrophenol } & \mathrm{UJ} & \mathrm{YI} & \mathrm{H} \\ \text { 4-Nitrophenol } & \mathrm{U} & \mathrm{Y} & 190 \\ \text { N-Nitrosodiphenylamine } & \mathrm{U} & \mathrm{Y} & 38 \\ \text { N-Nitrosodiphenylamine } & \mathrm{U} & \mathrm{Y} & 38 \\ \text { N-Nitrosodiphenylamine } & \mathrm{U} & \mathrm{Y} & 38 \\ \text { N-Nitrosodi-n-propylamine } & & 38\end{array}$

\begin{tabular}{|c|c|c|}
\hline$\mu \mathrm{g} / \mathrm{kg}$ & 38 & EPA8270 \\
\hline$\mu \mathrm{g} / \mathrm{kg}$ & 38 & EPA8270 \\
\hline$\mu \mathrm{g} / \mathrm{kg}$ & 38 & EPA8270 \\
\hline$\mu \mathrm{g} / \mathrm{kg}$ & 38 & EPA8270 \\
\hline$\mu \mathrm{g} / \mathrm{kg}$ & 38 & EPA8270 \\
\hline$\mu \mathrm{g} / \mathrm{kg}$ & 38 & EPA8270 \\
\hline$\mu \mathrm{g} / \mathrm{kg}$ & 38 & EPA8270 \\
\hline$\mu \mathrm{g} / \mathrm{kg}$ & 38 & EPA8270M \\
\hline$\mu \mathrm{g} / \mathrm{kg}$ & 38 & EPA8270M \\
\hline$\mu \mathrm{g} / \mathrm{kg}$ & 38 & EPA8270M \\
\hline$\mu \mathrm{g} / \mathrm{kg}$ & 38 & EPA8270M \\
\hline$\mu \mathrm{g} / \mathrm{kg}$ & 38 & EPA8270M \\
\hline$\mu \mathrm{g} / \mathrm{kg}$ & 38 & EPA8270M \\
\hline$\mu \mathrm{g} / \mathrm{kg}$ & 38 & EPA8270M \\
\hline$\mu \mathrm{g} / \mathrm{kg}$ & 38 & EPA8270M \\
\hline$\mu \mathrm{g} / \mathrm{kg}$ & 38 & EPA8270M \\
\hline$\mu \mathrm{g} / \mathrm{kg}$ & 38 & EPA8270M \\
\hline$\mu \mathrm{g} / \mathrm{kg}$ & 38 & EPA8270M \\
\hline$\mu \mathrm{g} / \mathrm{kg}$ & 38 & EPA8270M \\
\hline$\mu \mathrm{g} / \mathrm{kg}$ & 38 & EPA8270 \\
\hline$\mu \mathrm{g} / \mathrm{kg}$ & 38 & EPA8270 \\
\hline$\mu \mathrm{g} / \mathrm{kg}$ & 38 & EPA8270 \\
\hline$\mu \mathrm{g} / \mathrm{kg}$ & 38 & EPA8270 \\
\hline$\mu \mathrm{g} / \mathrm{kg}$ & 38 & EPA8270 \\
\hline$\mu \mathrm{g} / \mathrm{kg}$ & 38 & EPA8270 \\
\hline$\mu \mathrm{g} / \mathrm{kg}$ & 190 & EPA8270M \\
\hline$\mu \mathrm{g} / \mathrm{kg}$ & 190 & EPA8270M \\
\hline$\mu \mathrm{g} / \mathrm{kg}$ & 190 & EPA8270M \\
\hline$\mu \mathrm{g} / \mathrm{kg}$ & 38 & EPA8270 \\
\hline$\mu \mathrm{g} / \mathrm{kg}$ & 38 & EPA8270 \\
\hline$\mu \mathrm{g} / \mathrm{kg}$ & 38 & EPA8270 \\
\hline$\mu \mathrm{g} / \mathrm{kg}$ & 38 & EPA8270 \\
\hline$\mu \mathrm{g} / \mathrm{kg}$ & 38 & EPA8270 \\
\hline$\mu g / \mathrm{kg}$ & 38 & EPA8270 \\
\hline$\mu \mathrm{g} / \mathrm{kg}$ & 190 & EPA8270M \\
\hline$\mu g / k g$ & 190 & EPA8270M \\
\hline$\mu g / k g$ & 190 & EPA8270M \\
\hline$\mu g / \mathrm{kg}$ & 190 & EPA8270M \\
\hline$\mu g / \mathrm{kg}$ & 190 & EPA8270M \\
\hline$\mu \mathrm{g} / \mathrm{kg}$ & 190 & EPA8270M \\
\hline$\mu \mathrm{g} / \mathrm{kg}$ & 190 & EPA8270M \\
\hline$\mu \mathrm{g} / \mathrm{kg}$ & 190 & EPA8270M \\
\hline$\mu \mathrm{g} / \mathrm{kg}$ & 190 & EPA8270M \\
\hline$\mu \mathrm{g} / \mathrm{kg}$ & 38 & EPA8270 \\
\hline$\mu \mathrm{g} / \mathrm{kg}$ & 38 & EPA8270 \\
\hline$\mu \mathrm{g} / \mathrm{kg}$ & 38 & EPA8270 \\
\hline$\mu \mathrm{g} / \mathrm{kg}$ & 38 & EPA8270M \\
\hline$\mu \mathrm{g} / \mathrm{kg}$ & 38 & EPA8270M \\
\hline$\mu \mathrm{g} / \mathrm{kg}$ & 38 & EPA8270M \\
\hline$\mu \mathrm{g} / \mathrm{kg}$ & 190 & EPA8270M \\
\hline$\mu \mathrm{g} / \mathrm{kg}$ & 190 & EPA8270M \\
\hline$\mu g /$ & 38 & EPA8270M \\
\hline & 38 & EPA8270M \\
\hline$\mu \mathrm{g} / \mathrm{k}_{\mathrm{E}}$ & 38 & EPA8270M \\
\hline$\mu \mathrm{g} / \mathrm{kg}$ & 38 & EPA8270 \\
\hline
\end{tabular}


SURVEY ID: PPSC 0401D (continued)

Sample ID: 104647

Constituent

RQ AQ B Result

Unit

D. Limit Method

$B / N / A$ Extractables (including PAH and phenols)

N-Nitrosodi-n-propylamine

Pentachlorophenol

Pentachlorophenol

Phenanthrene

Phenanthrene

Phenanthrene

Phenol

Phenol

Pyrene

Pyrene

1,2,4-Trichlorobenzene

1,2,4-Trichlorobenzene

2,4,5-Trichlorophenol

2,4,5-Trichlorophenol

2,4,5-Trichlorophenol

2,4,6-Trichlorophenol

2,4,6-Trichlorophenol

2,4,6-Trichlorophenol

Unknown

Unknown

Unknown

Unknown

$\begin{array}{lll}U & \mathrm{Y} & 38 \\ \mathrm{U} & \mathrm{Y} & 190 \\ \mathrm{U} & \mathrm{Y} & 190 \\ \mathrm{U} & \mathrm{Y} & 38 \\ \mathrm{U} & \mathrm{Y} & 38 \\ \mathrm{U} & \mathrm{Y} & 38 \\ \mathrm{~J} & \mathrm{YE} & 4.88 \\ \mathrm{U} & \mathrm{Y} & 38 \\ \mathrm{U} & \mathrm{Y} & 38 \\ \mathrm{U} & \mathrm{Y} & 38 \\ \mathrm{U} & \mathrm{Y} & 38 \\ \mathrm{U} & \mathrm{Y} & 38 \\ \mathrm{U} & \mathrm{Y} & 190 \\ \mathrm{U} & \mathrm{Y} & 190 \\ \mathrm{U} & \mathrm{Y} & 190 \\ \mathrm{U} & \mathrm{Y} & 38 \\ \mathrm{U} & \mathrm{Y} & 38 \\ \mathrm{U} & \mathrm{Y} & 38 \\ \mathrm{~J} & \mathrm{~N} & 100 \\ \mathrm{UJJ} & \mathrm{NV} & 300 \\ \mathrm{~J} & \mathrm{~N} & 60 \\ \mathrm{UJ} & \mathrm{NV} & 100\end{array}$

Volatile Organic Compounds

$\begin{array}{lll}U & Y V & 8.22 \\ U & Y & 0.108 \\ U & Y & 0.22 \\ U & Y & 2.2 \\ U & Y & 0.66 \\ U & Y & 2.2 \\ U & Y & 0.12 \\ U & Y & 0.33 \\ U & Y & 0.22 \\ U & Y & 0.24 \\ U & Y & 0.22 \\ U & Y & 0.33 \\ U & Y & 0.77 \\ U & Y & 0.11 \\ U & Y & 0.22 \\ U & Y & 0.55 \\ U & Y & 2.2 \\ U & Y & 0.24 \\ U & Y & 0.072 \\ U & Y & 5.5 \\ U & Y & 5.5 \\ U & Y & 0.06 \\ U & Y & 11 \\ U & Y & 11 \\ U & Y & 11 \\ U & Y & 0.12 \\ U & Y & 0.12 \\ U & Y & 0.44 \\ & Y & 0.27 \\ U & Y & 0.33\end{array}$

$\begin{array}{lll}\mu \mathrm{g} / \mathrm{kg} & 38 & \text { EPA8270 } \\ \mu \mathrm{g} / \mathrm{kg} & 190 & \text { EPA8270M } \\ \mu \mathrm{g} / \mathrm{kg} & 190 & \text { EPA8270M } \\ \mu \mathrm{g} / \mathrm{kg} & 38 & \text { EPA8270 } \\ \mu \mathrm{g} / \mathrm{kg} & 38 & \text { EPA8270 } \\ \mu \mathrm{g} / \mathrm{kg} & 38 & \text { EPA8270 } \\ \mu \mathrm{g} / \mathrm{kg} & 38 & \text { EPA8270M } \\ \mu \mathrm{g} / \mathrm{kg} & 38 & \text { EPA8270M } \\ \mu \mathrm{g} / \mathrm{kg} & 38 & \text { EPA8270 } \\ \mu \mathrm{g} / \mathrm{kg} & 38 & \text { EPA8270 } \\ \mu \mathrm{g} / \mathrm{kg} & 38 & \text { EPA8270M } \\ \mu \mathrm{g} / \mathrm{kg} & 38 & \text { EPA8270M } \\ \mu \mathrm{g} / \mathrm{kg} & 190 & \text { EPA8270M } \\ \mu \mathrm{g} / \mathrm{kg} & 190 & \text { EPA8270M } \\ \mu \mathrm{g} / \mathrm{kg} & 190 & \text { EPA8270M } \\ \mu \mathrm{g} / \mathrm{kg} & 38 & \text { EPA8270 } \\ \mu \mathrm{g} / \mathrm{kg} & 38 & \text { EPA8270 } \\ \mu \mathrm{g} / \mathrm{kg} & 38 & \text { EPA8270 } \\ \mu \mathrm{g} / \mathrm{kg} & & \text { EPA8270 } \\ \mu \mathrm{g} / \mathrm{kg} & & \text { EPA8270 } \\ \mu \mathrm{g} / \mathrm{kg} & & \text { EPA8270 } \\ \mu \mathrm{g} / \mathrm{kg} & & \text { EPA8270 }\end{array}$

$\begin{array}{lll}\mu \mathrm{g} / \mathrm{kg} & 11 & \text { EPA8260 } \\ \mu \mathrm{g} / \mathrm{kg} & 0.108 & \text { EPA8021 } \\ \mu \mathrm{g} / \mathrm{kg} & 0.22 & \text { EPA8260 } \\ \mu \mathrm{g} / \mathrm{kg} & 2.2 & \text { EPA8260 } \\ \mu \mathrm{g} / \mathrm{kg} & 0.66 & \text { EPA8260 } \\ \mu \mathrm{g} / \mathrm{kg} & 2.2 & \text { EPA8260 } \\ \mu \mathrm{g} / \mathrm{kg} & 0.12 & \text { EPA8021 } \\ \mu \mathrm{g} / \mathrm{kg} & 0.33 & \text { EPA8260 } \\ \mu \mathrm{g} / \mathrm{kg} & 0.22 & \text { EPA8260 } \\ \mu \mathrm{g} / \mathrm{kg} & 0.24 & \text { EPA8021 } \\ \mu \mathrm{g} / \mathrm{kg} & 0.22 & \text { EPA8260 } \\ \mu \mathrm{g} / \mathrm{kg} & 0.33 & \text { EPA8260 } \\ \mu \mathrm{g} / \mathrm{kg} & 0.77 & \text { EPA8260 } \\ \mu \mathrm{g} / \mathrm{kg} & 0.11 & \text { EPA8260 } \\ \mu \mathrm{g} / \mathrm{kg} & 0.22 & \text { EPA8260 } \\ \mu \mathrm{g} / \mathrm{kg} & 0.55 & \text { EPA8260 } \\ \mu \mathrm{g} / \mathrm{kg} & 2.2 & \text { EPA8260 } \\ \mu \mathrm{g} / \mathrm{kg} & 0.24 & \text { EPA8021 } \\ \mu \mathrm{g} / \mathrm{kg} & 0.072 & \text { EPA8021 } \\ \mu \mathrm{g} / \mathrm{kg} & 5.5 & \text { EPA8260 } \\ \mu \mathrm{g} / \mathrm{kg} & 5.5 & \text { EPA8260 } \\ \mu \mathrm{g} / \mathrm{kg} & 0.06 & \text { EPA8021 } \\ \mu \mathrm{g} / \mathrm{kg} & 11 & \text { EPA8260 } \\ \mu \mathrm{g} / \mathrm{kg} & 11 & \text { EPA8260 } \\ \mu \mathrm{g} / \mathrm{kg} & 11 & \text { EPA8260 } \\ \mu \mathrm{g} / \mathrm{kg} & 0.12 & \text { EPA8021 } \\ \mu \mathrm{g} / \mathrm{kg} & 0.12 & \text { EPA8021 } \\ \mu \mathrm{g} / \mathrm{kg} & 0.44 & \text { EPA8260 } \\ \mu \mathrm{g} / \mathrm{kg} & 0.12 & \text { EPA8021 } \\ \mu \mathrm{g} / \mathrm{kg} & 0.33 & \text { EPA8260 }\end{array}$


SURVEY ID: PPSC $0401 D$ (continued)

Constituent

RQ AQ B Result

0.22

0.12

11

0.44

Volatile Organic Compounds

1,1,2-Trichloroethane

Trichloroethylene

Vinyl acetate

Xylenes

$\begin{array}{lll}\mathrm{U} & \mathrm{Y} & \mathbf{0 . 2 2} \\ \mathrm{U} & \mathrm{Y} & 0.12 \\ \mathrm{U} & \mathrm{Y} & 11 \\ \mathrm{U} & \mathrm{Y} & 0.44\end{array}$

Unit

D. Limit Method
Pesticides/PCBs and Dioxins/Furans

Aldrin

Aldrin

alpha-Benzene hexachloride

alpha-Benzene hexachloride

alpha-Benzene hexachloride

beta-Benzene hexachloride

beta-Benzene hexachloride

beta-Benzene hexachloride

delta-Benzene hexachloride

delta-Benzene hexachloride

delta-Benzene hexachloride

alpha-Chlordane

alpha-Chlordane

alpha-Chlordane

gamma-Chlordane

gamma-Chlordane

gamma-Chlordane

$p, p^{\prime}-D D D$

$p, p^{\prime}-D D D$

$p, p^{\prime}-D D D$

$p, p^{\prime}-D D E$

$p, p^{\prime}-D D E$

$p, p^{\circ}-D D E$

$p, p^{\prime}-D D T$

p,p'-DDT

Dieldrin

Dieldrin

Endosulfan sulfate

Endosulfan sulfate

Endosulfan sulfate

Endosulfan I

Endosulfan I

Endosulfan I

Endosulfan II

Endosulfan II

Endosulfan II

Endrin

Endrin

Endrin ketone

Endrin ketone

Endrin ketone

Heptachlor

Heptachlor

Heptachlor epoxide

Heptachlor epoxide

Heptachlor epoxide

Lindane

Lindane $\mu \mathrm{g} / \mathrm{kg}$

$\mu \mathrm{g} / \mathrm{kg}$

$\mu \mathrm{g} / \mathrm{kg}$

$\mu \mathrm{g} / \mathrm{kg}$

0.22

EPA8260

0.12

11

EPA8021

0.44

EPA8260

\begin{tabular}{|c|c|c|}
\hline$\mu \mathrm{g} / \mathrm{kg}$ & 1.99 & EPA8080 \\
\hline$\mu \mathrm{g} / \mathrm{kg}$ & 1.97 & EPA8080 \\
\hline$\mu \mathrm{g} / \mathrm{kg}$ & 1.99 & EPA8080 \\
\hline$\mu \mathrm{g} / \mathrm{kg}$ & 1.97 & EPA8080 \\
\hline$\mu \mathrm{g} / \mathrm{kg}$ & 1.98 & EPA8080 \\
\hline$\mu \mathrm{g} / \mathrm{kg}$ & 1.99 & EPA8080 \\
\hline$\mu \mathrm{g} / \mathrm{kg}$ & 1.97 & EPA8080 \\
\hline$\mu \mathrm{g} / \mathrm{kg}$ & 1.98 & EPA8080 \\
\hline$\mu \mathrm{g} / \mathrm{kg}$ & 1.99 & EPA8080 \\
\hline$\mu \mathrm{g} / \mathrm{kg}$ & 1.97 & EPA8080 \\
\hline$\mu \mathrm{g} / \mathrm{kg}$ & 1.98 & EPA8080 \\
\hline$\mu \mathrm{g} / \mathrm{kg}$ & 1.99 & EPA8080 \\
\hline$\mu \mathrm{g} / \mathrm{kg}$ & 1.97 & EPA8080 \\
\hline$\mu \mathrm{g} / \mathrm{kg}$ & 1.98 & EPA8080 \\
\hline$\mu \mathrm{g} / \mathrm{kg}$ & 1.99 & EPA8080 \\
\hline$\mu \mathrm{g} / \mathrm{kg}$ & 1.97 & EPA8080 \\
\hline$\mu \mathrm{g} / \mathrm{kg}$ & 1.98 & EPA8080 \\
\hline$\mu \mathrm{g} / \mathrm{kg}$ & 3.83 & EPA8080 \\
\hline$\mu \mathrm{g} / \mathrm{kg}$ & 3.79 & EPA8080 \\
\hline$\mu \mathrm{g} / \mathrm{kg}$ & 3.8 & EPA8080 \\
\hline$\mu \mathrm{g} / \mathrm{kg}$ & 3.06 & EPA8080 \\
\hline$\mu \mathrm{g} / \mathrm{kg}$ & 3.03 & EPA8080 \\
\hline$\mu \mathrm{g} / \mathrm{kg}$ & 3.04 & EPA8080 \\
\hline$\mu \mathrm{g} / \mathrm{kg}$ & 3.83 & EPA8080 \\
\hline$\mu \mathrm{g} / \mathrm{kg}$ & 3.79 & EPA8080 \\
\hline$\mu \mathrm{g} / \mathrm{kg}$ & 1.53 & EPA8080 \\
\hline$\mu \mathrm{g} / \mathrm{kg}$ & 1.52 & EPA8080 \\
\hline$\mu \mathrm{g} / \mathrm{kg}$ & 3.83 & EPA8080 \\
\hline$\mu \mathrm{g} / \mathrm{kg}$ & 3.79 & EPA8080 \\
\hline$\mu \mathrm{g} / \mathrm{kg}$ & 3.8 & EPA8080 \\
\hline$\mu \mathrm{g} / \mathrm{kg}$ & 1.99 & EPA8080 \\
\hline$\mu \mathrm{g} / \mathrm{kg}$ & 1.97 & EPA8080 \\
\hline$\mu \mathrm{g} / \mathrm{kg}$ & 1.98 & EPA8080 \\
\hline$\mu \mathrm{g} / \mathrm{kg}$ & 3.06 & EPA8080 \\
\hline$\mu \mathrm{g} / \mathrm{kg}$ & 3.03 & EPA8080 \\
\hline$\mu \mathrm{g} / \mathrm{kg}$ & 3.04 & EPA8080 \\
\hline$\mu \mathrm{g} / \mathrm{kg}$ & 3.83 & EPA8080 \\
\hline$\mu \mathrm{g} / \mathrm{kg}$ & 3.79 & EPA8080 \\
\hline$\mu \mathrm{g} / \mathrm{kg}$ & 3.83 & EPA8080 \\
\hline$\mu \mathrm{g} / \mathrm{kg}$ & 3.79 & EPA8080 \\
\hline$\mu \mathrm{g} / \mathrm{kg}$ & 3.8 & EPA8080 \\
\hline$\mu \mathrm{g} / \mathrm{kg}$ & 1.99 & EPA8080 \\
\hline$\mu \mathrm{g} / \mathrm{kg}$ & 1.97 & EPA8080 \\
\hline$\mu \mathrm{g} / \mathrm{kg}$ & 1.99 & EPA8080 \\
\hline$\mu \mathrm{g} / \mathrm{kg}$ & 1.97 & EPA8080 \\
\hline$\mu \mathrm{g} / \mathrm{kg}$ & 1.98 & EPA8080 \\
\hline$\mu \mathrm{g} / \mathrm{kg}$ & 1.99 & EPA8080 \\
\hline$\mu \mathrm{g} / \mathrm{kg}$ & 1.97 & EPA8080 \\
\hline
\end{tabular}


SURVEY ID: PPSC 0401D (continued)

Constituent

Pesticides/PCBs and Dioxins/Furans
RQ AQ B Result

Sample ID: 104647

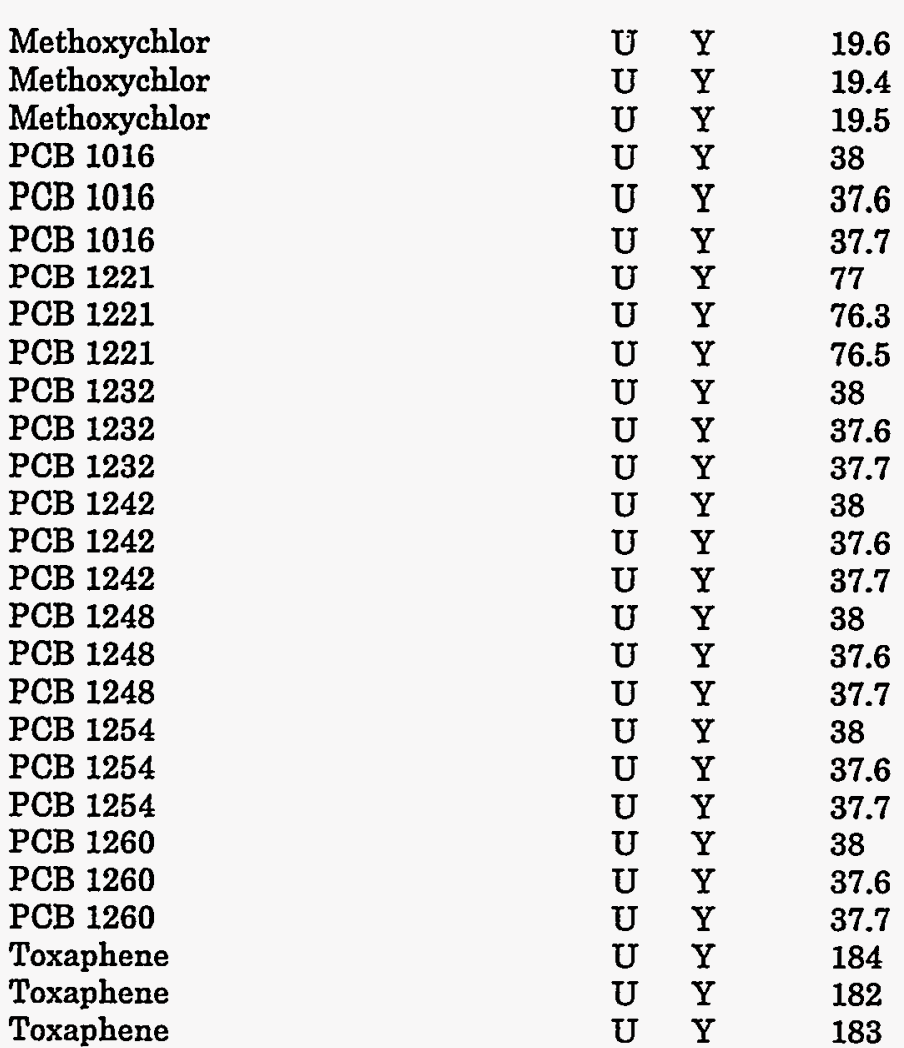

Radionuclides

Actinium-228

Antimony-124

Antimony-125

Barium-133

Cerium-144

Cesium-134

Cesium-137

Cobalt-57

Cobalt-58

Cobalt- 60

Europium-152

Europium-154

Europium-155

Gross alpha

Iodine-129

Lead-212

Manganese-54

Neptunium-239

Nonvolatile beta

Plutonium-238

Plutonium-239/240

Potassium-40

Promethium-144

Promethium-146

Ruthenium-106

$\begin{array}{ll}\text { UI } & \\ \text { UI } & \text { E } \\ \text { UI } & \\ \text { UI } & \\ \text { UI } & \\ \text { UI } & \\ \text { U } & \text { E } \\ & \\ \text { UI } & \\ \text { UI } & \\ \text { J } & \text { E }\end{array}$

UI

UI

UI

UI
$1.50 \mathrm{E}+00 \pm 2.90 \mathrm{E}-01$ $-2.00 \mathrm{E}-02 \pm 7.00 \mathrm{E}-02$ $-9.00 \mathrm{E}-02 \pm 1.50 \mathrm{E}-01$ 9.00E-02+7.00E-02

$-1.90 \mathrm{E}-01 \pm 3.40 \mathrm{E}-01$ $2.00 \mathrm{E}-02 \pm 6.00 \mathrm{E}-02$ $2.20 \mathrm{E}+00 \pm 1.80 \mathrm{E}-01$ $1.00 \mathrm{E}-02 \pm 4.00 \mathrm{E}-02$

$-1.00 \mathrm{E}-02 \pm 6.00 \mathrm{E}-02$ 5.00E-02 $\pm 5.00 \mathrm{E}-02$ 4.00E-01 $\pm 3.90 \mathrm{E}-01$ $-7.00 \mathrm{E}-02 \pm 1.50 \mathrm{E}-01$ $3.30 \mathrm{E}-01 \pm 1.70 \mathrm{E}-01$ $1.58 \mathrm{E}+01 \pm 3.20 \mathrm{E}+00$ 1.00E-02 $22.70 \mathrm{E}-01$

$1.40 \mathrm{E}+00 \pm 1.20 \mathrm{E}-01$

$-1.00 \mathrm{E}-02 \pm 6.00 \mathrm{E}-02$

$2.20 \mathrm{E}+00 \pm 2.00 \mathrm{E}+00$ $1.70 \mathrm{E}+01 \pm 5.30 \mathrm{E}+00$ $1.50 \mathrm{E}-01 \pm 1.50 \mathrm{E}-01$ 2.00E-02 $\pm 6.00 \mathrm{E} \cdot 02$ $1.00 \mathrm{E}+00 \pm 9.00 \mathrm{E}-01$ $3.00 \mathrm{E}-02 \pm 6.00 \mathrm{E}-02$ $-1.00 \mathrm{E}-02 \pm 7.00 \mathrm{E}-02$ 8.00E-02 $\pm 5.10 \mathrm{E}-01$
Unit

D. Limit Method

$\begin{array}{lll}\mu \mathrm{g} / \mathrm{kg} & 19.6 & \text { EPA8080 } \\ \mu \mathrm{g} / \mathrm{kg} & 19.4 & \text { EPA8080 } \\ \mu \mathrm{g} / \mathrm{kg} & 19.5 & \text { EPA8080 } \\ \mu \mathrm{g} / \mathrm{kg} & 38 & \text { EPA8080 } \\ \mu \mathrm{g} / \mathrm{kg} & 37.6 & \text { EPA8080 } \\ \mu \mathrm{g} / \mathrm{kg} & 37.7 & \text { EPA8080 } \\ \mu \mathrm{g} / \mathrm{kg} & 77 & \text { EPA8080 } \\ \mu \mathrm{g} / \mathrm{kg} & 76.3 & \text { EPA8080 } \\ \mu \mathrm{g} / \mathrm{kg} & 76.5 & \text { EPA8080 } \\ \mu \mathrm{g} / \mathrm{kg} & 38 & \text { EPA8080 } \\ \mu \mathrm{g} / \mathrm{kg} & 37.6 & \text { EPA8080 } \\ \mu \mathrm{g} / \mathrm{kg} & 37.7 & \text { EPA8080 } \\ \mu \mathrm{g} / \mathrm{kg} & 38 & \text { EPA8080 } \\ \mu \mathrm{g} / \mathrm{kg} & 37.6 & \text { EPA8080 } \\ \mu \mathrm{g} / \mathrm{kg} & 37.7 & \text { EPA8080 } \\ \mu \mathrm{g} / \mathrm{kg} & 38 & \text { EPA8080 } \\ \mu \mathrm{g} / \mathrm{kg} & 37.6 & \text { EPA8080 } \\ \mu \mathrm{g} / \mathrm{kg} & 37.7 & \text { EPA8080 } \\ \mu \mathrm{g} / \mathrm{kg} & 38 & \text { EPA8080 } \\ \mu \mathrm{g} / \mathrm{kg} & 37.6 & \text { EPA8080 } \\ \mu \mathrm{g} / \mathrm{kg} & 37.7 & \text { EPA8080 } \\ \mu \mathrm{g} / \mathrm{kg} & 38 & \text { EPA8080 } \\ \mu \mathrm{g} / \mathrm{kg} & 37.6 & \text { EPA8080 } \\ \mu \mathrm{g} / \mathrm{kg} & 37.7 & \text { EPA8080 } \\ \mu \mathrm{g} / \mathrm{kg} & 184 & \text { EPA8080 } \\ \mu \mathrm{g} / \mathrm{kg} & 182 & \text { EPA8080 } \\ \mu \mathrm{g} / \mathrm{kg} & 183 & \text { EPA8080 }\end{array}$

pCi/g 3.40E-01 LANLER130MOD

$\mathrm{pCi} / \mathrm{g}$ 1.00E-01 LANLER130MOD

pCi/g 2.40E-01 LANLERI30MOD

$\mathrm{pCi} / \mathrm{g}$ 1.20E-01 LANLER130MOD

pCi/g 5.30E-01 LANLER130MOD

pCi/g 1.00E-01 LANLER130MOD

pCi/g 1.00E-01 LANLER130MOD

$\mathrm{pCi} / \mathrm{g} \quad 7.00 \mathrm{E}-02$ LANLER130MOD

pCi/g 1.00E-01 LANLER130MOD

pCi/g 1.00E-01 LANLER130MOD

pCi/g 7.70E-01 LANLER130MOD

pCi/g 2.60E-01 LANLER130MOD

$\mathrm{pCi} / \mathrm{g}$ 3.30E-01 LANLER130MOD

$\mathrm{pCi} / \mathrm{g} \quad 2.64 \mathrm{E}+00$ LANLMLR100MOD

pCi/g 4.10E-01 LANLER130MOD

pCi/g 1.30E-01 LANLER130MOD

pCi/g 1.00E-01 LANLER130MOD

$\mathrm{pCi} / \mathrm{g} \quad 3.40 \mathrm{E}+00$ LANLER130MOD

$\mathrm{pCi} / \mathrm{g} \quad 1.70 \mathrm{E}+00$ LANLMIRI00MOD

$\mathrm{pCi} / \mathrm{g}$ 2.00E-01 EMLPUO2MOD

$\mathrm{pCi} / \mathrm{g}$ 1.60E-01 EMLPU02MOD

$\mathrm{pCi} / \mathrm{g}$ 9.20E-01 LANLER130MOD

pCi/g 1.10E-01 LANLER130MOD

$\mathrm{pCi} / \mathrm{g}$ 1.20E-01 LANLER130MOD

$\mathrm{pCi} / \mathrm{g}$ 8.90E-01 LANLER130MOD 
SURVEY ID: PPSC 0401D (continued)

Constituent

RQ AQ B Result

Unit

D. Limit Method

Radionuclides

Sodium-22

Strontium-90

Thorium-234

Tin-113

Yttrium-88

Zinc-65

Zirconium-95

UI
UI
UI
UI
UI
UI

UI

UI

UI

UI

\begin{abstract}
$-2.00 \mathrm{E}-02 \pm 5.00 \mathrm{E}-02$ $2.00 \mathrm{E}-02 \pm 3.90 \mathrm{E}-01$ $3.90 \mathrm{E}+00 \pm 2.10 \mathrm{E}+00$ $3.00 \mathrm{E} \cdot 02 \pm 7.00 \mathrm{E}-02$ $0.00 \mathrm{E}+00 \pm 5.00 \mathrm{E}-02$ $-2.00 \mathrm{E}-02 \pm 1.20 \mathrm{E}-01$ $-2.00 \mathrm{E}-02 \pm 1.10 \mathrm{E}-01$
\end{abstract}

Survey location: $45135 \mathrm{E}$ 3678345N (SRS coordinates)

Depth of core interval: 1.00 to $2.00 \mathrm{ft}$

Sample type: Normal 
SURVEY ID: PPSC 0402 (continued)

Constituent
Sample ID: 104644

RQ AQ B Result

Unit

D. Limit Method

Physical Parameters and Specified Analyses

Cation exchange capacity

Chloride

Cyanide

Nitrate-nitrite as nitrogen

Orthophosphate

Phenols

Sulfide

Total organic carbon

Total organic carbon

Total organic nitrogen

Total phosphates (as P)

Total phosphates (as P)

Metals (total recoverable)

$\begin{array}{llll} & & 48.3 \\ \text { U } & \text { E } & & 1.65 \\ J & \text { E } & & 1.1 \\ \text { U } & & & 0.812 \\ \text { UJ } & \text { C } & & 347 \\ \text { UJ } & \text { C } & & 291 \\ & & & 842 \\ & & & 871 \\ \text { C } & & & 103 \\ \text { J } & \text { EI } & \text { H } & 15 \\ \text { J } & \text { EI } & \text { H } & 17.3\end{array}$

Aluminum

Antimony

Arsenic

Barium

Beryllium

Cadmium

Calcium

Chromium

Cobalt

Copper

Iron

Lead

Magnesium

Manganese

Mercury

Nickel

Potassium

Selenium

Silver

Sodium

Thallium

Vanadium

Zinc

$\begin{array}{llll} & & 7480 \\ \text { UJ } & \mathrm{C} & 5.48 \\ & & 16.5 \\ \mathrm{~J} & \mathrm{E} & 15.7 \\ \mathrm{U} & & 0.0642 \\ & & 0.274 \\ & & 125 \\ \mathrm{~J} & \mathrm{E} & \mathbf{8 . 7 9} \\ & & 0.364 \\ \mathrm{~J} & \mathrm{C} & \mathbf{2 . 1 3} \\ & & \mathbf{1 1 2 0} \\ & & 14.03 \\ & & 17 \\ \mathrm{~J} & \mathrm{EV} & 0.0381 \\ & & 2.1 \\ \mathrm{~J} & \mathrm{E} & 102 \\ \mathrm{U} & & 16.5 \\ \mathrm{U} & & 1.37 \\ \mathrm{~J} & \mathrm{E} & 17.5 \\ \mathrm{UJ} & \mathrm{C} & 16.5 \\ & & 10.9 \\ & & 3.81\end{array}$

$B / N / A$ Extractables (including $P A H$ and phenols)

$\begin{array}{lcc}\text { Acenaphthene } & \mathrm{U} & 38.7 \\ \text { Acenaphthylene } & \mathrm{U} & 38.7 \\ \text { Anthracene } & \mathrm{U} & 38.7 \\ \text { Benzo[a]anthracene } & \mathrm{U} & 3.87 \\ \text { Benzo[b]fluoranthene } & \mathrm{U} & 3.87 \\ \text { Benzo[h]fluoranthene } & \mathrm{U} & 3.87 \\ \text { Benzoic acid } & \mathrm{U} & 38.7 \\ \text { Benzo[g,h,i]perylene } & \mathrm{U} & 38.7 \\ \text { Benzo[a]pyrene } & \mathrm{U} & 3.87 \\ \text { Benzyl alcohol } & \mathrm{U} & 38.7 \\ \text { Bis(2-chloroethoxy) methane } & \mathrm{U} & 38.7 \\ \text { Bis(2-chloroethyl) ether } & \mathrm{U} & 38.7 \\ \text { Bis(2-chloroisopropyl) ether } & \mathrm{U} & 38.7 \\ \text { Bis(2-ethylhexyl) phthalate } & \mathrm{U} & 38.7\end{array}$

$\begin{array}{lll}\mathrm{meq} / 100 \mathrm{~g} & 5 & \text { EPA9081 } \\ \mathrm{mg} / \mathrm{kg} & 2.79 & \text { EPA300.0 } \\ \mathrm{mg} / \mathrm{kg} & 1.1 & \text { EPA335.3 } \\ \mathrm{mg} / \mathrm{kg} & 1.16 & \text { EPA353.1 } \\ \mathrm{mg} / \mathrm{kg} & 11.3 & \text { EPA365.2 } \\ \mu \mathrm{g} / \mathrm{kg} & 347 & \text { EPA420.2 } \\ \mathrm{mg} / \mathrm{kg} & 291 & \text { EPA376.2 } \\ \mathrm{mg} / \mathrm{kg} & 116 & \text { EPA415.1 } \\ \mathrm{mg} / \mathrm{kg} & 116 & \text { EPA415.1 } \\ \mathrm{mg} / \mathrm{kg} & 0.233 & \text { EPA351.2 } \\ \mathrm{mg} / \mathrm{kg} & 87.2 & \text { EPA365.4 } \\ \mathrm{mg} / \mathrm{kg} & 87.6 & \text { EPA365.4 }\end{array}$

$\begin{array}{lll}\mathrm{mg} / \mathrm{kg} & 13.7 & \text { EPA6010A } \\ \mathrm{mg} / \mathrm{kg} & 5.48 & \text { EPA6010A } \\ \mathrm{mg} / \mathrm{kg} & 16.5 & \text { EPA6010A } \\ \mathrm{mg} / \mathrm{kg} & 1.37 & \text { EPA6010A } \\ \mathrm{mg} / \mathrm{kg} & 0.548 & \text { EPA6010A } \\ \mathrm{mg} / \mathrm{kg} & 0.274 & \text { EPA6010A } \\ \mathrm{mg} / \mathrm{kg} & 13.7 & \text { EPA6010A } \\ \mathrm{mg} / \mathrm{kg} & 1.37 & \text { EPA6010A } \\ \mathrm{mg} / \mathrm{kg} & 0.548 & \text { EPA6010A } \\ \mathrm{mg} / \mathrm{kg} & 1.37 & \text { EPA6010A } \\ \mathrm{mg} / \mathrm{kg} & 5.48 & \text { EPA6010A } \\ \mathrm{mg} / \mathrm{kg} & 5.48 & \text { EPA6010A } \\ \mathrm{mg} / \mathrm{kg} & 5.48 & \text { EPA6010A } \\ \mathrm{mg} / \mathrm{kg} & 0.548 & \text { EPA6010A } \\ \mathrm{mg} / \mathrm{kg} & 0.0383 & \text { EPA7471 } \\ \mathrm{mg} / \mathrm{kg} & 1.37 & \text { EPA6010A } \\ \mathrm{mg} / \mathrm{kg} & 110 & \text { EPA6010A } \\ \mathrm{mg} / \mathrm{kg} & 16.5 & \text { EPA6010A } \\ \mathrm{mg} / \mathrm{kg} & 1.37 & \text { EPA6010A } \\ \mathrm{mg} / \mathrm{kg} & 54.8 & \text { EPA6010A } \\ \mathrm{mg} / \mathrm{kg} & 16.5 & \text { EPA6010A } \\ \mathrm{mg} / \mathrm{kg} & 0.548 & \text { EPA6010A } \\ \mathrm{mg} / \mathrm{kg} & 1.1 & \text { EPA6010A }\end{array}$

$\begin{array}{lll}\mu \mathrm{g} / \mathrm{kg} & 38.7 & \text { EPA8270 } \\ \mu \mathrm{g} / \mathrm{kg} & 38.7 & \text { EPA8270 } \\ \mu \mathrm{g} / \mathrm{kg} & 38.7 & \text { EPA8270 } \\ \mu \mathrm{g} / \mathrm{kg} & 3.87 & \text { EPA8270 } \\ \mu \mathrm{g} / \mathrm{kg} & 3.87 & \text { EPA8270 } \\ \mu \mathrm{g} / \mathrm{kg} & 3.87 & \text { EPA8270 } \\ \mu \mathrm{g} / \mathrm{kg} & 38.7 & \text { EPA8270 } \\ \mu \mathrm{g} / \mathrm{kg} & 38.7 & \text { EPA8270 } \\ \mu \mathrm{g} / \mathrm{kg} & 3.87 & \text { EPA8270 } \\ \mu \mathrm{g} / \mathrm{kg} & 38.7 & \text { EPA8270 } \\ \mu \mathrm{g} / \mathrm{kg} & 38.7 & \text { EPA8270 } \\ \mu \mathrm{g} / \mathrm{kg} & 38.7 & \text { EPA8270 } \\ \mu \mathrm{g} / \mathrm{kg} & 38.7 & \text { EPA8270 } \\ \mu \mathrm{g} / \mathrm{kg} & 38.7 & \text { EPA8270 }\end{array}$


SURVEY ID: PPSC 0402 (continued)

Constituent
Sample ID: 104644

RQ AQ B Result
Unit

D. Limit Method

$B / N / A$ Extractables (including $P A H$ and phenols)

\begin{tabular}{|c|c|c|c|c|c|c|}
\hline 4-Bromophenyl phenyl ether & $\mathrm{U}$ & & 38.7 & $\mu \mathrm{g} / \mathrm{kg}$ & 38.7 & EPA8270 \\
\hline Butylbenzyl phthalate & $\mathrm{U}$ & & 38.7 & $\mu \mathrm{g} / \mathrm{kg}$ & 38.7 & EPA8270 \\
\hline 4-Chloroaniline & $\mathrm{U}$ & & 38.7 & $\mu \mathrm{g} / \mathrm{kg}$ & 38.7 & EPA8270 \\
\hline 4-Chloro-m-cresol & $\mathrm{U}$ & & 38.7 & $\mu \mathrm{g} / \mathrm{kg}$ & 38.7 & EPA8270 \\
\hline 2-Chloronaphthalene & $\mathrm{U}$ & & 38.7 & $\mu \mathrm{g} / \mathrm{kg}$ & 38.7 & EPA8270 \\
\hline 2-Chlorophenol & $\mathrm{U}$ & & 38.7 & $\mu \mathrm{g} / \mathrm{kg}$ & 38.7 & EPA8270 \\
\hline 4-Chlorophenyl phenyl ether & U & & 38.7 & $\mu \mathrm{g} / \mathrm{kg}$ & 38.7 & EPA8270 \\
\hline Chrysene & $\mathrm{U}$ & & 3.87 & $\mu \mathrm{g} / \mathrm{kg}$ & 3.87 & EPA8270 \\
\hline $\mathrm{m} / \mathrm{p}$-Cresol & $\mathrm{U}$ & & 387 & $\mu \mathrm{g} / \mathrm{kg}$ & 387 & EPA8270 \\
\hline o-Cresol (2-Methylphenol) & $\mathbf{U}$ & & 38.7 & $\mu \mathrm{g} / \mathrm{kg}$ & 38.7 & EPA8270 \\
\hline Dibenz $[a, h]$ anthracene & $\mathbf{U}$ & & 3.87 & $\mu \mathrm{g} / \mathrm{kg}$ & 3.87 & EPA8270 \\
\hline Dibenzofuran & $\mathbf{U}$ & & 38.7 & $\mu \mathrm{g} / \mathrm{kg}$ & 38.7 & EPA8270 \\
\hline Di-n-butyl phthalate & $\mathrm{U}$ & $\mathrm{V}$ & 42.9 & $\mu \mathrm{g} / \mathrm{kg}$ & 38.7 & EPA8270 \\
\hline 1,2-Dichlorobenzene & U & & 38.7 & $\mu \mathrm{g} / \mathrm{kg}$ & 38.7 & EPA8270 \\
\hline 1,3-Dichlorobenzene & U & & 38.7 & $\mu \mathrm{g} / \mathrm{kg}$ & 38.7 & EPA8270 \\
\hline 1,4-Dichlorobenzene & $\mathrm{U}$ & & 38.7 & $\mu \mathrm{g} / \mathrm{kg}$ & 38.7 & EPA8270 \\
\hline 3,3'-Dichlorobenzidine & $\mathrm{U}$ & & 38.7 & $\mu \mathrm{g} / \mathrm{kg}$ & 38.7 & EPA8270 \\
\hline 2,4-Dichlorophenol & $\mathrm{U}$ & & 38.7 & $\mu \mathrm{g} / \mathrm{kg}$ & 38.7 & EPA8270 \\
\hline Diethyl phthalate & U & & 38.7 & $\mu \mathrm{g} / \mathrm{kg}$ & 38.7 & EPA8270 \\
\hline 2,4-Dimethyl phenol & $\mathrm{U}$ & & 38.7 & $\mu \mathrm{g} / \mathrm{kg}$ & 38.7 & EPA8270 \\
\hline Dimethyl phthalate & $\mathrm{U}$ & & 38.7 & $\mu \mathrm{g} / \mathrm{kg}$ & 38.7 & EPA8270 \\
\hline 2,4-Dinitrophenol & $\mathrm{U}$ & & 387 & $\mu \mathrm{g} / \mathrm{kg}$ & 387 & EPA8270 \\
\hline 2,4-Dinitrotoluene & UJ & CI $\mathrm{H}$ & 3.87 & $\mu \mathrm{g} / \mathrm{kg}$ & 3.87 & EPA8270 \\
\hline 2,6-Dinitrotoluene & U & & 3.87 & $\mu \mathrm{g} / \mathrm{kg}$ & 3.87 & EPA8270 \\
\hline Di-n-octyl phthalate & $\mathrm{U}$ & & 38.7 & $\mu \mathrm{g} / \mathrm{kg}$ & 38.7 & EPA8270 \\
\hline Fluoranthene & U & & 38.7 & $\mu \mathrm{g} / \mathrm{kg}$ & 38.7 & EPA8270 \\
\hline Fluorene & $\mathrm{U}$ & & 38.7 & $\mu \mathrm{g} / \mathrm{kg}$ & 38.7 & EPA8270 \\
\hline Hexachlorobenzene & $\mathrm{U}$ & & 3.87 & $\mu \mathrm{g} / \mathrm{kg}$ & 3.87 & EPA8270 \\
\hline Hexachlorobutadiene & $\mathrm{U}$ & & 38.7 & $\mu \mathrm{g} / \mathrm{kg}$ & 38.7 & EPA8270 \\
\hline Hexachlorocyclopentadiene & $\mathbf{U}$ & & 38.7 & $\mu \mathrm{g} / \mathrm{kg}$ & 38.7 & EPA8270 \\
\hline Hexachloroethane & $\mathbf{U}$ & & 3.87 & $\mu \mathrm{g} / \mathrm{kg}$ & 3.87 & EPA8270 \\
\hline Indeno[1,2,3-c,d]pyrene & $\mathrm{U}$ & & 3.87 & $\mu \mathrm{g} / \mathrm{kg}$ & 3.87 & EPA8270 \\
\hline Isophorone & $\mathrm{U}$ & & 38.7 & $\mu \mathrm{g} / \mathrm{kg}$ & 38.7 & EPA8270 \\
\hline 2-Methyl-4,6-dinitrophenol & $\mathrm{U}$ & & 387 & $\mu \mathrm{g} / \mathrm{kg}$ & 387 & EPA8270 \\
\hline 2-Methylnaphthalene & $\mathrm{U}$ & & 38.7 & $\mu \mathrm{g} / \mathrm{kg}$ & 38.7 & EPA8270 \\
\hline Naphthalene & $\mathrm{U}$ & & 38.7 & $\mu \mathrm{g} / \mathrm{kg}$ & 38.7 & EPA8270 \\
\hline m-Nitroaniline & $\mathrm{U}$ & & 38.7 & $\mu \mathrm{g} / \mathrm{kg}$ & 38.7 & EPA8270 \\
\hline o-Nitroaniline & $\mathrm{U}$ & & 38.7 & $\mu \mathrm{g} / \mathrm{kg}$ & 38.7 & EPA8270 \\
\hline p-Nitroaniline & $\mathrm{U}$ & & 38.7 & $\mu \mathrm{g} / \mathrm{kg}$ & 38.7 & EPA8270 \\
\hline Nitrobenzene & $\mathrm{U}$ & & 38.7 & $\mu \mathrm{g} / \mathrm{kg}$ & 38.7 & EPA8270 \\
\hline 2-Nitrophenol & $\mathrm{U}$ & & 38.7 & $\mu \mathrm{g} / \mathrm{kg}$ & 38.7 & EPA8270 \\
\hline 4-Nitrophenol & $\mathrm{U}$ & & 38.7 & $\mu \mathrm{g} / \mathrm{kg}$ & 38.7 & EPA8270 \\
\hline N-Nitrosodiphenylamine & $\mathrm{U}$ & & 38.7 & $\mu \mathrm{g} / \mathrm{kg}$ & 38.7 & EPA8270 \\
\hline N-Nitrosodi-n-propylamine & $\mathrm{U}$ & & 38.7 & $\mu \mathrm{g} / \mathrm{kg}$ & 38.7 & EPA8270 \\
\hline Pentachlorophenol & UJ & $\mathrm{I}$ & 38.7 & $\mu \mathrm{g} / \mathrm{kg}$ & 38.7 & EPA8270 \\
\hline Phenanthrene & U & & 38.7 & $\mu \mathrm{g} / \mathrm{kg}$ & 38.7 & EPA8270 \\
\hline Phenol & $\mathrm{U}$ & & 38.7 & $\mu \mathrm{g} / \mathrm{kg}$ & 38.7 & EPA8270 \\
\hline Pyrene & $\mathrm{U}$ & & 38.7 & $\mu \mathrm{g} / \mathrm{kg}$ & 38.7 & EPA8270 \\
\hline 1,2,4-Trichlorobenzene & $\mathrm{U}$ & & 3.87 & $\mu \mathrm{g} / \mathrm{kg}$ & 3.87 & EPA8270 \\
\hline 2,4,5-Trichlorophenol & $\mathrm{U}$ & & 38.7 & $\mu g / \mathrm{kg}$ & 38.7 & EPA8270 \\
\hline 2,4,6-Trichlorophenol & $\mathrm{U}$ & & 38.7 & $\mu \mathrm{g} / \mathrm{kg}$ & 38.7 & EPA8270 \\
\hline
\end{tabular}


SURVEY ID: PPSC 0402 (continued)

Constituent

Volatile Organic Compounds
Sample ID: 104644

RQ AQ B Result

Unit

D. Limit Method

Acetone
Benzene
Bromodichloromethane
Bromoform
Bromomethane (Methyl bromide)
Carbon disulfide
Carbon tetrachloride
Chlorobenzene
Chloroethane
Chloroethene (Vinyl chloride)
Chloroform
Chloromethane (Methyl chloride)
Dibromochloromethane
1,1-Dichloroethane
1,2-Dichloroethane
1,1-Dichloroethylene
1,2-Dichloroethylene
Dichloromethane (Methylene chloride)
1,2-Dichloropropane
cis-1,3-Dichloropropene
trans-1,3-Dichloropropene
Ethylbenzene
2-Hexanone
Methyl ethyl ketone
Methyl isobutyl ketone
Styrene
1,1,2,2-Tetrachloroethane
Tetrachloroethylene
Toluene
1,1,1-Trichloroethane
1,1,2-Trichloroethane
Trichloroethylene
Vinyl acetate
Xylenes

Pesticides/PCBs and Dioxins/Furans

\begin{tabular}{|c|c|c|}
\hline U & 8 & 42.6 \\
\hline U & & 0.581 \\
\hline $\mathrm{U}$ & & 0.0581 \\
\hline $\mathrm{U}$ & & 0.0581 \\
\hline $\mathrm{U}$ & & 0.116 \\
\hline $\mathrm{J}$ & $E$ & 0.465 \\
\hline$U$ & & 0.0581 \\
\hline$U$ & & 0.0581 \\
\hline $\mathrm{U}$ & & 0.116 \\
\hline $\mathrm{U}$ & & 0.116 \\
\hline $\mathrm{U}$ & & 0.0581 \\
\hline U & & 0.116 \\
\hline U & & 0.0581 \\
\hline$U$ & & 0.0581 \\
\hline U & & 0.0581 \\
\hline $\mathrm{U}$ & & 0.0581 \\
\hline $\mathbf{U}$ & & 0.116 \\
\hline$U$ & V & 9.59 \\
\hline U & & 0.0581 \\
\hline U & & 0.0581 \\
\hline U & & 0.0581 \\
\hline U & & 0.0581 \\
\hline U & V & 0.256 \\
\hline U & V & 0.837 \\
\hline U & & 1.16 \\
\hline U & & 0.0581 \\
\hline U & & 0.0581 \\
\hline U & & 0.0581 \\
\hline$U$ & $\mathrm{~V}$ & 0.116 \\
\hline$U$ & & 0.0581 \\
\hline U & & 0.0581 \\
\hline$U$ & & 0.0581 \\
\hline U & & 1.16 \\
\hline U & & 0.174 \\
\hline
\end{tabular}

$\begin{array}{lll}\mu \mathrm{g} / \mathrm{kg} & 5.81 & \text { EPA8260 } \\ \mu \mathrm{g} / \mathrm{kg} & 0.581 & \text { EPA8260 } \\ \mu \mathrm{g} / \mathrm{kg} & 0.0581 & \text { EPA8260 } \\ \mu \mathrm{g} / \mathrm{kg} & 0.0581 & \text { EPA8260 } \\ \mu \mathrm{g} / \mathrm{kg} & 0.116 & \text { EPA8260 } \\ \mu \mathrm{g} / \mathrm{kg} & 1.16 & \text { EPA8260 } \\ \mu \mathrm{g} / \mathrm{kg} & 0.0581 & \text { EPA8260 } \\ \mu \mathrm{g} / \mathrm{kg} & 0.0581 & \text { EPA8260 } \\ \mu \mathrm{g} / \mathrm{kg} & 0.116 & \text { EPA8260 } \\ \mu \mathrm{g} / \mathrm{kg} & 0.116 & \text { EPA8260 } \\ \mu \mathrm{g} / \mathrm{kg} & 0.0581 & \text { EPA8260 } \\ \mu \mathrm{g} / \mathrm{kg} & 0.116 & \text { EPA8260 } \\ \mu \mathrm{g} / \mathrm{kg} & 0.0581 & \text { EPA8260 } \\ \mu \mathrm{g} / \mathrm{kg} & 0.0581 & \text { EPA8260 } \\ \mu \mathrm{g} / \mathrm{kg} & 0.0581 & \text { EPA8260 } \\ \mu \mathrm{g} / \mathrm{kg} & 0.0581 & \text { EPA8260 } \\ \mu \mathrm{g} / \mathrm{kg} & 0.116 & \text { EPA8260 } \\ \mu \mathrm{g} / \mathrm{kg} & 0.581 & \text { EPA8260 } \\ \mu \mathrm{g} / \mathrm{kg} & 0.0581 & \text { EPA8260 } \\ \mu \mathrm{g} / \mathrm{kg} & 0.0581 & \text { EPA8260 } \\ \mu \mathrm{g} / \mathrm{kg} & 0.0581 & \text { EPA8260 } \\ \mu \mathrm{g} / \mathrm{kg} & 0.0581 & \text { EPA8260 } \\ \mu \mathrm{g} / \mathrm{kg} & 1.16 & \text { EPA8260 } \\ \mu \mathrm{g} / \mathrm{kg} & 1.16 & \text { EPA8260 } \\ \mu \mathrm{g} / \mathrm{kg} & 1.16 & \text { EPA8260 } \\ \mu \mathrm{g} / \mathrm{kg} & 0.0581 & \text { EPA8260 } \\ \mu \mathrm{g} / \mathrm{kg} & 0.0581 & \text { EPA8260 } \\ \mu \mathrm{g} / \mathrm{kg} & 0.0581 & \text { EPA8260 } \\ \mu \mathrm{g} / \mathrm{kg} & 0.581 & \text { EPA8260 } \\ \mu \mathrm{g} / \mathrm{kg} & 0.0581 & \text { EPA8260 } \\ \mu \mathrm{g} / \mathrm{kg} & 0.0581 & \text { EPA8260 } \\ \mu \mathrm{g} / \mathrm{kg} & 0.0581 & \text { EPA8260 } \\ \mu \mathrm{g} / \mathrm{kg} & 1.16 & \text { EPA8260 } \\ \mu \mathrm{g} / \mathrm{kg} & 0.174 & \text { EPA8260 } \\ & & \end{array}$

$\begin{array}{ll}\mu \mathrm{g} / \mathrm{kg} & 4.82 \\ \mu \mathrm{g} / \mathrm{kg} & 4.82 \\ \mu \mathrm{g} / \mathrm{kg} & 9.64 \\ \mu \mathrm{g} / \mathrm{kg} & 9.64 \\ \mu \mathrm{g} / \mathrm{kg} & 9.64 \\ \mu \mathrm{g} / \mathrm{kg} & 9.64 \\ \mu \mathrm{g} / \mathrm{kg} & 19.3 \\ \mu \mathrm{g} / \mathrm{kg} & 9.64 \\ \mu \mathrm{g} / \mathrm{kg} & 19.3 \\ \mu \mathrm{g} / \mathrm{kg} & 9.64 \\ \mu \mathrm{g} / \mathrm{kg} & 19.3 \\ \mu \mathrm{g} / \mathrm{kg} & 9.64 \\ \mu \mathrm{g} / \mathrm{kg} & 19.3 \\ \mu \mathrm{g} / \mathrm{kg} & 9.64 \\ \mu \mathrm{g} / \mathrm{kg} & 19.3 \\ \mu \mathrm{g} / \mathrm{kg} & 9.64 \\ \mu \mathrm{g} / \mathrm{kg} & 9.64 \\ \mu \mathrm{g} / \mathrm{kg} & 4.82\end{array}$

EPA8080

EPA8080

EPA8080

EPA8080

EPA8080

EPA8080

EPA8080

EPA8080

EPA8080

EPA8080

EPA8080

EPA8080

EPA8080

EPA8080

EPA8080

EPA8080

EPA8080

EPA8080 
SURVEY ID: PPSC 0402 (continued)

Constituent

Pesticides/PCBs and Dioxins/Furans

Methoxychlor

PCB 1016

PCB 1221

PCB 1232

PCB 1242

PCB 1248

PCB 1254

PCB 1260

Toxaphene

\section{Radionuclides}

Actinium-228

Antimony-124

Antimony-125

Barium-133

Cerium-144

Cesium-134

Cesium-137

Cobalt-57

Cobalt-58

Cobalt-60

Europium-152

Europium-154

Europium-155

Gross alpha

Lead-212

Manganese-54

Neptunium-239

Nonvolatile beta

Plutonium-238

Plutonium-239/240

Potassium-40

Promethium-144

Promethium-146

Ruthenium-106

Sodium-22

Strontium-90

Thorium-234

Tin-113

Yttrium-88

Zinc-65

Zirconium-95

Sample ID: 104644
RQ AQ B Result
Unit
D. Limit Method
96.4
24.1
24.1
24.1
24.1
24.1
24.1
24.1
193

$\begin{array}{lll}\mu \mathrm{g} / \mathrm{kg} & 96.4 & \text { EPA8080 } \\ \mu \mathrm{g} / \mathrm{kg} & 24.1 & \text { EPA8080 } \\ \mu \mathrm{g} / \mathrm{kg} & 24.1 & \text { EPA8080 } \\ \mu \mathrm{g} / \mathrm{kg} & 24.1 & \text { EPA8080 } \\ \mu \mathrm{g} / \mathrm{kg} & 24.1 & \text { EPA8080 } \\ \mu \mathrm{g} / \mathrm{kg} & 24.1 & \text { EPA8080 } \\ \mu \mathrm{g} / \mathrm{kg} & 24.1 & \text { EPA8080 } \\ \mu \mathrm{g} / \mathrm{kg} & 24.1 & \text { EPA8080 } \\ \mu \mathrm{g} / \mathrm{kg} & 193 & \text { EPA8080 }\end{array}$

$1.08 \mathrm{E}+00 \pm 5.59 \mathrm{E}-02 \mathrm{pCi} / \mathrm{g}$ $-3.83 \mathrm{E}-03 \pm 6.70 \mathrm{E}-03 \mathrm{pCi} / \mathrm{g}$ 9.13E-03 $\pm 1.39 \mathrm{E}-02 \mathrm{pCi} / \mathrm{g}$ 2.41E-03 $\pm 7.05 \mathrm{E}-03 \mathrm{pCi} / \mathrm{g}$ $1.28 \mathrm{E}-02 \pm 4.44 \mathrm{E}-02 \mathrm{pCj} / \mathrm{g}$ $4.95 \mathrm{E}-03 \pm 5.05 \mathrm{E}-03 \mathrm{pCi} / \mathrm{g}$ 7.75E-02 $\pm 9.93 \mathrm{E}-03 \mathrm{pCi} / \mathrm{g}$ $3.33 \mathrm{E}-03 \pm 5.14 \mathrm{E}-03 \mathrm{pCi} / \mathrm{g}$ $-1.36 \mathrm{E}-03 \pm 6.83 \mathrm{E}-03 \mathrm{pCi} / \mathrm{g}$ $-3.76 \mathrm{E}-03 \pm 4.62 \mathrm{E}-03 \mathrm{pCj} / \mathrm{g}$ $-1.47 \mathrm{E}-02 \pm 1.50 \mathrm{E}-02 \mathrm{pCi} / \mathrm{g}$ $-3.31 \mathrm{E}-02 \pm 4.65 \mathrm{E}-02 \mathrm{pCi} / \mathrm{g}$ $0.00 \mathrm{E}+00 \quad \mathrm{pCi} / \mathrm{g}$ $1.67 \mathrm{E}+01 \pm 3.21 \mathrm{E}+00 \mathrm{pCi} / \mathrm{g}$ $1.11 \mathrm{E}+00 \pm 2.67 \mathrm{E}-02 \mathrm{pCi} / \mathrm{g}$ $1.36 \mathrm{E}-02 \pm 7.10 \mathrm{E}-03 \mathrm{pCi} / \mathrm{g}$ $-8.00 \mathrm{E}-03 \pm 3.66 \mathrm{E}-02 \mathrm{pCi} / \mathrm{g}$ $9.73 \mathrm{E}+00 \pm 1.98 \mathrm{E}+00 \mathrm{pCi} / \mathrm{g}$ $1.34 \mathrm{E}-03 \pm 1.59 \mathrm{E}-02 \mathrm{pCi} / \mathrm{g}$ $-7.44 \mathrm{E}-03 \pm 6.68 \mathrm{E}-03 \mathrm{pCi} / \mathrm{g}$ 9.12E-01 $\pm 1.11 \mathrm{E}-01 \mathrm{pCi} / \mathrm{g}$ $-1.88 \mathrm{E}-03 \pm 5.13 \mathrm{E}-03 \mathrm{pCi} / \mathrm{g}$ 6.51E-03 $18.81 \mathrm{E}-03 \mathrm{pCi} / \mathrm{g}$ 2.51E-02 $44.70 \mathrm{E}-02 \mathrm{pCi} / \mathrm{g}$ $-1.16 \mathrm{E}-03 \pm 4.61 \mathrm{E}-03 \mathrm{pCi} / \mathrm{g}$ 9.95E-01 $1.04 \mathrm{E}-01 \mathrm{pCi} / \mathrm{g}$ 8.23E-01 $\pm 6.83 \mathrm{E}-01 \mathrm{pCi} / \mathrm{g}$ $3.70 \mathrm{E}-03 \pm 8.38 \mathrm{E}-03 \mathrm{pCi} / \mathrm{g}$ $1.30 \mathrm{E}-03 \pm 5.42 \mathrm{E}-03 \mathrm{pCi} / \mathrm{g}$ $-4.96 \mathrm{E}-03 \pm 1.15 \mathrm{E}-02 \mathrm{pCi} / \mathrm{g}$ $1.60 \mathrm{E}-02 \pm 1.87 \mathrm{E}-02 \mathrm{pCi} / \mathrm{g}$
2.69E-02 EPIA-013B 1.12E-02 EPIA-013B 2.46E-02 EPIA-013B

1.04E-02 EPIA-013B

6.84E-02 EPIA-013B

8.04E-03 EPIA-013B

8.93E-03 EPIA-013B

8.89E-03 EPIA-013B

9.96E-03 EPIA-013B

7.60E-03 EPIA-013B

2.37E-02 EPIA-013B

6.75E-02 EPIA-013B

3.60E-02 EPIA-013B

$1.62 \mathrm{E}+00$ EPIA-001B

1.54E-02 EPIA-013B

9.48E-03 EPIA-013B

6.25E-02 EPIA-013B

2.72E+00 EPIA-001B

3.81E-02 EPIA-012B

3.06E-02 EPIA-012B

6.71E-02 EPIA-013B

8.57E-03 EPIA-013B

1.20E-02 EPIA-013B

8.23E-02 EPIA-013B

7.95E-03 EPIA-013B

5.95E-02 EPIA-004

4.69E-01 EPIA-013B

1.32E.02 EPIA-013B

1.01E-02 EPIA-013B

1.69E-02 EPIA-013B

2.37E-02 EPIA-013B

SURVEY ID: PPSC 0402A

Sample ID: 104648

Survey location: $45135 \mathrm{E} 3678345 \mathrm{~N}$ (SRS coordinates)

Depth of core interval: 1.00 to $2.00 \mathrm{ft}$

Sample type: Normal

Sample matrix: Other

Percent solids: $\mathbf{8 6 . 0 0}$ 
SURVEY ID: PPSC 0402A (continued)

Sample ID: 104648

Constituent

RQ AQ B Result

Unit

D. Limit Method

Physical Parameters and Specified Analyses

Cation exchange capacity

Cation exchange capacity

Chloride

Cyanide

Nitrate-nitrite as nitrogen

Orthophosphate

Phenols

Sulfide

Total organic carbon

Total organic nitrogen

Total phosphates (as P)

Total phosphates (as P)

Metals (total recoverable)

Aluminum

Antimony

Arsenic

Barium

Beryllium

Cadmium

Calcium

Chromium

Cobalt

Copper

Iron

Lead

Magnesium

Manganese

Mercury

Nickel

Potassium

Selenium

Silver

Sodium

Thallium

Vanadium

Zinc

\section{$B / N / A$ Extractables (including $P A H$ and phenols)}

Acenaphthene

Acenaphthylene

Anthracene

Benzo[a]anthracene

Benzo[b]fluoranthene

Benzo[k]fluoranthene

Benzoic acid

Benzo[g, $h, i]$ perylene

Benzo[a]pyrene

Benzyl alcohol

Bis(2-chloroethoxy) methane

Bis(2-chloroethyl) ether

Bis(2-chloroisopropyl) ether

Bis(2-ethylhexyl) phthalate
U

48.5

49.1

J $\mathrm{E} \quad 1.18$

U 1.16

J E $\quad 0.643$

U 11.6

UJ C 349

UJ C 291

C $\quad 98.3$

$\begin{array}{lll}\mathrm{J} & \mathrm{E} & \mathbf{5 4 . 9}\end{array}$

$\begin{array}{lll}\text { J } & \mathrm{E} & \mathbf{7 2 . 2}\end{array}$

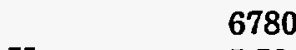

5.59

16.8

14.2

J $\quad \mathrm{E} \quad 0.0671$

U $\quad 0.28$

72.1

6.53

0.49

1.64

1260

6.21

160

12.2

J EV

0.0303

$\begin{array}{lll}\mathrm{J} & \mathrm{E} & 109\end{array}$

U $\quad 16.8$

U 1.4

J $\quad \mathrm{E} \quad 11.4$

16.8

9.16

5.09

38.5

38.5

38.5

3.85

3.85

3.85

38.5

38.5

3.85

38.5

38.5

38.5

38.5

38.5

$\begin{array}{ll}\mathrm{meq} / 100 \mathrm{~g} & 5 \\ \mathrm{meq} / 100 \mathrm{~g} & 5.81 \\ \mathrm{mg} / \mathrm{kg} & 2.72 \\ \mathrm{mg} / \mathrm{kg} & 1.16 \\ \mathrm{mg} / \mathrm{kg} & 1.07 \\ \mathrm{mg} / \mathrm{kg} & 11.6 \\ \mathrm{\mu g} / \mathrm{kg} & 349 \\ \mathrm{mg} / \mathrm{kg} & 291 \\ \mathrm{mg} / \mathrm{kg} & 116 \\ \mathrm{mg} / \mathrm{kg} & 0.233 \\ \mathrm{mg} / \mathrm{kg} & 172 \\ \mathrm{mg} / \mathrm{kg} & 223\end{array}$

EPA9081

EPA9081

EPA300.0

EPA335.3

EPA353.1

EPA365.2

EPA420.2

EPA376.2

EPA415.1

EPA351.2

EPA365.4

EPA365.4

$\begin{array}{lll}\mathrm{mg} / \mathrm{kg} & 14 & \text { EPA6010A } \\ \mathrm{mg} / \mathrm{kg} & 5.59 & \text { EPA6010A } \\ \mathrm{mg} / \mathrm{kg} & 16.8 & \text { EPA6010A } \\ \mathrm{mg} / \mathrm{kg} & 1.4 & \text { EPA6010A } \\ \mathrm{mg} / \mathrm{kg} & 0.559 & \text { EPA6010A } \\ \mathrm{mg} / \mathrm{kg} & 0.28 & \text { EPA6010A } \\ \mathrm{mg} / \mathrm{kg} & 14 & \text { EPA6010A } \\ \mathrm{mg} / \mathrm{kg} & 1.4 & \text { EPA6010A } \\ \mathrm{mg} / \mathrm{kg} & 0.559 & \text { EPA6010A } \\ \mathrm{mg} / \mathrm{kg} & 1.4 & \text { EPA6010A } \\ \mathrm{mg} / \mathrm{kg} & 5.59 & \text { EPA6010A } \\ \mathrm{mg} / \mathrm{kg} & 5.59 & \text { EPA6010A } \\ \mathrm{mg} / \mathrm{kg} & 5.59 & \text { EPA6010A } \\ \mathrm{mg} / \mathrm{kg} & 0.559 & \text { EPA6010A } \\ \mathrm{mg} / \mathrm{kg} & 0.0309 & \text { EPA7471 } \\ \mathrm{mg} / \mathrm{kg} & 1.4 & \text { EPA6010A } \\ \mathrm{mg} / \mathrm{kg} & 112 & \text { EPA6010A } \\ \mathrm{mg} / \mathrm{kg} & 16.8 & \text { EPA6010A } \\ \mathrm{mg} / \mathrm{kg} & 1.4 & \text { EPA6010A } \\ \mathrm{mg} / \mathrm{kg} & 55.9 & \text { EPA6010A } \\ \mathrm{mg} / \mathrm{kg} & 16.8 & \text { EPA6010A } \\ \mathrm{mg} / \mathrm{kg} & 0.559 & \text { EPA6010A } \\ \mathrm{mg} / \mathrm{kg} & 1.12 & \text { EPA6010A }\end{array}$

$\begin{array}{ll}\mu \mathrm{g} / \mathrm{kg} & 38.5 \\ \mu \mathrm{g} / \mathrm{kg} & 38.5 \\ \mu \mathrm{g} / \mathrm{kg} & 38.5 \\ \mu \mathrm{g} / \mathrm{kg} & 3.85 \\ \mu \mathrm{g} / \mathrm{kg} & 3.85 \\ \mu \mathrm{g} / \mathrm{kg} & 3.85 \\ \mu \mathrm{g} / \mathrm{kg} & 38.5 \\ \mu \mathrm{g} / \mathrm{kg} & 38.5 \\ \mu \mathrm{g} / \mathrm{kg} & 3.85 \\ \mu \mathrm{g} / \mathrm{kg} & 38.5 \\ \mu \mathrm{g} / \mathrm{kg} & 38.5 \\ \mu \mathrm{g} / \mathrm{kg} & 38.5 \\ \mu \mathrm{g} / \mathrm{kg} & 38.5 \\ \mu \mathrm{g} / \mathrm{kg} & 38.5\end{array}$

EPA8270

EPA8270

EPA8270

EPA8270

EPA8270

EPA8270

EPA8270

EPA8270

EPA8270

EPA8270

EPA8270

EPA8270

EPA8270

EPA8270 
SURVEY ID: PPSC 0402A (continued)

Constituent
Sample ID: 104648
RQ AQ B Result
Unit
D. Limit Method

$B / N / A$ Extractables (including $P A H$ and phenols)

\begin{tabular}{|c|c|c|c|c|c|}
\hline 4-Bromophenyl phenyl ether & $\mathrm{U}$ & 38.5 & $\mu \mathrm{g} / \mathrm{kg}$ & 38.5 & EPA8270 \\
\hline Butylbenzyl phthalate & $\mathrm{U}$ & 38.5 & $\mu g / \mathrm{kg}$ & 38.5 & EPA8270 \\
\hline 4-Chloroaniline & $\mathrm{U}$ & 38.5 & $\mu \mathrm{g} / \mathrm{kg}$ & 38.5 & EPA8270 \\
\hline 4-Chloro-m-cresol & $\mathrm{U}$ & 38.5 & $\mu \mathrm{g} / \mathrm{kg}$ & 38.5 & EPA8270 \\
\hline 2-Chloronaphthalene & U & 38.5 & $\mu \mathrm{g} / \mathrm{kg}$ & 38.5 & EPA8270 \\
\hline 2-Chlorophenol & $\mathrm{U}$ & 38.5 & $\mu \mathrm{g} / \mathrm{kg}$ & 38.5 & EPA8270 \\
\hline 4-Chlorophenyl phenyl ether & U & 38.5 & $\mu g / \mathrm{kg}$ & 38.5 & EPA8270 \\
\hline Chrysene & $\mathrm{U}$ & 3.85 & $\mu g / \mathrm{kg}$ & 3.85 & EPA8270 \\
\hline $\mathrm{m} / \mathrm{p}$-Cresol & $\mathrm{U}$ & 385 & $\mu g / k g$ & 385 & EPA8270 \\
\hline o-Cresol (2-Methylphenol) & $\mathrm{U}$ & 38.5 & $\mu \mathrm{g} / \mathrm{kg}$ & 38.5 & EPA8270 \\
\hline Dibenz $[a, h]$ anthracene & $\mathrm{U}$ & 3.85 & $\mu \mathrm{g} / \mathrm{kg}$ & 3.85 & EPA8270 \\
\hline Dibenzofuran & $\mathrm{U}$ & 38.5 & $\mu \mathrm{g} / \mathrm{kg}$ & 38.5 & EPA8270 \\
\hline Di-n-butyl phthalate & $\mathrm{U}$ & 45 & $\mu \mathrm{g} / \mathrm{kg}$ & 38.5 & EPA8270 \\
\hline 1,2-Dichlorobenzene & $\mathrm{U}$ & 38.5 & $\mu \mathrm{g} / \mathrm{kg}$ & 38.5 & EPA8270 \\
\hline 1,3-Dichlorobenzene & $\mathrm{U}$ & 38.5 & $\mu \mathrm{g} / \mathrm{kg}$ & 38.5 & EPA8270 \\
\hline 1,4-Dichlorobenzene & U & 38.5 & $\mu \mathrm{g} / \mathrm{kg}$ & 38.5 & EPA8270 \\
\hline 3,3'-Dichlorobenzidine & $\mathrm{U}$ & 38.5 & $\mu \mathrm{g} / \mathrm{kg}$ & 38.5 & EPA8270 \\
\hline 2,4-Dichlorophenol & $\mathrm{U}$ & 38.5 & $\mu \mathrm{g} / \mathrm{kg}$ & 38.5 & EPA8270 \\
\hline Diethyl phthalate & $\mathrm{U}$ & 38.5 & $\mu \mathrm{g} / \mathrm{kg}$ & 38.5 & EPA8270 \\
\hline 2,4-Dimethyl phenol & $\mathrm{U}$ & 38.5 & $\mu \mathrm{g} / \mathrm{kg}$ & 38.5 & EPA8270 \\
\hline Dimethyl phthalate & $\mathrm{U}$ & 38.5 & $\mu \mathrm{g} / \mathrm{kg}$ & 38.5 & EPA8270 \\
\hline 2,4-Dinitrophenol & $U$ & 385 & $\mu \mathrm{g} / \mathrm{kg}$ & 385 & EPA8270 \\
\hline 2,4-Dinitrotoluene & UJ & 3.85 & $\mu \mathrm{g} / \mathrm{kg}$ & 3.85 & EPA8270 \\
\hline 2,6-Dinitrotoluene & $\mathbf{U}$ & 3.85 & $\mu \mathrm{g} / \mathrm{kg}$ & 3.85 & EPA8270 \\
\hline Di-n-octyl phthalate & $\mathrm{U}$ & 38.5 & $\mu \mathrm{g} / \mathrm{kg}$ & 38.5 & EPA8270 \\
\hline Fluoranthene & $\mathrm{U}$ & 38.5 & $\mu g / \mathrm{kg}$ & 38.5 & EPA8270 \\
\hline Fluorene & $\mathrm{U}$ & 38.5 & $\mu \mathrm{g} / \mathrm{kg}$ & 38.5 & EPA8270 \\
\hline Hexachlorobenzene & $\mathrm{U}$ & 3.85 & $\mu g / \mathrm{kg}$ & 3.85 & EPA8270 \\
\hline Hexachlorobutadiene & $\mathrm{U}$ & 38.5 & $\mu g / \mathrm{kg}$ & 38.5 & EPA8270 \\
\hline Hexachlorocyclopentadiene & $\mathrm{U}$ & 38.5 & $\mu g / \mathrm{kg}$ & 38.5 & EPA8270 \\
\hline Hexachloroethane & $\mathrm{U}$ & 3.85 & $\mu \mathrm{g} / \mathrm{kg}$ & 3.85 & EPA8270 \\
\hline Indeno $[1,2,3-c, d]$ pyrene & $\mathrm{U}$ & 3.85 & $\mu \mathrm{g} / \mathrm{kg}$ & 3.85 & EPA8270 \\
\hline Isophorone & $\mathrm{U}$ & 38.5 & $\mu \mathrm{g} / \mathrm{kg}$ & 38.5 & EPA8270 \\
\hline 2-Methyl-4,6-dinitrophenol & $\mathrm{U}$ & 385 & $\mu \mathrm{g} / \mathrm{kg}$ & 385 & EPA8270 \\
\hline 2-Methylnaphthalene & $\mathrm{U}$ & 38.5 & $\mu \mathrm{g} / \mathrm{kg}$ & 38.5 & EPA8270 \\
\hline Naphthalene & $\mathrm{U}$ & 38.5 & $\mu \mathrm{g} / \mathrm{kg}$ & 38.5 & EPA8270 \\
\hline m-Nitroaniline & $\mathrm{U}$ & 38.5 & $\mu \mathrm{g} / \mathrm{kg}$ & 38.5 & EPA8270 \\
\hline o-Nitroaniline & $\mathrm{U}$ & 38.5 & $\mu \mathrm{g} / \mathrm{kg}$ & 38.5 & EPA8270 \\
\hline p-Nitroaniline & $\mathrm{U}$ & 38.5 & $\mu \mathrm{g} / \mathrm{kg}$ & 38.5 & EPA8270 \\
\hline Nitrobenzene & $\mathrm{U}$ & 38.5 & $\mu \mathrm{g} / \mathrm{kg}$ & 38.5 & EPA8270 \\
\hline 2-Nitrophenol & $\mathrm{U}$ & 38.5 & $\mu g / \mathrm{kg}$ & 38.5 & EPA8270 \\
\hline 4-Nitrophenol & $\mathrm{U}$ & 38.5 & $\mu g / \mathrm{kg}$ & 38.5 & EPA8270 \\
\hline N-Nitrosodiphenylamine & $\mathrm{U}$ & 38.5 & $\mu \mathrm{g} / \mathrm{kg}$ & 38.5 & EPA8270 \\
\hline N-Nitrosodi-n-propylamine & $\mathrm{U}$ & 38.5 & $\mu \mathrm{g} / \mathrm{kg}$ & 38.5 & EPA8270 \\
\hline Pentachlorophenol & $\mathrm{U}$ & 38.5 & $\mu \mathrm{g} / \mathrm{kg}$ & 38.5 & EPA8270 \\
\hline Phenanthrene & $\mathrm{U}$ & 38.5 & $\mu \mathrm{g} / \mathrm{kg}$ & 38.5 & EPA8270 \\
\hline Phenol & $\mathrm{U}$ & 38.5 & $\mu \mathrm{g} / \mathrm{kg}$ & 38.5 & EPA8270 \\
\hline Pyrene & $\mathrm{U}$ & 38.5 & $\mu \mathrm{g} / \mathrm{kg}$ & 38.5 & EPA8270 \\
\hline 1,2,4-Trichlorobenzene & $\mathrm{U}$ & 3.85 & $\mu g / \mathrm{kg}$ & 3.85 & EPA8270 \\
\hline 2,4,5-Trichlorophenol & U & 38.5 & $\mu \mathrm{g} / \mathrm{kg}$ & 38.5 & EPA8270 \\
\hline 2,4,6-Trichlorophenol & $\mathrm{U}$ & 38.5 & $\mu \mathrm{g} / \mathrm{kg}$ & 38.5 & EPA8270 \\
\hline
\end{tabular}




\section{Constituent}

RQ AQ B Result

Volatile Organic Compounds

\begin{tabular}{|c|c|c|}
\hline $\mathrm{U}$ & 8 & 14.8 \\
\hline U & & 0.581 \\
\hline $\mathrm{U}$ & & 0.0581 \\
\hline U & & 0.0581 \\
\hline$U$ & & 0.116 \\
\hline $\mathbf{J}$ & E & 0.233 \\
\hline $\mathbf{U}$ & & 0.0581 \\
\hline $\mathbf{U}$ & & 0.0581 \\
\hline U & & 0.116 \\
\hline $\mathbf{U}$ & & 0.116 \\
\hline U & & 0.058 \\
\hline $\mathrm{U}$ & & 0.116 \\
\hline $\mathbf{U}$ & & 0.0581 \\
\hline $\mathrm{U}$ & & 0.0581 \\
\hline $\mathrm{U}$ & & 0.0581 \\
\hline $\mathrm{U}$ & & 0.0581 \\
\hline$U$ & & 0.116 \\
\hline U & V & 9.2 \\
\hline U & & 0.0581 \\
\hline $\mathrm{U}$ & & 0.0581 \\
\hline U & & 0.0581 \\
\hline U & & 0.058 \\
\hline $\mathrm{U}$ & & 1.16 \\
\hline $\mathrm{U}$ & $\mathrm{V}$ & 0.779 \\
\hline U & & 1.16 \\
\hline $\mathrm{U}$ & & 0.0581 \\
\hline$U$ & & 0.058 \\
\hline $\mathrm{U}$ & & 0.058 \\
\hline U & V & 0.128 \\
\hline U & & 0.058 \\
\hline$U$ & & 0.058 \\
\hline $\mathrm{U}$ & & 0.058 \\
\hline U & & 1.16 \\
\hline & & 0.174 \\
\hline
\end{tabular}

Pesticides/PCBs and Dioxins/Furans

Acetone
Benzene
Bromodichloromethane
Bromoform
Bromomethane (Methyl bromide)
Carbon disulfide
Carbon tetrachloride
Chlorobenzene
Chloroethane
Chloroethene (Vinyl chloride)
Chloroform
Chloromethane (Methyl chloride)
Dibromochloromethane
1,1-Dichloroethane
1,2-Dichloroethane
1,1-Dichloroethylene
1,2-Dichloroethylene
Dichloromethane (Methylene chloride)
1,2-Dichloropropane
cis-1,3-Dichloropropene
trans-1,3-Dichloropropene
Ethylbenzene
2-Hexanone
Methyl ethyl-ketone
Methyl isobutyl ketone
Styrene
1,1,2,2-Tetrachloroethane
Tetrachloroethylene
Toluene
1,1,1-Trichloroethane
1,1,2-Trichloroethane
Trichloroethylene
Vinyl acetate
Xylenes

$\begin{array}{ll}U & 19.3 \\ U & 19.3 \\ U & 38.7 \\ U & 38.7 \\ U & 38.7 \\ U & 38.7 \\ U & 77.4 \\ U & 38.7 \\ U & 77.4 \\ U & 38.7 \\ U & 77.4 \\ U & 38.7 \\ U & 77.4 \\ U & 38.7 \\ U & 77.4 \\ U & 38.7 \\ U & 38.7 \\ U & 19.3\end{array}$

Unit

D. Limit Method

$\begin{array}{lll}\mu \mathrm{g} / \mathrm{kg} & 5.81 & \text { EPA8260 } \\ \mu \mathrm{g} / \mathrm{kg} & 0.581 & \text { EPA8260 } \\ \mu \mathrm{g} / \mathrm{kg} & 0.0581 & \text { EPA8260 } \\ \mu \mathrm{g} / \mathrm{kg} & 0.0581 & \text { EPA8260 } \\ \mu \mathrm{g} / \mathrm{kg} & 0.116 & \text { EPA8260 } \\ \mu \mathrm{g} / \mathrm{kg} & 1.16 & \text { EPA8260 } \\ \mu \mathrm{g} / \mathrm{kg} & 0.0581 & \text { EPA8260 } \\ \mu \mathrm{g} / \mathrm{kg} & 0.0581 & \text { EPA8260 } \\ \mu \mathrm{g} / \mathrm{kg} & 0.116 & \text { EPA8260 } \\ \mu \mathrm{g} / \mathrm{kg} & 0.116 & \text { EPA8260 } \\ \mu \mathrm{g} / \mathrm{kg} & 0.0581 & \text { EPA8260 } \\ \mu \mathrm{g} / \mathrm{kg} & 0.116 & \text { EPA8260 } \\ \mu \mathrm{g} / \mathrm{kg} & 0.0581 & \text { EPA8260 } \\ \mu \mathrm{g} / \mathrm{kg} & 0.0581 & \text { EPA8260 } \\ \mu \mathrm{g} / \mathrm{kg} & 0.0581 & \text { EPA8260 } \\ \mu \mathrm{g} / \mathrm{kg} & 0.0581 & \text { EPA8260 } \\ \mu \mathrm{g} / \mathrm{kg} & 0.116 & \text { EPA8260 } \\ \mu \mathrm{g} / \mathrm{kg} & 0.581 & \text { EPA8260 } \\ \mu \mathrm{g} / \mathrm{kg} & 0.0581 & \text { EPA8260 } \\ \mu \mathrm{g} / \mathrm{kg} & 0.0581 & \text { EPA8260 } \\ \mu \mathrm{g} / \mathrm{kg} & 0.0581 & \text { EPA8260 } \\ \mu \mathrm{g} / \mathrm{kg} & 0.0581 & \text { EPA8260 } \\ \mu \mathrm{g} / \mathrm{kg} & 1.16 & \text { EPA8260 } \\ \mu \mathrm{g} / \mathrm{kg} & 1.16 & \text { EPA8260 } \\ \mu \mathrm{g} / \mathrm{kg} & 1.16 & \text { EPA8260 } \\ \mu \mathrm{g} / \mathrm{kg} & 0.0581 & \text { EPA8260 } \\ \mu \mathrm{g} / \mathrm{kg} & 0.0581 & \text { EPA8260 } \\ \mu \mathrm{g} / \mathrm{kg} & 0.0581 & \text { EPA8260 } \\ \mu \mathrm{g} / \mathrm{kg} & 0.581 & \text { EPA8260 } \\ \mu \mathrm{g} / \mathrm{kg} & 0.0581 & \text { EPA8260 } \\ \mu \mathrm{g} / \mathrm{kg} & 0.0581 & \text { EPA8260 } \\ \mu \mathrm{g} / \mathrm{kg} & 0.0581 & \text { EPA8260 } \\ \mu \mathrm{g} / \mathrm{kg} & 1.16 & \text { EPA8260 } \\ \mu \mathrm{g} / \mathrm{kg} & 0.174 & \text { EPA8260 } \\ & & \end{array}$

$\begin{array}{lll}\mu \mathrm{g} / \mathrm{kg} & 19.3 & \text { EPA8080 } \\ \mu \mathrm{g} / \mathrm{kg} & 19.3 & \text { EPA8080 } \\ \mu \mathrm{g} / \mathrm{kg} & 38.7 & \text { EPA8080 } \\ \mu \mathrm{g} / \mathrm{kg} & 38.7 & \text { EPA8080 } \\ \mu \mathrm{g} / \mathrm{kg} & 38.7 & \text { EPA8080 } \\ \mu \mathrm{g} / \mathrm{kg} & 38.7 & \text { EPA8080 } \\ \mu \mathrm{g} / \mathrm{kg} & 77.4 & \text { EPA8080 } \\ \mu \mathrm{g} / \mathrm{kg} & 38.7 & \text { EPA8080 } \\ \mu \mathrm{g} / \mathrm{kg} & 77.4 & \text { EPA8080 } \\ \mu \mathrm{g} / \mathrm{kg} & 38.7 & \text { EPA8080 } \\ \mu \mathrm{g} / \mathrm{kg} & 77.4 & \text { EPA8080 } \\ \mu \mathrm{g} / \mathrm{kg} & 38.7 & \text { EPA8080 } \\ \mu \mathrm{g} / \mathrm{kg} & 77.4 & \text { EPA8080 } \\ \mu \mathrm{g} / \mathrm{kg} & 38.7 & \text { EPA8080 } \\ \mu \mathrm{g} / \mathrm{kg} & 77.4 & \text { EPA8080 } \\ \mu \mathrm{g} / \mathrm{kg} & 38.7 & \text { EPA8080 } \\ \mu \mathrm{g} / \mathrm{kg} & 38.7 & \text { EPA8080 } \\ \mu \mathrm{g} / \mathrm{kg} & 19.3 & \text { EPA8080 }\end{array}$


SURVEY ID: PPSC 0402A (continued)

Constituent

Pesticides/PCBs and Dioxins/Furans
RQ AQ B Result

Sample ID: 104648

Methoxychlor
PCB 1016
PCB 1221
PCB 1232
PCB 1242
PCB 1248
PCB 1254
PCB 1260
Toxaphene

$\begin{array}{ll}\text { U } & 387 \\ \mathrm{U} & 96.7 \\ \mathrm{U} & 96.7 \\ \mathrm{U} & 96.7 \\ \mathrm{U} & 96.7 \\ \mathrm{U} & 96.7 \\ \mathrm{U} & 96.7 \\ \mathrm{U} & \mathbf{9 6 . 7} \\ \mathrm{U} & \mathbf{7 7 4}\end{array}$

\section{Radionuclides}

Actinium-228

Antimony-124

Antimony-125

Barium-133

Cerium-144

Cesium-134

Cesium-137

Cobalt-57

Cobalt-58

Cobalt-60

Europium-152

Europium-154

Europium-155

Gross alpha

Lead-212

Manganese-54

Neptunium-239

Nonvolatile beta

Plutonium-238

Plutonium-239/240

Potassium-40

Promethium-144

Promethium-146

Ruthenium-106

Sodium-22

Strontium-90

Thorium-234

Tin-113

Yttrium-88

Zinc-65

Zirconium-95
387

6.7

96.7

96.7

96.7

96.7

774
Unit D. Limit Method

$\begin{array}{lll}\mu \mathrm{g} / \mathrm{kg} & 387 & \text { EPA8080 } \\ \mu \mathrm{g} / \mathrm{kg} & 96.7 & \text { EPA8080 } \\ \mu \mathrm{g} / \mathrm{kg} & 96.7 & \text { EPA8080 } \\ \mu \mathrm{g} / \mathrm{kg} & 96.7 & \text { EPA8080 } \\ \mu \mathrm{g} / \mathrm{kg} & 96.7 & \text { EPA8080 } \\ \mu \mathrm{g} / \mathrm{kg} & 96.7 & \text { EPA8080 } \\ \mu \mathrm{g} / \mathrm{kg} & 96.7 & \text { EPA8080 } \\ \mu \mathrm{g} / \mathrm{kg} & 96.7 & \text { EPA8080 } \\ \mu \mathrm{g} / \mathrm{kg} & 774 & \text { EPA8080 }\end{array}$

9.64E-01 $\pm 5.69 \mathrm{E}-02 \mathrm{pCi} / \mathrm{g}$ $1.17 \mathrm{E}-03 \pm 6.41 \mathrm{E}-03 \mathrm{pCi} / \mathrm{g}$ $1.37 \mathrm{E}-02 \pm 1.45 \mathrm{E}-02 \mathrm{pCi} / \mathrm{g}$ $-3.99 \mathrm{E}-02 \pm 7.65 \mathrm{E}-03 \mathrm{pCi} / \mathrm{g}$ $-3.50 \mathrm{E}-02 \pm 3.84 \mathrm{E}-02 \mathrm{pCi} / \mathrm{g}$ $-3.78 \mathrm{E}-03 \pm 5.03 \mathrm{E}-03 \mathrm{pCi} / \mathrm{g}$ $8.85 \mathrm{E}-02 \pm 1.02 \mathrm{E}-02 \mathrm{pCi} / \mathrm{g}$ 2.47E-03 $\pm 4.84 \mathrm{E}-03 \mathrm{pCi} / \mathrm{g}$ -3.43E-03 $\pm 6.29 \mathrm{E}-03 \mathrm{pCj} / \mathrm{g}$ $1.35 \mathrm{E}-02 \pm 6.69 \mathrm{E}-03 \mathrm{pCi} / \mathrm{g}$ $-5.83 \mathrm{E}-03 \pm 1.43 \mathrm{E}-02 \mathrm{pCi} / \mathrm{g}$ 1.72E-02 $\$ 3.41 \mathrm{E}-02 \mathrm{pCi} / \mathrm{g}$ $0.00 \mathrm{E}+00 \quad \mathrm{pCi} / \mathrm{g}$ $6.22 \mathrm{E}+01 \pm 5.83 \mathrm{E}+00 \mathrm{pCi} / \mathrm{g}$ $1.03 \mathrm{E}+00 \pm 2.66 \mathrm{E}-02 \mathrm{pCi} / \mathrm{g}$ $1.46 \mathrm{E}-02 \pm 6.70 \mathrm{E}-03 \mathrm{pCi} / \mathrm{g}$ $0.00 \mathrm{E}+00 \quad \mathrm{pCi} / \mathrm{g}$ $1.48 \mathrm{E}+01 \pm 2.24 \mathrm{E}+00 \mathrm{pCi} / \mathrm{g}$ $-1.15 \mathrm{E}-04 \pm 1.42 \mathrm{E}-02 \mathrm{pCi} / \mathrm{g}$ $0.00 \mathrm{E}+00 \quad \mathrm{pCi} / \mathrm{g}$ $6.54 \mathrm{E}-01 \pm 1.04 \mathrm{E}-01 \mathrm{pCi} / \mathrm{g}$ $3.69 \mathrm{E}-03 \pm 5.01 \mathrm{E}-03$ pCi/g 2.21E-03 7.84E-03 pCi/g 1.97E-02+4.68E-02 pCig $1.40 \mathrm{E}-03 \pm 4.09 \mathrm{E}-03 \mathrm{pCi} / \mathrm{g}$ 3.67E-01 $\pm 6.67 \mathrm{E}-02 \mathrm{pCi} / \mathrm{g}$ $1.26 \mathrm{E}+00 \pm 5.44 \mathrm{E}-01 \mathrm{pCi} / \mathrm{g}$ 2.47E-03 $\pm 7.93 \mathrm{E}-03 \mathrm{pCi} / \mathrm{g}$ $1.60 \mathrm{E}-03 \pm 5.81 \mathrm{E}-03 \mathrm{pCi} / \mathrm{g}$ $-3.00 \mathrm{E}-02 \pm 1.21 \mathrm{E}-02 \mathrm{pCi} / \mathrm{g}$ $0.00 \mathrm{E}+00 \quad \mathrm{pCi} / \mathrm{g}$
2.68E-02 EPIA-013B
1.13E-02 EPIA-013B
2.47E-02 EPIA-013B
1.05E-02 EPIA-013B
6.49E-02 EPIA-013B
7.39E-03 EPIA-013B
8.49E-03 EPIA-013B
8.41E-03 EPLA-013B
1.05E-02 EPIA-013B
8.24E-03 EPIA-013B
2.34E-02 EPIA-013B
6.95E-02 EPIA-013B
3.51E-02 EPIA-013B
2.32E+00 EPIA-001B
1.49E-02 EPIA-013B
8.98E-03 EPIA-013B
9.63E+00 EPLA-013B
2.53E+00 EPIA-001B
3.56E-02 EPIA-012B
8.66E-03 EPIA-012B
7.49E-02 EPIA-013B
8.97E-03 EPIA-013B
1.16E-02 EPIA-013B
8.31E-02 EPIA-013B
7.61E-03 EPIA-013B
5.78E-02 EPIA-004
4.56E-01 EPIA-013B
1.32E-02 EPIA-013B
1.07E-02 EPIA-013B
1.70E-02 EPIA-013B
2.43E-02 EPIA-013B

\section{SURVEY ID: PPSC 0403}

Sample ID: 104645

Survey location: $45135 \mathrm{E} 3678345 \mathrm{~N}$ (SRS coordinates)

Depth of core interval: 2.00 to $4.00 \mathrm{ft}$

Sample type: Normal

Sample matrix: Soil

Percent solids: $\mathbf{8 7 . 0 0}$ 
SURVEY ID: PPSC 0403 (continued)

Sample ID: 104645

Constituent

RQ AQ B Result

Unit

D. Limit Method

Physical Parameters and Specified Analyses

Cation exchange capacity

Chloride

Cyanide

Nitrate-nitrite as nitrogen

Orthophosphate

Phenols

Sulfide

Total organic carbon

Total organic nitrogen

Total phosphates (as P)

Metals (total recoverable)

$\begin{array}{lll}\mathrm{meq} / 100 \mathrm{~g} & 5 & \text { EPA9081 } \\ \mathrm{mg} / \mathrm{kg} & 2.86 & \text { EPA300.0 } \\ \mathrm{mg} / \mathrm{kg} & 1.12 & \text { EPA335.3 } \\ \mathrm{mg} / \mathrm{kg} & 1.06 & \text { EPA353.1 } \\ \mathrm{mg} / \mathrm{kg} & 11.1 & \text { EPA365.2 } \\ \mu \mathrm{g} / \mathrm{kg} & 342 & \text { EPA420.2 } \\ \mathrm{mg} / \mathrm{kg} & 287 & \text { EPA376.2 } \\ \mathrm{mg} / \mathrm{kg} & 115 & \text { EPA415.1 } \\ \mathrm{mg} / \mathrm{kg} & 0.23 & \text { EPA351.2 } \\ \mathrm{mg} / \mathrm{kg} & 115 & \text { EPA365.4 }\end{array}$

$\begin{array}{lll}\mathrm{mg} / \mathrm{kg} & 13.4 & \text { EPA6010A } \\ \mathrm{mg} / \mathrm{kg} & 5.37 & \text { EPA6010A } \\ \mathrm{mg} / \mathrm{kg} & 16.1 & \text { EPA6010A } \\ \mathrm{mg} / \mathrm{kg} & 1.34 & \text { EPA6010A } \\ \mathrm{mg} / \mathrm{kg} & 0.537 & \text { EPA6010A } \\ \mathrm{mg} / \mathrm{kg} & 0.269 & \text { EPA6010A } \\ \mathrm{mg} / \mathrm{kg} & 13.4 & \text { EPA6010A } \\ \mathrm{mg} / \mathrm{kg} & 1.34 & \text { EPA6010A } \\ \mathrm{mg} / \mathrm{kg} & 0.537 & \text { EPA6010A } \\ \mathrm{mg} / \mathrm{kg} & 1.34 & \text { EPA6010A } \\ \mathrm{mg} / \mathrm{kg} & 5.37 & \text { EPA6010A } \\ \mathrm{mg} / \mathrm{kg} & 5.37 & \text { EPA6010A } \\ \mathrm{mg} / \mathrm{kg} & 5.37 & \text { EPA6010A } \\ \mathrm{mg} / \mathrm{kg} & 0.537 & \text { EPA6010A } \\ \mathrm{mg} / \mathrm{kg} & 0.0366 & \text { EPA7471 } \\ \mathrm{mg} / \mathrm{kg} & 1.34 & \text { EPA6010A } \\ \mathrm{mg} / \mathrm{kg} & 107 & \text { EPA6010A } \\ \mathrm{mg} / \mathrm{kg} & 16.1 & \text { EPA6010A } \\ \mathrm{mg} / \mathrm{kg} & 1.34 & \text { EPA6010A } \\ \mathrm{mg} / \mathrm{kg} & 53.7 & \text { EPA6010A } \\ \mathrm{mg} / \mathrm{kg} & 16.1 & \text { EPA6010A } \\ \mathrm{mg} / \mathrm{kg} & 0.537 & \text { EPA6010A } \\ \mathrm{mg} / \mathrm{kg} & 1.07 & \text { EPA6010A }\end{array}$

$B / N / A$ Extractables (including $P A H$ and phenols)

\begin{tabular}{|c|c|c|c|c|c|}
\hline Acenaphthene & $\mathrm{U}$ & 37.7 & $\mu \mathrm{g} / \mathrm{kg}$ & 37.7 & EPA8270 \\
\hline Acenaphthene & $\mathrm{U}$ & 37.9 & $\mu \mathrm{g} / \mathrm{kg}$ & 37.9 & EPA8270 \\
\hline Acenaphthylene & U & 37.7 & $\mu \mathrm{g} / \mathrm{kg}$ & 37.7 & EPA8270 \\
\hline Acenaphthylene & $\mathrm{U}$ & 37.9 & $\mu g / \mathrm{kg}$ & 37.9 & EPA8270 \\
\hline Anthracene & $\mathrm{U}$ & 37.7 & $\mu \mathrm{g} / \mathrm{kg}$ & 37.7 & EPA8270 \\
\hline Anthracene & U & 37.9 & $\mu \mathrm{g} / \mathrm{kg}$ & 37.9 & EPA8270 \\
\hline Benzo[a]anthracene & $U$ & 3.77 & $\mu \mathrm{g} / \mathrm{kg}$ & 3.77 & EPA8270 \\
\hline Benzo[a]anthracene & $U$ & 3.79 & $\mu \mathrm{g} / \mathrm{kg}$ & 3.79 & EPA8270 \\
\hline Benzo $[b]$ fluoranthene & $\mathrm{U}$ & 3.77 & $\mu \mathrm{g} / \mathrm{kg}$ & 3.77 & EPA8270 \\
\hline Benzo[b]fluoranthene & $\mathbf{U}$ & 3.79 & $\mu \mathrm{g} / \mathrm{kg}$ & 3.79 & EPA8270 \\
\hline Benzo $[k]$ fluoranthene & U & 3.77 & $\mu \mathrm{g} / \mathrm{kg}$ & 3.77 & EPA8270 \\
\hline Benzo $[k]$ fluoranthene & U & 3.79 & $\mu \mathrm{g} / \mathrm{kg}$ & 3.79 & EPA8270 \\
\hline Benzoic acid & $\mathbf{U}$ & 37.7 & $\mu \mathrm{g} / \mathrm{kg}$ & 37.7 & EPA8270 \\
\hline Benzoic acid & $\mathrm{U}$ & 37.9 & $\mu \mathrm{g} / \mathrm{kg}$ & 37.9 & EPA8270 \\
\hline Benzo $[g, h, i]$ perylene & U & 37.7 & $\mu \mathrm{g} / \mathrm{kg}$ & 37.7 & EPA8270 \\
\hline Benzo $[g, h, i]$ perylene & $\mathrm{U}$ & 37.9 & $\mu \mathrm{g} / \mathrm{kg}$ & 37.9 & EPA8270 \\
\hline
\end{tabular}


Appendix D: Sediment Tables

SURVEY ID: PPSC 0403 (continued)

Sample ID: 104645

Constituent

RQ AQ B Result

Unit

D. Limit Method

$B / N / A$ Extractables (including PAH and phenols)

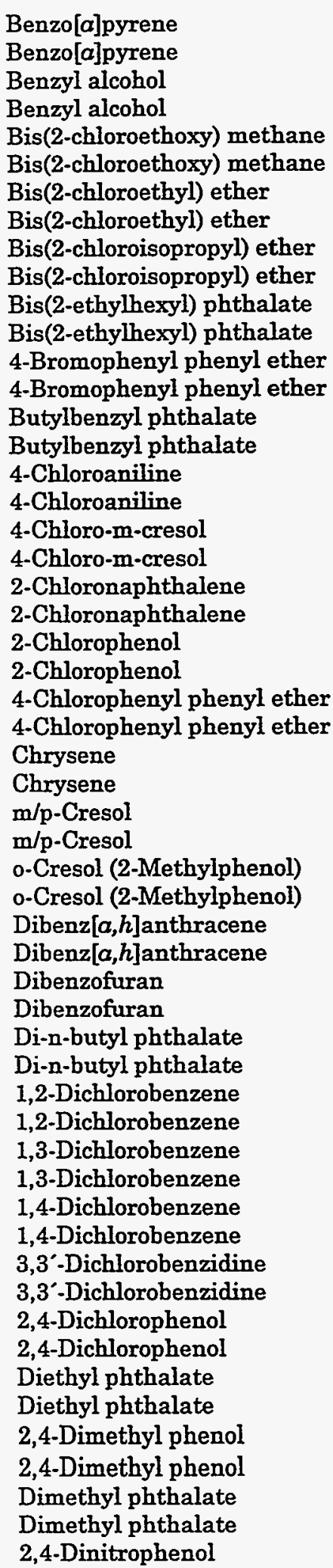

\begin{tabular}{|c|c|c|c|c|c|c|}
\hline $\mathrm{U}$ & & 3.77 & & $\mu \mathrm{g} / \mathrm{kg}$ & 3.77 & EPA8270 \\
\hline $\mathrm{U}$ & & 3.79 & & $\mu \mathrm{g} / \mathrm{kg}$ & 3.79 & EPA8270 \\
\hline $\mathrm{U}$ & & 37.7 & & $\mu \mathrm{g} / \mathrm{kg}$ & 37.7 & EPA8270 \\
\hline $\mathrm{U}$ & & 37.9 & & $\mu \mathrm{g} / \mathrm{kg}$ & 37.9 & EPA8270 \\
\hline $\mathrm{U}$ & & 37.7 & & $\mu \mathrm{g} / \mathrm{kg}$ & 37.7 & EPA8270 \\
\hline $\mathrm{U}$ & & 37.9 & & $\mu \mathrm{g} / \mathrm{kg}$ & 37.9 & EPA8270 \\
\hline $\mathbf{U}$ & & 37.7 & & $\mu \mathrm{g} / \mathrm{kg}$ & 37.7 & EPA8270 \\
\hline $\mathbf{U}$ & & 37.9 & & $\mu \mathrm{g} / \mathrm{kg}$ & 37.9 & EPA8270 \\
\hline $\mathrm{U}$ & & 37.7 & & $\mu \mathrm{g} / \mathrm{kg}$ & 37.7 & EPA8270 \\
\hline $\mathbf{U}$ & & 37.9 & & $\mu \mathrm{g} / \mathrm{kg}$ & 37.9 & EPA8270 \\
\hline $\mathrm{J}$ & $\mathrm{E}$ & 29.4 & & $\mu \mathrm{g} / \mathrm{kg}$ & 37.7 & EPA8270 \\
\hline $\mathrm{U}$ & & 37.9 & & $\mu \mathrm{g} / \mathrm{kg}$ & 37.9 & EPA8270 \\
\hline $\mathrm{U}$ & & 37.7 & & $\mu \mathrm{g} / \mathrm{kg}$ & 37.7 & EPA8270 \\
\hline $\mathrm{U}$ & & 37.9 & & $\mu \mathrm{g} / \mathrm{kg}$ & 37.9 & EPA8270 \\
\hline $\mathrm{U}$ & & 37.7 & & $\mu \mathrm{g} / \mathrm{kg}$ & 37.7 & EPA8270 \\
\hline $\mathrm{U}$ & & 37.9 & & $\mu \mathrm{g} / \mathrm{kg}$ & 37.9 & EPA8270 \\
\hline $\mathrm{U}$ & & 37.7 & & $\mu \mathrm{g} / \mathrm{kg}$ & 37.7 & EPA8270 \\
\hline $\mathrm{U}$ & & 37.9 & & $\mu \mathrm{g} / \mathrm{kg}$ & 37.9 & EPA8270 \\
\hline $\mathrm{U}$ & & 37.7 & & $\mu \mathrm{g} / \mathrm{kg}$ & 37.7 & EPA8270 \\
\hline $\mathrm{U}$ & & 37.9 & & $\mu \mathrm{g} / \mathrm{kg}$ & 37.9 & EPA8270 \\
\hline $\mathrm{U}$ & & 37.7 & & $\mu \mathrm{g} / \mathrm{kg}$ & 37.7 & EPA8270 \\
\hline $\mathrm{U}$ & & 37.9 & & $\mu \mathrm{g} / \mathrm{kg}$ & 37.9 & EPA8270 \\
\hline $\mathrm{U}$ & & 37.7 & & $\mu \mathrm{g} / \mathrm{kg}$ & 37.7 & EPA8270 \\
\hline $\mathrm{U}$ & & 37.9 & & $\mu \mathrm{g} / \mathrm{kg}$ & 37.9 & EPA8270 \\
\hline $\mathbf{U}$ & & 37.7 & & $\mu \mathrm{g} / \mathrm{kg}$ & 37.7 & EPA8270 \\
\hline $\mathbf{U}$ & & 37.9 & & $\mu \mathrm{g} / \mathrm{kg}$ & 37.9 & EPA8270 \\
\hline $\mathrm{U}$ & & 3.77 & & $\mu \mathrm{g} / \mathrm{kg}$ & 3.77 & EPA8270 \\
\hline $\mathrm{U}$ & & 3.79 & & $\mu \mathrm{g} / \mathrm{kg}$ & 3.79 & EPA8270 \\
\hline $\mathrm{U}$ & & 377 & & $\mu \mathrm{g} / \mathrm{kg}$ & 377 & EPA8270 \\
\hline$U$ & & 379 & & $\mu \mathrm{g} / \mathrm{kg}$ & 379 & EPA8270 \\
\hline $\mathrm{U}$ & & 37.7 & & $\mu \mathrm{g} / \mathrm{kg}$ & 37.7 & EPA8270 \\
\hline $\mathrm{U}$ & & 37.9 & & $\mu \mathrm{g} / \mathrm{kg}$ & 37.9 & EPA8270 \\
\hline $\mathrm{U}$ & & 3.77 & & $\mu \mathrm{g} / \mathrm{kg}$ & 3.77 & EPA8270 \\
\hline U & & 3.79 & & $\mu \mathrm{g} / \mathrm{kg}$ & 3.79 & EPA8270 \\
\hline $\mathrm{U}$ & & 37.7 & & $\mu \mathrm{g} / \mathrm{kg}$ & 37.7 & EPA8270 \\
\hline $\mathrm{U}$ & & 37.9 & & $\mu \mathrm{g} / \mathrm{kg}$ & 37.9 & EPA8270 \\
\hline $\mathbf{U}$ & $\mathrm{V}$ & 61.5 & & $\mu \mathrm{g} / \mathrm{kg}$ & 37.7 & EPA8270 \\
\hline $\mathrm{U}$ & $\mathrm{V}$ & 39.8 & ' & $\mu \mathrm{g} / \mathrm{kg}$ & 37.9 & EPA8270 \\
\hline $\mathrm{U}$ & & 37.7 & & $\mu \mathrm{g} / \mathrm{kg}$ & 37.7 & EPA8270 \\
\hline $\mathbf{U}$ & & 37.9 & & $\mu \mathrm{g} / \mathrm{kg}$ & 37.9 & EPA8270 \\
\hline $\mathrm{U}$ & & 37.7 & t & $\mu \mathrm{g} / \mathrm{kg}$ & 37.7 & EPA8270 \\
\hline $\mathrm{U}$ & & 37.9 & & $\mu \mathrm{g} / \mathrm{kg}$ & 37.9 & EPA8270 \\
\hline $\mathrm{U}$ & & 37.7 & & $\mu \mathrm{g} / \mathrm{kg}$ & 37.7 & EPA8270 \\
\hline $\mathrm{U}$ & & 37.9 & & $\mu \mathrm{g} / \mathrm{kg}$ & 37.9 & EPA8270 \\
\hline $\mathrm{U}$ & & 37.7 & & $\mu \mathrm{g} / \mathrm{kg}$ & 37.7 & EPA8270 \\
\hline $\mathrm{U}$ & & 37.9 & & $\mu \mathrm{g} / \mathrm{kg}$ & 37.9 & EPA8270 \\
\hline $\mathrm{U}$ & & 37.7 & & $\mu \mathrm{g} / \mathrm{kg}$ & 37.7 & EPA8270 \\
\hline $\mathrm{U}$ & & 37.9 & & $\mu \mathrm{g} / \mathrm{kg}$ & 37.9 & EPA8270 \\
\hline $\mathrm{U}$ & & 37.7 & & $\mu \mathrm{g} / \mathrm{kg}$ & 37.7 & EPA8270 \\
\hline $\mathrm{U}$ & & 37.9 & & $\mu \mathrm{g} / \mathrm{kg}$ & 37.9 & EPA8270 \\
\hline $\mathrm{U}$ & & 37.7 & & $\mu \mathrm{g} / \mathrm{kg}$ & 37.7 & EPA8270 \\
\hline $\mathrm{U}$ & & 37.9 & & $\mu \mathrm{g} / \mathrm{kg}$ & 37.9 & EPA8270 \\
\hline $\mathrm{U}$ & & 37.7 & & $\mu \mathrm{g} / \mathrm{kg}$ & 37.7 & EPA8270 \\
\hline $\mathrm{U}$ & & 37.9 & & $\mu \mathrm{g} / \mathrm{kg}$ & 37.9 & EPA8270 \\
\hline $\mathrm{U}$ & & 377 & & $\mu \mathrm{g} / \mathrm{kg}$ & 377 & EPA8270 \\
\hline
\end{tabular}


SURVEY ID: PPSC 0403 (continued)

Sample ID: 104645

Constituent

RQ AQ B Result

Unit

D. Limit Method

$B / N / A$ Extractables (including $P A H$ and phenols)

2,4-Dinitrophenol

2,4-Dinitrotoluene

2,4-Dinitrotoluene

2,6-Dinitrotoluene

2,6-Dinitrotoluene

Di-n-octyl phthalate

Di-n-octyl phthalate

Fluoranthene

Fluoranthene

Fluorene

Fluorene

Hexachlorobenzene

Hexachlorobenzene

Hexachlorobutadiene

Hexachlorobutadiene

Hexachlorocyclopentadiene

Hexachlorocyclopentadiene

Hexachloroethane

Hexachloroethane

Indeno[1,2,3-c,d]pyrene

Indeno[1,2,3-c,d]pyrene

Isophorone

Isophorone

2-Methyl-4,6-dinitrophenol

2-Methyl-4,6-dinitrophenol

2-Methylnaphthalene

2-Methylnaphthalene

Naphthalene

Naphthalene

m-Nitroaniline

m-Nitroaniline

o-Nitroaniline

o-Nitroaniline

p-Nitroaniline

p-Nitroaniline

Nitrobenzene

Nitrobenzene

2-Nitrophenol

2-Nitrophenol

4-Nitrophenol

4-Nitrophenol

N-Nitrosodiphenylamine

N-Nitrosodiphenylamine

N-Nitrosodi-n-propylamine

N-Nitrosodi-n-propylamine

Pentachlorophenol

Pentachlorophenol

Phenanthrene

Phenanthrene

Phenol

Phenol

Pyrene

Pyrene

1,2,4-Trichlorobenzene

1,2,4-Trichlorobenzene

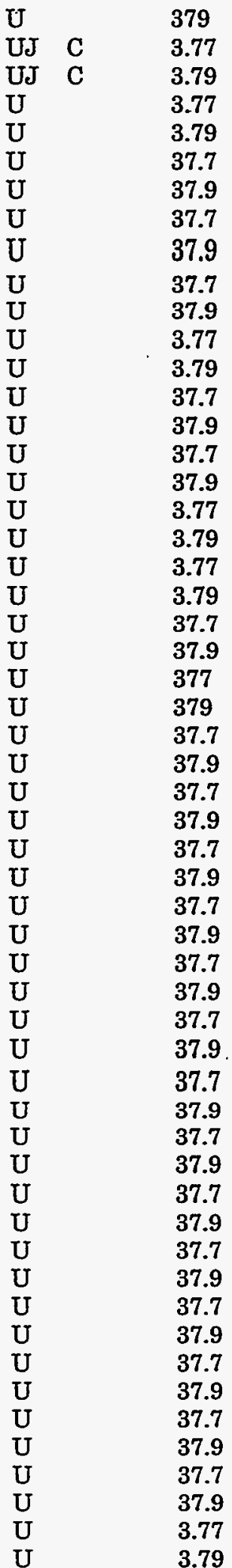

\begin{tabular}{|c|c|c|}
\hline$\mu \mathrm{g} / \mathrm{kg}$ & 379 & EPA8270 \\
\hline$\mu \mathrm{g} / \mathrm{kg}$ & 3.77 & EPA8270 \\
\hline$\mu \mathrm{g} / \mathrm{kg}$ & 3.79 & EPA8270 \\
\hline$\mu \mathrm{g} / \mathrm{kg}$ & 3.77 & EPA8270 \\
\hline$\mu \mathrm{g} / \mathrm{kg}$ & 3.79 & EPA8270 \\
\hline$\mu \mathrm{g} / \mathrm{kg}$ & 37.7 & EPA8270 \\
\hline$\mu \mathrm{g} / \mathrm{kg}$ & 37.9 & EPA8270 \\
\hline$\mu \mathrm{g} / \mathrm{kg}$ & 37.7 & EPA8270 \\
\hline$\mu g / k g$ & 37.9 & EPA8270 \\
\hline$\mu \mathrm{g} / \mathrm{kg}$ & 37.7 & EPA8270 \\
\hline$\mu \mathrm{g} / \mathrm{kg}$ & 37.9 & EPA8270 \\
\hline $\mathrm{Hg} / \mathrm{kg}$ & 3.77 & EPA8270 \\
\hline$\mu \mathrm{g} / \mathrm{kg}$ & 3.79 & EPA8270 \\
\hline$\mu \mathrm{g} / \mathrm{kg}$ & 37.7 & EPA8270 \\
\hline$\mu \mathrm{g} / \mathrm{kg}$ & 37.9 & EPA8270 \\
\hline$\mu g / \mathbf{k g}$ & 37.7 & EPA8270 \\
\hline$\mu \mathrm{g} / \mathrm{kg}$ & 37.9 & EPA8270 \\
\hline$\mu \mathrm{g} / \mathrm{kg}$ & 3.77 & EPA8270 \\
\hline$\mu \mathrm{g} / \mathrm{kg}$ & 3.79 & EPA8270 \\
\hline$\mu \mathrm{g} / \mathrm{kg}$ & 3.77 & EPA8270 \\
\hline$\mu \mathrm{g} / \mathrm{kg}$ & 3.79 & EPA8270 \\
\hline$\mu \mathrm{g} / \mathrm{kg}$ & 37.7 & EPA8270 \\
\hline$\mu \mathrm{g} / \mathrm{kg}$ & 37.9 & EPA8270 \\
\hline$\mu \mathrm{g} / \mathrm{kg}$ & 377 & EPA8270 \\
\hline$\mu g / k g$ & 379 & EPA8270 \\
\hline$\mu \mathrm{g} / \mathrm{kg}$ & 37.7 & EPA8270 \\
\hline$\mu \mathrm{g} / \mathrm{kg}$ & 37.9 & EPA8270 \\
\hline$\mu \mathrm{g} / \mathrm{kg}$ & 37.7 & EPA8270 \\
\hline$\mu \mathrm{g} / \mathrm{kg}$ & 37.9 & EPA8270 \\
\hline$\mu \mathrm{g} / \mathrm{kg}$ & 37.7 & EPA8270 \\
\hline$\mu \mathrm{g} / \mathrm{kg}$ & 37.9 & EPA8270 \\
\hline$\mu \mathrm{g} / \mathrm{kg}$ & 37.7 & EPA8270 \\
\hline$\mu \mathrm{g} / \mathrm{kg}$ & 37.9 & EPA8270 \\
\hline$\mu \mathrm{g} / \mathrm{kg}$ & 37.7 & EPA8270 \\
\hline$\mu \mathrm{g} / \mathrm{kg}$ & 37.9 & EPA8270 \\
\hline$\mu \mathrm{g} / \mathrm{kg}$ & 37.7 & EPA8270 \\
\hline$\mu g / k g$ & 37.9 & EPA8270 \\
\hline$\mu \mathrm{g} / \mathrm{kg}$ & 37.7 & EPA8270 \\
\hline$\mu \mathrm{g} / \mathrm{kg}$. & 37.9 & EPA8270 \\
\hline$\mu \mathrm{g} / \mathrm{kg}$ & 37.7 & EPA8270 \\
\hline$\mu \mathrm{g} / \mathrm{kg}$ & 37.9 & EPA8270 \\
\hline$\mu \mathrm{g} / \mathrm{kg}$ & 37.7 & EPA8270 \\
\hline$\mu \mathrm{g} / \mathrm{kg}$ & 37.9 & EPA8270 \\
\hline$\mu \mathrm{g} / \mathrm{kg}$ & 37.7 & EPA8270 \\
\hline$\mu \mathrm{g} / \mathrm{kg}$ & 37.9 & EPA8270 \\
\hline$\mu \mathrm{g} / \mathrm{kg}$ & 37.7 & EPA8270 \\
\hline$\mu \mathrm{g} / \mathrm{kg}$ & 37.9 & EPA8270 \\
\hline$\mu \mathrm{g} / \mathrm{kg}$ & 37.7 & EPA8270 \\
\hline$\mu \mathrm{g} / \mathrm{kg}$ & 37.9 & EPA8270 \\
\hline$\mu \mathrm{g} / \mathrm{kg}$ & 37.7 & EPA8270 \\
\hline$\mu \mathrm{g} / \mathrm{kg}$ & 37.9 & EPA8270 \\
\hline$\mu \mathrm{g} / \mathrm{kg}$ & 37.7 & EPA8270 \\
\hline$\mu \mathrm{g} / \mathrm{kg}$ & 37.9 & EPA8270 \\
\hline$\mu \mathrm{g} / \mathrm{kg}$ & 3.77 & EPA8270 \\
\hline$\mu g / \mathrm{kg}$ & 3.79 & EPA8270 \\
\hline
\end{tabular}


SURVEY ID: PPSC 0403 (continued)

Constituent

$B / N / A$ Extractables (including $P A H$ and phenols)
Sample ID: 104645

Unit

$\mu \mathrm{g} / \mathrm{kg}$

$\mu \mathrm{g} / \mathrm{kg}$

$\mu \mathrm{g} / \mathrm{kg}$

$\mu \mathrm{g} / \mathrm{kg}$
37.7

37.9

37.9
37.7

37.9

37.7

37.9
EPA8270

EPA8270

EPA8270

EPA8270

Volatile Organic Compounds

Acetone
Benzene
Bromodichloromethane
Bromoform
Bromomethane (Methyl bromide)
Carbon disulfide
Carbon tetrachloride
Chlorobenzene
Chloroethane
Chloroethene (Vinyl chloride)
Chloroform
Chloromethane (Methyl chloride)
Dibromochloromethane
1,1-Dichloroethane
1,2-Dichloroethane
1,1-Dichloroethylene
1,2-Dichloroethylene
Dichloromethane (Methylene chloride)
1,2-Dichloropropane
cis-1,3-Dichloropropene
trans-1,3-Dichloropropene
Ethylbenzene
2-Hexanone
Methyl ethyl ketone
Methyl isobutyl ketone
Styrene
1,1,2,2-Tetrachloroethane
Tetrachloroethylene
Toluene
1,1,1-Trichloroethane
1,1,2-Trichloroethane
Trichloroethylene
Vinyl acetate
Xylenes
(a)

$\begin{array}{lll}\text { U } & 8 & 19 \\ U & & 0.575 \\ U & & 0.0575 \\ U & & 0.0575 \\ U & & 0.115 \\ U & & 1.15 \\ U & & 0.0575 \\ U & & 0.0575 \\ U & & 0.115 \\ U & & 0.115 \\ U & & 0.0575 \\ U & & 0.115 \\ U & & 0.0575 \\ U & & 0.0575 \\ U & & 0.0575 \\ U & & 0.0575 \\ U & & 0.115 \\ U & V & 9.28 \\ U & & 0.0575 \\ U & & 0.0575 \\ U & & 0.0575 \\ U & & 0.0575 \\ U & V & 0.253 \\ U & V & 1.11 \\ U & & 1.15 \\ U & & 0.0575 \\ U & & 0.0575 \\ U & & 0.0575 \\ U & V & 0.115 \\ U & & 0.0575 \\ U & & 0.0575 \\ U & & 0.0575 \\ U & & 1.15 \\ U & & 0.172\end{array}$

$\mu \mathrm{g} / \mathrm{kg}$

$\mu \mathrm{g} / \mathrm{kg}$

5.75

EPA8260

$\mu \mathrm{g} / \mathrm{kg}$

$\mu \mathrm{g} / \mathrm{kg}$

$\mu \mathrm{g} / \mathrm{kg}$

$\mu \mathrm{g} / \mathrm{kg}$

$\mu \mathrm{g} / \mathrm{kg}$

$\mu \mathrm{g} / \mathrm{kg}$

$\mu \mathrm{g} / \mathrm{kg}$

$\mu \mathrm{g} / \mathrm{kg}$

$\mu \mathrm{g} / \mathrm{kg}$

$\mu \mathrm{g} / \mathrm{kg}$

$\mu \mathrm{g} / \mathrm{kg}$

$\mu \mathrm{g} / \mathrm{kg}$

$\mu \mathrm{g} / \mathrm{kg}$

$\mu \mathrm{g} / \mathrm{kg}$

$\mu \mathrm{g} / \mathrm{kg}$

$\mu \mathrm{g} / \mathrm{kg}$

$\mu \mathrm{g} / \mathrm{kg}$

$\mu \mathrm{g} / \mathrm{kg}$

$\mu \mathrm{g} / \mathrm{kg}$

$\mu \mathrm{g} / \mathrm{kg}$

$\mu \mathrm{g} / \mathrm{kg}$

$\mu \mathrm{g} / \mathrm{kg}$

$\mu \mathrm{g} / \mathrm{kg}$

$\mu \mathrm{g} / \mathrm{kg}$

$\mu \mathrm{g} / \mathrm{kg}$

$\mu \mathrm{g} / \mathrm{kg}$

$\mu \mathrm{g} / \mathrm{kg}$

$\mu \mathrm{g} / \mathrm{kg}$

$\mu \mathrm{g} / \mathrm{kg}$

$\mu \mathrm{g} / \mathrm{kg}$

$\mu \mathrm{g} / \mathrm{kg}$

$\mu \mathrm{g} / \mathrm{kg}$

0.575

0.0575

0.0575

0.115

1.15

0.0575

0.0575

0.115

0.115

0.0575

0.115

0.0575

0.0575

0.0575

0.0575

0.115

0.575

0.0575

0.0575

0.0575

0.0575

1.15

1.15

1.15

0.0575

0.0575

0.0575

0.575

0.0575

0.0575

0.0575

1.15

0.172

EPA8260

EPA8260

EPA8260

EPA8260

EPA8260

EPA8260

EPA8260

EPA8260

EPA8260

EPA8260

EPA8260

EPA8260

EPA8260

EPA8260

EPA8260

EPA8260

EPA8260

EPA8260

EPA8260

EPA8260

EPA8260

EPA8260

EPA8260

EPA8260

EPA8260

EPA8260

EPA8260

EPA8260

EPA8260

EPA8260

EPA8260

EPA8260

EPA8260

Pesticides/PCBs and Dioxins/Furans

Aldrin

alpha-Benzene hexachloride

beta-Benzene hexachloride

delta-Benzene hexachloride

alpha-Chlordane

gamma-Chlordane

p,p'-DDD

$\mathrm{p}, \mathrm{p}^{\prime}-\mathrm{DDE}$

$p, p^{\prime}-D D T$

Dieldrin

Endosulfan sulfate

$\begin{array}{lll}\mu \mathrm{g} / \mathrm{kg} & 0.945 & \text { EPA8080 } \\ \mu \mathrm{g} / \mathrm{kg} & 0.945 & \text { EPA8080 } \\ \mu \mathrm{g} / \mathrm{kg} & 1.89 & \text { EPA8080 } \\ \mu \mathrm{g} / \mathrm{kg} & 1.89 & \text { EPA8080 } \\ \mu \mathrm{g} / \mathrm{kg} & 1.89 & \text { EPA8080 } \\ \mu \mathrm{g} / \mathrm{kg} & 1.89 & \text { EPA8080 } \\ \mu \mathrm{g} / \mathrm{kg} & 3.78 & \text { EPA8080 } \\ \mu \mathrm{g} / \mathrm{kg} & 1.89 & \text { EPA8080 } \\ \mu \mathrm{g} / \mathrm{kg} & 3.78 & \text { EPA8080 } \\ \mu \mathrm{g} / \mathrm{kg} & 1.89 & \text { EPA8080 } \\ \mu \mathrm{g} / \mathrm{kg} & 3.78 & \text { EPA8080 }\end{array}$


SURVEY ID: PPSC 0403 (continued)

Constituent

Pesticides/PCBs and Dioxins/Furans

Endosulfan I

Endosulfan II

Endrin

Endrin ketone

Heptachlor

Heptachlor epoxide

Lindane

Methoxychior

PCB 1016

PCB 1221

PCB 1232

PCB 1242

PCB 1248

PCB 1254

PCB 1260

Toxaphene

Rádionuclides

Actinium-228

Antimony-124

Antimony-125

Barium-133

Cerium-144

Cesium-134

Cesium-137

Cobalt-57

Cobalt-58

Cobalt-60

Europium-152

Europium-154

Europium-155

Gross alpha

Lead-212

Manganese-54

Neptunium-239

Nonvolatile beta

Plutonium-238

Plutonium-239/240

Potassium-40

Promethium-144

Promethium-146

Ruthenium-106

Sodium-22

Strontium-90

Thorium-234

Tin-113

Yttrium-88

Zinc-65

Zirconium-95

Sample ID: 104645

RQ AQ B Result Unit D. Limit Method

$\begin{array}{lllllll}\text { UJ } & \text { O } & \text { L } & 1.89 & \mu \mathrm{g} / \mathrm{kg} & 1.89 & \text { EPA8080 } \\ \text { UJ } & \mathrm{O} & \mathrm{L} & 3.78 & \mu \mathrm{g} / \mathrm{kg} & 3.78 & \text { EPA8080 } \\ \text { UJ } & \mathrm{O} & \mathrm{L} & 1.89 & \mu \mathrm{g} / \mathrm{kg} & 1.89 & \text { EPA8080 } \\ \text { UJ } & \mathrm{O} & \mathrm{L} & 3.78 & \mu \mathrm{g} / \mathrm{kg} & 3.78 & \text { EPA8080 } \\ \text { UJ } & \mathrm{O} & \mathrm{L} & 1.89 & \mu \mathrm{g} / \mathrm{kg} & 1.89 & \text { EPA8080 } \\ \text { UJ } & \mathrm{O} & \mathrm{L} & 1.89 & \mu \mathrm{g} / \mathrm{kg} & 1.89 & \text { EPA8080 } \\ \text { UJ } & \mathrm{O} & \mathrm{L} & \mathbf{0 . 9 4 5} & \mu \mathrm{g} / \mathrm{kg} & \mathbf{0 . 9 4 5} & \text { EPA8080 } \\ \text { UJ } & \mathrm{O} & \mathrm{L} & 18.9 & \mu \mathrm{g} / \mathrm{kg} & 18.9 & \text { EPA8080 } \\ \text { UJ } & \mathrm{O} & \mathrm{L} & 4.73 & \mu \mathrm{g} / \mathrm{kg} & 4.73 & \text { EPA8080 } \\ \text { UJ } & \mathrm{O} & \mathrm{L} & 4.73 & \mu \mathrm{g} / \mathrm{kg} & 4.73 & \text { EPA8080 } \\ \text { UJ } & \mathrm{O} & \mathrm{L} & 4.73 & \mu \mathrm{g} / \mathrm{kg} & 4.73 & \text { EPA8080 } \\ \text { UJ } & \mathrm{O} & \mathrm{L} & 4.73 & \mu \mathrm{g} / \mathrm{kg} & 4.73 & \text { EPA8080 } \\ \text { UJ } & \mathrm{O} & \mathrm{L} & 4.73 & \mu \mathrm{g} / \mathrm{kg} & 4.73 & \text { EPA8080 } \\ \text { UJ } & \mathrm{O} & \mathrm{L} & 4.73 & \mu \mathrm{g} / \mathrm{kg} & 4.73 & \text { EPA8080 } \\ \text { UJ } & \mathrm{O} & \mathrm{L} & 4.73 & \mu \mathrm{g} / \mathrm{kg} & 4.73 & \text { EPA8080 } \\ \text { UJ } & \mathrm{O} & \mathrm{L} & 37.8 & \mu \mathrm{g} / \mathrm{kg} & 37.8 & \text { EPA8080 }\end{array}$

8.42E-01 $\pm 4.04 \mathrm{E}-02 \mathrm{pCi} / \mathrm{g}$ 4.70E-03 $\pm 3.67 \mathrm{E}-03 \mathrm{pCi} / \mathrm{g}$ $-7.11 \mathrm{E}-04 \pm 1.02 \mathrm{E}-02 \mathrm{pCi} / \mathrm{g}$ $6.29 \mathrm{E}-05 \pm 5.06 \mathrm{E}-03 \mathrm{pCi} / \mathrm{g}$ $-2.24 \mathrm{E}-02 \pm 3.49 \mathrm{E}-02 \mathrm{pCi} / \mathrm{g}$ $4.06 \mathrm{E}-03 \pm 3.46 \mathrm{E}-03 \mathrm{pCi} / \mathrm{g}$ $4.29 \mathrm{E}-03 \pm 4.79 \mathrm{E}-03 \mathrm{pCi} / \mathrm{g}$ $5.53 \mathrm{E}-03 \pm 3.86 \mathrm{E}-03 \mathrm{pCi} / \mathrm{g}$ $-4.26 \mathrm{E}-03 \pm 4.62 \mathrm{E}-03 \mathrm{pCi} / \mathrm{g}$ $-1.33 \mathrm{E}-05 \pm 3.15 \mathrm{E}-03 \mathrm{pCi} / \mathrm{g}$ $-9.04 \mathrm{E}-03 \pm 1.06 \mathrm{E}-02 \mathrm{pCi} / \mathrm{g}$ $-4.25 \mathrm{E}-02 \pm 3.10 \mathrm{E}-02 \mathrm{pCi} / \mathrm{g}$ $0.00 \mathrm{E}+00 \quad \mathrm{pCi} / \mathrm{g}$ $2.04 \mathrm{E}+01 \pm 3.39 \mathrm{E}+00 \mathrm{pCi} / \mathrm{g}$

$\mathrm{V} \quad 9.11 \mathrm{E}-01 \pm 2.00 \mathrm{E}-02 \mathrm{pCi} / \mathrm{g}$ $1.68 \mathrm{E}-02 \pm 5.17 \mathrm{E}-03 \mathrm{pCi} / \mathrm{g}$ $0.00 \mathrm{E}+00 \quad \mathrm{pCi} / \mathrm{g}$ $9.61 \mathrm{E}+00 \pm 2.01 \mathrm{E}+00 \mathrm{pCi} / \mathrm{g}$ $3.90 \mathrm{E}-01 \pm 7.74 \mathrm{E}-02 \mathrm{pCi} / \mathrm{g}$ $1.10 \mathrm{E}-01 \pm 3.74 \mathrm{E}-02 \mathrm{pCi} / \mathrm{g}$

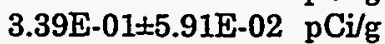
-2.51E-04 $\pm 3.78 \mathrm{E}-03 \mathrm{pCi} / \mathrm{g}$ 4.36E-04 $\pm 5.53 \mathrm{E}-03 \mathrm{pCi} / \mathrm{g}$ $-1.97 \mathrm{E}-03 \pm 3.51 \mathrm{E}-02 \mathrm{pCi} / \mathrm{g}$ $-1.61 \mathrm{E}-04 \pm 3.22 \mathrm{E}-03 \mathrm{pCj} / \mathrm{g}$ $1.49 \mathrm{E}+00 \pm 1.46 \mathrm{E}-01 \mathrm{pCi} / \mathrm{g}$ 9.50E-01 $44.40 \mathrm{E}-01 \mathrm{pCi} / \mathrm{g}$ $-3.19 \mathrm{E}-03 \pm 5.68 \mathrm{E}-03 \mathrm{pCi} / \mathrm{g}$ 5.88E-04 $13.86 \mathrm{E}-03 \mathrm{pCi} / \mathrm{g}$ 4.10E-03 $\pm 7.81 \mathrm{E}-03 \mathrm{pCi} / \mathrm{g}$

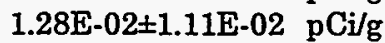

1.99E-02 EPIA-013B

8.54E.03 EPIA-013B

1.74E-02 EPIA-013B

7.70E-03 EPIA-013B

5.12E-02 EPIA-013B

5.28E-03 EPIA-013B

7.32E-03 EPIA-013B

6.58E-03 EPIA-013B

7.35E-03 EPIA-013B

5.45E-03 EPIA-013B

1.79E-02 EPIA-013B

5.03E-02 EPIA-013B

2.61E.02 EPIA-013B

1.47E+00 EPIA-001B

1.12E-02 EPIA-013B

5.92E-03 EPIA-013B

4.67E-02 EPIA-013B

2.80E+00 EPIA-001B

4.35E-02 EPIA-012B

2.17E-02 EPIA-012B

5.11E-02 EPIA-013B

6.30E-03 EPIA-013B

8.32E-03 EPIA-013B

5.90E-02 EPIA-013B

5.57E-03 EPIA-013B

7.90E-02 EPIA-004

3.36E-01 EPIA-013B

9.61E-03 EPIA-013B

7.02E-03 EPIA-013B

1.23E-02 EPIA-013B

1.71E-02 EPIA-013B 
SURVEY ID: PPSC 0403D

Sample type: Split

Constituent
Sample ID: 104649

Associated sample: 104645

RQ AQ B Result

Unit

D. Limit Method

Physical Parameters and Specified Analyses

Cation exchange capacity

Chloride

Cyanide

Nitrate-nitrite as nitrogen

Orthophosphate

Phenols

Total organic carbon

Total organic nitrogen

Total phosphates (as P)

Metals (total recoverable)

$\begin{array}{lll}\text { U } & \text { Y } & \mathbf{2 5 . 8} \\ J & \text { YE } & 16.5 \\ \text { U } & \text { YV } & 0.11 \\ & \text { Y } & 0.726 \\ J & \text { YE } & 0.161 \\ \text { J } & \text { YE } & 142 \\ & \text { YV } & 240 \\ J & \text { YE } & 39.2 \\ & \text { Y } & 16.5\end{array}$

$\begin{array}{lll} & \text { Y } & 1620 \\ \text { U } & \text { Y } & 48 \\ \text { UJ } & \text { YW } & 0.703 \\ \text { J } & \text { YE } & 4.2 \\ \text { J } & \text { YE } & 0.04 \\ \text { U } & \text { Y } & 2.72 \\ & \text { YV } & \mathbf{5 7 . 1} \\ \text { J } & \text { YE } & 2.4 \\ \text { U } & \text { Y } & 5.5 \\ J & \text { YE } & 0.925 \\ & \text { Y } & 254 \\ J & \text { YE } & 1.3 \\ & \text { Y } & \mathbf{5 0 . 6} \\ & \text { Y } & 5.6 \\ \text { U } & \text { Y } & 0.639 \\ \text { U } & \text { Y } & 8.66 \\ \text { U } & \text { Y } & 1030 \\ \text { U } & \text { Y } & 1.76 \\ \text { U } & \text { Y } & \mathbf{5 . 7 1} \\ \text { U } & \text { YV } & 18.2 \\ \text { U } & \text { YV } & 0.211 \\ \text { J } & \text { YE } & 1.9 \\ \text { J } & \text { YE } & 2.4\end{array}$

$\begin{array}{lll}\mathrm{mg} / \mathrm{kg} & 25.8 & \text { EPA9081 } \\ \mathrm{mg} / \mathrm{kg} & 20.3 & \text { EPA300.0 } \\ \mathrm{mg} / \mathrm{kg} & 1.08 & \text { EPA9010 } \\ \mathrm{mg} / \mathrm{kg} & 0.7 & \text { EPA353.2 } \\ \mathrm{mg} / \mathrm{kg} & 1.17 & \text { EPA365.2 } \\ \mu \mathrm{g} / \mathrm{kg} & 1190 & \text { EPA420.2 } \\ \mathrm{mg} / \mathrm{kg} & 8.4 & \text { LLOYDKAHN } \\ \mathrm{mg} / \mathrm{kg} & 125 & \text { EPA351.4 } \\ \mathrm{mg} / \mathrm{kg} & 3.48 & \text { EPA365.2 }\end{array}$

$\mathrm{mg} / \mathrm{kg}$

$\mathrm{mg} / \mathrm{kg}$

$\mathrm{mg} / \mathrm{kg}$

$\mathrm{mg} / \mathrm{kg}$

$\mathrm{mg} / \mathrm{kg}$

$\mathrm{mg} / \mathrm{kg}$

$\mathrm{mg} / \mathrm{kg}$

$\mathrm{mg} / \mathrm{kg}$

$\mathrm{mg} / \mathrm{kg}$

$\mathrm{mg} / \mathrm{kg}$

$\mathrm{mg} / \mathrm{kg}$

$\mathrm{mg} / \mathrm{kg}$

$\mathrm{mg} / \mathrm{kg}$

$\mathrm{mg} / \mathrm{kg}$

$\mathrm{mg} / \mathrm{kg}$

mg/kg

$\mathrm{mg} / \mathrm{kg}$

$\mathrm{mg} / \mathrm{kg}$

$\mathrm{mg} / \mathrm{kg}$

$\mathrm{mg} / \mathrm{kg}$

$\mathrm{mg} / \mathrm{kg}$

$\mathrm{mg} / \mathrm{kg}$

$\mathrm{mg} / \mathrm{kg}$
CLP-MOD

CLP-MOD

CLP-MOD

CLP-MOD

CLP.MOD

CLP-MOD

CLP-MOD

CLP-MOD

CLP-MOD

CLP-MOD

CLP-MOD

CLP-MOD

CLP-MOD

CLP-MOD

CLP-MOD

CLP-MOD

CLP-MOD

CLP-MOD

CLP-MOD

CLP-MOD

CLP-MOD

CLP-MOD

CLP-MOD 
SURVEY ID: PPSC 0403D (continued)

Sample ID: 104649

Constituent

RQ AQ B Result.

Unit

D. Limit Method

$B / N / A$ Extractables (including PAH and phenols)

Acenaphthene

Acenaphthylene

Aldol condensate

Aldol condensate

Aldol condensate

Anthracene

Benzo[a]anthracene

Benzo[b]fluoranthene

Benzo[k]fluoranthene

Benzoic acid

Benzo[g,h,i]perylene

Benzo[a]pyrene

Benzyl alcohol

Bis(2-chloroethoxy) methane

Bis(2-chloroethyl) ether.

$\mathrm{Bis}$ (2-chloroisopropyl) ether

Bis(2-ethylhexyl) phthalate

4-Bromophenyl phenyl ether

Butylbenzyl phthalate

4-Chloroaniline

4-Chloro-m-cresol

2-Chloronaphthalene

2-Chlorophenol

4-Chlorophenyl phenyl ether

Chrysene

o-Cresol (2-Methylphenol)

p-Cresol (4-Methylphenol)

Dibenz[a,h]anthracene

Dibenzofuran

Di-n-butyl phthalate

1,2-Dichlorobenzene

1,3-Dichlorobenzene

1,4-Dichlorobenzene

3,3'-Dichlorobenzidine

2,4-Dichlorophenol

Diethyl phthalate

2,4-Dimethyl phenol

Dimethyl phthalate

2,4-Dinitrophenol

2,4-Dinitrotoluene

2,6-Dinitrotoluene

Di-n-octyl phthalate

Fluoranthene

Fluorene

Hexachlorobenzene

Hexachlorobutadiene

Hexachlorocyclopentadiene

$\begin{array}{lll}\text { U } & \mathrm{Y} & \mathbf{3 9} \\ \mathrm{U} & \mathrm{Y} & \mathbf{3 9} \\ \mathrm{UJ} & \mathrm{NKV} & \mathbf{5 0 0} \\ \mathrm{UJ} & \mathrm{NKV} & \mathbf{8 0} \\ \mathrm{UJ} & \mathrm{NKV} & \mathbf{1 0 0} \\ \mathrm{U} & \mathrm{Y} & \mathbf{3 9} \\ \mathrm{U} & \mathrm{Y} & \mathbf{3 9} \\ \mathrm{U} & \mathrm{Y} & 39 \\ \mathrm{U} & \mathrm{Y} & \mathbf{3 9} \\ \mathrm{U} & \mathrm{YV} & \mathbf{5 . 1 9} \\ \mathrm{U} & \mathrm{Y} & \mathbf{3 9} \\ \mathrm{U} & \mathrm{Y} & \mathbf{3 9} \\ \mathrm{U} & \mathrm{Y} & \mathbf{3 9} \\ \mathrm{U} & \mathrm{Y} & \mathbf{3 9} \\ \mathrm{U} & \mathrm{Y} & \mathbf{3 9} \\ \mathrm{U} & \mathrm{Y} & \mathbf{3 9} \\ \mathrm{U} & \mathrm{YV} & \mathbf{2 2 . 3} \\ \mathrm{U} & \mathrm{Y} & \mathbf{3 9} \\ \mathrm{U} & \mathrm{Y} & \mathbf{3 9} \\ \mathrm{U} & \mathrm{Y} & \mathbf{3 9} \\ \mathrm{U} & \mathrm{Y} & \mathbf{3 9} \\ \mathrm{U} & \mathrm{Y} & \mathbf{3 9} \\ \mathrm{U} & \mathrm{Y} & \mathbf{3 9} \\ \mathrm{U} & \mathrm{Y} & \mathbf{3 9} \\ \mathrm{U} & \mathrm{Y} & \mathbf{3 9} \\ \mathrm{U} & \mathrm{Y} & \mathbf{3 9} \\ \mathrm{U} & \mathrm{Y} & \mathbf{3 9} \\ \mathrm{U} & \mathrm{Y} & \mathbf{3 9} \\ \mathrm{U} & \mathrm{Y} & \mathbf{3 9} \\ \mathrm{U} & \mathrm{YV} & \mathbf{1 5 8} \\ \mathrm{U} & \mathrm{Y} & \mathbf{3 9} \\ \mathrm{U} & \mathrm{Y} & \mathbf{3 9} \\ \mathrm{U} & \mathrm{Y} & \mathbf{3 9} \\ \mathrm{U} & \mathrm{Y} & \mathbf{7 8} \\ \mathrm{U} & \mathrm{Y} & 39 \\ J & \mathrm{YE} & 4.11 \\ \mathrm{U} & \mathrm{Y} & \mathbf{3 9} \\ \mathrm{U} & \mathrm{Y} & \mathbf{3 9} \\ \mathrm{U} & \mathrm{Y} & \mathbf{1 9 5} \\ \mathrm{U} & \mathrm{Y} & \mathbf{3 9} \\ \mathrm{U} & \mathrm{Y} & \mathbf{3 9} \\ \mathrm{U} & \mathrm{Y} & \mathbf{3 9} \\ \mathrm{U} & \mathrm{Y} & \mathbf{3 9} \\ \mathrm{U} & \mathrm{Y} & \mathbf{3 9} \\ \mathrm{U} & \mathrm{Y} & \mathbf{3 9} \\ \mathrm{U} & \mathrm{Y} & \mathbf{3 9} \\ \mathrm{U} & \mathrm{Y} & \mathbf{3 9} \\ & & \\ & & \end{array}$

\begin{tabular}{|c|c|c|}
\hline$\mu \mathrm{g} / \mathrm{kg}$ & 39 & EPA8270 \\
\hline$\mu \mathrm{g} / \mathrm{kg}$ & 39 & EPA8270 \\
\hline$\mu \mathrm{g} / \mathrm{kg}$ & & EPA8270 \\
\hline$\mu \mathrm{g} / \mathrm{kg}$ & & EPA8270 \\
\hline$\mu \mathrm{g} / \mathrm{kg}$ & & EPA8270 \\
\hline$\mu \mathrm{g} / \mathrm{kg}$ & 39 & EPA8270 \\
\hline$\mu \mathrm{g} / \mathrm{kg}$ & 39 & EPA8270 \\
\hline$\mu \mathrm{g} / \mathrm{kg}$ & 39 & EPA8270 \\
\hline$\mu \mathrm{g} / \mathrm{kg}$ & 39 & EPA8270 \\
\hline$\mu \mathrm{g} / \mathrm{kg}$ & 195 & EPA8270 \\
\hline$\mu \mathrm{g} / \mathrm{kg}$ & 39 & EPA8270 \\
\hline$\mu \mathrm{g} / \mathrm{kg}$ & 39 & EPA8270 \\
\hline$\mu \mathrm{g} / \mathrm{kg}$ & 39 & EPA8270 \\
\hline$\mu \mathrm{g} / \mathrm{kg}$ & 39 & EPA8270 \\
\hline$\mu \mathrm{g} / \mathrm{kg}$ & 39 & EPA8270 \\
\hline$\mu \mathrm{g} / \mathrm{kg}$ & 39 & EPA8270 \\
\hline$\mu \mathrm{g} / \mathrm{kg}$ & 39 & EPA8270 \\
\hline$\mu \mathrm{g} / \mathrm{kg}$ & 39 & EPA8270 \\
\hline$\mu \mathrm{g} / \mathrm{kg}$ & 39 & EPA8270M \\
\hline$\mu \mathrm{g} / \mathrm{kg}$ & 39 & EPA8270 \\
\hline$\mu \mathrm{g} / \mathrm{kg}$ & 39 & EPA8270M \\
\hline$\mu \mathrm{g} / \mathrm{kg}$ & 39 & EPA8270 \\
\hline$\mu \mathrm{g} / \mathrm{kg}$ & 39 & EPA8270M \\
\hline$\mu \mathrm{g} / \mathrm{kg}$ & 39 & EPA8270 \\
\hline$\mu \mathrm{g} / \mathrm{kg}$ & 39 & EPA8270 \\
\hline$\mu \mathrm{g} / \mathrm{kg}$ & 39 & EPA8270 \\
\hline$\mu \mathrm{g} / \mathrm{kg}$ & 39 & EPA8270 \\
\hline$\mu \mathrm{g} / \mathrm{kg}$ & 39 & EPA8270 \\
\hline$\mu \mathrm{g} / \mathrm{kg}$ & 39 & EPA8270 \\
\hline$\mu \mathrm{g} / \mathrm{kg}$ & 39 & EPA8270M \\
\hline$\mu \mathrm{g} / \mathrm{kg}$ & 39 & EPA8270 \\
\hline$\mu g / \mathbf{k g}$ & 39 & EPA8270 \\
\hline$\mu \mathrm{g} / \mathrm{kg}$ & 39 & EPA8270 \\
\hline$\mu g / \mathrm{kg}$ & 78 & EPA8270 \\
\hline$\mu \mathrm{g} / \mathrm{kg}$ & 39 & EPA8270M \\
\hline$\mu \mathrm{g} / \mathrm{kg}$ & 39 & EPA8270 \\
\hline$\mu \mathrm{g} / \mathrm{kg}$ & 39 & EPA8270M \\
\hline$\mu \mathrm{g} / \mathrm{kg}$ & 39 & EPA8270M \\
\hline$\mu \mathrm{g} / \mathrm{kg}$ & 195 & EPA8270M \\
\hline$\mu \mathrm{g} / \mathrm{kg}$ & 39 & EPA8270 \\
\hline$\mu \mathrm{g} / \mathrm{kg}$ & 39 & EPA8270 \\
\hline$\mu \mathrm{g} / \mathrm{kg}$ & 39 & EPA8270 \\
\hline$\mu \mathrm{g} / \mathrm{kg}$ & 39 & EPA8270 \\
\hline$\mu \mathrm{g} / \mathrm{kg}$ & 39 & EPA8270 \\
\hline$\mu \mathrm{g} / \mathrm{kg}$ & 39 & EPA8270M \\
\hline$\mu \mathrm{g} / \mathrm{kg}$ & 39 & EPA8270M \\
\hline$\mu \mathrm{g} / \mathrm{kg}$ & 39 & EPA8270M \\
\hline
\end{tabular}


SURVEY ID: PPSC 0403D (continued)

Constituent

$\mathbf{R Q}$

AQ B

B Result

Unit

D. Limit Method

$B / N / A$ Extractables (including $P A H$ and phenols)

Hexachloroethane

Indeno[1,2,3-c,d]pyrene

Isophorone

2-Methyl-4,6-dinitrophenol

2-Methylnaphthalene

Naphthalene

m-Nitroaniline

o-Nitroaniline

p-Nitroaniline

Nitrobenzene

2-Nitrophenol

4-Nitrophenol

N-Nitrosodiphenylamine

N-Nitrosodi-n-propylamine

Pentachlorophenol

Phenanthrene

Phenol

Pyrene

1,2,4-Trichlorobenzene

2,4,5-Trichlorophenol

2,4,6-Trichlorophenol

Unknown

Unknown

$\begin{array}{lll}U & Y & 39 \\ U & Y & 39 \\ U & Y & 39 \\ U & Y & 195 \\ U & Y & 39 \\ U & Y & 39 \\ U & Y & 195 \\ U & Y & 195 \\ U & Y & 195 \\ U & Y & 39 \\ U & Y & 39 \\ U & Y & 195 \\ U & Y & 39 \\ U & Y & 39 \\ U & Y & 195 \\ U & Y & 39 \\ U & Y & 39 \\ U & Y & 39 \\ U & Y & 39 \\ U & Y & 195 \\ U & Y & 39 \\ J & N & 50 \\ U J & N V & 90\end{array}$

Volatile Organic Compounds

$\begin{array}{lll}\mu \mathrm{g} / \mathrm{kg} & 39 & \text { EPA8270M } \\ \mu \mathrm{g} / \mathrm{kg} & 39 & \text { EPA8270 } \\ \mu \mathrm{g} / \mathrm{kg} & 39 & \text { EPA8270 } \\ \mu \mathrm{g} / \mathrm{kg} & 195 & \text { EPA8270M } \\ \mu \mathrm{g} / \mathrm{kg} & 39 & \text { EPA8270 } \\ \mu \mathrm{g} / \mathrm{kg} & 39 & \text { EPA8270 } \\ \mu \mathrm{g} / \mathrm{kg} & 195 & \text { EPA8270M } \\ \mu \mathrm{g} / \mathrm{kg} & 195 & \text { EPA8270M } \\ \mu \mathrm{g} / \mathrm{kg} & 195 & \text { EPA8270M } \\ \mu \mathrm{g} / \mathrm{kg} & 39 & \text { EPA8270 } \\ \mu \mathrm{g} / \mathrm{kg} & 39 & \text { EPA8270M } \\ \mu \mathrm{g} / \mathrm{kg} & 195 & \text { EPA8270M } \\ \mu \mathrm{g} / \mathrm{kg} & 39 & \text { EPA8270M } \\ \mu \mathrm{g} / \mathrm{kg} & 39 & \text { EPA8270 } \\ \mu \mathrm{g} / \mathrm{kg} & 195 & \text { EPA8270M } \\ \mu \mathrm{g} / \mathrm{kg} & 39 & \text { EPA8270 } \\ \mu \mathrm{g} / \mathrm{kg} & 39 & \text { EPA8270M } \\ \mu \mathrm{g} / \mathrm{kg} & 39 & \text { EPA8270 } \\ \mu \mathrm{g} / \mathrm{kg} & 39 & \text { EPA8270M } \\ \mu \mathrm{g} / \mathrm{kg} & 195 & \text { EPA8270M } \\ \mu \mathrm{g} / \mathrm{kg} & 39 & \text { EPA8270 } \\ \mu \mathrm{g} / \mathrm{kg} & & \text { EPA8270 } \\ \mu \mathrm{g} / \mathrm{kg} & & \text { EPA8270 }\end{array}$

$\begin{array}{lll}\mu \mathrm{g} / \mathrm{kg} & 12 & \text { EPA8260 } \\ \mu \mathrm{g} / \mathrm{kg} & 12 & \text { EPA8260 } \\ \mu \mathrm{g} / \mathrm{kg} & 0.108 & \text { EPA8021 } \\ \mu \mathrm{g} / \mathrm{kg} & 0.24 & \text { EPA8260 } \\ \mu \mathrm{g} / \mathrm{kg} & 0.24 & \text { EPA8260 } \\ \mu \mathrm{g} / \mathrm{kg} & 2.4 & \text { EPA8260 } \\ \mu \mathrm{g} / \mathrm{kg} & 2.4 & \text { EPA8260 } \\ \mu \mathrm{g} / \mathrm{kg} & 0.72 & \text { EPA8260 } \\ \mu \mathrm{g} / \mathrm{kg} & 0.72 & \text { EPA8260 } \\ \mu \mathrm{g} / \mathrm{kg} & 2.4 & \text { EPA8260 } \\ \mu \mathrm{g} / \mathrm{kg} & 2.4 & \text { EPA8260 } \\ \mu \mathrm{g} / \mathrm{kg} & 0.12 & \text { EPA8021 } \\ \mu \mathrm{g} / \mathrm{kg} & 0.36 & \text { EPA8260 } \\ \mu \mathrm{g} / \mathrm{kg} & 0.36 & \text { EPA8260 } \\ \mu \mathrm{g} / \mathrm{kg} & 0.24 & \text { EPA8260 } \\ \mu \mathrm{g} / \mathrm{kg} & 0.24 & \text { EPA8260 } \\ \mu \mathrm{g} / \mathrm{kg} & 0.24 & \text { EPA8021 } \\ \mu \mathrm{g} / \mathrm{kg} & 0.24 & \text { EPA8260 } \\ \mu \mathrm{g} / \mathrm{kg} & 0.24 & \text { EPA8260 } \\ \mu \mathrm{g} / \mathrm{kg} & 0.36 & \text { EPA8260 } \\ \mu \mathrm{g} / \mathrm{kg} & 0.36 & \text { EPA8260 } \\ \mu \mathrm{g} / \mathrm{kg} & 0.84 & \text { EPA8260 } \\ \mu \mathrm{g} / \mathrm{kg} & 0.84 & \text { EPA8260 } \\ \mu \mathrm{g} / \mathrm{kg} & 0.12 & \text { EPA8260 } \\ \mu \mathrm{g} / \mathrm{kg} & 0.12 & \text { EPA8260 } \\ \mu \mathrm{g} / \mathrm{kg} & 0.24 & \text { EPA8260 } \\ \mu \mathrm{g} / \mathrm{kg} & 0.24 & \text { EPA8260 } \\ \mu \mathrm{g} / \mathrm{kg} & 0.6 & \text { EPA8260 } \\ \mu \mathrm{g} / \mathrm{kg} & 0.6 & \text { EPA8260 }\end{array}$

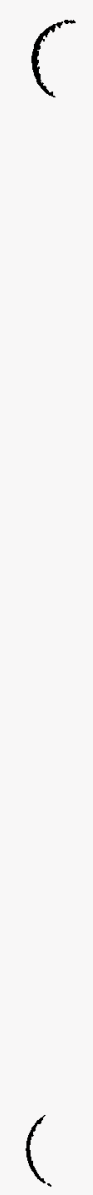


SURVEY ID: PPSC 0403D (continued)

Constituent

RQ AQ B Result

Sample ID: 104649

Volatile Organic Compounds

1,2-Dichloroethylene
1,2-Dichloroethylene
Dichloromethane (Methylene chloride)
1,2-Dichloropropane
cis-1,3-Dichloropropene
cis-1,3-Dichloropropene
trans-1,3-Dichloropropene
trans-1,3-Dichloropropene
Ethylbenzene
2-Hexanone
2-Hexanone
Methyl ethyl ketone
Methyl ethyl ketone
Methyl isobutyl ketone
Methyl isobutyl ketone
Styrene
1,1,2,2-Tetrachloroethane
Tetrachloroethylene
Tetrachloroethylene
Toluene
1,1,1-Trichloroethane
1,1,1-Trichloroethane
1,1,2-Trichloroethane
1,1,2-Trichloroethane
Trichloroethylene
Unknown
Vinyl acetate
Vinyl acetate
Xylenes
Xylenes

\begin{tabular}{|c|c|c|c|}
\hline $\mathrm{J}$ & $\mathrm{Y}$ & & 2.4 \\
\hline UJJ & YO & $\mathrm{H}$ & 2.4 \\
\hline & $\mathrm{Y}$ & & 0.24 \\
\hline & $Y$ & & 0.07 \\
\hline & $\mathrm{Y}$ & & 6 \\
\hline $\mathrm{JJ}$ & $\mathrm{YO}$ & $\mathrm{H}$ & 6 \\
\hline & $\mathrm{Y}$ & & 6 \\
\hline JJ & YO & $\mathrm{H}$ & 6 \\
\hline & $Y$ & & 0.06 \\
\hline & $\bar{Y}$ & & 12 \\
\hline & YO & $\mathrm{H}$ & 12 \\
\hline & $\mathrm{Y}$ & & 12 \\
\hline & YO & $\mathrm{H}$ & 12 \\
\hline & $\mathrm{Y}$ & & 12 \\
\hline & YO & $\mathrm{H}$ & 12 \\
\hline & $\mathrm{Y}$ & & 0.12 \\
\hline & $Y$ & & 0.12 \\
\hline & $\mathrm{Y}$ & & 0.48 \\
\hline $\mathrm{J}$ & YO & $\mathrm{H}$ & 0.48 \\
\hline & $\mathrm{Y}$ & & 0.12 \\
\hline & $\mathrm{Y}$ & & 0.36 \\
\hline 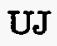 & YO & $\mathrm{H}$ & 0.36 \\
\hline $\mathrm{U}$ & $\mathrm{Y}$ & & 0.24 \\
\hline [JJ & YO & $\mathrm{H}$ & 0.24 \\
\hline & $\mathrm{Y}$ & & 0.12 \\
\hline & $\mathrm{N}$ & & 3 \\
\hline & $Y$ & & 12 \\
\hline & YO & $\mathrm{H}$ & 12 \\
\hline & $\bar{Y}$ & & 0.48 \\
\hline & YO & 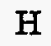 & 0.48 \\
\hline
\end{tabular}

Pesticides/PCBs and Dioxins/Furans

Aldrin
alpha-Benzene hexachloride
beta-Benzene hexachloride
delta-Benzene hexachloride
alpha-Chlordane
gamma-Chlordane
p,p'-DDD
p,p'-DDE
p,p'-DDT
Dieldrin
Endosulfan sulfate
Endosulfan I
Endosulfan II
Endrin
Endrin ketone
Heptachlor
Heptachlor epoxide
Lindane
Methoxychlor
PCB 1016
PCB 1221
PCB 1232

$\begin{array}{lll}U & Y & 2 \\ U & Y & 2 \\ U & Y & 2 \\ U & Y & 2 \\ U & Y & 2 \\ U & Y & 2 \\ U & Y & 3.85 \\ U & Y & 3.08 \\ U & Y & 3.85 \\ U & Y & 1.54 \\ U & Y & 3.85 \\ U & Y & 2 \\ U & Y & 3.08 \\ U & Y & 3.85 \\ U & Y & 3.85 \\ U & Y & 2 \\ U & Y & 2 \\ U & Y & 2 \\ U & Y & 19.7 \\ U & Y & 38.2 \\ U & Y & 77.5 \\ U & Y & 38.2\end{array}$

Unit D. Limit Method

$\begin{array}{lll}\mu \mathrm{g} / \mathrm{kg} & 2 & \text { EPA8080 } \\ \mu \mathrm{g} / \mathrm{kg} & 2 & \text { EPA8080 } \\ \mu \mathrm{g} / \mathrm{kg} & 2 & \text { EPA8080 } \\ \mu \mathrm{g} / \mathrm{kg} & 2 & \text { EPA8080 } \\ \mu \mathrm{g} / \mathrm{kg} & 2 & \text { EPA8080 } \\ \mu \mathrm{g} / \mathrm{kg} & 2 & \text { EPA8080 } \\ \mu \mathrm{g} / \mathrm{kg} & 3.85 & \text { EPA8080 } \\ \mu \mathrm{g} / \mathrm{kg} & 3.08 & \text { EPA8080 } \\ \mu \mathrm{g} / \mathrm{kg} & 3.85 & \text { EPA8080 } \\ \mu \mathrm{g} / \mathrm{kg} & 1.54 & \text { EPA8080 } \\ \mu \mathrm{g} / \mathrm{kg} & 3.85 & \text { EPA8080 } \\ \mu \mathrm{g} / \mathrm{kg} & 2 & \text { EPA8080 } \\ \mu \mathrm{g} / \mathrm{kg} & 3.08 & \text { EPA8080 } \\ \mu \mathrm{g} / \mathrm{kg} & 3.85 & \text { EPA8080 } \\ \mu \mathrm{g} / \mathrm{kg} & 3.85 & \text { EPA8080 } \\ \mu \mathrm{g} / \mathrm{kg} & 2 & \text { EPA8080 } \\ \mu \mathrm{g} / \mathrm{kg} & 2 & \text { EPA8080 } \\ \mu \mathrm{g} / \mathrm{kg} & 2 & \text { EPA8080 } \\ \mu \mathrm{g} / \mathrm{kg} & -19.7 & \text { EPA8080 } \\ \mu \mathrm{g} / \mathrm{kg} & 38.2 & \text { EPA8080 } \\ \mu \mathrm{g} / \mathrm{kg} & 77.5 & \text { EPA8080 } \\ \mu \mathrm{g} / \mathrm{kg} & 38.2 & \text { EPA8080 }\end{array}$


SURVEY ID: PPSC 0403D (continued)

Constituent

RQ AQ B Result

Unit

Pesticides/PCBs and Dioxins/Furans

PCB 1242

PCB 1248

PCB 1254

PCB 1260

Toxaphene

$\begin{array}{lll}\mathrm{U} & \mathrm{Y} & 38.2 \\ \mathrm{U} & \mathrm{Y} & 38.2 \\ \mathrm{U} & \mathrm{Y} & 38.2 \\ \mathrm{U} & \mathrm{Y} & 38.2 \\ \mathrm{U} & \mathrm{Y} & 185\end{array}$

38.2

38.2

38.2

185

$\begin{array}{lll}\mu \mathrm{g} / \mathrm{kg} & 38.2 & \text { EPA8080 } \\ \mu \mathrm{g} / \mathrm{kg} & 38.2 & \text { EPA8080 } \\ \mu \mathrm{g} / \mathrm{kg} & 38.2 & \text { EPA8080 } \\ \mu \mathrm{g} / \mathrm{kg} & 38.2 & \text { EPA8080 } \\ \mu \mathrm{g} / \mathrm{kg} & 185 & \text { EPA8080 }\end{array}$

Radionuclides

Actinium-228

Antimony-124 UI

Antimony-125 UI

Barium-133 UI

Cerium-144 UI

Cesium-134 UI

Cesium-137 UI

Cobalt-57 UI

Cobalt-58 UI

Cobalt-60 UI

Europium-152 UI

Europium-154 UI

Europium-155 J

Gross alpha

Iodine-129 UI

Lead-212

Manganese-54 UI

Neptunium-239 UI

Nonvolatile beta

Plutonium-238 UI

Plutonium-239/240 UI

Potassium-40 UI

Promethium-144 UI

Promethium-146 UI

Ruthenium-106 UI

Sodium-22 UI

Strontium-90 UI

Thorium-234

Tin-113

Yttrium-88 UI

Zinc-65 UI

Zirconium-95 UI $1.00 \mathrm{E}-02 \pm 4.00 \mathrm{E}-02$

$-1.00 \mathrm{E}-02 \pm 7.00 \mathrm{E}-02$ $2.00 \mathrm{E}-02 \pm 4.00 \mathrm{E}-02$ $8.00 \mathrm{E}-02 \pm 1.90 \mathrm{E}-01$ $1.00 \mathrm{E}-02 \pm 4.00 \mathrm{E}-02$ $1.00 \mathrm{E}-02 \pm 3.00 \mathrm{E}-02$ $-1.00 \mathrm{E}-02 \pm 2.00 \mathrm{E} \cdot 02$ $-1.00 \mathrm{E}-02 \pm 4.00 \mathrm{E}-02$ $3.00 \mathrm{E}-02 \pm 3.00 \mathrm{E}-02$ $1.20 \mathrm{E}-01 \pm 2.30 \mathrm{E}-01$ $-7.00 \mathrm{E}-02 \pm 8.00 \mathrm{E}-02$ $1.80 \mathrm{E}-01 \pm 1.20 \mathrm{E}-01$ $1.05 \mathrm{E}+01 \pm 2.70 \mathrm{E}+00$ $1.30 \mathrm{E}-01 \pm 1.40 \mathrm{E}-01$ $1.10 \mathrm{E}+00 \pm 9.00 \mathrm{E}-02$ $1.00 \mathrm{E}-02 \pm 3.00 \mathrm{E}-02$ $6.80 \mathrm{E}-01 \pm 1.20 \mathrm{E}+00$ $4.80 \mathrm{E}+00 \pm 4.40 \mathrm{E}+00$ $1.10 \mathrm{E}-01 \pm 1.40 \mathrm{E}-01$ $-1.00 \mathrm{E}-02 \pm 1.00 \mathrm{E}-02$ $3.30 \mathrm{E}-01 \pm 4.60 \mathrm{E}-01$ $2.00 \mathrm{E}-02 \pm 3.00 \mathrm{E}-02$ $1.00 \mathrm{E}-02 \pm 3.00 \mathrm{E}-02$ $0.00 \mathrm{E}+00 \pm 2.90 \mathrm{E} \cdot 01$ $-2.00 \mathrm{E}-02 \pm 3.00 \mathrm{E}-02$ $2.00 \mathrm{E}-02 \pm 3.70 \mathrm{E}-01$ $1.90 \mathrm{E}+00 \pm 1.30 \mathrm{E}+00$ $7.00 \mathrm{E}-02 \pm 5.00 \mathrm{E}-02$ $3.00 \mathrm{E}-02 \pm 3.00 \mathrm{E}-02$ $2.00 \mathrm{E}-02 \pm 7.00 \mathrm{E}-02$ $-3.00 \mathrm{E}-02 \pm 6.00 \mathrm{E}-02$

Sample ID: 104646
$9.70 \mathrm{E}-01 \pm 1.40 \mathrm{E}-01$
$\mathrm{pCi} / \mathrm{g}$

$\mathrm{pCi} / \mathrm{g}$

$\mathrm{pCi} / \mathrm{g}$

$\mathrm{pCi} / \mathrm{g}$

pCi/g

pCi/g

pCi/g

$\mathrm{pCi} / \mathrm{g}$

$\mathrm{pCi} / \mathrm{g}$

$\mathrm{pCi} / \mathrm{g}$

$\mathrm{pCi} / \mathrm{g}$

$\mathrm{pCi} / \mathrm{g}$

$\mathrm{pCi} / \mathrm{g}$

$\mathrm{pCi} / \mathrm{g}$

$\mathrm{pCi} / \mathrm{g}$

$\mathrm{pCi} / \mathrm{g}$

$\mathrm{pCi} / \mathrm{g}$

$\mathrm{pCi} / \mathrm{g}$

$\mathrm{pCi} / \mathrm{g}$

$\mathrm{pCi} / \mathrm{g}$

$\mathrm{pCi} / \mathrm{g}$

$\mathrm{pCi} / \mathrm{g}$

$\mathrm{pCi} / \mathrm{g}$

$\mathrm{pCi} / \mathrm{g}$

$\mathrm{pCi} / \mathrm{g}$

$\mathrm{pCi} / \mathrm{g}$

$\mathrm{pCi} / \mathrm{g}$

$\mathrm{pCi} / \mathrm{g}$

$\mathrm{pCi} / \mathrm{g}$

$\mathrm{pCi} / \mathrm{g}$

$\mathrm{pCi} / \mathrm{g}$

$\mathrm{pCi} / \mathrm{g}$
2.00E-01 LANLER130MOD 6.00E-02 LANLER130MOD

1.20E.01 LANLER130MOD

6.00E-02 LANLER130MOD

3.10E-01 LANLER130MOD

5.00E-02 LANLER130MOD

6.00E-02 LANLER130MOD

4.00E.02 LANLER130MOD

6.00E-02 LANLER130MOD

5.00E-02 LANLER 130MOD

4.50E-01 LANLERI30MOD

1.30E-01 LANLER130MOD

1.90E.01 LANLER130MOD

2.58E+00 LANLMLR100MOD

2.20E-01 LANLER130MOD

6.00E-02 LANLER130MOD

6.00E-02 LANLER130MOD

$1.90 \mathrm{E}+00$ LAANLER130MOD

$1.60 \mathrm{E}+00$ LANLMLRIOOMOD

2.20E-01 EMLPU02MOD

1.60E-01 EMLPU02MOD

5.00E-01 LANLER130MOD

6.00E-02 LANLER130MOD

6.00E-02 LANLER130MOD

4.90E-01 LANLER130MOD

5.00E-02 LANLER130MOD

9.60E-01 EMLSR02MOD

$1.40 \mathrm{E}+00$ LANLER130MOD

5.00E-02 LANLER130MOD

6.00E-02 LANLER130MOD

1.20E-01 LANLER130MOD

1.00E-01 LANLER130MOD
D. Limit Method

EPA8080

SURVEY ID: PPSC 0404

Survey location: 45135E $3678345 \mathrm{~N}$ (SRS coordinates)

Depth of core interval: 4.00 to $5.80 \mathrm{ft}$

Sample type: Normal

Sample matrix: Soil

Percent solids: $\mathbf{8 5 . 0 0}$

Constituent

RQ AQ B Result

Unit

D. Limit Method

Physical Parameters and Specified Analyses

Cation exchange capacity

Chloride

Cyanide

Cyanide

$\begin{array}{lll} & & 51.1 \\ \mathrm{~J} & \mathrm{E} & 1.64 \\ \mathrm{U} & & 1.13 \\ \mathrm{U} & & 1.11\end{array}$

$\begin{array}{ll}\mathrm{meq} / 100 \mathrm{~g} & 5 \\ \mathrm{mg} / \mathrm{kg} & 2.93 \\ \mathrm{mg} / \mathrm{kg} & 1.13 \\ \mathrm{mg} / \mathrm{kg} & 1.11\end{array}$

EPA9081

EPA300.0

EPA335.3

EPA335.3 
SURVEY ID: PPSC 0404 (continued) Sample ID: 104646

Constituent

RQ AQ B Result

Unit

D. Limit Method

Physical Parameters and Specified Analyses

Nitrate-nitrite as nitrogen

Orthophosphate

Phenols

Sulfide

Total organic carbon

Total organic nitrogen

Total phosphates (as P)

$\begin{array}{lll}\text { J } & \text { E } & 0.683 \\ J & \text { E } & 3.43 \\ \text { UJ } & \text { C } & 348 \\ \text { UJ } & \text { C } & 294 \\ & & 821 \\ \text { C } & & 41.8 \\ \text { J } & \text { E } & 91.3\end{array}$

Metals (total recoverable)
5160

UJ C $\quad 5.5$

$\mathrm{U} \quad \mathbf{1 6 . 5}$

J E $\quad 0.0869$

$\begin{array}{lll}J & E & 0.0869 \\ \mathrm{U} & & 0.275\end{array}$

24

7.34

$\begin{array}{lll}J & \mathrm{E} & 0.243\end{array}$

J $\mathrm{E} \quad 1.35$

J C 1420

J E 4.02

78.7

5.95

$\begin{array}{lll}\mathrm{U} & \mathrm{V} & \mathbf{0 . 0 1 7}\end{array}$

$\begin{array}{ll}\mathrm{J} & \mathrm{E}\end{array}$

$\mathrm{U} \quad 16.5$

U $\quad 1.37$

J $\mathrm{E} \quad 10.4$

UJ C 16.5

7.34

3.54

$\begin{array}{lll}\mathrm{mg} / \mathrm{kg} & 0.975 & \text { EPA353.1 } \\ \mathrm{mg} / \mathrm{kg} & 11.5 & \text { EPA365.2 } \\ \mu \mathrm{g} / \mathrm{kg} & 348 & \text { EPA420.2 } \\ \mathrm{mg} / \mathrm{kg} & 294 & \text { EPA376.2 } \\ \mathrm{mg} / \mathrm{kg} & 118 & \text { EPA415.1 } \\ \mathrm{mg} / \mathrm{kg} & 0.235 & \text { EPA351.2 } \\ \mathrm{mg} / \mathrm{kg} & 168 & \text { EPA365.4 }\end{array}$

$\begin{array}{lll}\mathrm{mg} / \mathrm{kg} & 13.7 & \text { EPA6010A } \\ \mathrm{mg} / \mathrm{kg} & 5.5 & \text { EPA6010A } \\ \mathrm{mg} / \mathrm{kg} & 16.5 & \text { EPA6010A } \\ \mathrm{mg} / \mathrm{kg} & 1.37 & \text { EPA6010A } \\ \mathrm{mg} / \mathrm{kg} & 0.55 & \text { EPA6010A } \\ \mathrm{mg} / \mathrm{kg} & 0.275 & \text { EPA6010A } \\ \mathrm{mg} / \mathrm{kg} & 13.7 & \text { EPA6010A } \\ \mathrm{mg} / \mathrm{kg} & 1.37 & \text { EPA6010A } \\ \mathrm{mg} / \mathrm{kg} & 0.55 & \text { EPA6010A } \\ \mathrm{mg} / \mathrm{kg} & 1.37 & \text { EPA6010A } \\ \mathrm{mg} / \mathrm{kg} & 5.5 & \text { EPA6010A } \\ \mathrm{mg} / \mathrm{kg} & 5.5 & \text { EPA6010A } \\ \mathrm{mg} / \mathrm{kg} & 5.5 & \text { EPA6010A } \\ \mathrm{mg} / \mathrm{kg} & 0.55 & \text { EPA6010A } \\ \mathrm{mg} / \mathrm{kg} & 0.039 & \text { EPA7471 } \\ \mathrm{mg} / \mathrm{kg} & 1.37 & \text { EPA6010A } \\ \mathrm{mg} / \mathrm{kg} & 110 & \text { EPA6010A } \\ \mathrm{mg} / \mathrm{kg} & 16.5 & \text { EPA6010A } \\ \mathrm{mg} / \mathrm{kg} & 1.37 & \text { EPA6010A } \\ \mathrm{mg} / \mathrm{kg} & 55 & \text { EPA6010A } \\ \mathrm{mg} / \mathrm{kg} & 16.5 & \text { EPA6010A } \\ \mathrm{mg} / \mathrm{kg} & 0.55 & \text { EPA6010A } \\ \mathrm{mg} / \mathrm{kg} & 1.1 & \text { EPA6010A }\end{array}$

$B / N / A$ Extractables (including $P A H$ and phenols)

$\begin{array}{lll}\text { Acenaphthene } & \mathrm{U} & 38.8 \\ \text { Acenaphthylene } & \mathrm{U} & 38.8 \\ \text { Anthracene } & \mathrm{U} & 38.8 \\ \text { Benzo[a]anthracene } & \mathrm{U} & 3.88 \\ \text { Benzo[b]fluoranthene } & \mathrm{U} & 3.88 \\ \text { Benzo[k]fluoranthene } & \mathrm{U} & 3.88 \\ \text { Benzoic acid } & \mathrm{U} & 38.8 \\ \text { Benzo[g,h,i]perylene } & \mathrm{U} & 38.8 \\ \text { Benzo[a]pyrene } & \mathrm{U} & 3.88 \\ \text { Benzyl alcohol } & \mathrm{U} & 38.8 \\ \text { Bis(2-chloroethoxy) methane } & \mathrm{U} & 38.8\end{array}$

$\begin{array}{lll}\mu \mathrm{g} / \mathrm{kg} & 38.8 & \text { EPA8270 } \\ \mu \mathrm{g} / \mathrm{kg} & 38.8 & \text { EPA8270 } \\ \mu \mathrm{g} / \mathrm{kg} & 38.8 & \text { EPA8270 } \\ \mu \mathrm{g} / \mathrm{kg} & 3.88 & \text { EPA8270 } \\ \mu \mathrm{g} / \mathrm{kg} & 3.88 & \text { EPA8270 } \\ \mu \mathrm{g} / \mathrm{kg} & 3.88 & \text { EPA8270 } \\ \mu \mathrm{g} / \mathrm{kg} & 38.8 & \text { EPA8270 } \\ \mu \mathrm{g} / \mathrm{kg} & 38.8 & \text { EPA8270 } \\ \mu \mathrm{g} / \mathrm{kg} & 3.88 & \text { EPA8270 } \\ \mu \mathrm{g} / \mathrm{kg} & 38.8 & \text { EPA8270 } \\ \mu \mathrm{g} / \mathrm{kg} & 38.8 & \text { EPA8270 }\end{array}$


Appendix D: Sediment Tables

D-62

SURVEY ID: PPSC 0404 (continued)

Constituent
Sample ID: 104646

RQ AQ B Result Unit D. Limit Method

$B / N / A$ Extractables (including $P A H$ and phenols)

\begin{tabular}{|c|c|c|c|c|c|}
\hline Bis(2-chloroethyl) ether & $\mathrm{U}$ & 38.8 & $\mu \mathrm{g} / \mathrm{kg}$ & 38.8 & EPA8270 \\
\hline Bis(2-chloroisopropyl) ether & $\mathrm{U}$ & 38.8 & $\mu \mathrm{g} / \mathrm{kg}$ & 38.8 & EPA8270 \\
\hline Bis(2-ethylhexyl) phthalate & $\mathbf{U}$ & 38.8 & $\mu \mathrm{g} / \mathrm{kg}$ & 38.8 & EPA8270 \\
\hline 4-Bromophenyl phenyl ether & $\mathbf{U}$ & 38.8 & $\mu \mathrm{g} / \mathrm{kg}$ & 38.8 & EPA8270 \\
\hline Butylbenzyl phthalate & $\mathrm{U}$ & 38.8 & $\mu \mathrm{g} / \mathrm{kg}$ & 38.8 & EPA8270 \\
\hline 4-Chloroaniline & $\mathrm{U}$ & 38.8 & $\mu \mathrm{g} / \mathrm{kg}$ & 38.8 & EPA8270 \\
\hline 4-Chloro-m-cresol & $\mathrm{U}$ & 38.8 & $\mu \mathrm{g} / \mathrm{kg}$ & 38.8 & EPA8270 \\
\hline 2-Chloronaphthalene & $\mathbf{U}$ & 38.8 & $\mu \mathrm{g} / \mathrm{kg}$ & 38.8 & EPA8270 \\
\hline 2-Chlorophenol & $\mathrm{U}$ & 38.8 & $\mu \mathrm{g} / \mathrm{kg}$ & 38.8 & EPA8270 \\
\hline 4-Chlorophenyl phenyl ether & $\mathrm{U}$ & 38.8 & $\mu \mathrm{g} / \mathrm{kg}$ & 38.8 & EPA8270 \\
\hline Chrysene & $\mathrm{U}$ & 3.88 & $\mu \mathrm{g} / \mathrm{kg}$ & 3.88 & EPA8270 \\
\hline $\mathrm{m} / \mathrm{p}-$ Cresol & $\mathrm{U}$ & 388 & $\mu \mathrm{g} / \mathrm{kg}$ & 388 & EPA8270 \\
\hline o-Cresol (2-Methylphenol) & $\mathrm{U}$ & 38.8 & $\mu \mathrm{g} / \mathrm{kg}$ & 38.8 & EPA8270 \\
\hline Dibenz $[a, h]$ anthracene & $\mathrm{U}$ & 3.88 & $\mu \mathrm{g} / \mathrm{kg}$ & 3.88 & EPA8270 \\
\hline Dibenzofuran & U & 38.8 & $\mu \mathrm{g} / \mathrm{kg}$ & 38.8 & EPA8270 \\
\hline Di-n-butyl phthalate & $\mathrm{U}$ & 44.3 & $\mu \mathrm{g} / \mathrm{kg}$ & 38.8 & EPA8270 \\
\hline 1,2-Dichlorobenzene & $\mathrm{U}$ & 38.8 & $\mu \mathrm{g} / \mathrm{kg}$ & 38.8 & EPA8270 \\
\hline 1,3-Dichlorobenzene & $\mathrm{U}$ & 38.8 & $\mu \mathrm{g} / \mathrm{kg}$ & 38.8 & EPA8270 \\
\hline 1,4-Dichlorobenzene & $\mathrm{U}$ & 38.8 & $\mu \mathrm{g} / \mathrm{kg}$ & 38.8 & EPA8270 \\
\hline 3,3'-Dichlorobenzidine & $\mathrm{U}$ & 38.8 & $\mu \mathrm{g} / \mathrm{kg}$ & 38.8 & EPA8270 \\
\hline 2,4-Dichlorophenol & $\mathrm{U}$ & 38.8 & $\mu \mathrm{g} / \mathrm{kg}$ & 38.8 & EPA8270 \\
\hline Diethyl phthalate & $U$ & 38.8 & $\mu \mathrm{g} / \mathrm{kg}$ & 38.8 & EPA8270 \\
\hline 2,4-Dimethyl phenol & $\mathrm{U}$ & 38.8 & $\mu \mathrm{g} / \mathrm{kg}$ & 38.8 & EPA8270 \\
\hline Dimethyl phthalate & $\mathrm{U}$ & 38.8 & $\mu \mathrm{g} / \mathrm{kg}$ & 38.8 & EPA8270 \\
\hline 2,4-Dinitrophenol & $\mathrm{U}$ & 388 & $\mu \mathrm{g} / \mathrm{kg}$ & 388 & EPA8270 \\
\hline 2,4-Dinitrotoluene & UJ & 3.88 & $\mu \mathrm{g} / \mathrm{kg}$ & 3.88 & EPA8270 \\
\hline 2,6-Dinitrotoluene & U & 3.88 & $\mu \mathrm{g} / \mathrm{kg}$ & 3.88 & EPA8270 \\
\hline Di-n-octyl phthalate & U & 38.8 & $\mu \mathrm{g} / \mathrm{kg}$ & 38.8 & EPA8270 \\
\hline Fluoranthene & U & 38.8 & $\mu \mathrm{g} / \mathrm{kg}$ & 38.8 & EPA8270 \\
\hline Fluorene & $\mathrm{U}$ & 38.8 & $\mu \mathrm{g} / \mathrm{kg}$ & 38.8 & EPA8270 \\
\hline Hexachlorobenzene & $\mathrm{U}$ & 3.88 & $\mu \mathrm{g} / \mathrm{kg}$ & 3.88 & EPA8270 \\
\hline Hexachlorobutadiene & $\mathbf{U}$ & 38.8 & $\mu \mathrm{g} / \mathrm{kg}$ & 38.8 & EPA8270 \\
\hline Hexachlorocyclopentadiene & $\mathrm{U}$ & 38.8 & $\mu \mathrm{g} / \mathrm{kg}$ & 38.8 & EPA8270 \\
\hline Hexachloroethane & $\mathrm{U}$ & 3.88 & $\mu \mathrm{g} / \mathrm{kg}$ & 3.88 & EPA8270 \\
\hline Indeno $[1,2,3-c, d]$ pyrene & $\mathrm{U}$ & 3.88 & $\mu \mathrm{g} / \mathrm{kg}$ & 3.88 & EPA8270 \\
\hline Isophorone & $\mathrm{U}$ & 38.8 & $\mu \mathrm{g} / \mathrm{kg}$ & 38.8 & EPA8270 \\
\hline 2-Methyl-4,6-dinitrophenol & $\mathrm{U}$ & 388 & $\mu \mathrm{g} / \mathrm{kg}$ & 388 & EPA8270 \\
\hline 2-Methylnaphthalene & $\mathrm{U}$ & 38.8 & $\mu \mathrm{g} / \mathrm{kg}$ & 38.8 & EPA8270 \\
\hline Naphthalene & $\mathrm{U}$ & 38.8 & $\mu \mathrm{g} / \mathrm{kg}$ & 38.8 & EPA8270 \\
\hline m-Nitroaniline & $\mathrm{U}$ & 38.8 & $\mu \mathrm{g} / \mathrm{kg}$ & 38.8 & EPA8270 \\
\hline o-Nitroaniline & $\mathrm{U}$ & 38.8 & $\mu \mathrm{g} / \mathrm{kg}$ & 38.8 & EPA8270 \\
\hline p-Nitroaniline & $\mathrm{U}$ & 38.8 & $\mu \mathrm{g} / \mathrm{kg}$ & 38.8 & EPA8270 \\
\hline Nitrobenzene & $\mathrm{U}$ & 38.8 & $\mu \mathrm{g} / \mathrm{kg}$ & 38.8 & EPA8270 \\
\hline 2-Nitrophenol & $\mathrm{U}$ & 38.8 & $\mu \mathrm{g} / \mathrm{kg}$ & 38.8 & EPA8270 \\
\hline 4-Nitrophenol & $\mathrm{U}$ & 38.8 & $\mu \mathrm{g} / \mathrm{kg}$ & 38.8 & EPA8270 \\
\hline N-Nitrosodiphenylamine & $\mathrm{U}$ & 38.8 & $\mu \mathrm{g} / \mathrm{kg}$ & 38.8 & EPA8270 \\
\hline N-Nitrosodi-n-propylamine & U & 38.8 & $\mu \mathrm{g} / \mathrm{kg}$ & 38.8 & EPA8270 \\
\hline Octadecanoic acid, butyl ester & $\mathbf{N}$ & 400 & $\mu \mathrm{g} / \mathrm{kg}$ & & EPA8270 \\
\hline Pentachlorophenol & $\mathrm{U}$ & 38.8 & $\mu \mathrm{g} / \mathrm{kg}$ & 38.8 & EPA8270 \\
\hline Phenanthrene & $\mathrm{U}$ & 38.8 & $\mu \mathrm{g} / \mathrm{kg}$ & 38.8 & EPA8270 \\
\hline Phenol & $\mathrm{U}$ & 38.8 & $\mu \mathrm{g} / \mathrm{kg}$ & 38.8 & EPA8270 \\
\hline Pyrene & $\mathrm{U}$ & 38.8 & $\mu \mathrm{g} / \mathrm{kg}$ & 38.8 & EPA8270 \\
\hline 1,2,4-Trichlorobenzene & U & 3.88 & $\mu \mathrm{g} / \mathrm{kg}$ & 3.88 & EPA8270 \\
\hline 2,4,5-Trichlorophenol & $\mathrm{U}$ & 38.8 & $\mu \mathrm{g} / \mathrm{kg}$ & 38.8 & EPA8270 \\
\hline 2,4,6-Trichlorophenol & $U$ & 38.8 & $\mu \mathrm{g} / \mathrm{kg}$ & 38.8 & EPA8270 \\
\hline
\end{tabular}


SURVEY ID: PPSC 0404 (continued)

\section{Constituent}

Volatile Organic Compounds

Acetone
Benzene
Bromodichloromethane
Bromoform
Bromomethane (Methyl bromide)
Carbon disulfide
Carbon tetrachloride
Chlorobenzene
Chloroethane
Chloroethene (Vinyl chloride)
Chloroform
Chloromethane (Methyl chloride)
Dibromochloromethane
1,1-Dichloroethane
1,2-Dichloroethane
1,1-Dichloroethylene
1,2-Dichloroethylene
Dichloromethane (Methylene chloride)
1,2-Dichloropropane
cis-1,3-Dichloropropene
trans-1,3-Dichloropropene
Ethylbenzene
2-Hexanone
Methyl ethyl ketone
Methyl isobutyl ketone
Styrene
1,1,2,2-Tetrachloroethane
Tetrachloroethylene
Toluene
1,1,1-Trichloroethane
1,1,2-Trichloroethane
Trichloroethylene
Vinyl acetate
Xylenes
(n)

Pesticides/PCBs and Dioxins/Furans
Sample ID: 104646

RQ AQ B Result Unit D. Limit Method

\begin{tabular}{|c|c|c|c|c|}
\hline $\mathrm{U}$ & 24.1 & $\mu \mathrm{g} / \mathrm{kg}$ & 5.88 & EPA8260 \\
\hline $\mathrm{U}$ & 0.588 & $\mu \mathrm{g} / \mathrm{kg}$ & 0.588 & EPA8260 \\
\hline $\mathrm{U}$ & 0.0588 & $\mu \mathrm{g} / \mathrm{kg}$ & 0.0588 & EPA8260 \\
\hline $\mathrm{U}$ & 0.0588 & $\mu \mathrm{g} / \mathrm{kg}$ & 0.0588 & EPA8260 \\
\hline $\mathrm{U}$ & 0.118 & $\mu \mathrm{g} / \mathrm{kg}$ & 0.118 & EPA8260 \\
\hline $\mathbf{U}$ & 1.18 & $\mu \mathrm{g} / \mathrm{kg}$ & 1.18 & EPA8260 \\
\hline $\mathrm{U}$ & 0.0588 & $\mu \mathrm{g} / \mathrm{kg}$ & 0.0588 & EPA8260 \\
\hline $\mathbf{U}$ & 0.0588 & $\mu \mathrm{g} / \mathrm{kg}$ & 0.0588 & EPA8260 \\
\hline $\mathrm{U}$ & 0.118 & $\mu \mathrm{g} / \mathrm{kg}$ & 0.118 & EPA8260 \\
\hline $\mathbf{U}$ & 0.118 & $\mu \mathrm{g} / \mathrm{kg}$ & 0.118 & EPA8260 \\
\hline$U$ & 0.0588 & $\mu g / k g$ & 0.0588 & EPA8260 \\
\hline $\mathrm{U}$ & 0.118 & $\mu \mathrm{g} / \mathrm{kg}$ & 0.118 & EPA8260 \\
\hline $\mathrm{U}$ & 0.0588 & $\mu g / \mathrm{kg}$ & 0.0588 & EPA8260 \\
\hline $\mathrm{U}$ & 0.0588 & $\mu \mathrm{g} / \mathrm{kg}$ & 0.0588 & EPA8260 \\
\hline $\mathrm{U}$ & 0.0588 & $\mu \mathrm{g} / \mathrm{kg}$ & 0.0588 & EPA8260 \\
\hline $\mathrm{U}$ & 0.0588 & $\mu \mathrm{g} / \mathrm{kg}$ & 0.0588 & EPA8260 \\
\hline $\mathrm{U}$ & 0.118 & $\mu \mathrm{g} / \mathrm{kg}$ & 0.118 & EPA8260 \\
\hline $\mathrm{U}$ & 9.66 & $\mu \mathrm{g} / \mathrm{kg}$ & 0.588 & EPA8260 \\
\hline $\mathrm{U}$ & 0.0588 & $\mu \mathrm{g} / \mathrm{kg}$ & 0.0588 & EPA8260 \\
\hline $\mathrm{U}$ & 0.0588 & $\mu \mathrm{g} / \mathrm{kg}$ & 0.0588 & EPA8260 \\
\hline $\mathrm{U}$ & 0.0588 & $\mu \mathrm{g} / \mathrm{kg}$ & 0.0588 & EPA8260 \\
\hline $\mathrm{U}$ & 0.0588 & $\mu \mathrm{g} / \mathrm{kg}$ & 0.0588 & EPA8260 \\
\hline $\mathrm{U}$ & 1.18 & $\mu \mathrm{g} / \mathrm{kg}$ & 1.18 & EPA8260 \\
\hline $\mathrm{U}$ & 1.16 & $\mu \mathrm{g} / \mathrm{kg}$ & 1.18 & EPA8260 \\
\hline $\mathrm{U}$ & 1.18 & $\mu \mathrm{g} / \mathrm{kg}$ & 1.18 & EPA8260 \\
\hline $\mathrm{U}$ & 0.0588 & $\mu \mathrm{g} / \mathrm{kg}$ & 0.0588 & EPA8260 \\
\hline $\mathrm{U}$ & 0.0588 & $\mu \mathrm{g} / \mathrm{kg}$ & 0.0588 & EPA8260 \\
\hline $\mathrm{U}$ & 0.0588 & $\mu \mathrm{g} / \mathrm{kg}$ & 0.0588 & EPA8260 \\
\hline $\mathbf{U}$ & 0.129 & $\mu \mathrm{g} / \mathrm{kg}$ & 0.588 & EPA8260 \\
\hline $\mathrm{U}$ & 0.0588 & $\mu \mathrm{g} / \mathrm{kg}$ & 0.0588 & EPA8260 \\
\hline $\mathrm{U}$ & 0.0588 & $\mu \mathrm{g} / \mathrm{kg}$ & 0.0588 & EPA8260 \\
\hline $\mathrm{U}$ & 0.0588 & $\mu g / \mathrm{kg}$ & 0.0588 & EPA8260 \\
\hline $\mathrm{U}$ & 1.18 & $\mu g / \mathrm{kg}$ & 1.18 & EPA8260 \\
\hline $\mathbf{U}$ & 0.176 & $\mu g / \mathrm{kg}$ & 0.176 & EPA8260 \\
\hline
\end{tabular}

Aldrin
alpha-Benzene hexachloride
beta-Benzene hexachloride
delta-Benzene hexachloride
alpha-Chlordane
gamma-Chlordane
p,p'-DDD
p,p'-DDE
p,p'-DDT
Dieldrin
Endosulfan sulfate
Endosulfan I
Endosulfan II
Endrin
Endrin ketone
Heptachlor
Heptachlor epoxide
Lindane

$\begin{array}{llll}\text { UJ } & \text { O } & \text { L } & \mathbf{0 . 9 6 9} \\ \text { UJ } & \text { O } & \text { L } & 0.969 \\ \text { UJ } & \text { O } & \text { L } & 1.94 \\ \text { UJ } & \text { O } & \text { L } & 1.94 \\ \text { UJ } & \text { O } & \text { L } & 1.94 \\ \text { UJ } & \text { O } & \text { L } & 1.94 \\ \text { UJ } & \text { O } & \text { L } & 3.88 \\ \text { UJ } & \text { O } & \text { L } & 1.94 \\ \text { UJ } & \text { O } & \text { L } & 3.88 \\ \text { UJ } & \text { O } & \text { L } & 1.94 \\ \text { UJ } & \text { O } & \text { L } & 3.88 \\ \text { UJ } & \text { O } & \text { L } & 1.94 \\ \text { UJ } & \text { O } & \text { L } & 3.88 \\ \text { UJ } & \text { O } & \text { L } & 1.94 \\ \text { UJ } & \text { O } & \text { L } & 3.88 \\ \text { UJ } & \text { O } & \text { L } & 1.94 \\ \text { UJ } & \text { O } & \text { L } & 1.94 \\ \text { UJ } & \text { O } & \text { L } & 0.969\end{array}$

$\begin{array}{rll}\mu \mathrm{g} / \mathrm{kg} & 0.969 & \text { EPA8080 } \\ \mu \mathrm{g} / \mathrm{kg} & 0.969 & \text { EPA8080 } \\ \mu \mathrm{g} / \mathrm{kg} & 1.94 & \text { EPA8080 } \\ \mu \mathrm{g} / \mathrm{kg} & 1.94 & \text { EPA8080 } \\ \mu \mathrm{g} / \mathrm{kg} & 1.94 & \text { EPA8080 } \\ \mu \mathrm{g} / \mathrm{kg} & 1.94 & \text { EPA8080 } \\ \mu \mathrm{g} / \mathrm{kg} & 3.88 & \text { EPA8080 } \\ \mu \mathrm{g} / \mathrm{kg} & 1.94 & \text { EPA8080 } \\ \mu \mathrm{g} / \mathrm{kg} & 3.88 & \text { EPA8080 } \\ \mu \mathrm{g} / \mathrm{kg} & 1.94 & \text { EPA8080 } \\ \mu \mathrm{g} / \mathrm{kg} & 3.88 & \text { EPA8080 } \\ \mu \mathrm{g} / \mathrm{kg} & 1.94 & \text { EPA8080 } \\ \mu \mathrm{g} / \mathrm{kg} & 3.88 & \text { EPA8080 } \\ \mu \mathrm{g} / \mathrm{kg} & 1.94 & \text { EPA8080 } \\ \mu \mathrm{g} / \mathrm{kg} & .3 .88 & \text { EPA8080 } \\ \mu \mathrm{g} / \mathrm{kg} & 1.94 & \text { EPA8080 } \\ \mu \mathrm{g} / \mathrm{kg} & 1.94 & \text { EPA8080 } \\ \mu \mathrm{g} / \mathrm{kg} & 0.969 & \text { EPA8080 }\end{array}$


SURVEY ID: PPSC 0404 (continued)

Constituent

Pesticides/PCBs and Dioxins/Furans

Methoxychlor

PCB 1016

PCB 1221

PCB 1232

PCB 1242

PCB 1248

PCB 1254

PCB 1260

Toxaphene

Radionuclides

Actinium-228

Antimony-124

Antimony-125

Barium-133

Cerium-144

Cesium-134

Cesium-137

Cobalt-57

Cobalt-58

Cobalt-60

Europium-152

Europium-154

Europium-155

Gross alpha

Lead-212

Manganese-54

Neptunium-239

Nonvolatile beta

Plutonium-238

Plutonium-239/240

Potassium-40

Promethium-144

Promethium-146

Ruthenium-106

Sodium-22

Strontium-90

Thorium-234

Tin-113

Yttrium-88

Zinc-65

Zirconium-95

Sample ID: 104646

RQ AQ B Result Unit D. Limịt Method

$\begin{array}{llll}\text { UJ } & \text { O } & \text { L } & 19.4 \\ \text { UJ } & \text { O } & \text { L } & 4.84 \\ \text { UJ } & \text { O } & \text { L } & 4.84 \\ \text { UJ } & \text { O } & \text { L } & 4.84 \\ \text { UJ } & \text { O } & \text { L } & 4.84 \\ \text { UJ } & \text { O } & \text { L } & 4.84 \\ \text { UJ } & \text { O } & \text { L } & 4.84 \\ \text { UJ } & \text { O } & \text { L } & 4.84 \\ \text { UJ } & \text { O } & \text { L } & 38.8\end{array}$

$\begin{array}{lll}\mu \mathrm{g} / \mathrm{kg} & 19.4 & \text { EPA8080 } \\ \mu \mathrm{g} / \mathrm{kg} & 4.84 & \text { EPA8080 } \\ \mu \mathrm{g} / \mathrm{kg} & 4.84 & \text { EPA8080 } \\ \mu \mathrm{g} / \mathrm{kg} & 4.84 & \text { EPA8080 } \\ \mu \mathrm{g} / \mathrm{kg} & 4.84 & \text { EPA8080 } \\ \mu \mathrm{g} / \mathrm{kg} & 4.84 & \text { EPA8080 } \\ \mu \mathrm{g} / \mathrm{kg} & 4.84 & \text { EPA8080 } \\ \mu \mathrm{g} / \mathrm{kg} & 4.84 & \text { EPA8080 } \\ \mu \mathrm{g} / \mathrm{kg} & 38.8 & \text { EPA8080 }\end{array}$

$1.71 \mathrm{E}+00 \pm 8.02 \mathrm{E}-02 \mathrm{pCi} / \mathrm{g}$

2.77E-03 $\pm 9.14 \mathrm{E}-03 \mathrm{pCi} / \mathrm{g}$ $-4.50 \mathrm{E}-03 \pm 1.91 \mathrm{E}-02 \mathrm{pCi} / \mathrm{g}$ $-4.93 \mathrm{E}-03 \pm 9.29 \mathrm{E}-03 \mathrm{pCi} / \mathrm{g}$ $-3.97 \mathrm{E}-02 \pm 5.20 \mathrm{E}-02 \mathrm{pCi} / \mathrm{g}$ $-9.15 \mathrm{E}-02 \pm 8.85 \mathrm{E}-03 \mathrm{pCi} / \mathrm{g}$ $-4.39 \mathrm{E}-03 \pm 7.88 \mathrm{E}-03 \mathrm{pCi} / \mathrm{g}$ $-2.61 \mathrm{E}-03 \pm 6.53 \mathrm{E}-03 \mathrm{pCi} / \mathrm{g}$ $-4.90 \mathrm{E}-03 \pm 8.12 \mathrm{E}-03 \mathrm{pCj} / \mathrm{g}$ $2.86 \mathrm{E}-03 \pm 5.65 \mathrm{E}-03 \mathrm{pCi} / \mathrm{g}$ $1.56 \mathrm{E}-03 \pm 1.96 \mathrm{E}-02 \mathrm{pCi} / \mathrm{g}$ $-8.51 \mathrm{E}-03 \pm 5.95 \mathrm{E}-02 \mathrm{pCi} / \mathrm{g}$ $0.00 \mathrm{E}+00 \quad \mathrm{pCi} / \mathrm{g}$ $5.21 \mathrm{E}+01 \pm 5.77 \mathrm{E}+00 \mathrm{pCi} / \mathrm{g}$ $1.75 \mathrm{E}+00 \pm 3.49 \mathrm{E}-02 \mathrm{pCi} / \mathrm{g}$ 4.30E-02+8.28E-03 pCi/g 8.17E-03 $\pm 5.06 \mathrm{E}-02 \mathrm{pCi} / \mathrm{g}$ $2.34 \mathrm{E}+01 \pm 2.77 \mathrm{E}+00 \mathrm{pCi} / \mathrm{g}$ 1.49E-02 $\pm 2.14 \mathrm{E}-02 \mathrm{pCi} / \mathrm{g}$ $1.20 \mathrm{E}-02 \pm 1.42 \mathrm{E}-02 \mathrm{pCi} / \mathrm{g}$ 7.60E-01 $1.48 \mathrm{E}-01 \mathrm{pCi} / \mathrm{g}$ 2.35E-03 $\pm 6.77 \mathrm{E}-03 \mathrm{pCi} / \mathrm{g}$ $1.27 \mathrm{E}-02 \pm 1.13 \mathrm{E}-02 \mathrm{pCi} / \mathrm{g}$ 6.03E-03 $16.25 \mathrm{E}-02 \mathrm{pCi} / \mathrm{g}$ $-5.71 \mathrm{E}-03 \pm 7.37 \mathrm{E}-03 \mathrm{pCi} / \mathrm{g}$ $6.79 \mathrm{E}-01 \pm 8.47 \mathrm{E}-02 \mathrm{pCi} / \mathrm{g}$ $1.57 \mathrm{E}+00 \pm 6.96 \mathrm{E}-01 \mathrm{pCi} / \mathrm{g}$ $-3.31 \mathrm{E}-03 \pm 1.04 \mathrm{E}-02 \mathrm{pCj} / \mathrm{g}$ $-1.34 \mathrm{E}-03 \pm 7.65 \mathrm{E}-03 \mathrm{pCi} / \mathrm{g}$ $-4.63 \mathrm{E}-03 \pm 1.43 \mathrm{E}-02 \mathrm{pCi} / \mathrm{g}$ $0.00 \mathrm{E}+00 \quad \mathrm{pCi} / \mathrm{g}$
3.61E-02 EPIA-013B

1.61E-02 EPIA-013B

3.11E-02 EPIA-013B

1.34E-02 EPIA-013B

8.83E-02 EPLA-013B

1.08E-02 EPIA-013B

1.17E-02 EPIA-013B

1.12E-02 EPIA-013B

1.36E-02 EPIA-013B

1.05E-02 EPIA-013B

3.26E-02 EPIA-013B

8.83E-02 EPIA-013B

4.65E-02 EPIA-013B

$1.56 \mathrm{E}+00$ EPIA-001B

2.06E-02 EPIA-013B

9.77E-03 EPIA-013B

7.89E-02 EPIA-013B

2.61E+00 EPIA-001B

4.05E-02 EPIA-012B

2.31E-02 EPIA-012B

9.53E-02 EPIA-013B

1.18E-02 EPIA-013B

1.55E-02 EPIA-013B

1.09E-01 EPIA-013B

1.00E-02 EPIA-013B

5.60E-02 EPIA-004

5.97E-01 EPIA-013B

1.69E-02 EPIA-013B

1.34E-02 EPIA-013B

2.07E-02 EPIA-013B

3.07E-02 EPIA-013B

SURVEY ID: PPSC 0404A

Sample ID: 104650

Survey location: $45135 \mathrm{E} 3678345 \mathrm{~N}$ (SRS coordinates)

Depth of core interval: 4.00 to $5.80 \mathrm{ft}$

Sample type: Normal

Sample matrix: Other

Percent solids: $\mathbf{8 6 . 0 0}$ 
SURVEY ID: PPSC 0404A (continued)

Sample ID: 104650

Constituent

RQ AQ B Result

Unit

D. Limit Method

Physical Parameters and Specified Analyses

Cation exchange capacity

Cation exchange capacity

Chloride

Cyanide

Nitrate-nitrite as nitrogen

Orthophosphate

Phenols

Sulfide

Total organic carbon

Total organic nitrogen

Total phosphates (as P)

Metals (total recoverable)

Aluminum

Aluminum

Antimony

Antimony

Arsenic

Arsenic

Barium

Barium

Beryllium

Beryllium

Cadmium

Cadmium

Calcium

Calcium

Chromium

Chromium

Cobalt

Cobalt

Copper

Copper

Iron

Iron

Lead

Lead

Magnesium

Magnesium

Manganese

Manganese

Mercury

Nickel

Nickel

Potassium

Potassium

Selenium

Selenium

Silver

Silver

Sodium

Sodium

Thallium

Thallium

$\begin{array}{lll} & & 68.8 \\ & & 73.9 \\ \text { J } & \text { E } & 1.45 \\ \text { U } & & 1.16 \\ & & 1.15 \\ \text { U } & & 11.3 \\ \text { UJ } & \text { C } & 346 \\ \text { UJ } & \text { C } & 291 \\ & & 551 \\ \text { C } & & 63.3 \\ \text { J } & \text { E } & 87.1\end{array}$

$\begin{array}{ll}\text { UJ } & \text { I } \\ \text { UJ } & \text { I } \\ \text { U } & \\ \text { U } & \\ & \text { X } \\ & \text { X } \\ \text { J } & \text { E } \\ \text { J } & \text { E } \\ \text { U } & \\ \text { U } & \end{array}$

2860

3500

5.43

$\begin{array}{ll}\text { L } & 5.43 \\ \text { L } & 4.76\end{array}$

16.3

14.3

14.56
$\times \quad 9.48$

$\mathrm{X} \quad 9.48$

0.0451

U $\quad 0.272$

0.238

26

20.6

4.03

4.34

$\begin{array}{ll}\mathrm{U} & 0.543 \\ \mathrm{~J} & \mathbf{0 . 2 2 8}\end{array}$

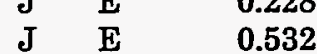

$\begin{array}{lll}J & \mathrm{E} & 0.728\end{array}$

450

623

$\mathrm{J} \quad \mathrm{E} \quad 4.27$

$\begin{array}{lll}\mathrm{J} & \mathrm{E} & 4.26\end{array}$

45.8

53.7

3.41

3.73

$\begin{array}{lll}\text { U } & \text { V } & \mathbf{0 . 0 2 1 4} \\ \text { J } & \text { E } & \mathbf{0 . 6 9 1}\end{array}$

$\begin{array}{lll}J & \mathrm{E} & 0.691 \\ J & \mathrm{E} & 0.996\end{array}$

J $\quad \mathrm{E} \quad 27.5$

$\begin{array}{lll}J & \mathrm{E} & 28.7\end{array}$

U $\quad 16.3$

$\mathrm{U} \quad 14.3$

U $\quad 1.36$

$\mathrm{U} \quad 1.19$

$\mathrm{U} \quad \mathbf{5 4 . 3}$

$\mathrm{U} \quad 47.6$

$\mathrm{U} \quad 16.3$

$\mathrm{U} \quad 14.3$

$\begin{array}{lll}\mathrm{meq} / 100 \mathrm{~g} & 5 & \text { EPA9081 } \\ \mathrm{meq} / 100 \mathrm{~g} & 5.81 & \text { EPA9081 } \\ \mathrm{mg} / \mathrm{kg} & 2.76 & \text { EPA300.0 } \\ \mathrm{mg} / \mathrm{kg} & 1.16 & \text { EPA335.3 } \\ \mathrm{mg} / \mathrm{kg} & 1.04 & \text { EPA353.1 } \\ \mathrm{mg} / \mathrm{kg} & 11.3 & \text { EPA365.2 } \\ \mu \mathrm{g} / \mathrm{kg} & 346 & \text { EPA420.2 } \\ \mathrm{mg} / \mathrm{kg} & 291 & \text { EPA376.2 } \\ \mathrm{mg} / \mathrm{kg} & 116 & \text { EPA415.1 } \\ \mathrm{mg} / \mathrm{kg} & 0.233 & \text { EPA351.2 } \\ \mathrm{mg} / \mathrm{kg} & 213 & \text { EPA365.4 }\end{array}$

$\mathrm{mg} / \mathrm{kg} \quad 13.6 \quad$ EPA6010A

$\mathrm{mg} / \mathrm{kg} \quad 11.9 \quad$ EPA6010A

$\mathrm{mg} / \mathrm{kg} \quad 5.43 \quad$ EPA6010A

$\mathrm{mg} / \mathrm{kg} \quad 4.76 \quad$ EPA6010A

$\mathrm{mg} / \mathrm{kg} \quad 16.3 \quad$ EPA6010A

$\mathrm{mg} / \mathrm{kg} \quad 14.3 \quad$ EPA6010A

$\mathrm{mg} / \mathrm{kg} \quad 1.36 \quad$ EPA6010A

$\begin{array}{lll}\mathrm{mg} / \mathrm{kg} & 1.19 \quad \text { EPA6010A }\end{array}$

$\mathrm{mg} / \mathrm{kg} \quad 0.543 \quad$ EPA6010A

$\mathrm{mg} / \mathrm{kg} \quad 0.476 \quad$ EPA6010A

$\mathrm{mg} / \mathrm{kg} \quad 0.272 \quad$ EPA6010A

$\begin{array}{lll}\mathrm{mg} / \mathrm{kg} & 0.238 \quad \text { EPA6010A }\end{array}$

$\mathrm{mg} / \mathrm{kg} \quad 13.6 \quad$ EPA6010A

$\begin{array}{lll}\mathrm{mg} / \mathrm{kg} & 11.9 \quad \text { EPA6010A }\end{array}$

$\begin{array}{lll}\mathrm{mg} / \mathrm{kg} & 1.36 \quad \text { EPA6010A }\end{array}$

$\mathrm{mg} / \mathrm{kg} \quad 1.19 \quad$ EPA6010A

$\mathrm{mg} / \mathrm{kg} \quad 0.543 \quad$ EPA6010A

$\mathrm{mg} / \mathrm{kg} \quad 0.476 \quad$ EPA6010A

$\mathrm{mg} / \mathrm{kg} \quad 1.36 \quad$ EPA6010A

$\begin{array}{lll}\mathrm{mg} / \mathrm{kg} & 1.19 \quad \text { EPA6010A }\end{array}$

$\mathrm{mg} / \mathrm{kg} \quad 5.43 \quad$ EPA6010A

$\mathrm{mg} / \mathrm{kg} \quad 4.76 \quad$ EPA6010A

$\mathrm{mg} / \mathrm{kg} \quad 5.43 \quad$ EPA6010A

$\mathrm{mg} / \mathrm{kg} \quad 4.76 \quad$ EPA6010A

$\mathrm{mg} / \mathrm{kg} \quad 5.43 \quad$ EPA6010A

$\mathrm{mg} / \mathrm{kg} \quad 4.76 \quad$ EPA6010A

$\mathrm{mg} / \mathrm{kg} \quad 0.543 \quad$ EPA6010A

$\mathrm{mg} / \mathrm{kg} \quad 0.476 \quad$ EPA6010A

$\mathrm{mg} / \mathrm{kg} \quad 0.0334 \quad$ EPA7471.

$\mathrm{mg} / \mathrm{kg} \quad 1.36 \quad$ EPA6010A

$\begin{array}{lll}\mathrm{mg} / \mathrm{kg} & 1.19 & \text { EPA6010A }\end{array}$

$\mathrm{mg} / \mathrm{kg} \quad 109 \quad$ EPA6010A

$\mathrm{mg} / \mathrm{kg} \quad 95.2 \quad$ EPA6010A

$\begin{array}{lll}\mathrm{mg} / \mathrm{kg} & 16.3 \quad \mathrm{EPA} 6010 \mathrm{~A}\end{array}$

$\mathrm{mg} / \mathrm{kg} \quad 14.3 \quad$ EPA6010A

$\mathrm{mg} / \mathrm{kg} \quad 1.36 \quad$ EPA6010A

$\mathrm{mg} / \mathrm{kg} \quad 1.19 \quad$ EPA6010A

$\begin{array}{lll}\mathrm{mg} / \mathrm{kg} & \mathbf{5 4 . 3} \quad \text { EPA6010A }\end{array}$

$\mathrm{mg} / \mathrm{kg} \quad 47.6 \quad$ EPA6010A

$\mathrm{mg} / \mathrm{kg} \quad 16.3 \quad$ EPA6010A

$\mathrm{mg} / \mathrm{kg} \quad 14.3 \quad$ EPA6010A 
SURVEY ID: PPSC 0404A (continued)

Constituent

RQ AQ B Result

3.68

Vanadium

Vanadium

Zinc

Zinc

$B / N / A$ Extractables (including $P A H$ and phenols)
4.28

1.81

2.03
Sample ID: 104650

Unit D. Limit Method

$\begin{array}{lll}\mathrm{mg} / \mathrm{kg} & 0.543 & \text { EPA6010A } \\ \mathrm{mg} / \mathrm{kg} & 0.476 & \text { EPA6010A } \\ \mathrm{mg} / \mathrm{kg} & 1.09 & \text { EPA6010A } \\ \mathrm{mg} / \mathrm{kg} & 0.952 & \text { EPA6010A }\end{array}$

\begin{tabular}{|c|c|c|c|c|c|}
\hline $\mathrm{U}$ & & 38.3 & $\mu \mathrm{g} / \mathrm{kg}$ & 38.3 & EPA8270 \\
\hline$U$ & & 38.3 & $\mu \mathrm{g} / \mathrm{kg}$ & 38.3 & EPA8270 \\
\hline $\mathrm{U}$ & & 38.3 & $\mu \mathrm{g} / \mathrm{kg}$ & 38.3 & EPA8270 \\
\hline$U$ & & 3.83 & $\mu \mathrm{g} / \mathrm{kg}$ & 3.83 & EPA8270 \\
\hline $\mathrm{U}$ & & 3.83 & $\mu \mathrm{g} / \mathrm{kg}$ & 3.83 & EPA8270 \\
\hline U & & 3.83 & $\mu \mathrm{g} / \mathrm{kg}$ & 3.83 & EPA8270 \\
\hline U & & 38.3 & $\mu \mathrm{g} / \mathrm{kg}$ & 38.3 & EPA8270 \\
\hline $\mathrm{U}$ & & 38.3 & $\mu \mathrm{g} / \mathrm{kg}$ & 38.3 & EPA8270 \\
\hline $\mathrm{U}$ & & 3.83 & $\mu \mathrm{g} / \mathrm{kg}$ & 3.83 & EPA8270 \\
\hline U & & 38.3 & $\mu \mathrm{g} / \mathrm{kg}$ & 38.3 & EPA8270 \\
\hline $\mathrm{U}$ & & 38.3 & $\mu \mathrm{g} / \mathrm{kg}$ & 38.3 & EPA8270 \\
\hline $\mathrm{U}$ & & 38.3 & $\mu \mathrm{g} / \mathrm{kg}$ & 38.3 & EPA8270 \\
\hline$U$ & & 38.3 & $\mu \mathrm{g} / \mathrm{kg}$ & 38.3 & EPA8270 \\
\hline$J$ & $\mathbf{E}$ & 20.7 & $\mu \mathrm{g} / \mathrm{kg}$ & 38.3 & EPA8270 \\
\hline $\mathrm{U}$ & & 38.3 & $\mu \mathrm{g} / \mathrm{kg}$ & 38.3 & EPA8270 \\
\hline $\mathbf{U}$ & & 38.3 & $\mu \mathrm{g} / \mathrm{kg}$ & 38.3 & EPA8270 \\
\hline U & & 38.3 & $\mu \mathrm{g} / \mathrm{kg}$ & 38.3 & EPA8270 \\
\hline $\mathrm{U}$ & & 38.3 & $\mu \mathrm{g} / \mathrm{kg}$ & 38.3 & EPA8270 \\
\hline U & & 38.3 & $\mu \mathrm{g} / \mathrm{kg}$ & 38.3 & EPA8270 \\
\hline $\mathrm{U}$ & & 38.3 & $\mu g / \mathrm{kg}$ & 38.3 & EPA8270 \\
\hline $\mathrm{U}$ & & 38.3 & $\mu \mathrm{g} / \mathrm{kg}$ & 38.3 & EPA8270 \\
\hline U & & 3.83 & $\mu \mathrm{g} / \mathrm{kg}$ & 3.83 & EPA8270 \\
\hline $\mathrm{U}$ & & 383 & $\mu \mathrm{g} / \mathrm{kg}$ & 383 & EPA8270 \\
\hline$U$ & & 38.3 & $\mu \mathrm{g} / \mathrm{kg}$ & 38.3 & EPA8270 \\
\hline $\mathrm{U}$ & & 3.83 & $\mu \mathrm{g} / \mathrm{kg}$ & 3.83 & EPA8270 \\
\hline U & & 38.3 & $\mu \mathrm{g} / \mathrm{kg}$ & 38.3 & EPA8270 \\
\hline $\mathrm{U}$ & V & 50.2 & $\mu \mathrm{g} / \mathrm{kg}$ & 38.3 & EPA8270 \\
\hline $\mathrm{U}$ & & 38.3 & $\mu \mathrm{g} / \mathrm{kg}$ & 38.3 & EPA8270 \\
\hline $\mathrm{U}$ & & 38.3 & $\mu \mathrm{g} / \mathrm{kg}$ & 38.3 & EPA8270 \\
\hline U & & 38.3 & $\mu \mathrm{g} / \mathrm{kg}$ & 38.3 & EPA8270 \\
\hline $\mathrm{U}$ & & 38.3 & $\mu \mathrm{g} / \mathrm{kg}$ & 38.3 & EPA8270 \\
\hline $\mathrm{U}$ & & 38.3 & $\mu \mathrm{g} / \mathrm{kg}$ & 38.3 & EPA8270 \\
\hline U & & 38.3 & $\mu \mathrm{g} / \mathrm{kg}$ & 38.3 & EPA8270 \\
\hline $\mathrm{U}$ & & 38.3 & $\mu \mathrm{g} / \mathrm{kg}$ & 38.3 & EPA8270 \\
\hline$U$ & & 38.3 & $\mu g / \mathrm{kg}$ & 38.3 & EPA8270 \\
\hline $\mathrm{U}$ & & 383 & $\mu g / \mathrm{kg}$ & 383 & EPA8270 \\
\hline UJ & $\mathrm{C}$ & 3.83 & $\mu g / \mathrm{kg}$ & 3.83 & EPA8270 \\
\hline $\mathrm{U}$ & & 3.83 & $\mu \mathrm{g} / \mathrm{kg}$ & 3.83 & EPA8270 \\
\hline U & & 38.3 & $\mu g / k g$ & 38.3 & EPA8270 \\
\hline $\mathrm{U}$ & & 38.3 & $\mu g / \mathrm{kg}$ & 38.3 & EPA8270 \\
\hline $\mathrm{U}$ & & 38.3 & $\mu \mathrm{g} / \mathrm{kg}$ & 38.3 & EPA8270 \\
\hline U & & 3.83 & $\mu \mathrm{g} / \mathrm{kg}$ & 3.83 & EPA8270 \\
\hline$U$ & & 38.3 & $\mu \mathrm{g} / \mathrm{kg}$ & 38.3 & EPA8270 \\
\hline $\mathrm{U}$ & & 38.3 & $\mu \mathrm{g} / \mathrm{kg}$ & 38.3 & EPA8270 \\
\hline $\mathrm{U}$ & & 3.83 & $\mu g / \mathrm{kg}$ & 3.83 & EPA8270 \\
\hline U & & 3.83 & $\mu \mathrm{g} / \mathrm{kg}$ & 3.83 & EPA8270 \\
\hline $\mathrm{U}$ & & 38.3 & $\mu g / \mathrm{kg}$ & 38.3 & EPA8270 \\
\hline U & & 383 & $\mu \mathrm{g} / \mathrm{kg}$ & 383 & EPA8270 \\
\hline
\end{tabular}


SURVEY ID: PPSC 0404A (continued)

Sample ID: 104650

Constituent

RQ AQ B Result

Unit

D. Limit Method

$B / N / A$ Extractables (including PAH and phenols)

$\begin{array}{lcc}\text { 2-Methylnaphthalene } & \mathrm{U} & 38.3 \\ \text { Naphthalene } & \mathrm{U} & 38.3 \\ \text { m-Nitroaniline } & \mathrm{U} & 38.3 \\ \text { 0-Nitroaniline } & \mathrm{U} & 38.3 \\ \text { p-Nitroaniline } & \mathrm{U} & 38.3 \\ \text { Nitrobenzene } & \mathrm{U} & 38.3 \\ \text { 2-Nitrophenol } & \mathrm{U} & 38.3 \\ \text { 4-Nitrophenol } & \mathrm{U} & 38.3 \\ \text { N-Nitrosodiphenylamine } & \mathrm{U} & 38.3 \\ \text { N-Nitrosodi-n-propylamine } & \mathrm{U} & 38.3 \\ \text { Pentachlorophenol } & \mathrm{U} & 38.3 \\ \text { Phenanthrene } & \mathrm{U} & 38.3 \\ \text { Phenol } & \mathrm{U} & 38.3 \\ \text { Pyrene } & \mathrm{U} & 38.3 \\ \text { 1,2,4-Trichlorobenzene } & \mathrm{U} & 3.83 \\ \text { 2,4,5-Trichlorophenol } & \mathrm{U} & 38.3 \\ \text { 2,4,6-Trichlorophenol } & \mathrm{U} & 38.3\end{array}$

$\begin{array}{lll}\mu \mathrm{g} / \mathrm{kg} & 38.3 & \text { EPA8270 } \\ \mu \mathrm{g} / \mathrm{kg} & 38.3 & \text { EPA8270 } \\ \mu \mathrm{g} / \mathrm{kg} & 38.3 & \text { EPA8270 } \\ \mu \mathrm{g} / \mathrm{kg} & 38.3 & \text { EPA8270 } \\ \mu \mathrm{g} / \mathrm{kg} & 38.3 & \text { EPA8270 } \\ \mu \mathrm{g} / \mathrm{kg} & 38.3 & \text { EPA8270 } \\ \mu \mathrm{g} / \mathrm{kg} & 38.3 & \text { EPA8270 } \\ \mu \mathrm{g} / \mathrm{kg} & 38.3 & \text { EPA8270 } \\ \mu \mathrm{g} / \mathrm{kg} & 38.3 & \text { EPA8270 } \\ \mu \mathrm{g} / \mathrm{kg} & 38.3 & \text { EPA8270 } \\ \mu \mathrm{g} / \mathrm{kg} & 38.3 & \text { EPA8270 } \\ \mu \mathrm{g} / \mathrm{kg} & 38.3 & \text { EPA8270 } \\ \mu \mathrm{g} / \mathrm{kg} & 38.3 & \text { EPA8270 } \\ \mu \mathrm{g} / \mathrm{kg} & 38.3 & \text { EPA8270 } \\ \mu \mathrm{g} / \mathrm{kg} & 3.83 & \text { EPA8270 } \\ \mu \mathrm{g} / \mathrm{kg} & 38.3 & \text { EPA8270 } \\ \mu \mathrm{g} / \mathrm{kg} & 38.3 & \text { EPA8270 }\end{array}$

Volatile Organic Compounds

Acetone
Benzene
Bromodichloromethane
Bromoform
Bromomethane (Methyl bromide)
Carbon disulfide
Carbon tetrachloride
Chlorobenzene
Chloroethane
Chloroethene (Vinyl chloride)
Chloroform
Chloromethane (Methyl chloride)
Dibromochloromethane
1,1-Dichloroethane
1,2-Dichloroethane
1,1-Dichloroethylene
1,2-Dichloroethylene
Dichloromethane (Methylene chloride)
1,2-Dichloropropane
cis-1,3-Dichloropropene
trans-1,3-Dichloropropene
Ethylbenzene
2-Hexanone
Methyl ethyl ketone
Methyl isobutyl ketone
Styrene
1,1,2,2-Tetrachloroethane
Tetrachloroethylene
Toluene
1,1,1-Trichloroethane
1,1,2-Trichloroethane
Trichloroethylene
Vinyl acetate
Xylenes

\begin{tabular}{|c|c|c|}
\hline $\begin{array}{l}\text { U } \\
\text { U }\end{array}$ & 8 & $\begin{array}{l}19.9 \\
0.581\end{array}$ \\
\hline $\mathrm{U}$ & & 0.0581 \\
\hline U & & 0.0581 \\
\hline$U$ & & 0.116 \\
\hline U & & 1.16 \\
\hline U & & 0.0581 \\
\hline U & & 0.0581 \\
\hline U & & 0.116 \\
\hline U & & 0.116 \\
\hline U & & 0.0581 \\
\hline U & & 0.116 \\
\hline U & & 0.0581 \\
\hline$U$ & & 0.0581 \\
\hline U & & 0.0581 \\
\hline $\mathrm{U}$ & & 0.0581 \\
\hline U & & 0.116 \\
\hline U & $\mathrm{V}$ & 8.84 \\
\hline IT & & 0.0581 \\
\hline U & & 0.0581 \\
\hline $\mathrm{U}$ & & 0.0581 \\
\hline U & & 0.058 \\
\hline $\mathrm{U}$ & & 1.16 \\
\hline U & V & 1.08 \\
\hline $\mathrm{U}$ & & 1.16 \\
\hline U & & 0.058 \\
\hline U & & 0.058 \\
\hline U & & 0.0581 \\
\hline U & & 0.581 \\
\hline $\mathrm{J}$ & & 0.058 \\
\hline & & 0.058 \\
\hline & & 0.058 \\
\hline & & 1.16 \\
\hline & & 0.174 \\
\hline
\end{tabular}

$\begin{array}{lll}\mu \mathrm{g} / \mathrm{kg} & 5.81 & \text { EPA8260 } \\ \mu \mathrm{g} / \mathrm{kg} & 0.581 & \text { EPA8260 } \\ \mu \mathrm{g} / \mathrm{kg} & 0.0581 & \text { EPA8260 } \\ \mu \mathrm{g} / \mathrm{kg} & 0.0581 & \text { EPA8260 } \\ \mu \mathrm{g} / \mathrm{kg} & 0.116 & \text { EPA8260 } \\ \mu \mathrm{g} / \mathrm{kg} & 1.16 & \text { EPA8260 } \\ \mu \mathrm{g} / \mathrm{kg} & 0.0581 & \text { EPA8260 } \\ \mu \mathrm{g} / \mathrm{kg} & 0.0581 & \text { EPA8260 } \\ \mu \mathrm{g} / \mathrm{kg} & 0.116 & \text { EPA8260 } \\ \mu \mathrm{g} / \mathrm{kg} & 0.116 & \text { EPA8260 } \\ \mu \mathrm{g} / \mathrm{kg} & 0.0581 & \text { EPA8260 } \\ \mu \mathrm{g} / \mathrm{kg} & 0.116 & \text { EPA8260 } \\ \mu \mathrm{g} / \mathrm{kg} & 0.0581 & \text { EPA8260 } \\ \mu \mathrm{g} / \mathrm{kg} & 0.0581 & \text { EPA8260 } \\ \mu \mathrm{g} / \mathrm{kg} & 0.0581 & \text { EPA8260 } \\ \mu \mathrm{g} / \mathrm{kg} & 0.0581 & \text { EPA8260 } \\ \mu \mathrm{g} / \mathrm{kg} & 0.116 & \text { EPA8260 } \\ \mu \mathrm{g} / \mathrm{kg} & 0.581 & \text { EPA8260 } \\ \mu \mathrm{g} / \mathrm{kg} & 0.0581 & \text { EPA8260 } \\ \mu \mathrm{g} / \mathrm{kg} & 0.0581 & \text { EPA8260 } \\ \mu \mathrm{g} / \mathrm{kg} & 0.0581 & \text { EPA8260 } \\ \mu \mathrm{g} / \mathrm{kg} & 0.0581 & \text { EPA8260 } \\ \mu \mathrm{g} / \mathrm{kg} & 1.16 & \text { EPA8260 } \\ \mu \mathrm{g} / \mathrm{kg} & 1.16 & \text { EPA8260 } \\ \mu \mathrm{g} / \mathrm{kg} & 1.16 & \text { EPA8260 } \\ \mu \mathrm{g} / \mathrm{kg} & 0.0581 & \text { EPA8260 } \\ \mu \mathrm{g} / \mathrm{kg} & 0.0581 & \text { EPA8260 } \\ \mu \mathrm{g} / \mathrm{kg} & 0.0581 & \text { EPA8260 } \\ \mu \mathrm{g} / \mathrm{kg} & 0.581 & \text { EPA8260 } \\ \mu \mathrm{g} / \mathrm{kg} & 0.0581 & \text { EPA8260 } \\ \mu \mathrm{g} / \mathrm{kg} & 0.0581 & \text { EPA8260 } \\ \mu \mathrm{g} / \mathrm{kg} & 0.0581 & \text { EPA8260 } \\ \mu \mathrm{g} / \mathrm{kg} & 1.16 & \text { EPA8260 } \\ \mu \mathrm{g} / \mathrm{kg} & 0.174 & \text { EPA8260 } \\ & & \end{array}$


SURVEY ID: PPSC 0404A (continued)

Constituent

RQ AQ B Result

Unit

D. Limit Method

Pesticides/PCBs and Dioxins/Furans

$\begin{array}{lllll}\text { Aldrin } & \text { UJ } & \text { O } & \text { L } & 0.957 \\ \text { alpha-Benzene hexachloride } & \text { UJ } & \text { O } & \text { L } & 0.957 \\ \text { beta-Benzene hexachloride } & \text { UJ } & \text { O } & \text { L } & 1.91 \\ \text { delta-Benzene hexachloride } & \text { UJ } & \text { O } & \text { L } & 1.91 \\ \text { alpha-Chlordane } & \text { UJ } & \text { O } & \text { L } & 1.91 \\ \text { gamma-Chlordane } & \text { UJ } & \text { O } & \text { L } & 1.91 \\ \text { p,p'-DDD } & \text { UJ } & \text { O } & \text { L } & 3.83 \\ \text { p,p'-DDE } & \text { UJ } & \text { O } & \text { L } & 1.91 \\ \text { p,p'-DDT } & \text { UJ } & \text { O } & \text { L } & 3.83 \\ \text { Dieldrin } & \text { UJ } & \text { O } & \text { L } & 1.91 \\ \text { Endosulfan sulfate } & \text { UJ } & \text { O } & \text { L } & 3.83 \\ \text { Endosulfan I } & \text { UJ } & \text { O } & \text { L } & 1.91 \\ \text { Endosulfan II } & \text { UJ } & \text { O } & \text { L } & 3.83 \\ \text { Endrin } & \text { UJ } & \text { O } & \text { L } & 1.91 \\ \text { Endrin ketone } & \text { UJ } & \text { O } & \text { L } & 3.83 \\ \text { Heptachlor } & \text { UJ } & \text { O } & \text { L } & 1.91 \\ \text { Heptachlor epoxide } & \text { UJ } & \text { O } & \text { L } & 1.91 \\ \text { Lindane } & \text { UJ } & \text { O } & \text { L } & 0.957 \\ \text { Methoxychlor } & \text { UJ } & \text { O } & \text { L } & 19.1 \\ \text { PCB 1016 } & \text { UJ } & \text { O } & \text { L } & 4.79 \\ \text { PCB 1221 } & \text { UJ } & \text { O } & \text { L } & 4.79 \\ \text { PCB 1232 } & \text { UJ } & \text { O } & \text { L } & 4.79 \\ \text { PCB 1242 } & \text { UJ } & \text { O } & \text { L } & 4.79 \\ \text { PCB 1248 } & \text { UJ } & \text { O } & \text { L } & 4.79 \\ \text { PCB 1254 } & \text { UJ } & \text { O } & \text { L } & 4.79 \\ \text { PCB 1260 } & \text { UJ } & \text { O } & \text { L } & 4.79 \\ \text { Toxaphene } & \text { UJ } & \text { O } & \text { L } & 38.3\end{array}$

$1.45 \mathrm{E}+00 \pm 6.23 \mathrm{E}-02 \mathrm{pCi} / \mathrm{g}$ $-1.79 \mathrm{E}-03 \pm 7.45 \mathrm{E}-03 \mathrm{pCi} / \mathrm{g}$ 9.26E-03 $\pm 1.45 \mathrm{E}-02 \mathrm{pCi} / \mathrm{g}$ $-2.22 \mathrm{E}-03 \pm 7.87 \mathrm{E}-03 \mathrm{pCi} / \mathrm{g}$ $-2.00 \mathrm{E}-02 \pm 4.46 \mathrm{E}-02 \mathrm{pCi} / \mathrm{g}$ $4.75 \mathrm{E}-03 \pm 5.70 \mathrm{E}-03 \mathrm{pCi} / \mathrm{g}$ 2.00E-04 $06.43 \mathrm{E}-03 \mathrm{pCj} / \mathrm{g}$ $-4.85 \mathrm{E}-03 \pm 5.64 \mathrm{E}-03 \mathrm{pCi} / \mathrm{g}$ $-2.55 \mathrm{E}-03 \pm 7.08 \mathrm{E}-03 \mathrm{pCi} / \mathrm{g}$ $1.88 \mathrm{E}-02 \pm 8.85 \mathrm{E}-03 \mathrm{pCi} / \mathrm{g}$ $1.81 \mathrm{E}-03 \pm 1.65 \mathrm{E}-02 \mathrm{pCi} / \mathrm{g}$ $-5.36 \mathrm{E}-02 \pm 4.50 \mathrm{E}-02 \mathrm{pCi} / \mathrm{g}$ $0.00 \mathrm{E}+00 \quad \mathrm{pCi} / \mathrm{g}$ $3.04 \mathrm{E}+01 \pm 4.40 \mathrm{E}+00 \mathrm{pCi} / \mathrm{g}$ $1.54 \mathrm{E}+00 \pm 3.01 \mathrm{E}-02 \mathrm{pCi} / \mathrm{g}$ $2.86 \mathrm{E}-02 \pm 9.08 \mathrm{E}-03 \mathrm{pCi} / \mathrm{g}$ $-1.18 \mathrm{E}-02 \pm 4.40 \mathrm{E}-02 \mathrm{pCi} / \mathrm{g}$ $1.07 \mathrm{E}+01 \pm 2.07 \mathrm{E}+00 \mathrm{pCi} / \mathrm{g}$ $2.36 \mathrm{E}-02 \pm 2.53 \mathrm{E}-02 \mathrm{pCi} / \mathrm{g}$ 6.30E-03 $\pm 1.19 \mathrm{E}-02 \mathrm{pCi} / \mathrm{g}$ $7.80 \mathrm{E}-01 \pm 1.23 \mathrm{E}-01 \mathrm{pCi} / \mathrm{g}$ $-2.14 \mathrm{E}-03 \pm 5.63 \mathrm{E}-03 \mathrm{pCi} / \mathrm{g}$ 8.20E-03 $\pm 8.42 \mathrm{E}-03 \mathrm{pCi} / \mathrm{g}$ $-4.39 \mathrm{E}-03 \pm 5.01 \mathrm{E}-02 \mathrm{pCi} / \mathrm{g}$ $4.52 \mathrm{E}-04 \pm 4.93 \mathrm{E}-03 \mathrm{pCi} / \mathrm{g}$
3.02E-02 EPIA-013B

1.26E-02 EPIA-013B

2.55E-02 EPIA-013B

1.13E-02 EPIA-013B

7.53E-02 EPIA-013B

8.84E-03 EPIA-013B

9.56E-03 EPIA-013B

9.51E-03 EPIA-013B

1.17E-02 EPIA-013B

7.77E-03 EPIA-013B

2.69E-02 EPIA-013B

7.46E-02 EPIA-013B

3.96E-02 EPIA-013B

$2.20 \mathrm{E}+00$ EPIA-001B

1.61E-02 EPIA-013B

9.55E-03 EPLA-013B

6.73E-02 EPIA-013B

2.79E+00 EPIA-001B

4.42E-02 EPIA-012B

2.43E-02 EPLA-012B

7.63E-02 EPIA-013B

9.35E-03 EPIA-013B

1.29E-02 EPIA-013B

8.49E-02 EPIA-013B

8.61E-03 EPIA-013B 
SURVEY ID: PPSC 0404A (continued)

Constituent

RQ AQ B Result

Unit

D. Limit Method

Radionuclides

Strontium-90

Thorium-234

Tin-113

Yttrium-88

Zinc-65

Zirconium-95

Sample ID: 104650

7.46E-02 EPIA-004

5.12E-01 EPLA-013B

1.36E-02 EPIA-013B

1.06E-02 EPIA-013B

1.82E-02 EPIA-013B

2.58E-02 EPIA-013B

$\begin{array}{llll} & \mathrm{V} & & 1.16 \mathrm{E}+00 \pm 1.26 \mathrm{E}-01 \mathrm{pCi} / \mathrm{g} \\ \text { U } & \mathrm{V} & 1.42 \mathrm{E}+00 \pm 6.49 \mathrm{E}-01 \mathrm{pCi} / \mathrm{g} \\ \text { UI } & & -2.39 \mathrm{E}-03 \pm 7.90 \mathrm{E}-03 \mathrm{pCi} / \mathrm{g} \\ \text { UI } & & -3.69 \mathrm{E}-04 \pm 5.94 \mathrm{E}-03 \mathrm{pCi} / \mathrm{g} \\ \text { UI } & & -6.02 \mathrm{E}-03 \pm 1.24 \mathrm{E}-02 \mathrm{pCi} / \mathrm{g} \\ \text { UI } & & 0.00 \mathrm{E}+00 & \mathrm{pCi} / \mathrm{g}\end{array}$

Sample ID: 104613

SURVEY ID: PPSC 0501

Survey location: 45166E 3678653N (SRS coordinates)

Depth of core interval: 0.00 to $1.00 \mathrm{ft}$

Sample type: Normal

Sample matrix: Soil

Percent solids: $\mathbf{8 2 . 0 0}$

Constituent

RQ AQ B Result

Unit

Physical Parameters and Specified Analyses

Cation exchange capacity
Chloride
Cyanide
Cyanide
Nitrate-nitrite as nitrogen
Nitrate-nitrite as nitrogen
Orthophosphate
Orthophosphate
Phenols
Sulfide
Total organic carbon
Total organic nitrogen
Total phosphates (as P)
Total phosphates (as P)

Metals (total recoverable)

$\begin{array}{llll}\text { J } & \text { E } & & 21 \\ \text { UJ } & \text { I } & \text { L } & 1.4 \\ \text { UJ } & \text { I } & \text { L } & 1.21 \\ \text { U } & & & 1.16 \\ \text { U } & & & 0.943 \\ \text { J } & \text { EI } & \text { L } & 9.57 \\ \text { J } & \text { EI } & \text { L } & 5.65 \\ \text { U } & & & 366 \\ \text { UJ } & \text { C } & & 267 \\ & & & 1920 \\ \text { C } & & & 67.9 \\ \text { J } & \text { E } & & 76.5 \\ \text { J } & \text { E } & & 71.3\end{array}$

$\begin{array}{lll} & & 2400 \\ \text { U } & & 5.92 \\ \text { U } & & 17.8 \\ & & 12.7 \\ \text { J } & \text { E } & 0.071 \\ \text { U } & & 0.296 \\ & \text { V } & 81.4 \\ \text { U } & \text { V } & 2.48 \\ \text { J } & \text { E } & 0.39 \\ \text { U } & \text { V } & 1.18 \\ & \text { V } & 1360 \\ \text { J } & \text { E } & 2.52 \\ & \text { V } & 64.7 \\ & \text { V } & 21.6 \\ \text { J } & \text { E } & 0.0161 \\ \text { J } & \text { E } & 0.00914 \\ \text { J } & \text { E } & 0.857 \\ \text { J } & \text { E } & 46.8\end{array}$

$\begin{array}{lll}\mathrm{meq} / 100 \mathrm{~g} & 5 & \text { EPA9081 } \\ \mathrm{mg} / \mathrm{kg} & 2.82 & \text { EPA300.0 } \\ \mathrm{mg} / \mathrm{kg} & 1.16 & \text { EPA335.3 } \\ \mathrm{mg} / \mathrm{kg} & 1.21 & \text { EPA335.3 } \\ \mathrm{mg} / \mathrm{kg} & 1.16 & \text { EPA353.1 } \\ \mathrm{mg} / \mathrm{kg} & 0.943 & \text { EPA353.1 } \\ \mathrm{mg} / \mathrm{kg} & 25.1 & \text { EPA365.2 } \\ \mathrm{mg} / \mathrm{kg} & 26.8 & \text { EPA365.2 } \\ \mu \mathrm{g} / \mathrm{kg} & 366 & \text { EPA420.2 } \\ \mathrm{mg} / \mathrm{kg} & 267 & \text { EPA376.2 } \\ \mathrm{mg} / \mathrm{kg} & 122 & \text { EPA415.1 } \\ \mathrm{mg} / \mathrm{kg} & 0.244 & \text { EPA351.2 } \\ \mathrm{mg} / \mathrm{kg} & 198 & \text { EPA365.4 } \\ \mathrm{mg} / \mathrm{kg} & 205 & \text { EPA365.4 }\end{array}$

$\begin{array}{lll}\mathrm{mg} / \mathrm{kg} & 14.8 & \text { EPA6010A } \\ \mathrm{mg} / \mathrm{kg} & 5.92 & \text { EPA6010A } \\ \mathrm{mg} / \mathrm{kg} & 17.8 & \text { EPA6010A } \\ \mathrm{mg} / \mathrm{kg} & 1.48 & \text { EPA6010A } \\ \mathrm{mg} / \mathrm{kg} & 0.592 & \text { EPA6010A } \\ \mathrm{mg} / \mathrm{kg} & 0.296 & \text { EPA6010A } \\ \mathrm{mg} / \mathrm{kg} & 14.8 & \text { EPA6010A } \\ \mathrm{mg} / \mathrm{kg} & 1.48 & \text { EPA6010A } \\ \mathrm{mg} / \mathrm{kg} & 0.592 & \text { EPA6010A } \\ \mathrm{mg} / \mathrm{kg} & 1.48 & \text { EPA6010A } \\ \mathrm{mg} / \mathrm{kg} & 5.92 & \text { EPA6010A } \\ \mathrm{mg} / \mathrm{kg} & 5.92 & \text { EPA6010A } \\ \mathrm{mg} / \mathrm{kg} & 5.92 & \text { EPA6010A } \\ \mathrm{mg} / \mathrm{kg} & 0.592 & \text { EPA6010A } \\ \mathrm{mg} / \mathrm{kg} & 0.0358 & \text { EPA7471 } \\ \mathrm{mg} / \mathrm{kg} & 0.0389 & \text { EPA7471 } \\ \mathrm{mg} / \mathrm{kg} & 1.48 & \text { EPA6010A } \\ \mathrm{mg} / \mathrm{kg} & 118 & \text { EPA6010A }\end{array}$


SURVEY ID: PPSC 0501 (continued)

Constituent

Metals (total recoverable)

Selenium

Silver

Sodium

Thallium

Vanadium

Zinc

\section{Radionuclides}

Actinium-228

Antimony-124

Antimony-125

Barium-133

Cerium-144

Cesium-134

Cesium-137

Cobalt-57

Cobalt-58

Cobalt-60

Europium-152

Europium-154

Europium-155

Gross alpha

Lead-212

Manganese-54

Neptunium-239

Nonvolatile beta

Plutonium-238

Plutonium-239/240

Potassium-40

Promethium-144

Promethium-146

Ruthenium-106

Sodium-22

Strontium-90

Thorium-234

Tin-113

Yttrium-88

Zinc-65

Zirconium-95
Sample ID: 104613
RQ AQ B Result
Unit
D. Limit Method

$\begin{array}{lll}\text { U } & & 17.8 \\ \text { U } & & 1.48 \\ \text { U } & & 59.2 \\ \text { UJ } & \text { C } & 17.8 \\ \text { J } & \text { C } & 3.59 \\ & \text { V } & 3.18\end{array}$

$\begin{array}{ll}\mathrm{mg} / \mathrm{kg} & 17.8 \\ \mathrm{mg} / \mathrm{kg} & 1.48 \\ \mathrm{mg} / \mathrm{kg} & 59.2 \\ \mathrm{mg} / \mathrm{kg} & 17.8 \\ \mathrm{mg} / \mathrm{kg} & 0.592 \\ \mathrm{mg} / \mathrm{kg} & 1.18\end{array}$

EPA6010A

EPA6010A

EPA6010A

EPA6010A

EPA6010A

EPA6010A

\section{SURVEY ID: PPSC 0502}

UI

UI

UI

UI

UI

UI

UI

UI

UI

UI

UI

UI

UI

UI

UI

UI

UI

UI

UI
UI
UI
UI

UI

UI

UI

UI

$$
\begin{aligned}
& \text { 7.49E-01 } 44.57 \mathrm{E}-02 \mathrm{pCi} / \mathrm{g} \\
& -4.62 \mathrm{E}-03 \pm 7.22 \mathrm{E}-03 \mathrm{pCi} / \mathrm{g} \\
& 4.16 \mathrm{E}-03 \pm 1.72 \mathrm{E}-02 \mathrm{pCi} / \mathrm{g} \\
& 6.86 \mathrm{E}-03 \pm 8.16 \mathrm{E}-03 \mathrm{pCi} / \mathrm{g} \\
& \text { 1.63E-02 } \pm 3.96 \mathrm{E}-02 \mathrm{pCi} / \mathrm{g} \\
& 3.03 \mathrm{E}-03 \pm 5.40 \mathrm{E}-03 \mathrm{pCi} / \mathrm{g} \\
& 2.01 \mathrm{E}+00 \pm 3.40 \mathrm{E}-02 \mathrm{pCi} / \mathrm{g} \\
& -2.51 \mathrm{E}-03 \pm 5.08 \mathrm{E}-03 \mathrm{pCi} / \mathrm{g} \\
& \text { 2.87E-03 } \pm 6.07 \mathrm{E}-03 \mathrm{pCi} / \mathrm{g} \\
& \text { 2.37E-03 } \pm 4.09 \mathrm{E}-03 \mathrm{pCi} / \mathrm{g} \\
& -1.58 \mathrm{E}-02 \pm 1.66 \mathrm{E}-02 \mathrm{pCi} / \mathrm{g} \\
& 3.99 \mathrm{E}-03 \pm 3.43 \mathrm{E}-02 \mathrm{pCi} / \mathrm{g} \\
& 0.00 \mathrm{E}+00 \quad \mathrm{pCi} / \mathrm{g} \\
& 1.02 \mathrm{E}+01 \pm 2.03 \mathrm{E}+00 \mathrm{pCi} / \mathrm{g} \\
& \text { 7.79E-01 } \pm 2.36 \mathrm{E}-02 \mathrm{pCi} / \mathrm{g} \\
& \text { 9.32E-03 } \pm 7.09 \mathrm{E}-03 \mathrm{pCi} / \mathrm{g} \\
& \text { 4.08E-03 } 44.01 \mathrm{E}-02 \mathrm{pCi} / \mathrm{g} \\
& 5.56 \mathrm{E}+00 \pm 1.39 \mathrm{E}+00 \mathrm{pCi} / \mathrm{g} \\
& 1.34 \mathrm{E}-02 \pm 1.81 \mathrm{E}-02 \mathrm{pCi} / \mathrm{g} \\
& 5.42 \mathrm{E}-03 \pm 7.68 \mathrm{E}-03 \mathrm{pCi} / \mathrm{g} \\
& 5.02 \mathrm{E}-01 \pm 9.28 \mathrm{E}-02 \mathrm{pCi} / \mathrm{g} \\
& -4.00 \mathrm{E}-04 \pm 4.38 \mathrm{E}-03 \mathrm{pCi} / \mathrm{g} \\
& \text { 5.75E-03 } \pm 8.70 \mathrm{E}-03 \mathrm{pCi} / \mathrm{g} \\
& 1.26 \mathrm{E}-02 \pm 4.74 \mathrm{E}-02 \mathrm{pCi} / \mathrm{g} \\
& \text { 6.34E-04t4.05E-03 pCi/g } \\
& \text { 9.21E-0244.24E-02 pCi/g } \\
& \text { 9.17E-01 } \pm 5.34 \mathrm{E}-01 \mathrm{pCj} / \mathrm{g} \\
& -5.39 \mathrm{E}-03 \pm 8.88 \mathrm{E}-03 \mathrm{pCi} / \mathrm{g} \\
& \text { 2.21E-03 } \pm 4.98 \mathrm{E}-03 \mathrm{pCi} / \mathrm{g} \\
& -4.84 \mathrm{E}-03 \pm 9.61 \mathrm{E}-03 \mathrm{pCi} / \mathrm{g} \\
& 0.00 \mathrm{E}+00 \\
& \mathrm{pCi} / \mathrm{g}
\end{aligned}
$$

2.42E-02 EPIA-013B

1.20E-02 EPIA-013B

2.98E-02 EPIA-013B

1.22E-02 EPIA-013B

6.79E-02 EPIA-013B

8.30E-03 EPLA-013B

9.60E-03 EPIA-013B

8.62E-03 EPIA-013B

9.39E-03 EPIA-013B

7.49E-03 EPIA-013B

2.63E-02 EPIA-013B

6.17E-02 EPIA-013B

3.53E-02 EPIA-013B

1.75E+00 EPIA-001B

1.61E-02 EPIA-013B

7.56E-03 EPIA-013B

6.19E-02 EPIA-013B

2.22E+00 EPIA-001B

3.35E-02 EPLA-012B

8.13E-03 EPIA-012B

6.33E-02 EPIA-013B

7.43E-03 EPIA-013B

1.52E-02 EPIA-013B

8.17E-02 EPIA-013B

6.37E-03 EPIA-013B

5.76E-02 EPIA-004

4.23E-01 EPIA-013B

1.52E-02 EPIA-013B

9.49E-03 EPIA-013B

1.41E-02 EPIA-013B

2.10E-02 EPLA-013B

Survey location: 45166E $3678653 N$ (SRS coordinates)

Depth of core interval: 1.00 to $2.00 \mathrm{ft}$

Sample type: Normal

Sample matrix: Soil

Percent solids: $\mathbf{8 8 . 0 0}$ 
SURVEY ID: PPSC 0502 (continued)

Sample ID: 104614

Constituent

RQ AQ B Result

Unit

D. Limit Method

Physical Parameters and Specified Analyses

Cation exchange capacity

Chloride

Chloride

Cyanide

Nitrate-nitrite as nitrogen

Orthophosphate

Phenols

Sulfide

Total organic carbon

Total organic nitrogen

Total phosphates (as P)

Metals (total recoverable)

Aluminum

Aluminum

Antimony

Antimony

Arsenic

Arsenic

Barium

Barium

Beryllium

Beryllium

Cadmium

Cadmium

Calcium

Calcium

Chromium

Chromium

Cobalt

Cobalt

Copper

Copper

Iron

Iron

Lead

Lead

Magnesium

Magnesium

Manganese

Manganese

Mercury

Nickel

Nickel

Potassium

Potassium

Selenium

Selenium

Silver

Silver

Sodium

Sodium

Thallium

Thallium

$\begin{array}{lll} & & 9 \\ \text { U } & & 2.62 \\ \text { U } & & 2.66 \\ U & & 0.955 \\ U & & 0.918 \\ \text { J } & \text { E } & 7.06 \\ \text { U } & \cdot & 341 \\ \text { UJ } & \text { C } & 294 \\ & & 890 \\ \text { C } & & 35.5 \\ \text { J } & \text { E } & 64.2\end{array}$

$\begin{array}{ll}\mathrm{meq} / 100 \mathrm{~g} & 5 \\ \mathrm{mg} / \mathrm{kg} & 2.62 \\ \mathrm{mg} / \mathrm{kg} & 2.66 \\ \mathrm{mg} / \mathrm{kg} & 0.955 \\ \mathrm{mg} / \mathrm{kg} & 0.918 \\ \mathrm{mg} / \mathrm{kg} & 27.2 \\ \mu \mathrm{g} / \mathrm{kg} & 341 \\ \mathrm{mg} / \mathrm{kg} & 294 \\ \mathrm{mg} / \mathrm{kg} & 114 \\ \mathrm{mg} / \mathrm{kg} & 0.227 \\ \mathrm{mg} / \mathrm{kg} & 223\end{array}$

EPA9081

EPA300.0

EPA300.0

EPA335.3

EPA353.1

EPA365.2

EPA420.2

EPA376.2

EPA415.1

EPA351.2

EPA365.4
EPA6010A EPA6010A EPA6010A

EPA6010A

EPA6010A

EPA6010A

EPA6010A

EPA6010A

EPA6010A

EPA6010A

EPA6010A

EPA6010A

EPA6010A

EPA6010A

EPA6010A

EPA6010A

EPA6010A

EPA6010A

EPA6010A

EPA6010A

EPA6010A

EPA6010A

EPA6010A

EPA6010A

EPA6010A

EPA6010A

EPA6010A

EPA6010A

EPA7471

EPA6010A

EPA6010A

EPA6010A

EPA6010A

EPA6010A

EPA6010A

EPA6010A

EPA6010A

EPA6010A

EPA6010A

EPA6010A

EPA6010A 
SURVEY ID: PPSC 0502 (continued)

Constituent

Metals (total recoverable)

Vanadium

Vanadium

Zinc

Zinc

Radionuclides

Actinium-228

Antimony-124

Antimony-125

Barium-133

Cerium-144

Cesium-134

Cesium-137

Cobalt-57

Cobalt-58

Cobalt-60

Europium-152

Europium-154

Europium-155

Gross alpha

Lead-212

Manganese-54

Neptunium-239

Nonvolatile beta

Plutonium-238

Plutonium-239/240

Potassium-40

Promethium-144

Promethium-146

Ruthenium-106

Sodium-22

Strontium-90

Thorium-234

Tin-113

Yttrium-88

Zinc-65

Zirconium-95

Sample ID: 104614
RQ AQ B Result
D. Limit Method

$\begin{array}{llll}2.7 & \mathrm{mg} / \mathrm{kg} & 0.442 & \text { EPA6010A } \\ 3.35 & \mathrm{mg} / \mathrm{kg} & 0.499 & \text { EPA6010A } \\ 1.73 & \mathrm{mg} / \mathrm{kg} & 0.884 & \text { EPA6010A } \\ 2.32 & \mathrm{mg} / \mathrm{kg} & 0.999 & \text { EPA6010A }\end{array}$

$8.52 \mathrm{E}-01 \pm 5.02 \mathrm{E}-02 \mathrm{pCi} / \mathrm{g}$
$6.63 \mathrm{E}-03 \pm 6.52 \mathrm{E}-03 \mathrm{pCi} / \mathrm{g}$
$1.64 \mathrm{E}-02 \pm 1.45 \mathrm{E}-02 \mathrm{pCi} / \mathrm{g}$
$1.86 \mathrm{E}-03 \pm 6.77 \mathrm{E}-03 \mathrm{pCi} / \mathrm{g}$
$2.50 \mathrm{E}-02 \pm 5.57 \mathrm{E}-02 \mathrm{pCi} / \mathrm{g}$
$2.14 \mathrm{E}-03 \pm 4.90 \mathrm{E}-03 \mathrm{pCi} / \mathrm{g}$
$5.02 \mathrm{E}-01 \pm 1.77 \mathrm{E}-02 \mathrm{pCi} / \mathrm{g}$
$-1.09 \mathrm{E}-03 \pm 4.61 \mathrm{E}-03 \mathrm{pCi} / \mathrm{g}$
$-3.34 \mathrm{E}-03 \pm 6.35 \mathrm{E}-03 \mathrm{pCi} / \mathrm{g}$
$-5.77 \mathrm{E}-03 \pm 3.97 \mathrm{E}-03 \mathrm{pCi} / \mathrm{g}$
$1.03 \mathrm{E}-03 \pm 1.41 \mathrm{E}-02 \mathrm{pCi} / \mathrm{g}$
$2.42 \mathrm{E}-03 \pm 4.11 \mathrm{E}-02 \mathrm{pCi} / \mathrm{g}$
$0.00 \mathrm{E}+00$
$1.05 \mathrm{E}+01 \pm 2.09 \mathrm{E}+00 \mathrm{pCi} / \mathrm{g}$
$8.76 \mathrm{E}-01 \pm 2.42 \mathrm{E}-02 \mathrm{pCi} / \mathrm{g}$
$1.29 \mathrm{E}-02 \pm 7.36 \mathrm{E}-03 \mathrm{pCi} / \mathrm{g}$
$8.92 \mathrm{E}-03 \pm 3.63 \mathrm{E}-02 \mathrm{pC} / \mathrm{g}$
$2.95 \mathrm{E}+00 \pm 1.27 \mathrm{E}+00 \mathrm{pCi} / \mathrm{g}$
$2.43 \mathrm{E}-03 \pm 7.78 \mathrm{E}-03 \mathrm{pCi} / \mathrm{g}$
$-4.96 \mathrm{E}-03 \pm 4.98 \mathrm{E}-03 \mathrm{pCi} / \mathrm{g}$
$5.18 \mathrm{E}-01 \pm 9.73 \mathrm{E}-02 \mathrm{pCi} / \mathrm{g}$
$-1.94 \mathrm{E}-03 \pm 4.46 \mathrm{E}-03 \mathrm{pCi} / \mathrm{g}$
$6.33 \mathrm{E}-03 \pm 6.54 \mathrm{E}-03 \mathrm{pCi} / \mathrm{g}$
$-1.08 \mathrm{E}-02 \pm 4.11 \mathrm{E}-02 \mathrm{pCi} / \mathrm{g}$
$-1.06 \mathrm{E}-03 \pm 3.73 \mathrm{E}-03 \mathrm{pCi} / \mathrm{g}$
$3.19 \mathrm{E}-02 \pm 3.08 \mathrm{E}-02 \mathrm{pCi} / \mathrm{g}$
$8.55 \mathrm{E}-01 \pm 5.16 \mathrm{E}-01 \mathrm{pCi} / \mathrm{g}$
$-6.93 \mathrm{E}-03 \pm 7.75 \mathrm{E}-03 \mathrm{pC} / \mathrm{g}$
$-6.00 \mathrm{E}-04 \pm 5.25 \mathrm{E}-03 \mathrm{pCi} / \mathrm{g}$
$-6.60 \mathrm{E}-03 \pm 1.48 \mathrm{E}-02 \mathrm{pCi} / \mathrm{g}$
$1.17 \mathrm{E}-02 \pm 1.36 \mathrm{E}-02 \mathrm{pCi} / \mathrm{g}$

2.41E-02 EPIA-013B

1.18E-02 EPLA-013B

2.48E-02 EPIA-013B

1.01E-02 EPIA-013B

6.42E-02 EPIA-013B

7.77E-03 EPIA-013B

8.30E-03 EPIA-013B

7.91E-03 EPIA-013B

9.23E-03 EPIA-013B

6.32E-03 EPIA-013B

2.34E-02 EPIA-013B

6.25E-02 EPIA-013B

3.25E-02 EPIA-013B

2.04E+00 EPIA-001B

1.43E-02 EPIA-013B

7.94E-03 EPIA-013B

5.67E-02 EPIA-013B

2.39E+00 EPIA-001B

1.88E-02 EPIA-012B

2.36E.02 EPIA.012B

6.46E-02 EPIA-013B

7.59E-03 EPIA-013B

1.11E-02 EPIA-013B

7.09E-02 EPIA-013B

6.61E-03 EPIA-013B

4.98E-02 EPIA-004

4.04E-01 EPIA-013B

1.24E-02 EPIA-013B

9.32E-03 EPIA-013B

1.45E-02 EPIA-013B

2.18E-02 EPIA-013B

SURVEY ID: PPSC 0503

Sample ID: 104615

Survey location: 45166E $3678653 N$ (SRS coordinates)

Depth of core interval: 2.00 to $4.00 \mathrm{ft}$

Sample type: Normal

Sample matrix: Soil

Percent solids: $\mathbf{8 6 . 0 0}$ 
SURVEY ID: PPSC 0503 (continued)

Constituent

Sample ID: 104615

RQ AQ B Result

Unit

D. Limit Method

Physical Parameters and Specified Analyses

Cation exchange capacity

Chloride

Cyanide

Nitrate-nitrite as nitrogen

Orthophosphate

Phenols

Sulfide

Sulfide

Total organic carbon

Total organic nitrogen

Total phosphates (as P)

Metals (total recoverable)

$\begin{array}{lll}\mathrm{meq} / 100 \mathrm{~g} & 5 & \text { EPA9081 } \\ \mathrm{mg} / \mathrm{kg} & 2.9 & \text { EPA300.0 } \\ \mathrm{mg} / \mathrm{kg} & 1.26 & \text { EPA335.3 } \\ \mathrm{mg} / \mathrm{kg} & 0.951 & \text { EPA353.1 } \\ \mathrm{mg} / \mathrm{kg} & 28.7 & \text { EPA365.2 } \\ \mu \mathrm{g} / \mathrm{kg} & 349 & \text { EPA420.2 } \\ \mathrm{mg} / \mathrm{kg} & 300 & \text { EPA376.2 } \\ \mathrm{mg} / \mathrm{kg} & 252 & \text { EPA376.2 } \\ \mathrm{mg} / \mathrm{kg} & 116 & \text { EPA415.1 } \\ \mathrm{mg} / \mathrm{kg} & 0.233 & \text { EPA351.2 } \\ \mathrm{mg} / \mathrm{kg} & 398 & \text { EPA365.4 }\end{array}$

$\mathrm{mg} / \mathrm{kg} \quad 11$

$\mathrm{mg} / \mathrm{kg} \quad 4.42$

$\mathrm{mg} / \mathrm{kg} \quad 13.3$

$\mathrm{mg} / \mathrm{kg} \quad 1.1$

$\mathrm{mg} / \mathrm{kg} \quad 0.442$

$\mathrm{mg} / \mathrm{kg} \quad 0.221$

$\mathrm{mg} / \mathrm{kg} \quad 11$

$\mathrm{mg} / \mathrm{kg} \quad 1.1$

$\mathrm{mg} / \mathrm{kg} \quad 0.442$

$\mathrm{mg} / \mathrm{kg} \quad 1.1$

$\mathrm{mg} / \mathrm{kg}$

$\mathrm{mg} / \mathrm{kg}$

$\mathrm{mg} / \mathrm{kg}$

$\mathrm{mg} / \mathrm{kg}$

$\mathrm{mg} / \mathrm{kg}$

$\mathrm{mg} / \mathrm{kg}$

$\mathrm{mg} / \mathrm{kg}$

$\mathrm{mg} / \mathrm{kg}$

$\mathrm{mg} / \mathrm{kg}$

$\mathrm{mg} / \mathrm{kg}$

$\mathrm{mg} / \mathrm{kg}$

$\mathrm{mg} / \mathrm{kg}$

$\mathrm{mg} / \mathrm{kg}$
EPA6010A

EPA6010A

EPA6010A

EPA6010A

EPA6010A

EPA6010A

EPA6010A

EPA6010A

EPA6010A

EPA6010A

EPA6010A

EPA6010A

$\begin{array}{ll}4.42 & \text { EPA6010A } \\ 0.442 & \text { EPA6010A }\end{array}$

0.0381 EPA7471

1.1 EPA6010A

88.4 EPA6010A

13.3 EPA6010A

1.1 EPA6010A

44.2 EPA6010A

13.3 EPA6010A

0.442 EPA6010A

0.884 EPA6010A

Radionuclides

Actinium-228

Antimony-124

Antimony-125

Barium-133

Cerium-144

Cesium-134

Cesium-137

Cobalt-57

Cobalt-58

Cobalt-60

Europium-152

Europium-154

Europium-155

Gross alpha

Lead-212
$1.23 \mathrm{E}+00 \pm 6.24 \mathrm{E}-02 \mathrm{pCi} / \mathrm{g}$

UI $\quad 4.24 \mathrm{E}-03 \pm 7.76 \mathrm{E}-03 \mathrm{pCi} / \mathrm{g}$

UI $\quad-4.54 \mathrm{E}-03 \pm 1.52 \mathrm{E} \cdot 02 \mathrm{pCi} / \mathrm{g}$

UI $\quad-1.86 \mathrm{E}-02 \pm 7.74 \mathrm{E}-03 \mathrm{pCi} / \mathrm{g}$

UI $\quad 1.83 \mathrm{E}-02 \pm 4.27 \mathrm{E}-02 \mathrm{pCi} / \mathrm{g}$

UI $\quad 3.26 \mathrm{E}-04 \pm 5.80 \mathrm{E}-03 \quad \mathrm{pCi} / \mathrm{g}$

$1.50 \mathrm{E}-02 \pm 8.55 \mathrm{E}-03 \mathrm{pCi} / \mathrm{g}$

$8.22 \mathrm{E}-04 \pm 5.41 \mathrm{E}-03 \mathrm{pCi} / \mathrm{g}$

$-1.06 \mathrm{E}-02 \pm 7.70 \mathrm{E}-03 \mathrm{pCi} / \mathrm{g}$

$-1.58 \mathrm{E}-03 \pm 4.47 \mathrm{E}-03 \mathrm{pCi} / \mathrm{g}$

$-3.92 \mathrm{E}-03 \pm 1.58 \mathrm{E}-02 \mathrm{pCi} / \mathrm{g}$

$-6.79 \mathrm{E}-02 \pm 4.71 \mathrm{E}-02 \mathrm{pCi} / \mathrm{g}$

$0.00 \mathrm{E}+00 \quad \mathrm{pCi} / \mathrm{g}$

$1.84 \mathrm{E}+01 \pm 2.95 \mathrm{E}+00 \mathrm{pCi} / \mathrm{g}$

$1.30 \mathrm{E}+00 \pm 2.88 \mathrm{E}-02 \mathrm{pCi} / \mathrm{g}$
2.99E-02 EPIA-013B

1.38E-02 EPIA-013B

2.47E-02 EPIA-013B

1.19E.02 EPIA.013B

7.39E-02 EPIA-013B

9.02E-03 EPIA-013B

8.52E-03 EPIA-013B

9.36E-03 EPIA-013B

1.05E-02 EPIA-013B

7.86E-03 EPIA-013B

2.60E-02 EPIA-013B

7.33E-02 EPIA-013B

3.78E-02 EPIA-013B

$1.83 \mathrm{E}+00$ EPIA-001B

1.64E-02 EPIA-013B 
SURVEY ID: PPSC 0503 (continued)

Constituent

Radionuclides

Manganese-54

Neptunium-239

Nonvolatile beta

Plutonium-238

Plutonium-239/240

Potassium-40

Promethium-144

Promethium-146

Ruthenium-106

Sodium-22

Strontium-90

Thorium-234

Tin-113

Yttrium-88

Zinc-65

Zirconium-95

Sample ID: 104615

RQ AQ B Result Unit D. Limit Method

$2.24 \mathrm{E}-02 \pm 6.68 \mathrm{E}-03 \mathrm{pCi} / \mathrm{g}$

1.11E-02 4 .11E-02 pCi/g

$9.54 \mathrm{E}+00 \pm 1.68 \mathrm{E}+00 \mathrm{pCi} / \mathrm{g}$

$-1.42 \mathrm{E}-03 \pm 1.43 \mathrm{E}-02 \mathrm{pCi} / \mathrm{g}$

$-3.90 \mathrm{E}-03 \pm 7.73 \mathrm{E}-03 \mathrm{pCi} / \mathrm{g}$

9.11E-01 $1.17 \mathrm{E}-01 \mathrm{pCi} / \mathrm{g}$

$-4.79 \mathrm{E}-03 \pm 5.69 \mathrm{E}-03 \mathrm{pCi} / \mathrm{g}$

$0.00 \mathrm{E}+00 \quad \mathrm{pCi} / \mathrm{g}$

$-3.26 \mathrm{E}-02 \pm 4.92 \mathrm{E}-02 \mathrm{pCi} / \mathrm{g}$

$3.95 \mathrm{E}-03 \pm 4.68 \mathrm{E}-03 \mathrm{pCi} / \mathrm{g}$

9.76E-02 $\div 3.83 \mathrm{E}-02 \mathrm{pCi} / \mathrm{g}$

$1.13 \mathrm{E}+00 \pm 6.62 \mathrm{E}-01 \mathrm{pCi} / \mathrm{g}$

$-5.70 \mathrm{E}-03 \pm 9.86 \mathrm{E}-03 \mathrm{pCi} / \mathrm{g}$

$3.93 \mathrm{E}-03 \pm 6.58 \mathrm{E}-03 \mathrm{pCi} / \mathrm{g}$

4.17E-03 $\pm 1.13 \mathrm{E}-02 \mathrm{pCi} / \mathrm{g}$

1.44E-02 $1.52 \mathrm{E}-02 \mathrm{pCi} / \mathrm{g}$
9.39E-03 EPIA-013B

6.44E-02 EPIA-013B

$2.36 \mathrm{E}+00$ EPIA-001B

3.57E-02 EPIA-012B

2.66E-02 EPIA-012B

8.03E-02 EPIA-013B

9.52E-03 EPIA-013B

1.27E-02 EPIA-013B

8.36E-02 EPIA-013B

8.56E-03 EPIA-013B

4.93E-02 EPIA-004

4.96E-01 EPIA-013B

1.41E-02 EPIA-013B

1.23E-02 EPIA-013B

1.77E-02 EPIA-013B

2.45E-02 EPIA-013B

SURVEY ID: PPSC 0601

Sample ID: 104616

Survey location: $45034 \mathrm{E} 3679647 \mathrm{~N}$ (SRS coordinates)

Depth of core interval: 0.00 to $1.00 \mathrm{ft}$

Sample type: Normal

Sample matrix: Soil

Percent solids: $\mathbf{8 2 . 0 0}$

Constituent

RQ AQ B Result

Unit

D. Limit Method

Physical Parameters and Specified Analyses

Cation exchange capacity

Chloride

Cyanide

Nitrate-nitrite as nitrogen

Orthophosphate

Phenols

Sulfide

Total organic carbon

Total organic nitrogen

Total phosphates (as P)

Metals (total recoverable)

Aluminum

Antimony

Arsenic

Barium

Beryllium

Cadmium

Calcium

Chromium

Cobalt

Copper

Iron

Lead

$\begin{array}{lll} & & 21.6 \\ \mathrm{U} & & 2.8 \\ \mathrm{U} & & 1.01 \\ \mathrm{U} & & 0.999 \\ \mathrm{U} & & 24.8 \\ \mathrm{U} & & 366 \\ \mathrm{UJ} & \mathrm{C} & 313 \\ & & 2870 \\ \mathrm{C} & & 21.7 \\ \mathrm{~J} & \mathrm{E} & 98.4\end{array}$

2140

U $\quad 3.97$

U $\quad 11.9$

12.1

J $\quad \mathrm{E} \quad 0.0591$

U 0.198

57.8

1.96

0.263

1.16

1330

2.77

$\begin{array}{ll}\mathrm{meq} / 100 \mathrm{~g} & 5 \\ \mathrm{mg} / \mathrm{kg} & 2.8 \\ \mathrm{mg} / \mathrm{kg} & 1.01 \\ \mathrm{mg} / \mathrm{kg} & 0.999 \\ \mathrm{mg} / \mathrm{kg} & 24.8 \\ \mu \mathrm{g} / \mathrm{kg} & 366 \\ \mathrm{mg} / \mathrm{kg} & 313 \\ \mathrm{mg} / \mathrm{kg} & 122 \\ \mathrm{mg} / \mathrm{kg} & 0.244 \\ \mathrm{mg} / \mathrm{kg} & 432\end{array}$

EPA9081

EPA300.0

EPA335.3

EPA353.1

EPA365.2

EPA420.2

EPA376.2

EPA415.1

EPA351.2

EPA365.4
EPA6010A EPA6010A EPA6010A EPA6010A EPA6010A EPA6010A EPA6010A EPA6010A EPA6010A EPA6010A EPA6010A EPA6010A 
SURVEY ID: PPSC 0601 (continued)

Constituent

Metals (total recoverable)

Magnesium

Manganese

Mercury

Nickel

Potassium

Selenium

Silver

Sodium

Thallium

Vanadium

Zinc

\section{Radionuclides}

Actinium-228

Antimony-124

Antimony-125

Barium-133

Cerium-144

Cesium-134

Cesium-137

Cobalt-57

Cobalt-58

Cobalt-60

Europium-152

Europium-154

Europium-155

Gross alpha

Lead-212

Manganese-54

Neptunium-239

Nonvolatile beta

Plutonium-238

Plutonium-239/240

Potassium-40

Promethium-144

Promethium-146

Ruthenium-106

Sodium-22

Strontium-90

Thorium-234

Tin-113

Yttrium-88

Zinc-65

Zirconium-95
Sample ID: 104616

RQ AQ B Result Unit D. Limit Method

$\begin{array}{llllll} & & 53 & \mathrm{mg} / \mathrm{kg} & 3.97 & \text { EPA6010A } \\ & & 33.1 & \mathrm{mg} / \mathrm{kg} & 0.397 & \text { EPA6010A } \\ \mathrm{J} & \mathrm{E} & 0.0165 & \mathrm{mg} / \mathrm{kg} & 0.036 & \text { EPA7471 } \\ \mathrm{J} & \mathrm{E} & 0.541 & \mathrm{mg} / \mathrm{kg} & 0.992 & \text { EPA6010A } \\ \mathrm{J} & \mathrm{E} & 28.7 & \mathrm{mg} / \mathrm{kg} & 79.3 & \text { EPA6010A } \\ \mathrm{U} & & 11.9 & \mathrm{mg} / \mathrm{kg} & 11.9 & \text { EPA6010A } \\ \mathrm{U} & 0.992 & \mathrm{mg} / \mathrm{kg} & 0.992 & \text { EPA6010A } \\ \mathrm{U} & 39.7 & \mathrm{mg} / \mathrm{kg} & 39.7 & \text { EPA6010A } \\ \mathrm{U} & 11.9 & \mathrm{mg} / \mathrm{kg} & 11.9 & \text { EPA6010A } \\ & 3.82 & \mathrm{mg} / \mathrm{kg} & 0.397 & \text { EPA6010A } \\ & 2.61 & \mathrm{mg} / \mathrm{kg} & 0.793 & \text { EPA6010A }\end{array}$

8.19E-01 $\pm 5.43 \mathrm{E}-02 \mathrm{pCi} / \mathrm{g}$

$-1.18 \mathrm{E}-03 \pm 6.68 \mathrm{E}-03 \mathrm{pCi} / \mathrm{g}$

$1.77 \mathrm{E}-02 \pm 2.02 \mathrm{E}-02 \mathrm{pCi} / \mathrm{g}$

7.37E-03 $\pm 6.86 \mathrm{E}-03 \mathrm{pCi} / \mathrm{g}$

$-1.81 \mathrm{E}-02 \pm 3.92 \mathrm{E}-02 \mathrm{pCi} / \mathrm{g}$

$2.14 \mathrm{E}-03 \pm 5.10 \mathrm{E}-03 \mathrm{pCi} / \mathrm{g}$

4.31E-01 $1.84 \mathrm{E}-02 \mathrm{pCi} / \mathrm{g}$

$1.30 \mathrm{E}-03 \pm 4.86 \mathrm{E}-03 \mathrm{pCi} / \mathrm{g}$

$-1.34 \mathrm{E}-03 \pm 6.06 \mathrm{E}-03 \mathrm{pCi} / \mathrm{g}$

6.22E-03 $\pm 8.92 \mathrm{E}-03 \mathrm{pCi} / \mathrm{g}$

$-1.28 \mathrm{E}-02 \pm 1.66 \mathrm{E}-02 \mathrm{pCi} / \mathrm{g}$

$-4.48 \mathrm{E}-02 \pm 3.92 \mathrm{E}-02 \mathrm{pCi} / \mathrm{g}$

$0.00 \mathrm{E}+00 \quad \mathrm{pCi} / \mathrm{g}$

$8.74 \mathrm{E}+00 \pm 1.81 \mathrm{E}+00 \mathrm{pCi} / \mathrm{g}$

8.96E-01 $\pm 2.43 \mathrm{E}-02 \mathrm{pCi} / \mathrm{g}$

9.22E-03 $\pm 8.29 \mathrm{E}-03 \mathrm{pCi} / \mathrm{g}$

UI

2.21E-02 $\pm 3.75 \mathrm{E}-02 \mathrm{pCi} / \mathrm{g}$

$4.93 \mathrm{E}+00 \pm 1.21 \mathrm{E}+00 \mathrm{pCi} / \mathrm{g}$

5.99E-03 $\pm 7.24 \mathrm{E}-03 \mathrm{pCi} / \mathrm{g}$

$1.48 \mathrm{E}-03 \pm 2.97 \mathrm{E}-03 \mathrm{pCj} / \mathrm{g}$

$4.26 \mathrm{E}-01 \pm 1.18 \mathrm{E}-01 \mathrm{pCi} / \mathrm{g}$

$-1.84 \mathrm{E}-03 \pm 4.91 \mathrm{E}-03 \mathrm{pCi} / \mathrm{g}$

7.75E-03 $\pm 6.92 \mathrm{E}-03 \mathrm{pCi} / \mathrm{g}$

$1.21 \mathrm{E}-02 \pm 4.50 \mathrm{E}-02 \mathrm{pCi} / \mathrm{g}$

$1.25 \mathrm{E}-03 \pm 4.26 \mathrm{E}-03 \mathrm{pCi} / \mathrm{g}$

$3.31 \mathrm{E}-02 \pm 2.89 \mathrm{E}-02 \mathrm{pCi} / \mathrm{g}$ 9.63E-01 $\pm 5.70 \mathrm{E}-01 \mathrm{pCi} / \mathrm{g}$

$4.62 \mathrm{E}-03 \pm 7.40 \mathrm{E}-03 \mathrm{pCi} / \mathrm{g}$

$5.49 \mathrm{E}-03 \pm 7.61 \mathrm{E}-03 \mathrm{pCi} / \mathrm{g}$ $4.82 \mathrm{E}-03 \pm 1.22 \mathrm{E}-02 \mathrm{pCi} / \mathrm{g}$ $1.04 \mathrm{E}-02 \pm 1.27 \mathrm{E}-02 \mathrm{pCi} / \mathrm{g}$
2.59E-02 EPIA-013B

1.14E-02 EPIA-013B

2.46E-02 EPIA-013B

1.05E-02 EPIA-013B

6.64E-02 EPIA-013B

7.84E-03 EPIA-013B

9.34E-03 EPIA-013B

8.37E-03 EPIA-013B

1.01E-02 EPIA-013B

8.48E.03 EPIA-013B

2.33E-02 EPIA-013B

6.48E-02 EPIA-013B

3.40E-02 EPIA-013B

1.10E+00 EPIA-001B

1.47E-02 EPIA-013B

7.31E-03 EPIA-013B

5.88E-02 EPIA-013B

1.82E+00 EPIA-001B

1.12E-02 EPIA-012B

4.45E-03 EPIA-012B

6.79E-02 EPIA-013B

8.20E-03 EPIA-013B

1.24E-02 EPIA-013B

7.79E-02 EPIA-013B

7.67E-03 EPIA-013B

4.61E-02 EPIA-004

4.37E-01 EPIA-013B

1.31E-02 EPIA-013B

9.61E-03 EPIA-013B

1.40E-02 EPIA-013B

2.24E-02 EPIA-013B

SURVEY ID: PPSC 0602

Sample ID: 104617

Survey location: 45034E 3679647N (SRS coordinates)

Depth of core interval: 1.00 to $2.00 \mathrm{ft}$

Sample type: Normal

Sample matrix: Soil

Percent solids: 89.00 
SURVEY ID: PPSC 0602 (continued)

Constituent
Sample ID: 104617
RQ AQ B Result
Unit
D. Limit Method

Physical Parameters and Specified Analyses
Cation exchange capacity

Chloride

Cyanide

Nitrate-nitrite as nitrogen

Orthophosphate

Phenols

Sulfide

Total organic carbon

Total organic nitrogen

Total phosphates (as P)

$\begin{array}{lll} & & 11.7 \\ \mathrm{U} & & 2.55 \\ \mathrm{U} & & 0.871 \\ \mathrm{U} & & 1.09 \\ \mathrm{~J} & \mathrm{E} & 4.97 \\ \mathrm{U} & & 337 \\ \mathrm{UJ} & \mathrm{C} & 283 \\ & & 1140 \\ \mathrm{C} & & 42.9 \\ \mathrm{~J} & \mathrm{E} & 80.3\end{array}$

Metals (total recoverable)

Aluminum
Antimony
Arsenic
Barium
Beryllium
Cadmium
Calcium
Chromium
Cobalt
Copper
Iron
Lead
Magnesium
Manganese
Mercury
Nickel
Potassium
Selenium
Silver
Sodium
Thallium
Vanadium
Zinc

\section{Radionuclides}

Actinium-228

Antimony-124

Antimony-125

Barium-133

Cerium-144

Cesium-134

Cesium-137

Cobalt-57

Cobalt-58

Cobalt-60

Europium-152

Europium-154

$\begin{array}{lll} & & 2150 \\ U & & 3.98 \\ U & & 11.9 \\ & & 8.01 \\ J & E & 0.0514 \\ U & & 0.199 \\ & & 28.3 \\ & & 2.85 \\ J & E & 0.338 \\ & & 1.23 \\ & & 1610 \\ J & E & 2.32 \\ & & 56 \\ & & 14.9 \\ J & E & 0.0119 \\ J & E & 0.677 \\ \text { J } & E & 31.9 \\ U & & 11.9 \\ U & & 0.995 \\ U & & 39.8 \\ U & & 11.9 \\ & & 5.34 \\ & & 2.27\end{array}$

$\begin{array}{ll}\mathrm{meq} / 100 \mathrm{~g} & 5 \\ \mathrm{mg} / \mathrm{kg} & 2.55 \\ \mathrm{mg} / \mathrm{kg} & 0.871 \\ \mathrm{mg} / \mathrm{kg} & 1.09 \\ \mathrm{mg} / \mathrm{kg} & 24.2 \\ \mu \mathrm{g} / \mathrm{kg} & 337 \\ \mathrm{mg} / \mathrm{kg} & 283 \\ \mathrm{mg} / \mathrm{kg} & 112 \\ \mathrm{mg} / \mathrm{kg} & 0.225 \\ \mathrm{mg} / \mathrm{kg} & 436\end{array}$

EPA9081

EPA300.0 EPA335.3 EPA353.1 EPA365.2 EPA420.2 EPA376.2 EPA415.1 EPA351.2 EPA365.4
EPA6010A EPA6010A EPA6010A EPA6010A EPA6010A EPA6010A EPA6010A EPA6010A EPA6010A EPA6010A EPA6010A EPA6010A EPA6010A EPA6010A EPA7471 EPA6010A EPA6010A EPA6010A EPA6010A EPA6010A EPA6010A EPA6010A EPA6010A $\begin{array}{ll}2.68 E-02 & \text { EPIA-013B } \\ 1.17 E-02 & \text { EPIA-013B } \\ 2.38 E-02 & \text { EPIA-013B } \\ 1.06 \mathrm{E}-02 & \text { EPIA-013B } \\ 6.97 \mathrm{E}-02 & \text { EPIA-013B } \\ 8.16 \mathrm{E}-03 & \text { EPIA-013B } \\ 8.98 \mathrm{E}-03 & \text { EPIA-013B } \\ 8.81 \mathrm{E}-03 & \text { EPIA-013B } \\ 1.07 \mathrm{E}-02 & \text { EPIA-013B } \\ 6.95 \mathrm{E}-03 & \text { EPIA-013B } \\ 2.27 \mathrm{E}-02 & \text { EPIA-013B } \\ 6.55 \mathrm{E}-02 & \text { EPIA-013B }\end{array}$

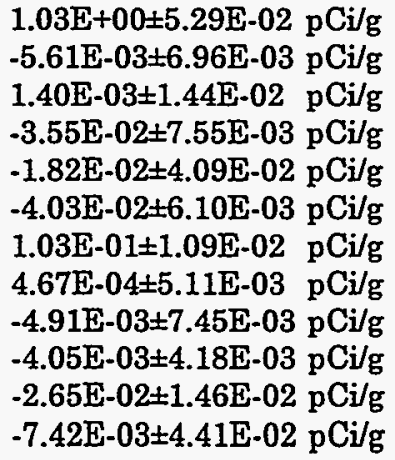


SURVEY ID: PPSC 0602 (continued)

Constituent

Radionuclides

Europium-155

Gross alpha

Lead-212

Manganese-54

Neptunium-239

Nonvolatile beta

Plutonium-238

Plutonium-239/240

Potassium-40

Promethium-144

Promethium-146

Ruthenium-106

Sodium-22

Strontium-90

Thorium-234

Tin-113

Yttrium-88

Zinc-65

Zirconium-95
Sample ID: 104617

RQ AQ B Result Unit D. Limit Method

\section{SURVEY ID: PPSC 0603}

$\begin{array}{llr}\text { UI } & 0.00 \mathrm{E}+00 \quad \mathrm{pCi} / \mathrm{g} \\ & 9.51 \mathrm{E}+00 \pm 1.96 \mathrm{E}+00 \mathrm{pCi} / \mathrm{g} \\ & 1.05 \mathrm{E}+00 \pm 2.68 \mathrm{E}-02 \mathrm{pCi} / \mathrm{g} \\ & 1.64 \mathrm{E}-02 \pm 7.85 \mathrm{E}-03 \mathrm{pCi} / \mathrm{g} \\ \text { UI } & 1.51 \mathrm{E}-02 \pm 4.00 \mathrm{E}-02 \mathrm{pCi} / \mathrm{g} \\ & 4.71 \mathrm{E}+00 \pm 1.33 \mathrm{E}+00 \mathrm{pCi} / \mathrm{g} \\ \text { UI } & 3.19 \mathrm{E}-03 \pm 1.15 \mathrm{E}-02 \mathrm{pCi} / \mathrm{g} \\ \text { UI } & -2.64 \mathrm{E}-03 \pm 3.74 \mathrm{E}-03 \mathrm{pCi} / \mathrm{g} \\ & 3.95 \mathrm{E}-01 \pm 1.03 \mathrm{E}-01 \mathrm{pCi} / \mathrm{g} \\ \text { UI } & 1.82 \mathrm{E}-03 \pm 5.11 \mathrm{E}-03 \mathrm{pCi} / \mathrm{g} \\ \text { UI } & 4.32 \mathrm{E}-03 \pm 6.87 \mathrm{E}-03 \mathrm{pCi} / \mathrm{g} \\ \text { UI } & -2.01 \mathrm{E}-02 \pm 4.60 \mathrm{E}-02 \mathrm{pCi} / \mathrm{g} \\ \text { UI } & -4.99 \mathrm{E}-04 \pm 4.07 \mathrm{E}-03 \mathrm{pCi} / \mathrm{g} \\ \text { UI } & -6.07 \mathrm{E}-02 \pm 1.44 \mathrm{E}-02 \mathrm{pCi} / \mathrm{g} \\ & 8.45 \mathrm{E}-01 \pm 4.41 \mathrm{E}-01 \mathrm{pCi} / \mathrm{g} \\ \text { UI } & 2.29 \mathrm{E}-03 \pm 8.28 \mathrm{E}-03 \mathrm{pCi} / \mathrm{g} \\ \text { UI } & 9.41 \mathrm{E}-04 \pm 5.85 \mathrm{E}-03 \mathrm{pCi} / \mathrm{g} \\ \text { UI } & -4.44 \mathrm{E}-03 \pm 1.12 \mathrm{E}-02 \mathrm{pCi} / \mathrm{g} \\ \text { UI } & 2.45 \mathrm{E}-02 \pm 1.46 \mathrm{E}-02 \mathrm{pCi} / \mathrm{g}\end{array}$

3.55E-02 EPLA-013B

1.47E+00 EPIA-001B

1.51E-02 EPIA-013B

9.50E-03 EPIA-013B

6.26E-02 EPIA-013B

2.21E+00 EPIA-001B

2.19E-02 EPIA-012B

1.30E-02 EPLA-012B

7.99E-02 EPIA-013B

8.98E-03 EPIA-013B

1.16E-02 EPIA-013B

7.87E-02 EPIA-013B

7.30E-03 EPIA-013B

5.12E-02 EPIA-004

4.56E-01 EPIA-013B

1.38E-02 EPIA-013B

1.07E-02 EPIA-013B

1.60E-02 EPIA-013B

2.46E-02 EPIA-013B

Survey location: $45034 \mathrm{E}$ 3679647N (SRS coordinates)

Depth of core interval: 2.00 to $4.00 \mathrm{ft}$

Sample type: Normal

Sample matrix: Soil

Percent solids: $\mathbf{8 7 . 0 0}$

\section{Constituent}

RQ AQ B Result

Unit

D. Limit Method

Physical Parameters and Specified Analyses

Cation exchange capacity

Cation exchange capacity

Chloride

Cyanide

Nitrate-nitrite as nitrogen

Orthophosphate

Phenols

Sulfide

Total organic carbon

Total organic nitrogen

Total phosphates (as P)

Metals (total recoverable)

Aluminum

Antimony

Arsenic

Barium

Beryllium

Cadmium

Calcium

Chromium

$\begin{array}{lll} & & 133 \\ & & 195 \\ J & E & 1.42 \\ \text { U } & & 1.05 \\ \text { U } & & 0.909 \\ \text { U } & & 26.5 \\ \text { U } & & 345 \\ \text { UJ } & \text { C } & 287 \\ & & 971 \\ \text { C } & & 68.7 \\ \text { J } & \text { E } & 78.2\end{array}$

$\begin{array}{lll} & & 5720 \\ \mathrm{U} & & 4.29 \\ \mathrm{~J} & \mathrm{E} & \mathbf{5 . 6 5} \\ & & 7.98 \\ \mathrm{~J} & \mathrm{E} & 0.0553 \\ \mathrm{U} & & 0.214 \\ & & 40 \\ & & 11.7\end{array}$

$\begin{array}{lll}\mathrm{meq} / 100 \mathrm{~g} & 5 & \text { EPA9081 } \\ \mathrm{meq} / 100 \mathrm{~g} & 5.75 & \text { EPA9081 } \\ \mathrm{mg} / \mathrm{kg} & 2.84 & \text { EPA300.0 } \\ \mathrm{mg} / \mathrm{kg} & 1.05 & \text { EPA335.3 } \\ \mathrm{mg} / \mathrm{kg} & 0.909 & \text { EPA353.1 } \\ \mathrm{mg} / \mathrm{kg} & 26.5 & \text { EPA365.2 } \\ \mu \mathrm{g} / \mathrm{kg} & 345 & \text { EPA420.2 } \\ \mathrm{mg} / \mathrm{kg} & 287 & \text { EPA376.2 } \\ \mathrm{mg} / \mathrm{kg} & 115 & \text { EPA415.1 } \\ \mathrm{mg} / \mathrm{kg} & 0.23 & \text { EPA351.2 } \\ \mathrm{mg} / \mathrm{kg} & 460 & \text { EPA365.4 }\end{array}$

$\mathrm{mg} / \mathrm{kg}$

$\mathrm{mg} / \mathrm{kg}$

$\mathrm{mg} / \mathrm{kg}$

$\mathrm{mg} / \mathrm{kg}$

$\mathrm{mg} / \mathrm{kg}$

$\mathrm{mg} / \mathrm{kg}$

$\mathrm{mg} / \mathrm{kg}$

$\mathrm{mg} / \mathrm{kg}$
10.7

4.29

12.9

1.07

0.429

.0 .214

10.7

1.07
EPA6010A

EPA6010A EPA6010A EPA6010A EPA6010A EPA6010A EPA6010A EPA6010A 
SURVEY ID: PPSC 0603 (continued)

Constituent

Metals (total recoverable)

Cobalt

Copper

Iron

Lead

Magnesium

Manganese

Mercury

Nickel

Potassium

Selenium

Silver

Sodium

Thallium

Vanadium

Zinc

\section{Radionuclides}

Actinium-228

Antimony-124

Antimony-125

Barium-133

Cerium-144

Cesium-134

Cesium-137

Cobalt-57

Cobalt-58

Cobalt-60

Europium-152

Europium-154

Europium-155

Gross alpha

Lead-212

Manganese-54

Neptunium-239

Nonvolatile beta

Plutonium-238

Plutonium-239/240

Potassium-40

Promethium-144

Promethium-146

Ruthenium-106

Sodium-22

Strontium-90

Thorium-234

Tin-113

Yttrium-88

Zinc-65

Zirconium-95

Sample ID: 104618
RQ AQ B Result
Unit
D. Limit Method

$\begin{array}{lll}\mathrm{mg} / \mathrm{kg} & 0.429 & \text { EPA6010A } \\ \mathrm{mg} / \mathrm{kg} & 1.07 & \text { EPA6010A } \\ \mathrm{mg} / \mathrm{kg} & 4.29 & \text { EPA6010A } \\ \mathrm{mg} / \mathrm{kg} & 4.29 & \text { EPA6010A } \\ \mathrm{mg} / \mathrm{kg} & 4.29 & \text { EPA6010A } \\ \mathrm{mg} / \mathrm{kg} & 0.429 & \text { EPA6010A } \\ \mathrm{mg} / \mathrm{kg} & 0.0363 & \text { EPA7471 } \\ \mathrm{mg} / \mathrm{kg} & 1.07 & \text { EPA6010A } \\ \mathrm{mg} / \mathrm{kg} & 85.8 & \text { EPA6010A } \\ \mathrm{mg} / \mathrm{kg} & 12.9 & \text { EPA6010A } \\ \mathrm{mg} / \mathrm{kg} & 1.07 & \text { EPA6010A } \\ \mathrm{mg} / \mathrm{kg} & 42.9 & \text { EPA6010A } \\ \mathrm{mg} / \mathrm{kg} & 12.9 & \text { EPA6010A } \\ \mathrm{mg} / \mathrm{kg} & 0.429 & \text { EPA6010A } \\ \mathrm{mg} / \mathrm{kg} & 0.858 & \text { EPA6010A }\end{array}$

$1.18 \mathrm{E}+00 \pm 5.50 \mathrm{E}-02 \mathrm{pCi} / \mathrm{g}$ $3.59 \mathrm{E}-03 \pm 7.25 \mathrm{E}-03 \mathrm{pCi} / \mathrm{g}$ 9.93E-03 $\pm 1.43 \mathrm{E}-02 \mathrm{pCi} / \mathrm{g}$ $1.75 \mathrm{E}-03 \pm 7.33 \mathrm{E}-03 \mathrm{pCi} / \mathrm{g}$ -2.62E-02 4.11E-02 pCi/g $-6.76 \mathrm{E}-02 \pm 6.90 \mathrm{E}-03 \mathrm{pCi} / \mathrm{g}$ 2.58E-02 $4.15 \mathrm{E}-03 \mathrm{pCi} / \mathrm{g}$ $-4.06 \mathrm{E}-03 \pm 5.15 \mathrm{E}-03 \mathrm{pCi} / \mathrm{g}$ $-3.12 \mathrm{E}-03 \pm 6.39 \mathrm{E}-03 \mathrm{pCi} / \mathrm{g}$ $1.16 \mathrm{E}-03 \pm 4.58 \mathrm{E}-03 \mathrm{pCi} / \mathrm{g}$ $-4.78 \mathrm{E}-03 \pm 1.53 \mathrm{E}-02 \mathrm{pCi} / \mathrm{g}$ $-3.28 \mathrm{E}-02 \pm 4.29 \mathrm{E}-02 \mathrm{pCi} / \mathrm{g}$ $0.00 \mathrm{E}+00 \quad \mathrm{pCi} / \mathrm{g}$ $1.54 \mathrm{E}+01 \pm 2.56 \mathrm{E}+00 \mathrm{pCi} / \mathrm{g}$ $1.25 \mathrm{E}+00 \pm 2.80 \mathrm{E}-02 \mathrm{pCi} / \mathrm{g}$ 2.23E-02 $\pm 1.01 \mathrm{E}-02 \mathrm{pCi} / \mathrm{g}$ 2.01E-02 4 .01E-02 pCi/g $9.74 \mathrm{E}+00 \pm 1.59 \mathrm{E}+00 \mathrm{pCi} / \mathrm{g}$ $-1.38 \mathrm{E}-03 \pm 9.50 \mathrm{E}-03 \mathrm{pCi} / \mathrm{g}$ $1.30 \mathrm{E}-03 \pm 5.88 \mathrm{E}-03 \mathrm{pCi} / \mathrm{g}$ 5.49E-01 $1.07 \mathrm{E}-01 \mathrm{pCi} / \mathrm{g}$ $-1.44 \mathrm{E}-03 \pm 5.11 \mathrm{E}-03 \mathrm{pCi} / \mathrm{g}$ 2.27E-03 $\pm 6.95 \mathrm{E}-03 \mathrm{pCi} / \mathrm{g}$ -7.05E-02 $4.82 \mathrm{E}-02 \mathrm{pCi} / \mathrm{g}$ $-1.11 \mathrm{E}-03 \pm 4.62 \mathrm{E}-03 \mathrm{pCi} / \mathrm{g}$ 5.95E-02 $44.03 \mathrm{E}-02 \mathrm{pCi} / \mathrm{g}$ 9.62E-01 $\pm 5.64 \mathrm{E}-01 \mathrm{pCi} / \mathrm{g}$ $-6.73 \mathrm{E}-03 \pm 8.34 \mathrm{E}-03 \mathrm{pCj} / \mathrm{g}$ $-1.33 \mathrm{E}-03 \pm 5.62 \mathrm{E}-03 \mathrm{pCi} / \mathrm{g}$ 2.83E-03 $\pm 1.03 \mathrm{E}-02 \mathrm{pCi} / \mathrm{g}$ 4.30E-03 $\pm 1.55 \mathrm{E}-02 \mathrm{pCj} / \mathrm{g}$
2.69E-02 EPIA-013B

1.29E-02 EPIA-013B

2.42E-02 EPIA-013B

1.10E-02 EPIA-013B

7.00E-02 EPIA-013B

8.34E-03 EPIA-013B

1.07E-02 EPIA-013B

8.77E-03 EPIA-013B

1.08E-02 EPIA-013B

8.36E-03 EPIA-013B

2.52E-02 EPIA-013B

7.01E-02 EPIA-013B

3.67E-02 EPIA-013B

1.48E+00 EPIA-001B

1.63E-02 EPIA-013B

7.92E-03 EPIA-013B

6.31E-02 EPIA-013B

2.12E+00 EPIA-001B

2.73E-02 EPIA-012B

1.65E-02 EPIA-012B

7.11E-02 EPIA-013B

8.75E-03 EPIA-013B

1.16E-02 EPIA-013B

7.90E-02 EPIA-013B

7.73E-03 EPIA-013B

6.09E-02 EPIA-004

4.41E-01 EPIA-013B

1.34E-02 EPIA-013B

9.85E-03 EPIA-013B

1.60E-02 EPIA-013B

2.39E-02 EPIA-013B 
Survey location: 45034E 3679647N (SRS coordinates)

Depth of core interval: 4.00 to $6.00 \mathrm{ft}$

Sample type: Normal

Sample matrix: Soil

Percent solids: 86.00

Constituent

RQ AQ B Result

Unit

D. Limit Method

Physical Parameters and Specified Analyses

Cation exchange capacity
Chloride
Cyanide
Nitrate-nitrite as nitrogen
Orthophosphate
Phenols
Phenols
Sulfide
Total organic carbon
Total organic nitrogen
Total phosphates (as P)

Metals (total recoverable)

$\begin{array}{lll} & & 87.4 \\ \text { J } & \text { E } & 1.5 \\ \text { U } & & 1.09 \\ \text { U } & & 0.941 \\ \text { U } & & 28.3 \\ \text { U } & & 349 \\ \text { U } & & 349 \\ \text { UJ } & \text { C } & 278 \\ & & 770 \\ \text { C } & & 26 \\ \text { J } & \text { E } & 86.8\end{array}$

$\begin{array}{ll}\text { U } & \\ J & E \\ J & E \\ U & \end{array}$

6160

4.48

7.43

5.73

0.0425

0.224

54.7

11.7

U

0.448

2.19

15100

6

64.2

3.07

$\begin{array}{lll}J & E & 0.0139 \\ J & E & 0.656\end{array}$

$\begin{array}{lll}\mathrm{J} & \mathrm{E} & 0.656 \\ \mathrm{~J} & \mathrm{E} & \mathbf{4 6 . 5}\end{array}$

$\begin{array}{lll}J & \mathrm{E} & 6.76\end{array}$

$\mathrm{U} \quad 1.12$

$\mathrm{U} \quad 44.8$

13.4

38.8

1.28

$\begin{array}{lll}\mathrm{meq} / 100 \mathrm{~g} & 5 & \text { EPA9081 } \\ \mathrm{mg} / \mathrm{kg} & 2.75 & \text { EPA300.0 } \\ \mathrm{mg} / \mathrm{kg} & 1.09 & \text { EPA335.3 } \\ \mathrm{mg} / \mathrm{kg} & 0.941 & \text { EPA353.1 } \\ \mathrm{mg} / \mathrm{kg} & 28.3 & \text { EPA365.2 } \\ \mu \mathrm{g} / \mathrm{kg} & 349 & \text { EPA420.2 } \\ \mu \mathrm{g} / \mathrm{kg} & 349 & \text { EPA420.2 } \\ \mathrm{mg} / \mathrm{kg} & 278 & \text { EPA376.2 } \\ \mathrm{mg} / \mathrm{kg} & 116 & \text { EPA415.1 } \\ \mathrm{mg} / \mathrm{kg} & 0.233 & \text { EPA351.2 } \\ \mathrm{mg} / \mathrm{kg} & 447 & \text { EPA365.4 }\end{array}$

Sodium

Thallium

Vanadium

Zinc

$1.11 \mathrm{E}+00 \pm 6.26 \mathrm{E}-02 \mathrm{pCi} / \mathrm{g}$ $-3.21 \mathrm{E}-03 \pm 7.75 \mathrm{E}-03 \mathrm{pCi} / \mathrm{g}$ $5.59 \mathrm{E}-03 \pm 1.55 \mathrm{E}-02 \mathrm{pCi} / \mathrm{g}$ $-2.33 \mathrm{E} \cdot 03 \pm 7.97 \mathrm{E}-03 \mathrm{pCi} / \mathrm{g}$ $-7.64 \mathrm{E}-03 \pm 4.40 \mathrm{E}-02 \mathrm{pCi} / \mathrm{g}$ $1.59 \mathrm{E}-03 \pm 5.70 \mathrm{E}-03 \mathrm{pCi} / \mathrm{g}$ $4.85 \mathrm{E}-04 \pm 7.13 \mathrm{E}-03 \mathrm{pCi} / \mathrm{g}$ $2.02 \mathrm{E}-03 \pm 5.47 \mathrm{E}-03 \mathrm{pCi} / \mathrm{g}$ 4.81E-03 $\pm 7.13 \mathrm{E}-03 \mathrm{pCi} / \mathrm{g}$ $1.70 \mathrm{E}-03 \pm 5.00 \mathrm{E}-03 \mathrm{pCi} / \mathrm{g}$ $-8.95 \mathrm{E}-03 \pm 1.62 \mathrm{E}-02 \mathrm{pCi} / \mathrm{g}$

$\begin{array}{ll}11.2 & \text { EPA6010A } \\ 4.48 & \text { EPA6010A } \\ 13.4 & \text { EPA6010A } \\ 1.12 & \text { EPA6010A } \\ 0.448 & \text { EPA6010A } \\ 0.224 & \text { EPA6010A } \\ 11.2 & \text { EPA6010A } \\ 1.12 & \text { EPA6010A } \\ 0.448 & \text { EPA6010A } \\ 1.12 & \text { EPA6010A } \\ 4.48 & \text { EPA6010A } \\ 4.48 & \text { EPA6010A } \\ 4.48 & \text { EPA6010A } \\ 0.448 & \text { EPA6010A } \\ 0.033 & \text { EPA7471 } \\ 1.12 & \text { EPA6010A } \\ 89.5 & \text { EPA6010A } \\ 13.4 & \text { EPA6010A } \\ 1.12 & \text { EPA6010A } \\ 44.8 & \text { EPA6010A } \\ 13.4 & \text { EPA6010A } \\ 0.448 & \text { EPA6010A } \\ 0.895 & \text { EPA6010A }\end{array}$

2.99E-02 EPIA-013B

1.31E-02 EPIA-013B

2.72E-02 EPIA-013B

1.14E-02 EPIA-013B

7.49E-02 EPIA-013B

8.77E-03 EPLA-013B

1.07E-02 EPIA-013B

- 9.43E-03 EPIA-013B

1.25E-02 EPIA-013B

9.02E-03 EPIA-013B

2.61E-02 EPIA-013B 
SURVEY ID: PPSC 0604 (continued)

Constituent

Radionuclides

Europium-154

Europium-155

Gross alpha

Lead-212

Manganese-54

Neptunium-239

Nonvolatile beta

Plutonium-238

Plutonium-239/240

Potassium-40

Promethium-144

Promethium-146

Ruthenium-106

Sodium-22

Strontium-90

Thorium-234

Tin-113

Yttrium-88

Zinc-65

Zirconium-95

Sample ID: 104619

RQ AQ B Result Unit D. Limit Method

UI

UI

UI

UI

UI

UI

UI

UI

UI

UI

UI

UI

UI

UI
$-1.50 \mathrm{E}-02 \pm 4.70 \mathrm{E}-02 \mathrm{pCi} / \mathrm{g}$ $0.00 \mathrm{E}+00 \quad \mathrm{pCi} / \mathrm{g}$

$1.64 \mathrm{E}+01 \pm 2.88 \mathrm{E}+00 \mathrm{pCi} / \mathrm{g}$

$1.18 \mathrm{E}+00 \pm 2.84 \mathrm{E}-02 \mathrm{pCi} / \mathrm{g}$ $2.39 \mathrm{E}-02 \pm 1.00 \mathrm{E}-02 \mathrm{pCi} / \mathrm{g}$ $2.15 \mathrm{E}-02 \pm 4.26 \mathrm{E}-02 \mathrm{pCi} / \mathrm{g}$ $1.06 \mathrm{E}+01 \pm 1.65 \mathrm{E}+00 \mathrm{pCi} / \mathrm{g}$ $1.10 \mathrm{E}-02 \pm 1.66 \mathrm{E}-02 \mathrm{pCi} / \mathrm{g}$ $-2.49 \mathrm{E}-03 \pm 3.52 \mathrm{E}-03 \mathrm{pCi} / \mathrm{g}$ $5.52 \mathrm{E}-01 \pm 9.67 \mathrm{E}-02 \mathrm{pCi} / \mathrm{g}$ $-6.00 \mathrm{E}-04 \pm 6.61 \mathrm{E}-03 \mathrm{pCi} / \mathrm{g}$ $7.25 \mathrm{E}-03 \pm 6.85 \mathrm{E}-03 \mathrm{pCi} / \mathrm{g}$ $-2.77 \mathrm{E}-02 \pm 5.31 \mathrm{E}-02 \mathrm{pCi} / \mathrm{g}$ $1.06 \mathrm{E}-03 \pm 5.60 \mathrm{E}-03 \mathrm{pCi} / \mathrm{g}$ $-1.55 \mathrm{E}-01 \pm 2.44 \mathrm{E}-02 \mathrm{pCi} / \mathrm{g}$ $8.43 \mathrm{E}-01 \pm 6.43 \mathrm{E}-01 \mathrm{pCi} / \mathrm{g}$ $-4.14 \mathrm{E}-03 \pm 8.09 \mathrm{E}-03 \mathrm{pCi} / \mathrm{g}$ $-2.94 \mathrm{E}-04 \pm 6.13 \mathrm{E}-03 \mathrm{pCi} / \mathrm{g}$ $-3.10 \mathrm{E}-03 \pm 1.33 \mathrm{E}-02 \mathrm{pCi} / \mathrm{g}$ $0.00 \mathrm{E}+00 \quad \mathrm{pCi} / \mathrm{g}$
7.09E-02 EPIA-013B

3.92E-02 EPIA-013B

$1.58 \mathrm{E}+00$ EPIA-001B

1.64E-02 EPIA-013B

8.96E-03 EPIA-013B

6.66E-02 EPIA-013B

2.13E+00 EPIA-001B

3.18E-02 EPIA-012B

1.89E-02 EPIA-012B

7.86E-02 EPIA-013B

9.78E-03 EPIA-013B

1.24E-02 EPIA-013B

8.88E-02 EPIA-013B

8.74E-03 EPIA-013B

4.10E-02 EPIA-004

5.08E-01 EPIA-013B

1.39E-02 EPIA.013B

1.12E-02 EPIA-013B

1.99E-02 EPIA-013B

2.78E-02 EPIA-013B

SURVEY ID: PPSC 0701

Sample ID: 104620

Survey location: $44980 \mathrm{E}$ 3680514N (SRS coordinates)

Depth of core interval: 0.00 to $1.00 \mathrm{ft}$

Sample type: Normal

Sample matrix: Soil

Percent solids: $\mathbf{6 9 . 0 0}$

Constituent

RQ AQ B Result

Unit

D. Limit Method

Physical Parameters and Specified Analyses

Cation exchange capacity

Chloride

Cyanide

Nitrate-nitrite as nitrogen

Orthophosphate

Phenols

Sulfide

Total organic carbon

Total organic nitrogen

Total phosphates (as P)

Metals (total recoverable)

Aluminum

Antimony

Arsenic

Barium

Beryllium

Cadmium

Calcium

Chromium

Cobalt

$\begin{array}{lll} & & 81.4 \\ \text { J } & \text { E } & 3.01 \\ \text { U } & & 1.01 \\ \text { J } & \text { E } & \mathbf{0 . 4 4 1} \\ \text { J } & \text { E } & 6.79 \\ \text { U } & & 435 \\ \text { UJ } & \text { C } & 349 \\ & & 14500 \\ \text { C } & & 332 \\ \text { J } & \text { E } & 161\end{array}$

$\mathrm{meq} / 100 \mathrm{~g}$ $\mathrm{mg} / \mathrm{kg} \quad 3.38$

$\mathrm{mg} / \mathrm{kg} \quad 1.01$

$\mathrm{mg} / \mathrm{kg} \quad 1.47$

$\mathrm{mg} / \mathrm{kg} \quad 31.7$

$\mu \mathrm{g} / \mathrm{kg} \quad 435$

$\mathrm{mg} / \mathrm{kg} \quad 349$

$\mathrm{mg} / \mathrm{kg} \quad 145$

$\mathrm{mg} / \mathrm{kg} \quad 0.29$

$\mathrm{mg} / \mathrm{kg} \quad 568$

EPA9081

EPA300.0

EPA335.3

EPA353.1

EPA365.2

EPA420.2

EPA376.2

EPA415.1

EPA351.2

EPA365.4

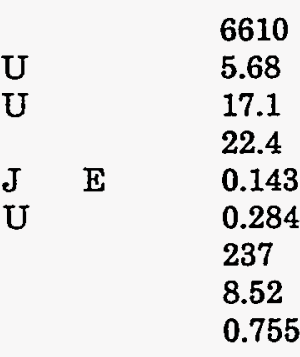

$\begin{array}{ll}\mathrm{mg} / \mathrm{kg} & 14.2 \\ \mathrm{mg} / \mathrm{kg} & 5.68 \\ \mathrm{mg} / \mathrm{kg} & 17.1 \\ \mathrm{mg} / \mathrm{kg} & 1.42 \\ \mathrm{mg} / \mathrm{kg} & 0.568 \\ \mathrm{mg} / \mathrm{kg} & 0.284 \\ \mathrm{mg} / \mathrm{kg} & 14.2 \\ \mathrm{mg} / \mathrm{kg} & 1.42 \\ \mathrm{mg} / \mathrm{kg} & 0.568\end{array}$

EPA6010A EPA6010A EPA6010A EPA6010A EPA6010A EPA6010A EPA6010A $\begin{array}{llll}8.52 & \mathrm{mg} / \mathrm{kg} & 1.42 & \text { EPA6010A } \\ 0.755 & \mathrm{mg} / \mathrm{kg} & 0.568 & \text { EPA6010A }\end{array}$ 
SURVEY ID: PPSC 0701 (continued)

Constituent

Metals (total recoverable)

Copper

Iron

Lead

Magnesium

Manganese

Mercury

Nickel

Potassium

Selenium

Silver

Sodium

Thallium

Vanadium

Zinc

Radionuclides

Actinium-228

Antimony-124

Antimony-125

Barium-133

Cerium-144

Cesium-134

Cesium-137

Cobalt-57

Cobalt-58

Cobalt-60

Europium-152

Europium-154

Europium-155

Gross alpha

Gross alpha

Lead-212

Manganese-54

Neptunium-239

Nonvolatile beta

Nonvolatile beta

Plutonium-238

Plutonium-239/240

Potassium-40

Promethium-144

Promethium-146

Ruthenium-106

Sodium-22

Strontium-90

Thorium-234

Tin-113

Yttrium-88

Zinc.65

Zirconium-95
Sample ID: 104620

RQ AQ B Result Unit D. Limit Method

$\begin{array}{llll}2.89 & \mathrm{mg} / \mathrm{kg} & 1.42 & \text { EPA6010A } \\ 5000 & \mathrm{mg} / \mathrm{kg} & 5.68 & \text { EPA6010A } \\ 6.3 & \mathrm{mg} / \mathrm{kg} & 5.68 & \text { EPA6010A } \\ 131 & \mathrm{mg} / \mathrm{kg} & 5.68 & \text { EPA6010A } \\ 60.8 & \mathrm{mg} / \mathrm{kg} & 0.568 & \text { EPA6010A } \\ 0.0533 & \mathrm{mg} / \mathrm{kg} & 0.043 & \text { EPA7471 } \\ 2.08 & \mathrm{mg} / \mathrm{kg} & 1.42 & \text { EPA6010A } \\ 95.1 & \mathrm{mg} / \mathrm{kg} & 114 & \text { EPA6010A } \\ 17.1 & \mathrm{mg} / \mathrm{kg} & 17.1 & \text { EPA6010A } \\ 1.42 & \mathrm{mg} / \mathrm{kg} & 1.42 & \text { EPA6010A } \\ 56.8 & \mathrm{mg} / \mathrm{kg} & 56.8 & \text { EPA6010A } \\ 17.1 & \mathrm{mg} / \mathrm{kg} & 17.1 & \text { EPA6010A } \\ 14.5 & \mathrm{mg} / \mathrm{kg} & 0.568 & \text { EPA6010A } \\ 6.35 & \mathrm{mg} / \mathrm{kg} & 1.14 & \text { EPA6010A }\end{array}$

$1.40 \mathrm{E}+00 \pm 6.91 \mathrm{E}-02 \mathrm{pCi} / \mathrm{g}$ $-3.77 \mathrm{E}-03 \pm 1.23 \mathrm{E}-02 \mathrm{pCi} / \mathrm{g}$ $1.46 \mathrm{E}-02 \pm 3.21 \mathrm{E}-02 \mathrm{pCi} / \mathrm{g}$

$-2.17 \mathrm{E}-01 \pm 1.58 \mathrm{E}-02 \mathrm{pCj} / \mathrm{g}$ $-4.81 \mathrm{E}-02 \pm 6.57 \mathrm{E}-02 \mathrm{pCi} / \mathrm{g}$ 9.74E-03 $\pm 9.10 \mathrm{E}-03 \mathrm{pCi} / \mathrm{g}$ $6.54 \mathrm{E}+00 \pm 6.51 \mathrm{E}-02 \mathrm{pCi} / \mathrm{g}$ $1.00 \mathrm{E}-03 \pm 8.31 \mathrm{E}-03 \mathrm{pCi} / \mathrm{g}$ $-7.50 \mathrm{E}-04 \pm 8.45 \mathrm{E}-03 \mathrm{pCi} / \mathrm{g}$ $3.98 \mathrm{E}-02 \pm 1.21 \mathrm{E}-02 \mathrm{pCi} / \mathrm{g}$ $-1.90 \mathrm{E}-02 \pm 2.81 \mathrm{E}-02 \mathrm{pCi} / \mathrm{g}$ 5.58E-02 $\pm 5.50 \mathrm{E}-02 \mathrm{pCi} / \mathrm{g}$ $0.00 \mathrm{E}+00 \quad \mathrm{pCi} / \mathrm{g}$

$2.13 \mathrm{E}+01 \pm 2.91 \mathrm{E}+00 \mathrm{pCi} / \mathrm{g}$ $2.15 \mathrm{E}+01 \pm 2.92 \mathrm{E}+00 \mathrm{pCi} / \mathrm{g}$ $1.48 \mathrm{E}+00 \pm 3.70 \mathrm{E}-02 \mathrm{pCi} / \mathrm{g}$ 2.73E-02+8.97E-03 pCi/g 2.38E-02 $\pm 6.44 \mathrm{E}-02 \mathrm{pCi} / \mathrm{g}$ $1.47 \mathrm{E}+01 \pm 1.85 \mathrm{E}+00 \mathrm{pCi} / \mathrm{g}$ $1.79 \mathrm{E}+01 \pm 2.00 \mathrm{E}+00 \mathrm{pCi} / \mathrm{g}$ $1.65 \mathrm{E}-02 \pm 1.76 \mathrm{E}-02 \mathrm{pCi} / \mathrm{g}$ $2.81 \mathrm{E}-02 \pm 1.82 \mathrm{E}-02 \mathrm{pCi} / \mathrm{g}$ 8.70E-01 $\pm 1.35 \mathrm{E}-01 \mathrm{pCi} / \mathrm{g}$ $-2.82 \mathrm{E}-03 \pm 6.73 \mathrm{E}-03 \mathrm{pCi} / \mathrm{g}$ $1.16 \mathrm{E}-02 \pm 1.61 \mathrm{E}-02 \mathrm{pCi} / \mathrm{g}$ $-1.54 \mathrm{E}-02 \pm 8.16 \mathrm{E}-02 \mathrm{pCi} / \mathrm{g}$ $5.70 \mathrm{E}-04 \pm 6.58 \mathrm{E}-03 \mathrm{pCi} / \mathrm{g}$ 3.26E-02 $\pm 3.34 \mathrm{E}-02 \mathrm{pCi} / \mathrm{g}$ $1.12 \mathrm{E}+00 \pm 6.61 \mathrm{E}-01 \mathrm{pCi} / \mathrm{g}$ $-1.30 \mathrm{E}-03 \pm 1.64 \mathrm{E}-02 \mathrm{pCi} / \mathrm{g}$ 7.20E-03 $\pm 6.65 \mathrm{E}-03 \mathrm{pCi} / \mathrm{g}$ $1.04 \mathrm{E}-02+2.11 \mathrm{E}-02 \mathrm{pCi} / \mathrm{g}$ $-7.60 \mathrm{E}-03 \pm 1.76 \mathrm{E}-02 \mathrm{pCi} / \mathrm{g}$
3.42E-02 EPIA-013B

2.05E-02 EPIA-013B

5.48E-02 EPIA-013B

2.17E-02 EPIA-013B

1.08E-01 EPIA-013B

1.38E-02 EPLA-013B

1.41E-02 EPIA-013B

1.39E-02 EPIA-013B

1.40E-02 EPIA-013B

9.23E-03 EPIA-013B

4.77E-02 EPIA-013B

9.07E-02 EPIA-013B

5.44E-02 EPIA-013B

1.31E+00 EPIA-001B

$1.48 \mathrm{E}+00$ EPIA-001B

2.73E-02 EPIA-013B

9.89E-03 EPIA-013B

9.68E-02 EPIA-013B

2.09E+00 EPIA-001B

1.97E+00 EPIA-001B

3.00E-02 EPIA-012B

1.96E-02 EPIA-012B

8.12E-02 EPIA-013B

1.11E-02 EPIA-013B

2.75E-02 EPIA-013B

1.36E-01 EPIA-013B

9.96E-03 EPIA-013B

5.46E-02 EPIA-004

6.75E-01 EPIA-013B

2.78E-02 EPIA-013B

1.31E-02 EPIA-013B

2.29E-02 EPIA-013B

2.88E-02 EPIA-013B 
Survey location: $44980 \mathrm{E} 3680514 \mathrm{~N}$ (SRS coordinates)

Depth of core interval: 1.00 to $2.00 \mathrm{ft}$

Sample type: Normal

Sample matrix: Soil

Percent solids: $\mathbf{7 9 . 0 0}$

Constituent

RQ AQ B Result

Unit

D. Limit Method

Physical Parameters and Specified Analyses

Cation exchange capacity

Chloride

Cyanide

Nitrate-nitrite as nitrogen

Orthophosphate

Phenols

Sulfide

Total organic carbon

Total organic carbon

Total organic nitrogen

Total phosphates (as P)

Metals (total recoverable)

Aluminum

Antimony

Arsenic

Barium

Beryllium

Cadmium

Calcium

Chromium

Cobalt

Copper

Iron

Lead

Magnesium

Manganese

Mercury

Nickel

Potassium

Selenium

Silver

Sodium

Thallium

Vanadium

Zinc

\section{Radionuclides}

Actinium-228

Antimony-124

Antimony-125

Barium-133

Cerium-144

Cesium-134

Cesium-137

Cobalt-57

Cobalt-58

Cobalt-60

Europium-152

$\begin{array}{lll} & & 55.6 \\ \mathrm{~J} & \mathrm{E} & 1.23 \\ \mathrm{U} & & 1.11 \\ \mathrm{U} & & 1.14 \\ \mathrm{U} & & 24.3 \\ \mathrm{UJ} & \mathrm{C} & 395 \\ & & 275 \\ & & 2820 \\ \mathrm{C} & & 3170 \\ \text { J } & \text { E } & 102 \\ & & 131\end{array}$

$\mathbf{7 8 6 0}$

$\mathrm{U} \quad \mathbf{5 . 6 6}$

U 17

$\begin{array}{lll} & \mathrm{E} & \mathbf{3 2 . 3} \\ \mathrm{U} & & 0.179\end{array}$

U 0.283

123

6.74

0.972

2.16

5020

6.24

180

38.5

J E $\quad 0.0365$

2.58

120

U $\quad 17$

U 1.41

$\begin{array}{lll}\mathrm{J} & \mathrm{E} & 8.91\end{array}$

2.81

14

4.52

$\begin{array}{lll}\mathrm{meq} / 100 \mathrm{~g} & 5 & \text { EPA9081 } \\ \mathrm{mg} / \mathrm{kg} & 2.9 & \text { EPA300.0 } \\ \mathrm{mg} / \mathrm{kg} & 1.11 & \text { EPA335.3 } \\ \mathrm{mg} / \mathrm{kg} & 1.14 & \text { EPA353.1 } \\ \mathrm{mg} / \mathrm{kg} & 24.3 & \text { EPA365.2 } \\ \mu \mathrm{g} / \mathrm{kg} & 395 & \text { EPA420.2 } \\ \mathrm{mg} / \mathrm{kg} & 275 & \text { EPA376.2 } \\ \mathrm{mg} / \mathrm{kg} & 127 & \text { EPA415.1 } \\ \mathrm{mg} / \mathrm{kg} & 127 & \text { EPA415.1 } \\ \mathrm{mg} / \mathrm{kg} & 0.253 & \text { EPA351.2 } \\ \mathrm{mg} / \mathrm{kg} & 465 & \text { EPA365.4 }\end{array}$

$\mathrm{mg} / \mathrm{kg}$

$\mathrm{mg} / \mathrm{kg}$

$\mathrm{mg} / \mathrm{kg}$

$\mathrm{mg} / \mathrm{kg}$

$\mathrm{mg} / \mathrm{kg}$

$\mathrm{mg} / \mathrm{kg}$

$\mathrm{mg} / \mathrm{kg}$

$\mathrm{mg} / \mathrm{kg}$

$\mathrm{mg} / \mathrm{kg}$

$\mathrm{mg} / \mathrm{kg}$

$\mathrm{mg} / \mathrm{kg}$

$\mathrm{mg} / \mathrm{kg}$

$\mathrm{mg} / \mathrm{kg}$

$\mathrm{mg} / \mathrm{kg}$

$\mathrm{mg} / \mathrm{kg}$

$\mathrm{mg} / \mathrm{kg}$

$\mathrm{mg} / \mathrm{kg}$

$\mathrm{mg} / \mathrm{kg}$

$\mathrm{mg} / \mathrm{kg}$

$\mathrm{mg} / \mathrm{kg}$

$\mathrm{mg} / \mathrm{kg}$

$\mathrm{mg} / \mathrm{kg}$

$\mathrm{mg} / \mathrm{kg}$

14.1

5.66

17

1.41

0.566

0.283

14.1

1.41

0.566

1.41

5.66

5.66

5.66

0.566

0.0414

1.41

113

17

1.41

56.6

17

0.566

1.13
EPA6010A EPA6010A EPA6010A EPA6010A EPA6010A EPA6010A EPA6010A EPA6010A EPA6010A EPA6010A EPA6010A EPA6010A EPA6010A EPA6010A EPA7471 EPA6010A EPA6010A EPA6010A EPA6010A EPA6010A EPA6010A EPA6010A EPA6010A
2.81E-02 EPLA-013B

1.31E-02 EPIA-013B

2.66E-02 EPIA-013B

1.11E-02 EPIA-013B

7.46E-02 EPIA-013B

8.68E-03 EPIA-013B

1.11E-02 EPIA-013B

9.58E-03 EPIA-013B

1.15E-02 EPIA-013B

8.40E-03 EPIA-013B

2.62E-02 EPIA-013B 
SURVEY ID: PPSC 0702 (continued)

Constituent

Radionuclides

Europium-154

Europium-155

Gross alpha

Lead-212

Manganese-54

Neptunium-239

Nonvolatile beta

Plutonium-238

Plutonium-239/240

Potassium-40

Promethium-144

Promethium-146

Ruthenium-106

Sodium-22

Strontium-90

Thorium-234

Tin-113

Yttrium-88

Zinc-65

Zirconium-95
Sample ID: 104621

RQ AQ B Result Unit D. Limit Method
7.49E-02 EPIA-013B

3.98E-02 EPIA-013B

1.32E+00 EPIA-001B

1.70E-02 EPIA-013B

8.74E-03 EPIA-013B

6.64E-02 EPIA-013B

2.11E+00 EPIA-001B

2.72E-02 EPIA-012B

1.65E-02 EPIA-012B

7.46E-02 EPIA-013B

9.03E-03 EPLA-013B

1.27E-02 EPIA-013B

8.54E-02 EPIA-013B

9.20E-03 EPIA-013B

5.12E-02 EPIA-004

5.02E-01 EPIA-013B

1.43E-02 EPIA-013B

1.11E-02 EPIA-013B

1.74E-02 EPIA-013B

2.69E-02 EPIA-013B

\section{SURVEY ID: PPSC 0703}

Sample ID: 104622

Survey location: 44980E $3680514 N$ (SRS coordinates)

Depth of core interval: 2.00 to $4.00 \mathrm{ft}$

Sample type: Normal

$\begin{array}{lllll}\text { Constituent } & \text { RQ AQ B Result D. Limit Method }\end{array}$

Physical Parameters and Specified Analyses

Cation exchange capacity

Chloride

Cyanide

Nitrate-nitrite as nitrogen

Orthophosphate

Phenols

Sulfide

Total organic carbon

Total organic nitrogen

Total phosphates (as P)

Metals (total recoverable)

$\begin{array}{lll} & & 68.1 \\ \text { U } & & 2.97 \\ \text { U } & & 1.23 \\ \text { U } & & 1.01 \\ \text { U } & & 20.9 \\ \text { U } & & 366 \\ \text { UJ } & \text { C } & 265 \\ & & 1120 \\ \text { C } & & 117 \\ \text { J } & \text { E } & 150\end{array}$

$\begin{array}{ll}\mathrm{meq} / 100 \mathrm{~g} & 5 \\ \mathrm{mg} / \mathrm{kg} & 2.97 \\ \mathrm{mg} / \mathrm{kg} & 1.23 \\ \mathrm{mg} / \mathrm{kg} & 1.01 \\ \mathrm{mg} / \mathrm{kg} & 20.9 \\ \mu \mathrm{gg} / \mathrm{kg} & 366 \\ \mathrm{mg} / \mathrm{kg} & 265 \\ \mathrm{mg} / \mathrm{kg} & 122 \\ \mathrm{mg} / \mathrm{kg} & 0.244 \\ \mathrm{mg} / \mathrm{kg} & 465\end{array}$

EPA9081 EPA300.0 EPA335.3 EPA353.1 EPA365.2 EPA420.2 EPA376.2 EPA415.1 EPA351.2 EPA365.4

$\begin{array}{llll}\text { Aluminum } & & & 8300 \\ \text { Antimony } & \mathrm{U} & & 5.44 \\ \text { Arsenic } & \mathrm{U} & & 16.3 \\ \text { Barium } & & & 19.4 \\ \text { Beryllium } & \mathrm{J} & \mathrm{E} & \mathbf{0 . 1 4 1} \\ \text { Cadmium } & \mathrm{U} & & 0.272 \\ \text { Calcium } & & & 69.9 \\ \text { Chromium } & & & 7.98 \\ \text { Cobalt } & & & \\ \end{array}$

$\begin{array}{lll}\mathrm{mg} / \mathrm{kg} & 13.6 & \text { EPA6010A } \\ \mathrm{mg} / \mathrm{kg} & 5.44 & \text { EPA6010A } \\ \mathrm{mg} / \mathrm{kg} & 16.3 & \text { EPA6010A } \\ \mathrm{mg} / \mathrm{kg} & 1.36 & \text { EPA6010A } \\ \mathrm{mg} / \mathrm{kg} & 0.544 & \text { EPA6010A } \\ \mathrm{mg} / \mathrm{kg} & 0.272 & \text { EPA6010A } \\ \mathrm{mg} / \mathrm{kg} & 13.6 & \text { EPA6010A } \\ \mathrm{mg} / \mathrm{kg} & 1.36 & \text { EPA6010A } \\ \mathrm{mg} / \mathrm{kg} & 0.544 & \text { EPA6010A }\end{array}$


SURVEY ID: PPSC 0703 (continued)

Constituent

Metals (total recoverable)
Sample ID: 104622
Unit
D. Limit Method

\begin{tabular}{|c|c|c|c|}
\hline $\begin{array}{l}\text { Copper } \\
\text { Iron }\end{array}$ & & & $\begin{array}{l}3.02 \\
7810\end{array}$ \\
\hline Lead & $J$ & $\mathrm{E}$ & 5.05 \\
\hline Magnesium & & & 139 \\
\hline Manganese & & & 22.3 \\
\hline Mercury & & & 0.055 \\
\hline Nickel & & & 2.11 \\
\hline Potassium & & & 117 \\
\hline Selenium & $\mathrm{U}$ & & 16.3 \\
\hline Silver & $\mathrm{U}$ & & 1.36 \\
\hline Sodium & $\mathbf{J}$ & $\mathbf{E}$ & 10.3 \\
\hline Thallium & $\mathrm{U}$ & & 16.3 \\
\hline Vanadium & & & 19.1 \\
\hline Zinc & & & 2.89 \\
\hline
\end{tabular}

Radionuclides

Actinium-228

Antimony-124

Antimony-125

Barium-133

Cerium-144

Cesium-134

Cesium-137

Cobalt-57

Cobalt-58

Cobalt -60

Europium-152

Europium-154

Europium-155

Gross alpha

Lead-212

Manganese-54

Neptunium-239

Nonvolatile beta

Plutonium-238

Plutonium-239/240

Potassium-40

Promethium-144

Promethium-146

Ruthenium-106

Sodium-22

Strontium-90

Thorium-234

Tin-113

Yttrium-88

Zinc-65

Zirconium-95

\begin{abstract}
1.47E $+00 \pm 6.12 \mathrm{E}-02 \mathrm{pCj} / \mathrm{g}$ $2.92 \mathrm{E}-03 \pm 8.59 \mathrm{E}-03 \mathrm{pCi} / \mathrm{g}$ $1.28 \mathrm{E}-02 \pm 1.63 \mathrm{E}-02 \mathrm{pCi} / \mathrm{g}$ $-7.58 \mathrm{E}-04 \pm 1.10 \mathrm{E}-02 \mathrm{pCi} / \mathrm{g}$ 7.38E-03 $\pm 4.98 \mathrm{E}-02 \mathrm{pCi} / \mathrm{g}$ $3.33 \mathrm{E}-03 \pm 6.50 \mathrm{E}-03 \mathrm{pCi} / \mathrm{g}$ $1.49 \mathrm{E}-02 \pm 9.98 \mathrm{E}-03 \mathrm{pCi} / \mathrm{g}$ $6.15 \mathrm{E}-04 \pm 6.21 \mathrm{E}-03 \mathrm{pCi} / \mathrm{g}$ $3.00 \mathrm{E}-03 \pm 8.28 \mathrm{E}-03 \mathrm{pCi} / \mathrm{g}$ $1.00 \mathrm{E}-03 \pm 5.07 \mathrm{E}-03 \mathrm{pCi} / \mathrm{g}$ $3.18 \mathrm{E}-04 \pm 1.92 \mathrm{E}-02 \mathrm{pCi} / \mathrm{g}$ $3.45 \mathrm{E}-02 \pm 5.54 \mathrm{E}-02 \mathrm{pCi} / \mathrm{g}$ $0.00 \mathrm{E}+00 \quad \mathrm{pCi} / \mathrm{g}$ $3.04 \mathrm{E}+01 \pm 3.55 \mathrm{E}+00 \mathrm{pCi} / \mathrm{g}$ $1.50 \mathrm{E}+00 \pm 3.23 \mathrm{E}-02 \mathrm{pCi} / \mathrm{g}$ 2.68E-0249.95E-03 pCi/g $-1.22 \mathrm{E}-02 \pm 4.47 \mathrm{E}-02 \mathrm{pCi} / \mathrm{g}$ $1.39 \mathrm{E}+01 \pm 1.87 \mathrm{E}+00 \mathrm{pCi} / \mathrm{g}$ $0.00 \mathrm{E}+00 \quad \mathrm{pCj} / \mathrm{g}$ $-2.57 \mathrm{E}-03 \pm 3.64 \mathrm{E}-03 \mathrm{pCi} / \mathrm{g}$ 9.67E-01 $\pm 1.24 \mathrm{E}-01 \mathrm{pCi} / \mathrm{g}$ $-3.83 \mathrm{E}-04 \pm 6.22 \mathrm{E}-03 \mathrm{pCi} / \mathrm{g}$ $0.00 \mathrm{E}+00 \quad \mathrm{pCi} / \mathrm{g}$ $-5.13 \mathrm{E}-05 \pm 5.66 \mathrm{E}-02 \mathrm{pCi} / \mathrm{g}$ $-6.90 \mathrm{E}-03 \pm 5.55 \mathrm{E}-03 \mathrm{pCi} / \mathrm{g}$ $5.58 \mathrm{E}-02 \pm 3.05 \mathrm{E}-02 \mathrm{pCi} / \mathrm{g}$ $1.46 \mathrm{E}+00 \pm 6.81 \mathrm{E}-01 \mathrm{pCi} / \mathrm{g}$ $-6.38 \mathrm{E}-03 \pm 9.24 \mathrm{E}-03 \mathrm{pCi} / \mathrm{g}$ $6.48 \mathrm{E}-03 \pm 6.27 \mathrm{E}-03 \mathrm{pCi} / \mathrm{g}$ $0.00 \mathrm{E}+00 \quad \mathrm{pCi} / \mathrm{g}$ 2.39E-02 $\pm 1.91 \mathrm{E}-02 \mathrm{pCi} / \mathrm{g}$
\end{abstract}

$\begin{array}{ll}1.36 & \text { EPA6010A } \\ 5.44 & \text { EPA6010A } \\ 5.44 & \text { EPA6010A } \\ 5.44 & \text { EPA6010A } \\ 0.544 & \text { EPA6010A } \\ 0.035 & \text { EPA7471 } \\ 1.36 & \text { EPA6010A } \\ 109 & \text { EPA6010A } \\ 16.3 & \text { EPA6010A } \\ 1.36 & \text { EPA6010A } \\ 54.4 & \text { EPA6010A } \\ 16.3 & \text { EPA6010A } \\ 0.544 & \text { EPA6010A } \\ 1.09 & \text { EPA6010A }\end{array}$

2.85E-02 EPIA-013B

1.47E-02 EPIA-013B

2.85E-02 EPIA-013B

1.22E-02 EPIA-013B

8.31E-02 EPIA-013B

9.84E-03 EPIA-013B

1.06E-02 EPIA-013B

1.04E-02 EPIA-013B

1.24E-02 EPIA-013B

8.91E-03 EPIA-013B

2.92E-02 EPIA-013B

8.55E-02 EPIA-013B

4.37E-02 EPLA-013B

1.26E+00 EPIA-001B

1.82E-02 EPIA-013B

9.84E-03 EPIA-013B

7.45E-02 EPIA-013B

$2.25 \mathrm{E}+00$ EPIA-001B

$0.00 \mathrm{E}+00$ EPIA-012B

1.97E-02 EPIA-012B

8.66E-02 EPIA-013B

1.04E-02 EPIA-013B

1.45E-02 EPIA-013B

9.54E-02 EPIA-013B

8.86E-03 EPIA-013B

4.36E-02 EPIA-004

5.50E-01 EPIA-013B

1.56E-02 EPIA-013B

1.23E-02 EPIA-013B

2.03E-02 EPIA-013B

2.97E-02 EPIA-013B

\section{SURVEY ID: PPSC 0704}

Sample ID: 104623

Survey location: 44980E 3680514N (SRS coordinates)

Depth of core interval: 4.00 to $5.80 \mathrm{ft}$

Sample type: Normal 
SURVEY ID: PPSC 0704 (continued)

Constituent
Sample ID: 104623

Unit D. Limit Method

Physical Parameters and Specified Analyses

Cation exchange capacity

Cation exchange capacity

Chloride

Cyanide

Nitrate-nitrite as nitrogen

Nitrate-nitrite as nitrogen

Orthophosphate

Orthophosphate

Phenols

Sulfide

Total organic carbon

Total organic nitrogen

Total phosphates (as P)

Total phosphates (as P)

Metals (total recoverable)

Aluminum

Antimony

Arsenic

Barium

Beryllium

Cadmium

Calcium

Chromium

Cobalt

Copper

Iron

Lead

Magnesium

Manganese

Mercury

Nickel

Potassium

Selenium

Silver

Sodium

Thallium

Vanadium

Zinc

Radionuclides

Actinium-228

Actinium-228

Antimony-124

Antimony-124

Antimony-125

Antimony-125

Barium-133

Barium-133

Cerium-144

Cerium-144

Cesium-134

Cesium-134
RQ AQ B Result

$\begin{array}{llll} & & 91.5 \\ & & 126 \\ J & \mathrm{E} & 1.81 \\ \mathrm{U} & & 1.2 \\ \mathrm{U} & & 1.23 \\ \mathrm{U} & & 1.09 \\ \mathrm{U} & & 30.4 \\ \mathrm{U} & & 29.6 \\ \mathrm{U} & & 366 \\ \mathrm{UJ} & \mathrm{C} & 302 \\ & & 904 \\ \mathrm{C} & & 76 \\ \text { J } & \mathrm{E} & 115 \\ \text { J } & \mathrm{E} & & 132\end{array}$

$\begin{array}{lll} & & 15000 \\ U & & 5.4 \\ J & E & 7.31 \\ & & 26.4 \\ J & E & 0.173 \\ U & & 0.27 \\ & & 76.4 \\ & & 18.9 \\ U & & 0.54 \\ & & 6.2 \\ & & 27800 \\ & & 11 \\ & & 118 \\ & & 20.3 \\ & & 0.111 \\ J & \cdot & 1.63 \\ J & E & 96.2 \\ J & E & 7.64 \\ J & E & 0.549 \\ U & & 10.7 \\ & & 16.2 \\ & & 57.1 \\ & & 2.29\end{array}$

$\mathrm{mg} / \mathrm{kg}$
$\mathrm{mg} / \mathrm{kg}$
$\mathrm{mg} / \mathrm{kg}$
$\mathrm{mg} / \mathrm{kg}$
$\mathrm{mg} / \mathrm{kg}$
$\mathrm{mg} / \mathrm{kg}$
$\mathrm{mg} / \mathrm{kg}$
$\mathrm{mg} / \mathrm{kg}$
$\mathrm{mg} / \mathrm{kg}$
$\mathrm{mg} / \mathrm{kg}$
$\mathrm{mg} / \mathrm{kg}$
$\mathrm{mg} / \mathrm{kg}$
$\mathrm{mg} / \mathrm{kg}$
$\mathrm{mg} / \mathrm{kg}$
$\mathrm{mg} / \mathrm{kg}$
$\mathrm{mg} / \mathrm{kg}$
$\mathrm{mg} / \mathrm{kg}$
$\mathrm{mg} / \mathrm{kg}$
$\mathrm{mg} / \mathrm{kg}$
$\mathrm{mg} / \mathrm{kg}$
$\mathrm{mg} / \mathrm{kg}$
$\mathrm{mg} / \mathrm{kg}$
$\mathrm{mg} / \mathrm{kg}$

13.5

5.4

16.2

1.35

0.54

0.27

13.5

1.35

0.54

1.35

5.4

5.4

5.4

0.54

0.0333

1.35

108

16.2

1.35

54

16.2

0.54

1.08
EPA9081

EPA9081

EPA300.0

EPA335.3

EPA353.1

EPA353.1

EPA365.2

EPA365.2

EPA420.2

EPA376.2

EPA415.1

EPA351.2

EPA365.4

EPA365.4
EPA6010A

EPA6010A

EPA6010A

EPA6010A

EPA6010A

EPA6010A

EPA6010A

EPA6010A

EPA6010A

EPA6010A

EPA6010A

EPA6010A

EPA6010A

EPA6010A

EPA7471

EPA6010A

EPA6010A

EPA6010A

EPA6010A

EPA6010A

EPA6010A

EPA6010A

EPA6010A
4.31E-02 EPIA-013B

4.43E-02 EPIA-013B

1.89E-02 EPIA-013B

1.96E-02 EPIA-013B

4.08E-02 EPIA-013B

3.91E-02 EPIA-013B

1.71E-02 EPIA-013B

1.76E-02 EPIA-013B

1.12E-01 EPIA-013B

1.09E-01 EPIA-013B

1.32E-02 EPIA-013B

1.29E-02 EPIA-013B 
SURVEY ID: PPSC 0704 (continued)

Constituent

Radionuclides

Cesium-137

Cesium-137

Cobalt-57

Cobalt-57

Cobalt-58

Cobalt-58

Cobalt-60

Cobalt-60

Europium-152

Europium-152

Europium-154

Europium-154

Europium-155

Europium-155

Gross alpha

Lead-212

Lead-212

Manganese-54

Manganese-54

Neptunium-239

Neptunium-239

Nonvolatile beta

Plutonium-238

Plutonium-238

Plutonium-239/240

Plutonium-239/240

Potassium -40

Potassium-40

Promethium-144

Promethium-144

Promethium-146

Promethium-146

Ruthenium-106

Ruthenium-106

Sodium-22

Sodium-22

Strontium-90

Strontium-90

Thorium-234

Thorium-234

Tin-113

Tin-113

Yttrium-88

Yttrium-88

Zinc-65

Zinc-65

Zirconium-95

Zirconium-95

UI

UI

UI

UI

UI

UI

UI

UI

UI

UI

UI

UI

UI

UI

UI
UI

UI

UI

UI

UI

UI

UI

UI

UI

UI

UI

UI

UI

SURVEY ID: PPSC 0801

RQ AQ B Result Unit

Sample ID: 104623

$-8.58 \mathrm{E}-04 \pm 1.03 \mathrm{E}-02 \mathrm{pCi} / \mathrm{g}$ $-3.53 \mathrm{E}-03 \pm 9.91 \mathrm{E}-03 \mathrm{pCi} / \mathrm{g}$ $3.08 \mathrm{E}-03 \pm 8.45 \mathrm{E}-03 \mathrm{pCi} / \mathrm{g}$ $-3.70 \mathrm{E}-03 \pm 7.91 \mathrm{E}-03 \mathrm{pCi} / \mathrm{g}$ $-2.75 \mathrm{E}-03 \pm 1.24 \mathrm{E}-02 \mathrm{pCi} / \mathrm{g}$ $4.69 \mathrm{E}-03 \pm 1.04 \mathrm{E}-02 \mathrm{pCi} / \mathrm{g}$ $4.13 \mathrm{E}-04 \pm 8.38 \mathrm{E}-03 \mathrm{pCi} / \mathrm{g}$ $3.48 \mathrm{E}-03 \pm 8.18 \mathrm{E}-03 \mathrm{pCi} / \mathrm{g}$ $-1.77 \mathrm{E}-02 \pm 2.38 \mathrm{E}-02 \mathrm{pCj} / \mathrm{g}$ $-9.79 \mathrm{E}-03 \pm 2.35 \mathrm{E}-02 \mathrm{pCi} / \mathrm{g}$ $4.30 \mathrm{E}-03 \pm 6.99 \mathrm{E}-02 \mathrm{pCi} / \mathrm{g}$ $1.84 \mathrm{E}-02 \pm 7.38 \mathrm{E}-02 \mathrm{pCi} / \mathrm{g}$ $0.00 \mathrm{E}+00 \quad \mathrm{pCi} / \mathrm{g}$ $0.00 \mathrm{E}+00 \quad \mathrm{pCi} / \mathrm{g}$ $2.02 \mathrm{E}+01 \pm 4.20 \mathrm{E}+00 \mathrm{pCi} / \mathrm{g}$ $2.29 \mathrm{E}+00 \pm 4.29 \mathrm{E}-02 \mathrm{pCi} / \mathrm{g}$ $2.34 \mathrm{E}+00 \pm 4.32 \mathrm{E}-02 \mathrm{pCj} / \mathrm{g}$ 2.89E-02 $\pm 1.17 \mathrm{E}-02 \mathrm{pCi} / \mathrm{g}$ $3.83 \mathrm{E}-02 \pm 1.30 \mathrm{E}-02 \mathrm{pCi} / \mathrm{g}$ $8.89 \mathrm{E}-02 \pm 7.67 \mathrm{E}-02 \mathrm{pCi} / \mathrm{g}$ 6.25E-02 $\pm 6.13 \mathrm{E}-02 \mathrm{pCi} / \mathrm{g}$ $9.50 \mathrm{E}+00 \pm 2.60 \mathrm{E}+00 \mathrm{pCi} / \mathrm{g}$ $1.66 \mathrm{E}-02 \pm 1.75 \mathrm{E}-02 \mathrm{pCi} / \mathrm{g}$ $1.47 \mathrm{E}-02 \pm 1.95 \mathrm{E}-02 \mathrm{pCi} / \mathrm{g}$ 5.09E-03 $49.51 \mathrm{E}-03 \mathrm{pCi} / \mathrm{g}$ 5.26E-03 7.45E-03 pCi/g $1.54 \mathrm{E}+00 \pm 1.93 \mathrm{E}-01 \mathrm{pCi} / \mathrm{g}$ $1.58 \mathrm{E}+00 \pm 1.65 \mathrm{E}-01 \mathrm{pCi} / \mathrm{g}$ $-1.70 \mathrm{E}-03 \pm 8.58 \mathrm{E}-03 \mathrm{pCi} / \mathrm{g}$ $3.58 \mathrm{E}-03 \pm 8.69 \mathrm{E}-03 \mathrm{pCi} / \mathrm{g}$ $1.87 \mathrm{E}-02 \pm 1.25 \mathrm{E}-02 \mathrm{pCi} / \mathrm{g}$ $1.30 \mathrm{E}-02 \pm 1.11 \mathrm{E}-02 \mathrm{pCi} / \mathrm{g}$ 2.17E-02 $\pm 7.97 \mathrm{E}-02 \mathrm{pCi} / \mathrm{g}$ $-2.16 \mathrm{E}-03 \pm 7.62 \mathrm{E}-02 \mathrm{pCi} / \mathrm{g}$ 7.54E-03 $1.04 \mathrm{E}-02 \mathrm{pCi} / \mathrm{g}$ $-1.73 \mathrm{E}-03 \pm 8.01 \mathrm{E}-03 \mathrm{pCi} / \mathrm{g}$ 7.11E-02 $\pm 3.78 \mathrm{E}-02 \mathrm{pCi} / \mathrm{g}$ $1.72 \mathrm{E}-01 \pm 5.25 \mathrm{E}-02 \mathrm{pCi} / \mathrm{g}$ $2.16 \mathrm{E}+00 \pm 8.66 \mathrm{E}-01 \mathrm{pCi} / \mathrm{g}$ $1.29 \mathrm{E}+00 \pm 7.43 \mathrm{E}-01 \mathrm{pCi} / \mathrm{g}$ $-8.35 \mathrm{E}-03 \pm 1.28 \mathrm{E}-02 \mathrm{pCi} / \mathrm{g}$ $-1.02 \mathrm{E}-03 \pm 1.30 \mathrm{E}-02 \mathrm{pCi} / \mathrm{g}$ 5.77E-03 $\pm 8.58 \mathrm{E}-03 \mathrm{pCi} / \mathrm{g}$ $3.12 \mathrm{E}-03 \pm 8.98 \mathrm{E}-03 \mathrm{pCi} / \mathrm{g}$ $0.00 \mathrm{E}+00 \quad \mathrm{pCi} / \mathrm{g}$ $3.85 \mathrm{E}-03 \pm 1.83 \mathrm{E}-02 \mathrm{pCi} / \mathrm{g}$ $3.09 \mathrm{E}-02 \pm 2.58 \mathrm{E}-02 \mathrm{pCi} / \mathrm{g}$ 2.94E-02 $2.41 \mathrm{E}-02 \mathrm{pCi} / \mathrm{g}$
1.50E-02 EPIA-013B
1.48E-02 EPIA-013B
1.42E-02 EPIA-013B
1.35E-02 EPIA-013B
1.77E-02 EPIA-013B
1.82E-02 EPIA-013B
1.25E-02 EPIA-013B
1.32E-02 EPIA-013B
4.04E-02 EPIA-013B
3.85E-02 EPIA-013B
1.07E-01 EPIA-013B
1.13E-01 EPIA-013B
5.75E-02 EPIA-013B
5.66E-02 EPIA-013B
$2.25 \mathrm{E}+00$ EPLA-001B
2.50E-02 EPIA-013B
2.39E-02 EPIA-013B
1.48E-02 EPIA-013B
1.36E-02 EPIA-013B
9.88E-02 EPIA-013B
9.72E-02 EPIA-013B
4.05E+00 EPIA-001B
2.97E-02 EPIA-012B
3.64E-02 EPIA-012B
1.94E-02 EPIA-012B
7.89E-03 EPIA-012B
1.23E-01 EPIA-013B
1.13E-01 EPIA-013B
1.42E-02 EPIA-013B
1.52E-02 EPIA-013B
1.97E-02 EPIA-013B
1.89E-02 EPIA-013B
1.35E-01 EPLA-013B
1.32E-01 EPIA-013B
1.19E-02 EPIA-013B
1.33E-02 EPIA-013B
5.37E-02 EPIA-004
6.09E-02 EPIA-004
7.49E-01 EPIA-013B
7.51E-01 EPIA-013B
2.16E-02 EPIA-013B
2.13E-02 EPIA-013B
1.61E-02 EPIA-013B
1.64E-02 EPIA-013B
3.05E-02 EPIA-013B
2.76E-02 EPLA-013B
3.96E-02 EPIA-013B
3.87E-02 EPIA-013B

Survey location: 45133E $3680562 N$ (SRS coordinates)

Depth of core interval: 0.00 to $1.00 \mathrm{ft}$

Sample type: Normal

Sample matrix: Soil

Percent solids: $\mathbf{7 3 . 0 0}$ 
SURVEY ID: PPSC 0801 (continued)

Constituent
Sample ID: 104630

RQ AQ B Result

Unit

D. Limit Method

Physical Parameters and Specified Analyses

Cation exchange capacity

Chloride

Cyanide

Nitrate-nitrite as nitrogen

Orthophosphate

Phenols

Sulfide

Sulfide

Total organic carbon

Total organic nitrogen

Total phosphates (as P)

Metals (total recoverable)

$\begin{array}{llllll} & & 97 & \mathrm{meq} / 100 \mathrm{~g} & 5 & \text { EPA9081 } \\ \mathrm{J} & \mathrm{E} & \mathbf{2 . 9 9} & \mathrm{mg} / \mathrm{kg} & 3.33 & \text { EPA300.0 } \\ \mathrm{U} & & 1.37 & \mathrm{mg} / \mathrm{kg} & 1.37 & \text { EPA335.3 } \\ \mathrm{U} & & 1.09 & \mathrm{mg} / \mathrm{kg} & 1.09 & \text { EPA353.1 } \\ \mathrm{U} & & 12.9 & \mathrm{mg} / \mathrm{kg} & 12.9 & \text { EPA365.2 } \\ \text { UJ } & \mathrm{C} & 403 & \mathrm{\mu g} / \mathrm{kg} & 403 & \text { EPA420.2 } \\ \text { UJ } & \mathrm{C} & 342 & \mathrm{mg} / \mathrm{kg} & 342 & \text { EPA376.2 } \\ \text { UJ } & \mathrm{C} & 342 & \mathrm{mg} / \mathrm{kg} & 342 & \text { EPA376.2 } \\ & & \mathbf{7 4 0 0} & \mathrm{mg} / \mathrm{kg} & 137 & \text { EPA415.1 } \\ \mathrm{C} & & 294 & \mathrm{mg} / \mathrm{kg} & \mathbf{0 . 2 7 4} & \text { EPA351.2 } \\ \mathrm{J} & \mathrm{E} & \mathbf{6 1 . 4} & \mathrm{mg} / \mathrm{kg} & \mathbf{2 8 2} & \text { EPA365.4 }\end{array}$

$\begin{array}{lll} & \text { V } & 12600 \\ \text { U } & & 6.72 \\ \text { U } & & 20.1 \\ & & 29.8 \\ \text { J } & E & 0.179 \\ \text { U } & & 0.336 \\ & \text { V } & 250 \\ & & 15.9 \\ & & 0.768 \\ \text { J } & \text { VC } & 4.52 \\ & & 7.8 \\ & \text { V } & 176 \\ & & 91 \\ & \text { V } & 0.0669 \\ \text { J } & \text { E } & 3.15 \\ \text { U } & & 20.1 \\ \text { U } & & 1.68 \\ \text { J } & \text { E } & 15.7 \\ \text { J } & \text { EC } & 6.89 \\ & & 29.9 \\ & & 8\end{array}$

$\begin{array}{lll}\mathrm{mg} / \mathrm{kg} & 16.8 & \text { EPA6010A } \\ \mathrm{mg} / \mathrm{kg} & 6.72 & \text { EPA6010A } \\ \mathrm{mg} / \mathrm{kg} & 20.1 & \text { EPA6010A } \\ \mathrm{mg} / \mathrm{kg} & 1.68 & \text { EPA6010A } \\ \mathrm{mg} / \mathrm{kg} & 0.672 & \text { EPA6010A } \\ \mathrm{mg} / \mathrm{kg} & 0.336 & \text { EPA6010A } \\ \mathrm{mg} / \mathrm{kg} & 16.8 & \text { EPA6010A } \\ \mathrm{mg} / \mathrm{kg} & 1.68 & \text { EPA6010A } \\ \mathrm{mg} / \mathrm{kg} & 0.672 & \text { EPA6010A } \\ \mathrm{mg} / \mathrm{kg} & 1.68 & \text { EPA6010A } \\ \mathrm{mg} / \mathrm{kg} & 6.72 & \text { EPA6010A } \\ \mathrm{mg} / \mathrm{kg} & 6.72 & \text { EPA6010A } \\ \mathrm{mg} / \mathrm{kg} & 6.72 & \text { EPA6010A } \\ \mathrm{mg} / \mathrm{kg} & 0.672 & \text { EPA6010A } \\ \mathrm{mg} / \mathrm{kg} & 0.0373 & \text { EPA7471 } \\ \mathrm{mg} / \mathrm{kg} & 1.68 & \text { EPA6010A } \\ \mathrm{mg} / \mathrm{kg} & 134 & \text { EPA6010A } \\ \mathrm{mg} / \mathrm{kg} & 20.1 & \text { EPA6010A } \\ \mathrm{mg} / \mathrm{kg} & 1.68 & \text { EPA6010A } \\ \mathrm{mg} / \mathrm{kg} & 67.2 & \text { EPA6010A } \\ \mathrm{mg} / \mathrm{kg} & 20.1 & \text { EPA6010A } \\ \mathrm{mg} / \mathrm{kg} & 0.672 & \text { EPA6010A } \\ \mathrm{mg} / \mathrm{kg} & 1.34 & \text { EPA6010A }\end{array}$

Radionuclides

Actinium-228

Antimony-124

Antimony-125

Barium-133

Cerium-144

Cesium-134

Cesium-137

Cobalt-57

Cobalt-58

Cobalt-60

Europium-152

Europium-154

Europium-155

Gross alpha
$1.43 \mathrm{E}+00 \pm 6.99 \mathrm{E}-02 \mathrm{pCi} / \mathrm{g}$

$-4.06 \mathrm{E}-03 \pm 1.56 \mathrm{E}-02 \mathrm{pCi} / \mathrm{g}$

$4.10 \mathrm{E}-02 \pm 4.07 \mathrm{E}-02 \mathrm{pCi} / \mathrm{g}$

$4.83 \mathrm{E}-03 \pm 1.76 \mathrm{E}-02 \quad \mathrm{pCi} / \mathrm{g}$

$-5.64 \mathrm{E}-02 \pm 7.58 \mathrm{E}-02 \mathrm{pCi} / \mathrm{g}$

$-6.81 \mathrm{E}-04 \pm 1.08 \mathrm{E}-02 \mathrm{pCi} / \mathrm{g}$

$1.15 \mathrm{E}+01 \pm 8.64 \mathrm{E}-02 \mathrm{pCi} / \mathrm{g}$

$1.16 \mathrm{E}-03 \pm 9.43 \mathrm{E}-03 \mathrm{pCi} / \mathrm{g}$

$-1.03 \mathrm{E}-02 \pm 9.59 \mathrm{E}-03 \mathrm{pCj} / \mathrm{g}$

$5.20 \mathrm{E}-02 \pm 1.14 \mathrm{E}-02 \mathrm{pCi} / \mathrm{g}$

$-1.85 \mathrm{E}-02 \pm 3.46 \mathrm{E}-02 \mathrm{pCi} / \mathrm{g}$

$-3.23 \mathrm{E}-02 \pm 6.25 \mathrm{E}-02 \mathrm{pCi} / \mathrm{g}$

$5.19 \mathrm{E}-02 \pm 4.39 \mathrm{E}-02 \mathrm{pCi} / \mathrm{g}$

$1.42 \mathrm{E}+01 \pm 2.45 \mathrm{E}+00 \mathrm{pCi} / \mathrm{g}$
3.69E-02 EPIA-013B

2.27E-02 EPIA-013B

6.97E-02 EPLA-013B

2.67E-02 EPIA-013B

1.25E-01 EPIA-013B

1.57E-02 EPIA-013B

1.57E-02 EPIA-013B

1.57E-02 EPIA-013B

1.29E-02 EPIA-013B

1.03E-02 EPIA-013B

5.87E-02 EPIA-013B

- 9.14E-02 EPLA-013B

6.31E-02 EPIA-013B

$1.44 \mathrm{E}+00$ EPIA-001B 
SURVEY ID: PPSC 0801 (continued)

Constituent

Radionuclides

Lead-212

Manganese-54

Neptunium-239

Nonvolatile beta

Plutonium-238

Plutonium-239/240

Potassium-40

Promethium-144

Promethium-146

Ruthenium-106

Sodium-22

Strontium-90

Thorium-234

Tin-113

Yttrium-88

Zinc-65

Zirconium-95
Sample ID: 104630
RQ AQ B Result
Unit
D. Limit Method

$\begin{array}{ll}3.27 \mathrm{E}-02 & \text { EPIA-013B } \\ 1.21 \mathrm{E}-02 & \text { EPIA-013B } \\ 1.10 \mathrm{E}-01 & \text { EPIA-013B } \\ 1.77 \mathrm{E}+00 & \text { EPIA-001B } \\ 2.41 \mathrm{E}-02 & \text { EPIA-012B } \\ 1.26 \mathrm{E}-02 & \text { EPIA-012B } \\ 9.84 \mathrm{E}-02 & \text { EPIA-013B } \\ 1.16 \mathrm{E}-02 & \text { EPIA-013B } \\ 3.54 \mathrm{E}-02 & \text { EPIA-013B } \\ 1.61 \mathrm{E}-01 & \text { EPIA-013B } \\ 1.09 \mathrm{E}-02 & \text { EPIA-013B } \\ 6.06 \mathrm{E}-02 & \text { EPIA-004 } \\ 7.66 \mathrm{E}-01 & \text { EPIA-013B } \\ 3.41 \mathrm{E}-02 & \text { EPIA-013B } \\ 1.27 \mathrm{E}-02 & \text { EPIA-013B } \\ 2.57 \mathrm{E}-02 & \text { EPIA-013B } \\ 2.68 \mathrm{E}-02 & \text { EPIA-013B }\end{array}$

3.27E-02 EPIA-013B

$1.51 \mathrm{E}+00 \pm 4.10 \mathrm{E}-02 \mathrm{pCi} / \mathrm{g}$ $2.83 \mathrm{E}-02 \pm 8.55 \mathrm{E}-03 \mathrm{pCi} / \mathrm{g}$ $8.58 \mathrm{E}-02 \pm 8.95 \mathrm{E}-02 \mathrm{pCi} / \mathrm{g}$ $1.82 \mathrm{E}+01 \pm 1.91 \mathrm{E}+00 \mathrm{pCi} / \mathrm{g}$ $-2.08 \mathrm{E}-03 \pm 1.17 \mathrm{E}-02 \mathrm{pCi} / \mathrm{g}$ $2.94 \mathrm{E}-02 \pm 1.37 \mathrm{E}-02 \mathrm{pCi} / \mathrm{g}$ $1.13 \mathrm{E}+00 \pm 1.51 \mathrm{E}-01 \mathrm{pCi} / \mathrm{g}$ $-9.62 \mathrm{E}-04 \pm 7.00 \mathrm{E}-03 \mathrm{pCi} / \mathrm{g}$ $3.07 \mathrm{E}-02 \pm 2.06 \mathrm{E}-02 \mathrm{pCi} / \mathrm{g}$ $-2.24 \mathrm{E}-02 \pm 9.70 \mathrm{E}-02 \mathrm{pCi} / \mathrm{g}$ $3.71 \mathrm{E}-03 \pm 6.95 \mathrm{E}-03 \mathrm{pCj} / \mathrm{g}$ 2.93E-02 $\pm 3.62 \mathrm{E}-02 \mathrm{pCi} / \mathrm{g}$ $1.50 \mathrm{E}+00 \pm 9.00 \mathrm{E}-01 \mathrm{pCi} / \mathrm{g}$ $9.38 \mathrm{E}-03 \pm 1.99 \mathrm{E}-02 \mathrm{pCi} / \mathrm{g}$ $5.38 \mathrm{E}-04 \pm 6.97 \mathrm{E}-03 \mathrm{pCi} / \mathrm{g}$ $2.15 \mathrm{E}-02 \pm 1.55 \mathrm{E}-02 \mathrm{pCi} / \mathrm{g}$ $6.98 \mathrm{E}-02 \pm 3.00 \mathrm{E}-02 \mathrm{pCi} / \mathrm{g}$

Sample ID: 104632

SURVEY ID: PPSC 0802

Survey location: 45133E 3680562N (SRS coordinates)

Depth of core interval: 1.00 to $2.00 \mathrm{ft}$

Sample type: Normal

Sample matrix: Soil

Percent solids: $\mathbf{8 1 . 0 0}$

Constituent

RQ AQ B Result

Unit

D. Limit Method

Physical Parameters and Specified Analyses

\begin{tabular}{|c|c|c|c|}
\hline Cation exchange capacity & & & 90.4 \\
\hline Chloride & $U$ & & 2.89 \\
\hline Cyanide & $\mathrm{U}$ & & 1.23 \\
\hline Nitrate-nitrite as nitrogen & $\mathrm{U}$ & & 1.19 \\
\hline Orthophosphate & $\mathrm{U}$ & & 12.3 \\
\hline Orthophosphate & $\mathrm{U}$ & & 12.1 \\
\hline Phenols & $J$ & EC & 97.6 \\
\hline Sulfide & UJ & C I & 309 \\
\hline Total organic carbon & & & 2310 \\
\hline Total organic nitrogen & $\mathrm{C}$ & & 49 \\
\hline Total phosphates (as P) & $\mathbf{J}$ & $\mathbf{E}$ & 81.7 \\
\hline \multicolumn{4}{|l|}{ Metals (total recoverable) } \\
\hline Aluminum & & $\mathrm{V}$ & 16500 \\
\hline Antimony & & & 11 \\
\hline Arsenic & $\mathrm{U}$ & & 18.2 \\
\hline Barium & & & 11.4 \\
\hline Beryllium & $J$ & $\mathbf{E}$ & 0.0829 \\
\hline Cadmium & $\mathrm{U}$ & & 0.303 \\
\hline Calcium & & $\mathrm{V}$ & 56.5 \\
\hline Chromium & & & 24.5 \\
\hline Cobalt & $\mathrm{U}$ & & 0.605 \\
\hline Copper & & & 4.07 \\
\hline
\end{tabular}

EPA9081

EPA300.0

EPA335.3

EPA353.1

EPA365.2

EPA365.2

EPA420.2

EPA376.2

EPA415.1

EPA365.4

$\begin{array}{lll}\mathrm{mg} / \mathrm{kg} & 15.1 & \text { EPA6010A } \\ \mathrm{mg} / \mathrm{kg} & 6.05 & \text { EPA6010A } \\ \mathrm{mg} / \mathrm{kg} & 18.2 & \text { EPA6010A } \\ \mathrm{mg} / \mathrm{kg} & 1.51 & \text { EPA6010A } \\ \mathrm{mg} / \mathrm{kg} & 0.605 & \text { EPA6010A } \\ \mathrm{mg} / \mathrm{kg} & 0.303 & \text { EPA6010A } \\ \mathrm{mg} / \mathrm{kg} & 15.1 & \text { EPA6010A } \\ \mathrm{mg} / \mathrm{kg} & -1.51 & \text { EPA6010A } \\ \mathrm{mg} / \mathrm{kg} & 0.605 & \text { EPA6010A } \\ \mathrm{mg} / \mathrm{kg} & 1.51 & \text { EPA6010A }\end{array}$

EPA351.2 
SURVEY ID: PPSC 0802 (continued)

Constituent

Metals (total recoverable)

Iron

Lead

Magnesium

Manganese

Mercury

Nickel

Potassium

Selenium

Silver

Sodium

Thallium

Vanadium

Zinc

Radionuclides

Actinium-228

Antimony-124

Antimony-125

Barium-133

Cerium-144

Cesium-134

Cesium-137

Cobalt-57

Cobalt-58

Cobalt-60

Europium-152

Europium-154

Europium-155

Gross alpha

Lead-212

Manganese-54

Neptunium-239

Nonvolatile beta

Plutonium-238

Plutonium-239/240

Potassium-40

Promethium-144

Promethium-146

Ruthenium-106

Sodium-22

Strontium-90

Thorium-234

Tin-113

Yttrium-88

Zinc-65

Zirconium-95
Sample ID: 104632

RQ AQ B Result

Unit

D. Limit Method

$\begin{array}{lll}J & \text { VC } & 25400 \\ & & 8.11 \\ & \text { V } & 141 \\ & & 15 \\ & \text { V } & 0.0695 \\ & & 2.65 \\ \text { J } & \text { E } & 118 \\ \text { J } & \text { E } & 7.37 \\ \text { J } & \text { E } & 0.903 \\ \text { J } & \text { E } & 13 \\ \text { UJ } & \text { C } & 18.2 \\ & & 53.2 \\ & & 3.71\end{array}$

$\begin{array}{lll}\mathrm{mg} / \mathrm{kg} & 6.05 & \text { EPA6010A } \\ \mathrm{mg} / \mathrm{kg} & 6.05 & \text { EPA6010A } \\ \mathrm{mg} / \mathrm{kg} & 6.05 & \text { EPA6010A } \\ \mathrm{mg} / \mathrm{kg} & 0.605 & \text { EPA6010A } \\ \mathrm{mg} / \mathrm{kg} & 0.0346 & \text { EPA7471 } \\ \mathrm{mg} / \mathrm{kg} & 1.51 & \text { EPA6010A } \\ \mathrm{mg} / \mathrm{kg} & 121 & \text { EPA6010A } \\ \mathrm{mg} / \mathrm{kg} & 18.2 & \text { EPA6010A } \\ \mathrm{mg} / \mathrm{kg} & 1.51 & \text { EPA6010A } \\ \mathrm{mg} / \mathrm{kg} & 60.5 & \text { EPA6010A } \\ \mathrm{mg} / \mathrm{kg} & 18.2 & \text { EPA6010A } \\ \mathrm{mg} / \mathrm{kg} & 0.605 & \text { EPA6010A } \\ \mathrm{mg} / \mathrm{kg} & 1.21 & \text { EPA6010A }\end{array}$

$1.51 \mathrm{E}+00 \pm 7.57 \mathrm{E}-02 \mathrm{pCi} / \mathrm{g}$ $-2.86 \mathrm{E}-03 \pm 8.95 \mathrm{E}-03 \mathrm{pCi} / \mathrm{g}$ $6.17 \mathrm{E}-03 \pm 1.76 \mathrm{E}-02 \mathrm{pCi} / \mathrm{g}$ 4.37E-03 $\pm 9.31 \mathrm{E}-03 \mathrm{pCi} / \mathrm{g}$ $-4.47 \mathrm{E}-02 \pm 5.08 \mathrm{E}-02 \mathrm{pCi} / \mathrm{g}$ $-4.95 \mathrm{E}-03 \pm 7.00 \mathrm{E}-03 \mathrm{pCi} / \mathrm{g}$ 9.50E-02 $\pm 1.33 \mathrm{E}-02 \mathrm{pCi} / \mathrm{g}$ $-4.65 \mathrm{E}-03 \pm 6.36 \mathrm{E}-03 \mathrm{pCi} / \mathrm{g}$ $-2.39 \mathrm{E}-03 \pm 8.17 \mathrm{E}-03 \mathrm{pCi} / \mathrm{g}$ $-1.67 \mathrm{E}-03 \pm 5.72 \mathrm{E}-03 \mathrm{pCi} / \mathrm{g}$ $-5.10 \mathrm{E}-03 \pm 1.95 \mathrm{E}-02 \mathrm{pCi} / \mathrm{g}$ $-4.03 \mathrm{E}-02 \pm 5.34 \mathrm{E}-02 \mathrm{pCi} / \mathrm{g}$ $0.00 \mathrm{E}+00 \quad \mathrm{pCi} / \mathrm{g}$ $1.81 \mathrm{E}+01 \pm 3.01 \mathrm{E}+00 \mathrm{pCi} / \mathrm{g}$ $\mathrm{V} \quad 1.53 \mathrm{E}+00 \pm 3.34 \mathrm{E}-02 \mathrm{pCi} / \mathrm{g}$ $2.61 \mathrm{E}-02 \pm 9.52 \mathrm{E}-03 \mathrm{pCi} / \mathrm{g}$ $0.00 \mathrm{E}+00 \quad \mathrm{pCi} / \mathrm{g}$ $1.01 \mathrm{E}+01 \pm 1.53 \mathrm{E}+00 \mathrm{pCi} / \mathrm{g}$ $7.96 \mathrm{E}-03 \pm 1.85 \mathrm{E}-02 \mathrm{pCi} / \mathrm{g}$ $3.77 \mathrm{E}-03 \pm 9.90 \mathrm{E}-03 \mathrm{pCi} / \mathrm{g}$ 9.79E-01 $1.41 \mathrm{E}-01 \mathrm{pCi} / \mathrm{g}$ 9.20E-05 $\pm 6.79 \mathrm{E}-03 \mathrm{pCi} / \mathrm{g}$ $7.05 \mathrm{E}-03 \pm 8.46 \mathrm{E}-03 \mathrm{pCi} / \mathrm{g}$ $-4.08 \mathrm{E}-02 \pm 6.07 \mathrm{E}-02 \mathrm{pCi} / \mathrm{g}$ $2.95 \mathrm{E}-03 \pm 6.69 \mathrm{E}-03 \mathrm{pCi} / \mathrm{g}$ $-6.28 \mathrm{E}-02 \pm 1.69 \mathrm{E}-02 \mathrm{pCi} / \mathrm{g}$ $1.31 \mathrm{E}+00 \pm 7.32 \mathrm{E}-01 \mathrm{pCi} / \mathrm{g}$ $-3.13 \mathrm{E}-03 \pm 9.87 \mathrm{E}-03 \mathrm{pCi} / \mathrm{g}$ $1.01 \mathrm{E}-03 \pm 6.85 \mathrm{E}-03 \mathrm{pCi} / \mathrm{g}$ $-1.45 \mathrm{E}-02 \pm 1.48 \mathrm{E}-02 \mathrm{pCi} / \mathrm{g}$ $0.00 \mathrm{E}+00 \quad \mathrm{pCi} / \mathrm{g}$
3.54E-02 EPIA-013B

1.51E-02 EPIA-013B

3.09E-02 EPIA-013B

8.54E-02 EPIA-013B

1.01E-02 EPIA-013B

1.18E-02 EPIA-013B

1.08E-02 EPIA-013B

1.36E-02 EPIA-013B

9.78E-03 EPIA-013B

3.15E-02 EPIA-013B

9.06E-02 EPIA-013B

4.57E-02 EPIA-013B

$1.50 \mathrm{E}+00$ EPIA-001B

1.96E-02 EPIA-013B

1.23E-02 EPIA-013B

7.64E-02 EPIA-013B

1.74E+00 EPIA-001B

3.34E-02 EPIA-012B

1.93E-02 EPIA-012B

9.67E-02 EPIA-013B

1.15E-02 EPIA-013B

1.50E-02 EPIA-013B

1.01E-01 EPIA-013B

1.06E.02 EPIA-013B

5.63E.02 EPIA.004

5.85E-01 EPIA-013B

1.70E-02 EPIA-013B

1.26E-02 EPIA-013B

2.08E-02 EPIA-013B

3.10E-02 EPIA-013B
1.38E-02 EPIA-013B

Survey location: $45133 \mathrm{E}$ 3680562N (SRS coordinates)

Depth of core interval: 2.00 to $3.00 \mathrm{ft}$

Sample type: Normal

Sample matrix: Soil

Percent solids: $\mathbf{8 5 . 0 0}$ 
SURVEY ID: PPSC 0803 (continued)

Constituent
Sample ID: 104633

RQ AQ B Result Unit D. Limit Method

Physical Parameters and Specified Analyses

Cation exchange capacity

Chloride

Cyanide

Nitrate-nitrite as nitrogen

Nitrate-nitrite as nitrogen

Orthophosphate

Phenols

Sulfide

Total organic carbon

Total organic nitrogen

Total phosphates (as P)

Metals (total recoverable)

$\begin{array}{llll}J & & 75.8 \\ \text { U } & \text { E } & 2.21 \\ \text { U } & & 1.18 \\ \text { U } & & 1.02 \\ \text { U } & & 1.09 \\ \text { UJ } & \text { C } & 11.7 \\ \text { UJ } & \text { C } & 351 \\ & & 294 \\ \text { C } & & 502 \\ \text { J } & \text { E } & 47.1 \\ & & 87.3\end{array}$

$\begin{array}{lll} & \mathrm{V} & \mathbf{8 7 3 0} \\ \mathrm{U} & & \mathbf{5 . 7 7} \\ \mathrm{J} & \mathrm{E} & \mathbf{6 . 7 5} \\ & & 4.11 \\ \mathrm{~J} & \mathrm{E} & \mathbf{0 . 0 3 1 7} \\ \mathrm{U} & & \mathbf{0 . 2 8 8} \\ \mathrm{J} & \mathrm{EV} & 14 \\ & & 18.8 \\ \mathrm{U} & & \mathbf{0 . 5 7 7} \\ & & \mathbf{2 . 6 1} \\ \mathrm{J} & \mathrm{VC} & \mathbf{2 1 1 0 0} \\ \mathrm{J} & \mathrm{E} & 4.58 \\ & \mathrm{~V} & \mathbf{5 3 . 1} \\ & & 4.88 \\ \mathrm{U} & \mathrm{V} & \mathbf{0 . 0 2 7 2} \\ \text { J } & \mathrm{E} & 1.22 \\ \mathrm{~J} & \mathrm{E} & \mathbf{3 5 . 7} \\ \mathrm{U} & & 17.3 \\ \mathrm{~J} & \mathrm{E} & \mathbf{0 . 8 5 6} \\ \mathrm{U} & & \mathbf{5 7 . 7} \\ \mathrm{UJ} & \mathrm{C} & 17.3 \\ & & 47.2 \\ & & 1.49\end{array}$

$\begin{array}{lll}\mathrm{meq} / 100 \mathrm{~g} & 5 & \text { EPA9081 } \\ \mathrm{mg} / \mathrm{kg} & 2.7 & \text { EPA300.0 } \\ \mathrm{mg} / \mathrm{kg} & 1.18 & \text { EPA335.3 } \\ \mathrm{mg} / \mathrm{kg} & 1.02 & \text { EPA353.1 } \\ \mathrm{mg} / \mathrm{kg} & 1.09 & \text { EPA353.1 } \\ \mathrm{mg} / \mathrm{kg} & 11.7 & \text { EPA365.2 } \\ \mu \mathrm{g} / \mathrm{kg} & 351 & \text { EPA420.2 } \\ \mathrm{mg} / \mathrm{kg} & 294 & \text { EPA376.2 } \\ \mathrm{mg} / \mathrm{kg} & 118 & \text { EPA415.1 } \\ \mathrm{mg} / \mathrm{kg} & 0.235 & \text { EPA351.2 } \\ \mathrm{mg} / \mathrm{kg} & 231 & \text { EPA365.4 }\end{array}$

$\begin{array}{lll}\mathrm{mg} / \mathrm{kg} & 14.4 & \text { EPA6010A } \\ \mathrm{mg} / \mathrm{kg} & 5.77 & \text { EPA6010A } \\ \mathrm{mg} / \mathrm{kg} & 17.3 & \text { EPA6010A } \\ \mathrm{mg} / \mathrm{kg} & 1.44 & \text { EPA6010A } \\ \mathrm{mg} / \mathrm{kg} & 0.577 & \text { EPA6010A } \\ \mathrm{mg} / \mathrm{kg} & 0.288 & \text { EPA6010A } \\ \mathrm{mg} / \mathrm{kg} & 14.4 & \text { EPA6010A } \\ \mathrm{mg} / \mathrm{kg} & 1.44 & \text { EPA6010A } \\ \mathrm{mg} / \mathrm{kg} & 0.577 & \text { EPA6010A } \\ \mathrm{mg} / \mathrm{kg} & 1.44 & \text { EPA6010A } \\ \mathrm{mg} / \mathrm{kg} & 5.77 & \text { EPA6010A } \\ \mathrm{mg} / \mathrm{kg} & 5.77 & \text { EPA6010A } \\ \mathrm{mg} / \mathrm{kg} & 5.77 & \text { EPA6010A } \\ \mathrm{mg} / \mathrm{kg} & 0.577 & \text { EPA6010A } \\ \mathrm{mg} / \mathrm{kg} & 0.0336 & \text { EPA7471 } \\ \mathrm{mg} / \mathrm{kg} & 1.44 & \text { EPA6010A } \\ \mathrm{mg} / \mathrm{kg} & 115 & \text { EPA6010A } \\ \mathrm{mg} / \mathrm{kg} & 17.3 & \text { EPA6010A } \\ \mathrm{mg} / \mathrm{kg} & 1.44 & \text { EPA6010A } \\ \mathrm{mg} / \mathrm{kg} & 57.7 & \text { EPA6010A } \\ \mathrm{mg} / \mathrm{kg} & 17.3 & \text { EPA6010A } \\ \mathrm{mg} / \mathrm{kg} & 0.577 & \text { EPA6010A } \\ \mathrm{mg} / \mathrm{kg} & 1.15 & \text { EPA6010A }\end{array}$

\section{Radionuclides}

Actinium-228

Actinium-228

Antimony-124

Antimony-124

Antimony-125

Antimony-125

Barium-133

Barium-133

Cerium-144

Cerium-144

Cesium-134

Cesium-134

Cesium-137

Cesium-137

Cobalt-57
$1.51 \mathrm{E}+00 \pm 6.99 \mathrm{E}-02 \mathrm{pCi} / \mathrm{g}$ $1.45 \mathrm{E}+00 \pm 8.30 \mathrm{E}-02 \mathrm{pCi} / \mathrm{g}$

UI

UI

UI

UI

UI

UI

UI

UI

UI

UI

UI

UI

UI
4.14E-03 $\pm 9.17 \mathrm{E}-03 \mathrm{pCi} / \mathrm{g}$

$1.02 \mathrm{E}-02 \pm 1.35 \mathrm{E}-02 \mathrm{pCi} / \mathrm{g}$

8.10E-03 $\pm 1.82 \mathrm{E}-02 \mathrm{pCi} / \mathrm{g}$ $-8.66 \mathrm{E}-03 \pm 1.98 \mathrm{E}-02 \mathrm{pCi} / \mathrm{g}$ $-7.28 \mathrm{E}-03 \pm 9.43 \mathrm{E}-03 \mathrm{pCi} / \mathrm{g}$ $-6.06 \mathrm{E}-04 \pm 9.39 \mathrm{E}-03 \mathrm{pCi} / \mathrm{g}$ $-2.06 \mathrm{E}-02 \pm 5.28 \mathrm{E}-02 \mathrm{pCi} / \mathrm{g}$ $-1.71 \mathrm{E}-02 \pm 5.15 \mathrm{E}-02 \mathrm{pCi} / \mathrm{g}$ $-1.50 \mathrm{E}-03 \pm 7.10 \mathrm{E}-03 \mathrm{pCi} / \mathrm{g}$ $6.99 \mathrm{E}-03 \pm 9.20 \mathrm{E}-03 \mathrm{pCi} / \mathrm{g}$ $-3.44 \mathrm{E}-03 \pm 7.76 \mathrm{E}-03 \mathrm{pCi} / \mathrm{g}$ 3.73E-03 $\pm 8.03 \mathrm{E}-03 \mathrm{pCi} / \mathrm{g}$ 4.37E-03 $\pm 6.33 \mathrm{E}-03 \mathrm{pCi} / \mathrm{g}$
3.65E-02 EPIA-013B

3.70E-02 EPIA-013B

1.60E-02 EPIA-013B

1.66E-02 EPIA-013B

3.21E-02 EPIA-013B

3.21E-02 EPIA-013B

1.32E-02 EPIA-013B

1.39E-02 EPIA-013B

8.95E-02 EPLA-013B

8.81E-02 EPIA-013B

1.06E-02 EPIA-013B

- 1.07E-02 EPIA-013B

1.29E-02 EPIA-013B

1.27E-02 EPIA-013B 1.10E-02 EPIA-013B 
SURVEY ID: PPSC 0803 (continued)

Constituent

Radionuclides

Cobalt-57

Cobalt-58

Cobalt-58

Cobalt-60

Cobalt-60

Europium-152

Europium-152

Europium-154

Europium-154

Europium-155

Europium-155

Gross alpha

Gross alpha

Lead-212

Lead-212

Manganese- 54

Manganese-54

Neptunium-239

Neptunium-239

Nonvolatile beta

Nonvolatile beta

Plutonium-238

Plutonium-238

Plutonium-239/240

Plutonium-239/240

Potassium-40

Potassium-40

Promethium-144

Promethium-144

Promethium-146

Promethium-146

Ruthenium-106

Ruthenium-106

Sodium-22

Sodium-22

Strontium-90

Strontium-90

Thorium-234

Thorium-234

Tin-113

Tin-113

Yttrium-88

Yttrium-88

Zinc-65

Zinc-65

Zirconium-95

Zirconium-95
Sample ID: 104633

RQ AQ B Result Unit

D. Limit Method
$1.94 \mathrm{E}-03 \pm 6.29 \mathrm{E}-03 \mathrm{pCi} / \mathrm{g}$ $-5.70 \mathrm{E}-03 \pm 8.69 \mathrm{E}-03 \mathrm{pCi} / \mathrm{g}$ $-3.45 \mathrm{E}-03 \pm 8.07 \mathrm{E}-03 \mathrm{pCj} / \mathrm{g}$ $1.98 \mathrm{E}-03 \pm 6.10 \mathrm{E}-03 \mathrm{pCi} / \mathrm{g}$ $-1.01 \mathrm{E}-03 \pm 5.97 \mathrm{E}-03 \mathrm{pCi} / \mathrm{g}$ $-1.61 \mathrm{E}-02 \pm 2.00 \mathrm{E}-02 \mathrm{pCi} / \mathrm{g}$ $-1.01 \mathrm{E}-02+1.93 \mathrm{E}-02 \mathrm{pCi} / \mathrm{g}$ $-3.57 \mathrm{E}-02 \pm 5.32 \mathrm{E}-02 \mathrm{pCi} / \mathrm{g}$ $-2.63 \mathrm{E}-02 \pm 5.80 \mathrm{E}-02 \mathrm{pCi} / \mathrm{g}$ $0.00 \mathrm{E}+00 \quad \mathrm{pCi} / \mathrm{g}$ $0.00 \mathrm{E}+00 \quad \mathrm{pCj} / \mathrm{g}$ $2.24 \mathrm{E}+01 \pm 3.25 \mathrm{E}+00 \mathrm{pCi} / \mathrm{g}$ $1.83 \mathrm{E}+01 \pm 3.03 \mathrm{E}+00 \mathrm{pCj} / \mathrm{g}$ $1.49 \mathrm{E}+00 \pm 3.38 \mathrm{E} .02 \mathrm{pCi} / \mathrm{g}$ $1.53 \mathrm{E}+00 \pm 3.49 \mathrm{E}-02 \mathrm{pCi} / \mathrm{g}$ 2.51E-02 $49.01 \mathrm{E}-03 \mathrm{pCj} / \mathrm{g}$ $2.37 \mathrm{E}-02 \pm 8.37 \mathrm{E}-03 \mathrm{pCi} / \mathrm{g}$ $0.00 \mathrm{E}+00 \quad \mathrm{pCi} / \mathrm{g}$ $3.99 \mathrm{E}-02 \pm 7.28 \mathrm{E}-02 \mathrm{pCi} / \mathrm{g}$ $1.01 \mathrm{E}+01 \pm 1.59 \mathrm{E}+00 \mathrm{pCi} / \mathrm{g}$ $1.10 \mathrm{E}+01 \pm 1.66 \mathrm{E}+00 \mathrm{pCi} / \mathrm{g}$ $3.28 \mathrm{E}-03 \pm 1.02 \mathrm{E}-02 \mathrm{pCi} / \mathrm{g}$ $4.72 \mathrm{E}-04 \pm 1.09 \mathrm{E}-02 \mathrm{pCi} / \mathrm{g}$ $1.49 \mathrm{E}-03 \pm 3.00 \mathrm{E}-03 \mathrm{pCi} / \mathrm{g}$ $1.05 \mathrm{E}-04 \pm 5.10 \mathrm{E}-03 \mathrm{pCj} / \mathrm{g}$ $8.70 \mathrm{E}-01 \pm 1.46 \mathrm{E}-01 \mathrm{pCi} / \mathrm{g}$ 8.05E-01 $1.48 \mathrm{E}-01 \mathrm{pCi} / \mathrm{g}$ $-3.97 \mathrm{E}-04 \pm 7.20 \mathrm{E}-03 \mathrm{pCj} / \mathrm{g}$ $-7.97 \mathrm{E}-04 \pm 6.88 \mathrm{E}-03 \mathrm{pCi} / \mathrm{g}$ $1.24 \mathrm{E} \cdot 02 \pm 8.96 \mathrm{E} \cdot 03 \mathrm{pCi} / \mathrm{g}$ $7.58 \mathrm{E} \cdot 03 \pm 9.41 \mathrm{E}-03 \mathrm{pCi} / \mathrm{g}$ $5.17 \mathrm{E}-02 \pm 6.27 \mathrm{E} .02 \mathrm{pCj} / \mathrm{g}$ $-2.31 \mathrm{E}-02 \pm 6.17 \mathrm{E}-02 \mathrm{pCi} / \mathrm{g}$ $5.26 \mathrm{E} \cdot 03 \pm 6.03 \mathrm{E}-03 \mathrm{pCj} / \mathrm{g}$ $2.05 \mathrm{E}-03 \pm 5.90 \mathrm{E}-03 \mathrm{pCi} / \mathrm{g}$ $-9.31 \mathrm{E}-03 \pm 3.17 \mathrm{E}-02 \mathrm{pCi} / \mathrm{g}$ $-9.89 \mathrm{E}-02 \pm 1.50 \mathrm{E}-02 \mathrm{pCi} / \mathrm{g}$ $1.75 \mathrm{E}+00 \pm 7.92 \mathrm{E}-01 \mathrm{pCi} / \mathrm{g}$ $1.06 \mathrm{E}+00 \pm 6.44 \mathrm{E}-01 \mathrm{pCi} / \mathrm{g}$ $-1.59 \mathrm{E} \cdot 03 \pm 9.92 \mathrm{E}-03 \mathrm{pCi} / \mathrm{g}$ $-1.45 \mathrm{E}-02 \pm 1.06 \mathrm{E}-02 \mathrm{pCi} / \mathrm{g}$ 9.67E-03 $\pm 7.72 \mathrm{E}-03 \mathrm{pCj} / \mathrm{g}$ $2.10 \mathrm{E}-03 \pm 7.77 \mathrm{E}-03 \mathrm{pCi} / \mathrm{g}$ $-1.60 \mathrm{E}-02 \pm 1.58 \mathrm{E}-02 \mathrm{pCi} / \mathrm{g}$ $1.56 \mathrm{E}-02 \pm 1.70 \mathrm{E}-02 \mathrm{pCi} / \mathrm{g}$ $2.43 \mathrm{E}-02 \pm 3.09 \mathrm{E}-02 \mathrm{pCi} / \mathrm{g}$ $5.37 \mathrm{E}-02 \pm 3.01 \mathrm{E}-02$ pCig $\begin{array}{ll}1.09 \mathrm{E}-02 & \text { EPIA-013B } \\ 1.42 \mathrm{E}-02 & \text { EPIA-013B } \\ 1.37 \mathrm{E}-02 & \text { EPIA-013B } \\ 1.10 \mathrm{E}-02 & \text { EPIA-013B } \\ 1.06 \mathrm{E}-02 & \text { EPIA-013B } \\ 3.18 \mathrm{E}-02 & \text { EPIA-013B } \\ 3.16 \mathrm{E}-02 & \text { EPIA-013B } \\ 9.07 \mathrm{E}-02 & \text { EPIA-013B } \\ 9.68 \mathrm{E}-02 & \text { EPIA-013B } \\ 4.65 \mathrm{E}-02 & \text { EPIA-013B } \\ 4.60 \mathrm{E}-02 & \text { EPIA-013B } \\ 1.94 \mathrm{E}+00 & \text { EPIA-001B } \\ 2.17 \mathrm{E}+00 & \text { EPIA-001B } \\ 1.97 \mathrm{E}-02 & \text { EPIA-013B } \\ 2.00 \mathrm{E}-02 & \text { EPIA-013B } \\ 9.82 \mathrm{E}-03 & \text { EPIA-013B } \\ 1.18 \mathrm{E}-02 & \text { EPIA-013B } \\ 7.59 \mathrm{E}-02 & \text { EPIA-013B } \\ 7.63 \mathrm{E}-02 & \text { EPIA-013B } \\ 2.01 \mathrm{E}+00 & \text { EPIA-001B } \\ 2.05 \mathrm{E}+00 & \text { EPIA-001B } \\ 1.97 \mathrm{E}-02 & \text { EPIA-012B } \\ 2.18 \mathrm{E}-02 & \text { EPIA-012B } \\ 4.47 \mathrm{E}-03 & \text { EPIA-012B } \\ 1.24 \mathrm{E}-02 & \text { EPIA-012B } \\ 1.04 \mathrm{E}-01 & \text { EPIA-013B } \\ 1.05 \mathrm{E}-01 & \text { EPIA-013B } \\ 1.22 \mathrm{E}-02 & \text { EPIA-013B } \\ 1.19 \mathrm{E}-02 & \text { EPIA-013B } \\ 1.62 \mathrm{E}-02 & \text { EPIA-013B } \\ 1.53 \mathrm{E}-02 & \text { EPIA-013B } \\ 1.05 \mathrm{E}-01 & \text { EPIA-013B } \\ 1.06 \mathrm{E}-01 & \text { EPIA-013B } \\ 1.13 \mathrm{E}-02 & \text { EPIA-013B } \\ 1.04 \mathrm{E}-02 & \text { EPIA-013B } \\ 6.33 \mathrm{E}-02 & \text { EPIA-004 } \\ 7.11 \mathrm{E}-02 & \text { EPIA-004 } \\ 6.04 \mathrm{E}-01 & \text { EPIA-013B } \\ 5.96 \mathrm{E}-01 & \text { EPIA-013B } \\ 1.72 \mathrm{E}-02 & \text { EPIA-013B } \\ 1.66 \mathrm{E}-02 & \text { EPIA-013B } \\ 1.55 \mathrm{E}-02 & \text { EPIA-013B } \\ 1.43 \mathrm{E}-02 & \text { EPIA-013B } \\ 2.19 \mathrm{E}-02 & \text { EPIA-013B } \\ 2.19 \mathrm{E}-02 & \text { EPIA-013B } \\ 3.28 \mathrm{E}-02 & \text { EPIA-013B } \\ 2.79 \mathrm{E}-02 & \text { EPIA-013B }\end{array}$

Sample ID: 104635

SURVEY ID: PPSC 0901
Sample matrix: Soil
Percent solids: 87.00

Survey location: $45224 \mathrm{E} 3680779 \mathrm{~N}$ (SRS coordinates)

Depth of core interval: 0.00 to $1.00 \mathrm{ft}$

Sample type: Normal 
SURVEY ID: PPSC 0901 (continued)

Constituent
Sample ID: 104635

RQ AQ B Result Unit D. Limit Method

Physical Parameters and Specified Analyses

Cation exchange capacity

Chloride

Cyanide

Nitrate-nitrite as nitrogen

Orthophosphate

Phenols

Sulfide

Total organic carbon

Total organic carbon

Total organic nitrogen

Total phosphates (as P)

Metals (total recoverable)

Aluminum
Antimony
Arsenic
Barium
Beryllium
Cadmium
Calcium
Chromium
Cobalt
Copper
Iron
Lead
Magnesium
Manganese
Mercury
Nickel
Potassium
Selenium
Silver
Sodium
Thallium
Vanadium
Zinc

$\begin{array}{lll} & V & 2270 \\ \mathrm{U} & & 5.63 \\ \mathrm{U} & & 16.9 \\ & & 17.2 \\ \mathrm{~J} & \mathrm{E} & 0.133 \\ \mathrm{U} & & 0.282 \\ & \mathrm{~V} & 92.6 \\ & & 1.83 \\ & & 0.643 \\ \mathrm{~J} & \mathrm{VC} & 1.8 \\ \mathrm{U} & & 5.63 \\ & \mathrm{~V} & 75.4 \\ & & 60.8 \\ \mathrm{U} & \mathrm{V} & 0.0206 \\ & & 1.48 \\ \mathrm{~J} & \mathrm{E} & 48.5 \\ \mathrm{U} & & 16.9 \\ \mathrm{U} & & 1.41 \\ \mathrm{U} & & 56.3 \\ \mathrm{~J} & \mathrm{EC} & 3.09 \\ & & 2.93 \\ & & 3.65\end{array}$

$B / N / A$ Extractables (including $P A H$ and phenols)

$\begin{array}{llll}\text { Acenaphthene } & \text { UJ } & \text { C } & 37.4 \\ \text { Acenaphthylene } & \text { U } & & 37.4 \\ \text { Alkylbenzene C15H24 } & \text { J } & \text { N } & 210 \\ \text { Anthracene } & \text { U } & & 37.4 \\ \text { Benzo[a]anthracene } & \text { U } & & 3.74 \\ \text { Benzo[b]fluoranthene } & \text { U } & & 3.74 \\ \text { Benzo[k]fluoranthene } & \text { U } & & 3.74 \\ \text { Benzoic acid } & \text { U } & & 37.4 \\ \text { Benzo[g,h,i]perylene } & \text { U } & & 37.4 \\ \text { Benzo[a]pyrene } & \text { U } & & 3.74 \\ \text { Benzyl alcohol } & \text { U } & & 37.4 \\ \text { Bis(2-chloroethoxy) methane } & \text { U } & & 37.4 \\ \text { Bis(2-chloroethyl) ether } & \text { U } & & 37.4 \\ \text { Bis(2-chloroisopropyl) ether } & \text { U } & & 37.4 \\ \text { Bis(2-ethylhexyl) phthalate } & \text { J } & \text { E } & 22.8\end{array}$

$\begin{array}{lll}\mathrm{meq} / 100 \mathrm{~g} & 5 & \text { EPA9081 } \\ \mathrm{mg} / \mathrm{kg} & 2.78 & \text { EPA300.0 } \\ \mathrm{mg} / \mathrm{kg} & 1.15 & \text { EPA335.3 } \\ \mathrm{mg} / \mathrm{kg} & 1.12 & \text { EPA353.1 } \\ \mathrm{mg} / \mathrm{kg} & 11.1 & \text { EPA365.2 } \\ \mu \mathrm{g} / \mathrm{kg} & 324 & \text { EPA420.2 } \\ \mathrm{mg} / \mathrm{kg} & 287 & \text { EPA376.2 } \\ \mathrm{mg} / \mathrm{kg} & 115 & \text { EPA415.1 } \\ \mathrm{mg} / \mathrm{kg} & 115 & \text { EPA415.1 } \\ \mathrm{mg} / \mathrm{kg} & 0.23 & \text { EPA351.2 } \\ \mathrm{mg} / \mathrm{kg} & 455 & \text { EPA365.4 }\end{array}$

$\begin{array}{lll}\mathrm{mg} / \mathrm{kg} & 14.1 & \text { EPA6010A } \\ \mathrm{mg} / \mathrm{kg} & 5.63 & \text { EPA6010A } \\ \mathrm{mg} / \mathrm{kg} & 16.9 & \text { EPA6010A } \\ \mathrm{mg} / \mathrm{kg} & 1.41 & \text { EPA6010A } \\ \mathrm{mg} / \mathrm{kg} & 0.563 & \text { EPA6010A } \\ \mathrm{mg} / \mathrm{kg} & 0.282 & \text { EPA6010A } \\ \mathrm{mg} / \mathrm{kg} & 14.1 & \text { EPA6010A } \\ \mathrm{mg} / \mathrm{kg} & 1.41 & \text { EPA6010A } \\ \mathrm{mg} / \mathrm{kg} & 0.563 & \text { EPA6010A } \\ \mathrm{mg} / \mathrm{kg} & 1.41 & \text { EPA6010A } \\ \mathrm{mg} / \mathrm{kg} & 5.63 & \text { EPA6010A } \\ \mathrm{mg} / \mathrm{kg} & 5.63 & \text { EPA6010A } \\ \mathrm{mg} / \mathrm{kg} & 5.63 & \text { EPA6010A } \\ \mathrm{mg} / \mathrm{kg} & 0.563 & \text { EPA6010A } \\ \mathrm{mg} / \mathrm{kg} & 0.0324 & \text { EPA7471 } \\ \mathrm{mg} / \mathrm{kg} & 1.41 & \text { EPA6010A } \\ \mathrm{mg} / \mathrm{kg} & 113 & \text { EPA6010A } \\ \mathrm{mg} / \mathrm{kg} & 16.9 & \text { EPA6010A } \\ \mathrm{mg} / \mathrm{kg} & 1.41 & \text { EPA6010A } \\ \mathrm{mg} / \mathrm{kg} & 56.3 & \text { EPA6010A } \\ \mathrm{mg} / \mathrm{kg} & 16.9 & \text { EPA6010A } \\ \mathrm{mg} / \mathrm{kg} & 0.563 & \text { EPA6010A } \\ \mathrm{mg} / \mathrm{kg} & 1.13 & \text { EPA6010A }\end{array}$

$\begin{array}{lll}\mu \mathrm{g} / \mathrm{kg} & 37.4 & \text { EPA8270 } \\ \mu \mathrm{g} / \mathrm{kg} & 37.4 & \text { EPA8270 } \\ \mu \mathrm{g} / \mathrm{kg} & & \text { EPA8270 } \\ \mu \mathrm{g} / \mathrm{kg} & 37.4 & \text { EPA8270 } \\ \mu \mathrm{g} / \mathrm{kg} & 3.74 & \text { EPA8270 } \\ \mu \mathrm{g} / \mathrm{kg} & 3.74 & \text { EPA8270 } \\ \mu \mathrm{g} / \mathrm{kg} & 3.74 & \text { EPA8270 } \\ \mu \mathrm{g} / \mathrm{kg} & 37.4 & \text { EPA8270 } \\ \mu \mathrm{g} / \mathrm{kg} & 37.4 & \text { EPA8270 } \\ \mu \mathrm{g} / \mathrm{kg} & 3.74 & \text { EPA8270 } \\ \mu \mathrm{g} / \mathrm{kg} & 37.4 & \text { EPA8270 } \\ \mu \mathrm{g} / \mathrm{kg} & 37.4 & \text { EPA8270 } \\ \mu \mathrm{g} / \mathrm{kg} & 37.4 & \text { EPA8270 } \\ \mu \mathrm{g} / \mathrm{kg} & 37.4 & \text { EPA8270 } \\ \mu \mathrm{g} / \mathrm{kg} & 37.4 & \text { EPA8270 }\end{array}$


SURVEY ID: PPSC 0901 (continued)

Constituent
Sample ID: 104635
RQ AQ B Result
Unit
D. Limit Method

$B / N / A$ Extractables (including $P A H$ and phenols)

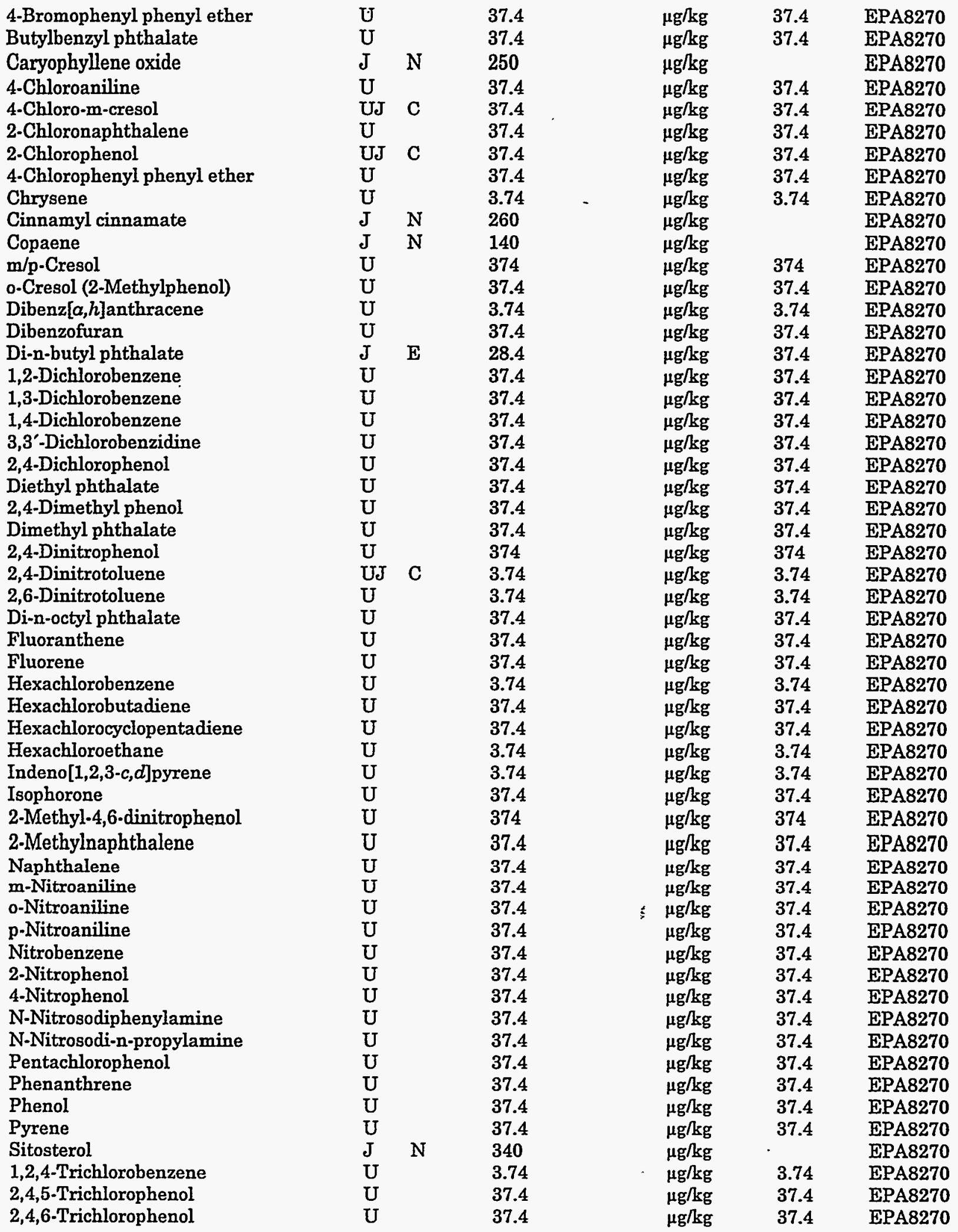


Appendix D: Sediment Tables

D-94

SURVEY ID: PPSC 0901 (continued)

Sample ID: 104635

Constituent

RQ AQ B Result

Unit

D. Limit Method

$B / N / A$ Extractables (including $P A H$ and phenols)

Unknown alkane

Unknown alkane

Unknown hydrocarbon

Unknown hydrocarbon

Unknown silane compound

Volatile Organic Compounds

Acetone
Acetone
Benzene
Benzene
Bromodichloromethane
Bromodichloromethane
Bromoform
Bromoform
Bromomethane (Methyl bromide)
Bromomethane (Methyl bromide)
Carbon disulfide
Carbon disulfide
Carbon tetrachloride
Carbon tetrachloride
Chlorobenzene
Chlorobenzene
Chloroethane
Chloroethane
Chloroethene (Vinyl chloride)
Chloroethene (Vinyl chloride)
Chloroform
Chloroform
Chloromethane (Methyl chloride)
Chloromethane (Methyl chloride)
Dibromochloromethane
Dibromochloromethane
1,1-Dichloroethane
1,1-Dichloroethane
1,2-Dichloroethane
1,2-Dichloroethane
1,1-Dichloroethylene
1,1-Dichloroethylene
1,2-Dichloroethylene
1,2-Dichloroethylene
Dichloromethane (Methylene chloride)
Dichloromethane (Methylene chloride)
1,2-Dichloropropane
1,2-Dichloropropane
cis-1,3-Dichloropropene
cis-1,3-Dichloropropene
trans-1,3-Dichloropropene
trans-1,3-Dichloropropene
Ethylbenzene
Ethylbenzene
2-Hexanone
2-Hexanone
Methyl cyclopentane
(n)

$\begin{array}{lll}\text { J } & \text { N } & 610 \\ \text { J } & \text { N } & 470 \\ J & N & 1000 \\ \text { J } & \text { N } & 1300 \\ \text { J } & \text { N } & 560\end{array}$

$\mu \mathrm{g} / \mathrm{kg}$

$\mu \mathrm{g} / \mathrm{kg}$

$\mu g / k g$

$\mu \mathrm{g} / \mathrm{kg}$

$\mu \mathrm{g} / \mathrm{kg}$

\begin{tabular}{|c|c|c|}
\hline$\mu \mathrm{g} / \mathrm{kg}$ & 5.75 & EPA8260 \\
\hline$\mu \mathrm{g} / \mathrm{kg}$ & 5.75 & EPA8260 \\
\hline$\mu \mathrm{g} / \mathrm{kg}$ & 0.575 & EPA8260 \\
\hline$\mu \mathrm{g} / \mathrm{kg}$ & 0.575 & EPA8260 \\
\hline$\mu \mathrm{g} / \mathrm{kg}$ & 0.0575 & EPA8260 \\
\hline$\mu \mathrm{g} / \mathrm{kg}$ & 0.0575 & EPA8260 \\
\hline$\mu \mathrm{g} / \mathrm{kg}$ & 0.0575 & EPA8260 \\
\hline$\mu \mathrm{g} / \mathrm{kg}$ & 0.0575 & EPA8260 \\
\hline$\mu \mathrm{g} / \mathrm{kg}$ & 0.115 & EPA8260 \\
\hline$\mu \mathrm{g} / \mathrm{kg}$ & 0.115 & EPA8260 \\
\hline$\mu \mathrm{g} / \mathrm{kg}$ & 1.15 & EPA8260 \\
\hline$\mu \mathrm{g} / \mathrm{kg}$ & 1.15 & EPA8260 \\
\hline$\mu \mathrm{g} / \mathrm{kg}$ & 0.0575 & EPA8260 \\
\hline$\mu \mathrm{g} / \mathrm{kg}$ & 0.0575 & EPA8260 \\
\hline$\mu \mathrm{g} / \mathrm{kg}$ & 0.0575 & EPA8260 \\
\hline$\mu \mathrm{g} / \mathrm{kg}$ & 0.0575 & EPA8260 \\
\hline$\mu \mathrm{g} / \mathrm{kg}$ & 0.115 & EPA8260 \\
\hline$\mu \mathrm{g} / \mathrm{kg}$ & 0.115 & EPA8260 \\
\hline$\mu \mathrm{g} / \mathrm{kg}$ & 0.115 & EPA8260 \\
\hline$\mu \mathrm{g} / \mathrm{kg}$ & 0.115 & EPA8260 \\
\hline$\mu \mathrm{g} / \mathrm{kg}$ & 0.0575 & EPA8260 \\
\hline$\mu \mathrm{g} / \mathrm{kg}$ & 0.0575 & EPA8260 \\
\hline$\mu \mathrm{g} / \mathrm{kg}$ & 0.115 & EPA8260 \\
\hline$\mu \mathrm{g} / \mathrm{kg}$ & 0.115 & EPA8260 \\
\hline$\mu \mathrm{g} / \mathrm{kg}$ & 0.0575 & EPA8260 \\
\hline$\mu \mathrm{g} / \mathrm{kg}$ & 0.0575 & EPA8260 \\
\hline$\mu \mathrm{g} / \mathrm{kg}$ & 0.0575 & EPA8260 \\
\hline$\mu \mathrm{g} / \mathrm{kg}$ & 0.0575 & EPA8260 \\
\hline$\mu \mathrm{g} / \mathrm{kg}$ & 0.0575 & EPA8260 \\
\hline$\mu \mathrm{g} / \mathrm{kg}$ & 0.0575 & EPA8260 \\
\hline$\mu \mathrm{g} / \mathrm{kg}$ & 0.0575 & EPA8260 \\
\hline$\mu \mathrm{g} / \mathrm{kg}$ & 0.0575 & EPA8260 \\
\hline$\mu \mathrm{g} / \mathrm{kg}$ & 0.115 & EPA8260 \\
\hline$\mu \mathrm{g} / \mathrm{kg}$ & 0.115 & EPA8260 \\
\hline$\mu \mathrm{g} / \mathrm{kg}$ & 0.575 & EPA8260 \\
\hline$\mu \mathrm{g} / \mathrm{kg}$ & 0.575 & EPA8260 \\
\hline$\mu \mathrm{g} / \mathrm{kg}$ & 0.0575 & EPA8260 \\
\hline$\mu \mathrm{g} / \mathrm{kg}$ & 0.0575 & EPA8260 \\
\hline$\mu \mathrm{g} / \mathrm{kg}$ & 0.0575 & EPA8260 \\
\hline$\mu \mathrm{g} / \mathrm{kg}$ & 0.0575 & EPA8260 \\
\hline$\mu g / k g$ & 0.0575 & EPA8260 \\
\hline$\mu g / \mathrm{kg}$ & 0.0575 & EPA8260 \\
\hline$\mu \mathrm{g} / \mathrm{kg}$ & 0.0575 & EPA8260 \\
\hline$\mu \mathrm{g} / \mathrm{kg}$ & 0.0575 & EPA8260 \\
\hline$\mu g / k g$ & 1.15 & EPA8260 \\
\hline$\mu \mathrm{g} / \mathrm{kg}$ & 1.15 & EPA8260 \\
\hline & & EPA8260 \\
\hline
\end{tabular}

EPA8270

EPA8270

EPA8270

EPA8270

EPA8270

\begin{tabular}{|c|c|c|}
\hline $\mathrm{U}$ & 8 & 44.2 \\
\hline & 8 & 62 \\
\hline$U$ & & 0.575 \\
\hline $\mathrm{U}$ & & 0.575 \\
\hline U & & 0.0575 \\
\hline $\mathrm{U}$ & & 0.0575 \\
\hline $\mathrm{U}$ & & 0.0575 \\
\hline U & & 0.0575 \\
\hline U & & 0.115 \\
\hline $\mathrm{U}$ & & 0.115 \\
\hline U & & 1.15 \\
\hline$U$ & & 1.15 \\
\hline U & & 0.0575 \\
\hline$U$ & & 0.0575 \\
\hline U & & 0.0575 \\
\hline U & & 0.0575 \\
\hline $\mathrm{U}$ & & 0.115 \\
\hline$U$ & & 0.115 \\
\hline $\mathrm{U}$ & & 0.115 \\
\hline $\mathrm{U}$ & & 0.115 \\
\hline $\mathrm{U}$ & & 0.0575 \\
\hline$U$ & & 0.0575 \\
\hline U & & 0.115 \\
\hline $\mathrm{U}$ & & 0.115 \\
\hline U & & 0.0575 \\
\hline $\mathrm{U}$ & & 0.0575 \\
\hline $\mathrm{U}$ & & 0.0575 \\
\hline U & & 0.0575 \\
\hline U & & 0.0575 \\
\hline U & & 0.0575 \\
\hline U & & 0.0575 \\
\hline $\mathrm{U}$ & & 0.0575 \\
\hline $\mathrm{U}$ & & 0.115 \\
\hline $\mathrm{U}$ & & 0.115 \\
\hline $\mathrm{U}$ & V & 11.9 \\
\hline $\mathrm{U}$ & V & 11.8 \\
\hline $\mathrm{U}$ & & 0.0575 \\
\hline $\mathbf{U}$ & & 0.0575 \\
\hline $\mathbf{U}$ & & 0.0575 \\
\hline $\mathrm{U}$ & & 0.0575 \\
\hline $\mathrm{U}$ & & 0.0575 \\
\hline U & & 0.0575 \\
\hline U & & 0.0575 \\
\hline $\mathrm{U}$ & & 0.0575 \\
\hline $\mathrm{U}$ & V & 3.03 \\
\hline$U$ & $\mathrm{~V}$ & 1.62 \\
\hline J & $\mathrm{N}$ & 2.83 \\
\hline
\end{tabular}


SURVEY ID: PPSC 0901 (continued)

Constituent

Volatile Organic Compounds

Methyl ethyl ketone

Methyl ethyl ketone

Methyl isobutyl ketone

Methyl isobutyl ketone

Styrene

Styrene

1,1,2,2-Tetrachloroethane

1,1,2,2-Tetrachloroethane

Tetrachloroethylene

Tetrachloroethylene

Toluene

Toluene

1,1,1-Trichloroethane

1,1,1-Trichloroethane

1,1,2-Trichloroethane

1,1,2-Trichloroethane

Trichloroethylene

Trichloroethylene

Vinyl acetate

Vinyl acetate

Xylenes

Xylenes

Pesticides/PCBs and Dioxins/Furans
Sample ID: 104635

RQ AQ B Result Unit D. Limit Method

$\begin{array}{lll}U & V & 0.782 \\ U & V & 1.51 \\ U & & 1.15 \\ U & & 1.15 \\ & & 0.138 \\ & & 0.276 \\ U & & 0.0575 \\ U & & 0.0575 \\ U & & 0.0575 \\ U & & 0.0575 \\ U & V & 0.276 \\ U & V & 0.264 \\ U & & 0.0575 \\ U & & 0.0575 \\ U & & 0.0575 \\ U & & 0.0575 \\ U & & 0.0575 \\ U & & 0.0575 \\ U & & 1.15 \\ U & & 1.15 \\ U & & 0.172 \\ U & & 0.172\end{array}$

\begin{tabular}{|c|c|c|c|}
\hline & OI & $\mathrm{H}$ & 0.951 \\
\hline JJ & 0 & L & 0.951 \\
\hline JJ & 0 & L & 1.9 \\
\hline $\mathrm{UJ}$ & O & L & 1.9 \\
\hline & 0 & L & 1.9 \\
\hline & 0 & L & 1.9 \\
\hline & 0 & L & 3.8 \\
\hline & 0 & L & 1.9 \\
\hline J & 0 & L & 3.8 \\
\hline J & OI & $\mathrm{H}$ & 1.9 \\
\hline & 0 & L & 3.8 \\
\hline & & & 1.9 \\
\hline & 0 & L & 3.8 \\
\hline e & OI & $\mathrm{H}$ & 1.9 \\
\hline & 0 & I & 3.8 \\
\hline$J$ & OI & $\mathrm{H}$ & 1.9 \\
\hline $\mathrm{JJ}$ & 0 & L & 1.9 \\
\hline Јر & OI & $\mathrm{H}$ & 0.951 \\
\hline $\mathrm{J}$ & 0 & L & 19 \\
\hline $\mathrm{JJ}$ & 0 & L & 4.75 \\
\hline U. & 0 & L & 4.75 \\
\hline UJ & & & 4.75 \\
\hline & 0 & L & 4.75 \\
\hline U & 0 & L & 4.75 \\
\hline & & & 4.75 \\
\hline & & & 4.75 \\
\hline & & & 38 \\
\hline
\end{tabular}

$\begin{array}{lll}\mu \mathrm{g} / \mathrm{kg} & 1.15 & \text { EPA8260 } \\ \mu \mathrm{g} / \mathrm{kg} & 1.15 & \text { EPA8260 } \\ \mu \mathrm{g} / \mathrm{kg} & 1.15 & \text { EPA8260 } \\ \mu \mathrm{g} / \mathrm{kg} & 1.15 & \text { EPA8260 } \\ \mu \mathrm{g} / \mathrm{kg} & 0.0575 & \text { EPA8260 } \\ \mu \mathrm{g} / \mathrm{kg} & 0.0575 & \text { EPA8260 } \\ \mu \mathrm{g} / \mathrm{kg} & 0.0575 & \text { EPA8260 } \\ \mu \mathrm{g} / \mathrm{kg} & 0.0575 & \text { EPA8260 } \\ \mu \mathrm{g} / \mathrm{kg} & 0.0575 & \text { EPA8260 } \\ \mu \mathrm{g} / \mathrm{kg} & 0.0575 & \text { EPA8260 } \\ \mu \mathrm{g} / \mathrm{kg} & 0.575 & \text { EPA8260 } \\ \mu \mathrm{g} / \mathrm{kg} & \mathbf{0 . 5 7 5} & \text { EPA8260 } \\ \mu \mathrm{g} / \mathrm{kg} & 0.0575 & \text { EPA8260 } \\ \mu \mathrm{g} / \mathrm{kg} & 0.0575 & \text { EPA8260 } \\ \mu \mathrm{g} / \mathrm{kg} & 0.0575 & \text { EPA8260 } \\ \mu \mathrm{g} / \mathrm{kg} & 0.0575 & \text { EPA8260 } \\ \mu \mathrm{g} / \mathrm{kg} & 0.0575 & \text { EPA8260 } \\ \mu \mathrm{g} / \mathrm{kg} & 0.0575 & \text { EPA8260 } \\ \mu \mathrm{g} / \mathrm{kg} & 1.15 & \text { EPA8260 } \\ \mu \mathrm{g} / \mathrm{kg} & 1.15 & \text { EPA8260 } \\ \mu \mathrm{g} / \mathrm{kg} & 0.172 & \text { EPA8260 } \\ \mu \mathrm{g} / \mathrm{kg} & 0.172 & \text { EPA8260 }\end{array}$

$\begin{array}{lll}\mu \mathrm{g} / \mathrm{kg} & 0.951 & \text { EPA8080 } \\ \mu \mathrm{g} / \mathrm{kg} & 0.951 & \text { EPA8080 } \\ \mu \mathrm{g} / \mathrm{kg} & 1.9 & \text { EPA8080 } \\ \mu \mathrm{g} / \mathrm{kg} & 1.9 & \text { EPA8080 } \\ \mu \mathrm{g} / \mathrm{kg} & 1.9 & \text { EPA8080 } \\ \mu \mathrm{g} / \mathrm{kg} & 1.9 & \text { EPA8080 } \\ \mu \mathrm{g} / \mathrm{kg} & 3.8 & \text { EPA8080 } \\ \mu \mathrm{g} / \mathrm{kg} & 1.9 & \text { EPA8080 } \\ \mu \mathrm{g} / \mathrm{kg} & 3.8 & \text { EPA8080 } \\ \mu \mathrm{g} / \mathrm{kg} & 1.9 & \text { EPA8080 } \\ \mu \mathrm{g} / \mathrm{kg} & 3.8 & \text { EPA8080 } \\ \mu \mathrm{g} / \mathrm{kg} & 1.9 & \text { EPA8080 } \\ \mu \mathrm{g} / \mathrm{kg} & 3.8 & \text { EPA8080 } \\ \mu \mathrm{g} / \mathrm{kg} & 1.9 & \text { EPA8080 } \\ \mu \mathrm{g} / \mathrm{kg} & 3.8 & \text { EPA8080 } \\ \mu \mathrm{g} / \mathrm{kg} & 1.9 & \text { EPA8080 } \\ \mu \mathrm{g} / \mathrm{kg} & 1.9 & \text { EPA8080 } \\ \mu \mathrm{g} / \mathrm{kg} & 0.951 & \text { EPA8080 } \\ \mu \mathrm{g} / \mathrm{kg} & 19 & \text { EPA8080 } \\ \mu \mathrm{g} / \mathrm{kg} & 4.75 & \text { EPA8080 } \\ \mu \mathrm{g} / \mathrm{kg} & 4.75 & \text { EPA8080 } \\ \mu \mathrm{g} / \mathrm{kg} & 4.75 & \text { EPA8080 } \\ \mu \mathrm{g} / \mathrm{kg} & 4.75 & \text { EPA8080 } \\ \mu \mathrm{g} / \mathrm{kg} & 4.75 & \text { EPA8080 } \\ \mu \mathrm{g} / \mathrm{kg} & 4.75 & \text { EPA8080 } \\ \mu \mathrm{g} / \mathrm{kg} & 4.75 & \text { EPA8080 } \\ \mu \mathrm{g} / \mathrm{kg} & 38 & \text { EPA8080 }\end{array}$


SURVEY ID: PPSC 0901 (continued)

Constituent

Radionuclides

Actinium-228

Antimony-124

Antimony-125

Barium-133

Cerium-144

Cesium-134

Cesium-137

Cobalt-57

Cobalt-58

Cobalt-60

Europium-152

Europium-154

Europium-155

Gross alpha

Lead-212

Manganese-54

Neptunium-239

Nonvolatile beta

Plutonium-238

Plutonium-239/240

Potassium-40

Promethium-144

Promethium-146

Ruthenium-106

Sodium-22

Strontium-90

Thorium-234

Tin-113

Yttrium-88

Zinc-65

Zirconium-95
Sample ID: 104635
$\mathbf{R Q}$
AQ
Result
Unit
D. Limit Method

$5.44 \mathrm{E}-01 \pm 3.94 \mathrm{E}-02 \mathrm{pCi} / \mathrm{g}$

4.01E-03 $\pm 8.32 \mathrm{E}-03 \mathrm{pCi} / \mathrm{g}$

$1.08 \mathrm{E}-02 \pm 1.74 \mathrm{E}-02 \mathrm{pCi} / \mathrm{g}$

$-4.59 \mathrm{E}-04 \pm 6.28 \mathrm{E}-03 \mathrm{pCi} / \mathrm{g}$

$4.62 \mathrm{E}-02 \pm 4.94 \mathrm{E}-02 \mathrm{pCi} / \mathrm{g}$

$-3.93 \mathrm{E}-03 \pm 6.50 \mathrm{E}-03 \mathrm{pCi} / \mathrm{g}$

$5.43 \mathrm{E}-01 \pm 1.84 \mathrm{E}-02 \mathrm{pCj} / \mathrm{g}$

$1.27 \mathrm{E}-03 \pm 4.09 \mathrm{E}-03 \mathrm{pCi} / \mathrm{g}$

$-5.46 \mathrm{E}-05 \pm 5.00 \mathrm{E}-03 \mathrm{pCi} / \mathrm{g}$

4.75E-03 $3.37 \mathrm{E}-03 \mathrm{pCi} / \mathrm{g}$

2.01E-02 $\pm 2.57 \mathrm{E}-02 \mathrm{pCi} / \mathrm{g}$

2.10E-02 $\pm 3.44 \mathrm{E}-02 \mathrm{pCi} / \mathrm{g}$

2.54E-02 $2.52 \mathrm{E}-02 \mathrm{pCi} / \mathrm{g}$

$1.39 \mathrm{E}+01 \pm 2.78 \mathrm{E}+00 \mathrm{pCi} / \mathrm{g}$

$5.45 \mathrm{E}-01 \pm 2.03 \mathrm{E}-02 \mathrm{pCi} / \mathrm{g}$

$9.50 \mathrm{E}-03 \pm 4.69 \mathrm{E}-03 \mathrm{pCi} / \mathrm{g}$

$1.25 \mathrm{E}-02 \pm 2.89 \mathrm{E}-02 \mathrm{pCi} / \mathrm{g}$

$7.97 \mathrm{E}+00 \pm 1.79 \mathrm{E}+00 \mathrm{pCi} / \mathrm{g}$

$1.59 \mathrm{E}-02 \pm 9.63 \mathrm{E}-03 \mathrm{pCi} / \mathrm{g}$

$2.88 \mathrm{E}-03 \pm 4.10 \mathrm{E}-03 \mathrm{pCi} / \mathrm{g}$

4.86E-01 $18.25 \mathrm{E}-02 \mathrm{pCi} / \mathrm{g}$

$7.62 \mathrm{E}-04 \pm 3.70 \mathrm{E}-03 \mathrm{pCi} / \mathrm{g}$

$8.52 \mathrm{E}-03 \pm 6.42 \mathrm{E}-03 \mathrm{pCi} / \mathrm{g}$ 2.03E-02 $\pm 3.89 \mathrm{E}-02 \mathrm{pCi} / \mathrm{g}$ $2.83 \mathrm{E}-03 \pm 3.60 \mathrm{E}-03 \mathrm{pCi} / \mathrm{g}$

$-7.60 \mathrm{E}-02 \pm 2.21 \mathrm{E}-02 \mathrm{pCi} / \mathrm{g}$

$6.28 \mathrm{E}-01 \pm 4.34 \mathrm{E}-01 \mathrm{pCi} / \mathrm{g}$

9.01E-04 7.17E-03 pCi/g

$1.92 \mathrm{E}-03 \pm 4.45 \mathrm{E}-03 \mathrm{pCi} / \mathrm{g}$

3.04E-03 $\pm 9.59 \mathrm{E}-03 \mathrm{pCi} / \mathrm{g}$

$1.40 \mathrm{E}-02 \pm 2.14 \mathrm{E}-02 \mathrm{pCi} / \mathrm{g}$
2.25E-02 EPIA-013B

1.04E-02 EPIA-013B

2.28E-02 EPIA-013B

9.29E-03 EPIA-013B

5.52E-02 EPIA-013B

6.71E-03 EPIA-013B

7.24E-03 EPIA-013B

7.12E-03 EPIA-013B

7.73E-03 EPIA-013B

6.98E-03 EPIA-013B

2.19E-02 EPIA-013B

6.29E-02 EPIA-013B

2.89E-02 EPIA-013B

1.80E+00 EPIA-001B

1.30E-02 EPIA-013B

7.06E-03 EPIA-013B

5.05E-02 EPIA-013B

2.53E+00 EPIA-001B

1.09E-02 EPIA-012B

4.32E-03 EPIA-012B

6.70E-02 EPLA-013B

6.58E-03 EPIA-013B

1.12E-02 EPIA-013B

7.01E-02 EPIA-013B

7.03E-03 EPIA-013B

7.09E-02 EPIA-004

3.63E-01 EPIA-013B

1.19E-02 EPIA-013B

8.62E-03 EPIA-013B

1.50E-02 EPIA-013B

1.96E-02 EPIA-013B

SURVEY ID: PPSC 0901A

Sample ID: 104638

Survey location: $45224 \mathrm{E} 3680779 \mathrm{~N}$ (SRS coordinates)

Depth of core interval: 0.00 to $1.00 \mathrm{ft}$

Sample type: Normal

Sample matrix: Other

Percent solids: $\mathbf{8 8 . 0 0}$

Constituent

RQ AQ B Result

Unit

D. Limit Method

Physical Parameters and Specified Analyses

Cation exchange capacity

Chloride

Cyanide

Nitrate-nitrite as nitrogen

Orthophosphate

Phenols

Sulfide

Total organic carbon

Total organic nitrogen

Total phosphates (as P)

$\begin{array}{llll} & & 12.1 \\ \text { U } & & 2.83 \\ \text { U } & & 1.14 \\ \text { U } & & 1.09 \\ \text { U } & & 11 \\ \text { UJ } & \text { C } & 338 \\ \text { UJ } & \text { C } & 284 \\ & & 1220 \\ \text { C } & & 49.2 \\ \text { J } & \text { E } & 94.5\end{array}$

$\begin{array}{ll}\mathrm{meq} / 100 \mathrm{~g} & 5 \\ \mathrm{mg} / \mathrm{kg} & 2.83 \\ \mathrm{mg} / \mathrm{kg} & 1.14 \\ \mathrm{mg} / \mathrm{kg} & 1.09 \\ \mathrm{mg} / \mathrm{kg} & 11 \\ \mu \mathrm{g} / \mathrm{kg} & 338 \\ \mathrm{mg} / \mathrm{kg} & 284 \\ \mathrm{mg} / \mathrm{kg} & 114 \\ \mathrm{mg} / \mathrm{kg} & 0.227 \\ \mathrm{mg} / \mathrm{kg} & 450\end{array}$

EPA9081

EPA300.0

EPA335.3

EPA353.1

EPA365.2

EPA420.2

EPA376.2

EPA415.1

EPA351.2

EPA365.4 
SURVEY ID: PPSC 0901A (continued)

Sample ID: 104638

Constituent

Metals (total recoverable)

Antimony

Arsenic

Barium

Beryllium

Cadmium

Calcium

Chromium

Cobalt

Copper

Iron

Lead

Magnesium

Manganese

Mercury

Nickel

Potassium

Selenium

Silver

Sodium

Thallium

Vanadium

Zinc
Aluminum

RQ AQ B Result

$\begin{array}{lll} & V & 3080 \\ \text { U } & & 5.57 \\ \mathrm{U} & & 16.7 \\ \mathrm{~J} & \mathrm{E} & \mathbf{1 3 . 1} \\ \mathrm{U} & & \mathbf{0 . 2 7 2 4} \\ & \mathrm{V} & 47.6 \\ \mathrm{~J} & \mathrm{E} & \mathbf{3 . 5 7} \\ & & 0.32 \\ \mathrm{~J} & \mathrm{VC} & \mathbf{3 . 7 1} \\ \mathrm{J} & \mathrm{E} & \mathbf{1 4 5 0} \\ & \mathrm{V} & \mathbf{7 3 . 7} \\ & & 47 \\ \mathrm{U} & \mathrm{V} & \mathbf{0 . 0 1 2} \\ \mathrm{J} & \mathrm{E} & \mathbf{0 . 9 7 6} \\ \mathrm{J} & \mathrm{E} & 39.9 \\ \mathrm{U} & & 16.7 \\ \mathrm{U} & & 1.39 \\ \mathrm{U} & & \mathbf{5 5 . 7} \\ \mathrm{UJ} & \mathrm{C} & 16.7 \\ & & 4.06 \\ & & 5.25\end{array}$

$B / N / A$ Extractables (including PAH and phenols)

Acenaphthene

Acenaphthylene

Anthracene

Benzo[a]anthracene

Benzo[b]fluoranthene

Benzo[k]fluoranthene

Benzoic acid

Benzo $[g, h, i]$ perylene

Benzo[a]pyrene

Benzyl alcohol

Bis(2-chloroethoxy) methane

Bis(2-chloroethyl) ether

Bis(2-chloroisopropyl) ether

Bis(2-ethylhexyl) phthalate

4-Bromophenyl phenyl ether

Butylbenzyl phthalate

4-Chloroaniline

4-Chloro-m-cresol

2.Chloronaphthalene

2-Chlorophenol

4-Chlorophenyl phenyl ether

Chrysene

$\mathrm{m} / \mathrm{p}$-Cresol

o-Cresol (2-Methylphenol)

Dibenz $[a, h]$ anthracene

Dibenzofuran

$\begin{array}{lll}\text { U } & 37.6 \\ U & 37.6 \\ U & 37.6 \\ U & 3.76 \\ U & 3.76 \\ U & 3.76 \\ U & 37.6 \\ U & 37.6 \\ U & 3.76 \\ U & 37.6 \\ U & & 37.6 \\ U & 37.6 \\ U & & 37.6 \\ J & E & 22.6 \\ U & 37.6 \\ U & 37.6 \\ U & 37.6 \\ U & 37.6 \\ U & 37.6 \\ U & 37.6 \\ U & 37.6 \\ U & 3.76 \\ U & 376 \\ U & 37.6 \\ U & 3.76 \\ U & 37.6\end{array}$

Unit

D. Limit Method

$\begin{array}{lll}\mathrm{mg} / \mathrm{kg} & 13.9 & \text { EPA6010A } \\ \mathrm{mg} / \mathrm{kg} & 5.57 & \text { EPA6010A } \\ \mathrm{mg} / \mathrm{kg} & 16.7 & \text { EPA6010A } \\ \mathrm{mg} / \mathrm{kg} & 1.39 & \text { EPA6010A } \\ \mathrm{mg} / \mathrm{kg} & 0.557 & \text { EPA6010A } \\ \mathrm{mg} / \mathrm{kg} & 0.279 & \text { EPA6010A } \\ \mathrm{mg} / \mathrm{kg} & 13.9 & \text { EPA6010A } \\ \mathrm{mg} / \mathrm{kg} & 1.39 & \text { EPA6010A } \\ \mathrm{mg} / \mathrm{kg} & 0.557 & \text { EPA6010A } \\ \mathrm{mg} / \mathrm{kg} & 1.39 & \text { EPA6010A } \\ \mathrm{mg} / \mathrm{kg} & 5.57 & \text { EPA6010A } \\ \mathrm{mg} / \mathrm{kg} & 5.57 & \text { EPA6010A } \\ \mathrm{mg} / \mathrm{kg} & 5.57 & \text { EPA6010A } \\ \mathrm{mg} / \mathrm{kg} & 0.557 & \text { EPA6010A } \\ \mathrm{mg} / \mathrm{kg} & 0.0338 & \text { EPA7471 } \\ \mathrm{mg} / \mathrm{kg} & 1.39 & \text { EPA6010A } \\ \mathrm{mg} / \mathrm{kg} & 111 & \text { EPA6010A } \\ \mathrm{mg} / \mathrm{kg} & 16.7 & \text { EPA6010A } \\ \mathrm{mg} / \mathrm{kg} & 1.39 & \text { EPA6010A } \\ \mathrm{mg} / \mathrm{kg} & \mathbf{5 5 . 7} & \text { EPA6010A } \\ \mathrm{mg} / \mathrm{kg} & 16.7 & \text { EPA6010A } \\ \mathrm{mg} / \mathrm{kg} & 0.557 & \text { EPA6010A } \\ \mathrm{mg} / \mathrm{kg} & 1.11 & \text { EPA6010A }\end{array}$

$\begin{array}{lcc}\mu \mathrm{g} / \mathrm{kg} & 37.6 & \text { EPA8270 } \\ \mu \mathrm{g} / \mathrm{kg} & 37.6 & \text { EPA8270 } \\ \mu \mathrm{g} / \mathrm{kg} & 37.6 & \text { EPA8270 } \\ \mu \mathrm{g} / \mathrm{kg} & 3.76 & \text { EPA8270 } \\ \mu \mathrm{g} / \mathrm{kg} & 3.76 & \text { EPA8270 } \\ \mu \mathrm{g} / \mathrm{kg} & 3.76 & \text { EPA8270 } \\ \mu \mathrm{g} / \mathrm{kg} & 37.6 & \text { EPA8270 } \\ \mu \mathrm{g} / \mathrm{kg} & 37.6 & \text { EPA8270 } \\ \mu \mathrm{g} / \mathrm{kg} & 3.76 & \text { EPA8270 } \\ \mu \mathrm{g} / \mathrm{kg} & 37.6 & \text { EPA8270 } \\ \mu \mathrm{g} / \mathrm{kg} & 37.6 & \text { EPA8270 } \\ \mu \mathrm{g} / \mathrm{kg} & 37.6 & \text { EPA8270 } \\ \mu \mathrm{g} / \mathrm{kg} & 37.6 & \text { EPA8270 } \\ \mu \mathrm{g} / \mathrm{kg} & 37.6 & \text { EPA8270 } \\ \mu \mathrm{g} / \mathrm{kg} & 37.6 & \text { EPA8270 } \\ \mu \mathrm{g} / \mathrm{kg} & 37.6 & \text { EPA8270 } \\ \mu \mathrm{g} / \mathrm{kg} & 37.6 & \text { EPA8270 } \\ \mu \mathrm{g} / \mathrm{kg} & 37.6 & \text { EPA8270 } \\ \mu \mathrm{g} / \mathrm{kg} & 37.6 & \text { EPA8270 } \\ \mu \mathrm{g} / \mathrm{kg} & 37.6 & \text { EPA8270 } \\ \mu \mathrm{g} / \mathrm{kg} & 37.6 & \text { EPA8270 } \\ \mu \mathrm{g} / \mathrm{kg} & 3.76 & \text { EPA8270 } \\ \mu \mathrm{g} / \mathrm{kg} & 376 & \text { EPA8270 } \\ \mu \mathrm{g} / \mathrm{kg} & 37.6 & \text { EPA8270 } \\ \mu \mathrm{g} / \mathrm{kg} & 3.76 & \text { EPA8270 } \\ \mu \mathrm{g} / \mathrm{kg} & 37.6 & \text { EPA8270 }\end{array}$


SURVEY ID: PPSC 0901A (continued)

Sample ID: 104638

Constituent

RQ AQ B Result

Unit

D. Limit Method

$B / N / A$ Extractables (including PAH and phenols)

Di-n-butyl phthalate

1,2-Dichlorobenzene

1,3-Dichlorobenzene

1,4-Dichlorobenzene

3,3'-Dichlorobenzidine

2,4-Dichlorophenol

Diethyl phthalate

2,4-Dimethyl phenol

Dimethyl phthalate

2,4-Dinitrophenol

2,4-Dinitrotoluene

2,6-Dinitrotoluene

Di-n-octyl phthalate

Fluoranthene

Fluorene

Hexachlorobenzene

Hexachlorobutadiene

Hexachlorocyclopentadiene

Hexachloroethane

Indeno[1,2,3-c,d]pyrene

Isophorone

2-Methyl-4,6-dinitrophenol

2-Methylnaphthalene

Naphthalene

m-Nitroaniline

o-Nitroaniline

p-Nitroaniline

Nitrobenzene

2-Nitrophenol

4-Nitrophenol

N-Nitrosodiphenylamine

N-Nitrosodi-n-propylamine

Pentachlorophenol

Phenanthrene

Phenol

Pyrene

1,2,4-Trichlorobenzene

2,4,5-Trichlorophenol

2,4,6-Trichlorophenol

Unknown alcohol

Unknown alcohol

Unknown alcohol

$\begin{array}{ccc}\text { J } & E & 24.8 \\ \mathrm{U} & & 37.6 \\ \mathrm{U} & & 37.6 \\ \mathrm{U} & & 37.6 \\ \mathrm{U} & & 37.6 \\ \mathrm{U} & & 37.6 \\ \mathrm{U} & & 37.6 \\ \mathrm{U} & & 37.6 \\ \mathrm{U} & & 37.6 \\ \mathrm{U} & & 376 \\ \mathrm{U} & & 3.76 \\ \mathrm{U} & & 3.76 \\ \mathrm{U} & & 37.6 \\ \mathrm{U} & & 37.6 \\ \mathrm{U} & & 37.6 \\ \mathrm{U} & & 3.76 \\ \mathrm{U} & & 37.6 \\ \mathrm{U} & & 37.6 \\ \mathrm{U} & & 3.76 \\ \mathrm{U} & & 3.76 \\ \mathrm{U} & & 37.6 \\ \mathrm{U} & & 376 \\ \mathrm{U} & & 37.6 \\ \mathrm{U} & & 37.6 \\ \mathrm{U} & & 37.6 \\ \mathrm{U} & & 37.6 \\ \mathrm{U} & & 37.6 \\ \mathrm{U} & & 37.6 \\ \mathrm{U} & & 37.6 \\ \mathrm{U} & & 37.6 \\ \mathrm{U} & & 37.6 \\ \mathrm{U} & & 37.6 \\ \mathrm{U} & & 37.6 \\ \mathrm{U} & & 37.6 \\ \mathrm{U} & & 37.6 \\ \mathrm{U} & & 37.6 \\ \mathrm{U} & & 37.6 \\ & & \end{array}$

$\mu \mathrm{g} / \mathrm{kg}$

$\mu \mathrm{g} / \mathrm{kg}$

$\mu \mathrm{g} / \mathrm{kg}$

$\mu \mathrm{g} / \mathrm{kg}$

$\mu \mathrm{g} / \mathrm{kg}$

$\mu \mathrm{g} / \mathrm{kg}$

$\mu \mathrm{g} / \mathrm{kg}$

$\mu \mathrm{g} / \mathrm{kg}$

$\mu \mathrm{g} / \mathrm{kg}$

$\mu \mathrm{g} / \mathrm{kg}$

$\mu \mathrm{g} / \mathrm{kg}$

$\mu \mathrm{g} / \mathrm{kg}$

$\mu \mathrm{g} / \mathrm{kg}$

$\mu \mathrm{g} / \mathrm{kg}$

$\mu \mathrm{g} / \mathrm{kg}$

$\mu \mathrm{g} / \mathrm{kg}$

$\mu \mathrm{g} / \mathrm{kg}$

$\mu \mathrm{g} / \mathrm{kg}$

$\mu \mathrm{g} / \mathrm{kg}$

$\mu \mathrm{g} / \mathrm{kg}$

$\mu \mathrm{g} / \mathrm{kg}$

$\mu \mathrm{g} / \mathrm{kg}$

$\mu \mathrm{g} / \mathrm{kg}$

$\mu \mathrm{g} / \mathrm{kg}$

$\mu \mathrm{g} / \mathrm{kg}$

$\mu \mathrm{g} / \mathrm{kg}$

$\mu \mathrm{g} / \mathrm{kg}$

$\mu \mathrm{g} / \mathrm{kg}$

$\mu \mathrm{g} / \mathrm{kg}$

$\mu \mathrm{g} / \mathrm{kg}$

$\mu \mathrm{g} / \mathrm{kg}$

$\mu \mathrm{g} / \mathrm{kg}$

$\mu \mathrm{g} / \mathrm{kg}$

$\mu \mathrm{g} / \mathrm{kg}$

$\mu \mathrm{g} / \mathrm{kg}$

$\mu \mathrm{g} / \mathrm{kg}$

$\mu \mathrm{g} / \mathrm{kg}$

$\mu \mathrm{g} / \mathrm{kg}$

$\mu \mathrm{g} / \mathrm{kg}$

$\mu \mathrm{g} / \mathrm{kg}$

$\mu \mathrm{g} / \mathrm{kg}$

$\mu \mathrm{g} / \mathrm{kg}$
$37.6 \quad$ EPA8270

$37.6 \quad$ EPA8270

$37.6 \quad$ EPA8270

$37.6 \quad$ EPA8270

37.6 EPA8270

$37.6 \quad$ EPA8270

$37.6 \quad$ EPA8270

$37.6 \quad$ EPA8270

$37.6 \quad$ EPA8270

$376 \quad$ EPA8270

$3.76 \quad$ EPA8270

$3.76 \quad$ EPA8270

$37.6 \quad$ EPA8270

$37.6 \quad$ EPA8270

$37.6 \quad$ EPA8270

$3.76 \quad$ EPA8270

$37.6 \quad$ EPA8270

37.6 EPA8270

$3.76 \quad$ EPA8270

$3.76 \quad$ EPA8270

$37.6 \quad$ EPA8270

$376 \quad$ EPA8270

$37.6 \quad$ EPA8270

$37.6 \quad$ EPA8270

$37.6 \quad$ EPA8270

$37.6 \quad$ EPA8270

$37.6 \quad$ EPA8270

$37.6 \quad$ EPA8270

$37.6 \quad$ EPA8270

$37.6 \quad$ EPA8270

$37.6 \quad$ EPA8270

$37.6 \quad$ EPA8270

$37.6 \quad$ EPA8270

$37.6 \quad$ EPA8270

$37.6 \quad$ EPA8270

$37.6 \quad$ EPA8270

$3.76 \quad$ EPA8270

$37.6 \quad$ EPA8270

$37.6 \quad$ EPA8270

EPA8270

EPA8270

EPA8270

Volatile Organic Compounds

Acetone
Benzene
Bromodichloromethane
Bromoform
Bromomethane (Methyl bromide)
Carbon disulfide
Carbon tetrachloride
Chlorobenzene
Chloroethane
Chloroethene (Vinyl chloride)

$\begin{array}{lll} & 8 & 40.1 \\ \mathrm{U} & & 0.568 \\ \mathrm{U} & & 0.0568 \\ \mathrm{U} & & 0.0568 \\ \mathrm{U} & & 0.114 \\ \mathrm{U} & & 1.14 \\ \mathrm{U} & & 0.0568 \\ \mathrm{U} & & 0.0568 \\ \mathrm{U} & & 0.114 \\ \mathrm{U} & & 0.114\end{array}$

$\begin{array}{ll}5.68 & \text { EPA8260 } \\ 0.568 & \text { EPA8260 } \\ 0.0568 & \text { EPA8260 } \\ 0.0568 & \text { EPA8260 } \\ 0.114 & \text { EPA8260 } \\ 1.14 & \text { EPA8260 } \\ 0.0568 & \text { EPA8260 } \\ 0.0568 & \text { EPA8260 } \\ 0.114 & \text { EPA8260 } \\ 0.114 & \text { EPA8260 }\end{array}$


SURVEY ID: PPSC 0901A (continued)

Constituent

RQ AQ B Result

Sample ID: 104638

Volatile Organic Compounds

Chloroform
Chloromethane (Methyl chloride)
Dibromochloromethane
1,1-Dichloroethane
1,2-Dichloroethane
1,1-Dichloroethylene
1,2-Dichloroethylene
Dichloromethane (Methylene chloride)
1,2-Dichloropropane
cis-1,3-Dichloropropene
trans-1,3-Dichloropropene
Ethylbenzene
2-Hexanone
Methyl cyclopentane
Methyl ethyl ketone
Methyl isobutyl ketone
Styrene
1,1,2,2-Tetrachloroethane
Tetrachloroethylene
Toluene
1,1,1-Trichloroethane
1,1,2-Trichloroethane
Trichloroethylene
Vinyl acetate
Xylenes

Pesticides/PCBs and Dioxins/Furans

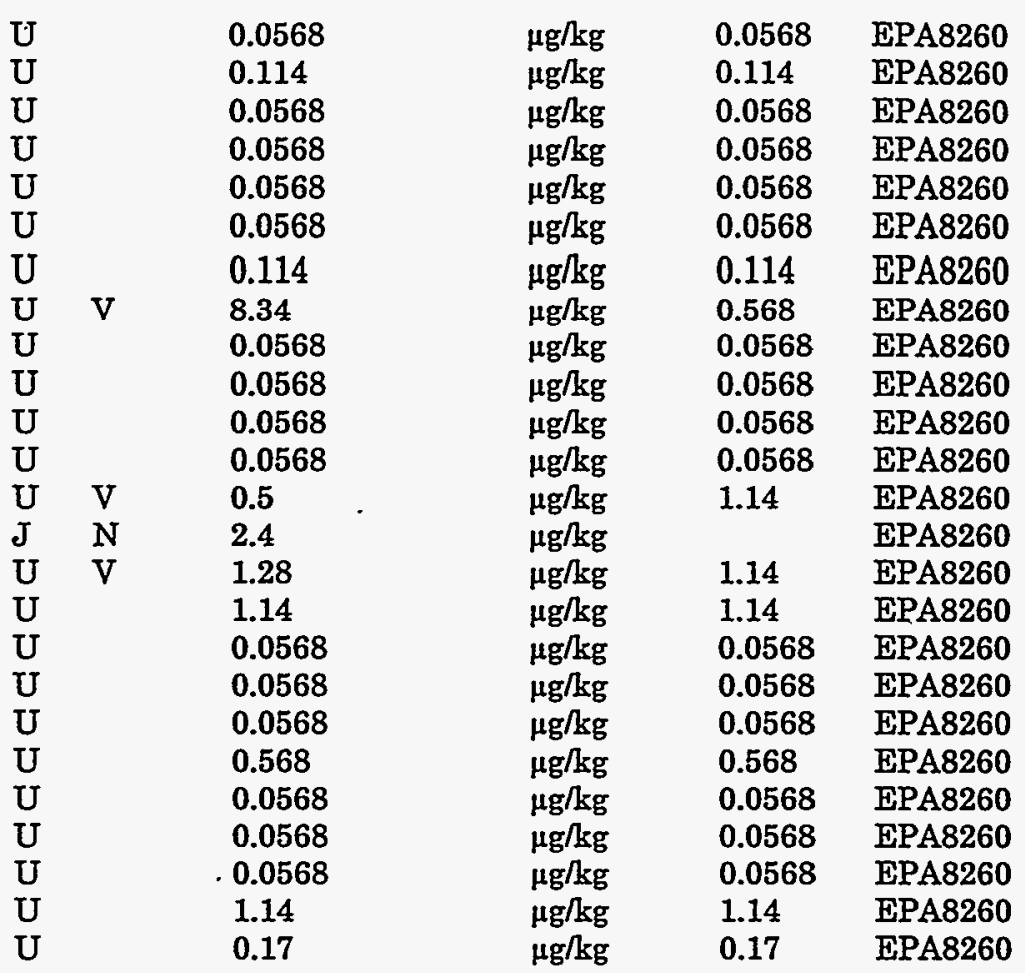

$\begin{array}{lcc}\text { Aldrin } & \mathrm{U} & 4.69 \\ \text { alpha-Benzene hexachloride } & \mathrm{U} & 4.69 \\ \text { beta-Benzene hexachloride } & \mathrm{U} & 9.38 \\ \text { delta-Benzene hexachloride } & \mathrm{U} & 9.38 \\ \text { alpha-Chlordane } & \mathrm{U} & 9.38 \\ \text { gamma-Chlordane } & \mathrm{U} & 9.38 \\ \text { p,p'-DDD } & \mathrm{U} & 18.8 \\ \text { p,p'-DDE } & \mathrm{U} & 9.38 \\ \text { p,p'-DDT } & \mathrm{U} & 18.8 \\ \text { Dieldrin } & \mathrm{U} & 9.38 \\ \text { Endosulfan sulfate } & \mathrm{U} & 18.8 \\ \text { Endosulfan I } & \mathrm{U} & 9.38 \\ \text { Endosulfan II } & \mathrm{U} & 18.8 \\ \text { Endrin } & \mathrm{U} & 9.38 \\ \text { Endrin ketone } & \mathrm{U} & 18.8 \\ \text { Heptachlor } & \mathrm{U} & 9.38 \\ \text { Heptachlor epoxide } & \mathrm{U} & 9.38 \\ \text { Lindane } & \mathrm{U} & 4.69 \\ \text { Methoxychlor } & \mathrm{U} & 93.8 \\ \text { PCB 1016 } & \mathrm{U} & 23.5 \\ \text { PCB 1221 } & \mathrm{U} & 23.5 \\ \text { PCB 1232 } & \mathrm{U} & 23.5 \\ \text { PCB 1242 } & \mathrm{U} & 23.5 \\ \text { PCB 1248 } & \mathrm{U} & 23.5 \\ \text { PCB 1254 } & \mathrm{U} & 23.5 \\ \text { PCB 1260 } & \mathrm{U} & 23.5 \\ \text { Toxaphene } & \mathrm{U} & 188\end{array}$

Unit D. Limit Method 
SURVEY ID: PPSC 0901A (continued)

\section{Constituent}

Radionuclides

Actinium-228

Antimony-124

Antimony-125

Barium-133

Cerium-144

Cesium-134

Cesium-137

Cobalt-57

Cobalt-58

Cobalt-60

Europium-152

Europium-154

Europium-155

Gross alpha

Lead-212

Manganese-54

Neptunium-239

Nonvolatile beta

Plutonium-238

Plutonium-239/240

Potassium-40

Promethium-144

Promethium-146

Ruthenium-106

Sodium-22

Strontium-90

Thorium-234

Tin-113

Yttrium-88

Zinc-65

Zirconium-95
Sample ID: 104638

RQ AQ B Result Unit D. Limit Method
2.10E-02 EPIA-013B
9.90E-03 EPIA-013B
2.12E-02 EPIA-013B
8.75E-03 EPIA-013B
5.29E-02 EPIA-013B
6.40E-03 EPIA-013B
7.90E-03 EPIA.013B
6.85E-03 EPIA-013B
8.15E-03 EPIA-013B
6.34E-03 EPIA-013B
2.00E-02 EPIA-013B
5.41E-02 EPIA-013B
2.93E-02 EPIA-013B
$1.58 \mathrm{E}+00$ EPIA-001B
1.19E-02 EPIA-013B
7.13E-03 EPIA-013B
4.89E-02 EPIA-013B
2.25E+00 EPIA-001B
3.00E-02 EPIA-012B
1.41E-02 EPIA-012B
5.86E-02 EPIA-013B
6.64E-03 EPIA-013B
1.07E-02 EPIA-013B
6.4IE-02 EPIA-013B
5.76E-03 EPIA-013B
6.10E-02 EPIA-004
3.55E-01 EPIA-013B
1.10E-02 EPIA-013B
7.43E-03 EPIA-013B
1.31E-02 EPIA-013B
1.71E-02 EPIA-013B

\section{SURVEY ID: PPSC 0902}

Sample ID: 104636

Survey location: 45224E $3680779 \mathrm{~N}$ (SRS coordinates)

Depth of core interval: 1.00 to $2.00 \mathrm{ft}$

Sample type: Normal

Sample matrix: Soil

Percent solids: $\mathbf{8 7 . 0 0}$

Constituent

RQ AQ B Result

Unit

D. Limit Method

Physical Parameters and Specified Analyses

$\begin{array}{lllll}\text { Cation exchange capacity } & & & & 12.3 \\ \text { Chloride } & \text { J } & \text { E } & & 1.19 \\ \text { Cyanide } & \text { U } & & & 1.15 \\ \text { Nitrate-nitrite as nitrogen } & \text { U } & & & 1.1 \\ \text { Orthophosphate } & \text { J } & \text { EI } & \text { L } & 4.1 \\ \text { Orthophosphate } & \text { J } & \text { EI } & \text { L } & 4.34 \\ \text { Phenols } & \text { J } & \text { EC } & & 89.5\end{array}$

$\begin{array}{lll}\mathrm{meq} / 100 \mathrm{~g} & 5 & \text { EPA9081 } \\ \mathrm{mg} / \mathrm{kg} & 2.64 & \text { EPA300.0 } \\ \mathrm{mg} / \mathrm{kg} & 1.15 & \text { EPA335.3 } \\ \mathrm{mg} / \mathrm{kg} & 1.1 & \text { EPA353.1 } \\ \mathrm{mg} / \mathrm{kg} & 11.1 & \text { EPA365.2 } \\ \mathrm{mg} / \mathrm{kg} & 11 & \text { EPA365.2 } \\ \mu \mathrm{g} / \mathrm{kg} & 345 & \text { EPA420.2 }\end{array}$


SURVEY ID: PPSC 0902 (continued)

Constituent
Sample ID: 104636

RQ AQ B Result

Unit

D. Limit Method

Physical Parameters and Specified Analyses

Phenols

Sulfide

Total organic carbon

Total organic nitrogen

Total phosphates (as P)

$\begin{array}{lll}\text { UJ } & \mathrm{C} & 343 \\ \text { UJ } & \mathrm{C} & 287 \\ & & 3110 \\ \mathrm{C} & & 29.4 \\ \mathrm{~J} & \mathrm{E} & 104\end{array}$

Metals (total recoverable)

$\begin{array}{lll} & \text { V } & 2200 \\ \text { U } & & 5.63 \\ \text { U } & & 16.9 \\ & & 16.1 \\ \text { J } & \text { E } & 0.0963 \\ \text { U } & & 0.282 \\ & \text { V } & 81.8 \\ & & 3.13 \\ \text { J } & \text { E } & 0.313 \\ & & 2.01 \\ \text { J } & \text { VC } & 2810 \\ \text { U } & & 5.63 \\ & \text { V } & 67.2 \\ & & 38.1 \\ \text { U } & \text { V } & 0.0127 \\ \text { J } & \text { E } & 0.939 \\ \text { J } & \text { E } & 52.7 \\ \text { U } & & 16.9 \\ \text { U } & & 1.41 \\ \text { U } & & 56.3 \\ \text { UJ } & \text { C } & 16.9 \\ & & 5.31 \\ & & 3.46\end{array}$

$B / N / A$ Extractables (including PAH and phenols)

Aluminum
Antimony
Arsenic
Barium
Beryllium
Cadmium
Calcium
Chromium
Cobalt
Copper
Iron
Lead
Magnesium
Manganese
Mercury
Nickel
Potassium
Selenium
Silver
Sodium
Thallium
Vanadium
Zinc

Acenaphthene Acenaphthylene Anthracene

Benzo[a]anthracene

Benzo[b]fluoranthene

Benzo[k]fluoranthene

Benzoic acid

Benzo[g, $h, i]$ perylene

Benzo[a]pyrene

Benzyl alcohol

Bis(2-chloroethoxy) methane

$\mathrm{Bis(2-chloroethyl)}$ ether

Bis(2-chloroisopropyl) ether

Bis(2-ethylhexyl) phthalate

4-Bromophenyl phenyl ether

Butylbenzyl phthalate

4-Chloroaniline

4-Chloro-m-cresol

2-Chloronaphthalene

2-Chlorophenol

4-Chlorophenyl phenyl ether

$\begin{array}{ll}\text { U } & 37.4 \\ U & 37.4 \\ U & 37.4 \\ U & 3.74 \\ U & 3.74 \\ U & 3.74 \\ U & 37.4 \\ U & 37.4 \\ U & 3.74 \\ U & 37.4 \\ U & 37.4 \\ U & 37.4 \\ U & 37.4 \\ U & 37.4 \\ U & 37.4 \\ U & 37.4 \\ U & 37.4 \\ U & 37.4 \\ U & 37.4 \\ U & 37.4 \\ U & 37.4\end{array}$

$\begin{array}{lll}\mu \mathrm{g} / \mathrm{kg} & 343 & \text { EPA420.2 } \\ \mathrm{mg} / \mathrm{kg} & 287 & \text { EPA376.2 } \\ \mathrm{mg} / \mathrm{kg} & 115 & \text { EPA415.1 } \\ \mathrm{mg} / \mathrm{kg} & 0.23 & \text { EPA351.2 } \\ \mathrm{mg} / \mathrm{kg} & 414 & \text { EPA365.4 }\end{array}$

$\begin{array}{lll}\mathrm{mg} / \mathrm{kg} & 14.1 & \text { EPA6010A } \\ \mathrm{mg} / \mathrm{kg} & 5.63 & \text { EPA6010A } \\ \mathrm{mg} / \mathrm{kg} & 16.9 & \text { EPA6010A } \\ \mathrm{mg} / \mathrm{kg} & 1.41 & \text { EPA6010A } \\ \mathrm{mg} / \mathrm{kg} & 0.563 & \text { EPA6010A } \\ \mathrm{mg} / \mathrm{kg} & 0.282 & \text { EPA6010A } \\ \mathrm{mg} / \mathrm{kg} & 14.1 & \text { EPA6010A } \\ \mathrm{mg} / \mathrm{kg} & 1.41 & \text { EPA6010A } \\ \mathrm{mg} / \mathrm{kg} & 0.563 & \text { EPA6010A } \\ \mathrm{mg} / \mathrm{kg} & 1.41 & \text { EPA6010A } \\ \mathrm{mg} / \mathrm{kg} & 5.63 & \text { EPA6010A } \\ \mathrm{mg} / \mathrm{kg} & 5.63 & \text { EPA6010A } \\ \mathrm{mg} / \mathrm{kg} & 5.63 & \text { EPA6010A } \\ \mathrm{mg} / \mathrm{kg} & 0.563 & \text { EPA6010A } \\ \mathrm{mg} / \mathrm{kg} & 0.0276 & \text { EPA7471 } \\ \mathrm{mg} / \mathrm{kg} & 1.41 & \text { EPA6010A } \\ \mathrm{mg} / \mathrm{kg} & 113 & \text { EPA6010A } \\ \mathrm{mg} / \mathrm{kg} & 16.9 & \text { EPA6010A } \\ \mathrm{mg} / \mathrm{kg} & 1.41 & \text { EPA6010A } \\ \mathrm{mg} / \mathrm{kg} & 56.3 & \text { EPA6010A } \\ \mathrm{mg} / \mathrm{kg} & 16.9 & \text { EPA6010A } \\ \mathrm{mg} / \mathrm{kg} & 0.563 & \text { EPA6010A } \\ \mathrm{mg} / \mathrm{kg} & 1.13 & \text { EPA6010A }\end{array}$

$\begin{array}{lll}\mu \mathrm{g} / \mathrm{kg} & 37.4 & \text { EPA8270 } \\ \mu \mathrm{g} / \mathrm{kg} & 37.4 & \text { EPA8270 } \\ \mu \mathrm{g} / \mathrm{kg} & 37.4 & \text { EPA8270 } \\ \mu \mathrm{g} / \mathrm{kg} & 3.74 & \text { EPA8270 } \\ \mu \mathrm{g} / \mathrm{kg} & 3.74 & \text { EPA8270 } \\ \mu \mathrm{g} / \mathrm{kg} & 3.74 & \text { EPA8270 } \\ \mu \mathrm{g} / \mathrm{kg} & 37.4 & \text { EPA8270 } \\ \mu \mathrm{g} / \mathrm{kg} & 37.4 & \text { EPA8270 } \\ \mu \mathrm{g} / \mathrm{kg} & 3.74 & \text { EPA8270 } \\ \mu \mathrm{g} / \mathrm{kg} & 37.4 & \text { EPA8270 } \\ \mu \mathrm{g} / \mathrm{kg} & 37.4 & \text { EPA8270 } \\ \mu \mathrm{g} / \mathrm{kg} & 37.4 & \text { EPA8270 } \\ \mu \mathrm{g} / \mathrm{kg} & 37.4 & \text { EPA8270 } \\ \mu \mathrm{g} / \mathrm{kg} & 37.4 & \text { EPA8270 } \\ \mu \mathrm{g} / \mathrm{kg} & 37.4 & \text { EPA8270 } \\ \mu \mathrm{g} / \mathrm{kg} & 37.4 & \text { EPA8270 } \\ \mu \mathrm{g} / \mathrm{kg} & 37.4 & \text { EPA8270 } \\ \mu \mathrm{g} / \mathrm{kg} & 37.4 & \text { EPA8270 } \\ \mu \mathrm{g} / \mathrm{kg} & 37.4 & \text { EPA8270 } \\ \mu \mathrm{g} / \mathrm{kg} & 37.4 & \text { EPA8270 } \\ \mu \mathrm{g} / \mathrm{kg} & 37.4 & \text { EPA8270 }\end{array}$


Constituent

RQ AQ B Result

Unit

D. Limit Method

$B / N / A$ Extractables (including $P A H$ and phenols)

Chrysene

$\mathrm{m} / \mathrm{p}$-Cresol

o-Cresol (2-Methylphenol)

Dibenz $[a, h]$ anthracene

Dibenzofuran

Di-n-butyl phthalate

1,2-Dichlorobenzene

1,3-Dichlorobenzene

1,4-Dichlorobenzene

3,3'-Dichlorobenzidine

2,4-Dichlorophenol

Diethyl phthalate

2,4-Dimethyl phenol

Dimethyl phthalate

2,4-Dinitrophenol

2,4-Dinitrotoluene

2,6-Dinitrotoluene

Di-n-octyl phthalate

Fluoranthene

Fluorene

Hexachlorobenzene

Hexachlorobutadiene

Hexachlorocyclopentadiene

Hexachloroethane

Indeno $[1,2,3-c, d]$ pyrene

Isophorone

2-Methyl-4,6-dinitrophenol

2-Methylnaphthalene

Naphthalene

m-Nitroaniline

0-Nitroaniline

p-Nitroaniline

Nitrobenzene

2-Nitrophenol

4-Nitrophenol

N-Nitrosodiphenylamine

N-Nitrosodi-n-propylamine

Pentachlorophenol

Phenanthrene

Phenol

Pyrene

1,2,4-Trichlorobenzene

2,4,5-Trichlorophenol

2,4,6-Trichlorophenol

Volatile Organic Compounds

Acetone

Benzene

Bromodichloromethane

Bromoform

Bromomethane (Methyl bromide)

Carbon disulfide

Carbon tetrachloride

Chlorobenzene
U $\quad 3.7$

U 374

U $\quad 37.4$

U 3.74

U 37.4

J $\quad \mathrm{E} \quad 26.9$

U 37.4

U 37.4

37.4

37.4

37.4

37.4

37.4

37.4

374

3.74

3.74

37.4

37.4

37.4

3.74

37.4

37.4

3.74

3.74

37.4

374

37.4

37.4

37.4

37.4

37.4

37.4

37.4

37.4

37.4

37.4

37.4

37.4

37.4

37.4

3.74

37.4

37.4

\begin{tabular}{|c|c|c|}
\hline$\mu \mathrm{g} / \mathrm{kg}$ & 3.74 & EPA8270 \\
\hline$\mu \mathrm{g} / \mathrm{kg}$ & 374 & EPA8270 \\
\hline$\mu \mathrm{g} / \mathrm{kg}$ & 37.4 & EPA8270 \\
\hline$\mu \mathrm{g} / \mathrm{kg}$ & 3.74 & EPA8270 \\
\hline$\mu \mathrm{g} / \mathrm{kg}$ & 37.4 & EPA8270 \\
\hline$\mu \mathrm{g} / \mathrm{kg}$ & 37.4 & EPA8270 \\
\hline$\mu \mathrm{g} / \mathrm{kg}$ & 37.4 & EPA8270 \\
\hline$\mu \mathrm{g} / \mathrm{kg}$ & 37.4 & EPA8270 \\
\hline$\mu \mathrm{g} / \mathrm{kg}$ & 37.4 & EPA8270 \\
\hline$\mu \mathrm{g} / \mathrm{kg}$ & 37.4 & EPA8270 \\
\hline$\mu \mathrm{g} / \mathrm{kg}$ & 37.4 & EPA8270 \\
\hline$\mu \mathrm{g} / \mathrm{kg}$ & 37.4 & EPA8270 \\
\hline$\mu \mathrm{g} / \mathrm{kg}$ & 37.4 & EPA8270 \\
\hline$\mu \mathrm{g} / \mathrm{kg}$ & 37.4 & EPA8270 \\
\hline$\mu \mathrm{g} / \mathrm{kg}$ & 374 & EPA8270 \\
\hline$\mu g / \mathrm{kg}$ & 3.74 & EPA8270 \\
\hline$\mu \mathrm{g} / \mathrm{kg}$ & 3.74 & EPA8270 \\
\hline$\mu \mathrm{g} / \mathrm{kg}$ & 37.4 & EPA8270 \\
\hline$\mu \mathrm{g} / \mathrm{kg}$ & 37.4 & EPA8270 \\
\hline$\mu \mathrm{g} / \mathrm{kg}$ & 37.4 & EPA8270 \\
\hline$\mu \mathrm{g} / \mathrm{kg}$ & 3.74 & EPA8270 \\
\hline$\mu \mathrm{g} / \mathrm{kg}$ & 37.4 & EPA8270 \\
\hline$\mu \mathrm{g} / \mathrm{kg}$ & 37.4 & EPA8270 \\
\hline$\mu \mathrm{g} / \mathrm{kg}$ & 3.74 & EPA8270 \\
\hline$\mu \mathrm{g} / \mathrm{kg}$ & 3.74 & EPA8270 \\
\hline$\mu \mathrm{g} / \mathrm{kg}$ & 37.4 & EPA8270 \\
\hline$\mu \mathrm{g} / \mathrm{kg}$ & 374 & EPA8270 \\
\hline$\mu \mathrm{g} / \mathrm{kg}$ & 37.4 & EPA8270 \\
\hline$\mu \mathrm{g} / \mathrm{kg}$ & 37.4 & EPA8270 \\
\hline$\mu \mathrm{g} / \mathrm{kg}$ & 37.4 & EPA8270 \\
\hline$\mu \mathrm{g} / \mathrm{kg}$ & 37.4 & EPA8270 \\
\hline$\mu \mathrm{g} / \mathrm{kg}$ & 37.4 & EPA8270 \\
\hline$\mu \mathrm{g} / \mathrm{kg}$ & 37.4 & EPA8270 \\
\hline$\mu \mathrm{g} / \mathrm{kg}$ & 37.4 & EPA8270 \\
\hline$\mu \mathrm{g} / \mathrm{kg}$ & 37.4 & EPA8270 \\
\hline$\mu \mathrm{g} / \mathrm{kg}$ & 37.4 & EPA8270 \\
\hline$\mu \mathrm{g} / \mathrm{kg}$ & 37.4 & EPA8270 \\
\hline$\mu \mathrm{g} / \mathrm{kg}$ & 37.4 & EPA8270 \\
\hline$\mu \mathrm{g} / \mathrm{kg}$ & 37.4 & EPA8270 \\
\hline$\mu g / \mathrm{kg}$ & 37.4 & EPA8270 \\
\hline$\mu \mathrm{g} / \mathrm{kg}$ & 37.4 & EPA8270 \\
\hline$\mu \mathrm{g} / \mathrm{kg}$ & 3.74 & EPA8270 \\
\hline$\mu \mathrm{g} / \mathrm{kg}$ & 37.4 & EPA8270 \\
\hline$\mu \mathrm{g} / \mathrm{kg}$ & 37.4 & EPA8270 \\
\hline
\end{tabular}

$\mu \mathrm{g} / \mathrm{kg}$

$\mu \mathrm{g} / \mathrm{kg}$

$\mu \mathrm{g} / \mathrm{kg}$

$\mu \mathrm{g} / \mathrm{kg}$

$\mu \mathrm{g} / \mathrm{kg}$

$\mu \mathrm{g} / \mathrm{kg}$

$\mu \mathrm{g} / \mathrm{kg}$

$\mu \mathrm{g} / \mathrm{kg}$

\subsection{5}

0.575

0.0575

0.0575

- 0.115

1.15

0.0575

0.0575
EPA8260

EPA8260

EPA8260

EPA8260

EPA8260

EPA8260

EPA8260

EPA8260 
SURVEY ID: PPSC 0902 (continued)

Constituent

RQ AQ B Result

Volatile Organic Compounds

Chloroethane

Chloroethene (Vinyl chloride)

Chloroform

Chloromethane (Methyl chloride)

Dibromochloromethane

1,1-Dichloroethane

1,2-Dichloroethane

1,1-Dichloroethylene

1,2-Dichloroethylene

Dichloromethane (Methylene chloride)

1,2-Dichloropropane

cis-1,3-Dichloropropene

trans-1,3-Dichloropropene

Ethylbenzene

2-Hexanone

Methyl cyclopentane

Methyl ethyl ketone

Methyl isobutyl ketone

Styrene

1,1,2,2-Tetrachloroethane

Tetrachloroethylene

Toluene

1,1,1-Trichloroethane

1,1,2-Trichloroethane

Trichloroethylene

Vinyl acetate

Xylenes

Pesticides/PCBs and Dioxins/Furans
Unit D. Limit Method

Unit D. Limit Method

Sample ID: 104636

\begin{tabular}{|c|c|c|c|c|c|}
\hline$U$ & & 0.115 & $\mu \mathrm{g} / \mathrm{kg}$ & 0.115 & EPA8260 \\
\hline$U$ & & 0.115 & $\mu \mathrm{g} / \mathrm{kg}$ & 0.115 & EPA8260 \\
\hline$U$ & & 0.0575 & $\mu \mathrm{g} / \mathrm{kg}$ & 0.0575 & EPA8260 \\
\hline$U$ & & 0.115 & $\mu \mathrm{g} / \mathrm{kg}$ & 0.115 & EPA8260 \\
\hline $\mathrm{U}$ & & 0.0575 & $\mu \mathrm{g} / \mathrm{kg}$ & 0.0575 & EPA8260 \\
\hline $\mathrm{U}$ & & 0.0575 & $\mu \mathrm{g} / \mathrm{kg}$ & 0.0575 & EPA8260 \\
\hline $\mathrm{U}$ & & 0.0575 & $\mu \mathrm{g} / \mathrm{kg}$ & 0.0575 & EPA8260 \\
\hline $\mathrm{U}$ & & 0.0575 & $\mu \mathrm{g} / \mathrm{kg}$ & 0.0575 & EPA8260 \\
\hline $\mathrm{U}$ & & 0.115 & $\mu \mathrm{g} / \mathrm{kg}$ & 0.115 & EPA8260 \\
\hline $\mathrm{U}$ & V & 9.07 & $\mu \mathrm{g} / \mathrm{kg}$ & 0.575 & EPA8260 \\
\hline $\mathrm{U}$ & & 0.0575 & $\mu \mathrm{g} / \mathrm{kg}$ & 0.0575 & EPA8260 \\
\hline $\mathbf{U}$ & & 0.0575 & $\mu \mathrm{g} / \mathrm{kg}$ & 0.0575 & EPA8260 \\
\hline U & & 0.0575 & $\mu \mathrm{g} / \mathrm{kg}$ & 0.0575 & EPA8260 \\
\hline U & & 0.0575 & $\mu \mathrm{g} / \mathrm{kg}$ & 0.0575 & EPA8260 \\
\hline $\mathrm{U}$ & V & 1.84 & $\mu \mathrm{g} / \mathrm{kg}$ & 1.15 & EPA8260 \\
\hline J & $\mathbf{N}$ & 2.45 & $\mu \mathrm{g} / \mathrm{kg}$ & & EPA8260 \\
\hline $\mathbf{U}$ & V & 5.89 & $\mu \mathrm{g} / \mathrm{kg}$ & 1.15 & EPA8260 \\
\hline $\mathrm{U}$ & & 1.15 & $\mu \mathrm{g} / \mathrm{kg}$ & 1.15 & EPA8260 \\
\hline $\mathrm{U}$ & & 0.0575 & $\mu \mathrm{g} / \mathrm{kg}$ & 0.0575 & EPA8260 \\
\hline $\mathrm{U}$ & & 0.0575 & $\mu \mathrm{g} / \mathrm{kg}$ & 0.0575 & EPA8260 \\
\hline $\mathrm{U}$ & & 0.0575 & $\mu \mathrm{g} / \mathrm{kg}$ & 0.0575 & EPA8260 \\
\hline$U$ & V & 0.138 & $\mu \mathrm{g} / \mathrm{kg}$ & 0.575 & EPA8260 \\
\hline U & & 0.0575 & $\mu \mathrm{g} / \mathrm{kg}$ & 0.0575 & EPA8260 \\
\hline $\mathrm{U}$ & & 0.0575 & $\mu \mathrm{g} / \mathrm{kg}$ & 0.0575 & EPA8260 \\
\hline $\mathrm{U}$ & & 0.0575 & $\mu \mathrm{g} / \mathrm{kg}$ & 0.0575 & EPA8260 \\
\hline U & & 1.15 & $\mu \mathrm{g} / \mathrm{kg}$ & 1.15 & EPA8260 \\
\hline U & & 0.172 & $\mu \mathrm{g} / \mathrm{kg}$ & 0.172 & EPA8260 \\
\hline
\end{tabular}

Aldrin
Aldrin
alpha-Benzene hexachloride
alpha-Benzene hexachloride
beta-Benzene hexachloride
beta-Benzene hexachloride
delta-Benzene hexachloride
delta-Benzene hexachloride
alpha-Chlordane
alpha-Chlordane
gamma-Chlordane
gamma-Chlordane
p,p'-DDD
p,p'-DDD
p,p'-DDE
p,p'-DDE
p,p'-DDT
p,p'-DDT
Dieldrin
Dieldrin
Endosulfan sulfate
Endosulfan sulfate
Endosulfan I
Endosulfan I
Endosulfan II

\begin{tabular}{|c|c|c|c|}
\hline T & 0 & L & 0.948 \\
\hline UJJ & 0 & L & 0.931 \\
\hline UJ & 0 & L & 0.948 \\
\hline UJ & 0 & L & 0.931 \\
\hline UJ & 0 & L & 1.9 \\
\hline UJ & 0 & L & 1.86 \\
\hline UJ & 0 & L & 1.9 \\
\hline UJ & 0 & L & 1.86 \\
\hline UJ & 0 & L & 1.9 \\
\hline UJ & 0 & L & 1.86 \\
\hline JJ & 0 & I & 1.9 \\
\hline JJ & 0 & L & 1.86 \\
\hline JJ & 0 & L & 3.79 \\
\hline $\mathrm{JJ}$ & 0 & L & 3.72 \\
\hline J & 0 & L & 1.9 \\
\hline J. & 0 & L & 1.86 \\
\hline$J J$ & 0 & L & 3.79 \\
\hline$J^{J}$ & 0 & L & 3.72 \\
\hline Je & 0 & L & 1.9 \\
\hline JJ & 0 & L & 1.86 \\
\hline$J$ & 0 & L & 3.79 \\
\hline J. & 0 & L & 3.72 \\
\hline $\mathrm{Jc}$ & 0 & L & 1.9 \\
\hline & 0 & L & 1.86 \\
\hline & 0 & $\mathrm{~L}$ & 3.79 \\
\hline
\end{tabular}

$\begin{array}{lll}\mu \mathrm{g} / \mathrm{kg} & 0.948 & \text { EPA8080 } \\ \mu \mathrm{g} / \mathrm{kg} & 0.931 & \text { EPA8080 } \\ \mu \mathrm{g} / \mathrm{kg} & 0.948 & \text { EPA8080 } \\ \mu \mathrm{g} / \mathrm{kg} & 0.931 & \text { EPA8080 } \\ \mu \mathrm{g} / \mathrm{kg} & 1.9 & \text { EPA8080 } \\ \mu \mathrm{g} / \mathrm{kg} & 1.86 & \text { EPA8080 } \\ \mu \mathrm{g} / \mathrm{kg} & 1.9 & \text { EPA8080 } \\ \mu \mathrm{g} / \mathrm{kg} & 1.86 & \text { EPA8080 } \\ \mu \mathrm{g} / \mathrm{kg} & 1.9 & \text { EPA8080 } \\ \mu \mathrm{g} / \mathrm{kg} & 1.86 & \text { EPA8080 } \\ \mu \mathrm{g} / \mathrm{kg} & 1.9 & \text { EPA8080 } \\ \mu \mathrm{g} / \mathrm{kg} & 1.86 & \text { EPA8080 } \\ \mu \mathrm{g} / \mathrm{kg} & 3.79 & \text { EPA8080 } \\ \mu \mathrm{g} / \mathrm{kg} & 3.72 & \text { EPA8080 } \\ \mu \mathrm{g} / \mathrm{kg} & 1.9 & \text { EPA8080 } \\ \mu \mathrm{g} / \mathrm{kg} & 1.86 & \text { EPA8080 } \\ \mu \mathrm{g} / \mathrm{kg} & 3.79 & \text { EPA8080 } \\ \mu \mathrm{g} / \mathrm{kg} & 3.72 & \text { EPA8080 } \\ \mu \mathrm{g} / \mathrm{kg} & 1.9 & \text { EPA8080 } \\ \mu \mathrm{g} / \mathrm{kg} & 1.86 & \text { EPA8080 } \\ \mu \mathrm{g} / \mathrm{kg} & 3.79 & \text { EPA8080 } \\ \mu \mathrm{g} / \mathrm{kg} & 3.72 & \text { EPA8080 } \\ \mu \mathrm{g} / \mathrm{kg} & 1.9 & \text { EPA8080 } \\ \mu \mathrm{g} / \mathrm{kg} & 1.86 & \text { EPA8080 } \\ \mu \mathrm{g} / \mathrm{kg} & 3.79 & \text { EPA8080 }\end{array}$


SURVEY ID: PPSC 0902 (continued)

Constituent

Pesticides/PCBs and Dioxins/Furans

$\begin{array}{lllll}\text { Endosulfan II } & \text { UJ } & \text { O } & \text { L } & 3.72 \\ \text { Endrin } & \text { UJ } & \text { O } & \text { L } & 1.9 \\ \text { Endrin } & \text { UJ } & \text { O } & \text { L } & 1.86 \\ \text { Endrin ketone } & \text { UJ } & \text { O } & \text { L } & 3.79 \\ \text { Endrin ketone } & \text { UJ } & \text { O } & \text { L } & 3.72 \\ \text { Heptachlor } & \text { UJ } & \text { O } & \text { L } & 1.9 \\ \text { Heptachlor } & \text { UJ } & \text { O } & \text { L } & 1.86 \\ \text { Heptachlor epoxide } & \text { UJ } & \text { OG } & \text { L } & 1.36 \\ \text { Heptachlor epoxide } & \text { UJ } & \text { O } & \text { L } & 1.86 \\ \text { Lindane } & \text { UJ } & \text { O } & \text { L } & 0.948 \\ \text { Lindane } & \text { UJ } & \text { O } & \text { L } & 0.931 \\ \text { Methoxychlor } & \text { UJ } & \text { O } & \text { L } & 19 \\ \text { Methoxychlor } & \text { UJ } & \text { O } & \text { L } & 18.6 \\ \text { PCB 1016 } & \text { UJ } & \text { O } & \text { L } & 4.74 \\ \text { PCB 1016 } & \text { UJ } & \text { O } & \text { L } & 4.65 \\ \text { PCB 1221 } & \text { UJ } & \text { O } & \text { L } & 4.74 \\ \text { PCB 1221 } & \text { UJ } & \text { O } & \text { L } & 4.65 \\ \text { PCB 1232 } & \text { UJ } & \text { O } & \text { L } & 4.74 \\ \text { PCB 1232 } & \text { UJ } & \text { O } & \text { L } & 4.65 \\ \text { PCB 1242 } & \text { UJ } & \text { O } & \text { L } & 4.74 \\ \text { PCB 1242 } & \text { UJ } & \text { O } & \text { L } & 4.65 \\ \text { PCB 1248 } & \text { UJ } & \text { O } & \text { L } & 4.74 \\ \text { PCB 1248 } & \text { UJ } & \text { O } & \text { L } & 4.65 \\ \text { PCB 1254 } & \text { UJ } & \text { O } & \text { L } & 4.74 \\ \text { PCB 1254 } & \text { UJ } & \text { O } & \text { L } & 4.65 \\ \text { PCB 1260 } & \text { UJ } & \text { O } & \text { L } & 4.74 \\ \text { PCB 1260 } & \text { UJ } & \text { O } & \text { L } & 4.65 \\ \text { Toxaphene } & \text { UJ } & \text { O } & \text { L } & 37.9 \\ \text { Toxaphene } & \text { UJ } & \text { O } & \text { L } & 37.2\end{array}$

Sample ID: 104636

RQ AQ B Result Unit D. Limit Method

$\begin{array}{lll}\mu \mathrm{g} / \mathrm{kg} & 3.72 & \text { EPA8080 } \\ \mu \mathrm{g} / \mathrm{kg} & 1.9 & \text { EPA8080 } \\ \mu \mathrm{g} / \mathrm{kg} & 1.86 & \text { EPA8080 } \\ \mu \mathrm{g} / \mathrm{kg} & 3.79 & \text { EPA8080 } \\ \mu \mathrm{g} / \mathrm{kg} & 3.72 & \text { EPA8080 } \\ \mu \mathrm{g} / \mathrm{kg} & 1.9 & \text { EPA8080 } \\ \mu \mathrm{g} / \mathrm{kg} & 1.86 & \text { EPA8080 } \\ \mu \mathrm{g} / \mathrm{kg} & 1.9 & \text { EPA8080 } \\ \mu \mathrm{g} / \mathrm{kg} & 1.86 & \text { EPA8080 } \\ \mu \mathrm{g} / \mathrm{kg} & 0.948 & \text { EPA8080 } \\ \mu \mathrm{g} / \mathrm{kg} & 0.931 & \text { EPA8080 } \\ \mu \mathrm{g} / \mathrm{kg} & 19 & \text { EPA8080 } \\ \mu \mathrm{g} / \mathrm{kg} & 18.6 & \text { EPA8080 } \\ \mu \mathrm{g} / \mathrm{kg} & 4.74 & \text { EPA8080 } \\ \mu \mathrm{g} / \mathrm{kg} & 4.65 & \text { EPA8080 } \\ \mu \mathrm{g} / \mathrm{kg} & 4.74 & \text { EPA8080 } \\ \mu \mathrm{g} / \mathrm{kg} & 4.65 & \text { EPA8080 } \\ \mu \mathrm{g} / \mathrm{kg} & 4.74 & \text { EPA8080 } \\ \mu \mathrm{g} / \mathrm{kg} & 4.65 & \text { EPA8080 } \\ \mu \mathrm{g} / \mathrm{kg} & 4.74 & \text { EPA8080 } \\ \mu \mathrm{g} / \mathrm{kg} & 4.65 & \text { EPA8080 } \\ \mu \mathrm{g} / \mathrm{kg} & 4.74 & \text { EPA8080 } \\ \mu \mathrm{g} / \mathrm{kg} & 4.65 & \text { EPA8080 } \\ \mu \mathrm{g} / \mathrm{kg} & 4.74 & \text { EPA8080 } \\ \mu \mathrm{g} / \mathrm{kg} & 4.65 & \text { EPA8080 } \\ \mu \mathrm{g} / \mathrm{kg} & 4.74 & \text { EPA8080 } \\ \mu \mathrm{g} / \mathrm{kg} & 4.65 & \text { EPA8080 } \\ \mu \mathrm{g} / \mathrm{kg} & 37.9 & \text { EPA8080 } \\ \mu \mathrm{g} / \mathrm{kg} & 37.2 & \text { EPA8080 }\end{array}$

Radionuclides

Actinium-228

Antimony-124

Antimony-125

Barium-133

Cerium-144

Cesium-134

Cesium-137

Cobalt-57

Cobalt-58

Cobalt-60

Europium-152

Europium-154

Europium-155

Gross alpha

Lead-212

Manganese-54

Neptunium-239

Nonvolatile beta

Plutonium-238

Plutonium-239/240

Potassium-40

Promethium-144

Promethium-146
5.46E-01 $\pm 3.67 \mathrm{E}-02 \mathrm{pCi} / \mathrm{g}$ 9.45E-04 $\pm 5.52 \mathrm{E}-03 \mathrm{pCi} / \mathrm{g}$ $-5.13 \mathrm{E}-03 \pm 1.13 \mathrm{E}-02 \mathrm{pCi} / \mathrm{g}$ $-9.37 \mathrm{E}-02 \pm 7.63 \mathrm{E}-03 \mathrm{pCj} / \mathrm{g}$ $1.36 \mathrm{E}-02 \pm 3.30 \mathrm{E}-02 \mathrm{pCi} / \mathrm{g}$ $7.56 \mathrm{E}-05 \pm 4.40 \mathrm{E}-03 \mathrm{pCi} / \mathrm{g}$ $1.27 \mathrm{E}-01 \pm 1.35 \mathrm{E}-02 \mathrm{pCi} / \mathrm{g}$ $-1.32 \mathrm{E}-03 \pm 4.14 \mathrm{E}-03 \mathrm{pCi} / \mathrm{g}$ $-1.96 \mathrm{E}-03 \pm 5.64 \mathrm{E}-03 \mathrm{pCi} / \mathrm{g}$ $-3.12 \mathrm{E}-03 \pm 3.67 \mathrm{E}-03 \mathrm{pCj} / \mathrm{g}$ $-1.02 \mathrm{E}-02 \pm 1.17 \mathrm{E}-02 \mathrm{pCi} / \mathrm{g}$ $-2.79 \mathrm{E}-02 \pm 3.44 \mathrm{E}-02 \mathrm{pCi} / \mathrm{g}$ $3.02 \mathrm{E}-02 \pm 2.43 \mathrm{E}-02 \mathrm{pCj} / \mathrm{g}$ $1.62 \mathrm{E}+01 \pm 2.92 \mathrm{E}+00 \mathrm{pCi} / \mathrm{g}$ $5.28 \mathrm{E}-01 \pm 1.95 \mathrm{E}-02 \mathrm{pCi} / \mathrm{g}$ 8.63E-03 $\pm 6.28 \mathrm{E}-03 \mathrm{pCi} / \mathrm{g}$ $\begin{array}{lll}\text { UI } & & 6.74 \mathrm{E}-03 \pm 3.02 \mathrm{E}-02 \mathrm{pCi} / \mathrm{g} \\ \mathrm{J} & \mathrm{C} & 8.63 \mathrm{E}+00 \pm 1.73 \mathrm{E}+00 \mathrm{pCi} / \mathrm{g}\end{array}$ UI $\quad 1.09 \mathrm{E}-02 \pm 1.64 \mathrm{E}-02 \mathrm{pCi} / \mathrm{g}$ UI $\quad-1.28 \mathrm{E}-03 \pm 6.30 \mathrm{E}-03 \mathrm{pCi} / \mathrm{g}$ $\mathrm{V} \quad 6.42 \mathrm{E}-01 \pm 8.18 \mathrm{E}-02 \mathrm{pCi} / \mathrm{g}$ UI $\quad 2.62 \mathrm{E}-03 \pm 3.71 \mathrm{E}-03 \mathrm{pCi} / \mathrm{g}$ UI $-3.14 \mathrm{E}-03 \pm 5.28 \mathrm{E}-03 \mathrm{pCi} / \mathrm{g}$
2.04E-02 EPIA-013B

9.47E-03 EPIA-013B

1.92E-02 EPIA-013B

8.90E-03 EPIA-013B

5.56E-02 EPIA-013B

6.58E-03 EPIA-013B

7.58E-03 EPIA-013B

6.90E-03 EPIA-013B

8.04E-03 EPIA-013B

5.98E-03 EPIA-013B

1.98E-02 EPIA-013B

5.77E-02 EPIA-013B

2.93E-02 EPIA-013B

$1.72 \mathrm{E}+00$ EPIA-001B

1.24E-02 EPIA-013B

6.55E-03 EPIA-013B

5.09E-02 EPIA-013B

2.27E+00 EPIA-001B

2.82E-02 EPIA-012B

1.58E-02 EPIA-012B

5.27E-02 EPIA-013B

7.40E-03 EPIA-013B

8.88E-03 EPIA-013B 
SURVEY ID: PPSC 0902 (continued)

Constituent

Radionuclides

Ruthenium-106

Sodium-22

Strontium-90

Thorium-234

Tin-113

Yttrium-88

Zinc-65

Zirconium-95
Sample ID: 104636

RQ AQ B Result Unit D. Limit Method

$\begin{array}{lllll}\text { UI } & & 1.16 \mathrm{E}-02 \pm 3.64 \mathrm{E}-02 \mathrm{pCi} / \mathrm{g} & 6.31 \mathrm{E}-02 & \text { EPIA-013B } \\ \text { UI } & & -1.75 \mathrm{E}-03 \pm 3.56 \mathrm{E}-03 \mathrm{pCi} / \mathrm{g} & 6.02 \mathrm{E}-03 & \text { EPIA-013B } \\ \text { UI } & & -2.03 \mathrm{E}-02 \pm 3.52 \mathrm{E}-02 \mathrm{pCi} / \mathrm{g} & 7.34 \mathrm{E}-02 & \text { EPIA-004 } \\ \text { U } & \text { V } & 6.40 \mathrm{E}-01 \pm 4.28 \mathrm{E}-01 \mathrm{pCi} / \mathrm{g} & 3.61 \mathrm{E}-01 & \text { EPIA-013B } \\ \text { UI } & & -4.20 \mathrm{E}-03 \pm 6.20 \mathrm{E}-03 \mathrm{pCi} / \mathrm{g} & 1.05 \mathrm{E}-02 & \text { EPIA-013B } \\ \text { UI } & & -4.39 \mathrm{E}-03 \pm 4.00 \mathrm{E}-03 \mathrm{pCi} / \mathrm{g} & 6.42 \mathrm{E}-03 & \text { EPIA-013B } \\ \text { UI } & & 8.42 \mathrm{E}-03 \pm 9.27 \mathrm{E}-03 \mathrm{pCi} / \mathrm{g} & 1.54 \mathrm{E}-02 & \text { EPIA-013B } \\ \text { UI } & & 1.57 \mathrm{E}-02 \pm 1.38 \mathrm{E}-02 \mathrm{pCi} / \mathrm{g} & 1.69 \mathrm{E}-02 & \text { EPIA-013B }\end{array}$


SURVEY ID: PPSC 0902D

Sample type: Split

Constituent
Sample ID: 104639

Associated sample: 104636

Unit

D. Limit Method

Physical Parameters and Specified Analyses

Cation exchange capacity
Cation exchange capacity
Chloride
Cyanide
Nitrate-nitrite as nitrogen
Orthophosphate
Phenols
Sulfide
Total organic carbon
Total organic carbon
Total organic nitrogen
Total phosphates (as P)

Metals (total recoverable)

$\begin{array}{lll}J & \text { YE } & 3.69 \\ J & \mathrm{YE} & 3.36 \\ \mathrm{U} & \mathrm{Y} & 20 \\ \mathrm{U} & \mathrm{YV} & 0.108 \\ & \mathrm{Y} & 0.714 \\ \mathrm{U} & \mathrm{Y} & 1.15 \\ J & \mathrm{YE} & 315 \\ \mathrm{U} & \mathrm{Y} & 28800 \\ & \mathrm{YV} & 330 \\ & \mathrm{YV} & 319 \\ J & \mathrm{YE} & \mathbf{6 2 . 4} \\ & \mathrm{Y} & 68.6\end{array}$

$\begin{array}{lll}\mathrm{mg} / \mathrm{kg} & 25.3 & \text { EPA9081 } \\ \mathrm{mg} / \mathrm{kg} & 25.3 & \text { EPA9081 } \\ \mathrm{mg} / \mathrm{kg} & 20 & \text { EPA300.0 } \\ \mathrm{mg} / \mathrm{kg} & 1.06 & \text { EPA9010 } \\ \mathrm{mg} / \mathrm{kg} & 0.69 & \text { EPA353.2 } \\ \mathrm{mg} / \mathrm{kg} & 1.15 & \text { EPA365.2 } \\ \mu \mathrm{g} / \mathrm{kg} & 1190 & \text { EPA420.2 } \\ \mu \mathrm{g} / \mathrm{kg} & 28800 & \text { SW9030 } \\ \mathrm{mg} / \mathrm{kg} & 8.4 & \text { LLOYDKAHN } \\ \mathrm{mg} / \mathrm{kg} & 8.4 & \text { LLOYDKAHN } \\ \mathrm{mg} / \mathrm{kg} & 125 & \text { EPA351.4 } \\ \mathrm{mg} / \mathrm{kg} & 6.69 & \text { EPA365.2 }\end{array}$

\begin{tabular}{|c|c|c|c|c|c|c|}
\hline Aluminum & $J$ & $\mathrm{YD}$ & 2230 & $\mathrm{mg} / \mathrm{kg}$ & 46 & CLP-MOD \\
\hline Aluminum & $J$ & $\mathrm{Y}$ & 2400 & $\mathrm{mg} / \mathrm{kg}$ & 46 & CLP-MOD \\
\hline Antimony & U & $\mathrm{Y}$ & 47 & $\mathrm{mg} / \mathrm{kg}$ & 47 & CLP-MOD \\
\hline Antimony & $\mathrm{U}$ & $\mathrm{Y}$ & 47 & $\mathrm{mg} / \mathrm{kg}$ & 47 & CLP-MOD \\
\hline Arsenic & $J$ & YEI & 0.472 & $\mathrm{mg} / \mathrm{kg}$ & 0.691 & CLP-MOD \\
\hline Arsenic & $J$ & YEI & 0.495 & $\mathrm{mg} / \mathrm{kg}$ & 0.691 & CLP-MOD \\
\hline Barium & & $\mathrm{Y}$ & 10.5 & $\mathrm{mg} / \mathrm{kg}$ & 4.39 & CLP-MOD \\
\hline Barium & & $\mathrm{Y}$ & 11.4 & $\mathrm{mg} / \mathrm{kg}$ & 4.39 & CLP-MOD \\
\hline Beryllium & $J$ & $\mathrm{YE}$ & 0.09 & $\mathrm{mg} / \mathrm{kg}$ & 0.346 & CLP-MOD \\
\hline Beryllium & $\mathrm{J}$ & $\mathrm{YE}$ & 0.1 & $\mathrm{mg} / \mathrm{kg}$ & 0.346 & CLP-MOD \\
\hline Cadmium & $U$ & $\mathrm{Y}$ & 2.67 & $\mathrm{mg} / \mathrm{kg}$ & 2.67 & CLP-MOD \\
\hline Cadmium & $\mathrm{U}$ & $\mathrm{Y}$ & 2.67 & $\mathrm{mg} / \mathrm{kg}$ & 2.67 & CLP-MOD \\
\hline Calcium & & YV & 71.5 & $\mathrm{mg} / \mathrm{kg}$ & 26 & CLP-MOD \\
\hline Calcium & & YV & 75.4 & $\mathrm{mg} / \mathrm{kg}$ & 26 & CLP-MOD \\
\hline Chromium & $\mathrm{J}$ & $\mathrm{YE}$ & 6.9 & $\mathrm{mg} / \mathrm{kg}$ & 7.26 & CLP-MOD \\
\hline Chromium & $\mathbf{J}$ & YE & 7.1 & $\mathrm{mg} / \mathrm{kg}$ & 7.26 & CLP-MOD \\
\hline Cobalt & $J$ & $\mathrm{YE}$ & 0.588 & $\mathrm{mg} / \mathrm{kg}$ & 5.41 & CLP-MOD \\
\hline Cobalt & $\mathrm{J}$ & $\mathrm{YE}$ & 0.737 & $\mathrm{mg} / \mathrm{kg}$ & 5.41 & CLP-MOD \\
\hline Copper & $J$ & $\mathrm{YE}$ & 1.5 & $\mathrm{mg} / \mathrm{kg}$ & 3.66 & CLP-MOD \\
\hline Copper & $J$ & YE & 1.8 & $\mathrm{mg} / \mathrm{kg}$ & 3.66 & CLP-MOD \\
\hline Iron & $J$ & YD & 6610 & $\mathrm{mg} / \mathrm{kg}$ & 23 & CLP-MOD \\
\hline Iron & $\mathrm{J}$ & $\mathrm{Y}$ & 6890 & $\mathrm{mg} / \mathrm{kg}$ & 23 & CLP-MOD \\
\hline Lead & $J$ & $\mathrm{YE}$ & 1.3 & $\mathrm{mg} / \mathrm{kg}$ & 3.8 & CLP-MOD \\
\hline Lead & $\mathrm{J}$ & $\mathrm{YE}$ & 0.991 & $\mathrm{mg} / \mathrm{kg}$ & 3.8 & CLP-MOD \\
\hline Magnesium & & $\mathrm{Y}$ & 60.6 & $\mathrm{mg} / \mathrm{kg}$ & 27 & CLP-MOD \\
\hline Magnesium & & $\mathrm{Y}$ & 64.2 & $\mathrm{mg} / \mathrm{kg}$ & 27 & CLP-MOD \\
\hline Manganese & $\mathrm{J}$ & $\mathrm{YD}$ & 25.2 & $\mathrm{mg} / \mathrm{kg}$ & 1.12 & CLP-MOD \\
\hline Manganese & & $\mathrm{Y}$ & 27.3 & $\mathrm{mg} / \mathrm{kg}$ & 1.12 & CLP-MOD \\
\hline Mercury & $\mathrm{U}$ & $\mathrm{Y}$ & 0.691 & $\mathrm{mg} / \mathrm{kg}$ & 0.691 & CLP-MOD \\
\hline Mercury & $\mathrm{U}$ & $\mathrm{Y}$ & 0.691 & $\mathrm{mg} / \mathrm{kg}$ & 0.691 & CLP-MOD \\
\hline Nickel & U & $\mathrm{Y}$ & 8.52 & $\mathrm{mg} / \mathrm{kg}$ & 8.52 & CLP-MOD \\
\hline Nickel & $\mathrm{U}$ & $\mathrm{Y}$ & 8.52 & $\mathrm{mg} / \mathrm{kg}$ & 8.52 & CLP-MOD \\
\hline Potassium & $U$ & $\mathrm{Y}$ & 1010 & $\mathrm{mg} / \mathrm{kg}$ & 1010 & CLP-MOD \\
\hline Potassium & $\mathrm{U}$ & $\mathrm{Y}$ & 1010 & $\mathrm{mg} / \mathrm{kg}$ & 1010 & CLP-MOD \\
\hline Selenium & UJ & YIW & 1.73 & $\mathrm{mg} / \mathrm{kg}$ & 1.73 & CLP-MOD \\
\hline Selenium & UJ & YI & 1.73 & $\mathrm{mg} / \mathrm{kg}$ & 1.73 & CLP-MOD \\
\hline Silver & U & $\mathrm{Y}$ & 5.62 & $\mathrm{mg} / \mathrm{kg}$ & 5.62 & CLP-MOD \\
\hline Silver & $\mathrm{U}$ & $\bar{Y}$ & 5.62 & $\mathrm{mg} / \mathrm{kg}$ & 5.62 & CLP-MOD \\
\hline Sodium & $\mathrm{U}$ & YV & 20.8 & $\mathrm{mg} / \mathrm{kg}$ & 69 & CLP-MOD \\
\hline
\end{tabular}


SURVEY ID: PPSC 0902D (continued)

Constituent

RQ AQ B Result

Unit

D. Limit Method

Metals (total recoverable)

Thallium

$\begin{array}{lll}\mathrm{mg} / \mathrm{kg} & 69 & \text { CLP-MOD } \\ \mathrm{mg} / \mathrm{kg} & 1.84 & \text { CLP-MOD } \\ \mathrm{mg} / \mathrm{kg} & 1.84 & \text { CLP-MOD } \\ \mathrm{mg} / \mathrm{kg} & 4.19 & \text { CLP-MOD } \\ \mathrm{mg} / \mathrm{kg} & 4.19 & \text { CLP-MOD } \\ \mathrm{mg} / \mathrm{kg} & 4.95 & \text { CLP-MOD } \\ \mathrm{mg} / \mathrm{kg} & 4.95 & \text { CLP-MOD }\end{array}$

Sodium

Thallium

Vanadium

Vanadium

Zinc

Zinc

$\begin{array}{lll}U & \text { YV } & 19.8 \\ U & Y V & 0.23 \\ U & Y V & 0.184 \\ & \text { Y } & 17.7 \\ & \text { Y } & 17.8 \\ J & \text { YE } & 2.1 \\ J & \text { YE } & 2.1\end{array}$

$B / N / A$ Extractables (including PAH and phenols)

\begin{tabular}{|c|c|c|c|c|c|c|}
\hline Acenaphthene & $\mathrm{U}$ & $\mathrm{Y}$ & 38 & $\mu \mathrm{g} / \mathrm{kg}$ & 38 & EPA8270 \\
\hline Acenaphthylene & $U$ & $\mathrm{Y}$ & 38 & $\mu \mathrm{g} / \mathrm{kg}$ & 38 & EPA8270 \\
\hline Anthracene & $\mathrm{U}$ & Y & 38 & $\mu \mathrm{g} / \mathrm{kg}$ & 38 & EPA8270 \\
\hline Benzo[a]anthracene & U & $\mathrm{Y}$ & 38 & $\mu \mathrm{g} / \mathrm{kg}$ & 38 & EPA8270 \\
\hline Benzo[b]fluoranthene & $U$ & $\mathrm{Y}$ & 38 & $\mu \mathrm{g} / \mathrm{kg}$ & 38 & EPA8270 \\
\hline Benzo[k]fluoranthene & $U$ & $\mathrm{Y}$ & 38 & $\mu \mathrm{g} / \mathrm{kg}$ & 38 & EPA8270 \\
\hline Benzoic acid & $\mathrm{U}$ & YV & 14 & $\mu \mathrm{g} / \mathrm{kg}$ & 190 & EPA8270 \\
\hline Benzo $[g, h, i]$ perylene & $\mathrm{U}$ & $\mathrm{Y}$ & 38 & $\mu \mathrm{g} / \mathrm{kg}$ & 38 & EPA8270 \\
\hline Benzo[a]pyrene & $\mathrm{U}$ & $\mathrm{Y}$ & 38 & $\mu \mathrm{g} / \mathrm{kg}$ & 38 & EPA8270 \\
\hline Benzyl alcohol & $\mathbf{U}$ & $\mathrm{Y}$ & 38 & $\mu \mathrm{g} / \mathrm{kg}$ & 38 & EPA8270 \\
\hline Bis(2-chloroethoxy) methane & $\mathrm{U}$ & $\mathrm{Y}$ & 38 & $\mu \mathrm{g} / \mathrm{kg}$ & 38 & EPA8270 \\
\hline Bis(2-chloroethyl) ether & U & $\mathbf{Y}$ & 38 & $\mu \mathrm{g} / \mathrm{kg}$ & 38 & EPA8270 \\
\hline Bis(2-chloroisopropyl) ether & $U$ & $\mathrm{Y}$ & 38 & $\mu \mathrm{g} / \mathrm{kg}$ & 38 & EPA8270 \\
\hline Bis(2-ethylhexyl) phthalate & $\mathbf{U}$ & YV & 27.5 & $\mu \mathrm{g} / \mathrm{kg}$ & 38 & EPA8270 \\
\hline 4-Bromophenyl phenyl ether & U & $\mathrm{Y}$ & 38 & $\mu \mathrm{g} / \mathrm{kg}$ & 38 & EPA8270 \\
\hline Butylbenzyl phthalate & $\mathrm{U}$ & $\mathrm{Y}$ & 38 & $\mu \mathrm{g} / \mathrm{kg}$ & 38 & EPA8270M \\
\hline 4-Chloroaniline & $\mathrm{U}$ & $\mathrm{Y}$ & 38 & $\mu g / \mathrm{kg}$ & 38 & EPA8270 \\
\hline 4-Chloro-m-cresol & $\mathrm{U}$ & $\mathrm{Y}$ & 38 & $\mu \mathrm{g} / \mathrm{kg}$ & 38 & EPA8270M \\
\hline 2-Chloronaphthalene & $\mathrm{U}$ & $\mathrm{Y}$ & 38 & $\mu \mathrm{g} / \mathrm{kg}$ & 38 & EPA8270 \\
\hline 2-Chlorophenol & $\mathrm{U}$ & $\mathrm{Y}$ & 38 & $\mu \mathrm{g} / \mathrm{kg}$ & 38 & EPA8270M \\
\hline 4-Chlorophenyl phenyl ether & $U$ & $\mathrm{Y}$ & 38 & $\mu g / \mathrm{kg}$ & 38 & EPA8270 \\
\hline Chrysene & $\mathrm{U}$ & $\mathrm{Y}$ & 38 & $\mu \mathrm{g} / \mathrm{kg}$ & 38 & EPA8270 \\
\hline o-Cresol (2-Methylphenol) & $\mathrm{U}$ & $\mathrm{Y}$ & 38 & $\mu g / \mathrm{kg}$ & 38 & EPA8270 \\
\hline p-Cresol (4-Methylphenol) & $\mathrm{U}$ & $\mathrm{Y}$ & 38 & $\mu \mathrm{g} / \mathrm{kg}$ & 38 & EPA8270 \\
\hline Dibenz $[a, h]$ anthracene & $\mathrm{U}$ & $\mathrm{Y}$ & 38 & $\mu \mathrm{g} / \mathrm{kg}$ & 38 & EPA8270 \\
\hline Dibenzofuran & $\mathrm{U}$ & $\mathrm{Y}$ & 38 & $\mu g / \mathrm{kg}$ & 38 & EPA8270 \\
\hline Di-n-butyl phthalate & $\mathrm{U}$ & YV & 98.5 & $\mu \mathrm{g} / \mathrm{kg}$ & 38 & EPA8270M \\
\hline 1,2-Dichlorobenzene & $\mathrm{U}$ & $\mathrm{Y}$ & 38 & $\mu \mathrm{g} / \mathrm{kg}$ & 38 & EPA8270 \\
\hline 1,3-Dichlorobenzene & $U$ & $\mathrm{Y}$ & 38 & $\mu \mathrm{g} / \mathrm{kg}$ & 38 & EPA8270 \\
\hline 1,4-Dichlorobenzene & $\mathrm{U}$ & $\mathrm{Y}$ & 38 & $\mu \mathrm{g} / \mathrm{kg}$ & 38 & EPA8270 \\
\hline 3,3'-Dichlorobenzidine & $\mathrm{U}$ & $\mathrm{Y}$ & 76 & $\mu \mathrm{g} / \mathrm{kg}$ & 76 & EPA8270 \\
\hline 2,4-Dichlorophenol & $\mathrm{U}$ & $\mathrm{Y}$ & 38 & $\mu g / k g$ & 38 & EPA8270M \\
\hline Diethyl phthalate & U & $\mathrm{Y}$ & 38 & $\mu \mathrm{g} / \mathrm{kg}$ & 38 & EPA8270 \\
\hline 2,4-Dimethyl phenol & $\mathbf{U}$ & $\mathrm{Y}$ & 38 & $\mu \mathrm{g} / \mathrm{kg}$ & 38 & EPA8270M \\
\hline Dimethyl phthalate & $\mathrm{U}$ & $\mathrm{Y}$ & 38 & $\mu \mathrm{g} / \mathrm{kg}$ & 38 & EPA8270M \\
\hline 2,4-Dinitrophenol & $\mathrm{U}$ & $\mathrm{Y}$ & 190 & $\mu \mathrm{g} / \mathrm{kg}$ & 190 & EPA8270M \\
\hline 2,4-Dinitrotoluene & $\mathrm{U}$ & $\mathrm{Y}$ & 38 & $\mu \mathrm{g} / \mathrm{kg}$ & 38 & EPA8270 \\
\hline 2,6-Dinitrotoluene & U & $\mathrm{Y}$ & 38 & $\mu \mathrm{g} / \mathrm{kg}$ & 38 & EPA8270 \\
\hline Di-n-octyl phthalate & U & $\mathrm{Y}$ & 38 & $\mu \mathrm{g} / \mathrm{kg}$ & 38 & EPA8270 \\
\hline Fluoranthene & $\mathrm{U}$ & $\mathrm{Y}$ & 38 & $\mu \mathrm{g} / \mathrm{kg}$ & 38 & EPA8270 \\
\hline Fluorene & $\mathrm{U}$ & $\mathrm{Y}$ & 38 & $\mu \mathrm{g} / \mathrm{kg}$ & 38 & EPA8270 \\
\hline Hexachlorobenzene & $\mathrm{U}$ & $\mathrm{Y}$ & 38 & $\mu \mathrm{g} / \mathrm{kg}$ & 38 & EPA8270M \\
\hline Hexachlorobutadiene & $\mathrm{U}$ & $\mathrm{Y}$ & 38 & $\mu \mathrm{g} / \mathrm{kg}$ & 38 & EPA8270M \\
\hline Hexachlorocyclopentadiene & $U$ & $\mathrm{Y}$ & 38 & $\mu \mathrm{g} / \mathrm{kg}$ & 38 & EPA8270M \\
\hline Hexachloroethane & U & $\mathrm{Y}$ & 38 & $\mu \mathrm{g} / \mathrm{kg}$ & 38 & EPA8270M \\
\hline Indeno $[1,2,3-c, d]$ pyrene & $U$ & $\mathrm{Y}$ & 38 & $\mu \mathrm{g} / \mathrm{kg}$ & 38 & EPA8270 \\
\hline
\end{tabular}


$B / N / A$ Extractables (including $P A H$ and phenols)

$\begin{array}{llll}\text { Isophorone } & \mathrm{U} & \mathrm{Y} & 38 \\ \text { 2-Methyl-4,6-dinitrophenol } & \mathrm{U} & \mathrm{Y} & 190 \\ \text { 2-Methylnaphthalene } & \mathrm{U} & \mathrm{Y} & 38 \\ \text { Naphthalene } & \mathrm{U} & \mathrm{Y} & 38 \\ \text { m-Nitroaniline } & \mathrm{U} & \mathrm{Y} & 190 \\ \text { o-Nitroaniline } & \mathrm{U} & \mathrm{Y} & 190 \\ \text { p-Nitroaniline } & \mathrm{U} & \mathrm{Y} & 190 \\ \text { Nitrobenzene } & \mathrm{U} & \mathrm{Y} & 38 \\ \text { 2-Nitrophenol } & \mathrm{U} & \mathrm{Y} & 38 \\ \text { 4-Nitrophenol } & \mathrm{U} & \mathrm{Y} & 190 \\ \text { N-Nitrosodiphenylamine } & \mathrm{U} & \mathrm{Y} & 38 \\ \text { N-Nitrosodi-n-propylamine } & \mathrm{U} & \mathrm{Y} & 38 \\ \text { Pentachlorophenol } & \mathrm{U} & \mathrm{Y} & 190 \\ \text { Phenanthrene } & \mathrm{U} & \mathrm{Y} & 38 \\ \text { Phenol } & \mathrm{U} & \mathrm{Y} & 38 \\ \text { Pyrene } & \mathrm{U} & \mathrm{Y} & 38 \\ \text { 1,2,4-Trichlorobenzene } & \mathrm{U} & \mathrm{Y} & 38 \\ \text { 2,4,5-Trichlorophenol } & \mathrm{U} & \mathrm{Y} & 190 \\ \text { 2,4,6-Trichlorophenol } & \mathrm{U} & \mathrm{Y} & 38\end{array}$

$\begin{array}{lll}\mu \mathrm{g} / \mathrm{kg} & 38 & \text { EPA8270 } \\ \mu \mathrm{g} / \mathrm{kg} & 190 & \text { EPA8270M } \\ \mu \mathrm{g} / \mathrm{kg} & 38 & \text { EPA8270 } \\ \mu \mathrm{g} / \mathrm{kg} & 38 & \text { EPA8270 } \\ \mu \mathrm{g} / \mathrm{kg} & 190 & \text { EPA8270M } \\ \mu \mathrm{g} / \mathrm{kg} & 190 & \text { EPA8270M } \\ \mu \mathrm{g} / \mathrm{kg} & 190 & \text { EPA8270M } \\ \mu \mathrm{g} / \mathrm{kg} & 38 & \text { EPA8270 } \\ \mu \mathrm{g} / \mathrm{kg} & 38 & \text { EPA8270M } \\ \mu \mathrm{g} / \mathrm{kg} & 190 & \text { EPA8270M } \\ \mu \mathrm{g} / \mathrm{kg} & 38 & \text { EPA8270M } \\ \mu \mathrm{g} / \mathrm{kg} & 38 & \text { EPA8270 } \\ \mu \mathrm{g} / \mathrm{kg} & 190 & \text { EPA8270M } \\ \mu \mathrm{g} / \mathrm{kg} & 38 & \text { EPA8270 } \\ \mu \mathrm{g} / \mathrm{kg} & 38 & \text { EPA8270M } \\ \mu \mathrm{g} / \mathrm{kg} & 38 & \text { EPA8270 } \\ \mu \mathrm{g} / \mathrm{kg} & 38 & \text { EPA8270M } \\ \mu \mathrm{g} / \mathrm{kg} & 190 & \text { EPA8270M } \\ \mu \mathrm{g} / \mathrm{kg} & 38 & \text { EPA8270 }\end{array}$

Volatile Organic Compounds

\begin{tabular}{|c|c|c|c|c|}
\hline Acetone & UJ & \multicolumn{2}{|c|}{ YVOH } & 3.68 \\
\hline Acetone & UJ & \multicolumn{2}{|c|}{ YVOH } & 3.51 \\
\hline Benzene & $U$ & \multicolumn{2}{|c|}{$\mathrm{Y}$} & 0.108 \\
\hline Bromodichloromethane & UJ & YO & $\mathrm{H}$ & 0.22 \\
\hline Bromodichloromethane & UJ & YO & $\mathrm{H}$ & 0.22 \\
\hline Bromoform & UJ & YO & $\mathrm{H}$ & 2.2 \\
\hline Bromoform & UJ & YO & $\mathrm{H}$ & 2.2 \\
\hline Bromomethane (Methyl bromide) & UJ & YO & $\mathrm{H}$ & 0.66 \\
\hline Bromomethane (Methyl bromide) & UJ & YO & $\mathrm{H}$ & 0.66 \\
\hline Carbon disulfide & UJ & YVO & $\mathrm{H}$ & 0.62 \\
\hline Carbon disulfide & & YV & $\mathrm{H}$ & 19.2 \\
\hline Carbon tetrachloride & $\mathrm{U}$ & $\mathrm{Y}$ & & 0.12 \\
\hline Chlorobenzene & UJ & YO & $\mathrm{H}$ & 0.33 \\
\hline Chlorobenzene & UJ & YO & $\mathrm{H}$ & 0.33 \\
\hline Chloroethane & UJ & YO & $\mathrm{H}$ & 0.22 \\
\hline Chloroethane & UJ & YO & $\mathrm{H}$ & 0.22 \\
\hline Chloroethene (Vinyl chloride) & $\mathrm{U}$ & $\mathrm{Y}$ & & 0.24 \\
\hline Chloroform & UJ & YO & $\mathrm{H}$ & 0.22 \\
\hline Chloroform & UJ & YO & $\mathrm{H}$ & 0.22 \\
\hline Chloromethane (Methyl chloride) & UJ & YO & $\mathrm{H}$ & 0.33 \\
\hline Chloromethane (Methyl chloride) & UJ & YO & $\mathrm{H}$ & 0.33 \\
\hline Dibromochloromethane & UJ & YO & $\mathrm{H}$ & 0.77 \\
\hline Dibromochloromethane & UJ & YO & $\mathrm{H}$ & 0.77 \\
\hline 1,1-Dichloroethane & UJ & YO & $\mathrm{H}$ & 0.11 \\
\hline 1,1-Dichloroethane & UJ & YO & $\mathrm{H}$ & 0.11 \\
\hline 1,2-Dichloroethane & UJ & YO & $\mathrm{H}$ & 0.22 \\
\hline 1,2-Dichloroethane & UJ & YO & $\mathrm{H}$ & 0.22 \\
\hline 1,1-Dichloroethylene & UJ & YO & $\mathrm{H}$ & 0.55 \\
\hline 1,1-Dichloroethylene & UJ & YO & $\mathrm{H}$ & 0.55 \\
\hline 1,2-Dichloroethylene & UJ & YO & $\mathrm{H}$ & 2.2 \\
\hline 1,2-Dichloroethylene & UJ & YO & $\mathrm{H}$ & 2.2 \\
\hline Dichloromethane (Methylene chloride) & $\mathrm{U}$ & $\mathrm{Y}$ & & 0.24 \\
\hline 1,2-Dichloropropane & $\mathrm{U}$ & $\hat{\mathrm{Y}}$ & & 0.072 \\
\hline cis-1,3-Dichloropropene & UJ & YO & $\mathrm{H}$ & 5.5 \\
\hline
\end{tabular}

\begin{tabular}{lll}
$\mu \mathrm{g} / \mathrm{kg}$ & 11 & EPA8260 \\
$\mu \mathrm{g} / \mathrm{kg}$ & 11 & EPA8260 \\
$\mu \mathrm{g} / \mathrm{kg}$ & 0.108 & EPA8021 \\
$\mu \mathrm{g} / \mathrm{kg}$ & 0.22 & EPA8260 \\
$\mu \mathrm{g} / \mathrm{kg}$ & 0.22 & EPA8260 \\
$\mu \mathrm{g} / \mathrm{kg}$ & 2.2 & EPA8260 \\
$\mu \mathrm{g} / \mathrm{kg}$ & 2.2 & EPA8260 \\
$\mu \mathrm{g} / \mathrm{kg}$ & 0.66 & EPA8260 \\
$\mu \mathrm{g} / \mathrm{kg}$ & 0.66 & EPA8260 \\
$\mu \mathrm{g} / \mathrm{kg}$ & 2.2 & EPA8260 \\
$\mu \mathrm{g} / \mathrm{kg}$ & 2.2 & EPA8260 \\
$\mu \mathrm{g} / \mathrm{kg}$ & 0.12 & EPA8021 \\
$\mu \mathrm{g} / \mathrm{kg}$ & 0.33 & EPA8260 \\
$\mu \mathrm{g} / \mathrm{kg}$ & 0.33 & EPA8260 \\
$\mu \mathrm{g} / \mathrm{kg}$ & 0.22 & EPA8260 \\
$\mu \mathrm{g} / \mathrm{kg}$ & 0.22 & EPA8260 \\
$\mu \mathrm{g} / \mathrm{kg}$ & 0.24 & EPA8021 \\
$\mu \mathrm{g} / \mathrm{kg}$ & 0.22 & EPA8260 \\
$\mu \mathrm{g} / \mathrm{kg}$ & 0.22 & EPA8260 \\
$\mu \mathrm{g} / \mathrm{kg}$ & 0.33 & EPA8260 \\
$\mu \mathrm{g} / \mathrm{kg}$ & 0.33 & EPA8260 \\
$\mu \mathrm{g} / \mathrm{kg}$ & 0.77 & EPA8260 \\
$\mu \mathrm{g} / \mathrm{kg}$ & 0.77 & EPA8260 \\
$\mu \mathrm{g} / \mathrm{kg}$ & 0.11 & EPA8260 \\
$\mu \mathrm{g} / \mathrm{kg}$ & 0.11 & EPA8260 \\
$\mu \mathrm{g} / \mathrm{kg}$ & 0.22 & EPA8260 \\
$\mu \mathrm{g} / \mathrm{kg}$ & 0.22 & EPA8260 \\
$\mu \mathrm{g} / \mathrm{kg}$ & 0.55 & EPA8260 \\
$\mu \mathrm{g} / \mathrm{kg}$ & 0.55 & EPA8260 \\
$\mu \mathrm{g} / \mathrm{kg}$ & 2.2 & EPA8260 \\
$\mu \mathrm{g} / \mathrm{kg}$ & 2.2 & EPA8260 \\
$\mu \mathrm{g} / \mathrm{kg}$ & 0.24 & EPA8021 \\
$\mu \mathrm{g} / \mathrm{kg}$ & 0.072 & EPA8021 \\
$\mu \mathrm{g} / \mathrm{kg}$ & 5.5 & EPA8260 \\
& & \\
\hline
\end{tabular}


SURVEY ID: PPSC 0902D (continued)

Sample ID: 104639

Constituent

RQ AQ B Result

Unit

D. Limit Method

Volatile Organic Compounds

cis-1,3-Dichloropropene

trans-1,3-Dichloropropene

trans-1,3-Dichloropropene

Ethylbenzene

2-Hexanone

2-Hexanone

Methyl ethyl ketone

Methyl ethyl ketone

Methyl isobutyl ketone

Methyl isobutyl ketone

Styrene

1,1,2,2-Tetrachloroethane

Tetrachloroethylene

Tetrachloroethylene

Toluene

1,1,1-Trichloroethane

1,1,1-Trichloroethane

1,1,2-Trichloroethane

1,1,2-Trichloroethane

Trichloroethylene

Vinyl acetate

Vinyl acetate

Xylenes

Xylenes
UJ YO $\mathrm{H} \quad 5.5$

UJ YO $\mathrm{H} \quad \mathbf{5 . 5}$

UJ YO $\mathrm{H} \quad 5.5$

$\begin{array}{llll}U & Y & 0.06\end{array}$

UJ YO $\mathrm{H} 11$

UJ YO $\mathrm{H} 11$

UJ YO $\mathrm{H} 11$

UJ YO $\mathrm{H} 11$

UJ YO $\mathrm{H} \quad 11$

UJ YO $\mathrm{H} 11$

$\begin{array}{lll}U & \mathrm{Y} & 0.12\end{array}$

$\begin{array}{llll}U & \mathrm{Y} & 0.12\end{array}$

UJ YO $\mathrm{H} \quad \mathbf{0 . 4 4}$

UJ $\quad$ YO $\mathrm{H} \quad \mathbf{0 . 4 4}$

$\begin{array}{llll}U & \mathrm{Y} & 0.12\end{array}$

UJ YO $\mathrm{H} \quad \mathbf{0 . 3 3}$

UJ YO $\mathrm{H} \quad \mathbf{0 . 3 3}$

UJ YO $\mathrm{H} \quad \mathbf{0 . 2 2}$

UJ YO $\mathrm{H} \quad \mathbf{0 . 2 2}$

$\begin{array}{llll}U & Y & 0.12\end{array}$

UJ YO $\mathrm{H} 11$

UJ YO $\mathrm{H} 11$

UJ YO $\mathrm{H} \quad \mathbf{0 . 4 4}$

UJ YO $\mathrm{H} \quad 0.44$

Pesticides/PCBs and Dioxins/Furans

Aldrin

alpha-Benzene hexachloride

beta-Benzene hexachloride

delta-Benzene hexachloride

alpha-Chlordane

gamma-Chlordane

p,p'-DDD

$\mathrm{p}, \mathrm{p}^{\prime} \cdot \mathrm{DDE}$

p, $\mathbf{p}^{\prime}$-DDT

Dieldrin

Endosulfan sulfate

Endosulfan I

Endosulfan II

Endrin

Endrin ketone

Heptachlor

Heptachlor epoxide

Lindane

Methoxychlor

PCB 1016

PCB 1221

PCB 1232

PCB 1242

PCB 1248

PCB 1254

PCB 1260

Toxaphene

$\begin{array}{lll}U & Y & 1.99 \\ U & Y & 1.99 \\ U & Y & 1.99 \\ U & Y & 1.99 \\ U & Y & 1.99 \\ U & Y & 1.99 \\ U & Y & 3.82 \\ U & Y & 3.06 \\ U & Y & 3.82 \\ U & Y & 1.53 \\ U & Y & 3.82 \\ U & Y & 1.99 \\ U & Y & 3.06 \\ U & Y & 3.82 \\ U & Y & 3.82 \\ U & Y & 1.99 \\ U & Y & 1.99 \\ U & Y & 1.99 \\ U & Y & 19.6 \\ U & Y & 37.9 \\ U & Y & 77 \\ U & Y & 37.9 \\ U & Y & 37.9 \\ U & Y & 37.9 \\ U & Y & 37.9 \\ U & Y & 37.9 \\ U & Y & 184\end{array}$

$\begin{array}{lll}\mu \mathrm{g} / \mathrm{kg} & 5.5 & \text { EPA8260 } \\ \mu \mathrm{g} / \mathrm{kg} & 5.5 & \text { EPA8260 } \\ \mu \mathrm{g} / \mathrm{kg} & 5.5 & \text { EPA8260 } \\ \mu \mathrm{g} / \mathrm{kg} & 0.06 & \text { EPA8021 } \\ \mu \mathrm{g} / \mathrm{kg} & 11 & \text { EPA8260 } \\ \mu \mathrm{g} / \mathrm{kg} & 11 & \text { EPA8260 } \\ \mu \mathrm{g} / \mathrm{kg} & 11 & \text { EPA8260 } \\ \mu \mathrm{g} / \mathrm{kg} & 11 & \text { EPA8260 } \\ \mu \mathrm{g} / \mathrm{kg} & 11 & \text { EPA8260 } \\ \mu \mathrm{g} / \mathrm{kg} & 11 & \text { EPA8260 } \\ \mu \mathrm{g} / \mathrm{kg} & 0.12 & \text { EPA8021 } \\ \mu \mathrm{g} / \mathrm{kg} & 0.12 & \text { EPA8021 } \\ \mu \mathrm{g} / \mathrm{kg} & 0.44 & \text { EPA8260 } \\ \mu \mathrm{g} / \mathrm{kg} & 0.44 & \text { EPA8260 } \\ \mu \mathrm{g} / \mathrm{kg} & 0.12 & \text { EPA8021 } \\ \mu \mathrm{g} / \mathrm{kg} & 0.33 & \text { EPA8260 } \\ \mu \mathrm{g} / \mathrm{kg} & 0.33 & \text { EPA8260 } \\ \mu \mathrm{g} / \mathrm{kg} & 0.22 & \text { EPA8260 } \\ \mu \mathrm{g} / \mathrm{kg} & 0.22 & \text { EPA8260 } \\ \mu \mathrm{g} / \mathrm{kg} & 0.12 & \text { EPA8021 } \\ \mu \mathrm{g} / \mathrm{kg} & 11 & \text { EPA8260 } \\ \mu \mathrm{g} / \mathrm{kg} & 11 & \text { EPA8260 } \\ \mu \mathrm{g} / \mathrm{kg} & 0.44 & \text { EPA8260 } \\ \mu \mathrm{g} / \mathrm{kg} & 0.44 & \text { EPA8260 }\end{array}$

$\begin{array}{lll}\mu \mathrm{g} / \mathrm{kg} & 1.99 & \text { EPA8080 } \\ \mu \mathrm{g} / \mathrm{kg} & 1.99 & \text { EPA8080 } \\ \mu \mathrm{g} / \mathrm{kg} & 1.99 & \text { EPA8080 } \\ \mu \mathrm{g} / \mathrm{kg} & 1.99 & \text { EPA8080 } \\ \mu \mathrm{g} / \mathrm{kg} & 1.99 & \text { EPA8080 } \\ \mu \mathrm{g} / \mathrm{kg} & 1.99 & \text { EPA8080 } \\ \mu \mathrm{g} / \mathrm{kg} & 3.82 & \text { EPA8080 } \\ \mu \mathrm{g} / \mathrm{kg} & 3.06 & \text { EPA8080 } \\ \mu \mathrm{g} / \mathrm{kg} & 3.82 & \text { EPA8080 } \\ \mu \mathrm{g} / \mathrm{kg} & 1.53 & \text { EPA8080 } \\ \mu \mathrm{g} / \mathrm{kg} & 3.82 & \text { EPA8080 } \\ \mu \mathrm{g} / \mathrm{kg} & 1.99 & \text { EPA8080 } \\ \mu \mathrm{g} / \mathrm{kg} & 3.06 & \text { EPA8080 } \\ \mu \mathrm{g} / \mathrm{kg} & 3.82 & \text { EPA8080 } \\ \mu \mathrm{g} / \mathrm{kg} & 3.82 & \text { EPA8080 } \\ \mu \mathrm{g} / \mathrm{kg} & 1.99 & \text { EPA8080 } \\ \mu \mathrm{g} / \mathrm{kg} & 1.99 & \text { EPA8080 } \\ \mu \mathrm{g} / \mathrm{kg} & 1.99 & \text { EPA8080 } \\ \mu \mathrm{g} / \mathrm{kg} & 19.6 & \text { EPA8080 } \\ \mu \mathrm{g} / \mathrm{kg} & 37.9 & \text { EPA8080 } \\ \mu \mathrm{g} / \mathrm{kg} & 77 & \text { EPA8080 } \\ \mu \mathrm{g} / \mathrm{kg} & 37.9 & \text { EPA8080 } \\ \mu \mathrm{g} / \mathrm{kg} & 37.9 & \text { EPA8080 } \\ \mu \mathrm{g} / \mathrm{kg} & 37.9 & \text { EPA8080 } \\ \mu \mathrm{g} / \mathrm{kg} & 37.9 & \text { EPA8080 } \\ \mu \mathrm{g} / \mathrm{kg} & 37.9 & \text { EPA8080 } \\ \mu \mathrm{g} / \mathrm{kg} & 184 & \text { EPA8080 }\end{array}$


Appendix D: Sediment Tables

D-110

SURVEY ID: PPSC 0902D (continued)

Constituent

Radionuclides

Actinium-228

Actinium-228

Antimony-124

Antimony-124

Antimony-125

Antimony-125

Barium-133

Barium-133

Cerium-144

Cerium-144

Cesium-134

Cesium-134

Cesium-137

Cesium-137

Cobalt-57

Cobalt-57

Cobalt-58

Cobalt-58

Cobalt-60

Cobalt-60

Europium-152

Europium-152

Europium-154

Europium-154

Europium-155

Europium-155

Gross alpha

Gross alpha

Iodine-129

Iodine-129

Lead-212

Lead-212

Manganese-54

Manganese-54

Neptunium-239

Neptunium-239

Nonvolatile beta

Nonvolatile beta

Plutonium-238

Plutonium-238

Plutonium-239/240

Plutonium-239/240

Potassium-40

Potassium-40

Promethium-144

Promethium-144

Promethium-146

Promethium-146

Ruthenium-106

Ruthenium-106

Sodium-22

Sodium-22

Strontium-90

Strontium-90

Thorium-234

Sample ID: 104639

RQ AQ B Result Unit

D. Limit Method

9.40E-01 $\pm 2.30 \mathrm{E}-01$

$1.00 \mathrm{E}+00 \pm 2.60 \mathrm{E}-01$

$4.00 \mathrm{E}-02 \pm 6.00 \mathrm{E}-02$

$2.00 \mathrm{E}-02 \pm 6.00 \mathrm{E}-02$

$-1.00 \mathrm{E}-01 \pm 1.40 \mathrm{E}-01$

$-2.00 \mathrm{E}-02 \pm 1.50 \mathrm{E}-01$

$2.50 \mathrm{E}-01 \pm 8.00 \mathrm{E}-02$

$2.70 \mathrm{E}-01 \pm 8.00 \mathrm{E}-02$

4.00E-02+3.50E-01

$3.20 \mathrm{E}-01 \pm 4.50 \mathrm{E}-01$

$1.90 \mathrm{E}-01 \pm 7.00 \mathrm{E}-02$

$1.30 \mathrm{E}-01 \pm 7.00 \mathrm{E}-02$

$1.60 \mathrm{E}-01 \pm 9.00 \mathrm{E}-02$

$1.30 \mathrm{E}-01 \pm 1.00 \mathrm{E}-01$

$-2.00 \mathrm{E}-02 \pm 5.00 \mathrm{E}-02$

$-3.00 \mathrm{E}-02 \pm 4.00 \mathrm{E}-02$

$-3.00 \mathrm{E}-02 \pm 5.00 \mathrm{E}-02$

$-1.00 \mathrm{E}-02 \pm 5.00 \mathrm{E}-02$

2.00E-02 $\pm 5.00 \mathrm{E}-02$

1.00E-02 $44.00 \mathrm{E}-02$

$2.60 \mathrm{E}-01 \pm 3.40 \mathrm{E}-01$

4.70E-01 $\$ 3.00 \mathrm{E}-01$

$-7.00 \mathrm{E}-02 \pm 1.40 \mathrm{E}-01$

$-1.40 \mathrm{E}-01 \pm 1.40 \mathrm{E}-01$

$-1.90 \mathrm{E}-01 \pm 1.80 \mathrm{E}-01$

$-1.70 \mathrm{E}-01 \pm 1.80 \mathrm{E}-01$

$1.29 \mathrm{E}+01 \pm 3.00 \mathrm{E}+00$

$1.38 \mathrm{E}+01 \pm 3.00 \mathrm{E}+00$.

$-2.10 \mathrm{E}-01 \pm 4.50 \mathrm{E}-01$

7.00E-02 $44.50 \mathrm{E}-01$

$1.10 \mathrm{E}+00 \pm 1.90 \mathrm{E}-01$

$1.10 \mathrm{E}+00 \pm 1.40 \mathrm{E}-01$

$6.00 \mathrm{E}-02 \pm 5.00 \mathrm{E}-02$

$5.00 \mathrm{E}-02 \pm 5.00 \mathrm{E}-02$

$-2.40 \mathrm{E}+00 \pm 2.40 \mathrm{E}+00$

$3.10 \mathrm{E}+00 \pm 2.00 \mathrm{E}+00$

$1.23 \mathrm{E}+01 \pm 5.20 \mathrm{E}+00$

$1.37 \mathrm{E}+01 \pm 5.10 \mathrm{E}+00$

2.90E-01 $\pm 2.20 \mathrm{E}-01$

$2.10 \mathrm{E}-01 \pm 1.70 \mathrm{E}-01$

$-2.00 \mathrm{E}-02 \pm 2.00 \mathrm{E}-02$

$0.00 \mathrm{E}+00$

$1.10 \mathrm{E}+00 \pm 8.50 \mathrm{E}-01$

9.80E-01 $\pm 7.70 \mathrm{E}-01$

$-2.00 \mathrm{E}-02 \pm 5.00 \mathrm{E}-02$

$1.00 \mathrm{E}-02+5.00 \mathrm{E}-02$

$-6.00 \mathrm{E}-02 \pm 7.00 \mathrm{E}-02$

3.00E-02 7.00E-02

2.50E-01 $\pm 5.10 \mathrm{E}-01$

$-3.30 \mathrm{E}-01 \pm 4.80 \mathrm{E}-01$

$-2.00 \mathrm{E}-02 \pm 5.00 \mathrm{E}-02$

$-5.00 \mathrm{E}-02 \pm 5.00 \mathrm{E}-02$

$0.00 \mathrm{E}+00 \pm 4.30 \mathrm{E}-01$

$-2.80 \mathrm{E}-01 \pm 3.70 \mathrm{E}-01$

$2.70 \mathrm{E}+00 \pm 2.20 \mathrm{E}+00$
$\mathrm{pCi} / \mathrm{g}$

$\mathrm{pCi} / \mathrm{g}$

$\mathrm{pCi} / \mathrm{g}$

$\mathrm{pCi} / \mathrm{g}$

$\mathrm{pCi} / \mathrm{g}$

$\mathrm{pCi} / \mathrm{g}$

$\mathrm{pCi} / \mathrm{g}$

pCíg

$\mathrm{pCi} / \mathrm{g}$

$\mathrm{pCi} / \mathrm{g}$

$\mathrm{pCi} / \mathrm{g}$

pCi/g

pCi/g

$\mathrm{pCi} / \mathrm{g}$

$\mathrm{pCi} / \mathrm{g}$

$\mathrm{pCi} / \mathrm{g}$

$\mathrm{pCi} / \mathrm{g}$

$\mathrm{pCi} / \mathrm{g}$

pCi/g

$\mathrm{pCi} / \mathrm{g}$

pCi/g

pCi/g

$\mathrm{pCi} / \mathrm{g}$

pCi/g

pCi/g

$\mathrm{pCi} / \mathrm{g}$

pCi/g

$\mathrm{pCi} / \mathrm{g}$

pCi/g

$\mathrm{pCi} / \mathrm{g}$

pCig

pCi/g

pCi/g

pCi/g

pCi/g

pCi/g

pCi'g

pCi/g

pCi/g

$\mathrm{pCi} / \mathrm{g}$

$\mathrm{pCi} / \mathrm{g}$

$\mathrm{pCi} / \mathrm{g}$

$\mathrm{pCi} / \mathrm{g}$

$\mathrm{pCi} / \mathrm{g}$

$\mathrm{pCi} / \mathrm{g}$

$\mathrm{pCi} / \mathrm{g}$

$\mathrm{pCi} / \mathrm{g}$

$\mathrm{pCi} / \mathrm{g}$

$\mathrm{pCj} / \mathrm{g}$

$\mathrm{pCi} / \mathrm{g}$

$\mathrm{pCi} / \mathrm{g}$

$\mathrm{pCi} / \mathrm{g}$

$\mathrm{pCi} / \mathrm{g}$

$\mathrm{pCi} / \mathrm{g}$

$\mathrm{pCi} / \mathrm{g}$
3.30E-01 LANLER130MOD

3.20E-01 LANLER130MOD

1.00E-01 LANLER130MOD

1.00E-01 LANLER130MOD

2.20E-01 LANLER130MOD

2.50E-01 LANLER130MOD

1.50E-01 LANLER130MOD

1.50E-01 LANLERI30MOD

5.30E.01 LANLER130MOD

5.20E-01 LANLER130MOD

1.30E-01 LANLER130MOD

1.20E-01 LANLER130MOD

9.00E-02 LANLER130MOD

8.00E-02 LANLER130MOD

7.00E-02 LANLER130MOD

6.00E-02 LANLER130MOD

9.00E-02 LANLER130MOD

9.00E-02 LANLER130MOD

9.00E-02 LANLER130MOD

8.00E-02 LANLER130MOD

7.00E-01 LANLER130MOD

6.60E-01 LAANLER130MOD

2.20E-01 LANLER130MOD

2.20E-01 LANLER130MOD

2.70E-01 LANLER130MOD

2.70E-01 LANLER130MOD

2.70E+00 LANLMLR100MOD

2.63E+00 LANLMLR100MOD

4.80E-01 LANLER130MOD

5.10E-01 LANLER130MOD

1.20E-01 LANLER130MOD

1.30E-01 LANLER130MOD

1.00E-01 LANLER130MOD

1.00E-01 LANLER130MOD

$3.90 \mathrm{E}+00$ LANLER130MOD

4.20E+00 LANLER130MOD

1.70E+00 LANLMLR100MOD

1.70E+00 LANLMUR100MOD

2.00E-01 EMLPUO2MOD

1.70E-01 EMLPU02MOD

2.30E-01 EMLPU02MOD

8.00E-02 EMLPU02MOD

7.70E-01 LANLER130MOD

7.90E-01 LANLER130MOD

9.00E-02 LANLER130MOD

9.00E-02 LANLER130MOD

1.10E-01 LANLER130MOD

1.20E-01 LAANLER130MOD

9.20E-01 LANLER130MOD

7.90E-01 LAANLER130MOD

8.00E-02 LANLER130MOD

8.00E-02 LANLER130MOD

1.10E+00 EMLSR02MOD

1.06E+00 EMLSR02MOD

2.00E+00 LANLER130MOD 
SURVEY ID: PPSC 0902D (continued)

Constituent

RQ AQ B Result

$9.00 \mathrm{E}-01 \pm 1.30 \mathrm{E}+00$ $-1.00 \mathrm{E}-02 \pm 6.00 \mathrm{E}-02$ $2.00 \mathrm{E}-02 \pm 6.00 \mathrm{E}-02$ $3.00 \mathrm{E}-02 \pm 5.00 \mathrm{E}-02$ $-2.00 \mathrm{E}-02 \pm 5.00 \mathrm{E}-02$ $1.90 \mathrm{E}-01 \pm 1.30 \mathrm{E}-01$ 1.60E-01 $\pm 1.20 \mathrm{E}-01$ $2.00 \mathrm{E}-02 \pm 1.00 \mathrm{E}-01$ $-1.00 \mathrm{E}-02 \pm 1.00 \mathrm{E}-01$

Radionuclides
Thorium-234

Tin-113

Yttrium-88

Yttrium-88

Zinc-65

Zinc-65

Zirconium-95

Zirconium-95
Unit

D. Limit Method

$\begin{array}{ll}\text { UI } & \\ \text { UI } & \\ \text { UI } & \\ \text { UI } & \\ \text { UI } & \\ \text { J } & \text { E } \\ \text { J } & \text { E } \\ \text { UI } & \\ \text { UI } & \end{array}$

SURVEY ID: PPSC 0903

Sample ID: 104637

Survey location: $45224 \mathrm{E} 3680779 \mathrm{~N}$ (SRS coordinates)

Depth of core interval: 2.00 to $4.00 \mathrm{ft}$

Sample type: Normal

Sample matrix: Soil $\begin{array}{lll}\text { pCi/g } & 1.90 \mathrm{E}+00 & \text { LANLER130MOD } \\ \text { pCi/g } & 1.10 \mathrm{E}-01 & \text { LANLER130MOD } \\ \text { pCi/g } & 1.10 \mathrm{E}-01 & \text { LANLER130MOD } \\ \text { pCi/g } & 1.00 \mathrm{E}-01 & \text { LANLER130MOD } \\ \text { pCi/g } & 1.00 \mathrm{E}-01 & \text { LANLER130MOD } \\ \text { pCi/g } & 2.40 \mathrm{E}-01 & \text { LANLER130MOD } \\ \text { pCi/g } & 2.20 \mathrm{E}-01 & \text { LANLER130MOD } \\ \text { pCi/g } & 1.70 \mathrm{E}-01 & \text { LANLER130MOD } \\ \text { pCi/g } & 1.70 \mathrm{E}-01 & \text { LANLER130MOD }\end{array}$
Constituent $\mathbf{R Q}$

Physical Parameters and Specified Analyses

Cation exchange capacity

Cation exchange capacity

Chloride

Cyanide

Cyanide

Nitrate-nitrite as nitrogen

Orthophosphate

Phenols

Sulfide

Sulfide

Total organic carbon

Total organic nitrogen

Total phosphates (as P)

Metals (total recoverable)

$\begin{array}{lll} & & 100 \\ & & 87 \\ \text { U } & & 2.72 \\ \text { U } & & 0.98 \\ \text { U } & & 1.18 \\ \text { U } & & 0.863 \\ \text { J } & \text { E } & 2.56 \\ \text { UJ } & \text { C } & 345 \\ \text { UJ } & \text { C } & 294 \\ \text { UJ } & \text { C } & 294 \\ & & 1060 \\ \text { C } & & 73.1 \\ \text { J } & \text { E } & 722\end{array}$

\begin{tabular}{|c|c|c|c|c|c|c|}
\hline Aluminum & & V & 5690 & $\mathrm{mg} / \mathrm{kg}$ & 14.4 & EPA6010A \\
\hline Antimony & U & & 5.77 & ; mg/kg & 5.77 & EPA6010A \\
\hline Arsenic & $\mathrm{U}$ & & 17.3 & $\mathrm{mg} / \mathrm{kg}$ & 17.3 & EPA6010A \\
\hline Barium & & & 147 & $\mathrm{mg} / \mathrm{kg}$ & 1.44 & EPA6010A \\
\hline Beryllium & $J$ & $\mathbf{E}$ & 0.486 & $\mathrm{mg} / \mathrm{kg}$ & 0.577 & EPA6010A \\
\hline Cadmium & U & & 0.288 & $\mathrm{mg} / \mathrm{kg}$ & 0.288 & EPA6010A \\
\hline Calcium & & V & 112 & $\mathrm{mg} / \mathrm{kg}$ & 14.4 & EPA6010A \\
\hline Chromium & & & 13.5 & $\mathrm{mg} / \mathrm{kg}$ & 1.44 & EPA6010A \\
\hline Cobalt & $\mathbf{J}$ & $\mathbf{E}$ & 0.206 & $\mathrm{mg} / \mathrm{kg}$ & 0.577 & EPA6010A \\
\hline Copper & & & 2.27 & $\mathrm{mg} / \mathrm{kg}$ & 1.44 & EPA6010A \\
\hline Iron & $J$ & VC & 4690 & $\mathrm{mg} / \mathrm{kg}$ & 5.77 & EPA6010A \\
\hline Lead & $J$ & $\mathrm{E}$ & 3.84 & $\mathrm{mg} / \mathrm{kg}$ & 5.77 & EPA6010A \\
\hline Magnesium & & V & 157 & $\mathrm{mg} / \mathrm{kg}$ & 5.77 & EPA6010A \\
\hline Manganese & & & 5.2 & $\mathrm{mg} / \mathrm{kg}$ & 0.577 & EPA6010A \\
\hline Mercury & $J$ & EV & 0.0357 & $\mathrm{mg} / \mathrm{kg}$ & 0.0392 & EPA7471 \\
\hline
\end{tabular}

Percent solids: $\mathbf{8 5 . 0 0}$

Unit D. Limit Method 
SURVEY ID: PPSC 0903 (continued)

Constituent

Metals (total recoverable)

Nickel

Potassium

Selenium

Silver

Sodium

Thallium

Vanadium

Zinc

\section{$B / N / A$ Extractables (including $P A H$ and phenols)}

Acenaphthene

Acenaphthylene

Anthracene

Benzo[a]anthracene

Benzo[b]fluoranthene

Benzo[k]fluoranthene

Benzoic acid

Benzo[g,h,i]perylene

Benzo[a]pyrene

Benzyl alcohol

Bis(2-chloroethoxy) methane

$\mathrm{Bis}$ (2-chloroethyl) ether

Bis(2-chloroisopropyl) ether

Bis(2-ethylhexyl) phthalate

4-Bromophenyl phenyl ether

Butylbenzyl phthalate

4-Chloroaniline

4-Chloro-m-cresol

2-Chloronaphthalene

2-Chlorophenol

4-Chlorophenyl phenyl ether

Chrysene

$\mathrm{m} / \mathrm{p}$-Cresol

o-Cresol (2-Methylphenol)

Dibenz $[a, h]$ anthracene

Dibenzofuran

Di-n-butyl phthalate

1,2-Dichlorobenzene

1,3-Dichlorobenzene

1,4-Dichlorobenzene

3,3'-Dichlorobenzidine

2,4-Dichlorophenol

Diethyl phthalate

2,4-Dimethyl phenol

Dimethyl phthalate

2,4-Dinitrophenol

2,4-Dinitrotoluene

2,6-Dinitrotoluene

Di-n-octyl phthalate

Fluoranthene

Fluorene

Hexachlorobenzene

Hexachlorobutadiene

Hexachlorocyclopentadiene

Hexachloroethane
Sample ID: 104637

RQ AQ B Result Unit D. Limit Method

\begin{tabular}{|c|c|c|c|c|}
\hline & 1.56 & $\mathrm{mg} / \mathrm{kg}$ & 1.44 & EPA6010A \\
\hline & 155 & $\mathrm{mg} / \mathrm{kg}$ & 115 & EPA6010A \\
\hline $\mathrm{U}$ & 17.3 & $\mathrm{mg} / \mathrm{kg}$ & 17.3 & EPA6010A \\
\hline$U$ & 1.44 & $\mathrm{mg} / \mathrm{kg}$ & 1.44 & EPA6010A \\
\hline$J$ & 8.99 & $\mathrm{mg} / \mathrm{kg}$ & 57.7 & EPA6010A \\
\hline UJ & 17.3 & $\mathrm{mg} / \mathrm{kg}$ & 17.3 & EPA6010A \\
\hline & 16.2 & $\mathrm{mg} / \mathrm{kg}$ & 0.577 & EPA6010A \\
\hline & 3.96 & $\mathrm{mg} / \mathrm{kg}$ & 1.15 & EPA6010A \\
\hline
\end{tabular}

$\mathrm{U} \quad 38.3$

U $\quad 38.3$

U $\quad 3.83$

U $\quad 3.83$

U $\quad 3.83$

U $\quad 38.3$

$\mathrm{U} \quad 38.3$

U $\quad 3.83$

U $\quad 38.3$

U $\quad 38.3$

U 38.3

38.3

38.3

38.3

38.3

38.3

38.3

38.3

38.3

3.83

383

38.3

3.83

38.3

E $\quad 21$

38.3

38.3

38.3

38.3

38.3

38.3

38.3

38.3

383

3.83

3.83

38.3

38.3

38.3

3.83

38.3

38.3

3.83
U $\quad 38.3$

U $\quad 38.3$

\begin{tabular}{lll}
$\mu \mathrm{g} / \mathrm{kg}$ & 38.3 & EPA8270 \\
$\mu \mathrm{g} / \mathrm{kg}$ & 38.3 & EPA8270 \\
$\mu \mathrm{g} / \mathrm{kg}$ & 38.3 & EPA8270 \\
$\mu \mathrm{g} / \mathrm{kg}$ & 3.83 & EPA8270 \\
$\mu \mathrm{g} / \mathrm{kg}$ & 3.83 & EPA8270 \\
$\mu \mathrm{g} / \mathrm{kg}$ & 3.83 & EPA8270 \\
$\mu \mathrm{g} / \mathrm{kg}$ & 38.3 & EPA8270 \\
$\mu \mathrm{g} / \mathrm{kg}$ & 38.3 & EPA8270 \\
$\mu \mathrm{g} / \mathrm{kg}$ & 3.83 & EPA8270 \\
$\mu \mathrm{g} / \mathrm{kg}$ & 38.3 & EPA8270 \\
$\mu \mathrm{g} / \mathrm{kg}$ & 38.3 & EPA8270 \\
$\mu \mathrm{g} / \mathrm{kg}$ & 38.3 & EPA8270 \\
$\mu \mathrm{g} / \mathrm{kg}$ & 38.3 & EPA8270 \\
$\mu \mathrm{g} / \mathrm{kg}$ & 38.3 & EPA8270 \\
$\mu \mathrm{g} / \mathrm{kg}$ & 38.3 & EPA8270 \\
$\mu \mathrm{g} / \mathrm{kg}$ & 38.3 & EPA8270 \\
$\mu \mathrm{g} / \mathrm{kg}$ & 38.3 & EPA8270 \\
$\mu \mathrm{g} / \mathrm{kg}$ & 38.3 & EPA8270 \\
$\mu \mathrm{g} / \mathrm{kg}$ & 38.3 & EPA8270 \\
$\mu \mathrm{g} / \mathrm{kg}$ & 38.3 & EPA8270 \\
$\mu \mathrm{g} / \mathrm{kg}$ & 38.3 & EPA8270 \\
$\mu \mathrm{g} / \mathrm{kg}$ & 3.83 & EPA8270 \\
$\mu \mathrm{g} / \mathrm{kg}$ & 383 & EPA8270 \\
$\mu \mathrm{g} / \mathrm{kg}$ & 38.3 & EPA8270 \\
$\mu \mathrm{g} / \mathrm{kg}$ & 3.83 & EPA8270 \\
$\mu \mathrm{g} / \mathrm{kg}$ & 38.3 & EPA8270 \\
$\mu \mathrm{g} / \mathrm{kg}$ & 38.3 & EPA8270 \\
$\mu \mathrm{g} / \mathrm{kg}$ & 38.3 & EPA8270 \\
$\mu \mathrm{g} / \mathrm{kg}$ & 38.3 & EPA8270 \\
$\mu \mathrm{g} / \mathrm{kg}$ & 38.3 & EPA8270 \\
$\mu \mathrm{g} / \mathrm{kg}$ & 38.3 & EPA8270 \\
$\mu \mathrm{g} / \mathrm{kg}$ & 38.3 & EPA8270 \\
$\mu \mathrm{g} / \mathrm{kg}$ & 38.3 & EPA8270 \\
$\mu \mathrm{g} / \mathrm{kg}$ & 38.3 & EPA8270 \\
$\mu \mathrm{g} / \mathrm{kg}$ & 38.3 & EPA8270 \\
$\mu \mathrm{g} / \mathrm{kg}$ & 383 & EPA8270 \\
$\mu \mathrm{g} / \mathrm{kg}$ & 3.83 & EPA8270 \\
$\mu \mathrm{g} / \mathrm{kg}$ & 3.83 & EPA8270 \\
$\mu \mathrm{g} / \mathrm{kg}$ & 38.3 & EPA8270 \\
$\mu \mathrm{g} / \mathrm{kg}$ & 38.3 & EPA8270 \\
$\mu \mathrm{g} / \mathrm{kg}$ & 38.3 & EPA8270 \\
$\mu \mathrm{g} / \mathrm{kg}$ & 3.83 & EPA8270 \\
$\mu \mathrm{g} / \mathrm{kg}$ & 38.3 & EPA8270 \\
$\mu \mathrm{g} / \mathrm{kg}$ & 38.3 & EPA8270 \\
$\mu \mathrm{g} / \mathrm{kg}$ & 3.83 & EPA8270 \\
& & \\
\hline
\end{tabular}


SURVEY ID: PPSC 0903 (continued)

Constituent
Sample ID: 104637

RQ AQ B Result
Unit

D. Limit Method

$B / N / A$ Extractables (including PAH and phenols)

$\begin{array}{lcc}\text { Indeno[1,2,3-c, d]pyrene } & \mathrm{U} & 3.83 \\ \text { Isophorone } & \mathrm{U} & 38.3 \\ \text { 2-Methyl-4,6-dinitrophenol } & \mathrm{U} & 383 \\ \text { 2-Methylnaphthalene } & \mathrm{U} & 38.3 \\ \text { Naphthalene } & \mathrm{U} & 38.3 \\ \text { m-Nitroaniline } & \mathrm{U} & 38.3 \\ \text { o-Nitroaniline } & \mathrm{U} & 38.3 \\ \text { p-Nitroaniline } & \mathrm{U} & 38.3 \\ \text { Nitrobenzene } & \mathrm{U} & 38.3 \\ \text { 2-Nitrophenol } & \mathrm{U} & 38.3 \\ \text { 4-Nitrophenol } & \mathrm{U} & 38.3 \\ \text { N-Nitrosodiphenylamine } & \mathrm{U} & 38.3 \\ \text { N-Nitrosodi-n-propylamine } & \mathrm{U} & 38.3 \\ \text { Pentachlorophenol } & \mathrm{U} & 38.3 \\ \text { Phenanthrene } & \mathrm{U} & 38.3 \\ \text { Phenol } & \mathrm{U} & 38.3 \\ \text { Pyrene } & \mathrm{U} & 38.3 \\ \text { 1,2,4-Trichlorobenzene } & \mathrm{U} & 3.83 \\ \text { 2,4,5-Trichlorophenol } & \mathrm{U} & 38.3 \\ \text { 2,4,6-Trichlorophenol } & \mathrm{U} & 38.3\end{array}$

Volatile Organic Compounds $\mu \mathrm{g} / \mathrm{kg}$

$\mu \mathrm{g} / \mathrm{kg}$

$\mu \mathrm{g} / \mathrm{kg}$

$\mu \mathrm{g} / \mathrm{kg}$

$\mu \mathrm{g} / \mathrm{kg}$

$\mu \mathrm{g} / \mathrm{kg}$

$\mu \mathrm{g} / \mathrm{kg}$

$\mu \mathrm{g} / \mathrm{kg}$

$\mu \mathrm{g} / \mathrm{kg}$

$\mu \mathrm{g} / \mathrm{kg}$

$\mu \mathrm{g} / \mathrm{kg}$

$\mu \mathrm{g} / \mathrm{kg}$

$\mu \mathrm{g} / \mathrm{kg}$

$\mu \mathrm{g} / \mathrm{kg}$

$\mu \mathrm{g} / \mathrm{kg}$

$\mu \mathrm{g} / \mathrm{kg}$

$\mu \mathrm{g} / \mathrm{kg}$

$\mu \mathrm{g} / \mathrm{kg}$

$\mu \mathrm{g} / \mathrm{kg}$

$\mu \mathrm{g} / \mathrm{kg}$

$\begin{array}{ll}3.83 & \text { EPA8270 } \\ 38.3 & \text { EPA8270 } \\ 383 & \text { EPA8270 } \\ 38.3 & \text { EPA8270 } \\ 38.3 & \text { EPA8270 } \\ 38.3 & \text { EPA8270 } \\ 38.3 & \text { EPA8270 } \\ 38.3 & \text { EPA8270 } \\ 38.3 & \text { EPA8270 } \\ 38.3 & \text { EPA8270 } \\ 38.3 & \text { EPA8270 } \\ 38.3 & \text { EPA8270 } \\ 38.3 & \text { EPA8270 } \\ 38.3 & \text { EPA8270 } \\ 38.3 & \text { EPA8270 } \\ 38.3 & \text { EPA8270 } \\ 38.3 & \text { EPA8270 } \\ 3.83 & \text { EPA8270 } \\ 38.3 & \text { EPA8270 } \\ 38.3 & \text { EPA8270 }\end{array}$

$\mu \mathrm{g} / \mathrm{kg}$

$\mu \mathrm{g} / \mathrm{kg}$

$\mu \mathrm{g} / \mathrm{kg}$

$\mu \mathrm{g} / \mathrm{kg}$

$\mu \mathrm{g} / \mathrm{kg}$

$\mu \mathrm{g} / \mathrm{kg}$

$\mu \mathrm{g} / \mathrm{kg}$

$\mu \mathrm{g} / \mathrm{kg}$

$\mu g / \mathrm{kg}$

$\mu \mathrm{g} / \mathrm{kg}$

$\mu \mathrm{g} / \mathrm{kg}$

$\mu \mathrm{g} / \mathrm{kg}$

$\mu \mathrm{g} / \mathrm{kg}$

$\mu \mathrm{g} / \mathrm{kg}$

$\mu \mathrm{g} / \mathrm{kg}$

$\mu \mathrm{g} / \mathrm{kg}$

$\mu \mathrm{g} / \mathrm{kg}$

$\mu \mathrm{g} / \mathrm{kg}$

$\mu \mathrm{g} / \mathrm{kg}$

$\mu \mathrm{g} / \mathrm{kg}$

$\mu \mathrm{g} / \mathrm{kg}$

$\mu \mathrm{g} / \mathrm{kg}$

$\mu \mathrm{g} / \mathrm{kg}$

$\mu \mathrm{g} / \mathrm{kg}$

$\mu \mathrm{g} / \mathrm{kg}$

$\mu \mathrm{g} / \mathrm{kg}$

$\mu \mathrm{g} / \mathrm{kg}$

$\mu \mathrm{g} / \mathrm{kg}$

$\mu \mathrm{g} / \mathrm{kg}$

$\mu \mathrm{g} / \mathrm{kg}$

$\mu \mathrm{g} / \mathrm{kg}$

$\mu \mathrm{g} / \mathrm{kg}$

$\mu \mathrm{g} / \mathrm{kg}$
5.88

0.588

0.0588

0.0588

0.118

1.18

0.0588

0.0588

0.118

0.118

0.0588

0.118

0.0588

0.0588

0.0588

0.0588

0.118

0.588

0.0588

0.0588

0.0588

1.18

1.18

1.18 .

0.0588

0.0588

0.0588

0.588

0.0588

0.0588

0.0588
0.0588
EPA8260

EPA8260

EPA8260

EPA8260

EPA8260

EPA8260

EPA8260

EPA8260

EPA8260

EPA8260

EPA8260

EPA8260

EPA8260

EPA8260

EPA8260

EPA8260

EPA8260

EPA8260

EPA8260

EPA8260

EPA8260

EPA8260

EPA8260

EPA8260

EPA8260

EPA8260

EPA8260

EPA8260

EPA8260

EPA8260

EPA8260

EPA8260

EPA8260 
SURVEY ID: PPSC 0903 (continued)

Constituent

Volatile Organic Compounds

Vinyl acetate

Xylenes

U

U

1.18

0.176

$\mu \mathrm{g} / \mathrm{kg}$

$\mu \mathrm{g} / \mathrm{kg}$

1.18

0.176

EPA8260

EPA8260

Pesticides/PCBs and Dioxins/Furans

Aldrin

alpha-Benzene hexachloride

beta-Benzene hexachloride

delta-Benzene hexachloride

alpha-Chlordane

gamma-Chlordane

p,p'-DDD

$p, p^{\prime}-D D E$

$\mathrm{p}, \mathrm{p}^{\prime}-\mathrm{DDT}$

Dieldrin

Endosulfan sulfate

Endosulfan I

Endosulfan II

Endrin

Endrin ketone

Heptachlor

Heptachlor epoxide

Lindane

Methoxychlor

PCB 1016

PCB 1221

PCB 1232

PCB 1242

PCB 1248

PCB 1254

PCB 1260

Toxaphene

Radionuclides

Actinium-228

Antimony-124

Antimony-125

Barium-133

Cerium-144

Cesium-134

Cesium-137

Cobalt-57

Cobalt-58

Cobalt-60

Europium-152

Europium-154

Europium-155

Gross alpha

Lead-212

Manganese-54

Neptunium-239

Nonvolatile beta

Plutonium-238

Plutonium-239/240

$\begin{array}{llll}\text { UJ } & \text { O } & \text { L } & 0.974 \\ \text { UJ } & \text { O } & \text { L } & 0.974 \\ \text { UJ } & \text { O } & \text { L } & 1.95 \\ \text { UJ } & \text { O } & \text { L } & 1.95 \\ \text { UJ } & \text { O } & \text { L } & 1.95 \\ \text { UJ } & \text { O } & \text { L } & 1.95 \\ \text { UJ } & \text { O } & \text { L } & 3.9 \\ \text { UJ } & \text { O } & \text { L } & 1.95 \\ \text { UJ } & \text { O } & \text { L } & 3.9 \\ \text { UJ } & \text { O } & \text { L } & 1.95 \\ \text { UJ } & \text { O } & \text { L } & 3.9 \\ \text { UJ } & \text { O } & \text { L } & 1.95 \\ \text { UJ } & \text { O } & \text { L } & 3.9 \\ \text { UJ } & \text { O } & \text { L } & 1.95 \\ \text { UJ } & \text { O } & \text { L } & 3.9 \\ \text { UJ } & \text { O } & \text { L } & 1.95 \\ \text { UJ } & \text { O } & \text { L } & 1.95 \\ \text { UJ } & \text { O } & \text { L } & 0.974 \\ \text { UJ } & \text { O } & \text { L } & 19.5 \\ \text { UJ } & \text { O } & \text { L } & 4.87 \\ \text { UJ } & \text { O } & \text { L } & 4.87 \\ \text { UJ } & \text { O } & \text { L } & 4.87 \\ \text { UJ } & \text { O } & \text { L } & 4.87 \\ \text { UJ } & \text { O } & \text { L } & 4.87 \\ \text { UJ } & \text { O } & \text { L } & 4.87 \\ \text { UJ } & \text { O } & \text { L } & 4.87 \\ \text { UJ } & \text { O } & \text { L } & 39\end{array}$

$\begin{array}{lll}\mu \mathrm{g} / \mathrm{kg} & 0.974 & \text { EPA8080 } \\ \mu \mathrm{g} / \mathrm{kg} & 0.974 & \text { EPA8080 } \\ \mu \mathrm{g} / \mathrm{kg} & 1.95 & \text { EPA8080 } \\ \mu \mathrm{g} / \mathrm{kg} & 1.95 & \text { EPA8080 } \\ \mu \mathrm{g} / \mathrm{kg} & 1.95 & \text { EPA8080 } \\ \mu \mathrm{g} / \mathrm{kg} & 1.95 & \text { EPA8080 } \\ \mu \mathrm{g} / \mathrm{kg} & 3.9 & \text { EPA8080 } \\ \mu \mathrm{g} / \mathrm{kg} & 1.95 & \text { EPA8080 } \\ \mu \mathrm{g} / \mathrm{kg} & 3.9 & \text { EPA8080 } \\ \mu \mathrm{g} / \mathrm{kg} & 1.95 & \text { EPA8080 } \\ \mu \mathrm{g} / \mathrm{kg} & 3.9 & \text { EPA8080 } \\ \mu \mathrm{g} / \mathrm{kg} & 1.95 & \text { EPA8080 } \\ \mu \mathrm{g} / \mathrm{kg} & 3.9 & \text { EPA8080 } \\ \mu \mathrm{g} / \mathrm{kg} & 1.95 & \text { EPA8080 } \\ \mu \mathrm{g} / \mathrm{kg} & 3.9 & \text { EPA8080 } \\ \mu \mathrm{g} / \mathrm{kg} & 1.95 & \text { EPA8080 } \\ \mu \mathrm{g} / \mathrm{kg} & 1.95 & \text { EPA8080 } \\ \mu \mathrm{g} / \mathrm{kg} & 0.974 & \text { EPA8080 } \\ \mu \mathrm{g} / \mathrm{kg} & 19.5 & \text { EPA8080 } \\ \mu \mathrm{g} / \mathrm{kg} & 4.87 & \text { EPA8080 } \\ \mu \mathrm{g} / \mathrm{kg} & 4.87 & \text { EPA8080 } \\ \mu \mathrm{g} / \mathrm{kg} & 4.87 & \text { EPA8080 } \\ \mu \mathrm{g} / \mathrm{kg} & 4.87 & \text { EPA8080 } \\ \mu \mathrm{g} / \mathrm{kg} & 4.87 & \text { EPA8080 } \\ \mu \mathrm{g} / \mathrm{kg} & 4.87 & \text { EPA8080 } \\ \mu \mathrm{g} / \mathrm{kg} & 4.87 & \text { EPA8080 } \\ \mu \mathrm{g} / \mathrm{kg} & 39 & \text { EPA8080 }\end{array}$

UI

UI

UI

UI

UI

UI

UI

UI

UI

UI

UI

$\mathrm{V}$

UI
UI

$1.05 \mathrm{E}+00 \pm 6.36 \mathrm{E}-02 \mathrm{pCi} / \mathrm{g}$ $-6.21 \mathrm{E}-04 \pm 8.30 \mathrm{E}-03 \mathrm{pCi} / \mathrm{g}$ $4.38 \mathrm{E}-03 \pm 1.76 \mathrm{E}-02 \mathrm{pCi} / \mathrm{g}$ $6.99 \mathrm{E}-03 \pm 8.17 \mathrm{E}-03 \mathrm{pCi} / \mathrm{g}$ $-2.20 \mathrm{E}-02 \pm 4.67 \mathrm{E}-02 \mathrm{pCi} / \mathrm{g}$ $-2.66 \mathrm{E}-03 \pm 6.50 \mathrm{E}-03 \mathrm{pCi} / \mathrm{g}$ 3.04E-03 \pm 7.21E-03 pCi/g 2.21E-03 $45.91 \mathrm{E}-03 \mathrm{pCj} / \mathrm{g}$ $-5.61 \mathrm{E}-03 \pm 7.31 \mathrm{E}-03 \mathrm{pCi} / \mathrm{g}$ $-2.25 \mathrm{E}-03 \pm 5.42 \mathrm{E}-03 \mathrm{pCi} / \mathrm{g}$ $-1.76 \mathrm{E}-02 \pm 1.83 \mathrm{E}-02 \mathrm{pCi} / \mathrm{g}$ 4.11E-02 $\pm 5.59 \mathrm{E}-02 \mathrm{pCi} / \mathrm{g}$ 7.97E-02+4.82E-02 pCi/g $3.08 \mathrm{E}+01 \pm 4.46 \mathrm{E}+00 \mathrm{pCi} / \mathrm{g}$ $1.12 \mathrm{E}+00 \pm 2.85 \mathrm{E}-02 \mathrm{pCi} / \mathrm{g}$ 2.33E-02 $1.01 \mathrm{E}-02 \mathrm{pCi} / \mathrm{g}$ $-6.27 \mathrm{E}-02 \pm 4.13 \mathrm{E}-02 \mathrm{pCi} / \mathrm{g}$ $1.17 \mathrm{E}+01 \pm 2.03 \mathrm{E}+00 \mathrm{pCi} / \mathrm{g}$ $1.60 \mathrm{E}-03 \pm 8.13 \mathrm{E}-03 \mathrm{pCi} / \mathrm{g}$ $5.51 \mathrm{E}-05 \pm 3.80 \mathrm{E}-03 \mathrm{pCi} / \mathrm{g}$
3.18E-02 EPIA-013B 1.44E-02 EPIA-013B 2.92E-02 EPLA-013B

1.26E-02 EPIA-013B

7.98E-02 EPIA-013B

9.76E-03 EPIA-013B

1.13E-02 EPIA-013B

1.03E-02 EPIA-013B

1.21E-02 EPIA-013B

9.46E-03 EPIA-013B

2.96E-02 EPIA-013B

8.98E-02 EPIA-013B

4.19E-02 EPIA-013B

$1.81 \mathrm{E}+00$ EPIA-001B

1.78E-02 EPIA-013B

1.00E-02 EPIA-013B

6.95E-02 EPIA-013B

2.42E+00 EPIA-001B

1.67E-02 EPLA-012B

1.04E-02 EPIA-012B 
SURVEY ID: PPSC 0903 (continued)

Constituent

Radionuclides

Potassium-40

Promethium-144

Promethium-146

Ruthenium-106

Sodium-22

Strontium-90

Thorium-234

Tin-113

Yttrium-88

Zinc-65

Zirconium-95
Sample ID: 104637
RQ AQ B Result
Unit
D. Limit Method

8.57E-02 EPIA-013B

1.01E-02 EPIA-013B

1.38E-02 EPIA-013B

9.60E-02 EPIA-013B

8.93E-03 EPIA-013B

6.47E-02 EPIA-004

5.30E-01 EPIA-013B

1.57E-02 EPIA-013B

1.20E-02 EPIA-013B

2.21E-02 EPIA-013B

2.30E-02 EPIA-013B

\section{SURVEY ID: PPSC 0903A}

Sample ID: 104640

Survey location: $45224 \mathrm{E} 3680779 \mathrm{~N}$ (SRS coordinates)

Depth of core interval: 2.00 to $4.00 \mathrm{ft}$

Sample type: Normal

Percent solids: $\mathbf{8 5 . 0 0}$

Constituent

RQ AQ B Result

Unit

D. Limit Method

Physical Parameters and Specified Analyses

Cation exchange capacity

Chloride

Cyanide

Nitrate-nitrite as nitrogen

Orthophosphate

Phenols

Sulfide

Total organic carbon

Total organic nitrogen

Total phosphates (as P)

Metals (total recoverable)

$\begin{array}{lll} & & 68.2 \\ \text { J } & E & 1.41 \\ U & & 1.18 \\ U & & \mathbf{0 . 8 9 3} \\ \text { U } & & 11.5 \\ \text { UJ } & \text { C } & 350 \\ \text { UJ } & \text { C } & 294 \\ & & 896 \\ \text { C } & & 86.8 \\ \text { J } & \text { E } & 254\end{array}$

V $\quad 3880$

$\begin{array}{ll}\mathrm{U} & \mathbf{5 . 7 7}\end{array}$

U $\quad 17.3$

28.2

$\begin{array}{lll}\mathrm{J} & \mathrm{E} & 0.165\end{array}$

$\begin{array}{lll}\text { U } & \mathrm{L} & 0.288\end{array}$

$\begin{array}{ll}\mathrm{V} & 40.2\end{array}$

11.7

$\mathrm{U} \quad 0.577$

2.34

J VC 3240

J $\quad \mathrm{E} \quad 2.36$

$\mathrm{V} \quad 93.2$

2.96

$\begin{array}{lll}J & \mathrm{EV} & 0.0318\end{array}$

$\begin{array}{lll}\mathrm{J} & \mathrm{E} & 1.27\end{array}$

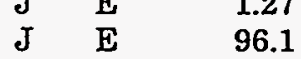

$\begin{array}{lll}\mathrm{meq} / 100 \mathrm{~g} & 5 & \text { EPA9081 } \\ \mathrm{mg} / \mathrm{kg} & 2.92 & \text { EPA300.0 } \\ \mathrm{mg} / \mathrm{kg} & 1.18 & \text { EPA335.3 } \\ \mathrm{mg} / \mathrm{kg} & 0.893 & \text { EPA353.1 } \\ \mathrm{mg} / \mathrm{kg} & 11.5 & \text { EPA365.2 } \\ \mu \mathrm{g} / \mathrm{kg} & 350 & \text { EPA420.2 } \\ \mathrm{mg} / \mathrm{kg} & 294 & \text { EPA376.2 } \\ \mathrm{mg} / \mathrm{kg} & 118 & \text { EPA415.1 } \\ \mathrm{mg} / \mathrm{kg} & 0.235 & \text { EPA351.2 } \\ \mathrm{mg} / \mathrm{kg} & 466 & \text { EPA365.4 }\end{array}$

$\begin{array}{lll}\mathrm{mg} / \mathrm{kg} & 14.4 & \text { EPA6010A } \\ \mathrm{mg} / \mathrm{kg} & 5.77 & \text { EPA6010A } \\ \mathrm{mg} / \mathrm{kg} & 17.3 & \text { EPA6010A } \\ \mathrm{mg} / \mathrm{kg} & 1.44 & \text { EPA6010A } \\ \mathrm{mg} / \mathrm{kg} & 0.577 & \text { EPA6010A } \\ \mathrm{mg} / \mathrm{kg} & 0.288 & \text { EPA6010A } \\ \mathrm{mg} / \mathrm{kg} & 14.4 & \text { EPA6010A } \\ \mathrm{mg} / \mathrm{kg} & 1.44 & \text { EPA6010A } \\ \mathrm{mg} / \mathrm{kg} & 0.577 & \text { EPA6010A } \\ \mathrm{mg} / \mathrm{kg} & 1.44 & \text { EPA6010A } \\ \mathrm{mg} / \mathrm{kg} & 5.77 & \text { EPA6010A } \\ \mathrm{mg} / \mathrm{kg} & 5.77 & \text { EPA6010A } \\ \mathrm{mg} / \mathrm{kg} & 5.77 & \text { EPA6010A } \\ \mathrm{mg} / \mathrm{kg} & 0.577 & \text { EPA6010A } \\ \mathrm{mg} / \mathrm{kg} & .0 .0369 & \text { EPA7471 } \\ \mathrm{mg} / \mathrm{kg} & 1.44 & \text { EPA6010A } \\ \mathrm{mg} / \mathrm{kg} & 115 & \text { EPA6010A } \\ & & \end{array}$


SURVEY ID: PPSC 0903A (continued)

Constituent

RQ AQ B Result

Sample ID: 104640

Metals (total recoverable)
Unit

D. Limit Method

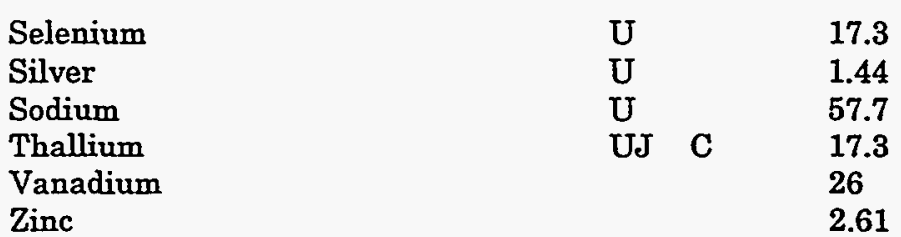

$B / N / A$ Extractables (including $P A H$ and phenols)

\begin{tabular}{|c|c|c|}
\hline U & & 39.2 \\
\hline$U$ & & 38.7 \\
\hline $\mathrm{U}$ & & 39.2 \\
\hline$U$ & & 38.7 \\
\hline $\mathrm{U}$ & & 39.2 \\
\hline$U$ & & 38.7 \\
\hline $\mathrm{U}$ & & 3.92 \\
\hline $\mathrm{U}$ & & 3.87 \\
\hline$U$ & & 3.92 \\
\hline$U$ & & 3.87 \\
\hline$U$ & & 3.92 \\
\hline U & & 3.87 \\
\hline$U$ & & 39.2 \\
\hline U & & 38.7 \\
\hline U & & 39.2 \\
\hline U & & 38.7 \\
\hline U & & 3.92 \\
\hline $\mathrm{U}$ & & 3.87 \\
\hline U & & 39.2 \\
\hline U & & 38.7 \\
\hline $\mathrm{U}$ & & 39.2 \\
\hline $\mathrm{U}$ & & 38.7 \\
\hline $\mathrm{U}$ & & 39.2 \\
\hline U & & 38.7 \\
\hline$U$ & & 39.2 \\
\hline$U$ & & 38.7 \\
\hline$U$ & & 39.2 \\
\hline & $\mathrm{E}$ & 22.1 \\
\hline $\mathrm{U}$ & & 39.2 \\
\hline U & & 38.7 \\
\hline $\mathrm{U}$ & & 39.2 \\
\hline $\mathrm{U}$ & & 38.7 \\
\hline U & & 39.2 \\
\hline U & & 38.7 \\
\hline $\mathrm{U}$ & & 39.2 \\
\hline U & & 38.7 \\
\hline U & & 39.2 \\
\hline $\mathrm{U}$ & & 38.7 \\
\hline U & & 39.2 \\
\hline U & & 38.7 \\
\hline U & & 39.2 \\
\hline U & & 38.7 \\
\hline $\mathrm{U}$ & & 3.92 \\
\hline U & & 3.87 \\
\hline $\mathrm{U}$ & & 392 \\
\hline$U$ & & 387 \\
\hline
\end{tabular}

39.2

39.2

38.7

38.7

3.87

3.92

3.92

3.87

39.2

39.2

3.92

3.87

38.2

39.2

39.2

38.7

38.7

22.1

38.7

38.7

39.2

39.2

38.7

39.2

39.2

38.7

38.7

3.92

392

387

$\begin{array}{lll}\mathrm{mg} / \mathrm{kg} & 17.3 & \text { EPA6010A } \\ \mathrm{mg} / \mathrm{kg} & 1.44 & \text { EPA6010A } \\ \mathrm{mg} / \mathrm{kg} & 57.7 & \text { EPA6010A } \\ \mathrm{mg} / \mathrm{kg} & 17.3 & \text { EPA6010A } \\ \mathrm{mg} / \mathrm{kg} & 0.577 & \text { EPA6010A } \\ \mathrm{mg} / \mathrm{kg} & 1.15 & \text { EPA6010A }\end{array}$

$\begin{array}{lll}\mu \mathrm{g} / \mathrm{kg} & 39.2 & \text { EPA8270 } \\ \mu \mathrm{g} / \mathrm{kg} & 38.7 & \text { EPA8270 } \\ \mu \mathrm{g} / \mathrm{kg} & 39.2 & \text { EPA8270 } \\ \mu \mathrm{g} / \mathrm{kg} & 38.7 & \text { EPA8270 } \\ \mu \mathrm{g} / \mathrm{kg} & 39.2 & \text { EPA8270 } \\ \mu \mathrm{g} / \mathrm{kg} & 38.7 & \text { EPA8270 } \\ \mu \mathrm{g} / \mathrm{kg} & 3.92 & \text { EPA8270 } \\ \mu \mathrm{g} / \mathrm{kg} & 3.87 & \text { EPA8270 } \\ \mu \mathrm{g} / \mathrm{kg} & 3.92 & \text { EPA8270 } \\ \mu \mathrm{g} / \mathrm{kg} & 3.87 & \text { EPA8270 } \\ \mu \mathrm{g} / \mathrm{kg} & 3.92 & \text { EPA8270 } \\ \mu \mathrm{g} / \mathrm{kg} & 3.87 & \text { EPA8270 } \\ \mu \mathrm{g} / \mathrm{kg} & 39.2 & \text { EPA8270 } \\ \mu \mathrm{g} / \mathrm{kg} & 38.7 & \text { EPA8270 } \\ \mu \mathrm{g} / \mathrm{kg} & 39.2 & \text { EPA8270 } \\ \mu \mathrm{g} / \mathrm{kg} & 38.7 & \text { EPA8270 } \\ \mu \mathrm{g} / \mathrm{kg} & 3.92 & \text { EPA8270 } \\ \mu \mathrm{g} / \mathrm{kg} & 3.87 & \text { EPA8270 } \\ \mu \mathrm{g} / \mathrm{kg} & 39.2 & \text { EPA8270 } \\ \mu \mathrm{g} / \mathrm{kg} & 38.7 & \text { EPA8270 } \\ \mu \mathrm{g} / \mathrm{kg} & 39.2 & \text { EPA8270 } \\ \mu \mathrm{g} / \mathrm{kg} & 38.7 & \text { EPA8270 } \\ \mu \mathrm{g} / \mathrm{kg} & 39.2 & \text { EPA8270 } \\ \mu \mathrm{g} / \mathrm{kg} & 38.7 & \text { EPA8270 } \\ \mu \mathrm{g} / \mathrm{kg} & 39.2 & \text { EPA8270 } \\ \mu \mathrm{g} / \mathrm{kg} & 38.7 & \text { EPA8270 } \\ \mu \mathrm{g} / \mathrm{kg} & 39.2 & \text { EPA8270 } \\ \mu \mathrm{g} / \mathrm{kg} & 38.7 & \text { EPA8270 } \\ \mu \mathrm{g} / \mathrm{kg} & 39.2 & \text { EPA8270 } \\ \mu \mathrm{g} / \mathrm{kg} & 38.7 & \text { EPA8270 } \\ \mu \mathrm{g} / \mathrm{kg} & 39.2 & \text { EPA8270 } \\ \mu \mathrm{g} / \mathrm{kg} & 38.7 & \text { EPA8270 } \\ \mu \mathrm{g} / \mathrm{kg} & 39.2 & \text { EPA8270 } \\ \mu \mathrm{g} / \mathrm{kg} & 38.7 & \text { EPA8270 } \\ \mu \mathrm{g} / \mathrm{kg} & 39.2 & \text { EPA8270 } \\ \mu \mathrm{g} / \mathrm{kg} & 38.7 & \text { EPA8270 } \\ \mu \mathrm{g} / \mathrm{kg} & 39.2 & \text { EPA8270 } \\ \mu \mathrm{g} / \mathrm{kg} & 38.7 & \text { EPA8270 } \\ \mu \mathrm{g} / \mathrm{kg} & 39.2 & \text { EPA8270 } \\ \mu \mathrm{g} / \mathrm{kg} & 38.7 & \text { EPA8270 } \\ \mu \mathrm{g} / \mathrm{kg} & 39.2 & \text { EPA8270 } \\ \mu \mathrm{g} / \mathrm{kg} & 38.7 & \text { EPA8270 } \\ \mu \mathrm{g} / \mathrm{kg} & 3.92 & \text { EPA8270 } \\ \mu \mathrm{g} / \mathrm{kg} & 3.87 & \text { EPA8270 } \\ \mu \mathrm{g} / \mathrm{kg} & 392 & \text { EPA8270 } \\ \mu \mathrm{g} / \mathrm{kg} & 387 & \text { EPA8270 } \\ & & \end{array}$

Acenaphthene

Acenaphthene

Acenaphthylene

Acenaphthylene

Anthracene

Benzo[a]anthracene

Benzo[b]fluoranthene

Benzoic acid

Benzo[g,h,i]perylene

Benzo[g, $h, i]$ perylene

Benzo[a]pyrene

Benzo[a]pyrene

Benzyl alcohol

Benzyl alcohol

Bis(2-chloroethoxy) methane

Bis(2-chloroethoxy) methane

Bis(2-chloroethyl) ether

Bis(2-chloroethyl) ether

Bis(2-chloroisopropyl) ether

Bis(2-ethylhexyl) phthalate

(2) phthalate

4-Bromophenyl phenyl ether

4-Bromophenyl phenyl ether

Butylbenzyl phthalate

phalate

4-Chloroaniline

oroaniline

4-Chloro-m-cresol

2-Chloronaphthalene

2-Chloronaphthalene

2-Chlorophenol

2-Chlorophenol

4-Chlorophenyl phenyl ether

4-Chlorophenyl phenyl ether

Chrysene

Chrysene

$\mathrm{m} / \mathrm{p}$-Cresol 
$B / N / A$ Extractables (including PAH and phenols)

o-Cresol (2-Methylphenol)

o-Cresol (2-Methylphenol)

Dibenz $[a, h]$ anthracene

Dibenz $[a, h]$ anthracene

Dibenzofuran

Dibenzofuran

Di-n-butyl phthalate

Di-n-butyl phthalate

1,2-Dichlorobenzene

1,2-Dichlorobenzene

1,3-Dichlorobenzene

1,3-Dichlorobenzene

1,4-Dichlorobenzene

1,4-Dichlorobenzene

3,3'-Dichlorobenzidine

3,3'-Dichlorobenzidine

2,4-Dichlorophenol

2,4-Dichlorophenol

Diethyl phthalate

Diethyl phthalate

2,4-Dimethyl phenol

2,4-Dimethyl phenol

Dimethyl phthalate

Dimethyl phthalate

2,4-Dinitrophenol

2,4-Dinitrophenol

2,4-Dinitrotoluene

2,4-Dinitrotoluene

2,6-Dinitrotoluene

2,6-Dinitrotoluene

Di-n-octyl phthalate

Di-n-octyl phthalate

Fluoranthene

Fluoranthene

Fluorene

Fluorene

Hexachlorobenzene

Hexachlorobenzene

Hexachlorobutadiene

Hexachlorobutadiene

Hexachlorocyclopentadiene

Hexachlorocyclopentadiene

Hexachloroethane

Hexachloroethane

Indeno $[1,2,3-c, d]$ pyrene

Indeno $[1,2,3-c, d]$ pyrene

Isophorone

Isophorone

2-Methyl-4,6-dinitrophenol

2-Methyl-4,6-dinitrophenol

2-Methylnaphthalene

2-Methylnaphthalene

Naphthalene

Naphthalene

m-Nitroaniline
U

U

$\mathrm{U}$

U

U

U

U

U

U

U

U

U

U

U

U

U

U

U

$U$

U

$\mathrm{U}$

U

U

U

U

U

U

U

$\mathrm{U}$

$\mathrm{U}$

U

U

U

U

U

U

U

U

U

U

U

U

U

U

U

U

U

U

U

U

U
39.2

38.7

3.92

3.87

39.2

38.7

25.1

23.2

39.2

38.7

39.2

38.7

39.2

38.7

39.2

38.7

39.2

38.7

20.7

38.7

39.2

38.7

39.2

38.7

392

387

3.92

3.87

3.92

3.87

39.2

38.7

39.2

38.7

39.2

38.7

3.92

3.87

39.2

38.7

39.2

38.7

3.92

3.87

3.92

3.87

39.2

38.7

392

387

39.2

38.7

39.2

38.7

39.2 $\begin{array}{lll}\mu \mathrm{g} / \mathrm{kg} & 39.2 & \text { EPA8270 } \\ \mu \mathrm{g} / \mathrm{kg} & 38.7 & \text { EPA8270 }\end{array}$

$\mu \mathrm{g} / \mathrm{kg} \quad 3.92 \quad$ EPA8270

$\mu \mathrm{g} / \mathrm{kg} \quad 3.87 \quad$ EPA8270

$\mu \mathrm{g} / \mathrm{kg} \quad 39.2 \quad$ EPA8270

$\mu \mathrm{g} / \mathrm{kg} \quad 38.7 \quad$ EPA8270

$\mu \mathrm{g} / \mathrm{kg} \quad 39.2 \quad$ EPA8270

$\mu \mathrm{g} / \mathrm{kg} \quad 38.7 \quad$ EPA8270

$\mu \mathrm{g} / \mathrm{kg} \quad 39.2 \quad$ EPA8270

$\mu \mathrm{g} / \mathrm{kg} \quad 38.7 \quad$ EPA8270

$\mu \mathrm{g} / \mathrm{kg} \quad 39.2 \quad$ EPA8270

$\mu \mathrm{g} / \mathrm{kg} \quad 38.7 \quad$ EPA8270

$\mu \mathrm{g} / \mathrm{kg} \quad 39.2 \quad$ EPA8270

$\mu \mathrm{g} / \mathrm{kg} \quad 38.7 \quad$ EPA8270

$\mu \mathrm{g} / \mathrm{kg} \quad 39.2 \quad$ EPA8270

$\mu \mathrm{g} / \mathrm{kg} \quad 38.7 \quad$ EPA8270

$\mu \mathrm{g} / \mathrm{kg} \quad 39.2 \quad$ EPA8270

$\mu \mathrm{g} / \mathrm{kg} \quad 38.7 \quad$ EPA8270

$\mu \mathrm{g} / \mathrm{kg} \quad 39.2 \quad$ EPA8270

$\mu \mathrm{g} / \mathrm{kg} \quad 38.7 \quad$ EPA8270

$\mu \mathrm{g} / \mathrm{kg} \quad 39.2 \quad$ EPA8270

$\mu \mathrm{g} / \mathrm{kg} \quad 38.7 \quad$ EPA8270

$\mu \mathrm{g} / \mathrm{kg} \quad 39.2 \quad$ EPA8270

$\mu \mathrm{g} / \mathrm{kg} \quad 38.7 \quad$ EPA8270

$\mu \mathrm{g} / \mathrm{kg} \quad 392 \quad$ EPA8270

$\mu \mathrm{g} / \mathrm{kg} \quad 387 \quad$ EPA8270

$\mu \mathrm{g} / \mathrm{kg} \quad 3.92 \quad$ EPA8270

$\mu \mathrm{g} / \mathrm{kg} \quad 3.87 \quad$ EPA8270

$\mu \mathrm{g} / \mathrm{kg} \quad 3.92 \quad$ EPA8270

$\mu \mathrm{g} / \mathrm{kg} \quad 3.87 \quad$ EPA8270

$\mu \mathrm{g} / \mathrm{kg} \quad 39.2 \quad$ EPA8270

$\mu \mathrm{g} / \mathrm{kg} \quad 38.7 \quad$ EPA8270

$\mu \mathrm{g} / \mathrm{kg} \quad 39.2 \quad$ EPA8270

$\mu \mathrm{g} / \mathrm{kg} \quad 38.7 \quad$ EPA8270

$\mu \mathrm{g} / \mathrm{kg} \quad 39.2 \quad$ EPA8270

$\mu \mathrm{g} / \mathrm{kg} \quad 38.7 \quad$ EPA8270

$\mu \mathrm{g} / \mathrm{kg} \quad 3.92 \quad$ EPA8270

$\mu \mathrm{g} / \mathrm{kg} \quad 3.87 \quad$ EPA8270

$\mu \mathrm{g} / \mathrm{kg} \quad 39.2 \quad$ EPA8270

$\mu \mathrm{g} / \mathrm{kg} \quad 38.7 \quad$ EPA8270

$\mu \mathrm{g} / \mathrm{kg} \quad 39.2 \quad$ EPA8270

$\mu \mathrm{g} / \mathrm{kg} \quad 38.7 \quad$ EPA8270

$\mu \mathrm{g} / \mathrm{kg} \quad 3.92 \quad$ EPA8270

$\mu \mathrm{g} / \mathrm{kg} \quad 3.87 \quad$ EPA8270

$\mu \mathrm{g} / \mathrm{kg} \quad 3.92 \quad$ EPA8270

$\mu \mathrm{g} / \mathrm{kg} \quad 3.87 \quad$ EPA8270

$\mu g / \mathrm{kg} \quad 39.2 \quad$ EPA8270

$\mu \mathrm{g} / \mathrm{kg} \quad 38.7 \quad$ EPA8270

$\mu \mathrm{g} / \mathrm{kg} \quad 392 \quad$ EPA8270

$\mu \mathrm{g} / \mathrm{kg} \quad 387 \quad$ EPA8270

$\mu \mathrm{g} / \mathrm{kg} \quad 39.2 \quad$ EPA8270

$\mu \mathrm{g} / \mathrm{kg} \quad \cdot 38.7 \quad$ EPA8270

$\mu \mathrm{g} / \mathrm{kg} \quad 39.2 \quad$ EPA8270

$\mu \mathrm{g} / \mathrm{kg} \quad 38.7 \quad$ EPA8270

$\mu \mathrm{g} / \mathrm{kg} \quad 39.2 \quad$ EPA8270 
SURVEY ID: PPSC 0903A (continued)

Constituent

RQ AQ B Result

$B / N / A$ Extractables (including PAH and phenols)

m-Nitroaniline

o-Nitroaniline

o-Nitroaniline

p-Nitroaniline

p-Nitroaniline

Nitrobenzene

Nitrobenzene

2-Nitrophenol

2-Nitrophenol

4-Nitrophenol

4-Nitrophenol

$\mathrm{N}$-Nitrosodiphenylamine

N-Nitrosodiphenylamine

N-Nitrosodi-n-propylamine

N-Nitrosodi-n-propylamine

Pentachlorophenol

Pentachlorophenol

Phenanthrene

Phenanthrene

Phenol

Phenol

Pyrene

Pyrene

1,2,4-Trichlorobenzene

2,4,5-Trichlorophenol

2,4,5-Trichlorophenol

2,4,6-Trichlorophenol

2,4,6-Trichlorophenol
1,2,4-Trichlorobenzene

U

$\quad 38.7$

U $\quad 38.7$

U

U

U

U

U

U

U

U

$\mathrm{U}$

U

U

$\mathrm{U}$

U

$\mathrm{U}$

U

$\mathrm{U}$

U

U

$\mathrm{U}$

U

U

$\mathrm{U}$

U

U

U

Volatile Organic Compounds

Acetone

Benzene

Bromodichloromethane

Bromoform

Bromomethane (Methyl bromide)

Carbon disulfide

Carbon tetrachloride

Chlorobenzene

Chloroethane

Chloroethene (Vinyl chloride)

Chloroform

Chloromethane (Methyl chloride)

Dibromochloromethane

1,1-Dichloroethane

1,2-Dichloroethane

1,1-Dichloroethylene

1,2-Dichloroethylene

Dichloromethane (Methylene chloride)

1,2-Dichloropropane

cis-1,3-Dichloropropene

trans-1,3-Dichloropropene

Ethylbenzene

2-Hexanone

U

U

$8 \quad 55.5$

1.18

V

8.44
Sample ID: 104640

Unit

D. Limit Method

$\begin{array}{llll}38.7 & \mu \mathrm{g} / \mathrm{kg} & 38.7 & \text { EPA8270 } \\ 39.2 & \mu \mathrm{g} / \mathrm{kg} & 39.2 & \text { EPA8270 } \\ 38.7 & \mu \mathrm{g} / \mathrm{kg} & 38.7 & \text { EPA8270 } \\ 39.2 & \mu \mathrm{g} / \mathrm{kg} & 39.2 & \text { EPA8270 } \\ 38.7 & \mu \mathrm{g} / \mathrm{kg} & 38.7 & \text { EPA8270 } \\ 39.2 & \mu \mathrm{g} / \mathrm{kg} & 39.2 & \text { EPA8270 } \\ 38.7 & \mu \mathrm{g} / \mathrm{kg} & 38.7 & \text { EPA8270 } \\ 39.2 & \mu \mathrm{g} / \mathrm{kg} & 39.2 & \text { EPA8270 } \\ 38.7 & \mu \mathrm{g} / \mathrm{kg} & 38.7 & \text { EPA8270 } \\ 39.2 & \mu \mathrm{g} / \mathrm{kg} & 39.2 & \text { EPA8270 } \\ 38.7 & \mu \mathrm{g} / \mathrm{kg} & 38.7 & \text { EPA8270 } \\ 39.2 & \mu \mathrm{g} / \mathrm{kg} & 39.2 & \text { EPA8270 } \\ 38.7 & \mu \mathrm{g} / \mathrm{kg} & 38.7 & \text { EPA8270 } \\ 39.2 & \mu \mathrm{gg} / \mathrm{kg} & 39.2 & \text { EPA8270 } \\ 38.7 & \mu \mathrm{g} / \mathrm{kg} & 38.7 & \text { EPA8270 } \\ 39.2 & \mu \mathrm{g} / \mathrm{kg} & 39.2 & \text { EPA8270 } \\ 38.7 & \mu \mathrm{g} / \mathrm{kg} & 38.7 & \text { EPA8270 } \\ 39.2 & \mu \mathrm{g} / \mathrm{kg} & 39.2 & \text { EPA8270 } \\ 38.7 & \mu \mathrm{g} / \mathrm{kg} & 38.7 & \text { EPA8270 } \\ 39.2 & \mu \mathrm{gg} / \mathrm{kg} & 39.2 & \text { EPA8270 } \\ 38.7 & \mu \mathrm{gg} / \mathrm{kg} & 38.7 & \text { EPA8270 } \\ 39.2 & \mu \mathrm{g} / \mathrm{kg} & 39.2 & \text { EPA8270 } \\ 38.7 & \mu \mathrm{gg} / \mathrm{kg} & 38.7 & \text { EPA8270 } \\ 3.92 & \mu \mathrm{g} / \mathrm{kg} & 3.92 & \text { EPA8270 } \\ 3.87 & \mu \mathrm{g} / \mathrm{kg} & 3.87 & \text { EPA8270 } \\ 39.2 & \mu \mathrm{g} / \mathrm{kg} & 39.2 & \text { EPA8270 } \\ 38.7 & \mu \mathrm{gg} / \mathrm{kg} & 38.7 & \text { EPA8270 } \\ 39.2 & \mu \mathrm{gg} / \mathrm{kg} & 39.2 & \text { EPA8270 } \\ 38.7 & \mu \mathrm{g} / \mathrm{kg} & 38.7 & \text { EPA8270 }\end{array}$

0.588

0.0588

0.0588

0.118

0.0588

0.0588

0.118

0.118

0.0588

0.118

0.0588

0.0588

0.0588

0.0588

0.118

0.0588

0.0588

0.0588

0.0588

$\mathrm{V} \quad 0.624$ $\mu \mathrm{g} / \mathrm{kg}$

$\mu \mathrm{g} / \mathrm{kg}$

$\mu \mathrm{g} / \mathrm{kg}$

$\mu \mathrm{g} / \mathrm{kg}$

$\mu \mathrm{g} / \mathrm{kg}$

$\mu \mathrm{g} / \mathrm{kg}$

$\mu \mathrm{g} / \mathrm{kg}$

$\mu \mathrm{g} / \mathrm{kg}$

$\mu \mathrm{g} / \mathrm{kg}$

$\mu \mathrm{g} / \mathrm{kg}$

$\mu \mathrm{g} / \mathrm{kg}$

$\mu \mathrm{g} / \mathrm{kg}$

$\mu \mathrm{g} / \mathrm{kg}$

$\mu \mathrm{g} / \mathrm{kg}$

$\mu \mathrm{g} / \mathrm{kg}$

$\mu \mathrm{g} / \mathrm{kg}$

$\mu \mathrm{g} / \mathrm{kg}$

$\mu \mathrm{g} / \mathrm{kg}$

$\mu \mathrm{g} / \mathrm{kg}$

$\mu \mathrm{g} / \mathrm{kg}$

$\mu \mathrm{g} / \mathrm{kg}$

$\mu \mathrm{g} / \mathrm{kg}$

$\mu \mathrm{g} / \mathrm{kg}$
5.88

0.588

0.0588

0.0588

0.118

1.18

0.0588

0.0588

0.118

0.118

0.0588

0.118

0.0588

0.0588

0.0588

0.0588

0.118

0.588

0.0588

.0 .0588

0.0588

0.0588

1.18
EPA8260

EPA8260

EPA8260

EPA8260

EPA8260

EPA8260

EPA8260

EPA8260

EPA8260

EPA8260

EPA8260

EPA8260

EPA8260

EPA8260

EPA8260

EPA8260

EPA8260

EPA8260

EPA8260

EPA8260

EPA8260

EPA8260

EPA8260 
SURVEY ID: PPSC 0903A (continued)

Constituent

Volatile Organic Compounds

Methyl cyclopentane

Methyl ethyl ketone

Methyl isobutyl ketone

Styrene

1,1,2,2-Tetrachloroethane

Tetrachloroethylene

Toluene

1,1,1-Trichloroethane

1,1,2-Trichloroethane

Trichloroethylene

Vinyl acetate

Xylenes

\section{Pesticides/PCBs and Dioxins/Furans}

Aldrin

alpha-Benzene hexachloride

beta-Benzene hexachloride

delta-Benzene hexachloride

alpha-Chlordane

gamma-Chlordane

p,p'-DDD

$\mathrm{p}, \mathrm{p}^{\circ}-\mathrm{DDE}$

p,p'-DDT

Dieldrin

Endosulfan sulfate

Endosulfan I

Endosulfan II

Endrin

Endrin ketone

Heptachlor

Heptachlor epoxide

Lindane

Methoxychlor

PCB 1016

PCB 1221

PCB 1232

PCB 1242

PCB 1248

PCB 1254

PCB 1260

Toxaphene

Radionuclides

Actinium-228

Antimony-124

Antimony-125

Barium-133

Cerium-144

Cesium-134

Cesium-137

Cobalt-57

Cobalt-58

Cobalt. 60
Sample ID: 104640

RQ AQ B Result Unit D. Limit Method

$\begin{array}{llllll}\mathrm{J} & \mathrm{N} & 2.19 & \mu \mathrm{g} / \mathrm{kg} & & \text { EPA8260 } \\ \mathrm{U} & \mathrm{V} & 3.25 & \mu \mathrm{g} / \mathrm{kg} & 1.18 & \text { EPA8260 } \\ \mathrm{U} & & 1.18 & \mu \mathrm{g} / \mathrm{kg} & 1.18 & \text { EPA8260 } \\ \mathrm{U} & & 0.0588 & \mu \mathrm{g} / \mathrm{kg} & 0.0588 & \text { EPA8260 } \\ \mathrm{U} & & 0.0588 & \mu \mathrm{g} / \mathrm{kg} & 0.0588 & \text { EPA8260 } \\ \mathrm{U} & & 0.0588 & \mu \mathrm{g} / \mathrm{kg} & 0.0588 & \text { EPA8260 } \\ \mathrm{U} & \mathrm{V} & 0.118 & \mu \mathrm{g} / \mathrm{kg} & 0.588 & \text { EPA8260 } \\ \mathrm{U} & & 0.0588 & \mu \mathrm{g} / \mathrm{kg} & 0.0588 & \text { EPA8260 } \\ \mathrm{U} & & 0.0588 & \mu \mathrm{g} / \mathrm{kg} & 0.0588 & \text { EPA8260 } \\ \mathrm{U} & & 0.0588 & \mu \mathrm{g} / \mathrm{kg} & 0.0588 & \text { EPA8260 } \\ \mathrm{U} & & 1.18 & \mu \mathrm{g} / \mathrm{kg} & 1.18 & \text { EPA8260 } \\ \mathrm{U} & & 0.176 & \mu \mathrm{g} / \mathrm{kg} & 0.176 & \text { EPA8260 }\end{array}$

$\begin{array}{llll}4.9 & \mu \mathrm{g} / \mathrm{kg} & 4.9 & \text { EPA8080 } \\ 4.9 & \mu \mathrm{g} / \mathrm{kg} & 4.9 & \text { EPA8080 } \\ 9.8 & \mu \mathrm{g} / \mathrm{kg} & 9.8 & \text { EPA8080 } \\ 9.8 & \mu \mathrm{g} / \mathrm{kg} & 9.8 & \text { EPA8080 } \\ 9.8 & \mu \mathrm{g} / \mathrm{kg} & 9.8 & \text { EPA8080 } \\ 9.8 & \mu \mathrm{g} / \mathrm{kg} & 9.8 & \text { EPA8080 } \\ 19.6 & \mu \mathrm{g} / \mathrm{kg} & 19.6 & \text { EPA8080 } \\ 9.8 & \mu \mathrm{g} / \mathrm{kg} & 9.8 & \text { EPA8080 } \\ 19.6 & \mu \mathrm{g} / \mathrm{kg} & 19.6 & \text { EPA8080 } \\ 9.8 & \mu \mathrm{g} / \mathrm{kg} & 9.8 & \text { EPA8080 } \\ 19.6 & \mu \mathrm{g} / \mathrm{kg} & 19.6 & \text { EPA8080 } \\ 9.8 & \mu \mathrm{g} / \mathrm{kg} & 9.8 & \text { EPA8080 } \\ 19.6 & \mu \mathrm{g} / \mathrm{kg} & 19.6 & \text { EPA8080 } \\ 9.8 & \mu \mathrm{g} / \mathrm{kg} & 9.8 & \text { EPA8080 } \\ 19.6 & \mu \mathrm{g} / \mathrm{kg} & 19.6 & \text { EPA8080 } \\ 9.8 & \mu \mathrm{g} / \mathrm{kg} & 9.8 & \text { EPA8080 } \\ 9.8 & \mu \mathrm{g} / \mathrm{kg} & 9.8 & \text { EPA8080 } \\ 4.9 & \mu \mathrm{g} / \mathrm{kg} & 4.9 & \text { EPA8080 } \\ 98 & \mu \mathrm{g} / \mathrm{kg} & 98 & \text { EPA8080 } \\ 24.5 & \mu \mathrm{g} / \mathrm{kg} & 24.5 & \text { EPA8080 } \\ 24.5 & \mu \mathrm{g} / \mathrm{kg} & 24.5 & \text { EPA8080 } \\ 24.5 & \mu \mathrm{g} / \mathrm{kg} & 24.5 & \text { EPA8080 } \\ 24.5 & \mu \mathrm{g} / \mathrm{kg} & 24.5 & \text { EPA8080 } \\ 24.5 & \mu \mathrm{g} / \mathrm{kg} & 24.5 & \text { EPA8080 } \\ 24.5 & \mu \mathrm{g} / \mathrm{kg} & 24.5 & \text { EPA8080 } \\ 24.5 & \mu \mathrm{g} / \mathrm{kg} & 24.5 & \text { EPA8080 } \\ 196 & \mu \mathrm{g} / \mathrm{kg} & 196 & \text { EPA8080 }\end{array}$

$1.09 \mathrm{E}+00 \pm 6.88 \mathrm{E}-02 \mathrm{pCi} / \mathrm{g}$ $-2.85 \mathrm{E}-03 \pm 8.00 \mathrm{E}-03 \mathrm{pCj} / \mathrm{g}$ $1.33 \mathrm{E}-02 \pm 1.68 \mathrm{E}-02 \mathrm{pCi} / \mathrm{g}$ $1.46 \mathrm{E}-03 \pm 8.15 \mathrm{E}-03 \mathrm{pCi} / \mathrm{g}$ $4.76 \mathrm{E}-02 \pm 5.56 \mathrm{E}-02 \mathrm{pCi} / \mathrm{g}$ $3.59 \mathrm{E}-04 \pm 6.30 \mathrm{E}-03 \mathrm{pCi} / \mathrm{g}$ $2.60 \mathrm{E}-03 \pm 7.05 \mathrm{E}-03 \mathrm{pCi} / \mathrm{g}$ $6.71 \mathrm{E}-04 \pm 5.87 \mathrm{E}-03 \mathrm{pCi} / \mathrm{g}$ $-3.07 \mathrm{E}-03 \pm 7.54 \mathrm{E}-03 \mathrm{pCi} / \mathrm{g}$ $-1.55 \mathrm{E}-03 \pm 5.86 \mathrm{E}-03 \mathrm{pCi} / \mathrm{g}$ $\begin{array}{cc}3.29 E-02 & \text { EPIA-013B } \\ 1.37 E-02 & \text { EPIA-013B } \\ 2.84 E-02 & \text { EPIA-013B } \\ 1.22 E-02 & \text { EPIA-013B } \\ 8.07 E-02 & \text { EPIA-013B } \\ 9.65 E-03 & \text { EPIA-013B } \\ -1.10 E-02 & \text { EPIA-013B } \\ 1.01 E-02 & \text { EPIA-013B } \\ 1.27 E-02 & \text { EPIA-013B } \\ 1.03 E-02 & \text { EPIA-013B }\end{array}$ 
SURVEY ID: PPSC 0903A (continued)

Constituent

Radionuclides

Europium-152

Europium-154

Europium-155

Gross alpha

Lead-212

Manganese-54

Neptunium-239

Nonvolatile beta

Plutonium-238

Plutonium-239/240

Potassium-40

Promethium-144

Promethium-146

Ruthenium-106

Sodium-22

Strontium-90

Thorium-234

Tin-113

Yttrium-88

Zinc-65

Zirconium-95
Sample ID: 104640

RQ AQ B Result Unit D. Limit Method
3.01E-02 EPIA-013B

8.60E-02 EPIA-013B

4.14E-02 EPIA-013B

$1.60 \mathrm{E}+00$ EPIA-001B

1.79E-02 EPIA-013B

9.66E-03 EPIA-013B

7.15E-02 EPIA-013B

2.14E+00 EPIA-001B

1.88E-02 EPIA-012B

3.98E-03 EPIA-012B

8.84E-02 EPIA-013B

1.02E-02 EPIA-013B

1.38E-02 EPIA-013B

9.32E-02 EPIA-013B

1.02E-02 EPIA-013B

6.32E-02 EPIA-004

5.66E-01 EPIA-013B

1.58E-02 EPIA-013B

1.34E-02 EPIA-013B

2.00E-02 EPIA-013B

2.87E-02 EPIA-013B

SURVEY ID: PPSC 1001

Sample ID: 104625

Survey location: $45176 \mathrm{E}$ 3684285N (SRS coordinates)

Depth of core interval: 0.00 to $1.00 \mathrm{ft}$

Sample type: Normal

Sample matrix: Soil

Percent solids: $\mathbf{4 1 . 0 0}$
Constituent
RQ AQ B Result
Unit
D. Limit Method

Physical Parameters and Specified Analyses

Cation exchange capacity
Chloride
Chloride
Cyanide
Cyanide
Nitrate-nitrite as nitrogen
Orthophosphate
Phenols
Sulfide
Total organic carbon
Total organic nitrogen
Total phosphates (as P)

$\begin{array}{llll} & & & 242 \\ & & & 7.8 \\ & & & 9.64 \\ \text { UJ } & \text { I } & \text { L } & 2.41 \\ \text { J } & \text { IE } & \text { L } & 0.601 \\ \text { J } & \text { E } & & 0.674 \\ \text { U } & & & 24.4 \\ \text { J } & \text { EC } & & 223 \\ \text { UJ } & \text { C } & & 610 \\ & & & 76300 \\ \text { C } & & & 466 \\ \text { J } & \text { E } & & 458\end{array}$

$\begin{array}{ll}\mathrm{meq} / 100 \mathrm{~g} & 5 \\ \mathrm{mg} / \mathrm{kg} & 5.77 \\ \mathrm{mg} / \mathrm{kg} & 5.93 \\ \mathrm{mg} / \mathrm{kg} & 2.41 \\ \mathrm{mg} / \mathrm{kg} & 2.37 \\ \mathrm{mg} / \mathrm{kg} & 2.25 \\ \mathrm{mg} / \mathrm{kg} & 24.4 \\ \mu \mathrm{g} / \mathrm{kg} & 729 \\ \mathrm{mg} / \mathrm{kg} & 610 \\ \mathrm{mg} / \mathrm{kg} & 2170 \\ \mathrm{mg} / \mathrm{kg} & 0.488 \\ \mathrm{mg} / \mathrm{kg} & 894\end{array}$

EPA9081

EPA300.0

EPA300.0

EPA335.3

EPA335.3

EPA353.1

EPA365.2

EPA420.2

EPA376.2

EPA415.1

EPA351.2

EPA365.4 
SURVEY ID: PPSC 1001 (continued)

Constituent

Metals (total recoverable)
Sample ID: 104625

RQ AQ B Result Unit D. Limit Method

\begin{tabular}{|c|c|}
\hline \multicolumn{2}{|l|}{ Aluminum } \\
\hline \multicolumn{2}{|l|}{ Aluminum } \\
\hline \multicolumn{2}{|l|}{ Antimony } \\
\hline \multicolumn{2}{|l|}{ Antimony } \\
\hline \multicolumn{2}{|l|}{ Arsenic } \\
\hline \multicolumn{2}{|l|}{ Arsenic } \\
\hline \multicolumn{2}{|l|}{ Barium } \\
\hline \multicolumn{2}{|l|}{ Barium } \\
\hline \multicolumn{2}{|l|}{ Beryllium } \\
\hline \multicolumn{2}{|l|}{ Beryllium } \\
\hline \multicolumn{2}{|l|}{ Cadmium } \\
\hline \multicolumn{2}{|l|}{ Cadmium } \\
\hline \multicolumn{2}{|l|}{ Calcium } \\
\hline \multicolumn{2}{|l|}{ Calcium } \\
\hline \multicolumn{2}{|l|}{ Chromium } \\
\hline \multicolumn{2}{|l|}{ Chromium } \\
\hline \multicolumn{2}{|l|}{ Cobalt } \\
\hline \multicolumn{2}{|l|}{ Cobalt } \\
\hline \multicolumn{2}{|l|}{ Copper } \\
\hline Copper & ' \\
\hline \multicolumn{2}{|l|}{ Iron } \\
\hline \multicolumn{2}{|l|}{ Iron } \\
\hline \multicolumn{2}{|l|}{ Lead } \\
\hline \multicolumn{2}{|l|}{ Lead } \\
\hline \multicolumn{2}{|l|}{ Magnesium } \\
\hline \multicolumn{2}{|l|}{ Magnesium } \\
\hline Manganese & \\
\hline Manganese & \\
\hline Mercury & \\
\hline Mercury & \\
\hline Nickel & \\
\hline Nickel & \\
\hline Potassium & \\
\hline Potassium & \\
\hline Selenium & \\
\hline Selenium & \\
\hline Silver & \\
\hline Silver & \\
\hline Sodium & \\
\hline Sodium & \\
\hline Thallium & \\
\hline Thallium & \\
\hline Vanadium & \\
\hline Vanadium & \\
\hline Zinc & \\
\hline Zinc & \\
\hline
\end{tabular}

Radionuclides

Actinium-228

Antimony-124

Antimony-125

Barium-133

Cerium-144

Cesium-134

\begin{tabular}{|c|c|c|c|c|c|}
\hline & $\mathrm{V}$ & 15900 & $\mathrm{mg} / \mathrm{kg}$ & 30.2 & EPA6010A \\
\hline & $\mathrm{V}$ & 11200 & $\mathrm{mg} / \mathrm{kg}$ & 30.2 & EPA6010A \\
\hline UJ & I. & 12.1 & $\mathrm{mg} / \mathrm{kg}$ & 12.1 & EPA6010A \\
\hline UJ & $\mathrm{L}$ & 12.1 & $\mathrm{mg} / \mathrm{kg}$ & 12.1 & EPA6010A \\
\hline $\mathrm{U}$ & & 36.2 & $\mathrm{mg} / \mathrm{kg}$ & 36.2 & EPA6010A \\
\hline \multirow[t]{4}{*}{$\mathbf{U}$} & & 36.2 & $\mathrm{mg} / \mathrm{kg}$ & 36.2 & EPA6010A \\
\hline & & 69.2 & $\mathrm{mg} / \mathrm{kg}$ & 3.02 & EPA6010A \\
\hline & & 53.2 & $\mathrm{mg} / \mathrm{kg}$ & 3.02 & EPA6010A \\
\hline & & 1.33 & $\mathrm{mg} / \mathrm{kg}$ & 1.21 & EPA6010A \\
\hline$J$ & $\mathbf{E}$ & 1.13 & $\mathrm{mg} / \mathrm{kg}$ & 1.21 & EPA6010A \\
\hline$U$ & & 0.604 & $\mathrm{mg} / \mathrm{kg}$ & 0.604 & EPA6010A \\
\hline \multirow[t]{9}{*}{$\mathbf{U}$} & & 0.604 & $\mathrm{mg} / \mathrm{kg}$ & 0.604 & EPA6010A \\
\hline & & 835 & $\mathrm{mg} / \mathrm{kg}$ & 30.2 & EPA6010A \\
\hline & & 759 & $\mathrm{mg} / \mathrm{kg}$ & 30.2 & EPA6010A \\
\hline & & 25.4 & $\mathrm{mg} / \mathrm{kg}$ & 3.02 & EPA6010A \\
\hline & & 20.5 & $\mathrm{mg} / \mathrm{kg}$ & 3.02 & EPA6010A \\
\hline & & 7.75 & $\mathrm{mg} / \mathrm{kg}$ & 1.21 & EPA6010A \\
\hline & & 6.78 & $\mathrm{mg} / \mathrm{kg}$ & 1.21 & EPA6010A \\
\hline & & 7.03 & $\mathrm{mg} / \mathrm{kg}$ & 3.02 & EPA6010A \\
\hline & & 5.13 & $\mathrm{mg} / \mathrm{kg}$ & 3.02 & EPA6010A \\
\hline $\mathbf{J}$ & $\mathrm{C}$ & 7570 & $\mathrm{mg} / \mathrm{kg}$ & 12.1 & EPA6010A \\
\hline \multirow[t]{2}{*}{$\mathbf{J}$} & $\mathrm{C}$ & 5750 & $\mathrm{mg} / \mathrm{kg}$ & 12.1 & EPA6010A \\
\hline & & 17.9 & $\mathrm{mg} / \mathrm{kg}$ & 12.1 & EPA6010A \\
\hline$J$ & $\mathrm{E}$ & 11 & $\mathrm{mg} / \mathrm{kg}$ & 12.1 & EPA6010A \\
\hline$J$ & IX & 256 & $\mathrm{mg} / \mathrm{kg}$ & 12.1 & EPA6010A \\
\hline \multirow[t]{7}{*}{$\mathrm{J}$} & IX & 171 & $\mathrm{mg} / \mathrm{kg}$ & 12.1 & EPA6010A \\
\hline & & 117 & $\mathrm{mg} / \mathrm{kg}$ & 1.21 & EPA6010A \\
\hline & & 101 & $\mathrm{mg} / \mathrm{kg}$ & 1.21 & EPA6010A \\
\hline & $\mathrm{V}$ & 0.0846 & $\mathrm{mg} / \mathrm{kg}$ & 0.0762 & EPA7471 \\
\hline & $V$ & 0.0838 & $\mathrm{mg} / \mathrm{kg}$ & 0.0752 & EPA7471 \\
\hline & & 7.82 & $\mathrm{mg} / \mathrm{kg}$ & 3.02 & EPA6010A \\
\hline & & 6.21 & $\mathrm{mg} / \mathrm{kg}$ & 3.02 & EPA6010A \\
\hline $\mathbf{J}$ & $\mathrm{E}$ & 185 & $\mathrm{mg} / \mathrm{kg}$ & 241 & EPA6010A \\
\hline$J$ & $\mathrm{E}$ & 118 & $\mathrm{mg} / \mathrm{kg}$ & 241 & EPA6010A \\
\hline $\mathrm{U}$ & & 36.2 & $\mathrm{mg} / \mathrm{kg}$ & 36.2 & EPA6010A \\
\hline $\mathrm{U}$ & & 36.2 & $\mathrm{mg} / \mathrm{kg}$ & 36.2 & EPA6010A \\
\hline $\mathrm{U}$ & & 3.02 & $\mathrm{mg} / \mathrm{kg}$ & 3.02 & EPA6010A \\
\hline $\mathrm{U}$ & & 3.02 & $\mathrm{mg} / \mathrm{kg}$ & 3.02 & EPA6010A \\
\hline$J$ & $\mathbf{E}$ & 30.7 & $\mathrm{mg} / \mathrm{kg}$ & 121 & EPA6010A \\
\hline $\mathrm{J}$ & $\mathbf{E}$ & 27.7 & $\mathrm{mg} / \mathrm{kg}$ & 121 & EPA6010A \\
\hline UJ & $\mathrm{C}$ & 36.2 & $\mathrm{mg} / \mathrm{kg}$ & 36.2 & EPA6010A \\
\hline \multirow[t]{5}{*}{ UJ } & $\mathrm{C}$ & 36.2 & $\mathrm{mg} / \mathrm{kg}$ & 36.2 & EPA6010A \\
\hline & & 30.2 & $\mathrm{mg} / \mathrm{kg}$ & 1.21 & EPA6010A \\
\hline & & 24 & $\mathrm{mg} / \mathrm{kg}$ & 1.21 & EPA6010A \\
\hline & $\mathrm{V}$ & 31.8 & $\mathrm{mg} / \mathrm{kg}$ & 2.41 & EPA6010A \\
\hline & $\mathrm{V}$ & 26.6 & $\mathrm{mg} / \mathrm{kg}$ & 2.41 & EPA6010A \\
\hline
\end{tabular}

6.60E-02 EPIA-013B 2.98E-02 EPIA-013B

8.64E-02 EPIA-013B

3.36E-02 EPIA-013B

1.60E-01 EPIA-013B

2.01E-02 EPIA-013B 
SURVEY ID: PPSC 1001 (continued)

\section{Constituent}

Radionuclides

Cesium-137

Cobalt-57

Cobalt-58

Cobalt-60

Europium-152

Europium-154

Europium-155

Gross alpha

Lead-212

Manganese-54

Neptunium-239

Nonvolatile beta

Plutonium-238

Plutonium-239/240

Potassium-40

Promethium-144

Promethium-146

Ruthenium-106

Sodium-22

Strontium-90

Thorium-234

Tin-113

Yttrium-88

Zinc-65

Zirconium-95
Sample ID: 104625

RQ AQ B Result Unit D. Limit Method
2.21E-02 EPIA-013B
2.08E-02 EPIA-013B
2.37E-02 EPLA-013B
1.79E-02 EPIA-013B
7.49E-02 EPLA-013B
1.63E-01 EPIA-013B
8.38E-02 EPIA-013B
1.75E+00 EPIA-001B
4.18E-02 EPLA-013B
2.08E-02 EPLA-013B
1.49E-01 EPIA-013B
2.63E+00 EPIA-001B
2.01E-02 EPIA-012B
1.01E-02 EPIA-012B
1.63E-01 EPIA-013B
1.94E-02 EPIA-013B
4.32E-02 EPIA-013B
2.20E-01 EPIA-013B
1.82E-02 EPIA-013B
8.74E-02 EPIA-004
$1.05 \mathrm{E}+00$ EPIA-013B
4.24E-02 EPIA-013B
2.21E-02 EPIA-013B
4.24E-02 EPIA-013B
5.26E-02 EPIA-013B

SURVEY ID: PPSC 1002

Survey location: $45176 \mathrm{E}$ 3684285N (SRS coordinates)

Depth of core interval: 1.00 to $2.00 \mathrm{ft}$

Sample type: Normal

Sample matrix: Soil

Sample ID: 104626

\section{Constituent}

RQ AQ B Result

Physical Parameters and Specified Analyses

\section{ses}

Percent solids: $\mathbf{5 8 . 0 0}$

Unit

D. Limit Method

Cation exchange capacity
Chloride
Cyanide
Nitrate-nitrite as nitrogen
Orthophosphate
Phenols
Sulfide
Total organic carbon
Total organic nitrogen
Total phosphates (as P)

Cation exchange capacity

Chloride

Nitrate-nitrite as nitrogen

Phenols

Total organic carbon

Total phosphates (as $\mathrm{P}$ )

$\begin{array}{lll} & & 140 \\ \text { J } & \text { E } & 3.17 \\ \text { U } & & 1.69 \\ \text { U } & & 1.89 \\ \text { U } & & 17 \\ \text { UJ } & \text { C } & 503 \\ \text { UJ } & \text { C } & 431 \\ & & 43300 \\ \text { C } & & 587 \\ \text { J } & \text { E } & 199\end{array}$

$\begin{array}{lll}\mathrm{meq} / 100 \mathrm{~g} & 5 & \text { EPA9081 } \\ \mathrm{mg} / \mathrm{kg} & 4.12 & \text { EPA300.0 } \\ \mathrm{mg} / \mathrm{kg} & 1.69 & \text { EPA335.3 } \\ \mathrm{mg} / \mathrm{kg} & 1.89 & \text { EPA353.1 } \\ \mathrm{mg} / \mathrm{kg} & 17 & \text { EPA365.2 } \\ \mu \mathrm{g} / \mathrm{kg} & 503 & \text { EPA420.2 } \\ \mathrm{mg} / \mathrm{kg} & 431 & \text { EPA376.2 } \\ \mathrm{mg} / \mathrm{kg} & 1480 & \text { EPA415.1 } \\ \mathrm{mg} / \mathrm{kg} & 0.345 & \text { EPA351.2 } \\ \mathrm{mg} / \mathrm{kg} & 563 & \text { EPA365.4 }\end{array}$


SURVEY ID: PPSC 1002 (continued)

Constituent

Metals (total recoverable)
Sample ID: 104626

RQ AQ B Result Unit D. Limit Method

\begin{tabular}{|c|c|c|c|c|c|c|}
\hline Aluminum & . & V & 3310 & $\mathrm{mg} / \mathrm{kg}$ & 21.3 & EPA6010A \\
\hline Antimony & U & & 8.54 & $\mathrm{mg} / \mathrm{kg}$ & 8.54 & EPA6010A \\
\hline Arsenic & $\mathrm{U}$ & & 25.6 & $\mathrm{mg} / \mathrm{kg}$ & 25.6 & EPA6010A \\
\hline Barium & & & 12.2 & $\mathrm{mg} / \mathrm{kg}$ & 2.13 & EPA6010A \\
\hline Beryllium & $\mathrm{J}$ & $\mathbf{E}$ & 0.563 & $\mathrm{mg} / \mathrm{kg}$ & 0.854 & EPA6010A \\
\hline Cadmium & $\mathrm{U}$ & & 0.427 & $\mathrm{mg} / \mathrm{kg}$ & 0.427 & EPA6010A \\
\hline Calcium & & & 232 & $\mathrm{mg} / \mathrm{kg}$ & 21.3 & EPA6010A \\
\hline Chromium & & & 6.6 & $\mathrm{mg} / \mathrm{kg}$ & 2.13 & EPA6010A \\
\hline Cobalt & $J$ & $\mathrm{E}$ & 0.302 & $\mathrm{mg} / \mathrm{kg}$ & 0.854 & EPA6010A \\
\hline Copper & U & & 2.13 & $\mathrm{mg} / \mathrm{kg}$ & 2.13 & EPA6010A \\
\hline Iron & $\mathrm{J}$ & C & 629 & $\mathrm{mg} / \mathrm{kg}$ & 8.54 & EPA6010A \\
\hline Lead & $J$ & $E$ & 4.97 & $\mathrm{mg} / \mathrm{kg}$ & 8.54 & EPA6010A \\
\hline Magnesium & & & 40.2 & $\mathrm{mg} / \mathrm{kg}$ & 8.54 & EPA6010A \\
\hline Manganese & & & 8.59 & $\mathrm{mg} / \mathrm{kg}$ & 0.854 & EPA6010A \\
\hline Mercury & $J$ & $\mathrm{EV}$ & 0.0348 & $\mathrm{mg} / \mathrm{kg}$ & 0.0544 & EPA7471 \\
\hline Nickel & $J$ & $\mathbf{E}$ & 1.57 & $\mathrm{mg} / \mathrm{kg}$ & 2.13 & EPA6010A \\
\hline Potassium & $\mathrm{J}$ & $\mathbf{E}$ & 33.4 & $\mathrm{mg} / \mathrm{kg}$ & 171 & EPA6010A \\
\hline Selenium & $\mathrm{U}$ & & 25.6 & $\mathrm{mg} / \mathrm{kg}$ & 25.6 & EPA6010A \\
\hline Silver & U & & 2.13 & $\mathrm{mg} / \mathrm{kg}$ & 2.13 & EPA6010A \\
\hline Sodium & $J$ & $\mathrm{E}$ & 14.3 & $\mathrm{mg} / \mathrm{kg}$ & 85.4 & EPA6010A \\
\hline Thallium & UJ & C & 25.6 & $\mathrm{mg} / \mathrm{kg}$ & 25.6 & EPA6010A \\
\hline Vanadium & & & 3.57 & $\mathrm{mg} / \mathrm{kg}$ & 0.854 & EPA6010A \\
\hline Zinc & & $\mathrm{V}$ & 6.98 & $\mathrm{mg} / \mathrm{kg}$ & 1.71 & EPA6010A \\
\hline
\end{tabular}

Radionuclides

Actinium-228

Antimony-124

Antimony-125

Barium-133

Cerium-144

Cesium-134

Cesium-137

Cobalt-57

Cobalt-58

Cobalt-60

Europium-152

Europium-154

Europium-155

Gross alpha

Lead-212

Manganese-54

Neptunium-239

Nonvolatile beta

Plutonium-238

Plutonium-239/240

Potassium-40

Promethium-144

Promethium-146

Ruthenium-106

Sodium-22

Strontium-90

Thorium-234

Tin-113

Yttrium-88
$1.73 \mathrm{E}+00 \pm 8.55 \mathrm{E}-02 \mathrm{pCi} / \mathrm{g}$ $-3.76 \mathrm{E}-03 \pm 9.97 \mathrm{E}-03 \mathrm{pCi} / \mathrm{g}$ $-4.00 \mathrm{E}-03 \pm 1.97 \mathrm{E}-02 \mathrm{pCi} / \mathrm{g}$ $-5.95 \mathrm{E}-04 \pm 1.08 \mathrm{E}-02 \mathrm{pCi} / \mathrm{g}$ $-4.90 \mathrm{E}-02 \pm 5.79 \mathrm{E}-02 \mathrm{pCi} / \mathrm{g}$ $1.89 \mathrm{E}-03 \pm 7.60 \mathrm{E}-03 \mathrm{pCi} / \mathrm{g}$ $1.63 \mathrm{E}-01 \pm 1.52 \mathrm{E}-02 \mathrm{pCi} / \mathrm{g}$ $-1.24 \mathrm{E}-03 \pm 7.17 \mathrm{E}-03 \mathrm{pCi} / \mathrm{g}$ $-1.18 \mathrm{E}-02 \pm 9.50 \mathrm{E}-03 \mathrm{pCi} / \mathrm{g}$ $4.46 \mathrm{E}-03 \pm 6.53 \mathrm{E}-03 \mathrm{pCi} / \mathrm{g}$ $1.87 \mathrm{E}-02 \pm 2.37 \mathrm{E}-02 \mathrm{pCj} / \mathrm{g}$ $-2.93 \mathrm{E}-02 \pm 6.26 \mathrm{E}-02 \mathrm{pCi} / \mathrm{g}$ $0.00 \mathrm{E}+00 \quad \mathrm{pCi} / \mathrm{g}$ $1.82 \mathrm{E}+01 \pm 3.14 \mathrm{E}+00 \mathrm{pCi} / \mathrm{g}$ $1.76 \mathrm{E}+00 \pm 3.66 \mathrm{E}-02 \mathrm{pCi} / \mathrm{g}$ $3.55 \mathrm{E}-02 \pm 8.99 \mathrm{E}-03 \mathrm{pCi} / \mathrm{g}$ $1.06 \mathrm{E}-01 \pm 8.95 \mathrm{E}-02 \mathrm{pCj} / \mathrm{g}$ $7.42 \mathrm{E}+00 \pm 1.76 \mathrm{E}+00 \mathrm{pCi} / \mathrm{g}$ $1.29 \mathrm{E}-02 \pm 1.51 \mathrm{E}-02 \mathrm{pCi} / \mathrm{g}$ $-1.15 \mathrm{E}-03 \pm 7.10 \mathrm{E}-03 \mathrm{pCi} / \mathrm{g}$ $5.75 \mathrm{E}-01 \pm 1.66 \mathrm{E}-01 \mathrm{pCi} / \mathrm{g}$ $2.73 \mathrm{E}-03 \pm 1.28 \mathrm{E}-02 \mathrm{pCi} / \mathrm{g}$ $1.52 \mathrm{E}-02 \pm 1.58 \mathrm{E}-02 \mathrm{pCi} / \mathrm{g}$ $-2.66 \mathrm{E}-02 \pm 7.00 \mathrm{E}-02 \mathrm{pCi} / \mathrm{g}$ $1.62 \mathrm{E}-03 \pm 6.49 \mathrm{E}-03 \mathrm{pCi} / \mathrm{g}$ $1.52 \mathrm{E}+00 \pm 1.37 \mathrm{E}-01 \mathrm{pCi} / \mathrm{g}$ $3.06 \mathrm{E}+00 \pm 1.00 \mathrm{E}+00 \mathrm{pCi} / \mathrm{g}$ $-8.03 \mathrm{E}-04 \pm 1.13 \mathrm{E}-02 \mathrm{pCi} / \mathrm{g}$ $4.53 \mathrm{E}-03 \pm 7.68 \mathrm{E}-03 \mathrm{pCi} / \mathrm{g}$
4.06E-02 EPIA-013B

$1.68 \mathrm{E}-02$ EPIA-013B

3.41E-02 EPIA-013B

1.56E-02 EPIA-013B

9.74E-02 EPIA-013B

1.15E-02 EPIA-013B

1.29E-02 EPIA-013B

1.22E-02 EPIA-013B

1.50E-02 EPIA-013B

1.19E-02 EPIA-013B

3.50E.02 EPIA.013B

9.28E-02 EPIA-013B

5.11E-02 EPIA-013B

1.28E+00 EPIA-001B

2.16E-02 EPIA-013B

1.34E-02 EPIA-013B

8.73E-02 EPIA-013B

2.50E +00 EPIA-001B

2.48E-02 EPIA-012B

1.66E-02 EPIA-012B

1.18E-01 EPIA-013B

1.34E-02 EPIA-013B

1.73E-02 EPIA-013B

1.18E-01 EPIA-013B

1.15E-02 EPIA-013B

6.93E.02 EPIA-004

6.46E-01 EPIA-013B

1.97E-02 EPLA-013B

1.46E-02 EPIA-013B 
SURVEY ID: PPSC 1002 (continued)

Constituent

Radionuclides
Sample ID: 104626
RQ AQ B Result
Unit
D. Limit Method

2.44E-02 EPIA-013B

3.74E-02 EPIA-013B

$\begin{array}{ll}-1.94 \mathrm{E}-02 \pm 2.70 \mathrm{E}-02 & \mathrm{pCi} / \mathrm{g} \\ 0.00 \mathrm{E}+00 & \mathrm{pCi} / \mathrm{g}\end{array}$
Zinc-65

Zirconium-95

SURVEY ID: PPSC 1003

UI

UI

3.74E-02 LPLA-013B

Sample ID: 104627

Survey location: $45176 \mathrm{E}$ 3684285N (SRS coordinates)

Depth of core interval: 2.00 to $4.00 \mathrm{ft}$

Sample type: Normal

Sample matrix: Soil

Percent solids: $\mathbf{6 3 . 0 0}$

Constituent

RQ AQ B Result

Unit

D. Limit Method

Physical Parameters and Specified Analyses

Cation exchange capacity

Chloride

Cyanide

Nitrate-nitrite as nitrogen

Orthophosphate

Phenols

Sulfide

Total organic carbon

Total organic nitrogen

Total phosphates (as P)

Metals (total recoverable)

$\begin{array}{lll} & & 143 \\ \text { J } & \mathbf{E} & 2.05 \\ \mathrm{U} & & 1.46 \\ \mathrm{U} & & 1.41 \\ \text { U } & & 15.7 \\ \text { UJ } & \mathrm{C} & 473 \\ \text { UJ } & \mathrm{C} & 397 \\ & & 44000 \\ \mathrm{C} & & 455 \\ \text { J } & \text { E } & 121\end{array}$

Aluminum

Antimony

Arsenic

Barium

Beryllium

Cadmium

Calcium

Chromium

Cobalt

Copper

Iron

Lead

Magnesium

Manganese

Mercury

Nickel

Potassium

Selenium

Silver

Sodium

Thallium

Vanadium

Zinc

$\begin{array}{lll} & \mathrm{V} & 1880 \\ \mathrm{U} & & 7.86 \\ \mathrm{U} & & 23.6 \\ & & 5.56 \\ \mathrm{~J} & \mathrm{E} & 0.493 \\ \mathrm{~J} & \mathrm{E} & 0.265 \\ & & 186 \\ \mathrm{U} & & 5.72 \\ \mathrm{~J} & \mathrm{E} & 0.786 \\ \mathrm{~J} & \mathrm{C} & 2.3 \\ \mathrm{~J} & \mathrm{E} & 4.33 \\ & & 11.3 \\ \mathrm{~J} & \mathrm{EV} & \mathbf{1 . 5 8} \\ \mathrm{J} & \mathrm{E} & 1.13 \\ \mathrm{U} & & 157 \\ \mathrm{U} & & 23.6 \\ \mathrm{U} & & 1.96 \\ \mathrm{~J} & \mathrm{E} & 12.8 \\ \mathrm{UJ} & \mathrm{C} & 23.6 \\ & & 2.66 \\ & \mathrm{~V} & 2.03\end{array}$

$\begin{array}{lll}\mathrm{meq} / 100 \mathrm{~g} & 5 & \text { EPA9081 } \\ \mathrm{mg} / \mathrm{kg} & 3.71 & \text { EPA300.0 } \\ \mathrm{mg} / \mathrm{kg} & 1.46 & \text { EPA335.3 } \\ \mathrm{mg} / \mathrm{kg} & 1.41 & \text { EPA353.1 } \\ \mathrm{mg} / \mathrm{kg} & 15.7 & \text { EPA365.2 } \\ \mu \mathrm{g} / \mathrm{kg} & 473 & \text { EPA420.2 } \\ \mathrm{mg} / \mathrm{kg} & \mathbf{3 9 7} & \text { EPA376.2 } \\ \mathrm{mg} / \mathrm{kg} & 1630 & \text { EPA415.1 } \\ \mathrm{mg} / \mathrm{kg} & \mathbf{0 . 3 1 7} & \text { EPA351.2 } \\ \mathrm{mg} / \mathrm{kg} & \mathbf{3 3 4} & \text { EPA365.4 }\end{array}$

$\begin{array}{lll}\mathrm{mg} / \mathrm{kg} & 19.6 & \text { EPA6010A } \\ \mathrm{mg} / \mathrm{kg} & 7.86 & \text { EPA6010A } \\ \mathrm{mg} / \mathrm{kg} & 23.6 & \text { EPA6010A } \\ \mathrm{mg} / \mathrm{kg} & 1.96 & \text { EPA6010A } \\ \mathrm{mg} / \mathrm{kg} & 0.786 & \text { EPA6010A } \\ \mathrm{mg} / \mathrm{kg} & 0.393 & \text { EPA6010A } \\ \mathrm{mg} / \mathrm{kg} & 19.6 & \text { EPA6010A } \\ \mathrm{mg} / \mathrm{kg} & 1.96 & \text { EPA6010A } \\ \mathrm{mg} / \mathrm{kg} & 0.786 & \text { EPA6010A } \\ \mathrm{mg} / \mathrm{kg} & 1.96 & \text { EPA6010A } \\ \mathrm{mg} / \mathrm{kg} & 7.86 & \text { EPA6010A } \\ \mathrm{mg} / \mathrm{kg} & 7.86 & \text { EPA6010A } \\ \mathrm{mg} / \mathrm{kg} & 7.86 & \text { EPA6010A } \\ \mathrm{mg} / \mathrm{kg} & 0.786 & \text { EPA6010A } \\ \mathrm{mg} / \mathrm{kg} & 0.0506 & \text { EPA7471 } \\ \mathrm{mg} / \mathrm{kg} & 1.96 & \text { EPA6010A } \\ \mathrm{mg} / \mathrm{kg} & 157 & \text { EPA6010A } \\ \mathrm{mg} / \mathrm{kg} & 23.6 & \text { EPA6010A } \\ \mathrm{mg} / \mathrm{kg} & 1.96 & \text { EPA6010A } \\ \mathrm{mg} / \mathrm{kg} & 78.6 & \text { EPA6010A } \\ \mathrm{mg} / \mathrm{kg} & 23.6 & \text { EPA6010A } \\ \mathrm{mg} / \mathrm{kg} & 0.786 & \text { EPA6010A } \\ \mathrm{mg} / \mathrm{kg} & 1.57 & \text { EPA6010A }\end{array}$


SURVEY ID: PPSC 1003 (continued)

Constituent

Radionuclides

Actinium-228

Antimony-124

Antimony-125

Barium-133

Cerium-144

Cesium-134

Cesium-137

Cobalt-57

Cobalt-58

Cobalt-60

Europium-152

Europium-154

Europium-155

Gross alpha

Lead-212

Manganese-54

Neptunium-239

Nonvolatile beta

Plutonium-238

Plutonium-239/240

Potassium-40

Promethium-144

Promethium-146

Ruthenium-106

Sodium-22

Strontium-90

Thorium-234

Tin-113

Yttrium-88

Zinc-65

Zirconium-95
Sample ID: 104627

RQ AQ B Result

Unit

D. Limit Method

3.79E-02 EPIA-013B

1.56E-02 EPIA-013B

3.12E-02 EPIA-013B

1.44E-02 EPIA-013B

9.03E-02 EPIA-013B

1.11E-02 EPIA-013B

1.28E-02 EPIA-013B

1.16E-02 EPIA-013B

1.43E-02 EPIA-013B

1.10E-02 EPIA-013B

3.21E-02 EPIA-013B

9.10E-02 EPIA-013B

4.71E-02 EPIA-013B

1.48E+00 EPIA-00IB

2.02E-02 EPIA-013B

1.20E-02 EPIA-013B

8.10E-02 EPIA-013B

2.52E+00 EPIA-001B

9.50E-03 EPIA-012B

$0.00 \mathrm{E}+00$ EPIA-012B

1.01E-01 EPIA-013B

1.27E-02 EPIA-013B

1.60E-02 EPIA-013B

1.04E-01 EPIA-013B

1.01E-02 EPIA-013B

6.07E-02 EPIA-004

6.17E-01 EPIA-013B

1.81E-02 EPIA-013B

1.41E-02 EPIA-013B

2.10E-02 EPIA-013B

2.95E-02 EPIA-013B

SURVEY ID: PPSC 1004

Sample ID: 104628

Survey location: $45176 \mathrm{E}$ 3684285N (SRS coordinates)

Depth of core interval: 4.00 to $6.00 \mathrm{ft}$

Sample type: Normal

Sample matrix: Soil

Percent solids: $\mathbf{5 0 . 0 0}$

Constituent

Physical Parameters and Specified Analyses

Cation exchange capacity

Chloride

Cyanide

Nitrate-nitrite as nitrogen

Orthophosphate

Phenols

Sulfide

Total organic carbon

Total organic nitrogen

Total phosphates (as P)
RQ AQ B Result

$\begin{array}{lll} & & 268 \\ J & E & 3.03 \\ U & & 1.98 \\ U & & 1.86 \\ J & E & 5.03 \\ J & E C & 260 \\ \text { UJ } & C & 500 \\ & & 75100 \\ \text { C } & & 1240 \\ J & E & 140\end{array}$

Unit

D. Limit Method

$\begin{array}{lll}\mathrm{meq} / 100 \mathrm{~g} & 5 & \text { EPA9081 } \\ \mathrm{mg} / \mathrm{kg} & 5.05 & \text { EPA300.0 } \\ \mathrm{mg} / \mathrm{kg} & 1.98 & \text { EPA335.3 } \\ \mathrm{mg} / \mathrm{kg} & 1.86 & \text { EPA353.1 } \\ \mathrm{mg} / \mathrm{kg} & 19.9 & \text { EPA365.2 } \\ \mu \mathrm{g} / \mathrm{kg} & 600 & \text { EPA420.2 } \\ \mathrm{mg} / \mathrm{kg} & 500 & \text { EPA376.2 } \\ \mathrm{mg} / \mathrm{kg} & 1950 & \text { EPA415.1 } \\ \mathrm{mg} / \mathrm{kg} & 0.4 & \text { EPA351.2 } \\ \mathrm{mg} / \mathrm{kg} & 503 & \text { EPA365.4 }\end{array}$


SURVEY ID: PPSC 1004 (continued)

Constituent

Metals (total recoverable)
Sample ID: 104628

D. Limit Method

Aluminum
Antimony
Arsenic
Barium
Beryllium
Cadmium
Calcium
Chromium
Cobalt
Copper
Iron
Lead
Magnesium
Manganese
Mercury
Nickel
Potassium
Selenium
Silver
Sodium
Thallium
Vanadium
Zinc

Radionuclides

Actinium-228

Antimony-124

Antimony-125

Barium-133

Cerium-144

Cesium-134

Cesium-137

Cobalt-57

Cobalt-58

Cobalt-60

Europium-152

Europium-154

Europium-155

Gross alpha

Lead-212

Manganese-54

Neptunium-239

Nonvolatile beta

Plutonium-238

Plutonium-239/240

Potassium-40

Promethium-144

Promethium-146

Ruthenium-106

Sodium-22

Strontium-90

$\begin{array}{llllll} & \mathrm{V} & 8070 & \mathrm{mg} / \mathrm{kg} & 24.8 & \text { EPA6010A } \\ \mathrm{U} & & 9.9 & \mathrm{mg} / \mathrm{kg} & 9.9 & \text { EPA6010A } \\ \mathrm{U} & & 29.7 & \mathrm{mg} / \mathrm{kg} & 29.7 & \text { EPA6010A } \\ & & 26.4 & \mathrm{mg} / \mathrm{kg} & 2.48 & \text { EPA6010A } \\ \mathrm{J} & \mathrm{E} & 0.96 & \mathrm{mg} / \mathrm{kg} & 0.99 & \text { EPA6010A } \\ & & 0.59 & \mathrm{mg} / \mathrm{kg} & 0.495 & \text { EPA6010A } \\ & & 1460 & \mathrm{mg} / \mathrm{kg} & 24.8 & \text { EPA6010A } \\ & & 13.9 & \mathrm{mg} / \mathrm{kg} & 2.48 & \text { EPA6010A } \\ & & 1.23 & \mathrm{mg} / \mathrm{kg} & 0.99 & \text { EPA6010A } \\ & & 5.08 & \mathrm{mg} / \mathrm{kg} & 2.48 & \text { EPA6010A } \\ \mathrm{J} & \mathrm{C} & 693 & \mathrm{mg} / \mathrm{kg} & 9.9 & \text { EPA6010A } \\ \mathrm{J} & \mathrm{E} & 9.29 & \mathrm{mg} / \mathrm{kg} & 9.9 & \text { EPA6010A } \\ & & 103 & \mathrm{mg} / \mathrm{kg} & 9.9 & \text { EPA6010A } \\ & & 2.6 & \mathrm{mg} / \mathrm{kg} & 0.99 & \text { EPA6010A } \\ \mathrm{J} & \mathrm{EV} & 0.0463 & \mathrm{mg} / \mathrm{kg} & 0.0565 & \text { EPA7471 } \\ & & 9.15 & \mathrm{mg} / \mathrm{kg} & 2.48 & \text { EPA6010A } \\ \mathrm{J} & \mathrm{E} & 102 & \mathrm{mg} / \mathrm{kg} & 198 & \text { EPA6010A } \\ \mathrm{U} & & 29.7 & \mathrm{mg} / \mathrm{kg} & 29.7 & \text { EPA6010A } \\ \mathrm{U} & & 2.48 & \mathrm{mg} / \mathrm{kg} & 2.48 & \text { EPA6010A } \\ \mathrm{J} & \mathrm{E} & 23.3 & \mathrm{mg} / \mathrm{kg} & 99 & \text { EPA6010A } \\ \mathrm{UJ} & \mathrm{C} & 29.7 & \mathrm{mg} / \mathrm{kg} & 29.7 & \text { EPA6010A } \\ & & 18.5 & \mathrm{mg} / \mathrm{kg} & 0.99 & \text { EPA6010A } \\ & \mathrm{V} & 8.48 & \mathrm{mg} / \mathrm{kg} & 1.98 & \text { EPA6010A }\end{array}$

5.31E-02 EPIA-013B

$1.45 \mathrm{E}+00 \pm 8.97 \mathrm{E}-02 \mathrm{pCi} / \mathrm{g}$ $-7.51 \mathrm{E}-03 \pm 1,27 \mathrm{E}-02 \mathrm{pCi} / \mathrm{g}$ $1.69 \mathrm{E}-02 \pm 2.50 \mathrm{E}-02 \mathrm{pCi} / \mathrm{g}$ $-4.67 \mathrm{E}-03 \pm 1.20 \mathrm{E}-02 \mathrm{pCi} / \mathrm{g}$ $1.17 \mathrm{E}-03 \pm 6.75 \mathrm{E}-02 \mathrm{pCi} / \mathrm{g}$ $1.33 \mathrm{E}-03 \pm 9.80 \mathrm{E}-03 \mathrm{pCi} / \mathrm{g}$ $2.34 \mathrm{E}-03 \pm 1.13 \mathrm{E}-02 \mathrm{pCi} / \mathrm{g}$ $-7.35 \mathrm{E}-04 \pm 8.53 \mathrm{E}-03 \mathrm{pCi} / \mathrm{g}$ $-2.39 \mathrm{E}-03 \pm 1.09 \mathrm{E}-02 \mathrm{pCi} / \mathrm{g}$ $-3.70 \mathrm{E}-03 \pm 7.39 \mathrm{E}-03 \mathrm{pCi} / \mathrm{g}$ $-8.21 \mathrm{E}-03 \pm 2.52 \mathrm{E}-02 \mathrm{pCi} / \mathrm{g}$ $3.47 \mathrm{E}-02 \pm 7.63 \mathrm{E}-02 \mathrm{pCi} / \mathrm{g}$ $5.88 \mathrm{E}-02 \pm 5.12 \mathrm{E}-02 \mathrm{pCi} / \mathrm{g}$ $1.28 \mathrm{E}+01 \pm 2.72 \mathrm{E}+00 \mathrm{pCi} / \mathrm{g}$ $1.44 \mathrm{E}+00 \pm 3.93 \mathrm{E}-02 \mathrm{pCi} / \mathrm{g}$ $2.33 \mathrm{E}-02 \pm 1.36 \mathrm{E}-02 \mathrm{pCi} / \mathrm{g}$ $6.45 \mathrm{E}-02 \pm 6.98 \mathrm{E}-02 \mathrm{pCi} / \mathrm{g}$ $9.16 \mathrm{E}+00 \pm 1.77 \mathrm{E}+00 \mathrm{pCi} / \mathrm{g}$ $7.24 \mathrm{E}-04 \pm 1.30 \mathrm{E}-02 \mathrm{pCi} / \mathrm{g}$ $-1.11 \mathrm{E}-03 \pm 4.10 \mathrm{E}-03 \mathrm{pCi} / \mathrm{g}$ $1.72 \mathrm{E}+00 \pm 1.96 \mathrm{E}-01 \mathrm{pCi} / \mathrm{g}$ $6.30 \mathrm{E}-03 \pm 9.03 \mathrm{E}-03 \mathrm{pCi} / \mathrm{g}$ $7.48 \mathrm{E}-03 \pm 1.13 \mathrm{E}-02 \mathrm{pCi} / \mathrm{g}$ $-3.80 \mathrm{E}-03 \pm 8.34 \mathrm{E}-02 \mathrm{pCi} / \mathrm{g}$ 1.32E-02 $7.61 \mathrm{E}-03 \mathrm{pCi} / \mathrm{g}$ $-5.03 \mathrm{E}-02 \pm 2.08 \mathrm{E}-02 \mathrm{pCi} / \mathrm{g}$
2.10E-02 EPIA-013B

4.39E-02 EPIA-013B

1.81E-02 EPIA-013B

1.13E-01 EPIA-013B

1.47E-02 EPIA-013B

1.69E-02 EPIA-013B

1.43E-02 EPIA-013B

1.82E-02 EPIA-013B

1.24E-02 EPIA-013B

4.32E-02 EPIA-013B

1.23E-01 EPIA-013B

5.95E-02 EPIA-013B

2.28E+00 EPIA-001B

2.60E-02 EPIA-013B

1.50E-02 EPIA-013B

1.02E-01 EPIA-013B

2.32E +00 EPIA-001B

2.49E-02 EPIA-012B

1.14E-02 EPIA-012B

1.19E-01 EPIA-013B

1.57E-02 EPIA-013B

1.99E-02 EPIA-013B

1.41E-01 EPIA-013B

1.43E-02 EPIA-013B

5.76E-02 EPIA-004 
SURVEY ID: PPSC 1004 (continued)

Constituent

RQ AQ B Result

D. Limit Method

Radionuclides

Thorium-234

Tin-113

$\begin{array}{ll}\text { UI } & \text { V } \\ \text { UI } \\ \text { UI }\end{array}$

Zirconium-95
Unit

Sample ID: 104628

$$
\begin{aligned}
& 1.92 \mathrm{E}+00 \pm 7.35 \mathrm{E}-01 \mathrm{pCi} / \mathrm{g} \\
& -7.62 \mathrm{E}-03 \pm 1.33 \mathrm{E}-02 \mathrm{pCi} / \mathrm{g} \\
& 3.55 \mathrm{E}-03 \pm 1.09 \mathrm{E}-02 \mathrm{pCi} / \mathrm{g} \\
& 1.43 \mathrm{E}-02 \pm 2.03 \mathrm{E}-02 \mathrm{pCi} / \mathrm{g} \\
& 4.46 \mathrm{E}-02 \pm 4.38 \mathrm{E}-02 \mathrm{pCi} / \mathrm{g}
\end{aligned}
$$

8.05E-01 EPIA-013B

2.25E-02 EPIA-013B

1.80E-02 EPIA-013B

3.28E-02 EPIA-013B

3.80E-02 EPIA-013B

SURVEY ID: PPSC 1005

Sample ID: 104629

Survey location: $45176 \mathrm{E} 3684285 \mathrm{~N}$ (SRS coordinates)

Depth of core interval: 6.00 to $8.00 \mathrm{ft}$

Sample type: Normal

Sample matrix: Soil

Percent solids: $\mathbf{7 1 . 0 0}$

Constituent

RQ AQ B Result

Unit

D. Limit Method

Physical Parameters and Specified Analyses

Cation exchange capacity

Chloride

Cyanide

Nitrate-nitrite as nitrogen

Orthophosphate

Orthophosphate

Phenols

Sulfide

Total organic carbon

Total organic carbon

Total organic nitrogen

Total phosphates (as P)

$\begin{array}{lll} & & 93.9 \\ \text { J } & \text { E } & 1.9 \\ \text { U } & & 1.41 \\ \text { U } & & 1.46 \\ \text { J } & \text { E } & 5 \\ \text { J } & \text { E } & 5.03 \\ \text { UJ } & \text { C } & 418 \\ \text { UJ } & \text { C } & 352 \\ & & 29000 \\ & & 32300 \\ \text { C } & & 355 \\ \text { J } & \text { E } & 113\end{array}$

$\begin{array}{ll}\mathrm{meg} / 100 \mathrm{~g} & 5 \\ \mathrm{mg} / \mathrm{kg} & 3.35 \\ \mathrm{mg} / \mathrm{kg} & 1.41 \\ \mathrm{mg} / \mathrm{kg} & 1.46 \\ \mathrm{mg} / \mathrm{kg} & 13.8 \\ \mathrm{mg} / \mathrm{kg} & 14.1 \\ \mu \mathrm{g} / \mathrm{kg} & 418 \\ \mathrm{mg} / \mathrm{kg} & 352 \\ \mathrm{mg} / \mathrm{kg} & 1080 \\ \mathrm{mg} / \mathrm{kg} & 1080 \\ \mathrm{mg} / \mathrm{kg} & 0.282 \\ \mathrm{mg} / \mathrm{kg} & 377\end{array}$

EPA9081

EPA300.0

EPA335.3

EPA353.1

EPA365.2

EPA365.2

EPA420.2

EPA376.2

EPA415.1

EPA415.1

EPA351.2

Metals (total recoverable)

$\begin{array}{llll}\text { Aluminum } & & \mathrm{V} & 4030 \\ \text { Antimony } & \mathrm{U} & & 6.9 \\ \text { Arsenic } & \mathrm{U} & & 20.7 \\ \text { Barium } & & & 14.1 \\ \text { Beryllium } & \mathrm{J} & \mathrm{E} & 0.36 \\ \text { Cadmium } & \mathrm{J} & \mathrm{E} & 0.281 \\ \text { Calcium } & & \mathrm{V} & 525 \\ \text { Chromium } & & & 7.06 \\ \text { Cobalt } & & & 0.775 \\ \text { Copper } & & & 2.02 \\ \text { Iron } & \mathrm{J} & \mathrm{VC} & 405 \\ \text { Lead } & \mathrm{J} & \mathrm{E} & 2.57 \\ \text { Magnesium } & & \mathrm{V} & 68.9 \\ \text { Manganese } & & & 3.49 \\ \text { Mercury } & \mathrm{U} & \mathrm{V} & \mathbf{0 . 0 1 8 8} \\ \text { Nickel } & & & 5.76 \\ \text { Potassium } & \mathrm{J} & \mathrm{E} & 63.2 \\ \text { Selenium } & \mathrm{U} & & 20.7 \\ \text { Silver } & \mathrm{U} & & 1.73 \\ \text { Sodium } & \mathrm{U} & & 69 \\ \text { Thallium } & \mathrm{UJ} & \mathrm{C} & 20.7\end{array}$

$\begin{array}{lll}\mathrm{mg} / \mathrm{kg} & 17.3 & \text { EPA6010A } \\ \mathrm{mg} / \mathrm{kg} & 6.9 & \text { EPA6010A } \\ \mathrm{mg} / \mathrm{kg} & 20.7 & \text { EPA6010A } \\ \mathrm{mg} / \mathrm{kg} & 1.73 & \text { EPA6010A } \\ \mathrm{mg} / \mathrm{kg} & 0.69 & \text { EPA6010A } \\ \mathrm{mg} / \mathrm{kg} & 0.345 & \text { EPA6010A } \\ \mathrm{mg} / \mathrm{kg} & 17.3 & \text { EPA6010A } \\ \mathrm{mg} / \mathrm{kg} & 1.73 & \text { EPA6010A } \\ \mathrm{mg} / \mathrm{kg} & 0.69 & \text { EPA6010A } \\ \mathrm{mg} / \mathrm{kg} & 1.73 & \text { EPA6010A } \\ \mathrm{mg} / \mathrm{kg} & 6.9 & \text { EPA6010A } \\ \mathrm{mg} / \mathrm{kg} & 6.9 & \text { EPA6010A } \\ \mathrm{mg} / \mathrm{kg} & 6.9 & \text { EPA6010A } \\ \mathrm{mg} / \mathrm{kg} & 0.69 & \text { EPA6010A } \\ \mathrm{mg} / \mathrm{kg} & 0.0414 & \text { EPA7471 } \\ \mathrm{mg} / \mathrm{kg} & 1.73 & \text { EPA6010A } \\ \mathrm{mg} / \mathrm{kg} & 138 & \text { EPA6010A } \\ \mathrm{mg} / \mathrm{kg} & 20.7 & \text { EPA6010A } \\ \mathrm{mg} / \mathrm{kg} & 1.73 & \text { EPA6010A } \\ \mathrm{mg} / \mathrm{kg} & 69 & \text { EPA6010A } \\ \mathrm{mg} / \mathrm{kg} & 20.7 & \text { EPA6010A }\end{array}$

Par Pond, Pond C, and L Lake, Revision 2 
SURVEY ID: PPSC 1005 (continued)

Constituent

Metals (total recoverable)

Vanadium

Zinc

Radionuclides

Actinium-228

Antimony-124

Antimony-125

Barium-133

Cerium-144

Cesium-134

Cesium-137

Cobalt-57

Cobalt-58

Cobalt-60

Europium-152

Europium-154

Europium-155

Gross alpha

Lead-212

Manganese-54

Neptunium-239

Nonvolatile beta

Plutonium-238

Plutonium-239/240

Potassium-40

Promethium-144

Promethium-146

Ruthenium-106

Sodium-22

Strontium-90

Thorium-234

Tin-113

Yttrium-88

Zinc-65

Zirconium-95
Sample ID: 104629

RQ AQ B Result Unit D. Limit Method

$\begin{array}{llll}7.87 & \mathrm{mg} / \mathrm{kg} & 0.69 & \text { EPA6010A } \\ 9.48 & \mathrm{mg} / \mathrm{kg} & 1.38 & \text { EPA6010A }\end{array}$

$\begin{array}{ll}2.72 \mathrm{E}-02 & \text { EPIA-013B } \\ 1.26 \mathrm{E}-02 & \text { EPIA-013B } \\ 2.58 \mathrm{E}-02 & \text { EPIA-013B } \\ 1.16 \mathrm{E}-02 & \text { EPIA-013B } \\ 7.42 \mathrm{E}-02 & \text { EPIA-013B } \\ 8.52 \mathrm{E}-03 & \text { EPIA-013B } \\ 1.03 \mathrm{E}-02 & \text { EPIA-013B } \\ 9.14 \mathrm{E}-03 & \text { EPIA-013B } \\ 1.08 \mathrm{E}-02 & \text { EPIA-013B } \\ 8.39 \mathrm{E}-03 & \text { EPIA-013B } \\ 2.56 \mathrm{E}-02 & \text { EPIA-013B } \\ 7.13 \mathrm{E}-02 & \text { EPIA-013B } \\ 3.91 \mathrm{E}-02 & \text { EPIA-013B } \\ 1.12 \mathrm{E}+00 & \text { EPIA-001B } \\ 1.61 \mathrm{E}-02 & \text { EPIA-013B } \\ 9.76 \mathrm{E}-03 & \text { EPIA-013B } \\ 6.58 \mathrm{E}-02 & \text { EPIA-013B } \\ 1.82 \mathrm{E}+00 & \text { EPIA-001B } \\ 2.37 \mathrm{E}-02 & \text { EPIA-012B } \\ 1.48 \mathrm{E}-02 & \text { EPIA-012B } \\ 8.58 \mathrm{E}-02 & \text { EPIA-013B } \\ 1.01 \mathrm{E}-02 & \text { EPIA-013B } \\ 1.23 \mathrm{E}-02 & \text { EPIA-013B } \\ 8.69 \mathrm{E}-02 & \text { EPIA-013B } \\ 8.09 \mathrm{E}-03 & \text { EPIA-013B } \\ 6.36 \mathrm{E}-02 & \text { EPIA-004 } \\ 5.02 \mathrm{E}-01 & \text { EPLA-013B } \\ 1.36 \mathrm{E}-02 & \text { EPIA-013B } \\ 1.18 \mathrm{E}-02 & \text { EPIA-013B } \\ 1.69 \mathrm{E}-02 & \text { EPIA-013B } \\ 2.71 \mathrm{E}-02 & \text { EPIA-013B }\end{array}$

2.72E-02 EPIA-013B

$1.06 \mathrm{E}+00 \pm 6.22 \mathrm{E}-02 \mathrm{pCi} / \mathrm{g}$ $4.25 \mathrm{E}-03 \pm 7.05 \mathrm{E}-03 \mathrm{pCi} / \mathrm{g}$ $-1.72 \mathrm{E}-03 \pm 1.58 \mathrm{E}-02 \mathrm{pCi} / \mathrm{g}$ 9.24E-04 7.79E-03 pCi/g 1.20E-02 $44.30 \mathrm{E}-02 \mathrm{pCi} / \mathrm{g}$ $-1.14 \mathrm{E}-03 \pm 5.60 \mathrm{E}-03 \mathrm{pCi} / \mathrm{g}$ $3.06 \mathrm{E}-04 \pm 6.70 \mathrm{E}-03 \mathrm{pCi} / \mathrm{g}$ $1.05 \mathrm{E}-04 \pm 5.30 \mathrm{E}-03 \mathrm{pCi} / \mathrm{g}$ 5.99E-04 7.04E-03 pCi/g 2.43E-04 $\pm 4.62 \mathrm{E}-03 \mathrm{pCi} / \mathrm{g}$ $-1.03 \mathrm{E}-02 \pm 1.57 \mathrm{E}-02 \mathrm{pCi} / \mathrm{g}$ $-3.01 \mathrm{E}-02 \pm 5.04 \mathrm{E}-02 \mathrm{pCi} / \mathrm{g}$ $0.00 \mathrm{E}+00 \quad \mathrm{pCi} / \mathrm{g}$ $1.00 \mathrm{E}+01 \pm 1.88 \mathrm{E}+00 \mathrm{pCi} / \mathrm{g}$

$\mathrm{V} \quad 1.09 \mathrm{E}+00 \pm 2.81 \mathrm{E}-02 \mathrm{pCi} / \mathrm{g}$ $1.65 \mathrm{E}-02 \pm 7.89 \mathrm{E}-03 \mathrm{pCi} / \mathrm{g}$ 6.53E-02 $\pm 5.74 \mathrm{E}-02 \mathrm{pCi} / \mathrm{g}$ $4.03 \mathrm{E}+00 \pm 1.13 \mathrm{E}+00 \mathrm{pCi} / \mathrm{g}$ $-4.27 \mathrm{E}-03 \pm 1.02 \mathrm{E}-02 \mathrm{pCi} / \mathrm{g}$

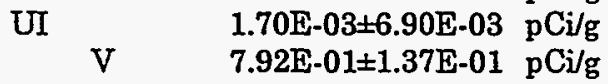

UI $\quad 4.60 \mathrm{E}-03 \pm 5.61 \mathrm{E}-03 \mathrm{pCi} / \mathrm{g}$ 6.21E-03 $\pm 1.02 \mathrm{E}-02 \mathrm{pCi} / \mathrm{g}$ $-1.67 \mathrm{E}-03 \pm 4.99 \mathrm{E}-02 \mathrm{pCi} / \mathrm{g}$ $3.99 \mathrm{E}-04 \pm 4.43 \mathrm{E}-03 \mathrm{pCi} / \mathrm{g}$ $-2.74 \mathrm{E}-01 \pm 4.22 \mathrm{E}-02 \mathrm{pCi} / \mathrm{g}$ $1.04 \mathrm{E}+00 \pm 5.78 \mathrm{E}-01 \mathrm{pCi} \mathrm{g}$ $-6.02 \mathrm{E}-03 \pm 8.44 \mathrm{E}-03 \mathrm{pCi} / \mathrm{g}$ $6.65 \mathrm{E}-03 \pm 5.84 \mathrm{E}-03 \mathrm{pCi} / \mathrm{g}$ $-4.46 \mathrm{E}-02 \pm 1.31 \mathrm{E}-02 \mathrm{pCi} / \mathrm{g}$ $0.00 \mathrm{E}+00$ $\mathrm{pCi} / \mathrm{g}$

SURVEY ID: PPSC 1101

Sample ID: 104652

Survey location: 44944E $3681248 \mathrm{~N}$ (SRS coordinates)

Depth of core interval: 0.00 to $1.00 \mathrm{ft}$

Sample type: Normal

Sample matrix: Soil

Percent solids: $\mathbf{4 8 . 0 0}$
Constituent
RQ AQ B Result
Unit
D. Limit Method

Physical Parameters and Specified Analyses

Ammonia nitrogen

Chloride

Cyanide

Nitrate-nitrite as nitrogen

Nitrate-nitrite as nitrogen

$\begin{array}{ll} & 64.3 \\ & 6.99 \\ U & 2.08 \\ U & 1.9 \\ U & 2.04\end{array}$

$\mathrm{mg} / \mathrm{kg}$

$\mathrm{mg} / \mathrm{kg}$

$\mathrm{mg} / \mathrm{kg}$

$\mathrm{mg} / \mathrm{kg}$

$\mathrm{mg} / \mathrm{kg}$
43.4

5.4

2.08

1.9

2.04
EPA350.1

EPA300.0

EPA335.3

EPA353.1

EPA353.1 
SURVEY ID: PPSC 1101 (continued)

Sample ID: 104652

Constituent

RQ AQ B Result

Unit

D. Limit Method

Physical Parameters and Specified Analyses

Nitrogen by Kjeldahl method

Orthophosphate

Phenols

Sulfide

Total organic carbon

Total organic nitrogen

Total phosphates (as P)

Metals (total recoverable)

$\begin{array}{lll} & & 1140 \\ \text { U } & & 20.8 \\ \text { UJ } & \text { C } & 609 \\ \text { UJ } & \text { C } & 521 \\ & & 31000 \\ \text { C } & & 1080 \\ \text { J } & \text { E } & 375\end{array}$

17300

$\begin{array}{ll}\mathrm{U} & 9.92 \\ \mathrm{U} & 29.8\end{array}$

$\begin{array}{lll} & & \mathbf{5 8 . 5} \\ \mathrm{J} & \mathrm{E} & \mathbf{0 . 5 7 3}\end{array}$

U $\quad 0.496$

482

32.7

5.5

14.1

17600

17.2

367

152

0.0766

0.101

6.29

244

$\begin{array}{ll}\mathrm{U} & 29.8\end{array}$

U $\quad 2.48$

J $\quad \mathrm{E} \quad 27.2$

U $\quad 29.8$

34

31.1

$\begin{array}{lll}\mathrm{mg} / \mathrm{kg} & 160 & \text { EPA351.2 } \\ \mathrm{mg} / \mathrm{kg} & 20.8 & \text { EPA365.2 } \\ \mu \mathrm{g} / \mathrm{kg} & 609 & \text { EPA420.2 } \\ \mathrm{mg} / \mathrm{kg} & 521 & \text { EPA376.2 } \\ \mathrm{mg} / \mathrm{kg} & 208 & \text { EPA415.1 } \\ \mathrm{mg} / \mathrm{kg} & 0.417 & \text { EPA351.2 } \\ \mathrm{mg} / \mathrm{kg} & 801 & \text { EPA365.4 }\end{array}$

\begin{tabular}{lll}
$\mathrm{mg} / \mathrm{kg}$ & 24.8 & EPA6010A \\
$\mathrm{mg} / \mathrm{kg}$ & 9.92 & EPA6010A \\
$\mathrm{mg} / \mathrm{kg}$ & 29.8 & EPA6010A \\
$\mathrm{mg} / \mathrm{kg}$ & 2.48 & EPA6010A \\
$\mathrm{mg} / \mathrm{kg}$ & 0.992 & EPA6010A \\
$\mathrm{mg} / \mathrm{kg}$ & 0.496 & EPA6010A \\
$\mathrm{mg} / \mathrm{kg}$ & 24.8 & EPA6010A \\
$\mathrm{mg} / \mathrm{kg}$ & 2.48 & EPA6010A \\
$\mathrm{mg} / \mathrm{kg}$ & 0.992 & EPA6010A \\
$\mathrm{mg} / \mathrm{kg}$ & 2.48 & EPA6010A \\
$\mathrm{mg} / \mathrm{kg}$ & 9.92 & EPA6010A \\
$\mathrm{mg} / \mathrm{kg}$ & 9.92 & EPA6010A \\
$\mathrm{mg} / \mathrm{kg}$ & 9.92 & EPA6010A \\
$\mathrm{mg} / \mathrm{kg}$ & 0.992 & EPA6010A \\
$\mathrm{mg} / \mathrm{kg}$ & 0.0638 & EPA7471 \\
$\mathrm{mg} / \mathrm{kg}$ & 0.0644 & EPA7471 \\
$\mathrm{mg} / \mathrm{kg}$ & 2.48 & EPA6010A \\
$\mathrm{mg} / \mathrm{kg}$ & 198 & EPA6010A \\
$\mathrm{mg} / \mathrm{kg}$ & 29.8 & EPA6010A \\
$\mathrm{mg} / \mathrm{kg}$ & 2.48 & EPA6010A \\
$\mathrm{mg} / \mathrm{kg}$ & 99.2 & EPA6010A \\
$\mathrm{mg} / \mathrm{kg}$ & 29.8 & EPA6010A \\
$\mathrm{mg} / \mathrm{kg}$ & 0.992 & EPA6010A \\
$\mathrm{mg} / \mathrm{kg}$ & 1.98 & EPA6010A \\
\hline
\end{tabular}

$B / N / A$ Extractables (including PAH and phenols)

Acenaphthene

Acenaphthylene

Anthracene

Benzo[a]anthracene

Benzo[b]fluoranthene

Benzo[k]fluoranthene

Benzoic acid

Benzo[g,h,i]perylene

Benzo[a]pyrene

Benzyl alcohol

Bis(2-chloroethoxy) methane

Bis(2-chloroethyl) ether

Bis(2-chloroisopropyl)-ether

Bis(2-ethylhexyl) phthalate

4-Bromophenyl phenyl ether

Butylbenzyl phthalate

4-Chloroaniline

4-Chloro-m-cresol
U

U

$\mathrm{U}$

U

U

U

U

U

U

U

U

U

U

$\mathrm{U}$

U

U

U

U
69.4

69.4

69.4

6.94

6.94

6.94

69.4

69.4

6.94

69.4

69.4

69.4

69.4

69.4

69.4

69.4

69.4

69.4 $\mu \mathrm{g} / \mathrm{kg}$

$\mu \mathrm{g} / \mathrm{kg}$

$\mu \mathrm{g} / \mathrm{kg}$

$\mu \mathrm{g} / \mathrm{kg}$

$\mu \mathrm{g} / \mathrm{kg}$

$\mu \mathrm{g} / \mathrm{kg}$

$\mu \mathrm{g} / \mathrm{kg}$

$\mu \mathrm{g} / \mathrm{kg}$

$\mu \mathrm{g} / \mathrm{kg}$

$\mu \mathrm{g} / \mathrm{kg}$

$\mu \mathrm{g} / \mathrm{kg}$

$\mu \mathrm{g} / \mathrm{kg}$

$\mu \mathrm{g} / \mathrm{kg}$

$\mu \mathrm{g} / \mathrm{kg}$

$\mu \mathrm{g} / \mathrm{kg}$

$\mu \mathrm{g} / \mathrm{kg}$

$\mu \mathrm{g} / \mathrm{kg}$

$\mu \mathrm{g} / \mathrm{kg}$
69.4

69.4

69.4

6.94

6.94

6.94

69.4

69.4

6.94

69.4

69.4

69.4

69.4

69.4

69.4

69.4

69.4

69.4
EPA8270

EPA8270

EPA8270

EPA8270

EPA8270

EPA8270

EPA8270

EPA8270

EPA8270

EPA8270

EPA8270

EPA8270

EPA8270

EPA8270

EPA8270

EPA8270

EPA8270

EPA8270 
SURVEY ID: PPSC 1101 (continued)

Sample ID: 104652

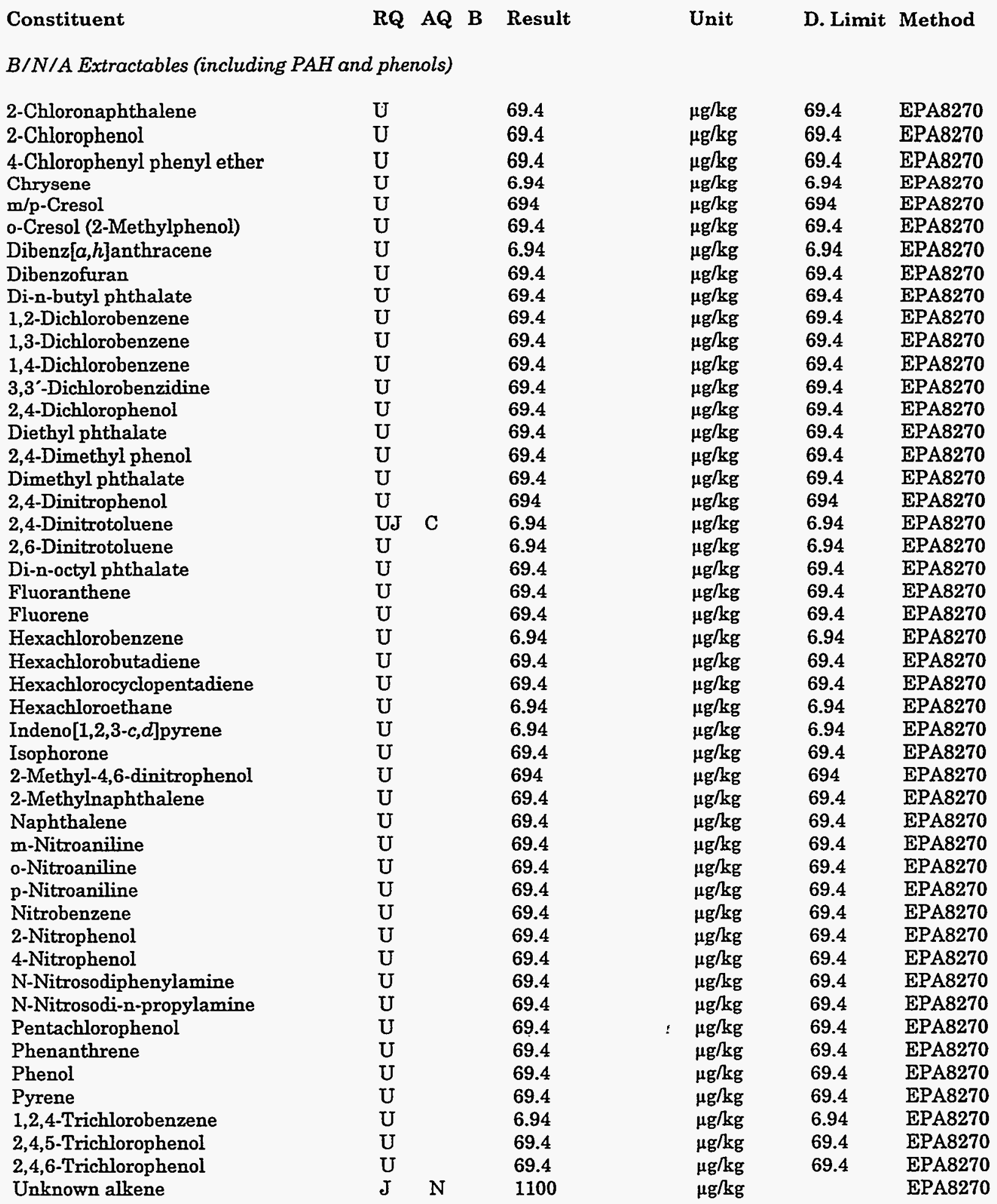


SURVEY ID: PPSC 1101 (continued)

RQ AQ B Result

\begin{tabular}{lll}
$\mu \mathrm{g} / \mathrm{kg}$ & 10.4 & EPA8260 \\
$\mu \mathrm{g} / \mathrm{kg}$ & 1.04 & EPA8260 \\
$\mu \mathrm{g} / \mathrm{kg}$ & 0.104 & EPA8260 \\
$\mu \mathrm{g} / \mathrm{kg}$ & 0.104 & EPA8260 \\
$\mu \mathrm{g} / \mathrm{kg}$ & 0.208 & EPA8260 \\
$\mu \mathrm{g} / \mathrm{kg}$ & 2.08 & EPA8260 \\
$\mu \mathrm{g} / \mathrm{kg}$ & 0.104 & EPA8260 \\
$\mu \mathrm{g} / \mathrm{kg}$ & 0.104 & EPA8260 \\
$\mu \mathrm{g} / \mathrm{kg}$ & 0.208 & EPA8260 \\
$\mu \mathrm{g} / \mathrm{kg}$ & 0.208 & EPA8260 \\
$\mu \mathrm{g} / \mathrm{kg}$ & 0.104 & EPA8260 \\
$\mu \mathrm{g} / \mathrm{kg}$ & 0.208 & EPA8260 \\
$\mu \mathrm{g} / \mathrm{kg}$ & 0.104 & EPA8260 \\
$\mu \mathrm{g} / \mathrm{kg}$ & 0.104 & EPA8260 \\
$\mu \mathrm{g} / \mathrm{kg}$ & 0.104 & EPA8260 \\
$\mu \mathrm{g} / \mathrm{kg}$ & 0.104 & EPA8260 \\
$\mu \mathrm{g} / \mathrm{kg}$ & 0.208 & EPA8260 \\
$\mu \mathrm{g} / \mathrm{kg}$ & 1.04 & EPA8260 \\
$\mu \mathrm{g} / \mathrm{kg}$ & 0.104 & EPA8260 \\
$\mu \mathrm{g} / \mathrm{kg}$ & 0.104 & EPA8260 \\
$\mu \mathrm{g} / \mathrm{kg}$ & 0.104 & EPA8260 \\
$\mu \mathrm{g} / \mathrm{kg}$ & 0.104 & EPA8260 \\
$\mu \mathrm{g} / \mathrm{kg}$ & 2.08 & EPA8260 \\
$\mu \mathrm{g} / \mathrm{kg}$ & & EPA8260 \\
$\mu \mathrm{g} / \mathrm{kg}$ & 2.08 & EPA8260 \\
$\mu \mathrm{g} / \mathrm{kg}$ & 2.08 & EPA8260 \\
$\mu \mathrm{g} / \mathrm{kg}$ & 0.104 & EPA8260 \\
$\mu \mathrm{g} / \mathrm{kg}$ & 0.104 & EPA8260 \\
$\mu \mathrm{g} / \mathrm{kg}$ & 0.104 & EPA8260 \\
$\mu \mathrm{g} / \mathrm{kg}$ & 1.04 & EPA8260 \\
$\mu \mathrm{g} / \mathrm{kg}$ & 0.104 & EPA8260 \\
$\mu \mathrm{g} / \mathrm{kg}$ & 0.104 & EPA8260 \\
$\mu \mathrm{g} / \mathrm{kg}$ & 0.104 & EPA8260 \\
$\mu \mathrm{g} / \mathrm{kg}$ & & EPA8260 \\
$\mu \mathrm{g} / \mathrm{kg}$ & 2.08 & EPA8260 \\
$\mu \mathrm{g} / \mathrm{kg}$ & 0.313 & EPA8260 \\
& & \\
\hline
\end{tabular}

Pesticides/PCBs and Dioxins/Furans

Aldrin
alpha-Benzene hexachloride
beta-Benzene hexachloride
delta-Benzene hexachloride
alpha-Chlordane
gamma-Chlordane
p,p'-DDD
p,p'-DDE
p,p'-DDT
Dieldrin
Endosulfan sulfate
Endosulfan I
Endosulfan II
Endrin
Endrin ketone
Heptachlor

$\begin{array}{ll}U & 173 \\ U & 173 \\ U & 346 \\ U & 346 \\ U & 346 \\ U & 346 \\ U & 692 \\ U & 346 \\ U & 692 \\ U & 346 \\ U & 692 \\ U & 346 \\ U & 692 \\ U & 346 \\ U & 692 \\ U & 346\end{array}$

Sample ID: 104652
Unit
D. Limit Method

Constituent

Volatile Organic Compounds

Acetone
Benzene
Bromodichloromethane
Bromoform
Bromomethane (Methyl bromide)
Carbon disulfide
Carbon tetrachloride
Chlorobenzene
Chloroethane
Chloroethene (Vinyl chloride)
Chloroform
Chloromethane (Methyl chloride)
Dibromochloromethane
1,1-Dichloroethane
1,2-Dichloroethane
1,1-Dichloroethylene
1,2-Dichloroethylene
Dichloromethane (Methylene chloride)
1,2-Dichloropropane
cis-1,3-Dichloropropene
trans-1,3-Dichloropropene
Ethylbenzene
2-Hexanone
Isocamphene
Methyl ethyl ketone
Methyl isobutyl ketone
Styrene
1,1,2,2-Tetrachloroethane
Tetrachloroethylene
Toluene
1,1,1-Trichloroethane
1,1,2-Trichloroethane
Trichloroethylene
Unknown alcohol
Vinyl acetate
Xylenes

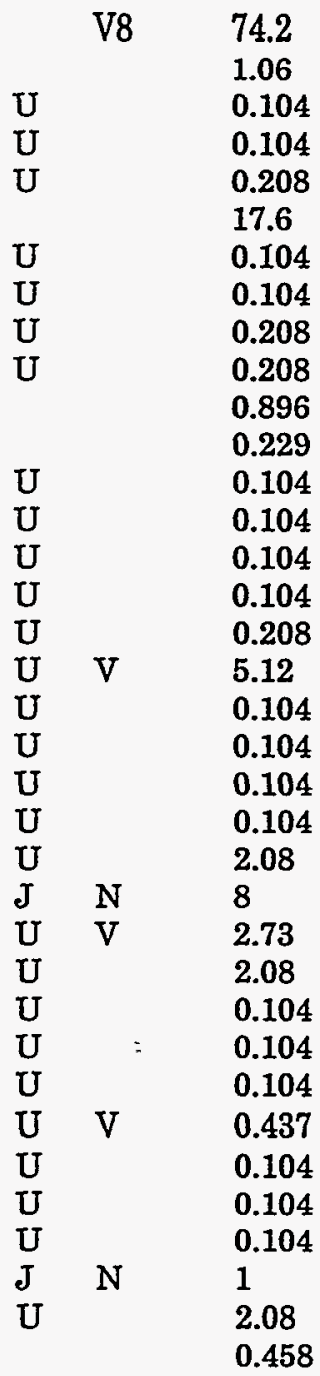

0.104

0.208

0.104

.208

0.896

0.104

0.104

0.104

5.12

0.104

0.104

0.104

.73

0.104

0.104

0.437

0.104

0.104

2.08
0.458

$\begin{array}{lrl}\mu \mathrm{g} / \mathrm{kg} & 173 & \text { EPA8080 } \\ \mu \mathrm{g} / \mathrm{kg} & 173 & \text { EPA8080 } \\ \mu \mathrm{g} / \mathrm{kg} & 346 & \text { EPA8080 } \\ \mu \mathrm{g} / \mathrm{kg} & 346 & \text { EPA8080 } \\ \mu \mathrm{g} / \mathrm{kg} & 346 & \text { EPA8080 } \\ \mu \mathrm{g} / \mathrm{kg} & 346 & \text { EPA8080 } \\ \mu \mathrm{g} / \mathrm{kg} & 692 & \text { EPA8080 } \\ \mu \mathrm{g} / \mathrm{kg} & 346 & \text { EPA8080 } \\ \mu \mathrm{g} / \mathrm{kg} & 692 & \text { EPA8080 } \\ \mu \mathrm{g} / \mathrm{kg} & 346 & \text { EPA8080 } \\ \mu \mathrm{g} / \mathrm{kg} & 692 & \text { EPA8080 } \\ \mu \mathrm{g} / \mathrm{kg} & 346 & \text { EPA8080 } \\ \mu \mathrm{g} / \mathrm{kg} & -692 & \text { EPA8080 } \\ \mu \mathrm{g} / \mathrm{kg} & 346 & \text { EPA8080 } \\ \mu \mathrm{g} / \mathrm{kg} & 692 & \text { EPA8080 } \\ \mu \mathrm{g} / \mathrm{kg} & 346 & \text { EPA8080 }\end{array}$


SURVEY ID: PPSC 1101 (continued)

Constituent

Pesticides/PCBs and Dioxins/Furans

Heptachlor epoxide

Lindane

Methoxychlor

PCB 1016

PCB 1221

PCB 1232

PCB 1242

PCB 1248

PCB 1254

PCB 1260

Toxaphene

Radionuclides

Actinium-228

Antimony-124

Antimony-125

Barium-133

Cerium-144

Cesium-134

Cesium-137

Cobalt-57

Cobalt-58

Cobalt-60

Europium-152

Europium-154

Europium-155

Gross alpha

Lead-212

Manganese-54

Neptunium-239

Nonvolatile beta

Plutonium-238

Plutonium-239/240

Potassium-40

Promethium-144

Promethium-146

Ruthenium-106

Sodium-22

Strontium-90

Thorium-234

Tin-113

Yttrium-88

Zinc-65

Zirconium-95

Sample ID: 104652

RQ AQ B Result Unit D. Limit Method

$\begin{array}{lllll}\mathrm{U} & 346 & \mu \mathrm{g} / \mathrm{kg} & 346 & \text { EPA8080 } \\ U & 173 & \mu \mathrm{g} / \mathrm{kg} & 173 & \text { EPA8080 } \\ U & 3460 & \mu \mathrm{g} / \mathrm{kg} & 3460 & \text { EPA8080 } \\ U & 865 & \mu \mathrm{g} / \mathrm{kg} & 865 & \text { EPA8080 } \\ U & 865 & \mu \mathrm{g} / \mathrm{kg} & 865 & \text { EPA8080 } \\ U & 865 & \mu \mathrm{kg} & 865 & \text { EPA8080 } \\ U & 865 & \mu \mathrm{g} / \mathrm{kg} & 865 & \text { EPA8080 } \\ U & 865 & \mu \mathrm{kg} & 865 & \text { EPA8080 } \\ U & 865 & \mu \mathrm{kg} / \mathrm{kg} & 865 & \text { EPA8080 } \\ \mathrm{U} & 865 & \mu \mathrm{g} / \mathrm{kg} & 865 & \text { EPA8080 } \\ U & 6920 & \mu \mathrm{g} / \mathrm{kg} & 6920 & \text { EPA8080 }\end{array}$

2.07E+00 $1.73 \mathrm{E}-01 \mathrm{pCi} / \mathrm{g}$ $-1.60 \mathrm{E}-02 \pm 2.80 \mathrm{E}-02 \mathrm{pCi} / \mathrm{g}$ $-8.71 \mathrm{E}-03 \pm 1.09 \mathrm{E}-01 \mathrm{pCi} / \mathrm{g}$ $1.04 \mathrm{E}-02 \pm 5.91 \mathrm{E}-02 \mathrm{pCi} / \mathrm{g}$ $-1.18 \mathrm{E}-02 \pm 1.59 \mathrm{E}-01 \mathrm{pCi} / \mathrm{g}$ $-8.96 \mathrm{E}-03 \pm 2.61 \mathrm{E}-02 \mathrm{pCi} / \mathrm{g}$ $1.99 \mathrm{E}+01 \pm 2.03 \mathrm{E}-01 \mathrm{pCi} / \mathrm{g}$ $-1.13 \mathrm{E}-02 \pm 1.93 \mathrm{E}-02 \mathrm{pCi} / \mathrm{g}$ $7.84 \mathrm{E}-03 \pm 1.94 \mathrm{E}-02 \mathrm{pCi} / \mathrm{g}$ $3.58 \mathrm{E}-01 \pm 4.62 \mathrm{E}-02 \mathrm{pCi} / \mathrm{g}$ $-5.50 \mathrm{E}-02 \pm 8.71 \mathrm{E}-02 \mathrm{pCi} / \mathrm{g}$ $-1.77 \mathrm{E}-01 \pm 1.73 \mathrm{E}-01 \mathrm{pCi} / \mathrm{g}$ $5.96 \mathrm{E}-02 \div 8.77 \mathrm{E}-02 \mathrm{pCi} / \mathrm{g}$ $2.53 \mathrm{E}+01 \pm 4.33 \mathrm{E}+00 \mathrm{pCi} / \mathrm{g}$ $2.33 \mathrm{E}+00 \pm 9.02 \mathrm{E}-02 \mathrm{pCi} / \mathrm{g}$ $4.76 \mathrm{E}-02 \pm 2.36 \mathrm{E}-02 \mathrm{pCi} / \mathrm{g}$ $-3.91 \mathrm{E}-02+1.46 \mathrm{E}-01 \mathrm{pCi} / \mathrm{g}$ $2.77 \mathrm{E}+01 \pm 3.06 \mathrm{E}+00 \mathrm{pCi} / \mathrm{g}$ $1.09 \mathrm{E}-02 \pm 2.52 \mathrm{E}-02 \mathrm{pCi} / \mathrm{g}$ 5.58E-02 $43.72 \mathrm{E}-02 \mathrm{pCi} / \mathrm{g}$ $2.38 \mathrm{E}+00 \pm 4.70 \mathrm{E}-01 \mathrm{pCi} / \mathrm{g}$ $1.03 \mathrm{E}-02 \pm 1.80 \mathrm{E}-02 \mathrm{pCi} / \mathrm{g}$ $3.80 \mathrm{E}-02 \pm 4.06 \mathrm{E}-02 \mathrm{pCi} / \mathrm{g}$ $2.50 \mathrm{E}-02 \pm 2.20 \mathrm{E}-01 \mathrm{pCi} / \mathrm{g}$ 9.08E-04 $1.76 \mathrm{E}-02 \mathrm{pCi} / \mathrm{g}$

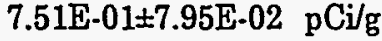
$3.83 \mathrm{E}+00 \pm 1.26 \mathrm{E}+00 \mathrm{pCi} / \mathrm{g}$ 2.95E-02 $\pm 4.39 \mathrm{E}-02 \mathrm{pCi} / \mathrm{g}$ $1.44 \mathrm{E}-02 \pm 1.78 \mathrm{E}-02 \mathrm{pCi} / \mathrm{g}$ $-1.11 \mathrm{E}-02 \pm 4.69 \mathrm{E}-02 \mathrm{pCi} / \mathrm{g}$ $-4.55 \mathrm{E}-03 \pm 3.57 \mathrm{E}-02 \mathrm{pCi} / \mathrm{g}$
1.08E-01 EPIA-013B
4.59E-02 EPIA-013B
1.62E-01 EPIA-013B
6.56E-02 EPIA-013B
2.67E-01 EPIA-013B
3.75E-02 EPIA-013B
4.12E-02 EPIA-013B
3.22E-02 EPIA-013B
3.49E-02 EPIA-013B
3.02E-02 EPIA-013B
1.48E-01 EPIA-013B
2.83E-01 EPLA-013B
1.28E-01 EPIA-013B
2.11E+00 EPIA-001B
8.08E-02 EPIA-013B
3.11E-02 EPIA-013B
2.46E-01 EPIA-013B
2.85E+00 EPIA-001B
5.72E-02 EPLA-012B
4.37E-02 EPIA-012B
2.77E-01 EPLA-013B
3.10E-02 EPLA-013B
8.08E-02 EPIA-013B
3.70E-01 EPIA-013B
3.06E-02 EPIA-013B
4.60E-02 EPIA-004
1.21E+00 EPIA-013B
7.58E-02 EPIA-013B
3.48E-02 EPIA-013B
6.86E-02 EPIA-013B
6.27E-02 EPIA-013B

\section{SURVEY ID: PPSC 1102}

Sample ID: 104653

Survey location: $44944 \mathrm{E}$ 3681248N (SRS coordinates)

Depth of core interval: 1.00 to $2.00 \mathrm{ft}$

Sample type: Normal

Sample matrix: Soil

Percent solids: $\mathbf{8 1 . 0 0}$ 
SURVEY ID: PPSC 1102 (continued)

Constituent
Sample ID: 104653
RQ AQ B Result
Unit
D. Limit Method

Physical Parameters and Specified Analyses

Cation exchange capacity

Chloride

Cyanide

Nitrate-nitrite as nitrogen

Orthophosphate

Phenols

Sulfide

Total organic carbon

Total organic nitrogen

Total phosphates (as P)

$\begin{array}{lll} & & 35.9 \\ \text { U } & & 3.19 \\ \text { U } & & 1.23 \\ \text { J } & \text { E } & 0.472 \\ \text { U } & & 12.3 \\ \text { UJ } & \text { C } & 359 \\ \text { UJ } & \text { C } & 309 \\ & & 4380 \\ \text { C } & & 87 \\ \text { J } & \text { E } & 119\end{array}$

Metals (total recoverable)

Aluminum

Antimony

Arsenic

Barium

Beryllium

Cadmium

Calcium

Chromium

Cobalt

Copper

Iron

Lead

Magnesium

Manganese

Mercury

Nickel

Potassium

Selenium

Silver

Sodium

Thallium

Vanadium

Zinc
3330

5.88

17.6

12.6

0.0905

0.294

70.5

6.02

0.636

1.01

4310

5.5

61.1

23.3

0.0283

1.35

44

17.6

1.47

58.8

17.6

9.28

3.63

$\begin{array}{ll}\mathrm{meq} / 100 \mathrm{~g} & 5 \\ \mathrm{mg} / \mathrm{kg} & 3.19 \\ \mathrm{mg} / \mathrm{kg} & 1.23 \\ \mathrm{mg} / \mathrm{kg} & 1.18 \\ \mathrm{mg} / \mathrm{kg} & 12.3 \\ \mu \mathrm{g} / \mathrm{kg} & 359 \\ \mathrm{mg} / \mathrm{kg} & 309 \\ \mathrm{mg} / \mathrm{kg} & 123 \\ \mathrm{mg} / \mathrm{kg} & 0.247 \\ \mathrm{mg} / \mathrm{kg} & 466\end{array}$

EPA9081

EPA300.0

EPA335.3

EPA353.1

EPA365.2

EPA420.2

EPA376.2

EPA415.1

EPA351.2

EPA365.4

$B / N / A$ Extractables (including PAH and phenols)

Acenaphthene

Acenaphthylene

Anthracene

Benzo[a]anthracene

Benzo[b] lluoranthene

Benzo[k]fluoranthene

Benzoic acid

Benzo[g,h,i]perylene

Benzo[a]pyrene

Benzyl alcohol

Bis(2-chloroethoxy) methane

Bis(2-chloroethyl) ether
$\mathrm{U}$

$\mathrm{U}$

$\mathrm{U}$

$\mathrm{U}$

$\mathrm{U}$

$\mathrm{U}$

$\mathrm{U}$

U

$U$

U

U

$\begin{array}{lll}\mathrm{mg} / \mathrm{kg} & 14.7 & \text { EPA6010A } \\ \mathrm{mg} / \mathrm{kg} & 5.88 & \text { EPA6010A } \\ \mathrm{mg} / \mathrm{kg} & 17.6 & \text { EPA6010A } \\ \mathrm{mg} / \mathrm{kg} & 1.47 & \text { EPA6010A } \\ \mathrm{mg} / \mathrm{kg} & 0.588 & \text { EPA6010A } \\ \mathrm{mg} / \mathrm{kg} & 0.294 & \text { EPA6010A } \\ \mathrm{mg} / \mathrm{kg} & 14.7 & \text { EPA6010A } \\ \mathrm{mg} / \mathrm{kg} & 1.47 & \text { EPA6010A } \\ \mathrm{mg} / \mathrm{kg} & 0.588 & \text { EPA6010A } \\ \mathrm{mg} / \mathrm{kg} & 1.47 & \text { EPA6010A } \\ \mathrm{mg} / \mathrm{kg} & 5.88 & \text { EPA6010A } \\ \mathrm{mg} / \mathrm{kg} & 5.88 & \text { EPA6010A } \\ \mathrm{mg} / \mathrm{kg} & 5.88 & \text { EPA6010A } \\ \mathrm{mg} / \mathrm{kg} & 0.588 & \text { EPA6010A } \\ \mathrm{mg} / \mathrm{kg} & 0.0283 & \text { EPA7471 } \\ \mathrm{mg} / \mathrm{kg} & 1.47 & \text { EPA6010A } \\ \mathrm{mg} / \mathrm{kg} & 118 & \text { EPA6010A } \\ \mathrm{mg} / \mathrm{kg} & 17.6 & \text { EPA6010A } \\ \mathrm{mg} / \mathrm{kg} & 1.47 & \text { EPA6010A } \\ \mathrm{mg} / \mathrm{kg} & 58.8 & \text { EPA6010A } \\ \mathrm{mg} / \mathrm{kg} & 17.6 & \text { EPA6010A } \\ \mathrm{mg} / \mathrm{kg} & 0.588 & \text { EPA6010A } \\ \mathrm{mg} / \mathrm{kg} & 1.18 & \text { EPA6010A }\end{array}$

40.2

40.2

40.2

4.02

4.02

4.02

40.2

40.2

4.02

40.2

40.2

40.2 $\mu \mathrm{g} / \mathrm{kg}$

$\leqslant \mu \mathrm{g} / \mathrm{kg}$

$\mu \mathrm{g} / \mathrm{kg}$

$\mu \mathrm{g} / \mathrm{kg}$

$\mu \mathrm{g} / \mathrm{kg}$

$\mu \mathrm{g} / \mathrm{kg}$

$\mu \mathrm{g} / \mathrm{kg}$

$\mu \mathrm{g} / \mathrm{kg}$

$\mu \mathrm{g} / \mathrm{kg}$

$\mu \mathrm{g} / \mathrm{kg}$

$\mu \mathrm{g} / \mathrm{kg}$

$\mu \mathrm{g} / \mathrm{kg}$

40.2

EPA8270

40.2

40.2

4.02

EPA8270

EPA8270

EPA8270

EPA8270

402

EPA8270

40.2

EPA8270

40.2

4.02

40.2

EPA8270

EPA8270

EPA8270

40.2

EPA8270

40.2

EPA8270 
$B / N / A$ Extractables (including PAH and phenols)

Bis(2-chloroisopropyl) ether

Bis(2-ethylhexyl) phthalate

4-Bromophenyl phenyl ether

Butylbenzyl phthalate

4-Chloroaniline

4-Chloro-m-cresol

2-Chloronaphthalene

2-Chlorophenol

4-Chlorophenyl phenyl ether

Chrysene

$\mathrm{m} / \mathrm{p}$-Cresol

o-Cresol (2-Methylphenol)

Dibenz $[a, h]$ anthracene

Dibenzofuran

Di-n-butyl phthalate

1,2-Dichlorobenzene

1,3-Dichlorobenzene

1,4-Dichlorobenzene

3,3'-Dichlorobenzidine

2,4-Dichlorophenol

Diethyl phthalate

2,4-Dimethyl phenol

Dimethyl phthalate

2,4-Dinitrophenol

2,4-Dinitrotoluene

2,6-Dinitrotoluene

Di-n-octyl phthalate

Fluoranthene

Fluorene

Hexachlorobenzene

Hexachlorobutadiene

Hexachlorocyclopentadiene

Hexachloroethane

Indeno[1,2,3-c,d]pyrene

Isophorone

2-Methyl-4,6-dinitrophenol

2-Methylnaphthalene

Naphthalene

m-Nitroaniline

o-Nitroaniline

p-Nitroaniline

Nitrobenzene

2-Nitrophenol

4-Nitrophenol

N-Nitrosodiphenylamine

N-Nitrosodi-n-propylamine

Pentachlorophenol

Phenanthrene

Phenol

Pyrene

1,2,4-Trichlorobenzene

2,4,5-Trichlorophenol

2,4,6-Trichlorophenol
U

$\mathrm{U}$

U

U

$\mathrm{U}$

U

$\mathrm{U}$

U

$\mathrm{U}$

U

U

U

U

U

$\mathrm{U}$

U

U

U

$\mathrm{U}$

U

U

U

U

Ue

$U$

U

U

U

U

U

U

U

U

U

U

U

U

U

U

U

U

U

U

U

U

U

U

U

U

U

U
40.2

40.2

40.2

40.2

40.2

40.2

40.2

40.2

40.2

4.02

402

40.2

4.02

40.2

34.1

40.2

40.2

40.2

40.2

40.2

40.2

40.2

40.2

402

4.02

4.02

40.2

40.2

40.2

4.02

40.2

40.2

4.02

4.02

40.2

402

40.2

40.2

40.2

40.2

40.2

40.2

40.2

40.2

40.2

40.2

40.2

40.2

40.2

40.2

4.02

40.2

40.2

\begin{tabular}{|c|c|c|}
\hline$\mu \mathrm{g} / \mathrm{kg}$ & 40.2 & EPA8270 \\
\hline$\mu g / \mathrm{kg}$ & 40.2 & EPA8270 \\
\hline$\mu g / \mathrm{kg}$ & 40.2 & EPA8270 \\
\hline$\mu \mathrm{g} / \mathrm{kg}$ & 40.2 & EPA8270 \\
\hline$\mu \mathrm{g} / \mathrm{kg}$ & 40.2 & EPA8270 \\
\hline$\mu \mathrm{g} / \mathrm{kg}$ & 40.2 & EPA8270 \\
\hline$\mu \mathrm{g} / \mathrm{kg}$ & 40.2 & EPA8270 \\
\hline$\mu \mathrm{g} / \mathrm{kg}$ & 40.2 & EPA8270 \\
\hline$\mu \mathrm{g} / \mathrm{kg}$ & 40.2 & EPA8270 \\
\hline$\mu \mathrm{g} / \mathrm{kg}$ & 4.02 & EPA8270 \\
\hline$\mu \mathrm{g} / \mathrm{kg}$ & 402 & EPA8270 \\
\hline$\mu \mathrm{g} / \mathrm{kg}$ & 40.2 & EPA8270 \\
\hline$\mu \mathrm{g} / \mathrm{kg}$ & 4.02 & EPA8270 \\
\hline$\mu g / \mathrm{kg}$ & 40.2 & EPA8270 \\
\hline$\mu \mathrm{g} / \mathrm{kg}$ & 40.2 & EPA8270 \\
\hline$\mu g / k g$ & 40.2 & EPA8270 \\
\hline$\mu \mathrm{g} / \mathrm{kg}$ & 40.2 & EPA8270 \\
\hline$\mu \mathrm{g} / \mathrm{kg}$ & 40.2 & EPA8270 \\
\hline$\mu \mathrm{g} / \mathrm{kg}$ & 40.2 & EPA8270 \\
\hline$\mu \mathrm{g} / \mathrm{kg}$ & 40.2 & EPA8270 \\
\hline$\mu \mathrm{g} / \mathrm{kg}$ & 40.2 & EPA8270 \\
\hline$\mu \mathrm{g} / \mathrm{kg}$ & 40.2 & EPA8270 \\
\hline$\mu \mathrm{g} / \mathrm{kg}$ & 40.2 & EPA8270 \\
\hline$\mu \mathrm{g} / \mathrm{kg}$ & 402 & EPA8270 \\
\hline$\mu \mathrm{g} / \mathrm{kg}$ & 4.02 & EPA8270 \\
\hline$\mu \mathrm{g} / \mathrm{kg}$ & 4.02 & EPA8270 \\
\hline$\mu \mathrm{g} / \mathrm{kg}$ & 40.2 & EPA8270 \\
\hline$\mu \mathrm{g} / \mathrm{kg}$ & 40.2 & EPA8270 \\
\hline$\mu \mathrm{g} / \mathrm{kg}$ & 40.2 & EPA8270 \\
\hline$\mu \mathrm{g} / \mathrm{kg}$ & 4.02 & EPA8270 \\
\hline$\mu \mathrm{g} / \mathrm{kg}$ & 40.2 & EPA8270 \\
\hline$\mu \mathrm{g} / \mathrm{kg}$ & 40.2 & EPA8270 \\
\hline$\mu \mathrm{g} / \mathrm{kg}$ & 4.02 & EPA8270 \\
\hline$\mu g / \mathrm{kg}$ & 4.02 & EPA8270 \\
\hline$\mu \mathrm{g} / \mathrm{kg}$ & 40.2 & EPA8270 \\
\hline$\mu \mathrm{g} / \mathrm{kg}$ & 402 & EPA8270 \\
\hline$\mu \mathrm{g} / \mathrm{kg}$ & 40.2 & EPA8270 \\
\hline$\mu \mathrm{g} / \mathrm{kg}$ & 40.2 & EPA8270 \\
\hline$\mu \mathrm{g} / \mathrm{kg}$ & 40.2 & EPA8270 \\
\hline$\mu \mathrm{g} / \mathrm{kg}$ & 40.2 & EPA 8270 \\
\hline$\mu \mathrm{g} / \mathrm{kg}$ & 40.2 & EPA8270 \\
\hline$\mu \mathrm{g} / \mathrm{kg}$ & 40.2 & EPA8270 \\
\hline$\mu \mathrm{g} / \mathrm{kg}$ & 40.2 & EPA8270 \\
\hline$\mu \mathrm{g} / \mathrm{kg}$ & 40.2 & EPA8270 \\
\hline$\mu \mathrm{g} / \mathrm{kg}$ & 40.2 & EPA8270 \\
\hline$\mu \mathrm{g} / \mathrm{kg}$ & 40.2 & EPA8270 \\
\hline$\mu \mathrm{g} / \mathrm{kg}$ & 40.2 & EPA8270 \\
\hline$\mu \mathrm{g} / \mathrm{kg}$ & 40.2 & EPA8270 \\
\hline$\mu g / k g$ & 40.2 & EPA8270 \\
\hline$\mu \mathrm{g} / \mathrm{kg}$ & 40.2 & EPA8270 \\
\hline$\mu \mathrm{g} / \mathrm{kg}$ & 4.02 & EPA8270 \\
\hline$\mu \mathrm{g} / \mathrm{kg}$ & 40.2 & EPA8270 \\
\hline$\mu \mathrm{g} / \mathrm{kg}$ & 40.2 & EPA8270 \\
\hline
\end{tabular}


SURVEY ID: PPSC 1102 (continued)

Constituent

Volatile Organic Compounds

Acetone
Benzene
Bromodichloromethane
Bromoform
Bromomethane (Methyl bromide)
Carbon disulfide
Carbon tetrachloride
Chlorobenzene
Chloroethane
Chloroethene (Vinyl chloride)
Chloroform
Chloromethane (Methyl chloride)
Dibromochloromethane
1,1-Dichloroethane
1,2-Dichloroethane
1,1-Dichloroethylene
1,2-Dichloroethylene
Dichloromethane (Methylene chloride)
1,2-Dichloropropane
cis-1,3-Dichloropropene
trans-1,3-Dichloropropene
Ethylbenzene
2-Hexanone
Methyl ethyl ketone
Methyl isobutyl ketone
Styrene
1,1,2,2-Tetrachloroethane
Tetrachloroethylene
Toluene
1,1,1-Trichloroethane
1,1,2-Trichloroethane
Trichloroethylene
Vinyl acetate
Xylenes
(a)

Pesticides/PCBs and Dioxins/Furans
Sample ID: 104653

RQ AQ B Result

Unit

D. Limit Method

\begin{tabular}{|c|c|c|}
\hline $\mathrm{U}$ & V8 & 47 \\
\hline $\mathbf{J}$ & \multirow[t]{16}{*}{$\mathbf{E}$} & 0.593 \\
\hline $\mathrm{U}$ & & 0.0617 \\
\hline $\mathrm{U}$ & & 0.0617 \\
\hline $\mathbf{U}$ & & 0.123 \\
\hline U & & 1.23 \\
\hline $\mathbf{U}$ & & 0.0617 \\
\hline $\mathbf{U}$ & & 0.0617 \\
\hline U & & 0.123 \\
\hline $\mathrm{U}$ & & 0.123 \\
\hline & & 0.506 \\
\hline $\mathrm{U}$ & & 0.123 \\
\hline$U$ & & 0.0617 \\
\hline $\mathbf{U}$ & & 0.0617 \\
\hline$U$ & & 0.0617 \\
\hline $\mathrm{U}$ & & 0.0617 \\
\hline $\mathrm{U}$ & & 0.123 \\
\hline $\mathrm{U}$ & \multirow[t]{5}{*}{$\mathrm{V}$} & 3.15 \\
\hline $\mathrm{U}$ & & 0.0617 \\
\hline $\mathrm{U}$ & & 0.0617 \\
\hline $\mathrm{U}$ & & 0.0617 \\
\hline U & & 0.0617 \\
\hline $\mathrm{U}$ & $\mathrm{V}$ & 0.975 \\
\hline $\mathrm{U}$ & \multirow[t]{5}{*}{$\mathrm{V}$} & 3.23 \\
\hline $\mathbf{U}$ & & 1.23 \\
\hline U & & 0.0617 \\
\hline $\mathbf{U}$ & & 0.0617 \\
\hline $\mathrm{U}$ & & 0.0617 \\
\hline $\mathrm{U}$ & \multirow[t]{6}{*}{$V$} & 0.173 \\
\hline $\mathrm{U}$ & & 0.0617 \\
\hline $\mathrm{U}$ & & 0.0617 \\
\hline $\mathrm{U}$ & & 0.0617 \\
\hline $\mathrm{U}$ & & 1.23 \\
\hline $\mathrm{U}$ & & 0.185 \\
\hline
\end{tabular}

$\begin{array}{lll}\mu \mathrm{g} / \mathrm{kg} & 6.17 & \text { EPA8260 } \\ \mu \mathrm{g} / \mathrm{kg} & 0.617 & \text { EPA8260 } \\ \mu \mathrm{g} / \mathrm{kg} & 0.0617 & \text { EPA8260 } \\ \mu \mathrm{g} / \mathrm{kg} & 0.0617 & \text { EPA8260 } \\ \mu \mathrm{g} / \mathrm{kg} & 0.123 & \text { EPA8260 } \\ \mu \mathrm{g} / \mathrm{kg} & 1.23 & \text { EPA8260 } \\ \mu \mathrm{g} / \mathrm{kg} & 0.0617 & \text { EPA8260 } \\ \mu \mathrm{g} / \mathrm{kg} & 0.0617 & \text { EPA8260 } \\ \mu \mathrm{g} / \mathrm{kg} & 0.123 & \text { EPA8260 } \\ \mu \mathrm{g} / \mathrm{kg} & 0.123 & \text { EPA8260 } \\ \mu \mathrm{g} / \mathrm{kg} & 0.0617 & \text { EPA8260 } \\ \mu \mathrm{g} / \mathrm{kg} & 0.123 & \text { EPA8260 } \\ \mu \mathrm{g} / \mathrm{kg} & 0.0617 & \text { EPA8260 } \\ \mu \mathrm{g} / \mathrm{kg} & 0.0617 & \text { EPA8260 } \\ \mu \mathrm{g} / \mathrm{kg} & 0.0617 & \text { EPA8260 } \\ \mu \mathrm{g} / \mathrm{kg} & 0.0617 & \text { EPA8260 } \\ \mu \mathrm{g} / \mathrm{kg} & 0.123 & \text { EPA8260 } \\ \mu \mathrm{g} / \mathrm{kg} & 0.617 & \text { EPA8260 } \\ \mu \mathrm{g} / \mathrm{kg} & 0.0617 & \text { EPA8260 } \\ \mu \mathrm{g} / \mathrm{kg} & 0.0617 & \text { EPA8260 } \\ \mu \mathrm{g} / \mathrm{kg} & 0.0617 & \text { EPA8260 } \\ \mu \mathrm{g} / \mathrm{kg} & 0.0617 & \text { EPA8260 } \\ \mu \mathrm{g} / \mathrm{kg} & 1.23 & \text { EPA8260 } \\ \mu \mathrm{g} / \mathrm{kg} & 1.23 & \text { EPA8260 } \\ \mu \mathrm{g} / \mathrm{kg} & 1.23 & \text { EPA8260 } \\ \mu \mathrm{g} / \mathrm{kg} & 0.0617 & \text { EPA8260 } \\ \mu \mathrm{g} / \mathrm{kg} & 0.0617 & \text { EPA8260 } \\ \mu \mathrm{g} / \mathrm{kg} & 0.0617 & \text { EPA8260 } \\ \mu \mathrm{g} / \mathrm{kg} & 0.617 & \text { EPA8260 } \\ \mu \mathrm{g} / \mathrm{kg} & 0.0617 & \text { EPA8260 } \\ \mu \mathrm{g} / \mathrm{kg} & 0.0617 & \text { EPA8260 } \\ \mu \mathrm{g} / \mathrm{kg} & 0.0617 & \text { EPA8260 } \\ \mu \mathrm{g} / \mathrm{kg} & 0.185 & \text { EPA8260 } \\ & & \end{array}$

$\mu \mathrm{g} / \mathrm{kg}$
$\mu \mathrm{g} / \mathrm{kg}$
$\mu \mathrm{g} / \mathrm{kg}$
$\mu \mathrm{g} / \mathrm{kg}$
$\mu \mathrm{g} / \mathrm{kg}$
$\mu \mathrm{g} / \mathrm{kg}$
$\mu \mathrm{g} / \mathrm{kg}$
$\mu \mathrm{g} / \mathrm{kg}$
$\mu \mathrm{g} / \mathrm{kg}$
$\mu \mathrm{g} / \mathrm{kg}$
$\mu \mathrm{g} / \mathrm{kg}$
$\mu \mathrm{g} / \mathrm{kg}$
$\mu \mathrm{g} / \mathrm{kg}$
$\mu \mathrm{g} / \mathrm{kg}$
$\mu \mathrm{g} / \mathrm{kg}$
$\mu \mathrm{g} / \mathrm{kg}$
$\mu \mathrm{g} / \mathrm{kg}$
$\mu \mathrm{g} / \mathrm{kg}$

10

EPA8080

EPA8080

EPA8080

EPA8080

EPA8080

EPA8080

EPA8080

EPA8080

EPA8080

EPA8080

EPA8080

EPA8080

EPA8080

EPA8080

EPA8080

EPA8080

EPA8080

EPA8080 
SURVEY ID: PPSC 1102 (continued)

Constituent

Pesticides/PCBs and Dioxins/Furans

Methoxychlor

PCB 1016

PCB 1221

PCB 1232

PCB 1242

PCB 1248

PCB 1254

PCB 1260

Toxaphene

Radionuclides

Actinium-228

Antimony-124

Antimony-125

Barium-133

Cerium-144

Cesium-134

Cesium-137

Cobalt-57

Cobalt-58

Cobalt-60

Europium-152

Europium-154

Europium-155

Gross alpha

Lead-212

Manganese-54

Neptunium-239

Nonvolatile beta

Plutonium-238

Plutonium-239/240

Potassium-40

Promethium-144

Promethium-146

Ruthenium-106

Sodium-22

Strontium-90

Thorium-234

Tin-113

Yttrium-88

Zinc-65

Zirconium-95

\section{RQ AQ B Result}

Unit
Sample ID: 104653

$\begin{array}{llll}201 & \mu g / k g & 201 & \text { EPA8080 } \\ 50.2 & \mu \mathrm{g} / \mathrm{kg} & 50.2 & \text { EPA8080 } \\ 50.2 & \mu \mathrm{g} / \mathrm{kg} & 50.2 & \text { EPA8080 } \\ 50.2 & \mu \mathrm{g} / \mathrm{kg} & 50.2 & \text { EPA8080 } \\ 50.2 & \mu \mathrm{g} / \mathrm{kg} & 50.2 & \text { EPA8080 } \\ 50.2 & \mu \mathrm{g} / \mathrm{kg} & 50.2 & \text { EPA8080 } \\ 50.2 & \mu \mathrm{g} / \mathrm{kg} & 50.2 & \text { EPA8080 } \\ 50.2 & \mu \mathrm{g} / \mathrm{kg} & \mathbf{5 0 . 2} & \text { EPA8080 } \\ 402 & \mu \mathrm{g} / \mathrm{kg} & 402 & \text { EPA8080 }\end{array}$

$2.07 \mathrm{E}+00 \pm 7.77 \mathrm{E}-02 \mathrm{pCi} / \mathrm{g}$ $4.77 \mathrm{E}-03 \pm 8.66 \mathrm{E}-03 \mathrm{pCi} / \mathrm{g}$ $-5.45 \mathrm{E}-03 \pm 2.10 \mathrm{E}-02 \mathrm{pCi} / \mathrm{g}$ $3.22 \mathrm{E}-03 \pm 9.87 \mathrm{E}-03 \mathrm{pCi} / \mathrm{g}$ $4.84 \mathrm{E}-02 \pm 5.37 \mathrm{E}-02 \mathrm{pCi} / \mathrm{g}$ $2.59 \mathrm{E}-03 \pm 6.80 \mathrm{E}-03 \mathrm{pCi} / \mathrm{g}$ $1.28 \mathrm{E}+00 \pm 2.74 \mathrm{E}-02 \mathrm{pCi} / \mathrm{g}$ $1.52 \mathrm{E}-03 \pm 6.72 \mathrm{E}-03 \mathrm{pCi} / \mathrm{g}$ $-1.92 \mathrm{E}-03 \pm 8.32 \mathrm{E}-03 \mathrm{pCi} / \mathrm{g}$ $7.84 \mathrm{E}-03 \pm 1.06 \mathrm{E}-02 \mathrm{pCi} / \mathrm{g}$ 9.30E-03 $\pm 2.04 \mathrm{E}-02 \mathrm{pCi} / \mathrm{g}$ $-3.00 \mathrm{E}-02 \pm 5.24 \mathrm{E}-02 \mathrm{pCi} / \mathrm{g}$ $0.00 \mathrm{E}+00 \quad \mathrm{pCi} / \mathrm{g}$ $1.25 \mathrm{E}+01 \pm 2.72 \mathrm{E}+00 \mathrm{pCi} / \mathrm{g}$ $2.20 \mathrm{E}+00 \pm 3.75 \mathrm{E}-02 \mathrm{pCi} / \mathrm{g}$ $3.55 \mathrm{E}-02 \pm 9.38 \mathrm{E}-03 \mathrm{pCi} / \mathrm{g}$ $2.23 \mathrm{E}-02 \pm 5.34 \mathrm{E}-02 \mathrm{pCi} / \mathrm{g}$ $7.32 \mathrm{E}+00 \pm 1.87 \mathrm{E}+00 \mathrm{pCi} / \mathrm{g}$ $2.69 \mathrm{E}-03 \pm 5.39 \mathrm{E}-03 \mathrm{pCi} / \mathrm{g}$ $0.00 \mathrm{E}+00 \quad \mathrm{pCj} / \mathrm{g}$ $8.35 \mathrm{E}-01 \pm 1.33 \mathrm{E}-01 \mathrm{pCi} / \mathrm{g}$ 2.83E-04 $16.35 \mathrm{E}-03 \mathrm{pCi} / \mathrm{g}$ $9.21 \mathrm{E}-03 \pm 1.03 \mathrm{E}-02 \mathrm{pCi} / \mathrm{g}$ $1.60 \mathrm{E}-02 \pm 6.21 \mathrm{E}-02 \mathrm{pCi} / \mathrm{g}$ $-2.88 \mathrm{E}-04 \pm 5.72 \mathrm{E}-03 \mathrm{pCi} / \mathrm{g}$ $1.62 \mathrm{E}+00 \pm 1.35 \mathrm{E}-01 \mathrm{pCi} / \mathrm{g}$ $1.32 \mathrm{E}+00 \pm 6.10 \mathrm{E}-01 \mathrm{pCi} / \mathrm{g}$ $-1.36 \mathrm{E}-02 \pm 1.12 \mathrm{E}-02 \mathrm{pCi} / \mathrm{g}$ $4.70 \mathrm{E}-03 \pm 6.83 \mathrm{E}-03 \mathrm{pCi} / \mathrm{g}$ $1.96 \mathrm{E}-03 \pm 1.23 \mathrm{E}-02 \mathrm{pCi} / \mathrm{g}$ $2.60 \mathrm{E}-02 \pm 1.77 \mathrm{E}-02 \mathrm{pCj} / \mathrm{g}$
3.44E-02 EPIA-013B
1.52E-02 EPIA-013B
3.40E-02 EPIA-013B
1.47E-02 EPIA-013B
9.29E-02 EPIA-013B
1.05E-02 EPIA-013B
1.16E-02 EPIA-013B
1.16E-02 EPIA-013B
1.24E.02 EPIA-013B
9.67E-03 EPIA-013B
3.39E-02 EPIA-013B
8.64E-02 EPIA-013B
4.63E-02 EPIA-013B
1.33E+00 EPIA-001B
2.07E-02 EPIA-013B
9.01E-03 EPIA-013B
8.33E-02 EPIA.013B
$2.91 \mathrm{E}+00$ EPIA-001B
8.08E-03 EPIA-012B
8.08E-03 EPIA-012B
8.65E-02 EPIA-013B
1.09E-02 EPIA-013B
1.69E-02 EPIA-013B
1.08E-01 EPIA-013B
9.57E-03 EPIA-013B
6.32E-02 EPIA-004
6.00E-01 EPIA-013B
1.78E-02 EPIA-013B
1.26E-02 EPIA-013B
1.85E-02 EPIA-013B
2.85E-02 EPIA-013B

\section{SURVEY ID: PPSC 1103}

Sample ID: 104655

Survey location: $44944 \mathrm{E}$ 3681248N (SRS coordinates)

Depth of core interval: 2.00 to $4.00 \mathrm{ft}$

Sample type: Normal

Sample matrix: Soil

Percent solids: $\mathbf{8 7 . 0 0}$ 
SURVEY ID: PPSC 1103 (continued)

Sample ID: 104655

Constituent

RQ AQ B Result

Unit

D. Limit Method

Physical Parameters and Specified Analyses

Cation exchange capacity

Chloride

Chloride

Cyanide

Nitrate-nitrite as nitrogen

Orthophosphate

Phenols

Sulfide

Sulfide

Total organic carbon

Total organic nitrogen

Total phosphates (as P)

Metals (total recoverable)

Aluminum

Antimony

Arsenic

Barium

Beryllium

Cadmium

Calcium

Chromium

Cobalt

Copper

Iron

Lead

Magnesium

Manganese

Mercury

Nickel

Potassium

Selenium

Silver

Sodium

Thallium

Vanadium

Zinc

$\begin{array}{llll} & & & 32.4 \\ \text { U } & & & 3.01 \\ U & & & 2.87 \\ \text { U } & & & 1.15 \\ \text { J } & \text { E } & & 1.15 \\ \text { U } & & & 11.5 \\ \text { UJ } & \text { C } & & 334 \\ \text { UJ } & \text { IC } & \text { P } & 287 \\ \text { UJ } & \text { IC } & \text { P } & 287 \\ & & & 1110 \\ \text { C } & & & 47.6 \\ \text { J } & \text { E } & & 100\end{array}$

6720

U

U $\quad 17.1$

14.4

J $\quad$ E $\quad 0.0859$

$\mathrm{U} \quad 0.285$

36.9

7.74

$\begin{array}{lll}J & \mathrm{E} & 0.409\end{array}$

1.48

6580

6.18

186

11.4

J $\quad \mathrm{E} \quad 0.00681$

2.12

129

$\begin{array}{ll}\mathrm{U} & 17.1 \\ \mathrm{U} & 1.42\end{array}$

$\begin{array}{ll}\mathrm{U} & 17.1 \\ \mathrm{U} & 1.42\end{array}$

J $\quad \mathrm{E} \quad 8.72$

$\mathrm{U} \quad 17.1$

14.7

5.45

$\begin{array}{lll}\mathrm{meq} / 100 \mathrm{~g} & 5 & \text { EPA9081 } \\ \mathrm{mg} / \mathrm{kg} & 3.01 & \text { EPA300.0 } \\ \mathrm{mg} / \mathrm{kg} & 2.87 & \text { EPA300.0 } \\ \mathrm{mg} / \mathrm{kg} & 1.15 & \text { EPA335.3 } \\ \mathrm{mg} / \mathrm{kg} & 1.15 & \text { EPA353.1 } \\ \mathrm{mg} / \mathrm{kg} & 11.5 & \text { EPA365.2 } \\ \mu \mathrm{g} / \mathrm{kg} & 334 & \text { EPA420.2 } \\ \mathrm{mg} / \mathrm{kg} & 287 & \text { EPA376.2 } \\ \mathrm{mg} / \mathrm{kg} & 287 & \text { EPA376.2 } \\ \mathrm{mg} / \mathrm{kg} & 115 & \text { EPA415.1 } \\ \mathrm{mg} / \mathrm{kg} & 0.23 & \text { EPA351.2 } \\ \mathrm{mg} / \mathrm{kg} & 451 & \text { EPA365.4 }\end{array}$

$B / N / A$ Extractables (including $P A H$ and phenols)

Acenaphthene

Acenaphthylene

Anthracene

Benzo[a]anthracene

Benzo[b]fluoranthene

Benzo[k]fluoranthene

Benzoic acid

Benzo[g, $h, i]$ perylene

Benzo[a]pyrene

Benzyl alcohol

Bis(2-chloroethoxy) methane

Bis(2-chloroethyl) ether

Bis(2-chloroisopropyl) ether

Bis(2-ethylhexyl) phthalate
$\mathrm{U}$

U

U

U

U

U

U

U

U

U

U

U

$\begin{array}{lll}\mathrm{J} & \mathrm{E} & 33.9\end{array}$
38.1

38.1

38.1

3.81

3.81

3.81

101

38.1

3.81

38.1

38.1

38.1

38.1

$\begin{array}{lll}\mathrm{mg} / \mathrm{kg} & 14.2 & \text { EPA6010A } \\ \mathrm{mg} / \mathrm{kg} & 5.69 & \text { EPA6010A } \\ \mathrm{mg} / \mathrm{kg} & 17.1 & \text { EPA6010A } \\ \mathrm{mg} / \mathrm{kg} & 1.42 & \text { EPA6010A } \\ \mathrm{mg} / \mathrm{kg} & 0.569 & \text { EPA6010A } \\ \mathrm{mg} / \mathrm{kg} & 0.285 & \text { EPA6010A } \\ \mathrm{mg} / \mathrm{kg} & 14.2 & \text { EPA6010A } \\ \mathrm{mg} / \mathrm{kg} & 1.42 & \text { EPA6010A } \\ \mathrm{mg} / \mathrm{kg} & 0.569 & \text { EPA6010A } \\ \mathrm{mg} / \mathrm{kg} & 1.42 & \text { EPA6010A } \\ \mathrm{mg} / \mathrm{kg} & 5.69 & \text { EPA6010A } \\ \mathrm{mg} / \mathrm{kg} & 5.69 & \text { EPA6010A } \\ \mathrm{mg} / \mathrm{kg} & 5.69 & \text { EPA6010A } \\ \mathrm{mg} / \mathrm{kg} & 0.569 & \text { EPA6010A } \\ \mathrm{mg} / \mathrm{kg} & 0.0303 & \text { EPA7471 } \\ \mathrm{mg} / \mathrm{kg} & 1.42 & \text { EPA6010A } \\ \mathrm{mg} / \mathrm{kg} & 114 & \text { EPA6010A } \\ \mathrm{mg} / \mathrm{kg} & 17.1 & \text { EPA6010A } \\ \mathrm{mg} / \mathrm{kg} & 1.42 & \text { EPA6010A } \\ \mathrm{mg} / \mathrm{kg} & 56.9 & \text { EPA6010A } \\ \mathrm{mg} / \mathrm{kg} & 17.1 & \text { EPA6010A } \\ \mathrm{mg} / \mathrm{kg} & 0.569 & \text { EPA6010A } \\ \mathrm{mg} / \mathrm{kg} & 1.14 & \text { EPA6010A }\end{array}$

$\mu \mathrm{g} / \mathrm{kg} \quad 38.1$

EPA8270

$\mu \mathrm{g} / \mathrm{kg} \quad 38.1$

$\mu \mathrm{g} / \mathrm{kg} \quad 38.1$

$\mu \mathrm{g} / \mathrm{kg} \quad 3.81$

$\mu \mathrm{g} / \mathrm{kg} \quad 3.81$

$\mu \mathrm{g} / \mathrm{kg} \quad 3.81$

$\mu \mathrm{g} / \mathrm{kg} \quad 38.1$

$\mu \mathrm{g} / \mathrm{kg} \quad 38.1$

$\mu \mathrm{g} / \mathrm{kg} \quad 3.81$

$\mu \mathrm{g} / \mathrm{kg} \quad 38.1$

$\mu \mathrm{g} / \mathrm{kg} \quad 38.1$

$\mu \mathrm{g} / \mathrm{kg} \quad 38.1$

$\mu \mathrm{g} / \mathrm{kg} \quad 38.1$

$\mu \mathrm{g} / \mathrm{kg} \quad 38.1$
EPA8270

EPA8270

EPA8270

EPA8270

EPA8270

EPA8270

EPA8270

EPA8270

EPA8270

EPA8270

EPA8270

EPA8270
EPA8270 
Appendix D: Sediment Tables

D-138

SURVEY ID: PPSC 1103 (continued)

Sample ID: 104655

Constituent

RQ AQ B Result

Unit

D. Limit Method

$B / N / A$ Extractables (including PAH and phenols)

4-Bromophenyl phenyl ether

Butylbenzyl phthalate

4-Chloroaniline

4-Chloro-m-cresol

2-Chloronaphthalene

2-Chlorophenol

4-Chlorophenyl phenyl ether

Chrysene

$\mathrm{m} / \mathrm{p}$-Cresol

o-Cresol (2-Methylphenol)

Dibenz $[a, h]$ anthracene

Dibenzofuran

Di-n-butyl phthalate

1,2-Dichlorobenzene

1,3-Dichlorobenzene

1,4-Dichlorobenzene

3,3'-Dichlorobenzidine

2,4-Dichlorophenol

Diethyl phthalate

2,4-Dimethyl phenol

Dimethyl phthalate

2,4-Dinitrophenol

2,4-Dinitrotoluene

2,6-Dinitrotoluene

Di-n-octyl phthalate

Fluoranthene

Fluorene

Hexachlorobenzene

Hexachlorobutadiene

Hexachlorocyclopentadiene

Hexachloroethane

Indeno[1,2,3-c,d]pyrene

Isophorone

2-Methyl-4,6-dinitrophenol

2-Methylnaphthalene

Naphthalene

m-Nitroaniline

o-Nitroaniline

p-Nitroaniline

Nitrobenzene

2-Nitrophenol

4-Nitrophenol

N-Nitrosodiphenylamine

N-Nitrosodi-n-propylamine

Pentachlorophenol

Phenanthrene

Phenol

Pyrene

1,2,4-Trichlorobenzene

2,4,5-Trichlorophenol

2,4,6-Trichlorophenol
38.1

38.1

38.1

38.1

38.1

38.1

38.1

3.81

381

38.1

3.81

38.1

48.4

38.1

38.1

38.1

38.1

38.1

38.1

38.1

38.1

381

3.81

3.81

38.1

38.1

38.1

3.81

38.1

38.1

3.81

3.81

38.1

381

38.1

38.1

38.1

38.1

38.1

38.1

38.1

38.1

38.1

38.1

38.1

38.1

38.1

38.1

3.81

38.1

38.1

\begin{tabular}{|c|c|c|}
\hline$\mu \mathrm{g} / \mathrm{kg}$ & 38.1 & EPA8270 \\
\hline$\mu \mathrm{g} / \mathrm{kg}$ & 38.1 & EPA8270 \\
\hline$\mu \mathrm{g} / \mathrm{kg}$ & 38.1 & EPA8270 \\
\hline$\mu \mathrm{g} / \mathrm{kg}$ & 38.1 & EPA8270 \\
\hline$\mu \mathrm{g} / \mathrm{kg}$ & 38.1 & EPA8270 \\
\hline$\mu \mathrm{g} / \mathrm{kg}$ & 38.1 & EPA8270 \\
\hline$\mu \mathrm{g} / \mathrm{kg}$ & 38.1 & EPA8270 \\
\hline$\mu \mathrm{g} / \mathrm{kg}$ & 3.81 & EPA8270 \\
\hline$\mu \mathrm{g} / \mathrm{kg}$ & 381 & EPA8270 \\
\hline$\mu \mathrm{g} / \mathrm{kg}$ & 38.1 & EPA8270 \\
\hline$\mu \mathrm{g} / \mathrm{kg}$ & 3.81 & EPA8270 \\
\hline$\mu \mathrm{g} / \mathrm{kg}$ & 38.1 & EPA8270 \\
\hline$\mu \mathrm{g} / \mathrm{kg}$ & 38.1 & EPA8270 \\
\hline$\mu \mathrm{g} / \mathrm{kg}$ & 38.1 & EPA8270 \\
\hline$\mu \mathrm{g} / \mathrm{kg}$ & 38.1 & EPA8270 \\
\hline$\mu \mathrm{g} / \mathrm{kg}$ & 38.1 & EPA8270 \\
\hline$\mu \mathrm{g} / \mathrm{kg}$ & 38.1 & EPA8270 \\
\hline$\mu \mathrm{g} / \mathrm{kg}$ & 38.1 & EPA8270 \\
\hline$\mu \mathrm{g} / \mathrm{kg}$ & 38.1 & EPA8270 \\
\hline$\mu \mathrm{g} / \mathrm{kg}$ & 38.1 & EPA8270 \\
\hline$\mu \mathrm{g} / \mathrm{kg}$ & 38.1 & EPA8270 \\
\hline$\mu \mathrm{g} / \mathrm{kg}$ & 381 & EPA8270 \\
\hline$\mu \mathrm{g} / \mathrm{kg}$ & 3.81 & EPA8270 \\
\hline$\mu \mathrm{g} / \mathrm{kg}$ & 3.81 & EPA8270 \\
\hline$\mu \mathrm{g} / \mathrm{kg}$ & 38.1 & EPA8270 \\
\hline$\mu \mathrm{g} / \mathrm{kg}$ & 38.1 & EPA8270 \\
\hline$\mu \mathrm{g} / \mathrm{kg}$ & 38.1 & EPA8270 \\
\hline$\mu \mathrm{g} / \mathrm{kg}$ & 3.81 & EPA8270 \\
\hline$\mu \mathrm{g} / \mathrm{kg}$ & 38.1 & EPA8270 \\
\hline$\mu \mathrm{g} / \mathrm{kg}$ & 38.1 & EPA8270 \\
\hline$\mu \mathrm{g} / \mathrm{kg}$ & 3.81 & EPA8270 \\
\hline$\mu \mathrm{g} / \mathrm{kg}$ & 3.81 & EPA8270 \\
\hline$\mu \mathrm{g} / \mathrm{kg}$ & 38.1 & EPA8270 \\
\hline$\mu \mathrm{g} / \mathrm{kg}$ & 381 & EPA8270 \\
\hline$\mu \mathrm{g} / \mathrm{kg}$ & 38.1 & EPA8270 \\
\hline$\mu \mathrm{g} / \mathrm{kg}$ & 38.1 & EPA8270 \\
\hline$\mu \mathrm{g} / \mathrm{kg}$ & 38.1 & EPA8270 \\
\hline$\mu \mathrm{g} / \mathrm{kg}$ & 38.1 & EPA8270 \\
\hline$\mu \mathrm{g} / \mathrm{kg}$ & 38.1 & EPA8270 \\
\hline$\mu \mathrm{g} / \mathrm{kg}$ & 38.1 & EPA8270 \\
\hline$\mu \mathrm{g} / \mathrm{kg}$ & 38.1 & EPA8270 \\
\hline$\mu \mathrm{g} / \mathrm{kg}$ & 38.1 & EPA8270 \\
\hline$\mu \mathrm{g} / \mathrm{kg}$ & 38.1 & EPA8270 \\
\hline$\mu \mathrm{g} / \mathrm{kg}$ & 38.1 & EPA8270 \\
\hline$\mu \mathrm{g} / \mathrm{kg}$ & 38.1 & EPA8270 \\
\hline$\mu \mathrm{g} / \mathrm{kg}$ & 38.1 & EPA8270 \\
\hline$\mu \mathrm{g} / \mathrm{kg}$ & 38.1 & EPA8270 \\
\hline$\mu \mathrm{g} / \mathrm{kg}$ & 38.1 & EPA8270 \\
\hline$\mu \mathrm{g} / \mathrm{kg}$ & 3.81 & EPA8270 \\
\hline & 38.1 & EPA8270 \\
\hline$\mu \mathrm{g} / \mathrm{kg}$ & 38.1 & EPA8270 \\
\hline
\end{tabular}


SURVEY ID: PPSC 1103 (continued)

Constituent

RQ AQ B Result

Unit

D. Limit Method

Volatile Organic Compounds

Acetone
Benzene
Bromodichloromethane
Bromoform
Bromomethane (Methyl bromide)
Carbon disulfide
Carbon tetrachloride
Chlorobenzene
Chloroethane
Chloroethene (Vinyl chloride)
Chloroform
Chloromethane (Methyl chloride)
Dibromochloromethane
1,1-Dichloroethane.
1,2-Dichloroethane
1,1-Dichloroethylene
1,2-Dichloroethylene
Dichloromethane (Methylene chloride)
1,2-Dichloropropane
cis-1,3-Dichloropropene
trans-1,3-Dichloropropene
Ethylbenzene
2-Hexanone
Methyl ethyl ketone
Methyl isobutyl ketone
Styrene
1,1,2,2-Tetrachloroethane
Tetrachloroethylene
Toluene
1,1,1-Trichloroethane
1,1,2-Trichloroethane
Trichloroethylene
Vinyl acetate
Xylenes

$\begin{array}{lll}\text { UJ } & \mathbf{Q} 8 & 30.8 \\ \text { UJ } & \mathbf{Q} & \mathbf{0 . 5 7 5} \\ \text { UJ } & \mathbf{Q} & \mathbf{0 . 0 5 7 5} \\ \text { UJ } & \mathbf{Q} & \mathbf{0 . 0 5 7 5} \\ \text { UJ } & \mathbf{Q} & \mathbf{0 . 1 1 5} \\ \text { UJ } & \mathbf{Q} & 1.15 \\ \text { UJ } & \mathbf{Q} & \mathbf{0 . 0 5 7 5} \\ \text { UJ } & \mathbf{Q} & \mathbf{0 . 0 5 7 5} \\ \text { UJ } & \mathbf{Q} & \mathbf{0 . 1 1 5} \\ \text { UJ } & \mathbf{Q} & \mathbf{0 . 1 1 5} \\ \text { UJ } & \mathbf{Q} & \mathbf{0 . 0 5 7 5} \\ \text { UJ } & \mathbf{Q} & \mathbf{0 . 1 1 5} \\ \text { UJ } & \mathbf{Q} & \mathbf{0 . 0 5 7 5} \\ \text { UJ } & \mathbf{Q} & \mathbf{0 . 0 5 7 5} \\ \text { UJ } & \mathbf{Q} & \mathbf{0 . 0 5 7 5} \\ \text { UJ } & \mathbf{Q} & \mathbf{0 . 0 5 7 5} \\ \text { UJ } & \mathbf{Q} & \mathbf{0 . 1 1 5} \\ \text { UJ } & \mathbf{Q V} & \mathbf{1 . 5 5} \\ \text { UJ } & \mathbf{Q} & \mathbf{0 . 0 5 7 5} \\ \text { UJ } & \mathbf{Q} & \mathbf{0 . 0 5 7 5} \\ \text { UJ } & \mathbf{Q} & \mathbf{0 . 0 5 7 5} \\ \text { UJ } & \mathbf{Q} & \mathbf{0 . 0 5 7 5} \\ \text { J } & \mathbf{Q E} & \mathbf{0 . 2 8 7} \\ \text { J } & \mathbf{Q} & \mathbf{1 . 3 3} \\ \text { UJ } & \mathbf{Q} & 1.15 \\ \text { UJ } & \mathbf{Q} & \mathbf{0 . 0 5 7 5} \\ \text { UJ } & \mathbf{Q} & \mathbf{0 . 0 5 7 5} \\ \text { UJ } & \mathbf{Q} & \mathbf{0 . 0 5 7 5} \\ \text { UJ } & \mathbf{Q V} & \mathbf{0 . 1 4 9} \\ \text { UJ } & \mathbf{Q} & \mathbf{0 . 0 5 7 5} \\ \text { UJ } & \mathbf{Q} & \mathbf{0 . 0 5 7 5} \\ \text { UJ } & \mathbf{Q} & \mathbf{0 . 0 5 7 5} \\ \text { UJ } & \mathbf{Q} & 1.15 \\ \text { UJ } & \mathbf{Q} & \mathbf{0 . 1 7 2} \\ & & \end{array}$

$\begin{array}{lll}\mu \mathrm{g} / \mathrm{kg} & 5.75 & \text { EPA8260 } \\ \mu \mathrm{g} / \mathrm{kg} & 0.575 & \text { EPA8260 } \\ \mu \mathrm{g} / \mathrm{kg} & 0.0575 & \text { EPA8260 } \\ \mu \mathrm{g} / \mathrm{kg} & 0.0575 & \text { EPA8260 } \\ \mu \mathrm{g} / \mathrm{kg} & 0.115 & \text { EPA8260 } \\ \mu \mathrm{g} / \mathrm{kg} & 1.15 & \text { EPA8260 } \\ \mu \mathrm{g} / \mathrm{kg} & 0.0575 & \text { EPA8260 } \\ \mu \mathrm{g} / \mathrm{kg} & 0.0575 & \text { EPA8260 } \\ \mu \mathrm{g} / \mathrm{kg} & 0.115 & \text { EPA8260 } \\ \mu \mathrm{g} / \mathrm{kg} & 0.115 & \text { EPA8260 } \\ \mu \mathrm{g} / \mathrm{kg} & 0.0575 & \text { EPA8260 } \\ \mu \mathrm{g} / \mathrm{kg} & 0.115 & \text { EPA8260 } \\ \mu \mathrm{g} / \mathrm{kg} & 0.0575 & \text { EPA8260 } \\ \mu \mathrm{g} / \mathrm{kg} & 0.0575 & \text { EPA8260 } \\ \mu \mathrm{g} / \mathrm{kg} & 0.0575 & \text { EPA8260 } \\ \mu \mathrm{g} / \mathrm{kg} & 0.0575 & \text { EPA8260 } \\ \mu \mathrm{g} / \mathrm{kg} & 0.115 & \text { EPA8260 } \\ \mu \mathrm{g} / \mathrm{kg} & 0.575 & \text { EPA8260 } \\ \mu \mathrm{g} / \mathrm{kg} & 0.0575 & \text { EPA8260 } \\ \mu \mathrm{g} / \mathrm{kg} & 0.0575 & \text { EPA8260 } \\ \mu \mathrm{g} / \mathrm{kg} & 0.0575 & \text { EPA8260 } \\ \mu \mathrm{g} / \mathrm{kg} & 0.0575 & \text { EPA8260 } \\ \mu \mathrm{g} / \mathrm{kg} & 1.15 & \text { EPA8260 } \\ \mu \mathrm{g} / \mathrm{kg} & 1.15 & \text { EPA8260 } \\ \mu \mathrm{g} / \mathrm{kg} & 1.15 & \text { EPA8260 } \\ \mu \mathrm{g} / \mathrm{kg} & 0.0575 & \text { EPA8260 } \\ \mu \mathrm{g} / \mathrm{kg} & 0.0575 & \text { EPA8260 } \\ \mu \mathrm{g} / \mathrm{kg} & 0.0575 & \text { EPA8260 } \\ \mu \mathrm{g} / \mathrm{kg} & 0.575 & \text { EPA8260 } \\ \mu \mathrm{g} / \mathrm{kg} & 0.0575 & \text { EPA8260 } \\ \mu \mathrm{g} / \mathrm{kg} & 0.0575 & \text { EPA8260 } \\ \mu \mathrm{g} / \mathrm{kg} & 0.0575 & \text { EPA8260 } \\ \mu \mathrm{g} / \mathrm{kg} & 1.15 & \text { EPA8260 } \\ \mu \mathrm{g} / \mathrm{kg} & 0.172 & \text { EPA8260 } \\ & & \end{array}$

Pesticides/PCBs and Dioxins/Furans

Aldrin
alpha-Benzene hexachloride
beta-Benzene hexachloride
delta-Benzene hexachloride
alpha-Chlordane
gamma-Chlordane
p,p'-DDD
p,p'-DDE
p,p'-DDT
Dieldrin
Endosulfan sulfate
Endosulfan I
Endosulfan II
Endrin
Endrin ketone
Heptachlor
Heptachlor epoxide
Lindane

$\begin{array}{ll}U & 9.52 \\ U & 9.52 \\ U & 19 \\ U & 19 \\ U & 19 \\ U & 19 \\ U & 38.1 \\ U & 19 \\ U & 38.1 \\ U & 19 \\ U & 38.1 \\ U & 19 \\ U & 38.1 \\ U & 19 \\ U & 38.1 \\ U & 19 \\ U & 19 \\ U & 9.52\end{array}$

$\begin{array}{lll}\mu \mathrm{g} / \mathrm{kg} & 9.52 & \text { EPA8080 } \\ \mu \mathrm{g} / \mathrm{kg} & 9.52 & \text { EPA8080 } \\ \mu \mathrm{g} / \mathrm{kg} & 19 & \text { EPA8080 } \\ \mu \mathrm{g} / \mathrm{kg} & 19 & \text { EPA8080 } \\ \mu \mathrm{g} / \mathrm{kg} & 19 & \text { EPA8080 } \\ \mu \mathrm{g} / \mathrm{kg} & 19 & \text { EPA8080 } \\ \mu \mathrm{g} / \mathrm{kg} & 38.1 & \text { EPA8080 } \\ \mu \mathrm{g} / \mathrm{kg} & 19 & \text { EPA8080 } \\ \mu \mathrm{g} / \mathrm{kg} & 38.1 & \text { EPA8080 } \\ \mu \mathrm{g} / \mathrm{kg} & 19 & \text { EPA8080 } \\ \mu \mathrm{g} / \mathrm{kg} & 38.1 & \text { EPA8080 } \\ \mu \mathrm{g} / \mathrm{kg} & 19 & \text { EPA8080 } \\ \mu \mathrm{g} / \mathrm{kg} & 38.1 & \text { EPA8080 } \\ \mu \mathrm{g} / \mathrm{kg} & 19 & \text { EPA8080 } \\ \mu \mathrm{g} / \mathrm{kg} & 38.1 & \text { EPA8080 } \\ \mu \mathrm{g} / \mathrm{kg} & 19 & \text { EPA8080 } \\ \mu \mathrm{g} / \mathrm{kg} & 19 & \text { EPA8080 } \\ \mu \mathrm{g} / \mathrm{kg} & 9.52 & \text { EPA8080 }\end{array}$


SURVEY ID: PPSC 1103 (continued)

\section{Constituent}

Pesticides/PCBs and Dioxins/Furans
$\mathbf{R Q}$

AQ B Result
Unit

$\begin{array}{ll}\mu \mathrm{g} / \mathrm{kg} & 190 \\ \mu \mathrm{g} / \mathrm{kg} & 47.6 \\ \mu \mathrm{g} / \mathrm{kg} & 47.6 \\ \mu \mathrm{g} / \mathrm{kg} & 47.6 \\ \mu \mathrm{g} / \mathrm{kg} & 47.6 \\ \mu \mathrm{g} / \mathrm{kg} & 47.6 \\ \mu \mathrm{g} / \mathrm{kg} & 47.6 \\ \mu \mathrm{g} / \mathrm{kg} & 47.6 \\ \mu \mathrm{g} / \mathrm{kg} & 381\end{array}$

EPA8080
EPA8080
EPA8080
EPA8080
EPA8080
EPA8080
EPA8080
EPA8080
EPA8080

EPA8080 EPA8080 EPA8080

47.6

47.6

47.6

47.6

381
3.90E-02 EPIA-013B
1.54E-02 EPIA-013B
3.43E-02 EPIA-013B
1.43E-02 EPIA-013B
9.10E-02 EPIA-013B
1.04E-02 EPIA-013B
1.25E-02 EPIA-013B
1.13E-02 EPIA-013B
1.34E-02 EPIA-013B
1.01E-02 EPIA-013B
3.27E-02 EPIA-013B
8.00E-02 EPIA-013B
4.69E-02 EPIA-013B
1.67E+00 EPIA-001B
2.13E-02 EPIA-013B
1.05E-02 EPIA-013B
8.23E-02 EPIA-013B
2.35E+00 EPIA-001B
2.84E-02 EPIA-012B
1.72E-02 EPIA-012B
9.97E-02 EPIA-013B
1.13E-02 EPIA-013B
1.56E-02 EPIA-013B
1.05E-01 EPIA-013B
1.18E-02 EPIA-013B
9.78E-02 EPIA-004
6.36E-01 EPIA-013B
1.73E-02 EPLA-013B
1.44E-02 EPIA-013B
2.24E-02 EPIA-013B
3.16E-02 EPIA-013B

SURVEY ID: PPSC 1201

Sample ID: 104656

Survey location: $44888 \mathrm{E} 3682027 \mathrm{~N}$ (SRS coordinates)

Depth of core interval: 0.00 to $1.00 \mathrm{ft}$

Sample type: Normal

Sample matrix: Soil

Percent solids: $\mathbf{5 2 . 0 0}$ 
SURVEY ID: PPSC 1201 (continued)

Sample ID: 104656

Constituent

RQ AQ B Result

Unit

D. Limit Method

Physical Parameters and Specified Analyses

$\begin{array}{llll}\text { Chloride } & \text { J } & \text { E } & 4.55 \\ \text { Cyanide } & \text { U } & & 1.92 \\ \text { Nitrate-nitrite as nitrogen } & \text { J } & \text { E } & 0.595 \\ \text { Orthophosphate } & \text { U } & & 19.2 \\ \text { Phenols } & \text { UJ } & \text { C } & 577 \\ \text { Sulfide } & \text { UJ } & \text { C } & 481 \\ \text { Total organic carbon } & & & 15100 \\ \text { Total organic nitrogen } & \text { C } & & 385 \\ \text { Total phosphates (as P) } & \text { J } & \text { E } & 227\end{array}$

$\begin{array}{lll}\mathrm{mg} / \mathrm{kg} & 4.84 & \text { EPA300.0 } \\ \mathrm{mg} / \mathrm{kg} & 1.92 & \text { EPA335.3 } \\ \mathrm{mg} / \mathrm{kg} & 1.98 & \text { EPA353.1 } \\ \mathrm{mg} / \mathrm{kg} & 19.2 & \text { EPA365.2 } \\ \mu \mathrm{g} / \mathrm{kg} & 577 & \text { EPA420.2 } \\ \mathrm{mg} / \mathrm{kg} & 481 & \text { EPA376.2 } \\ \mathrm{mg} / \mathrm{kg} & 192 & \text { EPA415.1 } \\ \mathrm{mg} / \mathrm{kg} & 0.385 & \text { EPA351.2 } \\ \mathrm{mg} / \mathrm{kg} & \mathbf{7 3 3} & \text { EPA365.4 }\end{array}$

Metals (total recoverable)

\begin{tabular}{|c|c|c|c|}
\hline Aluminum & & & 18000 \\
\hline Antimony & U & & 9.34 \\
\hline Arsenic & $\mathrm{U}$ & & 28 \\
\hline Barium & & & 86.1 \\
\hline Beryllium & $\mathrm{J}$ & $E$ & 0.772 \\
\hline Cadmium & U & & 0.467 \\
\hline Calcium & & & 817 \\
\hline Chromium & & & 23.7 \\
\hline Cobalt & & & 3.45 \\
\hline Copper & & & 7.92 \\
\hline Iron & & & 14000 \\
\hline Lead & & & 18.1 \\
\hline Magnesium & & & 376 \\
\hline Manganese & & & 181 \\
\hline Mercury & $J$ & $\mathrm{E}$ & 0.039 \\
\hline Nickel & & & 5.25 \\
\hline Potassium & & & 320 \\
\hline Selenium & $U$ & & 28 \\
\hline Silver & $\mathrm{U}$ & & 2.33 \\
\hline Sodium & $J$ & $\mathrm{E}$ & 20.8 \\
\hline Thallium & $J$ & $\mathbf{E}$ & 4.59 \\
\hline Vanadium & & & 40.4 \\
\hline Zinc & & & 19.5 \\
\hline
\end{tabular}

$\begin{array}{lll}\mathrm{mg} / \mathrm{kg} & 23.3 & \text { EPA6010A } \\ \mathrm{mg} / \mathrm{kg} & 9.34 & \text { EPA6010A } \\ \mathrm{mg} / \mathrm{kg} & 28 & \text { EPA6010A } \\ \mathrm{mg} / \mathrm{kg} & 2.33 & \text { EPA6010A } \\ \mathrm{mg} / \mathrm{kg} & 0.934 & \text { EPA6010A } \\ \mathrm{mg} / \mathrm{kg} & 0.467 & \text { EPA6010A } \\ \mathrm{mg} / \mathrm{kg} & 23.3 & \text { EPA6010A } \\ \mathrm{mg} / \mathrm{kg} & 2.33 & \text { EPA6010A } \\ \mathrm{mg} / \mathrm{kg} & \mathbf{0 . 9 3 4} & \text { EPA6010A } \\ \mathrm{mg} / \mathrm{kg} & 2.33 & \text { EPA6010A } \\ \mathrm{mg} / \mathrm{kg} & \mathbf{9 . 3 4} & \text { EPA6010A } \\ \mathrm{mg} / \mathrm{kg} & 9.34 & \text { EPA6010A } \\ \mathrm{mg} / \mathrm{kg} & 9.34 & \text { EPA6010A } \\ \mathrm{mg} / \mathrm{kg} & 0.934 & \text { EPA6010A } \\ \mathrm{mg} / \mathrm{kg} & 0.0525 & \text { EPA7471 } \\ \mathrm{mg} / \mathrm{kg} & 2.33 & \text { EPA6010A } \\ \mathrm{mg} / \mathrm{kg} & 187 & \text { EPA6010A } \\ \mathrm{mg} / \mathrm{kg} & 28 & \text { EPA6010A } \\ \mathrm{mg} / \mathrm{kg} & 2.33 & \text { EPA6010A } \\ \mathrm{mg} / \mathrm{kg} & 93.4 & \text { EPA6010A } \\ \mathrm{mg} / \mathrm{kg} & 28 & \text { EPA6010A } \\ \mathrm{mg} / \mathrm{kg} & 0.934 & \text { EPA6010A } \\ \mathrm{mg} / \mathrm{kg} & 1.87 & \text { EPA6010A }\end{array}$

$B / N / A$ Extractables (including $P A H$ and phenols)

$\begin{array}{lcc}\text { Acenaphthene } & \mathrm{U} & 63.3 \\ \text { Acenaphthylene } & \mathrm{U} & 63.3 \\ \text { Anthracene } & \mathrm{U} & 63.3 \\ \text { Benzo[a]anthracene } & \mathrm{U} & 6.33 \\ \text { Benzo[b]fluoranthene } & \mathrm{U} & 6.33 \\ \text { Benzo[k]fluoranthene } & \mathrm{U} & 6.33 \\ \text { Benzoic acid } & \mathrm{U} & 63.3 \\ \text { Benzo[g,h,i]perylene } & \mathrm{U} & 63.3 \\ \text { Benzo[a]pyrene } & \mathrm{U} & 6.33 \\ \text { Benzyl alcohol } & \mathrm{U} & 63.3 \\ \text { Bis(2-chloroethoxy) methane } & \mathrm{U} & 63.3 \\ \text { Bis(2-chloroethyl) ether } & \mathrm{U} & 63.3 \\ \text { Bis(2-chloroisopropyl) ether } & \mathrm{U} & 63.3 \\ \text { Bis(2-ethylhexyl) phthalate } & \mathrm{U} & 63.3 \\ \text { 4-Bromophenyl phenyl ether } & \mathrm{U} & 63.3 \\ \text { Butylbenzyl phthalate } & \mathrm{U} & 63.3 \\ \text { 4-Chloroaniline } & \mathrm{U} & 63.3\end{array}$

$\begin{array}{lll}\mu \mathrm{g} / \mathrm{kg} & 63.3 & \text { EPA8270 } \\ \mu \mathrm{g} / \mathrm{kg} & 63.3 & \text { EPA8270 } \\ \mu \mathrm{g} / \mathrm{kg} & 63.3 & \text { EPA8270 } \\ \mu \mathrm{g} / \mathrm{kg} & 6.33 & \text { EPA8270 } \\ \mu \mathrm{g} / \mathrm{kg} & 6.33 & \text { EPA8270 } \\ \mu \mathrm{g} / \mathrm{kg} & 6.33 & \text { EPA8270 } \\ \mu \mathrm{g} / \mathrm{kg} & 63.3 & \text { EPA8270 } \\ \mu \mathrm{g} / \mathrm{kg} & 63.3 & \text { EPA8270 } \\ \mu \mathrm{g} / \mathrm{kg} & 6.33 & \text { EPA8270 } \\ \mu \mathrm{g} / \mathrm{kg} & 63.3 & \text { EPA8270 } \\ \mu \mathrm{g} / \mathrm{kg} & 63.3 & \text { EPA8270 } \\ \mu \mathrm{g} / \mathrm{kg} & 63.3 & \text { EPA8270 } \\ \mu \mathrm{g} / \mathrm{kg} & 63.3 & \text { EPA8270 } \\ \mu \mathrm{g} / \mathrm{kg} & -63.3 & \text { EPA8270 } \\ \mu \mathrm{g} / \mathrm{kg} & 63.3 & \text { EPA8270 } \\ \mu \mathrm{g} / \mathrm{kg} & 63.3 & \text { EPA8270 } \\ \mu \mathrm{g} / \mathrm{kg} & 63.3 & \text { EPA8270 }\end{array}$


SURVEY ID: PPSC 1201 (continued)

Sample ID: 104656
Constituent
RQ AQ B Result
Unit
D. Limit Method

$B / N / A$ Extractables (including PAH and phenols)

\begin{tabular}{|c|c|c|c|c|c|}
\hline 4-Chloro-m-cresol & $\mathbf{U}$ & 63.3 & $\mu \mathrm{g} / \mathrm{kg}$ & 63.3 & EPA8270 \\
\hline 2-Chloronaphthalene & $\mathrm{U}$ & 63.3 & $\mu \mathrm{g} / \mathrm{kg}$ & 63.3 & EPA8270 \\
\hline 2-Chlorophenol & U & 63.3 & $\mu \mathrm{g} / \mathrm{kg}$ & 63.3 & EPA8270 \\
\hline 4-Chlorophenyl phenyl ether & $\mathrm{U}$ & 63.3 & $\mu \mathrm{g} / \mathrm{kg}$ & 63.3 & EPA8270 \\
\hline Chrysene & $\mathrm{U}$ & 6.33 & $\mu \mathrm{g} / \mathrm{kg}$ & 6.33 & EPA8270 \\
\hline $\mathrm{m} / \mathrm{p}$-Cresol & $\mathrm{U}$ & 633 & $\mu \mathrm{g} / \mathrm{kg}$ & 633 & EPA8270 \\
\hline o-Cresol (2-Methylphenol) & $\mathrm{U}$ & 63.3 & $\mu \mathrm{g} / \mathrm{kg}$ & 63.3 & EPA8270 \\
\hline Dibenz $[a, h]$ anthracene & $\mathrm{U}$ & 6.33 & $\mu \mathrm{g} / \mathrm{kg}$ & 6.33 & EPA8270 \\
\hline Dibenzofuran & U & 63.3 & $\mu \mathrm{g} / \mathrm{kg}$ & 63.3 & EPA8270 \\
\hline Di-n-butyl phthalate & $\mathrm{U}$ & 55.7 & $\mu \mathrm{g} / \mathrm{kg}$ & 63.3 & EPA8270 \\
\hline 1,2-Dichlorobenzene & $\mathrm{U}$ & 63.3 & $\mu \mathrm{g} / \mathrm{kg}$ & 63.3 & EPA8270 \\
\hline 1,3-Dichlorobenzene & $\mathrm{U}$ & 63.3 & $\mu \mathrm{g} / \mathrm{kg}$ & 63.3 & EPA8270 \\
\hline 1,4-Dichlorobenzene & $U$ & 63.3 & $\mu \mathrm{g} / \mathrm{kg}$ & 63.3 & EPA8270 \\
\hline 3,3'-Dichlorobenzidine & $\mathrm{U}$ & 63.3 & $\mu \mathrm{g} / \mathrm{kg}$ & 63.3 & EPA8270 \\
\hline 2,4-Dichlorophenol & $\mathrm{U}$ & 63.3 & $\mu \mathrm{g} / \mathrm{kg}$ & 63.3 & EPA8270 \\
\hline Diethyl phthalate & $\mathrm{U}$ & 63.3 & $\mu \mathrm{g} / \mathrm{kg}$ & 63.3 & EPA8270 \\
\hline 2,4-Dimethyl phenol & $\mathrm{U}$ & 63.3 & $\mu \mathrm{g} / \mathrm{kg}$ & 63.3 & EPA8270 \\
\hline Dimethyl phthalate & U & 63.3 & $\mu \mathrm{g} / \mathrm{kg}$ & 63.3 & EPA8270 \\
\hline 2,4-Dinitrophenol & $\mathrm{U}$ & 633 & $\mu \mathrm{g} / \mathrm{kg}$ & 633 & EPA8270 \\
\hline 2,4-Dinitrotoluene & UJ & 6.33 & $\mu \mathrm{g} / \mathrm{kg}$ & 6.33 & EPA8270 \\
\hline 2,6-Dinitrotoluene & $\mathrm{U}$ & 6.33 & $\mu \mathrm{g} / \mathrm{kg}$ & 6.33 & EPA8270 \\
\hline Di-n-octyl phthalate & $\mathrm{U}$ & 63.3 & $\mu \mathrm{g} / \mathrm{kg}$ & 63.3 & EPA8270 \\
\hline Fluoranthene & $\mathrm{U}$ & 63.3 & $\mu \mathrm{g} / \mathrm{kg}$ & 63.3 & EPA8270 \\
\hline Fluorene & U & 63.3 & $\mu \mathrm{g} / \mathrm{kg}$ & 63.3 & EPA8270 \\
\hline Hexachlorobenzene & $\mathrm{U}$ & 6.33 & $\mu \mathrm{g} / \mathrm{kg}$ & 6.33 & EPA8270 \\
\hline Hexachlorobutadiene & $\mathrm{U}$ & 63.3 & $\mu \mathrm{g} / \mathrm{kg}$ & 63.3 & EPA8270 \\
\hline Hexachlorocyclopentadiene & $\mathrm{U}$ & 63.3 & $\mu \mathrm{g} / \mathrm{kg}$ & 63.3 & EPA8270 \\
\hline Hexachloroethane & $\mathrm{U}$ & 6.33 & $\mu \mathrm{g} / \mathrm{kg}$ & 6.33 & EPA8270 \\
\hline Indeno $[1,2,3-c, d]$ pyrene & $\mathrm{U}$ & 6.33 & $\mu \mathrm{g} / \mathrm{kg}$ & 6.33 & EPA8270 \\
\hline Isophorone & $\mathrm{U}$ & 63.3 & $\mu \mathrm{g} / \mathrm{kg}$ & 63.3 & EPA8270 \\
\hline 2-Methyl-4,6-dinitrophenol & $\mathrm{U}$ & 633 & $\mu \mathrm{g} / \mathrm{kg}$ & 633 & EPA8270 \\
\hline 2-Methylnaphthalene & $\mathrm{U}$ & 63.3 & $\mu \mathrm{g} / \mathrm{kg}$ & 63.3 & EPA8270 \\
\hline Naphthalene & $U$ & 63.3 & $\mu \mathrm{g} / \mathrm{kg}$ & 63.3 & EPA8270 \\
\hline m-Nitroaniline & $\mathrm{U}$ & 63.3 & $\mu \mathrm{g} / \mathrm{kg}$ & 63.3 & EPA8270 \\
\hline o-Nitroaniline & $\mathrm{U}$ & 63.3 & $\mu \mathrm{g} / \mathrm{kg}$ & 63.3 & EPA8270 \\
\hline p-Nitroaniline & $\mathrm{U}$ & 63.3 & $\mu \mathrm{g} / \mathrm{kg}$ & 63.3 & EPA8270 \\
\hline Nitrobenzene & $\mathrm{U}$ & 63.3 & $\mu \mathrm{g} / \mathrm{kg}$ & 63.3 & EPA8270 \\
\hline 2-Nitrophenol & $\mathrm{U}$ & 63.3 & $\mu \mathrm{g} / \mathrm{kg}$ & 63.3 & EPA8270 \\
\hline 4-Nitrophenol & $\mathrm{U}$ & 63.3 & $\mu \mathrm{g} / \mathrm{kg}$ & 63.3 & EPA8270 \\
\hline N-Nitrosodiphenylamine & $\mathrm{U}$ & 63.3 & $\mu \mathrm{g} / \mathrm{kg}$ & 63.3 & EPA8270 \\
\hline N-Nitrosodi-n-propylamine & $\mathrm{U}$ & 63.3 & $\mu \mathrm{g} / \mathrm{kg}$ & 63.3 & EPA8270 \\
\hline Pentachlorophenol & $\mathrm{U}$ & 63.3 & $\mu g / \mathrm{kg}$ & 63.3 & EPA8270 \\
\hline Phenanthrene & $\mathrm{U}$ & 63.3 & $\mu \mathrm{g} / \mathrm{kg}$ & 63.3 & EPA8270 \\
\hline Phenol & $\mathrm{U}$ & 63.3 & $\mu \mathrm{g} / \mathrm{kg}$ & 63.3 & EPA8270 \\
\hline Pyrene & $\mathrm{U}$ & 63.3 & $\mu \mathrm{g} / \mathrm{kg}$ & 63.3 & EPA8270 \\
\hline 1,2,4-Trichlorobenzene & $\mathrm{U}$ & 6.33 & $\mu \mathrm{g} / \mathrm{kg}$ & 6.33 & EPA8270 \\
\hline 2,4,5-Trichlorophenol & $\mathrm{U}$ & 63.3 & $\mu g / \mathrm{kg}$ & 63.3 & EPA8270 \\
\hline 2,4,6-Trichlorophenol . & $\mathrm{U}$ & 63.3 & $\mu \mathrm{g} / \mathrm{kg}$ & 63.3 & EPA8270 \\
\hline
\end{tabular}


SURVEY ID: PPSC 1201 (continued)

\section{Constituent}

Volatile Organic Compounds
Sample ID: 104656

RQ AQ B Result

Unit

\begin{tabular}{|c|c|c|c|}
\hline Acetone & & V8 & $\begin{array}{l}73.1 \\
0.923\end{array}$ \\
\hline $\begin{array}{l}\text { Benzene } \\
\text { Bromodichloromethane }\end{array}$ & $\begin{array}{l}J \\
U\end{array}$ & $\mathrm{E}$ & $\begin{array}{l}0.923 \\
0.0962\end{array}$ \\
\hline Bromoform & U & & 0.0962 \\
\hline Bromomethane (Methyl bromide) & $\mathrm{U}$ & & 0.192 \\
\hline Carbon disulfide & $\mathrm{J}$ & $\mathrm{E}$ & 0.462 \\
\hline Carbon tetrachloride & $\mathrm{U}$ & & 0.0962 \\
\hline Chlorobenzene & $\mathrm{U}$ & & 0.0962 \\
\hline Chloroethane & $\mathrm{U}$ & & 0.192 \\
\hline Chloroethene (Vinyl chloride) & $\mathrm{U}$ & & 0.192 \\
\hline Chloroform & & & 0.75 \\
\hline Chloromethane (Methyl chloride) & $\mathrm{U}$ & & 0.192 \\
\hline Dibromochloromethane & $\mathrm{U}$ & & 0.0962 \\
\hline 1,1-Dichloroethane & $\mathrm{U}$ & & 0.0962 \\
\hline 1,2-Dichloroethane & $\mathrm{U}$ & & 0.0962 \\
\hline 1,1-Dichloroethylene & $\mathrm{U}$ & & 0.0962 \\
\hline 1,2-Dichloroethylene & $U$ & & 0.192 \\
\hline Dichloromethane (Methylene chloride) & $U$ & $\mathrm{~V}$ & 4.52 \\
\hline 1,2-Dichloropropane & U & & 0.0962 \\
\hline cis-1,3-Dichloropropene & $\mathrm{U}$ & & 0.0962 \\
\hline trans-1,3-Dichloropropene & $\mathrm{U}$ & & 0.0962 \\
\hline Ethylbenzene & $U$ & & 0.0962 \\
\hline 2-Hexanone & $\mathrm{U}$ & $\mathrm{V}$ & 0.865 \\
\hline Isocamphene & $\mathrm{J}$ & $\mathrm{N}$ & 3 \\
\hline Methyl ethyl ketone & $\mathrm{U}$ & $\mathrm{V}$ & 2.52 \\
\hline Methyl isobutyl ketone & $\mathrm{U}$ & & 1.92 \\
\hline Styrene & $\mathrm{U}$ & & 0.0962 \\
\hline 1,1,2,2-Tetrachloroethane & U & & 0.0962 \\
\hline Tetrachloroethylene & $\mathrm{U}$ & & 0.0962 \\
\hline Toluene & U & V & 0.269 \\
\hline 1,1,1-Trichloroethane & $\mathrm{U}$ & & 0.0962 \\
\hline 1,1,2-Trichloroethane & $U$ & & 0.0962 \\
\hline Trichloroethylene & U & & 0.0962 \\
\hline Vinyl acetate & $\mathrm{U}$ & & 1.92 \\
\hline Xylenes & $\mathbf{J}$ & F & 0.23 \\
\hline
\end{tabular}

Pesticides/PCBs and Dioxins/Furans

$\begin{array}{ll}U & 31.8 \\ U & 31.8 \\ U & 63.5 \\ U & 63.5 \\ U & 63.5 \\ U & 63.5 \\ U & 127 \\ U & 63.5 \\ U & 127 \\ U & 63.5 \\ U & 127 \\ U & 63.5 \\ U & 127 \\ U & 63.5 \\ U & 127 \\ U & 63.5 \\ U & 63.5\end{array}$

$\begin{array}{lll}\mu \mathrm{g} / \mathrm{kg} & 9.62 & \text { EPA8260 } \\ \mu \mathrm{g} / \mathrm{kg} & 0.962 & \text { EPA8260 } \\ \mu \mathrm{g} / \mathrm{kg} & 0.0962 & \text { EPA8260 } \\ \mu \mathrm{g} / \mathrm{kg} & 0.0962 & \text { EPA8260 } \\ \mu \mathrm{g} / \mathrm{kg} & 0.192 & \text { EPA8260 } \\ \mu \mathrm{g} / \mathrm{kg} & 1.92 & \text { EPA8260 } \\ \mu \mathrm{g} / \mathrm{kg} & 0.0962 & \text { EPA8260 } \\ \mu \mathrm{g} / \mathrm{kg} & 0.0962 & \text { EPA8260 } \\ \mu \mathrm{g} / \mathrm{kg} & 0.192 & \text { EPA8260 } \\ \mu \mathrm{g} / \mathrm{kg} & 0.192 & \text { EPA8260 } \\ \mu \mathrm{g} / \mathrm{kg} & 0.0962 & \text { EPA8260 } \\ \mu \mathrm{g} / \mathrm{kg} & 0.192 & \text { EPA8260 } \\ \mu \mathrm{g} / \mathrm{kg} & 0.0962 & \text { EPA8260 } \\ \mu \mathrm{g} / \mathrm{kg} & 0.0962 & \text { EPA8260 } \\ \mu \mathrm{g} / \mathrm{kg} & 0.0962 & \text { EPA8260 } \\ \mu \mathrm{g} / \mathrm{kg} & 0.0962 & \text { EPA8260 } \\ \mu \mathrm{g} / \mathrm{kg} & 0.192 & \text { EPA8260 } \\ \mu \mathrm{g} / \mathrm{kg} & 0.962 & \text { EPA8260 } \\ \mu \mathrm{g} / \mathrm{kg} & 0.0962 & \text { EPA8260 } \\ \mu \mathrm{g} / \mathrm{kg} & 0.0962 & \text { EPA8260 } \\ \mu \mathrm{g} / \mathrm{kg} & 0.0962 & \text { EPA8260 } \\ \mu \mathrm{g} / \mathrm{kg} & 0.0962 & \text { EPA8260 } \\ \mu \mathrm{g} / \mathrm{kg} & 1.92 & \text { EPA8260 } \\ \mu \mathrm{g} / \mathrm{kg} & & \text { EPA8260 } \\ \mu \mathrm{g} / \mathrm{kg} & 1.92 & \text { EPA8260 } \\ \mu \mathrm{g} / \mathrm{kg} & 1.92 & \text { EPA8260 } \\ \mu \mathrm{g} / \mathrm{kg} & 0.0962 & \text { EPA8260 } \\ \mu \mathrm{g} / \mathrm{kg} & 0.0962 & \text { EPA8260 } \\ \mu \mathrm{g} / \mathrm{kg} & 0.0962 & \text { EPA8260 } \\ \mu \mathrm{g} / \mathrm{kg} & 0.962 & \text { EPA8260 } \\ \mu \mathrm{g} / \mathrm{kg} & 0.0962 & \text { EPA8260 } \\ \mu \mathrm{g} / \mathrm{kg} & 0.0962 & \text { EPA8260 } \\ \mu \mathrm{g} / \mathrm{kg} & 0.0962 & \text { EPA8260 } \\ \mu \mathrm{g} / \mathrm{kg} & 1.92 & \text { EPA8260 } \\ \mu \mathrm{g} / \mathrm{kg} & 0.288 & \text { EPA8260 }\end{array}$

$\begin{array}{lll}\mu \mathrm{g} / \mathrm{kg} & 31.8 & \text { EPA8080 } \\ \mu \mathrm{g} / \mathrm{kg} & 31.8 & \text { EPA8080 } \\ \mu \mathrm{g} / \mathrm{kg} & 63.5 & \text { EPA8080 } \\ \mu \mathrm{g} / \mathrm{kg} & 63.5 & \text { EPA8080 } \\ \mu \mathrm{g} / \mathrm{kg} & 63.5 & \text { EPA8080 } \\ \mu \mathrm{g} / \mathrm{kg} & 63.5 & \text { EPA8080 } \\ \mu \mathrm{g} / \mathrm{kg} & 127 & \text { EPA8080 } \\ \mu \mathrm{g} / \mathrm{kg} & 63.5 & \text { EPA8080 } \\ \mu \mathrm{g} / \mathrm{kg} & 127 & \text { EPA8080 } \\ \mu \mathrm{g} / \mathrm{kg} & 63.5 & \text { EPA8080 } \\ \mu \mathrm{g} / \mathrm{kg} & 127 & \text { EPA8080 } \\ \mu \mathrm{g} / \mathrm{kg} & 63.5 & \text { EPA8080 } \\ \mu \mathrm{g} / \mathrm{kg} & 127 & \text { EPA8080 } \\ \mu \mathrm{g} / \mathrm{kg} & 63.5 & \text { EPA8080 } \\ \mu \mathrm{g} / \mathrm{kg} & 127 & \text { EPA8080 } \\ \mu \mathrm{g} / \mathrm{kg} & 63.5 & \text { EPA8080 } \\ \mu \mathrm{g} / \mathrm{kg} & 63.5 & \text { EPA8080 }\end{array}$


SURVEY ID: PPSC 1201 (continued)

Constituent

Pesticides/PCBs and Dioxins/Furans

Lindane

Methoxychlor

PCB 1016

PCB 1221

PCB 1232

PCB 1242

PCB 1248

PCB 1254

PCB 1260

Toxaphene

Radionuclides

Actinium-228

Antimony-124

Antimony-125

Barium-133

Cerium-144

Cesium-134

Cesium-137

Cobalt-57

Cobalt-58

Cobalt-60

Europium-152

Europium-154

Europium-155

Gross alpha

Lead-212

Manganese-54

Neptunium-239

Nonvolatile beta

Plutonium-238

Plutonium-239/240

Potassium-40

Promethium-144

Promethium-146

Ruthenium-106

Sodium-22

Strontium-90

Thorium-234

Tin-113

Yttrium-88

Zinc-65

Zirconium-95

Sample ID: 104656

RQ AQ B Result Unit D. Limit Method

$\begin{array}{lllll}\mathrm{U} & 31.8 & \mu \mathrm{g} / \mathrm{kg} & 31.8 & \text { EPA8080 } \\ \mathrm{U} & 635 & \mu \mathrm{g} / \mathrm{kg} & 635 & \text { EPA8080 } \\ \mathrm{U} & 159 & \mu \mathrm{g} / \mathrm{kg} & 159 & \text { EPA8080 } \\ \mathrm{U} & 159 & \mu \mathrm{g} / \mathrm{kg} & 159 & \text { EPA8080 } \\ \mathrm{U} & 159 & \mu \mathrm{g} / \mathrm{kg} & 159 & \text { EPA8080 } \\ \mathrm{U} & 159 & \mu \mathrm{g} / \mathrm{kg} & 159 & \text { EPA8080 } \\ \mathrm{U} & 159 & \mu \mathrm{g} / \mathrm{kg} & 159 & \text { EPA8080 } \\ \mathrm{U} & 159 & \mu \mathrm{g} / \mathrm{kg} & 159 & \text { EPA8080 } \\ \mathrm{U} & 159 & \mu \mathrm{g} / \mathrm{kg} & 159 & \text { EPA8080 } \\ \mathrm{U} & 1270 & \mu \mathrm{g} / \mathrm{kg} & 1270 & \text { EPA8080 }\end{array}$

$1.71 \mathrm{E}+00 \pm 2.03 \mathrm{E}-01 \mathrm{pCi} / \mathrm{g}$ $3.87 \mathrm{E}-03 \pm 4.01 \mathrm{E}-02 \mathrm{pCi} / \mathrm{g}$ $1.24 \mathrm{E}-01 \pm 1.45 \mathrm{E}-01 \mathrm{pCi} / \mathrm{g}$ $1.06 \mathrm{E}-02 \pm 6.49 \mathrm{E}-02 \mathrm{pCi} / \mathrm{g}$ 1.34E-02 $2.27 \mathrm{E}-01 \mathrm{pCi} / \mathrm{g}$ $-7.45 \mathrm{E}-03 \pm 3.76 \mathrm{E}-02 \mathrm{pCi} / \mathrm{g}$ $2.40 \mathrm{E}+01 \pm 2.97 \mathrm{E}-01 \mathrm{pCi} / \mathrm{g}$ $4.92 \mathrm{E}-03 \pm 2.52 \mathrm{E}-02 \mathrm{pCi} / \mathrm{g}$ 2.65E-02 $2.85 \mathrm{E}-02 \mathrm{pCi} / \mathrm{g}$ $4.15 \mathrm{E}-01 \pm 7.26 \mathrm{E}-02 \mathrm{pCi} / \mathrm{g}$ $-5.22 \mathrm{E}-02 \pm 1.28 \mathrm{E}-01 \mathrm{pCi} / \mathrm{g}$ $-2.47 \mathrm{E}-01 \pm 2.48 \mathrm{E}-01 \mathrm{pCi} / \mathrm{g}$ $7.03 \mathrm{E}-02 \pm 1.15 \mathrm{E}-01 \mathrm{pCi} / \mathrm{g}$ $1.78 \mathrm{E}+01 \pm 3.46 \mathrm{E}+00 \mathrm{pCi} / \mathrm{g}$ $1.78 \mathrm{E}+00 \pm 1.19 \mathrm{E}-01 \mathrm{pCi} / \mathrm{g}$ 1.44E-02 $2.74 \mathrm{E}-02 \mathrm{pCi} / \mathrm{g}$ $.7 .60 \mathrm{E}-02 \pm 1.85 \mathrm{E}-01 \mathrm{pCi} / \mathrm{g}$ $2.18 \mathrm{E}+01 \pm 2.59 \mathrm{E}+00 \mathrm{pCi} / \mathrm{g}$ 1.21E-02 $1.83 \mathrm{E}-02 \mathrm{pCi} / \mathrm{g}$ 1.71E-01 $44.60 \mathrm{E}-02 \mathrm{pCi} / \mathrm{g}$ $3.22 \mathrm{E}+00 \pm 6.11 \mathrm{E}-01 \mathrm{pCi} / \mathrm{g}$ $-5.46 \mathrm{E}-03 \pm 2.37 \mathrm{E}-02 \mathrm{pCi} / \mathrm{g}$ $-7.37 \mathrm{E}-03 \pm 8.69 \mathrm{E}-02 \mathrm{pCi} / \mathrm{g}$ $8.52 \mathrm{E}-02 \pm 3.25 \mathrm{E}-01 \mathrm{pCi} / \mathrm{g}$ $-2.42 \mathrm{E}-02 \pm 3.02 \mathrm{E}-02 \mathrm{pCi} / \mathrm{g}$ $1.49 \mathrm{E}+00 \pm 1.27 \mathrm{E}-01 \mathrm{pCi} / \mathrm{g}$ $2.77 \mathrm{E}+00 \pm 1.90 \mathrm{E}+00 \mathrm{pCi} / \mathrm{g}$ 2.40E-02 $\pm 6.71 \mathrm{E}-02 \mathrm{pCi} / \mathrm{g}$ 2.52E-02 $\pm 2.62 \mathrm{E}-02 \mathrm{pCi} / \mathrm{g}$ $-1.52 \mathrm{E}-03 \pm 6.31 \mathrm{E}-02 \mathrm{pCi} / \mathrm{g}$ $-4.63 \mathrm{E}-02 \pm 5.24 \mathrm{E}-02 \mathrm{pCi} g$
1.58E-01 EPIA-013B
6.82E-02 EPIA-013B
2.52E-01 EPIA-013B
9.91E-02 EPIA-013B
3.41E-01 EPIA-013B
5.51E-02 EPIA-013B
5.89E-02 EPIA-013B
4.26E-02 EPIA-013B
4.73E-02 EPIA-013B
4.43E-02 EPIA-013B
2.19E-01 EPIA-013B
3.39E-01 EPIA-013B
1.69E-01 EPIA-013B
2.28E +00 EPIA-001B
1.12E-01 EPIA-013B
4.81E-02 EPIA-013B
3.08E-01 EPIA-013B
2.34E+00 EPIA-001B
3.51E-02 EPIA-012B
2.09E-02 EPIA-012B
4.68E-01 EPIA-013B
4.00E-02 EPIA-013B
1.29E-01 EPIA-013B
5.56E-01 EPIA-013B
4.10E-02 EPIA-013B
6.08E-02 EPIA-004
2.04E+00 EPIA-013B
1.16E-01 EPIA-013B
5.48E-02 EPIA-013B
9.74E-02 EPIA-013B
8.35E-02 EPIA-013B

SURVEY ID: PPSC 1201A

Sample ID: 104657

Survey location: $44888 \mathrm{E}$ 3682027N (SRS coordinates)

Depth of core interval: 0.00 to $1.00 \mathrm{ft}$

Sample type: Normal

Sample matrix: Other

Percent solids: $\mathbf{5 6 . 0 0}$ 
SURVEY ID: PPSC 1201A (continued)

Sample ID: 104657

Constituent

RQ AQ B Result

Unit

D. Limit Method

Physical Parameters and Specified Analyses

Chloride

Cyanide

Nitrate-nitrite as nitrogen

Orthophosphate

Phenols

Sulfide

Total organic carbon

Total organic nitrogen

Total phosphates (as P)

Total phosphates (as P)

Metals (total recoverable)

$\begin{array}{lll}\text { J } & \text { E } & 3.1 \\ \text { U } & & 1.79 \\ \text { J } & E & 0.504 \\ \text { U } & & 17.9 \\ \text { UJ } & \text { C } & 536 \\ \text { UJ } & \text { C } & 446 \\ & & 13400 \\ \text { C } & & 434 \\ \text { J } & \text { E } & 195 \\ \text { J } & \text { E } & 193\end{array}$

$\begin{array}{lll} & & 12000 \\ U & & 8.75 \\ U & & 26.3 \\ & & 62.6 \\ \text { J } & E & 0.593 \\ \text { U } & & 0.438 \\ & & 743 \\ & & 20.6 \\ & & 2.44 \\ & & 7.68 \\ & & 12500 \\ & & 27.6 \\ & & 179 \\ & & 0.0512 \\ & & 4.3 \\ \text { U } & & 242 \\ \text { U } & & 26.3 \\ \text { J } & \text { E } & 2.19 \\ \text { U } & & 16 \\ & & 26.3 \\ & & 16.9 \\ & & \end{array}$

$B / N / A$ Extractables (including $P A H$ and phenols)
Acenaphthene

Acenaphthylene

Anthracene

Benzo[a]anthracene

Benzo[b]fluoranthene

Benzo[k]fluoranthene

Benzoic acid

Benzo[g,h,i]perylene

Benzo[a]pyrene

Benzyl alcohol

Bis(2-chloroethoxy) methane

Bis(2-chloroethyl) ether

Bis(2-chloroisopropyl) ether

Bis(2-ethylhexyl) phthalate

4-Bromophenyl phenyl ether

Butylbenzyl phthalate

$\begin{array}{ll}\text { U } & \mathbf{5 8 . 4} \\ \mathrm{U} & \mathbf{5 8 . 4} \\ \mathrm{U} & \mathbf{5 8 . 4} \\ \mathrm{U} & \mathbf{5 . 8 4} \\ \mathrm{U} & \mathbf{5 . 8 4} \\ \mathrm{U} & \mathbf{5 . 8 4} \\ & 308 \\ \mathrm{U} & 58.4 \\ \mathrm{U} & \mathbf{5 . 8 4} \\ \mathrm{U} & 58.4 \\ \mathrm{U} & 58.4 \\ \mathrm{U} & \mathbf{5 8 . 4} \\ \mathrm{U} & 58.4 \\ \mathrm{U} & \mathbf{5 8 . 4} \\ \mathrm{U} & \mathbf{5 8 . 4} \\ \mathrm{U} & \mathbf{5 8 . 4}\end{array}$

$\begin{array}{lll}\mathrm{mg} / \mathrm{kg} & 4.28 & \text { EPA300.0 } \\ \mathrm{mg} / \mathrm{kg} & 1.79 & \text { EPA335.3 } \\ \mathrm{mg} / \mathrm{kg} & 1.68 & \text { EPA353.1 } \\ \mathrm{mg} / \mathrm{kg} & 17.9 & \text { EPA365.2 } \\ \mu \mathrm{g} / \mathrm{kg} & 536 & \text { EPA420.2 } \\ \mathrm{mg} / \mathrm{kg} & 446 & \text { EPA376.2 } \\ \mathrm{mg} / \mathrm{kg} & 179 & \text { EPA415.1 } \\ \mathrm{mg} / \mathrm{kg} & \mathbf{0 . 3 5 7} & \text { EPA351.2 } \\ \mathrm{mg} / \mathrm{kg} & 700 & \text { EPA365.4 } \\ \mathrm{mg} / \mathrm{kg} & \mathbf{6 3 8} & \text { EPA365.4 }\end{array}$

$\begin{array}{lll}\mathrm{mg} / \mathrm{kg} & 21.9 & \text { EPA6010A } \\ \mathrm{mg} / \mathrm{kg} & 8.75 & \text { EPA6010A } \\ \mathrm{mg} / \mathrm{kg} & 26.3 & \text { EPA6010A } \\ \mathrm{mg} / \mathrm{kg} & 2.19 & \text { EPA6010A } \\ \mathrm{mg} / \mathrm{kg} & 0.875 & \text { EPA6010A } \\ \mathrm{mg} / \mathrm{kg} & 0.438 & \text { EPA6010A } \\ \mathrm{mg} / \mathrm{kg} & 21.9 & \text { EPA6010A } \\ \mathrm{mg} / \mathrm{kg} & 2.19 & \text { EPA6010A } \\ \mathrm{mg} / \mathrm{kg} & 0.875 & \text { EPA6010A } \\ \mathrm{mg} / \mathrm{kg} & 2.19 & \text { EPA6010A } \\ \mathrm{mg} / \mathrm{kg} & 8.75 & \text { EPA6010A } \\ \mathrm{mg} / \mathrm{kg} & 8.75 & \text { EPA6010A } \\ \mathrm{mg} / \mathrm{kg} & 8.75 & \text { EPA6010A } \\ \mathrm{mg} / \mathrm{kg} & 0.875 & \text { EPA6010A } \\ \mathrm{mg} / \mathrm{kg} & 0.0488 & \text { EPA7471 } \\ \mathrm{mg} / \mathrm{kg} & 2.19 & \text { EPA6010A } \\ \mathrm{mg} / \mathrm{kg} & 175 & \text { EPA6010A } \\ \mathrm{mg} / \mathrm{kg} & 26.3 & \text { EPA6010A } \\ \mathrm{mg} / \mathrm{kg} & 2.19 & \text { EPA6010A } \\ \mathrm{mg} / \mathrm{kg} & 87.5 & \text { EPA6010A } \\ \mathrm{mg} / \mathrm{kg} & 26.3 & \text { EPA6010A } \\ \mathrm{mg} / \mathrm{kg} & 0.875 & \text { EPA6010A } \\ \mathrm{mg} / \mathrm{kg} & 1.75 & \text { EPA6010A }\end{array}$

$\begin{array}{lll}\mu \mathrm{g} / \mathrm{kg} & 58.4 & \text { EPA8270 } \\ \mu \mathrm{g} / \mathrm{kg} & 58.4 & \text { EPA8270 } \\ \mu \mathrm{g} / \mathrm{kg} & 58.4 & \text { EPA8270 } \\ \mu \mathrm{g} / \mathrm{kg} & 5.84 & \text { EPA8270 } \\ \mu \mathrm{g} / \mathrm{kg} & 5.84 & \text { EPA8270 } \\ \mu \mathrm{g} / \mathrm{kg} & 5.84 & \text { EPA8270 } \\ \mu \mathrm{g} / \mathrm{kg} & 58.4 & \text { EPA8270 } \\ \mu \mathrm{g} / \mathrm{kg} & 58.4 & \text { EPA8270 } \\ \mu \mathrm{g} / \mathrm{kg} & 5.84 & \text { EPA8270 } \\ \mu \mathrm{g} / \mathrm{kg} & 58.4 & \text { EPA8270 } \\ \mu \mathrm{g} / \mathrm{kg} & 58.4 & \text { EPA8270 } \\ \mu \mathrm{g} / \mathrm{kg} & 58.4 & \text { EPA8270 } \\ \mu \mathrm{g} / \mathrm{kg} & 58.4 & \text { EPA8270 } \\ \mu \mathrm{g} / \mathrm{kg} & 58.4 & \text { EPA8270 } \\ \mu \mathrm{g} / \mathrm{kg} & 58.4 & \text { EPA8270 } \\ \mu \mathrm{g} / \mathrm{kg} & 58.4 & \text { EPA8270 }\end{array}$


SURVEY ID: PPSC 1201A (continued)

Constituent
Sample ID: 104657

RQ AQ B Result

Unit

D. Limit Method

$B / N / A$ Extractables (including $P A H$ and phenols)

4-Chloroaniline

4-Chloro-m-cresol

2-Chloronaphthalene

2-Chlorophenol

4-Chlorophenyl phenyl ether

Chrysene

$\mathrm{m} / \mathrm{p}$-Cresol

o-Cresol (2-Methylphenol)

Dibenz $[a, h]$ anthracene

Dibenzofuran

Di-n-butyl phthalate

1,2-Dichlorobenzene

1,3-Dichlorobenzene

1,4-Dichlorobenzene

$3,3^{\prime}$-Dichlorobenzidine

2,4-Dichlorophenol

Diethyl phthalate

2,4-Dimethyl phenol

Dimethyl phthalate

2,4-Dinitrophenol

2,4-Dinitrotoluene

2,6-Dinitrotoluene

Di-n-octyl phthalate

Fluoranthene

Fluorene

Hexachlorobenzene

Hexachlorobutadiene

Hexachlorocyclopentadiene

Hexachloroethane

Indeno[1,2,3-c,d]pyrene

Isophorone

2-Methyl-4,6-dinitrophenol

2-Methylnaphthalene

Naphthalene

m-Nitroaniline

o-Nitroaniline

p-Nitroaniline

Nitrobenzene

2-Nitrophenol

4-Nitrophenol

N-Nitrosodiphenylamine

N-Nitrosodi-n-propylamine

Pentachlorophenol

Phenanthrene

Phenol

Pyrene

1,2,4-Trichlorobenzene

2,4,5-Trichlorophenol

2,4,6-Trichlorophenol
U

U

$\mathrm{U}$

U

U

U

U

U

U

U

$\begin{array}{lll}\mathrm{U} & \mathrm{V} & \mathbf{5 2 . 6}\end{array}$

$\mathrm{U} \quad \mathbf{5 8 . 4}$

$\mathrm{U} \quad \mathbf{5 8 . 4}$

$\mathrm{U} \quad \mathbf{5 8 . 4}$

U $\quad \mathbf{5 8 . 4}$

$\mathrm{U} \quad \mathbf{5 8 . 4}$

$\mathrm{U} \quad \mathbf{5 8 . 4}$

U $\quad \mathbf{5 8 . 4}$

U $\quad 58.4$

U $\quad 584$

UJ C $\quad \mathbf{5 . 8 4}$

5.84

58.4

58.4

58.4

5.84

58.4

58.4

5.84

5.84

58.4

584

58.4

58.4

58.4

58.4

58.4

58.4

58.4

58.4

58.4

58.4

58.4

58.4

58.4

58.4

5.84

58.4

58.4

\begin{tabular}{lll}
$\mu \mathrm{g} / \mathrm{kg}$ & 58.4 & EPA8270 \\
$\mu \mathrm{g} / \mathrm{kg}$ & 58.4 & EPA8270 \\
$\mu \mathrm{g} / \mathrm{kg}$ & 58.4 & EPA8270 \\
$\mu \mathrm{g} / \mathrm{kg}$ & 58.4 & EPA8270 \\
$\mu \mathrm{g} / \mathrm{kg}$ & 58.4 & EPA8270 \\
$\mu \mathrm{g} / \mathrm{kg}$ & 5.84 & EPA8270 \\
$\mu \mathrm{g} / \mathrm{kg}$ & $\mathbf{5 8 4}$ & EPA8270 \\
$\mu \mathrm{g} / \mathrm{kg}$ & 58.4 & EPA8270 \\
$\mu \mathrm{g} / \mathrm{kg}$ & 5.84 & EPA8270 \\
$\mu \mathrm{g} / \mathrm{kg}$ & $\mathbf{5 8 . 4}$ & EPA8270 \\
$\mu \mathrm{g} / \mathrm{kg}$ & 58.4 & EPA8270 \\
$\mu \mathrm{g} / \mathrm{kg}$ & 58.4 & EPA8270 \\
$\mu \mathrm{g} / \mathrm{kg}$ & 58.4 & EPA8270 \\
$\mu \mathrm{g} / \mathrm{kg}$ & $\mathbf{5 8 . 4}$ & EPA8270 \\
$\mu \mathrm{g} / \mathrm{kg}$ & 58.4 & EPA8270 \\
$\mu \mathrm{g} / \mathrm{kg}$ & $\mathbf{5 8 . 4}$ & EPA8270 \\
$\mu \mathrm{g} / \mathrm{kg}$ & 58.4 & EPA8270 \\
$\mu \mathrm{g} / \mathrm{kg}$ & 58.4 & EPA8270 \\
$\mu \mathrm{g} / \mathrm{kg}$ & 58.4 & EPA8270 \\
$\mu \mathrm{g} / \mathrm{kg}$ & $\mathbf{5 8 4}$ & EPA8270 \\
$\mu \mathrm{g} / \mathrm{kg}$ & 5.84 & EPA8270 \\
$\mu \mathrm{g} / \mathrm{kg}$ & 5.84 & EPA8270 \\
$\mu \mathrm{g} / \mathrm{kg}$ & $\mathbf{5 8 . 4}$ & EPA8270 \\
$\mu \mathrm{g} / \mathrm{kg}$ & 58.4 & EPA8270 \\
$\mu \mathrm{g} / \mathrm{kg}$ & 58.4 & EPA8270 \\
$\mu \mathrm{g} / \mathrm{kg}$ & 5.84 & EPA8270 \\
$\mu \mathrm{g} / \mathrm{kg}$ & 58.4 & EPA8270 \\
$\mu \mathrm{g} / \mathrm{kg}$ & 58.4 & EPA8270 \\
$\mu \mathrm{g} / \mathrm{kg}$ & 5.84 & EPA8270 \\
$\mu \mathrm{g} / \mathrm{kg}$ & 5.84 & EPA8270 \\
$\mu \mathrm{g} / \mathrm{kg}$ & 58.4 & EPA8270 \\
$\mu \mathrm{g} / \mathrm{kg}$ & $\mathbf{5 8 4}$ & EPA8270 \\
$\mu \mathrm{g} / \mathrm{kg}$ & 58.4 & EPA8270 \\
$\mu \mathrm{g} / \mathrm{kg}$ & 58.4 & EPA8270 \\
$\mu \mathrm{g} / \mathrm{kg}$ & 58.4 & EPA8270 \\
$\mu \mathrm{g} / \mathrm{kg}$ & 58.4 & EPA8270 \\
$\mu \mathrm{g} / \mathrm{kg}$ & 58.4 & EPA8270 \\
$\mu \mathrm{g} / \mathrm{kg}$ & 58.4 & EPA8270 \\
$\mu \mathrm{g} / \mathrm{kg}$ & 58.4 & EPA8270 \\
$\mu \mathrm{g} / \mathrm{kg}$ & 58.4 & EPA8270 \\
$\mu \mathrm{g} / \mathrm{kg}$ & 58.4 & EPA8270 \\
$\mu \mathrm{g} / \mathrm{kg}$ & 58.4 & EPA8270 \\
$\mu \mathrm{g} / \mathrm{kg}$ & 58.4 & EPA8270 \\
$\mu \mathrm{g} / \mathrm{kg}$ & 58.4 & EPA8270 \\
$\mu \mathrm{g} / \mathrm{kg}$ & 58.4 & EPA8270 \\
$\mu \mathrm{g} / \mathrm{kg}$ & $\mathbf{5 8 . 4}$ & EPA8270 \\
$\mu \mathrm{g} / \mathrm{kg}$ & 5.84 & EPA8270 \\
$\mu \mathrm{g} / \mathrm{kg}$ & 58.4 & EPA8270 \\
$\mu \mathrm{g} / \mathrm{kg}$ & 58.4 & EPA8270 \\
& & \\
\hline
\end{tabular}


SURVEY ID: PPSC 1201A (continued)

Constituent

RQ AQ B Result
Sample ID: 104657

Unit D. Limit Method

Volatile Organic Compounds

\begin{tabular}{|c|c|c|c|c|}
\hline Acetone & UJ & OV8 & & 61.7 \\
\hline Benzene & $\mathbf{J}$ & 0 & $\mathrm{H}$ & 0.929 \\
\hline Bromodichloromethane & UJ & 0 & $\mathrm{H}$ & 0.0893 \\
\hline Bromoform & UJ & 0 & $\mathrm{H}$ & 0.0893 \\
\hline Bromomethane (Methyl bromide) & UJ & 0 & $\mathrm{H}$ & 0.179 \\
\hline Carbon disulfide & $\mathbf{J}$ & $\mathrm{OE}$ & $\mathrm{H}$ & 0.661 \\
\hline Carbon tetrachloride & UJ & 0 & $\mathrm{H}$ & 0.0893 \\
\hline Chlorobenzene & UJ & 0 & $\mathrm{H}$ & 0.0893 \\
\hline Chloroethane & UJ & 0 & $\mathrm{H}$ & 0.179 \\
\hline Chloroethene (Vinyl chloride) & UJ & 0 & $\mathrm{H}$ & 0.179 \\
\hline Chloroform & $\mathrm{J}$ & 0 & $\mathrm{H}$ & 0.768 \\
\hline Chloromethane (Methyl chloride) & UJ & 0 & $\mathrm{H}$ & 0.179 \\
\hline ochloromethane & UJ & 0 & $\mathrm{H}$ & 0.0893 \\
\hline 1,1-Dichloroethane & UJ & 0 & $\mathrm{H}$ & 0.0893 \\
\hline 1,2-Dichloroethane & UJ & 0 & $\mathrm{H}$ & 0.0893 \\
\hline loroethylene & UJ & 0 & $\mathrm{H}$ & 0.0893 \\
\hline chloroethylene & UJ & 0 & $\mathrm{H}$ & 0.179 \\
\hline Dichloromethane (Methylene chloride) & UJ & OV & $\mathrm{H}$ & 5.98 \\
\hline 1,2-Dichloropropane & UJ & 0 & $\mathrm{H}$ & 0.0893 \\
\hline ichloropropene & UJ & 0 & $\mathrm{H}$ & 0.0893 \\
\hline 1,3-Dichloropropene & UJ & 0 & $\mathrm{H}$ & 0.089 \\
\hline Ethy & UJ & 0 & $\mathrm{H}$ & 0.089 \\
\hline 2-Hexanone & UJ & 0 & $\mathrm{H}$ & 1.79 \\
\hline Isocamphene & $\mathbf{J}$ & $\mathbf{N}$ & & 3.5 \\
\hline Methyl ethyl ketone & UJ & OV & $\mathrm{H}$ & 2.61 \\
\hline 1 isobutyl ketone & UJ & 0 & $\mathrm{H}$ & 1.79 \\
\hline Styrene & UJ & 0 & $\mathrm{H}$ & 0.089 \\
\hline 1,1,2,2-Tetrachloroethane & UJ & 0 & $\mathrm{H}$ & 0.089 \\
\hline Tetrachloroethylene & UJ & 0 & $\mathrm{H}$ & 0.0893 \\
\hline Toluene & UJ & OV & $\mathrm{H}$ & 0.304 \\
\hline 1,1,1-Trichloroethane & UJ & 0 & $\mathrm{H}$ & 0.0893 \\
\hline 1,1,2-Trichloroethane & UJ & 0 & $\mathrm{H}$ & 0.0893 \\
\hline Trichloroethylene & UJ & 0 & $\mathrm{H}$ & 0.0893 \\
\hline Vinyl acetate & UJ & $\mathrm{O}$ & & 1.79 \\
\hline Xylenes & $\mathrm{J}$ & $\mathrm{OE}$ & $\mathrm{H}$ & 0.196 \\
\hline
\end{tabular}

$\begin{array}{lll}\mu \mathrm{g} / \mathrm{kg} & 8.93 & \text { EPA8260 } \\ \mu \mathrm{g} / \mathrm{kg} & 0.893 & \text { EPA8260 } \\ \mu \mathrm{g} / \mathrm{kg} & 0.0893 & \text { EPA8260 } \\ \mu \mathrm{g} / \mathrm{kg} & 0.0893 & \text { EPA8260 } \\ \mu \mathrm{g} / \mathrm{kg} & 0.179 & \text { EPA8260 } \\ \mu \mathrm{g} / \mathrm{kg} & 1.79 & \text { EPA8260 } \\ \mu \mathrm{g} / \mathrm{kg} & 0.0893 & \text { EPA8260 } \\ \mu \mathrm{g} / \mathrm{kg} & 0.0893 & \text { EPA8260 } \\ \mu \mathrm{g} / \mathrm{kg} & 0.179 & \text { EPA8260 } \\ \mu \mathrm{g} / \mathrm{kg} & 0.179 & \text { EPA8260 } \\ \mu \mathrm{g} / \mathrm{kg} & 0.0893 & \text { EPA8260 } \\ \mu \mathrm{g} / \mathrm{kg} & 0.179 & \text { EPA8260 } \\ \mu \mathrm{g} / \mathrm{kg} & 0.0893 & \text { EPA8260 } \\ \mu \mathrm{g} / \mathrm{kg} & 0.0893 & \text { EPA8260 } \\ \mu \mathrm{g} / \mathrm{kg} & 0.0893 & \text { EPA8260 } \\ \mu \mathrm{g} / \mathrm{kg} & 0.0893 & \text { EPA8260 } \\ \mu \mathrm{g} / \mathrm{kg} & 0.179 & \text { EPA8260 } \\ \mu \mathrm{g} / \mathrm{kg} & 0.893 & \text { EPA8260 } \\ \mu \mathrm{g} / \mathrm{kg} & 0.0893 & \text { EPA8260 } \\ \mu \mathrm{g} / \mathrm{kg} & 0.0893 & \text { EPA8260 } \\ \mu \mathrm{g} / \mathrm{kg} & 0.0893 & \text { EPA8260 } \\ \mu \mathrm{g} / \mathrm{kg} & 0.0893 & \text { EPA8260 } \\ \mu \mathrm{g} / \mathrm{kg} & 1.79 & \text { EPA8260 } \\ \mu \mathrm{g} / \mathrm{kg} & & \text { EPA8260 } \\ \mu \mathrm{g} / \mathrm{kg} & 1.79 & \text { EPA8260 } \\ \mu \mathrm{g} / \mathrm{kg} & 1.79 & \text { EPA8260 } \\ \mu \mathrm{g} / \mathrm{kg} & 0.0893 & \text { EPA8260 } \\ \mu \mathrm{g} / \mathrm{kg} & 0.0893 & \text { EPA8260 } \\ \mu \mathrm{g} / \mathrm{kg} & 0.0893 & \text { EPA8260 } \\ \mu \mathrm{g} / \mathrm{kg} & 0.893 & \text { EPA8260 } \\ \mu \mathrm{g} / \mathrm{kg} & 0.0893 & \text { EPA8260 } \\ \mu \mathrm{g} / \mathrm{kg} & 0.0893 & \text { EPA8260 } \\ \mu \mathrm{g} / \mathrm{kg} & 0.0893 & \text { EPA8260 } \\ \mu \mathrm{g} / \mathrm{kg} & 1.79 & \text { EPA8260 } \\ \mu \mathrm{g} / \mathrm{kg} & 0.268 & \text { EPA8260 }\end{array}$

Pesticides/PCBs and Dioxins/Furans

Aldrin
alpha-Benzene hexachloride
beta-Benzene hexachloride
delta-Benzene hexachloride
alpha-Chlordane
gamma-Chlordane
p,p'-DDD
p,p'-DDE
p, p $^{\prime}$-DDT
Dieldrin
Endosulfan sulfate
Endosulfan I
Endosulfan II
Endrin
Endrin ketone
Heptachlor
Heptachlor epoxide

$\begin{array}{ll}\text { U } & 29.1 \\ \text { U } & 29.1 \\ U & 58.2 \\ U & 58.2 \\ U & 58.2 \\ U & 58.2 \\ U & 116 \\ U & 58.2 \\ U & 116 \\ U & 58.2 \\ U & 116 \\ U & 58.2 \\ U & 116 \\ U & 58.2 \\ U & 116 \\ U & 58.2 \\ U & 58.2\end{array}$

$\begin{array}{lll}\mu \mathrm{g} / \mathrm{kg} & 29.1 & \text { EPA8080 } \\ \mu \mathrm{g} / \mathrm{kg} & 29.1 & \text { EPA8080 } \\ \mu \mathrm{g} / \mathrm{kg} & 58.2 & \text { EPA8080 } \\ \mu \mathrm{g} / \mathrm{kg} & 58.2 & \text { EPA8080 } \\ \mu \mathrm{g} / \mathrm{kg} & 58.2 & \text { EPA8080 } \\ \mu \mathrm{g} / \mathrm{kg} & \mathbf{5 8 . 2} & \text { EPA8080 } \\ \mu \mathrm{g} / \mathrm{kg} & 116 & \text { EPA8080 } \\ \mu \mathrm{g} / \mathrm{kg} & 58.2 & \text { EPA8080 } \\ \mu \mathrm{g} / \mathrm{kg} & 116 & \text { EPA8080 } \\ \mu \mathrm{g} / \mathrm{kg} & 58.2 & \text { EPA8080 } \\ \mu \mathrm{g} / \mathrm{kg} & 116 & \text { EPA8080 } \\ \mu \mathrm{g} / \mathrm{kg} & 58.2 & \text { EPA8080 } \\ \mu \mathrm{g} / \mathrm{kg} & 116 & \text { EPA8080 } \\ \mu \mathrm{g} / \mathrm{kg} & 58.2 & \text { EPA8080 } \\ \mu \mathrm{g} / \mathrm{kg} & 116 & \text { EPA8080 } \\ \mu \mathrm{g} / \mathrm{kg} & 58.2 & \text { EPA8080 } \\ \mu \mathrm{g} / \mathrm{kg} & 58.2 & \text { EPA8080 }\end{array}$


SURVEY ID: PPSC 1201A (continued)

Constituent

Pesticides/PCBs and Dioxins/Furans

Lindane

Methoxychlor

PCB 1016

PCB 1221

PCB 1232

PCB 1242

PCB 1248

PCB 1254

PCB 1260

Toxaphene

Radionuclides

Actinium-228

Antimony-124

Antimony-125

Barium-133

Cerium-144

Cesium-134

Cesium-137

Cobalt-57

Cobalt-58

Cobalt-60

Europium-152

Europium-154

Europium-155

Gross alpha

Lead-212

Manganese-54

Neptunium-239

Nonvolatile beta

Plutonium-238

Plutonium-239/240

Potassium-40

Promethium-144

Promethium-146

Ruthenium-106

Sodium-22

Strontium-90

Thorium-234

Tin-113

Yttrium-88

Zinc-65

Zirconium-95
RQ AQ B Result

$\mu \mathrm{g} / \mathrm{kg}$

$\mu \mathrm{g} / \mathrm{kg}$

$\mu \mathrm{g} / \mathrm{kg}$

$\mu \mathrm{g} / \mathrm{kg}$

$\mu \mathrm{g} / \mathrm{kg}$

$\mu \mathrm{g} / \mathrm{kg}$

$\mu \mathrm{g} / \mathrm{kg}$

$\mu \mathrm{g} / \mathrm{kg}$

$\mu \mathrm{g} / \mathrm{kg}$

$\mu \mathrm{g} / \mathrm{kg}$
$1.41 \mathrm{E}+00 \pm 2.32 \mathrm{E}-01 \mathrm{pCi} / \mathrm{g}$ $2.63 \mathrm{E}-03 \pm 3.82 \mathrm{E}-02 \mathrm{pCi} / \mathrm{g}$ $8.35 \mathrm{E}-02 \pm 1.45 \mathrm{E}-01 \mathrm{pCi} / \mathrm{g}$ $-1.63 \mathrm{E}-02 \pm 6.46 \mathrm{E}-02 \mathrm{pCi} / \mathrm{g}$ $-8.18 \mathrm{E}-02 \pm 1.90 \mathrm{E}-01 \mathrm{pCi} / \mathrm{g}$ $3.16 \mathrm{E}-03 \pm 3.49 \mathrm{E}-02 \mathrm{pCj} / \mathrm{g}$ $2.34 \mathrm{E}+01 \pm 3.04 \mathrm{E}-01 \mathrm{pCi} / \mathrm{g}$ $-1.30 \mathrm{E}-03 \pm 2.43 \mathrm{E}-02 \mathrm{pCi} / \mathrm{g}$ $-1.33 \mathrm{E}-02 \pm 2.63 \mathrm{E}-02 \mathrm{pCi} / \mathrm{g}$ $4.48 \mathrm{E}-01 \pm 5.90 \mathrm{E}-02 \mathrm{pCi} / \mathrm{g}$ $2.07 \mathrm{E}-01 \pm 1.27 \mathrm{E}-01 \mathrm{pCi} / \mathrm{g}$ $-2.19 \mathrm{E}-01 \pm 2.68 \mathrm{E}-01 \mathrm{pCi} / \mathrm{g}$ $5.25 \mathrm{E}-02 \pm 9.39 \mathrm{E}-02 \mathrm{pCi} / \mathrm{g}$ $2.38 \mathrm{E}+01 \pm 4.19 \mathrm{E}+00 \mathrm{pCi} / \mathrm{g}$ $1.62 \mathrm{E}+00 \pm 1.12 \mathrm{E}-01 \mathrm{pCi} / \mathrm{g}$ $-5.57 \mathrm{E}-03 \pm 2.68 \mathrm{E}-02 \mathrm{pCi} / \mathrm{g}$ $-1.06 \mathrm{E}-01 \pm 1.72 \mathrm{E}-01 \mathrm{pCi} / \mathrm{g}$ $3.07 \mathrm{E}+01 \pm 3.00 \mathrm{E}+00 \mathrm{pCi} / \mathrm{g}$ $1.72 \mathrm{E}-02 \pm 1.83 \mathrm{E}-02 \mathrm{pCi} / \mathrm{g}$ 9.30E-02 $\pm 3.31 \mathrm{E}-02 \mathrm{pCi} / \mathrm{g}$ $2.96 \mathrm{E}+00 \pm 6.19 \mathrm{E}-01 \mathrm{pCi} / \mathrm{g}$ $-3.99 \mathrm{E}-03 \pm 2.30 \mathrm{E}-02 \mathrm{pCi} / \mathrm{g}$ $1.09 \mathrm{E}-02 \pm 7.56 \mathrm{E}-02 \mathrm{pCj} / \mathrm{g}$ $-2.81 \mathrm{E}-02 \pm 2.97 \mathrm{E}-01 \mathrm{pCi} / \mathrm{g}$ $2.56 \mathrm{E}-02 \pm 3.08 \mathrm{E}-02 \mathrm{pCi} / \mathrm{g}$ $1.20 \mathrm{E}+00 \pm 1.15 \mathrm{E}-01 \mathrm{pCj} / \mathrm{g}$ $3.56 \mathrm{E}+00 \pm 2.12 \mathrm{E}+00 \mathrm{pCi} / \mathrm{g}$ $-2.41 \mathrm{E}-02 \pm 6.56 \mathrm{E}-02 \mathrm{pCi} / \mathrm{g}$ $6.08 \mathrm{E}-03 \pm 2.52 \mathrm{E}-02 \mathrm{pCi} / \mathrm{g}$ $3.86 \mathrm{E}-02 \pm 6.09 \mathrm{E}-02 \mathrm{pCi} / \mathrm{g}$ $-3.15 \mathrm{E}-02 \pm 4.91 \mathrm{E}-02 \mathrm{pCi} / \mathrm{g}$
1.60E-01 EPIA-013B
6.71E-02 EPIA-013B
2.41E-01 EPIA-013B
9.44E-02 EPIA-013B
3.26E-01 EPIA-013B
5.43E-02 EPIA-013B
6.13E-02 EPIA-013B
4.21E-02 EPIA-013B
4.47E-02 EPIA-013B
5.18E-02 EPIA-013B
2.21E-01 EPIA-013B
3.67E-01 EPIA-013B
1.66E-01 EPIA-013B
$1.77 \mathrm{E}+00$ EPIA-001B
1.08E-01 EPIA-013B
4.63E-02 EPIA-013B
2.95E-01 EPIA-013B
2.28E+00 EPIA-001B
3.12E-02 EPIA-012B
2.04E-02 EPIA-012B
3.92E-01 EPIA-013B
4.04E-02 EPIA-013B
1.24E-01 EPIA-013B
5.18E-01 EPIA-013B
3.95E-02 EPIA-013B
6.09E-02 EPIA-004
$1.95 \mathrm{E}+00$ EPIA-013B
1.07E-01 EPIA-013B
4.90E-02 EPIA-013B
1.01E-01 EPIA-013B
8.29E-02 EPIA-013B

SURVEY ID: PPSC 1202

Sample ID: 104658

Survey location: $44888 \mathrm{E} 3682027 \mathrm{~N}$ (SRS coordinates)

Depth of core interval: 1.00 to $2.00 \mathrm{ft}$

Sample type: Normal

Sample matrix: Soil

Percent solids: 84.00 
SURVEY ID: PPSC 1202 (continued)

Constituent
Sample ID: 104658

RQ AQ B Result

Unit

D. Limit Method

Physical Parameters and Specified Analyses

$\begin{array}{lllllll}\text { Chloride } & \mathrm{J} & \mathrm{E} & 1.13 & \mathrm{mg} / \mathrm{kg} & 2.78 & \text { EPA300.0 } \\ \text { Cyanide } & \mathrm{U} & & 1.19 & \mathrm{mg} / \mathrm{kg} & 1.19 & \text { EPA335.3 } \\ \text { Nitrate-nitrite as nitrogen } & \mathrm{J} & \mathrm{E} & 0.497 & \mathrm{mg} / \mathrm{kg} & 1.24 & \text { EPA353.1 } \\ \text { Orthophosphate } & \mathrm{J} & \mathrm{E} & 2.62 & \mathrm{mg} / \mathrm{kg} & 11.9 & \text { EPA365.2 } \\ \text { Phenols } & \mathrm{UJ} & \mathrm{C} & 357 & \mu \mathrm{g} / \mathrm{kg} & 357 & \text { EPA420.2 } \\ \text { Sulfide } & \mathrm{UJ} & \mathrm{C} & 298 & \mathrm{mg} / \mathrm{kg} & 298 & \text { EPA376.2 } \\ \text { Total organic carbon } & & & 11100 & \mathrm{mg} / \mathrm{kg} & 119 & \text { EPA415.1 } \\ \text { Total organic carbon } & & & 9740 & \mathrm{mg} / \mathrm{kg} & 119 & \text { EPA415.1 } \\ \text { Total organic nitrogen } & \mathrm{C} & & 150 & \mathrm{mg} / \mathrm{kg} & 0.238 & \text { EPA351.2 } \\ \text { Total phosphates (as P) } & \mathrm{J} & \mathrm{E} & 93.7 & \mathrm{mg} / \mathrm{kg} & 378 & \text { EPA365.4 }\end{array}$

Metals (total recoverable)

\begin{tabular}{|c|c|c|c|}
\hline Aluminum & & & 4380 \\
\hline Antimony & $\mathrm{U}$ & & 5.84 \\
\hline Arsenic & $\mathbf{U}$ & & 17.5 \\
\hline Barium & & & 24.3 \\
\hline Beryllium & $\mathbf{J}$ & $\mathbf{E}$ & 0.19 \\
\hline Cadmium & $U$ & & 0.292 \\
\hline Calcium & & & 166 \\
\hline Chromium & & & 5.76 \\
\hline Cobalt & & & 0.718 \\
\hline Copper & & & 1.72 \\
\hline Iron & & & 2570 \\
\hline Lead & & & 6.13 \\
\hline Magnesium & & & 100 \\
\hline Manganese & & & 38.5 \\
\hline Mercury & $J$ & $\mathbf{E}$ & 0.012 \\
\hline Nickel & $J$ & $\mathbf{E}$ & 1.41 \\
\hline Potassium & $\mathbf{J}$ & $\mathbf{E}$ & 75.3 \\
\hline Selenium & $\mathrm{U}$ & & 17.5 \\
\hline Silver & $\mathrm{U}$ & & 1.46 \\
\hline Sodium & $\mathrm{U}$ & & 58.4 \\
\hline Thallium & $\mathrm{U}$ & & 17.5 \\
\hline Vanadium & & & 8.86 \\
\hline Zinc & & & 4.69 \\
\hline
\end{tabular}

$\begin{array}{lll}\mathrm{mg} / \mathrm{kg} & 14.6 & \text { EPA6010A } \\ \mathrm{mg} / \mathrm{kg} & 5.84 & \text { EPA6010A } \\ \mathrm{mg} / \mathrm{kg} & 17.5 & \text { EPA6010A } \\ \mathrm{mg} / \mathrm{kg} & 1.46 & \text { EPA6010A } \\ \mathrm{mg} / \mathrm{kg} & 0.584 & \text { EPA6010A } \\ \mathrm{mg} / \mathrm{kg} & 0.292 & \text { EPA6010A } \\ \mathrm{mg} / \mathrm{kg} & 14.6 & \text { EPA6010A } \\ \mathrm{mg} / \mathrm{kg} & 1.46 & \text { EPA6010A } \\ \mathrm{mg} / \mathrm{kg} & 0.584 & \text { EPA6010A } \\ \mathrm{mg} / \mathrm{kg} & 1.46 & \text { EPA6010A } \\ \mathrm{mg} / \mathrm{kg} & 5.84 & \text { EPA6010A } \\ \mathrm{mg} / \mathrm{kg} & 5.84 & \text { EPA6010A } \\ \mathrm{mg} / \mathrm{kg} & 5.84 & \text { EPA6010A } \\ \mathrm{mg} / \mathrm{kg} & 0.584 & \text { EPA6010A } \\ \mathrm{mg} / \mathrm{kg} & 0.0299 & \text { EPA7471 } \\ \mathrm{mg} / \mathrm{kg} & 1.46 & \text { EPA6010A } \\ \mathrm{mg} / \mathrm{kg} & 117 & \text { EPA6010A } \\ \mathrm{mg} / \mathrm{kg} & 17.5 & \text { EPA6010A } \\ \mathrm{mg} / \mathrm{kg} & 1.46 & \text { EPA6010A } \\ \mathrm{mg} / \mathrm{kg} & 58.4 & \text { EPA6010A } \\ \mathrm{mg} / \mathrm{kg} & 17.5 & \text { EPA6010A } \\ \mathrm{mg} / \mathrm{kg} & 0.584 & \text { EPA6010A } \\ \mathrm{mg} / \mathrm{kg} & 1.17 & \text { EPA6010A }\end{array}$

$B / N / A$ Extractables (including $P A H$ and phenols)

Acenaphthene

Acenaphthylene

Anthracene

Benzo[a]anthracene

Benzo[b]fluoranthene

Benzo[ $k]$ fluoranthene

Benzoic acid

Benzo[g, $h, i]$ perylene

Benzo[a]pyrene

Benzyl alcohol

Bis(2-chloroethoxy) methane

$\mathrm{Bis}$ (2-chloroethyl) ether

Bis(2-chloroisopropyl) ether

Bis(2-ethylhexyl) phthalate

4-Bromophenyl phenyl ether

Butylbenzyl phthalate

$\begin{array}{ll}\mathrm{U} & 39.2 \\ \mathrm{U} & 39.2 \\ \mathrm{U} & 39.2 \\ \mathrm{U} & 3.92 \\ \mathrm{U} & 3.92 \\ \mathrm{U} & 3.92 \\ \mathrm{U} & 39.2 \\ \mathrm{U} & 39.2 \\ \mathrm{U} & 3.92 \\ \mathrm{U} & 39.2 \\ \mathrm{U} & 39.2 \\ \mathrm{U} & 39.2 \\ \mathrm{U} & 39.2 \\ \mathrm{U} & 39.2 \\ \mathrm{U} & 39.2 \\ \mathrm{U} & 39.2\end{array}$

$\begin{array}{lrr}\mu \mathrm{g} / \mathrm{kg} & 39.2 & \text { EPA8270 } \\ \mu \mathrm{g} / \mathrm{kg} & 39.2 & \text { EPA8270 } \\ \mu \mathrm{g} / \mathrm{kg} & 39.2 & \text { EPA8270 } \\ \mu \mathrm{g} / \mathrm{kg} & 3.92 & \text { EPA8270 } \\ \mu \mathrm{g} / \mathrm{kg} & 3.92 & \text { EPA8270 } \\ \mu \mathrm{g} / \mathrm{kg} & 3.92 & \text { EPA8270 } \\ \mu \mathrm{g} / \mathrm{kg} & 39.2 & \text { EPA8270 } \\ \mu \mathrm{g} / \mathrm{kg} & 39.2 & \text { EPA8270 } \\ \mu \mathrm{g} / \mathrm{kg} & 3.92 & \text { EPA8270 } \\ \mu \mathrm{g} / \mathrm{kg} & 39.2 & \text { EPA8270 } \\ \mu \mathrm{g} / \mathrm{kg} & 39.2 & \text { EPA8270 } \\ \mu \mathrm{g} / \mathrm{kg} & 39.2 & \text { EPA8270 } \\ \mu \mathrm{g} / \mathrm{kg} & 39.2 & \text { EPA8270 } \\ \mu \mathrm{g} / \mathrm{kg} & 39.2 & \text { EPA8270 } \\ \mu \mathrm{g} / \mathrm{kg} & 39.2 & \text { EPA8270 } \\ \mu \mathrm{g} / \mathrm{kg} & 39.2 & \text { EPA8270 }\end{array}$


Appendix D: Sediment Tables

D-150

SURVEY ID: PPSC 1202 (continued)

Sample ID: 104658

Constituent

RQ AQ B Result

Unit

D. Limit Method

$B / N / A$ Extractables (including $P A H$ and phenols)

4-Chloroaniline

4-Chloro-m-cresol

2-Chloronaphthalene

2-Chlorophenol

4-Chlorophenyl phenyl ether

Chrysene

$\mathrm{m} / \mathrm{p}$-Cresol

o-Cresol (2-Methylphenol)

Dibenz $[a, h]$ anthracene

Dibenzofuran

Di-n-butyl phthalate

1,2-Dichlorobenzene

1,3-Dichlorobenzene

1,4-Dichlorobenzene

3,3'-Dichlorobenzidine

2,4-Dichlorophenol

Diethyl phthalate

2,4-Dimethyl phenol

Dimethyl phthalate

2,4-Dinitrophenol

2,4-Dinitrotoluene

2,6-Dinitrotoluene

Di-n-octyl phthalate

Docosane

Fluoranthene

Fluorene

Hexachlorobenzene

Hexachlorobutadiene

Hexachlorocyclopentadiene

Hexachloroethane

Indeno[1,2,3-c,d]pyrene

Isophorone

2-Methyl-4,6-dinitrophenol

2-Methylnaphthalene

Naphthalene

m-Nitroaniline

o-Nitroaniline

p-Nitroaniline

Nitrobenzene

2-Nitrophenol

4-Nitrophenol

N-Nitrosodiphenylamine

N-Nitrosodi-n-propylamine

Pentachlorophenol

Phenanthrene

Phenol

Pyrene

1,2,4-Trichlorobenzene

2,4,5-Trichlorophenol

2,4,6-Trichlorophenol

Unknown alcohol

$\begin{array}{lll}\text { U } & & 39.2 \\ \text { U } & & 39.2 \\ \text { U } & & 39.2 \\ \text { U } & & 39.2 \\ \text { U } & & 39.2 \\ \text { U } & & 3.92 \\ \text { U } & & 392 \\ \text { U } & & 39.2 \\ \text { U } & & 3.92 \\ \text { U } & & 39.2 \\ \text { J } & \text { E } & 21.9 \\ \text { U } & & 39.2 \\ \text { U } & & 39.2 \\ \text { U } & & 39.2 \\ \text { U } & & 39.2 \\ \text { U } & & 39.2 \\ \text { U } & & 39.2 \\ \text { U } & & 39.2 \\ \text { U } & & 39.2 \\ \text { U } & & 392 \\ \text { UJ } & \text { C } & 3.92 \\ \text { U } & & 3.92 \\ \text { U } & & 39.2 \\ \text { J } & \text { N } & 540 \\ \text { U } & & 39.2 \\ \text { U } & & 39.2 \\ \text { U } & & 3.92 \\ \text { U } & & 39.2 \\ \text { U } & & 39.2 \\ \text { U } & & 3.92 \\ \text { U } & & 3.92 \\ \text { U } & & 39.2 \\ \text { U } & & 392 \\ \text { U } & & 39.2 \\ \text { U } & & 39.2 \\ \text { U } & & 39.2 \\ \text { U } & & 39.2 \\ \text { U } & & 39.2 \\ \text { U } & & 39.2 \\ \text { U } & & 39.2 \\ \text { U } & & 39.2 \\ \text { U } & & 39.2 \\ \text { U } & & 39.2 \\ \text { UJ } & \text { C } & 39.2 \\ \text { U } & & 39.2 \\ \text { U } & & 39.2 \\ \text { U } & & 39.2 \\ \text { U } & & 3.92 \\ \text { U } & & 39.2 \\ \text { U } & & 39.2 \\ \text { J } & \text { N } & 540 \\ & & \end{array}$

\begin{tabular}{lll}
$\mu \mathrm{g} / \mathrm{kg}$ & 39.2 & EPA8270 \\
$\mu \mathrm{g} / \mathrm{kg}$ & 39.2 & EPA8270 \\
$\mu \mathrm{g} / \mathrm{kg}$ & 39.2 & EPA8270 \\
$\mu \mathrm{g} / \mathrm{kg}$ & 39.2 & EPA8270 \\
$\mu \mathrm{g} / \mathrm{kg}$ & 39.2 & EPA8270 \\
$\mu \mathrm{g} / \mathrm{kg}$ & 3.92 & EPA8270 \\
$\mu \mathrm{g} / \mathrm{kg}$ & 392 & EPA8270 \\
$\mu \mathrm{g} / \mathrm{kg}$ & 39.2 & EPA8270 \\
$\mu \mathrm{g} / \mathrm{kg}$ & 3.92 & EPA8270 \\
$\mu \mathrm{g} / \mathrm{kg}$ & 39.2 & EPA8270 \\
$\mu \mathrm{g} / \mathrm{kg}$ & 39.2 & EPA8270 \\
$\mu \mathrm{g} / \mathrm{kg}$ & 39.2 & EPA8270 \\
$\mu \mathrm{g} / \mathrm{kg}$ & 39.2 & EPA8270 \\
$\mu \mathrm{g} / \mathrm{kg}$ & 39.2 & EPA8270 \\
$\mu \mathrm{g} / \mathrm{kg}$ & 39.2 & EPA8270 \\
$\mu \mathrm{g} / \mathrm{kg}$ & 39.2 & EPA8270 \\
$\mu \mathrm{g} / \mathrm{kg}$ & 39.2 & EPA8270 \\
$\mu \mathrm{g} / \mathrm{kg}$ & 39.2 & EPA8270 \\
$\mu \mathrm{g} / \mathrm{kg}$ & 39.2 & EPA8270 \\
$\mu \mathrm{g} / \mathrm{kg}$ & 392 & EPA8270 \\
$\mu \mathrm{g} / \mathrm{kg}$ & 3.92 & EPA8270 \\
$\mu \mathrm{g} / \mathrm{kg}$ & 3.92 & EPA8270 \\
$\mu \mathrm{g} / \mathrm{kg}$ & 39.2 & EPA8270 \\
$\mu \mathrm{g} / \mathrm{kg}$ & & EPA8270 \\
$\mu \mathrm{g} / \mathrm{kg}$ & 39.2 & EPA8270 \\
$\mu \mathrm{g} / \mathrm{kg}$ & 39.2 & EPA8270 \\
$\mu \mathrm{g} / \mathrm{kg}$ & 3.92 & EPA8270 \\
$\mu \mathrm{g} / \mathrm{kg}$ & 39.2 & EPA8270 \\
$\mu \mathrm{g} / \mathrm{kg}$ & 39.2 & EPA8270 \\
$\mu \mathrm{g} / \mathrm{kg}$ & 3.92 & EPA8270 \\
$\mu \mathrm{g} / \mathrm{kg}$ & 3.92 & EPA8270 \\
$\mu \mathrm{g} / \mathrm{kg}$ & 39.2 & EPA8270 \\
$\mu \mathrm{g} / \mathrm{kg}$ & .392 & EPA8270 \\
$\mu \mathrm{g} / \mathrm{kg}$ & 39.2 & EPA8270 \\
$\mu \mathrm{g} / \mathrm{kg}$ & 39.2 & EPA8270 \\
$\mu \mathrm{g} / \mathrm{kg}$ & 39.2 & EPA8270 \\
$\mu \mathrm{g} / \mathrm{kg}$ & 39.2 & EPA8270 \\
$\mu \mathrm{g} / \mathrm{kg}$ & 39.2 & EPA8270 \\
$\mu \mathrm{g} / \mathrm{kg}$ & 39.2 & EPA8270 \\
$\mu \mathrm{g} / \mathrm{kg}$ & 39.2 & EPA8270 \\
$\mu \mathrm{g} / \mathrm{kg} / \mathrm{kg}$ & 39.2 & EPA8270 \\
$\mu \mathrm{g} / \mathrm{kg}$ & 39.2 & EPA8270 \\
$\mu \mathrm{g} / \mathrm{kg}$ & 39.2 & EPA8270 \\
$\mu \mathrm{g} / \mathrm{kg}$ & 39.2 & EPA8270 \\
$\mu \mathrm{g} / \mathrm{kg}$ & 39.2 & EPA8270 \\
$\mu \mathrm{g} / \mathrm{kg}$ & 39.2 & EPA8270 \\
$\mu \mathrm{g} / \mathrm{kg}$ & 39.2 & EPA8270 \\
$\mu \mathrm{g} / \mathrm{kg}$ & 39.2 & EPA8270 \\
$\mu \mathrm{g} / \mathrm{kg}$ & 3.92 & EPA8270 \\
\hline & 39.2 & EPA8270 \\
\hline
\end{tabular}


SURVEY ID: PPSC 1202 (continued)

Constituent

RQ AQ B Result

Unit

D. Limit Method

Volatile Organic Compounds

Acetone
Benzene
Bromodichloromethane
Bromoform
Bromomethane (Methyl bromide)
Camphene
Carbon disulfide
Carbon tetrachloride
Chlorobenzene
Chloroethane
Chloroethene (Vinyl chloride)
Chloroform
Chloromethane (Methyl chloride)
Dibromochloromethane
1,1-Dichloroethane
1,2-Dichloroethane
1,1-Dichloroethylene
1,2-Dichloroethylene
Dichloromethane (Methylene chloride)
1,2-Dichloropropane
cis-1,3-Dichloropropene
trans-1,3-Dichloropropene
Ethylbenzene
2-Hexanone
Methyl ethyl ketone
Methyl isobutyl ketone
Styrene
1,1,2,2-Tetrachloroethane
Tetrachloroethylene
Toluene
1,1,1-Trichloroethane
1,1,2-Trichloroethane
Trichloroethylene
Unknown alkane
Vinyl acetate
Xylenes
(n)

$\begin{array}{lll}\mu \mathrm{g} / \mathrm{kg} & 5.95 & \text { EPA8260 } \\ \mu \mathrm{g} / \mathrm{kg} & 0.595 & \text { EPA8260 } \\ \mu \mathrm{g} / \mathrm{kg} & 0.0595 & \text { EPA8260 } \\ \mu \mathrm{g} / \mathrm{kg} & 0.0595 & \text { EPA8260 } \\ \mu \mathrm{g} / \mathrm{kg} & 0.119 & \text { EPA8260 } \\ \mu \mathrm{g} / \mathrm{kg} & & \text { EPA8260 } \\ \mu \mathrm{g} / \mathrm{kg} & 1.19 & \text { EPA8260 } \\ \mu \mathrm{g} / \mathrm{kg} & 0.0595 & \text { EPA8260 } \\ \mu \mathrm{g} / \mathrm{kg} & 0.0595 & \text { EPA8260 } \\ \mu \mathrm{g} / \mathrm{kg} & 0.119 & \text { EPA8260 } \\ \mu \mathrm{g} / \mathrm{kg} & 0.119 & \text { EPA8260 } \\ \mu \mathrm{g} / \mathrm{kg} & 0.0595 & \text { EPA8260 } \\ \mu \mathrm{g} / \mathrm{kg} & 0.119 & \text { EPA8260 } \\ \mu \mathrm{g} / \mathrm{kg} & 0.0595 & \text { EPA8260 } \\ \mu \mathrm{g} / \mathrm{kg} & 0.0595 & \text { EPA8260 } \\ \mu \mathrm{g} / \mathrm{kg} & 0.0595 & \text { EPA8260 } \\ \mu \mathrm{g} / \mathrm{kg} & 0.0595 & \text { EPA8260 } \\ \mu \mathrm{g} / \mathrm{kg} & 0.119 & \text { EPA8260 } \\ \mu \mathrm{g} / \mathrm{kg} & 0.595 & \text { EPA8260 } \\ \mu \mathrm{g} / \mathrm{kg} & 0.0595 & \text { EPA8260 } \\ \mu \mathrm{g} / \mathrm{kg} & 0.0595 & \text { EPA8260 } \\ \mu \mathrm{g} / \mathrm{kg} & 0.0595 & \text { EPA8260 } \\ \mu \mathrm{g} / \mathrm{kg} & 0.0595 & \text { EPA8260 } \\ \mu \mathrm{g} / \mathrm{kg} & 1.19 & \text { EPA8260 } \\ \mu \mathrm{g} / \mathrm{kg} & 1.19 & \text { EPA8260 } \\ \mu \mathrm{g} / \mathrm{kg} & 1.19 & \text { EPA8260 } \\ \mu \mathrm{g} / \mathrm{kg} & 0.0595 & \text { EPA8260 } \\ \mu \mathrm{g} / \mathrm{kg} & 0.0595 & \text { EPA8260 } \\ \mu \mathrm{g} / \mathrm{kg} & 0.0595 & \text { EPA8260 } \\ \mu \mathrm{g} / \mathrm{kg} & 0.595 & \text { EPA8260 } \\ \mu \mathrm{g} / \mathrm{kg} & 0.0595 & \text { EPA8260 } \\ \mu \mathrm{g} / \mathrm{kg} & 0.0595 & \text { EPA8260 } \\ \mu \mathrm{g} / \mathrm{kg} & 0.0595 & \text { EPA8260 } \\ \mu \mathrm{g} / \mathrm{kg} & & \text { EPA8260 } \\ \mu \mathrm{g} / \mathrm{kg} & 1.19 & \text { EPA8260 } \\ \mu \mathrm{g} / \mathrm{kg} & 0.179 & \text { EPA8260 }\end{array}$

Pesticides/PCBs and Dioxins/Furans

\begin{tabular}{|c|c|c|c|}
\hline $\mathbf{J}$ & OV8 & $\mathrm{H}$ & 70.3 \\
\hline $\boldsymbol{J}$ & 0 & $\mathrm{H}$ & 0.643 \\
\hline UJ & 0 & $\mathrm{H}$ & 0.0595 \\
\hline UJ & 0 & $\mathrm{H}$ & 595 \\
\hline UJ & 0 & $\mathrm{H}$ & 0.119 \\
\hline J & $N$ & & 2.5 \\
\hline J & EO & $\mathrm{H}$ & 0.393 \\
\hline UJ & 0 & $\mathrm{H}$ & 0.0595 \\
\hline UJ & 0 & $I$ & 0.0595 \\
\hline UJ & 0 & $\mathrm{H}$ & 0.119 \\
\hline UJ & 0 & $\mathrm{H}$ & 0.119 \\
\hline & 0 & $\mathrm{H}$ & 0.524 \\
\hline & 0 & $\mathrm{H}$ & 0.298 \\
\hline UJ & 0 & $\mathrm{H}$ & 0.0595 \\
\hline$J J$ & 0 & $\mathrm{H}$ & 0.0595 \\
\hline UJ & 0 & $\mathrm{H}$ & 0.0595 \\
\hline UJ & 0 & $\mathrm{H}$ & 0.0595 \\
\hline UJ & 0 & $\mathbf{H}$ & 0.119 \\
\hline UJ & OV & $\mathrm{H}$ & 4.1 \\
\hline UJ & 0 & $\mathrm{H}$ & 0.0595 \\
\hline IT & 0 & $\mathrm{H}$ & 0.0595 \\
\hline UJ & 0 & $\mathrm{H}$ & 0.0595 \\
\hline UJ & 0 & $\mathrm{H}$ & 0.0595 \\
\hline UJ & 0 & $\mathrm{H}$ & 1.19 \\
\hline UJ & OV & $\mathrm{H}$ & 5.99 \\
\hline UJ & 0 & $\mathrm{H}$ & 1.19 \\
\hline UJ & 0 & $\mathrm{H}$ & 0.0595 \\
\hline IJT & 0 & $\mathrm{H}$ & 0.0595 \\
\hline UJ & 0 & $\mathrm{H}$ & 0.0595 \\
\hline UJ & OV & $\mathrm{H}$ & 0.238 \\
\hline UJ & 0 & $\mathrm{H}$ & 0.0595 \\
\hline UJ & 0 & $\mathrm{H}$ & 0.0595 \\
\hline UJ & 0 & $\mathrm{H}$ & 0.0595 \\
\hline $\mathbf{J}$ & $\mathbf{N}$ & & 6 \\
\hline UJ & 0 & $F$ & 1.19 \\
\hline & $\mathrm{OE}$ & $\mathrm{H}$ & 0.155 \\
\hline
\end{tabular}

19.3

19.3

38.7

38.7

38.7

38.7

77.4

38.7

77.4

38.7

77.4

38.7

77.4 .

38.7

77.4

38.7

$\begin{array}{lll}\mu \mathrm{g} / \mathrm{kg} & 19.3 & \text { EPA8080 } \\ \mu \mathrm{g} / \mathrm{kg} & 19.3 & \text { EPA8080 } \\ \mu \mathrm{g} / \mathrm{kg} & 38.7 & \text { EPA8080 } \\ \mu \mathrm{g} / \mathrm{kg} & 38.7 & \text { EPA8080 } \\ \mu \mathrm{g} / \mathrm{kg} & 38.7 & \text { EPA8080 } \\ \mu \mathrm{g} / \mathrm{kg} & 38.7 & \text { EPA8080 } \\ \mu \mathrm{g} / \mathrm{kg} & 77.4 & \text { EPA8080 } \\ \mu \mathrm{g} / \mathrm{kg} & 38.7 & \text { EPA8080 } \\ \mu \mathrm{g} / \mathrm{kg} & 77.4 & \text { EPA8080 } \\ \mu \mathrm{g} / \mathrm{kg} & 38.7 & \text { EPA8080 } \\ \mu \mathrm{g} / \mathrm{kg} & 77.4 & \text { EPA8080 } \\ \mu \mathrm{g} / \mathrm{kg} & 38.7 & \text { EPA8080 } \\ \mu \mathrm{g} / \mathrm{kg} & -77.4 & \text { EPA8080 } \\ \mu \mathrm{g} / \mathrm{kg} & 38.7 & \text { EPA8080 } \\ \mu \mathrm{g} / \mathrm{kg} & 77.4 & \text { EPA8080 } \\ \mu \mathrm{g} / \mathrm{kg} & 38.7 & \text { EPA8080 }\end{array}$


SURVEY ID: PPSC 1202 (continued)

Constituent

Pesticides/PCBs and Dioxins/Furans

Heptachlor epoxide

Lindane

Methoxychlor

PCB 1016

PCB 1221

PCB 1232

PCB 1242

PCB 1248

PCB 1254

PCB 1260

Toxaphene

\section{Radionuclides}

Actinium-228

Antimony-124

Antimony-125

Barium-133

Cerium-144

Cesium-134

Cesium-137

Cobalt-57

Cobalt-58

Cobalt -60

Europium-152

Europium-154

Europium-155

Gross alpha

Lead-212

Manganese-54

Neptunium-239

Nonvolatile beta

Plutonium-238

Plutonium-239/240

Potassium-40

Promethium-144

Promethium-146

Ruthenium-106

Sodium-22

Strontium-90

Thorium-234

Tin-113

Yttrium-88

Zinc-65

Zirconium-95

Sample ID: 104658
RQ AQ B Result
Unit
D. Limit Method

38.7
19.3
387
96.7
96.7
96.7
96.7
96.7
96.7
96.7
774

7.41E-01 $5.20 \mathrm{E}-02 \mathrm{pCi} / \mathrm{g}$ $-1.12 \mathrm{E}-03 \pm 9.64 \mathrm{E}-03 \mathrm{pCi} / \mathrm{g}$ $-7.97 \mathrm{E}-03 \pm 2.32 \mathrm{E}-02 \mathrm{pCi} / \mathrm{g}$ $5.06 \mathrm{E}-03 \pm 1.04 \mathrm{E}-02 \mathrm{pCi} / \mathrm{g}$ 8.71E-03 $\pm 5.38 \mathrm{E}-02 \mathrm{pCi} / \mathrm{g}$ $.4 .47 \mathrm{E}-03 \pm 6.90 \mathrm{E}-03 \mathrm{pCi} / \mathrm{g}$ $3.18 \mathrm{E}+00 \pm 4.40 \mathrm{E}-02 \mathrm{pCi} / \mathrm{g}$ $-3.64 \mathrm{E}-03 \pm 6.14 \mathrm{E}-03 \mathrm{pCi} / \mathrm{g}$ $-3.10 \mathrm{E}-03 \pm 7.63 \mathrm{E}-03 \mathrm{pCi} / \mathrm{g}$ $8.88 \mathrm{E}-02 \pm 1.30 \mathrm{E}-02 \mathrm{pCj} / \mathrm{g}$ $-2.83 \mathrm{E}-03 \pm 2.07 \mathrm{E}-02 \mathrm{pCj} / \mathrm{g}$ $-1.03 \mathrm{E}-02 \pm 4.80 \mathrm{E}-02 \mathrm{pCi} / \mathrm{g}$ $0.00 \mathrm{E}+00 \quad \mathrm{pCi} / \mathrm{g}$ $7.34 \mathrm{E}+00 \pm 2.13 \mathrm{E}+00 \mathrm{pCi} / \mathrm{g}$ $\mathrm{V} \quad 7.82 \mathrm{E}-01 \pm 2.69 \mathrm{E}-02 \mathrm{pCi} / \mathrm{g}$ 1.32E-02 $\pm 7.32 \mathrm{E}-03 \mathrm{pCi} / \mathrm{g}$ $-5.34 \mathrm{E}-03 \pm 4.37 \mathrm{E}-02 \mathrm{pCi} / \mathrm{g}$ $7.04 \mathrm{E}+00 \pm 1.70 \mathrm{E}+00 \mathrm{pCi} / \mathrm{g}$ $3.12 \mathrm{E}-03 \pm 2.46 \mathrm{E}-02 \mathrm{pCi} / \mathrm{g}$ $1.18 \mathrm{E}-02 \pm 1.63 \mathrm{E}-02 \mathrm{pCi} / \mathrm{g}$ $6.95 \mathrm{E}-0 \mathrm{l} \pm 1.03 \mathrm{E}-01 \mathrm{pCi} / \mathrm{g}$ $5.05 \mathrm{E}-03 \pm 5.01 \mathrm{E}-03 \mathrm{pCi} / \mathrm{g}$ $1.52 \mathrm{E}-03 \pm 1.13 \mathrm{E}-02 \mathrm{pCi} / \mathrm{g}$ $-7.47 \mathrm{E}-03 \pm 5.89 \mathrm{E}-02 \mathrm{pCi} / \mathrm{g}$ $-2.50 \mathrm{E}-03 \pm 4.44 \mathrm{E}-03 \mathrm{pCi} / \mathrm{g}$ $1.58 \mathrm{E}+00 \pm 1.38 \mathrm{E}-01 \mathrm{pCi} / \mathrm{g}$ 8.17E-01 $\pm 5.01 \mathrm{E}-01 \mathrm{pCi} / \mathrm{g}$ $-1.73 \mathrm{E}-03 \pm 1.29 \mathrm{E}-02 \mathrm{pCj} / \mathrm{g}$ 5.16E-03 $\pm 6.85 \mathrm{E}-03 \mathrm{pCi} / \mathrm{g}$ 1.62E-02士1.21E-02 pCi/g $1.85 \mathrm{E}-02 \pm 1.44 \mathrm{E}-02 \mathrm{pCi} / \mathrm{g}$
2.99E-02 EPIA-013B

1.41E-02 EPIA-013B

3.91E-02 EPIA-013B

1.59E-02 EPIA-013B

8.04E.02 EPIA-013B

9.86E-03 EPIA-013B

1.16E-02 EPIA-013B

1.02E-02 EPIA-013B

1.08E-02 EPIA-013B

8.20E-03 EPIA-013B

3.55E-02 EPIA-013B

7.23E-02 EPIA-013B

4.18E-02 EPIA-013B

2.07E+00 EPIA-001B

1.98E-02 EPIA-013B

8.78E-03 EPIA-013B

7.31E-02 EPIA-013B

2.47E+00 EPLA-001B

5.50E-02 EPIA-012B

2.96E-02 EPIA-012B

6.80E-02 EPIA-013B

8.84E-03 EPIA-013B

1.93E-02 EPIA-013B

9.86E-02 EPLA-013B

7.45E-03 EPIA-013B

6.82E-02 EPIA-004

5.05E-01 EPIA-013B

1.93E-02 EPIA-013B

9.88E-03 EPIA-013B

2.03E-02 EPIA-013B

2.30E-02 EPIA-013B 
SURVEY ID: PPSC 1202D

Sample type: Split
Sample ID: 104659

Associated sample: 104658

\section{Constituent}

RQ AQ B Result - Unit

D. Limit Method

Physical Parameters and Specified Analyses

$\begin{array}{llll}\text { Cation exchange capacity } & \text { J } & \text { E } & 5.53 \\ \text { Chloride } & \text { J } & \text { E } & 15.1 \\ \text { Chloride } & \text { J } & \text { E } & 14.8 \\ \text { Cyanide } & \text { U } & \text { V } & 0.118 \\ \text { Nitrate-nitrite as nitrogen } & & \text { V } & 1.92 \\ \text { Orthophosphate } & \text { U } & & 1.26 \\ \text { Phenols } & \text { J } & \text { E } & 215 \\ \text { Phenols } & \text { J } & \text { E } & 275 \\ \text { Sulfide } & \text { U } & & 31.3 \\ \text { Total organic carbon } & & \text { V } & 2220 \\ \text { Total organic nitrogen } & & \text { V } & 228 \\ \text { Total organic nitrogen } & & \text { V } & 201 \\ \text { Total phosphates (as P) } & & & 86 \\ & & & \\ \text { Metals (total recoverable) } & & & \\ \text { Aluminum } & & & \\ \text { Antimony } & & & 2790 \\ \text { Arsenic } & \text { U } & & 51 \\ \text { Barium } & \text { J } & \text { E } & 0.578 \\ \text { Beryllium } & & & 17.1 \\ \text { Cadmium } & \text { J } & \text { E } & 0.15 \\ \text { Calcium } & \text { U } & & 2.91 \\ \text { Chromium } & & \text { V } & 164 \\ \text { Cobalt } & \text { J } & \text { E } & 4.7 \\ \text { Copper } & \text { J } & \text { E } & 1.1 \\ \text { Iron } & \text { J } & \text { E } & 2.2 \\ \text { Lead } & & & 2330 \\ \text { Magnesium } & \text { J } & \text { E } & 3.7 \\ \text { Manganese } & & & 79.5 \\ \text { Mercury } & & & 34.3 \\ \text { Nickel } & \text { U } & & 0.753 \\ \text { Potassium } & \text { U } & & 9.29 \\ \text { Selenium } & \text { U } & & 1100 \\ \text { Silver } & \text { UJ } & \text { W } & 1.88 \\ \text { Sodium } & \text { U } & & 6.13 \\ \text { Thallium } & \text { U } & \text { V } & 16.9 \\ \text { Vanadium } & \text { U } & \text { V } & 0.214 \\ \text { Zinc } & & & 7.7 \\ & \text { J } & \text { E } & 3.7\end{array}$

$\begin{array}{lll}\mathrm{mg} / \mathrm{kg} & 27.6 & \text { EPA9081 } \\ \mathrm{mg} / \mathrm{kg} & 21.8 & \text { EPA300.0 } \\ \mathrm{mg} / \mathrm{kg} & 21.8 & \text { EPA300.0 } \\ \mathrm{mg} / \mathrm{kg} & 1.15 & \text { EPA9010 } \\ \mathrm{mg} / \mathrm{kg} & 0.75 & \text { EPA353.2 } \\ \mathrm{mg} / \mathrm{kg} & 1.26 & \text { EPA365.2 } \\ \mu \mathrm{g} / \mathrm{kg} & 1190 & \text { EPA420.2 } \\ \mu \mathrm{g} / \mathrm{kg} & 1190 & \text { EPA420.2 } \\ \mathrm{mg} / \mathrm{kg} & 31.3 & \text { EPA376.2 } \\ \mathrm{mg} / \mathrm{kg} & 8.4 & \text { LLOYDKAHN } \\ \mathrm{mg} / \mathrm{kg} & 125 & \text { EPA351.4 } \\ \mathrm{mg} / \mathrm{kg} & 125 & \text { EPA351.4 } \\ \mathrm{mg} / \mathrm{kg} & 17.1 & \text { EPA365.2 }\end{array}$

$B / N / A$ Extractables (including PAH and phenols)

Acenaphthene

Acenaphthylene

Aldol condensate

Aldol condensate

Aldol condensate

Aldol condensate

Anthracene

Benzo[a]anthracene

Benzo[b]fluoranthene

Benzo[k]fluoranthene

Benzoic acid

Benzo $[g, h, i]$ perylene

$\begin{array}{llll} & & & \\ & \text { U } & & 42 \\ \text { U } & & 42 \\ \text { UJ } & \text { NKV } & 900 \\ \text { UJ } & \text { NKV } & 300 \\ \text { UJ } & \text { NKV } & 100 \\ \text { J } & \text { NK } & 200 \\ \text { U } & & 42 \\ \text { U } & & 42 \\ \text { J } & \text { E } & 5.45 \\ \text { U } & & 42 \\ \text { U } & & 210 \\ \text { J } & \text { E } & 4.24\end{array}$

$\begin{array}{lll}\mathrm{mg} / \mathrm{kg} & 51 & \text { CLP-MOD } \\ \mathrm{mg} / \mathrm{kg} & 51 & \text { CLP-MOD }\end{array}$

$\mathrm{mg} / \mathrm{kg} \quad 0.754 \quad$ CLP-MOD

$\mathrm{mg} / \mathrm{kg} \quad 4.78 \quad$ CLP-MOD

$\mathrm{mg} / \mathrm{kg} \quad 0.377 \quad$ CLP-MOD

$\mathrm{mg} / \mathrm{kg} \quad 2.91 \quad$ CLP-MOD

$\begin{array}{lll}\mathrm{mg} / \mathrm{kg} & 28 & \text { CLP-MOD }\end{array}$

$\begin{array}{lll}\mathrm{mg} / \mathrm{kg} & 7.91 & \text { CLP-MOD }\end{array}$

$\mathrm{mg} / \mathrm{kg} \quad 5.9 \quad$ CLP-MOD

$\begin{array}{lll}\mathrm{mg} / \mathrm{kg} & 3.99 & \text { CLP-MOD }\end{array}$

$\mathrm{mg} / \mathrm{kg} \quad 25 \quad$ CLP-MOD

$\mathrm{mg} / \mathrm{kg} \quad 4.14 \quad$ CLP-MOD

$\begin{array}{lll}\mathrm{mg} / \mathrm{kg} & 30 & \text { CLP-MOD }\end{array}$

$\begin{array}{lll}\mathrm{mg} / \mathrm{kg} & 1.22 \quad \text { CLP-MOD }\end{array}$

$\mathrm{mg} / \mathrm{kg} \quad 0.753 \quad$ CLP-MOD

$\mathrm{mg} / \mathrm{kg} \quad 9.29 \quad$ CLP-MOD

$\mathrm{mg} / \mathrm{kg} \quad 1100 \quad$ CLP-MOD

$\begin{array}{lll}\mathrm{mg} / \mathrm{kg} & 1.88 \quad \text { CLP-MOD }\end{array}$

$\mathrm{mg} / \mathrm{kg} \quad 6.13 \quad$ CLP-MOD

$\mathrm{mg} / \mathrm{kg} \quad 76 \quad$ CLP-MOD

$\begin{array}{lll}\mathrm{mg} / \mathrm{kg} & 2.01 & \text { CLP-MOD }\end{array}$

$\mathrm{mg} / \mathrm{kg} \quad 4.57 \quad$ CLP-MOD

$\begin{array}{lll}\mathrm{mg} / \mathrm{kg} & 5.4 & \text { CLP-MOD }\end{array}$

-

$\begin{array}{lll}\mu \mathrm{g} / \mathrm{kg} & 42 & \text { EPA8270 } \\ \mu \mathrm{g} / \mathrm{kg} & 42 & \text { EPA8270 } \\ \mu \mathrm{g} / \mathrm{kg} & & \text { EPA8270 } \\ \mu \mathrm{g} / \mathrm{kg} & & \text { EPA8270 } \\ \mu \mathrm{g} / \mathrm{kg} & & \text { EPA8270 } \\ \mu \mathrm{g} / \mathrm{kg} & & \text { EPA8270 } \\ \mu \mathrm{g} / \mathrm{kg} & 42 & \text { EPA8270 } \\ \mu \mathrm{g} / \mathrm{kg} & 42 & \text { EPA8270 } \\ \mu \mathrm{g} / \mathrm{kg} & 42 & \text { EPA8270 } \\ \mu \mathrm{g} / \mathrm{kg} & 42 & \text { EPA8270 } \\ \mu \mathrm{g} / \mathrm{kg} & 210 & \text { EPA8270 } \\ \mu \mathrm{g} / \mathrm{kg} & 42 & \text { EPA8270 }\end{array}$



Constituent
RQ AQ B Result
Unit
D. Limit Method

$B / N / A$ Extractables (including PAH and phenols)

Benzo[a]pyrene

Benzyl alcohol

Bis(2-chloroethoxy) methane

Bis(2-chloroethyl) ether

Bis(2-chloroisopropyl) ether

Bis(2-ethylhexyl) phthalate

4-Bromophenyl phenyl ether

Butylbenzyl phthalate

4-Chloroaniline

4-Chloro-m-cresol

2-Chloronaphthalene

2-Chlorophenol

4-Chlorophenyl phenyl ether

Chrysene

o-Cresol (2-Methylphenol)

p-Cresol (4-Methylphenol)

Dibenz $[a, h]$ anthracene

Dibenzofuran

Di-n-butyl phthalate

1,2-Dichlorobenzene

1,3-Dichlorobenzene

1,4-Dichlorobenzene

$3,3^{\circ}$-Dichlorobenzidine

2,4-Dichlorophenol

Diethyl phthalate

2,4-Dimethyl phenol

Dimethyl phthalate

2,4-Dinitrophenol

2,4-Dinitrotoluene

2,6-Dinitrotoluene

Di-n-octyl phthalate

Fluoranthene

Fluorene

Hexachlorobenzene

Hexachlorobutadiene

Hexachlorocyclopentadiene

Hexachloroethane

Indeno[1,2,3-c,d]pyrene

Isophorone

2-Methyl-4,6-dinitrophenol

2-Methylnaphthalene

Naphthalene

m-Nitroaniline

o-Nitroaniline

p-Nitroaniline

Nitrobenzene

2-Nitrophenol

4-Nitrophenol

$\mathrm{N}$-Nitrosodiphenylamine

$\mathrm{N}$-Nitrosodi-n-propylamine

Pentachlorophenol

Phenanthrene

Phenol

Pyrene

1,2,4-Trichlorobenzene

2,4,5-Trichlorophenol
$\begin{array}{lll}J & \mathrm{E} & 5.29 \\ \mathrm{U} & & 42\end{array}$

$\mathrm{U} \quad 42$

U 42

U 42

U $\quad \mathrm{V} \quad \mathbf{5 4}$

U 42

$\mathrm{U} \quad 42$

$\mathrm{U} \quad 42$

U 42

U 42

$\mathrm{U} \quad 42$

U 42

$\begin{array}{lll}J & \mathrm{E} & 4.38\end{array}$

$\mathrm{U} \quad 42$

U 42

U 42

$U \quad 42$

$\begin{array}{lll}U & V & 92.5\end{array}$

$\mathrm{U} \quad 42$

U 42

U 42

U $\quad 84$

U $\quad 42$

$\begin{array}{lll}J & E & 6.01\end{array}$

U 42

U 42

U 210

$\mathrm{U} \quad 42$

$\mathrm{U} \quad 42$

U 42

$\begin{array}{lll}\mathrm{J} & \mathrm{E} & 7.13\end{array}$

$\mathrm{U} \quad 42$

$\mathrm{U} \quad 42$

U 42

U 42

U 42

U 42

U 42

U 210

U 42

U 42

U 210

U 210

U 210

U 42

U 42

U 210

U 42

$\mathrm{U} \quad 42$

U $\quad 210$

U 42

J $\quad \mathrm{E} \quad 4.21$

$\begin{array}{lll}J & \mathrm{E} & 6.67\end{array}$

$\mathrm{U} \quad 42$

U $\quad 210$

\begin{tabular}{|c|c|c|}
\hline$\mu \mathrm{g} / \mathrm{kg}$ & 42 & EPA8270 \\
\hline$\mu \mathrm{g} / \mathrm{kg}$ & 42 & EPA8270 \\
\hline$\mu \mathrm{g} / \mathrm{kg}$ & 42 & EPA8270 \\
\hline$\mu \mathrm{g} / \mathrm{kg}$ & 42 & EPA8270 \\
\hline$\mu \mathrm{g} / \mathrm{kg}$ & 42 & EPA8270 \\
\hline$\mu \mathrm{g} / \mathrm{kg}$ & 42 & EPA8270 \\
\hline$\mu \mathrm{g} / \mathrm{kg}$ & 42 & EPA8270 \\
\hline$\mu \mathrm{g} / \mathrm{kg}$ & 42 & EPA8270M \\
\hline$\mu \mathrm{g} / \mathrm{kg}$ & 42 & EPA8270 \\
\hline$\mu \mathrm{g} / \mathrm{kg}$ & 42 & EPA8270M \\
\hline$\mu \mathrm{g} / \mathrm{kg}$ & 42 & EPA8270 \\
\hline$\mu \mathrm{g} / \mathrm{kg}$ & 42 & EPA8270M \\
\hline$\mu \mathrm{g} / \mathrm{kg}$ & 42 & EPA8270 \\
\hline$\mu \mathrm{g} / \mathrm{kg}$ & 42 & EPA8270 \\
\hline$\mu \mathrm{g} / \mathrm{kg}$ & 42 & EPA8270 \\
\hline$\mu \mathrm{g} / \mathrm{kg}$ & 42 & EPA8270 \\
\hline$\mu \mathrm{g} / \mathrm{kg}$ & 42 & EPA8270 \\
\hline$\mu \mathrm{g} / \mathrm{kg}$ & 42 & EPA8270 \\
\hline$\mu \mathrm{g} / \mathrm{kg}$ & 42 & EPA8270M \\
\hline$\mu \mathrm{g} / \mathrm{kg}$ & 42 & EPA8270 \\
\hline$\mu \mathrm{g} / \mathrm{kg}$ & 42 & EPA8270 \\
\hline$\mu \mathrm{g} / \mathrm{kg}$ & 42 & EPA8270 \\
\hline$\mu \mathrm{g} / \mathrm{kg}$ & 84 & EPA8270 \\
\hline$\mu \mathrm{g} / \mathrm{kg}$ & 42 & EPA8270M \\
\hline$\mu \mathrm{g} / \mathrm{kg}$ & 42 & EPA8270 \\
\hline$\mu \mathrm{g} / \mathrm{kg}$ & 42 & EPA8270M \\
\hline$\mu \mathrm{g} / \mathrm{kg}$ & 42 & EPA8270M \\
\hline$\mu \mathrm{g} / \mathrm{kg}$ & 210 & EPA8270M \\
\hline$\mu \mathrm{g} / \mathrm{kg}$ & 42 & EPA8270 \\
\hline$\mu \mathrm{g} / \mathrm{kg}$ & 42 & EPA8270 \\
\hline$\mu \mathrm{g} / \mathrm{kg}$ & 42 & EPA8270 \\
\hline$\mu \mathrm{g} / \mathrm{kg}$ & 42 & EPA8270 \\
\hline$\mu \mathrm{g} / \mathrm{kg}$ & 42 & EPA8270 \\
\hline$\mu \mathrm{g} / \mathrm{kg}$ & 42 & EPA8270M \\
\hline$\mu \mathrm{g} / \mathrm{kg}$ & 42 & EPA8270M \\
\hline$\mu \mathrm{g} / \mathrm{kg}$ & 42 & EPA8270M \\
\hline$\mu \mathrm{g} / \mathrm{kg}$ & 42 & EPA8270M \\
\hline$\mu \mathrm{g} / \mathrm{kg}$ & 42 & EPA8270 \\
\hline$\mu \mathrm{g} / \mathrm{kg}$ & 42 & EPA8270 \\
\hline$\mu \mathrm{g} / \mathrm{kg}$ & 210 & EPA8270M \\
\hline$\mu \mathrm{g} / \mathrm{kg}$ & 42 & EPA8270 \\
\hline$\mu \mathrm{g} / \mathrm{kg}$ & 42 & EPA8270 \\
\hline$\mu \mathrm{g} / \mathrm{kg}$ & 210 & EPA8270M \\
\hline$\mu \mathrm{g} / \mathrm{kg}$ & 210 & EPA8270M \\
\hline$\mu \mathrm{g} / \mathrm{kg}$ & 210 & EPA8270M \\
\hline$\mu \mathrm{g} / \mathrm{kg}$ & 42 & EPA8270 \\
\hline$\mu \mathrm{g} / \mathrm{kg}$ & 42 & EPA8270M \\
\hline$\mu \mathrm{g} / \mathrm{kg}$ & 210 & EPA8270M \\
\hline$\mu \mathrm{g} / \mathrm{kg}$ & 42 & EPA8270M \\
\hline$\mu \mathrm{g} / \mathrm{kg}$ & 42 & EPA8270 \\
\hline$\mu g / \mathrm{kg}$ & 210 & EPA8270M \\
\hline$\mu \mathrm{g} / \mathrm{kg}$ & 42 & EPA8270 \\
\hline$\mu \mathrm{g} / \mathrm{kg}$ & 42 & EPA8270M \\
\hline$\mu \mathrm{g} / \mathrm{kg}$ & 42 & EPA8270 \\
\hline$\mu \mathrm{g} / \mathrm{kg}$ & 42 & EPA8270M \\
\hline$\mu \mathrm{g} / \mathrm{kg}$ & 210 & EPA8270M \\
\hline
\end{tabular}


SURVEY ID: PPSC 1202D (continued)

Sample ID: 104659

Constituent

RQ AQ B Result

Unit

D. Limit Method

$B / N / A$ Extractables (including $P A H$ and phenols)

2,4,6-Trichlorophenol

Unknown

Unknown

Volatile Organic Compounds

Acetone

Benzene

Bromodichloromethane

Bromoform

Bromomethane (Methyl bromide)

Carbon disulfide

Carbon tetrachloride

Chlorobenzene

Chloroethane

Chloroethene (Vinyl chloride)

Chloroform

Chloromethane (Methyl chloride)

Dibromochloromethane

1,1-Dichloroethane

1,2-Dichloroethane

1,1-Dichloroethylene

1,2-Dichloroethylene

Dichloromethane (Methylene chloride)

1,2-Dichloropropane

cis-1,3-Dichloropropene

trans-1,3-Dichloropropene

Ethylbenzene

2-Hexanone $\mu \mathrm{g} / \mathrm{kg}$

$\mu \mathrm{g} / \mathrm{kg}$

$\mu \mathrm{g} / \mathrm{kg}$
42

EPA8270

EPA8270

EPA8270
24.3

0.117

0.24

2.4

0.72

2.4

0.13

0.36

0.24

0.26

0.24

0.36

0.84

0.12

0.24

0.6

2.4

0.26

0.078

6

6

0.065

12

$\begin{array}{lll}\mu \mathrm{g} / \mathrm{kg} & 12 & \text { EPA8260 } \\ \mu \mathrm{g} / \mathrm{kg} & 0.117 & \text { EPA8021 } \\ \mu \mathrm{g} / \mathrm{kg} & 0.24 & \text { EPA8260 } \\ \mu \mathrm{g} / \mathrm{kg} & 2.4 & \text { EPA8260 } \\ \mu \mathrm{g} / \mathrm{kg} & 0.72 & \text { EPA8260 } \\ \mu \mathrm{g} / \mathrm{kg} & 2.4 & \text { EPA8260 } \\ \mu \mathrm{g} / \mathrm{kg} & 0.13 & \text { EPA8021 } \\ \mu \mathrm{g} / \mathrm{kg} & 0.36 & \text { EPA8260 } \\ \mu \mathrm{g} / \mathrm{kg} & 0.24 & \text { EPA8260 } \\ \mu \mathrm{g} / \mathrm{kg} & 0.26 & \text { EPA8021 } \\ \mu \mathrm{g} / \mathrm{kg} & 0.24 & \text { EPA8260 } \\ \mu \mathrm{g} / \mathrm{kg} & 0.36 & \text { EPA8260 } \\ \mu \mathrm{g} / \mathrm{kg} & 0.84 & \text { EPA8260 } \\ \mu \mathrm{g} / \mathrm{kg} & 0.12 & \text { EPA8260 } \\ \mu \mathrm{g} / \mathrm{kg} & 0.24 & \text { EPA8260 } \\ \mu \mathrm{g} / \mathrm{kg} & 0.6 & \text { EPA8260 } \\ \mu \mathrm{g} / \mathrm{kg} & 2.4 & \text { EPA8260 } \\ \mu \mathrm{g} / \mathrm{kg} & 0.26 & \text { EPA8021 } \\ \mu \mathrm{g} / \mathrm{kg} & 0.078 & \text { EPA8021 } \\ \mu \mathrm{g} / \mathrm{kg} & 6 & \text { EPA8260 } \\ \mu \mathrm{g} / \mathrm{kg} & 6 & \text { EPA8260 } \\ \mu \mathrm{g} / \mathrm{kg} & 0.065 & \text { EPA8021 } \\ \mu \mathrm{g} / \mathrm{kg} & 12 & \text { EPA8260 }\end{array}$

Volatile Organic Compounds

Methyl ethyl ketone

Methyl isobutyl ketone

Styrene

1,1,2,2-Tetrachloroethane

Tetrachloroethylene

Toluene

1,1,1-Trichloroethane

1,1,2-Trichloroethane

Trichloroethylene

Unknown

Vinyl acetate

Xylenes

$\begin{array}{lll}\text { J } & \text { EV } & 3.47 \\ \text { U } & & 12 \\ U & & 0.13 \\ U & & 0.13 \\ U & & 0.48 \\ U & & 0.13 \\ U & & 0.36 \\ U & & 0.24 \\ U & & 0.13 \\ J & N & 3 \\ \text { U } & & \\ \text { U } & & 12 \\ & & 0.48\end{array}$

$\mu \mathrm{g} / \mathrm{kg}$

$\mu \mathrm{g} / \mathrm{kg}$

$\mu \mathrm{g} / \mathrm{kg}$

$\mu \mathrm{g} / \mathrm{kg}$

$\mu \mathrm{g} / \mathrm{kg}$

$\mu \mathrm{g} / \mathrm{kg}$

$\mu \mathrm{g} / \mathrm{kg}$

$\mu \mathrm{g} / \mathrm{kg}$

$\mu \mathrm{g} / \mathrm{kg}$

$\mu \mathrm{g} / \mathrm{kg}$

$\mu \mathrm{g} / \mathrm{kg}$

$\mu \mathrm{g} / \mathrm{kg}$

$\begin{array}{ll}12 & \text { EPA8260 } \\ 12 & \text { EPA8260 } \\ 0.13 & \text { EPA8021 } \\ 0.13 & \text { EPA8021 } \\ 0.48 & \text { EPA8260 } \\ 0.13 & \text { EPA8021 } \\ 0.36 & \text { EPA8260 } \\ 0.24 & \text { EPA8260 } \\ 0.13 & \text { EPA8021 } \\ & \text { EPA8260 } \\ 12 & \text { EPA8260 } \\ 0.48 & \text { EPA8260 }\end{array}$

Pesticides/PCBs and Dioxins/Furans

Aldrin

alpha-Benzene hexachloride

beta-Benzene hexachloride

delta-Benzene hexachloride

alpha-Chlordane

gamma-Chlordane

$p, p^{\prime} \cdot$ DDD

$p, p^{\prime}-\mathrm{DDE}$

p,p'-DDT

2.13
2.13
2.13
2.13
2.13
2.13
4.09
3.27
4.09

$\mu \mathrm{g} / \mathrm{kg}$

$\mu \mathrm{g} / \mathrm{kg}$

$\mu \mathrm{g} / \mathrm{kg}$

$\mu \mathrm{g} / \mathrm{kg}$

$\mu \mathrm{g} / \mathrm{kg}$

$\mu \mathrm{g} / \mathrm{kg}$

$\mu \mathrm{g} / \mathrm{kg}$

$\mu \mathrm{g} / \mathrm{kg}$

$\mu \mathrm{g} / \mathrm{kg}$

2.13

EPA8080

EPA8080

EPA8080

EPA8080

EPA8080

EPA8080

EPA8080

EPA8080

EPA8080 
SURVEY ID: PPSC 1202D (continued)

Constituent

Pesticides/PCBs and Dioxins/Furans

Dieldrin

Endosulfan sulfate

Endosulfan I

Endosulfan II

Endrin

Endrin ketone

Heptachlor

Heptachlor epoxide

Lindane

Methoxychlor

PCB 1016

PCB 1221

PCB 1232

PCB 1242

PCB 1248

PCB 1254

PCB 1260

Toxaphene

Radionuclides

\section{Gross alpha}

Gross alpha

Nonvolatile beta

Nonvolatile beta

Plutonium-238

Plutonium-238

Plutonium-239/240

Plutonium-239/240

Strontium-90

Strontium-90

SURVEY ID: PPSC 1203
Sample ID: 104659

RQ AQ B Result

Unit

D. Limit Method

$\begin{array}{lll}\mu \mathrm{g} / \mathrm{kg} & 1.64 & \text { EPA8080 } \\ \mu \mathrm{g} / \mathrm{kg} & 4.09 & \text { EPA8080 } \\ \mu \mathrm{g} / \mathrm{kg} & 2.13 & \text { EPA8080 } \\ \mu \mathrm{g} / \mathrm{kg} & 3.27 & \text { EPA8080 } \\ \mu \mathrm{g} / \mathrm{kg} & 4.09 & \text { EPA8080 } \\ \mu \mathrm{g} / \mathrm{kg} & 4.09 & \text { EPA8080 } \\ \mu \mathrm{g} / \mathrm{kg} & 2.13 & \text { EPA8080 } \\ \mu \mathrm{g} / \mathrm{kg} & 2.13 & \text { EPA8080 } \\ \mu \mathrm{g} / \mathrm{kg} & 2.13 & \text { EPA8080 } \\ \mu \mathrm{g} / \mathrm{kg} & 20.9 & \text { EPA8080 } \\ \mu \mathrm{g} / \mathrm{kg} & 40.6 & \text { EPA8080 } \\ \mu \mathrm{g} / \mathrm{kg} & 82.3 & \text { EPA8080 } \\ \mu \mathrm{g} / \mathrm{kg} & 40.6 & \text { EPA8080 } \\ \mu \mathrm{g} / \mathrm{kg} & 40.6 & \text { EPA8080 } \\ \mu \mathrm{g} / \mathrm{kg} & 40.6 & \text { EPA8080 } \\ \mu \mathrm{g} / \mathrm{kg} & 40.6 & \text { EPA8080 } \\ \mu \mathrm{g} / \mathrm{kg} & 40.6 & \text { EPA8080 } \\ \mu \mathrm{g} / \mathrm{kg} & 196 & \text { EPA8080 }\end{array}$

$\begin{array}{ll}U & 1.64 \\ U & 4.09 \\ U & 2.13 \\ U & 3.27 \\ U & 4.09 \\ U & 4.09 \\ U & 2.13 \\ U & 2.13 \\ U & 2.13 \\ U & 20.9 \\ U & 40.6 \\ U & 82.3 \\ U & 40.6 \\ U & 40.6 \\ U & 40.6 \\ U & 40.6 \\ U & 40.6 \\ U & 196\end{array}$

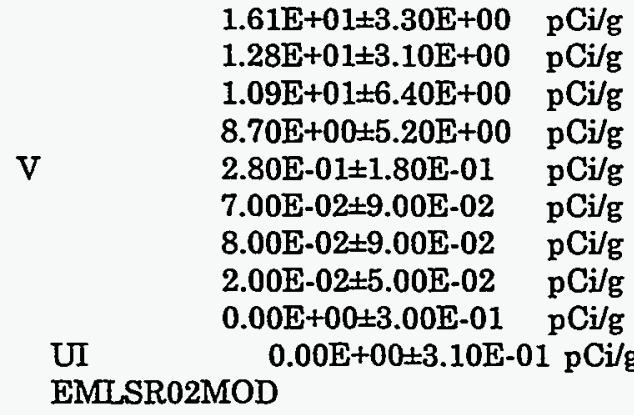

Sample ID: 104660

Survey location: 44888E $3682027 \mathrm{~N}$ (SRS coordinates)

Depth of core interval: 2.00 to $4.00 \mathrm{ft}$

Sample type: Normal

Sample matrix: Soil

2.80E+00 LANLMIR100MOD 2.82E+00 LANLMIR100MOD 1.79E+00 LANLMIR100MOD 1.79E+00 LANLMLR100MOD 1.20E-01 EMLPU02MOD 1.30E-01 EMLPUO2MOD 7.00E-02 EMLPU02MOD 1.10E-01 EMLPU02MOD

7.70E-01 EMLSR02MOD 8.00E-01

Percent solids: $\mathbf{8 8 . 0 0}$ 
SURVEY ID: PPSC 1203 (continued)

Constituent

Sample ID: 104660

RQ AQ B Result

Physical Parameters and Specified Analyses

Cation exchange capacity

Cyanide

Nitrate-nitrite as nitrogen

Orthophosphate

Phenols

Sulfide

Total organic carbon

Total organic nitrogen

Total phosphates (as P)
Chloride

$\begin{array}{lll} & & 58.1 \\ \text { J } & \text { E } & 1.28 \\ \text { U } & & 1.14 \\ \text { J } & \text { E } & 0.49 \\ \text { U } & & 11.4 \\ \text { UJ } & \text { C } & 338 \\ \text { UJ } & \text { C } & 284 \\ & & 969 \\ \text { C } & & 76.5 \\ \text { J } & \text { E } & 60.5\end{array}$

Metals (total recoverable)

Aluminum

Antimony

Arsenic

Barium

Beryllium

Cadmium

Calcium

Chromium

Cobalt

Copper

Iron

Lead

Magnesium

Manganese

Mercury

Nickel

Potassium

Selenium

Silver

Sodium

Thallium

Vanadium

Zinc

$B / N / A$ Extractables (including $P A H$ and phenols)

Acenaphthene

Acenaphthylene

Anthracene

Benzo[a]anthracene

Benzo[b]fluoranthene

Benzo[k]fluoranthene

Benzoic acid

Benzo[g,h,i]perylene

Benzo[a]pyrene

Benzyl alcohol

Bis(2-chloroethoxy) methane

Bis(2-chloroethyl) ether

$\begin{array}{ll}U & 36.9 \\ U & 36.9 \\ U & 36.9 \\ U & 3.69 \\ U & 3.69 \\ U & 3.69 \\ U & 36.9 \\ U & 36.9 \\ U & 3.69 \\ U & 36.9 \\ U & 36.9 \\ U & 36.9\end{array}$

Unit D. Limit Method

$\begin{array}{lll}\mathrm{meq} / 100 \mathrm{~g} & 5 & \text { EPA9081 } \\ \mathrm{mg} / \mathrm{kg} & 2.8 & \text { EPA300.0 } \\ \mathrm{mg} / \mathrm{kg} & 1.14 & \text { EPA335.3 } \\ \mathrm{mg} / \mathrm{kg} & 0.98 & \text { EPA353.1 } \\ \mathrm{mg} / \mathrm{kg} & 11.4 & \text { EPA365.2 } \\ \mu \mathrm{g} / \mathrm{kg} & 338 & \text { EPA420.2 } \\ \mathrm{mg} / \mathrm{kg} & 284 & \text { EPA376.2 } \\ \mathrm{mg} / \mathrm{kg} & 114 & \text { EPA415.1 } \\ \mathrm{mg} / \mathrm{kg} & 0.227 & \text { EPA351.2 } \\ \mathrm{mg} / \mathrm{kg} & 221 & \text { EPA365.4 }\end{array}$

$\begin{array}{lll}\mathrm{mg} / \mathrm{kg} & 13.9 & \text { EPA6010A } \\ \mathrm{mg} / \mathrm{kg} & 5.57 & \text { EPA6010A } \\ \mathrm{mg} / \mathrm{kg} & 16.7 & \text { EPA6010A } \\ \mathrm{mg} / \mathrm{kg} & 1.39 & \text { EPA6010A } \\ \mathrm{mg} / \mathrm{kg} & 0.557 & \text { EPA6010A } \\ \mathrm{mg} / \mathrm{kg} & 0.279 & \text { EPA6010A } \\ \mathrm{mg} / \mathrm{kg} & 13.9 & \text { EPA6010A } \\ \mathrm{mg} / \mathrm{kg} & 1.39 & \text { EPA6010A } \\ \mathrm{mg} / \mathrm{kg} & \mathbf{0 . 5 5 7} & \text { EPA6010A } \\ \mathrm{mg} / \mathrm{kg} & 1.39 & \text { EPA6010A } \\ \mathrm{mg} / \mathrm{kg} & 5.57 & \text { EPA6010A } \\ \mathrm{mg} / \mathrm{kg} & \mathbf{5 . 5 7} & \text { EPA6010A } \\ \mathrm{mg} / \mathrm{kg} & 5.57 & \text { EPA6010A } \\ \mathrm{mg} / \mathrm{kg} & 0.557 & \text { EPA6010A } \\ \mathrm{mg} / \mathrm{kg} & 0.0317 & \text { EPA7471 } \\ \mathrm{mg} / \mathrm{kg} & 1.39 & \text { EPA6010A } \\ \mathrm{mg} / \mathrm{kg} & 111 & \text { EPA6010A } \\ \mathrm{mg} / \mathrm{kg} & 16.7 & \text { EPA6010A } \\ \mathrm{mg} / \mathrm{kg} & 1.39 & \text { EPA6010A } \\ \mathrm{mg} / \mathrm{kg} & 55.7 & \text { EPA6010A } \\ \mathrm{mg} / \mathrm{kg} & 16.7 & \text { EPA6010A } \\ \mathrm{mg} / \mathrm{kg} & 0.557 & \text { EPA6010A } \\ \mathrm{mg} / \mathrm{kg} & 1.11 & \text { EPA6010A }\end{array}$

$\begin{array}{lll}\mu \mathrm{g} / \mathrm{kg} & 36.9 & \text { EPA8270 } \\ \mu \mathrm{g} / \mathrm{kg} & 36.9 & \text { EPA8270 } \\ \mu \mathrm{g} / \mathrm{kg} & 36.9 & \text { EPA8270 } \\ \mu \mathrm{g} / \mathrm{kg} & 3.69 & \text { EPA8270 } \\ \mu \mathrm{g} / \mathrm{kg} & 3.69 & \text { EPA8270 } \\ \mu \mathrm{g} / \mathrm{kg} & 3.69 & \text { EPA8270 } \\ \mu \mathrm{g} / \mathrm{kg} & 36.9 & \text { EPA8270 } \\ \mu \mathrm{g} / \mathrm{kg} & 36.9 & \text { EPA8270 } \\ \mu \mathrm{g} / \mathrm{kg} & 3.69 & \text { EPA8270 } \\ \mu \mathrm{g} / \mathrm{kg} & 36.9 & \text { EPA8270 } \\ \mu \mathrm{g} / \mathrm{kg} & 36.9 & \text { EPA8270 } \\ \mu \mathrm{g} / \mathrm{kg} & 36.9 & \text { EPA8270 }\end{array}$


Appendix D: Sediment Tables

D-158

SURVEY ID: PPSC 1203 (continued)

Sample ID: 104660

Constituent

RQ AQ B Result

Unit

D. Limit Method

$B / N / A$ Extractables (including PAH and phenols)

$\mathrm{Bis}$ (2-chloroisopropyl) ether

Bis(2-ethylhexyl) phthalate

4-Bromophenyl phenyl ether

Butylbenzyl phthalate

4-Chloroaniline

4-Chloro-m-cresol

2-Chloronaphthalene

2-Chlorophenol

4-Chlorophenyl phenyl ether

Chrysene

$\mathrm{m} / \mathrm{p}$-Cresol

o-Cresol (2-Methylphenol)

Dibenz $[a, h]$ anthracene

Dibenzofuran

Di-n-butyl phthalate

1,2-Dichlorobenzene

1,3-Dichlorobenzene

1,4-Dichlorobenzene

3,3'-Dichlorobenzidine

2,4-Dichlorophenol

Diethyl phthalate

2,4-Dimethyl phenol

Dimethyl phthalate

2,4-Dinitrophenol

2,4-Dinitrotoluene

2,6-Dinitrotoluene

Di-n-octyl phthalate

Fluoranthene

Fluorene

Hexachlorobenzene

Hexachlorobutadiene

Hexachlorocyclopentadiene

Hexachloroethane

Indeno[1,2,3-c,d]pyrene

Isophorone

2-Methyl-4,6-dinitrophenol

2-Methylnaphthalene

Naphthalene

m-Nitroaniline

o-Nitroaniline

p-Nitroaniline

Nitrobenzene

2-Nitrophenol

4-Nitrophenol

N-Nitrosodiphenylamine

N-Nitrosodi-n-propylamine

Pentachlorophenol

Phenanthrene

Phenol

Pyrene

1,2,4-Trichlorobenzene

2,4,5-Trichlorophenol

2,4,6-Trichlorophenol

Unknown acid

Unknown alcohol

\begin{tabular}{|c|c|c|}
\hline U & & 36.9 \\
\hline $\mathrm{U}$ & & 36.9 \\
\hline U & & 36.9 \\
\hline U & & 36.9 \\
\hline $\mathrm{U}$ & & 36.9 \\
\hline $\mathrm{U}$ & & 36.9 \\
\hline $\mathrm{U}$ & & 36.9 \\
\hline $\mathrm{U}$ & & 36.9 \\
\hline U & & 36.9 \\
\hline$U$ & & 3.69 \\
\hline $\mathrm{U}$ & & 369 \\
\hline U & & 36.9 \\
\hline $\mathrm{U}$ & & 3.69 \\
\hline U & & 36.9 \\
\hline $\mathbf{J}$ & $\mathbf{E}$ & 27.7 \\
\hline U & & 36.9 \\
\hline $\mathrm{U}$ & & 36.9 \\
\hline$U$ & & 36.9 \\
\hline $\mathrm{U}$ & & 36.9 \\
\hline U & & 36.9 \\
\hline $\mathrm{U}$ & & 36.9 \\
\hline $\mathrm{U}$ & & 36.9 \\
\hline U & & 36.9 \\
\hline $\mathrm{U}$ & & 369 \\
\hline UJ & C & 3.69 \\
\hline $\mathbf{U}$ & & 3.69 \\
\hline $\mathbf{U}$ & & 36.9 \\
\hline$U$ & & 36.9 \\
\hline U & & 36.9 \\
\hline U & & 3.69 \\
\hline U & & 36.9 \\
\hline U & & 36.9 \\
\hline $\mathrm{U}$ & & 3.69 \\
\hline $\mathrm{U}$ & & 3.69 \\
\hline U & & 36.9 \\
\hline U & & 369 \\
\hline $\mathrm{U}$ & & 36.9 \\
\hline $\mathrm{U}$ & & 36.9 \\
\hline$U$ & & 36.9 \\
\hline$U$ & & 36.9 \\
\hline$U$ & & 36.9 \\
\hline $\mathrm{U}$ & & 36.9 \\
\hline$U$ & & 36.9 \\
\hline $\mathrm{U}$ & & 36.9 \\
\hline$U$ & & 36.9 \\
\hline $\mathrm{U}$ & & 36.9 \\
\hline UJ & C & 36.9 \\
\hline U & & 36.9 \\
\hline $\mathrm{U}$ & & 36.9 \\
\hline $\mathrm{U}$ & & 36.9 \\
\hline $\mathrm{U}$ & & 3.69 \\
\hline $\mathrm{U}$ & & 36.9 \\
\hline $\mathrm{U}$ & & 36.9 \\
\hline$J$ & $N$ & 920 \\
\hline J & $\mathrm{N}$ & 500 \\
\hline
\end{tabular}

\begin{tabular}{|c|c|c|}
\hline$\mu \mathrm{g} / \mathrm{kg}$ & 36.9 & EPA8270 \\
\hline$\mu \mathrm{g} / \mathrm{kg}$ & 36.9 & EPA8270 \\
\hline$\mu \mathrm{g} / \mathrm{kg}$ & 36.9 & EPA8270 \\
\hline$\mu \mathrm{g} / \mathrm{kg}$ & 36.9 & EPA8270 \\
\hline$\mu \mathrm{g} / \mathrm{kg}$ & 36.9 & EPA8270 \\
\hline$\mu \mathrm{g} / \mathrm{kg}$ & 36.9 & EPA8270 \\
\hline$\mu \mathrm{g} / \mathrm{kg}$ & 36.9 & EPA8270 \\
\hline$\mu \mathrm{g} / \mathrm{kg}$ & 36.9 & EPA8270 \\
\hline$\mu \mathrm{g} / \mathrm{kg}$ & 36.9 & EPA8270 \\
\hline$\mu \mathrm{g} / \mathrm{kg}$ & 3.69 & EPA8270 \\
\hline$\mu \mathrm{g} / \mathrm{kg}$ & 369 & EPA8270 \\
\hline$\mu \mathrm{g} / \mathrm{kg}$ & 36.9 & EPA8270 \\
\hline$\mu \mathrm{g} / \mathrm{kg}$ & 3.69 & EPA8270 \\
\hline$\mu \mathrm{g} / \mathrm{kg}$ & 36.9 & EPA8270 \\
\hline$\mu \mathrm{g} / \mathrm{kg}$ & 36.9 & EPA8270 \\
\hline$\mu \mathrm{g} / \mathrm{kg}$ & 36.9 & EPA8270 \\
\hline$\mu \mathrm{g} / \mathrm{kg}$ & 36.9 & EPA8270 \\
\hline$\mu \mathrm{g} / \mathrm{kg}$ & 36.9 & EPA8270 \\
\hline$\mu \mathrm{g} / \mathrm{kg}$ & 36.9 & EPA8270 \\
\hline$\mu \mathrm{g} / \mathrm{kg}$ & 36.9 & EPA8270 \\
\hline$\mu \mathrm{g} / \mathrm{kg}$ & 36.9 & EPA8270 \\
\hline$\mu \mathrm{g} / \mathrm{kg}$ & 36.9 & EPA8270 \\
\hline$\mu \mathrm{g} / \mathrm{kg}$ & 36.9 & EPA8270 \\
\hline$\mu \mathrm{g} / \mathrm{kg}$ & 369 & EPA8270 \\
\hline$\mu \mathrm{g} / \mathrm{kg}$ & 3.69 & EPA8270 \\
\hline$\mu \mathrm{g} / \mathrm{kg}$ & 3.69 & EPA8270 \\
\hline$\mu \mathrm{g} / \mathrm{kg}$ & 36.9 & EPA8270 \\
\hline$\mu \mathrm{g} / \mathrm{kg}$ & 36.9 & EPA8270 \\
\hline$\mu \mathrm{g} / \mathrm{kg}$ & 36.9 & EPA8270 \\
\hline$\mu \mathrm{g} / \mathrm{kg}$ & 3.69 & EPA8270 \\
\hline$\mu \mathrm{g} / \mathrm{kg}$ & 36.9 & EPA8270 \\
\hline$\mu \mathrm{g} / \mathrm{kg}$ & 36.9 & EPA8270 \\
\hline$\mu \mathrm{g} / \mathrm{kg}$ & 3.69 & EPA8270 \\
\hline$\mu \mathrm{g} / \mathrm{kg}$ & 3.69 & EPA8270 \\
\hline$\mu \mathrm{g} / \mathrm{kg}$ & 36.9 & EPA8270 \\
\hline$\mu \mathrm{g} / \mathrm{kg}$ & 369 & EPA8270 \\
\hline$\mu \mathrm{g} / \mathrm{kg}$ & 36.9 & EPA8270 \\
\hline$\mu \mathrm{g} / \mathrm{kg}$ & 36.9 & EPA8270 \\
\hline$\mu \mathrm{g} / \mathrm{kg}$ & 36.9 & EPA8270 \\
\hline$\mu \mathrm{g} / \mathrm{kg}$ & 36.9 & EPA8270 \\
\hline$\mu \mathrm{g} / \mathrm{kg}$ & 36.9 & EPA8270 \\
\hline$\mu \mathrm{g} / \mathrm{kg}$ & 36.9 & EPA8270 \\
\hline$\mu \mathrm{g} / \mathrm{kg}$ & 36.9 & EPA8270 \\
\hline$\mu \mathrm{g} / \mathrm{kg}$ & 36.9 & EPA8270 \\
\hline$\mu \mathrm{g} / \mathrm{kg}$ & 36.9 & EPA8270 \\
\hline$\mu \mathrm{g} / \mathrm{kg}$ & 36.9 & EPA8270 \\
\hline$\mu \mathrm{g} / \mathrm{kg}$ & 36.9 & EPA8270 \\
\hline$\mu \mathrm{g} / \mathrm{kg}$ & 36.9 & EPA8270 \\
\hline$\mu \mathrm{g} / \mathrm{kg}$ & 36.9 & EPA8270 \\
\hline$\mu \mathrm{g} / \mathrm{kg}$ & 36.9 & EPA8270 \\
\hline$\mu \mathrm{g} / \mathrm{kg}$ & 3.69 & EPA8270 \\
\hline$\mu \mathrm{g} / \mathrm{kg}$ & 36.9 & EPA8270 \\
\hline$\mu \mathrm{g} / \mathrm{kg}$ & 36.9 & EPA8270 \\
\hline$\mu g / \mathrm{kg}$ & & EPA8270 \\
\hline & & EPA8270 \\
\hline
\end{tabular}


SURVEY ID: PPSC 1203 (continued)

Constituent

RQ AQ B Result

Sample ID: 104660

Volatile Organic Compounds

Acetone
Benzene
Bromodichloromethane
Bromoform
Bromomethane (Methyl bromide)
Carbon disulfide
Carbon tetrachloride
Chlorobenzene
Chloroethane
Chloroethene (Vinyl chloride)
Chloroform
Chloromethane (Methyl chloride)
Dibromochloromethane
1,1-Dichloroethane
1,2-Dichloroethane
1,1-Dichloroethylene
1,2-Dichloroethylene
Dichloromethane (Methylene chloride)
1,2-Dichloropropane
cis-1,3-Dichloropropene
trans-1,3-Dichloropropene
Ethylbenzene
Hexanol
2-Hexanone
Isocamphene
Methyl ethyl ketone
Methyl isobutyl ketone
Styrene
1,1,2,2-Tetrachloroethane
Tetrachloroethylene
Toluene
1,1,1-Trichloroethane
1,1,2-Trichloroethane
Trichloroethylene
Vinyl acetate
Xylenes

\begin{tabular}{|c|c|c|}
\hline & V8 & 111 \\
\hline & & 0.648 \\
\hline U & & 0.0568 \\
\hline $\mathrm{U}$ & & 0.0568 \\
\hline U & & 0.114 \\
\hline & & 2.05 \\
\hline $\mathrm{U}$ & & 0.0568 \\
\hline $\mathrm{U}$ & & 0.0568 \\
\hline $\mathrm{U}$ & & 0.114 \\
\hline $\mathrm{U}$ & & 0.114 \\
\hline & & 0.545 \\
\hline$U$ & & 0.114 \\
\hline U & & 0.0568 \\
\hline $\mathrm{U}$ & & 0.0568 \\
\hline U & & 0.0568 \\
\hline $\mathrm{U}$ & & 0.0568 \\
\hline$U$ & & 0.114 \\
\hline$U$ & $\mathrm{~V}$ & 3.59 \\
\hline$U$ & & 0.0568 \\
\hline$U$ & & 0.0568 \\
\hline U & & 0.0568 \\
\hline U & & 0.0568 \\
\hline $\mathbf{J}$ & $\mathrm{N}$ & 1 \\
\hline$U$ & $\mathrm{~V}$ & 1.11 \\
\hline $\mathbf{J}$ & $\mathrm{N}$ & 1.2 \\
\hline & V & 25.5 \\
\hline $\mathbf{J}$ & $\mathbf{E}$ & 0.557 \\
\hline$U$ & & 0.0568 \\
\hline$U$ & & 0.0568 \\
\hline U & & 0.0568 \\
\hline $\mathrm{U}$ & $\mathrm{V}$ & 0.193 \\
\hline $\mathrm{U}$ & & 0.0568 \\
\hline $\mathrm{U}$ & & 0.0568 \\
\hline $\mathrm{U}$ & & 0.0568 \\
\hline U & & 1.14 \\
\hline $\mathrm{U}$ & & 0.17 \\
\hline
\end{tabular}

Pesticides/PCBs and Dioxins/Furans

\author{
Aldrin \\ alpha-Benzene hexachloride \\ beta-Benzene hexachloride \\ delta-Benzene hexachloride \\ alpha-Chlordane \\ gamma-Chlordane \\ $p, p^{\prime}-D D D$ \\ p, $\mathrm{p}^{\prime}-\mathrm{DDE}$ \\ p,p'-DDT \\ Dieldrin \\ Endosulfan sulfate \\ Endosulfan I \\ Endosulfan II
}

$\begin{array}{ll}\text { U } & 3.77 \\ U & 3.77 \\ U & 7.53 \\ U & 7.53 \\ U & 7.53 \\ U & 7.53 \\ U & 15.1 \\ U & 7.53 \\ U & 15.1 \\ U & 7.53 \\ U & 15.1 \\ U & 7.53 \\ U & 15.1\end{array}$

$\begin{array}{lll}\mu \mathrm{g} / \mathrm{kg} & 5.68 & \text { EPA8260 } \\ \mu \mathrm{g} / \mathrm{kg} & 0.568 & \text { EPA8260 } \\ \mu \mathrm{g} / \mathrm{kg} & 0.0568 & \text { EPA8260 } \\ \mu \mathrm{g} / \mathrm{kg} & 0.0568 & \text { EPA8260 } \\ \mu \mathrm{g} / \mathrm{kg} & 0.114 & \text { EPA8260 } \\ \mu \mathrm{g} / \mathrm{kg} & 1.14 & \text { EPA8260 } \\ \mu \mathrm{g} / \mathrm{kg} & 0.0568 & \text { EPA8260 } \\ \mu \mathrm{g} / \mathrm{kg} & 0.0568 & \text { EPA8260 } \\ \mu \mathrm{g} / \mathrm{kg} & 0.114 & \text { EPA8260 } \\ \mu \mathrm{g} / \mathrm{kg} & 0.114 & \text { EPA8260 } \\ \mu \mathrm{g} / \mathrm{kg} & 0.0568 & \text { EPA8260 } \\ \mu \mathrm{g} / \mathrm{kg} & 0.114 & \text { EPA8260 } \\ \mu \mathrm{g} / \mathrm{kg} & 0.0568 & \text { EPA8260 } \\ \mu \mathrm{g} / \mathrm{kg} & 0.0568 & \text { EPA8260 } \\ \mu \mathrm{g} / \mathrm{kg} & 0.0568 & \text { EPA8260 } \\ \mu \mathrm{g} / \mathrm{kg} & 0.0568 & \text { EPA8260 } \\ \mu \mathrm{g} / \mathrm{kg} & 0.114 & \text { EPA8260 } \\ \mu \mathrm{g} / \mathrm{kg} & 0.568 & \text { EPA8260 } \\ \mu \mathrm{g} / \mathrm{kg} & 0.0568 & \text { EPA8260 } \\ \mu \mathrm{g} / \mathrm{kg} & 0.0568 & \text { EPA8260 } \\ \mu \mathrm{g} / \mathrm{kg} & 0.0568 & \text { EPA8260 } \\ \mu \mathrm{g} / \mathrm{kg} & 0.0568 & \text { EPA8260 } \\ \mu \mathrm{g} / \mathrm{kg} & & \text { EPA8260 } \\ \mu \mathrm{g} / \mathrm{kg} & 1.14 & \text { EPA8260 } \\ \mu \mathrm{g} / \mathrm{kg} & & \text { EPA8260 } \\ \mu \mathrm{g} / \mathrm{kg} & 1.14 & \text { EPA8260 } \\ \mu \mathrm{g} / \mathrm{kg} & 1.14 & \text { EPA8260 } \\ \mu \mathrm{g} / \mathrm{kg} & 0.0568 & \text { EPA8260 } \\ \mu \mathrm{g} / \mathrm{kg} & 0.0568 & \text { EPA8260 } \\ \mu \mathrm{g} / \mathrm{kg} & 0.0568 & \text { EPA8260 } \\ \mu \mathrm{g} / \mathrm{kg} & 0.568 & \text { EPA8260 } \\ \mu \mathrm{g} / \mathrm{kg} & 0.0568 & \text { EPA8260 } \\ \mu \mathrm{g} / \mathrm{kg} & 0.0568 & \text { EPA8260 } \\ \mu \mathrm{g} / \mathrm{kg} & 0.0568 & \text { EPA8260 } \\ \mu \mathrm{g} / \mathrm{kg} & 1.14 & \text { EPA8260 } \\ \mu \mathrm{g} / \mathrm{kg} & 0.17 & \text { EPA8260 } \\ & & \end{array}$

$\begin{array}{lrr}\mu \mathrm{g} / \mathrm{kg} & 3.77 & \text { EPA8080 } \\ \mu \mathrm{g} / \mathrm{kg} & 3.77 & \text { EPA8080 } \\ \mu \mathrm{g} / \mathrm{kg} & 7.53 & \text { EPA8080 } \\ \mu \mathrm{g} / \mathrm{kg} & 7.53 & \text { EPA8080 } \\ \mu \mathrm{g} / \mathrm{kg} & 7.53 & \text { EPA8080 } \\ \mu \mathrm{g} / \mathrm{kg} & 7.53 & \text { EPA8080 } \\ \mu \mathrm{g} / \mathrm{kg} & 15.1 & \text { EPA8080 } \\ \mu \mathrm{g} / \mathrm{kg} & 7.53 & \text { EPA8080 } \\ \mu \mathrm{g} / \mathrm{kg} & 15.1 & \text { EPA8080 } \\ \mu \mathrm{g} / \mathrm{kg} & 7.53 & \text { EPA8080 } \\ \mu \mathrm{g} / \mathrm{kg} & 15.1 & \text { EPA8080 } \\ \mu \mathrm{g} / \mathrm{kg} & 7.53 & \text { EPA8080 } \\ \mu \mathrm{g} / \mathrm{kg} & .15 .1 & \text { EPA8080 }\end{array}$


SURVEY ID: PPSC 1203 (continued)

\section{Constituent}

Pesticides/PCBs and Dioxins/Furans

Endrin

Endrin ketone

Heptachlor

Heptachlor epoxide

Lindane

Methoxychlor

PCB 1016

PCB 1221

PCB 1232

PCB 1242

PCB 1248

PCB 1254

PCB 1260

Toxaphene

\section{Radionuclides}

Actinium-228

Antimony-124

Antimony-125

Barium-133

Cerium-144

Cesium-134

Cesium-137

Cobalt-57

Cobalt-58

Cobalt-60

Europium-152

Europium-154

Europium-155

Gross alpha

Lead-212

Manganese-54

Neptunium-239

Nonvolatile beta

Plutonium-238

Plutonium-238

Plutonium-239/240

Plutonium-239/240

Potassium-40

Promethium-144

Promethium-146

Ruthenium-106

Sodium-22

Strontium-90

Thorium-234

Tin-113

Yttrium-88

Zinc-65

Zirconium-95
Sample ID: 104660

RQ AQ B Result

Unit

D. Limit Method

$\begin{array}{lll}\mu \mathrm{g} / \mathrm{kg} & 7.53 & \text { EPA8080 } \\ \mu \mathrm{g} / \mathrm{kg} & 15.1 & \text { EPA8080 } \\ \mu \mathrm{g} / \mathrm{kg} & 7.53 & \text { EPA8080 } \\ \mu \mathrm{g} / \mathrm{kg} & 7.53 & \text { EPA8080 } \\ \mu \mathrm{g} / \mathrm{kg} & 3.77 & \text { EPA8080 } \\ \mu \mathrm{g} / \mathrm{kg} & 75.3 & \text { EPA8080 } \\ \mu \mathrm{g} / \mathrm{kg} & 18.8 & \text { EPA8080 } \\ \mu \mathrm{g} / \mathrm{kg} & 18.8 & \text { EPA8080 } \\ \mu \mathrm{g} / \mathrm{kg} & 18.8 & \text { EPA8080 } \\ \mu \mathrm{g} / \mathrm{kg} & 18.8 & \text { EPA8080 } \\ \mu \mathrm{g} / \mathrm{kg} & 18.8 & \text { EPA8080 } \\ \mu \mathrm{g} / \mathrm{kg} & 18.8 & \text { EPA8080 } \\ \mu \mathrm{g} / \mathrm{kg} & 18.8 & \text { EPA8080 } \\ \mu \mathrm{g} / \mathrm{kg} & 151 & \text { EPA8080 }\end{array}$

8.34E-01 $44.98 \mathrm{E}-02 \mathrm{pCi} / \mathrm{g}$ 6.39E-04 $\pm 7.14 \mathrm{E}-03 \mathrm{pCi} / \mathrm{g}$ $-1.01 \mathrm{E}-03 \pm 1.48 \mathrm{E}-02 \mathrm{pCi} / \mathrm{g}$ $-1.37 \mathrm{E}-03 \pm 6.46 \mathrm{E}-03 \mathrm{pCi} / \mathrm{g}$ $-2.23 \mathrm{E}-02 \pm 3.77 \mathrm{E}-02 \mathrm{pCi} / \mathrm{g}$ 6.36E-04 $\pm 5.00 \mathrm{E}-03 \mathrm{pCi} / \mathrm{g}$ $5.69 \mathrm{E}-02 \pm 9.20 \mathrm{E}-03 \mathrm{pCi} / \mathrm{g}$

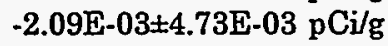
$-1.22 \mathrm{E}-03 \pm 6.21 \mathrm{E}-03 \mathrm{pCi} / \mathrm{g}$ $1.82 \mathrm{E}-03 \pm 3.61 \mathrm{E}-03 \mathrm{pCi} / \mathrm{g}$ $-4.45 \mathrm{E}-03 \pm 1.33 \mathrm{E}-02 \mathrm{pCi} / \mathrm{g}$ $-1.74 \mathrm{E}-02 \pm 3.70 \mathrm{E}-02 \mathrm{pCi} / \mathrm{g}$ $0.00 \mathrm{E}+00 \quad \mathrm{pCi} / \mathrm{g}$ $2.29 \mathrm{E}+01 \pm 3.82 \mathrm{E}+00 \mathrm{pCi} / \mathrm{g}$ $9.09 \mathrm{E}-01 \pm 2.43 \mathrm{E}-02 \mathrm{pCi} / \mathrm{g}$ 1.32E-02 $2.64 \mathrm{E}-03 \mathrm{pCi} / \mathrm{g}$ $-4.53 \mathrm{E}-03 \pm 3.83 \mathrm{E}-02 \mathrm{pCi} / \mathrm{g}$ $9.20 \mathrm{E}+00 \pm 1.85 \mathrm{E}+00 \mathrm{pCi} / \mathrm{g}$ $1.74 \mathrm{E}-02 \pm 2.08 \mathrm{E}-02 \mathrm{pCi} / \mathrm{g}$ $6.91 \mathrm{E}-03 \pm 1.72 \mathrm{E}-02 \mathrm{pCi} / \mathrm{g}$ $1.34 \mathrm{E}-03 \pm 6.10 \mathrm{E}-03 \mathrm{pCi} / \mathrm{g}$ $6.97 \mathrm{E}-03 \pm 1.41 \mathrm{E}-02 \mathrm{pCi} / \mathrm{g}$ $6.01 \mathrm{E}-01 \pm 7.34 \mathrm{E}-02 \mathrm{pCi} / \mathrm{g}$ $-2.31 \mathrm{E}-03 \pm 4.72 \mathrm{E}-03 \mathrm{pCi} / \mathrm{g}$ $7.41 \mathrm{E}-03 \pm 6.06 \mathrm{E}-03 \mathrm{pCi} / \mathrm{g}$ $5.24 \mathrm{E}-03 \pm 4.29 \mathrm{E}-02 \mathrm{pCi} / \mathrm{g}$ 8.83E-04 $4.07 \mathrm{E}-03 \mathrm{pCi} / \mathrm{g}$ $1.61 \mathrm{E}+00 \pm 1.52 \mathrm{E}-01 \mathrm{pCi} / \mathrm{g}$ $1.21 \mathrm{E}+00 \pm 5.44 \mathrm{E}-01 \mathrm{pCi} / \mathrm{g}$ $-3.06 \mathrm{E}-03 \pm 6.92 \mathrm{E}-03 \mathrm{pCi} / \mathrm{g}$ $-3.79 \mathrm{E}-03 \pm 5.01 \mathrm{E}-03 \mathrm{pCi} / \mathrm{g}$ $8.17 \mathrm{E}-03 \pm 1.09 \mathrm{E}-02 \mathrm{pCi} / \mathrm{g}$ 8.50E-03 $\pm 1.28 \mathrm{E}-02 \mathrm{pCi} / \mathrm{g}$
2.57E-02 EPIA-013B 1.06E-02 EPIA-013B 2.23E-02 EPIA-013B 9.78E-03 EPIA-013B 6.23E-02 EPIA-013B 7.42E-03 EPIA-013B

8.19E-03 EPIA-013B 7.86E-03 EPIA-013B 8.96E-03 EPLA-013B

7.39E-03 EPIA-013B 2.28E-02 EPIA-013B 5.45E-02 EPIA-013B

3.17E-02 EPIA-013B

2.53E+00 EPIA-001B 1.38E-02 EPIA-013B

8.48E-03 EPIA-013B

5.73E-02 EPIA-013B

$2.45 \mathrm{E}+00$ EPIA-001B

3.77E-02 EPIA-012B

3.64E-02 EPIA-012B

1.70E-02 EPIA-012B

2.92E.02 EPLA-012B

1.59E-01 EPIA-013B

7.76E-03 EPIA-013B

1.08E-02 EPIA-013B

7.31E-02 EPIA-013B

7.23E-03 EPIA-013B

8.00E-02 EPIA-004

4.20E-01 EPIA-013B

1.18E-02 EPIA-013B

8.44E-03 EPIA-013B

1.75E-02 EPIA-013B

1.96E-02 EPIA-013B 
Survey location: 44888E $3682027 \mathrm{~N}$ (SRS coordinates)

Depth of core interval: 2.00 to $4.00 \mathrm{ft}$

Sample type: Normal

Sample matrix: Other

Percent solids: $\mathbf{8 7 . 0 0}$

Constituent

RQ AQ B Result

Unit

D. Limit Method

Physical Parameters and Specified Analyses

Cation exchange capacity

Chloride

Cyanide

Cyanide

Nitrate-nitrite as nitrogen

Orthophosphate

Orthophosphate

Phenols

Sulfide

Total organic carbon

Total organic nitrogen

Total phosphates (as P)

Metals (total recoverable)

$\begin{array}{llll} & & & 58.3 \\ \text { U } & & & 2.71 \\ \text { U } & & & 1.15 \\ \text { U } & & & 1.15 \\ \text { J } & \text { E } & & 0.406 \\ \text { UJ } & \text { I } & \text { L } & 11.5 \\ \text { UJ } & \text { I } & \text { L } & 11.5 \\ \text { UJ } & \text { C } & & 343 \\ \text { UJ } & \text { C } & & 287 \\ & & & 907 \\ \text { C } & & & 45.2 \\ \text { J } & \text { E } & & 63.8\end{array}$

4570

$\mathrm{U} \quad 5.37$

$\mathrm{U} \quad 16.1$

J $\quad 9.05$

Beryllium

Cadmium

Calcium

Chromium

Cobalt

Copper

Iron

Lead

Magnesium

Manganese

Mercury

Nickel

Potassium

Selenium

Silver

Sodium

Thallium

Vanadium

Zinc

$B / N / A$ Extractables (including $P A H$ and phenols)

Acenaphthene

Acenaphthylene

Anthracene

Benzo[a]anthracene

Benzo[b]fluoranthene

Benzo[k]fluoranthene

Benzoic acid

Benzo[g,h,i]perylene

Benzo[a]pyrene

$\begin{array}{ll}\mathrm{U} & 37.6 \\ \mathrm{U} & 37.6 \\ \mathrm{U} & 37.6 \\ \mathrm{U} & 3.76 \\ \mathrm{U} & 3.76 \\ \mathrm{U} & 3.76 \\ \mathrm{U} & 37.6 \\ \mathrm{U} & 37.6 \\ \mathrm{U} & 3.76\end{array}$

$\begin{array}{lll}\mathrm{meq} / 100 \mathrm{~g} & 5 & \text { EPA9081 } \\ \mathrm{mg} / \mathrm{kg} & 2.71 & \text { EPA300.0 } \\ \mathrm{mg} / \mathrm{kg} & 1.15 & \text { EPA335.3 } \\ \mathrm{mg} / \mathrm{kg} & 1.15 & \text { EPA335.3 } \\ \mathrm{mg} / \mathrm{kg} & 1.02 & \text { EPA353.1 } \\ \mathrm{mg} / \mathrm{kg} & 11.5 & \text { EPA365.2 } \\ \mathrm{mg} / \mathrm{kg} & 11.5 & \text { EPA365.2 } \\ \mu \mathrm{g} / \mathrm{kg} & 343 & \text { EPA420.2 } \\ \mathrm{mg} / \mathrm{kg} & 287 & \text { EPA376.2 } \\ \mathrm{mg} / \mathrm{kg} & 115 & \text { EPA415.1 } \\ \mathrm{mg} / \mathrm{kg} & 0.23 & \text { EPA351.2 } \\ \mathrm{mg} / \mathrm{kg} & 223 & \text { EPA365.4 }\end{array}$

$\begin{array}{lll}\mathrm{mg} / \mathrm{kg} & 13.4 & \text { EPA6010A } \\ \mathrm{mg} / \mathrm{kg} & 5.37 & \text { EPA6010A } \\ \mathrm{mg} / \mathrm{kg} & 16.1 & \text { EPA6010A } \\ \mathrm{mg} / \mathrm{kg} & 1.34 & \text { EPA6010A } \\ \mathrm{mg} / \mathrm{kg} & 0.537 & \text { EPA6010A } \\ \mathrm{mg} / \mathrm{kg} & 0.269 & \text { EPA6010A } \\ \mathrm{mg} / \mathrm{kg} & 13.4 & \text { EPA6010A } \\ \mathrm{mg} / \mathrm{kg} & 1.34 & \text { EPA6010A } \\ \mathrm{mg} / \mathrm{kg} & 0.537 & \text { EPA6010A } \\ \mathrm{mg} / \mathrm{kg} & 1.34 & \text { EPA6010A } \\ \mathrm{mg} / \mathrm{kg} & 5.37 & \text { EPA6010A } \\ \mathrm{mg} / \mathrm{kg} & 5.37 & \text { EPA6010A } \\ \mathrm{mg} / \mathrm{kg} & 5.37 & \text { EPA6010A } \\ \mathrm{mg} / \mathrm{kg} & 0.537 & \text { EPA6010A } \\ \mathrm{mg} / \mathrm{kg} & 0.0308 & \text { EPA7471 } \\ \mathrm{mg} / \mathrm{kg} & 1.34 & \text { EPA6010A } \\ \mathrm{mg} / \mathrm{kg} & 107 & \text { EPA6010A } \\ \mathrm{mg} / \mathrm{kg} & 16.1 & \text { EPA6010A } \\ \mathrm{mg} / \mathrm{kg} & 1.34 & \text { EPA6010A } \\ \mathrm{mg} / \mathrm{kg} & 53.7 & \text { EPA6010A } \\ \mathrm{mg} / \mathrm{kg} & 16.1 & \text { EPA6010A } \\ \mathrm{mg} / \mathrm{kg} & 0.537 & \text { EPA6010A } \\ \mathrm{mg} / \mathrm{kg} & 1.07 & \text { EPA6010A }\end{array}$

$\begin{array}{lrr}\mu \mathrm{g} / \mathrm{kg} & 37.6 & \text { EPA8270 } \\ \mu \mathrm{g} / \mathrm{kg} & 37.6 & \text { EPA8270 } \\ \mu \mathrm{g} / \mathrm{kg} & 37.6 & \text { EPA8270 } \\ \mu \mathrm{g} / \mathrm{kg} & 3.76 & \text { EPA8270 } \\ \mu \mathrm{g} / \mathrm{kg} & 3.76 & \text { EPA8270 } \\ \mu \mathrm{g} / \mathrm{kg} & 3.76 & \text { EPA8270 } \\ \mu \mathrm{g} / \mathrm{kg} & 37.6 & \text { EPA8270 } \\ \mu \mathrm{g} / \mathrm{kg} & 37.6 & \text { EPA8270 } \\ \mu \mathrm{g} / \mathrm{kg} & 3.76 & \text { EPA8270 }\end{array}$


SURVEY ID: PPSC 1203A (continued)

Constituent

RQ

$A Q$

Sample ID: 104661

$B / N / A$ Extractables (including $P A H$ and phenols)
Benzyl alcohol

Bis(2-chloroethoxy) methane

Bis(2-chloroethyl) ether

Bis(2-chloroisopropyl) ether

Bis(2-ethylhexyl) phthalate

4-Bromophenyl phenyl ether

Butylbenzyl phthalate

4-Chloroaniline

4-Chloro-m-cresol

2-Chloronaphthalene

2-Chlorophenol

4-Chlorophenyl phenyl ether

Chrysene

$\mathrm{m} / \mathrm{p}$-Cresol

o-Cresol (2-Methylphenol)

Dibenz $[\alpha, h]$ anthracene

Dibenzofuran

Di-n-butyl phthalate

1,2-Dichlorobenzene

1,3-Dichlorobenzene

1,4-Dichlorobenzene

3,3'-Dichlorobenzidine

2,4-Dichlorophenol

Diethyl phthalate

2,4-Dimethyl phenol

Dimethyl phthalate

2,4-Dinitrophenol

2,4-Dinitrotoluene

2,6-Dinitrotoluene

Di-n-octyl phthalate

Fluoranthene

Fluorene

Hexachlorobenzene

Hexachlorobutadiene

Hexachlorocyclopentadiene

Hexachloroethane

Indeno $[1,2,3-c, d]$ pyrene

Isophorone

2-Methyl-4,6-dinitrophenol

2-Methylnaphthalene

Naphthalene

m-Nitroaniline

o-Nitroaniline

p-Nitroaniline

Nitrobenzene

2-Nitrophenol

4-Nitrophenol

N-Nitrosodiphenylamine

N-Nitrosodi-n-propylamine

Pentachlorophenol

Phenanthrene

Phenol

Pyrene

1,2,4-Trichlorobenzene

2,4,5-Trichlorophenol

2,4,6-Trichlorophenol

\begin{tabular}{|c|c|c|}
\hline $\mathrm{U}$ & & 37.6 \\
\hline $\mathrm{U}$ & & 37.6 \\
\hline $\mathrm{U}$ & & 37.6 \\
\hline $\mathrm{U}$ & & 37.6 \\
\hline $\mathrm{U}$ & & 37.6 \\
\hline$U$ & & 37.6 \\
\hline $\mathrm{U}$ & & 37.6 \\
\hline $\mathrm{U}$ & & 37.6 \\
\hline $\mathrm{U}$ & & 37.6 \\
\hline U & & 37.6 \\
\hline $\mathrm{U}$ & & 37.6 \\
\hline $\mathrm{U}$ & & 37.6 \\
\hline U & & 3.76 \\
\hline U & & 376 \\
\hline$U$ & & 37.6 \\
\hline U & & 3.76 \\
\hline $\mathrm{U}$ & & 37.6 \\
\hline U & & 37.6 \\
\hline$U$ & & 37.6 \\
\hline$U$ & & 37.6 \\
\hline $\mathrm{U}$ & & 37.6 \\
\hline U & & 37.6 \\
\hline $\mathrm{U}$ & & 37.6 \\
\hline $\mathrm{U}$ & & 37.6 \\
\hline $\mathrm{U}$ & & 37.6 \\
\hline U & & 37.6 \\
\hline$U$ & & 376 \\
\hline UJ & C & 3.76 \\
\hline$U$ & & 3.76 \\
\hline U & & 37.6 \\
\hline$U$ & & 37.6 \\
\hline U & & 37.6 \\
\hline$U$ & & 3.76 \\
\hline U & & 37.6 \\
\hline $\mathrm{U}$ & & 37.6 \\
\hline U & & 3.76 \\
\hline $\mathrm{U}$ & & 3.76 \\
\hline $\mathrm{U}$ & & 37.6 \\
\hline $\mathrm{U}$ & & 376 \\
\hline $\mathrm{U}$ & & 37.6 \\
\hline $\mathrm{U}$ & & 37.6 \\
\hline$U$ & & 37.6 \\
\hline U & & 37.6 \\
\hline$U$ & & 37.6 \\
\hline $\mathrm{U}$ & & 37.6 \\
\hline U & & 37.6 \\
\hline U & & 37.6 \\
\hline $\mathrm{U}$ & & 37.6 \\
\hline $\mathrm{U}$ & & 37.6 \\
\hline UJ & C & 37.6 \\
\hline $\mathrm{U}$ & & 37.6 \\
\hline$U$ & & 37.6 \\
\hline U & & 37.6 \\
\hline U & & 3.76 \\
\hline U & & 37.6 \\
\hline$U$ & & 37.6 \\
\hline
\end{tabular}

Unit

D. Limit Method

$\begin{array}{lll}\mu \mathrm{g} / \mathrm{kg} & 37.6 & \text { EPA8270 } \\ \mu \mathrm{g} / \mathrm{kg} & 37.6 & \text { EPA8270 } \\ \mu \mathrm{g} / \mathrm{kg} & 37.6 & \text { EPA8270 } \\ \mu \mathrm{g} / \mathrm{kg} & 37.6 & \text { EPA8270 } \\ \mu \mathrm{g} / \mathrm{kg} & 37.6 & \text { EPA8270 } \\ \mu \mathrm{g} / \mathrm{kg} & 37.6 & \text { EPA8270 } \\ \mu \mathrm{g} / \mathrm{kg} & 37.6 & \text { EPA8270 } \\ \mu \mathrm{g} / \mathrm{kg} & 37.6 & \text { EPA8270 } \\ \mu \mathrm{g} / \mathrm{kg} & 37.6 & \text { EPA8270 } \\ \mu \mathrm{g} / \mathrm{kg} & 37.6 & \text { EPA8270 } \\ \mu \mathrm{g} / \mathrm{kg} & 37.6 & \text { EPA8270 } \\ \mu \mathrm{g} / \mathrm{kg} & 37.6 & \text { EPA8270 } \\ \mu \mathrm{g} / \mathrm{kg} & 3.76 & \text { EPA8270 } \\ \mu \mathrm{g} / \mathrm{kg} & 376 & \text { EPA8270 } \\ \mu \mathrm{g} / \mathrm{kg} & 37.6 & \text { EPA8270 } \\ \mu \mathrm{g} / \mathrm{kg} & 3.76 & \text { EPA8270 } \\ \mu \mathrm{g} / \mathrm{kg} & 37.6 & \text { EPA8270 } \\ \mu \mathrm{g} / \mathrm{kg} & 37.6 & \text { EPA8270 } \\ \mu \mathrm{g} / \mathrm{kg} & 37.6 & \text { EPA8270 } \\ \mu \mathrm{g} / \mathrm{kg} & 37.6 & \text { EPA8270 } \\ \mu \mathrm{g} / \mathrm{kg} & 37.6 & \text { EPA8270 } \\ \mu \mathrm{g} / \mathrm{kg} & 37.6 & \text { EPA8270 } \\ \mu \mathrm{g} / \mathrm{kg} & 37.6 & \text { EPA8270 } \\ \mu \mathrm{g} / \mathrm{kg} & 37.6 & \text { EPA8270 } \\ \mu \mathrm{g} / \mathrm{kg} & 37.6 & \text { EPA8270 } \\ \mu \mathrm{g} / \mathrm{kg} & 37.6 & \text { EPA8270 } \\ \mu \mathrm{g} / \mathrm{kg} & 376 & \text { EPA8270 } \\ \mu \mathrm{g} / \mathrm{kg} & 3.6 & \text { EPA8270 }\end{array}$

EPA8270

EPA8270

EPA8270

EPA8270

EPA8270

EPA8270

EPA8270

EPA8270

EPA8270

EPA8270

EPA8270

EPA8270

EPA8270

EPA8270

EPA8270

EPA8270

EPA8270

EPA8270

EPA8270

EPA8270

EPA8270

EPA8270

EPA8270

EPA8270

EPA8270

EPA8270

EPA8270

EPA8270

EPA8270 
SURVEY ID: PPSC 1203A (continued)

Constituent

RQ AQ B Result

Volatile Organic Compounds

Acetone
Benzene
Bromodichloromethane
Bromoform
Bromomethane (Methyl bromide)
Carbon disulfide
Carbon tetrachloride
Chlorobenzene
Chloroethane
Chloroethene (Vinyl chloride)
Chloroform
Chloromethane (Methyl chloride)
Dibromochloromethane
1,1-Dichloroethane
1,2-Dichloroethane
1,1-Dichloroethylene
1,2-Dichloroethylene
Dichloromethane (Methylene chloride)
1,2-Dichloropropane
cis-1,3-Dichloropropene
trans-1,3-Dichloropropene
Ethylbenzene
2-Hexanone
Methyl ethyl ketone
Methyl isobutyl ketone
Styrene
1,1,2,2-Tetrachloroethane
Tetrachloroethylene
Toluene
1,1,1-Trichloroethane
1,1,2-Trichloroethane
Trichloroethylene
Vinyl acetate
Xylenes

\section{Pesticides/PCBs and Dioxins/Furans}

Unit

D. Limit Method
Sample ID: 104661

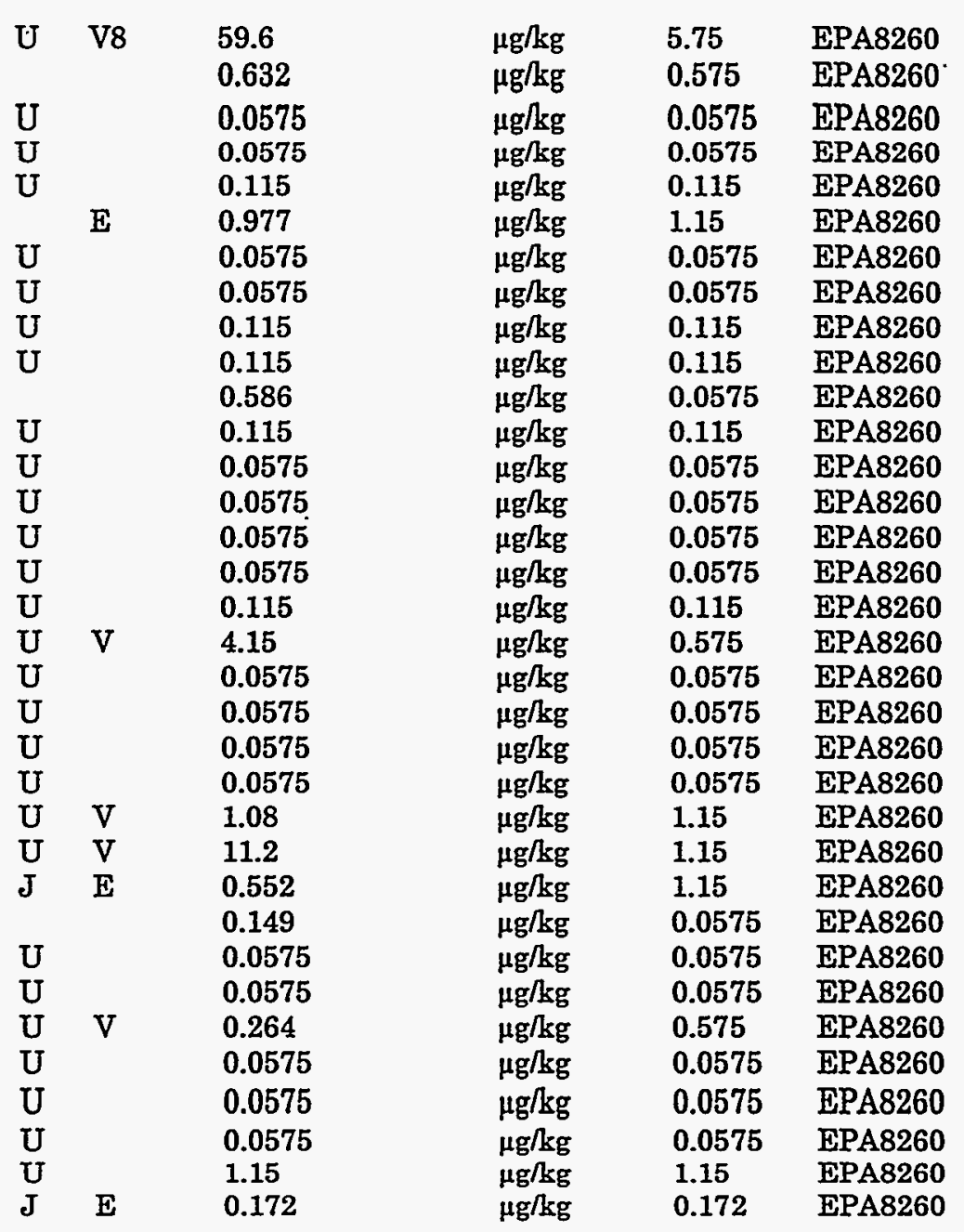

$\begin{array}{lll}\mu \mathrm{g} / \mathrm{kg} & 3.82 & \text { EPA8080 } \\ \mu \mathrm{g} / \mathrm{kg} & 3.82 & \text { EPA8080 } \\ \mu \mathrm{g} / \mathrm{kg} & 7.65 & \text { EPA8080 } \\ \mu \mathrm{g} / \mathrm{kg} & 7.65 & \text { EPA8080 } \\ \mu \mathrm{g} / \mathrm{kg} & 7.65 & \text { EPA8080 } \\ \mu \mathrm{g} / \mathrm{kg} & 7.65 & \text { EPA8080 } \\ \mu \mathrm{g} / \mathrm{kg} & 15.3 & \text { EPA8080 } \\ \mu \mathrm{g} / \mathrm{kg} & 7.65 & \text { EPA8080 } \\ \mu \mathrm{g} / \mathrm{kg} & 15.3 & \text { EPA8080 } \\ \mu \mathrm{g} / \mathrm{kg} & 7.65 & \text { EPA8080 } \\ \mu \mathrm{g} / \mathrm{kg} & 15.3 & \text { EPA8080 } \\ \mu \mathrm{g} / \mathrm{kg} & 7.65 & \text { EPA8080 } \\ \mu \mathrm{g} / \mathrm{kg} & 15.3 & \text { EPA8080 } \\ \mu \mathrm{g} / \mathrm{kg} & 7.65 & \text { EPA8080 } \\ \mu \mathrm{g} / \mathrm{kg} & -15.3 & \text { EPA8080 } \\ \mu \mathrm{g} / \mathrm{kg} & 7.65 & \text { EPA8080 } \\ \mu \mathrm{g} / \mathrm{kg} & 7.65 & \text { EPA8080 } \\ \mu \mathrm{g} / \mathrm{kg} & 3.82 & \text { EPA8080 }\end{array}$


SURVEY ID: PPSC 1203A (continued)

Constituent

RQ AQ B Result

Unit

D. Limit Method

Pesticides/PCBs and Dioxins/Furans

Methoxychlor
PCB 1016
PCB 1221
PCB 1232
PCB 1242
PCB 1248
PCB 1254
PCB 1260
Toxaphene

\section{Radionuclides}

Actinium-228

Antimony-124

Antimony-125

Barium-133

Cerium-144

Cesium-134

Cesium-137

Cobalt-57

Cobalt-58

Cobalt-60

Europium-152

Europium-154

Europium-155

Gross alpha

Lead-212

Manganese-54

Neptunium-239

Nonvolatile beta

Plutonium-238

Plutonium-239/240

Potassium-40

Promethium-144

Promethium-146

Ruthenium-106

Sodium-22

Strontium-90

Thorium-234

Tin-113

Yttrium-88

Zinc-65

Zirconium-95

$\begin{array}{ll}\mathrm{U} & 76.5 \\ \mathrm{U} & 19.1 \\ \mathrm{U} & 19.1 \\ \mathrm{U} & 19.1 \\ \mathrm{U} & 19.1 \\ \mathrm{U} & 19.1 \\ \mathrm{U} & 19.1 \\ \mathrm{U} & 19.1 \\ \mathrm{U} & 153\end{array}$

8.74E-01 $\pm 4.97 \mathrm{E}-02 \mathrm{pCi} / \mathrm{g}$ 7.01E-03 $\pm 1.29 \mathrm{E}-02 \mathrm{pCi} / \mathrm{g}$ $4.17 \mathrm{E}-03 \pm 1.20 \mathrm{E}-02 \mathrm{pCi} / \mathrm{g}$ $-2.32 \mathrm{E}-03 \pm 6.47 \mathrm{E}-03 \mathrm{pCi} / \mathrm{g}$ $-3.59 \mathrm{E}-02 \pm 3.50 \mathrm{E}-02 \mathrm{pCi} / \mathrm{g}$ $2.24 \mathrm{E}-03 \pm 6.20 \mathrm{E}-03 \mathrm{pCi} / \mathrm{g}$ 6.70E-02 $1.01 \mathrm{E}-02 \mathrm{pCi} / \mathrm{g}$ $-8.00 \mathrm{E}-04 \pm 4.44 \mathrm{E}-03 \mathrm{pCj} / \mathrm{g}$ 8.14E-04 $\pm 5.19 \mathrm{E}-03 \mathrm{pCi} / \mathrm{g}$ $3.19 \mathrm{E}-04 \pm 4.04 \mathrm{E}-03 \mathrm{pCi} / \mathrm{g}$ $-8.66 \mathrm{E}-03 \pm 1.31 \mathrm{E}-02 \mathrm{pCi} / \mathrm{g}$ $.1 .51 \mathrm{E}-02 \pm 3.91 \mathrm{E}-02 \mathrm{pCi} / \mathrm{g}$ $0.00 \mathrm{E}+00 \quad \mathrm{pCi} / \mathrm{g}$ $2.00 \mathrm{E}+01 \pm 3.75 \mathrm{E}+00 \mathrm{pCi} / \mathrm{g}$ 8.72E-01 $\pm 2.31 \mathrm{E}-02 \mathrm{pCi} / \mathrm{g}$ 1.41E-02士6.87E-03 pCi/g 4.79E-02 $\pm 4.80 \mathrm{E}-02 \mathrm{pCi} / \mathrm{g}$ $7.32 \mathrm{E}+00 \pm 1.88 \mathrm{E}+00 \mathrm{pCi} / \mathrm{g}$ $1.76 \mathrm{E}-01 \pm 5.60 \mathrm{E}-02 \mathrm{pCi} / \mathrm{g}$ $1.19 \mathrm{E}-01 \pm 4.18 \mathrm{E}-02 \mathrm{pCi} / \mathrm{g}$ 7.77E-01 $1.17 \mathrm{E}-01 \mathrm{pCi} / \mathrm{g}$ $-1.17 \mathrm{E}-03 \pm 4.51 \mathrm{E}-03 \mathrm{pCi} / \mathrm{g}$ 5.81E-03 $\pm 6.23 \mathrm{E}-03 \mathrm{pCi} / \mathrm{g}$ $7.40 \mathrm{E}-03 \pm 4.06 \mathrm{E}-02 \mathrm{pCi} / \mathrm{g}$ $4.92 \mathrm{E}-04 \pm 4.14 \mathrm{E}-03 \mathrm{pCi} / \mathrm{g}$ $1.55 \mathrm{E}+00 \pm 1.38 \mathrm{E}-01 \mathrm{pCi} / \mathrm{g}$ 7.06E-01 $\pm 5.56 \mathrm{E}-01 \mathrm{pCi} / \mathrm{g}$ $7.41 \mathrm{E}-04 \pm 4.88 \mathrm{E}-03 \mathrm{pCi} / \mathrm{g}$ $-1.65 \mathrm{E}-03 \pm 9.97 \mathrm{E}-03 \mathrm{pCi} / \mathrm{g}$ $0.00 \mathrm{E}+00 \quad \mathrm{pCi} / \mathrm{g}$ $-1.40 \mathrm{E}-03 \pm 7.62 \mathrm{E}-03 \mathrm{pCi} / \mathrm{g}$
2.31E-02 EPIA-013B

1.00E-02 EPIA-013B

2.02E-02 EPIA-013B

9.44E-03 EPIA-013B

5.92E-02 EPIA-013B

7.23E-03 EPIA-013B

8.13E-03 EPIA-013B

7.64E-03 EPIA-013B

9.11E-03 EPIA-013B

7.33E-03 EPIA-013B

2.13E-02 EPIA-013B

5.69E-02 EPIA-013B

3.18E-02 EPIA-013B

2.01E+00 EPIA-001B

1.35E-02 EPIA-013B

7.14E-03 EPIA-013B

5.02E-02 EPIA-013B

2.85E+00 EPIA-001B

5.48E-02 EPIA-012B

2.53E-02 EPIA-012B

6.36E-02 EPIA-013B

7.75E-03 EPIA-013B

1.06E-02 EPIA-013B

7.18E-02 EPIA-013B

7.16E-03 EPLA-013B

6.88E-02 EPIA-004

4.04E-01 EPIA-013B

1.11E-02 EPIA-013B

8.96E-03 EPIA-013B

1.47E-02 EPIA-013B

2.04E-02 EPIA-013B

SURVEY ID: PPSC 1301

Sample ID: 104664

Survey location: 44128E 3669963N (SRS coordinates)

Depth of core interval: 0.00 to $1.00 \mathrm{ft}$

Sample type: Normal

Sample matrix: Soil

Percent solids: $\mathbf{8 5 . 0 0}$ 
SURVEY ID: PPSC 1301 (continued)

Sample ID: 104664

Constituent

RQ AQ B Result

Unit

D. Limit Method

Physical Parameters and Specified Analyses

Cation exchange capacity

Chloride

Cyanide

Nitrate-nitrite as nitrogen

Nitrate-nitrite as nitrogen

Orthophosphate

Phenols

Phenols

Sulfide

Sulfide

Total organic carbon

Total organic nitrogen

Total phosphates (as P)

Metals (total recoverable)

Aluminum

Aluminum

Antimony

Antimony

Arsenic

Arsenic

Barium

Barium

Beryllium

Beryllium

Cadmium

Cadmium

Calcium

Calcium

Chromium

Chromium

Cobalt

Cobalt

Copper

Copper

Iron

Iron

Lead

Lead

Magnesium

Magnesium

Manganese

Manganese

Mercury

Mercury

Nickel

Nickel

Potassium

Potassium

Selenium

Selenium

Silver

Silver

Sodium

$\begin{array}{llll} & & & 33.5 \\ \text { J } & \text { E } & & 1.59 \\ \text { U } & & & \mathbf{1 . 1 5} \\ \text { J } & \text { E } & & \mathbf{0 . 6 0 2} \\ \text { J } & \text { E } & & \mathbf{0 . 6 2 4} \\ \text { U } & & & 11.4 \\ \text { UJ } & \text { C } & & \mathbf{3 4 7} \\ \text { UJ } & \text { C } & & \mathbf{3 4 7} \\ \text { UJ } & \text { IC } & \text { P } & 294 \\ \text { UJ } & \text { IC } & \text { P } & \mathbf{2 9 4} \\ & & & 4540 \\ \text { C } & & & \mathbf{9 0 . 8} \\ \text { J } & \text { E } & & \mathbf{6 4 . 9}\end{array}$

meq $/ 100 \mathrm{~g}$

$\mathrm{mg} / \mathrm{kg}$

$\mathrm{mg} / \mathrm{kg}$

$\mathrm{mg} / \mathrm{kg}$

$\mathrm{mg} / \mathrm{kg}$

$\mathrm{mg} / \mathrm{kg}$

$\mu \mathrm{g} / \mathrm{kg}$

$\mu \mathrm{g} / \mathrm{kg}$

$\mathrm{mg} / \mathrm{kg}$

$\mathrm{mg} / \mathrm{kg}$

$\mathrm{mg} / \mathrm{kg}$

$\mathrm{mg} / \mathrm{kg}$

$\mathrm{mg} / \mathrm{kg}$

2380

2190

UJ I $\quad$ L $\quad \mathbf{5 . 7 1}$

UJ I L 5.5

U $\quad 17.1$

$\mathrm{U} \quad 16.5$

14.8

15.6

J $\quad \mathrm{E} \quad 0.0811$

$\begin{array}{lll}J & \mathrm{E} & \mathbf{0 . 0 8 9 6}\end{array}$

U $\quad 0.286$

$\mathrm{U} \quad 0.275$

62.6

58.4

1.83

1.8

$\begin{array}{lll}\mathrm{J} & \mathrm{E} & 0.378\end{array}$

0.556

2.54

$\begin{array}{lll}J & E & 1.25\end{array}$

$\begin{array}{lll}J & C & 918\end{array}$

J $\quad \mathrm{C} \quad 1030$

J $\quad \mathrm{E} \quad 3.44$

$\begin{array}{lll}\mathrm{J} & \mathrm{E} & 2.12\end{array}$

44.2

59.5

$\begin{array}{llll}J & I & L & 55.7\end{array}$

$\begin{array}{llll}J & I & \text { L } & \mathbf{4 5 . 5}\end{array}$

$\begin{array}{lll}J & \mathrm{E} & \mathbf{0 . 0 0 4 3 6}\end{array}$

U $\quad 0.0378$

$\begin{array}{lll}J & \mathrm{E} & \mathbf{0 . 8 2 5}\end{array}$

$\begin{array}{lll}J & \mathrm{E} & 1.18\end{array}$

$\begin{array}{lll}J & \mathrm{E} & 39.4\end{array}$

J $\cdot \mathrm{E} \quad \mathbf{5 4 . 3}$

$\mathrm{U} \quad 17.1$

$\mathrm{U} \quad 16.5$

$\mathrm{U} \quad 1.43$

$\mathrm{U} \quad 1.37$

U $\quad 57.1$

$\begin{array}{lll}\mathrm{mg} / \mathrm{kg} & 14.3 & \text { EPA6010A } \\ \mathrm{mg} / \mathrm{kg} & 13.7 & \text { EPA6010A } \\ \mathrm{mg} / \mathrm{kg} & 5.71 & \text { EPA6010A } \\ \mathrm{mg} / \mathrm{kg} & 5.5 & \text { EPA6010A } \\ \mathrm{mg} / \mathrm{kg} & 17.1 & \text { EPA6010A } \\ \mathrm{mg} / \mathrm{kg} & 16.5 & \text { EPA6010A } \\ \mathrm{mg} / \mathrm{kg} & 1.43 & \text { EPA6010A } \\ \mathrm{mg} / \mathrm{kg} & 1.37 & \text { EPA6010A } \\ \mathrm{mg} / \mathrm{kg} & 0.571 & \text { EPA6010A } \\ \mathrm{mg} / \mathrm{kg} & 0.55 & \text { EPA6010A } \\ \mathrm{mg} / \mathrm{kg} & 0.286 & \text { EPA6010A } \\ \mathrm{mg} / \mathrm{kg} & 0.275 & \text { EPA6010A } \\ \mathrm{mg} / \mathrm{kg} & 14.3 & \text { EPA6010A } \\ \mathrm{mg} / \mathrm{kg} & 13.7 & \text { EPA6010A } \\ \mathrm{mg} / \mathrm{kg} & 1.43 & \text { EPA6010A } \\ \mathrm{mg} / \mathrm{kg} & 1.37 & \text { EPA6010A } \\ \mathrm{mg} / \mathrm{kg} & 0.571 & \text { EPA6010A } \\ \mathrm{mg} / \mathrm{kg} & 0.55 & \text { EPA6010A } \\ \mathrm{mg} / \mathrm{kg} & 1.43 & \text { EPA6010A } \\ \mathrm{mg} / \mathrm{kg} & 1.37 & \text { EPA6010A } \\ \mathrm{mg} / \mathrm{kg} & 5.71 & \text { EPA6010A } \\ \mathrm{mg} / \mathrm{kg} & 5.5 & \text { EPA6010A } \\ \mathrm{mg} / \mathrm{kg} & 5.71 & \text { EPA6010A } \\ \mathrm{mg} / \mathrm{kg} & 5.5 & \text { EPA6010A } \\ \mathrm{mg} / \mathrm{kg} & 5.71 & \text { EPA6010A } \\ \mathrm{mg} / \mathrm{kg} & 5.5 & \text { EPA6010A } \\ \mathrm{mg} / \mathrm{kg} & 0.571 & \text { EPA6010A } \\ \mathrm{mg} / \mathrm{kg} & 0.55 & \text { EPA6010A } \\ \mathrm{mg} / \mathrm{kg} & 0.038 & \text { EPA7471 } \\ \mathrm{mg} / \mathrm{kg} & 0.0378 & \text { EPA7471 } \\ \mathrm{mg} / \mathrm{kg} & 1.43 & \text { EPA6010A } \\ \mathrm{mg} / \mathrm{kg} & 1.37 & \text { EPA6010A } \\ \mathrm{mg} / \mathrm{kg} & 114 & \text { EPA6010A } \\ \mathrm{mg} / \mathrm{kg} & 110 & \text { EPA6010A } \\ \mathrm{mg} / \mathrm{kg} & 17.1 & \text { EPA6010A } \\ \mathrm{mg} / \mathrm{kg} & -16.5 & \text { EPA6010A } \\ \mathrm{mg} / \mathrm{kg} & 1.43 & \text { EPA6010A } \\ \mathrm{mg} / \mathrm{kg} & 1.37 & \text { EPA6010A } \\ \mathrm{mg} / \mathrm{kg} & 57.1 & \text { EPA6010A } \\ & & \\ & & \end{array}$

EPA9081

EPA300.0

EPA335.3

EPA353.1

EPA353.1

EPA365.2

EPA420.2

EPA420.2

EPA376.2

EPA376.2

EPA415.1

EPA351.2

EPA365.4

228

EPA6010A 
SURVEY ID: PPSC 1301 (continued)

Constituent

Metals (total recoverable)

Sodium

Thallium

Thallium

Vanadium

Vanadium

Zinc

Zinc

\section{Radionuclides}

Actinium-228

Antimony-124

Antimony-125

Barium-133

Cerium-144

Cesium-134

Cesium-137

Cobalt-57

Cobalt-58

Cobalt-60

Europium-152

Europium-154

Europium-155

Gross alpha

Lead-212

Manganese-54

Neptunium-239

Nonvolatile beta

Plutonium-238

Plutonium-239/240

Potassium-40

Promethium-144

Promethium-146

Ruthenium-106

Sodium-22

Strontium-90

Thorium-234

Tin-113

Yttrium-88

Zinc-65

Zirconium-95
Sample ID: 104664

RQ AQ B Result Unit D. Limit Method

$\begin{array}{llll}55 & \mathrm{mg} / \mathrm{kg} & 55 & \text { EPA6010A } \\ 17.1 & \mathrm{mg} / \mathrm{kg} & 17.1 & \text { EPA6010A } \\ 16.5 & \mathrm{mg} / \mathrm{kg} & 16.5 & \text { EPA6010A } \\ 2.42 & \mathrm{mg} / \mathrm{kg} & 0.571 & \text { EPA6010A } \\ 2.7 & \mathrm{mg} / \mathrm{kg} & 0.55 & \text { EPA6010A } \\ 3.18 & \mathrm{mg} / \mathrm{kg} & 1.14 & \text { EPA6010A } \\ 2.23 & \mathrm{mg} / \mathrm{kg} & 1.1 & \text { EPA6010A }\end{array}$

4.66E-01 $\pm 3.67 \mathrm{E}-02 \mathrm{pCi} / \mathrm{g}$

1.93E-02 EPIA-013B

8.21E-03 EPIA-013B

1.74E-02 EPIA-013B

7.59E-03 EPIA-013B

4.84E-02 EPIA-013B

5.73E-03 EPIA-013B

7.05E-03 EPIA-013B

6.32E-03 EPIA-013B

7.41E-03 EPIA-013B

5.59E-03 EPIA-013B

1.84E-02 EPLA-013B

5.36E-02 EPIA-013B

2.55E-02 EPLA-013B

1.87E+00 EPIA-001B

1.14E-02 EPIA-013B

6.47E-03 EPIA-013B

4.52E-02 EPIA-013B

2.54E+00 EPIA-001B

4.53E-02 EPIA-012B

1.95E-02 EPIA-012B

5.18E-02 EPIA-013B

6.05E-03 EPIA-013B

8.62E-03 EPIA-013B

5.85E-02 EPIA-013B

5.19E-03 EPIA-013B

9.72E-02 EPIA-004

2.91E-01 EPIA-013B

9.29E-03 EPIA-013B

6.52E-03 EPIA-013B

1.30E-02 EPIA-013B

1.49E-02 EPIA-013B

SURVEY ID: PPSC 1302

Sample ID: 104665

Survey location: $44128 \mathrm{E}$ 3669963N (SRS coordinates)

Depth of core interval: 1.00 to $2.00 \mathrm{ft}$

Sample type: Normal

Sample matrix: Soil

Percent solids: $\mathbf{8 6 . 0 0}$ 
SURVEY ID: PPSC 1302 (continued)

Constituent
Sample ID: 104665

RQ AQ B Result

Unit

D. Limit Method

Physical Parameters and Specified Analyses

Ammonia nitrogen

Cation exchange capacity

Chloride

Chloride

Cyanide

Nitrate-nitrite as nitrogen

Nitrogen by Kjeldahl method

Orthophosphate

Phenols

Sulfide

Total organic carbon

Total organic nitrogen

Total phosphates (as P)

Metals (total recoverable)

$\begin{array}{lll} & & 26.4 \\ & & 52.7 \\ J & Q & 4.24 \\ J & \mathrm{QE} & 2.45 \\ \mathrm{U} & & 1.14 \\ \mathrm{U} & & 1.08 \\ & & 119 \\ \mathrm{U} & & 11.5 \\ & & 340 \\ \mathrm{UJ} & \mathrm{C} & 291 \\ & & 6610 \\ \mathrm{C} & & 92.5 \\ \mathrm{~J} & \mathrm{E} & 80.1\end{array}$

$\begin{array}{lll}\text { J } & \text { C } & 2070 \\ \text { U } & & 5.59 \\ U & & 16.8 \\ & & 24 \\ J & E & 0.115 \\ \text { U } & & 0.28 \\ & V & 94.9 \\ & & 1.57 \\ & & 0.623 \\ \text { U } & \text { V } & 1.69 \\ \text { J } & \text { VC } & 1320 \\ J & E & 2.21 \\ & & 46 \\ & & 136 \\ \text { J } & E & 0.0103 \\ \text { J } & \text { E } & 1.11 \\ \text { J } & \text { E } & 39.6 \\ \text { U } & & 16.8 \\ \text { U } & & 1.4 \\ \text { U } & & 55.9 \\ \text { UJ } & \text { C } & 16.8 \\ & & 2.91 \\ & \text { V } & 2.64\end{array}$

$\begin{array}{lll}\mathrm{mg} / \mathrm{kg} & 21.4 & \text { EPA350.1 } \\ \mathrm{meq} / 100 \mathrm{~g} & 5 & \text { EPA9081 } \\ \mathrm{mg} / \mathrm{kg} & 2.87 & \text { EPA300.0 } \\ \mathrm{mg} / \mathrm{kg} & 2.85 & \text { EPA300.0 } \\ \mathrm{mg} / \mathrm{kg} & 1.14 & \text { EPA335.3 } \\ \mathrm{mg} / \mathrm{kg} & 1.08 & \text { EPA353.1 } \\ \mathrm{mg} / \mathrm{kg} & 46.6 & \text { EPA351.2 } \\ \mathrm{mg} / \mathrm{kg} & 11.5 & \text { EPA365.2 } \\ \mu \mathrm{g} / \mathrm{kg} & 332 & \text { EPA420.2 } \\ \mathrm{mg} / \mathrm{kg} & 291 & \text { EPA376.2 } \\ \mathrm{mg} / \mathrm{kg} & 116 & \text { EPA415.1 } \\ \mathrm{mg} / \mathrm{kg} & 0.233 & \text { EPA351.2 } \\ \mathrm{mg} / \mathrm{kg} & 461 & \text { EPA365.4 }\end{array}$

$\begin{array}{lll}\mathrm{mg} / \mathrm{kg} & 14 & \text { EPA6010A } \\ \mathrm{mg} / \mathrm{kg} & 5.59 & \text { EPA6010A } \\ \mathrm{mg} / \mathrm{kg} & 16.8 & \text { EPA6010A } \\ \mathrm{mg} / \mathrm{kg} & 1.4 & \text { EPA6010A } \\ \mathrm{mg} / \mathrm{kg} & 0.559 & \text { EPA6010A } \\ \mathrm{mg} / \mathrm{kg} & 0.28 & \text { EPA6010A } \\ \mathrm{mg} / \mathrm{kg} & 14 & \text { EPA6010A } \\ \mathrm{mg} / \mathrm{kg} & 1.4 & \text { EPA6010A } \\ \mathrm{mg} / \mathrm{kg} & 0.559 & \text { EPA6010A } \\ \mathrm{mg} / \mathrm{kg} & 1.4 & \text { EPA6010A } \\ \mathrm{mg} / \mathrm{kg} & 5.59 & \text { EPA6010A } \\ \mathrm{mg} / \mathrm{kg} & 5.59 & \text { EPA6010A } \\ \mathrm{mg} / \mathrm{kg} & 5.59 & \text { EPA6010A } \\ \mathrm{mg} / \mathrm{kg} & 0.559 & \text { EPA6010A } \\ \mathrm{mg} / \mathrm{kg} & 0.0376 & \text { EPA7471 } \\ \mathrm{mg} / \mathrm{kg} & 1.4 & \text { EPA6010A } \\ \mathrm{mg} / \mathrm{kg} & 112 & \text { EPA6010A } \\ \mathrm{mg} / \mathrm{kg} & 16.8 & \text { EPA6010A } \\ \mathrm{mg} / \mathrm{kg} & 1.4 & \text { EPA6010A } \\ \mathrm{mg} / \mathrm{kg} & 55.9 & \text { EPA6010A } \\ \mathrm{mg} / \mathrm{kg} & 16.8 & \text { EPA6010A } \\ \mathrm{mg} / \mathrm{kg} & 0.559 & \text { EPA6010A } \\ \mathrm{mg} / \mathrm{kg} & 1.12 & \text { EPA6010A }\end{array}$

Radionuclides

Actinium-228

Antimony-124

Antimony-125

Barium-133

Cerium-144

Cesium-134

Cesium-137

Cobalt-57

Cobalt-58

Cobalt-60

Europium-152

Europium-154

Europium-155
4.59E-01 $\pm 4.10 \mathrm{E}-02 \mathrm{pCi} / \mathrm{g}$

6.65E-04 $\pm 5.02 \mathrm{E}-03 \mathrm{pCi} / \mathrm{g}$

$-4.23 \mathrm{E}-03 \pm 1.14 \mathrm{E}-02 \mathrm{pCi} / \mathrm{g}$

1.42E-03 $\pm 5.77 \mathrm{E}-03 \mathrm{pCi} / \mathrm{g}$

$-2.24 \mathrm{E}-02 \pm 2.99 \mathrm{E}-02 \mathrm{pCi} / \mathrm{g}$

-6.80E-04 4 .10E-03 pCi/g

1.61E-01 $\pm 1.10 \mathrm{E}-02 \mathrm{pCi} / \mathrm{g}$

$-1.08 \mathrm{E}-03 \pm 3.77 \mathrm{E}-03 \mathrm{pCi} / \mathrm{g}$

$-4.98 \mathrm{E}-03 \pm 4.55 \mathrm{E}-03 \mathrm{pCi} / \mathrm{g}$

-2.01E-04 $13.32 \mathrm{E}-03 \mathrm{pCi} \mathrm{g}$

$-1.61 \mathrm{E}-02 \pm 1.18 \mathrm{E}-02 \mathrm{pCi} / \mathrm{g}$

$-7.40 \mathrm{E}-03 \pm 3.70 \mathrm{E}-02 \mathrm{pCi} / \mathrm{g}$

$0.00 \mathrm{E}+00 \quad \mathrm{pCi} / \mathrm{g}$
2.03E-02 EPIA-013B

8.91E.03 EPIA-013B

1.86E-02 EPLA-013B

8.70E-03 EPIA-013B

5.08E-02 EPIA-013B

6.34E-03 EPIA-013B

6.77E-03 EPIA-013B

6.48E-03 EPLA-013B

7.33E-03 EPIA-013B

6.07E.03 EPIA-013B

1.86E-02 EPIA-013B

5.52E-02 EPIA-013B

2.74E-02 EPLA-013B 
SURVEY ID: PPSC 1302 (continued)

Constituent

Radionuclides

Gross alpha

Lead-212

Manganese-54

Neptunium-239

Nonvolatile beta

Plutonium-238

Plutonium-239/240

Potassium-40

Promethium-144

Promethium-146

Ruthenium-106

Sodium-22

Strontium-90

Thorium-234

Tin-113

Yttrium-88

Zinc-65

Zirconium-95
Sample ID: 104665

RQ AQ B Result Unit D. Limit Method

$\begin{array}{ll}1.99 E+00 & \text { EPIA-001B } \\ 1.20 E-02 & \text { EPIA-013B } \\ 7.19 E-03 & \text { EPIA-013B } \\ 4.69 E-02 & \text { EPIA-013B } \\ 2.61 E+00 & \text { EPIA-001B } \\ 5.06 E-02 & \text { EPIA-012B } \\ 1.79 E-02 & \text { EPIA-012B } \\ 6.35 E-02 & \text { EPIA-013B } \\ 6.92 E-03 & \text { EPIA-013B } \\ 9.17 E-03 & \text { EPIA-013B } \\ 6.58 E-02 & \text { EPIA-013B } \\ 6.01 E-03 & \text { EPIA-013B } \\ 1.11 E-01 & \text { EPIA-004 } \\ 3.40 E-01 & \text { EPIA-013B } \\ 9.99 E-03 & \text { EPIA-013B } \\ 7.57 E-03 & \text { EPIA-013B } \\ 1.36 E-02 & \text { EPIA-013B } \\ 1.72 E-02 & \text { EPIA-013B }\end{array}$

1.99E +00 EPIA-001B

$9.79 \mathrm{E}+00 \pm 2.34 \mathrm{E}+00 \mathrm{pCi} / \mathrm{g}$ $4.82 \mathrm{E}-01 \pm 1.88 \mathrm{E}-02 \mathrm{pCj} / \mathrm{g}$ 2.56E-03 $\pm 3.00 \mathrm{E}-02 \mathrm{pCi} / \mathrm{g}$ $6.05 \mathrm{E}+00 \pm 1.64 \mathrm{E}+00 \mathrm{pCi} / \mathrm{g}$ $-1.17 \mathrm{E}-02 \pm 1.90 \mathrm{E}-02 \mathrm{pCi} / \mathrm{g}$ 9.94E-03 $\pm 1.18 \mathrm{E}-02 \mathrm{pCi} / \mathrm{g}$ $5.65 \mathrm{E}-01 \pm 9.87 \mathrm{E}-02$ pCi $/ \mathrm{g}$ 1.66E-03 $43.86 \mathrm{E}-03 \mathrm{pCi} / \mathrm{g}$ 2.48E-03 $\pm 5.42 \mathrm{E}-03 \mathrm{pCi} / \mathrm{g}$ $1.42 \mathrm{E}-02 \pm 3.66 \mathrm{E}-02 \mathrm{pCi} / \mathrm{g}$ -3.75E-04 $\pm 3.30 \mathrm{E}-03 \mathrm{pCi} / \mathrm{g}$ $2.18 \mathrm{E}+00 \pm 2.08 \mathrm{E}-01 \mathrm{pCi} / \mathrm{g}$ 5.98E-01 $\pm 3.25 \mathrm{E}-01 \mathrm{pCi} / \mathrm{g}$ $-3.59 \mathrm{E}-03 \pm 6.17 \mathrm{E}-03 \mathrm{pCi} / \mathrm{g}$ -1.92E-03+4.37E-03 pCi/g $-4.56 \mathrm{E}-03 \pm 9.61 \mathrm{E}-03 \mathrm{pCi} / \mathrm{g}$ $0.00 \mathrm{E}+00 \quad \mathrm{pCi} / \mathrm{g}$

Sample ID: 104666

SURVEY ID: PPSC 1303

ates)

Survey location: $44128 \mathrm{E} 3669963 \mathrm{~N}$ (

Sample type: Normal

Sample matrix: Soil

Percent solids: $\mathbf{8 7 . 0 0}$

Constituent

RQ AQ B Result

Unit

D. Limit Method

Physical Parameters and Specified Analyses

Cation exchange capacity

Chloride

Cyanide

Nitrate-nitrite as nitrogen

Orthophosphate

Phenols

Sulfide

Total organic carbon

Total organic nitrogen

Total phosphates (as P)

Metals (total recoverable)

Aluminum

Antimony

Arsenic

Barium

Beryllium

Cadmium

Calcium

Chromium

Cobalt

Copper

$\begin{array}{lll} & & 80.3 \\ \mathrm{~J} & \mathrm{QE} & 1.61 \\ \mathrm{U} & & 1.15 \\ \mathrm{U} & & 1.14 \\ \mathrm{U} & & 11.4 \\ \mathrm{~J} & \mathrm{E} & 191 \\ \mathrm{UJ} & \mathrm{C} & 287 \\ & & 5660 \\ \mathrm{C} & & 162 \\ \mathrm{~J} & \mathrm{E} & 99.2\end{array}$

$\mathrm{meq} / 100 \mathrm{~g}$

$\mathrm{mg} / \mathrm{kg}$

$\mathrm{mg} / \mathrm{kg}$

$\mathrm{mg} / \mathrm{kg}$

$\mathrm{mg} / \mathrm{kg}$

$\mu \mathrm{g} / \mathrm{kg}$

$\mathrm{mg} / \mathrm{kg}$

$\mathrm{mg} / \mathrm{kg}$

$\mathrm{mg} / \mathrm{kg}$

$\mathrm{mg} / \mathrm{kg}$

5

2.85

1.15

1.14

11.4

343

287

115

0.23

455

$\mathrm{mg} / \mathrm{kg}$

$\mathrm{mg} / \mathrm{kg}$

$\mathrm{mg} / \mathrm{kg}$

$\mathrm{mg} / \mathrm{kg}$

$\mathrm{mg} / \mathrm{kg}$

$\mathrm{mg} / \mathrm{kg}$

$\mathrm{mg} / \mathrm{kg}$

$\mathrm{mg} / \mathrm{kg}$

$\mathrm{mg} / \mathrm{kg}$

0.65

$\begin{array}{lll}\mathrm{U} & \mathrm{V} & 1.68\end{array}$

$\mathrm{mg} / \mathrm{kg}$
14.4

5.75

17.2

1.44

0.575

0.287

14.4

1.44

0.575

1.44
EPA9081

EPA300.0

EPA335.3

EPA353.1

EPA365.2

EPA420.2

EPA376.2

EPA415.1

EPA351.2

EPA365.4

EPA6010A EPA6010A EPA6010A

EPA6010A

EPA6010A

EPA6010A

EPA6010A

EPA6010A

EPA6010A

EPA6010A 
SURVEY ID: PPSC 1303 (continued)

\section{Constituent}

Metals (total recoverable)

Iron

Lead

Magnesium

Manganese

Mercury

Nickel

Potassium

Selenium

Silver

Sodium

Thallium

Vanadium

Zinc

\section{Radionuclides}

Actinium-228

Antimony-124

Antimony-125

Barium-133

Cerium-144

Cesium-134

Cesium-137

Cobalt-57

Cobalt-58

Cobalt-60

Europium-152

Europium-154

Europium-155

Gross alpha

Lead-212

Manganese-54

Neptunium-239

Nonvolatile beta

Plutonium-238

Plutonium-239/240

Potassium-40

Promethium-144

Promethium-146

Ruthenium-106

Sodium-22

Strontium-90

Thorium-234

Tin-113

Yttrium-88

Zinc-65

Zirconium-95
Sample ID: 104666

RQ AQ B Result

Unit

D. Limit Method

$\begin{array}{lll}\mathrm{mg} / \mathrm{kg} & 5.75 & \text { EPA6010A } \\ \mathrm{mg} / \mathrm{kg} & \mathbf{5 . 7 5} & \text { EPA6010A } \\ \mathrm{mg} / \mathrm{kg} & \mathbf{5 . 7 5} & \text { EPA6010A } \\ \mathrm{mg} / \mathrm{kg} & \mathbf{0 . 5 7 5} & \text { EPA6010A } \\ \mathrm{mg} / \mathrm{kg} & \mathbf{0 . 0 3 4 6} & \text { EPA7471 } \\ \mathrm{mg} / \mathrm{kg} & 1.44 & \text { EPA6010A } \\ \mathrm{mg} / \mathrm{kg} & 115 & \text { EPA6010A } \\ \mathrm{m} / \mathrm{kg} & 17.2 & \text { EPA6010A } \\ \mathrm{mg} / \mathrm{kg} & 1.44 & \text { EPA6010A } \\ \mathrm{mg} / \mathrm{kg} & \mathbf{5 7 . 5} & \text { EPA6010A } \\ \mathrm{mg} / \mathrm{kg} & 17.2 & \text { EPA6010A } \\ \mathrm{mg} / \mathrm{kg} & \mathbf{0 . 5 7 5} & \text { EPA6010A } \\ \mathrm{mg} / \mathrm{kg} & 1.15 & \text { EPA6010A }\end{array}$

2.36E-02 EPIA-013B

9.63E-03 EPIA-013B

2.04E-02 EPIA-013B

8.28E-03 EPIA-013B

5.47E-02 EPIA-013B

6.76E-03 EPIA-013B

7.14E-03 EPIA-013B

6.76E-03 EPIA.013B

7.52E-03 EPIA-013B

6.01E-03 EPIA-013B

1.93E-02 EPIA-013B

5.70E-02 EPLA-013B

2.81E-02 EPIA-013B

2.18E+00 EPIA-001B

1.21E-02 EPIA-013B

6.77E-03 EPIA-013B

4.82E-02 EPIA-013B

2.44E+00 EPIA-001B

4.02E-02 EPIA-012B

1.60E-02 EPIA-012B

6.21E-02 EPIA-013B

6.65E-03 EPIA-013B

1.05E-02 EPIA-013B

6.51E-02 EPIA-013B

6.15E-03 EPIA-013B

6.82E-02 EPIA-004

3.60E-01 EPIA-013B

1.04E-02 EPIA-013B

7.75E-03 EPIA-013B

1.38E-02 EPIA-013B

1.63E-02 EPIA.013B

SURVEY ID: PPSC 1304

Sample ID: 104667

Survey location: $44128 \mathrm{E} 3669963 \mathrm{~N}$ (SRS coordinates)

Depth of core interval: 4.00 to $6.00 \mathrm{ft}$

Sample type: Normal

Sample matrix: Soil

Percent solids: $\mathbf{8 6 . 0 0}$ 
SURVEY ID: PPSC 1304 (continued)

Constituent

RQ AQ B Result

Sample ID: 104667

Physical Parameters and Specified Analyses

$\begin{array}{lll} & & 47.4 \\ J & E & 1.5 \\ U & & 1.12 \\ J & E & 0.795 \\ J \cdot & E & 2.49 \\ \text { J } & \text { E } & 233 \\ \text { UJ } & \text { C } & 291 \\ & & 574 \\ \text { C } & & 67.3 \\ \text { J } & \text { E } & 130\end{array}$

Metals (total recoverable)
Unit

D. Limit Method
Cation exchange capacity

Chloride

Nitrate-nitrite as nitrogen

Orthophosphate

Phenols

Sulfide

Total organic carbon

Total organic nitrogen

Total phosphates (as P)

Aluminum

Aluminum

Antimony

Antimony

Arsenic

Arsenic

Barium

Barium

Beryllium

Beryllium

Cadmium

Cadmium

Calcium

Calcium

Chromium

Chromium

Cobalt

Cobalt

Copper

Copper

Iron

Iron

Lead

Lead

Magnesium

Magnesium

Manganese

Manganese

Mercury

Nickel

Nickel

Potassium

Potassium

Selenium

Selenium

Silver

Silver

Sodium

Sodium

Thallium

Thallium

Vanadium
J CX 3810

J CX 2510

UJ I $\quad$ L 5.48

UJ I $\quad$ L 5.48

U $\quad 16.5$

U $\quad 16.5$

25.6

23.1

J $\mathrm{E} \quad 0.123$

J E 0.105

U $\quad 0.274$

U $\quad 0.274$

. V 101

V $\quad 75.7$

4.14

2.32

0.891

0.861

$\begin{array}{lll}\text { U } & \mathrm{V} & 2.1\end{array}$

$\begin{array}{lll}U & V & 1.81\end{array}$

J VCX 4680

$J \quad V C X \quad 2650$

$\mathrm{U} \quad 5.48$

U $\quad 5.48$

$\begin{array}{llll}J & \text { IX } & \text { L } & 86\end{array}$

J IX L $\quad \mathbf{5 5 . 4}$

63.2

58.6

$\begin{array}{lll}\mathrm{J} & \mathrm{E} & \mathbf{0 . 0 2 9}\end{array}$

1.69

$\mathrm{J} \quad \mathrm{E} \quad 1.18$

$\mathrm{J} \quad \mathrm{E} \quad 54.6$

$\mathrm{U} \quad 16.5$

U $\quad 16.5$

U $\quad 1.37$

U $\quad 1.37$

$\begin{array}{lll}J & E & 8.83\end{array}$

U $\quad 54.8$

UJ $\mathrm{C} \quad 16.5$

UJ $\quad \mathrm{C} \quad 16.5$

$\begin{array}{lll}\mathrm{meq} / 100 \mathrm{~g} & 5 & \text { EPA9081 } \\ \mathrm{mg} / \mathrm{kg} & 2.98 & \text { EPA300.0 } \\ \mathrm{mg} / \mathrm{kg} & 1.12 & \text { EPA335.3 } \\ \mathrm{mg} / \mathrm{kg} & 1.14 & \text { EPA353.1 } \\ \mathrm{mg} / \mathrm{kg} & 11.6 & \text { EPA365.2 } \\ \mu \mathrm{g} / \mathrm{kg} & 348 & \text { EPA420.2 } \\ \mathrm{mg} / \mathrm{kg} & 291 & \text { EPA376.2 } \\ \mathrm{mg} / \mathrm{kg} & 116 & \text { EPA415.1 } \\ \mathrm{mg} / \mathrm{kg} & 0.233 & \text { EPA351.2 } \\ \mathrm{mg} / \mathrm{kg} & 456 & \text { EPA365.4 }\end{array}$

$\mathrm{mg} / \mathrm{kg}$

$13.7^{\circ}$

EPA6010A

$\mathrm{mg} / \mathrm{kg}$

$\mathrm{mg} / \mathrm{kg}$

$\mathrm{mg} / \mathrm{kg}$

$\mathrm{mg} / \mathrm{kg}$

$\mathrm{mg} / \mathrm{kg}$

$\mathrm{mg} / \mathrm{kg}$

$\mathrm{mg} / \mathrm{kg}$

$\mathrm{mg} / \mathrm{kg}$

$\mathrm{mg} / \mathrm{kg}$

$\mathrm{mg} / \mathrm{kg}$

$\mathrm{mg} / \mathrm{kg}$

$\mathrm{mg} / \mathrm{kg}$

$\mathrm{mg} / \mathrm{kg}$

$\mathrm{mg} / \mathrm{kg}$

$\mathrm{mg} / \mathrm{kg}$

$\mathrm{mg} / \mathrm{kg}$

$\mathrm{mg} / \mathrm{kg}$

$\mathrm{mg} / \mathrm{kg}$

$\mathrm{mg} / \mathrm{kg}$

$\mathrm{mg} / \mathrm{kg}$

$\mathrm{mg} / \mathrm{kg}$

$\mathrm{mg} / \mathrm{kg}$

$\mathrm{mg} / \mathrm{kg}$

$\mathrm{mg} / \mathrm{kg}$

$\mathrm{mg} / \mathrm{kg}$

$\mathrm{mg} / \mathrm{kg}$

$\mathrm{mg} / \mathrm{kg}$

$\mathrm{mg} / \mathrm{kg}$

$\mathrm{mg} / \mathrm{kg}$

$\mathrm{mg} / \mathrm{kg}$

$\mathrm{mg} / \mathrm{kg}$

$\mathrm{mg} / \mathrm{kg}$

$\mathrm{mg} / \mathrm{kg}$

$\mathrm{mg} / \mathrm{kg}$

$\mathrm{mg} / \mathrm{kg}$

$\mathrm{mg} / \mathrm{kg}$

$\mathrm{mg} / \mathrm{kg}$

$\mathrm{mg} / \mathrm{kg}$

$\mathrm{mg} / \mathrm{kg}$

$\mathrm{mg} / \mathrm{kg}$

$\mathrm{mg} / \mathrm{kg}$
13.7

5.48

5.48

16.5

16.5

1.37

1.37

0.548

0.548

0.274

0.274

13.7

13.7

1.37

1.37

0.548

0.548

1.37

1.37

5.48

5.48

5.48

5.48

5.48

5.48

0.548

0.548

0.0384

1.37

1.37

110

110

16.5

16.5

1.37

1.37

54.8

- 54.8

16.5

16.5

0.548
EPA6010A

EPA6010A

EPA6010A

EPA6010A

EPA6010A

EPA6010A

EPA6010A

EPA6010A

EPA6010A

EPA6010A

EPA6010A

EPA6010A

EPA6010A

EPA6010A

EPA6010A

EPA6010A

EPA6010A

EPA6010A

EPA6010A

EPA6010A

EPA6010A

EPA6010A

EPA6010A

EPA6010A

EPA6010A

EPA6010A

EPA6010A

EPA7471

EPA6010A

EPA6010A

EPA6010A

EPA6010A

EPA6010A

EPA6010A

EPA6010A

EPA6010A

EPA6010A

EPA6010A

EPA6010A

EPA6010A

EPA6010A 
SURVEY ID: PPSC 1304 (continued)

Constituent

Metals (total recoverable)

Vanadium

Zinc

Zinc

Radionuclides

Actinium-228

Antimony-124

Antimony-125

Barium-133

Cerium-144

Cesium-134

Cesium-137

Cobalt-57

Cobalt. 58

Cobalt-60

Europium-152

Europium-154

Europium-155

Gross alpha

Lead-212

Manganese-54

Neptunium-239

Nonvolatile beta

Plutonium-238

Plutonium-239/240

Potassium-40

Promethium-144

Promethium-146

Ruthenium-106

Sodium-22

Strontium-90

Thorium-234

Tin-113

Yttrium-88

Zinc-65

Zirconium-95
Sample ID: 104667

RQ AQ B Result

Unit

D. Limit Method

$\begin{array}{llllll} & \mathrm{X} & 5.94 & \mathrm{mg} / \mathrm{kg} & 0.548 & \text { EPA6010A } \\ & \mathrm{V} & 2.74 & \mathrm{mg} / \mathrm{kg} & 1.1 & \text { EPA6010A } \\ \mathrm{U} & \mathrm{V} & 2.29 & \mathrm{mg} / \mathrm{kg} & 1.1 & \text { EPA6010A }\end{array}$

8.06E-01 $\pm 4.71 \mathrm{E}-02 \mathrm{pCj} / \mathrm{g}$ 2.52E-05 $\pm 5.65 \mathrm{E}-03 \mathrm{pCi} / \mathrm{g}$ $3.81 \mathrm{E}-03 \pm 1.17 \mathrm{E}-02 \mathrm{pCi} / \mathrm{g}$ $4.56 \mathrm{E}-04 \pm 6.06 \mathrm{E}-03 \mathrm{pCi} / \mathrm{g}$ $2.28 \mathrm{E}-03 \pm 3.54 \mathrm{E}-02 \mathrm{pCi} / \mathrm{g}$ $1.83 \mathrm{E}-03 \pm 4.50 \mathrm{E}-03 \mathrm{pCi} / \mathrm{g}$ $1.60 \mathrm{E}-03 \pm 7.34 \mathrm{E}-03 \mathrm{pCi} / \mathrm{g}$ $-3.10 \mathrm{E}-03 \pm 4.44 \mathrm{E}-03 \mathrm{pCi} / \mathrm{g}$ 6.62E-04 $0.04 \mathrm{E}-03 \mathrm{pCi} / \mathrm{g}$ $-1.30 \mathrm{E}-03 \pm 3.78 \mathrm{E}-03 \mathrm{pCi} / \mathrm{g}$ $-1.40 \mathrm{E}-02 \pm 1.31 \mathrm{E}-02 \mathrm{pCi} / \mathrm{g}$ $-3.73 \mathrm{E}-02 \pm 3.80 \mathrm{E}-02 \mathrm{pCi} / \mathrm{g}$ $0.00 \mathrm{E}+00 \quad \mathrm{pCi} / \mathrm{g}$ $1.43 \mathrm{E}+01 \pm 2.89 \mathrm{E}+00 \mathrm{pCi} / \mathrm{g}$ 8.30E-01 $\pm 2.26 \mathrm{E}-02 \mathrm{pCi} / \mathrm{g}$ $6.83 \mathrm{E}-03 \pm 5.73 \mathrm{E}-03 \mathrm{pCi} / \mathrm{g}$ 3.53E-02 $\pm 5.09 \mathrm{E}-02 \mathrm{pCi} / \mathrm{g}$ $8.17 \mathrm{E}+00 \pm 1.80 \mathrm{E}+00 \mathrm{pCi} / \mathrm{g}$ $-6.92 \mathrm{E}-03 \pm 1.72 \mathrm{E}-02 \mathrm{pCi} / \mathrm{g}$ $-5.35 \mathrm{E}-05 \pm 9.30 \mathrm{E}-03 \mathrm{pCi} / \mathrm{g}$ 8.89E-01 $1.15 \mathrm{E}-01 \mathrm{pCi} / \mathrm{g}$ $-2.04 \mathrm{E}-03 \pm 4.57 \mathrm{E}-03 \mathrm{pCj} / \mathrm{g}$ 4.46E-03 $\pm 5.49 \mathrm{E}-03 \mathrm{pCi} / \mathrm{g}$ 7.33E-03 $\pm 4.01 \mathrm{E}-02 \mathrm{pCi} / \mathrm{g}$ $1.55 \mathrm{E}-03 \pm 3.89 \mathrm{E}-03 \mathrm{pCi} / \mathrm{g}$ $2.17 \mathrm{E}+00 \pm 2.08 \mathrm{E}-01 \mathrm{pCj} / \mathrm{g}$ $1.12 \mathrm{E}+00 \pm 5.74 \mathrm{E}-01 \mathrm{pCi} / \mathrm{g}$ $-3.25 \mathrm{E}-03 \pm 6.25 \mathrm{E}-03 \mathrm{pCi} / \mathrm{g}$ $-1.45 \mathrm{E}-03 \pm 4.57 \mathrm{E}-03 \mathrm{pCi} / \mathrm{g}$ $6.34 \mathrm{E}-04 \pm 1.45 \mathrm{E}-02 \mathrm{pCi} / \mathrm{g}$ $1.37 \mathrm{E}-02 \pm 1.26 \mathrm{E}-02 \mathrm{pCi} / \mathrm{g}$
2.50E-02 EPIA-013B

9.69E-03 EPIA-013B

2.07E-02 EPIA-013B

8.85E-03 EPIA-013B

6.05E-02 EPIA-013B

6.89E-03 EPIA-013B

7.52E-03 EPIA-013B

9.02E-03 EPIA.013B

6.48E-03 EPIA-013B

2.06E-02 EPIA-013B

6.35E-02 EPIA-013B

3.05E-02 EPIA-013B

1.46E+00 EPIA-001B

1.30E-02 EPIA-013B

8.53E-03 EPIA-013B

5.37E-02 EPIA-013B

2.40E+00 EPIA-001B

4.45E-02 EPIA-012B

2.57E-02 EPIA-012B

6.65E-02 EPIA-013B

7.61E-03 EPIA-013B

9.80E-03 EPIA-013B

6.93E-02 EPIA-013B

7.05E-03 EPIA-013B

1.10E-01 EPIA-004

3.99E-01 EPIA-013B

1.08E-02 EPIA-013B

8.10E-03 EPIA-013B

1.50E-02 EPIA-013B

1.99E-02 EPIA-013B
8.10E-03 EPIA-013B

SURVEY ID: PPSC 1401

Sample ID: 105109

Survey location: $44071 \mathrm{E} 3670210 \mathrm{~N}$ (SRS coordinates)

Depth of core interval: 0.00 to $1.00 \mathrm{ft}$

Sample type: Normal

Sample matrix: Soil

Percent solids: 91.00 
SURVEY ID: PPSC 1401 (continued)

Constituent

RQ AQ B Result

Physical Parameters and Specified Analyses

Ammonia nitrogen

Cation exchange capacity

Chloride

Cyanide

Nitrate-nitrite as nitrogen

Nitrogen by Kjeldahl method

Orthophosphate

Phenols

Sulfide

Total organic carbon

Total organic nitrogen

Total phosphates (as P)

Metals (total recoverable)

\begin{tabular}{|c|c|c|c|}
\hline Aluminum & & $\mathrm{V}$ & 1020 \\
\hline Antimony & $\mathrm{U}$ & & 5.02 \\
\hline Arsenic & $\mathrm{U}$ & & 15.1 \\
\hline Barium & $\mathbf{J}$ & $\mathrm{C}$ & 5.58 \\
\hline Beryllium & $\mathbf{J}$ & $\mathrm{EC}$ & 0.0437 \\
\hline Cadmium & $\mathrm{U}$ & & 0.251 \\
\hline Calcium & & $\mathrm{V}$ & 37.4 \\
\hline Chromium & & & 1.73 \\
\hline Cobalt & $\mathrm{J}$ & $\mathbf{E}$ & 0.199 \\
\hline Copper & J & $\mathrm{E}$ & 0.566 \\
\hline Iron & $J$ & $\mathrm{C}$ & 1040 \\
\hline Lead & $\mathrm{U}$ & & 5.02 \\
\hline Magnesium & & & 34.6 \\
\hline Manganese & & & 7.2 \\
\hline Mercury & $\mathrm{U}$ & & 0.0296 \\
\hline Nickel & $J$ & $\mathrm{E}$ & 0.568 \\
\hline Potassium & $\mathbf{J}$ & $\mathrm{E}$ & 30.7 \\
\hline Selenium & $\mathrm{U}$ & & 15.1 \\
\hline Silver & $\mathrm{U}$ & & 1.26 \\
\hline Sodium & $\mathrm{U}$ & & 50.2 \\
\hline Thallium & $\mathrm{U}$ & & 15.1 \\
\hline Vanadium & $\mathbf{J}$ & $\mathrm{C}$ & 2.95 \\
\hline Zinc & & $\mathrm{V}$ & 1.71 \\
\hline
\end{tabular}

$B / N / A$ Extractables (including $P A H$ and phenols)

Acenaphthene

Acenaphthylene

Anthracene

Benzo[a]anthracene

Benzo[b]fluoranthene

Benzo[k]fluoranthene

Benzoic acid

Benzo $[g, h, i]$ perylene

Benzo[a]pyrene

Benzyl alcohol

Bis(2-chloroethoxy) methane

Bis(2-chloroethyl) ether

Bis(2-chloroisopropyl) ether

Bis(2-ethylhexyl) phthalate
U

U

U

U V

U

UJ C

UJ C

$\begin{array}{ll}\mathrm{C} & 823 \\ & 27\end{array}$

$\begin{array}{lll}\mathrm{J} & \mathrm{E} & \mathbf{3 . 7 2}\end{array}$
21.7

10.6

2.78

30.9

11

216

275

823
36.3

36.3

36.3

3.63

3.63

3.63

36.3

36.3

3.63

36.3

36.3

36.3

36.3

20.7
Unit

D. Limit Method

$\begin{array}{lll}\mathrm{mg} / \mathrm{kg} & 21.7 & \text { EPA350.1 } \\ \mathrm{meq} / 100 \mathrm{~g} & 5 & \text { EPA9081 } \\ \mathrm{mg} / \mathrm{kg} & 2.78 & \text { EPA300.0 } \\ \mathrm{mg} / \mathrm{kg} & 1.04 & \text { EPA335.3 } \\ \mathrm{mg} / \mathrm{kg} & 1.06 & \text { EPA353.1 } \\ \mathrm{mg} / \mathrm{kg} & 4.39 & \text { EPA351.2 } \\ \mathrm{mg} / \mathrm{kg} & 11 & \text { EPA365.2 } \\ \mu \mathrm{g} / \mathrm{kg} & 216 & \text { EPA420.2 } \\ \mathrm{mg} / \mathrm{kg} & 275 & \text { EPA376.2 } \\ \mathrm{mg} / \mathrm{kg} & 110 & \text { EPA415.1 } \\ \mathrm{mg} / \mathrm{kg} & 0.22 & \text { EPA351.2 } \\ \mathrm{mg} / \mathrm{kg} & 10.9 & \text { EPA365.4 }\end{array}$

$\begin{array}{lll}\mathrm{mg} / \mathrm{kg} & 12.6 & \text { EPA6010A } \\ \mathrm{mg} / \mathrm{kg} & 5.02 & \text { EPA6010A } \\ \mathrm{mg} / \mathrm{kg} & 15.1 & \text { EPA6010A } \\ \mathrm{mg} / \mathrm{kg} & 1.26 & \text { EPA6010A } \\ \mathrm{mg} / \mathrm{kg} & 0.502 & \text { EPA6010A } \\ \mathrm{mg} / \mathrm{kg} & 0.251 & \text { EPA6010A } \\ \mathrm{mg} / \mathrm{kg} & 12.6 & \text { EPA6010A } \\ \mathrm{mg} / \mathrm{kg} & 1.26 & \text { EPA6010A } \\ \mathrm{mg} / \mathrm{kg} & 0.502 & \text { EPA6010A } \\ \mathrm{mg} / \mathrm{kg} & 1.26 & \text { EPA6010A } \\ \mathrm{mg} / \mathrm{kg} & 5.02 & \text { EPA6010A } \\ \mathrm{mg} / \mathrm{kg} & 5.02 & \text { EPA6010A } \\ \mathrm{mg} / \mathrm{kg} & 5.02 & \text { EPA6010A } \\ \mathrm{mg} / \mathrm{kg} & 0.502 & \text { EPA6010A } \\ \mathrm{mg} / \mathrm{kg} & 0.0296 & \text { EPA7471 } \\ \mathrm{mg} / \mathrm{kg} & 1.26 & \text { EPA6010A } \\ \mathrm{mg} / \mathrm{kg} & 100 & \text { EPA6010A } \\ \mathrm{mg} / \mathrm{kg} & 15.1 & \text { EPA6010A } \\ \mathrm{mg} / \mathrm{kg} & 1.26 & \text { EPA6010A } \\ \mathrm{mg} / \mathrm{kg} & 50.2 & \text { EPA6010A } \\ \mathrm{mg} / \mathrm{kg} & 15.1 & \text { EPA6010A } \\ \mathrm{mg} / \mathrm{kg} & 0.502 & \text { EPA6010A } \\ \mathrm{mg} / \mathrm{kg} & 1 & \text { EPA6010A }\end{array}$

$\mu \mathrm{g} / \mathrm{kg}$

$\mu \mathrm{g} / \mathrm{kg}$

$\mu \mathrm{g} / \mathbf{k g}$

$\mu \mathrm{g} / \mathrm{kg}$

$\mu \mathrm{g} / \mathrm{kg}$

$\mu \mathrm{g} / \mathrm{kg}$

$\mu \mathrm{g} / \mathrm{kg}$

$\mu \mathrm{g} / \mathrm{kg}$

$\mu \mathrm{g} / \mathrm{kg}$

$\mu \mathrm{g} / \mathrm{kg}$

$\mu \mathrm{g} / \mathrm{kg}$

$\mu \mathrm{g} / \mathrm{kg}$

$\mu \mathrm{g} / \mathrm{kg}$

$\mu \mathrm{g} / \mathrm{kg}$
36.3

36.3

36.3

3.63

3.63

3.63

36.3

36.3

3.63

36.3

36.3

36.3

36.3

36.3
EPA8270

EPA8270

EPA8270

EPA8270

EPA8270

EPA8270

EPA8270

EPA8270

EPA8270

EPA8270

EPA8270

EPA8270

EPA8270

EPA8270 
SURVEY ID: PPSC 1401 (continued)

Constituent

RQ AQ B Result

Sample ID: 105109

$B / N / A$ Extractables (including PAH and phenols)

4-Bromophenyl phenyl ether

Butylbenzyl phthalate

4-Chloroaniline

4-Chloro-m-cresol

2-Chloronaphthalene

2-Chlorophenol

4-Chlorophenyl phenyl ether

Chrysene

$\mathrm{m} / \mathrm{p}$-Cresol

o-Cresol (2-Methylphenol)

Dibenz $[a, h]$ anthracene

Dibenzofuran

Di-n-butyl phthalate

1,2-Dichlorobenzene

1,3-Dichlorobenzene

1,4-Dichlorobenzene

3,3'-Dichlorobenzidine

2,4-Dichlorophenol

Diethyl phthalate

2,4-Dimethyl phenol

Dimethyl phthalate

2,4-Dinitrophenol

2,4-Dinitrotoluene

2,6-Dinitrotoluene

Di-n-octyl phthalate

Fluoranthene

Fluorene

Hexachlorobenzene

Hexachlorobutadiene

Hexachlorocyclopentadiene

Hexachloroethane

Indeno[1,2,3-c,d]pyrene

Isophorone

2-Methyl-4,6-dinitrophenol

2-Methylnaphthalene

Naphthalene

m-Nitroaniline

o-Nitroaniline

p-Nitroaniline

Nitrobenzene

2-Nitrophenol

4-Nitrophenol

N-Nitrosodiphenylamine

N-Nitrosodi-n-propylamine

Pentachlorophenol

Phenanthrene

Phenol

Pyrene

1,2,4-Trichlorobenzene

2,4,5-Trichlorophenol

2,4,6-Trichlorophenol

\begin{tabular}{|c|c|c|}
\hline U & & 36.3 \\
\hline U & & 36.3 \\
\hline $\mathbf{U}$ & & 36.3 \\
\hline $\mathrm{U}$ & & 36.3 \\
\hline $\mathrm{U}$ & & 36.3 \\
\hline $\mathrm{U}$ & & 36.3 \\
\hline $\mathbf{U}$ & & 36.3 \\
\hline U & & 3.63 \\
\hline $\mathrm{U}$ & & 363 \\
\hline $\mathbf{U}$ & & 36.3 \\
\hline $\mathrm{U}$ & & 3.63 \\
\hline U & & 36.3 \\
\hline $\mathrm{U}$ & V & 28.3 \\
\hline U & & 36.3 \\
\hline $\mathrm{U}$ & & 36.3 \\
\hline$U$ & & 36.3 \\
\hline U & & 36.3 \\
\hline U & & 36.3 \\
\hline U & & 36.3 \\
\hline $\mathrm{U}$ & & 36.3 \\
\hline$U$ & & 36.3 \\
\hline$U$ & & 363 \\
\hline UJ & C & 3.63 \\
\hline U & & 3.63 \\
\hline U & & 36.3 \\
\hline$U$ & & 36.3 \\
\hline$U$ & & 36.3 \\
\hline U & & 3.63 \\
\hline $\mathrm{U}$ & & 36.3 \\
\hline$U$ & & 36.3 \\
\hline$U$ & & 3.63 \\
\hline$U$ & & 3.63 \\
\hline$U$ & & 36.3 \\
\hline U & & 363 \\
\hline$U$ & & 36.3 \\
\hline$U$ & & 36.3 \\
\hline U & & 36.3 \\
\hline$U$ & & 36.3 \\
\hline$U$ & & 36.3 \\
\hline$U$ & & 36.3 \\
\hline U & & 36.3 \\
\hline$U$ & & 36.3 \\
\hline $\mathrm{U}$ & & 36.3 \\
\hline U & & 36.3 \\
\hline$U$ & & 36.3 \\
\hline$U$ & & 36.3 \\
\hline$U$ & & 36.3 \\
\hline $\boldsymbol{U}$ & & 36.3 \\
\hline $\mathrm{U}$ & & 3.63 \\
\hline$U$ & & 36.3 \\
\hline U & & 36.3 \\
\hline
\end{tabular}

Unit

D. Limit Method

\begin{tabular}{|c|c|c|}
\hline$\mu \mathrm{g} / \mathrm{kg}$ & 36.3 & EPA8270 \\
\hline$\mu \mathrm{g} / \mathrm{kg}$ & 36.3 & EPA8270 \\
\hline$\mu \mathrm{g} / \mathrm{kg}$ & 36.3 & EPA8270 \\
\hline$\mu \mathrm{g} / \mathrm{kg}$ & 36.3 & EPA8270 \\
\hline$\mu \mathrm{g} / \mathrm{kg}$ & 36.3 & EPA8270 \\
\hline$\mu \mathrm{g} / \mathrm{kg}$ & 36.3 & EPA8270 \\
\hline$\mu \mathrm{g} / \mathrm{kg}$ & 36.3 & EPA8270 \\
\hline$\mu \mathrm{g} / \mathrm{kg}$ & 3.63 & EPA8270 \\
\hline$\mu \mathrm{g} / \mathrm{kg}$ & 363 & EPA8270 \\
\hline$\mu \mathrm{g} / \mathrm{kg}$ & 36.3 & EPA8270 \\
\hline$\mu \mathrm{g} / \mathrm{kg}$ & 3.63 & EPA8270 \\
\hline$\mu \mathrm{g} / \mathrm{kg}$ & 36.3 & EPA8270 \\
\hline$\mu \mathrm{g} / \mathrm{kg}$ & 36.3 & EPA8270 \\
\hline$\mu \mathrm{g} / \mathrm{kg}$ & 36.3 & EPA8270 \\
\hline$\mu \mathrm{g} / \mathrm{kg}$ & 36.3 & EPA8270 \\
\hline$\mu \mathrm{g} / \mathrm{kg}$ & 36.3 & EPA8270 \\
\hline$\mu \mathrm{g} / \mathrm{kg}$ & 36.3 & EPA8270 \\
\hline$\mu \mathrm{g} / \mathrm{kg}$ & 36.3 & EPA8270 \\
\hline$\mu \mathrm{g} / \mathrm{kg}$ & 36.3 & EPA8270 \\
\hline$\mu \mathrm{g} / \mathrm{kg}$ & 36.3 & EPA8270 \\
\hline$\mu \mathrm{g} / \mathrm{kg}$ & 36.3 & EPA8270 \\
\hline$\mu \mathrm{g} / \mathrm{kg}$ & 363 & EPA8270 \\
\hline$\mu \mathrm{g} / \mathrm{kg}$ & 3.63 & EPA8270 \\
\hline$\mu \mathrm{g} / \mathrm{kg}$ & 3.63 & EPA8270 \\
\hline$\mu \mathrm{g} / \mathrm{kg}$ & 36.3 & EPA8270 \\
\hline$\mu \mathrm{g} / \mathrm{kg}$ & 36.3 & EPA8270 \\
\hline$\mu \mathrm{g} / \mathrm{kg}$ & 36.3 & EPA8270 \\
\hline$\mu \mathrm{g} / \mathrm{kg}$ & 3.63 & EPA8270 \\
\hline$\mu \mathrm{g} / \mathrm{kg}$ & 36.3 & EPA8270 \\
\hline$\mu \mathrm{g} / \mathrm{kg}$ & 36.3 & EPA8270 \\
\hline$\mu \mathrm{g} / \mathrm{kg}$ & 3.63 & EPA8270 \\
\hline$\mu \mathrm{g} / \mathrm{kg}$ & 3.63 & EPA8270 \\
\hline$\mu \mathrm{g} / \mathrm{kg}$ & 36.3 & EPA8270 \\
\hline$\mu \mathrm{g} / \mathrm{kg}$ & 363 & EPA8270 \\
\hline$\mu \mathrm{g} / \mathrm{kg}$ & 36.3 & EPA8270 \\
\hline$\mu \mathrm{g} / \mathrm{kg}$ & 36.3 & EPA8270 \\
\hline$\mu \mathrm{g} / \mathrm{kg}$ & 36.3 & EPA8270 \\
\hline$\mu \mathrm{g} / \mathrm{kg}$ & 36.3 & EPA8270 \\
\hline$\mu \mathrm{g} / \mathrm{kg}$ & 36.3 & EPA8270 \\
\hline$\mu \mathrm{g} / \mathrm{kg}$ & 36.3 & EPA8270 \\
\hline$\mu \mathrm{g} / \mathrm{kg}$ & 36.3 & EPA8270 \\
\hline$\mu \mathrm{g} / \mathrm{kg}$ & 36.3 & EPA8270 \\
\hline$\mu \mathrm{g} / \mathrm{kg}$ & 36.3 & EPA8270 \\
\hline$\mu \mathrm{g} / \mathrm{kg}$ & 36.3 & EPA8270 \\
\hline$\mu \mathrm{g} / \mathrm{kg}$ & 36.3 & EPA8270 \\
\hline$\mu \mathrm{g} / \mathrm{kg}$ & 36.3 & EPA8270 \\
\hline$\mu \mathrm{g} / \mathrm{kg}$ & 36.3 & EPA8270 \\
\hline$\mu \mathrm{g} / \mathrm{kg}$ & 36.3 & EPA8270 \\
\hline$\mu \mathrm{g} / \mathrm{kg}$ & 3.63 & EPA8270 \\
\hline$\mu \mathrm{g} / \mathrm{kg}$ & 36.3 & EPA8270 \\
\hline$\mu \mathrm{g} / \mathrm{kg}$ & 36.3 & EPA8270 \\
\hline
\end{tabular}


SURVEY ID: PPSC 1401 (continued)

Constituent

Volatile Organic Compounds

Acetone

Benzene

Bromodichloromethane

Bromoform

Bromomethane (Methyl bromide)

Carbon disulfide

Carbon tetrachloride

Chlorobenzene

Chloroethane

Chloroethene (Vinyl chloride)

Chloroform

Chloromethane (Methyl chloride)

Dibromochloromethane

1,1-Dichloroethane

1,2-Dichloroethane

1,1-Dichloroethylene

1,2-Dichloroethylene

Dichloromethane (Methylene chloride)

1,2-Dichloropropane

cis-1,3-Dichloropropene

trans-1,3-Dichloropropene

Ethylbenzene

2-Hexanone

Methyl ethyl ketone

Methyl isobutyl ketone

Styrene

1,1,2,2-Tetrachloroethane

Tetrachloroethylene

Toluene

1,1,1-Trichloroethane

1,1,2-Trichloroethane

Trichloroethylene

Vinyl acetate

Xylenes

Pesticides/PCBs and Dioxins/Furans
Sample ID: 105109

RQ AQ B Result Unit D. Limit Method

\begin{tabular}{|c|c|c|c|c|c|}
\hline$J$ & \multirow[t]{17}{*}{ Q8 } & 302 & $\mu \mathrm{g} / \mathrm{kg}$ & 54.9 & EPA8260 \\
\hline $\mathrm{U}$ & & 0.549 & $\mu \mathrm{g} / \mathrm{kg}$ & 0.549 & EPA8260 \\
\hline $\mathrm{U}$ & & 0.0549 & $\mu \mathrm{g} / \mathrm{kg}$ & 0.0549 & EPA8260 \\
\hline $\mathbf{U}$ & & 0.0549 & $\mu \mathrm{g} / \mathrm{kg}$ & 0.0549 & EPA8260 \\
\hline $\mathrm{U}$ & & 0.11 & $\mu \mathrm{g} / \mathrm{kg}$ & 0.11 & EPA8260 \\
\hline $\mathrm{U}$ & & 1.1 & $\mu \mathrm{g} / \mathrm{kg}$ & 1.1 & EPA8260 \\
\hline $\mathrm{U}$ & & 0.0549 & $\mu \mathrm{g} / \mathrm{kg}$ & 0.0549 & EPA8260 \\
\hline $\mathrm{U}$ & & 0.0549 & $\mu \mathrm{g} / \mathrm{kg}$ & 0.0549 & EPA8260 \\
\hline $\mathrm{U}$ & & 0.11 & $\mu \mathrm{g} / \mathrm{kg}$ & 0.11 & EPA8260 \\
\hline $\mathrm{U}$ & & 0.11 & $\mu \mathrm{g} / \mathrm{kg}$ & 0.11 & EPA8260 \\
\hline $\mathrm{U}$ & & 0.0549 & $\mu \mathrm{g} / \mathrm{kg}$ & 0.0549 & EPA8260 \\
\hline $\mathrm{U}$ & & 0.11 & $\mu \mathrm{g} / \mathrm{kg}$ & 0.11 & EPA8260 \\
\hline $\mathbf{U}$ & & 0.0549 & $\mu \mathrm{g} / \mathrm{kg}$ & 0.0549 & EPA8260 \\
\hline U & & 0.0549 & $\mu \mathrm{g} / \mathrm{kg}$ & 0.0549 & EPA8260 \\
\hline $\mathrm{U}$ & & 0.0549 & $\mu \mathrm{g} / \mathrm{kg}$ & 0.0549 & EPA8260 \\
\hline $\mathrm{U}$ & & 0.0549 & $\mu \mathrm{g} / \mathrm{kg}$ & 0.0549 & EPA8260 \\
\hline $\mathbf{U}$ & & 0.11 & $\mu \mathrm{g} / \mathrm{kg}$ & 0.11 & EPA8260 \\
\hline $\mathrm{U}$ & \multirow[t]{5}{*}{$\mathrm{V}$} & 2.03 & $\mu \mathrm{g} / \mathrm{kg}$ & 0.549 & EPA8260 \\
\hline $\mathrm{U}$ & & $0.0549^{\circ}$ & $\mu \mathrm{g} / \mathrm{kg}$ & 0.0549 & EPA8260 \\
\hline U & & 0.0549 & $\mu \mathrm{g} / \mathrm{kg}$ & 0.0549 & EPA8260 \\
\hline \multirow[t]{2}{*}{$\mathrm{U}$} & & 0.0549 & $\mu \mathrm{g} / \mathrm{kg}$ & 0.0549 & EPA8260 \\
\hline & & 0.187 & $\mu \mathrm{g} / \mathrm{kg}$ & 0.0549 & EPA8260 \\
\hline $\mathrm{U}$ & V & 1.04 & $\mu \mathrm{g} / \mathrm{kg}$ & 1.1 & EPA8260 \\
\hline $\mathrm{U}$ & \multirow[t]{5}{*}{ V } & 2.24 & $\mu \mathrm{g} / \mathrm{kg}$ & 1.1 & EPA8260 \\
\hline $\mathrm{U}$ & & 1.1 & $\mu \mathrm{g} / \mathrm{kg}$ & 1.1 & EPA8260 \\
\hline $\mathrm{U}$ & & 0.0549 & $\mu \mathrm{g} / \mathrm{kg}$ & 0.0549 & EPA8260 \\
\hline $\mathrm{U}$ & & 0.0549 & $\mu \mathrm{g} / \mathrm{kg}$ & 0.0549 & EPA8260 \\
\hline $\mathrm{U}$ & & 0.0549 & $\mu \mathrm{g} / \mathrm{kg}$ & 0.0549 & EPA8260 \\
\hline $\mathrm{U}$ & \multirow[t]{4}{*}{8} & 0.165 & $\mu \mathrm{g} / \mathrm{kg}$ & 0.549 & EPA8260 \\
\hline $\mathbf{U}$ & & 0.0549 & $\mu \mathrm{g} / \mathrm{kg}$ & 0.0549 & EPA8260 \\
\hline $\mathbf{U}$ & & 0.0549 & $\mu \mathrm{g} / \mathrm{kg}$ & 0.0549 & EPA8260 \\
\hline $\mathbf{U}$ & & 0.0549 & $\mu \mathrm{g} / \mathrm{kg}$ & 0.0549 & EPA8260 \\
\hline \multirow[t]{2}{*}{ UJ } & $Q$ & 11 & $\mu \mathrm{g} / \mathrm{kg}$ & 11 & EPA8260 \\
\hline & & 0.692 & $\mu \mathrm{g} / \mathrm{kg}$ & 0.165 & EPA826 \\
\hline
\end{tabular}

\begin{tabular}{|c|c|c|c|c|c|}
\hline Aldrin & $\mathrm{U}$ & 4.49 & $\mu \mathrm{g} / \mathrm{kg}$ & 4.49 & EPA8080 \\
\hline alpha-Benzene hexachloride & $\mathrm{U}$ & 4.49 & $\mu \mathrm{g} / \mathrm{kg}$ & 4.49 & EPA8080 \\
\hline beta-Benzene hexachloride & $\mathrm{U}$ & 8.98 & $\mu \mathrm{g} / \mathrm{kg}$ & 8.98 & EPA8080 \\
\hline delta-Benzene hexachloride & $\mathrm{U}$ & 8.98 & $\mu \mathrm{g} / \mathrm{kg}$ & 8.98 & EPA8080 \\
\hline alpha-Chlordane & $\mathrm{U}$ & 8.98 & $\mu \mathrm{g} / \mathrm{kg}$ & 8.98 & EPA8080 \\
\hline gamma-Chlordane & $\mathbf{U}$ & 8.98 & $\mu \mathrm{g} / \mathrm{kg}$ & 8.98 & EPA8080 \\
\hline$p, p^{\prime}-D D D$ & $\mathrm{U}$ & 18 & $\mu \mathrm{g} / \mathrm{kg}$ & 18 & EPA8080 \\
\hline $\mathrm{p}, \mathrm{p}^{\prime}-\mathrm{DDE}$ & $\mathrm{U}$ & 8.98 & $\mu \mathrm{g} / \mathrm{kg}$ & 8.98 & EPA8080 \\
\hline $\mathrm{p}, \mathrm{p}^{\prime}-\mathrm{DDT}$ & $\mathrm{U}$ & 18 & $\mu \mathrm{g} / \mathrm{kg}$ & 18 & EPA8080 \\
\hline Dieldrin & $\mathrm{U}$ & 8.98 & $\mu \mathrm{g} / \mathrm{kg}$ & 8.98 & EPA8080 \\
\hline Endosulfan sulfate & $U$ & 18 & $\mu \mathrm{g} / \mathrm{kg}$ & 18 & EPA8080 \\
\hline Endosulfan I & $\mathrm{U}$ & 8.98 & $\mu \mathrm{g} / \mathrm{kg}$ & 8.98 & EPA8080 \\
\hline Endosulfan II & $\mathrm{U}$ & 18 & $\mu \mathrm{g} / \mathrm{kg}$ & 18 & EPA8080 \\
\hline Endrin & $\mathrm{U}$ & 8.98 & $\mu \mathrm{g} / \mathrm{kg}$ & 8.98 & EPA8080 \\
\hline Endrin ketone & $\mathrm{U}$ & 18 & $\mu \mathrm{g} / \mathrm{kg}$ & 18 & EPA8080 \\
\hline Heptachlor & U & 8.98 & $\mu \mathrm{g} / \mathrm{kg}$ & 8.98 & EPA8080 \\
\hline Heptachlor epoxide & $\mathrm{U}$ & 8.98 & $\mu \mathrm{g} / \mathrm{kg}$ & 8.98 & EPA8080 \\
\hline Lindane & U & 4.49 & $\mu \mathrm{g} / \mathrm{kg}$ & 4.49 & EPA8080 \\
\hline
\end{tabular}


SURVEY ID: PPSC 1401 (continued)

Constituent

Pesticides/PCBs and Dioxins/Furans

Methoxychlor

PCB 1016

PCB 1221

PCB 1232

PCB 1242

PCB 1248

PCB 1254

PCB 1260

Toxaphene

Radionuclides

Actinium-228

Antimony-124

Antimony-125

Barium-133

Cerium-144

Cesium-134

Cesium-137

Cobalt-57

Cobalt-58

Cobalt-60

Europium-152

Europium-154

Europium-155

Gross alpha

Lead-212

Manganese-54

Neptunium-239

Nonvolatile beta

Plutonium-238

Plutonium-239/240

Potassium- 40

Promethium-144

Promethium-146

Radium-226

Radium-228

Ruthenium-106

Sodium-22

Strontium-90

Thorium-234

Tin-113

Yttrium-88

Zinc-65

Zirconium-95
Sample ID: 105109

RQ AQ B Result Unit D. Limit Method
$5.26 \mathrm{E}-01 \pm 3.23 \mathrm{E}-02 \mathrm{pCi} / \mathrm{g}$ 7.63E-05 $\pm 3.59 \mathrm{E}-03 \mathrm{pCi} / \mathrm{g}$ $-5.49 \mathrm{E}-04 \pm 8.76 \mathrm{E} .03 \mathrm{pCi} / \mathrm{g}$ $4.48 \mathrm{E}-03 \pm 4.04 \mathrm{E}-03 \mathrm{pCi} / \mathrm{g}$ $-1.32 \mathrm{E}-02 \pm 2.25 \mathrm{E}-02 \mathrm{pCi} / \mathrm{g}$ $1.48 \mathrm{E}-03 \pm 3.00 \mathrm{E}-03 \mathrm{pCi} / \mathrm{g}$ 9.46E-03 $\pm 5.34 \mathrm{E}-03 \mathrm{pCi} / \mathrm{g}$ $1.02 \mathrm{E}-03 \pm 2.79 \mathrm{E}-03 \mathrm{pCi} / \mathrm{g}$ $-1.28 \mathrm{E}-03 \pm 3.64 \mathrm{E}-03 \mathrm{pCi} / \mathrm{g}$ $1.19 \mathrm{E}-03 \pm 2.49 \mathrm{E}-03 \mathrm{pCi} / \mathrm{g}$ 2.99E-03 $\pm 8.37 \mathrm{E}-03 \mathrm{pCi} / \mathrm{g}$ $-1.89 \mathrm{E}-03 \pm 2.87 \mathrm{E}-02 \mathrm{pCi} / \mathrm{g}$ $0.00 \mathrm{E}+00 \quad \mathrm{pCi} / \mathrm{g}$ $1.56 \mathrm{E}+01 \pm 2.80 \mathrm{E}+00 \mathrm{pCi} / \mathrm{g}$ $5.24 \mathrm{E}-01 \pm 1.51 \mathrm{E}-02 \mathrm{pCi} / \mathrm{g}$ $6.05 \mathrm{E}-03 \pm 4.90 \mathrm{E}-03 \mathrm{pCi} / \mathrm{g}$. $-2.00 \mathrm{E}-02 \pm 2.04 \mathrm{E}-02 \mathrm{pCi} / \mathrm{g}$ $8.00 \mathrm{E}+00 \pm 1.74 \mathrm{E}+00 \mathrm{pCi} / \mathrm{g}$ 9.65E-03 $\pm 1.14 \mathrm{E}-02 \mathrm{pCi} / \mathrm{g}$ 4.26E-02 $\pm 2.25 \mathrm{E}-02 \mathrm{pCi} / \mathrm{g}$

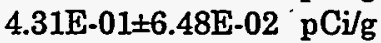
$-1.00 \mathrm{E}-03 \pm 2.94 \mathrm{E}-03 \mathrm{pCj} / \mathrm{g}$ 1.52E-03 $\pm 3.93 \mathrm{E}-03 \mathrm{pCi} / \mathrm{g}$ $3.69 \mathrm{E}-01 \pm 2.05 \mathrm{E}-02 \mathrm{pCi} / \mathrm{g}$ $5.26 \mathrm{E}-01 \pm 3.23 \mathrm{E}-02 \mathrm{pCi} / \mathrm{g}$ $-1.45 \mathrm{E}-02 \pm 2.58 \mathrm{E}-02 \mathrm{pCi} / \mathrm{g}$ $-5.58 \mathrm{E}-04 \pm 2.72 \mathrm{E}-03 \mathrm{pCi} / \mathrm{g}$ $-1.70 \mathrm{E}-01 \pm 5.30 \mathrm{E}-02 \mathrm{pCi} / \mathrm{g}$

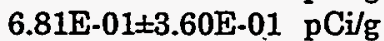
$-4.11 \mathrm{E}-04 \pm 4.35 \mathrm{E}-03 \mathrm{pCi} / \mathrm{g}$ $-9.33 \mathrm{E}-04 \pm 3.73 \mathrm{E}-03 \mathrm{pCi} / \mathrm{g}$

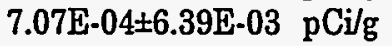

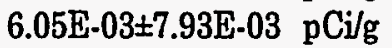
1.66E.02 EPIA.013B
6.29E-03 EPIA-013B
1.44E.02 EPIA.013B
6.32E-03 EPIA-013B
3.83E-02 EPIA-013B
4.70E-03 EPIA-013B
5.65E-03 EPIA-013B
4.86E-03 EPIA-013B
5.40E-03 EPIA-013B
4.67E-03 EPIA-013B
1.41E-02 EPIA-013B
4.30E-02 EPIA-013B
1.99E-02 EPIA-013B
$1.28 \mathrm{E}+00$ EPIA-001B
8.61E-03 EPIA-013B
5.20E-03 EPIA-013B
3.47E-02 EPIA-013B
2.38E+00 EPIA-001B
1.72E-02 EPIA-012B
1.72E-02 EPIA-012B
4.47E-02 EPIA-013B
5.04E-03 EPIA-013B
6.58E-03 EPIA-013B
9.15E-03 EPIA-013B
1.66E-02 EPIA-013B
4.40E-02 EPIA-013B
4.57E-03 EPIA-013B
1.28E-01 EPIA-004
2.56E-01 EPIA-013B
7.19E-03 EPLA-013B
5.59E-03 EPIA-013B
9.70E-03 EPIA-013B
1.27E-02 EPIA-013B

SURVEY ID: PPSC 1402

Sample ID: 105110

Survey location: 44071E $3670210 \mathrm{~N}$ (SRS coordinates)

Depth of core interval: 1.00 to $2.00 \mathrm{ft}$

Sample type: Normal

Sample matrix: Soil

Percent solids: $\mathbf{8 8 . 0 0}$ 
SURVEY ID: PPSC 1402 (continued)

Constituent
Sample ID: 105110

RQ AQ B Result

Unit

D. Limit Method

Physical Parameters and Specified Analyses

$\begin{array}{lllll}\text { Ammonia nitrogen } & \text { U } & & & 22.6 \\ \text { Cation exchange capacity } & & & 22 \\ \text { Chloride } & \text { J } & \text { E } & 2.62 \\ \text { Cyanide } & \text { U } & & & 1.05 \\ \text { Nitrate-nitrite as nitrogen } & \text { U } & \text { V } & & 1.12 \\ \text { Nitrogen by Kjeldahl method } & & & & 89.5 \\ \text { Orthophosphate } & \text { U } & & & 11.4 \\ \text { Phenols } & \text { UJ } & \text { C } & & 226 \\ \text { Sulfide } & \text { UJ } & \text { IC } & \text { L } & 284 \\ \text { Sulfide } & \text { UJ } & \text { IC } & \text { L } & 284 \\ \text { Total organic carbon } & & \text { V } & & 2730 \\ \text { Total organic carbon } & & \text { V } & 3140 \\ \text { Total organic nitrogen } & \text { C } & & & 80 \\ \text { Total phosphates (as P) } & \text { J } & \text { E } & 4.17 \\ \text { Total phosphates (as P) } & \text { J } & \text { E } & 5.34\end{array}$

$\begin{array}{lll}\mathrm{mg} / \mathrm{kg} & 22.6 & \text { EPA350.1 } \\ \mathrm{meq} / 100 \mathrm{~g} & 5 & \text { EPA9081 } \\ \mathrm{mg} / \mathrm{kg} & 2.74 & \text { EPA300.0 } \\ \mathrm{mg} / \mathrm{kg} & 1.05 & \text { EPA335.3 } \\ \mathrm{mg} / \mathrm{kg} & 1.12 & \text { EPA353.1 } \\ \mathrm{mg} / \mathrm{kg} & 44.7 & \text { EPA351.2 } \\ \mathrm{mg} / \mathrm{kg} & 11.4 & \text { EPA365.2 } \\ \mu \mathrm{g} / \mathrm{kg} & 226 & \text { EPA420.2 } \\ \mathrm{mg} / \mathrm{kg} & 284 & \text { EPA376.2 } \\ \mathrm{mg} / \mathrm{kg} & 284 & \text { EPA376.2 } \\ \mathrm{mg} / \mathrm{kg} & 114 & \text { EPA415.1. } \\ \mathrm{mg} / \mathrm{kg} & 114 & \text { EPA415.1 } \\ \mathrm{mg} / \mathrm{kg} & 0.227 & \text { EPA351.2 } \\ \mathrm{mg} / \mathrm{kg} & 11.3 & \text { EPA365.4 } \\ \mathrm{mg} / \mathrm{kg} & 10.9 & \text { EPA365.4 }\end{array}$

Metals (total recoverable)

\begin{tabular}{|c|c|c|c|c|c|c|}
\hline Aluminum & $J$ & C & 927 & $\mathrm{mg} / \mathrm{kg}$ & 14.1 & EPA6010A \\
\hline Aluminum & J & C & 1190 & $\mathrm{mg} / \mathrm{kg}$ & 14.1 & EPA6010A \\
\hline Antimony & UJ & I & 5.63 & $\mathrm{mg} / \mathrm{kg}$ & 5.63 & EPA6010A \\
\hline Antimony & UJ & I & 5.63 & $\mathrm{mg} / \mathrm{kg}$ & 5.63 & EPA6010A \\
\hline Arsenic & $\mathrm{U}$ & & 16.9 & $\mathrm{mg} / \mathrm{kg}$ & 16.9 & EPA6010A \\
\hline Arsenic & $\mathrm{U}$ & & 16.9 & $\mathrm{mg} / \mathrm{kg}$ & 16.9 & EPA6010A \\
\hline Barium & & & 5.6 & $\mathrm{mg} / \mathrm{kg}$ & 1.41 & EPA6010A \\
\hline Barium & & & 7.1 & $\mathrm{mg} / \mathrm{kg}$ & 1.41 & EPA6010A \\
\hline Beryllium & $\mathbf{J}$ & $\mathbf{E}$ & 0.0433 & $\mathrm{mg} / \mathrm{kg}$ & 0.563 & EPA6010A \\
\hline Beryllium & $J$ & $\mathbf{E}$ & 0.0467 & $\mathrm{mg} / \mathrm{kg}$ & 0.563 & EPA6010A \\
\hline Cadmium & U & & 0.281 & $\mathrm{mg} / \mathrm{kg}$ & 0.281 & EPA6010A \\
\hline Cadmium & U & & 0.281 & $\mathrm{mg} / \mathrm{kg}$ & 0.281 & EPA6010A \\
\hline Calcium & & V & 22 & $\mathrm{mg} / \mathrm{kg}$ & 14.1 & EPA6010A \\
\hline Calcium & & V & 21.7 & $\mathrm{mg} / \mathrm{kg}$ & 14.1 & EPA6010A \\
\hline Chromium & & & 1.83 & $\mathrm{mg} / \mathrm{kg}$ & 1.41 & EPA6010A \\
\hline Chromium & & & 2.32 & $\mathrm{mg} / \mathrm{kg}$ & 1.41 & EPA6010A \\
\hline Cobalt & $J$ & $E$ & 0.212 & $\mathrm{mg} / \mathrm{kg}$ & 0.563 & EPA6010A \\
\hline Cobalt & $\mathrm{J}$ & $\mathrm{E}$ & 0.221 & $\mathrm{mg} / \mathrm{kg}$ & 0.563 & EPA6010A \\
\hline Copper & $J$ & $\mathrm{E}$ & 0.804 & $\mathrm{mg} / \mathrm{kg}$ & 1.41 & EPA6010A \\
\hline Copper & $J$ & $\mathbf{E}$ & 0.778 & $\mathrm{mg} / \mathrm{kg}$ & 1.41 & EPA6010A \\
\hline Iron & $J$ & $\mathrm{VC}$ & 1210 & $\mathrm{mg} / \mathrm{kg}$ & 5.63 & EPA6010A \\
\hline Iron & $J$ & VC & 1490 & $\mathrm{mg} / \mathrm{kg}$ & 5.63 & EPA6010A \\
\hline Lead & $J$ & $\mathrm{E}$ & 2.44 & $\mathrm{mg} / \mathrm{kg}$ & 5.63 & EPA6010A \\
\hline Lead & $\mathrm{U}$ & & 5.63 & $\mathrm{mg} / \mathrm{kg}$ & 5.63 & EPA6010A \\
\hline Magnesium & & $\mathrm{X}$ & 24.4 & $\mathrm{mg} / \mathrm{kg}$ & 5.63 & EPA6010A \\
\hline Magnesium & & $\mathrm{X}$ & 36 & $\mathrm{mg} / \mathrm{kg}$ & 5.63 & EPA6010A \\
\hline Manganese & & & 10.3 & $\mathrm{mg} / \mathrm{kg}$ & 0.563 & EPA6010A \\
\hline Manganese & & & 10.2 & $\mathrm{mg} / \mathrm{kg}$ & 0.563 & EPA6010A \\
\hline Mercury & $\mathrm{U}$ & V & 0.00873 & $\mathrm{mg} / \mathrm{kg}$ & 0.0349 & EPA7471 \\
\hline Mercury & $\mathrm{U}$ & & 0.0345 & $\mathrm{mg} / \mathrm{kg}$ & 0.0345 & EPA7471 \\
\hline Nickel & $J$ & $\mathrm{E}$ & 0.639 & $\mathrm{mg} / \mathrm{kg}$ & 1.41 & EPA6010A \\
\hline Nickel & $J$ & $\mathrm{E}$ & 0.658 & $\mathrm{mg} / \mathrm{kg}$ & 1.41 & EPA6010A \\
\hline Potassium & $J$ & $\mathbf{E}$ & 18.2 & $\mathrm{mg} / \mathrm{kg}$ & 113 & EPA6010A \\
\hline
\end{tabular}


SURVEY ID: PPSC 1402 (continued)

Constituent

RQ AQ B Result

Potassium

Selenium

Selenium

Silver

Silver

Sodium

Sodium

Thallium

Thallium

Vanadium

Vanadium

Zinc

Zinc

$B / N / A$ Extractables (including $P A H$ and phenols)

Acenaphthene

Acenaphthylene

Anthracene

Benzo[a]anthracene

Benzo[b]fluoranthene

Benzo[ $k]$ fluoranthene

Benzoic acid

Benzo $[g, h, i]$ perylene

Benzo[a]pyrene

Benzyl alcohol

Bis(2-chloroethoxy) methane

Bis(2-chloroethyl) ether

Bis(2-chloroisopropyl) ether

Bis(2-ethylhexyl) phthalate

4-Bromophenyl phenyl ether

Butylbenzyl phthalate

4-Chloroaniline

4-Chloro-m-cresol

2-Chloronaphthalene

2-Chlorophenol

4-Chlorophenyl phenyl ether

Chrysene

$\mathrm{m} / \mathrm{p}$-Cresol

o-Cresol (2-Methylphenol)

Dibenz[a,h]anthracene

Dibenzofuran

Di-n-butyl phthalate

1,2-Dichlorobenzene

1,3-Dichlorobenzene

1,4-Dichlorobenzene

3,3'-Dichlorobenzidine

2,4-Dichlorophenol

Diethyl phthalate

2,4-Dimethyl phenol

Dimethyl phthalate

2,4-Dinitrophenol

2,4-Dinitrotoluene

2,6-Dinitrotoluene

Di-n-octyl phthalate
17.7

$\begin{array}{lll}J & E & 17.7 \\ U & & 16.9 \\ U & & 16.9 \\ U & & 1.41 \\ U & & 1.41 \\ U & & 56.3 \\ U & & 56.3 \\ U & & 16.9 \\ U & & 16.9 \\ & & 3.39 \\ & & 4.41 \\ & & 1.47 \\ & & 1.39\end{array}$

37.2

37.2

37.2

3.72

3.72

3.72

37.2

37.2

3.72

37.2

37.2

37.2

37.2

22.7

37.2

37.2

37.2

37.2

37.2

37.2

37.2

3.72

372

37.2

3.72

37.2

26.4

37.2

37.2

37.2

37.2

37.2

37.2

37.2

37.2

372

3.72

3.72

37.2
Unit

D. Limit Method

$\begin{array}{lll}\mathrm{mg} / \mathrm{kg} & 113 & \text { EPA6010A } \\ \mathrm{mg} / \mathrm{kg} & 16.9 & \text { EPA6010A } \\ \mathrm{mg} / \mathrm{kg} & 16.9 & \text { EPA6010A } \\ \mathrm{mg} / \mathrm{kg} & 1.41 & \text { EPA6010A } \\ \mathrm{mg} / \mathrm{kg} & 1.41 & \text { EPA6010A } \\ \mathrm{mg} / \mathrm{kg} & 56.3 & \text { EPA6010A } \\ \mathrm{mg} / \mathrm{kg} & 56.3 & \text { EPA6010A } \\ \mathrm{mg} / \mathrm{kg} & 16.9 & \text { EPA6010A } \\ \mathrm{mg} / \mathrm{kg} & 16.9 & \text { EPA6010A } \\ \mathrm{mg} / \mathrm{kg} & 0.563 & \text { EPA6010A } \\ \mathrm{mg} / \mathrm{kg} & 0.563 & \text { EPA6010A } \\ \mathrm{mg} / \mathrm{kg} & 1.13 & \text { EPA6010A } \\ \mathrm{mg} / \mathrm{kg} & 1.13 & \text { EPA6010A }\end{array}$

$\mu \mathrm{g} / \mathrm{kg}$

$\mu \mathrm{g} / \mathrm{kg}$

$\mu \mathrm{g} / \mathrm{kg}$

$\mu \mathrm{g} / \mathrm{kg}$

$\mu \mathrm{g} / \mathrm{kg}$

$\mu \mathrm{g} / \mathrm{kg}$

$\mu \mathrm{g} / \mathrm{kg}$

$\mu \mathrm{g} / \mathrm{kg}$

$\mu \mathrm{g} / \mathrm{kg}$

$\mu \mathrm{g} / \mathrm{kg}$

$\mu \mathrm{g} / \mathrm{kg}$

$\mu \mathrm{g} / \mathrm{kg}$

$\mu \mathrm{g} / \mathrm{kg}$

$\mu \mathrm{g} / \mathrm{kg}$

$\mu \mathrm{g} / \mathrm{kg}$

$\mu \mathrm{g} / \mathrm{kg}$

$\mu \mathrm{g} / \mathrm{kg}$

$\mu \mathrm{g} / \mathrm{kg}$

$\mu \mathrm{g} / \mathrm{kg}$

$\mu \mathrm{g} / \mathrm{kg}$

$\mu \mathrm{g} / \mathrm{kg}$

$\mu \mathrm{g} / \mathrm{kg}$

$\mu \mathrm{g} / \mathrm{kg}$

$\mu \mathrm{g} / \mathrm{kg}$

, $\mu \mathrm{g} / \mathrm{kg}$

$\mu \mathrm{g} / \mathrm{kg}$

$\mu \mathrm{g} / \mathrm{kg}$

$\mu \mathrm{g} / \mathrm{kg}$

$\mu \mathrm{g} / \mathrm{kg}$

$\mu \mathrm{g} / \mathrm{kg}$

$\mu \mathrm{g} / \mathrm{kg}$

$\mu \mathrm{g} / \mathrm{kg}$

$\mu \mathrm{g} / \mathrm{kg}$

$\mu \mathrm{g} / \mathrm{kg}$

$\mu \mathrm{g} / \mathrm{kg}$

$\mu \mathrm{g} / \mathrm{kg}$

$\mu \mathrm{g} / \mathrm{kg}$

$\mu \mathrm{g} / \mathrm{kg}$

$\mu \mathrm{g} / \mathrm{kg}$
37.2

EPA8270

EPA8270

EPA8270

EPA8270

EPA8270

EPA8270

EPA8270

EPA8270

EPA8270

EPA8270

EPA8270

EPA8270

EPA8270

EPA8270

EPA8270

EPA8270

EPA8270

EPA8270

EPA8270

EPA8270

EPA8270

EPA8270

EPA8270 -

EPA8270

EPA8270

EPA8270

EPA8270

EPA8270

EPA8270

EPA8270

EPA8270

EPA8270

EPA8270

EPA8270

EPA8270

EPA8270

EPA8270

EPA8270

EPA8270 
SURVEY ID: PPSC 1402 (continued)

Constituent
Sample ID: 105110

RQ AQ B Result

Unit

D. Limit Method

B/N/A Extractables (including PAH and phenols)

$\begin{array}{lcc}\text { Fluoranthene } & \mathrm{U} & 37.2 \\ \text { Fluorene } & \mathrm{U} & 37.2 \\ \text { Hexachlorobenzene } & \mathrm{U} & 3.72 \\ \text { Hexachlorobutadiene } & \mathrm{U} & 37.2 \\ \text { Hexachlorocyclopentadiene } & \mathrm{U} & 37.2 \\ \text { Hexachloroethane } & \mathrm{U} & 3.72 \\ \text { Indeno[1,2,3-c,d]pyrene } & \mathrm{U} & 3.72 \\ \text { Isophorone } & \mathrm{U} & 37.2 \\ \text { 2-Methyl-4,6-dinitrophenol } & \mathrm{U} & 372 \\ \text { 2-Methylnaphthalene } & \mathrm{U} & 37.2 \\ \text { Naphthalene } & \mathrm{U} & 37.2 \\ \text { m-Nitroaniline } & \mathrm{U} & 37.2 \\ \text { o-Nitroaniline } & \mathrm{U} & 37.2 \\ \text { p-Nitroaniline } & \mathrm{U} & 37.2 \\ \text { Nitrobenzene } & \mathrm{U} & 37.2 \\ \text { 2-Nitrophenol } & \mathrm{U} & 37.2 \\ \text { 4-Nitrophenol } & \mathrm{U} & 37.2 \\ \text { N-Nitrosodiphenylamine } & \mathrm{U} & 37.2 \\ \text { N-Nitrosodi-n-propylamine } & \mathrm{U} & 37.2 \\ \text { Pentachlorophenol } & \mathrm{U} & 37.2 \\ \text { Phenanthrene } & \mathrm{U} & 37.2 \\ \text { Phenol } & \mathrm{U} & 37.2 \\ \text { Pyrene } & \mathrm{U} & 37.2 \\ \text { 1,2,4-Trichlorobenzene } & \mathrm{U} & 3.72 \\ \text { 2,4,5-Trichlorophenol } & \mathrm{U} & 37.2 \\ \text { 2,4,6-Trichlorophenol } & \mathrm{U} & 37.2\end{array}$

Volatile Organic Compounds

Acetone
Benzene
Bromodichloromethane
Bromoform
Bromomethane (Methyl bromide)
Carbon disulfide
Carbon tetrachloride
Chlorobenzene
Chloroethane
Chloroethene (Vinyl chloride)
Chloroform
Chloromethane (Methyl chloride)
Dibromochloromethane
1,1-Dichloroethane
1,2-Dichloroethane
1,1-Dichloroethylene
1,2-Dichloroethylene
Dichloromethane (Methylene chloride)
1,2-Dichloropropane
cis-1,3-Dichloropropene
trans-1,3-Dichloropropene
Ethylbenzene
2-Hexanone
Isocamphene
Methyl ethyl ketone
Methyl isobutyl ketone

$\begin{array}{lll}\text { J } & \text { Q8 } & 244 \\ U & & 0.568 \\ U & & 0.0568 \\ U & & 0.0568 \\ U & & 0.114 \\ U & & 1.14 \\ U & & 0.0568 \\ U & & 0.0568 \\ U & & 0.114 \\ U & & 0.114 \\ U & & 0.0568 \\ U & & 0.114 \\ U & & 0.0568 \\ U & & 0.0568 \\ U & & 0.0568 \\ U & & 0.0568 \\ U & & 0.114 \\ U & V & 3.09 \\ U & & 0.0568 \\ U & & 0.0568 \\ U & & 0.0568 \\ & & 0.636 \\ U & V & 0.795 \\ J & N & 1 \\ & V & 4.56 \\ U & & 1.14\end{array}$

$\begin{array}{lll}\mu \mathrm{g} / \mathrm{kg} & 37.2 & \text { EPA8270 } \\ \mu \mathrm{g} / \mathrm{kg} & 37.2 & \text { EPA8270 } \\ \mu \mathrm{g} / \mathrm{kg} & 3.72 & \text { EPA8270 } \\ \mu \mathrm{g} / \mathrm{kg} & 37.2 & \text { EPA8270 } \\ \mu \mathrm{g} / \mathrm{kg} & 37.2 & \text { EPA8270 } \\ \mu \mathrm{g} / \mathrm{kg} & 3.72 & \text { EPA8270 } \\ \mu \mathrm{g} / \mathrm{kg} & 3.72 & \text { EPA8270 } \\ \mu \mathrm{g} / \mathrm{kg} & 37.2 & \text { EPA8270 } \\ \mu \mathrm{g} / \mathrm{kg} & 372 & \text { EPA8270 } \\ \mu \mathrm{g} / \mathrm{kg} & 37.2 & \text { EPA8270 } \\ \mu \mathrm{g} / \mathrm{kg} & 37.2 & \text { EPA8270 } \\ \mu \mathrm{g} / \mathrm{kg} & 37.2 & \text { EPA8270 } \\ \mu \mathrm{g} / \mathrm{kg} & 37.2 & \text { EPA8270 } \\ \mu \mathrm{g} / \mathrm{kg} & 37.2 & \text { EPA8270 } \\ \mu \mathrm{g} / \mathrm{kg} & 37.2 & \text { EPA8270 } \\ \mu \mathrm{g} / \mathrm{kg} & 37.2 & \text { EPA8270 } \\ \mu \mathrm{g} / \mathrm{kg} & 37.2 & \text { EPA8270 } \\ \mu \mathrm{g} / \mathrm{kg} & 37.2 & \text { EPA8270 } \\ \mu \mathrm{g} / \mathrm{kg} & 37.2 & \text { EPA8270 } \\ \mu \mathrm{g} / \mathrm{kg} & 37.2 & \text { EPA8270 } \\ \mu \mathrm{g} / \mathrm{kg} & 37.2 & \text { EPA8270 } \\ \mu \mathrm{g} / \mathrm{kg} & 37.2 & \text { EPA8270 } \\ \mu \mathrm{g} / \mathrm{kg} & 37.2 & \text { EPA8270 } \\ \mu \mathrm{g} / \mathrm{kg} & 3.72 & \text { EPA8270 } \\ \mu \mathrm{g} / \mathrm{kg} & 37.2 & \text { EPA8270 } \\ \mu \mathrm{g} / \mathrm{kg} & 37.2 & \text { EPA8270 }\end{array}$

$\begin{array}{lll}\mu \mathrm{g} / \mathrm{kg} & 28.4 & \text { EPA8260 } \\ \mu \mathrm{g} / \mathrm{kg} & 0.568 & \text { EPA8260 } \\ \mu \mathrm{g} / \mathrm{kg} & 0.0568 & \text { EPA8260 } \\ \mu \mathrm{g} / \mathrm{kg} & 0.0568 & \text { EPA8260 } \\ \mu \mathrm{g} / \mathrm{kg} & 0.114 & \text { EPA8260 } \\ \mu \mathrm{g} / \mathrm{kg} & 1.14 & \text { EPA8260 } \\ \mu \mathrm{g} / \mathrm{kg} & 0.0568 & \text { EPA8260 } \\ \mu \mathrm{g} / \mathrm{kg} & 0.0568 & \text { EPA8260 } \\ \mu \mathrm{g} / \mathrm{kg} & 0.114 & \text { EPA8260 } \\ \mu \mathrm{g} / \mathrm{kg} & 0.114 & \text { EPA8260 } \\ \mu \mathrm{g} / \mathrm{kg} & 0.0568 & \text { EPA8260 } \\ \mu \mathrm{g} / \mathrm{kg} & 0.114 & \text { EPA8260 } \\ \mu \mathrm{g} / \mathrm{kg} & 0.0568 & \text { EPA8260 } \\ \mu \mathrm{g} / \mathrm{kg} & 0.0568 & \text { EPA8260 } \\ \mu \mathrm{g} / \mathrm{kg} & 0.0568 & \text { EPA8260 } \\ \mu \mathrm{g} / \mathrm{kg} & 0.0568 & \text { EPA8260 } \\ \mu \mathrm{g} / \mathrm{kg} & 0.114 & \text { EPA8260 } \\ \mu \mathrm{g} / \mathrm{kg} & 0.568 & \text { EPA8260 } \\ \mu \mathrm{g} / \mathrm{kg} & 0.0568 & \text { EPA8260 } \\ \mu \mathrm{g} / \mathrm{kg} & 0.0568 & \text { EPA8260 } \\ \mu \mathrm{g} / \mathrm{kg} & 0.0568 & \text { EPA8260 } \\ \mu \mathrm{g} / \mathrm{kg} & 0.0568 & \text { EPA8260 } \\ \mu \mathrm{g} / \mathrm{kg} & 1.14 & \text { EPA8260 } \\ \mu \mathrm{g} / \mathrm{kg} & & \text { EPA8260 } \\ \mu \mathrm{g} / \mathrm{kg} & 1.14 & \text { EPA8260 } \\ \mu \mathrm{g} / \mathrm{kg} & 1.14 & \text { EPA8260 }\end{array}$


SURVEY ID: PPSC 1402 (continued)

Constituent

Volatile Organic Compounds

Methyl(methylethyl)cyclohexane

Styrene

1,1,2,2-Tetrachloroethane

Tetrachloroethylene

Toluene

1,1,1-Trichloroethane

1,1,2-Trichloroethane

Trichloroethylene

Vinyl acetate

Xylenes

Pesticides/PCBs and Dioxins/Furans

Aldrin

alpha-Benzene hexachloride

beta-Benzene hexachloride

delta-Benzene hexachloride

alpha-Chlordane

gamma-Chlordane

$\mathrm{p}, \mathrm{p}^{\prime}-\mathrm{DDD}$

$\mathrm{p}, \mathrm{p}^{\prime}-\mathrm{DDE}$

p,p'-DDT

Dieldrin

Endosulfan sulfate

Endosulfan I

Endosulfan II

Endrin

Endrin ketone

Heptachlor

Heptachlor epoxide

Lindane

Methoxychlor

PCB 1016

PCB 1221

PCB 1232

PCB 1242

PCB 1248

PCB 1254

PCB 1260

Toxaphene

\section{Radionuclides}

Actinium-228

Antimony-124

Antimony-125

Baxium-133

Cerium-144

Cesium-134

Cesium-137

Cobalt-57

Cobalt-58

Cobalt-60

Europium-152

Europium-154
Sample ID: 105110

RQ AQ B Result Unit D. Limit Method

$\begin{array}{llllll}J & N & 5.5 & \mu g / k g & & \text { EPA8260 } \\ U & & 0.0568 & \mu g / k g & 0.0568 & \text { EPA8260 } \\ U & & 0.0568 & \mu g / k g & 0.0568 & \text { EPA8260 } \\ & & 0.102 & \mu g / k g & 0.0568 & \text { EPA8260 } \\ U & 8 & 0.33 & \mu g / k g & 0.568 & \text { EPA8260 } \\ U & & 0.0568 & \mu g / k g & 0.0568 & \text { EPA8260 } \\ U & & 0.0568 & \mu g / k g & 0.0568 & \text { EPA8260 } \\ U & 8 & 0.0795 & \mu g / k g & 0.0568 & \text { EPA8260 } \\ U & & 1.14 & \mu g / k g & 1.14 & \text { EPA8260 } \\ & & 0.557 & \mu \mathrm{gg} / \mathrm{kg} & 0.17 & \text { EPA8260 }\end{array}$

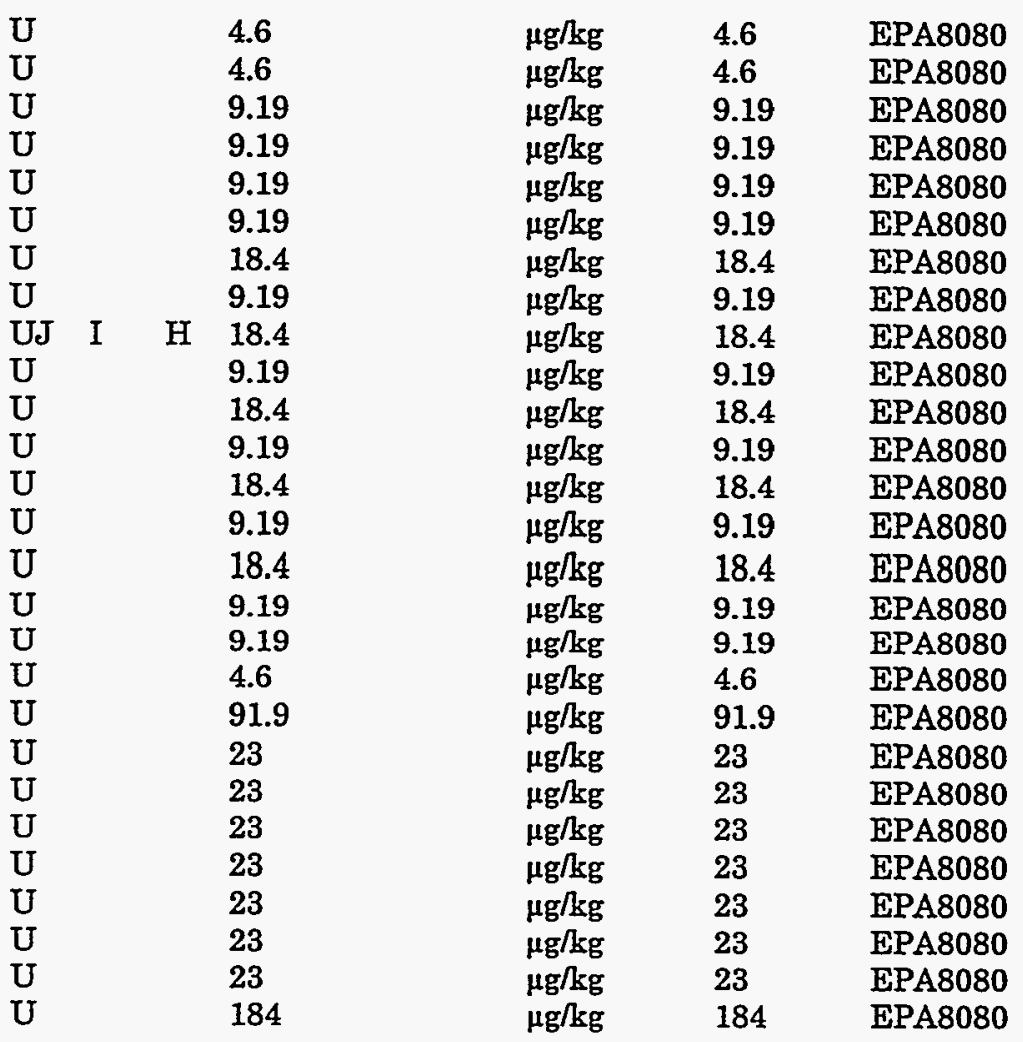

$\begin{array}{ll} & 5.65 \mathrm{E}-01 \pm 3.07 \mathrm{E}-02 \mathrm{pCi} / \mathrm{g} \\ \text { UI } & 2.51 \mathrm{E}-03 \pm 4.00 \mathrm{E}-03 \mathrm{pCi} / \mathrm{g} \\ \text { UI } & 3.74 \mathrm{E}-03 \pm 9.20 \mathrm{E}-03 \mathrm{pCi} / \mathrm{g} \\ \text { UI } & -4.04 \mathrm{E}-02 \pm 5.27 \mathrm{E}-03 \mathrm{pCi} / \mathrm{g} \\ \text { UI } & -3.54 \mathrm{E}-04 \pm 2.41 \mathrm{E}-02 \mathrm{pCi} / \mathrm{g} \\ \text { UI } & -1.88 \mathrm{E}-02 \pm 3.70 \mathrm{E}-03 \mathrm{pCi} / \mathrm{g} \\ & 2.03 \mathrm{E}-02 \pm 5.82 \mathrm{E}-03 \mathrm{pCi} / \mathrm{g} \\ \text { UI } & -4.35 \mathrm{E}-04 \pm 3.08 \mathrm{E}-03 \mathrm{pCi} / \mathrm{g} \\ \text { UI } & -2.57 \mathrm{E}-03 \pm 4.18 \mathrm{E}-03 \mathrm{pCi} / \mathrm{g} \\ \text { UI } & 1.49 \mathrm{E}-03 \pm 2.93 \mathrm{E}-03 \mathrm{pCi} / \mathrm{g} \\ \text { UI } & -7.62 \mathrm{E}-03 \pm 9.41 \mathrm{E}-03 \mathrm{pCi} / \mathrm{g} \\ \text { UI } & -1.31 \mathrm{E}-02 \pm 2.55 \mathrm{E}-02 \mathrm{pCi} / \mathrm{g}\end{array}$

1.64E-02 EPIA-013B

6.99E-03 EPIA-013B

1.44E-02 EPIA-013B

6.67E-03 EPIA-013B

4.11E-02 EPIA-013B

5.13E-03 EPIA-013B

5.58E-03 EPIA-013B

5.26E-03 EPIA-013B

- 5.88E-03 EPIA-013B

5.29E-03 EPIA-013B

1.50E-02 EPIA-013B

4.39E-02 EPIA-013B 
SURVEY ID: PPSC 1402 (continued)

Constituent

Radionuclides

Europium-155
Gross alpha
Lead-212
Manganese-54
Neptunium-239
Nonvolatile beta
Plutonium-238
Plutonium-239/240
Potassium-40
Promethium-144
Promethium-146
Radium-226
Radium-228
Ruthenium-106
Sodium-22
Strontium-90
Thorium-234
Tin-113
Yttrium-88
Zinc-65
Zirconium-95

RQ

AQ B Result

D. Limit Method
Sample ID: 105110

Unit
2.23E-02 EPLA-013B 2.09E+00 EPIA-001B

9.41E-03 EPIA-013B

4.16E-03 EPIA-013B

3.85E-02 EPIA-013B

2.50E+00 EPIA-001B

3.43E-02 EPIA-012B

0.00E+00 EPIA-012B

4.20E-02 EPIA-013B

5.04E-03 EPIA-013B

6.99E-03 EPIA-013B

1.02E-02 EPIA-013B

1.64E-02 EPIA-013B

4.93E-02 EPIA-013B

4.79E-03 EPIA-013B

1.48E-01 EPIA-004

2.76E-01 EPIA-013B

7.31E-03 EPLA-013B

6.21E-03 EPIA-013B

9.87E-03 EPIA-013B

1.34E-02 EPIA-013B

SURVEY ID: PPSC 1403

Sample ID: 105111

Survey location: 44071E 3670210N (SRS coordinates)

Depth of core interval: 2.00 to $2.70 \mathrm{ft}$

Sample type: Normal

Sample matrix: Soil

Percent solids: $\mathbf{8 5 . 0 0}$

\section{Constituent}

RQ AQ B Result

Unit

D. Limit Method

Physical Parameters and Specified Analyses

$\begin{array}{lllll}\text { Ammonia nitrogen } & \text { UJ } & \text { I } & \text { H } & 23.6 \\ \text { Ammonia nitrogen } & \text { UJ } & \text { I } & \text { H } & 23.6 \\ \text { Cation exchange capacity } & & & & 35.3 \\ \text { Chloride } & & & & 4.93 \\ \text { Cyanide } & \text { U } & & & 1.16 \\ \text { Cyanide } & \text { U } & & & 1.1 \\ \text { Nitrate-nitrite as nitrogen } & \text { U } & \text { V } & & 0.298 \\ \text { Nitrate-nitrite as nitrogen } & \text { U } & \text { V } & & 0.291 \\ \text { Nitrogen by Kjeldahl method } & & & & 209 \\ \text { Nitrogen by Kjeldahl method } & & & & 219 \\ \text { Orthophosphate } & \text { UJ } & \text { I } & \text { L } & 11.8 \\ \text { Orthophosphate } & \text { UJ } & \text { I } & \text { L } & 11.8 \\ \text { Phenols } & \text { UJ } & \text { C } & & 233 \\ \text { Phenols } & \text { UJ } & \text { C } & & 235 \\ \text { Sulfide } & \text { UJ } & \text { CI } & \text { L } & 294 \\ \text { Sulfide } & \text { UJ } & \text { CI } & \text { L } & 294 \\ \text { Total organic carbon } & \text { J } & \text { QV } & \text { L } & 7850 \\ \text { Total organic carbon } & \text { J } & \text { QV } & \text { L } & 8250 \\ \text { Total organic nitrogen } & \text { C } & & & 196 \\ \text { Total phosphates (as P) } & \text { J } & \text { EI } & \text { P } & 6.47\end{array}$

$\begin{array}{lll}\mathrm{mg} / \mathrm{kg} & 23.6 & \text { EPA350.1 } \\ \mathrm{mg} / \mathrm{kg} & 23.6 & \text { EPA350.1 } \\ \mathrm{meq} / \mathrm{l00g} & 5 & \text { EPA9081 } \\ \mathrm{mg} / \mathrm{kg} & 2.93 & \text { EPA300.0 } \\ \mathrm{mg} / \mathrm{kg} & 1.16 & \text { EPA335.3 } \\ \mathrm{mg} / \mathrm{kg} & 1.1 & \text { EPA335.3 } \\ \mathrm{mg} / \mathrm{kg} & 0.992 & \text { EPA353.1 } \\ \mathrm{mg} / \mathrm{kg} & 0.97 & \text { EPA353.1 } \\ \mathrm{mg} / \mathrm{kg} & 92.8 & \text { EPA351.2 } \\ \mathrm{mg} / \mathrm{kg} & 93.4 & \text { EPA351.2 } \\ \mathrm{mg} / \mathrm{kg} & 11.8 & \text { EPA365.2 } \\ \mathrm{mg} / \mathrm{kg} & 11.8 & \text { EPA365.2 } \\ \mu \mathrm{g} / \mathrm{kg} & 233 & \text { EPA420.2 } \\ \mu \mathrm{g} / \mathrm{kg} & 235 & \text { EPA420.2 } \\ \mathrm{mg} / \mathrm{kg} & 294 & \text { EPA376.2 } \\ \mathrm{mg} / \mathrm{kg} & 294 & \text { EPA376.2 } \\ \mathrm{mg} / \mathrm{kg} & 118 & \text { EPA415.1 } \\ \mathrm{mg} / \mathrm{kg} & 118 & \text { EPA415.1 } \\ \mathrm{mg} / \mathrm{kg} & 0.235 & \text { EPA351.2 } \\ \mathrm{mg} / \mathrm{kg} & 39.9 & \text { EPA365.4 }\end{array}$


SURVEY ID: PPSC 1403 (continued)

Constituent

Sample ID: 105111

RQ AQ B Result

Unit

D. Limit Method

Physical Parameters and Specified Analyses

Total phosphates (as P)

$\begin{array}{llll}J & \text { EI } & \text { P } & \mathbf{8 . 5 5}\end{array}$

$\mathrm{mg} / \mathrm{kg}$

43.2

EPA365.4

Metals (total recoverable)

Aluminum
Antimony
Arsenic
Barium
Beryllium
Cadmium
Calcium
Chromium
Cobalt
Copper
Iron
Lead
Magnesium
Manganese
Mercury
Nickel
Potassium
Selenium
Silver
Sodium
Thallium
Vanadium
Zinc

$\begin{array}{lll}J & C & 1730 \\ U & & 5.66 \\ U & & 17 \\ & & 10.5 \\ J & E & 0.0792 \\ U & & 0.283 \\ & V & 57.5 \\ & & 3.93 \\ U & & 0.566 \\ & & 1.54 \\ J & V C & 2770 \\ U & & 5.66 \\ & & 41 \\ & & 19.4 \\ U & & 0.0372 \\ J & E & 0.616 \\ J & E & 42.7 \\ U & & 17 \\ U & & 1.41 \\ U & & 56.6 \\ U & & 17 \\ & & 8.22 \\ & & 2.07\end{array}$

$B / N / A$ Extractables (including $P A H$ and phenols)

Acenaphthene

Acenaphthylene

Anthracene

Benzo[a]anthracene

Benzo[b]fluoranthene

Benzo $[k]$ fluoranthene

Benzoic acid

Benzo[g,h,i]perylene

Benzo[a]pyrene

Benzyl alcohol

Bis(2-chloroethoxy) methane

Bis(2-chloroethyl) ether

Bis(2-chloroisopropyl) ether

Bis(2-ethylhexyl) phthalate

4-Bromophenyl phenyl ether

Butylbenzyl phthalate

4-Chloroaniline

4-Chloro-m-cresol

2-Chloronaphthalene

2-Chlorophenol

4-Chlorophenyl phenyl ether

Chrysene

$\mathrm{m} / \mathrm{p}$-Cresol

o-Cresol (2-Methylphenol)

Dibenz $[a, h]$ anthracene

$\begin{array}{ll}U & 39.1 \\ U & 39.1 \\ U & 39.1 \\ U & 3.91 \\ U & 3.91 \\ U & 3.91 \\ U & 39.1 \\ U & 39.1 \\ U & 3.91 \\ U & 39.1 \\ U & 39.1 \\ U & 39.1 \\ U & 39.1 \\ & 65.7 \\ U & 39.1 \\ U & 39.1 \\ U & 39.1 \\ U & 39.1 \\ U & 39.1 \\ U & 39.1 \\ U & 39.1 \\ U & 3.91 \\ U & 391 \\ U & 39.1 \\ U & 3.91\end{array}$

$\begin{array}{lll}\mathrm{mg} / \mathrm{kg} & 14.1 & \text { EPA6010A } \\ \mathrm{mg} / \mathrm{kg} & 5.66 & \text { EPA6010A } \\ \mathrm{mg} / \mathrm{kg} & 17 & \text { EPA6010A } \\ \mathrm{mg} / \mathrm{kg} & 1.41 & \text { EPA6010A } \\ \mathrm{mg} / \mathrm{kg} & 0.566 & \text { EPA6010A } \\ \mathrm{mg} / \mathrm{kg} & 0.283 & \text { EPA6010A } \\ \mathrm{mg} / \mathrm{kg} & 14.1 & \text { EPA6010A } \\ \mathrm{mg} / \mathrm{kg} & 1.41 & \text { EPA6010A } \\ \mathrm{mg} / \mathrm{kg} & 0.566 & \text { EPA6010A } \\ \mathrm{mg} / \mathrm{kg} & 1.41 & \text { EPA6010A } \\ \mathrm{mg} / \mathrm{kg} & 5.66 & \text { EPA6010A } \\ \mathrm{mg} / \mathrm{kg} & 5.66 & \text { EPA6010A } \\ \mathrm{mg} / \mathrm{kg} & 5.66 & \text { EPA6010A } \\ \mathrm{mg} / \mathrm{kg} & 0.566 & \text { EPA6010A } \\ \mathrm{mg} / \mathrm{kg} & 0.0372 & \text { EPA7471 } \\ \mathrm{mg} / \mathrm{kg} & 1.41 & \text { EPA6010A } \\ \mathrm{mg} / \mathrm{kg} & 113 & \text { EPA6010A } \\ \mathrm{mg} / \mathrm{kg} & 17 & \text { EPA6010A } \\ \mathrm{mg} / \mathrm{kg} & 1.41 & \text { EPA6010A } \\ \mathrm{mg} / \mathrm{kg} & 56.6 & \text { EPA6010A } \\ \mathrm{mg} / \mathrm{kg} & 17 & \text { EPA6010A } \\ \mathrm{mg} / \mathrm{kg} & 0.566 & \text { EPA6010A } \\ \mathrm{mg} / \mathrm{kg} & 1.13 & \text { EPA6010A } \\ & & \end{array}$

$\begin{array}{lll}\mu \mathrm{g} / \mathrm{kg} & 39.1 & \text { EPA8270 } \\ \mu \mathrm{g} / \mathrm{kg} & 39.1 & \text { EPA8270 } \\ \mu \mathrm{g} / \mathrm{kg} & 39.1 & \text { EPA8270 } \\ \mu \mathrm{g} / \mathrm{kg} & 3.91 & \text { EPA8270 } \\ \mu \mathrm{g} / \mathrm{kg} & 3.91 & \text { EPA8270 } \\ \mu \mathrm{g} / \mathrm{kg} & 3.91 & \text { EPA8270 } \\ \mu \mathrm{g} / \mathrm{kg} & 39.1 & \text { EPA8270 } \\ \mu \mathrm{g} / \mathrm{kg} & 39.1 & \text { EPA8270 } \\ \mu \mathrm{g} / \mathrm{kg} & 3.91 & \text { EPA8270 } \\ \mu \mathrm{g} / \mathrm{kg} & 39.1 & \text { EPA8270 } \\ \mu \mathrm{g} / \mathrm{kg} & 39.1 & \text { EPA8270 } \\ \mu \mathrm{g} / \mathrm{kg} & 39.1 & \text { EPA8270 } \\ \mu \mathrm{g} / \mathrm{kg} & 39.1 & \text { EPA8270 } \\ \mu \mathrm{g} / \mathrm{kg} & 39.1 & \text { EPA8270 } \\ \mu \mathrm{g} / \mathrm{kg} & 39.1 & \text { EPA8270 } \\ \mu \mathrm{g} / \mathrm{kg} & 39.1 & \text { EPA8270 } \\ \mu \mathrm{g} / \mathrm{kg} & 39.1 & \text { EPA8270 } \\ \mu \mathrm{g} / \mathrm{kg} & 39.1 & \text { EPA8270 } \\ \mu \mathrm{g} / \mathrm{kg} & 39.1 & \text { EPA8270 } \\ \mu \mathrm{g} / \mathrm{kg} & 39.1 & \text { EPA8270 } \\ \mu \mathrm{g} / \mathrm{kg} & 39.1 & \text { EPA8270 } \\ \mu \mathrm{g} / \mathrm{kg} & 3.91 & \text { EPA8270 } \\ \mu \mathrm{g} / \mathrm{kg} & 391 & \text { EPA8270 } \\ \mu \mathrm{g} / \mathrm{kg} & 39.1 & \text { EPA8270 } \\ \mu \mathrm{g} / \mathrm{kg} & 3.91 & \text { EPA8270 }\end{array}$


SURVEY ID: PPSC 1403 (continued)

Constituent

Sample ID: 105111
$\mathbf{R Q} \mathbf{A Q}$
B Result
Unit
D. Limit Method

$B / N / A$ Extractables (including $P A H$ and phenols)

Dibenzofuran

Di-n-butyl phthalate

1,2-Dichlorobenzene

1,3-Dichlorobenzene

1,4-Dichlorobenzene

3,3'-Dichlorobenzidine

2,4-Dichlorophenol

Diethyl phthalate

2,4-Dimethyl phenol

Dimethyl phthalate

2,4-Dinitrophenol

2,4-Dinitrotoluene

Di-n-octyl phthalate

Docosane

Fluoranthene

Fluorene

Hexachlorobenzene

Hexachlorobutadiene

Hexachlorocyclopentadiene

Hexachloroethane

Hexyl pentadecane

Indeno $[1,2,3 \cdot c, d]$ pyrene

Isophorone

2-Methyl-4,6-dinitrophenol

2-Methylnaphthalene

Naphthalene

m-Nitroaniline

o-Nitroaniline

p-Nitroaniline

Nitrobenzene

2-Nitrophenol

4-Nitrophenol

N-Nitrosodiphenylamine

N-Nitrosodi-n-propylamine

Pentachlorophenol

Phenanthrene

Phenol

Pyrene

1,2,4-Trichlorobenzene

2,4,5-Trichlorophenol

2,4,6-Trichlorophenol

Unknown alcohol

Unknown hydrocarbon
2,6-Dinitrotoluene

\begin{tabular}{|c|c|c|}
\hline U & & 39.1 \\
\hline $\mathrm{U}$ & $\mathrm{V}$ & 33.6 \\
\hline $\mathrm{U}$ & & 39.1 \\
\hline $\mathrm{U}$ & & 39.1 \\
\hline $\mathrm{U}$ & & 39.1 \\
\hline$U$ & & 39.1 \\
\hline $\mathrm{U}$ & & 39.1 \\
\hline $\mathrm{U}$ & & 39.1 \\
\hline $\mathrm{U}$ & & 39.1 \\
\hline $\mathrm{U}$ & & 39.1 \\
\hline $\mathrm{U}$ & & 391 \\
\hline UJ & $\mathrm{C}$ & 3.91 \\
\hline $\mathrm{U}$ & & 3.91 \\
\hline $\mathrm{U}$ & & 39.1 \\
\hline $\mathrm{J}$ & $N$ & 630 \\
\hline $\mathrm{U}$ & & 39.1 \\
\hline$U$ & & 39.1 \\
\hline $\mathrm{U}$ & & 3.91 \\
\hline $\mathrm{U}$ & & 39.1 \\
\hline $\mathrm{U}$ & & 39.1 \\
\hline $\mathrm{U}$ & & 3.91 \\
\hline $\mathbf{J}$ & $\mathbf{N}$ & 640 \\
\hline $\mathrm{U}$ & & 3.91 \\
\hline $\mathrm{U}$ & & 39.1 \\
\hline $\mathrm{U}$ & & 391 \\
\hline $\mathbf{U}$ & & 39.1 \\
\hline $\mathrm{U}$ & & 39.1 \\
\hline $\mathrm{U}$ & & 39.1 \\
\hline $\mathrm{U}$ & & 39.1 \\
\hline $\mathrm{U}$ & & 39.1 \\
\hline $\mathrm{U}$ & & 39.1 \\
\hline $\mathrm{U}$ & & 39.1 \\
\hline $\mathrm{U}$ & & 39.1 \\
\hline $\mathrm{U}$ & & 39.1 \\
\hline $\mathrm{U}$ & & 39.1 \\
\hline $\mathrm{U}$ & & 39.1 \\
\hline $\mathrm{U}$ & & 39.1 \\
\hline $\mathrm{U}$ & & 39.1 \\
\hline $\mathrm{U}$ & & 39.1 \\
\hline $\mathrm{U}$ & & 3.91 \\
\hline $\mathrm{U}$ & & 39.1 \\
\hline $\mathrm{U}$ & & 39.1 \\
\hline$J$ & $N$ & 610 \\
\hline $\mathbf{J}$ & $N$ & 940 \\
\hline
\end{tabular}

Volatile Organic Compounds

Acetone

Benzene

Bromodichloromethane

Bromoform

Bromomethane (Methyl bromide)

Carbon disulfide

Carbon tetrachloride

Chlorobenzene
39.1

39.1

39.1

39.1

39.1

39.1

9.1

3.91

3.91

630

39.1

39.1

39.1

39.1

3.91

.91

39.1

391

39.1

39.1

39.1

39.1

39.1

39.1

39.1

39.1

39.1

39.1

39.1

3.91

39.1

39.1

940

\begin{tabular}{|c|c|c|}
\hline$\mu \mathrm{g} / \mathrm{kg}$ & 39.1 & EPA8270 \\
\hline$\mu \mathrm{g} / \mathrm{kg}$ & 39.1 & EPA8270 \\
\hline$\mu \mathrm{g} / \mathrm{kg}$ & 39.1 & EPA8270 \\
\hline$\mu \mathrm{g} / \mathrm{kg}$ & 39.1 & EPA8270 \\
\hline$\mu \mathrm{g} / \mathrm{kg}$ & 39.1 & EPA8270 \\
\hline$\mu \mathrm{g} / \mathrm{kg}$ & 39.1 & EPA8270 \\
\hline$\mu \mathrm{g} / \mathrm{kg}$ & 39.1 & EPA8270 \\
\hline$\mu \mathrm{g} / \mathrm{kg}$ & 39.1 & EPA8270 \\
\hline$\mu \mathrm{g} / \mathrm{kg}$ & 39.1 & EPA8270 \\
\hline$\mu \mathrm{g} / \mathrm{kg}$ & 39.1 & EPA8270 \\
\hline$\mu \mathrm{g} / \mathrm{kg}$ & 391 & EPA8270 \\
\hline$\mu \mathrm{g} / \mathrm{kg}$ & 3.91 & EPA8270 \\
\hline$\mu \mathrm{g} / \mathrm{kg}$ & 3.91 & EPA8270 \\
\hline$\mu \mathrm{g} / \mathrm{kg}$ & 39.1 & EPA8270 \\
\hline$\mu \mathrm{g} / \mathrm{kg}$ & & EPA8270 \\
\hline$\mu \mathrm{g} / \mathrm{kg}$ & 39.1 & EPA8270 \\
\hline$\mu \mathrm{g} / \mathrm{kg}$ & 39.1 & EPA8270 \\
\hline$\mu \mathrm{g} / \mathrm{kg}$ & 3.91 & EPA8270 \\
\hline$\mu \mathrm{g} / \mathrm{kg}$ & 39.1 & EPA8270 \\
\hline$\mu \mathrm{g} / \mathrm{kg}$ & 39.1 & EPA8270 \\
\hline$\mu \mathrm{g} / \mathrm{kg}$ & 3.91 & EPA8270 \\
\hline$\mu \mathrm{g} / \mathrm{kg}$ & & EPA8270 \\
\hline$\mu \mathrm{g} / \mathrm{kg}$ & 3.91 & EPA8270 \\
\hline$\mu \mathrm{g} / \mathrm{kg}$ & 39.1 & EPA8270 \\
\hline$\mu \mathrm{g} / \mathrm{kg}$ & 391 & EPA8270 \\
\hline$\mu \mathrm{g} / \mathrm{kg}$ & 39.1 & EPA8270 \\
\hline$\mu \mathrm{g} / \mathrm{kg}$ & 39.1 & EPA8270 \\
\hline$\mu \mathrm{g} / \mathrm{kg}$ & 39.1 & EPA8270 \\
\hline$\mu \mathrm{g} / \mathrm{kg}$ & 39.1 & EPA8270 \\
\hline$\mu \mathrm{g} / \mathrm{kg}$ & 39.1 & EPA8270 \\
\hline$\mu \mathrm{g} / \mathrm{kg}$ & 39.1 & EPA8270 \\
\hline$\mu \mathrm{g} / \mathrm{kg}$ & 39.1 & EPA8270 \\
\hline$\mu \mathrm{g} / \mathrm{kg}$ & 39.1 & EPA8270 \\
\hline$\mu \mathrm{g} / \mathrm{kg}$ & 39.1 & EPA8270 \\
\hline$\mu \mathrm{g} / \mathrm{kg}$ & 39.1 & EPA8270 \\
\hline$\mu \mathrm{g} / \mathrm{kg}$ & 39.1 & EPA8270 \\
\hline$\mu \mathrm{g} / \mathrm{kg}$ & 39.1 & EPA8270 \\
\hline$\mu \mathrm{g} / \mathrm{kg}$ & 39.1 & EPA8270 \\
\hline$\mu \mathrm{g} / \mathrm{kg}$ & 39.1 & EPA8270 \\
\hline$\mu \mathrm{g} / \mathrm{kg}$ & 3.91 & EPA8270 \\
\hline$\mu \mathrm{g} / \mathrm{kg}$ & 39.1 & EPA8270 \\
\hline$\mu \mathrm{g} / \mathrm{kg}$ & 39.1 & EPA8270 \\
\hline$\mu \mathrm{g} / \mathrm{kg}$ & & EPA8270 \\
\hline$\mu \mathrm{g} / \mathrm{kg}$ & & EPA8270 \\
\hline
\end{tabular}

$\mu \mathrm{g} / \mathrm{kg}$

$\mu \mathrm{g} / \mathrm{kg}$

$\mu \mathrm{g} / \mathrm{kg}$

$\mu \mathrm{g} / \mathrm{kg}$

$\mu \mathrm{g} / \mathrm{kg}$

$\mu \mathrm{g} / \mathrm{kg}$

$\mu \mathrm{g} / \mathrm{kg}$

$\mu \mathrm{g} / \mathrm{kg}$
58.8

0.588

0.0588

0.0588

0.118

1.18

0.0588

0.0588
EPA8260

EPA8260

EPA8260

EPA8260

EPA8260

EPA8260

EPA8260

EPA8260 
SUURVEY ID: PPSC 1403 (continued)

Constituent

Volatile Organic Compounds

Chloroethane

Chloroethene (Vinyl chloride)

Chloroform

Chloromethane (Methyl chloride)

Dibromochloromethane

1,1-Dichloroethane

1,2-Dichloroethane

1,1-Dichloroethylene

1,2-Dichloroethylene

Dichloromethane (Methylene chloride)

1,2-Dichloropropane

cis-1,3-Dichloropropene

trans-1,3-Dichloropropene

Ethylbenzene

2-Hexanone

Methyl ethyl ketone

Methyl isobutyl ketone

Methyl(methylethyl)cyclohexane

Propylbenzene

Styrene

1,1,2,2-Tetrachloroethane

Tetrachloroethylene

Toluene

1,1,1-Trichloroethane

1,1,2-Trichloroethane

Trichloroethylene

Unknown alkane

Vinyl acetate

Xylenes

\section{Pesticides/PCBs and Dioxins/Furans}

Aldrin

alpha-Benzene hexachloride

beta-Benzene hexachloride

delta-Benzene hexachloride

alpha-Chlordane

gamma-Chlordane

$\mathrm{p}, \mathrm{p}^{\prime}-\mathrm{DDD}$

$p, p^{\prime}-D D E$

p,p'-DDT

Dieldrin

Endosulfan sulfate

Endosulfan I

Endosulfan II

Endrin

Endrin ketone

Heptachlor

Heptachlor epoxide

Lindane

Methoxychlor

PCB 1016

PCB 1221

PCB 1232

PCB 1242
Sample ID: 105111

RQ AQ B Result

Unit

D. Limit Method

$\begin{array}{lll}\mu \mathrm{g} / \mathrm{kg} & 0.118 & \text { EPA8260 } \\ \mu \mathrm{g} / \mathrm{kg} & 0.118 & \text { EPA8260 } \\ \mu \mathrm{g} / \mathrm{kg} & 0.0588 & \text { EPA8260 } \\ \mu \mathrm{g} / \mathrm{kg} & 0.118 & \text { EPA8260 } \\ \mu \mathrm{g} / \mathrm{kg} & 0.0588 & \text { EPA8260 } \\ \mu \mathrm{g} / \mathrm{kg} & 0.0588 & \text { EPA8260 } \\ \mu \mathrm{g} / \mathrm{kg} & 0.0588 & \text { EPA8260 } \\ \mu \mathrm{g} / \mathrm{kg} & 0.0588 & \text { EPA8260 } \\ \mu \mathrm{g} / \mathrm{kg} & 0.118 & \text { EPA8260 } \\ \mu \mathrm{g} / \mathrm{kg} & 0.588 & \text { EPA8260 } \\ \mu \mathrm{g} / \mathrm{kg} & 0.0588 & \text { EPA8260 } \\ \mu \mathrm{g} / \mathrm{kg} & 0.0588 & \text { EPA8260 } \\ \mu \mathrm{g} / \mathrm{kg} & 0.0588 & \text { EPA8260 } \\ \mu \mathrm{g} / \mathrm{kg} & 0.0588 & \text { EPA8260 } \\ \mu \mathrm{g} / \mathrm{kg} & 1.18 & \text { EPA8260 } \\ \mu \mathrm{g} / \mathrm{kg} & 1.18 & \text { EPA8260 } \\ \mu \mathrm{g} / \mathrm{kg} & 1.18 & \text { EPA8260 } \\ \mu \mathrm{g} / \mathrm{kg} & & \text { EPA8260 } \\ \mu \mathrm{g} / \mathrm{kg} & & \text { EPA8260 } \\ \mu \mathrm{g} / \mathrm{kg} & 0.0588 & \text { EPA8260 } \\ \mu \mathrm{g} / \mathrm{kg} & 0.0588 & \text { EPA8260 } \\ \mu \mathrm{g} / \mathrm{kg} & 0.0588 & \text { EPA8260 } \\ \mu \mathrm{g} / \mathrm{kg} & 0.588 & \text { EPA8260 } \\ \mu \mathrm{g} / \mathrm{kg} & 0.0588 & \text { EPA8260 } \\ \mu \mathrm{g} / \mathrm{kg} & 0.0588 & \text { EPA8260 } \\ \mu \mathrm{g} / \mathrm{kg} & 0.0588 & \text { EPA8260 } \\ \mu \mathrm{g} / \mathrm{kg} & & \text { EPA8260 } \\ \mu \mathrm{g} / \mathrm{kg} & 1.18 & \text { EPA8260 } \\ \mu \mathrm{g} / \mathrm{kg} & 0.176 & \text { EPA8260 }\end{array}$

4.77

4.77

9.54

9.54

9.54

9.54

19.1

9.54

19.1

9.54

19.1

9.54

19.1

9.54

19.1

9.54

9.54

4.77

95.4

23.8

23.8

23.8

23.8

$\begin{array}{lrl}\mu \mathrm{g} / \mathrm{kg} & 4.77 & \text { EPA8080 } \\ \mu \mathrm{g} / \mathrm{kg} & 4.77 & \text { EPA8080 } \\ \mu \mathrm{g} / \mathrm{kg} & 9.54 & \text { EPA8080 } \\ \mu \mathrm{g} / \mathrm{kg} & 9.54 & \text { EPA8080 } \\ \mu \mathrm{g} / \mathrm{kg} & 9.54 & \text { EPA8080 } \\ \mu \mathrm{g} / \mathrm{kg} & 9.54 & \text { EPA8080 } \\ \mu \mathrm{g} / \mathrm{kg} & 19.1 & \text { EPA8080 } \\ \mu \mathrm{g} / \mathrm{kg} & 9.54 & \text { EPA8080 } \\ \mu \mathrm{g} / \mathrm{kg} & 19.1 & \text { EPA8080 } \\ \mu \mathrm{g} / \mathrm{kg} & 9.54 & \text { EPA8080 } \\ \mu \mathrm{g} / \mathrm{kg} & 19.1 & \text { EPA8080 } \\ \mu \mathrm{g} / \mathrm{kg} & 9.54 & \text { EPA8080 } \\ \mu \mathrm{g} / \mathrm{kg} & 19.1 & \text { EPA8080 } \\ \mu \mathrm{g} / \mathrm{kg} & 9.54 & \text { EPA8080 } \\ \mu \mathrm{g} / \mathrm{kg} & 19.1 & \text { EPA8080 } \\ \mu \mathrm{g} / \mathrm{kg} & 9.54 & \text { EPA8080 } \\ \mu \mathrm{g} / \mathrm{kg} & 9.54 & \text { EPA8080 } \\ \mu \mathrm{g} / \mathrm{kg} & 4.77 & \text { EPA8080 } \\ \mu \mathrm{g} / \mathrm{kg} & 95.4 & \text { EPA8080 } \\ \mu \mathrm{g} / \mathrm{kg} & 23.8 & \text { EPA8080 } \\ \mu \mathrm{g} / \mathrm{kg} & 23.8 & \text { EPA8080 } \\ \mu \mathrm{g} / \mathrm{kg} & 23.8 & \text { EPA8080 } \\ \mu \mathrm{g} / \mathrm{kg} & 23.8 & \text { EPA8080 }\end{array}$


SURVEY ID: PPSC 1403 (continued)

Constituent

Pesticides/PCBs and Dioxins/Furans

PCB 1248

PCB 1254

PCB 1260

Toxaphene

Radionuclides

Actinium-228

Antimony-124

Antimony-125

Barium-133

Cerium-144

Cesium-134

Cesium-137

Cobalt-57

Cobalt-58

Cobalt-60

Europium-152

Europium-154

Europium-155

Gross alpha

Gross alpha

Lead-212

Manganese-54

Neptunium-239

Nonvolatile beta

Nonvolatile beta

Plutonium-238

Plutonium-238

Plutonium-239/240

Plutonium-239/240

Potassium -40

Promethium-144

Promethium-146

Radium-226

Radium-228

Ruthenium-106

Sodium-22

Strontium-90

Strontium-90

Thorium-234

Tin-113

Yttrium-88

Zinc-65

Zirconium-95

Sample ID: 105111

RQ AQ B Result Unit D. Limit Method

23.8

23.8

23.8

191

$\begin{array}{lll}\mu \mathrm{g} / \mathrm{kg} & 23.8 & \text { EPA8080 } \\ \mu \mathrm{g} / \mathrm{kg} & 23.8 & \text { EPA8080 } \\ \mu \mathrm{g} / \mathrm{kg} & 23.8 & \text { EPA8080 } \\ \mu \mathrm{g} / \mathrm{kg} & 191 & \text { EPA8080 }\end{array}$

6.04E-01士4.42E-02 pCi/g $-4.59 \mathrm{E}-03 \pm 6.30 \mathrm{E}-03 \mathrm{pCi} / \mathrm{g}$ $1.64 \mathrm{E}-03 \pm 1.39 \mathrm{E}-02 \mathrm{pCi} / \mathrm{g}$ $1.22 \mathrm{E}-03 \pm 6.61 \mathrm{E}-03 \mathrm{pCi} / \mathrm{g}$ $-1.99 \mathrm{E}-02 \pm 3.53 \mathrm{E}-02 \mathrm{pCj} / \mathrm{g}$ 7.20E-04 $\pm 5.10 \mathrm{E}-03 \mathrm{pCi} / \mathrm{g}$ 8.59E-02 $\pm 1.30 \mathrm{E}-02 \mathrm{pCi} / \mathrm{g}$ $3.52 \mathrm{E}-03 \pm 4.47 \mathrm{E}-03 \mathrm{pCi} / \mathrm{g}$ $-2.61 \mathrm{E}-03 \pm 5.51 \mathrm{E}-03 \mathrm{pCi} / \mathrm{g}$ $1.59 \mathrm{E}-03 \pm 4.48 \mathrm{E}-03 \mathrm{pCi} / \mathrm{g}$ $-8.50 \mathrm{E}-03 \pm 1.48 \mathrm{E}-02 \mathrm{pCi} / \mathrm{g}$ $-5.32 \mathrm{E}-03 \pm 5.11 \mathrm{E}-02 \mathrm{pCi} / \mathrm{g}$ $0.00 \mathrm{E}+00 \quad \mathrm{pCi} / \mathrm{g}$ $1.28 \mathrm{E}+01 \pm 2.67 \mathrm{E}+00 \mathrm{pCi} / \mathrm{g}$ $2.07 \mathrm{E}+01 \pm 3.57 \mathrm{E}+00 \mathrm{pCi} / \mathrm{g}$ 6.11E-01 $2.01 \mathrm{E}-02 \mathrm{pCi} / \mathrm{g}$ 8.82E-03 $\pm 5.98 \mathrm{E}-03 \mathrm{pCi} / \mathrm{g}$ $3.86 \mathrm{E}-02 \pm 4.82 \mathrm{E}-02 \mathrm{pCi} / \mathrm{g}$ $6.81 \mathrm{E}+00 \pm 1.67 \mathrm{E}+00 \mathrm{pCi} / \mathrm{g}$ $9.86 \mathrm{E}+00 \pm 2.05 \mathrm{E}+00 \mathrm{pCi} / \mathrm{g}$

UIJ I L 9.05E-03 $1.76 \mathrm{E}-02 \mathrm{pCi} / \mathrm{g}$
UIJ I I $1.39 \mathrm{E}-02 \pm 1.72 \mathrm{E}-02 \mathrm{pCj} / \mathrm{g}$ UIJ I I $8.02 \mathrm{E}-03 \pm 1.11 \mathrm{E}-02 \mathrm{pCi} / \mathrm{g}$ UIJ I L 8.14E-03 $\pm 1.12 \mathrm{E}-02 \mathrm{pCi} / \mathrm{g}$ $5.64 \mathrm{E}-01 \pm 9.37 \mathrm{E}-02 \mathrm{pCi} / \mathrm{g}$ $\begin{array}{ll}\text { UI } & 7.03 \mathrm{E}-04 \pm 5.07 \mathrm{E}-03 \mathrm{pCi} / \mathrm{g} \\ \text { UI } & -2.53 \mathrm{E}-03 \pm 7.07 \mathrm{E}-03 \mathrm{pCi} / \mathrm{g}\end{array}$ 5.03E-01 $\pm 3.01 \mathrm{E}-02 \mathrm{pCj} / \mathrm{g}$ $6.04 \mathrm{E}-01 \pm 4.42 \mathrm{E}-02 \mathrm{pCi} / \mathrm{g}$ $-3.00 \mathrm{E}-02 \pm 4.42 \mathrm{E}-02 \mathrm{pCi} / \mathrm{g}$ $-4.23 \mathrm{E}-04 \pm 4.77 \mathrm{E}-03 \mathrm{pCi} / \mathrm{g}$ 1.79E-01 $\pm 5.57 \mathrm{E}-02 \mathrm{pCi} / \mathrm{g}$ 1.40E-01士4.34E-02 pCi/g $6.10 \mathrm{E}-01 \pm 3.67 \mathrm{E}-01 \mathrm{pCi} / \mathrm{g}$ $-1.67 \mathrm{E}-03 \pm 7.64 \mathrm{E}-03 \mathrm{pCi} / \mathrm{g}$ $6.53 \mathrm{E}-03 \pm 5.38 \mathrm{E}-03 \mathrm{pCi} / \mathrm{g}$ $-1.90 \mathrm{E}-03 \pm 1.14 \mathrm{E}-02 \mathrm{pCi} / \mathrm{g}$ $-1.24 \mathrm{E}-02 \pm 1.19 \mathrm{E}-02 \mathrm{pCi} / \mathrm{g}$

\begin{tabular}{|c|c|}
\hline & \\
\hline & \\
\hline & \\
\hline $9 \mathrm{E}-03$ & EPIA \\
\hline & EPIA \\
\hline & EPIA \\
\hline & \\
\hline & EPL \\
\hline & \\
\hline & \\
\hline & \\
\hline & \\
\hline & \\
\hline+00 & \\
\hline & EP. \\
\hline & \\
\hline 12E-03 & EP \\
\hline & \\
\hline-00 & $\mathrm{EP}$ \\
\hline & EP \\
\hline & \\
\hline & EP \\
\hline 02 & $\mathrm{EP}$ \\
\hline & \\
\hline 02 & $\mathrm{EF}$ \\
\hline-03 & EP \\
\hline-02 & EP \\
\hline 1E-02 & EP \\
\hline-02 & EPI \\
\hline 51E-02 & EPIA-013B \\
\hline 16E-03 & EPIA-013E \\
\hline $6.51 \mathrm{E}-02$ & EPIA-0 \\
\hline 5.06E-02 & EPLA-004 \\
\hline $4.18 \mathrm{E}-01$ & EPIA-013E \\
\hline & \\
\hline 1.11E-02 & EPIA-013E \\
\hline & \\
\hline $194 \mathrm{~F}-02$ & \\
\hline
\end{tabular}

2.77E-02 EPIA-013B

1.07E-02 EPIA-013B

2.32E-02 EPIA-013B

9.99E-03 EPIA-013B

1.10E-02 EPIA-013B

7.89E-03 EPIA-013B

2.41E-02 EPIA-013B

$1.55 \mathrm{E}+00$ EPIA-001B

1.57E+00 EPIA-001B

8.42E-03 FPIA-013B

5.68E-02 EPIA-013B

2.39E+00 EPIA-001B

3.54E-02

3.14E-02 EPIA-012B

2.01E-02 EPIA-012B

8.21E.02 EPIA-013B

8.92E-03 EPIA-013B

1.15E-02 EPLA-013B

1.61E-02 EPIA-013B

2.77E-02 EPIA-013B

7.51E-02 EPIA-013B

8.16E-03 EPIA-013B

6.51E-02 EPIA-004

5.06E-02 EPLA-004

4.18E-01 EPIA-013B

1.11E-02

1.94E-02 EPIA-013B

\section{SURVEY ID: PPSC 1501}

Sample ID: 105104

Survey location: 44072E 3671020N (SRS coordinates)

Depth of core interval: 0.00 to $1.00 \mathrm{ft}$

Sample type: Normal

Sample matrix: Soil

Pèrcent solids: $\mathbf{5 3 . 0 0}$ 
SURVEY ID: PPSC 1501 (continued)

Constituent

Sample ID: 105104

RQ AQ B Result

Unit

D. Limit Method

Physical Parameters and Specified Analyses

Ammonia nitrogen

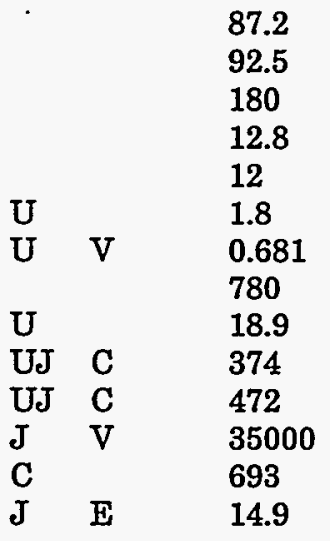

$\mathrm{mg} / \mathrm{kg}$

37.6

$\mathrm{mg} / \mathrm{kg} \quad 37.3$

meq $/ 100 \mathrm{~g} \quad 5$

Cation exchange capacity

$\mathrm{mg} / \mathrm{kg} \quad 4.71$

$\mathrm{mg} / \mathrm{kg} \quad 4.55$

$\mathrm{mg} / \mathrm{kg} \quad 1.8$

$\mathrm{mg} / \mathrm{kg} \quad 1.7$

$\mathrm{mg} / \mathrm{kg} \quad 150$

$\mathrm{mg} / \mathrm{kg} \quad 18.9$

$\mu \mathrm{g} / \mathrm{kg} \quad 374$

$\mathrm{mg} / \mathrm{kg} \quad 472$

$\mathrm{mg} / \mathrm{kg} \quad 189$

$\mathrm{mg} / \mathrm{kg} \quad 0.377$

$\mathrm{mg} / \mathrm{kg}$

65.3

EPA350.1

EPA350.1

EPA9081

EPA300.0

EPA300.0

EPA335.3

EPA353.1

EPA351.2

EPA365.2

EPA420.2

EPA376.2

EPA415.1

EPA351.2

EPA365.4

Metals (total recoverable)

\begin{tabular}{|c|c|c|c|c|}
\hline Aluminum & $J$ & C & 7730 & $\mathrm{mg} / \mathrm{k}$ \\
\hline Antimony & $\mathrm{U}$ & & 8.98 & $\mathrm{mg} / \mathrm{k}$ \\
\hline Arsenic & $U$ & & 27 & $\mathrm{mg} /$ \\
\hline Barium & & & 51 & $\mathrm{mg} /$ \\
\hline Beryllium & $\mathrm{J}$ & $\mathbf{E}$ & 0.813 & $\mathrm{mg} / \mathrm{s}$ \\
\hline Cadmium & $\mathrm{U}$ & & 0.449 & $\mathrm{mg} /$ \\
\hline Calcium & & $\mathrm{V}$ & 451 & $\mathrm{mg} /$ \\
\hline Chromium & & & 32.3 & $\mathbf{m g} /$ \\
\hline Cobalt & & & 3.91 & $\mathrm{mg} /$ \\
\hline Copper & & & 10.8 & $\mathrm{mg} /$ \\
\hline Iron & $J$ & VC & 14400 & $\mathrm{mg} /$ \\
\hline Lead & & & 19 & $\mathrm{mg} /$ \\
\hline Magnesium & & & 387 & $\mathrm{mg} /$ \\
\hline Manganese & & & 289 & $\mathrm{mg} /$ \\
\hline Mercury & $\mathrm{U}$ & $\mathrm{V}$ & 0.119 & \\
\hline Nickel & & & 3.65 & $\mathrm{mg} /$ \\
\hline Potassium & & & 228 & \\
\hline Selenium & $\mathrm{U}$ & & 27 & \\
\hline Silver & $\mathrm{U}$ & & 2.25 & \\
\hline Sodium & $\mathrm{J}$ & $\mathrm{E}$ & 15.3 & \\
\hline Thallium & $\mathrm{U}$ & & 27 & \\
\hline Vanadium & & . & 37.8 & \\
\hline Zinc & & & 21.4 & \\
\hline \multicolumn{5}{|l|}{ Radionuclides } \\
\hline Actinium-228 & & & $1.16 \mathrm{E}+00 \pm 7.84 \mathrm{E}-02$ & $\mathrm{pCi}$ \\
\hline Antimony-124 & UI & & $-3.03 \mathrm{E}-03 \pm 1.22 \mathrm{E}-02$ & $\mathrm{pCi}$ \\
\hline Antimony-125 & UI & & $2.21 \mathrm{E}-02 \pm 4.14 \mathrm{E}-02$ & $\mathrm{pCi}$ \\
\hline Barium-133 & UI & & $3.15 \mathrm{E}-03 \pm 1.77 \mathrm{E}-02$ & $\mathrm{pC}$ \\
\hline Cerium-144 & UI & & $8.35 \mathrm{E}-04 \pm 6.61 \mathrm{E}-02$ & \\
\hline Cesium-134 & UI & & $4.48 \mathrm{E}-03 \pm 1.00 \mathrm{E}-02$ & \\
\hline Cesium-137 & & & $1.25 \mathrm{E}+01 \pm 8.46 \mathrm{E}-02$ & $\mathrm{pCi}$ \\
\hline Cobalt-57 & UI & & $5.18 \mathrm{E}-03 \pm 8.59 \mathrm{E}-03$ & $\mathrm{pC}$ \\
\hline
\end{tabular}

4.89E-02 EPIA-013B

2.08E-02 EPIA-013B

6.73E-02 EPIA-013B

2.58E-02 EPIA-013B

1.13E-01 EPIA-013B

1.54E-02 EPIA-013B

1.79E-02 EPIA-013B

1.48E-02 EPIA-013B 
SURVEY ID: PPSC 1501 (continued)

\begin{tabular}{|c|c|c|c|c|}
\hline Constituent & $\mathbf{R Q}$ & AQ B & Result & Unit \\
\hline \multicolumn{5}{|l|}{ Radionuclides } \\
\hline Cobalt-58 & UT & & $-6.00 \mathrm{E}-03 \pm 9.68 \mathrm{E}-03$ & $\mathrm{pCi} / \mathrm{g}$ \\
\hline Cobalt-60 & & & $7.26 \mathrm{E}-01 \pm 2.77 \mathrm{E}-02$ & $\mathrm{pCi} / \mathrm{g}$ \\
\hline Europium-152 & UI & & $2.60 \mathrm{E}-02 \pm 3.51 \mathrm{E}-02$ & $\mathrm{pCi} / \mathrm{g}$ \\
\hline Europium-154 & UI & & $-5.37 \mathrm{E}-02 \pm 7.88 \mathrm{E}-02$ & $\mathrm{pCi} / \mathrm{g}$ \\
\hline Europium-155 & UI & & $5.41 \mathrm{E}-02 \pm 4.42 \mathrm{E}-02$ & $\mathrm{pCi} / \mathrm{g}$ \\
\hline Gross alpha & & & $1.76 \mathrm{E}+01 \pm 3.76 \mathrm{E}+00$ & $\mathrm{pCi} / \mathrm{g}$ \\
\hline Lead-212 & & & $1.08 \mathrm{E}+00 \pm 3.34 \mathrm{E}-02$ & $\mathrm{pCi} / \mathrm{g}$ \\
\hline Manganese-54 & & & $3.00 \mathrm{E}-02 \pm 1.10 \mathrm{E}-02$ & $\mathrm{pCi} / \mathrm{g}$ \\
\hline Neptunium-239 & UI & & $7.69 \mathrm{E}-02 \pm 8.17 \mathrm{E}-02$ & $\mathrm{pCi} / \mathrm{g}$ \\
\hline Nonvolatile beta & & & $1.75 \mathrm{E}+01 \pm 2.34 \mathrm{E}+00$ & $\mathrm{pCi} / \mathrm{g}$ \\
\hline Plutonium-238 & UI & & $-1.06 \mathrm{E}-03 \pm 1.24 \mathrm{E}-02$ & $\mathrm{pCi} / \mathrm{g}$ \\
\hline Plutonium-239/240 & & & $5.50 \mathrm{E}-02 \pm 2.46 \mathrm{E}-02$ & $\mathrm{pCi} / \mathrm{g}$ \\
\hline Potassium-40 & & V & $4.60 \mathrm{E}+00 \pm 2.27 \mathrm{E}-01$ & $\mathrm{pCi} / \mathrm{g}$ \\
\hline Promethium-144 & UI & & $-4.49 \mathrm{E}-04 \pm 7.21 \mathrm{E}-03$ & $\mathrm{pCi} / \mathrm{g}$ \\
\hline Promethium-146 & UI & & $2.04 \mathrm{E}-02 \pm 2.11 \mathrm{E}-02$ & $\mathrm{pCi} / \mathrm{g}$ \\
\hline Radium-226 & & & $1.43 \mathrm{E}+00 \pm 5.35 \mathrm{E}-02$ & $\mathrm{pCi} / \mathrm{g}$ \\
\hline Radium-228 & & & $1.16 \mathrm{E}+00 \pm 7.84 \mathrm{E}-02$ & $\mathrm{pCi} / \mathrm{g}$ \\
\hline Ruthenium-106 & UI & & $8.47 \mathrm{E}-02 \pm 9.17 \mathrm{E}-02$ & $\mathrm{pCi} / \mathrm{g}$ \\
\hline Sodium-22 & UI & & $2.55 \mathrm{E}-03 \pm 6.78 \mathrm{E}-03$ & $\mathrm{pCi} / \mathrm{g}$ \\
\hline Strontium-90 & & & $1.34 \mathrm{E}-01 \pm 4.97 \mathrm{E}-02$ & $\mathrm{pCi} / \mathrm{g}$ \\
\hline Thorium-234 & & $\mathrm{V}$ & $2.79 \mathrm{E}+00 \pm 7.55 \mathrm{E}-01$ & $\mathrm{pCi} / \mathrm{g}$ \\
\hline Tin-113 & UI & & $-1.30 \mathrm{E}-02 \pm 1.95 \mathrm{E}-02$ & $\mathrm{pCi} / \mathrm{g}$ \\
\hline Yttrium-88 & UI & & $4.99 \mathrm{E}-03 \pm 6.92 \mathrm{E}-03$ & $\mathrm{pCi} / \mathrm{g}$ \\
\hline Zinc-65 & UI & & $2.86 \mathrm{E}-02 \pm 1.40 \mathrm{E}-02$ & $\mathrm{pCi} / \mathrm{g}$ \\
\hline Zirconium-95 & UI & & $3.64 \mathrm{E}-03 \pm 1.82 \mathrm{E}-02$ & $\mathrm{pCi} / \mathrm{g}$ \\
\hline
\end{tabular}

Sample ID: 105104

SURVEY ID: PPSC 1502

Sample ID: 105105

Survey location: 44072E $3671020 \mathrm{~N}$ (SRS coordinates)

Depth of core interval: 1.00 to $2.00 \mathrm{ft}$

Sample type: Normal

Sample matrix: Soil

Percent solids: 65.00

$\begin{array}{lllll}\text { Constituent } & \text { RQ AQ B Result Unit } \quad \text { D. Limit Method }\end{array}$

Physical Parameters and Specified Analyses

Ammonia nitrogen

Cation exchange capacity

Chloride

Cyanide

Nitrate-nitrite as nitrogen

Nitrogen by Kjeldahl method

Orthophosphate

Phenols

Sulfide

Total organic carbon

Total organic nitrogen

Total phosphates (as P)

$\begin{array}{lll} & & 38.2 \\ & & 134 \\ & & 8.38 \\ \text { U } & & 1.41 \\ \text { U } & \mathrm{V} & 0.457 \\ & & 627 \\ \text { U } & & 15.4 \\ \text { UJ } & \text { C } & 307 \\ \text { UJ } & \mathrm{C} & 385 \\ \text { J } & \mathrm{V} & 19500 \\ \mathrm{C} & & 589 \\ \text { J } & \mathrm{E} & 10.7\end{array}$
1.61E-02 EPIA-013B
1.18E-02 EPIA-013B
5.80E-02 EPIA-013B
1.29E-01 EPLA-013B
5.94E-02 EPIA-013B
2.44E+00 EPIA-001B
3.09E-02 EPIA-013B
1.43E-02 EPIA-013B
1.06E-01 EPIA-013B
2.46E+00 EPIA-001B
3.19E-02 EPIA-012B
1.90E-02 EPIA-012B
9.19E-02 EPIA-013B
1.23E-02 EPIA-013B
3.44E-02 EPIA-013B
3.15E-02 EPIA-013B
4.89E-02 EPIA-013B
1.60E-01 EPIA-013B
1.22E-02 EPIA-013B
6.24E-02 EPIA-004
7.32E-01 EPIA-013B
3.14E-02 EPIA-013B
1.30E-02 EPIA-013B
3.07E-02 EPIA-013B
3.13E-02 EPIA-013B

Pexcent solids. 65.00
EPA350.1

EPA9081

EPA300.0

EPA335.3

EPA353.1

EPA351.2

EPA365.2

EPA420.2

EPA376.2

EPA415.1

EPA351.2

EPA365.4 
SURVEY ID: PPSC 1502 (continued)

Constituent

Metals (total recoverable)

\begin{tabular}{|c|c|c|c|}
\hline Aluminum & $J$ & $\mathrm{C}$ & 4990 \\
\hline Antimony & $\mathrm{U}$ & & 7.12 \\
\hline Arsenic & $\mathrm{U}$ & & 21.4 \\
\hline Barium & & & 23.9 \\
\hline Beryllium & $\mathbf{J}$ & $\mathbf{E}$ & 0.442 \\
\hline Cadmium & $\mathrm{U}$ & & 0.356 \\
\hline Calcium & & $\mathrm{V}$ & 230 \\
\hline Chromium & & & 13.8 \\
\hline Cobalt & & & 1.75 \\
\hline Copper & & & 4.55 \\
\hline Iron & $J$ & VC & 8690 \\
\hline Lead & & & 12 \\
\hline Magnesium & & & 222 \\
\hline Manganese & & & 86 \\
\hline Mercury & $\mathrm{U}$ & V & 0.0456 \\
\hline Nickel & & & 2.16 \\
\hline Potassium & & & 162 \\
\hline Selenium & $\mathrm{U}$ & & 21.4 \\
\hline Silver & U & & 1.78 \\
\hline Sodium & $\mathrm{U}$ & & 71.2 \\
\hline Thallium & $\mathrm{U}$ & & 21.4 \\
\hline Vanadium & & & 24.1 \\
\hline Zinc & & & 9.06 \\
\hline
\end{tabular}

\section{Radionuclides}

Actinium-228

Antimony-124

Antimony-125

Barium-133

Cerium-144

Cesium-134

Cesium-137

Cobalt-57

Cobalt-58

Cobalt- 60

Europium-152

Europium-154

Europium-155

Gross alpha

Lead-212

Manganese-54

Neptunium-239

Nonvolatile beta

Plutonium-238

Plutonium-239/240

Potassium-40

Promethium-144

Promethium-146

Radium-226

Radium-228

Ruthenium-106

Sodium-22

Strontium-90

Thorium-234

Sample ID: 105105

RQ AQ B Result Unit D. Limit Method

$\begin{array}{llllll}\mathrm{J} & \mathrm{C} & 4990 & \mathrm{mg} / \mathrm{kg} & 17.8 & \text { EPA6010A } \\ \mathrm{U} & & 7.12 & \mathrm{mg} / \mathrm{kg} & 7.12 & \text { EPA6010A } \\ \mathrm{U} & & 21.4 & \mathrm{mg} / \mathrm{kg} & 21.4 & \text { EPA6010A } \\ & & 23.9 & \mathrm{mg} / \mathrm{kg} & 1.78 & \text { EPA6010A } \\ \mathrm{J} & \mathrm{E} & \mathbf{0 . 4 4 2} & \mathrm{mg} / \mathrm{kg} & 0.712 & \text { EPA6010A } \\ \mathrm{U} & & 0.356 & \mathrm{mg} / \mathrm{kg} & 0.356 & \text { EPA6010A } \\ & \mathrm{V} & 230 & \mathrm{mg} / \mathrm{kg} & 17.8 & \text { EPA6010A } \\ & & 13.8 & \mathrm{mg} / \mathrm{kg} & 1.78 & \text { EPA6010A } \\ & & 1.75 & \mathrm{mg} / \mathrm{kg} & 0.712 & \text { EPA6010A } \\ & & 4.55 & \mathrm{mg} / \mathrm{kg} & 1.78 & \text { EPA6010A } \\ \mathrm{J} & \mathrm{VC} & 8690 & \mathrm{mg} / \mathrm{kg} & 7.12 & \text { EPA6010A } \\ & & 12 & \mathrm{mg} / \mathrm{kg} & 7.12 & \text { EPA6010A } \\ & & 222 & \mathrm{mg} / \mathrm{kg} & 7.12 & \text { EPA6010A } \\ & & 86 & \mathrm{mg} / \mathrm{kg} & 0.712 & \text { EPA6010A } \\ \mathrm{U} & \mathrm{V} & 0.0456 & \mathrm{mg} / \mathrm{kg} & 0.0413 & \text { EPA7471 } \\ & & 2.16 & \mathrm{mg} / \mathrm{kg} & 1.78 & \text { EPA6010A } \\ & & 162 & \mathrm{mg} / \mathrm{kg} & 142 & \text { EPA6010A } \\ \mathrm{U} & & 21.4 & \mathrm{mg} / \mathrm{kg} & 21.4 & \text { EPA6010A } \\ \mathrm{U} & & 1.78 & \mathrm{mg} / \mathrm{kg} & 1.78 & \text { EPA6010A } \\ \mathrm{U} & & \mathrm{mg} / \mathrm{kg} & 71.2 & \text { EPA6010A } \\ \mathrm{U} & & & \mathrm{mg} / \mathrm{kg} & 21.4 & \text { EPA6010A } \\ & & 21.2 & \mathrm{mg} / \mathrm{kg} & 0.712 & \text { EPA6010A } \\ & & 24.1 & \mathrm{mg} / \mathrm{kg} & 1.42 & \text { EPA6010A }\end{array}$

$1.09 \mathrm{E}+00 \pm 5.62 \mathrm{E}-02 \mathrm{pCi} / \mathrm{g}$ 4.09E-04 $\pm 8.60 \mathrm{E}-03 \mathrm{pCi} / \mathrm{g}$ 2.90E-02 $2.51 \mathrm{E}-02 \mathrm{pCi} / \mathrm{g}$ $5.27 \mathrm{E}-03 \pm 1.11 \mathrm{E}-02 \mathrm{pCi} \mathrm{g}$ $-2.31 \mathrm{E}-02 \pm 5.00 \mathrm{E}-02 \mathrm{pCi} / \mathrm{g}$ $-5.49 \mathrm{E}-05 \pm 7.20 \mathrm{E}-03 \mathrm{pCi} / \mathrm{g}$ $6.14 \mathrm{E}+00 \pm 5.05 \mathrm{E}-02 \mathrm{pCi} / \mathrm{g}$ $-1.21 \mathrm{E}-03 \pm 6.39 \mathrm{E}-03 \mathrm{pCi} / \mathrm{g}$ $-1.91 \mathrm{E}-03 \pm 6.33 \mathrm{E}-03 \mathrm{pCi} / \mathrm{g}$ 1.97E-01 $1.27 \mathrm{E}-02 \mathrm{pCi} / \mathrm{g}$ $-3.39 \mathrm{E}-02 \pm 2.24 \mathrm{E}-02 \mathrm{pCi} / \mathrm{g}$ $3.75 \mathrm{E}-02 \pm 5.36 \mathrm{E}-02 \mathrm{pCi} / \mathrm{g}$ $0.00 \mathrm{E}+00 \quad \mathrm{pCi} / \mathrm{g}$ $1.07 \mathrm{E}+01 \pm 2.70 \mathrm{E}+00 \mathrm{pCi} / \mathrm{g}$ $1.20 \mathrm{E}+00 \pm 2.78 \mathrm{E}: 02 \mathrm{pCi} / \mathrm{g}$ $1.36 \mathrm{E}-02 \pm 7.52 \mathrm{E}-03 \mathrm{pCi} / \mathrm{g}$ $5.98 \mathrm{E}-02 \pm 6.09 \mathrm{E}-02 \mathrm{pCi} / \mathrm{g}$ $1.52 \mathrm{E}+01 \pm 2.27 \mathrm{E}+00 \mathrm{pCi} / \mathrm{g}$ 1.22E-02 $1.49 \mathrm{E} \cdot 02 \cdot \mathrm{pCi} / \mathrm{g}$ $3.03 \mathrm{E}-02 \pm 1.78 \mathrm{E}-02 \mathrm{pCi} / \mathrm{g}$ $3.27 \mathrm{E}+00 \pm 1.64 \mathrm{E}-01 \mathrm{pCi} / \mathrm{g}$ $-3.28 \mathrm{E}-04 \pm 5.38 \mathrm{E}-03 \mathrm{pCi} / \mathrm{g}$ 1.97E-02 $1.76 \mathrm{E}-02 \mathrm{pCi} / \mathrm{g}$ $1.02 \mathrm{E}+00 \pm 3.54 \mathrm{E}-02 \mathrm{pCi} / \mathrm{g}$ $1.09 \mathrm{E}+00 \pm 5.62 \mathrm{E}-02 \mathrm{pCi} / \mathrm{g}$ $-1.81 \mathrm{E}-03 \pm 6.30 \mathrm{E}-02 \mathrm{pCi} / \mathrm{g}$ $-5.52 \mathrm{E}-03 \pm 5.20 \mathrm{E}-03 \mathrm{pCi} / \mathrm{g}$ $1.29 \mathrm{E}-01 \pm 8.66 \mathrm{E}-02 \mathrm{pCi} / \mathrm{g}$ $1.82 \mathrm{E}+00 \pm 5.87 \mathrm{E}-01 \mathrm{pCi} / \mathrm{g}$
3.11E-02 EPIA-013B 1.44E-02 EPIA-013B 4.31E-02 EPIA-013B 1.68E-02 EPIA-013B 8.25E-02 EPIA-013B 1.05E-02 EPIA-013B 1.19E-02 EPIA-013B 1.06E-02 EPIA-013B 1.03E-02 EPIA-013B 7.44E-03 EPIA-013B 3.75E-02 EPIA-013B 8.36E-02 EPIA-013B 4.36E-02 EPLA-013B 2.11E+00 EPIA-001B 2.09E-02 EPIA-013B 9.42E-03 EPIA-013B 7.51E-02 EPIA-013B $2.58 \mathrm{E}+00$ EPIA-001B 2.66E-02 EPIA-012B 1.52E-02 EPIA-012B 7.40E-02 EPIA-013B 8.93E-03 EPIA-013B 2.18E-02 EPIA-013B 2.15E-02 EPIA-013B 3.11E-02 EPLA-013B 1.05E-01 EPIA-013B 8.44E-03 EPIA-013B 1.37E-01 EPIA-004 5.29E-01 EPIA-013B 
SURVEY ID: PPSC 1502 (continued)

Constituent

RQ AQ B Result

$-1.49 \mathrm{E}-02+1.21 \mathrm{E}-02 \mathrm{pCi} / \mathrm{g}$ $6.62 \mathrm{E}-03 \pm 5.35 \mathrm{E}-03 \mathrm{pCi} / \mathrm{g}$

$1.97 \mathrm{E}-02 \pm 1.28 \mathrm{E}-02 \mathrm{pCi} / \mathrm{g}$

$0.00 \mathrm{E}+00 \quad \mathrm{pCi} / \mathrm{g}$

Sample ID: 105106

SURVEY ID: PPSC 1503

UI
UI
UI

Zinc-65

$\begin{array}{ll}2.02 \mathrm{E}-02 & \text { EPIA-013B } \\ 9.33 \mathrm{E}-03 & \text { EPIA-013B } \\ 2.07 \mathrm{E}-02 & \text { EPIA-013B } \\ 2.22 \mathrm{E}-02 & \text { EPIA-013B }\end{array}$

2.02E-02 EPIA-013B

Survey location: 44072E $3671020 \mathrm{~N}$ (SRS coordinates)

Depth of core interval: 2.00 to $4.00 \mathrm{ft}$

Sample type: Normal

Sample matrix: Soil

Percent solids: 63.00

Constituent

RQ AQ B Result

Unit

D. Limit Method

Physical Parameters and Specified Analyses

Ammonia nitrogen
Cation exchange capacity
Chloride
Cyanide
Nitrate-nitrite as nitrogen
Nitrogen by Kjeldahl method
Orthophosphate
Phenols
Sulfide
Total organic carbon
Total organic nitrogen
Total phosphates (as P)

Metals (total recoverable)

$\begin{array}{lll} & & 47.8 \\ & & 128 \\ & & 7.74 \\ \text { U } & & 1.28 \\ \text { U } & \text { V } & 0.431 \\ & & 1020 \\ \text { U } & & 15.9 \\ \text { J } & \text { EC } & 142 \\ \text { UJ } & \text { C } & 397 \\ \text { J } & \text { V } & 24200 \\ \text { C } & & 970 \\ \text { J } & \text { E } & 8.81\end{array}$

$\begin{array}{lll}\mathrm{mg} / \mathrm{kg} & 31.2 & \text { EPA350.1 } \\ \mathrm{meq} / 100 \mathrm{~g} & 5 & \text { EPA9081 } \\ \mathrm{mg} / \mathrm{kg} & 3.82 & \text { EPA300.0 } \\ \mathrm{mg} / \mathrm{kg} & 1.28 & \text { EPA335.3 } \\ \mathrm{mg} / \mathrm{kg} & 1.44 & \text { EPA353.1 } \\ \mathrm{mg} / \mathrm{kg} & 121 & \text { EPA351.2 } \\ \mathrm{mg} / \mathrm{kg} & 15.9 & \text { EPA365.2 } \\ \mu \mathrm{g} / \mathrm{kg} & 310 & \text { EPA420.2 } \\ \mathrm{mg} / \mathrm{kg} & 397 & \text { EPA376.2 } \\ \mathrm{mg} / \mathrm{kg} & 1560 & \text { EPA415.1 } \\ \mathrm{mg} / \mathrm{kg} & 0.317 & \text { EPA351.2 } \\ \mathrm{mg} / \mathrm{kg} & 51.2 & \text { EPA365.4 }\end{array}$

$\begin{array}{lll}\mathrm{mg} / \mathrm{kg} & 19.6 & \text { EPA6010A } \\ \mathrm{mg} / \mathrm{kg} & 7.86 & \text { EPA6010A } \\ \mathrm{mg} / \mathrm{kg} & 23.6 & \text { EPA6010A } \\ \mathrm{mg} / \mathrm{kg} & 1.96 & \text { EPA6010A } \\ \mathrm{mg} / \mathrm{kg} & 0.786 & \text { EPA6010A } \\ \mathrm{mg} / \mathrm{kg} & 0.393 & \text { EPA6010A } \\ \mathrm{mg} / \mathrm{kg} & 19.6 & \text { EPA6010A } \\ \mathrm{mg} / \mathrm{kg} & 1.96 & \text { EPA6010A } \\ \mathrm{mg} / \mathrm{kg} & 0.786 & \text { EPA6010A } \\ \mathrm{mg} / \mathrm{kg} & 1.96 & \text { EPA6010A } \\ \mathrm{mg} / \mathrm{kg} & 7.86 & \text { EPA6010A } \\ \mathrm{mg} / \mathrm{kg} & 7.86 & \text { EPA6010A } \\ \mathrm{mg} / \mathrm{kg} & 7.86 & \text { EPA6010A } \\ \mathrm{mg} / \mathrm{kg} & 0.786 & \text { EPA6010A } \\ \mathrm{mg} / \mathrm{kg} & 0.0513 & \text { EPA7471 } \\ \mathrm{mg} / \mathrm{kg} & 1.96 & \text { EPA6010A } \\ \mathrm{mg} / \mathrm{kg} & 157 & \text { EPA6010A } \\ \mathrm{mg} / \mathrm{kg} & 23.6 & \text { EPA6010A } \\ \mathrm{mg} / \mathrm{kg} & 1.96 & \text { EPA6010A } \\ \mathrm{mg} / \mathrm{kg} & 78.6 & \text { EPA6010A } \\ \mathrm{mg} / \mathrm{kg} & 23.6 & \text { EPA6010A } \\ \mathrm{mg} / \mathrm{kg} & 0.786 & \text { EPA6010A } \\ \mathrm{mg} / \mathrm{kg} & 1.57 & \text { EPA6010A }\end{array}$


SURVEY ID: PPSC 1503 (continued)

\section{Constituent}

Radionuclides

Actinium-228

Antimony-124

Antimony-125

Barium-133

Cerium-144

Cesium-134

Cesium-137

Cobalt-57

Cobalt-58

Cobalt-60

Europium-152

Europium-154

Europium-155

Gross alpha

Lead-212

Manganese-54

Neptunium-239

Nonvolatile beta

Plutonium-238

Plutonium-239/240

Potassium-40

Promethium-144

Promethium-146

Radium-226

Radium-228

Ruthenium-106

Sodium-22

Strontium-90

Thorium-234

Tin-113

Yttrium-88

Zinc-65

Zirconium-95
Sample ID: 105106

RQ AQ B Result

Unit

D. Limit Method
3.16E-02 EPIA-013B

1.40E-02 EPIA.013B

3.84E-02 EPIA-013B

1.56E-02 EPIA-013B

7.83E-02 EPIA-013B

1.04E-02 EPIA-013B

1.24E-02 EPIA-013B

1.02E-02 EPIA-013B

1.08E-02 EPIA-013B

8.64E-03 EPIA-013B

3.56E-02 EPIA-013B

8.39E-02 EPIA-013B

4.12E-02 EPIA-013B

2.18E+00 EPIA-001B

1.98E-02 EPIA-013B

9.46E-03 EPIA-013B

7.30E-02 EPIA-013B

2.56E+00 EPIA-001B

2.95E-02 EPIA-012B

2.80E-02 EPIA-012B

7.25E-02 EPIA-013B

9.26E-03 EPIA-013B

1.93E-02 EPIA-013B

2.10E-02 EPIA-013B

3.16E-02 EPIA-013B

1.02E-01 EPIA-013B

8.81E-03 EPIA-013B

1.25E-01 EPIA-004

5.11E-01 EPIA-013B

1.84E-02 EPIA-013B

1.03E-02 EPIA-013B

2.04E-02 EPIA-013B

2.22E-02 EPIA-013B

\section{SURVEY ID: PPSC 1504}

Sample ID: 105107

Survey location: 44072E 3671020N (SRS coordinates)

Depth of core interval: 4.00 to $6.00 \mathrm{ft}$

Sample type: Normal 
SURVEY ID: PPSC 1504 (continued)

Constituent

Sample ID: 105107
RQ AQ B Result
Unit
D. Limit Method

Physical Parameters and Specified Analyses

\begin{tabular}{|c|c|c|c|c|c|c|}
\hline Ammonia nitrogen & $J$ & $\mathbf{E}$ & 18.4 & $\mathrm{mg} / \mathrm{kg}$ & 27.4 & EPA350.1 \\
\hline Cation exchange capacity & & & 130 & $\mathrm{meq} / 100 \mathrm{~g}$ & 5 & EPA9081 \\
\hline Cation exchange capacity & & & 190 & $\mathrm{meq} / 100 \mathrm{~g}$ & 6.94 & EPA9081 \\
\hline Chloride & & & 6.11 & $\mathrm{mg} / \mathrm{kg}$ & 3.38 & EPA300.0 \\
\hline Cyanide & $\mathrm{U}$ & & 1.38 & $\mathrm{mg} / \mathrm{kg}$ & 1.38 & EPA335.3 \\
\hline Nitrate-nitrite as nitrogen & $\mathrm{U}$ & $\mathrm{V}$ & 0.4 & $\mathrm{mg} / \mathrm{kg}$ & 1.33 & EPA353.1 \\
\hline Nitrogen by Kjeldahl method & & & 286 & $\mathrm{mg} / \mathrm{kg}$ & 110 & EPA351.2 \\
\hline Orthophosphate & $\mathrm{U}$ & & 13.9 & $\mathrm{mg} / \mathrm{kg}$ & 13.9 & EPA365.2 \\
\hline Phenols & UJ & C & 278 & $\mu \mathrm{g} / \mathrm{kg}$ & 278 & EPA420.2 \\
\hline Sulfide & UJ & C & 347 & $\mathrm{mg} / \mathrm{kg}$ & 347 & EPA376.2 \\
\hline Total organic carbon & $\mathbf{J}$ & $\mathrm{V}$ & 17500 & $\mathrm{mg} / \mathrm{kg}$ & 139 & EPA415.1 \\
\hline Total organic nitrogen & $\mathrm{C}$ & & 268 & $\mathrm{mg} / \mathrm{kg}$ & 0.278 & EPA351.2 \\
\hline Total phosphates (as P) & $\mathrm{J}$ & $\mathbf{E}$ & 6.15 & $\mathrm{mg} / \mathrm{kg}$ & 24.8 & EPA365.4 \\
\hline \multicolumn{7}{|l|}{ Metals (total recoverable) } \\
\hline Aluminum & $J$ & $\mathbf{C}$ & 2300 & $\mathrm{mg} / \mathrm{kg}$ & 17 & EPA6010A \\
\hline Antimony & $\mathrm{U}$ & & 6.81 & $\mathrm{mg} / \mathrm{kg}$ & 6.81 & EPA6010A \\
\hline Arsenic & $\mathrm{U}$ & & 20.4 & $\mathrm{mg} / \mathrm{kg}$ & 20.4 & EPA6010A \\
\hline Barium & & & 7.96 & $\mathrm{mg} / \mathrm{kg}$ & 1.7 & EPA6010A \\
\hline Beryllium & J & $\mathbf{E}$ & 0.217 & $\mathrm{mg} / \mathrm{kg}$ & 0.681 & EPA6010A \\
\hline Cadmium & $\mathrm{U}$ & & 0.34 & $\mathrm{mg} / \mathrm{kg}$ & 0.34 & EPA6010A \\
\hline Calcium & & $\mathrm{V}$ & 76.6 & $\mathrm{mg} / \mathrm{kg}$ & 17 & EPA6010A \\
\hline Chromium & & & 8.56 & $\mathrm{mg} / \mathrm{kg}$ & 1.7 & EPA6010A \\
\hline Cobalt & $\boldsymbol{J}$ & $\mathbf{E}$ & 0.235 & $\mathrm{mg} / \mathrm{kg}$ & 0.681 & EPA6010A \\
\hline Copper & & & 2 & $\mathrm{mg} / \mathrm{kg}$ & 1.7 & EPA6010A \\
\hline Iron & $J$ & VC & 399 & $\mathrm{mg} / \mathrm{kg}$ & 6.81 & EPA6010A \\
\hline Lead & $\mathbf{J}$ & $\mathrm{E}$ & 5.03 & $\mathrm{mg} / \mathrm{kg}$ & 6.81 & EPA6010A \\
\hline Magnesium & & & 34.7 & $\mathrm{mg} / \mathrm{kg}$ & 6.81 & EPA6010A \\
\hline Manganese & & & 2.66 & $\mathrm{mg} / \mathrm{kg}$ & 0.681 & EPA6010A \\
\hline Mercury & $\mathrm{U}$ & $\mathrm{V}$ & 0.0124 & $\mathrm{mg} / \mathrm{kg}$ & 0.0353 & EPA7471 \\
\hline Nickel & $\mathrm{U}$ & & 1.7 & $\mathrm{mg} / \mathrm{kg}$ & 1.7 & EPA6010A \\
\hline Potassium & $\mathrm{U}$ & & 136 & $\mathrm{mg} / \mathrm{kg}$ & 136 & EPA6010A \\
\hline Selenium & $\mathrm{U}$ & & 20.4 & $\mathrm{mg} / \mathrm{kg}$ & 20.4 & EPA6010A \\
\hline Silver & $U$ & & 1.7 & $\mathrm{mg} / \mathrm{kg}$ & 1.7 & EPA6010A \\
\hline Sodium & $\mathrm{U}$ & & 68.1 & $\mathrm{mg} / \mathrm{kg}$ & 68.1 & EPA6010A \\
\hline Thallium & $\mathrm{U}$ & & 20.4 & $\mathrm{mg} / \mathrm{kg}$ & 20.4 & EPA6010A \\
\hline Vanadium & & & 18 & $\mathrm{mg} / \mathrm{kg}$ & 0.681 & EPA6010A \\
\hline Zinc & $J$ & $\mathrm{E}$ & 1.05 & $\mathrm{mg} / \mathrm{kg}$ & 1.36 & EPA6010A \\
\hline
\end{tabular}

Radionuclides

Actinium-228

Antimony-124

Antimony-125

Barium-133

Cerium-144

Cesium-134

Cesium-137

Cobalt-57

Cobalt-58

Cobalt-60

Europium-152

Europium-154

Europium-155
$1.48 \mathrm{E}+00 \pm 5.63 \mathrm{E}-02 \mathrm{pCi} / \mathrm{g}$ 8.36E-04 $\pm 6.19 \mathrm{E}-03 \mathrm{pCi} / \mathrm{g}$ $-9.77 \mathrm{E}-03 \pm 1.40 \mathrm{E}-02 \mathrm{pCi} / \mathrm{g}$ $4.39 \mathrm{E}-03 \pm 6.85 \mathrm{E}-03 \mathrm{pCi} / \mathrm{g}$ $-1.33 \mathrm{E}-03 \pm 3.84 \mathrm{E}-02 \mathrm{pCi} / \mathrm{g}$ $-6.31 \mathrm{E}-02 \pm 6.20 \mathrm{E}-03 \mathrm{pCi} / \mathrm{g}$ 1.13E-01 $\pm 1.02 \mathrm{E}-02 \mathrm{pCi} / \mathrm{g}$ 2.69E-03 $\pm 4.80 \mathrm{E}-03 \mathrm{pCi} / \mathrm{g}$ $3.47 \mathrm{E}-03 \pm 5.66 \mathrm{E}-03 \mathrm{pCi} / \mathrm{g}$ 5.25E-03 $\pm 4.47 \mathrm{E}-03 \mathrm{pCi} / \mathrm{g}$ $-1.36 \mathrm{E}-02 \pm 1.47 \mathrm{E}-02 \mathrm{pCi} / \mathrm{g}$ $-2.33 \mathrm{E}-02 \pm 3.84 \mathrm{E}-02 \mathrm{pCi} / \mathrm{g}$ $0.00 \mathrm{E}+00$ $\begin{array}{ll}2.64 \mathrm{E}-02 & \text { EPIA-013B } \\ 1.07 \mathrm{E}-02 & \text { EPIA-013B } \\ 2.24 \mathrm{E}-02 & \text { EPIA-013B } \\ 1.03 \mathrm{E}-02 & \text { EPIA-013B } \\ 6.57 \mathrm{E}-02 & \text { EPLA-013B } \\ 7.92 \mathrm{E}-03 & \text { EPIA-013B } \\ 8.96 \mathrm{E}-03 & \text { EPIA-013B } \\ 8.29 \mathrm{E}-03 & \text { EPIA-013B } \\ 9.91 \mathrm{E}-03 & \text { EPLA-013B } \\ 8.35 \mathrm{E}-03 & \text { EPIA-013B } \\ 2.38 \mathrm{E}-02 & \text { EPIA-013B } \\ 6.32 \mathrm{E}-02 & \text { EPIA-013B } \\ 3.50 \mathrm{E}-02 & \text { EPIA-013B }\end{array}$ 
SURVEY ID: PPSC 1504 (continued)

Constituent

Radionuclides

Gross alpha

Lead-212

Manganese-54

Neptunium-239

Nonvolatile beta

Plutonium-238

Plutonium-239/240

Potassium-40

Promethium-144

Promethium-146

Radium-226

Radium-228

Ruthenium-106

Sodium-22

Strontium-90

Thorium-234

Tin-113

Yttrium-88

Zinc-65

Zirconium-95
Sample ID: 105107

RQ AQ B Result Unit D. Limit Method

$1.47 \mathrm{E}+01 \pm 3.01 \mathrm{E}+00 \mathrm{pCi} / \mathrm{g}$ $1.57 \mathrm{E}+00 \pm 2.66 \mathrm{E}-02 \mathrm{pCi} / \mathrm{g}$

$3.17 \mathrm{E}-02 \pm 9.24 \mathrm{E}-03 \mathrm{pCi} / \mathrm{g}$ $8.82 \mathrm{E} \cdot 02 \pm 6.06 \mathrm{E}-02 \mathrm{pCi} / \mathrm{g}$ $8.05 \mathrm{E}+00 \pm 1.84 \mathrm{E}+00 \mathrm{pCi} / \mathrm{g}$

UI $\quad 3.26 \mathrm{E} \cdot 03 \pm 8.52 \mathrm{E}-03 \mathrm{pCi} / \mathrm{g}$

UI $1.09 \mathrm{E}-03 \pm 4.90 \mathrm{E}-03 \mathrm{pCi} / \mathrm{g}$

$\mathrm{V} \quad 1.48 \mathrm{E}+00 \pm 1.26 \mathrm{E}-01 \mathrm{pCi} / \mathrm{g}$

UI $\quad 5.64 \mathrm{E}-04 \pm 5.11 \mathrm{E}-03 \mathrm{pCi} / \mathrm{g}$

UI $\quad 0.00 \mathrm{E}+00 \quad \mathrm{pCi} / \mathrm{g}$

$8.94 \mathrm{E}-01 \pm 2.99 \mathrm{E}-02 \mathrm{pCi} / \mathrm{g}$ $1.48 \mathrm{E}+00 \pm 5.63 \mathrm{E}-02 \mathrm{pCi} / \mathrm{g}$

UI $\quad 6.23 \mathrm{E}-02 \pm 7.70 \mathrm{E}-02 \mathrm{pCi} / \mathrm{g}$

UI $\quad-1.87 \mathrm{E}-03 \pm 4.15 \mathrm{E}-03 \mathrm{pCi} / \mathrm{g}$

UI $3.92 \mathrm{E}-02 \pm 7.21 \mathrm{E}-02 \mathrm{pCi} / \mathrm{g}$

$\mathrm{V} \quad 1.23 \mathrm{E}+00 \pm 5.95 \mathrm{E}-01 \mathrm{pCi} / \mathrm{g}$

$-6.46 \mathrm{E}-04 \pm 8.38 \mathrm{E}-03 \mathrm{pCi} / \mathrm{g}$

$-1.45 \mathrm{E}-03 \pm 4.84 \mathrm{E}-03 \mathrm{pCi} / \mathrm{g}$

$1.33 \mathrm{E}-03 \pm 1.03 \mathrm{E}-02 \mathrm{pCi} / \mathrm{g}$

$0.00 \mathrm{E}+00$

$\mathrm{pCi} / \mathrm{g}$
2.21E+00 EPIA-001B

1.48E-02 EPIA-013B

7.63E-03 EPIA-013B

5.94E-02 EPIA-013B

2.69E+00 EPLA-001B

1.91E-02 EPIA-012B

1.38E-02 EPIA-012B

7.04E-02 EPIA-013B

8.77E-03 EPIA-013B

1.17E.02 EPLA-013B

1.60E-02 EPIA-013B

2.64E-02 EPIA-013B

7.43E-02 EPIA-013B

7.19E-03 EPIA-013B

1.50E-01 EPIA-004

4.62E-01 EPLA-013B

1.22E-02 EPIA-013B

8.32E-03 EPIA-013B

1.53E-02 EPIA-013B

2.19E-02 EPIA-013B

SURVEY ID: PPSC 1505

Sample ID: 105108

Survey location: $44072 \mathrm{E} 3671020 \mathrm{~N}$ (SRS coordinates)

Depth of core interval: 6.00 to $8.00 \mathrm{ft}$

Sample type: Normal

Sample matrix: Soil

Percent solids: 71.00

Constituent

RQ AQ B Result

Unit

D. Limit Method

Physical Parameters and Specified Analyses

Ammonia nitrogen

Cation exchange capacity

Chloride

Cyanide

Cyanide

Nitrate-nitrite as nitrogen

Nitrate-nitrite as nitrogen

Nitrogen by Kjeldahl method

Orthophosphäte

Phenols

Sulfide

Total organic carbon

Total organic nitrogen

Total phosphates (as P)
$\mathrm{U}$

$\begin{array}{lll}\text { U } & & 27.1 \\ & & 124 \\ & & 3.78 \\ \text { U } & & 1.41 \\ \text { U } & & 1.34 \\ \text { U } & \text { V } & 0.405 \\ \text { U } & \text { V } & 0.418 \\ & & 325 \\ \text { U } & & 14.1 \\ \text { J } & \text { EC } & 74.7 \\ \text { UJ } & \text { C } & 352 \\ & \text { V } & 19300 \\ \text { C } & & 312 \\ \text { J } & \text { E } & 6.96\end{array}$

$\begin{array}{lll}\mathrm{mg} / \mathrm{kg} & 27.1 & \text { EPA350.1 } \\ \mathrm{meq} / 100 \mathrm{~g} & 5 & \text { EPA9081 } \\ \mathrm{mg} / \mathrm{kg} & 3.33 & \text { EPA300.0 } \\ \mathrm{mg} / \mathrm{kg} & 1.41 & \text { EPA335.3 } \\ \mathrm{mg} / \mathrm{kg} & 1.34 & \text { EPA335.3 } \\ \mathrm{mg} / \mathrm{kg} & 1.35 & \text { EPA353.1 } \\ \mathrm{mg} / \mathrm{kg} & 1.39 & \text { EPA353.1 } \\ \mathrm{mg} / \mathrm{kg} & 112 & \text { EPA351.2 } \\ \mathrm{mg} / \mathrm{kg} & 14.1 & \text { EPA365.2 } \\ \mu \mathrm{g} / \mathrm{kg} & 280 & \text { EPA420.2 } \\ \mathrm{mg} / \mathrm{kg} & 352 & \text { EPA376.2 } \\ \mathrm{mg} / \mathrm{kg} & 141 & \text { EPA415.1 } \\ \mathrm{mg} / \mathrm{kg} & 0.282 & \text { EPA351.2 } \\ \mathrm{mg} / \mathrm{kg} & 25.8 & \text { EPA365.4 }\end{array}$


SURVEY ID: PPSC 1505 (continued)

Constituent

Metals (total recoverable)
Sample ID: 105108

RQ AQ B Result Unit D. Limit Method

Aluminum
Antimony
Arsenic
Barium
Beryllium
Cadmium
Calcium
Chromium
Cobalt
Copper
Iron
Lead
Magnesium
Manganese
Mercury
Nickel
Potassium
Selenium
Silver
Sodium
Thallium
Vanadium
Zinc

Radionuclides

Actinium-228

Actinium-228

Antimony-124

Antimony-124

Antimony-125

Antimony-125

Barium-133

Barium-133

Cerium-144

Cerium-144

Cesium-134

Cesium-134

Cesium-137

Cesium-137

Cobalt-57

Cobalt-57

Cobalt-58

Cobalt-58

Cobalt-60

Cobalt-60

Europium-152

Europium-152

Europium-154

Europium-154

Europium-155

Europium-155

Gross alpha

Lead-212

Lead-212

$\begin{array}{llllll}\mathrm{J} & \mathrm{C} & 1910 & \mathrm{mg} / \mathrm{kg} & 16.5 & \text { EPA6010A } \\ \mathrm{U} & & 6.58 & \mathrm{mg} / \mathrm{kg} & 6.58 & \text { EPA6010A } \\ \mathrm{U} & & 19.7 & \mathrm{mg} / \mathrm{kg} & 19.7 & \text { EPA6010A } \\ & & 6.68 & \mathrm{mg} / \mathrm{kg} & 1.65 & \text { EPA6010A } \\ \mathrm{J} & \mathrm{E} & 0.215 & \mathrm{mg} / \mathrm{kg} & 0.658 & \text { EPA6010A } \\ \mathrm{U} & & 0.329 & \mathrm{mg} / \mathrm{kg} & 0.329 & \text { EPA6010A } \\ & \mathrm{V} & 139 & \mathrm{mg} / \mathrm{kg} & 16.5 & \text { EPA6010A } \\ & & 12.7 & \mathrm{mg} / \mathrm{kg} & 1.65 & \text { EPA6010A } \\ \mathrm{U} & & 0.658 & \mathrm{mg} / \mathrm{kg} & 0.658 & \text { EPA6010A } \\ & & 1.92 & \mathrm{mg} / \mathrm{kg} & 1.65 & \text { EPA6010A } \\ \mathrm{J} & \mathrm{VC} & 264 & \mathrm{mg} / \mathrm{kg} & 6.58 & \text { EPA6010A } \\ \mathrm{J} & \mathrm{E} & 4.16 & \mathrm{mg} / \mathrm{kg} & 6.58 & \text { EPA6010A } \\ & & 29.3 & \mathrm{mg} / \mathrm{kg} & 6.58 & \text { EPA6010A } \\ & & 1.44 & \mathrm{mg} / \mathrm{kg} & 0.658 & \text { EPA6010A } \\ \mathrm{U} & \mathrm{V} & 0.0122 & \mathrm{mg} / \mathrm{kg} & 0.0451 & \text { EPA7471 } \\ \mathrm{J} & \mathrm{E} & 0.748 & \mathrm{mg} / \mathrm{kg} & 1.65 & \text { EPA6010A } \\ \mathrm{U} & & 132 & \mathrm{mg} / \mathrm{kg} & 132 & \text { EPA6010A } \\ \mathrm{U} & & 19.7 & \mathrm{mg} / \mathrm{kg} & 19.7 & \text { EPA6010A } \\ \mathrm{U} & & 1.65 & \mathrm{mg} / \mathrm{kg} & 1.65 & \text { EPA6010A } \\ \mathrm{U} & & 65.8 & \mathrm{mg} / \mathrm{kg} & 65.8 & \text { EPA6010A } \\ \mathrm{U} & & 19.7 & \mathrm{mg} / \mathrm{kg} & 19.7 & \text { EPA6010A } \\ & & 22.3 & \mathrm{mg} / \mathrm{kg} & 0.658 & \text { EPA6010A } \\ & 1.32 & \mathrm{mg} / \mathrm{kg} & 1.32 & \text { EPA6010A }\end{array}$

UI

UI

UI

UI

UI

UI

UI

UI

UI

UI

UI

UI

UI

UI

UI

UI

UI

UI

UI

UI

UI

UI

UI
1.45E $+00 \pm 5.56 \mathrm{E}-02 \mathrm{pCi} / \mathrm{g}$ $1.43 \mathrm{E}+00 \pm 5.69 \mathrm{E}-02 \mathrm{pCi} / \mathrm{g}$ 3.22E-04 $\pm 6.09 \mathrm{E}-03 \mathrm{pCi} / \mathrm{g}$ $6.18 \mathrm{E}-03 \pm 5.93 \mathrm{E}-03 \mathrm{pCi} / \mathrm{g}$ $6.22 \mathrm{E}-03 \pm 1.30 \mathrm{E}-02 \mathrm{pCi} / \mathrm{g}$ 4.93E-03 $\pm 1.37 \mathrm{E}-02 \mathrm{pCi} / \mathrm{g}$ 2.36E-03 $\pm 6.63 \mathrm{E}-03 \mathrm{pCi} / \mathrm{g}$ $-2.42 \mathrm{E}-03 \pm 6.84 \mathrm{E}-03 \mathrm{pCi} / \mathrm{g}$ $-4.04 \mathrm{E}-02 \pm 3.96 \mathrm{E}-02 \mathrm{pCi} / \mathrm{g}$ $-9.02 \mathrm{E}-03 \pm 3.76 \mathrm{E}-02 \mathrm{pCi} / \mathrm{g}$ 1.12E-03 $\pm 5.10 \mathrm{E}-03 \mathrm{pCi} / \mathrm{g}$ $1.50 \mathrm{E}-04 \pm 5.00 \mathrm{E}-03 \mathrm{pCi} / \mathrm{g}$ $1.50 \mathrm{E}-02 \pm 7.40 \mathrm{E}-03 \mathrm{pCi} / \mathrm{g}$ $0.00 \mathrm{E}+00 \quad \mathrm{pCi} / \mathrm{g}$ 6.83E-04t4.88E-03 $\mathrm{pCi} / \mathrm{g}$ $-4.20 \mathrm{E}-03 \pm 4.72 \mathrm{E}-03 \mathrm{pCi} / \mathrm{g}$ $-5.02 \mathrm{E}-04 \pm 5.51 \mathrm{E}-03 \mathrm{pCi} / \mathrm{g}$ $-3.19 \mathrm{E}-03 \pm 6.33 \mathrm{E}-03 \mathrm{pCi} / \mathrm{g}$ $-1.02 \mathrm{E}-03 \pm 4.30 \mathrm{E}-03 \mathrm{pCi} / \mathrm{g}$ $-2.83 \mathrm{E}-03 \pm 4.34 \mathrm{E}-03 \mathrm{pCi} / \mathrm{g}$ $-1.73 \mathrm{E}-02 \pm 1.39 \mathrm{E}-02 \mathrm{pCi} / \mathrm{g}$ $-3.94 \mathrm{E}-03 \pm 1.38 \mathrm{E}-02 \mathrm{pCi} / \mathrm{g}$ $5.26 \mathrm{E}-02 \pm 4.35 \mathrm{E}-02 \mathrm{pCi} / \mathrm{g}$ $-1.02 \mathrm{E}-02 \pm 4.33 \mathrm{E}-02 \mathrm{pCi} / \mathrm{g}$ $0.00 \mathrm{E}+00 \quad \mathrm{pCi} / \mathrm{g}$ $0.00 \mathrm{E}+00 \quad \mathrm{pCi} / \mathrm{g}$ $2.01 \mathrm{E}+01 \pm 3.49 \mathrm{E}+00 \mathrm{pCi} / \mathrm{g}$ $1.51 \mathrm{E}+00 \pm 2.59 \mathrm{E}-02 \mathrm{pCi} / \mathrm{g}$ $1.47 \mathrm{E}+00 \pm 2.62 \mathrm{E}-02 \mathrm{pCi} / \mathrm{g}$
2.59E-02 EPIA-013B 2.56E-02 EPIA-013B 1.02E-02 EPIA-013B 1.06E-02 EPIA-013B 2.24E-02 EPIA-013B 2.27E-02 EPIA-013B 1.02E-02 EPIA-013B 9.91E-03 EPIA-013B

6.49E-02 EPIA-013B 6.41E-02 EPIA-013B 7.51E-03 EPIA-013B

7.63E-03 EPIA-013B

8.72E-03 EPIA-013B

9.83E-03 EPIA-013B

8.14E-03 EPIA-013B

8.00E-03 EPIA-013B

9.09E-03 EPIA-013B

9.22E-03 EPIA-013B

7.26E-03 EPIA-013B

7.39E-03 EPIA-013B

2.33E-02 EPIA-013B

2.26E-02 EPIA-013B

7.04E-02 EPIA-013B

6.33E-02 EPLA-013B

3.48E-02 EPIA-013B

3.42E-02 EPIA-013B

1.63E+00 EPIA-001B

1.44E-02 EPIA-013B

1.51E-02 EPIA-013B 
SURVEY ID: PPSC 1505 (continued)

Constituent

Radionuclides

Manganese-54

Manganese-54

Neptunium-239

Neptunium-239

Nonvolatile beta

Plutonium-238

Plutonium-239/240

Potassium-40

Potassium-40

Promethium-144

Promethium-144

Promethium-146

Promethium-146

Radium-226

Radium-226

Radium-228

Radium-228

Ruthenium-106

Ruthenium-106

Sodium-22

Sodium-22

Strontium-90

Strontium-90

Thorium-234

Thorium-234

Tin-113

Tin-113

Yttrium-88

Yttrium-88

Zinc-65

Zinc-65

Zirconium-95

Zirconium-95
RQ AQ B Result

$\begin{array}{ll}\text { UI } \\ \\ \text { UI } & \\ \text { UI } & \\ & \text { V } \\ & \text { V } \\ \text { UI } & \\ \text { UI } & \\ \text { UI } & \\ \text { UI } & \end{array}$

UI

UI

UI

UI

UI

UI

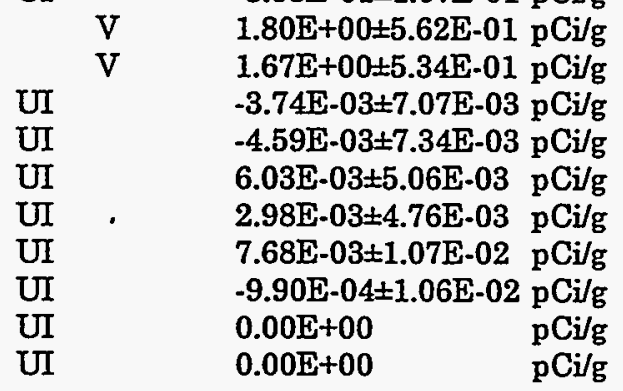

2.21E-02 $\pm 7.22 \mathrm{E}-03 \mathrm{pCi} / \mathrm{g}$ $2.48 \mathrm{E}-02 \pm 6.30 \mathrm{E}-03 \mathrm{pCi} / \mathrm{g}$ $0.00 \mathrm{E}+00 \quad \mathrm{pCi} / \mathrm{g}$ 7.65E-02 $\pm 5.88 \mathrm{E}-02 \mathrm{pCi} / \mathrm{g}$ $1.02 \mathrm{E}+01 \pm 1.98 \mathrm{E}+00 \mathrm{pCi} / \mathrm{g}$ $1.09 \mathrm{E}-02 \pm 1.64 \mathrm{E}-02 \mathrm{pCi} / \mathrm{g}$ $3.64 \mathrm{E}-03 \pm 7.30 \mathrm{E}-03 \mathrm{pCi} / \mathrm{g}$ $1.39 \mathrm{E}+00 \pm 1.09 \mathrm{E}-01 \mathrm{pCi} / \mathrm{g}$ $1.34 \mathrm{E}+00 \pm 1.13 \mathrm{E}-01 \mathrm{pCi} / \mathrm{g}$ $-1.14 \mathrm{E}-03 \pm 4.92 \mathrm{E}-03 \mathrm{pCi} / \mathrm{g}$ $1.60 \mathrm{E}-03 \pm 4.81 \mathrm{E}-03 \mathrm{pCj} / \mathrm{g}$ $0.00 \mathrm{E}+00 \quad \mathrm{pCi} / \mathrm{g}$ $0.00 \mathrm{E}+00 \quad \mathrm{pCi} / \mathrm{g}$ 9.08E-01 $\pm 2.86 \mathrm{E}-02 \mathrm{pCi} / \mathrm{g}$ 9.30E-01 $\pm 3.03 \mathrm{E}-02 \mathrm{pCi} / \mathrm{g}$ $1.45 \mathrm{E}+00 \pm 5.56 \mathrm{E}-02 \mathrm{pCi} / \mathrm{g}$ $1.43 \mathrm{E}+00 \pm 5.69 \mathrm{E}-02 \mathrm{pCi} / \mathrm{g}$ 2.08E-02士4.46E.02 pCi/g 7.00E-04 $\pm 4.43 \mathrm{E}-02 \mathrm{pCi} / \mathrm{g}$ $-6.35 \mathrm{E}-03 \pm 4.52 \mathrm{E}-03 \mathrm{pCi} / \mathrm{g}$ $-2.28 \mathrm{E}-04 \pm 4.17 \mathrm{E}-03 \mathrm{pCi} / \mathrm{g}$ $1.67 \mathrm{E}-01 \pm 1.16 \mathrm{E}-01 \mathrm{pCi} / \mathrm{g}$ $-3.53 \mathrm{E}-01 \pm 1.07 \mathrm{E}-01 \mathrm{pCi} / \mathrm{g}$ pCig $-3.74 \mathrm{E}-03 \pm 7.07 \mathrm{E}-03 \mathrm{pCi} / \mathrm{g}$ $-4.59 \mathrm{E}-03 \pm 7.34 \mathrm{E}-03 \mathrm{pCi} / \mathrm{g}$ $0.00 \mathrm{E}+00 \quad \mathrm{pCi} / \mathrm{g}$ $\mathrm{pCi} / \mathrm{g}$
8.08E-03 EPIA-013B

7.46E-03 EPIA-013B

5.77E-02 EPIA-013B

5.90E-02 EPIA-013B

2.58E+00 EPIA-001B

3.15E-02 EPIA-012B

1.54E-02 EPIA-012B

6.47E-02 EPIA-013B

7.02E-02 EPIA-013B

8.12E-03 EPIA-013B

8.33E-03 EPIA-013B

1.11E-02 EPIA-013B

1.09E-02 EPIA-013B

1.53E-02 EPIA-013B

1.60E-02 EPIA-013B

2.59E-02 EPIA-013B

2.56E-02 EPIA-013B

7.58E-02 EPIA-013B

7.65E-02 EPIA-013B

7.20E-03 EPIA-013B

7.36E-03 EPIA-013B

1.84E-01 EPIA-004

2.08E-01 EPIA-004

4.47E-01 EPIA-013B

4.42E-01 EPIA-013B

1.20E-02 EPLA-013B

1.18E-02 EPIA-013B

9.60E-03 EPIA-013B

8.82E-03 EPIA-013B

1.67E-02 EPIA-013B

1.55E-02 EPIA-013B

2.05E-02 EPIA-013B

2.18E-02 EPIA-013B

SURVEY ID: PPSC 1601

Sample ID: 104697

Survey location: $44074 \mathrm{E} 3671718 \mathrm{~N}$ (SRS coordinates)

Depth of core interval: 0.00 to $1.00 \mathrm{ft}$

Sample type: Normal

Sample matrix: Soil

Percent solids: 64.00

Constituent

RQ AQ B Result

Unit

D. Limit Method

Physical Parameters and Specified Analyses

Ammonia nitrogen

Cation exchange capacity

Chloride

Chloride

Cyanide

Nitrate-nitrite as nitrogen

Nitrogen by Kjeldahl method

Orthophosphate

$\begin{array}{lll} & & 56.2 \\ & & 147 \\ \text { J } & Q & 5.93 \\ \text { J } & \text { Q } & 7.29 \\ \text { U } & & 1.43 \\ \text { J } & \text { E } & 0.648 \\ & & 523 \\ \text { J } & \text { E } & 3.73\end{array}$

$\begin{array}{lll}\mathrm{mg} / \mathrm{kg} & 31.1 & \text { EPA350.1 } \\ \mathrm{meq} / 100 \mathrm{~g} & 5 & \text { EPA9081 } \\ \mathrm{mg} / \mathrm{kg} & 3.84 & \text { EPA300.0 } \\ \mathrm{mg} / \mathrm{kg} & 3.87 & \text { EPA300.0 } \\ \mathrm{mg} / \mathrm{kg} & 1.43 & \text { EPA335.3 } \\ \mathrm{mg} / \mathrm{kg} & 1.62 & \text { EPA353.1 } \\ \mathrm{mg} / \mathrm{kg} & 116 & \text { EPA351.2 } \\ \mathrm{mg} / \mathrm{kg} & 15.6 & \text { EPA365.2 }\end{array}$


SURVEY ID: PPSC 1601 (continued)

Constituent

Sample ID: 104697
RQ AQ B Result
Unit
D. Limit Method

Physical Parameters and Specified Analyses

$\begin{array}{llllllll}\text { Phenols } & \text { UJ } & \text { C } & & 467 & \mu \mathrm{g} / \mathrm{kg} & 467 & \text { EPA420.2 } \\ \text { Sulfide } & \text { UJ } & \text { IC } & \text { L } & 391 & \mathrm{mg} / \mathrm{kg} & 391 & \text { EPA376.2 } \\ \text { Sulfide } & \text { UJ } & \text { IC } & \text { L } & 391 & \mathrm{mg} / \mathrm{kg} & 391 & \text { EPA376.2 } \\ \text { Total organic carbon } & & \text { V } & & 15900 & \mathrm{mg} / \mathrm{kg} & 156 & \text { EPA415.1 } \\ \text { Total organic nitrogen } & \text { C } & & & 467 & \mathrm{mg} / \mathrm{kg} & \mathbf{0 . 3 1 3} & \text { EPA351.2 } \\ \text { Total phosphates (as P) } & \mathrm{J} & \mathrm{E} & & 163 & \mathrm{mg} / \mathrm{kg} & 625 & \text { EPA365.4 }\end{array}$

Metals (total recoverable)

\begin{tabular}{|c|c|c|c|}
\hline Aluminum & & & 10200 \\
\hline Antimony & $\mathrm{U}$ & & 7.17 \\
\hline Arsenic & $\mathrm{U}$ & & 21.5 \\
\hline Barium & & & 28.1 \\
\hline Beryllium & $J$ & $\mathrm{E}$ & 0.399 \\
\hline Cadmium & $\mathrm{U}$ & & 0.358 \\
\hline Calcium & & & 390 \\
\hline Chromium & & & 18.3 \\
\hline Cobalt & & & 2.32 \\
\hline Copper & & & 8 \\
\hline Iron & & $\mathrm{V}$ & 13100 \\
\hline Lead & & & 13.3 \\
\hline Magnesium & & & 208 \\
\hline Manganese & & & 168 \\
\hline Mercury & & & 0.101 \\
\hline Nickel & & & 2.66 \\
\hline Potassium & $\mathbf{J}$ & $\mathrm{E}$ & 137 \\
\hline Selenium & $\mathrm{U}$ & & 21.5 \\
\hline Silver & $\mathrm{U}$ & & 1.79 \\
\hline Sodium & $\bar{J}$ & $\mathrm{E}$ & 16.5 \\
\hline Thallium & $\mathrm{U}$ & & 21.5 \\
\hline Vanadium & & & 36 \\
\hline Zinc & & & 18.2 \\
\hline
\end{tabular}

$\begin{array}{lll}\mathrm{mg} / \mathrm{kg} & 17.9 & \text { EPA6010A } \\ \mathrm{mg} / \mathrm{kg} & 7.17 & \text { EPA6010A } \\ \mathrm{mg} / \mathrm{kg} & 21.5 & \text { EPA6010A } \\ \mathrm{mg} / \mathrm{kg} & 1.79 & \text { EPA6010A } \\ \mathrm{mg} / \mathrm{kg} & 0.717 & \text { EPA6010A } \\ \mathrm{mg} / \mathrm{kg} & 0.358 & \text { EPA6010A } \\ \mathrm{mg} / \mathrm{kg} & 17.9 & \text { EPA6010A } \\ \mathrm{mg} / \mathrm{kg} & 1.79 & \text { EPA6010A } \\ \mathrm{mg} / \mathrm{kg} & 0.717 & \text { EPA6010A } \\ \mathrm{mg} / \mathrm{kg} & 1.79 & \text { EPA6010A } \\ \mathrm{mg} / \mathrm{kg} & 7.17 & \text { EPA6010A } \\ \mathrm{mg} / \mathrm{kg} & 7.17 & \text { EPA6010A } \\ \mathrm{mg} / \mathrm{kg} & 7.17 & \text { EPA6010A } \\ \mathrm{mg} / \mathrm{kg} & 0.717 & \text { EPA6010A } \\ \mathrm{mg} / \mathrm{kg} & 0.0493 & \text { EPA7471 } \\ \mathrm{mg} / \mathrm{kg} & 1.79 & \text { EPA6010A } \\ \mathrm{mg} / \mathrm{kg} & 143 & \text { EPA6010A } \\ \mathrm{mg} / \mathrm{kg} & 21.5 & \text { EPA6010A } \\ \mathrm{mg} / \mathrm{kg} & 1.79 & \text { EPA6010A } \\ \mathrm{mg} / \mathrm{kg} & 71.7 & \text { EPA6010A } \\ \mathrm{mg} / \mathrm{kg} & 21.5 & \text { EPA6010A } \\ \mathrm{mg} / \mathrm{kg} & 0.717 & \text { EPA6010A } \\ \mathrm{mg} / \mathrm{kg} & 1.43 & \text { EPA6010A }\end{array}$

Radionuclides

Actinium-228

Antimony-124

Antimony-125

Barium-133

Cerium-144

Cesium-134

Cesium-137

Cobalt-57

Cobalt-58

Cobalt-60

Europium-152

Europium-154

Europium-155

Gross alpha

Lead-212

Manganese-54

Neptunium-239

Nonvolatile beta

Plutonium-238

Plutonium-239/240
$1.53 \mathrm{E}+00 \pm 7.67 \mathrm{E}-02 \mathrm{pCi} / \mathrm{g}$ 1.94E-02 $2.64 \mathrm{E}-02 \mathrm{pCi} / \mathrm{g}$ 2.06E-02 $\pm 8.64 \mathrm{E}-02 \mathrm{pCi} / \mathrm{g}$ 2.71E-02 $\pm 3.59 \mathrm{E}-02 \mathrm{pCi} / \mathrm{g}$ $3.39 \mathrm{E}-02 \pm 1.22 \mathrm{E}-01 \mathrm{pCi} / \mathrm{g}$ 1.43E-02 $1.95 \mathrm{E}-02 \mathrm{pCi} / \mathrm{g}$ $7.92 \mathrm{E}+01 \pm 1.98 \mathrm{E}-01 \mathrm{pCi} / \mathrm{g}$ 5.46E-03 $\pm 1.56 \mathrm{E}-02 \mathrm{pCi} / \mathrm{g}$ $-9.82 \mathrm{E}-03 \pm 9.21 \mathrm{E}-03 \mathrm{pCi} / \mathrm{g}$ $7.44 \mathrm{E}-01 \pm 2.72 \mathrm{E}-02 \mathrm{pCi} / \mathrm{g}$ $-1.88 \mathrm{E}-02 \pm 7.07 \mathrm{E}-02 \mathrm{pCi} / \mathrm{g}$ $-2.19 \mathrm{E}-02 \pm 8.63 \mathrm{E}-02 \mathrm{pCi} / \mathrm{g}$ 7.44E-02 $\pm 9.10 \mathrm{E}-02 \mathrm{pCi} / \mathrm{g}$ $1.80 \mathrm{E}+01 \pm 3.77 \mathrm{E}+00 \mathrm{pCi} / \mathrm{g}$ $1.54 \mathrm{E}+00 \pm 5.52 \mathrm{E}-02 \mathrm{pCi} / \mathrm{g}$ $2.28 \mathrm{E}-02 \pm 1.13 \mathrm{E}-02 \mathrm{pCi} / \mathrm{g}$ 6.91E-04 $1.12 \mathrm{E}-01 \mathrm{pCi} / \mathrm{g}$ $6.39 \mathrm{E}+01 \pm 4.20 \mathrm{E}+00 \mathrm{pCi} / \mathrm{g}$ 4.32E-01 $\pm 7.60 \mathrm{E}-02 \mathrm{pCi} / \mathrm{g}$ $1.36 \mathrm{E}-01 \pm 3.89 \mathrm{E}-02 \mathrm{pCi} / \mathrm{g}$
4.55E-02 EPIA-013B 3.71E-02 EPLA-013B 1.39E-01 EPIA-013B 5.23E-02 EPIA-013B 2.07E-01 EPIA-013B 2.58E-02 EPIA-013B 2.63E-02 EPIA-013B 2.66E-02 EPIA-013B 1.51E-02 EPIA-013B 1.03E-02 EPIA-013B 1.15E-01 EPIA-013B 1.25E-01 EPLA-013B 1.05E-01 EPIA-013B 3.18E+00 EPIA-001B 5.88E-02 EPIA-013B 1.38E-02 EPIA-013B 1.91E-01 EPIA-013B 2.52E+00 EPIA-001B 3.25E-02 EPIA-012B 2.11E-02 EPLA-012B 
SURVEY ID: PPSC 1601 (continued)

\begin{tabular}{|c|c|c|c|}
\hline Constituent & $\mathbf{R Q}$ & AQ $\mathbf{B}$ & Result \\
\hline \multicolumn{4}{|l|}{ Radionuclides } \\
\hline Potassium-40 & & & $3.62 \mathrm{E}+00 \pm 2.07 \mathrm{E}-01 \mathrm{pCi} / \mathrm{g}$ \\
\hline Promethium-144 & UI & & $-5.06 \mathrm{E}-03 \pm 7.27 \mathrm{E}-03 \mathrm{pCi} / \mathrm{g}$ \\
\hline Promethium-146 & UI & & $8.57 \mathrm{E}-03 \pm 4.50 \mathrm{E}-02 \mathrm{pCi} / \mathrm{g}$ \\
\hline Radium-226 & & $\mathrm{V}$ & $1.33 \mathrm{E}+00 \pm 7.38 \mathrm{E}-02 \mathrm{pCi} / \mathrm{g}$ \\
\hline Radium-228 & & & $1.53 \mathrm{E}+00 \pm 7.67 \mathrm{E}-02 \mathrm{pCi} / \mathrm{g}$ \\
\hline Ruthenium-106 & UI & & $4.73 \mathrm{E}-02 \pm 1.59 \mathrm{E}-01 \mathrm{pCi} / \mathrm{g}$ \\
\hline Sodium-22 & & & $1.47 \mathrm{E}-02 \pm 9.50 \mathrm{E}-03 \mathrm{pCi} / \mathrm{g}$ \\
\hline Strontium-90 & & & $1.82 \mathrm{E}-01 \pm 8.77 \mathrm{E}-02 \mathrm{pCi} / \mathrm{g}$ \\
\hline Thorium-234 & $\mathrm{U}$ & $\mathrm{V}$ & $1.49 \mathrm{E}+00 \pm 1.04 \mathrm{E}+00 \mathrm{pCi} / \mathrm{g}$ \\
\hline Tin-113 & UI & & $-2.93 \mathrm{E}-02 \pm 3.96 \mathrm{E}-02 \mathrm{pCj} / \mathrm{g}$ \\
\hline Yttrium-88 & UI & & $8.36 \mathrm{E}-03 \pm 6.77 \mathrm{E}-03 \mathrm{pCi} / \mathrm{g}$ \\
\hline Zinc-65 & UI & & $-1.77 \mathrm{E}-02 \pm 1.96 \mathrm{E}-02 \mathrm{pCi} / \mathrm{g}$ \\
\hline Zirconium-95 & UI & & $0.00 \mathrm{E}+00 \quad \mathrm{pCi} / \mathrm{g}$ \\
\hline
\end{tabular}

Sample ID: 104697

D. Limit Method

8.95E-02 EPIA-013B

$1.22 \mathrm{E}-02$ EPIA-013B

7.20E-02 EPIA-013B

5.48E-02 EPIA-013B

4.55E-02 EPIA-013B

2.70E-01 EPIA-013B

1.08E-02 EPIA-013B

1.18E-01 EPIA-004

$1.21 \mathrm{E}+00$ EPIA.013B

6.36E-02 EPIA-013B

1.18E-02 EPIA.013B

2.71E-02 EPIA-013B

3.27E.02 EPIA.013B

SURVEY ID: PPSC 1602

Sample ID: 104698

Survey location: $44074 \mathrm{E} 3671718 \mathrm{~N}$ (SRS coordinates)

Depth of core interval: 1.00 to $2.00 \mathrm{ft}$

Sample type: Normal

Sample matrix: Soil

Percent solids: 71.00

Constituent

RQ AQ B Result

Unit

D. Limit Method

Physical Parameters and Specified Analyses

Ammonia nitrogen

Cation exchange capacity

Chloride

Cyanide

Nitrate-nitrite as nitrogen

Nitrogen by Kjeldahl method

Orthophosphate

Phenols

Sulfide

Total organic carbon .

Total organic nitrogen

Total phosphates (as P)

Metals (total recoverable)

Aluminum

Antimony

Arsenic

Barium

Beryllium

Cadmium

Calcium

Chromium

Cobalt

Copper

Iron

Lead

Magnesium

$\begin{array}{llllll} & & 45.6 & \mathrm{mg} / \mathrm{kg} & 28.2 & \text { EPA350.1 } \\ & & 121 & \mathrm{meq} / 100 \mathrm{~g} & 5 & \text { EPA9081 } \\ & & 3.5 & \mathrm{mg} / \mathrm{kg} & 3.46 & \text { EPA300.0 } \\ \mathrm{U} & & 1.33 & \mathrm{mg} / \mathrm{kg} & 1.33 & \text { EPA335.3 } \\ \mathrm{J} & \mathrm{E} & 0.541 & \mathrm{mg} / \mathrm{kg} & 1.35 & \text { EPA353.1 } \\ & & 585 & \mathrm{mg} / \mathrm{kg} & 107 & \text { EPA351.2 } \\ \mathrm{U} & & 14.1 & \mathrm{mg} / \mathrm{kg} & 14.1 & \text { EPA365.2 } \\ \mathrm{UJ} & \mathrm{C} & 415 & \mu \mathrm{mg} / \mathrm{kg} & 415 & \text { EPA420.2 } \\ \mathrm{UJ} & \mathrm{C} & 352 & \mathrm{mg} / \mathrm{kg} & 352 & \text { EPA376.2 } \\ & \mathrm{V} & 16800 & \mathrm{mg} / \mathrm{kg} & 141 & \text { EPA415.1 } \\ \mathrm{C} & & 539 & \mathrm{mg} / \mathrm{kg} & 0.282 & \text { EPA351.2 } \\ \mathrm{J} & \mathrm{E} & 175 & \mathrm{mg} / \mathrm{kg} & 1330 & \text { EPA365.4 }\end{array}$

$\begin{array}{lll}\mathrm{mg} / \mathrm{kg} & 17.3 & \text { EPA6010A } \\ \mathrm{mg} / \mathrm{kg} & 6.9 & \text { EPA6010A } \\ \mathrm{mg} / \mathrm{kg} & 20.7 & \text { EPA6010A } \\ \mathrm{mg} / \mathrm{kg} & 1.73 & \text { EPA6010A } \\ \mathrm{mg} / \mathrm{kg} & 0.69 & \text { EPA6010A } \\ \mathrm{mg} / \mathrm{kg} & 0.345 & \text { EPA6010A } \\ \mathrm{mg} / \mathrm{kg} & 17.3 & \text { EPA6010A } \\ \mathrm{mg} / \mathrm{kg} & 1.73 & \text { EPA6010A } \\ \mathrm{mg} / \mathrm{kg} & 0.69 & \text { EPA6010A } \\ \mathrm{mg} / \mathrm{kg} & 1.73 & \text { EPA6010A } \\ \mathrm{mg} / \mathrm{kg} & 6.9 & \text { EPA6010A } \\ \mathrm{mg} / \mathrm{kg} & 6.9 & \text { EPA6010A } \\ \mathrm{mg} / \mathrm{kg} & 6.9 & \text { EPA6010A }\end{array}$


SURVEY ID: PPSC 1602 (continued)

Constituent

Metals (total recoverable)

Manganese

Mercury

Nickel

Potassium

Selenium

Silver

Sodium

Thallium

Vanadium

Zinc

\section{Radionuclides}

Actinium-228

Antimony-124

Antimony-125

Barium-133

Cerium-144

Cesium-134

Cesium-137

Cobalt-57

Cobalt-58

Cobalt-60

Europium-152

Europium-154

Europium-155

Gross alpha

Lead-212

Manganese-54

Neptunium-239

Nonvolatile beta

Plutonium-238

Plutonium-239/240

Potassium-40

Promethium-144

Promethium-146

Radium-226

Radium-228

Ruthenium-106

Sodium-22

Strontium-90

Thorium-234

Tin-113

Yttrium-88

Zinc-65

Zirconium-95

Sample ID: 104698

RQ AQ B Result Unit D. Limit Method

$\begin{array}{llllll} & & 66.4 & \mathrm{mg} / \mathrm{kg} & 0.69 & \text { EPA6010A } \\ & & 0.102 & \mathrm{mg} / \mathrm{kg} & 0.043 & \text { EPA7471 } \\ & & 1.76 & \mathrm{mg} / \mathrm{kg} & 1.73 & \text { EPA6010A } \\ \mathrm{J} & \mathrm{E} & 78.7 & \mathrm{mg} / \mathrm{kg} & 138 & \text { EPA6010A } \\ \mathrm{U} & & 20.7 & \mathrm{mg} / \mathrm{kg} & 20.7 & \text { EPA6010A } \\ \mathrm{U} & & 1.73 & \mathrm{mg} / \mathrm{kg} & 1.73 & \text { EPA6010A } \\ \mathrm{J} & \mathrm{E} & 16.2 & \mathrm{mg} / \mathrm{kg} & 69 & \text { EPA6010A } \\ \mathrm{U} & & 20.7 & \mathrm{mg} / \mathrm{kg} & 20.7 & \text { EPA6010A } \\ & 20.5 & \mathrm{mg} / \mathrm{kg} & 0.69 & \text { EPA6010A } \\ & & 8.37 & \mathrm{mg} / \mathrm{kg} & 1.38 & \text { EPA6010A }\end{array}$

$1.29 \mathrm{E}+00 \pm 6.54 \mathrm{E}-02 \mathrm{pCi} / \mathrm{g}$ $-1.16 \mathrm{E}-02 \pm 2.26 \mathrm{E}-02 \mathrm{pCi} / \mathrm{g}$ $-2.43 \mathrm{E}-02 \pm 8.31 \mathrm{E}-02 \mathrm{pCi} / \mathrm{g}$ 3.50E-02 $\pm 3.53 \mathrm{E}-02 \mathrm{pCi} / \mathrm{g}$ $1.01 \mathrm{E}-01 \pm 1.73 \mathrm{E}-01 \mathrm{pCi} / \mathrm{g}$ $1.59 \mathrm{E}-03 \pm 1.92 \mathrm{E}-02 \mathrm{pCi} / \mathrm{g}$ $1.03 \mathrm{E}+02 \pm 1.96 \mathrm{E}-01 \mathrm{pCi} / \mathrm{g}$ $3.51 \mathrm{E}-02 \pm 2.53 \mathrm{E}-02 \mathrm{pCi} / \mathrm{g}$ $-3.35 \mathrm{E}-03 \pm 8.17 \mathrm{E}-03 \mathrm{pCi} / \mathrm{g}$ 8.05E-01 $\pm 2.36 \mathrm{E}-02 \mathrm{pCi} / \mathrm{g}$ $-5.16 \mathrm{E}-02 \pm 6.87 \mathrm{E}-02 \mathrm{pCi} / \mathrm{g}$ 7.34E-02 $7.01 \mathrm{E}-02 \mathrm{pCi} / \mathrm{g}$ $0.00 \mathrm{E}+00 \quad \mathrm{pCi} / \mathrm{g}$ $1.47 \mathrm{E}+01 \pm 2.91 \mathrm{E}+00 \mathrm{pCi} / \mathrm{g}$ $1.35 \mathrm{E}+00 \pm 6.13 \mathrm{E}-02 \mathrm{pCi} / \mathrm{g}$ 1.57E-02 $\pm 1.09 \mathrm{E}-02 \mathrm{pCi} / \mathrm{g}$ 2.47E-02 $\pm 1.19 \mathrm{E}-01 \mathrm{pCi} / \mathrm{g}$ 8.37E+01+4.67E+00 pCi/g $4.04 \mathrm{E}-01 \pm 7.54 \mathrm{E}-02 \mathrm{pCi} / \mathrm{g}$ $3.00 \mathrm{E}-01 \pm 6.32 \mathrm{E}-02 \mathrm{pCi} / \mathrm{g}$ $1.42 \mathrm{E}+00 \pm 1.26 \mathrm{E}-01 \mathrm{pCi} / \mathrm{g}$ $-5.00 \mathrm{E}-03 \pm 6.62 \mathrm{E}-03 \mathrm{pCi} / \mathrm{g}$ $-1.89 \mathrm{E}-02 \pm 4.30 \mathrm{E}-02 \mathrm{pCi} / \mathrm{g}$ $1.05 \mathrm{E}+00 \pm 6.91 \mathrm{E}-02 \mathrm{pCi} / \mathrm{g}$ $1.29 \mathrm{E}+00 \pm 6.54 \mathrm{E}-02 \mathrm{pCi} / \mathrm{g}$ $6.85 \mathrm{E}-02 \pm 1.71 \mathrm{E}-01 \mathrm{pCi} / \mathrm{g}$ $0.00 \mathrm{E}+00 \quad \mathrm{pCi} / \mathrm{g}$ 9.22E-01 $1.85 \mathrm{E}-01 \mathrm{pCi} / \mathrm{g}$ $2.03 \mathrm{E}+00 \pm 1.19 \mathrm{E}+00 \mathrm{pCi} / \mathrm{g}$ 9.09E-03 $3.83 \mathrm{E}-02 \mathrm{pCi} / \mathrm{g}$ $2.72 \mathrm{E}-03 \pm 5.06 \mathrm{E}-03 \mathrm{pCi} / \mathrm{g}$ $-1.42 \mathrm{E}-03 \pm 1.63 \mathrm{E}-02 \mathrm{pCi} / \mathrm{g}$ 1.67E-02 $1.83 \mathrm{E}-02 \mathrm{pCi} / \mathrm{g}$
4.11E-02 EPIA-013B 3.72E-02 EPIA-013B

1.39E-01 EPIA-013B

5.30E-02 EPIA-013B

2.15E-01 EPIA-013B

2.77E-02 EPIA-013B

2.67E-02 EPIA-013B

2.73E-02 EPIA-013B

1.32E-02 EPIA-013B

8.28E-03 EPIA-013B

1.16E-01 EPIA-013B

1.09E-01 EPIA-013B

1.10E-01 EPIA-013B

1.69E+00 EPIA-001B

6.09E-02 EPIA-013B

1.14E-02 EPIA-013B

1.98E-01 EPIA-013B

2.39E+00 EPIA-001B

1.73E-02 EPIA-012B

1.73E-02 EPIA-012B

7.15E-02 EPIA-013B

1.07E-02 EPIA-013B

7.16E-02 EPIA-013B

5.68E-02 EPIA-013B

4.11E-02 EPIA-013B

2.82E-01 EPIA-013B

1.05E-02 EPIA-013B

1.34E-01 EPIA-004

$1.18 \mathrm{E}+00$ EPIA-013B

6.45E-02 EPIA-013B

9.28E-03 EPIA-013B

2.40E-02 EPIA-013B

2.71E-02 EPIA-013B

SURVEY ID: PPSC 1603

Sample ID: 105100

Survey location: $44074 \mathrm{E}$ 3671718N (SRS coordinates)

Depth of core interval: 2.00 to $4.00 \mathrm{ft}$

Sample type: Normal

Sample matrix: Soil

Percent solids: $\mathbf{8 6 . 0 0}$ 
SURVEY ID: PPSC 1603 (continued)

Constituent
Sample ID: 105100

RQ AQ B Result

Unit

D. Limit Method

Physical Parameters and Specified Analyses

Ammonia nitrogen

Cation exchange capacity

Cation exchange capacity

Chloride

Cyanide

Nitrate-nitrite as nitrogen

Nitrogen by Kjeldahl method

Orthophosphate

Phenols

Sulfide

Total organic carbon

Total organic nitrogen

Total phosphates (as P)

Metals (total recoverable)

Aluminum

Antimony

Arsenic

Barium

Beryllium

Cadmium

Calcium

Chromium

Cobalt

Copper

Iron

Lead

Magnesium

Manganese

Mercury

Mercury

Nickel

Potassium

Selenium

Silver

Sodium

Thallium

Vanadium

Zinc

Radionuclides

Actinium-228

Antimony-124

Antimony-125

Barium-133

Cerium-144

Cesium-134

Cesium-137

Cobalt-57

Cobalt-58

Cobalt-60

Europium-152

Europium-154

Europium-155

$\begin{array}{lll}J & E & 16.1 \\ & & 67.8 \\ & & 69 \\ U & & 2.87 \\ U & & 1.14 \\ U & & 1.19 \\ & & 126 \\ \text { U } & & 11.6 \\ \text { U } & & 330 \\ \text { UJ } & \text { C } & 291 \\ & \text { V } & 3680 \\ \text { C } & & 110 \\ \text { J } & \text { E } & 93\end{array}$

$\begin{array}{ll}\mathrm{mg} / \mathrm{kg} & 23.1 \\ \mathrm{meq} / 100 \mathrm{~g} & 5 \\ \mathrm{meq} / 100 \mathrm{~g} & 5.81 \\ \mathrm{mg} / \mathrm{kg} & 2.87 \\ \mathrm{mg} / \mathrm{kg} & 1.14 \\ \mathrm{mg} / \mathrm{kg} & 1.19 \\ \mathrm{mg} / \mathrm{kg} & 45.8 \\ \mathrm{mg} / \mathrm{kg} & 11.6 \\ \mu \mathrm{g} / \mathrm{kg} & 330 \\ \mathrm{mg} / \mathrm{kg} & 291 \\ \mathrm{mg} / \mathrm{kg} & 116 \\ \mathrm{mg} / \mathrm{kg} & 0.233 \\ \mathrm{mg} / \mathrm{kg} & 452\end{array}$

EPA350.1 EPA9081 EPA9081

EPA300.0 EPA335.3 EPA353.1 EPA351.2 EPA365.2 EPA420.2 EPA376.2 EPA415.1 EPA351.2 EPA365.4

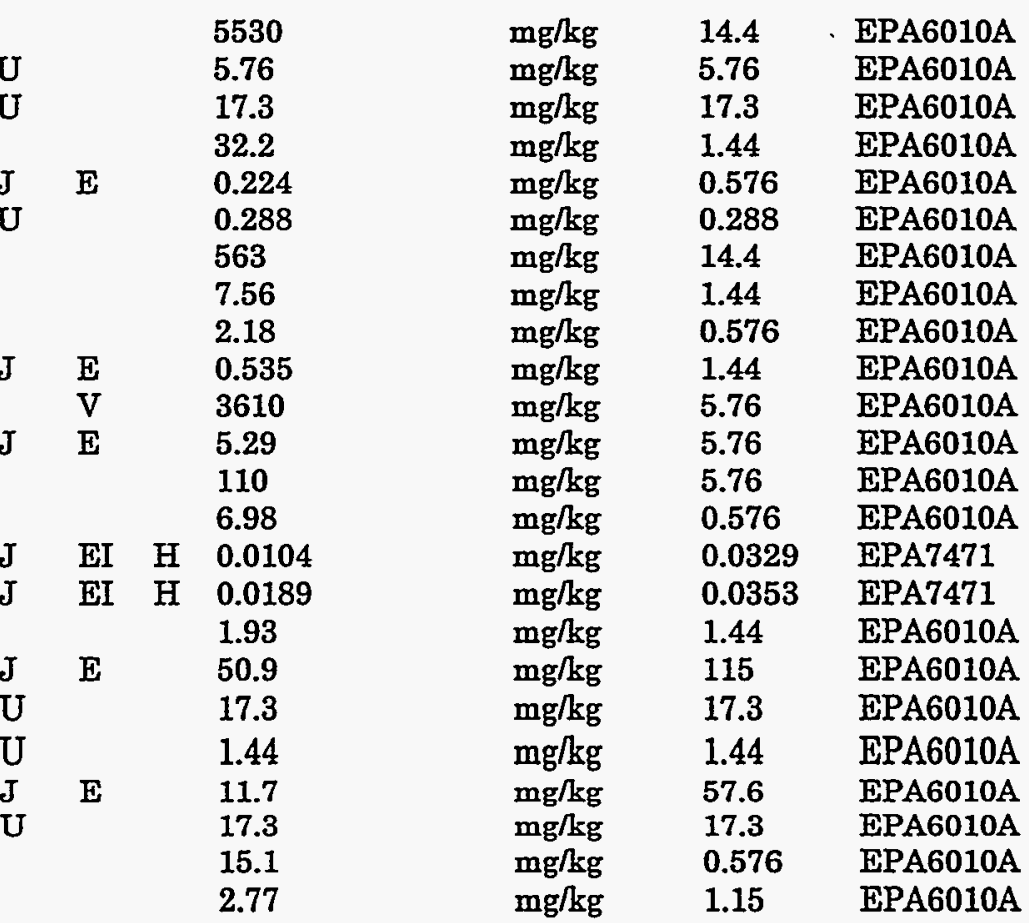

\begin{tabular}{|c|c|c|}
\hline & $1.22 \mathrm{E}+00 \pm 4.88 \mathrm{E}-02$ & $\mathrm{pCi} / \mathrm{g}$ \\
\hline UI & $3.36 \mathrm{E}-03 \pm 5.62 \mathrm{E}-03$ & $\mathrm{pCi} / \mathrm{g}$ \\
\hline UI & $1.27 \mathrm{E}-02 \pm 1.68 \mathrm{E}-02$ & $\mathrm{pCi} / \mathrm{g}$ \\
\hline UI & $2.48 \mathrm{E}-03 \pm 6.90 \mathrm{E}-03$ & $\mathrm{pCi} / g$ \\
\hline UI & $-8.13 \mathrm{E}-03 \pm 3.66 \mathrm{E}-02$ & $\mathrm{pCi} / \mathrm{g}$ \\
\hline U & 9.71E-04士4.70E-03 & $\mathrm{pCi}$ \\
\hline & 8.29E-01士1.90E-02 & \\
\hline UI & $-3.28 \mathrm{E}-03 \pm 4.54 \mathrm{E}-03$ & $\mathrm{pCi}$ \\
\hline U & $-1.58 \mathrm{E}-03 \pm 5.07 \mathrm{E}-03$ & $\mathrm{pCi}$ \\
\hline UI & $1.63 \mathrm{E}-03 \pm 3.98 \mathrm{E}-03$ & $\mathrm{pCi}$ \\
\hline & $-1.44 \mathrm{E}-02 \pm 1.39 \mathrm{E}-02$ & $\mathrm{pCi}$ \\
\hline $\mathrm{U}$ & $-4.59 \mathrm{E}-03 \pm 3.56 \mathrm{E}-02$ & $\mathrm{pCi}$ \\
\hline UI & $0.00 \mathrm{E}+00$ & $\mathrm{pCi}$ \\
\hline
\end{tabular}

2.36E-02 EPIA-013B 9.86E-03 EPIA-013B 2.39E-02 EPIA-013B 1.02E-02 EPIA-013B

6.24E-02 EPIA-013B

7.17E-03 EPIA-013B

8.08E-03 EPIA-013B

7.72E-03 EPIA-013B

8.55E-03 EPIA-013B

7.18E-03 EPIA-013B

2.24E-02 EPIA-013B

6.00E-02 EPIA-013B

3.29E-02 EPIA-013B 
SURVEY ID: PPSC 1603 (continued)

Constituent

Radionuclides

Gross alpha

Lead-212

Manganese-54

Neptunium-239

Nonvolatile beta

Plutonium-238

Plutonium-239/240

Potassium-40

Promethium-144

Promethium-146

Radium-226

Radium-228

Ruthenium-106

Sodium-22

Strontium-90

Thorium-234

Tin-113

Yttrium-88

Zinc-65

Zirconium-95
Sample ID: 105100

RQ AQ B Result Unit D. Limit Method
SURVEY ID: PPSC 1604

$1.73 \mathrm{E}+01 \pm 3.22 \mathrm{E}+00 \mathrm{pCi} / \mathrm{g}$
$1.27 \mathrm{E}+00 \pm 2.41 \mathrm{E}-02 \mathrm{pCi} / \mathrm{g}$
$3.15 \mathrm{E}-02 \pm 7.48 \mathrm{E}-03 \mathrm{pCi} / \mathrm{g}$
$0.00 \mathrm{E}+00 \quad \mathrm{pCi} / \mathrm{g}$
$1.00 \mathrm{E}+01 \pm 1.97 \mathrm{E}+00 \mathrm{pCi} / \mathrm{g}$
$5.00 \mathrm{E}-03 \pm 1.45 \mathrm{E}-02 \mathrm{pCi} / \mathrm{g}$
$6.36 \mathrm{E}-03 \pm 9.20 \mathrm{E}-03 \mathrm{pCi} / \mathrm{g}$
$9.25 \mathrm{E}-01 \pm 1.13 \mathrm{E}-01 \mathrm{pCi} / \mathrm{g}$
$-2.11 \mathrm{E}-03 \pm 4.38 \mathrm{E}-03 \mathrm{pCi} / \mathrm{g}$
$0.00 \mathrm{E}+00 \quad \mathrm{pCi} / \mathrm{g}$
$9.12 \mathrm{E}-01 \pm 2.91 \mathrm{E}-02 \mathrm{pCi} / \mathrm{g}$
$1.22 \mathrm{E}+00 \pm 4.88 \mathrm{E}-02 \mathrm{pCi} / \mathrm{g}$
$1.91 \mathrm{E}-02 \pm 4.22 \mathrm{E}-02 \mathrm{pC} / \mathrm{g}$
$-1.12 \mathrm{E}-03 \pm 3.94 \mathrm{E}-03 \mathrm{pCi} / \mathrm{g}$
$-9.02 \mathrm{E}-02 \pm 1.20 \mathrm{E}-02 \mathrm{pCi} / \mathrm{g}$
$1.10 \mathrm{E}+00 \pm 4.73 \mathrm{E}-01 \mathrm{pCi} / \mathrm{g}$
$-3.14 \mathrm{E}-03 \pm 7.31 \mathrm{E}-03 \mathrm{pCi} / \mathrm{g}$
$1.72 \mathrm{E}-03 \pm 4.76 \mathrm{E}-03 \mathrm{pCi} / \mathrm{g}$
$-4.25 \mathrm{E}-03 \pm 9.45 \mathrm{E}-03 \mathrm{pCi} / \mathrm{g}$
$0.00 \mathrm{E}+00 \quad \mathrm{pCi} / \mathrm{g}$

$1.73 \mathrm{E}+01 \pm 3.22 \mathrm{E}+00 \mathrm{pCi} / \mathrm{g}$ $1.27 \mathrm{E}+0012.41 \mathrm{~m}-02 \mathrm{pCi} / \mathrm{g}$

UI

UI

UI

UI

UI

$\mathrm{V}$

UI

UII

U V

UI

UI

UI

UI
1.41E+00 EPIA-001B

$1.43 \mathrm{E} \cdot 02$ EPIA-013B

6.92E-03 EPIA-013B

5.64E-02 EPIA-013B

$2.60 \mathrm{E}+00$ EPIA-001B

3.15E-02 EPIA-012B

1.61E-02 EPIA-012B

6.56E-02 EPIA-013B

7.40E-03 EPIA-013B

1.16E-02 EPIA-013B

1.45E-02 EPIA-013B

2.36E-02 EPIA-013B

7.38E-02 EPIA-013B

6.88E-03 EPIA-013B

1.32E-01 EPIA-004

4.21E-01 EPIA-013B

1.18E-02 EPIA-013B

8.58E-03 EPIA-013B

1.34E-02 EPIA.013B

1.92E-02 EPIA-013B

Survey location: 44074E $3671718 \mathrm{~N}$ (SRS coordinates)

Depth of core interval: 4.00 to $6.00 \mathrm{ft}$

Sample type: Normal

Sample matrix: Soil

Percent solids: $\mathbf{8 1 . 0 0}$

Constituent

RQ AQ B Result

Unit

D. Limit Method

Physical Parameters and Specified Analyses

Ammonia nitrogen

Cation exchange capacity

Chloride

Cyanide

Nitrate-nitrite as nitrogen

Nitrogen by Kjeldahl method

Nitrogen by Kjeldahl method

Orthophosphate

Phenols

Phenols

Sulfide

Total organic carbon

Total organic nitrogen

Total phosphates (as P)

$\begin{array}{lll}\text { U } & & 24.7 \\ & & 124 \\ \text { J } & E & 1.35 \\ \text { U } & & 1.19 \\ \text { J } & \text { E } & 0.366 \\ & & 141 \\ & & 129 \\ \text { U } & & 12.3 \\ \text { U } & & 367 \\ \text { U } & & 369 \\ \text { UJ } & \text { C } & 309 \\ & \text { V } & 2140 \\ \text { C } & & 130 \\ \text { J } & \text { E } & 268\end{array}$

$\begin{array}{lll}\mathrm{mg} / \mathrm{kg} & 24.7 & \text { EPA350.1 } \\ \mathrm{meq} / 100 \mathrm{~g} & 5 & \text { EPA9081 } \\ \mathrm{mg} / \mathrm{kg} & 3.05 & \text { EPA300.0 } \\ \mathrm{mg} / \mathrm{kg} & 1.19 & \text { EPA335.3 } \\ \mathrm{mg} / \mathrm{kg} & 1.22 & \text { EPA353.1 } \\ \mathrm{mg} / \mathrm{kg} & 48.7 & \text { EPA351.2 } \\ \mathrm{mg} / \mathrm{kg} & 48.7 & \text { EPA351.2 } \\ \mathrm{mg} / \mathrm{kg} & 12.3 & \text { EPA365.2 } \\ \mu \mathrm{g} / \mathrm{kg} & 367 & \text { EPA420.2 } \\ \mu \mathrm{g} / \mathrm{kg} & 369 & \text { EPA420.2 } \\ \mathrm{mg} / \mathrm{kg} & 309 & \text { EPA376.2 } \\ \mathrm{mg} / \mathrm{kg} & 123 & \text { EPA415.1 } \\ \mathrm{mg} / \mathrm{kg} & 0.247 & \text { EPA351.2 } \\ \mathrm{mg} / \mathrm{kg} & 1180 & \text { EPA365.4 }\end{array}$


SURVEY ID: PPSC 1604 (continued)

Constituent

Metals (total recoverable)
Sample ID: 105101
RQ AQ B Result
Unit
D. Limit Method

\begin{tabular}{|c|c|c|c|}
\hline Aluminum & & & 4120 \\
\hline Antimony & $U$ & & 5.99 \\
\hline Arsenic & $U$ & & 18 \\
\hline Barium & & & 120 \\
\hline Beryllium & $\mathrm{J}$ & $\mathbf{E}$ & 0.539 \\
\hline Cadmium & $\mathrm{U}$ & & 0.3 \\
\hline Calcium & & & 1150 \\
\hline Chromium & & & 6.94 \\
\hline Cobalt & & & 1.07 \\
\hline Copper & & & 2.21 \\
\hline Iron & & V & 1660 \\
\hline Lead & $J$ & $\mathrm{E}$ & 2.71 \\
\hline Magnesium & & & 353 \\
\hline Manganese & & & 3.5 \\
\hline Mercury & $J$ & $\mathbf{E}$ & 0.013 \\
\hline Nickel & & & 3.04 \\
\hline Potassium & & & 346 \\
\hline Selenium & $\mathrm{U}$ & & 18 \\
\hline Silver & $U$ & & 1.5 \\
\hline Sodium & $\mathrm{U}$ & & 59.9 \\
\hline Thallium & U & & 18 \\
\hline Vanadium & & & 10.6 \\
\hline Zinc & & & 10 \\
\hline
\end{tabular}

Radionuclides

Actinium-228

Antimony-124

Antimony-125

Barium-133

Cerium-144

Cesium-134

Cesium-137

Cobalt-57

Cobalt-58

Cobalt-60

Europium-152

Europium-154

Europium-155

Gross alpha

Lead-212

Manganese- 54

Neptunium-239

Nonvolatile beta

Plutonium-238

Plutonium-239/240

Potassium-40

Promethium-144

Promethium-146

Radium-226

Radium-228

Ruthenium-106

Sodium-22

Strontium-90

Thorium-234
$1.01 \mathrm{E}+00 \pm 4.71 \mathrm{E}-02 \mathrm{pCi} / \mathrm{g}$ $-4.24 \mathrm{E}-04 \pm 5.04 \mathrm{E}-03 \mathrm{pCi} / \mathrm{g}$ $-5.68 \mathrm{E}-03 \pm 1.14 \mathrm{E}-02 \mathrm{pCi} / \mathrm{g}$ $3.83 \mathrm{E}-03 \pm 5.67 \mathrm{E}-03 \mathrm{pCi} / \mathrm{g}$ $-5.50 \mathrm{E}-03 \pm 3.25 \mathrm{E}-02 \mathrm{pCi} / \mathrm{g}$ $1.08 \mathrm{E}-03 \pm 4.20 \mathrm{E}-03 \mathrm{pCj} / \mathrm{g}$ $1.66 \mathrm{E}-02 \pm 8.09 \mathrm{E}-03 \mathrm{pCi} / \mathrm{g}$ $1.82 \mathrm{E}-03 \pm 4.03 \mathrm{E}-03 \mathrm{pCi} / \mathrm{g}$ $1.51 \mathrm{E}-03 \pm 5.09 \mathrm{E}-03 \mathrm{pCi} / \mathrm{g}$ $1.80 \mathrm{E}-03 \pm 3.69 \mathrm{E}-03 \mathrm{pCi} / \mathrm{g}$ $-4.81 \mathrm{E}-03 \pm 1.20 \mathrm{E}-02 \mathrm{pCi} / \mathrm{g}$ $-4.99 \mathrm{E}-02 \pm 4.14 \mathrm{E}-02 \mathrm{pCi} / \mathrm{g}$ $0.00 \mathrm{E}+00 \quad \mathrm{pCi} / \mathrm{g}$ $1.40 \mathrm{E}+01 \pm 2.85 \mathrm{E}+00 \mathrm{pCi} / \mathrm{g}$ $1.08 \mathrm{E}+00 \pm 2.16 \mathrm{E}-02 \mathrm{pCi} / \mathrm{g}$ $1.86 \mathrm{E}-02 \pm 6.16 \mathrm{E}-03 \mathrm{pCi} / \mathrm{g}$ $2.87 \mathrm{E}-02 \pm 3.21 \mathrm{E}-02 \mathrm{pCi} / \mathrm{g}$ $1.32 \mathrm{E}+01 \pm 2.15 \mathrm{E}+00 \mathrm{pCi} / \mathrm{g}$ $3.03 \mathrm{E}-02 \pm 1.70 \mathrm{E}-02 \mathrm{pCi} / \mathrm{g}$ $2.33 \mathrm{E}-03 \pm 4.70 \mathrm{E}-03 \mathrm{pCi} / \mathrm{g}$ $1.72 \mathrm{E}+00 \pm 1.17 \mathrm{E}-01 \mathrm{pCi} / \mathrm{g}$ $2.99 \mathrm{E}-04 \pm 4.04 \mathrm{E}-03 \mathrm{pCj} / \mathrm{g}$ $4.94 \mathrm{E}-03 \pm 5.53 \mathrm{E}-03 \mathrm{pCi} / \mathrm{g}$ $7.63 \mathrm{E}-01 \pm 2.85 \mathrm{E}-02 \mathrm{pCi} / \mathrm{g}$ $1.01 \mathrm{E}+00 \pm 4.71 \mathrm{E}-02 \mathrm{pCi} / \mathrm{g}$ $5.50 \mathrm{E}-05 \pm 3.71 \mathrm{E}-02 \mathrm{pCi} / \mathrm{g}$ $1.27 \mathrm{E}-03 \pm 3.79 \mathrm{E}-03 \mathrm{pCi} / \mathrm{g}$ $1.20 \mathrm{E}-01 \pm 8.97 \mathrm{E}-02 \mathrm{pCi} / \mathrm{g}$ $1.32 \mathrm{E}+00 \pm 6.18 \mathrm{E}-01 \mathrm{pCi} / \mathrm{g}$
2.26E-02 EPIA-013B 8.69E-03 EPIA-013B

1.84E-02 EPIA-013B

8.55E-03 EPIA-013B

5.54E-02 EPIA-013B

6.47E-03 EPIA-013B

8.22E-03 'EPIA-013B

6.95E-03 EPIA-013B

7.80E-03 EPIA-013B

6.71E-03 EPIA-013B

1.95E-02 EPIA-013B

5.58E-02 EPIA-013B

2.88E-02 EPIA-013B

$1.39 \mathrm{E}+00$ EPIA.001B

1.21E-02 EPIA-013B

5.56E-03 EPIA-013B

5.04E-02 EPIA-013B

2.53E+00 EPIA-001B

3.69E-02 EPIA-012B

7.00E-03 EPIA-012B

5.90E-02 EPIA-013B

6.96E-03 EPIA-013B

9.27E-03 EPIA-013B

$1.29 \mathrm{E}-02$ EPIA-013B

2.26E-02 EPIA-013B

6.41E-02 EPIA-013B

6.83E-03 EPIA-013B

1.47E-01 EPIA-004

3.75E-01 EPIA-013B 
SURVEY ID: PPSC 1604 (continued)

Constituent

Radionuclides

Tin-113

Yttrium-88

Zinc-65

Zirconium-95
Sample ID: 105101

RQ AQ B Result Unit D. Limit Method

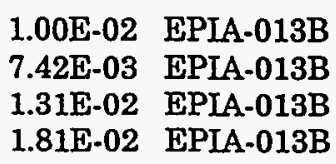

SURVEY ID: PPSC 1605

Sample ID: 105102

Survey location: $44074 \mathrm{E} 3671718 \mathrm{~N}$ (SRS coordinates)

Depth of core interval: 6.00 to $8.00 \mathrm{ft}$

Sample type: Normal

Sample matrix: Soil

Percent solids: $\mathbf{7 7 . 0 0}$

\section{Constituent}

RQ AQ B Result

Unit

D. Limit Method

Physical Parameters and Specified Analyses

$\begin{array}{llll}\text { Ammonia nitrogen } & \text { U } & & 25.8 \\ \text { Cation exchange capacity } & & & 139 \\ \text { Chloride } & \text { J } & \text { Q } & 6.77 \\ \text { Cyanide } & \text { U } & & 1.27 \\ \text { Nitrate-nitrite as nitrogen } & \text { J } & \text { E } & 0.4 \\ \text { Nitrogen by Kjeldahl method } & & & 23.6 \\ \text { Orthophosphate } & \text { J } & \text { E } & 3.19 \\ \text { Orthophosphate } & \text { J } & \text { E } & 2.69 \\ \text { Phenols } & \text { UJ } & \text { C } & 381 \\ \text { Sulfide } & \text { UJ } & \text { C } & 325 \\ \text { Total organic carbon } & & \text { V } & 255 \\ \text { Total organic nitrogen } & \text { C } & & 20.8 \\ \text { Total phosphates (as P) } & \text { J } & \text { E } & 408 \\ \text { Total phosphates (as P) } & \text { J } & \text { E } & 393\end{array}$

$\begin{array}{lll}\mathrm{mg} / \mathrm{kg} & 25.8 & \text { EPA350.1 } \\ \mathrm{meq} / 100 \mathrm{~g} & 5 & \text { EPA9081 } \\ \mathrm{mg} / \mathrm{kg} & 3.18 & \text { EPA300.0 } \\ \mathrm{mg} / \mathrm{kg} & 1.27 & \text { EPA335.3 } \\ \mathrm{mg} / \mathrm{kg} & 1.33 & \text { EPA353.1 } \\ \mathrm{mg} / \mathrm{kg} & 5.19 & \text { EPA351.2 } \\ \mathrm{mg} / \mathrm{kg} & 13 & \text { EPA365.2 } \\ \mathrm{mg} / \mathrm{kg} & 13 & \text { EPA365.2 } \\ \mu \mathrm{g} / \mathrm{kg} & 381 & \text { EPA420.2 } \\ \mathrm{mg} / \mathrm{kg} & 325 & \text { EPA376.2 } \\ \mathrm{mg} / \mathrm{kg} & 130 & \text { EPA415.1 } \\ \mathrm{mg} / \mathrm{kg} & 0.26 & \text { EPA351.2 } \\ \mathrm{mg} / \mathrm{kg} & 2430 & \text { EPA365.4 } \\ \mathrm{mg} / \mathrm{kg} & 2430 & \text { EPA365.4 }\end{array}$

Metals (total recoverable)

\begin{tabular}{|c|c|c|c|c|c|c|}
\hline Aluminum & & & 4890 & $\mathrm{mg} / \mathrm{kg}$ & 15.8 & EPA6010A \\
\hline Antimony & $U$ & & 6.3 & $\mathrm{mg} / \mathrm{kg}$ & 6.3 & EPA6010A \\
\hline Arsenic & $U$ & & 18.9 & $\mathrm{mg} / \mathrm{kg}$ & 18.9 & EPA6010A \\
\hline Barium & & & 79.5 & $\mathrm{mg} / \mathrm{kg}$ & 1.58 & EPA6010A \\
\hline Beryllium & $\mathrm{J}$ & $\mathbf{E}$ & 0.504 & $\mathrm{mg} / \mathrm{kg}$ & 0.63 & EPA6010A \\
\hline Cadmium & $\mathrm{U}$ & & 0.315 & $\mathrm{mg} / \mathrm{kg}$ & 0.315 & EPA6010A \\
\hline Calcium & & & 1100 & $\mathrm{mg} / \mathrm{kg}$ & 15.8 & EPA6010A \\
\hline Chromium & & & 9.95 & $\mathrm{mg} / \mathrm{kg}$ & 1.58 & EPA6010A \\
\hline Cobalt & & & 2.06 & $\mathrm{mg} / \mathrm{kg}$ & 0.63 & EPA6010A \\
\hline Copper & & & 2.22 & $\mathrm{mg} / \mathrm{kg}$ & 1.58 & EPA6010A \\
\hline Iron & & V & 6060 & $\mathrm{mg} / \mathrm{kg}$ & 6.3 & EPA6010A \\
\hline Lead & $J$ & $\mathrm{E}$ & 3.21 & $\mathrm{mg} / \mathrm{kg}$ & 6.3 & EPA6010A \\
\hline Magnesium & & & 634 & $\mathrm{mg} / \mathrm{kg}$ & 6.3 & EPA6010A \\
\hline Manganese & & & 16.7 & $\mathrm{mg} / \mathrm{kg}$ & 0.63 & EPA6010A \\
\hline Mercury & $J$ & $\mathrm{E}$ & 0.00463 & $\mathrm{mg} / \mathrm{kg}$ & 0.0421 & EPA7471 \\
\hline Nickel & & & 7.36 & $\mathrm{mg} / \mathrm{kg}$ & 1.58 & EPA6010A \\
\hline Potassium & & & 537 & $\mathrm{mg} / \mathrm{kg}$ & 126 & EPA6010A \\
\hline Selenium & $\mathrm{U}$ & & 18.9 & $\mathrm{mg} / \mathrm{kg}$ & 18.9 & EPA6010A \\
\hline Silver & U & & 1.58 & $\mathrm{mg} / \mathrm{kg}$ & 1.58 & EPA6010A \\
\hline Sodium & $\mathrm{U}$ & & 63 & $\mathrm{mg} / \mathrm{kg}$ & 63 & EPA6010A \\
\hline
\end{tabular}


SURVEY ID: PPSC 1605 (continued)

\section{Constituent}

Metals (total recoverable)

Thallium

Vanadium

Zinc

\section{Radionuclides}

Actinium-228

Actinium-228

Antimony-124

Antimony-124

Antimony-125

Antimony-125

Barium-133

Barium-133

Cerium-144

Cerium-144

Cesium-134

Cesium-134

Cesium-137

Cesium-137

Cobalt-57

Cobalt-57

Cobalt-58

Cobalt-58

Cobalt-60

Cobalt-60

Europium-152

Europium-152

Europium-154

Europium-154

Europium-155

Europium-155

Gross alpha

Lead-212

Lead-212

Manganese-54

Manganese-54

Neptunium-239

Neptunium-239

Nonvolatile beta

Plutonium-238

Plutonium-239/240

Potassium-40

Potassium-40

Promethium-144

Promethium-144

Promethium-146

Promethium-146

Radium-226

Radium-226

Radium-228

Radium-228

Ruthenium-106

Ruthenium-106

Sodium-22

Sample ID: 105102

RQ AQ B Result Unit D. Limit Method

U

UI

UI

UI

UI

UI

UI

UI

UI

UI

UI

UI

UI

UI

UI

UI

UI

UI

UI

UI

UI

UI

UI

UI

UI

UI

UI

UI

UI

UI

UI

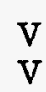

UI

UI
$1.22 \mathrm{E}+00 \pm 5.37 \mathrm{E}-02 \mathrm{pCi} / \mathrm{g}$ $1.14 \mathrm{E}+00 \pm 5.08 \mathrm{E}-02 \mathrm{pCi} / \mathrm{g}$ $-4.07 \mathrm{E}-04 \pm 5.34 \mathrm{E}-03 \mathrm{pCi} / \mathrm{g}$ $4.03 \mathrm{E}-04 \pm 5.36 \mathrm{E}-03 \mathrm{pCi} / \mathrm{g}$ $8.47 \mathrm{E}-03 \pm 1.25 \mathrm{E}-02 \mathrm{pCi} / \mathrm{g}$ $6.28 \mathrm{E}-03 \pm 1.18 \mathrm{E}-02 \mathrm{pCj} / \mathrm{g}$ $-5.03 \mathrm{E}-04 \pm 6.06 \mathrm{E}-03 \mathrm{pCi} / \mathrm{g}$ $-8.73 \mathrm{E}-04 \pm 6.30 \mathrm{E}-03 \mathrm{pCi} / \mathrm{g}$ $-2.81 \mathrm{E}-02 \pm 3.39 \mathrm{E}-02 \mathrm{pCi} / \mathrm{g}$ $-3.29 \mathrm{E}-02 \pm 3.45 \mathrm{E}-02 \mathrm{pCi} / \mathrm{g}$ $-1.36 \mathrm{E}-04 \pm 4.50 \mathrm{E}-03 \mathrm{pCi} / \mathrm{g}$ $3.08 \mathrm{E}-03 \pm 4.40 \mathrm{E}-03 \mathrm{pCi} / \mathrm{g}$ $1.01 \mathrm{E}-02 \pm 5.59 \mathrm{E}-03 \mathrm{pCi} / \mathrm{g}$ $1.44 \mathrm{E}-02 \pm 8.36 \mathrm{E}-03 \mathrm{pCi} / \mathrm{g}$ $1.80 \mathrm{E}-03 \pm 4.27 \mathrm{E}-03 \mathrm{pCj} / \mathrm{g}$ $2.29 \mathrm{E} \cdot 03 \pm 4.35 \mathrm{E}-03 \mathrm{pCi} / \mathrm{g}$ $-5.07 \mathrm{E}-03 \pm 4.89 \mathrm{E}-03 \mathrm{pCi} / \mathrm{g}$ $2.66 \mathrm{E}-03 \pm 5.79 \mathrm{E}-03 \mathrm{pCi} / \mathrm{g}$ $-1.88 \mathrm{E}-04 \pm 3.78 \mathrm{E}-03 \mathrm{pCi} / \mathrm{g}$ $-1.77 \mathrm{E}-03 \pm 4.07 \mathrm{E}-03 \mathrm{pCi} / \mathrm{g}$ $-5.12 \mathrm{E}-03 \pm 1.27 \mathrm{E}-02 \mathrm{pCi} / \mathrm{g}$ $-1.86 \mathrm{E}-02 \pm 1.32 \mathrm{E}-02 \mathrm{pCi} / \mathrm{g}$ $2.36 \mathrm{E}-03 \pm 3.95 \mathrm{E}-02 \mathrm{pCi} / \mathrm{g}$ $-4.04 \mathrm{E}-03 \pm 4.12 \mathrm{E}-02 \mathrm{pCi} / \mathrm{g}$ $0.00 \mathrm{E}+00 \quad \mathrm{pCi} / \mathrm{g}$ $0.00 \mathrm{E}+00 \quad \mathrm{pCi} / \mathrm{g}$ $1.89 \mathrm{E}+01 \pm 3.36 \mathrm{E}+00 \mathrm{pCi} / \mathrm{g}$ $1.22 \mathrm{E}+00 \pm 2.28 \mathrm{E}-02 \mathrm{pCi} / \mathrm{g}$ $1.23 \mathrm{E}+00 \pm 2.29 \mathrm{E}-02 \mathrm{pCi} / \mathrm{g}$ $1.57 \mathrm{E}-02 \pm 5.68 \mathrm{E}-03 \mathrm{pCi} / \mathrm{g}$ $2.55 \mathrm{E}-02 \pm 5.71 \mathrm{E}-03 \mathrm{pCi} / \mathrm{g}$ $-3.88 \mathrm{E}-03 \pm 3.39 \mathrm{E}-02 \mathrm{pCj} / \mathrm{g}$ $4.58 \mathrm{E}-02 \pm 5.32 \mathrm{E}-02 \mathrm{pCi} / \mathrm{g}$ $1.28 \mathrm{E}+01 \pm 2.05 \mathrm{E}+00 \mathrm{pCi} / \mathrm{g}$ $0.00 \mathrm{E}+00 \quad \mathrm{pCi} / \mathrm{g}$ $1.46 \mathrm{E}-02 \pm 1.50 \mathrm{E}-02 \mathrm{pCi} / \mathrm{g}$ $2.01 \mathrm{E}+00 \pm 1.30 \mathrm{E}-01 \mathrm{pCi} / \mathrm{g}$ $2.17 \mathrm{E}+00 \pm 1.22 \mathrm{E}-01 \mathrm{pCi} / \mathrm{g}$ $-5.41 \mathrm{E}-04 \pm 4.47 \mathrm{E}-03 \mathrm{pCi} / \mathrm{g}$ $8.65 \mathrm{E}-05 \pm 4.44 \mathrm{E}-03 \mathrm{pCi} / \mathrm{g}$ $0.00 \mathrm{E}+00 \quad \mathrm{pCi} / \mathrm{g}$ $0.00 \mathrm{E}+00 \quad \mathrm{pCi} / \mathrm{g}$ $7.68 \mathrm{E}-01 \pm 2.84 \mathrm{E}-02 \quad \mathrm{pCi} / \mathrm{g}$ $7.70 \mathrm{E}-01 \pm 2.76 \mathrm{E}-02 \mathrm{pCj} / \mathrm{g}$ $1.22 \mathrm{E}+00 \pm 5.37 \mathrm{E}-02 \mathrm{pCi} / \mathrm{g}$ $1.14 \mathrm{E}+00 \pm 5.08 \mathrm{E}-02 \mathrm{pCj} / \mathrm{g}$ $-3.10 \mathrm{E}-02 \pm 3.98 \mathrm{E}-02 \mathrm{pCi} / \mathrm{g}$ $6.32 \mathrm{E}-03 \pm 4.03 \mathrm{E}-02 \mathrm{pCi} / \mathrm{g}$ $-8.63 \mathrm{E}-06 \pm 4.19 \mathrm{E}-03 \mathrm{pCi} / \mathrm{g}$
2.32E-02 EPIA-013B 2.35E-02 EPIA-013B 9.24E-03 EPIA-013B 9.14E-03 EPIA-013B 2.08E.02 EPIA-013B 2.07E-02 EPIA-013B 8.91E-03 EPIA-013B 9.05E-03 EPLA-013B 5.75E-02 EPIA-013B 5.79E-02 EPIA-013B 6.82E-03 EPIA-013B 6.84E-03 EPIA-013B 7.81E-03 EPIA-013B 7.94E-03 EPIA-013B 7.39E-03 EPIA-013B 7.48E-03 EPLA-013B 8.02E-03 EPIA-013B 8.71E-03 EPIA-013B 6.71E-03 EPIA-013B 6.85E-03 EPIA-013B 2.08E-02 EPIA-013B 2.07E-02 EPIA-013B 5.94E-02 EPIA-013B 6.25E-02 EPIA-013B 3.09E-02 EPIA-013B 3.16E-02 EPIA-013B $2.02 \mathrm{E}+00$ EPIA-001B

1.34E-02 EPIA-013B

1.27E-02 EPIA-013B

7.75E-03 EPIA-013B

6.68E-03 EPIA.013B

5.26E-02 EPIA-013B

5.23E-02 EPIA-013B

2.41E+00 EPIA-001B

3.93E-02 EPIA-012B

2.25E-02 EPIA-012B

6.40E-02 EPIA-013B

6.20E-02 EPIA-013B

7.65E-03 EPIA-013B

$7.48 \mathrm{E}-03$ EPIA-013B

1.03E-02 EPIA-013B

1.01E-02 EPLA-013B

1.38E-02 EPIA-013B

1.39E-02 EPIA-013B

2.32E-02 EPIA-013B 2.35E-02 EPIA-013B 6.72E-02 EPIA-013B 6.87E-02 EPIA-013B 7.04E-03 EPIA-013B 
SURVEY ID: PPSC 1605 (continued)

Constituent

Radionuclides

Sodium-22

Strontium-90

Thorium-234

Thorium-234

Tin-113

Tin-113

Yttrium-88

Yttrium-88

Zinc-65

Zinc-65

Zirconium-95

Zirconium-95
Sample ID: 105102

RQ AQ B Result Unit D. Limit Method
7.61E-03 EPIA-013B

1.52E-01 EPIA-004

3.94E-01 EPIA-013B

4.02E-01 EPIA-013B

1.07E-02 EPIA-013B

1.02E-02 EPIA-013B

8.34E-03 EPIA-013B

8.64E-03 EPIA-013B

1.47E-02 EPIA-013B

1.54E-02 EPIA-013B

1.79E-02 EPIA-013B

2.03E-02 EPIA-013B

SURVEY ID: PPSC 1701

Sample ID: 104693

Survey location: 44049E 3671941N (SRS coordinates)

Depth of core interval: 0.00 to $1.00 \mathrm{ft}$

Sample type: Normal

Sample matrix: Soil

Percent solids: $\mathbf{8 8 . 0 0}$

Constituent

RQ AQ B Result

Unit

D. Limit Method

Physical Parameters and Specified Analyses

Ammonia nitrogen

Cation exchange capacity

Chloride

Cyanide

Nitrate-nitrite as nitrogen

Nitrogen by Kjeldahl method

Orthophosphate

Phenols

Sulfide

Total organic carbon

Total organic carbon

Total organic nitrogen

Total phosphates (as P)

Metals (total recoverable)

$\begin{array}{lll}\text { U } & & 22.5 \\ & & 31.2 \\ \mathrm{U} & & 2.83 \\ \mathrm{U} & & 1.1 \\ \mathrm{U} & & 1.12 \\ & & 86.4 \\ \mathrm{~J} & \mathrm{E} & 5.66 \\ \mathrm{U} & & 340 \\ \mathrm{UJ} & \mathrm{C} & 284 \\ & \mathrm{~V} & 1310 \\ & \mathrm{~V} & 1350 \\ \mathrm{C} & & 75.2 \\ \mathrm{~J} & \mathrm{E} & 131\end{array}$

$\begin{array}{llll} & & & 5100 \\ & & & 4280 \\ \text { UJ } & \text { I } & \text { L } & 5.46 \\ \text { UJ } & \text { I } & \text { L } & 5.46 \\ \text { U } & & & 16.4 \\ \text { U } & & & 16.4 \\ & & & 13.6 \\ & & & 12 \\ \text { J } & \text { E } & & 0.0459 \\ \text { J } & \text { E } & & 0.041 \\ \text { U } & & & 0.273 \\ \text { U } & & & 0.273\end{array}$

$\begin{array}{ll}\mathrm{mg} / \mathrm{kg} & 22.5 \\ \mathrm{meq} / 100 \mathrm{~g} & 5 \\ \mathrm{mg} / \mathrm{kg} & 2.83 \\ \mathrm{mg} / \mathrm{kg} & 1.1 \\ \mathrm{mg} / \mathrm{kg} & 1.12 \\ \mathrm{mg} / \mathrm{kg} & 45.5 \\ \mathrm{mg} / \mathrm{kg} & 11.4 \\ \mu \mathrm{g} / \mathrm{kg} & 340 \\ \mathrm{mg} / \mathrm{kg} & 284 \\ \mathrm{mg} / \mathrm{kg} & 114 \\ \mathrm{mg} / \mathrm{kg} & 114 \\ \mathrm{mg} / \mathrm{kg} & 0.227 \\ \mathrm{mg} / \mathrm{kg} & 450\end{array}$

EPA350.1

EPA9081

EPA300.0

EPA335.3

EPA353.1

EPA351.2

EPA365.2

EPA420.2

EPA376.2

EPA415.1

EPA415.1

EPA351.2

EPA365.4

\begin{abstract}
Aluminum
Aluminum

Antimony

Antimony

Arsenic

Arsenic

Barium

Barium

Beryllium

Beryllium

Cadmium

Cadmium

Calcium
\end{abstract}

$\begin{array}{lll}\mathrm{mg} / \mathrm{kg} & 13.7 & \text { EPA6010A } \\ \mathrm{mg} / \mathrm{kg} & 13.7 & \text { EPA6010A } \\ \mathrm{mg} / \mathrm{kg} & 5.46 & \text { EPA6010A } \\ \mathrm{mg} / \mathrm{kg} & 5.46 & \text { EPA6010A } \\ \mathrm{mg} / \mathrm{kg} & 16.4 & \text { EPA6010A } \\ \mathrm{mg} / \mathrm{kg} & 16.4 & \text { EPA6010A } \\ \mathrm{mg} / \mathrm{kg} & 1.37 & \text { EPA6010A } \\ \mathrm{mg} / \mathrm{kg} & 1.37 & \text { EPA6010A } \\ \mathrm{mg} / \mathrm{kg} & 0.546 & \text { EPA6010A } \\ \mathrm{mg} / \mathrm{kg} & 0.546 & \text { EPA6010A } \\ \mathrm{mg} / \mathrm{kg} & 0.273 & \text { EPA6010A } \\ \mathrm{mg} / \mathrm{kg} & 0.273 & \text { EPA6010A } \\ \mathrm{mg} / \mathrm{kg} & 13.7 & \text { EPA6010A }\end{array}$


SURVEY ID: PPSC 1701 (continued)

Sample ID: 104693

Constituent

RQ AQ B Result

Unit

D. Limit Method

Metals (total recoverable)

Calcium
Chromium
Chromium
Cobalt
Cobalt
Copper
Copper
Iron
Iron
Lead
Lead
Magnesium
Magnesium
Manganese
Manganese
Mercury
Nickel
Nickel
Potassium
Potassium
Selenium
Selenium
Silver
Silver
Sodium
Sodium
Thallium
Thallium
Vanadium
Vanadium
Zinc
Zinc
Tam

$\begin{array}{llll} & & & 42.8 \\ & & & 3.69 \\ & & & 3.11 \\ \text { J } & \text { E } & & 0.232 \\ \text { J } & \text { E } & & 0.209 \\ & & & 2.72 \\ \text { J } & \text { E } & & 1.31 \\ & \text { V } & & 1550 \\ & \text { V } & & 1380 \\ \text { J } & \text { E } & & 4.31 \\ \text { J } & \text { E } & & 2.04 \\ \text { J } & \text { I } & \text { L } & 147 \\ \text { J } & \text { I } & \text { L } & 132 \\ & \text { X } & & 21 \\ & \text { X } & & 12.9 \\ \text { J } & \text { E } & & 0.0285 \\ \text { J } & \text { E } & & 1.33 \\ & & & 1.41 \\ \text { J } & \text { EI } & \text { L } & 94.4 \\ \text { J } & \text { EI } & \text { L } & 97.9 \\ \text { U } & & & 16.4 \\ \text { U } & & & 16.4 \\ \text { U } & & & 1.37 \\ \text { U } & & & 1.37 \\ \text { U } & & & 54.6 \\ \text { U } & & & 54.6 \\ \text { U } & & & 16.4 \\ \text { U } & & & 16.4 \\ & & & 5.6 \\ & & & 5.09 \\ & & & 5.52 \\ & & & 3.78 \\ & & & \\ & & \end{array}$

$B / N / A$ Extractables (including $P A H$ and phenols)

Acenaphthene
Acenaphthylene
Anthracene
Benzo[a]anthracene
Benzo[b]fluoranthene
Benzo[k]fluoranthene
Benzoic acid
Benzo[g,h,i]perylene
Benzo[a]pyrene
Benzyl alcohol
Bis(2-chloroethoxy) methane
Bis(2-chloroethyl) ether
Bis(2-chloroisopropyl) ether
Bis(2-ethylhexyl) phthalate
4-Bromophenyl phenyl ether
Butylbenzyl phthalate
4-Chloroaniline
4-Chloro-m-cresol
2-Chloronaphthalene
2-Chlorophenol

$\begin{array}{lll}\text { UJ } & \text { Q } & 37.1 \\ \text { UJ } & Q & 37.1 \\ \text { UJ } & Q & 37.1 \\ \text { UJ } & Q & 3.71 \\ \text { UJ } & Q & 3.71 \\ \text { UJ } & Q & 3.71 \\ \text { UJ } & Q & 37.1 \\ \text { UJ } & Q & 37.1 \\ \text { UJ } & Q & 3.71 \\ \text { UJ } & Q & 37.1 \\ \text { UJ } & Q & 37.1 \\ \text { UJ } & Q & 37.1 \\ \text { UJ } & Q & 37.1 \\ \text { UJ } & Q & 37.1 \\ \text { UJ } & Q & 37.1 \\ \text { UJ } & Q & 37.1 \\ \text { UJ } & Q & 37.1 \\ \text { UJ } & Q & 37.1 \\ \text { UJ } & Q & 37.1 \\ \text { UJ } & Q & 37.1\end{array}$

$\begin{array}{lll}\mathrm{mg} / \mathrm{kg} & 13.7 & \text { EPA6010A } \\ \mathrm{mg} / \mathrm{kg} & 1.37 & \text { EPA6010A } \\ \mathrm{mg} / \mathrm{kg} & 1.37 & \text { EPA6010A } \\ \mathrm{mg} / \mathrm{kg} & 0.546 & \text { EPA6010A } \\ \mathrm{mg} / \mathrm{kg} & 0.546 & \text { EPA6010A } \\ \mathrm{mg} / \mathrm{kg} & 1.37 & \text { EPA6010A } \\ \mathrm{mg} / \mathrm{kg} & 1.37 & \text { EPA6010A } \\ \mathrm{mg} / \mathrm{kg} & 5.46 & \text { EPA6010A } \\ \mathrm{mg} / \mathrm{kg} & 5.46 & \text { EPA6010A } \\ \mathrm{mg} / \mathrm{kg} & 5.46 & \text { EPA6010A } \\ \mathrm{mg} / \mathrm{kg} & 5.46 & \text { EPA6010A } \\ \mathrm{mg} / \mathrm{kg} & 5.46 & \text { EPA6010A } \\ \mathrm{mg} / \mathrm{kg} & 5.46 & \text { EPA6010A } \\ \mathrm{mg} / \mathrm{kg} & 0.546 & \text { EPA6010A } \\ \mathrm{mg} / \mathrm{kg} & 0.546 & \text { EPA6010A } \\ \mathrm{mg} / \mathrm{kg} & 0.0333 & \text { EPA7471 } \\ \mathrm{mg} / \mathrm{kg} & 1.37 & \text { EPA6010A } \\ \mathrm{mg} / \mathrm{kg} & 1.37 & \text { EPA6010A } \\ \mathrm{mg} / \mathrm{kg} & 109 & \text { EPA6010A } \\ \mathrm{mg} / \mathrm{kg} & 109 & \text { EPA6010A } \\ \mathrm{mg} / \mathrm{kg} & 16.4 & \text { EPA6010A } \\ \mathrm{mg} / \mathrm{kg} & 16.4 & \text { EPA6010A } \\ \mathrm{mg} / \mathrm{kg} & 1.37 & \text { EPA6010A } \\ \mathrm{mg} / \mathrm{kg} & 1.37 & \text { EPA6010A } \\ \mathrm{mg} / \mathrm{kg} & 54.6 & \text { EPA6010A } \\ \mathrm{mg} / \mathrm{kg} & 54.6 & \text { EPA6010A } \\ \mathrm{mg} / \mathrm{kg} & 16.4 & \text { EPA6010A } \\ \mathrm{mg} / \mathrm{kg} & 16.4 & \text { EPA6010A } \\ \mathrm{mg} / \mathrm{kg} & 0.546 & \text { EPA6010A } \\ \mathrm{mg} / \mathrm{kg} & 0.546 & \text { EPA6010A } \\ \mathrm{mg} / \mathrm{kg} & 1.09 & \text { EPA6010A } \\ \mathrm{mg} / \mathrm{kg} & 1.09 & \text { EPA6010A } \\ & & \end{array}$

$\begin{array}{lll}\mu \mathrm{g} / \mathrm{kg} & 37.1 & \text { EPA8270 } \\ \mu \mathrm{g} / \mathrm{kg} & 37.1 & \text { EPA8270 } \\ \mu \mathrm{g} / \mathrm{kg} & 37.1 & \text { EPA8270 } \\ \mu \mathrm{g} / \mathrm{kg} & 3.71 & \text { EPA8270 } \\ \mu \mathrm{g} / \mathrm{kg} & 3.71 & \text { EPA8270 } \\ \mu \mathrm{g} / \mathrm{kg} & 3.71 & \text { EPA8270 } \\ \mu \mathrm{g} / \mathrm{kg} & 37.1 & \text { EPA8270 } \\ \mu \mathrm{g} / \mathrm{kg} & 37.1 & \text { EPA8270 } \\ \mu \mathrm{g} / \mathrm{kg} & 3.71 & \text { EPA8270 } \\ \mu \mathrm{g} / \mathrm{kg} & 37.1 & \text { EPA8270 } \\ \mu \mathrm{g} / \mathrm{kg} & 37.1 & \text { EPA8270 } \\ \mu \mathrm{g} / \mathrm{kg} & 37.1 & \text { EPA8270 } \\ \mu \mathrm{g} / \mathrm{kg} & 37.1 & \text { EPA8270 } \\ \mu \mathrm{g} / \mathrm{kg} & 37.1 & \text { EPA8270 } \\ \mu \mathrm{g} / \mathrm{kg} & 37.1 & \text { EPA8270 } \\ \mu \mathrm{g} / \mathrm{kg} & 37.1 & \text { EPA8270 } \\ \mu \mathrm{g} / \mathrm{kg} & 37.1 & \text { EPA8270 } \\ \mu \mathrm{g} / \mathrm{kg} & 37.1 & \text { EPA8270 } \\ \mu \mathrm{g} / \mathrm{kg} & 37.1 & \text { EPA8270 } \\ \mu \mathrm{g} / \mathrm{kg} & 37.1 & \text { EPA8270 }\end{array}$


SURVEY ID: PPSC 1701 (continued)

Sample ID: 104693

\begin{tabular}{|c|c|c|c|c|c|c|}
\hline \multirow{2}{*}{\multicolumn{7}{|c|}{$\begin{array}{l}\text { Constituent } \mathrm{RQ} \text { AQ B } \\
B / N / A \text { Extractables (including PAH and phenols) }\end{array}$}} \\
\hline & & & & & & \\
\hline 4-Chlorophenyl phenyl ether & UJ & $Q$ & 37.1 & $\mu \mathrm{g} / \mathrm{kg}$ & 37.1 & EPA8270 \\
\hline Chrysene & UJ & $\mathbf{Q}$ & 3.71 & $\mu \mathrm{g} / \mathrm{kg}$ & 3.71 & EPA8270 \\
\hline $\mathrm{m} / \mathrm{p}$-Cresol & UJ & $\mathrm{Q}$ & 371 & $\mu \mathrm{g} / \mathrm{kg}$ & 371 & EPA8270 \\
\hline o-Cresol (2-Methylphenol) & UJ & $\mathbf{Q}$ & 37.1 & $\mu \mathrm{g} / \mathrm{kg}$ & 37.1 & EPA8270 \\
\hline Dibenz $[a, h]$ anthracene & UJ & $\mathbf{Q}$ & 3.71 & $\mu g / k g$ & 3.71 & EPA8270 \\
\hline Dibenzofuran & UJ & $\mathbf{Q}$ & 37.1 & $\mu \mathrm{g} / \mathrm{kg}$ & 37.1 & EPA8270 \\
\hline Di-n-butyl phthalate & UJ & $\mathbf{Q}$ & 37.1 & $\mu \mathrm{g} / \mathrm{kg}$ & 37.1 & EPA8270 \\
\hline 1,2-Dichlorobenzene & UJ & $\dot{Q}$ & 37.1 & $\mu \mathrm{g} / \mathrm{kg}$ & 37.1 & EPA8270 \\
\hline 1,3-Dichlorobenzene & UJ & $\mathbf{Q}$ & 37.1 & $\mu \mathrm{g} / \mathrm{kg}$ & 37.1 & EPA8270 \\
\hline 1,4-Dichlorobenzene & UJ & $\mathbf{Q}$ & 37.1 & $\mu \mathrm{g} / \mathrm{kg}$ & 37.1 & EPA8270 \\
\hline 3,3'-Dichlorobenzidine & UJ & $Q$ & 37.1 & $\mu \mathrm{g} / \mathrm{kg}$ & 37.1 & EPA8270 \\
\hline 2,4-Dichlorophenol & UJ & $\mathrm{Q}$ & 37.1 & $\mu \mathrm{g} / \mathrm{kg}$ & 37.1 & EPA8270 \\
\hline Diethyl phthalate & UJ & $\mathbf{Q}$ & 37.1 & $\mu \mathrm{g} / \mathrm{kg}$ & 37.1 & EPA8270 \\
\hline 2,4-Dimethyl phenol & UJ & $\mathbf{Q}$ & 37.1 & $\mu \mathrm{g} / \mathrm{kg}$ & 37.1 & EPA8270 \\
\hline Dimethyl phthalate & UJ & $\mathbf{Q}$ & 37.1 & $\mu \mathrm{g} / \mathrm{kg}$ & 37.1 & EPA8270 \\
\hline 2,4-Dinitrophenol & UJ & $\mathbf{Q}$ & 371 & $\mu \mathrm{g} / \mathrm{kg}$ & 371 & EPA8270 \\
\hline 2,4-Dinitrotoluene & $\mathrm{UJ}$ & QC & 3.71 & $\mu \mathrm{g} / \mathrm{kg}$ & 3.71 & EPA8270 \\
\hline 2,6-Dinitrotoluene & UJ & Q & 3.71 & $\mu g / k g$ & 3.71 & EPA8270 \\
\hline Di-n-octyl phthalate & UJ & $\mathbf{Q}$ & 37.1 & $\mu g / \mathrm{kg}$ & 37.1 & EPA8270 \\
\hline Fluoranthene & UJ & $\mathrm{Q}$ & 37.1 & $\mu \mathrm{g} / \mathrm{kg}$ & 37.1 & EPA8270 \\
\hline Fluorene & UJ & $\mathbf{Q}$ & 37.1 & $\mu \mathrm{g} / \mathrm{kg}$ & 37.1 & EPA8270 \\
\hline Hexachlorobenzene & UJ & $Q$ & 3.71 & $\mu \mathrm{g} / \mathrm{kg}$ & 3.71 & EPA8270 \\
\hline Hexachlorobutadiene & UJ & $\mathbf{Q}$ & 37.1 & $\mu \mathrm{g} / \mathrm{kg}$ & 37.1 & EPA8270 \\
\hline Hexachlorocyclopentadiene & UJ & $Q$ & 37.1 & $\mu \mathrm{g} / \mathrm{kg}$ & 37.1 & EPA8270 \\
\hline Hexachloroethane & UJ & $Q$ & 3.71 & $\mu \mathrm{g} / \mathrm{kg}$ & 3.71 & EPA8270 \\
\hline Indeno $[1,2,3-c, d]$ pyrene & UJ & $\mathbf{Q}$ & 3.71 & $\mu \mathrm{g} / \mathrm{kg}$ & 3.71 & EPA8270 \\
\hline Isophorone & UJ & $\mathbf{Q}$ & 37.1 & $\mu \mathrm{g} / \mathrm{kg}$ & 37.1 & EPA8270 \\
\hline 2-Methyl-4,6-dinitrophenol & UJ & Q & 371 & $\mu \mathrm{g} / \mathrm{kg}$ & 371 & EPA8270 \\
\hline 2-Methylnaphthalene & UJ & $\mathbf{Q}$ & 37.1 & $\mu \mathrm{g} / \mathrm{kg}$ & 37.1 & EPA8270 \\
\hline Naphthalene & UJ & $Q$ & 37.1 & $\mu \mathrm{g} / \mathrm{kg}$ & 37.1 & EPA8270 \\
\hline m-Nitroaniline & UJ & $\mathbf{Q}$ & 37.1 & $\mu \mathrm{g} / \mathrm{kg}$ & 37.1 & EPA8270 \\
\hline o-Nitroaniline & UJ & $\mathbf{Q}$ & 37.1 & $\mu \mathrm{g} / \mathrm{kg}$ & 37.1 & EPA8270 \\
\hline p-Nitroaniline & UJ & $Q$ & 37.1 & $\mu \mathrm{g} / \mathrm{kg}$ & 37.1 & EPA8270 \\
\hline Nitrobenzene & UJ & $Q$ & 37.1 & $\mu \mathrm{g} / \mathrm{kg}$ & 37.1 & EPA8270 \\
\hline 2-Nitrophenol & UJ & Q & 37.1 & $\mu \mathrm{g} / \mathrm{kg}$ & 37.1 & EPA8270 \\
\hline 4-Nitrophenol & UJ & $Q$ & 37.1 & $\mu \mathrm{g} / \mathrm{kg}$ & 37.1 & EPA8270 \\
\hline N-Nitrosodiphenylamine & UJ & $\mathbf{Q}$ & 37.1 & $\mu \mathrm{g} / \mathrm{kg}$ & 37.1 & EPA8270 \\
\hline N-Nitrosodi-n-propylamine & UJ & $\mathrm{Q}$ & 37.1 & $\mu \mathrm{g} / \mathrm{kg}$ & 37.1 & EPA8270 \\
\hline Pentachlorophenol & UJ & $Q$ & 37.1 & $\mu \mathrm{g} / \mathrm{kg}$ & 37.1 & EPA8270 \\
\hline Phenanthrene & UJ & $\mathrm{Q}$ & 37.1 & $\mu \mathrm{g} / \mathrm{kg}$ & 37.1 & EPA8270 \\
\hline Phenol & UJ & $Q$ & 37.1 & $\mu \mathrm{g} / \mathrm{kg}$ & 37.1 & EPA8270 \\
\hline Pyrene & UJ & $\mathbf{Q}$ & 37.1 & $\mu \mathrm{g} / \mathrm{kg}$ & 37.1 & EPA8270 \\
\hline 1,2,4-Trichlorobenzene & UJ & $Q$ & 3.71 & $\mu g / \mathrm{kg}$ & 3.71 & EPA8270 \\
\hline 2,4,5-Trichlorophenol & UJ & $\mathbf{Q}$ & 37.1 & $\mu \mathrm{g} / \mathrm{kg}$ & 37.1 & EPA8270 \\
\hline 2,4,6-Trichlorophenol & UJ & $\mathbf{Q}$ & 37.1 & $\mu \mathrm{g} / \mathrm{kg}$ & 37.1 & EPA8270 \\
\hline
\end{tabular}

Volatile Organic Compounds

$\begin{array}{llll}\text { Acetone } & & 8 & 91.3 \\ \text { Benzene } & \mathrm{U} & & 0.568 \\ \text { Bromodichloromethane } & \mathrm{U} & & \mathbf{0 . 0 5 6 8} \\ \text { Bromoform } & \mathrm{U} & & \mathbf{0 . 0 5 6 8} \\ \text { Bromomethane (Methyl bromide) } & \mathrm{U} & & \mathbf{0 . 1 1 4} \\ \text { Carbon disulfide } & & & \mathbf{5 . 5 6} \\ \text { Carbon tetrachloride } & \mathrm{U} & & \mathbf{0 . 0 5 6 8}\end{array}$

$\begin{array}{lll}\mu \mathrm{g} / \mathrm{kg} & 11.4 & \text { EPA8260 } \\ \mu \mathrm{g} / \mathrm{kg} & 0.568 & \text { EPA8260 } \\ \mu \mathrm{g} / \mathrm{kg} & 0.0568 & \text { EPA8260 } \\ \mu \mathrm{g} / \mathrm{kg} & -0.0568 & \text { EPA8260 } \\ \mu \mathrm{g} / \mathrm{kg} & 0.114 & \text { EPA8260 } \\ \mu \mathrm{g} / \mathrm{kg} & 1.14 & \text { EPA8260 } \\ \mu \mathrm{g} / \mathrm{kg} & 0.0568 & \text { EPA8260 }\end{array}$


SURVEY ID: PPSC 1701 (continued)

Sample ID: 104693

Constituent

RQ AQ B Result

Unit

D. Limit Method

Volatile Organic Compounds

Chlorobenzene

Chloroethane

Chloroethene (Vinyl chloride)

Chloroform

Chloromethane (Methyl chloride)

Dibromochloromethane

1,1-Dichloroethane

1,2-Dichloroethane

1,1-Dichloroethylene

1,2-Dichloroethylene

Dichloromethane (Methylene chloride)

1,2-Dichloropropane

cis-1,3-Dichloropropene

trans-1,3-Dichloropropene

Ethylbenzene

2-Hexanone

Methyl ethyl ketone

Methyl isobutyl ketone

Styrene

1,1,2,2-Tetrachloroethane

Tetrachloroethylene

Toluene

1,1,1-Trichloroethane

1,1,2-Trichloroethane

Trichloroethylene

Unknown hydrocarbon

Vinyl acetate

Xylenes

0.0568
0.114
0.114
0.0568
0.114
0.0568
0.0568
0.0568
0.0568
0.114
1.38
0.0568
0.0568
0.0568
0.0568
0.341
1.3
1.14
0.0568
0.0568
0.0568
0.568
0.0568
0.0568
0.0568
2
1.14
0.17

$\begin{array}{lll}\mu \mathrm{g} / \mathrm{kg} & 0.0568 & \text { EPA8260 } \\ \mu \mathrm{g} / \mathrm{kg} & 0.114 & \text { EPA8260 } \\ \mu \mathrm{g} / \mathrm{kg} & 0.114 & \text { EPA8260 } \\ \mu \mathrm{g} / \mathrm{kg} & 0.0568 & \text { EPA8260 } \\ \mu \mathrm{g} / \mathrm{kg} & 0.114 & \text { EPA8260 } \\ \mu \mathrm{g} / \mathrm{kg} & 0.0568 & \text { EPA8260 } \\ \mu \mathrm{g} / \mathrm{kg} & 0.0568 & \text { EPA8260 } \\ \mu \mathrm{g} / \mathrm{kg} & 0.0568 & \text { EPA8260 } \\ \mu \mathrm{g} / \mathrm{kg} & 0.0568 & \text { EPA8260 } \\ \mu \mathrm{g} / \mathrm{kg} & 0.114 & \text { EPA8260 } \\ \mu \mathrm{g} / \mathrm{kg} & 0.568 & \text { EPA8260 } \\ \mu \mathrm{g} / \mathrm{kg} & 0.0568 & \text { EPA8260 } \\ \mu \mathrm{g} / \mathrm{kg} & 0.0568 & \text { EPA8260 } \\ \mu \mathrm{g} / \mathrm{kg} & 0.0568 & \text { EPA8260 } \\ \mu \mathrm{g} / \mathrm{kg} & 0.0568 & \text { EPA8260 } \\ \mu \mathrm{g} / \mathrm{kg} & 1.14 & \text { EPA8260 } \\ \mu \mathrm{g} / \mathrm{kg} & 1.14 & \text { EPA8260 } \\ \mu \mathrm{g} / \mathrm{kg} & 1.14 & \text { EPA8260 } \\ \mu \mathrm{g} / \mathrm{kg} & 0.0568 & \text { EPA8260 } \\ \mu \mathrm{g} / \mathrm{kg} & 0.0568 & \text { EPA8260 } \\ \mu \mathrm{g} / \mathrm{kg} & 0.0568 & \text { EPA8260 } \\ \mu \mathrm{g} / \mathrm{kg} & 0.568 & \text { EPA8260 } \\ \mu \mathrm{g} / \mathrm{kg} & 0.0568 & \text { EPA8260 } \\ \mu \mathrm{g} / \mathrm{kg} & 0.0568 & \text { EPA8260 } \\ \mu \mathrm{g} / \mathrm{kg} & 0.0568 & \text { EPA8260 } \\ \mu \mathrm{g} / \mathrm{kg} & & \text { EPA8260 } \\ \mu \mathrm{g} / \mathrm{kg} & 1.14 & \text { EPA8260 } \\ \mu \mathrm{g} / \mathrm{kg} & 0.17 & \text { EPA8260 }\end{array}$

Pesticides/PCBs and Dioxins/Furans

Aldrin
alpha-Benzene hexachloride
beta-Benzene hexachloride
delta-Benzene hexachloride
alpha-Chlordane
gamma-Chlordane
p,p'-DDD
p,p'-DDE
p,p'-DDT
Dieldrin
Endosulfan sulfate
Endosulfan I
Endosulfan II
Endrin
Endrin ketone
Heptachlor
Heptachlor epoxide
Lindane
Methoxychlor
PCB 1016
PCB 1221
PCB 1232
PCB 1242
PCB 1248

$\begin{array}{ll}U & 4.61 \\ U & 4.61 \\ U & 9.22 \\ U & 9.22 \\ U & 9.22 \\ U & 9.22 \\ U & 18.4 \\ U & 9.22 \\ U & 18.4 \\ U & 9.22 \\ U & 18.4 \\ U & 9.22 \\ U & 18.4 \\ U & 9.22 \\ U & 18.4 \\ U & 9.22 \\ U & 9.22 \\ U & 4.61 \\ U & 92.2 \\ U & 23.1 \\ U & 23.1 \\ U & 23.1 \\ U & 23.1 \\ U & 23.1\end{array}$

$\begin{array}{lcc}\mu \mathrm{g} / \mathrm{kg} & 4.61 & \text { EPA8080 } \\ \mu \mathrm{g} / \mathrm{kg} & 4.61 & \text { EPA8080 } \\ \mu \mathrm{g} / \mathrm{kg} & 9.22 & \text { EPA8080 } \\ \mu \mathrm{g} / \mathrm{kg} & 9.22 & \text { EPA8080 } \\ \mu \mathrm{g} / \mathrm{kg} & 9.22 & \text { EPA8080 } \\ \mu \mathrm{g} / \mathrm{kg} & 9.22 & \text { EPA8080 } \\ \mu \mathrm{g} / \mathrm{kg} & 18.4 & \text { EPA8080 } \\ \mu \mathrm{g} / \mathrm{kg} & 9.22 & \text { EPA8080 } \\ \mu \mathrm{g} / \mathrm{kg} & 18.4 & \text { EPA8080 } \\ \mu \mathrm{g} / \mathrm{kg} & 9.22 & \text { EPA8080 } \\ \mu \mathrm{g} / \mathrm{kg} & 18.4 & \text { EPA8080 } \\ \mu \mathrm{g} / \mathrm{kg} & 9.22 & \text { EPA8080 } \\ \mu \mathrm{g} / \mathrm{kg} & 18.4 & \text { EPA8080 } \\ \mu \mathrm{g} / \mathrm{kg} & 9.22 & \text { EPA8080 } \\ \mu \mathrm{g} / \mathrm{kg} & 18.4 & \text { EPA8080 } \\ \mu \mathrm{g} / \mathrm{kg} & 9.22 & \text { EPA8080 } \\ \mu \mathrm{g} / \mathrm{kg} & 9.22 & \text { EPA8080 } \\ \mu \mathrm{g} / \mathrm{kg} & 4.61 & \text { EPA8080 } \\ \mu \mathrm{g} / \mathrm{kg} & 92.2 & \text { EPA8080 } \\ \mu \mathrm{g} / \mathrm{kg} & 23.1 & \text { EPA8080 } \\ \mu \mathrm{g} / \mathrm{kg} & 23.1 & \text { EPA8080 } \\ \mu \mathrm{g} / \mathrm{kg} & 23.1 & \text { EPA8080 } \\ \mu \mathrm{g} / \mathrm{kg} & 23.1 & \text { EPA8080 } \\ \mu \mathrm{g} / \mathrm{kg} & 23.1 & \text { EPA8080 }\end{array}$


SURVEY ID: PPSC 1701 (continued)

Constituent

Pesticides/PCBs and Dioxins/Furans

PCB 1254

PCB 1260

Toxaphene

Radionuclides

Actinium-228

Antimony-124

Antimony-125

Barium-133

Cerium-144

Cesium-134

Cesium-137

Cobalt-57

Cobalt-58

Cobalt-60

Europium-152

Europium-154

Europium-155

Gross alpha

Lead-212

Manganese-54

Neptunium-239

Nonvolatile beta

Plutonium-238

Plutonium-239/240

Potassium-40

Promethium-144

Promethium-146

Radium-226

Radium-228

Ruthenium-106

Sodium-22

Strontium-90

Thorium-234

Tin-113

Yttrium-88

Zinc-65

Zirconium-95

Sample ID: 104693
$\mathbf{R Q}$
AQ
Result
Unit
D. Limit Method

23.1

$\begin{array}{ll}\mathrm{U} & 23.1 \\ \mathrm{U} & 184\end{array}$

$\mu \mathrm{g} / \mathrm{kg}$

$\mu \mathrm{g} / \mathrm{kg}$

$\mu \mathrm{g} / \mathrm{kg}$

23.1

EPA8080

23.1

EPA8080

EPA8080

7.44E-01 $\pm 3.87 \mathrm{E}-02 \mathrm{pCi} / \mathrm{g}$ 2.37E-03 $\pm 4.32 \mathrm{E}-03 \mathrm{pCi} / \mathrm{g}$ $-2.49 \mathrm{E}-03 \pm 9.43 \mathrm{E}-03 \mathrm{pCi} / \mathrm{g}$ $-1.88 \mathrm{E}-03 \pm 5.12 \mathrm{E}-03 \mathrm{pCi} / \mathrm{g}$ $-1.01 \mathrm{E}-02 \pm 2.78 \mathrm{E}-02 \mathrm{pCi} / \mathrm{g}$ $-2.24 \mathrm{E} \cdot 03 \pm 3.70 \mathrm{E}-03 \mathrm{pCi} / \mathrm{g}$ $6.46 \mathrm{E}-02 \pm 7.29 \mathrm{E}-03 \mathrm{pCi} / \mathrm{g}$ $-1.91 \mathrm{E}-03 \pm 3.47 \mathrm{E}-03 \mathrm{pCi} / \mathrm{g}$ $-1.88 \mathrm{E}-03 \pm 4.21 \mathrm{E}-03 \mathrm{pCi} / \mathrm{g}$ $1.13 \mathrm{E}-03 \pm 3.16 \mathrm{E}-03 \mathrm{pCi} / \mathrm{g}$ $-1.07 \mathrm{E}-02 \pm 1.03 \mathrm{E}-02 \mathrm{pCi} / \mathrm{g}$ $-1.60 \mathrm{E}-02 \pm 2.90 \mathrm{E}-02 \mathrm{pCi} / \mathrm{g}$ $0.00 \mathrm{E}+00 \quad \mathrm{pCi} / \mathrm{g}$ $6.59 \mathrm{E}+00 \pm 1.93 \mathrm{E}+00 \mathrm{pCi} / \mathrm{g}$ $7.90 \mathrm{E}-01 \pm 1.82 \mathrm{E}-02 \mathrm{pCi} / \mathrm{g}$ 7.61E-03 $\pm 5.62 \mathrm{E}-03 \mathrm{pCi} / \mathrm{g}$ $-4.25 \mathrm{E}-03 \pm 2.57 \mathrm{E}-02 \mathrm{pCi} / \mathrm{g}$ $3.55 \mathrm{E}+00 \pm 1.38 \mathrm{E}+00 \mathrm{pCi} / \mathrm{g}$ 5.11E-03 $\pm 1.48 \mathrm{E}-02 \mathrm{pCi} / \mathrm{g}$ $0.00 \mathrm{E}+00 \quad \mathrm{pCi} / \mathrm{g}$ 5.82E-01 \pm 7.29E-02 pCi/g 1.39E-04 $\pm 3.53 \mathrm{E}-03 \mathrm{pCi} / \mathrm{g}$ $5.00 \mathrm{E}-03 \pm 4.60 \mathrm{E}-03 \mathrm{pCi} / \mathrm{g}$ $5.36 \mathrm{E}-01 \pm 2.34 \mathrm{E}-02 \mathrm{pCi} / \mathrm{g}$ $7.44 \mathrm{E}-01 \pm 3.87 \mathrm{E}-02 \mathrm{pCi} / \mathrm{g}$ $1.90 \mathrm{E}-02 \pm 3.18 \mathrm{E}-02 \mathrm{pCi} / \mathrm{g}$ $-2.20 \mathrm{E}-04 \pm 3.00 \mathrm{E}-03 \mathrm{pCi} / \mathrm{g}$ 2.02E-01 $1.02 \mathrm{E}-01 \mathrm{pCi} / \mathrm{g}$ 7.06E-01 $13.59 \mathrm{E}-01 \mathrm{pCi} / \mathrm{g}$ $-1.45 \mathrm{E}-03 \pm 4.90 \mathrm{E}-03 \mathrm{pCi} / \mathrm{g}$ 2.22E-03 $43.62 \mathrm{E}-03 \mathrm{pCi} / \mathrm{g}$ $0.00 \mathrm{E}+00 \quad \mathrm{pCi} / \mathrm{g}$ $0.00 \mathrm{E}+00 \quad \mathrm{pCi} / \mathrm{g}$
1.99E-02 EPIA-013B
7.51E-03 EPIA-013B
1.63E-02 EPIA-013B
7.30E-03 EPIA-013B
4.70E-02 EPIA-013B
5.40E-03 EPIA-013B
6.54E-03 EPIA-013B
5.88E-03 EPIA-013B
6.93E-03 EPIA-013B
5.63E-03 EPIA-013B
1.64E-02 EPIA-013B
4.97E-02 EPIA-013B
2.54E-02 EPIA-013B
$1.35 \mathrm{E}+00$ EPIA-001B
1.08E-02 EPIA-013B
6.46E-03 EPIA-013B
4.39E-02 EPIA-013B
2.39E+00 EPIA-001B
3.22E-02 EPIA-012B
7.82E-03 EPIA-012B
5.16E-02 EPIA-013B
5.99E-03 EPIA-013B
8.19E-03 EPIA-013B
1.11E-02 EPIA-013B
1.99E-02 EPIA-013B
5.56E-02 EPIA-013B
5.23E-03 EPIA-013B
1.40E-01 EPIA-004
3.17E-01 EPIA-013B
8.48E-03 EPIA-013B
6.88E-03 EPIA-013B
1.15E-02 EPIA-013B
1.48E-02 EPIA-013B

SURVEY ID: PPSC 1702

Sample ID: 104694

Survey location: 44049E 3671941N (SRS coordinates)

Depth of core interval: 1.00 to $2.00 \mathrm{ft}$

Sample type: Normal

Sample matrix: Soil

Percent solids: $\mathbf{8 9 . 0 0}$ 
SURVEY ID: PPSC 1702 (continued)

Sample ID: 104694

Constituent

RQ AQ B Result

Unit

D. Limit Method

Physical Parameters and Specified Analyses

$\begin{array}{llll}\text { Ammonia nitrogen } & \text { U } & & 22.5 \\ \text { Cation exchange capacity } & & & 26.4 \\ \text { Chloride } & \text { J } & \text { E } & 2.12 \\ \text { Cyanide } & \text { U } & & 1.06 \\ \text { Nitrate-nitrite as nitrogen } & \text { U } & & 1.07 \\ \text { Nitrogen by Kjeldahl method } & & & 120 \\ \text { Orthophosphate } & \text { U } & & 22.5 \\ \text { Phenols } & \text { U } & & 337 \\ \text { Sulfide } & \text { UJ } & \text { C } & 281 \\ \text { Total organic carbon } & & \text { V } & 1400 \\ \text { Total organic carbon } & & \text { V } & 1510 \\ \text { Total organic nitrogen } & \text { C } & & 104 \\ \text { Total phosphates (as P) } & \text { J } & \text { E } & 114\end{array}$

$\begin{array}{lll}\mathrm{mg} / \mathrm{kg} & 22.5 & \text { EPA350.1 } \\ \mathrm{meq} / 100 \mathrm{~g} & 5 & \text { EPA9081 } \\ \mathrm{mg} / \mathrm{kg} & 2.77 & \text { EPA300.0 } \\ \mathrm{mg} / \mathrm{kg} & 1.06 & \text { EPA335.3 } \\ \mathrm{mg} / \mathrm{kg} & 1.07 & \text { EPA353.1 } \\ \mathrm{mg} / \mathrm{kg} & 44.4 & \text { EPA351.2 } \\ \mathrm{mg} / \mathrm{kg} & 22.5 & \text { EPA365.2 } \\ \mathrm{mg} / \mathrm{kg} & 337 & \text { EPA420.2 } \\ \mathrm{mg} / \mathrm{kg} & 281 & \text { EPA376.2 } \\ \mathrm{mg} / \mathrm{kg} & 112 & \text { EPA415.1 } \\ \mathrm{mg} / \mathrm{kg} & 112 & \text { EPA415.1 } \\ \mathrm{mg} / \mathrm{kg} & 0.225 & \text { EPA351.2 } \\ \mathrm{mg} / \mathrm{kg} & 424 & \text { EPA365.4 }\end{array}$

Metals (total recoverable)

\begin{tabular}{|c|c|c|c|c|c|c|}
\hline Aluminum & & & 4540 & $\mathrm{mg} / \mathrm{kg}$ & 13.5 & EPA6010A \\
\hline Antimony & $\mathrm{U}$ & & 5.4 & $\mathrm{mg} / \mathrm{kg}$ & 5.4 & EPA6010A \\
\hline Arsenic & U & & 16.2 & $\mathrm{mg} / \mathrm{kg}$ & 16.2 & EPA6010A \\
\hline Barium & & & 9.47 & $\mathrm{mg} / \mathrm{kg}$ & 1.35 & EPA6010A \\
\hline Beryllium & $J$ & $\mathrm{E}$ & 0.0281 & $\mathrm{mg} / \mathrm{kg}$ & 0.54 & EPA6010A \\
\hline Cadmium & $\mathrm{U}$ & & 0.27 & $\mathrm{mg} / \mathrm{kg}$ & 0.27 & EPA6010A \\
\hline Calcium & & & 31.6 & $\mathrm{mg} / \mathrm{kg}$ & 13.5 & EPA6010A \\
\hline Chromium & & & 2.36 & $\mathrm{mg} / \mathrm{kg}$ & 1.35 & EPA6010A \\
\hline Cobalt & $\mathrm{U}$ & & 0.54 & $\mathrm{mg} / \mathrm{kg}$ & 0.54 & EPA6010A \\
\hline Copper & & & 1.4 & $\mathrm{mg} / \mathrm{kg}$ & 1.35 & EPA6010A \\
\hline Iron & & V & 1270 & $\mathrm{mg} / \mathrm{kg}$ & 5.4 & EPA6010A \\
\hline Lead & $\mathrm{U}$ & & 5.4 & $\mathrm{mg} / \mathrm{kg}$ & 5.4 & EPA6010A \\
\hline Magnesium & & & 89.5 & $\mathrm{mg} / \mathrm{kg}$ & 5.4 & EPA6010A \\
\hline Manganese & & & 6.96 & $\mathrm{mg} / \mathrm{kg}$ & 0.54 & EPA6010A \\
\hline Mercury & & & 1.21 & $\mathrm{mg} / \mathrm{kg}$ & 0.0314 & EPA7471 \\
\hline Nickel & $\mathrm{J}$ & $\mathrm{E}$ & 0.939 & $\mathrm{mg} / \mathrm{kg}$ & 1.35 & EPA6010A \\
\hline Potassium & $J$ & $E$ & 63.2 & $\mathrm{mg} / \mathrm{kg}$ & 108 & EPA6010A \\
\hline Selenium & $U$ & & 16.2 & $\mathrm{mg} / \mathrm{kg}$ & 16.2 & EPA6010A \\
\hline Silver & $\mathrm{U}$ & & 1.35 & $\mathrm{mg} / \mathrm{kg}$ & 1.35 & EPA6010A \\
\hline Sodium & $\mathrm{U}$ & & 54 & $\mathrm{mg} / \mathrm{kg}$ & 54 & EPA6010A \\
\hline Thallium & $U$ & & 16.2 & $\mathrm{mg} / \mathrm{kg}$ & 16.2 & EPA6010A \\
\hline Vanadium & & & 4.32 & $\mathrm{mg} / \mathrm{kg}$ & 0.54 & EPA6010A \\
\hline Zinc & & & 2.1 & $\mathrm{mg} / \mathrm{kg}$ & 1.08 & EPA6010A \\
\hline$B / N / A$ Extractables (includin & $d$ phe & nols) & & & & \\
\hline Acenaphthene & UJ & $\mathbf{Q}$ & 37.4 & $\mu \mathrm{g} / \mathrm{kg}$ & 37.4 & EPA8270 \\
\hline Acenaphthylene & UJ & $\mathbf{Q}$ & 37.4 & $\mu g / \mathrm{kg}$ & 37.4 & EPA8270 \\
\hline Anthracene & UJ & $\mathbf{Q}$ & 37.4 & $\mu g / k g$ & 37.4 & EPA8270 \\
\hline Benzo[a]anthracene & UJ & $Q$ & 3.74 & $\mu \mathrm{g} / \mathrm{kg}$ & 3.74 & EPA8270 \\
\hline Benzo[b]fluoranthene & UJ & $\mathbf{Q}$ & 3.74 & $\mu \mathrm{g} / \mathrm{kg}$ & 3.74 & EPA8270 \\
\hline Benzo $[k]$ fluoranthene & UJ & $\mathbf{Q}$ & 3.74 & $\mu \mathrm{g} / \mathrm{kg}$ & 3.74 & EPA8270 \\
\hline Benzoic acid & $\mathrm{J}$ & $\mathbf{Q}$ & 81.5 & $\mu \mathrm{g} / \mathrm{kg}$ & 37.4 & EPA8270 \\
\hline Benzo $[g, h, i]$ perylene & UJ & $\mathbf{Q}$ & 37.4 & $\mu \mathrm{g} / \mathrm{kg}$ & 37.4 & EPA8270 \\
\hline Benzo[a]pyrene & UJ & $\mathbf{Q}$ & 3.74 & $\mu \mathrm{g} / \mathrm{kg}$ & 3.74 & EPA8270 \\
\hline Benzyl alcohol & UJ & $\dot{Q}$ & 37.4 & $\mu \mathrm{g} / \mathrm{kg}$ & 37.4 & EPA8270 \\
\hline Bis(2-chloroethoxy) methane & UJ & $\mathbf{Q}$ & 37.4 & $\mu \mathrm{g} / \mathrm{kg}$ & 37.4 & EPA8270 \\
\hline Bis(2-chloroethyl) ether & UJ & $\mathbf{Q}$ & 37.4 & $\mu \mathrm{g} / \mathrm{kg}$ & 37.4 & EPA8270 \\
\hline Bis(2-chloroisopropyl) ether & UJ & $Q$ & 37.4 & $\mu \mathrm{g} / \mathrm{kg}$ & 37.4 & EPA8270 \\
\hline
\end{tabular}


Constituent

RQ AQ B Result

$B / N / A$ Extractables (including $P A H$ and phenols)
Bis(2-ethylhexyl) phthalate

4-Bromophenyl phenyl ether

Butylbenzyl phthalate

4-Chloroaniline

4-Chloro-m-cresol

2-Chloronaphthalene

2-Chlorophenol

4-Chlorophenyl phenyl ether

Chrysene

$\mathrm{m} / \mathrm{p}$-Cresol

o-Cresol (2-Methylphenol)

Dibenz $[a, h]$ anthracene

Dibenzofuran

Di-n-butyl phthalate

1,2-Dichlorobenzene

1,3-Dichlorobenzene

1,4-Dichlorobenzene

$3,3^{\circ}$-Dichlorobenzidine

2,4-Dichlorophenol

2,4-Dimethyl phenol

Dimethyl phthalate

2,4-Dinitrophenol

2,4-Dinitrotoluene

2,6-Dinitrotoluene

Di-n-octyl phthalate

Fluoranthene

Fluorene

Hexachlorobenzene

Hexachlorobutadiene

Hexachlorocyclopentadiene

Hexachloroethane

Indeno $[1,2,3-c, d]$ pyrene

Isophorone

2-Methyl-4,6-dinitrophenol

2-Methylnaphthalene

Naphthalene

$\mathrm{m} \cdot$ Nitroaniline

o-Nitroaniline

p-Nitroaniline

Nitrobenzene

2-Nitrophenol

4-Nitrophenol

N-Nitrosodiphenylamine

N-Nitrosodi-n-propylamine

Pentachlorophenol

Phenanthrene

Phenol

Pyrene

1,2,4-Trichlorobenzene

2,4,5-Trichlorophenol

2,4,6-Trichlorophenol
Diethyl phthalate
UJ $Q$

UJ Q

UJ $Q$

UJ Q

UJ Q

UJ $Q$

UJ Q

UJ Q

UJ Q

UJ $Q$

UJ Q

UJ $Q$

UJ $Q$

UJ $Q$

UJ $Q$

UJ Q

UJ $Q$

UJ $Q$

UJ Q

UJ $\mathbf{Q}$

UJ $Q$

UJ QC

UJ $Q$

UJ $Q$

UJ Q

UJ Q

UJ Q

UJ $\mathbf{Q}$

UJ Q

UJ $Q$

UJ Q

UJ $Q$

UJ Q

UJ Q

UJ Q

UJ $Q$

UJ Q

UJ Q

UJ Q

UJ Q

UJ $Q$

UJ Q

UJ Q

UJ

UJ Q

UJ Q

UJ Q

UJ $Q$

UJ Q

UJ Q
UJ $Q$
Unit

D. Limit Method

$\begin{array}{lll}\mu \mathrm{g} / \mathrm{kg} & 37.4 & \text { EPA8270 } \\ \mu \mathrm{g} / \mathrm{kg} & 37.4 & \text { EPA8270 } \\ \mu \mathrm{g} / \mathrm{kg} & 37.4 & \text { EPA8270 } \\ \mu \mathrm{g} / \mathrm{kg} & 37.4 & \text { EPA8270 } \\ \mu \mathrm{g} / \mathrm{kg} & 37.4 & \text { EPA8270 } \\ \mu \mathrm{g} / \mathrm{kg} & 37.4 & \text { EPA8270 } \\ \mu \mathrm{g} / \mathrm{kg} & 37.4 & \text { EPA8270 } \\ \mu \mathrm{g} / \mathrm{kg} & 37.4 & \text { EPA8270 } \\ \mu \mathrm{g} / \mathrm{kg} & 3.74 & \text { EPA8270 } \\ \mu \mathrm{g} / \mathrm{kg} & 374 & \text { EPA8270 } \\ \mu \mathrm{g} / \mathrm{kg} & 37.4 & \text { EPA8270 } \\ \mu \mathrm{g} / \mathrm{kg} & 3.74 & \text { EPA8270 } \\ \mu \mathrm{g} / \mathrm{kg} & 37.4 & \text { EPA8270 } \\ \mu \mathrm{g} / \mathrm{kg} & 37.4 & \text { EPA8270 } \\ \mu \mathrm{g} / \mathrm{kg} & 37.4 & \text { EPA8270 } \\ \mu \mathrm{g} / \mathrm{kg} & 37.4 & \text { EPA8270 } \\ \mu \mathrm{g} / \mathrm{kg} & 37.4 & \text { EPA8270 } \\ \mu \mathrm{g} / \mathrm{kg} & 37.4 & \text { EPA8270 } \\ \mu \mathrm{g} / \mathrm{kg} & 37.4 & \text { EPA8270 } \\ \mu \mathrm{g} / \mathrm{kg} & 37.4 & \text { EPA8270 } \\ \mu \mathrm{g} / \mathrm{kg} & 37.4 & \text { EPA8270 } \\ \mu \mathrm{g} / \mathrm{kg} & 37.4 & \text { EPA8270 } \\ \mu \mathrm{g} / \mathrm{kg} & 374 & \text { EPA8270 } \\ \mu \mathrm{g} / \mathrm{kg} & 3.74 & \text { EPA8270 } \\ \mu \mathrm{g} / \mathrm{kg} & 3.74 & \text { EPA8270 } \\ \mu \mathrm{g} / \mathrm{kg} & 37.4 & \text { EPA8270 } \\ \mu \mathrm{g} / \mathrm{kg} & 37.4 & \text { EPA8270 } \\ \mu \mathrm{g} / \mathrm{kg} & 374 & \text { EPA8270 }\end{array}$

37.4

37.4

37.4

37.4

37.4

37.4

37.4

37.4

3.74

374

37.4

3.74

37.4

37.4

37.4

37.4

37.4

37.4

37.4

37.4

37.4

37.4

374

3.74

3.74

37.4

37.4

37.4

3.74

37.4

37.4

3.74

3.74

37.4

374

37.4

37.4

37.4

37.4

37.4

37.4

37.4

37.4

37.4

37.4

37.4

37.4

37.4

37.4

3.74

37.4

37.4 $\mu \mathrm{g} / \mathrm{kg}$

$\mu \mathrm{g} / \mathrm{kg}$

$\mu \mathrm{g} / \mathrm{kg}$

$\mu \mathrm{g} / \mathrm{kg}$

$\mu \mathrm{g} / \mathrm{kg}$

$\mu \mathrm{g} / \mathrm{kg}$

$\mu \mathrm{g} / \mathrm{kg}$

$\mu \mathrm{g} / \mathrm{kg}$

$\mu \mathrm{g} / \mathrm{kg}$

$\mu \mathrm{g} / \mathrm{kg}$

$\mu \mathrm{g} / \mathrm{kg}$

$\mu \mathrm{g} / \mathrm{kg}$

$\mu \mathrm{g} / \mathrm{kg}$

$\mu \mathrm{g} / \mathrm{kg}$

$\mu \mathrm{g} / \mathrm{kg}$

$\mu \mathrm{g} / \mathrm{kg}$

$\mu \mathrm{g} / \mathrm{kg}$

$\mu \mathrm{g} / \mathrm{kg}$

$\mu \mathrm{g} / \mathrm{kg}$

$\mu \mathrm{g} / \mathrm{kg}$

$\mu \mathrm{g} / \mathrm{kg}$

$\mu \mathrm{g} / \mathrm{kg}$

$\mu \mathrm{g} / \mathrm{kg}$

$\mu \mathrm{g} / \mathrm{kg}$

37.4

EPA8270

EPA8270

EPA8270

EPA8270

EPA8270

EPA8270

EPA8270

EPA8270

EPA8270

EPA8270

EPA8270

EPA8270

EPA8270

EPA8270

EPA8270

EPA8270

EPA8270

EPA8270

EPA8270

EPA8270

EPA8270

EPA8270

EPA8270

EPA8270

$\mu \mathrm{g} / \mathrm{kg}$

EPA8270 
SURVEY ID: PPSC 1702 (continued)

Sample ID: 104694

Constituent

RQ AQ B Result

Unit

D. Limit Method

Volatile Organic Compounds

Acetone

Alkylbenzene C11H160

Benzene

Bromodichloromethane

Bromoform

Bromomethane (Methyl bromide)

Carbon disulfide

Carbon tetrachloride

Chlorobenzene

Chloroethane

Chloroethene (Vinyl chloride)

Chloroform

Chloromethane (Methyl chloride)

Dibromochloromethane

1,1-Dichloroethane

1,2-Dichloroethane

1,1-Dichloroethylene

1,2-Dichloroethylene

Dichloromethane (Methylene chloride)

1,2-Dichloropropane

cis-1,3-Dichloropropene

trans-1,3-Dichloropropene

Ethylbenzene

2-Hexanone

Methyl ethyl ketone

Methyl isobutyl ketone

Styrene

1,1,2,2-Tetrachloroethane

Tetrachloroethylene

Toluene

1,1,1-Trichloroethane

1,1,2-Trichloroethane

Trichloroethylene

Unknown alcohol

Unknown alkylbenzene

Vinyl acetate

Xylenes

$\begin{array}{lll} & 8 & 161 \\ J & N & 1 \\ U & & 0.562 \\ U & & 0.0562 \\ U & & 0.0562 \\ U & & 0.112 \\ U & & 1.12 \\ U & & 0.0562 \\ U & & 0.0562 \\ U & & 0.112 \\ U & & 0.112 \\ U & & 0.0562 \\ U & & 0.112 \\ U & & 0.0562 \\ U & & 0.0562 \\ U & & 0.0562 \\ U & & 0.0562 \\ U & & 0.112 \\ U & V & 1.4 \\ U & & 0.0562 \\ U & & 0.0562 \\ U & & 0.0562 \\ U & & 0.0562 \\ U & V & 1.74 \\ U & V & \mathbf{2 . 7 9} \\ U & & 1.12 \\ U & & \mathbf{0 . 0 5 6 2} \\ U & & 0.0562 \\ U & & 0.0562 \\ U & & 0.562 \\ U & & 0.0562 \\ U & & 0.0562 \\ U & & 0.0562 \\ J & N & 2 \\ J & N & 1 \\ U & & 1.12 \\ U & & 0.169 \\ & & \end{array}$

$\begin{array}{lll}\mu \mathrm{g} / \mathrm{kg} & 28.1 & \text { EPA8260 } \\ \mu \mathrm{g} / \mathrm{kg} & & \text { EPA8260 } \\ \mu \mathrm{g} / \mathrm{kg} & 0.562 & \text { EPA8260 } \\ \mu \mathrm{g} / \mathrm{kg} & 0.0562 & \text { EPA8260 } \\ \mu \mathrm{g} / \mathrm{kg} & 0.0562 & \text { EPA8260 } \\ \mu \mathrm{g} / \mathrm{kg} & 0.112 & \text { EPA8260 } \\ \mu \mathrm{g} / \mathrm{kg} & 1.12 & \text { EPA8260 } \\ \mu \mathrm{g} / \mathrm{kg} & 0.0562 & \text { EPA8260 } \\ \mu \mathrm{g} / \mathrm{kg} & 0.0562 & \text { EPA8260 } \\ \mu \mathrm{g} / \mathrm{kg} & 0.112 & \text { EPA8260 } \\ \mu \mathrm{g} / \mathrm{kg} & 0.112 & \text { EPA8260 } \\ \mu \mathrm{g} / \mathrm{kg} & 0.0562 & \text { EPA8260 } \\ \mu \mathrm{g} / \mathrm{kg} & 0.112 & \text { EPA8260 } \\ \mu \mathrm{g} / \mathrm{kg} & 0.0562 & \text { EPA8260 } \\ \mu \mathrm{g} / \mathrm{kg} & 0.0562 & \text { EPA8260 } \\ \mu \mathrm{g} / \mathrm{kg} & 0.0562 & \text { EPA8260 } \\ \mu \mathrm{g} / \mathrm{kg} & 0.0562 & \text { EPA8260 } \\ \mu \mathrm{g} / \mathrm{kg} & 0.112 & \text { EPA8260 } \\ \mu \mathrm{g} / \mathrm{kg} & 0.562 & \text { EPA8260 } \\ \mu \mathrm{g} / \mathrm{kg} & 0.0562 & \text { EPA8260 } \\ \mu \mathrm{g} / \mathrm{kg} & 0.0562 & \text { EPA8260 } \\ \mu \mathrm{g} / \mathrm{kg} & 0.0562 & \text { EPA8260 } \\ \mu \mathrm{g} / \mathrm{kg} & 0.0562 & \text { EPA8260 } \\ \mu \mathrm{g} / \mathrm{kg} & 5.62 & \text { EPA8260 } \\ \mu \mathrm{g} / \mathrm{kg} & 1.12 & \text { EPA8260 } \\ \mu \mathrm{g} / \mathrm{kg} & 1.12 & \text { EPA8260 } \\ \mu \mathrm{g} / \mathrm{kg} & 0.0562 & \text { EPA8260 } \\ \mu \mathrm{g} / \mathrm{kg} & 0.0562 & \text { EPA8260 } \\ \mu \mathrm{g} / \mathrm{kg} & 0.0562 & \text { EPA8260 } \\ \mu \mathrm{g} / \mathrm{kg} & 0.562 & \text { EPA8260 } \\ \mu \mathrm{g} / \mathrm{kg} & 0.0562 & \text { EPA8260 } \\ \mu \mathrm{g} / \mathrm{kg} & 0.0562 & \text { EPA8260 } \\ \mu \mathrm{g} / \mathrm{kg} & 0.0562 & \text { EPA8260 } \\ \mu \mathrm{g} / \mathrm{kg} & & \text { EPA8260 } \\ \mu \mathrm{g} / \mathrm{kg} & & \text { EPA8260 } \\ \mu \mathrm{g} / \mathrm{kg} & 1.12 & \text { EPA8260 } \\ \mu \mathrm{g} / \mathrm{kg} & 0.169 & \text { EPA8260 } \\ & & \end{array}$

Pesticides/PCBs and Dioxins/Furans

Aldrin

alpha-Benzene hexachloride

beta-Benzene hexachloride

delta-Benzene hexachloride

alpha-Chlordane

gamma-Chlordane

$\mathrm{p}, \mathrm{p}^{\prime}-\mathrm{DDD}$

$p, p^{\prime}-\mathrm{DDE}$

p,p'-DDT

Dieldrin

Endosulfan sulfate

Endosulfan I

Endosulfan II

Endrin

Endrin ketone

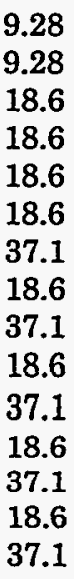

$\begin{array}{lll}\mu \mathrm{g} / \mathrm{kg} & 9.28 & \text { EPA8080 } \\ \mu \mathrm{g} / \mathrm{kg} & 9.28 & \text { EPA8080 } \\ \mu \mathrm{g} / \mathrm{kg} & 18.6 & \text { EPA8080 } \\ \mu \mathrm{g} / \mathrm{kg} & 18.6 & \text { EPA8080 } \\ \mu \mathrm{g} / \mathrm{kg} & 18.6 & \text { EPA8080 } \\ \mu \mathrm{g} / \mathrm{kg} & 18.6 & \text { EPA8080 } \\ \mu \mathrm{g} / \mathrm{kg} & 37.1 & \text { EPA8080 } \\ \mu \mathrm{g} / \mathrm{kg} & 18.6 & \text { EPA8080 } \\ \mu \mathrm{g} / \mathrm{kg} & 37.1 & \text { EPA8080 } \\ \mu \mathrm{g} / \mathrm{kg} & 18.6 & \text { EPA8080 } \\ \mu \mathrm{g} / \mathrm{kg} & 37.1 & \text { EPA8080 } \\ \mu \mathrm{g} / \mathrm{kg} & 18.6 & \text { EPA8080 } \\ \mu \mathrm{g} / \mathrm{kg} & 37.1 & \text { EPA8080 } \\ \mu \mathrm{g} / \mathrm{kg} & 18.6 & \text { EPA8080 } \\ \mu \mathrm{g} / \mathrm{kg} & 37.1 & \text { EPA8080 }\end{array}$


SURVEY ID: PPSC 1702 (continued)

Constituent

RQ AQ B Result

Unit

D. Limit Method

Pesticides/PCBs and Dioxins/Furans

Heptachlor

Heptachlor epoxide

Lindane

Methoxychlor

PCB 1016

PCB 1221

PCB 1232

PCB 1242

PCB 1248

PCB 1254

PCB 1260

Toxaphene

Radionuclides

Actinium-228

Antimony-124

Antimony-125

Barium-133

Cerium-144

Cesium-134

Cesium-137

Cobalt-57

Cobalt-58

Cobalt-60

Europium-152

Europium-154

Europium-155

Gross alpha

Lead-212

Manganese-54

Neptunium-239

Nonvolatile beta

Plutonium-238

Plutonium-239/240

Potassium-40

Promethium-144

Promethium-146

Radium-226

Radium-228

Ruthenium-106

Sodium-22

Strontium-90

Thorium-234

Tin-113

Yttrium-88

Zinc-65

Zirconium-95
18.6
18.6
9.28
186
46.4
46.4
46.4
46.4
46.4
46.4
46.4
371

$\mu \mathrm{g} / \mathrm{kg}$

$\mathrm{\mu g} / \mathrm{kg}$

$\mu \mathrm{g} / \mathrm{kg}$

$\mu \mathrm{g} / \mathrm{kg}$

$\mu \mathrm{g} / \mathrm{kg}$

$\mu \mathrm{g} / \mathrm{kg}$

$\mu \mathrm{g} / \mathrm{kg}$

$\mu \mathrm{g} / \mathrm{kg}$

$\mu \mathrm{g} / \mathrm{kg}$

$\mu \mathrm{g} / \mathrm{kg}$

$\mu \mathrm{g} / \mathrm{kg}$

$\mu \mathrm{g} / \mathrm{kg}$

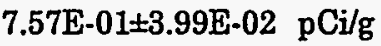
-3.52E-04t5.13E-03 pCi/g 3.41E-03 $\pm 9.48 \mathrm{E}-03 \mathrm{pCi} / \mathrm{g}$ $-1.12 \mathrm{E}-01 \pm 6.74 \mathrm{E}-03 \mathrm{pCi} / \mathrm{g}$ $6.87 \mathrm{E}-03 \pm 2.87 \mathrm{E}-02 \mathrm{pCi} / \mathrm{g}$ 1.11E-03 $\pm 3.80 \mathrm{E}-03 \mathrm{pCi} / \mathrm{g}$ 2.19E-02+5.76E-03 pCi/g $-1.55 \mathrm{E}-03 \pm 3.67 \mathrm{E}-03 \mathrm{pCi} / \mathrm{g}$ $-5.49 \mathrm{E}-04 \pm 4.08 \mathrm{E}-03 \mathrm{pCi} / \mathrm{g}$ $2.12 \mathrm{E}-03 \pm 2.96 \mathrm{E}-03 \mathrm{pCi} / \mathrm{g}$ $-9.89 \mathrm{E}-03 \pm 1.02 \mathrm{E}-02 \mathrm{pCi} / \mathrm{g}$ $6.96 \mathrm{E}-03 \pm 2.91 \mathrm{E}-02 \mathrm{pCi} / \mathrm{g}$ $0.00 \mathrm{E}+00 \quad \mathrm{pCi} / \mathrm{g}$ $1.02 \mathrm{E}+01 \pm 2.34 \mathrm{E}+00 \mathrm{pCi} / \mathrm{g}$ $7.77 \mathrm{E}-01 \pm 1.85 \mathrm{E}-02 \mathrm{pCi} / \mathrm{g}$ 1.12E-02 $\pm 5.93 \mathrm{E}-03 \mathrm{pCi} / \mathrm{g}$ $0.00 \mathrm{E}+00 \quad \mathrm{pCi} / \mathrm{g}$ $4.58 \mathrm{E}+00 \pm 1.45 \mathrm{E}+00 \mathrm{pCi} / \mathrm{g}$ $1.21 \mathrm{E}+00 \pm 1.66 \mathrm{E}-01 \mathrm{pCi} / \mathrm{g}$ $3.41 \mathrm{E}-02 \pm 2.08 \mathrm{E}-02 \mathrm{pCi} / \mathrm{g}$ 5.41E-01 $18.08 \mathrm{E}-02 \mathrm{pCi} / \mathrm{g}$ $\begin{array}{ll}\text { UI } & 1.89 \mathrm{E}-03 \pm 3.61 \mathrm{E}-03 \mathrm{pCi} / \mathrm{g} \\ \text { UI } & 7.12 \mathrm{E}-03 \pm 4.67 \mathrm{E}-03 \mathrm{pCi} / \mathrm{g}\end{array}$ 5.31E-01 $\pm 2.33 \mathrm{E}-02 \mathrm{pCi} / \mathrm{g}$ 7.57E-01 $\pm 3.99 \mathrm{E}-02 \mathrm{pCi} / \mathrm{g}$ $-1.06 \mathrm{E}-02 \pm 3.29 \mathrm{E}-02 \mathrm{pCi} / \mathrm{g}$ $-5.75 \mathrm{E}-03 \pm 3.26 \mathrm{E}-03 \mathrm{pCi} / \mathrm{g}$ $3.40 \mathrm{E}-02 \pm 6.20 \mathrm{E}-02 \mathrm{pCi} / \mathrm{g}$ $1.58 \mathrm{E}+00 \pm 5.28 \mathrm{E}-01 \mathrm{pCi} / \mathrm{g}$ 4.62E-04 $\pm 5.64 \mathrm{E}-03 \mathrm{pCi} / \mathrm{g}$ $3.35 \mathrm{E}-03 \pm 3.72 \mathrm{E}-03 \mathrm{pCi} / \mathrm{g}$ $6.55 \mathrm{E}-03 \pm 7.62 \mathrm{E}-03 \mathrm{pCi} / \mathrm{g}$ $1.04 \mathrm{E}-02 \pm 9.35 \mathrm{E}-03 \mathrm{pCi} / \mathrm{g}$
1.95E-02 EPIA-013B

7.54E-03 EPIA-013B

1.64E-02 EPLA-013B

7.73E-03 EPIA-013B

4.80E-02 EPIA-013B

5.75E-03 EPIA-013B

6.21E-03 EPIA-013B

6.09E-03 EPIA-013B

6.75E-03 EPIA-013B

5.38E-03 EPIA-013B

1.73E-02 EPIA-013B

4.55E-02 EPIA-013B

2.52E-02 EPIA-013B

1.21E+00 EPIA-001B

1.06E-02 EPIA-013B

5.99E-03 EPIA-013B

4.36E-02 EPIA-013B

2.38E+00 EPIA-001B

5.02E-02 EPIA-012B

9.29E-03 EPIA-012B

4.65E-02 EPIA-013B

6.16E-03 EPIA-013B

8.32E-03 EPIA-013B

1.16E-02 EPIA-013B

1.95E-02 EPIA-013B

5.48E-02 EPIA-013B

5.00E-03 EPIA-013B

1.29E-01 EPIA-004

3.22E-01 EPIA-013B

8.55E-03 EPIA-013B

7.12E-03 EPIA-013B

1.23E-02 EPIA-013B

1.45E-02 EPIA-013B

SURVEY ID: PPSC 1703

Sample ID: 104695

Survey location: $44049 \mathrm{E} 3671941 \mathrm{~N}$ (SRS coordinates)

Depth of core interval: 2.00 to $4.00 \mathrm{ft}$

Sample type: Normal

Sample matrix: Soil

Percent solids: 90.00 
SURVEY ID: PPSC 1703 (continued)

Constituent

RQ AQ B Result

Sample ID: 104695

Physical Parameters and Specified Analyses

Ammonia nitrogen

Cation exchange capacity

Chloride

Cyanide

Nitrate-nitrite as nitrogen

Nitrogen by Kjeldahl method

Orthophosphate

Phenols

Sulfide

Total organic carbon

Total organic nitrogen

Total phosphates (as P)

$\begin{array}{lll}\text { U } & & 22.2 \\ & & 32 \\ \text { J } & \text { E } & 1.28 \\ \text { U } & & 1.06 \\ \text { U } & & 1.02 \\ & & 114 \\ \text { U } & & 11.1 \\ \text { U } & & 329 \\ \text { UJ } & \text { C } & 278 \\ & \text { V } & 1520 \\ \text { C } & & 105 \\ \text { J } & \text { E } & 147\end{array}$

Metals (total recoverable)

Aluminum

Antimony

Arsenic

Barium

Beryllium

Cadmium

Calcium

Chromium

Cobalt

Copper

Iron

Lead

Magnesium

Manganese

Mercury

Nickel

Potassium

Selenium

Silver

Sodium

Thallium

Vanadium

Zinc

$B / N / A$ Extractables (including $P A H$ and phenols)

Acenaphthene

Acenaphthylene

Anthracene

Benzo[a]anthracene

Benzo[b]fluoranthene

Benzo[k]fluoranthene

Benzoic acid

Benzo[g,h,i]perylene

Benzo[a]pyrene

Benzyl alcohol

Bis(2-chloroethoxy) methane

Bis(2-chloroethyl) ether

Bis(2-chloroisopropyl) ether

\begin{tabular}{|c|c|c|}
\hline UJ & $\mathbf{Q}$ & 36 \\
\hline UJ & $\mathbf{Q}$ & 36 \\
\hline UJ & $\mathbf{Q}$ & 36 \\
\hline UJ & $\mathbf{Q}$ & 3.6 \\
\hline UJ & $\mathbf{Q}$ & 3.6 \\
\hline UJ & $\mathbf{Q}$ & 3.6 \\
\hline $\mathbf{J}$ & $\mathbf{Q}$ & 94.7 \\
\hline UJ & $\mathbf{Q}$ & 36 \\
\hline UJ & $\mathbf{Q}$ & 3.6 \\
\hline UJ & $\mathbf{Q}$ & 36 \\
\hline UJ & Q & 36 \\
\hline & $\mathbf{Q}$ & 36 \\
\hline & $\mathbf{Q}$ & 36 \\
\hline
\end{tabular}

4350
5.39
16.2
13.7
0.0453
0.27
36.8
3.03
0.539
1.04
1780
2.72
123
9.89
0.0344
1.05
73.7
16.2
1.35
53.9
16.2
5.88
3.24

Unit D. Limit Method

$\begin{array}{lll}\mathrm{mg} / \mathrm{kg} & 22.2 & \text { EPA350.1 } \\ \mathrm{meq} / 100 \mathrm{~g} & 5 & \text { EPA9081 } \\ \mathrm{mg} / \mathrm{kg} & 2.71 & \text { EPA300.0 } \\ \mathrm{mg} / \mathrm{kg} & 1.06 & \text { EPA335.3 } \\ \mathrm{mg} / \mathrm{kg} & 1.02 & \text { EPA353.1 } \\ \mathrm{mg} / \mathrm{kg} & 43.7 & \text { EPA351.2 } \\ \mathrm{mg} / \mathrm{kg} & 11.1 & \text { EPA365.2 } \\ \mu \mathrm{gg} & 329 & \text { EPA420.2 } \\ \mathrm{mg} / \mathrm{kg} & 278 & \text { EPA376.2 } \\ \mathrm{mg} / \mathrm{kg} & 111 & \text { EPA415.1 } \\ \mathrm{mg} / \mathrm{kg} & 0.222 & \text { EPA351.2 } \\ \mathrm{mg} / \mathrm{kg} & 1100 & \text { EPA365.4 }\end{array}$

$\mathrm{mg} / \mathrm{kg} \quad 13.5 \quad$ EPA6010A

$\mathrm{mg} / \mathrm{kg} \quad 5.39 \quad$ EPA6010A

$\mathrm{mg} / \mathrm{kg} \quad 16.2 \quad$ EPA6010A

$\mathrm{mg} / \mathrm{kg} \quad 1.35 \quad$ EPA6010A

$\begin{array}{lll}\mathrm{mg} / \mathrm{kg} & 0.539 & \text { EPA6010A }\end{array}$

$\begin{array}{lll}\mathrm{mg} / \mathrm{kg} & 0.27 \quad \text { EPA6010A }\end{array}$

$\mathrm{mg} / \mathrm{kg} \quad 13.5 \quad$ EPA6010A

$\mathrm{mg} / \mathrm{kg} \quad 1.35 \quad$ EPA6010A

$\begin{array}{lll}\mathrm{mg} / \mathrm{kg} & 0.539 & \text { EPA6010A }\end{array}$

$\mathrm{mg} / \mathrm{kg} \quad 1.35 \quad$ EPA6010A

$\mathrm{mg} / \mathrm{kg} \quad 5.39 \quad$ EPA6010A

$\mathrm{mg} / \mathrm{kg} \quad 5.39 \quad$ EPA6010A

$\mathrm{mg} / \mathrm{kg} \quad 5.39 \quad$ EPA6010A

$\begin{array}{lll}\mathrm{mg} / \mathrm{kg} & 0.539 \quad \text { EPA6010A }\end{array}$

$\mathrm{mg} / \mathrm{kg} \quad 0.0344 \quad$ EPA7471

$\mathrm{mg} / \mathrm{kg} \quad 1.35 \quad$ EPA6010A

$\mathrm{mg} / \mathrm{kg} \quad 108 \quad$ EPA6010A

$\mathrm{mg} / \mathrm{kg} \quad 16.2 \quad$ EPA6010A

$\mathrm{mg} / \mathrm{kg} \quad 1.35 \quad$ EPA6010A

$\mathrm{mg} / \mathrm{kg} \quad 53.9 \quad$ EPA6010A

$\mathrm{mg} / \mathrm{kg} \quad 16.2 \quad$ EPA6010A

$\mathrm{mg} / \mathrm{kg} \quad 0.539 \quad$ EPA6010A

$\begin{array}{lll}\mathrm{mg} / \mathrm{kg} & 1.08 \quad \text { EPA6010A }\end{array}$

$\begin{array}{lll}\mu \mathrm{g} / \mathrm{kg} & 36 & \text { EPA8270 } \\ \mu \mathrm{g} / \mathrm{kg} & 36 & \text { EPA8270 } \\ \mu \mathrm{g} / \mathrm{kg} & 36 & \text { EPA8270 } \\ \mu \mathrm{g} / \mathrm{kg} & 3.6 & \text { EPA8270 } \\ \mu \mathrm{g} / \mathrm{kg} & 3.6 & \text { EPA8270 } \\ \mu \mathrm{g} / \mathrm{kg} & 3.6 & \text { EPA8270 } \\ \mu \mathrm{g} / \mathrm{kg} & 36 & \text { EPA8270 } \\ \mu \mathrm{g} / \mathrm{kg} & 36 & \text { EPA8270 } \\ \mu \mathrm{g} / \mathrm{kg} & 3.6 & \text { EPA8270 } \\ \mu \mathrm{g} / \mathrm{kg} & 36 & \text { EPA8270 } \\ \mu \mathrm{g} / \mathrm{kg} & 36 & \text { EPA8270 } \\ \mu \mathrm{g} / \mathrm{kg} & 36 & \text { EPA8270 } \\ \mu \mathrm{g} / \mathrm{kg} & 36 & \text { EPA8270 }\end{array}$


Constituent

RQ AQ B Result

$B / N / A$ Extractables (including $P A H$ and phenols)

Bis(2-ethylhexyl) phthalate

4-Bromophenyl phenyl ether

Butylbenzyl phthalate

4-Chloroaniline

4-Chloro-m-cresol

2-Chloronaphthalene

2-Chlorophenol

4-Chlorophenyl phenyl ether

Chrysene

$\mathrm{m} / \mathrm{p}$-Cresol

o-Cresol (2-Methylphenol)

Dibenz $[a, h]$ anthracene

Dibenzofuran

Di-n-butyl phthalate

1,2-Dichlorobenzene

1,3-Dichlorobenzene

1,4-Dichlorobenzene

3,3'-Dichlorobenzidine

2,4-Dichlorophenol

Diethyl phthalate

2,4-Dimethyl phenol

Dimethyl phthalate

2,4-Dinitrophenol

2,4-Dinitrotoluene

2,6-Dinitrotoluene

Di-n-octyl phthalate

Fluoranthene

Fluorene

Hexachlorobenzene

Hexachlorobutadiene

Hexachlorocyclopentadiene

Hexachloroethane

Indeno[1,2,3-c,d]pyrene

Isophorone

2-Methyl-4,6-dinitrophenol

2-Methylnaphthalene

Naphthalene

m-Nitroaniline

o-Nitroaniline

p-Nitroaniline

Nitrobenzene

2-Nitrophenol

4-Nitrophenol

N-Nitrosodiphenylamine

N-Nitrosodi-n-propylamine

Pentachlorophenol

Phenanthrene

Phenol

Pyrene

1,2,4-Trichlorobenzene

2,4,5-Trichlorophenol

2,4,6-Trichlorophenol

$\begin{array}{lll}\text { UJ } & \mathbf{Q} & 36 \\ \text { UJ } & \mathbf{Q} & 36 \\ \text { UJ } & \mathbf{Q} & 36 \\ \text { UJ } & \mathbf{Q} & 36 \\ \text { UJ } & \mathbf{Q} & 36 \\ \text { UJ } & \mathbf{Q} & 36 \\ \text { UJ } & \mathbf{Q} & 36 \\ \text { UJ } & \mathbf{Q} & 36 \\ \text { UJ } & \mathbf{Q} & 3.6\end{array}$

UJ $Q \quad 360$

UJ $Q$

UJ $Q \quad 3.6$

UJ $Q \quad 36$

UJ $Q \quad 36$

UJ $Q \quad 36$

UJ Q 36

UJ $Q \quad 36$

UJ $Q \quad 36$

UJ $Q \quad 36$

UJ $Q \quad 36$

UJ $Q \quad 36$

UJ $Q \quad 360$

UJ QC $\quad 3.6$

UJ $Q \quad 3.6$

UJ Q 36

UJ $Q \quad 36$

UJ $Q \quad 36$

UJ $Q \quad 3.6$

UJ $Q \quad 36$

UJ $Q$

UJ Q $\quad 3.6$

UJ $Q \quad 3.6$

UJ $Q \quad 36$

UJ $Q \quad 360$

UJ $Q \quad 36$

UJ $Q \quad 36$

UJ $Q \quad 36$

UJ $Q \quad 36$

UJ $Q \quad 36$

UJ $Q \quad 36$

UJ $Q \quad 36$

UJ $\quad \mathrm{Q} \quad 36$

UJ $Q \quad 36$

UJ $Q \quad 36$

UJ $Q \quad 36$

UJ Q 36

UJ $Q \quad 36$

UJ $\quad \mathrm{Q} \quad 36$

UJ $Q \quad 3.6$

UJ $Q \quad 36$

UJ $Q \quad 36$
UJ $Q \quad 36$
Unit

D. Limit Method

$\begin{array}{lll}\mu \mathrm{g} / \mathrm{kg} & 36 & \text { EPA8270 } \\ \mu \mathrm{g} / \mathrm{kg} & 36 & \text { EPA8270 } \\ \mu \mathrm{g} / \mathrm{kg} & 36 & \text { EPA8270 } \\ \mu \mathrm{g} / \mathrm{kg} & 36 & \text { EPA8270 } \\ \mu \mathrm{g} / \mathrm{kg} & 36 & \text { EPA8270 } \\ \mu \mathrm{g} / \mathrm{kg} & 36 & \text { EPA8270 } \\ \mu \mathrm{g} / \mathrm{kg} & 36 & \text { EPA8270 } \\ \mu \mathrm{g} / \mathrm{kg} & 36 & \text { EPA8270 } \\ \mu \mathrm{g} / \mathrm{kg} & 3.6 & \text { EPA8270 } \\ \mu \mathrm{g} / \mathrm{kg} & 360 & \text { EPA8270 } \\ \mu \mathrm{g} / \mathrm{kg} & 36 & \text { EPA8270 } \\ \mu \mathrm{g} / \mathrm{kg} & 3.6 & \text { EPA8270 } \\ \mu \mathrm{g} / \mathrm{kg} & 36 & \text { EPA8270 } \\ \mu \mathrm{g} / \mathrm{kg} & 36 & \text { EPA8270 } \\ \mu \mathrm{g} / \mathrm{kg} & 36 & \text { EPA8270 } \\ \mu \mathrm{g} / \mathrm{kg} & 36 & \text { EPA8270 } \\ \mu \mathrm{g} / \mathrm{kg} & 36 & \text { EPA8270 } \\ \mu \mathrm{g} / \mathrm{kg} & 36 & \text { EPA8270 } \\ \mu \mathrm{g} / \mathrm{kg} & 36 & \text { EPA8270 } \\ \mu \mathrm{g} / \mathrm{kg} & 36 & \text { EPA8270 } \\ \mu \mathrm{g} / \mathrm{kg} & 36 & \text { EPA8270 } \\ \mu \mathrm{g} / \mathrm{kg} & 36 & \text { EPA8270 } \\ \mu \mathrm{g} / \mathrm{kg} & 360 & \text { EPA8270 } \\ \mu \mathrm{g} / \mathrm{kg} & 3.6 & \text { EPA8270 } \\ \mu \mathrm{g} / \mathrm{kg} & 3.6 & \text { EPA8270 } \\ \mu \mathrm{g} / \mathrm{kg} & 36 & \text { EPA8270 } \\ \mu \mathrm{g} / \mathrm{kg} & 36 & \text { EPA8270 } \\ \mu \mathrm{g} / \mathrm{kg} & 36 & \text { EPA8270 }\end{array}$

$\mu \mathrm{g} / \mathrm{kg} \quad 36 \quad$ EPA8270

$\mu \mathrm{g} / \mathrm{kg} \quad 3.6 \quad$ EPA8270

$\mu \mathrm{g} / \mathrm{kg} \quad 36 \quad$ EPA8270

$\mu \mathrm{g} / \mathrm{kg} \quad 36 \quad$ EPA8270

$\mu \mathrm{g} / \mathrm{kg} \quad 3.6 \quad$ EPA8270

$\mu \mathrm{g} / \mathrm{kg} \quad 3.6 \quad$ EPA8270

$\mu \mathrm{g} / \mathrm{kg} \quad 36 \quad$ EPA8270

$\mu \mathrm{g} / \mathrm{kg} \quad 360 \quad$ EPA8270

$\mu \mathrm{g} / \mathrm{kg} \quad 36 \quad$ EPA8270

$\mu \mathrm{g} / \mathrm{kg} \quad 36 \quad$ EPA8270

$\mu \mathrm{g} / \mathrm{kg} \quad 36 \quad$ EPA8270

$\mu \mathrm{g} / \mathrm{kg} \quad 36 \quad$ EPA8270

$\mu \mathrm{g} / \mathrm{kg} \quad 36 \quad$ EPA8270

$\mu \mathrm{g} / \mathrm{kg} \quad 36 \quad$ EPA8270

$\mu \mathrm{g} / \mathrm{kg} \quad 36 \quad$ EPA8270

$\mu \mathrm{g} / \mathrm{kg} \quad 36 \quad$ EPA8270

$\mu \mathrm{g} / \mathrm{kg} \quad 36 \quad$ EPA8270

$\mu g / \mathrm{kg} \quad 36 \quad$ EPA8270

$\mu g / k g \quad 36 \quad$ EPA8270

$\mu \mathrm{g} / \mathrm{kg} \quad 36 \quad$ EPA8270

$\mu \mathrm{g} / \mathrm{kg} \quad 36 \quad$ EPA8270

$\mu \mathrm{g} / \mathrm{kg} \quad 36 \quad$ EPA8270

$\mu \mathrm{g} / \mathrm{kg} \quad 3.6 \quad$ EPA8270

$\mu \mathrm{g} / \mathrm{kg} \quad 36 \quad$ EPA8270

$\mu \mathrm{g} / \mathrm{kg} \quad \cdot 36 \quad$ EPA8270 
SURVEY ID: PPSC 1703 (continued)

Constituent

Volatile Organic Compounds

Acetone

Alkane

Benzene

Bromodichloromethane

Bromoform

Bromomethane (Methyl bromide)

Carbon disulfide

Carbon tetrachloride

Chlorobenzene

Chloroethane

Chloroethene (Vinyl chloride)

Chloroform

Chloromethane (Methyl chloride)

Dibromochloromethane

1,1-Dichloroethane

1,2-Dichloroethane

1,1-Dichloroethylene

1,2-Dichloroethylene

Dichloromethane (Methylene chloride)

1,2-Dichloropropane

cis-1,3-Dichloropropene

trans-1,3-Dichloropropene

Ethylbenzene

2-Hexanone

Isocamphene

Methyl ethyl ketone

Methyl isobutyl ketone

Propylbenzene

Styrene

1,1,2,2-Tetrachloroethane

Tetrachloroethylene

Toluene

1,1,1-Trichloroethane

1,1,2-Trichloroethane

Trichloroethylene

Vinyl acetate

Xylenes

Pesticides/PCBs and Dioxins/Furans

Aldrin

alpha-Benzene hexachloride

beta-Benzene hexachloride

delta-Benzene hexachloride

alpha-Chlordane

gamma-Chlordane

p,p'-DDD

$p, p^{\prime}-D D E$

p,p'-DDT

Dieldrin

Endosulfan sulfate

Endosulfan I

Endosulfan II

Endrin

Endrin ketone
Sample ID: 104695

RQ AQ B Result

Unit

D. Limit Method

\begin{tabular}{|c|c|c|c|c|}
\hline 8 & 77.5 & $\mu \mathrm{g} / \mathrm{kg}$ & 27.8 & EPA8260 \\
\hline$N$ & 7 & $\mu \mathrm{g} / \mathrm{kg}$ & & EPA8260 \\
\hline$U$ & 0.556 & $\mu \mathrm{g} / \mathrm{kg}$ & 0.556 & EPA8260 \\
\hline $\mathrm{U}$ & 0.0556 & $\mu \mathrm{g} / \mathrm{kg}$ & 0.0556 & EPA8260 \\
\hline $\mathrm{U}$ & 0.0556 & $\mu \mathrm{g} / \mathrm{kg}$ & 0.0556 & EPA8260 \\
\hline $\mathrm{U}$ & 0.111 & $\mu \mathrm{g} / \mathrm{kg}$ & 0.111 & EPA8260 \\
\hline $\mathrm{U}$ & 1.11 & $\mu \mathrm{g} / \mathrm{kg}$ & 1.11 & EPA8260 \\
\hline $\mathrm{U}$ & 0.0556 & $\mu \mathrm{g} / \mathrm{kg}$ & 0.0556 & EPA8260 \\
\hline $\mathrm{U}$ & 0.0556 & $\mu \mathrm{g} / \mathrm{kg}$ & 0.0556 & EPA8260 \\
\hline $\mathrm{U}$ & 0.111 & $\mu \mathrm{g} / \mathrm{kg}$ & 0.111 & EPA8260 \\
\hline U & 0.111 & $\mu \mathrm{g} / \mathrm{kg}$ & 0.111 & EPA8260 \\
\hline $\mathrm{U}$ & 0.0556 & $\mu \mathrm{g} / \mathrm{kg}$ & 0.0556 & EPA8260 \\
\hline$U$ & 0.111 & $\mu \mathrm{g} / \mathrm{kg}$ & 0.111 & EPA8260 \\
\hline $\mathrm{U}$ & 0.0556 & $\mu \mathrm{g} / \mathrm{kg}$ & 0.0556 & EPA8260 \\
\hline $\mathrm{U}$ & 0.0556 & $\mu \mathrm{g} / \mathrm{kg}$ & 0.0556 & EPA8260 \\
\hline $\mathrm{U}$ & 0.0556 & $\mu \mathrm{g} / \mathrm{kg}$ & 0.0556 & EPA8260 \\
\hline $\mathrm{U}$ & 0.0556 & $\mu \mathrm{g} / \mathrm{kg}$ & 0.0556 & EPA8260 \\
\hline U & 0.111 & $\mu g / k g$ & 0.111 & EPA8260 \\
\hline $\mathbf{U}$ & 1.72 & $\mu \mathrm{g} / \mathrm{kg}$ & 0.556 & EPA8260 \\
\hline $\mathbf{U}$ & 0.0556 & $\mu \mathrm{g} / \mathrm{kg}$ & 0.0556 & EPA8260 \\
\hline $\mathbf{U}$ & 0.0556 & $\mu \mathrm{g} / \mathrm{kg}$ & 0.0556 & EPA8260 \\
\hline \multirow[t]{2}{*}{$\mathrm{U}$} & 0.0556 & $\mu \mathrm{g} / \mathrm{kg}$ & 0.0556 & EPA8260 \\
\hline & 9.56 & $\mu \mathrm{g} / \mathrm{kg}$ & 0.0556 & EPA8260 \\
\hline $\mathbf{U}$ & 0.311 & $\mu \mathrm{g} / \mathrm{kg}$ & 1.11 & EPA8260 \\
\hline \multirow{3}{*}{$\begin{array}{l}N \\
V\end{array}$} & 4 & $\mu \mathrm{g} / \mathrm{kg}$ & & EPA8260 \\
\hline & 7.54 & $\mu \mathrm{g} / \mathrm{kg}$ & 1.11 & EPA8260 \\
\hline & 1.11 & $\mu \mathrm{g} / \mathrm{kg}$ & 1.11 & EPA8260 \\
\hline$N$ & 75 & $\mu \mathrm{g} / \mathrm{kg}$ & & EPA8260 \\
\hline$U$ & 0.0556 & $\mu \mathrm{g} / \mathrm{kg}$ & 0.0556 & EPA8260 \\
\hline \multirow[t]{2}{*}{$\mathrm{U}$} & 0.0556 & $\mu \mathrm{g} / \mathrm{kg}$ & 0.0556 & EPA8260 \\
\hline & 0.111 & $\mu \mathrm{g} / \mathrm{kg}$ & 0.0556 & EPA8260 \\
\hline U & 0.311 & $\mu \mathrm{g} / \mathrm{kg}$ & 0.556 & EPA8260 \\
\hline $\mathrm{U}$ & 0.0556 & $\mu \mathrm{g} / \mathrm{kg}$ & 0.0556 & EPA8260 \\
\hline $\mathrm{U}$ & 0.0556 & $\mu \mathrm{g} / \mathrm{kg}$ & 0.0556 & EPA8260 \\
\hline $\mathrm{U}$ & 0.0889 & $\mu \mathrm{g} / \mathrm{kg}$ & 0.0556 & EPA8260 \\
\hline $\mathrm{E}$ & 0.622 & $\mu \mathrm{g} / \mathrm{kg}$ & 1.11 & EPA8260 \\
\hline $\mathbf{J} \quad \mathbf{E}$ & 0.667 & $\mu \mathrm{g} / \mathrm{kg}$ & 0.833 & EPA8260 \\
\hline
\end{tabular}

\begin{tabular}{|c|c|}
\hline U & 9.04 \\
\hline $\mathrm{U}$ & 9.04 \\
\hline $\mathrm{U}$ & 18.1 \\
\hline $\mathbf{U}$ & 18.1 \\
\hline $\mathrm{U}$ & 18.1 \\
\hline $\mathrm{U}$ & 18.1 \\
\hline $\mathbf{U}$ & 36.2 \\
\hline $\mathrm{U}$ & 18.1 \\
\hline $\mathrm{U}$ & 36.2 \\
\hline $\mathbf{U}$ & 18.1 \\
\hline $\mathrm{U}$ & 36.2 \\
\hline $\mathrm{U}$ & 18.1 \\
\hline $\mathrm{U}$ & 36.2 \\
\hline $\mathrm{U}$ & 18.1 \\
\hline $\mathrm{U}$ & 36.2 \\
\hline
\end{tabular}

$\begin{array}{ll}\mu \mathrm{g} / \mathrm{kg} & 9.04 \\ \mu \mathrm{g} / \mathrm{kg} & 9.04 \\ \mu \mathrm{g} / \mathrm{kg} & 18.1 \\ \mu \mathrm{g} / \mathrm{kg} & 18.1 \\ \mu \mathrm{g} / \mathrm{kg} & 18.1 \\ \mu \mathrm{g} / \mathrm{kg} & 18.1 \\ \mu \mathrm{g} / \mathrm{kg} & 36.2 \\ \mu \mathrm{g} / \mathrm{kg} & 18.1 \\ \mu \mathrm{g} / \mathrm{kg} & 36.2 \\ \mu \mathrm{g} / \mathrm{kg} & 18.1 \\ \mu \mathrm{g} / \mathrm{kg} & 36.2 \\ \mu \mathrm{g} / \mathrm{kg} & 18.1 \\ \mu \mathrm{g} / \mathrm{kg} & 36.2 \\ \mu \mathrm{g} / \mathrm{kg} & 18.1 \\ \mu \mathrm{g} / \mathrm{kg} & 36.2\end{array}$

EPA8080

EPA8080

EPA8080

EPA8080

EPA8080

EPA8080

EPA8080

EPA8080

EPA8080

EPA8080

EPA8080

EPA8080

EPA8080

EPA8080

EPA8080 
SURVEY ID: PPSC 1703 (continued)

Constituent

Pesticides/PCBs and Dioxins/Furans

Heptachlor

Heptachlor epoxide

Lindane

Methoxychlor

PCB 1016

PCB 1221

PCB 1232

PCB 1242

PCB 1248

PCB 1254

PCB 1260

Toxaphene

Radionuclides

Actinium-228

Antimony-124

Antimony-125

Barium-133

Cerium-144

Cesium-134

Cesium-137

Cobalt-57

Cobalt-58

Cobalt-60

Europium-152

Europium-154

Europium-155

Gross alpha

Lead-212

Manganese-54

Neptunium-239

Nonvolatile beta

Plutonium-238

Plutonium-239/240

Potassium-40

Promethium-144

Promethium-146

Radium-226

Radium-228

Ruthenium-106

Sodium-22

Strontium-90

Thorium-234

Tin-113

Yttrium-88

Zinc-65

Zirconium-95
Sample ID: 104695

RQ AQ B Result

Unit

D. Limit Method

18.1

$\mu g / k g \quad 18.1 \quad$ EPA8080

18.1

9.04

181

45.2

45.2

45.2

45.2

45.2

45.2

45.2

362

$\mu \mathrm{g} / \mathrm{kg} \quad 18.1 \quad$ EPA8080

$\mu \mathrm{g} / \mathrm{kg} \quad 9.04 \quad$ EPA8080

$\mu \mathrm{g} / \mathrm{kg} \quad 181 \quad$ EPA8080

$\mu \mathrm{g} / \mathrm{kg} \quad 45.2 \quad$ EPA8080

$\mu g / \mathrm{kg} \quad 45.2 \quad$ EPA8080

$\mu \mathrm{g} / \mathrm{kg} \quad 45.2 \quad$ EPA8080

$\mu \mathrm{g} / \mathrm{kg} \quad 45.2 \quad$ EPA8080

$\mu \mathrm{g} / \mathrm{kg} \quad 45.2 \quad$ EPA8080

$\mu \mathrm{g} / \mathrm{kg} \quad 45.2 \quad$ EPA8080

$\mu \mathrm{g} / \mathrm{kg} \quad 45.2 \quad \mathrm{EPA} 8080$

$\mu \mathrm{g} / \mathrm{kg} \quad 362 \quad$ EPA8080

7.67E-01 $\pm 4.10 \mathrm{E}-02 \mathrm{pCi} / \mathrm{g}$ $-2.32 \mathrm{E}-04 \pm 4.28 \mathrm{E}-03 \mathrm{pCi} / \mathrm{g}$ $3.67 \mathrm{E}-03 \pm 9.95 \mathrm{E}-03 \mathrm{pCi} / \mathrm{g}$ 6.67E-04 $\pm 4.99 \mathrm{E}-03 \mathrm{pCi} / \mathrm{g}$ $-2.41 \mathrm{E}-02 \pm 2.73 \mathrm{E}-02 \mathrm{pCi} / \mathrm{g}$ $-1.60 \mathrm{E}-03 \pm 3.70 \mathrm{E}-03 \mathrm{pCi} / \mathrm{g}$ $4.34 \mathrm{E}-03 \pm 7.05 \mathrm{E}-03 \mathrm{pCi} / \mathrm{g}$ $-2.27 \mathrm{E}-03 \pm 3.44 \mathrm{E}-03 \mathrm{pCi} / \mathrm{g}$ $2.05 \mathrm{E}-03 \pm 4.30 \mathrm{E}-03 \mathrm{pCi} / \mathrm{g}$ $-2.13 \mathrm{E}-04 \pm 3.10 \mathrm{E}-03 \mathrm{pCi} / \mathrm{g}$ $3.49 \mathrm{E}-03 \pm 1.03 \mathrm{E}-02 \mathrm{pCi} / \mathrm{g}$ $2.25 \mathrm{E}-02 \pm 3.08 \mathrm{E}-02 \mathrm{pCi} / \mathrm{g}$ $0.00 \mathrm{E}+00 \quad \mathrm{pCi} / \mathrm{g}$ $9.99 \mathrm{E}+00 \pm 2.27 \mathrm{E}+00 \mathrm{pCi} / \mathrm{g}$ 8.09E-01 $\pm 1.83 \mathrm{E}-02 \mathrm{pCi} / \mathrm{g}$ $1.26 \mathrm{E}-02 \pm 4.47 \mathrm{E}-03 \mathrm{pCi} / \mathrm{g}$ 2.57E-02 $\pm 3.43 \mathrm{E}-02 \mathrm{pCi} / \mathrm{g}$ $6.30 \mathrm{E}+00 \pm 1.62 \mathrm{E}+00 \mathrm{pCi} / \mathrm{g}$ $1.26 \mathrm{E}-02 \pm 1.80 \mathrm{E}-02 \mathrm{pCi} / \mathrm{g}$ $3.80 \mathrm{E}-03 \pm 7.60 \mathrm{E}-03 \mathrm{pCi} / \mathrm{g}$ $6.28 \mathrm{E}-01 \pm 8.77 \mathrm{E}-02 \mathrm{pCi} / \mathrm{g}$ $3.88 \mathrm{E}-03 \pm 4.65 \mathrm{E}-03 \mathrm{pCi} / \mathrm{g}$ 6.73E-03 $\pm 4.80 \mathrm{E}-03 \mathrm{pCi} / \mathrm{g}$ 5.48E-01 $\pm 2.28 \mathrm{E}-02 \mathrm{pCi} / \mathrm{g}$ 7.67E-01 $\pm 4.10 \mathrm{E}-02 \mathrm{pCi} / \mathrm{g}$ $-2.43 \mathrm{E}-03 \pm 3.23 \mathrm{E}-02 \mathrm{pCi} / \mathrm{g}$ 3.39E-04 $\pm 3.07 \mathrm{E}-03 \mathrm{pCi} / \mathrm{g}$ $-1.11 \mathrm{E}-01 \pm 2.93 \mathrm{E}-02 \mathrm{pCi} / \mathrm{g}$ 7.07E-01土4.05E-01 pCi/g $-1.49 \mathrm{E}-03 \pm 5.89 \mathrm{E}-03 \mathrm{pCi} / \mathrm{g}$ $-4.33 \mathrm{E}-04 \pm 3.88 \mathrm{E}-03 \mathrm{pCi} / \mathrm{g}$ $-3.76 \mathrm{E}-03 \pm 7.65 \mathrm{E}-03 \mathrm{pCi} / \mathrm{g}$ 1.05E-02 $48.93 \mathrm{E}-03 \mathrm{pCi} / \mathrm{g}$
1.98E-02 EPIA-013B

7.45E-03 EPIA-013B

1.66E-02 EPIA-013B

7.42E-03 EPIA-013B

4.63E-02 EPIA-013B

5.52E-03 EPIA-013B

7.00E-03 EPIA-013B

5.87E-03 EPIA-013B

6.76E-03 EPIA-013B

5.53E-03 EPIA-013B

1.72E-02 EPIA-013B

4.96E-02 EPIA-013B

2.47E-02 EPIA-013B

1.54E+00 EPIA-001B

1.10E-02 EPIA-013B

6.09E-03 EPIA-013B

4.32E-02 EPIA-013B

2.49E+00 EPIA-001B

3.42E-02 EPIA-012B

1.60E-02 EPIA-012B

5.21E-02 EPIA-013B

6.09E-03 EPIA-013B

8.27E-03 EPIA-013B

1.14E-02 EPIA-013B

1.98E-02 EPIA-013B

5.60E-02 EPIA-013B

5.28E-03 EPIA-013B

1.29E-01 EPIA-004

3.22E-01 EPIA-013B

8.57E-03 EPIA-013B

6.83E-03 EPIA-013B

1.09E-02 EPIA-013B

1.45E-02 EPIA-013B

Survey location: $44049 \mathrm{E}$ 3671941N (SRS coordinates)

Depth of core interval: 4.00 to $6.00 \mathrm{ft}$

Sample type: Normal 
SURVEY ID: PPSC 1704 (continued)

Constituent
Sample ID: 104696

RQ AQ B Result

Physical Parameters and Specified Analyses

Ammonia nitrogen

$\mathrm{U} \quad 22.5$

Cation exchange capacity

Chloride

Cyanide

Cyanide

Nitrate-nitrite as nitrogen

Nitrogen by Kjeldahl method

Orthophosphate

Phenols

Sulfide

Total organic carbon

Total organic nitrogen

Total phosphates (as P)

Metals (total recoverable)

$\begin{array}{lll} & & 55.6 \\ U & & 2.69 \\ \text { U } & & 1.14 \\ \text { U } & & 1.14 \\ \text { J } & \text { E } & 0.343 \\ & & 102 \\ \text { U } & & 11.4 \\ \text { U } & & 338 \\ \text { UJ } & \text { C } & 284 \\ & \text { V } & 1250 \\ \text { C } & & 96.9 \\ \text { J } & \text { E } & 126\end{array}$

Aluminum

Antimony

Arsenic

Barium

Beryllium .

Cadmium

Calcium

Chromium

Cobalt

Copper

Iron

Lead

Magnesium

Manganese

Mercury

Nickel

Potassium

Selenium

Silver

Sodium

Thallium

Vanadium

Zinc
10800

$\begin{array}{ll}\mathrm{U} & \mathbf{5 . 5 2}\end{array}$

U $\quad 16.5$

$\begin{array}{lll} & & 22.9 \\ U & \mathrm{E} & \mathbf{0 . 0 5 3}\end{array}$

$\begin{array}{lll}J & \mathrm{U} & 0.276\end{array}$

$\begin{array}{lll}\mathrm{J} & \mathrm{E} & \mathbf{0 . 3 6 5}\end{array}$

$\begin{array}{ll}\mathrm{E} & \mathbf{2 . 2 6} \\ \mathrm{V} & 2260\end{array}$

V 2260

7.14

328

7.18

$\begin{array}{lll}J & E & 0.0183\end{array}$

2.74

178

$\begin{array}{ll}\mathrm{U} & 16.5 \\ \mathrm{U} & 1.38\end{array}$

$\begin{array}{lll}\mathrm{J} & \mathrm{E} & \mathbf{9 . 8 6}\end{array}$

$\mathrm{U} \quad 16.5$

12.3

6.72
67.1

$\begin{array}{lll}\mathrm{mg} / \mathrm{kg} & 22.5 & \text { EPA350.1 } \\ \mathrm{meq} / 100 \mathrm{~g} & 5 & \text { EPA9081 } \\ \mathrm{mg} / \mathrm{kg} & 2.69 & \text { EPA300.0 } \\ \mathrm{mg} / \mathrm{kg} & 1.14 & \text { EPA335.3 } \\ \mathrm{mg} / \mathrm{kg} & 1.14 & \text { EPA335.3 } \\ \mathrm{mg} / \mathrm{kg} & 1.14 & \text { EPA353.1 } \\ \mathrm{mg} / \mathrm{kg} & 45.4 & \text { EPA351.2 } \\ \mathrm{mg} / \mathrm{kg} & 11.4 & \text { EPA365.2 } \\ \mu \mathrm{g} / \mathrm{kg} & 338 & \text { EPA420.2 } \\ \mathrm{mg} / \mathrm{kg} & 284 & \text { EPA376.2 } \\ \mathrm{mg} / \mathrm{kg} & 114 & \text { EPA415.1 } \\ \mathrm{mg} / \mathrm{kg} & 0.227 & \text { EPA351.2 } \\ \mathrm{mg} / \mathrm{kg} & 421 & \text { EPA365.4 }\end{array}$

$B / N / A$ Extractables (including $P A H$ and phenols)

$\begin{array}{llll}\text { Acenaphthene } & \text { UJ } & \text { Q } & 37.9 \\ \text { Acenaphthylene } & \text { UJ } & \text { Q } & 37.9 \\ \text { Anthracene } & \text { UJ } & \text { Q } & 37.9 \\ \text { Benzo[a]anthracene } & \text { UJ } & \text { Q } & 3.79 \\ \text { Benzo[b]fluoranthene } & \text { UJ } & \text { Q } & 3.79 \\ \text { Benzo[k]fluoranthene } & \text { UJ } & \text { Q } & 3.79 \\ \text { Benzoic acid } & \text { J } & \text { Q } & 60.2 \\ \text { Benzo[g,h,i]perylene } & \text { UJ } & \text { Q } & 37.9 \\ \text { Benzo[a]pyrene } & \text { UJ } & \text { Q } & 3.79 \\ \text { Benzyl alcohol } & \text { UJ } & \text { Q } & 37.9 \\ \text { Bis(2-chloroethoxy) methane } & \text { UJ } & \text { Q } & 37.9 \\ \text { Bis(2-chloroethyl) ether } & \text { UJ } & \text { Q } & 37.9\end{array}$

Unit D. Limit Method 
SURVEY ID: PPSC 1704 (continued)

Sample ID: 104696

$\begin{array}{lllll}\text { Constituent } & \text { RQ AQ B Result Unit D. Limit Method }\end{array}$

$B / N / A$ Extractables (including $P A H$ and phenols)

\begin{tabular}{|c|c|c|c|}
\hline Bis(2-chloroisopropyl) ether & UJ & Q & 37.9 \\
\hline Bis(2-ethylhexyl) phthalate & UJ & Q & 37.9 \\
\hline 4-Bromophenyl phenyl ether & UJ & $\mathbf{Q}$ & 37.9 \\
\hline Butylbenzyl phthalate & UJ & $\mathbf{Q}$ & 37.9 \\
\hline 4-Chloroaniline & UJ & Q & 37.9 \\
\hline 4-Chloro-m-cresol & UJ & Q & 37.9 \\
\hline 2.Chloronaphthalene & UJ & $\mathbf{Q}$ & 37.9 \\
\hline 2-Chlorophenol & UJ & $\mathbf{Q}$ & 37.9 \\
\hline 4-Chlorophenyl phenyl ether & UJ & $\mathbf{Q}$ & 37.9 \\
\hline Chrysene & UJ & $\mathbf{Q}$ & 3.79 \\
\hline $\mathrm{m} / \mathrm{p}$-Cresol & UJ & $\mathbf{Q}$ & 379 \\
\hline o-Cresol (2-Methylphenol) & UJ & $\mathbf{Q}$ & 37.9 \\
\hline Dibenz $[a, h]$ anthracene & UJ & $\mathbf{Q}$ & 3.79 \\
\hline Dibenzofuran & UJ & $\mathbf{Q}$ & 37.9 \\
\hline Di-n-butyl phthalate & $J$ & $\mathrm{QE}$ & 22.3 \\
\hline 1,2-Dichlorobenzene & UJ & $\mathbf{Q}$ & 37.9 \\
\hline 1,3-Dichlorobenzene & UJ & $\mathbf{Q}$ & 37.9 \\
\hline 1,4-Dichlorobenzene & UJ & $\mathbf{Q}$ & 37.9 \\
\hline 3,3'-Dichlorobenzidine & UJ & $\mathbf{Q}$ & 37.9 \\
\hline 2,4-Dichlorophenol & UJ & $\mathbf{Q}$ & 37.9 \\
\hline Diethyl phthalate & UJ & $\mathbf{Q}$ & 37.9 \\
\hline 2,4-Dimethyl phenol & UJ & $\mathbf{Q}$ & 37.9 \\
\hline Dimethyl phthalate & UJ & $\mathbf{Q}$ & 37.9 \\
\hline 2,4-Dinitrophenol & UJ & $\mathbf{Q}$ & 379 \\
\hline 2,4-Dinitrotoluene & UJ & QC & 3.79 \\
\hline 2,6-Dinitrotoluene & UJ & $\mathbf{Q}$ & 3.79 \\
\hline Di-n-octyl phthalate & UJ & $\mathbf{Q}$ & 37.9 \\
\hline Fluoranthene & UJ & $\mathbf{Q}$ & 37.9 \\
\hline Fluorene & UJ & Q & 37.9 \\
\hline Hexachlorobenzene & UJ & $Q$ & 3.79 \\
\hline Hexachlorobutadiene & UJ & $Q$ & 37.9 \\
\hline Hexachlorocyclopentadiene & UJ & Q & $\mathbf{3 7 . 9}$ \\
\hline Hexachloroethane & UJ & $\mathbf{Q}$ & 3.79 \\
\hline Indeno $[1,2,3-c, d]$ pyrene & UJ & Q & 3.79 \\
\hline Isophorone & UJ & Q & 37.9 \\
\hline 2-Methyl-4,6-dinitrophenol & UJ & Q & 379 \\
\hline 2-Methylnaphthalene & UJ & Q & 37.9 \\
\hline Naphthalene & UJ & Q & 37.9 \\
\hline m-Nitroaniline & UJ & Q & 37.9 \\
\hline o-Nitroaniline & UJ & $\mathrm{Q}$ & 37.9 \\
\hline p-Nitroaniline & UJ & Q & 37.9 \\
\hline Nitrobenzene & UJ & Q & 37.9 \\
\hline 2-Nitrophenol & UJ & $Q$ & 37.9 \\
\hline 4-Nitrophenol & UJ & Q & 37.9 \\
\hline N-Nitrosodiphenylamine & UJ & $\mathbf{Q}$ & 37.9 \\
\hline N-Nitrosodi-n-propylamine & UJ & $\mathbf{Q}$ & 37.9 \\
\hline Pentachlorophenol & UJ & $\mathbf{Q}$ & 37.9 \\
\hline Phenanthrene & UJ & $\mathbf{Q}$ & 37.9 \\
\hline Phenol & UJ & $\mathbf{Q}$ & 37.9 \\
\hline Pyrene & UJ & $\mathbf{Q}$ & 37.9 \\
\hline 1,2,4-Trichlorobenzene & UJ & Q & 3.79 \\
\hline 2,4,5-Trichlorophenol & UJ & $\mathbf{Q}$ & 37.9 \\
\hline 2,4,6-Trichlorophenol & UJ & $\mathbf{Q}$ & 37.9 \\
\hline
\end{tabular}

\begin{tabular}{|c|c|c|}
\hline$\mu \mathrm{g} / \mathrm{kg}$ & 37.9 & EPA8270 \\
\hline$\mu \mathrm{g} / \mathrm{kg}$ & 37.9 & EPA8270 \\
\hline$\mu \mathrm{g} / \mathrm{kg}$ & 37.9 & EPA8270 \\
\hline$\mu \mathrm{g} / \mathrm{kg}$ & 37.9 & EPA8270 \\
\hline$\mu \mathrm{g} / \mathrm{kg}$ & 37.9 & EPA8270 \\
\hline$\mu \mathrm{g} / \mathrm{kg}$ & 37.9 & EPA8270 \\
\hline$\mu \mathrm{g} / \mathrm{kg}$ & 37.9 & EPA8270 \\
\hline$\mu \mathrm{g} / \mathrm{kg}$ & 37.9 & EPA8270 \\
\hline$\mu \mathrm{g} / \mathrm{kg}$ & 37.9 & EPA8270 \\
\hline$\mu \mathrm{g} / \mathrm{kg}$ & 3.79 & EPA8270 \\
\hline$\mu \mathrm{g} / \mathrm{kg}$ & 379 & EPA8270 \\
\hline$\mu \mathrm{g} / \mathrm{kg}$ & 37.9 & EPA8270 \\
\hline$\mu \mathrm{g} / \mathrm{kg}$ & 3.79 & EPA8270 \\
\hline$\mu \mathrm{g} / \mathrm{kg}$ & 37.9 & EPA8270 \\
\hline$\mu \mathrm{g} / \mathrm{kg}$ & 37.9 & EPA8270 \\
\hline$\mu \mathrm{g} / \mathrm{kg}$ & 37.9 & EPA8270 \\
\hline$\mu \mathrm{g} / \mathrm{kg}$ & 37.9 & EPA8270 \\
\hline$\mu \mathrm{g} / \mathrm{kg}$ & 37.9 & EPA8270 \\
\hline$\mu \mathrm{g} / \mathrm{kg}$ & 37.9 & EPA8270 \\
\hline$\mu \mathrm{g} / \mathrm{kg}$ & 37.9 & EPA8270 \\
\hline$\mu \mathrm{g} / \mathrm{kg}$ & 37.9 & EPA8270 \\
\hline$\mu \mathrm{g} / \mathrm{kg}$ & 37.9 & EPA8270 \\
\hline$\mu \mathrm{g} / \mathrm{kg}$ & 37.9 & EPA8270 \\
\hline$\mu \mathrm{g} / \mathrm{kg}$ & 379 & EPA8270 \\
\hline$\mu \mathrm{g} / \mathrm{kg}$ & 3.79 & EPA8270 \\
\hline$\mu \mathrm{g} / \mathrm{kg}$ & 3.79 & EPA8270 \\
\hline$\mu \mathrm{g} / \mathrm{kg}$ & 37.9 & EPA8270 \\
\hline$\mu \mathrm{g} / \mathrm{kg}$ & 37.9 & EPA8270 \\
\hline$\mu \mathrm{g} / \mathrm{kg}$ & 37.9 & EPA8270 \\
\hline$\mu \mathrm{g} / \mathrm{kg}$ & 3.79 & EPA8270 \\
\hline$\mu \mathrm{g} / \mathrm{kg}$ & 37.9 & EPA8270 \\
\hline$\mu \mathrm{g} / \mathrm{kg}$ & 37.9 & EPA8270 \\
\hline$\mu \mathrm{g} / \mathrm{kg}$ & 3.79 & EPA8270 \\
\hline$\mu \mathrm{g} / \mathrm{kg}$ & 3.79 & EPA8270 \\
\hline$\mu \mathrm{g} / \mathrm{kg}$ & 37.9 & EPA8270 \\
\hline$\mu \mathrm{g} / \mathrm{kg}$ & 379 & EPA8270 \\
\hline$\mu \mathrm{g} / \mathrm{kg}$ & 37.9 & EPA8270 \\
\hline$\mu \mathrm{g} / \mathrm{kg}$ & 37.9 & EPA8270 \\
\hline$\mu \mathrm{g} / \mathrm{kg}$ & 37.9 & EPA8270 \\
\hline$\mu \mathrm{g} / \mathrm{kg}$ & 37.9 & EPA8270 \\
\hline$\mu \mathrm{g} / \mathrm{kg}$ & 37.9 & EPA8270 \\
\hline$\mu \mathrm{g} / \mathrm{kg}$ & 37.9 & EPA8270 \\
\hline$\mu \mathrm{g} / \mathrm{kg}$ & 37.9 & EPA8270 \\
\hline$\mu \mathrm{g} / \mathrm{kg}$ & 37.9 & EPA8270 \\
\hline$\mu \mathrm{g} / \mathrm{kg}$ & 37.9 & EPA8270 \\
\hline$\mu \mathrm{g} / \mathrm{kg}$ & 37.9 & EPA8270 \\
\hline$\mu \mathrm{g} / \mathrm{kg}$ & 37.9 & EPA8270 \\
\hline$\mu \mathrm{g} / \mathrm{kg}$ & 37.9 & EPA8270 \\
\hline$\mu \mathrm{g} / \mathrm{kg}$ & 37.9 & EPA8270 \\
\hline$\mu \mathrm{g} / \mathrm{kg}$ & 37.9 & EPA8270 \\
\hline$\mu \mathrm{g} / \mathrm{kg}$ & 3.79 & EPA8270 \\
\hline$\mu \mathrm{g} / \mathrm{kg}$ & 37.9 & EPA8270 \\
\hline$\mu \mathrm{g} / \mathrm{kg}$ & 37.9 & EPA8270 \\
\hline
\end{tabular}


SURVEY ID: PPSC 1704 (continued)

Constituent

Volatile Organic Compounds

Acetone
Benzene
Bromodichloromethane
Bromoform
Bromomethane (Methyl bromide)
Carbon disulfide
Carbon tetrachloride
Chlorobenzene
Chloroethane
Chloroethene (Vinyl chloride)
Chloroform
Chloromethane (Methyl chloride)
Dibromochloromethane
1,1-Dichloroethane
1,2-Dichloroethane
1,1-Dichloroethylene
1,2-Dichloroethylene
Dichloromethane (Methylene chloride)
1,2-Dichloropropane
cis-1,3-Dichloropropene
trans-1,3-Dichloropropene
Ethylbenzene
2-Hexanone
Methyl ethyl ketone
Methyl isobutyl ketone
Propylbenzene
Styrene
1,1,2,2-Tetrachloroethane
Tetrachloroethylene
Toluene
1,1,1-Trichloroethane
1,1,2-Trichloroethane
Trichloroethylene
Vinyl acetate
Xylenes
(n)

Sample ID: 104696

RQ AQ B Result Unit D. Limit Method

\begin{tabular}{|c|c|c|c|c|}
\hline $\mathrm{U}$ & 21.7 & $\mu \mathrm{g} / \mathrm{kg}$ & 28.4 & EPA8260 \\
\hline $\mathbf{U}$ & 0.568 & $\mu \mathrm{g} / \mathrm{kg}$ & 0.568 & EPA8260 \\
\hline U & 0.0568 & $\mu \mathrm{g} / \mathrm{kg}$ & 0.0568 & EPA8260 \\
\hline $\mathrm{U}$ & 0.0568 & $\mu \mathrm{g} / \mathrm{kg}$ & 0.0568 & EPA8260 \\
\hline $\mathrm{U}$ & 0.114 & $\mu \mathrm{g} / \mathrm{kg}$ & 0.114 & EPA8260 \\
\hline $\mathrm{U}$ & 1.14 & $\mu \mathrm{g} / \mathrm{kg}$ & 1.14 & EPA8260 \\
\hline $\mathrm{U}$ & 0.0568 & $\mu \mathrm{g} / \mathrm{kg}$ & 0.0568 & EPA8260 \\
\hline $\mathrm{U}$ & 0.0568 & $\mu \mathrm{g} / \mathrm{kg}$ & 0.0568 & EPA8260 \\
\hline $\mathrm{U}$ & 0.114 & $\mu \mathrm{g} / \mathrm{kg}$ & 0.114 & EPA8260 \\
\hline $\mathrm{U}$ & 0.114 & $\mu \mathrm{g} / \mathrm{kg}$ & 0.114 & EPA8260 \\
\hline $\mathrm{U}$ & 0.0568 & $\mu \mathrm{g} / \mathrm{kg}$ & 0.0568 & EPA8260 \\
\hline $\mathrm{U}$ & 0.114 & $\mu \mathrm{g} / \mathrm{kg}$ & 0.114 & EPA8260 \\
\hline $\mathrm{U}$ & 0.0568 & $\mu \mathrm{g} / \mathrm{kg}$ & 0.0568 & EPA8260 \\
\hline $\mathrm{U}$ & 0.0568 & $\mu \mathrm{g} / \mathrm{kg}$ & 0.0568 & EPA8260 \\
\hline $\mathrm{U}$ & 0.0568 & $\mu \mathrm{g} / \mathrm{kg}$ & 0.0568 & EPA8260 \\
\hline $\mathrm{U}$ & 0.0568 & $\mu \mathrm{g} / \mathrm{kg}$ & 0.0568 & EPA8260 \\
\hline $\mathrm{U}$ & 0.114 & $\mu \mathrm{g} / \mathrm{kg}$ & 0.114 & EPA8260 \\
\hline $\mathrm{U}$ & 1.52 & $\mu \mathrm{g} / \mathrm{kg}$ & 0.568 & EPA8260 \\
\hline $\mathrm{U}$ & 0.0568 & $\mu \mathrm{g} / \mathrm{kg}$ & 0.0568 & EPA8260 \\
\hline $\mathbf{U}$ & 0.0568 & $\mu \mathrm{g} / \mathrm{kg}$ & 0.0568 & EPA8260 \\
\hline \multirow[t]{2}{*}{$\mathbf{U}$} & 0.0568 & $\mu \mathrm{g} / \mathrm{kg}$ & 0.0568 & EPA8260 \\
\hline & 0.125 & $\mu \mathrm{g} / \mathrm{kg}$ & 0.0568 & EPA8260 \\
\hline $\mathrm{U}$ & 0.239 & $\mu \mathrm{g} / \mathrm{kg}$ & 1.14 & EPA8260 \\
\hline $\mathrm{V}$ & 4.07 & $\mu \mathrm{g} / \mathrm{kg}$ & 1.14 & EPA8260 \\
\hline$U$ & 1.14 & $\mu \mathrm{g} / \mathrm{kg}$ & 1.14 & EPA8260 \\
\hline$N$ & 3 & $\mu \mathrm{g} / \mathrm{kg}$ & & EPA 8260 \\
\hline $\mathrm{U}$ & 0.0568 & $\mu \mathrm{g} / \mathrm{kg}$ & 0.0568 & EPA8260 \\
\hline $\mathbf{U}$ & 0.0568 & $\mu \mathrm{g} / \mathrm{kg}$ & 0.0568 & EPA8260 \\
\hline $\mathbf{U}$ & 0.0568 & $\mu \mathrm{g} / \mathrm{kg}$ & 0.0568 & EPA8260 \\
\hline $\mathrm{U}$ & 0.568 & $\mu \mathrm{g} / \mathrm{kg}$ & 0.568 & EPA8260 \\
\hline $\mathrm{U}$ & 0.0568 & $\mu \mathrm{g} / \mathrm{kg}$ & 0.0568 & EPA8260 \\
\hline $\mathrm{U}$ & 0.0568 & $\mu \mathrm{g} / \mathrm{kg}$ & 0.0568 & EPA8260 \\
\hline $\mathbf{U}$ & 0.0568 & $\mu \mathrm{g} / \mathrm{kg}$ & 0.0568 & EPA8260 \\
\hline $\mathrm{U}$ & 1.14 & $\mu \mathrm{g} / \mathrm{kg}$ & 1.14 & EPA8260 \\
\hline $\mathrm{U}$ & 0.17 & $\mu \mathrm{g} / \mathrm{kg}$ & 0.17 & EPA8260 \\
\hline
\end{tabular}

Pesticides/PCBs and Dioxins/Furans

Aldrin
alpha-Benzene hexachloride
beta-Benzene hexachloride
delta-Benzene hexachloride
alpha-Chlordane
gamma-Chlordane
p,p'-DDD
p,p'-DDE
p,p'-DDT
Dieldrin
Endosulfan sulfate
Endosulfan I
Endosulfan II
Endrin
Endrin ketone
Heptachlor
Heptachlor epoxide

$\begin{array}{ll}\text { U } & 9.35 \\ \mathrm{U} & 9.35 \\ \mathrm{U} & 18.7 \\ \mathrm{U} & 18.7 \\ \mathrm{U} & 18.7 \\ \mathrm{U} & 18.7 \\ \mathrm{U} & 37.4 \\ \mathrm{U} & 18.7 \\ \mathrm{U} & 37.4 \\ \mathrm{U} & 18.7 \\ \mathrm{U} & 37.4 \\ \mathrm{U} & 18.7 \\ \mathrm{U} & 37.4 \\ \mathrm{U} & 18.7 \\ \mathrm{U} & 37.4 \\ \mathrm{U} & 18.7 \\ \mathrm{U} & 18.7\end{array}$

$\begin{array}{lll}\mu \mathrm{g} / \mathrm{kg} & 9.35 & \text { EPA8080 } \\ \mu \mathrm{g} / \mathrm{kg} & 9.35 & \text { EPA8080 } \\ \mu \mathrm{g} / \mathrm{kg} & 18.7 & \text { EPA8080 } \\ \mu \mathrm{g} / \mathrm{kg} & 18.7 & \text { EPA8080 } \\ \mu \mathrm{g} / \mathrm{kg} & 18.7 & \text { EPA8080 } \\ \mu \mathrm{g} / \mathrm{kg} & 18.7 & \text { EPA8080 } \\ \mu \mathrm{g} / \mathrm{kg} & 37.4 & \text { EPA8080 } \\ \mu \mathrm{g} / \mathrm{kg} & 18.7 & \text { EPA8080 } \\ \mu \mathrm{g} / \mathrm{kg} & 37.4 & \text { EPA8080 } \\ \mu \mathrm{g} / \mathrm{kg} & 18.7 & \text { EPA8080 } \\ \mu \mathrm{g} / \mathrm{kg} & 37.4 & \text { EPA8080 } \\ \mu \mathrm{g} / \mathrm{kg} & 18.7 & \text { EPA8080 } \\ \mu \mathrm{g} / \mathrm{kg} & 37.4 & \text { EPA8080 } \\ \mu \mathrm{g} / \mathrm{kg} & 18.7 & \text { EPA8080 } \\ \mu \mathrm{g} / \mathrm{kg} & 37.4 & \text { EPA8080 } \\ \mu \mathrm{g} / \mathrm{kg} & 18.7 & \text { EPA8080 } \\ \mu \mathrm{g} / \mathrm{kg} & 18.7 & \text { EPA8080 }\end{array}$


SURVEY ID: PPSC 1704 (continued)

Constituent

RQ AQ B Result

9.35

187

46.8

46.8

46.8

46.8

46.8

46.8

46.8

374

Toxaphene

Radionuclides

Actinium-228

Antimony-124

Antimony-125

Barium-133

Cerium-144

Cesium-134

Cesium-137

Cobalt-57

Cobalt-58

Cobalt-60

Europium-152

Europium-154

Europium-155

Gross alpha

Lead-212

Manganese-54

Neptunium-239

Nonvolatile beta

Plutonium-238

Plutonium-239/240

Potassium-40

Promethium-144

Promethium-146

Radium-226

Radium-228

Ruthenium-106

Sodium-22

Strontium-90

Thorium-234

Tin-113

Yttrium-88

Zinc-65

Zirconium-95
9.04E-01 $\pm 4.13 \mathrm{E}-02 \mathrm{pCi} / \mathrm{g}$ $1.73 \mathrm{E}-03 \pm 4.63 \mathrm{E}-03 \mathrm{pCi} / \mathrm{g}$ 9.38E-03 $\pm 9.90 \mathrm{E}-03 \mathrm{pCj} / \mathrm{g}$ $0.00 \mathrm{E}+00 \quad \mathrm{pCi} / \mathrm{g}$ 3.22E-02士4.37E-02 pCi/g $-1.21 \mathrm{E}-03 \pm 4.00 \mathrm{E}-03 \mathrm{pCi} / \mathrm{g}$ 6.19E-03 $\pm 4.34 \mathrm{E}-03 \mathrm{pCj} / \mathrm{g}$ $4.90 \mathrm{E}-04 \pm 3.65 \mathrm{E}-03 \mathrm{pCi} / \mathrm{g}$ $3.18 \mathrm{E}-06 \pm 4.79 \mathrm{E}-03 \mathrm{pCi} / \mathrm{g}$ 5.59E-04 $\$ 3.38 \mathrm{E}-03 \mathrm{pCi} / \mathrm{g}$ $-1.14 \mathrm{E}-02 \pm 1.11 \mathrm{E}-02 \mathrm{pCi} / \mathrm{g}$ $-1.71 \mathrm{E}-02 \pm 3.34 \mathrm{E}-02 \mathrm{pCi} / \mathrm{g}$ $0.00 \mathrm{E}+00 \quad \mathrm{pCi} / \mathrm{g}$ $3.35 \mathrm{E}+01 \pm 4.36 \mathrm{E}+00 \mathrm{pCi} / \mathrm{g}$ 9.42E-01 $\pm 1.95 \mathrm{E}-02 \mathrm{pCi} / \mathrm{g}$ $1.52 \mathrm{E}-02 \pm 4.85 \mathrm{E}-03 \mathrm{pCi} / \mathrm{g}$ 6.59E-02 $44.70 \mathrm{E}-02 \mathrm{pCi} / \mathrm{g}$ $1.00 \mathrm{E}+01 \pm 1.92 \mathrm{E}+00 \mathrm{pCi} / \mathrm{g}$ $1.09 \mathrm{E}-03 \pm 1.46 \mathrm{E}-02 \mathrm{pCi} / \mathrm{g}$ 9.81E-03 $\pm 9.80 \mathrm{E}-03 \mathrm{pCi} / \mathrm{g}$ 8.14E-01 $18.90 \mathrm{E}-02 \mathrm{pCi} / \mathrm{g}$ $-1.87 \mathrm{E}-03 \pm 3.78 \mathrm{E}-03 \mathrm{pCi} / \mathrm{g}$ 7.73E-03 $\pm 7.65 \mathrm{E}-03 \mathrm{pCi} / \mathrm{g}$ 6.17E-01 $\pm 2.51 \mathrm{E}-02 \mathrm{pCi} / \mathrm{g}$ 9.04E-01 $14.13 \mathrm{E}-02 \mathrm{pCj} / \mathrm{g}$ 2.78E-02 4 .07E-02 pCi/g $-1.72 \mathrm{E}-03 \pm 3.81 \mathrm{E}-03 \mathrm{pCi} / \mathrm{g}$ $1.61 \mathrm{E}-01 \pm 9.08 \mathrm{E}-02 \mathrm{pCi} / \mathrm{g}$ $1.27 \mathrm{E}+00 \pm 5.17 \mathrm{E}-01 \mathrm{pCj} / \mathrm{g}$ $-2.88 \mathrm{E}-03 \pm 5.16 \mathrm{E}=03 \mathrm{pCi} / \mathrm{g}$ $7.50 \mathrm{E}-05 \pm 3.72 \mathrm{E}-03 \mathrm{pCi} / \mathrm{g}$ $-7.99 \mathrm{E}-04 \pm 1.21 \mathrm{E}-02 \mathrm{pCi} / \mathrm{g}$ $0.00 \mathrm{E}+00 \quad \mathrm{pCi} / \mathrm{g}$
D. Limit Method

$\begin{array}{ll}9.35 & \text { EPA8080 } \\ 187 & \text { EPA8080 } \\ 46.8 & \text { EPA8080 } \\ 46.8 & \text { EPA8080 } \\ 46.8 & \text { EPA8080 } \\ 46.8 & \text { EPA8080 } \\ 46.8 & \text { EPA8080 } \\ 46.8 & \text { EPA8080 } \\ 46.8 & \text { EPA8080 } \\ 374 & \text { EPA8080 }\end{array}$

2.02E-02 EPIA-013B

7.97E-03 EPIA-013B

1.76E-02 EPIA-013B

8.07E-03 EPIA-013B

5.06E-02 EPIA-013B

5.86E-03 EPIA-013B

6.57E-03 EPIA-013B

6.25E-03 EPIA-013B

7.04E-03 EPLA-013B

5.93E-03 EPIA-013B

1.75E-02 EPIA-013B

4.93E-02 EPIA-013B

2.63E-02 EPIA-013B

1.73E+00 EPIA-001B

1.07E-02 EPIA-013B

6.78E-03 EPIA-013B

4.42E-02 EPIA-013B

2.43E+00 EPIA-001B

3.42E-02 EPIA-012B

7.35E-03 EPIA-012B

5.40E-02 EPIA-013B

6.26E-03 EPIA-013B

8.61E-03 EPIA-013B

1.18E-02 EPIA-013B

2.02E-02 EPIA-013B

5.65E-02 EPIA-013B

5.52E-03 EPIA-013B

1.32E-01 EPIA-004

3.44E-01 EPIA-013B

8.85E-03 EPLA-013B

6.72E-03 EPIA-013B

1.22E-02 EPIA-013B

1.66E-02 EPIA-013B

SURVEY ID: PPSC 1801

Sample ID: 104668

Survey location: 44086E $3672804 N$ (SRS coordinates)

Depth of core interval: 0.00 to $1.00 \mathrm{ft}$

Sample type: Normal

Sample matrix: Soil

Percent solids: $\mathbf{8 3 . 0 0}$ 
SURVEY ID: PPSC 1801 (continued)

Constituent

$A Q$

Sample ID: 104668

Physical Parameters and Specified Analyses

Ammonia nitrogen

Cation exchange capacity

Chloride

Cyanide

Nitrate-nitrite as nitrogen

Nitrogen by Kjeldahl method

Orthophosphate

Phenols

Sulfide

Total organic carbon

Total organic nitrogen

Total phosphates (as P)

Total phosphates (as P)

Metals (total recoverable)

$\begin{array}{llll} & & 29.8 \\ & & 56.8 \\ \mathrm{~J} & \mathrm{Q} & 7.34 \\ \mathrm{U} & & 1.2 \\ \mathrm{U} & & 1.08 \\ & & 308 \\ \mathrm{U} & & 1.2 \\ & & 643 \\ \mathrm{UJ} & \mathrm{C} & 301 \\ & & 8250 \\ \mathrm{C} & & 278 \\ \mathrm{~J} & \mathrm{E} & 151 \\ \mathrm{~J} & \mathrm{E} & 138\end{array}$

$\begin{array}{lll} & & 2450 \\ \text { U } & & 5.58 \\ \text { U } & & 16.7 \\ \text { J } & \text { E } & 8.44 \\ \text { U } & & 0.0491 \\ & & 0.279 \\ & & 41.1 \\ \text { U } & & 0.65 \\ \text { J } & \text { E } & 1.22 \\ \text { J } & \text { C } & 3250 \\ \text { J } & \text { E } & 3.8 \\ & & 47.1 \\ & & 30.7 \\ \text { J } & \text { E } & 0.0183 \\ \text { J } & \text { E } & 0.551 \\ \text { J } & \text { E } & 57 \\ \text { U } & & 16.7 \\ \text { U } & & 1.39 \\ \text { U } & & 55.8 \\ \text { U } & & 16.7 \\ & & 9.06 \\ & & 2.83\end{array}$

Unit

D. Limit Method

Aluminum
Antimony
Arsenic
Barium
Beryllium
Cadmium
Calcium
Chromium
Cobalt
Copper
Iron
Lead
Magnesium
Manganese
Mercury
Nickel
Potassium
Selenium
Silver
Sodium
Thallium
Vanadium
Zinc

Radionuclides

Actinium-228

Antimony-124

Antimony-125

Barium-133

Cerium-144

Cesium-134

Cesium-137

Cobalt-57

Cobalt-58

Cobalt-60

Europium-152

Europium-154

Europium-155

Gross alpha

$\begin{array}{lll}\mathrm{mg} / \mathrm{kg} & 22.6 & \text { EPA350.1 } \\ \mathrm{meq} / 100 \mathrm{~g} & 5 & \text { EPA9081 } \\ \mathrm{mg} / \mathrm{kg} & 2.87 & \text { EPA300.0 } \\ \mathrm{mg} / \mathrm{kg} & 1.2 & \text { EPA335.3 } \\ \mathrm{mg} / \mathrm{kg} & 1.08 & \text { EPA353.1 } \\ \mathrm{mg} / \mathrm{kg} & 96.2 & \text { EPA351.2 } \\ \mathrm{mg} / \mathrm{kg} & 1.2 & \text { EPA365.2 } \\ \mu \mathrm{g} / \mathrm{kg} & 361 & \text { EPA420.2 } \\ \mathrm{mg} / \mathrm{kg} & 301 & \text { EPA376.2 } \\ \mathrm{mg} / \mathrm{kg} & 120 & \text { EPA415.1 } \\ \mathrm{mg} / \mathrm{kg} & 0.241 & \text { EPA351.2 } \\ \mathrm{mg} / \mathrm{kg} & 1150 & \text { EPA365.4 } \\ \mathrm{mg} / \mathrm{kg} & 1150 & \text { EPA365.4 }\end{array}$

$\begin{array}{lll}\mathrm{mg} / \mathrm{kg} & 13.9 & \text { EPA6010A } \\ \mathrm{mg} / \mathrm{kg} & 5.58 & \text { EPA6010A } \\ \mathrm{mg} / \mathrm{kg} & 16.7 & \text { EPA6010A } \\ \mathrm{mg} / \mathrm{kg} & 1.39 & \text { EPA6010A } \\ \mathrm{mg} / \mathrm{kg} & 0.558 & \text { EPA6010A } \\ \mathrm{mg} / \mathrm{kg} & 0.279 & \text { EPA6010A } \\ \mathrm{mg} / \mathrm{kg} & 13.9 & \text { EPA6010A } \\ \mathrm{mg} / \mathrm{kg} & 1.39 & \text { EPA6010A } \\ \mathrm{mg} / \mathrm{kg} & 0.558 & \text { EPA6010A } \\ \mathrm{mg} / \mathrm{kg} & 1.39 & \text { EPA6010A } \\ \mathrm{mg} / \mathrm{kg} & 5.58 & \text { EPA6010A } \\ \mathrm{mg} / \mathrm{kg} & 5.58 & \text { EPA6010A } \\ \mathrm{mg} / \mathrm{kg} & 5.58 & \text { EPA6010A } \\ \mathrm{mg} / \mathrm{kg} & 0.558 & \text { EPA6010A } \\ \mathrm{mg} / \mathrm{kg} & 0.0398 & \text { EPA7471 } \\ \mathrm{mg} / \mathrm{kg} & 1.39 & \text { EPA6010A } \\ \mathrm{mg} / \mathrm{kg} & 112 & \text { EPA6010A } \\ \mathrm{mg} / \mathrm{kg} & 16.7 & \text { EPA6010A } \\ \mathrm{mg} / \mathrm{kg} & 1.39 & \text { EPA6010A } \\ \mathrm{mg} / \mathrm{kg} & 55.8 & \text { EPA6010A } \\ \mathrm{mg} / \mathrm{kg} & 16.7 & \text { EPA6010A } \\ \mathrm{mg} / \mathrm{kg} & 0.558 & \text { EPA6010A } \\ \mathrm{mg} / \mathrm{kg} & 1.12 & \text { EPA6010A }\end{array}$

$1.09 \mathrm{E}+00 \pm 5.67 \mathrm{E}-02 \mathrm{pCi} / \mathrm{g}$

$-1.26 \mathrm{E}-03 \pm 6.12 \mathrm{E}-03 \mathrm{pCi} / \mathrm{g}$

1.97E-04 $1.24 \mathrm{E}-02 \mathrm{pCi} / \mathrm{g}$

$7.45 \mathrm{E}-04 \pm 6.75 \mathrm{E}-03 \mathrm{pCi} / \mathrm{g}$

$1.72 \mathrm{E}-02 \pm 3.80 \mathrm{E}-02 \mathrm{pCi} / \mathrm{g}$ $-5.06 \mathrm{E}-04 \pm 5.00 \mathrm{E}-03 \mathrm{pCi} / \mathrm{g}$ 5.70E-02 $\pm 9.19 \mathrm{E}-03 \mathrm{pCi} / \mathrm{g}$ $3.61 \mathrm{E}-03 \pm 4.76 \mathrm{E}-03 \mathrm{pCi} / \mathrm{g}$ $-3.19 \mathrm{E}-04 \pm 6.29 \mathrm{E}-03 \mathrm{pCi} / \mathrm{g}$ $1.12 \mathrm{E}-03 \pm 4.26 \mathrm{E}-03 \mathrm{pCi} / \mathrm{g}$ $-6.27 \mathrm{E}-04 \pm 1.43 \mathrm{E}-02 \mathrm{pCi} / \mathrm{g}$ 2.73E-02 $44.01 \mathrm{E}-02 \mathrm{pCi} / \mathrm{g}$ $0.00 \mathrm{E}+00 \quad \mathrm{pCi} / \mathrm{g}$ $1.08 \mathrm{E}+01 \pm 2.40 \mathrm{E}+00 \mathrm{pCi} / \mathrm{g}$
2.39E-02 EPIA-013B

1.04E-02 EPIA-013B

2.17E-02 EPIA-013B

9.85E-03 EPIA-013B

6.53E-02 EPIA-013B

7.50E-03 EPIA-013B

8.40E-03 EPIA-013B

8.25E-03 EPIA-013B

9.27E-03 EPIA-013B

7.60E-03 EPLA-013B

2.33E-02 EPIA-013B

7.36E-02 EPIA-013B

3.41E-02 EPIA-013B

1.27E+00 EPIA-001B 
SURVEY ID: PPSC 1801 (continued)

Constituent

Radionuclides

Lead-212

Manganese-54

Neptunium-239

Nonvolatile beta

Plutonium-238

Plutonium-239/240

Potassium-40

Promethium-144

Promethium-146

Ruthenium-106

Sodium-22

Strontium-90

Thorium-234

Tin-113

Yttrium-88

Zinc-65

Zirconium-95
Sample ID: 104668
RQ AQ B Result
Unit
D. Limit Method

1.41E-02 EPIA-013B

$1.68 \mathrm{E}-02 \pm 7.59 \mathrm{E}-03 \mathrm{pCi} / \mathrm{g} \quad 8.97 \mathrm{E}-03 \quad \mathrm{EPIA}-013 \mathrm{~B}$

UI $\quad 0.00 \mathrm{E}+00 \quad \mathrm{pCi} / \mathrm{g} \quad 5.96 \mathrm{E}-02 \quad$ EPIA-013B

$8.55 \mathrm{E}+00 \pm 1.67 \mathrm{E}+00 \mathrm{pCi} / \mathrm{g} \quad 2.09 \mathrm{E}+00 \mathrm{EPIA}-001 \mathrm{~B}$

UI $\quad 0.00 \mathrm{E}+00 \quad \mathrm{pCi} / \mathrm{g} \quad 7.90 \mathrm{E}-03$ EPIA.012B

$7.94 \mathrm{E}-03$ EPIA-012B

6.56E-02 EPIA-013B

8.38E-03 EPIA-013B

1.10E-02 EPIA-013B

7.61E-02 EPIA-013B

7.61E-03 EPIA-013B

8.74E-02 EPIA-004

4.27E-01 EPIA-013B

1.18E-02 EPIA-013B

8.70E-03 EPIA-013B

1.49E-02 EPIA-013B

2.26E-02 EPIA-013B

SURVEY ID: PPSC 1802

Sample ID: 104669

Survey location: 44086E $3672804 \mathrm{~N}$ (SRS coordinates)

Depth of core interval: 1.00 to $2.00 \mathrm{ft}$

Sample type: Normal

Sample matrix: Soil

Percent solids: $\mathbf{8 5 . 0 0}$

Constituent

RQ AQ B Result

Unit

D. Limit Method

Physical Parameters and Specified Analyses

Ammonia nitrogen

Cation exchange capacity

Cation exchange capacity

Chloride

Cyanide

Nitrate-nitrite as nitrogen

Nitrogen by Kjeldahl method

Orthophosphate

Phenols

Sulfide

Total organic carbon

Total organic carbon

Total organic nitrogen

Total phosphates (as P)

Metals (total recoverable)
Aluminum
Antimony
Arsenic
Barium
Beryllium
Cadmium
Calcium

$\begin{array}{llll} & & 31.3 \\ & & & 58.7 \\ & & 60.9 \\ J & Q & & 4.97 \\ \text { U } & & & 1.14 \\ \text { U } & & & 1 \\ & & & 173 \\ \text { U } & & & 11.8 \\ & & & 364 \\ \text { UJ } & \text { C } & & 294 \\ & & & 3000 \\ & & 3440 \\ \text { C } & & & 142 \\ \text { J } & \text { E } & & 90.4\end{array}$

$\begin{array}{lll}\mathrm{J} & \mathrm{C} & 2000 \\ \mathrm{U} & & 5.4 \\ \mathrm{U} & & 16.2 \\ & & 4.24 \\ \mathrm{~J} & \mathrm{E} & 0.0216 \\ \mathrm{U} & & 0.27 \\ & \mathrm{~V} & 32.8\end{array}$

$\begin{array}{lll}\mathrm{mg} / \mathrm{kg} & 19.9 & \text { EPA350.1 } \\ \mathrm{meq} / 100 \mathrm{~g} & 5 & \text { EPA9081 } \\ \mathrm{meq} / 100 \mathrm{~g} & 5.88 & \text { EPA9081 } \\ \mathrm{mg} / \mathrm{kg} & 2.77 & \text { EPA300.0 } \\ \mathrm{mg} / \mathrm{kg} & 1.14 & \text { EPA335.3 } \\ \mathrm{mg} / \mathrm{kg} & 1 & \text { EPA353.1 } \\ \mathrm{mg} / \mathrm{kg} & 46.9 & \text { EPA351.2 } \\ \mathrm{mg} / \mathrm{kg} & 11.8 & \text { EPA365.2 } \\ \mu \mathrm{g} / \mathrm{kg} & 353 & \text { EPA420.2 } \\ \mathrm{mg} / \mathrm{kg} & 294 & \text { EPA376.2 } \\ \mathrm{mg} / \mathrm{kg} & 118 & \text { EPA415.1 } \\ \mathrm{mg} / \mathrm{kg} . & 118 & \text { EPA415.1 } \\ \mathrm{mg} / \mathrm{kg} & 0.235 & \text { EPA351.2 } \\ \mathrm{mg} / \mathrm{kg} & 471 & \text { EPA365.4 }\end{array}$

$\begin{array}{lll}\mathrm{mg} / \mathrm{kg} & 13.5 & \text { EPA6010A } \\ \mathrm{mg} / \mathrm{kg} & 5.4 & \text { EPA6010A } \\ \mathrm{mg} / \mathrm{kg} & 16.2 & \text { EPA6010A } \\ \mathrm{mg} / \mathrm{kg} & 1.35 & \text { EPA6010A } \\ \mathrm{mg} / \mathrm{kg} & 0.54 & \text { EPA6010A } \\ \mathrm{mg} / \mathrm{kg} & 0.27 & \text { EPA6010A } \\ \mathrm{mg} / \mathrm{kg} & 13.5 & \text { EPA6010A }\end{array}$


SURVEY ID: PPSC 1802 (continued)

Constituent

RQ AQ B Result

Sample ID: 104669

Metals (total recoverable)

Chromium

Cobalt

Copper

Iron

Lead

Magnesium

Manganese

Mercury

Nickel

Potassium

Selenium

Silver

Sodium

Thallium

Vanadium

Zinc

Radionuclides

Actinium-228

Antimony-124

Antimony-125

Barium-133

Cerium-144

Cesium-134

Cesium-137

Cobalt-57

Cobalt-58

Cobalt-60

Europium-152

Europium-154

- Europium-155

Gross alpha

Lead-212

Manganese-54

Neptunium-239

Nonvolatile beta

Plutonium-238

Plutonium-239/240

Potassium-40

Promethium-144

Promethium-146

Ruthenium-106

Sodium-22

Strontium-90

Thorium-234

Tin-113

Yttrium-88

Zinc-65

Zirconium-95

$\begin{array}{llllll} & & 6.58 & \mathrm{mg} / \mathrm{kg} & 1.35 & \text { EPA6010A } \\ \mathrm{U} & & \mathbf{0 . 5 4} & \mathrm{mg} / \mathrm{kg} & 0.54 & \text { EPA6010A } \\ \mathrm{U} & \mathrm{V} & 1.54 & \mathrm{mg} / \mathrm{kg} & 1.35 & \text { EPA6010A } \\ \mathrm{J} & \mathrm{VC} & 4330 & \mathrm{mg} / \mathrm{kg} & 5.4 & \text { EPA6010A } \\ \mathrm{U} & & 5.4 & \mathrm{mg} / \mathrm{kg} & 5.4 & \text { EPA6010A } \\ & & 33.5 & \mathrm{mg} / \mathrm{kg} & 5.4 & \text { EPA6010A } \\ & & 7.94 & \mathrm{mg} / \mathrm{kg} & 0.54 & \text { EPA6010A } \\ \mathrm{J} & \mathrm{E} & \mathbf{0 . 0 1 6 4} & \mathrm{mg} / \mathrm{kg} & 0.0385 & \text { EPA7471 } \\ \mathrm{J} & \mathrm{E} & \mathbf{0 . 5 6 9} & \mathrm{mg} / \mathrm{kg} & 1.35 & \text { EPA6010A } \\ \mathrm{J} & \mathrm{E} & 48.6 & \mathrm{mg} / \mathrm{kg} & 108 & \text { EPA6010A } \\ \mathrm{U} & & 16.2 & \mathrm{mg} / \mathrm{kg} & 16.2 & \text { EPA6010A } \\ \mathrm{U} & & 1.35 & \mathrm{mg} / \mathrm{kg} & 1.35 & \text { EPA6010A } \\ \mathrm{U} & & 54 & \mathrm{mg} / \mathrm{kg} & 54 & \text { EPA6010A } \\ \mathrm{UJJ} & \mathrm{C} & 16.2 & \mathrm{mg} / \mathrm{kg} & 16.2 & \text { EPA6010A } \\ & & 13 & \mathrm{mg} / \mathrm{kg} & 0.54 & \text { EPA6010A } \\ \mathrm{U} & \mathrm{V} & 1.7 & \mathrm{mg} / \mathrm{kg} & 1.08 & \text { EPA6010A }\end{array}$

$1.12 \mathrm{E}+00 \pm 5.98 \mathrm{E}-02 \mathrm{pCi} / \mathrm{g}$

$-1.61 \mathrm{E}-03 \pm 6.98 \mathrm{E}-03 \mathrm{pCi} / \mathrm{g}$

$-3.08 \mathrm{E}-03 \pm 1.40 \mathrm{E}-02 \mathrm{pCi} / \mathrm{g}$

$-1.58 \mathrm{E}-02 \pm 6.73 \mathrm{E}-03 \mathrm{pCi} / \mathrm{g}$

$-1.42 \mathrm{E}-02 \pm 3.92 \mathrm{E}-02 \mathrm{pCi} / \mathrm{g}$

$2.93 \mathrm{E}-03 \pm 6.50 \mathrm{E}-03 \mathrm{pCi} / \mathrm{g}$

$7.80 \mathrm{E}-03 \pm 8.68 \mathrm{E}-03 \mathrm{pCi} / \mathrm{g}$

$-2.99 \mathrm{E}-03 \pm 4.89 \mathrm{E}-03 \mathrm{pCi} / \mathrm{g}$

$1.51 \mathrm{E}-03 \pm 5.85 \mathrm{E}-03 \mathrm{pCi} / \mathrm{g}$

$5.35 \mathrm{E}-03 \pm 4.54 \mathrm{E}-03 \mathrm{pCi} / \mathrm{g}$

$-3.64 \mathrm{E}-03 \pm 1.46 \mathrm{E}-02 \mathrm{pCi} / \mathrm{g}$

$6.86 \mathrm{E}-03 \pm 4.29 \mathrm{E}-02 \mathrm{pCi} / \mathrm{g}$

$0.00 \mathrm{E}+00 \quad \mathrm{pCi} / \mathrm{g}$

$2.02 \mathrm{E}+01 \pm 3.42 \mathrm{E}+00 \mathrm{pCi} / \mathrm{g}$

$1.22 \mathrm{E}+00 \pm 2.78 \mathrm{E}-02 \mathrm{pCi} / \mathrm{g}$

$2.48 \mathrm{E}-02 \pm 7.80 \mathrm{E}-03 \mathrm{pCi} / \mathrm{g}$

$0.00 \mathrm{E}+00 \quad \mathrm{pCi} / \mathrm{g}$

$7.99 \mathrm{E}+00 \pm 1.65 \mathrm{E}+00 \mathrm{pCi} / \mathrm{g}$

$4.57 \mathrm{E}-02 \pm 2.84 \mathrm{E}-02 \mathrm{pCi} / \mathrm{g}$

$1.70 \mathrm{E}-02 \pm 1.42 \mathrm{E}-02 \mathrm{pCi} / \mathrm{g}$

$\mathrm{V} \quad 6.28 \mathrm{E}-01 \pm 1.24 \mathrm{E}-01 \mathrm{pCi} / \mathrm{g}$

$3.65 \mathrm{E}-03 \pm 4.96 \mathrm{E}-03 . \mathrm{pCi} / \mathrm{g}$

$6.40 \mathrm{E}-03 \pm 6.70 \mathrm{E}-03 \mathrm{pCi} / \mathrm{g}$

$-3.21 \mathrm{E}-02 \pm 4.42 \mathrm{E}=02 \mathrm{pCi} / \mathrm{g}$

$-1.20 \mathrm{E}-03 \pm 4.54 \mathrm{E}-03 \mathrm{pCi} / \mathrm{g}$

$1.48 \mathrm{E}+00 \pm 1.47 \mathrm{E}-01 \mathrm{pCi} / \mathrm{g}$

$8.59 \mathrm{E}-01 \pm 5.43 \mathrm{E}-01 \mathrm{pCi} / \mathrm{g}$

$-3.87 \mathrm{E}-03 \pm 7.74 \mathrm{E}-03 \mathrm{pCi} / \mathrm{g}$

$4.63 \mathrm{E}-04 \pm 5.19 \mathrm{E}-03 \mathrm{pCi} / \mathrm{g}$

$4.69 \mathrm{E}-03 \pm 1.03 \mathrm{E}-02 \mathrm{pCi} / \mathrm{g}$

$1.33 \mathrm{E}-02 \pm 1.33 \mathrm{E}-02 \mathrm{pCi} / \mathrm{g}$
2.65E-02 EPIA-013B

1.06E-02 EPIA-013B

1.03E-02 EPIA-013B

6.70E-02 EPIA-013B

7.67E-03 EPIA-013B

1.01E-02 EPIA-013B

8.35E-03 EPIA-013B

1.03E-02 EPIA-013B

8.79E-03 EPIA-013B

2.41E-02 EPIA-013B

6.62E-02 EPIA-013B

3.52E-02 EPIA-013B

2.00E+00 EPIA-001B

1.43E-02 EPIA-013B

8.08E-03 EPIA-013B

5.85E-02 EPIA-013B

2.13E+00 EPIA-001B

4.14E-02 EPIA-012B

1.65E-02 EPIA-012B

6.96E-02 EPIA-013B

8.88E-03 EPIA-013B

1.14E.02 EPIA-013B

7.48E-02 EPIA-013B

7.60E-03 EPIA.013B

8.06E-02 EPIA-004

4.52E-01 EPIA-013B

1.26E-02 EPIA-013B

9.43E-03 EPIA-013B

1.62E-02 EPIA-013B

2.16E-02 EPIA-013B
2.29E-02 EPIA-013B 
Survey location: 44086E $3672804 N$ (SRS coordinates)

Depth of core interval: 2.00 to $3.75 \mathrm{ft}$

Sample type: Normal

Constituent

RQ AQ B Result

Unit

D. Limit Method

Physical Parameters and Specified Analyses

Ammonia nitrogen

Cation exchange capacity

Chloride

Cyanide

Nitrate-nitrite as nitrogen

Nitrogen by Kjeldahl method

Orthophosphate

Phenols

Sulfide

Total organic carbon

Total organic nitrogen

Total phosphates (as P)

Metals (total recoverable)

Aluminum

Antimony

Arsenic

Barium

Beryllium

Cadmium

Calcium

Chromium

Cobalt

Copper

Iron

Lead

Magnesium

Manganese

Mercury

Nickel

Potassium

Selenium

Silver

Sodium

Thallium

Vanadium

Zinc

Radionuclides

Actinium-228

Antimony-124

Antimony-125

Barium-133

Cerium-144

Cesium-134

Cesium-137

Cobalt-57

Cobalt-58

$\begin{array}{llllll} & & 22.4 & \mathrm{mg} / \mathrm{kg} & 20.8 & \text { EPA350.1 } \\ & & 79.8 & \mathrm{meq} / \mathrm{l00g} & \mathbf{5} & \text { EPA9081 } \\ \mathrm{J} & \mathrm{Q} & \mathbf{5 . 7 3} & \mathrm{mg} / \mathrm{kg} & 2.66 & \text { EPA300.0 } \\ \mathrm{U} & & 1.12 & \mathrm{mg} / \mathrm{kg} & 1.12 & \text { EPA335.3 } \\ \mathrm{J} & \mathrm{E} & \mathbf{0 . 3 3 7} & \mathrm{mg} / \mathrm{kg} & 1.12 & \text { EPA353.1 } \\ & & 44.9 & \mathrm{mg} / \mathrm{kg} & 8.88 & \text { EPA351.2 } \\ \mathrm{U} & & 11.2 & \mathrm{mg} / \mathrm{kg} & 11.2 & \text { EPA365.2 } \\ \mathrm{U} & & 336 & \mu \mathrm{mg} / \mathrm{kg} & 336 & \text { EPA420.2 } \\ \mathrm{UJ} & \mathrm{C} & 281 & \mathrm{mg} / \mathrm{kg} & 281 & \text { EPA376.2 } \\ & & 365 & \mathrm{mg} / \mathrm{kg} & 112 & \text { EPA415.1 } \\ \mathrm{C} & & 22.4 & \mathrm{mg} / \mathrm{kg} & 0.225 & \text { EPA351.2 } \\ \mathrm{J} & \mathrm{E} & 9080 & \mathrm{mg} / \mathrm{kg} & 44900 & \text { EPA365.4 }\end{array}$

$\begin{array}{lll}\text { J } & \text { C } & 2770 \\ \text { U } & & 5.45 \\ \text { U } & & 16.4 \\ & & 2.77 \\ \text { J } & \text { E } & 0.0164 \\ \text { U } & & 0.273 \\ & \text { V } & 21.4 \\ & & 9.2 \\ \text { U } & & 0.545 \\ \text { U } & \text { V } & 2.03 \\ \text { J } & \text { VC } & 6010 \\ \text { J } & \text { E } & 2.16 \\ & & 39.3 \\ & & 4.06 \\ \text { J } & \text { E } & 0.0125 \\ \text { J } & \text { E } & 0.597 \\ \text { J } & \text { E } & 69.5 \\ \text { U } & & 16.4 \\ \text { U } & & 1.36 \\ \text { U } & & 54.5 \\ \text { UJ } & \text { C } & 16.4 \\ \text { U } & \text { V } & 17.4 \\ & & 1.63\end{array}$

9.41E-01 $\pm 5.14 \mathrm{E}-02 \mathrm{pCi} / \mathrm{g}$ 3.65E-03 $\pm 6.36 \mathrm{E}-03 \mathrm{pCi} / \mathrm{g}$ $1.29 \mathrm{E}-03 \pm 1.27 \mathrm{E}-02 \mathrm{pCj} / \mathrm{g}$ $-2.41 \mathrm{E}-03 \pm 6.46 \mathrm{E}-03 \mathrm{pCi} / \mathrm{g}$ 1.60E-02 $\pm 3.87 \mathrm{E}-02 \mathrm{pCi} / \mathrm{g}$ $-2.83 \mathrm{E}-03 \pm 5.20 \mathrm{E}-03 \mathrm{pCi} / \mathrm{g}$ $-3.43 \mathrm{E}-05 \pm 5.71 \mathrm{E}-03 \mathrm{pCi} / \mathrm{g}$ 1.27E-03 $44.76 \mathrm{E}-03 \mathrm{pCi} / \mathrm{g}$ 5.08E-04 $46.06 \mathrm{E}-03 \mathrm{pCi} / \mathrm{g}$
2.41E-02 EPIA-013B 1.10E-02 EPIA-013B 2.19E-02 EPIA-013B

9.71E-03 EPIA-013B

6.49E-02 EPIA-013B

7.49E-03 EPIA-013B

8.42E-03 EPIA-013B

8.01E-03 EPIA-013B 8.95E-03 EPIA-013B 
SURVEY ID: PPSC 1803 (continued)

Constituent

Radionuclides

Cobalt-60

Europium-152

Europium-154

Europium-155

Gross alpha

Lead-212

Manganese-54

Neptunium-239

Nonvolatile beta

Plutonium-238

Plutonium-239/240

Potassium-40

Promethium-144

Promethium-146

Ruthenium-106

Sodium-22

Strontium-90

Thorium-234

Tin-113

Yttrium-88

Zinc-65

Zirconium-95
Sample ID: 104670

RQ AQ B Result Unit D. Limit Method

\section{SURVEY ID: PPSC 1901}

$\begin{array}{ll}2.36 \mathrm{E}-03 \pm 3.95 \mathrm{E}-03 & \mathrm{pCi} / \mathrm{g} \\ -7.40 \mathrm{E}-03 \pm 1.36 \mathrm{E}-02 \mathrm{pCi} / \mathrm{g} \\ 9.12 \mathrm{E}-03 \pm 3.99 \mathrm{E}-02 \mathrm{pCi} / \mathrm{g} \\ 0.00 \mathrm{E}+00 & \mathrm{pCi} / \mathrm{g} \\ 1.84 \mathrm{E}+01 \pm 3.33 \mathrm{E}+00 \mathrm{pCi} / \mathrm{g} \\ 1.03 \mathrm{E}+00 \pm 2.53 \mathrm{E}-02 \mathrm{pCi} / \mathrm{g} \\ 1.37 \mathrm{E}-02 \pm 6.37 \mathrm{E}-03 \mathrm{pCi} / \mathrm{g} \\ 8.49 \mathrm{E}-03 \pm 3.76 \mathrm{E}-02 \mathrm{pCi} / \mathrm{g} \\ 8.18 \mathrm{E}+00 \pm 1.71 \mathrm{E}+00 \mathrm{pCi} / \mathrm{g} \\ 5.69 \mathrm{E}-03 \pm 1.85 \mathrm{E}-02 \mathrm{pCi} / \mathrm{g} \\ 1.17 \mathrm{E}-03 \pm 5.30 \mathrm{E}-03 \mathrm{pCi} / \mathrm{g} \\ 5.75 \mathrm{E}-01 \pm 9.90 \mathrm{E}-02 \mathrm{pCi} / \mathrm{g} \\ -1.58 \mathrm{E}-03 \pm 4.89 \mathrm{E}-03 \mathrm{pCi} / \mathrm{g} \\ 8.48 \mathrm{E}-03 \pm 8.64 \mathrm{E}-03 \mathrm{pCi} / \mathrm{g} \\ 2.87 \mathrm{E}-02 \pm 4.40 \mathrm{E}-02 \mathrm{pCi} / \mathrm{g} \\ 1.70 \mathrm{E}-03 \pm 3.91 \mathrm{E}-03 \mathrm{pCi} / \mathrm{g} \\ 1.35 \mathrm{E}+00 \pm 1.38 \mathrm{E}-01 \mathrm{pCi} / \mathrm{g} \\ 1.07 \mathrm{E}+00 \pm 5.40 \mathrm{E}-01 \mathrm{pCi} / \mathrm{g} \\ -2.43 \mathrm{E}-04 \pm 6.88 \mathrm{E}-03 \mathrm{pCi} / \mathrm{g} \\ 1.40 \mathrm{E}-04 \pm 5.16 \mathrm{E}-03 \mathrm{pCi} \mathrm{g} \\ 5.76 \mathrm{E}-03 \pm 8.03 \mathrm{E}-03 \mathrm{pCi} / \mathrm{g} \\ 1.62 \mathrm{E}-02 \pm 1.32 \mathrm{E}-02 \mathrm{pCi} / \mathrm{g}\end{array}$

2.36E-03 $\pm 3.95 \mathrm{E}-03 \mathrm{pCi} / \mathrm{g}$

UI

UI

UI

UI

UI

UI

UI

V

UI

UI

UI

U V

V

UI

UI

UI

UI $\begin{array}{ll}7.22 \mathrm{E}-03 & \text { EPIA-013B } \\ 2.32 \mathrm{E}-02 & \text { EPIA-013B } \\ 6.28 \mathrm{E}-02 & \text { EPIA-013B } \\ 3.39 \mathrm{E}-02 & \text { EPIA-013B } \\ 1.86 \mathrm{E}+00 & \text { EPIA-001B } \\ 1.40 \mathrm{E}-02 & \text { EPIA-013B } \\ 7.69 \mathrm{E}-03 & \text { EPIA-013B } \\ 5.68 \mathrm{E}-02 & \text { EPIA-013B } \\ 2.31 \mathrm{E}+00 & \text { EPIA-001B } \\ 3.92 \mathrm{E}-02 & \text { EPIA-012B } \\ 1.49 \mathrm{E}-02 & \text { EPIA-012B } \\ 6.02 \mathrm{E}-02 & \text { EPIA-013B } \\ 8.09 \mathrm{E}-03 & \text { EPIA-013B } \\ 1.07 \mathrm{E}-02 & \text { EPIA-013B } \\ 7.66 \mathrm{E}-02 & \text { EPIA-013B } \\ 7.07 \mathrm{E}-03 & \text { EPIA-013B } \\ 7.71 \mathrm{E}-02 & \text { EPIA-004 } \\ 4.23 \mathrm{E}-01 & \text { EPIA-013B } \\ 1.18 \mathrm{E}-02 & \text { EPIA-013B } \\ 9.35 \mathrm{E}-03 & \text { EPIA-013B } \\ 1.48 \mathrm{E}-02 & \text { EPIA-013B } \\ 2.09 \mathrm{E}-02 & \text { EPIA-013B }\end{array}$

Survey location: 44200E 3673595N (SRS coordinates)

Depth of core interval: 0.00 to $1.00 \mathrm{ft}$

Sample type: Normal

Sample matrix: Soil

RQ AQ B Result

Constituent

Physical Parameters and Specified Analyses

Ammonia nitrogen

Cation exchange capacity

Chloride

Cyanide

Cyanide

Nitrate-nitrite as nitrogen

Nitrogen by Kjeldahl method

Orthophosphate

Phenols

Sulfide

Total organic carbon

Total organic nitrogen

Total phosphates (as P)
Sample ID: 104671
Percent solids: $\mathbf{5 6 . 0 0}$

Unit

D. Limit Method

$\begin{array}{llllll} & & 58.5 & \mathrm{mg} / \mathrm{kg} & 38.2 & \text { EPA350.1 } \\ \mathrm{J} & \mathrm{Q} & 131 & \mathrm{meq} / \mathrm{lo0g} & 5 & \text { EPA9081 } \\ \mathrm{U} & 6.43 & \mathrm{mg} / \mathrm{kg} & 4.44 & \text { EPA300.0 } \\ \mathrm{U} & & 1.64 & \mathrm{mg} / \mathrm{kg} & 1.64 & \text { EPA335.3 } \\ \mathrm{U} & 1.61 & \mathrm{mg} / \mathrm{kg} & 1.61 & \text { EPA335.3 } \\ & & 1.75 & \mathrm{mg} / \mathrm{kg} & 1.75 & \text { EPA353.1 } \\ \mathrm{U} & & 455 & \mathrm{mg} / \mathrm{kg} & 142 & \text { EPA351.2 } \\ & & 17.9 & \mathrm{mg} / \mathrm{kg} & 17.9 & \text { EPA365.2 } \\ \mathrm{UJ} & \mathrm{C} & \mathbf{9 0 5} & \mathrm{\mu g} / \mathrm{kg} & 535 & \text { EPA420.2 } \\ & & 446 & \mathrm{mg} / \mathrm{kg} & 446 & \text { EPA376.2 } \\ \mathrm{C} & & 19300 & \mathrm{mg} / \mathrm{kg} & 179 & \text { EPA415.1 } \\ \mathrm{J} & \mathrm{E} & 397 & \mathrm{mg} / \mathrm{kg} & 0.357 & \text { EPA351.2 } \\ & 185 & \mathrm{mg} / \mathrm{kg} & 610 & \text { EPA365.4 }\end{array}$


SURVEY ID: PPSC 1901 (continued)

Constituent

Metals (total recoverable)

\begin{tabular}{|c|c|c|c|}
\hline Aluminum & & $\mathrm{V}$ & 11100 \\
\hline Aluminum & & $\mathrm{V}$ & 9140 \\
\hline Antimony & $J$ & $\mathrm{E}$ & 7.62 \\
\hline Antimony & UJ & L & 8.4 \\
\hline Arsenic & $J$ & $\mathbf{E}$ & 5.15 \\
\hline Arsenic & $J$ & $\mathbf{E}$ & 6.44 \\
\hline Barium & $\mathbf{J}$ & C & 106 \\
\hline Barium & $J$ & $\mathrm{C}$ & 96.9 \\
\hline Beryllium & $\mathbf{J}$ & $\mathrm{C}$ & 1.05 \\
\hline Beryllium & $\mathbf{J}$ & C & 1.04 \\
\hline Cadmium & U & & 0.44 \\
\hline Cadmium & $\mathrm{U}$ & & 0.42 \\
\hline Calcium & & V & 349 \\
\hline Calcium & & $\mathrm{V}$ & 349 \\
\hline Chromium & & & 34.1 \\
\hline Chromium & & & 31.3 \\
\hline Cobalt & & & 7.82 \\
\hline Cobalt & & & 6.97 \\
\hline Copper & & & 14.5 \\
\hline Copper & & & 12.8 \\
\hline Iron & $J$ & C & 23500 \\
\hline Iron & $J$ & C & 21900 \\
\hline Lead & & & 18.6 \\
\hline Lead & & & 16.1 \\
\hline Magnesium & & & 1410 \\
\hline Magnesium & & & 1250 \\
\hline Manganese & & & 360 \\
\hline Manganese & & & 347 \\
\hline Mercury & & & 0.114 \\
\hline Nickel & & & 7.74 \\
\hline Nickel & & & 6.34 \\
\hline Potassium & & & 796 \\
\hline Potassium & & & 691 \\
\hline Selenium & $\mathbf{J}$ & $\mathbf{E}$ & 7.54 \\
\hline Selenium & $\mathrm{U}$ & . & 25.2 \\
\hline Silver & $\mathbf{U}$ & & 2.2 \\
\hline Silver & $\mathrm{U}$ & & 2.1 \\
\hline Sodium & $J$ & $\mathrm{E}$ & 23.1 \\
\hline Sodium & $\mathrm{J}$ & $\mathrm{E}$ & 23 \\
\hline Thallium & $\mathrm{U}$ & & 26.4 \\
\hline Thallium & $\mathrm{U}$ & & 25.2 \\
\hline Vanadium & $J$ & $\mathrm{C}$ & 43.5 \\
\hline Vanadium & $J$ & $\mathrm{C}$ & 41.2 \\
\hline Zinc & & $\mathrm{V}$ & 38.8 \\
\hline Zinc & & $\mathrm{V}$ & 35.7 \\
\hline
\end{tabular}

$B / N / A$ Extractables (including $P A H$ and phenols)

Acenaphthene

Acenaphthylene

Anthracene

Benzo[a]anthracene

Benzo[b]fluoranthene

Benzo[k]fluoranthene

Benzoic acid
Sample ID: 104671

RQ AQ B Result Unit D. Limit Method

\begin{tabular}{|c|c|c|}
\hline $\mathrm{mg} / \mathrm{kg}$ & 22 & EPA6010A \\
\hline $\mathrm{mg} / \mathrm{kg}$ & 21 & EPA6010A \\
\hline $\mathrm{mg} / \mathrm{kg}$ & 8.8 & EPA6010A \\
\hline $\mathrm{mg} / \mathrm{kg}$ & 8.4 & EPA6010A \\
\hline $\mathrm{mg} / \mathrm{kg}$ & 26.4 & EPA6010A \\
\hline $\mathrm{mg} / \mathrm{kg}$ & 25.2 & EPA6010A \\
\hline $\mathrm{mg} / \mathrm{kg}$ & 2.2 & EPA6010A \\
\hline $\mathrm{mg} / \mathrm{kg}$ & 2.1 & EPA6010A \\
\hline $\mathrm{mg} / \mathrm{kg}$ & 0.88 & EPA6010A \\
\hline $\mathrm{mg} / \mathrm{kg}$ & 0.84 & EPA6010A \\
\hline $\mathrm{mg} / \mathrm{kg}$ & 0.44 & EPA6010A \\
\hline $\mathrm{mg} / \mathrm{kg}$ & 0.42 & EPA6010A \\
\hline $\mathrm{mg} / \mathrm{kg}$ & 22 & EPA6010A \\
\hline $\mathrm{mg} / \mathrm{kg}$ & 21 & EPA6010A \\
\hline $\mathrm{mg} / \mathrm{kg}$ & 2.2 & EPA6010A \\
\hline $\mathrm{mg} / \mathrm{kg}$ & 2.1 & EPA6010A \\
\hline $\mathrm{mg} / \mathrm{kg}$ & 0.88 & EPA6010A \\
\hline $\mathrm{mg} / \mathrm{kg}$ & 0.84 & EPA6010A \\
\hline $\mathrm{mg} / \mathrm{kg}$ & 2.2 & EPA6010A \\
\hline $\mathrm{mg} / \mathrm{kg}$ & 2.1 & EPA6010A \\
\hline $\mathrm{mg} / \mathrm{kg}$ & 8.8 & EPA6010A \\
\hline $\mathrm{mg} / \mathrm{kg}$ & 8.4 & EPA6010A \\
\hline $\mathrm{mg} / \mathrm{kg}$ & 8.8 & EPA6010A \\
\hline $\mathrm{mg} / \mathrm{kg}$ & 8.4 & EPA6010A \\
\hline $\mathrm{mg} / \mathrm{kg}$ & 8.8 & EPA6010A \\
\hline $\mathrm{mg} / \mathrm{kg}$ & 8.4 & EPA6010A \\
\hline $\mathrm{mg} / \mathrm{kg}$ & 0.88 & EPA6010A \\
\hline $\mathrm{mg} / \mathrm{kg}$ & 0.84 & EPA6010A \\
\hline $\mathrm{mg} / \mathrm{kg}$ & 0.0567 & EPA7471 \\
\hline $\mathrm{mg} / \mathrm{kg}$ & 2.2 & EPA6010A \\
\hline $\mathrm{mg} / \mathrm{kg}$ & 2.1 & EPA6010A \\
\hline $\mathrm{mg} / \mathrm{kg}$ & 176 & EPA6010A \\
\hline $\mathrm{mg} / \mathrm{kg}$ & 168 & EPA6010A \\
\hline $\mathrm{mg} / \mathrm{kg}$ & 26.4 & EPA6010A \\
\hline $\mathrm{mg} / \mathrm{kg}$ & 25.2 & EPA6010A \\
\hline $\mathrm{mg} / \mathrm{kg}$ & 2.2 & EPA6010A \\
\hline $\mathrm{mg} / \mathrm{kg}$ & 2.1 & EPA6010A \\
\hline $\mathrm{mg} / \mathrm{kg}$ & 88 & EPA6010A \\
\hline $\mathrm{mg} / \mathrm{kg}$ & 84 & EPA6010A \\
\hline $\mathrm{mg} / \mathrm{kg}$ & 26.4 & EPA6010A \\
\hline $\mathrm{mg} / \mathrm{kg}$ & 25.2 & EPA6010A \\
\hline $\mathrm{mg} / \mathrm{kg}$ & 0.88 & EPA6010A \\
\hline $\mathrm{mg} / \mathrm{kg}$ & 0.84 & EPA6010A \\
\hline $\mathrm{mg} / \mathrm{kg}$ & 1.76 & EPA6010A \\
\hline $\mathrm{g} / \mathrm{kg}$ & 1.68 & EPA6010A \\
\hline
\end{tabular}

59.2
59.2
59.2
5.92
5.92
5.92
59.2

$\begin{array}{lrr}\mu \mathrm{g} / \mathrm{kg} & 59.2 & \text { EPA8270 } \\ \mu \mathrm{g} / \mathrm{kg} & 59.2 & \text { EPA8270 } \\ \mu \mathrm{g} / \mathrm{kg} & 59.2 & \text { EPA8270 } \\ \mu \mathrm{g} / \mathrm{kg} & 5.92 & \text { EPA8270 } \\ \mu \mathrm{g} / \mathrm{kg} & 5.92 & \text { EPA8270 } \\ \mu \mathrm{g} / \mathrm{kg} & 5.92 & \text { EPA8270 } \\ \mu \mathrm{g} / \mathrm{kg} & 59.2 & \text { EPA8270 }\end{array}$




\section{Constituent}

RQ AQ B Result

Unit

D. Limit Method

$B / N / A$ Extractables (including $P A H$ and phenols)

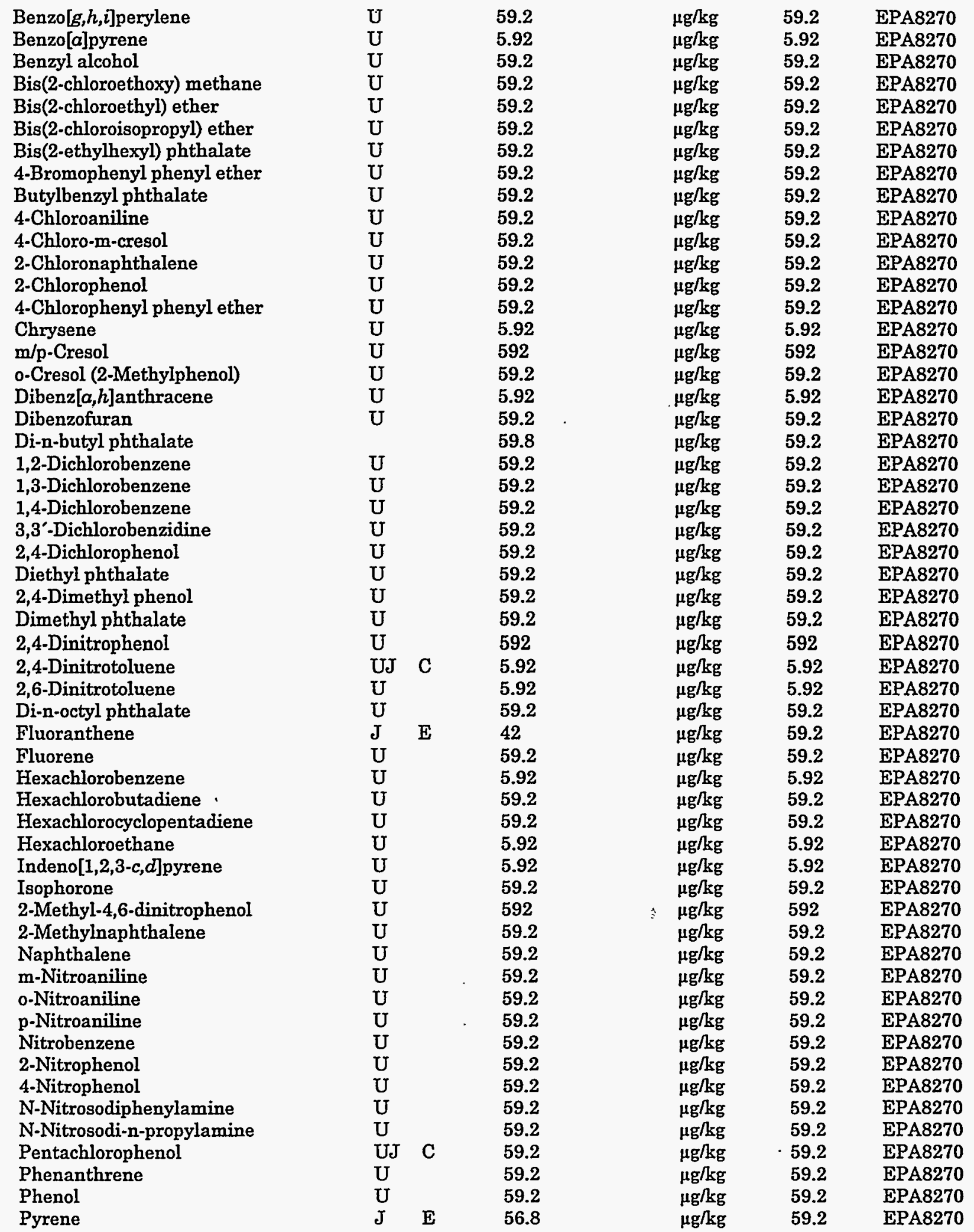


SURVEY ID: PPSC 1901 (continued)

Constituent

RQ AQ

Sample ID: 104671

$B / N / A$ Extractables (including $P A H$ and phenols)

1,2,4-Trichlorobenzene

2,4,5-Trichlorophenol

2,4,6-Trichlorophenol

Volatile Organic Compounds

$\begin{array}{ll}\text { U } & 5.92 \\ \text { U } & 59.2 \\ \text { U } & 59.2\end{array}$

\section{$U$}

Benzene

Bromodichloromethane

Bromoform

Bromomethane (Methyl bromide)

Carbon disulfide

Carbon tetrachloride

Chlorobenzene

Chloroethane

Chloroethene (Vinyl chloride)

Chloroform

Chloromethane (Methyl chloride)

Dibromochloromethane

1,1-Dichloroethane

1,2-Dichloroethane

1,1-Dichloroethylene

1,2-Dichloroethylene

Dichloromethane (Methylene chloride)

1,2-Dichloropropane

cis-1,3-Dichloropropene

trans-1,3-Dichloropropene

Ethylbenzene

2-Hexanone

Methyl ethyl ketone

Methyl isobutyl ketone

Styrene

1,1,2,2-Tetrachloroethane

Tetrachloroethylene

Toluene

1,1,1-Trichloroethane

1,1,2-Trichloroethane

Trichloroethylene

Unknown alcohol

Vinyl acetate

Xylenes

5.92

59.2

$\begin{array}{lll}U & 8 & 63.7 \\ U & & 0.893 \\ U & & 0.0893 \\ U & & 0.0893 \\ U & & 0.179 \\ & & 3.45 \\ U & & 0.0893 \\ U & & 0.0893 \\ U & & 0.179 \\ U & & 0.179 \\ U & & 0.0893 \\ U & & 0.179 \\ U & & 0.0893 \\ U & & 0.0893 \\ U & & 0.0893 \\ U & & 0.0893 \\ U & & 0.179 \\ U & 8 & 1.88 \\ U & & 0.0893 \\ U & & 0.0893 \\ U & & 0.0893 \\ U & & 0.0893 \\ U & & 1.79 \\ U & V & 1.66 \\ U & & 1.79 \\ U & & 0.0893 \\ U & & 0.0893 \\ U & & 0.0893 \\ U & & 0.893 \\ U & & 0.0893 \\ U & & 0.0893 \\ U & & 0.0893 \\ J & N & 3.5 \\ U & & 1.79 \\ U & & 0.268\end{array}$

Pesticides/PCBs and Dioxins/Furans

Aldrin

alpha-Benzene hexachloride

beta-Benzene hexachloride

delta-Benzene hexachloride

alpha-Chlordane

gamma-Chlordane

p,p'-DDD

$p, p^{\prime}-D D E$

p, $\mathrm{p}^{\prime}$-DDT

Dieldrin

Endosulfan sulfate $\mu \mathrm{g} / \mathrm{kg}$

$\mu \mathrm{g} / \mathrm{kg}$

$\mu \mathrm{g} / \mathrm{kg}$

5.92

59.2

59.2

EPA8270

EPA8270

EPA8270

$\begin{array}{lll}\mu \mathrm{g} / \mathrm{kg} & 8.93 & \text { EPA8260 } \\ \mu \mathrm{g} / \mathrm{kg} & 0.893 & \text { EPA8260 } \\ \mu \mathrm{g} / \mathrm{kg} & 0.0893 & \text { EPA8260 } \\ \mu \mathrm{g} / \mathrm{kg} & 0.0893 & \text { EPA8260 } \\ \mu \mathrm{g} / \mathrm{kg} & 0.179 & \text { EPA8260 } \\ \mu \mathrm{g} / \mathrm{kg} & 1.79 & \text { EPA8260 } \\ \mu \mathrm{g} / \mathrm{kg} & 0.0893 & \text { EPA8260 } \\ \mu \mathrm{g} / \mathrm{kg} & 0.0893 & \text { EPA8260 } \\ \mu \mathrm{g} / \mathrm{kg} & 0.179 & \text { EPA8260 } \\ \mu \mathrm{g} / \mathrm{kg} & 0.179 & \text { EPA8260 } \\ \mu \mathrm{g} / \mathrm{kg} & 0.0893 & \text { EPA8260 } \\ \mu \mathrm{g} / \mathrm{kg} & 0.179 & \text { EPA8260 } \\ \mu \mathrm{g} / \mathrm{kg} & 0.0893 & \text { EPA8260 } \\ \mu \mathrm{g} / \mathrm{kg} & 0.0893 & \text { EPA8260 } \\ \mu \mathrm{g} / \mathrm{kg} & 0.0893 & \text { EPA8260 } \\ \mu \mathrm{g} / \mathrm{kg} & 0.0893 & \text { EPA8260 } \\ \mu \mathrm{g} / \mathrm{kg} & 0.179 & \text { EPA8260 } \\ \mu \mathrm{g} / \mathrm{kg} & 0.893 & \text { EPA8260 } \\ \mu \mathrm{g} / \mathrm{kg} & 0.0893 & \text { EPA8260 } \\ \mu \mathrm{g} / \mathrm{kg} & 0.0893 & \text { EPA8260 } \\ \mu \mathrm{g} / \mathrm{kg} & 0.0893 & \text { EPA8260 } \\ \mu \mathrm{g} / \mathrm{kg} & 0.0893 & \text { EPA8260 } \\ \mu \mathrm{g} / \mathrm{kg} & 1.79 & \text { EPA8260 } \\ \mu \mathrm{g} / \mathrm{kg} & 1.79 & \text { EPA8260 } \\ \mu \mathrm{g} / \mathrm{kg} & 1.79 & \text { EPA8260 } \\ \mu \mathrm{g} / \mathrm{kg} & 0.0893 & \text { EPA8260 } \\ \mu \mathrm{g} / \mathrm{kg} & 0.0893 & \text { EPA8260 } \\ \mu \mathrm{g} / \mathrm{kg} & 0.0893 & \text { EPA8260 } \\ \mu \mathrm{g} / \mathrm{kg} & 0.893 & \text { EPA8260 } \\ \mu \mathrm{g} / \mathrm{kg} & 0.0893 & \text { EPA8260 } \\ \mu \mathrm{g} / \mathrm{kg} & 0.0893 & \text { EPA8260 } \\ \mu \mathrm{g} / \mathrm{kg} & 0.0893 & \text { EPA8260 } \\ \mu \mathrm{g} / \mathrm{kg} & & \text { EPA8260 } \\ \mu \mathrm{g} / \mathrm{kg} & 1.79 & \text { EPA8260 } \\ \mu \mathrm{g} / \mathrm{kg} & 0.268 & \text { EPA8260 } \\ & & \end{array}$

$\mu \mathrm{g} / \mathrm{kg}$

$\mu \mathrm{g} / \mathrm{kg}$

$\mu \mathrm{g} / \mathrm{kg}$

$\mu \mathrm{g} / \mathrm{kg}$

$\mu \mathrm{g} / \mathrm{kg}$

$\mu \mathrm{g} / \mathrm{kg}$

$\mu \mathrm{g} / \mathrm{kg}$

$\mu \mathrm{g} / \mathrm{kg}$

$\mu \mathrm{g} / \mathrm{kg}$

$\mu \mathrm{g} / \mathrm{kg}$

$\mu \mathrm{g} / \mathrm{kg}$
EPA8080

EPA8080

EPA8080

EPA8080

EPA8080

EPA8080

EPA8080

EPA8080

EPA8080

EPA8080

EPA8080 
SURVEY ID: PPSC 1901 (continued)

Constituent

RQ AQ B Result

Pesticides/PCBs and Dioxins/Furans

$\begin{array}{ll}\mathrm{U} & 148 \\ \mathrm{U} & 296 \\ \mathrm{U} & 148 \\ \mathrm{U} & 296 \\ \mathrm{U} & 148 \\ \mathrm{U} & 148 \\ \mathrm{U} & 74 \\ \mathrm{U} & \mathbf{1 4 8 0} \\ \mathrm{U} & \mathbf{3 7 0} \\ \mathrm{U} & 370 \\ \mathrm{U} & 370 \\ \mathrm{U} & 370 \\ \mathrm{U} & 370 \\ \mathrm{U} & 370 \\ \mathrm{U} & 370 \\ \mathrm{U} & 2960\end{array}$

Unit

D. Limit Method
Endosulfan I

Endosulfan II

Endrin

Endrin ketone

Heptachlor

Heptachlor epoxide

Lindane

Methoxychlor

PCB 1016

PCB 1221

PCB 1232

PCB 1242

PCB 1248

PCB 1254

PCB 1260

Toxaphene

\section{Radionuclides}

Actinium-228

Antimony-124

Antimony-125

Barium-133

Cerium-144

Cesium-134

Cesium-137

Cobalt-57

Cobalt-58

Cobalt-60

Europium-152

Europium-154

Europium-155

Gross alpha

Lead-212

Manganese-54

Neptunium-239

Nonvolatile beta

Plutonium-238

Plutonium-239/240

Potassium-40

Promethium-144

Promethium-146

Ruthenium-106

Sodium-22

Strontium-90

Thorium-234

Tin-113

Yttrium-88

Zinc-65

Zirconium-95
148

296

148

296

148

148

74

1480

370

370

370

370

370

370

370

2960
EPA8080

EPA8080

EPA8080

EPA8080

EPA8080

EPA8080

EPA8080

EPA8080

EPA8080

EPA8080

EPA8080

EPA8080

EPA8080

EPA8080

EPA8080

EPA8080
9.76E-01 $\pm 7.91 \mathrm{E}-02 \mathrm{pCi} / \mathrm{g}$ $-8.75 \mathrm{E}-04 \pm 1.95 \mathrm{E}-02 \mathrm{pCi} / \mathrm{g}$ 4.64E-02 $\pm 7.38 \mathrm{E}-02 \mathrm{pCi} / \mathrm{g}$ 1.01E-02 $\pm 3.04 \mathrm{E}-02 \mathrm{pCi} / \mathrm{g}$ $-1.83 \mathrm{E}-02 \pm 1.04 \mathrm{E}-01 \mathrm{pCi} / \mathrm{g}$ $-6.61 \mathrm{E}-03+1.59 \mathrm{E}-02 \mathrm{pCi} / \mathrm{g}$ $3.63 \mathrm{E}+01 \pm 1.57 \mathrm{E}-01 \mathrm{pCi} \mathrm{g}$ 2.80E-03 $\pm 1.33 \mathrm{E}-02 \mathrm{pCi} / \mathrm{g}$ $-6.87 \mathrm{E}-04 \pm 1.19 \mathrm{E}-02 \mathrm{pCi} / \mathrm{g}$ 8.77E-01 $\pm 3.55 \mathrm{E}-02 \mathrm{pCi} / \mathrm{g}$ 1.11E-02 $\pm 5.93 \mathrm{E}-02 \mathrm{pCi} / \mathrm{g}$ 6.06E-02 $49.59 \mathrm{E}-02 \mathrm{pCi} / \mathrm{g}$ $0.00 \mathrm{E}+00 \quad \mathrm{pCi} / \mathrm{g}$ $2.41 \mathrm{E}+01 \pm 4.86 \mathrm{E}+00 \mathrm{pCi} / \mathrm{g}$ 9.63E-01 $\pm 3.69 \mathrm{E}-02 \mathrm{pCi} / \mathrm{g}$ UI UI $1.38 \mathrm{E}-02 \pm 1.32 \mathrm{E}-02 \mathrm{pCi} / \mathrm{g}$ $-4.94 \mathrm{E}-02 \pm 9.43 \mathrm{E}-02 \mathrm{pCi} / \mathrm{g}$ $5.07 \mathrm{E}+01 \pm 3.93 \mathrm{E}+00 \mathrm{pCi} / \mathrm{g}$ $6.11 \mathrm{E}-02 \pm 2.70 \mathrm{E}-02 \mathrm{pCi} / \mathrm{g}$ 9.55E-02 $\pm 3.25 \mathrm{E}-02 \mathrm{pCi} / \mathrm{g}$ $8.41 \mathrm{E}+00 \pm 3.24 \mathrm{E}-01 \mathrm{pCi} / \mathrm{g}$ UI $\quad-3.40 \mathrm{E}-04 \pm 8.21 \mathrm{E}-03 \mathrm{pCi} / \mathrm{g}$ UI $\quad 9.48 \mathrm{E}-03 \pm 3.79 \mathrm{E}-02 \mathrm{pCi} / \mathrm{g}$ UI $4.52 \mathrm{E}-02 \pm 1.41 \mathrm{E}-01 \mathrm{pCi} / \mathrm{g}$ 1.80E-02 $\pm 1.37 \mathrm{E}-02 \mathrm{pCi} / \mathrm{g}$ 1.95E+00 $1.61 \mathrm{E}-01 \mathrm{pCi} / \mathrm{g}$ $1.48 \mathrm{E}+00 \pm 9.34 \mathrm{E}-01 \mathrm{pCi} / \mathrm{g}$ $-2.36 \mathrm{E}-02 \pm 3.41 \mathrm{E}-02 \mathrm{pCi} / \mathrm{g}$ 5.51E-03 $\pm 8.66 \mathrm{E}-03 \mathrm{pCi} / \mathrm{g}$ $-1.90 \mathrm{E}-02+2.58 \mathrm{E}-02 \mathrm{pCi} / \mathrm{g}$ 1.74E-02 $2.16 \mathrm{E}-02 \mathrm{pCi} / \mathrm{g}$
5.99E-02 EPIA-013B 3.33E-02 EPIA-013B

1.19E-01 EPIA-013B

4.44E-02 EPIA-013B

1.76E-01 EPIA-013B

2.37E-02 EPIA-013B

2.46E-02 EPIA-013B

2.28E-02 EPIA-013B

2.01E-02 EPIA-013B

1.36E-02 EPIA-013B

9.69E-02 EPIA-013B

1.65E-01 EPIA-013B

9.03E-02 EPIA-013B

2.69E+00 EPIA-001B

5.15E-02 EPIA-013B

1.72E-02 EPIA-013B

1.61E-01 EPIA-013B

2.77E+00 EPIA-001B

2.45E-02 EPLA-012B

1.61E-02 EPIA-012B

1.15E-01 EPIA-013B

1.41E.02 EPIA-013B

6.09E-02 EPIA-013B

2.41E-01 EPIA-013B

1.43E-02 EPIA-013B

7.53E-02 EPIA-004

1.07E+00 EPIA-013B

5.48E-02 EPIA-013B

1.61E-02 EPIA-013B

3.59E-02 EPIA-013B

3.79E-02 EPIA-013B 
Survey location: 44200E $3673595 \mathrm{~N}$ (SRS coordinates)

Depth of core interval: 1.00 to $2.00 \mathrm{ft}$

Sample type: Normal

Sample matrix: Soil

Percent solids: 84.00

Constituent

RQ AQ B Result

Physical Parameters and Specified Analyses

$\begin{array}{llll}\text { U } & & 22.2 \\ & & 14 \\ \text { J } & \text { Q } & & 2.96 \\ \text { UJ } & \text { C } & & 1.19 \\ \text { J } & \text { E } & & 0.34 \\ & & & 28.7 \\ \text { U } & & & 11.9 \\ \text { J } & \text { E } & & 251 \\ \text { UJ } & \text { IC } & \text { P } & 298 \\ \text { UJ } & \text { IC } & \text { P } & 298 \\ & & & \mathbf{5 3 6} \\ \text { C } & & & 20.9 \\ \text { J } & \text { E } & & \mathbf{7 9 . 7}\end{array}$

Metals (total recoverable)

$\begin{array}{lll}\mathrm{mg} / \mathrm{kg} & 22.2 & \text { EPA350.1 } \\ \mathrm{meq} / 100 \mathrm{~g} & 5 & \text { EPA9081 } \\ \mathrm{mg} / \mathrm{kg} & 2.74 & \text { EPA300.0 } \\ \mathrm{mg} / \mathrm{kg} & 1.19 & \text { EPA335.3 } \\ \mathrm{mg} / \mathrm{kg} & 1.13 & \text { EPA353.1 } \\ \mathrm{mg} / \mathrm{kg} & 4.74 & \text { EPA351.2 } \\ \mathrm{mg} / \mathrm{kg} & 11.9 & \text { EPA365.2 } \\ \mu \mathrm{g} / \mathrm{kg} & 356 & \text { EPA420.2 } \\ \mathrm{mg} / \mathrm{kg} & 298 & \text { EPA376.2 } \\ \mathrm{mg} / \mathrm{kg} & 298 & \text { EPA376.2 } \\ \mathrm{mg} / \mathrm{kg} & 119 & \text { EPA415.1 } \\ \mathrm{mg} / \mathrm{kg} & 0.238 & \text { EPA351.2 } \\ \mathrm{mg} / \mathrm{kg} & 390 & \text { EPA365.4 }\end{array}$

$\begin{array}{lll}\mathrm{mg} / \mathrm{kg} & 13.8 & \text { EPA6010A } \\ \mathrm{mg} / \mathrm{kg} & 5.51 & \text { EPA6010A } \\ \mathrm{mg} / \mathrm{kg} & 16.5 & \text { EPA6010A } \\ \mathrm{mg} / \mathrm{kg} & 1.38 & \text { EPA6010A } \\ \mathrm{mg} / \mathrm{kg} & 0.551 & \text { EPA6010A } \\ \mathrm{mg} / \mathrm{kg} & 0.276 & \text { EPA6010A } \\ \mathrm{mg} / \mathrm{kg} & 13.8 & \text { EPA6010A } \\ \mathrm{mg} / \mathrm{kg} & 1.38 & \text { EPA6010A } \\ \mathrm{mg} / \mathrm{kg} & 0.551 & \text { EPA6010A } \\ \mathrm{mg} / \mathrm{kg} & 1.38 & \text { EPA6010A } \\ \mathrm{mg} / \mathrm{kg} & 5.51 & \text { EPA6010A } \\ \mathrm{mg} / \mathrm{kg} & 5.51 & \text { EPA6010A } \\ \mathrm{mg} / \mathrm{kg} & 5.51 & \text { EPA6010A } \\ \mathrm{mg} / \mathrm{kg} & 0.551 & \text { EPA6010A } \\ \mathrm{mg} / \mathrm{kg} & 0.0388 & \text { EPA7471 } \\ \mathrm{mg} / \mathrm{kg} & 1.38 & \text { EPA6010A } \\ \mathrm{mg} / \mathrm{kg} & 110 & \text { EPA6010A } \\ \mathrm{mg} / \mathrm{kg} & 16.5 & \text { EPA6010A } \\ \mathrm{mg} / \mathrm{kg} & 1.38 & \text { EPA6010A } \\ \mathrm{mg} / \mathrm{kg} & \mathbf{5 5 . 1} & \text { EPA6010A } \\ \mathrm{mg} / \mathrm{kg} & 16.5 & \text { EPA6010A } \\ \mathrm{mg} / \mathrm{kg} & 0.551 & \text { EPA6010A } \\ \mathrm{mg} / \mathrm{kg} & 1.1 & \text { EPA6010A }\end{array}$

$B / N / A$ Extractables (including PAH and phenols)

$\begin{array}{lll}\text { Acenaphthene } & \mathrm{U} & 39.1 \\ \text { Acenaphthylene } & \mathrm{U} & 39.1 \\ \text { Anthracene } & \mathrm{U} & 39.1 \\ \text { Benzo[a]anthracene } & \mathrm{U} & 3.91 \\ \text { Benzo[b]fluoranthene } & \mathrm{U} & 3.91 \\ \text { Benzo[k]fluoranthene } & \mathrm{U} & 3.91 \\ \text { Benzoic acid } & & 140 \\ \text { Benzo[g, } h, i] \text { perylene } & \mathrm{U} & 39.1\end{array}$

1120

Barium

Calcium

Chromium

Cobalt

Copper

Iron

Magnesium

Manganese

Mercury

Nickel

Potassium

Selenium

Silver

Sodium

Thallium

Vanadium

Zinc
9.38

U

$\begin{array}{lll}\mathrm{J} & \mathrm{E} & 0.139\end{array}$

0.276

21.9

2.35

0.923

1120

3.71

67.2

15.3

0.0035

0.567

67.3

16.5

1.38

55.1

16.5

2.54

2.22
Unit

D. Limit Method

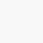


SURVEY ID: PPSC 1902 (continued)

Sample ID: 104672

Constituent

RQ AQ B Result

Unit

D. Limit Method

$B / N / A$ Extractables (including PAH and phenols)

\begin{tabular}{|c|c|c|c|c|c|}
\hline Benzo[a]pyrene & $\mathrm{U}$ & 3.91 & $\mu g / \mathrm{kg}$ & 3.91 & EPA8270 \\
\hline Benzyl alcohol & U & 39.1 & $\mu \mathrm{g} / \mathrm{kg}$ & 39.1 & EPA8270 \\
\hline Bis(2-chloroethoxy) methane & U & 39.1 & $\mu \mathrm{g} / \mathrm{kg}$ & 39.1 & EPA8270 \\
\hline Bis(2-chloroethyl) ether & $\mathrm{U}$ & 39.1 & $\mu \mathrm{g} / \mathrm{kg}$ & 39.1 & EPA8270 \\
\hline Bis(2-chloroisopropyl) ether & $\mathrm{U}$ & 39.1 & $\mu \mathrm{g} / \mathrm{kg}$ & 39.1 & EPA8270 \\
\hline Bis(2-ethylhexyl) phthalate & $\mathrm{U}$ & 39.1 & $\mu \mathrm{g} / \mathrm{kg}$ & 39.1 & EPA8270 \\
\hline 4-Bromophenyl phenyl ether & $\mathrm{U}$ & 39.1 & $\mu \mathrm{g} / \mathrm{kg}$ & 39.1 & EPA8270 \\
\hline Butylbenzyl phthalate & $\mathrm{U}$ & 39.1 & $\mu \mathrm{g} / \mathrm{kg}$ & 39.1 & EPA8270 \\
\hline 4-Chloroaniline & $\mathrm{U}$ & 39.1 & $\mu \mathrm{g} / \mathrm{kg}$ & 39.1 & EPA8270 \\
\hline 4-Chloro-m-cresol & $\mathrm{U}$ & 39.1 & $\mu \mathrm{g} / \mathrm{kg}$ & 39.1 & EPA8270 \\
\hline 2-Chloronaphthalene & $\mathrm{U}$ & 39.1 & $\mu \mathrm{g} / \mathrm{kg}$ & 39.1 & EPA8270 \\
\hline 2-Chlorophenol & $\mathrm{U}$ & 39.1 & $\mu \mathrm{g} / \mathrm{kg}$ & 39.1 & EPA8270 \\
\hline 4-Chlorophenyl phenyl ether & $\mathrm{U}$ & 39.1 & $\mu \mathrm{g} / \mathrm{kg}$ & 39.1 & EPA8270 \\
\hline Chrysene & $\mathrm{U}$ & 3.91 & $\mu \mathrm{g} / \mathrm{kg}$ & 3.91 & EPA8270 \\
\hline $\mathrm{m} / \mathrm{p} \cdot$ Cresol & $U$ & 391 & $\mu \mathrm{g} / \mathrm{kg}$ & 391 & EPA8270 \\
\hline o-Cresol (2-Methylphenol) & $\mathrm{U}$ & 39.1 & $\mu \mathrm{g} / \mathrm{kg}$ & 39.1 & EPA8270 \\
\hline Dibenz $[a, h]$ anthracene & $\mathrm{U}$ & 3.91 & $\mu \mathrm{g} / \mathrm{kg}$ & 3.91 & EPA8270 \\
\hline Dibenzofuran & $\mathrm{U}$ & 39.1 & $\mu \mathrm{g} / \mathrm{kg}$ & 39.1 & EPA8270 \\
\hline Di-n-butyl phthalate & & 41.1 & $\mu \mathrm{g} / \mathrm{kg}$ & 39.1 & EPA8270 \\
\hline 1,2-Dichlorobenzene & $\mathrm{U}$ & 39.1 & $\mu \mathrm{g} / \mathrm{kg}$ & 39.1 & EPA8270 \\
\hline 1,3-Dichlorobenzene & $\mathrm{U}$ & 39.1 & $\mu \mathrm{g} / \mathrm{kg}$ & 39.1 & EPA8270 \\
\hline 1,4-Dichlorobenzene & $U$ & 39.1 & $\mu \mathrm{g} / \mathrm{kg}$ & 39.1 & EPA8270 \\
\hline 3,3'-Dichlorobenzidine & $\mathrm{U}$ & 39.1 & $\mu \mathrm{g} / \mathrm{kg}$ & 39.1 & EPA8270 \\
\hline 2,4-Dichlorophenol & $\mathrm{U}$ & 39.1 & $\mu g / \mathrm{kg}$ & 39.1 & EPA8270 \\
\hline Diethyl phthalate & $\mathrm{U}$ & 39.1 & $\mu \mathrm{g} / \mathrm{kg}$ & 39.1 & EPA8270 \\
\hline 2,4-Dimethyl phenol & $\mathrm{U}$ & 39.1 & $\mu \mathrm{g} / \mathrm{kg}$ & 39.1 & EPA8270 \\
\hline Dimethyl phthalate & U & 39.1 & $\mu g / \mathrm{kg}$ & 39.1 & EPA8270 \\
\hline 2,4-Dinitrophenol & $\mathrm{U}$ & 391 & $\mu g / \mathrm{kg}$ & 391 & EPA8270 \\
\hline 2,4-Dinitrotoluene & UJ $\mathrm{C}$ & 3.91 & $\mu \mathrm{g} / \mathrm{kg}$ & 3.91 & EPA8270 \\
\hline 2,6-Dinitrotoluene & $\mathrm{U}$ & 3.91 & $\mu \mathrm{g} / \mathrm{kg}$ & 3.91 & EPA8270 \\
\hline Di-n-octyl phthalate & U & 39.1 & $\mu \mathrm{g} / \mathrm{kg}$ & 39.1 & EPA8270 \\
\hline Fluoranthene & $\mathrm{U}$ & 39.1 & $\mu \mathrm{g} / \mathrm{kg}$ & 39.1 & EPA8270 \\
\hline Fluorene & $U$ & 39.1 & $\mu \mathrm{g} / \mathrm{kg}$ & 39.1 & EPA8270 \\
\hline Hexachlorobenzene & $\mathrm{U}$ & 3.91 & $\mu \mathrm{g} / \mathrm{kg}$ & 3.91 & EPA8270 \\
\hline Hexachlorobutadiene & $\mathrm{U}$ & 39.1 & $\mu \mathrm{g} / \mathrm{kg}$ & 39.1 & EPA8270 \\
\hline Hexachlorocyclopentadiene & $\mathrm{U}$ & 39.1 & $\mu \mathrm{g} / \mathrm{kg}$ & 39.1 & EPA8270 \\
\hline Hexachloroethane & $\mathrm{U}$ & 3.91 & $\mu \mathrm{g} / \mathrm{kg}$ & 3.91 & EPA8270 \\
\hline Indeno $[1,2,3-c, d]$ pyrene & U & 3.91 & $\mu \mathrm{g} / \mathrm{kg}$ & 3.91 & EPA8270 \\
\hline Isophorone & $\mathrm{U}$ & 39.1 & $\mu \mathrm{g} / \mathrm{kg}$ & 39.1 & EPA8270 \\
\hline 2-Methyl-4,6-dinitrophenol & $U$ & 391 & $\mu \mathrm{g} / \mathrm{kg}$ & 391 & EPA8270 \\
\hline 2-Methylnaphthalene & U & 39.1 & $\mu \mathrm{g} / \mathrm{kg}$ & 39.1 & EPA8270 \\
\hline Naphthalene & $\mathrm{U}$ & 39.1 & $\mu \mathrm{g} / \mathrm{kg}$ & 39.1 & EPA8270 \\
\hline m-Nitroaniline & $\mathrm{U}$ & 39.1 & $\mu \mathrm{g} / \mathrm{kg}$ & 39.1 & EPA8270 \\
\hline o-Nitroaniline & U & 39.1 & $\mu \mathrm{g} / \mathrm{kg}$ & 39.1 & EPA8270 \\
\hline p-Nitroaniline & $\mathrm{U}$ & 39.1 & $\mu \mathrm{g} / \mathrm{kg}$ & 39.1 & EPA8270 \\
\hline Nitrobenzene & $\mathrm{U}$ & 39.1 & $\mu \mathrm{g} / \mathrm{kg}$ & 39.1 & EPA8270 \\
\hline 2-Nitrophenol & $\mathrm{U}$ & 39.1 & $\mu g / k g$ & 39.1 & EPA8270 \\
\hline 4-Nitrophenol & $\mathrm{U}$ & 39.1 & $\mu \mathrm{g} / \mathrm{kg}$ & 39.1 & EPA8270 \\
\hline N-Nitrosodiphenylamine & $\mathrm{U}$ & 39.1 & $\mu \mathrm{g} / \mathrm{kg}$ & 39.1 & EPA8270 \\
\hline N-Nitrosodi-n-propylamine & U & 39.1 & $\mu \mathrm{g} / \mathrm{kg}$ & 39.1 & EPA8270 \\
\hline Pentachlorophenol & UJ & 39.1 & $\mu \mathrm{g} / \mathrm{kg}$ & 39.1 & EPA8270 \\
\hline Phenanthrene & $\mathrm{U}$ & 39.1 & $\mu \mathrm{g} / \mathrm{kg}$ & 39.1 & EPA8270 \\
\hline Phenol & $\mathrm{U}$ & 39.1 & $\mu \mathrm{g} / \mathrm{kg}$ & 39.1 & EPA8270 \\
\hline Pyrene & U & 39.1 & $\mu \mathrm{g} / \mathrm{kg}$ & 39.1 & EPA8270 \\
\hline 1,2,4-Trichlorobenzene & $U$ & 3.91 & $\mu \mathrm{g} / \mathrm{kg}$ & 3.91 & EPA8270 \\
\hline 2,4,5-Trichlorophenol & $U$ & 39.1 & $\mu \mathrm{g} / \mathrm{kg}$ & 39.1 & EPA8270 \\
\hline
\end{tabular}


SURVEY ID: PPSC 1902 (continued)

Constituent

Sample ID: 104672

RQ AQ B Result

Unit

D. Limit Method

$B / N / A$ Extractables (including PAH and phenols)

2,4,6-Trichlorophenol

Unknown alcohol $\mu \mathrm{g} / \mathrm{kg}$

$\mu \mathrm{g} / \mathrm{kg}$
39.1

EPA8270

900

11.9

0.595

0.0595

0.0595

0.119

1.19

0.0595

0.0595

0.119

0.119

0.0595

0.119

0.0595

0.0595

0.0595

0.0595

0.119

1.57

0.0595

0.0595

0.0595

0.0595

1.19

V

14.1

1.19

0.0595

0.0595

0.0595

0.595

0.0595

0.0595

0.0595

N 2

1.19

0.179

$\begin{array}{lll}\mu \mathrm{g} / \mathrm{kg} & 11.9 & \text { EPA8260 } \\ \mu \mathrm{g} / \mathrm{kg} & 0.595 & \text { EPA8260 } \\ \mu \mathrm{g} / \mathrm{kg} & 0.0595 & \text { EPA8260 } \\ \mu \mathrm{g} / \mathrm{kg} & 0.0595 & \text { EPA8260 } \\ \mu \mathrm{g} / \mathrm{kg} & 0.119 & \text { EPA8260 } \\ \mu \mathrm{g} / \mathrm{kg} & 1.19 & \text { EPA8260 } \\ \mu \mathrm{g} / \mathrm{kg} & 0.0595 & \text { EPA8260 } \\ \mu \mathrm{g} / \mathrm{kg} & 0.0595 & \text { EPA8260 } \\ \mu \mathrm{g} / \mathrm{kg} & 0.119 & \text { EPA8260 } \\ \mu \mathrm{g} / \mathrm{kg} & 0.119 & \text { EPA8260 } \\ \mu \mathrm{g} / \mathrm{kg} & 0.0595 & \text { EPA8260 } \\ \mu \mathrm{g} / \mathrm{kg} & 0.119 & \text { EPA8260 } \\ \mu \mathrm{g} / \mathrm{kg} & 0.0595 & \text { EPA8260 } \\ \mu \mathrm{g} / \mathrm{kg} & 0.0595 & \text { EPA8260 } \\ \mu \mathrm{g} / \mathrm{kg} & 0.0595 & \text { EPA8260 } \\ \mu \mathrm{g} / \mathrm{kg} & 0.0595 & \text { EPA8260 } \\ \mu \mathrm{g} / \mathrm{kg} & 0.119 & \text { EPA8260 } \\ \mu \mathrm{g} / \mathrm{kg} & 0.595 & \text { EPA8260 } \\ \mu \mathrm{g} / \mathrm{kg} & 0.0595 & \text { EPA8260 } \\ \mu \mathrm{g} / \mathrm{kg} & 0.0595 & \text { EPA8260 } \\ \mu \mathrm{g} / \mathrm{kg} & 0.0595 & \text { EPA8260 } \\ \mu \mathrm{g} / \mathrm{kg} & 0.0595 & \text { EPA8260 } \\ \mu \mathrm{g} / \mathrm{kg} & 1.19 & \text { EPA8260 } \\ \mu \mathrm{g} / \mathrm{kg} & 1.19 & \text { EPA8260 } \\ \mu \mathrm{g} / \mathrm{kg} & 1.19 & \text { EPA8260 } \\ \mu \mathrm{g} / \mathrm{kg} & 0.0595 & \text { EPA8260 } \\ \mu \mathrm{g} / \mathrm{kg} & 0.0595 & \text { EPA8260 } \\ \mu \mathrm{g} / \mathrm{kg} & 0.0595 & \text { EPA8260 } \\ \mu \mathrm{g} / \mathrm{kg} & 0.595 & \text { EPA8260 } \\ \mu \mathrm{g} / \mathrm{kg} & 0.0595 & \text { EPA8260 } \\ \mu \mathrm{g} / \mathrm{kg} & 0.0595 & \text { EPA8260 } \\ \mu \mathrm{g} / \mathrm{kg} & 0.0595 & \text { EPA8260 } \\ \mu \mathrm{g} / \mathrm{kg} & & \text { EPA8260 } \\ \mu \mathrm{g} / \mathrm{kg} & 1.19 & \text { EPA8260 } \\ \mu \mathrm{g} / \mathrm{kg} & 0.179 & \text { EPA8260 }\end{array}$

0.179

EPA8260

Xylenes

U

Pesticides/PCBs and Dioxins/Furans

Aldrin

alpha-Benzene hexachloride

beta-Benzene hexachloride

delta-Benzene hexachloride

alpha-Chlordane

gamma-Chlordane

$\mathrm{p}, \mathrm{p}^{\prime}-\mathrm{DDD}$

$p, p^{\prime}-D D E$

p,p'DDT

Dieldrin

Endosulfan sulfate

Endosulfan I

Endosulfan II
49.6

49.6

99.2

99.2

99.2

99.2

198

99.2

198

99.2

198

99.2

198 $\mu \mathrm{g} / \mathrm{kg}$

$\mu \mathrm{g} / \mathrm{kg}$

$\mu \mathrm{g} / \mathrm{kg}$

$\mu \mathrm{g} / \mathrm{kg}$

$\mu \mathrm{g} / \mathrm{kg}$

$\mu \mathrm{g} / \mathrm{kg}$

$\mu \mathrm{g} / \mathrm{kg}$

$\mu \mathrm{g} / \mathrm{kg}$

$\mu \mathrm{g} / \mathrm{kg}$

$\mu \mathrm{g} / \mathrm{kg}$

$\mu \mathrm{g} / \mathrm{kg}$

$\mu \mathrm{g} / \mathrm{kg}$

$\mu \mathrm{g} / \mathrm{kg}$
49.6

49.6

99.2

99.2

99.2

99.2

198

99.2

198

99.2

198

99.2

198
EPA8080

EPA8080

EPA8080

EPA8080

EPA8080

EPA8080

EPA8080

EPA8080

EPA8080

EPA8080

EPA8080

EPA8080

EPA8080 
SURVEY ID: PPSC 1902 (continued)

Constituent

Pesticides/PCBs and Dioxins/Furans

Endrin

Endrin ketone

Heptachlor

Heptachlor epoxide

Lindane

Methoxychlor

PCB 1016

PCB 1221

PCB 1232

PCB 1242

PCB 1248

PCB 1254

PCB 1260

Toxaphene

\section{Radionuclides}

Actinium-228

Antimony-124

Antimony-125

Barium-133

Cerium-144

Cesium-134

Cesium-137

Cobalt-57

Cobalt-58

Cobalt-60

Europium-152

Europium-154

Europium-155

Gross alpha

Lead-212

Manganese-54

Neptunium-239

Nonvolatile beta

Plutonium-238

Plutonium-239/240

Potassium-40

Promethium-144

Promethium-146

Ruthenium-106

Sodium-22

Strontium-90

Thorium-234

Tin-113

Yttrium-88

Zinc-65

Zirconium-95
Sample ID: 104672

RQ AQ B Result

Unit

D. Limit Method

$\begin{array}{ll}\mathrm{U} & 99.2 \\ \mathrm{U} & 198 \\ \mathrm{U} & 99.2 \\ \mathrm{U} & 99.2 \\ \mathrm{U} & 49.6 \\ \mathrm{U} & 992 \\ \mathrm{U} & 248 \\ \mathrm{U} & 248 \\ \mathrm{U} & 248 \\ \mathrm{U} & 248 \\ \mathrm{U} & 248 \\ \mathrm{U} & 248 \\ \mathrm{U} & 248 \\ \mathrm{U} & 1980\end{array}$

$\mu \mathrm{g} / \mathrm{kg}$

$$
\mu g / k g
$$

$\mu \mathrm{g} / \mathrm{kg}$

$\mu \mathrm{g} / \mathrm{kg}$

$\mu \mathrm{g} / \mathrm{kg}$

$\mu \mathrm{g} / \mathrm{kg}$

$\mu \mathrm{g} / \mathrm{kg}$

$\mu \mathrm{g} / \mathrm{kg}$

$\mu \mathrm{g} / \mathrm{kg}$

$\mu \mathrm{g} / \mathrm{kg}$

$\mu \mathrm{g} / \mathrm{kg}$

$\mu \mathrm{g} / \mathrm{kg}$

$\mu \mathrm{g} / \mathrm{kg}$

$\mu \mathrm{g} / \mathrm{kg}$

2.69E-01 2.73E-02 pCi/g

$-1.76 \mathrm{E}-03 \pm 4.19 \mathrm{E}-03 \mathrm{pCi} / \mathrm{g}$

$-3.96 \mathrm{E}-03 \pm 1.01 \mathrm{E}-02 \mathrm{pCi} / \mathrm{g}$

$-4.60 \mathrm{E}-05 \pm 4.83 \mathrm{E}-03 \mathrm{pCi} / \mathrm{g}$

$1.29 \mathrm{E}-02 \pm 2.35 \mathrm{E}-02 \mathrm{pCi} / \mathrm{g}$

$6.85 \mathrm{E}-04 \pm 3.50 \mathrm{E}-03 \mathrm{pCi} / \mathrm{g}$

7.96E-01 1.81E-02 pCi/g

2.75E-03 $\pm 3.00 \mathrm{E}-03 \mathrm{pCi} / \mathrm{g}$

$-1.03 \mathrm{E}-03 \pm 3.48 \mathrm{E}-03 \mathrm{pCi} / \mathrm{g}$

8.86E-02 $\pm 7.45 \mathrm{E}-03 \mathrm{pCi} / \mathrm{g}$

$-1.05 \mathrm{E}-03 \pm 9.64 \mathrm{E}-03 \mathrm{pCi} / \mathrm{g}$

$-4.66 \mathrm{E}-04 \pm 2.81 \mathrm{E}-02 \mathrm{pCi} / \mathrm{g}$

4.44E-03 1.25E-02 pCi/g

$1.35 \mathrm{E}+01 \pm 2.83 \mathrm{E}+00 \mathrm{pCi} / \mathrm{g}$

2.78E-01 $1.31 \mathrm{E}-02 \mathrm{pCi} g$

$6.43 \mathrm{E}-03 \pm 5.85 \mathrm{E}-03 \mathrm{pCi} / \mathrm{g}$

UI $\quad 7.24 \mathrm{E}-03 \pm 2.16 \mathrm{E}-02 \mathrm{pCi} / \mathrm{g}$

$1.03 \mathrm{E}+01 \pm 2.01 \mathrm{E}+00 \mathrm{pCi} / \mathrm{g}$

UI $\quad 1.45 \mathrm{E}-02 \pm 1.98 \mathrm{E}-02 \mathrm{pCi} / \mathrm{g}$

UI $1.06 \mathrm{E}-02 \pm 1.41 \mathrm{E}-02 \mathrm{pCi} / \mathrm{g}$

$\mathrm{V} \quad 7.11 \mathrm{E}-01 \pm 7.48 \mathrm{E}-02 \mathrm{pCi} / \mathrm{g}$

UI $\quad-2.04 \mathrm{E}-03 \pm 2.93 \mathrm{E}-03 \mathrm{pCi} / \mathrm{g}$

UI $\quad 1.53 \mathrm{E}-03 \pm 4.90 \mathrm{E}-03 \mathrm{pCi} / \mathrm{g}$

UI $\quad 6.35 \mathrm{E}-03 \pm 3.01 \mathrm{E}-02 \mathrm{pCi} / \mathrm{g}$

UI 2.27E-03 $24.07 \mathrm{E}-03 \mathrm{pCi} / \mathrm{g}$

U V 1.59E+00 $1.47 \mathrm{E}-01 \mathrm{pCi} / \mathrm{g}$

$6.46 \mathrm{E}-01 \pm 2.67 \mathrm{E}-01 \mathrm{pCi} / \mathrm{g}$

$-4.45 \mathrm{E}-03 \pm 5.05 \mathrm{E}-03 \mathrm{pCi} / \mathrm{g}$

$-7.63 \mathrm{E}-04 \pm 2.48 \mathrm{E}-03 \mathrm{pCi} / \mathrm{g}$ $1.01 \mathrm{E}-02 \pm 6.79 \mathrm{E}-03 \mathrm{pCi} / \mathrm{g}$ $-4.35 \mathrm{E}-03 \pm 6.64 \mathrm{E}-03 \mathrm{pCj} / \mathrm{g}$
1.64E-02 EPLA-013B

6.96E-03 EPIA-013B

1.71E-02 EPIA-013B

7.35E-03 EPIA-013B

3.95E-02 EPIA-013B

5.21E-03 EPIA-013B

5.50E-03 EPIA-013B

5.11E-03 EPIA-013B

5.74E-03 EPIA-013B

4.33E-03 EPIA-013B

1.66E-02 EPIA-013B

4.30E-02 EPIA-013B

2.12E-02 EPIA-013B

1.34E+00 EPIA-001B

9.47E-03 EPIA-013B

4.57E-03 EPLA-013B

3.64E-02 EPIA-013B

2.76E+00 EPIA-001B

3.72E-02 EPIA-012B

2.56E-02 EPIA-012B

4.38E-02 EPIA-013B

4.76E-03 EPIA-013B

8.43E-03 EPIA-013B

5.13E-02 EPIA-013B

3.95E-03 EPIA-013B

7.55E-02 EPIA-004

2.51E-01 EPIA-013B

8.46E-03 EPIA-013B

4.44E-03 EPIA-013B

1.16E-02 EPIA-013B

1.08E-02 EPIA-013B

SURVEY ID: PPSC 1903

Sample ID: 104673

Survey location: $44200 \mathrm{E} 3673595 \mathrm{~N}$ (SRS coordinates)

Depth of core interval: 2.00 to $4.00 \mathrm{ft}$

Sample type: Normal

Sample matrix: Soil

Percent solids: 81.00 
SURVEY ID: PPSC 1903 (continued)

Constituent
Sample ID: 104673

RQ AQ B Result Unit D. Limit Method

Physical Parameters and Specified Analyses

Ammonia nitrogen
Ammonia nitrogen
Cation exchange capacity
Chloride
Cyanide
Nitrate-nitrite as nitrogen
Nitrogen by Kjeldahl method
Orthophosphate
Phenols
Sulfide
Total organic carbon
Total organic nitrogen
Total phosphates (as P)

Metals (total recoverable)

$\begin{array}{lll}\text { U } & & 24.7 \\ \text { U } & & 22.1 \\ & & 22.7 \\ J & \text { QE } & 1.97 \\ \text { UJ } & \text { C } & 1.23 \\ \text { J } & \text { E } & 0.359 \\ J & \text { E } & 4.13 \\ \text { U } & & 12.3 \\ & & 456 \\ \text { UJ } & \text { C } & 309 \\ & & 535 \\ \text { U } & & 0.247 \\ \text { J } & \text { E } & 135\end{array}$

$\begin{array}{lll}\mathrm{mg} / \mathrm{kg} & 24.7 & \text { EPA350.1 } \\ \mathrm{mg} / \mathrm{kg} & 22.1 & \text { EPA350.1 } \\ \mathrm{meq} / 100 \mathrm{~g} & 5 & \text { EPA9081 } \\ \mathrm{mg} / \mathrm{kg} & 2.98 & \text { EPA300.0 } \\ \mathrm{mg} / \mathrm{kg} & 1.23 & \text { EPA335.3 } \\ \mathrm{mg} / \mathrm{kg} & 1.2 & \text { EPA353.1 } \\ \mathrm{mg} / \mathrm{kg} & 4.86 & \text { EPA351.2 } \\ \mathrm{mg} / \mathrm{kg} & 12.3 & \text { EPA365.2 } \\ \mu \mathrm{g} / \mathrm{kg} & 370 & \text { EPA420.2 } \\ \mathrm{mg} / \mathrm{kg} & 309 & \text { EPA376.2 } \\ \mathrm{mg} / \mathrm{kg} & 123 & \text { EPA415.1 } \\ \mathrm{mg} / \mathrm{kg} & 0.247 & \text { EPA351.2 } \\ \mathrm{mg} / \mathrm{kg} & 449 & \text { EPA365.4 }\end{array}$

$\begin{array}{lll}\mathrm{mg} / \mathrm{kg} & 15.1 & \text { EPA6010A } \\ \mathrm{mg} / \mathrm{kg} & 6.05 & \text { EPA6010A } \\ \mathrm{mg} / \mathrm{kg} & 18.2 & \text { EPA6010A } \\ \mathrm{mg} / \mathrm{kg} & 1.51 & \text { EPA6010A } \\ \mathrm{mg} / \mathrm{kg} & 0.605 & \text { EPA6010A } \\ \mathrm{mg} / \mathrm{kg} & 0.303 & \text { EPA6010A } \\ \mathrm{mg} / \mathrm{kg} & 15.1 & \text { EPA6010A } \\ \mathrm{mg} / \mathrm{kg} & 1.51 & \text { EPA6010A } \\ \mathrm{mg} / \mathrm{kg} & 0.605 & \text { EPA6010A } \\ \mathrm{mg} / \mathrm{kg} & 1.51 & \text { EPA6010A } \\ \mathrm{mg} / \mathrm{kg} & 6.05 & \text { EPA6010A } \\ \mathrm{mg} / \mathrm{kg} & 6.05 & \text { EPA6010A } \\ \mathrm{mg} / \mathrm{kg} & 6.05 & \text { EPA6010A } \\ \mathrm{mg} / \mathrm{kg} & 0.605 & \text { EPA6010A } \\ \mathrm{mg} / \mathrm{kg} & 0.0395 & \text { EPA7471 } \\ \mathrm{mg} / \mathrm{kg} & 1.51 & \text { EPA6010A } \\ \mathrm{mg} / \mathrm{kg} & 121 & \text { EPA6010A } \\ \mathrm{mg} / \mathrm{kg} & 18.2 & \text { EPA6010A } \\ \mathrm{mg} / \mathrm{kg} & 1.51 & \text { EPA6010A } \\ \mathrm{mg} / \mathrm{kg} & 60.5 & \text { EPA6010A } \\ \mathrm{mg} / \mathrm{kg} & 18.2 & \text { EPA6010A } \\ \mathrm{mg} / \mathrm{kg} & 0.605 & \text { EPA6010A } \\ \mathrm{mg} / \mathrm{kg} & 1.21 & \text { EPA6010A }\end{array}$

$B / N / A$ Extractables (including $P A H$ and phenols)

$\begin{array}{lll}\text { Acenaphthene } & \mathrm{U} & 40 \\ \text { Acenaphthylene } & \mathrm{U} & 40 \\ \text { Anthracene } & \mathrm{U} & 40 \\ \text { Benzo[a]anthracene } & \mathrm{U} & 4 \\ \text { Benzo[b]fluoranthene } & \mathrm{U} & 4 \\ \text { Benzo[k]fluoranthene } & \mathrm{U} & 4 \\ \text { Benzoic acid } & \mathrm{U} & 40 \\ \text { Benzo[g,h,i]perylene } & \mathrm{U} & 40 \\ \text { Benzo[a]pyrene } & \mathrm{U} & 4 \\ \text { Benzyl alcohol } & \mathrm{U} & 40 \\ \text { Bis(2-chloroethoxy) methane } & \mathrm{U} & 40 \\ \text { Bis(2-chloroethyl) ether } & \mathrm{U} & 40\end{array}$

$\begin{array}{lll}\mu \mathrm{g} / \mathrm{kg} & 40 & \text { EPA8270 } \\ \mu \mathrm{g} / \mathrm{kg} & 40 & \text { EPA8270 } \\ \mu \mathrm{g} / \mathrm{kg} & 40 & \text { EPA8270 } \\ \mu \mathrm{g} / \mathrm{kg} & 4 & \text { EPA8270 } \\ \mu \mathrm{g} / \mathrm{kg} & 4 & \text { EPA8270 } \\ \mu \mathrm{g} / \mathrm{kg} & 4 & \text { EPA8270 } \\ \mu \mathrm{g} / \mathrm{kg} & 40 & \text { EPA8270 } \\ \mu \mathrm{g} / \mathrm{kg} & 40 & \text { EPA8270 } \\ \mu \mathrm{g} / \mathrm{kg} & 4 & \text { EPA8270 } \\ \mu \mathrm{g} / \mathrm{kg} & 40 & \text { EPA8270 } \\ \mu \mathrm{g} / \mathrm{kg} & 40 & \text { EPA8270 } \\ \mu \mathrm{g} / \mathrm{kg} & 40 & \text { EPA8270 }\end{array}$


Constituent

RQ AQ B Result
Unit

D. Limit Method

$B / N / A$ Extractables (including $P A H$ and phenols)

Bis(2-chloroisopropyl) ether

Bis(2-ethylhexyl) phthalate

4-Bromophenyl phenyl ether

Butylbenzyl phthalate

4-Chloroaniline

4-Chloro-m-cresol

2-Chloronaphthalene

2-Chlorophenol

4-Chlorophenyl phenyl ether

Chrysene

$\mathrm{m} / \mathrm{p}$-Cresol

o-Cresol (2-Methylphenol)

Dibenz $[a, h]$ anthracene

Dibenzofuran

Di-n-butyl phthalate

1,2-Dichlorobenzene

1,3-Dichlorobenzene

1,4-Dichlorobenzene

3,3'-Dichlorobenzidine

2,4-Dichlorophenol

Diethyl phthalate

2,4-Dimethyl phenol

Dimethyl phthalate

2,4-Dinitrophenol

2,4-Dinitrotoluene

2,6-Dinitrotoluene

Di-n-octyl phthalate

Fluoranthene

Fluorene

Hexachlorobenzene

Hexachlorobutadiene

Hexachlorocyclopentadiene

Hexachloroethane

Indeno $[1,2,3-c, d]$ pyrene

Isophorone

2-Methyl-4,6-dinitrophenol

2-Methylnaphthalene

Naphthalene

m-Nitroaniline

o-Nitroaniline

p-Nitroaniline

Nitrobenzene

2-Nitrophenol

4-Nitrophenol

N-Nitrosodiphenylamine

N-Nitrosodi-n-propylamine

Pentachlorophenol

Phenanthrene

Phenol

Pyrene

1,2,4-Trichlorobenzene

2,4,5-Trichlorophenol

2,4,6-Trichlorophenol

\begin{tabular}{|c|c|c|}
\hline$U$ & & \\
\hline $\mathrm{J}$ & $\mathbf{E}$ & 29.2 \\
\hline$U$ & & 40 \\
\hline$U$ & & 40 \\
\hline$U$ & & 40 \\
\hline U & & 40 \\
\hline U & & 40 \\
\hline $\mathrm{U}$ & & 40 \\
\hline $\mathrm{U}$ & & 40 \\
\hline $\mathrm{U}$ & & 4 \\
\hline $\mathrm{U}$ & & 400 \\
\hline $\mathrm{U}$ & & 40 \\
\hline $\mathrm{U}$ & & 4 \\
\hline $\mathrm{U}$ & & 40 \\
\hline $\mathrm{J}$ & $E$ & 38.4 \\
\hline $\mathrm{U}$ & & 40 \\
\hline $\mathrm{U}$ & & 40 \\
\hline$U$ & & 40 \\
\hline U & & 40 \\
\hline $\mathrm{U}$ & & 40 \\
\hline$U$ & & 40 \\
\hline $\mathrm{U}$ & & 40 \\
\hline$U$ & & 40 \\
\hline $\mathrm{U}$ & & 400 \\
\hline UJ & C & 4 \\
\hline $\mathrm{U}$ & & 4 \\
\hline $\mathrm{U}$ & & 40 \\
\hline $\mathrm{U}$ & & 40 \\
\hline$U$ & & 40 \\
\hline $\mathrm{U}$ & & 4 \\
\hline$U$ & & 40 \\
\hline $\mathrm{U}$ & & 40 \\
\hline $\mathrm{U}$ & & 4 \\
\hline $\mathrm{U}$ & & 4 \\
\hline $\mathrm{U}$ & & 40 \\
\hline$U$ & & 400 \\
\hline $\mathrm{U}$ & & 40 \\
\hline U & & 40 \\
\hline U & & 40 \\
\hline $\mathrm{U}$ & & 40 \\
\hline U & & 40 \\
\hline $\mathrm{U}$ & & 40 \\
\hline $\mathrm{U}$ & & 40 \\
\hline $\mathrm{U}$ & & 40 \\
\hline $\mathrm{U}$ & & 40 \\
\hline $\mathrm{U}$ & & 40 \\
\hline UJ & C & 40 \\
\hline $\mathrm{U}$ & & 40 \\
\hline$U$ & & 40 \\
\hline U & & 40 \\
\hline $\mathrm{U}$ & & 4 \\
\hline & & 40 \\
\hline $\mathbf{U}$ & & 40 \\
\hline
\end{tabular}

\begin{tabular}{|c|c|c|}
\hline$\mu \mathrm{g} / \mathrm{kg}$ & 40 & EPA8270 \\
\hline$\mu \mathrm{g} / \mathrm{kg}$ & 40 & EPA8270 \\
\hline$\mu \mathrm{g} / \mathrm{kg}$ & 40 & EPA8270 \\
\hline$\mu \mathrm{g} / \mathrm{kg}$ & 40 & EPA8270 \\
\hline$\mu \mathrm{g} / \mathrm{kg}$ & 40 & EPA8270 \\
\hline$\mu \mathrm{g} / \mathrm{kg}$ & 40 & EPA8270 \\
\hline$\mu \mathrm{g} / \mathrm{kg}$ & 40 & EPA8270 \\
\hline$\mu \mathrm{g} / \mathrm{kg}$ & 40 & EPA8270 \\
\hline$\mu \mathrm{g} / \mathrm{kg}$ & 40 & EPA8270 \\
\hline$\mu \mathrm{g} / \mathrm{kg}$ & 4 & EPA8270 \\
\hline$\mu \mathrm{g} / \mathrm{kg}$ & 400 & EPA8270 \\
\hline$\mu \mathrm{g} / \mathrm{kg}$ & 40 & EPA8270 \\
\hline$\mu \mathrm{g} / \mathrm{kg}$ & 4 & EPA8270 \\
\hline$\mu \mathrm{g} / \mathrm{kg}$ & 40 & EPA8270 \\
\hline$\mu \mathrm{g} / \mathrm{kg}$ & 40 & EPA8270 \\
\hline$\mu \mathrm{g} / \mathrm{kg}$ & 40 & EPA8270 \\
\hline$\mu \mathrm{g} / \mathrm{kg}$ & 40 & EPA8270 \\
\hline$\mu \mathrm{g} / \mathrm{kg}$ & 40 & EPA8270 \\
\hline$\mu \mathrm{g} / \mathrm{kg}$ & 40 & EPA8270 \\
\hline$\mu \mathrm{g} / \mathrm{kg}$ & 40 & EPA8270 \\
\hline$\mu \mathrm{g} / \mathrm{kg}$ & 40 & EPA8270 \\
\hline$\mu \mathrm{g} / \mathrm{kg}$ & 40 & EPA8270 \\
\hline$\mu \mathrm{g} / \mathrm{kg}$ & 40 & EPA8270 \\
\hline$\mu \mathrm{g} / \mathrm{kg}$ & 400 & EPA8270 \\
\hline$\mu \mathrm{g} / \mathrm{kg}$ & 4 & EPA8270 \\
\hline$\mu \mathrm{g} / \mathrm{kg}$ & 4 & EPA8270 \\
\hline$\mu \mathrm{g} / \mathrm{kg}$ & 40 & EPA8270 \\
\hline$\mu \mathrm{g} / \mathrm{kg}$ & 40 & EPA8270 \\
\hline$\mu \mathrm{g} / \mathrm{kg}$ & 40 & EPA8270 \\
\hline$\mu \mathrm{g} / \mathrm{kg}$ & 4 & EPA8270 \\
\hline$\mu \mathrm{g} / \mathrm{kg}$ & 40 & EPA8270 \\
\hline$\mu \mathrm{g} / \mathrm{kg}$ & 40 & EPA8270 \\
\hline$\mu \mathrm{g} / \mathrm{kg}$ & 4 & EPA8270 \\
\hline$\mu \mathrm{g} / \mathrm{kg}$ & 4 & EPA8270 \\
\hline$\mu \mathrm{g} / \mathrm{kg}$ & 40 & EPA8270 \\
\hline$\mu \mathrm{g} / \mathrm{kg}$ & 400 & EPA8270 \\
\hline$\mu \mathrm{g} / \mathrm{kg}$ & 40 & EPA8270 \\
\hline$\mu \mathrm{g} / \mathrm{kg}$ & 40 & EPA8270 \\
\hline$\mu \mathrm{g} / \mathrm{kg}$ & 40 & EPA8270 \\
\hline$\mu \mathrm{g} / \mathrm{kg}$ & 40 & EPA8270 \\
\hline$\mu \mathrm{g} / \mathrm{kg}$ & 40 & EPA8270 \\
\hline$\mu \mathrm{g} / \mathrm{kg}$ & 40 & EPA8270 \\
\hline$\mu \mathrm{g} / \mathrm{kg}$ & 40 & EPA8270 \\
\hline$\mu \mathrm{g} / \mathrm{kg}$ & 40 & EPA8270 \\
\hline$\mu \mathrm{g} / \mathrm{kg}$ & 40 & EPA8270 \\
\hline$\mu \mathrm{g} / \mathrm{kg}$ & 40 & EPA8270 \\
\hline$\mu \mathrm{g} / \mathrm{kg}$ & 40 & EPA8270 \\
\hline$\mu \mathrm{g} / \mathrm{kg}$ & 40 & EPA8270 \\
\hline$\mu \mathrm{g} / \mathrm{kg}$ & 40 & EPA8270 \\
\hline$\mu \mathrm{g} / \mathrm{kg}$ & 40 & EPA8270 \\
\hline$\mu \mathrm{g} / \mathrm{kg}$ & 4 & EPA8270 \\
\hline$\mu \mathrm{g} / \mathrm{kg}$ & 40 & EPA8270 \\
\hline$\mu \mathrm{g} / \mathrm{kg}$ & 40 & EPA8270 \\
\hline
\end{tabular}


SURVEY ID: PPSC 1903 (continued)

Constituent

Volatile Organic Compounds

Acetone

Benzene

Bromodichloromethane

Bromoform

Bromomethane (Methyl bromide)

Carbon disulfide

Carbon tetrachloride

Chlorobenzene

Chloroethane

Chloroethene (Vinyl chloride)

Chloroform

Chloromethane (Methyl chloride)

Dibromochloromethane

1,1-Dichloroethane

1,2-Dichloroethane

1,1-Dichloroethylene

1,2-Dichloroethylene

Dichloromethane (Methylene chloride)

1,2-Dichloropropane

cis-1,3-Dichloropropene

trans-1,3-Dichloropropene

Ethylbenzene

2-Hexanone

Methyl ethyl ketone

Methyl isobutyl ketone

Styrene

1,1,2,2-Tetrachloroethane

Tetrachloroethylene

Toluene

1,1,1-Trichloroethane

1,1,2-Trichloroethane

Trichloroethylene

Vinyl acetate

Xylenes

Pesticides/PCBs and Dioxins/Furans

Aldrin

alpha-Benzene hexachloride

beta-Benzene hexachloride

delta-Benzene hexachloride

alpha-Chlordane

gamma-Chlordane

p,p'-DDD

$p, p^{\prime}-D D E$

p,p'-DDT

Dieldrin

Endosulfan sulfate

Endosulfan I

Endosulfan II

Endrin

Endrin ketone

Heptachlor

Heptachlor epoxide

Lindane
Sample ID: 104673

RQ AQ B Result Unit $\quad$ D. Limit Method

$\begin{array}{lll}\mu \mathrm{g} / \mathrm{kg} & 6.17 & \text { EPA8260 } \\ \mu \mathrm{g} / \mathrm{kg} & 0.617 & \text { EPA8260 } \\ \mu \mathrm{g} / \mathrm{kg} & 0.0617 & \text { EPA8260 } \\ \mu \mathrm{g} / \mathrm{kg} & 0.0617 & \text { EPA8260 } \\ \mu \mathrm{g} / \mathrm{kg} & 0.123 & \text { EPA8260 } \\ \mu \mathrm{g} / \mathrm{kg} & 1.23 & \text { EPA8260 } \\ \mu \mathrm{g} / \mathrm{kg} & 0.0617 & \text { EPA8260 } \\ \mu \mathrm{g} / \mathrm{kg} & 0.0617 & \text { EPA8260 } \\ \mu \mathrm{g} / \mathrm{kg} & 0.123 & \text { EPA8260 } \\ \mu \mathrm{g} / \mathrm{kg} & 0.123 & \text { EPA8260 } \\ \mu \mathrm{g} / \mathrm{kg} & 0.0617 & \text { EPA8260 } \\ \mu \mathrm{g} / \mathrm{kg} & 0.123 & \text { EPA8260 } \\ \mu \mathrm{g} / \mathrm{kg} & 0.0617 & \text { EPA8260 } \\ \mu \mathrm{g} / \mathrm{kg} & 0.0617 & \text { EPA8260 } \\ \mu \mathrm{g} / \mathrm{kg} & 0.0617 & \text { EPA8260 } \\ \mu \mathrm{g} / \mathrm{kg} & 0.0617 & \text { EPA8260 } \\ \mu \mathrm{g} / \mathrm{kg} & 0.123 & \text { EPA8260 } \\ \mu \mathrm{g} / \mathrm{kg} & 0.617 & \text { EPA8260 } \\ \mu \mathrm{g} / \mathrm{kg} & 0.0617 & \text { EPA8260 } \\ \mu \mathrm{g} / \mathrm{kg} & 0.0617 & \text { EPA8260 } \\ \mu \mathrm{g} / \mathrm{kg} & 0.0617 & \text { EPA8260 } \\ \mu \mathrm{g} / \mathrm{kg} & 0.0617 & \text { EPA8260 } \\ \mu \mathrm{g} / \mathrm{kg} & 1.23 & \text { EPA8260 } \\ \mu \mathrm{g} / \mathrm{kg} & 1.23 & \text { EPA8260 } \\ \mu \mathrm{g} / \mathrm{kg} & 1.23 & \text { EPA8260 } \\ \mu \mathrm{g} / \mathrm{kg} & 0.0617 & \text { EPA8260 } \\ \mu \mathrm{g} / \mathrm{kg} & 0.0617 & \text { EPA8260 } \\ \mu \mathrm{g} / \mathrm{kg} & 0.0617 & \text { EPA8260 } \\ \mu \mathrm{g} / \mathrm{kg} & 0.617 & \text { EPA8260 } \\ \mu \mathrm{g} / \mathrm{kg} & 0.0617 & \text { EPA8260 } \\ \mu \mathrm{g} / \mathrm{kg} & 0.0617 & \text { EPA8260 } \\ \mu \mathrm{g} / \mathrm{kg} & 0.0617 & \text { EPA8260 } \\ \mu \mathrm{g} / \mathrm{kg} & 1.23 & \text { EPA8260 } \\ \mu \mathrm{g} / \mathrm{kg} & 0.185 & \text { EPA8260 } \\ & & \end{array}$

$\mu \mathrm{g} / \mathrm{kg}$

$\mu \mathrm{g} / \mathrm{kg}$

$\mu \mathrm{g} / \mathrm{kg}$

$\mu \mathrm{g} / \mathrm{kg}$

$\mu \mathrm{g} / \mathrm{kg}$

$\mu \mathrm{g} / \mathrm{kg}$

$\mu \mathrm{g} / \mathrm{kg}$

$\mu \mathrm{g} / \mathrm{kg}$

$\mu \mathrm{g} / \mathrm{kg}$

$\mu \mathrm{g} / \mathrm{kg}$

$\mu \mathrm{g} / \mathrm{kg}$

$\mu \mathrm{g} / \mathrm{kg}$

$\mu \mathrm{g} / \mathrm{kg}$

$\mu \mathrm{g} / \mathrm{kg}$

$\mu \mathrm{g} / \mathrm{kg}$

$\mu \mathrm{g} / \mathrm{kg}$

$\mu \mathrm{g} / \mathrm{kg}$

$\mu \mathrm{g} / \mathrm{kg}$
51.2

51.2

102

102

102

102

205

102

205

102

205

102

205

102

205

102

102

51.2
EPA8080

EPA8080

EPA8080

EPA8080

EPA8080

EPA8080

EPA8080

EPA8080

EPA8080

EPA8080

EPA8080

EPA8080

EPA8080

EPA8080

EPA8080

EPA8080

EPA8080

EPA8080 
SURVEY ID: PPSC 1903 (continued)

Constituent

Pesticides/PCBs and Dioxins/Furans

Methoxychlor

PCB 1016

PCB 1221

PCB 1232

PCB 1242

PCB 1248

PCB 1254

PCB 1260

Toxaphene

\section{Radionuclides}

Actinium-228

Antimony-124

Antimony-125

Barium-133

Cerium-144

Cesium-134

Cesium-137

Cobalt-57

Cobalt-58

Cobalt-60

Europium-152

Europium-154

Europium-155

Gross alpha

Lead-212

Manganese-54

Neptunium-239

Nonvolatile beta

Plutonium-238

Plutonium-239/240

Potassium-40

Promethium-144

Promethium-146

Ruthenium-106

Sodium-22

Strontium-90

Thorium-234

Tin-113

Yttrium-88

Zinc-65

Zirconium-95
Sample ID: 104673

RQ AQ B Result

Unit

D. Limit Method

$\begin{array}{lll}\mu \mathrm{g} / \mathrm{kg} & 1020 \cdot & \text { EPA8080 } \\ \mu \mathrm{g} / \mathrm{kg} & 256 & \text { EPA8080 } \\ \mu \mathrm{g} / \mathrm{kg} & 256 & \text { EPA8080 } \\ \mu \mathrm{g} / \mathrm{kg} & 256 & \text { EPA8080 } \\ \mu \mathrm{g} / \mathrm{kg} & 256 & \text { EPA8080 } \\ \mu \mathrm{g} / \mathrm{kg} & 256 & \text { EPA8080 } \\ \mu \mathrm{g} / \mathrm{kg} & 256 & \text { EPA8080 } \\ \mu \mathrm{g} / \mathrm{kg} & 256 & \text { EPA8080 } \\ \mu \mathrm{g} / \mathrm{kg} & 2050 & \text { EPA8080 }\end{array}$

1.66E-02 EPIA-013B 9.28E-03 EPIA-013B

1.41E-02 EPIA-013B

6.05E-03 EPIA-013B

3.79E-02 EPIA-013B

4.73E-03 EPIA-013B

5.60E-03 EPIA-013B

4.99E-03 EPIA-013B

7.81E-03 EPIA-013B

4.33E-03 EPIA-013B

1.43E-02 EPIA-013B

4.78E-02 EPIA-013B

1.94E-02 EPIA-013B

1.61E+00 EPIA-001B

9.60E-03 EPIA-013B

5.59E-03 EPIA-013B

3.31E-02 EPIA-013B

2.24E+00 EPIA-001B

3.67E-02 EPIA-012B

2.09E-02 EPIA-012B

4.50E-02 EPIA-013B

5.07E-03 EPIA-013B

6.86E-03 EPIA-013B

4.98E-02 EPIA-013B

4.80E-03 EPIA-013B

8.52E-02 EPIA-004

2.53E-01 EPIA-013B

8.50E-03 EPIA-013B

7.09E-03 EPIA-013B

1.19E-02 EPIA-013B

1.74E-02 EPIA-013B

SURVEY ID: PPSC 1904

Sample ID: 104674

Survey location: $44200 \mathrm{E} 3673595 \mathrm{~N}$ (SRS coordinates)

Depth of core interval: 4.00 to $6.00 \mathrm{ft}$

Sample type: Normal

Sample matrix: Soil

Percent solids: $\mathbf{7 9 . 0 0}$ 
SURVEY ID: PPSC 1904 (continued)

Sample ID: 104674

Constituent RQ AQ B Result Unit D. Limit Method

Physical Parameters and Specified Analyses

Ammonia nitrogen

Cation exchange capacity

Chloride

Cyanide

Nitrate-nitrite as nitrogen

Nitrate-nitrite as nitrogen

Nitrogen by Kjeldahl method

Orthophosphate

Phenols

Phenols

Sulfide

Total organic carbon

Total organic nitrogen

Total phosphates (as P)

Metals (total recoverable)

Aluminum

Antimony

Arsenic

Barium

Beryllium

Cadmium

Calcium

Chromium

Cobalt

Copper

Iron

Lead

Magnesium

Manganese

Mercury

Nickel

Potassium

Selenium

Silver

Sodium

Thallium

Vanadium

Zinc

$\begin{array}{lll}\text { U } & & 22.6 \\ J & Q E & 28 \\ \text { UJ } & \text { C } & 1.29 \\ J & \text { E } & 1.27 \\ J & \text { E } & 0.45 \\ & & 8.551 \\ \text { U } & & 12.7 \\ J & \text { E } & 114 \\ J & E & 123 \\ \text { UJ } & \text { C } & 316 \\ J & E & 56.5 \\ \text { C } & & 6.25 \\ J & E & 103\end{array}$

900

5.86

17.6

28.6

J $\quad \mathrm{E} \quad 0.165$

U 0.293

17.5

2.03

2.6

0.969

2500

3.23

99.9

23.7

0.00872

0.925

102

17.6

1.47

58.6

17.6

7.26

2.01

$\begin{array}{lll}\mathrm{mg} / \mathrm{kg} & 22.6 & \text { EPA350.1 } \\ \mathrm{meq} / 100 \mathrm{~g} & 5 & \text { EPA9081 } \\ \mathrm{mg} / \mathrm{kg} & 2.9 & \text { EPA300.0 } \\ \mathrm{mg} / \mathrm{kg} & 1.27 & \text { EPA335.3 } \\ \mathrm{mg} / \mathrm{kg} & 1.13 & \text { EPA353.1 } \\ \mathrm{mg} / \mathrm{kg} & 1.1 & \text { EPA353.1 } \\ \mathrm{mg} / \mathrm{kg} & 5.01 & \text { EPA351.2 } \\ \mathrm{mg} / \mathrm{kg} & 12.7 & \text { EPA365.2 } \\ \mu \mathrm{g} / \mathrm{kg} & 380 & \text { EPA420.2 } \\ \mu \mathrm{gg} / \mathrm{kg} & 379 & \text { EPA420.2 } \\ \mathrm{mg} / \mathrm{kg} & 316 & \text { EPA376.2 } \\ \mathrm{mg} / \mathrm{kg} & 127 & \text { EPA415.1 } \\ \mathrm{mg} / \mathrm{kg} & 0.253 & \text { EPA351.2 } \\ \mathrm{mg} / \mathrm{kg} & 443 & \text { EPA365.4 }\end{array}$

$\begin{array}{lll}\mathrm{mg} / \mathrm{kg} & 14.7 & \text { EPA6010A } \\ \mathrm{mg} / \mathrm{kg} & 5.86 & \text { EPA6010A } \\ \mathrm{mg} / \mathrm{kg} & 17.6 & \text { EPA6010A } \\ \mathrm{mg} / \mathrm{kg} & 1.47 & \text { EPA6010A } \\ \mathrm{mg} / \mathrm{kg} & 0.586 & \text { EPA6010A } \\ \mathrm{mg} / \mathrm{kg} & 0.293 & \text { EPA6010A } \\ \mathrm{mg} / \mathrm{kg} & 14.7 & \text { EPA6010A } \\ \mathrm{mg} / \mathrm{kg} & 1.47 & \text { EPA6010A } \\ \mathrm{mg} / \mathrm{kg} & 0.586 & \text { EPA6010A } \\ \mathrm{mg} / \mathrm{kg} & 1.47 & \text { EPA6010A } \\ \mathrm{mg} / \mathrm{kg} & 5.86 & \text { EPA6010A } \\ \mathrm{mg} / \mathrm{kg} & 5.86 & \text { EPA6010A } \\ \mathrm{mg} / \mathrm{kg} & 5.86 & \text { EPA6010A } \\ \mathrm{mg} / \mathrm{kg} & 0.586 & \text { EPA6010A } \\ \mathrm{mg} / \mathrm{kg} & 0.0415 & \text { EPA7471 } \\ \mathrm{mg} / \mathrm{kg} & 1.47 & \text { EPA6010A } \\ \mathrm{mg} / \mathrm{kg} & 117 & \text { EPA6010A } \\ \mathrm{mg} / \mathrm{kg} & 17.6 & \text { EPA6010A } \\ \mathrm{mg} / \mathrm{kg} & 1.47 & \text { EPA6010A } \\ \mathrm{mg} / \mathrm{kg} & 58.6 & \text { EPA6010A } \\ \mathrm{mg} / \mathrm{kg} & 17.6 & \text { EPA6010A } \\ \mathrm{mg} / \mathrm{kg} & 0.586 & \text { EPA6010A } \\ \mathrm{mg} / \mathrm{kg} & 1.17 & \text { EPA6010A }\end{array}$

$B / N / A$ Extractables (including $P A H$ and phenols)

$\begin{array}{lcc}\text { Acenaphthene } & \mathrm{U} & 41.3 \\ \text { Acenaphthylene } & \mathrm{U} & 41.3 \\ \text { Anthracene } & \mathrm{U} & 41.3 \\ \text { Benzo[a]anthracene } & \mathrm{U} & 4.13 \\ \text { Benzo[b]fluoranthene } & \mathrm{U} & 4.13 \\ \text { Benzo[k]fluoranthene } & \mathrm{U} & 4.13 \\ \text { Benzoic acid } & \mathrm{U} & 41.3 \\ \text { Benzo[g,h,i]perylene } & \mathrm{U} & 41.3 \\ \text { Benzo[a]pyrene } & \mathrm{U} & 4.13 \\ \text { Benzyl alcohol } & \mathrm{U} & 41.3 \\ \text { Bis(2-chloroethoxy) methane } & \mathrm{U} & 41.3 \\ \text { Bis(2-chloroethyl) ether } & \mathrm{U} & 41.3\end{array}$

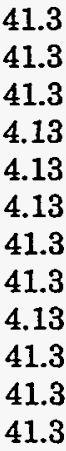

$\begin{array}{lll}\mu \mathrm{g} / \mathrm{kg} & 41.3 & \text { EPA8270 } \\ \mu \mathrm{g} / \mathrm{kg} & 41.3 & \text { EPA8270 } \\ \mu \mathrm{g} / \mathrm{kg} & 41.3 & \text { EPA8270 } \\ \mu \mathrm{g} / \mathrm{kg} & 4.13 & \text { EPA8270 } \\ \mu \mathrm{g} / \mathrm{kg} & 4.13 & \text { EPA8270 } \\ \mu \mathrm{g} / \mathrm{kg} & 4.13 & \text { EPA8270 } \\ \mu \mathrm{g} / \mathrm{kg} & 41.3 & \text { EPA8270 } \\ \mu \mathrm{g} / \mathrm{kg} & 41.3 & \text { EPA8270 } \\ \mu \mathrm{g} / \mathrm{kg} & -4.13 & \text { EPA8270 } \\ \mu \mathrm{g} / \mathrm{kg} & 41.3 & \text { EPA8270 } \\ \mu \mathrm{g} / \mathrm{kg} & 41.3 & \text { EPA8270 } \\ \mu \mathrm{g} / \mathrm{kg} & 41.3 & \text { EPA8270 }\end{array}$


SURVEY ID: PPSC 1904 (continued)

Sample ID: 104674

Constituent

RQ AQ B Result

Unit

D. Limit Method

$B / N / A$ Extractables (including PAH and phenols)

Bis(2-chloroisopropyl) ether

Bis(2-ethylhexyl) phthalate

4-Bromophenyl phenyl ether

Butylbenzyl phthalate

4-Chloroaniline

4-Chloro-m-cresol

2-Chloronaphthalene

2-Chlorophenol

4-Chlorophenyl phenyl ether

Chrysene

$\mathrm{m} / \mathrm{p}$-Cresol

o-Cresol (2-Methylphenol)

Dibenz $[a, h]$ anthracene

Dibenzofuran

Di-n-butyl phthalate

1,2-Dichlorobenzene

1,3-Dichlorobenzene

1,4-Dichlorobenzene

3,3'-Dichlorobenzidine

2,4-Dichlorophenol

Diethyl phthalate

2,4-Dimethyl phenol

Dimethyl phthalate

2,4-Dinitrophenol

2,4-Dinitrotoluene

2,6-Dinitrotoluene

Di-n-octyl phthalate

Fluoranthene

Fluorene

Hexachlorobenzene

Hexachlorobutadiene

Hexachlorocyclopentadiene

Hexachloroethane

Indeno[1,2,3-c,d]pyrene

Isophorone

2-Methyl-4,6-dinitrophenol

2-Methylnaphthalene

Naphthalene

m-Nitroaniline

0. Nitroaniline

p-Nitroaniline

Nitrobenzene

2-Nitrophenol

4-Nitrophenol

N-Nitrosodiphenylamine

N-Nitrosodi-n-propylamine

Pentachlorophenol

Phenanthrene

Phenol

Pyrene

1,2,4-Trichlorobenzene

2,4,5-Trichlorophenol

2,4,6-Trichlorophenol

\begin{tabular}{|c|c|c|}
\hline $\mathrm{U}$ & & 41.3 \\
\hline$U$ & & 41.3 \\
\hline $\mathrm{U}$ & & 41.3 \\
\hline $\mathrm{U}$ & & 41.3 \\
\hline U & & 41.3 \\
\hline $\mathrm{U}$ & & 41.3 \\
\hline $\mathrm{U}$ & & 41.3 \\
\hline $\mathrm{U}$ & & 41.3 \\
\hline$U$ & & 41.3 \\
\hline $\mathrm{U}$ & & 4.13 \\
\hline$U$ & & 413 \\
\hline U & & 41.3 \\
\hline $\mathrm{U}$ & & 4.13 \\
\hline $\mathrm{U}$ & & 41.3 \\
\hline$J$ & $\mathbf{E}$ & 32.6 \\
\hline U & & 41.3 \\
\hline U & & 41.3 \\
\hline U & & 41.3 \\
\hline U & & 41.3 \\
\hline $\mathrm{U}$ & & 41.3 \\
\hline U & & 41.3 \\
\hline $\mathrm{U}$ & & 41.3 \\
\hline U & & 41.3 \\
\hline $\mathrm{U}$ & & 413 \\
\hline UJ & C & 4.13 \\
\hline $\mathrm{U}$ & & 4.13 \\
\hline $\mathrm{U}$ & & 41.3 \\
\hline $\mathrm{U}$ & & 41.3 \\
\hline U & & 41.3 \\
\hline U & & 4.13 \\
\hline U & & 41.3 \\
\hline U & & 41.3 \\
\hline U & & 4.13 \\
\hline U & & 4.13 \\
\hline 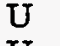 & & 41.3 \\
\hline U & & 413 \\
\hline U & & 41.3 \\
\hline$U$ & & 41.3 \\
\hline U & & 41.3 \\
\hline U & & 41.3 \\
\hline$U$ & & 41.3 \\
\hline U & & 41.3 \\
\hline U & & 41.3 \\
\hline U & & 41.3 \\
\hline U & & 41.3 \\
\hline U & & 41.3 \\
\hline UJ & C & 41.3 \\
\hline$U$ & & 41.3 \\
\hline U & & 41.3 \\
\hline U & & 41.3 \\
\hline U & & 4.13 \\
\hline U & & 41.3 \\
\hline $\mathrm{U}$ & & 41.3 \\
\hline
\end{tabular}

\begin{tabular}{|c|c|c|}
\hline$\mu \mathrm{g} / \mathrm{kg}$ & 41.3 & EPA8270 \\
\hline$\mu \mathrm{g} / \mathrm{kg}$ & 41.3 & EPA8270 \\
\hline$\mu \mathrm{g} / \mathrm{kg}$ & 41.3 & EPA8270 \\
\hline$\mu \mathrm{g} / \mathrm{kg}$ & 41.3 & EPA8270 \\
\hline$\mu \mathrm{g} / \mathrm{kg}$ & 41.3 & EPA8270 \\
\hline$\mu \mathrm{g} / \mathrm{kg}$ & 41.3 & EPA8270 \\
\hline$\mu \mathrm{g} / \mathrm{kg}$ & 41.3 & EPA8270 \\
\hline$\mu \mathrm{g} / \mathrm{kg}$ & 41.3 & EPA8270 \\
\hline$\mu \mathrm{g} / \mathrm{kg}$ & 41.3 & EPA8270 \\
\hline$\mu \mathrm{g} / \mathrm{kg}$ & 4.13 & EPA8270 \\
\hline$\mu \mathrm{g} / \mathrm{kg}$ & 413 & EPA8270 \\
\hline$\mu \mathrm{g} / \mathrm{kg}$ & 41.3 & EPA8270 \\
\hline$\mu \mathrm{g} / \mathrm{kg}$ & 4.13 & EPA8270 \\
\hline$\mu \mathrm{g} / \mathrm{kg}$ & 41.3 & EPA8270 \\
\hline$\mu \mathrm{g} / \mathrm{kg}$ & 41.3 & EPA8270 \\
\hline$\mu \mathrm{g} / \mathrm{kg}$ & 41.3 & EPA8270 \\
\hline$\mu \mathrm{g} / \mathrm{kg}$ & 41.3 & EPA8270 \\
\hline$\mu \mathrm{g} / \mathrm{kg}$ & 41.3 & EPA8270 \\
\hline$\mu \mathrm{g} / \mathrm{kg}$ & 41.3 & EPA8270 \\
\hline$\mu \mathrm{g} / \mathrm{kg}$ & 41.3 & EPA8270 \\
\hline$\mu \mathrm{g} / \mathrm{kg}$ & 41.3 & EPA8270 \\
\hline$\mu \mathrm{g} / \mathrm{kg}$ & 41.3 & EPA8270 \\
\hline$\mu \mathrm{g} / \mathrm{kg}$ & 41.3 & EPA8270 \\
\hline$\mu \mathrm{g} / \mathrm{kg}$ & 413 & EPA8270 \\
\hline$\mu \mathrm{g} / \mathrm{kg}$ & 4.13 & EPA8270 \\
\hline$\mu \mathrm{g} / \mathrm{kg}$ & 4.13 & EPA8270 \\
\hline$\mu \mathrm{g} / \mathrm{kg}$ & 41.3 & EPA8270 \\
\hline$\mu \mathrm{g} / \mathrm{kg}$ & 41.3 & EPA8270 \\
\hline$\mu \mathrm{g} / \mathrm{kg}$ & 41.3 & EPA8270 \\
\hline$\mu \mathrm{g} / \mathrm{kg}$ & 4.13 & EPA8270 \\
\hline$\mu \mathrm{g} / \mathrm{kg}$ & 41.3 & EPA8270 \\
\hline$\mu \mathrm{g} / \mathrm{kg}$ & 41.3 & EPA8270 \\
\hline$\mu \mathrm{g} / \mathrm{kg}$ & 4.13 & EPA8270 \\
\hline$\mu \mathrm{g} / \mathrm{kg}$ & 4.13 & EPA8270 \\
\hline$\mu \mathrm{g} / \mathrm{kg}$ & 41.3 & EPA8270 \\
\hline$\mu \mathrm{g} / \mathrm{kg}$ & 413 & EPA8270 \\
\hline$\mu \mathrm{g} / \mathrm{kg}$ & 41.3 & EPA8270 \\
\hline$\mu \mathrm{g} / \mathrm{kg}$ & 41.3 & EPA8270 \\
\hline$\mu \mathrm{g} / \mathrm{kg}$ & 41.3 & EPA8270 \\
\hline$\mu \mathrm{g} / \mathrm{kg}$ & 41.3 & EPA8270 \\
\hline$\mu \mathrm{g} / \mathrm{kg}$ & 41.3 & EPA8270 \\
\hline$\mu \mathrm{g} / \mathrm{kg}$ & 41.3 & EPA8270 \\
\hline$\mu \mathrm{g} / \mathrm{kg}$ & 41.3 & EPA8270 \\
\hline$\mu \mathrm{g} / \mathrm{kg}$ & 41.3 & EPA8270 \\
\hline$\mu \mathrm{g} / \mathrm{kg}$ & 41.3 & EPA8270 \\
\hline$\mu \mathrm{g} / \mathrm{kg}$ & 41.3 & EPA8270 \\
\hline$\mu \mathrm{g} / \mathrm{kg}$ & 41.3 & EPA8270 \\
\hline$\mu \mathrm{g} / \mathrm{kg}$ & 41.3 & EPA8270 \\
\hline$\mu \mathrm{g} / \mathrm{kg}$ & 41.3 & EPA8270 \\
\hline$\mu \mathrm{g} / \mathrm{kg}$ & 41.3 & EPA8270 \\
\hline$\mu \mathrm{g} / \mathrm{kg}$ & 4.13 & EPA8270 \\
\hline$\mu \mathrm{g} / \mathrm{kg}$ & 41.3 & EPA8270 \\
\hline$\mu \mathrm{g} / \mathrm{kg}$ & 41.3 & EPA8270 \\
\hline
\end{tabular}


SURVEY ID: PPSC 1904 (continued)

Constituent

Volatile Organic Compounds

Acetone
Benzene
Bromodichloromethane
Bromoform
Bromomethane (Methyl bromide)
Carbon disulfide
Carbon tetrachloride
Chlorobenzene
Chloroethane
Chloroethene (Vinyl chloride)
Chloroform
Chloromethane (Methyl chloride)
Dibromochloromethane
1,1-Dichloroethane
1,2-Dichloroethane
1,1-Dichloroethylene
1,2-Dichloroethylene
Dichloromethane (Methylene chloride)
1,2-Dichloropropane
cis-1,3-Dichloropropene
trans-1,3-Dichloropropene
Ethylbenzene
2-Hexanone
Methyl ethyl ketone
Methyl isobutyl ketone
Styrene
1,1,2,2-Tetrachloroethane
Tetrachloroethylene
Toluene
1,1,1-Trichloroethane
1,1,2-Trichloroethane
Trichloroethylene
Vinyl acetate
Xylenes
(n)

\begin{tabular}{|c|c|c|}
\hline $\mathrm{U}$ & \multirow[t]{5}{*}{8} & 23.7 \\
\hline $\mathrm{U}$ & & 0.633 \\
\hline $\mathbf{U}$ & & 0.0633 \\
\hline $\mathrm{U}$ & & 0.0633 \\
\hline U & & 0.127 \\
\hline $\mathrm{J}$ & \multirow[t]{12}{*}{$\mathbf{E}$} & 0.392 \\
\hline $\mathrm{U}$ & & 0.0633 \\
\hline $\mathrm{U}$ & & 0.0633 \\
\hline $\mathbf{U}$ & & 0.127 \\
\hline $\mathrm{U}$ & & 0.127 \\
\hline U & & 0.0633 \\
\hline $\mathrm{U}$ & & 0.127 \\
\hline $\mathrm{U}$ & & 0.0633 \\
\hline$U$ & & 0.0633 \\
\hline $\mathrm{U}$ & & 0.0633 \\
\hline $\mathrm{U}$ & & 0.0633 \\
\hline $\mathrm{U}$ & & 0.127 \\
\hline $\mathrm{U}$ & \multirow[t]{6}{*}{8} & 1.72 \\
\hline $\mathrm{U}$ & & 0.0633 \\
\hline $\mathbf{U}$ & & 0.0633 \\
\hline $\mathbf{U}$ & & 0.0633 \\
\hline $\mathrm{U}$ & & 0.0633 \\
\hline $\mathrm{U}$ & & 1.27 \\
\hline $\mathrm{U}$ & \multirow[t]{11}{*}{ V } & 0.747 \\
\hline $\mathrm{U}$ & & 1.27 \\
\hline $\mathrm{U}$ & & 0.0633 \\
\hline$U$ & & 0.0633 \\
\hline U & & 0.0633 \\
\hline$U$ & & 0.633 \\
\hline$U$ & & 0.0633 \\
\hline U & & 0.0633 \\
\hline $\mathrm{U}$ & & 0.0633 \\
\hline $\mathrm{U}$ & & 1.27 \\
\hline $\mathrm{U}$ & & 0.19 \\
\hline
\end{tabular}

Pesticides/PCBs and Dioxins/Furans

Sample ID: 104674
RQ
AQ B Result
Unit
D. Limit Method

$\begin{array}{lll}\mu \mathrm{g} / \mathrm{kg} & 6.33 & \text { EPA8260 } \\ \mu \mathrm{g} / \mathrm{kg} & 0.633 & \text { EPA8260 } \\ \mu \mathrm{g} / \mathrm{kg} & 0.0633 & \text { EPA8260 } \\ \mu \mathrm{g} / \mathrm{kg} & 0.0633 & \text { EPA8260 } \\ \mu \mathrm{g} / \mathrm{kg} & 0.127 & \text { EPA8260 } \\ \mu \mathrm{g} / \mathrm{kg} & 1.27 & \text { EPA8260 } \\ \mu \mathrm{g} / \mathrm{kg} & 0.0633 & \text { EPA8260 } \\ \mu \mathrm{g} / \mathrm{kg} & 0.0633 & \text { EPA8260 } \\ \mu \mathrm{g} / \mathrm{kg} & 0.127 & \text { EPA8260 } \\ \mu \mathrm{g} / \mathrm{kg} & 0.127 & \text { EPA8260 } \\ \mu \mathrm{g} / \mathrm{kg} & 0.0633 & \text { EPA8260 } \\ \mu \mathrm{g} / \mathrm{kg} & 0.127 & \text { EPA8260 } \\ \mu \mathrm{g} / \mathrm{kg} & 0.0633 & \text { EPA8260 } \\ \mu \mathrm{g} / \mathrm{kg} & 0.0633 & \text { EPA8260 } \\ \mu \mathrm{g} / \mathrm{kg} & 0.0633 & \text { EPA8260 } \\ \mu \mathrm{g} / \mathrm{kg} & 0.0633 & \text { EPA8260 } \\ \mu \mathrm{g} / \mathrm{kg} & 0.127 & \text { EPA8260 } \\ \mu \mathrm{g} / \mathrm{kg} & 0.633 & \text { EPA8260 } \\ \mu \mathrm{g} / \mathrm{kg} & 0.0633 & \text { EPA8260 } \\ \mu \mathrm{g} / \mathrm{kg} & 0.0633 & \text { EPA8260 } \\ \mu \mathrm{g} / \mathrm{kg} & 0.0633 & \text { EPA8260 } \\ \mu \mathrm{g} / \mathrm{kg} & 0.0633 & \text { EPA8260 } \\ \mu \mathrm{g} / \mathrm{kg} & 1.27 & \text { EPA8260 } \\ \mu \mathrm{g} / \mathrm{kg} & 1.27 & \text { EPA8260 } \\ \mu \mathrm{g} / \mathrm{kg} & 1.27 & \text { EPA8260 } \\ \mu \mathrm{g} / \mathrm{kg} & 0.0633 & \text { EPA8260 } \\ \mu \mathrm{g} / \mathrm{kg} & 0.0633 & \text { EPA8260 } \\ \mu \mathrm{g} / \mathrm{kg} & 0.0633 & \text { EPA8260 } \\ \mu \mathrm{g} / \mathrm{kg} & 0.633 & \text { EPA8260 } \\ \mu \mathrm{g} / \mathrm{kg} & 0.0633 & \text { EPA8260 } \\ \mu \mathrm{g} / \mathrm{kg} & 0.0633 & \text { EPA8260 } \\ \mu \mathrm{g} / \mathrm{kg} & 0.0633 & \text { EPA8260 } \\ \mu \mathrm{g} / \mathrm{kg} & 1.27 & \text { EPA8260 } \\ \mu \mathrm{g} / \mathrm{kg} & 0.19 & \text { EPA8260 } \\ & & \end{array}$

5.25

5.25

10.5

10.5

10.5

10.5

21

10.5

21

10.5

21

10.5

21

10.5

21

10.5

10.5

5.25

$\begin{array}{lll}\mu \mathrm{g} / \mathrm{kg} & 5.25 & \text { EPA8080 } \\ \mu \mathrm{g} / \mathrm{kg} & 5.25 & \text { EPA8080 } \\ \mu \mathrm{g} / \mathrm{kg} & 10.5 & \text { EPA8080 } \\ \mu \mathrm{g} / \mathrm{kg} & 10.5 & \text { EPA8080 } \\ \mu \mathrm{g} / \mathrm{kg} & 10.5 & \text { EPA8080 } \\ \mu \mathrm{g} / \mathrm{kg} & 10.5 & \text { EPA8080 } \\ \mu \mathrm{g} / \mathrm{kg} & 21 & \text { EPA8080 } \\ \mu \mathrm{g} / \mathrm{kg} & 10.5 & \text { EPA8080 } \\ \mu \mathrm{g} / \mathrm{kg} & 21 & \text { EPA8080 } \\ \mu \mathrm{g} / \mathrm{kg} & 10.5 & \text { EPA8080 } \\ \mu \mathrm{g} / \mathrm{kg} & 21 & \text { EPA8080 } \\ \mu \mathrm{g} / \mathrm{kg} & 10.5 & \text { EPA8080 } \\ \mu \mathrm{g} / \mathrm{kg} & 21 & \text { EPA8080 } \\ \mu \mathrm{g} / \mathrm{kg} & 10.5 & \text { EPA8080 } \\ \mu \mathrm{g} / \mathrm{kg} & 21 & \text { EPA8080 } \\ \mu \mathrm{g} / \mathrm{kg} & 10.5 & \text { EPA8080 } \\ \mu \mathrm{g} / \mathrm{kg} & 10.5 & \text { EPA8080 } \\ \mu \mathrm{g} / \mathrm{kg} & 5.25 & \text { EPA8080 }\end{array}$


SURVEY ID: PPSC 1904 (continued)

Constituent

Pesticides/PCBs and Dioxins/Furans

Methoxychlor

PCB 1016

PCB 1221

PCB 1232

PCB 1242

PCB 1248

PCB 1254

PCB 1260

Toxaphene

\section{Radionuclides}

Actinium-228

Antimony-124

Antimony-125

Barium-133

Cerium-144

Cesium-134

Cesium-137

Cobalt-57

Cobalt-58

Cobalt-60

Europium-152

Europium-154

Europium-155

Gross alpha

Gross alpha

Lead-212

Manganese-54

Neptunium-239

Nonvolatile beta

Nonvolatile beta

Plutonium-238

Plutonium-239/240

Potassium-40

Promethium-144

Promethium-146

Ruthenium-106

Sodium-22

Strontium-90

Thorium-234

Tin-113

Yttrium-88

Zinc-65

Zirconium-95
Sample ID: 104674

RQ AQ B Result

Unit

D. Limit Method

$\begin{array}{ll}\mathrm{U} & 105 \\ \mathrm{U} & 26.2 \\ \mathrm{U} & 26.2 \\ \mathrm{U} & 26.2 \\ \mathrm{U} & 26.2 \\ \mathrm{U} & 26.2 \\ \mathrm{U} & 26.2 \\ \mathrm{U} & 26.2 \\ \mathrm{U} & 210\end{array}$

$\begin{array}{ll}\mu \mathrm{g} / \mathrm{kg} & 105 \\ \mu \mathrm{g} / \mathrm{kg} & 26.2 \\ \mu \mathrm{g} / \mathrm{kg} & 26.2 \\ \mu \mathrm{g} / \mathrm{kg} & 26.2 \\ \mu \mathrm{g} / \mathrm{kg} & 26.2 \\ \mu \mathrm{g} / \mathrm{kg} & 26.2 \\ \mu \mathrm{g} / \mathrm{kg} & 26.2 \\ \mu \mathrm{g} / \mathrm{kg} & 26.2 \\ \mu \mathrm{g} / \mathrm{kg} & 210\end{array}$

EPA8080

EPA8080

EPA8080

EPA8080

EPA8080

EPA8080

EPA8080

EPA8080

EPA8080

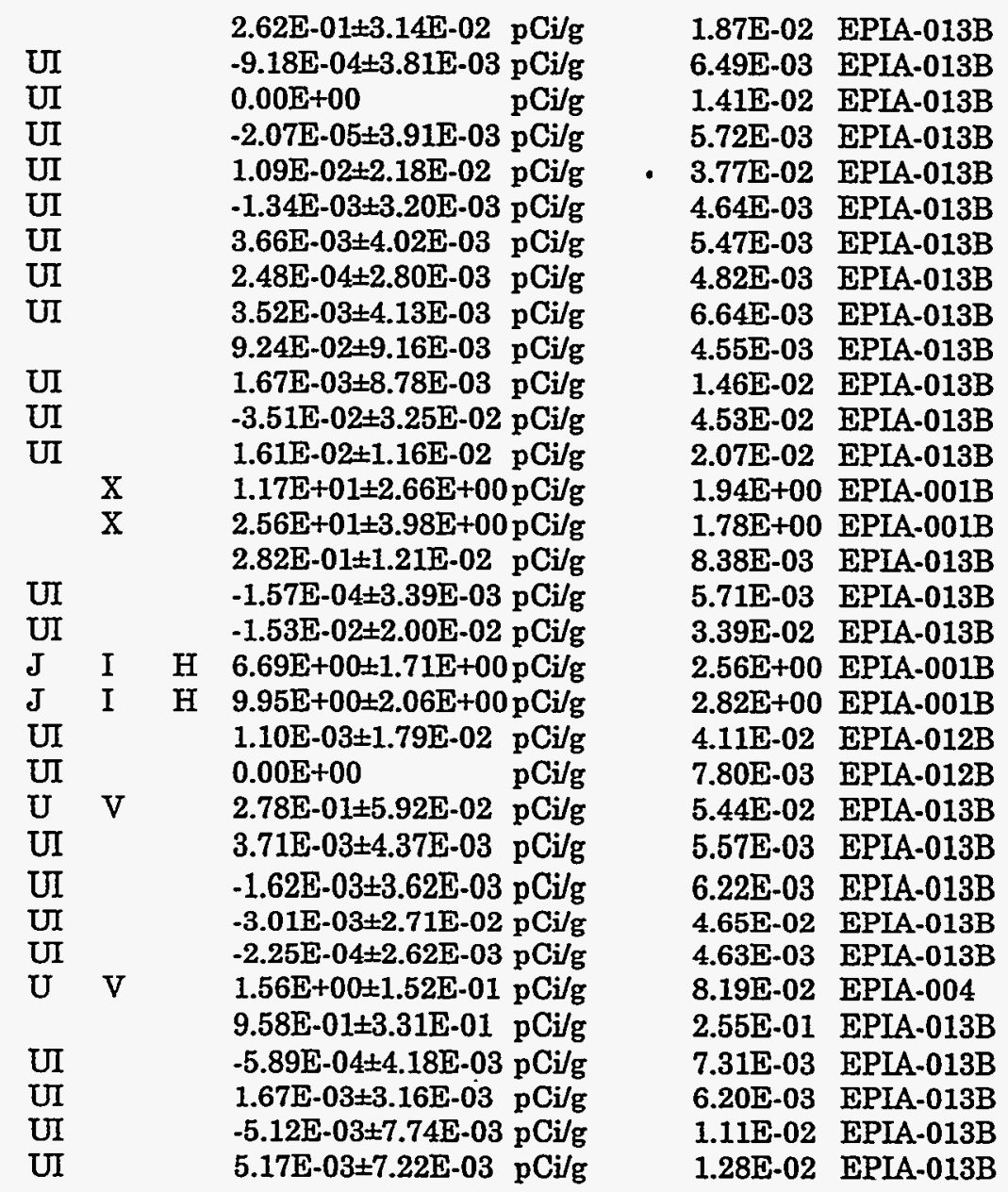

SURVEY ID: PPSC 1905
Sample ID: 104675

Survey location: $44200 \mathrm{E} 3673595 \mathrm{~N}$ (SRS coordinates)

Depth of core interval: 6.00 to $8.00 \mathrm{ft}$

Sample type: Normal

Sample matrix: Soil

Percent solids: $\mathbf{7 6 . 0 0}$ 
SURVEY ID: PPSC 1905 (continued)

Constituent
Sample ID: 104675

RQ AQ B Result

Unit

D. Limit Method

Physical Parameters and Specified Analyses

\begin{tabular}{|c|c|c|c|}
\hline Ammonia nitrogen & $\mathbf{U}$ & & 26 \\
\hline Cation exchange capacity & & & 53.9 \\
\hline Chloride & J & $\mathrm{QE}$ & 1.22 \\
\hline Cyanide & UJ & $\mathrm{C}$ & 1.32 \\
\hline Nitrate-nitrite as nitrogen & $J$ & $\mathbf{E}$ & 0.506 \\
\hline Nitrogen by Kjeldahl method & & & 8.01 \\
\hline Orthophosphate & $\mathrm{U}$ & & 13.2 \\
\hline Phenols & U & & 394 \\
\hline Sulfide & UJ & C & 329 \\
\hline Total organic carbon & $J$ & $\mathbf{E}$ & 37.9 \\
\hline Total organic nitrogen & $\mathrm{C}$ & & 6.2 \\
\hline Total phosphates (as P) & $\mathbf{J}$ & $\mathbf{E}$ & 85.5 \\
\hline Metals (total recoverable) & & & \\
\hline Aluminum & & $\mathrm{V}$ & 1500 \\
\hline Antimony & $\mathrm{U}$ & & 6.27 \\
\hline Arsenic & $\mathbf{U}$ & & 18.8 \\
\hline Barium & $\mathbf{J}$ & C & 61.7 \\
\hline Beryllium & $\mathrm{J}$ & EC & 0.352 \\
\hline Cadmium & $\mathbf{U}$ & & 0.313 \\
\hline Calcium & & $\mathrm{V}$ & 70.8 \\
\hline Chromium & & & 2.42 \\
\hline Cobalt & & & 11.6 \\
\hline Copper & $J$ & $\mathbf{E}$ & 1.05 \\
\hline Iron & $J$ & C & 2600 \\
\hline Lead & $\mathbf{J}$ & $\mathrm{E}$ & 2.7 \\
\hline Magnesium & & & 160 \\
\hline Manganese & & & 86.7 \\
\hline Mercury & $J$ & $\mathbf{E}$ & 0.00485 \\
\hline Nickel & & & 2.26 \\
\hline Potassium & & & 158 \\
\hline Selenium & $\mathrm{U}$ & & 18.8 \\
\hline Silver & $\mathrm{U}$ & & 1.57 \\
\hline Sodium & $\mathrm{U}$ & & 62.7 \\
\hline Thallium & $\mathbf{U}$ & & 18.8 \\
\hline Vanadium & $\mathbf{J}$ & C & 7.44 \\
\hline Zinc & & $\mathrm{V}$ & 3.31 \\
\hline
\end{tabular}

$\begin{array}{lll}\mathrm{mg} / \mathrm{kg} & 26 & \text { EPA350.1 } \\ \mathrm{meq} / 100 \mathrm{~g} & 5 & \text { EPA9081 } \\ \mathrm{mg} / \mathrm{kg} & 3.05 & \text { EPA300.0 } \\ \mathrm{mg} / \mathrm{kg} & 1.32 & \text { EPA335.3 } \\ \mathrm{mg} / \mathrm{kg} & 1.01 & \text { EPA353.1 } \\ \mathrm{mg} / \mathrm{kg} & 5.17 & \text { EPA351.2 } \\ \mathrm{mg} / \mathrm{kg} & 13.2 & \text { EPA365.2 } \\ \mu \mathrm{g} / \mathrm{kg} & 394 & \text { EPA420.2 } \\ \mathrm{mg} / \mathrm{kg} & 329 & \text { EPA376.2 } \\ \mathrm{mg} / \mathrm{kg} & 132 & \text { EPA415.1 } \\ \mathrm{mg} / \mathrm{kg} & 0.263 & \text { EPA351.2 } \\ \mathrm{mg} / \mathrm{kg} & 259 & \text { EPA365.4 }\end{array}$

$\begin{array}{lll}\mathrm{mg} / \mathrm{kg} & 15.7 & \text { EPA6010A } \\ \mathrm{mg} / \mathrm{kg} & 6.27 & \text { EPA6010A } \\ \mathrm{mg} / \mathrm{kg} & 18.8 & \text { EPA6010A } \\ \mathrm{mg} / \mathrm{kg} & 1.57 & \text { EPA6010A } \\ \mathrm{mg} / \mathrm{kg} & 0.627 & \text { EPA6010A } \\ \mathrm{mg} / \mathrm{kg} & 0.313 & \text { EPA6010A } \\ \mathrm{mg} / \mathrm{kg} & 15.7 & \text { EPA6010A } \\ \mathrm{mg} / \mathrm{kg} & 1.57 & \text { EPA6010A } \\ \mathrm{mg} / \mathrm{kg} & 0.627 & \text { EPA6010A } \\ \mathrm{mg} / \mathrm{kg} & 1.57 & \text { EPA6010A } \\ \mathrm{mg} / \mathrm{kg} & 6.27 & \text { EPA6010A } \\ \mathrm{mg} / \mathrm{kg} & 6.27 & \text { EPA6010A } \\ \mathrm{mg} / \mathrm{kg} & 6.27 & \text { EPA6010A } \\ \mathrm{mg} / \mathrm{kg} & 0.627 & \text { EPA6010A } \\ \mathrm{mg} / \mathrm{kg} & 0.0404 & \text { EPA7471 } \\ \mathrm{mg} / \mathrm{kg} & 1.57 & \text { EPA6010A } \\ \mathrm{mg} / \mathrm{kg} & 125 & \text { EPA6010A } \\ \mathrm{mg} / \mathrm{kg} & 18.8 & \text { EPA6010A } \\ \mathrm{mg} / \mathrm{kg} & 1.57 & \text { EPA6010A } \\ \mathrm{mg} / \mathrm{kg} & 62.7 & \text { EPA6010A } \\ \mathrm{mg} / \mathrm{kg} & 18.8 & \text { EPA6010A } \\ \mathrm{mg} / \mathrm{kg} & 0.627 & \text { EPA6010A } \\ \mathrm{mg} / \mathrm{kg} & 1.25 & \text { EPA6010A }\end{array}$

$B / N / A$ Extractables (including $P A H$ and phenols)

Acenaphthene

Acenaphthylene

Anthracene

Benzo[a]anthracene

Benzo[b]fluoranthene

Benzo[k]fluoranthene

Benzoic acid

Benzo[g, $h, l]$ perylene

Benzo[a]pyrene

Benzyl alcohol

$\begin{array}{ll}\mathrm{U} & 43.6 \\ \mathrm{U} & 43.6 \\ \mathrm{U} & 43.6 \\ \mathrm{U} & 4.36 \\ \mathrm{U} & 4.36 \\ \mathrm{U} & 4.36 \\ \mathrm{U} & 43.6 \\ \mathrm{U} & 43.6 \\ \mathrm{U} & 4.36 \\ \mathrm{U} & 43.6\end{array}$

$\begin{array}{lll}\mu \mathrm{g} / \mathrm{kg} & 43.6 & \text { EPA8270 } \\ \mu \mathrm{g} / \mathrm{kg} & 43.6 & \text { EPA8270 } \\ \mu \mathrm{g} / \mathrm{kg} & 43.6 & \text { EPA8270 } \\ \mu \mathrm{g} / \mathrm{kg} & 4.36 & \text { EPA8270 } \\ \mu \mathrm{g} / \mathrm{kg} & 4.36 & \text { EPA8270 } \\ \mu \mathrm{g} / \mathrm{kg} & 4.36 & \text { EPA8270 } \\ \mu \mathrm{g} / \mathrm{kg} & 43.6 & \text { EPA8270 } \\ \mu \mathrm{g} / \mathrm{kg} & 43.6 & \text { EPA8270 } \\ \mu \mathrm{g} / \mathrm{kg} & 4.36 & \text { EPA8270 } \\ \mu \mathrm{g} / \mathrm{kg} & 43.6 & \text { EPA8270 }\end{array}$


$B / N / A$ Extractables (including PAH and phenols)

\begin{tabular}{|c|c|c|c|}
\hline Bis(2-chloroethoxy) methane & U & & 43.6 \\
\hline Bis(2-chloroethyl) ether & U & & 43.6 \\
\hline Bis(2-chloroisopropyl) ether & $\mathrm{U}$ & & 43.6 \\
\hline Bis(2-ethylhexyl) phthalate & $\mathbf{U}$ & & 43.6 \\
\hline 4-Bromophenyl phenyl ether & $\mathrm{U}$ & & 43.6 \\
\hline Butylbenzyl phthalate & $\mathrm{U}$ & & 43.6 \\
\hline 4-Chloroaniline & U & & 43.6 \\
\hline 4-Chloro-m-cresol & $\mathrm{U}$ & & 43.6 \\
\hline 2-Chloronaphthalene & U & & 43.6 \\
\hline 2-Chlorophenol & $\mathrm{U}$ & & 43.6 \\
\hline 4-Chlorophenyl phenyl ether & U & & 43.6 \\
\hline Chrysene & $\mathrm{U}$ & & 4.36 \\
\hline $\mathrm{m} / \mathrm{p}$-Cresol & $\mathrm{U}$ & & 436 \\
\hline o-Cresol (2-Methylphenol) & $\mathrm{U}$ & & 43.6 \\
\hline Dibenz $[a, h]$ anthracene & $U$ & & 4.36 \\
\hline Dibenzofuran & $U$ & & 43.6 \\
\hline Di-n-butyl phthalate & $\mathrm{J}$ & $\mathbf{E}$ & 37.1 \\
\hline 1,2-Dichlorobenzene & $\mathrm{U}$ & & 43.6 \\
\hline 1,3-Dichlorobenzene & $\mathrm{U}$ & & 43.6 \\
\hline 1,4-Dichlorobenzene & $U$ & & 43.6 \\
\hline 3,3'-Dichlorobenzidine & $\mathrm{U}$ & & 43.6 \\
\hline 2,4-Dichlorophenol & $\mathrm{U}$ & & 43.6 \\
\hline Diethyl phthalate & $\mathrm{U}$ & & 43.6 \\
\hline 2,4-Dimethyl phenol & $\mathrm{U}$ & & 43.6 \\
\hline Dimethyl phthalate & $\mathrm{U}$ & & 43.6 \\
\hline 2,4-Dinitrophenol & $\mathrm{U}$ & & 436 \\
\hline 2,4-Dinitrotoluene & UJ & $\mathrm{C}$ & 4.36 \\
\hline 2,6-Dinitrotoluene & $U$ & & 4.36 \\
\hline Di-n-octyl phthalate & $U$ & & 43.6 \\
\hline Fluoranthene & U & & 43.6 \\
\hline Fluorene & $\mathrm{U}$ & & 43.6 \\
\hline Hexachlorobenzene & $\mathrm{U}$ & & 4.36 \\
\hline Hexachlorobutadiene & $U$ & & 43.6 \\
\hline Hexachlorocyclopentadiene & $\mathrm{U}$ & & 43.6 \\
\hline Hexachloroethane & $U$ & & 4.36 \\
\hline Indeno $[1,2,3-c, d]$ pyrene & $\mathrm{U}$ & & 4.36 \\
\hline Isophorone & U & & 43.6 \\
\hline 2-Methyl-4,6-dinitrophenol & $\mathrm{U}$ & & 436 \\
\hline 2-Methylnaphthalene & U & & 43.6 \\
\hline Naphthalene & $\mathbf{U}$ & & 43.6 \\
\hline m-Nitroaniline & U & & 43.6 \\
\hline o-Nitroaniline & $U$ & & 43.6 \\
\hline p-Nitroaniline & $\mathbf{U}$ & & 43.6 \\
\hline Nitrobenzene & $\mathrm{U}$ & & 43.6 \\
\hline 2-Nitrophenol & $\mathrm{U}$ & & 43.6 \\
\hline 4-Nitrophenol & $\mathrm{U}$ & & 43.6 \\
\hline N-Nitrosodiphenylamine & $\mathrm{U}$ & & 43.6 \\
\hline N-Nitrosodi-n-propylamine & $\mathrm{U}$ & & 43.6 \\
\hline Pentachlorophenol & UJ & C & 43.6 \\
\hline Phenanthrene & $\mathrm{U}$ & & 43.6 \\
\hline Phenol & $\mathrm{U}$ & & 43.6 \\
\hline Pyrene & $U$ & & 43.6 \\
\hline 1,2,4-Trichlorobenzene & $\mathrm{U}$ & & 4.36 \\
\hline 2,4,5-Trichlorophenol & $U$ & & 43.6 \\
\hline 2,4,6-Trichlorophenol & $\mathrm{U}$ & & 43.6 \\
\hline
\end{tabular}

\begin{tabular}{|c|c|c|}
\hline$\mu \mathrm{g} / \mathrm{kg}$ & 43.6 & EPA8270 \\
\hline$\mu \mathrm{g} / \mathrm{kg}$ & 43.6 & EPA8270 \\
\hline$\mu \mathrm{g} / \mathrm{kg}$ & 43.6 & EPA8270 \\
\hline$\mu \mathrm{g} / \mathrm{kg}$ & 43.6 & EPA8270 \\
\hline$\mu \mathrm{g} / \mathrm{kg}$ & 43.6 & EPA8270 \\
\hline$\mu \mathrm{g} / \mathrm{kg}$ & 43.6 & EPA8270 \\
\hline$\mu \mathrm{g} / \mathrm{kg}$ & 43.6 & EPA8270 \\
\hline$\mu \mathrm{g} / \mathrm{kg}$ & 43.6 & EPA8270 \\
\hline$\mu \mathrm{g} / \mathrm{kg}$ & 43.6 & EPA8270 \\
\hline$\mu \mathrm{g} / \mathrm{kg}$ & 43.6 & EPA8270 \\
\hline$\mu \mathrm{g} / \mathrm{kg}$ & 43.6 & EPA8270 \\
\hline$\mu \mathrm{g} / \mathrm{kg}$ & 4.36 & EPA8270 \\
\hline$\mu \mathrm{g} / \mathrm{kg}$ & 436 & EPA8270 \\
\hline$\mu \mathrm{g} / \mathrm{kg}$ & 43.6 & EPA8270 \\
\hline$\mu \mathrm{g} / \mathrm{kg}$ & 4.36 & EPA8270 \\
\hline$\mu \mathrm{g} / \mathrm{kg}$ & 43.6 & EPA8270 \\
\hline$\mu \mathrm{g} / \mathrm{kg}$ & 43.6 & EPA8270 \\
\hline$\mu \mathrm{g} / \mathrm{kg}$ & 43.6 & EPA8270 \\
\hline$\mu \mathrm{g} / \mathrm{kg}$ & 43.6 & EPA8270 \\
\hline$\mu \mathrm{g} / \mathrm{kg}$ & 43.6 & EPA8270 \\
\hline$\mu \mathrm{g} / \mathrm{kg}$ & 43.6 & EPA8270 \\
\hline$\mu \mathrm{g} / \mathrm{kg}$ & 43.6 & EPA8270 \\
\hline$\mu \mathrm{g} / \mathrm{kg}$ & 43.6 & EPA8270 \\
\hline$\mu \mathrm{g} / \mathrm{kg}$ & 43.6 & EPA8270 \\
\hline$\mu \mathrm{g} / \mathrm{kg}$ & 43.6 & EPA8270 \\
\hline$\mu \mathrm{g} / \mathrm{kg}$ & 436 & EPA8270 \\
\hline$\mu \mathrm{g} / \mathrm{kg}$ & 4.36 & EPA8270 \\
\hline$\mu \mathrm{g} / \mathrm{kg}$ & 4.36 & EPA8270 \\
\hline$\mu \mathrm{g} / \mathrm{kg}$ & 43.6 & EPA8270 \\
\hline$\mu \mathrm{g} / \mathrm{kg}$ & 43.6 & EPA8270 \\
\hline$\mu \mathrm{g} / \mathrm{kg}$ & 43.6 & EPA8270 \\
\hline$\mu \mathrm{g} / \mathrm{kg}$ & 4.36 & EPA8270 \\
\hline$\mu \mathrm{g} / \mathrm{kg}$ & 43.6 & EPA8270 \\
\hline$\mu \mathrm{g} / \mathrm{kg}$ & 43.6 & EPA8270 \\
\hline$\mu \mathrm{g} / \mathrm{kg}$ & 4.36 & EPA8270 \\
\hline$\mu \mathrm{g} / \mathrm{kg}$ & 4.36 & EPA8270 \\
\hline$\mu \mathrm{g} / \mathrm{kg}$ & 43.6 & EPA8270 \\
\hline$\mu \mathrm{g} / \mathrm{kg}$ & 436 & EPA8270 \\
\hline$\mu \mathrm{g} / \mathrm{kg}$ & 43.6 & EPA8270 \\
\hline$\mu \mathrm{g} / \mathrm{kg}$ & 43.6 & EPA8270 \\
\hline$\mu \mathrm{g} / \mathrm{kg}$ & 43.6 & EPA8270 \\
\hline$\mu \mathrm{g} / \mathrm{kg}$ & 43.6 & EPA8270 \\
\hline$\mu \mathrm{g} / \mathrm{kg}$ & 43.6 & EPA8270 \\
\hline$\mu \mathrm{g} / \mathrm{kg}$ & 43.6 & EPA8270 \\
\hline$\mu \mathrm{g} / \mathrm{kg}$ & 43.6 & EPA8270 \\
\hline$\mu \mathrm{g} / \mathrm{kg}$ & 43.6 & EPA8270 \\
\hline$\mu \mathrm{g} / \mathrm{kg}$ & 43.6 & EPA8270 \\
\hline$\mu \mathrm{g} / \mathrm{kg}$ & 43.6 & EPA8270 \\
\hline$\mu \mathrm{g} / \mathrm{kg}$ & 43.6 & EPA8270 \\
\hline$\mu \mathrm{g} / \mathrm{kg}$ & 43.6 & EPA8270 \\
\hline & 43.6 & EPA8270 \\
\hline$\mu$ & 43.6 & EPA8270 \\
\hline & 4.36 & EPA8270 \\
\hline$\mu$ & 43.6 & EPA8270 \\
\hline$\mu g \Omega$ & 43.6 & EPA8270 \\
\hline
\end{tabular}


SURVEY ID: PPSC 1905 (continued)

Constituent

RQ AQ B Result

Volatile Organic Compounds

\begin{tabular}{|c|c|c|c|}
\hline Acetone & $\mathrm{U}$ & 8 & 23.6 \\
\hline Benzene & $\mathrm{U}$ & & 0.658 \\
\hline Bromodichloromethane & $\mathrm{U}$ & & 0.0658 \\
\hline Bromoform & $\mathrm{U}$ & & 0.0658 \\
\hline Bromomethane (Methyl bromide) & $\mathrm{U}$ & & 0.132 \\
\hline Carbon disulfide & $\mathbf{J}$ & $\mathbf{E}$ & 0.461 \\
\hline Carbon tetrachloride & $\mathbf{U}$ & & 0.0658 \\
\hline Chlorobenzene & $\mathrm{U}$ & & 0.0658 \\
\hline Chloroethane & U & & 0.132 \\
\hline Chloroethene (Vinyl chloride) & $\mathrm{U}$ & & 0.132 \\
\hline Chloroform & $\mathrm{U}$ & & 0.0658 \\
\hline Chloromethane (Methyl chloride) & U & & 0.132 \\
\hline Dibromochloromethane & $\mathrm{U}$ & & 0.0658 \\
\hline 1,1-Dichloroethane & $\mathrm{U}$ & & 0.0658 \\
\hline 1,2-Dichloroethane & $\mathrm{U}$ & & 0.0658 \\
\hline 1,1-Dichloroethylene & $\mathrm{U}$ & & 0.0658 \\
\hline 1,2-Dichloroethylene & $\mathrm{U}$ & & 0.132 \\
\hline Dichloromethane (Methylene chloride) & $\mathrm{U}$ & 8 & 1.66 \\
\hline 1,2-Dichloropropane & $\mathrm{U}$ & & 0.0658 \\
\hline cis-1,3-Dichloropropene & $\mathrm{U}$ & & 0.0658 \\
\hline trans-1,3-Dichloropropene & $\mathrm{U}$ & & 0.0658 \\
\hline Ethylbenzene & $\mathrm{U}$ & & 0.0658 \\
\hline 2-Hexanone & $\mathrm{U}$ & V & 0.263 \\
\hline Methyl ethyl ketone & $\mathrm{U}$ & V & 0.526 \\
\hline Methyl isobutyl ketone & $\mathrm{U}$ & & 1.32 \\
\hline Styrene & $\mathrm{U}$ & & 0.0658 \\
\hline 1,1,2,2-Tetrachloroethane & $\mathrm{U}$ & & 0.0658 \\
\hline Tetrachloroethylene & $\mathrm{U}$ & & 0.0658 \\
\hline Toluene & $\mathrm{U}$ & & 0.658 \\
\hline 1,1,1-Trichloroethane & $\mathrm{U}$ & & 0.0658 \\
\hline 1,1,2-Trichloroethane & $\mathrm{U}$ & & 0.0658 \\
\hline Trichloroethylene &. $\mathrm{U}$ & & 0.0658 \\
\hline Unknown alcohol & Ј & $\mathrm{N}$ & 1 \\
\hline Vinyl acetate & U & & 1.32 \\
\hline Xylenes & $\mathrm{U}$ & & 0.197 \\
\hline
\end{tabular}

Pesticides/PCBs and Dioxins/Furans
Unit D. Limit Method

Unit D. Limit Method

Sample ID: 104675

$\begin{array}{lll}\mu \mathrm{g} / \mathrm{kg} & 6.58 & \text { EPA8260 } \\ \mu \mathrm{g} / \mathrm{kg} & 0.658 & \text { EPA8260 } \\ \mu \mathrm{g} / \mathrm{kg} & 0.0658 & \text { EPA8260 } \\ \mu \mathrm{g} / \mathrm{kg} & 0.0658 & \text { EPA8260 } \\ \mu \mathrm{g} / \mathrm{kg} & 0.132 & \text { EPA8260 } \\ \mu \mathrm{g} / \mathrm{kg} & 1.32 & \text { EPA8260 } \\ \mu \mathrm{g} / \mathrm{kg} & 0.0658 & \text { EPA8260 } \\ \mu \mathrm{g} / \mathrm{kg} & 0.0658 & \text { EPA8260 } \\ \mu \mathrm{g} / \mathrm{kg} & 0.132 & \text { EPA8260 } \\ \mu \mathrm{g} / \mathrm{kg} & 0.132 & \text { EPA8260 } \\ \mu \mathrm{g} / \mathrm{kg} & 0.0658 & \text { EPA8260 } \\ \mu \mathrm{g} / \mathrm{kg} & 0.132 & \text { EPA8260 } \\ \mu \mathrm{g} / \mathrm{kg} & 0.0658 & \text { EPA8260 } \\ \mu \mathrm{g} / \mathrm{kg} & 0.0658 & \text { EPA8260 } \\ \mu \mathrm{g} / \mathrm{kg} & 0.0658 & \text { EPA8260 } \\ \mu \mathrm{g} / \mathrm{kg} & 0.0658 & \text { EPA8260 } \\ \mu \mathrm{g} / \mathrm{kg} & 0.132 & \text { EPA8260 } \\ \mu \mathrm{g} / \mathrm{kg} & 0.658 & \text { EPA8260 } \\ \mu \mathrm{g} / \mathrm{kg} & 0.0658 & \text { EPA8260 } \\ \mu \mathrm{g} / \mathrm{kg} & 0.0658 & \text { EPA8260 } \\ \mu \mathrm{g} / \mathrm{kg} & 0.0658 & \text { EPA8260 } \\ \mu \mathrm{g} / \mathrm{kg} & 0.0658 & \text { EPA8260 } \\ \mu \mathrm{g} / \mathrm{kg} & 1.32 & \text { EPA8260 } \\ \mu \mathrm{g} / \mathrm{kg} & 1.32 & \text { EPA8260 } \\ \mu \mathrm{g} / \mathrm{kg} & 1.32 & \text { EPA8260 } \\ \mu \mathrm{g} / \mathrm{kg} & 0.0658 & \text { EPA8260 } \\ \mu \mathrm{g} / \mathrm{kg} & 0.0658 & \text { EPA8260 } \\ \mu \mathrm{g} / \mathrm{kg} & 0.0658 & \text { EPA8260 } \\ \mu \mathrm{g} / \mathrm{kg} & 0.658 & \text { EPA8260 } \\ \mu \mathrm{g} / \mathrm{kg} & 0.0658 & \text { EPA8260 } \\ \mu \mathrm{g} / \mathrm{kg} & 0.0658 & \text { EPA8260 } \\ \mu \mathrm{g} / \mathrm{kg} & 0.0658 & \text { EPA8260 } \\ \mu \mathrm{g} / \mathrm{kg} & & \text { EPA8260 } \\ \mu \mathrm{g} / \mathrm{kg} & 1.32 & \text { EPA8260 } \\ \mu \mathrm{g} / \mathrm{kg} & 0.197 & \text { EPA8260 } \\ & & \end{array}$

$\begin{array}{lll}\mu \mathrm{g} / \mathrm{kg} & 1.09 & \text { EPA8080 } \\ \mu \mathrm{g} / \mathrm{kg} & 1.09 & \text { EPA8080 } \\ \mu \mathrm{g} / \mathrm{kg} & 2.18 & \text { EPA8080 } \\ \mu \mathrm{g} / \mathrm{kg} & 2.18 & \text { EPA8080 } \\ \mu \mathrm{g} / \mathrm{kg} & 2.18 & \text { EPA8080 } \\ \mu \mathrm{g} / \mathrm{kg} & 2.18 & \text { EPA8080 } \\ \mu \mathrm{g} / \mathrm{kg} & 4.37 & \text { EPA8080 } \\ \mu \mathrm{g} / \mathrm{kg} & 2.18 & \text { EPA8080 } \\ \mu \mathrm{g} / \mathrm{kg} & 4.37 & \text { EPA8080 } \\ \mu \mathrm{g} / \mathrm{kg} & 2.18 & \text { EPA8080 } \\ \mu \mathrm{g} / \mathrm{kg} & 4.37 & \text { EPA8080 } \\ \mu \mathrm{g} / \mathrm{kg} & 2.18 & \text { EPA8080 } \\ \mu \mathrm{g} / \mathrm{kg} & 4.37 & \text { EPA8080 } \\ \mu \mathrm{g} / \mathrm{kg} & 2.18 & \text { EPA8080 }\end{array}$


SURVEY ID: PPSC 1905 (continued)

Constituent

Pesticides/PCBs and Dioxins/Furans

Endrin ketone

Heptachlor

Heptachlor epoxide

Lindane

Methoxychlor

PCB 1016

PCB 1221

PCB 1232

PCB 1242

PCB 1248

PCB 1254

PCB 1260

Toxaphene

Radionuclides

Actinium-228

Antimony-124

Antimony-125

Barium-133

Cerium-144

Cesium-134

Cesium-137

Cobalt-57

Cobalt-58

Cobalt-60

Europium-152

Europium-154

Europium-155

Gross alpha

Lead-212

Manganese-54

Neptunium-239

Nonvolatile beta

Plutonium-238

Plutonium-239/240

Potassium-40

Promethium-144

Promethium-146

Ruthenium-106

Sodium-22

Strontium-90

Thorium-234

Tin-113

Yttrium-88

Zinc-65

Zirconium-95
Sample ID: 104675

RQ AQ B Result

Unit

D. Limit Method

$\begin{array}{llll}\text { UJ } & \text { O } & \text { L } & 4.37 \\ \text { UJ } & \text { O } & \text { L } & 2.18 \\ \text { UJ } & \text { O } & \text { L } & 2.18 \\ \text { UJ } & \text { O } & \text { L } & 1.09 \\ \text { UJ } & \text { O } & \text { L } & 21.8 \\ \text { UJ } & \text { O } & \text { L } & 5.46 \\ \text { UJ } & \text { O } & \text { L } & 5.46 \\ \text { UJ. } & \text { O } & \text { L } & 5.46 \\ \text { UJ } & \text { O } & \text { L } & 5.46 \\ \text { UJ } & \text { O } & \text { L } & 5.46 \\ \text { UJ } & \text { O } & \text { L } & 5.46 \\ \text { UJ } & \text { O } & \text { L } & 5.46 \\ \text { UJ } & \text { O } & \text { L } & 43.7\end{array}$

2.78E-01 $\pm 3.09 \mathrm{E}-02 \mathrm{pCi} / \mathrm{g}$

UI

UI

UI

UI

UI

UI

UI

UI

UI

UI

UI

UI

UI

UI

UI

UI

UI

UI

UI

UI

U V

UI

UI

UI

UI
$1.01 \mathrm{E}-03 \pm 4.16 \mathrm{E}-03 \mathrm{pCi} / \mathrm{g}$

7.41E-04 9.29E-03 pCi/g

$.2 .38 \mathrm{E}-03 \pm 4.90 \mathrm{E}-03 \mathrm{pCi} / \mathrm{g}$

$-4.00 \mathrm{E}-03 \pm 2.57 \mathrm{E}-02 \mathrm{pCi} / \mathrm{g}$

$-2.01 \mathrm{E}-02 \pm 4.10 \mathrm{E}-03 \mathrm{pCi} / \mathrm{g}$

2.38E-03 $\pm 3.84 \mathrm{E}-03 \mathrm{pCi} / \mathrm{g}$

1.41E-03 $\pm 3.17 \mathrm{E}-03 \mathrm{pCi} / \mathrm{g}$

$3.82 \mathrm{E}-03 \pm 4.19 \mathrm{E}-03 \mathrm{pCi} / \mathrm{g}$ $-3.79 \mathrm{E}-04 \pm 2.87 \mathrm{E}-03 \mathrm{pCi} / \mathrm{g}$

$-7.90 \mathrm{E}-03 \pm 9.27 \mathrm{E}-03 \mathrm{pCi} / \mathrm{g}$

$3.98 \mathrm{E}-03 \pm 3.05 \mathrm{E}-02 \mathrm{pCi} / \mathrm{g}$ $0.00 \mathrm{E}+00 \quad \mathrm{pCi} / \mathrm{g}$

$1.82 \mathrm{E}+01 \pm 3.24 \mathrm{E}+00 \mathrm{pCi} / \mathrm{g}$

$2.85 \mathrm{E}-01 \pm 1.50 \mathrm{E}-02 \mathrm{pCi} / \mathrm{g}$

$6.93 \mathrm{E}-04 \pm 3.73 \mathrm{E}-03 \mathrm{pCi} / \mathrm{g}$

$-3.75 \mathrm{E}-03 \pm 2.30 \mathrm{E}-02 \mathrm{pCi} / \mathrm{g}$

$9.26 \mathrm{E}+00 \pm 1.90 \mathrm{E}+00 \mathrm{pCi} / \mathrm{g}$

$0.00 \mathrm{E}+00 \quad \mathrm{pCi} / \mathrm{g}$

4.24E-03 $\pm 1.32 \mathrm{E}-02 \mathrm{pCi} / \mathrm{g}$

$3.54 \mathrm{E}-01 \pm 9.65 \mathrm{E}-02 \mathrm{pCi} / \mathrm{g}$

$3.31 \mathrm{E}-03 \pm 3.16 \mathrm{E}-03 \mathrm{pCi} / \mathrm{g}$

1.72E-03 $\pm 4.36 \mathrm{E}-03 \mathrm{pCi} / \mathrm{g}$

4.22E-03 $\pm 3.18 \mathrm{E}-02 \mathrm{pCi} / \mathrm{g}$

2.26E-03 $\pm 3.03 \mathrm{E}-03 \mathrm{pCi} / \mathrm{g}$

$1.41 \mathrm{E}+00 \pm 1.39 \mathrm{E}-01 \mathrm{pCi} / \mathrm{g}$

$6.32 \mathrm{E}-01 \pm 3.51 \mathrm{E}-01 \mathrm{pCi} / \mathrm{g}$

-2.27E-04 $\pm 4.92 \mathrm{E}-03 \mathrm{pCi} / \mathrm{g}$

$-1.90 \mathrm{E}-03 \pm 4.87 \mathrm{E}-03 \mathrm{pCi} / \mathrm{g}$

$-5.18 \mathrm{E}-05 \pm 6.27 \mathrm{E}-03 \mathrm{pCi} / \mathrm{g}$

9.77E-04 $\pm 7.95 \mathrm{E}-03 \mathrm{pCj} / \mathrm{g}$
1.88E-02 EPIA-013B

7.50E-03 EPIA-013B

1.56E-02 EPIA-013B

7.08E-03 EPIA-013B

4.43E-02 EPIA-013B

5.28E-03 EPIA-013B

6.32E-03 EPIA-013B

5.58E-03 EPIA-013B

7.89E-03 EPIA-013B

5.26E-03 EPIA-013B

1.49E-02 EPIA-013B

4.82E-02 EPIA-013B

2.29E-02 EPIA-013B

$1.86 \mathrm{E}+00$ EPIA-001B

1.00E-02 EPIA-013B

6.60E-03 EPIA-013B

3.99E-02 EPIA-013B

2.59E+00 EPIA-001B

2.99E-02 EPIA-012B

2.99E-02 EPIA-012B

5.56E-02 EPIA-013B

5.97E-03 EPIA-013B

7.46E-03 EPIA-013B

5.06E-02 EPIA-013B

6.01E-03 EPIA-013B

7.54E-02 EPIA-004

2.95E-01 EPIA-013B

8.22E-03 EPIA-013B

8.43E-03 EPIA-013B

9.77E-03 EPIA-013B

1.41E-02 EPIA-013B

SURVEY ID: PPSC 2001

Sample ID: 104676

Survey location: $44285 \mathrm{E} 3674078 \mathrm{~N}$ (SRS coordinates)

Depth of core interval: 0.00 to $1.00 \mathrm{ft}$

Sample type: Normal

Sample matrix: Soil

Percent solids: $\mathbf{5 6 . 0 0}$ 
SURVEY ID: PPSC 2001 (continued)

Constituent

RQ AQ B Result

Unit

D. Limit Method

Physical Parameters and Specified Analyses

Ammonia nitrogen
Ammonia nitrogen
Cation exchange capacity
Chloride
Cyanide
Nitrate-nitrite as nitrogen

Nitrogen by Kjeldahl method

Nitrogen by Kjeldahl method

Orthophosphate

Phenols

Sulfide

Total organic carbon

Total organic nitrogen

Total phosphates (as P)

Metals (total recoverable)

\begin{tabular}{|c|c|c|c|c|}
\hline \multirow{2}{*}{\multicolumn{2}{|c|}{$\begin{array}{l}\text { Aluminum } \\
\text { Antimony }\end{array}$}} & & & 7320 \\
\hline & & \multicolumn{2}{|l|}{$\mathrm{U}$} & 8.34 \\
\hline Arsenic & & \multirow{2}{*}{\multicolumn{2}{|c|}{$\mathrm{U}$}} & 25 \\
\hline Barium & & & & 58.4 \\
\hline Beryllium & & $J$ & $\mathrm{E}$ & 0.695 \\
\hline Cadmium & & $\mathrm{U}$ & & 0.417 \\
\hline Calcium & & & & 572 \\
\hline Chromium & & & & 16.2 \\
\hline Cobalt & & & & 5.84 \\
\hline Copper & & & & 10.6 \\
\hline Iron & & $J$ & C & 12300 \\
\hline Lead & - & & & 12 \\
\hline Magnesium & & & & 473 \\
\hline Manganese & & & & 412 \\
\hline Mercury & & & & 0.0639 \\
\hline Nickel & & & & 3.74 \\
\hline Potassium & & & & 290 \\
\hline Selenium & & U & & 25 \\
\hline Silver & & $\mathrm{U}$ & & 2.09 \\
\hline Sodium & & $\boldsymbol{J}$ & $\mathbf{E}$ & 23.9 \\
\hline Thallium & & $\mathrm{U}$ & & 25 \\
\hline Vanadium & & & & 28.3 \\
\hline Zinc & & & & 32.6 \\
\hline
\end{tabular}

$\begin{array}{llllll}\mathrm{J} & \mathrm{E} & 24.2 & \mathrm{mg} / \mathrm{kg} & 35.1 & \text { EPA350.1 } \\ \mathrm{J} & \mathrm{E} & 28 & \mathrm{mg} / \mathrm{kg} & 33.3 & \text { EPA350.1 } \\ & & 159 & \mathrm{meq} / 100 \mathrm{~g} & 5 & \text { EPA9081 } \\ \mathrm{J} & \mathrm{Q} & 9.38 & \mathrm{mg} / \mathrm{kg} & 4.18 & \text { EPA300.0 } \\ \mathrm{J} & \mathrm{CE} & 0.42 & \mathrm{mg} / \mathrm{kg} & 1.79 & \text { EPA335.3 } \\ \mathrm{J} & \mathrm{E} & 0.456 & \mathrm{mg} / \mathrm{kg} & 1.52 & \text { EPA353.1 } \\ & & 831 & \mathrm{mg} / \mathrm{kg} & 142 & \text { EPA351.2 } \\ & & 906 & \mathrm{mg} / \mathrm{kg} & 134 & \text { EPA351.2 } \\ \mathrm{U} & & 17.9 & \mathrm{mg} / \mathrm{kg} & 17.9 & \text { EPA365.2 } \\ \mathrm{U} & & 525 & \mu \mathrm{mg} / \mathrm{kg} & 525 & \text { EPA420.2 } \\ \mathrm{UJ} & \mathrm{C} & 446 & \mathrm{mg} / \mathrm{kg} & 446 & \text { EPA376.2 } \\ & & 35400 & \mathrm{mg} / \mathrm{kg} & 179 & \text { EPA415.1 } \\ \mathrm{C} & & 807 & \mathrm{mg} / \mathrm{kg} & 0.357 & \text { EPA351.2 } \\ \mathrm{J} & \mathrm{E} & 326 & \mathrm{mg} / \mathrm{kg} & 1220 & \text { EPA365.4 }\end{array}$

$\begin{array}{lll}\mathrm{mg} / \mathrm{kg} & 20.9 & \text { EPA6010A } \\ \mathrm{mg} / \mathrm{kg} & 8.34 & \text { EPA6010A } \\ \mathrm{mg} / \mathrm{kg} & 25 & \text { EPA6010A } \\ \mathrm{mg} / \mathrm{kg} & 2.09 & \text { EPA6010A } \\ \mathrm{mg} / \mathrm{kg} & 0.834 & \text { EPA6010A } \\ \mathrm{mg} / \mathrm{kg} & 0.417 & \text { EPA6010A } \\ \mathrm{mg} / \mathrm{kg} & 20.9 & \text { EPA6010A } \\ \mathrm{mg} / \mathrm{kg} & 2.09 & \text { EPA6010A } \\ \mathrm{mg} / \mathrm{kg} & 0.834 & \text { EPA6010A } \\ \mathrm{mg} / \mathrm{kg} & 2.09 & \text { EPA6010A } \\ \mathrm{mg} / \mathrm{kg} & 8.34 & \text { EPA6010A } \\ \mathrm{mg} / \mathrm{kg} & 8.34 & \text { EPA6010A } \\ \mathrm{mg} / \mathrm{kg} & 8.34 & \text { EPA6010A } \\ \mathrm{mg} / \mathrm{kg} & 0.834 & \text { EPA6010A } \\ \mathrm{mg} / \mathrm{kg} & 0.0576 & \text { EPA7471 } \\ \mathrm{mg} / \mathrm{kg} & 2.09 & \text { EPA6010A } \\ \mathrm{mg} / \mathrm{kg} & 167 & \text { EPA6010A } \\ \mathrm{mg} / \mathrm{kg} & 25 & \text { EPA6010A } \\ \mathrm{mg} / \mathrm{kg} & 2.09 & \text { EPA6010A } \\ \mathrm{mg} / \mathrm{kg} & 83.4 & \text { EPA6010A } \\ \mathrm{mg} / \mathrm{kg} & 25 & \text { EPA6010A } \\ \mathrm{mg} / \mathrm{kg} & 0.834 & \text { EPA6010A } \\ \mathrm{mg} / \mathrm{kg} & 1.67 & \text { EPA6010A }\end{array}$

Radionuclides

Actinium-228

Antimony-124

Antimony-125

Barium-133

Cerium-144

Cesium-134

Cesium-137

Cobalt-57

Cobalt-58

Cobalt-60

Europium-152

Europium-154
$2.63 \mathrm{E}+00 \pm 1.03 \mathrm{E}-01 \mathrm{pCi} / \mathrm{g}$

$1.11 \mathrm{E}-02 \pm 2.19 \mathrm{E}-02 \mathrm{pCi} / \mathrm{g}$

3.61E-02 $\pm 8.63 \mathrm{E}-02 \mathrm{pCi} / \mathrm{g}$

$-3.37 \mathrm{E}-03 \pm 3.11 \mathrm{E}-02 \mathrm{pCi} / \mathrm{g}$

6.26E-03 $\pm 1.22 \mathrm{E}-01 \mathrm{pCi} / \mathrm{g}$

$5.78 \mathrm{E}-03 \pm 1.78 \mathrm{E}-02 \mathrm{pCi} / \mathrm{g}$

$3.43 \mathrm{E}+01 \pm 1.56 \mathrm{E}-01 \mathrm{pCi} / \mathrm{g}$

$-5.06 \mathrm{E}-03 \pm 1.54 \mathrm{E}-02 \mathrm{pCi} / \mathrm{g}$

$-6.38 \mathrm{E}-03 \pm 1.19 \mathrm{E}-02 \mathrm{pCi} / \mathrm{g}$

$1.37 \mathrm{E}-01 \pm 2.37 \mathrm{E}-02 \mathrm{pCi} / \mathrm{g}$

$-1.03 \mathrm{E}-02 \pm 6.03 \mathrm{E}-02 \mathrm{pCi} / \mathrm{g}$

$1.53 \mathrm{E}-02 \pm 9.16 \mathrm{E}-02 \mathrm{pCj} / \mathrm{g}$ $\begin{array}{ll}5.43 E-02 & \text { EPIA-013B } \\ 3.66 \mathrm{E}-02 & \text { EPIA-013B } \\ 1.21 \mathrm{E}-01 & \text { EPIA-013B } \\ 4.65 \mathrm{E}-02 & \text { EPIA-013B } \\ 2.02 \mathrm{E}-01 & \text { EPIA-013B } \\ 2.61 \mathrm{E}-02 & \text { EPIA-013B } \\ 2.66 \mathrm{E}-02 & \text { EPIA-013B } \\ 2.55 \mathrm{E}-02 & \text { EPIA-013B } \\ 1.93 \mathrm{E}-02 & \text { EPIA-013B } \\ 1.41 \mathrm{E}-02 & \text { EPIA-013B } \\ 1.03 \mathrm{E}-01 & \text { EPIA-013B } \\ 1.40 \mathrm{E}-01 & \text { EPIA-013B }\end{array}$ 
SURVEY ID: PPSC 2001 (continued)

Constituent

Radionuclides

Europium-155

Gross alpha

Lead-212

Manganese-54

Neptunium-239

Nonvolatile beta

Plutonium-238

Plutonium-239/240

Potassium-40

Promethium-144

Promethium-146

Ruthenium-106

Sodium-22

Strontium-90

Thorium-234

Tin-113

Yttrium-88

Zinc-65

Zirconium-95

Sample ID: 104676

D. Limit Method

UI

UI

SURVEY ID: PPSC 2002

$\begin{array}{lr}0.00 \mathrm{E}+00 & \mathrm{pCi} / \mathrm{g} \\ 2.95 \mathrm{E}+01 \pm 4.66 \mathrm{E}+00 \mathrm{pCi} / \mathrm{g} \\ 2.66 \mathrm{E}+00 \pm 6.17 \mathrm{E}-02 \mathrm{pCj} / \mathrm{g} \\ 4.50 \mathrm{E}-02 \pm 1.18 \mathrm{E}-02 \mathrm{pCi} / \mathrm{g} \\ -6.61 \mathrm{E}-02 \pm 1.21 \mathrm{E}-01 \mathrm{pCi} / \mathrm{g} \\ 5.25 \mathrm{E}+01 \pm 3.82 \mathrm{E}+00 \mathrm{pCi} / \mathrm{g} \\ 6.00 \mathrm{E}-02 \pm 2.93 \mathrm{E}-02 \mathrm{pCi} / \mathrm{g} \\ 5.76 \mathrm{E}-02 \pm 2.52 \mathrm{E}-02 \mathrm{pCi} / \mathrm{g} \\ 3.75 \mathrm{E}+00 \pm 2.65 \mathrm{E}-01 \mathrm{pCi} / \mathrm{g} \\ -2.35 \mathrm{E}-03 \pm 9.94 \mathrm{E}-03 \mathrm{pCi} / \mathrm{g} \\ 8.77 \mathrm{E}-03 \pm 3.70 \mathrm{E}-02 \mathrm{pCi} / \mathrm{g} \\ 2.94 \mathrm{E}-03 \pm 1.57 \mathrm{E}-01 \mathrm{pCi} / \mathrm{g} \\ -1.22 \mathrm{E}-02 \pm 9.06 \mathrm{E}-03 \mathrm{pCi} / \mathrm{g} \\ 1.20 \mathrm{E}+00 \pm 1.24 \mathrm{E}-01 \mathrm{pCi} / \mathrm{g} \\ 2.18 \mathrm{E}+00 \pm 1.10 \mathrm{E}+00 \mathrm{pCi} / \mathrm{g} \\ 2.61 \mathrm{E}-02 \pm 3.40 \mathrm{E}-02 \mathrm{pCi} / \mathrm{g} \\ 6.63 \mathrm{E}-03 \pm 1.03 \mathrm{E}-02 \mathrm{pCi} / \mathrm{g} \\ 1.48 \mathrm{E}-02 \pm 2.34 \mathrm{E}-02 \mathrm{pCi} / \mathrm{g} \\ 0.00 \mathrm{E}+00 \quad \mathrm{pCi} / \mathrm{g}\end{array}$

Sample ID: 104677

Survey location: $44285 \mathrm{E} 3674078 \mathrm{~N}$ (SRS coordinates)

Depth of core interval: 1.00 to $2.00 \mathrm{ft}$

Sample type: Normal

Sample matrix: Soil

RQ AQ B Result

Cation exchange capacity

Cation exchange capacity

Chloride

Cyanide

Nitrate-nitrite as nitrogen

Nitrogen by Kjeldahl method

Orthophosphate

Phenols

Sulfide

Total organic carbon

Total organic nitrogen

Total phosphates (as P)

Metals (total recoverable) .

Aluminum

Antimony

Arsenic

Barium

Beryllium

Cadmium
23.3

18.3

39

$\mathrm{U}$

J $\quad$ QE $\quad 2.63$

UJ C $\quad 1.25$

$\begin{array}{lll}J & \mathrm{E} & 0.289\end{array}$

98.4

$\mathrm{U} \quad 12.5$

UJ $\mathrm{C} \quad 313$

$\begin{array}{ll} & 3370 \\ \text { C } & 88.2\end{array}$

J $\quad$ E 183

$\begin{array}{lll} & \mathrm{V} & 1830 \\ \mathrm{U} & & 6.02 \\ \mathrm{U} & & 18 \\ \mathrm{~J} & \mathrm{C} & 9.97 \\ \mathrm{~J} & \mathrm{EC} & \mathbf{0 . 1 2 8} \\ \mathrm{U} & & 0.301\end{array}$

U 372
Percent solids: $\mathbf{8 0 . 0 0}$

Unit

D. Limit Method
1.03E-01 EPIA-013B

2.88E+00 EPIA-001B

5.43E-02 EPIA-013B

1.64E-02 EPIA-013B

1.79E-01 EPIA-013B

2.57E+00 EPIA-001B

3.57E-02 EPIA-012B

1.61E-02 EPIA-012B

1.37E-01 EPIA-013B

1.64E-02 EPIA-013B

6.22E-02 EPIA-013B

2.60E-01 EPIA-013B

$1.45 \mathrm{E}-02$ EPIA-013B

6.98E-02 EPIA-004

$1.21 \mathrm{E}+00$ EPIA-013B

5.79E-02 EPIA-013B

1.89E-02 EPIA-013B

3.62E-02 EPIA-013B

4.33E-02 EPIA-013B 
SURVEY ID: PPSC 2002 (continued)

Constituent

Metals (total recoverable)

Calcium

Chromium

Cobalt

Copper

Iron

Lead

Magnesium

Manganese

Mercury

Nickel

Potassium

Selenium

Silver

Sodium

Thallium

Vanadium

Zinc

Radionuclides

Actinium-228

Antimony-124

Antimony-125

Barium-133

Cerium-144

Cesium-134

Cesium-137

Cobalt-57

Cobalt-58

Cobalt-60

Europium-152

Europium-154

Europium-155

Gross alpha

Lead-212

Manganese-54

Neptunium-239

Nonvolatile beta

Plutonium-238

Plutonium-239/240

Potassium-40

Promethium-144

Promethium-146

Ruthenium-106

Sodium-22

Strontium-90

Thorium-234

Tin-113

Yttrium-88

Zinc-65

Zirconium-95
Sample ID: 104677
RQ AQ B Result
Unit
D. Limit Method

$\begin{array}{lll}\mathrm{mg} / \mathrm{kg} & 15 & \text { EPA6010A } \\ \mathrm{mg} / \mathrm{kg} & 1.5 & \text { EPA6010A } \\ \mathrm{mg} / \mathrm{kg} & 0.602 & \text { EPA6010A } \\ \mathrm{mg} / \mathrm{kg} & 1.5 & \text { EPA6010A } \\ \mathrm{mg} / \mathrm{kg} & 6.02 & \text { EPA6010A } \\ \mathrm{mg} / \mathrm{kg} & 6.02 & \text { EPA6010A } \\ \mathrm{mg} / \mathrm{kg} & 6.02 & \text { EPA6010A } \\ \mathrm{mg} / \mathrm{kg} & 0.602 & \text { EPA6010A } \\ \mathrm{mg} / \mathrm{kg} & 0.036 & \text { EPA7471 } \\ \mathrm{mg} / \mathrm{kg} & 1.5 & \text { EPA6010A } \\ \mathrm{mg} / \mathrm{kg} & 120 & \text { EPA6010A } \\ \mathrm{mg} / \mathrm{kg} & 18 & \text { EPA6010A } \\ \mathrm{mg} / \mathrm{kg} & 1.5 & \text { EPA6010A } \\ \mathrm{mg} / \mathrm{kg} & 60.2 & \text { EPA6010A } \\ \mathrm{mg} / \mathrm{kg} & 18 & \text { EPA6010A } \\ \mathrm{mg} / \mathrm{kg} & 0.602 & \text { EPA6010A } \\ \mathrm{mg} / \mathrm{kg} & 1.2 & \text { EPA6010A }\end{array}$

$3.44 \mathrm{E}+00 \pm 9.16 \mathrm{E}-02 \mathrm{pCi} / \mathrm{g}$ $1.52 \mathrm{E}-04 \pm 1.41 \mathrm{E}-02 \mathrm{pCi} / \mathrm{g}$ 8.70E-03 $44.34 \mathrm{E}-02 \mathrm{pCi} / \mathrm{g}$ $3.83 \mathrm{E}-04 \pm 1.92 \mathrm{E}-02 \mathrm{pCi} / \mathrm{g}$ $-6.74 \mathrm{E}-03 \pm 8.41 \mathrm{E}-02 \mathrm{pCi} / \mathrm{g}$ $-3.85 \mathrm{E}-03 \pm 1.14 \mathrm{E}-02 \mathrm{pCj} / \mathrm{g}$ $1.41 \mathrm{E}+01 \pm 9.13 \mathrm{E}-02 \mathrm{pCi} / \mathrm{g}$ $1.64 \mathrm{E}-02 \pm 1.38 \mathrm{E}-02 \mathrm{pCi} / \mathrm{g}$ $2.88 \mathrm{E}-03 \pm 9.30 \mathrm{E}-03 \mathrm{pCi} / \mathrm{g}$ $5.22 \mathrm{E}-02 \pm 1.60 \mathrm{E}-02 \mathrm{pCi} / \mathrm{g}$ $-2.78 \mathrm{E}-02 \pm 3.83 \mathrm{E}-02 \mathrm{pCi} / \mathrm{g}$ $-1.62 \mathrm{E}-03 \pm 7.21 \mathrm{E}-02 \mathrm{pCi} / \mathrm{g}$ $0.00 \mathrm{E}+00 \quad \mathrm{pCi} / \mathrm{g}$ $6.66 \mathrm{E}+01 \pm 6.02 \mathrm{E}+00 \mathrm{pCi} / \mathrm{g}$ $3.61 \mathrm{E}+00 \pm 5.11 \mathrm{E}-02 \mathrm{pCi} / \mathrm{g}$ $6.08 \mathrm{E}-02 \pm 9.50 \mathrm{E}-03 \mathrm{pCi} / \mathrm{g}$ $6.19 \mathrm{E}-02 \pm 8.35 \mathrm{E}-02 \mathrm{pCj} / \mathrm{g}$ $6.26 \mathrm{E}+01 \pm 4.08 \mathrm{E}+00 \mathrm{pCi} / \mathrm{g}$ $3.22 \mathrm{E}-02 \pm 1.99 \mathrm{E}-02 \mathrm{pCi} / \mathrm{g}$ 2.95E-02 $\pm 1.80 \mathrm{E}-02 \mathrm{pCi} / \mathrm{g}$ $\mathrm{V} \quad 1.40 \mathrm{E}+00 \pm 1.82 \mathrm{E}-01 \mathrm{pCi} / \mathrm{g}$ UI 2.52E-03 $2.43 \mathrm{E}-03 \mathrm{pCi} / \mathrm{g}$ UI $\quad 1.37 \mathrm{E}-02 \pm 2.21 \mathrm{E}-02 \mathrm{pCi} / \mathrm{g}$ UI $\quad 7.84 \mathrm{E}-03 \pm 1.01 \mathrm{E}-01 \mathrm{pCi} / \mathrm{g}$ UI $\quad-2.09 \mathrm{E}-03 \pm 7.44 \mathrm{E}-03 \mathrm{pCi} / \mathrm{g}$ U V $\quad 1.28 \mathrm{E}+00 \pm 1.45 \mathrm{E}-01 \mathrm{pCi} / \mathrm{g}$ $2.46 \mathrm{E}+00 \pm 9.81 \mathrm{E}-01 \mathrm{pCj} / \mathrm{g}$ $-1.01 \mathrm{E}-02 \pm 2.12 \mathrm{E}-02 \mathrm{pCi} / \mathrm{g}$ 9.11E-04 $\pm 1.03 \mathrm{E}-02 \mathrm{pCi} / \mathrm{g}$ $1.60 \mathrm{E}-02 \pm 1.61 \mathrm{E}-02 \mathrm{pCi} / \mathrm{g}$ $3.38 \mathrm{E}-02 \pm 2.31 \mathrm{E}-02 \mathrm{pCj} / \mathrm{g}$
4.05E-02 EPIA-013B

7.05E-02 EPIA-013B

2.79E-02 EPIA-013B

1.43E-01 EPIA-013B

1.71E-02 EPIA-013B

1.92E-02 EPIA-013B

1.82E-02 EPIA-013B

1.60E-02 EPIA-013B

1.21E-02 EPIA-013B

6.22E-02 EPIA-013B

1.07E-01 EPLA-013B

7.46E-02 EPIA-013B

1.85E+00 EPLA-001B

3.55E-02 EPIA-013B

1.36E-02 EPIA-013B

1.30E-01 EPIA-013B

2.47E+00 EPLA-001B

2.06E-02 EPIA-012B

8.05E-03 EPIA-012B

1.11E-01 EPIA-013B

1.45E-02 EPIA-013B

3.59E-02 EPIA-013B

1.73E-01 EPIA-013B

1.22E-02 EPIA-013B

8.79E-02 EPIA-004

8.86E-01 EPIA-013B

3.42E-02 EPIA-013B

1.56E-02 EPIA-013B

2.53E-02 EPIA-013B

3.65E-02 EPIA-013B
2.41E-02 EPIA-013B 
Survey location: $44285 \mathrm{E}$ 3674078N (SRS coordinates)

Depth of core interval: 2.00 to $4.00 \mathrm{ft}$

Sample type: Normal

Percent solids: 87.00

Constituent

RQ AQ B Result

Unit

D. Limit Method

Physical Parameters and Specified Analyses

Ammonia nitrogen

Cation exchange capacity

Cation exchange capacity

Chloride

Cyanide

Nitrate-nitrite as nitrogen

Nitrogen by Kjeldahl method

Orthophosphate

Orthophosphate

Phenols

Sulfide

Total organic carbon

Total organic nitrogen

Total phosphates (as P)

Metals (total recoverable)

Aluminum

Antimony

Arsenic

Barium

Beryllium

Cadmium

Calcium

Chromium

Cobalt

Copper

Iron

Lead

Magnesium

Manganese

Mercury

Nickel

Potassium

Selenium

Silver

Sodium

Thallium

Vanadium

Zinc

Radionuclides

Actinium-228

Antimony-124

Antimony-125

Barium-133

Cerium-144

Cesium-134

$\begin{array}{lll}\text { U } & & 23.3 \\ \text { U } & & 5 \\ & & 8.76 \\ \text { UJ } & \text { Q } & 2.62 \\ \text { UJ } & \text { C } & 1.15 \\ \text { J } & \text { E } & 0.40 \\ & & 10.5 \\ \text { U } & & 11.5 \\ \text { U } & & 11.5 \\ \text { U } & & 344 \\ \text { UJ } & \text { C } & 287 \\ & & 313 \\ \text { C } & & 7.93 \\ \text { J } & \text { E } & \mathbf{9 6 . 7}\end{array}$

$\mathrm{mg} / \mathrm{kg}$ meq $/ 100 \mathrm{~g}$

meq $/ 100 \mathrm{~g}$

$\mathrm{mg} / \mathrm{kg}$

$\mathrm{mg} / \mathrm{kg}$

$\mathrm{mg} / \mathrm{kg}$

$\mathrm{mg} / \mathrm{kg}$

$\mathrm{mg} / \mathrm{kg}$

$\mathrm{mg} / \mathrm{kg}$

$\mu \mathrm{g} / \mathrm{kg}$

$\mathrm{mg} / \mathrm{kg}$

$\mathrm{mg} / \mathrm{kg}$

$\mathrm{mg} / \mathrm{kg}$

$\mathrm{mg} / \mathrm{kg}$

$\mathrm{mg} / \mathrm{kg}$

$\mathrm{mg} / \mathrm{kg}$

$\mathrm{mg} / \mathrm{kg}$

$\mathrm{mg} / \mathrm{kg}$

$\mathrm{mg} / \mathrm{kg}$

$\mathrm{mg} / \mathrm{kg}$

$\mathrm{mg} / \mathrm{kg}$

$\mathrm{mg} / \mathrm{kg}$

$\mathrm{mg} / \mathrm{kg}$

$\mathrm{mg} / \mathrm{kg}$

$\mathrm{mg} / \mathrm{kg}$

$\mathrm{mg} / \mathrm{kg}$

$\mathrm{mg} / \mathrm{kg}$

$\mathrm{mg} / \mathrm{kg}$

$\mathrm{mg} / \mathrm{kg}$

$\mathrm{mg} / \mathrm{kg}$

$\mathrm{mg} / \mathrm{kg}$

$\mathrm{mg} / \mathrm{kg}$

; $\mathrm{mg} / \mathrm{kg}$

$\mathrm{mg} / \mathrm{kg}$

$\mathrm{mg} / \mathrm{kg}$

$\mathrm{mg} / \mathrm{kg}$

$\mathrm{mg} / \mathrm{kg}$
23.3 EPA350.1

5 EPA9081

5.75 EPA9081

2.62 EPA300.0

1.15 EPA335.3

1.01 EPA353.1

4.56 EPA351.2

$11.5 \quad$ EPA365.2

$11.5 \quad$ EPA365.2

$344 \quad$ EPA420.2

287 EPA376.2

$115 \quad$ EPA415.1

0.23 EPA351.2

$302 \quad$ EPA365.4

$\begin{array}{ll}14.2 & \text { EPA6010A } \\ 5.69 & \text { EPA6010A } \\ 17.1 & \text { EPA6010A } \\ 1.42 & \text { EPA6010A } \\ 0.569 & \text { EPA6010A } \\ 0.285 & \text { EPA6010A } \\ 14.2 & \text { EPA6010A } \\ 1.42 & \text { EPA6010A } \\ 0.569 & \text { EPA6010A } \\ 1.42 & \text { EPA6010A } \\ 5.69 & \text { EPA6010A } \\ 5.69 & \text { EPA6010A } \\ 5.69 & \text { EPA6010A } \\ 0.569 & \text { EPA6010A } \\ 0.0372 & \text { EPA7471 } \\ 1.42 & \text { EPA6010A } \\ 114 & \text { EPA6010A } \\ 17.1 & \text { EPA6010A } \\ 1.42 & \text { EPA6010A } \\ 56.9 & \text { EPA6010A } \\ 17.1 & \text { EPA6010A } \\ 0.569 & \text { EPA6010A } \\ 1.14 & \text { EPA6010A }\end{array}$

$3.58 \mathrm{E}+00 \pm 9.69 \mathrm{E} \cdot 02 \mathrm{pCi} / \mathrm{g}$

$-4.43 \mathrm{E}-03 \pm 1.15 \mathrm{E}-02 \mathrm{pCi} / \mathrm{g}$

$-2.42 \mathrm{E}-03 \pm 2.64 \mathrm{E}-02 \mathrm{pCi} / \mathrm{g}$

$1.63 \mathrm{E}-03 \pm 1.36 \mathrm{E}-02 \mathrm{pCi} / \mathrm{g}$

$-5.31 \mathrm{E}-02 \pm 7.09 \mathrm{E}-02 \mathrm{pCi} / \mathrm{g}$

$7.74 \mathrm{E}-03 \pm 9.20 \mathrm{E}-03 \mathrm{pCi} / \mathrm{g}$
4.20E-02 EPIA-013B

1.91E-02 EPIA-013B

4.52E-02 EPIA-013B

-1.96E-02 EPIA-013B

1.19E-01 EPIA-013B

1.40E-02 EPIA-013B 
SURVEY ID: PPSC 2003 (continued)

\begin{tabular}{|c|c|c|c|c|}
\hline Constituent & $\mathbf{R Q}$ & $\mathbf{A Q} \quad \mathbf{B}$ & Result & Unit \\
\hline \multicolumn{5}{|l|}{ Radionuclides } \\
\hline Cesium-137 & & & $2.84 \mathrm{E}+00 \pm 4.08 \mathrm{E}-02$ & $\mathrm{pCi} / \mathrm{g}$ \\
\hline Cobalt-57 & UI & & $-1.84 \mathrm{E}-04 \pm 8.88 \mathrm{E}-03$ & $\mathrm{pCi} / \mathrm{g}$ \\
\hline Cobalt-58 & UI & & $1.22 \mathrm{E}-03 \pm 1.05 \mathrm{E}-02$ & $\mathrm{pCi} / \mathrm{g}$ \\
\hline Cobalt-60 & & & $1.44 \mathrm{E}-02 \pm 1.04 \mathrm{E}-02$ & $\mathrm{pCi} / \mathrm{g}$ \\
\hline Europium-152 & UI & & $-4.79 \mathrm{E}-02 \pm 2.80 \mathrm{E}-02$ & $\mathrm{pCi} / \mathrm{g}$ \\
\hline Europium-154 & UI & & $-2.55 \mathrm{E}-02 \pm 6.19 \mathrm{E}-02$ & $\mathrm{pCi} / \mathrm{g}$ \\
\hline Europium-155 & UI & & $0.00 \mathrm{E}+00$ & $\mathrm{pCi} / \mathrm{g}$ \\
\hline Gross alpha & & & $3.53 \mathrm{E}+01 \pm 4.35 \mathrm{E}+00$ & $\mathrm{pCi} / \mathrm{g}$ \\
\hline Lead-212 & & & $3.70 \mathrm{E}+00 \pm 4.83 \mathrm{E}-02$ & $\mathrm{pCi} / \mathrm{g}$ \\
\hline Manganese-54 & & & 5.98E-02 $\pm 1.22 \mathrm{E}-02$ & $\mathrm{pCi} / \mathrm{g}$ \\
\hline Neptunium-239 & UI & & $3.33 \mathrm{E}-02 \pm 7.05 \mathrm{E}-02$ & $\mathrm{pCi} / \mathrm{g}$ \\
\hline Nonvolatile beta & & & $1.76 \mathrm{E}+01 \pm 2.34 \mathrm{E}+00$ & $\mathrm{pCi} / \mathrm{g}$ \\
\hline Plutonium-238 & UI & & $5.62 \mathrm{E}-03 \pm 1.06 \mathrm{E}-02$ & $\mathrm{pCi} / \mathrm{g}$ \\
\hline Plutonium-239/240 & & & $2.40 \mathrm{E}-02 \pm 1.73 \mathrm{E}-02$ & $\mathrm{pCi} / \mathrm{g}$ \\
\hline Potassium-40 & & $\mathrm{V}$ & $6.89 \mathrm{E}-01 \pm 1.75 \mathrm{E}-01$ & $\mathrm{pCi} / \mathrm{g}$ \\
\hline Promethium-144 & UI & & $1.49 \mathrm{E}-03 \pm 8.54 \mathrm{E}-03$ & $\mathrm{pCi} / \mathrm{g}$ \\
\hline Promethium-146 & UI & & $0.00 \mathrm{E}+00$ & $\mathrm{pCi} / \mathrm{g}$ \\
\hline Ruthenium-106 & UI & & $-2.72 \mathrm{E}-05 \pm 8.42 \mathrm{E}-02$ & $\mathrm{pCi} / \mathrm{g}$ \\
\hline Sodium-22 & UI & & $-3.42 \mathrm{E}-03 \pm 8.00 \mathrm{E}-03$ & $\mathrm{pCi} / \mathrm{g}$ \\
\hline Strontium-90 & $\mathbf{U}$ & $\mathrm{V}$ & $1.07 \mathrm{E}+00 \pm 1.20 \mathrm{E}-01$ & $\mathrm{pCi} / \mathrm{g}$ \\
\hline Thorium-234 & & & $2.30 \mathrm{E}+00 \pm 9.84 \mathrm{E}-01$ & $\mathrm{pCi} / \mathrm{g}$ \\
\hline Tin-113 & UI & & $-1.10 \mathrm{E}-02 \pm 1.55 \mathrm{E}-02$ & $\mathrm{pCi} / \mathrm{g}$ \\
\hline Yttrium-88 & UI & & $4.55 \mathrm{E}-03 \pm 1.06 \mathrm{E}-02$ & $\mathrm{pCi} / \mathrm{g}$ \\
\hline Zinc-65 & UI & & $1.32 \mathrm{E}-02 \pm 2.23 \mathrm{E}-02$ & $\mathrm{pCi} / \mathrm{g}$ \\
\hline Zirconium-95 & UI & & $0.00 \mathrm{E}+00$ & $\mathrm{p} C \mathrm{Ci} / \mathrm{g}$ \\
\hline
\end{tabular}

Sample ID: 104678

D. Limit Method

SURVEY ID: PPSC 2101

Sample ID: 104680

Survey location: 44387E $3669759 \mathrm{~N}$ (SRS coordinates)

Depth of core interval: 0.00 to $1.00 \mathrm{ft}$

Sample type: Normal

Sample matrix: Soil

Percent solids: $\mathbf{8 3 . 0 0}$

Constituent

RQ AQ B Result

Unit

D. Limit Method

Physical Parameters and Specified Analyses

$\begin{array}{lllll}\text { Ammonia nitrogen } & \text { U } & & & 22.1 \\ \text { Cation exchange capacity } & & & & 13.2 \\ \text { Chloride } & \text { J } & \text { E } & 1.46 \\ \text { Cyanide } & \text { UJ } & \text { C } & 1.11 \\ \text { Nitrate-nitrite as nitrogen } & \text { J } & \text { E } & & 0.307 \\ \text { Nitrogen by Kjeldahl method } & & & & 57.7 \\ \text { Orthophosphate } & \text { U } & & & 24.1 \\ \text { Phenols } & \text { U } & & & 361 \\ \text { Sulfide } & \text { UJ } & \text { CI } & \text { L } & 301 \\ \text { Sulfide } & \text { UJ } & \text { CI } & \text { L } & 301 \\ \text { Total organic carbon } & & & & 2090 \\ \text { Total organic nitrogen } & \text { C } & & & 48 \\ \text { Total phosphates (as P) } & \text { J } & \text { E } & & 47.2\end{array}$

$\begin{array}{lll}\mathrm{mg} / \mathrm{kg} & 22.1 & \text { EPA350.1 } \\ \mathrm{meq} / 100 \mathrm{~g} & 5 & \text { EPA9081 } \\ \mathrm{mg} / \mathrm{kg} & 2.89 & \text { EPA300.0 } \\ \mathrm{mg} / \mathrm{kg} & 1.11 & \text { EPA335.3 } \\ \mathrm{mg} / \mathrm{kg} & 1.02 & \text { EPA353.1 } \\ \mathrm{mg} / \mathrm{kg} & 9.62 & \text { EPA351.2 } \\ \mathrm{mg} / \mathrm{kg} & 24.1 & \text { EPA365.2 } \\ \mu \mathrm{g} / \mathrm{kg} & 361 & \text { EPA420.2 } \\ \mathrm{mg} / \mathrm{kg} & 301 & \text { EPA376.2 } \\ \mathrm{mg} / \mathrm{kg} & 301 & \text { EPA376.2 } \\ \mathrm{mg} / \mathrm{kg} & 120 & \text { EPA415.1 } \\ \mathrm{mg} / \mathrm{kg} & 0.241 & \text { EPA351.2 } \\ \mathrm{mg} / \mathrm{kg} & 197 & \text { EPA365.4 }\end{array}$


SURVEY ID: PPSC 2101 (continued)

Constituent

Metals (total recoverable)

Aluminum

Aluminum

Antimony

Antimony

Arsenic

Arsenic

Barium

Barium

Beryllium

Beryllium

Cadmium

Cadmium

Calcium

Calcium

Chromium

Chromium

Cobalt

Cobalt

Copper

Copper

Iron

Iron

Lead

Lead

Magnesium

Magnesium

Manganese

Manganese

Mercury

Mercury

Nickel

Nickel

Potassium

Potassium

Selenium

Selenium

Selenium

Selenium

Silver

Silver

Sodium

Sodium

Thallium

Thallium

Vanadium

Vanadium

Zinc

Zinc
RQ AQ B Result

Unit

D. Limit Method

Sample ID: 104680

\begin{tabular}{|c|c|c|c|c|c|c|}
\hline & & & 1060 & $\mathrm{mg} / \mathrm{kg}$ & 14.2 & EPA6010A \\
\hline & & & 924 & $\mathrm{mg} / \mathrm{kg}$ & 13.8 & EPA6010A \\
\hline U & & & 5.68 & $\mathrm{mg} / \mathrm{kg}$ & 5.68 & EPA6010A \\
\hline$U$ & & & 5.53 & $\mathrm{mg} / \mathrm{kg}$ & 5.53 & EPA6010A \\
\hline U & & & 17 & $\mathrm{mg} / \mathrm{kg}$ & 17 & EPA6010A \\
\hline U & & & 16.6 & $\mathrm{mg} / \mathrm{kg}$ & 16.6 & EPA6010A \\
\hline & & & 5.63 & $\mathrm{mg} / \mathrm{kg}$ & 1.42 & EPA6010A \\
\hline & & & 6.2 & $\mathrm{mg} / \mathrm{kg}$ & 1.38 & EPA6010A \\
\hline J & $\mathbf{E}$ & & 0.0494 & $\mathrm{mg} / \mathrm{kg}$ & 0.568 & EPA6010A \\
\hline $\mathrm{J}$ & $\mathbf{E}$ & & 0.0608 & $\mathrm{mg} / \mathrm{kg}$ & 0.553 & EPA6010A \\
\hline U & & & 0.284 & $\mathrm{mg} / \mathrm{kg}$ & 0.284 & EPA6010A \\
\hline $\mathrm{U}$ & & & 0.276 & $\mathrm{mg} / \mathrm{kg}$ & 0.276 & EPA6010A \\
\hline & & & 112 & $\mathrm{mg} / \mathrm{kg}$ & 14.2 & EPA6010A \\
\hline & & & 142 & $\mathrm{mg} / \mathrm{kg}$ & 13.8 & EPA6010A \\
\hline & $\mathrm{X}$ & & 7.02 & $\mathrm{mg} / \mathrm{kg}$ & 1.42 & EPA6010A \\
\hline & $\mathrm{X}$ & & 1.86 & $\mathrm{mg} / \mathrm{kg}$ & 1.38 & EPA6010A \\
\hline & & & 0.723 & $\mathrm{mg} / \mathrm{kg}$ & 0.568 & EPA6010A \\
\hline & & & 0.701 & $\mathrm{mg} / \mathrm{kg}$ & 0.553 & EPA6010A \\
\hline $\mathrm{J}$ & $\mathbf{E}$ & & 1.17 & $\mathrm{mg} / \mathrm{kg}$ & 1.42 & EPA6010A \\
\hline $\mathrm{J}$ & $E$ & & 0.854 & $\mathrm{mg} / \mathrm{kg}$ & 1.38 & EPA6010A \\
\hline $\mathrm{J}$ & C & & 887 & $\mathrm{mg} / \mathrm{kg}$ & 5.68 & EPA6010A \\
\hline$J$ & C & & 946 & $\mathrm{mg} / \mathrm{kg}$ & 5.53 & EPA6010A \\
\hline $\mathbf{J}$ & $\mathbf{E}$ & & 2.66 & $\mathrm{mg} / \mathrm{kg}$ & 5.68 & EPA6010A \\
\hline J & $\mathrm{E}$ & & 2.57 & $\mathrm{mg} / \mathrm{kg}$ & 5.53 & EPA6010A \\
\hline & & & 16.3 & $\mathrm{mg} / \mathrm{kg}$ & 5.68 & EPA6010A \\
\hline & & & 15.1 & $\mathrm{mg} / \mathrm{kg}$ & 5.53 & EPA6010A \\
\hline $\mathrm{J}$ & I & $\mathrm{H}$ & 65.1 & $\mathrm{mg} / \mathrm{kg}$ & 0.568 & EPA6010A \\
\hline$J$ & I & $\mathrm{H}$ & 65.3 & $\mathrm{mg} / \mathrm{kg}$ & 0.553 & EPA6010A \\
\hline UJ & I & $\mathrm{H}$ & 0.0325 & $\mathrm{mg} / \mathrm{kg}$ & 0.0325 & EPA7471 \\
\hline UJ & I & $\mathrm{H}$ & 0.0344 & $\mathrm{mg} / \mathrm{kg}$ & 0.0344 & EPA7471 \\
\hline & & & 2.49 & $\mathrm{mg} / \mathrm{kg}$ & 1.42 & EPA6010A \\
\hline $\mathbf{J}$ & $\mathrm{E}$ & & 0.503 & $\mathrm{mg} / \mathrm{kg}$ & 1.38 & EPA6010A \\
\hline J & $\mathrm{E}$ & & 21.4 & $\mathrm{mg} / \mathrm{kg}$ & 114 & EPA6010A \\
\hline J & $\mathrm{E}$ & & .17 .3 & $\mathrm{mg} / \mathrm{kg}$ & 111 & EPA6010A \\
\hline $\mathrm{U}$ & & & 17 & $\mathrm{mg} / \mathrm{kg}$ & 17 & EPA6010A \\
\hline $\mathbf{U}$ & & & 13.8 & $\mathrm{mg} / \mathrm{kg}$ & 13.8 & EPA6010A \\
\hline U & & & 13.8 & $\mathrm{mg} / \mathrm{kg}$ & 13.8 & EPA6010A \\
\hline $\mathrm{U}$ & & & 16.6 & $\mathrm{mg} / \mathrm{kg}$ & 16.6 & EPA6010A \\
\hline U & & & 1.42 & $\mathrm{mg} / \mathrm{kg}$ & 1.42 & EPA6010A \\
\hline$U$ & & & 1.38 & $\mathrm{mg} / \mathrm{kg}$ & 1.38 & EPA6010A \\
\hline $\mathrm{U}$ & & & 56.8 & $\mathrm{mg} / \mathrm{kg}$ & 56.8 & EPA6010A \\
\hline U & & & 55.3 & $\mathrm{mg} / \mathrm{kg}$ & 55.3 & EPA6010A \\
\hline $\mathrm{U}$ & & & 17 & $\mathrm{mg} / \mathrm{kg}$ & 17 & EPA6010A \\
\hline $\mathrm{U}$ & & & 16.6 & $\mathrm{mg} / \mathrm{kg}$ & 16.6 & EPA6010A \\
\hline & & & 1.67 & $\mathrm{mg} / \mathrm{kg}$ & 0.568 & EPA6010A \\
\hline & & & 1.51 & $\mathrm{mg} / \mathrm{kg}$ & 0.553 & EPA6010A \\
\hline & & & 2.57 & $\mathrm{mg} / \mathrm{kg}$ & 1.14 & EPA6010A \\
\hline & & & 2.47 & $\mathrm{mg} / \mathrm{kg}$ & 1.11 & EPA6010A \\
\hline
\end{tabular}


Appendix D: Sediment Tables

D-250

SURVEY ID: PPSC 2101 (continued)

Constituent
Sample ID: 104680
RQ AQ B Result
Unit
D. Limit Method

\section{$B / N / A$ Extractables (including $P A H$ and phenols)}

\begin{tabular}{|c|c|c|c|}
\hline Acenaphthene & $\mathrm{U}$ & & 39.5 \\
\hline Acenaphthylene & $U$ & & 39.5 \\
\hline Anthracene & $U$ & & 39.5 \\
\hline Benzo[a]anthracene & $\mathrm{U}$ & & 3.95 \\
\hline Benzo[b]fluoranthene & $\mathrm{U}$ & & 3.95 \\
\hline Benzo[k]fluoranthene & $\mathrm{U}$ & & 3.95 \\
\hline Benzoic acid & U & & 39.5 \\
\hline Benzo $[g, h, i]$ perylene & $\mathrm{U}$ & & 39.5 \\
\hline Benzo[a]pyrene & $\mathrm{U}$ & & 3.95 \\
\hline Benzyl alcohol & $\mathrm{U}$ & & 39.5 \\
\hline Bis(2-chloroethoxy) methane & U & & 39.5 \\
\hline Bis(2-chloroethyl) ether & $\mathrm{U}$ & & 39.5 \\
\hline Bis(2-chloroisopropyl) ether & $\mathrm{U}$ & & 39.5 \\
\hline Bis(2-ethylhexyl) phthalate & $\mathbf{J}$ & $\mathbf{E}$ & 21.7 \\
\hline 4-Bromophenyl phenyl ether & $\mathrm{U}$ & & 39.5 \\
\hline Butylbenzyl phthalate & $U$ & & 39.5 \\
\hline 4-Chloroaniline & $\mathrm{U}$ & & 39.5 \\
\hline 4-Chloro-m-cresol & $\mathrm{U}$ & & 39.5 \\
\hline 2-Chloronaphthalene & $\mathbf{U}$ & & 39.5 \\
\hline 2-Chlorophenol & $\mathbf{U}$ & & 39.5 \\
\hline 4-Chlorophenyl phenyl ether & $\mathrm{U}$ & & 39.5 \\
\hline Chrysene & $\mathrm{U}$ & & 3.95 \\
\hline $\mathrm{m} / \mathrm{p}$-Cresol & $\mathrm{U}$ & & 395 \\
\hline o-Cresol (2-Methylphenol) & $\mathrm{U}$ & & 39.5 \\
\hline Dibenz $[a, h]$ anthracene & $\mathrm{U}$ & & 3.95 \\
\hline Dibenzofuran & $\mathrm{U}$ & & 39.5 \\
\hline Di-n-butyl phthalate & $\mathbf{J}$ & $\mathbf{E}$ & 25.7 \\
\hline 1,2-Dichlorobenzene & $\mathrm{U}$ & & 39.5 \\
\hline 1,3-Dichlorobenzene & $\mathrm{U}$ & & 39.5 \\
\hline 1,4-Dichlorobenzene & $\mathrm{U}$ & & 39.5 \\
\hline 3,3'-Dichlorobenzidine & $\mathrm{U}$ & & 39.5 \\
\hline 2,4-Dichlorophenol & $U$ & & 39.5 \\
\hline Diethyl phthalate & $\mathrm{U}$ & & 39.5 \\
\hline 2,4-Dimethyl phenol & U & & 39.5 \\
\hline Dimethyl phthalate & $\mathrm{U}$ & & 39.5 \\
\hline 2,4-Dinitrophenol & $\mathrm{U}$ & & 395 \\
\hline 2,4-Dinitrotoluene & UJ & $\mathrm{C}$ & 3.95 \\
\hline 2,6-Dinitrotoluene & $\mathrm{U}$ & & 3.95 \\
\hline Di-n-octyl phthalate & $\mathrm{U}$ & & 39.5 \\
\hline Fluoranthene & $\mathrm{U}$ & & 39.5 \\
\hline Fluorene & $\mathrm{U}$ & & 39.5 \\
\hline Hexachlorobenzene & U & & 3.95 \\
\hline Hexachlorobutadiene & $\mathrm{U}$ & & 39.5 \\
\hline Hexachlorocyclopentadiene & $\mathrm{U}$ & & 39.5 \\
\hline Hexachloroethane & $\mathrm{U}$ & & 3.95 \\
\hline Indeno $[1,2,3-c, d]$ pyrene & $\mathrm{U}$ & & 3.95 \\
\hline Isophorone & $U$ & & 39.5 \\
\hline 2-Methyl-4,6-dinitrophenol & $\mathrm{U}$ & & 395 \\
\hline 2-Methylnaphthalene & $\mathrm{U}$ & & 39.5 \\
\hline Naphthalene & $\mathrm{U}$ & & 39.5 \\
\hline m-Nitroaniline & $\mathrm{U}$ & & 39.5 \\
\hline o-Nitroaniline & $\mathrm{U}$ & & 39.5 \\
\hline p-Nitroaniline & $\mathrm{U}$ & & 39.5 \\
\hline Nitrobenzene & $\mathrm{U}$ & & 39.5 \\
\hline 2-Nitrophenol & $\mathrm{U}$ & & 39.5 \\
\hline
\end{tabular}

\begin{tabular}{|c|c|c|}
\hline$\mu \mathrm{g} / \mathrm{kg}$ & 39.5 & EPA8270 \\
\hline$\mu \mathrm{g} / \mathrm{kg}$ & 39.5 & EPA8270 \\
\hline$\mu \mathrm{g} / \mathrm{kg}$ & 39.5 & EPA8270 \\
\hline$\mu \mathrm{g} / \mathrm{kg}$ & 3.95 & EPA8270 \\
\hline$\mu \mathrm{g} / \mathrm{kg}$ & 3.95 & EPA8270 \\
\hline$\mu \mathrm{g} / \mathrm{kg}$ & 3.95 & EPA8270 \\
\hline$\mu \mathrm{g} / \mathrm{kg}$ & 39.5 & EPA8270 \\
\hline$\mu \mathrm{g} / \mathrm{kg}$ & 39.5 & EPA8270 \\
\hline$\mu \mathrm{g} / \mathrm{kg}$ & 3.95 & EPA8270 \\
\hline$\mu \mathrm{g} / \mathrm{kg}$ & 39.5 & EPA8270 \\
\hline$\mu \mathrm{g} / \mathrm{kg}$ & 39.5 & EPA8270 \\
\hline$\mu \mathrm{g} / \mathrm{kg}$ & 39.5 & EPA8270 \\
\hline$\mu \mathrm{g} / \mathrm{kg}$ & 39.5 & EPA8270 \\
\hline$\mu \mathrm{g} / \mathrm{kg}$ & 39.5 & EPA8270 \\
\hline$\mu \mathrm{g} / \mathrm{kg}$ & 39.5 & EPA8270 \\
\hline$\mu \mathrm{g} / \mathrm{kg}$ & 39.5 & EPA8270 \\
\hline$\mu \mathrm{g} / \mathrm{kg}$ & 39.5 & EPA8270 \\
\hline$\mu \mathrm{g} / \mathrm{kg}$ & 39.5 & EPA8270 \\
\hline$\mu \mathrm{g} / \mathrm{kg}$ & 39.5 & EPA8270 \\
\hline$\mu \mathrm{g} / \mathrm{kg}$ & 39.5 & EPA8270 \\
\hline$\mu \mathrm{g} / \mathrm{kg}$ & 39.5 & EPA8270 \\
\hline$\mu \mathrm{g} / \mathrm{kg}$ & 3.95 & EPA8270 \\
\hline$\mu \mathrm{g} / \mathrm{kg}$ & 395 & EPA8270 \\
\hline$\mu \mathrm{g} / \mathrm{kg}$ & 39.5 & EPA8270 \\
\hline$\mu \mathrm{g} / \mathrm{kg}$ & 3.95 & EPA8270 \\
\hline$\mu \mathrm{g} / \mathrm{kg}$ & 39.5 & EPA8270 \\
\hline$\mu \mathrm{g} / \mathrm{kg}$ & 39.5 & EPA8270 \\
\hline$\mu \mathrm{g} / \mathrm{kg}$ & 39.5 & EPA8270 \\
\hline$\mu \mathrm{g} / \mathrm{kg}$ & 39.5 & EPA8270 \\
\hline$\mu \mathrm{g} / \mathrm{kg}$ & 39.5 & EPA8270 \\
\hline$\mu \mathrm{g} / \mathrm{kg}$ & 39.5 & EPA8270 \\
\hline$\mu \mathrm{g} / \mathrm{kg}$ & 39.5 & EPA8270 \\
\hline$\mu \mathrm{g} / \mathrm{kg}$ & 39.5 & EPA8270 \\
\hline$\mu \mathrm{g} / \mathrm{kg}$ & 39.5 & EPA8270 \\
\hline$\mu \mathrm{g} / \mathrm{kg}$ & 39.5 & EPA8270 \\
\hline$\mu \mathrm{g} / \mathrm{kg}$ & 395 & EPA8270 \\
\hline$\mu \mathrm{g} / \mathrm{kg}$ & 3.95 & EPA8270 \\
\hline$\mu \mathrm{g} / \mathrm{kg}$ & 3.95 & EPA8270 \\
\hline$\mu \mathrm{g} / \mathrm{kg}$ & 39.5 & EPA8270 \\
\hline$\mu \mathrm{g} / \mathrm{kg}$ & 39.5 & EPA8270 \\
\hline$\mu \mathrm{g} / \mathrm{kg}$ & 39.5 & EPA8270 \\
\hline$\mu \mathrm{g} / \mathrm{kg}$ & 3.95 & EPA8270 \\
\hline$\mu \mathrm{g} / \mathrm{kg}$ & 39.5 & EPA8270 \\
\hline$\mu \mathrm{g} / \mathrm{kg}$ & 39.5 & EPA8270 \\
\hline$\mu g / \mathrm{kg}$ & 3.95 & EPA8270 \\
\hline$\mu \mathrm{g} / \mathrm{kg}$ & 3.95 & EPA8270 \\
\hline$\mu \mathrm{g} / \mathrm{kg}$ & 39.5 & EPA8270 \\
\hline$\mu \mathrm{g} / \mathrm{kg}$ & 395 & EPA8270 \\
\hline$\mu \mathrm{g} / \mathrm{kg}$ & 39.5 & EPA8270 \\
\hline$\mu \mathrm{g} / \mathrm{kg}$ & 39.5 & EPA8270 \\
\hline$\mu \mathrm{g} / \mathrm{kg}$ & 39.5 & EPA8270 \\
\hline$\mu \mathrm{g} / \mathrm{kg}$ & 39.5 & EPA8270 \\
\hline$\mu \mathrm{g} / \mathrm{kg}$ & 39.5 & EPA8270 \\
\hline$\mu \mathrm{g} / \mathrm{kg}$ & 39.5 & EPA8270 \\
\hline$\mu \mathrm{g} / \mathrm{kg}$ & 39.5 & EPA8270 \\
\hline
\end{tabular}


SURVEY ID: PPSC 2101 (continued)

Sample ID: 104680

Constituent

RQ AQ B Result

Unit

D. Limit Method

$B / N / A$ Extractables (including $P A H$ and phenols)

4-Nitrophenol

N-Nitrosodiphenylamine

N-Nitrosodi-n-propylamine

Pentachlorophenol

Phenanthrene

Phenol

Pyrene

1,2,4-Trichlorobenzene

2,4,5-Trichlorophenol

2,4,6-Trichlorophenol

Volatile Organic Compounds

$\begin{array}{lll}\text { U } & & 39.5 \\ \text { U } & & 39.5 \\ \text { U } & & 39.5 \\ \text { UJ } & \text { C } & 39.5 \\ \text { U } & & 39.5 \\ \text { U } & & 39.5 \\ \text { U } & & 39.5 \\ \text { U } & & 3.95 \\ \text { U } & & 39.5 \\ \text { U } & & 39.5\end{array}$

U

Benzene

Bromodichloromethane

Bromoform

Bromomethane (Methyl bromide)

Carbon disulfide

Carbon tetrachloride

Chlorobenzene

Chloroethane

Chloroethene (Vinyl chloride)

Chloroform

Chloromethane (Methyl chloride)

Dibromochloromethane

1,1-Dichloroethane

1,2-Dichloroethane

1,1-Dichloroethylene

1,2-Dichloroethylene

Dichloromethane (Methylene chloride)

1,2-Dichloropropane

cis-1,3-Dichloropropene

trans-1,3-Dichloropropene

Ethylbenzene

2-Hexanone

Methyl ethyl ketone

Methyl isobutyl ketone

Styrene

1,1,2,2-Tetrachloroethane

Tetrachloroethylene

Toluene

1,1,1-Trichloroethane

1,1,2-Trichloroethane

Trichloroethylene

Vinyl acetate

Xylenes $\mu \mathrm{g} / \mathrm{kg}$

$\mu \mathrm{g} / \mathrm{kg}$

$\mu \mathrm{g} / \mathrm{kg}$

$\mu g / \mathrm{kg}$

$\mu \mathrm{g} / \mathrm{kg}$

$\mu \mathrm{g} / \mathrm{kg}$

$\mu \mathrm{g} / \mathrm{kg}$

$\mu \mathrm{g} / \mathrm{kg}$

$\mu \mathrm{g} / \mathrm{kg}$

$\mu g / \mathrm{kg}$

20.1

0.602

0.0602

0.0602

0.12

1.2

0.0602

0.0602

0.12

0.12

0.0602

0.12

0.0602

0.0602

0.0602

0.0602

0.12

$\mathrm{V} \quad \mathbf{1 . 2 5}$

0.0602

0.0602

0.0602

0.494

$\mathrm{V} \quad 1.99$

V8 $\quad 0.361$

1.2

0.0602

0.0602

0.145

$8 \quad 1.3$

0.0602

0.0602

$8 \quad 0.145$

1.2

$8 \quad 2.23$

$\begin{array}{lll}\mu \mathrm{g} / \mathrm{kg} & 6.02 & \text { EPA8260 } \\ \mu \mathrm{g} / \mathrm{kg} & 0.602 & \text { EPA8260 } \\ \mu \mathrm{g} / \mathrm{kg} & 0.0602 & \text { EPA8260 } \\ \mu \mathrm{g} / \mathrm{kg} & 0.0602 & \text { EPA8260 } \\ \mu \mathrm{g} / \mathrm{kg} & 0.12 & \text { EPA8260 } \\ \mu \mathrm{g} / \mathrm{kg} & 1.2 & \text { EPA8260 } \\ \mu \mathrm{g} / \mathrm{kg} & 0.0602 & \text { EPA8260 } \\ \mu \mathrm{g} / \mathrm{kg} & 0.0602 & \text { EPA8260 } \\ \mu \mathrm{g} / \mathrm{kg} & 0.12 & \text { EPA8260 } \\ \mu \mathrm{g} / \mathrm{kg} & 0.12 & \text { EPA8260 } \\ \mu \mathrm{g} / \mathrm{kg} & 0.0602 & \text { EPA8260 } \\ \mu \mathrm{g} / \mathrm{kg} & 0.12 & \text { EPA8260 } \\ \mu \mathrm{g} / \mathrm{kg} & 0.0602 & \text { EPA8260 } \\ \mu \mathrm{g} / \mathrm{kg} & 0.0602 & \text { EPA8260 } \\ \mu \mathrm{g} / \mathrm{kg} & 0.0602 & \text { EPA8260 } \\ \mu \mathrm{g} / \mathrm{kg} & 0.0602 & \text { EPA8260 } \\ \mu \mathrm{g} / \mathrm{kg} & 0.12 & \text { EPA8260 } \\ \mu \mathrm{g} / \mathrm{kg} & 0.602 & \text { EPA8260 } \\ \mu \mathrm{g} / \mathrm{kg} & 0.0602 & \text { EPA8260 } \\ \mu \mathrm{g} / \mathrm{kg} & 0.0602 & \text { EPA8260 } \\ \mu \mathrm{g} / \mathrm{kg} & 0.0602 & \text { EPA8260 } \\ \mu \mathrm{g} / \mathrm{kg} & 0.0602 & \text { EPA8260 } \\ \mu \mathrm{g} / \mathrm{kg} & 1.2 & \text { EPA8260 } \\ \mu \mathrm{g} / \mathrm{kg} & 1.2 & \text { EPA8260 } \\ \mu \mathrm{g} / \mathrm{kg} & 1.2 & \text { EPA8260 } \\ \mu \mathrm{g} / \mathrm{kg} & 0.0602 & \text { EPA8260 } \\ \mu \mathrm{g} / \mathrm{kg} & 0.0602 & \text { EPA8260 } \\ \mu \mathrm{g} / \mathrm{kg} & 0.0602 & \text { EPA8260 } \\ \mu \mathrm{g} / \mathrm{kg} & 0.602 & \text { EPA8260 } \\ \mu \mathrm{g} / \mathrm{kg} & 0.0602 & \text { EPA8260 } \\ \mu \mathrm{g} / \mathrm{kg} & 0.0602 & \text { EPA8260 } \\ \mu \mathrm{g} / \mathrm{kg} & 0.0602 & \text { EPA8260 } \\ \mu \mathrm{g} / \mathrm{kg} & 1.2 & \text { EPA8260 } \\ \mu \mathrm{g} / \mathrm{kg} & 0.181 & \text { EPA8260 } \\ & & \end{array}$

EPA8270

EPA8270

EPA8270

EPA8270

EPA8270

EPA8270

EPA8270

EPA8270

EPA8270

EPA8270 
SURVEY ID: PPSC 2101 (continued)

Constituent

Pesticides/PCBs and Dioxins/Furans

Aldrin

alpha-Benzene hexachloride

beta-Benzene hexachloride

delta-Benzene hexachloride

alpha-Chlordane

gamma-Chlordane

p,p'-DDD

$p, p^{\prime}-D D E$

$\mathrm{p}, \mathrm{p}^{\prime}-\mathrm{DDT}$

Dieldrin

Endosulfan sulfate

Endosulfan I

Endosulfan II

Endrin

Endrin ketone

Heptachlor

Heptachlor epoxide

Lindane

Methoxychlor

PCB 1016

PCB 1221

PCB 1232

PCB 1242

PCB 1248

PCB 1254

PCB 1260

Toxaphene

Radionuclides

Actinium-228

Antimony-124

Antimony-125

Barium-133

Cerium-144

Cesium-134

Cesium-137

Cobalt-57

Cobalt-58

Cobalt-60

Europium-152

Europium-154

Europium-155

Gross alpha

Lead-212

Manganese-54

Neptunium-239

Nonvolatile beta

Plutonium-238

Plutonium-239/240

Potassium-40

Promethium-144

Promethium-146

Radium-226

Radium-228
Sample ID: 104680

RQ AQ B Result

Unit

D. Limit Method

$\begin{array}{lll}\mu \mathrm{g} / \mathrm{kg} & 4.98 & \text { EPA8080 } \\ \mu \mathrm{g} / \mathrm{kg} & 4.98 & \text { EPA8080 } \\ \mu \mathrm{g} / \mathrm{kg} & 9.96 & \text { EPA8080 } \\ \mu \mathrm{g} / \mathrm{kg} & 9.96 & \text { EPA8080 } \\ \mu \mathrm{g} / \mathrm{kg} & 9.96 & \text { EPA8080 } \\ \mu \mathrm{g} / \mathrm{kg} & 9.96 & \text { EPA8080 } \\ \mu \mathrm{g} / \mathrm{kg} & 19.9 & \text { EPA8080 } \\ \mu \mathrm{g} / \mathrm{kg} & 9.96 & \text { EPA8080 } \\ \mu \mathrm{g} / \mathrm{kg} & 19.9 & \text { EPA8080 } \\ \mu \mathrm{g} / \mathrm{kg} & 9.96 & \text { EPA8080 } \\ \mu \mathrm{g} / \mathrm{kg} & 19.9 & \text { EPA8080 } \\ \mu \mathrm{g} / \mathrm{kg} & 9.96 & \text { EPA8080 } \\ \mu \mathrm{g} / \mathrm{kg} & 19.9 & \text { EPA8080 } \\ \mu \mathrm{g} / \mathrm{kg} & 9.96 & \text { EPA8080 } \\ \mu \mathrm{g} / \mathrm{kg} & 19.9 & \text { EPA8080 } \\ \mu \mathrm{g} / \mathrm{kg} & 9.96 & \text { EPA8080 } \\ \mu \mathrm{g} / \mathrm{kg} & 9.96 & \text { EPA8080 } \\ \mu \mathrm{g} / \mathrm{kg} & 4.98 & \text { EPA8080 } \\ \mu \mathrm{g} / \mathrm{kg} & 99.6 & \text { EPA8080 } \\ \mu \mathrm{g} / \mathrm{kg} & 24.9 & \text { EPA8080 } \\ \mu \mathrm{g} / \mathrm{kg} & 24.9 & \text { EPA8080 } \\ \mu \mathrm{g} / \mathrm{kg} & 24.9 & \text { EPA8080 } \\ \mu \mathrm{g} / \mathrm{kg} & 24.9 & \text { EPA8080 } \\ \mu \mathrm{g} / \mathrm{kg} & 24.9 & \text { EPA8080 } \\ \mu \mathrm{g} / \mathrm{kg} & 24.9 & \text { EPA8080 } \\ \mu \mathrm{g} / \mathrm{kg} & 24.9 & \text { EPA8080 } \\ \mu \mathrm{g} / \mathrm{kg} & 199 & \text { EPA8080 }\end{array}$

3.90E-01 $\pm 2.77 \mathrm{E}-02 \mathrm{pCi} / \mathrm{g}$

UI

UI

UI

UI

UI

UI

UI

UI

UI

UI

UI

UI

UI

UI

U V

UI

UI

$3.18 \mathrm{E}-04 \pm 3.30 \mathrm{E}-03 \mathrm{pCi} / \mathrm{g}$ 2.60E-03 $\pm 7.32 \mathrm{E}-03 \mathrm{pCi} / \mathrm{g}$ $-1.59 \mathrm{E}-02 \pm 3.88 \mathrm{E}-03 \mathrm{pCi} / \mathrm{g}$ 8.13E-03 $\pm 2.01 \mathrm{E}-02 \mathrm{pCi} / \mathrm{g}$ $-1.32 \mathrm{E}-03 \pm 2.80 \mathrm{E}-03 \mathrm{pCi} / \mathrm{g}$ 4.04E-02 $\pm 5.54 \mathrm{E}-03 \mathrm{pCi} / \mathrm{g}$ $-1.54 \mathrm{E}-03 \pm 2.50 \mathrm{E}-03 \mathrm{pCi} / \mathrm{g}$ $-8.29 \mathrm{E}-04 \pm 3.41 \mathrm{E}-03 \mathrm{pCi} / \mathrm{g}$ $-8.77 \mathrm{E}-04 \pm 2.32 \mathrm{E}-03 \mathrm{pCi} / \mathrm{g}$ $-4.38 \mathrm{E}-03 \pm 7.72 \mathrm{E}-03 \mathrm{pCi} / \mathrm{g}$ 9.82E-03 $\pm 2.14 \mathrm{E}-02 \mathrm{pCi} / \mathrm{g}$ $1.12 \mathrm{E}-02 \pm 1.64 \mathrm{E}-02 \mathrm{pCi} / \mathrm{g}$ $7.83 \mathrm{E}+00 \pm 2.19 \mathrm{E}+00 \mathrm{pCi} / \mathrm{g}$ $3.65 \mathrm{E}-01 \pm 1.28 \mathrm{E}-02 \mathrm{pCi} / \mathrm{g}$ $6.39 \mathrm{E}-03 \pm 3.97 \mathrm{E}-03 \mathrm{pCi} / \mathrm{g}$ $-9.35 \mathrm{E}-03 \pm 1.82 \mathrm{E}-02 \mathrm{pCi} / \mathrm{g}$ $3.25 \mathrm{E}+00 \pm 1.35 \mathrm{E}+00 \mathrm{pCi} / \mathrm{g}$ $-4.44 \mathrm{E}-03 \pm 8.78 \mathrm{E}-03 \mathrm{pCi} / \mathrm{g}$ 4.35E-03 $\pm 8.70 \mathrm{E}-03 \mathrm{pCi} / \mathrm{g}$ $1.36 \mathrm{E}-01 \pm 5.21 \mathrm{E}-02 \mathrm{pCi} / \mathrm{g}$ $-5.99 \mathrm{E}-04 \pm 2.53 \mathrm{E}-03 \mathrm{pCi} / \mathrm{g}$ $8.86 \mathrm{E}-04 \pm 3.54 \mathrm{E}-03 \mathrm{pCi} / \mathrm{g}$

4.98
4.98
9.96
9.96
9.96
9.96
19.9
9.96
19.9
9.96
19.9
9.96
19.9
9.96
19.9
9.96
9.96
4.98
99.6
24.9
24.9
24.9
24.9
24.9
24.9
24.9
199
$2.86 \mathrm{E}-01 \pm 1.79 \mathrm{E}-02 \mathrm{pCi} / \mathrm{g}$ $3.90 \mathrm{E}-01 \pm 2.77 \mathrm{E}-02 \mathrm{pCi} / \mathrm{g}$
1.42E-02 EPIA-013B
5.82E-03 EPLA-013B
1.23E-02 EPIA-013B
5.45E-03 EPIA-013B
3.48E-02 EPIA-013B
4.20E-03 EPLA-013B
4.40E-03 EPIA-013B
4.27E-03 EPIA-013B
5.11E-03 EPIA-013B
4.08E-03 EPIA.013B
1.26E-02 EPIA-013B
3.86E-02 EPIA-013B
1.84E-02 EPIA-013B
1.44E+00 EPIA-001B
7.73E-03 EPLA-013B
3.54E-03 EPIA-013B
3.12E-02 EPIA-013B
2.40E+00 EPIA-001B
3.02E-02 EPIA-012B
1.83E-02 EPIA-012B
4.74E-02 EPIA-013B
- 4.36E-03 EPIA-013B
5.91E-03 EPIA-013B
8.27E-03 EPIA-013B
1.42E-02 EPIA-013B 
SURVEY ID: PPSC 2101 (continued)

Constituent

Radionuclides

Ruthenium-106

Sodium-22

Strontium-90

Thorium-234

Tin-113

Yttrium-88

Zinc-65

Zirconium-95

Sample ID: 104680

RQ AQ B Result Unit $\quad$ D. Limit Method

UI

UI

\section{V}

UI

UI

UI

UI

$$
\begin{aligned}
& -5.28 \mathrm{E}-03 \pm 2.34 \mathrm{E}-02 \mathrm{pCi} / \mathrm{g} \\
& 1.99 \mathrm{E}-03 \pm 2.27 \mathrm{E}-03 \mathrm{pCi} / \mathrm{g} \\
& 2.54 \mathrm{E}+00 \pm 2.60 \mathrm{E}-01 \mathrm{pC} / \mathrm{g} \\
& 3.12 \mathrm{E}-01 \pm 2.90 \mathrm{E}-01 \mathrm{pCi} / \mathrm{g} \\
& 6.74 \mathrm{E}-04 \pm 4.41 \mathrm{E}-03 \mathrm{pCi} / \mathrm{g} \\
& 2.12 \mathrm{E}-03 \pm 3.47 \mathrm{E}-03 \mathrm{pCi} / \mathrm{g} \\
& -4.16 \mathrm{E}-03 \pm 5.95 \mathrm{E}-03 \mathrm{pCi} / \mathrm{g} \\
& 0.00 \mathrm{E}+00
\end{aligned}
$$

4.06E-02 EPIA-013B

4.43E-03 EPIA-013B

1.30E-01 EPIA-004

2.29E-01 EPIA-013B

6.56E-03 EPIA-013B

5.76E-03 EPIA-013B

8.20E-03 EPIA-013B

1.17E-02 EPIA-013B

SURVEY ID: PPSC 2102

Sample ID: 104681

Survey location: $44387 \mathrm{E}$ 3669759N (SRS coordinates)

Depth of core interval: 1.00 to $2.00 \mathrm{ft}$

Sample type: Normal

Sample matrix: Soil

Percent solids: $\mathbf{8 7 . 0 0}$

Constituent

RQ AQ B Result

Unit

D. Limit Method

Physical Parameters and Specified Analyses

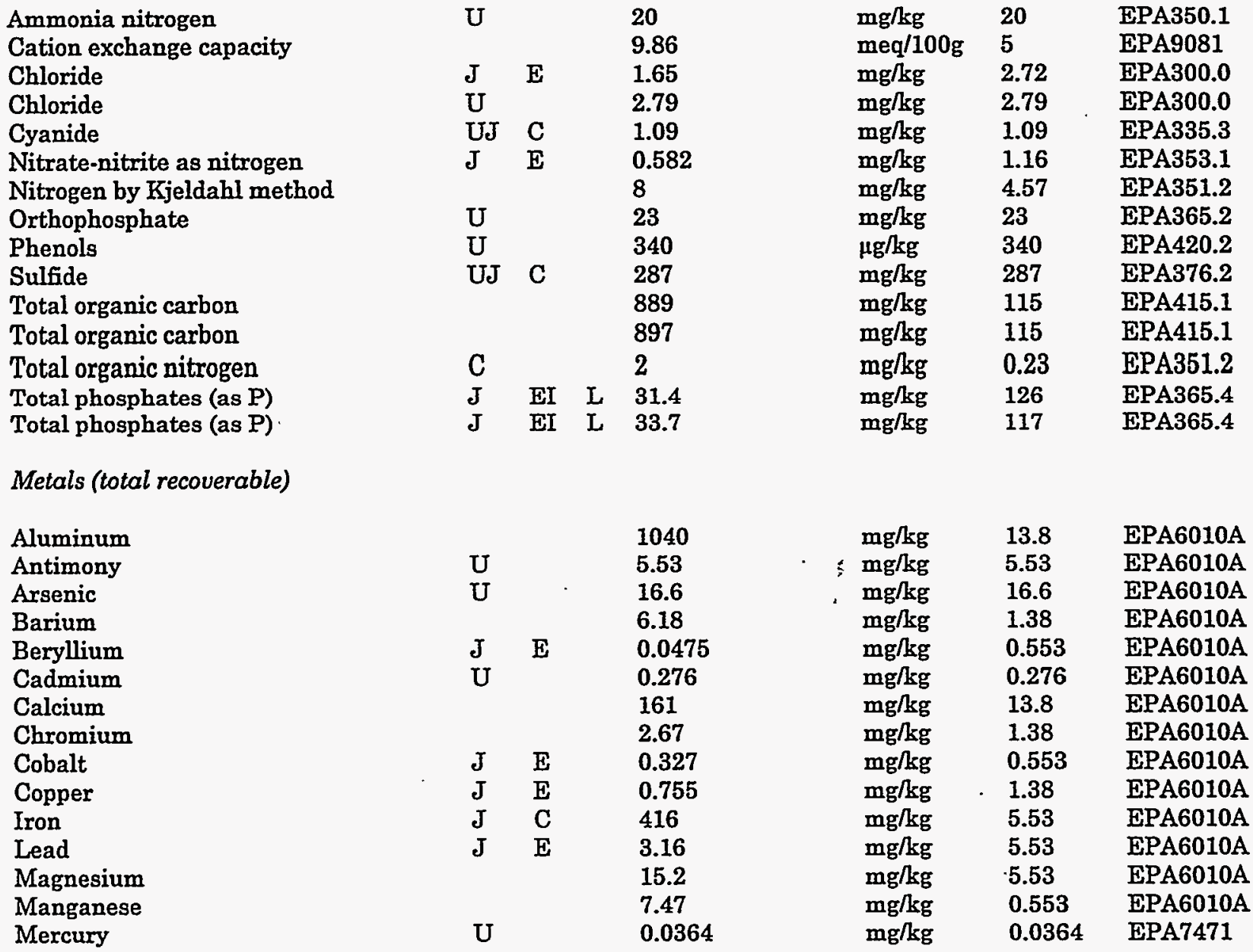


SURVEY ID: PPSC 2102 (continued)

Constituent

RQ AQ B Result

Unit D. Limit Method

Unit D. Limit Method

Sample ID: 104681

Metals (total recoverable)

Nickel

Potassium

Selenium

Silver

Sodium

Thallium

Vanadium

Zinc

$\begin{array}{lll}\text { U } & & 1.38 \\ \text { J } & \text { E } & 25.5 \\ \text { U } & & 16.6 \\ \text { U } & & 1.38 \\ \text { U } & & 55.3 \\ \text { U } & & 16.6 \\ & & 2.37 \\ \text { J } & \text { E } & 0.809\end{array}$

$B / N / A$ Extractables (including $P A H$ and phenols)

\begin{tabular}{|c|c|c|}
\hline UJ & $\mathbf{Q}$ & 37.3 \\
\hline UJ & Q & 37.3 \\
\hline UJ & Q & 37.3 \\
\hline UJ & $\mathbf{Q}$ & 3.73 \\
\hline UJ & $\mathbf{Q}$ & 3.73 \\
\hline UJ & $\mathbf{Q}$ & 3.73 \\
\hline UJ & $\mathbf{Q}$ & 37.3 \\
\hline UJ & $Q$ & 37.3 \\
\hline UJ & $\mathbf{Q}$ & 3.73 \\
\hline UJ & $\mathbf{Q}$ & 37.3 \\
\hline UJ & $\mathbf{Q}$ & 37.3 \\
\hline UJ & $\mathbf{Q}$ & 37.3 \\
\hline UJ & $\mathbf{Q}$ & 37.3 \\
\hline UJ & $\mathbf{Q}$ & 37.3 \\
\hline UJ & $\mathbf{Q}$ & 37.3 \\
\hline UJ & $\mathbf{Q}$ & 37.3 \\
\hline UJ & Q & 37.3 \\
\hline UJ & $\mathbf{Q}$ & 37.3 \\
\hline UJ & Q & 37.3 \\
\hline UJ & $\mathbf{Q}$ & 37.3 \\
\hline UJ & $\mathrm{Q}$ & 37.3 \\
\hline UJ & $\mathbf{Q}$ & 3.73 \\
\hline UJ & $\mathrm{Q}$ & 373 \\
\hline UJ & $Q$ & 37.3 \\
\hline UJ & $\mathbf{Q}$ & 3.73 \\
\hline UJ & $\mathbf{Q}$ & 37.3 \\
\hline UJ & $\mathbf{Q}$ & 37.3 \\
\hline UJ & $\mathbf{Q}$ & 37.3 \\
\hline UJ & $\mathbf{Q}$ & 37.3 \\
\hline UJ & $Q$ & 37.3 \\
\hline UJ & $\mathbf{Q}$ & 37.3 \\
\hline UJ & $Q$ & 37.3 \\
\hline UJ & $\dot{Q}$ & 37.3 \\
\hline UJ & $\mathbf{Q}$ & 37.3 \\
\hline UJ & $\mathbf{Q}$ & 37.3 \\
\hline UJ & $Q$ & 373 \\
\hline UJ & QC & 3.73 \\
\hline UJ & $\mathbf{Q}$ & 3.73 \\
\hline UJ & $\mathbf{Q}$ & 37.3 \\
\hline UJ & $\mathbf{Q}$ & 37.3 \\
\hline UJ & Q & 37.3 \\
\hline UJ & Q & 3.73 \\
\hline UJ & Q & 37.3 \\
\hline UJ & $\mathbf{Q}$ & 37.3 \\
\hline
\end{tabular}

$\begin{array}{lll}\mathrm{mg} / \mathrm{kg} & 1.38 & \text { EPA6010A } \\ \mathrm{mg} / \mathrm{kg} & 111 & \text { EPA6010A } \\ \mathrm{mg} / \mathrm{kg} & 16.6 & \text { EPA6010A } \\ \mathrm{mg} / \mathrm{kg} & 1.38 & \text { EPA6010A } \\ \mathrm{mg} / \mathrm{kg} & 55.3 & \text { EPA6010A } \\ \mathrm{mg} / \mathrm{kg} & 16.6 & \text { EPA6010A } \\ \mathrm{mg} / \mathrm{kg} & 0.553 & \text { EPA6010A } \\ \mathrm{mg} / \mathrm{kg} & 1.11 & \text { EPA6010A }\end{array}$

$\begin{array}{lll}\mu \mathrm{g} / \mathrm{kg} & 37.3 & \text { EPA8270 } \\ \mu \mathrm{g} / \mathrm{kg} & 37.3 & \text { EPA8270 } \\ \mu \mathrm{g} / \mathrm{kg} & 37.3 & \text { EPA8270 } \\ \mu \mathrm{g} / \mathrm{kg} & 3.73 & \text { EPA8270 } \\ \mu \mathrm{g} / \mathrm{kg} & 3.73 & \text { EPA8270 } \\ \mu \mathrm{g} / \mathrm{kg} & 3.73 & \text { EPA8270 } \\ \mu \mathrm{g} / \mathrm{kg} & 37.3 & \text { EPA8270 } \\ \mu \mathrm{g} / \mathrm{kg} & 37.3 & \text { EPA8270 } \\ \mu \mathrm{g} / \mathrm{kg} & 3.73 & \text { EPA8270 } \\ \mu \mathrm{g} / \mathrm{kg} & 37.3 & \text { EPA8270 } \\ \mu \mathrm{g} / \mathrm{kg} & 37.3 & \text { EPA8270 } \\ \mu \mathrm{g} / \mathrm{kg} & 37.3 & \text { EPA8270 } \\ \mu \mathrm{g} / \mathrm{kg} & 37.3 & \text { EPA8270 } \\ \mu \mathrm{g} / \mathrm{kg} & 37.3 & \text { EPA8270 } \\ \mu \mathrm{g} / \mathrm{kg} & 37.3 & \text { EPA8270 } \\ \mu \mathrm{g} / \mathrm{kg} & 37.3 & \text { EPA8270 } \\ \mu \mathrm{g} / \mathrm{kg} & 37.3 & \text { EPA8270 } \\ \mu \mathrm{g} / \mathrm{kg} & 37.3 & \text { EPA8270 } \\ \mu \mathrm{g} / \mathrm{kg} & 37.3 & \text { EPA8270 } \\ \mu \mathrm{g} / \mathrm{kg} & 37.3 & \text { EPA8270 } \\ \mu \mathrm{g} / \mathrm{kg} & 37.3 & \text { EPA8270 } \\ \mu \mathrm{g} / \mathrm{kg} & 3.73 & \text { EPA8270 } \\ \mu \mathrm{g} / \mathrm{kg} & 373 & \text { EPA8270 } \\ \mu \mathrm{g} / \mathrm{kg} & 37.3 & \text { EPA8270 } \\ \mu \mathrm{g} / \mathrm{kg} & 3.73 & \text { EPA8270 } \\ \mu \mathrm{g} / \mathrm{kg} & 37.3 & \text { EPA8270 } \\ \mu \mathrm{g} / \mathrm{kg} & 37.3 & \text { EPA8270 } \\ \mu \mathrm{g} / \mathrm{kg} & 37.3 & \text { EPA8270 } \\ \mu \mathrm{g} / \mathrm{kg} & 37.3 & \text { EPA8270 } \\ \mu \mathrm{g} / \mathrm{kg} & 37.3 & \text { EPA8270 } \\ \mu \mathrm{g} / \mathrm{kg} & 37.3 & \text { EPA8270 } \\ \mu \mathrm{g} / \mathrm{kg} & 37.3 & \text { EPA8270 } \\ \mu \mathrm{g} / \mathrm{kg} & 37.3 & \text { EPA8270 } \\ \mu \mathrm{g} / \mathrm{kg} & 37.3 & \text { EPA8270 } \\ \mu \mathrm{g} / \mathrm{kg} & 37.3 & \text { EPA8270 } \\ \mu \mathrm{g} / \mathrm{kg} & 373 & \text { EPA8270 } \\ \mu \mathrm{g} / \mathrm{kg} & 3.73 & \text { EPA8270 } \\ \mu \mathrm{g} / \mathrm{kg} & 3.73 & \text { EPA8270 } \\ \mu \mathrm{g} / \mathrm{kg} & 37.3 & \text { EPA8270 } \\ \mu \mathrm{g} / \mathrm{kg} & 37.3 & \text { EPA8270 } \\ \mu \mathrm{g} / \mathrm{kg} & 37.3 & \text { EPA8270 } \\ \mu \mathrm{g} / \mathrm{kg} & 3.73 & \text { EPA8270 } \\ \mu \mathrm{g} / \mathrm{kg} & 37.3 & \text { EPA8270 } \\ \mu \mathrm{gg} / \mathrm{kg} & 37.3 & \text { EPA8270 } \\ & & \end{array}$

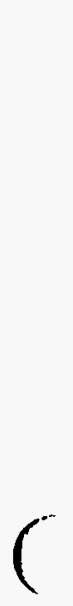

Butylbenzyl phthalate

4-Chloroaniline

4-Chloro-m-cresol

2-Chloronaphthalene

2-Chlorophenol

4-Chlorophenyl phenyl ether

Chrysene

$\mathrm{m} / \mathrm{p}$-Cresol

o-Cresol (2-Methylphenol)

Dibenz $[a, h]$ anthracene

Dibenzofuran

Di-n-butyl phthalate

1,2-Dichlorobenzene

1,3-Dichlorobenzene

1,4-Dichlorobenzene

3,3'-Dichlorobenzidine

2,4-Dichlorophenol

Diethyl phthalate

2,4-Dimethyl phenol

Dimethyl phthalate

2,4-Dinitrophenol

2,4-Dinitrotoluene

2,6-Dinitrotoluene

Di-n-octyl phthalate

Fluoranthene

Fluorene

Hexachlorobenzene

Hexachlorobutadiene

Hexachlorocyclopentadiene 
SURVEY ID: PPSC 2102 (continued)

Sample ID: 104681

Constituent

RQ AQ B Result

Unit

D. Limit Method

$B / N / A$ Extractables (including PAH and phenols)

Hexachloroethane

Indeno[1,2,3-c,d]pyrene

Isophorone

2-Methyl-4,6-dinitrophenol

2-Methylnaphthalene

Naphthalene

m-Nitroaniline

o-Nitroaniline

p-Nitroaniline

Nitrobenzene

2-Nitrophenol

4-Nitrophenol

N-Nitrosodiphenylamine

N-Nitrosodi-n-propylamine

Pentachlorophenol

Phenanthrene

Phenol

Pyrene

1,2,4-Trichlorobenzene

2,4,5-Trichlorophenol

2,4,6-Trichlorophenol
UJ Q

UJ $\mathbf{Q}$

UJ $Q$

UJ $Q$

UJ Q

UJ $Q$

UJ $Q$

UJ $Q$

UJ $\mathbf{Q}$

UJ $Q$

UJ $Q$

UJ $Q$

UJ $\mathbf{Q}$

UJ $Q$

UJ $Q$

UJ $Q$

UJ Q

UJ Q

UJ $Q$

UJ
3.73

3.73

37.3

373

37.3

37.3

37.3

37.3

37.3

37.3

37.3

37.3

37.3

37.3

37.3

37.3

37.3

37.3

3.73

37.3

37.3 $\mu \mathrm{g} / \mathrm{kg}$

$\mu \mathrm{g} / \mathrm{kg}$

$\mu \mathrm{g} / \mathrm{kg}$

$\mu g / \mathrm{kg}$

$\mu \mathrm{g} / \mathrm{kg}$

$\mu \mathrm{g} / \mathrm{kg}$

$\mu \mathrm{g} / \mathrm{kg}$

$\mu \mathrm{g} / \mathrm{kg}$

$\mu \mathrm{g} / \mathrm{kg}$

$\mu \mathrm{g} / \mathrm{kg}$

$\mu \mathrm{g} / \mathrm{kg}$

$\mu \mathrm{g} / \mathrm{kg}$

$\mu \mathrm{g} / \mathrm{kg}$

$\mu \mathrm{g} / \mathrm{kg}$

$\mu \mathrm{g} / \mathrm{kg}$

$\mu \mathrm{g} / \mathrm{kg}$

$\mu \mathrm{g} / \mathrm{kg}$

$\mu \mathrm{g} / \mathrm{kg}$

$\mu \mathrm{g} / \mathrm{kg}$

$\mu \mathrm{g} / \mathrm{kg}$

$\mu \mathrm{g} / \mathrm{kg}$
3.73

3.73

37.3

373

37.3

37.3

37.3

37.3

37.3

37.3

37.3

37.3

37.3

37.3

37.3

37.3

37.3

37.3

3.73

37,3

37.3
EPA8270

EPA8270

EPA8270

EPA8270

EPA8270

EPA8270

EPA8270

EPA8270

EPA8270

EPA8270

EPA8270

EPA8270

EPA8270

EPA8270

EPA8270

EPA8270

EPA8270

EPA8270

EPA8270

EPA8270

EPA8270

Volatile Organic Compounds

Acetone
Benzene
Bromodichloromethane
Bromoform
Bromomethane (Methyl bromide)
Carbon disulfide
Carbon tetrachloride
Chlorobenzene
Chloroethane
Chloroethene (Vinyl chloride)
Chloroform
Chloromethane (Methyl chloride)
Dibromochloromethane
1,1-Dichloroethane
1,2-Dichloroethane
1,1-Dichloroethylene
1,2-Dichloroethylene
Dichloromethane (Methylene chloride)
1,2-Dichloropropane
cis-1,3-Dichloropropene
trans-1,3-Dichloropropene
Ethylbenzene
2-Hexanone
Methyl ethyl ketone
Methyl isobutyl ketone
Styrene
1,1,2,2-Tetrachloroethane
Tetrachloroethylene
Toluene
1,1,1-Trichloroethane
1,1,2-Trichloroethane

$\begin{array}{lll}U & 8 & 9.39 \\ U & & 0.575 \\ U & & 0.0575 \\ U & & 0.0575 \\ U & & 0.115 \\ J & E & 0.195 \\ U & & 0.0575 \\ U & & 0.0575 \\ U & & 0.115 \\ U & & 0.115 \\ U & & 0.0575 \\ U & & 0.115 \\ U & & 0.0575 \\ U & & 0.0575 \\ U & & 0.0575 \\ U & & 0.0575 \\ U & & 0.115 \\ U & V & 1.01 \\ U & & 0.0575 \\ U & & 0.0575 \\ U & & 0.0575 \\ & & 0.207 \\ U & V & 1.21 \\ U & V 8 & \mathbf{0 . 6 4 4} \\ U & & 1.15 \\ U & & \mathbf{0 . 0 5 7 5} \\ U & & \mathbf{0 . 0 5 7 5} \\ U & & 0.0575 \\ U & 8 & 0.586 \\ U & & 0.0575 \\ U & & \mathbf{0 . 0 5 7 5}\end{array}$

$\begin{array}{lll}\mu \mathrm{g} / \mathrm{kg} & 5.75 & \text { EPA8260 } \\ \mu \mathrm{g} / \mathrm{kg} & 0.575 & \text { EPA8260 } \\ \mu \mathrm{g} / \mathrm{kg} & 0.0575 & \text { EPA8260 } \\ \mu \mathrm{g} / \mathrm{kg} & 0.0575 & \text { EPA8260 } \\ \mu \mathrm{g} / \mathrm{kg} & 0.115 & \text { EPA8260 } \\ \mu \mathrm{g} / \mathrm{kg} & 1.15 & \text { EPA8260 } \\ \mu \mathrm{g} / \mathrm{kg} & 0.0575 & \text { EPA8260 } \\ \mu \mathrm{g} / \mathrm{kg} & 0.0575 & \text { EPA8260 } \\ \mu \mathrm{g} / \mathrm{kg} & 0.115 & \text { EPA8260 } \\ \mu \mathrm{g} / \mathrm{kg} & 0.115 & \text { EPA8260 } \\ \mu \mathrm{g} / \mathrm{kg} & 0.0575 & \text { EPA8260 } \\ \mu \mathrm{g} / \mathrm{kg} & 0.115 & \text { EPA8260 } \\ \mu \mathrm{g} / \mathrm{kg} & 0.0575 & \text { EPA8260 } \\ \mu \mathrm{g} / \mathrm{kg} & 0.0575 & \text { EPA8260 } \\ \mu \mathrm{g} / \mathrm{kg} & 0.0575 & \text { EPA8260 } \\ \mu \mathrm{g} / \mathrm{kg} & 0.0575 & \text { EPA8260 } \\ \mu \mathrm{g} / \mathrm{kg} & 0.115 & \text { EPA8260 } \\ \mu \mathrm{gg} / \mathrm{kg} & 0.575 & \text { EPA8260 } \\ \mu \mathrm{g} / \mathrm{kg} & 0.0575 & \text { EPA8260 } \\ \mu \mathrm{g} / \mathrm{kg} & 0.0575 & \text { EPA8260 } \\ \mu \mathrm{g} / \mathrm{kg} & 0.0575 & \text { EPA8260 } \\ \mu \mathrm{g} / \mathrm{kg} & 0.0575 & \text { EPA8260 } \\ \mu \mathrm{g} / \mathrm{kg} & 1.15 & \text { EPA8260 } \\ \mu \mathrm{g} / \mathrm{kg} & 1.15 & \text { EPA8260 } \\ \mu \mathrm{g} / \mathrm{kg} & 1.15 & \text { EPA8260 } \\ \mu \mathrm{g} / \mathrm{kg} & 0.0575 & \text { EPA8260 } \\ \mu \mathrm{g} / \mathrm{kg} & 0.0575 & \text { EPA8260 } \\ \mu \mathrm{g} / \mathrm{kg} & 0.0575 & \text { EPA8260 } \\ \mu \mathrm{g} / \mathrm{kg} & 0.575 & \text { EPA8260 } \\ \mu \mathrm{g} / \mathrm{kg} & 0.0575 & \text { EPA8260 } \\ \mu \mathrm{g} / \mathrm{kg} & 0.0575 & \text { EPA8260 } \\ & & \end{array}$


SURVEY ID: PPSC 2102 (continued)

Constituent

Volatile Organic Compounds

Trichloroethylene

Vinyl acetate

Xylenes

Pesticides/PCBs and Dioxins/Furans

$\begin{array}{lc}\text { Aldrin } & \mathrm{U} \\ \text { alpha-Benzene hexachloride } & \mathrm{U} \\ \text { beta-Benzene hexachloride } & \mathrm{U} \\ \text { delta-Benzene hexachloride } & \mathrm{U} \\ \text { alpha-Chlordane } & \mathrm{U} \\ \text { gamma-Chlordane } & \mathrm{U} \\ \text { p,p'-DDD } & \mathrm{U} \\ \text { p,p'-DDE } & \mathrm{U} \\ \text { p,p'-DDT } & \mathrm{U} \\ \text { Dieldrin } & \mathrm{U} \\ \text { Endosulfan sulfate } & \mathrm{U} \\ \text { Endosulfan I } & \mathrm{U} \\ \text { Endosulfan II } & \mathrm{U} \\ \text { Endrin } & \mathrm{U} \\ \text { Endrin ketone } & \mathrm{U} \\ \text { Heptachlor } & \mathrm{U} \\ \text { Heptachlor epoxide } & \mathrm{U} \\ \text { Lindane } & \mathrm{U} \\ \text { Methoxychlor } & \mathrm{U} \\ \text { PCB 1016 } & \mathrm{U} \\ \text { PCB 1221 } & \mathrm{U} \\ \text { PCB 1232 } & \mathrm{U} \\ \text { PCB 1242 } & \mathrm{U} \\ \text { PCB 1248 } & \mathrm{U} \\ \text { PCB 1254 } & \mathrm{U} \\ \text { PCB 1260 } & \mathrm{U} \\ \text { Toxaphene } & \end{array}$

Radionuclides

Actinium-228

Actinium-228

Antimony-124

Antimony-124

Antimony-125

Antimony-125

Barium-133

Barium-133

Cerium-144

Cerium-144

Cesium-134

Cesium-134

Cesium-137

Cesium-137

Cobalt-57

Cobalt-57

Cobalt-58

Cobalt-58

Cobalt-60
Sample ID: 104681
RQ
AQ B Result
Unit
D. Limit Method

$\begin{array}{llllll}\mathrm{U} & & 0.0575 & \mu \mathrm{g} / \mathrm{kg} & 0.0575 & \text { EPA8260 } \\ \mathrm{U} & & 1.15 & \mu \mathrm{g} / \mathrm{kg} & 1.15 & \text { EPA8260 } \\ \mathrm{U} & 8 & 0.954 & \mu \mathrm{g} / \mathrm{kg} & 0.172 & \text { EPA8260 }\end{array}$

$\begin{array}{ll}U & 4.7 \\ U & 4.7 \\ U & 9.39 \\ U & 9.39 \\ U & 9.39 \\ U & 9.39 \\ U & 18.8 \\ U & 9.39 \\ U & 18.8 \\ U & 9.39 \\ U & 18.8 \\ U & 9.39 \\ U & 18.8 \\ U & 9.39 \\ U & 18.8 \\ U & 9.39 \\ U & 9.39 \\ U & 4.7 \\ U & 93.9 \\ U & 23.5 \\ U & 23.5 \\ U & 23.5 \\ U & 23.5 \\ U & 23.5 \\ U & 23.5 \\ U & 23.5 \\ U & 188\end{array}$

$\begin{array}{lll}\mu \mathrm{g} / \mathrm{kg} & 4.7 & \text { EPA8080 } \\ \mu \mathrm{g} / \mathrm{kg} & 4.7 & \text { EPA8080 } \\ \mu \mathrm{g} / \mathrm{kg} & 9.39 & \text { EPA8080 } \\ \mu \mathrm{g} / \mathrm{kg} & 9.39 & \text { EPA8080 } \\ \mu \mathrm{g} / \mathrm{kg} & 9.39 & \text { EPA8080 } \\ \mu \mathrm{g} / \mathrm{kg} & 9.39 & \text { EPA8080 } \\ \mu \mathrm{g} / \mathrm{kg} & 18.8 & \text { EPA8080 } \\ \mu \mathrm{g} / \mathrm{kg} & 9.39 & \text { EPA8080 } \\ \mu \mathrm{g} / \mathrm{kg} & 18.8 & \text { EPA8080 } \\ \mu \mathrm{g} / \mathrm{kg} & 9.39 & \text { EPA8080 } \\ \mu \mathrm{g} / \mathrm{kg} & 18.8 & \text { EPA8080 } \\ \mu \mathrm{g} / \mathrm{kg} & 9.39 & \text { EPA8080 } \\ \mu \mathrm{g} / \mathrm{kg} & 18.8 & \text { EPA8080 } \\ \mu \mathrm{g} / \mathrm{kg} & 9.39 & \text { EPA8080 } \\ \mu \mathrm{g} / \mathrm{kg} & 18.8 & \text { EPA8080 } \\ \mu \mathrm{g} / \mathrm{kg} & 9.39 & \text { EPA8080 } \\ \mu \mathrm{g} / \mathrm{kg} & 9.39 & \text { EPA8080 } \\ \mu \mathrm{g} / \mathrm{kg} & 4.7 & \text { EPA8080 } \\ \mu \mathrm{g} / \mathrm{kg} & 93.9 & \text { EPA8080 } \\ \mu \mathrm{g} / \mathrm{kg} & 23.5 & \text { EPA8080 } \\ \mu \mathrm{g} / \mathrm{kg} & 23.5 & \text { EPA8080 } \\ \mu \mathrm{g} / \mathrm{kg} & 23.5 & \text { EPA8080 } \\ \mu \mathrm{g} / \mathrm{kg} & 23.5 & \text { EPA8080 } \\ \mu \mathrm{g} / \mathrm{kg} & 23.5 & \text { EPA8080 } \\ \mu \mathrm{g} / \mathrm{kg} & 23.5 & \text { EPA8080 } \\ \mu \mathrm{g} / \mathrm{kg} & 23.5 & \text { EPA8080 } \\ \mu \mathrm{g} / \mathrm{kg} & 188 & \text { EPA8080 }\end{array}$

$5.22 \mathrm{E}-01 \pm 3.04 \mathrm{E}-02 \mathrm{pCi} / \mathrm{g}$

1.38E-02 EPIA-013B $4.91 \mathrm{E}-01 \pm 3.18 \mathrm{E}-02 \mathrm{pCj} / \mathrm{g}$ $-3.15 \mathrm{E}-03 \pm 3.20 \mathrm{E}-03 \mathrm{pCi} / \mathrm{g}$ $6.82 \mathrm{E}-05 \pm 3.40 \mathrm{E}-03 \mathrm{pCi} / \mathrm{g}$ $-2.65 \mathrm{E}-03 \pm 7.53 \mathrm{E}-03 \mathrm{pCi} / \mathrm{g}$ 4.92E-03 $\pm 7.02 \mathrm{E}-03 \mathrm{pCi} / \mathrm{g}$ $-9.53 \mathrm{E}-04 \pm 3.65 \mathrm{E}-03 \mathrm{pCi} / \mathrm{g}$ $1.78 \mathrm{E}-03 \pm 3.65 \mathrm{E}-03 \mathrm{pCi} / \mathrm{g}$ $-1.56 \mathrm{E}-02 \pm 2.04 \mathrm{E}-02 \mathrm{pCi} / \mathrm{g}$ 1.12E-02 $2.10 \mathrm{E}-02 \mathrm{pCi} / \mathrm{g}$ $-1.82 \mathrm{E}-02 \pm 3.10 \mathrm{E}-03 \mathrm{pCi} / \mathrm{g}$ $-4.78 \mathrm{E}-04 \pm 2.80 \mathrm{E}-03 \mathrm{pCi} / \mathrm{g}$ $1.52 \mathrm{E}-02 \pm 4.65 \mathrm{E}-03 \mathrm{pCi} / \mathrm{g}$ $1.63 \mathrm{E}-02 \pm 5.54 \mathrm{E}-03 \mathrm{pCi} / \mathrm{g}$ $2.92 \mathrm{E}-04 \pm 2.57 \mathrm{E}-03 \mathrm{pCi} / \mathrm{g}$ $-2.23 \mathrm{E}-04 \pm 2.89 \mathrm{E}-03 \mathrm{pCi} / \mathrm{g}$ $-1.45 \mathrm{E}-03 \pm 2.91 \mathrm{E}-03 \mathrm{pCi} / \mathrm{g}$ 8.35E-04 $\pm 3.43 \mathrm{E}-03 \mathrm{pCi} / \mathrm{g}$ 1.64E-03 $2.17 \mathrm{E}-03 \mathrm{pCi} / \mathrm{g}$
1.45E-02 EPIA-013B

5.37E-03 EPIA-013B

5.83E-03 EPIA-013B

1.23E-02 EPIA-013B

1.25E-02 EPIA-013B

5.36E-03 EPIA-013B

5.44E-03 EPIA-013B

3.47E-02 EPIA-013B

3.62E-02 EPIA-013B

4.26E.03 EPIA-013B

4.17E-03 EPIA-013B

3.92E-03 EPIA-013B

4.88E-03 EPIA-013B

4.45E-03 EPIA-013B

4.44E-03 EPIA-013B

4.91E-03 EPIA-013B

5.19E-03 EPIA-013B 4.15E-03 EPLA-013B 
SURVEY ID: PPSC 2102 (continued)

Constituent

Radionuclides

Cobalt-60

Europium-152

Europium-152

Europium-154

Europium-154

Europium-155

Europium-155

Gross alpha

Lead-212

Lead-212

Manganese-54

Manganese-54

Neptunium-239

Neptunium-239

Nonvolatile beta

Plutonium-238

Plutonium-239/240

Potassium-40

Potassium-40

Promethium-144

Promethium-144

Promethium-146

Promethium-146

Radium-226

Radium-226

Radium-228

Radium-228

Ruthenium-106

Ruthenium-106

Sodium-22

Sodium-22

Strontium-90

Strontium-90

Thorium-234

Thorium-234

Tin-113

Tin-113

Yttrium-88

Yttrium-88

Zinc-65

Zinc-65

Zirconium-95

Zirconium-95
Sample ID: 104681

RQ AQ B Result Unit D. Limit Method

\begin{tabular}{|c|c|c|c|c|}
\hline UI & & $-1.39 \mathrm{E}-03 \pm 2.24 \mathrm{E}-03 \mathrm{pCi} / \mathrm{g}$ & $3.74 \mathrm{E}-03$ & EPIA-013B \\
\hline UI & & $-5.77 \mathrm{E}-03 \pm 7.77 \mathrm{E}-03 \mathrm{pCi} / \mathrm{g}$ & 1.26E-02 & EPIA-013B \\
\hline UI & & $-5.87 \mathrm{E}-03 \pm 7.59 \mathrm{E}-03 \mathrm{pCi} / \mathrm{g}$ & 1.21E-02 & EPIA-013B \\
\hline UI & & $-1.00 \mathrm{E}-04 \pm 2.42 \mathrm{E}-02 \mathrm{pCi} / \mathrm{g}$ & 3.67E-02 & EPIA-013B \\
\hline UI & & $2.78 \mathrm{E}-02 \pm 2.31 \mathrm{E}-02 \mathrm{pCi} / \mathrm{g}$ & 3.97E-02 & EPLA-013B \\
\hline UI & & $0.00 \mathrm{E}+00$ & 1.91E-02 & EPIA-013B \\
\hline UI & & $0.00 \mathrm{E}+00 \quad \mathrm{pCi} / \mathrm{g}$ & 1.91E-02 & EPIA-013B \\
\hline & & $1.09 \mathrm{E}+01 \pm 2.54 \mathrm{E}+00 \mathrm{pCj} / \mathrm{g}$ & $1.83 E+00$ & EPIA-001B \\
\hline & & $5.45 \mathrm{E}-01 \pm 1.43 \mathrm{E}-02 \mathrm{pCi} / \mathrm{g}$ & 7.72E-03 & EPLA-013B \\
\hline & & 5.33E-01ะ1.41E.02 pCi/g & 7.53E-03 & EPIA-013B \\
\hline & & $1.02 \mathrm{E}-02 \pm 3.62 \mathrm{E}-03 \mathrm{pCi} / \mathrm{g}$ & $4.14 \mathrm{E}-03$ & EPIA-013B \\
\hline & & $5.85 \mathrm{E}-03 \pm 4.10 \mathrm{E}-03 \mathrm{pCi} / \mathrm{g}$ & 4.27E-03 & EPIA-013B \\
\hline UI & & $2.16 \mathrm{E}-03 \pm 1.90 \mathrm{E}-02 \mathrm{pCi} / \mathrm{g}$ & $3.29 \mathrm{E}-02$ & EPIA-013B \\
\hline UI & & $-9.81 \mathrm{E}-03 \pm 1.89 \mathrm{E}-02 \mathrm{pCi} / \mathrm{g}$ & 3.22E-02 & EPIA-013B \\
\hline & & $5.00 \mathrm{E}+00 \pm 1.59 \mathrm{E}+00 \mathrm{pCi} / \mathrm{g}$ & $2.69 \mathrm{E}+00$ & EPIA-001B \\
\hline UI & & $6.57 \mathrm{E}-03 \pm 1.16 \mathrm{E}-02 \mathrm{pCi} / \mathrm{g}$ & 2.31E-02 & EPIA-012B \\
\hline UI & & $-2.67 \mathrm{E}-03 \pm 3.80 \mathrm{E}-03 \mathrm{pCi} / \mathrm{g}$ & 2.03E-02 & EPIA-012B \\
\hline $\mathrm{U}$ & $\mathrm{V}$ & 2.20E-01 $\pm 5.54 \mathrm{E}-02 \mathrm{pCi} / \mathrm{g}$ & 3.40E-02 & EPIA-013B \\
\hline $\mathrm{U}$ & $\mathrm{V}$ & $2.15 \mathrm{E}-01 \pm 6.47 \mathrm{E}-02 \mathrm{pCi} / \mathrm{g}$ & 3.94E-02 & EPIA-013B \\
\hline UI & & $-1.83 \mathrm{E}-04 \pm 2.55 \mathrm{E}-03 \mathrm{pCi} / \mathrm{g}$ & 4.41E-03 & EPIA-013B \\
\hline UI & & $-2.67 \mathrm{E}-03 \pm 2.65 \mathrm{E}-03 \mathrm{pCi} / \mathrm{g}$ & 4.29E-03 & EPIA-013B \\
\hline UI & & $0.00 \mathrm{E}+00 \quad \mathrm{pCi} / \mathrm{g}$ & 5.97E-03 & EPIA-013B \\
\hline UI & & $4.97 \mathrm{E}-03 \pm 6.05 \mathrm{E}-03 \mathrm{pCi} / \mathrm{g}$ & 5.95E-03 & EPIA-013B \\
\hline & & $3.09 \mathrm{E}-01 \pm 1.61 \mathrm{E}-02 \mathrm{pCi} / \mathrm{g}$ & 8.68E-03 & EPLA-013B \\
\hline & & $3.05 \mathrm{E}-01 \pm 1.69 \mathrm{E}-02 \mathrm{pCi} / \mathrm{g}$ & 8.69E-03 & EPLA-013B \\
\hline & $\mathrm{X}$ & $5.22 \mathrm{E}-01 \pm 3.04 \mathrm{E}-02 \mathrm{pCi} / \mathrm{g}$ & 1.38E-02 & EPIA-013B \\
\hline & $\mathrm{X}$ & $4.91 \mathrm{E}+03 \pm 3.18 \mathrm{E}-02 \mathrm{pCi} / \mathrm{g}$ & $1.45 \mathrm{E}-02$ & EPIA-013B \\
\hline UI & & $-2.21 \mathrm{E}-02 \pm 2.38 \mathrm{E}-02 \mathrm{pCi} / \mathrm{g}$ & 3.98E-02 & EPLA-013B \\
\hline UI & & $-1.29 \mathrm{E}-02 \pm 2.46 \mathrm{E}-02 \mathrm{pCi} / \mathrm{g}$ & 4.12E-02 & EPIA-013B \\
\hline UI & & $-1.40 \mathrm{E}-03 \pm 2.69 \mathrm{E}-03 \mathrm{pCi} / \mathrm{g}$ & $3.77 \mathrm{E}-03$ & EPIA-013B \\
\hline UI & & $1.76 \mathrm{E}-03 \pm 2.38 \mathrm{E}-03 \mathrm{pCj} / \mathrm{g}$ & $4.41 \mathrm{E}-03$ & EPIA-013B \\
\hline $\mathbf{U}$ & $\mathrm{V}$ & $1.61 \mathrm{E}+00 \pm 1.90 \mathrm{E}-01 \mathrm{pCi} / \mathrm{g}$ & $1.10 \mathrm{E}-01$ & EPIA-004 \\
\hline $\mathbf{U}$ & $\mathrm{V}$ & $1.62 \mathrm{E}+00 \pm 2.51 \mathrm{E}-01 \mathrm{pCi} / \mathrm{g}$ & $1.45 \mathrm{E}-01$ & EPIA-004 \\
\hline & & $6.39 \mathrm{E}-01 \pm 3.88 \mathrm{E}-01 \mathrm{pCi} / \mathrm{g}$ & 2.35E-01 & EPIA-013B \\
\hline & & $5.59 \mathrm{E}-01 \pm 3.23 \mathrm{E}-01 \mathrm{pCi} / \mathrm{g}$ & 2.38E-01 & EPIA-013B \\
\hline UI & & $1.51 \mathrm{E}-04 \pm 3.92 \mathrm{E}-03 \mathrm{pCi} / \mathrm{g}$ & 6.50E-03 & EPIA-013B \\
\hline UI & & $-9.59 \mathrm{E}-04 \pm 4.20 \mathrm{E}-03 \mathrm{pCi} / \mathrm{g}$ & $6.42 \mathrm{E}-03$ & EPIA-013B \\
\hline UI & & $3.58 \mathrm{E}-03 \pm 3.03 \mathrm{E}-03 \mathrm{pCi} / \mathrm{g}$ & 6.00E-03 & EPIA-013B \\
\hline UI & & $-6.41 \mathrm{E}-04 \pm 2.69 \mathrm{E}-03 \mathrm{pCi} / \mathrm{g}$ & $4.82 \mathrm{E}-03$ & EPIA-013B \\
\hline UI & & $-3.72 \mathrm{E}-03 \pm 5.34 \mathrm{E}-03 \mathrm{pCi} / \mathrm{g}$ & 7.40E-03 & EPIA-013B \\
\hline UI & & $-2.39 \mathrm{E}-03 \pm 5.62 \mathrm{E}-03 \mathrm{pCi} / \mathrm{g}$ & $8.28 \mathrm{E}-03$ & EPIA-013B \\
\hline UI & & $2.75 \mathrm{E}-04 \pm 1.03 \mathrm{E}-02 \mathrm{pCi} / \mathrm{g}$ & 1.13E-02 & EPIA-013B \\
\hline UI & & $0.00 \mathrm{E}+00 \quad \mathrm{pCi} / \mathrm{g}$ & $1.21 \mathrm{E}-02$ & EPIA-013B \\
\hline
\end{tabular}

Sample ID: 104682

SURVEY ID: PPSC 2103

Survey location: 44387E $3669759 \mathrm{~N}$ (SRS coordinates)

Depth of core interval: 2.00 to $4.00 \mathrm{ft}$

Sample type: Normal

Sample matrix: Soil

Percent solids: 85.00 
SURVEY ID: PPSC 2103 (continued)

Sample ID: 104682

Constituent

RQ AQ B Result

Unit

D. Limit Method

Physical Parameters and Specified Analyses

Ammonia nitrogen

Cation exchange capacity

Chloride

Cyanide

Nitrate-nitrite as nitrogen

Nitrogen by Kjeldahl method

Orthophosphate

Phenols

Sulfide

Total organic carbon

Total organic nitrogen

Total phosphates (as P)

Metals (total recoverable)
U

19.4

52.6

3.23

UJ C 1.12

J E $\quad 0.475$

23

U $\quad 23.5$

U 347

UJ C 294

8420

C $\quad 15.1$

J E $\quad \mathbf{5 7 . 9}$

$\begin{array}{lll}\mathrm{mg} / \mathrm{kg} & 19.4 & \text { EPA350.1 } \\ \mathrm{meq} / 100 \mathrm{~g} & 5 & \text { EPA9081 } \\ \mathrm{mg} / \mathrm{kg} & 2.9 & \text { EPA300.0 } \\ \mathrm{mg} / \mathrm{kg} & 1.12 & \text { EPA335.3 } \\ \mathrm{mg} / \mathrm{kg} & 0.951 & \text { EPA353.1 } \\ \mathrm{mg} / \mathrm{kg} & 4.47 & \text { EPA351.2 } \\ \mathrm{mg} / \mathrm{kg} & 23.5 & \text { EPA365.2 } \\ \mu \mathrm{g} / \mathrm{kg} & 347 & \text { EPA420.2 } \\ \mathrm{mg} / \mathrm{kg} & 294 & \text { EPA376.2 } \\ \mathrm{mg} / \mathrm{kg} & 118 & \text { EPA415.1 } \\ \mathrm{mg} / \mathrm{kg} & 0.235 & \text { EPA351.2 } \\ \mathrm{mg} / \mathrm{kg} & 308 & \text { EPA365.4 }\end{array}$

1520

5.5

16.5

16.7

$\begin{array}{lll}\mathrm{J} & \mathrm{E} & 0.28\end{array}$

U $\quad 0.275$

784

10.5

0.55

0.844

326

3.89

33.6

3.3

0.00771

0.763

36

16.5

1.37

55

16.5

3.42

1.5

$B / N / A$ Extractables (including $P A H$ and phenols)

$\begin{array}{lll}\text { UJ } & \text { Q } & 38.9 \\ \text { UJ } & Q & 38.9 \\ \text { UJ } & \text { Q } & 38.9 \\ \text { UJ } & \text { Q } & 3.89 \\ \text { UJ } & \text { Q } & 3.89 \\ \text { UJJ } & \text { Q } & 3.89 \\ J & \text { QE } & 20.2 \\ \text { UJ } & \text { Q } & 38.9 \\ \text { UJ } & \text { Q } & 3.89 \\ \text { UJ } & \text { Q } & 38.9 \\ \text { UJ } & \text { Q } & 38.9 \\ \text { UJ } & \text { Q } & 38.9 \\ \text { UJ } & Q & 38.9 \\ \text { UJ } & Q & 38.9\end{array}$

$\begin{array}{lll}\mathrm{mg} / \mathrm{kg} & 13.7 & \text { EPA6010A } \\ \mathrm{mg} / \mathrm{kg} & 5.5 & \text { EPA6010A } \\ \mathrm{mg} / \mathrm{kg} & 16.5 & \text { EPA6010A } \\ \mathrm{mg} / \mathrm{kg} & 1.37 & \text { EPA6010A } \\ \mathrm{mg} / \mathrm{kg} & 0.55 & \text { EPA6010A } \\ \mathrm{mg} / \mathrm{kg} & 0.275 & \text { EPA6010A } \\ \mathrm{mg} / \mathrm{kg} & 13.7 & \text { EPA6010A } \\ \mathrm{mg} / \mathrm{kg} & 1.37 & \text { EPA6010A } \\ \mathrm{mg} / \mathrm{kg} & 0.55 & \text { EPA6010A } \\ \mathrm{mg} / \mathrm{kg} & 1.37 & \text { EPA6010A } \\ \mathrm{mg} / \mathrm{kg} & 5.5 & \text { EPA6010A } \\ \mathrm{mg} / \mathrm{kg} & 5.5 & \text { EPA6010A } \\ \mathrm{mg} / \mathrm{kg} & 5.5 & \text { EPA6010A } \\ \mathrm{mg} / \mathrm{kg} & 0.55 & \text { EPA6010A } \\ \mathrm{mg} / \mathrm{kg} & 0.0367 & \text { EPA7471 } \\ \mathrm{mg} / \mathrm{kg} & 1.37 & \text { EPA6010A } \\ \mathrm{mg} / \mathrm{kg} & 110 & \text { EPA6010A } \\ \mathrm{mg} / \mathrm{kg} & 16.5 & \text { EPA6010A } \\ \mathrm{mg} / \mathrm{kg} & 1.37 & \text { EPA6010A } \\ \mathrm{mg} / \mathrm{kg} & 55 & \text { EPA6010A } \\ \mathrm{mg} / \mathrm{kg} & 16.5 & \text { EPA6010A } \\ \mathrm{mg} / \mathrm{kg} & 0.55 & \text { EPA6010A } \\ \mathrm{mg} / \mathrm{kg} & 1.1 & \text { EPA6010A }\end{array}$

$\mu \mathrm{g} / \mathrm{kg}$ $\mu \mathrm{g} / \mathrm{kg}$

$\mu \mathrm{g} / \mathrm{kg}$

$\mu \mathrm{g} / \mathrm{kg}$

$\mu \mathrm{g} / \mathrm{kg}$

$\mu \mathrm{g} / \mathrm{kg}$

$\mu \mathrm{g} / \mathrm{kg}$

$\mu \mathrm{g} / \mathrm{kg}$

$\mu \mathrm{g} / \mathrm{kg}$

$\mu \mathrm{g} / \mathrm{kg}$

$\mu \mathrm{g} / \mathrm{kg}$

$\mu \mathrm{g} / \mathrm{kg}$

$\mu g / \mathrm{kg}$

$\mu \mathrm{g} / \mathrm{kg}$
38.9

38.9

38.9

3.89

3.89

3.89

38.9

38.9

3.89

38.9

38.9

38.9

38.9

38.9
EPA8270

EPA8270

EPA8270

EPA8270

EPA8270

EPA8270

EPA8270

EPA8270

EPA8270

EPA8270

EPA8270

EPA8270

EPA8270
EPA8270 
SURVEY ID: PPSC 2103 (continued)

Constituent
Sample ID: 104682

RQ AQ B Result

Unit

D. Limit Method

$B / N / A$ Extractables (including $P A H$ and phenols)

\begin{tabular}{|c|c|c|c|c|c|c|}
\hline 4-Bromophenyl phenyl ether & UJ & $\mathbf{Q}$ & 38.9 & $\mu \mathrm{g} / \mathrm{kg}$ & 38.9 & EPA8270 \\
\hline Butylbenzyl phthalate & UJ & $\mathbf{Q}$ & 38.9 & $\mu \mathrm{g} / \mathrm{kg}$ & 38.9 & EPA8270 \\
\hline 4-Chloroaniline & UJ & $\mathbf{Q}$ & 38.9 & $\mu \mathrm{g} / \mathrm{kg}$ & 38.9 & EPA8270 \\
\hline 4-Chloro-m-cresol & UJ & $\mathbf{Q}$ & 38.9 & $\mu g / \mathrm{kg}$ & 38.9 & EPA8270 \\
\hline 2-Chloronaphthalene & UJ & $\mathbf{Q}$ & 38.9 & $\mu \mathrm{g} / \mathrm{kg}$ & 38.9 & EPA8270 \\
\hline 2-Chlorophenol & UJ & $\mathbf{Q}$ & 38.9 & $\mu \mathrm{g} / \mathrm{kg}$ & 38.9 & EPA8270 \\
\hline 4-Chlorophenyl phenyl ether & UJ & $Q$ & 38.9 & $\mu \mathrm{g} / \mathrm{kg}$ & 38.9 & EPA8270 \\
\hline Chrysene & UJ & $Q$ & 3.89 & $\mu g / \mathrm{kg}$ & 3.89 & EPA8270 \\
\hline $\mathrm{m} / \mathrm{p}$-Cresol & UJ & $\mathbf{Q}$ & 389 & $\mu g / k g$ & 389 & EPA8270 \\
\hline 0-Cresol (2-Methylphenol) & UJ & $\mathbf{Q}$ & 38.9 & $\mu g / k g$ & 38.9 & EPA8270 \\
\hline Cyclotetracosane & J & $\mathrm{N}$ & 870 & $\mu \mathrm{g} / \mathrm{kg}$ & & EPA8270 \\
\hline Dibenz $[a, h]$ anthracene & UJ & $\mathbf{Q}$ & 3.89 & $\mu \mathrm{g} / \mathrm{kg}$ & 3.89 & EPA8270 \\
\hline Dibenzofuran & UJ & Q & 38.9 & $\mu g / \mathrm{kg}$ & 38.9 & EPA8270 \\
\hline Di-n-butyl phthalate & UJ & $\mathbf{Q}$ & 38.9 & $\mu g / \mathrm{kg}$ & 38.9 & EPA8270 \\
\hline 1,2-Dichlorobenzene & UJ & $\mathbf{Q}$ & 38.9 & $\mu g / k g$ & 38.9 & EPA8270 \\
\hline 1,3-Dichlorobenzene & UJ & $\mathbf{Q}$ & 38.9 & $\mu g / \mathrm{kg}$ & 38.9 & EPA8270 \\
\hline 1,4-Dichlorobenzene & UJ & $\mathbf{Q}$ & 38.9 & $\mu \mathrm{g} / \mathrm{kg}$ & 38.9 & EPA8270 \\
\hline 3,3'-Dichlorobenzidine & UJ & $\mathbf{Q}$ & 38.9 & $\mu g / \mathrm{kg}$ & 38.9 & EPA8270 \\
\hline 2,4-Dichlorophenol & UJ & $\mathbf{Q}$ & 38.9 & $\mu \mathrm{g} / \mathrm{kg}$ & 38.9 & EPA8270 \\
\hline Diethyl phthalate & UJ & $\mathbf{Q}$ & 38.9 & $\mu \mathrm{g} / \mathrm{kg}$ & 38.9 & EPA8270 \\
\hline 2,4-Dimethyl phenol & UJ & $\mathbf{Q}$ & 38.9 & $\mu \mathrm{g} / \mathrm{kg}$ & 38.9 & EPA8270 \\
\hline Dimethyl phthalate & UJ & Q & 38.9 & $\mu \mathrm{g} / \mathrm{kg}$ & 38.9 & EPA8270 \\
\hline 2,4-Dinitrophenol & UJ & $\mathbf{Q}$ & 389 & $\mu \mathrm{g} / \mathrm{kg}$ & 389 & EPA8270 \\
\hline 2,4-Dinitrotoluene & UJ & QC & 3.89 & $\mu g / \mathrm{kg}$ & 3.89 & EPA8270 \\
\hline 2,6-Dinitrotoluene & UJ & $\mathbf{Q}$ & 3.89 & $\mu \mathrm{g} / \mathrm{kg}$ & 3.89 & EPA8270 \\
\hline Di-n-octyl phthalate & UJ & $\mathbf{Q}$ & 38.9 & $\mu \mathrm{g} / \mathrm{kg}$ & 38.9 & EPA8270 \\
\hline Docosane & $\mathrm{J}$ & $\mathbf{N}$ & 1700 & $\mu g / \mathrm{kg}$ & & EPA8270 \\
\hline Docosane & $\mathrm{J}$ & $\mathrm{N}$ & 470 & $\mu \mathrm{g} / \mathrm{kg}$ & & EPA8270 \\
\hline Fluoranthene & UJ & $\mathbf{Q}$ & 38.9 & $\mu \mathrm{g} / \mathrm{kg}$ & 38.9 & EPA8270 \\
\hline Fluorene & UJ & $\mathbf{Q}$ & 38.9 & $\mu \mathrm{g} / \mathrm{kg}$ & 38.9 & EPA8270 \\
\hline Hexachlorobenzene & UJ & Q & 3.89 & $\mu \mathrm{g} / \mathrm{kg}$ & 3.89 & EPA8270 \\
\hline Hexachlorobutadiene & UJ & $\mathbf{Q}$ & 38.9 & $\mu \mathrm{g} / \mathrm{kg}$ & 38.9 & EPA8270 \\
\hline Hexachlorocyclopentadiene & UJ & $\mathbf{Q}$ & 38.9 & $\mu \mathrm{g} / \mathrm{kg}$ & 38.9 & EPA8270 \\
\hline Hexachloroethane & UJ & $\mathbf{Q}$ & 3.89 & $\mu \mathrm{g} / \mathrm{kg}$ & 3.89 & EPA8270 \\
\hline Indeno[1,2,3-c,d]pyrene & UJ & Q & 3.89 & $\mu \mathrm{g} / \mathrm{kg}$ & 3.89 & EPA8270 \\
\hline Isophorone & UJ & $\mathbf{Q}$ & 38.9 & $\mu \mathrm{g} / \mathrm{kg}$ & 38.9 & EPA8270 \\
\hline 2-Methyl-4,6-dinitrophenol & UJ & $\mathbf{Q}$ & 389 & $\mu \mathrm{g} / \mathrm{kg}$ & 389 & EPA8270 \\
\hline 2-Methylnaphthalene & UJ & $\mathbf{Q}$ & 38.9 & $\mu \mathrm{g} / \mathrm{kg}$ & 38.9 & EPA8270 \\
\hline Naphthalene & UJ & $\mathbf{Q}$ & 38.9 & $\mu \mathrm{g} / \mathrm{kg}$ & 38.9 & EPA8270 \\
\hline m-Nitroaniline & UJ & $\mathbf{Q}$ & 38.9 & $\mu \mathrm{g} / \mathrm{kg}$ & 38.9 & EPA8270 \\
\hline o-Nitroaniline & UJ & $\mathbf{Q}$ & 38.9 & $\mu \mathrm{g} / \mathrm{kg}$ & 38.9 & EPA8270 \\
\hline p-Nitroaniline & UJ & $\mathbf{Q}$ & 38.9 & $\mu \mathrm{g} / \mathrm{kg}$ & 38.9 & EPA8270 \\
\hline Nitrobenzene & UJ & $\mathbf{Q}$ & 38.9 & $\mu \mathrm{g} / \mathrm{kg}$ & 38.9 & EPA8270 \\
\hline 2-Nitrophenol & UJ & $\mathbf{Q}$ & 38.9 & $\mu \mathrm{g} / \mathrm{kg}$ & 38.9 & EPA8270 \\
\hline 4-Nitrophenol & UJ & Q & 38.9 & $\mu g / k g$ & 38.9 & EPA8270 \\
\hline N-Nitrosodiphenylamine & UJ & $\mathbf{Q}$ & 38.9 & $\mu \mathrm{g} / \mathrm{kg}$ & 38.9 & EPA8270 \\
\hline N-Nitrosodi-n-propylamine & UJ & $\mathbf{Q}$ & 38.9 & $\mu \mathrm{g} / \mathrm{kg}$ & 38.9 & EPA8270 \\
\hline Pentachlorophenol & UJ & $\mathbf{Q}$ & 38.9 & $\mu g / k g$ & 38.9 & EPA8270 \\
\hline Phenanthrene & UJ & $\mathbf{Q}$ & 38.9 & $\mu g / k g$ & 38.9 & EPA8270 \\
\hline Phenol & UJ & $\mathbf{Q}$ & 38.9 & $\mu g / k g$ & 38.9 & EPA8270 \\
\hline Pyrene & UJ & $\mathbf{Q}$ & 38.9 & $\mu g / k g$ & 38.9 & EPA8270 \\
\hline 1,2,4-Trichlorobenzene & UJ & $\mathbf{Q}$ & 3.89 & $\mu g / k g$ & $\cdot 3.89$ & EPA8270 \\
\hline 2,4,5-Trichlorophenol & UJ & Q & 38.9 & $\mu \mathrm{g} / \mathrm{kg}$ & 38.9 & EPA8270 \\
\hline 2,4,6-Trichlorophenol & UJ & $\mathbf{Q}$ & 38.9 & $\mu \mathrm{g} / \mathrm{kg}$ & 38.9 & EPA8270 \\
\hline Unknown alcohol & $J$ & $\mathbf{N}$ & 650 & $\mu \mathrm{g} / \mathrm{kg}$ & & EPA8270 \\
\hline
\end{tabular}


SURVEY ID: PPSC 2103 (continued)

Constituent

RQ AQ B Result

Unit

D. Limit Method

$B / N / A$ Extractables (including PAH and phenols)

Unknown amine

Unknown hydrocarbon

$\begin{array}{lll}J & N & 650 \\ J & N & 1000\end{array}$

Volatile Organic Compounds

Acetone
Benzene
Bromodichloromethane
Bromoform
Bromomethane (Methyl bromide)
Carbon disulfide
Carbon tetrachloride
Chlorobenzene
Chloroethane
Chloroethene (Vinyl chloride)
Chloroform
Chloromethane (Methyl chloride)
Dibromochloromethane
1,1-Dichloroethane
1,2-Dichloroethane
1,1-Dichloroethylene
1,2-Dichloroethylene
Dichloromethane (Methylene chloride)
1,2-Dichloropropane
cis-1,3-Dichloropropene
trans-1,3-Dichloropropene
Ethylbenzene
2-Hexanone
Methyl ethyl ketone
Methyl isobutyl ketone
Styrene
1,1,2,2-Tetrachloroethane
Tetrachloroethylene
Toluene
1,1,1-Trichloroethane
1,1,2-Trichloroethane
Trichloroethylene
Vinyl acetate
Xylenes
(n)

Pesticides/PCBs and Dioxins/Furans

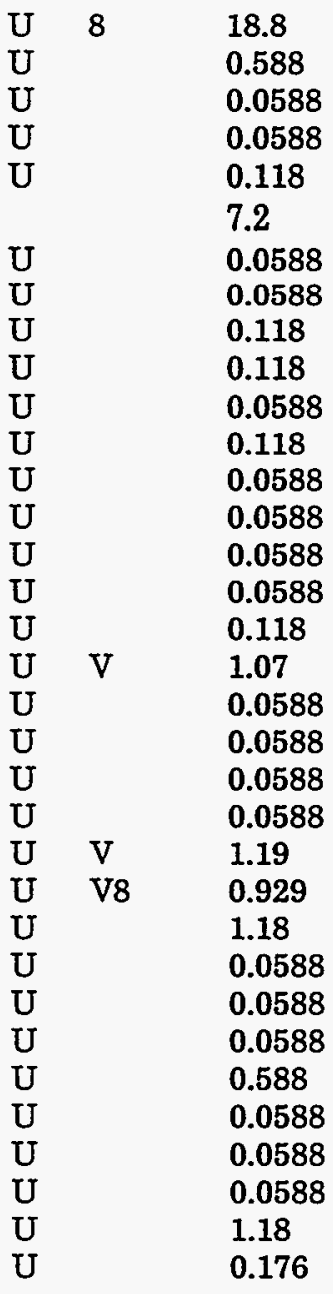

4.81

4.81

9.62

9.62

9.62

9.62

19.2

9.62

19.2

9.62

19.2

9.62

19.2 $\mu \mathrm{g} / \mathrm{kg}$

$\mu \mathrm{g} / \mathrm{kg}$

EPA8270

EPA8270

$\begin{array}{lll}\mu \mathrm{g} / \mathrm{kg} & 5.88 & \text { EPA8260 } \\ \mu \mathrm{g} / \mathrm{kg} & 0.588 & \text { EPA8260 } \\ \mu \mathrm{g} / \mathrm{kg} & 0.0588 & \text { EPA8260 } \\ \mu \mathrm{g} / \mathrm{kg} & 0.0588 & \text { EPA8260 } \\ \mu \mathrm{g} / \mathrm{kg} & 0.118 & \text { EPA8260 } \\ \mu \mathrm{g} / \mathrm{kg} & 1.18 & \text { EPA8260 } \\ \mu \mathrm{g} / \mathrm{kg} & 0.0588 & \text { EPA8260 } \\ \mu \mathrm{g} / \mathrm{kg} & 0.0588 & \text { EPA8260 } \\ \mu \mathrm{g} / \mathrm{kg} & 0.118 & \text { EPA8260 } \\ \mu \mathrm{g} / \mathrm{kg} & 0.118 & \text { EPA8260 } \\ \mu \mathrm{g} / \mathrm{kg} & 0.0588 & \text { EPA8260 } \\ \mu \mathrm{g} / \mathrm{kg} & 0.118 & \text { EPA8260 } \\ \mu \mathrm{g} / \mathrm{kg} & 0.0588 & \text { EPA8260 } \\ \mu \mathrm{g} / \mathrm{kg} & 0.0588 & \text { EPA8260 } \\ \mu \mathrm{g} / \mathrm{kg} & 0.0588 & \text { EPA8260 } \\ \mu \mathrm{g} / \mathrm{kg} & 0.0588 & \text { EPA8260 } \\ \mu \mathrm{g} / \mathrm{kg} & 0.118 & \text { EPA8260 } \\ \mu \mathrm{g} / \mathrm{kg} & 0.588 & \text { EPA8260 } \\ \mu \mathrm{g} / \mathrm{kg} & 0.0588 & \text { EPA8260 } \\ \mu \mathrm{g} / \mathrm{kg} & 0.0588 & \text { EPA8260 } \\ \mu \mathrm{g} / \mathrm{kg} & 0.0588 & \text { EPA8260 } \\ \mu \mathrm{g} / \mathrm{kg} & 0.0588 & \text { EPA8260 } \\ \mu \mathrm{g} / \mathrm{kg} & 1.18 & \text { EPA8260 } \\ \mu \mathrm{g} / \mathrm{kg} & 1.18 & \text { EPA8260 } \\ \mu \mathrm{g} / \mathrm{kg} & 1.18 & \text { EPA8260 } \\ \mu \mathrm{g} / \mathrm{kg} & 0.0588 & \text { EPA8260 } \\ \mu \mathrm{g} / \mathrm{kg} & 0.0588 & \text { EPA8260 } \\ \mu \mathrm{g} / \mathrm{kg} & 0.0588 & \text { EPA8260 } \\ \mu \mathrm{g} / \mathrm{kg} & 0.588 & \text { EPA8260 } \\ \mu \mathrm{g} / \mathrm{kg} & 0.0588 & \text { EPA8260 } \\ \mu \mathrm{g} / \mathrm{kg} & 0.0588 & \text { EPA8260 } \\ \mu \mathrm{g} / \mathrm{kg} & 0.0588 & \text { EPA8260 } \\ \mu \mathrm{g} / \mathrm{kg} & 1.18 & \text { EPA8260 } \\ \mu \mathrm{g} / \mathrm{kg} & 0.176 & \text { EPA8260 } \\ & & \end{array}$

$\mu \mathrm{g} / \mathrm{kg}$

$\mu \mathrm{g} / \mathrm{kg}$

$\mu \mathrm{g} / \mathrm{kg}$

$\mu \mathrm{g} / \mathrm{kg}$

$\mu \mathrm{g} / \mathrm{kg}$

$\mu \mathrm{g} / \mathrm{kg}$

$\mu \mathrm{g} / \mathrm{kg}$

$\mu \mathrm{g} / \mathrm{kg}$

$\mu \mathrm{g} / \mathrm{kg}$

$\mu \mathrm{g} / \mathrm{kg}$

$\mu \mathrm{g} / \mathrm{kg}$

$\mu \mathrm{g} / \mathrm{kg}$

$\mu \mathrm{g} / \mathrm{kg}$
4.81

4.81

9.62

9.62

9.62

9.62

19.2

9.62

19.2

9.62

19.2

9.62

19.2
EPA8080

EPA8080

EPA8080

EPA8080

EPA8080

EPA8080

EPA8080

EPA8080

EPA8080

EPA8080

EPA8080

EPA8080

EPA8080 
SURVEY ID: PPSC 2103 (continued)

Constituent

Pesticides/PCBs and Dioxins/Furans

Endrin

Endrin ketone

Heptachlor

Heptachlor epoxide

Lindane

Methoxychlor

PCB 1016

PCB 1221

PCB 1232

PCB 1242

PCB 1248

PCB 1254

PCB 1260

Toxaphene

Radionuclides

Actinium-228

Antimony-124

Antimony-125

Barium-133

Cexium-144

Cesium-134

Cesium-137

Cobalt-57

Cobalt-58

Cobalt-60

Europium-152

Europium-154

Europium-155

Gross alpha

Lead-212

Manganese-54

Neptunium-239

Nonvolatile beta

Plutonium-238

Plutonium-239/240

Potassium-40

Promethium-144

Promethium-146

Radium-226

Radium-228

Ruthenium-106

Sodium-22

Strontium-90

Thorium-234

Tin-113

Yttrium-88

Zinc-65

Zirconium-95
Sample ID: 104682

RQ AQ B Result

Unit

D. Limit Method

$\begin{array}{lll}\mu \mathrm{g} / \mathrm{kg} & 9.62 & \text { EPA8080 } \\ \mu \mathrm{g} / \mathrm{kg} & 19.2 & \text { EPA8080 } \\ \mu \mathrm{g} / \mathrm{kg} & 9.62 & \text { EPA8080 } \\ \mu \mathrm{g} / \mathrm{kg} & 9.62 & \text { EPA8080 } \\ \mu \mathrm{g} / \mathrm{kg} & 4.81 & \text { EPA8080 } \\ \mu \mathrm{g} / \mathrm{kg} & 96.2 & \text { EPA8080 } \\ \mu \mathrm{g} / \mathrm{kg} & 24 & \text { EPA8080 } \\ \mu \mathrm{g} / \mathrm{kg} & 24 & \text { EPA8080 } \\ \mu \mathrm{g} / \mathrm{kg} & 24 & \text { EPA8080 } \\ \mu \mathrm{g} / \mathrm{kg} & 24 & \text { EPA8080 } \\ \mu \mathrm{g} / \mathrm{kg} & 24 & \text { EPA8080 } \\ \mu \mathrm{g} / \mathrm{kg} & 24 & \text { EPA8080 } \\ \mu \mathrm{g} / \mathrm{kg} & 24 & \text { EPA8080 } \\ \mu \mathrm{g} / \mathrm{kg} & 192 & \text { EPA8080 }\end{array}$

$1.50 \mathrm{E}+00 \pm 5.97 \mathrm{E}-02 \mathrm{pCi} / \mathrm{g}$

2.97E-02 EPIA-013B

$-3.45 \mathrm{E}-03 \pm 7.32 \mathrm{E}-03 \mathrm{pCi} / \mathrm{g}$

$7.99 \mathrm{E}-03 \pm 1.69 \mathrm{E}-02 \mathrm{pCi} / \mathrm{g}$

$5.07 \mathrm{E}-04 \pm 7.77 \mathrm{E}-03 \mathrm{pCi} / \mathrm{g}$

$-7.43 \mathrm{E}-03 \pm 4.66 \mathrm{E}-02 \mathrm{pCi} / \mathrm{g}$

$1.24 \mathrm{E}-04 \pm 6.00 \mathrm{E}-03 \mathrm{pCi} / \mathrm{g}$

$1.55 \mathrm{E}-03 \pm 6.98 \mathrm{E}-03 \mathrm{pCi} / \mathrm{g}$

$-1.55 \mathrm{E}-03 \pm 5.79 \mathrm{E}-03 \mathrm{pCi} / \mathrm{g}$

8.07E-03 $\pm 6.22 \mathrm{E}-03 \mathrm{pCi} / \mathrm{g}$

$2.66 \mathrm{E} \cdot 03 \pm 4.73 \mathrm{E}-03 \mathrm{pCi} / \mathrm{g}$

$-2.59 \mathrm{E}-03 \pm 1.64 \mathrm{E}-02 \mathrm{pCi} / \mathrm{g}$

$9.82 \mathrm{E}-03 \pm 4.93 \mathrm{E}-02 \mathrm{pCi} / \mathrm{g}$

$0.00 \mathrm{E}+00 \quad \mathrm{pCi} / \mathrm{g}$

$2.54 \mathrm{E}+01 \pm 4.04 \mathrm{E}+00 \mathrm{pCi} / \mathrm{g}$

$1.60 \mathrm{E}+00 \pm 3.15 \mathrm{E}-02 \mathrm{pCi} / \mathrm{g}$

$3.12 \mathrm{E}-02 \pm 9.49 \mathrm{E}-03 \mathrm{pCi} / \mathrm{g}$

UI $\quad 1.53 \mathrm{E}-02 \pm 4.65 \mathrm{E}-02 \mathrm{pCi} / \mathrm{g}$

$1.11 \mathrm{E}+01 \pm 2.00 \mathrm{E}+00 \mathrm{pCi} / \mathrm{g}$

UI $\quad-1.52 \mathrm{E}-04 \pm 1.52 \mathrm{E}-02 \mathrm{pCi} / \mathrm{g}$

UI

$0.00 \mathrm{E}+00 \quad \mathrm{pCi} / \mathrm{g}$

$6.86 \mathrm{E}-01 \pm 1.15 \mathrm{E}-01 \mathrm{pCi} / \mathrm{g}$

UI $\quad-3.21 \mathrm{E}-04 \pm 5.84 \mathrm{E}-03 \mathrm{pCi} / \mathrm{g}$

$0.00 \mathrm{E}+00 \quad \mathrm{pCi} / \mathrm{g}$

$\mathrm{V} \quad 8.45 \mathrm{E}-01 \pm 3.52 \mathrm{E}-02 \mathrm{pCi} / \mathrm{g}$

$1.50 \mathrm{E}+00 \pm 5.97 \mathrm{E}-02 \mathrm{pCi} / \mathrm{g}$

$-1.82 \mathrm{E}-02 \pm 5.40 \mathrm{E}-02 \mathrm{pCi} / \mathrm{g}$

$-4.40 \mathrm{E} \cdot 05 \pm 4.76 \mathrm{E}-03 \mathrm{pCj} / \mathrm{g}$

$3.76 \mathrm{E}-01 \pm 1.24 \mathrm{E}-01 \mathrm{pCi} / \mathrm{g}$

U V $\quad 1.86 \mathrm{E}+00 \pm 7.47 \mathrm{E} .01 \mathrm{pCi} / \mathrm{g}$

2.05E-03 $\pm 8.38 \mathrm{E}-03 \mathrm{pCi} / \mathrm{g}$

$4.53 \mathrm{E}-03 \pm 5.65 \mathrm{E}-03 \mathrm{pCi} / \mathrm{g}$

$1.58 \mathrm{E}-02 \pm 1.15 \mathrm{E}-02 \mathrm{pCi} / \mathrm{g}$

4.61E-03 $\pm 1.61 \mathrm{E}-02 \mathrm{pCi} / \mathrm{g}$

1.21E-02 EPIA-013B

2.59E-02 EPIA-013B

1.18E-02 EPIA-013B

7.73E-02 EPIA-013B

8.89E-03 EPIA-013B

1.03E-02 EPIA-013B

9.62E-03 EPIA-013B

1.13E-02 EPIA-013B

8.48E-03 EPIA-013B

2.81E-02 EPIA-013B

7.62E-02 EPIA-013B

4.14E-02 EPIA-013B

$1.78 \mathrm{E}+00$ EPIA-001B

1.72E-02 EPIA-013B

8.41E-03 EPIA-013B

7.00E-02 EPIA-013B

$2.48 \mathrm{E}+00$ EPIA-001B

3.65E-02 EPIA.012B

$7.58 \mathrm{E}-03$ EPIA.012B

7.51E-02 EPIA-013B

9.72E-03 EPIA-013B

1.30E-02 EPIA-013B

1.80E-02 EPIA-013B

2.97E-02 EPIA-013B

8.98E-02 EPIA-013B

8.24E-03 EPIA-013B

1.32E-01 EPIA-004

5.22E-01 EPIA-013B

1.45E-02 EPIA-013B

1.08E-02 EPIA-013B

1.92E-02 EPIA-013B

2.37E-02 EPIA-013B 
Survey location: $44548 \mathrm{E} 3670160 \mathrm{~N}$ (SRS coordinates)

Depth of core interval: 0.00 to $1.00 \mathrm{ft}$

Sample type: Normal

Sample matrix: Soil

Percent solids: 82.00

Constituent

RQ AQ B Result

Unit

D. Limit Method

Physical Parameters and Specified Analyses

Ammonia nitrogen

Cation exchange capacity

Chloride

Cyanide

Nitrate-nitrite as nitrogen

Nitrate-nitrite as nitrogen

Nitrogen by Kjeldahl method

Orthophosphate

Phenols

Phenols

Sulfide

Total organic carbon

Total organic nitrogen

Total phosphates (as P)

Metals (total recoverable)

\begin{tabular}{|c|c|c|c|c|}
\hline Aluminum & & $\mathrm{X}$ & & 5270 \\
\hline Aluminum & & $\mathrm{X}$ & & 3590 \\
\hline Antimony & UJ & $I$ & $\mathrm{~L}$ & 5.65 \\
\hline Antimony & UJ & I & L & 5.7 \\
\hline Arsenic & $\mathbf{U}$ & & & 16.9 \\
\hline Arsenic & $\mathrm{U}$ & & & 17.1 \\
\hline Barium & & & & 12.2 \\
\hline Barium & & & & 9.71 \\
\hline Beryllium & $J$ & $\mathbf{E}$ & & 0.154 \\
\hline Beryllium & $\mathbf{J}$ & $\mathbf{E}$ & & 0.121 \\
\hline Cadmium & $\mathrm{U}$ & & & 0.282 \\
\hline Cadmium & $\mathrm{U}$ & & & 0.285 \\
\hline Calcium & $J$ & I & $L$ & 552 \\
\hline Calcium & $\boldsymbol{J}$ & I & $\mathrm{L}$ & 531 \\
\hline Chromium & & & & 8.86 \\
\hline Chromium & & & & 6.37 \\
\hline Cobalt & $\mathbf{J}$ & $\mathrm{E}$ & & 0.217 \\
\hline Cobalt & $\mathrm{U}$ & & & 0.57 \\
\hline Copper & & & & 1.42 \\
\hline Copper & $\mathbf{J}$ & $\mathbf{E}$ & & 0.499 \\
\hline Iron & & $\mathrm{V}$ & & 2000 \\
\hline Iron & & $\mathrm{V}$ & & 1630 \\
\hline Lead & $\mathbf{U}$ & & & 5.65 \\
\hline Lead & $\mathrm{U}$ & & & 5.7 \\
\hline Magnesium & & & & 627 \\
\hline Magnesium & & & & 450 \\
\hline Manganese & & & & 18.6 \\
\hline Manganese & & & & 14.3 \\
\hline Mercury & U & & & 0.0398 \\
\hline Nickel & & & & 1.79 \\
\hline Nickel & $J$ & $E$ & & 1.3 \\
\hline Potassium & $J$ & $\mathrm{I}$ & $\mathrm{L}$ & 379 \\
\hline Potassium & $\mathbf{J}$ & $\mathrm{I}$ & $\mathrm{L}$ & 292 \\
\hline
\end{tabular}

$\begin{array}{lll}\text { U } & & 23.3 \\ & & 45.6 \\ \text { J } & \text { E } & 2.8 \\ \text { UJ } & \text { C } & 1.22 \\ \text { J } & \text { E } & 0.354 \\ \text { J } & \text { E } & 0.422 \\ & & 23.4 \\ \text { U } & & 24.4 \\ \text { U } & & 364 \\ \text { U } & & 364 \\ \text { UJ } & \text { C } & 305 \\ & & 698 \\ \text { C } & & 15.5 \\ \text { J } & \text { E } & 86.6\end{array}$

$\begin{array}{ll}\mathrm{mg} / \mathrm{kg} & 23.3 \\ \mathrm{meq} / 100 \mathrm{~g} & 5 \\ \mathrm{mg} / \mathrm{kg} & 2.95 \\ \mathrm{mg} / \mathrm{kg} & 1.22 \\ \mathrm{mg} / \mathrm{kg} & 1.18 \\ \mathrm{mg} / \mathrm{kg} & 1.06 \\ \mathrm{mg} / \mathrm{kg} & 4.77 \\ \mathrm{mg} / \mathrm{kg} & 24.4 \\ \mu \mathrm{g} / \mathrm{kg} & 364 \\ \mu \mathrm{g} / \mathrm{kg} & 364 \\ \mathrm{mg} / \mathrm{kg} & 305 \\ \mathrm{mg} / \mathrm{kg} & 122 \\ \mathrm{mg} / \mathrm{kg} & 0.244 \\ \mathrm{mg} / \mathrm{kg} & 492\end{array}$

EPA350.1 EPA9081

EPA300.0

EPA335.3

EPA353.1

EPA353.1

EPA351.2

EPA365.2

EPA420.2

EPA420.2

EPA376.2

EPA415.1

EPA351.2

EPA365.4

\begin{tabular}{lll}
$\mathrm{mg} / \mathrm{kg}$ & 14.1 & EPA6010A \\
$\mathrm{mg} / \mathrm{kg}$ & 14.2 & EPA6010A \\
$\mathrm{mg} / \mathrm{kg}$ & 5.65 & EPA6010A \\
$\mathrm{mg} / \mathrm{kg}$ & 5.7 & EPA6010A \\
$\mathrm{mg} / \mathrm{kg}$ & 16.9 & EPA6010A \\
$\mathrm{mg} / \mathrm{kg}$ & 17.1 & EPA6010A \\
$\mathrm{mg} / \mathrm{kg}$ & 1.41 & EPA6010A \\
$\mathrm{mg} / \mathrm{kg}$ & 1.42 & EPA6010A \\
$\mathrm{mg} / \mathrm{kg}$ & 0.565 & EPA6010A \\
$\mathrm{mg} / \mathrm{kg}$ & 0.57 & EPA6010A \\
$\mathrm{mg} / \mathrm{kg}$ & 0.282 & EPA6010A \\
$\mathrm{mg} / \mathrm{kg}$ & 0.285 & EPA6010A \\
$\mathrm{mg} / \mathrm{kg}$ & 14.1 & EPA6010A \\
$\mathrm{mg} / \mathrm{kg}$ & 14.2 & EPA6010A \\
$\mathrm{mg} / \mathrm{kg}$ & 1.41 & EPA6010A \\
$\mathrm{mg} / \mathrm{kg}$ & 1.42 & EPA6010A \\
$\mathrm{mg} / \mathrm{kg}$ & 0.565 & EPA6010A \\
$\mathrm{mg} / \mathrm{kg}$ & 0.57 & EPA6010A \\
$\mathrm{mg} / \mathrm{kg}$ & 1.41 & EPA6010A \\
$\mathrm{mg} / \mathrm{kg}$ & 1.42 & EPA6010A \\
$\mathrm{mg} / \mathrm{kg}$ & 5.65 & EPA6010A \\
$\mathrm{mg} / \mathrm{kg}$ & 5.7 & EPA6010A \\
$\mathrm{mg} / \mathrm{kg}$ & 5.65 & EPA6010A \\
$\mathrm{mg} / \mathrm{kg}$ & 5.7 & EPA6010A \\
$\mathrm{mg} / \mathrm{kg}$ & 5.65 & EPA6010A \\
$\mathrm{mg} / \mathrm{kg}$ & 5.7 & EPA6010A \\
$\mathrm{mg} / \mathrm{kg}$ & 0.565 & EPA6010A \\
$\mathrm{mg} / \mathrm{kg}$ & 0.57 & EPA6010A \\
$\mathrm{mg} / \mathrm{kg}$ & 0.0398 & EPA7471 \\
$\mathrm{mg} / \mathrm{kg}$ & 1.41 & EPA6010A \\
\hline & 1.42 & EPA6010A \\
\hline
\end{tabular}


SURVEY ID: PPSC 2201 (continued)

Constituent

Metals (total recoverable)

Selenium

Selenium

Silver

Silver

Sodium

Sodium

Thallium

Thallium

Vanadium

Vanadium

Zinc

Zinc
Sample ID: 104683

RQ AQ B Result

Unit

D. Limit Method

\section{$B / N / A$ Extractables (including PAH and phenols)}

\begin{tabular}{|c|c|c|c|c|c|c|}
\hline Acenaphthene & UJ & $\mathbf{Q}$ & 40.2 & $\mu g / k g$ & 40.2 & EPA8270 \\
\hline Açenaphthylene & UJ & $\mathbf{Q}$ & 40.2 & $\mu \mathrm{g} / \mathrm{kg}$ & 40.2 & EPA8270 \\
\hline Anthracene & UJ & $\mathbf{Q}$ & 40.2 & $\mu \mathrm{g} / \mathrm{kg}$ & 40.2 & EPA8270 \\
\hline Benzo[a]anthracene & UJ & Q & 4.02 & $\mu \mathrm{g} / \mathrm{kg}$ & 4.02 & EPA8270 \\
\hline Benzo[b]fluoranthene & UJ & $\mathbf{Q}$ & 4.02 & $\mu \mathrm{g} / \mathrm{kg}$ & 4.02 & EPA8270 \\
\hline Benzo $[k]$ fluoranthene & UJ & $\mathbf{Q}$ & 4.02 & $\mu \mathrm{g} / \mathrm{kg}$ & 4.02 & EPA8270 \\
\hline Benzoic acid & UJ & $\mathbf{Q}$ & 40.2 & $\mu \mathrm{g} / \mathrm{kg}$ & 40.2 & EPA8270 \\
\hline Benzo $[g, h, i]$ perylene & UJ & Q & 40.2 & $\mu \mathrm{g} / \mathrm{kg}$ & 40.2 & EPA8270 \\
\hline Benzo[a]pyrene & UJ & $\mathbf{Q}$ & 4.02 & $\mu \mathrm{g} / \mathrm{kg}$ & 4.02 & EPA8270 \\
\hline Benzyl alcohol & UJ & Q & 40.2 & $\mu g / \mathrm{kg}$ & 40.2 & EPA8270 \\
\hline Bis(2-chloroethoxy) methane & UJ & Q & 40.2 & $\mu \mathrm{g} / \mathrm{kg}$ & 40.2 & EPA8270 \\
\hline Bis(2-chloroethyl) ether & UJ & $\mathbf{Q}$ & 40.2 & $\mu \mathrm{g} / \mathrm{kg}$ & 40.2 & EPA8270 \\
\hline Bis(2-chloroisopropyl) ether & UJ & $\mathbf{Q}$ & 40.2 & $\mu \mathrm{g} / \mathrm{kg}$ & 40.2 & EPA8270 \\
\hline Bis(2-ethylhexyl) phthalate & $\mathbf{J}$ & $\mathbf{Q}$ & 108 & $\mu \mathrm{g} / \mathrm{kg}$ & 40.2 & EPA8270 \\
\hline 4-Bromophenyl phenyl ether & UJ & $\mathbf{Q}$ & 40.2 & $\mu \mathrm{g} / \mathrm{kg}$ & 40.2 & EPA8270 \\
\hline Butylbenzyl phthalate & UJ & $\mathbf{Q}$ & 40.2 & $\mu \mathrm{g} / \mathrm{kg}$ & 40.2 & EPA8270 \\
\hline 4-Chloroaniline & UJ & $\mathbf{Q}$ & 40.2 & $\mu \mathrm{g} / \mathrm{kg}$ & 40.2 & EPA8270 \\
\hline 4-Chloro-m-cresol & UJ & Q & 40.2 & $\mu \mathrm{g} / \mathrm{kg}$ & 40.2 & EPA8270 \\
\hline 2-Chloronaphthalene & UJ & $\mathbf{Q}$ & 40.2 & $\mu g / k g$ & 40.2 & EPA8270 \\
\hline 2-Chlorophenol & UJ & $\mathbf{Q}$ & 40.2 & $\mu \mathrm{g} / \mathrm{kg}$ & 40.2 & EPA8270 \\
\hline 4-Chlorophenyl phenyl ether & UJ & $\mathbf{Q}$ & 40.2 & $\mu \mathrm{g} / \mathrm{kg}$ & 40.2 & EPA8270 \\
\hline Chrysene & UJ & $\mathbf{Q}$ & 4.02 & $\mu \mathrm{g} / \mathrm{kg}$ & 4.02 & EPA8270 \\
\hline $\mathrm{m} / \mathrm{p}$-Cresol & UJ & $\mathbf{Q}$ & 402 & $\mu \mathrm{g} / \mathrm{kg}$ & 402 & EPA8270 \\
\hline o-Cresol (2-Methylphenol) & UJ & $\mathbf{Q}$ & 40.2 & $\mu \mathrm{g} / \mathrm{kg}$ & 40.2 & EPA8270 \\
\hline Dibenz $[a, h]$ anthracene & UJ & Q & 4.02 & $\mu \mathrm{g} / \mathrm{kg}$ & 4.02 & EPA8270 \\
\hline Dibenzofuran & UJ & Q & 40.2 & $\mu \mathrm{g} / \mathrm{kg}$ & 40.2 & EPA8270 \\
\hline Di-n-butyl phthalate & $J$ & $\mathrm{QE}$ & 38.2 & $\mu \mathrm{g} / \mathrm{kg}$ & 40.2 & EPA8270 \\
\hline 1,2-Dichlorobenzene & UJ & $\mathbf{Q}$ & 40.2 & $\mu \mathrm{g} / \mathrm{kg}$ & 40.2 & EPA8270 \\
\hline 1,3-Dichlorobenzene & UJ & Q & 40.2 & $\mu \mathrm{g} / \mathrm{kg}$ & 40.2 & EPA8270 \\
\hline 1,4-Dichlorobenzene & UJ & $\mathbf{Q}$ & 40.2 & $\mu \mathrm{g} / \mathrm{kg}$ & 40.2 & EPA8270 \\
\hline 3,3'-Dichlorobenzidine & UJ & $\mathbf{Q}$ & 40.2 & $\mu \mathrm{g} / \mathrm{kg}$ & 40.2 & EPA8270 \\
\hline 2,4-Dichlorophenol & UJ & Q & 40.2 & $\mu g / \mathrm{kg}$ & 40.2 & EPA8270 \\
\hline Diethyl phthalate & UJ & Q & 40.2 & $\mu \mathrm{g} / \mathrm{kg}$ & 40.2 & EPA8270 \\
\hline 2,4-Dimethyl phenol & UJ & Q & 40.2 & $\mu g / \mathrm{kg}$ & 40.2 & EPA8270 \\
\hline Dimethyl phthalate & UJ & $\mathbf{Q}$ & 40.2 & $\mu \mathrm{g} / \mathrm{kg}$ & 40.2 & EPA8270 \\
\hline 2,4-Dinitrophenol & UJ & $\mathbf{Q}$ & 402 & $\mu g / \mathrm{kg}$ & 402 & EPA8270 \\
\hline 2,4-Dinitrotoluene & UJ & QC & 4.02 & $\mu g / \mathrm{kg}$ & -4.02 & EPA8270 \\
\hline 2,6-Dinitrotoluene & UJ & $\mathbf{Q}$ & 4.02 & $\mu g / \mathrm{kg}$ & 4.02 & EPA8270 \\
\hline Di-n-octyl phthalate & UJ & $\mathbf{Q}$ & 40.2 & $\mu g / \mathrm{kg}$ & 40.2 & EPA8270 \\
\hline Fluoranthene & $\mathrm{J}$ & $\mathbf{Q}$ & 43.9 & $\mu g / \mathrm{kg}$ & 40.2 & EPA8270 \\
\hline Fluorene & UJ & Q & 40.2 & $\mu \mathrm{g} / \mathrm{kg}$ & 40.2 & EPA8270 \\
\hline
\end{tabular}


SURVEY ID: PPSC 2201 (continued)

Constituent
Sample ID: 104683

RQ AQ B Result

Unit

D. Limit Method

$B / N / A$ Extractables (including $P A H$ and phenols)

\begin{tabular}{|c|c|c|c|}
\hline Hexachlorobenzene & UJ & Q & 4.02 \\
\hline Hexachlorobutadiene & UJ & Q & 40. \\
\hline Hexachlorocyclopentadiene & UJ & $\mathbf{Q}$ & 40 \\
\hline Hexachloroethane & UJ & $Q$ & \\
\hline Indeno $[1,2,3-c, d]$ pyrene & UJ & $\mathbf{Q}$ & 4. \\
\hline Isophorone & UJ & $\mathbf{Q}$ & 40 \\
\hline 2-Methyl-4,6-dinitrophenol & UJ & $\mathbf{Q}$ & \\
\hline 2-Methylnaphthalene & UJ & $\mathbf{Q}$ & \\
\hline Naphthalene & UJ & $\mathbf{Q}$ & 40 \\
\hline m-Nitroaniline & UJ & $\mathbf{Q}$ & \\
\hline o-Nitroaniline & UJ & $\mathbf{Q}$ & 40 \\
\hline p-Nitroaniline & UJ & $Q$ & \\
\hline Nitrobenzene & UJ & $\mathbf{Q}$ & \\
\hline 2-Nitrophenol & UJ & $Q$ & 40 \\
\hline 4-Nitrophenol & UJ & $\mathbf{Q}$ & \\
\hline N-Nitrosodiphenylamine & UJ & $Q$ & \\
\hline N-Nitrosodi-n-propylamine & UJ & $\mathbf{Q}$ & \\
\hline Pentachlorophenol & UJ & $\mathbf{Q}$ & \\
\hline Phenanthrene & $\boldsymbol{J}$ & Q & \\
\hline Phenol & UJ & $\mathbf{Q}$ & \\
\hline Pyrene & $J$ & $\mathbf{Q}$ & 12 \\
\hline 1,2,4-Trichlorobenzene & UJ & $Q$ & \\
\hline 2,4,5-Trichlorophenol & UJ & $Q$ & \\
\hline 2,4,6-Trichlorophenol & UJ & $\mathbf{Q}$ & \\
\hline
\end{tabular}

Volatile Organic Compounds

Acetone
Benzene
Bromodichloromethane
Bromoform
Bromomethane (Methyl bromide)
Carbon disulfide
Carbon tetrachloride
Chlorobenzene
Chloroethane
Chloroethene (Vinyl chloride)
Chloroform
Chloromethane (Methyl chloride)
Dibromochloromethane
1,1-Dichloroethane
1,2-Dichloroethane
1,1-Dichloroethylene
1,2-Dichloroethylene
Dichloromethane (Methylene chloride)
1,2-Dichloropropane
cis-1,3-Dichloropropene
trans-1,3-Dichloropropene
Ethylbenzene
2-Hexanone
Methyl ethyl ketone
Methyl isobutyl ketone
Styrene
1,1,2,2-Tetrachloroethane
Tetrachloroethylene
Toluene

$\begin{array}{lll}\text { U } & 8 & 36.9 \\ U & & 0.61 \\ U & & 0.061 \\ U & & 0.061 \\ U & & 0.122 \\ U & & 1.22 \\ U & & 0.061 \\ U & & 0.061 \\ U & & 0.122 \\ U & & 0.122 \\ U & & 0.061 \\ U & & 0.122 \\ U & & 0.061 \\ U & & 0.061 \\ U & & 0.061 \\ U & & 0.061 \\ U & & 0.122 \\ U & V & 0.976 \\ U & & 0.061 \\ U & & 0.061 \\ U & & 0.061 \\ U & & 0.061 \\ U & V & 0.939 \\ U & V 8 & 0.476 \\ U & & 1.22 \\ U & & 0.061 \\ U & & 0.061 \\ U & & 0.061 \\ U & & 0.61\end{array}$

$\begin{array}{lll}\mu \mathrm{g} / \mathrm{kg} & 4.02 & \text { EPA8270 } \\ \mu \mathrm{g} / \mathrm{kg} & 40.2 & \text { EPA8270 } \\ \mu \mathrm{g} / \mathrm{kg} & 40.2 & \text { EPA8270 } \\ \mu \mathrm{g} / \mathrm{kg} & 4.02 & \text { EPA8270 } \\ \mu \mathrm{g} / \mathrm{kg} & 4.02 & \text { EPA8270 } \\ \mu \mathrm{g} / \mathrm{kg} & 40.2 & \text { EPA8270 } \\ \mu \mathrm{g} / \mathrm{kg} & 402 & \text { EPA8270 } \\ \mu \mathrm{g} / \mathrm{kg} & 40.2 & \text { EPA8270 } \\ \mu \mathrm{g} / \mathrm{kg} & 40.2 & \text { EPA8270 } \\ \mu \mathrm{g} / \mathrm{kg} & 40.2 & \text { EPA8270 } \\ \mu \mathrm{g} / \mathrm{kg} & 40.2 & \text { EPA8270 } \\ \mu \mathrm{g} / \mathrm{kg} & 40.2 & \text { EPA8270 } \\ \mu \mathrm{g} / \mathrm{kg} & 40.2 & \text { EPA8270 } \\ \mu \mathrm{g} / \mathrm{kg} & 40.2 & \text { EPA8270 } \\ \mu \mathrm{g} / \mathrm{kg} & 40.2 & \text { EPA8270 } \\ \mu \mathrm{g} / \mathrm{kg} & 40.2 & \text { EPA8270 } \\ \mu \mathrm{g} / \mathrm{kg} & 40.2 & \text { EPA8270 } \\ \mu \mathrm{g} / \mathrm{kg} & 40.2 & \text { EPA8270 } \\ \mu \mathrm{g} / \mathrm{kg} & 40.2 & \text { EPA8270 } \\ \mu \mathrm{g} / \mathrm{kg} & 40.2 & \text { EPA8270 } \\ \mu \mathrm{g} / \mathrm{kg} & 40.2 & \text { EPA8270 } \\ \mu \mathrm{g} / \mathrm{kg} & 4.02 & \text { EPA8270 } \\ \mu \mathrm{g} / \mathrm{kg} & 40.2 & \text { EPA8270 } \\ \mu \mathrm{g} / \mathrm{kg} & 40.2 & \text { EPA8270 }\end{array}$

$\begin{array}{lll}\mu \mathrm{g} / \mathrm{kg} & 6.1 & \text { EPA8260 } \\ \mu \mathrm{g} / \mathrm{kg} & 0.61 & \text { EPA8260 } \\ \mu \mathrm{g} / \mathrm{kg} & 0.061 & \text { EPA8260 } \\ \mu \mathrm{g} / \mathrm{kg} & 0.061 & \text { EPA8260 } \\ \mu \mathrm{g} / \mathrm{kg} & 0.122 & \text { EPA8260 } \\ \mu \mathrm{g} / \mathrm{kg} & 1.22 & \text { EPA8260 } \\ \mu \mathrm{g} / \mathrm{kg} & 0.061 & \text { EPA8260 } \\ \mu \mathrm{g} / \mathrm{kg} & 0.061 & \text { EPA8260 } \\ \mu \mathrm{g} / \mathrm{kg} & 0.122 & \text { EPA8260 } \\ \mu \mathrm{g} / \mathrm{kg} & 0.122 & \text { EPA8260 } \\ \mu \mathrm{g} / \mathrm{kg} & 0.061 & \text { EPA8260 } \\ \mu \mathrm{g} / \mathrm{kg} & 0.122 & \text { EPA8260 } \\ \mu \mathrm{g} / \mathrm{kg} & 0.061 & \text { EPA8260 } \\ \mu \mathrm{g} / \mathrm{kg} & 0.061 & \text { EPA8260 } \\ \mu \mathrm{g} / \mathrm{kg} & 0.061 & \text { EPA8260 } \\ \mu \mathrm{g} / \mathrm{kg} & 0.061 & \text { EPA8260 } \\ \mu \mathrm{g} / \mathrm{kg} & 0.122 & \text { EPA8260 } \\ \mu \mathrm{g} / \mathrm{kg} & 0.61 & \text { EPA8260 } \\ \mu \mathrm{g} / \mathrm{kg} & 0.061 & \text { EPA8260 } \\ \mu \mathrm{g} / \mathrm{kg} & 0.061 & \text { EPA8260 } \\ \mu \mathrm{g} / \mathrm{kg} & 0.061 & \text { EPA8260 } \\ \mu \mathrm{g} / \mathrm{kg} & 0.061 & \text { EPA8260 } \\ \mu \mathrm{g} / \mathrm{kg} & 1.22 & \text { EPA8260 } \\ \mu \mathrm{g} / \mathrm{kg} & 1.22 & \text { EPA8260 } \\ \mu \mathrm{g} / \mathrm{kg} & 1.22 & \text { EPA8260 } \\ \mu \mathrm{g} / \mathrm{kg} & 0.061 & \text { EPA8260 } \\ \mu \mathrm{g} / \mathrm{kg} & 0.061 & \text { EPA8260 } \\ \mu \mathrm{g} / \mathrm{kg} & 0.061 & \text { EPA8260 } \\ \mu \mathrm{g} / \mathrm{kg} & 0.61 & \text { EPA8260 }\end{array}$


SURVEY ID: PPSC 2201 (continued)

Constituent

Volatile Organic Compounds
Sample ID: 104683
RQ AQ B Result
D. Limit Method

\begin{tabular}{|c|c|c|c|c|}
\hline $\mathrm{U}$ & 0.061 & $\mu g / \mathrm{kg}$ & 0.061 & EPA8260 \\
\hline $\mathrm{U}$ & 0.061 & $\mu \mathrm{g} / \mathrm{kg}$ & 0.061 & EPA8260 \\
\hline $\mathrm{U}$ & 0.061 & $\mu \mathrm{g} / \mathrm{kg}$ & 0.061 & EPA8260 \\
\hline $\mathrm{N}$ & 1 & $\mu \mathrm{g} / \mathrm{kg}$ & & EPA8260 \\
\hline $\mathbf{U}$ & 1.22 & $\mu \mathrm{g} / \mathrm{kg}$ & 1.22 & EPA8260 \\
\hline$U$ & 0.183 & $\mu \mathrm{g} / \mathrm{kg}$ & 0.183 & EPA8260 \\
\hline
\end{tabular}

Pesticides/PCBs and Dioxins/Furans

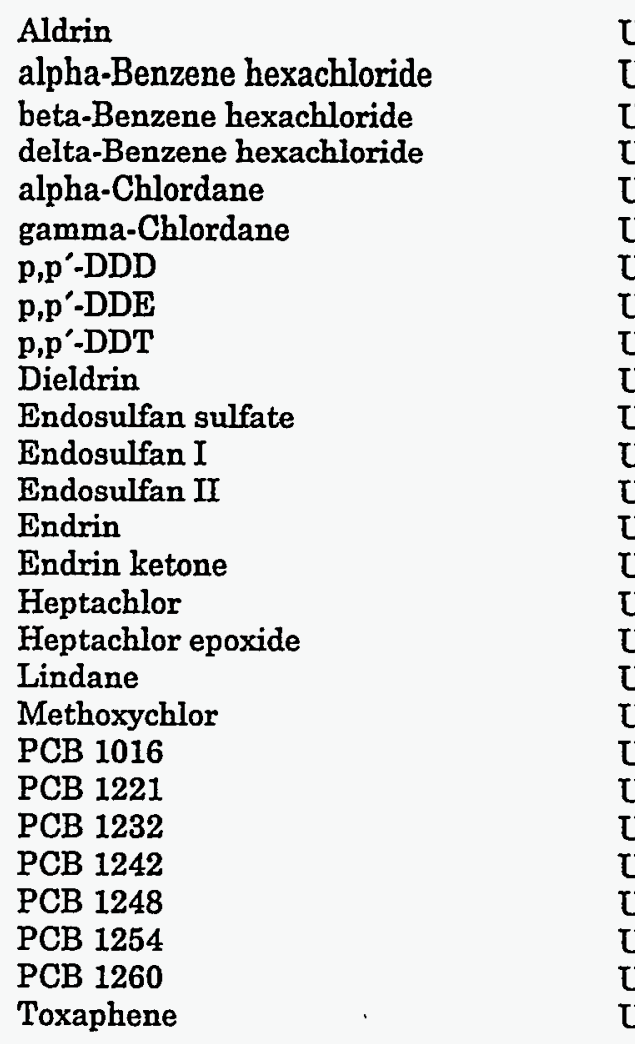

$\begin{array}{ll}U & 4.95 \\ U & 4.95 \\ U & 9.89 \\ U & 9.89 \\ U & 9.89 \\ U & 9.89 \\ U & 19.8 \\ U & 9.89 \\ U & 19.8 \\ U & 9.89 \\ U & 19.8 \\ U & 9.89 \\ U & 19.8 \\ U & 9.89 \\ U & 19.8 \\ U & 9.89 \\ U & 9.89 \\ U & 4.95 \\ U & 98.9 \\ U & 24.7 \\ U & 24.7 \\ U & 24.7 \\ U & 24.7 \\ U & 24.7 \\ U & 24.7 \\ U & 24.7 \\ U & 198\end{array}$

$\begin{array}{lll}\mu \mathrm{g} / \mathrm{kg} & 4.95 & \text { EPA8080 } \\ \mu \mathrm{g} / \mathrm{kg} & 4.95 & \text { EPA8080 } \\ \mu \mathrm{g} / \mathrm{kg} & 9.89 & \text { EPA8080 } \\ \mu \mathrm{g} / \mathrm{kg} & 9.89 & \text { EPA8080 } \\ \mu \mathrm{g} / \mathrm{kg} & 9.89 & \text { EPA8080 } \\ \mu \mathrm{g} / \mathrm{kg} & 9.89 & \text { EPA8080 } \\ \mu \mathrm{g} / \mathrm{kg} & 19.8 & \text { EPA8080 } \\ \mu \mathrm{g} / \mathrm{kg} & 9.89 & \text { EPA8080 } \\ \mu \mathrm{g} / \mathrm{kg} & 19.8 & \text { EPA8080 } \\ \mu \mathrm{g} / \mathrm{kg} & 9.89 & \text { EPA8080 } \\ \mu \mathrm{g} / \mathrm{kg} & 19.8 & \text { EPA8080 } \\ \mu \mathrm{g} / \mathrm{kg} & 9.89 & \text { EPA8080 } \\ \mu \mathrm{g} / \mathrm{kg} & 19.8 & \text { EPA8080 } \\ \mu \mathrm{g} / \mathrm{kg} & 9.89 & \text { EPA8080 } \\ \mu \mathrm{g} / \mathrm{kg} & 19.8 & \text { EPA8080 } \\ \mu \mathrm{g} / \mathrm{kg} & 9.89 & \text { EPA8080 } \\ \mu \mathrm{g} / \mathrm{kg} & 9.89 & \text { EPA8080 } \\ \mu \mathrm{g} / \mathrm{kg} & 4.95 & \text { EPA8080 } \\ \mu \mathrm{g} / \mathrm{kg} & 98.9 & \text { EPA8080 } \\ \mu \mathrm{g} / \mathrm{kg} & 24.7 & \text { EPA8080 } \\ \mu \mathrm{g} / \mathrm{kg} & 24.7 & \text { EPA8080 } \\ \mu \mathrm{g} / \mathrm{kg} & 24.7 & \text { EPA8080 } \\ \mu \mathrm{g} / \mathrm{kg} & 24.7 & \text { EPA8080 } \\ \mu \mathrm{g} / \mathrm{kg} & 24.7 & \text { EPA8080 } \\ \mu \mathrm{g} / \mathrm{kg} & 24.7 & \text { EPA8080 } \\ \mu \mathrm{g} / \mathrm{kg} & 24.7 & \text { EPA8080 } \\ \mu \mathrm{g} / \mathrm{kg} & 198 & \text { EPA8080 }\end{array}$

Radionuclides

Actinium-228

Antimony-124

Antimony-125

Barium-133

Cerium-144

Cesium-134

Cesium-137

Cobalt-57

Cobalt-58

Cobalt-60

Europium-152

Europium-154

Europium-155

Gross alpha

Lead-212

Manganese-54
7.78E-01士4.90E-02 pCi/g -2.68E-04 5.48E-03 pCi/g -3.01E-03+1.13E-02 pCi/g $-4.65 \mathrm{E}-03 \pm 5.84 \mathrm{E}-03 \mathrm{pCi} g$ $-4.49 \mathrm{E}-02 \pm 3.20 \mathrm{E}-02 \mathrm{pCi} / \mathrm{g}$ 1.32E-03 $\pm 4.50 \mathrm{E}-03 \mathrm{pCi} / \mathrm{g}$ 1.04E-02 $4.58 \mathrm{E}-03 \mathrm{pCi} / \mathrm{g}$ $-1.96 \mathrm{E}-03 \pm 3.98 \mathrm{E}-03 \mathrm{pCi} / \mathrm{g}$ 7.70E-04 $4.54 \mathrm{E}-03 \mathrm{pCi} / \mathrm{g}$ $-1.80 \mathrm{E}-04 \pm 3.53 \mathrm{E}-03 \mathrm{pCi} / \mathrm{g}$ $-6.24 \mathrm{E}-03 \pm 1.35 \mathrm{E}-02 \mathrm{pCi} / \mathrm{g}$ $-3.74 \mathrm{E}-03 \pm 3.80 \mathrm{E}-02 \mathrm{pCi} / \mathrm{g}$ $0.00 \mathrm{E}+00 \quad \mathrm{pCi} / \mathrm{g}$ $1.14 \mathrm{E}+01 \pm 2.59 \mathrm{E}+00 \mathrm{pCi} / \mathrm{g}$ 7.86E-01 $\pm 2.18 \mathrm{E}-02 \mathrm{pCi} / \mathrm{g}$ $0.00 \mathrm{E}+00 \quad \mathrm{pCi} / \mathrm{g}$
2.23E-02 EPIA-013B

9.59E-03 EPIA-013B

1.86E-02 EPIA-013B

8.32E-03 EPIA-013B

5.36E-02 EPIA-013B

7.06E.03 EPIA-013B

6.52E-03 EPLA-013B

6.81E-03 EPIA-013B

8.05E-03 EPIA-013B

6.40E-03 EPLA-013B

1.97E-02 EPIA-013B

5.74E-02 EPIA-013B

3.01E-02 EPIA-013B

1.29E+00 EPIA-001B

1.18E-02 EPIA-013B

8.08E-03 EPIA-013B 
SURVEY ID: PPSC 2201 (continued)

Constituent

Radionuclides

Neptunium-239

Nonvolatile beta

Plutonium-238

Plutonium-239/240

Potassium-40

Promethium-144

Promethium-146

Radium-226

Radium-228

Ruthenium-106

Sodium-22

Strontium-90

Thorium-234

Tin-113

Yttrium-88

Zinc-65

Zirconium-95
Sample ID: 104683
$\mathbf{R Q}$
AQ B Result
D. Limit Method

5.01E-02 EPIA-013B 2.37E+00 EPIA-001B

1.78E-02 EPIA-012B

1.78E-02 EPIA-012B

6.43E-02 EPIA-013B

6.94E-03 EPIA-013B

9.12E-03 EPIA-013B

1.37E-02 EPIA-013B

2.23E-02 EPIA-013B

6.59E-02 EPIA-013B

5.95E-03 EPIA-013B

1.36E-01 EPIA-004

3.62E-01 EPIA-013B

1.03E-02 EPIA-013B

7.97E-03 EPIA-013B

1.41E-02 EPIA-013B

1.76E-02 EPIA-013B

SURVEY ID: PPSC 2202

Sample ID: 104684

Survey location: 44548E 3670160N (SRS coordinates)

Depth of core interval: 1.00 to $2.00 \mathrm{ft}$

Sample type: Normal

Sample matrix: Soil

Percent solids: 79.00

Constituent

RQ AQ B Result

Unit

D. Limit Method

Physical Parameters and Specified Analyses

Ammonia nitrogen

Cation exchange capacity

Chloride

Cyanide

Nitrate-nitrite as nitrogen

Nitrogen by Kjeldahl method

Orthophosphate

Phenols

Sulfide

Total organic carbon

Total organic carbon

Total organic nitrogen

Total phosphates (as P)

Metals (total recoverable)

Aluminum

Antimony

Arsenic

Barium

Beryllium

Cadmium

Calcium

Chromium
$\mathbf{U}$

25.1
63.8

J $\quad \mathrm{E} \quad 2.74$

UJ C $\quad 1.25$

$\begin{array}{lll}J & \mathrm{E} & 0.6\end{array}$

J 24.4

$\begin{array}{lll} & \mathrm{E} & 8.2 \\ \mathrm{~J} & \mathrm{E} & 117\end{array}$

UJ C 316

$\mathrm{V} \quad 469$

V 439

C $\quad 16.4$

J E 115

$\begin{array}{lll}\mathrm{mg} / \mathrm{kg} & 25.1 & \text { EPA350.1 } \\ \mathrm{meq} / 100 \mathrm{~g} & 5 & \text { EPA9081 } \\ \mathrm{mg} / \mathrm{kg} & 3.03 & \text { EPA300.0 } \\ \mathrm{mg} / \mathrm{kg} & 1.25 & \text { EPA335.3 } \\ \mathrm{mg} / \mathrm{kg} & 1.2 & \text { EPA353.1 } \\ \mathrm{mg} / \mathrm{kg} & 5.03 & \text { EPA351.2 } \\ \mathrm{mg} / \mathrm{kg} & 25.3 & \text { EPA365.2 } \\ \mu \mathrm{g} / \mathrm{kg} & 379 & \text { EPA420.2 } \\ \mathrm{mg} / \mathrm{kg} & 316 & \text { EPA376.2 } \\ \mathrm{mg} / \mathrm{kg} & 127 & \text { EPA415.1 } \\ \mathrm{mg} / \mathrm{kg} & 127 & \text { EPA415.1 } \\ \mathrm{mg} / \mathrm{kg} & 0.253 & \text { EPA351.2 } \\ \mathrm{mg} / \mathrm{kg} & 518 & \text { EPA365.4 }\end{array}$

4770

6.27

18.8

27.6

$\begin{array}{lll}\mathrm{J} & \mathrm{E} & 0.236\end{array}$

U 0.313

854

10.3

$\begin{array}{lll}\mathrm{mg} / \mathrm{kg} & 15.7 & \text { EPA6010A } \\ \mathrm{mg} / \mathrm{kg} & 6.27 & \text { EPA6010A } \\ \mathrm{mg} / \mathrm{kg} & 18.8 & \text { EPA6010A } \\ \mathrm{mg} / \mathrm{kg} & 1.57 & \text { EPA6010A } \\ \mathrm{mg} / \mathrm{kg} & 0.627 & \text { EPA6010A } \\ \mathrm{mg} / \mathrm{kg} & 0.313 & \text { EPA6010A } \\ \mathrm{mg} / \mathrm{kg} & 15.7 & \text { EPA6010A } \\ \mathrm{mg} / \mathrm{kg} & 1.57 & \text { EPA6010A }\end{array}$


SURVEY ID: PPSC 2202 (continued)

Constituent

RQ AQ B Result

Unit

D. Limit Method

Metals (total recoverable)

Cobalt
Copper
Iron
Lead
Magnesium
Manganese
Mercury
Nickel
Potassium
Selenium
Silver
Sodium
Thallium
Vanadium
Zinc

$\begin{array}{lll}J & E & 0.271 \\ J & E & 0.69 \\ & V & 2150 \\ J & E & 3.7 \\ & & 683 \\ & & 2.61 \\ U & & 0.0324 \\ & & 1.88 \\ & & 448 \\ U & & 18.8 \\ U & & 1.57 \\ U & & 62.7 \\ U & & 18.8 \\ & & 5.57 \\ & & 5.72\end{array}$

\section{$B / N / A$ Extractables (including $P A H$ and phenols)}

\begin{tabular}{|c|c|c|}
\hline UJ & $Q$ & 41.9 \\
\hline UJ & $Q$ & 41.9 \\
\hline UJ & $Q$ & 41.9 \\
\hline UJ & $\mathbf{Q}$ & 4.19 \\
\hline UJ & $\mathbf{Q}$ & 4.19 \\
\hline UJ & $Q$ & 4.19 \\
\hline $\mathbf{J}$ & $Q$ & 59.9 \\
\hline UJ & $\mathbf{Q}$ & 41.9 \\
\hline UJ & $\mathbf{Q}$ & 4.19 \\
\hline UJ & $\mathbf{Q}$ & 41.9 \\
\hline UJJ & $\mathbf{Q}$ & 41.9 \\
\hline UJ & $\mathbf{Q}$ & 41.9 \\
\hline UJ & $\mathbf{Q}$ & 41.9 \\
\hline UJ & $\mathbf{Q}$ & 41.9 \\
\hline UJ & $\mathbf{Q}$ & 41.9 \\
\hline UJ & $\mathbf{Q}$ & 41.9 \\
\hline UJ & $\mathbf{Q}$ & 41.9 \\
\hline UJ & $\mathbf{Q}$ & 41.9 \\
\hline UJ & $\mathbf{Q}$ & 41.9 \\
\hline UJ & $\mathbf{Q}$ & 41.9 \\
\hline UJ & $\mathbf{Q}$ & 41.9 \\
\hline UJ & $\mathbf{Q}$ & 4.19 \\
\hline UJ & $\mathbf{Q}$ & 419 \\
\hline UJ & $Q$ & 41.9 \\
\hline UJ & $\mathrm{Q}$ & 4.19 \\
\hline UJ & $\mathbf{Q}$ & 41.9 \\
\hline UJ & $Q$ & 41.9 \\
\hline UJ & $Q$ & 41. \\
\hline UJ & $Q$ & 41. \\
\hline UJ & $\mathbf{Q}$ & 41.9 \\
\hline UJ & $\mathbf{Q}$ & 41. \\
\hline UJ & $\mathbf{Q}$ & 41. \\
\hline UJ & $\mathbf{Q}$ & 41.9 \\
\hline UJ & $\mathbf{Q}$ & 41 \\
\hline UJ & $\mathbf{Q}$ & 41.9 \\
\hline UJ & $\mathbf{Q}$ & 419 \\
\hline UJ & $\mathrm{QC}$ & 4.1 \\
\hline
\end{tabular}

$\begin{array}{lll}\mathrm{mg} / \mathrm{kg} & 0.627 & \text { EPA6010A } \\ \mathrm{mg} / \mathrm{kg} & 1.57 & \text { EPA6010A } \\ \mathrm{mg} / \mathrm{kg} & 6.27 & \text { EPA6010A } \\ \mathrm{mg} / \mathrm{kg} & 6.27 & \text { EPA6010A } \\ \mathrm{mg} / \mathrm{kg} & 6.27 & \text { EPA6010A } \\ \mathrm{mg} / \mathrm{kg} & 0.627 & \text { EPA6010A } \\ \mathrm{mg} / \mathrm{kg} & 0.0324 & \text { EPA7471 } \\ \mathrm{mg} / \mathrm{kg} & 1.57 & \text { EPA6010A } \\ \mathrm{mg} / \mathrm{kg} & 125 & \text { EPA6010A } \\ \mathrm{mg} / \mathrm{kg} & 18.8 & \text { EPA6010A } \\ \mathrm{mg} / \mathrm{kg} & 1.57 & \text { EPA6010A } \\ \mathrm{mg} / \mathrm{kg} & 62.7 & \text { EPA6010A } \\ \mathrm{mg} / \mathrm{kg} & 18.8 & \text { EPA6010A } \\ \mathrm{mg} / \mathrm{kg} & 0.627 & \text { EPA6010A } \\ \mathrm{mg} / \mathrm{kg} & 1.25 & \text { EPA6010A }\end{array}$

\begin{tabular}{|c|c|c|}
\hline$\mu \mathrm{g} / \mathrm{kg}$ & 41.9 & EPA8270 \\
\hline$\mu \mathrm{g} / \mathrm{kg}$ & 41.9 & EPA8270 \\
\hline$\mu \mathrm{g} / \mathrm{kg}$ & 41.9 & EPA8270 \\
\hline$\mu \mathrm{g} / \mathrm{kg}$ & 4.19 & EPA8270 \\
\hline$\mu \mathrm{g} / \mathrm{kg}$ & 4.19 & EPA8270 \\
\hline$\mu \mathrm{g} / \mathrm{kg}$ & 4.19 & EPA8270 \\
\hline$\mu \mathrm{g} / \mathrm{kg}$ & 41.9 & EPA8270 \\
\hline$\mu \mathrm{g} / \mathrm{kg}$ & 41.9 & EPA8270 \\
\hline$\mu \mathrm{g} / \mathrm{kg}$ & 4.19 & EPA8270 \\
\hline$\mu \mathrm{g} / \mathrm{kg}$ & 41.9 & EPA8270 \\
\hline$\mu \mathrm{g} / \mathrm{kg}$ & 41.9 & EPA8270 \\
\hline$\mu \mathrm{g} / \mathrm{kg}$ & 41.9 & EPA8270 \\
\hline$\mu \mathrm{g} / \mathrm{kg}$ & 41.9 & EPA8270 \\
\hline$\mu \mathrm{g} / \mathrm{kg}$ & 41.9 & EPA8270 \\
\hline$\mu \mathrm{g} / \mathrm{kg}$ & 41.9 & EPA8270 \\
\hline$\mu \mathrm{g} / \mathrm{kg}$ & 41.9 & EPA8270 \\
\hline$\mu \mathrm{g} / \mathrm{kg}$ & 41.9 & EPA8270 \\
\hline$\mu \mathrm{g} / \mathrm{kg}$ & 41.9 & EPA8270 \\
\hline$\mu \mathrm{g} / \mathrm{kg}$ & 41.9 & EPA8270 \\
\hline$\mu \mathrm{g} / \mathrm{kg}$ & 41.9 & EPA8270 \\
\hline$\mu \mathrm{g} / \mathrm{kg}$ & 41.9 & EPA8270 \\
\hline$\mu \mathrm{g} / \mathrm{kg}$ & 4.19 & EPA8270 \\
\hline$\mu \mathrm{g} / \mathrm{kg}$ & 419 & EPA8270 \\
\hline$\mu \mathrm{g} / \mathrm{kg}$ & 41.9 & EPA8270 \\
\hline$\mu \mathrm{g} / \mathrm{kg}$ & 4.19 & EPA8270 \\
\hline$\mu \mathrm{g} / \mathrm{kg}$ & 41.9 & EPA8270 \\
\hline$\mu \mathrm{g} / \mathrm{kg}$ & 41.9 & EPA8270 \\
\hline$\mu \mathrm{g} / \mathrm{kg}$ & 41.9 & EPA8270 \\
\hline$\mu \mathrm{g} / \mathrm{kg}$ & 41.9 & EPA8270 \\
\hline$\mu \mathrm{g} / \mathrm{kg}$ & 41.9 & EPA8270 \\
\hline$\mu \mathrm{g} / \mathrm{kg}$ & 41.9 & EPA8270 \\
\hline$\mu \mathrm{g} / \mathrm{kg}$ & 41.9 & EPA8270 \\
\hline$\mu \mathrm{g} / \mathrm{kg}$ & 41.9 & EPA8270 \\
\hline$\mu \mathrm{g} / \mathrm{kg}$ & 41.9 & EPA8270 \\
\hline$\mu \mathrm{g} / \mathrm{kg}$ & 41.9 & EPA8270 \\
\hline$\mu \mathrm{g} / \mathrm{kg}$ & 419 & EPA8270 \\
\hline$\mu \mathrm{g} / \mathrm{kg}$ & 4.19 & EPA8270 \\
\hline
\end{tabular}


SURVEY ID: PPSC 2202 (continued)

Sample ID: 104684

Constituent

RQ AQ B Result

Unit

D. Limit Method

$B / N / A$ Extractables (including PAH and phenols)

\begin{tabular}{|c|c|c|c|c|c|c|}
\hline 2,6-Dinitrotoluene & UJ & $Q$ & 4.19 & $\mu \mathrm{g} / \mathrm{kg}$ & 4.19 & EPA8270 \\
\hline Di-n-octyl phthalate & UJ & $Q$ & 41.9 & $\mu \mathrm{g} / \mathrm{kg}$ & 41.9 & EPA8270 \\
\hline Fluoranthene & UJ & Q & 41.9 & $\mu \mathrm{g} / \mathrm{kg}$ & 41.9 & EPA8270 \\
\hline Fluorene & UJ & $Q$ & 41.9 & $\mu \mathrm{g} / \mathrm{kg}$ & 41.9 & EPA8270 \\
\hline Hexachlorobenzene & UJ & $Q$ & 4.19 & $\mu \mathrm{g} / \mathrm{kg}$ & 4.19 & EPA8270 \\
\hline Hexachlorobutadiene & UJ & $\mathbf{Q}$ & 41.9 & $\mu \mathrm{g} / \mathrm{kg}$ & 41.9 & EPA8270 \\
\hline Hexachlorocyclopentadiene & UJ & $Q$ & 41.9 & $\mu \mathrm{g} / \mathrm{kg}$ & 41.9 & EPA8270 \\
\hline Hexachloroethane & UJ & $\mathbf{Q}$ & 4.19 & $\mu \mathrm{g} / \mathrm{kg}$ & 4.19 & EPA8270 \\
\hline Indeno $[1,2,3-c, d]$ pyrene & UJ & $Q$ & 4.19 & $\mu \mathrm{g} / \mathrm{kg}$ & 4.19 & EPA8270 \\
\hline Isophorone & UJ & $\vec{Q}$ & 41.9 & $\mu \mathrm{g} / \mathrm{kg}$ & 41.9 & EPA8270 \\
\hline 2-Methyl-4,6-dinitrophenol & UJ & $Q$ & 419 & $\mu g / \mathrm{kg}$ & 419 & EPA8270 \\
\hline 2-Methylnaphthalene & UJ & $Q$ & 41.9 & $\mu \mathrm{g} / \mathrm{kg}$ & 41.9 & EPA8270 \\
\hline Naphthalene & UJ & $\mathrm{Q}$ & 41.9 & $\mu \mathrm{g} / \mathrm{kg}$ & 41.9 & EPA8270 \\
\hline m-Nitroaniline & UJ & Q & 41.9 & $\mu \mathrm{g} / \mathrm{kg}$ & 41.9 & EPA8270 \\
\hline o-Nitroaniline & UJ & $Q$ & 41.9 & $\mu \mathrm{g} / \mathrm{kg}$ & 41.9 & EPA8270 \\
\hline p-Nitroaniline & UJ & $\mathrm{Q}$ & 41.9 & $\mu \mathrm{g} / \mathrm{kg}$ & 41.9 & EPA8270 \\
\hline Nitrobenzene & UJ & $Q$ & 41.9 & $\mu \mathrm{g} / \mathrm{kg}$ & 41.9 & EPA8270 \\
\hline 2-Nitrophenol & UJ & $\mathrm{Q}$ & 41.9 & $\mu \mathrm{g} / \mathrm{kg}$ & 41.9 & EPA8270 \\
\hline 4-Nitrophenol & UJ & $\vec{Q}$ & 41.9 & $\mu \mathrm{g} / \mathrm{kg}$ & 41.9 & EPA8270 \\
\hline N-Nitrosodiphenylamine & UJ & $\dot{Q}$ & 41.9 & $\mu \mathrm{g} / \mathrm{kg}$ & 41.9 & EPA8270 \\
\hline N-Nitrosodi-n-propylamine & UJ & $\mathrm{Q}$ & 41.9 & $\mu \mathrm{g} / \mathrm{kg}$ & 41.9 & EPA8270 \\
\hline Pentachlorophenol & UJ & $Q$ & 41.9 & $\mu \mathrm{g} / \mathrm{kg}$ & 41.9 & EPA8270 \\
\hline Phenanthrene & UJ & $\mathbf{Q}$ & 41.9 & $\mu \mathrm{g} / \mathrm{kg}$ & 41.9 & EPA8270 \\
\hline Phenol & UJ & $\dot{Q}$ & 41.9 & $\mu \mathrm{g} / \mathrm{kg}$ & 41.9 & EPA8270 \\
\hline Pyrene & UJ & $Q$ & 41.9 & $\mu \mathrm{g} / \mathrm{kg}$ & 41.9 & EPA8270 \\
\hline 1,2,4-Trichlorobenzene & UJ & $Q$ & 4.19 & $\mu \mathrm{g} / \mathrm{kg}$ & 4.19 & EPA8270 \\
\hline 2,4,5-Trichlorophenol & UJ & $\mathrm{Q}$ & 41.9 & $\mu \mathrm{g} / \mathrm{kg}$ & 41.9 & EPA8270 \\
\hline 2,4,6-Trichlorophenol & UJ & $Q$ & 41.9 & $\mu \mathrm{g} / \mathrm{kg}$ & 41.9 & EPA8270 \\
\hline
\end{tabular}

Volatile Organic Compounds

Acetone
Benzene
Bromodichloromethane
Bromoform
Bromomethane (Methyl bromide)
Carbon disulfide
Carbon tetrachloride
Chlorobenzene
Chloroethane
Chloroethene (Vinyl chloride)
Chloroform
Chloromethane (Methyl chloride)
Dibromochloromethane
1,1-Dichloroethane
1,2-Dichloroethane
1,1-Dichloroethylene
1,2-Dichloroethylene
Dichloromethane (Methylene chloride)
1,2-Dichloropropane
cis-1,3-Dichloropropene
trans-1,3-Dichloropropene
Ethylbenzene
2-Hexanone
Methyl ethyl ketone

$\begin{array}{lll}U & 8 & 43.7 \\ U & & 0.633 \\ U & & 0.0633 \\ U & & 0.0633 \\ U & & 0.127 \\ U & & 1.27 \\ U & & 0.0633 \\ U & & 0.0633 \\ U & & 0.127 \\ U & & 0.127 \\ U & & 0.0633 \\ U & & 0.127 \\ U & & 0.0633 \\ U & & 0.0633 \\ U & & 0.0633 \\ U & & 0.0633 \\ U & & 0.127 \\ U & V & 1.03 \\ U & & 0.0633 \\ U & & 0.0633 \\ U & & 0.0633 \\ U & & 0.0633 \\ U & V & 0.759 \\ U & V 8 & 0.506\end{array}$

$\begin{array}{lll}\mu \mathrm{g} / \mathrm{kg} & 6.33 & \text { EPA8260 } \\ \mu \mathrm{g} / \mathrm{kg} & 0.633 & \text { EPA8260 } \\ \mu \mathrm{g} / \mathrm{kg} & 0.0633 & \text { EPA8260 } \\ \mu \mathrm{g} / \mathrm{kg} & 0.0633 & \text { EPA8260 } \\ \mu \mathrm{g} / \mathrm{kg} & 0.127 & \text { EPA8260 } \\ \mu \mathrm{g} / \mathrm{kg} & 1.27 & \text { EPA8260 } \\ \mu \mathrm{g} / \mathrm{kg} & 0.0633 & \text { EPA8260 } \\ \mu \mathrm{g} / \mathrm{kg} & 0.0633 & \mathrm{EPA8260} \\ \mu \mathrm{g} / \mathrm{kg} & 0.127 & \text { EPA8260 } \\ \mu \mathrm{g} / \mathrm{kg} & 0.127 & \text { EPA8260 } \\ \mu \mathrm{g} / \mathrm{kg} & 0.0633 & \text { EPA8260 } \\ \mu \mathrm{g} / \mathrm{kg} & 0.127 & \text { EPA8260 } \\ \mu \mathrm{g} / \mathrm{kg} & 0.0633 & \text { EPA8260 } \\ \mu \mathrm{g} / \mathrm{kg} & 0.0633 & \text { EPA8260 } \\ \mu \mathrm{g} / \mathrm{kg} & 0.0633 & \text { EPA8260 } \\ \mu \mathrm{g} / \mathrm{kg} & 0.0633 & \text { EPA8260 } \\ \mu \mathrm{g} / \mathrm{kg} & 0.127 & \text { EPA8260 } \\ \mu \mathrm{g} / \mathrm{kg} & 0.633 & \text { EPA8260 } \\ \mu \mathrm{g} / \mathrm{kg} & 0.0633 & \text { EPA8260 } \\ \mu \mathrm{g} / \mathrm{kg} & 0.0633 & \text { EPA8260 } \\ \mu \mathrm{g} / \mathrm{kg} & -0.0633 & \text { EPA8260 } \\ \mu \mathrm{g} / \mathrm{kg} & 0.0633 & \text { EPA8260 } \\ \mu \mathrm{g} / \mathrm{kg} & 1.27 & \text { EPA8260 } \\ \mu \mathrm{g} / \mathrm{kg} & 1.27 & \text { EPA8260 }\end{array}$


SURVEY ID: PPSC 2202 (continued)

Constituent

Volatile Organic Compounds

Methyl isobutyl ketone

Styrene

1,1,2,2-Tetrachloroethane

Tetrachloroethylene

Toluene

1,1,1-Trichloroethane

1,1,2-Trichloroethane

Trichloroethylene

Unknown alcohol

Vinyl acetate

Xylenes

Pesticides/PCBs and Dioxins/Furans

Aldrin

alpha-Benzene hexachloride

beta-Benzene hexachloride

delta-Benzene hexachloride

alpha-Chlordane

gamma-Chlordane

$\mathrm{p}, \mathrm{p}^{\prime}-\mathrm{DDD}$

$p, p^{\prime}-D D E$

p,p'-DDT

Dieldrin

Endosulfan sulfate

Endosulfan I

Endosulfan II

Endrin

Endrin ketone

Heptachlor

Heptachlor epoxide

Lindane

Methoxychlor

PCB 1016

PCB 1221

PCB 1232

PCB 1242

PCB 1248

PCB 1254

PCB 1260

Toxaphene

Radionuclides

Actinium-228

Antimony-124

Antimony-125

Barium-133

Cerium-144

Cesium-134

Cesium-137

Cobalt-57

Cobalt-58

Cobalt-60

Europium-152
Sample ID: 104684

RQ AQ B Result Unit D. Limit Method

\begin{tabular}{|c|c|c|c|c|}
\hline$U$ & 1.27 & $\mu \mathrm{g} / \mathrm{kg}$ & 1.27 & EPA8260 \\
\hline $\mathrm{U}$ & 0.0633 & $\mu \mathrm{g} / \mathrm{kg}$ & 0.0633 & EPA8260 \\
\hline $\mathrm{U}$ & 0.0633 & $\mu \mathrm{g} / \mathrm{kg}$ & 0.0633 & EPA8260 \\
\hline $\mathrm{U}$ & 0.0633 & $\mu \mathrm{g} / \mathrm{kg}$ & 0.0633 & EPA8260 \\
\hline $\mathrm{U}$ & 0.633 & $\mu \mathrm{g} / \mathrm{kg}$ & 0.633 & EPA8260 \\
\hline$U$ & 0.0633 & $\mu \mathrm{g} / \mathrm{kg}$ & 0.0633 & EPA8260 \\
\hline U & 0.0633 & $\mu g / \mathrm{kg}$ & 0.0633 & EPA8260 \\
\hline $\mathbf{U}$ & 0.0633 & $\mu \mathrm{g} / \mathrm{kg}$ & 0.0633 & EPA8260 \\
\hline $\mathbf{N}$ & 1 & $\mu \mathrm{g} / \mathrm{kg}$ & & EPA8260 \\
\hline $\mathrm{U}$ & 1.27 & $\mu \mathrm{g} / \mathrm{kg}$ & 1.27 & EPA8260 \\
\hline $\mathrm{U}$ & 0.19 & $\mu \mathrm{g} / \mathrm{kg}$ & 0.19 & EPA8260 \\
\hline
\end{tabular}

$\begin{array}{llll}\text { UJ } & \text { O } & \text { L } & 1.03 \\ \text { UJ } & \text { O } & \text { L } & 1.03 \\ \text { UJ } & \text { O } & \text { L } & 2.06 \\ \text { UJ } & \text { O } & \text { L } & 2.06 \\ \text { UJ } & \text { O } & \text { L } & 2.06 \\ \text { UJ } & \text { O } & \text { L } & 2.06 \\ \text { UJ } & \text { O } & \text { L } & 4.11 \\ \text { UJ } & \text { O } & \text { L } & 2.06 \\ \text { UJ } & \text { O } & \text { L } & 4.11 \\ \text { UJ } & \text { O } & \text { L } & 2.06 \\ \text { UJ } & \text { O } & \text { L } & 4.11 \\ \text { UJ } & \text { O } & \text { L } & 2.06 \\ \text { UJ } & \text { O } & \text { L } & 4.11 \\ \text { UJ } & \text { O } & \text { L } & 2.06 \\ \text { UJ } & \text { O } & \text { L } & 4.11 \\ \text { UJ } & \text { O } & \text { L } & 2.06 \\ \text { UJ } & \text { O } & \text { L } & 2.06 \\ \text { UJ } & \text { O } & \text { L } & 1.03 \\ \text { UJ } & \text { O } & \text { L } & 20.6 \\ \text { UJ } & \text { O } & \text { L } & 5.14 \\ \text { UJ } & \text { O } & \text { L } & 5.14 \\ \text { UJ } & \text { O } & \text { L } & 5.14 \\ \text { UJ } & \text { O } & \text { L } & \mathbf{5 . 1 4} \\ \text { UJ } & \text { O } & \text { L } & 5.14 \\ \text { UJ } & \text { O } & \text { L } & 5.14 \\ \text { UJ } & \text { O } & \text { L } & \mathbf{5 . 1 4} \\ \text { UJ } & \text { O } & \text { L } & 41.1\end{array}$

$\begin{array}{lll}\mu \mathrm{g} / \mathrm{kg} & 1.03 & \text { EPA8080 } \\ \mu \mathrm{g} / \mathrm{kg} & 1.03 & \text { EPA8080 } \\ \mu \mathrm{g} / \mathrm{kg} & 2.06 & \text { EPA8080 } \\ \mu \mathrm{g} / \mathrm{kg} & 2.06 & \text { EPA8080 } \\ \mu \mathrm{g} / \mathrm{kg} & 2.06 & \text { EPA8080 } \\ \mu \mathrm{g} / \mathrm{kg} & 2.06 & \text { EPA8080 } \\ \mu \mathrm{g} / \mathrm{kg} & 4.11 & \text { EPA8080 } \\ \mu \mathrm{g} / \mathrm{kg} & 2.06 & \text { EPA8080 } \\ \mu \mathrm{g} / \mathrm{kg} & 4.11 & \text { EPA8080 } \\ \mu \mathrm{g} / \mathrm{kg} & 2.06 & \text { EPA8080 } \\ \mu \mathrm{g} / \mathrm{kg} & 4.11 & \text { EPA8080 } \\ \mu \mathrm{g} / \mathrm{kg} & 2.06 & \text { EPA8080 } \\ \mu \mathrm{g} / \mathrm{kg} & 4.11 & \text { EPA8080 } \\ \mu \mathrm{g} / \mathrm{kg} & 2.06 & \text { EPA8080 } \\ \mu \mathrm{g} / \mathrm{kg} & 4.11 & \text { EPA8080 } \\ \mu \mathrm{g} / \mathrm{kg} & 2.06 & \text { EPA8080 } \\ \mu \mathrm{g} / \mathrm{kg} & 2.06 & \text { EPA8080 } \\ \mu \mathrm{g} / \mathrm{kg} & 1.03 & \text { EPA8080 } \\ \mu \mathrm{g} / \mathrm{kg} & 20.6 & \text { EPA8080 } \\ \mu \mathrm{g} / \mathrm{kg} & 5.14 & \text { EPA8080 } \\ \mu \mathrm{g} / \mathrm{kg} & 5.14 & \text { EPA8080 } \\ \mu \mathrm{g} / \mathrm{kg} & 5.14 & \text { EPA8080 } \\ \mu \mathrm{g} / \mathrm{kg} & 5.14 & \text { EPA8080 } \\ \mu \mathrm{g} / \mathrm{kg} & 5.14 & \text { EPA8080 } \\ \mu \mathrm{g} / \mathrm{kg} & 5.14 & \text { EPA8080 } \\ \mu \mathrm{g} / \mathrm{kg} & 5.14 & \text { EPA8080 } \\ \mu \mathrm{g} / \mathrm{kg} & 41.1 & \text { EPA8080 }\end{array}$

$7.08 \mathrm{E}-01 \pm 5.60 \mathrm{E}-02 \mathrm{pCi} / \mathrm{g}$ $-3.42 \mathrm{E}-03 \pm 6.20 \mathrm{E}-03 \mathrm{pCi} / \mathrm{g}$ $-1.52 \mathrm{E}-03 \pm 1.46 \mathrm{E}-02 \mathrm{pCi} / \mathrm{g}$ $3.13 \mathrm{E}-04 \pm 6.48 \mathrm{E}-03 \mathrm{pCi} / \mathrm{g}$ $-2.23 \mathrm{E}-03 \pm 3.61 \mathrm{E}-02 \mathrm{pCi} / \mathrm{g}$ $2.33 \mathrm{E}-03 \pm 5.10 \mathrm{E}-03 \mathrm{pCi} / \mathrm{g}$ $1.53 \mathrm{E}-03 \pm 5.69 \mathrm{E}-03 \mathrm{pCi} / \mathrm{g}$ $-1.53 \mathrm{E}-03 \pm 4.57 \mathrm{E}-03 \mathrm{pCi} / \mathrm{g}$ $-2.00 \mathrm{E}-03 \pm 5.63 \mathrm{E}-03 \mathrm{pCi} / \mathrm{g}$ $-5.33 \mathrm{E}-04 \pm 4.42 \mathrm{E}-03 \mathrm{pCi} / \mathrm{g}$ $-6.79 \mathrm{E}-03 \pm 1.41 \mathrm{E}-02 \mathrm{pCi} / \mathrm{g}$
2.80E-02 EPIA-013B

1.04E-02 EPIA-013B

2.25E-02 EPIA-013B

9.51E-03 EPIA-013B

6.16E-02 EPIA-013B

7.94E-03 EPIA-013B

8.73E-03 EPIA-013B

7.79E-03 EPIA-013B

9.38E-03 EPIA-013B

7.75E-03 EPLA.013B

2.27E-02 EPIA-013B 
SURVEY ID: PPSC 2202 (continued)

Constituent

Radionuclides

Europium-154

Europium-155

Gross alpha

Lead-212

Manganese-54

Neptunium-239

Nonvolatile beta

Plutonium-238

Plutonium-238

Plutonium-239/240

Plutonium-239/240

Potassium-40

Promethium-144

Promethium-146

Radium-226

Radium-228

Ruthenium-106

Sodium-22

Strontium-90

Thorium-234

Tin-113

Yttrium-88

Zinc-65

Zirconium-95
Sample ID: 104684

RQ AQ B Result Unit D. Limit Method $\begin{array}{ll}7.14 \mathrm{E}-02 & \text { EPIA-013B } \\ 3.39 \mathrm{E}-02 & \text { EPIA-013B } \\ 1.56 \mathrm{E}+00 & \text { EPIA-001B } \\ 1.38 \mathrm{E}-02 & \text { EPIA-013B } \\ 8.22 \mathrm{E}-03 & \text { EPIA-013B } \\ 5.66 \mathrm{E}-02 & \text { EPIA-013B } \\ 2.49 \mathrm{E}+00 & \text { EPIA-001B } \\ 3.67 \mathrm{E}-02 & \text { EPIA-012B } \\ 2.64 \mathrm{E}-02 & \text { EPIA-012B } \\ 2.38 \mathrm{E}-02 & \text { EPIA-012B } \\ 1.60 \mathrm{E}-02 & \text { EPIA-012B } \\ 7.08 \mathrm{E}-02 & \text { EPIA-013B } \\ 8.45 \mathrm{E}-03 & \text { EPLA-013B } \\ 1.06 \mathrm{E}-02 & \text { EPIA-013B } \\ 1.58 \mathrm{E}-02 & \text { EPIA-013B } \\ 2.80 \mathrm{E}-02 & \text { EPIA-013B } \\ 7.41 \mathrm{E}-02 & \text { EPIA-013B } \\ 7.90 \mathrm{E}-03 & \text { EPIA-013B } \\ 1.58 \mathrm{E}-01 & \text { EPIA-004 } \\ 4.14 \mathrm{E}-01 & \text { EPIA-013B } \\ 1.14 \mathrm{E}-02 & \text { EPIA-013B } \\ 9.83 \mathrm{E}-03 & \text { EPIA-013B } \\ 1.67 \mathrm{E}-02 & \text { EPIA-013B } \\ 2.23 \mathrm{E}-02 & \text { EPIA-013B }\end{array}$ $-2.67 \mathrm{E}-02 \pm 4.17 \mathrm{E}-02 \mathrm{pCi} /$ $1.59 \mathrm{E}-02 \pm 2.78 \mathrm{E}-02 \mathrm{pCj} / \mathrm{g}$ $5.40 \mathrm{E}+00 \pm 1.79 \mathrm{E}+00 \mathrm{pCi} / \mathrm{g}$ 7.42E-01 $\pm 2.32 \mathrm{E}-02 \mathrm{pCi} / \mathrm{g}$ $7.00 \mathrm{E}-03 \pm 6.29 \mathrm{E}-03 \mathrm{pCi} / \mathrm{g}$ $-1.18 \mathrm{E}-02 \pm 3.31 \mathrm{E}-02 \mathrm{pCi} / \mathrm{g}$ $4.67 \mathrm{E}+00 \pm 1.50 \mathrm{E}+00 \mathrm{pCi} / \mathrm{g}$ 2.59E-03 $\pm 1.60 \mathrm{E}-02 \mathrm{pCi} / \mathrm{g}$ $6.30 \mathrm{E}-03 \pm 1.28 \mathrm{E}-02 \mathrm{pCi} / \mathrm{g}$ 4.05E-03 $\pm 1.06 \mathrm{E}-02 \mathrm{pCi} / \mathrm{g}$ $-1.28 \mathrm{E}-03 \pm 2.60 \mathrm{E}-03 \mathrm{pCi} / \mathrm{g}$ $1.60 \mathrm{E}+00 \pm 1.49 \mathrm{E}-01 \mathrm{pCi} / \mathrm{g}$ $-1.98 \mathrm{E}-03 \pm 5.05 \mathrm{E}-03 \mathrm{pCi} / \mathrm{g}$ $6.95 \mathrm{E}-03 \pm 7.40 \mathrm{E}-03 \mathrm{pCi} / \mathrm{g}$ 4.07E-01 $\pm 2.79 \mathrm{E}-02 \mathrm{pCi} / \mathrm{g}$ 7.08E-01 $\pm 5.60 \mathrm{E}-02 \mathrm{pCi} / \mathrm{g}$ $-1.25 \mathrm{E}-02 \pm 4.36 \mathrm{E}-02 \mathrm{pCi} / \mathrm{g}$ $-2.60 \mathrm{E}-03 \pm 4.68 \mathrm{E}-03 \mathrm{pCi} / \mathrm{g}$ $1.48 \mathrm{E}-01 \pm 1.00 \mathrm{E}-01 \mathrm{pCi} / \mathrm{g}$ 7.32E-01 4 .30E-01 pCi/g $-2.94 \mathrm{E}-03 \pm 6.61 \mathrm{E}-03 \mathrm{pCi} / \mathrm{g}$ $1.85 \mathrm{E}-03 \pm 5.12 \mathrm{E}-03 \mathrm{pCi} / \mathrm{g}$ $-8.30 \mathrm{E}-03 \pm 1.16 \mathrm{E}-02 \mathrm{pCi} / \mathrm{g}$ 1.50E-02士1.67E-02 pCi/g

Sample ID: 104685

SURVEY ID: PPSC 2203

Survey location: 44548E $3670160 \mathrm{~N}$ (SRS coordinates)

Depth of core interval: 2.00 to $4.00 \mathrm{ft}$

Sample type: Normal

Sample matrix: Soil

Percent solids: $\mathbf{7 6 . 0 0}$

Constituent

RQ AQ B Result

Unit

D. Limit Method

Physical Parameters and Specified Analyses

$\begin{array}{lllll}\text { Ammonia nitrogen } & \text { U } & & 25.6 \\ \text { Cation exchange capacity } & & & 59.6 \\ \text { Chloride } & & & 4.85 \\ \text { Cyanide } & \text { UJ } & \text { C } & 1.28 \\ \text { Nitrate-nitrite as nitrogen } & \text { J } & \text { E } & 0.455 \\ \text { Nitrogen by Kjeldahl method } & & & 16.1 \\ \text { Orthophosphate } & \text { J } & \text { E } & 8.16 \\ \text { Phenols } & \text { J } & \text { E } & 101 \\ \text { Sulfide } & \text { UJ } & \text { C } & 329 \\ \text { Total organic carbon } & & \text { V } & 310 \\ \text { Total organic nitrogen } & \text { C } & & 6.63 \\ \text { Total phosphates (as P) } & \text { J } & \text { E } & 83.9\end{array}$

$\begin{array}{lll}\mathrm{mg} / \mathrm{kg} & 25.6 & \text { EPA350.1 } \\ \mathrm{meq} / 100 \mathrm{~g} & 5 & \text { EPA9081 } \\ \mathrm{mg} / \mathrm{kg} & 3.19 & \text { EPA300.0 } \\ \mathrm{mg} / \mathrm{kg} & 1.28 & \text { EPA335.3 } \\ \mathrm{mg} / \mathrm{kg} & 1.14 & \text { EPA353.1 } \\ \mathrm{mg} / \mathrm{kg} & 5.19 & \text { EPA351.2 } \\ \mathrm{mg} / \mathrm{kg} & 26.3 & \text { EPA365.2 } \\ \mu \mathrm{g} / \mathrm{kg} & 393 & \text { EPA420.2 } \\ \mathrm{mg} / \mathrm{kg} & 329 & \text { EPA376.2 } \\ \mathrm{mg} / \mathrm{kg} & 132 & \text { EPA415.1 } \\ \mathrm{mg} / \mathrm{kg} & 0.263 & \text { EPA351.2 } \\ \mathrm{mg} / \mathrm{kg} & 287 & \text { EPA365.4 }\end{array}$


SURVEY ID: PPSC 2203 (continued)

Constituent

RQ AQ B Result

Metals (total recoverable)

\begin{tabular}{|c|c|c|c|}
\hline Aluminum & & & 4160 \\
\hline Antimony & $\mathrm{U}$ & & 6.15 \\
\hline Arsenic & $\mathrm{U}$ & & 18.4 \\
\hline Barium & & & 3.73 \\
\hline Beryllium & $\boldsymbol{J}$ & $\mathrm{E}$ & 0.172 \\
\hline Cadmium & $\mathrm{U}$ & & 0.307 \\
\hline Calcium & & & 869 \\
\hline Chromium & & & 10.2 \\
\hline Cobalt & $J$ & $\mathbf{E}$ & 0.443 \\
\hline Copper & $\mathrm{J}$ & $\mathbf{E}$ & 0.738 \\
\hline Iron & & $V$ & 3620 \\
\hline Lead & $\mathrm{U}$ & & 6.15 \\
\hline Magnesium & & & 624 \\
\hline Manganese & & & 9.85 \\
\hline Mercury & $\mathrm{U}$ & & 0.0408 \\
\hline Nickel & & & 2.26 \\
\hline Potassium & & & 402 \\
\hline Selenium & $\mathrm{U}$ & & 18.4 \\
\hline Silver & $\mathrm{U}$ & & 1.54 \\
\hline Sodium & $\mathrm{U}$ & & 61.5 \\
\hline Thallium & $\mathrm{U}$ & & 18.4 \\
\hline Vanadium & & & 6.3 \\
\hline Zinc & & & 6.85 \\
\hline
\end{tabular}

$B / N / A$ Extractables (including $P A H$ and phenols)
Unit D. Limit Method

Unit D. Limit Method
Sample ID: 104685

$\begin{array}{lll}\mathrm{mg} / \mathrm{kg} & 15.4 & \text { EPA6010A } \\ \mathrm{mg} / \mathrm{kg} & 6.15 & \text { EPA6010A } \\ \mathrm{mg} / \mathrm{kg} & 18.4 & \text { EPA6010A } \\ \mathrm{mg} / \mathrm{kg} & 1.54 & \text { EPA6010A } \\ \mathrm{mg} / \mathrm{kg} & 0.615 & \text { EPA6010A } \\ \mathrm{mg} / \mathrm{kg} & 0.307 & \text { EPA6010A } \\ \mathrm{mg} / \mathrm{kg} & 15.4 & \text { EPA6010A } \\ \mathrm{mg} / \mathrm{kg} & 1.54 & \text { EPA6010A } \\ \mathrm{mg} / \mathrm{kg} & 0.615 & \text { EPA6010A } \\ \mathrm{mg} / \mathrm{kg} & 1.54 & \text { EPA6010A } \\ \mathrm{mg} / \mathrm{kg} & 6.15 & \text { EPA6010A } \\ \mathrm{mg} / \mathrm{kg} & 6.15 & \text { EPA6010A } \\ \mathrm{mg} / \mathrm{kg} & 6.15 & \text { EPA6010A } \\ \mathrm{mg} / \mathrm{kg} & 0.615 & \text { EPA6010A } \\ \mathrm{mg} / \mathrm{kg} & 0.0408 & \text { EPA7471 } \\ \mathrm{mg} / \mathrm{kg} & 1.54 & \text { EPA6010A } \\ \mathrm{mg} / \mathrm{kg} & 123 & \text { EPA6010A } \\ \mathrm{mg} / \mathrm{kg} & 18.4 & \text { EPA6010A } \\ \mathrm{mg} / \mathrm{kg} & 1.54 & \text { EPA6010A } \\ \mathrm{mg} / \mathrm{kg} & 61.5 & \text { EPA6010A } \\ \mathrm{mg} / \mathrm{kg} & 18.4 & \text { EPA6010A } \\ \mathrm{mg} / \mathrm{kg} & 0.615 & \text { EPA6010A } \\ \mathrm{mg} / \mathrm{kg} & 1.23 & \text { EPA6010A } \\ & & \end{array}$

$\begin{array}{ll}U & 43.1 \\ U & 43.1 \\ U & 43.1 \\ U & 4.31 \\ U & 4.31 \\ U & 4.31 \\ U & 43.1 \\ U & 43.1 \\ U & 4.31 \\ U & 43.1 \\ U & 43.1 \\ U & 43.1 \\ U & 43.1 \\ U & 43.1 \\ U & 43.1 \\ U & 43.1 \\ U & 43.1 \\ U & 43.1 \\ U & 43.1 \\ U & 43.1 \\ U & 43.1 \\ U & 4.31 \\ U & 431 \\ U & 43.1 \\ U & 4.31 \\ U & 43.1 \\ U & 38 \\ U & 43.1 \\ U & 43.1\end{array}$

\begin{tabular}{lll}
$\mu \mathrm{g} / \mathrm{kg}$ & 43.1 & EPA8270 \\
$\mu \mathrm{g} / \mathrm{kg}$ & 43.1 & EPA8270 \\
$\mu \mathrm{g} / \mathrm{kg}$ & 43.1 & EPA8270 \\
$\mu \mathrm{g} / \mathrm{kg}$ & 4.31 & EPA8270 \\
$\mu \mathrm{g} / \mathrm{kg}$ & 4.31 & EPA8270 \\
$\mu \mathrm{g} / \mathrm{kg}$ & 4.31 & EPA8270 \\
$\mu \mathrm{g} / \mathrm{kg}$ & 43.1 & EPA8270 \\
$\mu \mathrm{g} / \mathrm{kg}$ & 43.1 & EPA8270 \\
$\mu \mathrm{g} / \mathrm{kg}$ & 4.31 & EPA8270 \\
$\mu \mathrm{g} / \mathrm{kg}$ & 43.1 & EPA8270 \\
$\mu \mathrm{g} / \mathrm{kg}$ & 43.1 & EPA8270 \\
$\mu \mathrm{g} / \mathrm{kg}$ & 43.1 & EPA8270 \\
$\mu \mathrm{g} / \mathrm{kg}$ & 43.1 & EPA8270 \\
$\mu \mathrm{g} / \mathrm{kg}$ & 43.1 & EPA8270 \\
$\mu \mathrm{g} / \mathrm{kg}$ & 43.1 & EPA8270 \\
$\mu \mathrm{g} / \mathrm{kg}$ & 43.1 & EPA8270 \\
$\mu \mathrm{g} / \mathrm{kg}$ & 43.1 & EPA8270 \\
$\mu \mathrm{g} / \mathrm{kg}$ & 43.1 & EPA8270 \\
$\mu \mathrm{g} / \mathrm{kg}$ & 43.1 & EPA8270 \\
$\mu \mathrm{g} / \mathrm{kg}$ & 43.1 & EPA8270 \\
$\mu \mathrm{g} / \mathrm{kg}$ & 43.1 & EPA8270 \\
$\mu \mathrm{g} / \mathrm{kg}$ & 4.31 & EPA8270 \\
$\mu \mathrm{g} / \mathrm{kg}$ & 431 & EPA8270 \\
$\mu \mathrm{g} / \mathrm{kg}$ & 43.1 & EPA8270 \\
$\mu \mathrm{g} / \mathrm{kg}$ & 4.31 & EPA8270 \\
$\mu \mathrm{g} / \mathrm{kg}$ & 43.1 & EPA8270 \\
$\mu \mathrm{g} / \mathrm{kg}$ & 43.1 & EPA8270 \\
$\mu \mathrm{g} / \mathrm{kg}$ & 43.1 & EPA8270 \\
$\mu \mathrm{g} / \mathrm{kg}$ & 43.1 & EPA8270 \\
& & \\
\hline
\end{tabular}


SURVEY ID: PPSC 2203 (continued)

Sample ID: 104685

Constituent

RQ AQ B Result

Unit

D. Limit Method

$B / N / A$ Extractables (including $P A H$ and phenols)

1,4-Dichlorobenzene

3,3'-Dichlorobenzidine

2,4-Dichlorophenol

Diethyl phthalate

2,4-Dimethyl phenol

Dimethyl phthalate

2,4-Dinitrophenol

2,4-Dinitrotoluene

2,6-Dinitrotoluene

Di-n-octyl phthalate

Fluoranthene

Fluorene

Hexachlorobenzene

Hexachlorobutadiene

Hexachlorocyclopentadiene

Hexachloroethane

Indeno[1,2,3-c,d]pyrene

Isophorone

2-Methyl-4,6-dinitrophenol

2-Methylnaphthalene

Naphthalene

m-Nitroaniline

o-Nitroaniline

p-Nitroaniline

Nitrobenzene

2-Nitrophenol

4-Nitrophenol

N-Nitrosodi-n-propylamine

Pentachlorophenol

Phenanthrene

Phenol

Pyrene

1,2,4-Trichlorobenzene

2,4,5-Trichlorophenol

2,4,6-Trichlorophenol

Volatile Organic Compounds
N-Nitrosodiphenylamine

\begin{tabular}{|c|c|}
\hline $\mathrm{U}$ & 43.1 \\
\hline $\mathrm{U}$ & 43.1 \\
\hline $\mathrm{U}$ & 43.1 \\
\hline $\mathrm{U}$ & 43.1 \\
\hline U & 43.1 \\
\hline$U$ & 43.1 \\
\hline $\mathrm{U}$ & 431 \\
\hline UJ $\quad \mathbf{C}$ & 4.31 \\
\hline $\mathrm{U}$ & 4.31 \\
\hline $\mathrm{U}$ & 43.1 \\
\hline $\mathrm{U}$ & 43.1 \\
\hline $\mathrm{U}$ & 43.1 \\
\hline$U$ & 4.31 \\
\hline $\mathrm{U}$ & 43.1 \\
\hline $\mathrm{U}$ & 43.1 \\
\hline $\mathrm{U}$ & 4.31 \\
\hline $\mathrm{U}$ & 4.31 \\
\hline $\mathrm{U}$ & 43.1 \\
\hline$U$ & 431 \\
\hline$U$ & 43.1 \\
\hline $\mathrm{U}$ & 43.1 \\
\hline $\mathrm{U}$ & 43.1 \\
\hline $\mathrm{U}$ & 43.1 \\
\hline $\mathrm{U}$ & 43.1 \\
\hline $\mathrm{U}$ & 43.1 \\
\hline $\mathrm{U}$ & 43.1 \\
\hline $\mathrm{U}$ & 43.1 \\
\hline $\mathrm{U}$ & 43.1 \\
\hline $\mathrm{U}$ & 43.1 \\
\hline $\mathrm{U}$ & 43.1 \\
\hline $\mathrm{U}$ & 43.1 \\
\hline $\mathrm{U}$ & 43.1 \\
\hline$U$ & 43.1 \\
\hline $\mathrm{U}$ & 4.31 \\
\hline $\mathrm{U}$ & 43.1 \\
\hline $\mathrm{U}$ & 43.1 \\
\hline
\end{tabular}

$\begin{array}{lll}\mu \mathrm{g} / \mathrm{kg} & 43.1 & \text { EPA8270 } \\ \mu \mathrm{g} / \mathrm{kg} & 43.1 & \text { EPA8270 } \\ \mu \mathrm{g} / \mathrm{kg} & 43.1 & \text { EPA8270 } \\ \mu \mathrm{g} / \mathrm{kg} & 43.1 & \text { EPA8270 } \\ \mu \mathrm{g} / \mathrm{kg} & 43.1 & \text { EPA8270 } \\ \mu \mathrm{g} / \mathrm{kg} & 43.1 & \text { EPA8270 } \\ \mu \mathrm{g} / \mathrm{kg} & 431 & \text { EPA8270 } \\ \mu \mathrm{g} / \mathrm{kg} & 4.31 & \text { EPA8270 } \\ \mu \mathrm{g} / \mathrm{kg} & 4.31 & \text { EPA8270 } \\ \mu \mathrm{g} / \mathrm{kg} & 43.1 & \text { EPA8270 } \\ \mu \mathrm{g} / \mathrm{kg} & 43.1 & \text { EPA8270 } \\ \mu \mathrm{g} / \mathrm{kg} & 43.1 & \text { EPA8270 } \\ \mu \mathrm{g} / \mathrm{kg} & 4.31 & \text { EPA8270 } \\ \mu \mathrm{g} / \mathrm{kg} & 43.1 & \text { EPA8270 } \\ \mu \mathrm{g} / \mathrm{kg} & 43.1 & \text { EPA8270 } \\ \mu \mathrm{g} / \mathrm{kg} & 4.31 & \text { EPA8270 } \\ \mu \mathrm{g} / \mathrm{kg} & 4.31 & \text { EPA8270 } \\ \mu \mathrm{g} / \mathrm{kg} & 43.1 & \text { EPA8270 } \\ \mu \mathrm{g} / \mathrm{kg} & 431 & \text { EPA8270 } \\ \mu \mathrm{g} / \mathrm{kg} & 43.1 & \text { EPA8270 } \\ \mu \mathrm{g} / \mathrm{kg} & 43.1 & \text { EPA8270 } \\ \mu \mathrm{g} / \mathrm{kg} & 43.1 & \text { EPA8270 } \\ \mu \mathrm{g} / \mathrm{kg} & 43.1 & \text { EPA8270 } \\ \mu \mathrm{g} / \mathrm{kg} & 43.1 & \text { EPA8270 } \\ \mu \mathrm{g} / \mathrm{kg} & 43.1 & \text { EPA8270 } \\ \mu \mathrm{g} / \mathrm{kg} & 43.1 & \text { EPA8270 } \\ \mu \mathrm{g} / \mathrm{kg} & 43.1 & \text { EPA8270 } \\ \mu \mathrm{g} / \mathrm{kg} & 43.1 & \text { EPA8270 } \\ \mu \mathrm{g} / \mathrm{kg} & 43.1 & \text { EPA8270 } \\ \mu \mathrm{g} / \mathrm{kg} & 43.1 & \text { EPA8270 } \\ \mu \mathrm{g} / \mathrm{kg} & 43.1 & \text { EPA8270 } \\ \mu \mathrm{g} / \mathrm{kg} & 43.1 & \text { EPA8270 } \\ \mu \mathrm{g} / \mathrm{kg} & 43.1 & \text { EPA8270 } \\ \mu \mathrm{g} / \mathrm{kg} & 4.31 & \text { EPA8270 } \\ \mu \mathrm{g} / \mathrm{kg} & 43.1 & \text { EPA8270 } \\ \mu \mathrm{g} / \mathrm{kg} & 43.1 & \text { EPA8270 }\end{array}$

\begin{tabular}{|c|c|c|}
\hline Acetone & U 8 & 9.21 \\
\hline Benzene & $\mathbf{U}$ & 0.658 \\
\hline Bromodichloromethane & $\mathrm{U}$ & 0.0658 \\
\hline Bromoform & $\mathrm{U}$ & 0.0658 \\
\hline Bromomethane (Methyl bromide) & $\mathrm{U}$ & 0.132 \\
\hline Carbon disulfide & & 2.2 \\
\hline Carbon tetrachloride & $\mathrm{U}$ & 0.0658 \\
\hline Chlorobenzene & $\mathrm{U}$ & 0.0658 \\
\hline Chloroethane & $\mathrm{U}$ & 0.132 \\
\hline Chloroethene (Vinyl chloride) & $\mathrm{U}$ & 0.132 \\
\hline Chloroform & $U$ & 0.0658 \\
\hline Chloromethane (Methyl chloride) & $\mathrm{U}$ & 0.132 \\
\hline Dibromochloromethane & $U$ & 0.0658 \\
\hline 1,1-Dichloroethane & $\mathrm{U}$ & 0.0658 \\
\hline 1,2-Dichloroethane & $\mathrm{U}$ & 0.0658 \\
\hline 1,1-Dichloroethylene & U & 0.0658 \\
\hline
\end{tabular}

43.1

43.1

43.1

43.1

4.31

43.1

43.1

4.31

43.1

4.31

4.31

431

43.1

43.1

43.1

43.1

43.1

43.1

43.1

43.1

43.1

43.1

4.31

43.1
EPA8270

EPA 8270

EPA8270

EPA8270

EPA8270

EPA 8270

EPA8270

EPA8270

PA 8270

EPA8270

EPA8270

EPA 8270

EPA8270

EPA 8270

EPA8270

EPA8270

EPA8270

EPA8270

EPA 8270

EPA8270

EPA8270

$\begin{array}{lll}\mu \mathrm{g} / \mathrm{kg} & 6.58 & \text { EPA8260 } \\ \mu \mathrm{g} / \mathrm{kg} & 0.658 & \text { EPA8260 } \\ \mu \mathrm{g} / \mathrm{kg} & 0.0658 & \text { EPA8260 } \\ \mu \mathrm{g} / \mathrm{kg} & 0.0658 & \text { EPA8260 } \\ \mu \mathrm{g} / \mathrm{kg} & 0.132 & \text { EPA8260 } \\ \mu \mathrm{g} / \mathrm{kg} & 1.32 & \text { EPA8260 } \\ \mu \mathrm{g} / \mathrm{kg} & 0.0658 & \text { EPA8260 } \\ \mu \mathrm{g} / \mathrm{kg} & 0.0658 & \text { EPA8260 } \\ \mu \mathrm{g} / \mathrm{kg} & 0.132 & \text { EPA8260 } \\ \mu \mathrm{g} / \mathrm{kg} & 0.132 & \text { EPA8260 } \\ \mu \mathrm{g} / \mathrm{kg} & 0.0658 & \text { EPA8260 } \\ \mu \mathrm{g} / \mathrm{kg} & 0.132 & \text { EPA8260 } \\ \mu \mathrm{g} / \mathrm{kg} & 0.0658 & \text { EPA8260 } \\ \mu \mathrm{g} / \mathrm{kg} & 0.0658 & \text { EPA8260 } \\ \mu \mathrm{g} / \mathrm{kg} & 0.0658 & \text { EPA8260 } \\ \mu \mathrm{g} / \mathrm{kg} & 0.0658 & \text { EPA8260 }\end{array}$


SURVEY ID: PPSC 2203 (continued)

Constituent

RQ AQ B Result

Sample ID: 104685

Volatile Organic Compounds

1,2-Dichloroethylene

Dichloromethane (Methylene chloride)

1,2-Dichloropropane

cis-1,3-Dichloropropene

trans-1,3-Dichloropropene

Ethylbenzene

2-Hexanone

Methyl ethyl ketone

Methyl isobutyl ketone

Styrene

1,1,2,2-Tetrachloroethane

Tetrachloroethylene

Toluene

1,1,1-Trichloroethane

1,1,2-Trichloroethane

Trichloroethylene

Vinyl acetate

Xylenes

Pesticides/PCBs and Dioxins/Furans

Aldrin

alpha-Benzene hexachloride

beta-Benzene hexachloride

delta-Benzene hexachloride

alpha-Chlordane

gamma-Chlordane

p, $\mathbf{p}^{\prime}-\mathrm{DDD}$

$p, p^{\prime}-\mathrm{DDE}$

p, $\mathrm{p}^{\prime}-\mathrm{DDT}$

Dieldrin

Endosulfan sulfate

Endosulfan I

Endosulfan II

Endrin

Endrin ketone

Heptachlor

Heptachlor epoxide

Lindane

Methoxychlor

PCB 1016

PCB 1221

PCB 1232

PCB 1242

PCB 1248

PCB 1254

PCB 1260

Toxaphene

$\begin{array}{lll}U & & 0.132 \\ U & V & 0.974 \\ U & & 0.0658 \\ U & & 0.0658 \\ U & & 0.0658 \\ U & & 0.0658 \\ U & V & 0.618 \\ U & V 8 & 0.303 \\ U & & 1.32 \\ U & & 0.0658 \\ U & & 0.0658 \\ U & & 0.0658 \\ U & & 0.658 \\ U & & 0.0658 \\ U & & 0.0658 \\ U & & 0.0658 \\ U & & 1.32 \\ U & & 0.197\end{array}$

$\mu \mathrm{g} / \mathrm{kg}$

$\mu \mathrm{g} / \mathrm{kg}$

$\mu \mathrm{g} / \mathrm{kg}$

$\mu \mathrm{g} / \mathrm{kg}$

$\mu \mathrm{g} / \mathrm{kg}$

$\mu \mathrm{g} / \mathrm{kg}$

$\mu \mathrm{g} / \mathrm{kg}$

$\mu \mathrm{g} / \mathrm{kg}$

$\mu g / \mathrm{kg}$

$\mu \mathrm{g} / \mathrm{kg}$

$\mu \mathrm{g} / \mathrm{kg}$

$\mu \mathrm{g} / \mathrm{kg}$

$\mu \mathrm{g} / \mathrm{kg}$

$\mu \mathrm{g} / \mathrm{kg}$

$\mu \mathrm{g} / \mathrm{kg}$

$\mu g / k g$

$\mu \mathrm{g} / \mathrm{kg}$

$\mu \mathrm{g} / \mathrm{kg}$

$\begin{array}{ll}\mathbf{0 . 1 3 2} & \text { EPA8260 } \\ \mathbf{0 . 6 5 8} & \text { EPA8260 } \\ \mathbf{0 . 0 6 5 8} & \text { EPA8260 } \\ \mathbf{0 . 0 6 5 8} & \text { EPA8260 } \\ 0.0658 & \text { EPA8260 } \\ \mathbf{0 . 0 6 5 8} & \text { EPA8260 } \\ \mathbf{1 . 3 2} & \text { EPA8260 } \\ \mathbf{1 . 3 2} & \text { EPA8260 } \\ \mathbf{1 . 3 2} & \text { EPA8260 } \\ \mathbf{0 . 0 6 5 8} & \text { EPA8260 } \\ \mathbf{0 . 0 6 5 8} & \text { EPA8260 } \\ \mathbf{0 . 0 6 5 8} & \text { EPA8260 } \\ \mathbf{0 . 6 5 8} & \text { EPA8260 } \\ \mathbf{0 . 0 6 5 8} & \text { EPA8260 } \\ \mathbf{0 . 0 6 5 8} & \text { EPA8260 } \\ \mathbf{0 . 0 6 5 8} & \text { EPA8260 } \\ \mathbf{1 . 3 2} & \text { EPA8260 } \\ \mathbf{0 . 1 9 7} & \text { EPA8260 }\end{array}$

$\mu \mathrm{g} / \mathrm{kg}$

$\mu g / \mathrm{kg}$

$\mu \mathrm{g} / \mathrm{kg}$

$\mu \mathrm{g} / \mathrm{kg}$

$\mu \mathrm{g} / \mathrm{kg}$

$\mu \mathrm{g} / \mathrm{kg}$

$\mu \mathrm{g} / \mathrm{kg}$

$\mu \mathrm{g} / \mathrm{kg}$

$\mu \mathrm{g} / \mathrm{kg}$

$\mu g / \mathrm{kg}$

$\mu \mathrm{g} / \mathrm{kg}$

$\mu \mathrm{g} / \mathrm{kg}$

$\mu \mathrm{g} / \mathrm{kg}$

$\mu \mathrm{g} / \mathrm{kg}$

$\mu \mathrm{g} / \mathrm{kg}$

$\mu \mathrm{g} / \mathrm{kg}$

$\mu \mathrm{g} / \mathrm{kg}$

$\mu \mathrm{g} / \mathrm{kg}$

$\mu \mathrm{g} / \mathrm{kg}$

$\mu \mathrm{g} / \mathrm{kg}$

$\mu \mathrm{g} / \mathrm{kg}$

$\mu \mathrm{g} / \mathrm{kg}$

$\mu \mathrm{g} / \mathrm{kg}$

$\mu \mathrm{g} / \mathrm{kg}$

$\mu \mathrm{g} / \mathrm{kg}$

$\mu \mathrm{g} / \mathrm{kg}$

$\mu \mathrm{g} / \mathrm{kg}$
D. Limit Method

0.197
UJ $O$ L 5.31

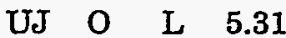

UJ $O \quad$ L $\quad 5.31$

$\begin{array}{llll}\text { UJ } & O & \text { L } & 42.5\end{array}$
1.06

1.06

2.13

2.13

2.13

2.13

4.25

2.13

4.25

2.13

4.25

2.13

4.25

2.13

4.25

2.13

2.13

1.06

21.3

5.31

5.31

5.31

5.31

5.31

5.31

5.31

42.5
EPA8080

EPA8080

EPA8080

EPA8080

EPA8080

EPA8080

EPA8080

EPA8080

EPA8080

EPA8080

EPA8080

EPA8080

EPA8080

EPA8080

EPA8080

EPA8080

EPA8080

EPA8080

EPA8080

EPA8080

EPA8080

EPA8080

EPA8080

EPA8080

EPA8080

EPA8080
EPA8080 
SURVEY ID: PPSC 2203 (continued)

Constituent

Radionuclides

Actinium-228

Antimony-124

Antimony-125

Barium-133

Cerium-144

Cesium-134

Cesium-137

Cobalt-57

Cobalt-58

Cobalt-60

Europium-152

Europium-154

Europium-155

Gross alpha

Lead-212

Manganese-54

Neptunium-239

Nonvolatile beta

Plutonium-238

Plutonium-239/240

Potassium-40

Promethium-144

Promethium-146

Radium-226

Radium-228

Ruthenium-106

Sodium-22

Strontium-90

Thorium-234

Tin-113

Yttrium-88

Zinc-65

Zirconium-95
Sample ID: 104685

RQ AQ B Result Unit D. Limit Method
1.98E-02 EPIA-013B

9.11E-03 EPIA-013B

1.72E-02 EPIA-013B

7.97E-03 EPIA-013B

5.27E-02 EPIA-013B

6.36E-03 EPIA-013B

6.83E-03 EPLA-013B

6.67E-03 EPIA-013B

7.42E-03 EPIA-013B

5.81E-03 EPLA-013B

1.85E-02 EPIA-013B

5.74E-02 EPIA-013B

2.75E-02 EPIA-013B

1.67E+00 EPIA-001B

1.15E-02 EPIA-013B

7.12E-03 EPLA-013B

4.69E-02 EPIA-013B

2.63E+00 EPIA-001B

4.57E-02 EPIA-012B

2.46E-02 EPIA-012B

5.86E-02 EPIA-013B

6.77E-03 EPIA-013B

8.41E-03 EPIA-013B

1.26E-02 EPIA-013B

1.98E-02 EPIA-013B

6.36E-02 EPIA-013B

6.04E-03 EPLA-013B

1.54E-01 EPIA-004

3.63E-01 EPIA-013B

9.59E-03 EPIA-013B

7.43E-03 EPIA-013B

1.37E-02 EPIA-013B

1.84E-02 EPIA-013B

SURVEY ID: PPSC 2204

Sample ID: 104686

Survey location: $44548 \mathrm{E} 3670160 \mathrm{~N}$ (SRS coordinates)

Depth of core interval: 4.00 to $6.00 \mathrm{ft}$

Sample type: Normal

Sample matrix: Soil

Percent solids: $\mathbf{6 9 . 0 0}$
Constituent
RQ AQ B Result
Unit
D. Limit Method

Physical Parameters and Specified Analyses

Ammonia nitrogen

Cation exchange capacity

Chloride

Cyanide

Nitrate-nitrite as nitrogen

Nitrogen by Kjeldahl method

Orthophosphate

Phenols

$\begin{array}{lll}\text { U } & & 26.3 \\ & & 107 \\ & & 3.73 \\ \text { UJ } & \text { C } & 1.35 \\ \text { J } & \text { E } & 0.538 \\ & & 10.9 \\ J & E & 11.7 \\ \text { U } & & 433\end{array}$

$\begin{array}{lll}\mathrm{mg} / \mathrm{kg} & 26.3 & \text { EPA350.1 } \\ \mathrm{meq} / 100 \mathrm{~g} & 5 & \text { EPA9081 } \\ \mathrm{mg} / \mathrm{kg} & 3.52 & \text { EPA300.0 } \\ \mathrm{mg} / \mathrm{kg} & 1.35 & \text { EPA335.3 } \\ \mathrm{mg} / \mathrm{kg} & 1.34 & \text { EPA353.1 } \\ \mathrm{mg} / \mathrm{kg} & -5.6 & \text { EPA351.2 } \\ \mathrm{mg} / \mathrm{kg} & 29 & \text { EPA365.2 } \\ \mu \mathrm{g} / \mathrm{kg} & 433 & \text { EPA420.2 }\end{array}$


SURVEY ID: PPSC 2204 (continued)

Sample ID: 104686

Constituent

RQ AQ B Result

Unit

D. Limit Method

Physical Parameters and Specified Analyses

Sulfide

Total organic carbon

Total organic nitrogen

Total phosphates (as P)

$\begin{array}{lll}\text { UJ } & \mathrm{C} & 362 \\ & \mathrm{~V} & 624 \\ \mathrm{C} & & 4.86 \\ \mathrm{~J} & \mathrm{E} & 186\end{array}$

Metals (total recoverable)

$\begin{array}{lll} & & 5270 \\ \mathrm{U} & & 6.71 \\ \mathrm{U} & & 20.1 \\ & & 9.58 \\ \mathrm{~J} & \mathrm{E} & \mathbf{0 . 4 2 5} \\ \mathrm{U} & & 0.335 \\ & & 1410 \\ & & 13 \\ & & 5.08 \\ \mathrm{~J} & \mathrm{E} & 1.55 \\ & \mathrm{~V} & 8910 \\ \mathrm{~J} & \mathrm{E} & 5.18 \\ & & 1170 \\ \mathrm{U} & & 374 \\ & & 0.0433 \\ & & 6.87 \\ \mathrm{U} & & 807 \\ \mathrm{U} & & 20.1 \\ \mathrm{U} & & 1.68 \\ \mathrm{U} & & 67.1 \\ & & 20.1 \\ & & 13.6 \\ & & 18.3\end{array}$

$B / N / A$ Extractables (including PAH and phenols)

$\begin{array}{llll}\text { UJ } & \text { Q } & 48.3 \\ \text { UJ } & \text { Q } & 48.3 \\ \text { UJ } & \text { Q } & 48.3 \\ \text { UJ } & \text { Q } & 4.83 \\ \text { UJ } & \text { Q } & 4.83 \\ \text { UJ } & \text { Q } & 4.83 \\ \text { J } & \text { Q } & 53.6 \\ \text { UJ } & \text { Q } & 48.3 \\ \text { UJ } & \text { Q } & 4.83 \\ \text { UJ } & \text { Q } & 48.3 \\ \text { UJ } & \text { Q } & 48.3 \\ \text { UJ } & \text { Q } & 48.3 \\ \text { UJ } & \text { Q } & 48.3 \\ \text { UJ } & \text { Q } & 48.3 \\ \text { UJ } & \text { Q } & 48.3 \\ \text { UJ } & \text { Q } & 48.3 \\ \text { UJ } & \text { Q } & 48.3 \\ \text { UJ } & \text { Q } & 48.3 \\ \text { UJ } & \text { Q } & 48.3 \\ \text { UJ } & \text { Q } & 48.3 \\ \text { UJ } & \text { Q } & 48.3 \\ \text { UJ } & \text { Q } & 4.83\end{array}$

$\begin{array}{lll}\mathrm{mg} / \mathrm{kg} & 362 & \text { EPA376.2 } \\ \mathrm{mg} / \mathrm{kg} & 145 & \text { EPA415.1 } \\ \mathrm{mg} / \mathrm{kg} & \mathbf{0 . 2 9} & \text { EPA351.2 } \\ \mathrm{mg} / \mathrm{kg} & 1000 & \text { EPA365.4 }\end{array}$

$\begin{array}{lll}\mathrm{mg} / \mathrm{kg} & 16.8 & \text { EPA6010A } \\ \mathrm{mg} / \mathrm{kg} & 6.71 & \text { EPA6010A } \\ \mathrm{mg} / \mathrm{kg} & 20.1 & \text { EPA6010A } \\ \mathrm{mg} / \mathrm{kg} & 1.68 & \text { EPA6010A } \\ \mathrm{mg} / \mathrm{kg} & 0.671 & \text { EPA6010A } \\ \mathrm{mg} / \mathrm{kg} & 0.335 & \text { EPA6010A } \\ \mathrm{mg} / \mathrm{kg} & 16.8 & \text { EPA6010A } \\ \mathrm{mg} / \mathrm{kg} & 1.68 & \text { EPA6010A } \\ \mathrm{mg} / \mathrm{kg} & 0.671 & \text { EPA6010A } \\ \mathrm{mg} / \mathrm{kg} & 1.68 & \text { EPA6010A } \\ \mathrm{mg} / \mathrm{kg} & 6.71 & \text { EPA6010A } \\ \mathrm{mg} / \mathrm{kg} & 6.71 & \text { EPA6010A } \\ \mathrm{mg} / \mathrm{kg} & 6.71 & \text { EPA6010A } \\ \mathrm{mg} / \mathrm{kg} & 0.671 & \text { EPA6010A } \\ \mathrm{mg} / \mathrm{kg} & 0.0433 & \text { EPA7471 } \\ \mathrm{mg} / \mathrm{kg} & 1.68 & \text { EPA6010A } \\ \mathrm{mg} / \mathrm{kg} & 134 & \text { EPA6010A } \\ \mathrm{mg} / \mathrm{kg} & 20.1 & \text { EPA6010A } \\ \mathrm{mg} / \mathrm{kg} & 1.68 & \text { EPA6010A } \\ \mathrm{mg} / \mathrm{kg} & 67.1 & \text { EPA6010A } \\ \mathrm{mg} / \mathrm{kg} & 20.1 & \text { EPA6010A } \\ \mathrm{mg} / \mathrm{kg} & 0.671 & \text { EPA6010A } \\ \mathrm{mg} / \mathrm{kg} & 1.34 & \text { EPA6010A }\end{array}$

$\begin{array}{lll}\mu \mathrm{g} / \mathrm{kg} & 48.3 & \text { EPA8270 } \\ \mu \mathrm{g} / \mathrm{kg} & 48.3 & \text { EPA8270 } \\ \mu \mathrm{g} / \mathrm{kg} & 48.3 & \text { EPA8270 } \\ \mu \mathrm{g} / \mathrm{kg} & 4.83 & \text { EPA8270 } \\ \mu \mathrm{g} / \mathrm{kg} & 4.83 & \text { EPA8270 } \\ \mu \mathrm{g} / \mathrm{kg} & 4.83 & \text { EPA8270 } \\ \mu \mathrm{g} / \mathrm{kg} & 48.3 & \text { EPA8270 } \\ \mu \mathrm{g} / \mathrm{kg} & 48.3 & \text { EPA8270 } \\ \mu \mathrm{g} / \mathrm{kg} & 4.83 & \text { EPA8270 } \\ \mu \mathrm{g} / \mathrm{kg} & 48.3 & \text { EPA8270 } \\ \mu \mathrm{g} / \mathrm{kg} & 48.3 & \text { EPA8270 } \\ \mu \mathrm{g} / \mathrm{kg} & 48.3 & \text { EPA8270 } \\ \mu \mathrm{g} / \mathrm{kg} & 48.3 & \text { EPA8270 } \\ \mu \mathrm{g} / \mathrm{kg} & 48.3 & \text { EPA8270 } \\ \mu \mathrm{g} / \mathrm{kg} & 48.3 & \text { EPA8270 } \\ \mu \mathrm{g} / \mathrm{kg} & 48.3 & \text { EPA8270 } \\ \mu \mathrm{g} / \mathrm{kg} & 48.3 & \text { EPA8270 } \\ \mu \mathrm{g} / \mathrm{kg} & 48.3 & \text { EPA8270 } \\ \mu \mathrm{g} / \mathrm{kg} & 48.3 & \text { EPA8270 } \\ \mu \mathrm{g} / \mathrm{kg} & 48.3 & \text { EPA8270 } \\ \mu \mathrm{g} / \mathrm{kg} & 48.3 & \text { EPA8270 } \\ \mu \mathrm{g} / \mathrm{kg} & 4.83 & \text { EPA8270 }\end{array}$


Constituent

RQ AQ

\section{B}

\section{$B / N / A$ Extractables (including $P A H$ and phenols)}

\begin{tabular}{|c|c|c|c|}
\hline $\mathrm{m} / \mathrm{p}-$ Cresol & UJ & $\mathbf{Q}$ & 483 \\
\hline o-Cresol (2-Methylphenol) & UJ & $\mathbf{Q}$ & 48.3 \\
\hline Dibenz $[a, h]$ anthracene & UJ & $Q$ & 4.83 \\
\hline Dibenzofuran & UJJ & $Q$ & 48.3 \\
\hline Di-n-butyl phthalate & $\mathrm{J}$ & $\mathrm{QE}$ & 25.6 \\
\hline 1,2-Dichlorobenzene & UJ & Q & 48.3 \\
\hline 1,3-Dichlorobenzene & UJ & $\mathbf{Q}$ & 48.3 \\
\hline 1,4-Dichlorobenzene & UJ & $\dot{Q}$ & 48.3 \\
\hline 3,3'-Dichlorobenzidine & UJ & $Q$ & 48.3 \\
\hline 2,4-Dichlorophenol & UJ & $\mathrm{Q}$ & 48.3 \\
\hline Diethyl phthalate & UJ & $Q$ & 48.3 \\
\hline 2,4-Dimethyl phenol & UJ & $Q$ & 48.3 \\
\hline Dimethyl phthalate & UJ & $\mathbf{Q}$ & 48.3 \\
\hline 2,4-Dinitrophenol & UJ & Q & 483 \\
\hline 2,4-Dinitrotoluene & UJ & $\mathrm{QC}$ & 4.83 \\
\hline 2,6-Dinitrotoluene & UJ & $\mathbf{Q}$ & 4.83 \\
\hline Di-n-octyl phthalate & UJ & $\mathbf{Q}$ & 48.3 \\
\hline Fluoranthene & UJ & $\mathbf{Q}$ & 48.3 \\
\hline Fluorene & UJ & $\mathbf{Q}$ & 48.3 \\
\hline Hexachlorobenzene & UJ & $\mathbf{Q}$ & 4.83 \\
\hline Hexachlorobutadiene & UJ & $\mathbf{Q}$ & 48.3 \\
\hline Hexachlorocyclopentadiene & UJ & $\mathbf{Q}$ & 48.3 \\
\hline Hexachloroethane & UJ & $\mathbf{Q}$ & 4.83 \\
\hline Indeno[1,2,3-c,d]pyrene & UJ & $\mathbf{Q}$ & 4.83 \\
\hline Isophorone & UJ & $\mathbf{Q}$ & 48.3 \\
\hline 2-Methyl-4,6-dinitrophenol & UJ & Q & 483 \\
\hline 2-Methylnaphthalene & UJ & Q & 48.3 \\
\hline Naphthalene & UJ & $Q$ & 48.3 \\
\hline m-Nitroaniline & UJ & $Q$ & 48.3 \\
\hline o-Nitroaniline & UJ & $Q$ & 48.3 \\
\hline p-Nitroaniline & UJ & $Q$ & 48.3 \\
\hline Nitrobenzene & UJ & $Q$ & 48.3 \\
\hline 2-Nitrophenol & UJ & $\mathbf{Q}$ & 48.3 \\
\hline 4-Nitrophenol & UJ & $\mathbf{Q}$ & 48.3 \\
\hline N-Nitrosodiphenylamine & UJ & $\mathrm{Q}$ & 48.3 \\
\hline N-Nitrosodi-n-propylamine & UJ & $\mathbf{Q}$ & 48.3 \\
\hline Pentachlorophenol & UJ & Q & 48.3 \\
\hline Phenanthrene & UJ & Q & 48.3 \\
\hline Phenol & UJ & $\mathbf{Q}$ & 48.3 \\
\hline Pyrene & UJ & $\mathbf{Q}$ & 48.3 \\
\hline 1,2,4-Trichlorobenzene & UJ & $Q$ & 4.83 \\
\hline 2,4,5-Trichlorophenol & UJ & $\mathbf{Q}$ & 48.3 \\
\hline 2,4,6-Trichlorophenol & UJ & $Q$ & 48.3 \\
\hline
\end{tabular}

Unit D. Limit Method

\begin{tabular}{|c|c|c|}
\hline$\mu \mathrm{g} / \mathrm{kg}$ & 483 & EPA8270 \\
\hline$\mu \mathrm{g} / \mathrm{kg}$ & 48.3 & EPA8270 \\
\hline$\mu \mathrm{g} / \mathrm{kg}$ & 4.83 & EPA8270 \\
\hline$\mu \mathrm{g} / \mathrm{kg}$ & 48.3 & EPA8270 \\
\hline$\mu \mathrm{g} / \mathrm{kg}$ & 48.3 & EPA8270 \\
\hline$\mu \mathrm{g} / \mathrm{kg}$ & 48.3 & EPA8270 \\
\hline$\mu \mathrm{g} / \mathrm{kg}$ & 48.3 & EPA8270 \\
\hline$\mu \mathrm{g} / \mathrm{kg}$ & 48.3 & EPA8270 \\
\hline$\mu \mathrm{g} / \mathrm{kg}$ & 48.3 & EPA8270 \\
\hline$\mu \mathrm{g} / \mathrm{kg}$ & 48.3 & EPA8270 \\
\hline$\mu \mathrm{g} / \mathrm{kg}$ & 48.3 & EPA8270 \\
\hline$\mu \mathrm{g} / \mathrm{kg}$ & 48.3 & EPA8270 \\
\hline$\mu \mathrm{g} / \mathrm{kg}$ & 48.3 & EPA8270 \\
\hline$\mu \mathrm{g} / \mathrm{kg}$ & 483 & EPA8270 \\
\hline$\mu \mathrm{g} / \mathrm{kg}$ & 4.83 & EPA8270 \\
\hline$\mu \mathrm{g} / \mathrm{kg}$ & 4.83 & EPA8270 \\
\hline$\mu \mathrm{g} / \mathrm{kg}$ & 48.3 & EPA8270 \\
\hline$\mu \mathrm{g} / \mathrm{kg}$ & 48.3 & EPA8270 \\
\hline$\mu \mathrm{g} / \mathrm{kg}$ & 48.3 & EPA8270 \\
\hline$\mu \mathrm{g} / \mathrm{kg}$ & 4.83 & EPA8270 \\
\hline$\mu \mathrm{g} / \mathrm{kg}$ & 48.3 & EPA8270 \\
\hline$\mu \mathrm{g} / \mathrm{kg}$ & 48.3 & EPA8270 \\
\hline$\mu \mathrm{g} / \mathrm{kg}$ & 4.83 & EPA8270 \\
\hline$\mu \mathrm{g} / \mathrm{kg}$ & 4.83 & EPA8270 \\
\hline$\mu \mathrm{g} / \mathrm{kg}$ & 48.3 & EPA8270 \\
\hline$\mu \mathrm{g} / \mathrm{kg}$ & 483 & EPA8270 \\
\hline$\mu \mathrm{g} / \mathrm{kg}$ & 48.3 & EPA8270 \\
\hline$\mu \mathrm{g} / \mathrm{kg}$ & 48.3 & EPA8270 \\
\hline$\mu \mathrm{g} / \mathrm{kg}$ & 48.3 & EPA8270 \\
\hline$\mu \mathrm{g} / \mathrm{kg}$ & 48.3 & EPA8270 \\
\hline$\mu \mathrm{g} / \mathrm{kg}$ & 48.3 & EPA8270 \\
\hline$\mu \mathrm{g} / \mathrm{kg}$ & 48.3 & EPA8270 \\
\hline$\mu \mathrm{g} / \mathrm{kg}$ & 48.3 & EPA8270 \\
\hline$\mu \mathrm{g} / \mathrm{kg}$ & 48.3 & EPA8270 \\
\hline$\mu \mathrm{g} / \mathrm{kg}$ & 48.3 & EPA8270 \\
\hline$\mu \mathrm{g} / \mathrm{kg}$ & 48.3 & EPA8270 \\
\hline$\mu \mathrm{g} / \mathrm{kg}$ & 48.3 & EPA8270 \\
\hline$\mu \mathrm{g} / \mathrm{kg}$ & 48.3 & EPA8270 \\
\hline$\mu \mathrm{g} / \mathrm{kg}$ & 48.3 & EPA8270 \\
\hline$\mu \mathrm{g} / \mathrm{kg}$ & 48.3 & EPA8270 \\
\hline$\mu \mathrm{g} / \mathrm{kg}$ & 4.83 & EPA8270 \\
\hline$\mu \mathrm{g} / \mathrm{kg}$ & 48.3 & EPA8270 \\
\hline$\mu \mathrm{g} / \mathrm{kg}$ & 48.3 & EPA8270 \\
\hline
\end{tabular}

Volatile Organic Compounds

$\begin{array}{llll}\text { Acetone } & \text { U } & 8 & 44 \\ \text { Benzene } & \text { U } & 0.725 \\ \text { Bromodichloromethane } & \text { U } & 0.0725 \\ \text { Bromoform } & \text { U } & 0.0725 \\ \text { Bromomethane (Methyl bromide) } & \text { U } & 0.145 \\ \text { Carbon disulfide } & \text { U } & 1.45 \\ \text { Carbon tetrachloride } & \text { U } & 0.0725 \\ \text { Chlorobenzene } & \text { U } & 0.0725 \\ \text { Chloroethane } & \text { U } & 0.145\end{array}$

$\begin{array}{lll}\mu \mathrm{g} / \mathrm{kg} & 7.25 & \text { EPA8260 } \\ \mu \mathrm{g} / \mathrm{kg} & 0.725 & \text { EPA8260 } \\ \mu \mathrm{g} / \mathrm{kg} & 0.0725 & \text { EPA8260 } \\ \mu \mathrm{g} / \mathrm{kg} & 0.0725 & \text { EPA8260 } \\ \mu \mathrm{g} / \mathrm{kg} & 0.145 & \text { EPA8260 } \\ \mu \mathrm{g} / \mathrm{kg} & 1.45 & \text { EPA8260 } \\ \mu \mathrm{g} / \mathrm{kg} & 0.0725 & \text { EPA8260 } \\ \mu \mathrm{g} / \mathrm{kg} & 0.0725 & \text { EPA8260 } \\ \mu \mathrm{g} / \mathrm{kg} & 0.145 & \text { EPA8260 }\end{array}$


SURVEY ID: PPSC 2204 (continued)

Constituent

Volatile Organic Compounds

Chloroethene (Vinyl chloride)

Chloroform

Chloromethane (Methyl chloride)

Dibromochloromethane

1,1-Dichloroethane

1,2-Dichloroethane

1,1-Dichloroethylene

1,2-Dichloroethylene

Dichloromethane (Methylene chloride)

1,2-Dichloropropane

cis-1,3-Dichloropropene

trans-1,3-Dichloropropene

Ethylbenzene

2.Hexanone

Methyl ethyl ketone

Methyl isobutyl ketone

Styrene

1,1,2,2-Tetrachloroethane

Tetrachloroethylene

Toluene

1,1,1-Trichloroethane

1,1,2-Trichloroethane

Trichloroethylene

Vinyl acetate

Xylenes

Pesticides/PCBs and Dioxins/Furans
Sample ID: 104686

RQ AQ B Result Unit D. Limit Method

\begin{tabular}{|c|c|c|c|c|}
\hline U & 0.145 & $\mu \mathrm{g} / \mathrm{kg}$ & 0.145 & EPA8260 \\
\hline $\mathrm{U}$ & 0.0725 & $\mu \mathrm{g} / \mathrm{kg}$ & 0.0725 & EPA8260 \\
\hline $\mathrm{U}$ & 0.145 & $\mu \mathrm{g} / \mathrm{kg}$ & 0.145 & EPA8260 \\
\hline$U$ & 0.0725 & $\mu \mathrm{g} / \mathrm{kg}$ & 0.0725 & EPA8260 \\
\hline $\mathbf{U}$ & 0.0725 & $\mu \mathrm{g} / \mathrm{kg}$ & 0.0725 & EPA8260 \\
\hline $\mathrm{U}$ & 0.0725 & $\mu \mathrm{g} / \mathrm{kg}$ & 0.0725 & EPA8260 \\
\hline $\mathrm{U}$ & 0.0725 & $\mu \mathrm{g} / \mathrm{kg}$ & 0.0725 & EPA8260 \\
\hline $\mathbf{U}$ & 0.145 & $\mu \mathrm{g} / \mathrm{kg}$ & 0.145 & EPA8260 \\
\hline $\mathrm{U}$ & 2.12 & $\mu \mathrm{g} / \mathrm{kg}$ & 0.725 & EPA8260 \\
\hline $\mathrm{U}$ & 0.0725 & $\mu \mathrm{g} / \mathrm{kg}$ & 0.0725 & EPA8260 \\
\hline $\mathrm{U}$ & 0.0725 & $\mu \mathrm{g} / \mathrm{kg}$ & 0.0725 & EPA8260 \\
\hline $\mathrm{U}$ & 0.0725 & $\mu \mathrm{g} / \mathrm{kg}$ & 0.0725 & EPA8260 \\
\hline $\mathrm{U}$ & 0.0725 & $\mu \mathrm{g} / \mathrm{kg}$ & 0.0725 & EPA8260 \\
\hline $\mathrm{U}$ & 0.464 & $\mu \mathrm{g} / \mathrm{kg}$ & 1.45 & EPA8260 \\
\hline V8 & 0.551 & $\mu \mathrm{g} / \mathrm{kg}$ & 1.45 & EPA8260 \\
\hline $\mathrm{U}$ & 1.45 & $\mu \mathrm{g} / \mathrm{kg}$ & 1.45 & EPA8260 \\
\hline $\mathrm{U}$ & 0.0725 & $\mu \mathrm{g} / \mathrm{kg}$ & 0.0725 & EPA8260 \\
\hline$U$ & 0.0725 & $\mu \mathrm{g} / \mathrm{kg}$ & 0.0725 & EPA8260 \\
\hline $\mathrm{U}$ & 0.0725 & $\mu \mathrm{g} / \mathrm{kg}$ & 0.0725 & EPA8260 \\
\hline $\mathrm{U}$ & 0.725 & $\mu \mathrm{g} / \mathrm{kg}$ & 0.725 & EPA8260 \\
\hline $\mathrm{U}$ & 0.0725 & $\mu \mathrm{g} / \mathrm{kg}$ & 0.0725 & EPA8260 \\
\hline $\mathbf{U}$ & 0.0725 & $\mu \mathrm{g} / \mathrm{kg}$ & 0.0725 & EPA8260 \\
\hline$U$ & 0.0725 & $\mu \mathrm{g} / \mathrm{kg}$ & 0.0725 & EPA8260 \\
\hline $\mathrm{U}$ & 1.45 & $\mu \mathrm{g} / \mathrm{kg}$ & 1.45 & EPA8260 \\
\hline $\mathrm{U}$ & 0.217 & $\mu \mathrm{g} / \mathrm{kg}$ & 0.217 & EPA8260 \\
\hline
\end{tabular}

$\begin{array}{lll}\mu \mathrm{g} / \mathrm{kg} & 1.17 & \text { EPA8080 } \\ \mu \mathrm{g} / \mathrm{kg} & 1.17 & \text { EPA8080 } \\ \mu \mathrm{g} / \mathrm{kg} & 2.35 & \text { EPA8080 } \\ \mu \mathrm{g} / \mathrm{kg} & 2.35 & \text { EPA8080 } \\ \mu \mathrm{g} / \mathrm{kg} & 2.35 & \text { EPA8080 } \\ \mu \mathrm{g} / \mathrm{kg} & 2.35 & \text { EPA8080 } \\ \mu \mathrm{g} / \mathrm{kg} & 4.7 & \text { EPA8080 } \\ \mu \mathrm{g} / \mathrm{kg} & 2.35 & \text { EPA8080 } \\ \mu \mathrm{g} / \mathrm{kg} & 4.7 & \text { EPA8080 } \\ \mu \mathrm{g} / \mathrm{kg} & 2.35 & \text { EPA8080 } \\ \mu \mathrm{g} / \mathrm{kg} & 4.7 & \text { EPA8080 } \\ \mu \mathrm{g} / \mathrm{kg} & 2.35 & \text { EPA8080 } \\ \mu \mathrm{g} / \mathrm{kg} & 4.7 & \text { EPA8080 } \\ \mu \mathrm{g} / \mathrm{kg} & 2.35 & \text { EPA8080 } \\ \mu \mathrm{g} / \mathrm{kg} & 4.7 & \text { EPA8080 } \\ \mu \mathrm{g} / \mathrm{kg} & 2.35 & \text { EPA8080 } \\ \mu \mathrm{g} / \mathrm{kg} & 2.35 & \text { EPA8080 } \\ \mu \mathrm{g} / \mathrm{kg} & 1.17 & \text { EPA8080 } \\ \mu \mathrm{g} / \mathrm{kg} & 23.5 & \text { EPA8080 } \\ \mu \mathrm{g} / \mathrm{kg} & 5.87 & \text { EPA8080 } \\ \mu \mathrm{g} / \mathrm{kg} & 5.87 & \text { EPA8080 } \\ \mu \mathrm{g} / \mathrm{kg} & 5.87 & \text { EPA8080 } \\ \mu \mathrm{g} / \mathrm{kg} & 5.87 & \text { EPA8080 } \\ \mu \mathrm{g} / \mathrm{kg} & -5.87 & \text { EPA8080 } \\ \mu \mathrm{g} / \mathrm{kg} & 5.87 & \text { EPA8080 } \\ \mu \mathrm{g} / \mathrm{kg} & 5.87 & \text { EPA8080 } \\ \mu \mathrm{g} / \mathrm{kg} & 47 & \text { EPA8080 }\end{array}$


SURVEY ID: PPSC 2204 (continued)

Constituent

Radionuclides

Actinium-228

Antimony-124

Antimony-125

Barium-133

Cerium-144

Cesium-134

Cesium-137

Cobalt-57

Cobalt-58

Cobalt-60

Europium-152

Europium-154

Europium-155

Gross alpha

Lead-212

Manganese-54

Neptunium-239

Nonvolatile beta

Plutonium-238

Plutonium-239/240

Potassium- 40

Promethium-144

Promethium-146

Radium-226

Radium-228

Ruthenium-106

Sodium-22

Strontium-90

Thorium-234

Tin-113

Yttrium-88

Zinc-65

Zirconium-95
Sample ID: 104686

RQ AQ B Result Unit D. Limit Method

$\begin{array}{ll} & 1.18 \mathrm{E}+00 \pm 5.46 \mathrm{E}-02 \mathrm{pCi} / \mathrm{g} \\ \text { UI } & 1.42 \mathrm{E}-03 \pm 6.69 \mathrm{E}-03 \mathrm{pCi} / \mathrm{g} \\ \text { UI } & -4.02 \mathrm{E}-05 \pm 1.29 \mathrm{E}-02 \mathrm{pCi} / \mathrm{g} \\ \text { UI } & -1.12 \mathrm{E}-03 \pm 6.73 \mathrm{E}-03 \mathrm{pCi} / \mathrm{g} \\ \text { UI } & 1.21 \mathrm{E}-03 \pm 4.01 \mathrm{E}-02 \mathrm{pCi} / \mathrm{g} \\ \text { UI } & -2.81 \mathrm{E}-03 \pm 5.50 \mathrm{E}-03 \mathrm{pCi} / \mathrm{g} \\ \text { UI } & 8.58 \mathrm{E}-04 \pm 6.14 \mathrm{E}-03 \mathrm{pCi} / \mathrm{g} \\ \text { UI } & 2.69 \mathrm{E}-04 \pm 4.92 \mathrm{E}-03 \mathrm{pCi} / \mathrm{g} \\ \text { UI } & -3.84 \mathrm{E}-03 \pm 6.09 \mathrm{E}-03 \mathrm{pCi} / \mathrm{g} \\ \text { UI } & 4.26 \mathrm{E}-03 \pm 4.79 \mathrm{E}-03 \mathrm{pCi} / \mathrm{g} \\ \text { UI } & -6.20 \mathrm{E}-03 \pm 1.40 \mathrm{E}-02 \mathrm{pCi} / \mathrm{g} \\ \text { UI } & -8.85 \mathrm{E}-03 \pm 4.47 \mathrm{E}-02 \mathrm{pCi} / \mathrm{g} \\ \text { UI } & 0.00 \mathrm{E}+00 \\ & 2.87 \mathrm{E}+01 \pm 4.13 \mathrm{E}+00 \mathrm{pCi} / \mathrm{g} \\ & 1.28 \mathrm{E}+00 \pm 2.63 \mathrm{E}-02 \mathrm{pCi} / \mathrm{g} \\ & 1.65 \mathrm{E}-02 \pm 6.53 \mathrm{E}-03 \mathrm{pCi} / \mathrm{g} \\ \text { UI } & 9.02 \mathrm{E}-03 \pm 3.56 \mathrm{E}-02 \mathrm{pCi} / \mathrm{g} \\ & 1.57 \mathrm{E}+01 \pm 2.25 \mathrm{E}+00 \mathrm{pCi} / \mathrm{g} \\ \text { UI } & -1.66 \mathrm{E}-03 \pm 1.67 \mathrm{E}-02 \mathrm{pCi} / \mathrm{g} \\ \text { UI } & 4.47 \mathrm{E}-03 \pm 9.00 \mathrm{E}-03 \mathrm{pCi} / \mathrm{g} \\ & 2.34 \mathrm{E}+00 \pm 1.44 \mathrm{E}-01 \mathrm{pCi} / \mathrm{g} \\ \text { UI } & 4.42 \mathrm{E}-04 \pm 4.98 \mathrm{E}-03 \mathrm{pCi} / \mathrm{g} \\ \text { UI } & 4.17 \mathrm{E}-03 \pm 9.91 \mathrm{E}-03 \mathrm{pCi} / \mathrm{g} \\ \text { UI } & 6.68 \mathrm{E}-01 \pm 2.90 \mathrm{E}-02 \mathrm{pCi} / \mathrm{g} \\ \text { UI } & 1.18 \mathrm{E}+00 \pm 5.46 \mathrm{E}-02 \mathrm{pCi} / \mathrm{g} \\ \text { UI } & 2.96 \mathrm{E}-02 \pm 4.65 \mathrm{E}-02 \mathrm{pCi} / \mathrm{g} \\ \text { UI } & -1.75 \mathrm{E}-03 \pm 4.81 \mathrm{E}-03 \mathrm{pCi} / \mathrm{g} \\ & 1.58 \mathrm{E}-01 \pm 9.93 \mathrm{E}-02 \mathrm{pCi} / \mathrm{g} \\ & 1.57 \mathrm{E}+00 \pm 5.04 \mathrm{E}-01 \mathrm{pCi} / \mathrm{g} \\ & 3.81 \mathrm{E}-03 \pm 8.21 \mathrm{E}-03 \mathrm{pCi} / \mathrm{g} \\ & 6.55 \mathrm{E}-03 \pm 7.63 \mathrm{E}-03 \mathrm{pCi} / \mathrm{g} \\ & 8.22 \mathrm{E}-03 \pm 1.15 \mathrm{E}-02 \mathrm{pCi} / \mathrm{g} \\ & 1.12 \mathrm{E}-02 \pm 1.40 \mathrm{E}-02 \mathrm{pCi} / \mathrm{g}\end{array}$

2.75E-02 EPIA-013B

1.13E-02 EPIA-013B

2.20E-02 EPIA-013B

1.02E-02 EPIA-013B

6.67E-02 EPIA-013B

7.96E-03 EPIA-013B

9.07E-03 EPLA-013B

8.23E-03 EPIA-013B

9.83E-03 EPIA-013B

7.76E-03 EPIA-013B

2.40E-02 EPIA-013B

6.71E-02 EPIA-013B

3.46E-02 EPIA-013B

$1.53 \mathrm{E}+00$ EPIA-001B

1.49E-02 EPIA-013B

8.57E-03 EPIA-013B

5.98E-02 EPIA-013B

$2.53 \mathrm{E}+00$ EPIA-001B

4.18E-02 EPIA-012B

1.89E-02 EPIA-012B

6.99E-02 EPIA-013B

8.36E-03 EPIA-013B

1.13E-02 EPIA-013B

1.63E-02 EPIA-013B

2.75E-02 EPIA-013B

8.01E-02 EPIA-013B

8.12E-03 EPIA-013B

1.52E-01 EPIA-004

4.52E-01 EPIA-013B

1.26E-02 EPIA-013B

9.81E-03 EPIA-013B

1.81E-02 EPLA-013B

2.13E-02 EPIA-013B

SURVEY ID: PPSC 2301

Sample ID: 104687

Survey location: $44336 \mathrm{E} 3688244 \mathrm{~N}$ (SRS coordinates)

Depth of core interval: 0.00 to $1.00 \mathrm{ft}$

Sample type: Normal

Sample matrix: Soil

Percent solids: 46.00

Constituent

RQ AQ B Result

Unit

D. Limit Method

Physical Parameters and Specified Analyses

Ammonia nitrogen

Cation exchange capacity

Chloride

Cyanide

Nitrate-nitrite as nitrogen

$\begin{array}{lll} & & 76.5 \\ & & 88.2 \\ & & 6.5 \\ \text { UJ } & \text { C } & 2.05 \\ \text { J } & \text { E } & 0.655\end{array}$

$\begin{array}{ll}\mathrm{mg} / \mathrm{kg} & 45.2 \\ \mathrm{meq} / 100 \mathrm{~g} & 5 \\ \mathrm{mg} / \mathrm{kg} & 5.13 \\ \mathrm{mg} / \mathrm{kg} & 2.05 \\ \mathrm{mg} / \mathrm{kg} & 2.18\end{array}$

EPA350.1 EPA9081 EPA300.0 EPA335.3 EPA353.1 
SURVEY ID: PPSC 2301 (continued)

Sample ID: 104687

Constituent

RQ AQ B Result

Unit

D. Limit Method

Physical Parameters and Specified Analyses

Nitrogen by Kjeldahl method

Nitrogen by Kjeldahl method

Orthophosphate

Phenols

Sulfide

Total organic carbon

Total organic nitrogen

Total phosphates (as P)

Metals (total recoverable)

$\begin{array}{llllll} & & 466 & \mathrm{mg} / \mathrm{kg} & 173 & \text { EPA351.2 } \\ & & 439 & \mathrm{mg} / \mathrm{kg} & 172 & \text { EPA351.2 } \\ \mathrm{U} & & 43.5 & \mathrm{mg} / \mathrm{kg} & 43.5 & \text { EPA365.2 } \\ \mathrm{J} & \mathrm{E} & 160 & \mu \mathrm{g} / \mathrm{kg} & 600 & \text { EPA420.2 } \\ \mathrm{UJ} & \mathrm{C} & 543 & \mathrm{mg} / \mathrm{kg} & 543 & \text { EPA376.2 } \\ & \mathrm{V} & 59900 & \mathrm{mg} / \mathrm{kg} & 2030 & \text { EPA415.1 } \\ \mathrm{C} & & 389 & \mathrm{mg} / \mathrm{kg} & 0.435 & \text { EPA351.2 } \\ \mathrm{J} & \mathrm{E} & 402 & \mathrm{mg} / \mathrm{kg} & 2010 & \text { EPA365.4 }\end{array}$

Aluminum
Antimony
Arsenic
Barium
Beryllium
Cadmium
Calcium
Chromium
Cobalt
Copper
Iron
Lead
Magnesium
Manganese
Mercury
Nickel
Potassium
Selenium
Silver
Sodium
Thallium
Vanadium
Zinc

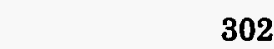

U $\quad 10.5$

U 31.4

J $\quad \mathrm{E} \quad \mathbf{0 . 2 8 6}$

U $\quad \mathrm{E} \quad 0.523$

669

5.07

4.75

2.69

$\begin{array}{lll} & \mathrm{V} & 2680 \\ \mathrm{U} & & 10.5\end{array}$

66.9

164

$\begin{array}{lll}J & \mathrm{E} & \mathbf{0 . 0 3 3 9}\end{array}$

J $\mathrm{E} \quad 2.45$

J E 35.1

U $\quad 31.4$

U $\quad 2.61$

U $\quad 105$

31.4

4.45

16.4

$\begin{array}{lll}\mathrm{mg} / \mathrm{kg} & 26.1 & \text { EPA6010A } \\ \mathrm{mg} / \mathrm{kg} & 10.5 & \text { EPA6010A } \\ \mathrm{mg} / \mathrm{kg} & 31.4 & \text { EPA6010A } \\ \mathrm{mg} / \mathrm{kg} & 2.61 & \text { EPA6010A } \\ \mathrm{mg} / \mathrm{kg} & 1.05 & \text { EPA6010A } \\ \mathrm{mg} / \mathrm{kg} & 0.523 & \text { EPA6010A } \\ \mathrm{mg} / \mathrm{kg} & 26.1 & \text { EPA6010A } \\ \mathrm{mg} / \mathrm{kg} & 2.61 & \text { EPA6010A } \\ \mathrm{mg} / \mathrm{kg} & 1.05 & \text { EPA6010A } \\ \mathrm{mg} / \mathrm{kg} & 2.61 & \text { EPA6010A } \\ \mathrm{mg} / \mathrm{kg} & 10.5 & \text { EPA6010A } \\ \mathrm{mg} / \mathrm{kg} & 10.5 & \text { EPA6010A } \\ \mathrm{mg} / \mathrm{kg} & 10.5 & \text { EPA6010A } \\ \mathrm{mg} / \mathrm{kg} & 1.05 & \text { EPA6010A } \\ \mathrm{mg} / \mathrm{kg} & 0.0606 & \text { EPA7471 } \\ \mathrm{mg} / \mathrm{kg} & 2.61 & \text { EPA6010A } \\ \mathrm{mg} / \mathrm{kg} & 209 & \text { EPA6010A } \\ \mathrm{mg} / \mathrm{kg} & 31.4 & \text { EPA6010A } \\ \mathrm{mg} / \mathrm{kg} & 2.61 & \text { EPA6010A } \\ \mathrm{mg} / \mathrm{kg} & 105 & \text { EPA6010A } \\ \mathrm{mg} / \mathrm{kg} & 31.4 & \text { EPA6010A } \\ \mathrm{mg} / \mathrm{kg} & 1.05 & \text { EPA6010A } \\ \mathrm{mg} / \mathrm{kg} & 2.09 & \text { EPA6010A }\end{array}$

$B / N / A$ Extractables (including $P A H$ and phenols)

$\begin{array}{lcc}\text { Acenaphthene } & \mathrm{U} & 71.2 \\ \text { Acenaphthylene } & \mathrm{U} & 71.2 \\ \text { Anthracene } & \mathrm{U} & 71.2 \\ \text { Benzo[a]anthracene } & \mathrm{U} & 7.12 \\ \text { Benzo[b]fluoranthene } & \mathrm{U} & 7.12 \\ \text { Benzo[k]fluoranthene } & \mathrm{U} & 7.12 \\ \text { Benzoic acid } & \mathrm{U} & 71.2 \\ \text { Benzo[g,h,i]perylene } & \mathrm{U} & 71.2 \\ \text { Benzo[a]pyrene } & \mathrm{U} & 7.12 \\ \text { Benzyl alcohol } & \mathrm{U} & 71.2 \\ \text { Beta-Amyrin } & \mathrm{J} & \mathrm{N} \\ \text { Bis(2-chloroethoxy) methane } & \mathrm{U} & 1000 \\ \text { Bis(2-chloroethyl) ether } & \mathrm{U} & 71.2 \\ \text { Bis(2-chloroisopropyl) ether } & \mathrm{U} & 71.2 \\ \text { Bis(2-ethylhexyl) phthalate } & \mathrm{U} & 71.2 \\ \text { 4-Bromophenyl phenyl ether } & \mathrm{U} & 71.2 \\ \text { Butylbenzyl phthalate } & \mathrm{U} & 71.2 \\ \text { 4-Chloroaniline } & \mathrm{U} & 71.2 \\ & & 71.2\end{array}$

$\begin{array}{lll}\mu \mathrm{g} / \mathrm{kg} & 71.2 & \text { EPA8270 } \\ \mu \mathrm{g} / \mathrm{kg} & 71.2 & \text { EPA8270 } \\ \mu \mathrm{g} / \mathrm{kg} & 71.2 & \text { EPA8270 } \\ \mu \mathrm{g} / \mathrm{kg} & 7.12 & \text { EPA8270 } \\ \mu \mathrm{g} / \mathrm{kg} & 7.12 & \text { EPA8270 } \\ \mu \mathrm{g} / \mathrm{kg} & 7.12 & \text { EPA8270 } \\ \mu \mathrm{g} / \mathrm{kg} & 71.2 & \text { EPA8270 } \\ \mu \mathrm{g} / \mathrm{kg} & 71.2 & \text { EPA8270 } \\ \mu \mathrm{g} / \mathrm{kg} & 7.12 & \text { EPA8270 } \\ \mu \mathrm{g} / \mathrm{kg} & 71.2 & \text { EPA8270 } \\ \mu \mathrm{g} / \mathrm{kg} & & \text { EPA8270 } \\ \mu \mathrm{g} / \mathrm{kg} & 71.2 & \text { EPA8270 } \\ \mu \mathrm{g} / \mathrm{kg} & 71.2 & \text { EPA8270 } \\ \mu \mathrm{g} / \mathrm{kg} & 71.2 & \text { EPA8270 } \\ \mu \mathrm{g} / \mathrm{kg} & 71.2 & \text { EPA8270 } \\ \mu \mathrm{g} / \mathrm{kg} & 71.2 & \text { EPA8270 } \\ \mu \mathrm{g} / \mathrm{kg} & 71.2 & \text { EPA8270 } \\ \mu \mathrm{g} / \mathrm{kg} & 71.2 & \text { EPA8270 }\end{array}$


SURVEY ID: PPSC 2301 (continued)

Constituent
Sample ID: 104687
RQ AQ B Result
Unit
D. Limit Method

$B / N / A$ Extractables (including $P A H$ and phenols)

\begin{tabular}{|c|c|c|c|c|c|}
\hline 4-Chloro-m-cresol & U & 71.2 & $\mu \mathrm{g} / \mathrm{kg}$ & 71.2 & EPA8270 \\
\hline 2-Chloronaphthalene & $\mathrm{U}$ & 71.2 & $\mu \mathrm{g} / \mathrm{kg}$ & 71.2 & EPA8270 \\
\hline 2-Chlorophenol & $\mathrm{U}$ & 71.2 & $\mu \mathrm{g} / \mathrm{kg}$ & 71.2 & EPA8270 \\
\hline 4-Chlorophenyl phenyl ether & $\mathrm{U}$ & 71.2 & $\mu \mathrm{g} / \mathrm{kg}$ & 71.2 & EPA8270 \\
\hline Chrysene & $\mathrm{U}$ & 7.12 & $\mu \mathrm{g} / \mathrm{kg}$ & 7.12 & EPA8270 \\
\hline $\mathrm{m} / \mathrm{p}$-Cresol & $\mathrm{U}$ & 712 & $\mu \mathrm{g} / \mathrm{kg}$ & 712 & EPA8270 \\
\hline o-Cresol (2-Methylphenol) & $\mathrm{U}$ & 71.2 & $\mu \mathrm{g} / \mathrm{kg}$ & 71.2 & EPA8270 \\
\hline Dibenz $[a, h]$ anthracene & $\mathrm{U}$ & 7.12 & $\mu \mathrm{g} / \mathrm{kg}$ & 7.12 & EPA8270 \\
\hline Dibenzofuran & U & 71.2 & $\mu \mathrm{g} / \mathrm{kg}$ & 71.2 & EPA8270 \\
\hline Di-n-butyl phthalate & $\mathbf{U}$ & 71.2 & $\mu \mathrm{g} / \mathrm{kg}$ & 71.2 & EPA8270 \\
\hline 1,2-Dichlorobenzene & $\mathrm{U}$ & 71.2 & $\mu \mathrm{g} / \mathrm{kg}$ & 71.2 & EPA8270 \\
\hline 1,3-Dichlorobenzene & $\mathbf{U}$ & 71.2 & $\mu \mathrm{g} / \mathrm{kg}$ & 71.2 & EPA8270 \\
\hline 1,4-Dichlorobenzene & $\mathbf{U}$ & 71.2 & $\mu \mathrm{g} / \mathrm{kg}$ & 71.2 & EPA8270 \\
\hline 3,3'-Dichlorobenzidine & $\mathrm{U}$ & 71.2 & $\mu \mathrm{g} / \mathrm{kg}$ & 71.2 & EPA8270 \\
\hline 2,4-Dichlorophenol & $\mathrm{U}$ & 71.2 & $\mu \mathrm{g} / \mathrm{kg}$ & 71.2 & EPA8270 \\
\hline Diethyl phthalate & U & 71.2 & $\mu \mathrm{g} / \mathrm{kg}$ & 71.2 & EPA8270 \\
\hline 2,4-Dimethyl phenol & $\mathrm{U}$ & 71.2 & $\mu \mathrm{g} / \mathrm{kg}$ & 71.2 & EPA8270 \\
\hline Dimethyl phthalate & $\mathrm{U}$ & 71.2 & $\mu \mathrm{g} / \mathrm{kg}$ & 71.2 & EPA8270 \\
\hline 2,4-Dinitrophenol & $\mathrm{U}$ & 712 & $\mu \mathrm{g} / \mathrm{kg}$ & 712 & EPA8270 \\
\hline 2,4-Dinitrotoluene & UJ $\quad \mathbf{c}$ & 7.12 & $\mu \mathrm{g} / \mathrm{kg}$ & 7.12 & EPA8270 \\
\hline 2,6-Dinitrotoluene & $\mathrm{U}$ & 7.12 & $\mu \mathrm{g} / \mathrm{kg}$ & 7.12 & EPA8270 \\
\hline Di-n-octyl phthalate & $U$ & 71.2 & $\mu \mathrm{g} / \mathrm{kg}$ & 71.2 & EPA8270 \\
\hline Ergostanol & $\mathrm{N}$ & 2000 & $\mu \mathrm{g} / \mathrm{kg}$ & & EPA8270 \\
\hline Fluoranthene & $\mathrm{U}$ & 71.2 & $\mu \mathrm{g} / \mathrm{kg}$ & 71.2 & EPA8270 \\
\hline Fluorene & $\mathrm{U}$ & 71.2 & $\mu \mathrm{g} / \mathrm{kg}$ & 71.2 & EPA8270 \\
\hline Friedoolean & $\mathbf{N}$ & 8800 & $\mu \mathrm{g} / \mathrm{kg}$ & & EPA8270 \\
\hline Heneicosane & $\mathrm{N}$ & 680 & $\mu \mathrm{g} / \mathrm{kg}$ & & EPA8270 \\
\hline Heptadecane & $\mathrm{N}$ & 3500 & $\mu \mathrm{g} / \mathrm{kg}$ & & EPA8270 \\
\hline Heptadecane, 9 octyl & $\mathrm{N}$ & 1700 & $\mu \mathrm{g} / \mathrm{kg}$ & & EPA8270 \\
\hline Hexachlorobenzene & $\mathrm{U}$ & 7.12 & $\mu \mathrm{g} / \mathrm{kg}$ & 7.12 & EPA8270 \\
\hline Hexachlorobutadiene & $\mathbf{U}$ & 71.2 & $\mu \mathrm{g} / \mathrm{kg}$ & 71.2 & EPA8270 \\
\hline Hexachlorocyclopentadiene & $\mathrm{U}$ & 71.2 & $\mu \mathrm{g} / \mathrm{kg}$ & 71.2 & EPA8270 \\
\hline Hexachloroethane & $\mathrm{U}$ & 7.12 & $\mu \mathrm{g} / \mathrm{kg}$ & 7.12 & EPA8270 \\
\hline Indeno $[1,2,3-c, d]$ pyrene & $\mathrm{U}$ & 7.12 & $\mu \mathrm{g} / \mathrm{kg}$ & 7.12 & EPA8270 \\
\hline Isophorone & $U$ & 71.2 & $\mu \mathrm{g} / \mathrm{kg}$ & 71.2 & EPA8270 \\
\hline 2-Methyl-4,6-dinitrophenol & $U$ & 712 & $\mu \mathrm{g} / \mathrm{kg}$ & 712 & EPA8270 \\
\hline 2-Methylnaphthalene & $\mathrm{U}$ & 71.2 & $\mu \mathrm{g} / \mathrm{kg}$ & 71.2 & EPA8270 \\
\hline Naphthalene & U & 71.2 & $\mu \mathrm{g} / \mathrm{kg}$ & 71.2 & EPA8270 \\
\hline m-Nitroaniline & $\mathrm{U}$ & 71.2 & $\mu \mathrm{g} / \mathrm{kg}$ & 71.2 & EPA8270 \\
\hline o-Nitroaniline & $\mathrm{U}$ & 71.2 & $\mu \mathrm{g} / \mathrm{kg}$ & 71.2 & EPA8270 \\
\hline p-Nitroaniline & $\mathrm{U}$ & 71.2 & $\mu \mathrm{g} / \mathrm{kg}$ & 71.2 & EPA8270 \\
\hline Nitrobenzene & $\mathrm{U}$ & 71.2 & $\mu \mathrm{g} / \mathrm{kg}$ & 71.2 & EPA8270 \\
\hline 2-Nitrophenol & $\mathrm{U}$ & 71.2 & $\mu \mathrm{g} / \mathrm{kg}$ & 71.2 & EPA8270 \\
\hline 4-Nitrophenol & $\mathrm{U}$ & 71.2 & $\mu \mathrm{g} / \mathrm{kg}$ & 71.2 & EPA8270 \\
\hline N-Nitrosodiphenylamine & $\mathrm{U}$ & 71.2 & $\mu \mathrm{g} / \mathrm{kg}$ & 71.2 & EPA8270 \\
\hline N-Nitrosodi-n-propylamine & $U$ & 71.2 & $\mu \mathrm{g} / \mathrm{kg}$ & 71.2 & EPA8270 \\
\hline Octadecanoic acid & $N$ & 980 & $\mu \mathrm{g} / \mathrm{kg}$ & & EPA8270 \\
\hline Pentachlorophenol & $U$ & 71.2 & $\mu \mathrm{g} / \mathrm{kg}$ & 71.2 & EPA8270 \\
\hline Phenanthrene & $\mathrm{U}$ & 71.2 & $\mu \mathrm{g} / \mathrm{kg}$ & 71.2 & EPA8270 \\
\hline Phenol & $\mathrm{U}$ & 71.2 & $\mu \mathrm{g} / \mathrm{kg}$ & 71.2 & EPA8270 \\
\hline Pyrene & $\mathrm{U}$ & 71.2 & $\mu \mathrm{g} / \mathrm{kg}$ & 71.2 & EPA8270 \\
\hline gamma-Sitosterol & $\mathrm{N}$ & 6000 & $\mu \mathrm{g} / \mathrm{kg}$ & & EPA8270 \\
\hline 1,2,4-Trichlorobenzene & U & 7.12 & $\mu \mathrm{g} / \mathrm{kg}$ & 7.12 & EPA8270 \\
\hline 2,4,5-Trichlorophenol & $\mathrm{U}$ & 71.2 & $\mu \mathrm{g} / \mathrm{kg}$ & 71.2 & EPA8270 \\
\hline 2,4,6-Trichlorophenol & $\mathrm{U}$ & 71.2 & $\mu \mathrm{g} / \mathrm{kg}$ & 71.2 & EPA8270 \\
\hline
\end{tabular}


SURVEY ID: PPSC 2301 (continued)

Constituent
Sample ID: 104687

RQ AQ B Result
Unit

D. Limit Method

$B / N / A$ Extractables (including $P A H$ and phenols)

Unknown aldehyde

Unknown alkene

Unknown hydrocarbon

Unknown naphthalene

Unknown vitamin

$\begin{array}{lll}\text { J } & \text { N } & 650 \\ J & N & 810 \\ J & N & 1300 \\ J & N & 400 \\ J & N & 1200\end{array}$

Volatile Organic Compounds

$\begin{array}{lll}\text { U } & 8 & 14.7 \\ U & & 1.09 \\ U & & 0.109 \\ U & & 0.109 \\ U & & 0.217 \\ U & & 2.17 \\ U & & 0.109 \\ U & & 0.109 \\ U & & 0.217 \\ U & & 0.217 \\ U & & 0.109 \\ U & & 0.217 \\ U & & 0.109 \\ U & & 0.109 \\ U & & 0.109 \\ U & & 0.109 \\ U & & 0.217 \\ U & V & 1.15 \\ U & & 0.109 \\ U & & 0.109 \\ U & & 0.109 \\ U & & 0.109 \\ U & V & 0.761 \\ U & V 8 & 0.5 \\ U & & 2.17 \\ U & & 0.109 \\ U & & 0.109 \\ & & 0.761 \\ U & & 1.09 \\ U & & 0.109 \\ U & & 0.109 \\ U & 8 & 0.478 \\ U & & 2.17 \\ U & 8 & 0.217\end{array}$

Benzene

Bromodichloromethane

Bromoform

Bromomethane (Methyl bromide)

Carbon disulfide

Carbon tetrachloride

Chlorobenzene

Chloroethane

Chloroethene (Vinyl chloride)

Chloroform

Chloromethane (Methyl chloride)

Dibromochloromethane

1,1-Dichloroethane

1,2-Dichloroethane

1,1-Dichloroethylene

1,2-Dichloroethylene

Dichloromethane (Methylene chloride)

1,2-Dichloropropane

cis-1,3-Dichloropropene

trans-1,3-Dichloropropene

Ethylbenzene

2-Hexanone

Methyl ethyl ketone

Methyl isobutyl ketone

Styrene

1,1,2,2-Tetrachloroethane

Tetrachloroethylene

Toluene

1,1,1-Trichloroethane

1,1,2-Trichloroethane

Trichloroethylene

Vinyl acetate

Xylenes

Pesticides/PCBs and Dioxins/Furans

\author{
Aldrin \\ alpha-Benzene hexachloride \\ beta-Benzene hexachloride \\ delta-Benzene hexachloride \\ alpha-Chlordane \\ gamma-Chlordane \\ p, $\mathbf{p}^{\prime} \cdot \mathrm{DDD}$ \\ $p, p^{\prime}-D D E$ \\ $\mathrm{p}, \mathrm{p}^{\prime}$-DDT \\ Dieldrin
}

$\begin{array}{ll}\text { U } & 17.8 \\ U & 17.8 \\ U & 35.6 \\ U & 35.6 \\ U & 35.6 \\ U & 35.6 \\ U & 71.1 \\ U & 35.6 \\ U & 71.1 \\ U & 35.6\end{array}$

EPA8270
EPA8270
EPA8270
EPA8270
EPA8270

$\begin{array}{lll}\mu \mathrm{g} / \mathrm{kg} & 10.9 & \text { EPA8260 } \\ \mu \mathrm{g} / \mathrm{kg} & 1.09 & \text { EPA8260 } \\ \mu \mathrm{g} / \mathrm{kg} & 0.109 & \text { EPA8260 } \\ \mu \mathrm{g} / \mathrm{kg} & 0.109 & \text { EPA8260 } \\ \mu \mathrm{g} / \mathrm{kg} & 0.217 & \text { EPA8260 } \\ \mu \mathrm{g} / \mathrm{kg} & 2.17 & \text { EPA8260 } \\ \mu \mathrm{g} / \mathrm{kg} & 0.109 & \text { EPA8260 } \\ \mu \mathrm{g} / \mathrm{kg} & 0.109 & \text { EPA8260 } \\ \mu \mathrm{g} / \mathrm{kg} & 0.217 & \text { EPA8260 } \\ \mu \mathrm{g} / \mathrm{kg} & 0.217 & \text { EPA8260 } \\ \mu \mathrm{g} / \mathrm{kg} & 0.109 & \text { EPA8260 } \\ \mu \mathrm{g} / \mathrm{kg} & 0.217 & \text { EPA8260 } \\ \mu \mathrm{g} / \mathrm{kg} & 0.109 & \text { EPA8260 } \\ \mu \mathrm{g} / \mathrm{kg} & 0.109 & \text { EPA8260 } \\ \mu \mathrm{g} / \mathrm{kg} & 0.109 & \text { EPA8260 } \\ \mu \mathrm{g} / \mathrm{kg} & 0.109 & \text { EPA8260 } \\ \mu \mathrm{g} / \mathrm{kg} & 0.217 & \text { EPA8260 } \\ \mu \mathrm{g} / \mathrm{kg} & 1.09 & \text { EPA8260 } \\ \mu \mathrm{g} / \mathrm{kg} & 0.109 & \text { EPA8260 } \\ \mu \mathrm{g} / \mathrm{kg} & 0.109 & \text { EPA8260 } \\ \mu \mathrm{g} / \mathrm{kg} & 0.109 & \text { EPA8260 } \\ \mu \mathrm{g} / \mathrm{kg} & 0.109 & \text { EPA8260 } \\ \mu \mathrm{g} / \mathrm{kg} & 2.17 & \text { EPA8260 } \\ \mu \mathrm{g} / \mathrm{kg} & 2.17 & \text { EPA8260 } \\ \mu \mathrm{g} / \mathrm{kg} & 2.17 & \text { EPA8260 } \\ \mu \mathrm{g} / \mathrm{kg} & 0.109 & \text { EPA8260 } \\ \mu \mathrm{g} / \mathrm{kg} & 0.109 & \text { EPA8260 } \\ \mu \mathrm{g} / \mathrm{kg} & 0.109 & \text { EPA8260 } \\ \mu \mathrm{g} / \mathrm{kg} & 1.09 & \text { EPA8260 } \\ \mu \mathrm{g} / \mathrm{kg} & 0.109 & \text { EPA8260 } \\ \mu \mathrm{g} / \mathrm{kg} & 0.109 & \text { EPA8260 } \\ \mu \mathrm{g} / \mathrm{kg} & 0.109 & \text { EPA8260 } \\ \mu \mathrm{g} / \mathrm{kg} & 2.17 & \text { EPA8260 } \\ \mu \mathrm{g} / \mathrm{kg} & 0.326 & \text { EPA8260 }\end{array}$


SURVEY ID: PPSC 2301 (continued)

Constituent

RQ AQ B Result

Unit

D. Limit Method

Pesticides/PCBs and Dioxins/Furans

$\begin{array}{lcl}\text { Endosulfan sulfate } & \mathrm{U} & 71.1 \\ \text { Endosulfan I } & \mathrm{U} & 35.6 \\ \text { Endosulfan II } & \mathrm{U} & 71.1 \\ \text { Endrin } & \mathrm{U} & 35.6 \\ \text { Endrin ketone } & \mathrm{U} & 71.1 \\ \text { Heptachlor } & \mathrm{U} & 35.6 \\ \text { Heptachlor epoxide } & \mathrm{U} & 35.6 \\ \text { Lindane } & \mathrm{U} & 17.8 \\ \text { Methoxychlor } & \mathrm{U} & 356 \\ \text { PCB 1016 } & \mathrm{U} & 88.9 \\ \text { PCB 1221 } & \mathrm{U} & 88.9 \\ \text { PCB 1232 } & \mathrm{U} & 88.9 \\ \text { PCB 1242 } & \mathrm{U} & 88.9 \\ \text { PCB 1248 } & \mathrm{U} & 88.9 \\ \text { PCB 1254 } & \mathrm{U} & 88.9 \\ \text { PCB 1260 } & \mathrm{U} & 88.9 \\ \text { Toxaphene } & \mathrm{U} & 711\end{array}$

Actinium-228

Antimony-124

Antimony-125

Barium-133

Cerium-144

Cesium-134

Cesium-137

Cobalt-57

Cobalt-58

Cobalt-60

Europium-152

Europium-154

Europium-155

Gross alpha

Lead-212

Manganese-54

Neptunium-239

Nonvolatile beta

Plutonium-238

Plutonium-239/240

Potassium -40

Promethium-144

Promethium-146

Radium-226

Radium-228

Ruthenium-106

Sodium-22

Strontium-90

Thorium-234

Tin-113

Yttrium-88

Zinc-65

Zirconium-95
6.20E-01 $\pm 5.46 \mathrm{E}-02 \mathrm{pCi} / \mathrm{g}$

$\mu \mathrm{g} / \mathrm{kg}$
$\mu \mathrm{g} / \mathrm{kg}$
$\mu \mathrm{g} / \mathrm{kg}$
$\mu \mathrm{g} / \mathrm{kg}$
$\mu \mathrm{g} / \mathrm{kg}$
$\mu \mathrm{g} / \mathrm{kg}$
$\mu \mathrm{g} / \mathrm{kg}$
$\mu \mathrm{g} / \mathrm{kg}$
$\mu \mathrm{g} / \mathrm{kg}$
$\mu \mathrm{g} / \mathrm{kg}$
$\mu \mathrm{g} / \mathrm{kg}$
$\mu \mathrm{g} / \mathrm{kg}$
$\mu \mathrm{g} / \mathrm{kg}$
$\mu \mathrm{g} / \mathrm{kg}$
$\mu \mathrm{g} / \mathrm{kg}$
$\mu \mathrm{g} / \mathrm{kg}$
$\mu \mathrm{g} / \mathrm{kg}$

71.1
35.6
71.1
35.6
71.1
35.6
35.6
17.8
356
88.9
88.9
88.9
88.9
88.9
88.9
88.9
711

EPA8080

EPA8080

EPA8080

EPA8080

EPA8080

EPA8080

EPA8080

EPA8080

EPA8080

EPA8080

EPA8080

EPA8080

EPA8080

EPA8080

EPA8080

EPA8080

EPA8080

3.02E-02 EPLA-013B

1.20E-02 EPIA-013B

2.52E-02 EPIA-013B

1.10E-02 EPIA-013B

6.12E-02 EPLA-013B

8.64E-03 EPIA-013B

1.05E-02 EPIA-013B

8.03E-03 EPIA-013B

1.08E-02 EPIA-013B

8.72E-03 EPIA-013B

2.56E-02 EPIA-013B

6.40E-02 EPIA-013B

3.36E-02 EPIA-013B

$1.38 \mathrm{E}+00$ EPIA $-001 \mathrm{~B}$

1.53E-02 EPIA-013B

1.04E-02 EPIA-013B

5.72E-02 EPIA-013B

2.42E+00 EPLA-001B

$0.00 \mathrm{E}+00$ EPIA-012B

2.02E-02 EPIA-012B

7.89E-02 EPIA-013B

9.54E-03 EPIA-013B

1.29E-02 EPIA-013B

1.66E-02 EPIA-013B

3.02E-02 EPIA-013B

8.60E-02 EPIA-013B

1.00E-02 EPIA-013B

1.67E-01 EPIA-004

4.24E-01 EPIA-013B

1.30E-02 EPIA-013B

1.21E-02 EPIA-013B

1.76E-02 EPIA-013B

2.33E-02 EPIA-013B 
SURVEY ID: PPSC 2302

Sample ID: 104688

Survey location: $44336 \mathrm{E} 3688244 \mathrm{~N}$ (SRS coordinates)

Depth of core interval: 1.00 to $2.00 \mathrm{ft}$

Sample type: Normal

Sample matrix: Soil

Percent solids: 72.00

$\begin{array}{lllll}\text { Constituent } & \text { RQ AQ B Result Unit D. Limit Method }\end{array}$

Physical Parameters and Specified Analyses

Ammonia nitrogen

Cation exchange capacity

Cation exchange capacity

Chloride

Cyanide

Nitrate-nitrite as nitrogen

Nitrogen by Kjeldahl method

Orthophosphate

Phenols

Sulfide

Total organic carbon

Total organic nitrogen

Total phosphates (as P)

Metals (total recoverable)

$\begin{array}{llllll} & & 39 & \mathrm{mg} / \mathrm{kg} & 28.9 & \text { EPA350.1 } \\ & & 77 & \mathrm{meq} / 100 \mathrm{~g} & 5 & \text { EPA9081 } \\ & & 82.1 & \mathrm{meq} / 100 \mathrm{~g} & 6.94 & \text { EPA9081 } \\ \mathrm{J} & \mathrm{E} & 3.08 & \mathrm{mg} / \mathrm{kg} & 3.38 & \text { EPA300.0 } \\ \mathrm{UJ} & \mathrm{C} & 1.27 & \mathrm{mg} / \mathrm{kg} & 1.27 & \text { EPA335.3 } \\ \mathrm{U} & & 1.41 & \mathrm{mg} / \mathrm{kg} & 1.41 & \text { EPA353.1 } \\ & & 470 & \mathrm{mg} / \mathrm{kg} & 219 & \text { EPA351.2 } \\ \mathrm{J} & \mathrm{E} & 6.61 & \mathrm{mg} / \mathrm{kg} & 27.8 & \text { EPA365.2 } \\ \mathrm{J} & \mathrm{E} & 114 & \mu \mathrm{g} / \mathrm{kg} & 411 & \text { EPA420.2 } \\ \mathrm{UJ} & \mathrm{C} & 347 & \mathrm{mg} / \mathrm{kg} & 347 & \text { EPA376.2 } \\ & \mathrm{V} & 22400 & \mathrm{mg} / \mathrm{kg} & 1240 & \text { EPA415.1 } \\ \mathrm{C} & & 431 & \mathrm{mg} / \mathrm{kg} & 0.278 & \text { EPA351.2 } \\ \text { J } & \mathrm{E} & 146 & \mathrm{mg} / \mathrm{kg} & 556 & \text { EPA365.4 }\end{array}$

\begin{tabular}{|c|c|c|c|c|c|c|}
\hline Aluminum & & & 2800 & $\mathrm{mg} / \mathrm{kg}$ & 16.7 & EPA6010A \\
\hline Antimony & $\mathrm{U}$ & & 6.68 & $\mathrm{mg} / \mathrm{kg}$ & 6.68 & EPA6010A \\
\hline Arsenic & $\mathrm{U}$ & & 20 & $\mathrm{mg} / \mathrm{kg}$ & 20 & EPA6010A \\
\hline Barium & & & 20.3 & $\mathrm{mg} / \mathrm{kg}$ & 1.67 & EPA6010A \\
\hline Beryllium & $J$ & $\mathrm{E}$ & 0.265 & $\mathrm{mg} / \mathrm{kg}$ & 0.668 & EPA6010A \\
\hline Cadmium & $U$ & & 0.334 & $\mathrm{mg} / \mathrm{kg}$ & 0.334 & EPA6010A \\
\hline Calcium & & & 564 & $\mathrm{mg} / \mathrm{kg}$ & 16.7 & EPA6010A \\
\hline Chromium & & & 5.45 & $\mathrm{mg} / \mathrm{kg}$ & 1.67 & EPA6010A \\
\hline Cobalt & & & 1.84 & $\mathrm{mg} / \mathrm{kg}$ & 0.668 & EPA6010A \\
\hline Copper & $\mathbf{U}$ & & 1.67 & $\mathrm{mg} / \mathrm{kg}$ & 1.67 & EPA6010A \\
\hline Iron & & V & 1190 & $\mathrm{mg} / \mathrm{kg}$ & 6.68 & EPA6010A \\
\hline Lead & $\mathrm{U}$ & & 6.68 & $\mathrm{mg} / \mathrm{kg}$ & 6.68 & EPA6010A \\
\hline Magnesium & & & 54.8 & $\mathrm{mg} / \mathrm{kg}$ & 6.68 & EPA6010A \\
\hline Manganese & & & 55.5 & $\mathrm{mg} / \mathrm{kg}$ & 0.668 & EPA6010A \\
\hline Mercury & $J$ & $\mathbf{E}$ & 0.0338 & $\mathrm{mg} / \mathrm{kg}$ & 0.0402 & EPA7471 \\
\hline Nickel & $J$ & $\mathbf{E}$ & 1.34 & $\mathrm{mg} / \mathrm{kg}$ & 1.67 & EPA6010A \\
\hline Potassium & $J$ & $\mathbf{E}$ & 27 & $\mathrm{mg} / \mathrm{kg}$ & 134 & EPA6010A \\
\hline Selenium & $\mathrm{U}$ & & 20 & $\mathrm{mg} / \mathrm{kg}$ & 20 . & EPA6010A \\
\hline Silver & $\mathbf{U}$ & & 1.67 & $\mathrm{mg} / \mathrm{kg}$ & 1.67 & EPA6010A \\
\hline Sodium & $\mathrm{U}$ & & 66.8 & $\mathrm{mg} / \mathrm{kg}$ & 66.8 & EPA6010A \\
\hline Thallium & $\mathrm{U}$ & & 20 & $\mathrm{mg} / \mathrm{kg}$ & 20 & EPA6010A \\
\hline Vanadium & & & 2.31 & $\mathrm{mg} / \mathrm{kg}$ & 0.668 & EPA6010A \\
\hline Zinc & & & 4.81 & $\mathrm{mg} / \mathrm{kg}$ & 1.34 & EPA6010A \\
\hline \multicolumn{7}{|c|}{$B / N / A$ Extractables (including $P A H$ and phenols) } \\
\hline Acenaphthene & UJ & $\mathbf{Q}$ & 45.8 & $\mu \mathrm{g} / \mathrm{kg}$ & 45.8 & EPA8270 \\
\hline Acenaphthylene & UJ & $\mathbf{Q}$ & 45.8 & $\mu \mathrm{g} / \mathrm{kg}$ & 45.8 & EPA8270 \\
\hline Anthracene & UJ & $Q$ & 45.8 & $\mu \mathrm{g} / \mathrm{kg}$ & 45.8 & EPA8270 \\
\hline Benzo[a]anthracene & UJ & $Q$ & 4.58 & $\mu \mathrm{g} / \mathrm{kg}$ & 4.58 & EPA8270 \\
\hline Benzo[b]fluoranthene & UJ & $Q$ & 4.58 & $\mu g / k g$ & 4.58 & EPA8270 \\
\hline Benzo[k]fluoranthene & UJ & $Q$ & 4.58 & $\mu \mathrm{g} / \mathrm{kg}$ & 4.58 & EPA8270 \\
\hline Benzoic acid & $\mathbf{J}$ & $\mathrm{Q}$ & 194 & $\mu \mathrm{g} / \mathrm{kg}$ & 45.8 & EPA8270 \\
\hline
\end{tabular}


SURVEY ID: PPSC 2302 (continued)

Constituent

RQ AQ B Result
Sample ID: 104688

Unit D. Limit Method

$B / N / A$ Extractables (including $P A H$ and phenols)

\begin{tabular}{|c|c|c|c|c|c|c|}
\hline Benzo $[g, h, i]$ perylene & UJ & $\mathbf{Q}$ & 45.8 & $\mu \mathrm{g} / \mathrm{kg}$ & 45.8 & EPA8270 \\
\hline Benzo $[a]$ pyrene & UJ & $Q$ & 4.58 & $\mu \mathrm{g} / \mathrm{kg}$ & 4.58 & EPA8270 \\
\hline Benzyl alcohol & UJ & $\mathbf{Q}$ & 45.8 & $\mu \mathrm{g} / \mathrm{kg}$ & 45.8 & EPA8270 \\
\hline Bis(2-chloroethoxy) methane & UJ & $Q$ & 45.8 & $\mu \mathrm{g} / \mathrm{kg}$ & 45.8 & EPA8270 \\
\hline Bis(2-chloroethyl) ether & UJ & $\mathbf{Q}$ & 45.8 & $\mu \mathrm{g} / \mathrm{kg}$ & 45.8 & EPA8270 \\
\hline Bis(2-chloroisopropyl) ether & UJ & $\mathbf{Q}$ & 45.8 & $\mu \mathrm{g} / \mathrm{kg}$ & 45.8 & EPA8270 \\
\hline Bis(2-ethylhexyl) phthalate & UJ & $\mathbf{Q}$ & 45.8 & $\mu \mathrm{g} / \mathrm{kg}$ & 45.8 & EPA8270 \\
\hline 4-Bromophenyl phenyl ether & UJ & $\mathbf{Q}$ & 45.8 & $\mu \mathrm{g} / \mathrm{kg}$ & 45.8 & EPA8270 \\
\hline Butylbenzyl phthalate & UJ & $\mathbf{Q}$ & 45.8 & $\mu \mathrm{g} / \mathrm{kg}$ & 45.8 & EPA8270 \\
\hline 4-Chloroaniline & UJ & $\mathbf{Q}$ & 45.8 & $\mu \mathrm{g} / \mathrm{kg}$ & 45.8 & EPA8270 \\
\hline 4-Chloro-m-cresol & UJ & $Q$ & 45.8 & $\mu \mathrm{g} / \mathrm{kg}$ & 45.8 & EPA8270 \\
\hline 2-Chloronaphthalene & UJ & $\mathbf{Q}$ & 45.8 & $\mu \mathrm{g} / \mathrm{kg}$ & 45.8 & EPA8270 \\
\hline 2-Chlorophenol & UJ & $\mathbf{Q}$ & 45.8 & $\mu \mathrm{g} / \mathrm{kg}$ & 45.8 & EPA8270 \\
\hline 4-Chlorophenyl phenyl ether & UJ & $\mathbf{Q}$ & 45.8 & $\mu \mathrm{g} / \mathrm{kg}$ & 45.8 & EPA8270 \\
\hline Chrysene & UJ & $Q$ & 4.58 & $\mu \mathrm{g} / \mathrm{kg}$ & 4.58 & EPA8270 \\
\hline $\mathrm{m} / \mathrm{p}$-Cresol & UJ & $\mathbf{Q}$ & 458 & $\mu \mathrm{g} / \mathrm{kg}$ & 458 & EPA8270 \\
\hline o-Cresol (2-Methylphenol) & UJ & $\mathbf{Q}$ & 45.8 & $\mu \mathrm{g} / \mathrm{kg}$ & 45.8 & EPA8270 \\
\hline Dibenz $[a, h]$ anthracene & UJ & $\mathbf{Q}$ & 4.58 & $\mu \mathrm{g} / \mathrm{kg}$ & 4.58 & EPA8270 \\
\hline Dibenzofuran & UJ & $Q$ & 45.8 & $\mu \mathrm{g} / \mathrm{kg}$ & 45.8 & EPA8270 \\
\hline Di-n-butyl phthalate & UJ & $Q$ & 45.8 & $\mu \mathrm{g} / \mathrm{kg}$ & 45.8 & EPA8270 \\
\hline 1,2-Dichlorobenzene & UJ & $Q$ & 45.8 & $\mu \mathrm{g} / \mathrm{kg}$ & 45.8 & EPA8270 \\
\hline 1,3-Dichlorobenzene & UJ & $\mathbf{Q}$ & 45.8 & $\mu \mathrm{g} / \mathrm{kg}$ & 45.8 & EPA8270 \\
\hline 1,4-Dichlorobenzene & UJ & $Q$ & 45.8 & $\mu \mathrm{g} / \mathrm{kg}$ & 45.8 & EPA8270 \\
\hline 3,3'-Dichlorobenzidine & UJ & $\mathbf{Q}$ & 45.8 & $\mu \mathrm{g} / \mathrm{kg}$ & 45.8 & EPA8270 \\
\hline 2,4-Dichlorophenol & UJ & $\mathbf{Q}$ & 45.8 & $\mu \mathrm{g} / \mathrm{kg}$ & 45.8 & EPA8270 \\
\hline Diethyl phthalate & UJ & $\mathbf{Q}$ & 45.8 & $\mu \mathrm{g} / \mathrm{kg}$ & 45.8 & EPA8270 \\
\hline 2,4-Dimethyl phenol & UJ & $\mathbf{Q}$ & 45.8 & $\mu \mathrm{g} / \mathrm{kg}$ & 45.8 & EPA8270 \\
\hline Dimethyl phthalate & UJ & $\mathbf{Q}$ & 45.8 & $\mu \mathrm{g} / \mathrm{kg}$ & 45.8 & EPA8270 \\
\hline 2,4-Dinitrophenol & UJ & $\mathbf{Q}$ & 458 & $\mu \mathrm{g} / \mathrm{kg}$ & 458 & EPA8270 \\
\hline 2,4-Dinitrotoluene & UJ & $\mathrm{QC}$ & 4.58 & $\mu \mathrm{g} / \mathrm{kg}$ & 4.58 & EPA8270 \\
\hline 2,6-Dinitrotoluene & UJ & $Q$ & 4.58 & $\mu \mathrm{g} / \mathrm{kg}$ & 4.58 & EPA8270 \\
\hline Di-n-octyl phthalate & UJ & $\mathbf{Q}$ & 45.8 & $\mu \mathrm{g} / \mathrm{kg}$ & 45.8 & EPA8270 \\
\hline Docosane & $\mathbf{J}$ & $N$ & 480 & $\mu \mathrm{g} / \mathrm{kg}$ & & EPA8270 \\
\hline Ergostanol & $\boldsymbol{J}$ & $\mathbf{N}$ & 600 & $\mu \mathrm{g} / \mathrm{kg}$ & & EPA8270 \\
\hline Fluoranthene & UJ & $Q$ & 45.8 & $\mu \mathrm{g} / \mathrm{kg}$ & 45.8 & EPA8270 \\
\hline Fluorene & UJ & $Q$ & 45.8 & $\mu \mathrm{g} / \mathrm{kg}$ & 45.8 & EPA8270 \\
\hline Hexachlorobenzene & UJ & $Q$ & 4.58 & $\mu \mathrm{g} / \mathrm{kg}$ & 4.58 & EPA8270 \\
\hline Hexachlorobutadiene & UJ & $Q$ & 45.8 & $\mu \mathrm{g} / \mathrm{kg}$ & 45.8 & EPA8270 \\
\hline Hexachlorocyclopentadiene & UJ & $\mathrm{Q}$ & 45.8 & $\mu \mathrm{g} / \mathrm{kg}$ & 45.8 & EPA8270 \\
\hline Hexachloroethane & UJ & $Q$ & 4.58 & $\mu \mathrm{g} / \mathrm{kg}$ & 4.58 & EPA8270 \\
\hline Indeno $[1,2,3-c, d]$ pyrene & UJ & $\mathbf{Q}$ & 4.58 & $\mu \mathrm{g} / \mathrm{kg}$ & 4.58 & EPA8270 \\
\hline Isophorone & UJ & $\mathbf{Q}$ & 45.8 & $\mu \mathrm{g} / \mathrm{kg}$ & 45.8 & EPA8270 \\
\hline 2-Methyl-4,6-dinitrophenol & UJ & $\mathbf{Q}$ & 458 & $\mu \mathrm{g} / \mathrm{kg}$ & 458 & EPA8270 \\
\hline 2-Methylnaphthalene & UJ & $\mathbf{Q}$ & 45.8 & $\mu \mathrm{g} / \mathrm{kg}$ & 45.8 & EPA8270 \\
\hline Naphthalene & UJ & $\mathbf{Q}$ & 45.8 & $\mu \mathrm{g} / \mathrm{kg}$ & 45.8 & EPA8270 \\
\hline m-Nitroaniline & UJ & $\mathbf{Q}$ & 45.8 & $\mu \mathrm{g} / \mathrm{kg}$ & 45.8 & EPA8270 \\
\hline o-Nitroaniline & UJ & $\mathbf{Q}$ & 45.8 & $\mu \mathrm{g} / \mathrm{kg}$ & 45.8 & EPA8270 \\
\hline p-Nitroaniline & UJ & Q & 45.8 & $\mu \mathrm{g} / \mathrm{kg}$ & 45.8 & EPA8270 \\
\hline Nitrobenzene & UJ & Q & 45.8 & $\mu \mathrm{g} / \mathrm{kg}$ & 45.8 & EPA8270 \\
\hline 2-Nitrophenol & UJ & Q & 45.8 & $\mu \mathrm{g} / \mathrm{kg}$ & 45.8 & EPA8270 \\
\hline 4-Nitrophenol & UJ & $Q$ & 45.8 & $\mu \mathrm{g} / \mathrm{kg}$ & 45.8 & EPA8270 \\
\hline N-Nitrosodiphenylamine & UJ & $Q$ & 45.8 & $\mu \mathrm{g} / \mathrm{kg}$ & 45.8 & EPA8270 \\
\hline N-Nitrosodi-n-propylamine & UJ & $Q$ & 45.8 & $\mu \mathrm{g} / \mathrm{kg}$ & 45.8 & EPA8270 \\
\hline Pentachlorophenol & UJ & $Q$ & 45.8 & $\mu \mathrm{g} / \mathrm{kg}$ & 45.8 & EPA8270 \\
\hline Phenanthrene & UJ & $\dot{Q}$ & 45.8 & $\mu \mathrm{g} / \mathrm{kg}$ & 45.8 & EPA8270 \\
\hline
\end{tabular}


SURVEY ID: PPSC 2302 (continued)

Sample ID: 104688
Constituent
RQ AQ B Result
Unit
D. Limit Method

$B / N / A$ Extractables (including PAH and phenols)

$\begin{array}{llll}\text { Phenol } & \text { UJ } & \text { Q } & 45.8 \\ \text { Pyrene } & \text { UJ } & \text { Q } & 45.8 \\ \text { gamma-Sitosterol } & \text { J } & \text { N } & 1300 \\ \text { 1,2,4-Trichlorobenzene } & \text { UJ } & \text { Q } & 4.58 \\ \text { 2,4,5-Trichlorophenol } & \text { UJ } & \text { Q } & 45.8 \\ \text { 2,4,6-Trichlorophenol } & \text { UJ } & \text { Q } & 45.8 \\ \text { Unknown alkane } & \text { J } & \text { N } & 480 \\ \text { Unknown azulene } & \text { J } & \text { N } & 680 \\ \text { Unknown hydrocarbon } & \text { J } & \text { N } & 600 \\ \text { Unknown hydrocarbon } & \text { J } & \text { N } & 470\end{array}$

$\begin{array}{lll}\mu \mathrm{g} / \mathrm{kg} & 45.8 & \text { EPA8270 } \\ \mu \mathrm{g} / \mathrm{kg} & 45.8 & \text { EPA8270 } \\ \mu \mathrm{g} / \mathrm{kg} & & \text { EPA8270 } \\ \mu \mathrm{g} / \mathrm{kg} & 4.58 & \text { EPA8270 } \\ \mu \mathrm{g} / \mathrm{kg} & 45.8 & \text { EPA8270 } \\ \mu \mathrm{g} / \mathrm{kg} & 45.8 & \text { EPA8270 } \\ \mu \mathrm{g} / \mathrm{kg} & & \text { EPA8270 } \\ \mu \mathrm{g} / \mathrm{kg} & & \text { EPA8270 } \\ \mu \mathrm{g} / \mathrm{kg} & & \text { EPA8270 } \\ \mu \mathrm{g} / \mathrm{kg} & & \text { EPA8270 }\end{array}$

Volatile Organic Compounds

\begin{tabular}{|c|c|c|c|c|c|c|}
\hline Acetone & U & 8 & 42.2 & $\mu \mathrm{g} / \mathrm{kg}$ & 6.94 & EPA8260 \\
\hline Benzene & $U$ & & 0.694 & $\mu \mathrm{g} / \mathrm{kg}$ & 0.694 & EPA8260 \\
\hline Bromodichloromethane & $\mathrm{U}$ & & 0.0694 & $\mu \mathrm{g} / \mathrm{kg}$ & 0.0694 & EPA8260 \\
\hline Bromoform & $U$ & & 0.0694 & $\mu \mathrm{g} / \mathrm{kg}$ & 0.0694 & EPA8260 \\
\hline Bromomethane (Methyl bromide) & $\mathrm{U}$ & & 0.139 & $\mu \mathrm{g} / \mathrm{kg}$ & 0.139 & EPA8260 \\
\hline Carbon disulfide & $\mathrm{U}$ & & 1.39 & $\mu \mathrm{g} / \mathrm{kg}$ & 1.39 & EPA8260 \\
\hline Carbon tetrachloride & $\mathrm{U}$ & & 0.0694 & $\mu \mathrm{g} / \mathrm{kg}$ & 0.0694 & EPA8260 \\
\hline Chlorobenzene & $U$ & & 0.0694 & $\mu \mathrm{g} / \mathrm{kg}$ & 0.0694 & EPA8260 \\
\hline Chloroethane & $\mathrm{U}$ & & 0.139 & $\mu \mathrm{g} / \mathrm{kg}$ & 0.139 & EPA8260 \\
\hline Chloroethene (Vinyl chloride) & $\mathrm{U}$ & & 0.139 & $\mu \mathrm{g} / \mathrm{kg}$ & 0.139 & EPA8260 \\
\hline Chloroform & $\mathrm{U}$ & & 0.0694 & $\mu \mathrm{g} / \mathrm{kg}$ & 0.0694 & EPA8260 \\
\hline Chloromethane (Methyl chloride) & $\mathrm{U}$ & & 0.139 & $\mu \mathrm{g} / \mathrm{kg}$ & 0.139 & EPA8260 \\
\hline Dibromochloromethane & U & & 0.0694 & $\mu \mathrm{g} / \mathrm{kg}$ & 0.0694 & EPA8260 \\
\hline 1,1-Dichloroethane & $\mathbf{U}$ & & 0.0694 & $\mu \mathrm{g} / \mathrm{kg}$ & 0.0694 & EPA8260 \\
\hline 1,2-Dichloroethane & $\mathrm{U}$ & & 0.0694 & $\mu \mathrm{g} / \mathrm{kg}$ & 0.0694 & EPA8260 \\
\hline 1,1-Dichloroethylene & $\mathrm{U}$ & & 0.0694 & $\mu g / k g$ & 0.0694 & EPA8260 \\
\hline 1,2-Dichloroethylene & U & & 0.139 & $\mu \mathrm{g} / \mathrm{kg}$ & 0.139 & EPA8260 \\
\hline Dichloromethane (Methylene chloride) & U & $\mathrm{V}$ & 1.13 & $\mu g / k g$ & 0.694 & EPA8260 \\
\hline 1,2-Dichloropropane & U & & 0.0694 & $\mu \mathrm{g} / \mathrm{kg}$ & 0.0694 & EPA8260 \\
\hline cis-1,3-Dichloropropene & U & & 0.0694 & $\mu \mathrm{g} / \mathrm{kg}$ & 0.0694 & EPA8260 \\
\hline trans-1,3-Dichloropropene & $U$ & & 0.0694 & $\mu \mathrm{g} / \mathrm{kg}$ & 0.0694 & EPA8260 \\
\hline Ethylbenzene & $\mathrm{U}$ & & 0.0694 & $\mu \mathrm{g} / \mathrm{kg}$ & 0.0694 & EPA8260 \\
\hline 2-Hexanone & $\mathrm{U}$ & $\mathrm{V}$ & 0.375 & $\mu \mathrm{g} / \mathrm{kg}$ & 1.39 & EPA8260 \\
\hline Methyl ethyl ketone & U & V8 & 0.847 & $\mu g / k g$ & 1.39 & EPA8260 \\
\hline Methyl isobutyl ketone & $\mathrm{U}$ & & 1.39 & $\mu \mathrm{g} / \mathrm{kg}$ & 1.39 & EPA8260 \\
\hline Styrene & U & & 0.0694 & $\mu \mathrm{g} / \mathrm{kg}$ & 0.0694 & EPA8260 \\
\hline 1,1,2,2-Tetrachloroethane & $\mathrm{U}$ & & 0.0694 & $\mu g / \mathrm{kg}$ & 0.0694 & EPA8260 \\
\hline Tetrachloroethylene & & & 0.333 & $\mu \mathrm{g} / \mathrm{kg}$ & 0.0694 & EPA8260 \\
\hline Toluene & $\mathrm{U}$ & 8 & 0.208 & . $\mu \mathrm{g} / \mathrm{kg}$ & 0.694 & EPA8260 \\
\hline 1,1,1-Trichloroethane & $\mathrm{U}$ & & 0.0694 & $\mu \mathrm{g} / \mathrm{kg}$ & 0.0694 & EPA8260 \\
\hline 1,1,2-Trichloroethane & $\mathrm{U}$ & & 0.0694 & $\mu \mathrm{g} / \mathrm{kg}$ & 0.0694 & EPA8260 \\
\hline Trichloroethylene & U & 8 & 0.167 & $\mu \mathrm{g} / \mathrm{kg}$ & 0.0694 & EPA8260 \\
\hline Vinyl acetate & $\mathrm{U}$ & & 1.39 & $\mu \mathrm{g} / \mathrm{kg}$ & 1.39 & EPA8260 \\
\hline Xylenes & $\mathrm{U}$ & 8 & 0.236 & $\mu \mathrm{g} / \mathrm{kg}$ & 0.208 & EPA8260 \\
\hline
\end{tabular}


SURVEY ID: PPSC 2302 (continued)

\section{Constituent}

Pesticides/PCBs and Dioxins/Furans

$\begin{array}{lcc}\text { Aldrin } & \mathrm{U} & 5.67 \\ \text { alpha-Benzene hexachloride } & \mathrm{U} & 5.67 \\ \text { beta-Benzene hexachloride } & \mathrm{U} & 11.3 \\ \text { delta-Benzene hexachloride } & \mathrm{U} & 11.3 \\ \text { alpha-Chlordane } & \mathrm{U} & 11.3 \\ \text { gamma-Chlordane } & \mathrm{U} & 11.3 \\ \text { p,p'-DDD } & \mathrm{U} & 22.7 \\ \text { p,p'-DDE } & \mathrm{U} & 11.3 \\ \text { p,p'-DDT } & \mathrm{U} & 22.7 \\ \text { Dieldrin } & \mathrm{U} & 11.3 \\ \text { Endosulfan sulfate } & \mathrm{U} & 22.7 \\ \text { Endosulfan I } & \mathrm{U} & 11.3 \\ \text { Endosulfan II } & \mathrm{U} & 22.7 \\ \text { Endrin } & \mathrm{U} & 11.3 \\ \text { Endrin ketone } & \mathrm{U} & 22.7 \\ \text { Heptachlor } & \mathrm{U} & 11.3 \\ \text { Heptachlor epoxide } & \mathrm{U} & 11.3 \\ \text { Lindane } & \mathrm{U} & 5.67 \\ \text { Methoxychlor } & \mathrm{U} & 113 \\ \text { PCB 1016 } & \mathrm{U} & 28.3 \\ \text { PCB 1221 } & \mathrm{U} & 28.3 \\ \text { PCB 1232 } & \mathrm{U} & 28.3 \\ \text { PCB 1242 } & \mathrm{U} & 28.3 \\ \text { PCB 1248 } & \mathrm{U} & 28.3 \\ \text { PCB 1254 } & \mathrm{U} & 28.3 \\ \text { PCB 1260 } & \mathrm{U} & 28.3 \\ \text { Toxaphene } & \mathrm{U} & 227\end{array}$

Radionuclides

Actinium-228

Antimony-124

Antimony-125

Barium-133

Cerium-144

Cesium-134

Cesium-137

Cobalt-57

Cobalt-58

Cobalt-60

Europium-152

Europium-154

Europium-155

Gross alpha

Lead-212

Manganese-54

Neptunium-239

Nonvolatile beta

Plutonium-238

Plutonium-239/240

Potassium-40

Promethium-144

Promethium-146

Radium-226

Radium-228
Sample ID: 104688

RQ AQ B Result Unit D. Limit Method

$1.19 \mathrm{E}+00 \pm 5.02 \mathrm{E}-02 \mathrm{pCi} / \mathrm{g}$ $3.56 \mathrm{E}-03 \pm 6.31 \mathrm{E}-03 \mathrm{pCi} / \mathrm{g}$ $8.23 \mathrm{E}-03 \pm 1.34 \mathrm{E}-02 \mathrm{pCi} / \mathrm{g}$ $-2.11 \mathrm{E}-03 \pm 6.82 \mathrm{E}-03 \mathrm{pCi} / \mathrm{g}$ $-3.50 \mathrm{E}-03 \pm 3.84 \mathrm{E}-02 \mathrm{pCi} / \mathrm{g}$ $-1.11 \mathrm{E}-03 \pm 5.10 \mathrm{E}-03 \mathrm{pCi} / \mathrm{g}$ $1.92 \mathrm{E}-02 \pm 9.37 \mathrm{E}-03 \mathrm{pCi} / \mathrm{g}$ $-1.82 \mathrm{E}-03 \pm 4.85 \mathrm{E}-03 \mathrm{pCi} / \mathrm{g}$ $7.29 \mathrm{E}-04 \pm 6.68 \mathrm{E}-03 \mathrm{pCi} / \mathrm{g}$ $-2.39 \mathrm{E}-03 \pm 4.53 \mathrm{E}-03 \mathrm{pCi} / \mathrm{g}$ $-7.65 \mathrm{E}-03 \pm 1.48 \mathrm{E}-02 \mathrm{pCi} / \mathrm{g}$ $2.10 \mathrm{E}-02 \pm 4.28 \mathrm{E}-02 \mathrm{pCi} / \mathrm{g}$ $0.00 \mathrm{E}+00 \quad \mathrm{pCi} / \mathrm{g}$ $2.22 \mathrm{E}+01 \pm 3.43 \mathrm{E}+00 \mathrm{pCi} / \mathrm{g}$ $1.29 \mathrm{E}+00 \pm 2.56 \mathrm{E}-02 \mathrm{pCi} / \mathrm{g}$ $1.95 \mathrm{E}-02 \pm 6.61 \mathrm{E}-03 \mathrm{pCi} / \mathrm{g}$ $0.00 \mathrm{E}+00 \quad \mathrm{pCj} / \mathrm{g}$ $8.65 \mathrm{E}+00 \pm 1.82 \mathrm{E}+00 \mathrm{pCi} / \mathrm{g}$ $-2.81 \mathrm{E}-05 \pm 6.91 \mathrm{E}-03 \mathrm{pCi} / \mathrm{g}$ $-4.26 \mathrm{E}-03 \pm 4.90 \mathrm{E}-03 \mathrm{pCi} / \mathrm{g}$ $1.46 \mathrm{E}+00 \pm 1.24 \mathrm{E}-01 \mathrm{pCi} / \mathrm{g}$ $4.27 \mathrm{E}-03 \pm 5.07 \mathrm{E}-03 \mathrm{pCi} / \mathrm{g}$ $7.79 \mathrm{E}-03 \pm 6.23 \mathrm{E}-03 \mathrm{pCi} / \mathrm{g}$ 8.03E-01 $+3.03 \mathrm{E}-02 \mathrm{pCi} / \mathrm{g}$ $1.19 \mathrm{E}+00 \pm 5.02 \mathrm{E}-02 \mathrm{pCi} / \mathrm{g}$
2.68E-02 EPIA-013B

1.10E-02 EPIA-013B

2.35E-02 EPIA-013B

9.75E-03 EPIA-013B

6.52E-02 EPIA-013B

7.61E-03 EPIA-013B

8.99E-03 EPIA-013B

8.25E-03 EPIA-013B

9.87E-03 EPIA-013B

7.60E-03 EPIA-013B

2.38E-02 EPLA-013B

6.81E-02 EPLA-013B

3.52E-02 EPIA-013B

$1.71 \mathrm{E}+00$ EPIA-001B

1.45E-02 EPIA-013B

9.04E-03 EPIA-013B

5.80E-02 EPIA-013B

2.51E+00 EPIA-001B

2.16E-02 EPIA-012B

2.45E-02 EPIA-012B

7.43E-02 EPIA-013B

8.81E-03 EPIA-013B

1.11E-02 EPIA-013B

1.55E-02 EPIA-013B

2.68E-02 EPIA-013B 
SURVEY ID: PPSC 2302 (continued)

Constituent

Radionuclides

Ruthenium-106

Sodium-22

Strontium-90

Thorium-234

Tin-113

Yttrium-88

Zinc-65

Zirconium-95
Sample ID: 104688

RQ AQ B Result

Unit

D. Limit Method

7.64E-02 EPIA-013B

7.93E-03 EPIA-013B

1.51E-01 EPIA-004

4.59E-01 EPIA-013B

1.21E-02 EPIA-013B

9.28E-03 EPIA-013B

1.58E-02 EPIA-013B

2.13E-02 EPIA-013B

SURVEY ID: PPSC 2303

Sample ID: 104689

Survey location: $44336 \mathrm{E} 3688244 \mathrm{~N}$ (SRS coordinates)

Depth of core interval: 2.00 to $4.00 \mathrm{ft}$

Sample type: Normal

Sample matrix: Soil

Percent solids: $\mathbf{7 1 . 0 0}$

Constituent

RQ AQ B Result

Unit

D. Limit Method

Physical Parameters and Specified Analyses

$\begin{array}{lllll}\text { Ammonia nitrogen } & \text { J } & \text { I } & \text { L } & 31.8 \\ \text { Ammonia nitrogen } & \text { J } & \text { I } & \text { L } & 32.3 \\ \text { Cation exchange capacity } & & & & 79.7 \\ \text { Chloride } & \text { J } & \text { E } & 2.58 \\ \text { Cyanide } & \text { UJ } & \text { C } & 1.39 \\ \text { Nitrate-nitrite as nitrogen } & \text { J } & \text { E } & & 0.371 \\ \text { Nitrogen by Kjeldahl method } & & & 443 \\ \text { Orthophosphate } & \text { J } & \text { E } & 7.41 \\ \text { Phenols } & \text { U } & & 417 \\ \text { Phenols } & \text { J } & \text { E } & 116 \\ \text { Sulfide } & \text { UJ } & \text { C } & 352 \\ \text { Total organic carbon } & & \text { V } & 29900 \\ \text { Total organic nitrogen } & \text { C } & & 411 \\ \text { Total phosphates (as P) } & \text { J } & \text { E } & 108 \\ & & & \\ \text { Metals (total recoverable) } & & & \\ & & & \\ \text { Aluminum } & & & 3660 \\ \text { Antimony } & \text { U } & & 6.46 \\ \text { Arsenic } & \text { U } & & 19.4 \\ \text { Barium } & & & 20.6 \\ \text { Beryllium } & \text { J } & \text { E } & 0.316 \\ \text { Cadmium } & \text { U } & & 0.323 \\ \text { Calcium } & & & 912 \\ \text { Chromium } & & & 7.02 \\ \text { Cobalt } & & & 1.03 \\ \text { Copper } & \text { J } & \text { E } & 1.4 \\ \text { Iron } & & \text { V } & 427 \\ \text { Lead } & \text { J } & \text { E } & 4.18 \\ \text { Magnesium } & & & 58.7 \\ \text { Manganese } & & & 12.5 \\ \text { Mercury } & \text { J } & \text { E } & 0.0134 \\ \text { Nickel } & \text { J } & \text { E } & 1.08 \\ & & & \end{array}$

$\begin{array}{lll}\mathrm{mg} / \mathrm{kg} & 25.7 & \text { EPA350.1 } \\ \mathrm{mg} / \mathrm{kg} & 24.5 & \text { EPA350.1 } \\ \mathrm{meq} / 100 \mathrm{~g} & 5 & \text { EPA9081 } \\ \mathrm{mg} / \mathrm{kg} & 3.43 & \text { EPA300.0 } \\ \mathrm{mg} / \mathrm{kg} & 1.39 & \text { EPA335.3 } \\ \mathrm{mg} / \mathrm{kg} & 1.24 & \text { EPA353.1 } \\ \mathrm{mg} / \mathrm{kg} & \mathbf{2 2 1} & \text { EPA351.2 } \\ \mathrm{mg} / \mathrm{kg} & 28.2 & \text { EPA365.2 } \\ \mu \mathrm{g} / \mathrm{kg} & 417 & \text { EPA420.2 } \\ \mu \mathrm{g} / \mathrm{kg} & 417 & \text { EPA420.2 } \\ \mathrm{mg} / \mathrm{kg} & 352 & \text { EPA376.2 } \\ \mathrm{mg} / \mathrm{kg} & \mathbf{7 0 2} & \text { EPA415.1 } \\ \mathrm{mg} / \mathrm{kg} & \mathbf{0 . 2 8 2} & \text { EPA351.2 } \\ \mathrm{mg} / \mathrm{kg} & \mathbf{5 5 2} & \text { EPA365.4 }\end{array}$

Metals (total recoverable)

$\begin{array}{ll}\mathrm{mg} / \mathrm{kg} & 16.2 \\ \mathrm{mg} / \mathrm{kg} & 6.46 \\ \mathrm{mg} / \mathrm{kg} & 19.4 \\ \mathrm{mg} / \mathrm{kg} & 1.62 \\ \mathrm{mg} / \mathrm{kg} & 0.646 \\ \mathrm{mg} / \mathrm{kg} & 0.323 \\ \mathrm{mg} / \mathrm{kg} & 16.2 \\ \mathrm{mg} / \mathrm{kg} & 1.62 \\ \mathrm{mg} / \mathrm{kg} & 0.646 \\ \mathrm{mg} / \mathrm{kg} & 1.62 \\ \mathrm{mg} / \mathrm{kg} & 6.46 \\ \mathrm{mg} / \mathrm{kg} & 6.46 \\ \mathrm{mg} / \mathrm{kg} & 6.46 \\ \mathrm{mg} / \mathrm{kg} & 0.646 \\ \mathrm{mg} / \mathrm{kg} & 0.0456 \\ \mathrm{mg} / \mathrm{kg} & 1.62\end{array}$

EPA6010A

EPA6010A

EPA6010A

EPA6010A

EPA6010A

EPA6010A

EPA6010A

EPA6010A

EPA6010A

EPA6010A

EPA6010A

EPA6010A

EPA6010A

EPA6010A

EPA7471

EPA6010A 
SURVEY ID: PPSC 2303 (continued)

Constituent

RQ AQ B Result

34.1

19.4

1.62

Selenium

Silver

$\begin{array}{lll}\mathrm{J} & \mathrm{E} & 34.1 \\ \mathrm{U} & & 19.4 \\ \mathrm{U} & & 1.62 \\ \mathrm{U} & & 64.6 \\ \mathrm{U} & & 19.4 \\ & & 2.92 \\ & & 3.08\end{array}$

$B / N / A$ Extractables (including $P A H$ and phenols)

Thallium

Vanadium

Zinc
Unit

D. Limit Method

\section{Acenaphthene}

Acenaphthylene

Anthracene

Benzo[a]anthracene

Benzo[b]fluoranthene

Benzo[k]fluoranthene

Benzoic acid

Benzo[g,h,i]perylene

Benzo[a]pyrene

Benzyl alcohol

Bis(2-chloroethoxy) methane

Bis(2-chloroethyl) ether

Bis(2-chloroisopropyl) ether

Bis(2-ethylhexyl) phthalate

4-Bromophenyl phenyl ether

Butylbenzyl phthalate

4-Chloroaniline

4-Chloro-m-cresol

2-Chloronaphthalene

2-Chlorophenol

4-Chlorophenyl phenyl ether

Chrysene

$\mathrm{m} / \mathrm{p}$-Cresol

o-Cresol (2-Methylphenol)

Dibenz $[a, h]$ anthracene

Dibenzofuran

Di-n-butyl phthalate

1,2-Dichlorobenzene

1,3-Dichlorobenzene

1,4-Dichlorobenzene

3,3'-Dichlorobenzidine

2,4-Dichlorophenol

Diethyl phthalate

2,4-Dimethyl phenol

Dimethyl phthalate

2,4-Dinitrophenol

2,4-Dinitrotoluene

2,6-Dinitrotoluene

Di-n-octyl phthalate

Docosane

Fluoranthene

Fluorene

Hexachlorobenzene

Hexachlorobutadiene

Hexachlorocyclopentadiene

\begin{tabular}{|c|c|c|}
\hline UJ & $Q$ & 45.8 \\
\hline UJ & $Q$ & 45.8 \\
\hline UJ & $Q$ & 45.8 \\
\hline UJ & $Q$ & 4.58 \\
\hline UJ & $\mathbf{Q}$ & 4.58 \\
\hline UJ & $\mathbf{Q}$ & 4.58 \\
\hline$J$ & $Q$ & 218 \\
\hline UJ & $\mathbf{Q}$ & 45.8 \\
\hline UJ & $\mathbf{Q}$ & 4.58 \\
\hline UJ & $\mathbf{Q}$ & 45.8 \\
\hline UJ & $\mathbf{Q}$ & 45.8 \\
\hline UJ & $\mathbf{Q}$ & 45.8 \\
\hline UJ & $\mathbf{Q}$ & 45.8 \\
\hline UJ & $\mathbf{Q}$ & 45.8 \\
\hline UJ & $Q$ & 45.8 \\
\hline $\mathrm{UJ}$ & $\mathbf{Q}$ & 45.8 \\
\hline UJ & $\mathbf{Q}$ & 45.8 \\
\hline UJ & $\mathbf{Q}$ & 45.8 \\
\hline UJ & $\mathbf{Q}$ & 45.8 \\
\hline UJ & $Q$ & 45.8 \\
\hline $\mathrm{UJ}$ & $\mathbf{Q}$ & 45.8 \\
\hline UJ & $Q$ & 4.58 \\
\hline UJ & $Q$ & 458 \\
\hline UJ & $Q$ & 45.8 \\
\hline UJ & $Q$ & 4.58 \\
\hline UJ & $Q$ & 45.8 \\
\hline UJ & $\mathbf{Q}$ & 45.8 \\
\hline UJ & $Q$ & 45.8 \\
\hline UJ & $\mathrm{Q}$ & 45.8 \\
\hline UJ & $Q$ & 45.8 \\
\hline UJ & $Q$ & 45.8 \\
\hline UJ & $Q$ & 45.8 \\
\hline UJ & $Q$ & 45.8 \\
\hline$J$ & $\mathrm{QE}$ & 25.2 \\
\hline UJ & $\mathrm{Q}$ & 45.8 \\
\hline UJ & $\mathrm{Q}$ & 458 \\
\hline UJ & $\mathrm{QC}$ & 4.58 \\
\hline UJ & $\mathbf{Q}$ & 4.58 \\
\hline UJ & $Q$ & 45.8 \\
\hline J & $\mathrm{N}$ & 780 \\
\hline UJ & $Q$ & 45.8 \\
\hline UJ & $Q$ & 45.8 \\
\hline UJ & $Q$ & 4.58 \\
\hline UJ & $\mathbf{Q}$ & 45.8 \\
\hline UJ & $Q$ & 45.8 \\
\hline
\end{tabular}

\begin{tabular}{|c|c|c|}
\hline$\mu \mathrm{g} / \mathrm{kg}$ & 45.8 & EPA8270 \\
\hline$\mu \mathrm{g} / \mathrm{kg}$ & 45.8 & EPA8270 \\
\hline$\mu \mathrm{g} / \mathrm{kg}$ & 45.8 & EPA8270 \\
\hline$\mu \mathrm{g} / \mathrm{kg}$ & 4.58 & EPA8270 \\
\hline$\mu \mathrm{g} / \mathrm{kg}$ & 4.58 & EPA8270 \\
\hline$\mu \mathrm{g} / \mathrm{kg}$ & 4.58 & EPA8270 \\
\hline$\mu \mathrm{g} / \mathrm{kg}$ & 45.8 & EPA8270 \\
\hline$\mu \mathrm{g} / \mathrm{kg}$ & 45.8 & EPA8270 \\
\hline$\mu \mathrm{g} / \mathrm{kg}$ & 4.58 & EPA8270 \\
\hline$\mu \mathrm{g} / \mathrm{kg}$ & 45.8 & EPA8270 \\
\hline$\mu \mathrm{g} / \mathrm{kg}$ & 45.8 & EPA8270 \\
\hline$\mu \mathrm{g} / \mathrm{kg}$ & 45.8 & EPA8270 \\
\hline$\mu \mathrm{g} / \mathrm{kg}$ & 45.8 & EPA8270 \\
\hline$\mu \mathrm{g} / \mathrm{kg}$ & 45.8 & EPA8270 \\
\hline$\mu g / \mathrm{kg}$ & 45.8 & EPA8270 \\
\hline$\mu \mathrm{g} / \mathrm{kg}$ & 45.8 & EPA8270 \\
\hline$\mu \mathrm{g} / \mathrm{kg}$ & 45.8 & EPA8270 \\
\hline$\mu \mathrm{g} / \mathrm{kg}$ & 45.8 & EPA8270 \\
\hline$\mu \mathrm{g} / \mathrm{kg}$ & 45.8 & EPA8270 \\
\hline$\mu \mathrm{g} / \mathrm{kg}$ & 45.8 & EPA8270 \\
\hline$\mu \mathrm{g} / \mathrm{kg}$ & 45.8 & EPA8270 \\
\hline$\mu \mathrm{g} / \mathrm{kg}$ & 4.58 & EPA8270 \\
\hline$\mu \mathrm{g} / \mathrm{kg}$ & 458 & EPA8270 \\
\hline$\mu \mathrm{g} / \mathrm{kg}$ & 45.8 & EPA8270 \\
\hline$\mu \mathrm{g} / \mathrm{kg}$ & 4.58 & EPA8270 \\
\hline$\mu \mathrm{g} / \mathrm{kg}$ & 45.8 & EPA8270 \\
\hline$\mu \mathrm{g} / \mathrm{kg}$ & 45.8 & EPA8270 \\
\hline$\mu \mathrm{g} / \mathrm{kg}$ & 45.8 & EPA8270 \\
\hline$\mu \mathrm{g} / \mathrm{kg}$ & 45.8 & EPA8270 \\
\hline$\mu \mathrm{g} / \mathrm{kg}$ & 45.8 & EPA8270 \\
\hline$\mu \mathrm{g} / \mathrm{kg}$ & 45.8 & EPA8270 \\
\hline$\mu \mathrm{g} / \mathrm{kg}$ & 45.8 & EPA8270 \\
\hline$\mu \mathrm{g} / \mathrm{kg}$ & 45.8 & EPA8270 \\
\hline$\mu \mathrm{g} / \mathrm{kg}$ & 45.8 & EPA8270 \\
\hline$\mu \mathrm{g} / \mathrm{kg}$ & 45.8 & EPA8270 \\
\hline$\mu \mathrm{g} / \mathrm{kg}$ & 458 & EPA8270 \\
\hline$\mu \mathrm{g} / \mathrm{kg}$ & 4.58 & EPA8270 \\
\hline$\mu \mathrm{g} / \mathrm{kg}$ & 4.58 & EPA8270 \\
\hline$\mu \mathrm{g} / \mathrm{kg}$ & 45.8 & EPA8270 \\
\hline$\mu \mathrm{g} / \mathrm{kg}$ & & EPA8270 \\
\hline$\mu \mathrm{g} / \mathrm{kg}$ & 45.8 & EPA8270 \\
\hline$\mu \mathrm{g} / \mathrm{kg}$ & 45.8 & EPA8270 \\
\hline$\mu \mathrm{g} / \mathrm{kg}$ & 4.58 & EPA8270 \\
\hline$\mu \mathrm{g} / \mathrm{kg}$ & 45.8 & EPA8270 \\
\hline$\mu \mathrm{g} / \mathrm{kg}$ & 45.8 & EPA8270 \\
\hline
\end{tabular}

EPA6010A EPA6010A EPA6010A EPA6010A EPA6010A EPA6010A EPA6010A 


\section{Constituent}

Unit

D. Limit Method

$B / N / A$ Extractables (including $P A H$ and phenols)

Hexachloroethane
Indeno[1,2,3-c,d]pyrene
Isophorone
2-Methyl-4,6-dinitrophenol
2-Methylnaphthalene
Naphthalene
m-Nitroaniline
o-Nitroaniline
p-Nitroaniline
Nitrobenzene
2-Nitrophenol
4-Nitrophenol
N-Nitrosodiphenylamine
N-Nitrosodi-n-propylamine
Pentachlorophenol
Phenanthrene
Phenol
Pyrene
1,2,4-Trichlorobenzene
2,4,5-Trichlorophenol
2,4,6-Trichlorophenol
Unknown alkane

Volatile Organic Compounds

Acetone
Benzene
Bromodichloromethane
Bromoform
Bromomethane (Methyl bromide)
Carbon disulfide
Carbon tetrachloride
Chlorobenzene
Chloroethane
Chloroethene (Vinyl chloride)
Chloroform
Chloromethane (Methyl chloride)
Dibromochloromethane
1,1-Dichloroethane
1,2-Dichloroethane
1,1-Dichloroethylene
1,2-Dichloroethylene
Dichloromethane (Methylene chloride)
1,2-Dichloropropane
cis-1,3-Dichloropropene
trans-1,3-Dichloropropene
Ethylbenzene
2-Hexanone
Methyl ethyl ketone
Methyl isobutyl ketone
Styrene
1,1,2,2-Tetrachloroethane
Tetrachloroethylene
Toluene
1,1,1-Trichloroethane

$\begin{array}{lll}\text { UJ } & \mathbf{Q} & 4.58 \\ \text { UJ } & \mathbf{Q} & 4.58 \\ \text { UJ } & \mathbf{Q} & 45.8 \\ \text { UJ } & \mathbf{Q} & 458 \\ \text { UJ } & \mathbf{Q} & 45.8 \\ \text { UJ } & \mathbf{Q} & 45.8 \\ \text { UJ } & \mathbf{Q} & 45.8 \\ \text { UJ } & \mathbf{Q} & 45.8 \\ \text { UJ } & \mathbf{Q} & 45.8 \\ \text { UJ } & \mathbf{Q} & 45.8 \\ \text { UJ } & \mathbf{Q} & 45.8 \\ \text { UJ } & \mathbf{Q} & 45.8 \\ \text { UJ } & \mathbf{Q} & 45.8 \\ \text { UJ } & \mathbf{Q} & 45.8 \\ \text { UJ } & \mathbf{Q} & 45.8 \\ \text { UJ } & \mathbf{Q} & 45.8 \\ \text { UJ } & \mathbf{Q} & 45.8 \\ \text { UJ } & \mathbf{Q} & 45.8 \\ \text { UJ } & \mathbf{Q} & 4.58 \\ \text { UJ } & \mathbf{Q} & 45.8 \\ \text { UJ } & \mathbf{Q} & 45.8 \\ \text { J } & \mathrm{N} & 420\end{array}$

$\begin{array}{llll}\text { UJ } & \text { O8 } & \text { H } & \mathbf{6 2 . 2} \\ \text { UJ } & \text { O } & \text { H } & 0.704 \\ \text { UJ } & \text { O } & \text { H } & \mathbf{0 . 0 7 0 4} \\ \text { UJ } & \text { O } & \text { H } & \mathbf{0 . 0 7 0 4} \\ \text { UJ } & \text { O } & \text { H } & \mathbf{0 . 1 4 1} \\ \text { J } & \text { OE } & \text { H } & \mathbf{0 . 7 1 8} \\ \text { UJ } & \text { O } & \text { H } & \mathbf{0 . 0 7 0 4} \\ \text { UJ } & \text { O } & \text { H } & \mathbf{0 . 0 7 0 4} \\ \text { UJ } & \text { O } & \text { H } & \mathbf{0 . 1 4 1} \\ \text { UJ } & \text { O } & \text { H } & \mathbf{0 . 1 4 1} \\ \text { UJ } & \text { O } & \text { H } & \mathbf{0 . 0 7 0 4} \\ \text { UJ } & \text { O } & \text { H } & \mathbf{0 . 1 4 1} \\ \text { UJ } & \text { O } & \text { H } & \mathbf{0 . 0 7 0 4} \\ \text { UJ } & \text { O } & \text { H } & \mathbf{0 . 0 7 0 4} \\ \text { UJ } & \text { O } & \text { H } & \mathbf{0 . 0 7 0 4} \\ \text { UJ } & \text { O } & \text { H } & \mathbf{0 . 0 7 0 4} \\ \text { UJ } & \text { O } & \text { H } & \mathbf{0 . 1 4 1} \\ \text { UJ } & \text { OV } & \text { H } & \mathbf{2 . 5 9} \\ \text { UJ } & \text { O } & \text { H } & \mathbf{0 . 0 7 0 4} \\ \text { UJ } & \text { O } & \text { H } & \mathbf{0 . 0 7 0 4} \\ \text { UJ } & \text { O } & \text { H } & \mathbf{0 . 0 7 0 4} \\ \text { UJ } & \text { O } & \text { H } & \mathbf{0 . 0 7 0 4} \\ \text { UJ } & \text { OV } & \text { H } & \mathbf{0 . 6 7 6} \\ \text { UJ } & \text { OV8 H } & \mathbf{2 . 6 5} \\ \text { UJ } & \text { O } & \text { H } & \mathbf{1 . 4 1} \\ \text { UJ } & \text { O } & \text { H } & \mathbf{0 . 0 7 0 4} \\ \text { UJ } & \text { O } & \text { H } & \mathbf{0 . 0 7 0 4} \\ \text { J } & \text { O } & \text { H } & \mathbf{0 . 2 1 1} \\ \text { UJ } & \text { O8 } & \text { H } & \mathbf{0 . 3 8} \\ \text { UJ } & \text { O } & \text { H } & \mathbf{0 . 0 7 0 4}\end{array}$

$\begin{array}{lll}\mu \mathrm{g} / \mathrm{kg} & 4.58 & \text { EPA8270 } \\ \mu \mathrm{g} / \mathrm{kg} & 4.58 & \text { EPA8270 } \\ \mu \mathrm{g} / \mathrm{kg} & 45.8 & \text { EPA8270 } \\ \mu \mathrm{g} / \mathrm{kg} & 458 & \text { EPA8270 } \\ \mu \mathrm{g} / \mathrm{kg} & 45.8 & \text { EPA8270 } \\ \mu \mathrm{g} / \mathrm{kg} & 45.8 & \text { EPA8270 } \\ \mu \mathrm{g} / \mathrm{kg} & 45.8 & \text { EPA8270 } \\ \mu \mathrm{g} / \mathrm{kg} & 45.8 & \text { EPA8270 } \\ \mu \mathrm{g} / \mathrm{kg} & 45.8 & \text { EPA8270 } \\ \mu \mathrm{g} / \mathrm{kg} & 45.8 & \text { EPA8270 } \\ \mu \mathrm{g} / \mathrm{kg} & 45.8 & \text { EPA8270 } \\ \mu \mathrm{g} / \mathrm{kg} & 45.8 & \text { EPA8270 } \\ \mu \mathrm{g} / \mathrm{kg} & 45.8 & \text { EPA8270 } \\ \mu \mathrm{g} / \mathrm{kg} & 45.8 & \text { EPA8270 } \\ \mu \mathrm{g} / \mathrm{kg} & 45.8 & \text { EPA8270 } \\ \mu \mathrm{g} / \mathrm{kg} & 45.8 & \text { EPA8270 } \\ \mu \mathrm{g} / \mathrm{kg} & 45.8 & \text { EPA8270 } \\ \mu \mathrm{g} / \mathrm{kg} & 45.8 & \text { EPA8270 } \\ \mu \mathrm{g} / \mathrm{kg} & 4.58 & \text { EPA8270 } \\ \mu \mathrm{g} / \mathrm{kg} & 45.8 & \text { EPA8270 } \\ \mu \mathrm{g} / \mathrm{kg} & 45.8 & \text { EPA8270 } \\ \mu \mathrm{g} / \mathrm{kg} & & \text { EPA8270 }\end{array}$

$\begin{array}{lll}\mu \mathrm{g} / \mathrm{kg} & 7.04 & \text { EPA8260 } \\ \mu \mathrm{g} / \mathrm{kg} & 0.704 & \text { EPA8260 } \\ \mu \mathrm{g} / \mathrm{kg} & 0.0704 & \text { EPA8260 } \\ \mu \mathrm{g} / \mathrm{kg} & 0.0704 & \text { EPA8260 } \\ \mu \mathrm{g} / \mathrm{kg} & 0.141 & \text { EPA8260 } \\ \mu \mathrm{g} / \mathrm{kg} & 1.41 & \text { EPA8260 } \\ \mu \mathrm{g} / \mathrm{kg} & 0.0704 & \text { EPA8260 } \\ \mu \mathrm{g} / \mathrm{kg} & 0.0704 & \text { EPA8260 } \\ \mu \mathrm{g} / \mathrm{kg} & 0.141 & \text { EPA8260 } \\ \mu \mathrm{g} / \mathrm{kg} & 0.141 & \text { EPA8260 } \\ \mu \mathrm{g} / \mathrm{kg} & 0.0704 & \text { EPA8260 } \\ \mu \mathrm{g} / \mathrm{kg} & 0.141 & \text { EPA8260 } \\ \mu \mathrm{g} / \mathrm{kg} & 0.0704 & \text { EPA8260 } \\ \mu \mathrm{g} / \mathrm{kg} & 0.0704 & \text { EPA8260 } \\ \mu \mathrm{g} / \mathrm{kg} & 0.0704 & \text { EPA8260 } \\ \mu \mathrm{g} / \mathrm{kg} & 0.0704 & \text { EPA8260 } \\ \mu \mathrm{g} / \mathrm{kg} & 0.141 & \text { EPA8260 } \\ \mu \mathrm{g} / \mathrm{kg} & 0.704 & \text { EPA8260 } \\ \mu \mathrm{g} / \mathrm{kg} & 0.0704 & \text { EPA8260 } \\ \mu \mathrm{g} / \mathrm{kg} & 0.0704 & \text { EPA8260 } \\ \mu \mathrm{g} / \mathrm{kg} & \mathbf{0 . 0 7 0 4} & \text { EPA8260 } \\ \mu \mathrm{g} / \mathrm{kg} & 0.0704 & \text { EPA8260 } \\ \mu \mathrm{g} / \mathrm{kg} & 1.41 & \text { EPA8260 } \\ \mu \mathrm{g} / \mathrm{kg} & 1.41 & \text { EPA8260 } \\ \mu \mathrm{g} / \mathrm{kg} & 1.41 & \text { EPA8260 } \\ \mu \mathrm{g} / \mathrm{kg} & 0.0704 & \text { EPA8260 } \\ \mu \mathrm{g} / \mathrm{kg} & 0.0704 & \text { EPA8260 } \\ \mu \mathrm{g} / \mathrm{kg} & 0.0704 & \text { EPA8260 } \\ \mu \mathrm{g} / \mathrm{kg} & 0.704 & \text { EPA8260 } \\ \mu \mathrm{g} / \mathrm{kg} & 0.0704 & \text { EPA8260 }\end{array}$


SURVEY ID: PPSC 2303 (continued)

Sample ID: 104689

Constituent

RQ AQ B Result

Unit

D. Limit Method

Volatile Organic Compounds

1,1,2-Trichloroethane

Trichloroethylene

Unknown alcohol

Vinyl acetate

Xylenes

$\begin{array}{llll}\text { UJ } & \mathrm{O} & \mathrm{H} & \mathbf{0 . 0 7 0 4} \\ \text { UJ } & \mathrm{O} 8 & \mathrm{H} & \mathbf{0 . 4 9 3} \\ \text { J } & \mathrm{N} & & \mathbf{2} \\ \text { UJ } & 0 & \mathrm{H} & 1.41 \\ \text { UJ } & \mathrm{O8} & \mathrm{H} & \mathbf{0 . 1 5 5}\end{array}$

$\begin{array}{lll}\mu \mathrm{g} / \mathrm{kg} & 0.0704 & \text { EPA8260 } \\ \mu \mathrm{g} / \mathrm{kg} & 0.0704 & \text { EPA8260 } \\ \mu \mathrm{g} / \mathrm{kg} & & \text { EPA8260 } \\ \mu \mathrm{g} / \mathrm{kg} & 1.41 & \text { EPA8260 } \\ \mu \mathrm{g} / \mathrm{kg} & 0.211 & \text { EPA8260 }\end{array}$

Pesticides/PCBs and Dioxins/Furans

Aldrin

alpha-Benzene hexachloride

beta-Benzene hexachloride

delta-Benzene hexachloride

alpha-Chlordane

gamma-Chlordane

p,p'-DDD

$p, p^{\prime}-\mathrm{DDE}$

p,p'-DDT

Dieldrin

Endosulfan sulfate

Endosulfan I

Endosulfan II

Endrin

Endrin ketone

Heptachlor

Heptachlor epoxide

Lindane

Methoxychlor

PCB 1016

PCB 1221

PCB 1232

PCB 1242

PCB 1248

PCB 1254

PCB 1260

Toxaphene

$\begin{array}{ll}\text { U } & 5.74 \\ U & 5.74 \\ U & 11.5 \\ U & 11.5 \\ U & 11.5 \\ U & 11.5 \\ U & 23 \\ U & 11.5 \\ U & 23 \\ U & 11.5 \\ U & 23 \\ U & 11.5 \\ U & 23 \\ U & 11.5 \\ U & 23 \\ U & 11.5 \\ U & 11.5 \\ U & 5.74 \\ U & 115 \\ U & 28.7 \\ U & 28.7 \\ U & 28.7 \\ U & 28.7 \\ U & 28.7 \\ U & 28.7 \\ U & 28.7 \\ U & 230\end{array}$

$$
\begin{aligned}
& \mu \mathrm{g} / \mathrm{kg} \\
& \mu \mathrm{g} / \mathrm{kg} \\
& \mu \mathrm{g} / \mathrm{kg} \\
& \mu \mathrm{g} / \mathrm{kg} \\
& \mu \mathrm{g} / \mathrm{kg} \\
& \mu \mathrm{g} / \mathrm{kg} \\
& \mu \mathrm{g} / \mathrm{kg} \\
& \mu \mathrm{g} / \mathrm{kg} \\
& \mu \mathrm{g} / \mathrm{kg} \\
& \mu \mathrm{g} / \mathrm{kg} \\
& \mu \mathrm{g} / \mathrm{kg} \\
& \mu \mathrm{g} / \mathrm{kg} \\
& \mu \mathrm{g} / \mathrm{kg} \\
& \mu \mathrm{g} / \mathrm{kg} \\
& \mu \mathrm{g} / \mathrm{kg} \\
& \mu \mathrm{g} / \mathrm{kg} \\
& \mu \mathrm{g} / \mathrm{kg} \\
& \mu \mathrm{g} / \mathrm{kg} \\
& \mu \mathrm{g} / \mathrm{kg} \\
& \mu \mathrm{g} / \mathrm{kg} \\
& \mu \mathrm{g} / \mathrm{kg} \\
& \mu \mathrm{g} / \mathrm{kg} \\
& \mu \mathrm{g} / \mathrm{kg} \\
& \mu \mathrm{g} / \mathrm{kg} \\
& \mu \mathrm{g} / \mathrm{kg} \\
& \mu \mathrm{g} / \mathrm{kg} \\
& \mu \mathrm{g} / \mathrm{kg}
\end{aligned}
$$

5.74

EPA8080

5.74

EPA8080

11.5 EPA8080

11.5 EPA8080

11.5 EPA8080

11.5 EPA8080

23 EPA8080

11.5 EPA8080

23 EPA8080

11.5 EPA8080

23 EPA8080

$11.5 \quad$ EPA8080

23 EPA8080

11.5 EPA8080

23 EPA8080

11.5 EPA8080

11.5 EPA8080

5.74 EPA8080

115 EPA8080

28.7 EPA8080

28.7 EPA8080

28.7 EPA8080

$28.7 \quad$ EPA8080

28.7 EPA8080

28.7 EPA8080

$28.7 \quad$ EPA8080

230 EPA8080

\section{Radionuclides}

Actinium-228

Antimony-124

Antimony-125

Barium-133

Cerium-144

Cesium-134

Cesium-137

Cobalt-57

Cobalt-58

Cobalt-60

Europium-152

Europium-154

Europium-155

Gross alpha

Lead-212

Manganese-54

Neptunium-239

$\begin{array}{ll} & 1.53 \mathrm{E}+00 \pm 5.57 \mathrm{E}-02 \mathrm{pCi} / \mathrm{g} \\ \text { UI } & 3.05 \mathrm{E}-03 \pm 6.18 \mathrm{E}-03 \mathrm{pCi} / \mathrm{g} \\ \text { UI } & -3.35 \mathrm{E}-03 \pm 1.40 \mathrm{E}-02 \mathrm{pCi} / \mathrm{g} \\ \text { UI } & -4.07 \mathrm{E}-02 \pm 7.18 \mathrm{E}-03 \mathrm{pCi} / \mathrm{g} \\ \text { UI } & -1.65 \mathrm{E}-02 \pm 3.80 \mathrm{E}-02 \mathrm{pCi} / \mathrm{g} \\ \text { UI } & -1.65 \mathrm{E}-03 \pm 5.10 \mathrm{E}-03 \mathrm{pCi} / \mathrm{g} \\ \text { UI } & -1.98 \mathrm{E}-03 \pm 6.10 \mathrm{E}-03 \mathrm{pCi} / \mathrm{g} \\ \text { UI } & 3.82 \mathrm{E}-05 \pm 4.78 \mathrm{E}-03 \mathrm{pCi} / \mathrm{g} \\ \text { UI } & -3.67 \mathrm{E}-03 \pm 5.73 \mathrm{E}-03 \mathrm{pCi} / \mathrm{g} \\ \text { UI } & -2.47 \mathrm{E}-03 \pm 4.36 \mathrm{E}-03 \mathrm{pCi} / \mathrm{g} \\ \text { UI } & 3.31 \mathrm{E}-03 \pm 1.44 \mathrm{E}-02 \mathrm{pCi} / \mathrm{g} \\ \text { UI } & 7.83 \mathrm{E}-03 \pm 4.27 \mathrm{E}-02 \mathrm{pCi} / \mathrm{g} \\ \text { UI } & 0.00 \mathrm{E}+00 \quad \mathrm{pCi} / \mathrm{g} \\ & 2.43 \mathrm{E}+01 \pm 3.66 \mathrm{E}+00 \mathrm{pCi} / \mathrm{g} \\ & 1.62 \mathrm{E}+00 \pm 2.69 \mathrm{E}-02 \mathrm{pCi} / \mathrm{g} \\ & 2.43 \mathrm{E}-02 \pm 8.72 \mathrm{E}-03 \mathrm{pCi} / \mathrm{g} \\ \text { UI } & 2.68 \mathrm{E}-02 \pm 3.81 \mathrm{E}-02 \mathrm{pCi} / \mathrm{g}\end{array}$

2.57E-02 EPIA-013B

1.08E-02 EPIA-013B

2.26E-02 EPIA-013B

1.03E-02 EPIA-013B

6.46E-02 EPIA-013B

7.63E-03 EPIA-013B

9.09E-03 EPLA-013B

8.20E-03 EPIA-013B

9.53E-03 EPIA-013B

7.46E-03 EPIA-013B

2.38E-02 EPIA-013B

6.44E-02 EPIA-013B

3.45E-02 EPIA-013B

$2.06 \mathrm{E}+00$ EPIA-001B

1.42E-02 EPIA-013B

7.91E-03 EPLA-013B

5.96E-02 EPIA-013B 
SURVEY ID: PPSC 2303 (continued)

Sample ID: 104689

\begin{tabular}{|c|c|c|c|c|c|c|}
\hline Constituent & $\mathbf{R Q}$ & $\mathbf{A Q} \mathbf{B}$ & Result & Unit & D. Limit & Method \\
\hline \multicolumn{7}{|l|}{ Radionuclides } \\
\hline Nonvolatile beta & & & $1.08 \mathrm{E}+01 \pm 1.94 \mathrm{E}+00$ & $0 \mathrm{pCi} / \mathrm{g}$ & $2.47 \mathrm{E}+00$ & EPIA-001B \\
\hline Plutonium-238 & UI & & $1.14 \mathrm{E}-02 \pm 1.73 \mathrm{E}-02$ & $\mathrm{pCi} / \mathrm{g}$ & $3.31 \mathrm{E}-02$ & EPIA.012B \\
\hline Plutonium-239/240 & UI & & $-1.35 \mathrm{E}-03 \pm 9.30 \mathrm{E}-03$ & $\mathrm{pCi} / \mathrm{g}$ & $2.66 \mathrm{E}-02$ & EPIA-012B \\
\hline Potassium-40 & & & $6.79 \mathrm{E}-01 \pm 9.86 \mathrm{E}-02$ & $\mathrm{pCi} / \mathrm{g}$ & $7.15 \mathrm{E}-02$ & EPIA.013B \\
\hline Promethium-144 & UI & & $4.97 \mathrm{E}-03 \pm 5.39 \mathrm{E}-03$ & $\mathrm{pCi} / \mathrm{g}$ & $8.62 \mathrm{E}-03$ & EPIA-013B \\
\hline Promethium-146 & UI & & $5.98 \mathrm{E}-03 \pm 7.58 \mathrm{E}-03$ & $\mathrm{pCi} / \mathrm{g}$ & $1.16 \mathrm{E} \cdot 02$ & EPIA-013B \\
\hline Radium-226 & & $\mathrm{V}$ & $9.49 \mathrm{E} \cdot 01 \pm 3.16 \mathrm{E}-02$ & $\mathrm{pCi} / \mathrm{g}$ & $1.58 \mathrm{E}-02$ & EPIA-013B \\
\hline Radium-228 & & & $1.53 \mathrm{E}+00 \pm 5.57 \mathrm{E}-02$ & $\mathrm{pCi} / \mathrm{g}$ & 2.57E-02 & EPIA-013B \\
\hline Ruthenium-106 & UI & & $1.17 \mathrm{E}-02 \pm 5.29 \mathrm{E}-02$ & $\mathrm{pCi} / \mathrm{g}$ & 8.11E-02 & EPIA-013B \\
\hline Sodium-22 & UI & & $2.82 \mathrm{E}-03 \pm 4.19 \mathrm{E}-03$ & $\mathrm{pCi} / \mathrm{g}$ & $7.69 \mathrm{E}-03$ & EPIA-013B \\
\hline Strontium-90 & UI & & $4.82 \mathrm{E}-02 \pm 5.96 \mathrm{E}-02$ & $\mathrm{pCi} / \mathrm{g}$ & $1.15 \mathrm{E}-01$ & EPIA-004 \\
\hline Thorium-234 & $\mathrm{U}$ & $\mathrm{V}$ & $1.66 \mathrm{E}+00 \pm 6.90 \mathrm{E}-01$ & $\mathrm{pCi} / \mathrm{g}$ & $4.38 \mathrm{E}-01$ & EPIA-013B \\
\hline Tin-113 & UI & & $3.08 \mathrm{E}-03 \pm 7.43 \mathrm{E}-03$ & $\mathrm{pCi} / \mathrm{g}$ & $1.23 \mathrm{E}-02$ & EPIA-013B \\
\hline Yttrium-88 & UI & & $0.00 \mathrm{E}+00$ & $\mathrm{pCi} / \mathrm{g}$ & $1.00 \mathrm{E}-02$ & EPIA-013B \\
\hline Zinc. 65 & UI & & $-5.65 \mathrm{E}-03 \pm 1.08 \mathrm{E}-02$ & $\mathrm{pCi} / \mathrm{g}$ & $1.52 \mathrm{E}-02$ & EPIA-013B \\
\hline Zirconium-95 & UT & & $0.00 \mathrm{E}+00$ & $\mathrm{pCi} / \mathrm{g}$ & 2.23E-02 & EPIA-013B \\
\hline
\end{tabular}

SURVEY ID: PPSC 2304

Sample ID: 104690

Survey location: $44336 \mathrm{E} 3688244 \mathrm{~N}$ (SRS coordinates)

Depth of core interval: 4.00 to $6.00 \mathrm{ft}$

Sample type: Normal

Physical Parameters and Specified Analyses

Ammonia nitrogen
Cation exchange capacity
Chloride
Cyanide
Nitrate-nitrite as nitroge
Nitrogen by Kjeldahl me
Orthophosphate
Orthophosphate
Phenols
Sulfide
Total organic carbon
Total organic nitrogen
Total phosphates (as P)

Metals (total recoverable)
Aluminum
Antimony
Arsenic
Barium
Beryllium
Cadmium
Calcium
Chromium
Cobalt

$\begin{array}{lll}\text { U } & & 21.2 \\ & & 9.08 \\ \text { UJ } & \text { Q } & 2.95 \\ \text { UJ } & \text { C } & 1.24 \\ \text { J } & \text { E } & 0.782 \\ & & 38.2 \\ \text { U } & & 25 \\ \text { U } & & 25 \\ \text { U } & & 365 \\ \text { UJ } & \text { C } & 313 \\ & \text { V } & 2550 \\ \text { C } & & 34.2 \\ \text { J } & \text { E } & 232\end{array}$

$\begin{array}{lll}\mathrm{mg} / \mathrm{kg} & 21.2 & \text { EPA350.1 } \\ \mathrm{meq} / 100 \mathrm{~g} & 5 & \text { EPA9081 } \\ \mathrm{mg} / \mathrm{kg} & 2.95 & \text { EPA300.0 } \\ \mathrm{mg} / \mathrm{kg} & 1.24 & \text { EPA335.3 } \\ \mathrm{mg} / \mathrm{kg} & 1.12 & \text { EPA353.1 } \\ \mathrm{mg} / \mathrm{kg} & 4.96 & \text { EPA351.2 } \\ \mathrm{mg} / \mathrm{kg} & 25 & \text { EPA365.2 } \\ \mathrm{mg} / \mathrm{kg} & 25 & \text { EPA365.2 } \\ \mu \mathrm{g} / \mathrm{kg} & 365 & \text { EPA420.2 } \\ \mathrm{mg} / \mathrm{kg} & 313 & \text { EPA376.2 } \\ \mathrm{mg} / \mathrm{kg} & 125 & \text { EPA415.1 } \\ \mathrm{mg} / \mathrm{kg} & 0.25 & \text { EPA351.2 } \\ \mathrm{mg} / \mathrm{kg} & 1110 & \text { EPA365.4 }\end{array}$

\begin{tabular}{|c|c|c|c|c|c|}
\hline & & 966 & $\mathrm{mg} / \mathrm{kg}$ & 15.2 & EPA6010A \\
\hline $\mathrm{U}$ & & 6.07 & $\mathrm{mg} / \mathrm{kg}$ & 6.07 & EPA6010A \\
\hline \multirow[t]{2}{*}{$\mathbf{U}$} & & 18.2 & $\mathrm{mg} / \mathrm{kg}$ & 18.2 & EPA6010A \\
\hline & & 4.19 & $\mathrm{mg} / \mathrm{kg}$ & 1.52 & EPA6010A \\
\hline J & $\mathrm{E}$ & 0.1 & $\mathrm{mg} / \mathrm{kg}$ & 0.607 & EPA6010A \\
\hline \multirow[t]{4}{*}{ U } & & 0.303 & $\mathrm{mg} / \mathrm{kg}$ & 0.303 & EPA6010A \\
\hline & & 128 & $\mathrm{mg} / \mathrm{kg}$ & 15.2 & EPA6010A \\
\hline & & 2.2 & $\mathrm{mg} / \mathrm{kg}$ & 1.52 & EPA6010A \\
\hline & & 0.607 & $\mathrm{mg} / \mathrm{kg}$ & 0.607 & EPA6010A \\
\hline
\end{tabular}


SURVEY ID: PPSC 2304 (continued)

Constituent

Metals (total recoverable)

$\begin{array}{llll}\text { Copper } & \mathrm{U} & & 1.52 \\ \text { Iron } & & \mathrm{V} & \mathbf{8 4 8} \\ \text { Lead } & \mathrm{U} & & 6.07 \\ \text { Magnesium } & & & 19.6 \\ \text { Manganese } & & & 5.33 \\ \text { Mercury } & \mathrm{U} & & 0.0351 \\ \text { Nickel } & \mathrm{U} & & 1.52 \\ \text { Potassium } & \mathrm{J} & \mathrm{E} & \mathbf{2 4 . 3} \\ \text { Selenium } & \mathrm{U} & & 18.2 \\ \text { Silver } & \mathrm{U} & & 1.52 \\ \text { Sodium } & \mathrm{U} & & 60.7 \\ \text { Thallium } & \mathrm{U} & & 18.2 \\ \text { Vanadium } & & & 1.61 \\ \text { Zinc } & & & 1.81\end{array}$

$B / N / A$ Extractables (including PAH and phenols)

Acenaphthene

Acenaphthylene

Anthracene

Benzo[a]anthracene

Benzo[b]fluoranthene

Benzo[k]fluoranthene

Benzoic acid

Benzo[g, $h, i]$ perylene

Benzo[a]pyrene

Benzyl alcohol

Bis(2-chloroethoxy) methane

Bis(2-chloroethyl) ether

Bis(2-chloroisopropyl) ether

Bis(2-ethylhexyl) phthalate

4-Bromophenyl phenyl ether

Butylbenzyl phthalate

4-Chloroaniline

4-Chloro-m-cresol

2-Chloronaphthalene

2-Chlorophenol

4-Chlorophenyl phenyl ether

Chrysene

$\mathrm{m} / \mathrm{p}$-Cresol

o-Cresol (2-Methylphenol)

Dibenz $[a, h]$ anthracene

Dibenzofuran

Di-n-butyl phthalate

1,2-Dichlorobenzene

1,3-Dichlorobenzene

1,4-Dichlorobenzene

$3,3^{\prime}$-Dichlorobenzidine

2,4-Dichlorophenol

Diethyl phthalate

2,4-Dimethyl phenol

Dimethyl phthalate

2,4-Dinitrophenol

2,4-Dinitrotoluene

2,6-Dinitrotoluene
40.8

40.8

40.8

4.08

4.08

4.08

74.3

40.8

4.08

40.8

40.8

40.8

40.8

40.8

40.8

40.8

$\begin{array}{llll}\text { UJ } & \text { Q } & & 40.8 \\ \text { UJ } & \text { QI } & \text { H } & 40.8\end{array}$

UJ Q 40.8

UJ $Q \quad 40.8$

UJ $\quad Q \quad 40.8$

UJ $Q \quad 4.08$

UJ $Q \quad 408$

UJ $Q \quad 40.8$

UJ $Q \quad 4.08$

UJ $Q \quad 40.8$

J $\quad \mathrm{QE} \quad 22.9$

UJ $Q \quad 40.8$

UJ $\quad \mathrm{Q} \quad 40.8$

UJ Q $\quad 40.8$

UJ $Q \quad 40.8$

UJ $\quad \mathrm{Q} \quad 40.8$

UJ $Q \quad 40.8$

UJ $Q$ Q 40.8

UJ Q $\quad 40.8$

UJ $Q \quad 408$

UJ QC 4.08

UJ $\mathrm{Q} \quad 4.08$

Sample ID: 104690
RQ AQ B Result
Unit
D. Limit Method

$\begin{array}{lll}\mathrm{mg} / \mathrm{kg} & 1.52 & \text { EPA6010A } \\ \mathrm{mg} / \mathrm{kg} & 6.07 & \text { EPA6010A } \\ \mathrm{mg} / \mathrm{kg} & 6.07 & \text { EPA6010A } \\ \mathrm{mg} / \mathrm{kg} & 6.07 & \text { EPA6010A } \\ \mathrm{mg} / \mathrm{kg} & 0.607 & \text { EPA6010A } \\ \mathrm{mg} / \mathrm{kg} & 0.0351 & \text { EPA7471 } \\ \mathrm{mg} / \mathrm{kg} & 1.52 & \text { EPA6010A } \\ \mathrm{mg} / \mathrm{kg} & 121 & \text { EPA6010A } \\ \mathrm{mg} / \mathrm{kg} & 18.2 & \text { EPA6010A } \\ \mathrm{mg} / \mathrm{kg} & 1.52 & \text { EPA6010A } \\ \mathrm{mg} / \mathrm{kg} & 60.7 & \text { EPA6010A } \\ \mathrm{mg} / \mathrm{kg} & 18.2 & \text { EPA6010A } \\ \mathrm{mg} / \mathrm{kg} & 0.607 & \text { EPA6010A } \\ \mathrm{mg} / \mathrm{kg} & 1.21 & \text { EPA6010A }\end{array}$

$\begin{array}{lll}\mu \mathrm{g} / \mathrm{kg} & 40.8 & \text { EPA8270 } \\ \mu \mathrm{g} / \mathrm{kg} & 40.8 & \text { EPA8270 } \\ \mu \mathrm{g} / \mathrm{kg} & 40.8 & \text { EPA8270 } \\ \mu \mathrm{g} / \mathrm{kg} & 4.08 & \text { EPA8270 } \\ \mu \mathrm{g} / \mathrm{kg} & 4.08 & \text { EPA8270 } \\ \mu \mathrm{g} / \mathrm{kg} & 4.08 & \text { EPA8270 } \\ \mu \mathrm{g} / \mathrm{kg} & 40.8 & \text { EPA8270 } \\ \mu \mathrm{g} / \mathrm{kg} & 40.8 & \text { EPA8270 } \\ \mu \mathrm{g} / \mathrm{kg} & 4.08 & \text { EPA8270 } \\ \mu \mathrm{g} / \mathrm{kg} & 40.8 & \text { EPA8270 } \\ \mu \mathrm{g} / \mathrm{kg} & 40.8 & \text { EPA8270 } \\ \mu \mathrm{g} / \mathrm{kg} & 40.8 & \text { EPA8270 } \\ \mu \mathrm{g} / \mathrm{kg} & 40.8 & \text { EPA8270 } \\ \mu \mathrm{g} / \mathrm{kg} & 40.8 & \text { EPA8270 } \\ \mu \mathrm{g} / \mathrm{kg} & 40.8 & \text { EPA8270 } \\ \mu \mathrm{g} / \mathrm{kg} & 40.8 & \text { EPA8270 } \\ \mu \mathrm{g} / \mathrm{kg} & 40.8 & \text { EPA8270 } \\ \mu \mathrm{g} / \mathrm{kg} & 40.8 & \text { EPA8270 } \\ \mu \mathrm{g} / \mathrm{kg} & 40.8 & \text { EPA8270 } \\ \mu \mathrm{g} / \mathrm{kg} & 40.8 & \text { EPA8270 } \\ \mu \mathrm{g} / \mathrm{kg} & 40.8 & \text { EPA8270 } \\ \mu \mathrm{g} / \mathrm{kg} & 4.08 & \text { EPA8270 } \\ \mu \mathrm{g} / \mathrm{kg} & 408 & \text { EPA8270 } \\ \mu \mathrm{g} / \mathrm{kg} & 40.8 & \text { EPA8270 } \\ \mu \mathrm{g} / \mathrm{kg} & 4.08 & \text { EPA8270 } \\ \mu \mathrm{g} / \mathrm{kg} & 40.8 & \text { EPA8270 } \\ \mu \mathrm{g} / \mathrm{kg} & 40.8 & \text { EPA8270 } \\ \mu \mathrm{g} / \mathrm{kg} & 40.8 & \text { EPA8270 } \\ \mu \mathrm{g} / \mathrm{kg} & 40.8 & \text { EPA8270 } \\ \mu \mathrm{g} / \mathrm{kg} & 40.8 & \text { EPA8270 } \\ \mu \mathrm{g} / \mathrm{kg} & 40.8 & \text { EPA8270 } \\ \mu \mathrm{g} / \mathrm{kg} & 40.8 & \text { EPA8270 } \\ \mu \mathrm{g} / \mathrm{kg} & 40.8 & \text { EPA8270 } \\ \mu \mathrm{g} / \mathrm{kg} & 40.8 & \text { EPA8270 } \\ \mu \mathrm{g} / \mathrm{kg} & 40.8 & \text { EPA8270 } \\ \mu \mathrm{g} / \mathrm{kg} & 408 & \text { EPA8270 } \\ \mu \mathrm{g} / \mathrm{kg} & 4.08 & \text { EPA8270 } \\ \mu \mathrm{kg} & 4.08 & \text { EPA8270 } \\ & & \end{array}$


SURVEY ID: PPSC 2304 (continued)

Sample ID: 104690

Constituent

RQ AQ B Result

Unit

D. Limit Method

$B / N / A$ Extractables (including PAH and phenols)

\begin{tabular}{|c|c|c|c|c|c|c|}
\hline Di-n-octyl phthalate & UJ & $\mathbf{Q}$ & 40.8 & $\mu \mathrm{g} / \mathrm{kg}$ & 40.8 & EPA8270 \\
\hline Fluoranthene & UJ & Q & 40.8 & $\mu \mathrm{g} / \mathrm{kg}$ & 40.8 & EPA8270 \\
\hline Fluorene & UJ & Q & 40.8 & $\mu \mathrm{g} / \mathrm{kg}$ & 40.8 & EPA8270 \\
\hline Hexachlorobenzene & UJ & $-\mathbf{Q}$ & 4.08 & $\mu g / k g$ & 4.08 & EPA8270 \\
\hline Hexachlorobutadiene & UJ & Q & 40.8 & $\mu \mathrm{g} / \mathrm{kg}$ & 40.8 & EPA8270 \\
\hline Hexachlorocyclopentadiene & UJ & $Q$ & 40.8 & $\mu g / k g$ & 40.8 & EPA8270 \\
\hline Hexachloroethane & UJ & $Q$ & 4.08 & $\mu \mathrm{g} / \mathrm{kg}$ & 4.08 & EPA8270 \\
\hline Indeno $[1,2,3-\dot{c}, d]$ pyrene & UJ & $\mathbf{Q}$ & 4.08 & $\mu \mathrm{g} / \mathrm{kg}$ & 4.08 & EPA8270 \\
\hline Isophorone & UJ & Q & 40.8 & $\mu \mathrm{g} / \mathrm{kg}$ & 40.8 & EPA8270 \\
\hline 2-Methyl-4,6-dinitrophenol & UJ & $\mathbf{Q}$ & 408 & $\mu \mathrm{g} / \mathrm{kg}$ & 408 & EPA8270 \\
\hline 2-Methylnaphthalene & UJ & $\mathbf{Q}$ & 40.8 & $\mu \mathrm{g} / \mathrm{kg}$ & 40.8 & EPA8270 \\
\hline Naphthalene & UJ & $\mathbf{Q}$ & 40.8 & $\mu \mathrm{g} / \mathrm{kg}$ & 40.8 & EPA8270 \\
\hline m-Nitroaniline & UJ & $\mathbf{Q}$ & 40.8 & $\mu \mathrm{g} / \mathrm{kg}$ & 40.8 & EPA8270 \\
\hline o-Nitroaniline & UJ & $\mathbf{Q}$ & 40.8 & $\mu \mathrm{g} / \mathrm{kg}$ & 40.8 & EPA8270 \\
\hline p-Nitroaniline & UJ & $\mathbf{Q}$ & 40.8 & $\mu g / k g$ & 40.8 & EPA8270 \\
\hline Nitrobenzene & UJ & $\mathbf{Q}$ & 40.8 & $\mu \mathrm{g} / \mathrm{kg}$ & 40.8 & EPA8270 \\
\hline 2-Nitrophenol & UJ & $\mathbf{Q}$ & 40.8 & $\mu g / k g$ & 40.8 & EPA8270 \\
\hline 4-Nitrophenol & UJ & QI & 40.8 & $\mu \mathrm{g} / \mathrm{kg}$ & 40.8 & EPA8270 \\
\hline N-Nitrosodiphenylamine & UJ & $\mathbf{Q}$ & 40.8 & $\mu g / k g$ & 40.8 & EPA8270 \\
\hline N-Nitrosodi-n-propylamine & UJ & $\mathbf{Q}$ & 40.8 & $\mu \mathrm{g} / \mathrm{kg}$ & 40.8 & EPA8270 \\
\hline Pentachlorophenol & UJ & QI & 40.8 & $\mu \mathrm{g} / \mathrm{kg}$ & 40.8 & EPA8270 \\
\hline Phenanthrene & UJ & $\mathbf{Q}$ & 40.8 & $\mu \mathrm{g} / \mathrm{kg}$ & 40.8 & EPA8270 \\
\hline Phenol · & UJ & $\mathbf{Q}$ & 40.8 & $\mu \mathrm{g} / \mathrm{kg}$ & 40.8 & EPA8270 \\
\hline Pyrene & UJ & $\mathbf{Q}$ & 40.8 & $\mu g / k g$ & 40.8 & EPA8270 \\
\hline 1,2,4-Trichlorobenzene & UJ & $Q$ & 4.08 & $\mu \mathrm{g} / \mathrm{kg}$ & 4.08 & EPA8270 \\
\hline 2,4,5-Trichlorophenol & UJ & Q & 40.8 & $\mu \mathrm{g} / \mathrm{kg}$ & 40.8 & EPA8270 \\
\hline 2,4,6-Trichlorophenol & UJ & $\mathbf{Q}$ & 40.8 & $\mu \mathrm{g} / \mathrm{kg}$ & 40.8 & EPA8270 \\
\hline
\end{tabular}

Volatile Organic Compounds

\begin{tabular}{|c|c|c|c|c|c|c|}
\hline Acetone & $\mathrm{U}$ & 8 & 11 & $\mu \mathrm{g} / \mathrm{kg}$ & 6.25 & EPA8260 \\
\hline Benzene & $U$ & & 0.625 & $\mu \mathrm{g} / \mathrm{kg}$ & 0.625 & EPA8260 \\
\hline Bromodichloromethane & $\mathrm{U}$ & & 0.0625 & $\mu \mathrm{g} / \mathrm{kg}$ & 0.0625 & EPA8260 \\
\hline Bromoform & $\mathrm{U}$ & & 0.0625 & $\mu \mathrm{g} / \mathrm{kg}$ & 0.0625 & EPA8260 \\
\hline Bromomethane (Methyl bromide) & $\mathrm{U}$ & & 0.125 & $\mu \mathrm{g} / \mathrm{kg}$ & 0.125 & EPA8260 \\
\hline Carbon disulfide & $\mathbf{J}$ & $\mathbf{E}$ & 0.25 & $\mu \mathrm{g} / \mathrm{kg}$ & 1.25 & EPA8260 \\
\hline Carbon tetrachloride & $\mathrm{U}$ & & 0.0625 & $\mu \mathrm{g} / \mathrm{kg}$ & 0.0625 & EPA8260 \\
\hline Chlorobenzene & $\mathrm{U}$ & & 0.0625 & $\mu \mathrm{g} / \mathrm{kg}$ & 0.0625 & EPA8260 \\
\hline Chloroethane & $\mathrm{U}$ & & 0.125 & $\mu \mathrm{g} / \mathrm{kg}$ & 0.125 & EPA8260 \\
\hline Chloroethene (Vinyl chloride) & $\mathrm{U}$ & & 0.125 & $\mu \mathrm{g} / \mathrm{kg}$ & 0.125 & EPA8260 \\
\hline Chloroform & $\mathrm{U}$ & & 0.0625 & $\mu \mathrm{g} / \mathrm{kg}$ & 0.0625 & EPA8260 \\
\hline Chloromethane (Methyl chloride) & $\mathbf{U}$ & & 0.125 & $\mu \mathrm{g} / \mathrm{kg}$ & 0.125 & EPA8260 \\
\hline Dibromochloromethane & $\mathrm{U}$ & & 0.0625 & $\mu \mathrm{g} / \mathrm{kg}$ & 0.0625 & EPA8260 \\
\hline 1,1-Dichloroethane & $\mathrm{U}$ & & 0.0625 & $\mu \mathrm{g} / \mathrm{kg}$ & 0.0625 & EPA8260 \\
\hline 1,2-Dichloroethane & $\mathrm{U}$ & & 0.0625 & $\mu \mathrm{g} / \mathrm{kg}$ & 0.0625 & EPA8260 \\
\hline 1,1-Dichloroethylene & $\mathrm{U}$ & & 0.0625 & $\mu \mathrm{g} / \mathrm{kg}$ & 0.0625 & EPA8260 \\
\hline 1,2-Dichloroethylene & $\mathrm{U}$ & & 0.125 & $\mu \mathrm{g} / \mathrm{kg}$ & 0.125 & EPA8260 \\
\hline Dichloromethane (Methylene chloride) & $\mathrm{U}$ & V & 1.25 & $\mu \mathrm{g} / \mathrm{kg}$ & 0.625 & EPA8260 \\
\hline 1,2-Dichloropropane & $\mathrm{U}$ & & 0.0625 & $\mu \mathrm{g} / \mathrm{kg}$ & 0.0625 & EPA8260 \\
\hline cis-1,3-Dichloropropene & U & & 0.0625 & $\mu \mathrm{g} / \mathrm{kg}$ & 0.0625 & EPA8260 \\
\hline trans-1,3-Dichloropropene & $\mathrm{U}$ & & 0.0625 & $\mu \mathrm{g} / \mathrm{kg}$ & 0.0625 & EPA8260 \\
\hline Ethylbenzene & $\mathrm{U}$ & & 0.0625 & $\mu \mathrm{g} / \mathrm{kg}$ & 0.0625 & EPA8260 \\
\hline 2-Hexanone & $\mathrm{U}$ & & 1.25 & $\mu \mathrm{g} / \mathrm{kg}$ & 1.25 & EPA8260 \\
\hline Methyl ethyl ketone & $\mathrm{U}$ & V8 & 1.11 & $\mu \mathrm{g} / \mathrm{kg}$ & 1.25 & EPA8260 \\
\hline Methyl isobutyl ketone & $\mathbf{U}$ & & 1.25 & $\mu \mathrm{g} / \mathrm{kg}$ & 1.25 & EPA8260 \\
\hline
\end{tabular}


SURVEY ID: PPSC 2304 (continued)

Constituent

RQ AQ B Result

1,1,2,2-Tetrachloroethane

Tetrachloroethylene

Toluene

1,1,1-Trichloroethane

1,1,2-Trichloroethane

Trichloroethylene

Vinyl acetate

Xylenes

Pesticides/PCBs and Dioxins/Furans

Aldrin
alpha-Benzene hexachloride
beta-Benzene hexachloride
delta-Benzene hexachloride
alpha-Chlordane
gamma-Chlordane
p,p'-DDD
p,p'-DDE
p,p'-DDT
Dieldrin
Endosulfan sulfate
Endosulfan I
Endosulfan II
Endrin
Endrin ketone
Heptachlor
Heptachlor epoxide
Lindane
Methoxychlor
PCB 1016
PCB 1221
PCB 1232
PCB 1242
PCB 1248
PCB 1254
PCB 1260
Toxaph

Toxaphene

Radionuclides

Actinium-228

Antimony-124

Antimony-125

Barium-133

Cerium-144

Cesium-134

Cesium-137

Cobalt-57

Cobalt-58

Cobalt-60

Europium-152

Europium-154

Europium-155
UI

UI

UI

UI

UI

UI

UI

UI

UI

UI

UI

UI

0.0625
0.0625
0.0625
0.625
0.0625
0.0625
0.0625
1.25
0.188

Sample ID: 104690

Unit - D. Limit Method

$\begin{array}{lll}\mu \mathrm{g} / \mathrm{kg} & 0.0625 & \text { EPA8260 } \\ \mu \mathrm{g} / \mathrm{kg} & 0.0625 & \text { EPA8260 } \\ \mu \mathrm{g} / \mathrm{kg} & 0.0625 & \text { EPA8260 } \\ \mu \mathrm{g} / \mathrm{kg} & 0.625 & \text { EPA8260 } \\ \mu \mathrm{g} / \mathrm{kg} & 0.0625 & \text { EPA8260 } \\ \mu \mathrm{g} / \mathrm{kg} & 0.0625 & \text { EPA8260 } \\ \mu \mathrm{g} / \mathrm{kg} & 0.0625 & \text { EPA8260 } \\ \mu \mathrm{g} / \mathrm{kg} & 1.25 & \text { EPA8260 } \\ \mu \mathrm{g} / \mathrm{kg} & 0.188 & \text { EPA8260 }\end{array}$

$\begin{array}{lll}\mu \mathrm{g} / \mathrm{kg} & 5.04 & \text { EPA8080 } \\ \mu \mathrm{g} / \mathrm{kg} & 5.04 & \text { EPA8080 } \\ \mu \mathrm{g} / \mathrm{kg} & 10.1 & \text { EPA8080 } \\ \mu \mathrm{g} / \mathrm{kg} & 10.1 & \text { EPA8080 } \\ \mu \mathrm{g} / \mathrm{kg} & 10.1 & \text { EPA8080 } \\ \mu \mathrm{g} / \mathrm{kg} & 10.1 & \text { EPA8080 } \\ \mu \mathrm{g} / \mathrm{kg} & 20.2 & \text { EPA8080 } \\ \mu \mathrm{g} / \mathrm{kg} & 10.1 & \text { EPA8080 } \\ \mu \mathrm{g} / \mathrm{kg} & 20.2 & \text { EPA8080 } \\ \mu \mathrm{g} / \mathrm{kg} & 10.1 & \text { EPA8080 } \\ \mu \mathrm{g} / \mathrm{kg} & 20.2 & \text { EPA8080 } \\ \mu \mathrm{g} / \mathrm{kg} & 10.1 & \text { EPA8080 } \\ \mu \mathrm{g} / \mathrm{kg} & 20.2 & \text { EPA8080 } \\ \mu \mathrm{g} / \mathrm{kg} & 10.1 & \text { EPA8080 } \\ \mu \mathrm{g} / \mathrm{kg} & 20.2 & \text { EPA8080 } \\ \mu \mathrm{g} / \mathrm{kg} & 10.1 & \text { EPA8080 } \\ \mu \mathrm{g} / \mathrm{kg} & 10.1 & \text { EPA8080 } \\ \mu \mathrm{g} / \mathrm{kg} & 5.04 & \text { EPA8080 } \\ \mu \mathrm{g} / \mathrm{kg} & 101 & \text { EPA8080 } \\ \mu \mathrm{g} / \mathrm{kg} & 25.2 & \text { EPA8080 } \\ \mu \mathrm{g} / \mathrm{kg} & 25.2 & \text { EPA8080 } \\ \mu \mathrm{g} / \mathrm{kg} & 25.2 & \text { EPA8080 } \\ \mu \mathrm{g} / \mathrm{kg} & 25.2 & \text { EPA8080 } \\ \mu \mathrm{g} / \mathrm{kg} & 25.2 & \text { EPA8080 } \\ \mu \mathrm{g} / \mathrm{kg} & 25.2 & \text { EPA8080 } \\ \mu \mathrm{g} / \mathrm{kg} & 25.2 & \text { EPA8080 } \\ \mu \mathrm{g} / \mathrm{kg} & 202 & \text { EPA8080 }\end{array}$
5.04
5.04
10.1
10.1
10.1
20.2
10.1
20.2
10.1
20.2
10.1
20.2
10.1
20.2
10.1
10.1
5.04
101
25.2
25.2
25.2
25.2
25.2
25.2
25.2
202

$9.22 \mathrm{E}+00 \pm 1.28 \mathrm{E}-01 \mathrm{pCi} / \mathrm{g}$ $-2.24 \mathrm{E}-03 \pm 1.35 \mathrm{E}-02 \mathrm{pCi} / \mathrm{g}$ $1.52 \mathrm{E}-02+2.81 \mathrm{E}-02 \mathrm{pCi} / \mathrm{g}$ 4.92E-03 $\pm 1.42 \mathrm{E}-02 \mathrm{pCi} / \mathrm{g}$ 2.15E-04 $\pm 9.68 \mathrm{E}-02 \mathrm{pCi} / \mathrm{g}$ 1.40E-02 $\pm 1.10 \mathrm{E}-02 \mathrm{pCi} / \mathrm{g}$ 6.77E-03 $1.27 \mathrm{E}-02 \mathrm{pCi} / \mathrm{g}$ 9.22E-03 $\pm 1.20 \mathrm{E}-02 \mathrm{pCi} / \mathrm{g}$ $-1.44 \mathrm{E}-02 \pm 1.19 \mathrm{E}-02 \mathrm{pCi} / \mathrm{g}$ $-3.67 \mathrm{E}-03 \pm 9.69 \mathrm{E}-03 \mathrm{pCi} / \mathrm{g}$ $-9.35 \mathrm{E}-02 \pm 2.93 \mathrm{E}-02 \mathrm{pCi} / \mathrm{g}$ $-1.98 \mathrm{E}-02 \pm 8.24 \mathrm{E}-02 \mathrm{pCi} / \mathrm{g}$ $0.00 \mathrm{E}+00$

$\mathrm{pCi} / \mathrm{g}$ $\begin{array}{ll}5.16 \mathrm{E}-02 & \text { EPIA-013B } \\ 2.23 \mathrm{E}-02 & \text { EPIA-013B } \\ 4.76 \mathrm{E}-02 & \text { EPIA-013B } \\ 2.13 \mathrm{E}-02 & \text { EPIA-013B } \\ 1.43 \mathrm{E}-01 & \text { EPIA-013B } \\ 1.63 \mathrm{E}-02 & \text { EPIA-013B } \\ 1.85 \mathrm{E}-02 & \text { EPLA-013B } \\ 1.78 \mathrm{E}-02 & \text { EPIA-013B } \\ 1.90 \mathrm{E}-02 & \text { EPIA-013B } \\ 1.38 \mathrm{E}-02 & \text { EPIA-013B } \\ 4.86 \mathrm{E}-02 & \text { EPIA-013B } \\ 1.21 \mathrm{E}-01 & \text { EPLA-013B } \\ 7.36 \mathrm{E}-02 & \text { EPIA-013B }\end{array}$ 
SURVEY ID: PPSC 2304 (continued)

Constituent

Radionuclides

Gross alpha

Lead-212

Manganese-54

Neptunium-239

Nonvolatile beta

Plutonium-238

Plutonium-239/240

Potassium-40

Promethium-144

Promethium-146

Radium-226

Radium-228

Ruthenium-106

Sodium-22

Strontium-90

Thorium-234

Tin-113

Yttrium-88

Zinc-65

Zirconium-95
Sample ID: 104690

RQ AQ B Result Unit D. Limit Method

SURVEY ID: PPSC 2305

$\begin{array}{ll}8.95 \mathrm{E}+01 \pm 6.37 \mathrm{E}+00 \mathrm{pCi} / \mathrm{g} \\ 1.00 \mathrm{E}+01 \pm 6.28 \mathrm{E}-02 \mathrm{pCi} / \mathrm{g} \\ 1.49 \mathrm{E}-01 \pm 1.66 \mathrm{E}-02 \mathrm{pCi} / \mathrm{g} \\ 0.00 \mathrm{E}+00 & \mathrm{pCi} / \mathrm{g} \\ 4.61 \mathrm{E}+01 \pm 3.52 \mathrm{E}+00 \mathrm{pCi} / \mathrm{g} \\ -1.09 \mathrm{E}-02 \pm 1.01 \mathrm{E}-02 \mathrm{pCi} / \mathrm{g} \\ -2.68 \mathrm{E}-05 \pm 6.60 \mathrm{E}-03 \mathrm{pCi} / \mathrm{g} \\ 0.00 \mathrm{E}+00 & \mathrm{pCi} / \mathrm{g} \\ -3.41 \mathrm{E}-03 \pm 1.08 \mathrm{E}-02 \mathrm{pCi} / \mathrm{g} \\ 0.00 \mathrm{E}+00 & \mathrm{pCi} / \mathrm{g} \\ 3.86 \mathrm{E}+00 \pm 6.44 \mathrm{E}-02 \mathrm{pCi} / \mathrm{g} \\ 9.22 \mathrm{E}+00 \pm 1.28 \mathrm{E}-01 \mathrm{pCi} / \mathrm{g} \\ -5.14 \mathrm{E}-02 \pm 1.12 \mathrm{E}-01 \mathrm{pCi} / \mathrm{g} \\ 1.21 \mathrm{E}-03 \pm 9.42 \mathrm{E}-03 \mathrm{pCi} / \mathrm{g} \\ 7.44 \mathrm{E}-02 \pm 8.20 \mathrm{E}-02 \mathrm{pCi} / \mathrm{g} \\ 3.82 \mathrm{E}+00 \pm 1.15 \mathrm{E}+00 \mathrm{pCi} / \mathrm{g} \\ -2.31 \mathrm{E}-02 \pm 1.52 \mathrm{E}-02 \mathrm{pCi} / \mathrm{g} \\ 1.66 \mathrm{E}-02 \pm 1.03 \mathrm{E}-02 \mathrm{pCi} / \mathrm{g} \\ 0.00 \mathrm{E}+00 & \mathrm{pCi} / \mathrm{g} \\ 0.00 \mathrm{E}+00 & \mathrm{pCi} / \mathrm{g}\end{array}$

$8.95 \mathrm{E}+01 \pm 6.37 \mathrm{E}+00 \mathrm{pCi} / \mathrm{g}$

1.46E+00 EPIA-001B

3.10E-02 EPIA-013B

1.53E-02 EPIA-013B

1.29E-01 EPIA-013B

2.36E+00 EPIA-001B

3.61E-02 EPIA-012B

2.06E-02 EPIA-012B

1.69E-01 EPIA-013B

1.76E-02 EPIA-013B

2.35E-02 EPIA-013B

3.27E-02 EPIA-013B

5.16E-02 EPIA-013B

1.60E-01 EPIA-013B

1.37E-02 EPIA-013B

1.53E-01 EPIA-004

9.35E-01 EPIA-013B

2.54E-02 EPIA-013B

1.84E-02 EPIA-013B

3.20E-02 EPIA-013B

4.49E-02 EPIA-013B

Survey location: $44336 \mathrm{E} 3688244 \mathrm{~N}$ (SRS coordinates)

Depth of core interval: 6.00 to $7.10 \mathrm{ft}$

Sample type: Normal

Sample matrix: Soil

Percent solids: $\mathbf{8 0 . 0 0}$

Constituent

RQ AQ B Result

Unit

D. Limit Method

Physical Parameters and Specified Analyses

Ammonia nitrogen
Cation exchange capacity
Chloride
Cyanide
Nitrate-nitrite as nitrogen
Nitrate-nitrite as nitrogen
Nitrogen by Kjeldahl method
Orthophosphate
Orthophosphate
Phenols
Sulfide
Total organic carbon
Total organic nitrogen
Total phosphates (as P)
Total phosphates (as P)

$\begin{array}{lll}\text { U } & & 24.2 \\ & & 67 \\ \text { UJ } & \text { Q } & 3.02 \\ \text { UJ } & \text { C } & 1.25 \\ \text { U } & & 1.04 \\ \text { U } & & 1.11 \\ & & 40.1 \\ \text { U } & & 25 \\ \text { U } & & 25 \\ \text { U } & & 371 \\ \text { UJ } & \text { C } & 313 \\ & \text { V } & 3540 \\ \text { C } & & 36.2 \\ \text { J } & \text { E } & 222 \\ \text { J } & \text { E } & 159\end{array}$

$\begin{array}{ll}\mathrm{mg} / \mathrm{kg} & 24.2 \\ \mathrm{meq} / 100 \mathrm{~g} & 5 \\ \mathrm{mg} / \mathrm{kg} & 3.02 \\ \mathrm{mg} / \mathrm{kg} & 1.25 \\ \mathrm{mg} / \mathrm{kg} & 1.04 \\ \mathrm{mg} / \mathrm{kg} & 1.11 \\ \mathrm{mg} / \mathrm{kg} & 4.92 \\ \mathrm{mg} / \mathrm{kg} & 25 \\ \mathrm{mg} / \mathrm{kg} & 25 \\ \mu \mathrm{g} / \mathrm{kg} & 371 \\ \mathrm{mg} / \mathrm{kg} & 313 \\ \mathrm{mg} / \mathrm{kg} & 125 \\ \mathrm{mg} / \mathrm{kg} & 0.25 \\ \mathrm{mg} / \mathrm{kg} & 1220 \\ \mathrm{mg} / \mathrm{kg} & 1030\end{array}$

EPA350.1 EPA9081 EPA300.0 EPA335.3 EPA353.1 EPA353.1 EPA351.2 EPA365.2 EPA365.2 EPA420.2 EPA376.2 EPA415.1 EPA351.2 EPA365.4 EPA365.4 
SURVEY ID: PPSC 2305 (continued)

Constituent

RQ AQ B Result

Metals (total recoverable)
Unit

D. Limit Method

\begin{tabular}{|c|c|c|c|}
\hline Aluminum & & & 4630 \\
\hline Antimony & $\mathrm{U}$ & & 5.95 \\
\hline Arsenic & $\mathbf{J}$ & $\mathrm{E}$ & 2.6 \\
\hline Barium & & & 33 \\
\hline Beryllium & & & 0.867 \\
\hline Cadmium & $\mathrm{U}$ & & 0.298 \\
\hline Calcium & & & 1730 \\
\hline Chromium & & & 6.72 \\
\hline Cobalt & & & 2.34 \\
\hline Copper & & & 2.08 \\
\hline Iron & & V & 6330 \\
\hline Lead & $\mathbf{J}$ & $\mathrm{E}$ & 3.76 \\
\hline Magnesium & & & 501 \\
\hline Manganese & & & 30.3 \\
\hline Mercury & $\mathrm{U}$ & & 0.036 \\
\hline Nickel & & & 4.2 \\
\hline Potassium & & & 941 \\
\hline Selenium & $\mathrm{U}$ & & 17.9 \\
\hline Silver & $\mathrm{U}$ & & 1.49 \\
\hline Sodium & $\mathbf{J}$ & $\mathbf{E}$ & 25 \\
\hline Thallium & $\mathrm{U}$ & & 17.9 \\
\hline Vanadium & & & 4.37 \\
\hline Zinc & & & 8.79 \\
\hline
\end{tabular}

$B / N / A$ Extractables (including $P A H$ and phenols)

Acenaphthene

Acenaphthene

Acenaphthylene

Acenaphthylene

Anthracene

Anthracene

Benzo[a]anthracene

Benzo[a]anthracene

Benzo[b]fluoranthene

Benzo[b]fluoranthene

Benzo[k]fluoranthene

Benzo[k]fluoranthene

Benzoic acid

Benzoic acid

Benzo[g,h,i]perylene

Benzo $[g, h, i]$ perylene

Benzo[a]pyrene

Benzo[a]pyrene

Benzyl alcohol

Benzyl alcohol

Bis(2-chloroethoxy) methane

$\mathrm{Bis}$ (2-chloroethoxy) methane

$\mathrm{Bis}$ (2-chloroethyl) ether

Bis(2-chloroethyl) ether

Bis(2-chloroisopropyl) ether

Bis(2-chloroisopropyl) ether

Bis(2-ethylhexyl) phthalate

Bis(2-ethylhexyl) phthalate

4-Bromophenyl phenyl ether

$\begin{array}{lll}\text { U } & & 33.2 \\ \text { UJ } & \text { Q } & 41.1 \\ \text { U } & & 33.2 \\ \text { UJ } & \text { Q } & 41.1 \\ \text { U } & & 33.2 \\ \text { UJ } & \text { Q } & 41.1 \\ \text { U } & & 3.32 \\ \text { UJ } & \text { Q } & 4.11 \\ \text { U } & & 3.32 \\ \text { UJ } & \text { Q } & 4.11 \\ \text { U } & & 3.32 \\ \text { UJ } & \text { Q } & 4.11 \\ \text { U } & & 33.2 \\ \text { J } & \text { QE } & 28.7 \\ \text { U } & & 33.2 \\ \text { UJ } & \text { Q } & 41.1 \\ \text { U } & & 3.32 \\ \text { UJ } & \text { Q } & 4.11 \\ \text { U } & & 33.2 \\ \text { UJ } & \text { Q } & 41.1 \\ \text { U } & & 33.2 \\ \text { UJ } & \text { Q } & 41.1 \\ \text { U } & & 33.2 \\ \text { UJ } & \text { Q } & 41.1 \\ \text { U } & & 33.2 \\ \text { UJ } & \text { Q } & 41.1 \\ \text { U } & & 33.2 \\ \text { UJ } & \text { Q } & 41.1 \\ \text { U } & & 33.2\end{array}$

$\begin{array}{lll}\mathrm{mg} / \mathrm{kg} & 14.9 & \text { EPA6010A } \\ \mathrm{mg} / \mathrm{kg} & 5.95 & \text { EPA6010A } \\ \mathrm{mg} / \mathrm{kg} & 17.9 & \text { EPA6010A } \\ \mathrm{mg} / \mathrm{kg} & 1.49 & \text { EPA6010A } \\ \mathrm{mg} / \mathrm{kg} & 0.595 & \text { EPA6010A } \\ \mathrm{mg} / \mathrm{kg} & 0.298 & \text { EPA6010A } \\ \mathrm{mg} / \mathrm{kg} & 14.9 & \text { EPA6010A } \\ \mathrm{mg} / \mathrm{kg} & 1.49 & \text { EPA6010A } \\ \mathrm{mg} / \mathrm{kg} & 0.595 & \text { EPA6010A } \\ \mathrm{mg} / \mathrm{kg} & 1.49 & \text { EPA6010A } \\ \mathrm{mg} / \mathrm{kg} & 5.95 & \text { EPA6010A } \\ \mathrm{mg} / \mathrm{kg} & 5.95 & \text { EPA6010A } \\ \mathrm{mg} / \mathrm{kg} & 5.95 & \text { EPA6010A } \\ \mathrm{mg} / \mathrm{kg} & 0.595 & \text { EPA6010A } \\ \mathrm{mg} / \mathrm{kg} & 0.0363 & \text { EPA7471 } \\ \mathrm{mg} / \mathrm{kg} & 1.49 & \text { EPA6010A } \\ \mathrm{mg} / \mathrm{kg} & 119 & \text { EPA6010A } \\ \mathrm{mg} / \mathrm{kg} & 17.9 & \text { EPA6010A } \\ \mathrm{mg} / \mathrm{kg} & 1.49 & \text { EPA6010A } \\ \mathrm{mg} / \mathrm{kg} & 59.5 & \text { EPA6010A } \\ \mathrm{mg} / \mathrm{kg} & 17.9 & \text { EPA6010A } \\ \mathrm{mg} / \mathrm{kg} & 0.595 & \text { EPA6010A } \\ \mathrm{mg} / \mathrm{kg} & 1.19 & \text { EPA6010A }\end{array}$

$\mu \mathrm{g} / \mathrm{kg}$

33.2

EPA8270

$\mu \mathrm{g} / \mathrm{kg}$

$\mu \mathrm{g} / \mathrm{kg}$

$\mu \mathrm{g} / \mathrm{kg}$

$\mu \mathrm{g} / \mathrm{kg}$

$\mu \mathrm{g} / \mathrm{kg}$

$\mu \mathrm{g} / \mathrm{kg}$

$\mu \mathrm{g} / \mathrm{kg}$

$\mu \mathrm{g} / \mathrm{kg}$.

$\mu \mathrm{g} / \mathrm{kg}$

$\mu \mathrm{g} / \mathrm{kg}$

$\mu \mathrm{g} / \mathrm{kg}$

$\mu \mathrm{g} / \mathrm{kg}$

$\mu \mathrm{g} / \mathrm{kg}$

$\mu \mathrm{g} / \mathrm{kg}$

$\mu \mathrm{g} / \mathrm{kg}$

$\mu \mathrm{g} / \mathrm{kg}$

$\mu \mathrm{g} / \mathrm{kg}$

$\mu \mathrm{g} / \mathrm{kg}$

$\mu \mathrm{g} / \mathrm{kg}$

$\mu \mathrm{g} / \mathrm{kg}$

$\mu \mathrm{g} / \mathrm{kg}$

$\mu \mathrm{g} / \mathrm{kg}$

$\mu \mathrm{g} / \mathrm{kg}$

$\mu \mathrm{g} / \mathrm{kg}$

$\mu \mathrm{g} / \mathrm{kg}$

$\mu \mathrm{g} / \mathrm{kg}$

$\mu \mathrm{g} / \mathrm{kg}$

$\mu \mathrm{g} / \mathrm{kg}$

\section{1}

33.2

41.1

33.2

41.1

3.32

4.11

3.32

4.11

3.32

4.11

33.2

41.1

33.2

41.1

3.32

4.11

33.2

41.1

33.2

41.1

33.2

41.1

33.2

41.1

33.2

41.1

33.2
EPA8270

EPA8270

EPA8270

EPA8270

EPA8270

EPA8270

EPA8270

EPA8270

EPA8270

EPA8270

EPA8270

EPA8270

EPA8270

EPA8270

EPA8270

EPA8270

EPA8270

EPA8270

EPA8270

EPA8270

EPA8270

EPA8270

EPA8270

EPA8270

EPA8270

EPA8270

EPA8270

EPA8270 
SURVEY ID: PPSC 2305 (continued)

Sample ID: 104691

Constituent

RQ AQ B Result

Unit

D. Limit Method

$B / N / A$ Extractables (including PAH and phenols)

\begin{tabular}{|c|c|c|c|c|c|c|}
\hline 4-Bromophenyl phenyl ether & UJ & $Q$ & 41.1 & $\mu \mathrm{g} / \mathrm{kg}$ & 41.1 & EPA8270 \\
\hline Butylbenzyl phthalate & $\mathrm{U}$ & & 33.2 & $\mu \mathrm{g} / \mathrm{kg}$ & 33.2 & EPA8270 \\
\hline Butylbenzyl phthalate & UJ & Q & 41.1 & $\mu \mathrm{g} / \mathrm{kg}$ & 41.1 & EPA8270 \\
\hline 4-Chloroaniline & $\mathrm{U}$ & & 33.2 & $\mu \mathrm{g} / \mathrm{kg}$ & 33.2 & EPA8270 \\
\hline 4-Chloroaniline & UJ & $\mathbf{Q}$ & 41.1 & $\mu \mathrm{g} / \mathrm{kg}$ & 41.1 & EPA8270 \\
\hline 4-Chloro-m-cresol & $\mathrm{U}$ & & 33.2 & $\mu \mathrm{g} / \mathrm{kg}$ & 33.2 & EPA8270 \\
\hline 4-Chloro-m-cresol & UJ & Q & 41.1 & $\mu \mathrm{g} / \mathrm{kg}$ & 41.1 & EPA8270 \\
\hline 2-Chloronaphthalene & $\mathrm{U}$ & & 33.2 & $\mu \mathrm{g} / \mathrm{kg}$ & 33.2 & EPA8270 \\
\hline 2-Chloronaphthalene & UJ & $\mathbf{Q}$ & 41.1 & $\mu \mathrm{g} / \mathrm{kg}$ & 41.1 & EPA8270 \\
\hline 2-Chlorophenol & U & & 33.2 & $\mu \mathrm{g} / \mathrm{kg}$ & 33.2 & EPA8270 \\
\hline 2-Chlorophenol & UJ & $\mathbf{Q}$ & 41.1 & $\mu \mathrm{g} / \mathrm{kg}$ & 41.1 & EPA8270 \\
\hline 4-Chlorophenyl phenyl ether & $\mathrm{U}$ & & 33.2 & $\mu g / \mathrm{kg}$ & 33.2 & EPA8270 \\
\hline 4-Chlorophenyl phenyl ether & UJ & $\mathbf{Q}$ & 41.1 & $\mu \mathrm{g} / \mathrm{kg}$ & 41.1 & EPA8270 \\
\hline Chrysene & $\mathrm{U}$ & & 3.32 & $\mu \mathrm{g} / \mathrm{kg}$ & 3.32 & EPA8270 \\
\hline Chrysene & UJ & $\mathbf{Q}$ & 4.11 & $\mu \mathrm{g} / \mathrm{kg}$ & 4.11 & EPA8270 \\
\hline $\mathrm{m} / \mathrm{p}$-Cresol & $\mathrm{U}$ & & 332 & $\mu \mathrm{g} / \mathrm{kg}$ & 332 & EPA8270 \\
\hline $\mathrm{m} / \mathrm{p}$-Cresol & UJ & $\mathbf{Q}$ & 411 & $\mu \mathrm{g} / \mathrm{kg}$ & 411 & EPA8270 \\
\hline o-Cresol (2-Methylphenol) & $\mathrm{U}$ & & 33.2 & $\mu g / k g$ & 33.2 & EPA8270 \\
\hline o-Cresol (2-Methylphenol) & UJ & $\mathbf{Q}$ & 41.1 & $\mu \mathrm{g} / \mathrm{kg}$ & 41.1 & EPA8270 \\
\hline $\operatorname{Dibenz}[\alpha, h]$ anthracene & $\mathrm{U}$ & & 3.32 & $\mu \mathrm{g} / \mathrm{kg}$ & 3.32 & EPA8270 \\
\hline Dibenz $[\alpha, h]$ anthracene & UJ & $\mathbf{Q}$ & 4.11 & $\mu \mathrm{g} / \mathrm{kg}$ & 4.11 & EPA8270 \\
\hline Dibenzofuran & $\mathrm{U}$ & & 33.2 & $\mu \mathrm{g} / \mathrm{kg}$ & 33.2 & EPA8270 \\
\hline Dibenzofuran & UJ & $\mathbf{Q}$ & 41.1 & $\mu \mathrm{g} / \mathrm{kg}$ & 41.1 & EPA8270 \\
\hline Di-n-butyl phthalate & $U$ & $\mathrm{~V}$ & 41.8 & $\mu \mathrm{g} / \mathrm{kg}$ & 33.2 & EPA8270 \\
\hline Di-n-butyl phthalate & $\mathrm{J}$ & $\mathrm{QE}$ & 23.8 & $\mu \mathrm{g} / \mathrm{kg}$ & 41.1 & EPA8270 \\
\hline 1,2-Dichlorobenzene & $\mathrm{U}$ & & 33.2 & $\mu \mathrm{g} / \mathrm{kg}$ & 33.2 & EPA8270 \\
\hline 1,2-Dichlorobenzene & UJ & Q & 41.1 & $\mu \mathrm{g} / \mathrm{kg}$ & 41.1 & EPA8270 \\
\hline 1,3-Dichlorobenzene & $\mathrm{U}$ & & 33.2 & $\mu \mathrm{g} / \mathrm{kg}$ & 33.2 & EPA8270 \\
\hline 1,3-Dichlorobenzene & UJ & Q & 41.1 & $\mu \mathrm{g} / \mathrm{kg}$ & 41.1 & EPA8270 \\
\hline 1,4-Dichlorobenzene & $\mathrm{U}$ & & 33.2 & $\mu \mathrm{g} / \mathrm{kg}$ & 33.2 & EPA8270 \\
\hline 1,4-Dichlorobenzene & UJ & $\mathbf{Q}$ & 41.1 & $\mu \mathrm{g} / \mathrm{kg}$ & 41.1 & EPA8270 \\
\hline 3,3'-Dichlorobenzidine & $\mathrm{U}$ & & 33.2 & $\mu \mathrm{g} / \mathrm{kg}$ & 33.2 & EPA8270 \\
\hline 3,3'-Dichlorobenzidine & UJ & Q & 41.1 & $\mu \mathrm{g} / \mathrm{kg}$ & 41.1 & EPA8270 \\
\hline 2,4-Dichlorophenol & $U$ & & 33.2 & $\mu \mathrm{g} / \mathrm{kg}$ & 33.2 & EPA8270 \\
\hline 2,4-Dichlorophenol & UJ & $\mathbf{Q}$ & 41.1 & $\mu \mathrm{g} / \mathrm{kg}$ & 41.1 & EPA8270 \\
\hline Diethyl phthalate & $\mathrm{U}$ & & 33.2 & $\mu \mathrm{g} / \mathrm{kg}$ & 33.2 & EPA8270 \\
\hline Diethyl phthalate & UJ & $\mathbf{Q}$ & 41.1 & $\mu \mathrm{g} / \mathrm{kg}$ & 41.1 & EPA8270 \\
\hline 2,4-Dimethyl phenol & $U$ & & 33.2 & $\mu \mathrm{g} / \mathrm{kg}$ & 33.2 & EPA8270 \\
\hline 2,4-Dimethyl phenol & UJ & $\mathbf{Q}$ & 41.1 & $\mu \mathrm{g} / \mathrm{kg}$ & 41.1 & EPA8270 \\
\hline Dimethyl phthalate & $\mathrm{U}$ & & 33.2 & $\mu \mathrm{g} / \mathrm{kg}$ & 33.2 & EPA8270 \\
\hline Dimethyl phthalate & UJ & $\mathbf{Q}$ & 41.1 & $\mu \mathrm{g} / \mathrm{kg}$ & 41.1 & EPA8270 \\
\hline 2,4-Dinitrophenol & $\mathrm{U}$ & & 332 & $\mu \mathrm{g} / \mathrm{kg}$ & 332 & EPA8270 \\
\hline 2,4-Dinitrophenol & UJ & $\mathbf{Q}$ & 411 & $\mu \mathrm{g} / \mathrm{kg}$ & 411 & EPA8270 \\
\hline 2,4-Dinitrotoluene & UJ & C & 3.32 & $\mu g / k g$ & 3.32 & EPA8270 \\
\hline 2,4-Dinitrotoluene & UJ & QCI H & 4.11 & $\mu \mathrm{g} / \mathrm{kg}$ & 4.11 & EPA8270 \\
\hline 2,6-Dinitrotoluene & $\mathrm{U}$ & & 3.32 & $\mu \mathrm{g} / \mathrm{kg}$ & 3.32 & EPA8270 \\
\hline 2,6-Dinitrotoluene & UJ & $\mathbf{Q}$ & 4.11 & $\mu \mathrm{g} / \mathrm{kg}$ & 4.11 & EPA8270 \\
\hline Di-n-octyl phthalate & $U$ & & 33.2 & $\mu \mathrm{g} / \mathrm{kg}$ & 33.2 & EPA8270 \\
\hline Di-n-octyl phthalate & UJ & $\mathbf{Q}$ & 41.1 & $\mu \mathrm{g} / \mathrm{kg}$ & 41.1 & EPA8270 \\
\hline Fluoranthene & $U$ & & 33.2 & $\mu \mathrm{g} / \mathrm{kg}$ & 33.2 & EPA8270 \\
\hline Fluoranthene & UJ & $\mathbf{Q}$ & 41.1 & $\mu \mathrm{g} / \mathrm{kg}$ & 41.1 & EPA8270 \\
\hline Fluorene & $U$ & & 33.2 & $\mu \mathrm{g} / \mathrm{kg}$ & $\cdot 33.2$ & EPA8270 \\
\hline Fluorene & UJ & $\mathbf{Q}$ & 41.1 & $\mu \mathrm{g} / \mathrm{kg}$ & 41.1 & EPA8270 \\
\hline Hexachlorobenzene & $\mathrm{U}$ & & 3.32 & $\mu \mathrm{g} / \mathrm{kg}$ & 3.32 & EPA8270 \\
\hline Hexachlorobenzene & UJ & $\mathbf{Q}$ & 4.11 & $\mu \mathrm{g} / \mathrm{kg}$ & 4.11 & EPA8270 \\
\hline
\end{tabular}


SURVEY ID: PPSC 2305 (continued)

Sample ID: 104691

Constituent

RQ AQ B Result

Unit

D. Limit Method

$B / N / A$ Extractables (including PAH and phenols)

Hexachlorobutadiene

Hexachlorobutadiene

Hexachlorocyclopentadiene

Hexachlorocyclopentadiene

Hexachloroethane

Hexachloroethane

Indeno[1,2,3-c,d]pyrene

Indeno[1,2,3-c,d]pyrene

Isophorone

Isophorone

2-Methyl-4,6-dinitrophenol

2-Methyl-4,6-dinitrophenol

2-Methylnaphthalene

2-Methylnaphthalene

Naphthalene

Naphthalene

m-Nitroaniline

m-Nitroaniline

o-Nitroaniline

o-Nitroaniline

p-Nitroaniline

p-Nitroaniline

Nitrobenzene

Nitrobenzene

2-Nitrophenol

2-Nitrophenol

4-Nitrophenol

4-Nitrophenol

N-Nitrosodiphenylamine

N-Nitrosodiphenylamine

N-Nitrosodi-n-propylamine

N-Nitrosodi-n-propylamine

Pentachlorophenol

Pentachlorophenol

Phenanthrene

Phenanthrene

Phenol

Phenol

Pyrene

Pyrene

1,2,4-Trichlorobenzene

1,2,4-Trichlorobenzene

2,4,5-Trichlorophenol

2,4,5-Trichlorophenol

2,4,6-Trichlorophenol

2,4,6-Trichlorophenol

Volatile Organic Compounds

Acetone

Benzene

Bromodichloromethane

Bromoform

Bromomethane (Methyl bromide)

Carbon disulfide

\begin{tabular}{|c|c|c|}
\hline $\mathrm{U}$ & & 33.2 \\
\hline UJ & $Q$ & 41.1 \\
\hline $\mathrm{U}$ & & 33.2 \\
\hline UJ & $Q$ & 41.1 \\
\hline $\mathrm{U}$ & & 3.32 \\
\hline UJ & $\mathbf{Q}$ & 4.11 \\
\hline $\mathrm{U}$ & & 3.32 \\
\hline UJ & $\mathbf{Q}$ & 4.11 \\
\hline U & & 33.2 \\
\hline UJ & $Q$ & 41.1 \\
\hline $\mathrm{U}$ & & 332 \\
\hline UJ & $\mathbf{Q}$ & 411 \\
\hline $\mathrm{U}$ & & 33.2 \\
\hline UJ & $\mathbf{Q}$ & 41.1 \\
\hline $\mathrm{U}$ & & 33.2 \\
\hline UJ & $Q$ & 41.1 \\
\hline $\mathrm{U}$ & & 33.2 \\
\hline UJ & $Q$ & 41.1 \\
\hline $\mathbf{U}$ & & 33.2 \\
\hline UJ & $\mathbf{Q}$ & 41.1 \\
\hline $\mathrm{U}$ & & 33.2 \\
\hline UJ & $Q$ & 41.1 \\
\hline $\mathrm{U}$ & & 33.2 \\
\hline UJ & $\mathbf{Q}$ & 41.1 \\
\hline $\mathrm{U}$ & & 33.2 \\
\hline UJ & $Q$ & 41.1 \\
\hline $\mathrm{U}$ & & 33.2 \\
\hline UJ & $Q$ & 41.1 \\
\hline $\mathrm{U}$ & & 33.2 \\
\hline UJ & $\mathbf{Q}$ & 41.1 \\
\hline $\mathrm{U}$ & & 33.2 \\
\hline UJ & $\mathbf{Q}$ & 41.1 \\
\hline $\mathrm{U}$ & & 33.2 \\
\hline UJ & $\mathbf{Q}$ & 41.1 \\
\hline $\mathrm{U}$ & & 33.2 \\
\hline UJ & $Q$ & 41.1 \\
\hline $\mathrm{U}$ & & 33.2 \\
\hline UJ & $Q$ & 41.1 \\
\hline $\mathrm{U}$ & & 33.2 \\
\hline UJ & $Q$ & 41.1 \\
\hline $\mathrm{U}$ & & $\mathbf{3 . 3 2}$ \\
\hline UJ & $Q$ & 4.11 \\
\hline $\mathrm{U}$ & & 33.2 \\
\hline UJ & $Q$ & 41.1 \\
\hline $\mathrm{U}$ & & 33.2 \\
\hline UJ & $Q$ & 41.1 \\
\hline
\end{tabular}

\begin{tabular}{lll}
$\mu \mathrm{g} / \mathrm{kg}$ & 33.2 & EPA8270 \\
$\mu \mathrm{g} / \mathrm{kg}$ & 41.1 & EPA8270 \\
$\mu \mathrm{g} / \mathrm{kg}$ & 33.2 & EPA8270 \\
$\mu \mathrm{g} / \mathrm{kg}$ & 41.1 & EPA8270 \\
$\mu \mathrm{g} / \mathrm{kg}$ & 3.32 & EPA8270 \\
$\mu \mathrm{g} / \mathrm{kg}$ & 4.11 & EPA8270 \\
$\mu \mathrm{g} / \mathrm{kg}$ & 3.32 & EPA8270 \\
$\mu \mathrm{g} / \mathrm{kg}$ & 4.11 & EPA8270 \\
$\mu \mathrm{g} / \mathrm{kg}$ & 33.2 & EPA8270 \\
$\mu \mathrm{g} / \mathrm{kg}$ & 41.1 & EPA8270 \\
$\mu \mathrm{g} / \mathrm{kg}$ & 332 & EPA8270 \\
$\mu \mathrm{g} / \mathrm{kg}$ & 411 & EPA8270 \\
$\mu \mathrm{g} / \mathrm{kg}$ & 33.2 & EPA8270 \\
$\mu \mathrm{g} / \mathrm{kg}$ & 41.1 & EPA8270 \\
$\mu \mathrm{g} / \mathrm{kg}$ & 33.2 & EPA8270 \\
$\mu \mathrm{g} / \mathrm{kg}$ & 41.1 & EPA8270 \\
$\mu \mathrm{g} / \mathrm{kg}$ & 33.2 & EPA8270 \\
$\mu \mathrm{g} / \mathrm{kg}$ & 41.1 & EPA8270 \\
$\mu \mathrm{g} / \mathrm{kg}$ & 33.2 & EPA8270 \\
$\mu \mathrm{g} / \mathrm{kg}$ & 41.1 & EPA8270 \\
$\mu \mathrm{g} / \mathrm{kg}$ & 33.2 & EPA8270 \\
$\mu \mathrm{g} / \mathrm{kg}$ & 41.1 & EPA8270 \\
$\mu \mathrm{g} / \mathrm{kg}$ & 33.2 & EPA8270 \\
$\mu \mathrm{g} / \mathrm{kg}$ & 41.1 & EPA8270 \\
$\mu \mathrm{g} / \mathrm{kg}$ & 33.2 & EPA8270 \\
$\mu \mathrm{g} / \mathrm{kg}$ & 41.1 & EPA8270 \\
$\mu \mathrm{g} / \mathrm{kg}$ & 33.2 & EPA8270 \\
$\mu \mathrm{g} / \mathrm{kg}$ & 41.1 & EPA8270 \\
$\mu \mathrm{g} / \mathrm{kg}$ & 33.2 & EPA8270 \\
$\mu \mathrm{g} / \mathrm{kg}$ & 41.1 & EPA8270 \\
$\mu \mathrm{g} / \mathrm{kg}$ & 33.2 & EPA8270 \\
$\mu \mathrm{g} / \mathrm{kg}$ & 41.1 & EPA8270 \\
$\mu \mathrm{g} / \mathrm{kg}$ & 33.2 & EPA8270 \\
$\mu \mathrm{g} / \mathrm{kg}$ & 41.1 & EPA8270 \\
$\mu \mathrm{g} / \mathrm{kg}$ & 33.2 & EPA8270 \\
$\mu \mathrm{g} / \mathrm{kg}$ & 41.1 & EPA8270 \\
$\mu \mathrm{g} / \mathrm{kg}$ & 33.2 & EPA8270 \\
$\mu \mathrm{g} / \mathrm{kg}$ & 41.1 & EPA8270 \\
$\mu \mathrm{g} / \mathrm{kg}$ & 33.2 & EPA8270 \\
$\mu \mathrm{g} / \mathrm{kg}$ & 41.1 & EPA8270 \\
$\mu \mathrm{g} / \mathrm{kg}$ & 3.32 & EPA8270 \\
$\mu \mathrm{g} / \mathrm{kg}$ & 4.11 & EPA8270 \\
$\mu \mathrm{g} / \mathrm{kg}$ & 33.2 & EPA8270 \\
$\mu \mathrm{g} / \mathrm{kg}$ & 41.1 & EPA8270 \\
$\mu \mathrm{g} / \mathrm{kg}$ & 33.2 & EPA8270 \\
$\mu \mathrm{g} / \mathrm{kg}$ & 41.1 & EPA8270 \\
& & \\
\hline
\end{tabular}

$\mu \mathrm{g} / \mathrm{kg}$

$\mu \mathrm{g} / \mathrm{kg}$

$\mu \mathrm{g} / \mathrm{kg}$

$\mu \mathrm{g} / \mathrm{kg}$

$\mu \mathrm{g} / \mathrm{kg}$

$\mu \mathrm{g} / \mathrm{kg}$
0.625

0.0625

0.125

1.25
6.25

0.625

0.0625

0.0625

0.125

1.25
EPA8260

EPA8260

EPA8260

EPA8260

EPA8260

EPA8260 
SURVEY ID: PPSC 2305 (continued)

\section{Constituent}

Volatile Organic Compounds

Carbon tetrachloride
Chlorobenzene
Chloroethane
Chloroethene (Vinyl chloride)
Chloroform
Chloromethane (Methyl chloride)
Dibromochloromethane
1,1-Dichloroethane
1,2-Dichloroethane
1,1-Dichloroethylene
1,2-Dichloroethylene
Dichloromethane (Methylene chloride)
1,2-Dichloropropane
cis-1,3-Dichloropropene
trans-1,3-Dichloropropene
Ethylbenzene
2-Hexanone
Methyl ethyl ketone
Methyl isobutyl ketone
Styrene
1,1,2,2-Tetrachloroethane
Tetrachloroethylene
Toluene
1,1,1-Trichloroethane
1,1,2-Trichloroethane
Trichloroethylene
Vinyl acetate
Xylenes

Pesticides/PCBs and Dioxins/Furans
Sample ID: 104691

RQ AQ B Result Unit D. Limit Method

\begin{tabular}{|c|c|c|c|c|}
\hline $\mathrm{U}$ & 0.0625 & $\mu \mathrm{g} / \mathrm{kg}$ & 0.0625 & EPA8260 \\
\hline $\mathrm{U}$ & 0.0625 & $\mu \mathrm{g} / \mathrm{kg}$ & 0.0625 & EPA8260 \\
\hline $\mathrm{U}$ & 0.125 & $\mu \mathrm{g} / \mathrm{kg}$ & 0.125 & EPA8260 \\
\hline U & 0.125 & $\mu \mathrm{g} / \mathrm{kg}$ & 0.125 & EPA8260 \\
\hline U & 0.0625 & $\mu \mathrm{g} / \mathrm{kg}$ & 0.0625 & EPA8260 \\
\hline $\mathrm{U}$ & 0.125 & $\mu \mathrm{g} / \mathrm{kg}$ & 0.125 & EPA8260 \\
\hline $\mathrm{U}$ & 0.0625 & $\mu \mathrm{g} / \mathrm{kg}$ & 0.0625 & EPA8260 \\
\hline $\mathbf{U}$ & 0.0625 & $\mu \mathrm{g} / \mathrm{kg}$ & 0.0625 & EPA8260 \\
\hline $\mathrm{U}$ & 0.0625 & $\mu \mathrm{g} / \mathrm{kg}$ & 0.0625 & EPA8260 \\
\hline $\mathbf{U}$ & 0.0625 & $\mu \mathrm{g} / \mathrm{kg}$ & 0.0625 & EPA8260 \\
\hline $\mathrm{U}$ & 0.125 & $\mu \mathrm{g} / \mathrm{kg}$ & 0.125 & EPA8260 \\
\hline $\mathrm{V}$ & 1.01 & $\mu \mathrm{g} / \mathrm{kg}$ & 0.625 & EPA8260 \\
\hline $\mathrm{U}$ & 0.0625 & $\mu \mathrm{g} / \mathrm{kg}$ & 0.0625 & EPA8260 \\
\hline $\mathrm{U}$ & 0.0625 & $\mu \mathrm{g} / \mathrm{kg}$ & 0.0625 & EPA8260 \\
\hline $\mathrm{U}$ & 0.0625 & $\mu \mathrm{g} / \mathrm{kg}$ & 0.0625 & EPA8260 \\
\hline $\mathrm{U}$ & 0.0625 & $\mu \mathrm{g} / \mathrm{kg}$ & 0.0625 & EPA8260 \\
\hline $\mathrm{U}$ & 1.25 & $\mu \mathrm{g} / \mathrm{kg}$ & 1.25 & EPA8260 \\
\hline V8 & 0.725 & $\mu \mathrm{g} / \mathrm{kg}$ & 1.25 & EPA8260 \\
\hline $\mathrm{U}$ & 1.25 & $\mu \mathrm{g} / \mathrm{kg}$ & 1.25 & EPA8260 \\
\hline $\mathrm{U}$ & 0.0625 & $\mu \mathrm{g} / \mathrm{kg}$ & 0.0625 & EPA8260 \\
\hline $\mathrm{U}$ & 0.0625 & $\mu \mathrm{g} / \mathrm{kg}$ & 0.0625 & EPA8260 \\
\hline U & 0.0625 & $\mu \mathrm{g} / \mathrm{kg}$ & 0.0625 & EPA8260 \\
\hline U & 0.625 & $\mu \mathrm{g} / \mathrm{kg}$ & 0.625 & EPA8260 \\
\hline $\mathrm{U}$ & 0.0625 & $\mu \mathrm{g} / \mathrm{kg}$ & 0.0625 & EPA8260 \\
\hline $\mathrm{U}$ & 0.0625 & $\mu \mathrm{g} / \mathrm{kg}$ & 0.0625 & EPA8260 \\
\hline $\mathrm{U}$ & 0.0625 & $\mu \mathrm{g} / \mathrm{kg}$ & 0.0625 & EPA8260 \\
\hline $\mathrm{U}$ & 1.25 & $\mu \mathrm{g} / \mathrm{kg}$ & 1.25 & EPA8260 \\
\hline $\mathrm{U}$ & 0.188 & $\mu \mathrm{g} / \mathrm{kg}$ & 0.188 & EPA8260 \\
\hline
\end{tabular}

Aldrin
Aldrin
alpha-Benzene hexachloride
alpha-Benzene hexachloride
beta-Benzene hexachloride
beta-Benzene hexachloride
delta-Benzene hexachloride
delta-Benzene hexachloride
alpha-Chlordane
alpha-Chlordane
gamma-Chlordane
gamma-Chlordane
p,p'-DDD
p,p'-DDD
p,p'-DDE
p,p'-DDE
p,p'-DDT
p,p'-DDT
Dieldrin
Dieldrin
Endosulfan sulfate
Endosulfan sulfate
Endosulfan I
Endosulfan I

$\begin{array}{ll}U & 5.06 \\ U & 4.08 \\ U & 5.06 \\ U & 4.08 \\ U & 10.1 \\ U & 8.16 \\ U & 10.1 \\ U & 8.16 \\ U & 10.1 \\ U & 8.16 \\ U & 10.1 \\ U & 8.16 \\ U & 20.2 \\ U & 16.3 \\ U & 10.1 \\ U & 8.16 \\ U & 20.2 \\ U & 16.3 \\ U & 10.1 \\ U & 8.16 \\ U & 20.2 \\ U & 16.3 \\ U & 10.1 \\ U & 8.16\end{array}$

$\begin{array}{lll}\mu \mathrm{g} / \mathrm{kg} & 5.06 & \text { EPA8080 } \\ \mu \mathrm{g} / \mathrm{kg} & 4.08 & \text { EPA8080 } \\ \mu \mathrm{g} / \mathrm{kg} & 5.06 & \text { EPA8080 } \\ \mu \mathrm{g} / \mathrm{kg} & 4.08 & \text { EPA8080 } \\ \mu \mathrm{g} / \mathrm{kg} & 10.1 & \text { EPA8080 } \\ \mu \mathrm{g} / \mathrm{kg} & 8.16 & \text { EPA8080 } \\ \mu \mathrm{g} / \mathrm{kg} & 10.1 & \text { EPA8080 } \\ \mu \mathrm{g} / \mathrm{kg} & 8.16 & \text { EPA8080 } \\ \mu \mathrm{g} / \mathrm{kg} & 10.1 & \text { EPA8080 } \\ \mu \mathrm{g} / \mathrm{kg} & 8.16 & \text { EPA8080 } \\ \mu \mathrm{g} / \mathrm{kg} & 10.1 & \text { EPA8080 } \\ \mu \mathrm{g} / \mathrm{kg} & 8.16 & \text { EPA8080 } \\ \mu \mathrm{g} / \mathrm{kg} & 20.2 & \text { EPA8080 } \\ \mu \mathrm{g} / \mathrm{kg} & 16.3 & \text { EPA8080 } \\ \mu \mathrm{g} / \mathrm{kg} & 10.1 & \text { EPA8080 } \\ \mu \mathrm{g} / \mathrm{kg} & 8.16 & \text { EPA8080 } \\ \mu \mathrm{g} / \mathrm{kg} & 20.2 & \text { EPA8080 } \\ \mu \mathrm{g} / \mathrm{kg} & 16.3 & \text { EPA8080 } \\ \mu \mathrm{g} / \mathrm{kg} & 10.1 & \text { EPA8080 } \\ \mu \mathrm{g} / \mathrm{kg} & 8.16 & \text { EPA8080 } \\ \mu \mathrm{g} / \mathrm{kg} & -20.2 & \text { EPA8080 } \\ \mu \mathrm{g} / \mathrm{kg} & 16.3 & \text { EPA8080 } \\ \mu \mathrm{g} / \mathrm{kg} & 10.1 & \text { EPA8080 } \\ \mu \mathrm{g} / \mathrm{kg} & 8.16 & \text { EPA8080 }\end{array}$


SURVEY ID: PPSC 2305 (continued)

Constituent

Pesticides/PCBs and Dioxins/Furans

Endosulfan II

Endosulfan II

Endrin

Endrin

Endrin ketone

Endrin ketone

Heptachlor

Heptachlor

Heptachlor epoxide

Heptachlor epoxide

Lindane

Lindane

Methoxychlor

Methoxychlor

PCB 1016

PCB 1016

PCB 1221

PCB 1221

PCB 1232

PCB 1232

PCB 1242

PCB 1242

PCB 1248

PCB 1248

PCB 1254

PCB 1254

PCB 1260

PCB 1260

Toxaphene

Toxaphene

Radionuclides

Actinium-228

Antimony-124

Antimony-125

Barium-133

Cerium-144

Cesium-134

Cesium-137

Cobalt-57

Cobalt-58

Cobalt-60

Europium-152

Europium-154

Europium-155

Gross alpha

Lead-212

Manganese-54

Neptunium-239

Nonvolatile beta

Plutonium-238

Plutonium-238

Plutonium-239/240

Plutonium-239/240
Sample ID: 104691
RQ
AQ B Result
Unit
D. Limit Method

$\begin{array}{lll}\mu \mathrm{g} / \mathrm{kg} & 20.2 & \text { EPA8080 } \\ \mu \mathrm{g} / \mathrm{kg} & 16.3 & \text { EPA8080 } \\ \mu \mathrm{g} / \mathrm{kg} & 10.1 & \text { EPA8080 } \\ \mu \mathrm{g} / \mathrm{kg} & 8.16 & \text { EPA8080 } \\ \mu \mathrm{g} / \mathrm{kg} & 20.2 & \text { EPA8080 } \\ \mu \mathrm{g} / \mathrm{kg} & 16.3 & \text { EPA8080 } \\ \mu \mathrm{g} / \mathrm{kg} & 10.1 & \text { EPA8080 } \\ \mu \mathrm{g} / \mathrm{kg} & 8.16 & \text { EPA8080 } \\ \mu \mathrm{g} / \mathrm{kg} & 10.1 & \text { EPA8080 } \\ \mu \mathrm{g} / \mathrm{kg} & 8.16 & \text { EPA8080 } \\ \mu \mathrm{g} / \mathrm{kg} & 5.06 & \text { EPA8080 } \\ \mu \mathrm{g} / \mathrm{kg} & 4.08 & \text { EPA8080 } \\ \mu \mathrm{g} / \mathrm{kg} & 101 & \text { EPA8080 } \\ \mu \mathrm{g} / \mathrm{kg} & 81.6 & \text { EPA8080 } \\ \mu \mathrm{g} / \mathrm{kg} & 25.3 & \text { EPA8080 } \\ \mu \mathrm{g} / \mathrm{kg} & 20.4 & \text { EPA8080 } \\ \mu \mathrm{g} / \mathrm{kg} & 25.3 & \text { EPA8080 } \\ \mu \mathrm{g} / \mathrm{kg} & 20.4 & \text { EPA8080 } \\ \mu \mathrm{g} / \mathrm{kg} & 25.3 & \text { EPA8080 } \\ \mu \mathrm{g} / \mathrm{kg} & 20.4 & \text { EPA8080 } \\ \mu \mathrm{g} / \mathrm{kg} & 25.3 & \text { EPA8080 } \\ \mu \mathrm{g} / \mathrm{kg} & 20.4 & \text { EPA8080 } \\ \mu \mathrm{g} / \mathrm{kg} & 25.3 & \text { EPA8080 } \\ \mu \mathrm{g} / \mathrm{kg} & 20.4 & \text { EPA8080 } \\ \mu \mathrm{g} / \mathrm{kg} & 25.3 & \text { EPA8080 } \\ \mu \mathrm{g} / \mathrm{kg} & 20.4 & \text { EPA8080 } \\ \mu \mathrm{g} / \mathrm{kg} & 25.3 & \text { EPA8080 } \\ \mu \mathrm{g} / \mathrm{kg} & 20.4 & \text { EPA8080 } \\ \mu \mathrm{g} / \mathrm{kg} & 202 & \text { EPA8080 } \\ \mu \mathrm{g} / \mathrm{kg} & 163 & \text { EPA8080 }\end{array}$

$3.96 \mathrm{E}+00 \pm 9.11 \mathrm{E}-02 \mathrm{pCi} / \mathrm{g}$ $2.62 \mathrm{E}-03 \pm 1.28 \mathrm{E}-02 \mathrm{pCi} / \mathrm{g}$ $-3.33 \mathrm{E}-04 \pm 1.99 \mathrm{E}-02 \mathrm{pCi} / \mathrm{g}$ 9.50E-03 $\pm 9.78 \mathrm{E}-03 \mathrm{pCi} / \mathrm{g}$ $-1.07 \mathrm{E}-01 \pm 5.52 \mathrm{E}-02 \mathrm{pCj} / \mathrm{g}$ $-4.30 \mathrm{E}-03 \pm 1.01 \mathrm{E}-02 \mathrm{pCi} / \mathrm{g}$ 1.41E-04 $\pm 8.62 \mathrm{E}-03 \mathrm{pCj} / \mathrm{g}$ $-2.45 \mathrm{E}-03 \pm 6.82 \mathrm{E}-03 \mathrm{pCi} / \mathrm{g}$ $-6.01 \mathrm{E}-03 \pm 8.08 \mathrm{E}-03 \mathrm{pCi} / \mathrm{g}$ 5.62E-04 $5.91 \mathrm{E}-03 \mathrm{pCi} / \mathrm{g}$ $-1.15 \mathrm{E}-02 \pm 2.04 \mathrm{E}-02 \mathrm{pCi} / \mathrm{g}$ 9.36E-03 $\pm 5.92 \mathrm{E}-02 \mathrm{pCi} / \mathrm{g}$ $0.00 \mathrm{E}+00 \quad \mathrm{pCi} / \mathrm{g}$ $6.03 \mathrm{E}+01 \pm 6.20 \mathrm{E}+00 \mathrm{pCi} / \mathrm{g}$ $4.24 \mathrm{E}+00 \pm 4.09 \mathrm{E}-02 \mathrm{pCi} / \mathrm{g}$ $6.75 \mathrm{E}-02 \pm 1.05 \mathrm{E}-02 \mathrm{pCi} / \mathrm{g}$ $-1.24 \mathrm{E}-02 \pm 5.42 \mathrm{E}-02 \mathrm{pCi} / \mathrm{g}$ $3.44 \mathrm{E}+01 \pm 3.17 \mathrm{E}+00 \mathrm{pCi} / \mathrm{g}$ 2.78E-02 $2.49 \mathrm{E}-02 \mathrm{pCi} / \mathrm{g}$ $-2.72 \mathrm{E}-03 \pm 1.58 \mathrm{E}-02 \mathrm{pCj} / \mathrm{g}$ 5.85E-03 $1.10 \mathrm{E}-02 \mathrm{pCi} / \mathrm{g}$ $3.78 \mathrm{E}-03 \pm 9.90 \mathrm{E}-03 \mathrm{pCi} / \mathrm{g}$
3.62E-02 EPIA-013B

1.51E-02 EPIA-013B

3.24E-02 EPIA-013B

1.46E-02 EPIA-013B

9.28E-02 EPIA-013B

1.09E-02 EPIA-013B

1.30E-02 EPIA-013B

1.17E-02 EPLA-013B

1.34E-02 EPIA-013B

1.04E-02 EPIA-013B

3.31E-02 EPIA-013B

8.78E-02 EPIA-013B

5.00E-02 EPLA-013B

2.26E+00 EPIA-001B

2.05E-02 EPIA-013B

1.13E-02 EPIA-013B

8.36E-02 EPIA-013B

2.45E+00 EPIA-001B

4.10E-02 EPIA-012B

3.91E-02 EPIA-012B

2.25E-02 EPIA-012B

2.22E-02 EPIA-012B 
SURVEY ID: PPSC 2305 (continued)

Sample ID: 104691

Constituent

RQ AQ B Result

Unit

D. Limit Method

Radionuclides

Potassium-40

Promethium-144

Promethium-146

Radium-226

Radium-228

Ruthenium-106

Sodium-22

Strontium-90

Thorium-234

Tin-113

Yttrium-88

Zinc-65

Zirconium-95

$\begin{array}{llll} & & 3.29 \mathrm{E}+00 \pm 1.77 \mathrm{E}-01 \mathrm{pCi} / \mathrm{g} \\ \text { UI } & & -6.82 \mathrm{E}-04 \pm 7.12 \mathrm{E}-03 \mathrm{pCi} / \mathrm{g} \\ \text { UI } & & 0.00 \mathrm{E}+00 & \mathrm{pCi} / \mathrm{g} \\ & \mathrm{V} & 1.80 \mathrm{E}+00 \pm 4.50 \mathrm{E}-02 \mathrm{pCi} / \mathrm{g} \\ & & 3.96 \mathrm{E}+00 \pm 9.11 \mathrm{E}-02 \mathrm{pCi} / \mathrm{g} \\ \text { UI } & & 3.65 \mathrm{E}-02 \pm 6.45 \mathrm{E}-02 \mathrm{pCi} / \mathrm{g} \\ \text { UI } & & -8.02 \mathrm{E}-03 \pm 7.28 \mathrm{E}-03 \mathrm{pCi} / \mathrm{g} \\ \text { UI } & & -1.93 \mathrm{E}-02 \pm 5.32 \mathrm{E}-02 \mathrm{pCi} / \mathrm{g} \\ & \mathrm{V} & 2.59 \mathrm{E}+00 \pm 7.00 \mathrm{E}-01 \mathrm{pCi} / \mathrm{g} \\ \text { UI } & & 2.65 \mathrm{E}-03 \pm 1.18 \mathrm{E}-02 \mathrm{pCi} / \mathrm{g} \\ \text { UI } & & 1.11 \mathrm{E}-02 \pm 1.01 \mathrm{E}-02 & \mathrm{pCi} / \mathrm{g} \\ \text { UI } & & 1.73 \mathrm{E}-02 \pm 1.75 \mathrm{E}-02 \mathrm{pCi} / \mathrm{g} \\ \text { UI } & & 0.00 \mathrm{E}+00 & \mathrm{pCi} / \mathrm{g}\end{array}$

9.22E-02 EPIA-013B

1.21E-02 EPIA-013B

1.60E-02 EPIA-013B

2.17E-02 EPIA-013B

3.62E-02 EPIA-013B

1.12E-01 EPIA-013B

9.82E-03 EPIA-013B

1.47E-01 EPIA-004

6.17E-01 EPIA-013B

1.72E-02 EPIA-013B

1.32E-02 EPIA-013B

2.02E-02 EPIA-013B

3.13E-02 EPIA-013B

SURVEY ID: PPSC 2401

Sample ID: 105117

Survey location: 44906E $3692181 \mathrm{~N}$ (SRS coordinates)

Depth of core interval: 0.00 to $1.00 \mathrm{ft}$

Sample type: Normal

Sample matrix: Soil

Percent solids: $\mathbf{7 8 . 0 0}$

Constituent

RQ AQ B Result

Unit

D. Limit Method

Physical Parameters and Specified Analyses

Ammonia nitrogen

Cation exchange capacity

Chloride

Cyanide

Cyanide

Nitrate-nitrite as nitrogen

Nitrate-nitrite as nitrogen

Nitrogen by Kjeldahl method

Orthophosphate

Phenols

Sulfide

Total organic carbon

Total organic nitrogen

Total phosphates (as P)

Metals (total recoverable)

Aluminum

Antimony

Arsenic

Barium

Beryllium

Cadmium

Calcium

Chromium

Cobalt

Copper

Iron

$\begin{array}{lll}\text { U } & & 25.6 \\ & & 7.04 \\ \text { J } & \text { E } & 2.59 \\ \text { U } & & 1.24 \\ \text { U } & & 1.24 \\ \text { U } & & 1.25 \\ \text { J } & \text { E } & 0.384 \\ & & 37.1 \\ \text { J } & \text { E } & 2.69 \\ \text { UJ } & \text { C } & 367 \\ \text { UJ } & \text { C } & 321 \\ & \text { V } & 754 \\ \text { C } & & 30.7 \\ \text { J } & \text { E } & 4.05\end{array}$

$\begin{array}{lll} & \mathrm{V} & 270 \\ \mathrm{U} & & 6.1 \\ \mathrm{U} & & 18.3 \\ \mathrm{~J} & \mathrm{C} & 1.7 \\ \mathrm{~J} & \mathrm{EC} & 0.0415 \\ \mathrm{U} & & 0.305 \\ & \mathrm{~V} & 27 \\ \mathrm{~J} & \mathrm{E} & 0.371 \\ \mathrm{U} & & 0.61 \\ \mathrm{U} & & 1.52 \\ \mathrm{~J} & \mathrm{C} & 160\end{array}$

$\begin{array}{lll}\mathrm{mg} / \mathrm{kg} & 25.6 & \text { EPA350.1 } \\ \mathrm{meq} / 100 \mathrm{~g} & 5 & \text { EPA9081 } \\ \mathrm{mg} / \mathrm{kg} & 3.29 & \text { EPA300.0 } \\ \mathrm{mg} / \mathrm{kg} & 1.24 & \text { EPA335.3 } \\ \mathrm{mg} / \mathrm{kg} & 1.24 & \text { EPA335.3 } \\ \mathrm{mg} / \mathrm{kg} & 1.25 & \text { EPA353.1 } \\ \mathrm{mg} / \mathrm{kg} & 1.28 & \text { EPA353.1 } \\ \mathrm{mg} / \mathrm{kg} & 5.01 & \text { EPA351.2 } \\ \mathrm{mg} / \mathrm{kg} & 12.8 & \text { EPA365.2 } \\ \mu \mathrm{g} / \mathrm{kg} & 367 & \text { EPA420.2 } \\ \mathrm{mg} / \mathrm{kg} & 321 & \text { EPA376.2 } \\ \mathrm{mg} / \mathrm{kg} & 128 & \text { EPA415.1 } \\ \mathrm{mg} / \mathrm{kg} & 0.256 & \text { EPA351.2 } \\ \mathrm{mg} / \mathrm{kg} & 12.7 & \text { EPA365.4 }\end{array}$

$\mathrm{mg} / \mathrm{kg}$

$\mathrm{mg} / \mathrm{kg}$

15.2

6.1

18.3

$\mathrm{mg} / \mathrm{kg}$

1.52

0.61

0.305

15.2

1.52

0.61

1.52

6.1
EPA6010A

EPA6010A

EPA6010A

EPA6010A

EPA6010A

EPA6010A

EPA6010A

EPA6010A

EPA6010A

EPA6010A

EPA6010A 
Appendix D: Sediment Tables

SURVEY ID: PPSC 2401 (continued)

Sample ID: 105117

Constituent

RQ AQ B Result

Unit

D. Limit Method

Metals (total recoverable)

Lead

Magnesium

Manganese

Mercury

Nickel

Potassium

Selenium

Silver

Sodium

Thallium

Vanadium

Zinc

$\begin{array}{lll}\text { U } & & 6.1 \\ & & 7.01 \\ & & 4.73 \\ \mathrm{U} & & 0.0328 \\ \mathrm{U} & & 1.52 \\ \mathrm{U} & & 122 \\ \mathrm{U} & & 18.3 \\ \mathrm{U} & & 1.52 \\ \mathrm{U} & & 61 \\ \mathrm{U} & & 18.3 \\ \mathrm{UJ} & \mathrm{C} & \mathbf{0 . 6 1} \\ \mathrm{J} & \mathrm{EV} & 1.15\end{array}$

$B / N / A$ Extractables (including $P A H$ and phenols)

Acenaphthene

Acenaphthylene

Anthracene

Benzo[a]anthracene

Benzo[b]fluoranthene

Benzo[k]fluoranthene

Benzoic acid

Benzo[g, $h, i]$ perylene

Benzo[a]pyrene

Benzyl alcohol

Bis(2-chloroethoxy) methane

Bis(2-chloroethyl) ether

Bis(2-chloroisopropyl) ether

Bis(2-ethylhexyl) phthalate

4-Bromophenyl phenyl ether

Butylbenzyl phthalate

4-Chloroaniline

4-Chloro-m-cresol

2-Chloronaphthalene

2-Chlorophenol

4-Chlorophenyl phenyl ether

Chrysene

$\mathrm{m} / \mathrm{p}$-Cresol

o-Cresol (2-Methylphenol)

Dibenz $[a, h]$ anthracene

Dibenzofuran

Di-n-butyl phthalate

1,2-Dichlorobenzene

1,3-Dichlorobenzene

1,4-Dichlorobenzene

3,3'-Dichlorobenzidine

2,4-Dichlorophenol

Diethyl phthalate

2,4-Dimethyl phenol

Dimethyl phthalate

2,4-Dinitrophenol

2,4-Dinitrotoluene

2,6-Dinitrotoluene

Di-n-octyl phthalate

Fluoranthene

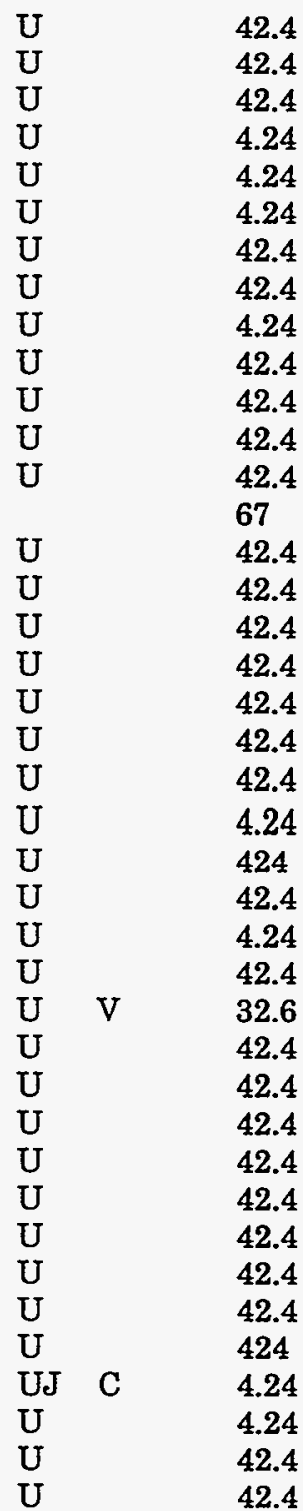

$\begin{array}{lll}\mathrm{mg} / \mathrm{kg} & 6.1 & \text { EPA6010A } \\ \mathrm{mg} / \mathrm{kg} & 6.1 & \text { EPA6010A } \\ \mathrm{mg} / \mathrm{kg} & 0.61 & \text { EPA6010A } \\ \mathrm{mg} / \mathrm{kg} & 0.0328 & \text { EPA7471 } \\ \mathrm{mg} / \mathrm{kg} & 1.52 & \text { EPA6010A } \\ \mathrm{mg} / \mathrm{kg} & 122 & \text { EPA6010A } \\ \mathrm{mg} / \mathrm{kg} & 18.3 & \text { EPA6010A } \\ \mathrm{mg} / \mathrm{kg} & 1.52 & \text { EPA6010A } \\ \mathrm{mg} / \mathrm{kg} & 61 & \text { EPA6010A } \\ \mathrm{mg} / \mathrm{kg} & 18.3 & \text { EPA6010A } \\ \mathrm{mg} / \mathrm{kg} & 0.61 & \text { EPA6010A } \\ \mathrm{mg} / \mathrm{kg} & 1.22 & \text { EPA6010A }\end{array}$

$\begin{array}{lll}\mu \mathrm{g} / \mathrm{kg} & 42.4 & \text { EPA8270 } \\ \mu \mathrm{g} / \mathrm{kg} & 42.4 & \text { EPA8270 } \\ \mu \mathrm{g} / \mathrm{kg} & 42.4 & \text { EPA8270 } \\ \mu \mathrm{g} / \mathrm{kg} & 4.24 & \text { EPA8270 } \\ \mu \mathrm{g} / \mathrm{kg} & 4.24 & \text { EPA8270 } \\ \mu \mathrm{g} / \mathrm{kg} & 4.24 & \text { EPA8270 } \\ \mu \mathrm{g} / \mathrm{kg} & 42.4 & \text { EPA8270 } \\ \mu \mathrm{g} / \mathrm{kg} & 42.4 & \text { EPA8270 } \\ \mu \mathrm{g} / \mathrm{kg} & 4.24 & \text { EPA8270 } \\ \mu \mathrm{g} / \mathrm{kg} & 42.4 & \text { EPA8270 } \\ \mu \mathrm{g} / \mathrm{kg} & 42.4 & \text { EPA8270 } \\ \mu \mathrm{g} / \mathrm{kg} & 42.4 & \text { EPA8270 } \\ \mu \mathrm{g} / \mathrm{kg} & 42.4 & \text { EPA8270 } \\ \mu \mathrm{g} / \mathrm{kg} & 42.4 & \text { EPA8270 } \\ \mu \mathrm{g} / \mathrm{kg} & 42.4 & \text { EPA8270 } \\ \mu \mathrm{g} / \mathrm{kg} & 42.4 & \text { EPA8270 } \\ \mu \mathrm{g} / \mathrm{kg} & 42.4 & \text { EPA8270 } \\ \mu \mathrm{g} / \mathrm{kg} & 42.4 & \text { EPA8270 } \\ \mu \mathrm{g} / \mathrm{kg} & 42.4 & \text { EPA8270 } \\ \mu \mathrm{g} / \mathrm{kg} & 42.4 & \text { EPA8270 } \\ \mu \mathrm{g} / \mathrm{kg} & 42.4 & \text { EPA8270 } \\ \mu \mathrm{g} / \mathrm{kg} & 4.24 & \text { EPA8270 } \\ \mu \mathrm{g} / \mathrm{kg} & 424 & \text { EPA8270 } \\ \mu \mathrm{g} / \mathrm{kg} & 42.4 & \text { EPA8270 } \\ \mu \mathrm{g} / \mathrm{kg} & 4.24 & \text { EPA8270 } \\ \mu \mathrm{g} / \mathrm{kg} & 42.4 & \text { EPA8270 } \\ \mu \mathrm{g} / \mathrm{kg} & 42.4 & \text { EPA8270 } \\ \mu \mathrm{g} / \mathrm{kg} & 42.4 & \text { EPA8270 } \\ \mu \mathrm{g} / \mathrm{kg} & 42.4 & \text { EPA8270 } \\ \mu \mathrm{g} / \mathrm{kg} & 42.4 & \text { EPA8270 } \\ \mu \mathrm{g} / \mathrm{kg} & 42.4 & \text { EPA8270 } \\ \mu \mathrm{g} / \mathrm{kg} & 42.4 & \text { EPA8270 } \\ \mu \mathrm{g} / \mathrm{kg} & 42.4 & \text { EPA8270 } \\ \mu \mathrm{g} / \mathrm{kg} & 42.4 & \text { EPA8270 } \\ \mu \mathrm{g} / \mathrm{kg} & 42.4 & \text { EPA8270 } \\ \mu \mathrm{g} / \mathrm{kg} & 424 & \text { EPA8270 } \\ \mu \mathrm{g} / \mathrm{kg} & 4.24 & \text { EPA8270 } \\ \mu \mathrm{g} / \mathrm{kg} & 4.24 & \text { EPA8270 } \\ \mu \mathrm{g} / \mathrm{kg} & 42.4 & \text { EPA8270 } \\ \mu \mathrm{g} / \mathrm{kg} & 42.4 & \text { EPA8270 } \\ & & \end{array}$


SURVEY ID: PPSC 2401 (continued)

Sample ID: 105117

Constituent

RQ AQ B Result

Unit

D. Limit Method

$B / N / A$ Extractables (including PAH and phenols)

Fluorene

Hexachlorobenzene

Hexachlorobutadiene

Hexachlorocyclopentadiene

Hexachloroethane

Indeno[1,2,3-c,d]pyrene

Isophorone

2-Methyl-4,6-dinitrophenol

2-Methylnaphthalene

Naphthalene

m-Nitroaniline

o-Nitroaniline

p-Nitroaniline

Nitrobenzene

2-Nitrophenol

4-Nitrophenol

N-Nitrosodiphenylamine

N-Nitrosodi-n-propylamine

Pentachlorophenol

Phenanthrene

Phenol

Pyrene

1,2,4-Trichlorobenzene

2,4,5-Trichlorophenol

2,4,6-Trichlorophenol

Volatile Organic Compounds

Acetone
Benzene
Bromodichloromethane
Bromoform
Bromomethane (Methyl bromide)
Carbon disulfide
Carbon tetrachloride
Chlorobenzene
Chloroethane
Chloroethene (Vinyl chloride)
Chloroform
Chloromethane (Methyl chloride)
Dibromochloromethane
1,1-Dichloroethane
1,2-Dichloroethane
1,1-Dichloroethylene
1,2-Dichloroethylene
Dichloromethane (Methylene chloride)
1,2-Dichloropropane
cis-1,3-Dichloropropene
trans-1,3-Dichloropropene
Ethylbenzene
2-Hexanone
Methyl ethyl ketone
Methyl isobutyl ketone
Styrene
1,1,2,2-Tetrachloroethane

$\begin{array}{ll}\text { U } & 42.4 \\ \mathrm{U} & 4.24 \\ \mathrm{U} & 42.4 \\ \mathrm{U} & 42.4 \\ \mathrm{U} & 4.24 \\ \mathrm{U} & 4.24 \\ \mathrm{U} & 42.4 \\ \mathrm{U} & 424 \\ \mathrm{U} & 42.4 \\ \mathrm{U} & 42.4 \\ \mathrm{U} & 42.4 \\ \mathrm{U} & 42.4 \\ \mathrm{U} & 42.4 \\ \mathrm{U} & 42.4 \\ \mathrm{U} & 42.4 \\ \mathrm{U} & 42.4 \\ \mathrm{U} & 42.4 \\ \mathrm{U} & 42.4 \\ \mathrm{U} & 42.4 \\ \mathrm{U} & 42.4 \\ \mathrm{U} & 42.4 \\ \mathrm{U} & 42.4 \\ \mathrm{U} & 4.24 \\ \mathrm{U} & 42.4 \\ \mathrm{U} & 42.4\end{array}$

$\begin{array}{lll}\text { U } & 8 & 10.7 \\ U & & 0.641 \\ U & & 0.0641 \\ U & & 0.0641 \\ U & & 0.128 \\ U & & 1.28 \\ U & & 0.0641 \\ U & & 0.0641 \\ U & & 0.128 \\ U & & 0.128 \\ U & & 0.0641 \\ U & & 0.128 \\ U & & 0.0641 \\ U & & 0.0641 \\ U & & 0.0641 \\ U & & 0.0641 \\ U & & 0.128 \\ U & V & 1.29 \\ U & & 0.0641 \\ U & & 0.0641 \\ U & & 0.0641 \\ U & & 0.0641 \\ U & & 1.28 \\ U & V & 1.36 \\ U & & 1.28 \\ U & & 0.0641 \\ U & & 0.0641 \\ \text { U } & & \end{array}$

$\begin{array}{lll}\mu \mathrm{g} / \mathrm{kg} & 42.4 & \text { EPA8270 } \\ \mu \mathrm{g} / \mathrm{kg} & 4.24 & \text { EPA8270 } \\ \mu \mathrm{g} / \mathrm{kg} & 42.4 & \text { EPA8270 } \\ \mu \mathrm{g} / \mathrm{kg} & 42.4 & \text { EPA8270 } \\ \mu \mathrm{g} / \mathrm{kg} & 4.24 & \text { EPA8270 } \\ \mu \mathrm{g} / \mathrm{kg} & 4.24 & \text { EPA8270 } \\ \mu \mathrm{g} / \mathrm{kg} & 42.4 & \text { EPA8270 } \\ \mu \mathrm{g} / \mathrm{kg} & 424 & \text { EPA8270 } \\ \mu \mathrm{g} / \mathrm{kg} & 42.4 & \text { EPA8270 } \\ \mu \mathrm{g} / \mathrm{kg} & 42.4 & \text { EPA8270 } \\ \mu \mathrm{g} / \mathrm{kg} & 42.4 & \text { EPA8270 } \\ \mu \mathrm{g} / \mathrm{kg} & 42.4 & \text { EPA8270 } \\ \mu \mathrm{g} / \mathrm{kg} & 42.4 & \text { EPA8270 } \\ \mu \mathrm{g} / \mathrm{kg} & 42.4 & \text { EPA8270 } \\ \mu \mathrm{g} / \mathrm{kg} & 42.4 & \text { EPA8270 } \\ \mu \mathrm{g} / \mathrm{kg} & 42.4 & \text { EPA8270 } \\ \mu \mathrm{g} / \mathrm{kg} & 42.4 & \text { EPA8270 } \\ \mu \mathrm{g} / \mathrm{kg} & 42.4 & \text { EPA8270 } \\ \mu \mathrm{g} / \mathrm{kg} & 42.4 & \text { EPA8270 } \\ \mu \mathrm{g} / \mathrm{kg} & 42.4 & \text { EPA8270 } \\ \mu \mathrm{g} / \mathrm{kg} & 42.4 & \text { EPA8270 } \\ \mu \mathrm{g} / \mathrm{kg} & 42.4 & \text { EPA8270 } \\ \mu \mathrm{g} / \mathrm{kg} & 4.24 & \text { EPA8270 } \\ \mu \mathrm{g} / \mathrm{kg} & 42.4 & \text { EPA8270 } \\ \mu \mathrm{g} / \mathrm{kg} & 42.4 & \text { EPA8270 }\end{array}$

$\begin{array}{lll}\mu \mathrm{g} / \mathrm{kg} & 6.41 & \text { EPA8260 } \\ \mu \mathrm{g} / \mathrm{kg} & 0.641 & \text { EPA8260 } \\ \mu \mathrm{g} / \mathrm{kg} & 0.0641 & \text { EPA8260 } \\ \mu \mathrm{g} / \mathrm{kg} & 0.0641 & \text { EPA8260 } \\ \mu \mathrm{g} / \mathrm{kg} & 0.128 & \text { EPA8260 } \\ \mu \mathrm{g} / \mathrm{kg} & 1.28 & \text { EPA8260 } \\ \mu \mathrm{g} / \mathrm{kg} & 0.0641 & \text { EPA8260 } \\ \mu \mathrm{g} / \mathrm{kg} & 0.0641 & \text { EPA8260 } \\ \mu \mathrm{g} / \mathrm{kg} & 0.128 & \text { EPA8260 } \\ \mu \mathrm{g} / \mathrm{kg} & 0.128 & \text { EPA8260 } \\ \mu \mathrm{g} / \mathrm{kg} & 0.0641 & \text { EPA8260 } \\ \mu \mathrm{g} / \mathrm{kg} & 0.128 & \text { EPA8260 } \\ \mu \mathrm{g} / \mathrm{kg} & 0.0641 & \text { EPA8260 } \\ \mu \mathrm{g} / \mathrm{kg} & 0.0641 & \text { EPA8260 } \\ \mu \mathrm{g} / \mathrm{kg} & 0.0641 & \text { EPA8260 } \\ \mu \mathrm{g} / \mathrm{kg} & 0.0641 & \text { EPA8260 } \\ \mu \mathrm{g} / \mathrm{kg} & 0.128 & \text { EPA8260 } \\ \mu \mathrm{g} / \mathrm{kg} & 0.641 & \text { EPA8260 } \\ \mu \mathrm{g} / \mathrm{kg} & 0.0641 & \text { EPA8260 } \\ \mu \mathrm{g} / \mathrm{kg} & 0.0641 & \text { EPA8260 } \\ \mu \mathrm{g} / \mathrm{kg} & 0.0641 & \text { EPA8260 } \\ \mu \mathrm{g} / \mathrm{kg} & 0.0641 & \text { EPA8260 } \\ \mu \mathrm{g} / \mathrm{kg} & 1.28 & \text { EPA8260 } \\ \mu \mathrm{g} / \mathrm{kg} & 1.28 & \text { EPA8260 } \\ \mu \mathrm{g} / \mathrm{kg} & 1.28 & \text { EPA8260 } \\ \mu \mathrm{g} / \mathrm{kg} & 0.0641 & \text { EPA8260 } \\ \mu \mathrm{g} / \mathrm{kg} & 0.0641 & \text { EPA8260 }\end{array}$


SURVEY ID: PPSC 2401 (continued)

Constituent

Volatile Organic Compounds

Tetrachloroethylene

Toluene

1,1,1-Trichloroethane

1,1,2-Trichloroethane

Trichloroethylene

Vinyl acetate

Xylenes

Pesticides/PCBs and Dioxins/Furans

$\begin{array}{lll}\text { Aldrin } & \mathrm{U} & 51.8 \\ \text { alpha-Benzene hexachloride } & \mathrm{U} & \mathbf{5 1 . 8} \\ \text { beta-Benzene hexachloride } & \mathrm{U} & 104 \\ \text { delta-Benzene hexachloride } & \mathrm{U} & 104 \\ \text { alpha-Chlordane } & \mathrm{U} & 104 \\ \text { gamma-Chlordane } & \mathrm{U} & 104 \\ \text { p,p'-DDD } & \mathrm{U} & 207 \\ \text { p,p'-DDE } & \mathrm{U} & 104 \\ \text { p,p'-DDT } & \mathrm{U} & 207 \\ \text { Dieldrin } & \mathrm{U} & 104 \\ \text { Endosulfan sulfate } & \mathrm{U} & 207 \\ \text { Endosulfan I } & \mathrm{U} & 104 \\ \text { Endosulfan II } & \mathrm{U} & 207 \\ \text { Endrin } & \mathrm{U} & 104 \\ \text { Endrin ketone } & \mathrm{U} & 207 \\ \text { Heptachlor } & \mathrm{U} & 104 \\ \text { Heptachlor epoxide } & \mathrm{U} & 104 \\ \text { Lindane } & \mathrm{U} & \mathbf{5 1 . 8} \\ \text { Methoxychlor } & \mathrm{U} & 1040 \\ \text { PCB 1016 } & \mathrm{U} & 259 \\ \text { PCB 1221 } & \mathrm{U} & 259 \\ \text { PCB 1232 } & \mathrm{U} & 259 \\ \text { PCB 1242 } & \mathrm{U} & 259 \\ \text { PCB 1248 } & \mathrm{U} & 259 \\ \text { PCB 1254 } & \mathrm{U} & 259 \\ \text { PCB 1260 } & \mathrm{U} & 259 \\ \text { Toxaphene } & \mathrm{U} & 2070\end{array}$

Radionuclides

Actinium-228

Antimony-124

Antimony-125

Barium-133

Cerium-144

Cesium-134

Cesium-137

Cobalt-57

Cobalt-58

Cobalt-60

Europium-152

Europium-154

Europium-155

Gross alpha

Lead-212
Sample ID: 105117
RQ AQ B Result
Unit
D. Limit Method

$\begin{array}{ll}U & 0.0641 \\ U & 0.641 \\ U & 0.0641 \\ U & 0.0641 \\ U & 0.0641 \\ U & 1.28 \\ U & 0.192\end{array}$

$\begin{array}{lll}\mu \mathrm{g} / \mathrm{kg} & 0.0641 & \text { EPA8260 } \\ \mu \mathrm{g} / \mathrm{kg} & 0.641 & \text { EPA8260 } \\ \mu \mathrm{g} / \mathrm{kg} & 0.0641 & \text { EPA8260 } \\ \mu \mathrm{g} / \mathrm{kg} & 0.0641 & \text { EPA8260 } \\ \mu \mathrm{g} / \mathrm{kg} & 0.0641 & \text { EPA8260 } \\ \mu \mathrm{g} / \mathrm{kg} & 1.28 & \text { EPA8260 } \\ \mu \mathrm{g} / \mathrm{kg} & 0.192 & \text { EPA8260 }\end{array}$

$\begin{array}{lll}\mu \mathrm{g} / \mathrm{kg} & 51.8 & \text { EPA8080 } \\ \mu \mathrm{g} / \mathrm{kg} & 51.8 & \text { EPA8080 } \\ \mu \mathrm{g} / \mathrm{kg} & 104 & \text { EPA8080 } \\ \mu \mathrm{g} / \mathrm{kg} & 104 & \text { EPA8080 } \\ \mu \mathrm{g} / \mathrm{kg} & 104 & \text { EPA8080 } \\ \mu \mathrm{g} / \mathrm{kg} & 104 & \text { EPA8080 } \\ \mu \mathrm{g} / \mathrm{kg} & 207 & \text { EPA8080 } \\ \mu \mathrm{g} / \mathrm{kg} & 104 & \text { EPA8080 } \\ \mu \mathrm{g} / \mathrm{kg} & 207 & \text { EPA8080 } \\ \mu \mathrm{g} / \mathrm{kg} & 104 & \text { EPA8080 } \\ \mu \mathrm{g} / \mathrm{kg} & 207 & \text { EPA8080 } \\ \mu \mathrm{g} / \mathrm{kg} & 104 & \text { EPA8080 } \\ \mu \mathrm{g} / \mathrm{kg} & 207 & \text { EPA8080 } \\ \mu \mathrm{g} / \mathrm{kg} & 104 & \text { EPA8080 } \\ \mu \mathrm{g} / \mathrm{kg} & 207 & \text { EPA8080 } \\ \mu \mathrm{g} / \mathrm{kg} & 104 & \text { EPA8080 } \\ \mu \mathrm{g} / \mathrm{kg} & 104 & \text { EPA8080 } \\ \mu \mathrm{g} / \mathrm{kg} & 51.8 & \text { EPA8080 } \\ \mu \mathrm{g} / \mathrm{kg} & 1040 & \text { EPA8080 } \\ \mu \mathrm{g} / \mathrm{kg} & 259 & \text { EPA8080 } \\ \mu \mathrm{g} / \mathrm{kg} & 259 & \text { EPA8080 } \\ \mu \mathrm{g} / \mathrm{kg} & 259 & \text { EPA8080 } \\ \mu \mathrm{g} / \mathrm{kg} & 259 & \text { EPA8080 } \\ \mu \mathrm{g} / \mathrm{kg} & 259 & \text { EPA8080 } \\ \mu \mathrm{g} / \mathrm{kg} & 259 & \text { EPA8080 } \\ \mu \mathrm{g} / \mathrm{kg} & 259 & \text { EPA8080 } \\ \mu \mathrm{g} / \mathrm{kg} & 2070 & \text { EPA8080 }\end{array}$

$3.50 \mathrm{E}-01 \pm 2.89 \mathrm{E}-02 \mathrm{pCi} / \mathrm{g}$ 5.82E-04 $\pm 3.60 \mathrm{E}-03 \mathrm{pCi} / \mathrm{g}$ 5.36E-04 $\pm 8.05 \mathrm{E}-03 \mathrm{pCi} / \mathrm{g}$ $-3.23 \mathrm{E}-03 \pm 4.05 \mathrm{E}-03 \mathrm{pCi} / \mathrm{g}$ $-6.65 \mathrm{E}-03 \pm 2.08 \mathrm{E}-02 \mathrm{pCi} / \mathrm{g}$ $1.64 \mathrm{E}-03 \pm 3.00 \mathrm{E}-03 \mathrm{pCi} / \mathrm{g}$ 9.34E-03 $\pm 5.53 \mathrm{E}-03 \mathrm{pCi} / \mathrm{g}$ $1.46 \mathrm{E}-03 \pm 2.56 \mathrm{E}-03 \mathrm{pCi} / \mathrm{g}$ $-1.20 \mathrm{E}-03 \pm 3.65 \mathrm{E}-03 \mathrm{pCi} / \mathrm{g}$ $1.26 \mathrm{E}-03 \pm 2.40 \mathrm{E}-03 \mathrm{pCi} / \mathrm{g}$ $-9.09 \mathrm{E}-03 \pm 8.35 \mathrm{E}-03 \mathrm{pCi} / \mathrm{g}$ $-1.62 \mathrm{E}-02 \pm 2.77 \mathrm{E}-02 \mathrm{pCi} / \mathrm{g}$ $0.00 \mathrm{E}+00 \quad \mathrm{pCi} / \mathrm{g}$ $3.45 \mathrm{E}+00 \pm 1.34 \mathrm{E}+00 \mathrm{pCi} / \mathrm{g}$ $3.47 \mathrm{E}-01 \pm 1.27 \mathrm{E}-02 \mathrm{pCi} / \mathrm{g}$ $\begin{array}{ll}1.62 \mathrm{E}-02 & \text { EPIA-013B } \\ 6.38 \mathrm{E}-03 & \text { EPIA-013B } \\ 1.34 \mathrm{E}-02 & \text { EPIA-013B } \\ 5.76 \mathrm{E}-03 & \text { EPIA-013B } \\ 3.58 \mathrm{E}-02 & \text { EPIA-013B } \\ 4.78 \mathrm{E}-03 & \text { EPIA-013B } \\ 5.78 \mathrm{E}-03 & \text { EPIA-013B } \\ 4.51 \mathrm{E}-03 & \text { EPIA-013B } \\ 5.45 \mathrm{E}-03 & \text { EPIA-013B } \\ 4.62 \mathrm{E}-03 & \text { EPIA-013B } \\ 1.34 \mathrm{E}-02 & \text { EPIA-013B } \\ 3.94 \mathrm{E}-02 & \text { EPIA-013B } \\ 1.98 \mathrm{E}-02 & \text { EPIA-013B } \\ 1.31 \mathrm{E}+00 & \text { EPIA-001B } \\ 8.35 \mathrm{E}-03 & \text { EPIA-013B }\end{array}$ 
SURVEY ID: PPSC 2401 (continued)

Constituent

Radionuclides

Manganese-54

Neptunium-239

Nonvolatile beta

Plutonium-238

Plutonium-239/240

Potassium-40

Promethium-144

Promethium-146

Radium-226

Radium-228

Ruthenium-106

Sodium-22

Strontium-90

Thorium-234

Tin-113

Yttrium-88

Zinc-65

Zirconium-95
Sample ID: 105117

RQ AQ B Result Unit D. Limit Method

\begin{tabular}{|c|c|c|}
\hline \multirow{3}{*}{ UI } & $1.11 \mathrm{E}-02 \pm 1.00 \mathrm{E}-02$ & $\mathrm{pCi}$ \\
\hline & 3.88E-04 $1.87 \mathrm{E}-02$ & $\mathrm{pCi} / \mathrm{g}$ \\
\hline & $2.99 \mathrm{E}+00 \pm 1.32 \mathrm{E}+00$ & $\mathrm{pCi} / \mathrm{g}$ \\
\hline UI & $1.53 \mathrm{E}-02 \pm 2.14 \mathrm{E}-02$ & $\mathrm{pCi} / \mathrm{g}$ \\
\hline UI & $1.55 \mathrm{E}-03 \pm 1.00 \mathrm{E}-02$ & $\mathrm{pCi} / \mathrm{g}$ \\
\hline V & $1.91 \mathrm{E}-01 \pm 5.55 \mathrm{E}-02$ & $\mathrm{pCi} / \mathrm{g}$ \\
\hline UI & $-1.87 \mathrm{E}-04 \pm 2.70 \mathrm{E}-03$ & $\mathrm{pCi} / \mathrm{g}$ \\
\hline & $1.72 \mathrm{E}-03 \pm 3.96 \mathrm{E}-03$ & $\mathrm{pCi} / \mathrm{g}$ \\
\hline & $2.56 \mathrm{E}-01 \pm 1.84 \mathrm{E}-02$ & $\mathrm{pCi} / \mathrm{g}$ \\
\hline & $3.50 \mathrm{E}-01 \pm 2.89 \mathrm{E}-02$ & $\mathrm{pCi} / \mathrm{g}$ \\
\hline$\pi$ & $1.74 \mathrm{E}-03 \pm 2.60 \mathrm{E}-02$ & $\mathrm{pCi} / \mathrm{g}$ \\
\hline UI & $2.84 \mathrm{E}-04 \pm 2.81 \mathrm{E}-03$ & $\mathrm{pCi}$ \\
\hline$\pi$ & $-1.29 \mathrm{E}-01 \pm 3.24 \mathrm{E}-02$ & $\mathrm{pCi} / \mathrm{g}$ \\
\hline UI & $1.81 \mathrm{E}-01 \pm 2.52 \mathrm{E}-01$ & $\mathrm{pCi} / \mathrm{g}$ \\
\hline UI & $-1.09 \mathrm{E}-03 \pm 4.05 \mathrm{E}-03$ & $\mathrm{pCi} / \mathrm{g}$ \\
\hline UI & $-7.19 \mathrm{E}-04 \pm 3.30 \mathrm{E}-03$ & pCi \\
\hline UI & $1.99 \mathrm{E}-03 \pm 6.73 \mathrm{E}-03$ & pCi \\
\hline UI & $1.00 \mathrm{E}-03 \pm 6.80 \mathrm{E}-03$ & $\mathrm{pCi}$ \\
\hline
\end{tabular}

4.85E-03 EPIA-013B

3.25E-02 EPIA-013B

2.41E+00 EPIA-001B

4.03E-02 EPIA-012B

2.62E-02 EPIA-012B

3.71E-02 EPIA-013B

4.72E-03 EPIA-013B

6.69E-03 EPIA-013B

9.26E-03 EPIA-013B

1.62E-02 EPIA-013B

4.61E-02 EPIA-013B

4.90E-03 EPIA-013B

1.58E-01 EPIA-004

2.49E-01 EPIA-013B

6.68E-03 EPIA-013B

5.85E-03 EPIA-013B

1.05E-02 EPIA-013B

1,20E-02 EPIA-013B

SURVEY ID: PPSC 2402

Sample ID: 105118

Survey location: 44906E 3692181N (SRS coordinates)

Depth of core interval: 1.00 to $2.00 \mathrm{ft}$

Sample type: Normal

Sample matrix: Soil

Percent solids: $\mathbf{8 3 . 0 0}$

Constituent

RQ AQ B Result

Unit

D. Limit Method

Physical Parameters and Specified Analyses

Ammonia nitrogen

Cation exchange capacity

Cation exchange capacity

Chloride

Cyanide

Nitrate-nitrite as nitrogen

Nitrogen by Kjeldahl method

Nitrogen by Kjeldahl method

Orthophosphate

Phenols

Phenols

Sulfide

Total organic carbon

Total organic nitrogen

Total phosphates (as P)
U 23.7

10.8

$\begin{array}{lll}\mathrm{J} & \mathrm{E} & 1.76 \\ \mathrm{U} & & 1.14\end{array}$

U $\quad \mathrm{V} \quad 0.343$

36.1

34.8

$\mathrm{U} \quad 12$

UJ C $\quad 358$

UJ C 354

UJ C 301

C 33.3

J $\quad \mathrm{E} \quad 1.75$

$\begin{array}{lll}\mathrm{mg} / \mathrm{kg} & 23.7 & \text { EPA350.1 } \\ \mathrm{meq} / 100 \mathrm{~g} & 5 & \text { EPA9081 } \\ \mathrm{meq} / 100 \mathrm{~g} & 6.02 & \text { EPA9081 } \\ \mathrm{mg} / \mathrm{kg} & 2.95 & \text { EPA300.0 } \\ \mathrm{mg} / \mathrm{kg} & 1.14 & \text { EPA335.3 } \\ \mathrm{mg} / \mathrm{kg} & 1.14 & \text { EPA353.1 } \\ \mathrm{mg} / \mathrm{kg} & 4.78 & \text { EPA351.2 } \\ \mathrm{mg} / \mathrm{kg} & 4.76 & \text { EPA351.2 } \\ \mathrm{mg} / \mathrm{kg} & 12 & \text { EPA365.2 } \\ \mu \mathrm{g} / \mathrm{kg} & 358 & \text { EPA420.2 } \\ \mu \mathrm{g} / \mathrm{kg} & 354 & \text { EPA420.2 } \\ \mathrm{mg} / \mathrm{kg} & 301 & \text { EPA376.2 } \\ \mathrm{mg} / \mathrm{kg} & 120 & \text { EPA415.1 } \\ \mathrm{mg} / \mathrm{kg} & 0.241 & \text { EPA351.2 } \\ \mathrm{mg} / \mathrm{kg} & 11.2 & \text { EPA365.4 }\end{array}$


SURVEY ID: PPSC 2402 (continued)

Constituent

RQ AQ B Result

Metals (total recoverable)

\begin{tabular}{|c|c|c|c|}
\hline Aluminum & & V & 348 \\
\hline Antimony & U & & 5.66 \\
\hline Arsenic & $\mathrm{U}$ & & 17 \\
\hline Barium & $\bar{J}$ & $\mathrm{C}$ & 1.95 \\
\hline Beryllium & $J$ & EC & 0.0571 \\
\hline Cadmium & $\mathrm{U}$ & & 0.283 \\
\hline Calcium & & $\mathrm{V}$ & 38.9 \\
\hline Chromium & $\mathbf{J}$ & E & 0.434 \\
\hline Cobalt & & & 0.567 \\
\hline Copper & $\mathrm{U}$ & & 1.41 \\
\hline Iron & $\mathrm{J}$ & $\mathrm{C}$ & 119 \\
\hline Lead & $\mathrm{U}$ & & 5.66 \\
\hline Magnesium & & & 7.35 \\
\hline Manganese & & & 5.37 \\
\hline Mercury & $\mathrm{U}$ & & 0.0379 \\
\hline Nickel & $\mathrm{U}$ & & 1.41 \\
\hline Potassium & $J$ & $\mathbf{E}$ & 15.7 \\
\hline Selenium & $\mathrm{U}$ & & 17 \\
\hline Silver & $\mathrm{U}$ & & 1.41 \\
\hline Sodium & $\mathrm{U}$ & & 56.6 \\
\hline Thallium & $\mathrm{U}$ & & 17 \\
\hline Vanadium & UJ & $\mathrm{C}$ & 0.566 \\
\hline Zinc & & $V$ & 1.4 \\
\hline
\end{tabular}

$B / N / A$ Extractables (including $P A H$ and phenols)
Sample ID: 105118

Unit D. Limit Method

$\begin{array}{lll}\mathrm{mg} / \mathrm{kg} & 14.1 & \text { EPA6010A } \\ \mathrm{mg} / \mathrm{kg} & 5.66 & \text { EPA6010A } \\ \mathrm{mg} / \mathrm{kg} & 17 & \text { EPA6010A } \\ \mathrm{mg} / \mathrm{kg} & 1.41 & \text { EPA6010A } \\ \mathrm{mg} / \mathrm{kg} & 0.566 & \text { EPA6010A } \\ \mathrm{mg} / \mathrm{kg} & 0.283 & \text { EPA6010A } \\ \mathrm{mg} / \mathrm{kg} & 14.1 & \text { EPA6010A } \\ \mathrm{mg} / \mathrm{kg} & 1.41 & \text { EPA6010A } \\ \mathrm{mg} / \mathrm{kg} & 0.566 & \text { EPA6010A } \\ \mathrm{mg} / \mathrm{kg} & 1.41 & \text { EPA6010A } \\ \mathrm{mg} / \mathrm{kg} & 5.66 & \text { EPA6010A } \\ \mathrm{mg} / \mathrm{kg} & 5.66 & \text { EPA6010A } \\ \mathrm{mg} / \mathrm{kg} & 5.66 & \text { EPA6010A } \\ \mathrm{mg} / \mathrm{kg} & 0.566 & \text { EPA6010A } \\ \mathrm{mg} / \mathrm{kg} & 0.0379 & \text { EPA7471 } \\ \mathrm{mg} / \mathrm{kg} & 1.41 & \text { EPA6010A } \\ \mathrm{mg} / \mathrm{kg} & 113 & \text { EPA6010A } \\ \mathrm{mg} / \mathrm{kg} & 17 & \text { EPA6010A } \\ \mathrm{mg} / \mathrm{kg} & 1.41 & \text { EPA6010A } \\ \mathrm{mg} / \mathrm{kg} & 56.6 & \text { EPA6010A } \\ \mathrm{mg} / \mathrm{kg} & 17 & \text { EPA6010A } \\ \mathrm{mg} / \mathrm{kg} & 0.566 & \text { EPA6010A } \\ \mathrm{mg} / \mathrm{kg} & 1.13 & \text { EPA6010A }\end{array}$

\begin{tabular}{|c|c|c|c|c|c|}
\hline Acenaphthene & $\mathrm{U}$ & 39.6 & $\mu \mathrm{g} / \mathrm{kg}$ & 39.6 & EPA8270 \\
\hline Acenaphthylene & $\mathrm{U}$ & 39.6 & $\mu \mathrm{g} / \mathrm{kg}$ & 39.6 & EPA8270 \\
\hline Anthracene & $\mathbf{U}$ & 39.6 & $\mu \mathrm{g} / \mathrm{kg}$ & 39.6 & EPA8270 \\
\hline Benzo[a]anthracene & $\mathrm{U}$ & 3.96 & $\mu \mathrm{g} / \mathrm{kg}$ & 3.96 & EPA8270 \\
\hline Benzo $[b]$ fluoranthene & $\mathrm{U}$ & 3.96 & $\mu \mathrm{g} / \mathrm{kg}$ & 3.96 & EPA8270 \\
\hline Benzo $[k]$ fluoranthene & $\mathrm{U}$ & 3.96 & $\mu \mathrm{g} / \mathrm{kg}$ & 3.96 & EPA8270 \\
\hline Benzoic acid & $\mathrm{U}$ & 39.6 & $\mu \mathrm{g} / \mathrm{kg}$ & 39.6 & EPA8270 \\
\hline Benzo[g, $h, i]$ perylene & $\mathrm{U}$ & 39.6 & $\mu \mathrm{g} / \mathrm{kg}$ & 39.6 & EPA8270 \\
\hline Benzo[a]pyrene & $\mathrm{U}$ & 3.96 & $\mu \mathrm{g} / \mathrm{kg}$ & 3.96 & EPA8270 \\
\hline Benzyl alcohol & $\mathbf{U}$ & 39.6 & $\mu \mathrm{g} / \mathrm{kg}$ & 39.6 & EPA8270 \\
\hline Bis(2-chloroethoxy) methane & $\mathrm{U}$ & 39.6 & $\mu g / \mathrm{kg}$ & 39.6 & EPA8270 \\
\hline Bis(2-chloroethyl) ether & $\mathrm{U}$ & 39.6 & $\mu \mathrm{g} / \mathrm{kg}$ & 39.6 & EPA8270 \\
\hline Bis(2-chloroisopropyl) ether & $\mathrm{U}$ & 39.6 & $\mu \mathrm{g} / \mathrm{kg}$ & 39.6 & EPA8270 \\
\hline Bis(2-ethylhexyl) phthalate & & 61.5 & $\mu \mathrm{g} / \mathrm{kg}$ & 39.6 & EPA8270 \\
\hline 4-Bromophenyl phenyl ether & $\mathrm{U}$ & 39.6 & $\mu \mathrm{g} / \mathrm{kg}$ & 39.6 & EPA8270 \\
\hline Butylbenzyl phthalate & $\mathrm{U}$ & 39.6 & $\mu \mathrm{g} / \mathrm{kg}$ & 39.6 & EPA8270 \\
\hline 4-Chloroaniline & U & 39.6 & $\mu \mathrm{g} / \mathrm{kg}$ & 39.6 & EPA8270 \\
\hline 4-Chloro-m-cresol & $\mathrm{U}$ & 39.6 & $\mu \mathrm{g} / \mathrm{kg}$ & 39.6 & EPA8270 \\
\hline 2-Chloronaphthalene & $\mathrm{U}$ & 39.6 & $\mu \mathrm{g} / \mathrm{kg}$ & 39.6 & EPA8270 \\
\hline 2-Chlorophenol & $\mathrm{U}$ & 39.6 & $\mu \mathrm{g} / \mathrm{kg}$ & 39.6 & EPA8270 \\
\hline 4-Chlorophenyl phenyl ether & $\mathrm{U}$ & 39.6 & $\mu \mathrm{g} / \mathrm{kg}$ & 39.6 & EPA8270 \\
\hline Chrysene & $\mathrm{U}$ & 3.96 & $\mu \mathrm{g} / \mathrm{kg}$ & 3.96 & EPA8270 \\
\hline $\mathrm{m} / \mathrm{p}$-Cresol & $\mathrm{U}$ & 396 & $\mu \mathrm{g} / \mathrm{kg}$ & 396 & EPA8270 \\
\hline o-Cresol (2-Methylphenol) & $\mathrm{U}$ & 39.6 & $\mu \mathrm{g} / \mathrm{kg}$ & 39.6 & EPA8270 \\
\hline Dibenz $[a, h]$ anthracene & U & 3.96 & $\mu \mathrm{g} / \mathrm{kg}$ & 3.96 & EPA8270 \\
\hline Dibenzofuran & $\mathrm{U}$ & 39.6 & $\mu \mathrm{g} / \mathrm{kg}$ & 39.6 & EPA8270 \\
\hline Di-n-butyl phthalate & $\mathrm{U}$ & 39.6 & $\mu \mathrm{g} / \mathrm{kg}$ & 39.6 & EPA8270 \\
\hline 1,2-Dichlorobenzene & $\mathrm{U}$ & 39.6 & $\mu \mathrm{g} / \mathrm{kg}$ & 39.6 & EPA8270 \\
\hline 1,3-Dichlorobenzene & $\mathrm{U}$ & 39.6 & $\mu \mathrm{g} / \mathrm{kg}$ & 39.6 & EPA8270 \\
\hline
\end{tabular}


SURVEY ID: PPSC 2402 (continued)

Sample ID: 105118

Constituent

RQ AQ B Result

Unit

D. Limit Method

$B / N / A$ Extractables (including $P A H$ and phenols)

1,4-Dichlorobenzene
3,3'-Dichlorobenzidine
2,4-Dichlorophenol
Diethyl phthalate
2,4-Dimethyl phenol
Dimethyl phthalate
2,4-Dinitrophenol
2,4-Dinitrotoluene
2,6-Dinitrotoluene
Di-n-octyl phthalate
Fluoranthene
Fluorene
Hexachlorobenzene
Hexachlorobutadiene
Hexachlorocyclopentadiene
Hexachloroethane
Indeno[1,2,3-c,d]pyrene
Isophorone
2-Methyl-4,6-dinitrophenol
2-Methylnaphthalene
Naphthalene
m-Nitroaniline
o-Nitroaniline
p-Nitroaniline
Nitrobenzene
2-Nitrophenol
4-Nitrophenol
N-Nitrosodiphenylamine
N-Nitrosodi-n-propylamine
Pentachlorophenol
Phenanthrene
Phenol
Pyrene
1,2,4-Trichlorobenzene
2,4,5-Trichlorophenol
2,4,6-Trichlorophenol

\begin{tabular}{|c|c|c|}
\hline U & & 39.6 \\
\hline U & & 39.6 \\
\hline $\mathrm{U}$ & & 39.6 \\
\hline U & & 39.6 \\
\hline $\mathrm{U}$ & & 39.6 \\
\hline $\mathrm{U}$ & & 39.6 \\
\hline $\mathbf{U}$ & & 396 \\
\hline & C & 3.96 \\
\hline $\mathrm{U}$ & & 3.96 \\
\hline $\mathrm{U}$ & & 39.6 \\
\hline $\mathrm{U}$ & & 39.6 \\
\hline U & & 39.6 \\
\hline U & & 3.96 \\
\hline$U$ & & 39.6 \\
\hline $\mathrm{U}$ & & 39.6 \\
\hline $\mathrm{U}$ & & 3.96 \\
\hline U & & 3.96 \\
\hline $\mathrm{U}$ & & 39.6 \\
\hline $\mathrm{U}$ & & 396 \\
\hline$U$ & & 39.6 \\
\hline $\mathbf{U}$ & & 39.6 \\
\hline $\mathbf{U}$ & & 39.6 \\
\hline U & & 39.6 \\
\hline $\mathbf{U}$ & & 39.6 \\
\hline $\mathbf{U}$ & & 39.6 \\
\hline U & & 39.6 \\
\hline$U$ & & 39.6 \\
\hline U & & 39.6 \\
\hline U & & 39.6 \\
\hline $\mathrm{U}$ & & 39.6 \\
\hline $\mathrm{U}$ & & 39.6 \\
\hline $\mathrm{U}$ & & 39.6 \\
\hline $\mathrm{U}$ & & 39.6 \\
\hline U & & 3.96 \\
\hline $\mathrm{U}$ & & 39.6 \\
\hline U & & 39.6 \\
\hline
\end{tabular}

$\begin{array}{lll}\mu \mathrm{g} / \mathrm{kg} & 39.6 & \text { EPA8270 } \\ \mu \mathrm{g} / \mathrm{kg} & 39.6 & \text { EPA8270 } \\ \mu \mathrm{g} / \mathrm{kg} & 39.6 & \text { EPA8270 } \\ \mu \mathrm{g} / \mathrm{kg} & 39.6 & \text { EPA8270 } \\ \mu \mathrm{g} / \mathrm{kg} & 39.6 & \text { EPA8270 } \\ \mu \mathrm{g} / \mathrm{kg} & 39.6 & \text { EPA8270 } \\ \mu \mathrm{g} / \mathrm{kg} & 396 & \text { EPA8270 } \\ \mu \mathrm{g} / \mathrm{kg} & 3.96 & \text { EPA8270 } \\ \mu \mathrm{g} / \mathrm{kg} & 3.96 & \text { EPA8270 } \\ \mu \mathrm{g} / \mathrm{kg} & 39.6 & \text { EPA8270 } \\ \mu \mathrm{g} / \mathrm{kg} & 39.6 & \text { EPA8270 } \\ \mu \mathrm{g} / \mathrm{kg} & 39.6 & \text { EPA8270 } \\ \mu \mathrm{g} / \mathrm{kg} & 3.96 & \text { EPA8270 } \\ \mu \mathrm{g} / \mathrm{kg} & 39.6 & \text { EPA8270 } \\ \mu \mathrm{g} / \mathrm{kg} & 39.6 & \text { EPA8270 } \\ \mu \mathrm{g} / \mathrm{kg} & 3.96 & \text { EPA8270 } \\ \mu \mathrm{g} / \mathrm{kg} & 3.96 & \text { EPA8270 } \\ \mu \mathrm{g} / \mathrm{kg} & 39.6 & \text { EPA8270 } \\ \mu \mathrm{g} / \mathrm{kg} & 396 & \text { EPA8270 } \\ \mu \mathrm{g} / \mathrm{kg} & 39.6 & \text { EPA8270 } \\ \mu \mathrm{g} / \mathrm{kg} & 39.6 & \text { EPA8270 } \\ \mu \mathrm{g} / \mathrm{kg} & 39.6 & \text { EPA8270 } \\ \mu \mathrm{g} / \mathrm{kg} & 39.6 & \text { EPA8270 } \\ \mu \mathrm{g} / \mathrm{kg} & 39.6 & \text { EPA8270 } \\ \mu \mathrm{g} / \mathrm{kg} & 39.6 & \text { EPA8270 } \\ \mu \mathrm{g} / \mathrm{kg} & 39.6 & \text { EPA8270 } \\ \mu \mathrm{g} / \mathrm{kg} & 39.6 & \text { EPA8270 } \\ \mu \mathrm{g} / \mathrm{kg} & 39.6 & \text { EPA8270 } \\ \mu \mathrm{g} / \mathrm{kg} & 39.6 & \text { EPA8270 } \\ \mu \mathrm{g} / \mathrm{kg} & 39.6 & \text { EPA8270 } \\ \mu \mathrm{g} / \mathrm{kg} & 39.6 & \text { EPA8270 } \\ \mu \mathrm{g} / \mathrm{kg} & 39.6 & \text { EPA8270 } \\ \mu \mathrm{g} / \mathrm{kg} & 39.6 & \text { EPA8270 } \\ \mu \mathrm{g} / \mathrm{kg} & 3.96 & \text { EPA8270 } \\ \mu \mathrm{g} / \mathrm{kg} & 39.6 & \text { EPA8270 } \\ \mu \mathrm{g} / \mathrm{kg} & 39.6 & \text { EPA8270 }\end{array}$

Volatile Organic Compounds

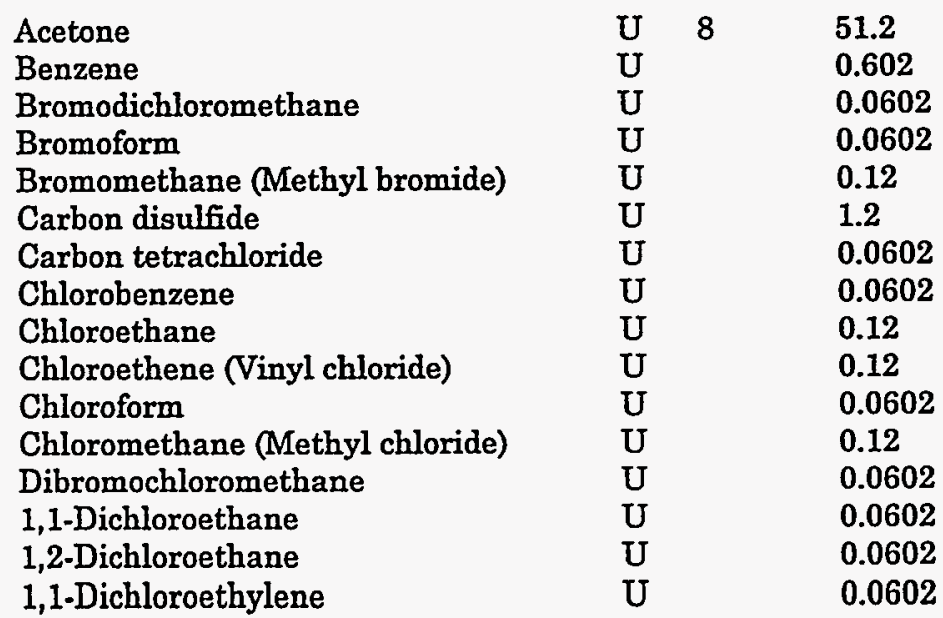

$\begin{array}{lll}\mu \mathrm{g} / \mathrm{kg} & 6.02 & \text { EPA8260 } \\ \mu \mathrm{ggg} & 0.602 & \text { EPA8260 } \\ \mu \mathrm{g} / \mathrm{kg} & 0.0602 & \text { EPA8260 } \\ \mu \mathrm{g} / \mathrm{kg} & 0.0602 & \text { EPA8260 } \\ \mu \mathrm{g} / \mathrm{kg} & 0.12 & \text { EPA8260 } \\ \mu \mathrm{g} / \mathrm{kg} & 1.2 & \text { EPA8260 } \\ \mu \mathrm{g} / \mathrm{kg} & 0.0602 & \text { EPA8260 } \\ \mu \mathrm{g} / \mathrm{kg} & 0.0602 & \text { EPA8260 } \\ \mu \mathrm{g} / \mathrm{kg} & 0.12 & \text { EPA8260 } \\ \mu \mathrm{g} / \mathrm{kg} & 0.12 & \text { EPA8260 } \\ \mu \mathrm{g} / \mathrm{kg} & 0.0602 & \text { EPA8260 } \\ \mu \mathrm{g} / \mathrm{kg} & 0.12 & \text { EPA8260 } \\ \mu \mathrm{g} / \mathrm{kg} & 0.0602 & \text { EPA8260 } \\ \mu \mathrm{g} / \mathrm{kg} & 0.0602 & \text { EPA8260 } \\ \mu \mathrm{g} / \mathrm{kg} & 0.0602 & \text { EPA8260 } \\ \mu \mathrm{g} / \mathrm{kg} & 0.0602 & \text { EPA8260 }\end{array}$


SURVEY ID: PPSC 2402 (continued)

Sample ID: 105118

Constituent

Volatile Organic Compounds

1,2-Dichloroethylene

Dichloromethane (Methylene chloride)

1,2-Dichloropropane

cis-1,3-Dichloropropene

trans-1,3-Dichloropropene

Ethylbenzene

2-Hexanone

Methyl ethyl ketone

Methyl isobutyl ketone

Styrene

1,1,2,2-Tetrachloroethane

Tetrachloroethylene

Toluene

1,1,1-Trichloroethane

1,1,2-Trichloroethane

Trichloroethylene

Unknown hydrocarbon

Vinyl acetate

Xylenes

Pesticides/PCBs and Dioxins/Furans

Aldrin

alpha-Benzene hexachloride

beta-Benzene hexachloride

delta-Benzene hexachloride

alpha-Chlordane

gamma-Chlordane

p,p'-DDD

$p, p^{\prime}-D D E$

p,p'-DDT

Dieldrin

Endosulfan sulfate

Endosulfan I

Endosulfan II

Endrin

Endrin ketone

Heptachlor

Heptachlor epoxide

Lindane

Methoxychlor

PCB 1016

PCB 1221

PCB 1232

PCB 1242

PCB 1248

PCB 1254

PCB 1260

Toxaphene
RQ AQ B Result

D. Limit Method

$\begin{array}{lll}\text { U } & & \mathbf{0 . 1 2} \\ \mathrm{U} & \mathrm{V} & \mathbf{1 . 3} \\ \mathrm{U} & & \mathbf{0 . 0 6 0 2} \\ \mathrm{U} & & \mathbf{0 . 0 6 0 2} \\ \mathrm{U} & & \mathbf{0 . 0 6 0 2} \\ \mathrm{U} & & \mathbf{0 . 0 6 0 2} \\ \mathrm{U} & & 1.2 \\ \mathrm{U} & \mathrm{V} & 1.17 \\ \mathrm{U} & & 1.2 \\ \mathrm{U} & & 0.0602 \\ \mathrm{U} & & \mathbf{0 . 0 6 0 2} \\ \mathrm{U} & & \mathbf{0 . 0 6 0 2} \\ \mathrm{U} & & \mathbf{0 . 6 0 2} \\ & & \mathbf{0 . 0 7 2 3} \\ \mathrm{U} & & \mathbf{0 . 0 6 0 2} \\ \mathrm{U} & & \mathbf{0 . 0 6 0 2} \\ \mathrm{J} & \mathrm{N} & \mathbf{2 . 5} \\ \mathrm{U} & & 1.2 \\ \mathrm{U} & & \mathbf{0 . 1 8 1}\end{array}$

$\mu g / \mathrm{kg}$
$\mu \mathrm{g} / \mathrm{kg}$
$\mu \mathrm{g} / \mathrm{kg}$
$\mu \mathrm{g} / \mathrm{kg}$
$\mu \mathrm{g} / \mathrm{kg}$
$\mu \mathrm{g} / \mathrm{kg}$
$\mu \mathrm{g} / \mathrm{kg}$
$\mu \mathrm{g} / \mathrm{kg}$
$\mu \mathrm{g} / \mathrm{kg}$
$\mu \mathrm{g} / \mathrm{kg}$
$\mu \mathrm{g} / \mathrm{kg}$
$\mu \mathrm{g} / \mathrm{kg}$
$\mu \mathrm{g} / \mathrm{kg}$
$\mu \mathrm{g} / \mathrm{kg}$
$\mu \mathrm{g} / \mathrm{kg}$
$\mu \mathrm{g} / \mathrm{kg}$
$\mu \mathrm{g} / \mathrm{kg}$
$\mu \mathrm{g} / \mathrm{kg}$
$\mu \mathrm{g} / \mathrm{kg}$

0.12

EPA8260

0.602

0.0602

0.0602

0.0602

0.0602

1.2

1.2

1.2

0.0602

0.0602

0.0602

0.602

0.0602

0.0602

0.0602

1.2

0.181

EPA8260

EPA8260

EPA8260

EPA8260

EPA8260

EPA8260

EPA8260

EPA8260

EPA8260

EPA8260

EPA8260

EPA8260

EPA8260

EPA8260

EPA8260

EPA8260

EPA8260

EPA8260

$\mu \mathrm{g} / \mathrm{kg}$

$\mu \mathrm{g} / \mathrm{kg}$

$\mu \mathrm{g} / \mathrm{kg}$

$\mu \mathrm{g} / \mathrm{kg}$

$\mu \mathrm{g} / \mathrm{kg}$

$\mu \mathrm{g} / \mathrm{kg}$

$\mu \mathrm{g} / \mathrm{kg}$

$\mu g / k g$

$\mu \mathrm{g} / \mathrm{kg}$

$\mu \mathrm{g} / \mathrm{kg}$

$\mu g / \mathrm{kg}$

$\mu \mathrm{g} / \mathrm{kg}$

$\mu \mathrm{g} / \mathrm{kg}$

$\mu \mathrm{g} / \mathrm{kg}$

$\mu \mathrm{g} / \mathrm{kg}$

$\mu \mathrm{g} / \mathrm{kg}$

$\mu \mathrm{g} / \mathrm{kg}$

$\mu \mathrm{g} / \mathrm{kg}$

$\mu \mathrm{g} / \mathrm{kg}$

$\mu \mathrm{g} / \mathrm{kg}$

$\mu \mathrm{g} / \mathrm{kg}$

$\mu \mathrm{g} / \mathrm{kg}$

$\mu \mathrm{g} / \mathrm{kg}$

$\mu \mathrm{g} / \mathrm{kg}$

$\mu \mathrm{g} / \mathrm{kg}$

$\mu \mathrm{g} / \mathrm{kg}$

$\mu \mathrm{g} / \mathrm{kg}$

$\begin{array}{ll}4.9 & \text { EPA8080 } \\ 4.9 & \text { EPA8080 } \\ 9.8 & \text { EPA8080 } \\ 9.8 & \text { EPA8080 } \\ 9.8 & \text { EPA8080 } \\ 9.8 & \text { EPA8080 } \\ 19.6 & \text { EPA8080 } \\ 9.8 & \text { EPA8080 } \\ 19.6 & \text { EPA8080 } \\ 9.8 & \text { EPA8080 } \\ 19.6 & \text { EPA8080 } \\ 9.8 & \text { EPA8080 } \\ 19.6 & \text { EPA8080 } \\ 9.8 & \text { EPA8080 } \\ 19.6 & \text { EPA8080 } \\ 9.8 & \text { EPA8080 } \\ 9.8 & \text { EPA8080 } \\ 4.9 & \text { EPA8080 } \\ 98 & \text { EPA8080 } \\ 24.5 & \text { EPA8080 } \\ 24.5 & \text { EPA8080 } \\ 24.5 & \text { EPA8080 } \\ 24.5 & \text { EPA8080 } \\ 24.5 & \text { EPA8080 } \\ 24.5 & \text { EPA8080 } \\ 24.5 & \text { EPA8080 } \\ 196 & \text { EPA8080 }\end{array}$


SURVEY ID: PPSC 2402 (continued)

Constituent

Radionuclides

Actinium-228

Antimony-124

Antimony-125

Barium-133

Cerium-144

Cesium-134

Cesium-137

Cobalt-57

Cobalt-58

Cobalt-60

Europium-152

Europium-154

Europium-155

Gross alpha

Lead-212

Manganese-54

Neptunium-239

Nonvolatile beta

Plutonium-238

Plutonium-239/240

Potassium-40

Promethium-144

Promethium-146

Radium-226

Radium-228

Ruthenium-106

Sodium-22

Strontium-90

Thorium-234

Tin-113

Yttrium-88

Zinc-65

Zirconium-95
Sample ID: 105118

RQ AQ B Result Unit D. Limit Method
2.55E-02 EPIA-013B
8.94E-03 EPIA-013B
1.97E-02 EPIA-013B
8.50E-03 EPIA-013B
5.38E-02 EPIA-013B
6.76E-03 EPIA-013B
7.63E-03 EPIA-013B
6.85E-03 EPIA-013B
8.31E-03 EPIA-013B
6.89E-03 EPIA-013B
1.93E-02 EPIA-013B
6.50E-02 EPIA-013B
2.86E-02 EPIA-013B
$1.56 \mathrm{E}+00$ EPIA-001B
1.20E-02 EPIA-013B
7.56E-03 EPIA-013B
4.97E-02 EPIA-013B
2.35E+00 EPLA-001B
3.69E-02 EPIA-012B
7.48E-03 EPIA-012B
6.63E-02 EPIA-013B
7.07E-03 EPIA-013B
9.20E-03 EPIA-013B
1.33E-02 EPIA-013B
2.55E-02 EPIA-013B
6.51E-02 EPIA-013B
7.43E-03 EPIA-013B
1.41E-01 EPIA-004
3.69E-01 EPIA-013B
9.95E-03 EPIA-013B
7.41E-03 EPIA-013B
1.53E-02 EPIA-013B
1.67E-02 EPIA-013B

SURVEY ID: PPSC 2403

Sample ID: 105119

Survey location: 44906E 3692181N (SRS coordinates)

Depth of core interval: 2.00 to $4.00 \mathrm{ft}$

Sample type: Normal

Sample matrix: Soil

Percent solids: $\mathbf{8 7 . 0 0}$

Constituent

RQ AQ B Result

Unit

D. Limit Method

Physical Parameters and Specified Analyses

$\begin{array}{lllll}\text { Ammonia nitrogen } & \text { U } & & & 22.8 \\ \text { Cation exchange capacity } & & & 18.6 \\ \text { Chloride } & \text { J } & \text { E } & 2.45 \\ \text { Cyanide } & \text { U } & & \\ \text { Nitrate-nitrite as nitrogen } & \text { U } & & 1.11 \\ \text { Nitrogen by Kjeldahl method } & & & & 1.03 \\ \text { Orthophosphate } & \text { UJ } & \text { I } & \text { L } & 11.8 \\ \text { Orthophosphate } & \text { UJ } & \text { I } & \text { L } & 11.5\end{array}$

$\begin{array}{lll}\mathrm{mg} / \mathrm{kg} & 22.8 & \text { EPA350.1 } \\ \mathrm{meq} / 100 \mathrm{~g} & 5 & \text { EPA9081 } \\ \mathrm{mg} / \mathrm{kg} & 2.77 & \text { EPA300.0 } \\ \mathrm{mg} / \mathrm{kg} & 1.11 & \text { EPA335.3 } \\ \mathrm{mg} / \mathrm{kg} & 1.03 & \text { EPA353.1 } \\ \mathrm{mg} / \mathrm{kg} & 4.56 & \text { EPA351.2 } \\ \mathrm{mg} / \mathrm{kg} & 11.5 & \text { EPA365.2 } \\ \mathrm{mg} / \mathrm{kg} & 11.5 & \text { EPA365.2 }\end{array}$


SURVEY ID: PPSC 2403 (continued)

Sample ID: 105119

Constituent

RQ AQ B Result

Unit

D. Limit Method

Physical Parameters and Specified Analyses

Phenols

Sulfide

Total organic carbon

Total organic nitrogen

Total phosphates (as P)

Metals (total recoverable)

Aluminum
Antimony
Arsenic
Barium
Beryllium
Cadmium
Calcium
Chromium
Cobalt
Copper
Iron
Lead
Magnesium
Manganese
Mercury
Nickel
Potassium
Selenium
Silver
Sodium
Thallium
Vanadium
Zinc

$\begin{array}{lll}\mu \mathrm{g} / \mathrm{kg} & 229 & \text { EPA420.2 } \\ \mathrm{mg} / \mathrm{kg} & 287 & \text { EPA376.2 } \\ \mathrm{mg} / \mathrm{kg} & 115 & \text { EPA415.1 } \\ \mathrm{mg} / \mathrm{kg} & \mathbf{0 . 2 3} & \text { EPA351.2 } \\ \mathrm{mg} / \mathrm{kg} & \mathbf{9 2 . 4} & \text { EPA365.4 }\end{array}$

$\begin{array}{lll}\mathrm{mg} / \mathrm{kg} & 13.6 & \text { EPA6010A } \\ \mathrm{mg} / \mathrm{kg} & 5.45 & \text { EPA6010A } \\ \mathrm{mg} / \mathrm{kg} & 16.3 & \text { EPA6010A } \\ \mathrm{mg} / \mathrm{kg} & 1.36 & \text { EPA6010A } \\ \mathrm{mg} / \mathrm{kg} & \mathbf{0 . 5 4 5} & \text { EPA6010A } \\ \mathrm{mg} / \mathrm{kg} & 0.272 & \text { EPA6010A } \\ \mathrm{mg} / \mathrm{kg} & 13.6 & \text { EPA6010A } \\ \mathrm{mg} / \mathrm{kg} & 1.36 & \text { EPA6010A } \\ \mathrm{mg} / \mathrm{kg} & 0.545 & \text { EPA6010A } \\ \mathrm{mg} / \mathrm{kg} & 1.36 & \text { EPA6010A } \\ \mathrm{mg} / \mathrm{kg} & 5.45 & \text { EPA6010A } \\ \mathrm{mg} / \mathrm{kg} & 5.45 & \text { EPA6010A } \\ \mathrm{mg} / \mathrm{kg} & 5.45 & \text { EPA6010A } \\ \mathrm{mg} / \mathrm{kg} & 0.545 & \text { EPA6010A } \\ \mathrm{mg} / \mathrm{kg} & 0.0304 & \text { EPA7471 } \\ \mathrm{mg} / \mathrm{kg} & 1.36 & \text { EPA6010A } \\ \mathrm{mg} / \mathrm{kg} & 109 & \text { EPA6010A } \\ \mathrm{mg} / \mathrm{kg} & 16.3 & \text { EPA6010A } \\ \mathrm{mg} / \mathrm{kg} & 1.36 & \text { EPA6010A } \\ \mathrm{mg} / \mathrm{kg} & 54.5 & \text { EPA6010A } \\ \mathrm{mg} / \mathrm{kg} & 16.3 & \text { EPA6010A } \\ \mathrm{mg} / \mathrm{kg} & 0.545 & \text { EPA6010A } \\ \mathrm{mg} / \mathrm{kg} & 1.09 & \text { EPA6010A }\end{array}$

$B / N / A$ Extractables (including $P A H$ and phenols)

$\begin{array}{llllll}\text { Acenaphthene } & \mathrm{U} & 37.9 & \mu \mathrm{g} / \mathrm{kg} & 37.9 & \text { EPA8270 } \\ \text { Acenaphthylene } & \mathrm{U} & 37.9 & \mu \mathrm{g} / \mathrm{kg} & 37.9 & \text { EPA8270 } \\ \text { Anthracene } & \mathrm{U} & 37.9 & \mu \mathrm{g} / \mathrm{kg} & 37.9 & \text { EPA8270 } \\ \text { Benzo[a]anthracene } & \mathrm{U} & 3.79 & \mu \mathrm{g} / \mathrm{kg} & 3.79 & \text { EPA8270 } \\ \text { Benzo[b]fluoranthene } & \mathrm{U} & 3.79 & \mu \mathrm{g} / \mathrm{kg} & 3.79 & \text { EPA8270 } \\ \text { Benzo[k]fluoranthene } & \mathrm{U} & 3.79 & \mu \mathrm{g} / \mathrm{kg} & 3.79 & \text { EPA8270 } \\ \text { Benzoic acid } & & 61.4 & \mu \mathrm{g} / \mathrm{kg} & 37.9 & \text { EPA8270 } \\ \text { Benzo[g,h,i]perylene } & \mathrm{U} & 37.9 & \mu \mathrm{g} / \mathrm{kg} & 37.9 & \text { EPA8270 } \\ \text { Benzo[a]pyrene } & \mathrm{U} & 3.79 & \mu \mathrm{g} / \mathrm{kg} & 3.79 & \text { EPA8270 } \\ \text { Benzyl alcohol } & \mathrm{U} & 37.9 & \mu \mathrm{g} / \mathrm{kg} & 37.9 & \text { EPA8270 } \\ \text { Bis(2-chloroethoxy) methane } & \mathrm{U} & 37.9 & \mu \mathrm{g} / \mathrm{kg} & 37.9 & \text { EPA8270 } \\ \text { Bis(2-chloroethyl) ether } & \mathrm{U} & 37.9 & \mu \mathrm{g} / \mathrm{kg} & 37.9 & \text { EPA8270 } \\ \text { Bis(2-chloroisopropyl) ether } & \mathrm{U} & 37.9 & \mu \mathrm{g} / \mathrm{kg} & 37.9 & \text { EPA8270 } \\ \text { Bis(2-ethylhexyl) phthalate } & & 66.7 & \mu \mathrm{g} / \mathrm{kg} & 37.9 & \text { EPA8270 } \\ \text { 4-Bromophenyl phenyl ether } & \mathrm{U} & 37.9 & \mu \mathrm{g} / \mathrm{kg} & 37.9 & \text { EPA8270 } \\ \text { Butylbenzyl phthalate } & \mathrm{U} & 37.9 & \mu \mathrm{g} / \mathrm{kg} & 37.9 & \text { EPA8270 } \\ \text { 4-Chloroaniline } & \mathrm{U} & 37.9 & \mu \mathrm{g} / \mathrm{kg} & 37.9 & \text { EPA8270 } \\ \text { 4-Chloro-m-cresol } & \mathrm{U} & 37.9 & \mu \mathrm{g} / \mathrm{kg} & 37.9 & \text { EPA8270 } \\ \text { 2-Chloronaphthalene } & \mathrm{U} & 37.9 & \mu \mathrm{g} / \mathrm{kg} & 37.9 & \text { EPA8270 } \\ \text { 2-Chlorophenol } & \mathrm{U} & 37.9 & \mu \mathrm{g} / \mathrm{kg} & 37.9 & \text { EPA8270 } \\ \text { 4-Chlorophenyl phenyl ether } & \mathrm{U} & 37.9 & \mu \mathrm{g} / \mathrm{kg} & 37.9 & \text { EPA8270 }\end{array}$


SURVEY ID: PPSC 2403 (continued)

Sample ID: 105119

Constituent

RQ AQ B Result

Unit

D. Limit Method

$B / N / A$ Extractables (including PAH and phenols)

\begin{tabular}{|c|c|c|c|}
\hline Chrysene & $U^{U}$ & & 3.79 \\
\hline $\mathrm{m} / \mathrm{p}$-Cresol & U & & 379 \\
\hline o-Cresol (2-Methylphenol) & $\mathrm{U}$ & & 37.9 \\
\hline Dibenz $[a, h]$ anthracene & $\mathrm{U}$ & & 3.79 \\
\hline Dibenzofuran & $\mathrm{U}$ & & 37.9 \\
\hline Di-n-butyl phthalate & $\mathrm{U}$ & $\mathrm{V}$ & 32.2 \\
\hline 1,2-Dichlorobenzene & $\mathrm{U}$ & & 37.9 \\
\hline 1,3-Dichlorobenzene & $\mathrm{U}$ & & 37.9 \\
\hline 1,4-Dichlorobenzene & $\mathrm{U}$ & & 37.9 \\
\hline 3,3'-Dichlorobenzidine & $U$ & & 37.9 \\
\hline 2,4-Dichlorophenol & $\mathrm{U}$ & & 37.9 \\
\hline Diethyl phthalate & $\mathrm{U}$ & & 37.9 \\
\hline 2,4-Dimethyl phenol & $\mathrm{U}$ & & 37.9 \\
\hline Dimethyl phthalate & $\mathbf{U}$ & & 37.9 \\
\hline 2,4-Dinitrophenol & U & & 379 \\
\hline 2,4-Dinitrotoluene & UJ & C & 3.79 \\
\hline 2,6-Dinitrotoluene & $\mathrm{U}$ & & 3.79 \\
\hline Di-n-octyl phthalate & $\mathrm{U}$ & & 37.9 \\
\hline Fluoranthene & U & & 37.9 \\
\hline Fluorene & $\mathrm{U}$ & & 37.9 \\
\hline Hexachlorobenzene & $\mathrm{U}$ & & 3.79 \\
\hline Hexachlorobutadiene & $\mathrm{U}$ & & 37.9 \\
\hline Hexachlorocyclopentadiene & U & & 37.9 \\
\hline Hexachloroethane & $\mathrm{U}$ & & 3.79 \\
\hline Indeno[1,2,3-c,d]pyrene & U & & 3.79 \\
\hline Isophorone & $\mathrm{U}$ & & 37.9 \\
\hline 2-Methyl-4,6-dinitrophenol & $\mathrm{U}$ & & 379 \\
\hline 2-Methylnaphthalene & $\mathrm{U}$ & & 37.9 \\
\hline Naphthalene & $\mathrm{U}$ & & 37.9 \\
\hline m-Nitroaniline & $\mathrm{U}$ & & 37.9 \\
\hline o-Nitroaniline & $\mathrm{U}$ & & 37.9 \\
\hline p-Nitroaniline & $\mathbf{U}$ & & 37.9 \\
\hline Nitrobenzene & $\mathrm{U}$ & & 37.9 \\
\hline 2-Nitrophenol & $\mathrm{U}$ & & 37.9 \\
\hline 4-Nitrophenol & $\mathrm{U}$ & & 37.9 \\
\hline N-Nitrosodiphenylamine & $U$ & & 37.9 \\
\hline N-Nitrosodi-n-propylamine & $\mathrm{U}$ & & 37.9 \\
\hline Pentachlorophenol & $\mathrm{U}$ & & 37.9 \\
\hline Phenanthrene & U & & 37.9 \\
\hline Phenol & $\mathrm{U}$ & & 37.9 \\
\hline Pyrene & $\mathrm{U}$ & & 37.9 \\
\hline 1,2,4-Trichlorobenzene & $\mathrm{U}$ & & 3.79 \\
\hline 2,4,5-Trichlorophenol & $\mathrm{U}$ & & 37.9 \\
\hline 2,4,6-Trichlorophenol & U & & 37.9 \\
\hline
\end{tabular}

$\begin{array}{lll}\mu \mathrm{g} / \mathrm{kg} & 3.79 & \text { EPA8270 } \\ \mu \mathrm{g} / \mathrm{kg} & 379 & \text { EPA8270 } \\ \mu \mathrm{g} / \mathrm{kg} & 37.9 & \text { EPA8270 } \\ \mu \mathrm{g} / \mathrm{kg} & 3.79 & \text { EPA8270 } \\ \mu \mathrm{g} / \mathrm{kg} & 37.9 & \text { EPA8270 } \\ \mu \mathrm{g} / \mathrm{kg} & 37.9 & \text { EPA8270 } \\ \mu \mathrm{g} / \mathrm{kg} & 37.9 & \text { EPA8270 } \\ \mu \mathrm{g} / \mathrm{kg} & 37.9 & \text { EPA8270 } \\ \mu \mathrm{g} / \mathrm{kg} & 37.9 & \text { EPA8270 } \\ \mu \mathrm{g} / \mathrm{kg} & 37.9 & \text { EPA8270 } \\ \mu \mathrm{g} / \mathrm{kg} & 37.9 & \text { EPA8270 } \\ \mu \mathrm{g} / \mathrm{kg} & 37.9 & \text { EPA8270 } \\ \mu \mathrm{g} / \mathrm{kg} & 37.9 & \text { EPA8270 } \\ \mu \mathrm{g} / \mathrm{kg} & 37.9 & \text { EPA8270 } \\ \mu \mathrm{g} / \mathrm{kg} & 379 & \text { EPA8270 } \\ \mu \mathrm{g} / \mathrm{kg} & 3.79 & \text { EPA8270 } \\ \mu \mathrm{g} / \mathrm{kg} & 3.79 & \text { EPA8270 } \\ \mu \mathrm{g} / \mathrm{kg} & 37.9 & \text { EPA8270 } \\ \mu \mathrm{g} / \mathrm{kg} & 37.9 & \text { EPA8270 } \\ \mu \mathrm{g} / \mathrm{kg} & 37.9 & \text { EPA8270 } \\ \mu \mathrm{g} / \mathrm{kg} & 3.79 & \text { EPA8270 } \\ \mu \mathrm{g} / \mathrm{kg} & 37.9 & \text { EPA8270 } \\ \mu \mathrm{g} / \mathrm{kg} & 37.9 & \text { EPA8270 } \\ \mu \mathrm{g} / \mathrm{kg} & 3.79 & \text { EPA8270 } \\ \mu \mathrm{g} / \mathrm{kg} & 3.79 & \text { EPA8270 } \\ \mu \mathrm{g} / \mathrm{kg} & 37.9 & \text { EPA8270 } \\ \mu \mathrm{g} / \mathrm{kg} & 379 & \text { EPA8270 } \\ \mu \mathrm{g} / \mathrm{kg} & 37.9 & \text { EPA8270 } \\ \mu \mathrm{g} / \mathrm{kg} & 37.9 & \text { EPA8270 } \\ \mu \mathrm{g} / \mathrm{kg} & 37.9 & \text { EPA8270 } \\ \mu \mathrm{g} / \mathrm{kg} & 37.9 & \text { EPA8270 } \\ \mu \mathrm{g} / \mathrm{kg} & 37.9 & \text { EPA8270 } \\ \mu \mathrm{g} / \mathrm{kg} & 37.9 & \text { EPA8270 } \\ \mu \mathrm{g} / \mathrm{kg} & 37.9 & \text { EPA8270 } \\ \mu \mathrm{g} / \mathrm{kg} & 37.9 & \text { EPA8270 } \\ \mu \mathrm{g} / \mathrm{kg} & 37.9 & \text { EPA8270 } \\ \mu \mathrm{g} / \mathrm{kg} & 37.9 & \text { EPA8270 } \\ \mu \mathrm{g} / \mathrm{kg} & 37.9 & \text { EPA8270 } \\ \mu \mathrm{g} / \mathrm{kg} & 37.9 & \text { EPA8270 } \\ \mu \mathrm{g} / \mathrm{kg} & 37.9 & \text { EPA8270 } \\ \mu \mathrm{g} / \mathrm{kg} & 37.9 & \text { EPA8270 } \\ \mu \mathrm{g} / \mathrm{kg} & 3.79 & \text { EPA8270 } \\ \mu \mathrm{g} / \mathrm{kg} & 37.9 & \text { EPA8270 } \\ \mu \mathrm{g} / \mathrm{kg} & 37.9 & \text { EPA8270 } \\ & & \end{array}$

Volatile Organic Compounds

\begin{tabular}{|c|c|c|c|c|c|}
\hline Acetone & UJ Q8 & 46.8 & $\mu \mathrm{g} / \mathrm{kg}$ & 11.5 & EPA8260 \\
\hline Benzene & $U$ & 0.575 & $\mu \mathrm{g} / \mathrm{kg}$ & 0.575 & EPA8260 \\
\hline Bromodichloromethane & $\mathrm{U}$ & 0.0575 & $\mu \mathrm{g} / \mathrm{kg}$ & 0.0575 & EPA8260 \\
\hline Bromoform & $U$ & 0.0575 & $\mu \mathrm{g} / \mathrm{kg}$ & 0.0575 & EPA8260 \\
\hline Bromomethane (Methyl bromide) & $\mathrm{U}$ & 0.115 & $\mu \mathrm{g} / \mathrm{kg}$ & 0.115 & EPA8260 \\
\hline Carbon disulfide & $U$ & 1.15 & $\mu \mathrm{g} / \mathrm{kg}$ & 1.15 & EPA8260 \\
\hline Carbon tetrachloride & U & 0.0575 & $\mu \mathrm{g} / \mathrm{kg}$ & 0.0575 & EPA8260 \\
\hline Chlorobenzene & $\mathrm{U}$ & 0.0575 & $\mu \mathrm{g} / \mathrm{kg}$ & 0.0575 & EPA8260 \\
\hline
\end{tabular}


SURVEY ID: PPSC 2403 (continued)

Constituent

Volatile Organic Compounds

Chloroethane
Chloroethene (Vinyl chloride)
Chloroform
Chloromethane (Methyl chloride)
Dibromochloromethane
1,1-Dichloroethane
1,2-Dichloroethane
1,1-Dichloroethylene
1,2-Dichloroethylene
Dichloromethane (Methylene chloride)
1,2-Dichloropropane
cis-1,3-Dichloropropene
trans-1,3-Dichloropropene
Ethylbenzene
2-Hexanone
Methyl ethyl ketone
Methyl isobutyl ketone
Styrene
1,1,2,2-Tetrachloroethane
Tetrachloroethylene
Toluene
1,1,1-Trichloroethane
1,1,2-Trichloroethane
Trichloroethylene
Unknown acid
Vinyl acetate
Xylenes

$\begin{array}{lll}U & & 0.115 \\ U & & 0.115 \\ U & & 0.0575 \\ U & & 0.115 \\ U & & 0.0575 \\ U & & 0.0575 \\ U & & 0.0575 \\ U & & 0.0575 \\ U & & 0.115 \\ U & V & 1.71 \\ U & & 0.0575 \\ U & & 0.0575 \\ U & & 0.0575 \\ U & & 0.0575 \\ U & & 1.15 \\ U & V & 1.7 \\ U & & 1.15 \\ U & & \mathbf{0 . 0 5 7 5} \\ U & & \mathbf{0 . 0 5 7 5} \\ U & & \mathbf{0 . 0 5 7 5} \\ U & 8 & \mathbf{0 . 0 6 9} \\ U & & \mathbf{0 . 0 5 7 5} \\ U & & \mathbf{0 . 0 5 7 5} \\ U & & \mathbf{0 . 0 5 7 5} \\ J & \mathrm{~N} & \mathbf{3} \\ U & & \mathbf{1 . 1 5} \\ U & & \mathbf{0 . 1 7 2}\end{array}$

Pesticides/PCBs and Dioxins/Furans
RQ AQ B Result

Unit

D. Limit Method

$\begin{array}{lll}\mu \mathrm{g} / \mathrm{kg} & 0.115 & \text { EPA8260 } \\ \mu \mathrm{g} / \mathrm{kg} & 0.115 & \text { EPA8260 } \\ \mu \mathrm{g} / \mathrm{kg} & 0.0575 & \text { EPA8260 } \\ \mu \mathrm{g} / \mathrm{kg} & 0.115 & \text { EPA8260 } \\ \mu \mathrm{g} / \mathrm{kg} & 0.0575 & \text { EPA8260 } \\ \mu \mathrm{g} / \mathrm{kg} & 0.0575 & \text { EPA8260 } \\ \mu \mathrm{g} / \mathrm{kg} & 0.0575 & \text { EPA8260 } \\ \mu \mathrm{g} / \mathrm{kg} & 0.0575 & \text { EPA8260 } \\ \mu \mathrm{g} / \mathrm{kg} & 0.115 & \text { EPA8260 } \\ \mu \mathrm{g} / \mathrm{kg} & 0.575 & \text { EPA8260 } \\ \mu \mathrm{g} / \mathrm{kg} & 0.0575 & \text { EPA8260 } \\ \mu \mathrm{g} / \mathrm{kg} & 0.0575 & \text { EPA8260 } \\ \mu \mathrm{g} / \mathrm{kg} & 0.0575 & \text { EPA8260 } \\ \mu \mathrm{g} / \mathrm{kg} & 0.0575 & \text { EPA8260 } \\ \mu \mathrm{g} / \mathrm{kg} & 1.15 & \text { EPA8260 } \\ \mu \mathrm{g} / \mathrm{kg} & 1.15 & \text { EPA8260 } \\ \mu \mathrm{g} / \mathrm{kg} & 1.15 & \text { EPA8260 } \\ \mu \mathrm{g} / \mathrm{kg} & 0.0575 & \text { EPA8260 } \\ \mu \mathrm{g} / \mathrm{kg} & 0.0575 & \text { EPA8260 } \\ \mu \mathrm{g} / \mathrm{kg} & 0.0575 & \text { EPA8260 } \\ \mu \mathrm{g} / \mathrm{kg} & 0.575 & \text { EPA8260 } \\ \mu \mathrm{g} / \mathrm{kg} & 0.0575 & \text { EPA8260 } \\ \mu \mathrm{g} / \mathrm{kg} & 0.0575 & \text { EPA8260 } \\ \mu \mathrm{g} / \mathrm{kg} & 0.0575 & \text { EPA8260 } \\ \mu \mathrm{g} / \mathrm{kg} & & \text { EPA8260 } \\ \mu \mathrm{g} / \mathrm{kg} & 1.15 & \text { EPA8260 } \\ \mu \mathrm{g} / \mathrm{kg} & 0.172 & \text { EPA8260 }\end{array}$

$\begin{array}{lll}\mu \mathrm{g} / \mathrm{kg} & 0.927 & \text { EPA8080 } \\ \mu \mathrm{g} / \mathrm{kg} & 0.927 & \text { EPA8080 } \\ \mu \mathrm{g} / \mathrm{kg} & 1.85 & \text { EPA8080 } \\ \mu \mathrm{g} / \mathrm{kg} & 1.85 & \text { EPA8080 } \\ \mu \mathrm{g} / \mathrm{kg} & 1.85 & \text { EPA8080 } \\ \mu \mathrm{g} / \mathrm{kg} & 1.85 & \text { EPA8080 } \\ \mu \mathrm{g} / \mathrm{kg} & 3.71 & \text { EPA8080 } \\ \mu \mathrm{g} / \mathrm{kg} & 1.85 & \text { EPA8080 } \\ \mu \mathrm{g} / \mathrm{kg} & 3.71 & \text { EPA8080 } \\ \mu \mathrm{g} / \mathrm{kg} & 1.85 & \text { EPA8080 } \\ \mu \mathrm{g} / \mathrm{kg} & 3.71 & \text { EPA8080 } \\ \mu \mathrm{g} / \mathrm{kg} & 1.85 & \text { EPA8080 } \\ \mu \mathrm{g} / \mathrm{kg} & 3.71 & \text { EPA8080 } \\ \mu \mathrm{g} / \mathrm{kg} & 1.85 & \text { EPA8080 } \\ \mu \mathrm{g} / \mathrm{kg} & 3.71 & \text { EPA8080 } \\ \mu \mathrm{g} / \mathrm{kg} & 1.85 & \text { EPA8080 } \\ \mu \mathrm{g} / \mathrm{kg} & 1.85 & \text { EPA8080 } \\ \mu \mathrm{g} / \mathrm{kg} & 0.927 & \text { EPA8080 } \\ \mu \mathrm{g} / \mathrm{kg} & 18.5 & \text { EPA8080 } \\ \mu \mathrm{g} / \mathrm{kg} & 4.64 & \text { EPA8080 } \\ \mu \mathrm{g} / \mathrm{kg} & 4.64 & \text { EPA8080 } \\ \mu \mathrm{g} / \mathrm{kg} & 4.64 & \text { EPA8080 } \\ \mu \mathrm{g} / \mathrm{kg} & 4.64 & \text { EPA8080 } \\ \mu \mathrm{g} / \mathrm{kg} & 4.64 & \text { EPA8080 } \\ \mu \mathrm{g} / \mathrm{kg} & 4.64 & \text { EPA8080 }\end{array}$


SURVEY ID: PPSC 2403 (continued)

Sample ID: 105119

Constituent

RQ AQ B Result

Unit

D. Limit Method

Pesticides/PCBs and Dioxins/Furans

PCB 1260

UJ $\quad \mathrm{O} \quad \mathrm{L} \quad 4.64$

$\mu \mathrm{g} / \mathrm{kg}$

4.64

EPA8080

Toxaphene

Radionuclides

Actinium-228

Antimony-124

Antimony-125

Barium-133

Cerium-144

Cesium-134

Cesium-137

Cobalt-57

Cobalt-58

Cobalt-60

Europium-152

Europium-154

Europium-155

Gross alpha

Lead-212

Manganese-54

Neptunium-239

Nonvolatile beta

Plutonium-238

Plutonium-239/240

Potassium-40

Promethium-144

Promethium-146

Radium-226

Radium-228

Ruthenium-106

Sodium-22

Strontium-90

Thorium-234

Tin-113

Yttrium-88

Zinc-65

Zirconium-95
$4.12 \mathrm{E}+00 \pm 8.76 \mathrm{E}-02 \mathrm{pCi} / \mathrm{g}$ $5.49 \mathrm{E}-03 \pm 8.31 \mathrm{E}-03 \mathrm{pCi} / \mathrm{g}$ $1.97 \mathrm{E}-02 \pm 1.98 \mathrm{E}-02 \mathrm{pCi} / \mathrm{g}$ $5.48 \mathrm{E}-03 \pm 9.56 \mathrm{E}-03 \mathrm{pCi} / \mathrm{g}$ $-1.86 \mathrm{E}-02 \pm 5.60 \mathrm{E}-02 \mathrm{pCj} / \mathrm{g}$ $1.05 \mathrm{E}-03 \pm 7.00 \mathrm{E}-03 \mathrm{pCj} / \mathrm{g}$ $-5.52 \mathrm{E}-03 \pm 8.50 \mathrm{E}-03 \mathrm{pCi} / \mathrm{g}$ $6.03 \mathrm{E}-04 \pm 7.00 \mathrm{E}-03 \mathrm{pCj} / \mathrm{g}$ $-7.79 \mathrm{E}-03 \pm 7.68 \mathrm{E}-03 \mathrm{pCi} / \mathrm{g}$ $1.22 \mathrm{E}-03 \pm 5.56 \mathrm{E}-03 \mathrm{pCi} / \mathrm{g}$ $-3.32 \mathrm{E}-02 \pm 2.01 \mathrm{E}-02 \mathrm{pCi} / \mathrm{g}$ $3.49 \mathrm{E}-02 \pm 5.27 \mathrm{E}-02 \mathrm{pCi} / \mathrm{g}$ $0.00 \mathrm{E}+00 \quad \mathrm{pCj} / \mathrm{g}$ $1.44 \mathrm{E}+02 \pm 8.82 \mathrm{E}+00 \mathrm{pCi} / \mathrm{g}$ $4.54 \mathrm{E}+00 \pm 4.22 \mathrm{E}-02 \mathrm{pCi} / \mathrm{g}$ $6.62 \mathrm{E}-02 \pm 9.94 \mathrm{E}-03 \mathrm{pCi} / \mathrm{g}$ 2.01E-01 $\pm 9.37 \mathrm{E}-02 \mathrm{pCi} / \mathrm{g}$ $6.27 \mathrm{E}+01 \pm 4.19 \mathrm{E}+00 \mathrm{pCi} / \mathrm{g}$

UI $\quad 1.14 \mathrm{E}-02 \pm 1.45 \mathrm{E}-02 \mathrm{pCj} / \mathrm{g}$

UI $\quad-1.25 \mathrm{E}-03 \pm 2.50 \mathrm{E}-03 \mathrm{pCi} / \mathrm{g}$

$\mathrm{V} \quad 8.33 \mathrm{E}-01 \pm 1.31 \mathrm{E} \cdot 01 \mathrm{pCj} / \mathrm{g}$

UI

UI $6.23 \mathrm{E}-04 \pm 7.00 \mathrm{E}-03 \mathrm{pCi} / \mathrm{g}$ $0.00 \mathrm{E}+00 \quad \mathrm{pCi} / \mathrm{g}$ $1.97 \mathrm{E}+00 \pm 4.66 \mathrm{E}-02 \mathrm{pCi} / \mathrm{g}$ $4.12 \mathrm{E}+00 \pm 8.76 \mathrm{E}-02 \mathrm{pCi} / \mathrm{g}$ UI $\quad 3.93 \mathrm{E}-05 \pm 6.37 \mathrm{E}-02 \mathrm{pCi} / \mathrm{g}$ UI $\quad-2.62 \mathrm{E}-03 \pm 5.64 \mathrm{E}-03 \mathrm{pCi} / \mathrm{g}$ $1.55 \mathrm{E}-01 \pm 9.69 \mathrm{E}-02 \mathrm{pCi} / \mathrm{g}$ $\mathrm{V} \quad 2.13 \mathrm{E}+00 \pm 7.53 \mathrm{E}-01 \mathrm{pCi} / \mathrm{g}$ $-1.56 \mathrm{E}-02 \pm 1.18 \mathrm{E}-02 \mathrm{pCi} / \mathrm{g}$ $1.30 \mathrm{E}-03 \pm 8.45 \mathrm{E}-03 \mathrm{pCi} / \mathrm{g}$ $-1.20 \mathrm{E}-02 \pm 1.40 \mathrm{E}-02 \mathrm{pCi} / \mathrm{g}$ $0.00 \mathrm{E}+00 \quad \mathrm{pCi} / \mathrm{g}$

UI $\begin{array}{ll}3.60 \mathrm{E}-02 & \text { EPIA-013B } \\ 1.44 \mathrm{E}-02 & \text { EPIA-013B } \\ 3.26 \mathrm{E}-02 & \text { EPIA-013B } \\ 1.41 \mathrm{E}-02 & \text { EPIA-013B } \\ 9.51 \mathrm{E}-02 & \text { EPIA-013B } \\ 1.06 \mathrm{E}-02 & \text { EPIA-013B } \\ 1.25 \mathrm{E}-02 & \text { EPIA-013B } \\ 1.20 \mathrm{E}-02 & \text { EPIA-013B } \\ 1.26 \mathrm{E}-02 & \text { EPIA-013B } \\ 9.74 \mathrm{E}-03 & \text { EPIA-013B } \\ 3.21 \mathrm{E}-02 & \text { EPIA-013B } \\ 9.01 \mathrm{E}-02 & \text { EPIA-013B } \\ 5.07 \mathrm{E}-02 & \text { EPIA-013B } \\ 1.48 \mathrm{E}+00 & \text { EPIA-001B } \\ 2.04 \mathrm{E}-02 & \text { EPIA-013B } \\ 1.09 \mathrm{E}-02 & \text { EPIA-013B } \\ 8.56 \mathrm{E}-02 & \text { EPIA-013B } \\ 2.49 \mathrm{E}+00 & \text { EPIA-001B } \\ 2.60 \mathrm{E}-02 & \text { EPIA-012B } \\ 1.58 \mathrm{E}-02 & \text { EPIA-012B } \\ 9.03 \mathrm{E}-02 & \text { EPIA-013B } \\ 1.19 \mathrm{E}-02 & \text { EPIA-013B } \\ 1.60 \mathrm{E}-02 & \text { EPIA-013B } \\ 2.20 \mathrm{E}-02 & \text { EPIA-013B } \\ 3.60 \mathrm{E}-02 & \text { EPIA-013B } \\ 1.09 \mathrm{E}-01 & \text { EPIA-013B } \\ 9.68 \mathrm{E}-03 & \text { EPIA-013B } \\ 1.48 \mathrm{E}-01 & \text { EPIA-004 } \\ 6.49 \mathrm{E}-01 & \text { EPIA-013B } \\ 1.67 \mathrm{E}-02 & \text { EPIA-013B } \\ 1.26 \mathrm{E}-02 & \text { EPIA-013B } \\ 1.93 \mathrm{E}-02 & \text { EPIA-013B } \\ 3.09 \mathrm{E}-02 & \text { EPIA-013B }\end{array}$

Sample ID: 105120

SURVEY ID: PPSC 2404

Survey location: $44906 \mathrm{E} 3692181 \mathrm{~N}$ (SRS coordinates)

Depth of core interval: 4.00 to $5.10 \mathrm{ft}$

Sample type: Normal

Sample matrix: Soil

Percent solids: 71.00 
Physical Parameters and Specified Analyses

\begin{tabular}{|c|c|c|c|c|c|c|}
\hline Ammonia nitrogen & $U$ & & 27.8 & $\mathrm{mg} / \mathrm{kg}$ & 27.8 & EPA350.1 \\
\hline Cation exchange capacity & & & 156 & meq $/ 100 \mathrm{~g}$ & 5 & EPA9081 \\
\hline Chloride & & & 7.24 & $\mathrm{mg} / \mathrm{kg}$ & 3.41 & EPA300.0 \\
\hline Cyanide & $U$ & & 1.29 & $\mathrm{mg} / \mathrm{kg}$ & 1.29 & EPA335.3 \\
\hline Nitrate-nitrite as nitrogen & $U$ & $\mathrm{~V}$ & 0.495 & $\mathrm{mg} / \mathrm{kg}$ & 1.24 & EPA353.1 \\
\hline Nitrogen by Kjeldahl method & & & 41 & $\mathrm{mg} / \mathrm{kg}$ & 5.47 & EPA351.2 \\
\hline Orthophosphate & $\mathrm{J}$ & $\mathbf{E}$ & 11.6 & $\mathrm{mg} / \mathrm{kg}$ & 28.2 & EPA365.2 \\
\hline Phenols & UJ & C & 282 & $\mu \mathrm{g} / \mathrm{kg}$ & 282 & EPA420.2 \\
\hline Sulfide & UJ & CI & 352 & $\mathrm{mg} / \mathrm{kg}$ & 352 & EPA376.2 \\
\hline Sulfide & UJ & CI & 352 & $\mathrm{mg} / \mathrm{kg}$ & 352 & EPA376.2 \\
\hline Total organic carbon & & $\mathrm{V}$ & 177 & $\mathrm{mg} / \mathrm{kg}$ & 141 & EPA415.1 \\
\hline Total organic nitrogen & $\mathrm{C}$ & & 39.6 & $\mathrm{mg} / \mathrm{kg}$ & 0.282 & EPA351.2 \\
\hline Total phosphates (as P) & $\mathbf{J}$ & $\mathbf{E}$ & 60.2 & $\mathrm{mg} / \mathrm{kg}$ & 273 & EPA365.4 \\
\hline
\end{tabular}

Metals (total recoverable)

\begin{tabular}{|c|c|c|c|c|c|c|}
\hline Aluminum & & V & 10500 & $\mathrm{mg} / \mathrm{kg}$ & 16.3 & EPA6010A \\
\hline Antimony & $\mathrm{U}$ & & 6.52 & $\mathrm{mg} / \mathrm{kg}$ & 6.52 & EPA6010A \\
\hline Arsenic & $\mathbf{J}$ & $\mathbf{E}$ & 4.55 & $\mathrm{mg} / \mathrm{kg}$ & 19.6 & EPA6010A \\
\hline Barium & $\mathbf{J}$ & C & 44 & $\mathrm{mg} / \mathrm{kg}$ & 1.63 & EPA6010A \\
\hline Beryllium & $\mathbf{J}$ & $\mathrm{C}$ & 2.53 & $\mathrm{mg} / \mathrm{kg}$ & 0.652 & EPA6010A \\
\hline Cadmium & $\mathrm{U}$ & & 0.326 & $\mathrm{mg} / \mathrm{kg}$ & 0.326 & EPA6010A \\
\hline Calcium & & $\mathrm{V}$ & 7450 & $\mathrm{mg} / \mathrm{kg}$ & 16.3 & EPA6010A \\
\hline Chromium & & & 14 & $\mathrm{mg} / \mathrm{kg}$ & 1.63 & EPA6010A \\
\hline Cobalt & & & 2.35 & $\mathrm{mg} / \mathrm{kg}$ & 0.652 & EPA6010A \\
\hline Copper & & & 2.39 & $\mathrm{mg} / \mathrm{kg}$ & 1.63 & EPA6010A \\
\hline Iron & $\mathbf{J}$ & C & 15600 & $\mathrm{mg} / \mathrm{kg}$ & 6.52 & EPA6010A \\
\hline Lead & $J$ & $\mathrm{E}$ & 2.53 & $\mathrm{mg} / \mathrm{kg}$ & 6.52 & EPA6010A \\
\hline Magnesium & & & 1400 & $\mathrm{mg} / \mathrm{kg}$ & 6.52 & EPA6010A \\
\hline Manganese & & & 70.9 & $\mathrm{mg} / \mathrm{kg}$ & 0.652 & EPA6010A \\
\hline Mercury & $J$ & $\mathrm{E}$ & 0.00588 & $\mathrm{mg} / \mathrm{kg}$ & 0.0392 & EPA7471 \\
\hline Nickel & & & 10.8 & $\mathrm{mg} / \mathrm{kg}$ & 1.63 & EPA6010A \\
\hline Potassium & & & 1220 & $\mathrm{mg} / \mathrm{kg}$ & 130 & EPA6010A \\
\hline Selenium & U & & 19.6 & $\mathrm{mg} / \mathrm{kg}$ & 19.6 & EPA6010A \\
\hline Silver & $U$ & & 1.63 & $\mathrm{mg} / \mathrm{kg}$ & 1.63 & EPA6010A \\
\hline Sodium & $J$ & $\mathbf{E}$ & 35.5 & $\mathrm{mg} / \mathrm{kg}$ & 65.2 & EPA6010A \\
\hline Thallium & $U$ & & 19.6 & $\mathrm{mg} / \mathrm{kg}$ & 19.6 & EPA6010A \\
\hline Vanadium & $J$ & C & 7.19 & $\mathrm{mg} / \mathrm{kg}$ & 0.652 & EPA6010A \\
\hline Zinc & & $\mathrm{V}$ & 38.5 & $\mathrm{mg} / \mathrm{kg}$ & 1.3 & EPA6010A \\
\hline \multicolumn{7}{|c|}{$B / N / A$ Extractables (including $P A H$ and phenols) } \\
\hline Acenaphthene & $U$ & & 46.9 & $\mu \mathrm{g} / \mathrm{kg}$ & 46.9 & EPA8270 \\
\hline Acenaphthylene & U & & 46.9 & $\mu \mathrm{g} / \mathrm{kg}$ & 46.9 & EPA8270 \\
\hline Anthracene & $\mathrm{U}$ & & 46.9 & $\mu \mathrm{g} / \mathrm{kg}$ & 46.9 & EPA8270 \\
\hline Benzo[a]anthracene & $U$ & & 4.69 & $\mu \mathrm{g} / \mathrm{kg}$ & 4.69 & EPA8270 \\
\hline Benzo[b]fluoranthene & $U$ & & 4.69 & $\mu \mathrm{g} / \mathrm{kg}$ & 4.69 & EPA8270 \\
\hline Benzo[k]fluoranthene & $\mathrm{U}$ & & 4.69 & $\mu \mathrm{g} / \mathrm{kg}$ & 4.69 & EPA8270 \\
\hline Benzoic acid & $\mathrm{U}$ & & 46.9 & $\mu \mathrm{g} / \mathrm{kg}$ & 46.9 & EPA8270 \\
\hline Benzo[g,h,i]perylene & $\mathrm{U}$ & & 46.9 & $\mu \mathrm{g} / \mathrm{kg}$ & 46.9 & EPA8270 \\
\hline Benzo[a]pyrene & $\mathrm{U}$ & & 4.69 & $\mu \mathrm{g} / \mathrm{kg}$ & 4.69 & EPA8270 \\
\hline Benzyl alcohol & $\mathrm{U}$ & & 46.9 & $\mu \mathrm{g} / \mathrm{kg}$ & 46.9 & EPA8270 \\
\hline Bis(2-chloroethoxy) methane & $\mathrm{U}$ & & 46.9 & $\mu \mathrm{g} / \mathrm{kg}$ & 46.9 & EPA8270 \\
\hline Bis(2-chloroethyl) ether & $\mathrm{U}$ & & 46.9 & $\mu \mathrm{g} / \mathrm{kg}$ & 46.9 & EPA8270 \\
\hline Bis(2-chloroisopropyl) ether & $\mathrm{U}$ & & 46.9 & $\mu \mathrm{g} / \mathrm{kg}$ & 46.9 & EPA8270 \\
\hline
\end{tabular}


SURVEY ID: PPSC 2404 (continued)

Constituent

RQ AQ B Result

Unit

D. Limit Method

$B / N / A$ Extractables (including PAH and phenols)

Bis(2-ethylhexyl) phthalate

4-Bromophenyl phenyl ether

Butylbenzyl phthalate

4-Chloroaniline

4-Chloro-m-cresol

2-Chloronaphthalene

2-Chlorophenol

4-Chlorophenyl phenyl-ether

Chrysene

$\mathrm{m} / \mathrm{p}$-Cresol

o-Cresol (2-Methylphenol)

Dibenz $[\alpha, h]$ anthracene

Dibenzofuran

Di-n-butyl phthalate

1,2-Dichlorobenzene

1,3-Dichlorobenzene

1,4-Dichlorobenzene

3,3'-Dichlorobenzidine

2,4-Dichlorophenol

Diethyl phthalate

2,4-Dimethyl phenol

Dimethyl phthalate

2,4-Dinitrophenol

2,4-Dinitrotoluene

2,6-Dinitrotoluene

Di-n-octyl phthalate

Fluoranthene

Fluorene

Hexachlorobenzene

Hexachlorobutadiene

Hexachlorocyclopentadiene

Hexachloroethane

Indeno $[1,2,3 \cdot c, d]$ pyrene

Isophorone

2-Methyl-4,6-dinitrophenol

2-Methylnaphthalene

Naphthalene

m-Nitroaniline

0. Nitroaniline

p-Nitroaniline

Nitrobenzene

2-Nitrophenol

4-Nitrophenol

N-Nitrosodiphenylamine

N-Nitrosodi-n-propylamine

Pentachlorophenol

Phenanthrene

Phenol

Pyrene

1,2,4-Trichlorobenzene

2,4,5-Trichlorophenol

2,4,6-Trichlorophenol

\begin{tabular}{|c|c|c|}
\hline U & & 46.9 \\
\hline $\mathbf{U}$ & & 46.9 \\
\hline U & & 46.9 \\
\hline $\mathrm{U}$ & & 46.9 \\
\hline U & & 46.9 \\
\hline$U$ & & 46.9 \\
\hline U & & 46.9 \\
\hline $\mathrm{U}$ & & 46.9 \\
\hline$U$ & & 4.69 \\
\hline$U$ & & 469 \\
\hline $\mathrm{U}$ & & 46.9 \\
\hline $\mathrm{U}$ & & 4.69 \\
\hline$U$ & & 46.9 \\
\hline $\mathrm{U}$ & $\mathrm{V}$ & 29.6 \\
\hline $\mathrm{U}$ & & 46.9 \\
\hline U & & 46.9 \\
\hline U & & 46.9 \\
\hline $\mathbf{U}$ & & 46.9 \\
\hline $\mathrm{U}$ & & 46.9 \\
\hline $\mathrm{U}$ & & 46.9 \\
\hline U & & 46.9 \\
\hline U & & 46.9 \\
\hline $\mathrm{U}$ & & 469 \\
\hline UJ & C & 4.69 \\
\hline$U$ & & 4.69 \\
\hline $\mathrm{U}$ & & 46.9 \\
\hline $\mathrm{U}$ & & 46.9 \\
\hline U & & 46.9 \\
\hline$U$ & & 4.69 \\
\hline $\mathrm{U}$ & & 46.9 \\
\hline U & & 46.9 \\
\hline$U$ & & 4.69 \\
\hline $\boldsymbol{U}$ & & 4.69 \\
\hline $\mathrm{U}$ & & 46.9 \\
\hline U & & 469 \\
\hline U & & 46.9 \\
\hline $\mathrm{U}$ & & 46.9 \\
\hline $\mathrm{U}$ & & 46.9 \\
\hline U & & 46.9 \\
\hline$U$ & & 46.9 \\
\hline $\mathrm{U}$ & & 46.9 \\
\hline $\mathrm{U}$ & & 46.9 \\
\hline U & & 46.9 \\
\hline U & & 46.9 \\
\hline U & & 46.9 \\
\hline $\mathrm{U}$ & & 46.9 \\
\hline $\mathrm{U}$ & & 46.9 \\
\hline $\mathrm{U}$ & & 46.9 \\
\hline $\mathrm{U}$ & & 46.9 \\
\hline $\mathrm{U}$ & & 4.69 \\
\hline $\mathrm{U}$ & & 46.9 \\
\hline $\mathrm{U}$ & & 46.9 \\
\hline
\end{tabular}

\begin{tabular}{|c|c|c|}
\hline$\mu \mathrm{g} / \mathrm{kg}$ & 46.9 & EPA8270 \\
\hline$\mu \mathrm{g} / \mathrm{kg}$ & 46.9 & EPA8270 \\
\hline$\mu \mathrm{g} / \mathrm{kg}$ & 46.9 & EPA8270 \\
\hline$\mu \mathrm{g} / \mathrm{kg}$ & 46.9 & EPA8270 \\
\hline$\mu \mathrm{g} / \mathrm{kg}$ & 46.9 & EPA8270 \\
\hline$\mu \mathrm{g} / \mathrm{kg}$ & 46.9 & EPA8270 \\
\hline$\mu \mathrm{g} / \mathrm{kg}$ & 46.9 & EPA8270 \\
\hline$\mu \mathrm{g} / \mathrm{kg}$ & 46.9 & EPA8270 \\
\hline$\mu \mathrm{g} / \mathrm{kg}$ & 4.69 & EPA8270 \\
\hline$\mu \mathrm{g} / \mathrm{kg}$ & 469 & EPA8270 \\
\hline$\mu \mathrm{g} / \mathrm{kg}$ & 46.9 & EPA8270 \\
\hline$\mu \mathrm{g} / \mathrm{kg}$ & 4.69 & EPA8270 \\
\hline$\mu \mathrm{g} / \mathrm{kg}$ & 46.9 & EPA8270 \\
\hline$\mu \mathrm{g} / \mathrm{kg}$ & 46.9 & EPA8270 \\
\hline$\mu \mathrm{g} / \mathrm{kg}$ & 46.9 & EPA8270 \\
\hline$\mu \mathrm{g} / \mathrm{kg}$ & 46.9 & EPA8270 \\
\hline$\mu \mathrm{g} / \mathrm{kg}$ & 46.9 & EPA8270 \\
\hline$\mu \mathrm{g} / \mathrm{kg}$ & 46.9 & EPA8270 \\
\hline$\mu \mathrm{g} / \mathrm{kg}$ & 46.9 & EPA8270 \\
\hline$\mu \mathrm{g} / \mathrm{kg}$ & 46.9 & EPA8270 \\
\hline$\mu \mathrm{g} / \mathrm{kg}$ & 46.9 & EPA8270 \\
\hline$\mu \mathrm{g} / \mathrm{kg}$ & 46.9 & EPA8270 \\
\hline$\mu \mathrm{g} / \mathrm{kg}$ & 469 & EPA8270 \\
\hline$\mu \mathrm{g} / \mathrm{kg}$ & 4.69 & EPA8270 \\
\hline$\mu \mathrm{g} / \mathrm{kg}$ & 4.69 & EPA8270 \\
\hline$\mu \mathrm{g} / \mathrm{kg}$ & 46.9 & EPA8270 \\
\hline$\mu \mathrm{g} / \mathrm{kg}$ & 46.9 & EPA8270 \\
\hline$\mu \mathrm{g} / \mathrm{kg}$ & 46.9 & EPA8270 \\
\hline$\mu \mathrm{g} / \mathrm{kg}$ & 4.69 & EPA8270 \\
\hline$\mu \mathrm{g} / \mathrm{kg}$ & 46.9 & EPA8270 \\
\hline$\mu \mathrm{g} / \mathrm{kg}$ & 46.9 & EPA8270 \\
\hline$\mu \mathrm{g} / \mathrm{kg}$ & -4.69 & EPA8270 \\
\hline$\mu \mathrm{g} / \mathrm{kg}$ & 4.69 & EPA8270 \\
\hline$\mu \mathrm{g} / \mathrm{kg}$ & 46.9 & EPA8270 \\
\hline$\mu \mathrm{g} / \mathrm{kg}$ & 469 & EPA8270 \\
\hline$\mu \mathrm{g} / \mathrm{kg}$ & 46.9 & EPA8270 \\
\hline$\mu \mathrm{g} / \mathrm{kg}$ & 46.9 & EPA8270 \\
\hline$\mu \mathrm{g} / \mathrm{kg}$ & 46.9 & EPA8270 \\
\hline$\mu \mathrm{g} / \mathrm{kg}$ & 46.9 & EPA8270 \\
\hline$\mu \mathrm{g} / \mathrm{kg}$ & 46.9 & EPA8270 \\
\hline$\mu \mathrm{g} / \mathrm{kg}$ & 46.9 & EPA8270 \\
\hline$\mu \mathrm{g} / \mathrm{kg}$ & 46.9 & EPA8270 \\
\hline$\mu \mathrm{g} / \mathrm{kg}$ & 46.9 & EPA8270 \\
\hline$\mu \mathrm{g} / \mathrm{kg}$ & 46.9 & EPA8270 \\
\hline$\mu \mathrm{g} / \mathrm{kg}$ & 46.9 & EPA8270 \\
\hline$\mu \mathrm{g} / \mathrm{kg}$ & 46.9 & EPA8270 \\
\hline$\mu \mathrm{g} / \mathrm{kg}$ & 46.9 & EPA8270 \\
\hline$\mu \mathrm{g} / \mathrm{kg}$ & 46.9 & EPA8270 \\
\hline$\mu \mathrm{g} / \mathrm{kg}$ & 46.9 & EPA8270 \\
\hline$\mu \mathrm{g} / \mathrm{kg}$ & 4.69 & EPA8270 \\
\hline$\mu \mathrm{g} / \mathrm{kg}$ & 46.9 & EPA8270 \\
\hline$\mu \mathrm{g} / \mathrm{kg}$ & 46.9 & EPA8270 \\
\hline
\end{tabular}


SURVEY ID: PPSC 2404 (continued)

Constituent

Volatile Organic Compounds

Acetone

Acetone

Benzene

Benzene

Bromodichloromethane

Bromodichloromethane

Bromoform

Bromoform

Bromomethane (Methyl bromide)

Bromomethane (Methyl bromide)

Carbon disulfide

Carbon disulfide

Carbon tetrachloride

Carbon tetrachloride

Chlorobenzene

Chlorobenzene

Chloroethane

Chloroethane

Chloroethene (Vinyl chloride)

Chloroethene (Vinyl chloride)

Chloroform

Chloroform

Chloromethane (Methyl chloride)

Chloromethane (Methyl chloride)

Dibromochloromethane

Dibromochloromethane

1,1-Dichloroethane

1,1-Dichloroethane

1,2-Dichloroethane

1,2-Dichloroethane

1,1-Dichloroethylene

1,1-Dichloroethylene

1,2-Dichloroethylene

1,2-Dichloroethylene

Dichloromethane (Methylene chloride)

Dichloromethane (Methylene chloride)

1,2-Dichloropropane

1,2-Dichloropropane

cis-1,3-Dichloropropene

cis-1,3-Dichloropropene

trans-1,3-Dichloropropene

trans-1,3-Dichloropropene

Ethylbenzene

Ethylbenzene

2-Hexanone

2-Hexanone

Methyl ethyl ketone

Methyl ethyl ketone

Methyl isobutyl ketone

Methyl isobutyl ketone

Styrene

Styrene

1,1,2,2-Tetrachloroethane

1,1,2,2-Tetrachloroethane

Tetrachloroethylene
Sample ID: 105120

RQ AQ B Result

Unit

D. Limit Method
39.2

76.2

0.704

0.704

0.0704

0.0704

0.0704

0.0704

0.141

0.141

1.41

0.324

0.0704

0.0704

0.0704

0.0704

0.141

0.141

0.141

0.141

0.0704

0.0704

0.141

0.141

0.0704

0.0704

0.0704

0.0704

0.0704

0.0704

0.0704

0.0704

0.141

0.141

$\begin{array}{ll}\mathrm{V} & 1.87\end{array}$

$\mathrm{V} \quad 4.48$

0.0704

0.0704

0.0704

0.0704

0.0704

0.0704

0.0704

0.0704

1.61

0.93

0.451

1.32

1.41

1.41

0.0704

0.0704

0.0704

0.0704

0.0704
EPA8260

EPA8260

$\begin{array}{lll}\mu \mathrm{g} / \mathrm{kg} & \mathbf{0 . 7 0 4} & \text { EPA8260 } \\ \mu \mathrm{g} / \mathrm{kg} & \mathbf{0 . 7 0 4} & \text { EPA8260 }\end{array}$

$\mu \mathrm{g} / \mathrm{kg} \quad 0.0704 \quad$ EPA8260

$\mu \mathrm{g} / \mathrm{kg} \quad 0.0704 \quad$ EPA8260

$\mu g / \mathrm{kg} \quad 0.0704 \quad$ EPA8260

$\mu \mathrm{g} / \mathrm{kg} \quad 0.141 \quad$ EPA8260

$\mu \mathrm{g} / \mathrm{kg} \quad 0.141 \quad$ EPA8260

$\mu \mathrm{g} / \mathrm{kg} \quad 1.41 \quad$ EPA8260

$\mu \mathrm{g} / \mathrm{kg} \quad 1.41 \quad$ EPA8260

$\mu \mathrm{g} / \mathrm{kg} \quad 0.0704 \quad$ EPA8260

$\mu \mathrm{g} / \mathrm{kg} \quad 0.0704 \quad$ EPA8260

$\mu \mathrm{g} / \mathrm{kg} \quad 0.0704 \quad$ EPA8260

$\mu \mathrm{g} / \mathrm{kg} \quad 0.141 \quad$ EPA8260

$\mu \mathrm{g} / \mathrm{kg} \quad 0.141 \quad$ EPA8260

$\mu \mathrm{g} / \mathrm{kg} \quad 0.141 \quad$ EPA8260

$\mu \mathrm{g} / \mathrm{kg} \quad 0.141 \quad$ EPA8260

$\mu \mathrm{g} / \mathrm{kg} \quad 0.0704 \quad$ EPA8260

$\mu \mathrm{g} / \mathrm{kg} \quad 0.141 \quad$ EPA8260

$\mu \mathrm{g} / \mathrm{kg} \quad 0.141 \quad$ EPA8260

$\mu \mathrm{g} / \mathrm{kg} \quad 0.0704 \quad$ EPA8260

$\mu \mathrm{g} / \mathrm{kg} \quad 0.0704 \quad$ EPA8260

$\mu \mathrm{g} / \mathrm{kg} \quad 0.0704 \quad$ EPA8260

$\mu \mathrm{g} / \mathrm{kg} \quad 0.0704 \quad$ EPA8260

$\mu g / k g \quad 0.0704 \quad$ EPA8260

$\mu \mathrm{g} / \mathrm{kg} \quad 0.0704 \quad$ EPA8260

$\mu \mathrm{g} / \mathrm{kg} \quad 0.0704 \quad$ EPA8260

$\mu \mathrm{g} / \mathrm{kg} \quad 0.0704 \quad$ EPA8260

$\mu \mathrm{g} / \mathrm{kg} \quad 0.141 \quad$ EPA8260

$\mu \mathrm{g} / \mathrm{kg} \quad 0.704 \quad$ EPA8260

$\mu \mathrm{g} / \mathrm{kg} \quad 0.704 \quad$ EPA8260

$\mu \mathrm{g} / \mathrm{kg} \quad 0.0704 \quad$ EPA8260

$\mu \mathrm{g} / \mathrm{kg} \quad 0.0704 \quad$ EPA8260

$\mu \mathrm{g} / \mathrm{kg} \quad 0.0704 \quad$ EPA8260

$\mu \mathrm{g} / \mathrm{kg} \quad 0.0704 \quad$ EPA8260

$\mu \mathrm{g} / \mathrm{kg} \quad 0.0704 \quad$ EPA8260

$\mu \mathrm{g} / \mathrm{kg} \quad 0.0704 \quad$ EPA8260

$\mu \mathrm{g} / \mathrm{kg} \quad 0.0704 \quad$ EPA8260

$\mu \mathrm{g} / \mathrm{kg} \quad 0.0704 \quad$ EPA8260

$\mu \mathrm{g} / \mathrm{kg} \quad 1.41 \quad$ EPA8260

$\mu \mathrm{g} / \mathrm{kg} \quad 1.41 \quad$ EPA8260

$\mu \mathrm{g} / \mathrm{kg} \quad 1.41 \quad$ EPA8260

$\mu \mathrm{g} / \mathrm{kg} \quad 1.41 \quad$ EPA8260

$\mu \mathrm{g} / \mathrm{kg} \quad 1.41 \quad$ EPA8260

$\mu \mathrm{g} / \mathrm{kg} \quad 1.41 \quad$ EPA8260

$\mu \mathrm{g} / \mathrm{kg} \quad 0.0704 \quad$ EPA8260

$\mu \mathrm{g} / \mathrm{kg} \quad 0.0704 \quad$ EPA8260

$\mu g / \mathrm{kg} \quad 0.0704 \quad$ EPA8260

$\mu g / k g \quad 0.0704 \quad$ EPA8260

$\mu \mathrm{g} / \mathrm{kg} \quad 0.0704$

EPA8260 $\mu \mathrm{g} / \mathrm{kg} \quad 0.0704 \quad$ EPA8260

$\mu \mathrm{g} / \mathrm{kg} \quad 0.0704 \quad$ EPA8260

$\mu \mathrm{g} / \mathrm{kg} \quad 0.0704 \quad$ EPA8260

$\mu \mathrm{g} / \mathrm{kg} \quad 0.141 \quad$ EPA8260 
SURVEY ID: PPSC 2404 (continued)

Constituent

Volatile Organic Compounds

Tetrachloroethylene

Toluene

Toluene

1,1,1-Trichloroethane

1,1,1-Trichloroethane

1,1,2-Trichloroethane

1,1,2-Trichloroethane

Trichloroethylene

Trichloroethylene

Vinyl acetate

Vinyl acetate

Xylenes

Xylenes

Pesticides/PCBs and Dioxins/Furans

$\begin{array}{lcccc}\text { Aldrin } & \text { UJ } & \text { O } & \text { L } & 1.14 \\ \text { alpha-Benzene hexachloride } & \text { UJ } & \text { O } & \text { L } & 1.14 \\ \text { beta-Benzene hexachloride } & \text { UJ } & \text { O } & \text { L } & 2.29 \\ \text { delta-Benzene hexachloride } & \text { UJ } & \text { O } & \text { L } & 2.29 \\ \text { alpha-Chlordane } & \text { UJ } & \text { O } & \text { L } & 2.29 \\ \text { gamma-Chlordane } & \text { UJ } & \text { O } & \text { L } & 2.29 \\ \text { p,p'-DDD } & \text { UJ } & \text { O } & \text { L } & 4.58 \\ \text { p,p'-DDE } & \text { UJ } & \text { O } & \text { L } & 2.29 \\ \text { p,p'-DDT } & \text { UJ } & \text { O } & \text { L } & 4.58 \\ \text { Dieldrin } & \text { UJ } & \text { O } & \text { L } & 2.29 \\ \text { Endosulfan sulfate } & \text { UJ } & \text { O } & \text { L } & 4.58 \\ \text { Endosulfan I } & \text { UJ } & \text { O } & \text { L } & 2.29 \\ \text { Endosulfan II } & \text { UJ } & \text { O } & \text { L } & 4.58 \\ \text { Endrin } & \text { UJ } & \text { O } & \text { L } & 2.29 \\ \text { Endrin ketone } & \text { UJ } & \text { O } & \text { L } & 4.58 \\ \text { Heptachlor } & \text { UJ } & \text { O } & \text { L } & 2.29 \\ \text { Heptachlor epoxide } & \text { UJ } & \text { O } & \text { L } & 2.29 \\ \text { Lindane } & \text { UJ } & \text { O } & \text { L } & 1.14 \\ \text { Methoxychlor } & \text { UJ } & \text { O } & \text { L } & 22.9 \\ \text { PCB 1016 } & \text { UJ } & \text { O } & \text { L } & 5.72 \\ \text { PCB 1221 } & \text { UJ } & \text { O } & \text { L } & 5.72 \\ \text { PCB 1232 } & \text { UJ } & \text { O } & \text { L } & 5.72 \\ \text { PCB 1242 } & \text { UJ } & \text { O } & \text { L } & 5.72 \\ \text { PCB 1248 } & \text { UJ } & \text { O } & \text { L } & \mathbf{5 . 7 2} \\ \text { PCB 1254 } & \text { UJ } & \text { O } & \text { L } & \mathbf{5 . 7 2} \\ \text { PCB 1260 } & \text { UJ } & \text { O } & \text { L } & 5.72 \\ \text { Toxaphene } & \text { UJ } & \text { O } & \text { L } & 45.8\end{array}$

Sample ID: 105120

RQ AQ B Result Unit D. Limit Method

$\begin{array}{llllll}U & & 0.0704 & \mu g / k g & 0.0704 & \text { EPA8260 } \\ U & 8 & 0.127 & \mu g / k g & 0.704 & \text { EPA8260 } \\ U & 8 & 0.155 & \mu g / k g & 0.704 & \text { EPA8260 } \\ U & & 0.0704 & \mu g / k g & 0.0704 & \text { EPA8260 } \\ U & 0.0704 & \mu g / k g & 0.0704 & \text { EPA8260 } \\ U & 0.0704 & \mu g / k g & 0.0704 & \text { EPA8260 } \\ U & 0.0704 & \mu g / k g & 0.0704 & \text { EPA8260 } \\ U & 0.0704 & \mu g / k g & 0.0704 & \text { EPA8260 } \\ U & 0.0704 & \mu g / k g & 0.0704 & \text { EPA8260 } \\ U & 1.41 & \mu g / k g & 1.41 & \text { EPA8260 } \\ U & 1.41 & \mu g / k g & 1.41 & \text { EPA8260 } \\ U & 0.211 & \mu g / k g & 0.211 & \text { EPA8260 } \\ U & 0.211 & \mu g / k g & 0.211 & \text { EPA8260 }\end{array}$

Radionuclides

Actinium-228

Antimony-124

Antimony-125

UI

UI

Barium-133

Cerium-144

Cesium-134

Cesium-137

UI

UI

UI

UI

Cobalt-57

Cobalt-58
9.57E-01士4.82E-02 pCi/g -3.14E-04 $\pm 5.76 \mathrm{E}-03 \mathrm{pCi} / \mathrm{g}$ $1.05 \mathrm{E}-03 \pm 1.20 \mathrm{E}-02 \mathrm{pCi} / \mathrm{g}$ 3.97E-03 $\pm 6.01 \mathrm{E}-03 \mathrm{pCi} / \mathrm{g}$ 1.36E-02 $\pm 3.55 \mathrm{E}-02 \mathrm{pCj} / \mathrm{g}$ 2.07E-03 $\pm 4.90 \mathrm{E}-03 \mathrm{pCi} / \mathrm{g}$ $-9.66 \mathrm{E}-04 \pm 5.55 \mathrm{E}-03 \mathrm{pCi} / \mathrm{g}$ 9.23E-04 $\pm 4.50 \mathrm{E}-03 \mathrm{pCi} / \mathrm{g}$ $-1.31 \mathrm{E}-03 \pm 6.24 \mathrm{E}-03 \mathrm{pCi} / \mathrm{g}$

$\begin{array}{lll}\mu \mathrm{g} / \mathrm{kg} & 1.14 & \text { EPA8080 } \\ \mu \mathrm{g} / \mathrm{kg} & 1.14 & \text { EPA8080 } \\ \mu \mathrm{g} / \mathrm{kg} & 2.29 & \text { EPA8080 } \\ \mu \mathrm{g} / \mathrm{kg} & 2.29 & \text { EPA8080 } \\ \mu \mathrm{g} / \mathrm{kg} & 2.29 & \text { EPA8080 } \\ \mu \mathrm{g} / \mathrm{kg} & 2.29 & \text { EPA8080 } \\ \mu \mathrm{g} / \mathrm{kg} & 4.58 & \text { EPA8080 } \\ \mu \mathrm{g} / \mathrm{kg} & 2.29 & \text { EPA8080 } \\ \mu \mathrm{g} / \mathrm{kg} & 4.58 & \text { EPA8080 } \\ \mu \mathrm{g} / \mathrm{kg} & 2.29 & \text { EPA8080 } \\ \mu \mathrm{g} / \mathrm{kg} & 4.58 & \text { EPA8080 } \\ \mu \mathrm{g} / \mathrm{kg} & 2.29 & \text { EPA8080 } \\ \mu \mathrm{g} / \mathrm{kg} & 4.58 & \text { EPA8080 } \\ \mu \mathrm{g} / \mathrm{kg} & 2.29 & \text { EPA8080 } \\ \mu \mathrm{g} / \mathrm{kg} & 4.58 & \text { EPA8080 } \\ \mu \mathrm{g} / \mathrm{kg} & 2.29 & \text { EPA8080 } \\ \mu \mathrm{g} / \mathrm{kg} & 2.29 & \text { EPA8080 } \\ \mu \mathrm{g} / \mathrm{kg} & 1.14 & \text { EPA8080 } \\ \mu \mathrm{g} / \mathrm{kg} & 22.9 & \text { EPA8080 } \\ \mu \mathrm{g} / \mathrm{kg} & 5.72 & \text { EPA8080 } \\ \mu \mathrm{g} / \mathrm{kg} & 5.72 & \text { EPA8080 } \\ \mu \mathrm{g} / \mathrm{kg} & 5.72 & \text { EPA8080 } \\ \mu \mathrm{g} / \mathrm{kg} & 5.72 & \text { EPA8080 } \\ \mu \mathrm{g} / \mathrm{kg} & 5.72 & \text { EPA8080 } \\ \mu \mathrm{g} / \mathrm{kg} & 5.72 & \text { EPA8080 } \\ \mu \mathrm{g} / \mathrm{kg} & 5.72 & \text { EPA8080 } \\ \mu \mathrm{g} / \mathrm{kg} & 45.8 & \text { EPA8080 }\end{array}$

2.68E-02 EPIA-013B

9.64E-03 EPIA-013B

2.06E-02 EPIA-013B

9.30E-03 EPIA-013B

5.93E-02 EPIA-013B

- 7.36E-03 EPIA-013B

8.04E-03 EPIA-013B

7.53E-03 EPIA-013B

8.91E-03 EPIA-013B 
SURVEY ID: PPSC 2404 (continued)

Constituent

Radionuclides

Cobalt- 60

Europium-152

Europium-154

Europium-155

Gross alpha

Lead-212

Manganese-54

Neptunium-239

Nonvolatile beta

Plutonium-238

Plutonium-239/240

Potassium -40

Promethium-144

Promethium-146

Radium-226

Radium-228

Ruthenium-106

Sodium-22

Strontium-90

Thorium-234

Tin-113

Yttrium-88

Zinc-65

Zirconium-95

Sample ID: 105120

RQ AQ B Result

Unit

D. Limit Method

7.07E-03 EPLA-013B

$-1.98 \mathrm{E}-03 \pm 4.23 \mathrm{E}-03 \mathrm{pCi} / \mathrm{g}$ $-1.96 \mathrm{E}-02 \pm 1.29 \mathrm{E}-02 \mathrm{pCi} / \mathrm{g}$ $-1.43 \mathrm{E}-04 \pm 4.60 \mathrm{E}-02 \mathrm{pCi} / \mathrm{g}$ $0.00 \mathrm{E}+00 \quad \mathrm{pCi} / \mathrm{g}$ $1.36 \mathrm{E}+01 \pm 2.83 \mathrm{E}+00 \mathrm{pCi} / \mathrm{g}$ $1.01 \mathrm{E}+00 \pm 2.17 \mathrm{E}-02 \mathrm{pCi} / \mathrm{g}$ 1.97E-02 $49.47 \mathrm{E}-03 \mathrm{pCi} / \mathrm{g}$ $0.00 \mathrm{E}+00 \quad \mathrm{pCi} / \mathrm{g}$ $1.49 \mathrm{E}+01 \pm 2.14 \mathrm{E}+00 \mathrm{pCi} / \mathrm{g}$ 2.62E-03 $\pm 1.19 \mathrm{E}-02 \mathrm{pCi} / \mathrm{g}$

$\begin{array}{lll}\text { UI } & & 2.62 \mathrm{E}-03 \pm 1.19 \mathrm{E}-02 \mathrm{pCi} / \mathrm{g} \\ \text { UI } & & 2.54 \mathrm{E}-03 \pm 5.10 \mathrm{E}-03 \mathrm{pCi} / \mathrm{g} \\ & \mathrm{V} & 6.32 \mathrm{E}+00 \pm 1.99 \mathrm{E}-01 \mathrm{pCi} / \mathrm{g}\end{array}$

UI $\quad-1.82 \mathrm{E}-03 \pm 4.63 \mathrm{E}-03 \mathrm{pCi} / \mathrm{g}$

UI $\quad 5.28 \mathrm{E}-03 \pm 5.82 \mathrm{E}-03 \mathrm{pCi} / \mathrm{g}$ 8.95E-01 $\pm 3.01 \mathrm{E}-02 \mathrm{pCi} / \mathrm{g}$ 9.57E-01 $\pm 4.82 \mathrm{E}-02 \mathrm{pCi} / \mathrm{g}$

UI $\quad-1.82 \mathrm{E}-02 \pm 4.17 \mathrm{E}-02 \mathrm{pCi} / \mathrm{g}$ $-5.58 \mathrm{E}-03 \pm 4.99 \mathrm{E}-03 \mathrm{pCi} / \mathrm{g}$ 2.94E-01 $1.25 \mathrm{E}-01 \mathrm{pCi} / \mathrm{g}$ $1.62 \mathrm{E}+00 \pm 5.12 \mathrm{E}-01 \mathrm{pCi} / \mathrm{g}$ $-2.01 \mathrm{E}-04 \pm 7.31 \mathrm{E}-03 \mathrm{pCi} / \mathrm{g}$ 3.69E-03 $44.45 \mathrm{E}-03 \mathrm{pCi} / \mathrm{g}$ 1.53E-02+1.15E-02 pCi/g $0.00 \mathrm{E}+00$

$\mathrm{pCi} / \mathrm{g}$

Sample ID: 105113

Survey location: $54602 E$ 3712523N (SRS coordinates)

Depth of core interval: 0.00 to $1.00 \mathrm{ft}$

Sample type: Normal

Sample matrix: Soil

Percent solids: $\mathbf{7 9 . 0 0}$

Constituent

RQ AQ B Result

Unit

D. Limit Method

Physical Parameters and Specified Analyses

Ammonia nitrogen

Ammonia nitrogen

Cation exchange capacity

Chloride

Cyanide

Nitrate-nitrite as nitrogen

Nitrogen by Kjeldahl method

Orthophosphate

Phenols

Sulfide

Total organic carbon

Total organic carbon

Total organic nitrogen

Total phosphates (as P)

$\begin{array}{lll}\text { U } & & 24.9 \\ \text { U } & & 25.3 \\ & & 23.6 \\ & & 5.61 \\ \text { U } & & 1.27 \\ \text { J } & \text { E } & 0.466 \\ & & 124 \\ \text { J } & \text { E } & 2.68 \\ \text { UJ } & \text { C } & 378 \\ \text { UJ } & \text { C } & 316 \\ & \text { V } & 3150 \\ & \text { V } & 2610 \\ \text { C } & & 114 \\ \text { J } & \text { E } & 3.53\end{array}$

2.15E-02 EPIA-013B

6.92E-02 EPIA-013B

3.08E-02 EPIA-013B

$1.81 \mathrm{E}+00$ EPLA-001B

1.33E-02 EPIA-013B

7.42E-03 EPIA-013B

5.42E-02 EPIA-013B

2.29E+00 EPIA-001B

2.80E-02 EPIA-012B

7.63E-03 EPIA-012B

6.29E-02 EPIA-013B

7.61E-03 EPIA-013B

1.01E-02 EPIA-013B

1.46E-02 EPIA-013B

2.68E-02 EPIA-013B

6.89E-02 EPIA-013B

8.08E-03 EPIA-013B

1.56E-01 EPIA-004

4.02E-01 EPIA-013B

1.10E-02 EPIA-013B

8.37E-03 EPIA-013B

1.84E-02 EPIA-013B

1.85E-02 EPIA-013B 
SURVEY ID: PPSC 2501 (continued)

Constituent

RQ AQ B Result

Sample ID: 105113

Metals (total recoverable)

Aluminum

Aluminum

Antimony

Antimony

Arsenic

Arsenic

Barium

Barium

Beryllium

Beryllium

Cadmium

Cadmium

Calcium

Calcium

Chromium

Chromium

Cobalt

Cobalt

Copper

Copper

Iron

Iron

Lead

Lead

Magnesium

Magnesium

Manganese

Manganese

Mercury

Nickel

Nickel

Potassium

Potassium

Selenium

Selenium

Silver

Silver

Sodium

Sodium

Thallium

Thallium

Vanadium

Vanadium

Zinc

Zinc

$\begin{array}{llll} & \text { VX } & & 1180 \\ & \text { VX } & & 2740 \\ \text { UJ } & \text { I } & \text { L } & 6.21 \\ \text { UJ } & \text { I } & \text { L } & 5.82 \\ \text { U } & & & 18.6 \\ \text { U } & & & 17.5 \\ \text { J } & \text { CX } & & 8.45 \\ \text { J } & \text { CX } & & 13.6 \\ \text { J } & \text { EC } & & 0.0615 \\ \text { J } & \text { EC } & & 0.101 \\ \text { U } & & & 0.311 \\ \text { U } & & & 0.291\end{array}$

$J \quad$ VIX H 276

J VIX H 437

1.62

2.84

$\begin{array}{lll}\text { J } & \mathrm{E} & 0.468\end{array}$

J E $\quad 1.03$

2.5

J $\quad$ CX 967

J $\quad$ CX 1490

U $\quad 6.21$

$\begin{array}{llll}J & \mathrm{E} & \mathbf{3 . 5 9}\end{array}$

$\begin{array}{llll}J & \text { IX } & \text { H } & 69.9\end{array}$

$\begin{array}{llll}J & \text { IX } & \text { H } & 137\end{array}$

$\mathrm{X} \quad 11.1$

$\mathrm{X} \quad 22.2$

U $\quad 0.0393$

$\begin{array}{lll}J & \mathrm{E} & \mathbf{0 . 8 6 7}\end{array}$

$\begin{array}{lll}J & \mathrm{E} & 1.39\end{array}$

$\begin{array}{lll}J & E & 49.1\end{array}$

$\begin{array}{lll}J & \mathrm{E} & 74.1\end{array}$

$\mathrm{U} \quad 18.6$

U $\quad 17.5$

$\mathrm{U} \quad 1.55$

$\mathrm{U} \quad 1.46$

U $\quad 62.1$

J $\quad \mathrm{E} \quad 9.2$

$\mathrm{U} \quad 18.6$

J $\quad$ CX $\quad 2.85$

J $\quad$ CX 4.59

VX 2.51

$\mathrm{VX} \quad 5.65$
U $\quad 17.5$
Unit

D. Limit Method

$B / N / A$ Extractables (including $P A H$ and phenols)

Acenaphthene

Acenaphthylene

Anthracene

Benzo[a]anthracene

Benzo[b]fluoranthene

Benzo[k]fluoranthene

Benzoic acid
U

U

U

U

$\mathrm{U}$

$U$

$\mathrm{U}$
41.7

41.7

41.7

4.17

4.17

4.17

41.7
EPA6010A EPA6010A

EPA6010A

EPA6010A

EPA6010A

EPA6010A

EPA6010A

EPA6010A

EPA6010A

EPA6010A

EPA6010A

EPA6010A

EPA6010A

EPA6010A

EPA6010A

EPA6010A

EPA6010A

EPA6010A

EPA6010A

EPA6010A

EPA6010A

EPA6010A

EPA6010A

EPA6010A

EPA6010A

EPA6010A

EPA6010A

EPA6010A

EPA7471

EPA6010A

EPA6010A

EPA6010A

EPA6010A

EPA6010A

EPA6010A

EPA6010A

EPA6010A

EPA6010A

EPA6010A

EPA6010A

EPA6010A

EPA6010A

EPA6010A

EPA6010A

EPA6010A

$\mathrm{mg} / \mathrm{kg}$

1.16

$\begin{array}{lll}\mu \mathrm{g} / \mathrm{kg} & 41.7 & \text { EPA8270 } \\ \mu \mathrm{g} / \mathrm{kg} & 41.7 & \text { EPA8270 } \\ \mu \mathrm{g} / \mathrm{kg} & 41.7 & \text { EPA8270 } \\ \mu \mathrm{g} / \mathrm{kg} & 4.17 & \text { EPA8270 } \\ \mu \mathrm{g} / \mathrm{kg} & 4.17 & \text { EPA8270 } \\ \mu \mathrm{g} / \mathrm{kg} & 4.17 & \text { EPA8270 } \\ \mu \mathrm{g} / \mathrm{kg} & 41.7 & \text { EPA8270 }\end{array}$


SURVEY ID: PPSC 2501 (continued)

Sample ID: 105113
Constituent
RQ AQ B Result
Unit
D. Limit Method

$B / N / A$ Extractables (including PAH and phenols)

\begin{tabular}{|c|c|c|c|c|c|}
\hline Benzo $[g, h, i]$ perylene & $\mathrm{U}$ & 41.7 & $\mu \mathrm{g} / \mathrm{kg}$ & 41.7 & EPA8270 \\
\hline Benzo[a]pyrene & $\mathrm{U}$ & 4.17 & $\mu \mathrm{g} / \mathrm{kg}$ & 4.17 & EPA8270 \\
\hline Benzyl alcohol & $\mathrm{U}$ & 41.7 & $\mu \mathrm{g} / \mathrm{kg}$ & 41.7 & EPA8270 \\
\hline Bis(2-chloroethoxy) methane & $\mathrm{U}$ & 41.7 & $\mu \mathrm{g} / \mathrm{kg}$ & 41.7 & EPA8270 \\
\hline Bis(2-chloroethyl) ether & $\mathrm{U}$ & 41.7 & $\mu \mathrm{g} / \mathrm{kg}$ & 41.7 & EPA8270 \\
\hline Bis(2-chloroisopropyl) ether & $\mathrm{U}$ & 41.7 & $\mu \mathrm{g} / \mathrm{kg}$ & 41.7 & EPA8270 \\
\hline Bis(2-ethylhexyl) phthalate & & 487 & $\mu \mathrm{g} / \mathrm{kg}$ & 41.7 & EPA8270 \\
\hline 4-Bromophenyl phenyl ether & $\mathrm{U}$ & 41.7 & $\mu \mathrm{g} / \mathrm{kg}$ & 41.7 & EPA8270 \\
\hline Butylbenzyl phthalate & $\mathrm{U}$ & 41.7 & $\mu \mathrm{g} / \mathrm{kg}$ & 41.7 & EPA8270 \\
\hline 4-Chloroaniline & $\mathrm{U}$ & 41.7 & $\mu \mathrm{g} / \mathrm{kg}$ & 41.7 & EPA8270 \\
\hline 4-Chloro-m-cresol & $\mathrm{U}$ & 41.7 & $\mu \mathrm{g} / \mathrm{kg}$ & 41.7 & EPA8270 \\
\hline 2.Chloronaphthalene & $\mathrm{U}$ & 41.7 & $\mu \mathrm{g} / \mathrm{kg}$ & 41.7 & EPA8270 \\
\hline 2-Chlorophenol & $U$ & 41.7 & $\mu \mathrm{g} / \mathrm{kg}$ & 41.7 & EPA8270 \\
\hline 4-Chlorophenyl phenyl ether & $\mathrm{U}$ & 41.7 & $\mu \mathrm{g} / \mathrm{kg}$ & 41.7 & EPA8270 \\
\hline Chrysene & $\mathrm{U}$ & 4.17 & $\mu \mathrm{g} / \mathrm{kg}$ & 4.17 & EPA8270 \\
\hline $\mathrm{m} / \mathrm{p}$-Cresol & $\mathrm{U}$ & 417 & $\mu \mathrm{g} / \mathrm{kg}$ & 417 & EPA8270 \\
\hline o-Cresol (2-Methylphenol) & $\mathrm{U}$ & 41.7 & $\mu \mathrm{g} / \mathrm{kg}$ & 41.7 & EPA8270 \\
\hline Dibenz $[a, h]$ anthracene & $\mathrm{U}$ & 4.17 & $\mu \mathrm{g} / \mathrm{kg}$ & 4.17 & EPA8270 \\
\hline Dibenzofuran & $U$ & 41.7 & $\mu \mathrm{g} / \mathrm{kg}$ & 41.7 & EPA8270 \\
\hline Di-n-butyl phthalate & $\mathrm{U}$ & 32.9 & $\mu \mathrm{g} / \mathrm{kg}$ & 41.7 & EPA8270 \\
\hline 1,2-Dichlorobenzene & U & 41.7 & $\mu \mathrm{g} / \mathrm{kg}$ & 41.7 & EPA8270 \\
\hline 1,3-Dichlorobenzene & $\mathrm{U}$ & 41.7 & $\mu \mathrm{g} / \mathrm{kg}$ & 41.7 & EPA8270 \\
\hline 1,4-Dichlorobenzene & $\mathrm{U}$ & 41.7 & $\mu \mathrm{g} / \mathrm{kg}$ & 41.7 & EPA8270 \\
\hline 3,3'-Dichlorobenzidine & U & 41.7 & $\mu \mathrm{g} / \mathrm{kg}$ & 41.7 & EPA8270 \\
\hline 2,4-Dichlorophenol & $\mathrm{U}$ & 41.7 & $\mu \mathrm{g} / \mathrm{kg}$ & 41.7 & EPA8270 \\
\hline Diethyl phthalate & $\mathbf{U}$ & 41.7 & $\mu \mathrm{g} / \mathrm{kg}$ & 41.7 & EPA8270 \\
\hline 2,4-Dimethyl phenol & U & 41.7 & $\mu \mathrm{g} / \mathrm{kg}$ & 41.7 & EPA8270 \\
\hline Dimethyl phthalate & $\mathrm{U}$ & 41.7 & $\mu \mathrm{g} / \mathrm{kg}$ & 41.7 & EPA8270 \\
\hline 2,4-Dinitrophenol & $U$ & 417 & $\mu \mathrm{g} / \mathrm{kg}$ & 417 & EPA8270 \\
\hline 2,4-Dinitrotoluene & UJ & 4.17 & $\mu \mathrm{g} / \mathrm{kg}$ & 4.17 & EPA8270 \\
\hline 2,6-Dinitrotoluene & $\mathrm{U}$ & 4.17 & $\mu \mathrm{g} / \mathrm{kg}$ & 4.17 & EPA8270 \\
\hline Di-n-octyl phthalate & & 240 & $\mu \mathrm{g} / \mathrm{kg}$ & 41.7 & EPA8270 \\
\hline Fluoranthene & $\mathrm{U}$ & 41.7 & $\mu g / k g$ & 41.7 & EPA8270 \\
\hline Fluorene & U & 41.7 & $\mu \mathrm{g} / \mathrm{kg}$ & 41.7 & EPA8270 \\
\hline Hexachlorobenzene & $U$ & 4.17 & $\mu \mathrm{g} / \mathrm{kg}$ & 4.17 & EPA8270 \\
\hline Hexachlorobutadiene & $\mathrm{U}$ & 41.7 & $\mu \mathrm{g} / \mathrm{kg}$ & 41.7 & EPA8270 \\
\hline Hexachlorocyclopentadiene & $\mathrm{U}$ & 41.7 & $\mu g / \mathrm{kg}$ & 41.7 & EPA8270 \\
\hline Hexachloroethane & $\mathrm{U}$ & 4.17 & $\mu \mathrm{g} / \mathrm{kg}$ & 4.17 & EPA8270 \\
\hline Indeno[1,2,3-c,d]pyrene & $\mathrm{U}$ & 4.17 & $\mu \mathrm{g} / \mathrm{kg}$ & 4.17 & EPA8270 \\
\hline Isophorone & $\mathrm{U}$ & 41.7 & $\mu \mathrm{g} / \mathrm{kg}$ & 41.7 & EPA8270 \\
\hline 2-Methyl-4,6-dinitrophenol & $\mathrm{U}$ & 417 & $\mu g / \mathrm{kg}$ & 417 & EPA8270 \\
\hline 2-Methylnaphthalene & U & 41.7 & $\mu \mathrm{g} / \mathrm{kg}$ & 41.7 & EPA8270 \\
\hline Naphthalene & U & 41.7 & $\mu \mathrm{g} / \mathrm{kg}$ & 41.7 & EPA8270 \\
\hline $\mathrm{m}$-Nitroaniline & $U$ & 41.7 & $\mu \mathrm{g} / \mathrm{kg}$ & 41.7 & EPA8270 \\
\hline o-Nitroaniline & $\mathrm{U}$ & 41.7 & $\mu \mathrm{g} / \mathrm{kg}$ & 41.7 & EPA8270 \\
\hline p-Nitroaniline & U & 41.7 & $\mu \mathrm{g} / \mathrm{kg}$ & 41.7 & EPA8270 \\
\hline Nitrobenzene & $\mathrm{U}$ & 41.7 & $\mu \mathrm{g} / \mathrm{kg}$ & 41.7 & EPA8270 \\
\hline 2-Nitrophenol & $\mathrm{U}$ & 41.7 & $\mu \mathrm{g} / \mathrm{kg}$ & 41.7 & EPA8270 \\
\hline 4-Nitrophenol & $\mathrm{U}$ & 41.7 & $\mu \mathrm{g} / \mathrm{kg}$ & 41.7 & EPA8270 \\
\hline N-Nitrosodiphenylamine & $U$ & 41.7 & $\mu \mathrm{g} / \mathrm{kg}$ & 41.7 & EPA8270 \\
\hline N-Nitrosodi-n-propylamine & $\mathrm{U}$ & 41.7 & $\mu g / \mathrm{kg}$ & 41.7 & EPA8270 \\
\hline Pentachlorophenol & $U$ & 41.7 & $\mu g / \mathrm{kg}$ & 41.7 & EPA8270 \\
\hline Phenanthrene & U & 41.7 & $\mu \mathrm{g} / \mathrm{kg}$ & 41.7 & EPA8270 \\
\hline Phenol & $\mathrm{U}$ & 41.7 & $\mu \mathrm{g} / \mathrm{kg}$ & 41.7 & EPA8270 \\
\hline Pyrene & $\mathrm{U}$ & 41.7 & $\mu \mathrm{g} / \mathrm{kg}$ & 41.7 & EPA8270 \\
\hline
\end{tabular}


SURVEY ID: PPSC 2501 (continued)

Sample ID: 105113

Constituent

RQ AQ B Result

Unit

D. Limit Method

$B / N / A$ Extractables (including PAH and phenols)

Tetradecane

1,2,4-Trichlorobenzene

2,4,6-Trichlorophenol

Unknown hydrocarbon
2,4,5-Trichlorophenol

$\begin{array}{lll}\mathrm{J} & \mathrm{N} & 380 \\ \mathrm{U} & & 4.17 \\ \mathrm{U} & & 41.7 \\ \mathrm{U} & & 41.7 \\ \mathrm{~J} & \mathrm{~N} & 720\end{array}$

Volatile Organic Compounds

Acetone

Benzene

Bromodichloromethane

Bromoform

Bromomethane (Methyl bromide)

Carbon disulfide

Carbon tetrachloride .

Chlorobenzene

Chloroethane

Chloroethene (Vinyl chloride)

Chloroform

Chloromethane (Methyl chloride)

Dibromochloromethane

1,1-Dichloroethane

1,2-Dichloroethane

1,1-Dichloroethylene

1,2-Dichloroethylene

Dichloromethane (Methylene chloride)

1,2-Dichloropropane

cis-1,3-Dichloropropene

trans-1,3-Dichloropropene

Ethylbenzene

2-Hexanone

Isopropyl alcohol

Methyl ethyl ketone

Methyl isobutyl ketone

Styrene

1,1,2,2-Tetrachloroethane

Tetrachloroethylene

Toluene

1,1,1-Trichloroethane

1,1,2-Trichloroethane

Trichloroethylene

Vinyl acetate

Xylenes

Pesticides/PCBs and Dioxins/Furans

Aldrin

alpha-Benzene hexachloride

beta-Benzene hexachloride

delta-Benzene hexachloride

alpha-Chlordane

gamma-Chlordane

p,p'-DDD

$p, p^{\prime}-\mathrm{DDE}$

$\mathrm{p}, \mathrm{p}^{\prime}-\mathrm{DDT}$
5.26
5.26
10.5
10.5
10.5
10.5
21
10.5
21

5.26
5.26
10.5
10.5
10.5
10.5
21
10.5
21

5.26
5.26
10.5
10.5
10.5
10.5
21
10.5
21

$\begin{array}{lll}\text { U } & 8 & 52.1 \\ U & & 0.633 \\ U & & 0.0633 \\ U & & 0.0633 \\ U & & 0.127 \\ U & & 1.27 \\ U & & 0.0633 \\ U & & 0.0633 \\ U & & 0.127 \\ U & & 0.127 \\ U & & 0.0633 \\ U & & 0.127 \\ U & & 0.0633 \\ U & & 0.0633 \\ U & & 0.0633 \\ U & & 0.0633 \\ U & & 0.127 \\ U & V & 1.29 \\ U & & 0.0633 \\ U & & 0.0633 \\ U & & 0.0633 \\ U & & 0.0633 \\ U & & 1.27 \\ J & N & 1.5 \\ U & V & 2.13 \\ U & & 1.27 \\ U & & 0.0633 \\ U & & 0.0633 \\ U & & 0.0633 \\ U & 8 & 0.987 \\ U & & 0.0633 \\ U & & 0.0633 \\ U & & 0.0633 \\ U & & 1.27 \\ U & & 0.19\end{array}$

$\mu g / \mathrm{kg}$

\& $\mu \mathrm{g} / \mathrm{kg}$ $\mu \mathrm{g} / \mathrm{kg}$

$\mu \mathrm{g} / \mathrm{kg}$

$\mu \mathrm{g} / \mathrm{kg}$

$\mu \mathrm{g} / \mathrm{kg}$

$\mu \mathrm{g} / \mathrm{kg}$

$\mu \mathrm{g} / \mathrm{kg}$

$\mu \mathrm{g} / \mathrm{kg}$

$\mu \mathrm{g} / \mathrm{kg}$

$\mu \mathrm{g} / \mathrm{kg}$

$\mu \mathrm{g} / \mathrm{kg}$

$\mu \mathrm{g} / \mathrm{kg}$

$\mu \mathrm{g} / \mathrm{kg}$

$\mu \mathrm{g} / \mathrm{kg}$

$\mu \mathrm{g} / \mathrm{kg}$

$\mu \mathrm{g} / \mathrm{kg}$

$\mu \mathrm{g} / \mathrm{kg}$

$\mu \mathrm{g} / \mathrm{kg}$

$\mu \mathrm{g} / \mathrm{kg}$

$\mu \mathrm{g} / \mathrm{kg}$

$\mu \mathrm{g} / \mathrm{kg}$

$\mu \mathrm{g} / \mathrm{kg}$

$\mu \mathrm{g} / \mathrm{kg}$

$\mu \mathrm{g} / \mathrm{kg}$

$\mu g / k g$

$\mu \mathrm{g} / \mathrm{kg}$

$\mu \mathrm{g} / \mathrm{kg}$

$\mu \mathrm{g} / \mathrm{kg}$

$\mu \mathrm{g} / \mathrm{kg}$

$\mu \mathrm{g} / \mathrm{kg}$

$\mu \mathrm{g} / \mathrm{kg}$

$\mu \mathrm{g} / \mathrm{kg}$

$\mu \mathrm{g} / \mathrm{kg}$

$\mu \mathrm{g} / \mathrm{kg}$

6.33

0.633

0.0633

0.0633

0.127

1.27

0.0633

0.0633

0.127

0.127

0.0633

0.127

0.0633

0.0633

0.0633

0.0633

0.127

0.633

0.0633

0.0633

0.0633

0.0633

1.27

1.27

1.27

0.0633

0.0633

0.0633

0.633

0.0633

0.0633

0.0633

1.27

0.19

$\mu \mathrm{g} / \mathrm{kg}$

$\mu \mathrm{g} / \mathrm{kg}$

$\mu \mathrm{g} / \mathrm{kg}$

$\mu \mathrm{g} / \mathrm{kg}$

$\mu \mathrm{g} / \mathrm{kg}$

$\mu \mathrm{g} / \mathrm{kg}$

$\mu \mathrm{g} / \mathrm{kg}$

$\mu \mathrm{g} / \mathrm{kg}$

$\mu \mathrm{g} / \mathrm{kg}$

$\begin{array}{ll}5.26 & \text { EPA8080 } \\ 5.26 & \text { EPA8080 } \\ 10.5 & \text { EPA8080 } \\ 10.5 & \text { EPA8080 } \\ 10.5 & \text { EPA8080 } \\ 10.5 & \text { EPA8080 } \\ 21 & \text { EPA8080 } \\ 10.5 & \text { EPA8080 } \\ 21 & \text { EPA8080 }\end{array}$

EPA8080
EPA8080
EPA8080
EPA8080
EPA8080
EPA8080
EPA8080
EPA8080
EPA8080
EPA8270

EPA8270

EPA8270

EPA8270

EPA8270
EPA8260

EPA8260

EPA8260

EPA8260

EPA8260

EPA8260

EPA8260

EPA8260

EPA8260

EPA8260

EPA8260

EPA8260

EPA8260

EPA8260

EPA8260

EPA8260

EPA8260

EPA8260

EPA8260

EPA8260

EPA8260

EPA8260

EPA8260

EPA8260

EPA8260

EPA8260

EPA8260

EPA8260

EPA8260

EPA8260

EPA8260

EPA8260

EPA8260

EPA8260

EPA8260 
SURVEY ID: PPSC 2501 (continued)

Constituent

Pesticides/PCBs and Dioxins/Furans

Dieldrin

Endosulfan sulfate

Endosulfan I

Endosulfan II

Endrin

Endrin ketone

Heptachlor

Heptachlor epoxide

Lindane

Methoxychlor

PCB 1016

PCB 1221

PCB 1232

PCB 1242

PCB 1248

PCB 1254

PCB 1260

Toxaphene

Radionuclides

Actinium-228

Antimony-124

Antimony-125

Barium-133

Cerium-144

Cesium-134

Cesium-137

Cobalt-57

Cobalt-58

Cobalt-60

Europium-152

Europium-154

Europium-155

Gross alpha

Lead-212

Manganese-54

Neptunium-239

Nonvolatile beta

Plutonium-238

Plutonium-239/240

Potassium.40

Promethium-144

Promethium-146

Radium-226

Radium-228

Ruthenium-106

Sodium-22

Strontium-90

Thorium-234

Tin-113

Yttrium-88

Zinc-65

Zirconium-95

Sample ID: 105113

RQ AQ B Result Unit D. Limit Method

$\begin{array}{llll}10.5 & \mu \mathrm{g} / \mathrm{kg} & 10.5 & \text { EPA8080 } \\ 21 & \mu \mathrm{g} / \mathrm{kg} & 21 & \text { EPA8080 } \\ 10.5 & \mu \mathrm{g} / \mathrm{kg} & 10.5 & \text { EPA8080 } \\ 21 & \mu \mathrm{g} / \mathrm{kg} & 21 & \text { EPA8080 } \\ 10.5 & \mu \mathrm{g} / \mathrm{kg} & 10.5 & \text { EPA8080 } \\ 21 & \mu \mathrm{g} / \mathrm{kg} & 21 & \text { EPA8080 } \\ 10.5 & \mu \mathrm{g} / \mathrm{kg} & 10.5 & \text { EPA8080 } \\ 10.5 & \mu \mathrm{g} / \mathrm{kg} & 10.5 & \text { EPA8080 } \\ 5.26 & \mu \mathrm{g} / \mathrm{kg} & 5.26 & \text { EPA8080 } \\ 105 & \mu \mathrm{g} / \mathrm{kg} & 105 & \text { EPA8080 } \\ 26.3 & \mu \mathrm{g} / \mathrm{kg} & 26.3 & \text { EPA8080 } \\ 26.3 & \mu \mathrm{g} / \mathrm{kg} & 26.3 & \text { EPA8080 } \\ 26.3 & \mu \mathrm{g} / \mathrm{kg} & 26.3 & \text { EPA8080 } \\ 26.3 & \mu \mathrm{g} / \mathrm{kg} & 26.3 & \text { EPA8080 } \\ 26.3 & \mu \mathrm{g} / \mathrm{kg} & 26.3 & \text { EPA8080 } \\ 26.3 & \mu \mathrm{g} / \mathrm{kg} & 26.3 & \text { EPA8080 } \\ 26.3 & \mu \mathrm{g} / \mathrm{kg} & 26.3 & \text { EPA8080 } \\ 210 & \mu \mathrm{g} / \mathrm{kg} & 210 & \text { EPA8080 }\end{array}$

$1.52 \mathrm{E}+00 \pm 5.23 \mathrm{E}-02 \mathrm{pCi} / \mathrm{g}$ 4.61E-04 $\pm 5.55 \mathrm{E}-03 \mathrm{pCi} / \mathrm{g}$ 2.26E-03 $\pm 1.30 \mathrm{E}-02 \mathrm{pCi} / \mathrm{g}$ $-1.08 \mathrm{E}-03 \pm 6.58 \mathrm{E}-03 \mathrm{pCi} / \mathrm{g}$ $-8.14 \mathrm{E}-04 \pm 3.63 \mathrm{E}-02 \mathrm{pCi} / \mathrm{g}$ $8.95 \mathrm{E}-05 \pm 4.70 \mathrm{E}-03 \mathrm{pCi} / \mathrm{g}$ $1.19 \mathrm{E}-02 \pm 7.60 \mathrm{E}-03 \mathrm{pCi} / \mathrm{g}$ $-1.06 \mathrm{E}-03 \pm 4.54 \mathrm{E}-03 \mathrm{pCi} / \mathrm{g}$ $3.79 \mathrm{E}-03 \pm 5.18 \mathrm{E}-03 \mathrm{pCi} / \mathrm{g}$ $2.02 \mathrm{E}-03 \pm 4.27 \mathrm{E}-03 \mathrm{pCi} / \mathrm{g}$ $6.50 \mathrm{E}-03 \pm 1.46 \mathrm{E}-02 \mathrm{pCj} / \mathrm{g}$ $-3.50 \mathrm{E}-03 \pm 4.52 \mathrm{E}-02 \mathrm{pCi} / \mathrm{g}$ $0.00 \mathrm{E}+00 \quad \mathrm{pCi} / \mathrm{g}$ $3.48 \mathrm{E}+01 \pm 4.63 \mathrm{E}+00 \mathrm{pCi} / \mathrm{g}$ $1.67 \mathrm{E}+00 \pm 2.61 \mathrm{E}-02 \mathrm{pCi} / \mathrm{g}$ $2.90 \mathrm{E}-02 \pm 6.50 \mathrm{E}-03 \mathrm{pCi} / \mathrm{g}$ $-2.90 \mathrm{E}-02 \pm 3.64 \mathrm{E}-02 \mathrm{pCi} / \mathrm{g}$ $2.53 \mathrm{E}+01 \pm 2.79 \mathrm{E}+00 \mathrm{pCi} / \mathrm{g}$ $-1.24 \mathrm{E}-03 \pm 1.26 \mathrm{E}-02 \mathrm{pCi} / \mathrm{g}$ $0.00 \mathrm{E}+00 \quad \mathrm{pCi} / \mathrm{g}$

$\begin{array}{llll}\text { UI } & 0.00 \mathrm{E}+00 \quad \mathrm{pCi} / \mathrm{g} \\ \mathrm{V} & 3.71 \mathrm{E}+00 \pm 1.59 \mathrm{E}-01 \mathrm{pCi} / \mathrm{g}\end{array}$
UI $\quad-2.12 \mathrm{E}-03 \pm 4.61 \mathrm{E}-03 \mathrm{pCi} / \mathrm{g}$ UI $\quad 0.00 \mathrm{E}+00 \quad \mathrm{pCi} / \mathrm{g}$ 8.63E-01 $\pm 2.90 \mathrm{E}-02 \mathrm{pCi} / \mathrm{g}$ $1.52 \mathrm{E}+00 \pm 5.23 \mathrm{E}-02 \mathrm{pCi} / \mathrm{g}$ $-7.12 \mathrm{E}-03 \pm 4.19 \mathrm{E}-02 \mathrm{pCi} / \mathrm{g}$ $-8.34 \mathrm{E}-04 \pm 4.42 \mathrm{E}-03 \mathrm{pCi} / \mathrm{g}$ $-8.53 \mathrm{E}-02 \pm 1.99 \mathrm{E}-02 \mathrm{pCi} / \mathrm{g}$ $9.86 \mathrm{E}-01 \pm 4.71 \mathrm{E}-01 \mathrm{pCi} / \mathrm{g}$ $-1.15 \mathrm{E}-03 \pm 6.80 \mathrm{E}-03 \mathrm{pCi} / \mathrm{g}$ 2.49E-03 $\pm 6.92 \mathrm{E}-03 \mathrm{pCi} / \mathrm{g}$ 5.33E-03 $1.09 \mathrm{E}-02 \mathrm{pCi} / \mathrm{g}$ $0.00 \mathrm{E}+00 \quad \mathrm{pCi} / \mathrm{g}$
2.59E-02 EPIA-013B

9.62E-03 EPIA-013B

2.14E-02 EPIA-013B

9.62E-03 EPIA-013B

6.22E-02 EPIA-013B

7.21E-03 EPIA-013B

7.46E-03 EPIA-013B

7.77E-03 EPIA-013B

9.09E-03 EPIA-013B

7.68E-03 EPIA-013B

2.18E-02 EPLA-013B

6.66E-02 EPIA-013B

3.23E-02 EPIA.013B

1.78E+00 EPIA-001B

1.36E-02 EPIA-013B

8.20E-03 EPIA-013B

5.56E-02 EPIA-013B

2.53E+00 EPIA-001B

3.24E-02 EPIA-012B

7.55E-03 EPIA-012B

6.35E-02 EPIA-013B

7.79E-03 EPIA-013B

1.07E-02 EPIA-013B

1.48E-02 EPIA-013B

2.59E-02 EPIA-013B

7.21E-02 EPIA-013B

7.31E-03 EPIA-013B

1.47E-01 EPLA-004

4.12E-01 EPIA-013B

1.11E-02 EPIA-013B

9.20E-03 EPIA-013B

1.65E-02 EPIA-013B

1.98E-02 EPIA-013B 
Survey location: $54602 \mathrm{E} 3712523 \mathrm{~N}$ (SRS coordinates)

Depth of core interval: 1.00 to $2.00 \mathrm{ft}$

Sample type: Normal

Constituent

RQ AQ B Result

Physical Parameters and Specified Analyses

$\begin{array}{llll}\text { Ammonia nitrogen } & \text { U } & & 23.4 \\ \text { Cation exchange capacity } & & & 16.2 \\ \text { Chloride } & & & 3.58 \\ \text { Cyanide } & \text { U } & & 1.15 \\ \text { Nitrate-nitrite as nitrogen } & \text { U } & & 1.01 \\ \text { Nitrogen by Kjeldahl method } & & & 96.5 \\ \text { Orthophosphate } & \text { J } & \text { E } & 2.64 \\ \text { Phenols } & \text { UJ } & \text { C } & 347 \\ \text { Sulfide } & \text { UJ } & \text { C } & 294 \\ \text { Total organic carbon } & & \text { V } & 2830 \\ \text { Total organic nitrogen } & \text { C } & & 90.4 \\ \text { Total phosphates (as P) } & \text { J } & \text { E } & 3.94\end{array}$

Metals (total recoverable)

$\begin{array}{lll}\mathrm{mg} / \mathrm{kg} & 23.4 & \text { EPA350.1 } \\ \mathrm{meq} / 100 \mathrm{~g} & 5 & \text { EPA9081 } \\ \mathrm{mg} / \mathrm{kg} & 2.81 & \text { EPA300.0 } \\ \mathrm{mg} / \mathrm{kg} & 1.15 & \text { EPA335.3 } \\ \mathrm{mg} / \mathrm{kg} & 1.01 & \text { EPA353.1 } \\ \mathrm{mg} / \mathrm{kg} & 22.7 & \text { EPA351.2 } \\ \mathrm{mg} / \mathrm{kg} & 11.8 & \text { EPA365.2 } \\ \mu \mathrm{g} / \mathrm{kg} & 347 & \text { EPA420.2 } \\ \mathrm{mg} / \mathrm{kg} & 294 & \text { EPA376.2 } \\ \mathrm{mg} / \mathrm{kg} & 118 & \text { EPA415.1 } \\ \mathrm{mg} / \mathrm{kg} & 0.235 & \text { EPA351.2 } \\ \mathrm{mg} / \mathrm{kg} & \mathbf{9 . 1 2} & \text { EPA365.4 }\end{array}$

\begin{tabular}{|c|c|c|c|c|c|c|}
\hline Aluminum & & V & 684 & $\mathrm{mg} / \mathrm{kg}$ & 13.7 & EPA6010A \\
\hline Antimony & $\mathrm{U}$ & & 5.46 & $\mathrm{mg} / \mathrm{kg}$ & 5.46 & EPA6010A \\
\hline Arsenic & U & & 16.4 & $\mathrm{mg} / \mathrm{kg}$ & 16.4 & EPA6010A \\
\hline Barium & $\mathbf{J}$ & C & 7.69 & $\mathrm{mg} / \mathrm{kg}$ & 1.37 & EPA6010A \\
\hline Beryllium & $\mathrm{J}$ & EC & 0.113 & $\mathrm{mg} / \mathrm{kg}$ & 0.546 & EPA6010A \\
\hline Cadmium & U & & 0.273 & $\mathrm{mg} / \mathrm{kg}$ & 0.273 & EPA6010A \\
\hline Calcium & & V & 245 & $\mathrm{mg} / \mathrm{kg}$ & 13.7 & EPA6010A \\
\hline Chromium & & & 2.72 & $\mathrm{mg} / \mathrm{kg}$ & 1.37 & EPA6010A \\
\hline Cobalt & $J$ & $\mathbf{E}$ & 0.332 & $\mathrm{mg} / \mathrm{kg}$ & 0.546 & EPA6010A \\
\hline Copper & $\mathrm{J}$ & $\mathrm{E}$ & 0.642 & $\mathrm{mg} / \mathrm{kg}$ & 1.37 & EPA6010A \\
\hline Iron & $J$ & C & 1390 & $\mathrm{mg} / \mathrm{kg}$ & 5.46 & EPA6010A \\
\hline Lead & $J$ & $\mathrm{E}$ & 2.54 & $\mathrm{mg} / \mathrm{kg}$ & 5.46 & EPA6010A \\
\hline Magnesium & & & 41.8 & $\mathrm{mg} / \mathrm{kg}$ & 5.46 & EPA6010A \\
\hline Manganese & & & 4.65 & $\mathrm{mg} / \mathrm{kg}$ & 0.546 & EPA6010A \\
\hline Mercury & $\mathrm{U}$ & & 0.0321 & $\mathrm{mg} / \mathrm{kg}$ & 0.0321 & EPA7471 \\
\hline Nickel & $J$ & $\mathbf{E}$ & 0.926 & $\mathrm{mg} / \mathrm{kg}$ & 1.37 & EPA6010A \\
\hline Potassium & $J$ & $E$ & 33.4 & $\mathrm{mg} / \mathrm{kg}$ & 109 & EPA6010A \\
\hline Selenium & $U$ & & 16.4 & $\mathrm{mg} / \mathrm{kg}$ & 16.4 & EPA6010A \\
\hline Silver & $\mathrm{U}$ & & 1.37 & $\mathrm{mg} / \mathrm{kg}$ & 1.37 & EPA6010A \\
\hline Sodium & $\mathrm{U}$ & & 54.6 & $\mathrm{mg} / \mathrm{kg}$ & 54.6 & EPA6010A \\
\hline Thallium & $\mathrm{U}$ & & 16.4 & $\mathrm{mg} / \mathrm{kg}$ & 16.4 & EPA6010A \\
\hline Vanadium & $J$ & $\mathrm{C}$ & 2.46 & $\mathrm{mg} / \mathrm{kg}$ & 0.546 & EPA6010A \\
\hline Zinc & & V & 1.98 & $\mathrm{mg} / \mathrm{kg}$ & 1.09 & EPA6010A \\
\hline
\end{tabular}

$B / N / A$ Extractables (including PAH and phenols)

Acenaphthene

Acenaphthylene

Anthracene

Benzo[a]anthracene

Benzo[b]fluoranthene

Benzo[k]fluoranthene

Benzoic acid

Benzo[g,h,i]perylene

$\begin{array}{ll}U & 38.8 \\ U & 38.8 \\ U & 38.8 \\ U & 3.88 \\ U & 3.88 \\ U & 3.88 \\ U & 38.8 \\ U & 38.8\end{array}$

Unit

D. Limit Method 
SURVEY ID: PPSC 2502 (continued)

Sample ID: 105114

Constituent

RQ AQ B Result

Unit

D. Limit Method

$B / N / A$ Extractables (including PAH and phenols)

Benzo[a]pyrene

Benzyl alcohol

Bis(2-chloroethoxy) methane

Bis(2-chloroethyl) ether

Bis(2-chloroisopropyl) ether

Bis(2-ethylhexyl) phthalate

4-Bromophenyl phenyl ether

Butylbenzyl phthalate

4-Chloroaniline

4-Chloro-m-cresol

2-Chloronaphthalene

2-Chlorophenol

4-Chlorophenyl phenyl ether

Chrysene

$\mathrm{m} / \mathrm{p}$-Cresol

o-Cresol (2-Methylphenol)

Dibenz $[a, h]$ anthracene

Dibenzofuran

Di-n-butyl phthalate

1,2-Dichlorobenzene

1,3-Dichlorobenzene

1,4-Dichlorobenzene

3,3'-Dichlorobenzidine

2,4-Dichlorophenol

Diethyl phthalate

2,4-Dimethyl phenol

Dimethyl phthalate

2,4-Dinitrophenol

2,4-Dinitrotoluene

2,6-Dinitrotoluene

Di-n-octyl phthalate

Fluoranthene

Fluorene

Hexachlorobenzene

Hexachlorobutadiene

Hexachlorocyclopentadiene

Hexachloroethane

Indeno $[1,2,3-c, d]$ pyrene

Isophorone

2-Methyl-4,6-dinitrophenol

2-Methylnaphthalene

Naphthalene

m-Nitroaniline

o-Nitroaniline

p-Nitroaniline

Nitrobenzene

2-Nitrophenol

4-Nitrophenol

N-Nitrosodiphenylamine

N-Nitrosodi-n-propylamine

Pentachlorophenol

Phenanthrene

Phenol

Pyrene

gamma-Sitosterol
$\mathrm{U}$

$\mathrm{U}$

U

U

38.8

216

38.8

38.8

38.8

38.8

38.8

38.8

38.8

3.88

388

38.8

3.88

38.8

$\mathrm{V}$

26

38.8

38.8

38.8

38.8

38.8

38.8

38.8

38.8

388

3.88

3.88

38.8

38.8

38.8

3.88

38.8

38.8

3.88

3.88

38.8

388

38.8

38.8

38.8

38.8

38.8

38.8

38.8

38.8

38.8

38.8

38.8

38.8

38.8

38.8

580

\begin{tabular}{|c|c|c|}
\hline$\mu \mathrm{g} / \mathrm{kg}$ & $\begin{array}{l}3.88 \\
38.8\end{array}$ & EPA8270 \\
\hline $\begin{array}{l}\mu \mathrm{g} / \mathrm{kg} \\
\mu \mathrm{g} / \mathrm{kg}\end{array}$ & $\begin{array}{l}38.8 \\
38.8\end{array}$ & $\begin{array}{l}\text { EPA8270 } \\
\text { EPA8270 }\end{array}$ \\
\hline $\begin{array}{l}\mu \mathrm{g} / \mathrm{kg} \\
\mu \mathrm{g} / \mathrm{kg}\end{array}$ & 38.8 & EPA8270 \\
\hline$\mu \mathrm{g} / \mathrm{kg}$ & 38.8 & EPA8270 \\
\hline$\mu \mathrm{g} / \mathrm{kg}$ & 38.8 & EPA8270 \\
\hline$\mu \mathrm{g} / \mathrm{kg}$ & 38.8 & EPA8270 \\
\hline$\mu \mathrm{g} / \mathrm{kg}$ & 38.8 & EPA8270 \\
\hline$\mu \mathrm{g} / \mathrm{kg}$ & 38.8 & EPA8270 \\
\hline$\mu \mathrm{g} / \mathrm{kg}$ & 38.8 & EPA8270 \\
\hline$\mu \mathrm{g} / \mathrm{kg}$ & 38.8 & EPA8270 \\
\hline$\mu \mathrm{g} / \mathrm{kg}$ & 38.8 & EPA8270 \\
\hline$\mu \mathrm{g} / \mathrm{kg}$ & 38.8 & EPA8270 \\
\hline$\mu \mathrm{g} / \mathrm{kg}$ & 3.88 & EPA8270 \\
\hline$\mu \mathrm{g} / \mathrm{kg}$ & 388 & EPA8270 \\
\hline$\mu \mathrm{g} / \mathrm{kg}$ & 38.8 & EPA8270 \\
\hline$\mu \mathrm{g} / \mathrm{kg}$ & 3.88 & EPA8270 \\
\hline$\mu \mathrm{g} / \mathrm{kg}$ & 38.8 & EPA8270 \\
\hline$\mu \mathrm{g} / \mathrm{kg}$ & 38.8 & EPA8270 \\
\hline$\mu \mathrm{g} / \mathrm{kg}$ & 38.8 & EPA8270 \\
\hline$\mu \mathrm{g} / \mathrm{kg}$ & 38.8 & EPA8270 \\
\hline$\mu \mathrm{g} / \mathrm{kg}$ & 38.8 & EPA8270 \\
\hline$\mu \mathrm{g} / \mathrm{kg}$ & 38.8 & EPA8270 \\
\hline$\mu \mathrm{g} / \mathrm{kg}$ & 38.8 & EPA8270 \\
\hline$\mu \mathrm{g} / \mathrm{kg}$ & 38.8 & EPA8270 \\
\hline$\mu \mathrm{g} / \mathrm{kg}$ & 38.8 & EPA8270 \\
\hline$\mu \mathrm{g} / \mathrm{kg}$ & 38.8 & EPA8270 \\
\hline$\mu \mathrm{g} / \mathrm{kg}$ & 388 & EPA8270 \\
\hline$\mu \mathrm{g} / \mathrm{kg}$ & 3.88 & EPA8270 \\
\hline$\mu \mathrm{g} / \mathrm{kg}$ & 3.88 & EPA8270 \\
\hline$\mu \mathrm{g} / \mathrm{kg}$ & 38.8 & EPA8270 \\
\hline$\mu \mathrm{g} / \mathrm{kg}$ & 38.8 & EPA8270 \\
\hline$\mu \mathrm{g} / \mathrm{kg}$ & 38.8 & EPA8270 \\
\hline$\mu \mathrm{g} / \mathrm{kg}$ & 3.88 & EPA8270 \\
\hline$\mu \mathrm{g} / \mathrm{kg}$ & 38.8 & EPA8270 \\
\hline$\mu \mathrm{g} / \mathrm{kg}$ & 38.8 & EPA8270 \\
\hline$\mu \mathrm{g} / \mathrm{kg}$ & 3.88 & EPA8270 \\
\hline$\mu \mathrm{g} / \mathrm{kg}$ & 3.88 & EPA8270 \\
\hline$\mu \mathrm{g} / \mathrm{kg}$ & 38.8 & EPA8270 \\
\hline$\mu \mathrm{g} / \mathrm{kg}$ & 388 & EPA8270 \\
\hline$\mu \mathrm{g} / \mathrm{kg}$ & 38.8 & EPA8270 \\
\hline$\mu \mathrm{g} / \mathrm{kg}$ & 38.8 & EPA8270 \\
\hline$\mu \mathrm{g} / \mathrm{kg}$ & 38.8 & EPA8270 \\
\hline$\mu \mathrm{g} / \mathrm{kg}$ & 38.8 & EPA8270 \\
\hline$\mu \mathrm{g} / \mathrm{kg}$ & 38.8 & EPA8270 \\
\hline$\mu \mathrm{g} / \mathrm{kg}$ & 38.8 & EPA8270 \\
\hline$\mu \mathrm{g} / \mathrm{kg}$ & 38.8 & EPA8270 \\
\hline$\mu \mathrm{g} / \mathrm{kg}$ & 38.8 & EPA8270 \\
\hline$\mu \mathrm{g} / \mathrm{kg}$ & 38.8 & EPA8270 \\
\hline$\mu \mathrm{g} / \mathrm{kg}$ & 38.8 & EPA8270 \\
\hline$\mu \mathrm{g} / \mathrm{kg}$ & 38.8 & EPA8270 \\
\hline$\mu \mathrm{g} / \mathrm{kg}$ & 38.8 & EPA8270 \\
\hline$\mu \mathrm{g} / \mathrm{kg}$ & 38.8 & EPA8270 \\
\hline$\mu \mathrm{g} / \mathrm{kg}$ & 38.8 & EPA8270 \\
\hline$\mu \mathrm{g} / \mathrm{kg}$ & & EPA8270 \\
\hline
\end{tabular}


SURVEY ID: PPSC 2502 (continued)

Constituent
Sample ID: 105114

RQ AQ B Result Unit D. Limit Method

$B / N / A$ Extractables (including $P A H$ and phenols)

1,2,4-Trichlorobenzene

2,4,5-Trichlorophenol

2,4,6-Trichlorophenol

Unknown hydrocarbon

Unknown naphthalene

$\begin{array}{lll}\mathrm{U} & & 3.88 \\ \mathrm{U} & & \mathbf{3 8 . 8} \\ \mathrm{U} & & \mathbf{3 8 . 8} \\ \mathrm{J} & \mathrm{N} & \mathbf{1 7 0 0} \\ \mathrm{J} & \mathrm{N} & \mathbf{8 7 0}\end{array}$

Volatile Organic Compounds

Acetone

Benzene

Bromodichloromethane

Bromoform

Bromomethane (Methyl bromide)

Carbon disulfide

Carbon tetrachloride

Chlorobenzene

Chloroethane

Chloroethene (Vinyl chloride)

Chloroform

Chloromethane (Methyl chloride)

Dibromochloromethane

1,1-Dichloroethane

1,2-Dichloroethane

1,1-Dichloroethylene

1,2-Dichloroethylene

Dichloromethane (Methylene chloride)

1,2-Dichloropropane

cis-1,3-Dichloropropene

trans-1,3-Dichloropropene

Ethylbenzene

2-Hexanone

Methyl ethyl ketone

Methyl isobutyl ketone

Styrene

1,1,2,2-Tetrachloroethane

Tetrachloroethylene

Toluene

1,1,1-Trichloroethane

1,1,2-Trichloroethane

Trichloroethylene

Unknown hydrocarbon

Vinyl acetate

Xylenes

Pesticides/PCBs and Dioxins/Furans

Aldrin

alpha-Benzene hexachloride

beta-Benzene hexachloride

delta-Benzene hexachloride

alpha-Chlordane

gamma-Chlordane

$p, p^{\prime}-D D D$

p,p'-DDE

$p, p^{\prime}-D D T$
$8 \quad 88.9$

U $\quad 0.588$

U $\quad 0.0588$

U $\quad 0.0588$

U $\quad 0.118$

U $\quad 1.18$

U $\quad 0.0588$

$\mathrm{U} \quad 0.0588$

U $\quad 0.118$

U $\quad 0.118$

U $\quad 0.0588$

$\begin{array}{lll}\mathrm{J} & \mathrm{E} & 0.106\end{array}$

U $\quad 0.0588$

U $\quad \mathbf{0 . 0 5 8 8}$

U $\quad 0.0588$

U $\quad 0.0588$

U $\quad 0.118$

$\begin{array}{lll}U & V & 1.58\end{array}$

U $\quad 0.0588$

U $\quad 0.0588$

U $\quad 0.0588$

$\begin{array}{ll}\mathrm{U} & 0.0588\end{array}$

$\begin{array}{lll}U & V & 0.294\end{array}$

$\begin{array}{lll}U & V & 3.04\end{array}$

$U \quad 1.18$

$\mathrm{U} \quad 0.0588$

U $\quad 0.0588$

U $\quad \mathbf{0 . 0 5 8 8}$

U $\quad 0.588$

$\mathrm{U} \quad 0.0588$

U $\quad 0.0588$

$\begin{array}{ll}U & 0.0588\end{array}$

J $\quad \mathrm{N} \quad 3$

U $\quad 1.18$

$\mathrm{U} \quad 0.176$ $\mu \mathrm{g} / \mathrm{kg}$

$\mu \mathrm{g} / \mathrm{kg}$

$\mu \mathrm{g} / \mathrm{kg}$

$\mu \mathrm{g} / \mathrm{kg}$

$\mu \mathrm{g} / \mathrm{kg}$

$\mu \mathrm{g} / \mathrm{kg}$

$\mu \mathrm{g} / \mathrm{kg}$

$\mu \mathrm{g} / \mathrm{kg}$

$\mu \mathrm{g} / \mathrm{kg}$

$\mu g / k g$

$\mu \mathrm{g} / \mathrm{kg}$

$\mu g / \mathrm{kg}$

$\mu \mathrm{g} / \mathrm{kg}$

$\mu \mathrm{g} / \mathrm{kg}$

$\mu \mathrm{g} / \mathrm{kg}$

$\mu \mathrm{g} / \mathrm{kg}$

$\mu \mathrm{g} / \mathrm{kg}$

$\mu \mathrm{g} / \mathrm{kg}$

$\mu \mathrm{g} / \mathrm{kg}$

$\mu \mathrm{g} / \mathrm{kg}$

$\mu \mathrm{g} / \mathrm{kg}$

$\mu \mathrm{g} / \mathrm{kg}$

$\mu \mathrm{g} / \mathrm{kg}$

$\mu \mathrm{g} / \mathrm{kg}$

$\mu \mathrm{g} / \mathrm{kg}$

$\mu \mathrm{g} / \mathrm{kg}$

$\mu \mathrm{g} / \mathrm{kg}$

$\mu \mathrm{g} / \mathrm{kg}$

$\mu \mathrm{g} / \mathrm{kg}$

$\mu \mathrm{g} / \mathrm{kg}$

$\mu \mathrm{g} / \mathrm{kg}$

$\mu \mathrm{g} / \mathrm{kg}$

$\mu \mathrm{g} / \mathrm{kg}$

$\mu \mathrm{g} / \mathrm{kg}$

$\mu \mathrm{g} / \mathrm{kg}$

$\mu \mathrm{g} / \mathrm{kg}$

$\mu \mathrm{g} / \mathrm{kg}$

$\mu \mathrm{g} / \mathrm{kg}$

$\mu \mathrm{g} / \mathrm{kg}$

$\mu \mathrm{g} / \mathrm{kg}$
3.88

38.8

EPA8270

38.8

EPA8270

EPA8270

EPA8270

EPA8270

29.4

0.588

0.0588

0.0588

0.118

1.18

0.0588

0.0588

0.118

0.118

0.0588

0.118

0.0588

0.0588

0.0588

0.0588

0.118

0.588

0.0588

0.0588

0.0588

0.0588

1.18

1.18

1.18

0.0588

0.0588

0.0588

0.588

0.0588

0.0588

0.0588

1.18

0.176
EPA8260

EPA8260

EPA8260

EPA8260

EPA8260

EPA8260

EPA8260

EPA8260

EPA8260

EPA8260

EPA8260

EPA8260

EPA8260

EPA8260

EPA8260

EPA8260

EPA8260

EPA8260

EPA8260

EPA8260

EPA8260

EPA8260

EPA8260

EPA8260

EPA8260

EPA8260

EPA8260

EPA8260

EPA8260

EPA8260

EPA8260

EPA8260

EPA8260

EPA8260

EPA8260

$\begin{array}{lll}\mu \mathrm{g} / \mathrm{kg} & 0.96 & \text { EPA8080 } \\ \mu \mathrm{g} / \mathrm{kg} & 0.96 & \text { EPA8080 } \\ \mu \mathrm{g} / \mathrm{kg} & 1.92 & \text { EPA8080 } \\ \mu \mathrm{g} / \mathrm{kg} & 1.92 & \text { EPA8080 } \\ \mu \mathrm{g} / \mathrm{kg} & 1.92 & \text { EPA8080 } \\ \mu \mathrm{g} / \mathrm{kg} & 1.92 & \text { EPA8080 } \\ \mu \mathrm{g} / \mathrm{kg} & 3.84 & \text { EPA8080 } \\ \mu \mathrm{g} / \mathrm{kg} & 1.92 & \text { EPA8080 } \\ \mu \mathrm{g} / \mathrm{kg} & 3.84 & \text { EPA8080 }\end{array}$


SURVEY ID: PPSC 2502 (continued)

Constituent

Pesticides/PCBs and Dioxins/Furans

Dieldrin

Endosulfan sulfate

Endosulfan I

Endosulfan II

Endrin

Endrin ketone

Heptachlor

Heptachlor epoxide

Lindane

Methoxychlor

PCB 1016

PCB 1221

PCB 1232

PCB 1242

PCB 1248

PCB 1254

PCB 1260

Toxaphene

Radionuclides

Actinium-228

Antimony-124

Antimony-125

Barium-133

Cerium-144

Cesium-134

Cesium-137

Cobalt-57

Cobalt-58

Cobalt-60

Europium-152

Europium-154

Europium-155

Gross alpha

Lead-212

Manganese-54

Neptunium-239

Nonvolatile beta

Plutonium-238

Plutonium-239/240

Potassium-40

Promethium-144

Promethium-146

Radium-226

Radium-228

Ruthenium-106

Sodium-22

Strontium-90

Thorium-234

Tin-113

Yttrium-88

Zinc-65

Zirconium-95
Sample ID: 105114
RQ AQ B Result
D. Limit Method

$\begin{array}{lll}\mu \mathrm{g} / \mathrm{kg} & 1.92 & \text { EPA8080 } \\ \mu \mathrm{g} / \mathrm{kg} & 3.84 & \text { EPA8080 } \\ \mu \mathrm{g} / \mathrm{kg} & 1.92 & \text { EPA8080 } \\ \mu \mathrm{g} / \mathrm{kg} & 3.84 & \text { EPA8080 } \\ \mu \mathrm{g} / \mathrm{kg} & 1.92 & \text { EPA8080 } \\ \mu \mathrm{g} / \mathrm{kg} & 3.84 & \text { EPA8080 } \\ \mu \mathrm{g} / \mathrm{kg} & 1.92 & \text { EPA8080 } \\ \mu \mathrm{g} / \mathrm{kg} & 1.92 & \text { EPA8080 } \\ \mu \mathrm{g} / \mathrm{kg} & 0.96 & \text { EPA8080 } \\ \mu \mathrm{g} / \mathrm{kg} & 19.2 & \text { EPA8080 } \\ \mu \mathrm{g} / \mathrm{kg} & 4.8 & \text { EPA8080 } \\ \mu \mathrm{g} / \mathrm{kg} & 4.8 & \text { EPA8080 } \\ \mu \mathrm{g} / \mathrm{kg} & 4.8 & \text { EPA8080 } \\ \mu \mathrm{g} / \mathrm{kg} & 4.8 & \text { EPA8080 } \\ \mu \mathrm{g} / \mathrm{kg} & 4.8 & \text { EPA8080 } \\ \mu \mathrm{g} / \mathrm{kg} & 4.8 & \text { EPA8080 } \\ \mu \mathrm{g} / \mathrm{kg} & 4.8 & \text { EPA8080 } \\ \mu \mathrm{g} / \mathrm{kg} & 38.4 & \text { EPA8080 }\end{array}$

5.96E-01 $\pm 3.88 \mathrm{E}-02 \mathrm{pCi} / \mathrm{g}$ $-1.97 \mathrm{E}-04 \pm 4.03 \mathrm{E}-03 \mathrm{pCi} / \mathrm{g}$ $-1.33 \mathrm{E}-04 \pm 9.31 \mathrm{E}-03 \mathrm{pCi} / \mathrm{g}$ $-2.20 \mathrm{E}-04 \pm 4.73 \mathrm{E}-03 \mathrm{pCi} / \mathrm{g}$ $-1.36 \mathrm{E}-03 \pm 2.57 \mathrm{E}-02 \mathrm{pCi} / \mathrm{g}$ $-5.43 \mathrm{E}-04 \pm 3.40 \mathrm{E}-03 \mathrm{pCi} / \mathrm{g}$ $3.22 \mathrm{E}-03 \pm 4.62 \mathrm{E}-03 \mathrm{pCi} / \mathrm{g}$ $-3.17 \mathrm{E}-04 \pm 3.24 \mathrm{E}-03 \mathrm{pCi} / \mathrm{g}$ $1.26 \mathrm{E}-04 \pm 4.58 \mathrm{E}-03 \mathrm{pCi} / \mathrm{g}$ $-2.11 \mathrm{E}-03 \pm 3.45 \mathrm{E}-03 \mathrm{pCi} / \mathrm{g}$ -7.04E-04 $\pm 9.67 \mathrm{E}-03 \mathrm{pCi} / \mathrm{g}$ $-2.19 \mathrm{E}-02 \pm 3.26 \mathrm{E}-02 \mathrm{pCi} / \mathrm{g}$ $0.00 \mathrm{E}+00 \quad \mathrm{pCi} / \mathrm{g}$ $1.40 \mathrm{E}+01 \pm 2.92 \mathrm{E}+00 \mathrm{pCi} / \mathrm{g}$ $6.27 \mathrm{E}-01 \pm 1.66 \mathrm{E}-02 \mathrm{pCi} / \mathrm{g}$ $1.06 \mathrm{E}-02 \pm 5.55 \mathrm{E}-03 \mathrm{pCi} / \mathrm{g}$

UI

UI

UI

UI

UI

V

UI

UI

U V

UI

UI

UI

UI
$8.44 \mathrm{E}-03 \pm 2.58 \mathrm{E}-02 \mathrm{pCi} / \mathrm{g}$ $1.78 \mathrm{E}+01 \pm 2.44 \mathrm{E}+00 \mathrm{pCi} / \mathrm{g}$ $-7.51 \mathrm{E}-03 \pm 1.38 \mathrm{E}-02 \mathrm{pCi} / \mathrm{g}$ $8.80 \mathrm{E}-03 \pm 1.21 \mathrm{E}-02 \mathrm{pCi} / \mathrm{g}$ $5.57 \mathrm{E}+00 \pm 1.76 \mathrm{E}-01 \mathrm{pCi} / \mathrm{g}$ 5.61E-03 $\pm 3.60 \mathrm{E}-03 \mathrm{pCi} / \mathrm{g}$ 5.55E-04 $44.46 \mathrm{E}-03 \mathrm{pCi} / \mathrm{g}$ 4.57E-01 $\pm 2.09 \mathrm{E}-02 \mathrm{pCi} / \mathrm{g}$ 5.96E-01 $\pm 3.88 \mathrm{E}-02 \mathrm{pCi} / \mathrm{g}$ 5.77E-03 $\pm 3.05 \mathrm{E}-02 \mathrm{pCi} / \mathrm{g}$ $3.62 \mathrm{E}-03 \pm 3.78 \mathrm{E}-03 \mathrm{pCi} / \mathrm{g}$ 2.63E-01 $1.29 \mathrm{E}-01 \mathrm{pCi} / \mathrm{g}$ $6.88 \mathrm{E}-01 \pm 3.40 \mathrm{E}-01 \mathrm{pCi} / \mathrm{g}$ 1.31E-03 $4.87 \mathrm{E}-03 \mathrm{pCi} / \mathrm{g}$ 2.10E-03 $\pm 3.51 \mathrm{E}-03 \mathrm{pCi} / \mathrm{g}$ 3.53E-05 $\pm 9.31 \mathrm{E}-03 \mathrm{pCi} / \mathrm{g}$ $4.16 \mathrm{E}-03 \pm 9.14 \mathrm{E}-03 \mathrm{pCi} / \mathrm{g}$
2.09E-02 EPIA-013B

6.98E-03 EPLA-013B

1.53E-02 EPIA-013B

6.96E-03 EPIA-013B

4.39E-02 EPIA-013B

5.11E-03 EPIA-013B

6.94E-03 EPIA-013B

5.56E-03 EPIA-013B

6.90E-03 EPIA-013B

5.89E-03 EPIA-013B

1.60E-02 EPIA-013B

5.34E-02 EPIA-013B

2.35E-02 EPIA-013B

1.97E+00 EPLA-001B

1.01E-02 EPIA-013B

6.09E-03 EPIA-013B

4.03E-02 EPIA-013B

2.69E+00 EPIA-001B

3.84E-02 EPIA-012B

2.18E-02 EPIA-012B

5.21E-02 EPIA-013B

5.91E-03 EPIA-013B

7.33E-03 EPIA-013B

1.04E-02 EPIA-013B

2.09E-02 EPIA-013B

5.33E-02 EPIA-013B

6.98E-03 EPIA-013B

1.75E-01 EPIA-004

2.93E-01 EPIA-013B

8.08E-03 EPIA-013B

6.58E-03 EPLA-013B

1.37E-02 EPIA-013B

1.41E-02 EPIA-013B 
Survey location: $54602 \mathrm{E} 3712523 \mathrm{~N}$ (SRS coordinates)

Depth of core interval: 2.00 to $4.00 \mathrm{ft}$

Sample type: Normal

Sample matrix: Soil

Percent solids: 90.00

Constituent

RQ AQ B Result

Unit

D. Limit Method

Physical Parameters and Specified Analyses

$\begin{array}{llll}\text { Ammonia nitrogen } & \text { U } & & 22.2 \\ \text { Cation exchange capacity } & & & 11.8 \\ \text { Chloride } & & & 2.88 \\ \text { Cyanide } & \text { U } & & 1.02 \\ \text { Nitrate-nitrite as nitrogen } & \text { U } & & 1.01 \\ \text { Nitrogen by Kjeldahl method } & & & 22.5 \\ \text { Orthophosphate } & \text { J } & \text { E } & 2.88 \\ \text { Phenols } & \text { J } & \text { C } & 382 \\ \text { Sulfide } & \text { UJ } & \text { C } & 278 \\ \text { Sulfide } & \text { UJ } & \text { C } & 278 \\ \text { Total organic carbon } & & \text { V } & 289 \\ \text { Total organic nitrogen } & \text { C } & & 20.7 \\ \text { Total phosphates (as P) } & \text { U } & & 11\end{array}$

$\begin{array}{lll}\mathrm{mg} / \mathrm{kg} & \mathbf{2 2 . 2} & \text { EPA350.1 } \\ \mathrm{meq} / 100 \mathrm{~g} & 5 & \text { EPA9081 } \\ \mathrm{mg} / \mathrm{kg} & 2.81 & \text { EPA300.0 } \\ \mathrm{mg} / \mathrm{kg} & 1.02 & \text { EPA335.3 } \\ \mathrm{mg} / \mathrm{kg} & 1.01 & \text { EPA353.1 } \\ \mathrm{mg} / \mathrm{kg} & 4.41 & \text { EPA351.2 } \\ \mathrm{mg} / \mathrm{kg} & 11.1 & \text { EPA365.2 } \\ \mu \mathrm{g} / \mathrm{kg} & 332 & \text { EPA420.2 } \\ \mathrm{mg} / \mathrm{kg} & 278 & \text { EPA376.2 } \\ \mathrm{mg} / \mathrm{kg} & 278 & \text { EPA376.2 } \\ \mathrm{mg} / \mathrm{kg} & 111 & \text { EPA415.1 } \\ \mathrm{mg} / \mathrm{kg} & 0.222 & \text { EPA351.2 } \\ \mathrm{mg} / \mathrm{kg} & 11 & \text { EPA365.4 }\end{array}$

Metals (total recoverable)

$\begin{array}{llll}\text { Aluminum } & & \mathrm{V} & \mathbf{8 3 1} \\ \text { Antimony } & \mathrm{U} & & \mathbf{5 . 2 9} \\ \text { Arsenic } & \mathrm{U} & & \mathbf{1 5 . 9} \\ \text { Barium } & \mathrm{J} & \mathrm{C} & 7.22 \\ \text { Beryllium } & \mathrm{J} & \mathrm{EC} & \mathbf{0 . 0 6 0 3} \\ \text { Cadmium } & \mathrm{U} & & 0.264 \\ \text { Calcium } & & \mathrm{V} & 108 \\ \text { Chromium } & \mathrm{J} & \mathrm{E} & 1.31 \\ \text { Cobalt } & & & \mathbf{0 . 7 9 2} \\ \text { Copper } & \mathrm{J} & \mathrm{E} & \mathbf{0 . 5 1 8} \\ \text { Iron } & \mathrm{J} & \mathrm{C} & \mathbf{8 4 2} \\ \text { Lead } & \mathrm{U} & & \mathbf{5 . 2 9} \\ \text { Magnesium } & & & \mathbf{5 7 . 5} \\ \text { Manganese } & & & 4.53 \\ \text { Mercury } & \mathrm{J} & \mathrm{E} & \mathbf{0 . 0 0 4 4 9} \\ \text { Nickel } & \mathrm{J} & \mathrm{E} & 1.03 \\ \text { Potassium } & \mathrm{J} & \mathrm{E} & 63.8 \\ \text { Selenium } & \mathrm{U} & & 15.9 \\ \text { Silver } & \mathrm{U} & & 1.32 \\ \text { Sodium } & \mathrm{U} & & \mathbf{5 2 . 9} \\ \text { Thallium } & \mathrm{U} & & 15.9 \\ \text { Vanadium } & \mathrm{J} & \mathrm{C} & 2.17 \\ \text { Zinc } & & \mathrm{V} & \mathbf{2 . 1 1}\end{array}$

$\begin{array}{lll}\mathrm{mg} / \mathrm{kg} & 13.2 & \text { EPA6010A } \\ \mathrm{mg} / \mathrm{kg} & 5.29 & \text { EPA6010A } \\ \mathrm{mg} / \mathrm{kg} & 15.9 & \text { EPA6010A } \\ \mathrm{mg} / \mathrm{kg} & 1.32 & \text { EPA6010A } \\ \mathrm{mg} / \mathrm{kg} & 0.529 & \text { EPA6010A } \\ \mathrm{mg} / \mathrm{kg} & 0.264 & \text { EPA6010A } \\ \mathrm{mg} / \mathrm{kg} & 13.2 & \text { EPA6010A } \\ \mathrm{mg} / \mathrm{kg} & 1.32 & \text { EPA6010A } \\ \mathrm{mg} / \mathrm{kg} & \mathbf{0 . 5 2 9} & \text { EPA6010A } \\ \mathrm{mg} / \mathrm{kg} & 1.32 & \text { EPA6010A } \\ \mathrm{mg} / \mathrm{kg} & \mathbf{5 . 2 9} & \text { EPA6010A } \\ \mathrm{mg} / \mathrm{kg} & 5.29 & \text { EPA6010A } \\ \mathrm{mg} / \mathrm{kg} & 5.29 & \text { EPA6010A } \\ \mathrm{mg} / \mathrm{kg} & 0.529 & \text { EPA6010A } \\ \mathrm{mg} / \mathrm{kg} & 0.031 & \text { EPA7471 } \\ \mathrm{mg} / \mathrm{kg} & 1.32 & \text { EPA6010A } \\ \mathrm{mg} / \mathrm{kg} & 106 & \text { EPA6010A } \\ \mathrm{mg} / \mathrm{kg} & 15.9 & \text { EPA6010A } \\ \mathrm{mg} / \mathrm{kg} & 1.32 & \text { EPA6010A } \\ \mathrm{mg} / \mathrm{kg} & 52.9 & \text { EPA6010A } \\ \mathrm{mg} / \mathrm{kg} & 15.9 & \text { EPA6010A } \\ \mathrm{mg} / \mathrm{kg} & 0.529 & \text { EPA6010A } \\ \mathrm{mg} / \mathrm{kg} & 1.06 & \text { EPA6010A }\end{array}$

$B / N / A$ Extractables (including $P A H$ and phenols)

Acenaphthene

Acenaphthylene

Anthracene

Benzo[a]anthracene

Benzo[b]fluoranthene

Benzo[k]fluoranthene

Benzoic acid

$\begin{array}{ll}\text { U } & 36.7 \\ \mathrm{U} & 36.7 \\ \mathrm{U} & 36.7 \\ \mathrm{U} & 3.67 \\ \mathrm{U} & 3.67 \\ \mathrm{U} & 3.67 \\ \mathrm{U} & 36.7\end{array}$

$\begin{array}{lll}\mu \mathrm{g} / \mathrm{kg} & 36.7 & \text { EPA8270 } \\ \mu \mathrm{g} / \mathrm{kg} & 36.7 & \text { EPA8270 } \\ \mu \mathrm{g} / \mathrm{kg} & 36.7 & \text { EPA8270 } \\ \mu \mathrm{g} / \mathrm{kg} & 3.67 & \text { EPA8270 } \\ \mu \mathrm{g} / \mathrm{kg} & \mathbf{3 . 6 7} & \text { EPA8270 } \\ \mu \mathrm{g} / \mathrm{kg} & 3.67 & \text { EPA8270 } \\ \mu \mathrm{g} / \mathrm{kg} & 36.7 & \text { EPA8270 }\end{array}$


SURVEY ID: PPSC 2503 (continued)

Sample ID: 105115

$\begin{array}{lllll}\text { Constituent } & \text { RQ AQ B Result D. Limit Method }\end{array}$

$B / N / A$ Extractables (including $P A H$ and phenols)

Benzo[g,h,i]perylene

Benzo[a]pyrene

Benzyl alcohol

$\mathrm{Bis}$ (2-chloroethoxy) methane

Bis(2-chloroethyl) ether

Bis(2-chloroisopropyl) ether

Bis(2-ethylhexyl) phthalate

4-Bromophenyl phenyl ether

Butylbenzyl phthalate

4-Chloroaniline

4-Chloro-m-cresol

2-Chloronaphthalene

2-Chlorophenol

4-Chlorophenyl phenyl ether

Chrysene

$\mathrm{m} / \mathrm{p}$-Cresol

o-Cresol (2-Methylphenol)

Dibenz $[a, h]$ anthracene

Dibenzofuran

Di-n-butyl phthalate

-1,2-Dichlorobenzene

1,3-Dichlorobenzene

1,4-Dichlorobenzene

3,3'-Dichlorobenzidine

2,4-Dichlorophenol

Diethyl phthalate

2,4-Dimethyl phenol

Dimethyl phthalate

2,4-Dinitrophenol

2,4-Dinitrotoluene

2,6-Dinitrotoluene

Di-n-octyl phthalate

Fluoranthene

Fluorene

Hexachlorobenzene

Hexachlorobutadiene

Hexachlorocyclopentadiene

Hexachloroethane

Indeno $[1,2,3-c, d]$ pyrene

Isophorone

2-Methyl-4,6-dinitrophenol

2-Methylnaphthalene

Naphthalene

m-Nitroaniline

o-Nitroaniline

p-Nitroaniline

Nitrobenzene

2-Nitrophenol

4-Nitrophenol

N-Nitrosodiphenylamine

N-Nitrosodi-n-propylamine

Pentachlorophenol

Phenanthrene

Phenol

Pyrene

\begin{tabular}{|c|c|c|}
\hline$U$ & & 36.7 \\
\hline $\mathrm{U}$ & & 3.67 \\
\hline $\mathrm{U}$ & & 36.7 \\
\hline $\mathrm{U}$ & & 36.7 \\
\hline$U$ & & 36.7 \\
\hline $\mathrm{U}$ & & 36.7 \\
\hline & & 160 \\
\hline $\mathrm{U}$ & & 36.7 \\
\hline $\mathrm{U}$ & & 36.7 \\
\hline $\mathrm{U}$ & & 36.7 \\
\hline $\mathrm{U}$ & & 36.7 \\
\hline$U$ & & 36.7 \\
\hline $\mathrm{U}$ & & 36.7 \\
\hline $\mathrm{U}$ & & 36.7 \\
\hline $\mathrm{U}$ & & 3.67 \\
\hline$U$ & & 367 \\
\hline $\mathrm{U}$ & & 36.7 \\
\hline $\mathbf{U}$ & & 3.67 \\
\hline $\mathrm{U}$ & & 36.7 \\
\hline$U$ & V & 27.9 \\
\hline $\mathrm{U}$ & & 36.7 \\
\hline $\mathrm{U}$ & & 36.7 \\
\hline $\mathrm{U}$ & & 36.7 \\
\hline U & & 36.7 \\
\hline $\mathrm{U}$ & & 36.7 \\
\hline $\mathrm{U}$ & & 36.7 \\
\hline $\mathrm{U}$ & & 36.7 \\
\hline $\mathrm{U}$ & & 36.7 \\
\hline $\mathrm{U}$ & & 367 \\
\hline UJ & C & 3.67 \\
\hline $\mathrm{U}$ & & 3.67 \\
\hline & & 73.7 \\
\hline $\mathrm{U}$ & & 36.7 \\
\hline $\mathrm{U}$ & & 36.7 \\
\hline $\mathrm{U}$ & & 3.67 \\
\hline U & & $36: 7$ \\
\hline $\mathrm{U}$ & & 36.7 \\
\hline $\mathbf{U}$ & & 3.67 \\
\hline $\mathrm{U}$ & & 3.67 \\
\hline $\mathrm{U}$ & & 36.7 \\
\hline $\mathrm{U}$ & & 367 \\
\hline $\mathrm{U}$ & & 36.7 \\
\hline $\mathrm{U}$ & & 36.7 \\
\hline $\mathrm{U}$ & & 36.7 \\
\hline $\mathrm{U}$ & & 36.7 \\
\hline $\mathrm{U}$ & & 36.7 \\
\hline $\mathrm{U}$ & & 36.7 \\
\hline $\mathrm{U}$ & & 36.7 \\
\hline $\mathrm{U}$ & & 36.7 \\
\hline $\mathrm{U}$ & & 36.7 \\
\hline $\mathrm{U}$ & & 36.7 \\
\hline $\mathrm{U}$ & & 36.7 \\
\hline $\mathrm{U}$ & & 36.7 \\
\hline $\mathrm{U}$ & & 36.7 \\
\hline $\mathrm{U}$ & & 36.7 \\
\hline
\end{tabular}

\begin{tabular}{|c|c|c|}
\hline$\mu \mathrm{g} / \mathrm{kg}$ & 36.7 & EPA8270 \\
\hline$\mu \mathrm{g} / \mathrm{kg}$ & 3.67 & EPA8270 \\
\hline$\mu \mathrm{g} / \mathrm{kg}$ & 36.7 & EPA8270 \\
\hline$\mu \mathrm{g} / \mathrm{kg}$ & 36.7 & EPA8270 \\
\hline$\mu \mathrm{g} / \mathrm{kg}$ & 36.7 & EPA8270 \\
\hline$\mu \mathrm{g} / \mathrm{kg}$ & 36.7 & EPA8270 \\
\hline$\mu \mathrm{g} / \mathrm{kg}$ & 36.7 & EPA8270 \\
\hline$\mu \mathrm{g} / \mathrm{kg}$ & 36.7 & EPA8270 \\
\hline$\mu \mathrm{g} / \mathrm{kg}$ & 36.7 & EPA8270 \\
\hline$\mu \mathrm{g} / \mathrm{kg}$ & 36.7 & EPA8270 \\
\hline$\mu \mathrm{g} / \mathrm{kg}$ & 36.7 & EPA8270 \\
\hline$\mu \mathrm{g} / \mathrm{kg}$ & 36.7 & EPA8270 \\
\hline$\mu \mathrm{g} / \mathrm{kg}$ & 36.7 & EPA8270 \\
\hline$\mu \mathrm{g} / \mathrm{kg}$ & 36.7 & EPA8270 \\
\hline$\mu g / k g$ & 3.67 & EPA8270 \\
\hline$\mu \mathrm{g} / \mathrm{kg}$ & 367 & EPA8270 \\
\hline$\mu \mathrm{g} / \mathrm{kg}$ & 36.7 & EPA8270 \\
\hline$\mu \mathrm{g} / \mathrm{kg}$ & 3.67 & EPA8270 \\
\hline$\mu g / k g$ & 36.7 & EPA8270 \\
\hline$\mu \mathrm{g} / \mathrm{kg}$ & 36.7 & EPA8270 \\
\hline$\mu \mathrm{g} / \mathrm{kg}$ & 36.7 & EPA8270 \\
\hline$\mu \mathrm{g} / \mathrm{kg}$ & 36.7 & EPA8270 \\
\hline$\mu g / \mathrm{kg}$ & 36.7 & EPA8270 \\
\hline$\mu \mathrm{g} / \mathrm{kg}$ & 36.7 & EPA8270 \\
\hline$\mu \mathrm{g} / \mathrm{kg}$ & 36.7 & EPA8270 \\
\hline$\mu \mathrm{g} / \mathrm{kg}$ & 36.7 & EPA8270 \\
\hline$\mu \mathrm{g} / \mathrm{kg}$ & 36.7 & EPA8270 \\
\hline$\mu \mathrm{g} / \mathrm{kg}$ & 36.7 & EPA8270 \\
\hline$\mu \mathrm{g} / \mathrm{kg}$ & 367 & EPA8270 \\
\hline$\mu \mathrm{g} / \mathrm{kg}$ & 3.67 & EPA8270 \\
\hline$\mu \mathrm{g} / \mathrm{kg}$ & 3.67 & EPA8270 \\
\hline$\mu \mathrm{g} / \mathrm{kg}$ & 36.7 & EPA8270 \\
\hline$\mu \mathrm{g} / \mathrm{kg}$ & 36.7 & EPA8270 \\
\hline$\mu \mathrm{g} / \mathrm{kg}$ & 36.7 & EPA8270 \\
\hline$\mu \mathrm{g} / \mathrm{kg}$ & 3.67 & EPA8270 \\
\hline$\mu \mathrm{g} / \mathrm{kg}$ & 36.7 & EPA8270 \\
\hline$\mu \mathrm{g} / \mathrm{kg}$ & 36.7 & EPA8270 \\
\hline$\mu \mathrm{g} / \mathrm{kg}$ & 3.67 & EPA8270 \\
\hline$\mu \mathrm{g} / \mathrm{kg}$ & 3.67 & EPA8270 \\
\hline$\mu \mathrm{g} / \mathrm{kg}$ & 36.7 & EPA8270 \\
\hline$\mu \mathrm{g} / \mathrm{kg}$ & 367 & EPA8270 \\
\hline$\mu \mathrm{g} / \mathrm{kg}$ & 36.7 & EPA8270 \\
\hline$\mu \mathrm{g} / \mathrm{kg}$ & 36.7 & EPA8270 \\
\hline$\mu \mathrm{g} / \mathrm{kg}$ & 36.7 & EPA8270 \\
\hline$\mu \mathrm{g} / \mathrm{kg}$ & 36.7 & EPA8270 \\
\hline$\mu \mathrm{g} / \mathrm{kg}$ & 36.7 & EPA8270 \\
\hline$\mu \mathrm{g} / \mathrm{kg}$ & 36.7 & EPA8270 \\
\hline$\mu \mathrm{g} / \mathrm{kg}$ & 36.7 & EPA8270 \\
\hline$\mu \mathrm{g} / \mathrm{kg}$ & 36.7 & EPA8270 \\
\hline$\mu \mathrm{g} / \mathrm{kg}$ & 36.7 & EPA8270 \\
\hline$\mu g / \mathrm{kg}$ & 36.7 & EPA8270 \\
\hline$\mu \mathrm{g} / \mathrm{kg}$ & 36.7 & EPA8270 \\
\hline$\mu \mathrm{g} / \mathrm{kg}$ & 36.7 & EPA8270 \\
\hline$\mu \mathrm{g} / \mathrm{kg}$ & 36.7 & EPA8270 \\
\hline$\mu \mathrm{g} / \mathrm{kg}$ & 36.7 & EPA8270 \\
\hline
\end{tabular}


SURVEY ID: PPSC 2503 (continued)

Constituent
Sample ID: 105115

RQ AQ B Result

Unit

D. Limit Method

$B / N / A$ Extractables (including PAH and phenols)

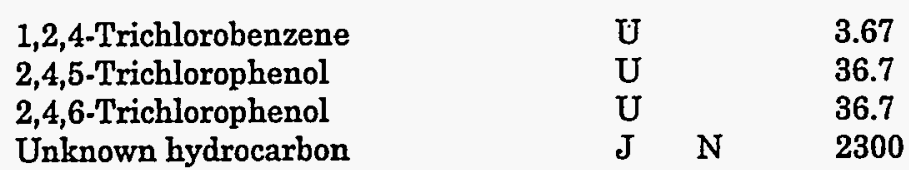

Volatile Organic Compounds

Acetone

Benzene

Bromodichloromethane

Bromoform

Bromomethane (Methyl bromide)

Carbon disulfide

Carbon tetrachloride

Chlorobenzene

Chloroethane

Chloroethene (Vinyl chloride)

Chloroform

Chloromethane (Methyl chloride)

Dibromochloromethane

1,1-Dichloroethane

1,2-Dichloroethane

1,1-Dichloroethylene

1,2-Dichloroethylene

Dichloromethane (Methylene chloride)

1,2-Dichloropropane

cis-1,3-Dichloropropene

trans-1,3-Dichloropropene

Ethylbenzene

2-Hexanone

Methyl ethyl ketone

Methyl isobutyl ketone

Styrene

1,1,2,2-Tetrachloroethane

Tetrachloroethylene

Toluene

1,1,1-Trichloroethane

1,1,2-Trichloroethane

Trichloroethylene

Unknown alcohol

Vinyl acetate

Xylenes

\section{Pesticides/PCBs and Dioxins/Furans}

Aldrin

alpha-Benzene hexachloride

beta-Benzene hexachloride

delta-Benzene hexachloride

alpha-Chlordane

gamma-Chlordane .

p, $p^{\prime}$-DDD

$p, p^{\prime}-D D E$

p,p'-DDT

Dieldrin

$\begin{array}{lll} & 8 & 152 \\ U & & 0.556 \\ U & & 0.0556 \\ U & & 0.0556 \\ U & & 0.111 \\ U & & 1.11 \\ U & & 0.0556 \\ U & & 0.0556 \\ U & & 0.111 \\ U & & 0.111 \\ U & & 0.0556 \\ U & & 0.111 \\ U & & 0.0556 \\ U & & 0.0556 \\ U & & 0.0556 \\ U & & 0.0556 \\ U & & 0.111 \\ U & V & 1.41 \\ U & & 0.0556 \\ U & & 0.0556 \\ U & & 0.0556 \\ U & & 0.0556 \\ U & & 1.11 \\ U & V & 1.59 \\ U & & 1.11 \\ U & & \mathbf{0 . 0 5 5 6} \\ U & & 0.0556 \\ U & & 0.0556 \\ U & 8 & \mathbf{0 . 0 5 5 6} \\ U & & 0.0556 \\ U & & 0.0556 \\ U & & 0.0556 \\ J & N & 6 \\ U & & 1.11 \\ U & & 0.167\end{array}$

3.67

36.7

36.7

$\mu \mathrm{g} / \mathrm{kg}$

$\mu \mathrm{g} / \mathrm{kg}$

$\mu \mathrm{g} / \mathrm{kg}$

$\mu \mathrm{g} / \mathrm{k}$

$\mu \mathrm{g} / \mathrm{kg}$

$\mu \mathrm{g} / \mathrm{kg}$

$\mu \mathrm{g} / \mathrm{kg}$

$\mu \mathrm{g} / \mathrm{kg}$

$\mu \mathrm{g} / \mathrm{kg}$

$\mu \mathrm{g} / \mathrm{kg}$

$\mu \mathrm{g} / \mathrm{kg}$

$\mu \mathrm{g} / \mathrm{kg}$

$\mu \mathrm{g} / \mathrm{kg}$

$\mu \mathrm{g} / \mathrm{kg}$

$\mu \mathrm{g} / \mathrm{kg}$

$\mu \mathrm{g} / \mathrm{kg}$

$\mu \mathrm{g} / \mathrm{kg}$

$\mu \mathrm{g} / \mathrm{kg}$

$\mu \mathrm{g} / \mathrm{kg}$

$\mu \mathrm{g} / \mathrm{kg}$

$\mu \mathrm{g} / \mathrm{kg}$

$\mu \mathrm{g} / \mathrm{kg}$

$\mu \mathrm{g} / \mathrm{kg}$

$\mu \mathrm{g} / \mathrm{kg}$

$\mu g / \mathrm{kg}$

$\mu \mathrm{g} / \mathrm{kg}$

$\mu \mathrm{g} / \mathrm{kg}$

$\mu \mathrm{g} / \mathrm{kg}$

$\mu g / \mathrm{kg}$

$\mu \mathrm{g} / \mathrm{kg}$

$\mu \mathrm{g} / \mathrm{kg}$

$\mu \mathrm{g} / \mathrm{kg}$

$\mu \mathrm{g} / \mathrm{kg}$

$\mu \mathrm{g} / \mathrm{kg}$

$\mu \mathrm{g} / \mathrm{kg}$

$\mu \mathrm{g} / \mathrm{kg}$

$\mu \mathrm{g} / \mathrm{kg}$

$\mu \mathrm{g} / \mathrm{kg}$
EPA8270

EPA8270

EPA8270

EPA8270

\section{$27.8 \quad$ EPA8260 \\ $0.556 \quad$ EPA8260 \\ $0.0556 \quad$ EPA8260 \\ 0.0556 EPA8260 \\ 0.111 EPA8260 \\ 1.11 EPA8260 \\ 0.0556 EPA8260 \\ 0.0556 EPA8260}

0.111 EPA8260

0.111 EPA8260

0.0556 EPA8260

0.111 EPA8260

0.0556 EPA8260

0.0556 EPA8260

0.0556 EPA8260

0.0556 EPA8260

0.111 EPA8260

0.556 EPA8260

0.0556 EPA8260

$0.0556 \quad$ EPA8260

0.0556 EPA8260

0.0556 EPA8260

1.11 EPA8260

1.11 EPA8260

1.11 EPA8260

0.0556 EPA8260

0.0556 EPA8260

$0.0556 \quad$ EPA8260

$0.556 \quad$ EPA8260

0.0556 EPA8260

$0.0556 \quad$ EPA8260

$0.0556 \quad$ EPA8260

EPA8260

$1.11 \quad$ EPA8260

0.167 EPA8260

$\begin{array}{lll}\mu \mathrm{g} / \mathrm{kg} & 0.921 & \text { EPA8080 } \\ \mu \mathrm{g} / \mathrm{kg} & 0.921 & \text { EPA8080 } \\ \mu \mathrm{g} / \mathrm{kg} & 1.84 & \text { EPA8080 } \\ \mu \mathrm{g} / \mathrm{kg} & 1.84 & \text { EPA8080 } \\ \mu \mathrm{g} / \mathrm{kg} & 1.84 & \text { EPA8080 } \\ \mu \mathrm{g} / \mathrm{kg} & 1.84 & \text { EPA8080 } \\ \mu \mathrm{g} / \mathrm{kg} & 3.69 & \text { EPA8080 } \\ \mu \mathrm{g} / \mathrm{kg} & 1.84 & \text { EPA8080 } \\ \mu \mathrm{g} / \mathrm{kg} & 3.69 & \text { EPA8080 } \\ \mu \mathrm{g} / \mathrm{kg} & 1.84 & \text { EPA8080 }\end{array}$


SURVEY ID: PPSC 2503 (continued)

Constituent

Pesticides/PCBs and Dioxins/Furans

Endosulfan sulfate

Endosulfan I

Endosulfan II

Endrin

Endrin ketone

Heptachlor

Heptachlor epoxide

Lindane

Methoxychlor

PCB 1016

PCB 1221

PCB 1232

PCB 1242

PCB 1248

PCB 1254

PCB 1260

Toxaphene

Radionuclides

Actinium-228

Antimony-124

Antimony-125

Barium-133

Cerium-144

Cesium-134

Cesium-137

Cobalt-57

Cobalt-58

Cobalt-60

Europium-152

Europium-154

Europium-155

Gross alpha

Lead-212

Manganese-54

Neptunium-239

Nonvolatile beta

Plutonium-238

Plutonium-239/240

Potassium-40

Promethium-144

Promethium-146

Radium-226

Radium-228

Ruthenium-106

Sodium-22

Strontium-90

Thorium-234

Tin-113

Yttrium-88

Zinc-65

Zirconium-95
Sample ID: 105115

RQ AQ B Result Unit D. Limit Method

$\begin{array}{lll}\mu g / k g & 3.69 & \text { EPA8080 } \\ \mu \mathrm{g} / \mathrm{kg} & 1.84 & \text { EPA8080 } \\ \mu \mathrm{g} / \mathrm{kg} & 3.69 & \text { EPA8080 } \\ \mu \mathrm{g} / \mathrm{kg} & 1.84 & \text { EPA8080 } \\ \mu \mathrm{g} / \mathrm{kg} & 3.69 & \text { EPA8080 } \\ \mu \mathrm{g} / \mathrm{kg} & 1.84 & \text { EPA8080 } \\ \mu \mathrm{g} / \mathrm{kg} & 1.84 & \text { EPA8080 } \\ \mu \mathrm{g} / \mathrm{kg} & 0.921 & \text { EPA8080 } \\ \mu \mathrm{g} / \mathrm{kg} & 18.4 & \text { EPA8080 } \\ \mu \mathrm{g} / \mathrm{kg} & 4.61 & \text { EPA8080 } \\ \mu \mathrm{g} / \mathrm{kg} & 4.61 & \text { EPA8080 } \\ \mu \mathrm{g} / \mathrm{kg} & 4.61 & \text { EPA8080 } \\ \mu \mathrm{g} / \mathrm{kg} & 4.61 & \text { EPA8080 } \\ \mu \mathrm{g} / \mathrm{kg} & 4.61 & \text { EPA8080 } \\ \mu \mathrm{g} / \mathrm{kg} & 4.61 & \text { EPA8080 } \\ \mu \mathrm{g} / \mathrm{kg} & 4.61 & \text { EPA8080 } \\ \mu \mathrm{g} / \mathrm{kg} & 36.9 & \text { EPA8080 }\end{array}$

2.33E-02 EPIA-013B 7.76E-03 EPIA-013B

1.63E-02 EPIA-013B

7.36E-03 EPIA-013B

4.69E-02 EPIA-013B

5.88E-03 EPIA-013B

6.75E-03 EPIA-013B

5.99E-03 EPIA-013B

7.38E-03 EPIA-013B

6.39E-03 EPIA-013B

1.76E-02 EPIA-013B

6.29E-02 EPIA-013B

2.51E-02 EPIA-013B

$1.55 \mathrm{E}+00$ EPIA-001B

1.05E-02 EPIA-013B

6.64E-03 EPIA-013B

4.37E-02 EPIA-013B

2.39E+00 EPIA-001B

1.38E-02 EPIA-012B

0.00E+00 EPLA-012B

4.72E-02 EPIA-013B

6.47E-03 EPLA-013B

8.31E-03 EPIA-013B

1.22E-02 EPIA-013B

2.33E-02 EPIA-013B

5.91E-02 EPIA-013B

7.77E-03 EPIA-013B

1.38E-01 EPIA-004

3.17E-01 EPIA-013B

8.50E-03 EPIA-013B

5.83E-03 EPIA-013B

1.68E-02 EPIA-013B

1.58E-02 EPLA-013B 
Survey location: 54602E 3712523N (SRS coordinates)

Depth of core interval: 4.00 to $5.70 \mathrm{ft}$

Sample type: Normal

\title{
Constituent
}

\author{
RQ AQ B Result
}

Unit

D. Limit Method

Physical Parameters and Specified Analyses

$\begin{array}{llll}\text { Ammonia nitrogen } & \text { U } & & 23.6 \\ \text { Cation exchange capacity } & & & 107 \\ \text { Chloride } & & & 6.65 \\ \text { Cyanide } & \text { U } & & 1.19 \\ \text { Nitrate-nitrite as nitrogen } & \text { J } & \text { E } & 0.365 \\ \text { Nitrogen by Kjeldahl method } & & & 77.1 \\ \text { Orthophosphate } & \text { J } & \text { E } & 2.51 \\ \text { Phenols } & \text { UJ } & \text { C } & 357 \\ \text { Sulfide } & \text { UJ } & \text { C } & 298 \\ \text { Total organic carbon } & & \text { V } & 1160 \\ \text { Total organic nitrogen } & \text { C } & & 71.5 \\ \text { Total phosphates (as P) } & \text { J } & \text { E } & 3.34\end{array}$

Metals (total recoverable)

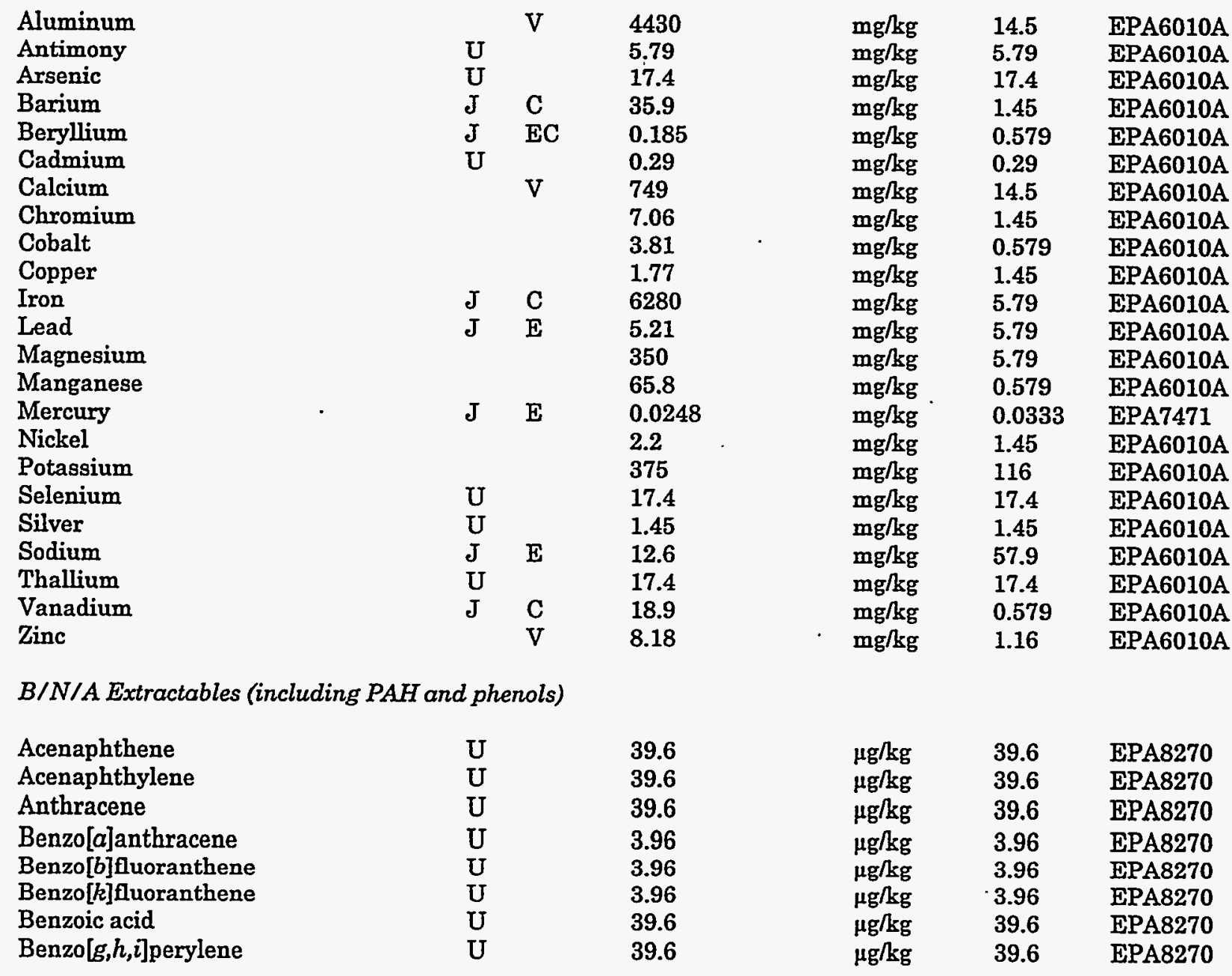


Appendix D: Sediment Tables

D-332

SURVEY ID: PPSC 2504 (continued)

Sample ID: 105116

Constituent

RQ AQ B Result

Unit

D. Limit Method

$B / N / A$ Extractables (including $P A H$ and phenols)

Benzo[a]pyrene

Benzyl alcohol

Bis(2-chloroethoxy) methane

Bis(2-chloroethyl) ether

Bis(2-chloroisopropyl) ether

Bis(2-ethylhexyl) phthalate

4-Bromophenyl phenyl ether

Butylbenzyl phthalate

4-Chloroaniline

4-Chloro-m-cresol

2-Chloronaphthalene

2-Chlorophenol

4-Chlorophenyl phenyl ether

Chrysene

$\mathrm{m} / \mathrm{p}$-Cresol

o-Cresol (2-Methylphenol)

Dibenz $[a, h]$ anthracene

Dibenzofuran

Di-n-butyl phthalate

1,2-Dichlorobenzene

1,3-Dichlorobenzene

1,4-Dichlorobenzene

3,3'-Dichlorobenzidine

2,4-Dichlorophenol

Diethyl phthalate

2,4-Dimethyl phenol

Dimethyl phthalate

2,4-Dinitrophenol

2,4-Dinitrotoluene

2,6-Dinitrotoluene

Di-n-octyl phthalate

Fluoranthene

Fluorene

Hexachlorobenzene

Hexachlorobutadiene

Hexachlorocyclopentadiene

Hexachloroethane

Indeno[1,2,3-c,d]pyrene

Isophorone

2-Methyl-4,6-dinitrophenol

2-Methylnaphthalene

Naphthalene

m-Nitroaniline

o-Nitroaniline

p-Nitroaniline

Nitrobenzene

2-Nitrophenol

4-Nitrophenol

N-Nitrosodiphenylamine

N-Nitrosodi-n-propylamine

Pentachlorophenol

Phenanthrene

Phenol

Pyrene

1,2,4-Trichlorobenzene

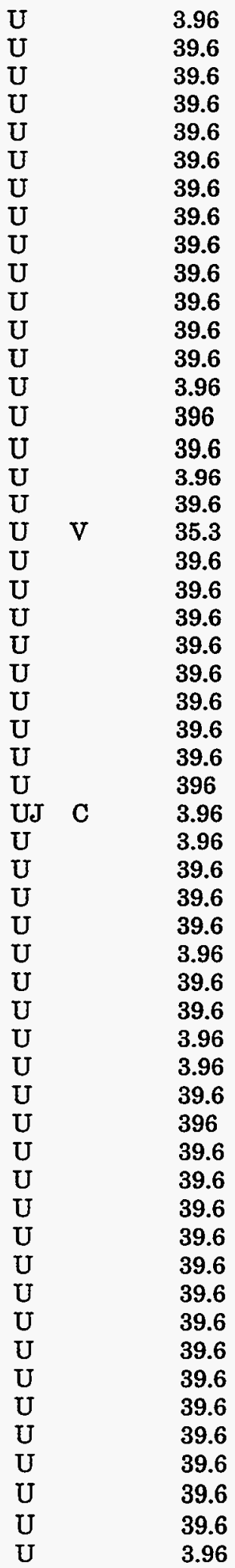

\begin{tabular}{|c|c|c|}
\hline$\mu \mathrm{g} / \mathrm{kg}$ & 3.96 & EPA8270 \\
\hline$\mu \mathrm{g} / \mathrm{kg}$ & 39.6 & EPA8270 \\
\hline$\mu \mathrm{g} / \mathrm{kg}$ & 39.6 & EPA8270 \\
\hline$\mu \mathrm{g} / \mathrm{kg}$ & 39.6 & EPA8270 \\
\hline$\mu \mathrm{g} / \mathrm{kg}$ & 39.6 & EPA8270 \\
\hline$\mu \mathrm{g} / \mathrm{kg}$ & 39.6 & EPA8270 \\
\hline$\mu \mathrm{g} / \mathrm{kg}$ & 39.6 & EPA8270 \\
\hline$\mu \mathrm{g} / \mathrm{kg}$ & 39.6 & EPA8270 \\
\hline$\mu \mathrm{g} / \mathrm{kg}$ & 39.6 & EPA8270 \\
\hline$\mu \mathrm{g} / \mathrm{kg}$ & 39.6 & EPA8270 \\
\hline$\mu \mathrm{g} / \mathrm{kg}$ & 39.6 & EPA8270 \\
\hline$\mu \mathrm{g} / \mathrm{kg}$ & 39.6 & EPA8270 \\
\hline$\mu \mathrm{g} / \mathrm{kg}$ & 39.6 & EPA8270 \\
\hline$\mu \mathrm{g} / \mathrm{kg}$ & 3.96 & EPA8270 \\
\hline$\mu \mathrm{g} / \mathrm{kg}$ & 396 & EPA8270 \\
\hline$\mu \mathrm{g} / \mathrm{kg}$ & 39.6 & EPA8270 \\
\hline$\mu \mathrm{g} / \mathrm{kg}$ & 3.96 & EPA8270 \\
\hline$\mu \mathrm{g} / \mathrm{kg}$ & 39.6 & EPA8270 \\
\hline$\mu \mathrm{g} / \mathrm{kg}$ & 39.6 & EPA8270 \\
\hline$\mu \mathrm{g} / \mathrm{kg}$ & 39.6 & EPA8270 \\
\hline$\mu \mathrm{g} / \mathrm{kg}$ & 39.6 & EPA8270 \\
\hline$\mu \mathrm{g} / \mathrm{kg}$ & 39.6 & EPA8270 \\
\hline$\mu \mathrm{g} / \mathrm{kg}$ & 39.6 & EPA8270 \\
\hline$\mu \mathrm{g} / \mathrm{kg}$ & 39.6 & EPA8270 \\
\hline$\mu \mathrm{g} / \mathrm{kg}$ & 39.6 & EPA8270 \\
\hline$\mu \mathrm{g} / \mathrm{kg}$ & 39.6 & EPA8270 \\
\hline$\mu \mathrm{g} / \mathrm{kg}$ & 39.6 & EPA8270 \\
\hline$\mu \mathrm{g} / \mathrm{kg}$ & 396 & EPA8270 \\
\hline$\mu \mathrm{g} / \mathrm{kg}$ & 3.96 & EPA8270 \\
\hline$\mu \mathrm{g} / \mathrm{kg}$ & 3.96 & EPA8270 \\
\hline$\mu \mathrm{g} / \mathrm{kg}$ & 39.6 & EPA8270 \\
\hline$\mu \mathrm{g} / \mathrm{kg}$ & 39.6 & EPA8270 \\
\hline$\mu \mathrm{g} / \mathrm{kg}$ & 39.6 & EPA8270 \\
\hline$\mu \mathrm{g} / \mathrm{kg}$ & 3.96 & EPA8270 \\
\hline$\mu \mathrm{g} / \mathrm{kg}$ & 39.6 & EPA8270 \\
\hline$\mu \mathrm{g} / \mathrm{kg}$ & 39.6 & EPA8270 \\
\hline$\mu \mathrm{g} / \mathrm{kg}$ & 3.96 & EPA8270 \\
\hline$\mu \mathrm{g} / \mathrm{kg}$ & 3.96 & EPA8270 \\
\hline$\mu \mathrm{g} / \mathrm{kg}$ & 39.6 & EPA8270 \\
\hline$\mu \mathrm{g} / \mathrm{kg}$ & 396 & EPA8270 \\
\hline$\mu \mathrm{g} / \mathrm{kg}$ & 39.6 & EPA8270 \\
\hline$\mu g / \mathrm{kg}$ & 39.6 & EPA8270 \\
\hline$\mu g / \mathrm{kg}$ & 39.6 & EPA8270 \\
\hline$\mu \mathrm{g} / \mathrm{kg}$ & 39.6 & EPA8270 \\
\hline$\mu \mathrm{g} / \mathrm{kg}$ & 39.6 & EPA8270 \\
\hline$\mu \mathrm{g} / \mathrm{kg}$ & 39.6 & EPA8270 \\
\hline$\mu g / \mathrm{kg}$ & 39.6 & EPA8270 \\
\hline$\mu \mathrm{g} / \mathrm{kg}$ & 39.6 & EPA8270 \\
\hline$\mu \mathrm{g} / \mathrm{kg}$ & 39.6 & EPA8270 \\
\hline$\mu \mathrm{g} / \mathrm{kg}$ & 39.6 & EPA8270 \\
\hline$\mu \mathrm{g} / \mathrm{kg}$ & 39.6 & EPA8270 \\
\hline$\mu \mathrm{g} / \mathrm{kg}$ & 39.6 & EPA8270 \\
\hline$\mu \mathrm{g} / \mathrm{kg}$ & 39.6 & EPA8270 \\
\hline$\mu \mathrm{g} / \mathrm{kg}$ & 39.6 & EPA8270 \\
\hline$\mu \mathrm{g} / \mathrm{kg}$ & 3.96 & EPA8270 \\
\hline
\end{tabular}


SURVEY ID: PPSC 2504 (continued)

Constituent

RQ

$A Q$

Sample ID: 105116

$B / N / A$ Extractables (including $P A H$ and phenols)
2,4,5-Trichlorophenol

2,4,6-Trichlorophenol

$\mathrm{U}$

U $\quad 39.6$

Volatile Organic Compounds

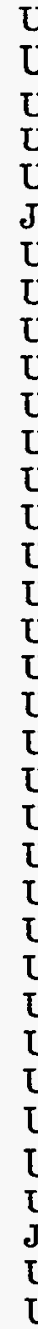

$\mathrm{U}$

Acetone

Bromodichloromethane

Bromoform

Bromomethane (Methyl bromide)

Carbon disulfide

Carbon tetrachloride

Chlorobenzene

Chloroethane

Chloroethene (Vinyl chloride)

Chloroform

Chloromethane (Methyl chloride)

Dibromochloromethane

1,1-Dichloroethane

1,2-Dichloroethane

1,1-Dichloroethylene

1,2-Dichloroethylene

Dichloromethane (Methylene chloride)

1,2-Dichloropropane

cis-1,3-Dichloropropene

trans-1,3-Dichloropropene

Ethylbenzene

2-Hexanone

Methyl ethyl ketone

Methyl isobutyl ketone

Styrene

1,1,2,2-Tetrachloroethane

Tetrachloroethylene

Toluene

1,1,1-Trichloroethane

1,1,2-Trichloroethane

Trichloroethylene

Unknown alcohol

Vinyl acetate

Xylenes

Aldrin

alpha-Benzene hexachloride

beta-Benzene hexachloride

delta-Benzene hexachloride

alpha-Chlordane

gamma-Chlordane

p,p'-DDD

$p, p^{\prime}-\mathrm{DDE}$

p,p'-DDT

Dieldrin

Endosulfan sulfate

Endosulfan I
Pesticides/PCBs and Dioxins/Furans
8

U

U

$\begin{array}{lll}\mathrm{J} & \mathrm{E} & \mathbf{0 . 3 3 3}\end{array}$

U $\quad 0.0595$

0.0595

0.119

0.119

0.0595

0.119

0.0595

0.0595

0.0595

0.0595

0.119

V

1.54

0.0595

0.0595

0.0595

0.0595

1.19

$\mathrm{V}$

2.24

1.19

0.0595

0.0595

0.0595

0.595

0.0595

0.0595

0.0595

$\mathrm{N}$

4

1.19

0.179
Unit

D. Limit Method $\mu \mathrm{g} / \mathrm{kg} \quad 39.6 \quad$ EPA8270 $\mu \mathrm{g} / \mathrm{kg} \quad 39.6 \quad \mathrm{EPA8270}$

$\begin{array}{lll}\mu \mathrm{g} / \mathrm{kg} & 11.9 & \text { EPA8260 } \\ \mu \mathrm{g} / \mathrm{kg} & 0.595 & \text { EPA8260 } \\ \mu \mathrm{g} / \mathrm{kg} & 0.0595 & \text { EPA8260 } \\ \mu \mathrm{g} / \mathrm{kg} & 0.0595 & \text { EPA8260 } \\ \mu \mathrm{g} / \mathrm{kg} & 0.119 & \text { EPA8260 } \\ \mu \mathrm{g} / \mathrm{kg} & 1.19 & \text { EPA8260 } \\ \mu \mathrm{g} / \mathrm{kg} & 0.0595 & \text { EPA8260 } \\ \mu \mathrm{g} / \mathrm{kg} & 0.0595 & \text { EPA8260 } \\ \mu \mathrm{g} / \mathrm{kg} & 0.119 & \text { EPA8260 } \\ \mu \mathrm{g} / \mathrm{kg} & 0.119 & \text { EPA8260 } \\ \mu \mathrm{g} / \mathrm{kg} & 0.0595 & \text { EPA8260 } \\ \mu \mathrm{g} / \mathrm{kg} & 0.119 & \text { EPA8260 } \\ \mu \mathrm{g} / \mathrm{kg} & 0.0595 & \text { EPA8260 } \\ \mu \mathrm{g} / \mathrm{kg} & 0.0595 & \text { EPA8260 } \\ \mu \mathrm{g} / \mathrm{kg} & 0.0595 & \text { EPA8260 } \\ \mu \mathrm{g} / \mathrm{kg} & 0.0595 & \text { EPA8260 } \\ \mu \mathrm{g} / \mathrm{kg} & 0.119 & \text { EPA8260 } \\ \mu \mathrm{g} / \mathrm{kg} & 0.595 & \text { EPA8260 } \\ \mu \mathrm{g} / \mathrm{kg} & 0.0595 & \text { EPA8260 } \\ \mu \mathrm{g} / \mathrm{kg} & 0.0595 & \text { EPA8260 } \\ \mu \mathrm{g} / \mathrm{kg} & 0.0595 & \text { EPA8260 } \\ \mu \mathrm{g} / \mathrm{kg} & 0.0595 & \text { EPA8260 } \\ \mu \mathrm{g} / \mathrm{kg} & 1.19 & \text { EPA8260 } \\ \mu \mathrm{g} / \mathrm{kg} & 1.19 & \text { EPA8260 } \\ \mu \mathrm{g} / \mathrm{kg} & 1.19 & \text { EPA8260 } \\ \mu \mathrm{g} / \mathrm{kg} & 0.0595 & \text { EPA8260 } \\ \mu \mathrm{g} / \mathrm{kg} & 0.0595 & \text { EPA8260 } \\ \mu \mathrm{g} / \mathrm{kg} & 0.0595 & \text { EPA8260 } \\ \mu \mathrm{g} / \mathrm{kg} & 0.595 & \text { EPA8260 } \\ \mu \mathrm{g} / \mathrm{kg} & 0.0595 & \text { EPA8260 } \\ \mu \mathrm{g} / \mathrm{kg} & 0.0595 & \text { EPA8260 } \\ \mu \mathrm{g} / \mathrm{kg} & 0.0595 & \text { EPA8260 } \\ \mu \mathrm{g} / \mathrm{kg} & & \text { EPA8260 } \\ \mu \mathrm{g} / \mathrm{kg} & 1.19 & \text { EPA8260 } \\ \mu \mathrm{g} / \mathrm{kg} & 0.179 & \text { EPA8260 } \\ & & \end{array}$

$\mu \mathrm{g} / \mathrm{kg}$

$\mu \mathrm{g} / \mathrm{kg}$

$\mu \mathrm{g} / \mathrm{kg}$

$\mu \mathrm{g} / \mathrm{kg}$

$\mu \mathrm{g} / \mathrm{kg}$

$\mu \mathrm{g} / \mathrm{kg}$

$\mu \mathrm{g} / \mathrm{kg}$

$\mu \mathrm{g} / \mathrm{kg}$

$\mu \mathrm{g} / \mathrm{kg}$

$\mu \mathrm{g} / \mathrm{kg}$

$\mu \mathrm{g} / \mathrm{kg}$

$\mu \mathrm{g} / \mathrm{kg}$

$\begin{array}{ll}0.982 & \text { EPA8080 } \\ 0.982 & \text { EPA8080 } \\ 1.96 & \text { EPA8080 } \\ 1.96 & \text { EPA8080 } \\ 1.96 & \text { EPA8080 } \\ 1.96 & \text { EPA8080 } \\ 3.93 & \text { EPA8080 } \\ 1.96 & \text { EPA8080 } \\ 3.93 & \text { EPA8080 } \\ 1.96 & \text { EPA8080 } \\ 3.93 & \text { EPA8080 } \\ 1.96 & \text { EPA8080 }\end{array}$


Appendix D: Sediment Tables

D-334

SURVEY ID: PPSC 2504 (continued)

Constituent

Pesticides/PCBs and Dioxins/Furans

$\begin{array}{lllll}\text { Endosulfan II } & \text { UJ } & \text { O } & \text { L } & 3.93 \\ \text { Endrin } & \text { UJ } & \text { O } & \text { L } & 1.96 \\ \text { Endrin ketone } & \text { UJ } & \text { O } & \text { L } & 3.93 \\ \text { Heptachlor } & \text { UJ } & \text { O } & \text { L } & 1.96 \\ \text { Heptachlor epoxide } & \text { UJ } & \text { O } & \text { L } & 1.96 \\ \text { Lindane } & \text { UJ } & \text { O } & \text { L } & 0.982 \\ \text { Methoxychlor } & \text { UJ } & \text { O } & \text { L } & 19.6 \\ \text { PCB 1016 } & \text { UJ } & \text { O } & \text { L } & 4.91 \\ \text { PCB 1221 } & \text { UJ } & \text { O } & \text { L } & 4.91 \\ \text { PCB 1232 } & \text { UJ } & \text { O } & \text { L } & 4.91 \\ \text { PCB 1242 } & \text { UJ } & \text { O } & \text { L } & 4.91 \\ \text { PCB 1248 } & \text { UJ } & \text { O } & \text { L } & 4.91 \\ \text { PCB 1254 } & \text { UJ } & \text { O } & \text { L } & 4.91 \\ \text { PCB 1260 } & \text { UJ } & \text { O } & \text { L } & 4.91 \\ \text { Toxaphene } & \text { UJ } & \text { O } & \text { L } & 39.3\end{array}$

Sample ID: 105116

RQ AQ B Result Unit D. Limit Method

\section{Radionuclides}

Actinium-228

Antimony-124

Antimony-125

Barium-133

Cerium-144

Cesium-134

Cesium-137

Cobalt-57

Cobalt-58

Cobalt-60

Europium-152

Europium-154

Europium-155

Gross alpha

Lead-212

Manganese-54

Neptunium-239

Nonvolatile beta

Plutonium-238

Plutonium-239/240

Potassium- 40

Promethium-144

Promethium-146

Radium-226

Radium-228

Ruthenium-106

Sodium-22

Strontium-90

Thorium-234

Tin-113

Yttrium-88

Zinc-65

Zirconium-95
$1.26 \mathrm{E}+00 \pm 5.60 \mathrm{E} .02 \mathrm{pCi} / \mathrm{g}$ 2.56E-03 $\pm 5.92 \mathrm{E}-03 \mathrm{pCi} / \mathrm{g}$ 1.21E-02 $\pm 2.24 \mathrm{E}-02 \mathrm{pCi} / \mathrm{g}$ $-6.62 \mathrm{E}-04 \pm 6.74 \mathrm{E}-03 \mathrm{pCi} / \mathrm{g}$ $6.96 \mathrm{E}-03 \pm 3.66 \mathrm{E}-02 \mathrm{pCi} / \mathrm{g}$ 3.83E-03 $\pm 5.00 \mathrm{E}-03 \mathrm{pCi} / \mathrm{g}$ $-1.97 \mathrm{E}-03 \pm 6.14 \mathrm{E}-03 \mathrm{pCi} / \mathrm{g}$ $1.04 \mathrm{E}-03 \pm 4.65 \mathrm{E}-03 \mathrm{pCi} / \mathrm{g}$ $-4.58 \mathrm{E}-04 \pm 6.65 \mathrm{E}-03 \mathrm{pCi} / \mathrm{g}$ $-2.86 \mathrm{E}-03 \pm 5.04 \mathrm{E}-03 \mathrm{pCi} / \mathrm{g}$ $-2.05 \mathrm{E}-02 \pm 1.40 \mathrm{E}-02 \mathrm{pCi} / \mathrm{g}$ $-6.58 \mathrm{E}-02 \pm 4.75 \mathrm{E}-02 \mathrm{pCi} / \mathrm{g}$ $0.00 \mathrm{E}+00 \quad \mathrm{pCi} / \mathrm{g}$ $1.90 \mathrm{E}+01 \pm 3.75 \mathrm{E}+00 \mathrm{pCi} / \mathrm{g}$ $1.31 \mathrm{E}+00 \pm 2.37 \mathrm{E}-02 \mathrm{pCi} / \mathrm{g}$ 2.04E-02 $56.80 \mathrm{E}-03 \mathrm{pCi} / \mathrm{g}$ $0.00 \mathrm{E}+00 \quad \mathrm{pCi} / \mathrm{g}$ $2.80 \mathrm{E}+01 \pm 2.94 \mathrm{E}+00 \mathrm{pCi} / \mathrm{g}$ $-6.72 \mathrm{E}-02 \pm 2.53 \mathrm{E}-02 \mathrm{pCi} / \mathrm{g}$ $-2.55 \mathrm{E}-02 \pm 1.37 \mathrm{E}-02 \mathrm{pCi} / \mathrm{g}$ $1.12 \mathrm{E}+01 \pm 2.60 \mathrm{E}-01 \mathrm{pCi} / \mathrm{g}$ $3.69 \mathrm{E}-03 \pm 4.92 \mathrm{E}-03 \mathrm{pCi} \mathrm{g}$ $0.00 \mathrm{E}+00 \quad \mathrm{pCi} / \mathrm{g}$ 7.73E-01士2.90E-02 pCi/g $1.26 \mathrm{E}+00 \pm 5.60 \mathrm{E}-02 \mathrm{pCi} / \mathrm{g}$ $3.16 \mathrm{E}-02 \pm 4.93 \mathrm{E}-02 \mathrm{pCi} / \mathrm{g}$ -3.93E-04 5 .78E-03 pCi/g $-4.61 \mathrm{E}-01 \pm 1.39 \mathrm{E}-01 \mathrm{pCi} / \mathrm{g}$ $1.75 \mathrm{E}+00 \pm 6.19 \mathrm{E}-01 \mathrm{pCi} / \mathrm{g}$ $5.16 \mathrm{E}-03 \pm 7.27 \mathrm{E}-03 \mathrm{pCi} / \mathrm{g}$ $1.81 \mathrm{E}-03 \pm 4.52 \mathrm{E}-03 \mathrm{pCj} / \mathrm{g}$ $-3.01 \mathrm{E}-03 \pm 1.38 \mathrm{E}-02 \mathrm{pCi} / \mathrm{g}$ 1.21E-02 $\pm 1.81 \mathrm{E}-02 \mathrm{pCi} / \mathrm{g}$
2.90E-02 EPIA-013B 1.01E-02 EPIA-013B

2.17E-02 EPIA-013B

9.68E-03 EPIA-013B

6.24E-02 EPIA-013B

7.70E-03 EPIA-013B

8.94E-03 EPIA-013B

7.94E-03 EPIA-013B

9.64E-03 EPIA-013B

8.39E-03 EPIA-013B

2.19E-02 EPIA-013B

7.86E-02 EPIA-013B

3.32E-02 EPIA-013B

1.63E+00 EPIA-001B

1.38E-02 EPIA-013B

7.59E-03 EPIA-013B

5.70E-02 EPIA-013B

2.62E+00 EPIA-001B

7.40E-02 EPIA-012B

4.62E-02 EPIA-012B

6.66E-02 EPIA-013B

8.43E-03 EPIA-013B

1.08E-02 EPLA-013B

1.54E-02 EPIA-013B

2.90E-02 EPIA-013B

7.52E-02 EPIA-013B

9.83E-03 EPIA-013B

2.67E-01 EPIA-004

4.20E-01 EPIA-013B

1.14E-02 EPIA-013B

8.31E-03 EPIA-013B

2.05E-02 EPIA-013B

2.19E-02 EPIA-013B 
SURVEY ID: PPSC FB01C

Sample type: Field Blank

Constituent
Sample ID: 104603

Associated sample: 104604

Physical Parameters and Specified Analyses

Cyanide

$\mathrm{U}$

Metals (total recoverable)
RQ AQ B Result Unit D. Limit Method

RQ AQ B Result Unit D. Limit Method

\begin{tabular}{|c|c|c|c|c|c|c|c|}
\hline Aluminum & & U & & 0.025 & $\mathrm{mg} / \mathrm{L}$ & 0.025 & EPA6010A \\
\hline Antimony & " & U & & 0.005 & $\mathrm{mg} / \mathrm{L}$ & 0.005 & EPA7041 \\
\hline Arsenic & & $\mathrm{U}$ & & 0.005 & $\mathrm{mg} / \mathrm{L}$ & 0.005 & EPA7060 \\
\hline Barium & & $\mathrm{U}$ & & 0.025 & $\mathrm{mg} / \mathrm{L}$ & 0.025 & EPA6010A \\
\hline Beryllium & & $\mathrm{U}$ & & 0.01 & $\mathrm{mg} / \mathrm{L}$ & 0.01 & EPA6010A \\
\hline Cadmium & & U & & 0.005 & $\mathrm{mg} / \mathrm{L}$ & 0.005 & EPA6010A \\
\hline Calcium & & $\mathbf{J}$ & $\mathrm{E}$ & 0.0357 & $\mathrm{mg} / \mathrm{L}$ & 0.25 & EPA6010A \\
\hline Chromium & & U & & 0.025 & $\mathrm{mg} / \mathrm{L}$ & 0.025 & EPA6010A \\
\hline Cobalt & & $\mathrm{U}$ & & 0.01 & $\mathrm{mg} / \mathrm{L}$ & 0.01 & EPA6010A \\
\hline Copper & & $\mathrm{U}$ & & 0.025 & $\mathrm{mg} / \mathrm{L}$ & 0.025 & EPA6010A \\
\hline Iron & & $\mathrm{J}$ & $\mathbf{E}$ & 0.013 & $\mathrm{mg} / \mathrm{L}$ & 0.1 & EPA6010A \\
\hline Lead & & $\mathrm{U}$ & & 0.005 & $\mathrm{mg} / \mathrm{L}$ & 0.005 & EPA7421 \\
\hline Magnesium & & $\mathrm{U}$ & & 0.1 & $\mathrm{mg} / \mathrm{L}$ & 0.1 & EPA6010A \\
\hline Manganese & & $\mathbf{J}$ & $\mathrm{E}$ & 0.00915 & $\mathrm{mg} / \mathrm{L}$ & 0.01 & EPA6010A \\
\hline Mercury & & U & & 0.0002 & $\mathrm{mg} / \mathrm{L}$ & 0.0002 & EPA7470 \\
\hline Nickel & & $\mathrm{U}$ & & 0.025 & $\mathrm{mg} / \mathrm{L}$ & 0.025 & EPA6010A \\
\hline Potassium. & & U & & 2 & $\mathrm{mg} / \mathrm{L}$ & 2 & EPA6010A \\
\hline Selenium & & U & & 0.005 & $\mathrm{mg} / \mathrm{L}$ & 0.005 & EPA7740 \\
\hline Silver & & $\mathrm{U}$ & & 0.025 & $\mathrm{mg} / \mathrm{L}$ & 0.025 & EPA6010A \\
\hline Sodium & & U & & 1 & $\mathrm{mg} / \mathrm{L}$ & 1 & EPA6010A \\
\hline Thallium & & U & & 0.005 & $\mathrm{mg} / \mathrm{L}$ & 0.005 & EPA7841 \\
\hline Vanadium & & U & & 0.01 & $\mathrm{mg} / \mathrm{L}$ & 0.01 & EPA6010A \\
\hline Zinc & & $J$ & $\mathrm{E}$ & 0.00469 & $\mathrm{mg} / \mathrm{L}$ & 0.02 & EPA6010A \\
\hline
\end{tabular}

$B / N / A$ Extractables (including $P A H$ and phenols)

\begin{tabular}{|c|c|c|}
\hline Acenaphthene & UJ & $\mathbf{Q}$ \\
\hline Acenaphthylene & UJ & $\mathbf{Q}$ \\
\hline Anthracene & UJ & $\mathbf{Q}$ \\
\hline Benzo[a]anthracene & UJ & $\mathbf{Q}$ \\
\hline Benzo[b]fluoranthene & UJ & $\mathbf{Q}$ \\
\hline Benzo $[k]$ fluoranthene & UJ & $\mathbf{Q}$ \\
\hline Benzoic acid & UJ & $\mathbf{Q}$ \\
\hline Benzo $[g, h, i]$ perylene & UJ & $\mathbf{Q}$ \\
\hline Benzo[a]pyrene & UJ & 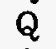 \\
\hline Benzyl alcohol & UJ & $\mathbf{Q}$ \\
\hline Bis(2-chloroethoxy) methane & UJ & $\mathbf{Q}$ \\
\hline Bis(2-chloroethyl) ether & UJ & $\mathbf{Q}$ \\
\hline Bis(2-chloroisopropyl) ether & UJ & $Q$ \\
\hline Bis(2-ethylhexyl) phthalate & UJ & $Q$ \\
\hline 4-Bromophenyl phenyl ether & UJ & $\mathbf{Q}$ \\
\hline Butylbenzyl phthalate & UJ & $Q$ \\
\hline 4-Chloroaniline & UJ & $\mathbf{Q}$ \\
\hline 4-Chloro-m-cresol & UJ & $\mathbf{Q}$ \\
\hline 2-Chloronaphthalene & UJ & $\mathbf{Q}$ \\
\hline 2-Chlorophenol & UJ & $\mathbf{Q}$ \\
\hline 4-Chlorophenyl phenyl ether & UJ & $\mathbf{Q}$ \\
\hline Chrysene & UJ & $\mathbf{Q}$ \\
\hline $\mathrm{m} / \mathrm{p}$-Cresol & UJ & $\mathrm{Q}$ \\
\hline
\end{tabular}

$\begin{array}{lll}\mu \mathrm{g} / \mathrm{L} & 1 & \text { EPA8270 } \\ \mu \mathrm{g} / \mathrm{L} & 1 & \text { EPA8270 } \\ \mu \mathrm{g} / \mathrm{L} & 1 & \text { EPA8270 } \\ \mu \mathrm{g} / \mathrm{L} & 0.1 & \text { EPA8270 } \\ \mu \mathrm{g} / \mathrm{L} & 0.1 & \text { EPA8270 } \\ \mu \mathrm{g} / \mathrm{L} & 0.1 & \text { EPA8270 } \\ \mu \mathrm{g} / \mathrm{L} & 1 & \text { EPA8270 } \\ \mu \mathrm{g} / \mathrm{L} & 1 & \text { EPA8270 } \\ \mu \mathrm{g} / \mathrm{L} & 0.1 & \text { EPA8270 } \\ \mu \mathrm{g} / \mathrm{L} & 1 & \text { EPA8270 } \\ \mu \mathrm{g} / \mathrm{L} & 1 & \text { EPA8270 } \\ \mu \mathrm{g} / \mathrm{L} & 1 & \text { EPA8270 } \\ \mu \mathrm{g} / \mathrm{L} & 1 & \text { EPA8270 } \\ \mu \mathrm{g} / \mathrm{L} & 1 & \text { EPA8270 } \\ \mu \mathrm{g} / \mathrm{L} & 1 & \text { EPA8270 } \\ \mu \mathrm{g} / \mathrm{L} & 1 & \text { EPA8270 } \\ \mu \mathrm{g} / \mathrm{L} & 1 & \text { EPA8270 } \\ \mu \mathrm{g} / \mathrm{L} & 1 & \text { EPA8270 } \\ \mu \mathrm{g} / \mathrm{L} & 1 & \text { EPA8270 } \\ \mu \mathrm{g} / \mathrm{L} & 1 & \text { EPA8270 } \\ \mu \mathrm{g} / \mathrm{L} & 1 & \text { EPA8270 } \\ \mu \mathrm{g} / \mathrm{L} & 0.1 & \text { EPA8270 } \\ \mu \mathrm{g} / \mathrm{L} & 10 & \text { EPA8270 }\end{array}$


SURVEY ID: PPSC FB01C (continued)

Sample ID: 104603

Constituent RQ AQ B Result Unit D. Limit Method

$B / N / A$ Extractables (including $P A H$ and phenols)

o-Cresol (2-Methylphenol)

Dibenz $[a, h]$ anthracene

Dibenzofuran

Di-n-butyl phthalate

1,2-Dichlorobenzene

1,3-Dichlorobenzene

1,4-Dichlorobenzene

3,3'-Dichlorobenzidine

2,4-Dichlorophenol

Diethyl phthalate

2,4-Dimethyl phenol

Dimethyl phthalate

2,4-Dinitrophenol

2,4-Dinitrotoluene

2,6-Dinitrotoluene

Di-n-octyl phthalate

Fluoranthene

Fluorene

Hexachlorobenzene

Hexachlorobutadiene

Hexachlorocyclopentadiene

Hexachloroethane

Indeno[1,2,3-c,d]pyrene

Isophorone

2-Methyl-4,6-dinitrophenol

2-Methylnaphthalene

Naphthalene

m-Nitroaniline

o-Nitroaniline

p-Nitroaniline

Nitrobenzene

2-Nitrophenol

4-Nitrophenol

N-Nitrosodiphenylamine

N-Nitrosodi-n-propylamine

Pentachlorophenol

Phenanthrene

Phenol

Pyrene

1,2,4-Trichlorobenzene

2,4,5-Trichlorophenol

2,4,6-Trichlorophenol

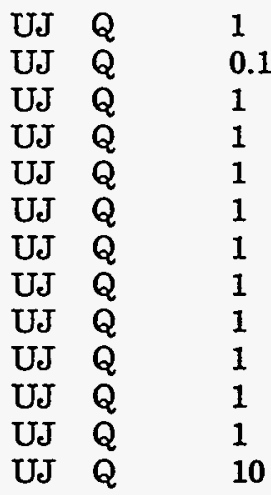

UJ $Q \quad 0.1$

UJ Q $\quad 0.1$

UJ $Q \quad 1$

UJ $Q \quad 1$

UJ $Q \quad 1$

UJ $Q \quad 0.1$

UJ $Q \quad 1$

UJ $Q \quad 1$

UJ $Q \quad 0.1$

UJ $Q \quad 0.1$

UJ $Q \quad 1$

UJ $Q \quad 10$

UJ Q 1

UJ $Q$

UJ $Q \quad 1$

UJ Q 1

UJ Q 1

UJ Q 1

UJ $Q \quad 1$

UJ $Q \quad 1$

UJ $\quad$ Q 1

UJ $Q \quad 1$

UJ $Q \quad 1$

UJ $Q \quad 1$

UJ $\quad \mathrm{Q} \quad 1$

$\begin{array}{lll}\text { UJ } & \mathrm{Q} & 1 \\ \text { UJ } & \mathrm{Q} & \mathbf{0 . 1}\end{array}$

UJ $Q \quad 1$

UJ Q 1 $\mu \mathrm{g}$

$\mu \mathrm{g} / \mathrm{L} \quad 1 \quad$ EPA8270

$\mu \mathrm{g} / \mathrm{L} \quad 1 \quad$ EPA8270

$\mu \mathrm{g} / \mathrm{L} \quad 1 \quad$ EPA8270

$\mu \mathrm{g} / \mathrm{L} \quad 1 \quad$ EPA8270

$\mu \mathrm{g} / \mathrm{L} \quad 1 \quad$ EPA8270

$\mu \mathrm{g} / \mathrm{L} \quad 1 \quad$ EPA8270

$\mu \mathrm{g} / \mathrm{L} \quad 1 \quad$ EPA8270

$\mu \mathrm{g} / \mathrm{L} \quad 1 \quad$ EPA8270

$\mu \mathrm{g} / \mathrm{L} \quad 1 \quad$ EPA8270

$\mu \mathrm{g} / \mathrm{L} \quad 1 \quad$ EPA8270

$\mu \mathrm{g} / \mathrm{L} \quad 10 \quad$ EPA8270

$\mu \mathrm{g} / \mathrm{L} \quad 0.1 \quad$ EPA8270

$\begin{array}{lll}\mu \mathrm{g} / \mathrm{L} & 0.1 & \mathrm{EPA8270}\end{array}$

$\mu \mathrm{g} / \mathrm{L} \quad 1 \quad$ EPA8270

$\mu \mathrm{g} / \mathrm{L} \quad 1 \quad$ EPA8270

$\mu \mathrm{g} / \mathrm{L} \quad 1 \quad$ EPA8270

$\mu \mathrm{g} / \mathrm{L} \quad 0.1 \quad$ EPA8270

$\mu \mathrm{g} / \mathrm{L} \quad 1 \quad$ EPA8270

$\mu \mathrm{g} / \mathrm{L} \quad 1 \quad$ EPA8270

$\mu \mathrm{g} / \mathrm{L} \quad 0.1 \quad$ EPA8270

$\mu \mathrm{g} / \mathrm{L} \quad 0.1 \quad$ EPA8270

$\mu \mathrm{g} / \mathrm{L} \quad 1 \quad$ EPA8270

$\mu \mathrm{g} / \mathrm{L} \quad 10 \quad$ EPA8270

$\mu \mathrm{g} / \mathrm{L} \quad 1 \quad$ EPA8270

$\mu \mathrm{g} / \mathrm{L} \quad 1 \quad$ EPA8270

$\mu \mathrm{g} / \mathrm{L} \quad 1 \quad$ EPA8270

$\mu \mathrm{g} / \mathrm{L} \quad 1 \quad$ EPA8270

$\mu g / L \quad 1 \quad$ EPA8270

$\mu \mathrm{g} / \mathrm{L} \quad 1 \quad$ EPA8270

$\mu \mathrm{g} / \mathrm{L} \quad 1 \quad$ EPA8270

$\mu \mathrm{g} / \mathrm{L} \quad 1 \quad$ EPA8270

$\mu \mathrm{g} / \mathrm{L} \quad 1 \quad$ EPA8270

$\mu \mathrm{g} / \mathrm{L} \quad 1 \quad$ EPA8270

$\mu \mathrm{g} / \mathrm{L} \quad 1 \quad$ EPA8270

$\mu \mathrm{g} / \mathrm{L} \quad 1 \quad$ EPA8270

$\mu \mathrm{g} / \mathrm{L} \quad 1 \quad$ EPA8270

$\mu \mathrm{g} / \mathrm{L} \quad 1 \quad$ EPA8270

$\mu g / L \quad 0.1 \quad$ EPA8270

$\mu g / L \quad 1 \quad$ EPA8270

$\mu \mathrm{g} / \mathrm{L} \quad 1 \quad$ EPA8270

Volatile Organic Compounds

$\begin{array}{lllll}\text { Acetone } & \text { U } & 8 & & 3.41 \\ \text { Acetone } & \text { U } & 8 & & 3.18 \\ \text { Benzene } & \text { UJ } & \text { I } & \text { L } & 0.5 \\ \text { Benzene } & \text { UJ } & \text { I } & \text { L } & 0.5 \\ \text { Bromodichloromethane } & \text { U } & & & 0.05 \\ \text { Bromodichloromethane } & \text { U } & & & 0.05 \\ \text { Bromoform } & \text { U } & & & 0.05 \\ \text { Bromoform } & \text { U } & & & 0.05 \\ \text { Bromomethane (Methyl bromide) } & \text { U } & & & 0.1 \\ \text { Bromomethane (Methyl bromide) } & \text { U } & & & 0.1\end{array}$

$\begin{array}{lll}\mu \mathrm{g} / \mathrm{L} & 5 & \text { EPA8260 } \\ \mu \mathrm{g} / \mathrm{L} & 5 & \text { EPA8260 } \\ \mu \mathrm{g} / \mathrm{L} & 0.5 & \text { EPA8260 } \\ \mu \mathrm{g} / \mathrm{L} & 0.5 & \text { EPA8260 } \\ \mu \mathrm{g} / \mathrm{L} & 0.05 & \text { EPA8260 } \\ \mu \mathrm{g} / \mathrm{L} & 0.05 & \text { EPA8260 } \\ \mu \mathrm{g} / \mathrm{L} & 0.05 & \text { EPA8260 } \\ \mu \mathrm{g} / \mathrm{L} & 0.05 & \text { EPA8260 } \\ \mu \mathrm{g} / \mathrm{L} & 0.1 & \text { EPA8260 } \\ \mu \mathrm{g} / \mathrm{L} & 0.1 & \text { EPA8260 }\end{array}$


SURVEY ID: PPSC FB01C (continued)

Sample ID: 104603

Constituent

RQ AQ B Result

Unit

D. Limit Method

Volatile Organic Compounds

Carbon disulfide
Carbon disulfide
Carbon tetrachloride
Carbon tetrachloride
Chlorobenzene
Chlorobenzene
Chloroethane
Chloroethane
Chloroethene (Vinyl chloride)
Chloroethene (Vinyl chloride)
Chloroform
Chloroform
Chloromethane (Methyl chloride)
Chloromethane (Methyl chloride)
Dibromochloromethane
Dibromochloromethane
1,1-Dichloroethane
1,1-Dichloroethane
1,2-Dichloroethane
1,2-Dichloroethane
1,1-Dichloroethylene
1,1-Dichloroethylene
1,2-Dichloroethylene
1,2-Dichloroethylene
Dichloromethane (Methylene chloride)
Dichloromethane (Methylene chloride)
1,2-Dichloropropane
1,2-Dichloropropane
cis-1,3-Dichloropropene
cis-1,3-Dichloropropene
trans-1,3-Dichloropropene
trans-1,3-Dichloropropene
Ethylbenzene
Ethylbenzene
2-Hexanone
2-Hexanone
Methyl ethyl ketone
Methyl ethyl ketone
Methyl isobutyl ketone
Methyl isobutyl ketone
Styrene
Styrene
1,1,2,2-Tetrachloroethane
1,1,2,2-Tetrachloroethane
Tetrachloroethylene
Tetrachloroethylene
Toluene
Toluene
1,1,1-Trichloroethane
1,1,1-Trichloroethane
1,1,2-Trichloroethane
1,1,2-Trichloroethane
Trichloroethylene
Trichloroethylene
Vinyl acetate

\begin{tabular}{|c|c|c|}
\hline U & & 1 \\
\hline U & & 1 \\
\hline$U$ & & 0.05 \\
\hline $\mathrm{U}$ & & 0.05 \\
\hline$J$ & & 0.17 \\
\hline & & \\
\hline $\begin{array}{l}U \\
U\end{array}$ & & 0.1 \\
\hline $\mathrm{U}$ & & 0.1 \\
\hline $\begin{array}{l}U \\
U\end{array}$ & & 0.1 \\
\hline U & & $\begin{array}{l}0.1 \\
0.05\end{array}$ \\
\hline U & & 0.05 \\
\hline $\mathrm{U}$ & & 0.1 \\
\hline U & & 0.1 \\
\hline U & & 0.05 \\
\hline U & & 0.05 \\
\hline$U$ & & 0.05 \\
\hline U & & 0.05 \\
\hline $\mathrm{U}$ & & 0.05 \\
\hline $\mathrm{U}$ & & 0.05 \\
\hline $\mathrm{U}$ & & 0.05 \\
\hline$U$ & & 0.05 \\
\hline U & & 0.1 \\
\hline U & & 0.1 \\
\hline $\mathbf{U}$ & & 0.5 \\
\hline U & 8 & 0.35 \\
\hline $\mathbf{U}$ & & 0.05 \\
\hline$U$ & & 0.05 \\
\hline$U$ & & 0.05 \\
\hline$U$ & & 0.05 \\
\hline U & & 0.05 \\
\hline $\mathbf{U}$ & & 0.05 \\
\hline U & & 0.05 \\
\hline$U$ & & 0.05 \\
\hline $\mathrm{U}$ & 8 & 0.94 \\
\hline $\mathrm{U}$ & 8 & 0.72 \\
\hline $\mathrm{U}$ & V & 0.82 \\
\hline $\mathrm{U}$ & V & 0.81 \\
\hline $\mathrm{J}$ & $\mathbf{E}$ & 0.27 \\
\hline U & & 1 \\
\hline $\mathrm{U}$ & & 0.05 \\
\hline $\mathrm{U}$ & & 0.05 \\
\hline$U$ & & 0.05 \\
\hline $\mathrm{U}$ & & 0.05 \\
\hline $\mathrm{U}$ & & 0.05 \\
\hline $\mathrm{U}$ & & 0.05 \\
\hline$U$ & V & 0.28 \\
\hline $\mathrm{U}$ & V & 0.3 \\
\hline $\mathrm{U}$ & & 0.05 \\
\hline $\mathrm{U}$ & & 0.05 \\
\hline $\mathrm{U}$ & & 0.05 \\
\hline U & & 0.05 \\
\hline $\mathrm{U}$ & & 0.05 \\
\hline $\mathrm{U}$ & & 0.05 \\
\hline $\mathrm{U}$ & & 1 \\
\hline
\end{tabular}

\begin{tabular}{|c|c|c|}
\hline$\mu \mathrm{g} / \mathrm{L}$ & 1 & EPA8260 \\
\hline$\mu \mathrm{g} / \mathrm{L}$ & 1 & EPA8260 \\
\hline$\mu \mathrm{g} / \mathrm{L}$ & 0.05 & EPA8260 \\
\hline$\mu \mathrm{g} / \mathrm{L}$ & 0.05 & EPA8260 \\
\hline$\mu g / L$ & 0.05 & EPA8260 \\
\hline$\mu \mathrm{g} / \mathrm{L}$ & 0.05 & EPA8260 \\
\hline$\mu \mathrm{g} / \mathrm{L}$ & 0.1 & EPA8260 \\
\hline$\mu \mathrm{g} / \mathrm{L}$ & 0.1 & EPA8260 \\
\hline$\mu \mathrm{g} / \mathrm{L}$ & 0.1 & EPA8260 \\
\hline$\mu \mathrm{g} / \mathrm{L}$ & 0.1 & EPA8260 \\
\hline$\mu \mathrm{g} / \mathrm{L}$ & 0.05 & EPA8260 \\
\hline$\mu \mathrm{g} / \mathrm{L}$ & 0.05 & EPA8260 \\
\hline$\mu \mathrm{g} / \mathrm{L}$ & 0.1 & EPA8260 \\
\hline$\mu \mathrm{g} / \mathrm{L}$ & 0.1 & EPA8260 \\
\hline$\mu g / L$ & 0.05 & EPA8260 \\
\hline$\mu \mathrm{g} / \mathrm{L}$ & 0.05 & EPA8260 \\
\hline$\mu \mathrm{g} / \mathrm{L}$ & 0.05 & EPA8260 \\
\hline$\mu g / L$ & 0.05 & EPA8260 \\
\hline$\mu \mathrm{g} / \mathrm{L}$ & 0.05 & EPA8260 \\
\hline$\mu \mathrm{g} / \mathrm{L}$ & 0.05 & EPA8260 \\
\hline$\mu \mathrm{g} / \mathrm{L}$ & 0.05 & EPA8260 \\
\hline$\mu \mathrm{g} / \mathrm{L}$ & 0.05 & EPA8260 \\
\hline$\mu \mathrm{g} / \mathrm{L}$ & 0.1 & EPA8260 \\
\hline$\mu \mathrm{g} / \mathrm{L}$ & 0.1 & EPA8260 \\
\hline$\mu \mathrm{g} / \mathrm{L}$ & 0.5 & EPA8260 \\
\hline$\mu \mathrm{g} / \mathrm{L}$ & 0.5 & EPA8260 \\
\hline$\mu \mathrm{g} / \mathrm{L}$ & 0.05 & EPA8260 \\
\hline$\mu \mathrm{g} / \mathrm{L}$ & 0.05 & EPA8260 \\
\hline$\mu \mathrm{g} / \mathrm{L}$ & 0.05 & EPA8260 \\
\hline$\mu \mathrm{g} / \mathrm{L}$ & 0.05 & EPA8260 \\
\hline$\mu g / L$ & 0.05 & EPA8260 \\
\hline$\mu \mathrm{g} / \mathrm{L}$ & 0.05 & EPA8260 \\
\hline$\mu g / L$ & 0.05 & EPA8260 \\
\hline$\mu \mathrm{g} / \mathrm{L}$ & 0.05 & EPA8260 \\
\hline$\mu \mathrm{g} / \mathrm{L}$ & 1 & EPA8260 \\
\hline$\mu \mathrm{g} / \mathrm{L}$ & 1 & EPA8260 \\
\hline$\mu \mathrm{g} / \mathrm{L}$ & 1 & EPA8260 \\
\hline$\mu g / L$ & 1 & EPA8260 \\
\hline$\mu \mathrm{g} / \mathrm{L}$ & 1 & EPA8260 \\
\hline$\mu \mathrm{g} / \mathrm{L}$ & 1 & EPA8260 \\
\hline$\mu \mathrm{g} / \mathrm{L}$ & 0.05 & EPA8260 \\
\hline$\mu \mathrm{g} / \mathrm{L}$ & 0.05 & EPA8260 \\
\hline$\mu \mathrm{g} / \mathrm{L}$ & 0.05 & EPA8260 \\
\hline$\mu \mathrm{g} / \mathrm{L}$ & 0.05 & EPA8260 \\
\hline$\mu \mathrm{g} / \mathrm{L}$ & 0.05 & EPA8260 \\
\hline$\mu \mathrm{g} / \mathrm{L}$ & 0.05 & EPA8260 \\
\hline$\mu \mathrm{g} / \mathrm{L}$ & 0.5 & EPA8260 \\
\hline$\mu \mathrm{g} / \mathrm{L}$ & 0.5 & EPA8260 \\
\hline$\mu \mathrm{g} / \mathrm{L}$ & 0.05 & EPA8260 \\
\hline$\mu \mathrm{g} / \mathrm{L}$ & 0.05 & EPA8260 \\
\hline$\mu \mathrm{g} / \mathrm{L}$ & 0.05 & EPA8260 \\
\hline$\mu \mathrm{g} / \mathrm{L}$ & 0.05 & EPA8260 \\
\hline$\mu \mathrm{g} / \mathrm{L}$ & 0.05 & EPA8260 \\
\hline$\mu g / L$ & 0.05 & EPA8260 \\
\hline & 1 & EPA8260 \\
\hline
\end{tabular}


SURVEY ID: PPSC FBOIC (continued)

Constituent

Volatile Organic Compounds

Vinyl acetate

Xylenes

Xylenes

U

U

U

Pesticides/PCBs and Dioxins/Furans

Aldrin

alpha-Benzene hexachloride

beta-Benzene hexachloride

delta-Benzene hexachloride

alpha-Chlordane

gamma-Chlordane

p,p'-DDD

p,p'-DDE

p,p'-DDT

Dieldrin

Endosulfan sulfate

Endosulfan I

Endosulfan II

Endrin

Endrin ketone

Heptachlor

Heptachlor epoxide

Lindane

Methoxychlor

PCB 1016

PCB 1221

PCB 1232

PCB 1242

PCB 1248

PCB 1254

PCB 1260

Toxaphene
Sample ID: 104603

RQ AQ B Result

Unit

D. Limit Method

1

0.15

0.15

$\mu \mathrm{g} / \mathrm{L}$

$\mu \mathrm{g} / \mathrm{L}$

$\mu \mathrm{g} / \mathrm{L}$

1

0.15

EPA8260

EPA8260

EPA8260

0.15

EPA8080

0.025

0.025

0.05

0.05

0.05

0.05

0.1

0.05

0.1

0.05

0.1

0.05

0.1

0.05

0.1

0.05

0.05

0.025

0.5

0.125

0.125

0.125

0.125

0.125

0.125

0.125

1

$\begin{array}{lll}\mu \mathrm{g} / \mathrm{L} & 0.025 & \text { EPA8080 } \\ \mu \mathrm{g} / \mathrm{L} & 0.025 & \text { EPA8080 } \\ \mu \mathrm{g} / \mathrm{L} & 0.05 & \text { EPA8080 } \\ \mu \mathrm{g} / \mathrm{L} & 0.05 & \text { EPA8080 } \\ \mu \mathrm{g} / \mathrm{L} & 0.05 & \text { EPA8080 } \\ \mu \mathrm{g} / \mathrm{L} & 0.05 & \text { EPA8080 } \\ \mu \mathrm{g} / \mathrm{L} & 0.1 & \text { EPA8080 } \\ \mu \mathrm{g} / \mathrm{L} & 0.05 & \text { EPA8080 } \\ \mu \mathrm{g} / \mathrm{L} & 0.1 & \text { EPA8080 } \\ \mu \mathrm{g} / \mathrm{L} & 0.05 & \text { EPA8080 } \\ \mu \mathrm{g} / \mathrm{L} & 0.1 & \text { EPA8080 } \\ \mu \mathrm{g} / \mathrm{L} & 0.05 & \text { EPA8080 } \\ \mu \mathrm{g} / \mathrm{L} & 0.1 & \text { EPA8080 } \\ \mu \mathrm{g} / \mathrm{L} & 0.05 & \text { EPA8080 } \\ \mu \mathrm{g} / \mathrm{L} & 0.1 & \text { EPA8080 } \\ \mu \mathrm{g} / \mathrm{L} & 0.05 & \text { EPA8080 } \\ \mu \mathrm{g} / \mathrm{L} & 0.05 & \text { EPA8080 } \\ \mu \mathrm{g} / \mathrm{L} & 0.025 & \text { EPA8080 } \\ \mu \mathrm{g} / \mathrm{L} & 0.5 & \text { EPA8080 } \\ \mu \mathrm{g} / \mathrm{L} & 0.125 & \text { EPA8080 } \\ \mu \mathrm{g} / \mathrm{L} & 0.125 & \text { EPA8080 } \\ \mu \mathrm{g} / \mathrm{L} & 0.125 & \text { EPA8080 } \\ \mu \mathrm{g} / \mathrm{L} & 0.125 & \text { EPA8080 } \\ \mu \mathrm{g} / \mathrm{L} & 0.125 & \text { EPA8080 } \\ \mu \mathrm{g} / \mathrm{L} & 0.125 & \text { EPA8080 } \\ \mu \mathrm{g} / \mathrm{L} & 0.125 & \text { EPA8080 } \\ \mu \mathrm{g} / \mathrm{L} & 1 & \text { EPA8080 }\end{array}$

SURVEY ID: PPSC FB02C

Sample type: Field Blank

Constituent
RQ AQ B Result

0.02
Unit

$\mathrm{mg} / \mathrm{L}$

0.02

EPA335.3

Metals (total recoverable)

Aluminum

Antimony

Arsenic

Barium

Beryllium

Cadmium

Calcium

Chromium

$\begin{array}{lll}U & X & 0.025 \\ U & & 0.005 \\ U & & 0.005 \\ U & & .0 .025 \\ U & & 0.01 \\ U & & 0.005 \\ U & & 0.25 \\ U & & 0.025\end{array}$

$\begin{array}{lll}\mathrm{mg} / \mathrm{L} & 0.025 & \text { EPA6010A } \\ \mathrm{mg} / \mathrm{L} & 0.005 & \text { EPA7041 } \\ \mathrm{mg} / \mathrm{L} & 0.005 & \text { EPA7060 } \\ \mathrm{mg} / \mathrm{L} & 0.025 & \text { EPA6010A } \\ \mathrm{mg} / \mathrm{L} & 0.01 & \text { EPA6010A } \\ \mathrm{mg} / \mathrm{L} & 0.005 & \text { EPA6010A } \\ \mathrm{mg} / \mathrm{L} & 0.25 & \text { EPA6010A } \\ \mathrm{mg} / \mathrm{L} & 0.025 & \text { EPA6010A }\end{array}$


SURVEY ID: PPSC FB02C (continued)

Constituent

RQ AQ B Result

Sample ID: 104654

Metals (total recoverable)

\begin{tabular}{|c|c|c|c|c|}
\hline Cobalt & $\mathrm{U}$ & & & 0.01 \\
\hline Copper & $\mathrm{U}$ & & & 0.025 \\
\hline Iron & $\mathrm{U}$ & & & 0.1 \\
\hline Lead & $\mathrm{U}$ & & & 0.005 \\
\hline Magnesium & $\mathrm{U}$ & & & 0.1 \\
\hline Manganese & $\mathrm{U}$ & & & 0.01 \\
\hline Mercury & $\mathrm{U}$ & V & & 0.000053 \\
\hline Nickel & $\mathrm{U}$ & & & 0.025 \\
\hline Potassium & $U$ & & & 2 \\
\hline Selenium & UJ & I & $\mathbf{L}$ & 0.005 \\
\hline Silver & $\mathrm{U}$ & & & 0.025 \\
\hline Sodium & UJ & I & $\mathrm{H}$ & 1 \\
\hline Thallium & $\mathrm{U}$ & & & 0.005 \\
\hline Vanadium & $\mathrm{U}$ & & & 0.01 \\
\hline Zinc & $\mathbf{J}$ & $E$ & & 0.0174 \\
\hline
\end{tabular}

$B / N / A$ Extractables (including $P A H$ and phenols)

Acenaphthene

Acenaphthylene

Anthracene

Benzo[a]anthracene

Benzo[b]fluoranthene

Benzo[k]fluoranthene

Benzoic acid

Benzo[g,h,i]perylene

Benzo[a]pyrene

Benzyl alcohol

Bis(2-chloroethoxy) methane

$\mathrm{Bis}$ (2-chloroethyl) ether

Bis(2-chloroisopropyl) ether

Bis(2-ethylhexyl) phthalate

4-Bromophenyl phenyl ether

Butylbenzyl phthalate

4-Chloroaniline

4-Chloro-m-cresol

2-Chloronaphthalene

2-Chlorophenol

4-Chlorophenyl phenyl ether

Chrysene

$\mathrm{m} / \mathrm{p}$-Cresol

o-Cresol (2-Methylphenol)

Dibenz[$[a, h]$ anthracene

- Dibenzofuran

Di-n-butyl phthalate

1,2-Dichlorobenzene

1,3-Dichlorobenzene

1,4-Dichlorobenzene

3,3'-Dichlorobenzidine

2,4-Dichlorophenol

Diethyl phthalate

2,4-Dimethyl phenol

Dimethyl phthalate

2,4-Dinitrophenol

2,4-Dinitrotoluene

$\begin{array}{lll}\text { U } & 1 \\ U & & 1 \\ U & & 1 \\ U & & 0.1 \\ U & & 0.1 \\ U & & 0.1 \\ U & & 1 \\ U & & 1 \\ U & & 0.1 \\ U & & 1 \\ U & & 1 \\ U & & 1 \\ U & & 1 \\ J & E & 0.5 \\ U & & 1 \\ U & & 1 \\ U & & 1 \\ U & & 1 \\ U & & 1 \\ U & & 1 \\ U & & 1 \\ U & & 0.1 \\ U & & 10 \\ U & & 1 \\ U & & 0.1 \\ U & & 1 \\ J & E & 0.9 \\ U & & 1 \\ U & & 1 \\ U & & 1 \\ U & & 1 \\ U & & 1 \\ J & E & 0.57 \\ U & & 1 \\ U & & 1 \\ U & & 10 \\ U & & 0.1 \\ & & \end{array}$

Unit

D. Limit Method

$\begin{array}{lll}\mathrm{mg} / \mathrm{L} & 0.01 & \text { EPA6010A } \\ \mathrm{mg} / \mathrm{L} & 0.025 & \text { EPA6010A } \\ \mathrm{mg} / \mathrm{L} & 0.1 & \text { EPA6010A } \\ \mathrm{mg} / \mathrm{L} & 0.005 & \text { EPA7421 } \\ \mathrm{mg} / \mathrm{L} & 0.1 & \text { EPA6010A } \\ \mathrm{mg} / \mathrm{L} & 0.01 & \text { EPA6010A } \\ \mathrm{mg} / \mathrm{L} & 0.0002 & \text { EPA7470 } \\ \mathrm{mg} / \mathrm{L} & 0.025 & \text { EPA6010A } \\ \mathrm{mg} / \mathrm{L} & 2 & \text { EPA6010A } \\ \mathrm{mg} / \mathrm{L} & 0.005 & \text { EPA7740 } \\ \mathrm{mg} / \mathrm{L} & 0.025 & \text { EPA6010A } \\ \mathrm{mg} / \mathrm{L} & 1 & \text { EPA6010A } \\ \mathrm{mg} / \mathrm{L} & 0.005 & \text { EPA7841 } \\ \mathrm{mg} / \mathrm{L} & 0.01 & \text { EPA6010A } \\ \mathrm{mg} / \mathrm{L} & 0.02 & \text { EPA6010A }\end{array}$

\begin{tabular}{lll}
$\mu \mathrm{g} / \mathrm{L}$ & 1 & EPA8270 \\
$\mu \mathrm{g} / \mathrm{L}$ & 1 & EPA8270 \\
$\mu \mathrm{g} / \mathrm{L}$ & 1 & EPA8270 \\
$\mu \mathrm{g} / \mathrm{L}$ & 0.1 & EPA8270 \\
$\mu \mathrm{g} / \mathrm{L}$ & 0.1 & EPA8270 \\
$\mu \mathrm{g} / \mathrm{L}$ & 0.1 & EPA8270 \\
$\mu \mathrm{g} / \mathrm{L}$ & 1 & EPA8270 \\
$\mu \mathrm{g} / \mathrm{L}$ & 1 & EPA8270 \\
$\mu \mathrm{g} / \mathrm{L}$ & 0.1 & EPA8270 \\
$\mu \mathrm{g} / \mathrm{L}$ & 1 & EPA8270 \\
$\mu \mathrm{g} / \mathrm{L}$ & 1 & EPA8270 \\
$\mu \mathrm{g} / \mathrm{L}$ & 1 & EPA8270 \\
$\mu \mathrm{g} / \mathrm{L}$ & 1 & EPA8270 \\
$\mu \mathrm{g} / \mathrm{L}$ & 1 & EPA8270 \\
$\mu \mathrm{g} / \mathrm{L}$ & 1 & EPA8270 \\
$\mu \mathrm{g} / \mathrm{L}$ & 1 & EPA8270 \\
$\mu \mathrm{g} / \mathrm{L}$ & 1 & EPA8270 \\
$\mu \mathrm{g} / \mathrm{L}$ & 1 & EPA8270 \\
$\mu \mathrm{g} / \mathrm{L}$ & 1 & EPA8270 \\
$\mu \mathrm{g} / \mathrm{L}$ & 1 & EPA8270 \\
$\mu \mathrm{g} / \mathrm{L}$ & 1 & EPA8270 \\
$\mu \mathrm{g} / \mathrm{L}$ & 0.1 & EPA8270 \\
$\mu \mathrm{g} / \mathrm{L}$ & 10 & EPA8270 \\
$\mu \mathrm{g} / \mathrm{L}$ & 1 & EPA8270 \\
$\mu \mathrm{g} / \mathrm{L}$ & 0.1 & EPA8270 \\
$\mu \mathrm{g} / \mathrm{L}$ & 1 & EPA8270 \\
$\mu \mathrm{g} / \mathrm{L}$ & 1 & EPA8270 \\
$\mu \mathrm{g} / \mathrm{L}$ & 1 & EPA8270 \\
$\mu \mathrm{g} / \mathrm{L}$ & 1 & EPA8270 \\
$\mu \mathrm{g} / \mathrm{L}$ & 1 & EPA8270 \\
$\mu \mathrm{g} / \mathrm{L}$ & 1 & EPA8270 \\
$\mu \mathrm{g} / \mathrm{L}$ & 1 & EPA8270 \\
$\mu \mathrm{g} / \mathrm{L}$ & 1 & EPA8270 \\
$\mu \mathrm{g} / \mathrm{L}$ & 1 & EPA8270 \\
$\mu \mathrm{g} / \mathrm{L}$ & 1 & EPA8270 \\
$\mu \mathrm{g} / \mathrm{L}$ & 10 & EPA8270 \\
$\mu \mathrm{g} / \mathrm{L}$ & 0.1 & EPA8270 \\
& & \\
\hline
\end{tabular}


SURVEY ID: PPSC FB02C (continued)

Sample ID: 104654

Constituent

RQ AQ B Result

Unit

D. Limit Method

$B / N / A$ Extractables (including PAH and phenols)

\begin{tabular}{|c|c|c|c|}
\hline 2,6-Dinitrotoluene & $\mathrm{U}$ & & \\
\hline Di-n-octyl phthalate & $\mathrm{U}$ & & \\
\hline Fluoranthene & $\mathrm{U}$ & & \\
\hline Fluorene & $\mathrm{U}$ & & \\
\hline Hexachlorobenzene & $\mathrm{U}$ & & \\
\hline Hexachlorobutadiene & $\mathrm{U}$ & & \\
\hline Hexachlorocyclopentadiene & $\mathrm{U}$ & & \\
\hline Hexachloroethane & $\mathrm{U}$ & & \\
\hline Indeno $[1,2,3 \cdot c, d]$ pyrene & $\mathrm{U}$ & & \\
\hline Isophorone & $\mathrm{U}$ & & \\
\hline 2-Methyl-4,6-dinitrophenol & $\mathrm{U}$ & & \\
\hline 2-Methylnaphthalene & $\mathrm{U}$ & & \\
\hline Naphthalene & $\mathrm{U}$ & & \\
\hline m-Nitroaniline & $\mathrm{U}$ & & \\
\hline o-Nitroaniline & $U$ & & \\
\hline p-Nitroaniline & $\mathrm{U}$ & & \\
\hline Nitrobenzene & $\mathrm{U}$ & & \\
\hline 2-Nitrophenol & $\mathrm{U}$ & & \\
\hline 4-Nitrophenol & $\mathrm{U}$ & & \\
\hline N-Nitrosodiphenylamine & $\mathrm{U}$ & & \\
\hline N-Nitrosodi-n-propylamine & $\mathrm{U}$ & & \\
\hline Pentachlorophenol & UJ & I & $\mathrm{H}$ \\
\hline Phenanthrene & $\mathrm{U}$ & & \\
\hline Phenol & U & & \\
\hline Pyrene & $\mathrm{U}$ & & \\
\hline 1,2,4-Trichlorobenzene & $\mathrm{U}$ & & \\
\hline 2,4,5-Trichlorophenol & $\mathrm{U}$ & & \\
\hline 2,4,6-Trichlorophenol & $\mathrm{U}$ & & \\
\hline
\end{tabular}

$\begin{array}{lll}\mu \mathrm{g} / \mathrm{L} & 0.1 & \text { EPA8270 } \\ \mu \mathrm{g} / \mathrm{L} & 1 & \text { EPA8270 } \\ \mu \mathrm{g} / \mathrm{L} & 1 & \text { EPA8270 } \\ \mu \mathrm{g} / \mathrm{L} & 1 & \text { EPA8270 } \\ \mu \mathrm{g} / \mathrm{L} & 0.1 & \text { EPA8270 } \\ \mu \mathrm{g} / \mathrm{L} & 1 & \text { EPA8270 } \\ \mu \mathrm{g} / \mathrm{L} & 1 & \text { EPA8270 } \\ \mu \mathrm{g} / \mathrm{L} & 0.1 & \text { EPA8270 } \\ \mu \mathrm{g} / \mathrm{L} & 0.1 & \text { EPA8270 } \\ \mu \mathrm{g} / \mathrm{L} & 1 & \text { EPA8270 } \\ \mu \mathrm{g} / \mathrm{L} & 10 & \text { EPA8270 } \\ \mu \mathrm{g} / \mathrm{L} & 1 & \text { EPA8270 } \\ \mu \mathrm{g} / \mathrm{L} & 1 & \text { EPA8270 } \\ \mu \mathrm{g} / \mathrm{L} & 1 & \text { EPA8270 } \\ \mu \mathrm{g} / \mathrm{L} & 1 & \text { EPA8270 } \\ \mu \mathrm{g} / \mathrm{L} & 1 & \text { EPA8270 } \\ \mu \mathrm{g} / \mathrm{L} & 1 & \text { EPA8270 } \\ \mu \mathrm{g} / \mathrm{L} & 1 & \text { EPA8270 } \\ \mu \mathrm{g} / \mathrm{L} & 1 & \text { EPA8270 } \\ \mu \mathrm{g} / \mathrm{L} & 1 & \text { EPA8270 } \\ \mu \mathrm{g} / \mathrm{L} & 1 & \text { EPA8270 } \\ \mu \mathrm{g} / \mathrm{L} & 1 & \text { EPA8270 } \\ \mu \mathrm{g} / \mathrm{L} & 1 & \text { EPA8270 } \\ \mu \mathrm{g} / \mathrm{L} & 1 & \text { EPA8270 } \\ \mu \mathrm{g} / \mathrm{L} & 1 & \text { EPA8270 } \\ \mu \mathrm{g} / \mathrm{L} & 0.1 & \text { EPA8270 } \\ \mu \mathrm{g} / \mathrm{L} & 1 & \text { EPA8270 } \\ \mu \mathrm{g} / \mathrm{L} & 1 & \text { EPA8270 }\end{array}$

Volatile Organic Compounds

\begin{tabular}{|c|c|c|c|c|c|c|}
\hline Acetone & U & 8 & 5.19 & $\mu \mathrm{g} / \mathrm{L}$ & 5 & EPA8260 \\
\hline Benzene & $U$ & & 0.5 & $\mu \mathrm{g} / \mathrm{L}$ & 0.5 & EPA8260 \\
\hline Bromodichloromethane & U & & 0.05 & $\mu \mathrm{g} / \mathrm{L}$ & 0.05 & EPA8260 \\
\hline Bromoform & $\mathrm{U}$ & & 0.05 & $\mu \mathrm{g} / \mathrm{L}$ & 0.05 & EPA8260 \\
\hline Bromomethane (Methyl bromide) & $\mathrm{U}$ & & 0.1 & $\mu \mathrm{g} / \mathrm{L}$ & 0.1 & EPA8260 \\
\hline Carbon disulfide & U & & 1 & $\mu \mathrm{g} / \mathrm{L}$ & 1 & EPA8260 \\
\hline Carbon tetrachloride & $\mathrm{U}$ & & 0.05 & $\mu \mathrm{g} / \mathrm{L}$ & 0.05 & EPA8260 \\
\hline Chlorobenzene & & & 0.21 & $\mu \mathrm{g} / \mathrm{L}$ & 0.05 & EPA8260 \\
\hline Chloroethane & $\mathrm{U}$ & & 0.1 & $\mu \mathrm{g} / \mathrm{L}$ & 0.1 & EPA8260 \\
\hline Chloroethene (Vinyl chloride) & $\mathrm{U}$ & & 0.1 & $\mu \mathrm{g} / \mathrm{L}$ & 0.1 & EPA8260 \\
\hline Chloroform & U & & 0.05 & $\mu \mathrm{g} / \mathrm{L}$ & 0.05 & EPA8260 \\
\hline Chloromethane (Methyl chloride) & $\mathrm{U}$ & & 0.1 & $\mu \mathrm{g} / \mathrm{L}$ & 0.1 & EPA8260 \\
\hline Dibromochloromethane & $\mathrm{U}$ & & 0.05 & $\mu \mathrm{g} / \mathrm{L}$ & 0.05 & EPA8260 \\
\hline 1,1-Dichloroethane & $\mathrm{U}$ & & 0.05 & $\mu \mathrm{g} / \mathrm{L}$ & 0.05 & EPA8260 \\
\hline 1,2-Dichloroethane & $\mathrm{U}$ & & 0.05 & $\mu \mathrm{g} / \mathrm{L}$ & 0.05 & EPA8260 \\
\hline 1,1-Dichloroethylene & $\mathrm{U}$ & & 0.05 & $\mu \mathrm{g} / \mathrm{L}$ & 0.05 & EPA8260 \\
\hline 1,2-Dichloroethylene & $U$ & & 0.1 & $\mu g / L$ & 0.1 & EPA8260 \\
\hline Dichloromethane (Methylene chloride) & $U$ & $\mathrm{~V}$ & 0.64 & $\mu \mathrm{g} / \mathrm{L}$ & 0.5 & EPA8260 \\
\hline 1,2-Dichloropropane & $\mathrm{U}$ & & 0.05 & $\mu \mathrm{g} / \mathrm{L}$ & 0.05 & EPA8260 \\
\hline cis-1,3-Dichloropropene & $\mathrm{U}$ & & 0.05 & $\mu \mathrm{g} / \mathrm{L}$ & 0.05 & EPA8260 \\
\hline trans-1,3-Dichloropropene & $\mathrm{U}$ & & 0.05 & $\mu \mathrm{g} / \mathrm{L}$ & 0.05 & EPA8260 \\
\hline Ethylbenzene & $\mathrm{U}$ & & 0.05 & $\mu \mathrm{g} / \mathrm{L}$ & 0.05 & EPA8260 \\
\hline 2-Hexanone & $\mathrm{U}$ & $\mathrm{V}$ & 0.89 & $\mu \mathrm{g} / \mathrm{L}$ & 1 & EPA8260 \\
\hline Methyl ethyl ketone & $\mathrm{U}$ & $\mathrm{V}$ & 1.49 & $\mu \mathrm{g} / \mathrm{L}$ & 1 & EPA8260 \\
\hline
\end{tabular}


SURVEY ID: PPSC FB02C (continued)

Constituent

Volatile Organic Compounds

Methyl isobutyl ketone

Styrene

1,1,2,2-Tetrachloroethane

Tetrachloroethylene

Toluene

1,1,1-Trichloroethane

1,1,2-Trichloroethane

Trichloroethylene

Vinyl acetate

Xylenes

Pesticides/PCBs and Dioxins/Furans
Sample ID: 104654

RQ AQ B Result

1

0.05

0.05

0.05

0.27

0.05

0.05

0.05

1

0.15

Unit

D. Limit Method

$\begin{array}{lll}\mu \mathrm{g} / \mathrm{L} & 1 & \text { EPA8260 } \\ \mu \mathrm{g} / \mathrm{L} & 0.05 & \text { EPA8260 } \\ \mu \mathrm{g} / \mathrm{L} & 0.05 & \text { EPA8260 } \\ \mu \mathrm{g} / \mathrm{L} & 0.05 & \text { EPA8260 } \\ \mu \mathrm{g} / \mathrm{L} & 0.5 & \text { EPA8260 } \\ \mu \mathrm{g} / \mathrm{L} & 0.05 & \text { EPA8260 } \\ \mu \mathrm{g} / \mathrm{L} & 0.05 & \text { EPA8260 } \\ \mu \mathrm{g} / \mathrm{L} & 0.05 & \text { EPA8260 } \\ \mu \mathrm{g} / \mathrm{L} & 1 & \text { EPA8260 } \\ \mu \mathrm{g} / \mathrm{L} & 0.15 & \text { EPA8260 }\end{array}$

0.025

0.025

0.05

0.05

0.05

0.05

0.1

0.05

0.1

0.05

0.1

0.05

0.1

0.05

0.1

0.05

0.05

0.025

0.5

0.125

0.125

0.125

0.125

0.125

0.125

0.125

1

$\begin{array}{lll}\mu \mathrm{g} / \mathrm{L} & 0.025 & \text { EPA8080 } \\ \mu \mathrm{g} / \mathrm{L} & 0.025 & \text { EPA8080 } \\ \mu \mathrm{g} / \mathrm{L} & 0.05 & \text { EPA8080 } \\ \mu \mathrm{g} / \mathrm{L} & 0.05 & \text { EPA8080 } \\ \mu \mathrm{g} / \mathrm{L} & 0.05 & \text { EPA8080 } \\ \mu \mathrm{g} / \mathrm{L} & 0.05 & \text { EPA8080 } \\ \mu \mathrm{g} / \mathrm{L} & 0.1 & \text { EPA8080 } \\ \mu \mathrm{g} / \mathrm{L} & 0.05 & \text { EPA8080 } \\ \mu \mathrm{g} / \mathrm{L} & 0.1 & \text { EPA8080 } \\ \mu \mathrm{g} / \mathrm{L} & 0.05 & \text { EPA8080 } \\ \mu \mathrm{g} / \mathrm{L} & 0.1 & \text { EPA8080 } \\ \mu \mathrm{g} / \mathrm{L} & 0.05 & \text { EPA8080 } \\ \mu \mathrm{g} / \mathrm{L} & 0.1 & \text { EPA8080 } \\ \mu \mathrm{g} / \mathrm{L} & 0.05 & \text { EPA8080 } \\ \mu \mathrm{g} / \mathrm{L} & 0.1 & \text { EPA8080 } \\ \mu \mathrm{g} / \mathrm{L} & 0.05 & \text { EPA8080 } \\ \mu \mathrm{g} / \mathrm{L} & 0.05 & \text { EPA8080 } \\ \mu \mathrm{g} / \mathrm{L} & 0.025 & \text { EPA8080 } \\ \mu \mathrm{g} / \mathrm{L} & 0.5 & \text { EPA8080 } \\ \mu \mathrm{g} / \mathrm{L} & 0.125 & \text { EPA8080 } \\ \mu \mathrm{g} / \mathrm{L} & 0.125 & \text { EPA8080 } \\ \mu \mathrm{g} / \mathrm{L} & 0.125 & \text { EPA8080 } \\ \mu \mathrm{g} / \mathrm{L} & 0.125 & \text { EPA8080 } \\ \mu \mathrm{g} / \mathrm{L} & 0.125 & \text { EPA8080 } \\ \mu \mathrm{g} / \mathrm{L} & 0.125 & \text { EPA8080 } \\ \mu \mathrm{g} / \mathrm{L} & 0.125 & \text { EPA8080 } \\ \mu \mathrm{g} / \mathrm{L} & 1 & \text { EPA8080 }\end{array}$

SURVEY ID: PPSC FB03C

Sample type: Field Blank

Constituent
Sample ID: 104631

Associated sample: 104630

RQ AQ B Result

Unit

D. Limit Method

Physical Parameters and Specified Analyses

Cyanide

J $\mathrm{E}$

0.00393

$\mathrm{mg} / \mathrm{L}$

0.02

EPA335.3 
SURVEY ID: PPSC FB03C (continued)

Sample ID: 104631

Constituent

Metals (total recoverable)

Aluminum

Antimony

Arsenic

Barium

Barium

Beryllium

Beryllium

Cadmium

Cadmium

Calcium

Calcium

Chromium

Chromium

Cobalt

Cobalt

Copper

Copper

Iron

Iron

Lead

Lead

Magnesium

Manganese

Manganese

Mercury

Nickel

Nickel

Potassium

Potassium

Selenium

Silver

Silver

Sodium

Sodium

Thallium

Vanadium

Zinc

Zinc
Aluminum

Magnesium

Vanadium

RQ AQ B Result

$\begin{array}{lll}J & E & \mathbf{0 . 0 2 3 2} \\ \mathrm{U} & & \mathbf{0 . 0 2 5} \\ \mathrm{U} & & \mathbf{0 . 0 0 5} \\ \mathrm{J} & \mathrm{E} & \mathbf{0 . 0 0 1 1 6}\end{array}$

U

U

U

U$$
\text { d }
$$$$
\mathrm{J}
$$$$
\text { U }
$$$$
\text { U }
$$$$
\text { U }
$$$$
\text { U }
$$$$
\text { U }
$$$$
\text { U }
$$$$
U
$$$$
U
$$$$
\text { U }
$$

$$
\text { ư }
$$$$
\text { U }
$$

J $\quad$ E $\quad 0.00395$

$\begin{array}{lll}J & \mathrm{E} & \mathbf{0 . 0 0 3 9 2}\end{array}$

$\begin{array}{lll}\mathrm{J} & \mathbf{E} & \mathbf{0 . 0 0 3 9 2} \\ \mathrm{U} & \mathrm{V} & \mathbf{0 . 0 0 0 0 5 1}\end{array}$

U $\quad 0.025$

U $\quad 0.025$

$\mathrm{U} 2$

U 2

U $\quad 0.005$

U $\quad 0.025$

$\begin{array}{ll}\mathrm{U} & \mathbf{0 . 0 2 5}\end{array}$

U 1

$\begin{array}{ll}\mathrm{U} & 1 \\ \mathrm{U} & \mathbf{0 . 0 0 5}\end{array}$

$\mathrm{U} \quad 0.01$

$\begin{array}{lll}\mathrm{U} & & 0.01 \\ \mathrm{~J} & \mathrm{E} & \mathbf{0 . 0 1 2 4}\end{array}$

$B / N / A$ Extractables (including PAH and phenols)

$\begin{array}{lll}\text { Acenaphthene } & \mathrm{U} & 1 \\ \text { Acenaphthene } & \mathrm{U} & 1 \\ \text { Acenaphthylene } & \mathrm{U} & 1 \\ \text { Acenaphthylene } & \mathrm{U} & 1 \\ \text { Anthracene } & \mathrm{U} & 1 \\ \text { Anthracene } & \mathrm{U} & 1 \\ \text { Benzo[a]anthracene } & \mathrm{U} & 0.1 \\ \text { Benzo[a]anthracene } & \mathrm{U} & 0.1 \\ \text { Benzo[b]fluoranthene } & \mathrm{U} & 0.1 \\ \text { Benzo[b]fluoranthene } & \mathrm{U} & 0.1 \\ \text { Benzo[k]fluoranthene } & \mathrm{U} & 0.1\end{array}$

Unit

D. Limit Method

\begin{tabular}{lll}
$\mathrm{mg} / \mathrm{L}$ & 0.025 & EPA6010A \\
$\mathrm{mg} / \mathrm{L}$ & 0.025 & EPA6010A \\
$\mathrm{mg} / \mathrm{L}$ & 0.005 & EPA7041 \\
$\mathrm{mg} / \mathrm{L}$ & 0.005 & EPA7060 \\
$\mathrm{mg} / \mathrm{L}$ & 0.025 & EPA6010A \\
$\mathrm{mg} / \mathrm{L}$ & 0.025 & EPA6010A \\
$\mathrm{mg} / \mathrm{L}$ & 0.01 & EPA6010A \\
$\mathrm{mg} / \mathrm{L}$ & 0.01 & EPA6010A \\
$\mathrm{mg} / \mathrm{L}$ & 0.005 & EPA6010A \\
$\mathrm{mg} / \mathrm{L}$ & 0.005 & EPA6010A \\
$\mathrm{mg} / \mathrm{L}$ & 0.25 & EPA6010A \\
$\mathrm{mg} / \mathrm{L}$ & 0.25 & EPA6010A \\
$\mathrm{mg} / \mathrm{L}$ & 0.025 & EPA6010A \\
$\mathrm{mg} / \mathrm{L}$ & 0.025 & EPA6010A \\
$\mathrm{mg} / \mathrm{L}$ & 0.01 & EPA6010A \\
$\mathrm{mg} / \mathrm{L}$ & 0.01 & EPA6010A \\
$\mathrm{mg} / \mathrm{L}$ & 0.025 & EPA6010A \\
$\mathrm{mg} / \mathrm{L}$ & 0.025 & EPA6010A \\
$\mathrm{mg} / \mathrm{L}$ & 0.1 & EPA6010A \\
$\mathrm{mg} / \mathrm{L}$ & 0.1 & EPA6010A \\
$\mathrm{mg} / \mathrm{L}$ & 0.1 & EPA6010A \\
$\mathrm{mg} / \mathrm{L}$ & 0.005 & EPA7421 \\
$\mathrm{mg} / \mathrm{L}$ & 0.1 & EPA6010A \\
$\mathrm{mg} / \mathrm{L}$ & 0.1 & EPA6010A \\
$\mathrm{mg} / \mathrm{L}$ & 0.01 & EPA6010A \\
$\mathrm{mg} / \mathrm{L}$ & 0.01 & EPA6010A \\
$\mathrm{mg} / \mathrm{L}$ & 0.0002 & EPA7470 \\
$\mathrm{mg} / \mathrm{L}$ & 0.025 & EPA6010A \\
$\mathrm{mg} / \mathrm{L}$ & 0.025 & EPA6010A \\
$\mathrm{mg} / \mathrm{L}$ & 2 & EPA6010A \\
$\mathrm{mg} / \mathrm{L}$ & 2 & EPA6010A \\
$\mathrm{mg} / \mathrm{L}$ & 0.005 & EPA7740 \\
$\mathrm{mg} / \mathrm{L}$ & 0.025 & EPA6010A \\
$\mathrm{mg} / \mathrm{L}$ & 0.025 & EPA6010A \\
$\mathrm{mg} / \mathrm{L}$ & 1 & EPA6010A \\
$\mathrm{mg} / \mathrm{L}$ & 1 & EPA6010A \\
$\mathrm{mg} / \mathrm{L}$ & 0.005 & EPA7841 \\
$\mathrm{mg} / \mathrm{L}$ & 0.01 & EPA6010A \\
$\mathrm{mg} / \mathrm{L}$ & 0.01 & EPA6010A \\
$\mathrm{mg} / \mathrm{L}$ & 0.02 & EPA6010A \\
$\mathrm{mg} / \mathrm{L}$ & 0.02 & EPA6010A \\
& & \\
\hline
\end{tabular}

EPA8270

EPA8270

EPA8270

EPA8270

EPA8270

EPA8270

EPA8270

EPA8270

EPA8270

EPA8270

EPA8270 
SURVEY ID: PPSC FB03C (continued)

Sample ID: 104631

$\begin{array}{lllll}\text { Constituent UQ AQ B Result Dit Limit Method } & \text { Un }\end{array}$

$B / N / A$ Extractables (including PAH and phenols)

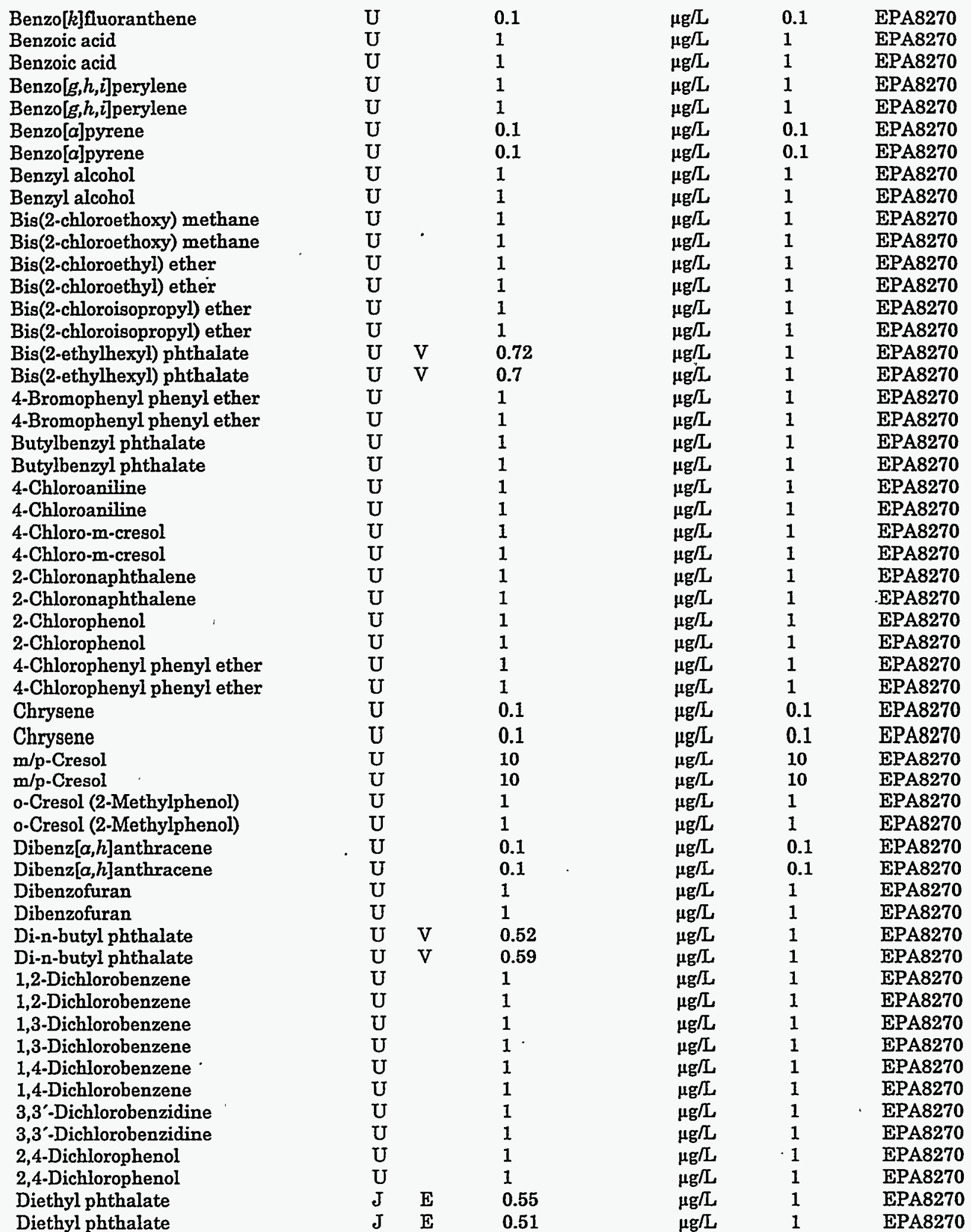


SURVEY ID: PPSC FB03C (continued)

Constituent
Sample ID: 104631
Unit
D. Limit Method

B/N/A Extractables (including PAH and phenols)

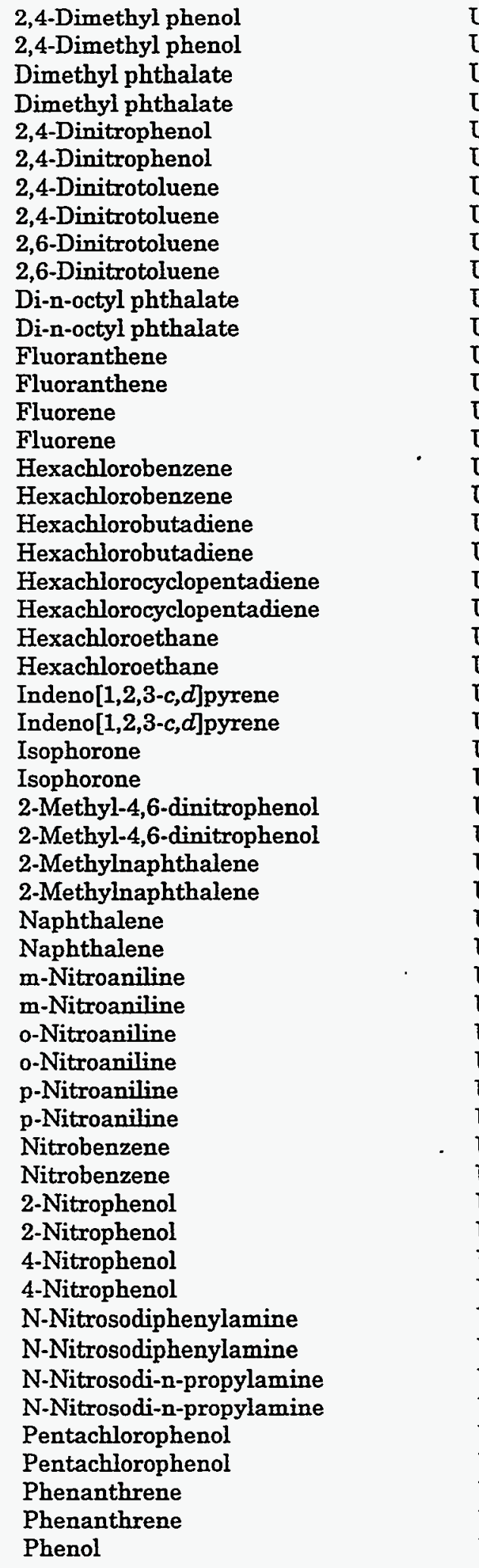

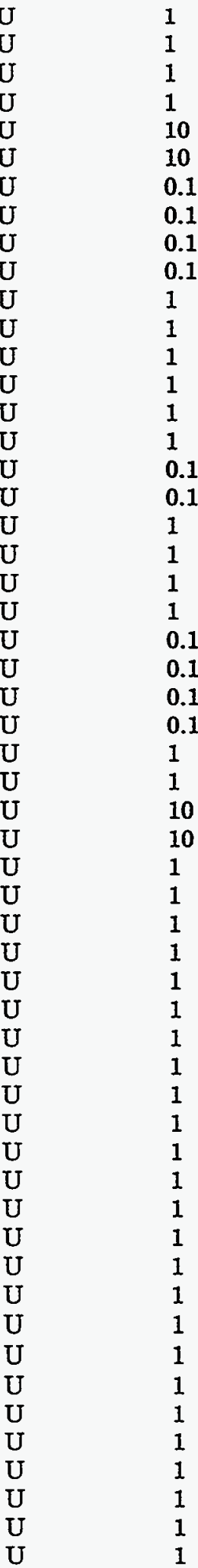

\begin{tabular}{|c|c|c|}
\hline$\mu \mathrm{g} / \mathrm{L}$ & 1 & EPA8270 \\
\hline$\mu \mathrm{g} / \mathrm{L}$ & 1 & EPA 8270 \\
\hline$\mu \mathrm{g} / \mathrm{L}$ & 1 & EPA8270 \\
\hline$\mu g / L$ & 1 & EPA8270 \\
\hline$\mu \mathrm{g} / \mathrm{L}$ & 10 & EPA8270 \\
\hline$\mu \mathrm{g} / \mathrm{L}$ & 10 & EPA8270 \\
\hline$\mu \mathrm{g} / \mathrm{L}$ & 0.1 & EPA8270 \\
\hline$\mu g / L$ & 0.1 & EPA8270 \\
\hline$\mu g / L$ & 0.1 & EPA8270 \\
\hline$\mu g / L$ & 0.1 & EPA8270 \\
\hline$\mu \mathrm{g} / \mathrm{L}$ & 1 & EPA8270 \\
\hline$\mu \mathrm{g} / \mathrm{L}$ & 1 & EPA8270 \\
\hline$\mu \mathrm{g} / \mathrm{L}$ & 1 & EPA8270 \\
\hline$\mu \mathrm{g} / \mathrm{L}$ & 1 & EPA8270 \\
\hline$\mu g / L$ & 1 & EPA8270 \\
\hline$\mu \mathrm{g} / \mathrm{L}$ & 1 & EPA8270 \\
\hline$\mu \mathrm{g} / \mathrm{L}$ & 0.1 & EPA8270 \\
\hline$\mu g / L$ & 0.1 & EPA8270 \\
\hline$\mu \mathrm{g} / \mathrm{L}$ & 1 & EPA8270 \\
\hline$\mu \mathrm{g} / \mathrm{L}$ & 1 & EPA8270 \\
\hline$\mu \mathrm{g} / \mathrm{L}$ & 1 & EPA8270 \\
\hline$\mu g / L$ & 1 & EPA8270 \\
\hline$\mu g / L$ & 0.1 & EPA8270 \\
\hline$\mu g / L$ & 0.1 & EPA8270 \\
\hline$\mu \mathrm{g} / \mathrm{L}$ & 0.1 & EPA8270 \\
\hline$\mu \mathrm{g} / \mathrm{L}$ & 0.1 & EPA8270 \\
\hline$\mu \mathrm{g} / \mathrm{L}$ & 1 & EPA8270 \\
\hline$\mu \mathrm{g} / \mathrm{L}$ & 1 & EPA8270 \\
\hline$\mu g / L$ & 10 & EPA8270 \\
\hline$\mu \mathrm{g} / \mathrm{L}$ & 10 & EPA8270 \\
\hline$\mu \mathrm{g} / \mathrm{L}$ & 1 & EPA8270 \\
\hline$\mu \mathrm{g} / \mathrm{L}$ & 1 & EPA8270 \\
\hline$\mu g / L$ & 1 & EPA8270 \\
\hline$\mu \mathrm{g} / \mathrm{L}$ & 1 & EPA 8270 \\
\hline$\mu \mathrm{g} / \mathrm{L}$ & 1 & EPA8270 \\
\hline$\mu \mathrm{g} / \mathrm{L}$ & 1 & EPA8270 \\
\hline$\mu \mathrm{g} / \mathrm{L}$ & 1 & EPA8270 \\
\hline$\mu g / L$ & 1 & EPA8270 \\
\hline$\mu \mathrm{g} / \mathrm{L}$ & 1 & EPA8270 \\
\hline$\mu g / L$ & 1 & EPA8270 \\
\hline$\mu \mathrm{g} / \mathrm{L}$ & 1 & EPA8270 \\
\hline$\mu g / L$ & 1 & EPA8270 \\
\hline$\mu \mathrm{g} / \mathrm{L}$ & 1 & EPA8270 \\
\hline$\mu \mathrm{g} / \mathrm{L}$ & 1 & EPA8270 \\
\hline$\mu \mathrm{g} / \mathrm{L}$ & 1 & EPA8270 \\
\hline$\mu \mathrm{g} / \mathrm{L}$ & 1 & EPA8270 \\
\hline$\mu g / L$ & 1 & EPA8270 \\
\hline$\mu \mathrm{g} / \mathrm{L}$ & 1 & EPA8270 \\
\hline$\mu \mathrm{g} / \mathrm{L}$ & 1 & EPA8270 \\
\hline$\mu g / L$ & 1 & EPA8270 \\
\hline$\mu \mathrm{g} / \mathrm{L}$ & 1 & EPA8270 \\
\hline$\mu g / L$ & 1 & EPA8270 \\
\hline$\mu \mathrm{g} / \mathrm{L}$ & 1 & EPA8270 \\
\hline$\mu g / L$ & 1 & EPA8270 \\
\hline$\mu \mathrm{g} / \mathrm{L}$ & 1 & EPA8270 \\
\hline
\end{tabular}


SURVEY ID: PPSC FB03C (continued)

Sample ID: 104631

$\begin{array}{lllll}\text { Constituent } & \text { RQ } & \text { U Result } & \text { D. Limit Method }\end{array}$

$B / N / A$ Extractables (including PAH and phenols)

Phenol

Pyrene

Pyrene

1,2,4-Trichlorobenzene

1,2,4-Trichlorobenzene

2,4,5-Trichlorophenol

2,4,5-Trichlorophenol

2,4,6-Trichlorophenol

2,4,6-Trichlorophenol

$\begin{array}{ll}\mathrm{U} & 1 \\ \mathrm{U} & 1 \\ \mathrm{U} & 1 \\ \mathrm{U} & 0.1 \\ \mathrm{U} & 0.1 \\ \mathrm{U} & 1 \\ \mathrm{U} & 1 \\ \mathrm{U} & 1 \\ \mathrm{U} & 1\end{array}$

Volatile Organic Compounds

\begin{tabular}{|c|c|c|}
\hline $\mathrm{U}$ & \multirow[t]{8}{*}{8} & 4.71 \\
\hline $\mathbf{U}$ & & 0.5 \\
\hline $\mathrm{U}$ & & 0.05 \\
\hline $\mathbf{U}$ & & 0.05 \\
\hline $\mathrm{U}$ & & 0.1 \\
\hline $\mathrm{U}$ & & 1 \\
\hline $\mathrm{U}$ & & 0.05 \\
\hline & & 0.17 \\
\hline $\mathrm{U}$ & & 0.1 \\
\hline $\mathrm{U}$ & & 0.1 \\
\hline $\mathbf{U}$ & & 0.05 \\
\hline $\mathrm{U}$ & & 0.1 \\
\hline $\mathbf{U}$ & & 0.05 \\
\hline $\mathrm{U}$ & & 0.05 \\
\hline $\mathrm{U}$ & & 0.05 \\
\hline $\mathbf{U}$ & & 0.05 \\
\hline $\mathrm{U}$ & & 0.1 \\
\hline $\mathrm{U}$ & V & 0.38 \\
\hline $\mathrm{U}$ & & 0.05 \\
\hline $\mathrm{U}$ & & 0.05 \\
\hline $\mathrm{U}$ & & 0.05 \\
\hline $\mathrm{U}$ & & 0.05 \\
\hline $\mathrm{U}$ & V & 1.33 \\
\hline $\mathrm{U}$ & $\mathrm{V}$ & 1.63 \\
\hline $\mathrm{U}$ & & 1 \\
\hline $\mathrm{U}$ & & 0.05 \\
\hline $\mathbf{U}$ & & 0.05 \\
\hline $\mathrm{U}$ & & 0.05 \\
\hline $\mathrm{U}$ & V & 0.19 \\
\hline $\mathrm{U}$ & & 0.05 \\
\hline $\mathrm{U}$ & & 0.05 \\
\hline $\mathrm{U}$ & & 0.05 \\
\hline U & & 1 \\
\hline $\mathrm{U}$ & & 0.15 \\
\hline
\end{tabular}

Pesticides/PCBs and Dioxins/Furans

Aldrin

alpha-Benzene hexachloride

beta-Benzene hexachloride

delta-Benzene hexachloride

alpha-Chlordane

gamma-Chlordane

$\begin{array}{ll}U & 0.025 \\ U & 0.025 \\ U & 0.05 \\ U & 0.05 \\ U & 0.05 \\ U & 0.05\end{array}$

$\begin{array}{lll}\mu \mathrm{g} / \mathrm{L} & 1 & \text { EPA8270 } \\ \mu \mathrm{g} / \mathrm{L} & 1 & \text { EPA8270 } \\ \mu \mathrm{g} / \mathrm{L} & 1 & \text { EPA8270 } \\ \mu \mathrm{g} / \mathrm{L} & 0.1 & \text { EPA8270 } \\ \mu \mathrm{g} / \mathrm{L} & 0.1 & \text { EPA8270 } \\ \mu \mathrm{g} / \mathrm{L} & 1 & \text { EPA8270 } \\ \mu \mathrm{g} / \mathrm{L} & 1 & \text { EPA8270 } \\ \mu \mathrm{g} / \mathrm{L} & 1 & \text { EPA8270 } \\ \mu \mathrm{g} / \mathrm{L} & 1 & \text { EPA8270 }\end{array}$

\begin{tabular}{|c|c|c|}
\hline$\mu \mathrm{g} / \mathrm{L}$ & 5 & EPA8260 \\
\hline$\mu \mathrm{g} / \mathrm{L}$ & 0.5 & EPA8260 \\
\hline$\mu \mathrm{g} / \mathrm{L}$ & 0.05 & EPA8260 \\
\hline$\mu \mathrm{g} / \mathrm{L}$ & 0.05 & EPA8260 \\
\hline$\mu \mathrm{g} / \mathrm{L}$ & 0.1 & EPA8260 \\
\hline$\mu g / L$ & 1 & EPA8260 \\
\hline$\mu \mathrm{g} / \mathrm{L}$ & 0.05 & EPA8260 \\
\hline$\mu \mathrm{g} / \mathrm{L}$ & 0.05 & EPA8260 \\
\hline$\mu \mathrm{g} / \mathrm{L}$ & 0.1 & EPA8260 \\
\hline$\mu \mathrm{g} / \mathrm{L}$ & 0.1 & EPA8260 \\
\hline$\mu \mathrm{g} / \mathrm{L}$ & 0.05 & EPA8260 \\
\hline$\mu \mathrm{g} / \mathrm{L}$ & 0.1 & EPA8260 \\
\hline$\mu g / L$ & 0.05 & EPA8260 \\
\hline$\mu \mathrm{g} / \mathrm{L}$ & 0.05 & EPA8260 \\
\hline$\mu \mathrm{g} / \mathrm{L}$ & 0.05 & EPA8260 \\
\hline$\mu \mathrm{g} / \mathrm{L}$ & 0.05 & EPA8260 \\
\hline$\mu g / L$ & 0.1 & EPA8260 \\
\hline$\mu \mathrm{g} / \mathrm{L}$ & 0.5 & EPA8260 \\
\hline$\mu g / L$ & 0.05 & EPA8260 \\
\hline$\mu g / L$ & 0.05 & EPA8260 \\
\hline$\mu g / L$ & 0.05 & EPA8260 \\
\hline$\mu \mathrm{g} / \mathrm{L}$ & 0.05 & EPA8260 \\
\hline$\mu \mathrm{g} / \mathrm{L}$ & 1 & EPA8260 \\
\hline$\mu \mathrm{g} / \mathrm{L}$ & 1 & EPA8260 \\
\hline$\mu \mathrm{g} / \mathrm{L}$ & 1 & EPA8260 \\
\hline$\mu \mathrm{g} / \mathrm{L}$ & 0.05 & EPA8260 \\
\hline$\mu \mathrm{g} / \mathrm{L}$ & 0.05 & EPA8260 \\
\hline$\mu \mathrm{g} / \mathrm{L}$ & 0.05 & EPA8260 \\
\hline$\mu \mathrm{g} / \mathrm{L}$ & 0.5 & EPA8260 \\
\hline$\mu \mathrm{g} / \mathrm{L}$ & 0.05 & EPA8260 \\
\hline$\mu \mathrm{g} / \mathrm{L}$ & 0.05 & EPA8260 \\
\hline$\mu \mathrm{g} / \mathrm{L}$ & 0.05 & EPA8260 \\
\hline$\mu \mathrm{g} / \mathrm{L}$ & 1 & EPA8260 \\
\hline$\mu \mathrm{g} / \mathrm{L}$ & 0.15 & EPA8260 \\
\hline
\end{tabular}

$\begin{array}{lll}\mu \mathrm{g} / \mathrm{L} & 0.025 & \text { EPA8080 } \\ \mu \mathrm{g} / \mathrm{L} & 0.025 & \text { EPA8080 } \\ \mu \mathrm{g} / \mathrm{L} & 0.05 & \text { EPA8080 } \\ \mu \mathrm{g} / \mathrm{L} & 0.05 & \text { EPA8080 } \\ \mu \mathrm{g} / \mathrm{L} & 0.05 & \text { EPA8080 } \\ \mu \mathrm{g} / \mathrm{L} & 0.05 & \text { EPA8080 }\end{array}$


SURVEY ID: PPSC FB03C (continued)

Constituent

RQ AQ B Result

\begin{tabular}{|c|c|c|c|c|c|}
\hline$p, p^{\prime}-D D D$ & $U$ & 0.1 & $\mu \mathrm{g} / \mathrm{L}$ & 0.1 & EPA8080 \\
\hline p,p'-DDE & $\mathrm{U}$ & 0.05 & $\mu \mathrm{g} / \mathrm{L}$ & 0.05 & EPA8080 \\
\hline $\mathrm{p}, \mathrm{p}^{\prime}-\mathrm{DDT}$ & $\mathrm{U}$ & 0.1 & $\mu \mathrm{g} / \mathrm{L}$ & 0.1 & EPA8080 \\
\hline Dieldrin & $\mathrm{U}$ & 0.05 & $\mu \mathrm{g} / \mathrm{L}$ & 0.05 & EPA8080 \\
\hline Endosulfan sulfate & $\mathrm{U}$ & 0.1 & $\mu \mathrm{g} / \mathrm{L}$ & 0.1 & EPA8080 \\
\hline Endosulfan I & $\mathrm{U}$ & 0.05 & $\mu g / L$ & 0.05 & EPA8080 \\
\hline Endosulfan II & $\mathrm{U}$ & 0.1 & $\mu \mathrm{g} / \mathrm{L}$ & 0.1 & EPA 8080 \\
\hline Endrin & $\mathrm{U}$ & 0.05 & $\mu g / L$ & 0.05 & EPA8080 \\
\hline Endrin ketone & $\mathrm{U}$ & 0.1 & $\mu \mathrm{g} / \mathrm{L}$ & 0.1 & EPA8080 \\
\hline Heptachlor & $\mathrm{U}$ & 0.05 & $\mu \mathrm{g} / \mathrm{L}$ & 0.05 & EPA8080 \\
\hline Heptachlor epoxide & $\mathrm{U}$ & 0.05 & $\mu \mathrm{g} / \mathrm{L}$ & 0.05 & EPA8080 \\
\hline Lindane & $\mathrm{U}$ & 0.025 & $\mu \mathrm{g} / \mathrm{L}$ & 0.025 & EPA8080 \\
\hline Methoxychlor & $\mathrm{U}$ & 0.5 & $\mu \mathrm{g} / \mathrm{L}$ & 0.5 & EPA8080 \\
\hline PCB 1016 & $\mathrm{U}$ & 0.125 & $\mu \mathrm{g} / \mathrm{L}$ & 0.125 & EPA8080 \\
\hline PCB 1221 & $\mathrm{U}$ & 0.125 & $\mu \mathrm{g} / \mathrm{L}$ & 0.125 & EPA8080 \\
\hline PCB 1232 & $\mathrm{U}$ & 0.125 & $\mu \mathrm{g} / \mathrm{L}$ & 0.125 & EPA 8080 \\
\hline РCB 1242 & $\mathrm{U}$ & 0.125 & $\mu \mathrm{g} / \mathrm{L}$ & 0.125 & EPA8080 \\
\hline PCB 1248 & $\mathrm{U}$ & 0.125 & $\mu \mathrm{g} / \mathrm{L}$ & 0.125 & EPA8080 \\
\hline PCB 1254 & $\mathrm{U}$ & 0.125 & $\mu \mathrm{g} / \mathrm{L}$ & 0.125 & EPA8080 \\
\hline PCB 1260 & $\mathrm{U}$ & 0.125 & $\mu \mathrm{g} / \mathrm{L}$ & 0.125 & EPA8080 \\
\hline Toxaphene & $\mathrm{U}$ & 1 & $\mu \mathrm{g} / \mathrm{L}$ & 1 & EPA8080 \\
\hline
\end{tabular}

Sample ID: 104699

Associated sample: 104698

Sample type: Field Blank

Constituent

RQ AQ B Result

Unit

D. Limit Method

Physical Parameters and Specified Analyses

Cyanide

$\mathbf{U}$

0.02

$\mathbf{m g} / \mathbf{L}$

0.02

EPA335.3

Metals (total recoverable)

Aluminum
Antimony
Arsenic
Barium
Beryllium
Cadmium
Calcium
Chromium
Cobalt
Copper
Iron
Lead
Magnesium
Manganese
Mercury
Nickel
Potassium
Selenium
Silver
Sodium

$\begin{array}{lll}\text { U } & V & 0.0558 \\ U & & 0.005 \\ U & & 0.005 \\ J & E V & 0.00114 \\ J & E & 0.0000669 \\ U & V & 0.000171 \\ & & 0.0632 \\ U & & 0.004 \\ U & & 0.004 \\ \text { J } & E & 0.00213 \\ U & & 0.018 \\ U & & 0.005 \\ J & E & 0.00464 \\ J & E & 0.000596 \\ U & & 0.0002 \\ U & & 0.01 \\ U & & 0.5 \\ U & & 0.005 \\ U & & 0.002 \\ J & E & 0.054\end{array}$

\begin{tabular}{|c|c|c|c|c|c|}
\hline$p, p^{\prime}-D D D$ & $U$ & 0.1 & $\mu g / L$ & 0.1 & EPA8080 \\
\hline p,p'-DDE & $\mathrm{U}$ & 0.05 & $\mu \mathrm{g} / \mathrm{L}$ & 0.05 & EPA8080 \\
\hline $\mathrm{p}, \mathrm{p}^{\prime}-\mathrm{DDT}$ & $\mathrm{U}$ & 0.1 & $\mu \mathrm{g} / \mathrm{L}$ & 0.1 & EPA8080 \\
\hline Dieldrin & $\mathrm{U}$ & 0.05 & $\mu \mathrm{g} / \mathrm{L}$ & 0.05 & EPA8080 \\
\hline Endosulfan sulfate & $\mathrm{U}$ & 0.1 & $\mu \mathrm{g} / \mathrm{L}$ & 0.1 & EPA8080 \\
\hline Endosulfan I & $\mathrm{U}$ & 0.05 & $\mu \mathrm{g} / \mathrm{L}$ & 0.05 & EPA8080 \\
\hline Endosulfan II & $\mathrm{U}$ & 0.1 & $\mu \mathrm{g} / \mathrm{L}$ & 0.1 & EPA8080 \\
\hline Endrin & $\mathrm{U}$ & 0.05 & $\mu \mathrm{g} / \mathrm{L}$ & 0.05 & EPA8080 \\
\hline Endrin ketone & $\mathrm{U}$ & 0.1 & $\mu \mathrm{g} / \mathrm{L}$ & 0.1 & EPA8080 \\
\hline Heptachlor & $\mathrm{U}$ & 0.05 & $\mu \mathrm{g} / \mathrm{L}$ & 0.05 & EPA8080 \\
\hline Heptachlor epoxide & U & 0.05 & $\mu \mathrm{g} / \mathrm{L}$ & 0.05 & EPA8080 \\
\hline Lindane & $\mathrm{U}$ & 0.025 & $\mu \mathrm{g} / \mathrm{L}$ & 0.025 & EPA8080 \\
\hline Methoxychlor & $\mathrm{U}$ & 0.5 & $\mu \mathrm{g} / \mathrm{L}$ & 0.5 & EPA8080 \\
\hline PCB 1016 & $\mathrm{U}$ & 0.125 & $\mu \mathrm{g} / \mathrm{L}$ & 0.125 & EPA8080 \\
\hline PCB 1221 & $\mathrm{U}$ & 0.125 & $\mu \mathrm{g} / \mathrm{L}$ & 0.125 & EPA8080 \\
\hline PCB 1232 & $\mathrm{U}$ & 0.125 & $\mu \mathrm{g} / \mathrm{L}$ & 0.125 & EPA8080 \\
\hline РCB 1242 & $\mathrm{U}$ & 0.125 & $\mu \mathrm{g} / \mathrm{L}$ & 0.125 & EPA8080 \\
\hline PCB 1248 & $\mathrm{U}$ & 0.125 & $\mu \mathrm{g} / \mathrm{L}$ & 0.125 & EPA8080 \\
\hline PCB 1254 & $\mathrm{U}$ & 0.125 & $\mu \mathrm{g} / \mathrm{L}$ & 0.125 & EPA8080 \\
\hline PCB 1260 & $\mathrm{U}$ & 0.125 & $\mu \mathrm{g} / \mathrm{L}$ & 0.125 & EPA8080 \\
\hline Toxaphene & $\mathrm{U}$ & 1 & $\mu \mathrm{g} / \mathrm{L}$ & 1 & EPA8080 \\
\hline
\end{tabular}

$\begin{array}{lll}\mathrm{mg} / \mathrm{L} & 0.02 & \text { EPA6010A } \\ \mathrm{mg} / \mathrm{L} & 0.005 & \text { EPA6010A } \\ \mathrm{mg} / \mathrm{L} & 0.005 & \text { EPA6010A } \\ \mathrm{mg} / \mathrm{L} & 0.003 & \text { EPA6010A } \\ \mathrm{mg} / \mathrm{L} & 0.003 & \text { EPA6010A } \\ \mathrm{mg} / \mathrm{L} & 0.002 & \text { EPA6010A } \\ \mathrm{mg} / \mathrm{L} & 0.02 & \text { EPA6010A } \\ \mathrm{mg} / \mathrm{L} & 0.004 & \text { EPA6010A } \\ \mathrm{mg} / \mathrm{L} & 0.004 & \text { EPA6010A } \\ \mathrm{mg} / \mathrm{L} & 0.004 & \text { EPA6010A } \\ \mathrm{mg} / \mathrm{L} & 0.018 & \text { EPA6010A } \\ \mathrm{mg} / \mathrm{L} & 0.005 & \text { EPA6010A } \\ \mathrm{mg} / \mathrm{L} & 0.02 & \text { EPA6010A } \\ \mathrm{mg} / \mathrm{L} & 0.002 & \text { EPA6010A } \\ \mathrm{mg} / \mathrm{L} & 0.0002 & \text { EPA7470 } \\ \mathrm{mg} / \mathrm{L} & 0.01 & \text { EPA6010A } \\ \mathrm{mg} / \mathrm{L} & 0.5 & \text { EPA6010A } \\ \mathrm{mg} / \mathrm{L} & 0.005 & \text { EPA6010A } \\ \mathrm{mg} / \mathrm{L} & 0.002 & \text { EPA6010A } \\ \mathrm{mg} / \mathrm{L} & 0.1 & \text { EPA6010A }\end{array}$


SURVEY ID: PPSC FB05C (continued)

Constituent

RQ AQ B Result

Sample ID: 104699

Metals (total recoverable)

Vanadium
Thallium

Zinc

$\begin{array}{lll}U & & 0.005 \\ U & & 0.01 \\ U & X & 0.005\end{array}$

B/N/A Extractables (including PAH and phenols)

\begin{tabular}{|c|c|c|c|}
\hline Acenaphthene & U & & 1 \\
\hline Acenaphthylene & $\mathrm{U}$ & & 1 \\
\hline Anthracene & $\mathrm{U}$ & & 1 \\
\hline Benzo[a]anthracene & $\mathrm{U}$ & & 0.1 \\
\hline Benzo[b]fluoranthene & $\mathrm{U}$ & & 0.1 \\
\hline Benzo[k]fluoranthene & $\mathrm{U}$ & & 0.1 \\
\hline Benzoic acid & $\mathrm{U}$ & & 1 \\
\hline Benzo $[g, h, i]$ perylene & $\mathrm{U}$ & & 1 \\
\hline Benzo[a]pyrene & U & & 0.1 \\
\hline Benzyl alcohol & U & & 1 \\
\hline Bis(2-chloroethoxy) methane & $\mathrm{U}$ & & 1 \\
\hline Bis(2-chloroethyl) ether & U & & 1 \\
\hline Bis(2-chloroisopropyl) ether & $\mathrm{U}$ & & 1 \\
\hline Bis(2-ethylhexyl) phthalate & $\mathrm{U}$ & $\mathrm{V}$ & 0.53 \\
\hline 4-Bromophenyl phenyl ether & $U$ & & 1 \\
\hline Butylbenzyl phthalate & $\mathrm{U}$ & & 1 \\
\hline 4-Chloroaniline & $U$ & & 1 \\
\hline 4-Chloro-m-cresol & U & & 1 \\
\hline 2-Chloronaphthalene & $\mathrm{U}$ & & 1 \\
\hline 2-Chlorophenol & $\mathrm{U}$ & & 1 \\
\hline 4-Chlorophenyl phenyl ether & $\mathrm{U}$ & & 1 \\
\hline Chrysene & $\mathrm{U}$ & & 0.1 \\
\hline m/p-Cresol & $U$ & & 10 \\
\hline o-Cresol (2-Methylphenol) & U & & 1 \\
\hline Dibenz $[a, h]$ anthracene & $\mathrm{U}$ & & 0.1 \\
\hline Dibenzofuran & $\mathbf{U}$ & & 1 \\
\hline Di-n-butyl phthalate & U & V & 0.71 \\
\hline 1,2-Dichlorobenzene & $\mathrm{U}$ & & 1 \\
\hline 1,3-Dichlorobenzene & $\mathrm{U}$ & & 1 \\
\hline 1,4-Dichlorobenzene & $\mathrm{U}$ & & 1 \\
\hline 3,3'-Dichlorobenzidine & $\mathrm{U}$ & & 1 \\
\hline 2,4-Dichlorophenol & $\mathrm{U}$ & & 1 \\
\hline Diethyl phthalate & $\mathrm{U}$ & & 1 \\
\hline 2,4-Dimethyl phenol & $\mathrm{U}$ & & 1 \\
\hline Dimethyl phthalate & $\mathrm{U}$ & & 1 \\
\hline 2,4-Dinitrophenol & $\mathrm{U}$ & & 10 \\
\hline 2,4-Dinitrotoluene & $\mathrm{U}$ & & 0.1 \\
\hline 2,6-Dinitrotoluene & $\mathrm{U}$ & & 0.1 \\
\hline Di-n-octyl phthalate & $\mathbf{U}$ & & 1 \\
\hline Fluoranthene & $U$ & & 1 \\
\hline Fluorene & $\mathrm{U}$ & & 1 \\
\hline Hexachlorobenzene & $\mathrm{U}$ & & 0.1 \\
\hline Hexachlorobutadiene & $\mathrm{U}$ & & 1 \\
\hline Hexachlorocyclopentadiene & $\mathrm{U}$ & & 1 \\
\hline Hexachloroethane & $\mathrm{U}$ & & 0.1 \\
\hline Indeno $[1,2,3-c, d]$ pyrene & $\mathrm{U}$ & & 0.1 \\
\hline Isophorone & $U$ & & 1 \\
\hline 2-Methyl-4,6-dinitrophenol & $\mathrm{U}$ & & 10 \\
\hline 2-Methylnaphthalene & $U$ & & 1 \\
\hline
\end{tabular}

Unit D. Limit Method

$\begin{array}{lll}\mathrm{mg} / \mathrm{L} & 0.005 & \text { EPA6010A } \\ \mathrm{mg} / \mathrm{L} & 0.01 & \text { EPA6010A } \\ \mathrm{mg} / \mathrm{L} & 0.005 & \text { EPA6010A }\end{array}$

EPA8270

EPA8270

EPA8270

EPA8270

EPA8270

EPA8270

EPA8270

EPA8270

EPA8270

EPA8270

EPA8270

EPA8270

EPA8270

EPA8270

EPA8270

EPA8270

EPA8270

EPA8270

EPA8270

EPA8270

EPA8270

EPA8270

EPA8270

EPA8270

EPA8270

EPA8270

EPA8270

EPA8270

EPA8270

EPA8270

EPA8270

EPA8270

EPA8270

EPA8270

EPA8270

EPA8270

EPA8270

EPA8270

EPA8270

EPA8270

EPA8270

EPA8270

EPA8270

EPA8270

EPA8270

EPA8270

EPA8270

EPA8270

EPA8270 


\section{B/N/A Extractables (including PAH and phenols)}

Naphthalene
m-Nitroaniline
o-Nitroaniline
p-Nitroaniline
Nitrobenzene
2-Nitrophenol
4-Nitrophenol
N-Nitrosodiphenylamine
N-Nitrosodi-n-propylamine
Pentachlorophenol
Phenanthrene
Phenol
Pyrene
1,2,4-Trichlorobenzene
2,4,5-Trichlorophenol
2,4,6-Trichlorophenol

Volatile Organic Compounds

Acetone
Benzene
Bromodichloromethane
Bromoform
Bromomethane (Methyl bromide)
Carbon disulfide
Carbon tetrachloride
Chlorobenzene
Chloroethane
Chloroethene (Vinyl chloride)
Chloroform
Chloromethane (Methyl chloride)
Dibromochloromethane
1,1-Dichloroethane
1,2-Dichloroethane
1,1-Dichloroethylene
1,2-Dichloroethylene
Dichloromethane (Methylene chloride)
1,2-Dichloropropane
cis-1,3-Dichloropropene
trans-1,3-Dichloropropene
Ethylbenzene
2-Hexanone
Methyl ethyl ketone
Methyl isobutyl ketone
Styrene
1,1,2,2-Tetrachloroethane
Tetrachloroethylene
Toluene
1,1,1-Trichloroethane
1,1,2-Trichloroethane
Trichloroethylene
Vinyl acetate
Xylenes

$\begin{array}{ll}\mathrm{U} & 1 \\ \mathrm{U} & 1 \\ \mathrm{U} & 1 \\ \mathrm{U} & 1 \\ \mathrm{U} & 1 \\ \mathrm{U} & 1 \\ \mathrm{U} & 1 \\ \mathrm{U} & 1 \\ \mathrm{U} & \mathbf{1} \\ \mathrm{U} & 1 \\ \mathrm{U} & 1 \\ \mathrm{U} & 1 \\ \mathrm{U} & 1 \\ \mathrm{U} & \mathbf{0 . 1} \\ \mathrm{U} & 1 \\ \mathrm{U} & 1\end{array}$

$\begin{array}{lll}\mu \mathrm{g} / \mathrm{L} & 1 & \text { EPA8270 } \\ \mu \mathrm{g} / \mathrm{L} & 1 & \text { EPA8270 } \\ \mu \mathrm{g} / \mathrm{L} & 1 & \text { EPA8270 } \\ \mu \mathrm{g} / \mathrm{L} & 1 & \text { EPA8270 } \\ \mu \mathrm{g} / \mathrm{L} & 1 & \text { EPA8270 } \\ \mu \mathrm{g} / \mathrm{L} & 1 & \text { EPA8270 } \\ \mu \mathrm{g} / \mathrm{L} & 1 & \text { EPA8270 } \\ \mu \mathrm{g} / \mathrm{L} & 1 & \text { EPA8270 } \\ \mu \mathrm{g} / \mathrm{L} & 1 & \text { EPA8270 } \\ \mu \mathrm{g} / \mathrm{L} & 1 & \text { EPA8270 } \\ \mu \mathrm{g} / \mathrm{L} & 1 & \text { EPA8270 } \\ \mu \mathrm{g} / \mathrm{L} & 1 & \text { EPA8270 } \\ \mu \mathrm{g} / \mathrm{L} & 1 & \text { EPA8270 } \\ \mu \mathrm{g} / \mathrm{L} & 0.1 & \text { EPA8270 } \\ \mu \mathrm{g} / \mathrm{L} & 1 & \text { EPA8270 } \\ \mu \mathrm{g} / \mathrm{L} & 1 & \text { EPA8270 }\end{array}$

$\begin{array}{lll}U & 8 & 6.71 \\ U & & 0.5 \\ U & & 0.05 \\ U & & 0.05 \\ U & & 0.1 \\ U & & 1 \\ U & & 0.05 \\ U & & 0.05 \\ U & & 0.1 \\ U & & 0.1 \\ U & & 0.05 \\ U & & 0.1 \\ U & & 0.05 \\ U & & 0.05 \\ U & & 0.05 \\ U & & 0.05 \\ U & & 0.1 \\ U & V & 0.48 \\ U & & 0.05 \\ U & & 0.05 \\ U & & 0.05 \\ U & & 0.05 \\ U & & 1 \\ U & V & 1.31 \\ U & & 1 \\ U & & 0.05 \\ U & & 0.05 \\ U & & 0.05 \\ U & & 0.5 \\ U & & 0.05 \\ U & & 0.05 \\ U & & 0.05 \\ U & & 1 \\ U & & 0.15\end{array}$

$\begin{array}{lll}\mu \mathrm{g} / \mathrm{L} & 5 & \text { EPA8260 } \\ \mu \mathrm{g} / \mathrm{L} & 0.5 & \text { EPA8260 } \\ \mu \mathrm{g} / \mathrm{L} & 0.05 & \text { EPA8260 } \\ \mu \mathrm{g} / \mathrm{L} & 0.05 & \text { EPA8260 } \\ \mu \mathrm{g} / \mathrm{L} & 0.1 & \text { EPA8260 } \\ \mu \mathrm{g} / \mathrm{L} & 1 & \text { EPA8260 } \\ \mu \mathrm{g} / \mathrm{L} & 0.05 & \text { EPA8260 } \\ \mu \mathrm{g} / \mathrm{L} & 0.05 & \text { EPA8260 } \\ \mu \mathrm{g} / \mathrm{L} & 0.1 & \text { EPA8260 } \\ \mu \mathrm{g} / \mathrm{L} & 0.1 & \text { EPA8260 } \\ \mu \mathrm{g} / \mathrm{L} & 0.05 & \text { EPA8260 } \\ \mu \mathrm{g} / \mathrm{L} & 0.1 & \text { EPA8260 } \\ \mu \mathrm{g} / \mathrm{L} & 0.05 & \text { EPA8260 } \\ \mu \mathrm{g} / \mathrm{L} & 0.05 & \text { EPA8260 } \\ \mu \mathrm{g} / \mathrm{L} & 0.05 & \text { EPA8260 } \\ \mu \mathrm{g} / \mathrm{L} & 0.05 & \text { EPA8260 } \\ \mu \mathrm{g} / \mathrm{L} & 0.1 & \text { EPA8260 } \\ \mu \mathrm{g} / \mathrm{L} & 0.5 & \text { EPA8260 } \\ \mu \mathrm{g} / \mathrm{L} & 0.05 & \text { EPA8260 } \\ \mu \mathrm{g} / \mathrm{L} & 0.05 & \text { EPA8260 } \\ \mu \mathrm{g} / \mathrm{L} & 0.05 & \text { EPA8260 } \\ \mu \mathrm{g} / \mathrm{L} & 0.05 & \text { EPA8260 } \\ \mu \mathrm{g} / \mathrm{L} & 1 & \text { EPA8260 } \\ \mu \mathrm{g} / \mathrm{L} & 1 & \text { EPA8260 } \\ \mu \mathrm{g} / \mathrm{L} & 1 & \text { EPA8260 } \\ \mu \mathrm{g} / \mathrm{L} & 0.05 & \text { EPA8260 } \\ \mu \mathrm{g} / \mathrm{L} & 0.05 & \text { EPA8260 } \\ \mu \mathrm{g} / \mathrm{L} & 0.05 & \text { EPA8260 } \\ \mu \mathrm{g} / \mathrm{L} & 0.5 & \text { EPA8260 } \\ \mu \mathrm{g} / \mathrm{L} & 0.05 & \text { EPA8260 } \\ \mu \mathrm{g} / \mathrm{L} & 0.05 & \text { EPA8260 } \\ \mu \mathrm{g} / \mathrm{L} & 0.05 & \text { EPA8260 } \\ \mu \mathrm{g} / \mathrm{L} & 1 & \text { EPA8260 } \\ \mu \mathrm{g} / \mathrm{L} & 0.15 & \text { EPA8260 }\end{array}$


SURVEY ID: PPSC FB05C (continued)

Constituent

RQ AQ B Result

Pesticides/PCBs and Dioxins/Furans

\begin{tabular}{|c|c|c|}
\hline $\mathrm{JJ}$ & 0 & 0.0258 \\
\hline $\mathrm{JJ}$ & 0 & 0.0258 \\
\hline UJ & 0 & 0.0515 \\
\hline $\mathrm{JJ}$ & 0 & 0.0515 \\
\hline UJ & 0 & 0.0515 \\
\hline UJ & 0 & 0.0515 \\
\hline UJ & 0 & 0.103 \\
\hline UJ & 0 & 0.0515 \\
\hline UJ & OCI & 0.103 \\
\hline UJ & OCI & 0.0515 \\
\hline $\mathrm{UJ}$ & 0 & 0.103 \\
\hline UJJ & 0 & 0.0515 \\
\hline UJ & 0 & 0.103 \\
\hline UJ & 0 & 0.0515 \\
\hline UJ & 0 & 0.103 \\
\hline Ue & 0 & 0.0515 \\
\hline UJ & 0 & 0.0515 \\
\hline UJ & 0 & 0.0258 \\
\hline UJ & 0 & 0.515 \\
\hline UJ & 0 & 0.129 \\
\hline UJ & 0 & 0.129 \\
\hline UJ & 0 & 0.129 \\
\hline UJ & 0 & 0.129 \\
\hline UJ & 0 & 0.129 \\
\hline UJ & 0 & 0.129 \\
\hline UJ & 0 & 0.129 \\
\hline UJ & 0 & L $\quad 1.03$ \\
\hline
\end{tabular}

Unit

D. Limit Method

Aldrin
alpha-Benzene hexachloride
beta-Benzene hexachloride
delta-Benzene hexachloride
alpha-Chlordane
gamma-Chlordane
p,p'-DDD
p,p'-DDE
p,p'-DDT
Dieldrin
Endosulfan sulfate
Endosulfan I
Endosulfan II
Endrin
Endrin ketone
Heptachlor
Heptachlor epoxide
Lindane
Methoxychlor
PCB 1016
PCB 1221
PCB 1232
PCB 1242
PCB 1248
PCB 1254
PCB 1260
Toxaphene

SURVEY ID: PPSC TB01B

Sample type: Trip Blank

Constituent

Volatile Organic Compounds
Sample ID: 104624

Associated sample: 104623

RQ AQ B Result

Unit

D. Limit Method

\begin{tabular}{|c|c|c|c|c|c|c|}
\hline Acetone & $J$ & E8 & 4.71 & $\mu \mathrm{g} / \mathrm{L}$ & 5 & EPA8260 \\
\hline Benzene & UJ & I & 0.5 & $\mu \mathrm{g} / \mathrm{L}$ & 0.5 & EPA8260 \\
\hline Bromodichloromethane & $\mathrm{U}$ & & 0.05 & $\mu \mathrm{g} / \mathrm{L}$ & 0.05 & EPA8260 \\
\hline Bromoform & $\mathrm{U}$ & & 0.05 & $\mu \mathrm{g} / \mathrm{L}$ & 0.05 & EPA8260 \\
\hline Bromomethane (Methyl bromide) & $\mathrm{U}$ & & 0.1 & $\mu \mathrm{g} / \mathrm{L}$ & 0.1 & EPA8260 \\
\hline Carbon disulfide & $U$ & & 1 & $\mu \mathrm{g} / \mathrm{L}$ & 1 & EPA8260 \\
\hline Carbon tetrachloride & $\mathrm{U}$ & & 0.05 & $\mu \mathrm{g} / \mathrm{L}$ & 0.05 & EPA8260 \\
\hline Chlorobenzene & $\mathrm{U}$ & & 0.05 & $\mu \mathrm{g} / \mathrm{L}$ & 0.05 & EPA8260 \\
\hline Chloroethane & $\mathrm{U}$ & & 0.1 & $\mu \mathrm{g} / \mathrm{L}$ & 0.1 & EPA8260 \\
\hline Chloroethene (Vinyl chloride) & U & & 0.1 & $\mu \mathrm{g} / \mathrm{L}$ & 0.1 & EPA8260 \\
\hline Chloroform & $\mathrm{U}$ & & 0.05 & $\mu \mathrm{g} / \mathrm{L}$ & 0.05 & EPA8260 \\
\hline Chloromethane (Methyl chloride) & $\mathrm{U}$ & & 0.1 & $\mu \mathrm{g} / \mathrm{L}$ & 0.1 & EPA8260 \\
\hline Dibromochloromethane & $\mathrm{U}$ & & 0.05 & $\mu g / L$ & 0.05 & EPA8260 \\
\hline 1,1-Dichloroethane & $\mathrm{U}$ & & 0.05 & $\mu \mathrm{g} / \mathrm{L}$ & 0.05 & EPA8260 \\
\hline 1,2-Dichloroethane & $\mathrm{U}$ & & 0.05 & $\mu \mathrm{g} / \mathrm{L}$ & 0.05 & EPA8260 \\
\hline 1,1-Dichloroethylene & $U^{*}$ & & 0.05 & $\mu \mathrm{g} / \mathrm{L}$ & 0.05 & EPA8260 \\
\hline 1,2-Dichloroethylene & $\mathrm{U}$ & & 0.1 & $\mu \mathrm{g} / \mathrm{L}$ & 0.1 & EPA8260 \\
\hline Dichloromethane (Methylene chloride) & & 8 & 0.83 & $\mu \mathrm{g} / \mathrm{L}$ & 0.5 & EPA8260 \\
\hline
\end{tabular}


SURVEY ID: PPSC TB01B (continued)

Constituent

Volatile Organic Compounds

1,2-Dichloropropane
cis-1,3-Dichloropropene
trans-1,3-Dichloropropene
Ethylbenzene
2-Hexanone
Methyl ethyl ketone
Methyl isobutyl ketone
Styrene
1,1,2,2-Tetrachloroethane
Tetrachloroethylene
Toluene
1,1,1-Trichloroethane
1,1,2-Trichloroethane
Trichloroethylene
Vinyl acetate
Xylenes

SURVEY ID: PPSC TB02B

Sample type: Trip Blank

Constituent

Volatile Organic Compounds

Acetone

Benzene

Bromodichloromethane

Bromoform

Bromomethane (Methyl bromide)

Carbon disulfide

Carbon tetrachloride

Chlorobenzene

Chloroethane

Chloroethene (Vinyl chloride)

Chloroform

Chloromethane (Methyl chloride)

Dibromochloromethane

1,1-Dichloroethane

1,2-Dichloroethane

1,1-Dichloroethylene

1,2-Dichloroethylene

Dichloromethane (Methylene chloride)

1,2-Dichloropropane

cis-1,3-Dichloropropene

trans-1,3-Dichloropropene

Ethylbenzene

2-Hexanone

Methyl ethyl ketone

Methyl isobutyl ketone

Styrene

1,1,2,2-Tetrachloroethane

Tetrachloroethylene

Toluene
Sample ID: 104624

RQ AQ B Result Unit D. Limit Method

\begin{tabular}{|c|c|c|c|c|c|}
\hline$U$ & & 0.05 & $\mu \mathrm{g} / \mathrm{L}$ & 0.05 & EPA8260 \\
\hline $\mathrm{U}$ & & 0.05 & $\mu \mathrm{g} / \mathrm{L}$ & 0.05 & EPA8260 \\
\hline $\mathrm{U}$ & & 0.05 & $\mu \mathrm{g} / \mathrm{L}$ & 0.05 & EPA8260 \\
\hline U & & 0.05 & $\mu \mathrm{g} / \mathrm{L}$ & 0.05 & EPA8260 \\
\hline $\mathrm{J}$ & E8 & 0.32 & $\mu \mathrm{g} / \mathrm{L}$ & 1 & EPA8260 \\
\hline U & V & 1.42 & $\mu \mathrm{g} / \mathrm{L}$ & 1 & EPA8260 \\
\hline $\mathrm{U}$ & & 1 & $\mu \mathrm{g} / \mathrm{L}$ & 1 & EPA8260 \\
\hline $\mathrm{U}$ & & 0.05 & $\mu \mathrm{g} / \mathrm{L}$ & 0.05 & EPA8260 \\
\hline$U$ & & 0.05 & $\mu \mathrm{g} / \mathrm{L}$ & 0.05 & EPA8260 \\
\hline$U$ & & 0.05 & $\mu \mathrm{g} / \mathrm{L}$ & 0.05 & EPA8260 \\
\hline $\mathrm{U}$ & V & 0.18 & $\mu \mathrm{g} / \mathrm{L}$ & 0.5 & EPA8260 \\
\hline U & & 0.05 & $\mu \mathrm{g} / \mathrm{L}$ & 0.05 & EPA8260 \\
\hline $\mathrm{U}$ & & 0.05 & $\mu g / L$ & 0.05 & EPA8260 \\
\hline & 8 & 0.11 & $\mu \mathrm{g} / \mathrm{L}$ & 0.05 & EPA8260 \\
\hline $\mathrm{U}$ & & 1 & $\mu \mathrm{g} / \mathrm{L}$ & 1 & EPA8260 \\
\hline U & & 0.15 & $\mu g / L$ & 0.15 & \\
\hline
\end{tabular}

Sample ID: 104634

Associated sample: 104633

RQ AQ B Result Unit D. Limit Method

\begin{tabular}{|c|c|c|c|c|}
\hline 8 & 5.79 & $\mu \mathrm{g} / \mathrm{L}$ & 5 & EPA8260 \\
\hline U & 0.5 & $\mu \mathrm{g} / \mathrm{L}$ & 0.5 & EPA8260 \\
\hline U & 0.05 & $\mu \mathrm{g} / \mathrm{L}$ & 0.05 & EPA8260 \\
\hline$U$ & 0.05 & $\mu \mathrm{g} / \mathrm{L}$ & 0.05 & EPA8260 \\
\hline U & 0.1 & $\mu \mathrm{g} / \mathrm{L}$ & 0.1 & EPA8260 \\
\hline $\mathrm{U}$ & 1 & $\mu \mathrm{g} / \mathrm{L}$ & 1 & EPA8260 \\
\hline $\mathrm{U}$ & 0.05 & $\mu \mathrm{g} / \mathrm{L}$ & 0.05 & EPA8260 \\
\hline $\mathrm{U}$ & 0.05 & $\mu \mathrm{g} / \mathrm{L}$ & 0.05 & EPA8260 \\
\hline $\mathrm{U}$ & 0.1 & $\mu \mathrm{g} / \mathrm{L}$ & 0.1 & EPA8260 \\
\hline $\mathrm{U}$ & 0.1 & $\mu \mathrm{g} / \mathrm{L}$ & 0.1 & EPA8260 \\
\hline U & 0.05 & $\mu \mathrm{g} / \mathrm{L}$ & 0.05 & EPA8260 \\
\hline U & 0.1 & $\mu \mathrm{g} / \mathrm{L}$ & 0.1 & EPA8260 \\
\hline $\mathrm{U}$ & 0.05 & $\mu \mathrm{g} / \mathrm{L}$ & 0.05 & EPA8260 \\
\hline $\mathrm{U}$ & 0.05 & $\mu \mathrm{g} / \mathrm{L}$ & 0.05 & EPA8260 \\
\hline$U$ & 0.05 & $\mu \mathrm{g} / \mathrm{L}$ & 0.05 & EPA8260 \\
\hline$U$ & 0.05 & $\mu \mathrm{g} / \mathrm{L}$ & 0.05 & EPA8260 \\
\hline $\mathrm{U}$ & 0.1 & $\mu \mathrm{g} / \mathrm{L}$ & 0.1 & EPA8260 \\
\hline V & 1.05 & $\mu \mathrm{g} / \mathrm{L}$ & 0.5 & EPA8260 \\
\hline$U$ & 0.05 & $\mu \mathrm{g} / \mathrm{L}$ & 0.05 & EPA8260 \\
\hline $\mathrm{U}$ & 0.05 & $\mu \mathrm{g} / \mathrm{L}$ & 0.05 & EPA8260 \\
\hline U & 0.05 & $\mu \mathrm{g} / \mathrm{L}$ & 0.05 & EPA8260 \\
\hline U & 0.05 & $\mu g / L$ & 0.05 & EPA8260 \\
\hline V & 1.65 & $\mu \mathrm{g} / \mathrm{L}$ & 1 & EPA8260 \\
\hline V & 1.72 & $\mu \mathrm{g} / \mathrm{L}$ & 1 & EPA8260 \\
\hline$U$ & 1 & $\mu \mathrm{g} / \mathrm{L}$ & 1 & EPA8260 \\
\hline $\mathrm{U}$ & 0.05 & $\mu \mathrm{g} / \mathrm{L}$ & 0.05 & EPA8260 \\
\hline $\mathrm{U}$ & 0.05 & $\mu g / L$ & 0.05 & EPA8260 \\
\hline$U$ & 0.05 & $\mu g / L$ & 0.05 & EPA8260 \\
\hline $\mathrm{V}$ & 0.22 & $\mu g / L$ & 0.5 & EPA8260 \\
\hline
\end{tabular}


SURVEY ID: PPSC TB02B (continued)

Constituent

Volatile Organic Compounds

1,1,1-Trichloroethane

1,1,2-Trichloroethane

Trichloroethylene

Vinyl acetate

Xylenes

\section{SURVEY ID: PPSC TB03B}

Sample type: Trip Blank

\section{Constituent}

Volatile Organic Compounds

Acetone
Benzene
Bromodichloromethane
Bromoform
Bromomethane (Methyl bromide)
Carbon disulfide
Carbon tetrachloride
Chlorobenzene
Chloroethane
Chloroethene (Vinyl chloride)
Chloroform
Chloromethane (Methyl chloride)
Dibromochloromethane
1,1-Dichloroethane
1,2-Dichloroethane
1,1-Dichloroethylene
1,2-Dichloroethylene
Dichloromethane (Methylene chloride)
1,2-Dichloropropane
cis-1,3-Dichloropropene
trans-1,3-Dichloropropene
Ethylbenzene
2-Hexanone
Methyl ethyl ketone
Methyl isobutyl ketone
Styrene
1,1,2,2-Tetrachloroethane
Tetrachloroethylene
Toluene
1,1,1-Trichloroethane
1,1,2-Trichloroethane
Trichloroethylene
Vinyl acetate
Xylenes
(n)

Sample ID: 104634

RQ AQ B Result Unit D. Limit Method

$\begin{array}{llllll}\mathrm{U} & & 0.05 & \mu \mathrm{g} / \mathrm{L} & 0.05 & \text { EPA8260 } \\ \mathrm{U} & & 0.05 & \mu \mathrm{g} / \mathrm{L} & 0.05 & \text { EPA8260 } \\ & 8 & 0.13 & \mu \mathrm{L} / \mathrm{L} & 0.05 & \text { EPA8260 } \\ \mathrm{U} & & 1 & \mu \mathrm{g} / \mathrm{L} & 1 & \text { EPA8260 } \\ \mathrm{U} & & 0.15 & \mu \mathrm{g} / \mathrm{L} & 0.15 & \text { EPA8260 }\end{array}$

Sample ID: 104641

Associated sample: 104635

Unit

D. Limit Method

\begin{tabular}{|c|c|c|c|c|}
\hline E8 & 3.25 & $\mu \mathrm{g} / \mathrm{L}$ & 5 & EPA8260 \\
\hline$U$ & 0.5 & $\mu \mathrm{g} / \mathrm{L}$ & 0.5 & EPA8260 \\
\hline $\mathrm{U}$ & 0.05 & $\mu g / L$ & 0.05 & EPA8260 \\
\hline $\mathrm{U}$ & 0.05 & $\mu \mathrm{g} / \mathrm{L}$ & 0.05 & EPA8260 \\
\hline $\mathrm{U}$ & 0.1 & $\mu \mathrm{g} / \mathrm{L}$ & 0.1 & EPA8260 \\
\hline $\mathrm{U}$ & 1 & $\mu \mathrm{g} / \mathrm{L}$ & 1 & EPA8260 \\
\hline U & 0.05 & $\mu \mathrm{g} / \mathrm{L}$ & 0.05 & EPA8260 \\
\hline $\mathrm{U}$ & 0.05 & $\mu \mathrm{g} / \mathrm{L}$ & 0.05 & EPA8260 \\
\hline $\mathrm{U}$ & 0.1 & $\mu \mathrm{g} / \mathrm{L}$ & 0.1 & EPA8260 \\
\hline U & 0.1 & $\mu \mathrm{g} / \mathrm{L}$ & 0.1 & EPA8260 \\
\hline $\mathrm{U}$ & 0.05 & $\mu \mathrm{g} / \mathrm{L}$ & 0.05 & EPA8260 \\
\hline $\mathrm{U}$ & 0.1 & $\mu \mathrm{g} / \mathrm{L}$ & 0.1 & EPA8260 \\
\hline $\mathrm{U}$ & 0.05 & $\mu \mathrm{g} / \mathrm{L}$ & 0.05 & EPA8260 \\
\hline $\mathrm{U}$ & 0.05 & $\mu \mathrm{g} / \mathrm{L}$ & 0.05 & EPA8260 \\
\hline $\mathrm{U}$ & 0.05 & $\mu \mathrm{g} / \mathrm{L}$ & 0.05 & EPA8260 \\
\hline $\mathrm{U}$ & 0.05 & $\mu g / L$ & 0.05 & EPA8260 \\
\hline U & 0.1 & $\mu \mathrm{g} / \mathrm{L}$ & 0.1 & EPA8260 \\
\hline $\mathrm{U}$ & 1.16 & $\mu \mathrm{g} / \mathrm{L}$ & 0.5 & EPA8260 \\
\hline U & 0.05 & $\mu \mathrm{g} / \mathrm{L}$ & 0.05 & EPA8260 \\
\hline $\mathrm{U}$ & 0.05 & $\mu \mathrm{g} / \mathrm{L}$ & 0.05 & EPA8260 \\
\hline $\mathrm{U}$ & 0.05 & $\mu g / L$ & 0.05 & EPA8260 \\
\hline $\mathrm{U}$ & 0.05 & $\mu \mathrm{g} / \mathrm{L}$ & 0.05 & EPA8260 \\
\hline $\mathrm{U}$ & 1.03 & $\mu g / L$ & 1 & EPA8260 \\
\hline $\mathrm{U}$ & 1.33 & $\mu \mathrm{g} / \mathrm{L}$ & 1 & EPA8260 \\
\hline $\mathrm{U}$ & 1 & $\mu \mathrm{g} / \mathrm{L}$ & 1 & EPA8260 \\
\hline $\mathrm{U}$ & 0.05 & $\mu \mathrm{g} / \mathrm{L}$ & 0.05 & EPA8260 \\
\hline U & 0.05 & $\mu g / L$ & 0.05 & EPA8260 \\
\hline$U$ & 0.05 & $\mu \mathrm{g} / \mathrm{L}$ & 0.05 & EPA8260 \\
\hline $\mathrm{U}$ & 0.17 & $\mu \mathrm{g} / \mathrm{L}$ & 0.5 & EPA8260 \\
\hline U & 0.05 & $\mu \mathrm{g} / \mathrm{L}$ & 0.05 & EPA8260 \\
\hline $\mathrm{U}$ & 0.05 & $\mu \mathrm{g} / \mathrm{L}$ & 0.05 & EPA8260 \\
\hline 8 & 0.14 & $\mu \mathrm{g} / \mathrm{L}$ & 0.05 & EPA8260 \\
\hline $\mathrm{U}$ & 1 & $\mu \mathrm{g} / \mathrm{L}$ & 1 & EPA8260 \\
\hline $\mathrm{U}$ & 0.15 & $\mu \mathrm{g} / \mathrm{L}$ & 0.15 & EPA8260 \\
\hline
\end{tabular}


SURVEY ID: PPSC TB04B

Sample type: Trip Blank

Constituent

Volatile Organic Compounds

\begin{tabular}{|c|c|c|c|}
\hline Acetone & $\mathrm{J}$ & Q8 & 5.07 \\
\hline Benzene & UJ & QI & 0.5 \\
\hline Bromodichloromethane & UJ & $\mathbf{Q}$ & 0.05 \\
\hline Bromoform & UJ & Q & 0.05 \\
\hline Bromomethane (Methyl bromide) & UJ & $\mathbf{Q}$ & 0.1 \\
\hline Carbon disulfide & UJ & $\mathbf{Q}$ & 1 \\
\hline Carbon tetrachloride & UJ & $\mathbf{Q}$ & 0.05 \\
\hline Chlorobenzene & UJ & $\mathbf{Q}$ & 0.05 \\
\hline Chloroethane & UJ & $\mathbf{Q}$ & 0.1 \\
\hline Chloroethene (Vinyl chloride) & UJ & Q & 0.1 \\
\hline Chloroform & UJ & $\mathbf{Q}$ & 0.05 \\
\hline Chloromethane (Methyl chloride) & UJ & Q & 0.1 \\
\hline Dibromochloromethane & UJ & $\mathbf{Q}$ & 0.05 \\
\hline 1,1-Dichloroethane & UJ & $\mathbf{Q}$ & 0.05 \\
\hline 1,2-Dichloroethane & UJ & Q & 0.05 \\
\hline 1,1-Dichloroethylene & UJ & Q & 0.05 \\
\hline 1,2-Dichloroethylene & UJ & $\mathrm{Q}$ & 0.1 \\
\hline Dichloromethane (Methylene chloride) & $\mathbf{J}$ & Q8 & 0.87 \\
\hline 1,2-Dichloropropane & UJ & $\mathbf{Q}$ & 0.05 \\
\hline cis-1,3-Dichloropropene & UJ & $\mathbf{Q}$ & 0.05 \\
\hline trans-1,3-Dichloropropene & UJ & $\mathbf{Q}$ & 0.05 \\
\hline Ethylbenzene & UJ & $\mathbf{Q}$ & 0.05 \\
\hline 2-Hexanone & $\mathbf{J}$ & QE8 & 0.25 \\
\hline Methyl ethyl ketone & UJ & QV & 1.44 \\
\hline Methyl isobutyl ketone & UJ & $\mathrm{Q}$ & 1 \\
\hline Styrene & UJ & Q & 0.05 \\
\hline 1,1,2,2-Tetrachloroethane & UJ & Q & 0.05 \\
\hline Tetrachloroethylene & UJ & $\mathrm{Q}$ & 0.05 \\
\hline Toluene & UJ & QV & 0.19 \\
\hline 1,1,1-Trichloroethane & UJ & $\mathbf{Q}$ & 0.05 \\
\hline 1,1,2-Trichloroethane & UJ & $\mathbf{Q}$ & 0.05 \\
\hline Trichloroethylene & J & Q8 & 0.1 \\
\hline Vinyl acetate & UJ & $\mathbf{Q}$ & 1 \\
\hline Xylenes & UJ & $\mathbf{Q}$ & 0.15 \\
\hline
\end{tabular}

Sample ID: 104651

Associated sample: 104650

RQ AQ B Result

Unit

D. Limit Method

$\begin{array}{lll}\mu \mathrm{g} / \mathrm{L} & 5 & \text { EPA8260 } \\ \mu \mathrm{g} / \mathrm{L} & 0.5 & \text { EPA8260 } \\ \mu \mathrm{g} / \mathrm{L} & 0.05 & \text { EPA8260 } \\ \mu \mathrm{g} / \mathrm{L} & 0.05 & \text { EPA8260 } \\ \mu \mathrm{g} / \mathrm{L} & 0.1 & \text { EPA8260 } \\ \mu \mathrm{g} / \mathrm{L} & 1 & \text { EPA8260 } \\ \mu \mathrm{g} / \mathrm{L} & 0.05 & \text { EPA8260 } \\ \mu \mathrm{g} / \mathrm{L} & 0.05 & \text { EPA8260 } \\ \mu \mathrm{g} / \mathrm{L} & 0.1 & \text { EPA8260 } \\ \mu \mathrm{g} / \mathrm{L} & 0.1 & \text { EPA8260 } \\ \mu \mathrm{g} / \mathrm{L} & 0.05 & \text { EPA8260 } \\ \mu \mathrm{g} / \mathrm{L} & 0.1 & \text { EPA8260 } \\ \mu \mathrm{g} / \mathrm{L} & 0.05 & \text { EPA8260 } \\ \mu \mathrm{g} / \mathrm{L} & 0.05 & \text { EPA8260 } \\ \mu \mathrm{g} / \mathrm{L} & 0.05 & \text { EPA8260 } \\ \mu \mathrm{g} / \mathrm{L} & 0.05 & \text { EPA8260 } \\ \mu \mathrm{g} / \mathrm{L} & 0.1 & \text { EPA8260 } \\ \mu \mathrm{g} / \mathrm{L} & 0.5 & \text { EPA8260 } \\ \mu \mathrm{g} / \mathrm{L} & 0.05 & \text { EPA8260 } \\ \mu \mathrm{g} / \mathrm{L} & 0.05 & \text { EPA8260 } \\ \mu \mathrm{g} / \mathrm{L} & 0.05 & \text { EPA8260 } \\ \mu \mathrm{g} / \mathrm{L} & 0.05 & \text { EPA8260 } \\ \mu \mathrm{g} / \mathrm{L} & 1 & \text { EPA8260 } \\ \mu \mathrm{g} / \mathrm{L} & 1 & \text { EPA8260 } \\ \mu \mathrm{g} / \mathrm{L} & 1 & \text { EPA8260 } \\ \mu \mathrm{g} / \mathrm{L} & 0.05 & \text { EPA8260 } \\ \mu \mathrm{g} / \mathrm{L} & 0.05 & \text { EPA8260 } \\ \mu \mathrm{g} / \mathrm{L} & 0.05 & \text { EPA8260 } \\ \mu \mathrm{g} / \mathrm{L} & 0.5 & \text { EPA8260 } \\ \mu \mathrm{g} / \mathrm{L} & 0.05 & \text { EPA8260 } \\ \mu \mathrm{g} / \mathrm{L} & 0.05 & \text { EPA8260 } \\ \mu \mathrm{g} / \mathrm{L} & 0.05 & \text { EPA8260 } \\ \mu \mathrm{g} / \mathrm{L} & 1 & \text { EPA8260 } \\ \mu \mathrm{g} / \mathrm{L} & 0.15 & \text { EPA8260 } \\ & & \end{array}$

Sample ID: 104662

Associated sample: 104660

RQ AQ B Result Unit D. Limit Method

Constituent

Volatile Organic Compounds

Acetone

Benzene

Bromodichloromethane

Bromoform

Bromomethane (Methyl bromide)

Carbon disulfide

Carbon tetrachloride

$\begin{array}{lll}\mathrm{J} & \mathrm{E} 8 & 4.04 \\ \mathrm{U} & & 0.5 \\ \mathrm{U} & & 0.05 \\ \mathrm{U} & & 0.05 \\ \mathrm{U} & & 0.1 \\ \mathrm{U} & & 1 \\ \mathrm{U} & & 0.05\end{array}$

$\begin{array}{lll}\mu \mathrm{g} / \mathrm{L} & 5 & \text { EPA8260 } \\ \mu \mathrm{g} / \mathrm{L} & 0.5 & \text { EPA8260 } \\ \mu \mathrm{g} / \mathrm{L} & 0.05 & \text { EPA8260 } \\ \mu \mathrm{g} / \mathrm{L} & 0.05 & \text { EPA8260 } \\ \mu \mathrm{g} / \mathrm{L} & 0.1 & \text { EPA8260 } \\ \mu \mathrm{g} / \mathrm{L} & 1 & \text { EPA8260 } \\ \mu \mathrm{g} / \mathrm{L} & 0.05 & \text { EPA8260 }\end{array}$


SURVEY ID: PPSC TB05B (continued)

Constituent

RQ AQ B Result

Sample ID: 104662

Volatile Organic Compounds

\begin{tabular}{|c|c|c|}
\hline Chlorobenzene & $\mathrm{U}$ & 0.05 \\
\hline Chloroethane & $\mathrm{U}$ & 0.1 \\
\hline Chloroethene (Vinyl chloride) & $\mathrm{U}$ & 0.1 \\
\hline Chloroform & $\mathrm{U}$ & 0.05 \\
\hline Chloromethane (Methyl chloride) & $\mathrm{U}$ & 0.1 \\
\hline Dibromochloromethane & $\mathrm{U}$ & 0.05 \\
\hline 1,1-Dichloroethane & $\mathrm{U}$ & 0.05 \\
\hline 1,2-Dichloroethane & $\mathrm{U}$ & 0.05 \\
\hline 1,1-Dichloroethylene & $\mathrm{U}$ & 0.05 \\
\hline 1,2-Dichloroethylene & $\mathrm{U}$ & 0.1 \\
\hline Dichloromethane (Methylene chloride) & $\mathrm{U}$ & 1.13 \\
\hline 1,2-Dichloropropane & $\mathrm{U}$ & 0.05 \\
\hline cis-1,3-Dichloropropene & $\mathrm{U}$ & 0.05 \\
\hline trans-1,3-Dichloropropene & $\mathrm{U}$ & 0.05 \\
\hline Ethylbenzene & $\mathrm{U}$ & 0.05 \\
\hline 2-Hexanone & $\mathrm{U}$ & 0.95 \\
\hline Methyl ethyl ketone & $\mathrm{U}$ & 1.34 \\
\hline Methyl isobutyl ketone & U & 1 \\
\hline Styrene & $\mathrm{U}$ & 0.05 \\
\hline 1,1,2,2-Tetrachloroethane & $\mathrm{U}$ & 0.05 \\
\hline Tetrachloroethylene & $\mathrm{U}$ & 0.05 \\
\hline Toluene & $\mathrm{U}$ & 0.13 \\
\hline 1,1,1-Trichloroethane & $\mathrm{U}$ & 0.05 \\
\hline 1,1,2-Trichloroethane & $\mathrm{U}$ & 0.05 \\
\hline Trichloroethylene & 8 & 0.11 \\
\hline Vinyl acetate & $\mathrm{U}$ & 1 \\
\hline Xylenes & $\mathrm{U}$ & 0.15 \\
\hline
\end{tabular}

Sample ID: 104679

Associated sample: 104675

Sample type: Trip Blank

Constituent

RQ AQ B Result

Unit

D. Limit Method

Volatile Organic Compounds

Acetone
Benzene
Bromodichloromethane
Bromoform
Bromomethane (Methyl bromide)
Carbon disulfide
Carbon tetrachloride
Chlorobenzene
Chloroethane
Chloroethene (Vinyl chloride)
Chloroform
Chloromethane (Methyl chloride)
Dibromochloromethane
1,1-Dichloroethane
1,2-Dichloroethane
1,1-Dichloroethylene
1,2-Dichloroethylene
Dichloromethane (Methylene chloride)

$\begin{array}{lll} & 8 & 10.6 \\ U & & 0.5 \\ U & & 0.05 \\ U & & 0.05 \\ U & & 0.1 \\ U & & 1 \\ U & & 0.05 \\ U & & 0.05 \\ U & & 0.1 \\ U & & 0.1 \\ U & & 0.05 \\ U & & 0.1 \\ U & & 0.05 \\ U & & 0.05 \\ U & & 0.05 \\ U & & 0.05 \\ U & & 0.1 \\ & 8 & 1.28\end{array}$

$\begin{array}{lll}\mu \mathrm{g} / \mathrm{L} & 0.05 & \text { EPA8260 } \\ \mu \mathrm{g} / \mathrm{L} & 0.1 & \text { EPA8260 } \\ \mu \mathrm{g} / \mathrm{L} & 0.1 & \text { EPA8260 } \\ \mu \mathrm{g} / \mathrm{L} & 0.05 & \text { EPA8260 } \\ \mu \mathrm{g} / \mathrm{L} & 0.1 & \text { EPA8260 } \\ \mu \mathrm{g} / \mathrm{L} & 0.05 & \text { EPA8260 } \\ \mu \mathrm{g} / \mathrm{L} & 0.05 & \text { EPA8260 } \\ \mu \mathrm{g} / \mathrm{L} & 0.05 & \text { EPA8260 } \\ \mu \mathrm{g} / \mathrm{L} & 0.05 & \text { EPA8260 } \\ \mu \mathrm{g} / \mathrm{L} & 0.1 & \text { EPA8260 } \\ \mu \mathrm{g} / \mathrm{L} & 0.5 & \text { EPA8260 } \\ \mu \mathrm{g} / \mathrm{L} & 0.05 & \text { EPA8260 } \\ \mu \mathrm{g} / \mathrm{L} & 0.05 & \text { EPA8260 } \\ \mu \mathrm{g} / \mathrm{L} & 0.05 & \text { EPA8260 } \\ \mu \mathrm{g} / \mathrm{L} & 0.05 & \text { EPA8260 } \\ \mu \mathrm{g} / \mathrm{L} & 1 & \text { EPA8260 } \\ \mu \mathrm{g} / \mathrm{L} & 1 & \text { EPA8260 } \\ \mu \mathrm{g} / \mathrm{L} & 1 & \text { EPA8260 } \\ \mu \mathrm{g} / \mathrm{L} & 0.05 & \text { EPA8260 } \\ \mu \mathrm{g} / \mathrm{L} & 0.05 & \text { EPA8260 } \\ \mu \mathrm{g} / \mathrm{L} & 0.05 & \text { EPA8260 } \\ \mu \mathrm{g} / \mathrm{L} & 0.5 & \text { EPA8260 } \\ \mu \mathrm{g} / \mathrm{L} & 0.05 & \text { EPA8260 } \\ \mu \mathrm{g} / \mathrm{L} & 0.05 & \text { EPA8260 } \\ \mu \mathrm{g} / \mathrm{L} & 0.05 & \text { EPA8260 } \\ \mu \mathrm{g} / \mathrm{L} & 1 & \text { EPA8260 } \\ \mu \mathrm{g} / \mathrm{L} & 0.15 & \text { EPA8260 }\end{array}$


SURVEY ID: PPSC TB06B (continued)

Constituent

Volatile Organic Compounds

1,2-Dichloropropane cis-1,3-Dichloropropene trans-1,3-Dichloropropene

Ethylbenzene

2-Hexanone

Methyl ethyl ketone

Methyl isobutyl ketone

Styrene

1,1,2,2-Tetrachloroethane

Tetrachloroethylene

Toluene

1,1,1-Trichloroethane

1,1,2-Trichloroethane

Trichloroethylene

Vinyl acetate

Xylenes

\section{SURVEY ID: PPSC TB07B}

Sample type: Trip Blank

\section{Constituent}

Volatile Organic Compounds

Acetone
Acetone
Benzene
Benzene
Bromodichloromethane
Bromodichloromethane
Bromoform
Bromoform
Bromomethane (Methyl bromide)
Bromomethane (Methyl bromide)
Carbon disulfide
Carbon disulfide
Carbon tetrachloride
Carbon tetrachloride
Chlorobenzene
Chlorobenzene
Chloroethane
Chloroethane
Chloroethene (Vinyl chloride)
Chloroethene (Vinyl chloride)
Chloroform
Chloroform
Chloromethane (Methyl chloride)
Chloromethane (Methyl chloride)
Dibromochloromethane
Dibromochloromethane
1,1-Dichloroethane
1,1-Dichloroethane
1,2-Dichloroethane

Sample ID: 104679

RQ AQ B Result Unit D. Limit Method

\begin{tabular}{|c|c|c|c|c|}
\hline U & 0.05 & $\mu \mathrm{g} / \mathrm{L}$ & 0.05 & EPA8260 \\
\hline $\mathrm{U}$ & 0.05 & $\mu \mathrm{g} / \mathrm{L}$ & 0.05 & EPA8260 \\
\hline $\mathrm{U}$ & 0.05 & $\mu \mathrm{g} / \mathrm{L}$ & 0.05 & EPA8260 \\
\hline $\mathrm{U}$ & 0.05 & $\mu \mathrm{g} / \mathrm{L}$ & 0.05 & EPA8260 \\
\hline U & 3.69 & $\mu \mathrm{g} / \mathrm{L}$ & 1 & EPA8260 \\
\hline $\mathrm{U}$ & 2.89 & $\mu \mathrm{g} / \mathrm{L}$ & 1 & EPA8260 \\
\hline$U$ & 1 & $\mu \mathrm{g} / \mathrm{L}$ & 1 & EPA8260 \\
\hline$U$ & 0.05 & $\mu \mathrm{g} / \mathrm{L}$ & 0.05 & EPA8260 \\
\hline $\mathbf{U}$ & 0.05 & $\mu \mathrm{g} / \mathrm{L}$ & 0.05 & EPA8260 \\
\hline $\mathbf{U}$ & 0.05 & $\mu \mathrm{g} / \mathrm{L}$ & 0.05 & EPA8260 \\
\hline E8 & 0.14 & $\mu \mathrm{g} / \mathrm{L}$ & 0.5 & EPA8260 \\
\hline U & 0.05 & $\mu \mathrm{g} / \mathrm{L}$ & 0.05 & EPA8260 \\
\hline $\mathrm{U}$ & 0.05 & $\mu g / L$ & 0.05 & EPA8260 \\
\hline 8 & 0.18 & $\mu g / L$ & 0.05 & EPA8260 \\
\hline $\mathrm{U}$ & 1 & $\mu g / L$ & 1 & EPA8260 \\
\hline E8 & 0.12 & $\mu g / L$ & 0.15 & EPA8260 \\
\hline
\end{tabular}

Sample ID: 104692

RQ AQ B Result

Unit

D. Limit Method

$\begin{array}{lll} & 8 & 7.36 \\ U & 8 & 4.63 \\ U & & 0.5 \\ U & & 0.5 \\ U & & 0.05 \\ U & & 0.05 \\ U & & 0.05 \\ U & & 0.05 \\ U & & 0.1 \\ U & & 0.1 \\ U & & 1 \\ U & & 1 \\ U & & 0.05 \\ U & & 0.05 \\ U & & 0.05 \\ U & & 0.05 \\ U & & 0.1 \\ U & & 0.1 \\ U & & 0.1 \\ U & & 0.1 \\ U & & 0.05 \\ U & & 0.05 \\ U & & 0.1 \\ U & & 0.1 \\ U & & 0.05 \\ U & & 0.05 \\ U & & 0.05 \\ U & & 0.05 \\ U & & 0.05\end{array}$

$\begin{array}{lll}\mu \mathrm{g} / \mathrm{L} & 5 & \text { EPA8260 } \\ \mu \mathrm{g} / \mathrm{L} & 5 & \text { EPA8260 } \\ \mu \mathrm{g} / \mathrm{L} & 0.5 & \text { EPA8260 } \\ \mu \mathrm{g} / \mathrm{L} & 0.5 & \text { EPA8260 } \\ \mu \mathrm{g} / \mathrm{L} & 0.05 & \text { EPA8260 } \\ \mu \mathrm{g} / \mathrm{L} & 0.05 & \text { EPA8260 } \\ \mu \mathrm{g} / \mathrm{L} & 0.05 & \text { EPA8260 } \\ \mu \mathrm{g} / \mathrm{L} & 0.05 & \text { EPA8260 } \\ \mu \mathrm{g} / \mathrm{L} & 0.1 & \text { EPA8260 } \\ \mu \mathrm{g} / \mathrm{L} & 0.1 & \text { EPA8260 } \\ \mu \mathrm{g} / \mathrm{L} & 1 & \text { EPA8260 } \\ \mu \mathrm{g} / \mathrm{L} & 1 & \text { EPA8260 } \\ \mu \mathrm{g} / \mathrm{L} & 0.05 & \text { EPA8260 } \\ \mu \mathrm{g} / \mathrm{L} & 0.05 & \text { EPA8260 } \\ \mu \mathrm{g} / \mathrm{L} & 0.05 & \text { EPA8260 } \\ \mu \mathrm{g} / \mathrm{L} & 0.05 & \text { EPA8260 } \\ \mu \mathrm{g} / \mathrm{L} & 0.1 & \text { EPA8260 } \\ \mu \mathrm{g} / \mathrm{L} & 0.1 & \text { EPA8260 } \\ \mu \mathrm{g} / \mathrm{L} & 0.1 & \text { EPA8260 } \\ \mu \mathrm{g} / \mathrm{L} & 0.1 & \text { EPA8260 } \\ \mu \mathrm{g} / \mathrm{L} & 0.05 & \text { EPA8260 } \\ \mu \mathrm{g} / \mathrm{L} & 0.05 & \text { EPA8260 } \\ \mu \mathrm{g} / \mathrm{L} & 0.1 & \text { EPA8260 } \\ \mu \mathrm{g} / \mathrm{L} & 0.1 & \text { EPA8260 } \\ \mu \mathrm{g} / \mathrm{L} & 0.05 & \text { EPA8260 } \\ \mu \mathrm{g} / \mathrm{L} & 0.05 & \text { EPA8260 } \\ \mu \mathrm{g} / \mathrm{L} & 0.05 & \text { EPA8260 } \\ \mu \mathrm{g} / \mathrm{L} & 0.05 & \text { EPA8260 } \\ \mu \mathrm{g} / \mathrm{L} & 0.05 & \text { EPA8260 }\end{array}$


SURVEY ID: PPSC TB07B (continued)

Constituent

RQ AQ B Result

Unit

D. Limit Method

Volatile Organic Compounds

\begin{tabular}{|c|c|c|c|}
\hline 1,2-Dichloroethane & $\mathrm{U}$ & & 0.05 \\
\hline 1,1-Dichloroethylene & $U$ & & 0.05 \\
\hline 1,1-Dichloroethylene & $\mathrm{U}$ & & 0.05 \\
\hline 1,2-Dichloroethylene & $\mathrm{U}$ & & 0.1 \\
\hline 1,2-Dichloroethylene & $\mathrm{U}$ & & 0.1 \\
\hline Dichloromethane (Methylene chloride) & $\mathrm{U}$ & $\mathrm{V}$ & 1.25 \\
\hline Dichloromethane (Methylene chloride) & $\mathrm{U}$ & $\mathrm{V}$ & 1.12 \\
\hline 1,2-Dichloropropane & $\mathrm{U}$ & & 0.05 \\
\hline 1,2-Dichloropropane & $\mathrm{U}$ & & 0.05 \\
\hline cis-1,3-Dichloropropene & $\mathrm{U}$ & & 0.05 \\
\hline cis-1,3-Dichloropropene & $\mathrm{U}$ & & 0.05 \\
\hline trans-1,3-Dichloropropene & U & & 0.05 \\
\hline trans-1,3-Dichloropropene & $\mathrm{U}$ & & 0.05 \\
\hline Ethylbenzene & $\mathrm{U}$ & & 0.05 \\
\hline Ethylbenzene & $U$ & & 0.05 \\
\hline 2-Hexanone & U & $\mathrm{V}$ & 1.23 \\
\hline 2-Hexanone & U & & 1 \\
\hline Methyl ethyl ketone & & V8 & 8.34 \\
\hline Methyl ethyl ketone & U & V8 & 1.01 \\
\hline Methyl isobutyl ketone & U & & 1 \\
\hline Methyl isobutyl ketone & $\mathrm{U}$ & & 1 \\
\hline Styrene & $\mathrm{U}$ & & 0.05 \\
\hline Styrene & $\mathrm{U}$ & & 0.05 \\
\hline 1,1,2,2-Tetrachloroethane & $\mathrm{U}$ & & 0.05 \\
\hline 1,1,2,2-Tetrachloroethane & $\mathrm{U}$ & & 0.05 \\
\hline Tetrachloroethylene & U & & 0.05 \\
\hline Tetrachloroethylene & $U$ & & 0.05 \\
\hline Toluene & $\mathrm{J}$ & E8 & 0.21 \\
\hline Toluene & $\mathrm{U}$ & 8 & 0.11 \\
\hline 1,1,1-Trichloroethane & $\mathrm{U}$ & & 0.05 \\
\hline 1,1,1-Trichloroethane & U & & 0.05 \\
\hline 1,1,2-Trichloroethane & $\mathrm{U}$ & & 0.05 \\
\hline 1,1,2-Trichloroethane & $\mathrm{U}$ & & 0.05 \\
\hline Trichloroethylene & & 8 & 0.23 \\
\hline Trichloroethylene & $\mathrm{U}$ & 8 & 0.22 \\
\hline Vinyl acetate & U & & 1 \\
\hline Vinyl acetate & $\mathrm{U}$ & & 1 \\
\hline Xylenes & & 8 & 0.23 \\
\hline Xylenes & $\mathrm{U}$ & & 0.15 \\
\hline
\end{tabular}

\begin{tabular}{lll}
$\mu \mathrm{g} / \mathrm{L}$ & 0.05 & EPA8260 \\
$\mu \mathrm{g} / \mathrm{L}$ & 0.05 & EPA8260 \\
$\mu \mathrm{g} / \mathrm{L}$ & 0.05 & EPA8260 \\
$\mu \mathrm{g} / \mathrm{L}$ & 0.1 & EPA8260 \\
$\mu \mathrm{g} / \mathrm{L}$ & 0.1 & EPA8260 \\
$\mu \mathrm{g} / \mathrm{L}$ & 0.5 & EPA8260 \\
$\mu \mathrm{g} / \mathrm{L}$ & 0.5 & EPA8260 \\
$\mu \mathrm{g} / \mathrm{L}$ & 0.05 & EPA8260 \\
$\mu \mathrm{g} / \mathrm{L}$ & 0.05 & EPA8260 \\
$\mu \mathrm{g} / \mathrm{L}$ & 0.05 & EPA8260 \\
$\mu \mathrm{g} / \mathrm{L}$ & 0.05 & EPA8260 \\
$\mu \mathrm{g} / \mathrm{L}$ & 0.05 & EPA8260 \\
$\mu \mathrm{g} / \mathrm{L}$ & 0.05 & EPA8260 \\
$\mu \mathrm{g} / \mathrm{L}$ & 0.05 & EPA8260 \\
$\mu \mathrm{g} / \mathrm{L}$ & 0.05 & EPA8260 \\
$\mu \mathrm{g} / \mathrm{L}$ & 1 & EPA8260 \\
$\mu \mathrm{g} / \mathrm{L}$ & 1 & EPA8260 \\
$\mu \mathrm{g} / \mathrm{L}$ & 1 & EPA8260 \\
$\mu \mathrm{g} / \mathrm{L}$ & 1 & EPA8260 \\
$\mu \mathrm{g} / \mathrm{L}$ & 1 & EPA8260 \\
$\mu \mathrm{g} / \mathrm{L}$ & 1 & EPA8260 \\
$\mu \mathrm{g} / \mathrm{L}$ & 0.05 & EPA8260 \\
$\mu \mathrm{g} / \mathrm{L}$ & 0.05 & EPA8260 \\
$\mu \mathrm{g} / \mathrm{L}$ & 0.05 & EPA8260 \\
$\mu \mathrm{g} / \mathrm{L}$ & 0.05 & EPA8260 \\
$\mu \mathrm{g} / \mathrm{L}$ & 0.05 & EPA8260 \\
$\mu \mathrm{g} / \mathrm{L}$ & 0.05 & EPA8260 \\
$\mu \mathrm{g} / \mathrm{L}$ & 0.5 & EPA8260 \\
$\mu \mathrm{g} / \mathrm{L}$ & 0.5 & EPA8260 \\
$\mu \mathrm{g} / \mathrm{L}$ & 0.05 & EPA8260 \\
$\mu \mathrm{g} / \mathrm{L}$ & 0.05 & EPA8260 \\
$\mu \mathrm{g} / \mathrm{L}$ & 0.05 & EPA8260 \\
$\mu \mathrm{g} / \mathrm{L}$ & 0.05 & EPA8260 \\
$\mu \mathrm{g} / \mathrm{L}$ & 0.05 & EPA8260 \\
$\mu \mathrm{g} / \mathrm{L}$ & 0.05 & EPA8260 \\
$\mu \mathrm{g} / \mathrm{L}$ & 1 & EPA8260 \\
$\mu \mathrm{g} / \mathrm{L}$ & 1 & EPA8260 \\
$\mu \mathrm{g} / \mathrm{L}$ & 0.15 & EPA8260 \\
$\mu \mathrm{g} / \mathrm{L}$ & 0.15 & EPA8260 \\
& & \\
\hline
\end{tabular}

Sample ID: 105103

Associated sample: 104696

RQ AQ B Result

Unit

D. Limit Method

Volatile Organic Compounds

Acetone

Benzene

Bromodichloromethane

Bromoform

Bromomethane (Methyl bromide)

Carbon disulfide

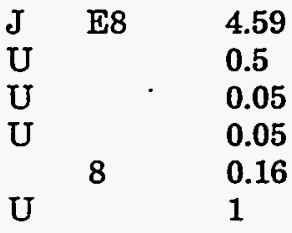

$\begin{array}{lll}\mu \mathrm{g} / \mathrm{L} & 5 & \text { EPA8260 } \\ \mu \mathrm{g} / \mathrm{L} & 0.5 & \text { EPA8260 } \\ \mu \mathrm{g} / \mathrm{L} & 0.05 & \text { EPA8260 } \\ \mu \mathrm{g} / \mathrm{L} & 0.05 & \text { EPA8260 } \\ \mu \mathrm{g} / \mathrm{L} & 0.1 & \text { EPA8260 } \\ \mu \mathrm{g} / \mathrm{L} & 1 & \text { EPA8260 }\end{array}$


SURVEY ID: PPSC TB08B (continued)

Sample ID: 105103

\begin{tabular}{|c|c|c|c|c|c|c|}
\hline Constituent & $\mathbf{R Q}$ & $\mathbf{A Q} \mathbf{B}$ & Result & Unit & D. Limit & Method \\
\hline \multicolumn{7}{|l|}{ Volatile Organic Compounds } \\
\hline Carbon tetrachloride & $\mathrm{U}$ & & 0.05 & $\mu g / L$ & 0.05 & EPA8260 \\
\hline Chlorobenzene & $\mathrm{U}$ & & 0.05 & $\mu \mathrm{g} / \mathrm{L}$ & 0.05 & EPA8260 \\
\hline Chloroethane & $\mathrm{U}$ & & 0.1 & $\mu g / L$ & 0.1 & EPA8260 \\
\hline Chloroethene (Vinyl chloride) & $\mathrm{U}$ & & 0.1 & $\mu g / L$ & 0.1 & EPA8260 \\
\hline Chloroform & $U$ & & 0.05 & $\mu \mathrm{g} / \mathrm{L}$ & 0.05 & EPA8260 \\
\hline Chloromethane (Methyl chloride) & & 8 & 0.11 & $\mu \mathrm{g} / \mathrm{L}$ & 0.1 & EPA8260 \\
\hline Dibromochloromethane & $\mathrm{U}$ & & 0.05 & $\mu g / L$ & 0.05 & EPA8260 \\
\hline 1,1-Dichloroethane & U & & 0.05 & $\mu \mathrm{g} / \mathrm{L}$ & 0.05 & EPA8260 \\
\hline 1,2-Dichloroethane & $U$ & & 0.05 & $\mu g / L$ & 0.05 & EPA8260 \\
\hline 1,1-Dichloroethylene & $\mathrm{U}$ & & 0.05 & $\mu \mathrm{g} / \mathrm{L}$ & 0.05 & EPA8260 \\
\hline 1,2-Dichloroethylene & $\mathrm{U}$ & & 0.1 & $\mu \mathrm{g} / \mathrm{L}$ & 0.1 & EPA8260 \\
\hline Dichloromethane (Methylene chloride) & $\mathrm{U}$ & $\mathrm{V}$ & 1.26 & $\mu \mathrm{g} / \mathrm{L}$ & 0.5 & EPA8260 \\
\hline 1,2-Dichloropropane & $\mathrm{U}$ & & 0.05 & $\mu g / L$ & 0.05 & EPA8260 \\
\hline cis-1,3-Dichloropropene & $\mathrm{U}$ & & 0.05 & $\mu \mathrm{g} / \mathrm{L}$ & 0.05 & EPA8260 \\
\hline trans-1,3-Dichloropropene & $\mathrm{U}$ & & 0.05 & $\mu \mathrm{g} / \mathrm{L}$ & 0.05 & EPA8260 \\
\hline Ethylbenzene & $\mathbf{U}$ & & 0.05 & $\mu \mathrm{g} / \mathrm{L}$ & 0.05 & EPA8260 \\
\hline 2-Hexanone & $\mathrm{U}$ & & 1 & $\mu \mathrm{g} / \mathrm{L}$ & 1 & EPA8260 \\
\hline Methyl ethyl ketone & $\mathrm{U}$ & $\mathrm{V}$ & 1.39 & $\mu g / L$ & 1 & EPA8260 \\
\hline Methyl isobutyl ketone & U & & 1 & $\mu \mathrm{g} / \mathrm{L}$ & 1 & EPA8260 \\
\hline Styrene & $\mathrm{U}$ & & 0.05 & $\mu \mathrm{g} / \mathrm{L}$ & 0.05 & EPA8260 \\
\hline 1,1,2,2-Tetrachloroethane & $\mathrm{U}$ & & 0.05 & $\mu g / L$ & 0.05 & EPA8260 \\
\hline Tetrachloroethylene & $U$ & & 0.05 & $\mu \mathrm{g} / \mathrm{L}$ & 0.05 & EPA8260 \\
\hline Toluene & $\mathbf{J}$ & E8 & 0.12 & $\mu \mathrm{g} / \mathrm{L}$ & 0.5 & EPA8260 \\
\hline 1,1,1-Trichloroethane & $U$ & & 0.05 & $\mu \mathrm{g} / \mathrm{L}$ & 0.05 & EPA8260 \\
\hline 1,1,2-Trichloroethane & $\mathrm{U}$ & & 0.05 & $\mu \mathrm{g} / \mathrm{L}$ & 0.05 & EPA8260 \\
\hline Trichloroethylene & & 8 & 0.27 & $\mu \mathrm{g} / \mathrm{L}$ & 0.05 & EPA8260 \\
\hline Vinyl acetate & $\mathrm{U}$ & & 1 & $\mu \mathrm{g} / \mathrm{L}$ & 1 & EPA8260 \\
\hline Xylenes & $\mathrm{U}$ & & 0.15 & $\mu \mathrm{g} / \mathrm{L}$ & 0.15 & EPA8260 \\
\hline
\end{tabular}

SURVEY ID: PPSC TB09B

Sample ID: 105112

Sample type: Trip Blank

Constituent

RQ AQ B Result

Unit

D. Limit Method

Volatile Organic Compounds

Acetone
Acetone
Benzene
Benzene
Bromodichloromethane
Bromodichloromethane
Bromoform
Bromoform
Bromomethane (Methyl bromide)
Bromomethane (Methyl bromide)
Carbon disulfide
Carbon disulfide
Carbon tetrachloride
Carbon tetrachloride
Chlorobenzene
Chlorobenzene
Chloroethane

$\begin{array}{lll} & 8 & 5.65 \\ & 8 & 9.22 \\ \text { U } & & 0.5 \\ \text { J } & \text { E8 } & 0.14 \\ \text { U } & & 0.05 \\ \text { U } & & 0.05 \\ \text { U } & & 0.05 \\ U & & 0.05 \\ \text { U } & & 0.1 \\ \text { U } & & 0.1 \\ \text { U } & & 1 \\ \text { U } & & 1 \\ \text { U } & & 0.05 \\ \text { U } & & 0.05 \\ \text { U } & & 0.05 \\ U & & 0.05 \\ \text { U } & & 0.1\end{array}$

$\begin{array}{lll}\mu \mathrm{g} / \mathrm{L} & 5 & \text { EPA8260 } \\ \mu \mathrm{g} / \mathrm{L} & 5 & \text { EPA8260 } \\ \mu \mathrm{g} / \mathrm{L} & 0.5 & \text { EPA8260 } \\ \mu \mathrm{g} / \mathrm{L} & 0.5 & \text { EPA8260 } \\ \mu \mathrm{g} / \mathrm{L} & 0.05 & \text { EPA8260 } \\ \mu \mathrm{g} / \mathrm{L} & 0.05 & \text { EPA8260 } \\ \mu \mathrm{g} / \mathrm{L} & 0.05 & \text { EPA8260 } \\ \mu \mathrm{g} / \mathrm{L} & 0.05 & \text { EPA8260 } \\ \mu \mathrm{g} / \mathrm{L} & 0.1 & \text { EPA8260 } \\ \mu \mathrm{g} / \mathrm{L} & 0.1 & \text { EPA8260 } \\ \mu \mathrm{g} / \mathrm{L} & 1 & \text { EPA8260 } \\ \mu \mathrm{g} / \mathrm{L} & 1 & \text { EPA8260 } \\ \mu \mathrm{g} / \mathrm{L} & 0.05 & \text { EPA8260 } \\ \mu \mathrm{g} / \mathrm{L} & 0.05 & \text { EPA8260 } \\ \mu \mathrm{g} / \mathrm{L} & 0.05 & \text { EPA8260 } \\ \mu \mathrm{g} / \mathrm{L} & 0.05 & \text { EPA8260 } \\ \mu \mathrm{g} / \mathrm{L} & 0.1 & \text { EPA8260 }\end{array}$


SURVEY ID: PPSC TB09B (continued)

Constituent

Volatile Organic Compounds

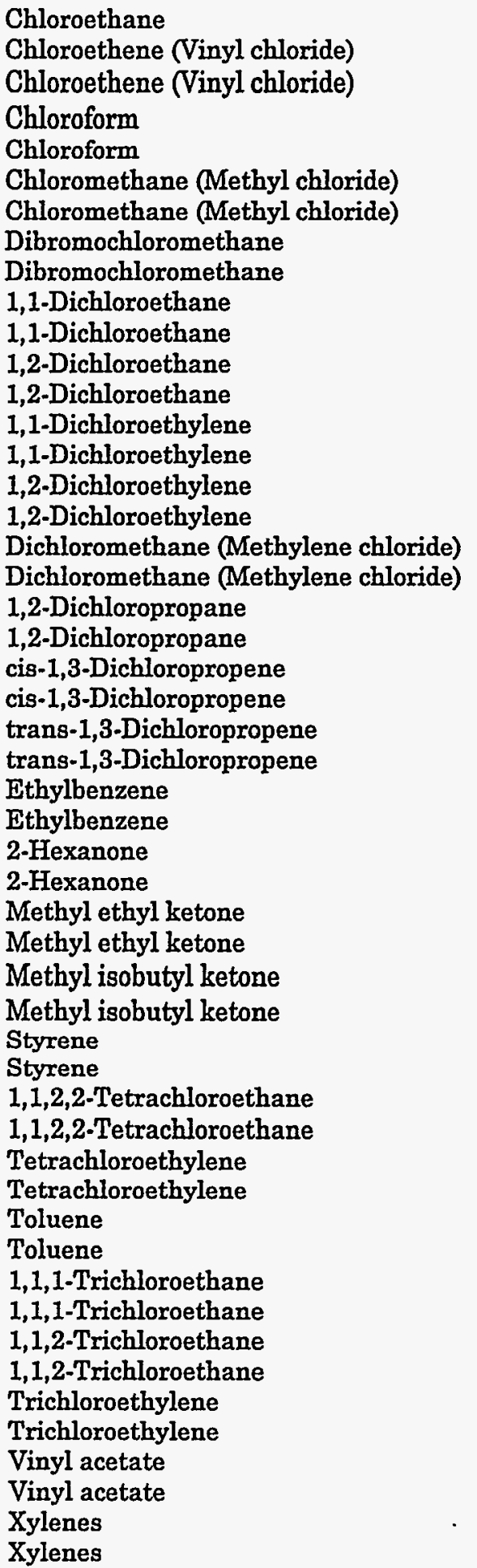

Sample ID: 105112

RQ AQ B Result Unit D. Limit Method

\begin{tabular}{|c|c|c|c|c|}
\hline & 0.1 & $\mu \mathrm{g} / \mathrm{L}$ & 0.1 & EPA8260 \\
\hline & 0.1 & $\mu \mathrm{g} / \mathrm{L}$ & 0.1 & EPA8260 \\
\hline & 0.1 & $\mu g / L$ & 0.1 & EPA8260 \\
\hline & 0.05 & $\mu \mathrm{g} / \mathrm{L}$ & 0.05 & EPA8260 \\
\hline & 0.05 & $\mu \mathrm{g} / \mathrm{L}$ & 0.05 & EPA8260 \\
\hline & 0.1 & $\mu \mathrm{g} / \mathrm{L}$ & 0.1 & EPA8260 \\
\hline & 0.1 & $\mu \mathrm{g} / \mathrm{L}$ & 0.1 & EPA8260 \\
\hline & 0.05 & $\mu \mathrm{g} / \mathrm{L}$ & 0.05 & EPA8260 \\
\hline & 0.05 & $\mu \mathrm{g} / \mathrm{L}$ & 0.05 & EPA8260 \\
\hline & 0.05 & $\mu \mathrm{g} / \mathrm{L}$ & 0.05 & EPA8260 \\
\hline & 0.05 & $\mu \mathrm{g} / \mathrm{L}$ & 0.05 & EPA8260 \\
\hline & 0.05 & $\mu \mathrm{g} / \mathrm{L}$ & 0.05 & EPA8260 \\
\hline & 0.05 & $\mu g / L$ & 0.05 & EPA8260 \\
\hline & 0.05 & $\mu \mathrm{g} / \mathrm{L}$ & 0.05 & EPA8260 \\
\hline & 0.05 & $\mu \mathrm{g} / \mathrm{L}$ & 0.05 & EPA8260 \\
\hline & 0.1 & $\mu \mathrm{g} / \mathrm{L}$ & 0.1 & EPA8260 \\
\hline & 0.1 & $\mu \mathrm{g} / \mathrm{L}$ & 0.1 & EPA8260 \\
\hline V & 1.73 & $\mu g / L$ & 0.5 & EPA8260 \\
\hline V & 2.08 & $\mu \mathrm{g} / \mathrm{L}$ & 0.5 & EPA8260 \\
\hline & 0.05 & $\mu \mathrm{g} / \mathrm{L}$ & 0.05 & EPA8260 \\
\hline & 0.05 & $\mu \mathrm{g} / \mathrm{L}$ & 0.05 & EPA8260 \\
\hline & 0.05 & $\mu \mathrm{g} / \mathrm{L}$ & 0.05 & EPA8260 \\
\hline & 0.05 & $\mu \mathrm{g} / \mathrm{L}$ & 0.05 & EPA8260 \\
\hline & 0.05 & $\mu \mathrm{g} / \mathrm{L}$ & 0.05 & EPA8260 \\
\hline & 0.05 & $\mu \mathrm{g} / \mathrm{L}$ & 0.05 & EPA8260 \\
\hline & 0.05 & $\mu \mathrm{g} / \mathrm{L}$ & 0.05 & EPA8260 \\
\hline & 0.05 & $\mu \mathrm{g} / \mathrm{L}$ & 0.05 & EPA8260 \\
\hline & 1 & $\mu \mathrm{g} / \mathrm{L}$ & 1 & EPA8260 \\
\hline V & 2 & $\mu \mathrm{g} / \mathrm{L}$ & 1 & EPA8260 \\
\hline V & 1.53 & $\mu \mathrm{g} / \mathrm{L}$ & 1 & EPA8260 \\
\hline $\mathrm{V}$ & 3.46 & $\mu \mathrm{g} / \mathrm{L}$ & 1 & EPA8260 \\
\hline & 1 & $\mu \mathrm{g} / \mathrm{L}$ & 1 & EPA8260 \\
\hline & 1 & $\mu \mathrm{g} / \mathrm{L}$ & 1 & EPA8260 \\
\hline & 0.05 & $\mu \mathrm{g} / \mathrm{L}$ & 0.05 & EPA8260 \\
\hline & 0.05 & $\mu \mathrm{g} / \mathrm{L}$ & 0.05 & EPA8260 \\
\hline & 0.05 & $\mu \mathrm{g} / \mathrm{L}$ & 0.05 & EPA8260 \\
\hline & 0.05 & $\mu \mathrm{g} / \mathrm{L}$ & 0.05 & EPA8260 \\
\hline & 0.05 & $\mu \mathrm{g} / \mathrm{L}$ & 0.05 & EPA8260 \\
\hline & 0.05 & $\mu \mathrm{g} / \mathrm{L}$ & 0.05 & EPA8260 \\
\hline EI8 L & 0.12 & $\mu \mathrm{g} / \mathrm{L}$ & 0.5 & EPA8260 \\
\hline EI8 L & 0.13 & $\mu \mathrm{g} / \mathrm{L}$ & 0.5 & EPA8260 \\
\hline & 0.05 & $\mu \mathrm{g} / \mathrm{L}$ & 0.05 & EPA8260 \\
\hline & 0.05 & $\mu \mathrm{g} / \mathrm{L}$ & 0.05 & EPA8260 \\
\hline & 0.05 & $\mu \mathrm{g} / \mathrm{L}$ & 0.05 & EPA8260 \\
\hline & 0.05 & $\mu \mathrm{g} / \mathrm{L}$ & 0.05 & EPA8260 \\
\hline 8 & 0.24 & $\mu \mathrm{g} / \mathrm{L}$ & 0.05 & EPA8260 \\
\hline 8 & 0.25 & $\mu \mathrm{g} / \mathrm{L}$ & 0.05 & EPA8260 \\
\hline & 1 & $\mu \mathrm{g} / \mathrm{L}$ & 1 & EPA8260 \\
\hline & 1 & $\mu \mathrm{g} / \mathrm{L}$ & 1 & EPA8260 \\
\hline & 0.15 & $\mu \mathrm{g} / \mathrm{L}$ & 0.15 & EPA8260 \\
\hline & 0.15 & $\mu g / L$ & 0.15 & EPA8260 \\
\hline
\end{tabular}


SURVEY ID: PPSC TB15B

Sample type: Trip Blank

Constituent

Volatile Organic Compounds

Acetone

Acetone

Benzene

Bromodichloromethane

Bromodichloromethane

Bromoform

Bromoform

Bromomethane (Methyl bromide)

Bromomethane (Methyl bromide)

Carbon disulfide

Carbon disulfide

Carbon tetrachloride

Chlorobenzene

Chlorobenzene

Chloroethane

Chloroethane

Chloroethene (Vinyl chloride)

Chloroform

Chloroform

Chloromethane (Methyl chloride)

Chloromethane (Methyl chloride)

Dibromochloromethane

Dibromochloromethane

1,1-Dichloroethane

1,1-Dichloroethane

1,2-Dichloroethane

1,2-Dichloroethane

1,1-Dichloroethylene

1,1-Dichloroethylene

1,2-Dichloroethylene

1,2-Dichloroethylene

Dichloromethane (Methylene chloride)

1,2-Dichloropropane

cis-1,3-Dichloropropene .

cis-1,3-Dichloropropene

trans-1,3-Dichloropropene

trans-1,3-Dichloropropene

Ethylbenzene

2-Hexanone

2-Hexanone

Methyl ethyl ketone

Methyl ethyl ketone .

Methyl isobutyl ketone

Methyl isobutyl ketone

Styrene

1,1,2,2-Tetrachloroethane

Tetrachloroethylene

Tetrachloroethylene

Toluene

1,1,1-Trichloroethane

1,1,1-Trichloroethane

1,1,2-Trichloroethane

1,1,2-Trichloroethane
Sample ID: 104642

Associated sample: 104639

RQ AQ B Result

Unit

D. Limit Method

\begin{tabular}{|c|c|c|c|}
\hline UJ & \multicolumn{2}{|c|}{ YVO H } & 2.82 \\
\hline UJ & YVO & & 1.3 \\
\hline & $\mathrm{Y}$ & & 0.09 \\
\hline UJ & Yo & $\mathrm{H}$ & \\
\hline UJ & YO & $\mathrm{H}$ & 0.2 \\
\hline $\mathrm{JJ}$ & YO & $\mathrm{H}$ & 2 \\
\hline UJ & YO & $\mathrm{H}$ & \\
\hline & YO & $\mathrm{H}$ & 0.6 \\
\hline & YO & $\mathrm{H}$ & 0.6 \\
\hline & YO & $\mathrm{H}$ & 2 \\
\hline & YO & $\mathrm{H}$ & 2 \\
\hline & & & 0.1 \\
\hline J & YO & $\mathrm{H}$ & 0.3 \\
\hline & YO & $\mathrm{H}$ & 0.3 \\
\hline & YO & $\mathrm{H}$ & 0.2 \\
\hline & YO & $\mathrm{H}$ & 0.2 \\
\hline & $\mathrm{Y}$ & & 0.2 \\
\hline & YO & $\mathrm{H}$ & 0.2 \\
\hline & YO & $\mathrm{H}$ & 0.2 \\
\hline & YO & $\mathrm{H}$ & 0.3 \\
\hline & YO & $\mathrm{H}$ & 0.3 \\
\hline & YO & $\mathrm{H}$ & 0.7 \\
\hline & YO & $\mathrm{H}$ & 0.7 \\
\hline UJ & YO & $\mathrm{H}$ & 0.1 \\
\hline & YO & $\mathrm{H}$ & 0.1 \\
\hline & YO & $\mathrm{H}$ & 0.2 \\
\hline & YO & $\mathrm{H}$ & 0.2 \\
\hline UJ & YO & $\mathrm{H}$ & 0.5 \\
\hline & YO & $\mathrm{H}$ & 0.5 \\
\hline & YO & $\mathrm{H}$ & 2 \\
\hline & YO & $\mathrm{H}$ & 2 \\
\hline & $8 Y$ & & 1.16 \\
\hline & $\mathrm{Y}$ & & 0.06 \\
\hline JJ & YO & $\mathrm{H}$ & 5 \\
\hline & YO & $\mathrm{H}$ & 5 \\
\hline & YO & $\underline{H}$ & 5 \\
\hline JJ & YO & $\mathrm{H}$ & 5 \\
\hline & $Y$ & & 0.05 \\
\hline & YO & $\mathrm{H}$ & 10 \\
\hline & YO & $\mathrm{H}$ & 10 \\
\hline JJ & YO & $\mathrm{H}$ & 10 \\
\hline JJ & YO & $\mathrm{H}$ & 10 \\
\hline UJ & YO & $\mathrm{H}$ & 10 \\
\hline UJ & YO & $\mathrm{H}$ & 10 \\
\hline & $\mathrm{Y}$ & & 0.1 \\
\hline 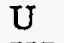 & Y & & 0.1 \\
\hline UJ & YO & $\mathrm{H}$ & 0.4 \\
\hline UJ & YO & $\mathrm{H}$ & 0.4 \\
\hline & $Y$ & & 0.1 \\
\hline UJ & YO & $\mathrm{H}$ & 0.3 \\
\hline & YO & $\mathrm{H}$ & 0.3 \\
\hline & YO & $\mathrm{H}$ & 0.2 \\
\hline & YO & $\mathrm{H}$ & 0.2 \\
\hline
\end{tabular}

\begin{tabular}{|c|c|c|}
\hline$\mu \mathrm{g} / \mathrm{L}$ & 10 & EPA8260 \\
\hline$\mu \mathrm{g} / \mathrm{L}$ & 10 & EPA8260 \\
\hline$\mu \mathrm{g} / \mathrm{L}$ & 0.09 & EPA8021 \\
\hline$\mu \mathrm{g} / \mathrm{L}$ & 0.2 & EPA8260 \\
\hline$\mu \mathrm{g} / \mathrm{L}$ & 0.2 & EPA8260 \\
\hline$\mu \mathrm{g} / \mathrm{L}$ & 2 & EPA8260 \\
\hline$\mu \mathrm{g} / \mathrm{L}$ & 2 & EPA8260 \\
\hline$\mu \mathrm{g} / \mathrm{L}$ & 0.6 & EPA8260 \\
\hline$\mu \mathrm{g} / \mathrm{L}$ & 0.6 & EPA8260 \\
\hline$\mu \mathrm{g} / \mathrm{L}$ & 2 & EPA8260 \\
\hline$\mu \mathrm{g} / \mathrm{L}$ & 2 & EPA8260 \\
\hline$\mu \mathrm{g} / \mathrm{L}$ & 0.1 & EPA8021 \\
\hline$\mu \mathrm{g} / \mathrm{L}$ & 0.3 & EPA8260 \\
\hline$\mu g / L$ & 0.3 & EPA8260 \\
\hline$\mu g / L$ & 0.2 & EPA8260 \\
\hline$\mu \mathrm{g} / \mathrm{L}$ & 0.2 & EPA8260 \\
\hline$\mu \mathrm{g} / \mathrm{L}$ & 0.2 & EPA8021 \\
\hline$\mu \mathrm{g} / \mathrm{L}$ & 0.2 & EPA8260 \\
\hline$\mu \mathrm{g} / \mathrm{L}$ & 0.2 & EPA8260 \\
\hline$\mu g / L$ & 0.3 & EPA8260 \\
\hline$\mu \mathrm{g} / \mathrm{L}$ & 0.3 & EPA8260 \\
\hline$\mu \mathrm{g} / \mathrm{L}$ & 0.7 & EPA8260 \\
\hline$\mu g / L$ & 0.7 & EPA8260 \\
\hline$\mu g / L$ & 0.1 & EPA8260 \\
\hline$\mu g / L$ & 0.1 & EPA8260 \\
\hline$\mu \mathrm{g} / \mathrm{L}$ & 0.2 & EPA8260 \\
\hline$\mu \mathrm{g} / \mathrm{L}$ & 0.2 & EPA8260 \\
\hline$\mu \mathrm{g} / \mathrm{L}$ & 0.5 & EPA8260 \\
\hline$\mu \mathrm{g} / \mathrm{L}$ & 0.5 & EPA8260 \\
\hline$\mu g / L$ & 2 & EPA8260 \\
\hline$\mu \mathrm{g} / \mathrm{L}$ & 2 & EPA8260 \\
\hline$\mu g / L$ & 0.2 & EPA8021 \\
\hline$\mu \mathrm{g} / \mathrm{L}$ & 0.06 & EPA8021 \\
\hline$\mu \mathrm{g} / \mathrm{L}$ & 5 & EPA8260 \\
\hline$\mu g / L$ & 5 & EPA8260 \\
\hline$\mu \mathrm{g} / \mathrm{L}$ & 5 & EPA8260 \\
\hline$\mu \mathrm{g} / \mathrm{L}$ & 5 & EPA8260 \\
\hline$\mu \mathrm{g} / \mathrm{L}$ & 0.05 & EPA8021 \\
\hline$\mu \mathrm{g} / \mathrm{L}$ & 10 & EPA8260 \\
\hline$\mu \mathrm{g} / \mathrm{L}$ & 10 & EPA8260 \\
\hline$\mu \mathrm{g} / \mathrm{L}$ & 10 & EPA8260 \\
\hline$\mu g / L$ & 10 & EPA8260 \\
\hline$\mu \mathrm{g} / \mathrm{L}$ & 10 & EPA8260 \\
\hline$\mu \mathrm{g} / \mathrm{L}$ & 10 & EPA8260 \\
\hline$\mu \mathrm{g} / \mathrm{L}$ & 0.1 & EPA8021 \\
\hline$\mu \mathrm{g} / \mathrm{L}$ & 0.1 & EPA8021 \\
\hline$\mu g / L$ & 0.4 & EPA8260 \\
\hline$\mu \mathrm{g} / \mathrm{L}$ & 0.4 & EPA8260 \\
\hline$\mu \mathrm{g} / \mathrm{L}$ & 0.1 & EPA8021 \\
\hline$\mu \mathrm{g} / \mathrm{L}$ & 0.3 & EPA8260 \\
\hline$\mu \mathrm{g} / \mathrm{L}$ & 0.3 & EPA8260 \\
\hline$\mu \mathrm{g} / \mathrm{L}$ & 0.2 & EPA8260 \\
\hline & 0.2 & \\
\hline
\end{tabular}


SURVEY ID: PPSC TB15B (continued)

Constituent

Volatile Organic Compounds

Trichloroethylene

Unknown

Unknown

Unknown

Unknown

Unknown

Vinyl acetate

Vinyl acetate

Xylenes

Xylenes

\section{SURVEY ID: PPSC TB16B}

Sample type: Trip Blank

Constituent

Volatile Organic Compounds

Acetone
Benzene
Bromodichloromethane
Bromoform
Bromomethane (Methyl bromide)
Carbon disulfide
Carbon tetrachloride
Chlorobenzene
Chloroethane
Chloroethene (Vinyl chloride)
Chloroform
Chloromethane (Methyl chloride)
Dibromochloromethane
1,1-Dichloroethane
1,2-Dichloroethane
1,1-Dichloroethylene
1,2-Dichloroethylene
Dichloromethane (Methylene chloride)
1,2-Dichloropropane
cis-1,3-Dichloropropene
trans-1,3-Dichloropropene
Ethylbenzene
2-Hexanone
Methyl ethyl ketone
Methyl isobutyl ketone
Styrene
1,1,2,2-Tetrachloroethane
Tetrachloroethylene
Toluene
1,1,1-Trichloroethane
1,1,2-Trichloroethane
Trichloroethylene
Vinyl acetate
Xylenes

Sample ID: 104642

RQ AQ B Result

Unit

D. Limit Method

$\begin{array}{lll}\mu \mathrm{g} / \mathrm{L} & 0.1 & \text { EPA8021 } \\ \mu \mathrm{g} / \mathrm{L} & & \text { EPA8260 } \\ \mu \mathrm{g} / \mathrm{L} & & \text { EPA8260 } \\ \mu \mathrm{g} / \mathrm{L} & & \text { EPA8260 } \\ \mu \mathrm{g} / \mathrm{L} & & \text { EPA8260 } \\ \mu \mathrm{g} / \mathrm{L} & & \text { EPA8260 } \\ \mu \mathrm{g} / \mathrm{L} & 10 & \text { EPA8260 } \\ \mu \mathrm{g} / \mathrm{L} & 10 & \text { EPA8260 } \\ \mu \mathrm{g} / \mathrm{L} & 0.4 & \text { EPA8260 } \\ \mu \mathrm{g} / \mathrm{L} & 0.4 & \text { EPA8260 }\end{array}$

Sample ID: 104663

Associated sample: 104659

RQ AQ B Result

Unit

D. Limit Method

\begin{tabular}{|c|c|c|c|c|}
\hline $\begin{array}{ll}\mathrm{U} & \mathrm{V}\end{array}$ & 0.71 & $\mu \mathrm{g} / \mathrm{L}$ & 10 & EPA8260 \\
\hline $\mathbf{U}$ & 0.09 & $\mu \mathrm{g} / \mathrm{L}$ & 0.09 & EPA8021 \\
\hline $\mathbf{U}$ & 0.2 & $\mu \mathrm{g} / \mathrm{L}$ & 0.2 & EPA8260 \\
\hline $\mathbf{U}$ & 2 & $\mu \mathrm{g} / \mathrm{L}$ & 2 & EPA8260 \\
\hline U & 0.6 & $\mu \mathrm{g} / \mathrm{L}$ & 0.6 & EPA8260 \\
\hline $\mathbf{U}$ & 2 & $\mu \mathrm{g} / \mathrm{L}$ & 2 & EPA8260 \\
\hline $\mathrm{U}$ & 0.1 & $\mu \mathrm{g} / \mathrm{L}$ & 0.1 & EPA8021 \\
\hline $\mathbf{U}$ & 0.3 & $\mu \mathrm{g} / \mathrm{L}$ & 0.3 & EPA8260 \\
\hline $\mathrm{U}$ & 0.2 & $\mu \mathrm{g} / \mathrm{L}$ & 0.2 & EPA8260 \\
\hline $\mathbf{U}$ & 0.2 & $\mu \mathrm{g} / \mathrm{L}$ & 0.2 & EPA8021 \\
\hline $\mathbf{U}$ & 0.2 & $\mu \mathrm{g} / \mathrm{L}$ & 0.2 & EPA8260 \\
\hline $\mathbf{U}$ & 0.3 & $\mu \mathrm{g} / \mathrm{L}$ & 0.3 & EPA8260 \\
\hline $\mathrm{U}$ & 0.7 & $\mu \mathrm{g} / \mathrm{L}$ & 0.7 & EPA8260 \\
\hline $\mathbf{U}$ & 0.1 & $\mu \mathrm{g} / \mathrm{L}$ & 0.1 & EPA8260 \\
\hline U & 0.2 & $\mu \mathrm{g} / \mathrm{L}$ & 0.2 & EPA8260 \\
\hline $\mathbf{U}$ & 0.5 & $\mu \mathrm{g} / \mathrm{L}$ & 0.5 & EPA8260 \\
\hline $\mathbf{U}$ & 2 & $\mu \mathrm{g} / \mathrm{L}$ & 2 & EPA8260 \\
\hline & 1.33 & $\mu \mathrm{g} / \mathrm{L}$ & 0.2 & EPA8021 \\
\hline $\mathrm{U}$ & 0.06 & $\mu \mathrm{g} / \mathrm{L}$ & 0.06 & EPA8021 \\
\hline $\mathrm{U}$ & 5 & $\mu \mathrm{g} / \mathrm{L}$ & 5 & EPA8260 \\
\hline $\mathrm{U}$ & 5 & $\mu \mathrm{g} / \mathrm{L}$ & 5 & EPA8260 \\
\hline $\mathrm{U}$ & 0.05 & $\mu \mathrm{g} / \mathrm{L}$ & 0.05 & EPA8021 \\
\hline $\mathrm{U}$ & 10 & $\mu g / L$ & 10 & EPA8260 \\
\hline $\mathrm{U}$ & 10 & $\mu \mathrm{g} / \mathrm{L}$ & 10 & EPA8260 \\
\hline $\mathrm{U}$ & 10 & $\mu \mathrm{g} / \mathrm{L}$ & 10 & EPA8260 \\
\hline U & 0.1 & $\mu \mathrm{g} / \mathrm{L}$ & 0.1 & EPA8021 \\
\hline U & 0.1 & $\mu \mathrm{g} / \mathrm{L}$ & 0.1 & EPA8021 \\
\hline $\mathrm{U}$ & 0.4 & $\mu \mathrm{g} / \mathrm{L}$ & 0.4 & EPA8260 \\
\hline $\mathrm{U}$ & 0.1 & $\mu \mathrm{g} / \mathrm{L}$ & 0.1 & EPA8021 \\
\hline $\mathrm{U}$ & 0.3 & $\mu \mathrm{g} / \mathrm{L}$ & 0.3 & EPA8260 \\
\hline $\mathrm{U}$ & 0.2 & $\mu \mathrm{g} / \mathrm{L}$ & 0.2 & EPA8260 \\
\hline $\mathrm{U}$ & 0.1 & $\mu \mathrm{g} / \mathrm{L}$ & 0.1 & EPA8021 \\
\hline $\mathrm{U}$ & 10 & $\mu \mathrm{g} / \mathrm{L}$ & 10 & EPA8260 \\
\hline $\mathrm{U}$ & 0.4 & $\mu \mathrm{g} / \mathrm{L}$ & 0.4 & EPA8260 \\
\hline
\end{tabular}




\section{Appendix E: Data Management/Administrative Files}

This section discusses the generation, processing, and storage of paper and computer records produced during the completion of this project. The processing of the materials follows methodology prescribed by the Environmental Protection Department//Environmental Monitoring Section (EPD/EMS).

EPD/EMS establishes administrative files for each project that are maintained as permanent Westinghouse Savannah River Company (WSRC) records. The following materials are placed in the administrative files:

- Bound field logbooks

- Original chain-of-custody forms

- Analytical narratives from the laboratories

- Data validation logbooks

- Data entry quality assurance task cover sheets for data files created by EPD/EMS

- Copies of all correspondence between the data validation personnel and the laboratories

EPD/EMS generates computer data files from paper records using double-entry methods to ensure a low transcription error rate. Computer data files for analytical data also are received from the laboratories. Changes to the data are recorded in the data validation logbook, approved by the project technical manager, and confirmed through a computer program by comparing the modified and original data files. EPD/EMS stores the final computer data files, original source files, and computer listings of all changes made to the original files in the administrative files.

Access to the administrative files is maintained by the manager of EPD/EMS. Paper materials eventually will be transferred to permanent box storage; however, EPD/EMS plans to make the final data files available to WSRC computer users indefinitely. Please contact the manager of EPD/EMS to obtain access to these data. 
This page was left blank intentionally. 


\section{Appendix F: Analytical Methods and Quantitation Limits}

In the following tables of analytical methods and quantitation limits, the estimated quantitation limit (EQL) column reports the minimum concentration of a substance that can be reliably measured and reported within specified limits of precision and accuracy during routine laboratory operating conditions. The Reference column provides the published source for the method, except for the radiological analyses that are performed by the laboratories' established methods. See the References section of this report for further information on the method sources.

Table F-1. Analytical Methods and Quantitation Limits for General Engineering Laboratories, Inc.

\begin{tabular}{|c|c|c|c|c|}
\hline \multicolumn{5}{|c|}{ Soil and Sediment Analyses } \\
\hline Analyte & Method & Units & EQL & Reference \\
\hline \multicolumn{5}{|c|}{ Target Analyte List (TAL): Inorganics } \\
\hline Aluminum & EPA6010A & $\mu \mathrm{g} / \mathrm{kg}$ & 250 & SW-846 \\
\hline Antimony & EPA6010A & $\mu \mathrm{g} / \mathrm{kg}$ & 100 & SW-846 \\
\hline Arsenic & EPA6010A & $\mu \mathrm{g} / \mathrm{kg}$ & 300 & SW-846 \\
\hline Barium & EPA6010A & $\mu \mathrm{g} / \mathrm{kg}$ & 25 & SW-846 \\
\hline Beryllium & EPA6010A & $\mu \mathrm{g} / \mathrm{kg}$ & 10 & SW-846 \\
\hline Cadmium & EPA6010A & $\mu \mathrm{g} / \mathrm{kg}$ & 5 & SW-846 \\
\hline Calcium & EPA6010A & $\mu \mathrm{g} / \mathrm{kg}$ & 250 & SW-846 \\
\hline Chromium & EPA6010A & $\mu \mathrm{g} / \mathrm{kg}$ & 25 & SW-846 \\
\hline Cobalt & EPA6010A & $\mu \mathrm{g} / \mathrm{kg}$ & 10 & SW-846 \\
\hline Copper & EPA6010A & $\mu \mathrm{g} / \mathrm{kg}$ & 25 & SW-846 \\
\hline Cyanide & EPA335.3 & $\mu \mathrm{g} / \mathrm{kg}$ & 20 & $600 / 4-79-020$ \\
\hline Iron & EPA6010A & $\mu \mathrm{g} / \mathrm{kg}$ & 100 & SW-846 \\
\hline Lead & EPA6010A & $\mu \mathrm{g} / \mathrm{kg}$ & 100 & SW-846 \\
\hline Magnesium & EPA6010A & $\mu \mathrm{g} / \mathrm{kg}$ & 100 & SW-846 \\
\hline Manganese & EPA6010A & $\mu \mathrm{g} / \mathrm{kg}$ & 10 & SW-846 \\
\hline Mercury & EPA7471 & $\mu \mathrm{g} / \mathrm{kg}$ & 0.2 & SW-846 \\
\hline Nickel & EPA6010A & $\mu \mathrm{g} / \mathrm{kg}$ & 25 & SW-846 \\
\hline Potassium & EPA6010A & $\mu \mathrm{g} / \mathrm{kg}$ & 2000 & SW-846 \\
\hline Selenium & EPA6010A & $\mu \mathrm{g} / \mathrm{kg}$ & 300 & SW-846 \\
\hline $\begin{array}{l}\text { Silver } \\
\end{array}$ & EPA6010A & $\mu \mathrm{g} / \mathrm{kg}$ & 25 & SW-846 \\
\hline Sodium & EPA6010A & $\mu \mathrm{g} / \mathrm{kg}$ & 1000 & SW-846 \\
\hline Thallium & EPA6010A & $\mu \mathrm{g} / \mathrm{kg}$ & 300 & SW-846 \\
\hline
\end{tabular}


Table F-1. Analytical Methods and Quantitation Limits for General Engineering Laboratories, Inc., Continued

\begin{tabular}{|c|c|c|c|c|}
\hline Analyte & Method & Units & EQL & Reference \\
\hline Vanadium & EPA6010A & $\mu \mathrm{g} / \mathrm{kg}$ & 10 & SW-846 \\
\hline Zinc & EPA6010A & $\mu \mathrm{g} / \mathrm{kg}$ & 20 & SW-846 \\
\hline \multicolumn{5}{|c|}{$\begin{array}{l}\text { Target Compound List (TCL): Pesticides/Polychlorinated biphenyls (PCBs) with } \\
\text { Tentatively Identified Compounds (TICs) }\end{array}$} \\
\hline Aldrin & EPA8080 & $\mu \mathrm{g} / \mathrm{kg}$ & 0.83 & SW-846 \\
\hline Aroclor 1016 & EPA8080 & $\mu \mathrm{g} / \mathrm{kg}$ & 4.16 & SW-846 \\
\hline Aroclor 1221 & EPA8080 & $\mu \mathrm{g} / \mathrm{kg}$ & 4.16 & SW-846 \\
\hline Aroclor 1232 & EPA8080 & $\mu \mathrm{g} / \mathrm{kg}$ & 4.16 & SW-846 \\
\hline Aroclor 1242 & EPA8080 & $\mu \mathrm{g} / \mathrm{kg}$ & 4.16 & SW-846 \\
\hline Aroclor 1248 & EPA8080 & $\mu \mathrm{g} / \mathrm{kg}$ & 4.16 & SW-846 \\
\hline Aroclor 1254 & EPA8080 & $\mu \mathrm{g} / \mathrm{kg}$ & 4.16 & SW-846 \\
\hline Aroclor 1260 & EPA8080 & $\mu \mathrm{g} / \mathrm{kg}$ & 4.16 & SW-846 \\
\hline alpha-BHC & EPA8080 & $\mu \mathrm{g} / \mathrm{kg}$ & 0.83 & SW-846 \\
\hline beta-BHC & EPA8080 & $\mu \mathrm{g} / \mathrm{kg}$ & 1.66 & SW-846 \\
\hline delta-BHC & EPA8080 & $\mu \mathrm{g} / \mathrm{kg}$ & 1.66 & SW-846 \\
\hline gamma-BHC (Lindane) & EPA8080 & $\mu \mathrm{g} / \mathrm{kg}$ & 0.83 & SW-846 \\
\hline alpha-Chlordane & EPA8080 & $\mu \mathrm{g} / \mathrm{kg}$ & 1.66 & SW-846 \\
\hline gamma-Chlordane & EPA8080 & $\mu \mathrm{g} / \mathrm{kg}$ & 1.66 & SW-846 \\
\hline p,p'-DDD & EPA8080 & $\mu \mathrm{g} / \mathrm{kg}$ & 3.33 & SW-846 \\
\hline $\mathrm{p}, \mathrm{p}^{\prime}-\mathrm{DDE}$ & EPA8080 & $\mu \mathrm{g} / \mathrm{kg}$ & 1.66 & SW-846 \\
\hline p,p'-DDT & EPA8080 & $\mu \mathrm{g} / \mathrm{kg}$ & 3.33 & SW-846 \\
\hline Dieldrin & EPA8080 & $\mu \mathrm{g} / \mathrm{kg}$ & 1.66 & SW-846 \\
\hline Endosulfan I & EPA8080 & $\mu \mathrm{g} / \mathrm{kg}$ & 1.66 & SW-846 \\
\hline Endosulfan II & EPA8080 & $\mu \mathrm{g} / \mathrm{kg}$ & 3.33 & SW-846 \\
\hline Endosulfan sulfate & EPA8080 & $\mu \mathrm{g} / \mathrm{kg}$ & 3.33 & SW-846 \\
\hline Endrin & EPA8080 & $\mu \mathrm{g} / \mathrm{kg}$ & 1.66 & SW-846 \\
\hline Endrin ketone ${ }^{1}$ & EPA8080 & $\mu \mathrm{g} / \mathrm{kg}$ & 3.3 & SW-846 \\
\hline Heptachlor & EPA8080 & $\mu \mathrm{g} / \mathrm{kg}$ & 1.66 & SW-846 \\
\hline Heptachlor epoxide & EPA8080 & $\mu \mathrm{g} / \mathrm{kg}$ & 1.66 & SW-846 \\
\hline Methoxychlor & EPA8080 & $\mu \mathrm{g} / \mathrm{kg}$ & 16.6 & SW-846 \\
\hline Toxaphene & EPA8080 & $\mu \mathrm{g} / \mathrm{kg}$ & 33 & SW-846 \\
\hline \multicolumn{5}{|c|}{ TCL: Volatile Organic Compounds with TIC } \\
\hline Acetone & EPA8260 & $\mu \mathrm{g} / \mathrm{kg}$ & 5 & SW-846 \\
\hline
\end{tabular}

1This compound is not specifically listed under this method in the cited reference. It is included here because the laboratory uses this method for its analysis. 
Table F-1. Analytical Methods and Quantitation Limits for General Engineering Laboratories, Inc., Continued

\begin{tabular}{|c|c|c|c|c|}
\hline Analyte & Method & Units & EQL & Reference \\
\hline Benzene & EPA8260 & $\mu \mathrm{g} / \mathrm{kg}$ & 0.5 & SW-846 \\
\hline Bromodichloromethane & EPA8260 & $\mu \mathrm{g} / \mathrm{kg}$ & 0.05 & SW-846 \\
\hline Bromoform & EPA8260 & $\mu \mathrm{g} / \mathrm{kg}$ & 0.05 & SW-846 \\
\hline $\begin{array}{l}\text { Bromomethane (Methyl } \\
\text { bromide) }\end{array}$ & EPA8260 & $\mu \mathrm{g} / \mathrm{kg}$ & 0.1 & SW-846 \\
\hline 2-Butanone (MEK) & EPA8260 & $\mu \mathrm{g} / \mathrm{kg}$ & 1 & SW-846 \\
\hline Carbon disulfide & EPA8260 & $\mu \mathrm{g} / \mathrm{kg}$ & 1 & SW-846 \\
\hline Carbon tetrachloride & EPA8260 & $\mu \mathrm{g} / \mathrm{kg}$ & 0.05 & SW-846 \\
\hline Chlorobenzene & EPA8260 & $\mu \mathrm{g} / \mathrm{kg}$ & 0.05 & SW-846 \\
\hline Chlorodibromomethane . & EPA8260 & $\mu \mathrm{g} / \mathrm{kg}$ & 0.05 & SW-846 \\
\hline Chloroethane & EPA8260 & $\mu \mathrm{g} / \mathrm{kg}$ & 0.1 & SW-846 \\
\hline Chloroethene (Vinyl chloride) & EPA8260 & $\mu \mathrm{g} / \mathrm{kg}$ & 0.1 & SW-846 \\
\hline Chloroform & EPA8260 & $\mu \mathrm{g} / \mathrm{kg}$ & 0.05 & SW-846 \\
\hline Chloromethane (Methyl chloride) & EPA8260 & $\mu \mathrm{g} / \mathrm{kg}$ & 0.1 & SW-846 \\
\hline 1,1-Dichloroethane & EPA8260 & $\mu \mathrm{g} / \mathrm{kg}$ & 0.05 & SW-846 \\
\hline 1,2-Dichloroethane & EPA8260 & $\mu \mathrm{g} / \mathrm{kg}$ & 0.05 & SW-846 \\
\hline 1,1-Dichloroethylene & EPA8260 & $\mu \mathrm{g} / \mathrm{kg}$ & 0.05 & SW-846 \\
\hline 1,2-Dichloroethylene (total) & EPA8260 & $\mu \mathrm{g} / \mathrm{kg}$ & 0.1 & SW-846 \\
\hline $\begin{array}{l}\text { Dichloromethane } \\
\text { (Methylene chloride) }\end{array}$ & EPA8260 & $\mu \mathrm{g} / \mathrm{kg}$ & 0.5 & SW-846 \\
\hline 1,2-Dichloropropane & EPA8260 & $\mu \mathrm{g} / \mathrm{kg}$ & 0.05 & SW-846 \\
\hline cis-1,3-Dichloropropene & EPA8260 & $\mu \mathrm{g} / \mathrm{kg}$ & 0.05 & SW-846 \\
\hline trans-1,3-Dichloropropene & EPA8260 & $\mu \mathrm{g} / \mathrm{kg}$ & 0.05 & SW-846 \\
\hline Ethylbenzene & EPA8260 & $\mu \mathrm{g} / \mathrm{kg}$ & 0.05 & SW-846 \\
\hline 2-Hexanone & EPA8260 & $\mu \mathrm{g} / \mathrm{kg}$ & 1 & SW-846 \\
\hline 4-Methyl-2-pentanone & EPA8260 & $\mu \mathrm{g} / \mathrm{kg}$ & 1 & SW-846 \\
\hline Styrene & EPA8260 & $\mu \mathrm{g} / \mathrm{kg}$ & 0.05 & SW-846 \\
\hline 1,1,2,2-Tetrachloroethane & EPA8260 & $\mu \mathrm{g} / \mathrm{kg}$ & 0.05 & SW-846 \\
\hline Tetrachloroethylene & EPA8260 & $\mu \mathrm{g} / \mathrm{kg}$ & 0.05 & SW-846 \\
\hline Toluene & EPA8260 & $\mu \mathrm{g} / \mathrm{kg}$ & 0.5 & SW-846 \\
\hline 1,1,1-Trichloroethane & EPA8260 & $\mu \mathrm{g} / \mathrm{kg}$ & 0.05 & SW-846 \\
\hline 1,1,2-Trichloroethane & EPA8260 & $\mu \mathrm{g} / \mathrm{kg}$ & 0.05 & SW-846 \\
\hline Trichloroethylene & EPA8260 & $\mu \mathrm{g} / \mathrm{kg}$ & 0.05 & SW-846 \\
\hline Vinyl acetate & EPA8260 & $\mu \mathrm{g} / \mathrm{kg}$ & 1 & SW-846 \\
\hline Xylenes (total) & EPA8260 & $\mu \mathrm{g} / \mathrm{kg}$ & 0.15 & SW-846 \\
\hline
\end{tabular}


Table F-1. Analytical Methods and Quantitation Limits for General Engineering Laboratories, Inc., Continued

\begin{tabular}{|c|c|c|c|c|}
\hline Analyte & Method & Units & EQL & Reference \\
\hline \multicolumn{5}{|c|}{$\begin{array}{l}\text { TCL: Semivolatile Organic Compounds (B/N/A Extractables), including } \\
\text { Polycyclic Aromatic Hydrocarbons (PAHs) and Phenols with TIC }\end{array}$} \\
\hline Acenaphthene & EPA8270 & $\mathrm{mg} / \mathrm{kg}$ & 0.0333 & SW-846 \\
\hline Acenaphthylene & EPA8270 & $\mathrm{mg} / \mathrm{kg}$ & 0.0333 & SW-846 \\
\hline Anthracene & EPA8270 & $\mathrm{mg} / \mathrm{kg}$ & 0.0333 & SW-846 \\
\hline Benzo $[a]$ anthracene & EPA8270 & $\mathrm{mg} / \mathrm{kg}$ & 0.00333 & SW-846 \\
\hline Benzo[b]fluoranthene & EPA8270 & $\mathrm{mg} / \mathrm{kg}$ & 0.00333 & SW-846 \\
\hline Benzo[k]fluoranthene & EPA8270 & $\mathrm{mg} / \mathrm{kg}$ & 0.00333 & SW-846 \\
\hline Benzoic acid & EPA8270 & $\mathrm{mg} / \mathrm{kg}$ & 0.0333 & $\mathrm{SW}-846$ \\
\hline Benzo[g,h,i]perylene & EPA8270 & $\mathrm{mg} / \mathrm{kg}$ & 0.0333 & SW-846 \\
\hline Benzo[a]pyrene & EPA8270 & $\mathrm{mg} / \mathrm{kg}$ & 0.00333 & SW-846 \\
\hline Benzyl alcohol & EPA8270 & $\mathrm{mg} / \mathrm{kg}$ & 0.0333 & SW-846 \\
\hline Bis(2-chloroethoxy) methane & EPA8270 & $\mathrm{mg} / \mathrm{kg}$ & 0.0333 & SW-846 \\
\hline Bis(2-chloroethyl) ether & EPA8270 & $\mathrm{mg} / \mathrm{kg}$ & 0.0333 & SW-846 \\
\hline Bis(2-chloroisopropyl) ether & EPA8270 & $\mathrm{mg} / \mathrm{kg}$ & 0.0333 & SW-846 \\
\hline Bis(2-ethylhexyl) phthalate & EPA8270 & $\mathrm{mg} / \mathrm{kg}$ & 0.0333 & SW-846 \\
\hline 4-Bromophenyl phenyl ether & EPA8270 & $\mathrm{mg} / \mathrm{kg}$ & 0.0333 & SW-846 \\
\hline Butylbenzyl phthalate & EPA8270 & $\mathrm{mg} / \mathrm{kg}$ & 0.0333 & SW-846 \\
\hline 4-Chloroaniline & EPA8270 & $\mathrm{mg} / \mathrm{kg}$ & 0.0333 & SW-846 \\
\hline $\begin{array}{l}\text { 4-Chloro-3-methylphenol } \\
\text { (p-Chloro-m-cresol) }\end{array}$ & EPA8270 & $\mathrm{mg} / \mathrm{kg}$ & 0.0333 & SW-846 \\
\hline 2-Chloronaphthalene & EPA8270 & $\mathrm{mg} / \mathrm{kg}$ & 0.0333 & SW-846 \\
\hline 2-Chlorophenol & EPA8270 & $\mathrm{mg} / \mathrm{kg}$ & 0.0333 & SW-846 \\
\hline 4-Chlorophenyl phenyl ether & EPA8270 & $\mathrm{mg} / \mathrm{kg}$ & 0.0333 & SW-846 \\
\hline Chrysene & EPA8270 & $\mathrm{mg} / \mathrm{kg}$ & 0.00333 & SW-846 \\
\hline m-Cresol(3-Methylphenol) & EPA8270 & $\mathrm{mg} / \mathrm{kg}$ & 0.330 & SW-846 \\
\hline o-Cresol (2-Methylphenol) & EPA8270 & $\mathrm{mg} / \mathrm{kg}$ & 0.0333 & SW-846 \\
\hline p-Cresol (4-Methylphenol) & EPA8270 & $\mathrm{mg} / \mathrm{kg}$ & 0.0333 & SW-846 \\
\hline Dibenzo $[a, h]$ anthracene & EPA8270 & $\mathrm{mg} / \mathrm{kg}$ & 0.00333 & SW-846 \\
\hline Dibenzofuran & EPA8270 & $\mathrm{mg} / \mathrm{kg}$ & 0.0333 & SW-846 \\
\hline Di-n-butyl phthalate & EPA8270 & $\mathrm{mg} / \mathrm{kg}$ & 0.0333 & SW-846 \\
\hline 1,2-Dichlorobenzene & EPA8270 & $\mathrm{mg} / \mathrm{kg}$ & 0.0333 & SW-846 \\
\hline 1,3-Dichlorobenzene & EPA8270 & $\mathrm{mg} / \mathrm{kg}$ & 0.0333 & SW-846 \\
\hline 1,4-Dichlorobenzene & EPA8270 & $\mathrm{mg} / \mathrm{kg}$ & 0.0333 & SW-846 \\
\hline 3,3'-Dichlorobenzidine & EPA8270 & $\mathrm{mg} / \mathrm{kg}$ & 0.0333 & SW-846 \\
\hline 2,4-Dichlorophenol & EPA8270 & $\mathrm{mg} / \mathrm{kg}$ & 0.0333 & SW-846 \\
\hline
\end{tabular}


Table F-1. Analytical Methods and Quantitation Limits for General Engineering Laboratories, Inc., Continued

\begin{tabular}{|c|c|c|c|c|}
\hline Analyte & Method & Units & EQL & Reference \\
\hline Diethyl phthalate & EPA8270 & $\mathrm{mg} / \mathrm{kg}$ & 0.0333 & SW-846 \\
\hline 2,4-Dimethylphenol & EPA8270 & $\mathrm{mg} / \mathrm{kg}$ & 0.0333 & SW-846 \\
\hline Dimethyl phthalate & EPA8270 & $\mathrm{mg} / \mathrm{kg}$ & 0.0333 & SW-846 \\
\hline 2,4-Dinitrophenol & EPA8270 & $\mathrm{mg} / \mathrm{kg}$ & 0.333 & SW-846 \\
\hline 2,4-Dinitrotoluene & EPA8270 & $\mathrm{mg} / \mathrm{kg}$ & 0.00333 & SW-846 \\
\hline 2,6-Dinitrotoluene & EPA8270 & $\mathrm{mg} / \mathrm{kg}$ & 0.00333 & SW-846 \\
\hline Di-n-octyl phthalate & EPA8270 & $\mathrm{mg} / \mathrm{kg}$ & 0.0333 & SW-846 \\
\hline Fluoranthene & EPA8270 & $\mathrm{mg} / \mathrm{kg}$ & 0.0333 & SW-846 \\
\hline Fluorene & EPA8270 & $\mathrm{mg} / \mathrm{kg}$ & 0.0333 & SW-846 \\
\hline Hexachlorobenzene & EPA8270. & $\mathrm{mg} / \mathrm{kg}$ & 0.00333 & SW-846 \\
\hline Hexachlorobutadiene & EPA8270 & $\mathrm{mg} / \mathrm{kg}$ & 0.0333 & SW-846 \\
\hline Hexachlorocyclopentadiene & EPA8270 & $\mathrm{mg} / \mathrm{kg}$ & 0.0333 & SW-846 \\
\hline Hexachloroethane & EPA8270 & $\mathrm{mg} / \mathrm{kg}$ & 0.00333 & SW-846 \\
\hline Indeno $[1,2,3-c, d]$ pyrene & EPA8270 & $\mathrm{mg} / \mathrm{kg}$ & 0.00333 & SW-846 \\
\hline Isophorone & EPA8270 & $\mathrm{mg} / \mathrm{kg}$ & 0.0333 & SW-846 \\
\hline $\begin{array}{l}\text { 2-Methyl-4,6-dinitrophenol } \\
\text { (4,6-Dinitro-ortho-cresol) }\end{array}$ & EPA8270 & $\mathrm{mg} / \mathrm{kg}$ & 0.333 & SW-846 \\
\hline 2-Methylnaphthalene & EPA8270 & $\mathrm{mg} / \mathrm{kg}$ & 0.0333 & SW-846 \\
\hline Naphthalene & EPA8270 & $\mathrm{mg} / \mathrm{kg}$ & 0.0333 & SW-846 \\
\hline 2-Nitroaniline & EPA8270 & $\mathrm{mg} / \mathrm{kg}$ & 0.0333 & SW-846 \\
\hline 3-Nitroaniline & EPA8270 & $\mathrm{mg} / \mathrm{kg}$ & 0.0333 & SW-846 \\
\hline 4-Nitroaniline & EPA8270 & $\mathrm{mg} / \mathrm{kg}$ & 0.0333 & SW-846 \\
\hline Nitrobenzene & EPA8270 & $\mathrm{mg} / \mathrm{kg}$ & 0.0333 & SW-846 \\
\hline 2-Nitrophenol & EPA8270 & $\mathrm{mg} / \mathrm{kg}$ & 0.0333 & SW-846 \\
\hline 4-Nitrophenol & EPA8270 & $\mathrm{mg} / \mathrm{kg}$ & 0.0333 & SW-846 \\
\hline N-Nitrosodipropylamine & EPA8270 & $\mathrm{mg} / \mathrm{kg}$ & 0.0333 & SW-846 \\
\hline N-Nitrosodiphenylamine & EPA8270 & $\mathrm{mg} / \mathrm{kg}$ & 0.0333 & SW-846 \\
\hline Pentachlorophenol & EPA8270 & mg/kg & 0.0333 & SW-846 \\
\hline Phenanthrene & EPA8270 & $\mathrm{mg} / \mathrm{kg}$ & 0.0333 & SW-846 \\
\hline Phenol & EPA8270 & $\mathrm{mg} / \mathrm{kg}$ & 0.0333 & SW-846 \\
\hline Pyrene & EPA8270 & $\mathrm{mg} / \mathrm{kg}$ & 0.0333 & SW-846 \\
\hline 1,$2 ; 4$-Trichlorobenzene & EPA8270 & $\mathrm{mg} / \mathrm{kg}$ & 0.00333 & SW-846 \\
\hline 2,4,5-Trichlorophenol & EPA8270 & $\mathrm{mg} / \mathrm{kg}$ & 0.0333 & SW-846 \\
\hline 2,4,6-Trichlorophenol & EPA8270 & $\mathrm{mg} / \mathrm{kg}$ & 0.0333 & SW-846 \\
\hline \multicolumn{5}{|l|}{ Specified Analyses } \\
\hline Cation exchange capacity & EPA9081 & meq/5 & 5 & SW-846 \\
\hline
\end{tabular}


Table F-1. Analytical Methods and Quantitation Limits for General Engineering Laboratories, Inc., Continued

\begin{tabular}{|c|c|c|c|c|}
\hline Analyte & Method & Units & EQL & Reference \\
\hline Chloride & EPA300.0 & $\mathrm{mg} / \mathrm{kg}$ & 2.5 & $600 / 4-79-020$ \\
\hline Nitrate/Nitrite & EPA353.1 & $\mathrm{mg} / \mathrm{kg}$ & 0.1 & $600 / 4-79-020$ \\
\hline Total organic nitrogen & EPA351.2 & $\mathrm{mg} / \mathrm{kg}$ & 0.2 & $600 / 4-79-020$ \\
\hline Orthophosphate & EPA365.2 & $\mathrm{mg} / \mathrm{kg}$ & 1 & 600/4-79-020 \\
\hline Particle size distribution & ACOE 1906 & & NA & \\
\hline Phenols & EPA420.2 & $\mu \mathrm{g} / \mathrm{kg}$ & 20 & $600 / 4-79-020$ \\
\hline Sulfide & EPA376.2 & $\mathrm{mg} / \mathrm{kg}$ & 2.5 & $600 / 4-79-020$ \\
\hline Total organic carbon & EPA415.1 & $\mathrm{mg} / \mathrm{kg}$ & 100 & $600 / 4-79-020$ \\
\hline Total phosphate & EPA365.4 & $\mathrm{mg} / \mathrm{kg}$ & $5 \quad$ & $600 / 4-79-020$ \\
\hline \multicolumn{5}{|c|}{ Water Analyses } \\
\hline \multicolumn{5}{|l|}{ TAL: Inorganics } \\
\hline Aluminum & EPA6010A & $\mu \mathrm{g} / \mathrm{L}$ & 20 & SW-846 \\
\hline Antimony & EPA6010A & $\mu \mathrm{g} / \mathrm{L}$ & 5 & SW-846 \\
\hline Arsenic & EPA6010A & $\mu \mathrm{g} / \mathrm{L}$ & 5 & SW-846 \\
\hline Barium & EPA6010A & $\mu \mathrm{g} / \mathrm{L}$ & 3 & SW-846 \\
\hline Beryllium & EPA6010A & $\mu \mathrm{g} / \mathrm{L}$ & 3 & SW-846 \\
\hline Cadmium & EPA6010A & $\mu \mathrm{g} / \mathrm{L}$ & 2 & SW-846 \\
\hline Calcium & EPA6010A & $\mu \mathrm{g} / \mathrm{L}$ & 20 & SW-846 \\
\hline Chromium & EPA6010A & $\mu \mathrm{g} / \mathrm{L}$ & 4 & SW-846 \\
\hline Cobalt & EPA6010A & $\mu \mathrm{g} / \mathrm{L}$ & 4 & SW-846 \\
\hline Copper & EPA6010A & $\mu \mathrm{g} / \mathrm{L}$ & 4 & SW-846 \\
\hline Cyanide & EPA335.3 & $\mu \mathrm{g} / \mathrm{L}$ & 20 & $600 / 4-79-020$ \\
\hline Iron & EPA6010A & $\mu \mathrm{g} / \mathrm{L}$ & 18 & SW-846 \\
\hline Lead & EPA6010A & $\mu \mathrm{g} / \mathrm{L}$ & 5 & SW-846 \\
\hline Magnesium & EPA6010A & $\mu \mathrm{g} / \mathrm{L}$ & 20 & SW-846 \\
\hline Manganese & EPA6010A & $\mu \mathrm{g} / \mathrm{L}$ & 2 & SW-846 \\
\hline Mercury & EPA7470 & $\mu \mathrm{g} / \mathrm{L}$ & 1 & SW-846 \\
\hline Nickel & EPA6010A & $\mu \mathrm{g} / \mathrm{L}$ & 10 & SW-846 \\
\hline Potassium & EPA6010A & $\mu \mathrm{g} / \mathrm{L}$ & 500 & SW-846 \\
\hline Selenium & EPA6010A & $\mu \mathrm{g} / \mathrm{L}$ & 50 & SW-846 \\
\hline Silver & EPA6010A & $\mu \mathrm{g} / \mathrm{L}$ & 2 & SW-846 \\
\hline Sodium & EPA6010A & $\mu \mathrm{g} / \mathrm{L}$ & 100 & SW-846 \\
\hline Thallium & EPA6010A & $\mu \mathrm{g} / \mathrm{L}$ & 5 & SW-846 \\
\hline Vanadium & EPA6010A & $\mu \mathrm{g} / \mathrm{L}$ & 10 & SW-846 \\
\hline Zinc & EPA6010A & $\mu \mathrm{g} / \mathrm{L}$ & 5 & SW-846 \\
\hline
\end{tabular}


Table F-1. Analytical Methods and Quantitation Limits for General Engineering Laboratories, Inc., Continued

\begin{tabular}{|c|c|c|c|c|}
\hline Analyte & Method & Units & EQL & Reference \\
\hline \multicolumn{5}{|l|}{ TCL: Pesticides/PCBs } \\
\hline Aldrin & EPA8080 & $\mu \mathrm{g} / \mathrm{L}$ & 0.025 & SW-846 \\
\hline Aroclor 1016 & EPA8080 & $\mu \mathrm{g} / \mathrm{L}$ & 0.1 & SW-846 \\
\hline Aroclor 1221 & EPA8080 & $\mu \mathrm{g} / \mathrm{L}$ & 0.1 & SW-846 \\
\hline Aroclor 1232 & EPA8080 & $\mu \mathrm{g} / \mathrm{L}$ & 0.1 & SW-846 \\
\hline Aroclor 1242 & EPA8080 & $\mu \mathrm{g} / \mathrm{L}$ & 0.1 & SW-846 \\
\hline Aroclor 1248 & EPA8080 & $\mu \mathrm{g} / \mathrm{L}$ & 0.1 & SW-846 \\
\hline Aroclor 1254 & EPA8080 & $\mu \mathrm{g} / \mathrm{L}$ & 0.1 & SW-846 \\
\hline Aroclor 1260 & EPA8080 & $\mu \mathrm{g} / \mathrm{L}$ & 0.1 & SW-846 \\
\hline alpha-BHC & EPA8080 & $\mu \mathrm{g} / \mathrm{L}$ & 0.025 & SW-846 \\
\hline beta-BHC & EPA8080 & $\mu \mathrm{g} / \mathrm{L}$ & 0.05 & SW-846 \\
\hline delta-BHC & EPA8080 & $\mu \mathrm{g} / \mathrm{L}$ & 0.05 & SW-846 \\
\hline gamma-BHC (Lindane) & EPA8080 & $\mu \mathrm{g} / \mathrm{L}$ & 0.025 & SW-846 \\
\hline alpha-Chlordane & EPA8080 & $\mu \mathrm{g} / \mathrm{L}$ & 0.05 & SW-846 \\
\hline gamma-Chlordane & EPA8080 & $\mu \mathrm{g} / \mathrm{L}$ & 0.05 & SW-846 \\
\hline$p, p^{\prime}-D D D$ & EPA8080 & $\mu \mathrm{g} / \mathrm{L}$ & 0.1 & SW-846 \\
\hline$p, p^{\prime}-D D E$ & EPA8080 & $\mu \mathrm{g} / \mathrm{L}$ & 0.05 & SW-846 \\
\hline $\mathrm{p}, \mathrm{p}^{\prime}-\mathrm{DDT}$ & EPA8080 & $\mu \mathrm{g} / \mathrm{L}$ & 0.1 & SW-846 \\
\hline Dieldrin & EPA8080 & $\mu g / L$ & 0.05 & SW-846 \\
\hline Endosulfan I & EPA8080 & $\mu \mathrm{g} / \mathrm{L}$ & 0.05 & SW-846 \\
\hline Endosulfan II & EPA8080 & $\mu \mathrm{g} / \mathrm{L}$ & 0.1 & SW-846 \\
\hline Endosulfan sulfate & EPA8080 & $\mu \mathrm{g} / \mathrm{L}$ & 0.1 & SW-846 \\
\hline Endrin & EPA8080 & $\mu \mathrm{g} / \mathrm{L}$ & 0.05 & SW-846 \\
\hline Endrin ketone & EPA8080 & $\mu \mathrm{g} / \mathrm{L}$ & 0.1 & SW-846 \\
\hline Heptachlor & EPA8080 & $\mu \mathrm{g} / \mathrm{L}$ & 0.05 & SW-846 \\
\hline Heptachlor epoxide & EPA8080 & $\mu \mathrm{g} / \mathrm{L}$ & 0.05 & SW-846 \\
\hline Methoxychlor & EPA8080 & $\mu \mathrm{g} / \mathrm{L}$ & 0.5 & SW-846 \\
\hline Toxaphene & EPA8080 & $\mu \mathrm{g} / \mathrm{L}$ & 1 & SW-846 \\
\hline \multicolumn{5}{|c|}{ TCL: Volatile Organic Compounds } \\
\hline Acetone & EPA8260 & $\mu \mathrm{g} / \mathrm{L}$ & 5 & SW-846 \\
\hline Benzene & EPA8260 & $\mu \mathrm{g} / \mathrm{L}$ & 0.5 & SW-846 \\
\hline Bromodichloromethane & EPA8260 & $\mu \mathrm{g} / \mathrm{L}$ & 0.05 & SW-846 \\
\hline Bromoform & EPA8260 & $\mu \mathrm{g} / \mathrm{L}$ & 0.05 & SW-846 \\
\hline $\begin{array}{l}\text { Bromomethane } \\
\text { (Methyl bromide) }\end{array}$ & ÉPA8260 & $\mu \mathrm{g} / \mathrm{L}$ & 0.1 & SW-846 \\
\hline Carbon disulfide & EPA8260 & $\mu \mathrm{g} / \mathrm{L}$ & 1 & SW-846 \\
\hline
\end{tabular}


Table F-1. Analytical Methods and Quantitation Limits for General Engineering Laboratories, Inc., Continued

\begin{tabular}{|c|c|c|c|c|}
\hline Analyte & Method & Units & EQL & Reference \\
\hline Carbon tetrachloride & EPA8260 & $\mu \mathrm{g} / \mathrm{L}$ & 0.05 & SW-846 \\
\hline Chlorobenzene & EPA8260 & $\mu \mathrm{g} / \mathrm{L}$ & 0.05 & SW-846 \\
\hline Chlorodibromomethane & EPA8260 & $\mu \mathrm{g} / \mathrm{L}$ & 0.05 & SW-846 \\
\hline Chloroethane & EPA8260 & $\mu \mathrm{g} / \mathrm{L}$ & 0.1 & SW-846 \\
\hline Chloroethene (Vinyl chloride) & EPA8260 & $\mu \mathrm{g} / \mathrm{L}$ & 0.1 & SW-846 \\
\hline Chloroform & EPA8260 & $\mu \mathrm{g} / \mathrm{L}$ & 0.05 & SW-846 \\
\hline Chloromethane (Methyl chloride) & EPA8260 & $\mu \mathrm{g} / \mathrm{L}$ & 0.1 & SW-846 \\
\hline 1,1-Dichloroethane & EPA8260 & $\mu \mathrm{g} / \mathrm{L}$ & 0.05 & SW-846 \\
\hline 1,2-Dichloroethane & EPA8260 & $\mu \mathrm{g} / \mathrm{L}$ & 0.05 & SW-846 \\
\hline 1,1-Dichloroethylene & EPA8260 & $\mu \mathrm{g} / \mathrm{L}$ & 0.05 & SW-846 \\
\hline 1,2-Dichloroethylene (total) & EPA8260 & $\mu \mathrm{g} / \mathrm{L}$ & 0.1 & SW-846 \\
\hline $\begin{array}{l}\text { Dichloromethane } \\
\text { (Methylene chloride) }\end{array}$ & EPA8260 & $\mu \mathrm{g} / \mathrm{L}$ & 0.5 & SW-846 \\
\hline 1,2-Dichloropropane & EPA8260 & $\mu \mathrm{g} / \mathrm{L}$ & 0.05 & SW-846 \\
\hline cis-1,3-Dichloropropene & EPA8260 & $\mu \mathrm{g} / \mathrm{L}$ & 0.05 & SW-846 \\
\hline trans-1,3-Dichloropropene & EPA8260 & $\mu \mathrm{g} / \mathrm{L}$ & 0.05 & SW-846 \\
\hline Ethylbenzene & EPA8260 & $\mu \mathrm{g} / \mathrm{L}$ & 0.05 & SW-846 \\
\hline 2-Hexanone & EPA8260 & $\mu \mathrm{g} / \mathrm{L}$ & 1 & SW-846 \\
\hline Methyl ethyl ketone & EPA8260 & $\mu \mathrm{g} / \mathrm{L}$ & 1 & SW-846 \\
\hline Methyl isobutyl ketone & EPA8260 & $\mu \mathrm{g} / \mathrm{L}$ & 1 & SW-846 \\
\hline Styrene & EPA8260 & $\mu \mathrm{g} / \mathrm{L}$ & 0.05 & SW-846 \\
\hline 1,1,2,2-Tetrachloroethane & EPA8260 & $\mu \mathrm{g} / \mathrm{L}$ & 0.05 & SW-846 \\
\hline Tetrachloroethylene & EPA8260 & $\mu \mathrm{g} / \mathrm{L}$ & 0.05 & SW-846 \\
\hline Toluene & EPA8260 & $\mu g / \mathrm{L}$ & 0.5 & SW-846 \\
\hline 1,1,1-Trichloroethane & EPA8260 & $\mu \mathrm{g} / \mathrm{L}$ & 0.05 & SW-846 \\
\hline 1,1,2-Trichloroethane & EPA8260 & $\mu \mathrm{g} / \mathrm{L}$ & 0.05 & SW-846 \\
\hline Trichloroethylene & EPA8260 & $\mu \mathrm{g} / \mathrm{L}$ & 0.05 & SW-846 \\
\hline Vinyl acetate & EPA8260 & $\mu \mathrm{g} / \mathrm{L}$ & 1 & SW-846 \\
\hline Xylenes (total) & EPA8260 & $\mu \mathrm{g} / \mathrm{L}$ & 0.15 & SW-846 \\
\hline \multicolumn{5}{|c|}{$\begin{array}{l}\text { TCL: Semivolatile Organic Compounds (B/N/A Extractables), including PAHs and } \\
\text { Phenols }\end{array}$} \\
\hline Acenaphthene & EPA8270 & $\mathrm{mg} / \mathrm{L}$ & 0.001 & SW-846 \\
\hline Acenaphthylene & EPA8270 & $\mathrm{mg} / \mathrm{L}$ & 0.001 & SW-846 \\
\hline Anthracene & EPA8270 & $\mathrm{mg} / \mathrm{L}$ & 0.001 & SW-846 \\
\hline Benzo $[\alpha]$ anthracene & EPA8270 & $\mathrm{mg} / \mathrm{L}$ & 0.0001 & SW-846 \\
\hline Benzo[b]fluoranthene & EPA8270 & $\mathrm{mg} / \mathrm{L}$ & 0.0001 & SW-846 \\
\hline Benzo $[k]$ fluoranthene & EPA8270 & $\mathrm{mg} / \mathrm{L}$ & 0.0001 & SW-846 \\
\hline
\end{tabular}


Table F-1. Analytical Methods and Quantitation Limits for General Engineering Laboratories, Inc., Continued

\begin{tabular}{|c|c|c|c|c|}
\hline Analyte & Method & Units & EQL & Reference \\
\hline Benzoic acid & EPA8270 & $\mathrm{mg} / \mathrm{L}$ & 0.001 & SW-846 \\
\hline Benzo[g,h,i]perylene & EPA8270 & $\mathrm{mg} / \mathrm{L}$ & 0.001 & SW-846 \\
\hline Benzo[a]pyrene & EPA8270 & $\mathrm{mg} / \mathrm{L}$ & 0.0001 & SW-846 \\
\hline Benzyl alcohol & EPA8270 & $\mathrm{mg} / \mathrm{L}$ & 0.001 & SW-846 \\
\hline Bis(2-chloroethoxy) methane & EPA8270 & $\mathrm{mg} / \mathrm{L}$ & 0.001 & SW-846 \\
\hline Bis(2-chloroethyl) ether & EPA8270 & $\mathrm{mg} / \mathrm{L}$ & 0.001 & SW-846 \\
\hline Bis(2-chloroisopropyl) ether & EPA8270 & $m g / L$ & 0.001 & SW-846 \\
\hline Bis(2-ethylhexyl) phthalate & EPA8270 & $\mathrm{mg} / \mathrm{L}$ & 0.001 & SW-846 \\
\hline 4-Bromophenyl phenyl ether & EPA8270 & $\mathrm{mg} / \mathrm{L}$ & 0.001 & SW-846 \\
\hline Butylbenzyl phthalate & EPA8270 & $\mathrm{mg} / \mathrm{L}$ & 0.001 & SW-846 \\
\hline 4-Chloroaniline & EPA8270 & $\mathrm{mg} / \mathrm{L}$ & 0.001 & SW-846 \\
\hline $\begin{array}{l}\text { 4-Chloro-3-methylphenol } \\
\text { (p-Chloro-m-cresol) }\end{array}$ & EPA8270 & $\mathrm{mg} / \mathrm{L}$ & 0.001 & SW-846 \\
\hline 2-Chloronaphthalene & EPA8270 & $\mathrm{mg} / \mathrm{L}$ & 0.001 & SW-846 \\
\hline 2-Chlorophenol & EPA8270 & $\mathrm{mg} / \mathrm{L}$ & 0.001 & SW-846 \\
\hline 4-Chlorophenyl phenyl ether & EPA8270 & $\mathrm{mg} / \mathrm{L}$ & 0.001 & SW-846 \\
\hline Chrysene & EPA8270 & $\mathrm{mg} / \mathrm{L}$ & 0.0001 & SW-846 \\
\hline Dibenzo $[a, h]$ anthracene & EPA8270 & $\mathrm{mg} / \mathrm{L}$ & 0.0001 & SW-846 \\
\hline Dibenzofuran & EPA8270 & $\mathrm{mg} / \mathrm{L}$ & 0.001 & SW-846 \\
\hline Di-n-butyl phthalate & EPA8270 & $\mathrm{mg} / \mathrm{L}$ & 0.001 & SW-846 \\
\hline 1,2-Dichlorobenzene & EPA8270 & $\mathrm{mg} / \mathrm{L}$ & 0.001 & SW-846 \\
\hline 1,3-Dichlorobenzene & EPA8270 & $\mathrm{mg} / \mathrm{L}$ & 0.001 & SW-846 \\
\hline 1,4-Dichlorobenzene & EPA8270 & $\mathrm{mg} / \mathrm{L}$ & 0.001 & SW-846 \\
\hline 3,3'-Dichlorobenzidine & EPA8270 & $\mathrm{mg} / \mathrm{L}$ & 0.001 & SW-846 \\
\hline 2,4-Dichlorophenol & EPA8270 & $\mathrm{mg} / \mathrm{L}$ & 0.001 & SW-846 \\
\hline Diethyl phthalate & EPA8270 & $\mathrm{mg} / \mathrm{L}$ & 0.001 & SW-846 \\
\hline 2,4-Dimethylphenol & EPA8270 & $\mathrm{mg} / \mathrm{L}$ & 0.001 & SW-846 \\
\hline Dimethyl phthalate & EPA8270 & $\mathrm{mg} / \mathrm{L}$ & 0.001 & SW-846 \\
\hline $\begin{array}{r}\text { 2-Methyl-4,6-dinitrophenol } \\
\text { (4,6-Dinitro-ortho-cresol) }\end{array}$ & EPA8270 & $\mathrm{mg} / \mathrm{L}$ & 0.01 & SW-846 \\
\hline 2,4-Dinitrophenol & EPA8270 & $\mathrm{mg} / \mathrm{L}$ & 0.01 & SW-846 \\
\hline 2,4-Dinitrotoluene & EPA8270 & $\mathrm{mg} / \mathrm{L}$ & 0.0001 & SW-846 \\
\hline 2,6-Dinitrotoluene & EPA8270 & $\mathrm{mg} / \mathrm{L}$ & 0.0001 & SW-846 \\
\hline Di-n-octyl phthalate & EPA8270 & $\mathrm{mg} / \mathrm{L}$ & 0.001 & SW-846 \\
\hline \begin{tabular}{|l} 
Fluoranthene. \\
\end{tabular} & EPA8270 & $\mathrm{mg} / \mathrm{L}$ & 0.001 & SW-846 \\
\hline Fluorene & EPA8270 & $\mathrm{mg} / \mathrm{L}$ & 0.001 & SW-846 \\
\hline Hexachlorobenzene & EPA8270 & $\mathrm{mg} / \mathrm{L}$ & 0.0001 & SW-846 \\
\hline
\end{tabular}


Table F-1. Analytical Methods and Quantitation Limits for General Engineering Laboratories, Inc., Continued

\begin{tabular}{|l|l|l|l|l|}
\hline Analyte & Method & Units & EQL & Reference \\
\hline Hexachlorobutadiene & EPA8270 & $\mathrm{mg} / \mathrm{L}$ & 0.001 & $\mathrm{SW}-846$ \\
\hline Hexachlorocyclopentadiene & EPA8270 & $\mathrm{mg} / \mathrm{L}$ & 0.001 & $\mathrm{SW}-846$ \\
\hline Hexachloroethane & EPA8270 & $\mathrm{mg} / \mathrm{L}$ & 0.0001 & $\mathrm{SW}-846$ \\
\hline Indeno[1,2,3-c,d]pyrene & EPA8270 & $\mathrm{mg} / \mathrm{L}$ & 0.0001 & $\mathrm{SW}-846$ \\
\hline Isophorone & EPA8270 & $\mathrm{mg} / \mathrm{L}$ & 0.001 & $\mathrm{SW}-846$ \\
\hline 2-Methylnaphthalene & EPA8270 & $\mathrm{mg} / \mathrm{L}$ & 0.001 & $\mathrm{SW}-846$ \\
\hline m-Cresol (3-Methylphenol) & EPA8270 & $\mathrm{mg} / \mathrm{L}$ & 0.01 & SW-846 \\
\hline o-Cresol (2-Methylphenol) & EPA8270 & $\mathrm{mg} / \mathrm{L}$ & 0.001 & $\mathrm{SW}-846$ \\
\hline p-Cresol (4-Methylphenol) & EPA8270 & $\mathrm{mg} / \mathrm{L}$ & 0.001 & $\mathrm{SW}-846$ \\
\hline Naphthalene & EPA8270 & $\mathrm{mg} / \mathrm{L}$ & 0.001 & $\mathrm{SW}-846$ \\
\hline 2-Nitroaniline & EPA8270 & $\mathrm{mg} / \mathrm{L}$ & 0.001 & SW-846 \\
\hline 3-Nitroaniline & EPA8270 & $\mathrm{mg} / \mathrm{L}$ & 0.001 & $\mathrm{SW}-846$ \\
\hline 4-Nitroaniline & EPA8270 & $\mathrm{mg} / \mathrm{L}$ & 0.001 & $\mathrm{SW}-846$ \\
\hline Nitrobenzene & EPA8270 & $\mathrm{mg} / \mathrm{L}$ & 0.001 & $\mathrm{SW}-846$ \\
\hline 2-Nitrophenol & EPA8270 & $\mathrm{mg} / \mathrm{L}$ & 0.001 & $\mathrm{SW}-846$ \\
\hline 4-Nitrophenol & EPA8270 & $\mathrm{mg} / \mathrm{L}$ & 0.001 & $\mathrm{SW}-846$ \\
\hline N-Nitrosodiphenylamine & EPA8270 & $\mathrm{mg} / \mathrm{L}$ & 0.001 & $\mathrm{SW}-846$ \\
\hline N-Nitrosodipropylamine & EPA8270 & $\mathrm{mg} / \mathrm{L}$ & 0.001 & $\mathrm{SW}-846$ \\
\hline Pentachlorophenol & EPA8270 & $\mathrm{mg} / \mathrm{L}$ & 0.001 & $\mathrm{SW}-846$ \\
\hline Phenanthrene & EPA8270 & $\mathrm{mg} / \mathrm{L}$ & 0.001 & $\mathrm{SW}-846$ \\
\hline Phenol & EPA8270 & $\mathrm{mg} / \mathrm{L}$ & 0.001 & $\mathrm{SW}-846$ \\
\hline Pyrene & EPA8270 & $\mathrm{mg} / \mathrm{L}$ & 0.001 & $\mathrm{SW}-846$ \\
\hline 1,2,4-Trichlorobenzene & EPA8270 & $\mathrm{mg} / \mathrm{L}$ & 0.0001 & $\mathrm{SW}-846$ \\
\hline 2,4,5-Trichlorophenol & EPA8270 & $\mathrm{mg} / \mathrm{L}$ & 0.001 & $\mathrm{SW}-846$ \\
\hline 2,4,6-Trichlorophenol & EPA8270 & $\mathrm{mg} / \mathrm{L}$ & 0.0001 & $\mathrm{SW}-846$ \\
\hline
\end{tabular}

Table F-2. Analytical Methods and Quantitation Limits for Roy F. Weston Laboratories, Inc.

\begin{tabular}{|l|l|l|l|l|}
\hline \multicolumn{5}{|c|}{ Soil and Sediment Analyses } \\
\hline Analyte & Method & Units & EQL & Reference \\
\hline TCL: Pesticides/PCBs with TIC & EPA8080 & $\mu \mathrm{g} / \mathrm{kg}$ & 1.7 & CLP \\
\hline Aldrin & EPA8080 & $\mu \mathrm{g} / \mathrm{kg}$ & 33 & CLP \\
\hline Aroclor 1016 & & & \\
\hline
\end{tabular}


Table F-2. Analytical Methods and Quantitation Limits for Roy F. Weston Laboratories, Inc., Continued

\begin{tabular}{|c|c|c|c|c|}
\hline Analyte & Method & Units & EQL & Reference \\
\hline Aroclor 1221 & EPA8080 & $\mu \mathrm{g} / \mathrm{kg}$ & 67 & CLP \\
\hline Aroclor 1232 & EPA8080 & $\mu \mathrm{g} / \mathrm{kg}$ & 33 & CLP \\
\hline Aroclor 1242 & EPA8080 & $\mu \mathrm{g} / \mathrm{kg}$ & 33 & CLP \\
\hline Aroclor 1248 & EPA8080 & $\mu \mathrm{g} / \mathrm{kg}$ & 33 & CLP \\
\hline Aroclor 1254 & EPA8080 & $\mu \mathrm{g} / \mathrm{kg}$ & 33 & CLP \\
\hline Aroclor 1260 & EPA8080 & $\mu \mathrm{g} / \mathrm{kg}$ & 33 & CLP \\
\hline alpha-BHC & EPA8080 & $\mu \mathrm{g} / \mathrm{kg}$ & 1.7 & CLP \\
\hline beta-BHC & EPA8080 & $\mu \mathrm{g} / \mathrm{kg}$ & 1.7 & CLP \\
\hline delta-BHC & EPA8080 & $\mu \mathrm{g} / \mathrm{kg}$ & 1.7 & CLP \\
\hline gamma-BHC (Lindane) & EPA8080 & $\mu \mathrm{g} / \mathrm{kg}$ & 1.7 & CLP \\
\hline alpha-Chlordane & EPA8080 & $\mu \mathrm{g} / \mathrm{kg}$ & 1.7 & CLP \\
\hline gamma-Chlordane & EPA8080 & $\mu \mathrm{g} / \mathrm{kg}$ & 1.7 & CLP \\
\hline $\mathrm{p}, \mathrm{p}^{\prime}-\mathrm{DDD}$ & EPA8080 & $\mu \mathrm{g} / \mathrm{kg}$ & 3.3 & CLP \\
\hline$p, p^{\prime}-D D E$ & EPA8080 & $\mu \mathrm{g} / \mathrm{kg}$ & 2.7 & CLP \\
\hline$p, p^{\prime}-D D T$ & EPA8080 & $\mu \mathrm{g} / \mathrm{kg}$ & 3.3 & CLP \\
\hline Dieldrin & EPA8080 & $\mu \mathrm{g} / \mathrm{kg}$ & 1.3 & CLP \\
\hline Endosulfan I & EPA8080 & $\mu \mathrm{g} / \mathrm{kg}$ & 1.7 & CLP \\
\hline Endosulfan II & EPA8080 & $\mu \mathrm{g} / \mathrm{kg}$ & 2.7 & CLP \\
\hline Endosulfan sulfate & EPA8080 & $\mu \mathrm{g} / \mathrm{kg}$ & 3.3 & CLP \\
\hline Endrin & EPA8080 & $\mu \mathrm{g} / \mathrm{kg}$ & 3.3 & CLP \\
\hline Endrin ketone & EPA8080 & $\mu \mathrm{g} / \mathrm{kg}$ & 3.3 & CLP \\
\hline Heptachlor & EPA8080 & $\mu \mathrm{g} / \mathrm{kg}$ & 1.7 & CLP \\
\hline Heptachlor epoxide & EPA8080 & $\mu \mathrm{g} / \mathrm{kg}$ & 1.7 & CLP \\
\hline Methoxychlor & EPA8080 & $\mu \mathrm{g} / \mathrm{kg}$ & 17 & CLP \\
\hline Toxaphene & EPA8080 & $\mu \mathrm{g} / \mathrm{kg}$ & 160 & CLP \\
\hline \multicolumn{5}{|c|}{ TCL: Volatile Organic Compounds with TIC } \\
\hline Acetone & EPA8260 & $\mu \mathrm{g} / \mathrm{kg}$ & 10 & SW-846 \\
\hline Benzene & EPA8021 & $\mu \mathrm{g} / \mathrm{kg}$ & 0.09 & SW-846 \\
\hline Bromodichloromethane & EPA8260 & $\mu \mathrm{g} / \mathrm{kg}$ & 0.2 & SW-846 \\
\hline Bromoform & EPA8260 & $\mu \mathrm{g} / \mathrm{kg}$ & 2.0 & SW-846 \\
\hline $\begin{array}{l}\text { Bromomethane (Methyl } \\
\text { bromide) }\end{array}$ & EPA8260 & $\mu \mathrm{g} / \mathrm{kg}$ & 0.6 & SW-846 \\
\hline Carbon disulfide & EPA8260 & $\mu \mathrm{g} / \mathrm{kg}$ & 5 & SW-846 \\
\hline Carbon tetrachloride & EPA8021 & $\mu \mathrm{g} / \mathrm{kg}$ & 0.1 & SW-846 \\
\hline Chlorobenzene & EPA8260 & $\mu \mathrm{g} / \mathrm{kg}$ & 0.3 & SW-846 \\
\hline Chlorodibromomethane & EPA8260 & $\mu \mathrm{g} / \mathrm{kg}$ & 0.7 & SW-846 \\
\hline
\end{tabular}


Table F-2. Analytical Methods and Quantitation Limits for Roy F. Weston Laboratories, Inc., Continued

\begin{tabular}{|c|c|c|c|c|}
\hline Analyte & Method & Units & EQL & Reference \\
\hline Chloroethane & EPA8260 & $\mu \mathrm{g} / \mathrm{kg}$ & 0.2 & SW-846 \\
\hline Chloroethene (Vinyl chloride) & EPA8021 & $\mu \mathrm{g} / \mathrm{kg}$ & 0.2 & SW-846 \\
\hline Chloroform & EPA8260 & $\mu \mathrm{g} / \mathrm{kg}$ & 0.2 & SW-846 \\
\hline Chloromethane (Methyl chloride) & EPA8260 & $\mu \mathrm{g} / \mathrm{kg}$ & 0.3 & SW-846 \\
\hline 1,1-Dichloroethane & EPA8260 & $\mu \mathrm{g} / \mathrm{kg}$ & 0.3 & SW-846 \\
\hline 1,2-Dichloroethane & EPA8260 & $\mu \mathrm{g} / \mathrm{kg}$ & 0.2 & SW-846 \\
\hline 1,1-Dichloroethylene & EPA8260 & $\mu \mathrm{g} / \mathrm{kg}$ & 0.5 & SW-846 \\
\hline 1,2-Dichloroethylene (total) & EPA8260 & $\mu \mathrm{g} / \mathrm{kg}$ & 0.3 & SW-846 \\
\hline $\begin{array}{l}\text { Dichloromethane } \\
\text { (Methylene chloride) }\end{array}$ & EPA8021 & $\mu \mathrm{g} / \mathrm{kg}$ & 0.2 & $\mathrm{SW}-846$ \\
\hline 1,2-Dichloropropane & EPA8021 & $\mu \mathrm{g} / \mathrm{kg}$ & 0.06 & SW-846 \\
\hline cis-1,3-Dichloropropene & EPA8260 & $\mu \mathrm{g} / \mathrm{kg}$ & 5 & SW-846 \\
\hline trans-1,3-Dichloropropene & EPA8260 & $\mu \mathrm{g} / \mathrm{kg}$ & 5 & SW-846 \\
\hline Ethylbenzene & EPA8021 & $\mu \mathrm{g} / \mathrm{kg}$ & 0.05 & SW-846 \\
\hline 2-Hexanone & EPA8260 & $\mu \mathrm{g} / \mathrm{kg}$ & 10 & SW-846 \\
\hline Methyl ethyl ketone & EPA8260 & $\mu \mathrm{g} / \mathrm{kg}$ & 10 & SW-846 \\
\hline Methyl isobutyl ketone & EPA8260 & $\mathrm{ug} / \mathrm{kg}$ & 5 & SW-846 \\
\hline Styrene & EPA8021 & $\mu \mathrm{g} / \mathrm{kg}$ & 0.1 & SW-846 \\
\hline 1,1,2,2-Tetrachloroethane & EPA8021 & $\mu \mathrm{g} / \mathrm{kg}$ & 0.1 & SW-846 \\
\hline Tetrachloroethylene & EPA8260 & $\mu \mathrm{g} / \mathrm{kg}$ & 0.4 & SW-846 \\
\hline Toluene & EPA8021 & $\mu \mathrm{g} / \mathrm{kg}$ & 0.1 & SW-846 \\
\hline 1,1,1-Trichloroethane & EPA8260 & $\mu \mathrm{g} / \mathrm{kg}$ & 0.3 & SW-846 \\
\hline 1,1,2-Trichloroethane & EPA8260 & $\mu \mathrm{g} / \mathrm{kg}$ & 0.2 & SW-846 \\
\hline Trichloroethylene & EPA8021 & $\mu \mathrm{g} / \mathrm{kg}$ & 0.1 & SW-846 \\
\hline Vinyl acetate & EPA8260 & $\mu \mathrm{g} / \mathrm{kg}$ & 5 & SW-846 \\
\hline Xylenes (total) & EPA8260 & $\mu \mathrm{g} / \mathrm{kg}$ & 0.4 & SW-846 \\
\hline \multicolumn{5}{|c|}{$\begin{array}{l}\text { TCL: Semivolatile Organic Compounds (B/N/A Extractables), including PAHs and } \\
\text { Phenols with TIC }\end{array}$} \\
\hline Acenaphthene & EPA8270 & $\mu \mathrm{g} / \mathrm{kg}$ & 330 & SW-846 \\
\hline Acenaphthylene & EPA8270 & $\mu \mathrm{g} / \mathrm{kg}$ & 330 & SW-846 \\
\hline Anthracene & EPA8270 & $\mu \mathrm{g} / \mathrm{kg}$ & 330 & SW-846 \\
\hline Benzo $[a]$ anthracene & EPA8270 & $\mu \mathrm{g} / \mathrm{kg}$ & 330 & SW-846 \\
\hline Benzo[b]fluoranthene & EPA8270 & $\mu \mathrm{g} / \mathrm{kg}$ & 330 & SW-846 \\
\hline Benzo[k]fluoranthene & EPA8270 & $\mu \mathrm{g} / \mathrm{kg}$ & 330 & SW-846 \\
\hline Benzoic acid & EPA8270 & $\mu \mathrm{g} / \mathrm{kg}$ & 330 & SW-846 \\
\hline Benzo[g,h,i]perylene & EPA8270 & $\mu \mathrm{g} / \mathrm{kg}$ & 330 & SW-846 \\
\hline
\end{tabular}


Table F-2. Analytical Methods and Quantitation Limits for Roy F. Weston Laboratories, Inc., Continued

\begin{tabular}{|c|c|c|c|c|}
\hline Analyte & Method & Units & EQL & Reference \\
\hline Benzo[a]pyrene & EPA8270 & $\mu \mathrm{g} / \mathrm{kg}$ & 330 & SW-846 \\
\hline Benzyl alcohol & EPA8270 & $\mu \mathrm{g} / \mathrm{kg}$ & 330 & SW-846 \\
\hline Bis(2-chloroethoxy) methane & EPA8270 & $\mu \mathrm{g} / \mathrm{kg}$ & 330 & SW-846 \\
\hline Bis(2-chloroethyl) ether & EPA8270 & $\mu \mathrm{g} / \mathrm{kg}$ & 330 & SW-846 \\
\hline Bis(2-chloroisopropyl) ether & EPA8270 & $\mu \mathrm{g} / \mathrm{kg}$ & 330 & SW-846 \\
\hline Bis(2-ethylhexyl) phthalate & EPA8270 & $\mu \mathrm{g} / \mathrm{kg}$ & 330 & SW-846 \\
\hline 4-Bromophenyl phenyl ether & EPA8270 & $\mu \mathrm{g} / \mathrm{kg}$ & 330 & SW-846 \\
\hline Butylbenzyl phthalate & EPA8270M² & $\mu \mathrm{g} / \mathrm{kg}$ & 230 & SW-846 \\
\hline 4-Chloroaniline & EPA8270 & $\mu \mathrm{g} / \mathrm{kg}$ & 330 & SW-846 \\
\hline $\begin{array}{c}\text { 4-Chloro-3-methylphenol } \\
\text { (p-Chloro-m-cresol) }\end{array}$ & EPA8270M & $\mu \mathrm{g} / \mathrm{kg}$ & 240 & SW-846 \\
\hline 2-Chloronaphthalene & EPA8270 & $\mu \mathrm{g} / \mathrm{kg}$ & 330 & SW-846 \\
\hline 2-Chlorophenol & EPA8270M & $\mu \mathrm{g} / \mathrm{kg}$ & 210 & SW-846 \\
\hline 4-Chlorophenyl phenyl ether & EPA8270 & $\mu \mathrm{g} / \mathrm{kg}$ & 330 & SW-846 \\
\hline Chrysene & EPA8270 & $\mu \mathrm{g} / \mathrm{kg}$ & 330 & SW-846 \\
\hline m-Cresol (3-Methylphenol) & EPA8270 & $\mu \mathrm{g} / \mathrm{kg}$ & 330 & SW-846 \\
\hline o-Cresol (2-Methylphenol) & EPA8270 & $\mu \mathrm{g} / \mathrm{kg}$ & 330 & SW-846 \\
\hline p-Cresol (4-Methylphenol) & EPA8270 & $\mu \mathrm{g} / \mathrm{kg}$ & 330 & SW-846 \\
\hline Dibenzo $[a, h]$ anthracene & EPA8270 & $\mu \mathrm{g} / \mathrm{kg}$ & 330 & SW-846 \\
\hline Dibenzofuran & EPA8270 & $\mu \mathrm{g} / \mathrm{kg}$ & 330 & SW-846 \\
\hline Di-n-butyl phthalate & EPA8270M & $\mu \mathrm{g} / \mathrm{kg}$ & 240 & SW-846 \\
\hline 1,2-Dichlorobenzene & EPA8270 & $\mu \mathrm{g} / \mathrm{kg}$ & 330 & SW-846 \\
\hline 1,3-Dichlorobenzene & EPA8270 & $\mu \mathrm{g} / \mathrm{kg}$ & 330 & SW-846 \\
\hline 1,4-Dichlorobenzene & EPA8270 & $\mu \mathrm{g} / \mathrm{kg}$ & 330 & SW-846 \\
\hline 3,3'-Dichlorobenzidine & EPA8270 & $\mu \mathrm{g} / \mathrm{kg}$ & 660 & SW-846 \\
\hline 2,4-Dichlorophenol & EPA8270M & $\mu \mathrm{g} / \mathrm{kg}$ & 260 & SW-846 \\
\hline Diethyl phthalate & EPA8270 & $\mu \mathrm{g} / \mathrm{kg}$ & 330 & SW-846 \\
\hline 2,4-Dimethylphenol & EPA8270M & $\mu \mathrm{g} / \mathrm{kg}$ & 220 & SW-846 \\
\hline Dimethyl phthalate & EPA8270M & $\mu \mathrm{g} / \mathrm{kg}$ & 200 & SW-846 \\
\hline 2,4-Dinitrophenol & EPA8270M & $\mu \mathrm{g} / \mathrm{kg}$ & 800 & SW-846 \\
\hline 2,4-Dinitrotoluene & EPA8270 & $\mu \mathrm{g} / \mathrm{kg}$ & 14 & SW-846 \\
\hline 2,6-Dinitrotoluene & EPA8270 & $\mu \mathrm{g} / \mathrm{kg}$ & 7 & SW-846 \\
\hline Di-n-octyl phthalate & EPA8270 & $\mu \mathrm{g} / \mathrm{kg}$ & 330 & SW-846 \\
\hline Fluoranthene & EPA8270 & $\mu \mathrm{g} / \mathrm{kg}$ & 330 & SW-846 \\
\hline Fluorene & EPA8270 & $\mu \mathrm{g} / \mathrm{kg}$ & 330 & SW-846 \\
\hline
\end{tabular}

${ }^{2}$ The letter $M$ following a method indicates a modification to the method by the laboratory. 
Table F-2. Analytical Methods and Quantitation Limits for Roy F. Weston Laboratories, Inc., Continued

\begin{tabular}{|c|c|c|c|c|}
\hline Analyte & Method & Units & EQL & Reference \\
\hline Hexachlorobenzene & EPA8270M & $\mu \mathrm{g} / \mathrm{kg}$ & 34 & SW-846 \\
\hline Hexachlorobutadiene & EPA8270M & $\mu \mathrm{g} / \mathrm{kg}$ & 230 & SW-846 \\
\hline Hexachlorocyclopentadiene & EPA8270M & $\mu \mathrm{g} / \mathrm{kg}$ & 270 & SW-846 \\
\hline Hexachloroethane & EPA8270M & $\mu \mathrm{g} / \mathrm{kg}$ & 48 & SW-846 \\
\hline Indeno $[1,2,3-c, d]$ pyrene & EPA8270 & $\mu \mathrm{g} / \mathrm{kg}$ & 330 & SW-846 \\
\hline Isophorone & EPA8270 & $\mu \mathrm{g} / \mathrm{kg}$ & 330 & SW-846 \\
\hline 2-Methylnaphthalene & EPA8270 & $\mu \mathrm{g} / \mathrm{kg}$ & 330 & SW-846 \\
\hline $\begin{array}{r}\text { 2-Methyl-4,6-dinitrophenol } \\
\text { (4,6-Dinitro-ortho-cresol) }\end{array}$ & EPA8270M & $\mu \mathrm{g} / \mathrm{kg}$ & 800 & SW-846 \\
\hline Naphthalene & EPA8270 & $\mu \mathrm{g} / \mathrm{kg}$ & 330 & SW-846 \\
\hline 2-Nitroaniline & EPA8270M & $\mu \mathrm{g} / \mathrm{kg}$ & 800 & SW-846 \\
\hline 3-Nitroaniline & EPA8270M & $\mu \mathrm{g} / \mathrm{kg}$ & 800 & SW-846 \\
\hline 4-Nitroaniline & EPA8270M & $\mu \mathrm{g} / \mathrm{kg}$ & 800 & SW-846 \\
\hline Nitrobenzene & EPA8270 & $\mu \mathrm{g} / \mathrm{kg}$ & 330 & SW-846 \\
\hline 2-Nitrophenol & EPA8270M & $\mu \mathrm{g} / \mathrm{kg}$ & 300 & SW-846 \\
\hline 4-Nitrophenol & EPA8270M & $\mu \mathrm{g} / \mathrm{kg}$ & 800 & SW-846 \\
\hline N-Nitrosodiphenylamine & EPA8270M & $\mu \mathrm{g} / \mathrm{kg}$ & 330 & SW-846 \\
\hline N-Nitrosodipropylamine & EPA8270 & $\mu \mathrm{g} / \mathrm{kg}$ & 330 & SW-846 \\
\hline Pentachlorophenol & EPA8270M & $\mu \mathrm{g} / \mathrm{kg}$ & 800 & SW-846 \\
\hline Phenanthrene & EPA8270 & $\mu \mathrm{g} / \mathrm{kg}$ & 330. & SW-846 \\
\hline Phenol & EPA8270M & $\mu \mathrm{g} / \mathrm{kg}$ & 100 & SW-846 \\
\hline Pyrene & EPA8270 & $\mu \mathrm{g} / \mathrm{kg}$ & 330 & SW-846 \\
\hline 1,2,4-Trichlorobenzene & EPA8270M & $\mu \mathrm{g} / \mathrm{kg}$ & 54 & SW-846 \\
\hline 2,4,5-Trichlorophenol & EPA8270M & $\mu \mathrm{g} / \mathrm{kg}$ & 330 & SW-846 \\
\hline 2,4,6-Trichlorophenol & EPA8270 & $\mu \mathrm{g} / \mathrm{kg}$ & 330 & SW-846 \\
\hline \multicolumn{5}{|l|}{ Specified Analyses } \\
\hline Cation exchange capacity & EPA9081 & $\mathrm{mg} / \mathrm{kg}$ & 2.2 & SW-846 \\
\hline Chloride & EPA300.0 & $\mathrm{mg} / \mathrm{kg}$ & 17.4 & $600 / 4-79-020$ \\
\hline Nitrate/Nitrite & EPA353.2 & $\mathrm{mg} / \mathrm{kg}$ & 0.6 & $600 / 4-79-020$ \\
\hline Total organic nitrogen & EPA351.4 & $\mathrm{mg} / \mathrm{kg}$ & 125 & $600 / 4-79-020$ \\
\hline Orthophosphate & EPA365.2 & $\mathrm{mg} / \mathrm{kg}$ & 1.0 & $600 / 4-79-020$ \\
\hline Particle size distribution & ACOE1906 & NA & $\mathrm{NA}$ & \\
\hline Phenols & EPA420.2 & $\mathrm{mg} / \mathrm{kg}$ & 1.19 & $600 / 4-79-020$ \\
\hline Sulfide & EPA376.2 & $\mathrm{mg} / \mathrm{kg}$ & 25 & $600 / 4-79-020$ \\
\hline Total organic carbon & LLOYDKAHN & $\mathrm{mg} / \mathrm{kg}$ & 8.4 & \\
\hline Total phosphate & EPA365.2 & $\mathrm{mg} / \mathrm{kg}$ & 1.5 & $600 / 4-79-020$ \\
\hline
\end{tabular}


Table F-2. Analytical Methods and Quantitation Limits for Roy F. Weston Laboratories, Inc., Continued

\begin{tabular}{|c|c|c|c|c|}
\hline \multicolumn{5}{|c|}{ Water Analyses } \\
\hline Analyte & Method & Units & EQL & Reference \\
\hline \multicolumn{5}{|c|}{ TCL: Volatile Organic Compounds } \\
\hline Acetone & EPA8260 & $\mu \mathrm{g} / \mathrm{L}$ & 10 & SW-846 \\
\hline Benzene & EPA8021 & $\mu \mathrm{g} / \mathrm{L}$ & 0.09 & SW-846 \\
\hline Bromodichloromethane & EPA8260 & $\mu \mathrm{g} / \mathrm{L}$ & 0.2 & SW-846 \\
\hline Bromoform & EPA8260 & $\mu \mathrm{g} / \mathrm{L}$ & 2 & SW-846 \\
\hline Bromomethane (Methyl bromide) & EPA8260 & $\mu \mathrm{g} / \mathrm{L}$ & 0.6 & SW-846 \\
\hline Carbon disulfide & EPA8260 & $\mu \mathrm{g} / \mathrm{L}$ & 5 & SW-846 \\
\hline Carbon tetrachloride & EPA8021 & $\mu \mathrm{g} / \mathrm{L}$ & 0.1 & SW-846 \\
\hline Chlorobenzene & EPA8260 & $\mu \mathrm{g} / \mathrm{L}$ & 0.3 & SW-846 \\
\hline Chlorodibromomethane & EPA8260 & $\mu \mathrm{g} / \mathrm{L}$ & 0.7 & SW-846 \\
\hline Chloroethane & EPA8260 & $\mu \mathrm{g} / \mathrm{L}$ & 0.2 & SW-846 \\
\hline Chloroethene (Vinyl chloride) & EPA8021 & $\mu \mathrm{g} / \mathrm{L}$ & 0.2 & SW-846 \\
\hline Chloroform & EPA8260 & $\mu \mathrm{g} / \mathrm{L}$ & 0.2 & SW-846 \\
\hline Chloromethane (Methyl chloride) & EPA8260 & $\mu \mathrm{g} / \mathrm{L}$ & 0.3 & SW-846 \\
\hline 1,1-Dichloroethane & EPA8260 & $\mu \mathrm{g} / \mathrm{L}$ & 0.3 & SW-846 \\
\hline 1,2-Dichloroethane & EPA8260 & $\mu \mathrm{g} / \mathrm{L}$ & 0.2 & SW-846 \\
\hline 1,1-Dichloroethylene & EPA8260 & $\mu \mathrm{g} / \mathrm{L}$ & 0.5 & SW-846 \\
\hline 1,2-Dichloroethylene (total) & EPA8260 & $\mu \mathrm{g} / \mathrm{L}$ & 17 & SW-846 \\
\hline $\begin{array}{l}\text { Dichloromethane } \\
\text { (Methylene chloride) }\end{array}$ & EPA8021 & $\mu \mathrm{g} / \mathrm{L}$ & 0.2 & SW-846 \\
\hline 1,2-Dichloropropane & EPA8021 & $\mu \mathrm{g} / \mathrm{L}$ & 0.06 & SW-846 \\
\hline cis-1,3-Dichloropropene & EPA8260 & $\mu \mathrm{g} / \mathrm{L}$ & 5 & SW-846 \\
\hline trans-1,3-Dichloropropene & EPA8260 & $\mu \mathrm{g} / \mathrm{L}$ & 5 & SW-846 \\
\hline Ethylbenzene & EPA8021 & $\mu \mathrm{g} / \mathrm{L}$ & 0.05 & SW-846 \\
\hline 2-Hexanone & EPA8260 & $\mu \mathrm{g} / \mathrm{L}$ & 10 & SW-846 \\
\hline Methyl ethyl ketone & EPA8260 & $\mu \mathrm{g} / \mathrm{L}$ & 10 & SW-846 \\
\hline Methyl isobutyl ketone & EPA8260 & $\mu \mathrm{g} / \mathrm{L}$ & 5 & SW-846 \\
\hline Styrene & EPA8021 & $\mu \mathrm{g} / \mathrm{L}$ & 0.1 & SW-846 \\
\hline 1,1,2,2-Tetrachloroethane & EPA8021 & $\mu \mathrm{g} / \mathrm{L}$ & 0.1 & SW-846 \\
\hline Tetrachloroethylene & EPA8260 & $\mu \mathrm{g} / \mathrm{L}$ & 0.4 & SW-846 \\
\hline Toluene & EPA8021 & $\mu g / L$ & 0.1 & SW-846 \\
\hline 1,1,1-Trichloroethane & EPA8260 & $\mu \mathrm{g} / \mathrm{L}$ & 0.3 & SW-846 \\
\hline 1,1,2-Trichloroethane & EPA8260 & $\mu \mathrm{g} / \mathrm{L}$ & 0.2 & SW-846 \\
\hline \begin{tabular}{|l|} 
Trichloroethylene \\
\end{tabular} & EPA8021 & $\mu \mathrm{g} / \mathrm{L}$ & 0.1 & SW-846 \\
\hline
\end{tabular}


Table F-2. Analytical Methods and Quantitation Limits for Roy F. Weston Laboratories, Inc., Continued

\begin{tabular}{|l|l|l|l|l|}
\hline Analyte & Method & Units & EQL & Reference \\
\hline Vinyl acetate & EPA8260 & $\mu \mathrm{g} / \mathrm{L}$ & 5 & $\mathrm{SW}-846$ \\
\hline Xylenes (total) & EPA8260 & $\mu \mathrm{g} / \mathrm{L}$ & 0.4 & $\mathrm{SW}-846$ \\
\hline
\end{tabular}

Table F-3. Radiological Methods and Quantitation Limits for General Engineering Laboratories, Inc.

\begin{tabular}{|l|l|l|l|l|}
\hline \multicolumn{4}{|c|}{ Soil and Sediment Analyses } \\
\hline Analyte & Method & Units & MDC & Reference \\
\hline Radionuclides and Radionuclide Indicators & pCi/g & NA & GE Method \\
\hline Gamma PHA & EPIA-013B & pCi/g & 4.0 & GE Method \\
\hline Gross alpha & EPIA-001B & pCi/g & 10 & GE Method \\
\hline Nonvolatile beta & EPIA-001B &
\end{tabular}

Table F-4. Radiological Methods and Quantitation Limits for Roy F. Weston Laboratories, Inc.

\begin{tabular}{|c|c|c|c|c|}
\hline \multicolumn{5}{|c|}{ Soil and Sediment Analyses } \\
\hline Analyte & Method & Units & MDC & Reference \\
\hline \multicolumn{5}{|c|}{ Radionuclides and Radionuclide Indicators } \\
\hline Gamma PHA & LANLER130MOD & $\mathrm{pCi} / \mathrm{g}$ & NA & LANL \\
\hline Gross alpha & LANLMLR100MOD & $\mathrm{pCi} / \mathrm{g}$ & 4.0 & LANL $^{4}$ \\
\hline Nonvolatile beta & LANLMLR100MOD & $\mathrm{pCi} / \mathrm{g}$ & 10 & LANL \\
\hline
\end{tabular}

${ }^{3}$ Radionuclides reported by this method vary. Typically, cesium-134, cesium-137, cobalt-60, iodine-129, and potassium-40 may be reported if positive.

Los Alamos National Laboratories, Los Alamos, NM 


\section{Appendix G: Particle Size Distribution Charts}

The following charts show the particle size distribution for sediment samples taken at Par Pond, L Lake, and associated areas. Most of the samples have a textural classification of sand to loamy sand according to the Unified Soil Classification System and Soil Taxonomy Textural Triangle in the Keys to Soil Taxonomy (Soil Survey Staff, 1990).

The following samples do not have corresponding charts: $104620,104622,104625,104626$, $104627,104628,104635,104636,104637,104638,104640,104643,104644,104645,104646$, $104648,104650,104652,104653,104656,104657,104658,104659,104664,104665,104666$, $104667,104668,104669,104670,104671,104673,104674,104675,104676,104677,104678$, 104697, and 104698. 


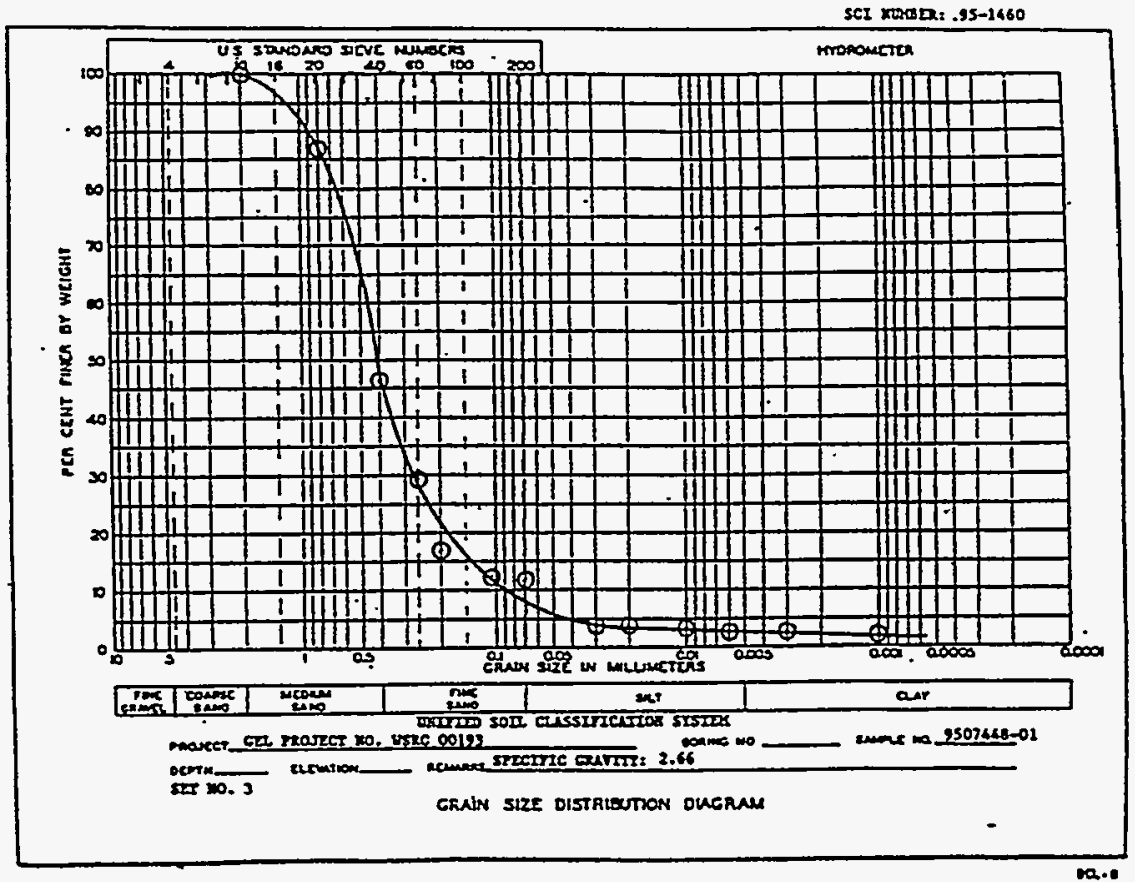

Survey ID: PPSC2101

Sample ID: 104680

SCI Merak: . 95-1660

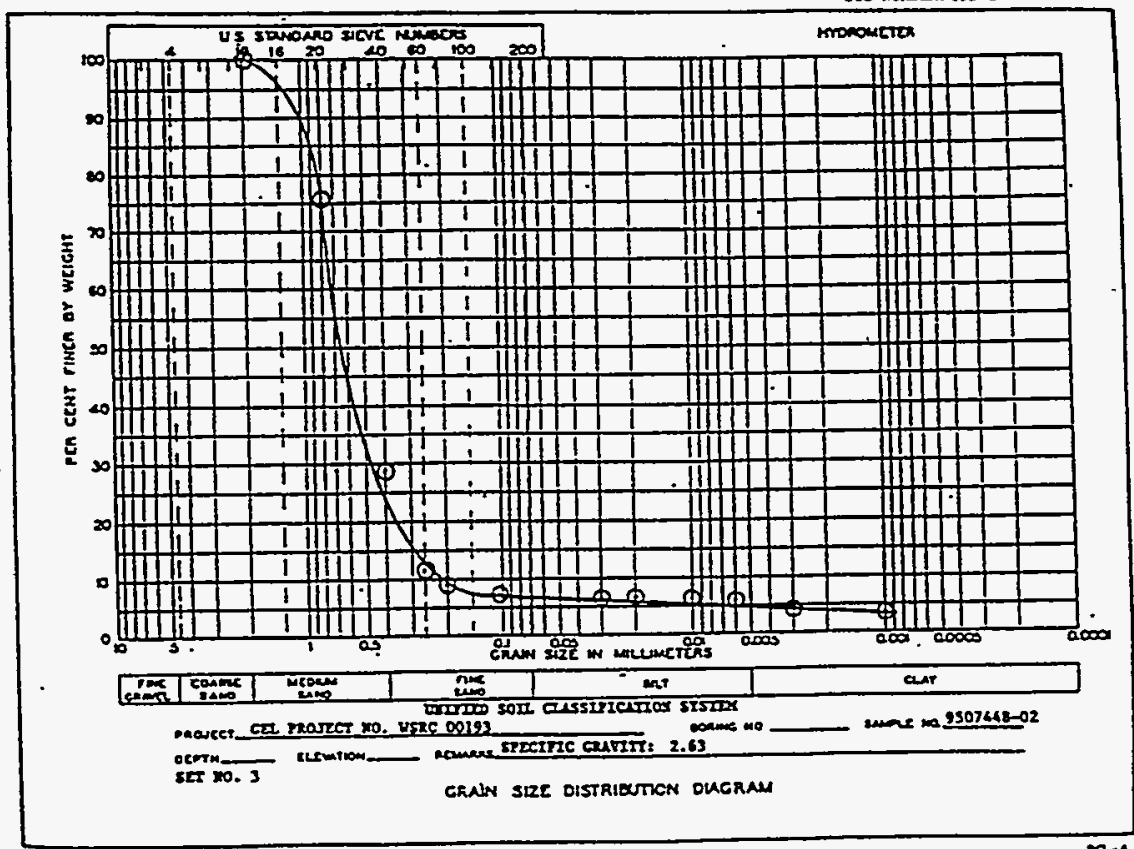

Survey ID: PPSC2102

Sample ID: 104681 


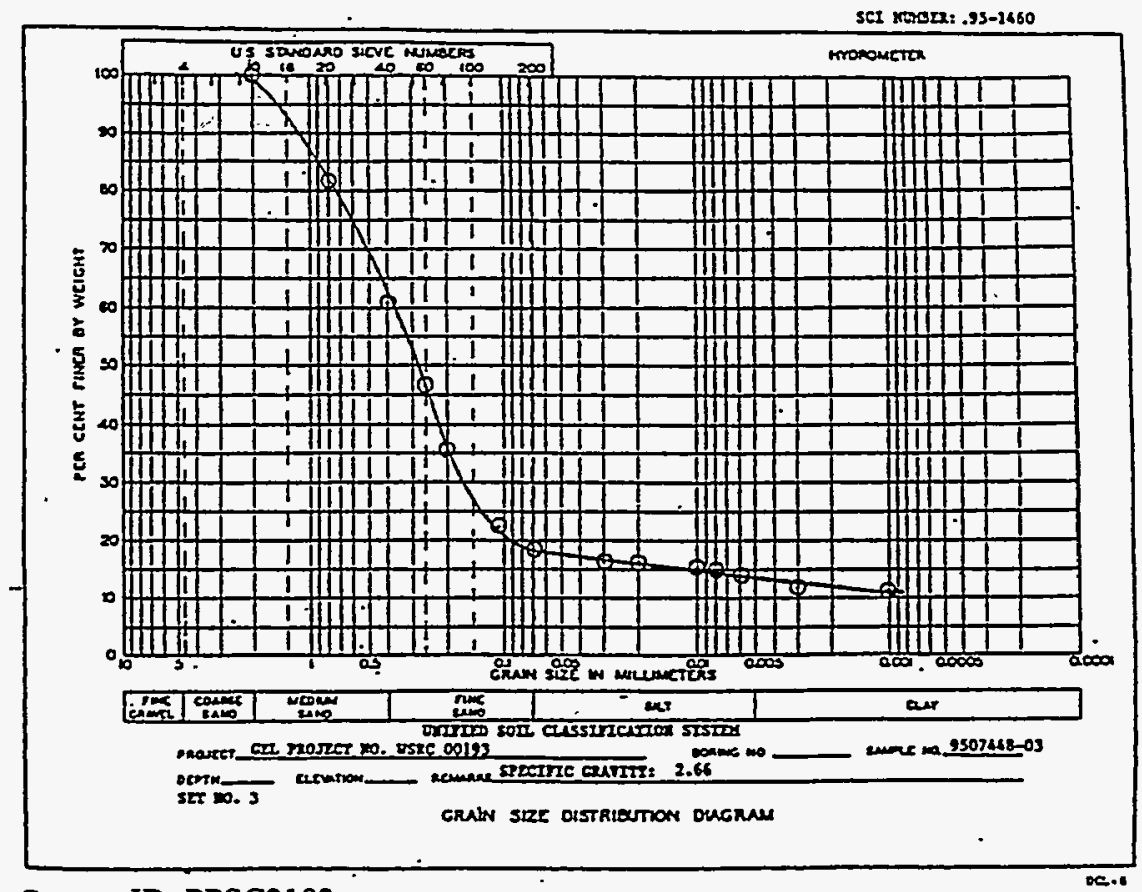

Survey ID: PPSC2103

Sample ID: 104682

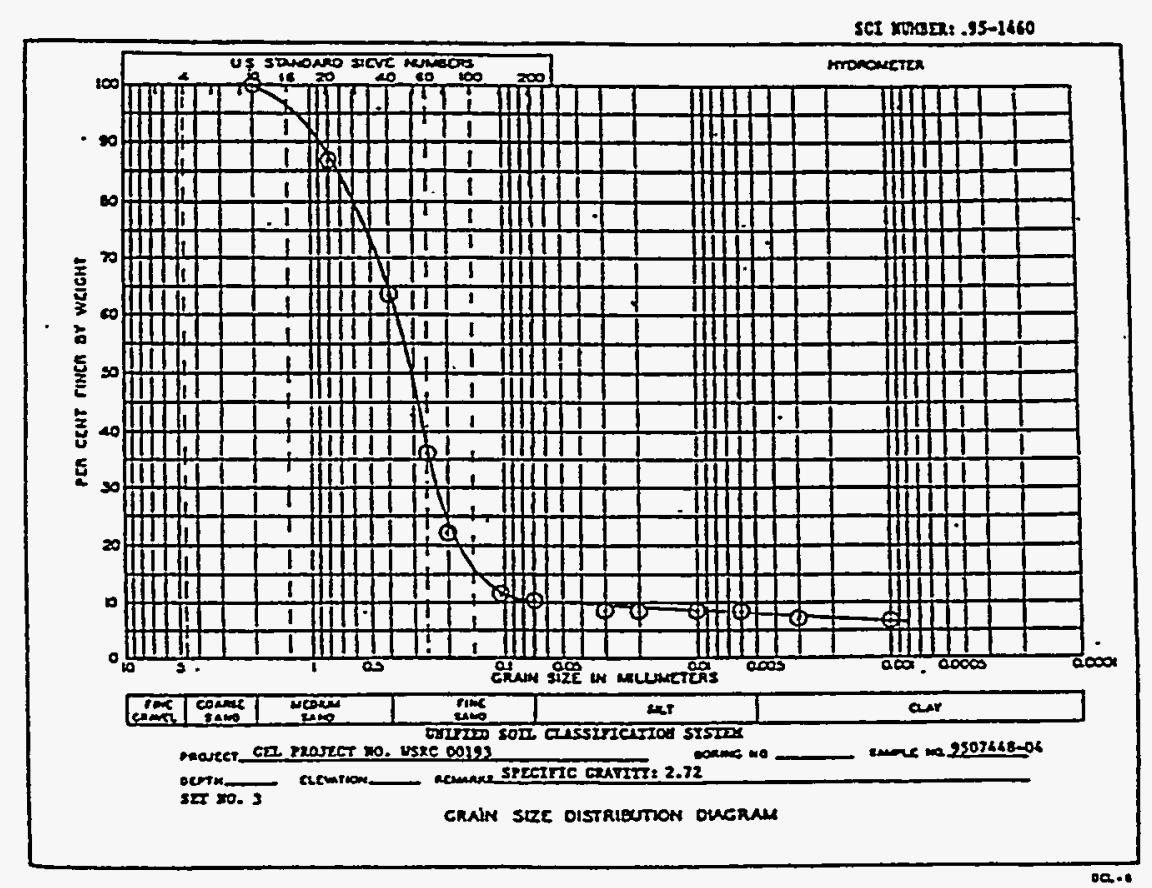

Survey ID: PPSC2201

Sample ID: 104683

Par Pond, Pond C, and L Lake, Revision 2 
SCt rasace: .95-1460

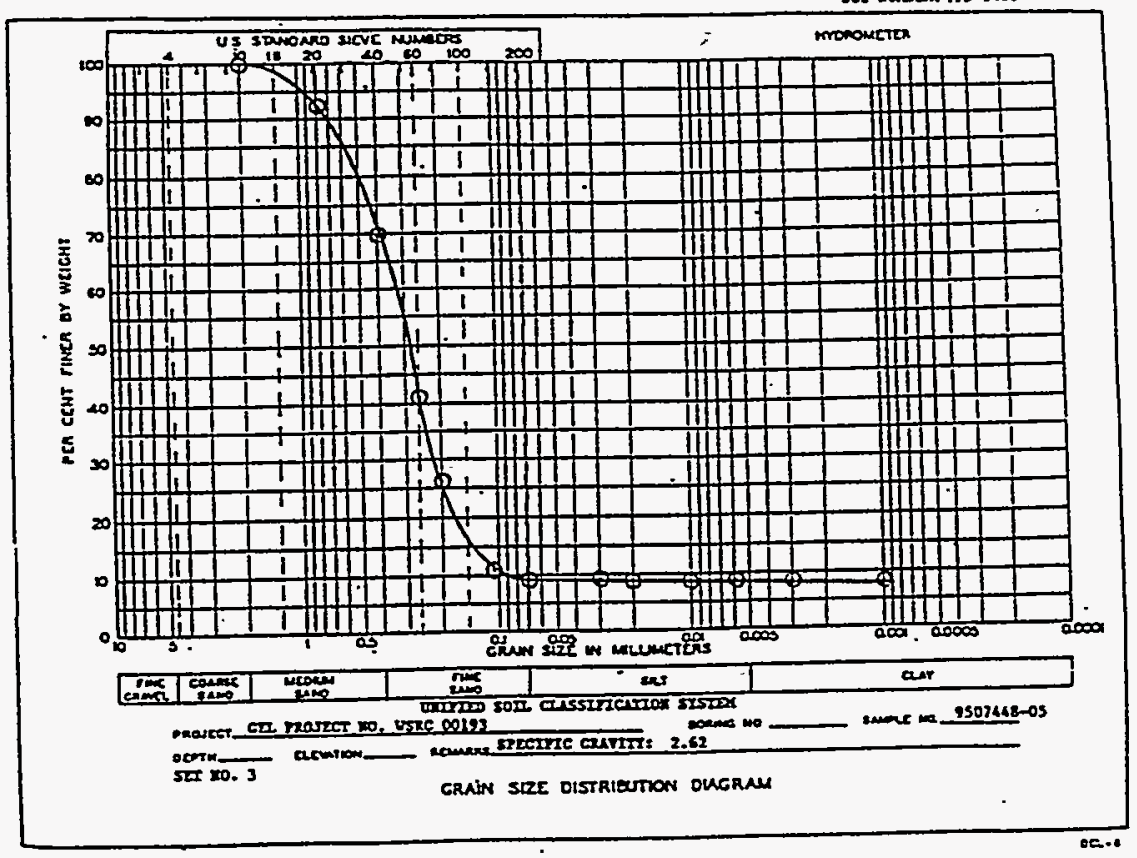

Survey ID: PPSC2202

Sample ID: 104684

set xrean: .95-2460

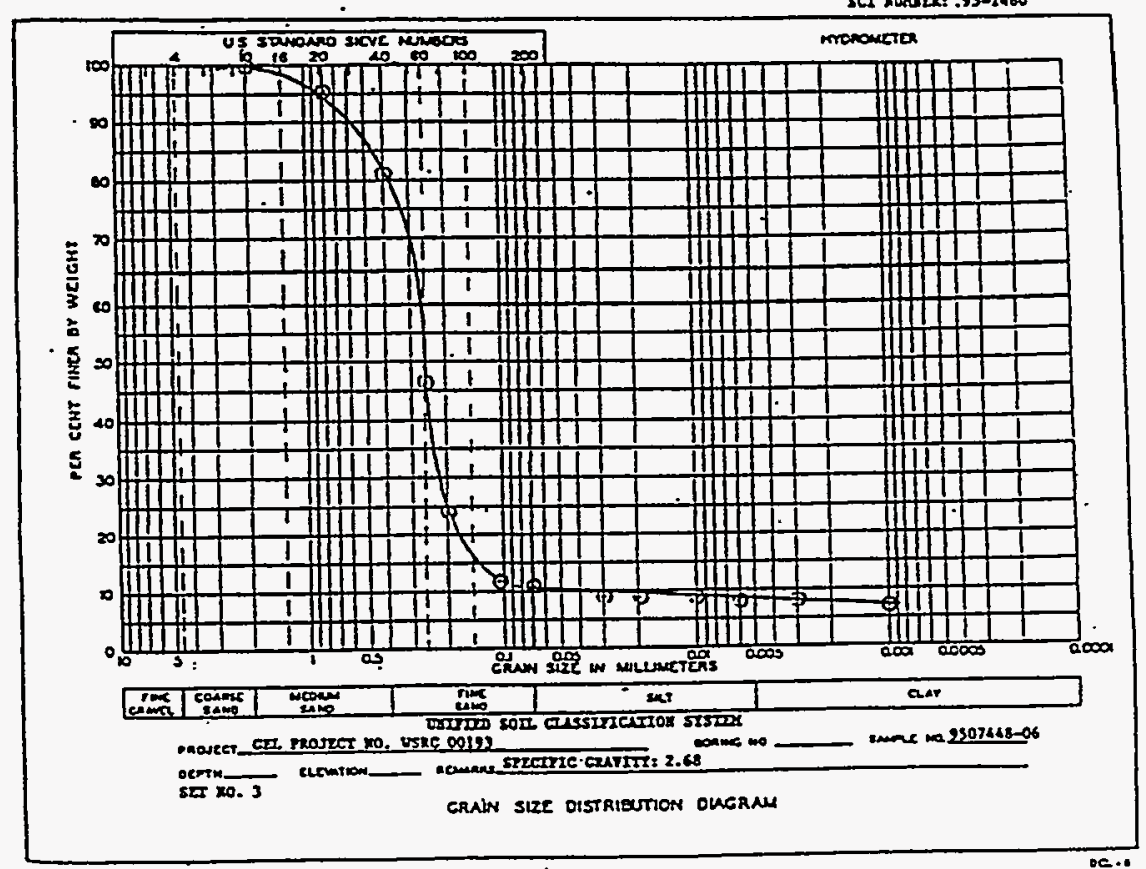

Survey ID: PPSC2203

Sample ID: 104685 


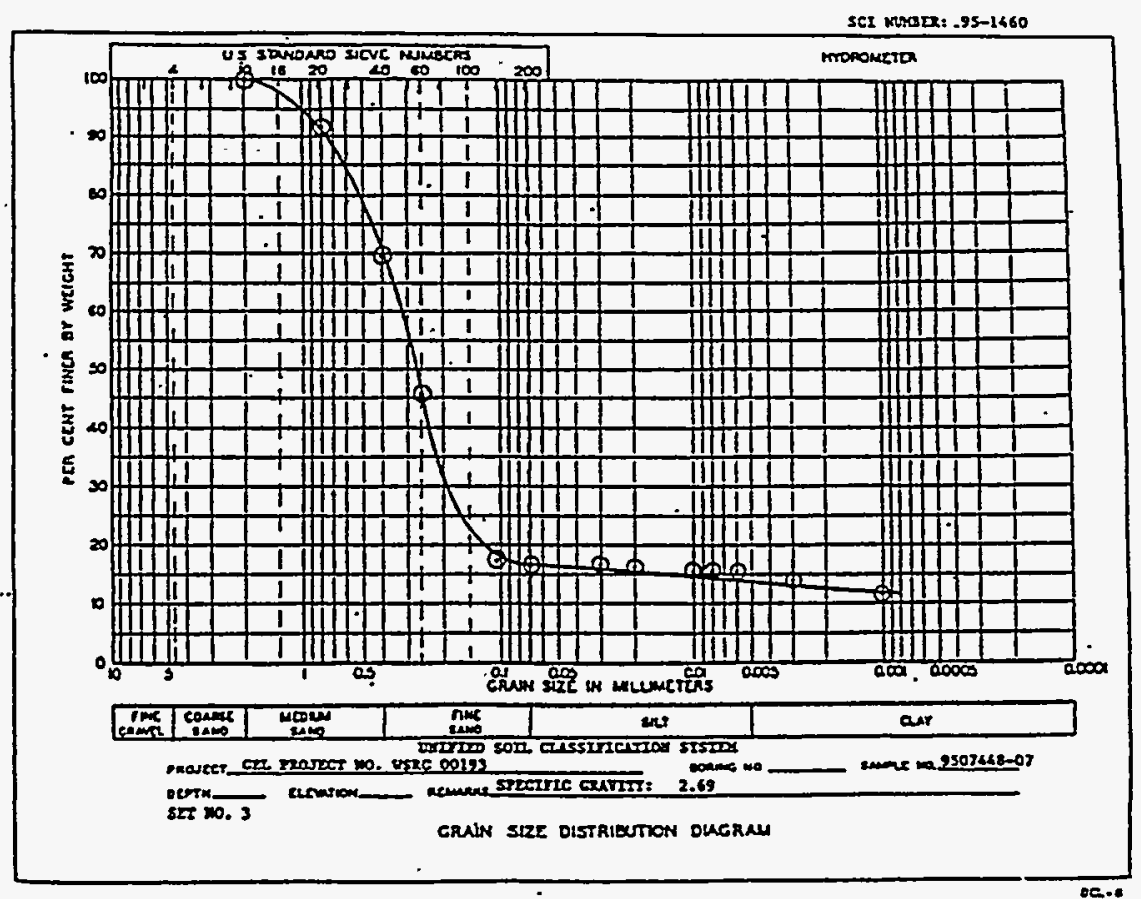

Survey ID: PPSC2204

Sample ID: 104686

Set x002023. .95-1460

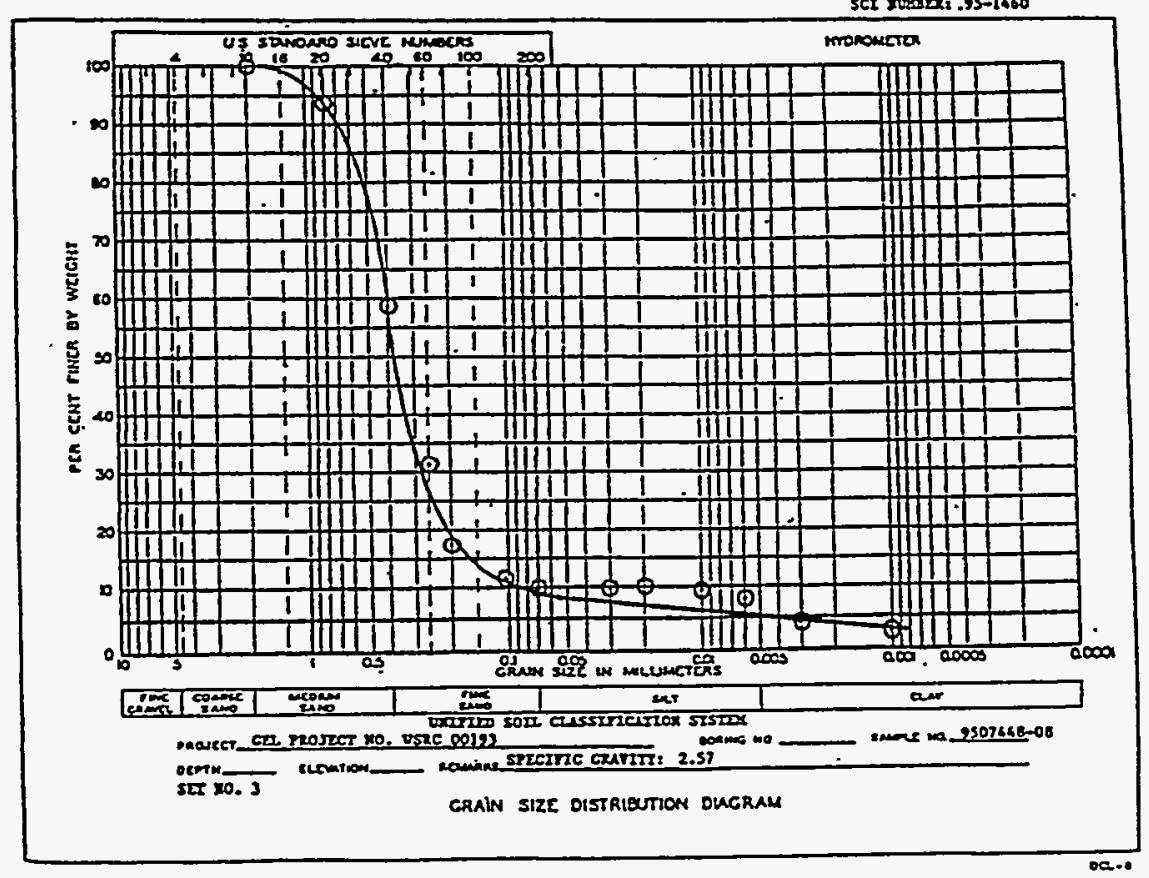

Survey ID: PPSC2301

Sample ID: 104687 


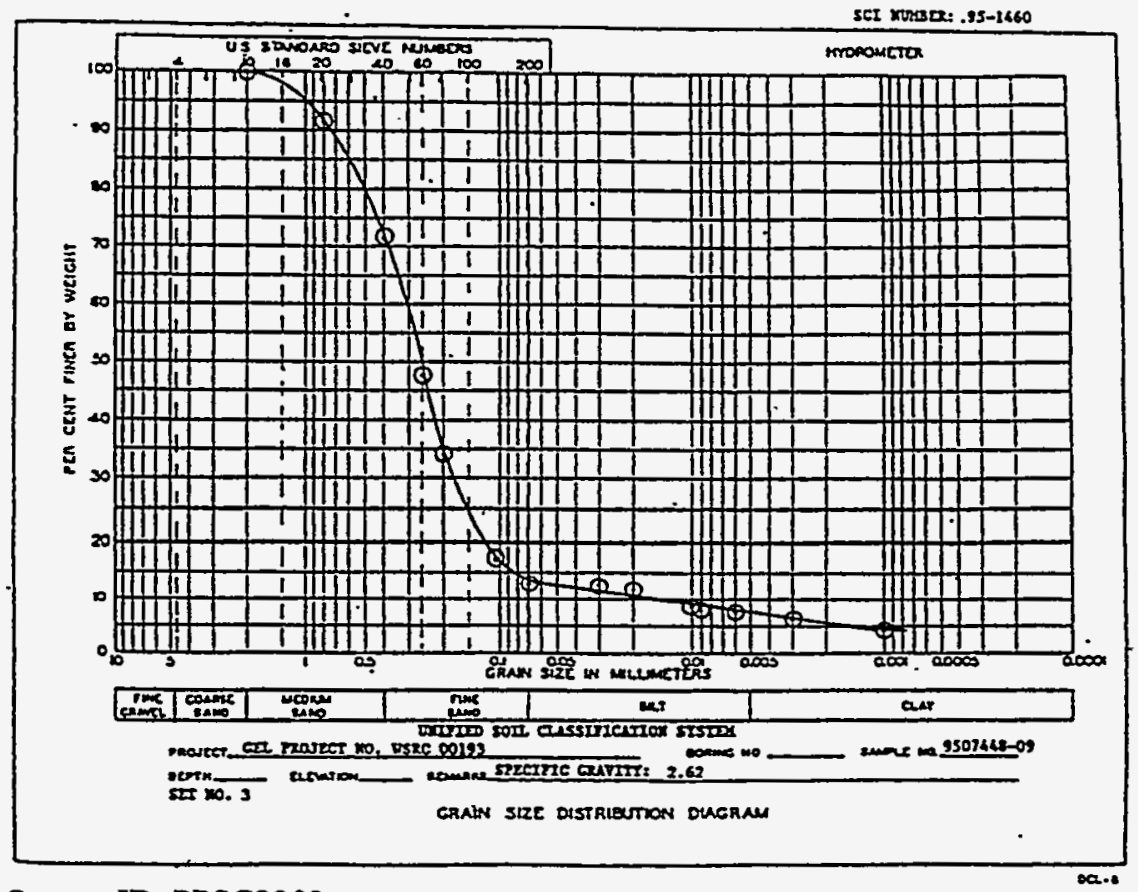

Survey ID: PPSC2302

Sample ID: 104688

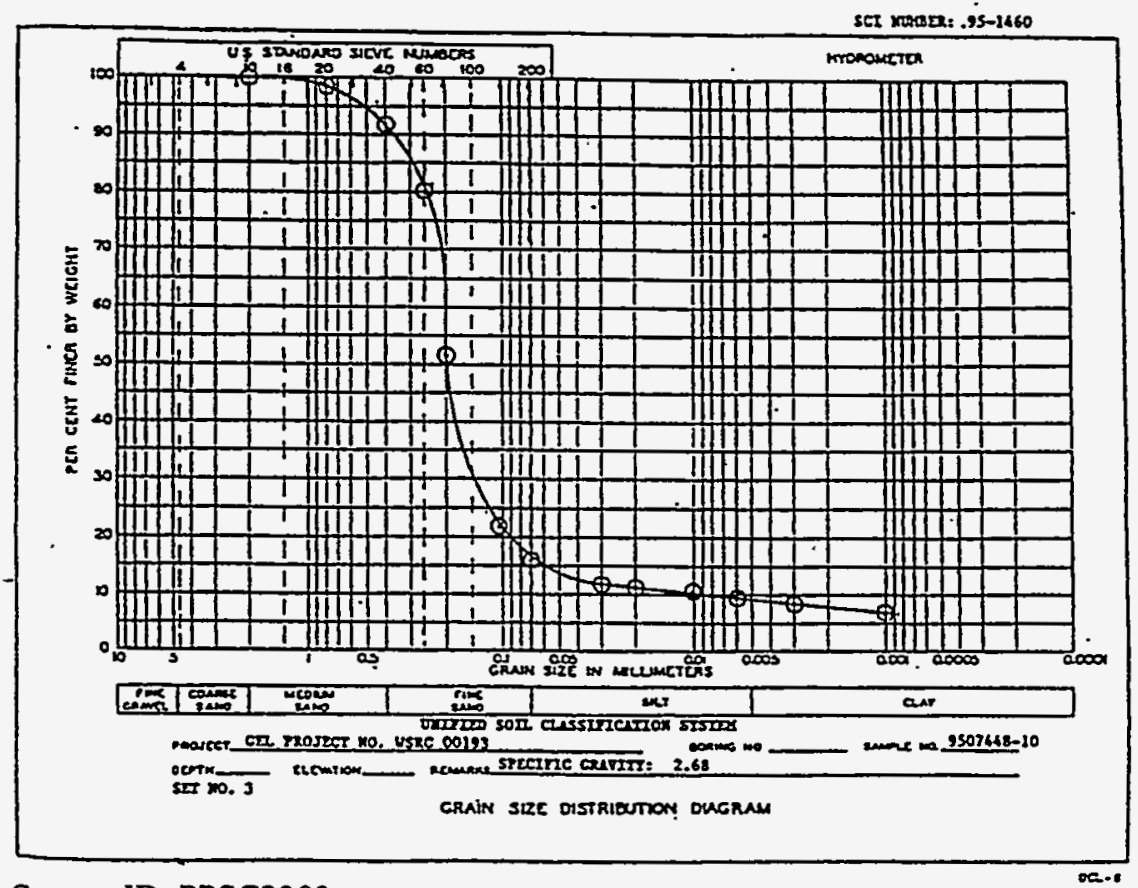

Survey ID: PPSC2303

Sample ID: 104689 


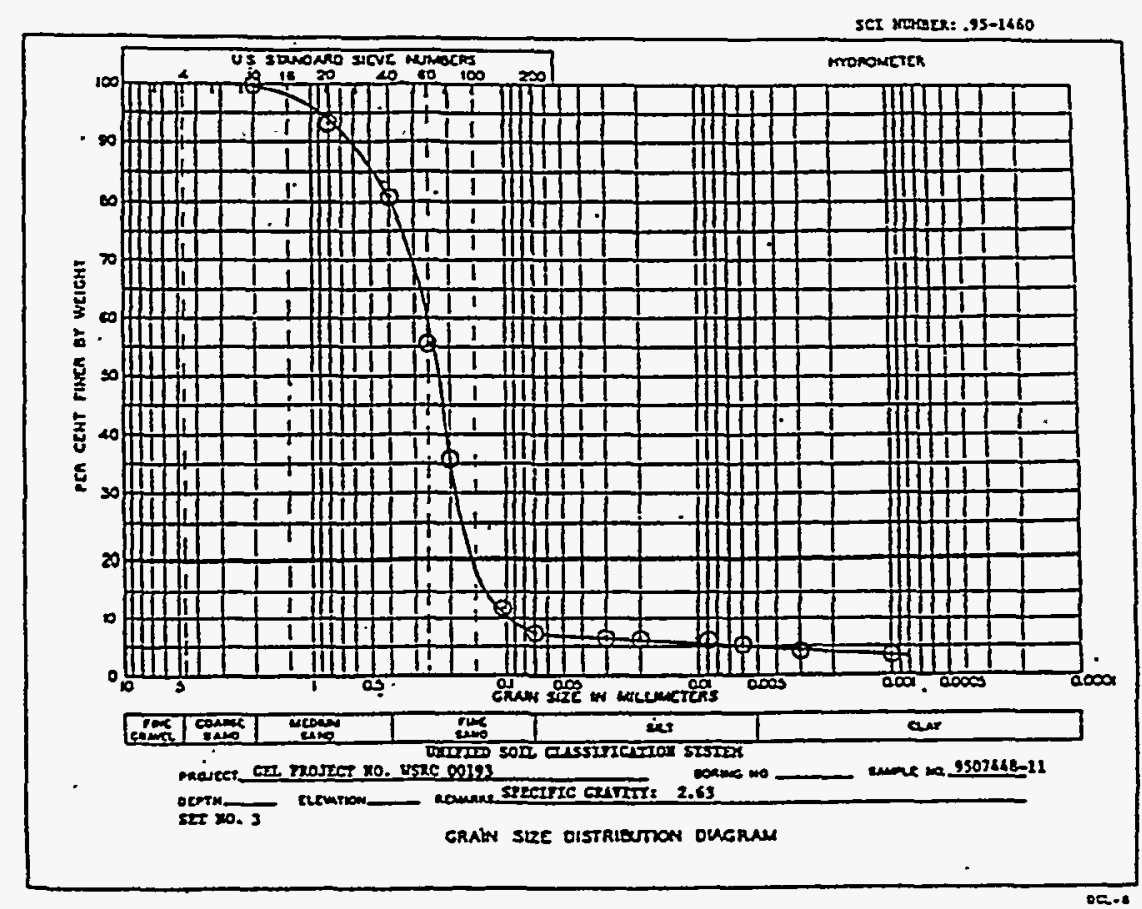

Survey ID: PPSC2304

Sample ID: 104690

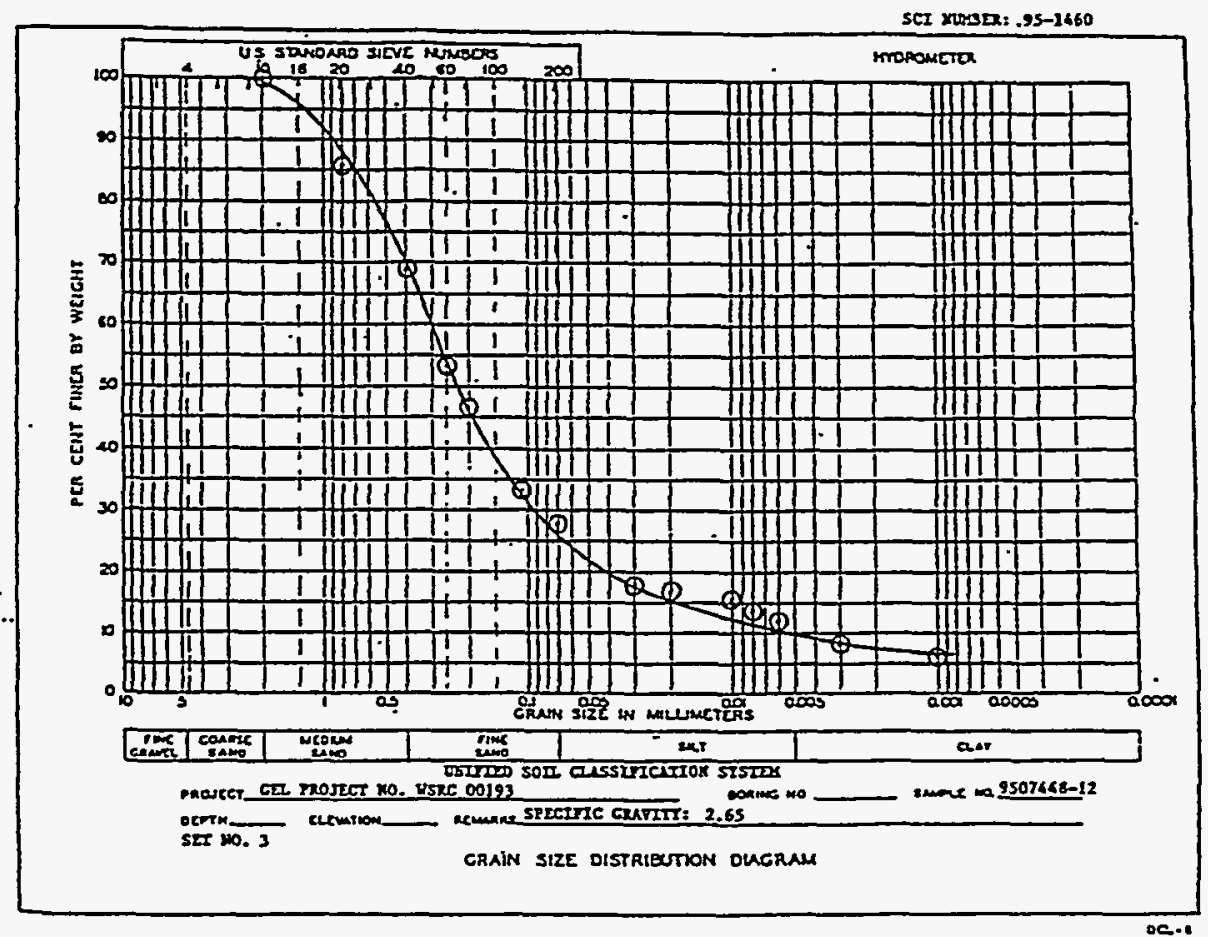

Survey ID: PPSC2305

Sample ID: 104691 


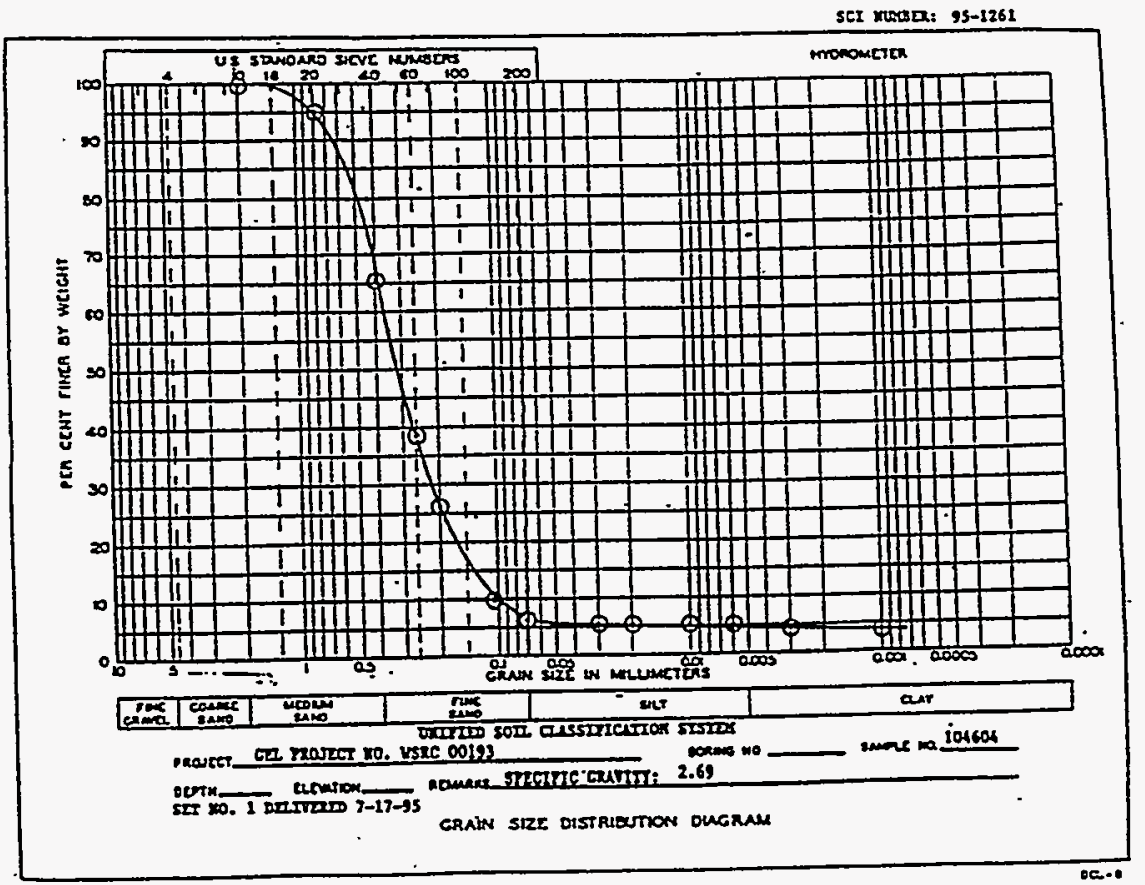

Sample ID: PPSC0201

Sample ID: 104604

SCI vorasx: 95-1261

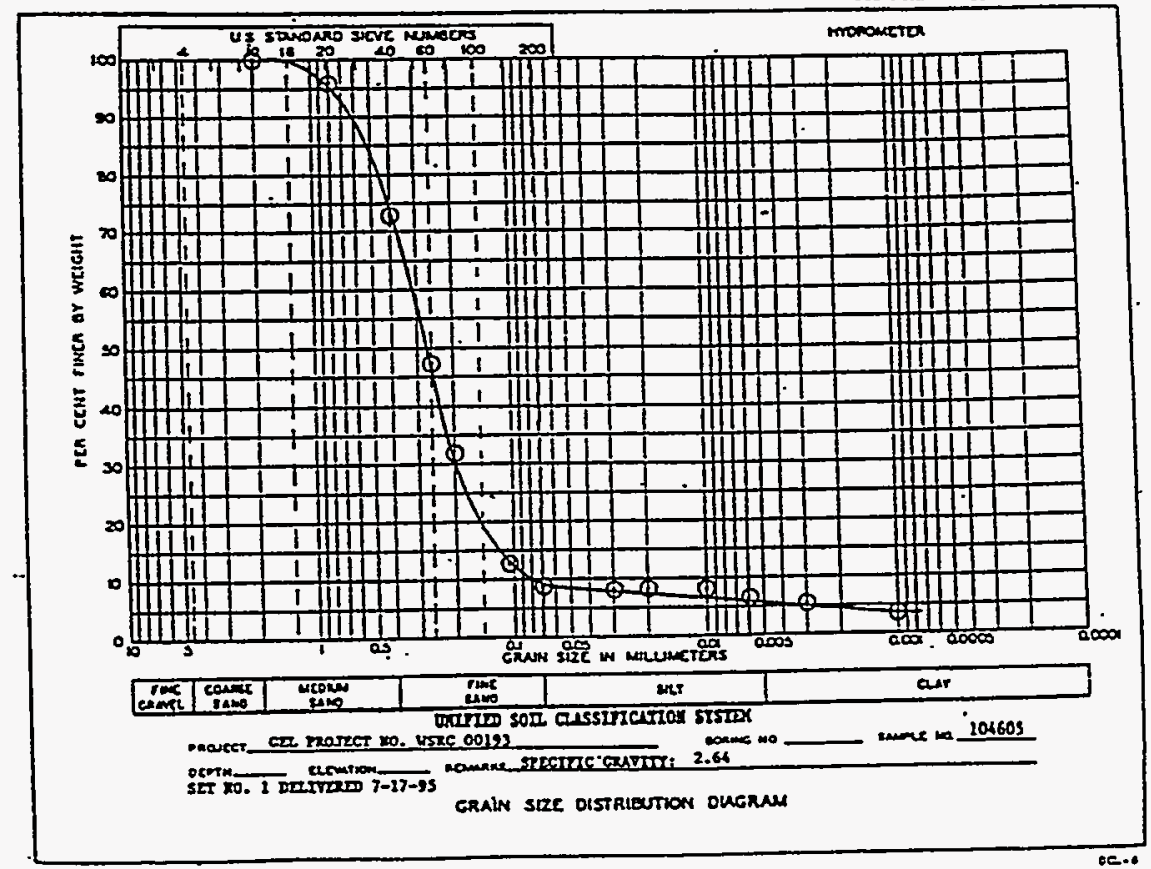

Survey ID: PPSC0202

Sample ID: 104605 


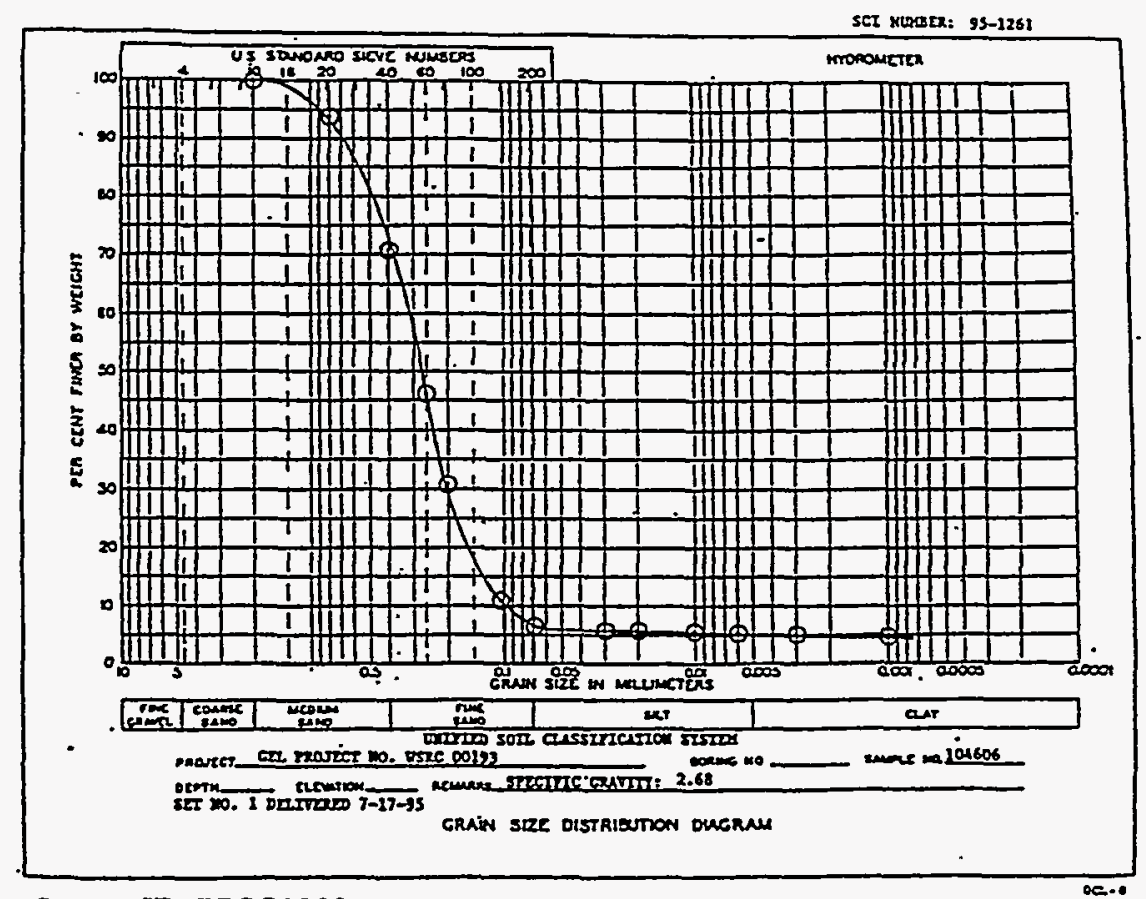

Survey ID: PPSC0203

Sample ID: 104606

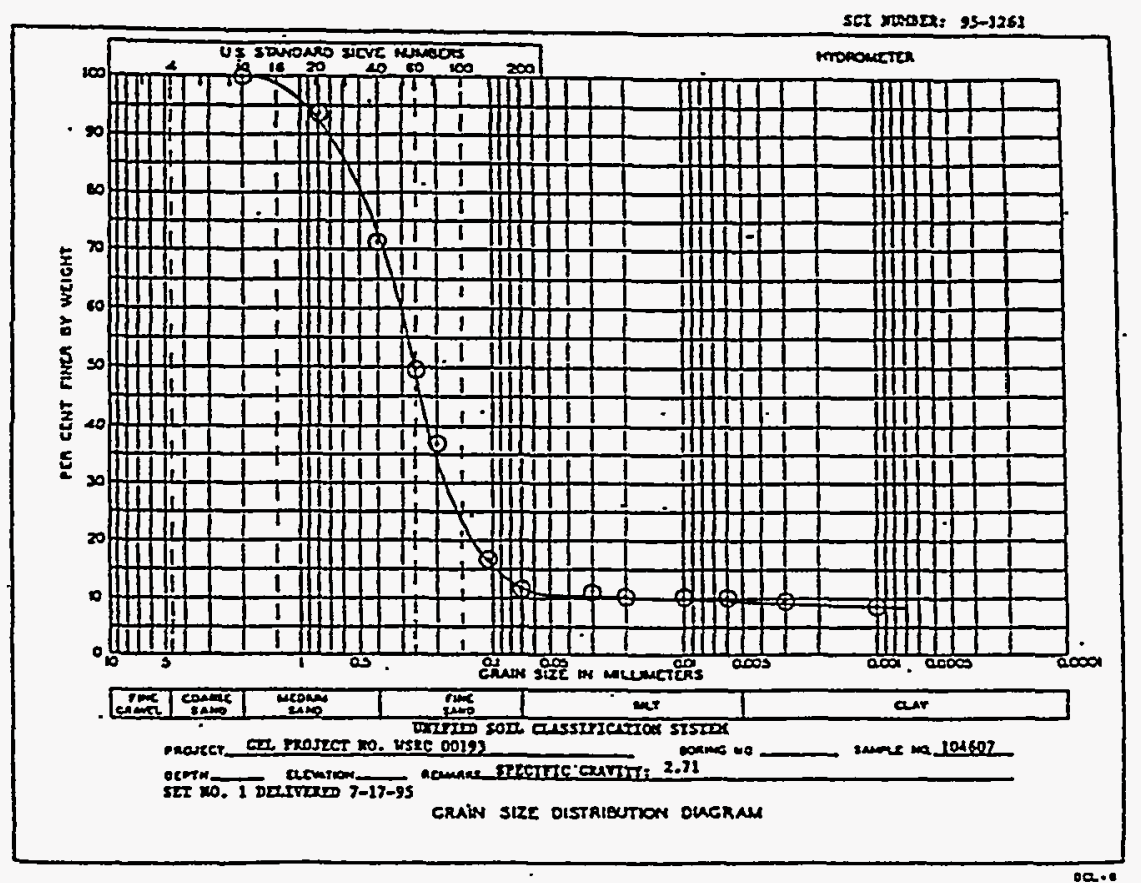

Survey ID: PPSC0204

Sample ID: 104607 
SCI Kunaz: 93-1261

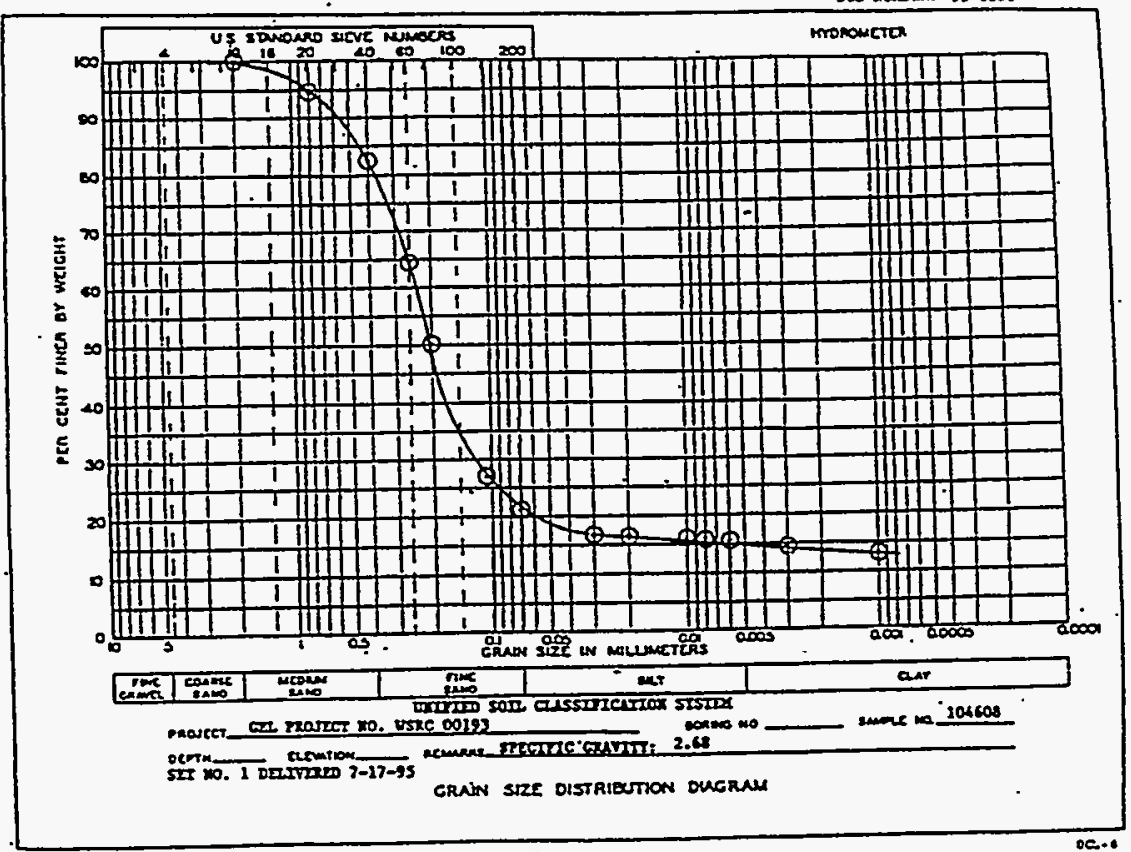

Survey ID: PPSC0205

Sample ID: 104608

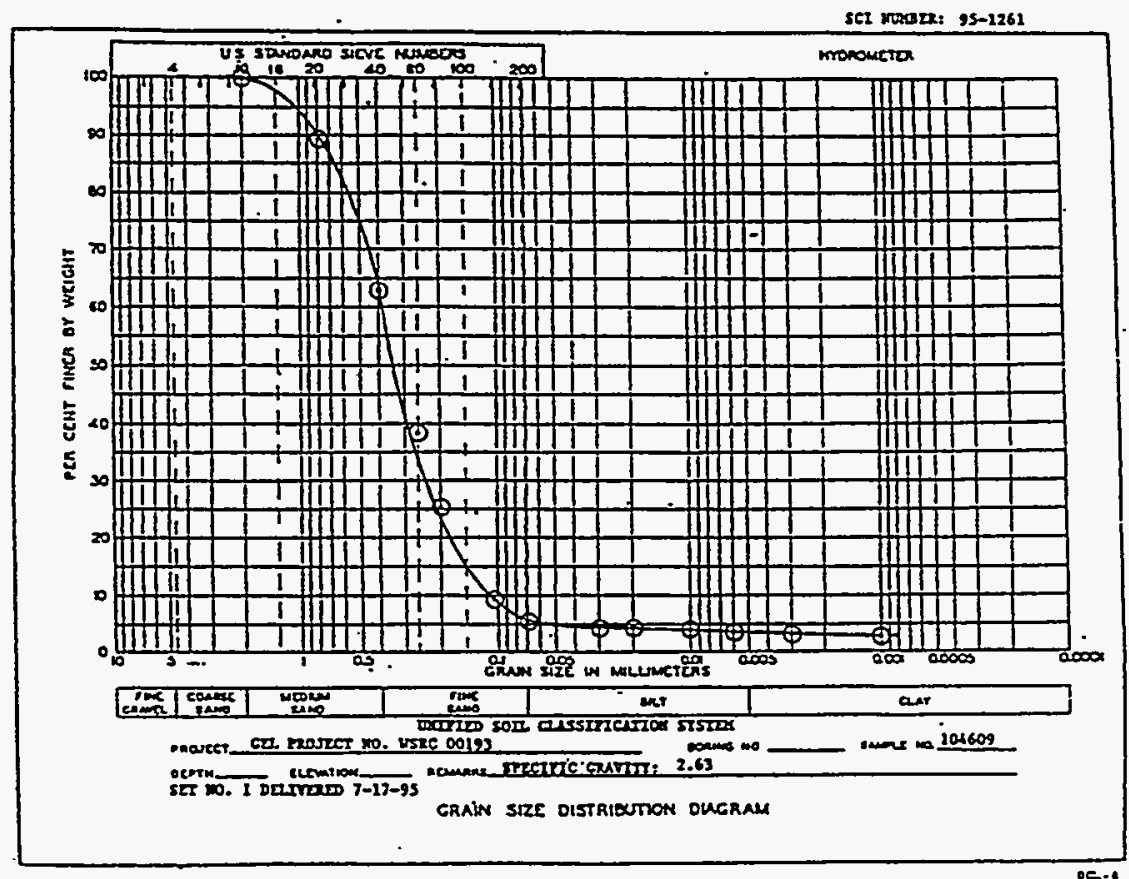

Survey ID: PPSC0301

Sample ID: 104609 


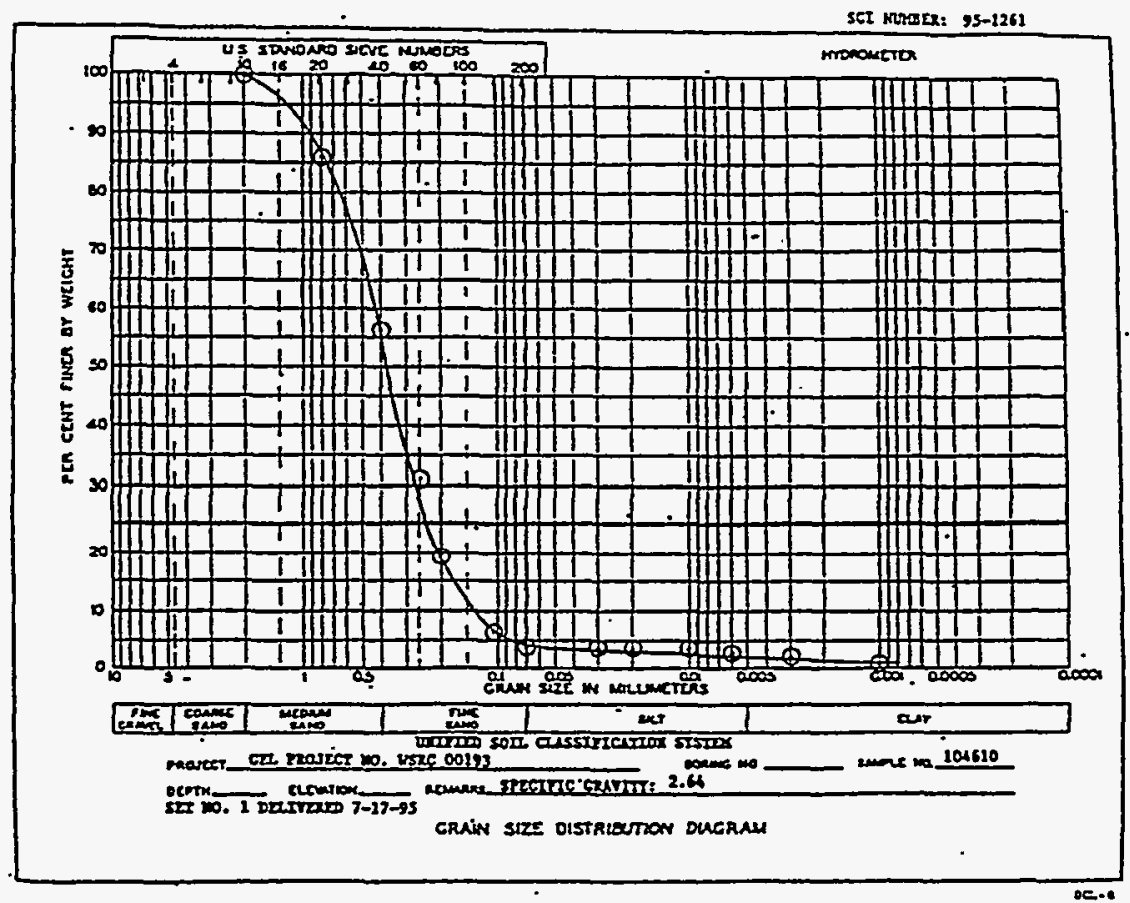

Survey ID: PPSC0302

Sample ID: 104610

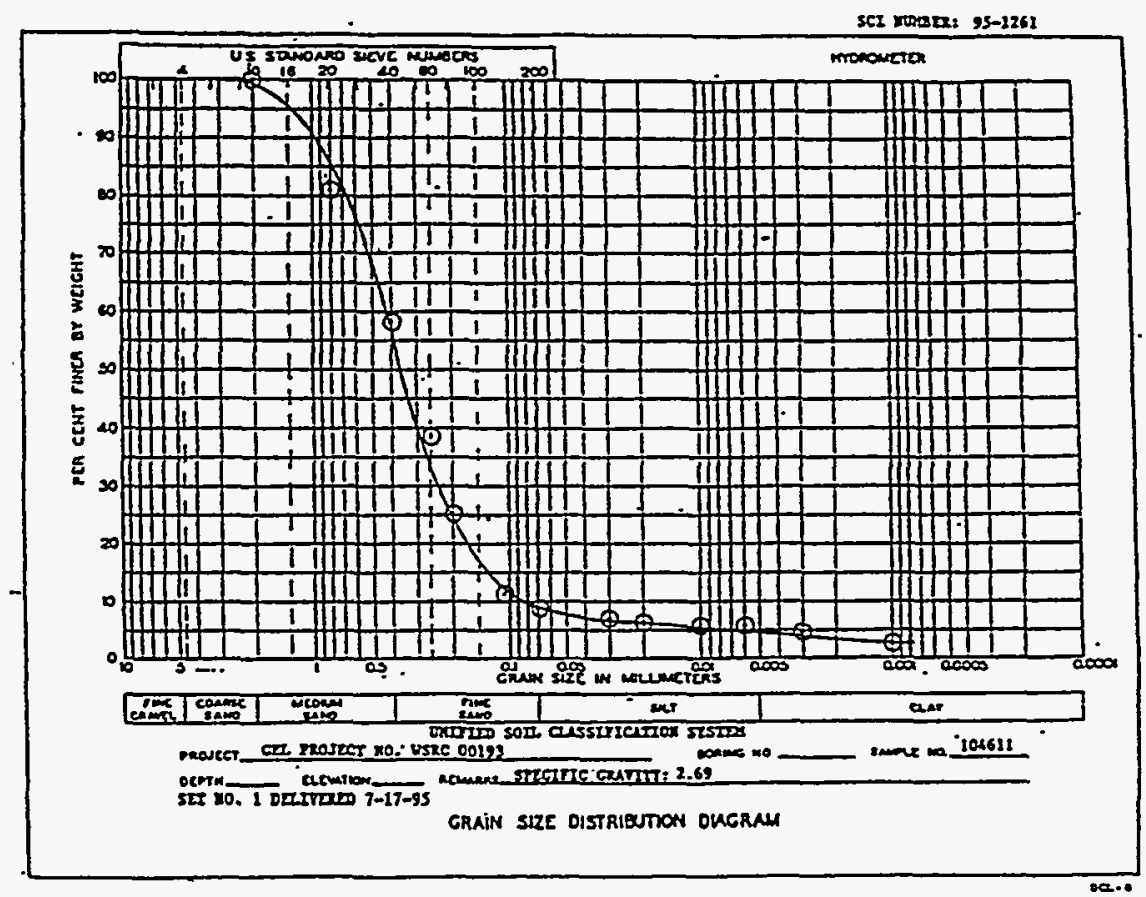

Survey ID: PPSC0303

Sample ID: 104611 


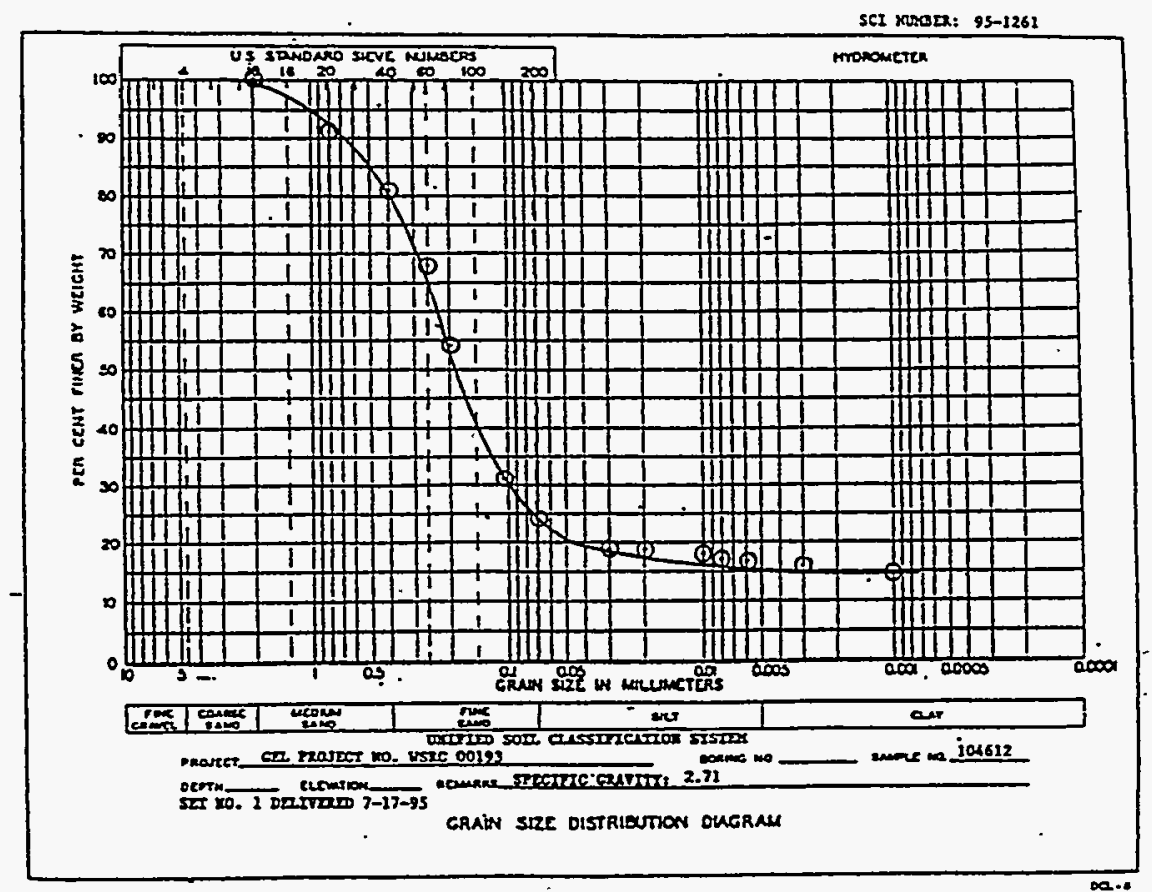

Survey ID: PPSC0304

Sample ID: 104612

SEI xwara: 95-3261

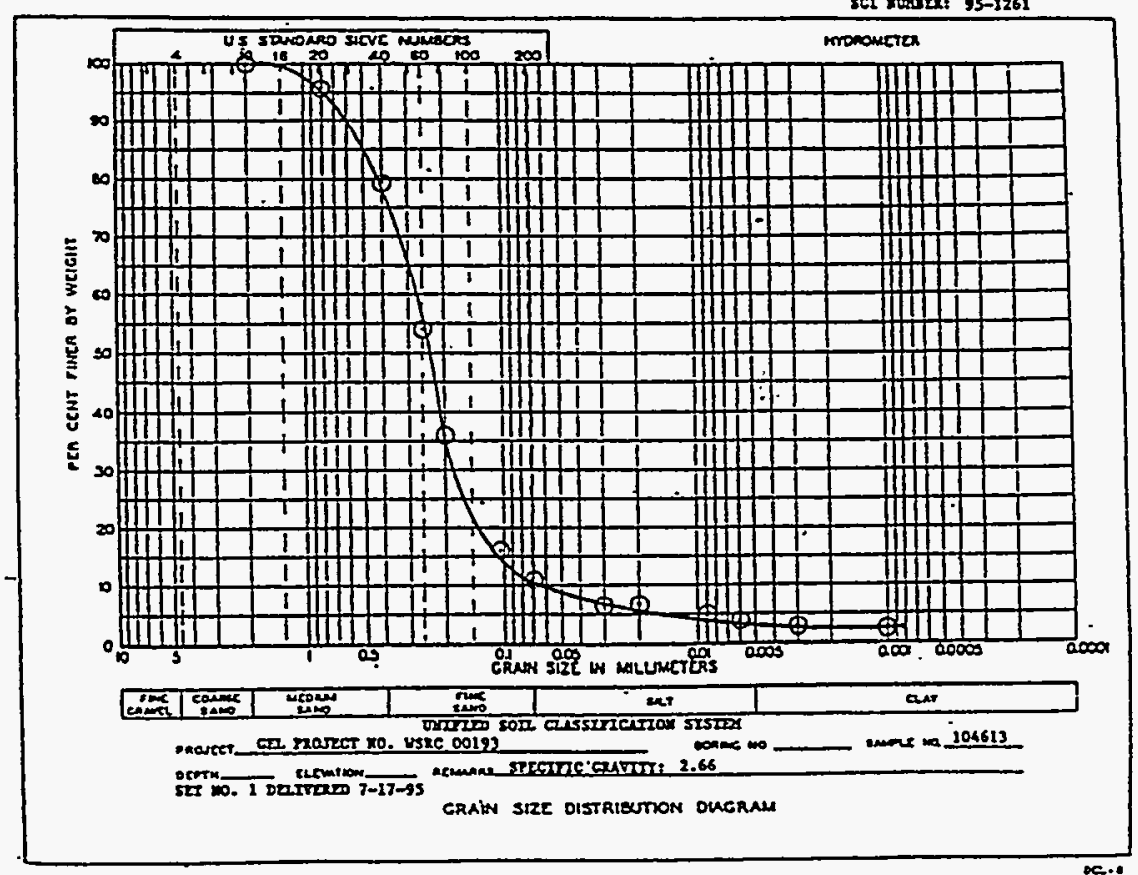

Survey ID: PPSC0501

Sample ID: 104613 


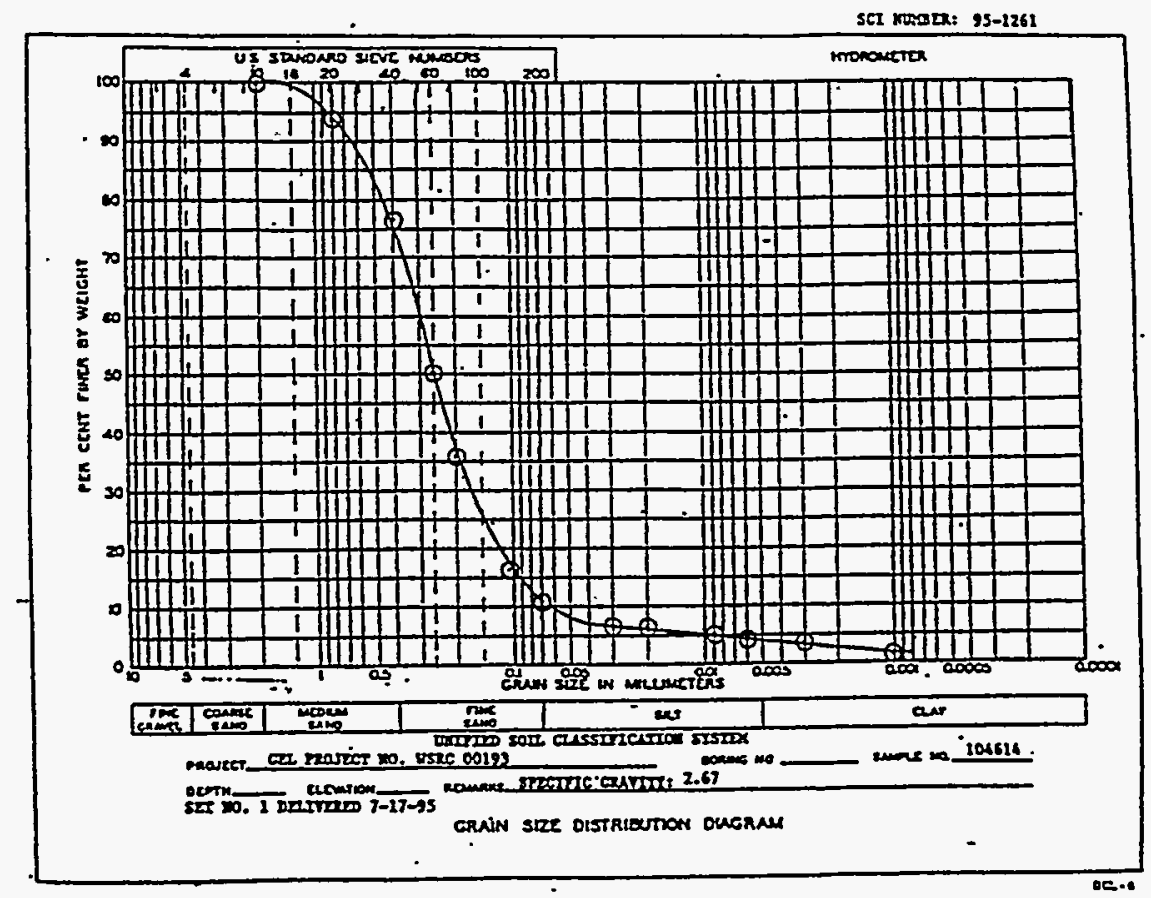

Survey ID: PPSC0502

Sample ID: 104614

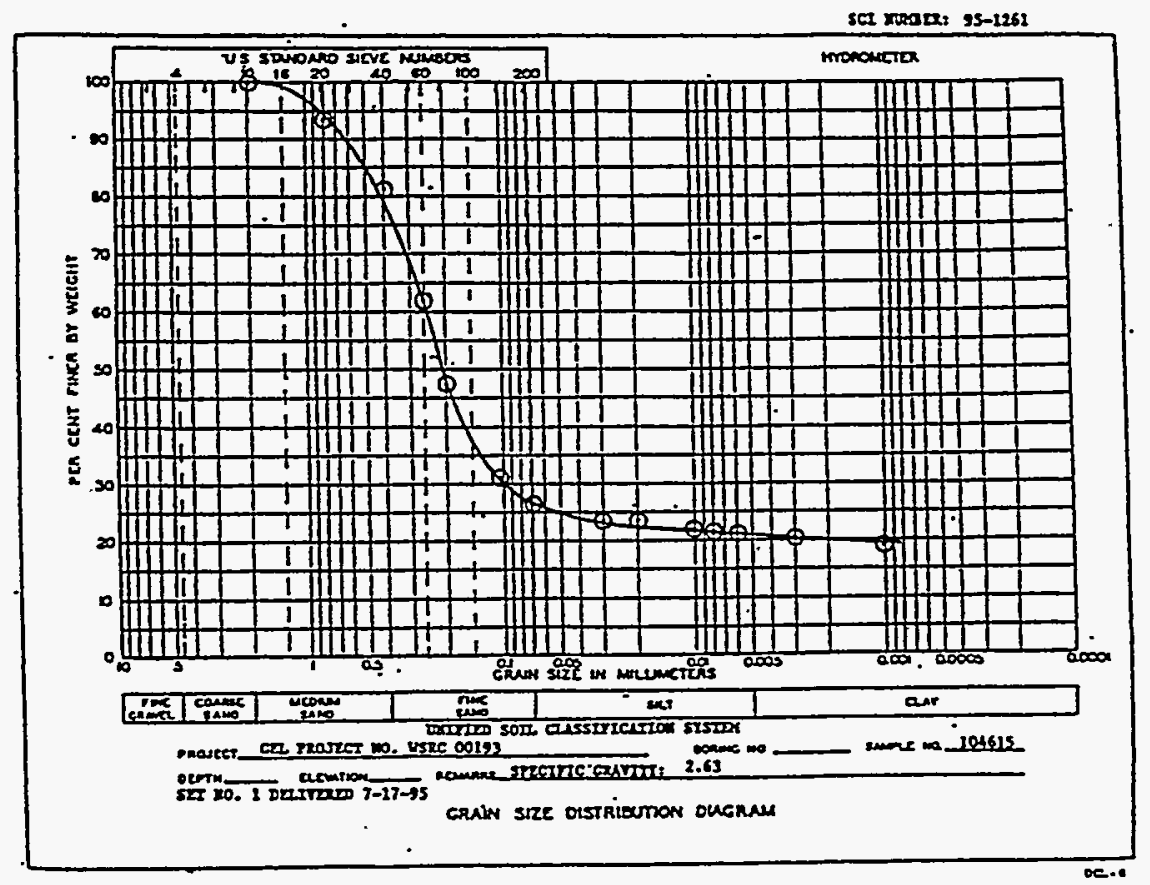

Survey ID: PPSC0503

Sample ID: 104615 


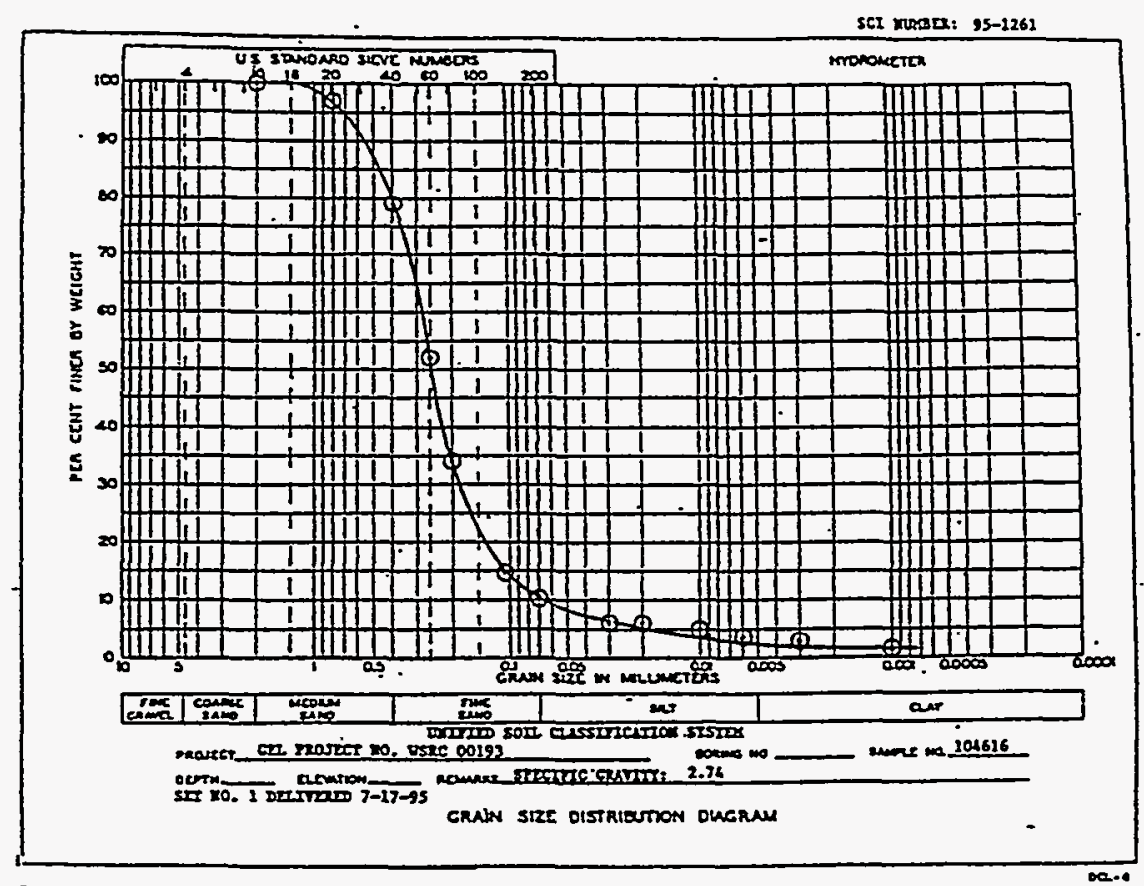

Survey ID: PPSC0601

Sample ID: 104616

SCI Mtrocks: 95-1261

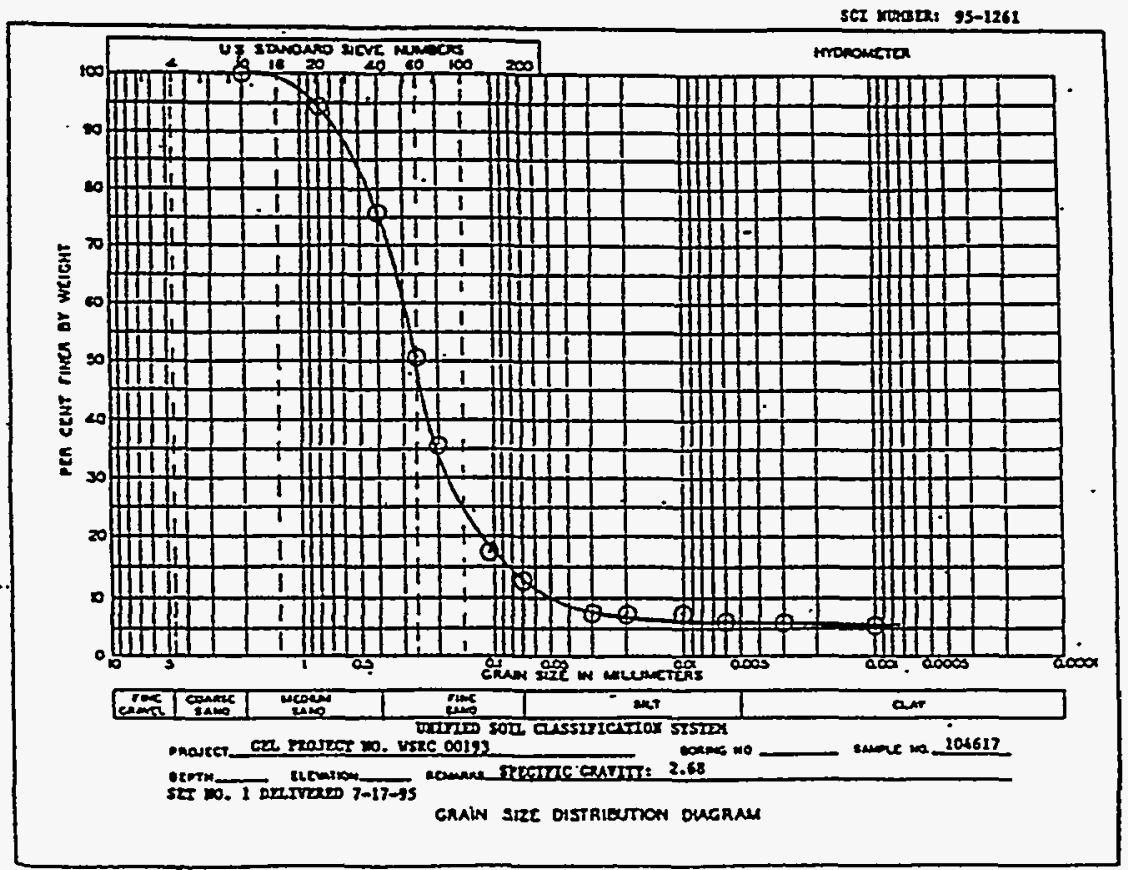

Survey ID: PPSC0602

Sample ID: 104617 


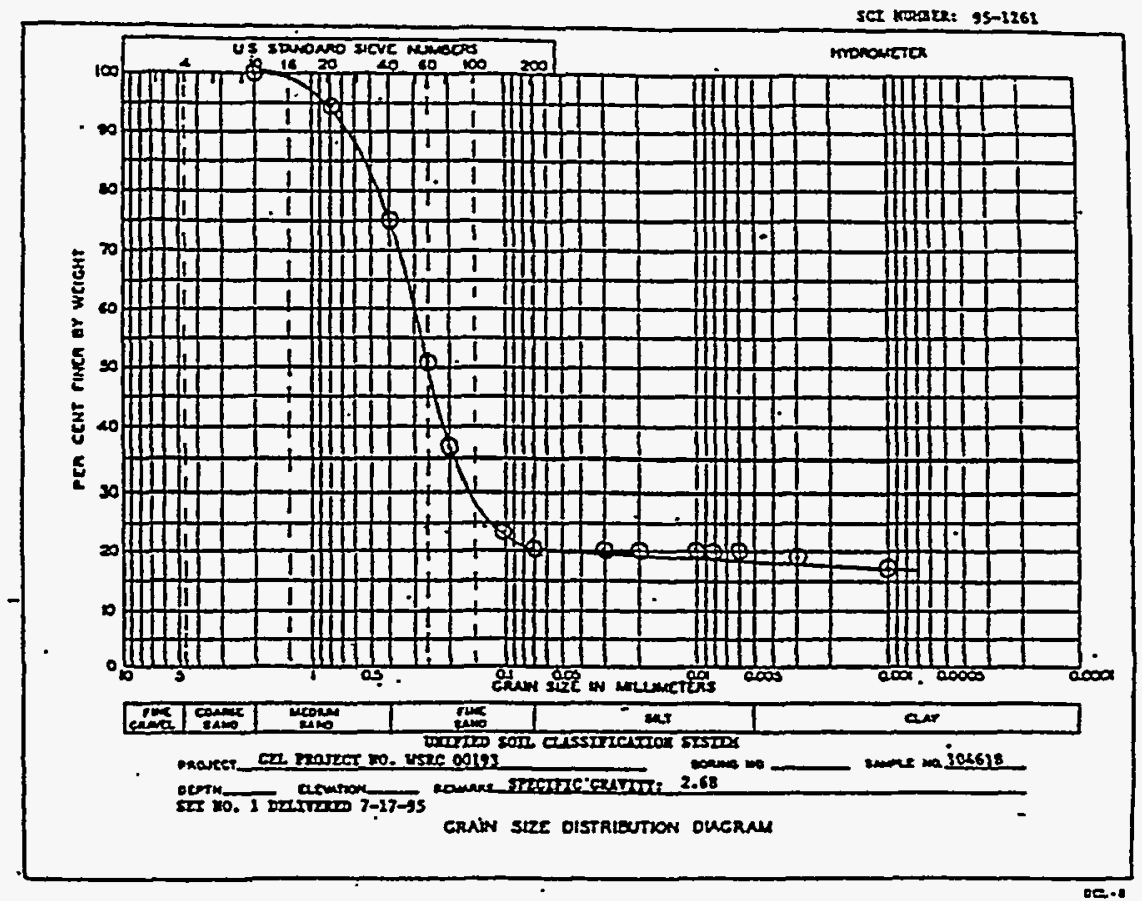

Survey ID: PPSC0603

Sample ID: 104618

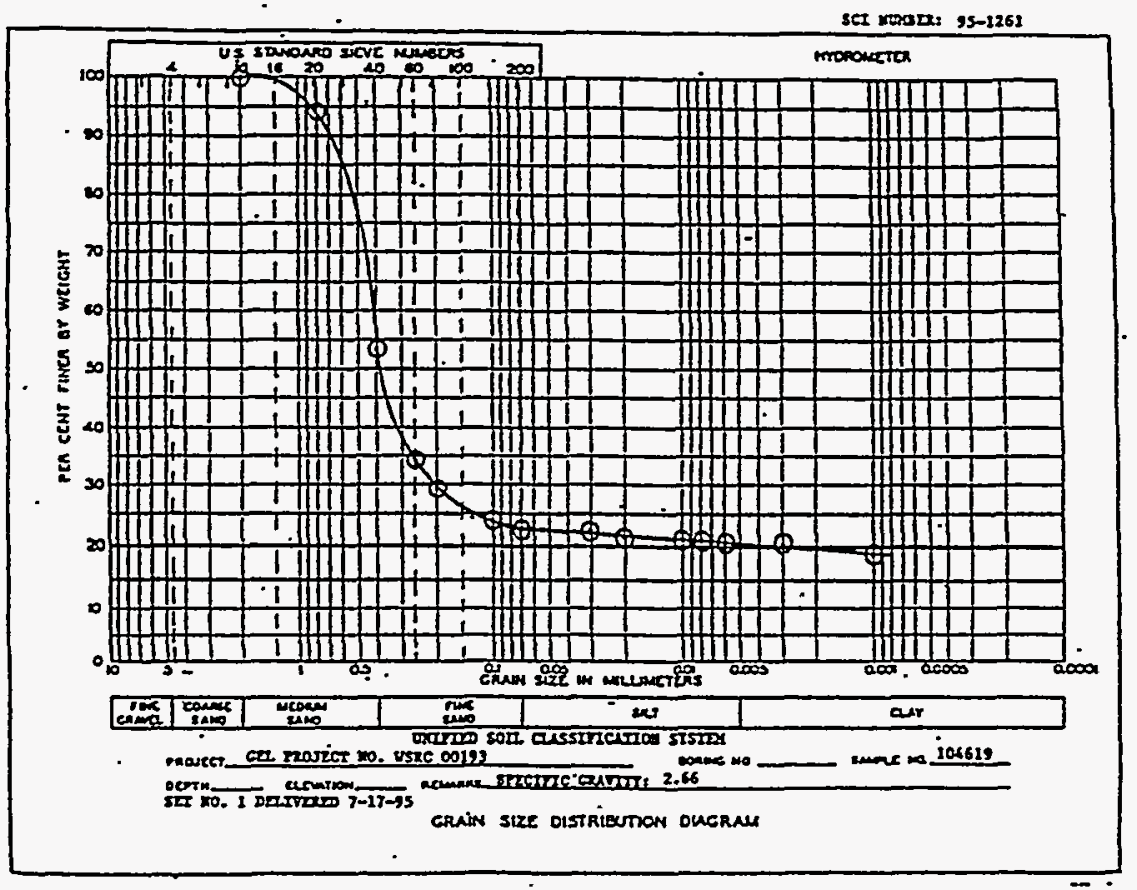

Survey ID: PPSC0604

Sample ID: 104619 


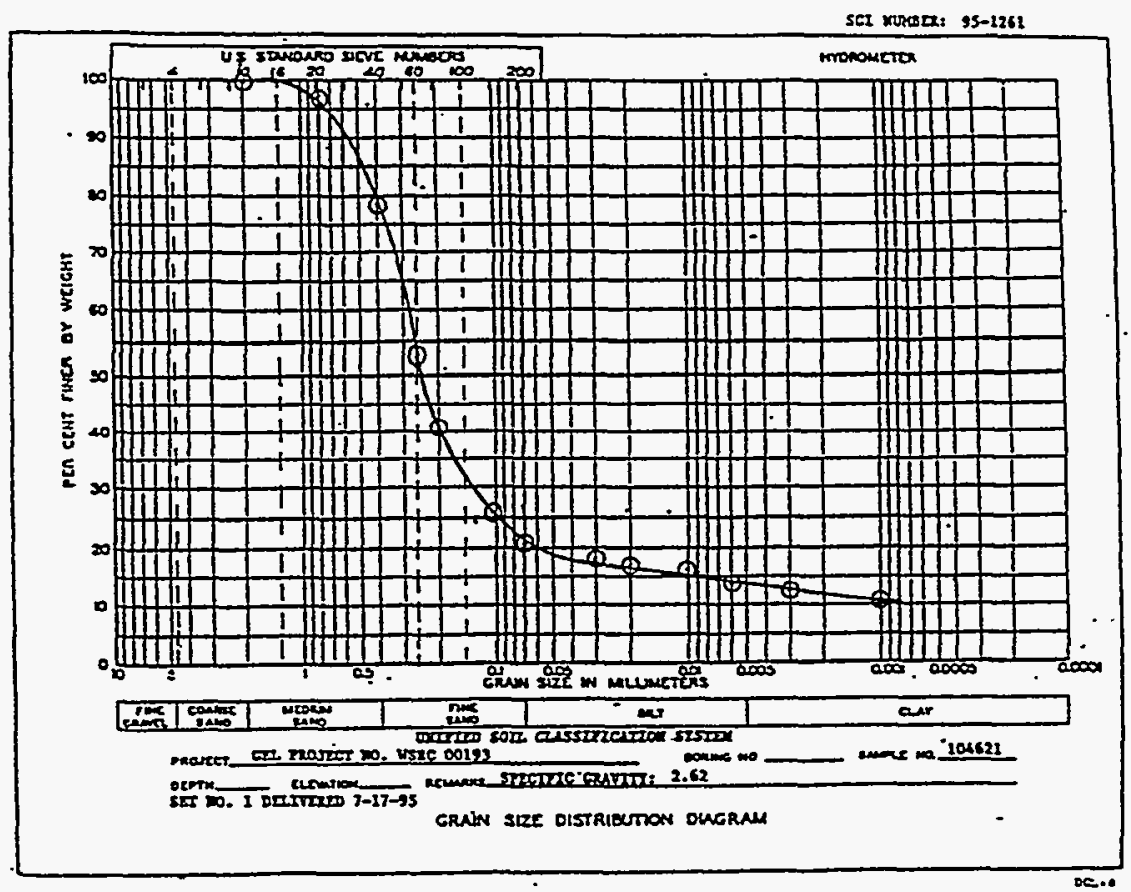

Survey ID: PPSC0702

Sample ID: 104621

sct xorsan: 95-1261

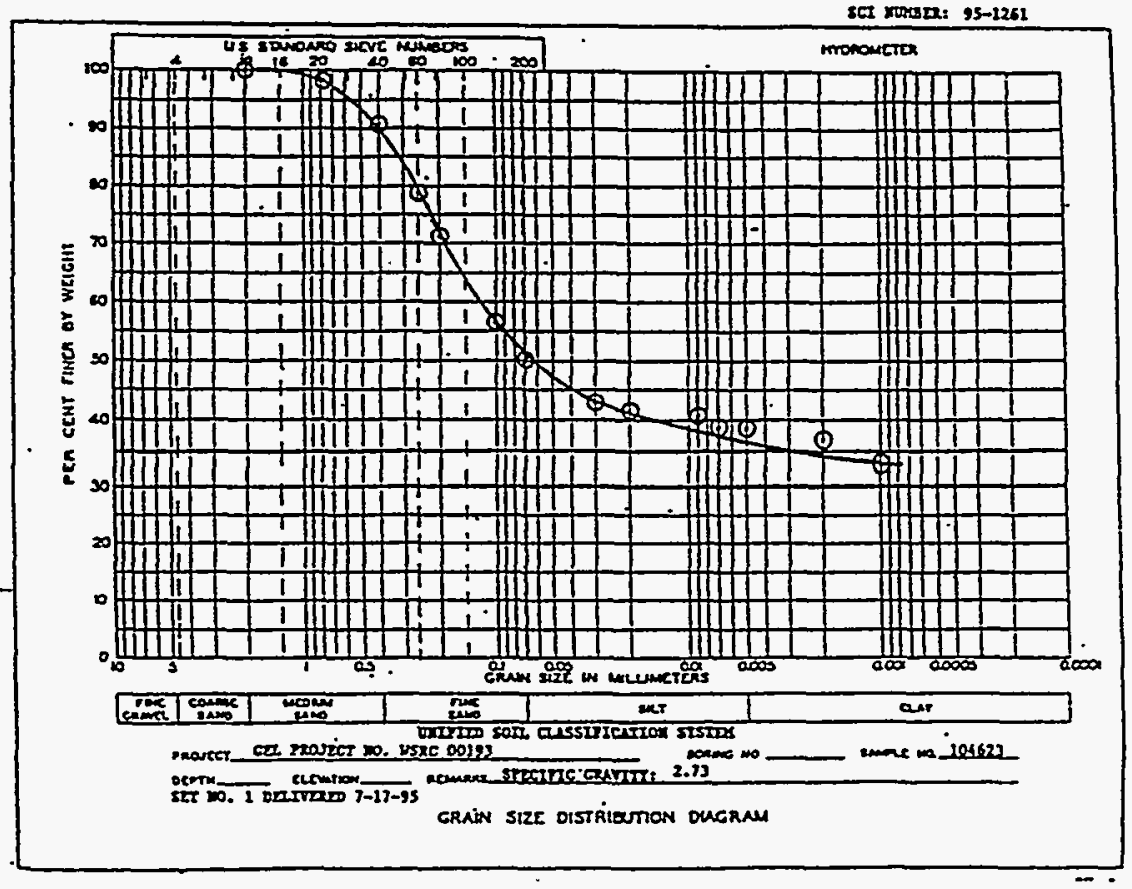

Survey ID: PPSC0704

Sample ID: 104623 


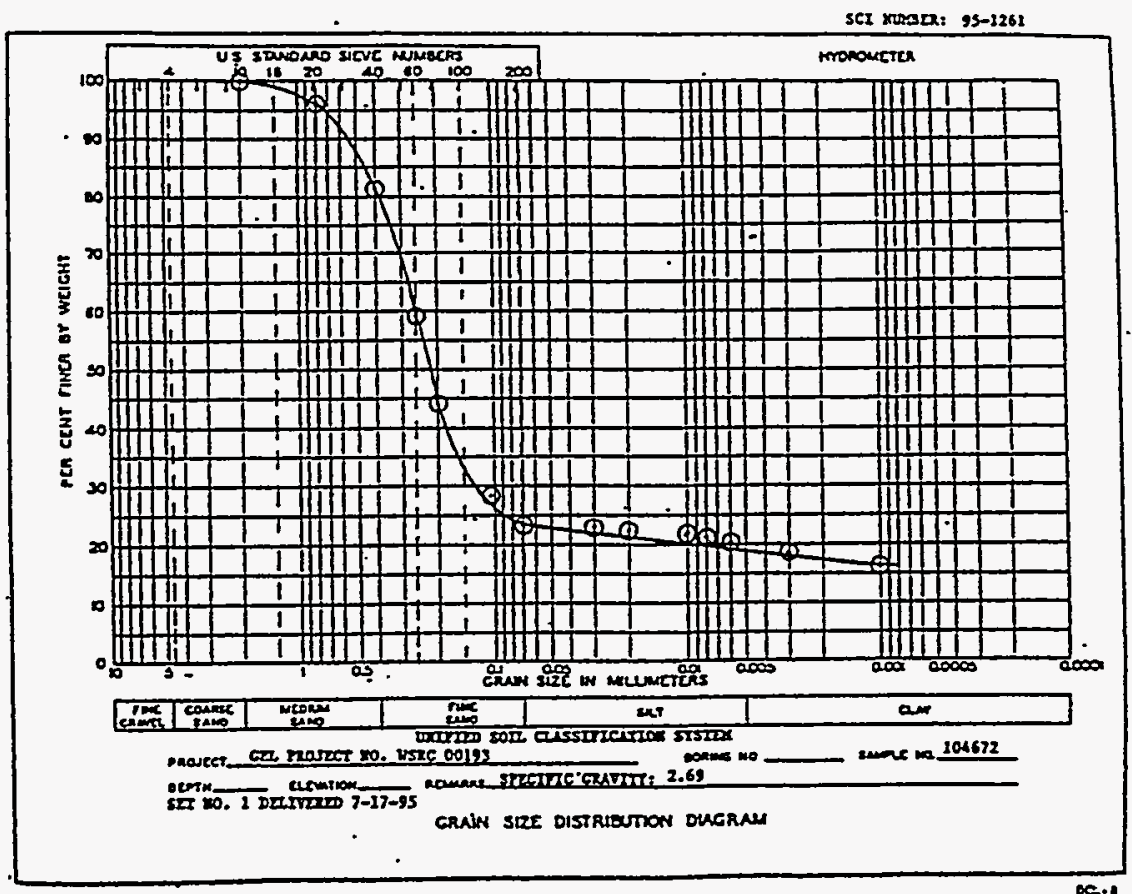

Survey ID: PPSC1902

Sample ID: 104672

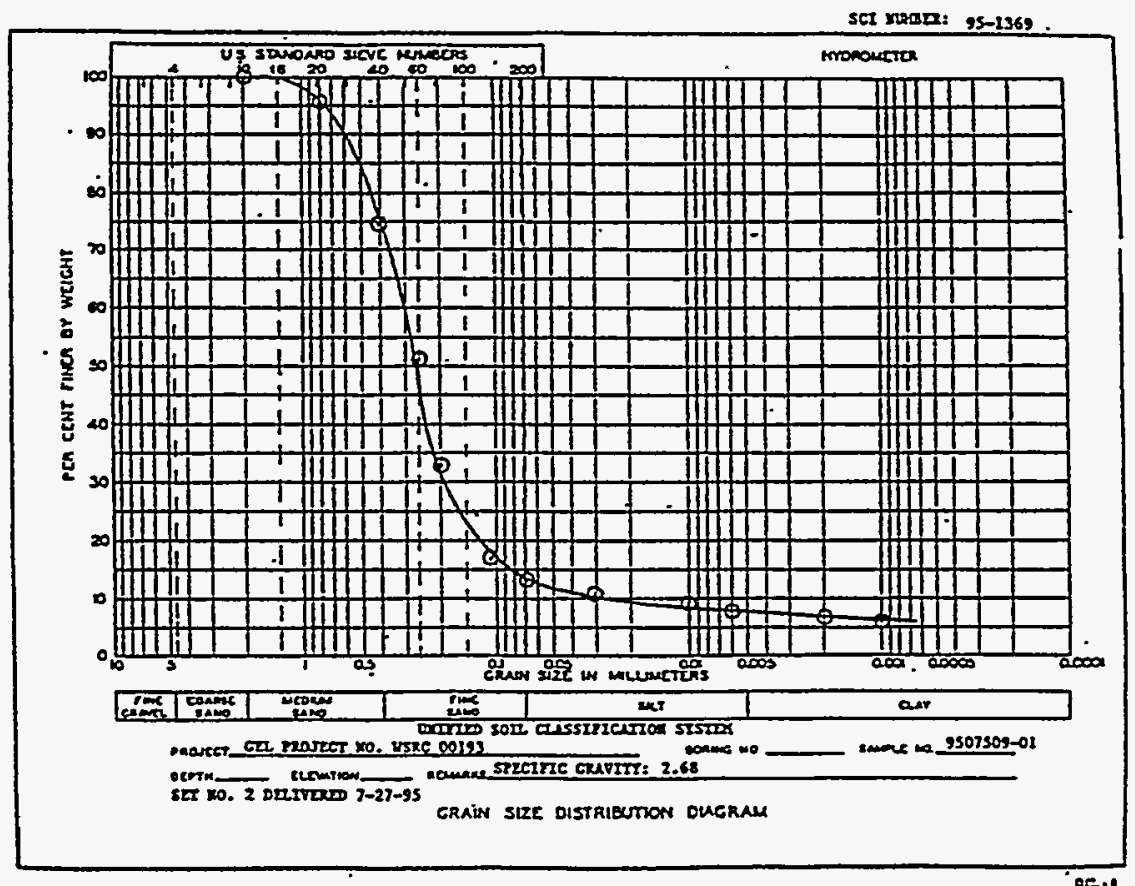

Survey ID: PPSC1701

Sample ID: 104693 


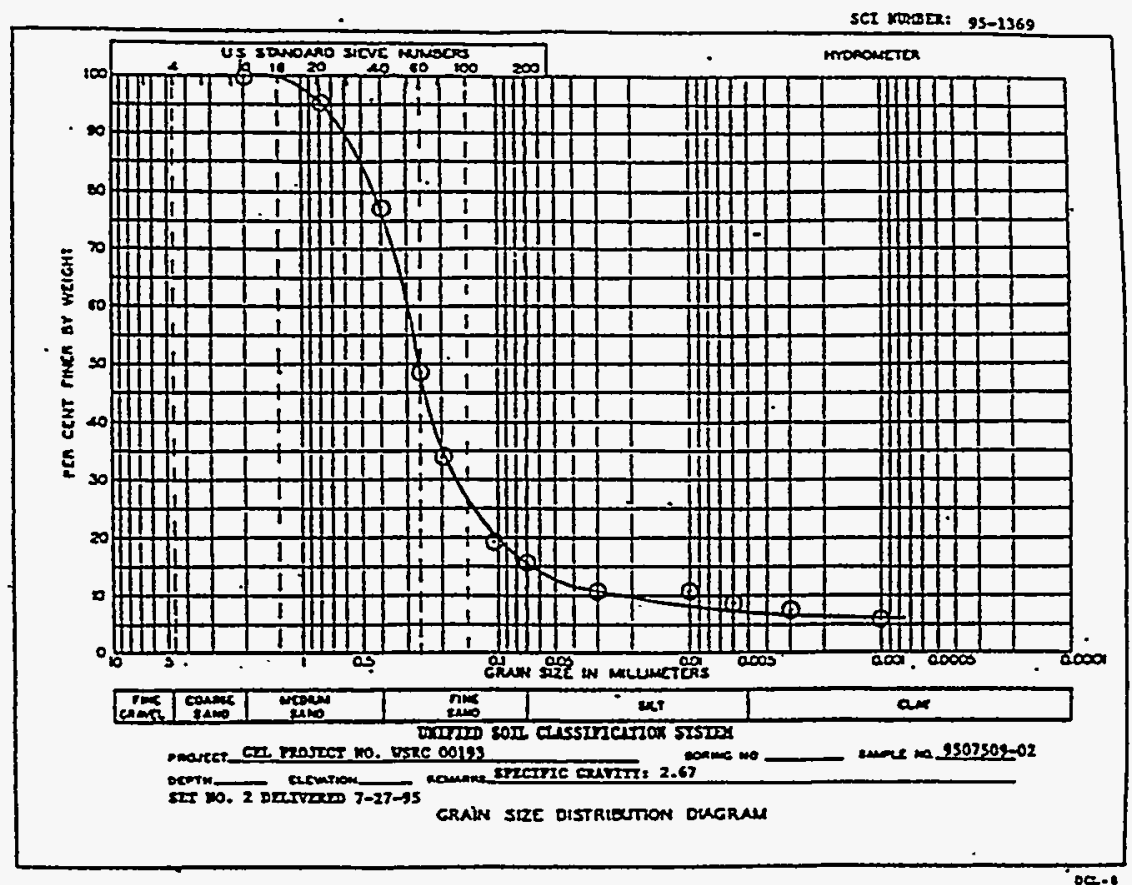

Survey ID: PPSC1702

Sample ID: 104694

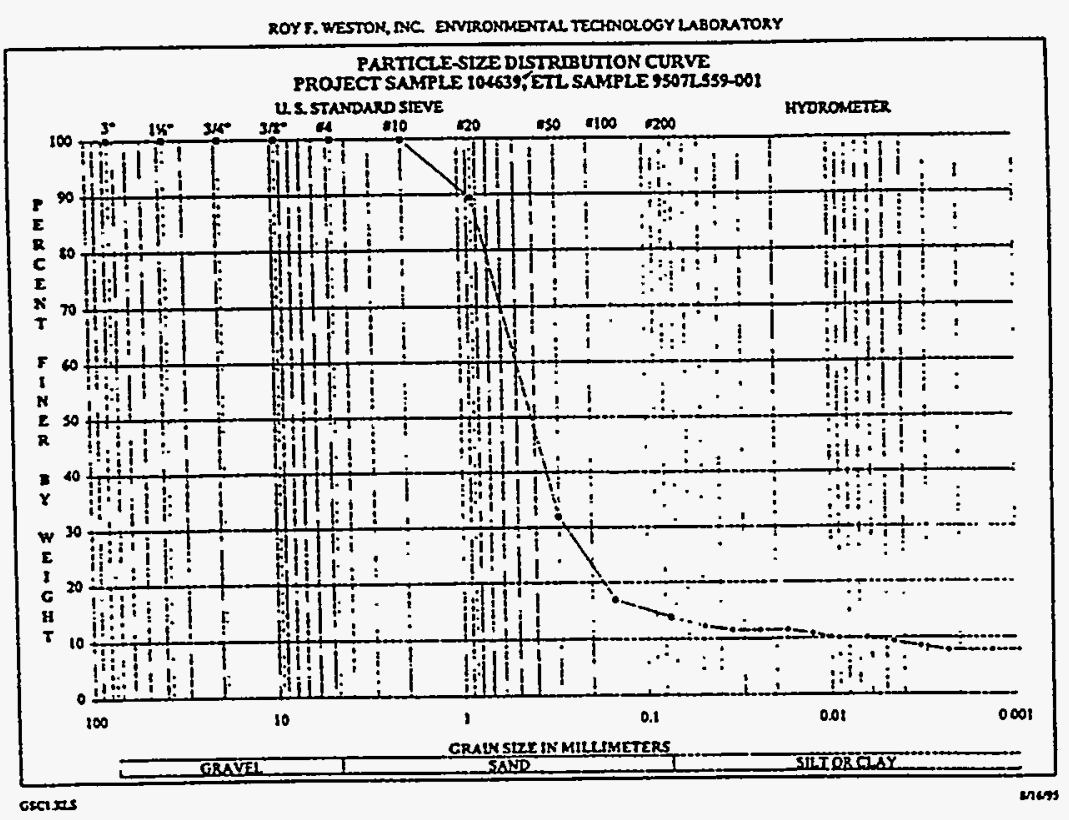

Survey ID: PPSC2602D

Sample ID: 104639 

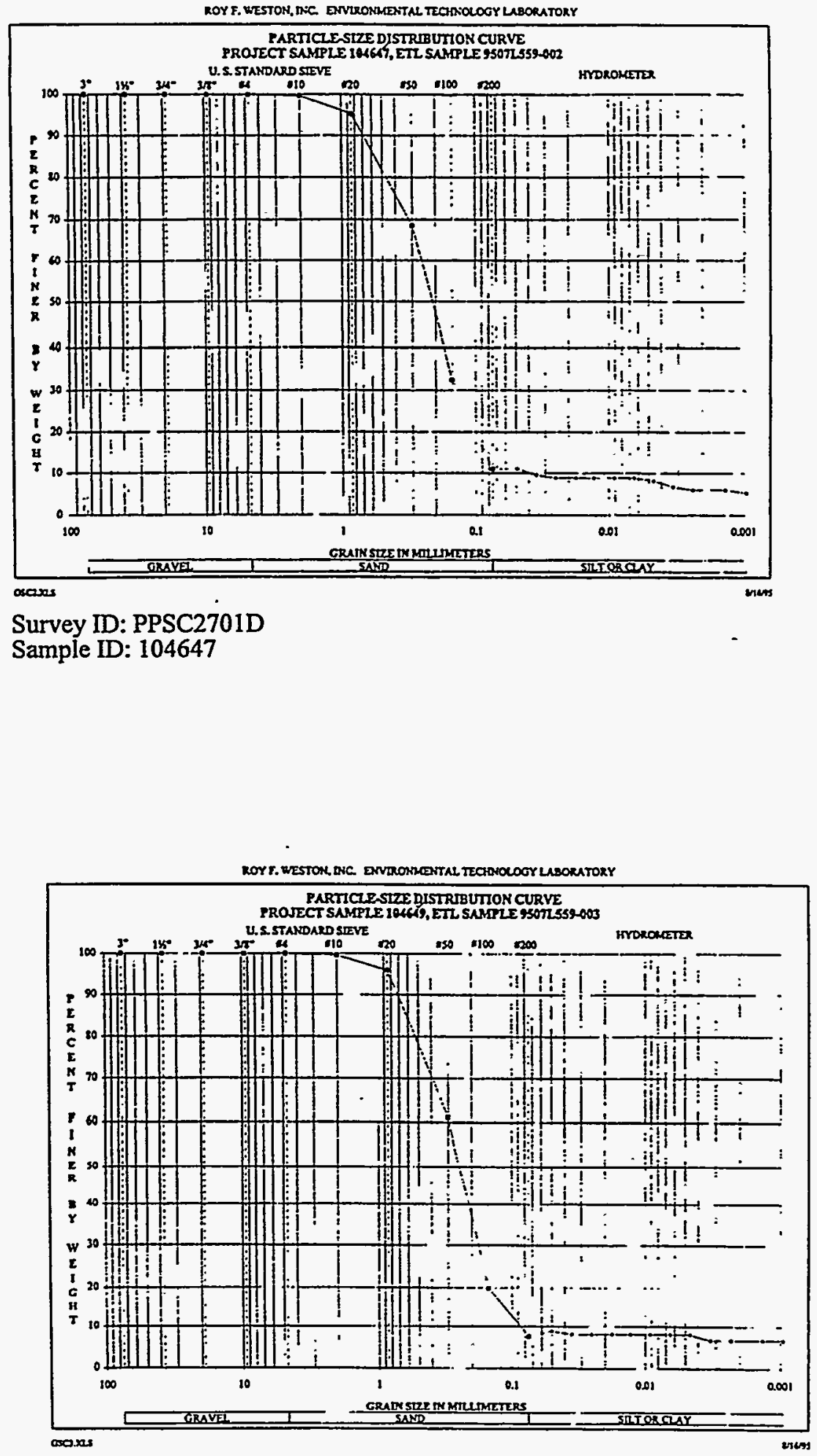

Survey ID: PPSC2703D

Sample ID: 104649 


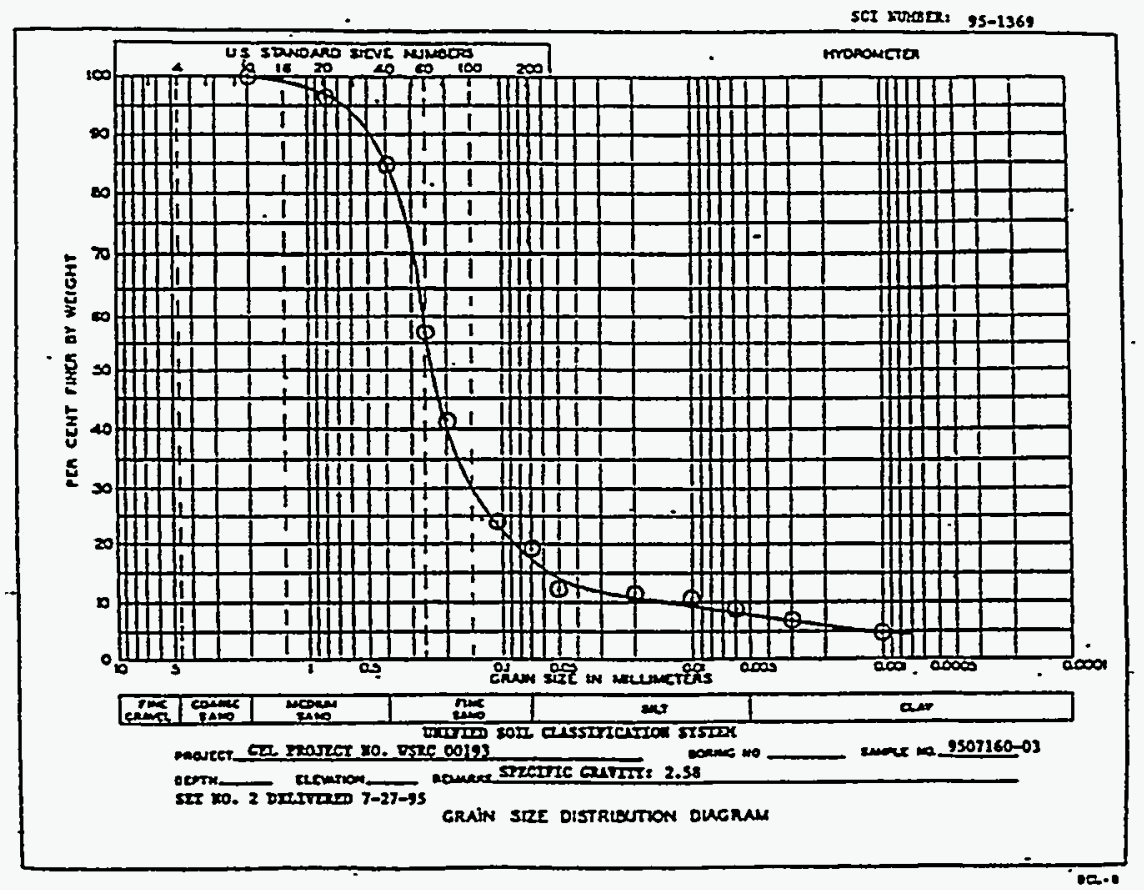

Survey ID: PPSC1005

Sample ID: 104629

SeI xurapa: 95-1369

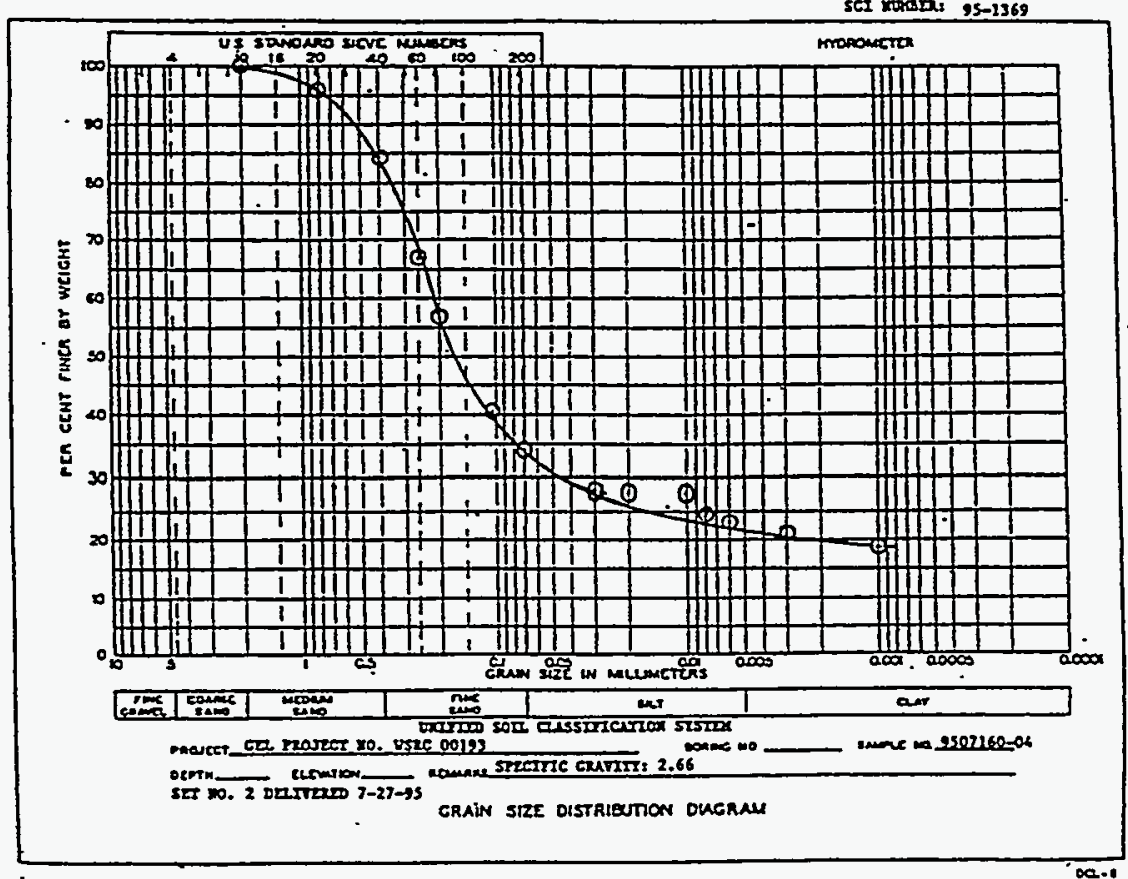

Survey ID: PPSC0801

Sample ID: 104630 


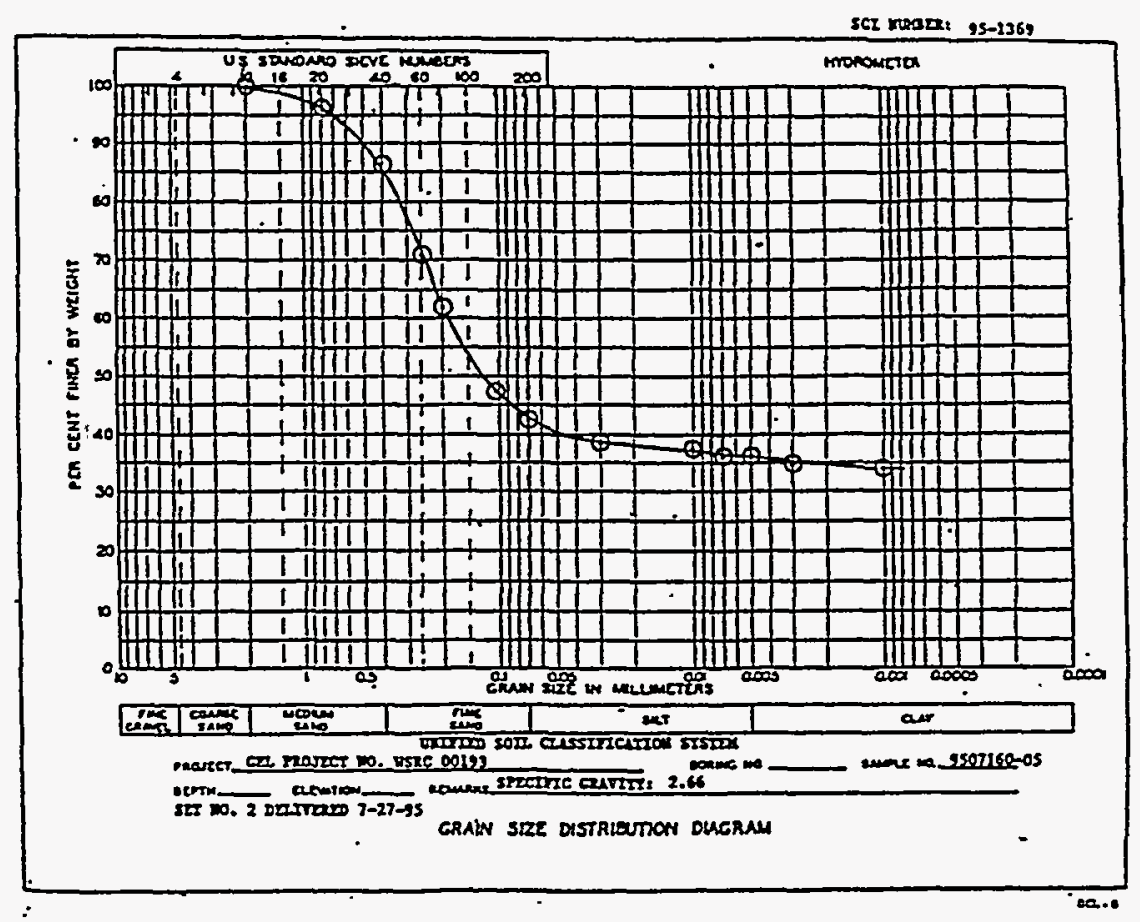

Survey ID: PPSC0802

Sample ID: 104632

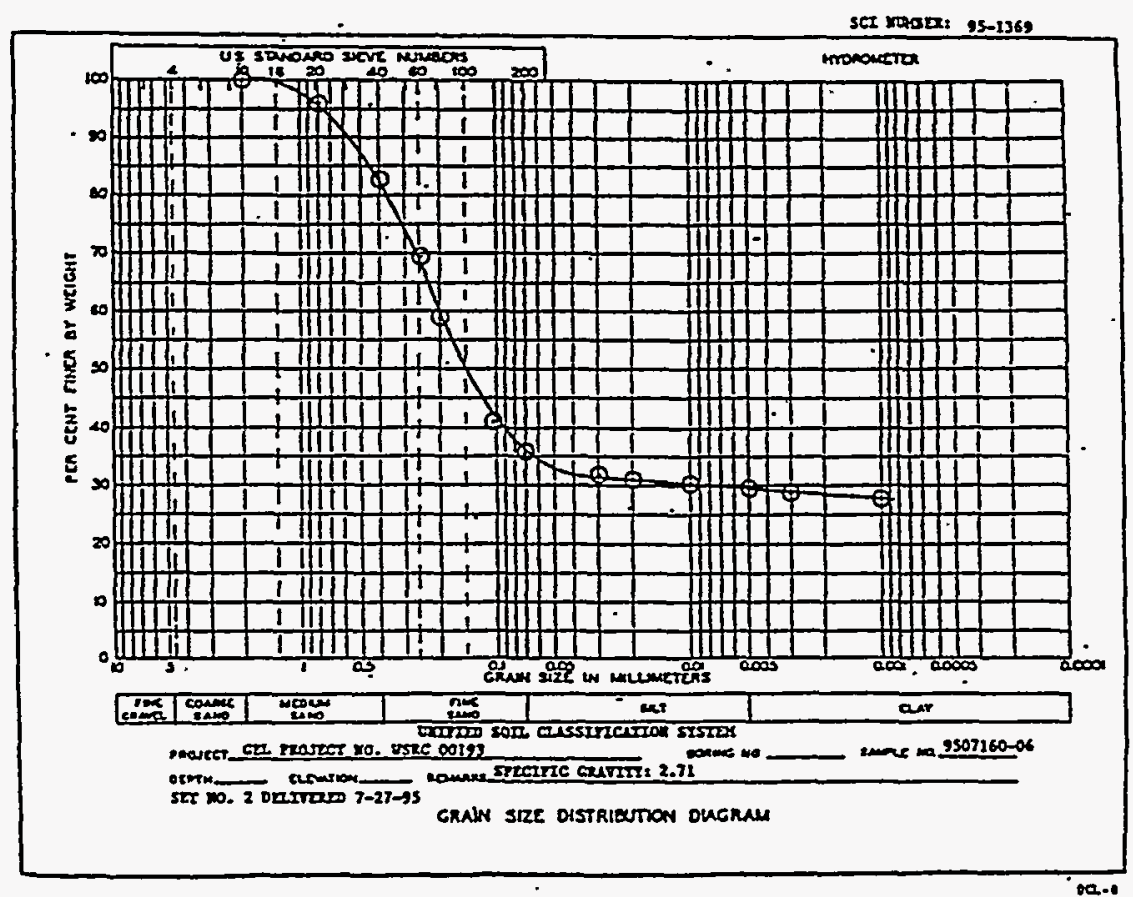

Survey ID: PPSC0803

Sample ID: 104633 


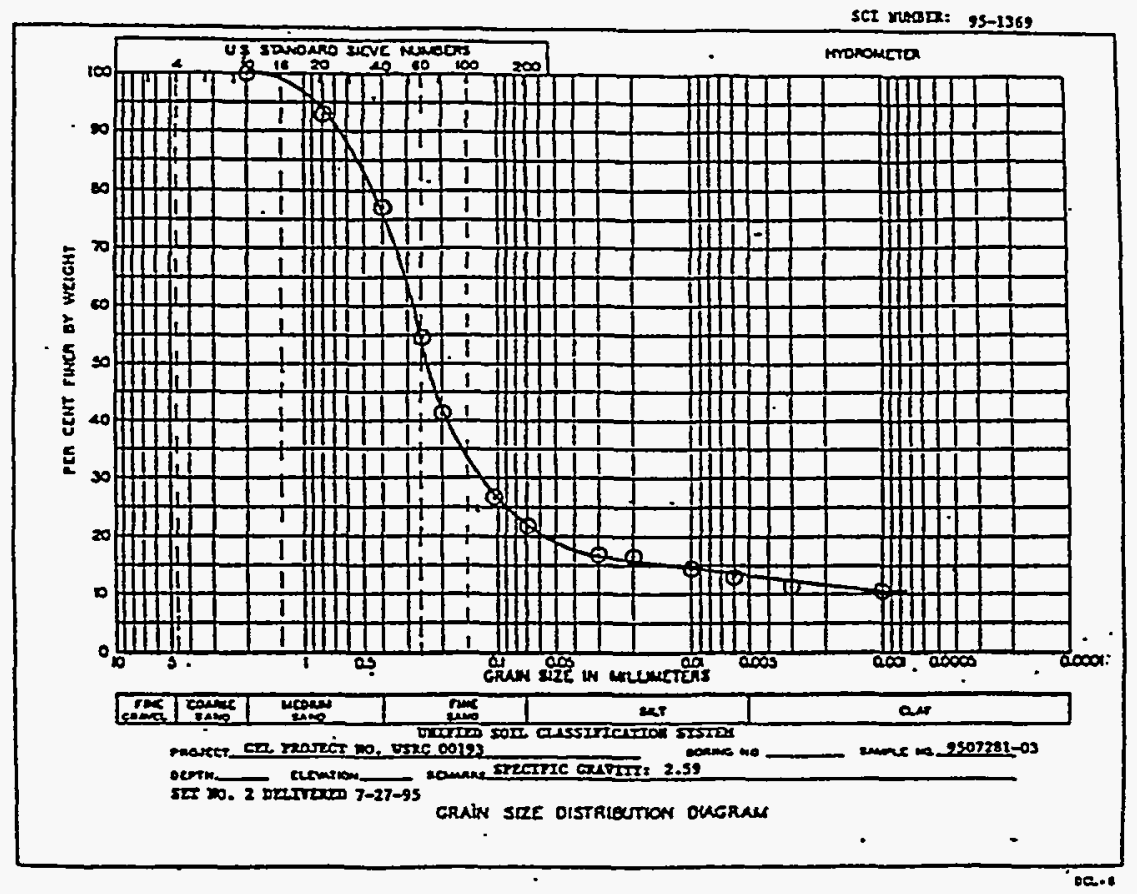

Survey ID: PPSC1103

Sample ID: 104655

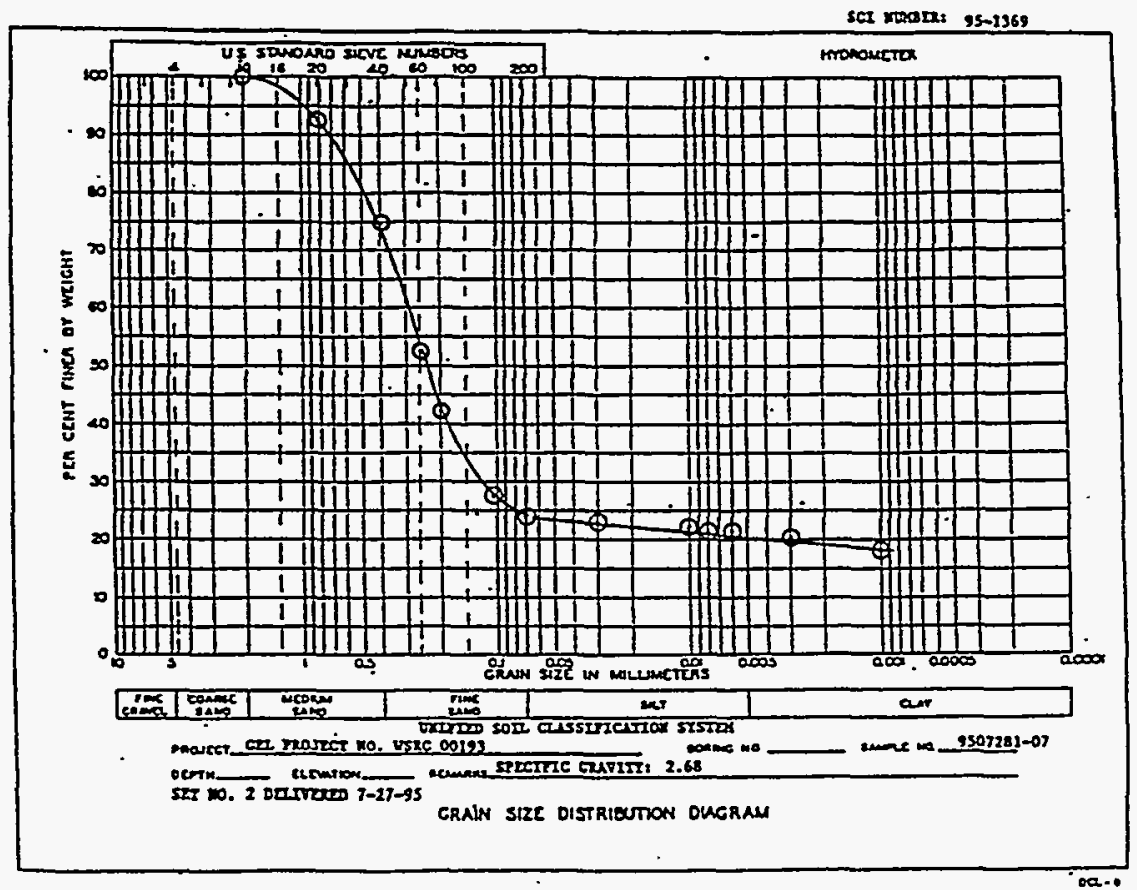

Survey ID: PPSC1203

Sample ID: 104660

Par Pond, Pond C, and L Lake, Revision 2 


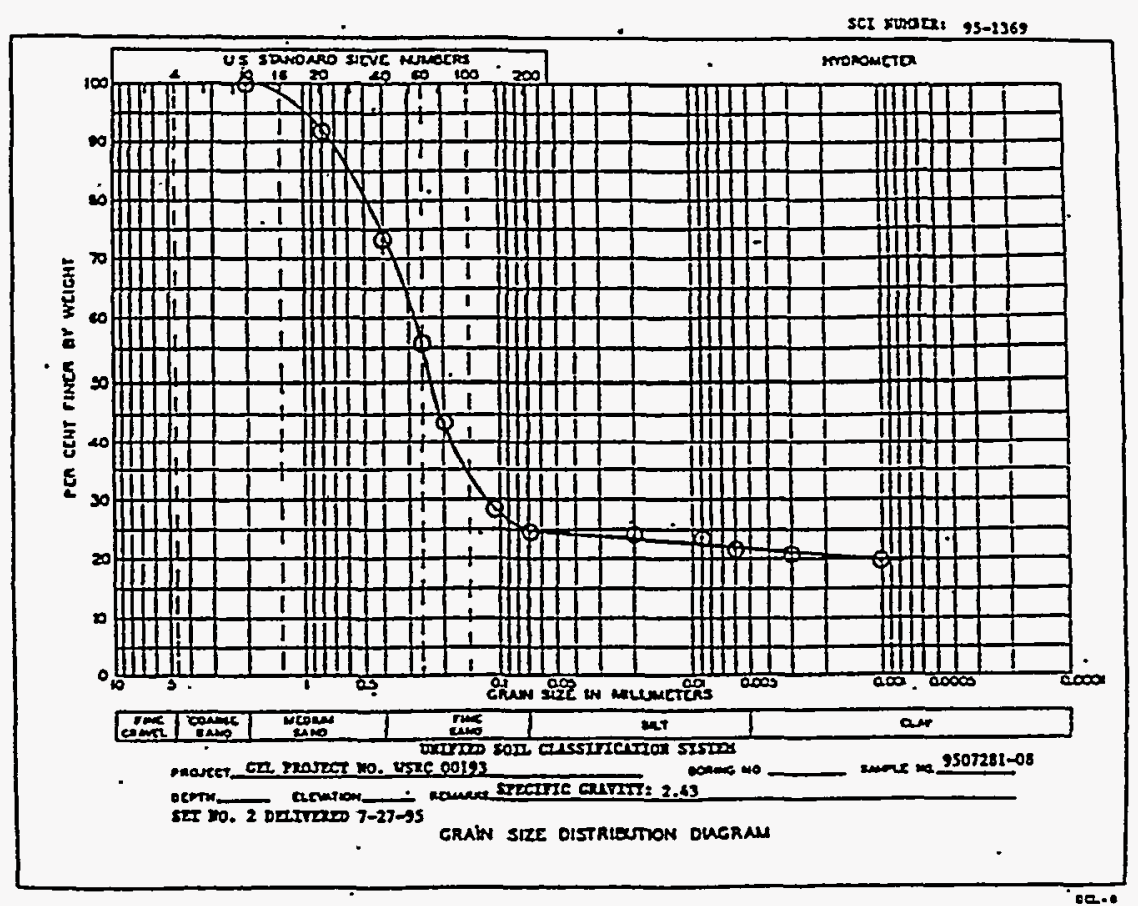

Survey ID: PPSC2803A

Sample ID: 104661

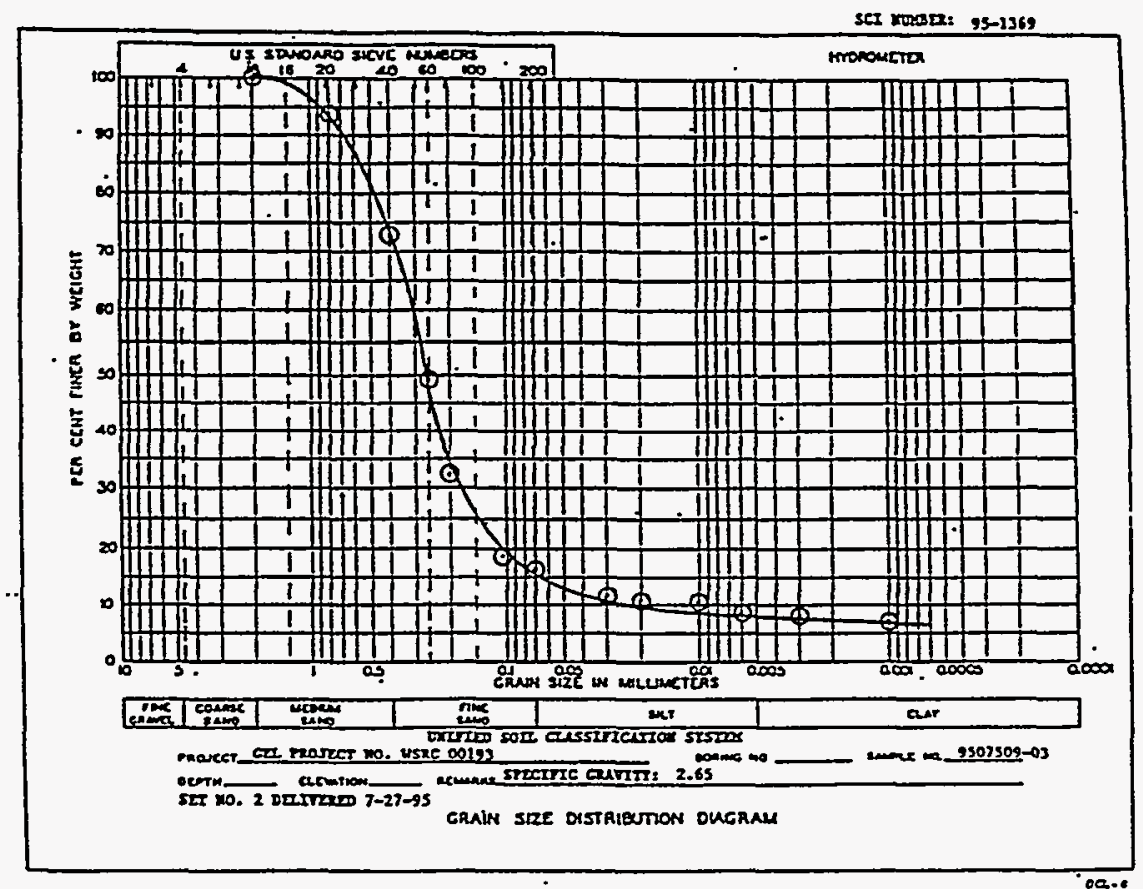

Survey ID: PPSC1703

Sample ID: 104695 


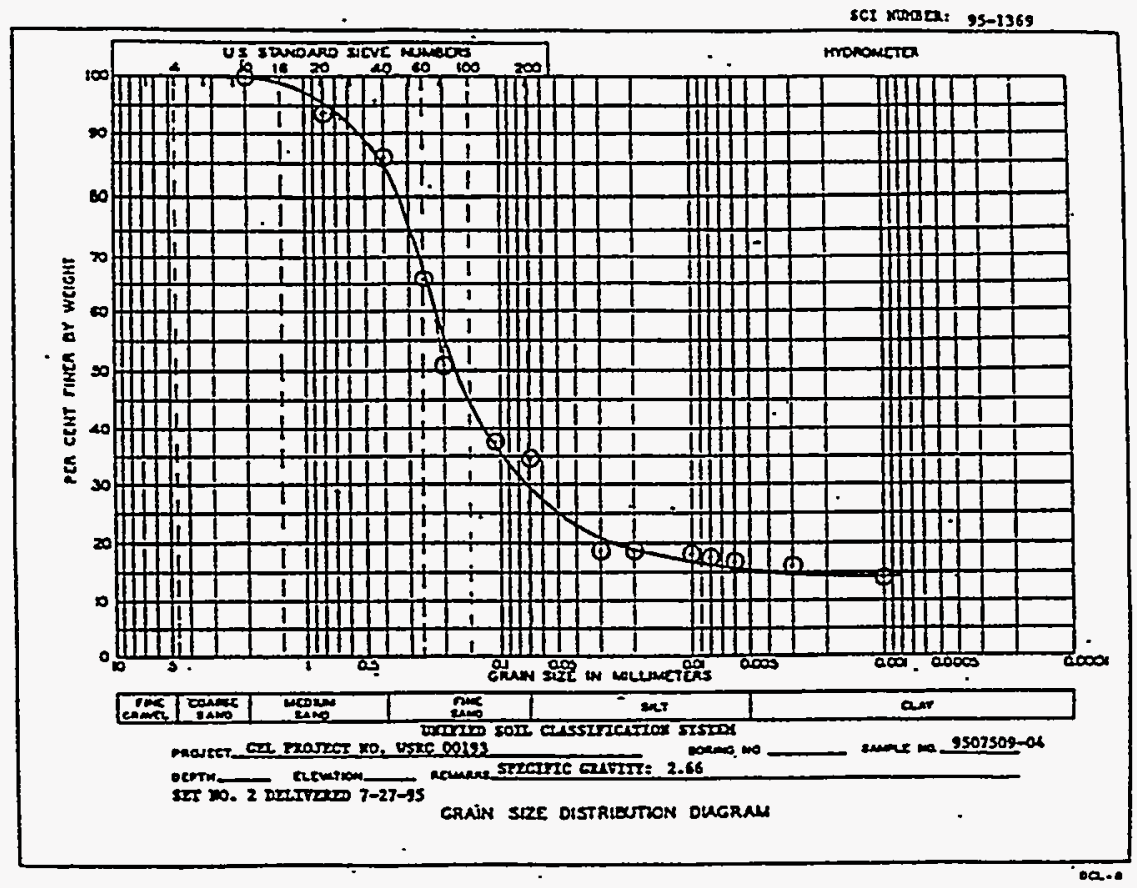

Survey ID: PPSC1704

Sample ID: 104696

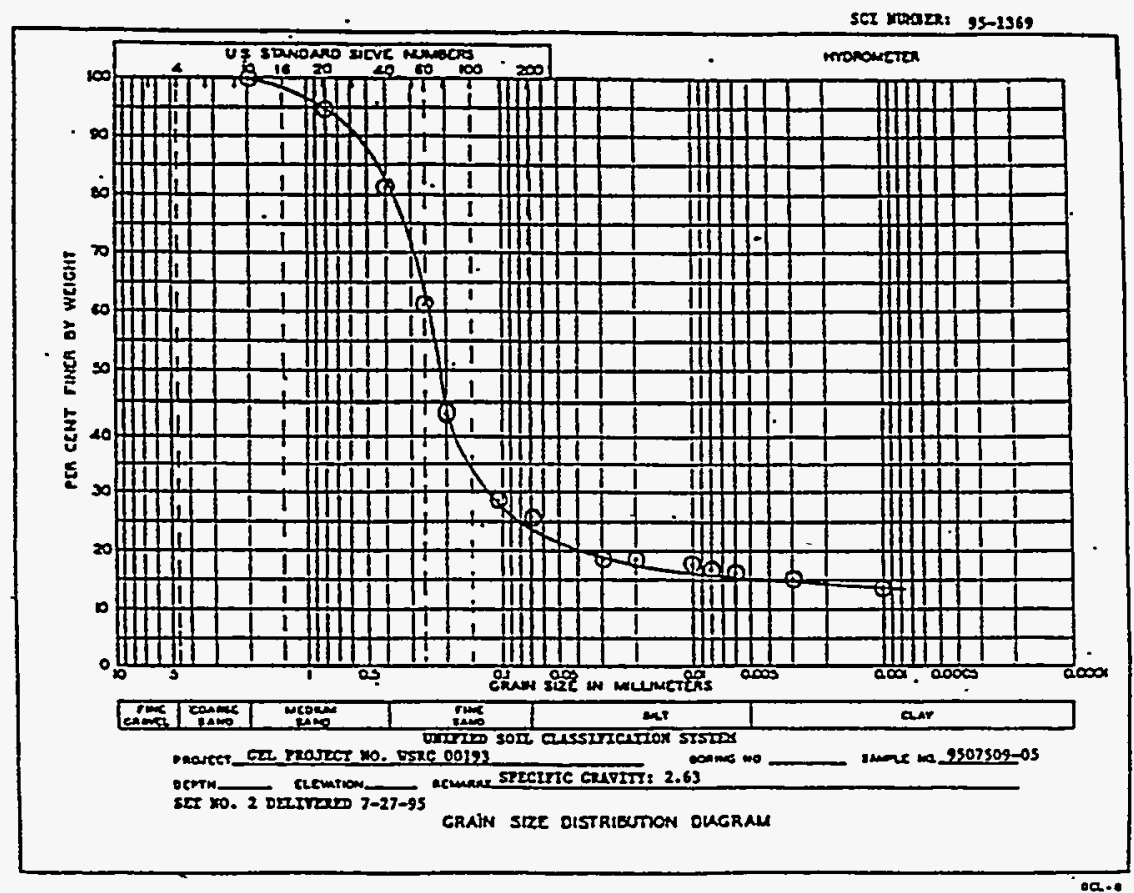

Survey ID: PPSC1603

Sample ID: 105100 


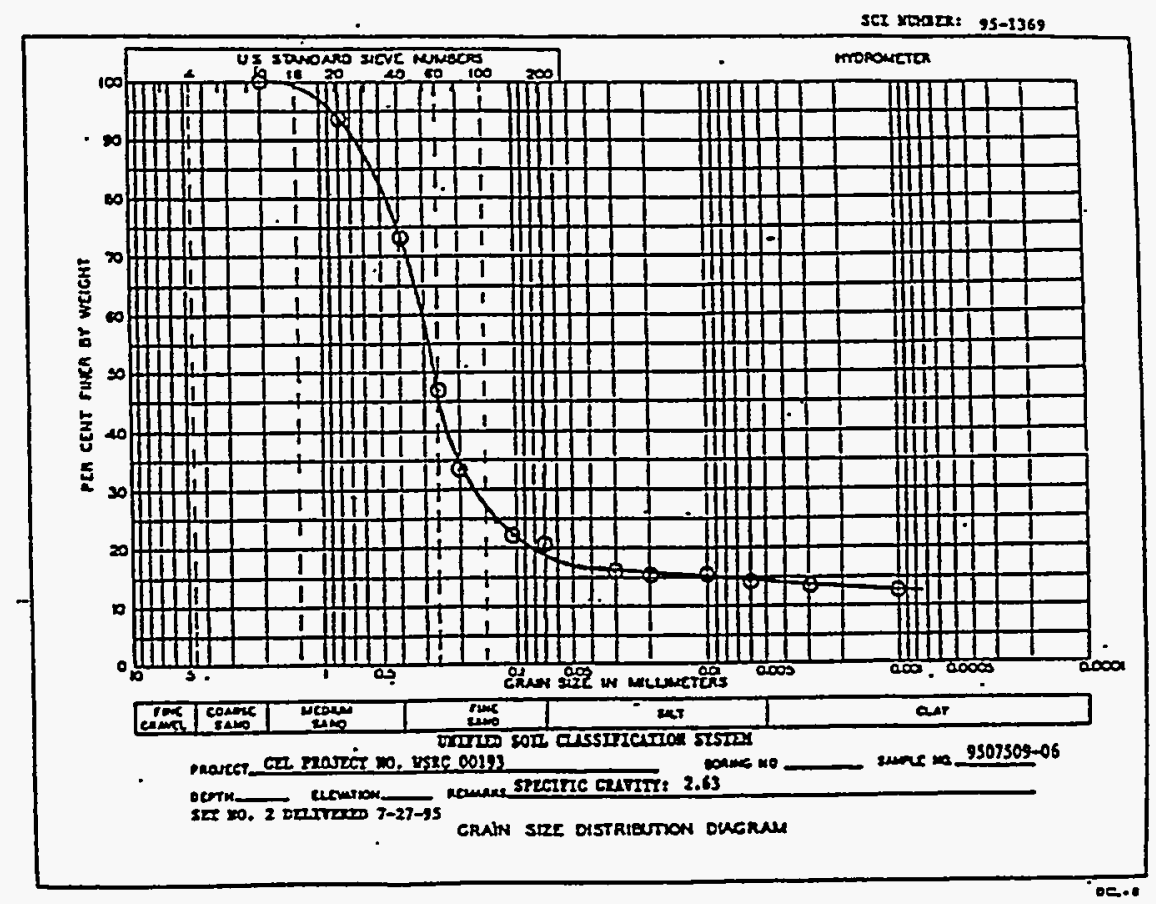

Survey ID: PPSC1604

Sample ID: 105101

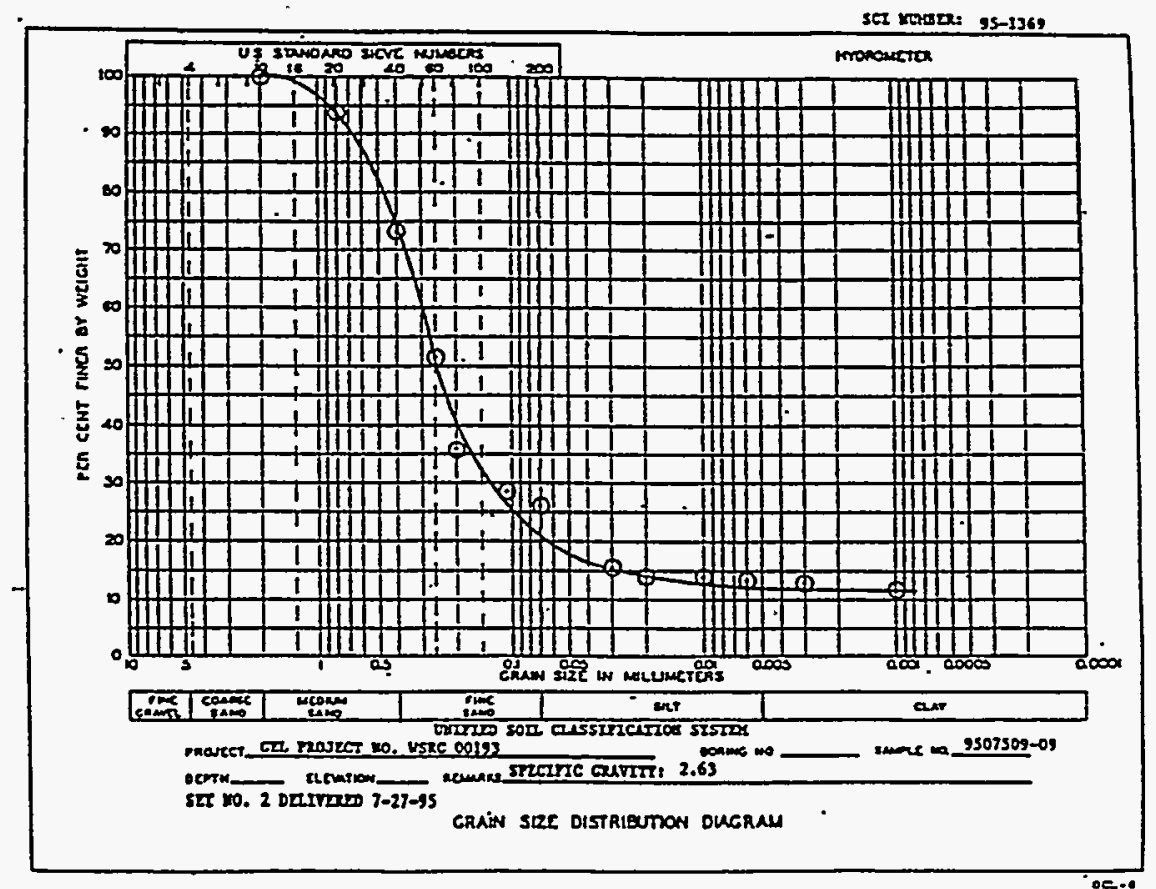

Survey ID: PPSCl605

Sample ID: 105102 


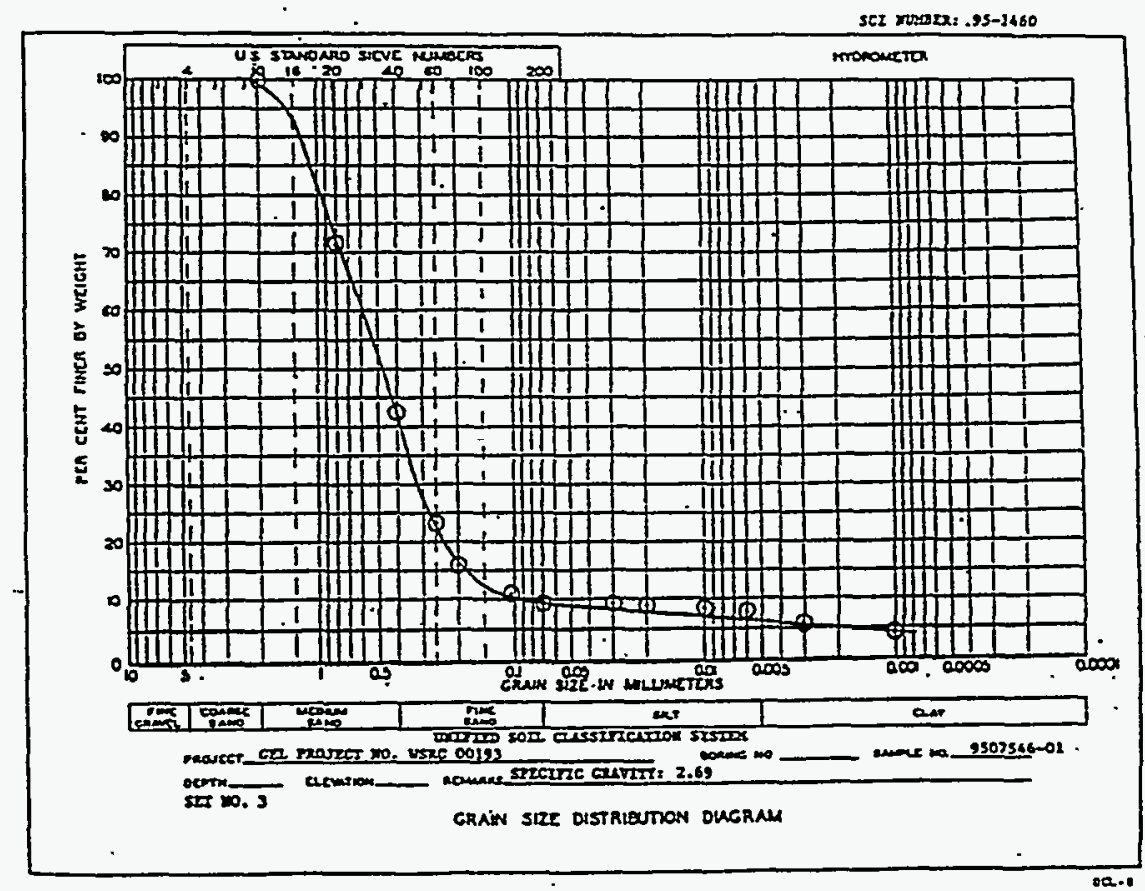

Survey ID: PPSC2501

Sample ID: 105113

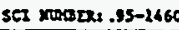

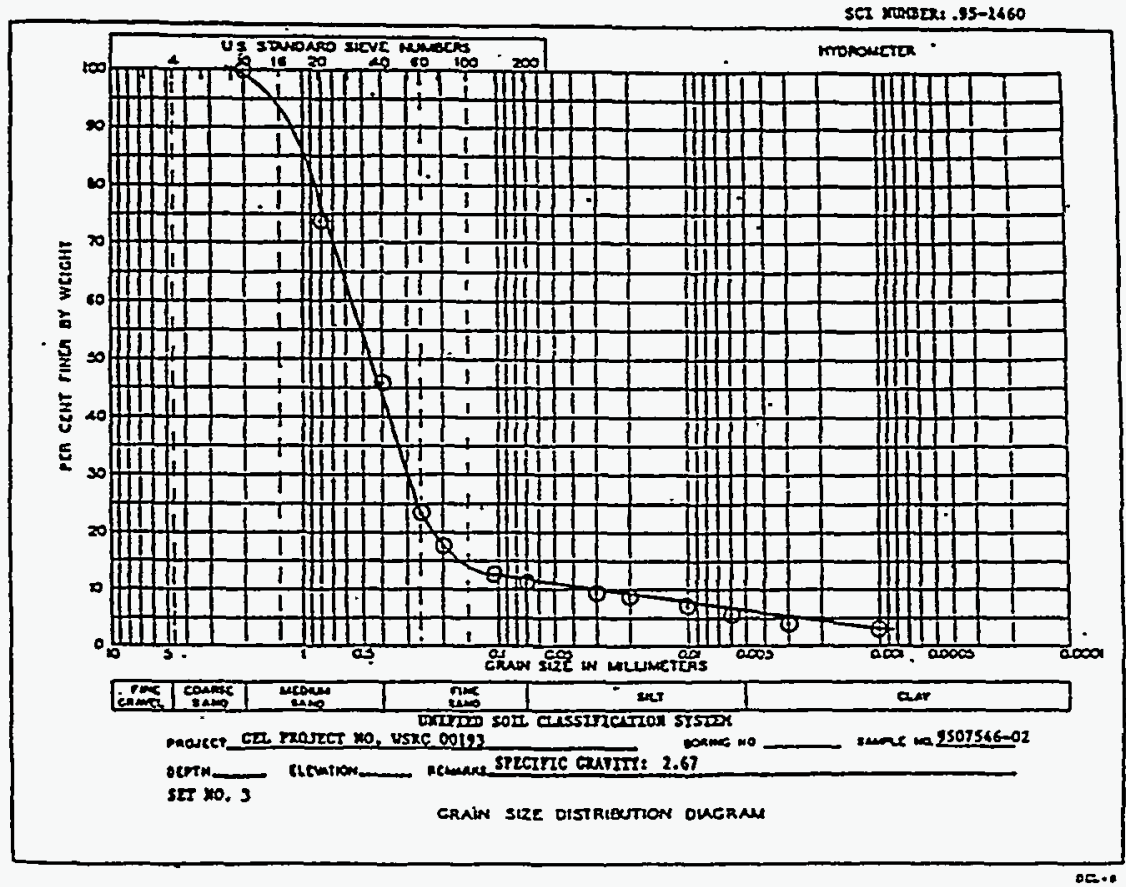

Survey ID: PPSC2502

Sample ID: 105114 


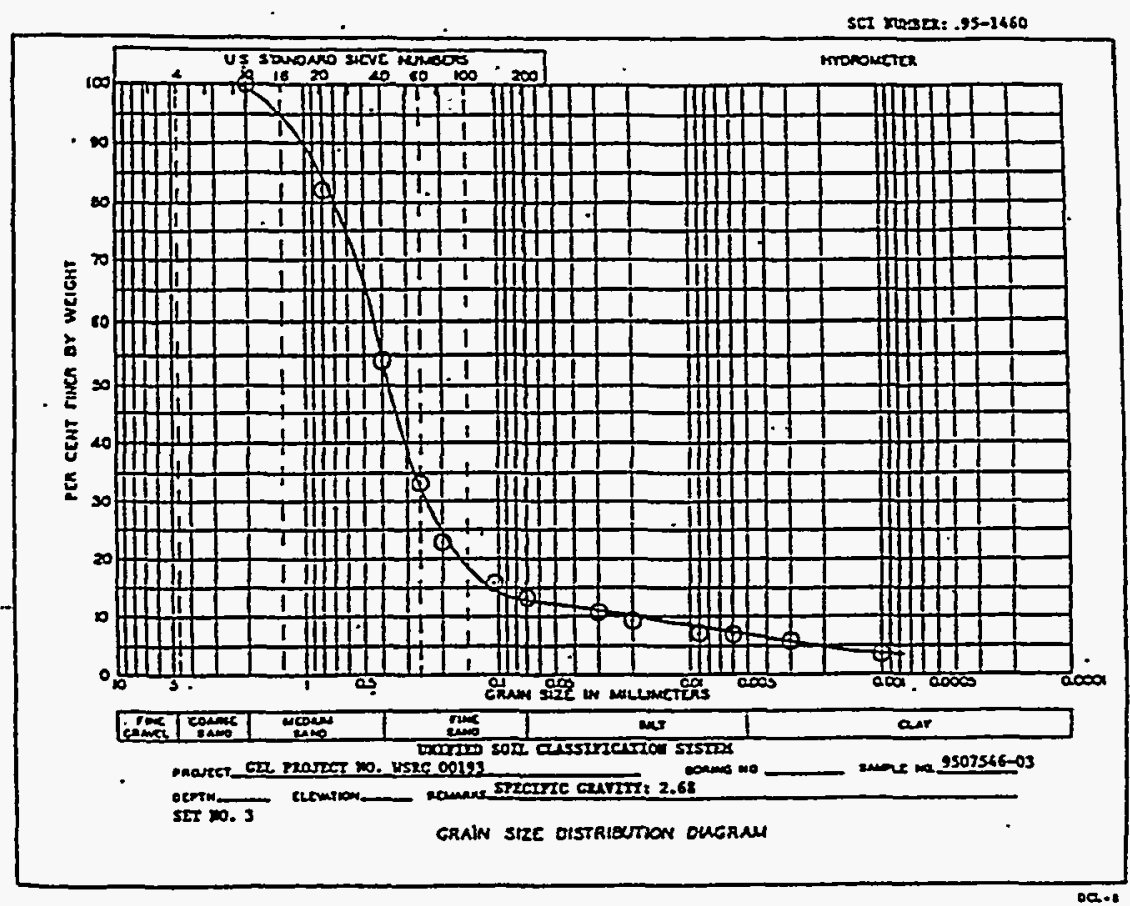

Survey ID: PPSC2503

Sample ID: 105115

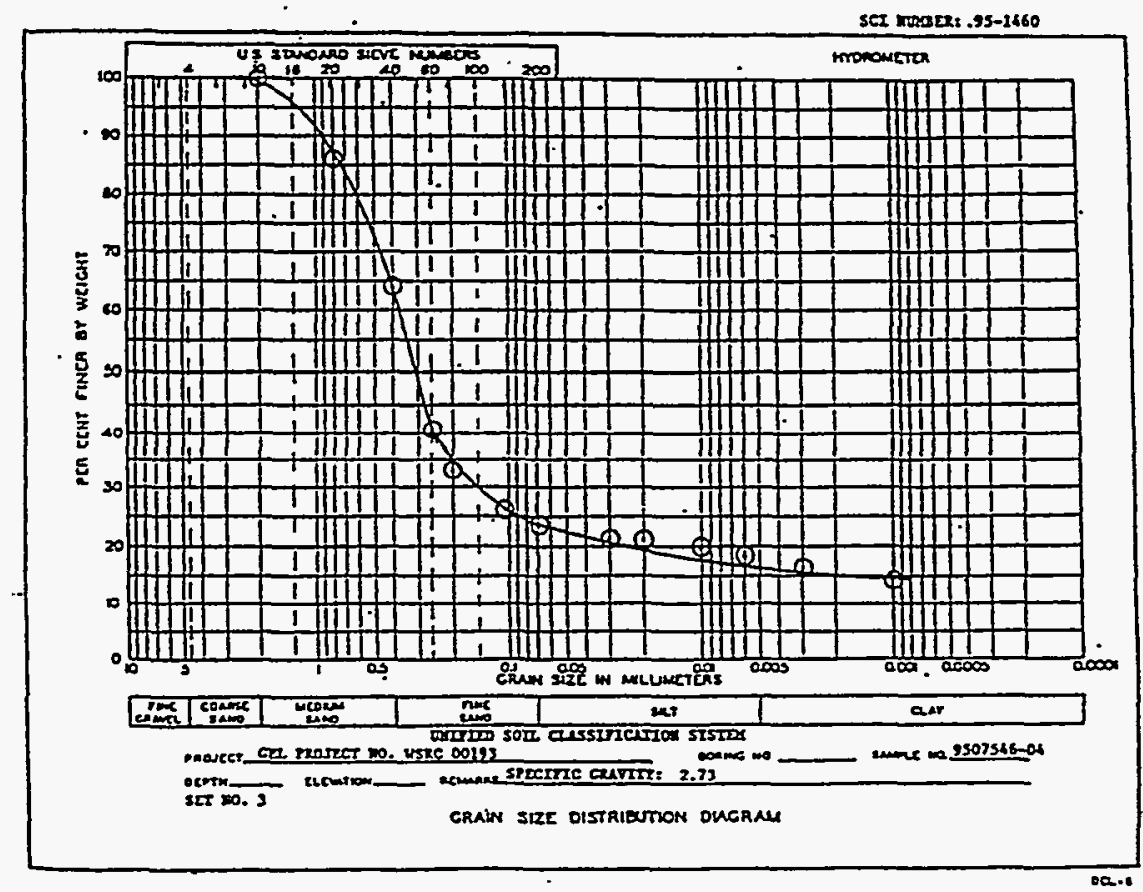

Survey ID: PPSC2504

Sample ID: 105116 


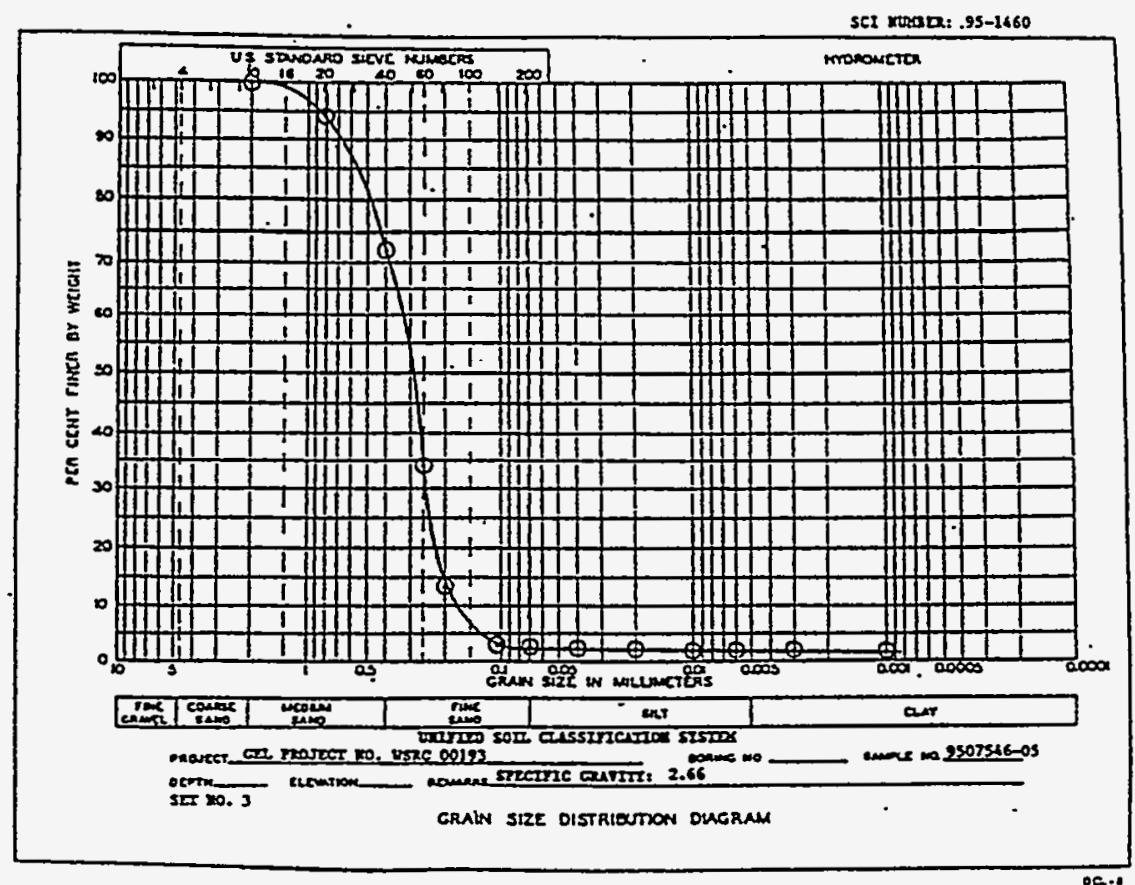

Survey ID: PPSC2401

Sample ID: 105117

SCI xotasexi .95-1460

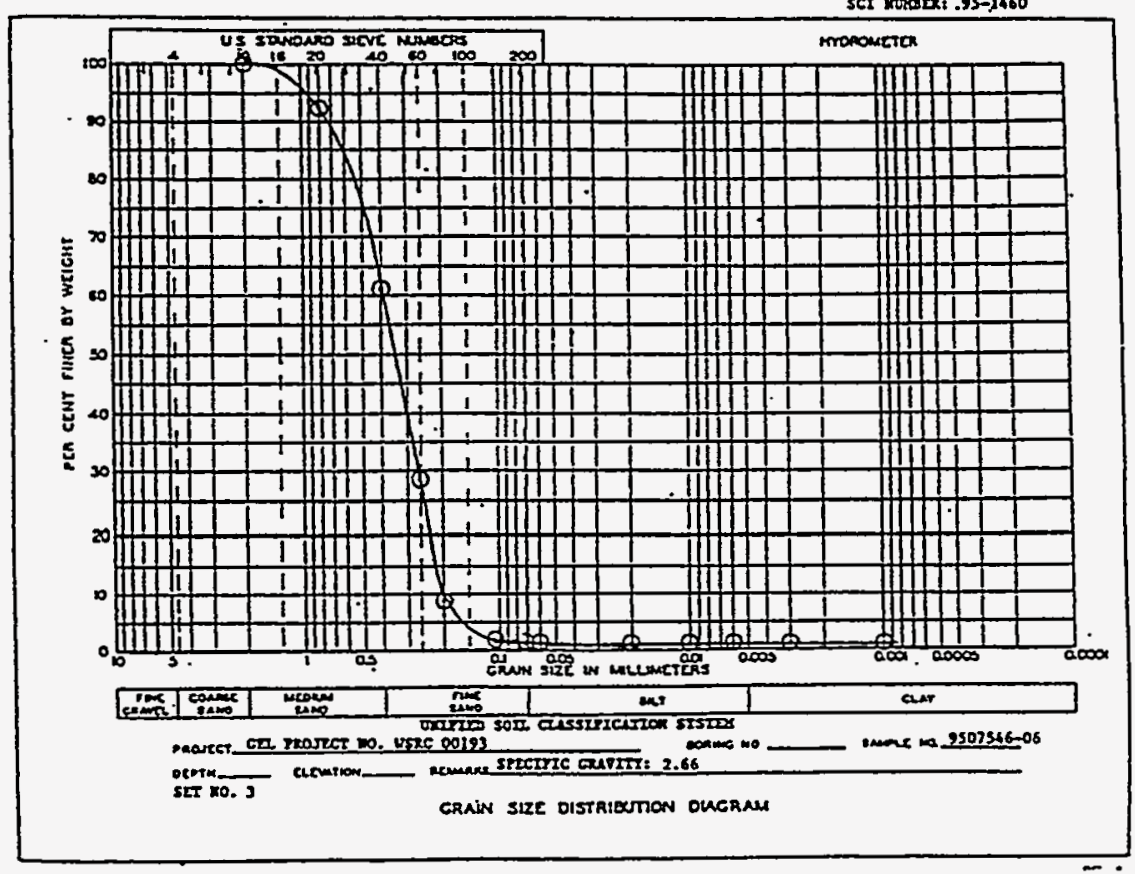

Survey ID: PPSC2402

Sample ID: 105118 


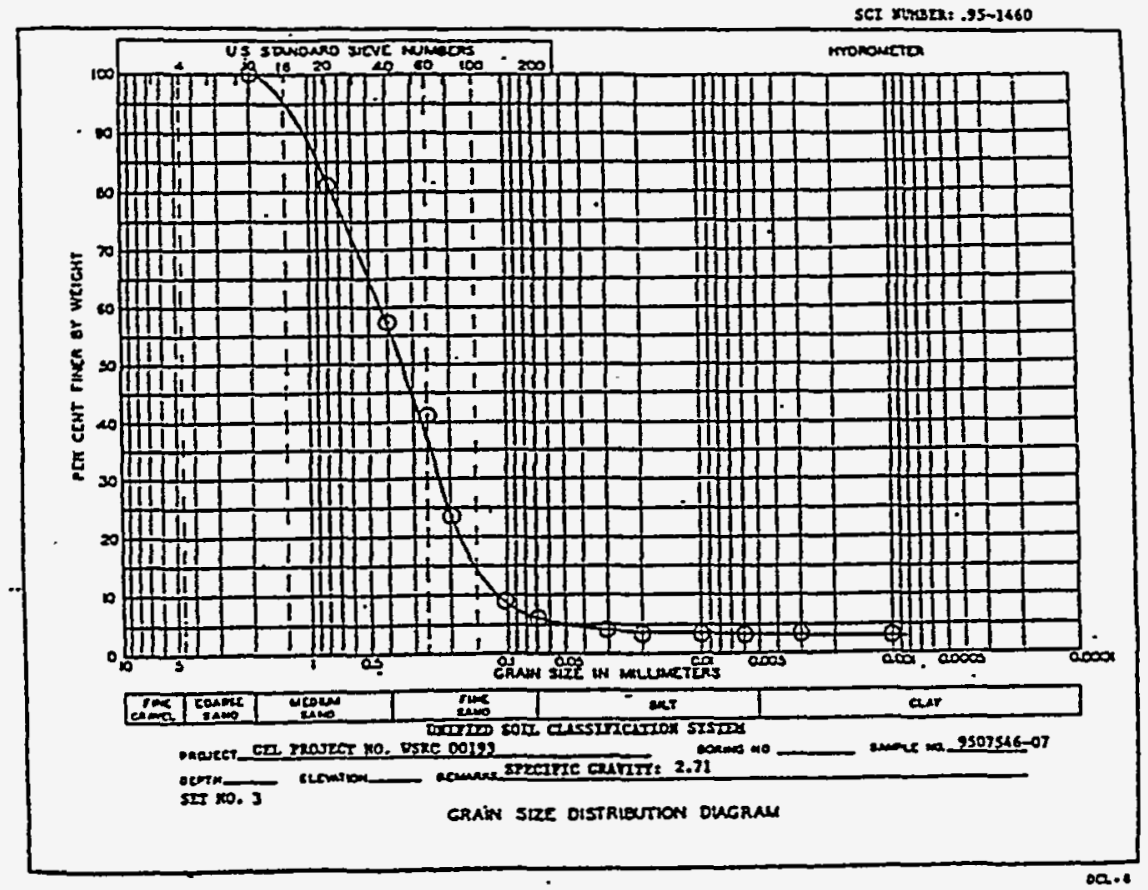

Survey ID: PPSC2403

Sample ID: 105119

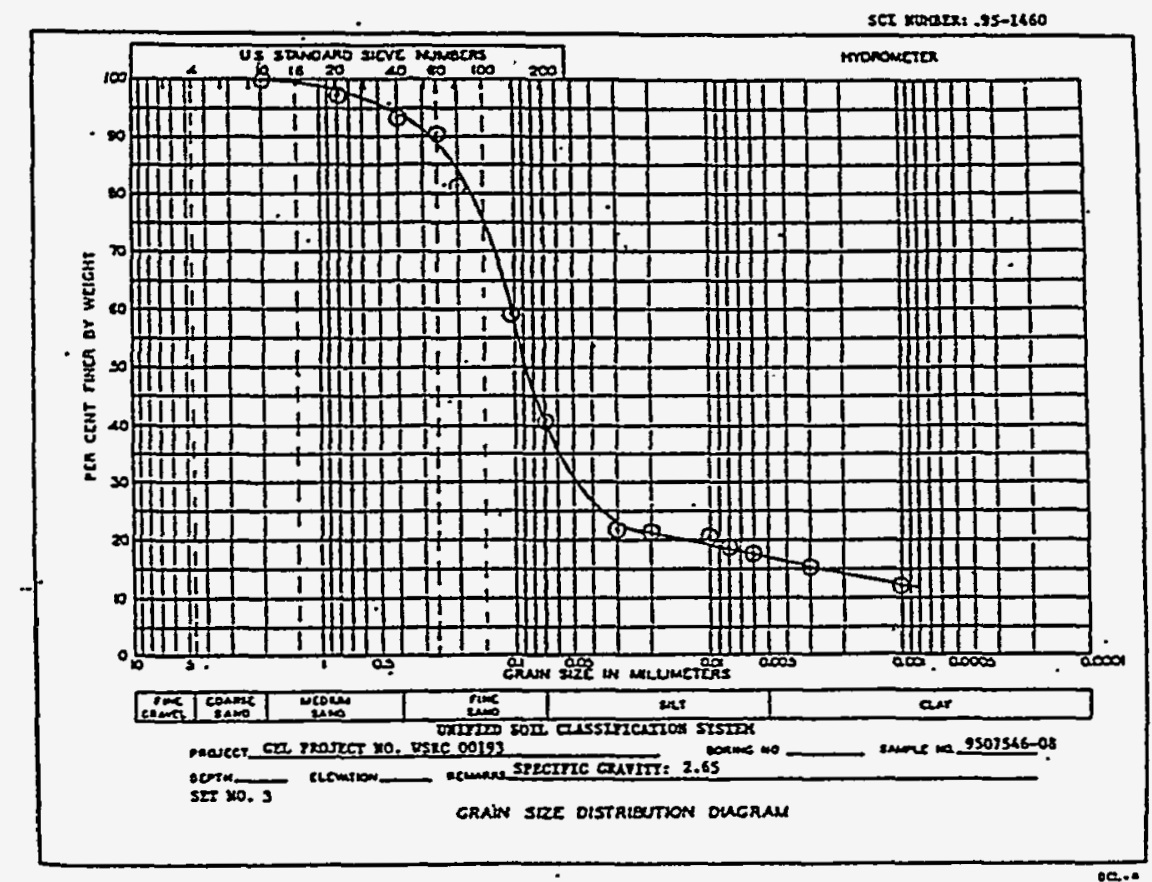

Survey ID: PPSC2404

Sample ID: 105120 


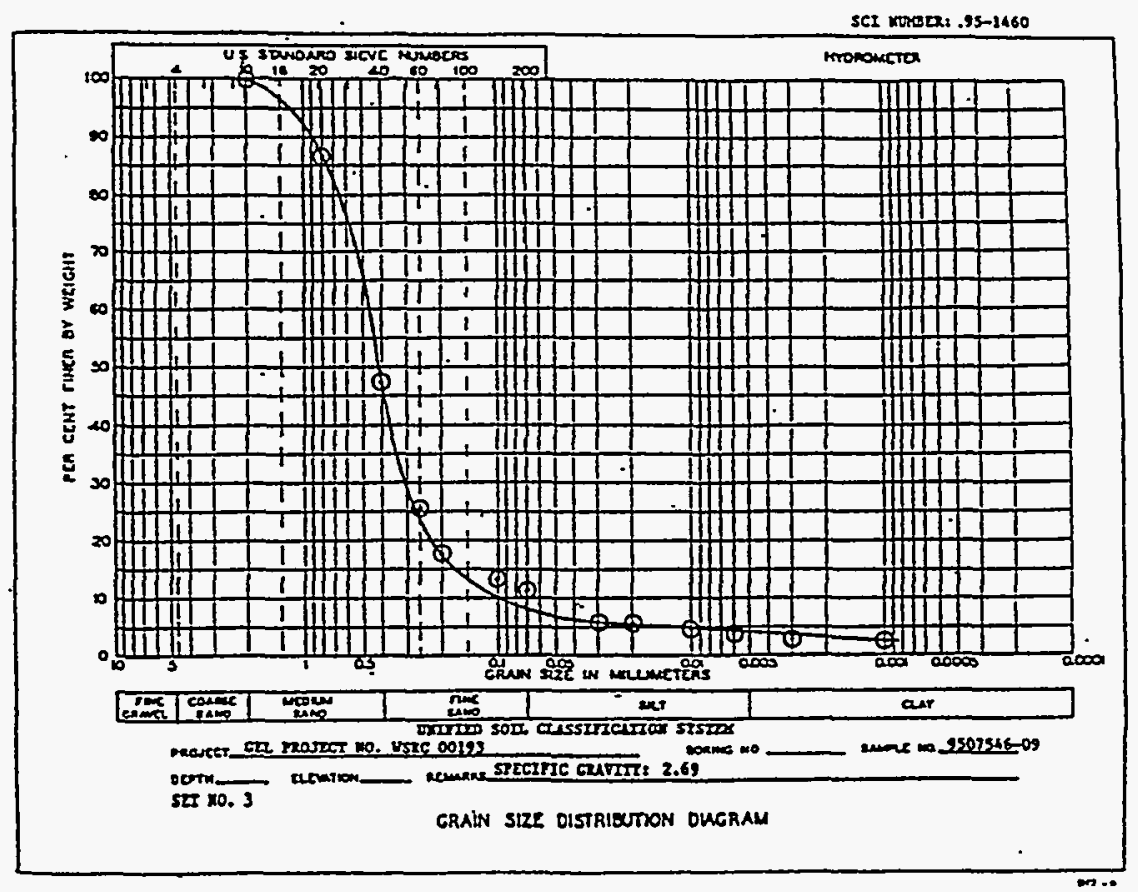

Survey ID: PPSC1401

Sample ID: 105109

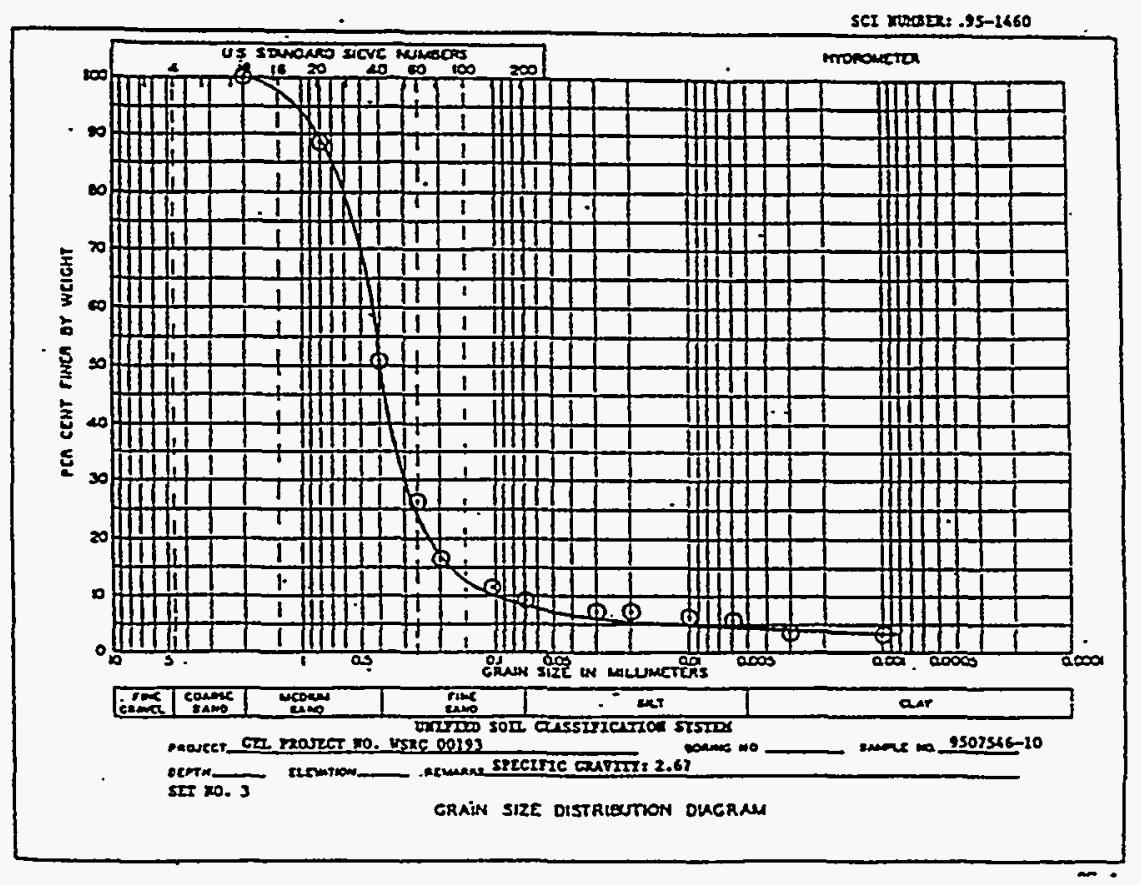

Survey ID: PPSC1402

Sample ID: 105110 


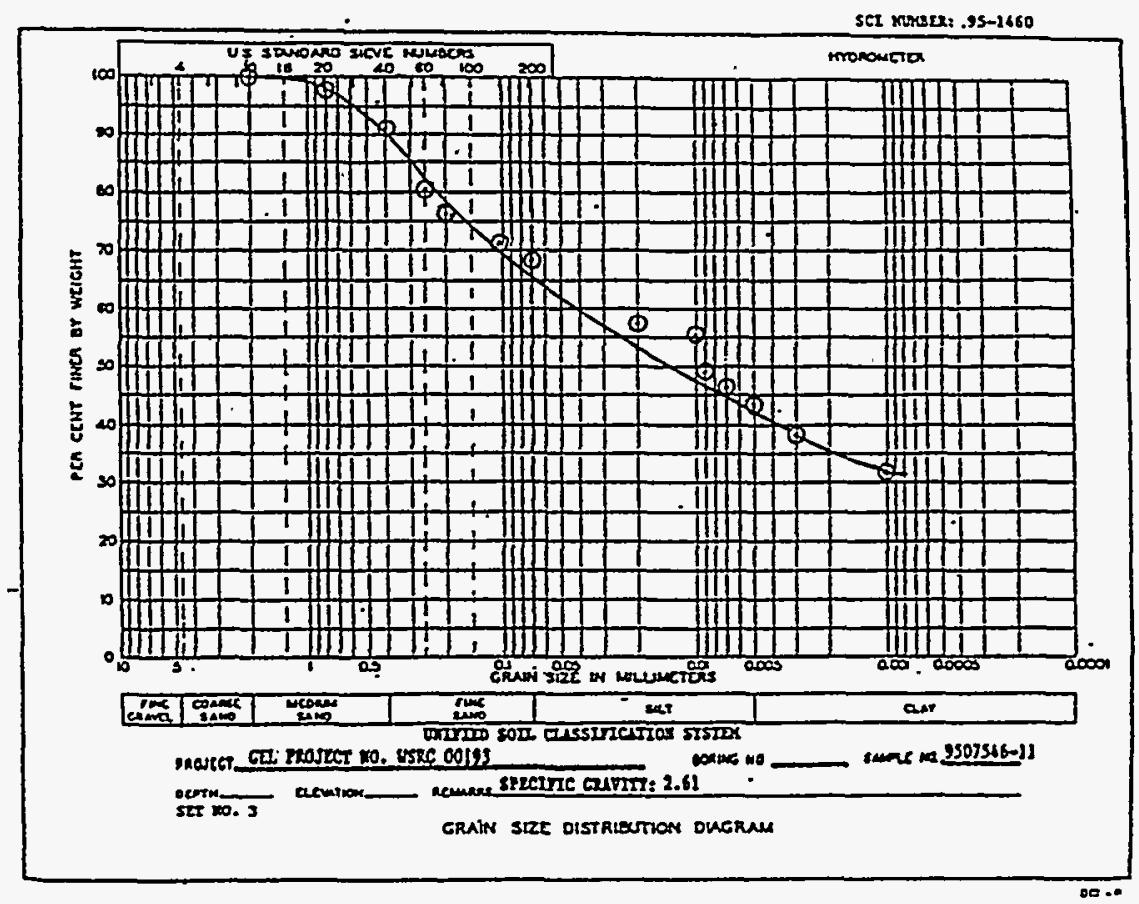

Survey ID: PPSC1501

Sample ID: 105104

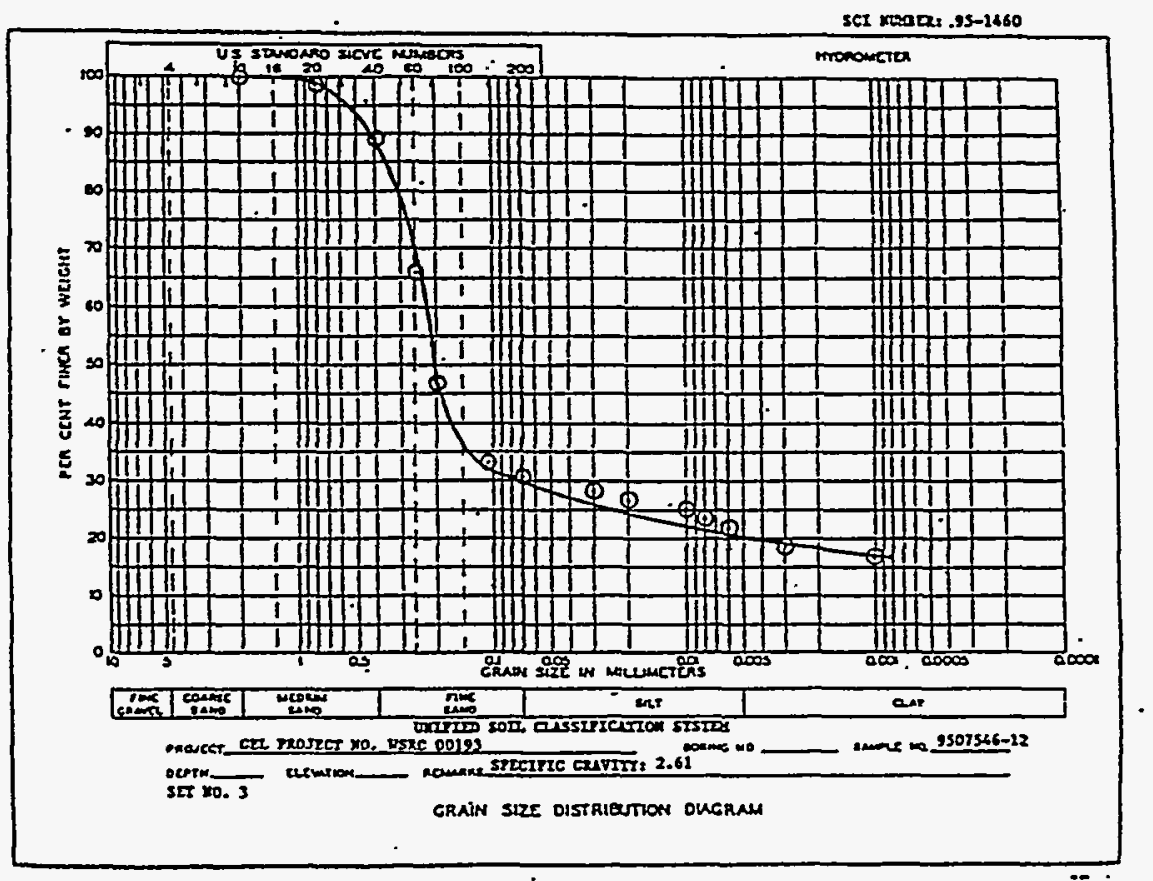

Survey ID: PPSC1503

Sample ID: 105106 


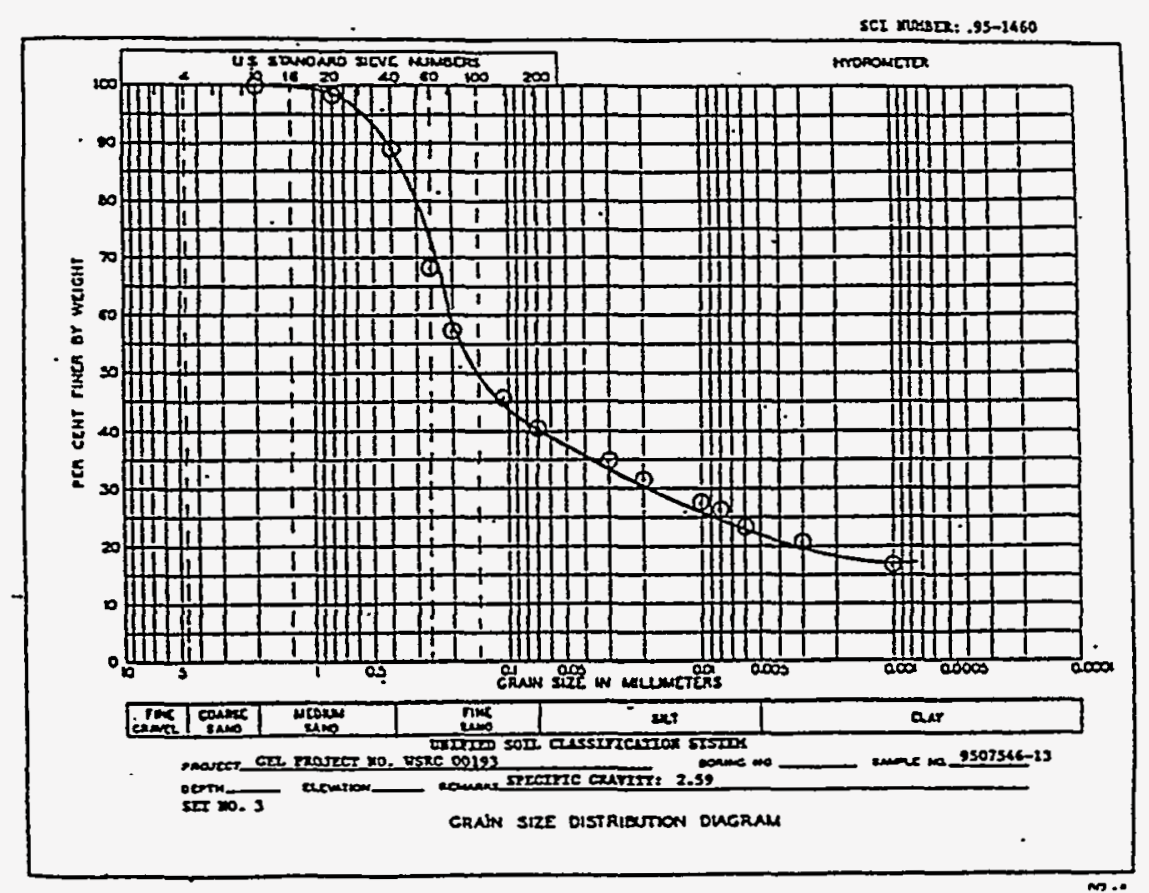

Survey ID: PPSC1502

Sample ID: 105105

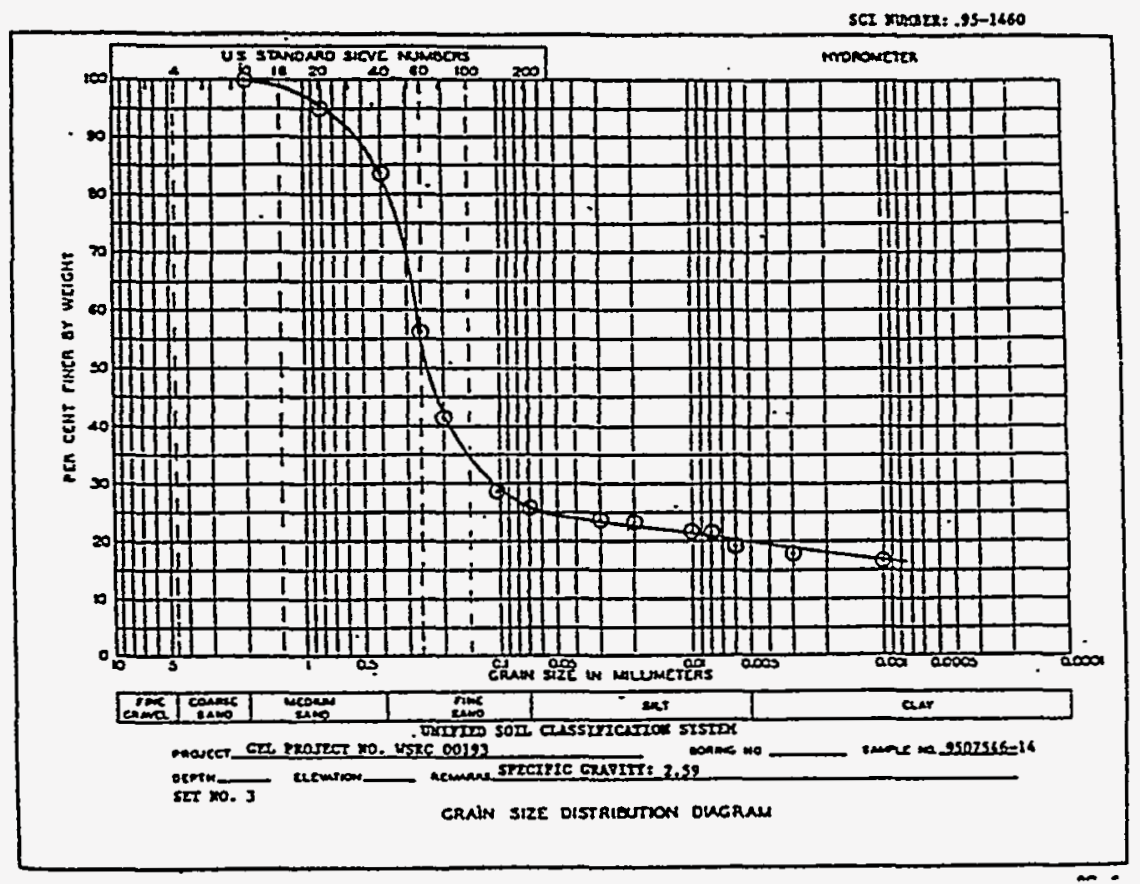

Survey ID: PPSC1504

Sample ID: 105107

Par Pond, Pond C, and L Lake, Revision 2 


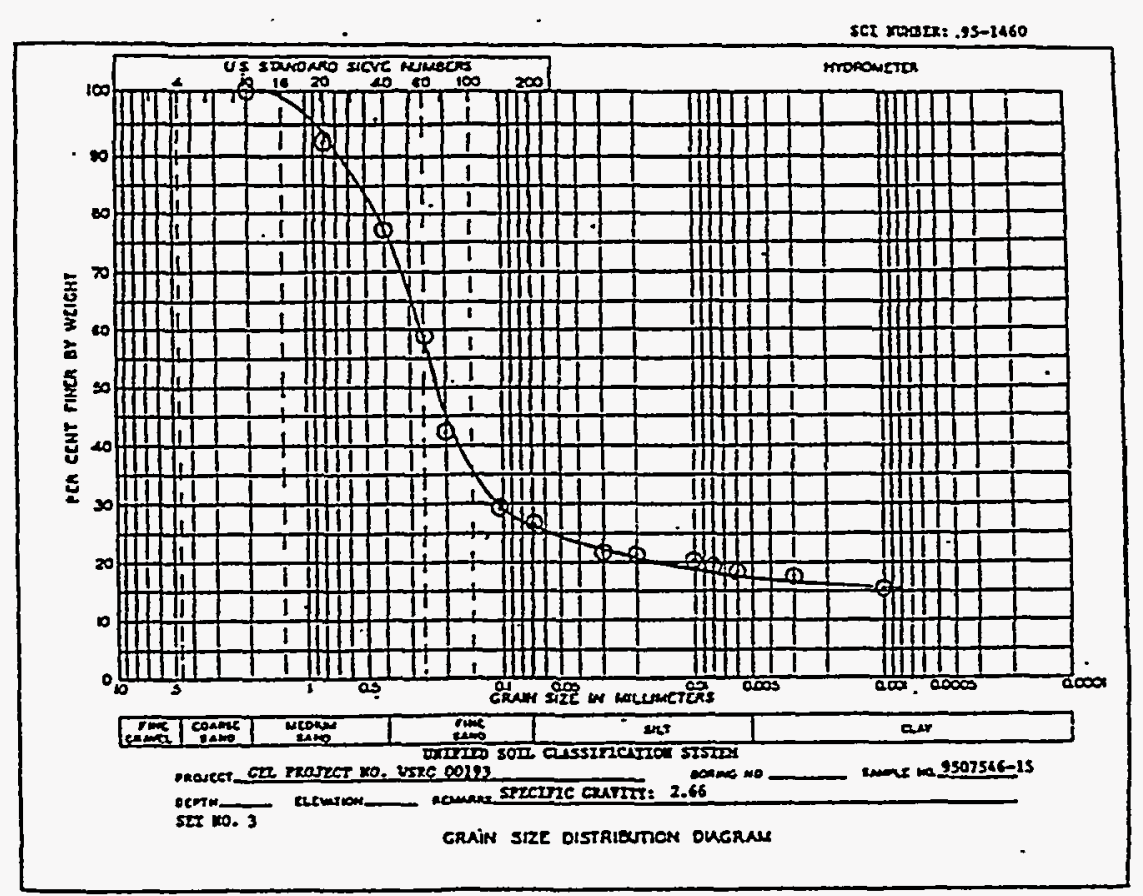

Survey ID: PPSC1505

Sample ID: 105108

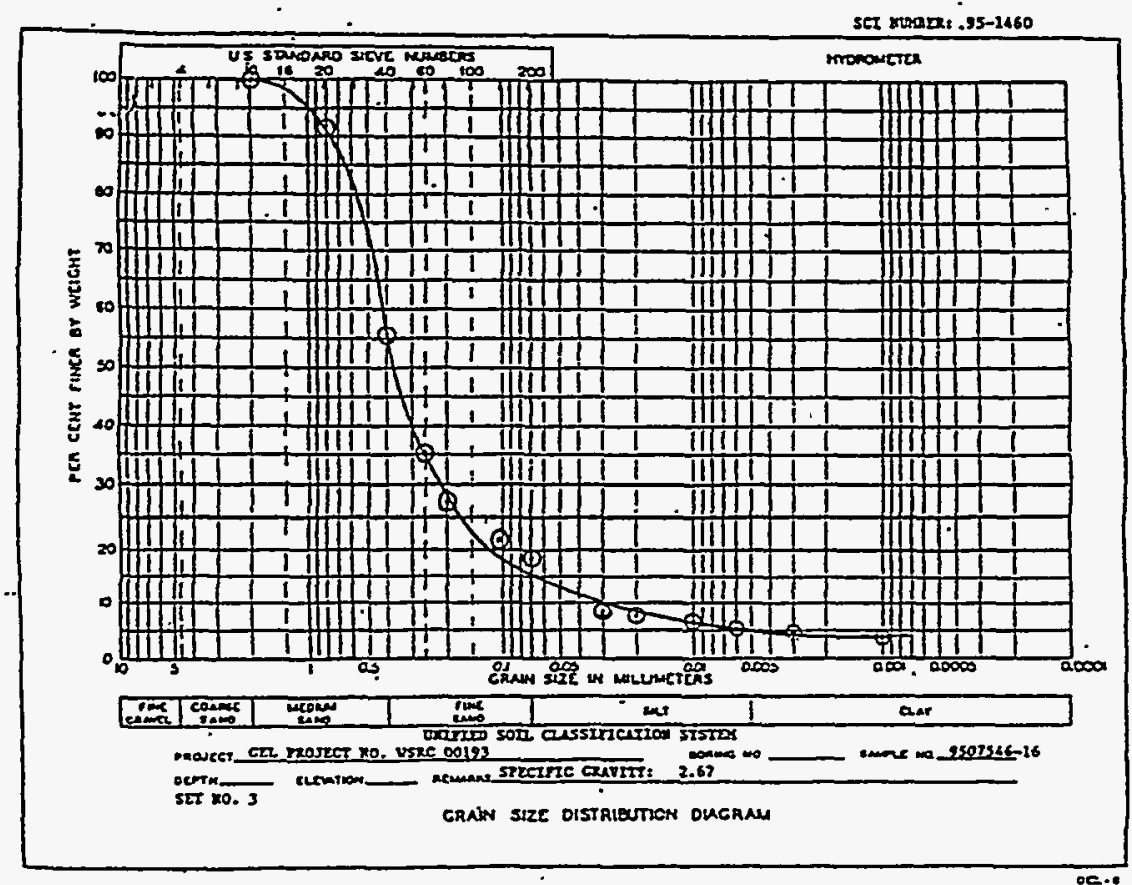

Survey ID: PPSC1403

Sample ID: 105111 


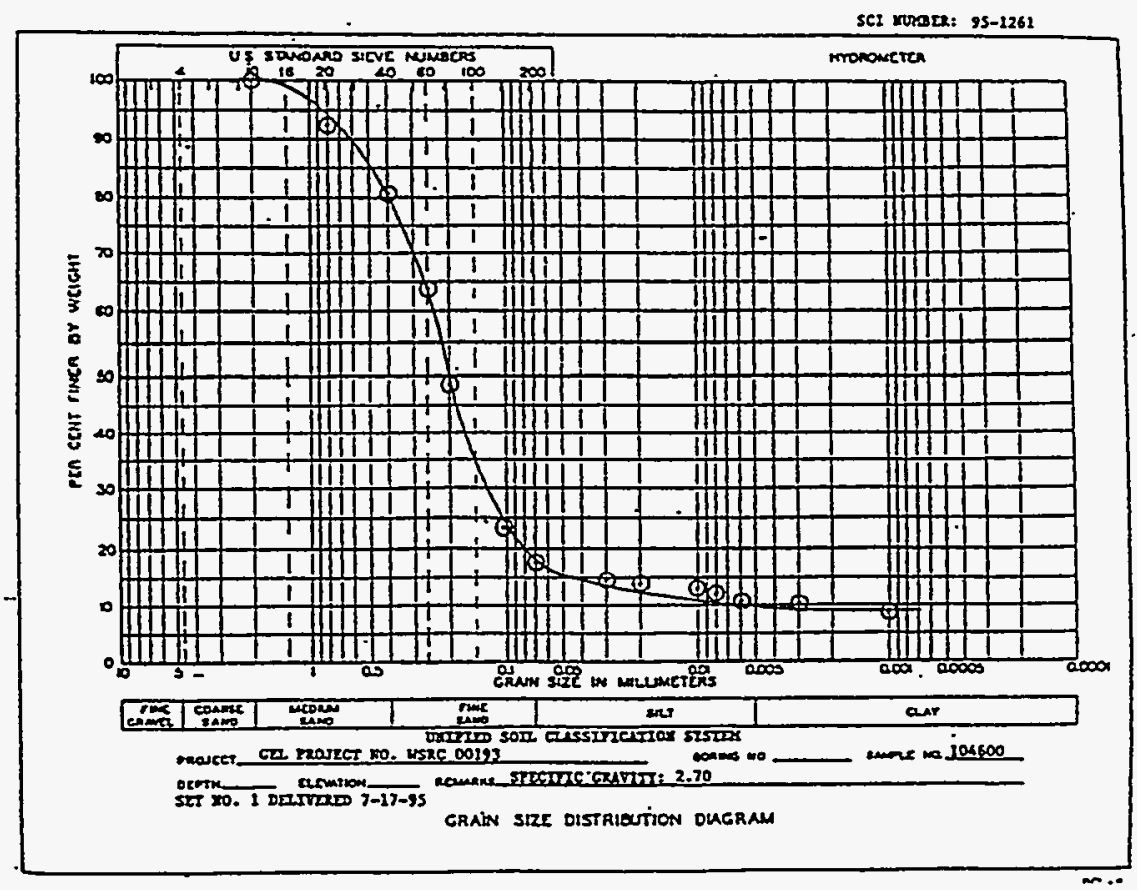

Survey ID: PPSC0101

Sample ID: 104600

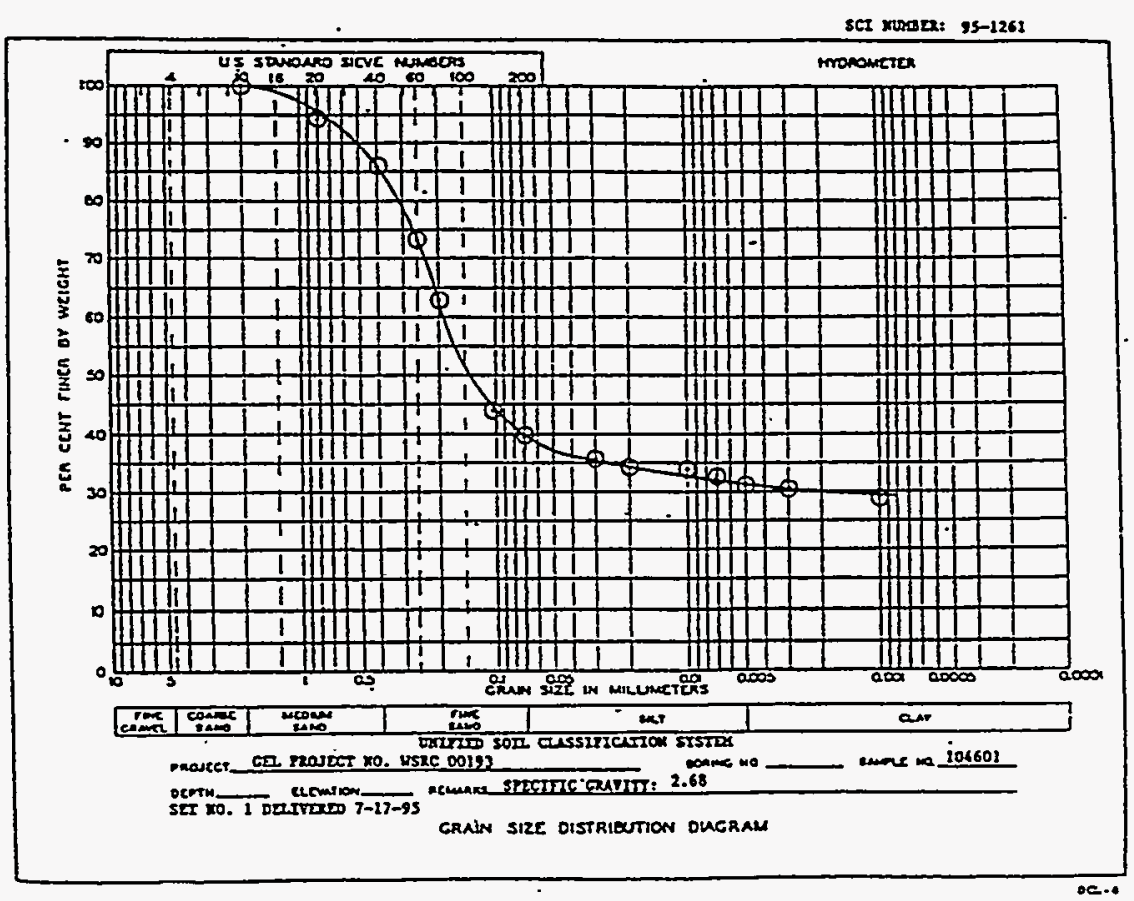

Survey ID: PPSC0102

Sample ID: 104601 
SCI xtosed: 92-1262

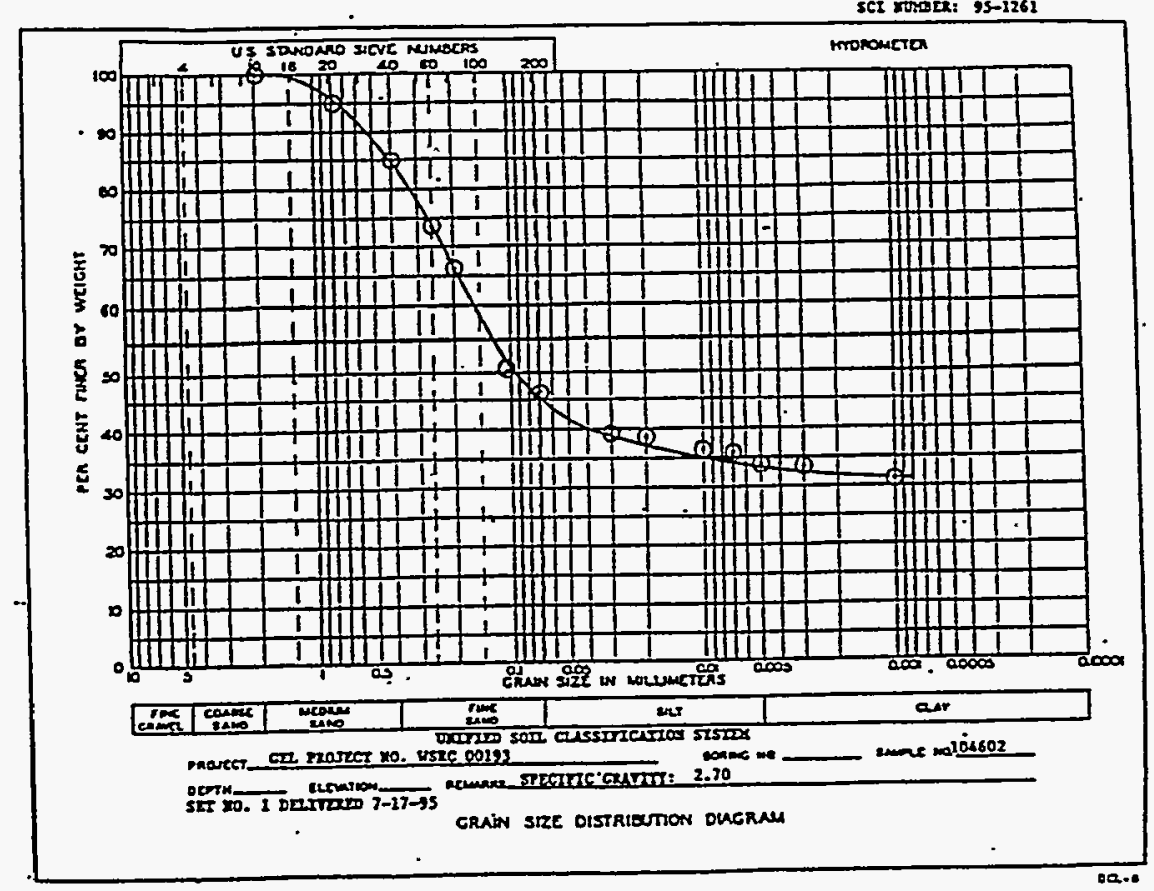

Survey ID: PPSC0103

Sample ID: 104602 
This page was left blank intentionallly.

$($ : 